WCH-91

Rev. 0

River Corridor Closure Contract

\title{
Columbia River Component Data Evaluation Summary Report
}

\section{July 2006}


TRADEMARK DISCLAIMER

Reference herein to any specific commercial product, process, or service by trade name, trademark, manufacturer, or otherwise, does not necessarily constitute or imply its endorsement, recommendation, or favoring by the United States Government or any agency thereof or its contractors or subcontractors.

This report has been reproduced from the best available copy. Available in paper copy and microfiche.

Available for a processing fee to U.S. Department of Energy and its contractors from:

U.S. Department of Energy

Office of Scientific and Technical Information

P.O. Box 62

Oak Ridge, TN 37831-0062

(865) $576-8401$

fax: (865) 576-5728

email: reports@adonis.osti.gov

online ordering: http://www.doe.gov/bridge

Available for sale to the public, in paper, from:

U.S. Department of Commerce

National Technical Information Service

5285 Port Royal Road

Springfield, VA 22161

(800) 553-6847

fax: (703) 605.6900

email: orders@ntis.fedworld.gov

online ordering: http://www.ntis.gov/ordering.htm

Printed in the United States of America

DISCLM-5.CHP (11/99) 


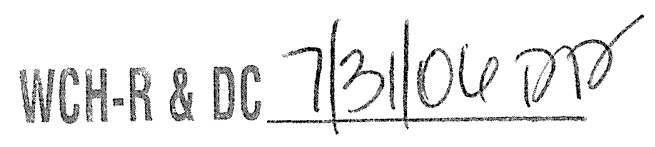

WCH-91

Rev. 0

OU: N/A

TSD: N/A

ERA: N/A

\section{STANDARD APPROVAL PAGE}

Title: Columbia River Component Data Evaluation Summary Report

Author Name: C. S. Cearlock

Approval: $\quad$ E. T. Feist, Director

End State and Final Closure

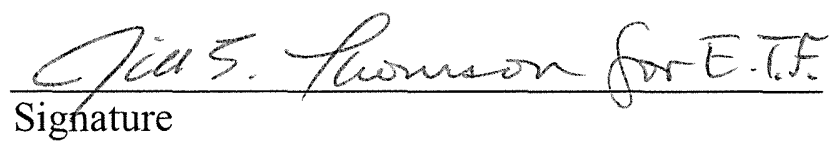

$7 / 27 / 06$

Date

The approval signatures on this page indicate that this document has been authorized for information release to the public through appropriate channels. No other forms or signatures are required to document this information release. 

WCH-91

Rev. 0

\section{River Corridor Closure Contract}

\section{Columbia River Component Data Evaluation Summary Report}

July 2006

Author:

C. S. Cearlock 



\section{TABLE OF CONTENTS}

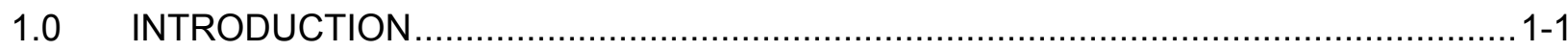

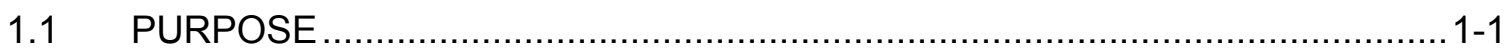

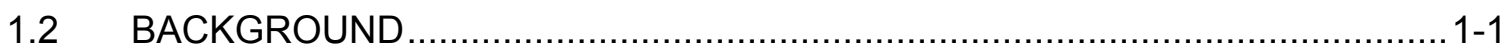

1.2.1 Lateral Shoreline Boundaries. ...................................................... 1-2

1.2.2 Focus on Hanford Site Related Contaminants. ................................ 1-3

1.2.3 Environmental Media of Interest. ................................................. 1-3

1.3 DESCRIPTION OF THE STUDY AREA …................................................. 1-3

1.4 SIMILAR DATA COMPILATION AND EVALUATION EFFORTS ......................1-4

1.4.1 Columbia River Comprehensive Impact Assessment......................... 1-5

1.4 .2 Upper Columbia River. ............................................................ 1-5

1.4 .3 Portland Harbor, RI/FS ........................................................ 1-5

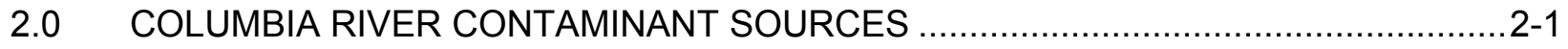

2.1 NON-HANFORD CONTAMINANT SOURCES ....................................... $2-1$

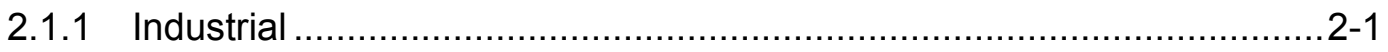

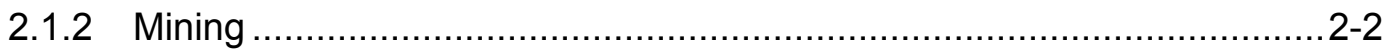

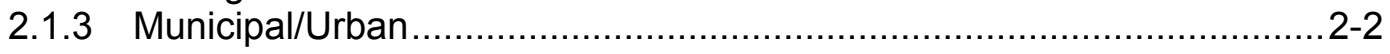

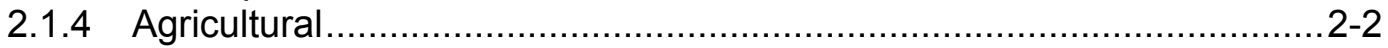

2.1.5 Nuclear Weapons Production \& Testing ......................................... 2-3

2.1.6 Commercial/Recreational Vessels ............................................ 2-3

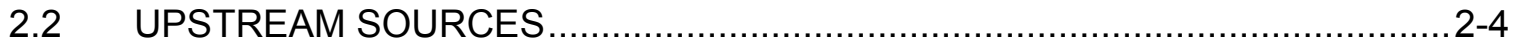

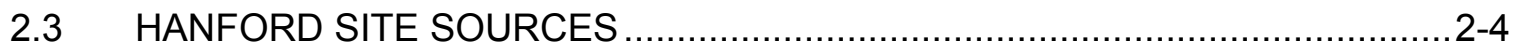

2.3.1 Historic Hanford Reactor Releases to the Columbia River.....................2-4

2.3.2 Current Hanford Site Groundwater Sources................................... 2-5

2.3.3 Summary of 100 Area Groundwater Contaminant Sources. .................... 2-5

2.3.4 Summary of 300 Area Groundwater Contaminant Sources. ................... 2-6

2.3.5 Summary of 200 Areas Groundwater Contaminant Sources................... $2-7$

2.3.6 Columbia River Shoreline Springs ........................................... 2-7

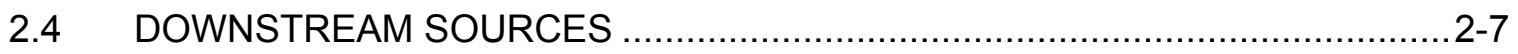

3.0 HISTORICAL DATA QUALITY REVIEW PROCEDURES ….................................. 3-1

3.1 REVIEW OF BIBLIOGRAPHICAL SOURCE INFORMATION .........................

3.2 QUALITY ASSURANCE/QUALITY CONTROL REQUIREMENTS .....................

3.3 ANALYTICAL DATA ENTRY INTO THE CRC DATABASE ............................ $3-5$ 
3.3.1 Standardization of Regional Datum

3.3.2 Methodology of Converting Descriptive Information into Sample

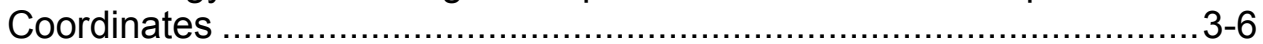

3.3.3 Verification of Sample Coordinates .................................................

3.3.4 Standardization of Concentration Units and Activity Levels ...................3-7

3.3.5 Decay of Radiological Results .........................................................

3.3.6 Uncertainties.........................................................................

3.4 DESCRIPTION OF HISTORICAL DATA USED IN THE EVALUATION .............3-8

3.4.1 Summary of Samples Used in Evaluation ..........................................3-9

3.4.2 Sample Summary - Segment 1 1.................................................. $3-9$

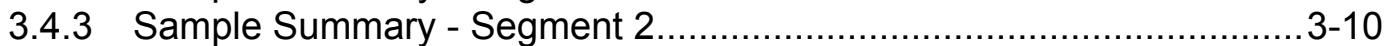

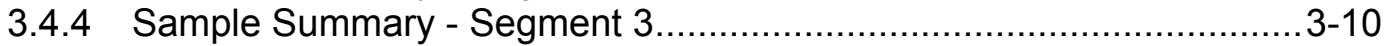

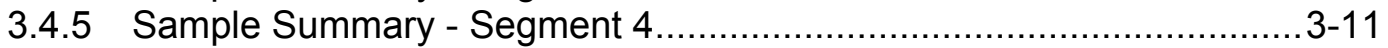

3.4.6 Sample Summary - Segment 5............................................... $3-11$

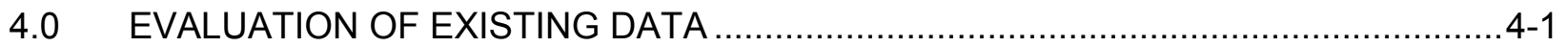

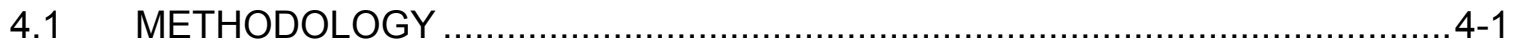

4.1.1 Sediment Benchmark Values ...........................................................4-1

4.1.2 Surface Water Benchmark Values......................................................4-2

4.2 CONTAMINANT DISTRIBUTIONS IN SEGMENT $1 \ldots \ldots \ldots \ldots \ldots \ldots \ldots \ldots \ldots \ldots \ldots . . . . .4-3$

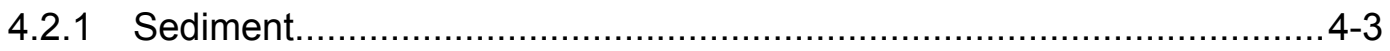

4.2.2 Surface Water .......................................................................... 4 -4

4.3 CONTAMINANT DISTRIBUTIONS IN SEGMENT 2 …................................4-4

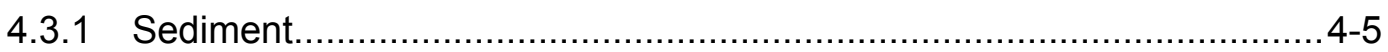

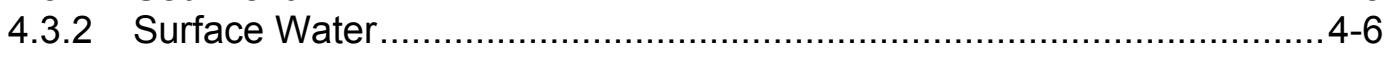

4.4 CONTAMINANT DISTRIBUTIONS IN SEGMENT 3 ................................. $4-8$

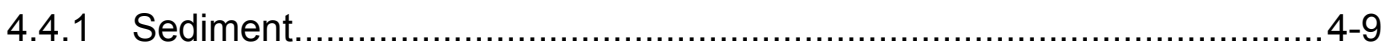

4.4.2 Surface Water ...................................................................... 4

4.5 CONTAMINANT DISTRIBUTIONS IN SEGMENT 4 ….............................4-12

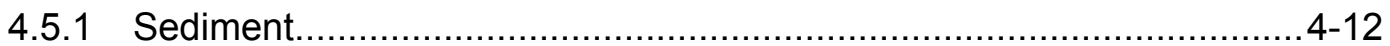

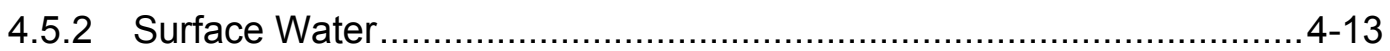

4.6 CONTAMINANT DISTRIBUTIONS IN SEGMENT 5 .................................4-15

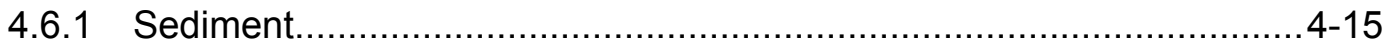

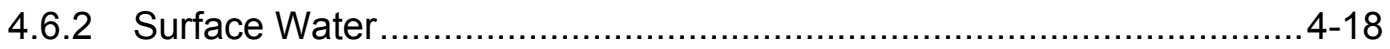

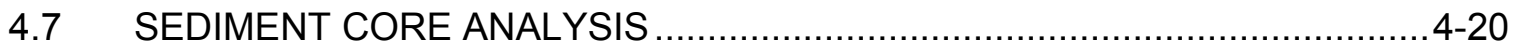


4.7.1 Core Samples - Cobalt-60 Analyses.

4-21

4.7.2 Core Samples - Cesium-137 Analysis

4.7.3 Core Samples - Plutonium-239/240 Analysis

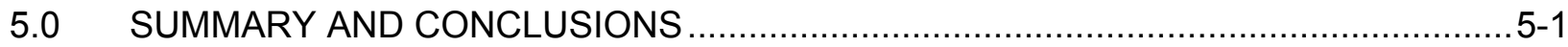

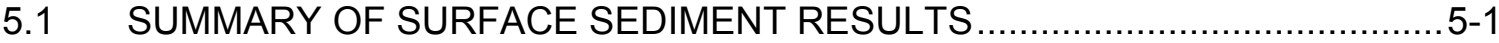

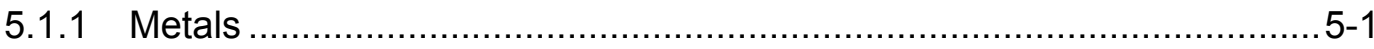

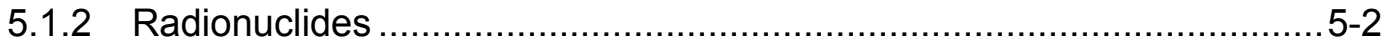

5.1.3 PCB Aroclors ............................................................................... 5

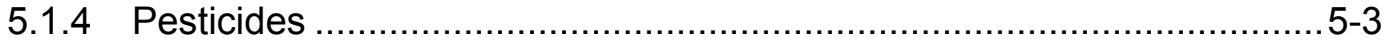

5.1.5 Polynuclear Aromatic Hydrocarbons ........................................... 5-4

5.2 SUMMARY OF SURFACE WATER RESULTS ....................................... $5-4$

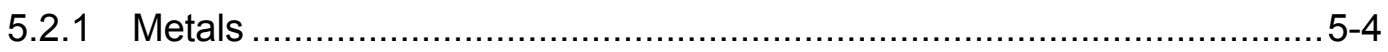

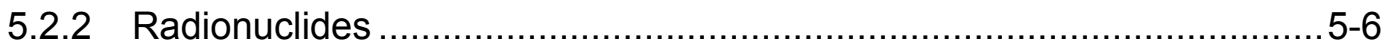

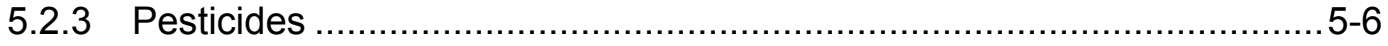

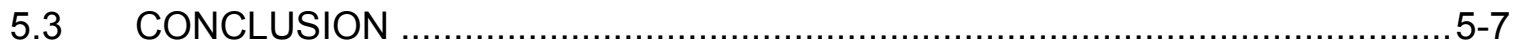

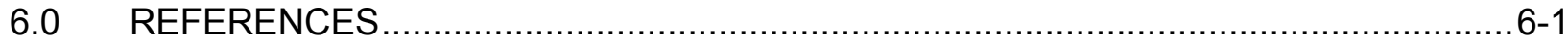

\section{APPENDICES}

A SEGMENT 1 SUMMARY STATISTICS, BENCHMARK COMPARISON, AND INDIVIDUAL SAMPLE EXCEEDENCES

B SEGMENT 2 SUMMARY STATISTICS, BENCHMARK COMPARISON, AND INDIVIDUAL SAMPLE EXCEEDENCES

C SEGMENT 3 SUMMARY STATISTICS, BENCHMARK COMPARISON, AND INDIVIDUAL SAMPLE EXCEEDENCES

D SEGMENT 4 SUMMARY STATISTICS, BENCHMARK COMPARISON, AND INDIVIDUAL SAMPLE EXCEEDENCES

E SEGMENT 5 SUMMARY STATISTICS, BENCHMARK COMPARISON, AND INDIVIDUAL SAMPLE EXCEEDENCES

F BIOTA SUMMARY STATISTICS TABLES 


\section{FIGURES}

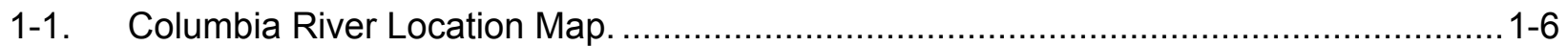

1-2. Columbia River Component Segments................................................................. 1-7

2-1. Hanford Site 2005 Groundwater Plume Contamination Maps for Radionuclides (Hartman et al. 2006) ................................................................................2-9

2-2. Hanford Site 2005 Groundwater Plume Contamination Maps for Chemicals (Hartman et al. 2006) .................................................................................. 2-10

3-1. Distribution of Sediment Samples by Segment.....................................................

3-2. Distribution of Surface Water Samples by Segment. ……..................................... 3-14

3-3. Grand Coulee Dam to Vernita Bridge - Segment 1 (All Samples)............................3-15

3-4. Vernita Bridge to Yakima River Confluence - Segment 2 (All Samples)......................3-16

3-5. Yakima River Confluence to McNary Dam - Segment 3 (All Samples)........................3-17

3-6. McNary Dam to Bonneville Dam - Segment 4 (All Samples)......................................3-18

3-7. Bonneville Dam to Astoria, Oregon - Segment 2 (All Samples) ................................3-19

4-1. Coblalt-60 Concentrations Behind Columbia River Dams from 1959 to 1975 .............4-24

4-2. Coblalt-60 Concentrations Behind Columbia River Dams from the 1940s to the

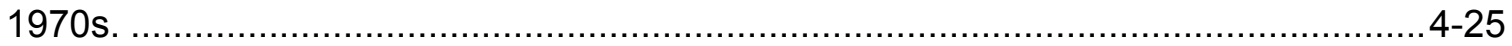

4-3. Cesium-137 Concentrations Behind Columbia River Dams from 1959 to 1975 .........4-26

4-4. Cesium-137 Concentrations Behind Columbia River Dams from 1940's to 1970's. ...4-27

4-5. Cesium-137 Concentrations at Depth Intervals Behind Columbia River Dams. ......... 4-28

4-6. Coblalt-60 and Cesium-137 Measurements Behind McNary Dam from 1942 to Present.................................................................................................. 4-29

4-7. Plutonium-239/240 Concentrations Behind Columbia River Dams from the 1940s

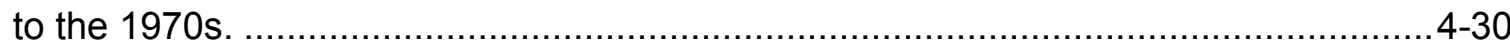

\section{TABLES}

3-1. Summary of Laboratory Qualifiers and Definitions............................................... 3-20

3-2. Summary of Documents with Segment 1 Sediment Data. .......................................3-22

3-3. Summary of Documents with Segment 1 Surface Water Data. ..................................3-24

3-4. Summary of Documents with Sediment Data in Segment 2. .................................3-26

3-5. Segment 2 Sediment Location Summary ...................................................... 3-32

3-6. Summary of Documents with Segment 2 Surface Water Data. ...............................3-34

3-7. Segment 2 Surface Water Location Summary .................................................. $3-40$

3-8. Summary of Documents with Segment 3 Sediment Data. ......................................3-42

3-9. Summary of Documents with Segment 3 Surface Water Data. .............................3-45

3-10. Summary of Documents with Segment 4 Sediment Data...................................... 3-47

3-11. Summary of Documents with Segment 4 Surface Water Data. .................................3-48

3-12. Summary of Documents with Segment 5 Sediment Data. ......................................3-49

3-13. Summary of Documents with Segment 5 Surface Water Data. ................................. 3-53

4-1. Summary of Hanford Site Soil Background Concentrations. …….............................4-31

4-2. Summary of Sediment Benchmark Values for the Protection of Human Health. .........4-33

4-3. Summary of Sediment Benchmark Values for the Protection of Ecological

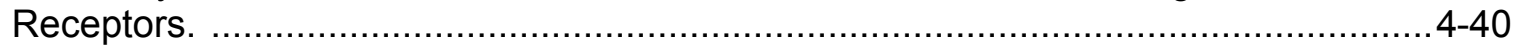

4-4. Summary of Surface Water Benchmark Values for the Protection of

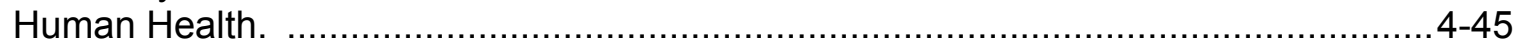

4-5. Summary of Surface Water Benchmark Values for the Protection of Ecological

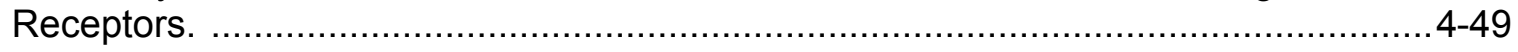


4-6. Summary of Segment 1 Sediment Benchmark Exceedances for All Categories of Data.

4-7. Summary of Segment 1 Surface Water Benchmark Exceedances for All Categories of Data..................................................................................................... 4-57

4-8. Summary of Segment 2 Sediment Benchmark Exceedances for All Categories of Data.

4-9. Summary of Segment 2 Surface Water Benchmark Exceedances for All Categories of Data.

4-10. Summary of Segment 3 Sediment Benchmark Exceedances for All Categories of Data.

4-11. Summary of Segment 3 Surface Water Benchmark Exceedances for All Categories of

Data.

4-12. Summary of Segment 4 Sediment Benchmark Exceedances for All Categories of Data.

4-13. Summary of Segment 4 Surface Water Benchmark Exceedances for All Categories of Data.

4-14. Summary of Segment 5 Sediment Benchmark Exceedances for All Categories of Data.

4-15. Summary of Segment 5 Surface Water Benchmark Exceedances for All Categories of

Data.

4-16. Summary of Contaminant Classes Reported with Sediment Core Samples. ..............4-66

4-17. Summary of Sediment Core Samples Collected in Segment 1 of the Columbia River Component.

4-18. Summary of Sediment Core Samples Collected in Segment 2 of the Columbia River Component.

4-19. Summary of Sediment Core Samples Collected in Segment 3 of the Columbia River Component.

4-20. Summary of Sediment Core Samples Collected in Segment 4 of the Columbia River Component.

4-21. Summary of Sediment Core Samples Collected in Segment 5 of the Columbia River Component.

5-1. Summary of Sediment Exceedences. ........................................................... $5-9$

5-2. Summary of Surface Water Exceedences. ................................................... 5-10 
WCH-91

Table of Contents

Rev. 0 


\section{ACRONYMS}

\begin{tabular}{ll} 
BHC & hexachlorocyclohexane \\
CERCLA & Comprehensive Environmental Response, Compensation, and Liability Act of \\
& 1980 \\
CRC & Columbia River Component \\
CRCIA & Columbia River Comprehensive Impact Assessment \\
DOE & U.S. Department of Energy \\
DDD & dichlorodiphenyldichloroethane \\
DDE & dichlorodiphenyldichloroethylene \\
DDT & dichlorodiphenyltrichloroethane \\
Ecology & Washington State Department of Ecology \\
EIM & Environmental Information Management \\
EPA & U.S. Environmental Protection Agency \\
ERAMS & Environmental Radiation Ambient Monitoring System \\
ESRI & Environmental Systems Research Institute \\
FS & feasibility study \\
GIS & Geographic Information System \\
HEIS & Hanford Environmental Information System \\
MCL & maximum contaminant level \\
Nadcon & North American Datum Conversion \\
NGS & National Geodetic Survey \\
NPDES & National Pollutant Discharge Elimination System \\
OU & Operable Unit \\
PAH & polyaromatic hydrocarbons \\
PCB & polychlorinated biphenyl \\
PRG & Preliminary Remediation Goal \\
QA & quality assurance \\
QC & quality control \\
RESRAD & RESidual RADioactivity \\
RI & remedial investigation \\
RL & DOE Richland Operations Office \\
RM & river mile \\
SEDQUAL & Sediment Quality Information System \\
SVOC & semivolatile organic compound \\
USGS & U.S. Geological Survey \\
VOC & volatile organic compound \\
& \\
\hline
\end{tabular}


WCH-91

Rev. 0 


\subsection{INTRODUCTION}

\subsection{PURPOSE}

The purpose of the Columbia River Component (CRC) Data Compilation and Evaluation task was to compile, review, and evaluate existing information for constituents that may have been released to the Columbia River due to Hanford Site operations (i.e., radionuclides and hazardous constituents). Through this effort an extensive compilation of information pertaining to Hanford Site-related contaminants released to the Columbia River has been completed for almost $965 \mathrm{~km}$ (600 miles) of the river. A summary of the process used to compile, classify relevant source documents, and manage the data is provided in the Existing Source Information Summary Report Compilation/Evaluation Effort: December 2004 to September 2005 (WCH 2006). The data compilation and evaluation task was initiated on behalf of the U.S. Department of Energy (DOE), Richland Operations Office (RL) under the Environmental Restoration Contract managed by Bechtel Hanford, Inc. in December 2004. However, this task was concluded under the River Corridor Closure Contract managed by Washington Closure Hanford following a contract transition from the Environmental Restoration Contract to the River Corridor Closure Contract on August 29, 2005.

The CRC data evaluation summary report is the second of several inter-related tasks that will culminate in assessing potential threats to human health and the environment in the Columbia River from Hanford Site releases. The purpose of the CRC data evaluation summary report is to describe the activities that were undertaken to evaluate the data collected in the compilation effort and to assist in defining the extent of Hanford Site-related contamination within the defined boundaries of the CRC (below Grand Coulee Dam to Astoria, Oregon) (Figure 1-1). This information will also be used to develop an initial understanding of river conditions and contaminant distribution within the river and several of the major tributaries.

A primary objective of this data evaluation was to determine if sufficient data was available to adequately characterize the downriver extent of Hanford Site-related contaminants. This information can also be used to identify data gaps and information that are needed to gain a better understanding of existing levels of contaminants in river sediments; assist in the development of a preliminary conceptual site model; and identify information to better understand the location, character, and long-term fate of contaminants in sediment and surface water, as well as the current threat to people, plants, and animals that may contact these media.

\subsection{BACKGROUND}

The Columbia River Component of the River Corridor Baseline Risk Assessment: Basis and Assumptions on Project Scope (DOE-RL 2004) broadly defined what constitutes the CRC in terms of its length and width, and specified that the results of the data evaluation would assist in defining where the study boundaries of the CRC should be drawn. For the purposes of helping to define the final boundary, information on contaminant releases was gathered from below Grand Coulee Dam to the upriver boundary of the Hanford Site to evaluate "upgradient" conditions, as well as information on releases from the Hanford Site that may have migrated as 
far downriver as Astoria, Oregon, to identify where contaminants may have come to reside within or adjacent to the Columbia River. ${ }^{1}$ The data evaluation report provides the following:

- Characterizes the lower boundary of the study area

- Describes the quality of the existing data used in the evaluation

- Describes the geographic and temporal extent of available data

- Compares abiotic media concentrations to available state and federal benchmarks for protecting human health and the environment

- Provides a preliminary assessment of data gaps that should to be addressed prior to conducting a risk assessment.

The scope of this report is constrained to characterizing available information and data relative to the broad definition of the CRC (upstream jurisdictional boundary of the Hanford Site downstream to Astoria, Oregon). Data and information from Grand Coulee Dam to the upriver boundary of the Hanford Site were also collected to support background conditions as well as contributions from upstream sources. Investigation areas along the shoreline of the Columbia River being evaluated as part of the 100 Area and 300 Area Component of the River Corridor Baseline Risk Assessment were excluded from this evaluation. The following subsections provide the general scope of the CRC data compilation and evaluation effort.

\subsubsection{Lateral Shoreline Boundaries}

The lateral shoreline boundaries of the CRC were bounded by the upper elevation of the third Holocene-age river terrace $(\mathrm{H}-3)$. Terrace $\mathrm{H}-3$ coincides with the terrace system situated immediately above the greatest lateral extent of flood waters outside the current river channel since the Hanford Site began operations in 1944. The largest flood on the Columbia River during Hanford Site operations occurred in 1948. In general, shoreline areas are primarily limited to the Hanford Reach of the Columbia River, as it is the last free-flowing stretch of the Columbia River. The Hanford Reach begins at the foot of the Priest Rapids Dam and extends $82 \mathrm{~km}$ (51 miles) to the slack waters of McNary Dam, just north of the City of Richland, Washington. The lateral boundaries of remaining downstream areas are limited to the "in water" portion of the Columbia River.

To the extent that information was available, data from major tributaries feeding into the Columbia River were also collected and evaluated. Boundaries for tributaries were loosely defined as the confluence of the tributary with the Columbia River, or the first upriver sampling location that would provide information on the contribution of contaminants made to the Columbia River by the respective tributary. However, in some instances data were collected further upstream within the tributaries when data were readily available.

\footnotetext{
${ }^{1}$ In this report, the term "Columbia River Component" is approximately defined as below Grand Coulee Dam at river mile 596.6 to Astoria, Oregon at river mile 12.
} 


\subsubsection{Focus on Hanford Site-Related Contaminants}

The goal of the data compilation effort was to primarily focus on collecting current and historical data for those contaminants released from Hanford Site activities. Although Hanford Site-related contaminants were the focus, all data from each source of information were obtained regardless of the source of contamination or analyses performed. The data evaluation effort attempts to identify current and historic sources of radiological and hazardous constituents within the boundaries of the CRC evaluation. This information potentially can be used to develop an approach for distinguishing the contribution of hazardous substances from the Hanford Site versus non-Hanford Site sources.

\subsubsection{Environmental Media of Interest}

The primary environmental media of interest during data collection included sediment, surface water (including river seeps), and aquatic biota.

\subsection{DESCRIPTION OF STUDY AREA}

The CRC study area was divided into five segments during the data compilation phase based on considerations of geologic and hydrologic information, as well as on the potential to obtain relevant information (Figure 1-2). Segments are described in terms of river miles (RMs), which are a statute mile measured along the center line of a river. The RM measurements start at the stream mouth (RM 0). Descriptions of each of the five segments are provided below.

Segment 1 (below Grand Coulee Dam to Vernita Bridge) - Segment 1 represents a 336-km (209-mile)-long stretch of the Columbia River ranging from RM 597 to RM 388. This segment includes the Upper Columbia River drainage above the Hanford Site. The Rock Island Dam, Wanapum Dam, and Priest Rapids Dam are located downstream from Grand Coulee Dam at RM 453.4, RM 415.8, and RM 397.1, respectively. Water quality in this portion of the Columbia River and associated tributaries (Wenatchee River and Lower Crab Creek) is influenced by the plutonic terrain of the Canadian Rocky Mountains, as well as mining waste in British Columbia, northeastern Washington, and northern Idaho. Information from this segment will assist in determining "upgradient" river conditions entering the Hanford Site. As discussed previously, the Upper Columbia River above Grand Coulee Dam was excluded from the CRC study area because it is currently under investigation by the U.S. Environmental Protection Agency (EPA).

Segment 2 (Vernita Bridge to the confluence of the Yakima River) - Segment 2 represents a 76-km (47-mile)-long stretch of the Columbia River ranging from RM 388 to RM 333). This segment includes the portion of the Columbia River primarily within the Hanford Site boundaries, referred to as the Hanford Reach. Information from this segment of the river will provide an indication of the condition of the river adjacent to or immediately downstream from the Hanford Site.

Segment 3 (Yakima River to McNary Dam) - Segment 3 represents a 66-km (41-mile)-long stretch of the Columbia River ranging from RM 333 to RM 292. This segment includes two major tributaries (Yakima River and Snake River) and one minor tributary (Walla Walla River) to the Columbia River and includes the drainage above McNary Dam. Ice Harbor Dam is located 
on the Snake River near the confluence of the Columbia River at Snake RM 9.7. Lake Wallula lies directly behind McNary Dam and extends $103 \mathrm{~km}$ (64 miles) upstream to the DOE's Hanford Site on the Columbia River to Ice Harbor Dam on the Snake River. This segment of the Columbia River is characterized by high turbidity (i.e., silt, clay) associated with extensive erosion in the eastern and western portions of the Columbia Plateau. In addition to the high turbidity, water quality in this section is impacted by agricultural practices in the Yakima and Kittitas Valleys and in the Palouse region.

Segment 4 (McNary Dam to Bonneville Dam) - Segment 4 represents a 235-km (146-mile)-long stretch of the Columbia River ranging from RM 292 to RM 146. This section includes the restricted flow of the Columbia River through the Columbia Gorge and represents the main stream that transports water from McNary Dam down to Bonneville Dam. The Umatilla, John Day, Deschutes, and Klickitat Rivers enter the Columbia River within this section. The John Day Dam, The Dalles Dam, and the Bonneville Dam are located downstream of the McNary Dam at RMs 215.6, 191.5, and 146.1, respectively. Behind each of the three dams are associated reservoirs: the John Day reservoir, Lake Celilo,; and Lake Bonneville. Each reservoir extends to the base of the respective upstream dams. A variety of minor industrial, agriculture, and forestry activities are conducted along this stretch of the Columbia River.

Segment 5 (Bonneville Dam to Astoria, Oregon) - Segment 5 represents a 216-km (134-mile)-long stretch of the Columbia River ranging from RM 146 to RM 12. This section does not contain any dams and includes the freshwater/saltwater interface of the Columbia River with the Pacific Ocean. The White Salmon, Willamette, Lewis, and Cowlitz Rivers enter the Columbia River within this section. This section is heavily influenced by the Cowlitz River because of extremely high turbidity from sediments being eroded out of the Mount St. Helens region. Sixty percent of the bedload and suspended load in the Columbia River is associated with the Cowlitz discharge (Meade and Parker 1984). This section has extensive floodplain areas due to the very low gradient that occurs immediately above the Columbia River estuary. This segment of the river contains some heavily industrialized areas between Vancouver, Washington and Portland, Oregon. However, this segment is also used for commercial, recreational, and agricultural purposes.

\subsection{SIMILAR DATA COMPILATION AND EVALUATION EFFORTS}

A number of similar data compilation and evaluation efforts have been conducted along the Columbia River for the purpose of evaluating the impact to the river from various contaminant sources. These studies were used as models for conducting the CRC data evaluation. The data compilation and evaluation approach used for the CRC was designed to maintain consistency between river segments along the entire stretch of the Columbia River. The following studies were used as models for the CRC:

- Screening Assessment and Requirements for a Comprehensive Assessment: Columbia River Comprehensive Impact Assessment (DOE-RL 1998b)

- Portland Harbor Remedial Investigation/Feasibility Study Programmatic Work Plan (Lower Willamette Group 2004) 
- Sediment Sampling Approach and Rationale, Upper Columbia River, Technical Briefing Sheet (EPA 2004a).

\subsubsection{Columbia River Comprehensive Impact Assessment}

The EPA, DOE, and Washington State Department of Ecology (Ecology) (otherwise known as the Tri-Parties) initiated the Columbia River Comprehensive Impact Assessment (CRCIA) to evaluate the impact to the Columbia River from Hanford Site-derived contaminants. The scope of the CRCIA screening assessment was to evaluate the potential impact to the river, resulting from current levels of Hanford Site-derived contaminants to support decisions on remedial actions under interim records of decisions. The study area for the screening assessment extended from the upstream boundary of the Hanford Site down to McNary Dam. An extensive compilation effort obtained sample data from Columbia River water, river seep, pore water, sediment, riparian soils, and riparian and aquatic biota representing "current conditions" from January 1990 to June 1996. Contaminants of interest included 12 radionuclides, 2 carcinogenic chemicals, and 13 toxic chemicals. Approximately 60 key documents contained data or described analyses important to evaluating the health of the Columbia River. Section 3.0 of the CRCIA report describes the approach used to gather data for the screening assessment.

\subsubsection{Upper Columbia River}

The EPA is studying hazardous waste contamination in the Columbia River from the United States/Canadian border to the Grand Coulee Dam (RM 744 to RM 597). Several investigations and studies over the past 20 years have identified the presence of heavy metals (arsenic, cadmium, copper, lead, mercury, and zinc) and organic contaminants in the sediments of the Upper Columbia River. The data and findings from these past studies and investigations have been used to develop a preliminary understanding of site conditions and contaminant distribution within the lake, river, and local tributaries.

\subsubsection{Portland Harbor Superfund Site}

The Lower Willamette Group, comprised of 10 parties that potentially share some of the responsibility for contamination at the site, developed and is currently implementing a remedial investigation and feasibility study (RI/FS) for the Portland Harbor Superfund Site in Portland, Oregon. The purpose of the Rl/FS is to "investigate the nature and extent of contamination for the in-water portion of the site, to assess the potential risk to human health and the environment, to develop and evaluate potential remedial alternatives, and to recommend a preferred alternative" (EPA 1998). The RI/FS was initiated with an extensive compilation of existing information, where approximately 700 documents and data sets were obtained that address conditions in the lower Willamette River and its confluences with the Columbia River. 
Figure 1-1. Columbia River Location Map.

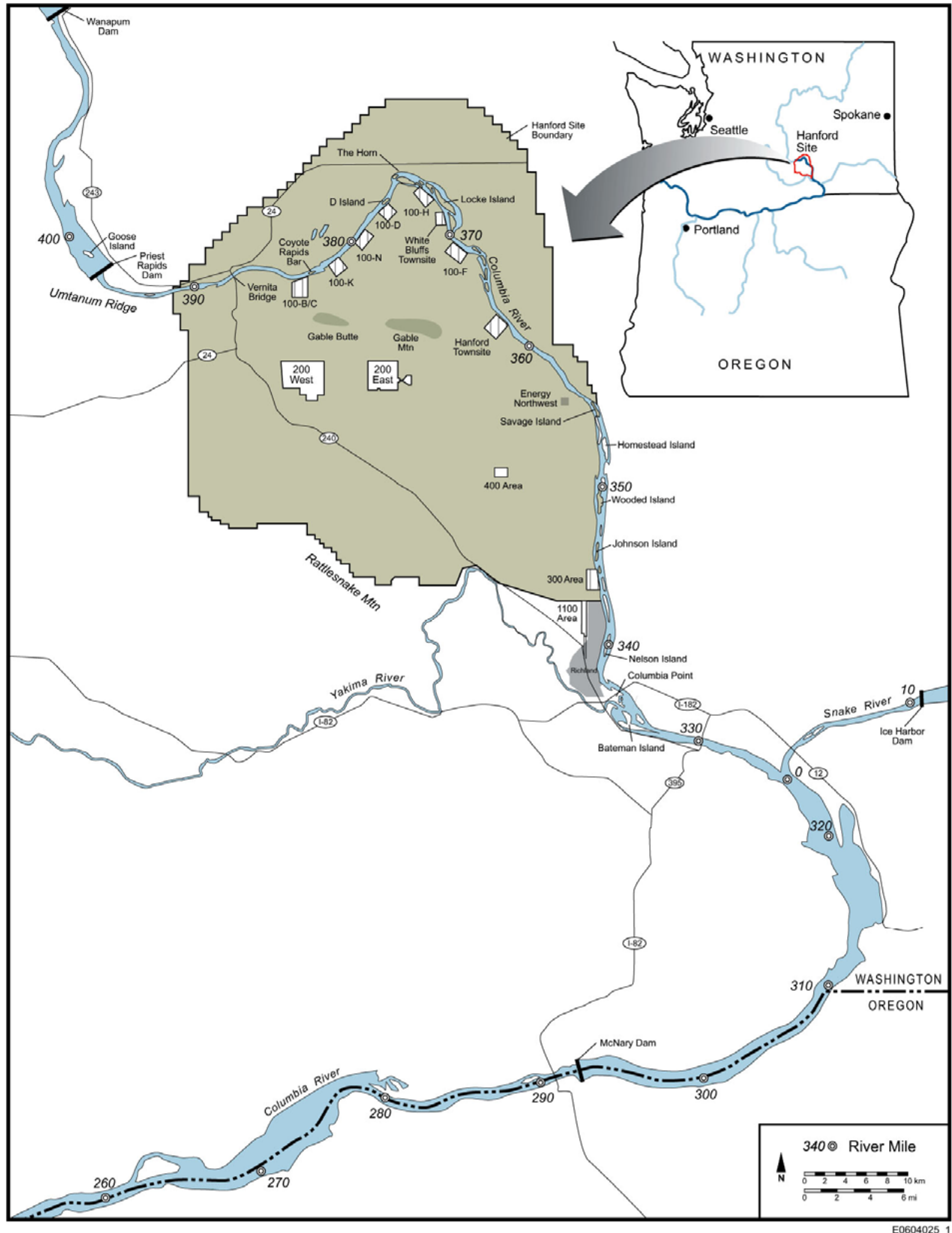


Figure 1-2. Columbia River Component Segments.

$\frac{1}{n}$

Not to scale

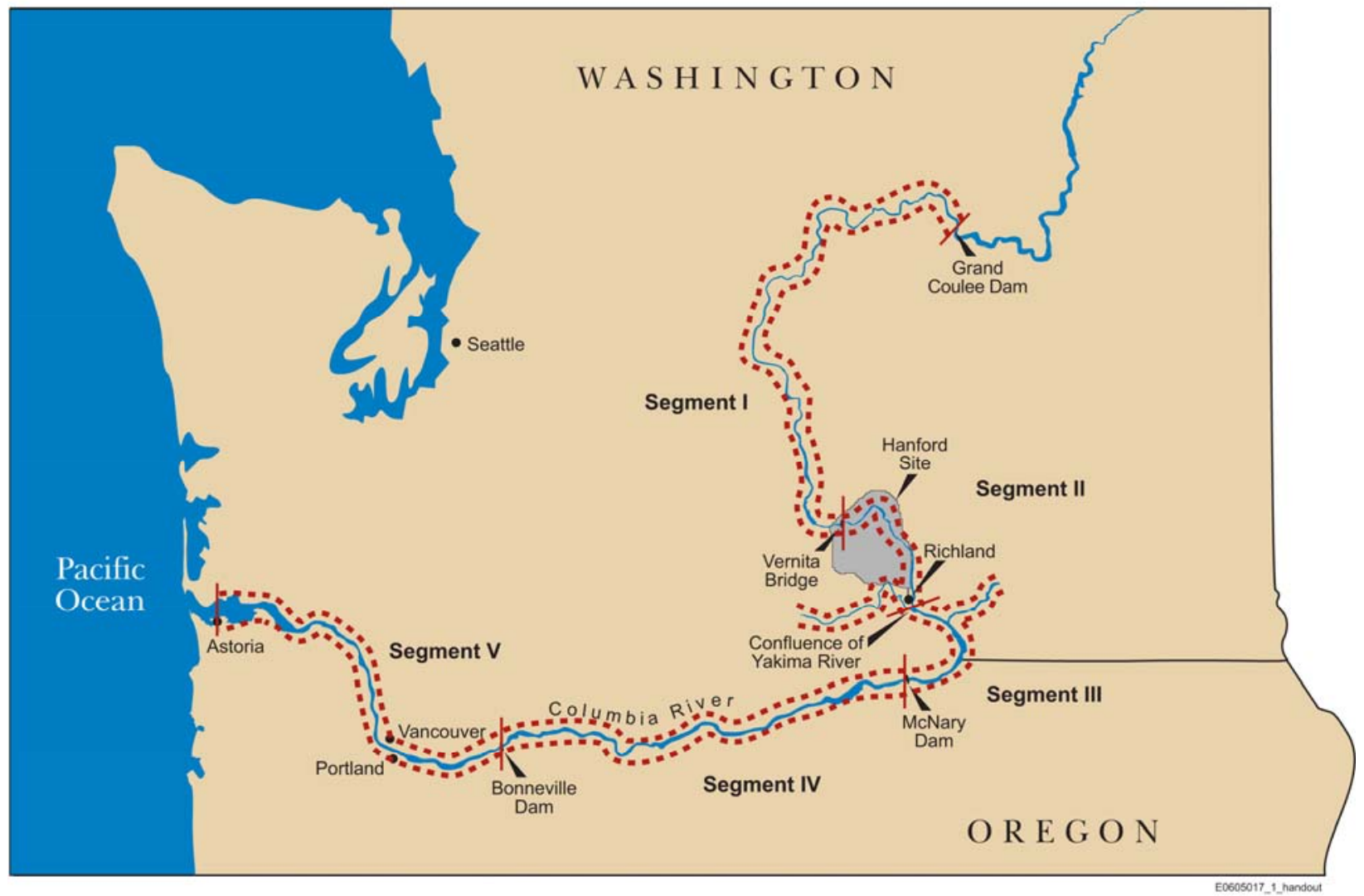


WCH-91

Introduction

Rev. 0 


\subsection{COLUMBIA RIVER CONTAMINANT SOURCES}

\section{$2.1 \quad$ NON-HANFORD SITE CONTAMINANT SOURCES}

Contaminant sources are defined as existing or potential sources of hazardous or radiological substances released to the environment that may pose a threat of adverse effects to human health or the environment. The purpose of this section is to identify the most important current and historic sources of contamination (both Hanford Site and non-Hanford Site) that have been released within the boundaries of the CRC. This is a summary of potential contaminant sources based on information obtained during the data compilation effort; it does not attempt to identify all possible sources of contaminants.

Six primary contaminant source types have been identified:

- Industrial

- Mining

- Municipal

- Agricultural

- Nuclear weapons production and testing

- Commercial/recreational vessels.

\subsubsection{Industrial}

Current and historical industrial activities and processes that may lead or may have led to point source releases to the Columbia River include aluminum production, pulp and paper mills, chemical production, and electrical power generation. Types of contaminants that may have been (or are being) released from facilities within the CRC include fluoride, aluminum, ammonia, nitrate, organic halides, dioxin, volatile organic compounds (VOCs), and semivolatile organic compounds (SVOCs). These facilities include National Pollutant Discharge Elimination System (NPDES) permitted, nonpermitted, and Comprehensive Environmental Response, Compensation, and Liability Act of 1980 (CERCLA) facilities.

There were over 200 NPDES permitted discharges to the Columbia River identified in 2005 from Grand Coulee Dam to the mouth of the river (http://www.ecy.wa.gov/programs/wq/permits/wplcs/index.html). Of these, 14 permits are classified as major industrial discharges of which only one of these point sources are located upstream of the Hanford Site (http://apps.ecy.wa.gov/industrial/final.asp; http://www.deq.state.or.us/wq/sisdata/basinsearchnew.asp). The majority of these major industrial discharge permits are associated with either pulp and paper, or aluminum facilities.

Additionally, eight CERCLA sites were identified that are located downstream from the Hanford Site along the CRC. These sites may have released, or may continue to release, wastes from aluminum, wood treatment, plating, and land disposal facilities. The types of contamination associated with these sites include ammonia, aluminum, arsenic, chromium, cadmium, copper, nickel, lead, cyanide, fluorides, fuel oil, polychlorinated biphenyls (PCBs), creosote components, pentachlorophenol, polyaromatic hydrocarbons (PAHs), VOCs, and SVOCs. 


\subsubsection{Mining}

Runoff from mining and milling operations along the tributaries feeding the Columbia River upstream of the Hanford Site is a potential source of metals contaminants. Large amounts of ore process wastes containing arsenic, cadmium, copper, lead, mercury, and zinc were produced in Washington, Idaho, and Canada from these operations. Previous investigations have detected elevated levels of these contaminants in the Upper Columbia River. The potential for particle transport and deposition of these contaminants into portions of the middle and lower Columbia River from these sources is likely.

\subsubsection{Municipal/Urban}

Municipal and urban activities contribute as point and nonpoint sources of contamination to the river. Other NPDES permitted discharges to the Columbia River include storm water, minor industrial process wastewater, contact and noncontact cooling waters, treated waters, construction sites, and domestic facilities. Effluents from municipal sewage treatment plants also contribute to waste loading within the Columbia River system. A total of 41 municipal sewage treatment plants were identified in 2005 that discharge effluent to the Columbia River within the study area.

Urban contributions including nonpermitted residential and commercial storm water runoff, residential use of fertilizers and pesticides, and septic sewage systems are some of the potential sources of contamination from communities along the banks of the Columbia River. Storm water runoff can contain a number of contaminants such as pesticide and weed control products, contamination from leaking transformers, hydraulic and lubricating fluids, petroleum products, metals, PAHs, and deicing salts. Runoff containing naturally occurring contaminants such as uranium also contributes to surface water contamination.

\subsubsection{Agricultural}

Agricultural activities are a potential source of contamination to the Columbia River via transport mechanisms such as runoff or wasteways that discharge irrigation returns to the Columbia River. An example is the Columbia Basin Irrigation Project, which began transporting water by canal in 1948 to more than 234,000 hectares (600,000 acres) of land in central Washington. It is estimated that the wasteway discharges account for approximately $0.4 \%$ of average river flows (Schneider 2002). Water from the wasteways in the Hanford Reach has been sampled to evaluate nutrient contributions from agriculture affects to surface waters. Nutrient contributions from these agriculture activities primarily consist of nitrogen, phosphate, and suspended solids. Sampling conducted in 1980 indicated that nitrate (as nitrogen) is the primary nutrient affecting the wasteways with loading, taken collectively from wasteways located in the South Columbia Irrigation District, near 50\% during the irrigation season (Ecology 1981). A larger study of water quality of the Columbia Basin Project irrigation return flows was initiated in 2002. This 5-year study will identify potential biological affects to the Endangered Species Act of 1973 listed species.

Sampling of irrigation return water from Franklin County and associated seeps resulting from irrigation practices entering the Columbia River, opposite the Hanford Site, have measured total uranium values of $8.6 \mathrm{pCi} / \mathrm{L}$ (PNL 1990). Uranium is commonly present in phosphate-based fertilizers and is a natural constituent that weathers from some types of rocks in the region. 
In recent years, total uranium concentrations in the Hanford Reach have been elevated along the Franklin County shoreline. Previous studies have indicated these elevated concentrations are likely the result of groundwater seepage and water from irrigation returns that contain natural occurring uranium.

The U.S. Geological Survey (USGS) has also documented nitrate groundwater contamination in Franklin County, which also seeps into the river along the Hanford Reach (USGS 1995). However, the USGS concluded that because of dilution in the river, river water sampling downstream from these sources show negligible differences in nutrient concentrations. The affects of nutrients to surface waters from agriculture activities are likely more substantial from downriver tributaries such as the Yakima, Snake, and Walla Walla Rivers.

Chloride, nitrate, and sulfate concentrations were also elevated along the Franklin County shoreline during annual monitoring in 2003 and also likely resulted from groundwater seepage associated with extensive irrigation (Poston et al. 2004).

\subsubsection{Nuclear Weapons Production and Testing}

Worldwide atmospheric nuclear testing contributed to radionuclide contaminants in surface waters and ultimately to sediments throughout the Pacific Northwest. Fallout from atmospheric testing by the United States, Russia, and China contributed significantly to radionuclide levels in the environment until 1963 (OHD 1994). The Nuclear Test Ban Treaty ended aboveground testing by the United States and Russia in 1964, and China continued limited aboveground testing until 1977 . The fallout materials consisted primarily of long-lived radionuclides such as cesium-137 and strontium-90, along with shorter-lived radionuclides such as cerium-141, zirconium-95/niobium-95, and ruthenium-103/106 (OHD 1994). Strontium and cesium are also associated with Hanford Site operations. The Soviet nuclear reactor accident at Chernobyl in 1986 also produced detectable levels of iodine-131 and cesium-137 in precipitation in Oregon (OHD 1994).

Past Hanford Site facility exhaust stack air emissions included both radiological and nonradionuclide emissions. It has been shown that residual levels along the Hanford Reach of radiologic, hazardous, or toxic materials from Hanford Site stack air emissions are negligible (DOE-RL 2005a).

\subsubsection{Commercial/Recreational Vessels}

Recreation and commercial activities on the Columbia River also contribute to some contamination to surface water and sediments via marinas, boats, or other recreational watercraft. Discharge of bilge and ballast water, spills, and materials associated with boat and shipyard maintenance are potential sources of contamination. These sources may contain old paint scrapings, anti-foulants, solvents, oil and grease, fuels, and cleaning agents. Vessel traffic and dredging activities within the marinas or shallow navigation channels can also resuspend sediments that may contain metals, nutrients, organic matter, and toxins into the water. Pilings, docks, and bulkheads associated with marine structures treated with creosote, chromated copper arsenate, or copper zinc arsenate are other sources of contamination. 


\subsection{UPSTREAM SOURCES}

The upstream segment of the CRC spans from below Grand Coulee Dam to the Vernita Bridge. Major tributaries along this stretch include the Okanogan and Wenatchee Rivers.

A major point source of metals contamination to the Columbia River is the Teck Cominco industrial/metallurgical complex in Trail, British Columbia. The primary source of historical loading of dioxins and furans to the Upper Columbia River was the Celgar pulp mill in Castlegar, British Columbia. Specific PCB point sources have not yet been identified (EPA 2004a).

As mentioned previously, only 1 of the 14 major industrial NPDES permitted facilities is located upstream of the Hanford Site, an aluminum smelter located near Wenatchee, Washington. Contaminants of interest associated with aluminum facilities include: fluoride, aluminum, benzo(a)pyrene, cyanide, and oil and grease. Nine of the 41 municipal treatment plants that discharge effluent to the CRC are located upstream of the Hanford Site. No CERCLA sites are located in the upstream segment of the CRC.

\subsection{HANFORD SITE SOURCES}

This segment of the Columbia River spans from the Vernita Bridge to the confluence of the Yakima River; and does not include any major tributaries. The purpose of this section is to discuss the historical releases to the Columbia River and summarize the current releases from groundwater contamination through seeps.

\subsubsection{Historic Hanford Reactor Releases to the Columbia River}

Eight nuclear reactors (105-B, 105-C, 105-KE, 105-KW, 105-D, 105-DR, 105-H, and 105-F) released contaminants to the Columbia River between 1944 and 1971 . These reactors are commonly referred to as single-pass reactors. The cooling water from these eight reactors was discharged through large pipes into retention basins and held for short periods of time to allow for decay of short-lived radionuclides and thermal cooling. After a period of decay, the water was then discharged directly into the Columbia River via outfall structures. The discharged cooling water contained radionuclides from the activation of impurities in the river and treated water. Cooling water also contained radioactive materials (fission products) that escaped from the fuel elements during the irradiation process (DOE-RL 1997). Although a variety of radionuclides were discharged, most of them had very small inventories or very short half-lives.

Nonradioactive contaminants in the cooling water discharges included lead and chromium. Lead entered the discharged water from process tubes, which were capped with lead-shield plugs that corroded over time. Chromium, in the form of sodium dichromate, was added to the reactor coolant to inhibit corrosion in the aluminum process tubes (DOE-RL 2005b).

The Hanford Site's ninth reactor, 105-N, used a closed-loop cooling system that did not discharge cooling water directly to the Columbia River. As cooling water became contaminated, it was bled off and routed to a covered trench for disposal to the soil. This provided opportunities for ion exchange with the soil and additional decay before the effluents eventually reached the Columbia River through the soil (DOE-RL 2002). 
The Hanford Site has one NPDES permit (WA-002591-7) that covers the DOE's nonradioactive discharges to the Columbia River from the 300 Area Treated Effluent Disposal Facility, 100-K Area discharges from secondary cooling water, and shoreline seepage of groundwater in the 100-N Area. Energy Northwest also discharges cooling tower blowdown water and treated effluents to the Columbia River within the Hanford Site boundary.

\subsubsection{Current Hanford Site Groundwater Sources}

Groundwater plumes serve as the current and future sources of contamination to the Columbia River. The following subsections summarize the current groundwater contaminants that are present in the 100 Areas and 300 Area and identify those that exceed drinking water standards as identified in Hanford Site Groundwater Monitoring for Fiscal Year 2005 (Hartman et al. 2006). In addition, seep information affecting the Columbia River and sediment monitoring are also presented. The 2005 groundwater plume contamination maps for both radionuclides and chemicals on the Hanford Site are shown in Figures 2-1 and 2-2, respectively.

\subsubsection{Summary of 100 Area Groundwater Contaminant Sources}

2.3.3.1 100-B/C Area. Groundwater flows primarily to the north toward the Columbia River beneath the 100-B/C Area. Groundwater contaminants in this area, commonly referred to as the 100-BC-5 Operable Unit (OU), include strontium-90, tritium, nitrate, and chromium. Of the four contaminants identified in the 100-B/C Area, only strontium-90 and tritium have been detected above the drinking water standards.

2.3.3.2 100-K Area. Groundwater beneath the 100-K Area generally flows to the northwest, with discharge to the Columbia River occurring through the riverbed sediments and, to a limited degree, as riverbank seeps during periods of low river stage (Hartman et al. 2006). Groundwater contaminants in this area, commonly referred to as the 100-KR-4 OU, include carbon-14, strontium-90, technetium 99, tritium, chromium, nitrate, and trichloroethene.

Of the seven groundwater contaminants identified in the 100-K Area, all but one (technetium-99) has been observed at levels above drinking water standards. A groundwater pump and treat system is currently operating in the $100-\mathrm{K}$ Area to reduce the chromium plume. The system began operation in 1997.

2.3.3.3 100-N Area. Groundwater flows primarily to the north and northwest, towards the Columbia River. Groundwater carrying mobile radioactive contaminants enters the Columbia River via a series of riverbank seeps, referred to as the N Springs. Groundwater contaminants in this area, commonly referred to as the 100-NR-2 OU, include strontium-90, tritium, nitrate, sulfate, petroleum hydrocarbons, manganese, and chromium.

Strontium-90 is the contaminant of greatest concern in this area. Aquifer tubes set in the gravel at the rivershore to monitor contamination have shown strontium-90 concentrations in excess of the drinking water standard. In addition to stronium-90, nitrate, chromium, and tritium have been identified at levels that exceed the drinking water standards in the 100-N Area. Tritium was not detected in the 100-N shoreline spring water during 2005.

A groundwater pump and treat system is currently operating in the $100-\mathrm{N}$ Area to treat strontium-90. The system began operation in 1995. 
2.3.3.4 100-D Area. Groundwater flows primarily to the north and northwest toward the Columbia River. Groundwater contaminants in this area, commonly referred to as the 100-HR-3-D OU, include chromium, strontium-90, tritium, nitrate, and sulfate.

Of the five groundwater contaminants identified in the 100-D Area, only chromium, nitrate, and tritium have been identified at levels above their respective drinking water standards. Hexavalent chromium is the contaminant of greatest concern in the groundwater. Aquifer tube sampling provides monitoring along the shoreline of the 100-D Area.

Groundwater remedial actions in the 100-D Area include two pump and treat systems and an in-situ redox manipulation system both operating to reduce chromium plumes. The first system began operation in 1997.

2.3.3.5 100-H Area. Groundwater flows from the southwest to northeast towards the Columbia River. Groundwater contaminants in this area, commonly referred to as the $100-\mathrm{HR}-3-\mathrm{H} \mathrm{OU}$, include chromium, nitrate, strontium-90, technetium-99, tritium, and uranium. Of the six contaminants identified in this area, all except tritium have been detected at concentrations above drinking water standards.

Chromium is the contaminant of greatest concern in the groundwater. A portion of the plume is thought to be migrating along the river based on aquifer tube results (PNNL 2003). A groundwater pump and treat system is currently operating in the $100-\mathrm{H}$ Area to reduce the chromium plume. The system began operation in 1997.

2.3.3.6 100-F Area. Groundwater flows primarily to the east and southeast beneath the $100-F$ Area. Groundwater contaminants in this area, also known as the 100-FR-3 OU, include chromium, nitrate, strontium-90, tritium, trichloroethene, and uranium. Only nitrate and trichloroethene have been detected in concentrations above the drinking water standards. Nitrate is the most widely distributed groundwater contaminant in the 100-F Area.

\subsubsection{Summary of $\mathbf{3 0 0}$ Area Groundwater Contaminant Sources}

The groundwater in this area, also known as the $300-F F-5$ OU, is divided into two areas: the 300 Area and the north region. Groundwater flow in the 300-FF-5 OU is generally to the east and southeast. However, in the 300 Area, flow is to the southeast and combines with flow from regions to the northwest, west, and southwest before discharging to the Columbia River. Groundwater flow in this area is strongly affected by changes in river elevation. Groundwater contaminants in this area include uranium, VOCs (cis-1,2-dichlroroethene, trichloroethene, tetrachloroethene), and strontium-90.

Tritium, nitrate, trichloroethene, cis-1,2-dichloroethene, and uranium are the contaminants that exceed their respective drinking water standards. However, several constituents migrate from sources outside of the 300 Area and include tritium and nitrate migrating from the 200 East Area. Nitrate and trichloroethene are also migrating into the 300 Area from offsite sources. The uranium plume in the 300 Area exceeds the drinking water standard in an area adjacent to the Columbia River. Uranium is monitored along the shoreline and in river water for this area. 


\subsubsection{Summary of 200 Areas Groundwater Contaminant Sources}

There are no groundwater contaminants from the 200 West Area that reach the Columbia River. Groundwater from the 200 East Area flows to the Columbia River along two separate paths. One path in the eastern portion of the site flows to the southeast, with a more eastern flow as it approaches the Columbia River. The other path is through the Gable Gap flowing to the northwest towards the Columbia River in an area between the 100-K and 100-B/C Areas. The primary contaminants include technetium-99, tritium, uranium, iodine-129, cobalt-60, cyanide, strontium-90, cesium-137, plutonium-239/240, and nitrate. Tritium and nitrate are the only two groundwater contaminants that currently reach the Columbia River from the 200 East Area. Of these two contaminants, only tritium is detected at concentrations above drinking water standards discharging to the Columbia River south of the Hanford Townsite.

\subsubsection{Columbia River Shoreline Springs}

Several contaminants entering the Columbia River from the Hanford Site continue to be detected in shoreline springs (seep) water. Radionuclides detected in spring water include tritium, strontium-90, technetium-99, iodine-129, uranium-234, uranium-235, and uranium-238. However, all radiological contaminant concentrations measured in the shoreline springs in 2004 were less than DOE-derived concentration guidelines (DOE Order 5400.5, Appendix D, Table D-5). Tritium in shoreline springs at the Hanford Townsite was the only radionuclide that was above the Washington State ambient surface-water quality criterion. Gross alpha ambient surface water quality criterion was also exceeded in the 300 Area. Nonradiological contaminants detected in spring water include metals and anions (chloride, fluoride, nitrate, and sulfate). However, the concentrations measured were below ambient surface water chronic toxicity levels with the exception of dissolved chromium.

Sediment samples are collected at shoreline springs in the 100-B, 100-F, 100-K, 300 Areas and the Hanford Townsite. During 2004, radionuclide concentrations in shoreline spring sediment were similar to those observed in the Columbia River sediment, with the exception of the 300 Area. In the 300 Area, uranium concentrations were two to four times the background concentrations measured from the Priest Rapids Dam. Concentrations of metals were similar to those from the Hanford Reach samples.

\subsection{DOWNSTREAM SOURCES}

This portion of the CRC spans from the confluence of the Yakima River to Astoria, Oregon. Major tributaries along this section of the Columbia River include the Yakima, Snake, John Day, Deschutes, Willamette, Lewis, and Cowlitz Rivers.

The downstream portion of the CRC consists of segments III, IV, and V. The Umatilla Army Depot (Lagoons) is the only CERCLA site located in Segment III. Contaminants of interest that might be released from this facility are heavy metals, trinitrotoluene, and cyclonite. Three of the 14 NPDES major industrial discharge permits are for facilities located in Segment III. The three facilities are AGRIUM, Boise Paper Solutions, and Goldendale Aluminum. AGRIUM is a fertilizer facility located in Kennewick, Washington, and might release ammonia and nitrate (Permit No.: WA-000367-1). Boise Paper Solutions is a pulp and paper mill located in Wallula, Washington, and does not operate with contaminants of interest (Permit No. WA-000369-7). 
Goldendale Aluminum is an aluminum smelter located in Goldendale, Washington.

Contaminants of interest that might be released include fluoride, oil and grease, benzopyrene, nickel, and arsenic (Permit No. WA-000054-0).

Union Pacific Railroad Tie Treatment and Martin Marietta Aluminum Company are the two CERCLA sites both located in The Dalles, Oregon, which falls within Segment IV of the CRC. Contaminants of interest that might be released from these facilities are pentachlorophenol, fuel oil, ammonia, arsenic, cyanide, fluoride, asbestos, and PAHs. Northwest Aluminum is the only NPDES major industrial discharge permit in Segment IV, also located in The Dalles, Oregon. Contaminants of interest that might be released from this facility include fluoride, oil and grease, benzopyrene, nickel, and arsenic.

The five remaining CERCLA sites are located in Segment $\mathrm{V}$. They include Reynolds Metals in Troutdale, Oregon; Allied Plating, Inc. in Portland, Oregon; Alcoa, an aluminum smelter, in Vancouver, Washington; Frontier Hard Chrome, Inc. in Vancouver, Washington; and Hamilton Island Landfill in North Bonneville, Washington. The combined contaminants of interest for these facilities include PAHs, aluminum, cyanide, fluoride, PCBs, arsenic, heavy metals including chromium, nickel, lead, and VOC and SVOCs.

The majority of agricultural land along the Columbia River is located in the downstream region. Agricultural activities historically contribute runoff containing fertilizers, sediments, pesticides, and herbicides to the surface waters

The higher populated downstream region of the Columbia River contains more commercial and recreational vessel use than the upstream region. The majority of possible contaminants, mentioned in the above section, are introduced to the Columbia River in this region. 
Figure 2-1. Hanford Site 2005 Groundwater Plume Contamination Maps for Radionuclides (Hartman et al. 2006).

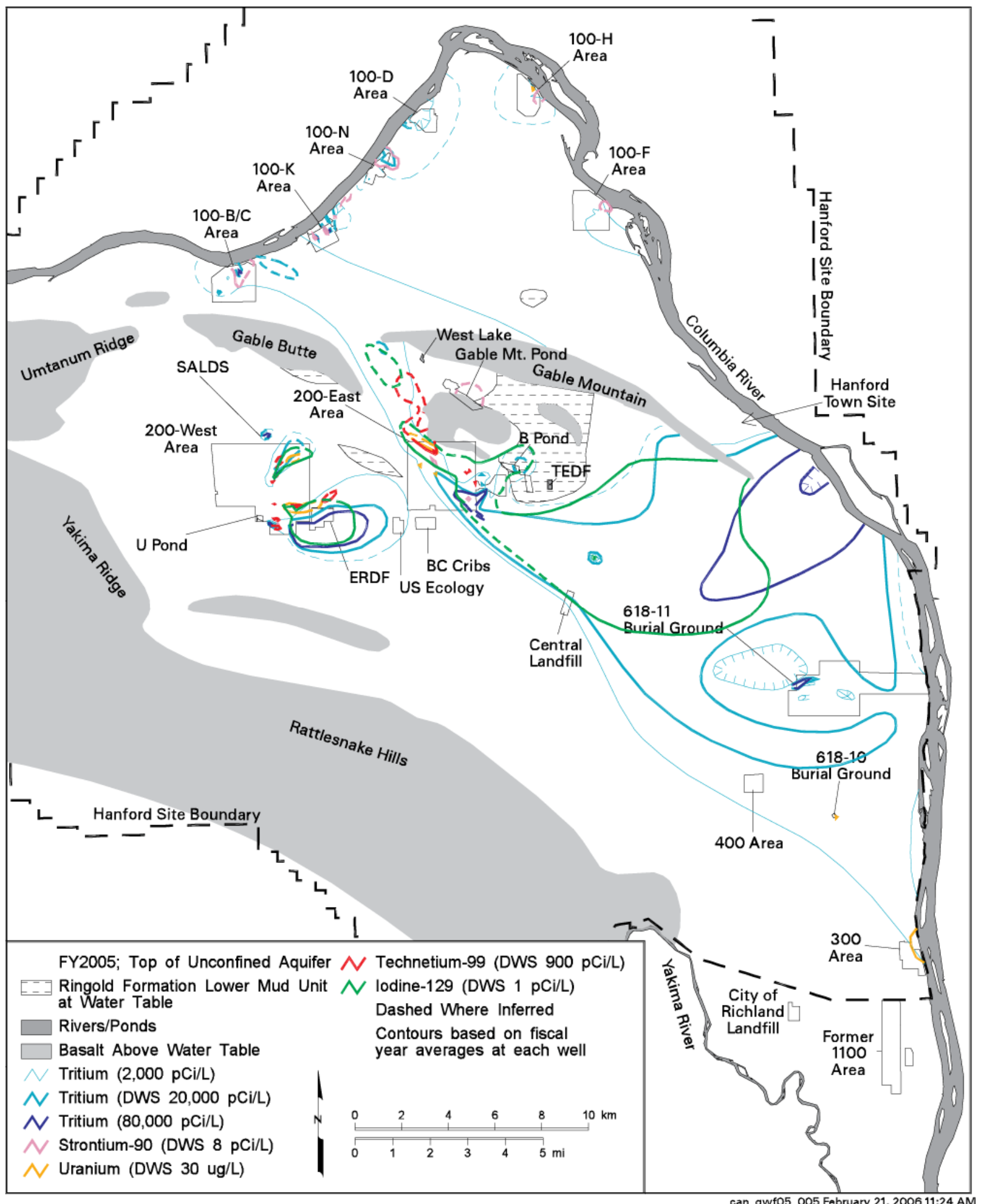


Figure 2-2. Hanford Site 2005 Groundwater Plume Contamination Maps for Chemicals (Hartman et al. 2006).

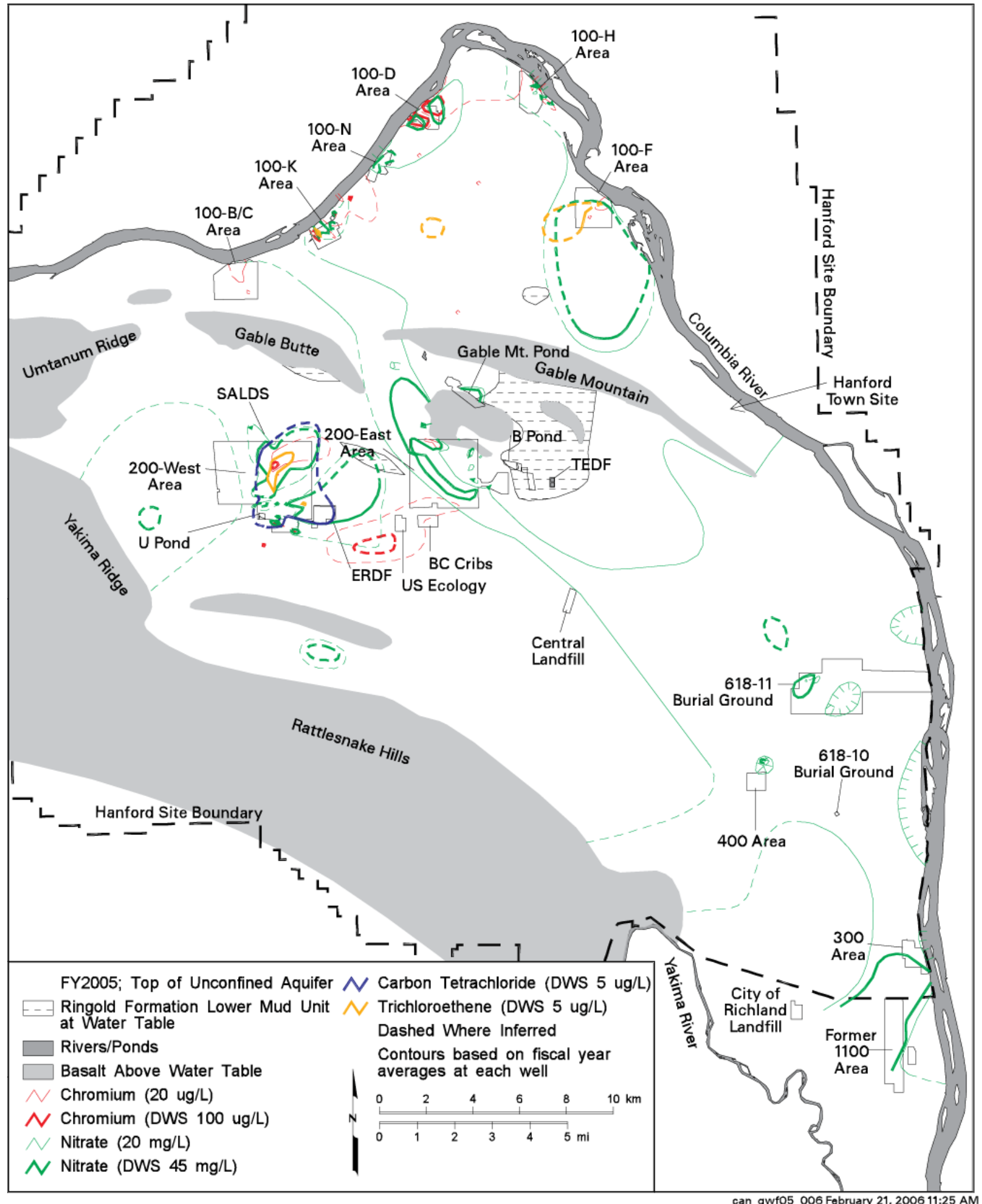




\subsection{HISTORICAL DATA QUALITY REVIEW PROCEDURES}

The primary purpose of the CRC data compilation and evaluation task was to conduct a thorough and comprehensive review of all appropriate and available information, past and present, to characterize the extent of Hanford Site-related contamination within the defined boundaries of the CRC defined in DOE-RL (2004). This section focuses on the procedures used to perform data quality reviews of all environmental media of interest entered into the CRC database. A data quality review was conducted for compiled historical chemistry and radiological data for sediment, water, and biota samples. The purpose of this review was to determine the relative quality of chemical and radiological data associated with each bibliographical source.

The steps for the review of all documents and their associated data include an initial evaluation to confirm the source of the information prior to database entry, confirmation of the quality assurance (QA) category assigned to the data in each document, review of the associated QA/quality control (QC) information associated with the analytical data provided in the source prior to data entry, and assignment of the initial analytical data by sample media to each CRC segment. If, during the course of the review, additional QA/QC data were obtained, the data were then reevaluated and the data category was reassigned as appropriate.

\subsection{REVIEW OF BIBLIOGRAPHICAL SOURCE INFORMATION}

The types of bibliographical resources that were identified and reviewed include databases, government publications, technical reports, newspapers, professional journals, books, and Internet web sites of local, state, and federal agencies.

A preliminary bibliographical source information analysis, providing a summary description of the source documents submitted for the CRC Bibliographic Database, is provided in WCH-64 (WCH 2006). The document states there were 1,540 source documents gathered for the database. The first step of the data evaluation was to confirm these sources and ensure that the bibliographic information for each source was complete and could be verified prior to finalizing the entries into the database.

The minimum information required for bibliographic source entry included the document title, publication date, author, organization responsible for sampling, document revision (if applicable), document type, program type, document repository, geographic region(s) covered by the source, description of the document contents, abstract or main points and finding of the document, data category, and website if the document was available online at the time of initial entry. Each input was confirmed for each bibliographic source, either with the hardcopy data package provided by the subcontractor or by reviewing the online document when it was available through a website.

As a result of reviewing the bibliographical database, some sources of information were eliminated. The number of sources was reduced from 1,540 sources to 1,448 sources. Instances where sources were eliminated included the following:

- Bibliographic source entries that were entered more than once 
- Bibliographical sources that had no relevance to the Columbia River or tributaries associated with the Columbia River

- Bibliographical sources listed as photographs of the study site taken during various times in history because the photographs were not included in the release of hardcopy reports to the $\mathrm{CRC}$ staff and the entry was deemed incomplete

- Bibliographical sources where hardcopy reports or online sources could not be confirmed by accessing the source repository listed in the bibliography or through an independent search by the CRC staff.

Databases maintained by government entities were among the sources of bibliographic entries in the CRC database, though they did not meet the minimum requirements for information entry as stated above. These sources typically have no document number, author, program type, or abstract information for the database. However, each database source maintains its own QA/QC program, and the data obtained from these databases were directly uploaded into the $\mathrm{CRC}$ database because this information is of value to the data evaluation effort. These databases were included as bibliographic sources and include the following examples:

- Ecology's Environmental Information Management (EIM) System database

- Washington Department of Health Environmental Radiation Oversite Program database

- EPA's Environmental Radiation Ambient Monitoring System (ERAMS) database

- Sediment Quality Information System (SEDQUAL).

Often a database is cited as the source of analytical data associated within a listed document. In these instances, the database that "houses" the analytical data is noted within each individual bibliographic entry. Data downloaded from the Hanford Environmental Information System (HEIS) database was placed in the individual bibliographic entry associated with the data.

Each bibliographic source can be classified as either primary or secondary, depending on whether the data contained within are original. Primary sources were defined as original sources of information or data, whereas secondary sources included information or data that was interpreted, analyzed, or summarized from the original source. Both primary and secondary sources were included in the database; however, secondary sources include a reference to the primary source within the database. When data were provided with a primary and secondary source, only analytical data from the primary source was entered.

\subsection{QUALITY ASSURANCEIQUALITY CONTROL REQUIREMENTS}

For each bibliographical source of information containing analytical data, a hardcopy of the report (i.e., data package) was reviewed to either confirm or update the QA category that was assigned to the source document (QA categories are described below). Categories indicate the extent and pedigree of the numerical information for the CRC data evaluation/scoping report and future efforts. The following descriptions indicate the differences between each of the data quality categories. 
Category 1 (QA1) - Data are of known quality and are considered to be acceptable for decision making in the data evaluation/scoping report. These data contained evidence of or documented the QA/QC criteria listed below. Data are not rejected as Category 1 based solely on the absence of a chain of custody (i.e., traceability). If a data set satisfies all other criteria except chain-of-custody documentation, there may be other documentation that demonstrates compliance with industry standard field collection and data documentation requirements. This other documentation will be assessed on a case-by-case basis with project personnel to make a final determination on the data category.

Category 2 (QA2) - Data are of partially known or suspect quality because QA/QC criteria are either incomplete or were not discussed or found. These data are considered suitable for qualitative use and may be considered suitable for further evaluation based on project-specific data quality objectives and intended end uses.

Category 3 (QA3) - Data that are of unknown quality and may have sample concentrations, but lack an adequate level of supporting QA/QC information, or are summarized and only provide ranges of concentrations. These data can be used on a limited or provisional basis for qualitative comparisons with other Category 1 or 2 data.

Category 4 (QA4) - No analytical data were reported.

The quality of the analytical data determines how the information was used within this CRC data evaluation summary report and future efforts. Category 1 data are of known quality and can be used to confidently characterize the extent of contamination with in the Columbia River (where contaminants have come to reside). Category 1 data may also be used for potential future risk assessment purposes. Category 2 data are of partially known or suspect quality and can be used to identify constituents of interest, data gaps, and general trends in contaminant concentrations and can help define future sampling needs. As previously indicated, Category 3 data can be used on a limited or provisional basis for qualitative comparisons with other Category 1 or 2 data.

The data quality categories are based on the QA/QC criteria described below. The quality of the data was determined using EPA's QA/QC criteria (EPA 2001). Performance criteria for establishing acceptable data quality included evidence of each of the following factors:

1. Traceability: The chain-of-custody is documented and complete, and attached to the report of supporting documentation package. However, the data is not rejected as Category 1 based entirely on the absence of chain-of-custody documentation. If a high-quality data set satisfies all criteria except chain-of-custody documentation, there may be references to chain-of-custody forms in the text of a report or the appendix. There may be other documentation that demonstrates compliance with the industry standard field collection and data documentation requirements, and implies chain-of-custody forms were used.

2. Comparability: Analytical procedures or methods are identified and are accepted as "standard" or "universal." These acceptable methods are identified in WCH-64 (WCH 2006).

3. Sample Integrity: Sample holding times, preservation, and conditions between collection and analysis meet established criteria, which are generally identified by the EPA. 
4. Potential Measurement Bias: The potential for introduction of positive biases in the reported results exists due to the presence of background or laboratory contamination. The evaluation of precision and accuracy is used to determine the extent of measurement bias caused by laboratory or background contamination. Generally, a goal of $\pm 30 \%$ is specified for both accuracy and precision.

Laboratory Contamination - Each analytical batch contains a laboratory (method) blank (material of similar composition as the samples with $\mathrm{known} /$ minimal contamination of the analytes of interest) carried through the complete analytical process. The method blank is used to evaluate false-positive results in samples due to contamination during handling at the laboratory.

Accuracy - Matrix spikes, laboratory control samples, and surrogate recoveries are performance measurements for evaluating accuracy. For most analytes, a known quantity of representative analytes of interest and matrix spike is added to a separate aliquot of a sample from an analytical batch. The known amount added is compared to the actual measured amount to calculate the percent recovery. The recovery percentage of the added matrix spike is used to evaluate analytical accuracy. For analyses not amenable to matrix spike techniques (e.g., gamma energy analysis or where analytical recovery is corrected via internal standards (e.g., alpha energy analyses), accuracy is evaluated from recovery of tracers or carriers.

In addition to the matrix spike recovery, surrogate compounds are used to evaluate accuracy in the VOC, SVOC, and PCB compound analyses. Surrogates are compounds with instrumental responses that are typical of the other analytes. The surrogates are added to the blanks, samples, and matrix spikes, and the recovery is evaluated.

The accuracy of the laboratory preparation and analysis is evaluated via $Q C$ reference samples (e.g., laboratory control spike). A laboratory control spike is prepared from an independent standard at a concentration other than that used for calibration, but within the calibration range. The laboratory control spike or QC reference sample measures the accuracy of the analytical process.

Precision - Separate aliquots removed from the same sample container (duplicate samples) are analyzed for each analytical batch for radionuclides and metals. The duplicate sample results are compared to the original sample results, are evaluated as relative percent differences, and are used to assess analytical precision. Alternately, a matrix spike duplicate may be used for assessing precision of metals and PCB. For a matrix spike duplicate, a separate aliquot is removed from the same sample container, and spiked in the same manner as the matrix spike. The recoveries from the matrix spike and matrix spike duplicate are used to calculate a relative percent difference and thus to assess precision.

5. Sample Bias: Evidence of field duplicates, field splits, or standards, or evidence of data qualifiers based on review of the above-mentioned criteria.

All of these factors require supporting information for placement into the Category 1 designation type. If supporting documentation for each of these factors were not available or were not reinforced by the availability of other high-quality QA/QC information, data were assigned to Category 2 designation type. If the acceptance criteria for any of the above factors were not 
satisfied for either the entire data set or a specific analyte group, data for that data set of group are qualified and determined to have limited usefulness.

\subsection{ANALYTICAL DATA ENTRY INTO THE CRC DATABASE}

The quality of the contents of the database is maintained by several means. A primary key is a type of table constraint that prevents duplicate entries in a table. The relationship between the bibliographic information and the sample information and between the sample information and the analytical results is maintained through the use of foreign keys and triggers. Foreign keys are table constraints that require that entries to a table be related to another table. For example, the following rules are enforced through the use of foreign keys in the database:

- All results have sample numbers

- All sample numbers have results

- All sample numbers have bibliographic sources

- A significant number of bibliographic sources will not have sample numbers and results.

When analytical results for a sample were entered manually or uploaded from an electronic source, the primary fields for each result were the document title and publication date; ensuring the sample result was unique to each source. For manual data entry, the constituent identification number (usually the CAS Registry number assigned to the chemical) selected from the pick list within the database was linked to the constituent name as reported in the source. The laboratory that performed the analyses, analytical method, analysis date, analytical value, analytical units, and lab qualifier(s) (if applicable) were entered exactly as reported. All reports entered manually were independently reviewed for accuracy.

For electronic sources of analytical data, data were uploaded exactly as reported. When a hardcopy was available, electronic results were reviewed against the hardcopy report.

For both manual and electronic sources, all results reported as nondetect concentrations were assigned with a "U" qualifier. In all cases, only one value was reported for nondetects and no distinction was made to determine the type (method detection limit, reporting limit, or quantitation limit).

It should be noted that the analytical data used in this evaluation were not independently validated. The qualifiers included in the database were carried forward from their source. A list of all data qualifiers included in the database and their definitions are found in Table 3-1.

\subsubsection{Standardization of Regional Datum}

Many different types of sample coordinates and descriptive information were obtained during the data compilation effort, resulting in considerable variability in the quality and type of this information provided in each bibliographic source. Regional datum (i.e., northings and eastings) were standardized for the purpose of obtaining an understanding of the spatial coverage of sample locations sampled and analyzed within the boundaries of the CRC. Each unique sample location was assigned two regional datum coordinate pairs to ensure that each location could be mapped using the Environmental Systems Research Institute (ESRI) Arclnfo Geographic Information System (GIS). The two regional datum coordinate pairs include 
(1) longitude/latitude and (2) State Plane Washington South (4602). When sample locations were not reported in the two selected regional datum formats, then they were converted into that format. Sample coordinate pairs were also reported in the following regional datum formats including: Decimal degrees, North American Datum of 1983/World Geodetic System of 1984 (NAD83/WGS84) easting and northing and Oregon State Plane.

In many cases no sample coordinates (in any format) were provided, but descriptive information about its information was provided. Descriptive information could include RMs, text descriptions, or a map or figure. It should be noted that when no coordinates and no descriptive information could be obtained, then these sample locations could not be mapped or included in the data evaluation.

\subsubsection{Methodology of Converting Descriptive Information into Sample Coordinates}

The following subsections provide a description of how descriptive information (RM, text, or maps) was converted to the selected regional datum formats.

3.3.2.1 River Miles. Transforming RM descriptions into sample coordinates (Cartesian x-coordinate, y-coordinate) required an electronic copy of National Geographic's Seamless USGS Topographic Maps for the States of Washington and Oregon. These USGS topographic maps were used to set waypoints where the USGS Topographic Maps identified RM locations. When the RM location was missing from the topographic maps (above Priest Rapids Dam), the RM was estimated. Setting waypoints allowed a set of points in latitude-longitude to be developed for the length of the Columbia River. This set of points was subsequently converted to Washington State Plane (South Zone) using version 6 of Corpscon, the U.S. Army Corps of Engineers Topographic Center software program for conversion of various coordinate systems. Corpscon uses the National Geodetic Survey (NGS) North American Datum Conversion (Nadcon) algorithms to convert between data.

River miles were shown on the electronic USGS Topographic Maps when the RM descriptions were from the tributaries feeding into the Columbia River (Snake River, the Yakima River, or the Willamette River). The same process was used to set waypoints and acquire the latitude-longitude values for the point when the RM was from a tributary.

3.3.2.2 Definitive Text Descriptions. When a text description was available for a sample location, then the location was estimated using the electronic USGS Topographic Maps. It was possible to locate the item (e.g., Cummins Road Bridge) by searching the internal gazetteer. When the item was found, a waypoint was established, the latitude-longitude coordinates preserved, and added to the database.

3.3.2.3 Hardcopy Maps. When only a map was provided with the bibliographic source a location was estimated using the GIS coverage developed for the CRC. A sample location could be estimated because the ESRI Arclnfo GIS coverage included tributaries, and named points, such as cities, allowing a location to be estimated.

3.3.2.4 Miscellaneous. In some cases, similar sample locations were common between more than one bibliographical source. When sample coordinates for similar locations were not reported in each bibliographic source, then the coordinates were applied to the common location. When sample coordinates were applied to similar locations, the location from which 
the coordinate was obtained was signified as "actual" in the accuracy field of the database. This process was also followed for several biota, surface water, and springs samples located on the Hanford Site.

\subsubsection{Verification of Sample Coordinates}

Sample coordinates were verified by visually inspecting each GIS map to ensure sample locations were in the proper location (or general area) based on the descriptions in the attribute information. Coordinates that were in error were re-assigned (moved) to their new location based on the location descriptions (signified as "moved on map" in the database). In instances where both a latitude-longitude and State Plane coordinate were provided, the conversion verified that both coordinates referenced the same location.

\subsubsection{Standardization of Concentration Units and Activity Levels}

All concentrations were standardized to $\mathrm{mg} / \mathrm{kg}$ for solid samples and to $\mathrm{mg} / \mathrm{L}$ for all liquid samples. Similarly, all activities were standardized to $\mathrm{pCi} / \mathrm{g}$ for solid samples and to $\mathrm{pCi} / \mathrm{L}$ for liquid samples. Units also reflect when a sample was reported on an "as received" or on a "dry weight". As received sample units represents measurements that were reported in the standard wet-weight reporting criteria. Dry-weight samples represent measurements reported in dry-weight criteria that were converted to the standard wet-weight unit. The dry-weight conversions were done using the following formulas:

Percent solids $=100-$ percent moisture

Wet-weight $=$ dry-weight $x($ percent solids $/ 100)$

\subsubsection{Decay of Radiological Results}

Sediment sample results included in this evaluation were analyzed between 1976 and 2004 . Radionuclides with short half-lives have decayed to insignificant levels during this 28-year time frame. All radionuclide results for sediment samples were decayed through 2005 to reflect current activity levels. The fraction of activity remaining after 29 years of decay was calculated using the following equation:

where:

$$
A_{r}=A_{0} e^{-\lambda t}
$$

$$
\begin{aligned}
& A_{0}=\text { activity at year of sample collection } \\
& A_{r}=\text { remaining activity (fraction of original amount) } \\
& \lambda=\text { radioactive decay constant, defined as: } L N(2) / T_{1 / 2} \\
& T_{1 / 2}=\text { radioactive half-life (radionuclide-specific) } \\
& t \quad=\text { elapsed time (29 years) }
\end{aligned}
$$

\subsubsection{Uncertainties}

Numerous uncertainties exist as a result of evaluating analytical data that were collected for purposes other than that intended for this report. As described earlier, analytical data were obtained from many different sources and information was reported in a variety of different methods and formats. Most sources of information reported data concentration differently (e.g., electronic, tabular, or appendices), provided different levels of supporting QA/QC 
information, and most reports were written to address the needs of a specific audience. The following paragraphs identify some of the uncertainties that were identified during the evaluation process and how the uncertainties were minimized where appropriate.

Based on the requirements set by the procedures for manual and electronic data entry, all database fields required population regardless of whether the information was provided in the report or electronic deliverable. However, in some instances this information was not provided. For example, the name and the location of the laboratory conducting the analysis and the analytical method were not reported on a consistent basis. When the name and location of the laboratory was not provided, it was entered as unknown in the database. If the name of the laboratory was not provided but supporting QA/QC information was provided, it did not affect the categorization of the associated data. Another example is when the analytical method numbers were not documented in the data source. A method number was assigned based on the description provided in the report or it was documented as an unknown method. Efforts were taken to obtain sampling and analysis plans and QA plans to provide complete information in the bibliographic source. For sources that could be verified, the analytical methods employed were appropriately assigned to the analytical result(s) in the source.

For a limited number of samples, the same sample number was assigned to more than one sample. For evaluation purposes, each individual sample was reassigned a unique sample number.

A number of samples were reported without coordinates documenting their location along the Columbia River. This practice was primarily observed for water and biota samples collected because they are mobile and were generally evaluated based on the general segment the sample was collected in. For sediment samples, sample coordinates were required because the results are used to determine where contaminants have come to reside. For those samples without reported coordinates, a map or other descriptor was used to estimate the approximate location. A comment was added in the database to indicate when a sample coordinate was estimated. When inadequate sample location information was available, the associated analytical data were retained in the CRC database, but was not included in the data evaluation and scoping effort.

Surface sediment data were reported using two different conventions, the first reporting the concentration on the basis of the actual weight of the sample ("as received") and the second reporting the concentration on a "dry-weight" basis. Surface sediment results reported on an "as received" basis represent the concentration of contaminant that the receptor comes in contact with or what is environmentally available. Surface sediment results reported on a "dry-weight" basis are considered biased high because the concentration reported represents only the contamination present in the dried sample.

\subsection{DESCRIPTION OF HISTORICAL DATA USED IN THE EVALUATION}

A substantial amount of historical data has been compiled using the quality review procedures discussed above. The extent to which these data can be used to characterize the extent of contamination within the Columbia River is determined by its QA category. 
For purposes of this evaluation, all Category 1 and Category 2 sediment data were used to characterize the extent of contamination within the Columbia River. To a limited extent, Category 3 sediment data were entered into the database when analytical data of higher quality were not available from a similar location. The majority of sediment core samples collected and analyzed were reported without supporting QA/QC documentation. Because a limited number of core samples were collected and analyzed, this information was entered into the database and used in the evaluation.

All Category 1 and 2 water sample data collected and analyzed since January 1, 1999, were considered representative of current contaminant concentrations potentially being transported to adjacent and downstream locations. Similarly, all Category 1 and 2 biota sample data collected and analyzed since 1995 were selected to represent current concentrations. Substantial amounts of analytical data were available for water and biota samples collected prior to these dates, but were not uploaded into the CRC database for this evaluation. However, the reports that contain these data are included as bibliographical sources of information.

\subsubsection{Summary of Samples Used in Evaluation}

As discussed earlier, a total of 1,448 source documents were entered into the CRC database that contained information pertinent to the CRC. Many of the sources entered into the bibliographical database did not contain analytical data representing contaminant concentrations measured in the sediment surface water or biota. Consequently, significantly fewer documents reported analytical data that could be used for the purpose of evaluating where contaminants have come to reside in the Columbia River. Sediment analytical data were acquired from a total of 72 sources meeting Category 1 QA requirements and 16 meeting Category $2 \mathrm{QA}$ requirements. Surface water analytical data were acquired from a total of 49 sources meeting Category 1 QA requirements and five meeting Category 2 QA requirements. From these 142 sources, a total of 1,578 sediment samples and 4,779 surface water samples were included in the data evaluation. A breakout of the sediment and surface water samples by segment is shown in Figures 3-1 and 3-2, respectively. Contaminant concentrations measured in biota were not reviewed against the QA categories. However, available biota data from 125 bibliographical sources that resulted in 8,606 samples were uploaded into the database and may require additional review to evaluate their quality and relevance to the health of the Columbia River.

The spatial distribution of all samples (including biota) included in the data evaluation for each of the respective segments is shown in Figures 3-3 through 3-7. Note that numerous surface water samples are collected at the same locations over the 5-year evaluation period. When numerous samples were collected from one location, the sample location is only represented by a single location on the figures.

\subsubsection{Sample Summary - Segment 1}

A total of 26 sources reported sediment analytical data associated with Segment 1 of the Columbia River. These sources include 136 Category 1 surface sediment samples collected between October 1992 and September 2004; 33 Category 2 surface sediment samples collected between September 1984 and October 1999, and four Category 3 sediment core samples. The majority of the surface sediment samples were collected behind the Priest Rapids Dam (121 samples); eight surface sediments collected near Vernita Bridge, four near 
Wanapum Dam, and the remaining eight samples were collected from other locations within Segment 1. One core was collected from the Okanogan River and the remaining three cores were collected behind the Priest Rapids Dam. A summary of the sources reporting sediment data within Segment 1 is provided in Table 3-2. Additionally, the data usability category, sample collection dates, number of samples, sample area, and contaminant classes that were analyzed are also included in Table 3-2.

A total of 19 sources reported surface water analytical data associated with Segment 1 of the Columbia River. These sources include 754 Category 1 surface water samples and 142 Category 2 surface water samples. The majority of the surface water samples were collected near the Priest Rapids Dam (300 samples) and near Vernita Bridge (451 samples), two samples were collected from the Methow River, and the remaining samples were collected from other locations along Segment 1 of the Columbia River. A summary of the sources reporting sediment data within Segment 1 is provided in Table 3-3. Additionally, the data usability category, sample collection dates, number of samples, sample area, and contaminant classes that were analyzed are also included in Table 3-3.

\subsubsection{Sample Summary - Segment 2}

A total of 55 sources reported sediment analytical data associated with Segment 2 of the Columbia River. These sources include 439 Category 1 surface sediment samples collected between October 1984 and March 2005; 36 Category 2 surface sediment samples collected between May 1978 and October 1991; 4 Category 3 samples collected in August 1986; and one Category 3 core sample collected from the Hanford Ferry area. Four Category 3 composite core samples were also collected in the Hanford Reach at RMs 393, 378.5, 373, and 365. A summary of the sources reporting sediment data within Segment 2 is provided in Table 3-4. Additionally, the data usability category, sample collection dates, number of samples, sample area, and contaminant classes that were analyzed are also included in Table 3-4. Surface sediment sample locations were primarily collected from the areas identified in Table 3-5.

A total of 24 sources reported surface water analytical data associated with Segment 2 of the Columbia River. These sources include 3,203 Category 1 surface water samples collected between January 1999 and February 2005 and 14 Category 2 surface water samples collected between May 2002 and January 2004. A summary of the sources reporting sediment data within Segment 2 is provided in Table 3-6. Additionally, the data usability category, sample collection dates, number of samples, sample area, and contaminant classes that were analyzed are also included in Table 3-6. Surface water samples were primarily collected from the areas identified in Table 3-7.

\subsubsection{Sample Summary - Segment 3}

A total of 42 sources reported sediment analytical data associated with Segment 3 of the Columbia River. These sources include 269 Category 1 surface sediment samples collected between September 1988 and September 2004; 14 Category 2 surface sediment samples collected between June 1987 and October 1991; 32 Category 3 samples collected between December 1976 and May 1993; and 18 Category 3 core samples collected and, one from Wallula, two from Port Kelly, two from Refuge Island, one behind the Ice Harbor Dam, and 12 from behind McNary Dam. Four Category 3 composite core samples were also collected above McNary Dam. 
A total of 227 surface sediment samples were collected from the Columbia River, 16 from Wallula Lake, 170 from McNary Dam, and the remainder between RM 274 and RM 340 . In addition, sediment samples were collected from the tributaries including 43 samples from the Snake River (32 behind Ice Harbor Dam); 13 samples from Walla Walla River, and 20 samples from the Yakima River. A summary of the sources reporting sediment data within Segment 3 is provided in Table 3-8. Additionally, the data usability category, sample collection dates, number of samples, sample area, and contaminant classes that were analyzed are also included in Table 3-8.

A total of 14 sources reported surface water analytical data associated with Segment 3 of the Columbia River. These sources include 178 Category 1 surface water samples and 220 Category 2 surface water samples. A total of 18 samples were collected near McNary Dam, 308 samples were collected from Yakima River primarily near Kiona, 20 samples were collected from Snake River near Hook Park, and 35 samples were collected from the confluence of the Walla Walla River. A summary of the sources reporting surface water data within Segment 3 is provided in Table 3-9. Additionally, the data usability category, sample collection dates, number of samples, sample area, and contaminant classes that were analyzed are also included in Table 3-9.

\subsubsection{Sample Summary - Segment 4}

A total of 10 sources reported sediment analytical data associated with Segment 4 of the Columbia River. These sources include 56 Category 1 surface sediment samples collected between March 1992 and September 2004; 91 Category 3 samples collected between February 1965 and August 1993; and six Category 3 core samples were collected, one from Irrigon, Oregon, one from behind John Day Dam, and three behind The Dalles Dam. Surface sediment samples were primarily collected from The Dalles Dam (101 samples), John Day Dam (23 samples), Bonneville Dam (17 samples), and the remainder were collected from RM 145. A summary of the sources reporting sediment data within Segment 4 is provided in Table 3-10. Additionally, the data usability category, sample collection dates, number of samples, sample area, and contaminant classes that were analyzed are also included in Table 3-10.

A total of five sources reported surface water analytical data associated with Segment 4 of the Columbia River. These sources include 112 Category 1 surface water samples collected between February 1999 and June 2005. A total of 11 samples were collected near Umatilla, Oregon; 71 samples were collected near The Dalles, Oregon; and 30 samples were collected near Bonneville Dam. A summary of the sources reporting surface water data within Segment 4 is provided in Table 3-11. Additionally, the data usability category, sample collection dates, number of samples, sample area, and contaminant classes that were analyzed are also included in Table 3-11.

\subsubsection{Sample Summary - Segment 5}

A total of 23 sources reported sediment analytical data associated with Segment 5 of the Columbia River. These sources include 210 Category 1 surface sediment samples collected between February 1990 and February 2005; 54 Category 2 samples collected between July 1987 and November 1997; 204 Category 3 samples collected between January 1963 and November 1993; and eight Category 3 core samples were collected, two from RM 21, three 
from the Skipanon Entrance Channel, one from Clatskanie Flats, one from Young's Bay, and one from Gray's Bay. Surface sediment samples were collected in focused areas but represent the Columbia River from RM 9 through RM 128. Additionally, sediment samples were collected from several tributaries including four samples from the mouth of the Cowlitz River, four samples from the mouth of the Willamette River, and 11 samples from the Skipanon Federal Channel. A summary of the sources reporting sediment data within Segment 5 is provided in Table 3-12. Additionally, the data usability category, sample collection dates, number of samples, sample area, and contaminant classes that were analyzed are also included in Table 3-12.

A total of 15 sources reported surface water analytical data associated with Segment 5 of the Columbia River. These sources include 234 Category 1 surface water samples collected between January 1999 and June 2004. A total of 133 samples were collected from Beaver Army Terminal, 12 samples near Longview, Washington, 14 samples from Columbia Slough, 16 samples collected near Kalama, Washington, three samples from the Cowlitz River, three samples from the Lewis River, 18 samples from the Willamette River, and 24 samples from near Vancouver, Washington. A summary of the sources reporting surface water data within Segment 5 is provided in Table 3-13. Additionally, the data usability category, sample collection dates, number of samples, sample area, and contaminant classes that were analyzed are also included in Table 3-13. 


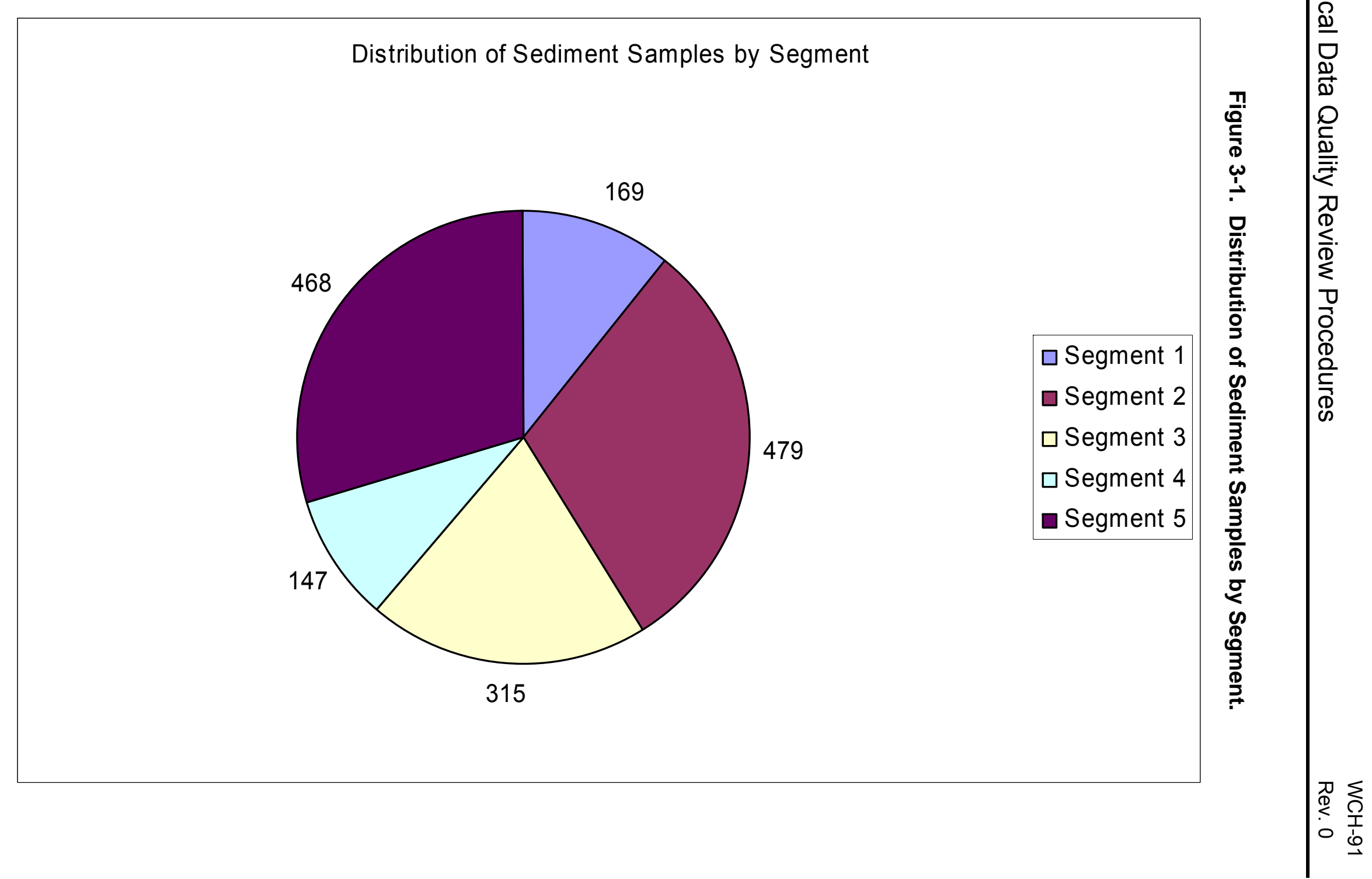




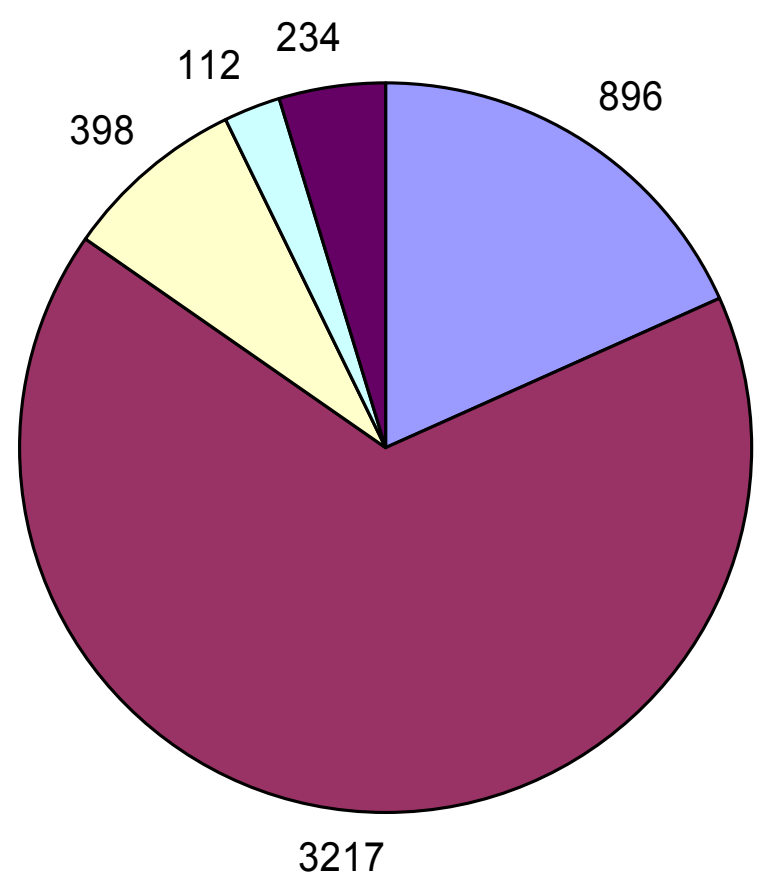

$\square$ Segment 1 $\square$ Segment 2 $\square$ Segment 3 $\square$ Segment 4 $\square$ Segment 5

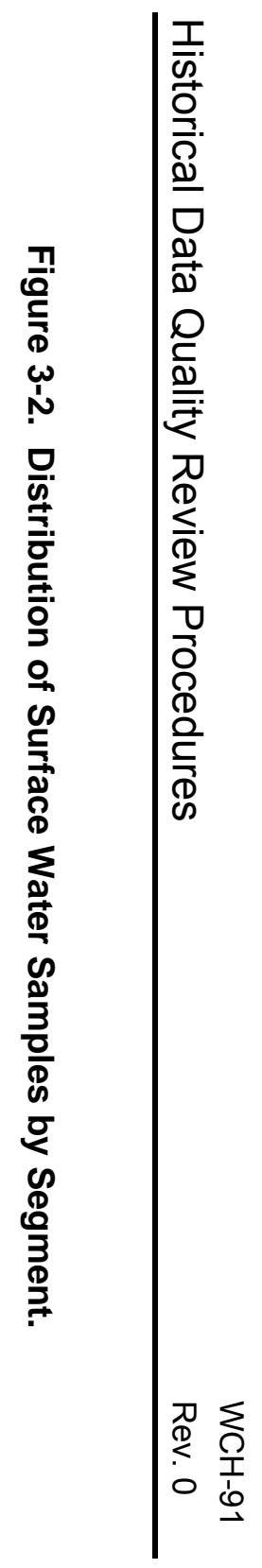




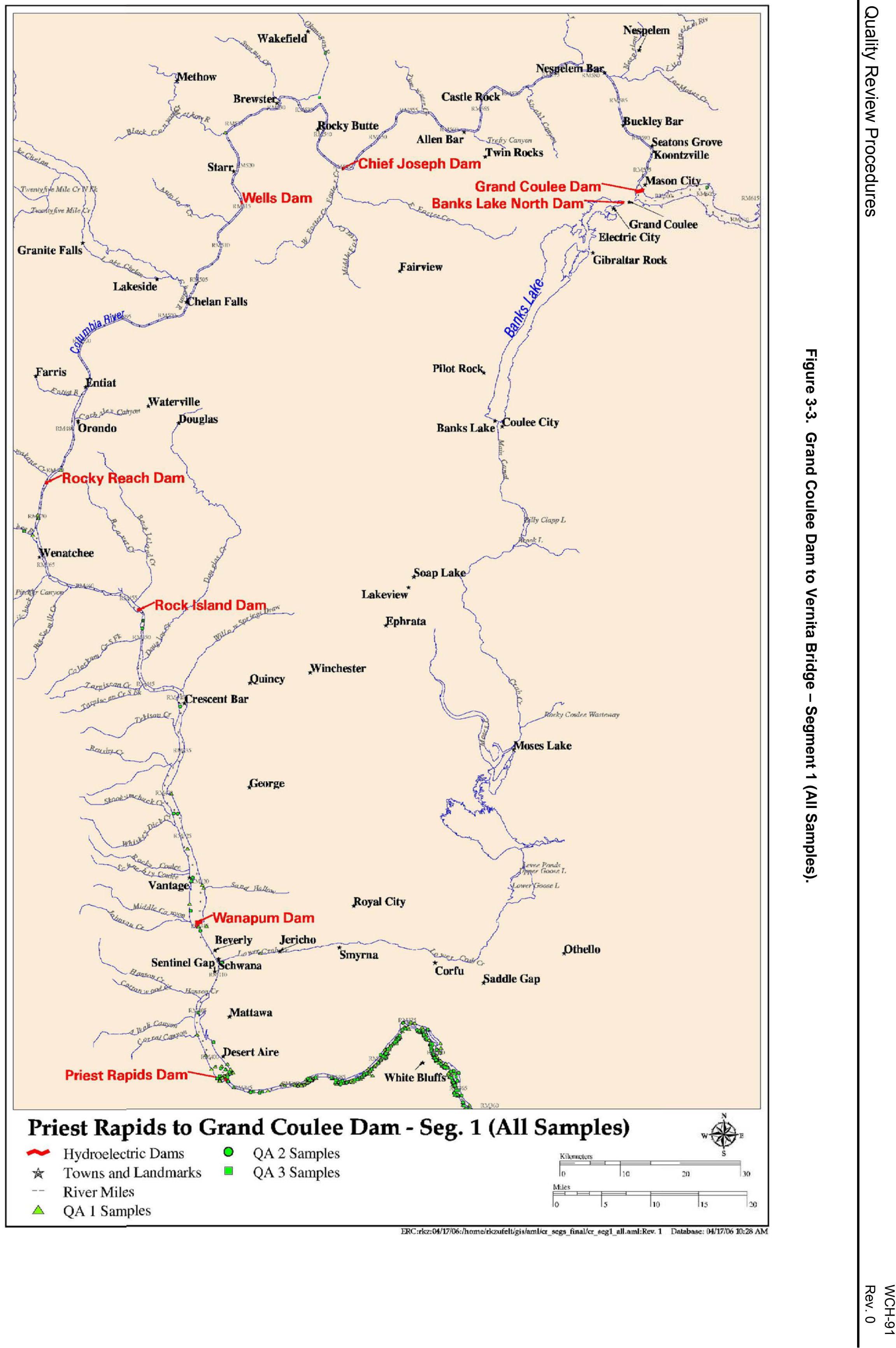


Figure 3-4. Vernita Bridge to Yakima River Confluence - Segment 2 (All Samples)

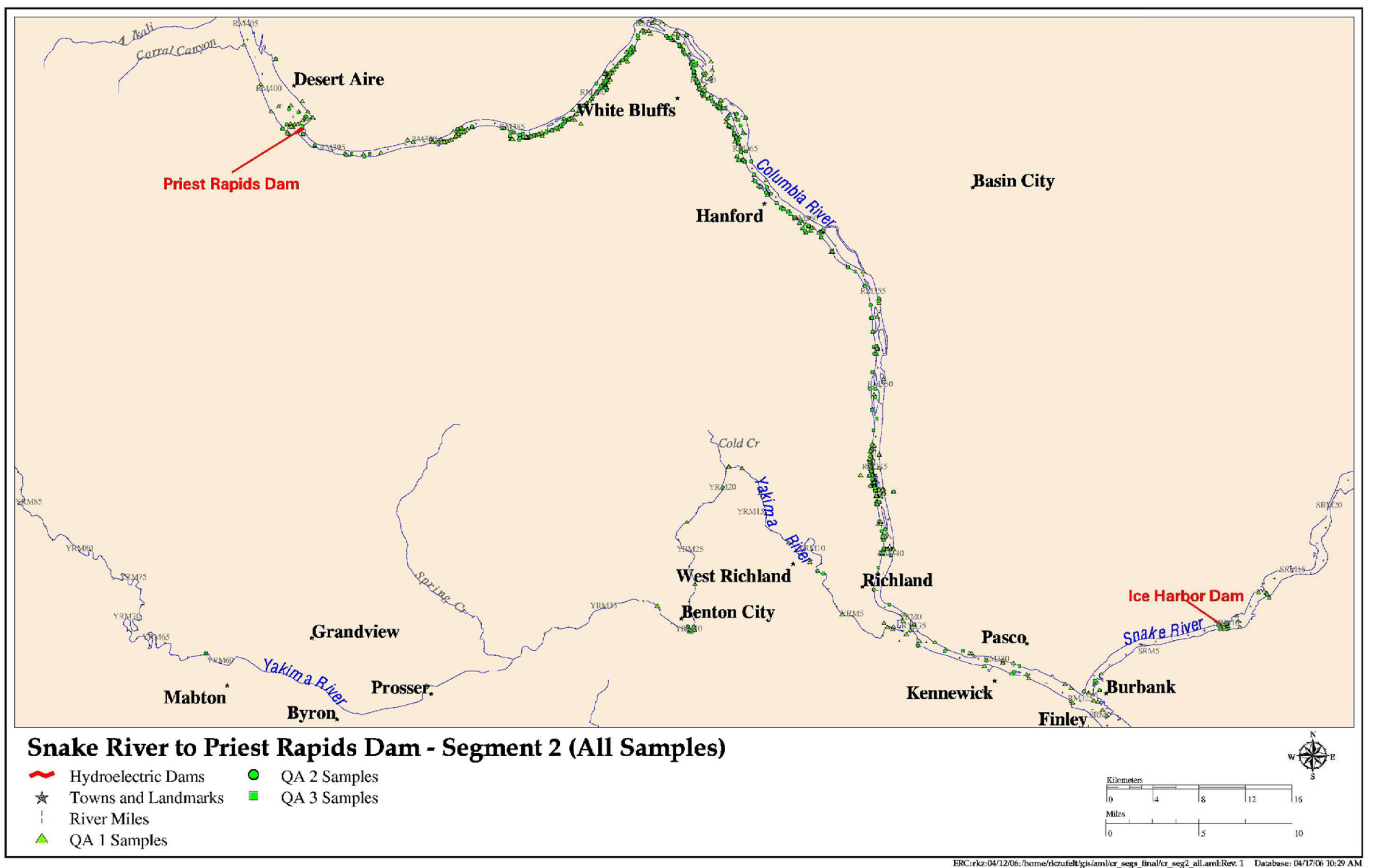


Figure 3-5. Yakima River Confluence to McNary Dam - Segment 3 (All Samples).

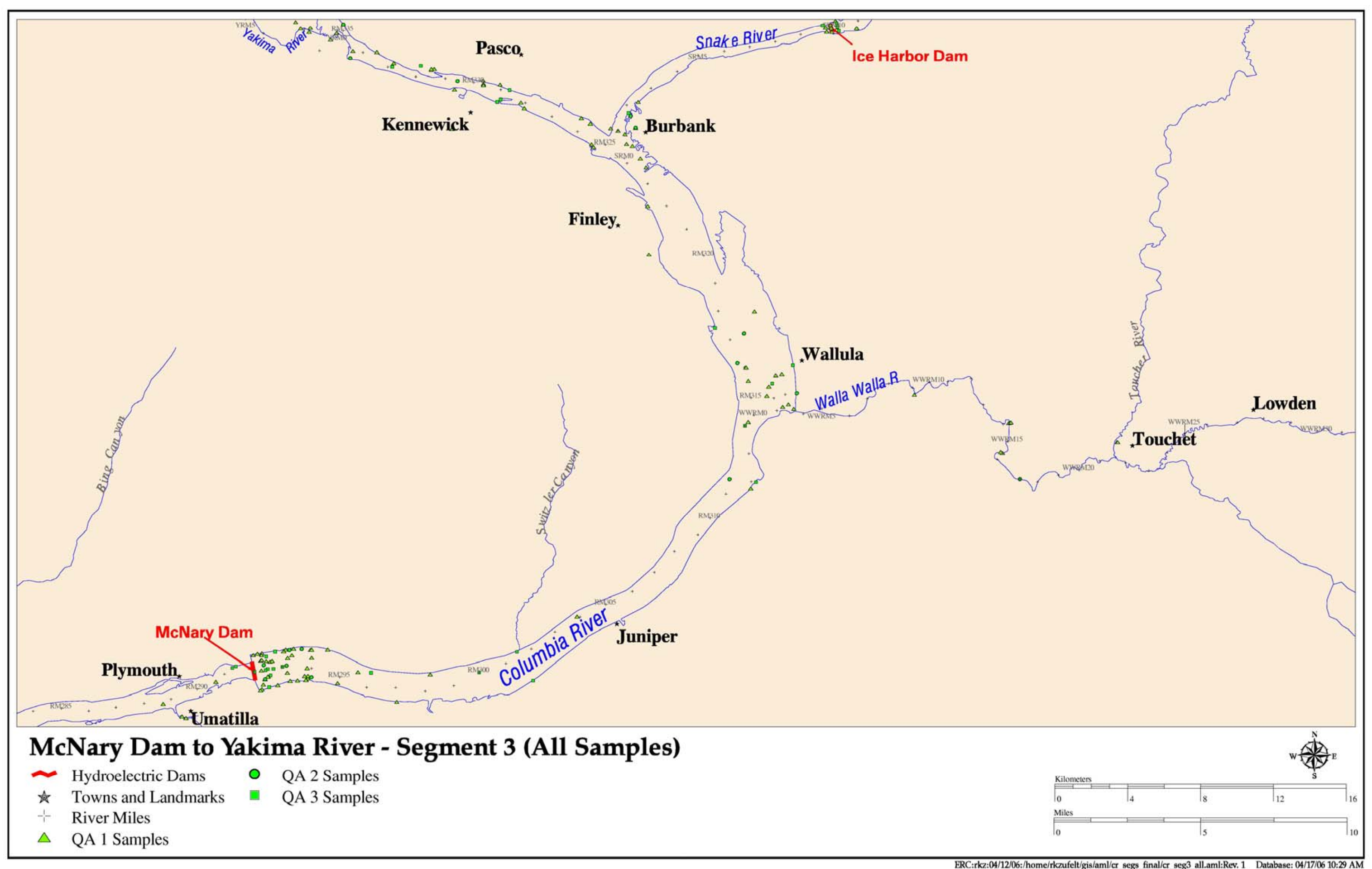




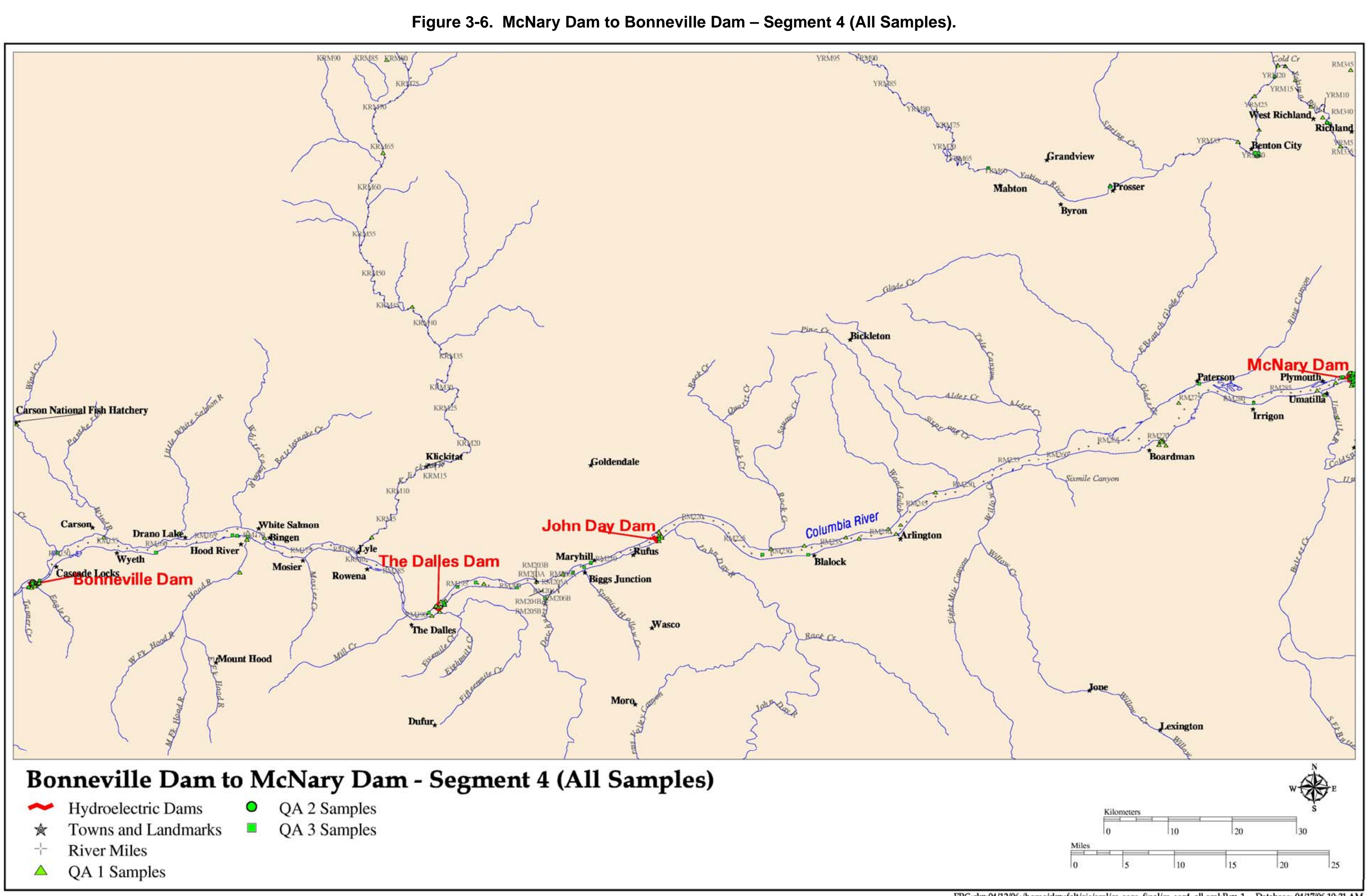


Figure 3-7. Bonneville Dam to Astoria, Oregon - Segment 2 (All Samples).

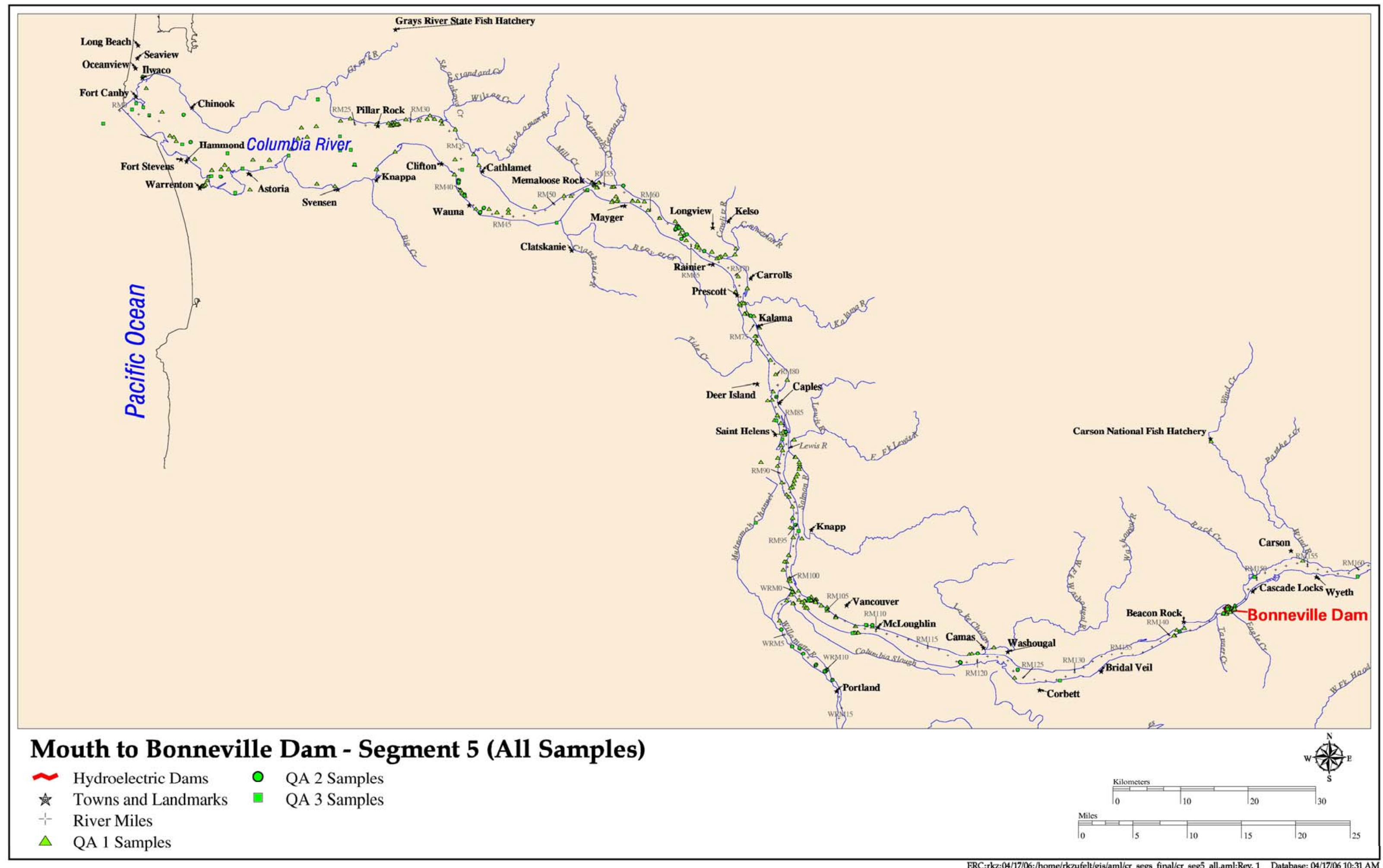


Table 3-1. Summary of Laboratory Qualifiers and Definitions.

\begin{tabular}{|c|c|}
\hline Qualifier & Definition \\
\hline * & Inorganics: Duplicate analysis not within control limits. \\
\hline+ & Inorganics: Correlation coefficient for method of standard additions is $<0.995$. \\
\hline$>$ & $\begin{array}{l}\text { Wet chemistry: Result greater than quantifiable range or greater than upper limit of the } \\
\text { analysis range. }\end{array}$ \\
\hline A & Organics: Valid for TICs only; the TIC is suspected aldol-condensation product. \\
\hline $\mathrm{B}$ & $\begin{array}{l}\text { Inorganics and wet chemistry: The analyte was detected at a value less than the contract } \\
\text { RDL, but greater than or equal to the IDL or MDL (as appropriate). }\end{array}$ \\
\hline $\mathrm{C}$ & $\begin{array}{l}\text { Inorganics and wet chemistry: The analyte was detected in the associated quality control } \\
\text { blank above the IDL/MDL. }\end{array}$ \\
\hline $\mathrm{D}$ & $\begin{array}{l}\text { Organics and wet chemistry: Analyte was identified in an analysis at a secondary dilution } \\
\text { factor (i.e., dilution factor different than 1.0). }\end{array}$ \\
\hline $\mathrm{E}$ & $\begin{array}{l}\text { Inorganics: Reported value is estimated because of interference. See comment on cover } \\
\text { page, hardcopy case narrative on specific Form I. }\end{array}$ \\
\hline $\mathrm{J}$ & $\begin{array}{l}\text { All: The reported value is an estimate. Analyte was analyzed for and detected but has } \\
\text { potentially larger associated error factors in the result. If this qualifier is applied for any } \\
\text { reason other than results reported close to the analytical detection limit, an explanation must } \\
\text { be provided in the associated hardcopy sample data summary package and/or case narrative. }\end{array}$ \\
\hline M & Duplicate injection precision not met. \\
\hline $\mathrm{N}$ & All: Spike sample recovery is outside control limits. \\
\hline$P$ & $\begin{array}{l}\text { Organics (polychlorinated biphenyl only): Aroclor target analyte with greater than } 25 \% \\
\text { difference between column analyses. }\end{array}$ \\
\hline Q & $\begin{array}{l}\text { Organics (dioxins only): Estimated maximum concentration. Used if one of the qualitative } \\
\text { identification criteria is not met (e.g., C1 isotopic rations outside theoretical range.). }\end{array}$ \\
\hline S & Inorganics: Reported value determined by the method of standard additions. \\
\hline$U$ & $\begin{array}{l}\text { All: Analyzed for but not detected above limiting criteria. NOTE: Limiting criteria may be any } \\
\text { of the following: value reported }<0 \text {; value reported }<\text { counting error; value reported }<\text { total } \\
\text { analytical error; value reported }<\text { MDL/IDL/MDA/PQL. }\end{array}$ \\
\hline W & $\begin{array}{l}\text { Inorganics: Post-digestion spike recovery for GFAA out-of-control limit. Sample absorbency } \\
<50 \% \text { of spike absorbency. }\end{array}$ \\
\hline $\mathrm{X}$ & $\begin{array}{l}\text { All: Other specific flags and notes required to properly qualify the result are described in the } \\
\text { hardcopy sample data summary package and/or case narrative. }\end{array}$ \\
\hline $\mathrm{Y}$ & Same as $\mathrm{X}$, if more than one flag is required. \\
\hline Z & Same as $\mathrm{X}$ and $\mathrm{Y}$ if more than two flags are required. \\
\hline $\begin{array}{ll}\text { GFAA } & = \\
\text { IDL } & = \\
\mathrm{MDA} & = \\
\mathrm{MDL} & = \\
\mathrm{PQL} & = \\
\mathrm{RDL} & = \\
\mathrm{TIC} & =\end{array}$ & $\begin{array}{l}\text { graphite furnace atomic absorption } \\
\text { nstrument detection limit } \\
\text { nethod detection analysis } \\
\text { method detection limit } \\
\text { oractical quantitation limit } \\
\text { eliable detection limit } \\
\text { entatively identified compounds }\end{array}$ \\
\hline
\end{tabular}


WCH-91

Historical Data Quality Review Procedures

Rev. 0 
Table 3-2. Summary of Documents with Segment 1 Sediment Data. (2 Pages)

\begin{tabular}{|c|c|c|c|c|c|c|c|c|c|c|c|}
\hline Source Title & $\begin{array}{l}\text { Data } \\
\text { Usability } \\
\text { Category }\end{array}$ & Begin Date & End Date & $\begin{array}{l}\text { Number of } \\
\text { Samples }\end{array}$ & Sample Area & RADs & METs & svoc & Pest & PCBs & $\begin{array}{c}\text { GEN } \\
\text { CHEM }\end{array}$ \\
\hline 1999 Hanford Environmental Oversight Program Data Summary Report & 1 & $8 / 3 / 1999$ & $8 / 3 / 1999$ & 1 & Columbia River RM 397.1 - Priest Rapids Dam & $\mathrm{x}$ & & & & & \\
\hline $\begin{array}{l}\text { Citizens Monitoring and Technical Assessment - Analysis of Chemical } \\
\text { Contaminants in Hanford Reach Biota and Environmental Materials at the } \\
\text { Perimeter of the Hanford Nuclear Reservation and on the Columbia River }\end{array}$ & 1 & 10/7/2003 & $10 / 7 / 2003$ & 1 & Columbia River - Vantage, Washington & $\mathrm{x}$ & $\mathrm{x}$ & & & & \\
\hline $\begin{array}{l}\text { Environmental Monitoring of Columbia River Sediments: Grain-Size } \\
\text { Distribution and Contaminant Association }\end{array}$ & 1 & 4/14/1994 & 4/14/1994 & 4 & Columbia River RM 397.1 - Priest Rapids Dam & $\mathrm{x}$ & & & & & \\
\hline Environmental Radiation Oversight Program - Database & 1 & $7 / 24 / 1995$ & $8 / 10 / 1998$ & 15 & Columbia River RM 397.1 - Priest Rapids Dam & $\mathrm{x}$ & & & & & $\mathrm{x}$ \\
\hline Environmental Radiation Program 1992-1994 Annual Report & 1 & 10/1/1992 & $9 / 22 / 1994$ & 8 & Columbia River RM 397.1 - Priest Rapids Dam & $\mathrm{x}$ & & & & & $\mathrm{x}$ \\
\hline Hanford Environmental Oversight Program 2000 Data Summary Report & 1 & $8 / 7 / 2000$ & $8 / 7 / 2000$ & 2 & Columbia River RM 397.1 - Priest Rapids Dam & $\mathrm{x}$ & & & & & $\mathrm{x}$ \\
\hline Hanford Environmental Oversight Program 2001 Data Summary Report & 1 & 7/19/2001 & $8 / 28 / 2001$ & $\begin{array}{l}2 \\
1\end{array}$ & $\begin{array}{l}\text { Columbia River RM } 397.1 \text { - Priest Rapids Dam } \\
\text { Columbia River RM } 388 \text { - Vernita Bridge }\end{array}$ & $\mathrm{x}$ & & & & & $\mathrm{x}$ \\
\hline Hanford Environmental Oversight Program 2002 Data Summary Report & 1 & $7 / 23 / 2002$ & $7 / 23 / 2002$ & 2 & Columbia River RM 397.1 - Priest Rapids Dam & $\mathrm{x}$ & $\mathrm{x}$ & & & & \\
\hline Hanford Environmental Oversight Program 2003 Data Summary Report & 1 & $7 / 14 / 2003$ & $7 / 14 / 2003$ & 2 & Columbia River RM 397.1 - Priest Rapids Dam & & & & & & \\
\hline Hanford Site Environmental Report for Calendar Year 1994 & 1 & 9/22/1994 & 9/24/1994 & 3 & Columbia River RM 397.1 - Priest Rapids Dam & & & & & & \\
\hline Hanford Site Environmental Report for Calendar Year 1995 & 1 & $7 / 24 / 1995$ & $7 / 24 / 1995$ & 4 & Columbia River RM 397.1 - Priest Rapids Dam & & & & & & \\
\hline Hanford Site Environmental Report for Calendar Year 1996 & 1 & 8/10/1996 & 8/15/1996 & 5 & Columbia River RM 397.1 - Priest Rapids Dam & & & & & & \\
\hline Hanford Site Environmental Report for Calendar Year 1999 & 1 & $8 / 3 / 1999$ & $8 / 3 / 1999$ & 6 & Columbia River RM 397.1 - Priest Rapids Dam & & & & & & \\
\hline Hanford Site Environmental Report for Calendar Year 2000 & 1 & $8 / 7 / 2000$ & $8 / 14 / 2000$ & $\begin{array}{l}2 \\
1\end{array}$ & $\begin{array}{l}\text { Columbia River RM } 397.1 \text { - Priest Rapids Dam } \\
\text { Wanapum Dam }\end{array}$ & $\mathrm{x}$ & $\mathrm{x}$ & & & & $\mathrm{x}$ \\
\hline Hanford Site Environmental Report for Calendar Year 2001 & 1 & $7 / 19 / 2001$ & $7 / 19 / 2001$ & 2 & Columbia River RM 388 - Vernita Bridge & $\mathrm{x}$ & $\mathrm{x}$ & & & & $\mathrm{x}$ \\
\hline Hanford Site Environmental Report for Calendar Year 2002 & 1 & $7 / 23 / 2002$ & $7 / 23 / 2002$ & 2 & Columbia River RM 397.1 - Priest Rapids Dam & $\mathrm{x}$ & $\mathrm{x}$ & & & & $\mathrm{x}$ \\
\hline Hanford Site Environmental Report for Calendar Year 2003 & 1 & $7 / 14 / 2003$ & $7 / 14 / 2003$ & 3 & Columbia River RM 397.1 - Priest Rapids Dam & $\mathrm{x}$ & $\mathrm{x}$ & & & & $\mathrm{x}$ \\
\hline $\begin{array}{l}\text { Mid-Columbia River Basin Sediment Chemical Contaminants Study June } \\
2004 \text { to October } 2004 \text { addendum }\end{array}$ & 1 & 6/1/2004 & $6 / 1 / 2004$ & $\begin{array}{l}1 \\
4 \\
3 \\
1\end{array}$ & $\begin{array}{l}\text { Columbia River RM } 391 \text { - Irrigation Returns } \\
\text { Columbia River RM } 397.1 \text { - Priest Rapids Dam } \\
\text { Columbia River RM 415.8 - Wanapum Dam } \\
\text { Lower Crab Creek - RM 5 - Above Delta } \\
\end{array}$ & & $\mathrm{x}$ & & & & $\mathrm{x}$ \\
\hline $\begin{array}{l}\text { Survey of Potential Hanford Site Contaminants in the Upper Sediment for } \\
\text { the Reservoirs at McNary, John Day, The Dalles, and Bonneville Dams, } \\
2003\end{array}$ & 1 & $7 / 14 / 2003$ & $7 / 14 / 2003$ & 6 & $\begin{array}{l}\text { Columbia River } \\
\text { RM 397.1 - Priest Rapids Dam }\end{array}$ & $\mathrm{x}$ & $\mathrm{x}$ & $\mathrm{x}$ & $\mathrm{x}$ & $\mathrm{x}$ & $\mathrm{x}$ \\
\hline $\begin{array}{l}\text { An Evaluation of Water Quality and Limnology for the Priest Rapids Project } \\
\text { Area }\end{array}$ & 2 & 10/1/1999 & 10/1/1999 & $\begin{array}{c}6 \\
1 \\
5 \\
5 \\
13 \\
1\end{array}$ & $\begin{array}{l}\text { RM 398.5 - Goose Island } \\
\text { RM 405.5 - Hansen Creek } \\
\text { RM 417.5 - Ginkgo Park } \\
\text { RM 428- Scammon's Landing } \\
\text { RM 440- Crescent Bar } \\
\text { RM 452- Rock Island Tailrace } \\
\end{array}$ & & $\mathrm{x}$ & $\mathrm{x}$ & & & $\mathrm{x}$ \\
\hline An Investigation of the Origin of 152 Eu in Columbia River Sediment & 2 & $8 / 3 / 1999$ & $8 / 3 / 1999$ & 1 Core & Columbia River RM 397.1 - Priest Rapids Dam & $\mathrm{x}$ & & & & & \\
\hline $\begin{array}{l}\text { Basic Water Monitoring Program Fish Tissue and Sediment Sampling for } \\
1984\end{array}$ & 2 & 9/1/1984 & $9 / 1 / 1984$ & 1 & Wenatchee, Washington & & $\mathrm{x}$ & & $\mathrm{x}$ & $\mathrm{x}$ & $\mathrm{x}$ \\
\hline
\end{tabular}


Table 3-2. Summary of Documents with Segment 1 Sediment Data. (2 Pages)

\begin{tabular}{|c|c|c|c|c|c|c|c|c|c|c|c|}
\hline Source Title & $\begin{array}{l}\text { Data } \\
\text { Usability } \\
\text { Category }\end{array}$ & Begin Date & End Date & $\begin{array}{l}\text { Number of } \\
\text { Samples }\end{array}$ & Sample Area & RADs & METs & svoc & Pest & PCBs & $\begin{array}{l}\text { GEN } \\
\text { CHEM }\end{array}$ \\
\hline $\begin{array}{l}\text { Reconnaissance Investigation of Water Quality, Bottom Sediment, and Biota } \\
\text { Associated with Irrigation Drainage in the Columbia Basin Project, } \\
\text { Washington, 1991-92 }\end{array}$ & 2 & 7/16/1992 & 7/16/1992 & 1 & Columbia River RM 354.4 - Irrigation Return & & $\mathrm{x}$ & $\mathrm{x}$ & & $\mathrm{x}$ & \\
\hline Association of Hanford Origin Radionuclides with Columbia River Sediment & 3 & $8 / 3 / 1976$ & $8 / 3 / 1976$ & 1 Core & Columbia River RM 397.1 - Priest Rapids Dam & $x$ & & & & & \\
\hline Heavy Metal Transport and Behavior in the Lower Columbia River, USA & 3 & $8 / 1 / 1999$ & 8/1/1999 & 1 Core & Columbia River RM 397.1 - Priest Rapids Dam & & $\mathrm{x}$ & & & & $\mathrm{x}$ \\
\hline Mercury Levels in the Lower Columbia River Watershed & 3 & 9/1/1971 & 9/1/1971 & 1 & Columbia River RM 397.1 - Priest Rapids Dam & \multicolumn{6}{|c|}{$\begin{array}{l}\text { Results not provided for samples collected at these } \\
\text { locations. }\end{array}$} \\
\hline TMDL Technical Assessment of DDT and PCBs in the Okanogan River & 3 & $8 / 21 / 2001$ & $8 / 21 / 2001$ & 1 Core & Okanogan River RM 2.3 - Mouth & & & & $\mathrm{x}$ & & $\mathrm{x}$ \\
\hline
\end{tabular}

DDT = dichlorodiphenyltrichloroethane

PCBs = metals

$\begin{array}{ll}\text { PCBS } & =\text { polychlorin } \\ \text { Pest } & =\text { pesticides }\end{array}$

RADs = radionuclides

$\mathrm{RM} \quad=$ river mile

SVOCs = semivolatile organic compounds 
Table 3-3. Summary of Documents with Segment 1 Surface Water Data. (2 Pages)

\begin{tabular}{|c|c|c|c|c|c|c|c|c|c|c|c|}
\hline Source Title & $\begin{array}{l}\text { Data } \\
\text { Usability } \\
\text { Category }\end{array}$ & $\begin{array}{l}\text { Begin } \\
\text { Date }\end{array}$ & End Date & $\begin{array}{c}\text { Number } \\
\text { of } \\
\text { Samples }\end{array}$ & Sample Area & RADs & METs & svoc & Pest & PCBs & $\begin{array}{l}\text { GEN } \\
\text { CHEM }\end{array}$ \\
\hline 1999 Hanford Environmental Oversight Program Data Summary Report & 1 & $5 / 5 / 1999$ & 11/3/1999 & 2 & $\begin{array}{l}\text { Columbia River - } \\
\text { RM } 397.1 \text { - Priest Rapids Dam }\end{array}$ & $\mathrm{x}$ & & & & & \\
\hline $\begin{array}{l}\text { Citizens Monitoring and Technical Assessment - Analysis of Chemical } \\
\text { Contaminants in Hanford Reach Biota and Environmental Materials at } \\
\text { the Perimeter of the Hanford Nuclear Reservation and on the Columbia } \\
\text { River }\end{array}$ & 1 & 10/7/2003 & 10/7/2003 & 1 & $\begin{array}{l}\text { Columbia River - } \\
\text { Vantage, Washington at } 190 \text { Bridge }\end{array}$ & $\mathrm{x}$ & $\mathrm{x}$ & & & & \\
\hline $\begin{array}{l}\text { Columbia Generating Station } 2004 \text { Annual Radiological Environmental } \\
\text { Operating Report }\end{array}$ & 1 & $2 / 4 / 2004$ & 7/8/2004 & 2 & $\begin{array}{l}\text { Columbia River } \\
\text { RM } 397.1 \text { - Priest Rapids Dam }\end{array}$ & $\mathrm{x}$ & & & & & \\
\hline EIM Database - Columbia River at Vernita & 1 & 1/13/1999 & 6/6/2005 & 75 & $\begin{array}{l}\text { Columbia River } \\
\text { RM } 388.1 \text { - Vernita Bridge }\end{array}$ & & & & & & $\mathrm{x}$ \\
\hline Hanford Environmental Oversight Program 2000 Data Summary Report & 1 & 2/2/2000 & $6 / 29 / 2000$ & 2 & $\begin{array}{l}\text { Columbia River } \\
\text { RM } 397.1 \text { - Priest Rapids Dam }\end{array}$ & $\mathrm{x}$ & & & & & \\
\hline Hanford Environmental Oversight Program 2001 Data Summary Report & 1 & $1 / 31 / 2001$ & $8 / 27 / 2001$ & $\begin{array}{l}2 \\
4\end{array}$ & $\begin{array}{l}\text { Columbia River } \\
\text { RM 397.1 - Priest Rapids Dam } \\
\text { RM 388.1 - Vernita Bridge }\end{array}$ & $\mathrm{x}$ & & & & & \\
\hline Hanford Environmental Oversight Program 2002 Data Summary Report & 1 & 2/6/2002 & 6/27/2002 & 2 & $\begin{array}{l}\text { Columbia River } \\
\text { RM } 397.1 \text { - Priest Rapids Dam }\end{array}$ & $\mathrm{x}$ & & & & & \\
\hline Hanford Environmental Oversight Program 2003 Data Summary Report & 1 & 2/5/2003 & $7 / 10 / 2003$ & 2 & $\begin{array}{l}\text { Columbia River } \\
\text { RM } 397.1 \text { - Priest Rapids Dam }\end{array}$ & $\mathrm{x}$ & & & & & \\
\hline Hanford Site Environmental Report for Calendar Year 1999 & 1 & 1/27/1999 & 12/29/1999 & $\begin{array}{l}48 \\
46\end{array}$ & $\begin{array}{l}\text { Columbia River - } \\
\text { RM } 397.1 \text { - Priest Rapids Dam } \\
\text { Vernita }\end{array}$ & $\mathrm{x}$ & & & & & $\mathrm{x}$ \\
\hline Hanford Site Environmental Report for Calendar Year 2000 & 1 & $1 / 26 / 2000$ & $12 / 28 / 2000$ & $\begin{array}{l}46 \\
64\end{array}$ & $\begin{array}{l}\text { Columbia River - } \\
\text { RM } 397.1 \text { - Priest Rapids } \\
\text { Vernita }\end{array}$ & $\mathrm{x}$ & $\mathrm{x}$ & & & & \\
\hline Hanford Site Environmental Report for Calendar Year 2001 & 1 & 1/4/2001 & $12 / 27 / 2001$ & $\begin{array}{l}48 \\
73\end{array}$ & $\begin{array}{l}\text { Columbia River - } \\
\text { RM 397.1-Priest Rapids Dam } \\
\text { RM 388.1 - Vernita Bridge }\end{array}$ & $\mathrm{x}$ & $\mathrm{x}$ & & & & \\
\hline Hanford Site Environmental Report for Calendar Year 2002 & 1 & 1/3/2002 & $11 / 26 / 2002$ & $\begin{array}{l}46 \\
56\end{array}$ & $\begin{array}{l}\text { Columbia River - } \\
\text { RM } 397.1 \text { - Priest Rapids Dam } \\
\text { Vernita }\end{array}$ & $\mathrm{x}$ & & & & & \\
\hline Hanford Site Environmental Report for Calendar Year 2003 & 1 & $1 / 8 / 2003$ & $11 / 25 / 2003$ & $\begin{array}{l}56 \\
64\end{array}$ & $\begin{array}{l}\text { Columbia River - } \\
\text { RM } 397.1 \text { - Priest Rapids Dam } \\
\text { Vernita }\end{array}$ & $\mathrm{x}$ & & & & & $\mathrm{x}$ \\
\hline Hanford Site Environmental Report for 2004 & 1 & 2/4/2004 & $1 / 5 / 2005$ & 32 & $\begin{array}{l}\text { Columbia River - } \\
\text { RM } 397.1 \text { - Priest Rapids }\end{array}$ & $\mathrm{x}$ & & & & & \\
\hline
\end{tabular}


Table 3-3. Summary of Documents with Segment 1 Surface Water Data. (2 Pages)

\begin{tabular}{|c|c|c|c|c|c|c|c|c|c|c|c|}
\hline Source Title & $\begin{array}{l}\text { Data } \\
\text { Usability } \\
\text { Category }\end{array}$ & $\begin{array}{l}\text { Begin } \\
\text { Date }\end{array}$ & End Date & $\begin{array}{c}\text { Number } \\
\text { of } \\
\text { Samples }\end{array}$ & Sample Area & RADs & METs & svoc & Pest & PCBs & $\begin{array}{l}\text { GEN } \\
\text { CHEM }\end{array}$ \\
\hline $\begin{array}{l}\text { Hydrogeology of the Unconsolidated Sediments, Water Quality, and } \\
\text { Ground-Water/Surface-Water Exchanges in the Methow River Basin, } \\
\text { Okanogan County, Washington }\end{array}$ & 1 & 9/21/2001 & $9 / 21 / 2001$ & 2 & $\begin{array}{l}\text { Columbia River - } \\
\text { Methow River - Pateros, Washington }\end{array}$ & & $\mathrm{x}$ & & $\mathrm{x}$ & & \\
\hline $\begin{array}{l}\text { Investigation into the Recurring Toxicity of the Chinet Company } \\
\text { Columbia River Intake }\end{array}$ & 1 & $3 / 6 / 2001$ & 6/20/2001 & 3 & $\begin{array}{l}\text { Columbia River - } \\
\text { Wenatchee, Washington@Chinet Co. } \\
\text { Intake } \\
\text { East Bank near Wenatchee }\end{array}$ & & $\mathrm{x}$ & & & & \\
\hline Statewide Arsenic Sampling in Selected Rivers & 1 & 7/9/2001 & 6/3/2002 & 12 & $\begin{array}{l}\text { Columbia River - } \\
\text { RM } 735.1 \text { - Northport, Washington }\end{array}$ & & $\mathrm{x}$ & & & & \\
\hline $\begin{array}{l}\text { Survey of Radiological and Chemical Contaminants in the Near-Shore } \\
\text { Environment at the Hanford Site } 300 \text { Area }\end{array}$ & 1 & 8/27/2001 & 8/27/2001 & 5 & $\begin{array}{l}\text { Columbia River - } \\
\text { RM } 388.1 \text { - Vernita Bridge }\end{array}$ & $\mathrm{x}$ & & & & & \\
\hline $\begin{array}{l}\text { An Evaluation of Water Quality and Limnology for the Priest Rapids } \\
\text { Project Area }\end{array}$ & 2 & $3 / 27 / 1999$ & 12/5/1999 & $\begin{array}{c}10 \\
10 \\
11 \\
6 \\
10 \\
10 \\
6 \\
6\end{array}$ & $\begin{array}{l}\text { Columbia River - } \\
\text { RM 397.1 - Priest Rapids Dam } \\
\text { RM 415..8 - Below Wanapum Dam } \\
\text { RM 440 - Crescent Bar } \\
\text { Dry Gulch } \\
\text { RM } 417.5 \text { - Ginko Park } \\
\text { RM 398.5 - Goose Island } \\
\text { RM } 405.5 \text { - Hanson Creek } \\
\text { RM } 428 \text { - Scammon's Landing }\end{array}$ & & & & & & $x$ \\
\hline $\begin{array}{ll}\text { METS } & =\text { metals } \\
\text { PCBs } & \text { = polychlorinated biphenyls } \\
\text { Pest } & \text { = pesticides } \\
\text { RADs } & \text { r radionuclides } \\
\text { RM } & \text { = river mile } \\
\text { SVOCs } & \text { = semivolatile organic compounds }\end{array}$ & & & & & & & & & & & \\
\hline
\end{tabular}


Table 3-4. Summary of Documents with Sediment Data in Segment 2. (6 Pages)

\begin{tabular}{|c|c|c|c|c|c|c|c|c|c|c|c|}
\hline Source Title & $\begin{array}{c}\text { Data } \\
\text { Usability } \\
\text { Category }\end{array}$ & Begin Date & End Date & $\begin{array}{l}\text { Number of } \\
\text { Samples }\end{array}$ & Sample Area & RADs & METs & svoc & Pest & PCBs & $\begin{array}{l}\text { GEN } \\
\text { CHEM }\end{array}$ \\
\hline 100 Area Columbia River Sediment Sampling & 1 & 11/12/1992 & 11/24/1992 & 45 & $\begin{array}{l}\text { Vernita Area } \\
\text { Horn Area } \\
100 \text { B/C Area } \\
100 \text { D Area } \\
\text { D Island } \\
100 \text { F Slough } \\
100 \mathrm{H} \text { Area } \\
100 \text { K Area } \\
\text { Hanford Townsite }\end{array}$ & $x$ & $\mathrm{x}$ & & & & \\
\hline $\begin{array}{l}1984 \text { Annual Report Radiological Environmental Monitoring Program } \\
\text { Nuclear Plant } 2\end{array}$ & 1 & $10 / 26 / 1984$ & 10/26/1984 & 2 & $\begin{array}{l}\text { CGS (WNP-2) STA } 33 \\
\text { CGS (WNP-2) STA } 34\end{array}$ & $\mathrm{x}$ & & & & & \\
\hline $\begin{array}{l}1985 \text { Annual Report Radiological Environmental Monitoring Program } \\
\text { Nuclear Plant } 2\end{array}$ & 1 & $4 / 16 / 1985$ & 10/10/1985 & 4 & $\begin{array}{l}\text { CGS (WNP-2) STA } 33 \\
\text { CGS (WNP-2) STA } 34\end{array}$ & $x$ & & & & & \\
\hline $\begin{array}{l}1986 \text { Radiological Environmental Monitoring Program Annual Report - } \\
\text { Plant } 2\end{array}$ & 1 & $1 / 21 / 1986$ & $10 / 21 / 1986$ & 8 & $\begin{array}{l}\text { CGS (WNP-2) STA } 33 \\
\text { CGS (WNP-2) STA } 34\end{array}$ & $x$ & & & & & \\
\hline $\begin{array}{l}1999 \text { Hanford Environmental Oversight Program Data Summary } \\
\text { Report }\end{array}$ & 1 & $8 / 2 / 1999$ & 11/1/1999 & 9 & $\begin{array}{l}\text { F Slough } \\
\text { CGS (WNP-2) STA } 33 \\
\text { CGS (WNP-2) STA } 34 \\
\text { 100F SPRING 207-1 }\end{array}$ & $\mathrm{x}$ & & & & & \\
\hline $\begin{array}{l}\text { Citizens Monitoring and Technical Assessment - Analysis of Chemical } \\
\text { Contaminants in Hanford Reach Biota and Environmental Materials at } \\
\text { the Perimeter of the Hanford Nuclear Reservation and on the } \\
\text { Columbia River }\end{array}$ & 1 & $10 / 7 / 2003$ & $8 / 4 / 2004$ & 12 & $\begin{array}{l}\text { 100-B/C Reactor Area - } \\
100-D \text { Reactor } \\
100-\mathrm{N} \text { Reactor Area } \\
100-\mathrm{H} \text { Reactor Area } \\
\text { 100-F Reactor Area } \\
300 \text { Area }\end{array}$ & $x$ & $\mathrm{x}$ & & & & \\
\hline $\begin{array}{l}\text { Columbia Generating Station } 2000 \text { Annual Radiological Environmental } \\
\text { Operating Report }\end{array}$ & 1 & $3 / 22 / 2000$ & $10 / 25 / 2000$ & 4 & $\begin{array}{l}\text { CGS (WNP-2) STA } 33 \\
\text { CGS (WNP-2) STA } 34\end{array}$ & \multicolumn{6}{|c|}{$\begin{array}{l}\text { Results not provided for samples collected at these } \\
\text { locations. }\end{array}$} \\
\hline $\begin{array}{l}\text { Columbia Generating Station } 2001 \text { Annual Radiological Environmental } \\
\text { Operating Report }\end{array}$ & 1 & $3 / 21 / 2001$ & $10 / 31 / 2001$ & 4 & $\begin{array}{l}\text { CGS (WNP-2) STA } 33 \\
\text { CGS (WNP-2) STA } 34\end{array}$ & $\mathrm{x}$ & & & & & \\
\hline $\begin{array}{l}\text { Columbia Generating Station } 2002 \text { Annual Radiological Environmental } \\
\text { Operating Report }\end{array}$ & 1 & 3/20/2002 & 10/29/2002 & 4 & $\begin{array}{l}\text { CGS (WNP-2) STA } 33 \\
\text { CGS (WNP-2) STA } 34\end{array}$ & $x$ & & & & & \\
\hline $\begin{array}{l}\text { Columbia Generating Station } 2003 \text { Annual Radiological Environmental } \\
\text { Operating Report }\end{array}$ & 1 & $3 / 13 / 2003$ & 10/30/2003 & 4 & $\begin{array}{l}\text { CGS (WNP-2) STA } 33 \\
\text { CGS (WNP-2) STA } 34\end{array}$ & $x$ & & & & & \\
\hline $\begin{array}{l}\text { Columbia Generating Station } 2004 \text { Annual Radiological Environmental } \\
\text { Operating Report }\end{array}$ & 1 & $3 / 25 / 2004$ & $3 / 10 / 2005$ & 9 & $\begin{array}{l}\text { CGS (WNP-2) STA } 33 \\
\text { CGS (WNP-2) STA } 34\end{array}$ & $\mathrm{x}$ & & & & & \\
\hline $\begin{array}{l}\text { Energy Northwest Nuclear Plant } 21999 \text { Annual Radiological } \\
\text { Environmental Operating Report }\end{array}$ & 1 & 10/19/1999 & 10/19/1999 & 2 & $\begin{array}{l}\text { CGS (WNP-2) STA } 33 \\
\text { CGS (WNP-2) STA } 34\end{array}$ & $x$ & & & & & \\
\hline $\begin{array}{l}\text { Environmental Monitoring of Columbia River Sediments: Grain-Size } \\
\text { Distribution and Contaminant Association }\end{array}$ & 1 & 4/15/1994 & 4/15/1994 & 3 & $\begin{array}{l}\text { White Bluffs Slough } \\
\text { F Slough } \\
\text { Hanford Slough }\end{array}$ & $x$ & & & & & \\
\hline
\end{tabular}


Table 3-4. Summary of Documents with Sediment Data in Segment 2. (6 Pages)

\begin{tabular}{|c|c|c|c|c|c|c|c|c|c|c|c|}
\hline Source Title & $\begin{array}{c}\text { Data } \\
\text { Usability } \\
\text { Category }\end{array}$ & Begin Date & End Date & $\begin{array}{l}\text { Number of } \\
\text { Samples }\end{array}$ & Sample Area & RADs & METs & svoc & Pest & PCBs & $\begin{array}{l}\text { GEN } \\
\text { CHEM }\end{array}$ \\
\hline Environmental Radiation Oversight Program - Database & 1 & 4/6/1995 & $10 / 25 / 2004$ & 21 & $\begin{array}{l}\text { CGS (WNP-2) STA } 33 \\
\text { CGS (WNP-2) STA } 34 \\
100-B \text { Area } \\
\text { D Island } \\
100-K \text { Area } \\
100-F \text { Area } \\
100 \text { F 1/10 } \\
\text { F Slough } \\
\text { Hanford Slough } \\
\text { White Bluffs Slough } \\
\text { Hanford Spring 28-2 } \\
\text { 300 Spring Mile 42.2 } \\
\text { Richland, Washington }\end{array}$ & $x$ & & & & & \\
\hline Environmental Radiation Program 1992-1994 Annual Report & 1 & 4/9/1992 & 9/26/1994 & 24 & $\begin{array}{l}\text { Horn Area } \\
\text { CGS (WNP-2) STA } 33 \\
\text { CGS (WNP-2) STA } 34 \\
100-B / C \text { Area } \\
100-F \text { Area } \\
100-N \text { Area } \\
300 \text { FF-5 } \\
\text { F Slough } \\
\text { H Slough } \\
\text { Hanford Slough } \\
\text { D Island } \\
\text { Shot Rock } \\
\text { Spit Tip }\end{array}$ & $x$ & & & & & \\
\hline $\begin{array}{l}\text { Environmental Radiation Program 23rd Annual Report January } 1984 \text { - } \\
\text { December } 1984\end{array}$ & 1 & $5 / 1 / 1984$ & 6/14/1984 & 3 & \begin{tabular}{|l} 
CGS (WNP-2) STA 33 \\
CGS (WNP-2) STA 34 \\
\end{tabular} & \multicolumn{6}{|c|}{$\begin{array}{l}\text { Results not provided for samples collected at these } \\
\text { locations. }\end{array}$} \\
\hline $\begin{array}{l}\text { Environmental Radiation Program 28th Annual Report January } \\
\text { through December } 1989\end{array}$ & 1 & 4/11/1989 & 6/7/1989 & 6 & $\begin{array}{l}\text { CGS (WNP-2) STA } 33 \\
\text { CGS (WNP-2) STA } 34\end{array}$ & $x$ & & & & & \\
\hline $\begin{array}{l}\text { Environmental Radiation Program 29th Annual Report January } \\
\text { through December } 1990\end{array}$ & 1 & 4/19/1990 & 4/19/1990 & 2 & $\begin{array}{l}\text { CGS (WNP-2) STA } 33 \\
\text { CGS (WNP-2) STA } 34\end{array}$ & $x$ & & & & & \\
\hline $\begin{array}{l}\text { Hanford Environmental Oversight Program } 2000 \text { Data Summary } \\
\text { Report }\end{array}$ & 1 & $3 / 22 / 2000$ & $11 / 7 / 2000$ & 7 & $\begin{array}{l}\text { CGS (WNP-2) STA } 33 \\
\text { CGS (WNP-2) STA } 34 \\
\text { 100-N Springs } \\
\text { F Slough } \\
\text { Hanford Spring 28-2 } \\
300 \text { Spring Mile 42.2 } \\
\text { 100-F Spring 207-1 }\end{array}$ & $x$ & & & & & \\
\hline
\end{tabular}


Table 3-4. Summary of Documents with Sediment Data in Segment 2. (6 Pages)

\begin{tabular}{|c|c|c|c|c|c|c|c|c|c|c|c|}
\hline Source Title & $\begin{array}{c}\text { Data } \\
\text { Usability } \\
\text { Category }\end{array}$ & Begin Date & End Date & $\begin{array}{l}\text { Number of } \\
\text { Samples }\end{array}$ & Sample Area & RADs & METS & svoc & Pest & PCBs & $\begin{array}{l}\text { GEN } \\
\text { CHEM }\end{array}$ \\
\hline $\begin{array}{l}\text { Hanford Environmental Oversight Program } 2001 \text { Data Summary } \\
\text { Report }\end{array}$ & 1 & $3 / 21 / 2001$ & 10/22/2001 & 12 & $\begin{array}{l}\text { CGS (WNP-2) STA } 33 \\
\text { CGS (WNP-2) STA } 34 \\
\text { 100B Spring 38-3 } \\
\text { 100F Spring 207-1 } \\
\text { F Slough } \\
300 \text { Spring Mile } 42.2 \\
300 \text { Spring } 11 \\
\text { 300 Spring 14 } \\
\text { Richland, Washington }\end{array}$ & $\mathrm{x}$ & & & & & \\
\hline $\begin{array}{l}\text { Hanford Environmental Oversight Program } 2002 \text { Data Summary } \\
\text { Report }\end{array}$ & 1 & 3/29/2002 & 10/29/2002 & 7 & $\begin{array}{l}\text { CGS (WNP-2) STA } 33 \\
\text { CGS (WNP-2) STA } 34 \\
\text { 100-N Spring } \\
\text { F Slough } \\
\text { 100K Spring 63-2 } \\
\text { Hanford Spring 28-2 } \\
\text { 100-F Spring 207-1 } \\
\end{array}$ & $\mathrm{x}$ & & & & & \\
\hline $\begin{array}{l}\text { Hanford Environmental Oversight Program } 2003 \text { Data Summary } \\
\text { Report }\end{array}$ & 1 & $3 / 13 / 2003$ & $12 / 8 / 2003$ & 9 & $\begin{array}{l}\text { CGS (WNP-2) STA } 33 \\
\text { CGS (WNP-2) STA } 34 \\
\text { F Slough } \\
300 \text { Spring Mile 42.2 } \\
\text { 100-B Spring 37-1 } \\
\text { 100-K Spring 63-1 } \\
\text { 100-F Spring 207-1 } \\
\text { 300 Spring 6 } \\
\end{array}$ & $x$ & & & & & \\
\hline Hanford Site Environmental Report for Calendar Year 1994 & 1 & 9/2/1994 & 9/25/1994 & $\begin{array}{l}1 \\
1 \\
1 \\
2 \\
2 \\
2 \\
1 \\
1 \\
1\end{array}$ & $\begin{array}{l}\text { North } 300 \text { Area } \\
300 \text { Spring 42-2 } \\
\text { F Slough } \\
\text { Hanford Spring 28-2 } \\
\text { Hanford Slough } \\
\text { White Bluffs Slough } \\
\text { Ringold } \\
\text { Richland, Washington } \\
\end{array}$ & & $\mathrm{x}$ & & & & \\
\hline Hanford Site Environmental Report for Calendar Year 1995 & 1 & 9/2/1994 & 9/25/1994 & $\begin{array}{l}1 \\
1 \\
1 \\
1 \\
1 \\
1 \\
1 \\
1 \\
1 \\
1 \\
1\end{array}$ & $\begin{array}{l}\text { 100-B Spring } \\
\text { 100-F Spring } \\
100-K \text { Spring } \\
300 \text { Spring 42-2 } \\
\text { F Slough } \\
\text { Hanford Spring 28-2 } \\
\text { Hanford Slough } \\
\text { White Bluffs Slough } \\
\text { Richland, Washington }\end{array}$ & & $\mathrm{x}$ & & & & \\
\hline
\end{tabular}


Table 3-4. Summary of Documents with Sediment Data in Segment 2. (6 Pages)

\begin{tabular}{|c|c|c|c|c|c|c|c|c|c|c|c|}
\hline Source Title & $\begin{array}{l}\text { Data } \\
\text { Usability } \\
\text { Category }\end{array}$ & Begin Date & End Date & $\begin{array}{l}\text { Number of } \\
\text { Samples }\end{array}$ & Sample Area & RADs & METs & svoc & Pest & PCBs & $\begin{array}{c}\text { GEN } \\
\text { CHEM }\end{array}$ \\
\hline Hanford Site Environmental Report for Calendar Year 1996 & 1 & 8/20/1996 & 11/21/1996 & $\begin{array}{l}1 \\
1 \\
1 \\
1 \\
1 \\
1 \\
1 \\
1 \\
1\end{array}$ & $\begin{array}{l}\text { 100-B Spring } \\
\text { 100-F Spring } \\
\text { 300 Spring 42-2 } \\
\text { F Slough } \\
\text { Hanford Spring 28-2 } \\
\text { Hanford Slough } \\
\text { White Bluffs Slough } \\
\text { Richland, Washington } \\
\end{array}$ & & $x$ & & & & \\
\hline Hanford Site Environmental Report for Calendar Year 1997 & 1 & 8/20/1997 & 11/3/1997 & $\begin{array}{l}1 \\
1 \\
1 \\
1 \\
1\end{array}$ & $\begin{array}{l}\text { North } 300 \text { Area } \\
100-B \text { Spring } \\
100-\text { F Spring } \\
300 \text { Spring 42-2 } \\
\text { Hanford Spring 28-2 }\end{array}$ & & $\mathrm{x}$ & & & & \\
\hline Hanford Site Environmental Report for Calendar Year 1998 & 1 & 7/24/1998 & 10/20/1998 & $\begin{array}{l}1 \\
1 \\
1 \\
1\end{array}$ & $\begin{array}{l}\text { North } 300 \text { Area } \\
100-B \text { Spring } \\
100-F \text { Spring } \\
\text { Ringold }\end{array}$ & & $\mathrm{x}$ & & & & \\
\hline Hanford Site Environmental Report for Calendar Year 1999 & 1 & $8 / 9 / 1999$ & 11/1/1999 & $\begin{array}{l}2 \\
1 \\
2 \\
2 \\
2 \\
2 \\
1 \\
1 \\
1\end{array}$ & $\begin{array}{l}\text { 100-B Spring } \\
100-\text { Spring } \\
300 \text { Spring 42-2 } \\
\text { F Slough } \\
\text { Hanford Spring 28-2 } \\
\text { Hanford Slough } \\
\text { White Bluffs Slough } \\
\text { Richland, Washington }\end{array}$ & & $x$ & & & & \\
\hline Hanford Site Environmental Report for Calendar Year 2000 & 1 & $8 / 8 / 2000$ & $11 / 02 / 2000$ & $\begin{array}{l}1 \\
1 \\
2 \\
3 \\
4 \\
1 \\
2 \\
2\end{array}$ & $\begin{array}{l}\text { 100-B Spring } \\
100-\text { F Spring } \\
300 \text { Spring 42-2 } \\
\text { F Slough } \\
\text { Hanford Spring 28-2 } \\
\text { Hanford Slough } \\
\text { White Bluffs Slough } \\
\text { Richland, Washington }\end{array}$ & & $\mathrm{x}$ & & & & $\mathrm{x}$ \\
\hline Hanford Site Environmental Report for Calendar Year 2001 & 1 & $3 / 5 / 2001$ & 10/22/2001 & $\begin{array}{l}2 \\
1 \\
1 \\
1 \\
4 \\
2 \\
2 \\
1 \\
1 \\
1 \\
1 \\
2\end{array}$ & $\begin{array}{l}\text { Vernita Bridge } \\
100-B \text { Spring } \\
100-\text { F Spring } \\
\text { North 300 Area } \\
300 \text { Spring 42-2 } \\
\text { F Slough } \\
\text { Hanford Slough } \\
\text { Hanford Townsite } \\
\text { White Bluffs Slough } \\
\text { Ringold } \\
\text { Richland, Washington }\end{array}$ & & $\mathrm{x}$ & & & & \\
\hline
\end{tabular}


Table 3-4. Summary of Documents with Sediment Data in Segment 2. (6 Pages)

\begin{tabular}{|c|c|c|c|c|c|c|c|c|c|c|c|}
\hline Source Title & $\begin{array}{l}\text { Data } \\
\text { Usability } \\
\text { Category }\end{array}$ & Begin Date & End Date & $\begin{array}{l}\text { Number of } \\
\text { Samples }\end{array}$ & Sample Area & RADs & METs & svoc & Pest & PCBs & $\begin{array}{l}\text { GEN } \\
\text { CHEM }\end{array}$ \\
\hline Hanford Site Environmental Report for Calendar Year 2002 & 1 & 7/19/2002 & 10/7/2002 & $\begin{array}{l}1 \\
1 \\
1 \\
2 \\
2 \\
2 \\
1 \\
1 \\
1 \\
2\end{array}$ & $\begin{array}{l}\text { 100-B Spring 37-1 } \\
\text { 100-F Spring 207-1 } \\
100-K \text { Spring 63-2 } \\
300 \text { Spring 42-2 } \\
\text { F Slough } \\
\text { Hanford Slough } \\
\text { Hanford Spring DR 28-2 } \\
\text { White Bluffs Slough } \\
\text { Richland, Washington }\end{array}$ & & $\mathrm{x}$ & & & & $\mathrm{x}$ \\
\hline Hanford Site Environmental Report for Calendar Year 2003 & 1 & 7/14/2003 & $11 / 13 / 2003$ & $\begin{array}{l}5 \\
1 \\
1 \\
1 \\
2 \\
1 \\
1 \\
1 \\
1 \\
1 \\
1\end{array}$ & $\begin{array}{l}\text { 100-B Spring } \\
\text { 100-F Spring } \\
\text { 100-H Spring } \\
100-K \text { Spring } \\
300 \text { Spring 42-2 } \\
\text { F Slough } \\
\text { Hanford Slough } \\
\text { Hanford Townsite } \\
\text { White Bluffs Slough } \\
\text { Richland, Washington }\end{array}$ & & & & & & \\
\hline $\begin{array}{l}\text { Mid-Columbia River Basin Sediment Chemical Contaminants Study } \\
\text { June } 2004 \text { to October } 2004 \text { addendum }\end{array}$ & 1 & 6/1/2004 & 6/1/2004 & $\begin{array}{l}5 \\
3\end{array}$ & $\begin{array}{l}\text { Columbia River RM } 343.5 \text { to } 371 \\
\text { - Irrigation Returns } \\
300 \text { Area } \\
\text { Hanford Slough } \\
\text { F Slough }\end{array}$ & & $\mathrm{x}$ & & & & $\mathrm{x}$ \\
\hline $\begin{array}{l}\text { Washington Public Power Supply System Nuclear Plant } 21993 \\
\text { Annual Report }\end{array}$ & 1 & 4/15/1993 & 10/6/1993 & 4 & $\begin{array}{l}\text { CGS (WNP-2) STA } 33 \\
\text { CGS (WNP-2) STA } 34\end{array}$ & $\mathrm{x}$ & & & & & \\
\hline $\begin{array}{l}\text { Washington Public Power Supply System Nuclear Plant } 21994 \\
\text { Annual Radiological Environmental Operating Report }\end{array}$ & 1 & 4/7/1994 & 10/5/1994 & 4 & $\begin{array}{l}\text { CGS (WNP-2) STA } 33 \\
\text { CGS (WNP-2) STA } 34\end{array}$ & $\mathrm{x}$ & & & & & \\
\hline $\begin{array}{l}\text { Washington Public Power Supply System Nuclear Plant } 21995 \\
\text { Annual Radiological Environmental Operating Report }\end{array}$ & 1 & 4/6/1995 & 10/25/1995 & 4 & $\begin{array}{l}\text { CGS (WNP-2) STA } 33 \\
\text { CGS (WNP-2) STA } 34\end{array}$ & $\mathrm{x}$ & & & & & \\
\hline $\begin{array}{l}\text { Washington Public Power Supply System Nuclear Plant } 21996 \\
\text { Annual Radiological Environmental Operating Report }\end{array}$ & 1 & 10/9/1996 & 10/9/1996 & 2 & $\begin{array}{l}\text { CGS (WNP-2) STA } 33 \\
\text { CGS (WNP-2) STA } 34\end{array}$ & $\mathrm{x}$ & & & & & \\
\hline $\begin{array}{l}\text { Washington Public Power Supply System Nuclear Plant } 21997 \\
\text { Annual Radiological Environmental Operating Report }\end{array}$ & 1 & 10/29/1997 & 10/29/1997 & 2 & $\begin{array}{l}\text { CGS (WNP-2) STA } 33 \\
\text { CGS (WNP-2) STA } 34\end{array}$ & $\mathrm{x}$ & & & & & \\
\hline $\begin{array}{l}\text { Washington Public Power Supply System Nuclear Plant } 21998 \\
\text { Annual Radiological Environmental Operating Report }\end{array}$ & 1 & 4/8/1998 & 10/21/1998 & 4 & $\begin{array}{l}\text { CGS (WNP-2) STA } 33 \\
\text { CGS (WNP-2) STA } 34\end{array}$ & $x$ & & & & & \\
\hline $\begin{array}{l}\text { Washington Public Power Supply System Nuclear Plant Number } 2 \\
\text { Radiological Environmental Monitoring Program } 1987 \text { Annual Report }\end{array}$ & 1 & 4/9/1987 & 10/14/1987 & 4 & $\begin{array}{l}\text { CGS (WNP-2) STA } 33 \\
\text { CGS (WNP-2) STA } 34\end{array}$ & $\mathrm{x}$ & & & & & \\
\hline $\begin{array}{l}\text { Washington Public Power Supply System Nuclear Plant Number } 2 \\
\text { Radiological Environmental Monitoring Program } 1988 \text { Annual Report }\end{array}$ & 1 & 4/21/1988 & 10/26/1988 & 4 & $\begin{array}{l}\text { CGS (WNP-2) STA } 33 \\
\text { CGS (WNP-2) STA } 34\end{array}$ & $x$ & & & & & \\
\hline
\end{tabular}


Table 3-4. Summary of Documents with Sediment Data in Segment 2. (6 Pages)

\begin{tabular}{|c|c|c|c|c|c|c|c|c|c|c|c|}
\hline Source Title & $\begin{array}{c}\text { Data } \\
\text { Usability } \\
\text { Category }\end{array}$ & Begin Date & End Date & $\begin{array}{l}\text { Number of } \\
\text { Samples }\end{array}$ & Sample Area & RADs & METs & svoc & Pest & PCBs & $\begin{array}{l}\text { GEN } \\
\text { CHEM }\end{array}$ \\
\hline $\begin{array}{l}\text { Washington Public Power Supply System Nuclear Plant Number } 2 \\
\text { Radiological Environmental Monitoring Program } 1989 \text { Annual Report }\end{array}$ & 1 & 4/11/1989 & 10/19/1989 & 4 & $\begin{array}{l}\text { CGS (WNP-2) STA } 33 \\
\text { CGS (WNP-2) STA } 34\end{array}$ & $x$ & & & & & \\
\hline $\begin{array}{l}\text { Washington Public Power Supply System Nuclear Plant Number } 2 \\
\text { Radiological Environmental Monitoring Program } 1990 \text { Annual Report }\end{array}$ & 1 & 4/19/1990 & 10/16/1990 & 4 & $\begin{array}{l}\text { CGS (WNP-2) STA } 33 \\
\text { CGS (WNP-2) STA } 34\end{array}$ & $x$ & & & & & \\
\hline $\begin{array}{l}\text { Washington Public Power Supply System Nuclear Plant Number } 2 \\
\text { Radiological Environmental Monitoring Program } 1991 \text { Annual Report }\end{array}$ & 1 & 4/10/1991 & 10/3/1991 & 4 & $\begin{array}{l}\text { CGS (WNP-2) STA } 33 \\
\text { CGS (WNP-2) STA } 34\end{array}$ & $x$ & & & & & \\
\hline $\begin{array}{l}\text { Washington Public Power Supply System Nuclear Plant Number } 2 \\
\text { Radiological Environmental Monitoring Program } 1992 \text { Annual Report } \\
\end{array}$ & 1 & 4/9/1992 & 10/20/1992 & 4 & $\begin{array}{l}\text { CGS (WNP-2) STA } 33 \\
\text { CGS (WNP-2) STA } 34\end{array}$ & $x$ & & & & & \\
\hline $\begin{array}{l}\text { Environmental Radiation Program 24th Annual Report January } 1985 \text { - } \\
\text { December } 1985\end{array}$ & 2 & 4/16/1985 & 4/16/1985 & 2 & $\begin{array}{l}\text { CGS (WNP-2) STA } 33 \\
\text { CGS (WNP-2) STA } 34\end{array}$ & $x$ & & & & & \\
\hline $\begin{array}{l}\text { Environmental Radiation Program 25th Annual Report January } 1986 \text { - } \\
\text { December } 1986\end{array}$ & 2 & 4/16/1986 & 4/16/1986 & 2 & $\begin{array}{l}\text { CGS (WNP-2) STA } 33 \\
\text { CGS (WNP-2) STA } 34\end{array}$ & $x$ & & & & & \\
\hline $\begin{array}{l}\text { Environmental Radiation Program 26th Annual Report January } 1987 \text { - } \\
\text { December } 1987\end{array}$ & 2 & 4/9/1987 & 6/25/1987 & 7 & $\begin{array}{l}\text { CGS (WNP-2) STA } 33 \\
\text { CGS (WNP-2) STA } 34 \\
100-H \text { Area } \\
\text { 100-D Area } \\
\text { Richland Near Hanford House } \\
\text { Locke Island }\end{array}$ & $x$ & & & & & \\
\hline $\begin{array}{l}\text { Environmental Radiation Program 27th Annual Report January } \\
\text { through December } 1988\end{array}$ & 2 & 4/21/1988 & 9/6/1988 & 3 & $\begin{array}{l}\text { CGS (WNP-2) STA } 33 \\
\text { CGS (WNP-2) STA } 34 \\
\text { 100-F Area }\end{array}$ & $x$ & & & & & \\
\hline $\begin{array}{l}\text { Hanford Environmental Oversight Program } 1991 \text { Data Summary } \\
\text { Report }\end{array}$ & 2 & 4/10/1991 & 4/10/1991 & 2 & $\begin{array}{l}\text { CGS (WNP-2) STA } 33 \\
\text { CGS (WNP-2) STA } 34\end{array}$ & $x$ & & & & & \\
\hline $\begin{array}{l}\text { Preoperational Environmental Radiological Monitoring Program } \\
\text { WNP-2 March } 1978 \text { Through January 19, } 1984\end{array}$ & 2 & 5/17/1978 & 11/1/1983 & 21 & $\begin{array}{l}\text { CGS (WNP-2) STA } 33 \\
\text { CGS (WNP-2) STA } 34 \\
\end{array}$ & $x$ & & & & & \\
\hline $\begin{array}{l}\text { Association of Hanford Origin Radionuclides with Columbia River } \\
\text { Sediment }\end{array}$ & 3 & 8/4/1976 & $8 / 4 / 1976$ & 1 Core & Hanford Ferry & $x$ & & & & & \\
\hline $\begin{array}{l}\text { Mid-Columbia River Feasibility Study: Physical And Chemical } \\
\text { Analyses Of Hanford Reach Sediments }\end{array}$ & 3 & 8/1/1986 & & $\begin{array}{c}4 \text { Cores } \\
\text { (Composite) }\end{array}$ & $\begin{array}{l}\text { Hanford Reach RM 393, } \\
\text { RM 378.5, RM 373, RM } 365\end{array}$ & & $x$ & $x$ & $x$ & & \\
\hline
\end{tabular}

CGS = Columbia Generating Station

METS $=$ metals
PCBs $=$ polychlorinated biphenyls

Pest $=$ pesticides
RADs $=$ radionuclides

RM = river mile

=

WNP-2 = Washington Public Power Supply System Nuclear Plant Number 2 
Table 3-5. Segment 2 Sediment Location Summary.

\begin{tabular}{|l|l|c|}
\hline \multicolumn{1}{|c|}{ Sample Area } & \multicolumn{1}{|c|}{ Sample Collection Dates } & No. of Samples \\
\hline $\begin{array}{l}\text { 100-B/C Area including } \\
\text { 100-B Spring 37-1 and 100-B Spring 38-3 }\end{array}$ & November 1992 - October 2004 & 23 \\
\hline 100-D Area & June 1987 - October 2005 & 5 \\
\hline D Island & November 1992 - September 1995 & 11 \\
\hline 100-F Area including 100-F Spring 207-1 & September 1988 - October 2004 & 22 \\
\hline 100-F Slough & November 1992 - July 2004 & 47 \\
\hline 100-H Area including 100-H Spring 145-1 & June 1987 - October 2004 & 12 \\
\hline $\begin{array}{l}\text { 100-K Area including 100-K Spring 63-1 and } \\
\text { Spring 63-2 }\end{array}$ & November 1992 - October 2003 & 12 \\
\hline $\begin{array}{l}\text { 100-N Area including HGP, N-Springs, and } \\
\text { 100N-51 }\end{array}$ & June 1992 - October 2003 & 7 \\
\hline $\begin{array}{l}\text { 300 Area including 300 Spring 42-2, 300-FF-5, } \\
\text { 300 Spring 11, 300 Spring 14, 300 Spring 42-2, } \\
\text { and 300 Spring 6 }\end{array}$ & September 1992 - October 2004 \\
\hline Hanford Slough & November 1992 - July 2004 & 7 \\
\hline Hanford Spring 28-2 & September 1994 - October 2004 & \\
\hline Hanford Townsite & November 1992 & 18 \\
\hline Horn Area & November 1992 & 7 \\
\hline White Bluffs Slough & April 1994 - July 2004 & 6 \\
\hline $\begin{array}{l}\text { Columbia Generation Station 33 } \\
\text { Columbia Generation Station 34 }\end{array}$ & May 1978 - March 2005 & 29 \\
\hline Richland, Washington & June 1987 - July 2004 & 70 \\
\hline $\begin{array}{l}\text { Other locations including Locke Island, Ringold } \\
\text { Wasteway, Esquatel Canal }\end{array}$ & August 1986 - June 2004 & 71 \\
\hline
\end{tabular}

HGP = Hanford Generating Plant 
WCH-91

Historical Data Quality Review Procedures

Rev. 0 
Table 3-6. Summary of Documents with Segment 2 Surface Water Data. (6 Pages)

\begin{tabular}{|c|c|c|c|c|c|c|c|c|c|c|c|}
\hline Source Title & $\begin{array}{c}\text { Data } \\
\text { Usability } \\
\text { Category }\end{array}$ & Begin Date & End Date & $\begin{array}{c}\text { Number } \\
\text { of } \\
\text { Samples }\end{array}$ & Sample Area & RADs & METs & svoc & Pest & PCBs & $\begin{array}{c}\text { GEN } \\
\text { CHEM }\end{array}$ \\
\hline $\begin{array}{l}1999 \text { Hanford Environmental Oversight Program Data Summary } \\
\text { Report }\end{array}$ & 1 & 1/5/1999 & 12/16/1999 & $\begin{array}{c}1 \\
1 \\
5 \\
1 \\
1 \\
1 \\
8 \\
2 \\
1 \\
16 \\
4 \\
1 \\
12 \\
2 \\
5\end{array}$ & $\begin{array}{l}100 \text { N-1 HRM 9.5 } \\
\text { 100F SPRING 207-1 } \\
100 \text { N SPR 1/10 } \\
100 \text { SPRING 8-13 } \\
100 \text { S SPRING 153-1 } \\
\text { 300 Area } \\
\text { 100N SPR STA 3 } \\
\text { Benton Pumphouse } \\
\text { Butterfield WTP, Pasco, Washington } \\
\text { CGS (WNP-2) STA 26, INTAKE } \\
\text { CGS (WNP-2) STA 29, WTP } \\
\text { Hip Deep Creek, Richland, } \\
\text { Washington } \\
\text { Richland Water Treatment Plant } \\
\text { RL PUMPHOUSE } \\
\text { TOWNSITE }\end{array}$ & $\mathrm{x}$ & & & & & \\
\hline $\begin{array}{l}\text { Citizens Monitoring and Technical Assessment - Analysis of } \\
\text { Chemical Contaminants in Hanford Reach Biota and } \\
\text { Environmental Materials at the Perimeter of the Hanford Nuclear } \\
\text { Reservation and on the Columbia River }\end{array}$ & 1 & $10 / 7 / 2003$ & $8 / 4 / 2004$ & $\begin{array}{l}1 \\
1 \\
1 \\
1 \\
1\end{array}$ & $\begin{array}{l}\text { K Reactor Area - Water } \\
\text { D Reactor Area - Water @ HR023D } \\
\text { Richland Water Intake } \\
\text { N Reactor Area-seep water } \\
\text { H Reactor Area }\end{array}$ & $\mathrm{x}$ & $\mathrm{x}$ & & & & \\
\hline City of Richland Drinking Water data & 2 & 10/7/2003 & $1 / 15 / 2004$ & 2 & City of Richland Water Intake & & $\mathrm{x}$ & & & & $\mathrm{x}$ \\
\hline Columbia Basin Crop and Water Quality Monitoring Study & 1 & $4 / 17 / 2000$ & 10/6/2003 & $\begin{array}{l}8 \\
8\end{array}$ & $\begin{array}{l}\text { At Road } 56 \\
\text { Esquatzel Coulee Wasteway at Road } \\
68\end{array}$ & $\mathrm{x}$ & & & & & \\
\hline $\begin{array}{l}\text { Columbia Generating Station } 2000 \text { Annual Radiological } \\
\text { Environmental Operating Report }\end{array}$ & 1 & $1 / 4 / 2000$ & $11 / 1 / 2000$ & $\begin{array}{l}12 \\
12\end{array}$ & $\begin{array}{l}\text { CGS (WNP-2) STA 26, INTAKE } \\
\text { CGS (WNP-2) STA } 27\end{array}$ & $\mathrm{x}$ & & & & & \\
\hline $\begin{array}{l}\text { Columbia Generating Station } 2001 \text { Annual Radiological } \\
\text { Environmental Operating Report }\end{array}$ & 1 & $1 / 3 / 2001$ & $11 / 6 / 2001$ & $\begin{array}{l}15 \\
15\end{array}$ & $\begin{array}{l}\text { CGS (WNP-2) STA 26, INTAKE } \\
\text { CGS (WNP-2) STA } 27\end{array}$ & $\mathrm{x}$ & & & & & \\
\hline $\begin{array}{l}\text { Columbia Generating Station } 2002 \text { Annual Radiological } \\
\text { Environmental Operating Report }\end{array}$ & 1 & $1 / 2 / 2002$ & $11 / 5 / 2002$ & $\begin{array}{l}14 \\
13\end{array}$ & $\begin{array}{l}\text { CGS (WNP-2) STA 26, INTAKE } \\
\text { CGS (WNP-2) STA } 27\end{array}$ & $\mathrm{x}$ & & & & & \\
\hline $\begin{array}{l}\text { Columbia Generating Station } 2003 \text { Annual Radiological } \\
\text { Environmental Operating Report }\end{array}$ & 1 & 1/2/2003 & $11 / 4 / 2003$ & $\begin{array}{l}16 \\
16\end{array}$ & $\begin{array}{l}\text { CGS (WNP-2) STA 26, INTAKE } \\
\text { CGS (WNP-2) STA } 27\end{array}$ & $\mathrm{x}$ & & & & & \\
\hline $\begin{array}{l}\text { Columbia Generating Station } 2004 \text { Annual Radiological } \\
\text { Environmental Operating Report }\end{array}$ & 1 & $1 / 6 / 2004$ & 2/1/2005 & $\begin{array}{l}35 \\
17\end{array}$ & $\begin{array}{l}\text { CGS (WNP-2) STA 26, INTAKE } \\
\text { CGS (WNP-2) STA } 27\end{array}$ & $\mathrm{x}$ & & & & & \\
\hline $\begin{array}{l}\text { Columbia Generating Station, } 2000 \text { Annual Environmental } \\
\text { Operating Report }\end{array}$ & 2 & $2 / 1 / 2000$ & $2 / 1 / 2000$ & 1 & CGS (WNP-2) STA 26, INTAKE & $\mathrm{x}$ & & & & & \\
\hline
\end{tabular}


Table 3-6. Summary of Documents with Segment 2 Surface Water Data. (6 Pages)

\begin{tabular}{|c|c|c|c|c|c|c|c|c|c|c|c|}
\hline Source Title & $\begin{array}{c}\text { Data } \\
\text { Usability } \\
\text { Category }\end{array}$ & Begin Date & End Date & $\begin{array}{c}\text { Number } \\
\text { of } \\
\text { Samples }\end{array}$ & Sample Area & RADs & METs & svoc & Pest & PCBs & $\begin{array}{c}\text { GEN } \\
\text { CHEM }\end{array}$ \\
\hline Environmental Radiation Oversight Program - Database & 1 & $5 / 7 / 2004$ & $10 / 25 / 2004$ & $\begin{array}{l}3 \\
3 \\
4 \\
1 \\
1 \\
2 \\
4 \\
1\end{array}$ & $\begin{array}{l}100 \text { F Shore HRM } 18-23 \\
100 \text { N Shore HRM } 8.4-9.8 \\
300 \text { Area Sr HRM } 41.5-42.9 \\
100 \text { K SPRING 77-2 } \\
100 \text { N SPRING 8-13 } \\
\text { Hanford Townsite HRM } 27-28 \\
\text { Rich.Pmphs HRM 43.5 - 45.8 } \\
\text { RIVERVIEW }\end{array}$ & $\mathrm{x}$ & & & & & $\mathrm{x}$ \\
\hline $\begin{array}{l}\text { Hanford Environmental Oversight Program } 2000 \text { Data Summary } \\
\text { Report }\end{array}$ & 1 & $1 / 4 / 2000$ & $12 / 5 / 2000$ & $\begin{array}{c}6 \\
1 \\
1 \\
1 \\
1 \\
5 \\
17 \\
4 \\
1 \\
12 \\
1\end{array}$ & $\begin{array}{l}\text { 100N SPR } \\
\text { 100H SPRING 153-1 } \\
\text { 100K SPRINGS } \\
\text { 100F SPRING 207-1 } \\
\text { SPRING 28.2 } \\
\text { 300 AREA } \\
\text { CGS (WNP-2) STA 26, INTAKE } \\
\text { CGS (WNP-2) STA 29, WTP } \\
\text { Powerline } \\
\text { Richland Water Treatment Plant } \\
\text { Wooded Island }\end{array}$ & $\mathrm{x}$ & & & & & \\
\hline $\begin{array}{l}\text { Hanford Environmental Oversight Program } 2001 \text { Data Summary } \\
\text { Report }\end{array}$ & 1 & $1 / 3 / 2001$ & 12/4/2001 & $\begin{array}{c}1 \\
2 \\
1 \\
10 \\
34 \\
4 \\
16 \\
11 \\
10 \\
1 \\
4\end{array}$ & $\begin{array}{l}\text { 100B SPRING 37-1 } \\
\text { 100H SPRING 145-1 } \\
\text { 100N SPRING 8-13 } \\
300 \text { AREA } \\
300 \text { SPR } \\
\text { CGS (WNP-2) STA 26, INTAKE } \\
\text { CGS (WNP-2) STA 29, WTP } \\
\text { Richland Water Treatment Plant } \\
\text { RL Pumphouse } \\
\text { SPRING 28.2 } \\
\text { VERNITA }\end{array}$ & $\mathrm{x}$ & & & & & \\
\hline $\begin{array}{l}\text { Hanford Environmental Oversight Program } 2002 \text { Data Summary } \\
\text { Report }\end{array}$ & 1 & $1 / 2 / 2002$ & $12 / 3 / 2002$ & $\begin{array}{c}3 \\
5 \\
1 \\
1 \\
1 \\
1 \\
16 \\
4 \\
3 \\
12 \\
1 \\
6\end{array}$ & $\begin{array}{l}100 \text { F Shore HRM 18-23 } \\
100 F \\
100 F \text { SPRING 207-1 } \\
\text { 100H SPRING 145-1 } \\
\text { 100K SPRING 63-2 } \\
\text { CGS (WNP-2) STA 26, INTAKE } \\
\text { CGS (WNP-2) STA 29, WTP } \\
\text { Hanfrd Twnsite HRM 26-30 } \\
\text { Richland Water Treatment Plant } \\
\text { RIVERVIEW } \\
\text { TOWNSITE }\end{array}$ & $\mathrm{x}$ & & & & & \\
\hline
\end{tabular}


Table 3-6. Summary of Documents with Segment 2 Surface Water Data. (6 Pages)

\begin{tabular}{|c|c|c|c|c|c|c|c|c|c|c|c|}
\hline Source Title & $\begin{array}{c}\text { Data } \\
\text { Usability } \\
\text { Category }\end{array}$ & Begin Date & End Date & \begin{tabular}{|c|}
$\begin{array}{c}\text { Number } \\
\text { of } \\
\text { Samples }\end{array}$ \\
\end{tabular} & Sample Area & RADs & METs & svoc & Pest & PCBs & $\begin{array}{l}\text { GEN } \\
\text { CHEM }\end{array}$ \\
\hline $\begin{array}{l}\text { Hanford Environmental Oversight Program } 2003 \text { Data Summary } \\
\text { Report }\end{array}$ & 1 & 1/2/2003 & $12 / 8 / 2003$ & $\begin{array}{c}4 \\
6 \\
6 \\
1 \\
1 \\
1 \\
1 \\
4 \\
1 \\
1 \\
1 \\
16 \\
4 \\
12 \\
1\end{array}$ & $\begin{array}{l}100 \text { N Shore HRM } 8.4-9.8 \\
100 N \text { SPR } \\
\text { 300 AREA } \\
100 \mathrm{~B} \text { SPRING } \\
100 \mathrm{~F} \text { SPRING } \\
\text { 100 SPRING } \\
100 \mathrm{~K} \text { SPRING } \\
300 \text { Area Sr HRM } 41.5-42.9 \\
\text { SPR DR 28.2 } \\
\text { 300 SPR 14 } \\
\text { 300 SPRING 6 } \\
\text { CGS (WNP-2) STA 26, INTAKE } \\
\text { CGS (WNP-2) STA 29, WTP } \\
\text { Richland Water Treatment Plant } \\
\text { RIVERVIEW }\end{array}$ & $\mathrm{x}$ & & & & & \\
\hline Hanford Site Environmental Report for 1999 & 1 & 1/27/1999 & 12/29/1999 & $\begin{array}{c}26 \\
12 \\
18 \\
3 \\
2 \\
5 \\
2 \\
2 \\
6 \\
2 \\
12 \\
6 \\
1 \\
18 \\
6 \\
30 \\
160 \\
44 \\
2\end{array}$ & $\begin{array}{l}\text { HRM } 19.0 \\
100 \text { N Shore HRM } 8.4-9.8 \\
\text { HRM 9.5 } \\
\text { 100B Area } \\
\text { 100BC Area } \\
\text { 100D Area } \\
\text { 100F Area } \\
\text { 100H Area } \\
\text { 100K Area } \\
\text { 100N Area } \\
300 \text { Area Sr HRM 41.5-42.9 } \\
\text { 300 Area SPRING 42.2 } \\
\text { Hanford Reach - 300 Area } \\
\text { 300 HRM 43.1 } \\
\text { HANFORD SPRING 28-2 } \\
\text { Hanfrd Twnsite HRM 26-30 } \\
\text { Rich.Pmphs HRM 43.5-46.4 } \\
\text { Vernita } \\
\text { WSU-Richland }\end{array}$ & $\mathrm{x}$ & & & & & \\
\hline
\end{tabular}


Table 3-6. Summary of Documents with Segment 2 Surface Water Data. (6 Pages)

\begin{tabular}{|c|c|c|c|c|c|c|c|c|c|c|c|}
\hline Source Title & $\begin{array}{c}\text { Data } \\
\text { Usability } \\
\text { Category }\end{array}$ & Begin Date & End Date & $\begin{array}{c}\begin{array}{c}\text { Number } \\
\text { of } \\
\text { Samples }\end{array} \\
\end{array}$ & Sample Area & RADs & METS & svoc & Pest & PCBs & $\begin{array}{c}\text { GEN } \\
\text { CHEM }\end{array}$ \\
\hline Hanford Site Environmental Report for Calendar Year 2000 & 1 & $1 / 26 / 2000$ & $12 / 28 / 2000$ & $\begin{array}{c}13 \\
24 \\
20 \\
27 \\
4 \\
4 \\
4 \\
7 \\
6 \\
8 \\
12 \\
9 \\
4 \\
46 \\
6 \\
23 \\
19 \\
213\end{array}$ & $\begin{array}{l}100 \text { F Shore HRM } 18-23 \\
100 \text { F-10 HRM } 19.0 \\
100 \text { N Shore HRM } 8.4-9.8 \\
\text { HRM 9.5 } \\
\text { 100B Area } \\
\text { 100D Area } \\
\text { 100K Area } \\
\text { 100B SPRING } \\
\text { 100F SPRING } \\
\text { 100K SPRING } \\
\text { 100D SPRING } \\
\text { 100 SPRING } \\
\text { 100N SPRING } \\
\text { 300 Area-10 HRM } 41.5-43.1 \\
\text { HANFORD SPRING 28-2 } \\
\text { Hanford TS-HRM 28.7 } \\
\text { Hanfrd Twnsite HRM 26-30 } \\
\text { Rich.Pmphs HRM 43.5-46.4 }\end{array}$ & $\mathrm{x}$ & & & & & \\
\hline Hanford Site Environmental Report for Calendar Year 2001 & 1 & $1 / 4 / 2001$ & 12/27/2001 & $\begin{array}{c}8 \\
24 \\
12 \\
24 \\
4 \\
4 \\
4 \\
9 \\
3 \\
8 \\
10 \\
3 \\
9 \\
12 \\
24 \\
6 \\
4 \\
24 \\
4 \\
213 \\
1\end{array}$ & $\begin{array}{l}\text { 100 F Shore HRM } 18-23 \\
100 \text { F-10 HRM } 19.0 \\
100 \text { N Shore HRM } 8.4-9.8 \\
100 \mathrm{~N} \text { - HRM } 9.5 \\
\text { 100B Area } \\
\text { 100D Area } \\
\text { 100K Area } \\
\text { 100B SPRING } \\
\text { 100D SPRING } \\
\text { 100F SPRING } \\
\text { 100H SPRING } \\
\text { 100N SPRING } \\
\text { 100K SPRRING } \\
\text { 300 Area Sr HRM 41.5 - } 42.9 \\
\text { 300 Area - HRM 43.1 } \\
\text { 300 SPR } \\
\text { HANFORD SPRING 28-2 } \\
\text { Hanfrd TS - HRM 28.7 } \\
\text { Hanfrd Twnsite HRM } 26-30 \\
\text { Rich.Pmphs HRM 43.5-46.4 } \\
\text { Richland Spr HRM 44.4 }\end{array}$ & $\mathrm{x}$ & & & & & \\
\hline
\end{tabular}


Table 3-6. Summary of Documents with Segment 2 Surface Water Data. (6 Pages)

\begin{tabular}{|c|c|c|c|c|c|c|c|c|c|c|c|}
\hline Source Title & $\begin{array}{c}\text { Data } \\
\text { Usability } \\
\text { Category }\end{array}$ & Begin Date & End Date & \begin{tabular}{|c|}
$\begin{array}{c}\text { Number } \\
\text { of } \\
\text { Samples }\end{array}$ \\
\end{tabular} & Sample Area & RADs & METs & svoc & Pest & PCBs & $\begin{array}{c}\text { GEN } \\
\text { CHEM }\end{array}$ \\
\hline Hanford Site Environmental Report for Calendar Year 2002 & 1 & $1 / 3 / 2002$ & $12 / 12 / 2002$ & $\begin{array}{c}12 \\
24 \\
16 \\
25 \\
5 \\
5 \\
10 \\
16 \\
6 \\
21 \\
4 \\
11 \\
16 \\
24 \\
7 \\
4 \\
40 \\
186\end{array}$ & 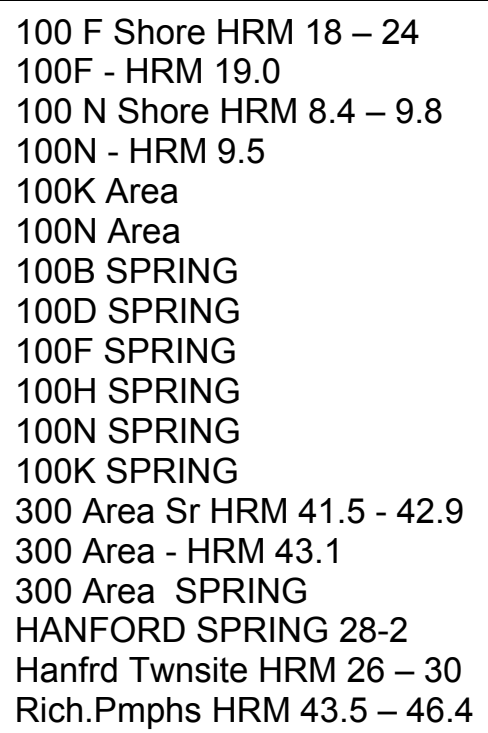 & $\mathrm{x}$ & & & & & \\
\hline Hanford Site Environmental Report for Calendar Year 2003 & 1 & $1 / 8 / 2003$ & $12 / 9 / 2003$ & $\begin{array}{c}8 \\
12 \\
21 \\
16 \\
25 \\
9 \\
9 \\
34 \\
16 \\
3 \\
3 \\
11 \\
16 \\
12 \\
10 \\
30 \\
213 \\
12\end{array}$ & $\begin{array}{l}100 \text { B/C Area } \\
100 \text { F Shore HRM } 18-23 \\
100 f \text { - HRM } 19.0 \\
100 \text { N Shore HRM } 8.4-9.8 \\
100 F \text { - HRM } 9.5 \\
100 \mathrm{~K} \text { Area } \\
\text { 100N Area } \\
\text { 100B SPRING } \\
\text { 100D SPRING } \\
\text { 100F SPRING } \\
\text { 100H SPRING } \\
\text { 100N SPRING } \\
\text { 100K SPRING } \\
\text { 300 Area Sr HRM } 41.5-42.9 \\
\text { 300 AREA SPRING } \\
\text { Hanfrd Twnsite HRM } 26-30 \\
\text { Rich.Pmphs HRM 43.5- } 46.4 \\
\text { Ringold Wasteway }\end{array}$ & $\mathrm{x}$ & & & & & \\
\hline
\end{tabular}


Table 3-6. Summary of Documents with Segment 2 Surface Water Data. (6 Pages)

\begin{tabular}{|c|c|c|c|c|c|c|c|c|c|c|c|}
\hline Source Title & $\begin{array}{l}\text { Data } \\
\text { Usability } \\
\text { Category }\end{array}$ & Begin Date & End Date & $\begin{array}{c}\text { Number } \\
\text { of } \\
\text { Samples }\end{array}$ & Sample Area & RADs & METs & svoc & Pest & PCBs & $\begin{array}{l}\text { GEN } \\
\text { CHEM }\end{array}$ \\
\hline Hanford Site Environmental Report for 2004 & 1 & $2 / 4 / 2004$ & $1 / 5 / 2005$ & $\begin{array}{c}40 \\
44 \\
6 \\
8 \\
8 \\
3 \\
6 \\
8 \\
4 \\
8 \\
3 \\
3 \\
61 \\
15 \\
40 \\
134 \\
1\end{array}$ & $\begin{array}{l}100 \mathrm{~F} \text { Area } \\
100 \text { N Area } \\
100 \text { B Area } \\
100 \text { D Area } \\
100 \text { H Area } \\
100 \text { K Area } \\
100 B \text { SPRING } \\
100 D \text { SPRING } \\
100 F \text { SPRING } \\
100 H \text { SPRING } \\
100 N \text { SPRING } \\
100 \mathrm{~K} \text { SPRING } \\
\text { 300 AREA } \\
\text { HANFORD SPRING } \\
\text { Hanfrd Twnsite HRM } 26-30 \\
\text { Rich Pmphs HRM 43-5 } 46.4 \\
\text { RICHLAND SPR(SRL 437-1) }\end{array}$ & $\mathrm{x}$ & & & & & \\
\hline $\begin{array}{l}\text { Survey of Radiological and Chemical Contaminants in the Near- } \\
\text { Shore Environment at the Hanford Site } 300 \text { Area }\end{array}$ & 1 & $8 / 27 / 2001$ & 8/27/2001 & $\begin{array}{c}1 \\
42\end{array}$ & $\begin{array}{l}100 \mathrm{~N}-1 \mathrm{HRM} 9.5 \\
300 \mathrm{SPR}\end{array}$ & $\mathrm{x}$ & & & & & \\
\hline $\begin{array}{l}\text { Biogeochemical Processes and Microbial Characteristics across } \\
\text { Groundwater-Surface Water Boundaries of the Hanford Reach of } \\
\text { the Columbia River }\end{array}$ & 3 & $3 / 23 / 2000$ & $11 / 2 / 2000$ & $\begin{array}{c}1 \\
10 \\
1 \\
1\end{array}$ & $\begin{array}{l}\text { RM } 366 \\
\text { RM } 372 \\
\text { RM } 373 \\
\text { RM } 374\end{array}$ & \multicolumn{6}{|c|}{ Results not available for these samples. } \\
\hline Water Resource Data Washington Water Year 2003 & 3 & 12/12/2002 & 9/26/2003 & 3 & Richland, Washington & \multicolumn{6}{|c|}{ Results not available for these samples. } \\
\hline
\end{tabular}

CGS = Columbia Generating Station

HRM = Hanford River Miles

$\begin{array}{ll}\text { METS } & =\text { metals } \\ \text { PCBs } & =\text { polychlo }\end{array}$

ated biphenyls

$\mathrm{RADs}=$ radionuclides

$\mathrm{RL} \quad=$ Richland Operations Office

$\mathrm{RM}=$ river mile

WNOCs $=$ semithitile organic compounds

WTP $=$ Water Treatment Plant 
Table 3-7. Segment 2 Surface Water Location Summary.

\begin{tabular}{|c|c|c|}
\hline Sample Area & Sample Collection Dates & No. of Samples \\
\hline $\begin{array}{l}\text { 100-B/C Area including } \\
\text { 100-B Spring 37-1,100-B Spring 38-3, 100-B- } \\
\text { Spring 39-2 }\end{array}$ & April 1999 - October 2004 & 156 \\
\hline $\begin{array}{l}\text { 100-D Area including 100-D Spring 102-1, 110-1, } \\
\text { and 98-1 }\end{array}$ & April 1999 - October 2004 & 85 \\
\hline $\begin{array}{l}\text { 100-F Area, F Area River Transect locations, and } \\
100-F \text { Spring } 207-1 \text {, Spring } 211-1 \text {, and F Spring }\end{array}$ & June 1999 - October 2004 & 212 \\
\hline $\begin{array}{l}\text { 100-H Area including 100-H Spring 144-1, 145-1, } \\
150-1,152-2 \text {, and } 153-1\end{array}$ & June 1999 - October 2004 & 80 \\
\hline $\begin{array}{l}\text { 100-K Area including 100-K Spring 63-1, 63-2, } \\
77-1,77-2 \text {, and } 82-2\end{array}$ & January 1999 - October 2004 & 87 \\
\hline 100-N Area, N Area River Transect s and Springs & June 1992 - October 2003 & 332 \\
\hline $\begin{array}{l}300 \text { Area and } 300 \text { Area River Transects and } \\
\text { Springs }\end{array}$ & July 1992 - December 2004 & 410 \\
\hline Hanford Spring 28-2 & November 1999 - October 2004 & 79 \\
\hline Hanford Townsite River Transects & $\begin{array}{l}\text { September } 1999- \\
\text { September } 2004\end{array}$ & 249 \\
\hline Hanford River Miles (HRM) - 3.6 - 45.6 & June 2001 & 21 \\
\hline $\begin{array}{l}\text { Richland, Washington } \\
\text { Spring } \\
\text { Pumphouse } \\
\text { WTNK } \\
\text { WTP }\end{array}$ & January 1999 - February 2005 & $\begin{array}{c}1 \\
1,131 \\
3 \\
77\end{array}$ \\
\hline Ringold & May 2003 & 12 \\
\hline Riverview & May 2002 - May 2004 & 3 \\
\hline $\begin{array}{l}\text { Columbia Generation Station } 26 \\
\text { Columbia Generation Station } 29\end{array}$ & January 1999 - February 2005 & $\begin{array}{c}173 \\
20\end{array}$ \\
\hline $\begin{array}{l}\text { Other locations including Road 56, WSU, Benton } \\
\text { Co. Pumphouse, Esquatel Canal }\end{array}$ & July 1999 - October 2003 & 33 \\
\hline
\end{tabular}

WSU = Washington State University

WTNK = water tank

WTP $=$ Water Treatment Plant 
Table 3-8. Summary of Documents with Segment 3 Sediment Data. (3 Pages)

\begin{tabular}{|c|c|c|c|c|c|c|c|c|c|c|c|}
\hline Source Title & $\begin{array}{c}\text { Data } \\
\text { Usability } \\
\text { Category }\end{array}$ & Begin Date & End Date & $\begin{array}{l}\text { Number of } \\
\text { Samples }\end{array}$ & Sample Area & RADs & METs & svoc & Pest & PCBs & $\begin{array}{l}\text { GEN } \\
\text { CHEM }\end{array}$ \\
\hline $\begin{array}{l}1999 \text { Hanford Environmental Oversight Program Data } \\
\text { Summary Report }\end{array}$ & 1 & 8/2/1999 & 8/2/1999 & 4 & Columbia River -Above McNary Dam & $\mathrm{x}$ & & & & & \\
\hline $\begin{array}{l}\text { Class II Inspection of the Boise Cascade Pulp \& Paper } \\
\text { Mill, Wallula, Washington- April } 1992\end{array}$ & 1 & 4/13/1992 & 4/14/1992 & 4 & $\begin{array}{l}\text { RM } 311 \text { - } 1.5 \text { mile below Port Kelly } \\
\text { RM } 298-1 \text { mile below Hat Rock State Park } \\
\text { RM } 318 \text { - Badger Island } \\
\text { RM } 316 \text { - Old Outfall }\end{array}$ & & $\mathrm{x}$ & $\mathrm{x}$ & $\mathrm{x}$ & $\mathrm{x}$ & \\
\hline $\begin{array}{l}\text { Environmental Contaminants in Aquatic Resources from } \\
\text { the Columbia River }\end{array}$ & 1 & 11/1/1991 & 11/1/1991 & 1 & RM 274-286 - Umatilla, Oregon & & $\mathrm{x}$ & $\mathrm{x}$ & & $\mathrm{x}$ & \\
\hline $\begin{array}{l}\text { Environmental Monitoring of Columbia River Sediments: } \\
\text { Grain-Size Distribution and Contaminant Association }\end{array}$ & 1 & 4/11/1994 & 4/11/1994 & 4 & Columbia River -Above McNary Dam & $\mathrm{x}$ & & & & & \\
\hline Environmental Radiation Oversight Program - Database & 1 & 7/28/1995 & 9/21/2004 & 19 & Columbia River -Above McNary Dam & $\mathrm{x}$ & & & & & \\
\hline $\begin{array}{l}\text { Environmental Radiation Program 1992-1994 Annual } \\
\text { Report }\end{array}$ & 1 & 9/29/1992 & 9/23/1994 & 8 & Columbia River -Above McNary Dam & $\mathrm{x}$ & & & & & \\
\hline $\begin{array}{l}\text { Hanford Environmental Oversight Program } 2000 \text { Data } \\
\text { Summary Report }\end{array}$ & 1 & 8/8/2000 & & 2 & Columbia River -Above McNary Dam & $\mathrm{x}$ & & & & & \\
\hline $\begin{array}{l}\text { Hanford Environmental Oversight Program } 2001 \text { Data } \\
\text { Summary Report }\end{array}$ & 1 & 7/20/2001 & 7/23/2001 & 2 & Columbia River -Above McNary Dam & $\mathrm{x}$ & & & & & \\
\hline $\begin{array}{l}\text { Hanford Environmental Oversight Program } 2002 \text { Data } \\
\text { Summary Report }\end{array}$ & 1 & 7/19/2002 & & 2 & Columbia River -Above McNary Dam & $\mathrm{x}$ & & & & & \\
\hline $\begin{array}{l}\text { Hanford Environmental Oversight Program } 2003 \text { Data } \\
\text { Summary Report }\end{array}$ & 1 & $7 / 16 / 2003$ & 8/8/2003 & 8 & Columbia River -Above McNary Dam & $\mathrm{x}$ & & & & & \\
\hline $\begin{array}{l}\text { Hanford Site Environmental Report for Calendar Year } \\
1994\end{array}$ & 1 & 9/23/1994 & 9/23/1994 & 7 & Columbia River -Above McNary Dam & & $\mathrm{x}$ & & & & \\
\hline $\begin{array}{l}\text { Hanford Site Environmental Report for Calendar Year } \\
1995\end{array}$ & 1 & 7/28/1995 & 7/28/1995 & 4 & Columbia River -Above McNary Dam & & & & & & \\
\hline $\begin{array}{l}\text { Hanford Site Environmental Report for Calendar Year } \\
1996\end{array}$ & 1 & 8/16/1996 & 8/16/1996 & 4 & Columbia River -Above McNary Dam & & & & & & \\
\hline $\begin{array}{l}\text { Hanford Site Environmental Report for Calendar Year } \\
1999\end{array}$ & 1 & 8/2/1999 & 8/16/1999 & $\begin{array}{l}3 \\
6\end{array}$ & $\begin{array}{l}\text { Snake River - Above Ice Harbor Dam } \\
\text { Columbia River - Above McNary Dam }\end{array}$ & & $\mathrm{x}$ & & & & $\mathrm{x}$ \\
\hline $\begin{array}{l}\text { Hanford Site Environmental Report for Calendar Year } \\
2000\end{array}$ & 1 & 8/8/2000 & $8 / 8 / 2000$ & 2 & Columbia River - Above McNary Dam & & $\mathrm{x}$ & & & & $x$ \\
\hline $\begin{array}{l}\text { Hanford Site Environmental Report for Calendar Year } \\
2001\end{array}$ & 1 & $3 / 26 / 2001$ & $7 / 23 / 2001$ & $\begin{array}{l}3 \\
9\end{array}$ & $\begin{array}{l}\text { Snake River - Above Ice Harbor Dam- } \\
\text { Columbia River - Above McNary Dam }\end{array}$ & & $\mathrm{x}$ & & & & $\mathrm{x}$ \\
\hline $\begin{array}{l}\text { Hanford Site Environmental Report for Calendar Year } \\
2002\end{array}$ & 1 & 7/19/2002 & $7 / 19 / 2002$ & 2 & Columbia River - Above McNary Dam & & $x$ & & & & $\mathrm{x}$ \\
\hline $\begin{array}{l}\text { Hanford Site Environmental Report for Calendar Year } \\
2003\end{array}$ & 1 & 7/19/2002 & $7 / 19 / 2002$ & $\begin{array}{l}6 \\
2 \\
2\end{array}$ & $\begin{array}{l}\text { Columbia River - Above McNary Dam } \\
\text { Yakima River } \\
\text { Walla Walla River }\end{array}$ & & $\mathrm{x}$ & & & & $x$ \\
\hline
\end{tabular}


Table 3-8. Summary of Documents with Segment 3 Sediment Data. (3 Pages)

\begin{tabular}{|c|c|c|c|c|c|c|c|c|c|c|c|}
\hline Source Title & $\begin{array}{c}\text { Data } \\
\text { Usability } \\
\text { Category }\end{array}$ & Begin Date & End Date & $\begin{array}{l}\text { Number of } \\
\text { Samples }\end{array}$ & Sample Area & RADs & METS & svoc & Pest & PCBs & $\begin{array}{l}\text { GEN } \\
\text { CHEM }\end{array}$ \\
\hline $\begin{array}{l}\text { Mercury in Edible Fish Tissue and Sediments from } \\
\text { Selected Lakes and Rivers of Washington State }\end{array}$ & 1 & 9/27/2002 & 9/27/2002 & 2 & Walla Walla River - Walla Walla, Washington & & $\mathrm{x}$ & & & & $\mathrm{x}$ \\
\hline $\begin{array}{l}\text { Mid-Columbia River Basin Sediment Chemical } \\
\text { Contaminants Study June } 2004 \text { to October } 2004 \\
\text { addendum }\end{array}$ & 1 & 6/1/2004 & 6/1/2004 & $\begin{array}{l}8 \\
3 \\
4 \\
7 \\
2 \\
4 \\
4 \\
2 \\
1\end{array}$ & $\begin{array}{l}\text { Columbia River - } \\
\text { Lake Wallula - above/below Snake River } \\
\text { Confluence } \\
\text { RM } 316 \text { - Lake Wallula } \\
\text { Above McNary Dam } \\
\text { Snake River - } \\
\text { RM10 - Above Ice Harbor Dam } \\
\text { Lower River Delta } \\
\text { Yakima River } \\
\text { RM } 1 \text { - Lower River Delta } \\
\text { RM } 2 \text { - Lower River (Below Highway } 240 \\
\text { Bridge) } \\
\text { Walla Walla River - Lower Delta }\end{array}$ & & $\mathrm{x}$ & & & & $\mathrm{x}$ \\
\hline $\begin{array}{l}\text { Occurrence and Significance of DDT Compounds and } \\
\text { Other Contaminants in Fish, Water, and Sediment from } \\
\text { the Yakima River Basin }\end{array}$ & 1 & 9/24/1985 & 9/24/1985 & 1 & $\begin{array}{l}\text { Yakima River } \\
\text { RM } 18 \text { - Horn Rapids Dam }\end{array}$ & & $\mathrm{x}$ & $\mathrm{x}$ & $\mathrm{x}$ & $\mathrm{x}$ & $\mathrm{x}$ \\
\hline $\begin{array}{l}\text { Pesticides Detected in the Walla Walla Drainage, April } \\
\text { and June } 1996\end{array}$ & 1 & 6/18/1996 & 6/18/1996 & 1 & Walla Walla River -Cummins Road Bridge & & & $\mathrm{x}$ & $\mathrm{x}$ & & $\mathrm{x}$ \\
\hline $\begin{array}{l}\text { Screening Survey of Contaminants in Groundwater and } \\
\text { Surface Water Drainage at the Port of Pasco }\end{array}$ & 1 & 9/10/1985 & 9/30/1986 & 11 & Port of Pasco & & $\mathrm{x}$ & $\mathrm{x}$ & & $\mathrm{x}$ & $\mathrm{x}$ \\
\hline Screening Survey of Mercury Levels in Fish Tissue & 1 & 9/27/2002 & 9/27/2002 & 1 & Walla Walla, Washington & & $\mathrm{x}$ & & & & $\mathrm{x}$ \\
\hline Snake and Columbia Rivers Sediment Sampling Project & 1 & 8/19/1991 & 8/19/1991 & $\begin{array}{l}1 \\
1 \\
2 \\
1\end{array}$ & $\begin{array}{l}\text { Columbia River - } \\
\text { RM 328- Port of Kennewick } \\
\text { RM 314-315 } \\
\text { RM 315-316 } \\
\text { Snake River - RM } 2 \text { - Port of Burbank }\end{array}$ & & $\mathrm{x}$ & $\mathrm{x}$ & $\mathrm{x}$ & $\mathrm{x}$ & $\mathrm{x}$ \\
\hline $\begin{array}{l}\text { Surface Water Quality Assessment of the Yakima River } \\
\text { Basin, Washington: Pesticide and Other Trace-Organic- } \\
\text { Compound Data for Water, Sediment, Soil, and Aquatic } \\
\text { Biota, 1987-91 }\end{array}$ & 1 & $8 / 31 / 1988$ & 11/10/1990 & 3 & Yakima River - Kiona, Washington & & & $\mathrm{x}$ & & $\mathrm{x}$ & \\
\hline $\begin{array}{l}\text { Surface-Water Quality Assessment of the Yakima River } \\
\text { Basin in Washington: Major and Minor Element Data for } \\
\text { Sediment, Water and Aquatic Biota, 1987-91 }\end{array}$ & 1 & 8/25/1987 & $8 / 25 / 1987$ & 1 & Yakima River - Kiona, Washington & & $\mathrm{x}$ & & & & \\
\hline $\begin{array}{l}\text { Survey of Potential Hanford Site Contaminants in the } \\
\text { Upper Sediment for the Reservoirs at McNary, John } \\
\text { Day, The Dalles, and Bonneville Dams, } 2003\end{array}$ & 1 & 7/14/2003 & $8 / 8 / 2003$ & 11 & Columbia River - Above McNary Dam & $\mathrm{x}$ & $\mathrm{x}$ & $\mathrm{x}$ & $\mathrm{x}$ & $\mathrm{x}$ & $\mathrm{x}$ \\
\hline $\begin{array}{l}\text { Washington State Pesticide Monitoring Program } \\
\text { Reconnaissance Sampling of Fish Tissue and } \\
\text { Sediments (1992) }\end{array}$ & 1 & 9/16/1992 & 9/16/1992 & $\begin{array}{l}1 \\
2\end{array}$ & $\begin{array}{l}\text { Walla Walla River - Near mouth } \\
\text { Yakima River - Horn Rapids Dam }\end{array}$ & & & & $\mathrm{x}$ & & $\mathrm{x}$ \\
\hline
\end{tabular}


Table 3-8. Summary of Documents with Segment 3 Sediment Data. (3 Pages)

\begin{tabular}{|c|c|c|c|c|c|c|c|c|c|c|c|}
\hline Source Title & $\begin{array}{l}\text { Data } \\
\text { Usability } \\
\text { Category }\end{array}$ & Begin Date & End Date & $\begin{array}{l}\text { Number of } \\
\text { Samples }\end{array}$ & Sample Area & RADs & METs & svoc & Pest & PCBs & $\begin{array}{c}\text { GEN } \\
\text { CHEM }\end{array}$ \\
\hline WSPMP 1994 Pesticides in Sediment & 1 & 9/14/1994 & 9/14/1994 & 1 & Snake River - near Lake Sacajawea & & & & $\mathrm{x}$ & & $\mathrm{x}$ \\
\hline $\begin{array}{l}\text { An Investigation of the Origin of } 152 \text { Eu in Columbia } \\
\text { River Sediment }\end{array}$ & 2 & 7/28/1999 & 8/16/1999 & 22 & $\begin{array}{l}\text { Columbia River - Above McNary Dam } \\
\text { Snake River - Above Ice Harbor Dam }\end{array}$ & $\mathrm{x}$ & & & & & \\
\hline $\begin{array}{l}\text { Basic Water Monitoring Program Fish Tissue and } \\
\text { Sediment Sampling for } 1984\end{array}$ & 2 & 9/12/1984 & 9/12/1984 & $\begin{array}{l}1 \\
1\end{array}$ & $\begin{array}{l}\text { Yakima River - Below Kiona, Washington } \\
\text { Walla Walla River - Below Warm Springs }\end{array}$ & & $\mathrm{x}$ & $\mathrm{x}$ & $\mathrm{x}$ & $\mathrm{x}$ & \\
\hline $\begin{array}{l}\text { Environmental Radiation Program 26th Annual Report } \\
\text { January } 1987 \text { - December } 1987\end{array}$ & 2 & 6/24/1987 & 6/24/1987 & 3 & Columbia River - Above McNary Dam & $\mathrm{x}$ & & & & & \\
\hline $\begin{array}{l}\text { Environmental Radiation Program 27th Annual Report } \\
\text { January through December } 1988\end{array}$ & 2 & 9/7/1988 & 9/7/1988 & 1 & Columbia River -Above McNary Dam & $\mathrm{x}$ & & & & & \\
\hline Oregon Department of Environmental Quality *a & 2 & 9/1/1991 & 10/23/1991 & 4 & $\begin{array}{l}\text { Columbia River - Wallula Mill Site - } 1 \mathrm{Mi} \\
\text { downstream }\end{array}$ & & & $\mathrm{x}$ & $\mathrm{x}$ & $x$ & $\mathrm{x}$ \\
\hline Oregon Department of Environmental Quality *b & 2 & 9/1/1991 & 10/23/1991 & 4 & Columbia River - Wallula Mill Site & & & $\mathrm{x}$ & $\mathrm{x}$ & $\mathrm{x}$ & $\mathrm{x}$ \\
\hline Oregon Department of Environmental Quality ${ }^{*} \mathrm{C}$ & 2 & 10/23/1991 & 10/23/1991 & 1 & Columbia River - RM 312 & & & $\mathrm{x}$ & $\mathrm{x}$ & $x$ & \\
\hline $243,244 \mathrm{Cm}$ in Columbia River Sediments & 3 & 8/1/1977 & 8/1/1977 & 1 Core & Columbia River - McNary Dam Reservoir & $\mathrm{x}$ & & & & & \\
\hline $\begin{array}{l}\text { Association of Hanford Origin Radionuclides with } \\
\text { Columbia River Sediment }\end{array}$ & 3 & 8/5/1976 & $8 / 1 / 1977$ & $\begin{array}{c}1 \text { Core } \\
2 \text { Cores } \\
10 \text { Cores }\end{array}$ & $\begin{array}{l}\text { Columbia River - } \\
\text { Wallula } \\
\text { Port Kelly } \\
\text { Above McNary Dam }\end{array}$ & $x$ & & & & & \\
\hline $\begin{array}{l}\text { Environmental Surveillance Report on Oregon Surface } \\
\text { Waters 1961- 1993, Vol. } 2\end{array}$ & 3 & $12 / 14 / 1976$ & $5 / 26 / 1993$ & 28 & Columbia River - RM 300 - McNary Dam & $\mathrm{x}$ & & & & & \\
\hline $\begin{array}{l}\text { Heavy Metal Transport and Behavior in the Lower } \\
\text { Columbia River, USA }\end{array}$ & 3 & $8 / 1 / 1999$ & 8/1/1999 & 2 Cores & Columbia River -Above McNary Dam & & $\mathrm{x}$ & & & & $\mathrm{x}$ \\
\hline $\begin{array}{l}\text { Mid-Columbia River Feasibility Study: Physical And } \\
\text { Chemical Analyses Of Hanford Reach Sediments }\end{array}$ & 3 & 10/1/1986 & 10/1/1986 & $\begin{array}{c}4 \text { Cores } \\
\text { (Composite) }\end{array}$ & Columbia River - Above McNary Dam & & $\mathrm{x}$ & $\mathrm{x}$ & $\mathrm{x}$ & & \\
\hline $\begin{array}{l}\text { Nickel-63 in Columbia River Sediments below the } \\
\text { Hanford Reservation }\end{array}$ & 3 & 8/1/1977 & 8/1/1977 & 1 Core & Columbia River - McNary Dam Reservoir & $\mathrm{x}$ & & & & & \\
\hline
\end{tabular}

DDT = dichlorodiphenyltrichloroethane

$\begin{array}{ll}\mathrm{DDT} & =\text { dichlor } \\ \mathrm{METS} & =\text { metals }\end{array}$

PCBS = polychlorinated biphenyls

Pest = pesticides

$\begin{array}{ll}\text { RL } & =\text { radionuclides } \\ \text { = Richland Operations Office }\end{array}$

$\mathrm{RM}=$ river mile

WSPNP = Washington State Pesticide Monitoring Program 
Table 3-9. Summary of Documents with Segment 3 Surface Water Data. (2 pages)

\begin{tabular}{|c|c|c|c|c|c|c|c|c|c|c|c|}
\hline Source Title & Data Usability Category & Begin Date & End Date & $\begin{array}{c}\text { Number } \\
\text { of } \\
\text { Samples }\end{array}$ & Sample Area & RADs & METs & svoc & Pest & PCBs & GEN CHEM \\
\hline $\begin{array}{l}\text { A Total Maximum Daily Load Evaluation for Chlorinated Pesticides } \\
\text { and PCBs in the Walla Walla River }\end{array}$ & 1 & $5 / 30 / 2002$ & 6/9/2003 & $\begin{array}{l}17 \\
4\end{array}$ & $\begin{array}{l}\text { Touchet River - } \\
\text { At Hwy } 12 \text { bridge } \\
\text { Walla Walla River } \\
\text { RM } 15.6 \text { Cummins } \\
\text { Bridge }\end{array}$ & & & & $x$ & $\mathrm{x}$ & $\mathrm{x}$ \\
\hline Columbia Basin Crop and Water Quality Monitoring Study & 1 & $4 / 17 / 2000$ & 10/6/2003 & 8 & $\begin{array}{l}\text { Snake River - } \\
\text { At Hood Park Boat } \\
\text { Launch }\end{array}$ & & & & & & $\mathrm{x}$ \\
\hline Hanford Site Environmental Report for Calendar Year 2003 & 1 & $5 / 14 / 2003$ & $5 / 15 / 2003$ & $\begin{array}{l}1 \\
12 \\
15 \\
15 \\
10\end{array}$ & $\begin{array}{l}\text { Columbia River - } \\
\text { Above McNary Dam } \\
\text { mid-river } \\
\text { Snake River } \\
\text { Yakima River } \\
\text { Walla Walla River }\end{array}$ & $\mathrm{x}$ & $\mathrm{x}$ & & & & $\mathrm{x}$ \\
\hline $\begin{array}{l}\text { Mercury in Edible Fish Tissue and Sediments from Selected Lakes } \\
\text { and Rivers of Washington State }\end{array}$ & 1 & 9/27/2002 & 9/27/2002 & 2 & Walla Walla River & & & & & & $\mathrm{x}$ \\
\hline $\begin{array}{l}\text { Occurrence and Distribution of Dissolved Trace Elements in the } \\
\text { Surface Waters of the Yakima River Basin, Washington, 1999-2000 }\end{array}$ & 1 & 5/19/1999 & $1 / 13 / 2000$ & 16 & $\begin{array}{l}\text { Yakima River } \\
\text { Kiona, Washington }\end{array}$ & \multicolumn{6}{|c|}{ Results not provided for samples from these locations. } \\
\hline $\begin{array}{l}\text { Results of Sampling to Verify } 303(\mathrm{~d}) \text { Metals Listings for Selected } \\
\text { Washington State Rivers and Creeks }\end{array}$ & 1 & 7/25/2001 & $5 / 16 / 2002$ & 6 & $\begin{array}{l}\text { Yakima River } \\
\text { Kiona, Washington }\end{array}$ & \multicolumn{6}{|c|}{ Results not provided for samples from these locations. } \\
\hline Statewide Arsenic Sampling in Selected Rivers & 1 & 7/9/2001 & $6 / 12 / 2002$ & 12 & $\begin{array}{l}\text { Yakima River } \\
\text { Kiona, Washington }\end{array}$ & & $\mathrm{x}$ & & & & \\
\hline Statewide Metals in Selected Rivers \& Creeks & 1 & 7/25/2001 & $5 / 16 / 2002$ & 14 & $\begin{array}{l}\text { Yakima River } \\
\text { Kiona, Washington }\end{array}$ & & $\mathrm{x}$ & & & & \\
\hline $\begin{array}{l}\text { USBR Columbia River Pump Exchange Project, Potential Water } \\
\text { Quality Impacts on the Lower Yakima River }\end{array}$ & 1 & 9/28/1999 & $7 / 26 / 2000$ & 26 & $\begin{array}{l}\text { Yakima River - } \\
\text { Kiona Washington and } \\
\text { lower Yakima River } \\
\text { areas }\end{array}$ & & & & & & $\mathrm{x}$ \\
\hline Walla Walla Bacteria and pH TMDL & 1 & 6/26/2002 & 6/26/2003 & 20 & $\begin{array}{l}\text { Walla Walla River } \\
\text { RM } 15.6, \text { Cummins } \\
\text { Bridge } \\
\text { Hwy } 12 \text { bridge }\end{array}$ & \multicolumn{6}{|c|}{ Results not provided for samples from these locations. } \\
\hline Walla Walla River Chlorinated Pesticide and PCB TMDL & 1 & $5 / 14 / 2002$ & 6/9/2003 & 16 & $\begin{array}{l}\text { Walla Walla River } \\
\text { Lower Walla Walla } \\
\text { River (32WAL-14.3) }\end{array}$ & & & & $x$ & $\mathrm{x}$ & $\mathrm{x}$ \\
\hline $\begin{array}{l}\text { Concentrations and Loads of Suspended Sediment and Nutrients in } \\
\text { Surface Water of the Yakima River Basin, Washington, 1999-2000- } \\
\text { With an Analysis of Trends of Concentrations }\end{array}$ & 2 & 1/13/1999 & 6/7/2005 & 185 & $\begin{array}{l}\text { Yakima River - } \\
\text { Kiona, Washington }\end{array}$ & & & & $\mathrm{x}$ & & $\mathrm{x}$ \\
\hline
\end{tabular}


Table 3-9. Summary of Documents with Segment 3 Surface Water Data. (2 pages)

\begin{tabular}{|c|c|c|c|c|c|c|c|c|c|c|c|}
\hline Source Title & Data Usability Category & Begin Date & End Date & $\begin{array}{c}\text { Number } \\
\text { of } \\
\text { Samples }\end{array}$ & Sample Area & RADs & METs & svoc & Pest & PCBs & GEN CHEM \\
\hline $\begin{array}{l}\text { Pesticides in Surface Water of the Yakima River Basin, Washington, } \\
\text { 1999-2000- Their Occurrence and an Assessment of Factors } \\
\text { Affecting Concentrations and Loads }\end{array}$ & 2 & $5 / 19 / 1999$ & $1 / 13 / 2000$ & 14 & $\begin{array}{l}\text { Yakima River } \\
\text { Kiona, Washington }\end{array}$ & \multicolumn{6}{|c|}{ Results not provided for samples from these locations. } \\
\hline Yakima Mainstem Monitoring and BMP Implementation Project & 2 & 6/3/2003 & $11 / 3 / 2004$ & 92 & $\begin{array}{l}\text { Yakima River - } \\
\text { Kiona Washington and } \\
\text { lower Yakima River } \\
\text { areas }\end{array}$ & & & & & & $\mathrm{x}$ \\
\hline
\end{tabular}

BMP = Baseline Monitoring Program

METs $=$ metals
PCBs $=$ polychlorinated biphenyls

Pest = pesticides

RADs = radionuclides

RMOCs = remivolatile organic compounds

TMDL = Total Maximum Daily Load

USBR =U.S. Bureau of Reclamation 
Table 3-10. Summary of Documents with Segment 4 Sediment Data.

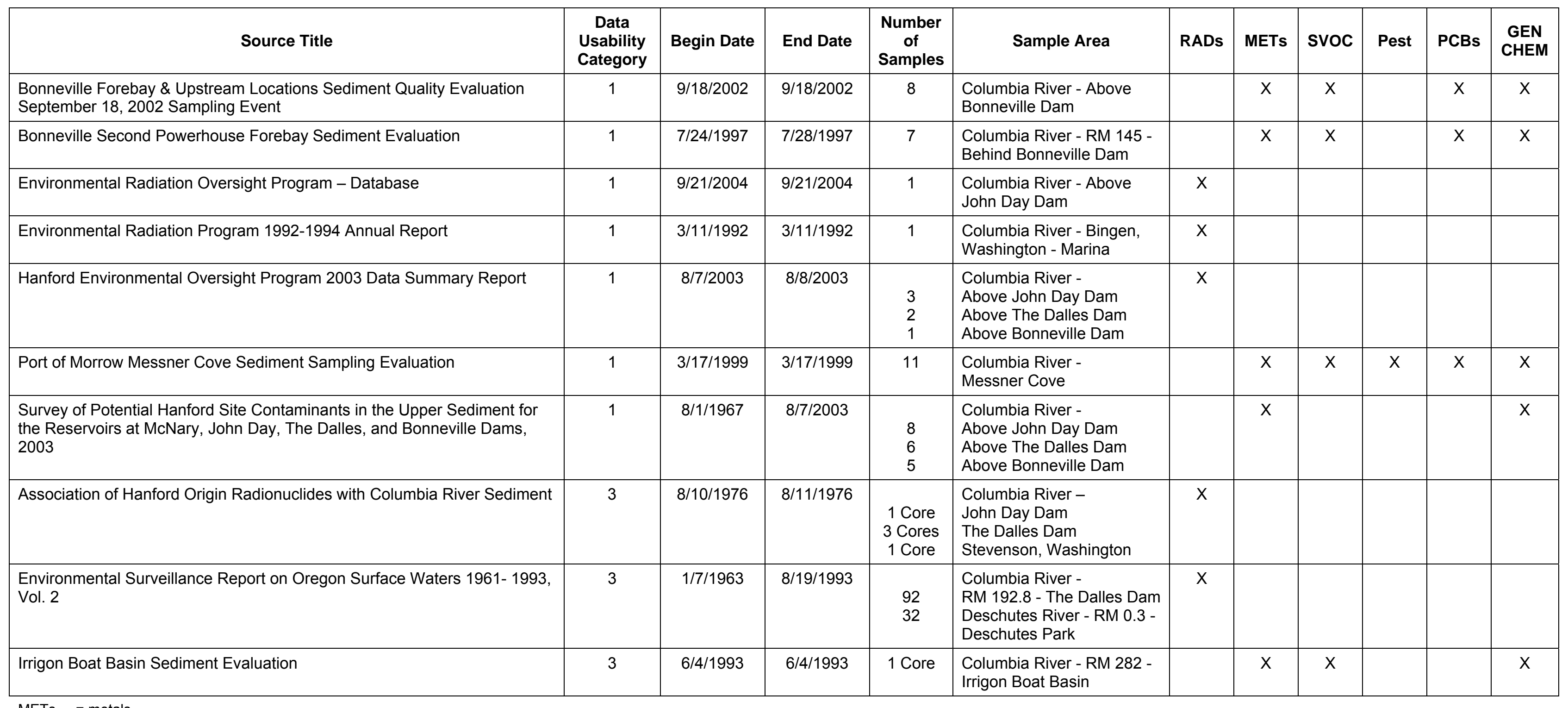

METs $=$ metals

PCBs = polychlorinated biphenyls

RADs = radionuclides

$\mathrm{RM} \quad$ = river mile

SVOCs = semivolatile organic compounds 
Table 3-11. Summary of Documents with Segment 4 Surface Water Data.

\begin{tabular}{|c|c|c|c|c|c|c|c|c|c|c|c|}
\hline Source Title & $\begin{array}{l}\text { Data Usability } \\
\text { Category }\end{array}$ & $\begin{array}{c}\text { Begin } \\
\text { Date }\end{array}$ & End Date & $\begin{array}{l}\text { Number of } \\
\text { Samples }\end{array}$ & Sample Area & RADs & METs & svoc & Pest & PCBs & $\begin{array}{l}\text { GEN } \\
\text { CHEM }\end{array}$ \\
\hline $\begin{array}{l}\text { Concentrations of 303(d) Listed Pesticides, PCBs, and PAHs Measured With } \\
\text { Passive Samplers Deployed in the Lower Columbia River }\end{array}$ & 1 & 9/1/2003 & 6/1/2004 & 3 & $\begin{array}{l}\text { Columbia River - } \\
\text { RM 147, above Bonneville Dam } \\
\end{array}$ & & & $\mathrm{x}$ & $\mathrm{x}$ & & \\
\hline EIM Database - Columbia River above Bonneville Dam & 1 & $8 / 27 / 2003$ & 6/22/2004 & 12 & $\begin{array}{l}\text { Columbia River - } \\
\text { Above Bonneville }\end{array}$ & & & $\mathrm{x}$ & & $\mathrm{x}$ & $\mathrm{x}$ \\
\hline Statewide Arsenic Sampling in Selected Rivers & 1 & $7 / 11 / 2001$ & $5 / 15 / 2002$ & 11 & $\begin{array}{l}\text { Columbia River - } \\
\text { Umatilla, Oregon }\end{array}$ & & $\mathrm{x}$ & & & & $\mathrm{x}$ \\
\hline The City of The Dalles Quarterly Testing Data & 1 & $2 / 8 / 2000$ & $5 / 10 / 2005$ & 22 & $\begin{array}{l}\text { Columbia River - } \\
\text { The Dalles Bridge, 12th pier west of the } \\
\text { bridge }\end{array}$ & $\mathrm{x}$ & $\mathrm{x}$ & & & & $\mathrm{x}$ \\
\hline Upper Deschutes Basin Spring Water Quality Survey 2002 & 1 & 2/22/1999 & $6 / 13 / 2005$ & 49 & $\begin{array}{l}\text { Columbia River - At footbridge downstream } \\
\text { of } \mathrm{l}-84 \text {, Hood River, Oregon }\end{array}$ & & & & & & $x$ \\
\hline
\end{tabular}

EIM = Environmental Information Management

$\begin{array}{ll}\text { METS } & =\text { metals } \\ \text { PAH } & \text { polyaromatic hydrocarbons }\end{array}$

PCBs = polychlorinated biphenyls

Pest = pesticides

RADs = radionuclides

RM = river mile

政 
Table 3-12. Summary of Documents with Segment 5 Sediment Data. (4 Pages)

\begin{tabular}{|c|c|c|c|c|c|c|c|c|c|c|c|}
\hline Source Title & $\begin{array}{l}\text { Data Usability } \\
\text { Category }\end{array}$ & $\begin{array}{c}\text { Begin } \\
\text { Date }\end{array}$ & End Date & $\begin{array}{c}\text { Number of } \\
\text { Samples }\end{array}$ & Sample Area & RADs & METs & svoc & Pest & PCBs & $\begin{array}{l}\text { GEN } \\
\text { CHEM }\end{array}$ \\
\hline Bachelor Slough Ecosystem Restoration Sediment Quality Evaluation Report & 1 & 6/3/2003 & 6/3/2003 & 10 & $\begin{array}{l}\text { Columbia River - Bachelor } \\
\text { Slough }\end{array}$ & & $\mathrm{x}$ & $\mathrm{x}$ & $\mathrm{x}$ & & $\mathrm{x}$ \\
\hline $\begin{array}{l}\text { Columbia and Willamette River Sediment Quality Evaluation for the Columbia River } \\
\text { Channel Deepening Feasibility Report }\end{array}$ & 1 & 6/3/1997 & 7/24/1997 & $\begin{array}{l}2 \\
1 \\
5 \\
2 \\
4 \\
2 \\
3 \\
1 \\
2 \\
2 \\
4 \\
4 \\
1 \\
4 \\
2 \\
4 \\
1 \\
4 \\
2 \\
1 \\
4 \\
1 \\
4 \\
2 \\
7 \\
2 \\
6 \\
7 \\
4 \\
3\end{array}$ & $\begin{array}{l}\text { Columbia River - } \\
\text { RM } 6 \text { Desdemona Shoal } \\
\text { RM } 6 \text { Off Buoy } 22 \\
\text { RM 11-12 Flavel Bar } \\
\text { RM 15-16 Upper Sands } \\
\text { RM 18-22 Tongue Pt X-ing } \\
\text { RM 23-24 Miller Sands } \\
\text { RM 27-29 Pillar Rock } \\
\text { RM 32 Brookfield-Welch } \\
\text { RM 33- Skamokawa Bar } \\
\text { RM 38-39 Puget Is. Bar } \\
\text { RM 40-45- Wanna-Driscoll } \\
\text { RM 46-51- West Port Bar } \\
\text { RM 54 - Island Bar } \\
\text { RM 56-59- Stella-Fisher Bar } \\
\text { RM 60-62- Walker Is. } \\
\text { RM 63-65 - Slaughters Bar } \\
\text { RM 66 R Turning Basin } \\
\text { Upper } \\
\text { RM 67-73 Dowelbower Bar } \\
\text { RM 74 -75- Kalama } \\
\text { RM 76 E8 on BiState } \\
\text { RM 79-83 - Martin Is Bar } \\
\text { RM 83 - E9D on BiState } \\
\text { RM 84-86- St Helens Bar } \\
\text { RM 88-89 Warrior Rock Bar } \\
\text { RM 90-96 Henrici Bar } \\
\text { RM 9-98 Willow Bar } \\
\text { RM 99-101 - Morgan Bar } \\
\text { RM 102-104 Vancouver, } \\
\text { Washington } \\
\text { RM 105-106 } \\
\text { Willamette River } \\
\text { RM 0.1-0.4 mouth }\end{array}$ & & $\mathrm{x}$ & $\mathrm{x}$ & $\mathrm{x}$ & $\mathrm{x}$ & $\mathrm{x}$ \\
\hline $\begin{array}{l}\text { Columbia River Channel Deepening (CRCD) Station \#76 (CR-BC-76) Sediment Quality } \\
\text { Evaluation }\end{array}$ & 1 & 6/1/1997 & 6/1/1997 & 3 & Columbia River & & $\mathrm{x}$ & $\mathrm{x}$ & & & $\mathrm{x}$ \\
\hline Columbia River Mile 29-34 Brookfield Mound and Skamakawa Turn Sediment Evaluation & 1 & 8/9/2000 & 9/7/2000 & 25 & $\begin{array}{l}\text { Columbia River - } \\
\text { RM 29-34 - Brookfield } \\
\text { Mound }\end{array}$ & & $\mathrm{x}$ & $x$ & & $x$ & $x$ \\
\hline Crims Island Ecosystem Restoration Sediment Quality Evaluation Report & 1 & $7 / 15 / 2003$ & $7 / 15 / 2003$ & 5 & $\begin{array}{l}\text { Columbia River - Crims } \\
\text { Island }\end{array}$ & & $\mathrm{x}$ & $x$ & & & $\mathrm{x}$ \\
\hline
\end{tabular}


Table 3-12. Summary of Documents with Segment 5 Sediment Data. (4 Pages)

\begin{tabular}{|c|c|c|c|c|c|c|c|c|c|c|c|}
\hline Source Title & $\begin{array}{l}\text { Data Usability } \\
\text { Category }\end{array}$ & $\begin{array}{c}\text { Begin } \\
\text { Date }\end{array}$ & End Date & $\begin{array}{c}\text { Number of } \\
\text { Samples }\end{array}$ & Sample Area & RADs & METs & svoc & Pest & PCBs & $\begin{array}{l}\text { GEN } \\
\text { CHEM }\end{array}$ \\
\hline Environmental Contaminants in Aquatic Resources from the Columbia River & 1 & 8/1/1991 & 11/1/1991 & $\begin{array}{l}1 \\
1 \\
1 \\
1 \\
1 \\
1 \\
1\end{array}$ & $\begin{array}{l}\text { Columbia River - } \\
\text { RM 2-6 - Baker Bay } \\
\text { RM 111-121 - Camas } \\
\text { Slough } \\
\text { RM 21 - Cathlamet Bay } \\
\text { RM 37-47 - Julia Butler } \\
\text { Hansen NWR } \\
\text { RM 64-72 - Longview, } \\
\text { Washington } \\
\text { RM 21-37 - Lewis and Clark } \\
\text { NWR } \\
\text { RM 87-102 - Ridgefield }\end{array}$ & & & $\mathrm{x}$ & & $\mathrm{x}$ & \\
\hline $\begin{array}{l}\text { Kalama Turning Basin (Columbia River Mile 73.9-74.8) Sediment Quality Evaluation Report } \\
\text { December } 2003\end{array}$ & 1 & 9/10/2003 & 9/10/2003 & 3 & $\begin{array}{l}\text { Columbia River - } \\
\text { RM } 73.9 \text { \& } 74.8\end{array}$ & & $\mathrm{x}$ & $\mathrm{x}$ & & & $\mathrm{x}$ \\
\hline Lower Columbia River Backwater Reconnaissance Survey & 1 & 6/24/1993 & 7/1/1993 & $\begin{array}{l}1 \\
1 \\
1 \\
1 \\
1 \\
1 \\
1 \\
1 \\
1 \\
1 \\
1 \\
1 \\
1 \\
1 \\
1 \\
1\end{array}$ & $\begin{array}{l}\text { Columbia River - } \\
\text { RM } 14 \text { - Young's Bay } \\
\text { RM } 21 \text { - Cathlamet Bay } \\
\text { RM } 23 \text {-Svensen Landing } \\
\text { RM } 26 \text { - Knappa Slough } \\
\text { RM } 29 \text { - Lewis \& Clark } \\
\text { NWR (Bug Hole) } \\
\text { RM } 36 \text { - Elochman Slough } \\
\text { RM } 59 \text { - Fisher Island } \\
\text { Slough } \\
\text { RM } 68 \text { - Carrolls Channel } \\
\text { RM } 81 \text { - Burke Slough } \\
\text { RM } 88 \text { - Scappoose Bay } \\
\text { RM } 90 \text { - Bachelor Island } \\
\text { Slough } \\
\text { RM 95 - Willow Bar Islands } \\
\text { RM } 120 \text { - Camas Slough } \\
\text { RM } 124 \text { - Gary \& Flag } \\
\text { Islands } \\
\text { RM } 141 \text { - Skamania } \\
\text { Landing }\end{array}$ & & $\mathrm{x}$ & $\mathrm{x}$ & $\mathrm{x}$ & $\mathrm{x}$ & $\mathrm{x}$ \\
\hline Old Mouth of the Cowlitz River Federal Project Sediment Quality Evaluation Report & 1 & 9/10/2003 & 9/10/2003 & 4 & Old Mouth of Cowlitz River & & $\mathrm{x}$ & $\mathrm{x}$ & & & $\mathrm{x}$ \\
\hline Oregon Slough Entrance Channel Sediment Quality Evaluation & 1 & $6 / 19 / 2001$ & 6/19/2001 & 4 & $\begin{array}{l}\text { Columbia River - } \\
\text { Oregon Slough }\end{array}$ & & $\mathrm{x}$ & $\mathrm{x}$ & & & $\mathrm{x}$ \\
\hline Reynolds Metal Company Class II Inspection February 1990 & 1 & 2/23/1990 & 2/23/1990 & 6 & $\begin{array}{l}\text { Columbia River - } \\
\text { Longview, Washington- }\end{array}$ & & $\mathrm{x}$ & $\mathrm{x}$ & $\mathrm{x}$ & $\mathrm{x}$ & $\mathrm{x}$ \\
\hline Sediment Quality Evaluation - 2005 Data from Oregon Slough & 1 & 2/17/2005 & 2/17/2005 & 8 & $\begin{array}{l}\text { Columbia River - } \\
\text { Oregon Slough }\end{array}$ & & $\mathrm{x}$ & $\mathrm{x}$ & $\mathrm{x}$ & $\mathrm{x}$ & $\mathrm{x}$ \\
\hline Skipanon Channel \& Boat Basin Sediment Evaluation Report & 1 & 6/24/2003 & $6 / 24 / 2003$ & 4 & $\begin{array}{l}\text { Skipanon Federal Channel - } \\
\text { Boat Basin }\end{array}$ & & $\mathrm{x}$ & $\mathrm{x}$ & & & $\mathrm{x}$ \\
\hline
\end{tabular}


Table 3-12. Summary of Documents with Segment 5 Sediment Data. (4 Pages)

\begin{tabular}{|c|c|c|c|c|c|c|c|c|c|c|c|}
\hline Source Title & $\begin{array}{c}\text { Data Usability } \\
\text { Category }\end{array}$ & $\begin{array}{c}\text { Begin } \\
\text { Date }\end{array}$ & End Date & $\begin{array}{c}\text { Number of } \\
\text { Samples }\end{array}$ & Sample Area & RADs & METS & svoc & Pest & PCBs & $\begin{array}{r}\text { GEN } \\
\text { CHEM }\end{array}$ \\
\hline Skipanon Entrance Channel and Boat Basin Sediment Quality Evaluation & 1 & 9/12/2001 & 9/12/2001 & 7 & $\begin{array}{l}\text { Skipanon Federal Channel - } \\
\text { Boat Basin }\end{array}$ & & $\mathrm{x}$ & $\mathrm{x}$ & & & $\mathrm{x}$ \\
\hline VANALCO Aluminum (former ALCOA Aluminum Plant) Sediment Quality Evaluation & 1 & 6/20/2001 & 6/20/2001 & 26 & \begin{tabular}{|l|} 
Columbia River - \\
RM 103-N. Side Federal \\
Navigation Channel \\
\end{tabular} & & $\mathrm{x}$ & $\mathrm{x}$ & $\mathrm{x}$ & $\mathrm{x}$ & \\
\hline $\begin{array}{l}\text { Investigation of the Distribution of Organochlorine and Polycyclic Aromatic Hydrocarbon } \\
\text { Compounds in the Lower Columbia River Using Semipermeable Membrane Devices }\end{array}$ & 2 & 9/8/1997 & 11/20/1997 & 3 & $\begin{array}{l}\text { Columbia River - } \\
\text { RM } 54 \text { - Beaver Army } \\
\text { Terminal } \\
\text { RM } 82 \text { - Columbia City } \\
\text { RM } 141 \text { - Warrendale, } \\
\text { Oregon }\end{array}$ & & & $\mathrm{x}$ & $\mathrm{x}$ & $\mathrm{x}$ & \\
\hline $\begin{array}{l}\text { Screening Survey for Chemical Contaminants and Toxicity in Sediments at Five Lower } \\
\text { Columbia River Ports September 22-24, } 1987\end{array}$ & 2 & 7/22/1987 & $7 / 24 / 1987$ & 12 & $\begin{array}{l}\text { Columbia River - } \\
\text { Reed Island } \\
\text { Camas Slough } \\
\text { Vanc. Lower Turning Basin } \\
\text { Vancouver below } \\
\text { VANALCO } \\
\text { Kalama Chemical Pier } \\
\text { Below Longview Fibre } \\
\text { Longview --below Weyco } \\
\text { Longview --below Weyco } \\
\text { Longview below Reynolds } \\
\text { Coal Creek Slough } \\
\text { Ilwaco Boat Basin } \\
\end{array}$ & & $\mathrm{x}$ & $\mathrm{x}$ & & & $\mathrm{x}$ \\
\hline Sediment Evaluation of Government Island South Channel & 2 & -- & -- & -- & & \multicolumn{6}{|c|}{$\begin{array}{l}\text { Results not provided for samples collected at these } \\
\text { locations. }\end{array}$} \\
\hline Sediment Quality Evaluation Lower Columbia and Willamette Rivers May 1990 & 2 & $5 / 3 / 1990$ & $5 / 10 / 1990$ & 39 & $\begin{array}{l}\text { Columbia River - } \\
\text { Willamette River }\end{array}$ & & & & & & $\mathrm{x}$ \\
\hline Environmental Surveillance Report on Oregon Surface Waters 1961- 1993, Vol. 2 & 3 & 1/19/1963 & 11/3/1993 & $\begin{array}{l}109 \\
16 \\
91\end{array}$ & \begin{tabular}{|l} 
Columbia River - \\
RM 13.7 - Astoria, Oregon \\
Portland, Oregon \\
RM 128.4 - Rooster Rock \\
\end{tabular} & $\mathrm{x}$ & & & & & \\
\hline St. Helens, Oregon Cross Channel, Sediment Quality Evaluation Report & 3 & 4/13/1989 & $5 / 4 / 1989$ & 12 & \begin{tabular}{|l|} 
Columbia River - \\
RM 86.5 - St.Helens Cross \\
Channel
\end{tabular} & & $\mathrm{x}$ & $\mathrm{x}$ & & & $\mathrm{x}$ \\
\hline $\begin{array}{l}\text { The Vertical Distribution of Selected Trace Metals and Organic Compounds in Bottom } \\
\text { Materials in the Proposed Lower Columbia River Export Channel, Oregon }\end{array}$ & 3 & 9/27/1984 & 9/27/1984 & 5 Cores & $\begin{array}{l}\text { Columbia River - } \\
\text { RM } 21 \text { - Cathlamet Bay }\end{array}$ & & $\mathrm{x}$ & & $\mathrm{x}$ & & $\mathrm{x}$ \\
\hline
\end{tabular}


Table 3-12. Summary of Documents with Segment 5 Sediment Data. (4 Pages)

\begin{tabular}{|c|c|c|c|c|c|c|c|c|c|c|c|}
\hline Source Title & $\begin{array}{c}\text { Data Usability } \\
\text { Category }\end{array}$ & $\begin{array}{c}\text { Begin } \\
\text { Date }\end{array}$ & End Date & $\begin{array}{c}\text { Number of } \\
\text { Samples }\end{array}$ & Sample Area & RADs & METs & svoc & Pest & PCBs & $\begin{array}{c}\text { GEN } \\
\text { CHEM }\end{array}$ \\
\hline $\begin{array}{l}\text { Historical Changes in the Columbia River Estuary Based on Sediment Cores: Feasibility } \\
\text { Studies }\end{array}$ & 3 & 12/1/1997 & $10 / 1 / 2001$ & 3 Cores & $\begin{array}{l}\text { Columbia River- } \\
\text { RM } 49 \text { - Clatskanie Flats } \\
\text { RM } 12 \text { - Young's Bay } \\
\text { RM } 24 \text { - Gray's Bay }\end{array}$ & $x$ & $x$ & & & & $x$ \\
\hline
\end{tabular}

METs = metals

NWR = National Wildlife Refuge

PCBs $=$ polychlorinated biphenyls

RADs = radionuclides

$\mathrm{RM} \quad=$ river mile

SVOCs = semivolatile organic compounds 
Table 3-13. Summary of Documents with Segment 5 Surface Water Data. (2 Pages)

\begin{tabular}{|c|c|c|c|c|c|c|c|c|c|c|c|}
\hline Source Title & $\begin{array}{l}\text { Data } \\
\text { Usability } \\
\text { Category }\end{array}$ & Begin Date & End Date & \begin{tabular}{|c|}
$\begin{array}{c}\text { Number } \\
\text { of } \\
\text { Samples }\end{array}$ \\
\end{tabular} & Sample Area & RADs & METs & SVOC & Pest & PCBs & $\begin{array}{l}\text { GEN } \\
\text { CHEN }\end{array}$ \\
\hline $\begin{array}{l}\text { Concentrations and Annual Fluxes for Selected Water Quality } \\
\text { Constituents from the USGS National Stream Quality Accounting } \\
\text { Network (NASQAN) 1996-02 (electronic resource) }\end{array}$ & 1 & 1/5/1999 & 2/9/2002 & 40 & $\begin{array}{l}\text { Columbia River - } \\
\text { Beaver Army Terminal, Quincy, } \\
\text { Oregon }\end{array}$ & & $\mathrm{x}$ & & $\mathrm{x}$ & $\mathrm{x}$ & $x$ \\
\hline $\begin{array}{l}\text { Concentrations of 303(d) Listed Pesticides, PCBs, and PAHs } \\
\text { Measured With Passive Samplers Deployed in the Lower } \\
\text { Columbia River }\end{array}$ & 1 & 9/1/2003 & $6 / 1 / 2004$ & $\begin{array}{l}3 \\
6 \\
3 \\
6 \\
2 \\
3 \\
5 \\
3 \\
3 \\
3\end{array}$ & $\begin{array}{l}\text { Columbia River } \\
\text { RM } 102 \text { - Columbia Slough } \\
\text { RM } 102-103 \text {-Near Willamette River } \\
\text { RM } 121 \text { - Washougal River } \\
\text { RM } 142 \text { - near Bonneville Dam } \\
\text { RM } 54 \text { - below Longview } \\
\text { RM } 68 \text { - at Cowlitz River } \\
\text { RM } 73-75 \text { - Kalama River } \\
\text { RM } 86 \text { - Multnomah Channel } \\
\text { RM } 87 \text { - at Lewis River } \\
\text { RM } 88 \text { - at Lake River }\end{array}$ & & & $\mathrm{x}$ & $\mathrm{x}$ & $\mathrm{x}$ & \\
\hline EIM Database - Columbia River above Kalama - location \#1 & 1 & $8 / 28 / 2003$ & $1 / 13 / 2004$ & 6 & $\begin{array}{l}\text { Columbia River - } \\
\text { Above Kalama, Washington }\end{array}$ & & & $\mathrm{x}$ & $\mathrm{x}$ & $\mathrm{x}$ & $\mathrm{x}$ \\
\hline EIM Database - Columbia River above Kalama - location \#2 & 1 & $5 / 25 / 2004$ & 6/23/2004 & 3 & $\begin{array}{l}\text { Columbia River - } \\
\text { Above Kalama, Washington }\end{array}$ & & & $\mathrm{x}$ & & $\mathrm{x}$ & $\mathrm{x}$ \\
\hline EIM Database - Columbia River above Willamette Influent & 1 & $6 / 25 / 2003$ & 6/22/2004 & 9 & $\begin{array}{l}\text { Columbia River - } \\
\text { Above Willamette River }\end{array}$ & & & $\mathrm{x}$ & $\mathrm{X}$ & $\mathrm{x}$ & $\mathrm{x}$ \\
\hline EIM Database - Columbia River at Vancouver & 1 & 10/30/2002 & 9/24/2003 & 12 & $\begin{array}{l}\text { Columbia River - } \\
\text { At Vancouver (28A100) }\end{array}$ & & $\mathrm{x}$ & & & & $\mathrm{x}$ \\
\hline $\begin{array}{l}\text { EIM Database - Columbia River below Bonneville Dam - location } \\
\# 2\end{array}$ & 1 & 8/27/2003 & 6/22/2004 & 9 & $\begin{array}{l}\text { Columbia River - } \\
\text { Below Bonneville Dam }\end{array}$ & & & $\mathrm{x}$ & $\mathrm{x}$ & $\mathrm{x}$ & $\mathrm{x}$ \\
\hline EIM Database - Columbia River below Longview & 1 & $5 / 25 / 2004$ & 6/23/2004 & 3 & $\begin{array}{l}\text { Columbia River } \\
\text { Below Longview, Washington }\end{array}$ & & & $\mathrm{x}$ & $\mathrm{X}$ & $\mathrm{x}$ & $\mathrm{x}$ \\
\hline EIM Database - Columbia River below Longview (Location 2) & 1 & $8 / 28 / 2003$ & $1 / 13 / 2004$ & 5 & $\begin{array}{l}\text { Columbia River } \\
\text { Below Longview, Washington }\end{array}$ & & & $\mathrm{x}$ & $x$ & $\mathrm{x}$ & $\mathrm{x}$ \\
\hline EIM Database - Columbia Slough & 1 & 8/27/2003 & $6 / 22 / 2004$ & 8 & $\begin{array}{l}\text { Columbia River - } \\
\text { Columbia Slough }\end{array}$ & & & $\mathrm{x}$ & $\mathrm{x}$ & $\mathrm{x}$ & $\mathrm{x}$ \\
\hline Statewide Arsenic Sampling in Selected Rivers & 1 & 9/25/2001 & 9/25/2001 & $\begin{array}{l}1 \\
1 \\
2 \\
1\end{array}$ & $\begin{array}{l}\text { Columbia River - } \\
\text { Beacon Rock, Washington } \\
\text { Columbia City, Oregon } \\
\text { County Line Park, Washington } \\
\text { Ryan Pt., Washington }\end{array}$ & & $\mathrm{x}$ & & & & \\
\hline USGS 14128910 - Columbia River at Warrendale & 1 & 1/27/1999 & 9/15/2004 & -- & Warrendale, Oregon & \multicolumn{6}{|c|}{ Results not available for these samples. } \\
\hline $\begin{array}{l}\text { USGS } 14246900 \text { - COLUMBIA RIVER @ BEAVER ARMY } \\
\text { TERMINAL NR QUINCY,Oregon }\end{array}$ & 1 & 1/5/1999 & 9/13/2004 & -- & $\begin{array}{l}\text { Beaver Army Terminal, Quincy, } \\
\text { Oregon }\end{array}$ & \multicolumn{6}{|c|}{ Results not available for these samples. } \\
\hline Water Resource Data Washington Water Year 2003 & 3 & 10/16/2002 & 9/8/2003 & -- & $\begin{array}{l}\text { Beaver Army Terminal, Quincy, } \\
\text { Oregon }\end{array}$ & \multicolumn{6}{|c|}{ Results not available for these samples. } \\
\hline
\end{tabular}


Table 3-13. Summary of Documents with Segment 5 Surface Water Data. (2 Pages)

\begin{tabular}{|c|c|c|c|c|c|c|c|c|c|c|c|}
\hline Source Title & $\begin{array}{c}\text { Data } \\
\text { Usability } \\
\text { Category }\end{array}$ & Begin Date & End Date & $\begin{array}{c}\begin{array}{c}\text { Number } \\
\text { of } \\
\text { Samples }\end{array} \\
\end{array}$ & Sample Area & RADs & METs & svoc & Pest & PCBs & $\begin{array}{l}\text { GEN } \\
\text { CHEM }\end{array}$ \\
\hline $\begin{array}{l}\text { Results and Recommendations from Monitoring Arsenic Levels in } \\
303 \text { (d) Listed Rivers in Washington }\end{array}$ & 1 & 7/25/2001 & 4/25/2002 & -- & $\begin{array}{l}\text { At Kelso, Allen Main Street Bridge } \\
\text { At Kelso, boat launch at Gerhart } \\
\text { Garden Park }\end{array}$ & \multicolumn{6}{|c|}{ Results not available for these samples. } \\
\hline
\end{tabular}

EIM = Environmental Information Management

METs $=$ metals

PAH = polyaromatic hydrocarbons

PCBS = polychlorinated biphenyls

RADs = radionuclides

$\mathrm{RM}=$ river mile

SVOCs = semivolatle organic compounds

USGS = U.S. Geological Survey 


\subsection{EVALUATION OF EXISTING DATA}

\subsection{METHODOLOGY}

As described previously, the purpose of the CRC data evaluation summary report is to evaluate the nature and extent of Hanford Site-related contamination within the defined boundaries of the CRC (below Grand Coulee Dam to Astoria, Oregon). This information will be used to develop an initial understanding of river conditions and contaminant distribution within the river and local tributaries. This was accomplished by comparing measured concentrations of radiological contaminants and hazardous constituents in sediment and surface water to available benchmarks. When more than one benchmark value was available for a medium and receptor, the lowest benchmark value was selected for comparison. A description of the benchmark values included in this evaluation is provided below. Sediment samples were also compared to the 90th percentile of the background concentrations from Hanford Site Background: Part 1, Soil Background for Nonradioactive Analytes (DOE-RL 2001); Hanford Site Background: Part 2, Soil Background for Radionuclides (DOE-RL 1996); and Natural Background Soil Metals Concentrations in Washington State (Ecology 1994). Although there are no sediment background values available for comparison, it is the general consensus that soil background values would be comparable to those observed in the sediments. A summary of the background values used in this evaluation are provided in Table 4-1.

To simplify the evaluation process, all sample results for a particular environmental medium (e.g., surface water) were grouped by river segment (e.g., Segment 1) regardless of the QA category. Summary statistics were calculated for each sample group that includes the frequency of detection, minimum and maximum nondetected values; and minimum, average, and maximum concentrations. The maximum detected concentration for each contaminant was compared to the lowest benchmark value for each receptor. When the maximum detected concentrations exceeded its respective benchmark value, all individual sample concentrations were compared to the benchmark value to determine where concentrations greater than benchmark values spatially reside within the river segment. If the benchmark value for sediments was lower than the soil background values, the benchmark defaulted to the background value.

It is important to note that when concentrations are below the selected benchmark value, an adverse health effect is not anticipated for the identified receptor. Conversely, when concentrations are above the selected benchmark value, the potential exists for an adverse health effect to occur. Although, the most protective value was selected for comparison purposes it may not be representative of actual river conditions. For example, freshwater sediment benchmark values have not been derived for all detected contaminants; therefore, marine sediment benchmark values were selected as a surrogate. Similarly, total chromium analyses were conducted for the majority of abiotic media. Therefore, measured concentrations of total chromium were compared to the hexavalent chromium benchmark value that assumes that all chromium is present in the hexavalent form.

\subsubsection{Sediment Benchmark Values}

Sediment benchmark values were selected to evaluate the protection of human and ecological receptors that could potentially come into contact with surface sediment from the Columbia 
River. Benchmark values have not been developed for the protection of human health from potential direct contact exposure to surface sediment. Therefore, the WAC 173-340-740 cleanup levels for unrestricted land use and the 2004 EPA Region 9 Preliminary Remediation Goals (PRGs) for residential land use were used to evaluate nonradiological contaminants and the cleanup levels developed in the Remedial Design Report/Remedial Action Work Plan for the 100 Area (DOE-RL 1998a) were used to evaluate radiological contaminants. A summary of the surface sediment benchmark values used to evaluate protection of human health are listed in Table 4-2.

Surface sediment benchmark values selected to evaluate protection of ecological receptors include the Development and Evaluation of Consensus-Based Sediment Quality Guidelines for Freshwater Ecosystems (MacDonald et al. 2000), the Creation and Analysis of Freshwater Sediment Quality Values in Washington State (Ecology 1997), and the Guidance for Ecological Risk Assessment Level II Screening Level Values (ODEQ 2001) for nonradiological contaminants. Although currently there are no specific statewide standards for freshwater sediment quality as discussed in WAC 173-204-340 (reserved for use by Ecology on a case-by-case-basis), the marine sediment quality standards have been included in this evaluation for completeness. The benchmark values selected for radiological contaminants to evaluate the protection of ecological receptors include the Biota Concentration Guides that were derived using the RESidual RADioactivity (RESRAD) Biota Version 1.0. A summary of the surface sediment benchmark values used to evaluate protection of ecological receptors are listed in Table 4-3.

\subsubsection{Surface Water Benchmark Values}

Surface water benchmark values were selected to evaluate the protection of human and ecological receptors that could potentially come into contact with surface water from the Columbia River. The surface water benchmark values selected to evaluate protection of human health were the EPA's National Recommended Water Quality Criteria for nonradiological contaminants. State standards for surface water protection are based on this EPA criteria. These water quality criteria include the protection of human health for the consumption of water and organism and for the consumption of the organism only (EPA 2006). The benchmark values selected for radiological contaminants to evaluate the protection of human health include the radiological drinking water maximum contaminant levels (MCLs). The drinking water MCLs derived for radiological contaminants were selected as a surrogate because surface water values have not been developed to evaluate direct exposure. A summary of the surface water benchmark values used to evaluate protection of human health are listed in Table 4-4.

Surface water benchmark values selected to evaluate protection of ecological receptors (including fish and water column organisms) include the Washington Chronic Water Quality standards (WAC 173-201A), the Oregon Aquatic Biota Screening Values (ODEQ 2001), and the National Ambient Water Quality Standards (EPA 2002) for nonradiological contaminants. The benchmark values selected for radiological contaminants to evaluate the protection of ecological receptors were the water concentration guides that were derived using RESRAD Biota Version 1.0. A summary of the surface water benchmark values used to evaluate protection of ecological receptors are listed in Table 4-5. 


\section{$4.2 \quad$ CONTAMINANT DISTRIBUTIONS IN SEGMENT 1}

A list of the surface sediment and surface water samples used to evaluate Segment 1 of the Columbia River is provided in Appendix A, Tables A-1 and A-2, respectively. All unique surface sediment sampling locations within Segment 1 are provided in Figure A-1. Summary statistics for detected and nondetected surface sediment sample results reported on an "as received" and "dry-weight" basis are presented in Appendix A, Tables A-3 through A-5. A comparison of all detected surface sediment concentrations to available human health and ecological benchmark values is presented in Appendix A, Tables A-6 and A-7. Individual surface sediment samples exceeding respective human health and ecological benchmark values and their sampling location are presented in Appendix A, Table A-8 and shown in Figure A-2.

Summary statistics for detected and nondetected surface water sample results are presented in Appendix A, Tables A-9 and A-10. All unique surface water sampling locations within Segment 1 are provided in Figure A-3. A comparison of all detected surface water concentrations to available human health and ecological benchmark values is presented in Appendix A, Table A-11. Individual surface water samples exceeding respective human health and ecological benchmark values and their sampling location are presented in Appendix A, Table A-12 and shown in Figure A-4.

\subsubsection{Sediment}

Sediment samples collected within this segment of the river were analyzed for metals, pesticide/PCBs, radionuclides, and SVOCs. Except for 11 metals, dichlorodiphenyldichloroethylene [DDE], and three radiological contaminants, all contaminant concentrations were less than their respective ecological or human health benchmark value. A summary of those contaminants that exceed their respective sediment benchmark values is presented in Table 4-6.

4.2.1.1 Comparison to Ecological Benchmark Values. Maximum concentrations of all 11 metals and DDE were reported above their ecological benchmark value. With the exception of cadmium, the majority of contaminants were only slightly greater than their ecological benchmark value with the factor of exceedance ranging from slightly greater than 1 to 7 . Cadmium was measured in 55 of 66 surface sediment samples (90\%) at concentrations that are greater than the ecological benchmark value of $0.60 \mathrm{mg} / \mathrm{kg}$ with concentrations ranging between $0.3 \mathrm{mg} / \mathrm{kg}$ and $16 \mathrm{mg} / \mathrm{kg}$. Cadmium occurs naturally in the environment with a $90^{\text {th }}$ percentile Washington State background value reported as $0.81 \mathrm{mg} / \mathrm{kg}$. All cadmium concentrations measured within this segment are greater than the background value. Results indicate that cadmium is present at levels greater than background and greater than the ecological benchmark value throughout Segment 1 suggesting an upgradient source of cadmium.

4.2.1.2 Comparison to Human Health Benchmark Values. Maximum concentrations of four metals and three radiological contaminants were reported above their human health benchmark value. With the exception of arsenic, the majority of contaminants were only slightly greater than their benchmark value with the factor of exceedance ranging from slightly greater than 1 to slightly greater than 3 . All arsenic concentrations measured in sediment are greater than the human health benchmark value of $0.39 \mathrm{mg} / \mathrm{kg}$ with concentrations ranging from $0.42 \mathrm{mg} / \mathrm{kg}$ to $12 \mathrm{mg} / \mathrm{kg}$. Arsenic occurs naturally in the environment with the $90^{\text {th }}$ percentile Hanford Site background value reported as $6.5 \mathrm{mg} / \mathrm{kg}$. Greater than one-half of all arsenic concentrations 
measured ( 28 of $51,55 \%$ ) were also greater than the Hanford Site background value (see Table A-8 for individual locations exceeding benchmark). The majority of sediment samples with arsenic concentrations greater than background were located near Priest Rapids Dam (22 of 28, 79\%). The remaining samples were collected near Vernita Bridge, RM 417.5 and RM 428. Results suggest that arsenic is present at naturally occurring levels and concentrations greater than naturally occurring levels may be contributed from an upgradient source.

Chromium was measured in 42 of $67(63 \%)$ at concentrations greater than the human health benchmark value of $30 \mathrm{mg} / \mathrm{kg}$ with concentrations ranging from $3.2 \mathrm{mg} / \mathrm{kg}$ to $93 \mathrm{mg} / \mathrm{kg}$. Locations where chromium was measured above the benchmark value include the Priest Rapids Dam and Columbia RMs 398.5, 405.5 417.5, 428, and 440.

Uranium-234 was measured in seven of 38 surface sediment samples (18\%) at concentrations greater than the human health benchmark value of $1.1 \mathrm{pCi} / \mathrm{g}$ with concentrations ranging from $0.064 \mathrm{pCi} / \mathrm{g}$ to $1.6 \mathrm{pCi} / \mathrm{g}$. Uranium-238 was measured in five of 69 surface sediment samples $(7 \%)$ at concentrations greater than the human health benchmark value of $1.1 \mathrm{pCi} / \mathrm{g}$ with concentrations ranging from $0.063 \mathrm{pCi} / \mathrm{g}$ to $1.3 \mathrm{pCi} / \mathrm{g}$. Uranium-234 and uranium-238 were measured above the benchmark value at Priest Rapids Dam (see Appendix A, Table A-8 for a list of individual locations).

\subsubsection{Surface Water}

Surface water samples collected within this segment of the river were analyzed for general chemistry parameters, metals, pesticides, radionuclides, and VOCs. Except for arsenic and barium, all contaminant concentrations were less than their respective ecological or human health benchmark value. The factor of exceedance for arsenic and barium are 94 and 7 fold, respectively. A summary of those contaminants that are greater than their respective sediment benchmark values is presented in Table 4-7.

4.2.2.1 Comparison to Ecological Benchmark Values. Barium was measured in all surface water (five samples) at concentrations that are greater than the ecological benchmark value of $0.004 \mathrm{mg} / \mathrm{L}$ with concentrations ranging from $0.028 \mathrm{mg} / \mathrm{L}$ to $0.029 \mathrm{mg} / \mathrm{L}$. Barium was measured once at Wenatchee, Washington, and four samples were collected from Vernita Bridge in 2001.

4.2.2.2 Comparison to Human Health Benchmark Values. Arsenic was measured in all surface water (173 samples) at concentrations that are greater than the human health benchmark value of $0.000018 \mathrm{mg} / \mathrm{L}$ with concentrations ranging from $0.00026 \mathrm{mg} / \mathrm{L}$ to $0.0017 \mathrm{mg} / \mathrm{L}$. Results indicate that arsenic is present at all locations sampled within Segment 1 of the river.

\subsection{CONTAMINANT DISTRIBUTIONS IN SEGMENT 2}

A list of the surface sediment and surface water samples used to evaluate Segment 2 of the Columbia River is provided in Appendix B, Tables B-1 and B-2, respectively. All unique surface sediment sampling locations within Segment 2 are provided in Figure B-1. Summary statistics for detected and nondetected surface sediment sample results reported on an "as received" and "dry-weight" basis are presented in Appendix B, Tables B-3 and B-4. A comparison of all 
detected surface sediment concentrations to available human health and ecological benchmark values is presented in Appendix B, Tables B-5 and B-6. Individual surface sediment samples with concentrations greater than their respective human health and ecological benchmark values and their sampling location are presented in Appendix B, Table B-7 and shown in Figure B-2.

Summary statistics for detected and nondetected surface water sample results are presented in Appendix B, Table B-8. All unique surface water sampling locations within Segment 2 are provided in Figure B-3. A comparison of all detected surface water concentrations to available human health and ecological benchmark values is presented in Appendix B, Table B-9. Individual surface water samples with concentrations greater than their respective human health and ecological benchmark values and their sampling location are presented in Appendix B, Table B-10 and shown in Figure B-4.

\subsubsection{Sediment}

Sediment samples collected within this segment of the river were analyzed for only metals and radionuclides. Maximum concentrations of 13 metals and five radionuclides were reported above their respective ecological or human health benchmark values. A summary of those contaminants that are greater than their respective sediment benchmark values is presented in Table 4-8.

4.3.1.1 Comparison to Ecological Benchmark Values. Maximum concentrations of 11 metals were reported above their ecological benchmark value. Similar to Segment 1, the majority of contaminants were only slightly greater than their ecological benchmark value with the factor of exceedance ranging from slightly greater than 1 to less than 7 , with the exception of cadmium. Cadmium was measured in 49 of 91 surface sediment samples (54\%) at concentrations that are greater than the ecological benchmark value of $0.60 \mathrm{mg} / \mathrm{kg}$ with concentrations ranging from $0.0004 \mathrm{mg} / \mathrm{kg}$ to $5.6 \mathrm{mg} / \mathrm{kg}$. Slightly less than one-half of cadmium concentrations greater than the ecological benchmark value are also greater than the Washington State background value of $0.81 \mathrm{mg} / \mathrm{kg}(37 \%)$. Note that the maximum concentration of cadmium measured within Segment $2(5.6 \mathrm{mg} / \mathrm{kg})$ is less than the maximum concentration measured in Segment $1(16 \mathrm{mg} / \mathrm{kg})$. Thus, elevated cadmium concentrations in Segment 2 are likely contributed from a source upgradient from the Hanford Site.

4.3.1.2 Comparison to Human Health Benchmark Values. Maximum concentrations of four metals and four radionuclides were reported above their human health benchmark value. With the exception of arsenic, uranium-234, and uranium-238, the majority of contaminants are only slightly greater than their human health benchmark value with the factor of exceedance ranging from slightly greater than 1 to 6 . Measured concentrations of benzo(a)pyrene were greater than both the ecological and human health benchmark values and were discussed in the previous section.

Arsenic was measured in 105 of 118 surface sediment samples (89\%) at concentrations that are greater than the human health benchmark value of $0.39 \mathrm{mg} / \mathrm{kg}$ with concentrations ranging from $0.002 \mathrm{mg} / \mathrm{kg}$ to $20 \mathrm{mg} / \mathrm{kg}$ (50 fold factor of exceedance). With the exception of arsenic measured at one location at a concentration of $20 \mathrm{mg} / \mathrm{kg}$, only 23 measurements were greater than the background value of $6.5 \mathrm{mg} / \mathrm{kg}$ with concentrations ranging from $6.6 \mathrm{mg} / \mathrm{kg}$ to $11 \mathrm{mg} / \mathrm{kg}$. Locations where arsenic was measured above background include the F Slough, 
Hanford Slough, White Bluffs Slough, 100-B Spring 38-3, 100-F Spring 207-1, 100-H Spring 145-1, 300 Spring 42-2, Hanford Spring 28-2, and the Richland, Washington, Columbia Generating Station sample station (see Appendix B, Table B-7 for a list of individual locations). Note that arsenic concentrations measured in Segment 2 are similar to those measured in Segment 1.

Cobalt-60 was measured in one of 40 surface sediment samples (3\%) at a concentration of $2.4 \mathrm{pCi} / \mathrm{g}$, which is slightly greater than the human health benchmark value of $1.4 \mathrm{pCi} / \mathrm{g}$. Cobalt-60 was measured above the human health benchmark value at Columbia Generating Station 34.

Thorium-232 was measured in 10 of 48 surface sediment samples (21\%) at concentrations greater than the human health benchmark value of $1.3 \mathrm{pCi} / \mathrm{g}$ with concentrations ranging from $0.5 \mathrm{pCi} / \mathrm{g}$ to $3.2 \mathrm{pCi} / \mathrm{g}$. Locations where thorium-232 was measured above the benchmark value include 300 Spring 42-2, F-Slough, 100-H Area, 100-K Area, Horn Area, and the Hanford Townsite (see Appendix B, Table B-7 for a list of individual locations).

Uranium-234 was measured in 28 of 145 surface sediment samples (19\%) at concentrations greater than the human health benchmark value of $1.1 \mathrm{pCi} / \mathrm{g}$ with concentrations ranging from $0.071 \mathrm{pCi} / \mathrm{g}$ to $11 \mathrm{pCi} / \mathrm{g}$. Locations where uranium-234 was measured above the benchmark value include 300 Spring 42-2 and 300 Spring 11 (see Appendix B, Table B-7 for a list of individual locations).

Uranium-238 was measured in 35 of 186 surface sediment samples (19\%) at concentrations greater than the human health benchmark value of $1.1 \mathrm{pCi} / \mathrm{g}$ with concentrations ranging from $0.051 \mathrm{pCi} / \mathrm{g}$ to $10 \mathrm{pCi} / \mathrm{g}$. Locations where uranium-238 was measured above the benchmark value include 300 Spring 42-2, 300 Spring 11, and the F Slough (see Appendix B, Table B-7 for a list of individual locations).

\subsubsection{Surface Water}

Surface water samples collected within this segment of the river were analyzed for general chemistry parameters, metals, pesticides, radionuclides, and VOCs. Maximum concentrations of 16 metals and five radionuclides were reported above their respective ecological or human health benchmark values. A summary of those contaminants that are greater than their respective surface water benchmark values is presented in Table 4-9.

4.3.2.1 Comparison to Ecological Benchmark Values. Maximum concentrations of 14 metals were reported above their ecological benchmark value. Five metals (copper, mercury, nickel, uranium, and vanadium) only slightly exceeded their ecological benchmark value with the factor of exceedance ranging from slightly greater than 1 to 7 . Maximum concentrations of nine metals (aluminum, barium, cadmium, chromium, iron, lead, manganese, silver, and zinc) exceed their ecological benchmark value with the factor of exceedance ranging from 8 to 243 fold.

Aluminum was measured in 18 of 57 surface water samples (32\%) at concentrations greater than the ecological benchmark value of $0.087 \mathrm{mg} / \mathrm{L}$ with concentrations ranging from $0.0003 \mathrm{mg} / \mathrm{L}$ to $21 \mathrm{mg} / \mathrm{L}$. Locations where aluminum was measured above the benchmark 
value include 100-D Spring 98-1, 102-1, and 110-1; 100-H Spring 150-1 and 152-2; 100-K Spring 77-1 and 82-2; and 300 Spring 11 and 42-2.

Barium was measured in 121 surface water samples (100\%) at concentrations greater than the ecological benchmark value of $0.004 \mathrm{mg} / \mathrm{L}$ with concentrations ranging from $0.017 \mathrm{mg} / \mathrm{L}$ to $0.29 \mathrm{mg} / \mathrm{L}$. Locations where barium was measured above the benchmark value include 100-B Spring 37-2, 38-3, 39-2; 100-D Spring 98-1, 102-1 and 110-1; 100-F Spring 207-1; 100-H Spring 144-1, 145-1, 150-1, 152-2, and 153-1; 100-K Spring 77-1, and 82-2; and 300 Spring 7, 9, 11, and 42-2.

Cadmium was measured in 15 of 795 surface water samples (2\%) at concentrations greater than the ecological benchmark value of $0.00025 \mathrm{mg} / \mathrm{L}$ with concentrations ranging from $0.000006 \mathrm{mg} / \mathrm{L}$ to $0.0035 \mathrm{mg} / \mathrm{L}$. Locations where cadmium was measured above the benchmark include 100-D Spring 98-1, 102-1, 110-1; 100-F Spring 207-1; 100-H Spring 144-1 and 145-1; 100-K Spring 77-1; 100-N Spring 8-13; 300 Spring 42-2, and Hanford Spring 28-2 (see Appendix B, Table B-10 for a list of individual locations).

Chromium was measured in 116 of 843 chromium surface water samples (14\%) at concentrations greater than the ecological benchmark value of $0.01 \mathrm{mg} / \mathrm{L}$ with concentrations ranging from $0.00002 \mathrm{mg} / \mathrm{L}$ to $0.22 \mathrm{mg} / \mathrm{L}$. Locations where chromium was measured above the benchmark include 100-B Spring 38-3 and 39-2; 100-D Spring 102-1, 110-1, and 98-1; 100-F Spring 207-1; $100-\mathrm{H}$ Spring 145-1 and 152-2; 100-K Spring 63-1 and 82-2; 100-N Spring 8-13; and 300 Spring 42-2 (see Appendix B, Table B-10 for a list of individual locations).

Iron was measured in five of 45 surface water samples (11\%) at concentrations greater than the ecological benchmark value of $1.0 \mathrm{mg} / \mathrm{L}$ and measured in nine of 45 samples $(20 \%)$ at concentrations that exceed the human health benchmark value of $0.30 \mathrm{mg} / \mathrm{L}$. Iron concentrations range from $0.016 \mathrm{mg} / \mathrm{L}$ to $20 \mathrm{mg} / \mathrm{L}$. Locations where iron was measured above the benchmark include 100-D Spring 98-2, 102-1, 110-1; 100-H Spring 150-1; and 100-K Spring 82-2 (see Appendix B, Table B-10 for a list of individual locations).

Lead was measured in 34 of 900 surface water samples (4\%) at concentrations greater than the ecological benchmark value of $0.00087 \mathrm{mg} / \mathrm{L}$ with concentrations ranging from $0.000001 \mathrm{mg} / \mathrm{L}$ to $0.066 \mathrm{mg} / \mathrm{L}$. Locations where lead was measured above the benchmark include 100-D Spring 102-1, 110-1; 100-F Spring 207-1; 100-K Spring 77-1; 300 Spring 42-2; and Hanford Spring 28-2 (see Appendix B, Table B-10 for a list of individual locations).

Manganese was measured in four of 103 surface water samples (4\%) at concentrations greater than the ecological benchmark value of $0.12 \mathrm{mg} / \mathrm{L}$ and measured in seven of $103 \mathrm{samples}$ at concentrations that exceed the human health benchmark value of $0.05 \mathrm{mg} / \mathrm{L}(7 \%)$. Manganese concentrations range from $0.00004 \mathrm{mg} / \mathrm{L}$ to $0.94 \mathrm{mg} / \mathrm{L}$. Locations where manganese was measured above the benchmarks include 100-D Spring 98-1, 102-1, 110-1; 100-H Spring 150-1; and 100-K Spring 77-1 (see Appendix B, Table B-10 for a list of individual locations).

Silver was measured in seven of 355 surface water samples (2\%) at concentrations greater than the ecological benchmark value of $0.00012 \mathrm{mg} / \mathrm{L}$ with concentrations ranging from $0.0000009 \mathrm{mg} / \mathrm{L}$ to $0.003 \mathrm{mg} / \mathrm{L}$. Locations where silver was measured above the benchmarks include 100-D Spring 98-1; 100-H Spring 144-1 and 145-1; 100-K Spring 77-1; and 300 Spring 42-2 (see Appendix B, Table B-10 for a list of individual locations). 
Zinc was measured in 12 of 1,038 surface water samples $(1 \%)$ at concentrations greater than the ecological benchmark value of $0.055 \mathrm{mg} / \mathrm{L}$ with concentrations ranging from $0.0004 \mathrm{mg} / \mathrm{L}$ to $0.42 \mathrm{mg} / \mathrm{L}$. Locations where zinc was measured above the benchmark include 100-D Spring 98-1 and 110-1; 100-F Spring 207-1; 100-H Spring 145-1 and 150-1; and 300 Spring 42-2; and Hanford Spring 28-2 (see Appendix B, Table B-10 for a list of individual locations).

4.3.2.2 Comparison to Human Health Benchmark Values. Maximum concentrations of four metals and three radionuclides were reported above their human health benchmark value. Thallium slightly exceeds its human health benchmark value with the factor of exceedance of approximately 2. Maximum concentrations of three metals (arsenic, iron, and manganese) and three radionuclides (strontium-90, tritium, and uranium) exceed their human health benchmark value with a factor of exceedance ranging from 3 to 14 fold. Measured concentrations of iron and manganese were greater than both the ecological and human health benchmark values and were discussed in the previous section.

Arsenic was measured in 978 of 979 surface water samples (>99\%) at concentrations greater than the human health benchmark value of $0.000018 \mathrm{mg} / \mathrm{L}$ with concentrations ranging from $0.000006 \mathrm{mg} / \mathrm{L}$ to $0.0085 \mathrm{mg} / \mathrm{L}$. Arsenic results are similar to Segment 1 indicating that arsenic is present at concentrations greater than the human health benchmark value at all locations sampled.

Strontium-90 was measured in six of 636 surface water samples $(1 \%)$ at concentrations greater than the human health benchmark value of $8 \mathrm{pCi} / \mathrm{L}$ with concentrations ranging from $0.022 \mathrm{pCi} / \mathrm{L}$ to $109 \mathrm{pCi} / \mathrm{L}$. Locations where strontium-90 was measured above the benchmark include 100-H Spring 153-1 and 100-N Spring 3.

Tritium was measured in 19 of 970 surface water samples $(2 \%)$ at concentrations that exceed the human health benchmark value of $20,000 \mathrm{pCi} / \mathrm{L}$ with concentrations ranging from $17 \mathrm{pCi} / \mathrm{L}$ and $112,000 \mathrm{pCi} / \mathrm{L}$. Hanford Spring $28-2$ is the primary location where tritium was measured above the benchmark.

\subsection{CONTAMINANT DISTRIBUTIONS IN SEGMENT 3}

A list of the surface sediment and surface water samples used to evaluate Segment 3 of the Columbia River is provided in Appendix C, Tables C-1 and C-2, respectively. All unique surface sediment sampling locations within Segment 3 are provided in Figure C-1. Summary statistics for detected and nondetected surface sediment sample results reported on an "as received" and "dry-weight" basis are presented in Appendix C, Tables C-3 through C-8. A comparison of all detected surface sediment concentrations to available human health and ecological benchmark values is presented in Appendix C, Tables C-9 and C-10. Individual surface sediment samples with concentrations greater than their respective human health and ecological benchmark values and their sampling location are presented in Appendix C, Table C-11 and shown in Figure C-2.

Summary statistics for detected and nondetected surface water sample results are presented in Appendix C, Tables $\mathrm{C}-12$ through 16. All unique surface water sampling locations within Segment 3 are provided in Figure C-3. A comparison of all detected surface water 
concentrations to available human health and ecological benchmark values is presented in Appendix C, Table C-17. Individual surface water samples with concentrations greater than their respective human health and ecological benchmark values and their sampling location are presented in Appendix C, Table C-18 and shown in Figure C-4.

\subsubsection{Sediment}

Sediment samples collected within this segment of the river were analyzed for metals, pesticide/PCBs, radionuclides, and SVOCs. Maximum concentrations of 13 metals, five pesticides, two radionuclides, eight PAHs, arochlor-1254, and bis(2-ethylhexyl)phthalate were reported above their respective ecological or human health benchmark values. A summary of those contaminants that are greater than their respective sediment benchmark values is presented in Table 4-10.

4.4.1.1 Comparison to Ecological Benchmark Values. Maximum concentrations of 11 metals, eight PAHs, five pesticides (dichlorodiphenyldichloroethane [DDD], DDE, dichlorodiphenyltrichloroethane [DDT], dieldrin, and gamma-Hexachlorocyclohexane [BHC]), arochlor-1254, and bis(2-ethylhexyl)phthalate were reported above their ecological benchmark value. With the exception of cadmium, DDE, DDT, and gamma-BHC, the majority of contaminants were only slightly greater than their ecological benchmark value with the factor of exceedance ranging from slightly greater than 1 to less than 7 . Cadmium was measured in 48 of 64 surface sediment samples $(75 \%)$ at concentrations that are greater than the ecological benchmark value of $0.60 \mathrm{mg} / \mathrm{kg}$ with concentrations ranging from $0.067 \mathrm{mg} / \mathrm{kg}$ to $6.0 \mathrm{mg} / \mathrm{kg}$. The majority of cadmium concentrations greater than the ecological benchmark value are also greater than the Washington State background value of $0.81 \mathrm{mg} / \mathrm{kg}(61 \%)$. Note that the maximum concentration of cadmium measured within Segment $3(6.0 \mathrm{mg} / \mathrm{kg})$ is only slightly greater than that measured in Segment $2(5.6 \mathrm{mg} / \mathrm{kg})$ and less than the maximum concentration measured in Segment $1(16 \mathrm{mg} / \mathrm{kg})$. Elevated cadmium concentrations are present in Segment 3 and are likely contributed from a source upgradient from the Hanford Site.

All three surface sediment samples with detected aroclor-1254 results were at concentrations greater than the ecological benchmark value of $0.007 \mathrm{mg} / \mathrm{kg}$, with concentrations ranging from $0.01 \mathrm{mg} / \mathrm{kg}$ to $0.013 \mathrm{mg} / \mathrm{kg}$. The location where aroclor-1254 was measured above the benchmark value is at the Port of Pasco (see Appendix C, Table C-11 for a list of individual locations).

Acenaphthene was measured in one surface sediment sample with a concentration of $0.021 \mathrm{mg} / \mathrm{kg}$, which is greater than the ecological benchmark value of $0.0067 \mathrm{mg} / \mathrm{kg}$. The location where acenaphthene was measured above the benchmark value was RM 328 (see Appendix C, Table C-11 for a list of individual locations).

Anthracene was measured in one of three surface sediment samples (33\%) at a concentration greater than the ecological benchmark value of $0.057 \mathrm{mg} / \mathrm{kg}$ with concentrations ranging from $0.006 \mathrm{mg} / \mathrm{kg}$ to $0.088 \mathrm{mg} / \mathrm{kg}$. The location where anthracene was measured above the benchmark value was the Port of Pasco (see Appendix C, Table C-11 for a list of individual locations).

Benzo(a)anthracene was measured in one of two surface sediment samples (50\%) at a concentration greater than the ecological benchmark value of $0.032 \mathrm{mg} / \mathrm{kg}$ with concentrations 
ranging from $0.013 \mathrm{mg} / \mathrm{kg}$ to $0.12 \mathrm{mg} / \mathrm{kg}$. The location where benzo(a)anthracene was measured above the benchmark value was the Port of Pasco (see Appendix C, Table C-11 for a list of individual locations).

Benzo(a)pyrene was measured in two of three surface sediment samples (67\%) at concentrations greater than the ecological benchmark value of $0.032 \mathrm{mg} / \mathrm{kg}$ and greater than the human health benchmark value of $0.062 \mathrm{mg} / \mathrm{kg}$. Benzo(a)pyrene concentrations ranged from $0.013 \mathrm{mg} / \mathrm{kg}$ to $0.21 \mathrm{mg} / \mathrm{kg}$. The location where benzo(a)pyrene was measured above the benchmark value was the Port of Pasco (see Appendix C, Table C-11 for a list of individual locations).

Chrysene was measured in one of three surface sediment samples (33\%) at concentrations greater than the ecological benchmark value of $0.057 \mathrm{mg} / \mathrm{kg}$ with the concentrations ranging from $0.013 \mathrm{mg} / \mathrm{kg}$ to $0.13 \mathrm{mg} / \mathrm{kg}$. The location where chrysene was measured above the benchmark value was the Port of Pasco (see Appendix C, Table C-11 for a list of individual locations).

Fluoranthene was measured in three of nine surface sediment samples (33\%) at concentrations greater than the ecological benchmark value of $0.11 \mathrm{mg} / \mathrm{kg}$ with concentrations ranging from $0.004 \mathrm{mg} / \mathrm{kg}$ to $0.2 \mathrm{mg} / \mathrm{kg}$. The location where fluoranthene was measured above the benchmark value was the Port of Pasco (see Appendix C, Table C-11 for a list of individual locations).

Phenanthrene was measured in one surface sediment sample at a concentration of $0.057 \mathrm{mg} / \mathrm{kg}$, which is greater than the ecological benchmark value of $0.042 \mathrm{mg} / \mathrm{kg}$. The location where phenanthrene was measured was at RM 328 (see Appendix C, Table C-11 for a list of individual locations).

Pyrene was measured in three of seven surface sediment samples (43\%) at concentrations greater than the ecological benchmark value of $0.053 \mathrm{mg} / \mathrm{kg}$ with concentrations ranging from $0.003 \mathrm{mg} / \mathrm{kg}$ to $0.22 \mathrm{mg} / \mathrm{kg}$. The location where pyrene was measured above the benchmark value was the Port of Pasco (see Appendix C, Table C-11 for a list of individual locations).

DDE was measured in 21 of 35 surface sediment samples $(60 \%)$ at concentrations that are greater than the ecological benchmark value of $0.0015 \mathrm{mg} / \mathrm{kg}$ with concentrations ranging from $0.00001 \mathrm{mg} / \mathrm{kg}$ to $0.058 \mathrm{mg} / \mathrm{kg}$. Locations where DDE was measured above the benchmark include the Yakima River near Kiona and Horn Rapids Dam, the Walla Walla River near Cummins Bridge and at the confluence, the Snake River in Lake Sacajawea, and in the Columbia River near McNary Dam.

DDT was measured in two of six surface sediment samples (33\%) at concentrations that are greater than the ecological benchmark value of $0.0042 \mathrm{mg} / \mathrm{kg}$ with concentrations ranging from $0.0003 \mathrm{mg} / \mathrm{kg}$ to $0.035 \mathrm{mg} / \mathrm{kg}$. The Yakima River near Kiona, Washington, was the primary location where DDT was measured above the ecological benchmark value.

4.4.1.2 Comparison to Human Health Benchmark Values. Maximum concentrations of four metals (arsenic, chromium, iron, and vanadium), two radionuclides (uranium-234 and uranium-238) and benzo(a)pyrene were reported above their human health benchmark value. With the exception of arsenic, the majority of contaminants are only slightly greater than their 
human health benchmark value with the factor of exceedance ranging from slightly greater than 1.0 to less than 3.0. Arsenic was measured in 67 of 68 surface sediment samples $(>99 \%)$ at concentrations that are greater than the human health benchmark value of $0.39 \mathrm{mg} / \mathrm{kg}$ with concentrations ranging from $0.37 \mathrm{mg} / \mathrm{kg}$ to $12 \mathrm{mg} / \mathrm{kg}$. Arsenic was measured in 33 of 67 surface sediment samples $(49 \%)$ at concentrations greater than the Hanford Site background value of $6.5 \mathrm{mg} / \mathrm{kg}$. Locations where arsenic was measured above background include the Snake River near the Ice Harbor Dam and at Snake RMs 1 and 10, and in the Columbia River near McNary Dam (see Appendix C, Table C-11 for a list of individual locations). Note that arsenic concentrations measured in Segment 3 are similar to those measured in Segments 1 and 2.

Benzo(a)pyrene was measured in two of three surface sediment samples (67\%) at concentrations greater than the human health benchmark value of $0.062 \mathrm{mg} / \mathrm{kg}$ with concentrations ranging from $0.013 \mathrm{mg} / \mathrm{kg}$ to $0.21 \mathrm{mg} / \mathrm{kg}$. The location where benzo(a)pyrene was measured above the benchmark value was the Port of Pasco (see Appendix C, Table C-11 for a list of individual locations).

Uranium-234 was measured in 38 of 83 surface sediment samples (45\%) at concentrations that are greater than the human health benchmark value of $1.1 \mathrm{pCi} / \mathrm{g}$ with concentrations ranging from $0.09 \mathrm{pCi} / \mathrm{g}$ to $1.9 \mathrm{pCi} / \mathrm{g}$. The primary locations where uranium-234 was measured above the benchmark value include the Snake River near the Ice Harbor Dam and the Columbia River near the McNary Dam.

Uranium-238 was measured in 23 of 86 surface sediment samples $(27 \%)$ at concentrations that are greater than the human health benchmark value of $1.1 \mathrm{pCi} / \mathrm{g}$ with concentrations ranging from $0.21 \mathrm{pCi} / \mathrm{g}$ to $1.6 \mathrm{pCi} / \mathrm{g}$. The primary locations where uranium-234 was measured above the benchmark value include the Snake River near the Ice Harbor Dam and the Columbia River near the McNary Dam.

\subsubsection{Surface Water}

Surface water samples collected within this segment of the river were analyzed for general chemistry parameters, metals, pesticides/PCBs, radionuclides, and VOCs. Maximum concentrations of one metal, and four pesticides (DDD, DDE, dieldrin, and heptachlor epoxide), were reported above their respective human health benchmark values. No contaminants were detected at concentrations exceeding available ecological benchmark values. A summary of those contaminants that are greater than their respective surface water benchmark values is presented in Table 4-11.

4.4.2.1 Comparison to Human Health Benchmark Values. Maximum concentrations of arsenic, DDD, DDT, dieldrin, and heptachlor epoxide were reported above their human health benchmark value. The pesticides slightly exceed their human health benchmark value with the factor of exceedance ranging from 3 to 6 fold and the factor of exceedance for arsenic was 156 fold.

Arsenic was measured in all surface water samples (48 samples) at concentrations greater than the human health benchmark value of $0.000018 \mathrm{mg} / \mathrm{L}$ with concentrations ranging from $0.0005 \mathrm{~g} / \mathrm{L}$ to $0.00028 \mathrm{mg} / \mathrm{L}$. Arsenic results are similar to Segments 1 and 2 indicating that 
arsenic is present at concentrations greater than the human health benchmark value at all locations sampled.

DDE was measured in eight of 18 surface water samples (44\%) at concentrations greater than the human health benchmark value of $0.00000022 \mathrm{mg} / \mathrm{L}$ with concentrations ranging from $0.000000071 \mathrm{mg} / \mathrm{L}$ to $0.0000012 \mathrm{mg} / \mathrm{L}$. DDT was measured in one of five surface water samples $(20 \%)$ at concentrations greater than the human health benchmark value of $0.00000022 \mathrm{mg} / \mathrm{L}$ with concentrations ranging from $0.0000001 \mathrm{mg} / \mathrm{L}$ to $0.00000065 \mathrm{mg} / \mathrm{L}$. Dieldrin was measured in all four surface water samples (100\%) at concentrations greater than the human health benchmark value of $0.000000058 \mathrm{mg} / \mathrm{L}$ with concentrations ranging from $0.000000076 \mathrm{mg} / \mathrm{L}$ to $0.0000003 \mathrm{mg} / \mathrm{L}$. Heptachlor epoxide was measured in four of six surface water samples $(66 \%)$ at concentrations greater than the human health benchmark value of $0.000000039 \mathrm{mg} / \mathrm{L}$ with concentrations ranging from $0.00000012 \mathrm{mg} / \mathrm{L}$ to $0.000000033 \mathrm{mg} / \mathrm{L}$. The Walla Walla River is the only location where DDE, DDT, dieldrin, and heptachlor epoxide were measured above the benchmarks (see Appendix C, Table C-18 for individual locations exceeding benchmark value).

\subsection{CONTAMINANT DISTRIBUTIONS IN SEGMENT 4}

A list of the surface sediment and surface water samples used to evaluate Segment 4 of the Columbia River is provided in Appendix D, Tables D-1 and D-2, respectively. All unique surface sediment sampling locations within Segment 4 are provided in Figure D-1. Summary statistics for detected and nondetected surface sediment sample results reported on an "as received" and "dry-weight" basis are presented in Appendix D, Tables D-3 and D-4. A comparison of all detected surface sediment concentrations to available human health and ecological benchmark values is presented in Appendix D, Tables D-5 and D-6. Individual surface sediment samples with concentrations greater than their respective human health and ecological benchmark values and their sampling location are presented in Appendix D, Table D-7 and shown in Figure D-2.

Summary statistics for detected and nondetected surface water sample results are presented in Appendix D, Table D-8. All unique surface water sampling locations within Segment 4 are provided in Figure D-3. A comparison of all detected surface water concentrations to available human health and ecological benchmark values is presented in Appendix D, Table D-9. Individual surface water samples with concentrations greater than their respective human health and ecological benchmark values and their sampling location are presented in Appendix D, Table D-10 and shown in Figure D-4.

\subsubsection{Sediment}

Limited sediment samples collected within this segment of the river were analyzed for metals, pesticide/PCBs, radionuclides, and SVOCs. Maximum concentrations of 12 metals, 2 PAHs, PCB aroclor-1254, di-n-butyl phthalate, and 2 radionuclides were reported above their respective ecological or human health benchmark values. A summary of those contaminants that are greater than their respective sediment benchmark values is presented in Table 4-12.

4.5.1.1 Comparison to Ecological Benchmark Values. Maximum concentrations of nine metals, PCB aroclor-1254, and two PAHs (acenaphthene and acenaphthylene) were reported 
above their ecological benchmark value. With the exception of antimony and lead, all contaminants were only slightly greater than their ecological benchmark value with the factor of exceedance ranging from slightly greater than 1 to less than 4.

Cadmium was measured in 17 of 35 surface sediment samples (49\%) at concentrations greater than the ecological benchmark value of $0.6 \mathrm{mg} / \mathrm{kg}$ with concentrations ranging from $0.003 \mathrm{mg} / \mathrm{kg}$ to $2.5 \mathrm{mg} / \mathrm{kg}$. The majority of cadmium concentrations greater than the ecological benchmark value are also greater than the Washington State background value of $0.81 \mathrm{mg} / \mathrm{kg}(76 \%)$.

Antimony was measured in 7 of 15 surface sediment samples $(47 \%)$ at concentrations greater than the ecological benchmark value of $2.0 \mathrm{mg} / \mathrm{kg}$ with concentrations ranging from $0.34 \mathrm{mg} / \mathrm{kg}$ to $65 \mathrm{mg} / \mathrm{kg}$. Antimony was measured above the ecological benchmark value of $2.0 \mathrm{mg} / \mathrm{kg}$ at Messner Cove and behind John Day and the Bonneville Dams.

Lead was measured in one of 32 surface sediment samples (3\%) at a concentration of $250 \mathrm{mg} / \mathrm{kg}$, which is greater than the ecological benchmark value of $35 \mathrm{mg} / \mathrm{kg}$. Lead was measured above the ecological benchmark value of $35 \mathrm{mg} / \mathrm{kg}$ at Messner Cove.

4.5.1.2 Comparison to Human Health Benchmark Values. Maximum concentration of six metals (antimony, arsenic, chromium, iron, lead, and vanadium), and two uranium isotopes were reported above their human health benchmark value. With the exception of arsenic, all metals and the uranium isotopes were only slightly greater than their human health benchmark value with the factor of exceedance ranging from slightly greater than 1 to 3 .

Arsenic was measured in all surface sediment samples at concentrations greater than the human health benchmark value of $0.39 \mathrm{mg} / \mathrm{kg}$ with concentrations ranging from $1.5 \mathrm{mg} / \mathrm{kg}$ to $15 \mathrm{~g} / \mathrm{kg}$. Arsenic was measured in six of 18 surface sediment samples (33\%) at concentrations greater than the Hanford Site background value of $6.5 \mathrm{mg} / \mathrm{kg}$. Arsenic was measured above the background value of $6.5 \mathrm{mg} / \mathrm{kg}$ behind the John Day Dam (see Appendix D, Table D-7 for a list of individual locations). Note that arsenic concentrations measured in Segment 4 are similar to those measured in Segments 1 through 3.

Uranium-234 was measured in 8 of 13 surface sediment samples $(62 \%)$ at concentrations that are greater than the human health benchmark value of $1.1 \mathrm{pCi} / \mathrm{g}$ with concentrations ranging from $0.50 \mathrm{pCi} / \mathrm{g}$ to $1.7 \mathrm{pCi} / \mathrm{g}$. Uranium-238 was measured in 4 of 12 surface sediment samples $(33 \%)$ at concentrations that are greater than the human health benchmark value of $1.1 \mathrm{pCi} / \mathrm{g}$ with concentrations ranging from $0.38 \mathrm{pCi} / \mathrm{g}$ to $1.7 \mathrm{pCi} / \mathrm{g}$. The primary locations where uranium-234 and uranium-238 were measured above the benchmark value include behind the John Day and The Dalles Dams on the Columbia River.

\subsubsection{Surface Water}

Surface water samples collected within this segment of the river were analyzed for general chemistry parameters, metals, pesticides/PCBs, radionuclides, and VOCs. Maximum concentrations of five metals, total PCBs, four pesticides (DDD, DDE, dieldrin, and heptachlor epoxide), and hexachlorobenzene were reported above their respective ecological or human health benchmark values. A summary of those contaminants that are greater than their respective surface water benchmark values is presented in Table 4-13. 
4.5.2.1 Comparison to Ecological Benchmark Values. Maximum concentrations of barium, cadmium, lead, and DDD were reported above their ecological benchmark value. Cadmium, lead, and DDE slightly exceed their ecological benchmark value with the factor of exceedance ranging from slightly greater than 1.2 to 4.0. Maximum concentration of barium was greater than the ecological benchmark value by an exceedance factor of 11 fold.

All barium surface water samples (21 samples) were reported with concentrations greater than the ecological benchmark value of $0.004 \mathrm{mg} / \mathrm{L}$ with concentrations ranging from $0.027 \mathrm{mg} / \mathrm{L}$ to $0.043 \mathrm{mg} / \mathrm{L}$. Barium was measured above the benchmark value near The Dalles Bridge in Oregon.

Cadmium was measured in 1 of 21 surface water samples (4\%) at a concentration of $0.0003 \mathrm{mg} / \mathrm{L}$, which is slightly greater than the ecological benchmark value of $0.00025 \mathrm{mg} / \mathrm{L}$. Cadmium was measured above the benchmark value near The Dalles Bridge in Oregon.

Lead was measured in one sample at a concentration of $0.0036 \mathrm{mg} / \mathrm{L}$, which is greater than the ecological benchmark value of $0.00086 \mathrm{mg} / \mathrm{L}$. Lead was measured near The Dalles Bridge in Oregon.

DDD was measured in two of six surface water samples (33\%) at concentrations greater than the ecological benchmark value of $1.0 \mathrm{E}-06 \mathrm{mg} / \mathrm{L}$ and measured in six samples at concentrations greater than the human health benchmark value of $3.1 \mathrm{E}-07 \mathrm{mg} / \mathrm{L}$. DDD concentrations in surface water ranged from $3.9 \mathrm{E}-07 \mathrm{mg} / \mathrm{L}$ to $2.9 \mathrm{E}-05 \mathrm{mg} / \mathrm{L}$. DDD was measured above the benchmark value at locations near the Bonneville Dam (see Appendix D, Table D-10 for individual locations exceeding benchmark value).

4.5.2.2 Comparison to Human Health Benchmark Values. Maximum concentrations of arsenic, iron, DDD, DDE, dieldrin, heptachlor epoxide, and hexachlorobenzene were reported above their human health benchmark value. Iron, DDE, heptachlor epoxide, hexachlorobenzene, and total PCBs slightly exceed their human health benchmark value with the factor of exceedance ranging from slightly greater than 1 to 6 . Maximum concentrations of DDD and dieldrin were greater than their human health benchmark value by a factor of 9 fold and arsenic was greater than the benchmark by a factor of 117 fold. Measured concentrations of DDD were greater than both the ecological and human health benchmark values and were discussed in the previous section.

Arsenic was measured in 12 surface water samples (100\%) at concentrations greater than the human health benchmark value of $0.000018 \mathrm{mg} / \mathrm{L}$ with concentrations ranging from $0.00042 \mathrm{mg} / \mathrm{L}$ to $0.0021 \mathrm{mg} / \mathrm{L}$. Arsenic results in this segment are similar to Segments 1 through 3 indicating that arsenic is present at concentrations greater than the human health benchmark value at all locations sampled.

Iron was measured in six of 27 surface water samples $(22 \%)$ at concentrations greater than the human health benchmark value of $0.3 \mathrm{mg} / \mathrm{L}$ with concentrations ranging from $0.072 \mathrm{mg} / \mathrm{L}$ to $0.89 \mathrm{mg} / \mathrm{L}$. Iron was measured near The Dalles Bridge in Oregon (see Appendix, Table D-10 for individual locations exceeding benchmark value).

Total PCBs were measured in one of three surface water samples (3\%) at a concentration $3.7 \mathrm{E}-7 \mathrm{mg} / \mathrm{L}$, which is greater than the human health benchmark value of $6.4 \mathrm{E}-08 \mathrm{mg} / \mathrm{L}$. 
DDE were measured in all six surface water samples (44\%) at concentrations greater than the human health benchmark values of $2.2 \mathrm{E}-07 \mathrm{mg} / \mathrm{L}$ with concentrations ranging from $2.7 \mathrm{E}-07$ $\mathrm{mg} / \mathrm{L}$ to $1.0 \mathrm{E}-06 \mathrm{mg} / \mathrm{L}$. Dieldrin was measured in five of six surface water samples $(83 \%)$ at concentrations greater than the human health benchmark value of $5.2 \mathrm{E}-08 \mathrm{mg} / \mathrm{L}$ with concentrations ranging from 4.6E-08 mg/L to 4.9E-07 mg/L. Heptachlor epoxide was measured in two surface water samples at concentrations greater than the human health benchmark value $3.9 \mathrm{E}-08 \mathrm{mg} / \mathrm{L}$ at a concentration of $6.5 \mathrm{E}-08 \mathrm{mg} / \mathrm{L}$. Hexachlorobenzene was measured in two of six surface water samples $(33 \%)$ at concentrations greater than the human health benchmark value $2.8 \mathrm{E}-07 \mathrm{mg} / \mathrm{L}$ at a concentration of $3.5 \mathrm{E}-07 \mathrm{mg} / \mathrm{L}$. Total PCBs, DDE, dieldrin, heptachlor epoxide, and hexachlorobenzene were all measured in locations near the Bonneville Dam (see Appendix D, Table D-10 for individual locations exceeding benchmark value).

\subsection{CONTAMINANT DISTRIBUTIONS IN SEGMENT 5}

A list of the surface sediment and surface water samples used to evaluate Segment 5 of the Columbia River is provided in Appendix E, Tables E-1 and E-2, respectively. All unique surface sediment sampling locations within Segment 5 are provided in Figure E-1. Summary statistics for detected and nondetected surface sediment sample results reported on an "as received" and "dry-weight" basis are presented in Appendix E, Tables E-3 through E-7. A comparison of all detected surface sediment concentrations to available human health and ecological benchmark values is presented in Appendix E, Tables E-8 and E-9. Individual surface sediment samples with concentrations greater than their respective human health and ecological benchmark values and their sampling location are presented in Appendix E, Table E-10 and shown in Figure E-2.

Summary statistics for detected and nondetected surface water sample results are presented in Appendix E, Table E-11. All unique surface water sampling locations within Segment 5 are provided in Figure E-3. A comparison of all detected surface water concentrations to available human health and ecological benchmark values is presented in Appendix E, Table E-12. Individual surface water samples with concentrations greater than their respective human health and ecological benchmark values and their sampling location are presented in Appendix E, Table E-13 and shown in Figure E-4.

\subsubsection{Sediment}

Sediment samples collected within this segment of the river were analyzed for metals, pesticide/PCBs, radionuclides, and SVOCs. Maximum concentrations of 10 metals, three PCB aroclors, two pesticides, 16 PAHs, and two phthalates were reported above their respective ecological or human health benchmark values. A summary of those contaminants that are greater than their respective sediment benchmark values is presented in Table 4-14.

4.6.1.1 Comparison to Ecological Benchmark Values. Maximum concentrations of 10 metals, three PCB aroclors, two pesticides (DDD and DDE), 16 PAHs, and two phthalates were reported above their ecological benchmark value. All metals, two PCB aroclors (aroclor-1254 and aroclor-1260), one pesticide (DDD), three PAHs, and bis(2-ethylhexyl)phthalate were only slightly greater than their ecological benchmark value with the factor of exceedance ranging from slightly greater than 1 to less than 8 . PCB aroclor-1248, $\mathrm{DDE}$, and the remaining PAHs were reported with concentrations that result in a factor of 
exceedance ranging from 12 to 67 fold. When a contaminant measured in sediment exceeded both the ecological and human health benchmark value, they are discussed together.

Iron was measured in 12 of 27 surface sediment samples (44\%) at concentrations that are greater than the ecological benchmark value of $20,000 \mathrm{mg} / \mathrm{kg}$ and measured in six of 27 samples $(22 \%)$ at concentrations greater than the human health benchmark value of $23,500 \mathrm{mg} / \mathrm{kg}$. Surface sediment concentrations range from $12,000 \mathrm{mg} / \mathrm{kg}$ to $39,000 \mathrm{mg} / \mathrm{kg}$. Locations where iron was measured above the benchmark value include Camas Slough, Ilwaco Boat Basin, and Columbia RMs 14, 21, 29, 36, 68, 81, 88, 95, and 120.

Aroclor-1248 was measured in six of seven surface sediment samples (85\%) at concentrations that are greater than the ecological benchmark value of $0.021 \mathrm{mg} / \mathrm{kg}$ and measured in three of seven samples (43\%) at concentrations greater than the human health benchmark value of $0.22 \mathrm{~g} / \mathrm{kg}$. Surface sediment concentrations ranging from $0.011 \mathrm{mg} / \mathrm{kg}$ to $1.4 \mathrm{mg} / \mathrm{kg}$. Aroclor-248 was only measured above the benchmark value at Columbia RM 103.

DDE was measured in nine of 27 surface sediment samples $(31 \%)$ at concentrations that are greater than the ecological benchmark value of $0.0015 \mathrm{mg} / \mathrm{kg}$ with concentrations ranging from $0.0004 \mathrm{mg} / \mathrm{kg}$ to $0.03 \mathrm{mg} / \mathrm{kg}$. DDE was measured above the benchmark value at Warrenton, Oregon; and Columbia RMs 37-47, 82, and 100.

2-Methylnaphthalene was measured in three of 72 surface sediment samples (4\%) at concentrations that are greater than the ecological benchmark value of $0.020 \mathrm{mg} / \mathrm{kg}$ with concentrations ranging from $0.00036 \mathrm{mg} / \mathrm{kg}$ to $0.41 \mathrm{mg} / \mathrm{kg}$. The locations where 2-ethylnaphthalene was measured above the benchmark value include the Oregon Slough and Columbia RM 86.5.

Acenaphthene was measured in eight of 59 surface sediment samples (14\%) at concentrations that are greater than the ecological benchmark value of $0.0067 \mathrm{mg} / \mathrm{kg}$ with concentrations ranging from $0.0005 \mathrm{mg} / \mathrm{kg}$ to $0.11 \mathrm{mg} / \mathrm{kg}$. The locations where acenaphthene was measured above the benchmark value include the Oregon Slough; Crims Island; Ilwaco Boat Basin; Longview and Vancouver, Washington; and Columbia RMs 82 and 86.5.

Benzo(a)anthracene was measured in 16 of 74 surface sediment samples (21\%) at concentrations that are greater than the ecological benchmark value of $0.032 \mathrm{mg} / \mathrm{kg}$ and measured in one of 75 samples $(1 \%)$ at concentrations greater than the human health benchmark value of $0.62 \mathrm{mg} / \mathrm{kg}$. Surface sediment concentrations range from $0.0007 \mathrm{mg} / \mathrm{kg}$ to $2.2 \mathrm{mg} / \mathrm{kg}$. The locations where benzo(a)anthracene was measured above the benchmark value include the Crims Island; Ilwaco Boat Basin; Longview and Vancouver, Washington; and Columbia RMs 21, 81, 82, 83, 86.5, and 88.

Benzo(a)pyrene was measured in 17 of 76 surface sediment samples (22\%) at concentrations that are greater than the ecological benchmark value of $0.032 \mathrm{mg} / \mathrm{kg}$ and measured in six of 76 surface sediment samples $(8 \%)$ at concentrations greater than the human health benchmark value of $0.062 \mathrm{mg} / \mathrm{kg}$. Surface sediment concentrations range from $0.0006 \mathrm{mg} / \mathrm{kg}$ to $1.5 \mathrm{mg} / \mathrm{kg}$. The locations where benzo(a)pyrene was measured above the benchmark value include the Oregon Slough; Crims Island; Longview and Vancouver, Washington; and Columbia RMs 21, 82, 83, $86.588,100$, and 124. 
Benzo(b)fluoranthene was measured in one of 36 surface sediment samples (3\%) at concentrations that are greater than the ecological benchmark value of $0.24 \mathrm{mg} / \mathrm{kg}$ and measured in one of 36 surface sediment samples (3\%) at concentrations greater than the human health benchmark value of $0.62 \mathrm{mg} / \mathrm{kg}$. Surface sediment concentrations range from $0.0016 \mathrm{mg} / \mathrm{kg}$ to $1.8 \mathrm{mg} / \mathrm{kg}$. The only location where benzo(b)fluoranthene was measured above the benchmark value was Longview, Washington.

Benzo(k)fluoranthene was measured in five of 33 surface sediment samples (15\%) at concentrations that are greater than the ecological benchmark value of $0.027 \mathrm{mg} / \mathrm{kg}$ with concentrations ranging from $0.00057 \mathrm{mg} / \mathrm{kg}$ to $0.91 \mathrm{mg} / \mathrm{kg}$. The locations where benzo(k)fluoranthene was measured above the benchmark value include the Camas Slough; Ilwaco Boat Basin; Longview, Washington; and Columbia RMs 21 and 82.

Chrysene was measured in 12 of 83 surface sediment samples (14\%) at concentrations that are greater than the ecological benchmark value of $0.057 \mathrm{mg} / \mathrm{kg}$ with concentrations ranging from $0.0006 \mathrm{mg} / \mathrm{kg}$ to $4.1 \mathrm{mg} / \mathrm{kg}$. The locations where chrysene was measured above the benchmark value include the Camas Slough; Crims Island; Ilwaco Boat Basin; Longview and Vancouver, Washington; and Columbia RMs 21, 81, 82, and 86.5.

Dibenz(a,h)anthracene was measured in one of 32 surface sediment samples $(3 \%)$ at concentrations that are greater than the ecological benchmark value of $0.033 \mathrm{mg} / \mathrm{kg}$ and measured in one of 32 surface sediment samples (3\%) greater than the human health benchmark value of $0.062 \mathrm{mg} / \mathrm{kg}$. Surface sediment concentrations range from $0.0009 \mathrm{mg} / \mathrm{kg}$ to $0.4 \mathrm{mg} / \mathrm{kg}$. The only locations where dibenz(a,h)anthracene was measured above the benchmark value is Longview, Washington.

Di-n-butylphthalate was measured in 14 of 47 surface sediment samples (30\%) at concentrations that are greater than the ecological benchmark value of $0.043 \mathrm{mg} / \mathrm{kg}$ with concentrations ranging from $0.0006 \mathrm{mg} / \mathrm{kg}$ to $0.84 \mathrm{mg} / \mathrm{kg}$. Although di-n-butylphthalate was present in sediment samples analyzed from this segment of the river, phthalate compounds are considered common laboratory contaminants and are not likely contributed as a result of a potential release of contaminants to the Columbia River.

Fluoranthene was measured in two of 84 surface sediment samples (2\%) at concentrations that are greater than the ecological benchmark value of $0.11 \mathrm{mg} / \mathrm{kg}$ with concentrations ranging from $0.0003 \mathrm{mg} / \mathrm{kg}$ to $2.1 \mathrm{mg} / \mathrm{kg}$. Locations where fluoranthene was measured above the benchmark value are Longview and Vancouver, Washington.

Indeno(1,2,3-cd)pyrene was measured in 19 of 61 surface sediment samples (31\%) at concentrations that are greater than the ecological benchmark value of $0.017 \mathrm{mg} / \mathrm{kg}$ and measured in one of 61 samples $(2 \%)$ greater than the human health benchmark value of $0.62 \mathrm{mg} / \mathrm{kg}$. Surface sediment concentrations range from $0.0007 \mathrm{mg} / \mathrm{kg}$ to $0.92 \mathrm{mg} / \mathrm{kg}$. Locations where indeno(1,2,3-cd)pyrene was measured above the benchmark value include Crims Island; Ilwaco Boat Basin; Warrenton, Oregon; Longview and Vancouver, Washington; and Columbia RMs 14, 26, 29, 82, 83, 88,95,100, and 124.

Phenanthrene was measured in 11 of 82 surface sediment samples (13\%) at concentrations that are greater than the ecological benchmark value of $0.042 \mathrm{mg} / \mathrm{kg}$ with concentrations ranging from $0.0004 \mathrm{mg} / \mathrm{kg}$ to $0.58 \mathrm{mg} / \mathrm{kg}$. Locations where phenanthrene was measured 
above the benchmark value include Camas Slough; Oregon Slough; Ilwaco Boat Basin; Longview and Vancouver, Washington; and Columbia RMs 82, 86.5, and 100.

Pyrene was measured in 18 of 81 surface sediment samples $(21 \%)$ at concentrations that are greater than the ecological benchmark value of $0.053 \mathrm{mg} / \mathrm{kg}$ with concentrations ranging from $0.0004 \mathrm{mg} / \mathrm{kg}$ to $2.5 \mathrm{mg} / \mathrm{kg}$. Locations where pyrene was measured above the benchmark value include Camas Slough; Oregon Slough; Ilwaco Boat Basin; Crims Island; Longview and Vancouver, Washington; and Columbia RMs 68, 81, 82, 83, 86.5, 88, and 100.

4.6.1.2 Comparison to Human Health Benchmark Values. Maximum concentrations of three metals, one PCB aroclor, DDD, and five PAHs were reported above their human health benchmark value. Three metals (arsenic, chromium, and iron), aroclor-1248, and five PAHs were only slightly greater than their human health benchmark value with the factor of exceedance ranging from slightly greater than 1 to less than 7 . Arsenic, DDE, and benzo(a)pyrene were reported with concentrations that result in a factor of exceedance ranging from 17 to 35 fold. With the exception of arsenic and chromium, measured concentrations of the contaminants listed above were greater than both the ecological and human health benchmark values and were discussed in the previous section.

Arsenic was measured in 116 of 119 surface sediment samples $(97 \%)$ at concentrations that are greater than the human health benchmark value of $0.39 \mathrm{mg} / \mathrm{kg}$ with concentrations ranging from $0.054 \mathrm{mg} / \mathrm{kg}$ to $14 \mathrm{mg} / \mathrm{kg}$. Arsenic was measured in 20 of the 116 surface sediment samples (17\%) at concentrations greater than the Hanford Site background value of $6.5 \mathrm{mg} / \mathrm{kg}$. Locations where arsenic was measured above background include the Camas Slough; Bachelor Slough; Warrenton, Oregon; Crims Island; Ilwaco Boat Basin; and Columbia RMs 21, 88, and 95 (see Appendix E, Table E-10 for a list of individual locations). Note that arsenic concentrations measured in Segment 5 are similar to those measured in Segments 1 through 4.

Chromium was measured in three of 87 surface sediment samples $(3 \%)$ at concentrations greater than the human health benchmark value of $30 \mathrm{mg} / \mathrm{kg}$ with concentrations ranging from $0.17 \mathrm{mg} / \mathrm{kg}$ to $35 \mathrm{mg} / \mathrm{kg}$. Locations where chromium was measured above the benchmark value include Crims Island; Warrenton, Oregon; and Columbia RM 36.

\subsubsection{Surface Water}

Surface water samples collected within this segment of the river were analyzed for general chemistry parameters, metals, pesticides/PCBs, radionuclides, and VOCs. Maximum concentrations of three metals, total PCBs, five pesticides (DDD, DDE, DDT, dieldrin, and heptachlor epoxide), and hexachlorobenzene were reported above their respective ecological or human health benchmark values. A summary of those contaminants that are greater than their respective surface water benchmark values is presented in Table 4-15.

4.6.2.1 Comparison to Ecological Benchmark Values. Maximum concentrations of barium, boron, total PCBs, DDD, DDT, and dieldrin were reported above their ecological benchmark value. Lead and DDE slightly exceed their ecological benchmark value with the factor of exceedance ranging from slightly greater than 3 to 4 . Maximum concentrations of barium and boron were greater than their ecological benchmark value with the factor of exceedance ranging from 5 to 12 fold. 
Barium was measured in all surface water samples (100\%) at concentrations greater than the ecological benchmark value of $0.004 \mathrm{mg} / \mathrm{L}$ with concentrations ranging from $0.011 \mathrm{mg} / \mathrm{L}$ to $0.021 \mathrm{mg} / \mathrm{L}$. Boron was measured in 16 of 18 surface water samples (89\%) at concentrations greater than the ecological benchmark value of $0.0016 \mathrm{mg} / \mathrm{L}$ with concentrations ranging from $0.0081 \mathrm{mg} / \mathrm{L}$ to $0.020 \mathrm{mg} / \mathrm{L}$. Barium and boron were only analyzed at locations near the Beaver Army Terminal in Oregon.

Total PCBs were measured in one of eight surface water samples (3\%) at a concentration greater than the ecological benchmark value of $0.000014 \mathrm{mg} / \mathrm{L}$ and measured in all eight samples greater than the human health benchmark value of $6.4 \mathrm{E}-08 \mathrm{mg} / \mathrm{L}$. Total PCB concentrations range from 3.3E- $07 \mathrm{mg} / \mathrm{L}$ to $0.000021 \mathrm{mg} / \mathrm{L}$. Total PCBs were measured at levels above the benchmark value in the Columbia Slough (see Appendix E, Table E-13 for individual locations exceeding benchmark value).

DDD was measured in four of 34 surface water samples (12\%) at concentrations greater than the ecological benchmark value of $1 \mathrm{E}-06 \mathrm{mg} / \mathrm{L}$ and measured in 27 of 34 surface water samples $(79 \%)$ at concentrations greater than the human health benchmark value of $31 . \mathrm{E}-07 \mathrm{mg} / \mathrm{L}$. DDD concentrations range from $1.9 \mathrm{E}-07 \mathrm{mg} / \mathrm{L}$ to $5.6 \mathrm{E}-06 \mathrm{mg} / \mathrm{L}$. DDD was measured above the benchmark value at locations below the Bonneville Dam and the Columbia Slough (see Appendix E, Table E-13 for individual locations exceeding benchmark value).

DDT was measured in three of 39 surface water samples (8\%) at concentrations greater than the ecological benchmark value of $1 \mathrm{E}-06 \mathrm{mg} / \mathrm{L}$ and measured in eight of 39 samples $(21 \%)$ at concentrations greater than the human health benchmark value of $2.2 \mathrm{E}-07 \mathrm{mg} / \mathrm{L}$.

DDT concentrations range from $1.3 \mathrm{E}-08 \mathrm{mg} / \mathrm{L}$ to $1.5 \mathrm{E}-06 \mathrm{mg} / \mathrm{L}$. DDT was measured above the benchmark value at locations near the Columbia Slough and the Willamette River (see Appendix E, Table E-13 for individual locations exceeding benchmark value).

Dieldrin was measured in three of 39 surface water samples $(8 \%)$ at concentrations greater than the ecological benchmark value of $1 \mathrm{E}-06 \mathrm{mg} / \mathrm{L}$ and measured in 36 of 39 samples $(92 \%)$ at concentrations greater than the human health benchmark value of $5.2 \mathrm{E}-08 \mathrm{mg} / \mathrm{L}$ Dieldrin concentrations range from $1.4 \mathrm{E}-08 \mathrm{mg} / \mathrm{L}$ to $5.4 \mathrm{E}-06 \mathrm{mg} / \mathrm{L}$. DDT was measured above the benchmark value at locations near the Columbia Slough and the Willamette River (see Appendix E, Table E-13 for individual locations exceeding benchmark value).

4.6.2.2 Comparison to Human Health Benchmark Values. Maximum concentrations of arsenic, total PCBs, DDD, DDE, DDT, dieldrin, heptachlor epoxide, and hexachlorobenzene were reported above their human health benchmark value. Heptachlor epoxide and DDT slightly exceed their human health benchmark value with the factor of exceedance ranging from slightly greater than 2 to 7 . Maximum concentrations of hexachlorobenzene and the remaining pesticides were greater than their human health benchmark value by a factor of exceedance ranging from 8 to 104 fold. Arsenic was greater than the benchmark by a factor of 328 fold. Measured concentrations of total PCBs, DDD, DDT, and dieldrin were also greater than ecological benchmark value and were discussed in the previous section.

Arsenic was measured in 27 surface water samples at concentrations greater than the human health benchmark value of $0.000018 \mathrm{mg} / \mathrm{L}$ with concentrations ranging from $0.0007 \mathrm{mg} / \mathrm{L}$ to $0.0015 \mathrm{mg} / \mathrm{L}$. Arsenic results in this segment are similar to Segments 1 through 4 indicating that 
arsenic is present at concentrations greater than the human health benchmark value at all locations sampled.

DDE was measured in 29 of 42 surface water samples $(69 \%)$ at concentrations greater than the human health benchmark value of $2.2 \mathrm{E}-07 \mathrm{mg} / \mathrm{L}$ with concentrations ranging from $1.5 \mathrm{E}-08 \mathrm{mg} / \mathrm{L}$ to $2.1 \mathrm{E}-06 \mathrm{mg} / \mathrm{L}$. DDE was measured above the benchmark value at locations below the Bonneville Dam; below Longview, Washington; Willamette River; and the Columbia Slough (see Appendix E, Table E-13 for individual locations exceeding benchmark value).

Heptachlor epoxide was measured in three of seven surface water samples (43\%) at concentrations greater than the human health benchmark value $3.9 \mathrm{E}-08 \mathrm{mg} / \mathrm{L}$ with concentrations ranging from $2.5 \mathrm{E}-08 \mathrm{mg} / \mathrm{L}$ to $6.7 \mathrm{E}-08 \mathrm{mg} / \mathrm{L}$. Heptachlor epoxide was measured above the benchmark value in the Willamette River (see Appendix E, Table E-13 for individual locations exceeding benchmark value).

Hexachlorobenzene was measured in 14 of 45 surface water samples (31\%) at concentrations greater than the human health benchmark value $2.8 \mathrm{E}-07 \mathrm{mg} / \mathrm{L}$ with concentrations ranging from 5.7E-08 mg/L to 2.3E-06 mg/L. Hexachlorobenzene was measured above the benchmark value at locations below the Bonneville Dam; Kalama, Washington; Kalama River; Willamette River; Cowlitz River; and the Columbia Slough (see Appendix E, Table E-13 for individual locations exceeding benchmark value).

\subsection{SEDIMENT CORE ANALYSIS}

The purpose of collecting and analyzing core samples is to determine the concentrations of select contaminants deposited at set points in time and to estimate the sediment depositional rate. Core samples are typically collected at a location where sediments are known to be deposited and where sediment deposition is thought to be the deepest. When a core sample is collected, it is divided into predetermined lengths (e.g., $2 \mathrm{~cm}[0.8 \mathrm{in}$.]) or depth intervals and each interval is analyzed for the contaminants of interest. The concentration measured at each interval can then be correlated to the date at which it was deposited.

Through the data compilation effort, nine bibliographical sources were obtained that reported the results of core samples collected within the boundaries of the CRC. Four core samples were collected and analyzed from Segment 1, three from behind Priest Rapids Dam, and one from the Okanogan River. One core sample was collected and analyzed from Segment 2, which was located near the Hanford Ferry. Eighteen core samples were collected and analyzed from Segment 3, one from Wallula, two from Port Kelly, two from Refuge Island, one behind Ice Harbor Dam, and 12 from behind McNary Dam. Six core samples were collected from Segment 4, one from Irrigon, Oregon; one from behind John Day Dam; and three behind The Dalles Dam. Eight core samples were collected from Segment 5, two from RM 21, three from the Skipanon Entrance Channel, one from Clatskanie Flats, one from Young's Bay, and one from Gray's Bay. A summary of the radionuclides and other contaminant classes each core sample was analyzed for is presented in Table 4-16. A summary of the core samples collected from each segment and the number of intervals (and their depths) are presented in Tables 4-17 through 4-21 for Segments 1 through 5, respectively. 
The purpose of evaluating the core samples is to determine if the levels of radiological contaminants deposited from past Hanford Site operations are at levels above the select ecological and human health benchmark values. As shown in Table 4-16, each of the core samples obtained for evaluation were not analyzed for the same suite of radiological contaminants. As a result of this inconsistency, the following discussion focuses on cesium-137, cobalt-60, and plutonium-239/240 as they were analyzed and reported in most of the core samples. Cesium-137 and cobalt-60 were selected to determine if the levels observed downstream from the Hanford Site can be distinguished from atmospheric fallout levels. Plutonium-239/240 was selected as its presence cannot be attributed to atmospheric deposition and was produced in the past during Hanford Site operations. Europium-152, strontium-90, and uranium isotopes are radiological contaminants that are analyzed for in current monitoring programs as they have been or are currently being released through Hanford Site operations. However, europium-152 was only analyzed in four cores and strontium-90 and uranium isotopes were not analyzed in any core sample.

An additional bibliographical source was obtained that reported results of composite cores samples for Segments 2 and 3. Four composite core samples each were collected and analyzed for nonradionuclides within the Hanford Reach and behind McNary Dam.

\subsubsection{Core Samples - Cobalt-60 Analyses}

Cobalt-60 was analyzed in 18 of the 36 core samples collected. Concentrations of cobalt-60 measured in sediments deposited during the same year behind the five dams along the Columbia River are shown in Figure 4-1. The core samples shown on this graph were collected during 1976 using the gravity coring method to reflect current concentrations; all results have been decayed to 2005 . The half-life of cobalt- 60 is 5.26 years.

Priest Rapids Dam is located upstream from the Hanford Site and shows no significant amounts of cobalt-60 $(<0.0007 \mathrm{pCi} / \mathrm{g})$ during 1959 , suggesting that the cobalt-60 measured at the dams located below the Hanford Site may have been contributed from Hanford Site operations. Concentrations of cobalt-60 measured behind McNary and The Dalles dams both show decreasing concentrations from 1959 to 1975, which coincides with a decrease in production activities at the Hanford Site. Concentrations measured in 1959 and 1964 behind John Day Dam do not correlate with the McNary and The Dalles dams because the John Day Dam was under construction from 1958 to 1972 with the filling of the reservoir occurring in 1968. Concentrations of cobalt-60 behind Bonneville Dam are much lower than the dams above it, but have increased slightly over time, which may indicate contaminated sediments being washed downstream from upstream sources.

Figure 4-2 shows a core collected behind each dam and the concentrations of cobalt-60 corresponding to the year at which it was deposited. Also noted in Figure 4-2 is the year at which the construction was completed for each dam. At McNary Dam, cobalt-60 concentrations correspond to years ranging from 1942 through 1974. Cobalt-60 concentrations peak around $1960(0.37 \mathrm{pCi} / \mathrm{g})$ and decrease thereafter to a concentration of approximately $0.02 \mathrm{pCi} / \mathrm{g}$ in 1974. 


\subsubsection{Core Samples - Cesium-137 Analysis}

Cesium-137 was analyzed in 24 of the 36 core samples collected. Concentrations of cesium-137 measured in sediments deposited during the same year behind five of the dams along the Columbia River are shown in Figure 4-3. The core samples shown on this graph were collected during 1976 using a gravity coring method to reflect current concentrations; all results have been decayed to 2005 to reflect current concentrations. The half-life of cesium-137 is 30.17 years.

The concentrations of cesium-137 measured behind Priest Rapids Dam are relatively low during $1959(<0.017 \mathrm{pCi} / \mathrm{g})$ and increase substantially $(\sim 1.8 \mathrm{pCi} / \mathrm{g})$ during 1964 , suggesting that cesium-137 may have been contributed from a source other than Hanford Site operations. It is likely that the cesium-137 measured behind the Priest Rapids Dam is from atmospheric fallout from nuclear testing that took place in the early 1960s. Concentrations of cesium-137 measured behind McNary Dam and The Dalles Dam during 1959 are higher than those concentrations observed at Priest Rapids Dam. Cesium-137 concentrations are similar at McNary Dam and The Dalles Dam ( 1.6 pCi/g), suggesting that cesium-137 may be contributed from Hanford Site operations. However, cesium-137 decreased from 1959 to 1975 to levels less than those observed behind Priest Rapids Dam for the same time period. Concentrations of cesium-137 behind Bonneville Dam are similar to those measured at Priest Rapids Dam during 1959. Concentrations of cesium-137 measured between 1964 and 1975 increase, but are less than those observed at Priest Rapids Dam.

Figure 4-4 shows a core collected behind each dam and the concentrations of cesium-137 corresponding to the year at which it was deposited. Also noted in Figure 4-4 is the year at which the construction was completed for each dam. At McNary Dam, cesium-137 concentrations correspond to years ranging from 1942 through 1974 . Cesium-137 concentrations peak around $1948(2.9 \mathrm{pCi} / \mathrm{g})$ and slowly decrease thereafter to concentrations that are less than the Hanford Site background value of $1.1 \mathrm{pCi} / \mathrm{g}$. Figure $4-5$ shows cesium-137 concentrations (same core as that presented in Figure 4-4) at each depth interval and the approximate year at which it was deposited.

Cesium-137 concentrations have been measured infrequently along the Columbia River since the mid-1970s. However, some recent cesium-137 measurements have been taken from behind Priest Rapids Dam, Ice Harbor Dam, McNary Dam, and the John Day Dam. Cesium-137 concentrations measured behind Priest Rapids Dam from 1999 to 2004 range between $0.32 \mathrm{pCi} / \mathrm{g}$ and $0.61 \mathrm{pCi} / \mathrm{g}$. Cesium-137 concentrations measured behind lce Harbor Dam from 1999 to 2004 range between $0.25 \mathrm{pCi} / \mathrm{g}$ and $0.32 \mathrm{pCi} / \mathrm{g}$. Cesium-137 concentrations measured behind the McNary Dam from 1999 to 2004 range between $0.04 \mathrm{pCi} / \mathrm{g}$ and $1.0 \mathrm{pCi} / \mathrm{g}$. Cesium-137 concentrations measured behind the John Day Dam from 2003 and 2004 range between $0.29 \mathrm{pCi} / \mathrm{g}$ and $0.41 \mathrm{pCi} / \mathrm{g}$. Recent cesium-137 concentrations are similar between Priest Rapids, Ice Harbor, and John Day dams suggesting the cesium-137 was not contributed from Hanford Site operations and are only slightly higher at McNary Dam. Cesium-137 concentrations measured behind the McNary Dam between 1942 and 2002 are presented in Figure 4-6. It is important to note that all recent cesium-137 measurements are less than or equal to the Hanford Site background value of $1.1 \mathrm{pCi} / \mathrm{g}$. 


\subsubsection{Core Samples - Plutonium-239/240 Analysis}

Plutonium-239/240 was analyzed in nine of the 36 core samples collected. Individual core samples collected behind each dam and the concentrations of plutonium-239/240 corresponding to the year at which it was deposited is presented in Figure 4-7. Approximately 35 years of time (1942 to 1974) is represented by the core collected behind McNary Dam. The highest plutonium-239/240 concentrations deposited are measured between 1942 and 1947 (0.1 pCi/g to $0.07 \mathrm{pCi} / \mathrm{g})$ and the levels deposited in 1967 and 1974 decrease to less than Hanford Site background of $0.025 \mathrm{pCi} / \mathrm{g}$. Approximately 13 years of time (1962 to 1975) is represented by the core collected behind The Dalles Dam. The highest plutonium-239/240 concentrations deposited are measured between 1962 and 1964 (0.04 pCi/g to $0.05 \mathrm{pCi} / \mathrm{g})$ and decrease to levels less than Hanford Site Background in 1975. Approximately 15 years of time (1959 to 1975) is represented by the core collected behind Bonneville Dam. The highest plutonium-239/240 concentrations deposited are measured in $1969(0.1 \mathrm{pCi} / \mathrm{g})$ and decrease to below background levels in 1975. Approximately 14 years are represented by the core collected behind Priest Rapids Dam. The highest plutonium-239/240 concentrations deposited are measured between 1962 and $1964(0.04 \mathrm{pCi} / \mathrm{g}$ to $0.05 \mathrm{pCi} / \mathrm{g})$ and decrease to levels below background from 1967 to 1972.

Based on the core data available, plutonium-239/240 is present at the highest levels behind McNary Dam. The levels of plutonium deposited to the dams located downstream of the Hanford Site between 1962 and 1967 ranged between $0.04 \mathrm{pCi} / \mathrm{g}$ and $0.05 \mathrm{pCi} / \mathrm{g}$. However, $0.1 \mathrm{pCi} / \mathrm{g}$ plutonium-239/240 was deposited behind Bonneville Dam in 1969, which may be a result of contaminated sediments being washed downstream from upstream sources. Plutonium-239/240 was deposited at levels above Hanford Site background between 1962 and 1967. Plutonium-239/240 is not naturally occurring; therefore, its presence could be contributed from an upstream source or as a result of atmospheric deposition.

\subsection{BIOTA SUMMARY}

As stated in previous sections, all Category 1 and 2 biota sample data collected and summarized since 1995 were selected to represent current concentrations. A total of 1,355 biota samples were identified that met this criterion. Summary statistic tables of these biota samples based on type and analysis are provided in Appendix $F$. The summary tables includes the frequency of detection, maximum and minimum concentrations (both detected and nondetected values), and average concentrations for approximately 20 different species.

It is important to note that contaminant concentrations measured in biota were not reviewed against the QA categories and may require additional review to evaluate their quality and relevance to the health of the Columbia River. 

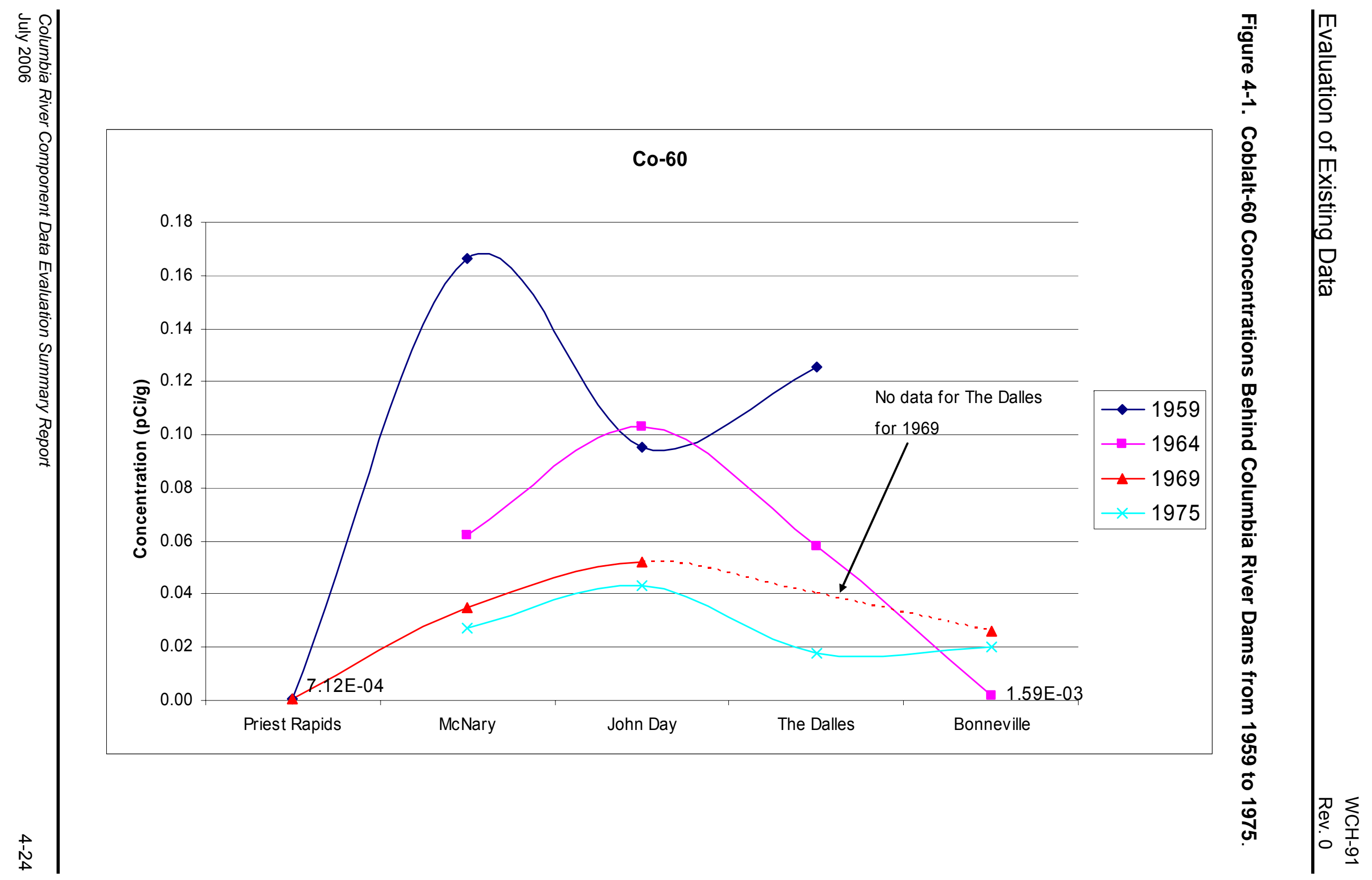

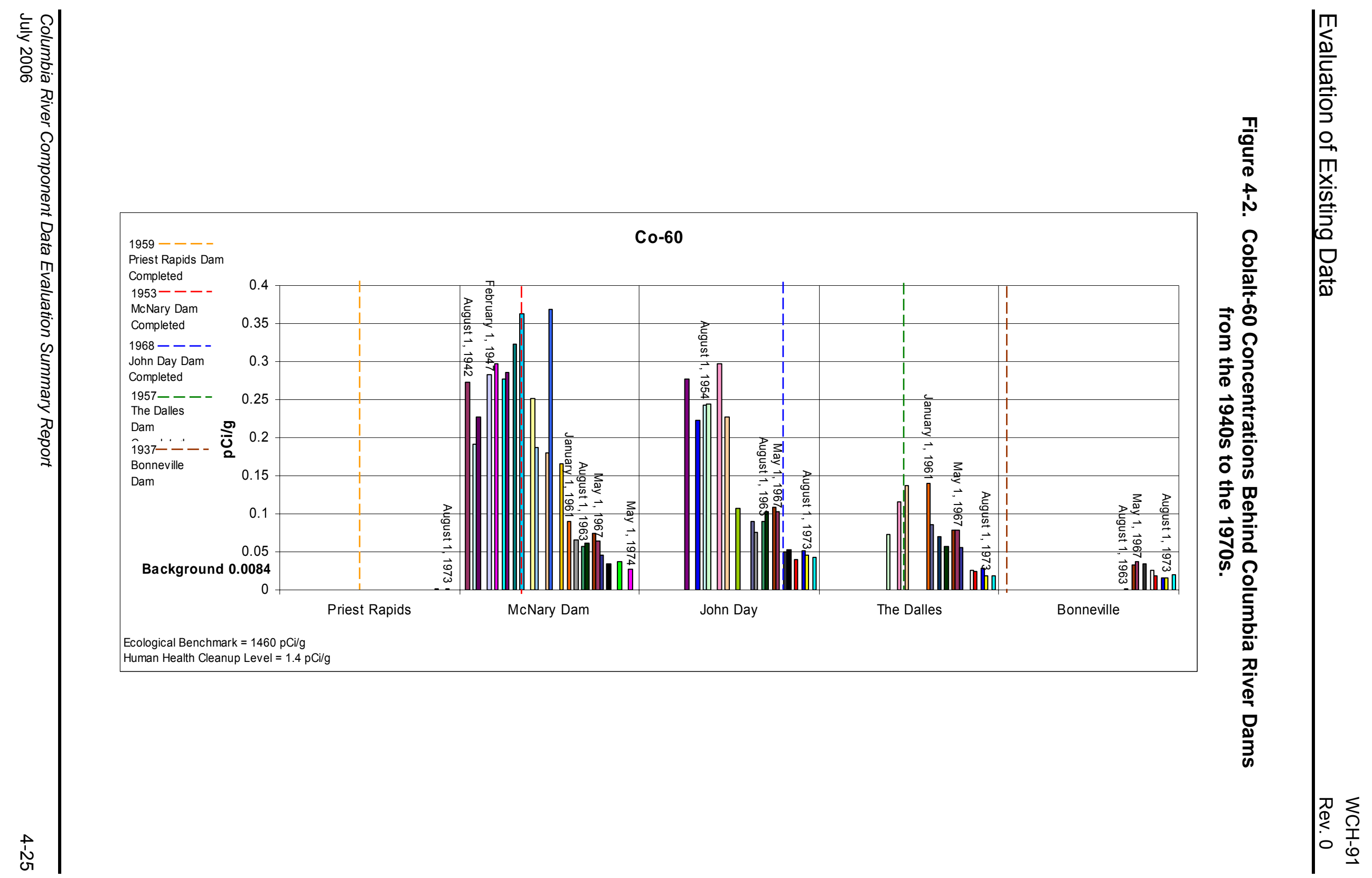

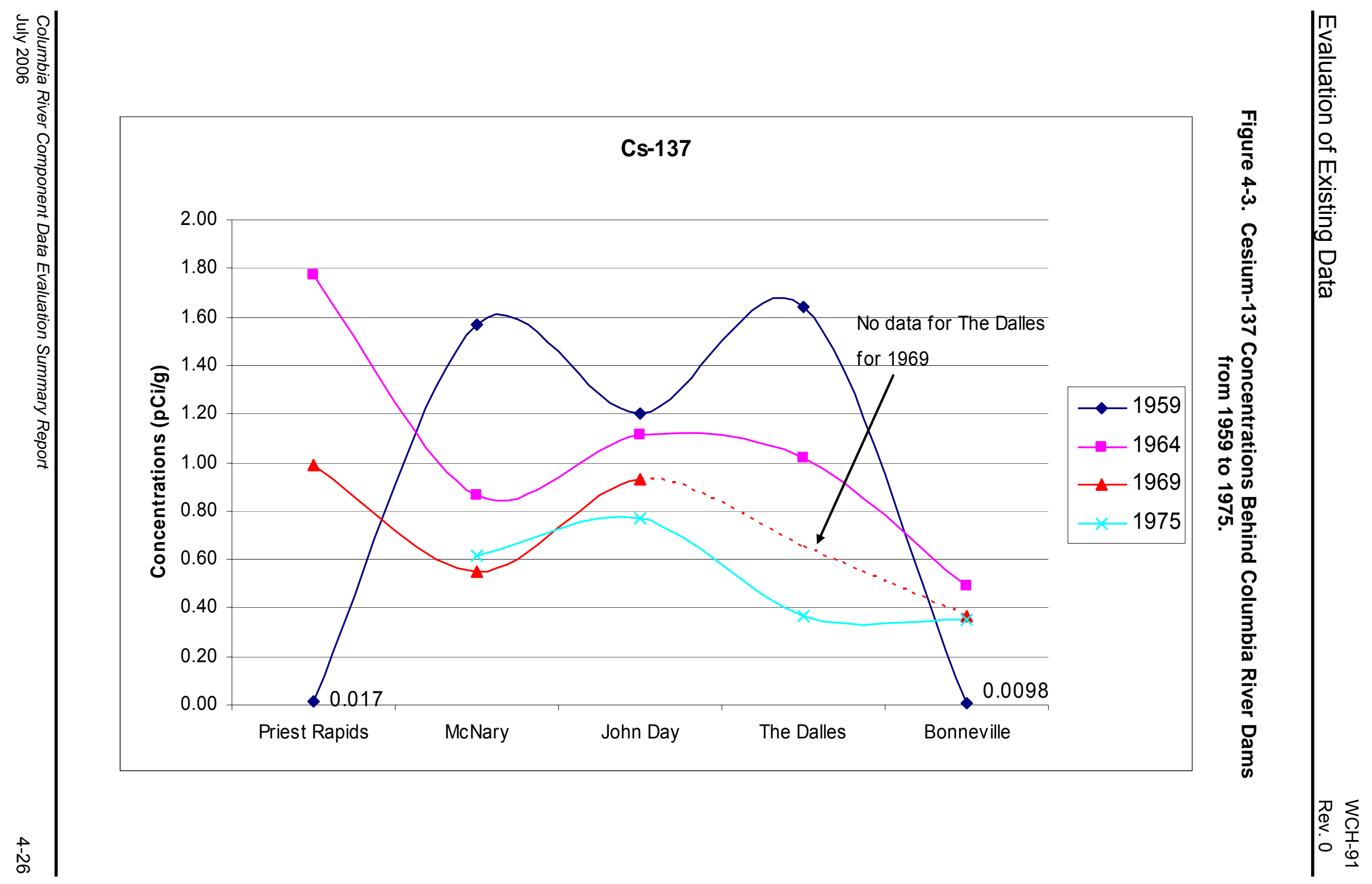

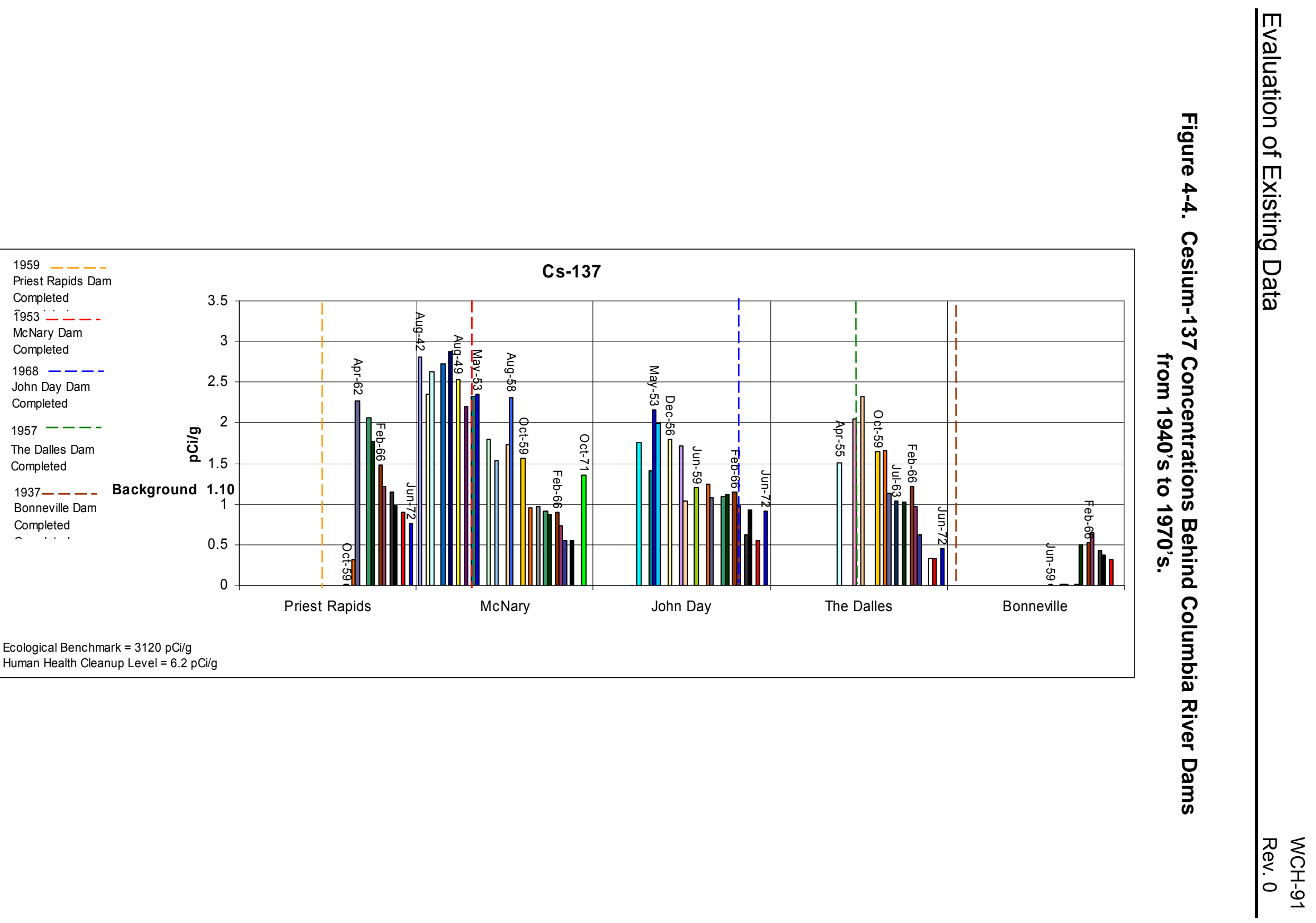

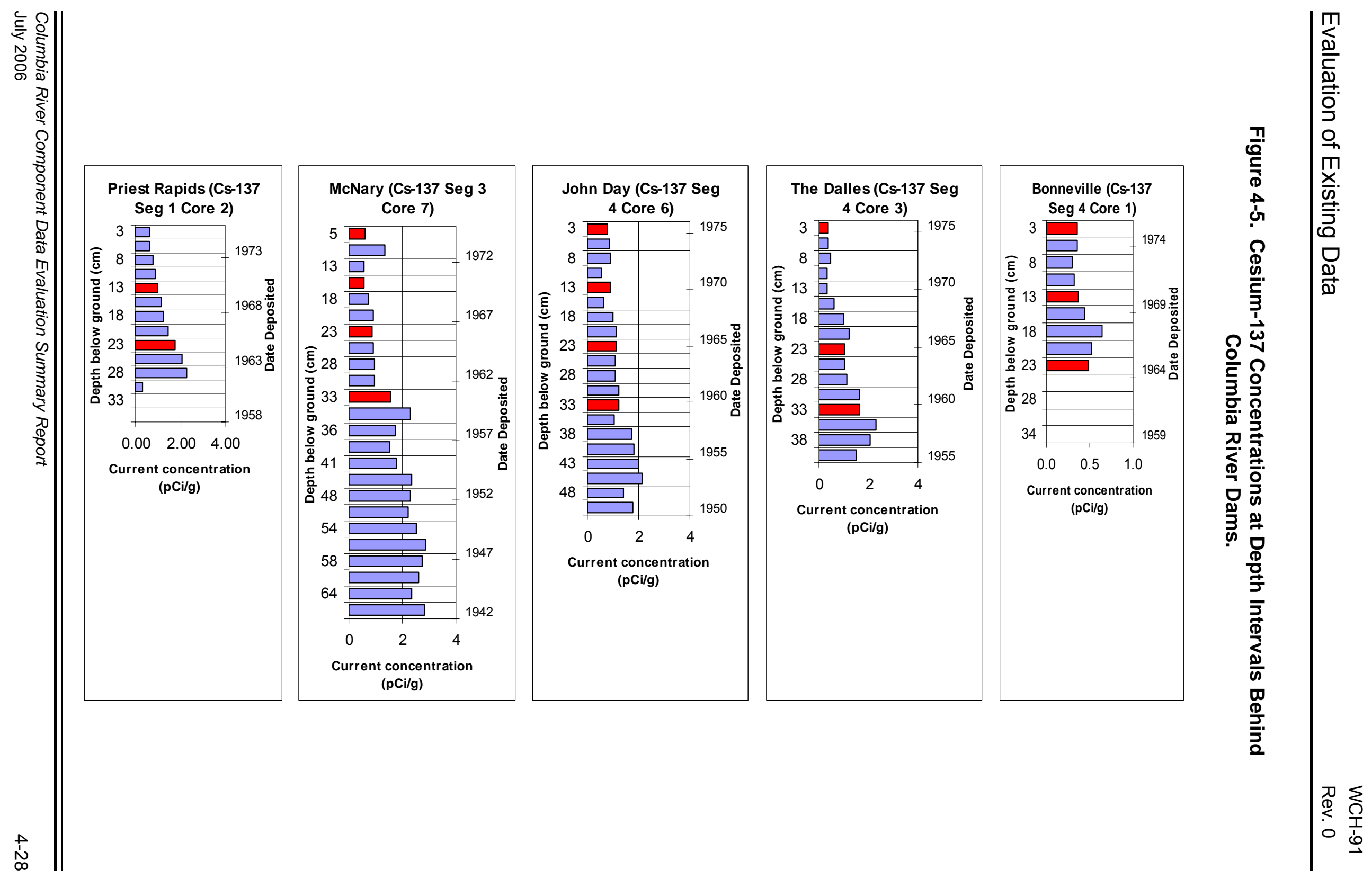

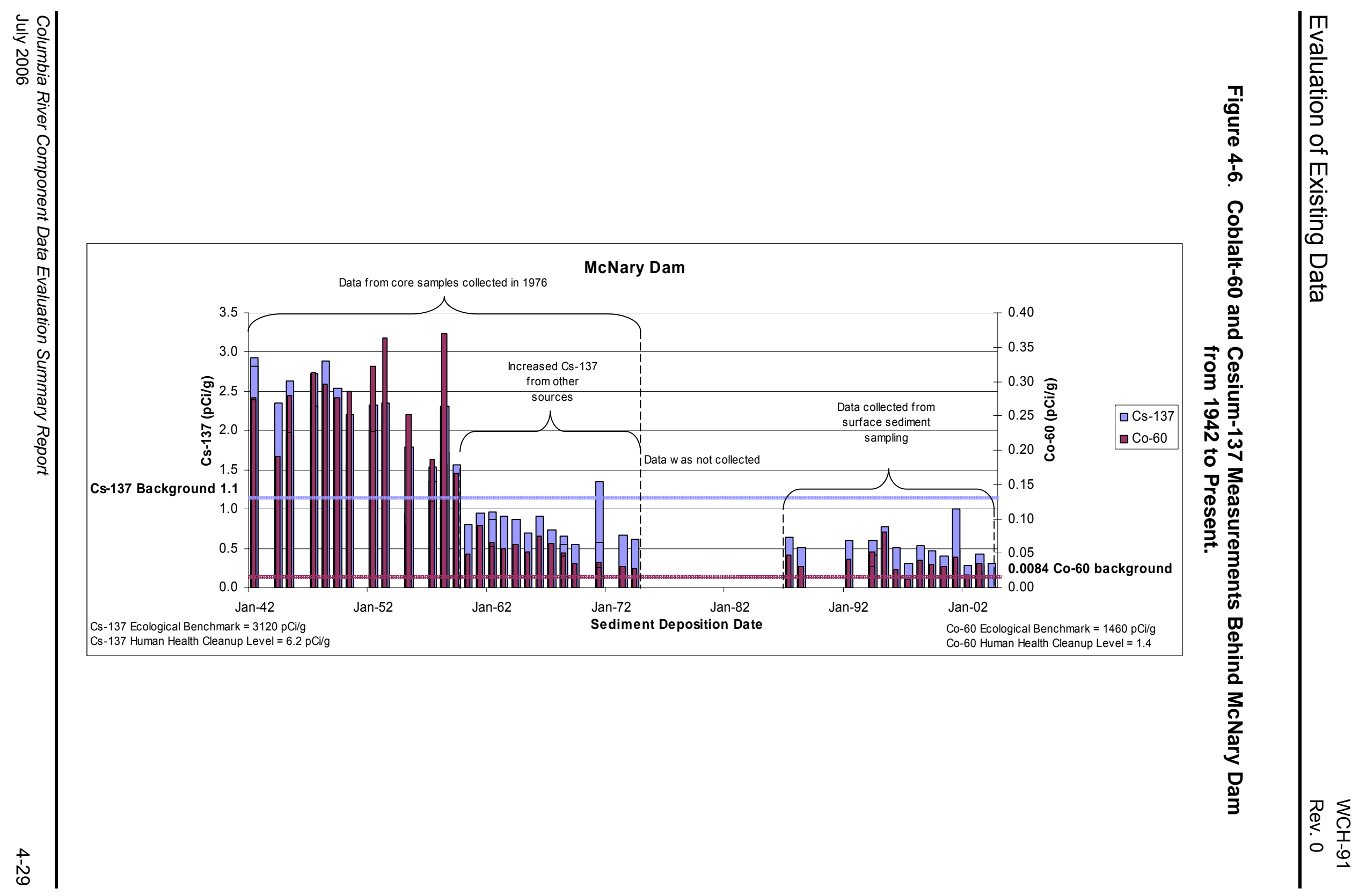

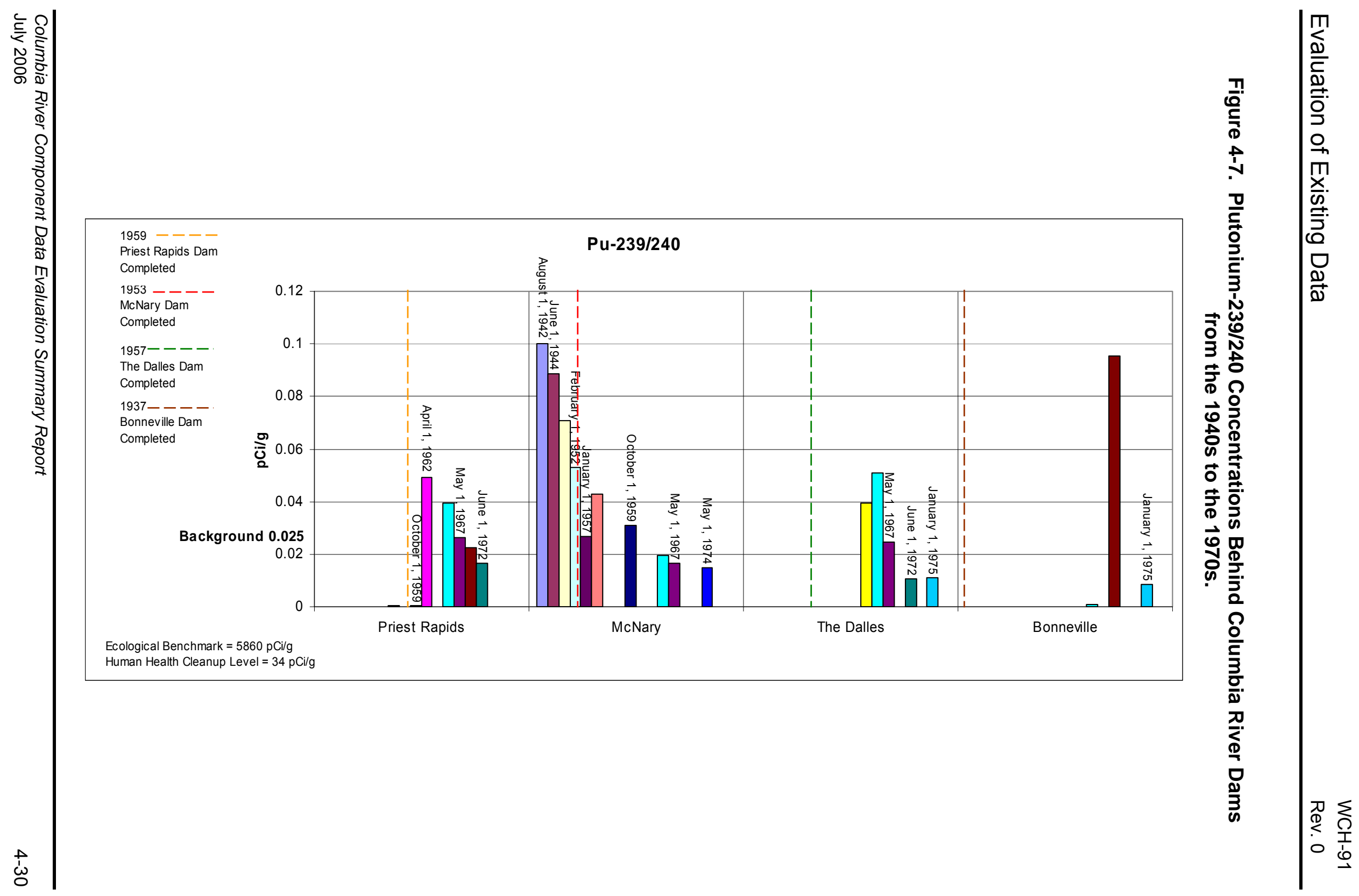
Table 4-1. Summary of Hanford Site Soil Background Concentrations. (2 Pages)

\begin{tabular}{|c|c|c|}
\hline Constituent Name & $\begin{array}{l}\text { 90th Percentile } \\
\text { Values of Background } \\
\text { Concentration }\end{array}$ & Source \\
\hline \multicolumn{3}{|l|}{ Metals (mg/kg) } \\
\hline Antimony & 5.0 & c \\
\hline Aluminum & 12,000 & $\mathrm{~b}$ \\
\hline Arsenic & 6.5 & $\mathrm{~b}$ \\
\hline Barium & 130 & $\mathrm{~b}$ \\
\hline Beryllium & 1.5 & $\mathrm{~b}$ \\
\hline Cadmium & 0.81 & c \\
\hline Calcium & 17,000 & $b$ \\
\hline Chromium & 19 & $b$ \\
\hline Cobalt & 16 & $b$ \\
\hline Copper & 22 & $b$ \\
\hline Iron & 33,000 & $b$ \\
\hline Lead & 10 & $b$ \\
\hline Lithium & 34 & $b$ \\
\hline Manganese & 510 & $b$ \\
\hline Magnesium & 7100 & $b$ \\
\hline Mercury & 0.33 & $b$ \\
\hline Nickel & 19 & $\mathrm{~b}$ \\
\hline Potassium & 2,200 & $b$ \\
\hline Selenium & 0.78 & $\mathrm{~b}$ \\
\hline Silver & 0.73 & c \\
\hline Sodium & 690 & $\mathrm{~b}$ \\
\hline Thallium & NA & $d$ \\
\hline Uranium & 3.2 & a \\
\hline Vanadium & 85 & $b$ \\
\hline Zinc & 68 & $\mathrm{~b}$ \\
\hline Zirconium & 40 & $b$ \\
\hline \multicolumn{3}{|c|}{ Radionuclides (pCi/g) } \\
\hline Americium-241 & NA & $d$ \\
\hline Carbon-14 & NA & $d$ \\
\hline Cesium-137 & 1.1 & $b$ \\
\hline
\end{tabular}


Table 4-1. Summary of Hanford Site Soil Background Concentrations. (2 Pages)

\begin{tabular}{|c|c|c|}
\hline Constituent Name & $\begin{array}{c}\text { 90th Percentile } \\
\text { Values of Background } \\
\text { Concentration }\end{array}$ & Source \\
\hline Cobalt-60 & 0.0084 & $a$ \\
\hline Europium-152 & NA & $d$ \\
\hline Europium-154 & 0.033 & $a$ \\
\hline Europium-155 & 0.054 & $a$ \\
\hline Nickel-63 & NA & $d$ \\
\hline Plutonium-238 & 0.0038 & $a$ \\
\hline Plutonium-239/240 & 0.025 & a \\
\hline Potassium-40 & 17 & $a$ \\
\hline Radium-226 & 0.82 & a \\
\hline Strontium-90 & 0.18 & a \\
\hline Technetium-99 & NA & $d$ \\
\hline Thorium-232 & 1.3 & a \\
\hline Tritium $(\mathrm{H}-3)$ & NA & $d$ \\
\hline Uranium-233/234 & 1.1 & a \\
\hline Uranium-235 & 0.11 & a \\
\hline Uranium-238 & 1.1 & a \\
\hline \multicolumn{3}{|c|}{$\begin{array}{l}\text { a Hanford Site Background: Part 2, Soil Background for Radionuclides, } \\
\text { (DOE-RL 1996). } \\
\text { "Hanford Site Background: Part } 1 \text { Soil Background for Nonradioactive } \\
\text { Analytes, DOEE/RL-92-94, Rev. 4, U.S. Department of Energy, Richland } \\
\text { Operations Office, Richland, Washington (DOE-RL 2001). } \\
{ }^{\circ} \text { Hanford Site-specific background not available; not evaluated during } \\
\text { background study. Value is from Ecology (1994), Natural Background Soil } \\
\text { Metals Concentrations in Washington State, Publication 94-115, } \\
\text { Washington State Department of Ecology, Olympia, Washington } \\
{ }^{\mathrm{d}} \text { Contaminant not evaluated during Hanford Site background study. }\end{array}$} \\
\hline NA = not applicable & & \\
\hline
\end{tabular}


Table 4-2. Summary of Sediment Benchmark Values for the Protection of Human Health. (7 Pages)

\begin{tabular}{|c|c|c|c|c|}
\hline Analyte & Units & $\begin{array}{c}\text { EPA Region } 9 \\
\text { Residential Soil } \\
\text { Preliminary } \\
\text { Remediation } \\
\text { Goals }^{\text {a }}\end{array}$ & $\begin{array}{c}\text { Soil Method B } \\
\text { Unrestricted } \\
\text { Land Use }\end{array}$ & $\begin{array}{l}\text { Protection from } \\
\text { Direct Exposure } \\
\text { Remedial Action } \\
\quad \text { Goal for } \\
\text { Radionuclides }\end{array}$ \\
\hline \multicolumn{5}{|l|}{ General Chemistry } \\
\hline Cyanide & $\mathrm{mg} / \mathrm{kg}$ & 1,200 & 1,600 & -- \\
\hline Soluble Fluoride & $\mathrm{mg} / \mathrm{kg}$ & 3,700 & 4,800 & -- \\
\hline \multicolumn{5}{|l|}{ Herbicide } \\
\hline $\begin{array}{l}\text { 2-(2,4,5- } \\
\text { Trichlorophenoxy)propionic acid }\end{array}$ & $\mathrm{mg} / \mathrm{kg}$ & 490 & 640 & -- \\
\hline $\begin{array}{l}\text { 2-(2-methyl-4-chlorophenoxy) } \\
\text { propionic acid }\end{array}$ & $\mathrm{mg} / \mathrm{kg}$ & 61 & 80 & -- \\
\hline 2,4,5-Trichlorophenoxyacetic acid & $\mathrm{mg} / \mathrm{kg}$ & 610 & 800 & -- \\
\hline 2,4-Dichlorophenoxyacetic acid & $\mathrm{mg} / \mathrm{kg}$ & 690 & 800 & -- \\
\hline $\begin{array}{l}\text { 2-Methyl-4 chlorophenoxyacetic } \\
\text { acid }\end{array}$ & $\mathrm{mg} / \mathrm{kg}$ & 31 & 40 & -- \\
\hline 3,5-dibromo-4-hydroxybenzonitrile & $\mathrm{mg} / \mathrm{kg}$ & 1,200 & 1,600 & -- \\
\hline $\begin{array}{l}\text { 4-(2,4-Dichlorophenoxy)butanoic } \\
\text { acid }\end{array}$ & $\mathrm{mg} / \mathrm{kg}$ & 490 & 640 & -- \\
\hline Dacthal & $\mathrm{mg} / \mathrm{kg}$ & 610 & 800 & -- \\
\hline Dicamba & $\mathrm{mg} / \mathrm{kg}$ & 1,800 & 2,400 & -- \\
\hline Dichloroprop & $\mathrm{mg} / \mathrm{kg}$ & -- & 800 & -- \\
\hline \multicolumn{5}{|l|}{ Metals } \\
\hline Aluminum & $\mathrm{mg} / \mathrm{kg}$ & 76,000 & -- & -- \\
\hline Antimony & $\mathrm{mg} / \mathrm{kg}$ & 31 & 32 & -- \\
\hline Arsenic & $\mathrm{mg} / \mathrm{kg}$ & 0.39 & 0.67 & -- \\
\hline Barium & $\mathrm{mg} / \mathrm{kg}$ & 5,400 & 16,000 & -- \\
\hline Beryllium & $\mathrm{mg} / \mathrm{kg}$ & 150 & 160 & -- \\
\hline Boron & $\mathrm{mg} / \mathrm{kg}$ & 16,000 & 16,000 & -- \\
\hline Cadmium & $\mathrm{mg} / \mathrm{kg}$ & 37 & 40 & -- \\
\hline Hexavalent chromium $^{d}$ & $\mathrm{mg} / \mathrm{kg}$ & 30 & 240 & -- \\
\hline Cobalt & $\mathrm{mg} / \mathrm{kg}$ & 900 & 1,600 & -- \\
\hline Copper & $\mathrm{mg} / \mathrm{kg}$ & 3,100 & 3,000 & -- \\
\hline Iron & $\mathrm{mg} / \mathrm{kg}$ & 23,000 & -- & -- \\
\hline Lead & $\mathrm{mg} / \mathrm{kg}$ & 400 & $250^{\mathrm{e}}$ & -- \\
\hline Lithium & $\mathrm{mg} / \mathrm{kg}$ & 1,600 & 1,600 & -- \\
\hline Manganese & $\mathrm{mg} / \mathrm{kg}$ & 1,800 & 11,000 & -- \\
\hline Mercury & $\mathrm{mg} / \mathrm{kg}$ & 23 & 24 & -- \\
\hline Molybdenum & $\mathrm{mg} / \mathrm{kg}$ & 390 & 400 & -- \\
\hline Nickel & $\mathrm{mg} / \mathrm{kg}$ & 1,600 & 1,600 & -- \\
\hline
\end{tabular}


Table 4-2. Summary of Sediment Benchmark Values for the Protection of Human Health. (7 Pages)

\begin{tabular}{|c|c|c|c|c|}
\hline Analyte & Units & $\begin{array}{l}\text { EPA Region } 9 \\
\text { Residential Soil } \\
\text { Preliminary } \\
\text { Remediation } \\
\text { Goals }^{\text {a }}\end{array}$ & $\begin{array}{c}\text { Soil Method B } \\
\text { Unrestricted } \\
\text { Land Use }^{\mathbf{b}}\end{array}$ & $\begin{array}{c}\text { Protection from } \\
\text { Direct Exposure } \\
\text { Remedial Action } \\
\text { Goal for } \\
\text { Radionuclides }^{c}\end{array}$ \\
\hline Phosphorus & $\mathrm{mg} / \mathrm{kg}$ & 1.6 & 1.6 & -- \\
\hline Selenium & $\mathrm{mg} / \mathrm{kg}$ & 390 & 400 & -- \\
\hline Silver & $\mathrm{mg} / \mathrm{kg}$ & 390 & 400 & -- \\
\hline Strontium & $\mathrm{mg} / \mathrm{kg}$ & 47,000 & 48,000 & -- \\
\hline Thallium & $\mathrm{mg} / \mathrm{kg}$ & 5.2 & 5.6 & -- \\
\hline Tin & $\mathrm{mg} / \mathrm{kg}$ & 47,000 & 48,000 & -- \\
\hline Titanium & $\mathrm{mg} / \mathrm{kg}$ & 100,000 & 320,000 & -- \\
\hline Uranium & $\mathrm{mg} / \mathrm{kg}$ & 16 & 240 & -- \\
\hline Vanadium & $\mathrm{mg} / \mathrm{kg}$ & 78 & 560 & -- \\
\hline Zinc & $\mathrm{mg} / \mathrm{kg}$ & 23,000 & 24,000 & -- \\
\hline \multicolumn{5}{|l|}{ Polychlorinated Biphenyls } \\
\hline Aroclor-1016 & $\mathrm{mg} / \mathrm{kg}$ & 3.9 & 5.6 & -- \\
\hline Aroclor-1221 & $\mathrm{mg} / \mathrm{kg}$ & -- & 0.50 & -- \\
\hline Aroclor-1232 & $\mathrm{mg} / \mathrm{kg}$ & -- & 0.50 & -- \\
\hline Aroclor-1242 & $\mathrm{mg} / \mathrm{kg}$ & -- & 0.50 & -- \\
\hline Aroclor-1248 & $\mathrm{mg} / \mathrm{kg}$ & -- & 0.50 & -- \\
\hline Aroclor-1254 & $\mathrm{mg} / \mathrm{kg}$ & 0.22 & 0.50 & -- \\
\hline Aroclor-1260 & $\mathrm{mg} / \mathrm{kg}$ & -- & 0.50 & -- \\
\hline Total polychlorinated biphenyl & $\mathrm{mg} / \mathrm{kg}$ & -- & 0.50 & -- \\
\hline \multicolumn{5}{|l|}{ Pesticides } \\
\hline $2,4^{\prime}-\mathrm{DDD}$ & $\mathrm{mg} / \mathrm{kg}$ & 2.4 & 4.2 & -- \\
\hline 2,4'-DDE & $\mathrm{mg} / \mathrm{kg}$ & 1.7 & 2.9 & -- \\
\hline $2,4^{\prime}-\mathrm{DDT}$ & $\mathrm{mg} / \mathrm{kg}$ & 1.7 & 2.9 & -- \\
\hline Alachlor & $\mathrm{mg} / \mathrm{kg}$ & 6.0 & 12 & -- \\
\hline Aldrin & $\mathrm{mg} / \mathrm{kg}$ & 0.03 & 0.06 & -- \\
\hline Alpha-BHC & $\mathrm{mg} / \mathrm{kg}$ & 0.09 & 0.16 & -- \\
\hline alpha-Chlordane & $\mathrm{mg} / \mathrm{kg}$ & 1.6 & 2.9 & -- \\
\hline Ametryn & $\mathrm{mg} / \mathrm{kg}$ & 550 & 720 & -- \\
\hline Atrazine & $\mathrm{mg} / \mathrm{kg}$ & 2.2 & 4.6 & -- \\
\hline Benefin (Benfluralin) & $\mathrm{mg} / \mathrm{kg}$ & 18,000 & 24,000 & -- \\
\hline $\begin{array}{l}\text { beta- } 1,2,3,4,5,6- \\
\text { Hexachlorocyclohexane }\end{array}$ & $\mathrm{mg} / \mathrm{kg}$ & 0.32 & 0.56 & -- \\
\hline Butylate & $\mathrm{mg} / \mathrm{kg}$ & 3,000 & 4,000 & -- \\
\hline Captan & $\mathrm{mg} / \mathrm{kg}$ & 140 & 290 & -- \\
\hline Carbethoxy malathion & $\mathrm{mg} / \mathrm{kg}$ & 1,200 & 1,600 & -- \\
\hline Carbophenothion & $\mathrm{mg} / \mathrm{kg}$ & -- & 10 & -- \\
\hline
\end{tabular}


Table 4-2. Summary of Sediment Benchmark Values for the Protection of Human Health. (7 Pages)

\begin{tabular}{|c|c|c|c|c|}
\hline Analyte & Units & $\begin{array}{l}\text { EPA Region } 9 \\
\text { Residential Soil } \\
\text { Preliminary } \\
\text { Remediation } \\
\text { Goals }^{\text {a }}\end{array}$ & $\begin{array}{l}\text { Soil Method B } \\
\text { Unrestricted } \\
\text { Land Use }^{b}\end{array}$ & $\begin{array}{c}\text { Protection from } \\
\text { Direct Exposure } \\
\text { Remedial Action } \\
\text { Goal for } \\
\text { Radionuclides }^{c}\end{array}$ \\
\hline Carboxin & $\mathrm{mg} / \mathrm{kg}$ & 6,100 & 8,000 & -- \\
\hline Chlordane & $\mathrm{mg} / \mathrm{kg}$ & 1.6 & 2.9 & -- \\
\hline Chlorine & $\mathrm{mg} / \mathrm{kg}$ & -- & 8,000 & -- \\
\hline Chlorothalonil & $\mathrm{mg} / \mathrm{kg}$ & 44 & 91 & -- \\
\hline Chlorpropham & $\mathrm{mg} / \mathrm{kg}$ & 12,000 & 16,000 & -- \\
\hline Chlorpyrifos & $\mathrm{mg} / \mathrm{kg}$ & 180 & 240 & -- \\
\hline Cyanazine & $\mathrm{mg} / \mathrm{kg}$ & 0.58 & 1.2 & -- \\
\hline Diallate & $\mathrm{mg} / \mathrm{kg}$ & 8.0 & 16 & -- \\
\hline Diazinon & $\mathrm{mg} / \mathrm{kg}$ & 55 & 72 & -- \\
\hline Dichlorodiphenyldichloroethane & $\mathrm{mg} / \mathrm{kg}$ & 2.4 & 4.2 & -- \\
\hline Dichlorodiphenyldichloroethylene & $\mathrm{mg} / \mathrm{kg}$ & 1.7 & 2.9 & -- \\
\hline Dichlorodiphenyltrichloroethane & $\mathrm{mg} / \mathrm{kg}$ & 1.7 & 2.9 & -- \\
\hline Dichlorvos & $\mathrm{mg} / \mathrm{kg}$ & 1.7 & 3.4 & -- \\
\hline Dicofol & $\mathrm{mg} / \mathrm{kg}$ & 1.1 & -- & -- \\
\hline Dieldrin & $\mathrm{mg} / \mathrm{kg}$ & 0.03 & 0.06 & -- \\
\hline Dimethoate & $\mathrm{mg} / \mathrm{kg}$ & 12 & 16 & -- \\
\hline Diphenamid & $\mathrm{mg} / \mathrm{kg}$ & 1,800 & 2,400 & -- \\
\hline Disulfoton & $\mathrm{mg} / \mathrm{kg}$ & 2.0 & 3.2 & -- \\
\hline Diuron & $\mathrm{mg} / \mathrm{kg}$ & 120 & 160 & -- \\
\hline Endosulfan I & $\mathrm{mg} / \mathrm{kg}$ & 370 & 480 & -- \\
\hline Endrin & $\mathrm{mg} / \mathrm{kg}$ & 18 & 24 & -- \\
\hline Eptam & $\mathrm{mg} / \mathrm{kg}$ & 1,500 & 2,000 & -- \\
\hline $\begin{array}{l}\text { Ethyl methylene } \\
\text { phosphorodithioate }\end{array}$ & $\mathrm{mg} / \mathrm{kg}$ & 31 & 40 & -- \\
\hline $\begin{array}{l}\text { Ethyl O-(p-nitrophenyl) } \\
\text { phenylphosphonothionate }\end{array}$ & $\mathrm{mg} / \mathrm{kg}$ & 0.61 & 0.80 & -- \\
\hline Fensulfothion & $\mathrm{mg} / \mathrm{kg}$ & -- & 20 & -- \\
\hline Fluridone & $\mathrm{mg} / \mathrm{kg}$ & 4,900 & 6,400 & -- \\
\hline Fonofos & $\mathrm{mg} / \mathrm{kg}$ & 120 & 160 & -- \\
\hline Gamma-BHC (Lindane) & $\mathrm{mg} / \mathrm{kg}$ & 0.44 & 0.77 & -- \\
\hline Heptachlor & $\mathrm{mg} / \mathrm{kg}$ & 0.11 & 0.22 & -- \\
\hline Heptachlor epoxide & $\mathrm{mg} / \mathrm{kg}$ & 0.05 & 0.11 & -- \\
\hline Hexazinone & $\mathrm{mg} / \mathrm{kg}$ & 2,000 & 2,600 & -- \\
\hline Metalaxyl & $\mathrm{mg} / \mathrm{kg}$ & 3,700 & 4,800 & -- \\
\hline Methoxychlor & $\mathrm{mg} / \mathrm{kg}$ & 310 & 400 & -- \\
\hline Methyl Chlorpyrifos & $\mathrm{mg} / \mathrm{kg}$ & 610 & 800 & -- \\
\hline
\end{tabular}


Table 4-2. Summary of Sediment Benchmark Values for the Protection of Human Health. (7 Pages)

\begin{tabular}{|c|c|c|c|c|}
\hline Analyte & Units & $\begin{array}{c}\text { EPA Region } 9 \\
\text { Residential Soil } \\
\text { Preliminary } \\
\text { Remediation }^{\text {Goals }}\end{array}$ & $\begin{array}{l}\text { Soil Method B } \\
\text { Unrestricted } \\
\text { Land Use }^{b}\end{array}$ & $\begin{array}{c}\text { Protection from } \\
\text { Direct Exposure } \\
\text { Remedial Action } \\
\text { Goal for } \\
\text { Radionuclides }^{c}\end{array}$ \\
\hline Methyl parathion & $\mathrm{mg} / \mathrm{kg}$ & 15 & 20 & -- \\
\hline Metolachlor & $\mathrm{mg} / \mathrm{kg}$ & 9,200 & 12,000 & -- \\
\hline Metribuzin & $\mathrm{mg} / \mathrm{kg}$ & 1,500 & 2,000 & -- \\
\hline Mevinphos & $\mathrm{mg} / \mathrm{kg}$ & -- & 20 & -- \\
\hline Mirex & $\mathrm{mg} / \mathrm{kg}$ & 0.27 & 0.56 & -- \\
\hline Molinate & $\mathrm{mg} / \mathrm{kg}$ & 120 & 160 & -- \\
\hline Napropamide & $\mathrm{mg} / \mathrm{kg}$ & 6,100 & 8,000 & -- \\
\hline Norflurazon & $\mathrm{mg} / \mathrm{kg}$ & 2,400 & 3,200 & -- \\
\hline Oxyfluorfen & $\mathrm{mg} / \mathrm{kg}$ & 180 & 240 & -- \\
\hline Parathion & $\mathrm{mg} / \mathrm{kg}$ & 370 & 480 & - \\
\hline Pebulate & $\mathrm{mg} / \mathrm{kg}$ & 3,100 & 4,000 & -- \\
\hline Pendimethalin & $\mathrm{mg} / \mathrm{kg}$ & 2,400 & 3,200 & -- \\
\hline Perthane & $\mathrm{mg} / \mathrm{kg}$ & -- & 240 & -- \\
\hline Phorate & $\mathrm{mg} / \mathrm{kg}$ & 12 & 16 & -- \\
\hline Phosmet & $\mathrm{mg} / \mathrm{kg}$ & 1,200 & 1,600 & -- \\
\hline Pramitol (Prometon) & $\mathrm{mg} / \mathrm{kg}$ & 920 & 1,200 & -- \\
\hline Profluralin & $\mathrm{mg} / \mathrm{kg}$ & 370 & 480 & -- \\
\hline Prometryn & $\mathrm{mg} / \mathrm{kg}$ & 240 & 320 & -- \\
\hline Pronamide & $\mathrm{mg} / \mathrm{kg}$ & 4,600 & 6,000 & -- \\
\hline Propachlor (Ramrod) & $\mathrm{mg} / \mathrm{kg}$ & 790 & 1,000 & -- \\
\hline Propazine & $\mathrm{mg} / \mathrm{kg}$ & 1,200 & 1,600 & -- \\
\hline Ronnel & $\mathrm{mg} / \mathrm{kg}$ & 3,100 & 4,000 & -- \\
\hline Simazine & $\mathrm{mg} / \mathrm{kg}$ & 4.0 & 8.0 & -- \\
\hline Terbacil & $\mathrm{mg} / \mathrm{kg}$ & 790 & 1,000 & -- \\
\hline Terbutryn & $\mathrm{mg} / \mathrm{kg}$ & 61 & 80 & -- \\
\hline Tetraethyl dithiopyrophosphate & $\mathrm{mg} / \mathrm{kg}$ & 31 & 40 & -- \\
\hline Toxaphene & $\mathrm{mg} / \mathrm{kg}$ & 0.44 & 0.91 & -- \\
\hline Triadimefon & $\mathrm{mg} / \mathrm{kg}$ & 1,800 & 2,400 & -- \\
\hline Triallate & $\mathrm{mg} / \mathrm{kg}$ & 790 & 1,000 & -- \\
\hline Tributylphosphorotrithioate & $\mathrm{mg} / \mathrm{kg}$ & 1.8 & 2.4 & - \\
\hline Trifluralin (Treflan) & $\mathrm{mg} / \mathrm{kg}$ & 63 & 130 & -- \\
\hline Vernolate & $\mathrm{mg} / \mathrm{kg}$ & 61 & 80 & -- \\
\hline \multicolumn{5}{|l|}{ Radionuclides } \\
\hline Americium-241 & $\mathrm{pCi} / \mathrm{g}$ & -- & -- & 31 \\
\hline Cesium-137 & $\mathrm{pCi} / \mathrm{g}$ & -- & -- & 6.2 \\
\hline
\end{tabular}


Table 4-2. Summary of Sediment Benchmark Values for the Protection of Human Health. (7 Pages)

\begin{tabular}{|c|c|c|c|c|}
\hline Analyte & Units & $\begin{array}{l}\text { EPA Region } 9 \\
\text { Residential Soil } \\
\text { Preliminary } \\
\text { Remediation } \\
\text { Goals }^{\text {a }}\end{array}$ & $\begin{array}{c}\text { Soil Method B } \\
\text { Unrestricted } \\
\text { Land Use }^{\mathbf{b}}\end{array}$ & $\begin{array}{c}\text { Protection from } \\
\text { Direct Exposure } \\
\text { Remedial Action } \\
\text { Goal for } \\
\text { Radionuclides }^{c}\end{array}$ \\
\hline Cobalt-60 & $\mathrm{pCi} / \mathrm{g}$ & -- & -- & 1.4 \\
\hline Europium-152 & $\mathrm{pCi} / \mathrm{g}$ & -- & -- & 3.3 \\
\hline Europium-154 & $\mathrm{pCi} / \mathrm{g}$ & -- & -- & 3.0 \\
\hline Europium-155 & $\mathrm{pCi} / \mathrm{g}$ & -- & -- & 130 \\
\hline Plutonium-238 & $\mathrm{pCi} / \mathrm{g}$ & -- & -- & 37 \\
\hline Plutonium-239/240 & $\mathrm{pCi} / \mathrm{g}$ & -- & -- & 34 \\
\hline Strontium-90 & $\mathrm{pCi} / \mathrm{g}$ & -- & -- & 4.5 \\
\hline Technetium-99 & $\mathrm{pCi} / \mathrm{g}$ & -- & -- & 15 \\
\hline Thorium-232 & $\mathrm{pCi} / \mathrm{g}$ & -- & -- & 1.3 \\
\hline Tritium & $\mathrm{pCi} / \mathrm{g}$ & -- & -- & 510 \\
\hline Uranium-234 & $\mathrm{pCi} / \mathrm{g}$ & -- & -- & 1.1 \\
\hline Uranium-235 & $\mathrm{pCi} / \mathrm{g}$ & -- & -- & 0.84 \\
\hline Uranium-238 & $\mathrm{pCi} / \mathrm{g}$ & -- & -- & 1.1 \\
\hline \multicolumn{5}{|c|}{ Semivolatile Organic Compounds } \\
\hline 1,2,4-Trichlorobenzene & $\mathrm{mg} / \mathrm{kg}$ & 62 & 800 & -- \\
\hline 1,2-Dichlorobenzene & $\mathrm{mg} / \mathrm{kg}$ & 600 & 7,200 & -- \\
\hline 1,2-Diphenylhydrazine & $\mathrm{mg} / \mathrm{kg}$ & 0.61 & 1.3 & -- \\
\hline 1,3-Dichlorobenzene & $\mathrm{mg} / \mathrm{kg}$ & 530 & 2,400 & -- \\
\hline 1,4-Dichlorobenzene & $\mathrm{mg} / \mathrm{kg}$ & 3.5 & 42 & -- \\
\hline 2,4,5-Trichlorophenol & $\mathrm{mg} / \mathrm{kg}$ & 6,100 & 8,000 & -- \\
\hline 2,4,6-Trichlorophenol & $\mathrm{mg} / \mathrm{kg}$ & 6.0 & 91 & -- \\
\hline 2,4-Dichlorophenol & $\mathrm{mg} / \mathrm{kg}$ & 180 & 240 & -- \\
\hline 2,4-Dimethylphenol & $\mathrm{mg} / \mathrm{kg}$ & 1,200 & 1,600 & -- \\
\hline 2,4-Dinitrophenol & $\mathrm{mg} / \mathrm{kg}$ & 120 & 160 & -- \\
\hline 2,4-Dinitrotoluene & $\mathrm{mg} / \mathrm{kg}$ & 120 & 160 & -- \\
\hline 2,6-Dinitrotoluene & $\mathrm{mg} / \mathrm{kg}$ & 61 & 80 & -- \\
\hline 2-Chloronaphthalene & $\mathrm{mg} / \mathrm{kg}$ & 4,900 & 6,400 & -- \\
\hline 2-Chlorophenol & $\mathrm{mg} / \mathrm{kg}$ & 63 & 400 & -- \\
\hline 2-Methylphenol (cresol, o-) & $\mathrm{mg} / \mathrm{kg}$ & 3,100 & 4,000 & -- \\
\hline 2-Nitroaniline & $\mathrm{mg} / \mathrm{kg}$ & 180 & 240 & -- \\
\hline $\begin{array}{l}\text { 2-secButyl-4,6-dinitrophenol } \\
\text { (DNBP) }\end{array}$ & $\mathrm{mg} / \mathrm{kg}$ & 61 & 80 & -- \\
\hline 3,3'-Dichlorobenzidine & $\mathrm{mg} / \mathrm{kg}$ & 1.0 & 2.0 & -- \\
\hline 3-Nitroaniline & $\mathrm{mg} / \mathrm{kg}$ & 18 & 24 & -- \\
\hline 4,6-Dinitro-2-methylphenol & $\mathrm{mg} / \mathrm{kg}$ & 6.0 & 8.0 & -- \\
\hline 4-Chloro-3-methylphenol & $\mathrm{mg} / \mathrm{kg}$ & -- & 4,000 & -- \\
\hline
\end{tabular}


Table 4-2. Summary of Sediment Benchmark Values for the Protection of Human Health. (7 Pages)

\begin{tabular}{|c|c|c|c|c|}
\hline Analyte & Units & $\begin{array}{c}\text { EPA Region } 9 \\
\text { Residential Soil } \\
\text { Preliminary } \\
\text { Remediation }^{\text {Goals }}\end{array}$ & $\begin{array}{l}\text { Soil Method B } \\
\text { Unrestricted } \\
\text { Land Use }^{b}\end{array}$ & $\begin{array}{c}\text { Protection from } \\
\text { Direct Exposure } \\
\text { Remedial Action } \\
\text { Goal for } \\
\text { Radionuclides }^{c}\end{array}$ \\
\hline 4-Chloroaniline & $\mathrm{mg} / \mathrm{kg}$ & 240 & 320 & -- \\
\hline 4-Methylphenol (cresol, p-) & $\mathrm{mg} / \mathrm{kg}$ & 310 & 400 & -- \\
\hline 4-Nitroaniline & $\mathrm{mg} / \mathrm{kg}$ & 23 & 48 & -- \\
\hline 4-Nitrophenol & $\mathrm{mg} / \mathrm{kg}$ & -- & 640 & -- \\
\hline Acenaphthene & $\mathrm{mg} / \mathrm{kg}$ & 3,700 & 4,800 & -- \\
\hline Acenaphthylene & $\mathrm{mg} / \mathrm{kg}$ & -- & 4,800 & -- \\
\hline Aniline & $\mathrm{mg} / \mathrm{kg}$ & 85 & 180 & -- \\
\hline Anthracene & $\mathrm{mg} / \mathrm{kg}$ & 22,000 & 24,000 & -- \\
\hline Azobenzene & $\mathrm{mg} / \mathrm{kg}$ & 4.0 & 9.0 & -- \\
\hline Benzidine & $\mathrm{mg} / \mathrm{kg}$ & 0.00 & 0.00 & -- \\
\hline Benzo(a)anthracene & $\mathrm{mg} / \mathrm{kg}$ & 0.62 & 1.37 & -- \\
\hline Benzo(a)pyrene & $\mathrm{mg} / \mathrm{kg}$ & 0.06 & 0.14 & -- \\
\hline Benzo(b)fluoranthene & $\mathrm{mg} / \mathrm{kg}$ & 0.62 & 1.0 & -- \\
\hline Benzo(ghi)perylene & $\mathrm{mg} / \mathrm{kg}$ & -- & 2,400 & -- \\
\hline Benzo(k)fluoranthene & $\mathrm{mg} / \mathrm{kg}$ & 6.0 & 13 & -- \\
\hline Benzoic acid & $\mathrm{mg} / \mathrm{kg}$ & 100,000 & 320,000 & -- \\
\hline Benzyl alcohol & $\mathrm{mg} / \mathrm{kg}$ & 18,000 & 24,000 & -- \\
\hline Biphenyl & $\mathrm{mg} / \mathrm{kg}$ & 3,000 & 4,000 & -- \\
\hline Bis(2-chloro-1-methylethyl)ether & $\mathrm{mg} / \mathrm{kg}$ & 3.0 & 14 & -- \\
\hline Bis(2-Chloroethoxy)methane & $\mathrm{mg} / \mathrm{kg}$ & -- & 0.91 & -- \\
\hline Bis(2-chloroethyl) ether & $\mathrm{mg} / \mathrm{kg}$ & 0.22 & 0.91 & -- \\
\hline Bis(2-chloroisopropyl) & $\mathrm{mg} / \mathrm{kg}$ & -- & 3,200 & -- \\
\hline Bis(2-ethylhexyl) phthalate & $\mathrm{mg} / \mathrm{kg}$ & 35 & 71 & -- \\
\hline Butylbenzylphthalate & $\mathrm{mg} / \mathrm{kg}$ & 12,000 & 16,000 & -- \\
\hline Carbazole & $\mathrm{mg} / \mathrm{kg}$ & 24 & 50 & -- \\
\hline Chrysene & $\mathrm{mg} / \mathrm{kg}$ & 62 & 137 & -- \\
\hline Dibenz[a,h]anthracene & $\mathrm{mg} / \mathrm{kg}$ & 0.06 & 0.14 & -- \\
\hline Dibenzofuran & $\mathrm{mg} / \mathrm{kg}$ & 150 & 160 & -- \\
\hline Diethylphthalate & $\mathrm{mg} / \mathrm{kg}$ & 49,000 & 64,000 & -- \\
\hline Dimethyl phthalate & $\mathrm{mg} / \mathrm{kg}$ & 100,000 & 80,000 & -- \\
\hline Di-n-butylphthalate & $\mathrm{mg} / \mathrm{kg}$ & 6,100 & 8,000 & -- \\
\hline Di-n-octylphthalate & $\mathrm{mg} / \mathrm{kg}$ & 2,400 & 1,600 & -- \\
\hline Diphenylamine & $\mathrm{mg} / \mathrm{kg}$ & 1,500 & 2,000 & -- \\
\hline Fluoranthene & $\mathrm{mg} / \mathrm{kg}$ & 2,300 & 3,200 & -- \\
\hline Fluorene & $\mathrm{mg} / \mathrm{kg}$ & 2,700 & 3,200 & -- \\
\hline
\end{tabular}


Table 4-2. Summary of Sediment Benchmark Values for the Protection of Human Health. (7 Pages)

\begin{tabular}{|c|c|c|c|c|}
\hline Analyte & Units & $\begin{array}{c}\text { EPA Region } 9 \\
\text { Residential Soil } \\
\text { Preliminary } \\
\text { Remediation } \\
\text { Goals }^{\text {a }}\end{array}$ & $\begin{array}{l}\text { Soil Method B } \\
\text { Unrestricted } \\
\text { Land Use }^{b}\end{array}$ & $\begin{array}{c}\text { Protection from } \\
\text { Direct Exposure } \\
\text { Remedial Action } \\
\text { Goal for } \\
\text { Radionuclides }\end{array}$ \\
\hline Hexachlorobenzene & $\mathrm{mg} / \mathrm{kg}$ & 0.30 & 0.63 & -- \\
\hline Hexachlorobutadiene & $\mathrm{mg} / \mathrm{kg}$ & 6.2 & 13 & -- \\
\hline Hexachlorocyclopentadiene & $\mathrm{mg} / \mathrm{kg}$ & 370 & 480 & -- \\
\hline Hexachloroethane & $\mathrm{mg} / \mathrm{kg}$ & 35 & 71 & - \\
\hline Indeno(1,2,3-cd)pyrene & $\mathrm{mg} / \mathrm{kg}$ & 0.62 & 1.4 & - \\
\hline Isophorone & $\mathrm{mg} / \mathrm{kg}$ & 510 & 1,100 & -- \\
\hline Naphthalene & $\mathrm{mg} / \mathrm{kg}$ & 56 & 320 & -- \\
\hline Nitrobenzene & $\mathrm{mg} / \mathrm{kg}$ & 20 & 40 & - \\
\hline N-Nitrosodimethylamine & $\mathrm{mg} / \mathrm{kg}$ & 0.01 & 0.02 & - \\
\hline N-Nitrosodi-n-dipropylamine & $\mathrm{mg} / \mathrm{kg}$ & 0.07 & 0.14 & - \\
\hline N-Nitrosodiphenylamine & $\mathrm{mg} / \mathrm{kg}$ & 99 & 200 & - \\
\hline Pentachlorophenol & $\mathrm{mg} / \mathrm{kg}$ & 3.0 & 8.0 & -- \\
\hline Phenol & $\mathrm{mg} / \mathrm{kg}$ & 18,000 & 24,000 & - \\
\hline Pyrene & $\mathrm{mg} / \mathrm{kg}$ & 2,300 & 2,400 & - \\
\hline Pyridine & $\mathrm{mg} / \mathrm{kg}$ & 61 & 80 & -- \\
\hline
\end{tabular}

${ }^{a}$ EPA Region 9 Soil Preliminary Remediation Goals for Residential Land use October 2004.

${ }^{\mathrm{b}}$ Cleanup Levels And Risk Calculations (CLARC) database Soil Method B Unrestricted Land Use Table January 2006

(https://fortress.wa.gov/ecy/clarc/CLARCHome.aspx)

${ }^{c}$ Remedial Design Report/Remedial Action Work Plan for the 100 Area, (DOE-RL 1998a).

${ }^{d}$ Hexavalent chromium was not analyzed in sediment samples collected, therefore all total chromium was assumed to be in the hexavalent form.

${ }^{\mathrm{e}}$ CLARC database Soil Method A Limit Table January 2006 (https://fortress.wa.gov/ecy/clarc/CLARCHome.aspx

$\mathrm{BHC}=$ Hexachlorocyclohexane

DDD = dichlorodiphenyldichloroethane

DDE = dichlorodiphenyldichloroethylene

DDT = dichlorodiphenyltrichloroethane

EPA $=$ U.S. Environmental Protection Agency 


\begin{tabular}{|c|c|c|c|c|c|c|c|}
\hline Analyte & Units & $\begin{array}{l}\text { Consensus- } \\
\text { based TEC }^{\mathrm{a}}\end{array}$ & $\begin{array}{l}\text { Source of } \\
\text { Consensus- } \\
\text { based TEC }\end{array}$ & $\begin{array}{l}\text { Washington } \\
\text { Sediment } \\
\text { Values }^{\mathbf{b}}\end{array}$ & $\begin{array}{c}\text { Source of } \\
\text { Washington } \\
\text { Sediment } \\
\text { Values }\end{array}$ & $\begin{array}{l}\text { Oregon } \\
\text { Sediment } \\
\text { Biota } \\
\text { Screening } \\
\text { Value }^{c}\end{array}$ & $\begin{array}{l}\text { Sediment Biota } \\
\text { Concentration } \\
\text { Guides (BCG) from } \\
\text { RESRAD Biota } \\
\text { Version } 1.0^{\mathrm{d}}\end{array}$ \\
\hline \multicolumn{8}{|l|}{ Metals } \\
\hline Antimony & $\mathrm{mg} / \mathrm{kg}$ & 2.0 & NOAA & 3.0 & LAET & 3.0 & -- \\
\hline Arsenic & $\mathrm{mg} / \mathrm{kg}$ & 9.8 & CBSQG & 57 & SMS & 6.0 & -- \\
\hline Cadmium & $\mathrm{mg} / \mathrm{kg}$ & 0.99 & CBSQG & 5.1 & SMS & 0.6 & -- \\
\hline Chromium & $\mathrm{mg} / \mathrm{kg}$ & 43 & CBSQG & 260 & SMS & 37 & -- \\
\hline Copper & $\mathrm{mg} / \mathrm{kg}$ & 32 & CBSQG & 390 & SMS & 36 & -- \\
\hline Iron & $\mathrm{mg} / \mathrm{kg}$ & 20,000 & Ontario & -- & -- & -- & -- \\
\hline Lead & $\mathrm{mg} / \mathrm{kg}$ & 36 & CBSQG & 450 & SMS & 35 & -- \\
\hline Manganese & $\mathrm{mg} / \mathrm{kg}$ & 460 & Ontario & 1,800 & LAET & 1,100 & -- \\
\hline Mercury & $\mathrm{mg} / \mathrm{kg}$ & 0.18 & CBSQG & 0.41 & SMS & 0.2 & -- \\
\hline Nickel & $\mathrm{mg} / \mathrm{kg}$ & 23 & CBSQG & 46 & LAET & 18 & -- \\
\hline Silver & $\mathrm{mg} / \mathrm{kg}$ & -- & -- & 6.1 & LAET & 4.5 & -- \\
\hline Zinc & $\mathrm{mg} / \mathrm{kg}$ & 120 & CBSQG & 410 & SMS & 120 & -- \\
\hline \multicolumn{8}{|c|}{ Polychlorinated Biphenyls } \\
\hline Aroclor-1016 & $\mathrm{mg} / \mathrm{kg}$ & 0.060 & -- & 0.021 & FSQV & -- & -- \\
\hline Aroclor-1221 & $\mathrm{mg} / \mathrm{kg}$ & 0.060 & -- & 0.021 & FSQV & -- & -- \\
\hline Aroclor-1232 & $\mathrm{mg} / \mathrm{kg}$ & 0.060 & -- & 0.021 & FSQV & -- & -- \\
\hline Aroclor-1242 & $\mathrm{mg} / \mathrm{kg}$ & 0.060 & -- & 0.021 & FSQV & -- & -- \\
\hline Aroclor-1248 & $\mathrm{mg} / \mathrm{kg}$ & 0.060 & -- & 0.021 & FSQV & 0.021 & -- \\
\hline Aroclor-1254 & $\mathrm{mg} / \mathrm{kg}$ & 0.060 & -- & 0.0073 & FSQV & 0.0070 & -- \\
\hline Aroclor-1260 & $\mathrm{mg} / \mathrm{kg}$ & 0.060 & -- & 0.021 & FSQV & -- & -- \\
\hline
\end{tabular}




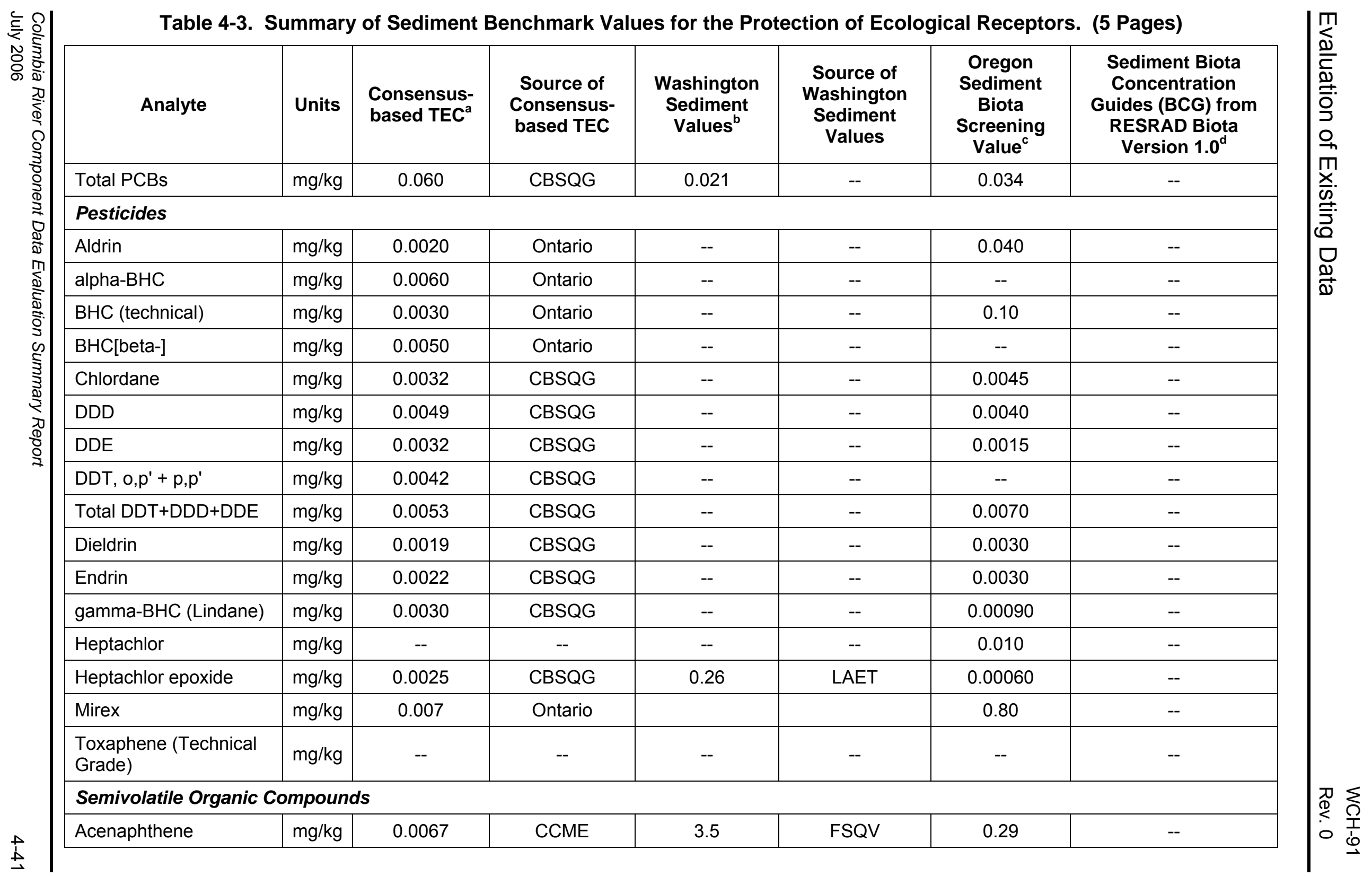




\begin{tabular}{|c|c|c|c|c|c|c|c|}
\hline Analyte & Units & $\begin{array}{l}\text { Consensus- } \\
\text { based TEC }^{a}\end{array}$ & $\begin{array}{c}\text { Source of } \\
\text { Consensus- } \\
\text { based TEC }\end{array}$ & $\begin{array}{l}\text { Washington } \\
\text { Sediment } \\
\text { Values }^{b}\end{array}$ & $\begin{array}{c}\text { Source of } \\
\text { Washington } \\
\text { Sediment } \\
\text { Values }\end{array}$ & $\begin{array}{l}\text { Oregon } \\
\text { Sediment } \\
\text { Biota } \\
\text { Screening } \\
\text { Value }^{c} \\
\end{array}$ & $\begin{array}{l}\text { Sediment Biota } \\
\text { Concentration } \\
\text { Guides (BCG) from } \\
\text { RESRAD Biota } \\
\text { Version } 1.0^{\mathrm{d}}\end{array}$ \\
\hline Acenaphthylene & $\mathrm{mg} / \mathrm{kg}$ & 0.0059 & CCME & 1.9 & FSQV & 0.16 & -- \\
\hline Anthracene & $\mathrm{mg} / \mathrm{kg}$ & 0.057 & CBSQG & 2.1 & FSQV & 0.057 & -- \\
\hline Benzo(a)anthracene & $\mathrm{mg} / \mathrm{kg}$ & 0.11 & CBSQG & 5.0 & FSQV & 0.032 & -- \\
\hline Benzo(a)pyrene & $\mathrm{mg} / \mathrm{kg}$ & 0.15 & CBSQG & 7.0 & FSQV & 0.032 & -- \\
\hline Benzo(b)fluoranthene & $\mathrm{mg} / \mathrm{kg}$ & 0.24 & Ontario & -- & -- & -- & -- \\
\hline Benzo(e)pyrene & $\mathrm{mg} / \mathrm{kg}$ & 0.15 & CBSQG & -- & -- & -- & -- \\
\hline Benzo(g,h,i)perylene & $\mathrm{mg} / \mathrm{kg}$ & 0.17 & Ontario & 1.2 & FSQV & 0.3 & -- \\
\hline Benzo(k)fluoranthene & $\mathrm{mg} / \mathrm{kg}$ & 0.24 & Ontario & -- & -- & 0.027 & -- \\
\hline $\begin{array}{l}\text { Bis(2- } \\
\text { ethylhexyl)phthalate } \\
\text { (DEHP) }\end{array}$ & $\mathrm{mg} / \mathrm{kg}$ & -- & -- & 0.64 & FSQV & 0.75 & -- \\
\hline Carbazole & $\mathrm{mg} / \mathrm{kg}$ & -- & -- & 0.14 & FSQV & 0.14 & -- \\
\hline Chrysene & $\mathrm{mg} / \mathrm{kg}$ & 0.17 & CBSQG & 7.4 & FSQV & 0.057 & -- \\
\hline Dibenz(a,h)anthracene & $\mathrm{mg} / \mathrm{kg}$ & 0.033 & CBSQG & 0.23 & FSQV & 0.033 & -- \\
\hline Dibenzofuran & $\mathrm{mg} / \mathrm{kg}$ & -- & -- & 32 & LAET & 5.1 & -- \\
\hline Di-n-Butyl Phthalate & $\mathrm{mg} / \mathrm{kg}$ & -- & -- & 0.043 & LAET & 0.11 & -- \\
\hline Fluoranthene & $\mathrm{mg} / \mathrm{kg}$ & 0.42 & CBSQG & 11 & FSQV & 0.11 & -- \\
\hline Fluorene & $\mathrm{mg} / \mathrm{kg}$ & 0.077 & CBSQG & 3.6 & FSQV & 0.077 & -- \\
\hline Hexachlorobenzene & $\mathrm{mg} / \mathrm{kg}$ & -- & -- & -- & -- & 0.1 & -- \\
\hline Indeno(1,2,3-cd)pyrene & $\mathrm{mg} / \mathrm{kg}$ & 0.2 & CBSQG & 0.73 & FSQV & 0.017 & -- \\
\hline Methylnaphthalene[2-] & $\mathrm{mg} / \mathrm{kg}$ & 0.02 & CCME & -- & -- & -- & -- \\
\hline Naphthalene & $\mathrm{mg} / \mathrm{kg}$ & 0.18 & CBSQG & 37 & FSQV & 0.18 & -- \\
\hline
\end{tabular}




\begin{tabular}{|c|c|c|c|c|c|c|c|}
\hline Analyte & Units & $\begin{array}{l}\text { Consensus- } \\
\text { based TEC }^{a}\end{array}$ & $\begin{array}{c}\text { Source of } \\
\text { Consensus- } \\
\text { based TEC }\end{array}$ & $\begin{array}{l}\text { Washington } \\
\text { Sediment } \\
\text { Values }^{b}\end{array}$ & $\begin{array}{l}\text { Source of } \\
\text { Washington } \\
\text { Sediment } \\
\text { Values }\end{array}$ & $\begin{array}{l}\text { Oregon } \\
\text { Sediment } \\
\text { Biota } \\
\text { Screening } \\
\text { Value }^{c}\end{array}$ & $\begin{array}{l}\text { Sediment Biota } \\
\text { Concentration } \\
\text { Guides (BCG) from } \\
\text { RESRAD Biota } \\
\text { Version } 1.0^{\mathrm{d}}\end{array}$ \\
\hline Phenanthrene & $\mathrm{mg} / \mathrm{kg}$ & 0.20 & CBSQG & 5.7 & FSQV & 0.042 & -- \\
\hline Pyrene & $\mathrm{mg} / \mathrm{kg}$ & 0.20 & CBSQG & 9.6 & FSQV & 0.053 & -- \\
\hline PAH, Total LMW & $\mathrm{mg} / \mathrm{kg}$ & -- & -- & 27 & FSQV & 0.076 & -- \\
\hline PAH, Total HMW & $\mathrm{mg} / \mathrm{kg}$ & -- & -- & 36 & FSQV & 0.19 & -- \\
\hline Total PAHs & $\mathrm{mg} / \mathrm{kg}$ & 1.6 & CBSQG & 60 & -- & 1.6 & -- \\
\hline \multicolumn{8}{|l|}{ Radionuclides } \\
\hline Americium-241 & $\mathrm{pCi} / \mathrm{g}$ & -- & -- & -- & -- & -- & 5,200 \\
\hline Cesium-137 & $\mathrm{pCi} / \mathrm{g}$ & -- & -- & -- & -- & -- & 3,100 \\
\hline Cobalt-60 & $\mathrm{pCi} / \mathrm{g}$ & -- & -- & -- & -- & -- & 1,500 \\
\hline Europium-154 & $\mathrm{pCi} / \mathrm{g}$ & -- & -- & -- & -- & -- & 2,600 \\
\hline Europium-155 & $\mathrm{pCi} / \mathrm{g}$ & -- & -- & -- & -- & -- & 32,000 \\
\hline Nickel-63 & $\mathrm{pCi} / \mathrm{g}$ & -- & -- & -- & -- & -- & $\mathrm{Na}$ \\
\hline Plutonium-239 & $\mathrm{pCi} / \mathrm{g}$ & -- & -- & -- & -- & -- & 5,900 \\
\hline Radium-226 & $\mathrm{pCi} / \mathrm{g}$ & -- & -- & -- & -- & -- & 100 \\
\hline Radium-228 & $\mathrm{pCi} / \mathrm{g}$ & -- & -- & -- & -- & -- & 88 \\
\hline Strontium-90 & $\mathrm{pCi} / \mathrm{g}$ & -- & -- & -- & -- & -- & 5.8 \\
\hline Technicium-99 & $\mathrm{pCi} / \mathrm{g}$ & -- & -- & -- & -- & -- & 42,000 \\
\hline Thorium-232 & $\mathrm{pCi} / \mathrm{g}$ & -- & -- & -- & -- & -- & 1,300 \\
\hline Tritium & $\mathrm{pCi} / \mathrm{g}$ & -- & -- & -- & -- & -- & 370,000 \\
\hline Uranium-234 & $\mathrm{pCi} / \mathrm{g}$ & -- & -- & -- & -- & -- & 5,300 \\
\hline Uranium-235 & $\mathrm{pCi} / \mathrm{g}$ & -- & -- & -- & -- & -- & 3,700 \\
\hline
\end{tabular}




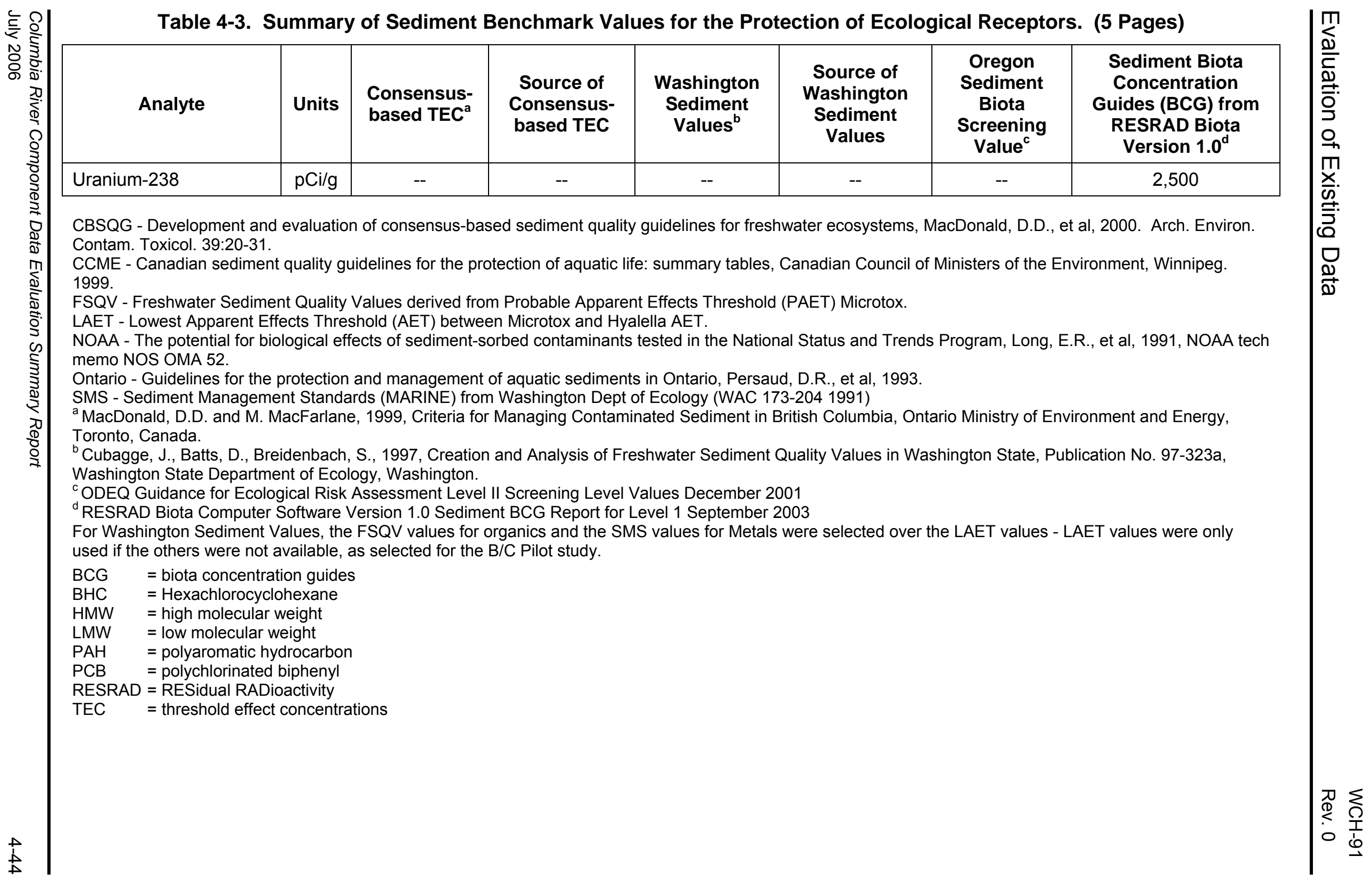


Table 4-4. Summary of Surface Water Benchmark Values for the Protection of Human Health. (4 Pages)

\begin{tabular}{|c|c|c|c|c|}
\hline Analyte & Units & $\begin{array}{c}\text { National } \\
\text { Recommended } \\
\text { Water Quality } \\
\text { Criteria - Water + } \\
\text { Organism }^{\mathrm{a}}\end{array}$ & $\begin{array}{c}\text { National } \\
\text { Recommended } \\
\text { Water Quality } \\
\text { Criteria - Organism } \\
\text { Only }^{\mathrm{a}}\end{array}$ & $\begin{array}{c}\text { Radionuclide } \\
\text { Drinking Water } \\
\text { MCLs }^{\mathbf{b}}\end{array}$ \\
\hline \multicolumn{5}{|l|}{ General Chemistry } \\
\hline Cyanide & $\mathrm{mg} / \mathrm{L}$ & 0.14 & 0.14 & -- \\
\hline Nitrate & $\mathrm{mg} / \mathrm{L}$ & 10 & -- & -- \\
\hline \multicolumn{5}{|l|}{ Metals } \\
\hline Antimony & $\mathrm{mg} / \mathrm{L}$ & 0.0056 & 0.64 & -- \\
\hline Arsenic & $\mathrm{mg} / \mathrm{L}$ & 0.000018 & $0.00014^{c}$ & -- \\
\hline Barium & $\mathrm{mg} / \mathrm{L}$ & 1.0 & -- & -- \\
\hline Iron & $\mathrm{mg} / \mathrm{L}$ & 0.3 & -- & -- \\
\hline Manganese & $\mathrm{mg} / \mathrm{L}$ & 0.050 & 0.1 & -- \\
\hline Nickel & $\mathrm{mg} / \mathrm{L}$ & 0.61 & 4.6 & -- \\
\hline Selenium & $\mathrm{mg} / \mathrm{L}$ & 0.17 & 4.2 & -- \\
\hline Thallium & $\mathrm{mg} / \mathrm{L}$ & 0.00024 & 0.00047 & -- \\
\hline Uranium & $\mathrm{mg} / \mathrm{L}$ & -- & & \\
\hline Zinc & $\mathrm{mg} / \mathrm{L}$ & 7.4 & 26 & -- \\
\hline \multicolumn{5}{|l|}{ Pesticides } \\
\hline Aldrin & $\mathrm{mg} / \mathrm{L}$ & 0.000000049 & 0.00000005 & -- \\
\hline Alpha-BHC & $\mathrm{mg} / \mathrm{L}$ & 0.0000026 & 0.0000049 & -- \\
\hline Beta-BHC & $\mathrm{mg} / \mathrm{L}$ & 0.0000091 & 0.000017 & -- \\
\hline Gamma-BHC & $\mathrm{mg} / \mathrm{L}$ & 0.00098 & .0018 & -- \\
\hline Chlordane & $\mathrm{mg} / \mathrm{L}$ & 0.0000008 & 0.00000081 & -- \\
\hline DDT & $\mathrm{mg} / \mathrm{L}$ & 0.00000022 & 0.00000022 & -- \\
\hline DDE & $\mathrm{mg} / \mathrm{L}$ & 0.00000022 & 0.00000022 & -- \\
\hline DDD & $\mathrm{mg} / \mathrm{L}$ & 0.00000031 & 0.00000031 & -- \\
\hline Dieldrin & $\mathrm{mg} / \mathrm{L}$ & 0.000000052 & 0.000000054 & -- \\
\hline Endosulfan & $\mathrm{mg} / \mathrm{L}$ & 0.062 & 0.089 & -- \\
\hline Endosulfan Sulfate & $\mathrm{mg} / \mathrm{L}$ & 0.062 & 0.089 & -- \\
\hline Endrin & $\mathrm{mg} / \mathrm{L}$ & 0.000059 & 0.00006 & -- \\
\hline Endrin Aldehyde & $\mathrm{mg} / \mathrm{L}$ & 0.00029 & 0.0003 & -- \\
\hline Heptachlor & $\mathrm{mg} / \mathrm{L}$ & 0.000000079 & 0.000000079 & -- \\
\hline
\end{tabular}


Table 4-4. Summary of Surface Water Benchmark Values for the Protection of Human Health. (4 Pages)

\begin{tabular}{|c|c|c|c|c|}
\hline Analyte & Units & $\begin{array}{c}\text { National } \\
\text { Recommended } \\
\text { Water Quality } \\
\text { Criteria - Water + } \\
\text { Organism }^{\mathrm{a}}\end{array}$ & $\begin{array}{c}\text { National } \\
\text { Recommended } \\
\text { Water Quality } \\
\text { Criteria - Organism } \\
\text { Only }^{\text {a }}\end{array}$ & $\begin{array}{c}\text { Radionuclide } \\
\text { Drinking Water } \\
\text { MCLs }^{\mathbf{b}}\end{array}$ \\
\hline Heptachlor Epoxide & $\mathrm{mg} / \mathrm{L}$ & 0.000000039 & 0.000000039 & -- \\
\hline $\begin{array}{l}\text { Hexachlorocyclohexane } \\
\text { (Lindane) }\end{array}$ & $\mathrm{mg} / \mathrm{L}$ & 0.000012 & 0.000041 & -- \\
\hline Toxaphene & $\mathrm{mg} / \mathrm{L}$ & 0.00000028 & 0.00000028 & -- \\
\hline \multicolumn{5}{|l|}{ Polychlorinated Biphenyls } \\
\hline $\begin{array}{l}\text { Polychlorinated biphenyls } \\
\text { (*PCBs) }\end{array}$ & $\mathrm{mg} / \mathrm{L}$ & 0.000000064 & 0.000000064 & -- \\
\hline \multicolumn{5}{|c|}{ Semivolatile Organic Compounds } \\
\hline 1,2-dichlorobenzene & $\mathrm{mg} / \mathrm{L}$ & 0.42 & 1.3 & -- \\
\hline 1,2-diphenyhydrazine & $\mathrm{mg} / \mathrm{L}$ & 0.000036 & 0.0002 & -- \\
\hline 1,2,4-trichlorobenzene & $\mathrm{mg} / \mathrm{L}$ & 0.035 & 0.070 & -- \\
\hline 1,2,4,5-tetrachlorobenzene & $\mathrm{mg} / \mathrm{L}$ & 0.00097 & .0011 & -- \\
\hline 1,3-dichlorobenzene & $\mathrm{mg} / \mathrm{L}$ & 0.32 & 0.96 & -- \\
\hline 1,4-Dichlorobenzene & $\mathrm{mg} / \mathrm{L}$ & 0.063 & 0.19 & -- \\
\hline 2-chloronaphthalene & $\mathrm{mg} / \mathrm{L}$ & 1.0 & 1.6 & -- \\
\hline 2-chlorophenol & $\mathrm{mg} / \mathrm{L}$ & 0.081 & 0.15 & -- \\
\hline 2-methyl-4,6-dinitrophenol & $\mathrm{mg} / \mathrm{L}$ & 0.013 & 0.28 & -- \\
\hline 2,4-dichlorphenol & $\mathrm{mg} / \mathrm{L}$ & 0.077 & 0.29 & -- \\
\hline 2,4-dimethylphenol & $\mathrm{mg} / \mathrm{L}$ & 0.38 & 0.85 & -- \\
\hline 2,4-dinitrophenol & $\mathrm{mg} / \mathrm{L}$ & 0.069 & 5.3 & -- \\
\hline 2,4-dinitrotoluene & $\mathrm{mg} / \mathrm{L}$ & 0.00011 & 0.0034 & -- \\
\hline 2,4,5-trichlorophenol & $\mathrm{mg} / \mathrm{L}$ & 1.8 & 3.6 & -- \\
\hline 2,4,6-trichlorophenol & $\mathrm{mg} / \mathrm{L}$ & 0.014 & 0.024 & -- \\
\hline 3,3-dichlorobenzidine & $\mathrm{mg} / \mathrm{L}$ & 0.000021 & 0.000028 & -- \\
\hline Acenaphthene & $\mathrm{mg} / \mathrm{L}$ & 0.67 & 0.99 & -- \\
\hline Anthracene & $\mathrm{mg} / \mathrm{L}$ & 8.3 & 40 & -- \\
\hline Benzidine & $\mathrm{mg} / \mathrm{L}$ & 0.000000086 & 0.0000002 & -- \\
\hline Benzo(a)anthracene & $\mathrm{mg} / \mathrm{L}$ & 0.0000038 & 0.000018 & -- \\
\hline Benzo(a)pyrene & $\mathrm{mg} / \mathrm{L}$ & 0.0000038 & 0.000018 & -- \\
\hline Benzo(b)fluoranthene & $\mathrm{mg} / \mathrm{L}$ & 0.0000038 & 0.0000018 & -- \\
\hline
\end{tabular}


Table 4-4. Summary of Surface Water Benchmark Values for the Protection of Human Health. (4 Pages)

\begin{tabular}{|c|c|c|c|c|}
\hline Analyte & Units & $\begin{array}{c}\text { National } \\
\text { Recommended } \\
\text { Water Quality } \\
\text { Criteria - Water + } \\
\text { Organism }^{\mathrm{a}}\end{array}$ & $\begin{array}{c}\text { National } \\
\text { Recommended } \\
\text { Water Quality } \\
\text { Criteria - Organism } \\
\text { Only }^{\mathrm{a}}\end{array}$ & $\begin{array}{c}\text { Radionuclide } \\
\text { Drinking Water } \\
\text { MCLs }^{\mathbf{b}}\end{array}$ \\
\hline Benzo(k)fluoranthene & $\mathrm{mg} / \mathrm{L}$ & 0.0000038 & 0.0000018 & -- \\
\hline Bis(2-chloroethyl) ether & $\mathrm{mg} / \mathrm{L}$ & 0.00003 & 0.00053 & -- \\
\hline Bis(2-chloroisopropyl) ether & $\mathrm{mg} / \mathrm{L}$ & 1.4 & 65 & -- \\
\hline Bis(2-ethylhexyl) phthalate & $\mathrm{mg} / \mathrm{L}$ & 0.0012 & 0.0022 & -- \\
\hline Butyl Benzyl Phthalate & $\mathrm{mg} / \mathrm{L}$ & 1.5 & 1.9 & -- \\
\hline Chrysene & $\mathrm{mg} / \mathrm{L}$ & 0.0000038 & 0.000018 & -- \\
\hline Dibenzo(a,h)anthracene & $\mathrm{mg} / \mathrm{L}$ & 0.0000038 & 0.000018 & -- \\
\hline Diethyl Phthalate & $\mathrm{mg} / \mathrm{L}$ & 17 & 44 & -- \\
\hline Dimethyl Phthalate & $\mathrm{mg} / \mathrm{L}$ & 270 & 1,100 & -- \\
\hline Di-n-butyl Phthalate & $\mathrm{mg} / \mathrm{L}$ & 2 & 4.5 & -- \\
\hline Fluoranthene & $\mathrm{mg} / \mathrm{L}$ & 0.13 & 0.14 & -- \\
\hline Fluorene & $\mathrm{mg} / \mathrm{L}$ & 1.1 & 5.3 & -- \\
\hline Hexachlorobenzene & $\mathrm{mg} / \mathrm{L}$ & 0.00000028 & 0.00000029 & -- \\
\hline Hexachlorobutadiene & $\mathrm{mg} / \mathrm{L}$ & 0.00044 & 0.018 & -- \\
\hline Hexachlorocyclopentadiene & $\mathrm{mg} / \mathrm{L}$ & 0.04 & 1.1 & -- \\
\hline Hexachloroethane & $\mathrm{mg} / \mathrm{L}$ & 0.0014 & 0.0033 & -- \\
\hline Indeno-(1,2,3-cd)pyrene & $\mathrm{mg} / \mathrm{L}$ & 0.0000038 & 0.000018 & -- \\
\hline Isophorone & $\mathrm{mg} / \mathrm{L}$ & 0.035 & 0.96 & -- \\
\hline Nitrobenzene & $\mathrm{mg} / \mathrm{L}$ & 0.017 & 0.69 & -- \\
\hline $\mathrm{N}$-nitrosodiethylamine & $\mathrm{mg} / \mathrm{L}$ & 0.00000069 & 0.003 & -- \\
\hline $\mathrm{N}$-nitrosodimethylamine & $\mathrm{mg} / \mathrm{L}$ & 0.0000008 & 0.00124 & -- \\
\hline N-nitrosodi-n-propylamine & $\mathrm{mg} / \mathrm{L}$ & 0.000005 & 0.00051 & -- \\
\hline $\mathrm{N}$-nitrosodiphenylamine & $\mathrm{mg} / \mathrm{L}$ & 0.0033 & 0.006 & -- \\
\hline Pentachlorobenzene & $\mathrm{mg} / \mathrm{L}$ & 0.0014 & 0.0015 & -- \\
\hline Pentachlorophenol & $\mathrm{mg} / \mathrm{L}$ & 0.00027 & 0.003 & -- \\
\hline Phenol & $\mathrm{mg} / \mathrm{L}$ & 21 & 1,700 & -- \\
\hline Pyrene & $\mathrm{mg} / \mathrm{L}$ & 0.83 & 4 & -- \\
\hline \multicolumn{5}{|l|}{ Radionuclides } \\
\hline Americium-241 & $\mathrm{pCi} / \mathrm{L}$ & -- & -- & 15 \\
\hline
\end{tabular}


Table 4-4. Summary of Surface Water Benchmark Values for the Protection of Human Health. (4 Pages)

\begin{tabular}{|c|c|c|c|c|}
\hline Analyte & Units & $\begin{array}{c}\text { National } \\
\text { Recommended } \\
\text { Water Quality } \\
\text { Criteria - Water + } \\
\text { Organism }^{\mathrm{a}}\end{array}$ & $\begin{array}{c}\text { National } \\
\text { Recommended } \\
\text { Water Quality } \\
\text { Criteria - Organism } \\
\text { Only }^{\text {a }}\end{array}$ & $\begin{array}{c}\text { Radionuclide } \\
\text { Drinking Water } \\
\text { MCLs }{ }^{\mathbf{b}}\end{array}$ \\
\hline Carbon-14 & $\mathrm{pCi} / \mathrm{L}$ & -- & -- & 2,000 \\
\hline Cesium-137 & $\mathrm{pCi} / \mathrm{L}$ & -- & -- & 200 \\
\hline Cobalt-60 & $\mathrm{pCi} / \mathrm{L}$ & -- & -- & 100 \\
\hline Europium-152 & $\mathrm{pCi} / \mathrm{L}$ & -- & -- & 200 \\
\hline Europium-154 & $\mathrm{pCi} / \mathrm{L}$ & -- & -- & 60 \\
\hline Europium-155 & $\mathrm{pCi} / \mathrm{L}$ & -- & -- & 600 \\
\hline Nickel-63 & $\mathrm{pCi} / \mathrm{L}$ & -- & -- & 50 \\
\hline Plutonium-238 & $\mathrm{pCi} / \mathrm{L}$ & -- & -- & 15 \\
\hline Plutonium-239/240 & $\mathrm{pCi} / \mathrm{L}$ & -- & -- & 15 \\
\hline Strontium-90 & $\mathrm{pCi} / \mathrm{L}$ & -- & -- & 8 \\
\hline Technicium-99 & $\mathrm{pCi} / \mathrm{L}$ & -- & -- & 900 \\
\hline Thorium-232 & $\mathrm{pCi} / \mathrm{L}$ & -- & -- & 15 \\
\hline Tritium & $\mathrm{pCi} / \mathrm{L}$ & -- & -- & 20,000 \\
\hline Uranium-234 & $\mathrm{pCi} / \mathrm{L}$ & -- & -- & 30 \\
\hline Uranium-235 & $\mathrm{pCi} / \mathrm{L}$ & -- & -- & 30 \\
\hline Uranium-238 & $\mathrm{pCi} / \mathrm{L}$ & -- & -- & 30 \\
\hline
\end{tabular}

a EPA National Recommended Water Quality Criteria Table Brochure, EPA/822-F-04-101, 2004.

${ }^{b}$ EPA Soil Screening Guidance for Radionuclides: Users Guide, Attachment D, Regulatory and Human Health Benchmarks Used for Radionuclides SSL Development, EPA/540-R-00-007, October 2000.

${ }^{\mathrm{c}}$ Refers to the inorganic form only.

$\mathrm{BHC}=$ hexachlorocyclohexane

DDD = dichlorodiphenyldichloroethane

$\mathrm{DDE}=$ dichlorodiphenyldichloroethylene

DDT = dichlorodiphenyltrichloroethane

EPA $=$ U.S. Environmental Protection Agency

$\mathrm{MCL}=$ maximum contaminant level 


\begin{tabular}{|c|c|c|c|c|c|c|c|}
\hline Analyte & Units & $\begin{array}{c}\text { Washington } \\
\text { Chronic Water } \\
\text { Quality } \\
\text { Standards }^{a}\end{array}$ & $\begin{array}{l}\text { Washington } \\
\text { Chronic Water } \\
\text { Quality } \\
\text { Standards } \\
\text { Notes }\end{array}$ & $\begin{array}{l}\text { Oregon } \\
\text { Aquatic } \\
\text { Biota } \\
\text { Screening } \\
\text { Value }^{b}\end{array}$ & $\begin{array}{c}\text { National } \\
\text { Ambient } \\
\text { Water Quality } \\
\text { Standards - } \\
\text { CCC }^{c}\end{array}$ & $\begin{array}{c}\text { National } \\
\text { Ambient } \\
\text { Water Quality } \\
\text { Standards - } \\
\text { Notes }\end{array}$ & $\begin{array}{c}\text { Water Biota } \\
\text { Concentration } \\
\text { Guides (BCG) from } \\
\text { RESRAD Biota } \\
\text { Version } 1.0^{\mathrm{d}}\end{array}$ \\
\hline \multicolumn{8}{|l|}{ General Chemistry } \\
\hline Ammonia & $\mathrm{mg} / \mathrm{L}$ & -- & -- & 0.017 & -- & -- & -- \\
\hline Chloride & $\mathrm{mg} / \mathrm{L}$ & 230 & -- & -- & 230 & -- & -- \\
\hline Cyanide & $\mathrm{mg} / \mathrm{L}$ & 0.0052 & -- & 0.0052 & 0.022 & -- & -- \\
\hline \multicolumn{8}{|l|}{ Metals } \\
\hline Aluminum & $\mathrm{mg} / \mathrm{L}$ & -- & -- & 0.087 & 0.087 & -- & -- \\
\hline Antimony & $\mathrm{mg} / \mathrm{L}$ & -- & -- & 1.6 & -- & -- & -- \\
\hline Arsenic & $\mathrm{mg} / \mathrm{L}$ & 0.19 & -- & 0.15 & 0.15 & -- & -- \\
\hline Barium & $\mathrm{mg} / \mathrm{L}$ & -- & -- & 0.004 & -- & -- & -- \\
\hline Beryllium & $\mathrm{mg} / \mathrm{L}$ & -- & -- & 0.0053 & -- & -- & -- \\
\hline Boron & $\mathrm{mg} / \mathrm{L}$ & -- & -- & 0.0016 & -- & -- & -- \\
\hline Cadmium & $\mathrm{mg} / \mathrm{L}$ & 0.00054 & $g$ & 0.0022 & 0.00025 & $g$ & -- \\
\hline Calcium & $\mathrm{mg} / \mathrm{L}$ & -- & -- & 120 & -- & -- & -- \\
\hline Hexavalent chromium & $\mathrm{mg} / \mathrm{L}$ & 0.001 & e & 0.011 & 0.011 & -- & -- \\
\hline Chromium & $\mathrm{mg} / \mathrm{L}$ & 0.01 & -- & 0.074 & 0.074 & -- & -- \\
\hline Cobalt & $\mathrm{mg} / \mathrm{L}$ & -- & -- & 0.023 & -- & -- & -- \\
\hline Copper & $\mathrm{mg} / \mathrm{L}$ & 0.0060 & g & 0.009 & 0.009 & g & -- \\
\hline Iron & $\mathrm{mg} / \mathrm{L}$ & -- & -- & 1 & 1 & -- & -- \\
\hline Lead & $\mathrm{mg} / \mathrm{L}$ & 0.00087 & g & 0.0025 & 0.0025 & g & -- \\
\hline Lithium & $\mathrm{mg} / \mathrm{L}$ & -- & -- & 0.014 & -- & -- & -- \\
\hline Magnesium & $\mathrm{mg} / \mathrm{L}$ & -- & -- & 82 & -- & -- & -- \\
\hline
\end{tabular}




\begin{tabular}{|c|c|c|c|c|c|c|c|}
\hline Analyte & Units & $\begin{array}{c}\text { Washington } \\
\text { Chronic Water } \\
\text { Quality } \\
\text { Standards }^{a}\end{array}$ & $\begin{array}{c}\text { Washington } \\
\text { Chronic Water } \\
\text { Quality } \\
\text { Standards } \\
\text { Notes }\end{array}$ & $\begin{array}{c}\text { Oregon } \\
\text { Aquatic } \\
\text { Biota } \\
\text { Screening } \\
\text { Value }^{b}\end{array}$ & $\begin{array}{c}\text { National } \\
\text { Ambient } \\
\text { Water Quality } \\
\text { Standards - } \\
\text { CCC }^{c}\end{array}$ & $\begin{array}{c}\text { National } \\
\text { Ambient } \\
\text { Water Quality } \\
\text { Standards - } \\
\text { Notes }\end{array}$ & $\begin{array}{c}\text { Water Biota } \\
\text { Concentration } \\
\text { Guides (BCG) from } \\
\text { RESRAD Biota } \\
\text { Version } 1.0^{\mathrm{d}}\end{array}$ \\
\hline Manganese & $\mathrm{mg} / \mathrm{L}$ & -- & -- & 0.12 & -- & -- & -- \\
\hline Mercury & $\mathrm{mg} / \mathrm{L}$ & 0.000012 & $\mathrm{~h}$ & 0.00077 & 0.00077 & -- & -- \\
\hline Molybdenum & $\mathrm{mg} / \mathrm{L}$ & -- & -- & 0.37 & -- & -- & -- \\
\hline Nickel & $\mathrm{mg} / \mathrm{L}$ & 0.083 & $g$ & 0.052 & 0.028 & $g$ & -- \\
\hline Potassium & $\mathrm{mg} / \mathrm{L}$ & -- & -- & 53 & -- & -- & -- \\
\hline Selenium & $\mathrm{mg} / \mathrm{L}$ & 0.005 & $\mathrm{~h}$ & 0.005 & 0.005 & -- & -- \\
\hline Silver & $\mathrm{mg} / \mathrm{L}$ & -- & $f$ & 0.00012 & -- & -- & -- \\
\hline Sodium & $\mathrm{mg} / \mathrm{L}$ & -- & -- & 680 & -- & -- & -- \\
\hline Strontium & $\mathrm{mg} / \mathrm{L}$ & -- & -- & 1.5 & -- & -- & -- \\
\hline Thallium & $\mathrm{mg} / \mathrm{L}$ & -- & -- & 0.04 & -- & -- & -- \\
\hline Tin & $\mathrm{mg} / \mathrm{L}$ & -- & -- & 0.073 & -- & -- & -- \\
\hline Uranium & $\mathrm{mg} / \mathrm{L}$ & -- & -- & 0.0026 & -- & -- & -- \\
\hline Vanadium & $\mathrm{mg} / \mathrm{L}$ & -- & -- & 0.02 & -- & -- & -- \\
\hline Zinc & $\mathrm{mg} / \mathrm{L}$ & 0.055 & g & 0.12 & 0.062 & g & -- \\
\hline Zirconium & $\mathrm{mg} / \mathrm{L}$ & -- & -- & 0.017 & 0.017 & -- & -- \\
\hline \multicolumn{8}{|l|}{ Pesticides } \\
\hline Methoxychlor & $\mathrm{mg} / \mathrm{L}$ & -- & -- & 0.00003 & 0.00003 & -- & -- \\
\hline Aldrin & $\mathrm{mg} / \mathrm{L}$ & 0.0000019 & -- & 0.00006 & -- & -- & -- \\
\hline Alpha-BHC & $\mathrm{mg} / \mathrm{L}$ & -- & -- & 0.0022 & -- & -- & -- \\
\hline Beta-BHC & $\mathrm{mg} / \mathrm{L}$ & -- & -- & 0.0022 & -- & -- & -- \\
\hline Gamma-BHC & $\mathrm{mg} / \mathrm{L}$ & 0.00008 & -- & 0.00008 & -- & -- & -- \\
\hline
\end{tabular}




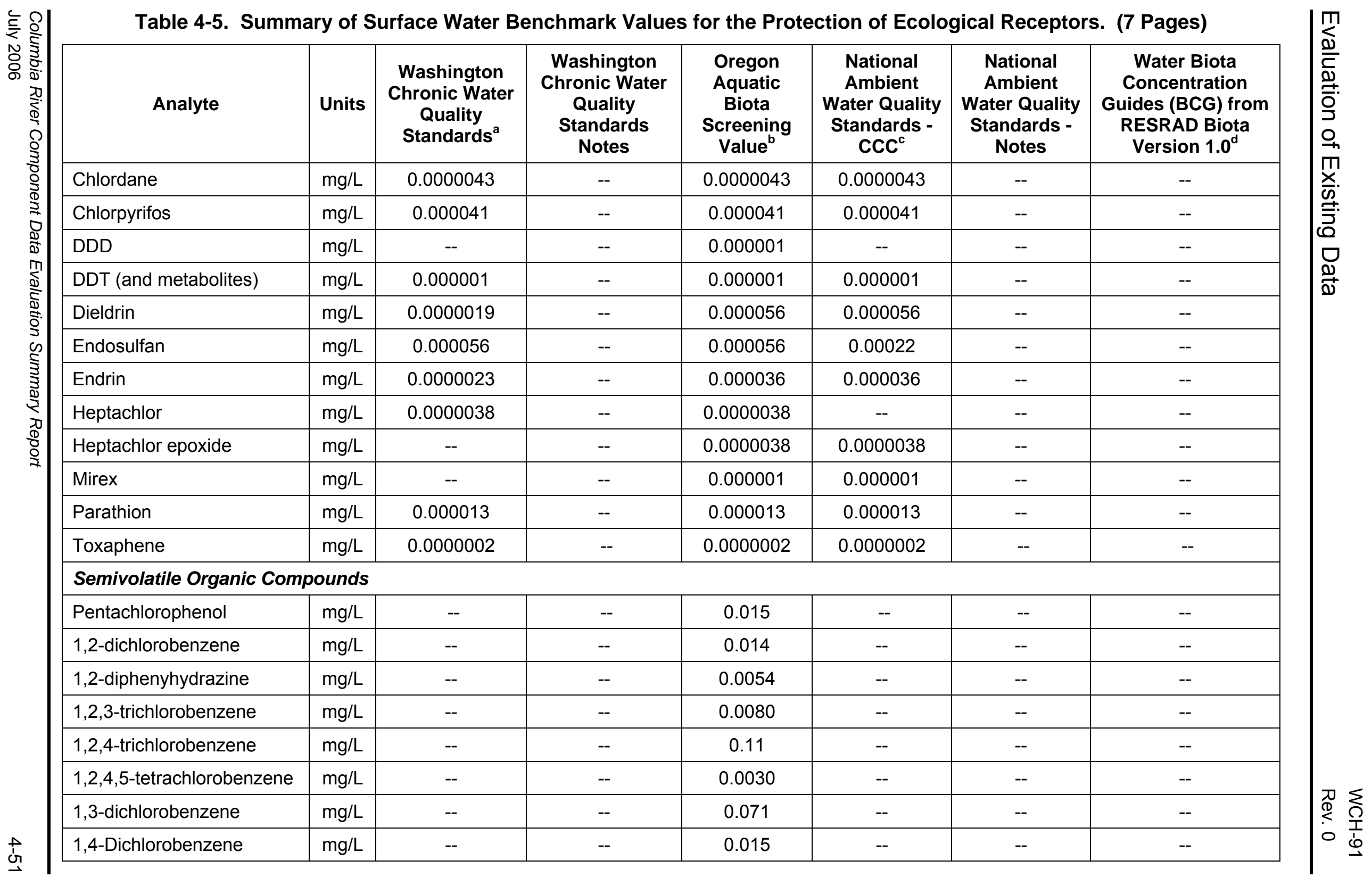




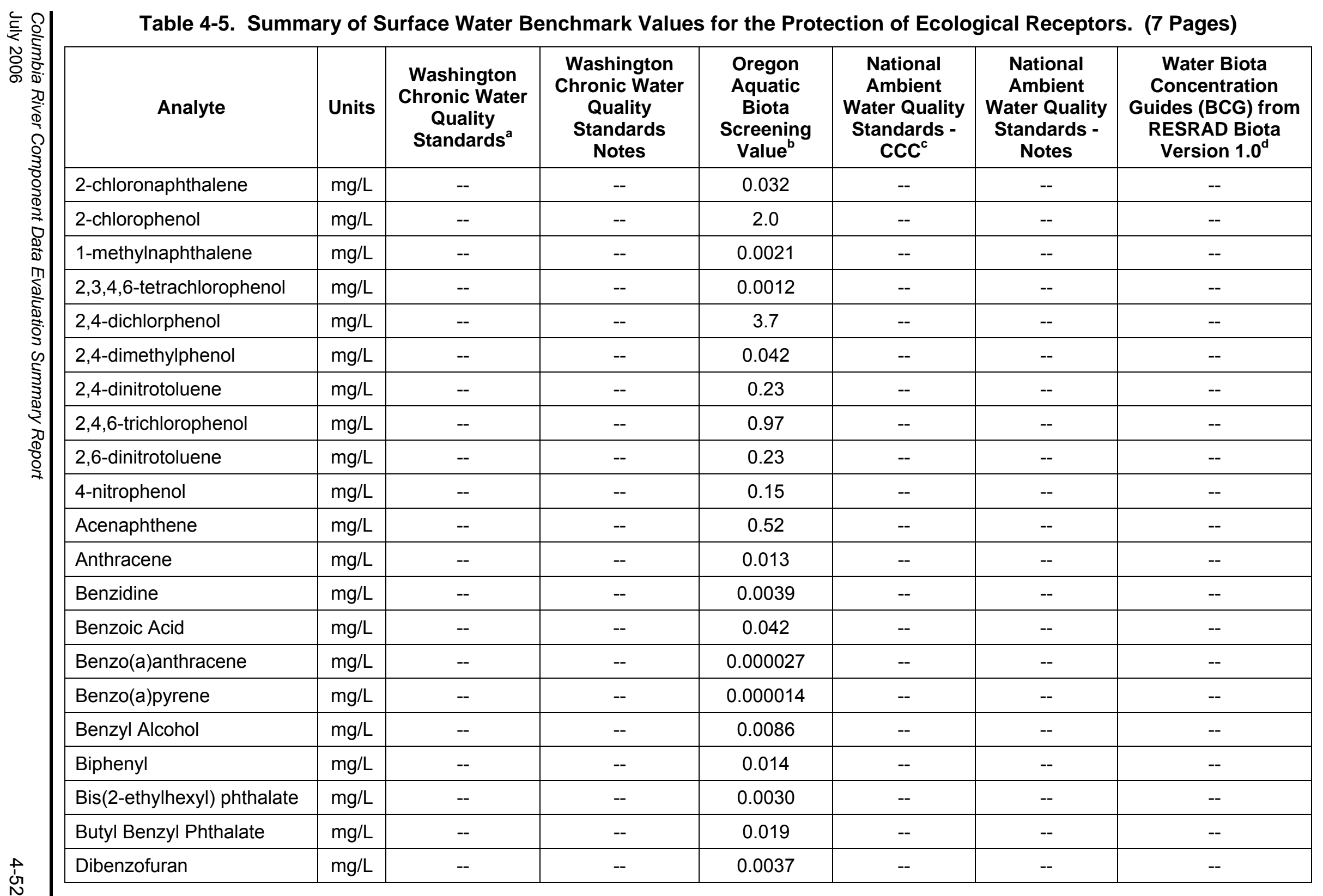




\begin{tabular}{|c|c|c|c|c|c|c|c|}
\hline Analyte & Units & $\begin{array}{c}\text { Washington } \\
\text { Chronic Water } \\
\text { Quality } \\
\text { Standards }^{\mathrm{a}}\end{array}$ & $\begin{array}{l}\text { Washington } \\
\text { Chronic Water } \\
\text { Quality } \\
\text { Standards } \\
\text { Notes }\end{array}$ & $\begin{array}{c}\text { Oregon } \\
\text { Aquatic } \\
\text { Biota } \\
\text { Screening } \\
\text { Value }^{b}\end{array}$ & $\begin{array}{c}\text { National } \\
\text { Ambient } \\
\text { Water Quality } \\
\text { Standards - } \\
\text { CCC }^{c}\end{array}$ & $\begin{array}{c}\text { National } \\
\text { Ambient } \\
\text { Water Quality } \\
\text { Standards - } \\
\text { Notes }\end{array}$ & $\begin{array}{l}\text { Water Biota } \\
\text { Concentration } \\
\text { Guides (BCG) from } \\
\text { RESRAD Biota } \\
\text { Version } 1.0^{\mathrm{d}}\end{array}$ \\
\hline Diethyl Phthalate & $\mathrm{mg} / \mathrm{L}$ & -- & -- & 0.21 & -- & -- & -- \\
\hline Dimethyl Phthalate & $\mathrm{mg} / \mathrm{L}$ & -- & -- & 0.0030 & -- & -- & -- \\
\hline Di-n-butyl Phthalate & $\mathrm{mg} / \mathrm{L}$ & -- & -- & 0.035 & -- & -- & -- \\
\hline Di-n-octyl Phthalate & $\mathrm{mg} / \mathrm{L}$ & -- & -- & 0.71 & -- & -- & -- \\
\hline Fluoranthene & $\mathrm{mg} / \mathrm{L}$ & -- & -- & 0.0062 & -- & -- & -- \\
\hline Fluorene & $\mathrm{mg} / \mathrm{L}$ & -- & -- & 0.0039 & -- & -- & -- \\
\hline Hexachlorobutadiene & $\mathrm{mg} / \mathrm{L}$ & -- & -- & 0.0093 & -- & -- & -- \\
\hline Hexachlorocyclopentadiene & $\mathrm{mg} / \mathrm{L}$ & -- & -- & 0.0052 & -- & -- & -- \\
\hline Hexachloroethane & $\mathrm{mg} / \mathrm{L}$ & -- & -- & 0.54 & -- & -- & -- \\
\hline Isophorone & $\mathrm{mg} / \mathrm{L}$ & -- & -- & 2.3 & -- & -- & -- \\
\hline Naphthalene & $\mathrm{mg} / \mathrm{L}$ & -- & -- & 0.62 & -- & -- & -- \\
\hline Nitrobenzene & $\mathrm{mg} / \mathrm{L}$ & -- & -- & 0.54 & - & -- & -- \\
\hline $\mathrm{N}$-nitrosodiethylamine & $\mathrm{mg} / \mathrm{L}$ & -- & -- & 0.12 & -- & -- & -- \\
\hline N-nitrosodimethylamine & $\mathrm{mg} / \mathrm{L}$ & -- & -- & 0.12 & -- & -- & -- \\
\hline N-nitrosodi-n-butylamine & $\mathrm{mg} / \mathrm{L}$ & -- & -- & 0.12 & -- & -- & -- \\
\hline N-nitrosodi-n-propylamine & $\mathrm{mg} / \mathrm{L}$ & -- & -- & 0.12 & -- & -- & -- \\
\hline N-nitrosodiphenylamine & $\mathrm{mg} / \mathrm{L}$ & -- & -- & 0.21 & - & -- & -- \\
\hline N-nitrosomethyl-ethylamine & $\mathrm{mg} / \mathrm{L}$ & -- & -- & 0.12 & -- & -- & -- \\
\hline O-cresol & $\mathrm{mg} / \mathrm{L}$ & -- & -- & 0.013 & -- & -- & -- \\
\hline Pentachlorobenzene & $\mathrm{mg} / \mathrm{L}$ & -- & -- & 0.00047 & -- & -- & -- \\
\hline Pentachlorophenol & $\mathrm{mg} / \mathrm{L}$ & -- & -- & 0.015 & 0.015 & -- & -- \\
\hline
\end{tabular}




\begin{tabular}{|c|c|c|c|c|c|c|c|}
\hline Analyte & Units & $\begin{array}{c}\text { Washington } \\
\text { Chronic Water } \\
\text { Quality } \\
\text { Standards }^{a}\end{array}$ & $\begin{array}{c}\text { Washington } \\
\text { Chronic Water } \\
\text { Quality } \\
\text { Standards } \\
\text { Notes }\end{array}$ & $\begin{array}{l}\text { Oregon } \\
\text { Aquatic } \\
\text { Biota } \\
\text { Screening } \\
\text { Value }^{b}\end{array}$ & $\begin{array}{c}\text { National } \\
\text { Ambient } \\
\text { Water Quality } \\
\text { Standards - } \\
\text { CCC }^{c}\end{array}$ & $\begin{array}{c}\text { National } \\
\text { Ambient } \\
\text { Water Quality } \\
\text { Standards - } \\
\text { Notes }\end{array}$ & $\begin{array}{c}\text { Water Biota } \\
\text { Concentration } \\
\text { Guides (BCG) from } \\
\text { RESRAD Biota } \\
\text { Version } 1.0^{\mathrm{d}}\end{array}$ \\
\hline Phenanthrene & $\mathrm{mg} / \mathrm{L}$ & -- & -- & 0.0063 & -- & -- & -- \\
\hline Phenol & $\mathrm{mg} / \mathrm{L}$ & -- & -- & 0.11 & -- & -- & -- \\
\hline \multicolumn{8}{|l|}{ Polychlorinated Biphenyls } \\
\hline Polychlorinated biphenyls * & $\mathrm{mg} / \mathrm{L}$ & 0.000014 & -- & 0.000014 & 0.000014 & -- & -- \\
\hline Aroclor-1221 & $\mathrm{mg} / \mathrm{L}$ & -- & -- & 0.00028 & -- & -- & -- \\
\hline Aroclor-1232 & $\mathrm{mg} / \mathrm{L}$ & -- & -- & 0.00058 & -- & -- & -- \\
\hline Aroclor-1242 & $\mathrm{mg} / \mathrm{L}$ & - & -- & 0.000053 & -- & -- & -- \\
\hline Aroclor-1248 & $\mathrm{mg} / \mathrm{L}$ & -- & -- & 0.000081 & -- & -- & -- \\
\hline Aroclor-1254 & $\mathrm{mg} / \mathrm{L}$ & -- & -- & 0.000033 & -- & -- & -- \\
\hline Aroclor-1260 & $\mathrm{mg} / \mathrm{L}$ & -- & -- & 0.094 & -- & -- & -- \\
\hline \multicolumn{8}{|l|}{ Radionuclides } \\
\hline Americium-241 & $\mathrm{pCi} / \mathrm{L}$ & -- & -- & -- & -- & -- & 440 \\
\hline Cesium-137 & $\mathrm{pCi} / \mathrm{L}$ & -- & -- & -- & -- & -- & 43 \\
\hline Cobalt-60 & $\mathrm{pCi} / \mathrm{L}$ & -- & -- & -- & -- & -- & 3800 \\
\hline Europium-154 & $\mathrm{pCi} / \mathrm{L}$ & -- & -- & -- & -- & -- & 22,000 \\
\hline Europium-155 & $\mathrm{pCi} / \mathrm{L}$ & -- & -- & -- & -- & -- & 260,000 \\
\hline Nickel-63 & $\mathrm{pCi} / \mathrm{L}$ & -- & -- & -- & -- & -- & -- \\
\hline Plutonium-239 & $\mathrm{pCi} / \mathrm{L}$ & -- & -- & -- & -- & -- & 190 \\
\hline Radium-226 & $\mathrm{pCi} / \mathrm{L}$ & -- & -- & -- & -- & -- & 4.0 \\
\hline Radium-228 & $\mathrm{pCi} / \mathrm{L}$ & -- & -- & -- & -- & -- & 3.4 \\
\hline Strontium-90 & $\mathrm{pCi} / \mathrm{L}$ & -- & -- & -- & -- & -- & 280 \\
\hline
\end{tabular}




\begin{tabular}{|c|c|c|c|c|c|c|c|}
\hline Analyte & Units & $\begin{array}{c}\text { Washington } \\
\text { Chronic Water } \\
\text { Quality } \\
\text { Standards }^{\mathrm{a}}\end{array}$ & $\begin{array}{l}\text { Washington } \\
\text { Chronic Water } \\
\text { Quality } \\
\text { Standards } \\
\text { Notes }\end{array}$ & $\begin{array}{c}\text { Oregon } \\
\text { Aquatic } \\
\text { Biota } \\
\text { Screening } \\
\text { Value }^{b}\end{array}$ & $\begin{array}{c}\text { National } \\
\text { Ambient } \\
\text { Water Quality } \\
\text { Standards - } \\
\text { CCC }^{c}\end{array}$ & $\begin{array}{c}\text { National } \\
\text { Ambient } \\
\text { Water Quality } \\
\text { Standards - } \\
\text { Notes }\end{array}$ & $\begin{array}{l}\text { Water Biota } \\
\text { Concentration } \\
\text { Guides (BCG) from } \\
\text { RESRAD Biota } \\
\text { Version } 1.0^{\mathrm{d}}\end{array}$ \\
\hline Technicium-99 & $\mathrm{pCi} / \mathrm{L}$ & -- & -- & -- & -- & -- & 670,000 \\
\hline Thorium-232 & $\mathrm{pCi} / \mathrm{L}$ & -- & -- & -- & -- & -- & 300 \\
\hline Tritium & $\mathrm{pCi} / \mathrm{L}$ & -- & -- & -- & -- & -- & $270,000,000$ \\
\hline Uranium-234 & $\mathrm{pCi} / \mathrm{L}$ & -- & -- & -- & -- & -- & 200 \\
\hline Uranium-235 & $\mathrm{pCi} / \mathrm{L}$ & -- & -- & -- & -- & -- & 220 \\
\hline Uranium-238 & $\mathrm{pCi} / \mathrm{L}$ & -- & -- & -- & -- & -- & 220 \\
\hline \multicolumn{8}{|c|}{$\begin{array}{l}\text { a USDOE "Water Quality Standards for Surface Waters" of } \\
\text { b ODEQ Guidance for Ecological Risk Assessment Level II } \\
\text { cEPA National Recommended Water Quality Criteria Table } \\
\text { d RESRAD Biota Computer Software Version 1.0 Aquatic } \\
\text { e Based on dissolved fraction. } \\
\text { f } \\
\text { Based on dissolved fraction / no chronic value. } \\
\text { g Based on dissolved fraction and lowest site-specific hardr } \\
\text { h Based on total-recoverable fraction of the metal. } \\
\text { BHC = hexachlorocyclohexane } \\
\text { CCC = criterion continuous concentration } \\
\text { EPA = U.S. Environmental Protection Agency } \\
\text { RESRAD = RESidual RADioactivity } \\
\text { WAC = Washington Administrative Code }\end{array}$} \\
\hline
\end{tabular}


Table 4-6. Summary of Segment 1 Sediment Benchmark Exceedances for All Categories of Data.

\begin{tabular}{|c|c|c|c|c|c|c|c|c|c|c|c|c|c|}
\hline Constituent Name & Units & $\begin{array}{c}\text { Number } \\
\text { Analyzed }\end{array}$ & $\begin{array}{l}\text { Number } \\
\text { Detected }\end{array}$ & $\begin{array}{l}\text { Frequency of } \\
\text { Detection }\end{array}$ & $\underset{\text { Detect }}{\text { Maximum }}$ & $\begin{array}{l}\text { Background } \\
\text { Value }\end{array}$ & $\begin{array}{c}\text { Is Max > } \\
\text { Background? }\end{array}$ & $\begin{array}{c}\text { Ecological } \\
\text { Benchmark } \\
\text { Value }\end{array}$ & $\begin{array}{c}\text { Factor of } \\
\text { Exceedance }\end{array}$ & $\begin{array}{c}\# \text { of } \\
\text { Exceedances }\end{array}$ & $\begin{array}{c}\text { Human Health } \\
\text { Benchmark } \\
\text { Value }\end{array}$ & $\begin{array}{c}\text { Factor of } \\
\text { Exceedance }\end{array}$ & $\begin{array}{c}\# \text { of } \\
\text { Exceedances }\end{array}$ \\
\hline \multicolumn{14}{|l|}{ Metals } \\
\hline Arsenic & $\mathrm{mg} / \mathrm{kg}$ & 47 & 44 & $94 \%$ & 12 & 6.5 & Yes & 6.0 & 2.0 & 27 & 0.39 & 31 & 44 \\
\hline Arsenic & $\begin{array}{l}\mathrm{mg} / \mathrm{kg} \\
\mathrm{dry}-\mathrm{wt}\end{array}$ & 4 & 4 & $100 \%$ & 9.6 & 6.5 & Yes & 6.0 & 1.6 & 3 & 0.39 & 25 & 4 \\
\hline Cadmium & $\mathrm{mg} / \mathrm{kg}$ & 62 & 57 & $92 \%$ & 16 & 0.81 & Yes & 0.60 & 27 & 53 & 37 & -- & -- \\
\hline Cadmium & $\begin{array}{l}\mathrm{mg} / \mathrm{kg} \\
\mathrm{dry}-\mathrm{wt}\end{array}$ & 4 & 4 & $100 \%$ & 8.0 & 0.81 & Yes & 0.60 & 13 & 2 & 37 & 0.22 & -- \\
\hline Chromium & $\mathrm{mg} / \mathrm{kg}$ & 63 & 63 & $100 \%$ & 92 & 19 & Yes & 37 & 2.5 & 39 & 30 & 3.1 & 41 \\
\hline Chromium & $\begin{array}{l}\mathrm{mg} / \mathrm{kg} \\
\mathrm{dry}-\mathrm{wt}\end{array}$ & 4 & 4 & $100 \%$ & 93 & 19 & Yes & 37 & 2.5 & 3 & 30 & 3.1 & 3 \\
\hline Copper & $\mathrm{mg} / \mathrm{kg}$ & 63 & 63 & $100 \%$ & 70 & 22 & Yes & 32 & 2.2 & 23 & 2,960 & -- & -- \\
\hline Copper & $\begin{array}{l}\mathrm{mg} / \mathrm{kg} \\
\mathrm{dry}-\mathrm{wt}\end{array}$ & 4 & 4 & $100 \%$ & 59 & 22 & Yes & 32 & 1.9 & 2 & 2,960 & 0.020 & -- \\
\hline Iron & $\mathrm{mg} / \mathrm{kg}$ & 9 & 9 & $100 \%$ & 40,000 & 32,600 & Yes & 20,000 & 2.0 & 1 & 23,463 & 1.7 & 1 \\
\hline Lead & $\mathrm{mg} / \mathrm{kg}$ & 59 & 59 & $100 \%$ & 140 & 10 & Yes & 35 & 4.0 & 37 & 250 & -- & -- \\
\hline Lead & $\begin{array}{l}\mathrm{mg} / \mathrm{kg} \\
\mathrm{dry}-\mathrm{wt}\end{array}$ & 4 & 3 & $75 \%$ & 56 & 10 & Yes & 35 & 1.6 & 2 & 250 & 0.23 & -- \\
\hline Manganese & $\mathrm{mg} / \mathrm{kg}$ & 9 & 9 & $100 \%$ & 660 & 512 & Yes & 460 & 1.4 & 1 & 1,762 & -- & -- \\
\hline Mercury & $\mathrm{mg} / \mathrm{kg}$ & 47 & 37 & $71 \%$ & 0.31 & 0.33 & No & 0.18 & 1.7 & 12 & 23 & -- & -- \\
\hline Nickel & $\mathrm{mg} / \mathrm{kg}$ & 33 & 33 & $100 \%$ & 53 & 19 & Yes & 18 & 2.9 & 14 & 1,564 & -- & -- \\
\hline Nickel & $\begin{array}{l}\mathrm{mg} / \mathrm{kg} \\
\mathrm{dry}-\mathrm{wt}\end{array}$ & 3 & 3 & $100 \%$ & 46 & 19 & Yes & 18 & 2.6 & 3 & 1,564 & 0.030 & - \\
\hline Vanadium & $\mathrm{mg} / \mathrm{kg}$ & 9 & 9 & $100 \%$ & 130 & 85 & Yes & -- & -- & -- & 78 & 1.7 & 1 \\
\hline Zinc & $\mathrm{mg} / \mathrm{kg}$ & 63 & 63 & $100 \%$ & 850 & 68 & Yes & 120 & 7.1 & 49 & 23,463 & -- & -- \\
\hline Zinc & $\begin{array}{l}\mathrm{mg} / \mathrm{kg} \\
\mathrm{dry}-\mathrm{wt}\end{array}$ & 4 & 4 & $100 \%$ & 616 & 68 & Yes & 120 & 5.1 & 3 & 23,463 & 0.026 & -- \\
\hline \multicolumn{14}{|l|}{ Pesticides } \\
\hline Dichlorodiphenyldichloroethylene & $\begin{array}{l}\mathrm{mg} / \mathrm{kg} \\
\mathrm{dry}-\mathrm{wt}\end{array}$ & 1 & 1 & $100 \%$ & 0.0070 & -- & -- & 0.0015 & 4.7 & 1 & 1.7 & 0.0041 & -- \\
\hline \multicolumn{14}{|l|}{ Radionuclides } \\
\hline Thorium-232 & $\mathrm{pCi} / \mathrm{g}$ & 3 & 3 & $100 \%$ & 2.5 & 1.3 & Yes & -- & -- & -- & 1.3 & 1.9 & 1 \\
\hline Thorium-232 & $\begin{array}{l}\mathrm{pCi} / \mathrm{g} \\
\text { dry-wt }\end{array}$ & 1 & 1 & $100 \%$ & 1.5 & 1.3 & Yes & 1,300 & 0.0012 & -- & 1.3 & 1.2 & 1 \\
\hline Uranium-234 & $\begin{array}{l}\text { pCi/g } \\
\text { dry-wt }\end{array}$ & 38 & 38 & $100 \%$ & 1.6 & 1.1 & Yes & 5,270 & 3.05E-04 & -- & 1.1 & 1.5 & 7 \\
\hline Uranium-238 & $\mathrm{pCi} / \mathrm{g}$ & 31 & 31 & $100 \%$ & 1.4 & 1.1 & Yes & -- & -- & -- & 1.1 & 1.2 & 1 \\
\hline Uranium-238 & $\begin{array}{l}\mathrm{pCi} / \mathrm{g} \\
\text { dry-wt }\end{array}$ & 38 & 38 & $100 \%$ & 1.3 & 3.2 & No & 2,490 & $5.26 \mathrm{E}-04$ & - & 1.1 & 1.2 & 4 \\
\hline
\end{tabular}


Table 4-7. Summary of Segment 1 Surface Water Benchmark Exceedances for All Categories of Data.

\begin{tabular}{|c|c|c|c|c|c|c|c|c|c|c|c|}
\hline $\begin{array}{c}\text { Constituent } \\
\text { Name }\end{array}$ & Units & $\begin{array}{c}\text { Number } \\
\text { Analyzed }\end{array}$ & $\begin{array}{c}\text { Number } \\
\text { Detected }\end{array}$ & $\begin{array}{l}\text { Frequency of } \\
\text { Detection }\end{array}$ & $\begin{array}{c}\text { Maximum } \\
\text { Detect }\end{array}$ & $\begin{array}{l}\text { Ecological Benchmark } \\
\text { Value }\end{array}$ & $\begin{array}{c}\text { Factor of } \\
\text { Exceedance }\end{array}$ & $\begin{array}{c}\text { \# of } \\
\text { Exceedances }\end{array}$ & $\begin{array}{c}\text { Human Health } \\
\text { Benchmark Value }\end{array}$ & $\begin{array}{c}\text { Factor of } \\
\text { Exceedance }\end{array}$ & $\begin{array}{c}\# \text { of } \\
\text { Exceedances }\end{array}$ \\
\hline Barium & $\mathrm{mg} / \mathrm{L}$ & 5 & 5 & $100 \%$ & 0.029 & 0.0040 & 7.4 & 5 & 1.0 & -- & -- \\
\hline Arsenic & $\mathrm{mg} / \mathrm{L}$ & 173 & 173 & $100 \%$ & 0.0017 & 0.15 & -- & -- & $1.80 \mathrm{E}-05$ & 96 & 173 \\
\hline
\end{tabular}

Table 4-8. Summary of Segment 2 Sediment Benchmark Exceedances for All Categories of Data. (2 Pages)

\begin{tabular}{|c|c|c|c|c|c|c|c|c|c|c|c|c|c|}
\hline $\begin{array}{l}\text { Constituent } \\
\text { Name }\end{array}$ & Units & $\begin{array}{c}\text { Number } \\
\text { Analyzed }\end{array}$ & $\begin{array}{l}\text { Number } \\
\text { Detected }\end{array}$ & $\begin{array}{l}\text { Frequency of } \\
\text { Detection }\end{array}$ & $\underset{\text { Detect }}{\text { Maximum }}$ & $\begin{array}{l}\text { Background } \\
\text { Value }\end{array}$ & $\begin{array}{c}\text { Is Max > } \\
\text { Background? }\end{array}$ & $\begin{array}{c}\text { Ecological } \\
\text { Benchmark } \\
\text { Value }\end{array}$ & $\begin{array}{c}\text { Factor of } \\
\text { Exceedance }\end{array}$ & $\begin{array}{c}\text { \# of } \\
\text { Exceedances }\end{array}$ & $\begin{array}{l}\text { Human Health } \\
\text { Benchmark Value }\end{array}$ & $\begin{array}{c}\text { Factor of } \\
\text { Exceedance }\end{array}$ & $\begin{array}{c}\text { \# of } \\
\text { Exceedances }\end{array}$ \\
\hline \multicolumn{14}{|l|}{ Metals } \\
\hline Antimony & $\mathrm{mg} / \mathrm{kg}$ & 99 & 61 & $62 \%$ & 6.8 & 5.0 & Yes & 2.0 & 3.4 & 3 & 31 & 0.22 & -- \\
\hline Arsenic & $\mathrm{mg} / \mathrm{kg}$ & 108 & 104 & $96 \%$ & 20 & 6.5 & Yes & 6.0 & 3.3 & 25 & 0.39 & 51 & 96 \\
\hline Arsenic & $\begin{array}{l}\mathrm{mg} / \mathrm{kg} \\
\mathrm{dry}-\mathrm{wt}\end{array}$ & 14 & 14 & $100 \%$ & 7.8 & 6.5 & Yes & 6.0 & 1.3 & 3 & 0.39 & 20 & 9 \\
\hline Cadmium & $\mathrm{mg} / \mathrm{kg}$ & 112 & 73 & $65 \%$ & 5.6 & 0.81 & Yes & 0.60 & 9.3 & 42 & 37 & 0.15 & -- \\
\hline Cadmium & $\begin{array}{l}\mathrm{mg} / \mathrm{kg} \\
\text { dry-wt }\end{array}$ & 18 & 18 & $100 \%$ & 1.6 & 0.81 & Yes & 0.60 & 2.7 & 7 & 37 & 0.043 & -- \\
\hline Chromium & $\mathrm{mg} / \mathrm{kg}$ & 120 & 119 & $99 \%$ & 112 & 19 & Yes & 37 & 3.0 & 48 & 30 & 3.7 & 50 \\
\hline Chromium & $\begin{array}{l}\mathrm{mg} / \mathrm{kg} \\
\mathrm{dry}-\mathrm{wt}\end{array}$ & 17 & 13 & $76 \%$ & 88 & 19 & Yes & 37 & 2.4 & 9 & 30 & 2.9 & 12 \\
\hline Copper & $\mathrm{mg} / \mathrm{kg}$ & 119 & 119 & $100 \%$ & 70 & 22 & Yes & 32 & 2.2 & 10 & 2,960 & 0.024 & -- \\
\hline Iron & $\mathrm{mg} / \mathrm{kg}$ & 45 & 45 & $100 \%$ & 31,100 & 32,600 & No & 20,000 & 1.6 & 2 & 23,463 & 1.3 & 1 \\
\hline Lead & $\mathrm{mg} / \mathrm{kg}$ & 108 & 108 & $100 \%$ & 76 & 10 & Yes & 35 & 2.2 & 24 & 250 & 0.30 & -- \\
\hline Lead & $\begin{array}{l}\mathrm{mg} / \mathrm{kg} \\
\text { dry-wt }\end{array}$ & 18 & 18 & $100 \%$ & 57 & 10 & Yes & 35 & 1.7 & 1 & 250 & 0.23 & -- \\
\hline Manganese & $\mathrm{mg} / \mathrm{kg}$ & 48 & 48 & $100 \%$ & 603 & 512 & Yes & 460 & 1.3 & 3 & 1,762 & 0.34 & -- \\
\hline Mercury & $\mathrm{mg} / \mathrm{kg}$ & 108 & 67 & $62 \%$ & 0.68 & 0.33 & Yes & 0.18 & 3.8 & 1 & 23 & 0.029 & -- \\
\hline Nickel & $\mathrm{mg} / \mathrm{kg}$ & 112 & 112 & $100 \%$ & 30 & 19 & Yes & 18 & 1.7 & 33 & 1,564 & 0.019 & -- \\
\hline Nickel & $\begin{array}{l}\mathrm{mg} / \mathrm{kg} \\
\text { dry-wt }\end{array}$ & 9 & 9 & $100 \%$ & 21 & 19 & Yes & 18 & 1.2 & 5 & 1,564 & 0.014 & -- \\
\hline Silver & $\mathrm{mg} / \mathrm{kg}$ & 99 & 58 & $59 \%$ & 5.3 & 0.73 & Yes & 4.5 & 1.2 & 1 & 391 & 0.014 & -- \\
\hline Vanadium & $\mathrm{mg} / \mathrm{kg}$ & 45 & 45 & $100 \%$ & 86 & 85 & Yes & -- & -- & -- & 78 & 1.1 & 1 \\
\hline Zinc & $\mathrm{mg} / \mathrm{kg}$ & 112 & 112 & $100 \%$ & 477 & 68 & Yes & 120 & 4.0 & 74 & 23,463 & 0.020 & -- \\
\hline Zinc & $\begin{array}{l}\mathrm{mg} / \mathrm{kg} \\
\mathrm{dry}-\mathrm{wt}\end{array}$ & 13 & 13 & $100 \%$ & 366 & 68 & Yes & 120 & 3.1 & 8 & 23,463 & 0.016 & -- \\
\hline \multicolumn{14}{|l|}{ Radionuclides } \\
\hline Cobalt-60 & $\mathrm{pCi} / \mathrm{g}$ & 40 & 40 & $100 \%$ & 2.4 & 0.0084 & Yes & 1,460 & 0.0017 & -- & 1.4 & 1.7 & 1 \\
\hline Thorium-232 & $\mathrm{pCi} / \mathrm{g}$ & 40 & 40 & $100 \%$ & 3.2 & 1.3 & Yes & 1,300 & 0.0025 & -- & 1.3 & 2.5 & 9 \\
\hline Thorium-232 & $\begin{array}{l}\text { pCi/g } \\
\text { dry-wt }\end{array}$ & 8 & 8 & $100 \%$ & 1.5 & 1.3 & Yes & 1,300 & 0.0012 & -- & 1.3 & 1.2 & 1 \\
\hline Uranium-234 & $\mathrm{pCi} / \mathrm{g}$ & 90 & 90 & $100 \%$ & 11 & 1.1 & Yes & 5,270 & 0.0021 & -- & 1.1 & 10 & 16 \\
\hline
\end{tabular}


Table 4-8. Summary of Segment 2 Sediment Benchmark Exceedances for All Categories of Data. (2 Pages)

\begin{tabular}{|c|c|c|c|c|c|c|c|c|c|c|c|c|c|}
\hline $\begin{array}{l}\text { Constituent } \\
\text { Name }\end{array}$ & Units & $\begin{array}{l}\text { Number } \\
\text { Analyzed }\end{array}$ & $\begin{array}{l}\text { Number } \\
\text { Detected }\end{array}$ & $\begin{array}{l}\text { Frequency of } \\
\text { Detection }\end{array}$ & $\underset{\text { Detect }}{\text { Maximum }}$ & $\begin{array}{l}\text { Background } \\
\text { Value }\end{array}$ & $\begin{array}{l}\text { Is Max > } \\
\text { Background? }\end{array}$ & $\begin{array}{c}\text { Ecological } \\
\text { Benchmark } \\
\text { Value }\end{array}$ & $\begin{array}{c}\text { Factor of } \\
\text { Exceedance }\end{array}$ & $\begin{array}{c}\text { \# of } \\
\text { Exceedances }\end{array}$ & $\begin{array}{c}\text { Human Health } \\
\text { Benchmark Value }\end{array}$ & $\begin{array}{c}\text { Factor of } \\
\text { Exceedance }\end{array}$ & $\begin{array}{c}\# \text { of } \\
\text { Exceedances }\end{array}$ \\
\hline Uranium-234 & $\begin{array}{l}\mathrm{pCi} / \mathrm{g} \\
\mathrm{dry}-\mathrm{wt}\end{array}$ & 55 & 55 & $100 \%$ & 4.5 & 1.1 & Yes & 5,270 & $8.50 \mathrm{E}-04$ & -- & 1.1 & 4.1 & 12 \\
\hline Uranium-238 & $\mathrm{pCi} / \mathrm{g}$ & 129 & 129 & $100 \%$ & 10.0 & 1.1 & Yes & 2,490 & 0.0040 & -- & 1.1 & 9.1 & 22 \\
\hline Uranium-238 & $\begin{array}{l}\mathrm{pCi} / \mathrm{g} \\
\mathrm{dry}-\mathrm{wt}\end{array}$ & 57 & 57 & $100 \%$ & 4.3 & 1.1 & Yes & 2,490 & 0.0017 & -- & 1.1 & 3.9 & 13 \\
\hline
\end{tabular}

Table 4-9. Summary of Segment 2 Surface Water Benchmark Exceedances for All Categories of Data.

\begin{tabular}{|c|c|c|c|c|c|c|c|c|c|c|c|}
\hline $\begin{array}{c}\text { Constituent } \\
\text { Name }\end{array}$ & Units & $\begin{array}{c}\text { Number } \\
\text { Analyzed }\end{array}$ & $\begin{array}{l}\text { Number } \\
\text { Detected }\end{array}$ & $\begin{array}{l}\text { Frequency of } \\
\text { Detection }\end{array}$ & $\begin{array}{c}\text { Maximum } \\
\text { Detect }\end{array}$ & $\begin{array}{l}\text { Ecological Benchmark } \\
\text { Value }\end{array}$ & $\begin{array}{c}\text { Factor of } \\
\text { Exceedance }\end{array}$ & $\begin{array}{c}\text { \# of } \\
\text { Exceedances }\end{array}$ & $\begin{array}{c}\text { Human Health } \\
\text { Benchmark Value }\end{array}$ & $\begin{array}{l}\text { Factor of } \\
\text { Exceedance }\end{array}$ & $\begin{array}{c}\text { \# of } \\
\text { Exceedances }\end{array}$ \\
\hline \multicolumn{12}{|l|}{ Metals } \\
\hline Aluminum & $\mathrm{mg} / \mathrm{L}$ & 109 & 57 & $52 \%$ & 21 & 0.087 & 243 & 18 & -- & -- & -- \\
\hline Arsenic & $\mathrm{mg} / \mathrm{L}$ & 980 & 979 & $100 \%$ & 0.0085 & 0.15 & 0.057 & -- & $1.80 \mathrm{E}-05$ & 473 & 978 \\
\hline Barium & $\mathrm{mg} / \mathrm{L}$ & 122 & 121 & $99 \%$ & 0.29 & 0.0040 & 73 & 121 & 1.0 & -- & -- \\
\hline Cadmium & $\mathrm{mg} / \mathrm{L}$ & 1057 & 795 & $75 \%$ & 0.0035 & 2.50E-04 & 14 & 15 & -- & -- & -- \\
\hline Chromium & $\mathrm{mg} / \mathrm{L}$ & 1065 & 843 & $79 \%$ & 0.22 & 0.010 & 22 & 116 & -- & -- & -- \\
\hline Copper & $\mathrm{mg} / \mathrm{L}$ & 1060 & 1001 & $94 \%$ & 0.037 & 0.0060 & 6.3 & 17 & -- & -- & -- \\
\hline Iron & $\mathrm{mg} / \mathrm{L}$ & 82 & 45 & $55 \%$ & 20 & 1.0 & 20 & 5 & 0.30 & 68 & 9 \\
\hline Lead & $\mathrm{mg} / \mathrm{L}$ & 980 & 900 & $92 \%$ & 0.066 & 8.67E-04 & 76 & 34 & -- & -- & -- \\
\hline Manganese & $\mathrm{mg} / \mathrm{L}$ & 134 & 103 & $77 \%$ & 0.94 & 0.12 & 7.8 & 4 & 0.050 & 19 & 7 \\
\hline Mercury & $\mathrm{mg} / \mathrm{L}$ & 398 & 397 & $100 \%$ & 4.07E-05 & $1.20 \mathrm{E}-05$ & 3.4 & 5 & -- & -- & -- \\
\hline Nickel & $\mathrm{mg} / \mathrm{L}$ & 1061 & 988 & $93 \%$ & 0.032 & 0.028 & 1.2 & 2 & 0.61 & -- & -- \\
\hline Silver & $\mathrm{mg} / \mathrm{L}$ & 1060 & 355 & $33 \%$ & 0.0030 & $1.20 \mathrm{E}-04$ & 25 & 7 & -- & -- & -- \\
\hline Thallium & $\mathrm{mg} / \mathrm{L}$ & 899 & 802 & $89 \%$ & 4.07E-04 & 0.040 & 0.010 & -- & $2.40 \mathrm{E}-04$ & 1.7 & 2 \\
\hline Uranium & $\mathrm{mg} / \mathrm{L}$ & 54 & 54 & $100 \%$ & 0.0087 & 0.0026 & 3.3 & 12 & -- & -- & -- \\
\hline Vanadium & $\mathrm{mg} / \mathrm{L}$ & 81 & 38 & $47 \%$ & 0.049 & 0.020 & 2.4 & 4 & -- & -- & -- \\
\hline Zinc & $\mathrm{mg} / \mathrm{L}$ & 1061 & 1038 & $98 \%$ & 0.42 & 0.055 & 7.5 & 12 & 7.4 & -- & -- \\
\hline \multicolumn{12}{|l|}{ Radionuclides } \\
\hline Strontium-90 & $\mathrm{pCi} / \mathrm{L}$ & 784 & 636 & $81 \%$ & 109 & 278 & 0.39 & -- & 8.0 & 14 & 6 \\
\hline Tritium & $\mathrm{pCi} / \mathrm{L}$ & 1136 & 970 & $85 \%$ & 112,000 & $2.65 \mathrm{E}+08$ & 0.00042 & -- & 20000 & 6 & 19 \\
\hline
\end{tabular}


Table 4-10. Summary of Segment 3 Sediment Benchmark Exceedances for All Categories of Data. (2 Pages)

\begin{tabular}{|c|c|c|c|c|c|c|c|c|c|c|c|c|c|}
\hline Constituent Name & Units & $\begin{array}{c}\text { Number } \\
\text { Analyzed }\end{array}$ & $\begin{array}{l}\text { Number } \\
\text { Detected }\end{array}$ & $\begin{array}{c}\text { Frequency of } \\
\text { Detection }\end{array}$ & $\underset{\text { Detect }}{\text { Maximum }}$ & $\begin{array}{l}\text { Background } \\
\text { Value }\end{array}$ & $\begin{array}{c}\text { Is Max > } \\
\text { Background? }\end{array}$ & $\begin{array}{c}\text { Ecological } \\
\text { Benchmark } \\
\text { Value }\end{array}$ & $\begin{array}{c}\text { Factor of } \\
\text { Exceedance }\end{array}$ & $\begin{array}{c}\# \text { of } \\
\text { Exceedances }\end{array}$ & $\begin{array}{l}\text { Human Health } \\
\text { Benchmark } \\
\text { Value }\end{array}$ & $\begin{array}{c}\text { Factor of } \\
\text { Exceedance }\end{array}$ & $\begin{array}{c}\text { \# of } \\
\text { Exceedances }\end{array}$ \\
\hline \multicolumn{14}{|l|}{ Metals } \\
\hline Antimony & $\begin{array}{l}\mathrm{mg} / \mathrm{kg} \\
\mathrm{dry}-\mathrm{wt}\end{array}$ & 17 & 13 & $76 \%$ & 5.1 & 5.0 & Yes & 2.0 & 2.6 & 4 & 31 & 0.16 & -- \\
\hline Arsenic & $\mathrm{mg} / \mathrm{kg}$ & 55 & 45 & $82 \%$ & 10 & 6.5 & Yes & 6.0 & 1.7 & 25 & 0.39 & 26 & 44 \\
\hline Arsenic & $\begin{array}{l}\mathrm{mg} / \mathrm{kg} \\
\mathrm{dry}-\mathrm{wt}\end{array}$ & 23 & 23 & $100 \%$ & 12 & 6.5 & Yes & 6.0 & 2.0 & 9 & 0.39 & 31 & 24 \\
\hline Cadmium & $\mathrm{mg} / \mathrm{kg}$ & 58 & 43 & $74 \%$ & 6.0 & 0.81 & Yes & 0.60 & 9.9 & 31 & 37 & 0.16 & -- \\
\hline Cadmium & $\begin{array}{l}\mathrm{mg} / \mathrm{kg} \\
\mathrm{dry}-\mathrm{wt}\end{array}$ & 23 & 21 & $91 \%$ & 4.3 & 0.81 & Yes & 0.60 & 7.2 & 17 & 37 & 0.12 & -- \\
\hline Chromium & $\mathrm{mg} / \mathrm{kg}$ & 59 & 59 & $100 \%$ & 74 & 19 & Yes & 37 & 2.0 & 20 & 30 & 2.5 & 21 \\
\hline Chromium & $\begin{array}{l}\mathrm{mg} / \mathrm{kg} \\
\mathrm{dry}-\mathrm{wt}\end{array}$ & 23 & 21 & $91 \%$ & 73 & 19 & Yes & 37 & 2.0 & 9 & 30 & 2.4 & 9 \\
\hline Copper & $\mathrm{mg} / \mathrm{kg}$ & 59 & 59 & $100 \%$ & 37 & 22 & Yes & 32 & 1.2 & 10 & 2,960 & 0.013 & -- \\
\hline Copper & $\begin{array}{l}\mathrm{mg} / \mathrm{kg} \\
\mathrm{dry}-\mathrm{wt}\end{array}$ & 23 & 23 & $100 \%$ & 39 & 22 & Yes & 32 & 1.2 & 7 & 2,960 & 0.013 & -- \\
\hline Iron & $\mathrm{mg} / \mathrm{kg}$ & 5 & 5 & $100 \%$ & 51,000 & 32,600 & Yes & 20,000 & 2.6 & 2 & 23,463 & 2.2 & 1 \\
\hline Iron & $\begin{array}{l}\mathrm{mg} / \mathrm{kg} \\
\mathrm{dry}-\mathrm{wt}\end{array}$ & 4 & 4 & $100 \%$ & 34,000 & 32,600 & Yes & 20,000 & 1.7 & 4 & 23,463 & 1.4 & -- \\
\hline Lead & $\begin{array}{l}\mathrm{mg} / \mathrm{kg} \\
\mathrm{dry}-\mathrm{wt}\end{array}$ & 23 & 22 & $96 \%$ & 58 & 10 & Yes & 35 & 1.7 & 4 & 250 & 0.23 & -- \\
\hline Manganese & $\mathrm{mg} / \mathrm{kg}$ & 5 & 5 & $100 \%$ & 1,500 & 512 & Yes & 460 & 3.3 & 1 & 1,762 & 0.85 & -- \\
\hline Manganese & $\begin{array}{l}\mathrm{mg} / \mathrm{kg} \\
\mathrm{dry}-\mathrm{wt}\end{array}$ & 4 & 4 & $100 \%$ & 640 & 512 & Yes & 460 & 1.4 & 2 & 1,762 & 0.36 & - \\
\hline Mercury & $\begin{array}{l}\mathrm{mg} / \mathrm{kg} \\
\mathrm{dry}-\mathrm{wt}\end{array}$ & 26 & 20 & $77 \%$ & 0.31 & 0.33 & No & 0.18 & 1.7 & 3 & 23 & 0.013 & -- \\
\hline Nickel & $\mathrm{mg} / \mathrm{kg}$ & 59 & 59 & $100 \%$ & 32 & 19 & Yes & 18 & 1.8 & 30 & 1,564 & 0.020 & -- \\
\hline Nickel & $\begin{array}{l}\mathrm{mg} / \mathrm{kg} \\
\text { dry-wt }\end{array}$ & 17 & 17 & $100 \%$ & 32 & 19 & Yes & 18 & 1.8 & 12 & 1,564 & 0.020 & -- \\
\hline Vanadium & $\mathrm{mg} / \mathrm{kg}$ & 5 & 5 & $100 \%$ & 140 & 85 & Yes & -- & -- & -- & 78 & 1.8 & 1 \\
\hline Zinc & $\mathrm{mg} / \mathrm{kg}$ & 59 & 59 & $100 \%$ & 558 & 68 & Yes & 120 & 4.7 & 24 & 23,463 & 0.024 & -- \\
\hline Zinc & $\begin{array}{l}\mathrm{mg} / \mathrm{kg} \\
\mathrm{dry}-\mathrm{wt}\end{array}$ & 23 & 22 & $96 \%$ & 375 & 68 & Yes & 120 & 3.1 & 12 & 23,463 & 0.016 & - \\
\hline \multicolumn{14}{|l|}{ Polychlorinated Biphenyls } \\
\hline Aroclor-1254 & $\begin{array}{l}\mathrm{mg} / \mathrm{kg} \\
\mathrm{dry}-\mathrm{wt}\end{array}$ & 6 & 3 & $50 \%$ & 0.013 & -- & -- & 0.0070 & 1.9 & 3 & 0.22 & 0.059 & -- \\
\hline \multicolumn{14}{|c|}{ Semivolatile Organic Compounds } \\
\hline Acenaphthene & $\begin{array}{l}\mathrm{mg} / \mathrm{kg} \\
\mathrm{dry}-\mathrm{wt}\end{array}$ & 6 & 1 & $17 \%$ & 0.021 & -- & - & 0.0067 & 3.1 & 1 & 3,682 & $5.70 \mathrm{E}-06$ & - \\
\hline
\end{tabular}


Table 4-10. Summary of Segment 3 Sediment Benchmark Exceedances for All Categories of Data. (2 Pages)

\begin{tabular}{|c|c|c|c|c|c|c|c|c|c|c|c|c|c|}
\hline Constituent Name & Units & $\begin{array}{c}\text { Number } \\
\text { Analyzed }\end{array}$ & $\begin{array}{l}\text { Number } \\
\text { Detected }\end{array}$ & $\begin{array}{l}\text { Frequency of } \\
\text { Detection }\end{array}$ & $\underset{\text { Detect }}{\text { Maximum }}$ & $\begin{array}{l}\text { Background } \\
\text { Value }\end{array}$ & $\begin{array}{c}\text { Is Max > } \\
\text { Background? }\end{array}$ & $\begin{array}{c}\text { Ecological } \\
\text { Benchmark } \\
\text { Value }\end{array}$ & $\begin{array}{c}\text { Factor of } \\
\text { Exceedance }\end{array}$ & $\begin{array}{c}\# \text { of } \\
\text { Exceedances }\end{array}$ & $\begin{array}{l}\text { Human Health } \\
\text { Benchmark } \\
\text { Value }\end{array}$ & $\begin{array}{c}\text { Factor of } \\
\text { Exceedance }\end{array}$ & $\begin{array}{c}\text { \# of } \\
\text { Exceedances }\end{array}$ \\
\hline Anthracene & $\begin{array}{l}\mathrm{mg} / \mathrm{kg} \\
\mathrm{dry}-\mathrm{wt}\end{array}$ & 20 & 3 & $15 \%$ & 0.088 & -- & -- & 0.057 & 1.5 & 1 & 21,896 & $4.02 \mathrm{E}-06$ & -- \\
\hline Benzo(a)anthracene & $\begin{array}{l}\mathrm{mg} / \mathrm{kg} \\
\text { dry-wt }\end{array}$ & 9 & 2 & $22 \%$ & 0.12 & -- & -- & 0.032 & 3.8 & 1 & 0.62 & 0.19 & -- \\
\hline Benzo(a)pyrene & $\begin{array}{l}\mathrm{mg} / \mathrm{kg} \\
\mathrm{dry}-\mathrm{wt}\end{array}$ & 9 & 3 & $33 \%$ & 0.21 & -- & -- & 0.032 & 6.6 & 2 & 0.062 & 3.4 & 2 \\
\hline Bis(2-ethylhexyl) phthalate & $\begin{array}{l}\mathrm{mg} / \mathrm{kg} \\
\mathrm{dry}-\mathrm{wt}\end{array}$ & 9 & 5 & $56 \%$ & 0.78 & -- & -- & 0.64 & 1.2 & 1 & 35 & 0.022 & -- \\
\hline Chrysene & $\begin{array}{l}\mathrm{mg} / \mathrm{kg} \\
\mathrm{dry}-\mathrm{wt}\end{array}$ & 22 & 3 & $14 \%$ & 0.13 & -- & -- & 0.057 & 2.3 & 1 & 62 & 0.0021 & -- \\
\hline Fluoranthene & $\begin{array}{l}\mathrm{mg} / \mathrm{kg} \\
\mathrm{dry}-\mathrm{wt}\end{array}$ & 22 & 9 & $41 \%$ & 0.20 & -- & -- & 0.11 & 1.8 & 3 & 2,294 & $8.72 \mathrm{E}-05$ & -- \\
\hline Phenanthrene & $\begin{array}{l}\mathrm{mg} / \mathrm{kg} \\
\mathrm{dry}-\mathrm{wt}\end{array}$ & 19 & 4 & $21 \%$ & 0.057 & -- & -- & 0.042 & 1.4 & 1 & -- & -- & -- \\
\hline Pyrene & $\begin{array}{l}\mathrm{mg} / \mathrm{kg} \\
\text { dry-wt }\end{array}$ & 22 & 7 & $32 \%$ & 0.22 & -- & -- & 0.053 & 4.2 & 3 & 2,316 & $9.50 \mathrm{E}-05$ & -- \\
\hline \multicolumn{14}{|l|}{ Pesticides } \\
\hline Dichlorodiphenyldichloroethane & $\begin{array}{l}\mathrm{mg} / \mathrm{kg} \\
\mathrm{dry}-\mathrm{wt}\end{array}$ & 26 & 15 & $58 \%$ & 0.023 & -- & -- & 0.0040 & 5.8 & 6 & 2.4 & 0.0094 & -- \\
\hline Dichlorodiphenyldichloroethylene & $\mathrm{mg} / \mathrm{kg}$ & 6 & 3 & $50 \%$ & 0.016 & -- & -- & 0.0015 & 11 & 3 & 1.7 & 0.0093 & -- \\
\hline Dichlorodiphenyldichloroethylene & $\begin{array}{l}\mathrm{mg} / \mathrm{kg} \\
\mathrm{dry}-\mathrm{wt}\end{array}$ & 28 & 15 & $54 \%$ & 0.051 & -- & -- & 0.0015 & 34 & 12 & 1.7 & 0.030 & -- \\
\hline Dichlorodiphenyltrichloroethane & $\begin{array}{l}\mathrm{mg} / \mathrm{kg} \\
\text { dry-wt }\end{array}$ & 26 & 6 & $23 \%$ & 0.035 & -- & -- & 0.0042 & 8.4 & 2 & 1.7 & 0.020 & -- \\
\hline Dieldrin & $\begin{array}{l}\mathrm{mg} / \mathrm{kg} \\
\mathrm{dry}-\mathrm{wt}\end{array}$ & 23 & 3 & $13 \%$ & 0.0050 & -- & -- & 0.0019 & 2.6 & 1 & 0.030 & 0.16 & -- \\
\hline Gamma-BHC (Lindane) & $\mathrm{mg} / \mathrm{kg}$ & 6 & 1 & $17 \%$ & 0.0080 & -- & -- & $9.00 \mathrm{E}-04$ & 8.9 & 1 & 0.44 & 0.018 & - \\
\hline \multicolumn{14}{|l|}{ Radionuclides } \\
\hline Uranium-234 & $\mathrm{pCi} / \mathrm{g}$ & 38 & 38 & $100 \%$ & 1.9 & 1.1 & Yes & 5,270 & $3.62 E-04$ & -- & 1.1 & 1.7 & 1 \\
\hline Uranium-234 & $\begin{array}{l}\text { pCi/g } \\
\text { dry-wt }\end{array}$ & 45 & 45 & $100 \%$ & 1.8 & 1.1 & Yes & 5,270 & $3.43 \mathrm{E}-04$ & -- & 1.1 & 1.6 & 30 \\
\hline Uranium-238 & $\mathrm{pCi} / \mathrm{g}$ & 41 & 41 & $100 \%$ & 1.6 & 1.1 & No & 2,490 & $6.47 \mathrm{E}-04$ & -- & 1.1 & 1.5 & 4 \\
\hline Uranium-238 & $\begin{array}{l}\mathrm{pCi} / \mathrm{g} \\
\text { dry-wt }\end{array}$ & 45 & 45 & $100 \%$ & 1.6 & 1.1 & Yes & 2,490 & $6.43 \mathrm{E}-04$ & -- & 1.1 & 1.5 & 19 \\
\hline
\end{tabular}


Table 4-11. Summary of Segment 3 Surface Water Benchmark Exceedances for All Categories of Data.

\begin{tabular}{|c|c|c|c|c|c|c|c|c|c|c|c|}
\hline Constituent Name & Units & $\begin{array}{c}\text { Number } \\
\text { Analyzed }\end{array}$ & $\begin{array}{c}\text { Number } \\
\text { Detected }\end{array}$ & $\begin{array}{c}\text { Frequency of } \\
\text { Detection }\end{array}$ & $\begin{array}{c}\text { Maximum } \\
\text { Detect }\end{array}$ & $\begin{array}{c}\text { Ecological } \\
\text { Benchmark Value } \\
\end{array}$ & $\begin{array}{c}\text { Factor of } \\
\text { Exceedance }\end{array}$ & $\begin{array}{c}\# \text { of } \\
\text { Exceedances }\end{array}$ & $\begin{array}{c}\text { Human Health } \\
\text { Benchmark Value }\end{array}$ & $\begin{array}{c}\text { Factor of } \\
\text { Exceedance }\end{array}$ & $\begin{array}{c}\text { \# of } \\
\text { Exceedances }\end{array}$ \\
\hline \multicolumn{12}{|l|}{ Metals } \\
\hline Arsenic & $\mathrm{mg} / \mathrm{L}$ & 48 & 48 & $100 \%$ & 0.0028 & 0.15 & -- & -- & $1.80 \mathrm{E}-05$ & 156 & 48 \\
\hline \multicolumn{12}{|l|}{ Pesticides } \\
\hline Dichlorodiphenyldichloroethylene & $\mathrm{mg} / \mathrm{L}$ & 21 & 18 & $86 \%$ & $1.20 \mathrm{E}-06$ & -- & -- & -- & $2.20 \mathrm{E}-07$ & 5 & 8 \\
\hline Dichlorodiphenyltrichloroethane & $\mathrm{mg} / \mathrm{L}$ & 21 & 5 & $24 \%$ & $6.50 \mathrm{E}-07$ & $1.00 \mathrm{E}-06$ & -- & -- & $2.20 \mathrm{E}-07$ & 3 & 1 \\
\hline Dieldrin & $\mathrm{mg} / \mathrm{L}$ & 21 & 4 & $19 \%$ & $3.00 \mathrm{E}-07$ & $1.90 \mathrm{E}-06$ & -- & -- & $5.20 \mathrm{E}-08$ & 6 & 4 \\
\hline Heptachlor epoxide & $\mathrm{mg} / \mathrm{L}$ & 21 & 6 & $29 \%$ & $1.20 \mathrm{E}-07$ & $3.80 \mathrm{E}-06$ & -- & - & $3.90 \mathrm{E}-08$ & 3 & 4 \\
\hline
\end{tabular}

Table 4-12. Summary of Segment 4 Sediment Benchmark Exceedances for All Categories of Data. (2 Pages)

\begin{tabular}{|c|c|c|c|c|c|c|c|c|c|c|c|c|c|}
\hline Constituent Name & Units & $\begin{array}{c}\text { Number } \\
\text { Analyzed }\end{array}$ & $\begin{array}{l}\text { Number } \\
\text { Detected }\end{array}$ & $\begin{array}{l}\text { Frequency of } \\
\text { Detection }\end{array}$ & $\underset{\text { Detect }}{\text { Maximum }}$ & $\begin{array}{l}\text { Background } \\
\text { Value }\end{array}$ & $\begin{array}{c}\text { Is Max > } \\
\text { Background? }\end{array}$ & $\begin{array}{c}\text { Ecological } \\
\text { Benchmark } \\
\text { Value }\end{array}$ & $\begin{array}{c}\text { Factor of } \\
\text { Exceedance }\end{array}$ & $\begin{array}{c}\# \text { of } \\
\text { Exceedances }\end{array}$ & $\begin{array}{l}\text { Human Health } \\
\text { Benchmark } \\
\text { Value }\end{array}$ & $\begin{array}{c}\text { Factor of } \\
\text { Exceedance }\end{array}$ & $\begin{array}{c}\# \text { of } \\
\text { Exceedances }\end{array}$ \\
\hline \multicolumn{14}{|l|}{ Metals } \\
\hline Antimony & $\mathrm{mg} / \mathrm{kg}$ & 19 & 10 & $53 \%$ & 65 & 5.0 & Yes & 2.0 & 33 & 2 & 31 & 2.1 & 2 \\
\hline Antimony & $\begin{array}{l}\mathrm{mg} / \mathrm{kg} \\
\mathrm{dry}-\mathrm{wt}\end{array}$ & 5 & 5 & $100 \%$ & 6.1 & 5.0 & Yes & 2.0 & 3.1 & 5 & 31 & 0.19 & -- \\
\hline Arsenic & $\mathrm{mg} / \mathrm{kg}$ & 24 & 13 & $54 \%$ & 15 & 6.5 & Yes & 6.0 & 2.5 & 2 & 0.39 & 38 & 12 \\
\hline Arsenic & $\begin{array}{l}\mathrm{mg} / \mathrm{kg} \\
\mathrm{dry}-\mathrm{wt}\end{array}$ & 5 & 5 & $100 \%$ & 9.5 & 6.5 & Yes & 6.0 & 1.6 & 3 & 0.39 & 24 & 5 \\
\hline Cadmium & $\mathrm{mg} / \mathrm{kg}$ & 24 & 18 & $75 \%$ & 2.2 & 0.81 & Yes & 0.60 & 3.7 & 8 & 37 & 0.059 & -- \\
\hline Cadmium & $\begin{array}{l}\mathrm{mg} / \mathrm{kg} \\
\mathrm{dry}-\mathrm{wt}\end{array}$ & 5 & 5 & $100 \%$ & 2.5 & 0.81 & Yes & 0.60 & 4.2 & 5 & 37 & 0.068 & -- \\
\hline Chromium & $\mathrm{mg} / \mathrm{kg}$ & 13 & 13 & $100 \%$ & 69 & 19 & Yes & 37 & 1.9 & 3 & 30 & 2.1 & 3 \\
\hline Copper & $\mathrm{mg} / \mathrm{kg}$ & 24 & 24 & $100 \%$ & 46 & 22 & Yes & 32 & 1.4 & 4 & 2,960 & 0.015 & 0 \\
\hline Copper & $\begin{array}{l}\mathrm{mg} / \mathrm{kg} \\
\mathrm{dry}-\mathrm{wt}\end{array}$ & 5 & 5 & $100 \%$ & 46 & 22 & Yes & 32 & 1.5 & 3 & 2,960 & 0.016 & 0 \\
\hline Iron & $\mathrm{mg} / \mathrm{kg}$ & 4 & 4 & $100 \%$ & 26,100 & 32,600 & No & 20,000 & 1.3 & 4 & 23,463 & 1.1 & 3 \\
\hline Iron & $\begin{array}{l}\mathrm{mg} / \mathrm{kg} \\
\mathrm{dry}-\mathrm{wt}\end{array}$ & 5 & 5 & $100 \%$ & 44,000 & 32,600 & Yes & 20,000 & 2.2 & 5 & 23,463 & 1.9 & 4 \\
\hline Lead & $\mathrm{mg} / \mathrm{kg}$ & 27 & 20 & $74 \%$ & 250 & 10 & Yes & 35 & 7.1 & 1 & 250 & 1.0 & 1 \\
\hline Lead & $\begin{array}{l}\mathrm{mg} / \mathrm{kg} \\
\mathrm{dry}-\mathrm{wt}\end{array}$ & 5 & 5 & $100 \%$ & 27 & 10 & Yes & 35 & 0.77 & 0 & 250 & 0.14 & 0 \\
\hline Manganese & $\mathrm{mg} / \mathrm{kg}$ & 4 & 4 & $100 \%$ & 716 & 512 & Yes & 460 & 1.6 & 3 & 1,762 & 0.41 & 0 \\
\hline Manganese & $\begin{array}{l}\mathrm{mg} / \mathrm{kg} \\
\mathrm{dry}-\mathrm{wt}\end{array}$ & 5 & 5 & $100 \%$ & 840 & 512 & Yes & 460 & 1.8 & 3 & 1,762 & 0.48 & 0 \\
\hline Mercury & $\mathrm{mg} / \mathrm{kg}$ & 28 & 13 & $46 \%$ & 0.51 & 0.33 & Yes & 0.18 & 2.8 & 3 & 23 & 0.022 & 0 \\
\hline
\end{tabular}


Table 4-12. Summary of Segment 4 Sediment Benchmark Exceedances for All Categories of Data. (2 Pages)

\begin{tabular}{|c|c|c|c|c|c|c|c|c|c|c|c|c|c|}
\hline Constituent Name & Units & $\begin{array}{c}\text { Number } \\
\text { Analyzed }\end{array}$ & $\begin{array}{l}\text { Number } \\
\text { Detected }\end{array}$ & $\begin{array}{l}\text { Frequency of } \\
\text { Detection }\end{array}$ & $\underset{\text { Detect }}{\text { Maximum }}$ & $\begin{array}{l}\text { Background } \\
\text { Value }\end{array}$ & $\begin{array}{c}\text { Is Max > } \\
\text { Background? }\end{array}$ & $\begin{array}{l}\text { Ecological } \\
\text { Benchmark } \\
\text { Value }\end{array}$ & $\begin{array}{c}\text { Factor of } \\
\text { Exceedance }\end{array}$ & $\begin{array}{c}\# \text { of } \\
\text { Exceedances }\end{array}$ & $\begin{array}{l}\text { Human Health } \\
\text { Benchmark } \\
\text { Value }\end{array}$ & $\begin{array}{c}\text { Factor of } \\
\text { Exceedance }\end{array}$ & $\begin{array}{c}\# \text { of } \\
\text { Exceedances }\end{array}$ \\
\hline Mercury & $\begin{array}{l}\mathrm{mg} / \mathrm{kg} \\
\mathrm{dry}-\mathrm{wt}\end{array}$ & 5 & 5 & $100 \%$ & 0.30 & 0.33 & No & 0.18 & 1.7 & 3 & 23 & 0.013 & 0 \\
\hline Nickel & $\mathrm{mg} / \mathrm{kg}$ & 24 & 23 & $96 \%$ & 33 & 19 & Yes & 18 & 1.8 & 6 & 1,564 & 0.021 & 0 \\
\hline Nickel & $\begin{array}{l}\mathrm{mg} / \mathrm{kg} \\
\mathrm{dry}-\mathrm{wt}\end{array}$ & 5 & 5 & $100 \%$ & 30 & 19 & Yes & 18 & 1.7 & 4 & 1,564 & 0.019 & 0 \\
\hline Vanadium & $\begin{array}{l}\mathrm{mg} / \mathrm{kg} \\
\mathrm{dry}-\mathrm{wt}\end{array}$ & 5 & 5 & $100 \%$ & 120 & 85 & Yes & -- & -- & -- & 78 & 1.5 & 2 \\
\hline Zinc & $\mathrm{mg} / \mathrm{kg}$ & 24 & 24 & $100 \%$ & 293 & 68 & Yes & 120 & 2.4 & 4 & 23,463 & 0.012 & 0 \\
\hline Zinc & $\begin{array}{l}\mathrm{mg} / \mathrm{kg} \\
\mathrm{dry}-\mathrm{wt}\end{array}$ & 5 & 5 & $100 \%$ & 270 & 68 & Yes & 120 & 2.3 & 3 & 23,463 & 0.012 & 0 \\
\hline
\end{tabular}

Polychlorinated Biphenyls

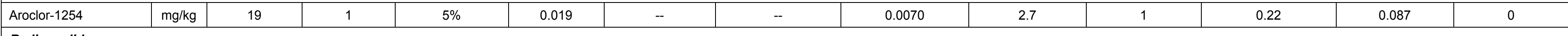
Radionuclides

\begin{tabular}{|c|c|c|c|c|c|c|c|c|c|c|c|c|c|}
\hline Uranium-234 & $\begin{array}{c}\mathrm{pCi} / \mathrm{g} \\
\mathrm{dry}-\mathrm{wt}\end{array}$ & 9 & 9 & $100 \%$ & 1.7 & 1.1 & Yes & 5,270 & $3.30 \mathrm{E}-04$ & -- & 1.1 & 1.6 & 8 \\
\hline Uranium-238 & $\begin{array}{l}\mathrm{pCi} / \mathrm{g} \\
\mathrm{dry}-\mathrm{wt}\end{array}$ & 8 & 8 & $100 \%$ & 1.7 & 1.1 & Yes & 2,490 & $6.95 \mathrm{E}-04$ & -- & 1.1 & 1.6 & 4 \\
\hline
\end{tabular}

Polyaromatic Hydrocarbons

\begin{tabular}{|c|c|c|c|c|c|c|c|c|c|c|c|c|c|}
\hline Acenaphthene & $\mathrm{mg} / \mathrm{kg}$ & 20 & 7 & $35 \%$ & 0.018 & -- & -- & 0.0067 & 2.7 & 1 & 3,682 & $4.89 \mathrm{E}-06$ & 0 \\
\hline Acenaphthylene & $\mathrm{mg} / \mathrm{kg}$ & 20 & 5 & $25 \%$ & 0.0060 & -- & -- & 0.0059 & 1.0 & 1 & 4,800 & $1.25 \mathrm{E}-06$ & 0 \\
\hline
\end{tabular}

Semivolatile Organic Compounds

Table 4-13. Summary of Segment 4 Surface Water Benchmark Exceedances for All Categories of Data. (2 Pages)

\begin{tabular}{|c|c|c|c|c|c|c|c|c|c|c|c|}
\hline Constituent Name & Units & $\begin{array}{c}\text { Number } \\
\text { Analyzed }\end{array}$ & $\begin{array}{l}\text { Number } \\
\text { Detected }\end{array}$ & $\begin{array}{l}\text { Frequency of } \\
\text { Detection }\end{array}$ & $\underset{\text { Detect }}{\text { Maximum }}$ & $\begin{array}{c}\text { Ecological } \\
\text { Benchmark Value }\end{array}$ & $\begin{array}{c}\text { Factor of } \\
\text { Exceedance }\end{array}$ & $\begin{array}{c}\text { \# of } \\
\text { Exceedances }\end{array}$ & $\begin{array}{c}\text { Human Health } \\
\text { Benchmark Value }\end{array}$ & $\begin{array}{c}\text { Factor of } \\
\text { Exceedance }\end{array}$ & $\begin{array}{c}\# \text { of } \\
\text { Exceedances }\end{array}$ \\
\hline \multicolumn{12}{|l|}{ Metals } \\
\hline Arsenic & $\mathrm{mg} / \mathrm{L}$ & 32 & 12 & $38 \%$ & 0.0021 & 0.15 & -- & -- & $1.80 \mathrm{E}-05$ & 117 & 12 \\
\hline Barium & $\mathrm{mg} / \mathrm{L}$ & 21 & 21 & $100 \%$ & 0.043 & 0.0040 & 11 & 21 & 1.0 & -- & -- \\
\hline Cadmium & $\mathrm{mg} / \mathrm{L}$ & 27 & 1 & $3.7 \%$ & 0.0003 & $2.50 \mathrm{E}-04$ & 1.2 & 1 & -- & -- & -- \\
\hline Lead & $\mathrm{mg} / \mathrm{L}$ & 27 & 1 & $4 \%$ & 0.0036 & $8.67 \mathrm{E}-04$ & 4.2 & 1 & -- & -- & -- \\
\hline Iron & $\mathrm{mg} / \mathrm{L}$ & 27 & 27 & $100 \%$ & 0.89 & 1.0 & -- & -- & 0.30 & 3.0 & 6 \\
\hline \multicolumn{12}{|l|}{ Polychlorinated Biphenyls } \\
\hline Total polychlorinated biphenyl & $\mathrm{mg} / \mathrm{L}$ & 3 & 1 & $33 \%$ & $3.70 \mathrm{E}-07$ & $1.40 \mathrm{E}-05$ & -- & -- & $6.40 \mathrm{E}-08$ & 5.8 & 1 \\
\hline
\end{tabular}


Table 4-13. Summary of Segment 4 Surface Water Benchmark Exceedances for All Categories of Data. (2 Pages)

\begin{tabular}{|c|c|c|c|c|c|c|c|c|c|c|c|}
\hline Constituent Name & Units & $\begin{array}{c}\text { Number } \\
\text { Analyzed }\end{array}$ & $\begin{array}{l}\text { Number } \\
\text { Detected }\end{array}$ & $\begin{array}{l}\text { Frequency of } \\
\text { Detection }\end{array}$ & $\begin{array}{c}\text { Maximum } \\
\text { Detect }\end{array}$ & $\begin{array}{c}\text { Ecological } \\
\text { Benchmark Value }\end{array}$ & $\begin{array}{c}\text { Factor of } \\
\text { Exceedance }\end{array}$ & $\begin{array}{c}\text { \# of } \\
\text { Exceedances }\end{array}$ & $\begin{array}{c}\text { Human Health } \\
\text { Benchmark Value }\end{array}$ & $\begin{array}{c}\text { Factor of } \\
\text { Exceedance }\end{array}$ & $\begin{array}{c}\text { \# of } \\
\text { Exceedances }\end{array}$ \\
\hline 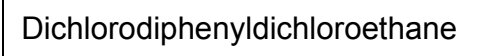 & $\mathrm{mg} / \mathrm{L}$ & 6 & 6 & $100 \%$ & $2.90 \mathrm{E}-06$ & $1.00 \mathrm{E}-06$ & 2.9 & 2 & $3.10 \mathrm{E}-07$ & 9.4 & 6 \\
\hline Dichlorodiphenyldichloroethylene & $\mathrm{mg} / \mathrm{L}$ & 6 & 6 & $100 \%$ & $1.00 \mathrm{E}-06$ & -- & -- & -- & $2.20 \mathrm{E}-07$ & 4.5 & 6 \\
\hline Dieldrin & $\mathrm{mg} / \mathrm{L}$ & 6 & 6 & $100 \%$ & 4.90E-07 & $1.90 \mathrm{E}-06$ & -- & -- & $5.20 \mathrm{E}-08$ & 9.4 & 5 \\
\hline Heptachlor epoxide & $\mathrm{mg} / \mathrm{L}$ & 6 & 2 & $33 \%$ & $6.50 \mathrm{E}-08$ & $3.80 \mathrm{E}-06$ & -- & -- & $3.90 \mathrm{E}-08$ & 1.7 & 2 \\
\hline \multicolumn{12}{|l|}{ Semivolatile Organic Compounds } \\
\hline Hexachlorobenzene & $\mathrm{mg} / \mathrm{L}$ & 6 & 6 & $100 \%$ & $3.50 \mathrm{E}-07$ & -- & -- & -- & $2.80 \mathrm{E}-07$ & 1.3 & 2 \\
\hline
\end{tabular}

Table 4-14. Summary of Segment 5 Sediment Benchmark Exceedances for All Categories of Data. (3 Pages)

\begin{tabular}{|c|c|c|c|c|c|c|c|c|c|c|c|c|c|}
\hline Constituent Name & Units & $\begin{array}{c}\text { Number } \\
\text { Analyzed }\end{array}$ & $\begin{array}{l}\text { Number } \\
\text { Detected }\end{array}$ & $\begin{array}{l}\text { Frequency of } \\
\text { Detection }\end{array}$ & $\begin{array}{c}\text { Maximum } \\
\text { Detect }\end{array}$ & $\begin{array}{l}\text { Background } \\
\text { Value }\end{array}$ & $\begin{array}{c}\text { Is Max > } \\
\text { Background? }\end{array}$ & $\begin{array}{c}\text { Ecological } \\
\text { Benchmark Value }\end{array}$ & $\begin{array}{c}\text { Factor of } \\
\text { Exceedance }\end{array}$ & $\begin{array}{c}\text { \# of } \\
\text { Exceedances }\end{array}$ & $\begin{array}{c}\text { Human Health } \\
\text { Benchmark Value }\end{array}$ & $\begin{array}{c}\text { Factor of } \\
\text { Exceedance }\end{array}$ & $\begin{array}{c}\text { \# of } \\
\text { Exceedances }\end{array}$ \\
\hline \multicolumn{14}{|l|}{ Metals } \\
\hline Antimony & $\mathrm{mg} / \mathrm{kg}$ & 36 & 25 & $69 \%$ & 3.6 & 5.0 & Yes & 2.0 & 1.8 & 3 & 31 & 0.12 & 0 \\
\hline Antimony & $\begin{array}{l}\mathrm{mg} / \mathrm{kg} \\
\mathrm{dry}-\mathrm{wt}\end{array}$ & 45 & 12 & $27 \%$ & 3.9 & 5.0 & No & 2.0 & 2.0 & 1 & 31 & 0.12 & 0 \\
\hline Arsenic & $\mathrm{mg} / \mathrm{kg}$ & 72 & 72 & $100 \%$ & 11 & 6.5 & Yes & 6.0 & 1.8 & 12 & 0.39 & 28 & 69 \\
\hline Arsenic & $\begin{array}{l}\mathrm{mg} / \mathrm{kg} \\
\mathrm{dry}-\mathrm{wt}\end{array}$ & 48 & 47 & $98 \%$ & 14 & 6.5 & Yes & 6.0 & 2.3 & 6 & 0.39 & 35 & 47 \\
\hline Cadmium & $\mathrm{mg} / \mathrm{kg}$ & 71 & 31 & $44 \%$ & 3.3 & 0.81 & Yes & 0.60 & 5.5 & 33 & 37 & 0.089 & 0 \\
\hline Cadmium & $\begin{array}{l}\mathrm{mg} / \mathrm{kg} \\
\mathrm{dry}-\mathrm{wt}\end{array}$ & 48 & 37 & $77 \%$ & 1.9 & 0.81 & Yes & 0.60 & 3.2 & 16 & 37 & 0.051 & 0 \\
\hline Chromium & $\mathrm{mg} / \mathrm{kg}$ & 49 & 49 & $100 \%$ & 35 & 19 & Yes & 37 & 0.93 & 0 & 30 & 1.1 & 1 \\
\hline Chromium & $\begin{array}{l}\mathrm{mg} / \mathrm{kg} \\
\mathrm{dry}-\mathrm{wt}\end{array}$ & 38 & 38 & $100 \%$ & 31 & 19 & Yes & 37 & 0.84 & 0 & 30 & 1.0 & 1 \\
\hline Copper & $\mathrm{mg} / \mathrm{kg}$ & 71 & 71 & $100 \%$ & 76 & 22 & Yes & 32 & 2.4 & 16 & 2,960 & 0.026 & 0 \\
\hline Copper & $\begin{array}{l}\mathrm{mg} / \mathrm{kg} \\
\mathrm{dry}-\mathrm{wt}\end{array}$ & 48 & 47 & $98 \%$ & 75 & 22 & Yes & 32 & 2.4 & 6 & 2,960 & 0.025 & 0 \\
\hline Iron & $\begin{array}{l}\mathrm{mg} / \mathrm{kg} \\
\mathrm{dry}-\mathrm{wt}\end{array}$ & 27 & 27 & $100 \%$ & 39,000 & 32,600 & Yes & 20,000 & 2.0 & 12 & 23,463 & 1.7 & 6 \\
\hline Lead & $\mathrm{mg} / \mathrm{kg}$ & 73 & 73 & $100 \%$ & 140 & 10 & Yes & 35 & 4.0 & 2 & 250 & 1.0 & 0 \\
\hline Lead & $\begin{array}{l}\mathrm{mg} / \mathrm{kg} \\
\mathrm{dry}-\mathrm{wt}\end{array}$ & 46 & 34 & $74 \%$ & 38 & 10 & Yes & 35 & 1.1 & 1 & 250 & 0.15 & 0 \\
\hline Mercury & $\mathrm{mg} / \mathrm{kg}$ & 72 & 38 & $53 \%$ & 0.56 & 0.33 & Yes & 0.18 & 3.1 & 3 & 23 & 0.024 & 0 \\
\hline Mercury & $\begin{array}{l}\mathrm{mg} / \mathrm{kg} \\
\mathrm{dry}-\mathrm{wt}\end{array}$ & 48 & 34 & $71 \%$ & 0.51 & 0.33 & Yes & 0.18 & 2.9 & 1 & 23 & 0.022 & 0 \\
\hline Nickel & $\mathrm{mg} / \mathrm{kg}$ & 71 & 71 & $100 \%$ & 27 & 19 & Yes & 18 & 1.5 & 15 & 1,564 & 0.017 & 0 \\
\hline Nickel & $\begin{array}{l}\mathrm{mg} / \mathrm{kg} \\
\mathrm{dry}-\mathrm{wt}\end{array}$ & 48 & 47 & $98 \%$ & 25 & 19 & Yes & 18 & 1.4 & 4 & 1,564 & 0.016 & 0 \\
\hline Zinc & $\mathrm{mg} / \mathrm{kg}$ & 72 & 71 & $99 \%$ & 200 & 68 & Yes & 120 & 1.7 & 13 & 23,463 & 0.0085 & 0 \\
\hline Zinc & $\begin{array}{l}\mathrm{mg} / \mathrm{kg} \\
\mathrm{dry}-\mathrm{wt}\end{array}$ & 48 & 47 & $98 \%$ & 155 & 68 & Yes & 120 & 1.3 & 8 & 23,463 & 0.0066 & 0 \\
\hline
\end{tabular}


Table 4-14. Summary of Segment 5 Sediment Benchmark Exceedances for All Categories of Data. (3 Pages)

\begin{tabular}{|c|c|c|c|c|c|c|c|c|c|c|c|c|c|}
\hline Constituent Name & Units & $\begin{array}{c}\text { Number } \\
\text { Analyzed }\end{array}$ & $\begin{array}{l}\text { Number } \\
\text { Detected }\end{array}$ & $\begin{array}{l}\text { Frequency of } \\
\text { Detection }\end{array}$ & $\begin{array}{c}\text { Maximum } \\
\text { Detect }\end{array}$ & $\begin{array}{l}\text { Background } \\
\text { Value }\end{array}$ & $\begin{array}{c}\text { Is Max > } \\
\text { Background? }\end{array}$ & \begin{tabular}{|c|} 
Ecological \\
Benchmark Value
\end{tabular} & $\begin{array}{c}\text { Factor of } \\
\text { Exceedance }\end{array}$ & $\begin{array}{c}\text { \# of } \\
\text { Exceedances }\end{array}$ & $\begin{array}{c}\text { Human Health } \\
\text { Benchmark Value }\end{array}$ & $\begin{array}{c}\text { Factor of } \\
\text { Exceedance }\end{array}$ & $\begin{array}{c}\# \text { of } \\
\text { Exceedances }\end{array}$ \\
\hline \multicolumn{14}{|l|}{ Polychlorinated Biphenyls } \\
\hline \begin{tabular}{|l|} 
Aroclor-1248 \\
\end{tabular} & $\mathrm{mg} / \mathrm{kg}$ & 37 & 6 & $16 \%$ & 1.4 & -- & -- & 0.021 & 67 & 6 & 0.22 & 6.3 & 2 \\
\hline Aroclor-1248 & $\begin{array}{l}\mathrm{mg} / \mathrm{kg} \\
\mathrm{dry}-\mathrm{wt}\end{array}$ & 25 & 1 & $4 \%$ & 0.011 & -- & -- & 0.021 & 0.46 & 0 & 0.22 & 0.05 & 0 \\
\hline \begin{tabular}{|l|} 
Aroclor-1254 \\
\end{tabular} & $\mathrm{mg} / \mathrm{kg}$ & 38 & 1 & $3 \%$ & 0.024 & -- & -- & 0.0070 & 3.4 & 1 & 0.22 & 0.11 & - \\
\hline Aroclor-1260 & $\mathrm{mg} / \mathrm{kg}$ & 38 & 2 & $5 \%$ & 0.037 & -- & -- & 0.021 & 1.8 & 1 & 0.22 & 0.17 & -- \\
\hline \multicolumn{14}{|l|}{ Pesticides } \\
\hline Dichlorodiphenyldichloroethane & $\mathrm{mg} / \mathrm{kg}$ & 97 & 13 & $13 \%$ & 0.0048 & -- & -- & 0.0040 & 1.2 & 2 & 2.4 & 0.0020 & -- \\
\hline Dichlorodiphenyldichloroethylene & $\mathrm{mg} / \mathrm{kg}$ & 97 & 20 & $21 \%$ & 0.0039 & -- & -- & 0.0015 & 2.6 & 7 & 1.7 & 0.0023 & -- \\
\hline Dichlorodiphenyldichloroethylene & $\begin{array}{l}\mathrm{mg} / \mathrm{kg} \\
\mathrm{dry}-\mathrm{wt}\end{array}$ & 33 & 7 & $21 \%$ & 0.30 & -- & -- & 0.0015 & 20 & 2 & 1.7 & 0.017 & -- \\
\hline \multicolumn{14}{|l|}{ Semivolatile Organic Compounds } \\
\hline 2-Methylnaphthalene & $\mathrm{mg} / \mathrm{kg}$ & 80 & 33 & $41 \%$ & 0.41 & -- & -- & 0.020 & 20 & 3 & -- & -- & -- \\
\hline Acenaphthene & $\mathrm{mg} / \mathrm{kg}$ & 80 & 28 & $35 \%$ & 0.11 & -- & -- & 0.0067 & 16 & 4 & 3,682 & $2.99 \mathrm{E}-05$ & -- \\
\hline Acenaphthene & $\begin{array}{l}\mathrm{mg} / \mathrm{kg} \\
\mathrm{dry}-\mathrm{wt}\end{array}$ & 40 & 23 & $58 \%$ & 0.094 & -- & -- & 0.0067 & 14 & 5 & 3,682 & $2.55 \mathrm{E}-05$ & -- \\
\hline Acenaphthylene & $\mathrm{mg} / \mathrm{kg}$ & 80 & 30 & $38 \%$ & 0.021 & -- & -- & 0.0059 & 3.6 & 3 & 4,800 & $4.38 \mathrm{E}-06$ & -- \\
\hline Acenaphthylene & $\begin{array}{l}\mathrm{mg} / \mathrm{kg} \\
\mathrm{dry}-\mathrm{wt}\end{array}$ & 38 & 13 & $34 \%$ & 0.012 & -- & -- & 0.0059 & 2.0 & 1 & 4,800 & $2.50 \mathrm{E}-06$ & -- \\
\hline Anthracene & $\mathrm{mg} / \mathrm{kg}$ & 80 & 34 & $43 \%$ & 0.41 & -- & -- & 0.057 & 7.2 & 1 & 21,896 & $1.88 \mathrm{E}-05$ & -- \\
\hline Anthracene & $\begin{array}{l}\mathrm{mg} / \mathrm{kg} \\
\mathrm{dry}-\mathrm{wt}\end{array}$ & 40 & 23 & $58 \%$ & 0.46 & -- & -- & 0.057 & 8.1 & 1 & 21,896 & $2.10 \mathrm{E}-05$ & -- \\
\hline Benzo(a)anthracene & $\mathrm{mg} / \mathrm{kg}$ & 76 & 47 & $62 \%$ & 0.096 & -- & -- & 0.032 & 3.0 & 7 & 0.62 & 0.15 & -- \\
\hline Benzo(a)anthracene & $\begin{array}{l}\mathrm{mg} / \mathrm{kg} \\
\mathrm{dry}-\mathrm{wt}\end{array}$ & 40 & 27 & $68 \%$ & 2.2 & -- & -- & 0.032 & 69 & 9 & 0.62 & 3.5 & 1 \\
\hline Benzo(a)pyrene & $\mathrm{mg} / \mathrm{kg}$ & 79 & 52 & $66 \%$ & 0.10 & -- & -- & 0.032 & 3.2 & 10 & 0.062 & 1.7 & 2 \\
\hline Benzo(a)pyrene & $\begin{array}{l}\mathrm{mg} / \mathrm{kg} \\
\mathrm{dry}-\mathrm{wt}\end{array}$ & 40 & 24 & $60 \%$ & 1.5 & -- & -- & 0.032 & 47 & 7 & 0.062 & 24 & 4 \\
\hline Benzo(b)fluoranthene & $\begin{array}{l}\mathrm{mg} / \mathrm{kg} \\
\mathrm{dry}-\mathrm{wt}\end{array}$ & 25 & 15 & $60 \%$ & 1.8 & -- & -- & 0.24 & 7.5 & 1 & 0.62 & 2.9 & 1 \\
\hline Benzo(ghi)perylene & $\begin{array}{l}\mathrm{mg} / \mathrm{kg} \\
\mathrm{dry}-\mathrm{wt}\end{array}$ & 40 & 18 & $45 \%$ & 1.1 & -- & -- & 0.17 & 6.5 & 1 & 2,400 & $4.58 \mathrm{E}-04$ & -- \\
\hline Benzo(k)fluoranthene & $\mathrm{mg} / \mathrm{kg}$ & 30 & 17 & $57 \%$ & 0.027 & -- & -- & 0.027 & 1.0 & 0 & 6.2 & 0.004 & 0 \\
\hline Benzo(k)fluoranthene & $\begin{array}{l}\mathrm{mg} / \mathrm{kg} \\
\mathrm{dry}-\mathrm{wt}\end{array}$ & 25 & 16 & $64 \%$ & 0.91 & -- & -- & 0.027 & 34 & 5 & 6.2 & 0.15 & 0 \\
\hline Bis(2-ethylhexyl) phthalate & $\begin{array}{l}\mathrm{mg} / \mathrm{kg} \\
\mathrm{dry}-\mathrm{wt}\end{array}$ & 38 & 33 & $87 \%$ & 1.9 & -- & -- & 0.64 & 3.0 & 1 & 35 & 0.055 & 0 \\
\hline Chrysene & $\mathrm{mg} / \mathrm{kg}$ & 80 & 54 & $68 \%$ & 0.13 & -- & -- & 0.057 & 2.3 & 4 & 62 & 0.0021 & 0 \\
\hline Chrysene & $\begin{array}{l}\mathrm{mg} / \mathrm{kg} \\
\mathrm{dry}-\mathrm{wt}\end{array}$ & 40 & 29 & $73 \%$ & 4.1 & -- & -- & 0.057 & 72 & 8 & 62 & 0.066 & -- \\
\hline Dibenz[a,h]anthracene & $\begin{array}{l}\mathrm{mg} / \mathrm{kg} \\
\mathrm{dry}-\mathrm{wt}\end{array}$ & 39 & 14 & $36 \%$ & 0.40 & -- & -- & 0.033 & 12 & 1 & 0.062 & 6.4 & 1 \\
\hline
\end{tabular}


Table 4-14. Summary of Segment 5 Sediment Benchmark Exceedances for All Categories of Data. (3 Pages)

\begin{tabular}{|c|c|c|c|c|c|c|c|c|c|c|c|c|c|}
\hline Constituent Name & Units & $\begin{array}{c}\text { Number } \\
\text { Analyzed }\end{array}$ & $\begin{array}{l}\text { Number } \\
\text { Detected }\end{array}$ & $\begin{array}{l}\text { Frequency of } \\
\text { Detection }\end{array}$ & $\begin{array}{l}\text { Maximum } \\
\text { Detect }\end{array}$ & $\begin{array}{l}\text { Background } \\
\text { Value }\end{array}$ & $\begin{array}{l}\text { Is Max > } \\
\text { Background? }\end{array}$ & $\begin{array}{c}\text { Ecological } \\
\text { Benchmark Value }\end{array}$ & $\begin{array}{c}\text { Factor of } \\
\text { Exceedance }\end{array}$ & $\begin{array}{c}\text { \# of } \\
\text { Exceedances }\end{array}$ & $\begin{array}{c}\text { Human Health } \\
\text { Benchmark Value }\end{array}$ & $\begin{array}{c}\text { Factor of } \\
\text { Exceedance }\end{array}$ & $\begin{array}{c}\text { \# of } \\
\text { Exceedances }\end{array}$ \\
\hline Di-n-butylphthalate & $\mathrm{mg} / \mathrm{kg}$ & 51 & 23 & $45 \%$ & 0.32 & -- & -- & 0.043 & 7.4 & 10 & 6,110 & $5.20 \mathrm{E}-05$ & -- \\
\hline Di-n-butylphthalate & $\begin{array}{l}\mathrm{mg} / \mathrm{kg} \\
\mathrm{dry}-\mathrm{wt}\end{array}$ & 74 & 24 & $32 \%$ & 0.84 & -- & -- & 0.043 & 20 & 1 & 6,110 & $1.37 \mathrm{E}-04$ & -- \\
\hline Fluoranthene & $\begin{array}{l}\mathrm{mg} / \mathrm{kg} \\
\mathrm{dry}-\mathrm{wt}\end{array}$ & 40 & 29 & $73 \%$ & 2.1 & -- & -- & 0.11 & 19 & 2 & 2,294 & $9.16 \mathrm{E}-04$ & -- \\
\hline Fluoranthene & $\mathrm{mg} / \mathrm{kg}$ & 75 & 55 & $73 \%$ & 0.087 & -- & -- & 0.11 & .013 & 0 & 2,294 & $3.17 \mathrm{E}-05$ & -- \\
\hline Fluorene & $\mathrm{mg} / \mathrm{kg}$ & 80 & 31 & $39 \%$ & 0.10 & -- & -- & 0.077 & 1.3 & 2 & 2,747 & $3.71 \mathrm{E}-05$ & -- \\
\hline Indeno(1,2,3-cd)pyrene & $\mathrm{mg} / \mathrm{kg}$ & 79 & 39 & $49 \%$ & 0.076 & -- & -- & 0.017 & 4.5 & 8 & 0.62 & 0.12 & -- \\
\hline Indeno(1,2,3-cd)pyrene & $\begin{array}{l}\mathrm{mg} / \mathrm{kg} \\
\mathrm{dry}-\mathrm{wt}\end{array}$ & 40 & 22 & $55 \%$ & 0.92 & -- & -- & 0.017 & 54 & 11 & 0.62 & 1.5 & 1 \\
\hline Phenanthrene & $\mathrm{mg} / \mathrm{kg}$ & 79 & 52 & $66 \%$ & 0.33 & -- & -- & 0.042 & 7.9 & 5 & -- & -- & -- \\
\hline Phenanthrene & $\begin{array}{l}\mathrm{mg} / \mathrm{kg} \\
\mathrm{dry}-\mathrm{wt}\end{array}$ & 40 & 30 & $75 \%$ & 0.58 & - & -- & 0.042 & 14 & 6 & -- & -- & -- \\
\hline Pyrene & $\mathrm{mg} / \mathrm{kg}$ & 80 & 52 & $65 \%$ & 0.17 & -- & -- & 0.053 & 3.1 & 8 & 2,316 & 7.12E-05 & -- \\
\hline Pyrene & $\begin{array}{l}\mathrm{mg} / \mathrm{kg} \\
\mathrm{dry}-\mathrm{wt}\end{array}$ & 40 & 29 & $73 \%$ & 2.5 & -- & - & 0.053 & 47 & 10 & 2,316 & 0.0011 & - \\
\hline
\end{tabular}

Table 4-15. Summary of Segment 5 Surface Water Benchmark Exceedances for All Categories of Data.

\begin{tabular}{|c|c|c|c|c|c|c|c|c|c|c|c|}
\hline Constituent Name & Units & $\begin{array}{c}\text { Number } \\
\text { Analyzed }\end{array}$ & $\begin{array}{l}\text { Number } \\
\text { Detected }\end{array}$ & $\begin{array}{l}\text { Frequency of } \\
\text { Detection }\end{array}$ & $\underset{\text { Detect }}{\text { Maximum }}$ & $\begin{array}{c}\text { Ecological } \\
\text { Benchmark Value }\end{array}$ & $\begin{array}{c}\text { Factor of } \\
\text { Exceedance }\end{array}$ & $\begin{array}{c}\# \text { of } \\
\text { Exceedances }\end{array}$ & $\begin{array}{c}\text { Human Health } \\
\text { Benchmark Value }\end{array}$ & $\begin{array}{c}\text { Factor of } \\
\text { Exceedance }\end{array}$ & $\begin{array}{c}\text { \# of } \\
\text { Exceedances }\end{array}$ \\
\hline \multicolumn{12}{|l|}{ Metals } \\
\hline Arsenic & $\mathrm{mg} / \mathrm{L}$ & 35 & 27 & $77 \%$ & 0.0015 & 0.15 & -- & -- & $1.80 \mathrm{E}-05$ & 83 & 27 \\
\hline Barium & $\mathrm{mg} / \mathrm{L}$ & 18 & 18 & $100 \%$ & 0.021 & 0.0040 & 5.2 & 18 & 1.0 & -- & -- \\
\hline Boron & $\mathrm{mg} / \mathrm{L}$ & 18 & 16 & $89 \%$ & 0.020 & 0.0016 & 12 & 16 & -- & -- & -- \\
\hline \multicolumn{12}{|l|}{ Polychlorinated biphenyls } \\
\hline Total polychlorinated biphenyl & $\mathrm{mg} / \mathrm{L}$ & 13 & 8 & $62 \%$ & $2.10 \mathrm{E}-05$ & $1.40 \mathrm{E}-05$ & 1.5 & 1 & $6.40 \mathrm{E}-08$ & 328 & 8 \\
\hline \multicolumn{12}{|l|}{ Pesticides } \\
\hline Dichlorodiphenyldichloroethane & $\mathrm{mg} / \mathrm{L}$ & 45 & 34 & $76 \%$ & $5.60 \mathrm{E}-06$ & $1.00 \mathrm{E}-06$ & 5.6 & 4 & $3.10 \mathrm{E}-07$ & 18 & 27 \\
\hline Dichlorodiphenyldichloroethylene & $\mathrm{mg} / \mathrm{L}$ & 93 & 42 & $45 \%$ & $2.10 \mathrm{E}-06$ & -- & -- & -- & $2.20 \mathrm{E}-07$ & 9.5 & 29 \\
\hline Dichlorodiphenyltrichloroethane & $\mathrm{mg} / \mathrm{L}$ & 45 & 39 & $87 \%$ & $1.50 \mathrm{E}-06$ & $1.00 \mathrm{E}-06$ & 1.5 & 3 & $2.20 \mathrm{E}-07$ & 6.8 & 8 \\
\hline Dieldrin & $\mathrm{mg} / \mathrm{L}$ & 88 & 39 & $44 \%$ & $5.40 \mathrm{E}-06$ & $1.90 \mathrm{E}-06$ & 2.8 & 3 & $5.20 \mathrm{E}-08$ & 104 & 37 \\
\hline Heptachlor epoxide & $\mathrm{mg} / \mathrm{L}$ & 45 & 7 & $16 \%$ & $6.70 \mathrm{E}-08$ & $3.80 \mathrm{E}-06$ & - & -- & $3.90 \mathrm{E}-08$ & 1.7 & 3 \\
\hline \multicolumn{12}{|l|}{ Semivolatile Organic Compounds } \\
\hline Hexachlorobenzene & $\mathrm{mg} / \mathrm{L}$ & 45 & 45 & $100 \%$ & $2.30 \mathrm{E}-06$ & -- & -- & -- & $2.80 \mathrm{E}-07$ & 8.2 & 14 \\
\hline
\end{tabular}


Table 4-16. Summary of Contaminant Classes Reported with Sediment Core Samples. (3 Pages)

\begin{tabular}{|c|c|c|c|c|c|c|c|c|c|c|c|c|c|c|c|c|c|}
\hline \multirow[b]{2}{*}{ Source Title } & \multirow[b]{2}{*}{ Core ID } & \multirow[b]{2}{*}{$\begin{array}{l}\text { River Segment/Core } \\
\text { Location/Collection Date }\end{array}$} & \multicolumn{11}{|c|}{ Radionuclides } & \multirow[b]{2}{*}{ Metals } & \multirow[b]{2}{*}{ Pesticides } & \multirow[b]{2}{*}{ PCBs } & \multirow[b]{2}{*}{ svoc } \\
\hline & & & 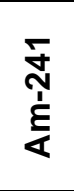 & $\begin{array}{l}\hat{m} \\
\dot{\tilde{u}}\end{array}$ & $\begin{array}{l}8 \\
\dot{d} \\
\dot{0}\end{array}$ & 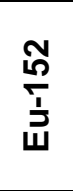 & 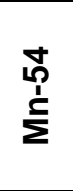 & 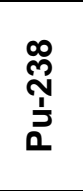 & 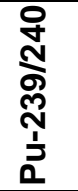 & $\underset{\dot{q}}{\dot{q}}$ & 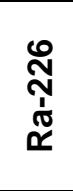 & $\begin{array}{l}\underset{N}{N} \\
\stackrel{\Sigma}{F}\end{array}$ & 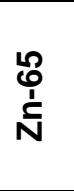 & & & & \\
\hline $\begin{array}{l}\text { TMDL Technical Assessment of DDT and PCBs in the } \\
\text { Okanogan River }\end{array}$ & Seg1_Core1 & $\begin{array}{l}\text { Segment } 1 \text { Core } 1 \text { from Okanogan River } \\
\text { (RM 2.3) }\end{array}$ & & & & & & & & & & & & & $x$ & $x$ & \\
\hline $\begin{array}{l}\text { Association of Hanford Origin Radionuclides with Columbia } \\
\text { River Sediment }\end{array}$ & Seg1_Core2 & $\begin{array}{l}\text { Segment } 1 \text { Core } 1 \text { from Priest Rapids Dam } \\
(8-3-76)\end{array}$ & $x$ & $\mathrm{x}$ & $x$ & & & $x$ & $x$ & $x$ & $x$ & $x$ & $x$ & & & & \\
\hline $\begin{array}{l}\text { An Investigation of the Origin of } 152 \mathrm{Eu} \text { in Columbia River } \\
\text { Sediment }\end{array}$ & Seg1_Core3 & $\begin{array}{l}\text { Segment } 1 \text { Core } 2 \text { from Priest Rapids Dam } \\
(8-3-99)\end{array}$ & & $x$ & & $x$ & & & & & & & & & & & \\
\hline $\begin{array}{l}\text { Heavy Metal Transport and Behavior in the Lower Columbia } \\
\text { River, USA }\end{array}$ & Seg1_Core4 & $\begin{array}{l}\text { Segment } 1 \text { Core } 3 \text { from Priest Rapids Dam } \\
(8-1-99)\end{array}$ & & & & & & & & & & & & $x$ & & & \\
\hline $\begin{array}{l}\text { Association of Hanford Origin Radionuclides with Columbia } \\
\text { River Sediment }\end{array}$ & Seg2_Core1 & $\begin{array}{l}\text { Segment } 2 \text { Core from Hanford Ferry } \\
(8-4-76)\end{array}$ & $x$ & $x$ & $x$ & & $x$ & $x$ & $x$ & $x$ & $x$ & $x$ & & & & & \\
\hline $\begin{array}{l}\text { Association of Hanford Origin Radionuclides with Columbia } \\
\text { River Sediment }\end{array}$ & Seg3_Core1 & $\begin{array}{l}\text { Segment } 3 \text { Core } 1 \text { from Wallula } \\
(8-5-76)\end{array}$ & & $x$ & $x$ & & & & & $x$ & $x$ & $x$ & & & & & \\
\hline $\begin{array}{l}\text { Association of Hanford Origin Radionuclides with Columbia } \\
\text { River Sediment }\end{array}$ & Seg3_Core2 & $\begin{array}{l}\text { Segment } 3 \text { Core } 1 \text { from Port Kelly } \\
(8-5-76)\end{array}$ & & $x$ & $x$ & & $x$ & & & $x$ & $x$ & $x$ & $x$ & & & & \\
\hline $\begin{array}{l}\text { Association of Hanford Origin Radionuclides with Columbia } \\
\text { River Sediment }\end{array}$ & Seg3_Core3 & $\begin{array}{l}\text { Segment } 3 \text { Core } 2 \text { from Port Kelly } \\
(9-9-76)\end{array}$ & $x$ & $x$ & $x$ & & $x$ & $x$ & $x$ & $x$ & $x$ & $x$ & $x$ & & & & \\
\hline $\begin{array}{l}\text { Association of Hanford Origin Radionuclides with Columbia } \\
\text { River Sediment }\end{array}$ & Seg3_Core4 & $\begin{array}{l}\text { Segment } 3 \text { Core } 1 \text { from Refuge Island (8-5- } \\
76 \text { ) }\end{array}$ & & $x$ & $x$ & & $x$ & & & $x$ & $x$ & $x$ & & & & & \\
\hline $\begin{array}{l}\text { Association of Hanford Origin Radionuclides with Columbia } \\
\text { River Sediment }\end{array}$ & Seg3_Core5 & $\begin{array}{l}\text { Segment } 3 \text { Core } 2 \text { from Refuge Island (8-5- } \\
76 \text { ) }\end{array}$ & & $x$ & $x$ & & $x$ & & & $x$ & $x$ & $x$ & $x$ & & & & \\
\hline $\begin{array}{l}\text { Association of Hanford Origin Radionuclides with Columbia } \\
\text { River Sediment }\end{array}$ & Seg3_Core6 & $\begin{array}{l}\text { Segment } 3 \text { Core } 1 \text { from McNary Dam } \\
(8-18-76)\end{array}$ & & $x$ & $x$ & & $x$ & & & $x$ & $x$ & $x$ & & & & & \\
\hline $\begin{array}{l}\text { Association of Hanford Origin Radionuclides with Columbia } \\
\text { River Sediment }\end{array}$ & Seg3_Core7 & $\begin{array}{l}\text { Segment } 3 \text { Core } 2 \text { from McNary Dam } \\
(8-5-76)\end{array}$ & $x$ & $x$ & $x$ & & $x$ & $x$ & $x$ & $x$ & $x$ & $x$ & $x$ & & & & \\
\hline $\begin{array}{l}\text { Association of Hanford Origin Radionuclides with Columbia } \\
\text { River Sediment }\end{array}$ & Seg3_Core8 & $\begin{array}{l}\text { Segment } 3 \text { Core } 3 \text { from McNary Dam } \\
(9-9-76)\end{array}$ & $x$ & $x$ & $x$ & & $x$ & $x$ & $x$ & $x$ & $x$ & $x$ & & & & & \\
\hline $\begin{array}{l}\text { Association of Hanford Origin Radionuclides with Columbia } \\
\text { River Sediment }\end{array}$ & Seg3_Core9 & $\begin{array}{l}\text { Segment } 3 \text { Core } 4 \text { from McNary Dam } \\
(9-9-76)\end{array}$ & & $\mathrm{x}$ & $x$ & & $x$ & & & $x$ & $x$ & $x$ & $x$ & & & & \\
\hline $\begin{array}{l}\text { Association of Hanford Origin Radionuclides with Columbia } \\
\text { River Sediment }\end{array}$ & Seg3_Core16 & $\begin{array}{l}\text { Segment } 3 \text { Core } 5 \text { from McNary Dam } \\
(9-9-76)\end{array}$ & $x$ & $x$ & $x$ & & $x$ & $x$ & $x$ & $x$ & $x$ & $x$ & & & & & \\
\hline $\begin{array}{l}\text { Association of Hanford Origin Radionuclides with Columbia } \\
\text { River Sediment }\end{array}$ & Seg3_Core17 & $\begin{array}{l}\text { Segment } 3 \text { Core } 6 \text { from McNary Dam } \\
(8-18-76)\end{array}$ & & $\mathrm{x}$ & $x$ & & $x$ & & & $x$ & $x$ & $x$ & & & & & \\
\hline $243,244 \mathrm{Cm}$ in Columbia River Sediments & Seg3_Core10 & $\begin{array}{l}\text { Segment } 3 \text { Core } 7 \text { from McNary Dam } \\
(8-1-77)^{\mathrm{a}}\end{array}$ & $x$ & & & & & $x$ & $x$ & & & & & & & & \\
\hline $\begin{array}{l}\text { An Investigation of the Origin of } 152 \mathrm{Eu} \text { in Columbia River } \\
\text { Sediment }\end{array}$ & Seg3_Core11 & $\begin{array}{l}\text { Segment } 3 \text { Core } 8 \text { from McNary Dam } \\
(7-28-99)\end{array}$ & & & & $x$ & & & & & & & & & & & \\
\hline $\begin{array}{l}\text { An Investigation of the Origin of } 152 \mathrm{Eu} \text { in Columbia River } \\
\text { Sediment }\end{array}$ & Seg3_Core12 & $\begin{array}{l}\text { Segment } 3 \text { Core } 9 \text { from McNary Dam } \\
(7-28-99)\end{array}$ & & & & $x$ & & & & & & & & & & & \\
\hline
\end{tabular}


Table 4-16. Summary of Contaminant Classes Reported with Sediment Core Samples. (3 Pages)

\begin{tabular}{|c|c|c|c|c|c|c|c|c|c|c|c|c|c|c|c|c|c|}
\hline \multirow[b]{2}{*}{ Source Title } & \multirow[b]{2}{*}{ Core ID } & \multirow[b]{2}{*}{$\begin{array}{l}\text { River Segment/Core } \\
\text { Location/Collection Date }\end{array}$} & \multicolumn{11}{|c|}{ Radionuclides } & \multirow[b]{2}{*}{ Metals } & \multirow[b]{2}{*}{ Pesticides } & \multirow[b]{2}{*}{ PCBs } & \multirow[b]{2}{*}{ svoc } \\
\hline & & & 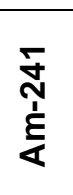 & 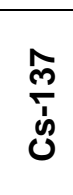 & $\begin{array}{l}8 \\
\vdots \\
\dot{0}\end{array}$ & 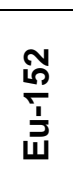 & 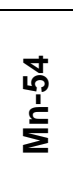 & $\underset{\substack{N \\
\vdots}}{\stackrel{0}{a}}$ & 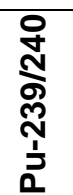 & $\underset{\dot{q}}{\dot{q}}$ & 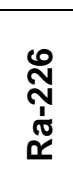 & $\underset{\substack{N \\
\\
亡}}{F}$ & $\begin{array}{l}\stackrel{L}{\grave{L}} \\
\stackrel{N}{N}\end{array}$ & & & & \\
\hline $\begin{array}{l}\text { Heavy Metal Transport and Behavior in the Lower Columbia } \\
\text { River, USA }\end{array}$ & Seg3_Core13 & $\begin{array}{l}\text { Segment } 3 \text { Core } 10 \text { from McNary Dam (8-1- } \\
\text { 99) }\end{array}$ & & & & & & & & & & & & $x$ & & & \\
\hline $\begin{array}{l}\text { Nickel-63 in Columbia River Sediments below the Hanford } \\
\text { Reservation }\end{array}$ & Seg3_Core14 & $\begin{array}{l}\text { Segment } 3 \text { Core } 11 \text { from McNary Dam (8-1- } \\
\text { 77) }\end{array}$ & & & & & & & & & & & & & & & \\
\hline $\begin{array}{l}\text { Association of Hanford Origin Radionuclides with Columbia } \\
\text { River Sediment }\end{array}$ & Seg3_Core15 & $\begin{array}{l}\text { Segment } 3 \text { Core } 12 \text { from McNary Dam (8-18- } \\
76 \text { ) }\end{array}$ & & $\mathrm{x}$ & $\mathrm{x}$ & & $x$ & & & $\mathrm{x}$ & $\mathrm{x}$ & $\mathrm{x}$ & & & & & \\
\hline Hanford-derived Plutonium in Columbia River Sediments & SR_Core1 & $\begin{array}{l}\text { Snake River Core from Ice Harbor Dam (8- } \\
16-99)\end{array}$ & & $x$ & & $x$ & & & & & & & & & & & \\
\hline $\begin{array}{l}\text { Association of Hanford Origin Radionuclides with Columbia } \\
\text { River Sediment }\end{array}$ & Seg4_Core1 & $\begin{array}{l}\text { Segment } 4 \text { Core } 1 \text { from Stevenson WA (8- } \\
11-76)\end{array}$ & $x$ & $x$ & $x$ & & $\mathrm{x}$ & $\mathrm{x}$ & $\mathrm{x}$ & $x$ & $\mathrm{x}$ & $x$ & $x$ & & & & \\
\hline $\begin{array}{l}\text { Association of Hanford Origin Radionuclides with Columbia } \\
\text { River Sediment }\end{array}$ & Seg4_Core2 & $\begin{array}{l}\text { Segment } 4 \text { Core } 1 \text { from The Dalles Dam (8- } \\
\text { 10-76) }\end{array}$ & & $\mathrm{x}$ & $\mathrm{x}$ & & $\mathrm{x}$ & & & $\mathrm{x}$ & $\mathrm{x}$ & $\mathrm{x}$ & & & & & \\
\hline $\begin{array}{l}\text { Association of Hanford Origin Radionuclides with Columbia } \\
\text { River Sediment }\end{array}$ & Seg4_Core3 & $\begin{array}{l}\text { Segment } 4 \text { Core } 2 \text { from The Dalles Dam (8- } \\
\text { 10-76) }\end{array}$ & & $\mathrm{x}$ & $\mathrm{x}$ & & $x$ & & & $\mathrm{x}$ & $x$ & $\mathrm{x}$ & & & & & \\
\hline $\begin{array}{l}\text { Association of Hanford Origin Radionuclides with Columbia } \\
\text { River Sediment }\end{array}$ & Seg4_Core4 & $\begin{array}{l}\text { Segment } 4 \text { Core } 3 \text { from The Dalles Dam (8- } \\
\text { 11-76) }\end{array}$ & $\mathrm{x}$ & $\mathrm{x}$ & $\mathrm{x}$ & & $x$ & $\mathrm{x}$ & $\mathrm{x}$ & $x$ & $\mathrm{x}$ & $\mathrm{x}$ & & & & & \\
\hline Irrigon Boat Basin Sediment Evaluation & Seg4_Core5 & $\begin{array}{l}\text { Segment } 4 \text { Core } 1 \text { from Irrigon Oregon } \\
(6-4-93)\end{array}$ & & & & & & & & & & & & $x$ & $\mathrm{x}$ & $x$ & $\mathrm{x}$ \\
\hline $\begin{array}{l}\text { Association of Hanford Origin Radionuclides with Columbia } \\
\text { River Sediment }\end{array}$ & Seg4_Core6 & $\begin{array}{l}\text { Segment } 4 \text { Core from John Day Dam } \\
(8-10-76)\end{array}$ & & $x$ & $\mathrm{x}$ & & $x$ & & & $\mathrm{x}$ & $\mathrm{x}$ & $\mathrm{x}$ & & & & & \\
\hline $\begin{array}{l}\text { The vertical distribution of selected trace metals and organic } \\
\text { compounds in bottom materials in the proposed lower Columbia } \\
\text { River export channel, Oregon. }\end{array}$ & Seg5_Core1 & Segment 5 Core 1 from RM 21 (9-27-84) & & & & & & & & & & & & $x$ & $\mathrm{x}$ & & \\
\hline $\begin{array}{l}\text { The vertical distribution of selected trace metals and organic } \\
\text { compounds in bottom materials in the proposed lower Columbia } \\
\text { River export channel, Oregon. }\end{array}$ & Seg5_Core2 & Segment 5 Core 2 from RM 21 (9-27-84) & & & & & & & & & & & & & $\mathrm{x}$ & & $\mathrm{x}$ \\
\hline $\begin{array}{l}\text { Historical Changes in the Columbia River Estuary based on } \\
\text { Sediment Cores: Feasibility Studies }\end{array}$ & Seg5_Core3 & $\begin{array}{l}\text { Segment } 5 \text { Core from Clatskanie Flats } \\
(2000)^{\circ}\end{array}$ & & $x$ & & & & & & & $x$ & & & $x$ & & & \\
\hline $\begin{array}{l}\text { Historical Changes in the Columbia River Estuary based on } \\
\text { Sediment Cores: Feasibility Studies }\end{array}$ & Seg5_Core4 & Segment 5 Core from Gray's Bay $(2000)^{c}$ & & $\mathrm{x}$ & & & & & & & $\mathrm{x}$ & & & $\mathrm{x}$ & & & \\
\hline $\begin{array}{l}\text { Historical Changes in the Columbia River Estuary based on } \\
\text { Sediment Cores: Feasibility Studies }\end{array}$ & Seg5_Core5 & Segment 5 Core from Young's Bay $(2000)^{c}$ & & $\mathrm{x}$ & & & & & & & $\mathrm{x}$ & & & $\mathrm{x}$ & & & \\
\hline $\begin{array}{l}\text { The vertical distribution of selected trace metals and organic } \\
\text { compounds in bottom materials in the proposed lower Columbia } \\
\text { River export channel, Oregon. }\end{array}$ & Seg5_Core6 & $\begin{array}{l}\text { Segment } 5 \text { Core } 1 \text { from Skipanon River (9- } \\
\text { 25-1984) }\end{array}$ & & & & & & & & & & & & $\mathrm{x}$ & $\mathrm{x}$ & & $\mathrm{x}$ \\
\hline $\begin{array}{l}\text { The vertical distribution of selected trace metals and organic } \\
\text { compounds in bottom materials in the proposed lower Columbia } \\
\text { River export channel, Oregon. }\end{array}$ & Seg5_Core7 & $\begin{array}{l}\text { Segment } 5 \text { Core } 2 \text { from Skipanon River (9- } \\
\text { 26-1984) }\end{array}$ & & & & & & & & & & & & $\mathrm{x}$ & $\mathrm{x}$ & & $\mathrm{x}$ \\
\hline
\end{tabular}


Table 4-16. Summary of Contaminant Classes Reported with Sediment Core Samples. (3 Pages)

\begin{tabular}{|c|c|c|c|c|c|c|c|c|c|c|c|c|c|c|c|c|c|}
\hline \multirow[b]{2}{*}{ Source Title } & \multirow[b]{2}{*}{ Core ID } & \multirow[b]{2}{*}{$\begin{array}{l}\text { River Segment/Core } \\
\text { Location/Collection Date }\end{array}$} & \multicolumn{11}{|c|}{ Radionuclides } & \multirow[b]{2}{*}{ Metals } & \multirow[b]{2}{*}{ Pesticides } & \multirow[b]{2}{*}{ PCBs } & \multirow[b]{2}{*}{ sVoc } \\
\hline & & & 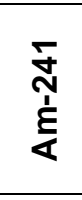 & 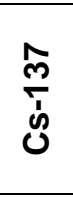 & $\begin{array}{l}8 \\
0 \\
\dot{1} \\
0\end{array}$ & 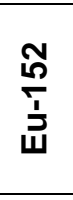 & 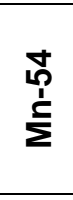 & 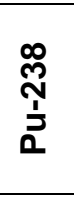 & 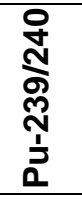 & 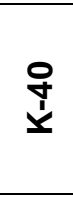 & 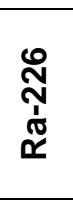 & $\begin{array}{c}\stackrel{\text { ND}}{\grave{F}} \\
\stackrel{F}{F}\end{array}$ & $\begin{array}{l}\text { 草 } \\
\text { ஸे } \\
\text { N }\end{array}$ & & & & \\
\hline $\begin{array}{l}\text { The vertical distribution of selected trace metals and organic } \\
\text { compounds in bottom materials in the proposed lower Columbia } \\
\text { River export channel, Oregon. }\end{array}$ & Seg5_Core8 & $\begin{array}{l}\text { Segment } 5 \text { Core } 3 \text { from Skipanon River (10- } \\
\text { 1-1984) }\end{array}$ & & & & & & & & & & & & $\mathrm{x}$ & & & \\
\hline
\end{tabular}

nnel, Oregon.

Radiological analysisi included only $\mathrm{Ni}-63$.
Radiological analysis also includes $\mathrm{Bi}-214, \mathrm{~Pb}-210$, and Pb-214.

$\mathrm{DDT}=$ dichlorodiphenyltrichloroethan

$\begin{array}{ll}\text { DDT } & =\text { dichlorodiphenyltrichloroe } \\ \text { PCB } & =\text { polychlorinated biphenyl }\end{array}$

$\mathrm{RM}=$ river mile

$\begin{array}{ll}\text { SVOC } & =\text { semivolatile organic compoun } \\ \text { TMDL } & =\text { Total Maximum Daily L Load }\end{array}$

Table 4-17. Summary of Sediment Core Samples Collected in Segment 1 of the Columbia River Component. (3 Pages)

\begin{tabular}{|c|c|c|c|c|c|c|c|c|c|}
\hline Source Title & Core ID & Sample ID & Sample Area & Sample Site & $\begin{array}{l}\text { Interval Depth } \\
\text { Units }\end{array}$ & $\begin{array}{l}\text { Upper } \\
\text { Depth }\end{array}$ & $\begin{array}{l}\text { Lower } \\
\text { Depth }\end{array}$ & $\begin{array}{l}\text { Collection } \\
\text { Date }\end{array}$ & $\begin{array}{l}\text { Estimated Sediment Deposition } \\
\text { Date }\end{array}$ \\
\hline \multirow[t]{7}{*}{ TMDL Technical Assessment of DDT and PCBs in the Okanogan River } & Seg1_Core1 & 1378105 & $\begin{array}{c}\text { Okanogan } \\
\text { River }\end{array}$ & RM 2.3 & $\mathrm{~cm}$ & 0 & 2 & August 21, 2001 & January 1, 2001 \\
\hline & Seg1_Core1 & 1378106 & $\begin{array}{l}\text { Okanogan } \\
\text { River }\end{array}$ & RM 2.3 & $\mathrm{~cm}$ & 6 & 8 & August 21, 2001 & January 1, 1998 \\
\hline & Seg1_Core1 & 1378107 & $\begin{array}{c}\text { Okanogan } \\
\text { River }\end{array}$ & RM 2.3 & $\mathrm{~cm}$ & 12 & 14 & August 21, 2001 & January 1, 1995 \\
\hline & Seg1_Core1 & 1378108 & $\begin{array}{l}\text { Okanogan } \\
\text { River }\end{array}$ & RM 2.3 & $\mathrm{~cm}$ & 18 & 20 & August 21, 2001 & January 1, 1992 \\
\hline & Seg1_Core1 & 1378109 & $\begin{array}{c}\text { Okanogan } \\
\text { River }\end{array}$ & RM 2.3 & $\mathrm{~cm}$ & 24 & 26 & August 21, 2001 & January 1, 1988 \\
\hline & Seg1_Core1 & 1378110 & $\begin{array}{l}\text { Okanogan } \\
\text { River }\end{array}$ & RM 2.3 & $\mathrm{~cm}$ & 28 & 30 & August 21, 2001 & January 1, 1984 \\
\hline & Seg1_Core1 & 1378111 & $\begin{array}{c}\text { Okanogan } \\
\text { River }\end{array}$ & RM 2.3 & $\mathrm{~cm}$ & 30 & 32 & August 21, 2001 & January 1, 1981 \\
\hline \multirow[t]{5}{*}{ Association of Hanford Origin Radionuclides with Columbia River Sediment } & Seg1_Core2 & PR-A-0-2.5 & Columbia River & $\begin{array}{l}\text { Priest Rapids } \\
\text { Dam }\end{array}$ & $\mathrm{cm}$ & 0 & 2.5 & August 3, 1976 & January 1,1975 \\
\hline & Seg1_Core2 & PR-A-2.5-5.1 & Columbia River & $\begin{array}{l}\text { Priest Rapids } \\
\text { Dam }\end{array}$ & $\mathrm{cm}$ & 2.5 & 5.1 & August 3, 1976 & August 1, 1973 \\
\hline & Seg1_Core2 & PR-A-5.1-7.6 & Columbia River & $\begin{array}{l}\text { Priest Rapids } \\
\text { Dam }\end{array}$ & $\mathrm{cm}$ & 5.1 & 7.6 & August 3, 1976 & June 1, 1972 \\
\hline & Seg1_Core2 & PR-A-7.6-10.2 & Columbia River & $\begin{array}{l}\text { Priest Rapids } \\
\text { Dam }\end{array}$ & $\mathrm{cm}$ & 7.6 & 10.2 & August 3, 1976 & February 1, 1971 \\
\hline & Seg1_Core2 & PR-A-10.2-12.7 & Columbia River & $\begin{array}{l}\text { Priest Rapids } \\
\text { Dam }\end{array}$ & $\mathrm{cm}$ & 10.2 & 12.7 & August 3, 1976 & November 1, 1969 \\
\hline
\end{tabular}


Table 4-17. Summary of Sediment Core Samples Collected in Segment 1 of the Columbia River Component. (3 Pages)

\begin{tabular}{|c|c|c|c|c|c|c|c|c|c|}
\hline Source Title & Core ID & Sample ID & Sample Area & Sample Site & $\begin{array}{l}\text { Interval Depth } \\
\text { Units }\end{array}$ & $\begin{array}{l}\text { Upper } \\
\text { Depth }\end{array}$ & $\begin{array}{l}\text { Lower } \\
\text { Depth }\end{array}$ & $\begin{array}{l}\text { Collection } \\
\text { Date }\end{array}$ & $\begin{array}{l}\text { Estimated Sediment Deposition } \\
\text { Date }\end{array}$ \\
\hline & Seg1_Core2 & PR-A-12.7-15.2 & Columbia River & $\begin{array}{l}\text { Priest Rapids } \\
\text { Dam }\end{array}$ & $\mathrm{cm}$ & 12.7 & 15.2 & August 3, 1976 & September 1, 1968 \\
\hline & Seg1_Core2 & PR-A-15.2-17.8 & Columbia River & $\begin{array}{l}\text { Priest Rapids } \\
\text { Dam }\end{array}$ & $\mathrm{cm}$ & 15.2 & 17.8 & August 3, 1976 & May 1,1967 \\
\hline & Seg1_Core2 & PR-A-17.8-20.3 & Columbia River & $\begin{array}{l}\text { Priest Rapids } \\
\text { Dam }\end{array}$ & $\mathrm{cm}$ & 17.8 & 20.3 & August 3,1976 & February 1, 1966 \\
\hline & Seg1_Core2 & PR-A-20.3-22.9 & Columbia River & $\begin{array}{l}\text { Priest Rapids } \\
\text { Dam }\end{array}$ & $\mathrm{cm}$ & 20.3 & 22.9 & August 3, 1976 & November 1, 1964 \\
\hline & Seg1_Core2 & PR-A-22.9-25.4 & Columbia River & $\begin{array}{l}\text { Priest Rapids } \\
\text { Dam }\end{array}$ & $\mathrm{cm}$ & 22.9 & 25.4 & August 3, 1976 & August 1, 1963 \\
\hline & Seg1_Core2 & PR-A-25.4-27.9 & Columbia River & $\begin{array}{l}\text { Priest Rapids } \\
\text { Dam }\end{array}$ & $\mathrm{cm}$ & 25.4 & 27.9 & August 3, 1976 & April 1, 1962 \\
\hline & Seg1_Core2 & PR-A-27.9-30.5 & Columbia River & $\begin{array}{l}\text { Priest Rapids } \\
\text { Dam }\end{array}$ & $\mathrm{cm}$ & 27.9 & 30.5 & August 3,1976 & January 1, 1961 \\
\hline & Seg1_Core2 & PR-A-30.5-33.0 & Columbia River & $\begin{array}{l}\text { Priest Rapids } \\
\text { Dam }\end{array}$ & $\mathrm{cm}$ & 30.5 & 33 & August 3, 1976 & October 1, 1959 \\
\hline & Seg1_Core2 & PR-A-33.0-38.1 & Columbia River & $\begin{array}{l}\text { Priest Rapids } \\
\text { Dam }\end{array}$ & $\mathrm{cm}$ & 33 & 38.1 & August 3,1976 & November 1, 1958 \\
\hline \multirow[t]{12}{*}{\begin{tabular}{|l|} 
An Investigation of the Origin of 152-Eu in Columbia River Sediment \\
Heavy Metal Transport and Behavior in the Lower Columbia River, USA
\end{tabular}} & Seg1_Core3 & $\begin{array}{l}\text { Priest Rapids-1- } \\
\quad 11\end{array}$ & Columbia River & $\begin{array}{l}\text { Priest Rapids } \\
\text { Dam }\end{array}$ & in & 0 & 1 & August 3, 1999 & October 31, 1997 \\
\hline & Seg1_Core3 & $\begin{array}{l}\text { Priest Rapids-1- } \\
10\end{array}$ & Columbia River & $\begin{array}{l}\text { Priest Rapids } \\
\text { Dam }\end{array}$ & in & 1 & 2 & August 3, 1999 & April 29, 1994 \\
\hline & Seg1_Core3 & Priest Rapids-1-9 & Columbia River & $\begin{array}{l}\text { Priest Rapids } \\
\text { Dam }\end{array}$ & in & 2 & 3 & August 3, 1999 & October 26, 1990 \\
\hline & Seg1_Core3 & Priest Rapids-1-8 & Columbia River & $\begin{array}{l}\text { Priest Rapids } \\
\text { Dam }\end{array}$ & in & 3 & 4 & August 3, 1999 & April 23, 1987 \\
\hline & Seg1_Core3 & Priest Rapids-1-7 & Columbia River & $\begin{array}{l}\text { Priest Rapids } \\
\text { Dam }\end{array}$ & in & 4 & 5 & August 3, 1999 & October 20, 1983 \\
\hline & Seg1_Core3 & Priest Rapids-1-6 & Columbia River & $\begin{array}{l}\text { Priest Rapids } \\
\text { Dam }\end{array}$ & in & 5 & 6 & August 3, 1999 & April 17, 1980 \\
\hline & Seg1_Core3 & Priest Rapids-1-5 & Columbia River & $\begin{array}{l}\text { Priest Rapids } \\
\text { Dam }\end{array}$ & in & 6 & 7 & August 3, 1999 & October 13, 1976 \\
\hline & Seg1_Core3 & Priest Rapids-1-4 & Columbia River & $\begin{array}{l}\text { Priest Rapids } \\
\text { Dam }\end{array}$ & in & 7 & 8 & August 3, 1999 & April 11, 1973 \\
\hline & Seg1_Core3 & Priest Rapids-1-3 & Columbia River & $\begin{array}{l}\text { Priest Rapids } \\
\text { Dam }\end{array}$ & in & 8 & 9 & August 3, 1999 & October 8, 1969 \\
\hline & Seg1_Core3 & Priest Rapids-1-2 & Columbia River & $\begin{array}{l}\text { Priest Rapids } \\
\text { Dam }\end{array}$ & in & 9 & 10 & August 3, 1999 & July 5, 1964 \\
\hline & Seg1_Core4 & PRC_1.2 & Columbia River & $\begin{array}{l}\text { Priest Rapids } \\
\text { Dam }\end{array}$ & $\mathrm{cm}$ & 1.2 & 3.8 & August 1, 1999 & January 1, 1997 \\
\hline & Seg1_Core4 & PRC_3.8 & Columbia River & $\begin{array}{l}\text { Priest Rapids } \\
\text { Dam }\end{array}$ & $\mathrm{cm}$ & 3.8 & 6.4 & August 1, 1999 & January 1, 1994 \\
\hline
\end{tabular}


Table 4-17. Summary of Sediment Core Samples Collected in Segment 1 of the Columbia River Component. (3 Pages)

\begin{tabular}{|c|c|c|c|c|c|c|c|c|c|}
\hline Source Title & Core ID & Sample ID & Sample Area & Sample Site & $\begin{array}{l}\text { Interval Depth } \\
\text { Units }\end{array}$ & $\begin{array}{l}\text { Upper } \\
\text { Depth }\end{array}$ & $\begin{array}{l}\text { Lower } \\
\text { Depth }\end{array}$ & $\begin{array}{l}\text { Collection } \\
\text { Date }\end{array}$ & $\begin{array}{l}\text { Estimated Sediment Deposition } \\
\text { Date }\end{array}$ \\
\hline & Seg1_Core4 & PRC_6.4 & Columbia River & $\begin{array}{l}\text { Priest Rapids } \\
\text { Dam }\end{array}$ & $\mathrm{cm}$ & 6.4 & 9 & August 1, 1999 & January 1, 1990 \\
\hline & Seg1_Core4 & PRC_9.0 & Columbia River & $\begin{array}{l}\text { Priest Rapids } \\
\text { Dam }\end{array}$ & $\mathrm{cm}$ & 9 & 11.4 & August 1, 1999 & January 1, 1986 \\
\hline & Seg1_Core4 & PRC_11.4 & Columbia River & $\begin{array}{l}\text { Priest Rapids } \\
\text { Dam }\end{array}$ & $\mathrm{cm}$ & 11.4 & 14 & August 1, 1999 & January 1, 1983 \\
\hline & Seg1_Core4 & PRC_14.0 & Columbia River & $\begin{array}{l}\text { Priest Rapids } \\
\text { Dam }\end{array}$ & $\mathrm{cm}$ & 14 & 16.6 & August 1, 1999 & January 1, 1980 \\
\hline & Seg1_Core4 & PRC_16.6 & Columbia River & $\begin{array}{l}\text { Priest Rapids } \\
\text { Dam }\end{array}$ & $\mathrm{cm}$ & 16.6 & 19.1 & August 1, 1999 & January 1, 1976 \\
\hline & Seg1_Core4 & PRC_19.1 & Columbia River & $\begin{array}{l}\text { Priest Rapids } \\
\text { Dam }\end{array}$ & $\mathrm{cm}$ & 19.1 & 21.7 & August 1, 1999 & January 1, 1972 \\
\hline & Seg1_Core4 & PRC_21.7 & Columbia River & $\begin{array}{l}\text { Priest Rapids } \\
\text { Dam }\end{array}$ & $\mathrm{cm}$ & 21.7 & 25.7 & August 1, 1999 & January 1,1969 \\
\hline & Seg1_Core4 & PRC_25.4 & Columbia River & $\begin{array}{l}\text { Priest Rapids } \\
\text { Dam }\end{array}$ & $\mathrm{cm}$ & 25.4 & 27.4 & August 1, 1999 & January 1, 1966 \\
\hline
\end{tabular}

$\begin{array}{ll}\text { DDT } & =\text { dichlorodiphenyltrichloroethane } \\ \text { PCB = polychlorinated biphenyl }\end{array}$

$\begin{array}{ll}\text { RM } & =\text { river mile } \\ \text { TMDL } & =\text { Total Maximum Daily Load }\end{array}$

Table 4-18. Summary of Sediment Core Samples Collected in Segment 2 of the Columbia River Component.

\begin{tabular}{|c|c|c|c|c|c|c|c|c|c|}
\hline Source Title & Core ID & Sample ID & Sample Area & Sample Site & $\begin{array}{l}\text { Interval Depth } \\
\text { Units }\end{array}$ & $\begin{array}{l}\text { Upper } \\
\text { Depth }\end{array}$ & $\begin{array}{l}\text { Lower } \\
\text { Depth }\end{array}$ & $\begin{array}{c}\text { Collection } \\
\text { Date }\end{array}$ & $\begin{array}{l}\text { Estimated Sediment Deposition } \\
\text { Date }\end{array}$ \\
\hline \multirow[t]{5}{*}{ Association of Hanford Origin Radionuclides with Columbia River Sediment } & Seg2_Core1 & $\mathrm{H}-4-0-5.1$ & Columbia River & Hanford Ferry & $\mathrm{cm}$ & 0 & 5.1 & August 4, 1976 & May 1, 1974 \\
\hline & Seg2_Core1 & $\mathrm{H}-4-5.1-10.2$ & Columbia River & Hanford Ferry & $\mathrm{cm}$ & 5.1 & 10.2 & August 4, 1976 & November 1, 1971 \\
\hline & Seg2_Core1 & $\mathrm{H}-4-10.2-12.7$ & Columbia River & Hanford Ferry & $\mathrm{cm}$ & 10.2 & 12.7 & August 4, 1976 & November 1, 1969 \\
\hline & Seg2_Core1 & $\mathrm{H}-4-12.7-17.8$ & Columbia River & Hanford Ferry & $\mathrm{cm}$ & 12.7 & 17.8 & August 4, 1976 & December 1, 1967 \\
\hline & Seg2_Core1 & $\mathrm{H}-4-17.8-22.9$ & Columbia River & Hanford Ferry & $\mathrm{cm}$ & 17.8 & 22.9 & August 4, 1976 & June 1, 1965 \\
\hline
\end{tabular}


Table 4-19. Summary of Sediment Core Samples Collected in Segment 3 of the Columbia River Component. (10 Pages)

\begin{tabular}{|c|c|c|c|c|c|c|c|c|c|}
\hline Source Title & Core ID & Sample ID & Sample Area & Sample Site & $\begin{array}{l}\text { Interval Depth } \\
\text { Units }\end{array}$ & $\begin{array}{l}\text { Upper } \\
\text { Depth }\end{array}$ & $\begin{array}{l}\text { Lower } \\
\text { Depth }\end{array}$ & Collection Date & $\begin{array}{l}\text { Estimated Sediment Deposition } \\
\text { Date }\end{array}$ \\
\hline \multirow{15}{*}{$\begin{array}{l}\text { Association of Hanford Origin Radionuclides with Columbia River } \\
\text { Sediment }\end{array}$} & Seg3_Core1 & M-A-0-2.5 & Columbia River & Wallula & $\mathrm{cm}$ & 0 & 2.5 & August 5, 1976 & January 1, 1975 \\
\hline & Seg3_Core1 & M-A-2.5-5.1 & Columbia River & Wallula & $\mathrm{cm}$ & 2.5 & 5.1 & August 5, 1976 & August 1, 1973 \\
\hline & Seg3_Core1 & M-A-5.1-7.6 & Columbia River & Wallula & $\mathrm{cm}$ & 5.1 & 7.6 & August 5, 1976 & June 1, 1972 \\
\hline & Seg3_Core1 & M-A-7.6-10.2 & Columbia River & Wallula & $\mathrm{cm}$ & 7.6 & 10.2 & August 5, 1976 & February 1, 1971 \\
\hline & Seg3_Core1 & M-A-10.2-12.7 & Columbia River & Wallula & $\mathrm{cm}$ & 10.2 & 12.7 & August 5, 1976 & November 1, 1969 \\
\hline & Seg3_Core1 & M-A-12.7-15.2 & Columbia River & Wallula & $\mathrm{cm}$ & 12.7 & 15.2 & August 5, 1976 & September 1, 1968 \\
\hline & Seg3_Core1 & M-A-15.2-17.8 & Columbia River & Wallula & $\mathrm{cm}$ & 15.2 & 17.8 & August 5, 1976 & May 1,1967 \\
\hline & Seg3_Core1 & M-A-17.8-20.3 & Columbia River & Wallula & $\mathrm{cm}$ & 17.8 & 20.3 & August 5, 1976 & February 1, 1966 \\
\hline & Seg3_Core1 & M-A-20.3-22.9 & \begin{tabular}{|l|} 
Columbia River \\
\end{tabular} & Wallula & $\mathrm{cm}$ & 20.3 & 22.9 & August 5, 1976 & November 1, 1964 \\
\hline & Seg3_Core1 & M-A-22.9-25.4 & Columbia River & Wallula & $\mathrm{cm}$ & 22.9 & 25.4 & August 5, 1976 & August 1, 1963 \\
\hline & Seg3_Core1 & M-A-25.4-27.9 & Columbia River & Wallula & $\mathrm{cm}$ & 25.4 & 27.9 & August 5, 1976 & April 1, 1962 \\
\hline & Seg3_Core1 & M-A-27.9-30.5 & Columbia River & Wallula & $\mathrm{cm}$ & 27.9 & 30.5 & August 5, 1976 & January 1, 1961 \\
\hline & Seg3_Core1 & M-A-30.5-33.0 & Columbia River & Wallula & $\mathrm{cm}$ & 30.5 & 33 & August 5, 1976 & June 1, 1959 \\
\hline & Seg3_Core1 & M-A-33.0-35.6 & Columbia River & Wallula & $\mathrm{cm}$ & 33 & 35.6 & August 5, 1976 & June 1, 1958 \\
\hline & Seg3_Core1 & M-A-35.6-40.6 & Columbia River & Wallula & $\mathrm{cm}$ & 35.6 & 40.6 & August 5, 1976 & August 1, 1956 \\
\hline \multirow{18}{*}{$\begin{array}{l}\text { Association of Hanford Origin Radionuclides with Columbia River } \\
\text { Sediment }\end{array}$} & Seg3_Core2 & M-B-1-0-2.5 & Columbia River & Port Kelly & $\mathrm{cm}$ & 0 & 2.5 & August 5, 1976 & January 1, 1975 \\
\hline & Seg3_Core2 & M-B-1-2.5-5.1 & Columbia River & Port Kelly & $\mathrm{cm}$ & 2.5 & 5.1 & August 5, 1976 & August 1, 1973 \\
\hline & Seg3_Core2 & M-B-1-5.1-7.6 & Columbia River & Port Kelly & $\mathrm{cm}$ & 5.1 & 7.6 & August 5, 1976 & June 1, 1972 \\
\hline & Seg3_Core2 & M-B-1-7.6-10.2 & Columbia River & Port Kelly & $\mathrm{cm}$ & 7.6 & 10.2 & August 5, 1976 & February 1, 1971 \\
\hline & Seg3_Core2 & M-B-1-10.2-12.7 & Columbia River & Port Kelly & $\mathrm{cm}$ & 10.2 & 12.7 & August 5, 1976 & November 1, 1969 \\
\hline & Seg3_Core2 & M-B-1-12.7-15.2 & Columbia River & Port Kelly & $\mathrm{cm}$ & 12.7 & 15.2 & August 5, 1976 & September 1, 1968 \\
\hline & Seg3_Core2 & M-B-1-15.2-17.8 & Columbia River & Port Kelly & $\mathrm{cm}$ & 15.2 & 17.8 & August 5, 1976 & May 1, 1967 \\
\hline & Seg3_Core2 & M-B-1-17.8-20.3 & Columbia River & Port Kelly & $\mathrm{cm}$ & 17.8 & 20.3 & August 5, 1976 & February 1, 1966 \\
\hline & Seg3_Core2 & M-B-1-20.3-22.9 & Columbia River & Port Kelly & $\mathrm{cm}$ & 20.3 & 22.9 & August 5, 1976 & November 1, 1964 \\
\hline & Seg3_Core2 & M-B-1-22.9-25.4 & Columbia River & Port Kelly & $\mathrm{cm}$ & 22.9 & 25.4 & August 5, 1976 & August 1,1963 \\
\hline & Seg3_Core2 & M-B-1-25.4-27.6 & Columbia River & Port Kelly & $\mathrm{cm}$ & 25.4 & 27.6 & August 5, 1976 & April 1, 1962 \\
\hline & Seg3_Core2 & M-B-1-27.6-30.5 & Columbia River & Port Kelly & $\mathrm{cm}$ & 27.6 & 30.5 & August 5, 1976 & January 1, 1961 \\
\hline & Seg3_Core2 & M-B-1-30.5-33.0 & Columbia River & Port Kelly & $\mathrm{cm}$ & 30.5 & 33 & August 5, 1976 & June 1, 1959 \\
\hline & Seg3_Core2 & M-B-1-33.0-35.6 & Columbia River & Port Kelly & $\mathrm{cm}$ & 33 & 35.6 & August 5, 1976 & June 1, 1958 \\
\hline & Seg3_Core2 & M-B-1-35.6-38.1 & Columbia River & Port Kelly & $\mathrm{cm}$ & 35.6 & 38.1 & August 5, 1976 & January 1, 1957 \\
\hline & Seg3_Core2 & M-B-1-38.1-40.6 & \begin{tabular}{|l|} 
Columbia River \\
\end{tabular} & Port Kelly & $\mathrm{cm}$ & 38.1 & 40.6 & August 5, 1976 & December 1, 1955 \\
\hline & Seg3_Core2 & M-B-1-40.6-43.2 & Columbia River & Port Kelly & $\mathrm{cm}$ & 40.6 & 43.2 & August 5, 1976 & August 1, 1954 \\
\hline & Seg3_Core2 & M-B-1-43.2-47.0 & Columbia River & Port Kelly & $\mathrm{cm}$ & 43.2 & 47 & August 5, 1976 & February 1, 1953 \\
\hline
\end{tabular}


Table 4-19. Summary of Sediment Core Samples Collected in Segment 3 of the Columbia River Component. (10 Pages)

\begin{tabular}{|c|c|c|c|c|c|c|c|c|c|}
\hline Source Title & Core ID & Sample ID & Sample Area & Sample Site & $\begin{array}{l}\text { Interval Depth } \\
\text { Units }\end{array}$ & $\begin{array}{l}\text { Upper } \\
\text { Depth }\end{array}$ & $\begin{array}{l}\text { Lower } \\
\text { Depth }\end{array}$ & Collection Date & $\begin{array}{l}\text { Estimated Sediment Deposition } \\
\text { Date }\end{array}$ \\
\hline & Seg3_Core2 & M-B-1-47.0-52.1 & Columbia River & Port Kelly & $\mathrm{cm}$ & 47 & 52.1 & August 5, 1976 & November 1, 1950 \\
\hline \multirow{23}{*}{$\begin{array}{l}\text { Association of Hanford Origin Radionuclides with Columbia River } \\
\text { Sediment }\end{array}$} & Seg3_Core3 & M-B-2-0-10.2 & Columbia River & Port Kelly & $\mathrm{cm}$ & 0 & 10.2 & September 9, 1976 & February 1, 1973 \\
\hline & Seg3_Core3 & M-B-2-10.2-20.3 & Columbia River & Port Kelly & $\mathrm{cm}$ & 10.2 & 20.3 & September 9, 1976 & January 1, 1968 \\
\hline & Seg3_Core3 & M-B-2-20.3-30.5 & Columbia River & Port Kelly & $\mathrm{cm}$ & 20.3 & 30.5 & September 9, 1976 & November 1, 1962 \\
\hline & Seg3_Core3 & M-B-2-30.5-40.6 & Columbia River & Port Kelly & $\mathrm{cm}$ & 30.5 & 40.6 & September 9, 1976 & November 1, 1957 \\
\hline & Seg3_Core3 & M-B-2-40.6-50.8 & Columbia River & Port Kelly & $\mathrm{cm}$ & 40.6 & 50.8 & September 9, 1976 & October 1, 1952 \\
\hline & Seg3_Core3 & M-B-2-50.8-61.0 & Columbia River & Port Kelly & $\mathrm{cm}$ & 50.8 & 61 & September 9, 1976 & August 1, 1947 \\
\hline & Seg3_Core3 & M-B-2-61.0-71.1 & Columbia River & Port Kelly & $\mathrm{cm}$ & 61 & 71.1 & September 9, 1976 & August 1, 1942 \\
\hline & Seg3_Core3 & M-B-2-71.1-81.3 & Columbia River & Port Kelly & $\mathrm{cm}$ & 71.1 & 81.3 & September 9, 1976 & pre-1942 \\
\hline & Seg3_Core3 & M-B-2-81.3-91.4 & Columbia River & Port Kelly & $\mathrm{cm}$ & 81.3 & 91.4 & September 9, 1976 & pre-1942 \\
\hline & Seg3_Core3 & M-B-2-91.4-101.6 & Columbia River & Port Kelly & $\mathrm{cm}$ & 91.4 & 101.6 & September 9, 1976 & pre-1942 \\
\hline & Seg3_Core3 & M-B-2-101.6-111.8 & Columbia River & Port Kelly & $\mathrm{cm}$ & 101.6 & 111.8 & September 9, 1976 & pre-1942 \\
\hline & Seg3_Core3 & M-B-2-111.8-121.9 & Columbia River & Port Kelly & $\mathrm{cm}$ & 111.8 & 121.9 & September 9, 1976 & pre-1942 \\
\hline & Seg3_Core3 & M-B-2-121.9-132.1 & Columbia River & Port Kelly & $\mathrm{cm}$ & 121.9 & 132.1 & September 9, 1976 & pre-1942 \\
\hline & Seg3_Core3 & M-B-2-132.1-142.2 & Columbia River & Port Kelly & $\mathrm{cm}$ & 132.1 & 142.2 & September 9, 1976 & pre-1942 \\
\hline & Seg3_Core3 & M-B-2-142.2-152.4 & Columbia River & Port Kelly & $\mathrm{cm}$ & 142.2 & 152.4 & September 9, 1976 & pre-1942 \\
\hline & Seg3_Core3 & M-B-2-152.4-162.6 & Columbia River & Port Kelly & $\mathrm{cm}$ & 152.4 & 162.6 & September 9, 1976 & pre-1942 \\
\hline & Seg3_Core3 & M-B-2-162.6-172.7 & Columbia River & Port Kelly & $\mathrm{cm}$ & 162.6 & 172.7 & September 9, 1976 & pre-1942 \\
\hline & Seg3_Core3 & M-B-2-172.7-182.9 & Columbia River & Port Kelly & $\mathrm{cm}$ & 172.7 & 182.9 & September 9, 1976 & pre-1942 \\
\hline & Seg3_Core3 & M-B-2-182.9-193.0 & Columbia River & Port Kelly & $\mathrm{cm}$ & 182.9 & 193 & September 9, 1976 & pre-1942 \\
\hline & Seg3_Core3 & M-B-2-193.0-203.2 & Columbia River & Port Kelly & $\mathrm{cm}$ & 193 & 203.2 & September 9, 1976 & pre-1942 \\
\hline & Seg3_Core3 & M-B-2-203.2-213.4 & Columbia River & Port Kelly & $\mathrm{cm}$ & 203.2 & 213.4 & September 9, 1976 & pre-1942 \\
\hline & Seg3_Core3 & M-B-2-213.4-223.5 & Columbia River & Port Kelly & $\mathrm{cm}$ & 213.4 & 223.5 & September 9, 1976 & pre-1942 \\
\hline & Seg3_Core3 & M-B-2-223.5-233.7 & Columbia River & Port Kelly & $\mathrm{cm}$ & 223.5 & 233.7 & September 9, 1976 & pre-1942 \\
\hline \multirow{10}{*}{$\begin{array}{l}\text { Association of Hanford Origin Radionuclides with Columbia River } \\
\text { Sediment }\end{array}$} & Seg3_Core4 & M-C-5.1-7.6 & Columbia River & Refuge Island & $\mathrm{cm}$ & 5.1 & 7.6 & August 5, 1976 & June 1,1972 \\
\hline & Seg3_Core4 & M-C-7.6-10.2 & Columbia River & Refuge Island & $\mathrm{cm}$ & 7.6 & 10.2 & August 5, 1976 & February 1, 1971 \\
\hline & Seg3_Core4 & M-C-10.2-12.7 & Columbia River & Refuge Island & $\mathrm{cm}$ & 10.2 & 12.7 & August 5, 1976 & November 1, 1965 \\
\hline & Seg3_Core4 & M-C-12.7-15.2 & Columbia River & Refuge Island & $\mathrm{cm}$ & 12.7 & 15.2 & August 5, 1976 & August 1, 1968 \\
\hline & Seg3_Core4 & M-C-15.2-17.8 & Columbia River & Refuge Island & $\mathrm{cm}$ & 15.2 & 17.8 & August 5, 1976 & May 1, 1967 \\
\hline & Seg3_Core4 & M-C-17.8-20.3 & \begin{tabular}{|l} 
Columbia River \\
\end{tabular} & Refuge Island & $\mathrm{cm}$ & 17.8 & 20.3 & August 5, 1976 & February 1, 1966 \\
\hline & Seg3_Core4 & M-C-20.3-22.9 & Columbia River & Refuge Island & $\mathrm{cm}$ & 20.3 & 22.9 & August 5, 1976 & November 1, 1964 \\
\hline & Seg3_Core4 & M-C-22.9-25.4 & Columbia River & Refuge Island & $\mathrm{cm}$ & 22.9 & 25.4 & August 5, 1976 & August 1, 1963 \\
\hline & Seg3_Core4 & M-C-25.4-27.9 & \begin{tabular}{|l} 
Columbia River \\
\end{tabular} & Refuge Island & $\mathrm{cm}$ & 25.4 & 27.9 & August 5, 1976 & May 1, 1962 \\
\hline & Seg3_Core4 & M-C-27.9-30.5 & Columbia River & Refuge Island & $\mathrm{cm}$ & 27.9 & 30.5 & August 5, 1976 & January 1, 1961 \\
\hline
\end{tabular}


Table 4-19. Summary of Sediment Core Samples Collected in Segment 3 of the Columbia River Component. (10 Pages)

\begin{tabular}{|c|c|c|c|c|c|c|c|c|c|}
\hline Source Title & Core ID & Sample ID & Sample Area & Sample Site & $\begin{array}{l}\text { Interval Depth } \\
\text { Units }\end{array}$ & $\begin{array}{l}\text { Upper } \\
\text { Depth }\end{array}$ & $\begin{array}{l}\text { Lower } \\
\text { Depth }\end{array}$ & Collection Date & $\begin{array}{l}\text { Estimated Sediment Deposition } \\
\text { Date }\end{array}$ \\
\hline & Seg3_Core4 & M-C-30.5-33.0 & Columbia River & Refuge Island & $\mathrm{cm}$ & 30.5 & 33 & August 5, 1976 & October 1, 1959 \\
\hline & Seg3_Core4 & M-C-33.0-35.6 & Columbia River & Refuge Island & $\mathrm{cm}$ & 33 & 35.6 & August 5, 1976 & June 1, 1958 \\
\hline & Seg3_Core4 & $M-C-35.6-38.1$ & Columbia River & Refuge Island & $\mathrm{cm}$ & 35.6 & 38.1 & August 5, 1976 & January 1, 1957 \\
\hline & Seg3_Core4 & M-C-38.1-40.6 & Columbia River & Refuge Island & $\mathrm{cm}$ & 38.1 & 40.6 & August 5, 1976 & December 1, 1955 \\
\hline & Seg3_Core4 & M-C-40.6-43.2 & \begin{tabular}{|l|} 
Columbia River \\
\end{tabular} & Refuge Island & $\mathrm{cm}$ & 40.6 & 43.2 & August 5, 1976 & August 1, 1954 \\
\hline & Seg3_Core4 & M-C-43.2-45.7 & \begin{tabular}{|l|l} 
Columbia River \\
\end{tabular} & Refuge Island & $\mathrm{cm}$ & 43.2 & 45.7 & August 5, 1976 & May 1, 1953 \\
\hline & Seg3_Core4 & M-C-45.7-48.3 & Columbia River & Refuge Island & $\mathrm{cm}$ & 45.7 & 48.3 & August 5, 1976 & February 1, 1952 \\
\hline & Seg3_Core4 & M-C-48.3-50.8 & Columbia River & Refuge Island & $\mathrm{cm}$ & 48.3 & 50.8 & August 5, 1976 & November 1, 1951 \\
\hline & Seg3_Core4 & M-C-50.8-53.5 & Columbia River & Refuge Island & $\mathrm{cm}$ & 50.8 & 53.5 & August 5, 1976 & August 1,1949 \\
\hline & Seg3_Core4 & M-C-53.5-55.9 & Columbia River & Refuge Island & $\mathrm{cm}$ & 53.5 & 55.9 & August 5, 1976 & April 1, 1948 \\
\hline & Seg3_Core4 & M-C-55.9-58.4 & Columbia River & Refuge Island & $\mathrm{cm}$ & 55.9 & 58.4 & August 5, 1976 & January 1, 1947 \\
\hline & Seg3_Core4 & M-C-58.4-63.5 & Columbia River & Refuge Island & $\mathrm{cm}$ & 58.4 & 63.5 & August 5, 1976 & February 1, 1945 \\
\hline \multirow{15}{*}{$\begin{array}{l}\text { Association of Hanford Origin Radionuclides with Columbia River } \\
\text { Sediment }\end{array}$} & Seg3_Core5 & M-D-0-2.5 & Columbia River & Refuge Island & $\mathrm{cm}$ & 0 & 2.5 & August 5, 1976 & January 1,1975 \\
\hline & Seg3_Core5 & M-D-2.5-5.1 & \begin{tabular}{|l|} 
Columbia River \\
\end{tabular} & Refuge Island & $\mathrm{cm}$ & 2.5 & 5.1 & August 5, 1976 & September 1, 1974 \\
\hline & Seg3_Core5 & M-D-5.1-7.6 & Columbia River & Refuge Island & $\mathrm{cm}$ & 5.1 & 7.6 & August 5, 1976 & June 1,1972 \\
\hline & Seg3_Core5 & M-D-7.6-10.2 & Columbia River & Refuge Island & $\mathrm{cm}$ & 7.6 & 10.2 & August 5, 1976 & February 1, 1971 \\
\hline & Seg3_Core5 & M-D-10.2-12.7 & Columbia River & Refuge Island & $\mathrm{cm}$ & 10.2 & 12.7 & August 5, 1976 & November 1, 1969 \\
\hline & Seg3_Core5 & M-D-12.7 & Columbia River & Refuge Island & $\mathrm{cm}$ & 12.7 & 15.2 & August 5, 1976 & April 1, 1969 \\
\hline & Seg3_Core5 & M-D-15.2 & Columbia River & Refuge Island & $\mathrm{cm}$ & 15.2 & 17.8 & August 5, 1976 & January 1, 1968 \\
\hline & Seg3_Core5 & M-D-17.8 & Columbia River & Refuge Island & $\mathrm{cm}$ & 17.8 & 20.3 & August 5, 1976 & July 1, 1966 \\
\hline & Seg3_Core5 & M-D-20.3 & Columbia River & Refuge Island & $\mathrm{cm}$ & 20.3 & 22.9 & August 5, 1976 & August 1,1970 \\
\hline & Seg3_Core5 & M-D-22.9 & Columbia River & Refuge Island & $\mathrm{cm}$ & 22.9 & 25.4 & August 5, 1976 & November 1, 1969 \\
\hline & Seg3_Core5 & M-D-25.4 & Columbia River & Refuge Island & $\mathrm{cm}$ & 25.4 & 27.9 & August 5, 1976 & November 1, 1962 \\
\hline & Seg3_Core5 & M-D-27.9 & Columbia River & Refuge Island & $\mathrm{cm}$ & 27.9 & 30.5 & August 5, 1976 & August 1, 1961 \\
\hline & Seg3_Core5 & M-D-30.5-34.3 & Columbia River & Refuge Island & $\mathrm{cm}$ & 30.5 & 34.3 & August 5, 1976 & May 1, 1959 \\
\hline & Seg3_Core5 & M-D-34.3-39.4 & \begin{tabular}{|l|l} 
Columbia River \\
\end{tabular} & Refuge Island & $\mathrm{cm}$ & 34.3 & 39.4 & August 5, 1976 & February 1, 1957 \\
\hline & Seg3_Core5 & M-D-39.4-44.5 & Columbia River & Refuge Island & $\mathrm{cm}$ & 39.4 & 44.5 & August 5, 1976 & August 1, 1954 \\
\hline \multirow{7}{*}{$\begin{array}{l}\text { Association of Hanford Origin Radionuclides with Columbia River } \\
\text { Sediment }\end{array}$} & Seg3_Core6 & M-F-5.1-7.6 & Columbia River & McNary Dam & $\mathrm{cm}$ & 5.1 & 7.6 & August 18,1976 & June 1,1972 \\
\hline & Seg3_Core6 & M-F-7.6-10.2 & Columbia River & McNary Dam & $\mathrm{cm}$ & 7.6 & 10.2 & August 18,1976 & February 1, 1971 \\
\hline & Seg3_Core6 & M-F-40.6-43.2 & Columbia River & McNary Dam & $\mathrm{cm}$ & 40.6 & 43.2 & August 18,1976 & August 1, 1954 \\
\hline & Seg3_Core6 & M-F-43.2-45.7 & \begin{tabular}{|l|} 
Columbia River \\
\end{tabular} & McNary Dam & $\mathrm{cm}$ & 43.2 & 45.7 & August 18, 1976 & November 1, 1952 \\
\hline & Seg3_Core6 & M-F-45.7-48.3 & Columbia River & McNary Dam & $\mathrm{cm}$ & 45.7 & 48.3 & August 18,1976 & February 1, 1952 \\
\hline & Seg3_Core6 & M-F-48.3-50.8 & Columbia River & McNary Dam & $\mathrm{cm}$ & 48.3 & 50.8 & August 18, 1976 & November 1, 1950 \\
\hline & Seg3_Core6 & M-F-50.8-53.5 & Columbia River & McNary Dam & $\mathrm{cm}$ & 50.8 & 53.5 & August 18,1976 & August 1, 1949 \\
\hline
\end{tabular}


Table 4-19. Summary of Sediment Core Samples Collected in Segment 3 of the Columbia River Component. (10 Pages)

\begin{tabular}{|c|c|c|c|c|c|c|c|c|c|}
\hline Source Title & Core ID & Sample ID & Sample Area & Sample Site & $\begin{array}{l}\text { Interval Depth } \\
\text { Units }\end{array}$ & $\begin{array}{l}\text { Upper } \\
\text { Depth }\end{array}$ & $\begin{array}{l}\text { Lower } \\
\text { Depth }\end{array}$ & Collection Date & $\begin{array}{c}\text { Estimated Sediment Deposition } \\
\text { Date }\end{array}$ \\
\hline & Seg3_Core6 & M-F-53.5-55.9 & Columbia River & McNary Dam & $\mathrm{cm}$ & 53.5 & 55.9 & August 18, 1976 & April 1, 1948 \\
\hline & Seg3_Core6 & M-F-55.9-58.4 & Columbia River & McNary Dam & $\mathrm{cm}$ & 55.9 & 58.4 & August 18, 1976 & February 1, 1947 \\
\hline & Seg3_Core6 & M-F-58.4-60.0 & Columbia River & McNary Dam & $\mathrm{cm}$ & 58.4 & 60 & August 18,1976 & January 1, 1946 \\
\hline \multirow{24}{*}{$\begin{array}{l}\text { Association of Hanford Origin Radionuclides with Columbia River } \\
\text { Sediment }\end{array}$} & Seg3_Core7 & M-G-1-0-5.1 & Columbia River & McNary Dam & $\mathrm{cm}$ & 0 & 5.1 & August 5, 1976 & May 1, 1974 \\
\hline & Seg3_Core7 & M-G-1-5.1-10.2 & Columbia River & McNary Dam & $\mathrm{cm}$ & 5.1 & 10.2 & August 5, 1976 & October 1, 1971 \\
\hline & Seg3_Core7 & M-G-1-10.2-12.7 & Columbia River & McNary Dam & $\mathrm{cm}$ & 10.2 & 12.7 & August 5, 1976 & November 1, 1969 \\
\hline & Seg3_Core7 & M-G-1-12.7-15.2 & Columbia River & McNary Dam & $\mathrm{cm}$ & 12.7 & 15.2 & August 5, 1976 & August 1,1968 \\
\hline & Seg3_Core7 & M-G-1-15.2-17.8 & Columbia River & McNary Dam & $\mathrm{cm}$ & 15.2 & 17.8 & August 5, 1976 & May 1, 1967 \\
\hline & Seg3_Core7 & M-G-1-17.8-20.3 & Columbia River & McNary Dam & $\mathrm{cm}$ & 17.8 & 20.3 & August 5, 1976 & February 1, 1966 \\
\hline & Seg3_Core7 & M-G-1-20.3-22.9 & Columbia River & McNary Dam & $\mathrm{cm}$ & 20.3 & 22.9 & August 5, 1976 & November 1, 1964 \\
\hline & Seg3_Core7 & M-G-1-22.9-25.4 & Columbia River & McNary Dam & $\mathrm{cm}$ & 22.9 & 25.4 & August 5, 1976 & August 1,1963 \\
\hline & Seg3_Core7 & M-G-1-25.4-27.9 & Columbia River & McNary Dam & $\mathrm{cm}$ & 25.4 & 27.9 & August 5, 1976 & May 1, 1962 \\
\hline & Seg3_Core7 & M-G-1-27.9-30.5 & Columbia River & McNary Dam & $\mathrm{cm}$ & 27.9 & 30.5 & August 5, 1976 & January 1, 1961 \\
\hline & Seg3_Core7 & M-G-1-30.5-33.0 & Columbia River & McNary Dam & $\mathrm{cm}$ & 30.5 & 33 & August 5, 1976 & October 1, 1959 \\
\hline & Seg3_Core7 & M-G-1-33.0-35.6 & Columbia River & McNary Dam & $\mathrm{cm}$ & 33 & 35.6 & August 5, 1976 & June 1, 1958 \\
\hline & Seg3_Core7 & M-G-1-35.6-38.1 & Columbia River & McNary Dam & $\mathrm{cm}$ & 35.6 & 38.1 & August 5, 1976 & January 1, 1957 \\
\hline & Seg3_Core7 & M-G-1-38.1-40.6 & Columbia River & McNary Dam & $\mathrm{cm}$ & 38.1 & 40.6 & August 5, 1976 & December 1, 1955 \\
\hline & Seg3_Core7 & M-G-1-40.6-43.2 & Columbia River & McNary Dam & $\mathrm{cm}$ & 40.6 & 43.2 & August 5, 1976 & August 1, 1958 \\
\hline & Seg3_Core7 & M-G-1-43.2-45.7 & Columbia River & McNary Dam & $\mathrm{cm}$ & 43.2 & 45.7 & August 5, 1976 & May 1, 1953 \\
\hline & Seg3_Core7 & M-G-1-45.7-48.3 & Columbia River & McNary Dam & $\mathrm{cm}$ & 45.7 & 48.3 & August 5, 1976 & February 1,1952 \\
\hline & Seg3_Core7 & M-G-1-48.3-50.8 & Columbia River & McNary Dam & $\mathrm{cm}$ & 48.3 & 50.8 & August 5, 1976 & November 1, 1950 \\
\hline & Seg3_Core7 & M-G-1-50.8-53.5 & Columbia River & McNary Dam & $\mathrm{cm}$ & 50.8 & 53.5 & August 5, 1976 & August 1, 1949 \\
\hline & Seg3_Core7 & M-G-1-53.5-55.9 & Columbia River & McNary Dam & $\mathrm{cm}$ & 53.5 & 55.9 & August 5, 1976 & April 1, 1948 \\
\hline & Seg3_Core7 & M-G-1-55.9-58.4 & Columbia River & McNary Dam & $\mathrm{cm}$ & 55.9 & 58.4 & August 5, 1976 & February 1, 1947 \\
\hline & Seg3_Core7 & M-G-1-58.4-61.0 & Columbia River & McNary Dam & $\mathrm{cm}$ & 58.4 & 61 & August 5, 1976 & October 1, 1945 \\
\hline & Seg3_Core7 & M-G-1-61.0-63.5 & Columbia River & McNary Dam & $\mathrm{cm}$ & 61 & 63.5 & August 5, 1976 & June 1, 1944 \\
\hline & Seg3_Core7 & M-G-1-63.5-68.6 & Columbia River & McNary Dam & $\mathrm{cm}$ & 63.5 & 68.6 & August 5, 1976 & August 1, 1942 \\
\hline \multirow{7}{*}{$\begin{array}{l}\text { Association of Hanford Origin Radionuclides with Columbia River } \\
\text { Sediment }\end{array}$} & Seg3_Core8 & M-G-2-0-10.2 & Columbia River & McNary Dam & $\mathrm{cm}$ & 0 & 10.2 & September 9, 1976 & February 1,1973 \\
\hline & Seg3_Core8 & M-G-2-10.2-20.3 & Columbia River & McNary Dam & $\mathrm{cm}$ & 10.2 & 20.3 & September 9, 1976 & January 1, 1968 \\
\hline & Seg3_Core8 & M-G-2-20.3-30.5 & Columbia River & McNary Dam & $\mathrm{cm}$ & 20.3 & 30.5 & September 9, 1976 & November 1, 1962 \\
\hline & Seg3_Core8 & M-G-2-30.5-40.6 & Columbia River & McNary Dam & $\mathrm{cm}$ & 30.5 & 40.6 & September 9, 1976 & November 1, 1957 \\
\hline & Seg3_Core8 & M-G-2-40.6-50.8 & Columbia River & McNary Dam & $\mathrm{cm}$ & 40.6 & 50.8 & September 9, 1976 & October 1, 1952 \\
\hline & Seg3_Core8 & M-G-2-50.8-61.0 & Columbia River & McNary Dam & $\mathrm{cm}$ & 50.8 & 61 & September 9, 1976 & August 1, 1947 \\
\hline & Seg3_Core8 & M-G-2-61.0-71.1 & Columbia River & McNary Dam & $\mathrm{cm}$ & 61 & 71.7 & September 9, 1976 & June 1, 1942 \\
\hline
\end{tabular}


Table 4-19. Summary of Sediment Core Samples Collected in Segment 3 of the Columbia River Component. (10 Pages)

\begin{tabular}{|c|c|c|c|c|c|c|c|c|c|}
\hline Source Title & Core ID & Sample ID & Sample Area & Sample Site & $\begin{array}{l}\text { Interval Depth } \\
\text { Units }\end{array}$ & $\begin{array}{l}\text { Upper } \\
\text { Depth }\end{array}$ & $\begin{array}{l}\text { Lower } \\
\text { Depth }\end{array}$ & Collection Date & $\begin{array}{c}\text { Estimated Sediment Deposition } \\
\text { Date }\end{array}$ \\
\hline & Seg3_Core8 & M-G-2-71.1-81.3 & Columbia River & McNary Dam & $\mathrm{cm}$ & 71.1 & 81.3 & September 9, 1976 & pre-1942 \\
\hline & Seg3_Core8 & M-G-2-81.3-91.4 & Columbia River & McNary Dam & $\mathrm{cm}$ & 81.3 & 91.4 & September 9, 1976 & pre-1942 \\
\hline & Seg3_Core8 & M-G-2-91.4-101.6 & Columbia River & McNary Dam & $\mathrm{cm}$ & 91.4 & 101.6 & September 9, 1976 & pre-1942 \\
\hline & Seg3_Core8 & M-G-2-101.6-111.8 & Columbia River & McNary Dam & $\mathrm{cm}$ & 101.6 & 111.8 & September 9, 1976 & pre-1942 \\
\hline & Seg3_Core8 & M-G-2-111.8-121.9 & Columbia River & McNary Dam & $\mathrm{cm}$ & 111.8 & 121.9 & September 9, 1976 & pre-1942 \\
\hline & Seg3_Core8 & M-G-2-121.9-132.1 & Columbia River & McNary Dam & $\mathrm{cm}$ & 121.9 & 132.1 & September 9, 1976 & pre-1942 \\
\hline & Seg3_Core8 & M-G-2-132.1-142.2 & Columbia River & McNary Dam & $\mathrm{cm}$ & 132.1 & 142.2 & September 9, 1976 & pre-1942 \\
\hline & Seg3_Core8 & M-G-2-142.2-152.4 & \begin{tabular}{|l|} 
Columbia River \\
\end{tabular} & McNary Dam & $\mathrm{cm}$ & 142.2 & 152.4 & September 9, 1976 & pre-1942 \\
\hline & Seg3_Core8 & M-G-2-152.4-162.6 & Columbia River & McNary Dam & $\mathrm{cm}$ & 152.4 & 162.6 & September 9, 1976 & pre-1942 \\
\hline & Seg3_Core8 & M-G-2-162.6-172.7 & Columbia River & McNary Dam & $\mathrm{cm}$ & 162.6 & 172.7 & September 9, 1976 & pre-1942 \\
\hline & Seg3_Core8 & M-G-2-172.7-182.9 & Columbia River & McNary Dam & $\mathrm{cm}$ & 172.7 & 182.9 & September 9, 1976 & pre-1942 \\
\hline & Seg3_Core8 & M-G-2-182.9-193.0 & \begin{tabular}{|l|} 
Columbia River \\
\end{tabular} & McNary Dam & $\mathrm{cm}$ & 182.9 & 193 & September 9, 1976 & pre-1942 \\
\hline & Seg3_Core8 & M-G-2-193.0-203.2 & Columbia River & McNary Dam & $\mathrm{cm}$ & 193 & 203.2 & September 9, 1976 & pre-1942 \\
\hline & Seg3_Core8 & M-G-2-203.2-213.4 & Columbia River & McNary Dam & $\mathrm{cm}$ & 203.2 & 213.4 & September 9, 1976 & pre-1942 \\
\hline & Seg3_Core8 & M-G-2-213.4-223.5 & Columbia River & McNary Dam & $\mathrm{cm}$ & 213.4 & 223.5 & September 9, 1976 & pre-1942 \\
\hline & Seg3_Core8 & M-G-2-223.5-233.7 & Columbia River & McNary Dam & $\mathrm{cm}$ & 223.5 & 233.7 & September 9, 1976 & pre-1942 \\
\hline & Seg3_Core8 & M-G-2-233.7-243.8 & Columbia River & McNary Dam & $\mathrm{cm}$ & 233.7 & 243.8 & September 9, 1976 & pre-1942 \\
\hline & Seg3_Core8 & M-G-2-243.8-254.0 & Columbia River & McNary Dam & $\mathrm{cm}$ & 243.8 & 254 & September 9, 1976 & pre-1942 \\
\hline & Seg3_Core8 & M-G-2-254.0-264.2 & Columbia River & McNary Dam & $\mathrm{cm}$ & 254 & 264.2 & September 9, 1976 & pre-1942 \\
\hline & Seg3_Core8 & M-G-2-264.2-274.3 & Columbia River & McNary Dam & $\mathrm{cm}$ & 264.2 & 274.3 & September 9, 1976 & pre-1942 \\
\hline \multirow{14}{*}{$\begin{array}{l}\text { Association of Hanford Origin Radionuclides with Columbia River } \\
\text { Sediment }\end{array}$} & Seg3_Core9 & M-G-3-0-15.2 & Columbia River & McNary Dam & $\mathrm{cm}$ & 0 & 15.2 & September 9, 1976 & October 1, 1971 \\
\hline & Seg3_Core9 & M-G-3-25.4-35.6 & Columbia River & McNary Dam & $\mathrm{cm}$ & 25.4 & 35.6 & September 9, 1976 & May 1, 1960 \\
\hline & Seg3_Core9 & M-G-3-35.6-45.7 & Columbia River & McNary Dam & $\mathrm{cm}$ & 35.6 & 45.7 & September 9, 1976 & April 1, 1955 \\
\hline & Seg3_Core9 & M-G-3-45.7-55.9 & Columbia River & McNary Dam & $\mathrm{cm}$ & 45.7 & 55.9 & September 9, 1976 & March 1, 1950 \\
\hline & Seg3_Core9 & M-G-3-55.9-66.0 & Columbia River & McNary Dam & $\mathrm{cm}$ & 55.9 & 66 & September 9, 1976 & February 1, 1945 \\
\hline & Seg3_Core9 & M-G-3-66.0-76.2 & Columbia River & McNary Dam & $\mathrm{cm}$ & 66 & 76.2 & September 9, 1976 & pre-1942 \\
\hline & Seg3_Core9 & M-G-3-76.2-86.4 & Columbia River & McNary Dam & $\mathrm{cm}$ & 76.2 & 86.4 & September 9, 1976 & pre-1942 \\
\hline & Seg3_Core9 & M-G-3-86.4-96.5 & \begin{tabular}{|l|} 
Columbia River \\
\end{tabular} & McNary Dam & $\mathrm{cm}$ & 86.4 & 96.5 & September 9, 1976 & pre-1942 \\
\hline & Seg3_Core9 & M-G-3-96.5-106.7 & Columbia River & McNary Dam & $\mathrm{cm}$ & 96.5 & 106.7 & September 9, 1976 & pre-1942 \\
\hline & Seg3_Core9 & M-G-3-106.7-116.8 & Columbia River & McNary Dam & $\mathrm{cm}$ & 106.7 & 116.8 & September 9, 1976 & pre-1942 \\
\hline & Seg3_Core9 & M-G-3-116.8-127.0 & Columbia River & McNary Dam & $\mathrm{cm}$ & 116.8 & 127 & September 9, 1976 & pre-1942 \\
\hline & Seg3_Core9 & M-G-3-127.0-137.2 & Columbia River & McNary Dam & $\mathrm{cm}$ & 127 & 137.2 & September 9, 1976 & pre-1942 \\
\hline & Seg3_Core9 & M-G-3-137.2-147.3 & Columbia River & McNary Dam & $\mathrm{cm}$ & 137.2 & 147.3 & September 9, 1976 & pre-1942 \\
\hline & Seg3_Core9 & M-G-3-147.3-157.4 & Columbia River & McNary Dam & $\mathrm{cm}$ & 147.3 & 157.4 & September 9, 1976 & pre-1942 \\
\hline
\end{tabular}


Table 4-19. Summary of Sediment Core Samples Collected in Segment 3 of the Columbia River Component. (10 Pages)

\begin{tabular}{|c|c|c|c|c|c|c|c|c|c|}
\hline Source Title & Core ID & Sample ID & Sample Area & Sample Site & $\begin{array}{l}\text { Interval Depth } \\
\text { Units }\end{array}$ & $\begin{array}{l}\text { Upper } \\
\text { Depth }\end{array}$ & $\begin{array}{l}\text { Lower } \\
\text { Depth }\end{array}$ & Collection Date & $\begin{array}{l}\text { Estimated Sediment Deposition } \\
\text { Date }\end{array}$ \\
\hline & Seg3_Core9 & M-G-3-15.2-25.4 & Columbia River & McNary Dam & $\mathrm{cm}$ & 15.2 & 25.4 & September 9, 1976 & June 1, 1965 \\
\hline & Seg3_Core9 & M-G-3-157.4-167.6 & Columbia River & McNary Dam & $\mathrm{cm}$ & 157.4 & 167.6 & September 9, 1976 & pre-1942 \\
\hline & Seg3_Core9 & M-G-3-167.6-177.8 & Columbia River & McNary Dam & $\mathrm{cm}$ & 167.6 & 177.8 & September 9, 1976 & pre-1942 \\
\hline & Seg3_Core9 & M-G-3-177.8-188.0 & Columbia River & McNary Dam & $\mathrm{cm}$ & 177.8 & 188 & September 9, 1976 & pre-1942 \\
\hline & Seg3_Core9 & M-G-3-188.0-198.1 & Columbia River & McNary Dam & $\mathrm{cm}$ & 188 & 198.1 & September 9, 1976 & pre-1942 \\
\hline & Seg3_Core9 & M-G-3-198.1-208.3 & Columbia River & McNary Dam & $\mathrm{cm}$ & 198.1 & 208.3 & September 9, 1976 & pre-1942 \\
\hline & Seg3_Core9 & M-G-3-208.3-218.4 & Columbia River & McNary Dam & $\mathrm{cm}$ & 208.3 & 218.4 & September 9, 1976 & pre-1942 \\
\hline \multirow[t]{20}{*}{$243,244 \mathrm{Cm}$ in Columbia River Sediments } & Seg3_Core10 & MC- 77 0-2cm & Columbia River & McNary Dam & $\mathrm{cm}$ & 0 & 2 & August 1, 1977 & not reported \\
\hline & Seg3_Core10 & MC-77 2-4cm & Columbia River & McNary Dam & $\mathrm{cm}$ & 2 & 4 & August 1, 1977 & not reported \\
\hline & Seg3_Core10 & MC-77 4-6cm & Columbia River & McNary Dam & $\mathrm{cm}$ & 4 & 6 & August 1, 1977 & not reported \\
\hline & Seg3_Core10 & MC-77 8-9cm & Columbia River & McNary Dam & $\mathrm{cm}$ & 8 & 9 & August 1, 1977 & not reported \\
\hline & Seg3_Core10 & MC-77 9-10cm & \begin{tabular}{|l|} 
Columbia River \\
\end{tabular} & McNary Dam & $\mathrm{cm}$ & 9 & 10 & August 1, 1977 & not reported \\
\hline & Seg3_Core 10 & MC-77 $10-11 \mathrm{~cm}$ & Columbia River & McNary Dam & $\mathrm{cm}$ & 10 & 11 & August 1, 1977 & not reported \\
\hline & Seg3_Core10 & MC-77 $13-14 \mathrm{~cm}$ & Columbia River & McNary Dam & $\mathrm{cm}$ & 13 & 14 & August 1, 1977 & January 1,1970 \\
\hline & Seg3_Core10 & MC-77 $14-15 \mathrm{~cm}$ & Columbia River & McNary Dam & $\mathrm{cm}$ & 14 & 15 & August 1, 1977 & January 1, 1970 \\
\hline & Seg3_Core10 & MC-77 $15-16 \mathrm{~cm}$ & Columbia River & McNary Dam & $\mathrm{cm}$ & 15 & 16 & August 1, 1977 & January 1, 1969 \\
\hline & Seg3_Core10 & MC-77 $16-17 \mathrm{~cm}$ & Columbia River & McNary Dam & $\mathrm{cm}$ & 16 & 17 & August 1, 1977 & January 1, 1969 \\
\hline & Seg3_Core10 & MC-77 $19-20 \mathrm{~cm}$ & Columbia River & McNary Dam & $\mathrm{cm}$ & 19 & 20 & August 1, 1977 & January 1, 1967 \\
\hline & Seg3_Core10 & MC-77 22-23cm & Columbia River & McNary Dam & $\mathrm{cm}$ & 22 & 23 & August 1, 1977 & January 1, 1967 \\
\hline & Seg3_Core10 & MC-77 $23-24 \mathrm{~cm}$ & Columbia River & McNary Dam & $\mathrm{cm}$ & 23 & 24 & August 1, 1977 & June 1,1964 \\
\hline & Seg3_Core10 & MC-77 24-25cm & Columbia River & McNary Dam & $\mathrm{cm}$ & 24 & 25 & August 1, 1977 & June 1,1964 \\
\hline & Seg3_Core10 & MC-77 25-26cm & Columbia River & McNary Dam & $\mathrm{cm}$ & 25 & 26 & August 1, 1977 & June 1, 1963 \\
\hline & Seg3_Core10 & MC-77 $26-27 \mathrm{~cm}$ & Columbia River & McNary Dam & $\mathrm{cm}$ & 26 & 27 & August 1, 1977 & June 1,1963 \\
\hline & Seg3_Core10 & MC-77 $34-35 \mathrm{~cm}$ & Columbia River & McNary Dam & $\mathrm{cm}$ & 34 & 35 & August 1, 1977 & June 1, 1958 \\
\hline & Seg3_Core 10 & MC-77 $39-40 \mathrm{~cm}$ & Columbia River & McNary Dam & $\mathrm{cm}$ & 39 & 40 & August 1,1977 & January 1,1956 \\
\hline & Seg3_Core 10 & MC-77 $53-54 \mathrm{~cm}$ & Columbia River & McNary Dam & $\mathrm{cm}$ & 53 & 54 & August 1, 1977 & January 1,1956 \\
\hline & Seg3_Core10 & MC-77 56-57cm & Columbia River & McNary Dam & $\mathrm{cm}$ & 56 & 57 & August 1,1977 & January 1, 1956 \\
\hline \multirow[t]{6}{*}{ An Investigation of the Origin of 152 Eu in Columbia River Sediment } & Seg3_Core11 & McNary-1-6 & Columbia River & McNary Dam & in & 24 & 25 & July 28, 1999 & March 12, 1971 \\
\hline & Seg3_Core11 & McNary-1-5 & Columbia River & McNary Dam & in & 25 & 26 & July 28, 1999 & January 25, 1970 \\
\hline & Seg3_Core11 & McNary-1-4 & Columbia River & McNary Dam & in & 26 & 27 & July 28, 1999 & December 10, 1968 \\
\hline & Seg3_Core11 & McNary-1-3 & Columbia River & McNary Dam & in & 27 & 28 & July 28, 1999 & October 25, 1967 \\
\hline & Seg3_Core11 & McNary-1-2 & Columbia River & McNary Dam & in & 28 & 29 & July 28, 1999 & September 9, 1966 \\
\hline & Seg3_Core11 & McNary-1-1 & Columbia River & McNary Dam & in & 29 & 30 & July 29, 1999 & July 24, 1965 \\
\hline
\end{tabular}


Table 4-19. Summary of Sediment Core Samples Collected in Segment 3 of the Columbia River Component. (10 Pages)

\begin{tabular}{|c|c|c|c|c|c|c|c|c|c|}
\hline Source Title & Core ID & Sample ID & Sample Area & Sample Site & $\begin{array}{l}\text { Interval Depth } \\
\text { Units }\end{array}$ & $\begin{array}{l}\text { Upper } \\
\text { Depth }\end{array}$ & $\begin{array}{l}\text { Lower } \\
\text { Depth }\end{array}$ & Collection Date & $\begin{array}{l}\text { Estimated Sediment Deposition } \\
\text { Date }\end{array}$ \\
\hline \multirow[t]{10}{*}{ An Investigation of the Origin of 152 Eu in Columbia River Sediment } & Seg3_Core12 & McNary-2-27.5 & Columbia River & McNary Dam & in & 0 & 1 & July 28,1999 & "post Hanford" \\
\hline & Seg3_Core12 & McNary-2-26.5 & Columbia River & McNary Dam & in & 1 & 2.5 & July 28, 1999 & "post Hanford" \\
\hline & Seg3_Core12 & McNary-2-25 & Columbia River & McNary Dam & in & 2.5 & 3.5 & July 28, 1999 & "post Hanford" \\
\hline & Seg3_Core12 & McNary-2-16 & Columbia River & McNary Dam & in & 11.5 & 12.5 & July 28, 1999 & "post Hanford" \\
\hline & Seg3_Core12 & McNary-2-15 & Columbia River & McNary Dam & in & 12.5 & 13.5 & July 28, 1999 & "post Hanford" \\
\hline & Seg3_Core12 & McNary-2-14 & Columbia River & McNary Dam & in & 13.5 & 14.5 & July 28, 1999 & "post Hanford" \\
\hline & Seg3_Core12 & McNary-2-4 & Columbia River & McNary Dam & in & 23.5 & 24.5 & July 28, 1999 & "post Hanford" \\
\hline & Seg3_Core12 & McNary-2-3 & \begin{tabular}{|l} 
Columbia River \\
\end{tabular} & McNary Dam & in & 24.5 & 25.5 & July 28, 1999 & "post Hanford" \\
\hline & Seg3_Core12 & McNary-2-2 & Columbia River & McNary Dam & in & 25.5 & 26.5 & July 28, 1999 & "post Hanford" \\
\hline & Seg3_Core12 & McNary-2-1 & Columbia River & McNary Dam & in & 26.5 & 27.5 & July 28, 1999 & "post Hanford" \\
\hline \multirow{24}{*}{$\begin{array}{l}\text { Heavy Metal Transport and Behavior in the Lower Columbia River, } \\
\text { USA }\end{array}$} & Seg3_Core13 & MDC_1.2 & Columbia River & McNary Dam & $\mathrm{cm}$ & 1.2 & 3.8 & August 1, 1999 & January 1, 1999 \\
\hline & Seg3_Core13 & MDC_3.8 & \begin{tabular}{|l} 
Columbia River \\
\end{tabular} & McNary Dam & $\mathrm{cm}$ & 3.8 & 6.4 & August 1, 1999 & January 1, 1998 \\
\hline & Seg3_Core13 & MDC_6.4 & Columbia River & McNary Dam & $\mathrm{cm}$ & 6.4 & 8.9 & August 1, 1999 & January 1, 1997 \\
\hline & Seg3_Core13 & MDC_8.9 & Columbia River & McNary Dam & $\mathrm{cm}$ & 8.9 & 11.4 & August 1, 1999 & January 1,1996 \\
\hline & Seg3_Core13 & MDC_11.4 & Columbia River & McNary Dam & $\mathrm{cm}$ & 11.4 & 14 & August 1, 1999 & January 1, 1995 \\
\hline & Seg3_Core13 & MDC_14.0 & Columbia River & McNary Dam & $\mathrm{cm}$ & 14 & 16.5 & August 1, 1999 & January 1, 1994 \\
\hline & Seg3_Core13 & MDC_16.5 & Columbia River & McNary Dam & $\mathrm{cm}$ & 16.5 & 19.1 & August 1, 1999 & January 1, 1992 \\
\hline & Seg3_Core13 & MDC_19.1 & Columbia River & McNary Dam & $\mathrm{cm}$ & 19.1 & 21.6 & August 1, 1999 & January 1, 1991 \\
\hline & Seg3_Core13 & MDC_21.6 & Columbia River & McNary Dam & $\mathrm{cm}$ & 21.6 & 24.2 & August 1, 1999 & January 1,1990 \\
\hline & Seg3_Core13 & MDC_24.2 & Columbia River & McNary Dam & $\mathrm{cm}$ & 24.2 & 26.2 & August 1, 1999 & January 1, 1989 \\
\hline & Seg3_Core13 & MDC_26.2 & Columbia River & McNary Dam & $\mathrm{cm}$ & 26.2 & 29.2 & August 1, 1999 & January 1,1987 \\
\hline & Seg3_Core13 & MDC_29.2 & Columbia River & McNary Dam & $\mathrm{cm}$ & 29.2 & 31.8 & August 1, 1999 & January 1, 1986 \\
\hline & Seg3_Core13 & MDC_31.8 & Columbia River & McNary Dam & $\mathrm{cm}$ & 31.8 & 34.3 & August 1, 1999 & January 1,1985 \\
\hline & Seg3_Core13 & MDC_34.3 & Columbia River & McNary Dam & $\mathrm{cm}$ & 34.3 & 36.8 & August 1, 1999 & January 1, 1984 \\
\hline & Seg3_Core13 & MDC_36.8 & Columbia River & McNary Dam & $\mathrm{cm}$ & 36.8 & 39.4 & August 1, 1999 & January 1,1982 \\
\hline & Seg3_Core13 & MDC_39.4 & Columbia River & McNary Dam & $\mathrm{cm}$ & 39.4 & 41.9 & August 1, 1999 & January 1,1981 \\
\hline & Seg3_Core13 & MDC_41.9 & Columbia River & McNary Dam & $\mathrm{cm}$ & 41.9 & 44.5 & August 1, 1999 & January 1,1980 \\
\hline & Seg3_Core13 & MDC_44.5 & \begin{tabular}{|l} 
Columbia River \\
\end{tabular} & McNary Dam & $\mathrm{cm}$ & 44.5 & 47 & August 1, 1999 & January 1, 1979 \\
\hline & Seg3_Core13 & MDC_47.0 & Columbia River & McNary Dam & $\mathrm{cm}$ & 47 & 49.6 & August 1, 1999 & January 1,1978 \\
\hline & Seg3_Core13 & MDC_49.6 & \begin{tabular}{|l} 
Columbia River \\
\end{tabular} & McNary Dam & $\mathrm{cm}$ & 49.6 & 52.1 & August 1, 1999 & January 1, 1977 \\
\hline & Seg3_Core13 & MDC_52.1 & Columbia River & McNary Dam & $\mathrm{cm}$ & 52.1 & 54.6 & August 1, 1999 & January 1,1976 \\
\hline & Seg3_Core13 & MDC_54.6 & \begin{tabular}{|l} 
Columbia River \\
\end{tabular} & McNary Dam & $\mathrm{cm}$ & 54.6 & 57.2 & August 1, 1999 & January 1,1975 \\
\hline & Seg3_Core13 & MDC_57.2 & \begin{tabular}{|l} 
Columbia River \\
\end{tabular} & McNary Dam & $\mathrm{cm}$ & 57.2 & 59.7 & August 1, 1999 & January 1,1974 \\
\hline & Seg3_Core13 & MDC_59.7 & Columbia River & McNary Dam & $\mathrm{cm}$ & 59.7 & 66.1 & August 1, 1999 & January 1,1973 \\
\hline
\end{tabular}


Table 4-19. Summary of Sediment Core Samples Collected in Segment 3 of the Columbia River Component. (10 Pages)

\begin{tabular}{|c|c|c|c|c|c|c|c|c|c|}
\hline Source Title & Core ID & Sample ID & Sample Area & Sample Site & $\begin{array}{l}\text { Interval Depth } \\
\text { Units }\end{array}$ & $\begin{array}{l}\text { Upper } \\
\text { Depth }\end{array}$ & $\begin{array}{l}\text { Lower } \\
\text { Depth }\end{array}$ & Collection Date & $\begin{array}{l}\text { Estimated Sediment Deposition } \\
\text { Date }\end{array}$ \\
\hline & Seg3_Core13 & MDC_62.2 & Columbia River & McNary Dam & $\mathrm{cm}$ & 62.2 & 66.1 & August 1, 1999 & January 1,1970 \\
\hline & Seg3_Core13 & MDC_66.1 & Columbia River & McNary Dam & $\mathrm{cm}$ & 66.1 & 68.6 & August 1, 1999 & January 1,1969 \\
\hline & Seg3_Core13 & MDC_68.6 & Columbia River & McNary Dam & $\mathrm{cm}$ & 68.6 & 70.6 & August 1, 1999 & January 1, 1968 \\
\hline \multirow{12}{*}{$\begin{array}{l}\text { Nickel-63 in Columbia River Sediments below the Hanford } \\
\text { Reservation }\end{array}$} & Seg3_Core14 & MR-86 2-4cm & Columbia River & McNary Dam & $\mathrm{cm}$ & 2 & 4 & August 1, 1977 & June 1,1975 \\
\hline & Seg3_Core14 & MR-86 4-6cm & Columbia River & McNary Dam & $\mathrm{cm}$ & 4 & 6 & August 1, 1977 & February 1, 1974 \\
\hline & Seg3_Core14 & MR-86 7-8cm & Columbia River & McNary Dam & $\mathrm{cm}$ & 7 & 8 & August 1, 1977 & February 1, 1972 \\
\hline & Seg3_Core14 & MR-86 $10-11 \mathrm{~cm}$ & Columbia River & McNary Dam & $\mathrm{cm}$ & 10 & 11 & August 1, 1977 & February 1, 1970 \\
\hline & Seg3_Core14 & MR-86 $13-14 \mathrm{~cm}$ & Columbia River & McNary Dam & $\mathrm{cm}$ & 13 & 14 & August 1, 1977 & February 1, 1968 \\
\hline & Seg3_Core14 & MR-86 $16-17 \mathrm{~cm}$ & Columbia River & McNary Dam & $\mathrm{cm}$ & 16 & 17 & August 1, 1977 & November 1, 1965 \\
\hline & Seg3_Core14 & MR-86 19-20cm & Columbia River & McNary Dam & $\mathrm{cm}$ & 19 & 20 & August 1, 1977 & June 1,1965 \\
\hline & Seg3_Core14 & MR-86 22-23cm & Columbia River & McNary Dam & $\mathrm{cm}$ & 22 & 23 & August 1, 1977 & June 1, 1961 \\
\hline & Seg3_Core14 & MR-86 $25-26 \mathrm{~cm}$ & Columbia River & McNary Dam & $\mathrm{cm}$ & 25 & 26 & August 1, 1977 & June 1, 1959 \\
\hline & Seg3_Core14 & MR-86 28-29cm & Columbia River & McNary Dam & $\mathrm{cm}$ & 28 & 29 & August 1, 1977 & February 1, 1957 \\
\hline & Seg3_Core14 & MR-86 $31-32 \mathrm{~cm}$ & Columbia River & McNary Dam & $\mathrm{cm}$ & 31 & 32 & August 1, 1977 & February 1, 1955 \\
\hline & Seg3_Core14 & MR-86 $34-35 \mathrm{~cm}$ & Columbia River & McNary Dam & $\mathrm{cm}$ & 34 & 35 & August 1, 1977 & February 1,1953 \\
\hline \multirow{13}{*}{$\begin{array}{l}\text { Association of Hanford Origin Radionuclides with Columbia River } \\
\text { Sediment }\end{array}$} & Seg3_Core15 & $M-F-0-5.1$ & Columbia River & McNary Dam & $\mathrm{cm}$ & 0 & 5.1 & August 18, 1976 & May 1, 1974 \\
\hline & Seg3_Core15 & M-F-10.2-12.7 & Columbia River & McNary Dam & $\mathrm{cm}$ & 10.2 & 12.7 & August 18, 1976 & January 1,1975 \\
\hline & Seg3_Core15 & M-F-12.7-15.2 & Columbia River & McNary Dam & $\mathrm{cm}$ & 12.7 & 15.2 & August 18,1976 & November 1, 1969 \\
\hline & Seg3_Core15 & M-F-15.2-17.9 & Columbia River & McNary Dam & $\mathrm{cm}$ & 15.2 & 17.9 & August 18, 1976 & September 1, 1968 \\
\hline & Seg3_Core15 & M-F-17.9-20.3 & Columbia River & McNary Dam & $\mathrm{cm}$ & 17.9 & 20.3 & August 18, 1976 & May 1,1967 \\
\hline & Seg3_Core15 & M-F-20.3-22.9 & Columbia River & McNary Dam & $\mathrm{cm}$ & 20.3 & 22.9 & August 18, 1976 & February 1, 1966 \\
\hline & Seg3_Core15 & M-F-22.9-25.4 & Columbia River & McNary Dam & $\mathrm{cm}$ & 22.9 & 25.4 & August 18, 1976 & August 1, 1963 \\
\hline & Seg3_Core15 & M-F-25.4-27.9 & Columbia River & McNary Dam & $\mathrm{cm}$ & 25.4 & 27.9 & August 18, 1976 & April 1, 1962 \\
\hline & Seg3_Core15 & M-F-27.9-30.5 & Columbia River & McNary Dam & $\mathrm{cm}$ & 27.9 & 30.5 & August 18, 1976 & January 1, 1961 \\
\hline & Seg3_Core15 & M-F-30.5-33.0 & Columbia River & McNary Dam & $\mathrm{cm}$ & 30.5 & 33 & August 18,1976 & October 1, 1959 \\
\hline & Seg3_Core15 & M-F-33.0-35.6 & Columbia River & McNary Dam & $\mathrm{cm}$ & 33 & 35.6 & August 18, 1976 & June 1,1958 \\
\hline & Seg3_Core15 & M-F-35.6-38.1 & Columbia River & McNary Dam & $\mathrm{cm}$ & 35.6 & 38.1 & August 18, 1976 & January 1,1957 \\
\hline & Seg3_Core15 & M-F-38.1-40.6 & Columbia River & McNary Dam & $\mathrm{cm}$ & 38.1 & 40.6 & August 18, 1976 & December 1, 1955 \\
\hline \multirow{6}{*}{$\begin{array}{l}\text { Association of Hanford Origin Radionuclides with Columbia River } \\
\text { Sediment }\end{array}$} & Seg3_Core16 & M-E-2-0-10.2 & Columbia River & McNary Dam & $\mathrm{cm}$ & 0 & 10.2 & September 9, 1976 & February 1, 1973 \\
\hline & Seg3_Core16 & M-E-2-10.2-20.3 & \begin{tabular}{|l} 
Columbia River \\
\end{tabular} & McNary Dam & $\mathrm{cm}$ & 10.2 & 20.3 & September 9, 1976 & January 1, 1968 \\
\hline & Seg3_Core16 & M-E-2-20.3-30.5 & Columbia River & McNary Dam & $\mathrm{cm}$ & 20.3 & 30.5 & September 9, 1976 & pre-1942 \\
\hline & Seg3_Core16 & M-E-2-30.5-40.6 & Columbia River & McNary Dam & $\mathrm{cm}$ & 30.5 & 40.6 & September 9, 1976 & November 1, 1957 \\
\hline & Seg3_Core16 & M-E-2-40.6-50.8 & Columbia River & McNary Dam & $\mathrm{cm}$ & 40.6 & 50.8 & September 9, 1976 & October 1,1952 \\
\hline & Seg3_Core16 & M-E-2-50.8-61.0 & Columbia River & McNary Dam & $\mathrm{cm}$ & 50.8 & 61 & September 9, 1976 & August 1,1947 \\
\hline
\end{tabular}


Table 4-19. Summary of Sediment Core Samples Collected in Segment 3 of the Columbia River Component. (10 Pages)

\begin{tabular}{|c|c|c|c|c|c|c|c|c|c|}
\hline Source Title & Core ID & Sample ID & Sample Area & Sample Site & $\begin{array}{l}\text { Interval Depth } \\
\text { Units }\end{array}$ & $\begin{array}{l}\text { Upper } \\
\text { Depth }\end{array}$ & $\begin{array}{l}\text { Lower } \\
\text { Depth }\end{array}$ & Collection Date & $\begin{array}{c}\text { Estimated Sediment Deposition } \\
\text { Date }\end{array}$ \\
\hline & Seg3_Core16 & M-E-2-61.0-71.1 & Columbia River & McNary Dam & $\mathrm{cm}$ & 61 & 71.1 & September 9, 1976 & August 1, 1942 \\
\hline & Seg3_Core16 & M-E-2-71.1-81.3 & Columbia River & McNary Dam & $\mathrm{cm}$ & 71.1 & 81.3 & September 9, 1976 & pre-1942 \\
\hline & Seg3_Core16 & M-E-2-81.3-91.4 & Columbia River & McNary Dam & $\mathrm{cm}$ & 81.3 & 91.4 & September 9, 1976 & pre-1942 \\
\hline & Seg3_Core16 & M-E-2-91.4-101.6 & Columbia River & McNary Dam & $\mathrm{cm}$ & 91.4 & 101.6 & September 9, 1976 & pre-1942 \\
\hline & Seg3_Core16 & M-E-2-101.6-111.8 & Columbia River & McNary Dam & $\mathrm{cm}$ & 101.6 & 111.8 & September 9, 1976 & pre-1942 \\
\hline & Seg3_Core16 & M-E-2-111.8-121.9 & Columbia River & McNary Dam & $\mathrm{cm}$ & 111.8 & 121.9 & September 9, 1976 & pre-1942 \\
\hline & Seg3_Core16 & M-E-2-121.9-132.1 & Columbia River & McNary Dam & $\mathrm{cm}$ & 121.9 & 132.1 & September 9, 1976 & pre-1942 \\
\hline & Seg3_Core16 & M-E-2-132.1-142.2 & \begin{tabular}{|l|} 
Columbia River \\
\end{tabular} & McNary Dam & $\mathrm{cm}$ & 132.1 & 142.2 & September 9, 1976 & pre-1942 \\
\hline & Seg3_Core16 & M-E-2-142.2-152.4 & Columbia River & McNary Dam & $\mathrm{cm}$ & 142.2 & 152.4 & September 9, 1976 & pre-1942 \\
\hline & Seg3_Core16 & M-E-2-152.4-162.6 & \begin{tabular}{|l|} 
Columbia River \\
\end{tabular} & McNary Dam & $\mathrm{cm}$ & 152.4 & 162.6 & September 9, 1976 & pre-1942 \\
\hline & Seg3_Core16 & M-E-2-162.6-172.7 & Columbia River & McNary Dam & $\mathrm{cm}$ & 162.6 & 172.7 & September 9, 1976 & pre-1942 \\
\hline & Seg3_Core16 & M-E-2-172.7-182.9 & \begin{tabular}{|l|} 
Columbia River \\
\end{tabular} & McNary Dam & $\mathrm{cm}$ & 172.7 & 182.9 & September 9, 1976 & pre-1942 \\
\hline & Seg3_Core 16 & M-E-2-182.9-193.0 & Columbia River & McNary Dam & $\mathrm{cm}$ & 182.9 & 193 & September 9, 1976 & pre-1942 \\
\hline & Seg3_Core16 & M-E-2-193.0-203.2 & Columbia River & McNary Dam & $\mathrm{cm}$ & 193 & 203.2 & September 9, 1976 & pre-1942 \\
\hline & Seg3_Core16 & M-E-2-203.2-213.4 & Columbia River & McNary Dam & $\mathrm{cm}$ & 203.2 & 213.4 & September 9, 1976 & pre-1942 \\
\hline & Seg3_Core16 & M-E-2-213.4-223.5 & Columbia River & McNary Dam & $\mathrm{cm}$ & 213.4 & 223.5 & September 9, 1976 & pre-1942 \\
\hline & Seg3_Core16 & M-E-2-223.5-233.7 & Columbia River & McNary Dam & $\mathrm{cm}$ & 223.5 & 233.7 & September 9, 1976 & pre-1942 \\
\hline & Seg3_Core16 & M-E-2-233.7-243.8 & Columbia River & McNary Dam & $\mathrm{cm}$ & 233.7 & 243.8 & September 9, 1976 & pre-1942 \\
\hline & Seg3_Core16 & M-E-2-243.8-254.0 & Columbia River & McNary Dam & $\mathrm{cm}$ & 243.8 & 254 & September 9, 1976 & pre-1942 \\
\hline & Seg3_Core16 & M-E-2-254.0-264.2 & Columbia River & McNary Dam & $\mathrm{cm}$ & 254 & 264.2 & September 9, 1976 & pre-1942 \\
\hline & Seg3_Core16 & M-E-2-264.2-274.3 & Columbia River & McNary Dam & $\mathrm{cm}$ & 264.2 & 274.3 & September 9, 1976 & pre-1942 \\
\hline & Seg3_Core16 & M-E-2-274.0-284.5 & Columbia River & McNary Dam & $\mathrm{cm}$ & 274 & 284.5 & September 9, 1976 & pre-1942 \\
\hline & Seg3_Core16 & M-E-2-284.5-294.6 & Columbia River & McNary Dam & $\mathrm{cm}$ & 284.5 & 294.6 & September 9, 1976 & pre-1942 \\
\hline \multirow{11}{*}{$\begin{array}{l}\text { Association of Hanford Origin Radionuclides with Columbia River } \\
\text { Sediment }\end{array}$} & Seg3_Core17 & M-E-1-0-5.1 & Columbia River & McNary Dam & $\mathrm{cm}$ & 0 & 5.1 & August 18, 1976 & May 1, 1974 \\
\hline & Seg3_Core17 & M-E-1-5.1-12.7 & Columbia River & McNary Dam & $\mathrm{cm}$ & 5.1 & 12.7 & August 18,1976 & February 1, 1971 \\
\hline & Seg3_Core17 & $M-E-1-12.7-15.2$ & Columbia River & McNary Dam & $\mathrm{cm}$ & 12.7 & 15.2 & August 18, 1976 & August 1, 1968 \\
\hline & Seg3_Core17 & M-E-1-15.2-17.8 & Columbia River & McNary Dam & $\mathrm{cm}$ & 15.2 & 17.8 & August 18, 1976 & May 1,1967 \\
\hline & Seg3_Core17 & M-E-1-17.8-20.0 & \begin{tabular}{|l|} 
Columbia River \\
\end{tabular} & McNary Dam & $\mathrm{cm}$ & 17.8 & 20 & August 18,1976 & February 1, 1966 \\
\hline & Seg3_Core17 & M-E-1-20.0-22.9 & Columbia River & McNary Dam & $\mathrm{cm}$ & 20 & 22.9 & August 18, 1976 & December 1, 1964 \\
\hline & Seg3_Core17 & M-E-1-22.9-25.4 & \begin{tabular}{|l|} 
Columbia River \\
\end{tabular} & McNary Dam & $\mathrm{cm}$ & 22.9 & 25.4 & August 18, 1976 & July 1, 1963 \\
\hline & Seg3_Core17 & M-E-1-25.4-27.9 & Columbia River & McNary Dam & $\mathrm{cm}$ & 25.4 & 27.9 & August 18, 1976 & May 1, 1962 \\
\hline & Seg3_Core17 & M-E-1-27.9-30.5 & \begin{tabular}{|l|} 
Columbia River \\
\end{tabular} & McNary Dam & $\mathrm{cm}$ & 27.9 & 30.5 & August 18, 1976 & January 1, 1961 \\
\hline & Seg3_Core17 & M-E-1-30.5-33.0 & Columbia River & McNary Dam & $\mathrm{cm}$ & 30.5 & 33 & August 18, 1976 & November 1, 1959 \\
\hline & Seg3_Core17 & M-E-1-33.0-35.6 & Columbia River & McNary Dam & $\mathrm{cm}$ & 33 & 35.6 & August 18, 1976 & June 1, 1958 \\
\hline
\end{tabular}


Table 4-19. Summary of Sediment Core Samples Collected in Segment 3 of the Columbia River Component. (10 Pages)

\begin{tabular}{|c|c|c|c|c|c|c|c|c|c|}
\hline Source Title & Core ID & Sample ID & Sample Area & Sample Site & $\begin{array}{l}\text { Interval Depth } \\
\text { Units }\end{array}$ & $\begin{array}{l}\text { Upper } \\
\text { Depth }\end{array}$ & $\begin{array}{l}\text { Lower } \\
\text { Depth }\end{array}$ & Collection Date & $\begin{array}{l}\text { Estimated Sediment Deposition } \\
\text { Date }\end{array}$ \\
\hline & Seg3_Core17 & M-E-1-35.6-38.1 & Columbia River & McNary Dam & $\mathrm{cm}$ & 35.6 & 38.1 & August 18,1976 & March 1, 1957 \\
\hline & Seg3_Core17 & M-E-1-38.1-40.6 & Columbia River & McNary Dam & $\mathrm{cm}$ & 38.1 & 40.6 & August 18, 1976 & December 1, 1955 \\
\hline & Seg3_Core17 & M-E-1-40.6-43.2 & Columbia River & McNary Dam & $\mathrm{cm}$ & 40.6 & 43.2 & August 18,1976 & August 1,1954 \\
\hline & Seg3_Core17 & M-E-1-43.2-45.7 & Columbia River & McNary Dam & $\mathrm{cm}$ & 43.2 & 45.7 & August 18, 1976 & May 1, 1953 \\
\hline & Seg3_Core17 & M-E-1-45.7-48.3 & Columbia River & McNary Dam & $\mathrm{cm}$ & 45.7 & 48.3 & August 18,1976 & February 1, 1952 \\
\hline & Seg3_Core17 & M-E-1-48.3-50.8 & Columbia River & McNary Dam & $\mathrm{cm}$ & 48.3 & 50.8 & August 18, 1976 & November 1, 1951 \\
\hline & Seg3_Core17 & M-E-1-50.8-53.5 & Columbia River & McNary Dam & $\mathrm{cm}$ & 50.8 & 53.5 & August 18, 1976 & July 1, 1949 \\
\hline & Seg3_Core17 & M-E-1-53.5-55.9 & \begin{tabular}{|l|} 
Columbia River \\
\end{tabular} & McNary Dam & $\mathrm{cm}$ & 53.5 & 55.9 & August 18, 1976 & March 1, 1948 \\
\hline & Seg3_Core17 & M-E-1-55.9-58.4 & Columbia River & McNary Dam & $\mathrm{cm}$ & 55.9 & 58.4 & August 18, 1976 & January 1, 1947 \\
\hline & Seg3_Core17 & M-E-1-58.4-60.0 & \begin{tabular}{|l|} 
Columbia River \\
\end{tabular} & McNary Dam & $\mathrm{cm}$ & 58.4 & 60 & August 18, 1976 & January 1, 1946 \\
\hline & Seg3_Core17 & M-E-1-60.0-63.5 & Columbia River & McNary Dam & $\mathrm{cm}$ & 60 & 63.5 & August 18, 1976 & October 1, 1945 \\
\hline & Seg3_Core17 & M-E-1-63.5-66.0 & \begin{tabular}{|l|} 
Columbia River \\
\end{tabular} & McNary Dam & $\mathrm{cm}$ & 63.5 & 66 & August 18, 1976 & April 1, 1943 \\
\hline & Seg3_Core17 & M-E-1-66.0-71.1 & Columbia River & McNary Dam & $\mathrm{cm}$ & 66 & 71.1 & August 18, 1976 & May 1, 1941 \\
\hline \multirow[t]{6}{*}{ Hanford-derived Plutonium in Columbia River Sediments } & SR_Core1 & Ice Harbor 1-1 & Columbia River & Ice Harbor Dam & $\mathrm{cm}$ & 0 & 2 & August 16, 1977 & February 1, 1977 \\
\hline & SR_Core1 & Ice Harbor 1-5 & Columbia River & Ice Harbor Dam & $\mathrm{cm}$ & 6 & 7 & August 16, 1977 & June 1,1974 \\
\hline & SR_Core1 & Ice Harbor 1-11 & Columbia River & Ice Harbor Dam & $\mathrm{cm}$ & 14 & 15 & August 16, 1977 & June 1,1970 \\
\hline & SR_Core1 & Ice Harbor 1-13 & Columbia River & Ice Harbor Dam & $\mathrm{cm}$ & 16 & 17 & August 16, 1977 & June 1,1969 \\
\hline & SR_Core1 & Ice Harbor 1-17 & Columbia River & Ice Harbor Dam & $\mathrm{cm}$ & 26.5 & 27.5 & August 16, 1977 & February 1, 1964 \\
\hline & SR_Core1 & Ice Harbor 1-20 & Columbia River & Ice Harbor Dam & $\mathrm{cm}$ & 27.5 & 28.5 & August 16, 1977 & June 1, 1963 \\
\hline
\end{tabular}

Table 4-20. Summary of Sediment Core Samples Collected in Segment 4 of the Columbia River Component. (3 Pages)

\begin{tabular}{|c|c|c|c|c|c|c|c|c|c|}
\hline Source Title & Core ID & Sample ID & Sample Area & Sample Site & $\begin{array}{l}\text { Interval Depth } \\
\text { Units }\end{array}$ & $\begin{array}{l}\text { Upper } \\
\text { Depth }\end{array}$ & $\begin{array}{l}\text { Lower } \\
\text { Depth }\end{array}$ & $\begin{array}{l}\text { Collection } \\
\text { Date }\end{array}$ & $\begin{array}{l}\text { Estimated Sediment Deposition } \\
\text { Date }\end{array}$ \\
\hline \multirow{11}{*}{$\begin{array}{l}\text { Association of Hanford Origin Radionuclides with Columbia River } \\
\text { Sediment }\end{array}$} & Seg4_Core1 & B-A-0-2.5 & Columbia River & Stevenson, WA & $\mathrm{cm}$ & 0 & 2.5 & August 11, 1976 & January 1,1975 \\
\hline & Seg4_Core1 & B-A-2.5-5.1 & Columbia River & Stevenson, WA & $\mathrm{cm}$ & 2.5 & 5.1 & August 11, 1976 & August 1, 1973 \\
\hline & Seg4_Core1 & B-A-5.1-7.6 & Columbia River & Stevenson, WA & $\mathrm{cm}$ & 5.1 & 7.6 & August 11, 1976 & July 1, 1972 \\
\hline & Seg4_Core1 & B-A-7.6-10.2 & Columbia River & Stevenson, WA & $\mathrm{cm}$ & 7.6 & 10.2 & August 11, 1976 & February 1, 1971 \\
\hline & Seg4_Core1 & B-A-10.2-12.7 & Columbia River & Stevenson, WA & $\mathrm{cm}$ & 10.2 & 12.7 & August 11, 1976 & November 1, 1969 \\
\hline & Seg4_Core1 & B-A-12.7-15.2 & Columbia River & Stevenson, WA & $\mathrm{cm}$ & 12.7 & 15.2 & August 11, 1976 & September 1, 1968 \\
\hline & Seg4_Core1 & B-A-15.2-17.8 & Columbia River & Stevenson, WA & $\mathrm{cm}$ & 15.2 & 17.8 & August 11, 1976 & May 1, 1967 \\
\hline & Seg4_Core1 & B-A-17.8-20.3 & Columbia River & Stevenson, WA & $\mathrm{cm}$ & 17.8 & 20.3 & August 11, 1976 & February 1, 1966 \\
\hline & Seg4_Core1 & B-A-20.3-22.9 & Columbia River & Stevenson, WA & $\mathrm{cm}$ & 20.3 & 22.9 & August 11, 1976 & November 1, 1964 \\
\hline & Seg4_Core1 & B-A-22.9-25.4 & Columbia River & Stevenson, WA & $\mathrm{cm}$ & 22.9 & 25.4 & August 11, 1976 & August 1, 1963 \\
\hline & Seg4_Core1 & B-A-25.4-27.9 & Columbia River & Stevenson, WA & $\mathrm{cm}$ & 25.4 & 27.9 & August 11, 1976 & April 1, 1962 \\
\hline
\end{tabular}


Table 4-20. Summary of Sediment Core Samples Collected in Segment 4 of the Columbia River Component. (3 Pages)

\begin{tabular}{|c|c|c|c|c|c|c|c|c|c|}
\hline Source Title & Core ID & Sample ID & Sample Area & Sample Site & $\begin{array}{l}\text { Interval Depth } \\
\text { Units }\end{array}$ & $\begin{array}{l}\text { Upper } \\
\text { Depth }\end{array}$ & $\begin{array}{l}\text { Lower } \\
\text { Depth }\end{array}$ & $\begin{array}{l}\text { Collection } \\
\text { Date }\end{array}$ & $\begin{array}{c}\text { Estimated Sediment Deposition } \\
\text { Date }\end{array}$ \\
\hline & Seg4_Core1 & B-A-27.9-30.5 & Columbia River & Stevenson, WA & $\mathrm{cm}$ & 27.9 & 30.5 & August 11,1976 & January 1, 1961 \\
\hline & Seg4_Core1 & B-A-30.5-34.3 & Columbia River & Stevenson, WA & $\mathrm{cm}$ & 30.5 & 34.3 & August 11, 1976 & June 1, 1959 \\
\hline & Seg4_Core1 & B-A-34.3-39.4 & Columbia River & Stevenson, WA & $\mathrm{cm}$ & 34.3 & 39.4 & August 11,1976 & February 1, 1957 \\
\hline \multirow{11}{*}{$\begin{array}{l}\text { Association of Hanford Origin Radionuclides with Columbia River } \\
\text { Sediment }\end{array}$} & Seg4_Core2 & D-A-0-2.5 & Columbia River & The Dalles Dam & $\mathrm{cm}$ & 0 & 2.5 & August 10, 1976 & January 1,1975 \\
\hline & Seg4_Core2 & D-A-2.5-5.1 & Columbia River & The Dalles Dam & $\mathrm{cm}$ & 2.5 & 5.1 & August 10, 1976 & August 1,1973 \\
\hline & Seg4_Core2 & D-A-5.1-7.6 & Columbia River & The Dalles Dam & $\mathrm{cm}$ & 5.1 & 7.6 & August 10, 1976 & July 1, 1972 \\
\hline & Seg4_Core2 & D-A-7.6-10.2 & Columbia River & The Dalles Dam & $\mathrm{cm}$ & 7.6 & 10.2 & August 10, 1976 & February 1, 1971 \\
\hline & Seg4_Core2 & D-A-10.2-12.7 & Columbia River & The Dalles Dam & $\mathrm{cm}$ & 10.2 & 12.7 & August 10, 1976 & November 1, 1970 \\
\hline & Seg4_Core2 & D-A-12.7-15.2 & Columbia River & The Dalles Dam & $\mathrm{cm}$ & 12.7 & 15.2 & August 10, 1976 & August 1, 1968 \\
\hline & Seg4_Core2 & D-A-15.2-17.8 & Columbia River & The Dalles Dam & $\mathrm{cm}$ & 15.2 & 20.3 & August 10, 1976 & May 1, 1963 \\
\hline & Seg4_Core2 & D-A-17.8-20.3 & Columbia River & The Dalles Dam & $\mathrm{cm}$ & 17.8 & 20.3 & August 10, 1976 & December 1, 1961 \\
\hline & Seg4_Core2 & D-A-20.3-22.9 & Columbia River & The Dalles Dam & $\mathrm{cm}$ & 20.3 & 22.9 & August 10,1976 & November 1, 1964 \\
\hline & Seg4_Core2 & D-A-22.7-25.4 & Columbia River & The Dalles Dam & $\mathrm{cm}$ & 22.7 & 25.4 & August 10, 1976 & August 1, 1963 \\
\hline & Seg4_Core2 & D-A-25.4-30.5 & Columbia River & The Dalles Dam & $\mathrm{cm}$ & 25.4 & 30.5 & August 10, 1976 & August 1, 1961 \\
\hline \multirow{16}{*}{$\begin{array}{l}\text { Association of Hanford Origin Radionuclides with Columbia River } \\
\text { Sediment }\end{array}$} & Seg4_Core3 & D-B-0-2.5 & Columbia River & The Dalles Dam & $\mathrm{cm}$ & 0 & 2.5 & August 10, 1976 & January 1, 1975 \\
\hline & Seg4_Core3 & D-B-2.5-5.1 & Columbia River & The Dalles Dam & $\mathrm{cm}$ & 2.5 & 5.1 & August 10, 1976 & August 1, 1973 \\
\hline & Seg4_Core3 & D-B-5.1-7.6 & Columbia River & The Dalles Dam & $\mathrm{cm}$ & 5.1 & 7.6 & August 10, 1976 & June 1, 1972 \\
\hline & Seg4_Core3 & D-B-7.6-10.2 & Columbia River & The Dalles Dam & $\mathrm{cm}$ & 7.6 & 10.2 & August 10, 1976 & February 1, 1971 \\
\hline & Seg4_Core3 & D-B-10.2-12.7 & Columbia River & The Dalles Dam & $\mathrm{cm}$ & 10.2 & 12.7 & August 10, 1976 & November 1, 1970 \\
\hline & Seg4_Core3 & D-B-12.7-15.2 & Columbia River & The Dalles Dam & $\mathrm{cm}$ & 12.7 & 15.2 & August 10, 1976 & August 1, 1968 \\
\hline & Seg4_Core3 & D-B-15.2-17.8 & Columbia River & The Dalles Dam & $\mathrm{cm}$ & 15.2 & 17.8 & August 10, 1976 & May 1, 1967 \\
\hline & Seg4_Core3 & D-B-17.8-20.3 & Columbia River & The Dalles Dam & $\mathrm{cm}$ & 17.8 & 20.3 & August 10, 1976 & February 1, 1966 \\
\hline & Seg4_Core3 & D-B-20.3-22.9 & Columbia River & The Dalles Dam & $\mathrm{cm}$ & 20.3 & 22.9 & August 10, 1976 & November 1, 1964 \\
\hline & Seg4_Core3 & D-B-22.9-25.4 & Columbia River & The Dalles Dam & $\mathrm{cm}$ & 22.9 & 25.4 & August 10, 1976 & July 1, 1963 \\
\hline & Seg4_Core3 & D-B-25.4-27.9 & Columbia River & The Dalles Dam & $\mathrm{cm}$ & 25.4 & 27.9 & August 10, 1976 & April 1, 1962 \\
\hline & Seg4_Core3 & D-B-27.9-30.5 & Columbia River & The Dalles Dam & $\mathrm{cm}$ & 27.9 & 30.5 & August 10, 1976 & January 1, 1961 \\
\hline & Seg4_Core3 & D-B-30.5-33.0 & Columbia River & The Dalles Dam & $\mathrm{cm}$ & 30.5 & 33 & August 10, 1976 & October 1, 1959 \\
\hline & Seg4_Core3 & D-B-33.0-35.6 & Columbia River & The Dalles Dam & $\mathrm{cm}$ & 33 & 35.6 & August 10, 1976 & June 1, 1958 \\
\hline & Seg4_Core3 & D-B-35.6-38.1 & Columbia River & The Dalles Dam & $\mathrm{cm}$ & 35.6 & 38.1 & August 10, 1976 & February 1, 1957 \\
\hline & Seg4_Core3 & D-B-38.1-43.2 & Columbia River & The Dalles Dam & $\mathrm{cm}$ & 38.1 & 43.2 & August 10, 1976 & April 1, 1955 \\
\hline \multirow{4}{*}{$\begin{array}{l}\text { Association of Hanford Origin Radionuclides with Columbia River } \\
\text { Sediment }\end{array}$} & Seg4_Core4 & D-C-0-2.5 & Columbia River & The Dalles Dam & $\mathrm{cm}$ & 0 & 2.5 & August 11, 1976 & January 1,1975 \\
\hline & Seg4_Core4 & D-C-2.5-5.1 & Columbia River & The Dalles Dam & $\mathrm{cm}$ & 2.5 & 5.1 & August 11,1976 & August 1, 1973 \\
\hline & Seg4_Core4 & D-C-5.1-7.6 & Columbia River & The Dalles Dam & $\mathrm{cm}$ & 5.1 & 7.6 & August 11, 1976 & June 1, 1972 \\
\hline & Seg4_Core4 & D-C-7.6-10.2 & Columbia River & The Dalles Dam & $\mathrm{cm}$ & 7.6 & 10.2 & August 11,1976 & February 1, 1971 \\
\hline
\end{tabular}


Table 4-20. Summary of Sediment Core Samples Collected in Segment 4 of the Columbia River Component. (3 Pages)

\begin{tabular}{|c|c|c|c|c|c|c|c|c|c|}
\hline Source Title & Core ID & Sample ID & Sample Area & Sample Site & $\begin{array}{l}\text { Interval Depth } \\
\text { Units }\end{array}$ & $\begin{array}{l}\text { Upper } \\
\text { Depth }\end{array}$ & $\begin{array}{l}\text { Lower } \\
\text { Depth }\end{array}$ & $\begin{array}{l}\text { Collection } \\
\text { Date }\end{array}$ & $\begin{array}{c}\text { Estimated Sediment Deposition } \\
\text { Date }\end{array}$ \\
\hline & Seg4_Core4 & D-C-10.2-12.7 & Columbia River & The Dalles Dam & $\mathrm{cm}$ & 10.2 & 12.7 & August 11,1976 & November 1, 1970 \\
\hline & Seg4_Core4 & D-C-12.7-15.2 & Columbia River & The Dalles Dam & $\mathrm{cm}$ & 12.7 & 15.2 & August 11, 1976 & August 1, 1968 \\
\hline & Seg4_Core4 & D-C-15.2-17.8 & Columbia River & The Dalles Dam & $\mathrm{cm}$ & 15.2 & 17.8 & August 11,1976 & May 1, 1967 \\
\hline & Seg4_Core4 & D-C-17.8-20.3 & Columbia River & The Dalles Dam & $\mathrm{cm}$ & 17.8 & 20.3 & August 11, 1976 & February 1, 1966 \\
\hline & Seg4_Core4 & D-C-20.3-22.9 & Columbia River & The Dalles Dam & $\mathrm{cm}$ & 20.3 & 22.9 & August 11, 1976 & November 1, 1964 \\
\hline & Seg4_Core4 & D-C-22.9-27.9 & Columbia River & The Dalles Dam & $\mathrm{cm}$ & 22.9 & 27.9 & August 11,1976 & November 1, 1962 \\
\hline \multirow[t]{3}{*}{ Irrigon Boat Basin Sediment Evaluation } & Seg4_Core5 & IR-GC-1 & Columbia River & RM 282 & -- & a & -- & June 4, 1993 & -- \\
\hline & Seg4_Core5 & IR-GC-2 & Columbia River & RM 282 & -- & a & -- & June 4, 1993 & -- \\
\hline & Seg4_Core5 & IR-GC-3 & Columbia River & RM 282 & - & $\mathrm{a}$ & -- & June 4, 1993 & - \\
\hline \multirow{20}{*}{$\begin{array}{l}\text { Association of Hanford Origin Radionuclides with Columbia River } \\
\text { Sediment }\end{array}$} & Seg4_Core6 & JD-A-0-2.5 & Columbia River & John Day Dam & $\mathrm{cm}$ & 0 & 2.5 & August 10, 1976 & January 1,1975 \\
\hline & Seg4_Core6 & JD-A-2.5-5.1 & Columbia River & John Day Dam & $\mathrm{cm}$ & 2.5 & 5.1 & August 10, 1976 & August 1, 1973 \\
\hline & Seg4_Core6 & JD-A-5.1-7.6 & Columbia River & John Day Dam & $\mathrm{cm}$ & 5.1 & 7.6 & August 10, 1976 & June 1,1972 \\
\hline & Seg4_Core6 & JD-A-7.6-10.2 & Columbia River & John Day Dam & $\mathrm{cm}$ & 7.6 & 10.2 & August 10, 1976 & February 1, 1971 \\
\hline & Seg4_Core6 & JD-A-10.2-12.7 & Columbia River & John Day Dam & $\mathrm{cm}$ & 10.2 & 12.7 & August 10, 1976 & November 1, 1969 \\
\hline & Seg4_Core6 & JD-A-12.7-15.2 & Columbia River & John Day Dam & $\mathrm{cm}$ & 12.7 & 15.2 & August 10, 1976 & September 1, 1968 \\
\hline & Seg4_Core6 & JD-A-15.2-17.8 & Columbia River & John Day Dam & $\mathrm{cm}$ & 15.2 & 17.8 & August 10, 1976 & May 1,1967 \\
\hline & Seg4_Core6 & JD-A-17.8-20.3 & Columbia River & John Day Dam & $\mathrm{cm}$ & 17.8 & 20.3 & August 10, 1976 & February 1, 1966 \\
\hline & Seg4_Core6 & JD-A-20.3-22.9 & Columbia River & John Day Dam & $\mathrm{cm}$ & 20.3 & 22.9 & August 10, 1976 & November 1, 1964 \\
\hline & Seg4_Core6 & JD-A-22.9-25.4 & Columbia River & John Day Dam & $\mathrm{cm}$ & 22.9 & 25.4 & August 10, 1976 & August 1, 1963 \\
\hline & Seg4_Core6 & JD-A-25.4-27.9 & Columbia River & John Day Dam & $\mathrm{cm}$ & 25.4 & 27.9 & August 10, 1976 & April 1, 1962 \\
\hline & Seg4_Core6 & JD-A-27.9-30.5 & Columbia River & John Day Dam & $\mathrm{cm}$ & 27.9 & 30.5 & August 10, 1976 & January 1,1961 \\
\hline & Seg4_Core6 & JD-A-30.5-33.0 & Columbia River & John Day Dam & $\mathrm{cm}$ & 30.5 & 33 & August 10, 1976 & June 1,1959 \\
\hline & Seg4_Core6 & JD-A-33.0-35.6 & Columbia River & John Day Dam & $\mathrm{cm}$ & 33 & 35.6 & August 10, 1976 & June 1, 1958 \\
\hline & Seg4_Core6 & JD-A-35.6-38.1 & Columbia River & John Day Dam & $\mathrm{cm}$ & 35.6 & 38.1 & August 10, 1976 & March 1, 1957 \\
\hline & Seg4_Core6 & JD-A-38.1-40.6 & Columbia River & John Day Dam & $\mathrm{cm}$ & 38.1 & 40.6 & August 10, 1976 & December 1, 1956 \\
\hline & Seg4_Core6 & JD-A-40.6-43.2 & Columbia River & John Day Dam & $\mathrm{cm}$ & 40.6 & 43.2 & August 10, 1976 & August 1, 1954 \\
\hline & Seg4_Core6 & JD-A-43.2-45.7 & Columbia River & John Day Dam & $\mathrm{cm}$ & 43.2 & 45.7 & August 10, 1976 & May 1, 1953 \\
\hline & Seg4_Core6 & JD-A-45.7-48.3 & Columbia River & John Day Dam & $\mathrm{cm}$ & 45.7 & 48.3 & August 10, 1976 & February 1, 1952 \\
\hline & Seg4_Core6 & JD-A-48.3-53.5 & Columbia River & John Day Dam & $\mathrm{cm}$ & 48.3 & 53.5 & August 10, 1976 & February 1, 1950 \\
\hline
\end{tabular}

${ }^{a}$ Entire core length was composited; no depth was given

\begin{tabular}{l|l|l|l|}
\hline Seg4_Core6 & JD-A-48.3-53.5 & Columbia River & John Day Dam \\
\hline
\end{tabular} 
Table 4-21. Summary of Sediment Core Samples Collected in Segment 5 of the Columbia River Component. (3 Pages)

\begin{tabular}{|c|c|c|c|c|c|c|c|c|c|}
\hline Source Title & Core ID & Sample ID & Sample Area & $\begin{array}{l}\text { Sample } \\
\text { Site }\end{array}$ & $\begin{array}{l}\text { Interval Depth } \\
\text { Units }\end{array}$ & $\begin{array}{l}\text { Upper } \\
\text { Depth }\end{array}$ & $\begin{array}{l}\text { Lower } \\
\text { Depth }\end{array}$ & Collection Date & $\begin{array}{l}\text { Estimated Sediment } \\
\text { Deposition Date }\end{array}$ \\
\hline \multirow{3}{*}{$\begin{array}{l}\text { The vertical distribution of selected trace metals and organic compounds in bottom } \\
\text { materials in the proposed lower Columbia River export channel, Oregon. }\end{array}$} & CB-S6a $0-0.9 \mathrm{~m}$ & Seg5_Core1 & Columbia River & RM 21 & $\mathrm{~cm}$ & 0 & 90 & September 27, 1984 & January 1, 1953 \\
\hline & CB-S6a 2.1-2-2 & Seg5_Core1 & Columbia River & RM 21 & $\mathrm{~cm}$ & 210 & 220 & September 27, 1984 & August 1, 1958 \\
\hline & CB-S6a 0.9-3.7m & Seg5_Core1 & Columbia River & RM 21 & $\mathrm{~cm}$ & 90 & 370 & September 27, 1984 & October 1,1956 \\
\hline \multirow{8}{*}{$\begin{array}{l}\text { The vertical distribution of selected trace metals and organic compounds in bottom } \\
\text { materials in the proposed lower Columbia River export channel, Oregon. }\end{array}$} & CB-S6b .56-.76m & Seg5_Core2 & Columbia River & RM 21 & $\mathrm{~cm}$ & 56 & 76 & September 27, 1984 & October 1, 1976 \\
\hline & CB-S6b $1.17-1.40 \mathrm{~m}$ & Seg5_Core2 & Columbia River & RM 21 & $\mathrm{~cm}$ & 117 & 140 & September 27, 1984 & March 1, 1969 \\
\hline & CB-S6b $1.78-1.98 m$ & Seg5_Core2 & Columbia River & RM 21 & $\mathrm{~cm}$ & 178 & 198 & September 27, 1984 & September 1, 1961 \\
\hline & CB-S6b 2.58-2.76m & Seg5_Core2 & Columbia River & RM 21 & $\mathrm{~cm}$ & 258 & 276 & September 27, 1984 & March 1, 1952 \\
\hline & CB-S6b 2.64-3.82m & Seg5_Core2 & Columbia River & RM 21 & $\mathrm{~cm}$ & 264 & 382 & September 27, 1984 & April 1, 1947 \\
\hline & CB-S6b 3.10-3.20m & Seg5_Core2 & Columbia River & RM 21 & $\mathrm{~cm}$ & 310 & 320 & September 27, 1984 & March 1, 1946 \\
\hline & CB-S6b 3.23-3.44m & Seg5_Core2 & Columbia River & RM 21 & $\mathrm{~cm}$ & 323 & 344 & September 27, 1984 & January 1, 1944 \\
\hline & CB-S6b 4.22-4.42m & Seg5_Core2 & Columbia River & RM 21 & $\mathrm{~cm}$ & 422 & 442 & September 27, 1984 & February 1, 1932 \\
\hline \multirow{18}{*}{$\begin{array}{l}\text { Historical Changes in the Columbia River Estuary based on Sediment Cores: Feasibility } \\
\text { Studies. }\end{array}$} & CRCF-04_02 & Seg5_Core3 & Columbia River & Clatskanie FIt & $\mathrm{cm}$ & 2 & 4 & January 1, 2000 & December 1, 1997 \\
\hline & CRCF-04_06 & Seg5_Core3 & Columbia River & Clatskanie Flt & $\mathrm{cm}$ & 6 & 8 & January 1, 1997 & January 1, 1997 \\
\hline & CRCF-04_10 & Seg5_Core3 & Columbia River & Clatskanie Flt & $\mathrm{cm}$ & 10 & 12 & January 1,2000 & December 1, 1995 \\
\hline & CRCF-04_15 & Seg5_Core3 & Columbia River & Clatskanie Flt & $\mathrm{cm}$ & 15 & 17 & January 1, 2000 & March 1, 1994 \\
\hline & CRCF-04_20 & Seg5_Core3 & Columbia River & Clatskanie Flt & $\mathrm{cm}$ & 20 & 22 & January 1,2000 & March 1, 1992 \\
\hline & CRCF-04_25 & Seg5_Core3 & Columbia River & Clatskanie Flt & $\mathrm{cm}$ & 25 & 27 & January 1, 2000 & January 1, 1990 \\
\hline & CRCF-04_30 & Seg5_Core3 & Columbia River & Clatskanie Flt & $\mathrm{cm}$ & 30 & 32 & January 1, 2000 & June 1, 1987 \\
\hline & CRCF-04_40 & Seg5_Core3 & Columbia River & Clatskanie Flt & $\mathrm{cm}$ & 40 & 42 & January 1, 2000 & June 1, 1981 \\
\hline & CRCF-04_50 & Seg5_Core3 & Columbia River & Clatskanie Flt & $\mathrm{cm}$ & 50 & 52 & January 1,2000 & March 1, 1974 \\
\hline & CRCF-04_60 & Seg5_Core3 & Columbia River & Clatskanie Flt & $\mathrm{cm}$ & 60 & 62 & January 1, 2000 & October 1, 1965 \\
\hline & CRCF-04_70 & Seg5_Core3 & Columbia River & Clatskanie Flt & $\mathrm{cm}$ & 70 & 72 & January 1, 2000 & March 1, 1956 \\
\hline & CRCF-04_80 & Seg5_Core3 & Columbia River & Clatskanie Flt & $\mathrm{cm}$ & 80 & 82 & January 1, 2000 & May 1, 1945 \\
\hline & CRCF-04_90 & Seg5_Core3 & Columbia River & Clatskanie Flt & $\mathrm{cm}$ & 90 & 92 & January 1, 2000 & May 1, 1933 \\
\hline & CRCF-04_100 & Seg5_Core3 & Columbia River & Clatskanie Flt & $\mathrm{cm}$ & 100 & 102 & January 1,2000 & February 1,1920 \\
\hline & CRCF-04_110 & Seg5_Core3 & Columbia River & Clatskanie Flt & $\mathrm{cm}$ & 110 & 112 & January 1, 2000 & October 1, 1905 \\
\hline & CRCF-04_120 & Seg5_Core3 & Columbia River & Clatskanie FIt & $\mathrm{cm}$ & 120 & 122 & January 1, 2000 & February 1, 1890 \\
\hline & CRCF-04_130 & Seg5_Core3 & Columbia River & Clatskanie Flt & $\mathrm{cm}$ & 130 & 132 & January 1,2000 & May 1,1873 \\
\hline & CRCF-04_140 & Seg5_Core3 & Columbia River & Clatskanie Flt & $\mathrm{cm}$ & 140 & 142 & January 1,2000 & May 1,1855 \\
\hline
\end{tabular}


Table 4-21. Summary of Sediment Core Samples Collected in Segment 5 of the Columbia River Component. (3 Pages)

\begin{tabular}{|c|c|c|c|c|c|c|c|c|c|}
\hline Source Title & Core ID & Sample ID & Sample Area & $\begin{array}{c}\text { Sample } \\
\text { Site }\end{array}$ & $\begin{array}{l}\text { Interval Depth } \\
\text { Units }\end{array}$ & $\begin{array}{l}\text { Upper } \\
\text { Depth }\end{array}$ & $\begin{array}{l}\text { Lower } \\
\text { Depth }\end{array}$ & Collection Date & $\begin{array}{l}\text { Estimated Sediment } \\
\text { Deposition Date }\end{array}$ \\
\hline \multirow{19}{*}{$\begin{array}{l}\text { Historical Changes in the Columbia River Estuary based on Sediment Cores: Feasibility } \\
\text { Studies. }\end{array}$} & CRGB-06_02 & Seg5_Core4 & Columbia River & Gray's Bay & $\mathrm{cm}$ & 2 & 4 & January 1,2000 & October 1, 2001 \\
\hline & CRGB-06_06 & Seg5_Core4 & Columbia River & Gray's Bay & $\mathrm{cm}$ & 6 & 8 & January 1,2000 & January 1, 1998 \\
\hline & CRGB-06_10 & Seg5_Core4 & Columbia River & Gray's Bay & $\mathrm{cm}$ & 10 & 12 & January 1,2000 & February 1, 1994 \\
\hline & CRGB-06_15 & Seg5_Core4 & Columbia River & Gray's Bay & $\mathrm{cm}$ & 15 & 17 & January 1,2000 & April 1, 1989 \\
\hline & CRGB-06_150 & Seg5_Core4 & Columbia River & Gray's Bay & $\mathrm{cm}$ & 150 & 152 & January 1,2000 & 10/1/1852 \\
\hline & CRGB-06_20 & Seg5_Core4 & Columbia River & Gray's Bay & $\mathrm{cm}$ & 20 & 22 & January 1, 2000 & May 1, 1984 \\
\hline & CRGB-06_25 & Seg5_Core4 & Columbia River & Gray's Bay & $\mathrm{cm}$ & 25 & 27 & January 1,2000 & July 1, 1979 \\
\hline & CRGB-06_30 & Seg5_Core4 & Columbia River & Gray's Bay & $\mathrm{cm}$ & 30 & 32 & January 1,2000 & September 1, 1974 \\
\hline & CRGB-06_40 & Seg5_Core4 & Columbia River & Gray's Bay & $\mathrm{cm}$ & 40 & 42 & January 1,2000 & November 1, 1964 \\
\hline & CRGB-06_50 & Seg5_Core4 & Columbia River & Gray's Bay & $\mathrm{cm}$ & 50 & 52 & January 1,2000 & January 1, 1955 \\
\hline & CRGB-06_60 & Seg5_Core4 & Columbia River & Gray's Bay & $\mathrm{cm}$ & 60 & 62 & January 1,2000 & February 1, 1945 \\
\hline & CRGB-06_70 & Seg5_Core4 & Columbia River & Gray's Bay & $\mathrm{cm}$ & 70 & 72 & January 1, 2000 & February 1, 1935 \\
\hline & CRGB-06_80 & Seg5_Core4 & Columbia River & Gray's Bay & $\mathrm{cm}$ & 80 & 82 & January 1,2000 & January 1,1925 \\
\hline & CRGB-06_90 & Seg5_Core4 & Columbia River & Gray's Bay & $\mathrm{cm}$ & 90 & 92 & January 1,2000 & November 1, 1914 \\
\hline & CRGB-06_100 & Seg5_Core4 & Columbia River & Gray's Bay & $\mathrm{cm}$ & 100 & 102 & January 1,2000 & September 1, 1904 \\
\hline & CRGB-06_110 & Seg5_Core4 & Columbia River & Gray's Bay & $\mathrm{cm}$ & 110 & 112 & January 1, 2000 & 6/1/1894 \\
\hline & CRGB-06_120 & Seg5_Core4 & Columbia River & Gray's Bay & $\mathrm{cm}$ & 120 & 122 & January 1,2000 & 2/1/1884 \\
\hline & CRGB-06_130 & Seg5_Core4 & Columbia River & Gray's Bay & $\mathrm{cm}$ & 130 & 132 & January 1,2000 & 9/1/1873 \\
\hline & CRGB-06_140 & Seg5_Core4 & Columbia River & Gray's Bay & $\mathrm{cm}$ & 140 & 142 & January 1,2000 & 4/1/1863 \\
\hline \multirow{13}{*}{$\begin{array}{l}\text { Historical Changes in the Columbia River Estuary based on Sediment Cores: Feasibility } \\
\text { Studies. }\end{array}$} & CRYB-02_02 & Seg5_Core5 & Columbia River & Young's Bay & $\mathrm{cm}$ & 2 & 4 & January 1,2000 & September 1, 1999 \\
\hline & CRYB-02_06 & Seg5_Core5 & Columbia River & Young's Bay & $\mathrm{cm}$ & 6 & 8 & January 1,2000 & January 1, 1991 \\
\hline & CRYB-02_10 & Seg5_Core5 & Columbia River & Young's Bay & $\mathrm{cm}$ & 10 & 12 & January 1, 2000 & January 1, 1983 \\
\hline & CRYB-02_15 & Seg5_Core5 & Columbia River & Young's Bay & $\mathrm{cm}$ & 15 & 17 & January 1,2000 & June 1,1973 \\
\hline & CRYB-02_17 & Seg5_Core5 & Columbia River & Young's Bay & $\mathrm{cm}$ & 17 & 19 & January 1,2000 & October 1, 1969 \\
\hline & CRYB-02_20 & Seg5_Core5 & Columbia River & Young's Bay & $\mathrm{cm}$ & 20 & 22 & January 1,2000 & July 1, 1964 \\
\hline & CRYB-02_25 & Seg5_Core5 & Columbia River & Young's Bay & $\mathrm{cm}$ & 25 & 27 & January 1,2000 & April 1, 1956 \\
\hline & CRYB-02_30 & Seg5_Core5 & Columbia River & Young's Bay & $\mathrm{cm}$ & 30 & 32 & January 1, 2000 & September 1, 1948 \\
\hline & CRYB-02_40 & Seg5_Core5 & Columbia River & Young's Bay & $\mathrm{cm}$ & 40 & 42 & January 1,2000 & July 1, 1935 \\
\hline & CRYB-02_50 & Seg5_Core5 & Columbia River & Young's Bay & $\mathrm{cm}$ & 50 & 52 & January 1, 2000 & January 1, 1925 \\
\hline & CRYB-02_60 & Seg5_Core5 & Columbia River & Young's Bay & $\mathrm{cm}$ & 60 & 62 & January 1,2000 & April 1, 1917 \\
\hline & CRYB-02_70 & Seg5_Core5 & Columbia River & Young's Bay & $\mathrm{cm}$ & 70 & 72 & January 1, 2000 & February 1, 1912 \\
\hline & CRYB-02_80 & Seg5_Core5 & Columbia River & Young's Bay & $\mathrm{cm}$ & 80 & 82 & January 1,2000 & August 1, 1909 \\
\hline
\end{tabular}


Table 4-21. Summary of Sediment Core Samples Collected in Segment 5 of the Columbia River Component. (3 Pages)

\begin{tabular}{|c|c|c|c|c|c|c|c|c|c|}
\hline Source Title & Core ID & Sample ID & Sample Area & $\begin{array}{c}\text { Sample } \\
\text { Site }\end{array}$ & $\begin{array}{l}\text { Interval Depth } \\
\text { Units }\end{array}$ & $\begin{array}{l}\text { Upper } \\
\text { Depth }\end{array}$ & $\begin{array}{l}\text { Lower } \\
\text { Depth }\end{array}$ & Collection Date & $\begin{array}{l}\text { Estimated Sediment } \\
\text { Deposition Date }\end{array}$ \\
\hline \multirow{5}{*}{$\begin{array}{l}\text { The vertical distribution of selected trace metals and organic compounds in bottom } \\
\text { materials in the proposed lower Columbia River export channel, Oregon. }\end{array}$} & SR-S2 $0-0.9 \mathrm{~m}$ & Seg5_Core6 & Skipanon River & SR-S2 & $\mathrm{cm}$ & 0 & 90 & September 25, 1984 & January 1, 1953 \\
\hline & SR-S2 .9-4.1m & Seg5_Core6 & Skipanon River & SR-S2 & $\mathrm{cm}$ & 90 & 410 & September 25, 1984 & March 1, 1954 \\
\hline & SR-S2 1.1-1.15 & Seg5_Core6 & Skipanon River & SR-S2 & $\mathrm{cm}$ & 110 & 115 & September 25, 1984 & February 1,1971 \\
\hline & SR-S2 2.7-2.78 & Seg5_Core6 & Skipanon River & SR-S2 & $\mathrm{cm}$ & 270 & 278 & September 25, 1984 & April 1, 1951 \\
\hline & SR-S2 4.1-5.7m & Seg5_Core6 & Skipanon River & SR-S2 & $\mathrm{cm}$ & 410 & 570 & September 25, 1984 & January 1, 1925 \\
\hline \multirow{4}{*}{$\begin{array}{l}\text { The vertical distribution of selected trace metals and organic compounds in bottom } \\
\text { materials in the proposed lower Columbia River export channel, Oregon. }\end{array}$} & CR-S1 0-1.9m & Seg5_Core7 & Columbia River & CR-S1 & $\mathrm{cm}$ & 0 & 190 & September 26, 1984 & February 1,1973 \\
\hline & CR-S1 0.5-0.6 & Seg5_Core7 & Columbia River & CR-S1 & $\mathrm{cm}$ & 50 & 60 & September 26, 1984 & March 1, 1978 \\
\hline & CR-S1 1.9-6.1m & Seg5_Core7 & Columbia River & CR-S1 & $\mathrm{cm}$ & 190 & 610 & September 26, 1984 & January 1, 1936 \\
\hline & CR-S1 4.7 & Seg5_Core7 & Columbia River & CR-S1 & $\mathrm{cm}$ & 470 & 472 & September 26, 1984 & June 1,1927 \\
\hline \multirow{2}{*}{$\begin{array}{l}\text { The vertical distribution of selected trace metals and organic compounds in bottom } \\
\text { materials in the proposed lower Columbia River export channel, Oregon. }\end{array}$} & CR-S5 .08-1.0m & Seg5_Core8 & Columbia River & CR-S5 & $\mathrm{cm}$ & 8 & 100 & October 1, 1984 & October 1, 1984 \\
\hline & CR-S5 1.8-3.8m & Seg5_Core8 & Columbia River & CR-S5 & $\mathrm{cm}$ & 180 & 380 & October 1, 1984 & October 1, 1984 \\
\hline
\end{tabular}




\subsection{SUMMARY AND CONCLUSIONS}

The following subsections present a summary of the results for the data evaluated within this report and provides some preliminary observations. Tables 5-1 and 5-2 provide a summary of constituents that exceed either a human health or ecological benchmark by segment for sediment and surface water samples, respectively. The discussion that follows focuses on the primary contaminants with concentrations that are above a human health or ecological benchmark value.

It is important to note that when concentrations are below the selected benchmark value, an adverse health effect is not anticipated for the identified receptor. Conversely, when concentrations are above the selected benchmark value, the potential exists for an adverse health effect to occur. All benchmark values selected for comparison are the most protective values available for the identified receptor.

\subsection{SUMMARY OF SURFACE SEDIMENT RESULTS}

\subsubsection{Metals}

The primary metals that greater than available background soil concentrations (background sediment values are not available) and exceed either a human health or ecological benchmark were arsenic, cadmium, and chromium. A summary of the results for each of these three constituents is provided in the following subsections.

5.1.1.1 Arsenic. Arsenic was measured in surface sediment from all segments of the Columbia River at concentrations above the human health benchmark value of $0.39 \mathrm{mg} / \mathrm{kg}$ and the Hanford Site background value of $6.5 \mathrm{mg} / \mathrm{kg}$. Detected concentrations of arsenic in all samples analyzed ranged from $0.002 \mathrm{mg} / \mathrm{kg}$ to $12 \mathrm{mg} / \mathrm{kg}$; however, there were three measurements greater than $15 \mathrm{mg} / \mathrm{kg}$. Because arsenic was measured in all segments at similar concentration ranges, these results suggest that arsenic is present at naturally occurring levels and concentrations greater than naturally occurring levels may be contributed from sources located upgradient from the boundaries considered in this evaluation. Also note that arsenic is present at higher naturally occurring levels with a background value of $16 \mathrm{mg} / \mathrm{kg}$ for Oregon.

5.1.1.2 Cadmium. Cadmium was measured in surface sediment from all segments of the Columbia River at concentrations above the ecological benchmark value of $0.6 \mathrm{mg} / \mathrm{kg}$ and the Hanford Site background value of $0.81 \mathrm{mg} / \mathrm{kg}$. Detected concentrations of cadmium in all samples analyzed ranged from $0.0004 \mathrm{mg} / \mathrm{kg}$ to $16 \mathrm{mg} / \mathrm{kg}$. Cadmium was measured in all segments at concentrations above the Hanford Site background value of $0.81 \mathrm{mg} / \mathrm{kg}$; however, the highest cadmium concentrations were observed in Segment 1. Similar to arsenic, these results suggest that cadmium is present at naturally occurring levels and concentrations greater than naturally occurring levels may be contributed from sources located upgradient from the boundaries considered in this evaluation.

5.1.1.3 Total Chromium. As discussed previously, all sediment samples that were analyzed for total chromium were compared to the hexavalent chromium benchmark value. Chromium 
was measured in surface sediment from all segments of the Columbia River at concentrations ranging from $0.056 \mathrm{mg} / \mathrm{kg}$ to $112 \mathrm{mg} / \mathrm{kg}$. Chromium was measured above the human health benchmark value of $30 \mathrm{mg} / \mathrm{kg}$ and the ecological benchmark value of $37 \mathrm{mg} / \mathrm{kg}$. It should be noted that all sediment concentrations were below the WAC-173-340-740 benchmark value of $240 \mathrm{mg} / \mathrm{kg}$ for unrestricted land use. Chromium was measured at Priest Rapids Dam and other locations along Segment 1 with concentrations above these benchmarks ranging from $32 \mathrm{mg} / \mathrm{kg}$ to $93 \mathrm{mg} / \mathrm{kg}$; various locations along Segment 2 with concentrations ranging from $32 \mathrm{mg} / \mathrm{kg}$ to $112 \mathrm{mg} / \mathrm{kg}$; at locations near McNary Dam and Yakima River in Segment 3 with concentrations ranging from $35 \mathrm{mg} / \mathrm{kg}$ to $73 \mathrm{mg} / \mathrm{kg}$; locations behind John Day and The Dalles dams in Segment 4 with concentrations ranging from $57 \mathrm{mg} / \mathrm{kg}$ to $69 \mathrm{mg} / \mathrm{kg}$; and at three locations along Segment 5 with concentrations ranging from $32 \mathrm{mg} / \mathrm{kg}$ to $35 \mathrm{mg} / \mathrm{kg}$. Chromium has been measured at elevated in all segments of the Columbia River; however, noticeably higher concentrations are measured in Segments 1 and 2. These results indicate sources of chromium located upgradient from the boundaries considered in this evaluation and potential Hanford Site contribution within Segment 2.

\subsubsection{Radionuclides}

The three primary radionuclides that were greater than available background soil concentrations (background sediment values are not available) and exceeded either a human health or ecological benchmark were thorium-232, uranium-234, and uranium-238. A summary of the results for each of these three constituents is provided in the following subsections.

5.1.2.1 Thorium-232. Thorium-232 was measured in surface sediment from all segments of the Columbia River with concentrations ranging from $0.24 \mathrm{pCi} / \mathrm{g}$ to $3.1 \mathrm{pCi} / \mathrm{g}$. Thorium-232 was only reported above the human health benchmark value of $1.3 \mathrm{pCi} / \mathrm{g}$ along several areas of the river adjacent from the Hanford Site.

5.1.2.2 Uranium-234 and Uranium-238. Uranium-234 and uranium-238 was measured in surface sediment from Segments 1 through 4 of the Columbia River at concentrations above the human health benchmark value of $1.1 \mathrm{pCi} / \mathrm{g}$. Uranium-234 and uranium-238 were not analyzed in any of the samples collected from Segment 5.

Detected concentrations of uranium-234 in all samples analyzed ranged from $0.064 \mathrm{pCi} / \mathrm{g}$ to $11 \mathrm{pCi} / \mathrm{g}$. The highest uranium-234 concentrations were measured in Segment 2 near the 300 Area where concentrations above the benchmark ranged from $1.3 \mathrm{pCi} / \mathrm{g}$ to $11 \mathrm{pCi} / \mathrm{g}$. Uranium-234 was measured in Segment 1 near Priest Rapids at concentrations between $1.1 \mathrm{pCi} / \mathrm{g}$ and $1.6 \mathrm{pCi} / \mathrm{g}$; in Segment 3 primarily near McNary Dam at concentrations ranging from $1.1 \mathrm{pCi} / \mathrm{g}$ to $1.9 \mathrm{pCi} / \mathrm{g}$; and in Segment 4 primarily near the John Day Dam and The Dalles Dam at concentrations ranging from $1.1 \mathrm{pCi} / \mathrm{g}$ to $1.7 \mathrm{pCi} / . \mathrm{g}$.

Detected concentrations of uranium-238 in all samples analyzed ranged from $0.05 \mathrm{pCi} / \mathrm{g}$ to $9.9 \mathrm{pCi} / \mathrm{g}$. The highest uranium-238 concentrations were measured in Segment 2 near the 300 Area where concentrations above the benchmark ranged from $1.2 \mathrm{pCi} / \mathrm{g}$ to $9.9 \mathrm{pCi} / \mathrm{g}$. Uranium-238 was measured in Segment 1 near Priest Rapids at concentrations between $1.2 \mathrm{pCi} / \mathrm{g}$ and $1.4 \mathrm{pCi} / \mathrm{g}$; in Segment 3 primarily near McNary Dam at concentrations ranging from $1.1 \mathrm{pCi} / \mathrm{g}$ to $1.6 \mathrm{pCi} / \mathrm{g}$; and in Segment 4 primarily near the John Day Dam and The Dalles Dam at concentrations ranging from $1.1 \mathrm{pCi} / \mathrm{g}$ to $1.7 \mathrm{pCi} / \mathrm{g}$. 
Concentration of both uranium-234 and uranium-238 are generally near or slightly above background levels in all segments of the river, except for portions of Segment 2 near the 300 Area discussed above. Similar concentrations to those observed in the vicinity of McNary Dam and downstream locations were also observed at Ice Harbor Dam in the Snake River. This suggests some natural occurring levels of these constituents may be depositing behind the downstream dams.

\subsubsection{PCB Aroclors}

PCB aroclors were primarily analyzed in Segments 1, 3, and 5 of the Columbia River. No PCB aroclors were analyzed within the Hanford Site portion of Segment 2 and only at few locations within Segment 4. Because PCB aroclors were not analyzed in surface sediment within the Hanford Site portion of Segment 2, it is difficult to determine if there may be a contribution of this contaminant from Hanford Site operations.

5.1.3.1 Aroclor-1254. Aroclor-1254 was reported above the ecological benchmark value of $0.007 \mathrm{mg} / \mathrm{kg}$ at locations within Segments 2, 4, and 5 with concentrations ranging from $0.01 \mathrm{mg} / \mathrm{kg}$ to $0.024 \mathrm{mg} / \mathrm{kg}$. Aroclor-1254 was measured above the benchmark value at the Port of Pasco, behind Bonneville Dam, and at Columbia RM 100.

5.1.3.2 Aroclor-1248 and -1260. Aroclor-1248 and -1260 were reported above the ecological benchmark value of $0.021 \mathrm{mg} / \mathrm{kg}$ with concentrations ranging from $0.024 \mathrm{mg} / \mathrm{kg}$ to $1.4 \mathrm{mg} / \mathrm{kg}$. These aroclors were only detected above the benchmark value in Segment 5 of the Columbia River.

\subsubsection{Pesticides}

Pesticides were primarily analyzed in Segments 1, 3, and 5 of the Columbia River. No pesticides were analyzed within the Hanford Site portion of Segment 2 and only at few locations within Segment 4. Because pesticides were not analyzed in surface sediments within the Hanford Site portion of Segment 2, it is difficult to determine if there may be a contribution of these contaminants from Hanford Site operations. However, it is apparent that the majority of the samples that exceeded the benchmark values were associated with tributaries that flow into the Columbia River downstream of the Hanford Site.

5.1.4.1 DDD. DDD was reported above the ecological benchmark value of $0.004 \mathrm{mg} / \mathrm{kg}$ primarily within the Yakima River and Segment 5 of the Columbia River. DDD concentrations above the benchmark ranged from $0.0043 \mathrm{mg} / \mathrm{kg}$ to $0.023 \mathrm{mg} / \mathrm{kg}$.

5.1.4.2 DDE. DDE was reported above the ecological benchmark value of $0.0015 \mathrm{mg} / \mathrm{kg}$ primarily within the Yakima River and Segment 5 of the Columbia River. DDE concentrations above the benchmark ranged from $0.0015 \mathrm{mg} / \mathrm{kg}$ to $0.05 \mathrm{mg} / \mathrm{kg}$.

5.1.4.3 DDT. DDT was reported above the ecological benchmark value of $0.0042 \mathrm{mg} / \mathrm{kg}$ within the Yakima River with concentrations ranging from $0.01 \mathrm{mg} / \mathrm{kg}$ to $0.035 \mathrm{mg} / \mathrm{kg}$.

5.1.4.4 Dieldrin. Dieldrin was reported once in the Yakima River $(0.005 \mathrm{mg} / \mathrm{kg})$ above the ecological benchmark value of $0.0019 \mathrm{mg} / \mathrm{kg}$. 
5.1.4.5 Gamma-BHC. Gamma-BHC was reported once in the Walla Walla River $(0.008 \mathrm{mg} / \mathrm{kg})$ above the ecological benchmark value of $0.0009 \mathrm{mg} / \mathrm{kg}$.

\subsubsection{Polynuclear Aromatic Hydrocarbons}

PAHs were primarily analyzed in Segments 1, 3, 4, and 5 of the Columbia River. No PAHs were analyzed within the Hanford Site portion of Segment 2 and only at few locations within Segment 4. Because PAHs were not analyzed in surface sediments within the Hanford Site portion of Segment 2, it is difficult to determine if there may be a contribution of these contaminants from Hanford Site operations.

Acenaphthene, acenaphthylene, benzo(b)fluoranthene, benzo(ghi)perylene, benzo(k)fluoranthene, dibenz(a,h)anthrancene, indeno(1,2,3-cd)pyrene, and phenanthrene were reported above their respective ecological benchmark values in Segment 5 of the Columbia River. Anthracene, benzo(a)anthracene, benzo(a)pyrene, chrysene, fluoranthene, fluorene, and pyrene were reported above their ecological benchmark values at the Port of Pasco and Segment 5 of the Columbia River. However, it is apparent that the majority of the sediment samples that exceeded the benchmark values were located in the vicinity or downstream of the Portland/Vancouver metropolitan area.

\subsection{SUMMARY OF SURFACE WATER RESULTS}

\subsubsection{Metals}

A total of 15 metals exceeded either a human health or ecological benchmark. A summary of the results for each of the primary constituents is provided in the following subsections.

5.2.1.1 Aluminum. Aluminum was only analyzed in surface water from Segments 1 and 2 of the Columbia River with concentrations ranging from $0.0004 \mathrm{mg} / \mathrm{L}$ to $21.2 \mathrm{mg} / \mathrm{L}$. Aluminum was reported above the ecological benchmark value of $0.087 \mathrm{mg} / \mathrm{L}$ along several areas of the river adjacent from the Hanford Site. The ecological benchmark value of $0.087 \mathrm{mg} / \mathrm{L}$ is the freshwater chronic national recommended water quality criteria for protection of aquatic life.

5.2.1.2 Arsenic. Arsenic was measured in surface water from all segments of the Columbia River at concentrations above the human health benchmark value of $0.000018 \mathrm{mg} / \mathrm{L}$. Detected concentrations of arsenic in all samples analyzed ranged from $0.000006 \mathrm{mg} / \mathrm{L}$ to $0.0085 \mathrm{mg} / \mathrm{L}$. The highest arsenic concentrations were observed in Segment 1 (maximum value $0.0017 \mathrm{mg} / \mathrm{L}$ ) and Segment 2 (maximum value of $0.0085 \mathrm{mg} / \mathrm{L}$ ). It is important to note that the benchmark value of $0.000018 \mathrm{mg} / \mathrm{L}$ is the national recommended water quality criteria to protect human health assuming the consumption of surface water as a drinking water source and the consumption of fish. Columbia River surface water is used as a public and domestic water supply, but undergoes treatment prior to consumption. Therefore, the national recommended water quality criteria of $0.00014 \mathrm{mg} / \mathrm{L}$ based on fish consumption only may be more appropriate. Additionally, no arsenic concentrations were greater than the federal drinking water MCL of $0.010 \mathrm{mg} / \mathrm{L}$.

5.2.1.3 Barium. Barium was measured in all surface water from all segments (except Segment 3) of the Columbia River at concentrations above the ecological benchmark value of 
$0.004 \mathrm{mg} / \mathrm{L}$. Detected concentrations of barium in all samples analyzed ranged from $0.017 \mathrm{mg} / \mathrm{L}$ to $0.29 \mathrm{mg} / \mathrm{L}$. Barium was not analyzed in any surface water samples in Segment 3 of the river. The only benchmark value available for barium was from the Oregon Department of Environmental Quality Level II Screening Level Values. This screening value is considered a Tier II secondary chronic value and was developed by the Oak Ridge National Laboratory.

5.2.1.4 Cadmium. Cadmium was analyzed in surface water samples from all segments of the Columbia River with concentrations of ranging from $5.6 \mathrm{E}-06 \mathrm{mg} / \mathrm{L}$ to $0.0048 \mathrm{mg} / \mathrm{L}$. Cadmium was reported above the ecological benchmark value of $0.00025 \mathrm{mg} / \mathrm{L}$ in samples collected from along areas of the river adjacent from the Hanford Site of Segment 2 and near The Dalles, Oregon, in Segment 4. The ecological benchmark value of $0.00025 \mathrm{mg} / \mathrm{L}$ is the freshwater chronic national recommended water quality criteria for protection of aquatic life.

5.2.1.5 Chromium. Chromium was primarily analyzed in surface water samples from Segments 1 and 2 of the Columbia River with concentrations ranging from $0.000019 \mathrm{mg} / \mathrm{L}$ to $0.22 \mathrm{mg} / \mathrm{L}$. Chromium was analyzed in surface water from Segments 3 through 5, but at very few locations and on an infrequent basis. Chromium was only reported above the ecological benchmark value of $0.01 \mathrm{mg} / \mathrm{L}$ in several areas along the river adjacent from the Hanford Site of Segment 2. The ecological benchmark value of $0.01 \mathrm{mg} / \mathrm{L}$ is the Washington chronic water quality standards for protection of aquatic life.

5.2.1.6 Iron. Iron was measured in surface water from Segments 1, 2, and 4 of the Columbia River with concentrations ranging from $0.015 \mathrm{mg} / \mathrm{L}$ to $20.4 \mathrm{mg} / \mathrm{L}$. Iron was not analyzed in any surface water samples collected with Segments 3 and 5 of the Columbia River. Iron was reported above the ecological benchmark value of $1.0 \mathrm{mg} / \mathrm{L}$ near the 100-D Area of Segment 2. The ecological benchmark value of $1.0 \mathrm{mg} / \mathrm{L}$ is the freshwater chronic national recommended water quality criteria for protection of aquatic life.

5.2.1.7 Lead. Lead was primarily analyzed in surface water samples from Segments 1 and 2 of the Columbia River with concentrations ranging from $1.1 \mathrm{E}-06 \mathrm{mg} / \mathrm{L}$ to $0.066 \mathrm{mg} / \mathrm{L}$. Lead was analyzed surface water from Segments 3 through 5 but at very few locations and on an infrequent basis. Lead was only reported above the ecological benchmark value of $0.00087 \mathrm{mg} / \mathrm{L}$ in several areas along the river adjacent from the Hanford Site of Segment 2. The ecological benchmark value of $0.00087 \mathrm{mg} / \mathrm{L}$ is the Washington chronic water quality standards for protection of aquatic life.

5.2.1.8 Manganese. Manganese was measured in surface water from Segments 1, 2, and 4 of the Columbia River with concentrations ranging from $0.000037 \mathrm{mg} / \mathrm{L}$ to $0.94 \mathrm{mg} / \mathrm{L}$. Manganese was not analyzed in any surface water samples collected with Segments 3 and 5 of the Columbia River. Manganese was reported above the ecological benchmark value of $0.12 \mathrm{mg} / \mathrm{L}$ and the human health benchmark value of $0.05 \mathrm{mg} / \mathrm{L}$ near the 100-D Area of Segment 2. The only benchmark value available for manganese was from the Oregon Department of Environmental Quality Level II Screening Level Values. This screening value is considered a Tier II secondary chronic value and was developed by the Oak Ridge National Laboratory.

5.2.1.9 Silver. Silver was primarily analyzed in surface water samples from Segments 1 and 2 of the Columbia River with concentrations ranging from $8.3 \mathrm{E}-07 \mathrm{mg} / \mathrm{L}$ to $0.003 \mathrm{mg} / \mathrm{L}$. Silver was analyzed in surface water from Segments 3 through 5 , but at very few locations and on an infrequent basis. Silver was only reported above the ecological benchmark value of 
$0.00012 \mathrm{mg} / \mathrm{L}$ in several areas along the river adjacent from the Hanford Site of Segment 2. The only benchmark value available for silver was from the Oregon Department of Environmental Quality Level II Screening Level Values. This screening value is a freshwater chronic value from the Oregon Water Quality Criteria (Oregon Administrative Record [OAR] 340-41).

5.2.1.10 Zinc. Zinc was primarily analyzed in surface water samples from Segments 1 and 2 of the Columbia River with concentrations ranging from $0.00035 \mathrm{mg} / \mathrm{L}$ to $0.42 \mathrm{mg} / \mathrm{L}$. Zinc was analyzed surface water from Segments 3 through 5 but at very few locations and on an infrequent basis. Zinc was only reported above the ecological benchmark value of $0.055 \mathrm{mg} / \mathrm{L}$ in several areas along the river adjacent from the Hanford Site of Segment 2. The ecological benchmark value of $0.055 \mathrm{mg} / \mathrm{L}$ is the Washington chronic water quality standards for protection of aquatic life.

\subsubsection{Radionuclides}

The two primary radionuclides that exceeded either a human health or ecological benchmark were strontium-90 and tritium. A summary of the results for each of these constituents is provided in the following subsections.

5.2.2.1 Strontium-90. Strontium-90 was only analyzed in surface water samples from Segments 1, 2, and 3 of the Columbia River. However, strontium-90 was only reported above the human health benchmark value of $8 \mathrm{pCi} / \mathrm{L}$ in several samples collected near the $100-\mathrm{H}$ Area and 100-N Area in Segment 2. It should be noted that surface water screening values for radionuclides have not been developed for the protection of human health; therefore, the derived MCL concentration was used for comparison purposes.

5.2.2.2 Tritium. Tritium was only analyzed in surface water samples from Segments 1, 2, and 3 of the Columbia River. However, tritium was only reported above the human health benchmark value of 20,000 pCi/L in samples collected near the Hanford Spring 28-2 in Segment 2. It should be noted that surface water screening values for radionuclides have not been developed for the protection of human health; therefore, the derived MCL concentration was used for comparison purposes.

\subsubsection{Pesticides}

Pesticides were primarily analyzed in Segments 3 , and 5 of the Columbia River. No pesticides were analyzed within the Hanford Site portion of Segment 2 and only a few samples analyzed from Segments 1 and 4 of the Columbia River. Because pesticides were infrequently analyzed in surface water for most segments, it is difficult to determine if there is a contribution of these contaminants from Hanford Site operations. However, similar to the sediment exceedences, it is apparent that the majority of the surface water samples that exceeded the benchmark values were associated with tributaries that flow into the Columbia River downstream of the Hanford Site.

5.2.3.1 DDE. DDE was reported above the human health benchmark value of $2.2 \mathrm{E}-07 \mathrm{mg} / \mathrm{L}$ in the Walla Walla River and various locations within Segment 5 of the Columbia River. DDE concentrations above the benchmark value ranged from $2.4 \mathrm{E}-06 \mathrm{mg} / \mathrm{L}$ to $2.1 \mathrm{E}-05 \mathrm{mg} / \mathrm{L}$. 
5.2.3.2 DDT. DDT was reported above the human health benchmark value of $2.2 \mathrm{E}-07 \mathrm{mg} / \mathrm{L}$ in the Walla Walla River and various locations within Segment 5 of the Columbia River. DDT concentrations above the benchmark value ranged from $2.7 \mathrm{E}-06 \mathrm{mg} / \mathrm{L}$ to $1.5 \mathrm{E}-05 \mathrm{mg} / \mathrm{L}$.

5.2.3.3 Dieldrin. Dieldrin was reported above the human health benchmark value of 5.2E-08 mg/L in the Walla Walla River, above the Bonneville Dam, and various locations within Segment 5 of the Columbia River. Dieldrin concentrations above the benchmark value ranged from 3.0E-06 mg/L to 3.6E-06 mg/L.

5.2.3.4 Heptachlor Epoxide. Heptachlor epoxide was reported above the human health benchmark value of 3.9E-08 mg/L in the Walla Walla River, above the Bonneville Dam, and various locations within Segment 5 of the Columbia River. Heptachlor epoxide concentrations above the benchmark value ranged from $6.7 \mathrm{E}-08 \mathrm{mg} / \mathrm{L}$ to $4.1 \mathrm{E}-08 \mathrm{mg} / \mathrm{L}$.

5.2.3.5 Hexachlorobenzene. Hexachlorobenzene was reported above the human health benchmark value of $2.8 \mathrm{E}-07 \mathrm{mg} / \mathrm{L}$ above the Bonneville Dam and various locations within Segment 5 of the Columbia River. Hexachlorobenzene concentrations above the benchmark value ranged from $3.4 \mathrm{E}-07 \mathrm{mg} / \mathrm{L}$ to $2.3 \mathrm{E}-06 \mathrm{mg} / \mathrm{L}$.

\subsection{CONCLUSION}

In general, contaminants of Hanford Site origin would be indicated by a particular trend in concentrations in river sediments or surface water. An increase in concentrations downstream of the Hanford Site above the concentrations observed at upstream locations (i.e., Segment 1) could indicate contamination of Hanford Site origin. The trend in concentrations would likely show peaks at the McNary Dam pool (closest downstream sediment reservoir to the Hanford Site), and gradually decline in the lower reservoirs due to dilution from other sediment sources. Based on findings of this evaluation mentioned above, very few of the contaminants that exceed a benchmark value match this trending.

It is also important to note that analysis for some constituents that may be indicators of Hanford Site origin contaminants had limited constituent analyses, especially for radionuclides in downstream segments. In addition, for some segments (i.e., sediment in Segment 4) there was limited sampling over a $245-\mathrm{km}$ (146-mile) stretch of river, thus a potential data gap in addition to the limited constituent analyses.

The results from this evaluation are consistent with the most recent study of Hanford Site-related contaminants in the Columbia River sediments, river water, and sediment porewater that was conducted in 2003. This multi-agency effort (Oregon Department of Energy, Washington Department of Health, Ecology, DOE, and Pacific Northwest National Laboratory) was conducted in the Priest Rapid, McNary, John Day, The Dalles, and Bonneville Dam Reservoirs. In this study they concluded that radionuclides detected in low concentrations in the upper layer of sediment were similar to background concentrations in the Priest Rapids Reservoir for most radionuclides. Only cesium-137 and europium-152 values were slightly elevated at McNary Dam, which may indicate some Hanford Site contribution (Priddy et al. 2005). 
Radionuclides with long half-lives deposited during the operational period of the single-pass reactors may be present in deeper sediments of the lower reservoirs that are now covered with layers of more recently deposited sediment. Although data collected and analyzed from core investigations and decayed to 2005 did not indicate any significant problems, the data set contained a limited number of radionuclides.

Hanford Site contribution for metals in the Columbia River does not appear to be significant. This is consistent with the 2003 study were there was no indication of a unique Hanford Site contribution for metals in Columbia River sediments (Priddy et al. 2005). The source of some metals may be associated with upstream contributions (i.e., mining) where previous and ongoing investigations have indicated the presence of contaminated sediments with arsenic, cadmium, copper, lead, mercury, and zinc.

Evaluation for sediment sample analyzed for PAH, PCBs, pesticides, and SVOCs were inconclusive. However, sampling conducted in 2003 resulted in concentrations that were all below analytical detection limits.

Surface water exceedences identified during this evaluation were primarily associated with ecological benchmark values. These values are considered conservative as the majority of these exceedences are tied to seeps located along Columbia River within the Hanford Site. In addition, the water concentrations observed for non-seep areas are consistent with the 2003 data where concentrations at McNary Dam were similar to levels reported by the Hanford Site Surface Environmental Surveillance Project for the Richland Pumphouse and Priest Rapids Dam. 
Table 5-1. Summary of Sediment Exceedences.

\begin{tabular}{|c|c|c|c|c|c|}
\hline \multirow{2}{*}{$\begin{array}{l}\text { Constituent } \\
\text { Name }\end{array}$} & \multicolumn{5}{|c|}{ Columbia River Component Segment } \\
\hline & Segment 1 & Segment 2 & Segment 3 & Segment 4 & Segment 5 \\
\hline Antimony & $\mathrm{a}$ & $x$ & $\mathrm{X}$ & $\mathrm{x}$ & $x$ \\
\hline Arsenic & $x$ & $x$ & $x$ & $x$ & $x$ \\
\hline Cadmium & $\mathrm{x}$ & $\mathrm{X}$ & $\mathrm{X}$ & $\mathrm{X}$ & $\mathrm{X}$ \\
\hline Total Chromium & $x$ & $x$ & $x$ & $x$ & $x$ \\
\hline Copper & $\mathrm{x}$ & $\mathrm{X}$ & $\mathrm{X}$ & $\mathrm{X}$ & $\mathrm{X}$ \\
\hline Iron & $x$ & $\mathrm{x}$ & $\mathrm{x}$ & $\mathrm{x}$ & $x$ \\
\hline Lead & $x$ & $x$ & $\mathrm{X}$ & $x$ & $x$ \\
\hline Manganese & $x$ & $x$ & $x$ & $x$ & a \\
\hline Mercury & $x$ & $x$ & $x$ & $x$ & $x$ \\
\hline Nickel & $x$ & $x$ & $x$ & $x$ & $x$ \\
\hline Silver & a & $x$ & $\mathrm{a}$ & a & a \\
\hline Vanadium & $x$ & $x$ & $x$ & $x$ & NA \\
\hline Zinc & $x$ & $x$ & $x$ & $x$ & $x$ \\
\hline Thorium-232 & $x$ & $x$ & a & a & a \\
\hline Cobalt-60 & $a$ & $x$ & $a$ & $\mathrm{a}$ & $\mathrm{a}$ \\
\hline Uranium-234 & $x$ & $x$ & $x$ & $x$ & NA \\
\hline Uranium-238 & $x$ & $x$ & $x$ & $x$ & NA \\
\hline Aroclor-1248 & a & NA & a & NA & $x$ \\
\hline Aroclor-1254 & a & NA & $x$ & $x$ & $x$ \\
\hline Arochlor-1260 & a & NA & a & NA & $x$ \\
\hline DDE & a & NA & $x$ & NA & $x$ \\
\hline DDT & a & NA & $x$ & a & $x$ \\
\hline Dieldrin & a & NA & $x$ & NA & a \\
\hline Gamma-BHC & a & NA & $x$ & a & a \\
\hline PAHs/SVOCs & a & NA & $x$ & $x$ & $x$ \\
\hline
\end{tabular}

${ }^{a}$ Not detected above human health or ecological benchmarks.

$\mathrm{BHC}=$ Hexachlorocyclohexane

DDE = dichlorodiphenyldichloroethylene

DDT = dichlorodiphenyltrichloroethane

NA = Constituent not analyzed

$\mathrm{PAH}=$ polyaromatic hydrocarbon

SVOC = semivolatile organic compound 
Table 5-2. Summary of Surface Water Exceedences.

\begin{tabular}{|c|c|c|c|c|c|}
\hline \multirow{2}{*}{$\begin{array}{l}\text { Constituent } \\
\text { Name }\end{array}$} & \multicolumn{5}{|c|}{ Columbia River Component Segment } \\
\hline & Segment 1 & Segment 2 & Segment 3 & Segment 4 & Segment 5 \\
\hline Aluminum & a & $\mathrm{x}$ & NA & NA & NA \\
\hline Arsenic & $x$ & $\mathrm{x}$ & $x$ & $\mathrm{x}$ & $x$ \\
\hline Barium & $x$ & $\mathrm{X}$ & NA & $\mathrm{x}$ & $x$ \\
\hline Boron & NA & NA & NA & NA & $x$ \\
\hline Cadmium & a & $x$ & $\mathrm{a}$ & $x$ & $\mathrm{a}$ \\
\hline Chromium & a & $\mathrm{x}$ & a & a & a \\
\hline Copper & a & $\mathrm{X}$ & a & a & a \\
\hline Iron & $a$ & $x$ & NA & $x$ & NA \\
\hline Lead & $\mathrm{a}$ & $x$ & $a$ & $x$ & $a$ \\
\hline Manganese & a & $x$ & NA & a & NA \\
\hline Silver & $\mathrm{a}$ & $x$ & $\mathrm{a}$ & $a$ & $\mathrm{a}$ \\
\hline Thallium & a & $x$ & a & a & NA \\
\hline Uranium & $a$ & $x$ & $a$ & NA & $a$ \\
\hline Vanadium & $\mathrm{a}$ & $x$ & NA & NA & NA \\
\hline Zinc & $\mathrm{a}$ & $x$ & $a$ & $\mathrm{a}$ & $\mathrm{a}$ \\
\hline Strontium-90 & a & $x$ & a & NA & NA \\
\hline Tritium & a & $x$ & a & NA & NA \\
\hline Total PCBs & NA & NA & a & $x$ & $x$ \\
\hline DDE & NA & NA & $x$ & $x$ & $x$ \\
\hline DDT & NA & NA & $x$ & $x$ & $x$ \\
\hline Dieldrin & NA & NA & $x$ & $x$ & $a$ \\
\hline Heptachlor Epoxide & NA & NA & $x$ & $x$ & a \\
\hline PAHs/SVOCs & NA & NA & & $x$ & $x$ \\
\hline $\begin{array}{ll}{ }^{a} \text { Not detected above } h \\
\text { DDE } & =\text { dichlorodiph } \\
\text { DDT } & =\text { dichlorodiph } \\
\text { NA } & =\text { Constituent } \\
\text { PAH } & =\text { polyaromati } \\
\text { PCB } & =\text { polychlorina } \\
\text { SVOC } & =\text { semivolatile }\end{array}$ & $\begin{array}{l}\text { iman health or } \\
\text { enyldichloroeth } \\
\text { enyltrichloroeth } \\
\text { iot analyzed } \\
\text { hydrocarbon } \\
\text { ed biphenyl. } \\
\text { organic compo }\end{array}$ & $\begin{array}{l}\text { ecological beno } \\
\text { lene } \\
\text { ane }\end{array}$ & & & \\
\hline
\end{tabular}




\subsection{REFERENCES}

Comprehensive Environmental Response, Compensation, and Liability Act of 1980, 42 U.S.C. 9601 , et seq.

DOE Order 5400.5, Radiation Protection of the Public and the Environment, U.S. Department of Energy, Office of Environment, Safety, and Health, Washington, D.C.

DOE-RL, 1995, Hanford Site Background: Part 1 Soil Background for Nonradioactive Analytes, DOE/RL-92-94, Rev. 4, U.S. Department of Energy, Richland Operations Office, Richland, Washington.

DOE-RL, 1996, Hanford Site Background: Part 2, Soil Background for Radionuclides, DOE/RL-96-12, Rev. 0, U.S. Department of Energy, Richland Operations Office, Richland, Washington.

DOE-RL, 1997, National Register of Historic Places Multiple Property Documentation Form Historic, Archeological and Traditional Cultural Properties of the Hanford Site, Washington, DOE/RL-97-02, Rev. 0, U.S. Department of Energy, Richland Operations Office, Richland, Washington.

DOE-RL, 1998a, Remedial Design Report/Remedial Action Work Plan for the 100 Area, DOE/RL-96-17, Rev. 5, U.S. Department of Energy, Richland Operations Office, Richland, Washington.

DOE-RL, 1998b, Screening Assessment and Requirements for a Comprehensive Assessment: Columbia River Comprehensive Impact Assessment, DOE/RL-96-16, Rev. 1, U.S. Department of Energy, Richland Operations Office, Richland, Washington.

DOE-RL, 2001, Hanford Site Background: Part 1, Soil Background for Nonradioactive Analytes, DOE/RL-92-24, Rev. 4, U.S. Department of Energy, Richland Operations Office, Richland, Washington.

DOE-RL, 2002, History of the Plutonium Production Facilities at the Hanford Site Historic District, 1943-1990, DOE/RL-97-1047, U.S. Department of Energy, Richland Operations Office, Richland, Washington.

DOE-RL, 2004, Columbia River Component of the River Corridor Baseline Risk Assessment: Basis and Assumptions on Project Scope, DOE/RL-2004-49, Rev. 0, U.S. Department of Energy, Richland Operations Office, Richland, Washington.

DOE-RL, 2005a, RCBRA Stack Air Emissions Deposition Scoping Document, DOE/RL-2005-49, Rev. 0, U.S. Department of Energy, Richland Operations Office, Richland, Washington.

DOE-RL, 2005b, 100 Area Remedial Action Sampling and Analysis Plan, DOE/RL-96-22, Rev. 4, U.S. Department of Energy, Richland Operations Office, Richland, Washington. 
Ecology, 1981, Irrigation Return Flow Quality-South Columbia Basin Irrigation District, Publication No. 81-E16 (WA-36-1010), Washington State Department of Ecology, Olympia, Washington.

Ecology, 1994, Natural Background Soil Metals Concentrations in Washington State, Publication No. 94-115, Washington State Department of Ecology, Toxics Cleanup Program, Olympia, Washington.

Ecology, 1997, Creation and Analysis of Freshwater Sediment Quality Values in Washington State, Publication No. 97-323a, Washington State Department of Ecology, Environmental Investigations and Laboratory Services Program, Olympia, Washington.

Endangered Species Act of 1973, 16 U.S.C 1531-1544.

EPA, 1998, Portland Harbor Sediment Investigation Report, EPA910/R-98-006,

U.S. Environmental Protection Agency, Office of Environmental Cleanup, Seattle, Washington.

EPA, 2001, EPA Requirements for Quality Assurance Project Plans, EPA QA/R-5, U.S. Environmental Protection Agency, Washington, D.C.

EPA, 2002, National Recommended Water Quality Criteria: 2002, EPA 822-R-02-047, Office of Water and Office of Science and Technology, U.S. Environmental Protection Agency, Washington, D.C.

EPA, 2004a, Sediment Sampling Approach and Rationale, Upper Columbia River, Technical Briefing Sheet, U.S. Environmental Protection Agency, Region 10, Olympia, Washington.

EPA, 2004b, National Recommended Water Quality Criteria Table: Brochure, EPA-822-F-04-010, Office of Water and Office of Science and Technology, U.S. Environmental Protection Agency, Washington, D.C.

EPA, 2006, Current National Recommended Water Quality Criteria, U.S. EPA, Washington, D.C. http://www.epa.gov/waterscience/criteria/wqcriteria.html

Hartman, M. J., L. F. Morasch, and W. D. Webber (eds.), 2006, Hanford Site Groundwater Monitoring for Fiscal Year 2005, PNNL-15670, Pacific Northwest National Laboratory, Richland, Washington.

Lower Willamette Group, 2004, Portland Harbor Remedial Investigation/Feasibility Study Programmatic Work Plan, Lower Willamette Group, Portland, Oregon.

MacDonald, D. D., C. G. Ingersoll, and T. A. Berger, 2000, Development and Evaluation of Consensus-Based Sediment Quality Guidelines for Freshwater Ecosystems, Arch. Environ. Contam. Toxicol. 39: 20-31.

Meade, R. H. and R. S. Parker, 1985, Sediments in Rivers of the United States, USGS Water-Supply Paper 2275, National Water Summary 1984, U.S. Geological Survey, Washington, D.C. 
NAD83, North American Datum of 1983.

OAR 340-41, "Oregon Water Quality Criteria," Oregon Administrative Record, as amended

ODEQ, 2001, Guidance for Ecological Risk Assessment Level II Screening Level Values, Oregon Department of Environmental Quality, Portland, Oregon.

OHD, 1994, Environmental Radiological Surveillance Report on Oregon Surface Waters 1961-1993, Volume I, Oregon Health Division Radiation Protection Services, Portland, Oregon.

PNL, 1990, 1988 Hanford Riverbank Springs Characterization Report, PNL-7500, Pacific Northwest Laboratory, Richland, Washington.

PNNL, 2003, Aquifer Sampling Tube Results for Fiscal Year 2003, PNNL-14444, Pacific Northwest National Laboratory, Richland, Washington.

Poston, T. M., R. W. Hanf, R. L. Dirkes, and L. F. Morasch, 2004, Hanford Site Environmental Report for Calendar Year 2003, PNNL-14687, Pacific Northwest National Laboratory, Richland, Washington.

Priddy, M., G. Patton, J. Yokel, D. Delistraty, and T. Stoops, 2005, Survey of Potential Hanford Site Contaminants in the Upper Sediment for the Reservoirs at McNary, John Day, The Dalles and Bonneville Dams, 2003, PNNL-14878, Pacific Northwest National Laboratory, Richland, Washington.

Schneider, M., 2002, Mainstem/Systemwide Water Quality Program Summary-Draft, Northwest Power Planning Council, Portland, Oregon.

USGS, 1995, Nitrate Concentrations in Groundwater of the Central Columbia Plateau, Open-File Report 95-445, U.S. Geological Survey, Tacoma, Washington.

WAC 173-201A, "Water Quality Standards for Surface Waters of the State of Washington," Washington Administrative Code, as amended.

WAC 173-204-340, "Freshwater Sediment Quality Standards," Washington Administrative Code, as amended

WAC 173-340-740, "Model Toxics Control Act - Cleanup, Unrestricted Land Use Soil Cleanup Standards," Washington Administrative Code, as amended.

WCH, 2006, Existing Source Information Summary Report Compilation/Evaluation Effort: December 2004 to September 2005, Columbia River Component of the River Corridor Baseline Risk Assessment, WCH-64, Rev. 0, Washington Closure Hanford, Richland, Washington.

WGS84, World Geodetic System of 1984. 
WCH-91

References

Rev. 0 


\section{DISTRIBUTION}

U. S. Department of Energy

Richland Operations Office

J. P. Sands

A3-04

D. C. Ward

A2-17

J. H. Zeisloft

A3-04

Washington Closure Hanford

C. S. Cearlock (5)

$\mathrm{HO}-23$

L. C. Hulstrom

$\mathrm{HO}-23$

S. K. Muns

$\mathrm{HO}-23$

J. E. Thomson

$\mathrm{HO}-23$

S. G. Weiss

$\mathrm{HO}-23$

Pacific Northwest National Laboratory

T. M. Poston

K6-75

U. S. Environmental Protection Agency

L. E. Gabois

B1-46

Washington State Department of Ecology

J. Price

$\mathrm{HO}-57$

E. Rochette

$\mathrm{HO}-57$

Records and Document Control

$\mathrm{HO}-30$ 
WCH-91

Distribution

Rev. 0 


\section{APPENDIX A \\ SEGMENT 1 \\ SUMMARY STATISTICS, BENCHMARK COMPARISON, AND INDIVIDUAL SAMPLE EXCEEDENCES}


WCH-91

Rev. 0

Columbia River Component Data Evaluation Summary Report

July 2006 


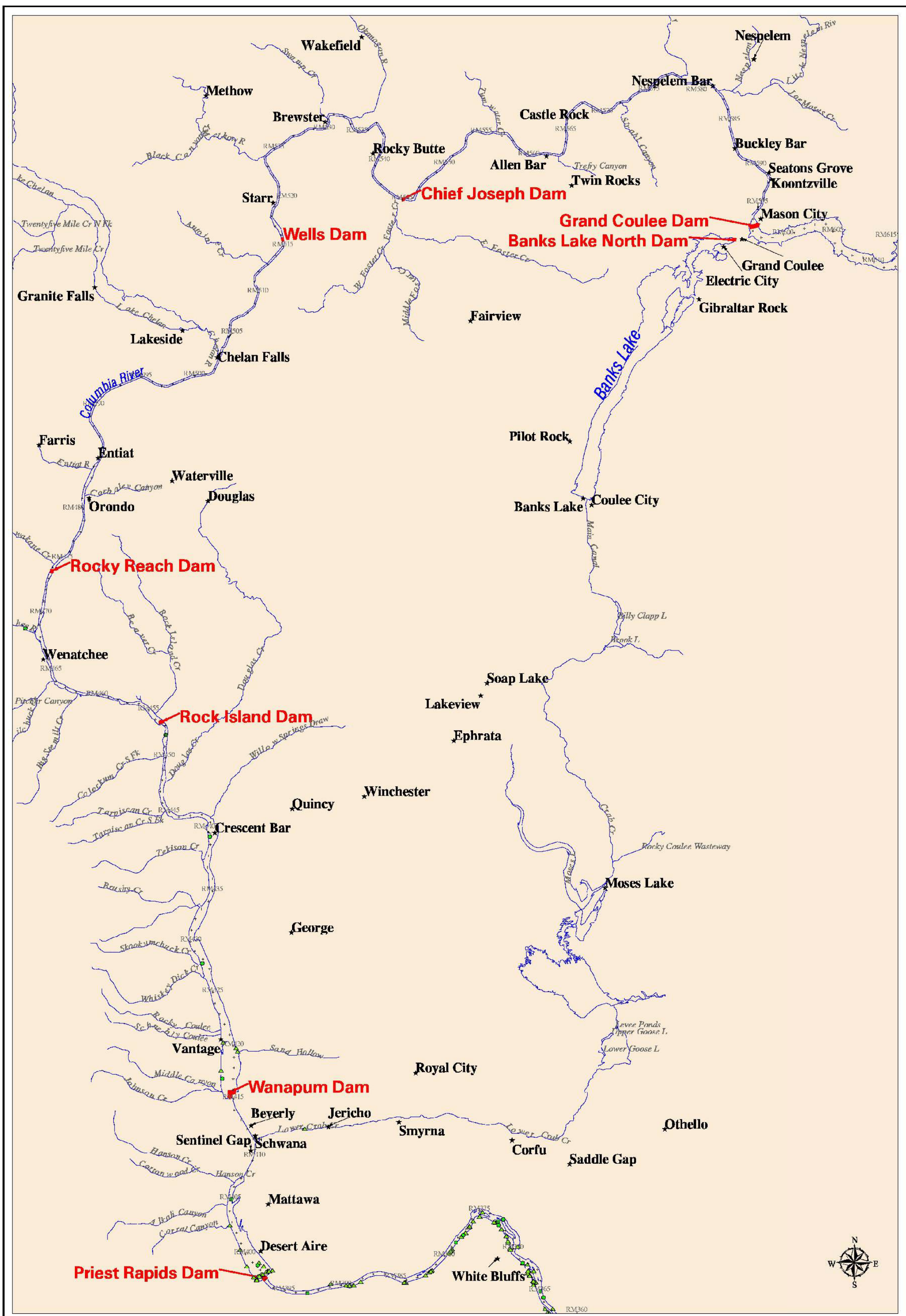

Priest Rapids to Grand Coulee Dam - Seg. 1 ( Sediment Samples)
Hydroelectric Dams
* Towns and Landmarks
River Miles
$\triangle$ QA 1 Samples

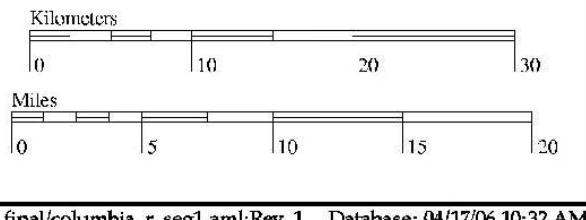




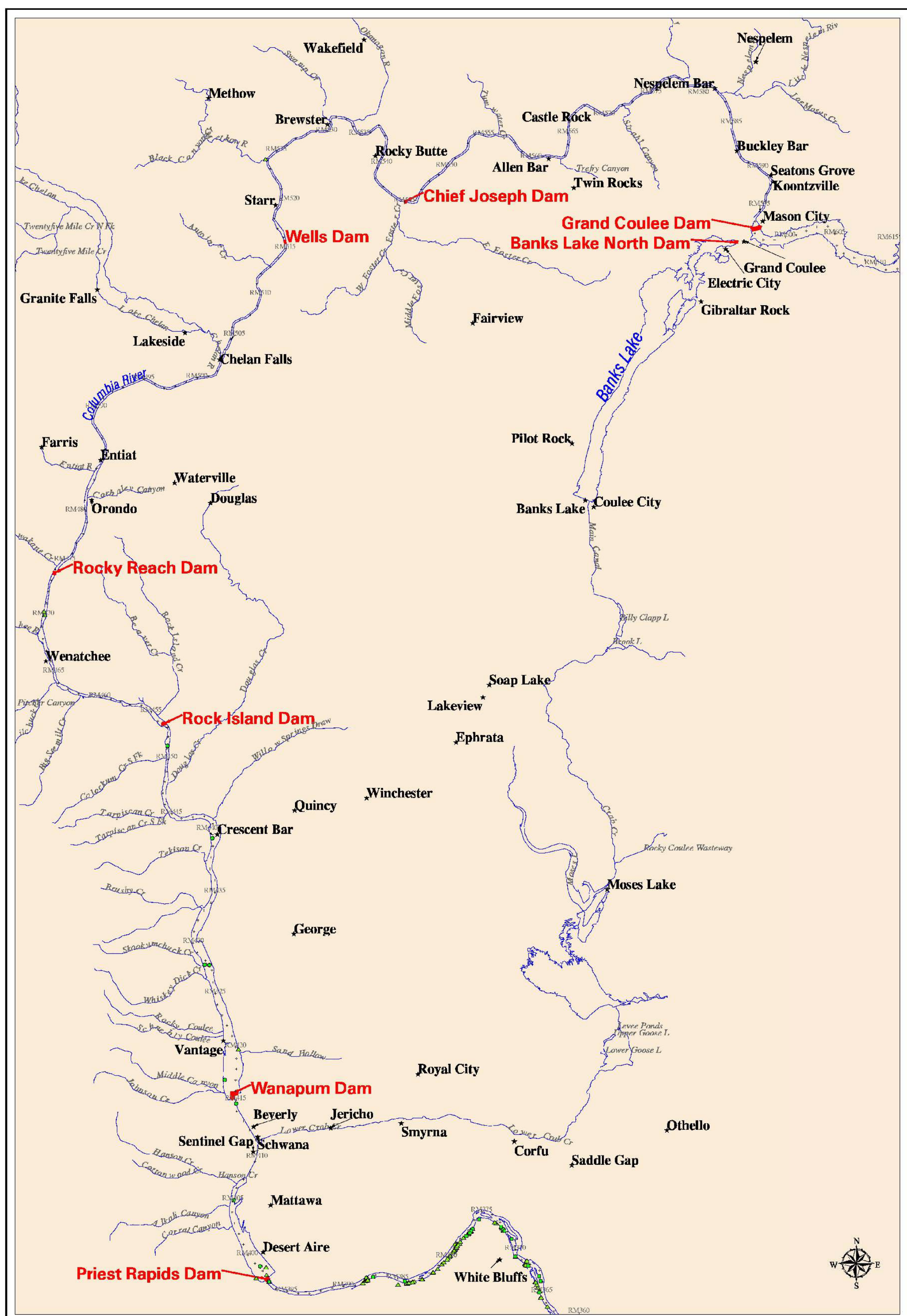

Priest Rapids to Grand Coulee Dam - Seg. 1 ( Surface Water Samples)
Hydroelectric Dams
* Towns and Landmarks
River Miles
$\triangle$ QA 1 Samples

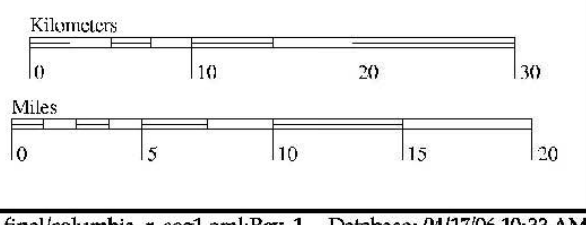




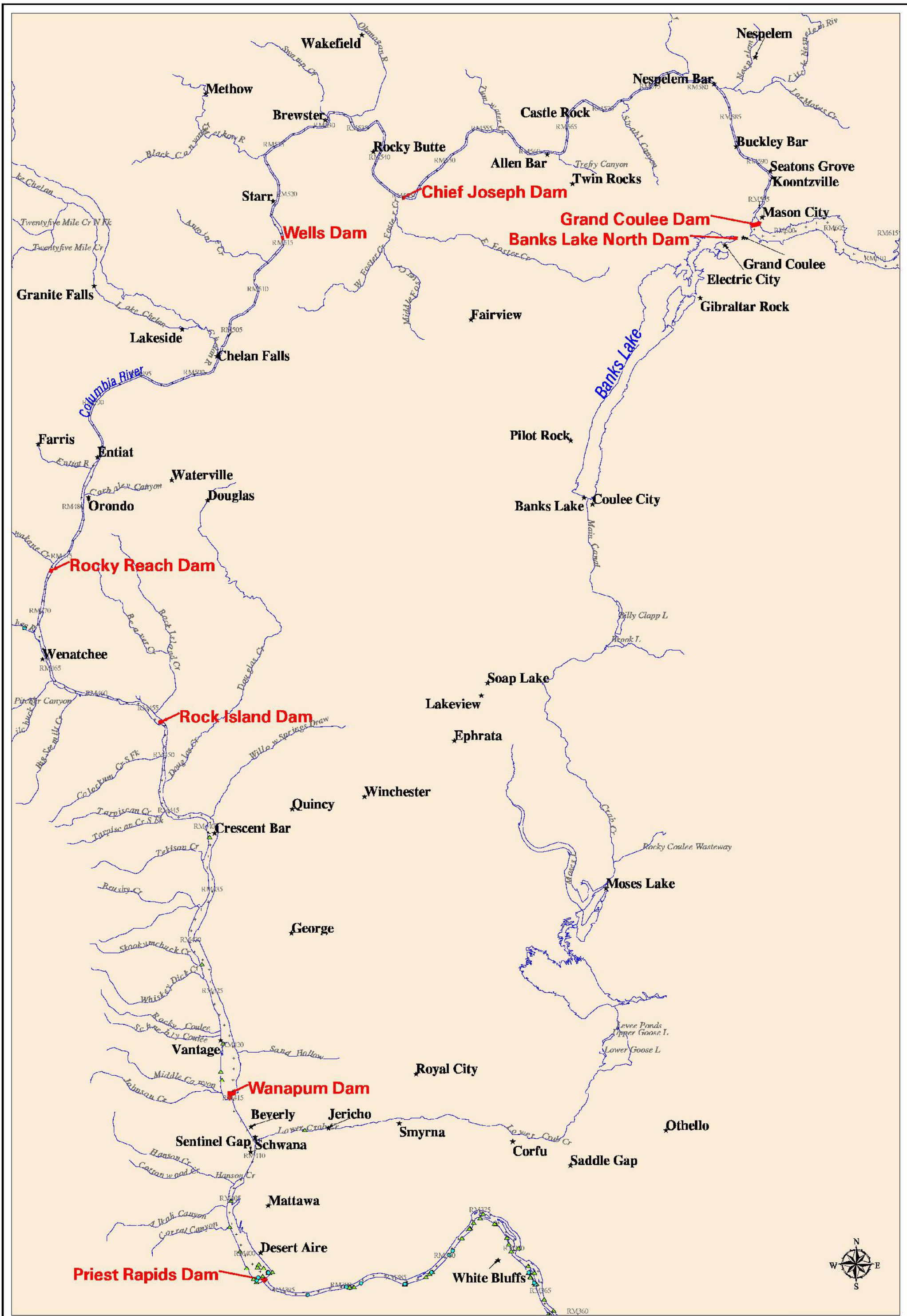

Priest Rapids to Grand Coulee Dam - Seg. 1 ( Sediment ) - Exceed.

Hydroelectric Dams

* Towns and Landmarks

River Miles

$\triangle$ Wet-Weight Basis 


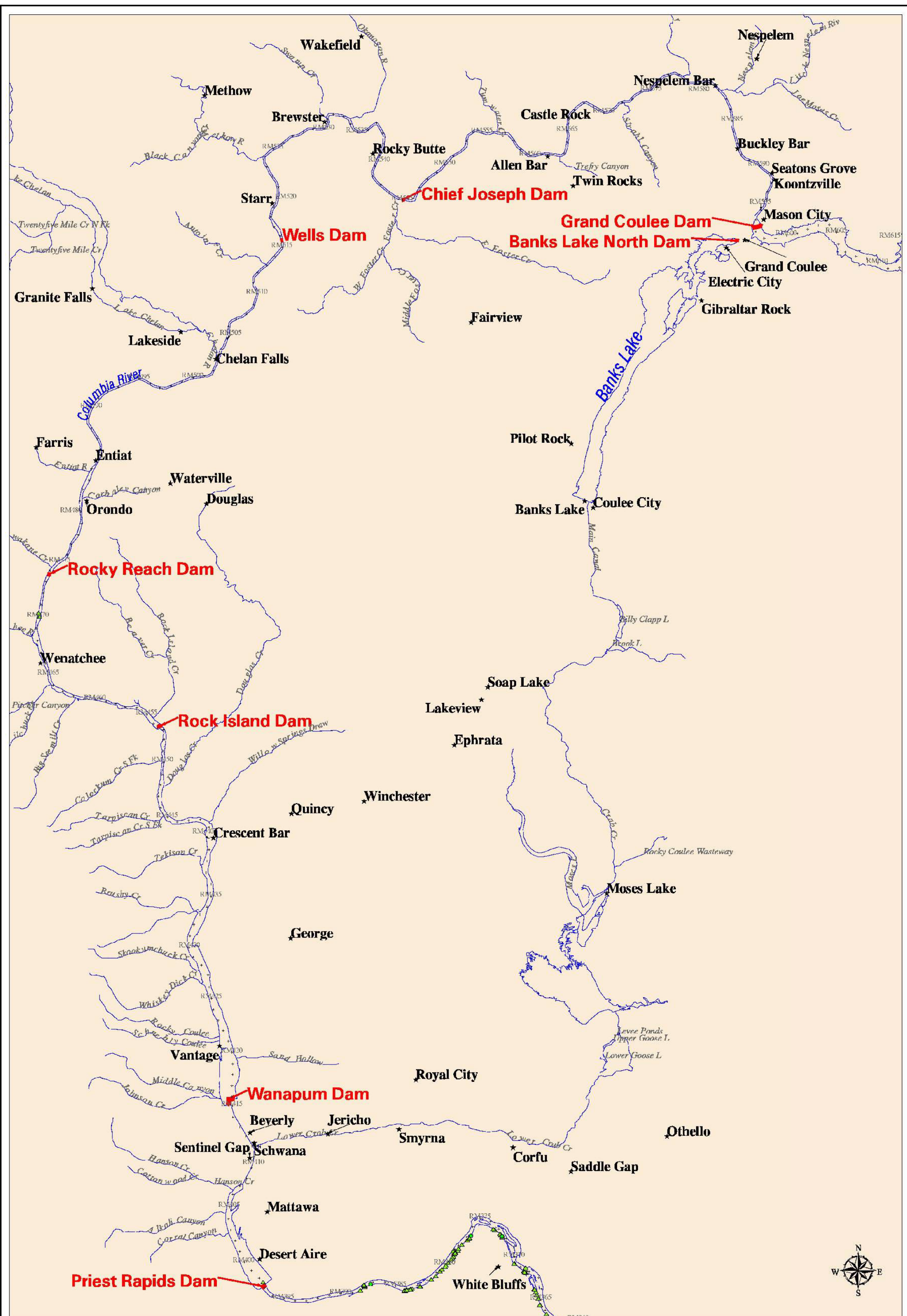

Priest Rapids to Grand Coulee Dam - Seg. 1 ( Surface Water ) - Exceed.

Hydroelectric Dams

* Towns and Landmarks

- QA 2 Samples

- River Miles

$\triangle$ QA 1 Samples

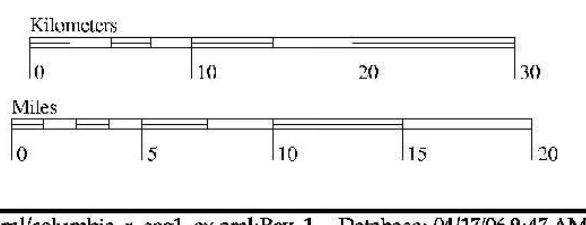


Table A-1

\section{Summary Table of Segment 1 Sediment Samples By Area, Site, Date, and Category}

\begin{tabular}{|c|c|c|c|c|}
\hline Sample ID & Sample Area & Sample Site & Sample Date & Category \\
\hline 12989 & Columbia River & Pr Rapids Dam & 01-Oct-92 & 1 \\
\hline 12990 & Columbia River & Pr Rapids Dam & 01-Oct-92 & 1 \\
\hline 12991 & Columbia River & Pr Rapids Dam & 01-Oct-92 & 1 \\
\hline 12992 & Columbia River & Pr Rapids Dam & 01-Oct-92 & 1 \\
\hline 15824 & Columbia River & Pr Rapids Dam & 22-Sep-94 & 1 \\
\hline 15825 & Columbia River & Pr Rapids Dam & 22-Sep-94 & 1 \\
\hline 15826 & Columbia River & Pr Rapids Dam & 22-Sep-94 & 1 \\
\hline 15827 & Columbia River & Pr Rapids Dam & 22-Sep-94 & 1 \\
\hline 16956 & Columbia River & Pr Rapids Dam & 24-Jul-95 & 1 \\
\hline 16957 & Columbia River & Pr Rapids Dam & 24-Jul-95 & 1 \\
\hline 16958 & Columbia River & Pr Rapids Dam & 24-Jul-95 & 1 \\
\hline 16959 & Columbia River & Pr Rapids Dam & 24-Jul-95 & 1 \\
\hline 18293 & Columbia River & Pr Rapids Dam & 15-Aug-96 & 1 \\
\hline 18294 & Columbia River & Pr Rapids Dam & 15-Aug-96 & 1 \\
\hline 18295 & Columbia River & Pr Rapids Dam & 15-Aug-96 & 1 \\
\hline 18296 & Columbia River & Pr Rapids Dam & 15-Aug-96 & 1 \\
\hline 19683 & Columbia River & Pr Rapids Dam & 18-Aug-97 & 1 \\
\hline 19684 & Columbia River & Pr Rapids Dam & 18-Aug-97 & 1 \\
\hline 19685 & Columbia River & Pr Rapids Dam & 18-Aug-97 & 1 \\
\hline 20753 & Columbia River & Pr Rapids Dam & 10-Aug-98 & 1 \\
\hline 20754 & Columbia River & Pr Rapids Dam & 10-Aug-98 & 1 \\
\hline 20755 & Columbia River & Pr Rapids Dam & 10-Aug-98 & 1 \\
\hline 20756 & Columbia River & Pr Rapids Dam & 10-Aug-98 & 1 \\
\hline 23519 & Columbia River & Pr Rapids Dam & 03-Aug-99 & 1 \\
\hline 23520 & Columbia River & Pr Rapids Dam & 03-Aug-99 & 1 \\
\hline 23521 & Columbia River & Pr Rapids Dam & 03-Aug-99 & 1 \\
\hline 23522 & Columbia River & Pr Rapids Dam & 03-Aug-99 & 1 \\
\hline 24748 & Columbia River & Pr Rapids Dam & 07-Aug-00 & 1 \\
\hline 24749 & Columbia River & Pr Rapids Dam & 07-Aug-00 & 1 \\
\hline 26250 & Columbia River & Pr Rapids Dam & 19-Jul-01 & 1 \\
\hline 26251 & Columbia River & Pr Rapids Dam & 19-Jul-01 & 1 \\
\hline 27652 & Columbia River & Vernita Bridge & 28-Aug-01 & 1 \\
\hline 29045 & Columbia River & Pr Rapids Dam & 23-Jul-02 & 1 \\
\hline 29046 & Columbia River & Pr Rapids Dam & 23-Jul-02 & 1 \\
\hline 30872 & Columbia River & Pr Rapids Dam & 14-Jul-03 & 1 \\
\hline 30873 & Columbia River & Pr Rapids Dam & 14-Jul-03 & 1 \\
\hline 4434247 & Columbia River & RM 391 & 01-Jun-04 & 1 \\
\hline 4444203 & Columbia River & Pr Rapids Dam & 01-Jun-04 & 1 \\
\hline 4444204 & Columbia River & Pr Rapids Dam & 01-Jun-04 & 1 \\
\hline 4444205 & Columbia River & Pr Rapids Dam & 01-Jun-04 & 1 \\
\hline 4444206 & Columbia River & Pr Rapids Dam & 01-Jun-04 & 1 \\
\hline 4444208 & Columbia River & Wanapum Dam & 01-Jun-04 & 1 \\
\hline 4444209 & Columbia River & Wanapum Dam & 01-Jun-04 & 1 \\
\hline 4444210 & Columbia River & Wanapum Dam & 01-Jun-04 & 1 \\
\hline B07NF5 & Columbia River & Vernita Area & 20-Nov-92 & 1 \\
\hline B07NF6 & Columbia River & Vernita Area & 20-Nov-92 & 1 \\
\hline B07NF7 & Columbia River & Vernita Area & 20-Nov-92 & 1 \\
\hline B07NF8 & Columbia River & Vernita Area & 20-Nov-92 & 1 \\
\hline
\end{tabular}


Table A-1

\section{Summary Table of Segment 1 Sediment Samples By Area, Site, Date, and Category}

\begin{tabular}{|c|c|c|c|c|}
\hline Sample ID & Sample Area & Sample Site & Sample Date & Category \\
\hline B0BRT6 & Columbia River & Pr Rapids Dam & 14-Apr-94 & 1 \\
\hline B0BRT8 & Columbia River & Pr Rapids Dam & 14-Apr-94 & 1 \\
\hline BOBRVO & Columbia River & Pr Rapids Dam & 14-Apr-94 & 1 \\
\hline BOBRV2 & Columbia River & Pr Rapids Dam & 14-Apr-94 & 1 \\
\hline B0CSG9 & Columbia River & Pr Rapids Dam & 22-Sep-94 & 1 \\
\hline BOCSHO & Columbia River & Pr Rapids Dam & 22-Sep-94 & 1 \\
\hline B0CSH1 & Columbia River & Pr Rapids Dam & 22-Sep-94 & 1 \\
\hline $\mathrm{BOCSH} 2$ & Columbia River & Pr Rapids Dam & 22-Sep-94 & 1 \\
\hline B0W3D5 & Columbia River & Pr Rapids Dam & 03-Aug-99 & 1 \\
\hline B0W3D6 & Columbia River & Pr Rapids Dam & 03-Aug-99 & 1 \\
\hline B0W3D7 & Columbia River & Pr Rapids Dam & 03-Aug-99 & 1 \\
\hline B0W3D8 & Columbia River & Pr Rapids Dam & 03-Aug-99 & 1 \\
\hline B0W3D9 & Columbia River & Pr Rapids Dam & 03-Aug-99 & 1 \\
\hline BOW3F0 & Columbia River & Pr Rapids Dam & 03-Aug-99 & 1 \\
\hline B0W3F1 & Columbia River & Pr Rapids Dam & 03-Aug-99 & 1 \\
\hline BoW3F2 & Columbia River & Pr Rapids Dam & 03-Aug-99 & 1 \\
\hline B0W3L1 & Columbia River & Pr Rapids Dam & 03-Aug-99 & 1 \\
\hline BOW3L3 & Columbia River & Pr Rapids Dam & 03-Aug-99 & 1 \\
\hline BOW3L5 & Columbia River & Pr Rapids Dam & 03-Aug-99 & 1 \\
\hline BOW3L7 & Columbia River & Pr Rapids Dam & 03-Aug-99 & 1 \\
\hline BOW3N2 & Columbia River & Pr Rapids Dam & 03-Aug-99 & 1 \\
\hline BOW3N3 & Columbia River & Pr Rapids Dam & 03-Aug-99 & 1 \\
\hline BOW3N4 & Columbia River & Pr Rapids Dam & 03-Aug-99 & 1 \\
\hline B0W3N5 & Columbia River & Pr Rapids Dam & 03-Aug-99 & 1 \\
\hline BoW3N6 & Columbia River & Pr Rapids Dam & 03-Aug-99 & 1 \\
\hline BOW3N7 & Columbia River & Pr Rapids Dam & 03-Aug-99 & 1 \\
\hline Bow3W2 & Columbia River & Pr Rapids Dam & 03-Aug-99 & 1 \\
\hline Bow3W3 & Columbia River & Pr Rapids Dam & 03-Aug-99 & 1 \\
\hline B0W3W4 & Columbia River & Pr Rapids Dam & 03-Aug-99 & 1 \\
\hline Bow3W5 & Columbia River & Pr Rapids Dam & 03-Aug-99 & 1 \\
\hline Bow3W6 & Columbia River & Pr Rapids Dam & 03-Aug-99 & 1 \\
\hline Bow3W7 & Columbia River & Pr Rapids Dam & 03-Aug-99 & 1 \\
\hline BOWFK9 & Columbia River & Pr Rapids Dam & 03-Aug-99 & 1 \\
\hline BOWFLO & Columbia River & Pr Rapids Dam & 03-Aug-99 & 1 \\
\hline B0WFL1 & Columbia River & Pr Rapids Dam & 03-Aug-99 & 1 \\
\hline BOWFL2 & Columbia River & Pr Rapids Dam & 03-Aug-99 & 1 \\
\hline BOWFL3 & Columbia River & Pr Rapids Dam & 03-Aug-99 & 1 \\
\hline B0YWC1 & Columbia River & Pr Rapids Dam & 07-Aug-00 & 1 \\
\hline BOYWC2 & Columbia River & Pr Rapids Dam & 07-Aug-00 & 1 \\
\hline B0YWC3 & Columbia River & Pr Rapids Dam & 07-Aug-00 & 1 \\
\hline BOYWC4 & Columbia River & Pr Rapids Dam & 07-Aug-00 & 1 \\
\hline B0YWC5 & Columbia river & Pr Rapids Dam & 07-Aug-00 & 1 \\
\hline BOYWC7 & Columbia river & Pr Rapids Dam & 07-Aug-00 & 1 \\
\hline BOYXP6 & Columbia river & Pr Rapids Dam & 07-Aug-00 & 1 \\
\hline BOYXP7 & Columbia river & Pr Rapids Dam & 07-Aug-00 & 1 \\
\hline B12CL9 & Columbia River & Pr Rapids Dam & 19-Jul-01 & 1 \\
\hline B12CM1 & Columbia River & Pr Rapids Dam & 19-Jul-01 & 1 \\
\hline B12CM2 & Columbia River & Pr Rapids Dam & 19-Jul-01 & 1 \\
\hline
\end{tabular}


Table A-1

\section{Summary Table of Segment 1 Sediment Samples By Area, Site, Date, and Category}

\begin{tabular}{|c|c|c|c|c|}
\hline Sample ID & Sample Area & Sample Site & Sample Date & Category \\
\hline B12CM4 & Columbia River & Pr Rapids Dam & 19-Jul-01 & 1 \\
\hline B12CV1 & Columbia River & Pr Rapids Dam & 19-Jul-01 & 1 \\
\hline B12CV2 & Columbia River & Pr Rapids Dam & 19-Jul-01 & 1 \\
\hline B12RY5 & Columbia River & Vernita BRG & 28-Aug-01 & 1 \\
\hline B12RY7 & Columbia River & Vernita BRG & 28-Aug-01 & 1 \\
\hline В12Т03 & Columbia River & Vernita BRG & 28-Aug-01 & 1 \\
\hline B14WF8 & Columbia River & Pr Rapids Dam & 23-Jul-02 & 1 \\
\hline B14WF9 & Columbia River & Pr Rapids Dam & 23-Jul-02 & 1 \\
\hline B14YM9 & Columbia River & Pr Rapids Dam & 23-Jul-02 & 1 \\
\hline B14YN0 & Columbia River & Pr Rapids Dam & 23-Jul-02 & 1 \\
\hline B14YN1 & Columbia River & Pr Rapids Dam & 23-Jul-02 & 1 \\
\hline B14YN2 & Columbia River & Pr Rapids Dam & 23-Jul-02 & 1 \\
\hline B14YN3 & Columbia River & Pr Rapids Dam & 23-Jul-02 & 1 \\
\hline B14YN5 & Columbia River & Pr Rapids Dam & 23-Jul-02 & 1 \\
\hline B176V1 & Columbia River & Pr Rapids Dam & 14-Jul-03 & 1 \\
\hline B176V2 & Columbia River & Pr Rapids Dam & 14-Jul-03 & 1 \\
\hline B17701 & Columbia River & Pr Rapids Dam & 14-Jul-03 & 1 \\
\hline B17702 & Columbia River & Pr Rapids Dam & 14-Jul-03 & 1 \\
\hline B17703 & Columbia River & Pr Rapids Dam & 14-Jul-03 & 1 \\
\hline B17704 & Columbia River & Pr Rapids Dam & 14-Jul-03 & 1 \\
\hline B17705 & Columbia River & Pr Rapids Dam & 14-Jul-03 & 1 \\
\hline B17707 & Columbia River & Pr Rapids Dam & 14-Jul-03 & 1 \\
\hline B199N9 & Columbia River & Wanapum Dam & 04-Jun-04 & 1 \\
\hline B19PK8 & Columbia River & Pr Rapids Dam & 12-Jul-04 & 1 \\
\hline B19PK9 & Columbia River & Pr Rapids Dam & 12-Jul-04 & 1 \\
\hline B19PL0 & Columbia River & Pr Rapids Dam & 12-Jul-04 & 1 \\
\hline B19PL1 & Columbia River & Pr Rapids Dam & 12-Jul-04 & 1 \\
\hline B19PL2 & Columbia River & Pr Rapids Dam & 12-Jul-04 & 1 \\
\hline B19PL4 & Columbia River & Pr Rapids Dam & 12-Jul-04 & 1 \\
\hline B19TF7 & Columbia River & Pr Rapids Dam & 12-Jul-04 & 1 \\
\hline B19TF8 & Columbia River & Pr Rapids Dam & 12-Jul-04 & 1 \\
\hline B1B7R2 & Columbia River & Pr Rapids Dam & 20-Sep-04 & 1 \\
\hline B1B7R3 & Columbia River & Pr Rapids Dam & 20-Sep-04 & 1 \\
\hline B1B7R4 & Columbia River & Pr Rapids Dam & 20-Sep-04 & 1 \\
\hline B1B7W2 & Columbia River & Pr Rapids Dam & 20-Sep-04 & 1 \\
\hline B1B7W3 & Columbia River & Pr Rapids Dam & 20-Sep-04 & 1 \\
\hline B1B7W4 & Columbia River & Pr Rapids Dam & 20-Sep-04 & 1 \\
\hline B1B7W5 & Columbia River & Pr Rapids Dam & 20-Sep-04 & 1 \\
\hline CRE-3 & Columbia River & RM 440 & 01-Oct-99 & 2 \\
\hline GHI-1 & Columbia River & RM 417.5 & 01-Oct-99 & 2 \\
\hline GHI-2 & Columbia River & RM 417.5 & 01-Oct-99 & 2 \\
\hline GHI-3 & Columbia River & RM 417.5 & 01-Oct-99 & 2 \\
\hline GHI-4 & Columbia River & RM 417.5 & 01-Oct-99 & 2 \\
\hline GHI-5 & Columbia River & RM 417.5 & 01-Oct-99 & 2 \\
\hline GOO-1 & Columbia River & RM 398.5 & 01-Oct-99 & 2 \\
\hline GOO-2 & Columbia River & RM 398.5 & 01-Oct-99 & 2 \\
\hline GOO-3A & Columbia River & RM 398.5 & 01-Oct-99 & 2 \\
\hline GOO-3B & Columbia River & RM 398.5 & 01-Oct-99 & 2 \\
\hline
\end{tabular}


Table A-1

Summary Table of Segment 1 Sediment Samples By Area, Site, Date, and Category

\begin{tabular}{|c|c|c|c|c|}
\hline Sample ID & Sample Area & Sample Site & Sample Date & Category \\
\hline GOO-3C & Columbia River & RM 398.5 & 01-Oct-99 & 2 \\
\hline GOO-4 & Columbia River & RM 398.5 & 01-Oct-99 & 2 \\
\hline HAN-5 & Columbia River & RM 405.5 & 01-Oct-99 & 2 \\
\hline HR002V & Columbia River & Vantage WA & 07-Oct-03 & 1 \\
\hline PEW-1sed & Columbia River & RM 354.4 & 16-Jul-92 & 2 \\
\hline RIT dep-1 & Columbia River & RM 452 & 01-Oct-99 & 2 \\
\hline SCA-3A & Columbia River & RM 428 & 01-Oct-99 & 2 \\
\hline SCA-3B & Columbia River & RM 428 & 01-Oct-99 & 2 \\
\hline SCA-3C & Columbia River & RM 428 & 01-Oct-99 & 2 \\
\hline SCA-4 & Columbia River & RM 428 & 01-Oct-99 & 2 \\
\hline SCA-5 & Columbia River & RM 428 & 01-Oct-99 & 2 \\
\hline SS-1-1 & Columbia River & RM 440 & 01-Oct-99 & 2 \\
\hline SS-1-2 & Columbia River & RM 440 & 01-Oct-99 & 2 \\
\hline SS-1-3 & Columbia River & RM 440 & 01-Oct-99 & 2 \\
\hline SS-2-1 & Columbia River & RM 440 & 01-Oct-99 & 2 \\
\hline SS-2-2 & Columbia River & RM 440 & 01-Oct-99 & 2 \\
\hline SS-2-3 & Columbia River & RM 440 & 01-Oct-99 & 2 \\
\hline SS-3-1 & Columbia River & RM 440 & 01-Oct-99 & 2 \\
\hline SS-3-2 & Columbia River & RM 440 & 01-Oct-99 & 2 \\
\hline SS-3-3 & Columbia River & RM 440 & 01-Oct-99 & 2 \\
\hline SS-4-1 & Columbia River & RM 440 & 01-Oct-99 & 2 \\
\hline SS-4-2 & Columbia River & RM 440 & 01-Oct-99 & 2 \\
\hline SS-4-3 & Columbia River & RM 440 & 01-Oct-99 & 2 \\
\hline 4444202 & Lower Crab Creek & RM 5 & 01-Jun-04 & 1 \\
\hline 45A070-7 & Wenatchee River & Wenatchee & 01-Sep-84 & 2 \\
\hline
\end{tabular}


Table A-2

Summary Table of Segment 1 Surface Water Samples By Area, Site, Date, and Category

\begin{tabular}{|c|c|c|c|c|}
\hline Sample Area & Sample Site & Sample ID & Sample Date & Category \\
\hline Columbia River & EB NR Wenatchee & 01258001 & June 20,2001 & 1 \\
\hline Columbia River & Near Vernita & 133303 & May 5, 2004 & 1 \\
\hline Columbia River & Near Vernita & 41949 & May 10, 2000 & 1 \\
\hline Columbia River & Near Vernita & 41908 & July 12,2000 & 1 \\
\hline Columbia River & Near Vernita & 41886 & April 12, 2000 & 1 \\
\hline Columbia River & Near Vernita & 41840 & June 14, 2000 & 1 \\
\hline Columbia River & Near Vernita & 41555 & October 3, 2000 & 1 \\
\hline Columbia River & Near Vernita & 41466 & August 9, 2000 & 1 \\
\hline Columbia River & Near Vernita & 41313 & September 13, 2000 & 1 \\
\hline Columbia River & Near Vernita & 406008 & October 3, 2000 & 1 \\
\hline Columbia River & Near Vernita & 376169 & September 13, 2000 & 1 \\
\hline Columbia River & Near Vernita & 132999 & February 11, 2004 & 1 \\
\hline Columbia River & Near Vernita & 286169 & July 12, 2000 & 1 \\
\hline Columbia River & Near Vernita & 66169 & February 9, 2000 & 1 \\
\hline Columbia River & Near Vernita & 26169 & January 12, 2000 & 1 \\
\hline Columbia River & Near Vernita & 246169 & June 14, 2000 & 1 \\
\hline Columbia River & Near Vernita & 196169 & May 10,2000 & 1 \\
\hline Columbia River & Near Vernita & 156169 & April 12, 2000 & 1 \\
\hline Columbia River & Near Vernita & 1366008 & September 4, 2001 & 1 \\
\hline Columbia River & Near Vernita & 134536 & June 6, 2005 & 1 \\
\hline Columbia River & Near Vernita & 134458 & May 2, 2005 & 1 \\
\hline Columbia River & Near Vernita & 133238 & April 14, 2004 & 1 \\
\hline Columbia River & Near Vernita & 133090 & March 10, 2004 & 1 \\
\hline Columbia River & Near Vernita & 326169 & August 9, 2000 & 1 \\
\hline Columbia River & Near Vernita & 99026169 & January 13, 1999 & 1 \\
\hline Columbia River & Near Vernita & 99506169 & December 15, 1999 & 1 \\
\hline Columbia River & Near Vernita & 99466169 & November 17, 1999 & 1 \\
\hline Columbia River & Near Vernita & 99416169 & October 13, 1999 & 1 \\
\hline Columbia River & Near Vernita & 99376169 & September 15, 1999 & 1 \\
\hline Columbia River & Near Vernita & 99326169 & August 11, 1999 & 1 \\
\hline Columbia River & Near Vernita & 99286169 & July 14, 1999 & 1 \\
\hline Columbia River & Near Vernita & 99236169 & June 9, 1999 & 1 \\
\hline Columbia River & Near Vernita & 99186169 & May 5, 1999 & 1 \\
\hline Columbia River & Near Vernita & 99146169 & April 7, 1999 & 1 \\
\hline Columbia River & Near Vernita & 456008 & November 7, 2000 & 1 \\
\hline Columbia River & Near Vernita & 99066169 & February 10, 1999 & 1 \\
\hline Columbia River & Near Vernita & 496008 & December 5, 2000 & 1 \\
\hline Columbia River & Near Vernita & 87081 & August 14, 2001 & 1 \\
\hline Columbia River & Near Vernita & 86895 & June 5, 2001 & 1 \\
\hline Columbia River & Near Vernita & 86779 & May 8, 2001 & 1 \\
\hline Columbia River & Near Vernita & 86688 & April 3, 2001 & 1 \\
\hline Columbia River & Near Vernita & 86594 & March 6, 2001 & 1 \\
\hline Columbia River & Near Vernita & 86500 & February 6, 2001 & 1 \\
\hline Columbia River & Near Vernita & 86430 & January 16, 2001 & 1 \\
\hline Columbia River & Near Vernita & 86341 & December 5, 2000 & 1 \\
\hline Columbia River & Near Vernita & 85847 & November 7, 2000 & 1 \\
\hline Columbia River & Near Vernita & 86994 & July 10, 2001 & 1 \\
\hline Columbia River & Near Vernita & 99106169 & March 10, 1999 & 1 \\
\hline
\end{tabular}


Table A-2

Summary Table of Segment 1 Surface Water Samples By Area, Site, Date, and Category

\begin{tabular}{|c|c|c|c|c|}
\hline Sample Area & Sample Site & Sample ID & Sample Date & Category \\
\hline Columbia River & Near Vernita & 131036 & May 8,2002 & 1 \\
\hline Columbia River & Near Vernita & 130522 & October 10, 2001 & 1 \\
\hline Columbia River & Near Vernita & 130655 & November 14, 2001 & 1 \\
\hline Columbia River & Near Vernita & 130674 & December 5, 2001 & 1 \\
\hline Columbia River & Near Vernita & 130696 & January 9, 2002 & 1 \\
\hline Columbia River & Near Vernita & 130766 & February 6, 2002 & 1 \\
\hline Columbia River & Near Vernita & 131675 & December 4, 2002 & 1 \\
\hline Columbia River & Near Vernita & 130967 & April 3, 2002 & 1 \\
\hline Columbia River & Near Vernita & 1196008 & May 8, 2001 & 1 \\
\hline Columbia River & Near Vernita & 131197 & June 12, 2002 & 1 \\
\hline Columbia River & Near Vernita & 131218 & July 10, 2002 & 1 \\
\hline Columbia River & Near Vernita & 131381 & August 7, 2002 & 1 \\
\hline Columbia River & Near Vernita & 131401 & September 11, 2002 & 1 \\
\hline Columbia River & Near Vernita & 131493 & October 9, 2002 & 1 \\
\hline Columbia River & Near Vernita & 131586 & November 6, 2002 & 1 \\
\hline Columbia River & Near Vernita & 130878 & March 6, 2002 & 1 \\
\hline Columbia River & Near Vernita & 133824 & November 3, 2004 & 1 \\
\hline Columbia River & Near Vernita & 87126 & September 4, 2001 & 1 \\
\hline Columbia River & Near Vernita & 132910 & January 14, 2004 & 1 \\
\hline Columbia River & Near Vernita & 133373 & June 9, 2004 & 1 \\
\hline Columbia River & Near Vernita & 133483 & July 14, 2004 & 1 \\
\hline Columbia River & Near Vernita & 133571 & August 4, 2004 & 1 \\
\hline Columbia River & Near Vernita & 1336008 & August 14, 2001 & 1 \\
\hline Columbia River & Near Vernita & 1286008 & July 10, 2001 & 1 \\
\hline Columbia River & Near Vernita & 133716 & October 4, 2004 & 1 \\
\hline Columbia River & Near Vernita & 1236008 & June 5, 2001 & 1 \\
\hline Columbia River & Near Vernita & 133923 & December 6, 2004 & 1 \\
\hline Columbia River & Near Vernita & 134126 & February 7, 2005 & 1 \\
\hline Columbia River & Near Vernita & 1066008 & February 6, 2001 & 1 \\
\hline Columbia River & Near Vernita & 1146008 & April 3, 2001 & 1 \\
\hline Columbia River & Near Vernita & 116169 & March 15, 2000 & 1 \\
\hline Columbia River & Near Vernita & 1106008 & March 6, 2001 & 1 \\
\hline Columbia River & Near Vernita & 133663 & September 15, 2004 & 1 \\
\hline Columbia River & Near Vernita & 131879 & February 5, 2003 & 1 \\
\hline Columbia River & Near Vernita & 132260 & June 4, 2003 & 1 \\
\hline Columbia River & Near Vernita & 132111 & April 9, 2003 & 1 \\
\hline Columbia River & Near Vernita & 131946 & March 5, 2003 & 1 \\
\hline Columbia River & Near Vernita & 132170 & May 7, 2003 & 1 \\
\hline Columbia River & Near Vernita & 132818 & December 9, 2003 & 1 \\
\hline Columbia River & Near Vernita & 132372 & July 9, 2003 & 1 \\
\hline Columbia River & Near Vernita & 132439 & August 6, 2003 & 1 \\
\hline Columbia River & Near Vernita & 132528 & September 10, 2003 & 1 \\
\hline Columbia River & Near Vernita & 131788 & January 8, 2003 & 1 \\
\hline Columbia River & Near Vernita & 132641 & October 8, 2003 & 1 \\
\hline Columbia River & Near Vernita & 132728 & November 5, 2003 & 1 \\
\hline Columbia River & Near Vernita & 1036008 & January 16, 2001 & 1 \\
\hline Columbia River & NORTHPORT WA & 1408080 & September 10, 2001 & 1 \\
\hline Columbia River & NORTHPORT WA & 2118105 & February 11, 2002 & 1 \\
\hline
\end{tabular}


Table A-2

Summary Table of Segment 1 Surface Water Samples By Area, Site, Date, and Category

\begin{tabular}{|c|c|c|c|c|}
\hline Sample Area & Sample Site & Sample ID & Sample Date & Category \\
\hline Columbia River & NORTHPORT WA & 2068080 & January 14,2002 & 1 \\
\hline Columbia River & NORTHPORT WA & 2158155 & March 11, 2002 & 1 \\
\hline Columbia River & NORTHPORT WA & 2018080 & December 3, 2001 & 1 \\
\hline Columbia River & NORTHPORT WA & 1468155 & October 15, 2001 & 1 \\
\hline Columbia River & NORTHPORT WA & 2178180 & April 8, 2002 & 1 \\
\hline Columbia River & NORTHPORT WA & 1368080 & August 6, 2001 & 1 \\
\hline Columbia River & NORTHPORT WA & 1498100 & November 5, 2001 & 1 \\
\hline Columbia River & NORTHPORT WA & 2228080 & May 12, 2002 & 1 \\
\hline Columbia River & NORTHPORT WA & 2268155 & June 3, 2002 & 1 \\
\hline Columbia River & NORTHPORT WA & 1286057 & July 9, 2001 & 1 \\
\hline Columbia River & Pr Rapids Dam & 24568 & February 2, 2000 & 1 \\
\hline Columbia River & Pr Rapids Dam & 29027 & February 6, 2002 & 1 \\
\hline Columbia River & Pr Rapids Dam & 30845 & July 10, 2003 & 1 \\
\hline Columbia River & Pr Rapids Dam & 30855 & February 5, 2003 & 1 \\
\hline Columbia River & Pr Rapids Dam & 33136 & July 8, 2004 & 1 \\
\hline Columbia River & Pr Rapids Dam & 33142 & February 4, 2004 & 1 \\
\hline Columbia River & Pr Rapids Dam & 26202 & June 23, 2001 & 1 \\
\hline Columbia River & Pr Rapids Dam & 24557 & June 29, 2000 & 1 \\
\hline Columbia River & Pr Rapids Dam & 23425 & November 3, 1999 & 1 \\
\hline Columbia River & Pr Rapids Dam & 23423 & May 5, 1999 & 1 \\
\hline Columbia River & Pr Rapids Dam & 29017 & June 27, 2002 & 1 \\
\hline Columbia River & Pr Rapids Dam & 26213 & January 31, 2001 & 1 \\
\hline Columbia River & Pr Rapids River & B18VH5 & July 8, 2004 & 1 \\
\hline Columbia River & Pr Rapids River & В1ВСТ8 & January 5, 2005 & 1 \\
\hline Columbia River & Pr Rapids River & B1BD44 & October 28, 2004 & 1 \\
\hline Columbia River & Pr Rapids River & B1BD45 & November 23, 2004 & 1 \\
\hline Columbia River & Pr Rapids River & B1BD46 & January 5, 2005 & 1 \\
\hline Columbia River & Pr Rapids River & B1BMR0 & December 1, 2004 & 1 \\
\hline Columbia River & Pr Rapids River & B1BD31 & October 28, 2004 & 1 \\
\hline Columbia River & Pr Rapids River & B1BD43 & January 5, 2005 & 1 \\
\hline Columbia River & Pr Rapids River & B18VT0 & May 6, 2004 & 1 \\
\hline Columbia River & Pr Rapids River & B18VV2 & July 8, 2004 & 1 \\
\hline Columbia River & Pr Rapids River & B18VV3 & April 28, 2004 & 1 \\
\hline Columbia River & Pr Rapids River & B18VV4 & June 8, 2004 & 1 \\
\hline Columbia River & Pr Rapids River & B18VV5 & July 8, 2004 & 1 \\
\hline Columbia River & Pr Rapids River & B19223 & June 8, 2004 & 1 \\
\hline Columbia River & Pr Rapids River & В19B08 & July 8, 2004 & 1 \\
\hline Columbia River & Pr Rapids River & B1BW13 & January 5, 2005 & 1 \\
\hline Columbia River & Pr Rapids River & B1B604 & October 1, 2004 & 1 \\
\hline Columbia River & Pr Rapids River & B18761 & April 1, 2004 & 1 \\
\hline Columbia River & Pr Rapids River & B19XX9 & August 31, 2004 & 1 \\
\hline Columbia River & Pr Rapids River & В19M38 & October 1, 2004 & 1 \\
\hline Columbia River & Pr Rapids River & В19M37 & August 31, 2004 & 1 \\
\hline Columbia River & Pr Rapids River & В19м36 & August 4, 2004 & 1 \\
\hline Columbia River & Pr Rapids River & B19M35 & October 1, 2004 & 1 \\
\hline Columbia River & Pr Rapids River & B19M25 & August 4, 2004 & 1 \\
\hline Columbia River & Pr Rapids River & B161R0 & January 8, 2003 & 1 \\
\hline Columbia River & Pr Rapids River & B18D84 & March 2, 2004 & 1 \\
\hline
\end{tabular}


Table A-2

Summary Table of Segment 1 Surface Water Samples By Area, Site, Date, and Category

\begin{tabular}{|c|c|c|c|c|}
\hline Sample Area & Sample Site & Sample ID & Sample Date & Category \\
\hline Columbia River & Pr Rapids River & B19LT2 & October 6, 2004 & 1 \\
\hline Columbia River & Pr Rapids River & B18763 & March 2, 2004 & 1 \\
\hline Columbia River & Pr Rapids River & B183H6 & January 8, 2004 & 1 \\
\hline Columbia River & Pr Rapids River & B186W3 & April 1, 2004 & 1 \\
\hline Columbia River & Pr Rapids River & B18746 & February 4, 2004 & 1 \\
\hline Columbia River & Pr Rapids River & B18764 & April 1, 2004 & 1 \\
\hline Columbia River & Pr Rapids River & B15JY4 & January 8, 2003 & 1 \\
\hline Columbia River & Pr Rapids River & B18K74 & April 1, 2004 & 1 \\
\hline Columbia River & Pr Rapids River & B17P39 & January 8, 2004 & 1 \\
\hline Columbia River & Pr Rapids River & B17NT2 & January 8, 2004 & 1 \\
\hline Columbia River & Pr Rapids River & B17P42 & January 8, 2004 & 1 \\
\hline Columbia River & Pr Rapids River & B18762 & February 4, 2004 & 1 \\
\hline Columbia River & Priest Rapids & B114V6 & April 4, 2001 & 1 \\
\hline Columbia River & Priest Rapids & B11C04 & March 1, 2001 & 1 \\
\hline Columbia River & Priest Rapids & B11LH3 & June 28, 2001 & 1 \\
\hline Columbia River & Priest Rapids & B114V4 & February 7, 2001 & 1 \\
\hline Columbia River & Priest Rapids & $\mathrm{B} 11 \mathrm{~J} 40$ & April 4, 2001 & 1 \\
\hline Columbia River & Priest Rapids & B114T8 & March 7, 2001 & 1 \\
\hline Columbia River & Priest Rapids & B114T9 & April 4, 2001 & 1 \\
\hline Columbia River & Priest Rapids & B114V3 & April 4, 2001 & 1 \\
\hline Columbia River & Priest Rapids & B15948 & October 2, 2002 & 1 \\
\hline Columbia River & Priest Rapids & B103W8 & October 5, 2000 & 1 \\
\hline Columbia River & Priest Rapids & B114V5 & March 7, 2001 & 1 \\
\hline Columbia River & Priest Rapids & B15VN2 & November 26, 2002 & 1 \\
\hline Columbia River & Priest Rapids & B15K74 & October 30, 2002 & 1 \\
\hline Columbia River & Priest Rapids & В15K91 & October 30, 2002 & 1 \\
\hline Columbia River & Priest Rapids & B15K84 & January 8, 2003 & 1 \\
\hline Columbia River & Priest Rapids & B15K85 & October 30, 2002 & 1 \\
\hline Columbia River & Priest Rapids & B15K86 & November 26, 2002 & 1 \\
\hline Columbia River & Priest Rapids & B15K87 & January 8, 2003 & 1 \\
\hline Columbia River & Priest Rapids & B15K90 & January 8, 2003 & 1 \\
\hline Columbia River & Priest Rapids & B15K92 & November 26, 2002 & 1 \\
\hline Columbia River & Priest Rapids & В15K93 & January 8, 2003 & 1 \\
\hline Columbia River & Priest Rapids & B16PK6 & May 28, 2003 & 1 \\
\hline Columbia River & Priest Rapids & $\mathrm{B} 10 \mathrm{C} 13$ & November 2, 2000 & 1 \\
\hline Columbia River & Priest Rapids & B10C26 & November 2, 2000 & 1 \\
\hline Columbia River & Priest Rapids & B10C25 & December 28, 2000 & 1 \\
\hline Columbia River & Priest Rapids & B10BR0 & January 4, 2001 & 1 \\
\hline Columbia River & Priest Rapids & B0YW65 & September 7, 2000 & 1 \\
\hline Columbia River & Priest Rapids & BOYKX8 & October 5, 2000 & 1 \\
\hline Columbia River & Priest Rapids & B16Р81 & July 10, 2003 & 1 \\
\hline Columbia River & Priest Rapids & B16PJ4 & April 30, 2003 & 1 \\
\hline Columbia River & Priest Rapids & B10C28 & December 28, 2000 & 1 \\
\hline Columbia River & Priest Rapids & B16PK5 & April 30, 2003 & 1 \\
\hline Columbia River & Priest Rapids & В10C31 & December 28, 2000 & 1 \\
\hline Columbia River & Priest Rapids & B16PK7 & July 10, 2003 & 1 \\
\hline Columbia River & Priest Rapids & B16PL0 & July 10, 2003 & 1 \\
\hline Columbia River & Priest Rapids & B11LR6 & May 2, 2001 & 1 \\
\hline
\end{tabular}


Table A-2

Summary Table of Segment 1 Surface Water Samples By Area, Site, Date, and Category

\begin{tabular}{|c|c|c|c|c|}
\hline Sample Area & Sample Site & Sample ID & Sample Date & Category \\
\hline Columbia River & Priest Rapids & B14408 & April 3, 2002 & 1 \\
\hline Columbia River & Priest Rapids & B16WD8 & May 28, 2003 & 1 \\
\hline Columbia River & Priest Rapids & B16PL1 & April 30, 2003 & 1 \\
\hline Columbia River & Priest Rapids & B16PL2 & May 28, 2003 & 1 \\
\hline Columbia River & Priest Rapids & B16PL3 & July 10, 2003 & 1 \\
\hline Columbia River & Priest Rapids & B16PK4 & July 10, 2003 & 1 \\
\hline Columbia River & Priest Rapids & B16876 & April 2, 2003 & 1 \\
\hline Columbia River & Priest Rapids & B114T6 & April 4, 2001 & 1 \\
\hline Columbia River & Priest Rapids & B114P8 & January 31, 2001 & 1 \\
\hline Columbia River & Priest Rapids & B114F5 & April 4, 2001 & 1 \\
\hline Columbia River & Priest Rapids & B110Y4 & January 4, 2001 & 1 \\
\hline Columbia River & Priest Rapids & B10MP5 & November 29, 2000 & 1 \\
\hline Columbia River & Priest Rapids & В10С34 & December 28, 2000 & 1 \\
\hline Columbia River & Priest Rapids & B167W7 & April 9, 2003 & 1 \\
\hline Columbia River & Priest Rapids & B16858 & February 5, 2003 & 1 \\
\hline Columbia River & Priest Rapids & B10C27 & November 29, 2000 & 1 \\
\hline Columbia River & Priest Rapids & B16875 & March 5, 2003 & 1 \\
\hline Columbia River & Priest Rapids & B114T7 & February 7, 2001 & 1 \\
\hline Columbia River & Priest Rapids & B16879 & April 2, 2003 & 1 \\
\hline Columbia River & Priest Rapids & B16880 & February 5, 2003 & 1 \\
\hline Columbia River & Priest Rapids & B16881 & March 5, 2003 & 1 \\
\hline Columbia River & Priest Rapids & B16882 & April 2, 2003 & 1 \\
\hline Columbia River & Priest Rapids & B16BY2 & March 5, 2003 & 1 \\
\hline Columbia River & Priest Rapids & B16JL8 & April 2, 2003 & 1 \\
\hline Columbia River & Priest Rapids & В10С33 & November 29, 2000 & 1 \\
\hline Columbia River & Priest Rapids & В10С32 & November 2, 2000 & 1 \\
\hline Columbia River & Priest Rapids & B16874 & February 5, 2003 & 1 \\
\hline Columbia River & Priest Rapids & B14B49 & June 27, 2002 & 1 \\
\hline Columbia River & Priest Rapids & B14B58 & June 27, 2002 & 1 \\
\hline Columbia River & Priest Rapids & B13VK3 & April 3, 2002 & 1 \\
\hline Columbia River & Priest Rapids & B13VK4 & February 6, 2002 & 1 \\
\hline Columbia River & Priest Rapids & B13VK5 & March 7, 2002 & 1 \\
\hline Columbia River & Priest Rapids & B13VK6 & April 3, 2002 & 1 \\
\hline Columbia River & Priest Rapids & B140P7 & March 7, 2002 & 1 \\
\hline Columbia River & Priest Rapids & B13VJ8 & March 7, 2002 & 1 \\
\hline Columbia River & Priest Rapids & B16WL1 & April 30, 2003 & 1 \\
\hline Columbia River & Priest Rapids & B13VJ7 & February 6, 2002 & 1 \\
\hline Columbia River & Priest Rapids & B14B50 & May 1, 2002 & 1 \\
\hline Columbia River & Priest Rapids & B14B51 & May 29, 2002 & 1 \\
\hline Columbia River & Priest Rapids & B14B52 & June 27, 2002 & 1 \\
\hline Columbia River & Priest Rapids & B14B55 & June 27, 2002 & 1 \\
\hline Columbia River & Priest Rapids & B14B56 & May 1, 2002 & 1 \\
\hline Columbia River & Priest Rapids & B14B37 & May 1, 2002 & 1 \\
\hline Columbia River & Priest Rapids & В149T6 & July 2, 2002 & 1 \\
\hline Columbia River & Priest Rapids & B13109 & December 27, 2001 & 1 \\
\hline Columbia River & Priest Rapids & В13CC6 & November 27, 2001 & 1 \\
\hline Columbia River & Priest Rapids & B13L96 & January 3, 2002 & 1 \\
\hline Columbia River & Priest Rapids & B13117 & November 27, 2001 & 1 \\
\hline
\end{tabular}


Table A-2

Summary Table of Segment 1 Surface Water Samples By Area, Site, Date, and Category

\begin{tabular}{|c|c|c|c|c|}
\hline Sample Area & Sample Site & Sample ID & Sample Date & Category \\
\hline Columbia River & Priest Rapids & B13116 & November 1, 2001 & 1 \\
\hline Columbia River & Priest Rapids & B13115 & December 27, 2001 & 1 \\
\hline Columbia River & Priest Rapids & B13112 & December 27, 2001 & 1 \\
\hline Columbia River & Priest Rapids & B13VJ9 & April 3, 2002 & 1 \\
\hline Columbia River & Priest Rapids & B13110 & November 1, 2001 & 1 \\
\hline Columbia River & Priest Rapids & B14JF2 & May 29, 2002 & 1 \\
\hline Columbia River & Priest Rapids & B130Y7 & November 1, 2001 & 1 \\
\hline Columbia River & Priest Rapids & B130M9 & January 3, 2002 & 1 \\
\hline Columbia River & Priest Rapids & В12Т28 & October 2, 2001 & 1 \\
\hline Columbia River & Priest Rapids & B13V74 & April 3, 2002 & 1 \\
\hline Columbia River & Priest Rapids & B13VF8 & February 6, 2002 & 1 \\
\hline Columbia River & Priest Rapids & B13VJ6 & April 3, 2002 & 1 \\
\hline Columbia River & Priest Rapids & B13111 & November 27, 2001 & 1 \\
\hline Columbia River & Priest Rapids & В153Т9 & September 4, 2002 & 1 \\
\hline Columbia River & Priest Rapids & B14B57 & May 29, 2002 & 1 \\
\hline Columbia River & Priest Rapids & B14WY0 & August 7, 2002 & 1 \\
\hline Columbia River & Priest Rapids & B14WY1 & September 4, 2002 & 1 \\
\hline Columbia River & Priest Rapids & B14WY2 & October 2, 2002 & 1 \\
\hline Columbia River & Priest Rapids & B14WY6 & October 2, 2002 & 1 \\
\hline Columbia River & Priest Rapids & B14WY7 & August 7, 2002 & 1 \\
\hline Columbia River & Priest Rapids & B14WW5 & August 7, 2002 & 1 \\
\hline Columbia River & Priest Rapids & B14WY9 & October 2, 2002 & 1 \\
\hline Columbia River & Priest Rapids & B14WJ9 & October 2, 2002 & 1 \\
\hline Columbia River & Priest Rapids & B11LV6 & May 30, 2001 & 1 \\
\hline Columbia River & Priest Rapids & B11LV5 & May 2, 2001 & 1 \\
\hline Columbia River & Priest Rapids & B11LV4 & June 28, 2001 & 1 \\
\hline Columbia River & Priest Rapids & B11LV1 & June 28, 2001 & 1 \\
\hline Columbia River & Priest Rapids & B11LV0 & May 30, 2001 & 1 \\
\hline Columbia River & Priest Rapids & B11LT9 & May 2, 2001 & 1 \\
\hline Columbia River & Priest Rapids & B14WY8 & September 4, 2002 & 1 \\
\hline Columbia River & Priest Rapids & B12849 & August 9, 2001 & 1 \\
\hline Columbia River & Priest Rapids & B12JR7 & September 5, 2001 & 1 \\
\hline Columbia River & Priest Rapids & B12858 & October 2, 2001 & 1 \\
\hline Columbia River & Priest Rapids & B12857 & September 5, 2001 & 1 \\
\hline Columbia River & Priest Rapids & B14RL3 & June 27, 2002 & 1 \\
\hline Columbia River & Priest Rapids & B12856 & August 9, 2001 & 1 \\
\hline Columbia River & Priest Rapids & B12855 & October 2, 2001 & 1 \\
\hline Columbia River & Priest Rapids & B14WX9 & October 2, 2002 & 1 \\
\hline Columbia River & Priest Rapids & B12850 & September 5, 2001 & 1 \\
\hline Columbia River & Priest Rapids & B11LT8 & June 28, 2001 & 1 \\
\hline Columbia River & Priest Rapids & B12848 & October 2, 2001 & 1 \\
\hline Columbia River & Priest Rapids & B12834 & August 9, 2001 & 1 \\
\hline Columbia River & Priest Rapids & B127T9 & October 2, 2001 & 1 \\
\hline Columbia River & Priest Rapids & B124L7 & June 28, 2001 & 1 \\
\hline Columbia River & Priest Rapids & B11WR8 & May 30, 2001 & 1 \\
\hline Columbia River & Priest Rapids & B11LV7 & June 28, 2001 & 1 \\
\hline Columbia River & Priest Rapids & B12851 & October 2, 2001 & 1 \\
\hline Columbia River & Priest Rapids & B0X7L3 & March 8, 2000 & 1 \\
\hline
\end{tabular}


Table A-2

Summary Table of Segment 1 Surface Water Samples By Area, Site, Date, and Category

\begin{tabular}{|c|c|c|c|c|}
\hline Sample Area & Sample Site & Sample ID & Sample Date & Category \\
\hline Columbia River & Priest Rapids & B0WJB2 & December 29, 1999 & 1 \\
\hline Columbia River & Priest Rapids & B0WJB3 & November 3, 1999 & 1 \\
\hline Columbia River & Priest Rapids & B0WJB4 & December 1, 1999 & 1 \\
\hline Columbia River & Priest Rapids & B0WJB5 & December 29, 1999 & 1 \\
\hline Columbia River & Priest Rapids & B17P41 & November 25, 2003 & 1 \\
\hline Columbia River & Priest Rapids & B0TM54 & March 3, 1999 & 1 \\
\hline Columbia River & Priest Rapids & B16WL3 & May 7, 2003 & 1 \\
\hline Columbia River & Priest Rapids & В0ТНJ8 & April 7, 1999 & 1 \\
\hline Columbia River & Priest Rapids & ВотHJ7 & February 24, 1999 & 1 \\
\hline Columbia River & Priest Rapids & B0X469 & December 29, 1999 & 1 \\
\hline Columbia River & Priest Rapids & B0X775 & April 5, 2000 & 1 \\
\hline Columbia River & Priest Rapids & B0X7J0 & February 2, 2000 & 1 \\
\hline Columbia River & Priest Rapids & B0X7M1 & April 5, 2000 & 1 \\
\hline Columbia River & Priest Rapids & B0X7L2 & January 26, 2000 & 1 \\
\hline Columbia River & Priest Rapids & BoWJ97 & November 3, 1999 & 1 \\
\hline Columbia River & Priest Rapids & B0X7L4 & April 5, 2000 & 1 \\
\hline Columbia River & Priest Rapids & В0ТНJ6 & January 27, 1999 & 1 \\
\hline Columbia River & Priest Rapids & B0X7L8 & April 5, 2000 & 1 \\
\hline Columbia River & Priest Rapids & B0V535 & June 30, 1999 & 1 \\
\hline Columbia River & Priest Rapids & B0X7L9 & January 26, 2000 & 1 \\
\hline Columbia River & Priest Rapids & BOWPR4 & December 1, 1999 & 1 \\
\hline Columbia River & Priest Rapids & B17VB4 & November 25, 2003 & 1 \\
\hline Columbia River & Priest Rapids & B17P47 & November 25, 2003 & 1 \\
\hline Columbia River & Priest Rapids & B17P46 & October 29, 2003 & 1 \\
\hline Columbia River & Priest Rapids & B13118 & December 27, 2001 & 1 \\
\hline Columbia River & Priest Rapids & B17P40 & October 29, 2003 & 1 \\
\hline Columbia River & Priest Rapids & B17P27 & October 29, 2003 & 1 \\
\hline Columbia River & Priest Rapids & B0X7L1 & April 5, 2000 & 1 \\
\hline Columbia River & Priest Rapids & BOVTY8 & October 6, 1999 & 1 \\
\hline Columbia River & Priest Rapids & B0V536 & May 5, 1999 & 1 \\
\hline Columbia River & Priest Rapids & B0V537 & June 2, 1999 & 1 \\
\hline Columbia River & Priest Rapids & B0V538 & June 30, 1999 & 1 \\
\hline Columbia River & Priest Rapids & B0V541 & June 30, 1999 & 1 \\
\hline Columbia River & Priest Rapids & B0V542 & May 5, 1999 & 1 \\
\hline Columbia River & Priest Rapids & B0V543 & June 2, 1999 & 1 \\
\hline Columbia River & Priest Rapids & B0V544 & June 30, 1999 & 1 \\
\hline Columbia River & Priest Rapids & BovCX9 & June 2, 1999 & 1 \\
\hline Columbia River & Priest Rapids & BOVL42 & June 30, 1999 & 1 \\
\hline Columbia River & Priest Rapids & BOVTL8 & October 6, 1999 & 1 \\
\hline Columbia River & Priest Rapids & BOVTX3 & July 28, 1999 & 1 \\
\hline Columbia River & Priest Rapids & B0VTY5 & October 6, 1999 & 1 \\
\hline Columbia River & Priest Rapids & BoWJ99 & December 29, 1999 & 1 \\
\hline Columbia River & Priest Rapids & BOVTY7 & September 8, 1999 & 1 \\
\hline Columbia River & Priest Rapids & BoWJ98 & December 1, 1999 & 1 \\
\hline Columbia River & Priest Rapids & B0VV01 & October 6, 1999 & 1 \\
\hline Columbia River & Priest Rapids & B0VV02 & July 28, 1999 & 1 \\
\hline Columbia River & Priest Rapids & B0VV03 & September 8, 1999 & 1 \\
\hline Columbia River & Priest Rapids & BOVV04 & October 6, 1999 & 1 \\
\hline
\end{tabular}


Table A-2

Summary Table of Segment 1 Surface Water Samples By Area, Site, Date, and Category

\begin{tabular}{|c|c|c|c|c|}
\hline Sample Area & Sample Site & Sample ID & Sample Date & Category \\
\hline Columbia River & Priest Rapids & B0W388 & September 1, 1999 & 1 \\
\hline Columbia River & Priest Rapids & Bow8Y9 & October 6, 1999 & 1 \\
\hline Columbia River & Priest Rapids & B0V520 & May 5, 1999 & 1 \\
\hline Columbia River & Priest Rapids & B0V4R7 & June 30, 1999 & 1 \\
\hline Columbia River & Priest Rapids & B0TW33 & April 7, 1999 & 1 \\
\hline Columbia River & Priest Rapids & BOWJ08 & December 29, 1999 & 1 \\
\hline Columbia River & Priest Rapids & B0WJ81 & November 3, 1999 & 1 \\
\hline Columbia River & Priest Rapids & BoWJ96 & December 29, 1999 & 1 \\
\hline Columbia River & Priest Rapids & B0X7M0 & March 8, 2000 & 1 \\
\hline Columbia River & Priest Rapids & BOVTY6 & July 28, 1999 & 1 \\
\hline Columbia River & Priest Rapids & BOYKV4 & August 10, 2000 & 1 \\
\hline Columbia River & Priest Rapids & $\mathrm{B} 175 \mathrm{H} 7$ & September 30, 2003 & 1 \\
\hline Columbia River & Priest Rapids & $\mathrm{B} 175 \mathrm{H} 6$ & September 3, 2003 & 1 \\
\hline Columbia River & Priest Rapids & B175H5 & August 8, 2003 & 1 \\
\hline Columbia River & Priest Rapids & $\mathrm{B} 175 \mathrm{H} 4$ & September 30, 2003 & 1 \\
\hline Columbia River & Priest Rapids & B175F4 & August 8, 2003 & 1 \\
\hline Columbia River & Priest Rapids & B17561 & September 30, 2003 & 1 \\
\hline Columbia River & Priest Rapids & $\mathrm{B} 172 \mathrm{KO}$ & June 25, 2003 & 1 \\
\hline Columbia River & Priest Rapids & B0XVR5 & June 29, 2000 & 1 \\
\hline Columbia River & Priest Rapids & B0YB64 & June 29, 2000 & 1 \\
\hline Columbia River & Priest Rapids & B0XH22 & March 8, 2000 & 1 \\
\hline Columbia River & Priest Rapids & B175J0 & September 30, 2003 & 1 \\
\hline Columbia River & Priest Rapids & B0YKJ9 & October 5, 2000 & 1 \\
\hline Columbia River & Priest Rapids & $\mathrm{B} 172 \mathrm{~F} 1$ & June 18, 2003 & 1 \\
\hline Columbia River & Priest Rapids & BOYKW8 & October 5, 2000 & 1 \\
\hline Columbia River & Priest Rapids & BOYKW9 & August 10, 2000 & 1 \\
\hline Columbia River & Priest Rapids & BOYKXO & September 7, 2000 & 1 \\
\hline Columbia River & Priest Rapids & BOYKX1 & October 5, 2000 & 1 \\
\hline Columbia River & Priest Rapids & B0YKX5 & October 5, 2000 & 1 \\
\hline Columbia River & Priest Rapids & BOYKX6 & August 10, 2000 & 1 \\
\hline Columbia River & Priest Rapids & BOYKX7 & September 7, 2000 & 1 \\
\hline Columbia River & Priest Rapids & $\mathrm{B} 171 \mathrm{~K} 6$ & June 5, 2003 & 1 \\
\hline Columbia River & Priest Rapids & B171D2 & July 10, 2003 & 1 \\
\hline Columbia River & Priest Rapids & B17140 & May 28, 2003 & 1 \\
\hline Columbia River & Priest Rapids & $\mathrm{B} 170 \times 7$ & May 21, 2003 & 1 \\
\hline Columbia River & Priest Rapids & B17214 & June 12, 2003 & 1 \\
\hline Columbia River & Priest Rapids & ВОТНJO & January 27, 1999 & 1 \\
\hline Columbia River & Priest Rapids & B0XVP8 & May 31, 2000 & 1 \\
\hline Columbia River & Priest Rapids & B0Y231 & May 31, 2000 & 1 \\
\hline Columbia River & Priest Rapids & B0XVP7 & May 3, 2000 & 1 \\
\hline Columbia River & Priest Rapids & B175J1 & August 8, 2003 & 1 \\
\hline Columbia River & Priest Rapids & B0XVP6 & June 29, 2000 & 1 \\
\hline Columbia River & Priest Rapids & B0XVN6 & May 3, 2000 & 1 \\
\hline Columbia River & Priest Rapids & B0XVP9 & June 29, 2000 & 1 \\
\hline Columbia River & Priest Rapids & B0XVD3 & June 29, 2000 & 1 \\
\hline Columbia River & Priest Rapids & В0THJ1 & February 24, 1999 & 1 \\
\hline Columbia River & Priest Rapids & В0THJ2 & April 7, 1999 & 1 \\
\hline Columbia River & Priest Rapids & B17DJ7 & September 30, 2003 & 1 \\
\hline
\end{tabular}


Table A-2

Summary Table of Segment 1 Surface Water Samples By Area, Site, Date, and Category

\begin{tabular}{|c|c|c|c|c|}
\hline Sample Area & Sample Site & Sample ID & Sample Date & Category \\
\hline Columbia River & Priest Rapids & $\mathrm{B} 170 \mathrm{P} 7$ & May 13,2003 & 1 \\
\hline Columbia River & Priest Rapids & ВОТНН9 & April 7, 1999 & 1 \\
\hline Columbia River & Priest Rapids & B0XP16 & April 5, 2000 & 1 \\
\hline Columbia River & Priest Rapids & В0THF7 & February 3, 1999 & 1 \\
\hline Columbia River & Priest Rapids & В0TH62 & April 7, 1999 & 1 \\
\hline Columbia River & Priest Rapids & BOXVR2 & June 29, 2000 & 1 \\
\hline Columbia River & Priest Rapids & B0XVR3 & May 3, 2000 & 1 \\
\hline Columbia River & Priest Rapids & BOXVR4 & May 31, 2000 & 1 \\
\hline Columbia River & Priest Rapids & B179R2 & September 3, 2003 & 1 \\
\hline Columbia River & Priest Rapids & B175J3 & September 30, 2003 & 1 \\
\hline Columbia River & Priest Rapids & B175J2 & September 3, 2003 & 1 \\
\hline Columbia River & Priest Rapids & В0THJ5 & April 7, 1999 & 1 \\
\hline Columbia River & Vantage WA I90B & HR001V & October 7, 2003 & 1 \\
\hline Columbia River & Vernita & BovWV8 & July 1, 1999 & 1 \\
\hline Columbia River & Vernita & BoVWW4 & July 1, 1999 & 1 \\
\hline Columbia River & VERNITA 1 & 27645 & August 27, 2001 & 1 \\
\hline Columbia River & VERNITA 2 & 27646 & August 27, 2001 & 1 \\
\hline Columbia River & VERNITA 3 & 27647 & August 27, 2001 & 1 \\
\hline Columbia River & VERNITA 4 & 27648 & August 27, 2001 & 1 \\
\hline Columbia River & Vernita BRG1 & B13J18 & November 14, 2001 & 1 \\
\hline Columbia River & Vernita BRG1 & B12RN9 & August 27, 2001 & 1 \\
\hline Columbia River & Vernita BRG1 & B12RL5 & August 27, 2001 & 1 \\
\hline Columbia River & Vernita BRG1 & B12RN4 & August 27, 2001 & 1 \\
\hline Columbia River & Vernita BRG1 & B13J17 & November 14, 2001 & 1 \\
\hline Columbia River & Vernita BRG1 & B12RP0 & August 27, 2001 & 1 \\
\hline Columbia River & Vernita BRG1 & B13J19 & November 14, 2001 & 1 \\
\hline Columbia River & Vernita BRG1 & B12RP9 & August 27, 2001 & 1 \\
\hline Columbia River & Vernita BRG2 & B12RN7 & August 27, 2001 & 1 \\
\hline Columbia River & Vernita BRG2 & B12RP4 & August 27, 2001 & 1 \\
\hline Columbia River & Vernita BRG2 & B12RP3 & August 27, 2001 & 1 \\
\hline Columbia River & Vernita BRG2 & B12RL3 & August 27, 2001 & 1 \\
\hline Columbia River & Vernita BRG2 & B12RR1 & August 27, 2001 & 1 \\
\hline Columbia River & Vernita BRG3 & B12RR3 & August 27, 2001 & 1 \\
\hline Columbia River & Vernita BRG4 & B12T11 & August 27, 2001 & 1 \\
\hline Columbia River & Vernita1 HRM0.3 & B0V228 & June 11, 1999 & 1 \\
\hline Columbia River & Vernita1 HRM0.3 & B0V256 & June 11, 1999 & 1 \\
\hline Columbia River & Vernita1 HRM0.3 & B12T87 & September 7, 2001 & 1 \\
\hline Columbia River & Vernita1 HRM0.3 & B0V229 & June 11, 1999 & 1 \\
\hline Columbia River & Vernita1 HRM0.3 & B12Т84 & September 7, 2001 & 1 \\
\hline Columbia River & Vernita1 HRM0.3 & B12TH6 & September 7, 2001 & 1 \\
\hline Columbia River & Vernita1 HRM0.3 & B12VM3 & September 7, 2001 & 1 \\
\hline Columbia River & Vernita1 HRM0.3 & B0V231 & June 11, 1999 & 1 \\
\hline Columbia River & Vernita1 HRM0.3 & B0YCD5 & June 19, 2000 & 1 \\
\hline Columbia River & Vernita1 HRM0.3 & B0X3X0 & December 7, 1999 & 1 \\
\hline Columbia River & Vernita1 HRM0.3 & B0XRW6 & March 27, 2000 & 1 \\
\hline Columbia River & Vernita1 HRM0.3 & B11241 & December 4, 2000 & 1 \\
\hline Columbia River & Vernita1 HRM0.3 & B11135 & December 4, 2000 & 1 \\
\hline Columbia River & Vernita1 HRM0.3 & B11125 & December 4, 2000 & 1 \\
\hline
\end{tabular}


Table A-2

Summary Table of Segment 1 Surface Water Samples By Area, Site, Date, and Category

\begin{tabular}{|c|c|c|c|c|}
\hline Sample Area & Sample Site & Sample ID & Sample Date & Category \\
\hline Columbia River & Vernita1 HRM0.3 & BOXRK2 & March 27, 2000 & 1 \\
\hline Columbia River & Vernita1 HRM0.3 & B0YC92 & June 19, 2000 & 1 \\
\hline Columbia River & Vernita1 HRM0.3 & B0XRJ9 & March 27, 2000 & 1 \\
\hline Columbia River & Vernita1 HRM0.3 & BOYCD7 & June 19, 2000 & 1 \\
\hline Columbia River & Vernita1 HRM0.3 & B106N8 & September 14, 2000 & 1 \\
\hline Columbia River & Vernita1 HRM0.3 & B10688 & September 14, 2000 & 1 \\
\hline Columbia River & Vernita1 HRM0.3 & B10673 & September 14, 2000 & 1 \\
\hline Columbia River & Vernita1 HRM0.3 & B10671 & September 14, 2000 & 1 \\
\hline Columbia River & Vernita1 HRM0.3 & B0YCJ3 & June 19, 2000 & 1 \\
\hline Columbia River & Vernita1 HRM0.3 & B11123 & December 4, 2000 & 1 \\
\hline Columbia River & Vernita1 HRM0.3 & B0X3Y2 & December 7, 1999 & 1 \\
\hline Columbia River & Vernita1 HRM0.3 & B12521 & June 14, 2001 & 1 \\
\hline Columbia River & Vernita1 HRM0.3 & B12501 & June 14, 2001 & 1 \\
\hline Columbia River & Vernita1 HRM0.3 & B124Y9 & June 14, 2001 & 1 \\
\hline Columbia River & Vernita1 HRM0.3 & B124W1 & June 14, 2001 & 1 \\
\hline Columbia River & Vernita1 HRM0.3 & Bow969 & September 10, 1999 & 1 \\
\hline Columbia River & Vernita1 HRM0.3 & B0XRM8 & March 27, 2000 & 1 \\
\hline Columbia River & Vernita1 HRM0.3 & B0X3X2 & December 7, 1999 & 1 \\
\hline Columbia River & Vernita1 HRM0.3 & Bow932 & September 10, 1999 & 1 \\
\hline Columbia River & Vernita1 HRM0.3 & B0X455 & December 7, 1999 & 1 \\
\hline Columbia River & Vernita1 HRM0.3 & $\mathrm{B} 11 \mathrm{~J} 26$ & February 27, 2001 & 1 \\
\hline Columbia River & Vernita1 HRM0.3 & B11H49 & February 27, 2001 & 1 \\
\hline Columbia River & Vernita1 HRM0.3 & B11H39 & February 27, 2001 & 1 \\
\hline Columbia River & Vernita1 HRM0.3 & B11H37 & February 27, 2001 & 1 \\
\hline Columbia River & Vernita1 HRM0.3 & B13LF3 & December 3, 2001 & 1 \\
\hline Columbia River & Vernita1 HRM0.3 & BOWBH8 & September 10, 1999 & 1 \\
\hline Columbia River & Vernita1 HRM0.3 & B16352 & December 9, 2002 & 1 \\
\hline Columbia River & Vernita1 HRM0.3 & B147T3 & March 25, 2002 & 1 \\
\hline Columbia River & Vernita1 HRM0.3 & B16L97 & March 24, 2003 & 1 \\
\hline Columbia River & Vernita1 HRM0.3 & B16L95 & March 24, 2003 & 1 \\
\hline Columbia River & Vernita1 HRM0.3 & B1B701 & September 13, 2004 & 1 \\
\hline Columbia River & Vernita1 HRM0.3 & B1B6Y9 & September 13, 2004 & 1 \\
\hline Columbia River & Vernita1 HRM0.3 & B184P5 & December 9, 2003 & 1 \\
\hline Columbia River & Vernita1 HRM0.3 & B16KD6 & March 24, 2003 & 1 \\
\hline Columbia River & Vernita1 HRM0.3 & B14RV3 & June 10, 2002 & 1 \\
\hline Columbia River & Vernita1 HRM0.3 & B14RV4 & June 10, 2002 & 1 \\
\hline Columbia River & Vernita1 HRM0.3 & B19J66 & June 25, 2004 & 1 \\
\hline Columbia River & Vernita1 HRM0.3 & B14RW4 & June 10, 2002 & 1 \\
\hline Columbia River & Vernita1 HRM0.3 & B164J6 & December 9, 2002 & 1 \\
\hline Columbia River & Vernita1 HRM0.3 & В17СM3 & September 4, 2003 & 1 \\
\hline Columbia River & Vernita1 HRM0.3 & B183R5 & December 9, 2003 & 1 \\
\hline Columbia River & Vernita1 HRM0.3 & B1B460 & September 13, 2004 & 1 \\
\hline Columbia River & Vernita1 HRM0.3 & B19HN4 & June 25, 2004 & 1 \\
\hline Columbia River & Vernita1 HRM0.3 & B183R7 & December 9, 2003 & 1 \\
\hline Columbia River & Vernita1 HRM0.3 & B14RY6 & June 10, 2002 & 1 \\
\hline Columbia River & Vernita1 HRM0.3 & B16342 & December 9, 2002 & 1 \\
\hline Columbia River & Vernita1 HRM0.3 & B18V15 & March 29, 2004 & 1 \\
\hline Columbia River & Vernita1 HRM0.3 & B16340 & December 9, 2002 & 1 \\
\hline
\end{tabular}


Table A-2

Summary Table of Segment 1 Surface Water Samples By Area, Site, Date, and Category

\begin{tabular}{|c|c|c|c|c|}
\hline Sample Area & Sample Site & Sample ID & Sample Date & Category \\
\hline Columbia River & Vernita1 HRM0.3 & B183T7 & December 9, 2003 & 1 \\
\hline Columbia River & Vernita1 HRM0.3 & $\mathrm{B} 158 \mathrm{H} 2$ & September 5, 2002 & 1 \\
\hline Columbia River & Vernita1 HRM0.3 & B18TH5 & March 29, 2004 & 1 \\
\hline Columbia River & Vernita1 HRM0.3 & $\mathrm{B} 158 \mathrm{H} 7$ & September 5, 2002 & 1 \\
\hline Columbia River & Vernita1 HRM0.3 & B158P2 & September 5, 2002 & 1 \\
\hline Columbia River & Vernita1 HRM0.3 & B15991 & September 5, 2002 & 1 \\
\hline Columbia River & Vernita1 HRM0.3 & B17HL9 & September 4, 2003 & 1 \\
\hline Columbia River & Vernita1 HRM0.3 & B13LF1 & December 3, 2001 & 1 \\
\hline Columbia River & Vernita1 HRM0.3 & B171W1 & June 9, 2003 & 1 \\
\hline Columbia River & Vernita1 HRM0.3 & B13LH3 & December 3, 2001 & 1 \\
\hline Columbia River & Vernita1 HRM0.3 & B17286 & June 9, 2003 & 1 \\
\hline Columbia River & Vernita1 HRM0.3 & B16LB7 & March 24, 2003 & 1 \\
\hline Columbia River & Vernita1 HRM0.3 & B171V9 & June 9, 2003 & 1 \\
\hline Columbia River & Vernita1 HRM0.3 & B17CD6 & September 4, 2003 & 1 \\
\hline Columbia River & Vernita1 HRM0.3 & B1BX61 & December 6, 2004 & 1 \\
\hline Columbia River & Vernita1 HRM0.3 & B171Y1 & June 9, 2003 & 1 \\
\hline Columbia River & Vernita1 HRM0.3 & B13LV4 & December 3, 2001 & 1 \\
\hline Columbia River & Vernita1 HRM0.3 & B1B747 & September 13, 2004 & 1 \\
\hline Columbia River & Vernita1 HRM0.3 & B1BW38 & December 6, 2004 & 1 \\
\hline Columbia River & Vernita1 HRM0.3 & B17CD8 & September 4, 2003 & 1 \\
\hline Columbia River & Vernita1 HRM0.3 & B146Y3 & March 25, 2002 & 1 \\
\hline Columbia River & Vernita2 HRM0.3 & B171V0 & June 9, 2003 & 1 \\
\hline Columbia River & Vernita2 HRM0.3 & BOYCC6 & June 19, 2000 & 1 \\
\hline Columbia River & Vernita2 HRM0.3 & B183R8 & December 9, 2003 & 1 \\
\hline Columbia River & Vernita2 HRM0.3 & B0YC93 & June 19, 2000 & 1 \\
\hline Columbia River & Vernita2 HRM0.3 & B16LB8 & March 24, 2003 & 1 \\
\hline Columbia River & Vernita2 HRM0.3 & B16343 & December 9, 2002 & 1 \\
\hline Columbia River & Vernita2 HRM0.3 & B16331 & December 9, 2002 & 1 \\
\hline Columbia River & Vernita2 HRM0.3 & B0YCD8 & June 19, 2000 & 1 \\
\hline Columbia River & Vernita2 HRM0.3 & B11136 & December 4, 2000 & 1 \\
\hline Columbia River & Vernita2 HRM0.3 & В183T8 & December 9, 2003 & 1 \\
\hline Columbia River & Vernita2 HRM0.3 & B13LD2 & December 3, 2001 & 1 \\
\hline Columbia River & Vernita2 HRM0.3 & $\mathrm{B} 11 \mathrm{H} 28$ & February 27, 2001 & 1 \\
\hline Columbia River & Vernita2 HRM0.3 & B15992 & September 5, 2002 & 1 \\
\hline Columbia River & Vernita2 HRM0.3 & B171Y2 & June 9, 2003 & 1 \\
\hline Columbia River & Vernita2 HRM0.3 & $\mathrm{B} 11 \mathrm{H} 40$ & February 27, 2001 & 1 \\
\hline Columbia River & Vernita2 HRM0.3 & В0X3Y3 & December 7, 1999 & 1 \\
\hline Columbia River & Vernita2 HRM0.3 & B171W2 & June 9, 2003 & 1 \\
\hline Columbia River & Vernita2 HRM0.3 & B11114 & December 4, 2000 & 1 \\
\hline Columbia River & Vernita2 HRM0.3 & B106N9 & September 14, 2000 & 1 \\
\hline Columbia River & Vernita2 HRM0.3 & B16L98 & March 24, 2003 & 1 \\
\hline Columbia River & Vernita2 HRM0.3 & B17CD9 & September 4, 2003 & 1 \\
\hline Columbia River & Vernita2 HRM0.3 & B0XRW7 & March 27, 2000 & 1 \\
\hline Columbia River & Vernita2 HRM0.3 & B16L86 & March 24, 2003 & 1 \\
\hline Columbia River & Vernita2 HRM0.3 & B17CM4 & September 4, 2003 & 1 \\
\hline Columbia River & Vernita2 HRM0.3 & B16KD7 & March 24, 2003 & 1 \\
\hline Columbia River & Vernita2 HRM0.3 & B10689 & September 14, 2000 & 1 \\
\hline Columbia River & Vernita2 HRM0.3 & $\mathrm{B} 11 \mathrm{H} 50$ & February 27, 2001 & 1 \\
\hline
\end{tabular}


Table A-2

Summary Table of Segment 1 Surface Water Samples By Area, Site, Date, and Category

\begin{tabular}{|c|c|c|c|c|}
\hline Sample Area & Sample Site & Sample ID & Sample Date & Category \\
\hline Columbia River & Vernita2 HRM0.3 & B16353 & December 9, 2002 & 1 \\
\hline Columbia River & Vernita2 HRM0.3 & B164J7 & December 9, 2002 & 1 \\
\hline Columbia River & Vernita2 HRM0.3 & B10668 & September 14, 2000 & 1 \\
\hline Columbia River & Vernita2 HRM0.3 & B10674 & September 14, 2000 & 1 \\
\hline Columbia River & Vernita2 HRM0.3 & BOXRK3 & March 27, 2000 & 1 \\
\hline Columbia River & Vernita2 HRM0.3 & B17287 & June 9, 2003 & 1 \\
\hline Columbia River & Vernita2 HRM0.3 & B11126 & December 4, 2000 & 1 \\
\hline Columbia River & Vernita2 HRM0.3 & BOXRJO & March 27, 2000 & 1 \\
\hline Columbia River & Vernita2 HRM0.3 & B17HM0 & September 4, 2003 & 1 \\
\hline Columbia River & Vernita2 HRM0.3 & B183P6 & December 9, 2003 & 1 \\
\hline Columbia River & Vernita2 HRM0.3 & B0XRM9 & March 27, 2000 & 1 \\
\hline Columbia River & Vernita2 HRM0.3 & B11242 & December 4, 2000 & 1 \\
\hline Columbia River & Vernita2 HRM0.3 & $\mathrm{B} 17 \mathrm{CC} 7$ & September 4, 2003 & 1 \\
\hline Columbia River & Vernita2 HRM0.3 & B1B $\times 62$ & December 6, 2004 & 1 \\
\hline Columbia River & Vernita2 HRM0.3 & B14RT4 & June 10, 2002 & 1 \\
\hline Columbia River & Vernita2 HRM0.3 & B12502 & June 14, 2001 & 1 \\
\hline Columbia River & Vernita2 HRM0.3 & B12T75 & September 7, 2001 & 1 \\
\hline Columbia River & Vernita2 HRM0.3 & Bow970 & September 10, 1999 & 1 \\
\hline Columbia River & Vernita2 HRM0.3 & B0V257 & June 11, 1999 & 1 \\
\hline Columbia River & Vernita2 HRM0.3 & B14RW5 & June 10, 2002 & 1 \\
\hline Columbia River & Vernita2 HRM0.3 & B12522 & June 14, 2001 & 1 \\
\hline Columbia River & Vernita2 HRM0.3 & Bow933 & September 10, 1999 & 1 \\
\hline Columbia River & Vernita2 HRM0.3 & B12TH7 & September 7, 2001 & 1 \\
\hline Columbia River & Vernita2 HRM0.3 & B19J67 & June 25, 2004 & 1 \\
\hline Columbia River & Vernita2 HRM0.3 & B19HN5 & June 25, 2004 & 1 \\
\hline Columbia River & Vernita2 HRM0.3 & B0YCJ4 & June 19, 2000 & 1 \\
\hline Columbia River & Vernita2 HRM0.3 & B12VM4 & September 7, 2001 & 1 \\
\hline Columbia River & Vernita2 HRM0.3 & B0X3X3 & December 7, 1999 & 1 \\
\hline Columbia River & Vernita2 HRM0.3 & B1B461 & September 13, 2004 & 1 \\
\hline Columbia River & Vernita2 HRM0.3 & B1B6Y0 & September 13, 2004 & 1 \\
\hline Columbia River & Vernita2 HRM0.3 & B12T88 & September 7, 2001 & 1 \\
\hline Columbia River & Vernita2 HRM0.3 & B1B702 & September 13, 2004 & 1 \\
\hline Columbia River & Vernita2 HRM0.3 & B147T4 & March 25, 2002 & 1 \\
\hline Columbia River & Vernita2 HRM0.3 & B13LV5 & December 3, 2001 & 1 \\
\hline Columbia River & Vernita2 HRM0.3 & B1BW39 & December 6, 2004 & 1 \\
\hline Columbia River & Vernita2 HRM0.3 & B146Y4 & March 25, 2002 & 1 \\
\hline Columbia River & Vernita2 HRM0.3 & B1B748 & September 13, 2004 & 1 \\
\hline Columbia River & Vernita2 HRM0.3 & B14RV5 & June 10, 2002 & 1 \\
\hline Columbia River & Vernita2 HRM0.3 & $\mathrm{B} 158 \mathrm{H} 5$ & September 5, 2002 & 1 \\
\hline Columbia River & Vernita2 HRM0.3 & B184P6 & December 9, 2003 & 1 \\
\hline Columbia River & Vernita2 HRM0.3 & B0V202 & June 11, 1999 & 1 \\
\hline Columbia River & Vernita2 HRM0.3 & B0X456 & December 7, 1999 & 1 \\
\hline Columbia River & Vernita2 HRM0.3 & $\mathrm{B} 11 \mathrm{~J} 27$ & February 27, 2001 & 1 \\
\hline Columbia River & Vernita2 HRM0.3 & B0V203 & June 11, 1999 & 1 \\
\hline Columbia River & Vernita2 HRM0.3 & B0X3W1 & December 7, 1999 & 1 \\
\hline Columbia River & Vernita2 HRM0.3 & B13LF4 & December 3, 2001 & 1 \\
\hline Columbia River & Vernita2 HRM0.3 & B158P3 & September 5, 2002 & 1 \\
\hline Columbia River & Vernita2 HRM0.3 & BOWBH9 & September 10, 1999 & 1 \\
\hline
\end{tabular}


Table A-2

Summary Table of Segment 1 Surface Water Samples By Area, Site, Date, and Category

\begin{tabular}{|c|c|c|c|c|}
\hline Sample Area & Sample Site & Sample ID & Sample Date & Category \\
\hline Columbia River & Vernita2 HRM0.3 & B18TH6 & March 29, 2004 & 1 \\
\hline Columbia River & Vernita2 HRM0.3 & B14RY7 & June 10, 2002 & 1 \\
\hline Columbia River & Vernita2 HRM0.3 & B0V232 & June 11, 1999 & 1 \\
\hline Columbia River & Vernita2 HRM0.3 & B124Y0 & June 14, 2001 & 1 \\
\hline Columbia River & Vernita2 HRM0.3 & B124W2 & June 14, 2001 & 1 \\
\hline Columbia River & Vernita2 HRM0.3 & B13LH4 & December 3, 2001 & 1 \\
\hline Columbia River & Vernita2 HRM0.3 & B18V16 & March 29, 2004 & 1 \\
\hline Columbia River & Vernita2 HRM0.3 & B158F3 & September 5, 2002 & 1 \\
\hline Columbia River & Vernita3 HRM0.3 & B0YC94 & June 19, 2000 & 1 \\
\hline Columbia River & Vernita3 HRM0.3 & B171Y3 & June 9, 2003 & 1 \\
\hline Columbia River & Vernita3 HRM0.3 & B17288 & June 9, 2003 & 1 \\
\hline Columbia River & Vernita3 HRM0.3 & B0V258 & June 11, 1999 & 1 \\
\hline Columbia River & Vernita3 HRM0.3 & B0V204 & June 11, 1999 & 1 \\
\hline Columbia River & Vernita3 HRM0.3 & B17CC8 & September 4, 2003 & 1 \\
\hline Columbia River & Vernita3 HRM0.3 & B1BX63 & December 6, 2004 & 1 \\
\hline Columbia River & Vernita3 HRM0.3 & BOYCC7 & June 19, 2000 & 1 \\
\hline Columbia River & Vernita3 HRM0.3 & BOYCD9 & June 19, 2000 & 1 \\
\hline Columbia River & Vernita3 HRM0.3 & B0V205 & June 11, 1999 & 1 \\
\hline Columbia River & Vernita3 HRM0.3 & B0V233 & June 11, 1999 & 1 \\
\hline Columbia River & Vernita3 HRM0.3 & B1BW40 & December 6, 2004 & 1 \\
\hline Columbia River & Vernita3 HRM0.3 & B17HM1 & September 4, 2003 & 1 \\
\hline Columbia River & Vernita3 HRM0.3 & B0X3X4 & December 7, 1999 & 1 \\
\hline Columbia River & Vernita3 HRM0.3 & B0X3Y4 & December 7, 1999 & 1 \\
\hline Columbia River & Vernita3 HRM0.3 & B0X3W2 & December 7, 1999 & 1 \\
\hline Columbia River & Vernita3 HRM0.3 & В183T9 & December 9, 2003 & 1 \\
\hline Columbia River & Vernita3 HRM0.3 & B0X457 & December 7, 1999 & 1 \\
\hline Columbia River & Vernita3 HRM0.3 & $\mathrm{B} 184 \mathrm{P} 7$ & December 9, 2003 & 1 \\
\hline Columbia River & Vernita3 HRM0.3 & B18TH7 & March 29, 2004 & 1 \\
\hline Columbia River & Vernita3 HRM0.3 & B183R9 & December 9, 2003 & 1 \\
\hline Columbia River & Vernita3 HRM0.3 & B18V17 & March 29, 2004 & 1 \\
\hline Columbia River & Vernita3 HRM0.3 & BOWBJO & September 10, 1999 & 1 \\
\hline Columbia River & Vernita3 HRM0.3 & B19J68 & June 25, 2004 & 1 \\
\hline Columbia River & Vernita3 HRM0.3 & Bow971 & September 10, 1999 & 1 \\
\hline Columbia River & Vernita3 HRM0.3 & B1B749 & September 13, 2004 & 1 \\
\hline Columbia River & Vernita3 HRM0.3 & B19HN6 & June 25, 2004 & 1 \\
\hline Columbia River & Vernita3 HRM0.3 & B0XRJ1 & March 27, 2000 & 1 \\
\hline Columbia River & Vernita3 HRM0.3 & Bow934 & September 10, 1999 & 1 \\
\hline Columbia River & Vernita3 HRM0.3 & B0XRK4 & March 27, 2000 & 1 \\
\hline Columbia River & Vernita3 HRM0.3 & B17CM5 & September 4, 2003 & 1 \\
\hline Columbia River & Vernita3 HRM0.3 & BOXRNO & March 27, 2000 & 1 \\
\hline Columbia River & Vernita3 HRM0.3 & B1B462 & September 13, 2004 & 1 \\
\hline Columbia River & Vernita3 HRM0.3 & B1B6Y1 & September 13, 2004 & 1 \\
\hline Columbia River & Vernita3 HRM0.3 & B0XRW8 & March 27, 2000 & 1 \\
\hline Columbia River & Vernita3 HRM0.3 & B1B703 & September 13, 2004 & 1 \\
\hline Columbia River & Vernita3 HRM0.3 & B17CF0 & September 4, 2003 & 1 \\
\hline Columbia River & Vernita3 HRM0.3 & B183P7 & December 9, 2003 & 1 \\
\hline Columbia River & Vernita3 HRM0.3 & B15993 & September 5, 2002 & 1 \\
\hline Columbia River & Vernita3 HRM0.3 & B158F4 & September 5, 2002 & 1 \\
\hline
\end{tabular}


Table A-2

Summary Table of Segment 1 Surface Water Samples By Area, Site, Date, and Category

\begin{tabular}{|c|c|c|c|c|}
\hline Sample Area & Sample Site & Sample ID & Sample Date & Category \\
\hline Columbia River & Vernita3 HRM0.3 & B158H6 & September 5, 2002 & 1 \\
\hline Columbia River & Vernita3 HRM0.3 & B11J28 & February 27, 2001 & 1 \\
\hline Columbia River & Vernita3 HRM0.3 & B158P4 & September 5, 2002 & 1 \\
\hline Columbia River & Vernita3 HRM0.3 & B11H51 & February 27, 2001 & 1 \\
\hline Columbia River & Vernita3 HRM0.3 & B11115 & December 4, 2000 & 1 \\
\hline Columbia River & Vernita3 HRM0.3 & B11H29 & February 27, 2001 & 1 \\
\hline Columbia River & Vernita3 HRM0.3 & B124Y1 & June 14, 2001 & 1 \\
\hline Columbia River & Vernita3 HRM0.3 & B16332 & December 9, 2002 & 1 \\
\hline Columbia River & Vernita3 HRM0.3 & B16344 & December 9, 2002 & 1 \\
\hline Columbia River & Vernita3 HRM0.3 & B11243 & December 4, 2000 & 1 \\
\hline Columbia River & Vernita3 HRM0.3 & B11137 & December 4, 2000 & 1 \\
\hline Columbia River & Vernita3 HRM0.3 & B16354 & December 9, 2002 & 1 \\
\hline Columbia River & Vernita3 HRM0.3 & B11127 & December 4, 2000 & 1 \\
\hline Columbia River & Vernita3 HRM0.3 & $\mathrm{B} 11 \mathrm{H} 41$ & February 27, 2001 & 1 \\
\hline Columbia River & Vernita3 HRM0.3 & B146Y5 & March 25, 2002 & 1 \\
\hline Columbia River & Vernita3 HRM0.3 & B0YCJ5 & June 19, 2000 & 1 \\
\hline Columbia River & Vernita3 HRM0.3 & B13LD3 & December 3, 2001 & 1 \\
\hline Columbia River & Vernita3 HRM0.3 & B13LF5 & December 3, 2001 & 1 \\
\hline Columbia River & Vernita3 HRM0.3 & B12VM5 & September 7, 2001 & 1 \\
\hline Columbia River & Vernita3 HRM0.3 & B13LH5 & December 3, 2001 & 1 \\
\hline Columbia River & Vernita3 HRM0.3 & B12TH8 & September 7, 2001 & 1 \\
\hline Columbia River & Vernita3 HRM0.3 & B14RY8 & June 10, 2002 & 1 \\
\hline Columbia River & Vernita3 HRM0.3 & B13LV6 & December 3, 2001 & 1 \\
\hline Columbia River & Vernita3 HRM0.3 & B124W3 & June 14, 2001 & 1 \\
\hline Columbia River & Vernita3 HRM0.3 & B147T5 & March 25, 2002 & 1 \\
\hline Columbia River & Vernita3 HRM0.3 & B14RV6 & June 10, 2002 & 1 \\
\hline Columbia River & Vernita3 HRM0.3 & B12523 & June 14, 2001 & 1 \\
\hline Columbia River & Vernita3 HRM0.3 & B12503 & June 14, 2001 & 1 \\
\hline Columbia River & Vernita3 HRM0.3 & B14RW6 & June 10, 2002 & 1 \\
\hline Columbia River & Vernita3 HRM0.3 & В12Т76 & September 7, 2001 & 1 \\
\hline Columbia River & Vernita3 HRM0.3 & В12Т89 & September 7, 2001 & 1 \\
\hline Columbia River & Vernita3 HRM0.3 & B171W3 & June 9, 2003 & 1 \\
\hline Columbia River & Vernita3 HRM0.3 & B106P0 & September 14, 2000 & 1 \\
\hline Columbia River & Vernita3 HRM0.3 & B10690 & September 14, 2000 & 1 \\
\hline Columbia River & Vernita3 HRM0.3 & B16L87 & March 24, 2003 & 1 \\
\hline Columbia River & Vernita3 HRM0.3 & B16L99 & March 24, 2003 & 1 \\
\hline Columbia River & Vernita3 HRM0.3 & B16LB9 & March 24, 2003 & 1 \\
\hline Columbia River & Vernita3 HRM0.3 & B171V1 & June 9, 2003 & 1 \\
\hline Columbia River & Vernita3 HRM0.3 & B10669 & September 14, 2000 & 1 \\
\hline Columbia River & Vernita3 HRM0.3 & B164J8 & December 9, 2002 & 1 \\
\hline Columbia River & Vernita3 HRM0.3 & B10675 & September 14, 2000 & 1 \\
\hline Columbia River & Vernita3 HRM0.3 & B16KD8 & March 24, 2003 & 1 \\
\hline Columbia River & Vernita4 HRM0.3 & B16LB0 & March 24, 2003 & 1 \\
\hline Columbia River & Vernita4 HRM0.3 & B146Y6 & March 25, 2002 & 1 \\
\hline Columbia River & Vernita4 HRM0.3 & B16LC0 & March 24, 2003 & 1 \\
\hline Columbia River & Vernita4 HRM0.3 & B147T6 & March 25, 2002 & 1 \\
\hline Columbia River & Vernita4 HRM0.3 & B1B6Y2 & September 13, 2004 & 1 \\
\hline Columbia River & Vernita4 HRM0.3 & B1B463 & September 13, 2004 & 1 \\
\hline
\end{tabular}


Table A-2

Summary Table of Segment 1 Surface Water Samples By Area, Site, Date, and Category

\begin{tabular}{|c|c|c|c|c|}
\hline Sample Area & Sample Site & Sample ID & Sample Date & Category \\
\hline Columbia River & Vernita4 HRM0.3 & B19J69 & June 25,2004 & 1 \\
\hline Columbia River & Vernita4 HRM0.3 & B14RV7 & June 10, 2002 & 1 \\
\hline Columbia River & Vernita4 HRM0.3 & B14RT6 & June 10, 2002 & 1 \\
\hline Columbia River & Vernita4 HRM0.3 & B10670 & September 14, 2000 & 1 \\
\hline Columbia River & Vernita4 HRM0.3 & B1B704 & September 13, 2004 & 1 \\
\hline Columbia River & Vernita4 HRM0.3 & B12504 & June 14, 2001 & 1 \\
\hline Columbia River & Vernita4 HRM0.3 & B19HN7 & June 25, 2004 & 1 \\
\hline Columbia River & Vernita4 HRM0.3 & B12524 & June 14, 2001 & 1 \\
\hline Columbia River & Vernita4 HRM0.3 & Bow935 & September 10, 1999 & 1 \\
\hline Columbia River & Vernita4 HRM0.3 & Bow972 & September 10, 1999 & 1 \\
\hline Columbia River & Vernita4 HRM0.3 & В12ТH9 & September 7, 2001 & 1 \\
\hline Columbia River & Vernita4 HRM0.3 & B0X458 & December 7, 1999 & 1 \\
\hline Columbia River & Vernita4 HRM0.3 & BOYCFO & June 19, 2000 & 1 \\
\hline Columbia River & Vernita4 HRM0.3 & B0YCJ6 & June 19, 2000 & 1 \\
\hline Columbia River & Vernita4 HRM0.3 & В12Т77 & September 7, 2001 & 1 \\
\hline Columbia River & Vernita4 HRM0.3 & B13LF6 & December 3, 2001 & 1 \\
\hline Columbia River & Vernita4 HRM0.3 & B0XRK5 & March 27, 2000 & 1 \\
\hline Columbia River & Vernita4 HRM0.3 & B0V207 & June 11, 1999 & 1 \\
\hline Columbia River & Vernita4 HRM0.3 & B1BX64 & December 6, 2004 & 1 \\
\hline Columbia River & Vernita4 HRM0.3 & B12VM6 & September 7, 2001 & 1 \\
\hline Columbia River & Vernita4 HRM0.3 & B0YC95 & June 19, 2000 & 1 \\
\hline Columbia River & Vernita4 HRM0.3 & B171Y4 & June 9, 2003 & 1 \\
\hline Columbia River & Vernita4 HRM0.3 & B14RW7 & June 10, 2002 & 1 \\
\hline Columbia River & Vernita4 HRM0.3 & BOYCC8 & June 19, 2000 & 1 \\
\hline Columbia River & Vernita4 HRM0.3 & B0V259 & June 11, 1999 & 1 \\
\hline Columbia River & Vernita4 HRM0.3 & B13LH6 & December 3, 2001 & 1 \\
\hline Columbia River & Vernita4 HRM0.3 & В12Т90 & September 7, 2001 & 1 \\
\hline Columbia River & Vernita4 HRM0.3 & B1BW41 & December 6, 2004 & 1 \\
\hline Columbia River & Vernita4 HRM0.3 & B171V2 & June 9, 2003 & 1 \\
\hline Columbia River & Vernita4 HRM0.3 & B1B750 & September 13, 2004 & 1 \\
\hline Columbia River & Vernita4 HRM0.3 & B0V206 & June 11, 1999 & 1 \\
\hline Columbia River & Vernita4 HRM0.3 & B13LV7 & December 3, 2001 & 1 \\
\hline Columbia River & Vernita4 HRM0.3 & B0V234 & June 11, 1999 & 1 \\
\hline Columbia River & Vernita4 HRM0.3 & B11138 & December 4, 2000 & 1 \\
\hline Columbia River & Vernita4 HRM0.3 & B0X3Y5 & December 7, 1999 & 1 \\
\hline Columbia River & Vernita4 HRM0.3 & B11H30 & February 27, 2001 & 1 \\
\hline Columbia River & Vernita4 HRM0.3 & B13LD4 & December 3, 2001 & 1 \\
\hline Columbia River & Vernita4 HRM0.3 & B106P1 & September 14, 2000 & 1 \\
\hline Columbia River & Vernita4 HRM0.3 & B15994 & September 5, 2002 & 1 \\
\hline Columbia River & Vernita4 HRM0.3 & B16L88 & March 24, 2003 & 1 \\
\hline Columbia River & Vernita4 HRM0.3 & В183Т0 & December 9, 2003 & 1 \\
\hline Columbia River & Vernita4 HRM0.3 & B16333 & December 9, 2002 & 1 \\
\hline Columbia River & Vernita4 HRM0.3 & B16345 & December 9, 2002 & 1 \\
\hline Columbia River & Vernita4 HRM0.3 & B11244 & December 4, 2000 & 1 \\
\hline Columbia River & Vernita4 HRM0.3 & B17CF1 & September 4, 2003 & 1 \\
\hline Columbia River & Vernita4 HRM0.3 & B16KD9 & March 24, 2003 & 1 \\
\hline Columbia River & Vernita4 HRM0.3 & BoXRW9 & March 27, 2000 & 1 \\
\hline Columbia River & Vernita4 HRM0.3 & B17HM2 & September 4, 2003 & 1 \\
\hline
\end{tabular}


Table A-2

Summary Table of Segment 1 Surface Water Samples By Area, Site, Date, and Category

\begin{tabular}{|c|c|c|c|c|}
\hline Sample Area & Sample Site & Sample ID & Sample Date & Category \\
\hline Columbia River & Vernita4 HRM0.3 & B0XRN1 & March 27, 2000 & 1 \\
\hline Columbia River & Vernita4 HRM0.3 & B11128 & December 4, 2000 & 1 \\
\hline Columbia River & Vernita4 HRM0.3 & B164J9 & December 9, 2002 & 1 \\
\hline Columbia River & Vernita4 HRM0.3 & BOXRJ2 & March 27, 2000 & 1 \\
\hline Columbia River & Vernita4 HRM0.3 & B16355 & December 9, 2002 & 1 \\
\hline Columbia River & Vernita4 HRM0.3 & B17CM6 & September 4, 2003 & 1 \\
\hline Columbia River & Vernita4 HRM0.3 & B11116 & December 4, 2000 & 1 \\
\hline Columbia River & Vernita4 HRM0.3 & B171W4 & June 9, 2003 & 1 \\
\hline Columbia River & Vernita4 HRM0.3 & B124Y2 & June 14, 2001 & 1 \\
\hline Columbia River & Vernita4 HRM0.3 & B183P8 & December 9, 2003 & 1 \\
\hline Columbia River & Vernita4 HRM0.3 & B0WBJ1 & September 10, 1999 & 1 \\
\hline Columbia River & Vernita4 HRM0.3 & B184P8 & December 9, 2003 & 1 \\
\hline Columbia River & Vernita4 HRM0.3 & B11J29 & February 27, 2001 & 1 \\
\hline Columbia River & Vernita4 HRM0.3 & B18V18 & March 29, 2004 & 1 \\
\hline Columbia River & Vernita4 HRM0.3 & B11H42 & February 27, 2001 & 1 \\
\hline Columbia River & Vernita4 HRM0.3 & B158F5 & September 5, 2002 & 1 \\
\hline Columbia River & Vernita4 HRM0.3 & B17CC9 & September 4, 2003 & 1 \\
\hline Columbia River & Vernita4 HRM0.3 & B10691 & September 14, 2000 & 1 \\
\hline Columbia River & Vernita4 HRM0.3 & B158H4 & September 5, 2002 & 1 \\
\hline Columbia River & Vernita4 HRM0.3 & B14RY9 & June 10, 2002 & 1 \\
\hline Columbia River & Vernita4 HRM0.3 & B0X3W3 & December 7, 1999 & 1 \\
\hline Columbia River & Vernita4 HRM0.3 & B10676 & September 14, 2000 & 1 \\
\hline Columbia River & Vernita4 HRM0.3 & B11H52 & February 27, 2001 & 1 \\
\hline Columbia River & Vernita4 HRM0.3 & B17289 & June 9, 2003 & 1 \\
\hline Columbia River & Vernita4 HRM0.3 & B124W4 & June 14, 2001 & 1 \\
\hline Columbia River & Vernita4 HRM0.3 & B158P5 & September 5, 2002 & 1 \\
\hline Columbia River & Vernita4 HRM0.3 & B0X3X5 & December 7, 1999 & 1 \\
\hline Columbia River & Vernita4 HRM0.3 & B183V0 & December 9, 2003 & 1 \\
\hline Columbia River & Vernita4 HRM0.3 & В18ТH8 & March 29, 2004 & 1 \\
\hline Columbia River & Wenatchee @ CHN & 01148005 & April 3, 2001 & 1 \\
\hline Columbia River & Wenatchee @ CHN & 01258000 & June 20, 2001 & 1 \\
\hline Columbia River & Wenatchee @ CHN & 01108030 & March 6, 2001 & 1 \\
\hline Columbia River & Crescent Bar & CRE-3.99-20 & October 1, 1999 & 2 \\
\hline Columbia River & Crescent Bar & CRE-4.99-1 & April 19, 1999 & 2 \\
\hline Columbia River & Crescent Bar & CRE-3.99-1 & March 28, 1999 & 2 \\
\hline Columbia River & Crescent Bar & CRE-12.99-20 & December 4, 1999 & 2 \\
\hline Columbia River & Crescent Bar & CRE-4.99-20 & April 19, 1999 & 2 \\
\hline Columbia River & Crescent Bar & CRE-11.99-20 & November 13, 1999 & 2 \\
\hline Columbia River & Crescent Bar & CRE-10.99-20 & October 10, 1999 & 2 \\
\hline Columbia River & Crescent Bar & CRE-5.99-1 & May 13, 1999 & 2 \\
\hline Columbia River & Crescent Bar & CRE-5.99-20 & May 13, 1999 & 2 \\
\hline Columbia River & Crescent Bar & CRE-6.99-1 & June 23, 1999 & 2 \\
\hline Columbia River & Crescent Bar & CRE-7.99-20 & July 13, 1999 & 2 \\
\hline Columbia River & Crescent Bar & CRE-7.99-1 & July 13, 1999 & 2 \\
\hline Columbia River & Crescent Bar & CRE-9.99-20 & September 11, 1999 & 2 \\
\hline Columbia River & Crescent Bar & CRE-10.99-1 & October 10, 1999 & 2 \\
\hline Columbia River & Crescent Bar & CRE-9.99-1 & September 11, 1999 & 2 \\
\hline Columbia River & Crescent Bar & CRE-11.99-1 & November 13, 1999 & 2 \\
\hline
\end{tabular}


Table A-2

Summary Table of Segment 1 Surface Water Samples By Area, Site, Date, and Category

\begin{tabular}{|c|c|c|c|c|}
\hline Sample Area & Sample Site & Sample ID & Sample Date & Category \\
\hline Columbia River & Crescent Bar & CRE-8.99-1 & August 18, 1999 & 2 \\
\hline Columbia River & Crescent Bar & CRE-8.99-20 & August 18, 1999 & 2 \\
\hline Columbia River & Crescent Bar & CRE-6.99-20 & June 23, 1999 & 2 \\
\hline Columbia River & Crescent Bar & CRE-12.99-1 & December 4, 1999 & 2 \\
\hline Columbia River & Dry Gulch & DG-4.99 & April 19, 1999 & 2 \\
\hline Columbia River & Dry Gulch & DG-11.99 & November 13, 1999 & 2 \\
\hline Columbia River & Dry Gulch & DG-6.99 & June 23, 1999 & 2 \\
\hline Columbia River & Dry Gulch & DG-7.99 & July 13, 1999 & 2 \\
\hline Columbia River & Dry Gulch & DG-8.99 & August 18, 1999 & 2 \\
\hline Columbia River & Dry Gulch & DG-9.99 & September 11, 1999 & 2 \\
\hline Columbia River & Ginkgo Park & GHI-5.99-1 & May 14, 1999 & 2 \\
\hline Columbia River & Ginkgo Park & GHI-11.99-40 & November 14, 1999 & 2 \\
\hline Columbia River & Ginkgo Park & GHI-11.99 & November 14, 1999 & 2 \\
\hline Columbia River & Ginkgo Park & GHI-12.99-20 & December 3, 1999 & 2 \\
\hline Columbia River & Ginkgo Park & GHI-12.99-40 & December 3, 1999 & 2 \\
\hline Columbia River & Ginkgo Park & GHI-3.99-15 & March 29, 1999 & 2 \\
\hline Columbia River & Ginkgo Park & GHI-11.99-20 & November 14, 1999 & 2 \\
\hline Columbia River & Ginkgo Park & GHI-4.99-1 & April 18, 1999 & 2 \\
\hline Columbia River & Ginkgo Park & GHI-12.99 & December 3, 1999 & 2 \\
\hline Columbia River & Ginkgo Park & GHI-7.99-1 & July 15, 1999 & 2 \\
\hline Columbia River & Ginkgo Park & GHI-9.99-40 & September 11, 1999 & 2 \\
\hline Columbia River & Ginkgo Park & GHI-9.99-20 & September 11, 1999 & 2 \\
\hline Columbia River & Ginkgo Park & GHI-9.99-1 & September 11, 1999 & 2 \\
\hline Columbia River & Ginkgo Park & GHI-8.99-40 & August 18, 1999 & 2 \\
\hline Columbia River & Ginkgo Park & GHI-8.99-20 & August 18, 1999 & 2 \\
\hline Columbia River & Ginkgo Park & GHI-8.99-1 & August 18, 1999 & 2 \\
\hline Columbia River & Ginkgo Park & GHI-4.99-20 & April 18, 1999 & 2 \\
\hline Columbia River & Ginkgo Park & GHI-7.99-20 & July 15, 1999 & 2 \\
\hline Columbia River & Ginkgo Park & GHI-4.99-40 & April 18, 1999 & 2 \\
\hline Columbia River & Ginkgo Park & GHI-6.99-40 & June 23, 1999 & 2 \\
\hline Columbia River & Ginkgo Park & GHI-6.99-20 & June 23, 1999 & 2 \\
\hline Columbia River & Ginkgo Park & GHI-6.99-1 & June 23, 1999 & 2 \\
\hline Columbia River & Ginkgo Park & GHI-5.99-40 & May 14, 1999 & 2 \\
\hline Columbia River & Ginkgo Park & GHI-5.99-20 & May 14, 1999 & 2 \\
\hline Columbia River & Ginkgo Park & GHI-3.99-1 & March 29, 1999 & 2 \\
\hline Columbia River & Ginkgo Park & GHI-7.99-40 & July 15, 1999 & 2 \\
\hline Columbia River & Ginkgo Park & GHI-10.99-20 & October 10, 1999 & 2 \\
\hline Columbia River & Ginkgo Park & GHI-10.99 & October 10, 1999 & 2 \\
\hline Columbia River & Ginkgo Park & GHI-10.99-40 & October 10, 1999 & 2 \\
\hline Columbia River & Ginkgo Park & GHI-3.99-30 & March 29, 1999 & 2 \\
\hline Columbia River & Goose Island & GOO-3.99-12 & March 27, 1999 & 2 \\
\hline Columbia River & Goose Island & GOO-7.99-11 & July 15, 1999 & 2 \\
\hline Columbia River & Goose Island & GOO-10.99-1 & October 8, 1999 & 2 \\
\hline Columbia River & Goose Island & GOO-10.99-10 & October 8, 1999 & 2 \\
\hline Columbia River & Goose Island & GOO-10.99-20 & October 8, 1999 & 2 \\
\hline Columbia River & Goose Island & GOO-11.99-1 & November 11, 1999 & 2 \\
\hline Columbia River & Goose Island & GOO-11.99-12.5 & November 11, 1999 & 2 \\
\hline Columbia River & Goose Island & GOO-11.99-25 & November 11, 1999 & 2 \\
\hline
\end{tabular}


Table A-2

Summary Table of Segment 1 Surface Water Samples By Area, Site, Date, and Category

\begin{tabular}{|c|c|c|c|c|}
\hline Sample Area & Sample Site & Sample ID & Sample Date & Category \\
\hline Columbia River & Goose Island & GOO-12.99-1 & December 3, 1999 & 2 \\
\hline Columbia River & Goose Island & GOO-12.99-12.5 & December 3, 1999 & 2 \\
\hline Columbia River & Goose Island & GOO-3.99-1 & March 27, 1999 & 2 \\
\hline Columbia River & Goose Island & GOO-8.99-12.5 & August 17, 1999 & 2 \\
\hline Columbia River & Goose Island & GOO-3.99-25 & March 27, 1999 & 2 \\
\hline Columbia River & Goose Island & GOO-4.99-1 & April 17, 1999 & 2 \\
\hline Columbia River & Goose Island & GOO-4.99-12 & April 17, 1999 & 2 \\
\hline Columbia River & Goose Island & GOO-4.99-25 & April 17, 1999 & 2 \\
\hline Columbia River & Goose Island & GOO-5.99-1 & May 14, 1999 & 2 \\
\hline Columbia River & Goose Island & GOO-5.99-12.5 & May 14, 1999 & 2 \\
\hline Columbia River & Goose Island & GOO-5.99-25 & May 14, 1999 & 2 \\
\hline Columbia River & Goose Island & GOO-6.99-1 & June 24, 1999 & 2 \\
\hline Columbia River & Goose Island & GOO-12.99-25 & December 3, 1999 & 2 \\
\hline Columbia River & Goose Island & GOO-8.99-1 & August 17, 1999 & 2 \\
\hline Columbia River & Goose Island & GOO-8.99-25 & August 17, 1999 & 2 \\
\hline Columbia River & Goose Island & GOO-9.99-1 & September 10, 1999 & 2 \\
\hline Columbia River & Goose Island & GOO-9.99-12 & September 10, 1999 & 2 \\
\hline Columbia River & Goose Island & GOO-9.99-25 & September 10, 1999 & 2 \\
\hline Columbia River & Goose Island & GOO-7.99-22 & July 15, 1999 & 2 \\
\hline Columbia River & Goose Island & GOO-7.99-1 & July 15, 1999 & 2 \\
\hline Columbia River & Goose Island & GOO-6.99-12 & June 24, 1999 & 2 \\
\hline Columbia River & Goose Island & GOO-6.99-25 & June 24, 1999 & 2 \\
\hline Columbia River & Hanson Creek & HAN-6.99-1 & June 24, 1999 & 2 \\
\hline Columbia River & Hanson Creek & HAN-9.99-10 & September 10, 1999 & 2 \\
\hline Columbia River & Hanson Creek & HAN-9.99-1 & September 10, 1999 & 2 \\
\hline Columbia River & Hanson Creek & HAN-8.99-10 & August 16, 1999 & 2 \\
\hline Columbia River & Hanson Creek & HAN-8.99-1 & August 16, 1999 & 2 \\
\hline Columbia River & Hanson Creek & HAN-7.99-10 & July 15, 1999 & 2 \\
\hline Columbia River & Hanson Creek & HAN-6.99-10 & June 24, 1999 & 2 \\
\hline Columbia River & Hanson Creek & HAN-4.99-10 & April 17, 1999 & 2 \\
\hline Columbia River & Hanson Creek & HAN-4.99-1 & April 17, 1999 & 2 \\
\hline Columbia River & Hanson Creek & HAN-11.99-10 & November 11, 1999 & 2 \\
\hline Columbia River & Hanson Creek & HAN-11.99-1 & November 11, 1999 & 2 \\
\hline Columbia River & Hanson Creek & HAN-7.99-1 & July 15, 1999 & 2 \\
\hline Columbia River & Pr Rapids Dam-B & BPRD-3.99 & March 28, 1999 & 2 \\
\hline Columbia River & Pr Rapids Dam-B & BPRD-4.99 & April 18, 1999 & 2 \\
\hline Columbia River & Pr Rapids Dam-B & BPRD-5.99 & May 15, 1999 & 2 \\
\hline Columbia River & Pr Rapids Dam-B & BPRD-10.99-1 & October 9, 1999 & 2 \\
\hline Columbia River & Pr Rapids Dam-B & BPRD-11.99 & November 12, 1999 & 2 \\
\hline Columbia River & Pr Rapids Dam-B & BPRD-12.99 & December 5, 1999 & 2 \\
\hline Columbia River & Pr Rapids Dam-B & BPRD-6.99 & June 22, 1999 & 2 \\
\hline Columbia River & Pr Rapids Dam-B & BPRD-7.99 & July 16, 1999 & 2 \\
\hline Columbia River & Pr Rapids Dam-B & BPRD-8.99 & August 19, 1999 & 2 \\
\hline Columbia River & Pr Rapids Dam-B & BPRD-9.99 & September 12, 1999 & 2 \\
\hline Columbia River & SCAMMON LDG & SCA-8.99-12.5 & August 18, 1999 & 2 \\
\hline Columbia River & SCAMMON LDG & SCA-4.99-12 & April 19, 1999 & 2 \\
\hline Columbia River & SCAMMON LDG & SCA-4.99-1 & April 19, 1999 & 2 \\
\hline Columbia River & SCAMMON LDG & SCA-11.99-25 & November 13, 1999 & 2 \\
\hline
\end{tabular}


Table A-2

Summary Table of Segment 1 Surface Water Samples By Area, Site, Date, and Category

\begin{tabular}{|c|c|c|c|c|}
\hline Sample Area & Sample Site & Sample ID & Sample Date & Category \\
\hline Columbia River & SCAMMON LDG & SCA-11.99-12.5 & November 13, 1999 & 2 \\
\hline Columbia River & SCAMMON LDG & SCA-11.99-1 & November 13, 1999 & 2 \\
\hline Columbia River & SCAMMON LDG & SCA-8.99-25 & August 18, 1999 & 2 \\
\hline Columbia River & SCAMMON LDG & SCA-9.99-1 & September 11, 1999 & 2 \\
\hline Columbia River & SCAMMON LDG & SCA-6.99-1 & June 23, 1999 & 2 \\
\hline Columbia River & SCAMMON LDG & SCA-9.99-25 & September 11, 1999 & 2 \\
\hline Columbia River & SCAMMON LDG & SCA-6.99-13 & June 23, 1999 & 2 \\
\hline Columbia River & SCAMMON LDG & SCA-8.99-1 & August 18, 1999 & 2 \\
\hline Columbia River & SCAMMON LDG & SCA-4.99-25 & April 19, 1999 & 2 \\
\hline Columbia River & SCAMMON LDG & SCA-7.99-28 & July 16, 1999 & 2 \\
\hline Columbia River & SCAMMON LDG & SCA-9.99-12.5 & September 11, 1999 & 2 \\
\hline Columbia River & SCAMMON LDG & SCA-7.99-14 & July 16, 1999 & 2 \\
\hline Columbia River & SCAMMON LDG & SCA-7.99-1 & July 16, 1999 & 2 \\
\hline Columbia River & SCAMMON LDG & SCA-6.99-26 & June 23, 1999 & 2 \\
\hline Columbia River & Vernita BRG & B14PX1 & June 10, 2002 & 2 \\
\hline Columbia River & Vernita BRG & B14PX2 & June 10, 2002 & 2 \\
\hline Columbia River & Vernita BRG & B14L11 & May 17, 2002 & 2 \\
\hline Columbia River & Vernita BRG & B14K65 & May 3, 2002 & 2 \\
\hline Columbia River & Vernita BRG & B14L10 & May 17, 2002 & 2 \\
\hline Columbia River & Vernita BRG & B14K64 & May 3, 2002 & 2 \\
\hline Columbia River & Wanapum Dam & BWD-10.99 & October 9, 1999 & 2 \\
\hline Columbia River & Wanapum Dam & BWD-11.99 & November 12, 1999 & 2 \\
\hline Columbia River & Wanapum Dam & BWD-12.99 & December 3, 1999 & 2 \\
\hline Columbia River & Wanapum Dam & BWD-3.99 & March 27, 1999 & 2 \\
\hline Columbia River & Wanapum Dam & BWD-4.99 & April 17, 1999 & 2 \\
\hline Columbia River & Wanapum Dam & BWD-5.99 & May 14, 1999 & 2 \\
\hline Columbia River & Wanapum Dam & BWD-6.99 & June 24, 1999 & 2 \\
\hline Columbia River & Wanapum Dam & BWD-7.99 & July 15, 1999 & 2 \\
\hline Columbia River & Wanapum Dam & BWD-8.99 & August 17, 1999 & 2 \\
\hline Columbia River & Wanapum Dam & BWD-9.99 & September 10, 1999 & 2 \\
\hline Methow River & PATEROS WA & 12449950-F & September 21, 2001 & 1 \\
\hline Methow River & PATEROS WA & 12449950 & September 21, 2001 & 1 \\
\hline
\end{tabular}


Table A-3

Summary Statistics for Sediment Collected from Segment 1 of the Columbia River Including All Categories of Data (units reported "as received")

\begin{tabular}{|c|c|c|c|c|c|c|c|c|c|c|}
\hline $\begin{array}{l}\text { Constituent } \\
\text { Class }\end{array}$ & Constituent Name & Units & $\begin{array}{l}\text { Number } \\
\text { Analyzed }\end{array}$ & $\begin{array}{l}\text { Number } \\
\text { Detected }\end{array}$ & $\begin{array}{c}\text { Frequency of } \\
\text { Detection }\end{array}$ & $\begin{array}{l}\text { Minimum } \\
\text { Nondetect }\end{array}$ & $\begin{array}{l}\text { Maximum } \\
\text { Nondetect }\end{array}$ & Minimum Detect & $\begin{array}{l}\text { Maximum } \\
\text { Detect }\end{array}$ & $\begin{array}{c}\text { Average } \\
\text { Concentration }\end{array}$ \\
\hline$\overline{\text { CONV }}$ & Percent Solids & $\%$ & 23 & 23 & $100 \%$ & $\overline{--}$ & $\overline{--}$ & 35 & 70 & 50 \\
\hline CONV & Total Organic Carbon & $\%$ & 9 & 9 & $100 \%$ & -- & -- & 0.27 & 4.0 & 1.7 \\
\hline CONV & Total Organic Carbon & $\mathrm{mg} / \mathrm{kg}$ & 16 & 16 & $100 \%$ & -- & -- & 2,069 & 32,710 & 13,363 \\
\hline METAL & Aluminum & $\mathrm{mg} / \mathrm{kg}$ & 9 & 9 & $100 \%$ & -- & -- & 4,790 & 69,000 & 13,443 \\
\hline METAL & Antimony & $\mathrm{mg} / \mathrm{kg}$ & 19 & 15 & $79 \%$ & 4.0 & 4.2 & 0.031 & 1.1 & 1.0 \\
\hline METAL & Arsenic & $\mathrm{mg} / \mathrm{kg}$ & 47 & 44 & $94 \%$ & 4.4 & 4.5 & 0.42 & 12 & 6.9 \\
\hline METAL & Barium & $\mathrm{mg} / \mathrm{kg}$ & 9 & 9 & $100 \%$ & -- & -- & 33 & 710 & 125 \\
\hline METAL & Beryllium & $\mathrm{mg} / \mathrm{kg}$ & 24 & 20 & $83 \%$ & 0.10 & 0.10 & 0.062 & 2.0 & 0.99 \\
\hline METAL & Bismuth & $\mathrm{mg} / \mathrm{kg}$ & 1 & 0 & $0 \%$ & 10 & 10 & -- & -- & 5.0 \\
\hline METAL & Boron & $\mathrm{mg} / \mathrm{kg}$ & 1 & 1 & $100 \%$ & -- & -- & 0.20 & 0.20 & 0.20 \\
\hline METAL & Cadmium & $\mathrm{mg} / \mathrm{kg}$ & 62 & 57 & $92 \%$ & 0.25 & 2.0 & 0.30 & 16 & 5.5 \\
\hline METAL & Calcium & $\mathrm{mg} / \mathrm{kg}$ & 9 & 9 & $100 \%$ & -- & -- & 2,760 & 31,000 & 6,442 \\
\hline METAL & Cerium & $\mathrm{mg} / \mathrm{kg}$ & 1 & 1 & $100 \%$ & -- & -- & 58 & 58 & 58 \\
\hline METAL & Chromium & $\mathrm{mg} / \mathrm{kg}$ & 63 & 63 & $100 \%$ & -- & -- & 3.3 & 92 & 48 \\
\hline METAL & Cobalt & $\mathrm{mg} / \mathrm{kg}$ & 9 & 9 & $100 \%$ & -- & -- & 4.0 & 15 & 7.0 \\
\hline METAL & Copper & $\mathrm{mg} / \mathrm{kg}$ & 63 & 63 & $100 \%$ & -- & -- & 0.034 & 70 & 31 \\
\hline METAL & Europium & $\mathrm{mg} / \mathrm{kg}$ & 1 & 0 & $0 \%$ & 2.0 & 2.0 & -- & -- & 1.0 \\
\hline METAL & Gallium & $\mathrm{mg} / \mathrm{kg}$ & 1 & 1 & $100 \%$ & -- & -- & 16 & 16 & 16 \\
\hline METAL & Gold & $\mathrm{mg} / \mathrm{kg}$ & 1 & 0 & $0 \%$ & 8.0 & 8.0 & -- & -- & 4.0 \\
\hline METAL & Iron & $\mathrm{mg} / \mathrm{kg}$ & 9 & 9 & $100 \%$ & -- & -- & 9,100 & 40,000 & 16,578 \\
\hline METAL & Lanthanum & $\mathrm{mg} / \mathrm{kg}$ & 1 & 1 & $100 \%$ & -- & -- & 31 & 31 & 31 \\
\hline METAL & Lead & $\mathrm{mg} / \mathrm{kg}$ & 59 & 59 & $100 \%$ & -- & -- & 1.9 & 140 & 48 \\
\hline METAL & Lithium & $\mathrm{mg} / \mathrm{kg}$ & 1 & 1 & $100 \%$ & -- & -- & 22 & 22 & 22 \\
\hline METAL & Magnesium & $\mathrm{mg} / \mathrm{kg}$ & 9 & 9 & $100 \%$ & -- & -- & 2,700 & 13,000 & 4,581 \\
\hline METAL & Manganese & $\mathrm{mg} / \mathrm{kg}$ & 9 & 9 & $100 \%$ & -- & -- & 110 & 660 & 238 \\
\hline METAL & Mercury & $\mathrm{mg} / \mathrm{kg}$ & 47 & 37 & $79 \%$ & 0.020 & 0.060 & 0.0062 & 0.31 & 0.12 \\
\hline METAL & Molybdenum & $\mathrm{mg} / \mathrm{kg}$ & 10 & 0 & $0 \%$ & 0.59 & 2.0 & -- & -- & 0.37 \\
\hline METAL & Neodymium & $\mathrm{mg} / \mathrm{kg}$ & 1 & 1 & $100 \%$ & -- & -- & 28 & 28 & 28 \\
\hline METAL & Nickel & $\mathrm{mg} / \mathrm{kg}$ & 33 & 33 & $100 \%$ & -- & -- & 1.7 & 53 & 23 \\
\hline METAL & Niobium & $\mathrm{mg} / \mathrm{kg}$ & 1 & 1 & $100 \%$ & -- & -- & 4.0 & 4.0 & 4.0 \\
\hline METAL & Phosphorus & $\mathrm{mg} / \mathrm{Kg}$ & 1 & 1 & $100 \%$ & -- & -- & 800 & 800 & 800 \\
\hline METAL & Potassium & $\mathrm{mg} / \mathrm{kg}$ & 9 & 9 & $100 \%$ & -- & -- & 565 & 19,000 & 2,868 \\
\hline METAL & Scandium & $\mathrm{mg} / \mathrm{kg}$ & 1 & 1 & $100 \%$ & -- & -- & 14 & 14 & 14 \\
\hline METAL & Selenium & $\mathrm{mg} / \mathrm{kg}$ & 29 & 8 & $28 \%$ & 0.47 & 5.0 & 0.016 & 1.9 & 1.1 \\
\hline METAL & Silver & $\mathrm{mg} / \mathrm{kg}$ & 50 & 14 & $28 \%$ & 0.013 & 75 & 0.0062 & 0.57 & 23 \\
\hline METAL & Sodium & $\mathrm{mg} / \mathrm{kg}$ & 9 & 9 & $100 \%$ & -- & -- & 102 & 20,000 & 2,440 \\
\hline METAL & Strontium & $\mathrm{mg} / \mathrm{kg}$ & 1 & 1 & $100 \%$ & -- & -- & 410 & 410 & 410 \\
\hline METAL & Tantalum & $\mathrm{mg} / \mathrm{kg}$ & 1 & 0 & $0 \%$ & 40 & 40 & -- & -- & 20 \\
\hline METAL & Thallium & $\mathrm{mg} / \mathrm{kg}$ & 19 & 17 & $89 \%$ & 0.31 & 0.32 & 0.049 & 1.9 & 0.95 \\
\hline METAL & Thorium & $\mathrm{mg} / \mathrm{kg}$ & 1 & 1 & $100 \%$ & -- & -- & 9.4 & 9.4 & 9.4 \\
\hline METAL & Tin & $\mathrm{mg} / \mathrm{kg}$ & 1 & 0 & $0 \%$ & 5.0 & 5.0 & -- & -- & 2.5 \\
\hline METAL & Titanium & $\mathrm{mg} / \mathrm{Kg}$ & 1 & 1 & $100 \%$ & -- & -- & 5,100 & 5,100 & 5,100 \\
\hline METAL & Uranium & $\mathrm{mg} / \mathrm{kg}$ & 1 & 1 & $100 \%$ & -- & -- & 2.5 & 2.5 & 2.5 \\
\hline METAL & Vanadium & $\mathrm{mg} / \mathrm{kg}$ & 9 & 9 & $100 \%$ & -- & -- & 23 & 130 & 44 \\
\hline METAL & Ytterbium & $\mathrm{mg} / \mathrm{kg}$ & 1 & 1 & $100 \%$ & -- & -- & 2.0 & 2.0 & 2.0 \\
\hline METAL & Yttrium & $\mathrm{mg} / \mathrm{kg}$ & 1 & 1 & $100 \%$ & -- & -- & 18 & 18 & 18 \\
\hline
\end{tabular}


Table A-3

Summary Statistics for Sediment Collected from Segment 1 of the Columbia River Including All Categories of Data (units reported "as received")

\begin{tabular}{|c|c|c|c|c|c|c|c|c|c|c|}
\hline $\begin{array}{c}\text { Constituent } \\
\text { Class }\end{array}$ & Constituent Name & Units & $\begin{array}{l}\text { Number } \\
\text { Analyzed }\end{array}$ & $\begin{array}{l}\text { Number } \\
\text { Detected }\end{array}$ & $\begin{array}{c}\text { Frequency of } \\
\text { Detection }\end{array}$ & $\begin{array}{l}\text { Minimum } \\
\text { Nondetect }\end{array}$ & $\begin{array}{l}\text { Maximum } \\
\text { Nondetect }\end{array}$ & Minimum Detect & $\begin{array}{l}\text { Maximum } \\
\text { Detect }\end{array}$ & $\begin{array}{c}\text { Average } \\
\text { Concentration }\end{array}$ \\
\hline METAL & Zinc & $\mathrm{mg} / \mathrm{kg}$ & 63 & 63 & $100 \%$ & -- & -- & 24 & 850 & 344 \\
\hline PCB & Aroclor-1016 & $\mathrm{mg} / \mathrm{kg}$ & 31 & 0 & $0 \%$ & 5.0 & 5.0 & -- & -- & 2.5 \\
\hline PCB & Aroclor-1221 & $\mathrm{mg} / \mathrm{kg}$ & 31 & 0 & $0 \%$ & 5.0 & 5.0 & -- & -- & 2.5 \\
\hline РCB & Aroclor-1232 & $\mathrm{mg} / \mathrm{kg}$ & 31 & 0 & $0 \%$ & 5.0 & 5.0 & -- & -- & 2.5 \\
\hline РСB & Aroclor-1242 & $\mathrm{mg} / \mathrm{kg}$ & 31 & 0 & $0 \%$ & 5.0 & 5.0 & -- & -- & 2.5 \\
\hline PCB & Aroclor-1248 & $\mathrm{mg} / \mathrm{kg}$ & 31 & 0 & $0 \%$ & 5.0 & 5.0 & -- & -- & 2.5 \\
\hline PCB & Aroclor-1254 & $\mathrm{mg} / \mathrm{kg}$ & 31 & 0 & $0 \%$ & 5.0 & 5.0 & -- & -- & 2.5 \\
\hline РСB & Aroclor-1260 & $\mathrm{mg} / \mathrm{kg}$ & 31 & 0 & $0 \%$ & 5.0 & 5.0 & -- & -- & 2.5 \\
\hline PCB & Total polychlorinated biphenyl & $\mathrm{mg} / \mathrm{kg}$ & 1 & 0 & $0 \%$ & 0.0010 & 0.0010 & -- & -- & $5.00 \mathrm{E}-04$ \\
\hline PEST & Aldrin & $\mathrm{mg} / \mathrm{Kg}$ & 21 & 0 & $0 \%$ & $1.00 \mathrm{E}-04$ & 0.66 & -- & -- & 0.30 \\
\hline PEST & Alpha-BHC & $\mathrm{mg} / \mathrm{kg}$ & 19 & 0 & $0 \%$ & 0.66 & 0.66 & -- & -- & 0.33 \\
\hline PEST & beta-1,2,3,4,5,6-Hexachlorocyclohexane & $\mathrm{mg} / \mathrm{kg}$ & 19 & 0 & $0 \%$ & 0.66 & 0.66 & -- & -- & 0.33 \\
\hline PEST & Chlordane & $\mathrm{mg} / \mathrm{kg}$ & 1 & 0 & $0 \%$ & 0.0010 & 0.0010 & -- & -- & $5.00 \mathrm{E}-04$ \\
\hline PEST & Delta-BHC & $\mathrm{mg} / \mathrm{kg}$ & 19 & 0 & $0 \%$ & 0.66 & 0.66 & -- & -- & 0.33 \\
\hline PEST & Dichlorodiphenyldichloroethane & $\mathrm{mg} / \mathrm{kg}$ & 20 & 0 & $0 \%$ & $1.00 \mathrm{E}-04$ & 0.66 & -- & -- & 0.31 \\
\hline PEST & Dichlorodiphenyldichloroethylene & $\mathrm{mg} / \mathrm{kg}$ & 20 & 1 & $5 \%$ & 0.66 & 0.66 & $1.00 \mathrm{E}-04$ & $1.00 \mathrm{E}-04$ & 0.31 \\
\hline PEST & Dichlorodiphenyltrichloroethane & $\mathrm{mg} / \mathrm{kg}$ & 20 & 0 & $0 \%$ & $1.00 \mathrm{E}-04$ & 0.66 & -- & -- & 0.31 \\
\hline PEST & Dieldrin & $\mathrm{mg} / \mathrm{Kg}$ & 21 & 1 & $5 \%$ & 0.0010 & 0.66 & $1.00 \mathrm{E}-04$ & $1.00 \mathrm{E}-04$ & 0.30 \\
\hline PEST & Endosulfan I & $\mathrm{mg} / \mathrm{kg}$ & 20 & 0 & $0 \%$ & $1.00 \mathrm{E}-04$ & 0.66 & -- & -- & 0.31 \\
\hline PEST & Endosulfan II & $\mathrm{mg} / \mathrm{kg}$ & 19 & 0 & $0 \%$ & 0.66 & 0.66 & -- & -- & 0.33 \\
\hline PEST & Endosulfan sulfate & $\mathrm{mg} / \mathrm{kg}$ & 19 & 0 & $0 \%$ & 0.66 & 0.66 & -- & -- & 0.33 \\
\hline PEST & Endrin & $\mathrm{mg} / \mathrm{kg}$ & 20 & 0 & $0 \%$ & $1.00 \mathrm{E}-04$ & 0.66 & -- & -- & 0.31 \\
\hline PEST & Endrin aldehyde & $\mathrm{mg} / \mathrm{kg}$ & 19 & 0 & $0 \%$ & 0.66 & 0.66 & -- & -- & 0.33 \\
\hline PEST & Gamma-BHC (Lindane) & $\mathrm{mg} / \mathrm{kg}$ & 20 & 0 & $0 \%$ & $1.00 \mathrm{E}-04$ & 0.66 & -- & -- & 0.31 \\
\hline PEST & Heptachlor & $\mathrm{mg} / \mathrm{kg}$ & 20 & 0 & $0 \%$ & $1.00 \mathrm{E}-04$ & 0.66 & -- & -- & 0.31 \\
\hline PEST & Heptachlor epoxide & $\mathrm{mg} / \mathrm{kg}$ & 20 & 0 & $0 \%$ & $1.00 \mathrm{E}-04$ & 0.66 & -- & -- & 0.31 \\
\hline PEST & Methoxychlor & $\mathrm{mg} / \mathrm{Kg}$ & 2 & 0 & $0 \%$ & $1.00 \mathrm{E}-04$ & 0.0010 & -- & -- & $2.75 \mathrm{E}-04$ \\
\hline PEST & Mirex & $\mathrm{mg} / \mathrm{kg}$ & 1 & 0 & $0 \%$ & $1.00 \mathrm{E}-04$ & $1.00 \mathrm{E}-04$ & -- & -- & $5.00 \mathrm{E}-05$ \\
\hline PEST & Perthane & $\mathrm{mg} / \mathrm{kg}$ & 1 & 0 & $0 \%$ & 0.0010 & 0.0010 & -- & -- & $5.00 \mathrm{E}-04$ \\
\hline PEST & Toxaphene & $\mathrm{mg} / \mathrm{kg}$ & 1 & 0 & $0 \%$ & 0.010 & 0.010 & -- & -- & 0.0050 \\
\hline RAD_D & Cesium-134, Decayed & $\mathrm{pCi} / \mathrm{g}$ & 1 & 1 & $100 \%$ & -- & -- & 1.13E-04 & $1.13 \mathrm{E}-04$ & 1.13E-04 \\
\hline RAD_D & Cesium-137, Decayed & $\mathrm{pCi} / \mathrm{g}$ & 49 & 49 & $100 \%$ & -- & -- & 0.023 & 0.78 & 0.32 \\
\hline RAD_D & Cobalt-60, Decayed & $\mathrm{pCi} / \mathrm{g}$ & 2 & 2 & $100 \%$ & -- & -- & 0.0047 & 0.0070 & 0.0059 \\
\hline RAD_D & Europium-155, Decayed & $\mathrm{pCi} / \mathrm{g}$ & 3 & 3 & $100 \%$ & -- & -- & 0.0096 & 0.018 & 0.013 \\
\hline RAD_D & Plutonium-238, Decayed & $\mathrm{pCi} / \mathrm{g}$ & 15 & 15 & $100 \%$ & -- & -- & $1.54 \mathrm{E}-04$ & $6.27 \mathrm{E}-04$ & 3.56E-04 \\
\hline RAD_D & Plutonium-239/240, Decayed & $\mathrm{pCi} / \mathrm{g}$ & 23 & 23 & $100 \%$ & -- & -- & 0.0045 & 0.015 & 0.0092 \\
\hline RAD_D & Potassium-40, Decayed & $\mathrm{pCi} / \mathrm{g}$ & 30 & 30 & $100 \%$ & -- & -- & 11 & 17 & 14 \\
\hline RAD D & Radium-226, Decayed & $\mathrm{pCi} / \mathrm{g}$ & 3 & 3 & $100 \%$ & -- & -- & 0.60 & 1.2 & 0.81 \\
\hline RAD_D & Strontium-90, Decayed & $\mathrm{pCi} / \mathrm{g}$ & 10 & 10 & $100 \%$ & -- & -- & 0.0041 & 0.019 & 0.012 \\
\hline RAD_D & Thorium-228, Decayed & $\mathrm{pCi} / \mathrm{g}$ & 3 & 3 & $100 \%$ & -- & -- & 0.0098 & 0.022 & 0.015 \\
\hline RAD_D & Thorium-232, Decayed & $\mathrm{pCi} / \mathrm{g}$ & 3 & 3 & $100 \%$ & -- & -- & 0.99 & 2.5 & 1.5 \\
\hline RAD_D & Uranium-233/234, Decayed & $\mathrm{pCi} / \mathrm{g}$ & 3 & 3 & $100 \%$ & -- & -- & 0.53 & 1.00 & 0.84 \\
\hline RAD_D & Uranium-234, Decayed & $\mathrm{pCi} / \mathrm{g}$ & 25 & 25 & $100 \%$ & -- & -- & 0.064 & 0.87 & 0.50 \\
\hline RAD_D & Uranium-235, Decayed & $\mathrm{pCi} / \mathrm{g}$ & 24 & 24 & $100 \%$ & -- & -- & 0.0027 & 0.16 & 0.030 \\
\hline RAD_D & Uranium-238, Decayed & $\mathrm{pCi} / \mathrm{g}$ & 31 & 31 & $100 \%$ & -- & -- & 0.063 & 1.4 & 0.54 \\
\hline RAD_D & Zirconium/Niobium-95, Decayed & $\mathrm{pCi} / \mathrm{g}$ & 1 & 1 & $100 \%$ & -- & -- & $2.15 \mathrm{E}-36$ & $2.15 \mathrm{E}-36$ & $2.15 \mathrm{E}-36$ \\
\hline
\end{tabular}


Table A-3

Summary Statistics for Sediment Collected from Segment 1 of the Columbia River Including All Categories of Data (units reported "as received")

\begin{tabular}{|c|c|c|c|c|c|c|c|c|c|c|}
\hline $\begin{array}{l}\text { Constituent } \\
\text { Class }\end{array}$ & Constituent Name & Units & $\begin{array}{l}\text { Number } \\
\text { Analyzed }\end{array}$ & $\begin{array}{l}\text { Number } \\
\text { Detected }\end{array}$ & $\begin{array}{l}\text { Frequency of } \\
\text { Detection }\end{array}$ & $\begin{array}{l}\text { Minimum } \\
\text { Nondetect }\end{array}$ & $\begin{array}{l}\text { Maximum } \\
\text { Nondetect }\end{array}$ & Minimum Detect & $\begin{array}{l}\text { Maximum } \\
\text { Detect }\end{array}$ & $\begin{array}{c}\text { Average } \\
\text { Concentration }\end{array}$ \\
\hline SVOC & 1,2,4-Trichlorobenzene & $\mathrm{mg} / \mathrm{Kg}$ & 32 & 0 & $0 \%$ & 0.010 & 0.66 & -- & -- & 0.32 \\
\hline SVOC & 1,2-Dichlorobenzene & $\mathrm{mg} / \mathrm{Kg}$ & 32 & 0 & $0 \%$ & 0.010 & 0.66 & -- & -- & 0.32 \\
\hline SVOC & 1,2-Diphenylhydrazine & $\mathrm{mg} / \mathrm{Kg}$ & 1 & 0 & $0 \%$ & 0.010 & 0.010 & -- & -- & 0.0050 \\
\hline SVOC & 1,3-Dichlorobenzene & $\mathrm{mg} / \mathrm{Kg}$ & 32 & 0 & $0 \%$ & 0.010 & 0.66 & -- & -- & 0.32 \\
\hline SVOC & 1,4-Dichlorobenzene & $\mathrm{mg} / \mathrm{Kg}$ & 32 & 0 & $0 \%$ & 0.010 & 0.66 & -- & -- & 0.32 \\
\hline SVOC & 1-Chloronaphthalene & $\mathrm{mg} / \mathrm{kg}$ & 19 & 0 & $0 \%$ & 0.66 & 0.66 & -- & -- & 0.33 \\
\hline SVOC & 2,4,5-Trichlorophenol & $\mathrm{mg} / \mathrm{Kg}$ & 20 & 0 & $0 \%$ & 0.010 & 0.66 & -- & -- & 0.31 \\
\hline SVOC & 2,4,6-Trichlorophenol & $\mathrm{mg} / \mathrm{Kg}$ & 20 & 0 & $0 \%$ & 0.010 & 0.66 & -- & -- & 0.31 \\
\hline SVOC & 2,4-Dichlorophenol & $\mathrm{mg} / \mathrm{Kg}$ & 20 & 0 & $0 \%$ & 0.010 & 0.66 & -- & -- & 0.31 \\
\hline SVOC & 2,4-Dimethylphenol & $\mathrm{mg} / \mathrm{Kg}$ & 32 & 0 & $0 \%$ & 0.010 & 0.66 & -- & -- & 0.32 \\
\hline SVOC & 2,4-Dinitrophenol & $\mathrm{mg} / \mathrm{Kg}$ & 20 & 0 & $0 \%$ & 0.025 & 3.3 & -- & -- & 1.6 \\
\hline SVOC & 2,4-Dinitrotoluene & $\mathrm{mg} / \mathrm{Kg}$ & 20 & 0 & $0 \%$ & 0.010 & 0.66 & -- & -- & 0.31 \\
\hline SVOC & 2,6-Dinitrotoluene & $\mathrm{mg} / \mathrm{Kg}$ & 19 & 0 & $0 \%$ & 0.010 & 0.66 & -- & -- & 0.31 \\
\hline SVOC & 2-Chloronaphthalene & $\mathrm{mg} / \mathrm{Kg}$ & 20 & 0 & $0 \%$ & 0.010 & 0.66 & -- & -- & 0.31 \\
\hline SVOC & 2-Chlorophenol & $\mathrm{mg} / \mathrm{Kg}$ & 20 & 0 & $0 \%$ & 0.010 & 0.66 & -- & -- & 0.31 \\
\hline SVOC & 2-Methylnaphthalene & $\mathrm{mg} / \mathrm{kg}$ & 31 & 0 & $0 \%$ & 0.66 & 0.66 & -- & -- & 0.33 \\
\hline SVOC & 2-Nitroaniline & $\mathrm{mg} / \mathrm{Kg}$ & 20 & 0 & $0 \%$ & 0.010 & 3.3 & -- & -- & 1.6 \\
\hline SVOC & 2-Nitrophenol & $\mathrm{mg} / \mathrm{Kg}$ & 20 & 0 & $0 \%$ & 0.010 & 0.66 & -- & -- & 0.31 \\
\hline SVOC & 3,3'-Dichlorobenzidine & $\mathrm{mg} / \mathrm{Kg}$ & 20 & 0 & $0 \%$ & 0.020 & 1.3 & -- & -- & 0.62 \\
\hline SVOC & 3-Nitroaniline & $\mathrm{mg} / \mathrm{Kg}$ & 20 & 0 & $0 \%$ & 0.010 & 3.3 & -- & -- & 1.6 \\
\hline SVOC & 4,6-Dinitro-2-methylphenol & $\mathrm{mg} / \mathrm{Kg}$ & 20 & 0 & $0 \%$ & 0.025 & 3.3 & -- & -- & 1.6 \\
\hline SVOC & 4-Bromophenylphenyl ether & $\mathrm{mg} / \mathrm{Kg}$ & 20 & 0 & $0 \%$ & 0.010 & 0.66 & -- & -- & 0.31 \\
\hline SVOC & 4-Chloro-3-methylphenol & $\mathrm{mg} / \mathrm{kg}$ & 19 & 0 & $0 \%$ & 1.3 & 1.3 & -- & -- & 0.65 \\
\hline SVOC & 4-Chloroaniline & $\mathrm{mg} / \mathrm{Kg}$ & 1 & 0 & $0 \%$ & 0.020 & 0.020 & -- & -- & 0.010 \\
\hline SVOC & 4-Chlorophenylphenyl ether & $\mathrm{mg} / \mathrm{Kg}$ & 20 & 0 & $0 \%$ & 0.010 & 0.66 & -- & -- & 0.31 \\
\hline SVOC & 4-Methylphenol (cresol, p-) & $\mathrm{mg} / \mathrm{Kg}$ & 1 & 0 & $0 \%$ & 0.010 & 0.010 & -- & -- & 0.0050 \\
\hline SVOC & 4-Nitroaniline & $\mathrm{mg} / \mathrm{Kg}$ & 20 & 0 & $0 \%$ & 0.010 & 3.3 & -- & -- & 1.6 \\
\hline SVOC & 4-Nitrophenol & $\mathrm{mg} / \mathrm{Kg}$ & 20 & 0 & $0 \%$ & 0.020 & 3.3 & -- & -- & 1.6 \\
\hline SVOC & Acenaphthene & $\mathrm{mg} / \mathrm{Kg}$ & 32 & 0 & $0 \%$ & 0.0050 & 0.66 & -- & -- & 0.32 \\
\hline SVOC & Acenaphthylene & $\mathrm{mg} / \mathrm{kg}$ & 31 & 0 & $0 \%$ & 0.66 & 0.66 & -- & -- & 0.33 \\
\hline SVOC & Aniline & $\mathrm{mg} / \mathrm{Kg}$ & 20 & 0 & $0 \%$ & 0.010 & 0.66 & -- & -- & 0.31 \\
\hline SVOC & Anthracene & $\mathrm{mg} / \mathrm{Kg}$ & 32 & 0 & $0 \%$ & 0.0050 & 0.66 & -- & -- & 0.32 \\
\hline SVOC & Benzidine & $\mathrm{mg} / \mathrm{Kg}$ & 1 & 0 & $0 \%$ & 0.050 & 0.050 & -- & -- & 0.025 \\
\hline SVOC & Benzo(a)anthracene & $\mathrm{mg} / \mathrm{kg}$ & 31 & 0 & $0 \%$ & 0.66 & 0.66 & -- & -- & 0.33 \\
\hline SVOC & Benzo(a)pyrene & $\mathrm{mg} / \mathrm{kg}$ & 31 & 0 & $0 \%$ & 0.66 & 0.66 & -- & -- & 0.33 \\
\hline SVOC & Benzo(b)fluoranthene & $\mathrm{mg} / \mathrm{kg}$ & 31 & 0 & $0 \%$ & 0.66 & 0.66 & -- & -- & 0.33 \\
\hline SVOC & Benzo(ghi)perylene & $\mathrm{mg} / \mathrm{Kg}$ & 32 & 0 & $0 \%$ & 0.010 & 0.66 & -- & -- & 0.32 \\
\hline SVOC & Benzo(k)fluoranthene & $\mathrm{mg} / \mathrm{kg}$ & 31 & 0 & $0 \%$ & 0.66 & 0.66 & -- & -- & 0.33 \\
\hline SVOC & Benzoic acid & $\mathrm{mg} / \mathrm{Kg}$ & 32 & 0 & $0 \%$ & 0.010 & 3.3 & -- & -- & 1.6 \\
\hline SVOC & Benzyl alcohol & $\mathrm{mg} / \mathrm{Kg}$ & 32 & 0 & $0 \%$ & 0.010 & 3.3 & -- & -- & 1.6 \\
\hline SVOC & Bis(2-chloro-1-methylethyl)ether & $\mathrm{mg} / \mathrm{Kg}$ & 1 & 0 & $0 \%$ & 0.010 & 0.010 & -- & -- & 0.0050 \\
\hline SVOC & Bis(2-Chloroethoxy)methane & $\mathrm{mg} / \mathrm{Kg}$ & 20 & 0 & $0 \%$ & 0.010 & 0.66 & -- & -- & 0.31 \\
\hline SVOC & Bis(2-chloroethyl) ether & $\mathrm{mg} / \mathrm{Kg}$ & 20 & 0 & $0 \%$ & 0.010 & 0.66 & -- & -- & 0.31 \\
\hline SVOC & Bis(2-chloroisopropyl) & $\mathrm{mg} / \mathrm{kg}$ & 19 & 0 & $0 \%$ & 0.66 & 0.66 & -- & -- & 0.33 \\
\hline SVOC & Bis(2-ethylhexyl) phthalate & $\mathrm{mg} / \mathrm{kg}$ & 31 & 0 & $0 \%$ & 0.66 & 0.66 & -- & -- & 0.33 \\
\hline SVOC & Butylbenzylphthalate & $\mathrm{mg} / \mathrm{kg}$ & 31 & 0 & $0 \%$ & 0.66 & 0.66 & -- & -- & 0.33 \\
\hline
\end{tabular}


Table A-3

Summary Statistics for Sediment Collected from Segment 1 of the Columbia River Including All Categories of Data (units reported "as received")

\begin{tabular}{|c|c|c|c|c|c|c|c|c|c|c|}
\hline $\begin{array}{l}\text { Constituent } \\
\text { Class }\end{array}$ & Constituent Name & Units & $\begin{array}{l}\text { Number } \\
\text { Analyzed }\end{array}$ & $\begin{array}{l}\text { Number } \\
\text { Detected }\end{array}$ & $\begin{array}{c}\text { Frequency of } \\
\text { Detection }\end{array}$ & $\begin{array}{l}\text { Minimum } \\
\text { Nondetect }\end{array}$ & $\begin{array}{l}\text { Maximum } \\
\text { Nondetect }\end{array}$ & Minimum Detect & $\begin{array}{l}\text { Maximum } \\
\text { Detect }\end{array}$ & $\begin{array}{c}\text { Average } \\
\text { Concentration }\end{array}$ \\
\hline SVOC & Chrysene & $\mathrm{mg} / \mathrm{kg}$ & 31 & 0 & $0 \%$ & 0.66 & 0.66 & -- & -- & 0.33 \\
\hline SVOC & Dibenz[a,h]anthracene & $\mathrm{mg} / \mathrm{Kg}$ & 32 & 0 & $0 \%$ & 0.010 & 0.66 & -- & -- & 0.32 \\
\hline SVOC & Dibenzofuran & $\mathrm{mg} / \mathrm{Kg}$ & 32 & 0 & $0 \%$ & 0.025 & 0.66 & -- & -- & 0.32 \\
\hline SVOC & Diethylphthalate & $\mathrm{mg} / \mathrm{kg}$ & 31 & 0 & $0 \%$ & 0.66 & 0.66 & -- & -- & 0.33 \\
\hline SVOC & Dimethyl phthalate & $\mathrm{mg} / \mathrm{Kg}$ & 31 & 0 & $0 \%$ & 0.010 & 0.66 & -- & -- & 0.32 \\
\hline SVOC & Di-n-butylphthalate & $\mathrm{mg} / \mathrm{Kg}$ & 32 & 0 & $0 \%$ & 0.0050 & 0.66 & -- & -- & 0.32 \\
\hline SVOC & Di-n-octylphthalate & $\mathrm{mg} / \mathrm{kg}$ & 31 & 0 & $0 \%$ & 0.66 & 0.66 & -- & -- & 0.33 \\
\hline SVOC & Diphenylamine & $\mathrm{mg} / \mathrm{kg}$ & 19 & 0 & $0 \%$ & 1.3 & 1.3 & -- & -- & 0.65 \\
\hline SVOC & Fluoranthene & $\mathrm{mg} / \mathrm{kg}$ & 31 & 0 & $0 \%$ & 0.66 & 0.66 & -- & -- & 0.33 \\
\hline SVOC & Fluorene & $\mathrm{mg} / \mathrm{Kg}$ & 32 & 0 & $0 \%$ & 0.010 & 0.66 & -- & -- & 0.32 \\
\hline SVOC & Hexachlorobenzene & $\mathrm{mg} / \mathrm{Kg}$ & 32 & 0 & $0 \%$ & 0.010 & 0.66 & -- & -- & 0.32 \\
\hline SVOC & Hexachlorobutadiene & $\mathrm{mg} / \mathrm{Kg}$ & 32 & 0 & $0 \%$ & 0.010 & 0.66 & -- & -- & 0.32 \\
\hline SVOC & Hexachlorocyclopentadiene & $\mathrm{mg} / \mathrm{Kg}$ & 20 & 0 & $0 \%$ & 0.010 & 0.66 & -- & -- & 0.31 \\
\hline SVOC & Hexachloroethane & $\mathrm{mg} / \mathrm{Kg}$ & 20 & 0 & $0 \%$ & 0.010 & 0.66 & -- & -- & 0.31 \\
\hline SVOC & Indeno(1,2,3-cd)pyrene & $\mathrm{mg} / \mathrm{Kg}$ & 32 & 0 & $0 \%$ & 0.010 & 0.66 & -- & -- & 0.32 \\
\hline SVOC & Isophorone & $\mathrm{mg} / \mathrm{kg}$ & 19 & 0 & $0 \%$ & 0.66 & 0.66 & -- & -- & 0.33 \\
\hline SVOC & Naphthalene & $\mathrm{mg} / \mathrm{kg}$ & 31 & 0 & $0 \%$ & 0.66 & 0.66 & -- & -- & 0.33 \\
\hline SVOC & Nitrobenzene & $\mathrm{mg} / \mathrm{Kg}$ & 19 & 0 & $0 \%$ & 0.010 & 0.66 & -- & -- & 0.31 \\
\hline SVOC & N-Nitrosodimethylamine & $\mathrm{mg} / \mathrm{kg}$ & 19 & 0 & $0 \%$ & 0.66 & 0.66 & -- & -- & 0.33 \\
\hline SVOC & N-Nitrosodi-n-dipropylamine & $\mathrm{mg} / \mathrm{Kg}$ & 20 & 0 & $0 \%$ & 0.020 & 0.66 & -- & -- & 0.31 \\
\hline SVOC & N-Nitrosodiphenylamine & $\mathrm{mg} / \mathrm{kg}$ & 31 & 0 & $0 \%$ & 0.66 & 0.66 & -- & -- & 0.33 \\
\hline SVOC & Pentachlorophenol & $\mathrm{mg} / \mathrm{Kg}$ & 32 & 0 & $0 \%$ & 0.020 & 3.3 & -- & -- & 1.6 \\
\hline SVOC & Phenanthrene & $\mathrm{mg} / \mathrm{kg}$ & 31 & 0 & $0 \%$ & 0.66 & 0.66 & -- & -- & 0.33 \\
\hline SVOC & Phenol & $\mathrm{mg} / \mathrm{kg}$ & 31 & 0 & $0 \%$ & 0.66 & 0.66 & -- & -- & 0.33 \\
\hline SVOC & Pyrene & $\mathrm{mg} / \mathrm{kg}$ & 30 & 0 & $0 \%$ & 0.66 & 0.66 & -- & -- & 0.33 \\
\hline
\end{tabular}

CONV - Conventional parameter

PEST - Pesticide

PCB - Polychlorinated biphenyl

RAD_D - Decayed radionuclide

SVOC - Semivolatile Organic Compound 
Table A-4

Summary Statistics for Category 1 Sediment Samples Collected From the Lower Crab Creek Tributary to the Columbia River (units reported "as received")

\begin{tabular}{|c|c|c|c|c|c|c|c|c|c|c|}
\hline Constituent Class & Constituent Name & Units & $\begin{array}{l}\text { Number } \\
\text { Analyzed }\end{array}$ & $\begin{array}{l}\text { Number } \\
\text { Detected }\end{array}$ & $\begin{array}{c}\text { Frequency of } \\
\text { Detection }\end{array}$ & $\begin{array}{c}\text { Minimum } \\
\text { Nondetect }\end{array}$ & $\begin{array}{l}\text { Maximum } \\
\text { Nondetect }\end{array}$ & $\begin{array}{l}\text { Minimum } \\
\text { Detect }\end{array}$ & $\begin{array}{l}\text { Maximum } \\
\text { Detect }\end{array}$ & $\begin{array}{c}\text { Average } \\
\text { Concentration }\end{array}$ \\
\hline$\overline{\text { METAL }}$ & Arsenic & $\mathrm{mg} / \mathrm{kg}$ & 1 & 1 & $100 \%$ & -- & -- & 5.9 & 5.9 & 5.9 \\
\hline METAL & Cadmium & $\mathrm{mg} / \mathrm{kg}$ & 1 & 0 & $0 \%$ & 0.49 & 0.49 & -- & -- & 0.25 \\
\hline METAL & Chromium & $\mathrm{mg} / \mathrm{kg}$ & 1 & 1 & $100 \%$ & -- & -- & 10 & 10 & 10 \\
\hline METAL & Copper & $\mathrm{mg} / \mathrm{kg}$ & 1 & 1 & $100 \%$ & -- & -- & 13 & 13 & 13 \\
\hline METAL & Lead & $\mathrm{mg} / \mathrm{kg}$ & 1 & 1 & $100 \%$ & -- & -- & 3.4 & 3.4 & 3.4 \\
\hline METAL & Mercury & $\mathrm{mg} / \mathrm{kg}$ & 1 & 1 & $100 \%$ & -- & -- & 0.044 & 0.044 & 0.044 \\
\hline METAL & Molybdenum & $\mathrm{mg} / \mathrm{kg}$ & 1 & 0 & $0 \%$ & 0.59 & 0.59 & -- & -- & 0.30 \\
\hline METAL & Nickel & $\mathrm{mg} / \mathrm{kg}$ & 1 & 1 & $100 \%$ & -- & -- & 11 & 11 & 11 \\
\hline METAL & Selenium & $\mathrm{mg} / \mathrm{kg}$ & 1 & 0 & $0 \%$ & 4.9 & 4.9 & -- & -- & 2.5 \\
\hline METAL & Zinc & $\mathrm{mg} / \mathrm{kg}$ & 1 & 1 & $100 \%$ & -- & -- & 38 & 38 & 38 \\
\hline
\end{tabular}


Table A-5

Summary Statistics for Sediment Collected from Segment 1 of the Columbia River Including All Categories of Data (units reported "dry-weight")

\begin{tabular}{|c|c|c|c|c|c|c|c|c|c|c|}
\hline $\begin{array}{l}\text { Constituent } \\
\text { Class }\end{array}$ & Constituent Name & Units & $\begin{array}{l}\text { Number } \\
\text { Analyzed }\end{array}$ & $\begin{array}{l}\text { Number } \\
\text { Detected }\end{array}$ & $\begin{array}{l}\text { Frequency of } \\
\text { Detection }\end{array}$ & $\begin{array}{l}\text { Minimum } \\
\text { Nondetect }\end{array}$ & $\begin{array}{l}\text { Maximum } \\
\text { Nondetect }\end{array}$ & $\begin{array}{c}\text { Minimum } \\
\text { Detect }\end{array}$ & $\begin{array}{l}\text { Maximum } \\
\text { Detect }\end{array}$ & $\begin{array}{c}\text { Average } \\
\text { Concentration }\end{array}$ \\
\hline$\overline{\text { METAL }}$ & Antimony & $\mathrm{mg} / \mathrm{kg}$ dry-wt & 3 & 3 & $100 \%$ & $\overline{--}$ & $\overline{--}$ & 0.73 & 1.1 & 0.87 \\
\hline METAL & Arsenic & $\mathrm{mg} / \mathrm{kg}$ dry-wt & 4 & 4 & $100 \%$ & -- & -- & 0.80 & 9.6 & 6.3 \\
\hline METAL & Beryllium & $\mathrm{mg} / \mathrm{kg}$ dry-wt & 3 & 3 & $100 \%$ & -- & -- & 1.5 & 1.7 & 1.6 \\
\hline METAL & Cadmium & $\mathrm{mg} / \mathrm{kg}$ dry-wt & 4 & 4 & $100 \%$ & -- & -- & 0.40 & 8.0 & 3.5 \\
\hline METAL & Chromium & $\mathrm{mg} / \mathrm{kg}$ dry-wt & 4 & 4 & $100 \%$ & -- & -- & 21 & 93 & 65 \\
\hline METAL & Copper & $\mathrm{mg} / \mathrm{kg}$ dry-wt & 4 & 4 & $100 \%$ & -- & -- & 20 & 59 & 36 \\
\hline METAL & Lead & $\mathrm{mg} / \mathrm{kg}$ dry-wt & 4 & 3 & $75 \%$ & 0.10 & 0.10 & 33 & 56 & 36 \\
\hline METAL & Mercury & $\mathrm{mg} / \mathrm{kg}$ dry-wt & 4 & 3 & $75 \%$ & 0.0080 & 0.0080 & 0.014 & 0.17 & 0.081 \\
\hline METAL & Nickel & $\mathrm{mg} / \mathrm{kg}$ dry-wt & 3 & 3 & $100 \%$ & -- & -- & 22 & 46 & 35 \\
\hline METAL & Selenium & $\mathrm{mg} / \mathrm{kg}$ dry-wt & 3 & 1 & $33 \%$ & 0.35 & 0.35 & 1.7 & 1.7 & 0.68 \\
\hline METAL & Silver & $\mathrm{mg} / \mathrm{kg}$ dry-wt & 3 & 3 & $100 \%$ & -- & -- & 0.33 & 0.50 & 0.41 \\
\hline METAL & Thallium & $\mathrm{mg} / \mathrm{kg}$ dry-wt & 3 & 3 & $100 \%$ & -- & -- & 0.49 & 1.9 & 1.1 \\
\hline METAL & Zinc & $\mathrm{mg} / \mathrm{kg}$ dry-wt & 4 & 4 & $100 \%$ & -- & -- & 42 & 616 & 329 \\
\hline PCB & Aroclor-1260 & $\mathrm{mg} / \mathrm{kg}$ dry-wt & 1 & 0 & $0 \%$ & 0.010 & 0.010 & -- & -- & 0.0050 \\
\hline PEST & Alpha-BHC & $\mathrm{mg} / \mathrm{kg}$ dry-wt & 1 & 0 & $0 \%$ & 0.0010 & 0.0010 & -- & -- & $5.00 \mathrm{E}-04$ \\
\hline PEST & Dichlorodiphenyldichloroethane & $\mathrm{mg} / \mathrm{kg}$ dry-wt & 1 & 0 & $0 \%$ & 0.0010 & 0.0010 & -- & -- & $5.00 \mathrm{E}-04$ \\
\hline PEST & Dichlorodiphenyldichloroethylene & $\mathrm{mg} / \mathrm{kg}$ dry-wt & 1 & 1 & $100 \%$ & -- & -- & 0.0070 & 0.0070 & 0.0070 \\
\hline PEST & Dichlorodiphenyltrichloroethane & $\mathrm{mg} / \mathrm{kg}$ dry-wt & 1 & 0 & $0 \%$ & 0.0010 & 0.0010 & -- & -- & $5.00 \mathrm{E}-04$ \\
\hline RAD D & Americium-241 & $\mathrm{pCi} / \mathrm{g}$ dry-wt & 1 & 1 & $100 \%$ & -- & -- & 0.0030 & 0.0030 & 0.0030 \\
\hline RAD_D & Beryllium-7 & $\mathrm{pCi} / \mathrm{g}$ dry-wt & 3 & 3 & $100 \%$ & -- & -- & 8.39E-20 & 8.54E-16 & $2.89 \mathrm{E}-16$ \\
\hline RAD_D & Cesium-134 & $\mathrm{pCi} / \mathrm{g}$ dry-wt & 2 & 2 & $100 \%$ & -- & -- & 0.018 & 0.023 & 0.020 \\
\hline RAD D & Cesium-137 & $\mathrm{pCi} / \mathrm{g}$ dry-wt & 16 & 16 & $100 \%$ & -- & -- & 0.0055 & 0.59 & 0.31 \\
\hline RAD_D & Cobalt-60 & pCi/g dry-wt & 1 & 1 & $100 \%$ & -- & -- & 0.0053 & 0.0053 & 0.0053 \\
\hline RAD D & Plutonium-238 & $\mathrm{pCi} / \mathrm{g}$ dry-wt & 10 & 10 & $100 \%$ & -- & -- & $2.17 \mathrm{E}-04$ & 0.0076 & 0.0039 \\
\hline RAD_D & Plutonium-239/240 & $\mathrm{pCi} / \mathrm{g}$ dry-wt & 32 & 32 & $100 \%$ & -- & -- & 0.0020 & 0.029 & 0.0097 \\
\hline RAD D $D$ & Potassium-40 & $\mathrm{pCi} / \mathrm{g}$ dry-wt & 38 & 38 & $100 \%$ & -- & -- & 11 & 21 & 14 \\
\hline RAD D & Strontium-90 & $\mathrm{pCi} / \mathrm{g}$ dry-wt & 35 & 35 & $100 \%$ & -- & -- & 0.0035 & 0.19 & 0.015 \\
\hline RAD_D & Thorium-228 & $\mathrm{pCi} / \mathrm{g}$ dry-wt & 1 & 1 & $100 \%$ & -- & -- & 0.33 & 0.33 & 0.33 \\
\hline RAD_D & Thorium-230 & $\mathrm{pCi} / \mathrm{g}$ dry-wt & 1 & 1 & $100 \%$ & -- & -- & 1.1 & 1.1 & 1.1 \\
\hline RAD D $D$ & Thorium-232 & $\mathrm{pCi} / \mathrm{g}$ dry-wt & 1 & 1 & $100 \%$ & -- & -- & 1.5 & 1.5 & 1.5 \\
\hline RAD_D & Uranium-234 & pCi/g dry-wt & 38 & 38 & $100 \%$ & -- & -- & 0.51 & 1.6 & 0.95 \\
\hline RAD_D & Uranium-235 & $\mathrm{pCi} / \mathrm{g}$ dry-wt & 12 & 12 & $100 \%$ & -- & -- & 0.015 & 0.050 & 0.036 \\
\hline RAD D & Uranium-238 & $\mathrm{pCi} / \mathrm{g}$ dry-wt & 38 & 38 & $100 \%$ & -- & -- & 0.49 & 1.3 & 0.89 \\
\hline SVOC & 2-Methylnaphthalene & $\mathrm{mg} / \mathrm{kg}$ dry-wt & 1 & 0 & $0 \%$ & 0.010 & 0.010 & -- & -- & 0.0050 \\
\hline SVOC & 2-Methylphenol (cresol, o-) & $\mathrm{mg} / \mathrm{kg}$ dry-wt & 1 & 0 & $0 \%$ & 0.010 & 0.010 & -- & -- & 0.0050 \\
\hline SVOC & Acenaphthylene & $\mathrm{mg} / \mathrm{kg}$ dry-wt & 1 & 0 & $0 \%$ & 0.010 & 0.010 & -- & -- & 0.0050 \\
\hline SVOC & Benzo(a)anthracene & $\mathrm{mg} / \mathrm{kg}$ dry-wt & 1 & 0 & $0 \%$ & 0.0050 & 0.0050 & -- & -- & 0.0025 \\
\hline SVOC & Benzo(a)pyrene & $\mathrm{mg} / \mathrm{kg}$ dry-wt & 1 & 0 & $0 \%$ & 0.010 & 0.010 & -- & -- & 0.0050 \\
\hline SVOC & Benzo(k)fluoranthene & $\mathrm{mg} / \mathrm{kg}$ dry-wt & 1 & 0 & $0 \%$ & 0.050 & 0.050 & -- & -- & 0.025 \\
\hline SVOC & Bis(2-ethylhexyl) phthalate & $\mathrm{mg} / \mathrm{kg}$ dry-wt & 1 & 0 & $0 \%$ & 0.0050 & 0.0050 & -- & -- & 0.0025 \\
\hline SVOC & Butylbenzylphthalate & $\mathrm{mg} / \mathrm{kg}$ dry-wt & 1 & 0 & $0 \%$ & 0.0050 & 0.0050 & -- & -- & 0.0025 \\
\hline SVOC & Chrysene & $\mathrm{mg} / \mathrm{kg}$ dry-wt & 1 & 0 & $0 \%$ & 0.0050 & 0.0050 & -- & -- & 0.0025 \\
\hline SVOC & Diethylphthalate & $\mathrm{mg} / \mathrm{kg}$ dry-wt & 1 & 0 & $0 \%$ & 0.010 & 0.010 & -- & -- & 0.0050 \\
\hline
\end{tabular}


Table A-5

Summary Statistics for Sediment Collected from Segment 1 of the Columbia River Including All Categories of Data (units reported "dry-weight")

\begin{tabular}{|c|c|c|c|c|c|c|c|c|c|c|}
\hline $\begin{array}{l}\text { Constituent } \\
\text { Class }\end{array}$ & Constituent Name & Units & $\begin{array}{l}\text { Number } \\
\text { Analyzed }\end{array}$ & $\begin{array}{l}\text { Number } \\
\text { Detected }\end{array}$ & $\begin{array}{l}\text { Frequency of } \\
\text { Detection }\end{array}$ & $\begin{array}{l}\text { Minimum } \\
\text { Nondetect }\end{array}$ & $\begin{array}{l}\text { Maximum } \\
\text { Nondetect }\end{array}$ & $\begin{array}{l}\text { Minimum } \\
\text { Detect }\end{array}$ & $\begin{array}{l}\text { Maximum } \\
\text { Detect }\end{array}$ & $\begin{array}{c}\text { Average } \\
\text { Concentration }\end{array}$ \\
\hline SVOC & Di-n-octylphthalate & $\mathrm{mg} / \mathrm{kg}$ dry-wt & 1 & 0 & $0 \%$ & 0.0050 & 0.0050 & -- & -- & 0.0025 \\
\hline SVOC & Fluoranthene & $\mathrm{mg} / \mathrm{kg}$ dry-wt & 1 & 0 & $0 \%$ & 0.0050 & 0.0050 & -- & -- & 0.0025 \\
\hline SVOC & Isophorone & $\mathrm{mg} / \mathrm{kg}$ dry-wt & 1 & 0 & $0 \%$ & 0.010 & 0.010 & -- & -- & 0.0050 \\
\hline SVOC & Naphthalene & $\mathrm{mg} / \mathrm{kg}$ dry-wt & 1 & 0 & $0 \%$ & 0.010 & 0.010 & -- & -- & 0.0050 \\
\hline SVOC & Phenanthrene & $\mathrm{mg} / \mathrm{kg}$ dry-wt & 1 & 0 & $0 \%$ & 0.0050 & 0.0050 & -- & -- & 0.0025 \\
\hline SVOC & Phenol & $\mathrm{mg} / \mathrm{kg}$ dry-wt & 1 & 0 & $0 \%$ & 0.010 & 0.010 & -- & -- & 0.0050 \\
\hline SVOC & Pyrene & $\mathrm{mg} / \mathrm{kg}$ dry-wt & 1 & 0 & $0 \%$ & 0.0050 & 0.0050 & -- & -- & 0.0025 \\
\hline
\end{tabular}

PEST - Pesticide

PCB - Polychlorinated biphenyl

RAD D - Decayed radionuclide

SVOC - Semivolatile Organic Compound 
Table A-6
Comparison of Segment 1 Detected Sediment Concentrations to Ecological and Human Health Benchmark Values (units reported "as received")

\begin{tabular}{|c|c|c|c|c|c|c|c|c|c|c|c|c|c|c|c|c|c|c|}
\hline $\begin{array}{c}\text { Constituent } \\
\text { Class }\end{array}$ & Constituent Name & Units & $\begin{array}{l}\text { Number } \\
\text { Analyzed }\end{array}$ & $\begin{array}{l}\text { Number } \\
\text { Detected }\end{array}$ & $\begin{array}{c}\text { Frequency of } \\
\text { Detection }\end{array}$ & $\begin{array}{c}\text { Minimum } \\
\text { Detect } \\
\end{array}$ & $\begin{array}{c}\text { Maximum } \\
\text { Detect }\end{array}$ & $\begin{array}{c}\text { Average } \\
\text { Concentration }\end{array}$ & $\begin{array}{c}\text { Background } \\
\text { value }\end{array}$ & $\begin{array}{c}\text { Is Max > } \\
\text { Background? }\end{array}$ & $\begin{array}{c}\text { Ecological } \\
\text { Benchmark Value }\end{array}$ & $\begin{array}{l}\text { Is Max > Eco } \\
\text { Benchmark? }\end{array}$ & $\begin{array}{c}\begin{array}{c}\text { Factor of } \\
\text { Exceedance }\end{array} \\
\end{array}$ & $\begin{array}{c}\text { Number of } \\
\text { Exceedances }\end{array}$ & $\begin{array}{c}\text { Human Health } \\
\text { Benchmark Value } \\
\end{array}$ & $\begin{array}{l}\text { Is Max }>\mathrm{HH} \\
\text { Benchmark? }\end{array}$ & $\begin{array}{c}\begin{array}{c}\text { Factor of } \\
\text { Exceedance }\end{array} \\
\end{array}$ & $\begin{array}{c}\text { Number of } \\
\text { Exceedances }\end{array}$ \\
\hline & Percent Solids & $\%$ & 23 & 23 & $100 \%$ & 35 & 70 & 50 & -- & - & -- & -- & $=$ & -- & $=$ & -- & -- & -- \\
\hline $\begin{array}{l}\text { CONV } \\
\text { CNV }\end{array}$ & $\begin{array}{l}\text { Total Organic C Crbono } \\
\text { Totat Oragain Carbon }\end{array}$ & $\begin{array}{l}\% \\
\mathrm{makg}\end{array}$ & $\stackrel{9}{16}$ & $\begin{array}{c}9 \\
16\end{array}$ & $\begin{array}{l}100 \% \\
100 \%\end{array}$ & $\begin{array}{c}0.27 \\
2.069\end{array}$ & $\begin{array}{c}4.0 \\
32.710\end{array}$ & $\begin{array}{c}1.7 \\
13.363\end{array}$ & - & - &.- &.- & - &.- &.- & - &.- &.- \\
\hline $\begin{array}{l}\text { CONV } \\
\text { METAL }\end{array}$ & $\begin{array}{l}\text { Total Orgagnic Carbon } \\
\text { Aluminum }\end{array}$ & $\begin{array}{l}\mathrm{mg} / \mathrm{kg} \\
\mathrm{mg} / \mathrm{kg}\end{array}$ & $\begin{array}{c}16 \\
9\end{array}$ & $\begin{array}{c}16 \\
9\end{array}$ & $\begin{array}{l}100 \% \\
100 \%\end{array}$ & $\begin{array}{l}2,069 \\
4,790\end{array}$ & $\begin{array}{l}32,110 \\
69,000\end{array}$ & $\begin{array}{l}13,363 \\
13,443\end{array}$ & 11,800 & Yes & - & -- & $\ddot{--}$ & -- & $\ddot{76.142}$ & No & $\begin{array}{l}-.91 \\
0.91\end{array}$ & $\overline{0}$ \\
\hline METAL & Antimony & $\mathrm{mg} / \mathrm{kg}$ & 19 & 15 & $79 \%$ & 0.031 & 1.1 & 1.0 & 5.0 & No & 2.0 & No & 0.53 & 0 & 31 & No & 0.034 & 0 \\
\hline METAL & Arsenic & $\mathrm{mg} / \mathrm{kg}$ & 47 & 44 & $94 \%$ & 0.42 & 12 & 6.9 & 6.5 & Yes & 6.0 & Yes & 2.0 & 27 & 0.39 & Yes & 31 & 45 \\
\hline $\begin{array}{l}\text { METAL } \\
\text { MTTL }\end{array}$ & $\begin{array}{l}\text { Barium } \\
\text { Bavilum }\end{array}$ & $\mathrm{mg} / \mathrm{kg}$ & 9 & 9 & 年00\% & 33 & 710 & ${ }_{125}^{125}$ & 132 & Yes & - & - & - & - & 5,375 & No & 0.13 & 0 \\
\hline $\begin{array}{l}\text { MUIALL } \\
\text { METAL }\end{array}$ & $\begin{array}{l}\text { Beyrlllum } \\
\text { Boron }\end{array}$ & $\begin{array}{l}\mathrm{mg} / \mathrm{kg} \\
\mathrm{m} / \mathrm{kg}\end{array}$ & ${ }_{1}^{24}$ & ${ }_{1}^{20}$ & $\begin{array}{l}8390 \% \\
100 \%\end{array}$ & $\begin{array}{l}0.062 \\
020\end{array}$ & $\begin{array}{l}2.0 \\
0.20\end{array}$ & $\begin{array}{l}0.99 \\
020\end{array}$ & 1.5 & res & $\because$ & -- & $\because$ & -- & $\begin{array}{l}154 \\
16000\end{array}$ & No & 0.013 & 0 \\
\hline METAL & Cadmium & makg & $62>02$ & 57 & $\begin{array}{l}92 \% \\
902 \%\end{array}$ & 0.30 & 16 & 5.5 & 0.81 & Yes & 0.60 & Yes & 27 & 53 & $\begin{array}{l}16,000 \\
37\end{array}$ & $\begin{array}{ll}\text { No } \\
\text { No }\end{array}$ & $\begin{array}{l}1.25 \mathrm{E}-05 \\
0.43\end{array}$ & 0 \\
\hline METAL & Calcium & $\mathrm{mg} / \mathrm{kg}$ & 9 & 9 & $100 \%$ & 2,760 & 31,000 & 6,442 & 17,200 & Yes & & & & & & No & 0.43 & $\begin{array}{l}0 \\
-.\end{array}$ \\
\hline METAL & Cerium & $\mathrm{mg} / \mathrm{kg}$ & 1 & 1 & $100 \%$ & 58 & 58 & 58 & -- & & -- & -- & -- & -- & -- & -- & - & $\cdots$ \\
\hline $\begin{array}{l}\text { METAL } \\
\text { METAL }\end{array}$ & Chromium & $\mathrm{mg} / \mathrm{kg}$ & 63 & 63 & $100 \%$ & 3.3 & 92 & 48 & 19 & Yes & 37 & Yes & 2.5 & 39 & 30 & Yes & 3.1 & 41 \\
\hline $\begin{array}{l}\text { MUIALEL } \\
\text { METAL }\end{array}$ & $\begin{array}{l}\text { Cobalt } \\
\text { Copper }\end{array}$ & $\begin{array}{l}\mathrm{mg} / \mathrm{kg} \\
\mathrm{mg} / \mathrm{kg}\end{array}$ & $\begin{array}{c}9 \\
63\end{array}$ & $\begin{array}{c}9 \\
63\end{array}$ & $\begin{array}{l}100 \% \\
100 \%\end{array}$ & $\begin{array}{l}4.0 \\
0.034\end{array}$ & $\begin{array}{l}15 \\
70\end{array}$ & $\begin{array}{l}7.0 \\
31\end{array}$ & $\begin{array}{l}16 \\
22\end{array}$ & $\begin{array}{l}\text { No } \\
\text { Yes }\end{array}$ & 32 & Yes & $\ddot{2}$ & 3 & $\begin{array}{l}903 \\
2060\end{array}$ & No & 0.017 & 0 \\
\hline METAL & Gallium & $\begin{array}{l}m g / k g \\
\mathrm{mg} / \mathrm{kg}\end{array}$ & $\begin{array}{l}{ }_{0}^{3} \\
1\end{array}$ & 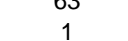 & $\begin{array}{l}100 \% \\
100 \%\end{array}$ & $\begin{array}{l}0.034 \\
16\end{array}$ & 16 & $\begin{array}{l}\underbrace{}_{10} \\
16\end{array}$ & 2 & $r_{-\infty}$ & 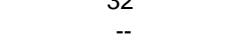 & ${ }_{-\infty}$ & $x_{i=2}^{2}$ & 23 & , & No & 0.024 & 0 \\
\hline METAL & Iron & $\mathrm{mg} / \mathrm{kg}$ & 9 & 9 & $100 \%$ & 9,100 & 40,000 & 16,578 & 32,600 & Yes & 20,000 & Yes & 2.0 & 1 & 23,463 & Yes & 1.7 & 1 \\
\hline METAL & Lanthanum & $\mathrm{mglkg}$ & 1 & 1 & $100 \%$ & 31 & 31 & 31 & & & & & & & & & 1.1 & \\
\hline METAL & Lead & $\mathrm{mg} / \mathrm{kg}$ & 59 & 59 & $100 \%$ & 1.9 & 140 & 48 & 10 & Yes & 35 & Yes & 4.0 & 37 & 250 & No & 0.56 & 0 \\
\hline 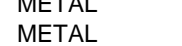 & Manim sium & $\begin{array}{l}\text { mglkg } \\
\text { maka }\end{array}$ & $\begin{array}{l}1 \\
9\end{array}$ & $\begin{array}{l}1 \\
9\end{array}$ & $\begin{array}{l}100 \% \\
100 \%\end{array}$ & 2700 & 13000 & 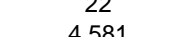 & 74 & No & $\because-$ & - & $\because$ & $\because-$ & 1,564 & No & 0.014 & 0 \\
\hline $\begin{array}{l}\text { MUIALE } \\
\text { METAL }\end{array}$ & $\begin{array}{l}\text { Magnesulum } \\
\text { Manganese }\end{array}$ & $\begin{array}{l}\mathrm{mg} / \mathrm{kg} \\
\mathrm{m} / \mathrm{kg}\end{array}$ & $\begin{array}{l}9 \\
9\end{array}$ & $\begin{array}{l}9 \\
9\end{array}$ & $\begin{array}{l}100 \% \\
100 \%\end{array}$ & $\begin{array}{l}2,100 \\
110\end{array}$ & $\begin{array}{c}13,000 \\
660\end{array}$ & $\begin{array}{l}4,581 \\
238\end{array}$ & $\begin{array}{l}7,060 \\
512\end{array}$ & $\begin{array}{l}\text { res } \\
\text { Yes }\end{array}$ & 460 & Yes & 1.4 & 1 & 1.762 & No & 037 & 0 \\
\hline METAL & Mercury & $\mathrm{mg} / \mathrm{kg}$ & 47 & 37 & $79 \%$ & 0.0062 & 0.31 & 0.12 & 0.33 & No & 0.18 & Yes & $\begin{array}{l}1.4 \\
1.7\end{array}$ & 12 & ${ }_{23}$ & No & $\begin{array}{l}0.07 \\
0.013\end{array}$ & $\begin{array}{l}0 \\
0\end{array}$ \\
\hline METAL & Neodymium & $\mathrm{mg} / \mathrm{kg}$ & 1 & 1 & $100 \%$ & 28 & 28 & 28 & & & & & & & & & . & 0 \\
\hline METAL & Nickel & $\mathrm{mg} / \mathrm{kg}$ & 33 & 33 & $100 \%$ & 1.7 & 53 & 23 & 19 & Yes & 18 & Yes & 2.9 & 14 & 1,564 & No & 0.034 & 0 \\
\hline $\begin{array}{l}\text { METALL } \\
\text { METAL }\end{array}$ & $\begin{array}{l}\text { Noobium } \\
\text { Potassium }\end{array}$ & $\mathrm{mg} / \mathrm{kg}$ & $\begin{array}{l}1 \\
9\end{array}$ & $\begin{array}{l}1 \\
9\end{array}$ & $\begin{array}{l}100 \% \\
100 \%\end{array}$ & $\begin{array}{l}4.0 \\
556\end{array}$ & 4.0 & $\begin{array}{l}4.0 \\
7090\end{array}$ & & & - & - & - & - & - & - & 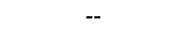 & \\
\hline $\begin{array}{l}\text { MEIALC } \\
\text { METAL }\end{array}$ & $\begin{array}{l}\text { Potasssumum } \\
\text { Scandium }\end{array}$ & $\begin{array}{l}\mathrm{mg} / \mathrm{kg} \\
\mathrm{mg} / \mathrm{kg}\end{array}$ & $\begin{array}{l}9 \\
1\end{array}$ & $\begin{array}{l}9 \\
1\end{array}$ & $\begin{array}{l}100 \% \\
100 \%\end{array}$ & $\begin{array}{c}565 \\
14\end{array}$ & $\begin{array}{l}19,000 \\
14\end{array}$ & 2,868 & $\begin{array}{l}2,150 \\
-.20\end{array}$ & res & $\ldots$ & - & $\therefore$ & $\ldots$ & - & - & - & - \\
\hline METAL & Selenium & $\mathrm{mg} / \mathrm{kg}$ & $\begin{array}{l}1 \\
29\end{array}$ & 8 & $28 \%$ & $\begin{array}{l}14 \\
0.016\end{array}$ & $\begin{array}{l}14 \\
1.9\end{array}$ & $\begin{array}{l}14.1 \\
1.1\end{array}$ & 0.78 & Yes & $\ldots$ &.- &.- & -- & 391 & No & 0.0049 & o \\
\hline METAL & Silver & $\mathrm{mg} / \mathrm{kg}$ & 50 & 14 & $28 \%$ & 0.0062 & 0.57 & 23 & 0.73 & No & 4.5 & No & 0.13 & 0 & 391 & No & 0.0014 & 0 \\
\hline METAL & Sodium & $\mathrm{mg} / \mathrm{kg}$ & 9 & 9 & $100 \%$ & 102 & 20,000 & 2,440 & 690 & Yes & -- & -- & -- & -- & & & & \\
\hline $\begin{array}{l}\text { METAL } \\
\text { METAL }\end{array}$ & Thallium & $\mathrm{mg} / \mathrm{kg}$ & 1 & 17 & $100 \%$ & 8000 & $\begin{array}{l}410 \\
10\end{array}$ & $\begin{array}{l}410 \\
410\end{array}$ & -- & - & - & - & -- & - & 46,924 & No & 0.0087 & 0 \\
\hline $\begin{array}{l}\text { MEIALC } \\
\text { METAL }\end{array}$ & $\begin{array}{l}\text { Inalum } \\
\text { Thorium }\end{array}$ & $\begin{array}{l}\mathrm{mg} / \mathrm{kg} \\
\mathrm{m} / \mathrm{kg}\end{array}$ & $\begin{array}{c}19 \\
1\end{array}$ & $\begin{array}{l}17 \\
1\end{array}$ & $\begin{array}{l}89 \% \\
100 \%\end{array}$ & $\begin{array}{l}0.049 \\
9.4\end{array}$ & $\begin{array}{l}1.9 \\
9.4\end{array}$ & $\begin{array}{l}0.95 \\
9.4\end{array}$ & 1.3 & Yes & 1300 & No & 0 & 0 & ${ }_{13}^{5.2}$ & $\begin{array}{l}\text { Noo } \\
\text { yes }\end{array}$ & 0.37 & 0 \\
\hline $\begin{array}{l}\text { METAL } \\
\text { METAL }\end{array}$ & $\begin{array}{l}\text { Titanium } \\
\text { Thium }\end{array}$ & $\begin{array}{l}m g / k g \\
\mathrm{mg} / \mathrm{kg}\end{array}$ & $\begin{array}{l}1 \\
1\end{array}$ & 1 & $100 \%$ & $\begin{array}{l}5.40 \\
5.100\end{array}$ & $\begin{array}{l}5,140 \\
5,100\end{array}$ & $\begin{array}{l}5.40 \\
5.100\end{array}$ & (1.5 & res & 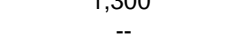 & $\ldots$ & 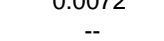 & $\ldots$ & $\begin{array}{l}100.000 \\
1000\end{array}$ & No & $\begin{array}{l}7.2 \\
0.051\end{array}$ & $\begin{array}{l}1 \\
0\end{array}$ \\
\hline METAL & Uranium & $\mathrm{mg} / \mathrm{kg}$ & 1 & 1 & $100 \%$ & 2.5 & 2.5 & 2.5 & 3.2 & No & 2,490 & No & 0.0010 & 0 & 240.0 & No & $\begin{array}{l}0.051 \\
0.010\end{array}$ & 0 \\
\hline METAL & Vanadium & $\mathrm{mg} / \mathrm{kg}$ & 9 & 9 & $100 \%$ & 23 & 130 & 44 & 85 & Yes & - & -- & -- & 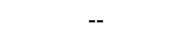 & 78 & Yes & $\begin{array}{l}0.010 \\
1.7\end{array}$ & 1 \\
\hline $\begin{array}{l}\text { METAL } \\
\text { META }\end{array}$ & Ytterbium & $\mathrm{mg} / \mathrm{kg}$ & 1 & 1 & 10 & 2.0 & 2.0 & 2.0 & - & - & - & - & - & - & - & - & -- & $\ldots$ \\
\hline $\begin{array}{l}\text { METAL } \\
\text { METAL }\end{array}$ & $\begin{array}{l}\text { Yttrium } \\
\text { Zinc }\end{array}$ & $\begin{array}{l}\mathrm{mg} / \mathrm{kg} \\
\mathrm{mg} / \mathrm{kg}\end{array}$ & $\begin{array}{c}1 \\
63\end{array}$ & $\begin{array}{c}1 \\
63\end{array}$ & $100 \%$ & $\begin{array}{l}18 \\
24\end{array}$ & $\begin{array}{l}18 \\
880\end{array}$ & ${ }_{344}^{18}$ & 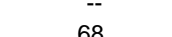 & $\because$ & $\ddot{1}$ & 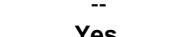 & 7 & $\ddot{0}$ & $376^{2}$ & No & -- & - \\
\hline $\begin{array}{l}\text { MUIIAL } \\
\text { PEST }\end{array}$ & $\begin{array}{l}\text { Lnc } \\
\text { Dichlorodiphenyldichloroethylene }\end{array}$ & $\begin{array}{l}\mathrm{mg} / \mathrm{kg} \\
\mathrm{m} / \mathrm{kg}\end{array}$ & $\begin{array}{l}63 \\
20\end{array}$ & $\begin{array}{c}63 \\
1\end{array}$ & $\begin{array}{l}100 \% \\
5 \%\end{array}$ & $\begin{array}{l}1.00 \mathrm{E}-04 \\
-125\end{array}$ & $\begin{array}{l}850 \\
1.00 E-04\end{array}$ & $\begin{array}{l}34.31 \\
0.31\end{array}$ & 68 & res & 0.0015 & $\begin{array}{l}\text { Yes } \\
\text { No }\end{array}$ & 0.067 & 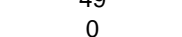 & $\begin{array}{l}23,463 \\
1.7\end{array}$ & $\begin{array}{l}\text { No } \\
\text { No }\end{array}$ & $\begin{array}{c}0.036 \\
581-05\end{array}$ & 0 \\
\hline PEST & Dieldrin & $\mathrm{mg} / \mathrm{kg}$ & 21 & 1 & $5 \%$ & $1.00 \mathrm{E}-04$ & $1.00 \mathrm{E}-04$ & 0.30 & -- & -- & 0.0019 & No & 0.053 & 0 & 0.030 & No & $\begin{array}{l}5.81 E-05 \\
0.0033\end{array}$ & $\begin{array}{l}0 \\
0\end{array}$ \\
\hline RAD_D & Cesium-134 & pCilg & 1 & 1 & $100 \%$ & $1.13 \mathrm{E}-04$ & $1.13 \mathrm{E}-04$ & $1.13 \mathrm{E}-04$ & & 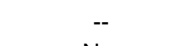 & & & & & & & 0.0005 & 0 \\
\hline $\begin{array}{l}\text { RAD_D } \\
\text { RAD }\end{array}$ & Cesium-137 & $\begin{array}{l}\mathrm{pC} / \mathrm{g} / \mathrm{g} \\
\mathrm{nc} / \mathrm{i} \mathrm{g}\end{array}$ & 49 & 49 & $\begin{array}{l}100 \% \\
100 \%\end{array}$ & 0.023 & 0.78 & 0.32 & 1.1 & No & 3,120 & No & 2.49E-04 & 0 & 6.2 & No & 0.13 & 0 \\
\hline $\begin{array}{l}\text { RADD } \\
\text { RAD_D }\end{array}$ & $\begin{array}{l}\text { Cobalt-60 } \\
\text { Europium-155 }\end{array}$ & $\begin{array}{l}\mathrm{pCClg} \\
\mathrm{pCi/g}\end{array}$ & $\begin{array}{l}2 \\
3\end{array}$ & $\begin{array}{l}2 \\
3\end{array}$ & $\begin{array}{l}100 \% \% \\
100 \%\end{array}$ & $\begin{array}{l}0.0041 \\
0.0096\end{array}$ & $\begin{array}{l}0.0070 \\
0.018\end{array}$ & & & $\begin{array}{l}\text { No } \\
\text { No }\end{array}$ & $\begin{array}{l}1,460 \\
31600\end{array}$ & No & $\begin{array}{l}4.800-06 \\
5.80-50\end{array}$ & & $\begin{array}{l}1.4 \\
125\end{array}$ & No & $\begin{array}{l}0.05050 \\
1.475 .04\end{array}$ & 0 \\
\hline RADDD & $\begin{array}{l}\text { Europium-155 } \\
\text { Plutonium-238 }\end{array}$ & $\begin{array}{l}\mathrm{pCClg} \\
\mathrm{pCi/g}\end{array}$ & 15 & $\begin{array}{l}3 \\
15\end{array}$ & $\begin{array}{l}100 \% \\
100 \%\end{array}$ & $\begin{array}{l}0.0006 \\
1.54 \mathrm{E}-04\end{array}$ & $\begin{array}{l}0.018 \\
6.27 \mathrm{E}-04\end{array}$ & $\begin{array}{c}0.013 \\
3.56 \mathrm{E}-04\end{array}$ & $\begin{array}{l}0.0044 \\
0.0038\end{array}$ & No & $\begin{array}{l}31,600 \\
-\end{array}$ & $\ldots$ & $5.80 E-0 r$ & .. & 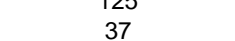 & No & 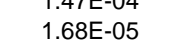 & $\begin{array}{l}0 \\
0\end{array}$ \\
\hline RAD_D & Plutonium-239/240 & $\begin{array}{l}\text { pCi/g } \\
\text { pCisa }\end{array}$ & 23 & 23 & $100 \%$ & 0.0045 & 0.015 & 0.0092 & 0.025 & No & 5,860 & No & $2.56 \mathrm{E}-06$ & 0 & 34 & No & $4.42 E-04$ & 0 \\
\hline RAD-D & Potassium-40 & $\mathrm{pCi} / \mathrm{g}$ & 30 & 30 & $100 \%$ & 11 & 17 & 14 & 17 & Yes & 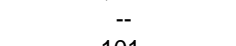 & & & & & & 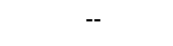 & \\
\hline $\begin{array}{l}\text { RAD_D } \\
\text { RAD D }\end{array}$ & $\begin{array}{l}\text { Radium-226 } \\
\text { Ctrotiummon }\end{array}$ & pCilg & 3 & 3 & $100 \%$ & 0.60 & 1.2 & 0.81 & 0.82 & Yes & 101 & No & $\begin{array}{l}0.012 \\
3.25 E-05\end{array}$ & 0 & -- & 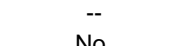 & $\therefore 0$ & $\ddot{0}$ \\
\hline $\begin{array}{l}\text { RADDD } \\
\text { RAD_D }\end{array}$ & $\begin{array}{l}\text { Strontumm-90 } \\
\text { Thorium-228 }\end{array}$ & $\begin{array}{l}\text { pCilg } \\
\text { pCi/g }\end{array}$ & $\begin{array}{l}100 \\
3\end{array}$ & $\begin{array}{l}10 \\
3\end{array}$ & $\begin{array}{l}100 \% \\
100 \%\end{array}$ & $\begin{array}{l}0.0041 \\
0.0098\end{array}$ & $\begin{array}{l}0.0019 \\
0.022\end{array}$ & $\begin{array}{l}0.012 \\
0.015\end{array}$ & 0.18 & $\begin{array}{l}\text { No } \\
\text { No }\end{array}$ & 582 & No. & $\begin{array}{l}3.25 E-05 \\
--\end{array}$ & $\frac{0}{-}$ & 4.5 & No. & $\begin{array}{l}0.0042 \\
-.\end{array}$ & $\begin{array}{l}0 \\
-.\end{array}$ \\
\hline RAD_D & Thorium-232 & pCilg & 3 & 3 & $100 \%$ & 0.99 & 2.5 & 1.5 & 1.3 & Yes & 1,300 & No & 0.0019 & 0 & 1.3 & Yes & 1.9 & 1 \\
\hline $\begin{array}{l}\text { RADDD } \\
\text { RAD D }\end{array}$ & Uranium-233/234 & $\begin{array}{l}\text { pCClig } \\
\text { pCigen }\end{array}$ & $\begin{array}{l}3 \\
35 \\
25\end{array}$ & $\begin{array}{l}3 \\
35 \\
3\end{array}$ & 年 $100 \%$ & $\begin{array}{l}0.53 \\
0.064\end{array}$ & $\begin{array}{l}1.00 \\
1.08 \\
\end{array}$ & 0.84 & $\because 1$ & No & & & $16 \mathrm{cos}$ & $=$ & 11 & 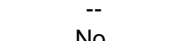 & & - \\
\hline $\begin{array}{l}\text { RAD D } \\
\text { RADD }\end{array}$ & $\begin{array}{l}\text { Uranumim-234 } \\
\text { Uranium-235 }\end{array}$ & $\begin{array}{l}\text { pCl/g } \\
\text { pCi/g }\end{array}$ & $\begin{array}{l}25 \\
24\end{array}$ & 24 & $\begin{array}{l}100 \% \\
100 \%\end{array}$ & $\begin{array}{l}0.004 \\
0.0027\end{array}$ & 0.16 & $\begin{array}{l}0.030 \\
0.030\end{array}$ & $\begin{array}{l}1.1 \\
0.11\end{array}$ & $\begin{array}{l}\text { No } \\
\text { Yes }\end{array}$ & 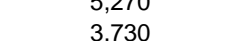 & 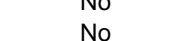 & $\begin{array}{l}1.060-04 \\
4.40-50\end{array}$ & 0 & $\begin{array}{l}1.14 \\
0.84\end{array}$ & 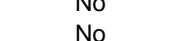 & 0.20 & $\begin{array}{l}0 \\
0\end{array}$ \\
\hline RAD_D & Uranium-238 & pCilg & 31 & & $100 \%$ & & & & 3.2 & No & 2,490 & No & $5.46 \mathrm{E}-04$ & 0 & 1.1 & Yes & 1.2 & 1 \\
\hline RAD_D & Zirconium/Niobium-95 & $\mathrm{pCi} / \mathrm{g}$ & 1 & 1 & $100 \%$ & $2.15 E-36$ & $2.15 E-36$ & $2.15 E-36$ & & +1 & 2,40 & - & -- & - & - & -- & - & 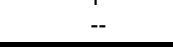 \\
\hline
\end{tabular}

CONV - Conventional parameter
PEST - Pesiciction

RAD_D - Decayed radionuclide 
Table A-7
Comparison of Segment 1 Detected Sediment Concentrations to Ecological and Human Health Benchmark Values (units reported "dry weight")

\begin{tabular}{|c|c|c|c|c|c|c|c|c|c|c|c|c|c|c|c|c|c|c|}
\hline $\begin{array}{c}\text { Constituent } \\
\text { Class } \\
\end{array}$ & Constituent Name & Units & $\begin{array}{c}\text { Number } \\
\text { Analyzed } \\
\end{array}$ & $\begin{array}{l}\text { Number } \\
\text { Detected }\end{array}$ & $\begin{array}{c}\text { Frequency of } \\
\text { Detection }\end{array}$ & $\begin{array}{c}\text { Minimum } \\
\text { Detect }\end{array}$ & $\begin{array}{c}\text { Maximum } \\
\text { Detect }\end{array}$ & $\begin{array}{c}\text { Average } \\
\text { Concentration }\end{array}$ & $\begin{array}{c}\text { Background } \\
\text { Value }\end{array}$ & $\begin{array}{c}\begin{array}{c}\text { Is Max } \\
\text { Background? }\end{array} \\
\text { Bats }\end{array}$ & $\begin{array}{c}\text { Ecological } \\
\text { Benchmark Value } \\
\end{array}$ & $\begin{array}{l}\text { Is Max > Eco } \\
\text { Benchmark? }\end{array}$ & $\begin{array}{c}\text { Factor of } \\
\text { Exceedance }\end{array}$ & $\begin{array}{c}\text { Number of } \\
\text { Exceedances } \\
\end{array}$ & $\begin{array}{l}\text { Human Health } \\
\text { Benchmark } \\
\text { value } \\
\end{array}$ & $\begin{array}{l}\text { Is Max> } \mathrm{HH} \\
\text { Benchmark? }\end{array}$ & $\begin{array}{c}\text { Factor of } \\
\text { Exceedance } \\
\end{array}$ & $\begin{array}{c}\text { Number of } \\
\text { Exceedances }\end{array}$ \\
\hline $\begin{array}{l}\text { METAL } \\
\text { METL }\end{array}$ & Antimony & $m g / k g$ dry-wt & 3 & 3 & $100 \%$ & 0.73 & 1.1 & 0.87 & 5.0 & No & 2.0 & No & 0.54 & 0 & & & 0.035 & 0 \\
\hline METAL & Arsenic & $\mathrm{mg} / \mathrm{kg}$ dry-wt & 4 & 4 & $100 \%$ & 0.80 & 9.6 & 6.3 & 6.5 & Yes & 6.0 & Yes & 1.6 & 3 & 0.39 & Yes & 24.562 & 4 \\
\hline $\begin{array}{l}\text { METAL } \\
\text { METAL }\end{array}$ & $\begin{array}{l}\text { Beryllium } \\
\text { Campium }\end{array}$ & $\begin{array}{l}\text { mg/kg dry-wt } \\
\text { makgadr-wt }\end{array}$ & $\begin{array}{l}3 \\
4\end{array}$ & $\begin{array}{l}3 \\
4\end{array}$ & $\begin{array}{l}100 \% \\
100 \%\end{array}$ & 1.5 & $\begin{array}{l}1.7 \\
8 .\end{array}$ & 1.6 & $\begin{array}{l}1.5 \\
0.81\end{array}$ & $\begin{array}{l}\text { Yres } \\
\text { S }\end{array}$ & 060 & $v_{o c}$ & 12 & $\ddot{z}$ & $\begin{array}{l}154 \\
{ }_{27}\end{array}$ & No & $\begin{array}{l}0.011 \\
0.11\end{array}$ & 0 \\
\hline $\begin{array}{l}\text { METAL } \\
\text { METAL }\end{array}$ & $\begin{array}{l}\text { Cadmimum } \\
\text { Chromium }\end{array}$ & $\begin{array}{l}\text { mg/kg dry-wt } \\
m a k \text { ka d d-wt }\end{array}$ & $\begin{array}{l}4 \\
4\end{array}$ & $\begin{array}{l}4 \\
4\end{array}$ & $\begin{array}{l}100 \% \\
100 \%\end{array}$ & $\begin{array}{l}0.40 \\
21\end{array}$ & $\begin{array}{l}8.0 \\
93\end{array}$ & $\begin{array}{l}3.5 \\
65 \\
65\end{array}$ & $\begin{array}{l}0.81 \\
19\end{array}$ & 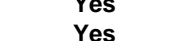 & $\begin{array}{l}0.60 \\
37\end{array}$ & $\begin{array}{l}\text { Yes } \\
\text { Yes }\end{array}$ & $\begin{array}{l}13 \\
25\end{array}$ & $\begin{array}{l}2 \\
3\end{array}$ & $\begin{array}{l}31 \\
30\end{array}$ & $\begin{array}{l}\text { No } \\
\text { Yes }\end{array}$ & $\begin{array}{l}0.215 \\
.0909\end{array}$ & $\begin{array}{l}0 \\
3\end{array}$ \\
\hline $\begin{array}{l}\text { MEIAL } \\
\text { METAL }\end{array}$ & $\begin{array}{l}\text { nromium } \\
\text { Copper }\end{array}$ & $\begin{array}{l}m \text { mg/kg ary-wt } \\
\text { mglkg dry-wt }\end{array}$ & $\begin{array}{l}4 \\
4\end{array}$ & $\begin{array}{l}4 \\
4\end{array}$ & $\begin{array}{l}100 \% \\
100 \%\end{array}$ & ${ }_{20}^{21}$ & $\begin{array}{l}93 \\
59\end{array}$ & 36 & ${ }_{22}^{19}$ & $\begin{array}{l}\text { Yes } \\
\text { Yes }\end{array}$ & 32 & $\begin{array}{l}\text { Yes } \\
\text { Yes }\end{array}$ & $\begin{array}{l}2.5 \\
1.9\end{array}$ & 2 & $\begin{array}{r}30 \\
2.960\end{array}$ & $\begin{array}{l}\text { Yes } \\
\text { No }\end{array}$ & $\begin{array}{l}3.090 \\
0.020\end{array}$ & $\begin{array}{l}3 \\
0\end{array}$ \\
\hline METAL & Lead & $\mathrm{mg} / \mathrm{kg}$ dry-wt & 4 & 3 & $75 \%$ & 33 & 56 & 36 & 10 & Yes & 35 & $\begin{array}{l}\text { Yes } \\
\text { Yos }\end{array}$ & 1.6 & 2 & 250 & No & 0.225 & 0 \\
\hline METAL & Mercury & $\mathrm{mg} / \mathrm{kg}$ dry-wt & 4 & 3 & $75 \%$ & 0.014 & 0.17 & 0.081 & 0.33 & No & 0.18 & No & 0.94 & 0 & 23 & No & 0.007 & 0 \\
\hline $\begin{array}{l}\text { METAL } \\
\text { METAA }\end{array}$ & $\begin{array}{l}\text { Nickel } \\
\text { Selenim }\end{array}$ & $\begin{array}{l}\mathrm{mg} / \mathrm{kg} \mathrm{dry}-\mathrm{wt} \\
\mathrm{mg} / \mathrm{kg} \mathrm{d}-\mathrm{wt}\end{array}$ & 3 & 3 & $100 \%$ & 22 & 46 & $\begin{array}{l}35 \\
068\end{array}$ & $\begin{array}{l}19 \\
078\end{array}$ & $\begin{array}{l}\text { Yes } \\
\text { Yes }\end{array}$ & 18 & Yes & 2.6 & 3 & 1,564 & No & 0.030 & 0 \\
\hline $\begin{array}{l}\text { METAL } \\
\text { METAL }\end{array}$ & $\begin{array}{l}\text { Selenium } \\
\text { Silver }\end{array}$ & $\begin{array}{l}m \mathrm{~m} / \mathrm{kg} \text { d dry-wt } \\
\mathrm{mg} / \mathrm{kg} \text { dry-wt }\end{array}$ & $\begin{array}{l}3 \\
3\end{array}$ & $\begin{array}{l}1 \\
3\end{array}$ & $\begin{array}{l}33 \% \\
130 \%\end{array}$ & $\begin{array}{l}1.7 \\
0.33\end{array}$ & $\begin{array}{l}1.7 \\
0.50\end{array}$ & $\begin{array}{l}0.68 \\
0.41\end{array}$ & $\begin{array}{l}0.78 \\
0.73\end{array}$ & $\begin{array}{l}\text { Yes } \\
\text { No }\end{array}$ & 4.5 & No & 011 & 0 & 391 & $\begin{array}{l}\text { No } \\
\text { No }\end{array}$ & 44 & $\begin{array}{l}0 \\
0\end{array}$ \\
\hline METAL & $\begin{array}{l}\text { Siver } \\
\text { Thallium }\end{array}$ & $\begin{array}{l}m \text { mg/kg dry-wt } \\
m g / k g \text { dry-wt }\end{array}$ & 3 & $\begin{array}{l}3 \\
3\end{array}$ & $\begin{array}{l}100 \% \% \\
100 \%\end{array}$ & $\begin{array}{l}0.33 \\
0.49\end{array}$ & $\begin{array}{l}0.50 \\
1.9\end{array}$ & 0.41 & 0.13 & № & 4.5 & No & 0.11 & 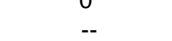 & $\begin{array}{l}391 \\
5.2\end{array}$ & $\begin{array}{l}\text { No } \\
\text { No }\end{array}$ & 0.001 & 0 \\
\hline METAL & Zinc & mglkg dry-wt & 4 & 4 & $100 \%$ & 42 & 616 & $\begin{array}{l}1.1 \\
329\end{array}$ & 68 & Yes & 120 & Yes & 5.1 & 3 & 23,463 & No & $\begin{array}{l}0.026 \\
0.026\end{array}$ & 0 \\
\hline PEST & Dichlorodiphenyldichloroethylene & $\mathrm{mg} / \mathrm{kg}$ dry-wt & 1 & 1 & $100 \%$ & 0.0070 & 0.0070 & 0.0070 & -- & -- & 0.0015 & Yes & 4.7 & 1 & 1.7 & No & 0.004 & 0 \\
\hline RAD_D & Americium-241 & pCi/g dry-wt & $\frac{1}{3}$ & $\frac{1}{3}$ & $100 \%$ & 0.0030 & & 0.0 & -- & -- & 5,150 & No & $5.81 \mathrm{E}-07$ & 0 & 31 & No & 0.000 & 0 \\
\hline $\begin{array}{l}\text { RAD_D } \\
\text { RADD }\end{array}$ & $\begin{array}{l}\text { Beryllium-7 } \\
\text { Cesium-134 }\end{array}$ & 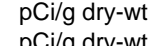 & 3 & 3 & $100 \%$ & $8.39 E-20$ & $8.54 E-16$ & $2.89 E-16$ & $-\overline{-}$ & 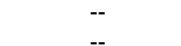 & $\ddot{-}$ & - & - & -- & $-\overline{-}$ & $\overline{-}$ & $\ddot{-}$ & -- \\
\hline $\begin{array}{ll}\text { RaD } \\
\text { RADDD }\end{array}$ & $\begin{array}{l}\text { Cesium-134 } \\
\text { Cesium }\end{array}$ & $\begin{array}{l}\text { pcllig ary-we } \\
\text { pcilg dry-wt }\end{array}$ & $\begin{array}{c}2 \\
16\end{array}$ & $\begin{array}{c}2 \\
16\end{array}$ & $\begin{array}{l}100 \% \% \\
100 \%\end{array}$ & $\begin{array}{l}0.018 \\
0.0055\end{array}$ & $\begin{array}{l}0.023 \\
0.59\end{array}$ & 0.020 & 1.1. & No & 3,120 & No & $1.88 \mathrm{E}-04$ & 0 & 6.2 & № & 0.095 & $\overline{0}$ \\
\hline $\begin{array}{l}\text { RADD } \\
\text { RAD_D }\end{array}$ & $\begin{array}{l}\text { Cesium-13t } \\
\text { Cobalt-60 }\end{array}$ & $\begin{array}{l}\text { plig ary-wt } \\
\text { pCilg dry-wt }\end{array}$ & $\begin{array}{c}16 \\
1\end{array}$ & $\begin{array}{c}16 \\
1\end{array}$ & $\begin{array}{l}100 \% \% \\
100 \%\end{array}$ & $\begin{array}{l}0.0055 \\
0.053\end{array}$ & $\begin{array}{l}0.59 \\
0.0053\end{array}$ & $\begin{array}{l}0.31 \\
0.0053\end{array}$ & $\begin{array}{l}1.1 \\
0.0084\end{array}$ & $\begin{array}{l}\text { No } \\
\text { No }\end{array}$ & $\begin{array}{l}3,120 \\
1,460\end{array}$ & $\begin{array}{l}\text { No } \\
\text { No }\end{array}$ & $\begin{array}{l}1.88 \mathrm{EE}-04 \\
3.64 \mathrm{E}-06\end{array}$ & $\begin{array}{l}0 \\
0\end{array}$ & $\begin{array}{l}6.2 \\
1.4\end{array}$ & $\begin{array}{l}\text { No } \\
\text { No }\end{array}$ & $\begin{array}{l}0.095 \\
0.004\end{array}$ & 0 \\
\hline RAD_D & Plutonium-238 & pcilg dry-wt & 10 & 10 & $100 \%$ & $2.17 \mathrm{E}-04$ & 0.0076 & 0.0039 & 0.0038 & Yes & & No & & & 37 & No & 0.000 & 0 \\
\hline RAD=D & Plutonium-239/240 & dry-wt & 32 & 32 & $100 \%$ & 0.0020 & 0.029 & 0.0097 & 0.025 & Yes & 5,860 & No & $4.95 \mathrm{E}-06$ & 0 & 34 & No & 0.001 & 0 \\
\hline $\begin{array}{l}\text { RADDD } \\
\text { RAD D }\end{array}$ & $\begin{array}{l}\text { Potatassium-40 } \\
\text { Strontium-90 }\end{array}$ & 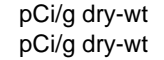 & $\begin{array}{l}38 \\
35 \\
35\end{array}$ & $\begin{array}{l}38 \\
35 \\
35\end{array}$ & $\begin{array}{l}100 \% \\
100 \%\end{array}$ & $\begin{array}{l}11 \\
0.0035\end{array}$ & $\begin{array}{l}21 \\
0.19\end{array}$ & $\begin{array}{c}14 \\
0.015\end{array}$ & $\begin{array}{l}17 \\
0.18\end{array}$ & $\begin{array}{l}\text { Yes } \\
\text { Yes }\end{array}$ & 582 & No & $3.27 \mathrm{E}-04$ & 0 & 4.5 & No & 0.042 & 0 \\
\hline RAD_D & Thorium-228 & pcilg dry-wt & 1 & 1 & $100 \%$ & 0.33 & 0.33 & 0.33 & - & $\cdots$ & $\ldots$ & - & -- & -. & $-\cdots$ & -- & -. & .. \\
\hline RAD_D & ium-230 & CCilg dry-wt & 1 & 1 & & 1 & 1.1 & 1.1 & $\therefore$ & $\cdots$ & $\cdots$ & 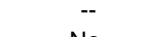 & $\cdots$ & - & $\therefore$ & - & 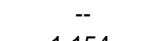 & \\
\hline RADD & 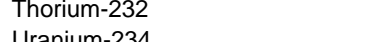 & 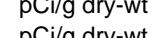 & $\begin{array}{l}1 \\
38\end{array}$ & $\begin{array}{l}1 \\
38\end{array}$ & 年 $100 \%$ & $\begin{array}{l}1.5 \\
0.51\end{array}$ & 1.5 & $\begin{array}{l}1.5 \\
09\end{array}$ & 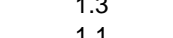 & $\begin{array}{l}\text { Yes } \\
\text { Yos }\end{array}$ & $\begin{array}{l}1,300 \\
1,300\end{array}$ & No & 0.0012 & 0 & 1.3 & Yes & 1.154 & 1 \\
\hline $\begin{array}{l}\text { RADDD } \\
\text { RADD }\end{array}$ & $\begin{array}{l}\text { Uraniumm-234 } \\
\text { Uraniumm-235 }\end{array}$ & $\begin{array}{l}\text { pCig dry-wt } \\
\text { ciligaryowt }\end{array}$ & $\begin{array}{l}38 \\
12\end{array}$ & $\begin{array}{l}38 \\
12\end{array}$ & $\begin{array}{l}100 \% 0 \\
100 \%\end{array}$ & $\begin{array}{l}0.011 \\
0.015\end{array}$ & $\begin{array}{l}1.6 \\
0.050\end{array}$ & $\begin{array}{l}0.035 \\
0.036\end{array}$ & $\begin{array}{l}1.1 \\
0.11\end{array}$ & Yes & $\begin{array}{l}5,210 \\
3,70\end{array}$ & No & $\begin{array}{l}3.00 E-04 \\
134.05\end{array}$ & 0 & $\begin{array}{ll}1.1 \\
0.84\end{array}$ & Yes & $\begin{array}{l}1.4646 \\
0.060\end{array}$ & 0 \\
\hline RAD_D & $\begin{array}{l}\text { Uranium-235 }-238 \\
\text { Uranim }\end{array}$ & 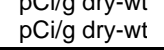 & $\begin{array}{l}14 \\
38\end{array}$ & 38 & $100 \%$ & 0.49 & 1.3 & 0.89 & 3.2 & No & $\begin{array}{l}2,490 \\
2,400\end{array}$ & No & $5.26 \mathrm{E}-04$ & 0 & $\begin{array}{l}0.14 \\
1.1\end{array}$ & Yes & $\begin{array}{l}0.191 \\
1.191\end{array}$ & 4 \\
\hline
\end{tabular}

PEST-Pesticide 


\section{Table A-8}

Summary of Surface Sediment Results From Segment 1 Exceeding Select Ecological or Human Health Benchmark Values

\begin{tabular}{|c|c|c|c|c|c|c|c|c|c|c|}
\hline Sample Area & Sample Site & Sample ID & Date Collected & Constituent Name & Units & $\begin{array}{c}\text { QA } \\
\text { Category }\end{array}$ & $\begin{array}{c}\text { Analytical } \\
\text { Value }\end{array}$ & Qualifier & $\begin{array}{c}\text { Ecological } \\
\text { Benchmark } \\
\text { Value }\end{array}$ & $\begin{array}{c}\text { Human Health } \\
\text { Benchmark } \\
\text { Value }\end{array}$ \\
\hline Columbia River & Pr Rapids Dam & B14YN2 & July 23, 2002 & Arsenic & $\mathrm{mg} / \mathrm{kg}$ & 1 & 0.42 & $=$ & 6.0 & 0.39 \\
\hline Columbia River & Pr Rapids Dam & B14YNO & July 23,2002 & Arsenic & $\mathrm{mg} / \mathrm{kg}$ & 1 & 0.53 & $=$ & 6.0 & 0.39 \\
\hline Wenatchee River & Wenatchee & 45A070-7 & September 1, 1984 & Arsenic & $\mathrm{mg} / \mathrm{kg}$ dry-wt & 2 & 0.80 & $=$ & 6.0 & 0.39 \\
\hline Columbia River & Vernita Area & B07NF6 & November 20, 1992 & Arsenic & $\mathrm{mg} / \mathrm{kg}$ & 1 & 1.9 & BJN & 6.0 & 0.39 \\
\hline Columbia River & RM 417.5 & GHI-1 & October 1, 1999 & Arsenic & $\mathrm{mg} / \mathrm{kg}$ & 2 & 2.3 & $=$ & 6.0 & 0.39 \\
\hline Columbia River & Vernita Area & B07NF7 & November 20, 1992 & Arsenic & $\mathrm{mg} / \mathrm{kg}$ & 1 & 2.4 & $\mathrm{JN}$ & 6.0 & 0.39 \\
\hline Columbia River & RM 440 & CRE-3 & October 1, 1999 & Arsenic & $\mathrm{mg} / \mathrm{kg}$ & 2 & 3.5 & $=$ & 6.0 & 0.39 \\
\hline Columbia River & RM 405.5 & HAN-5 & October 1, 1999 & Arsenic & $\mathrm{mg} / \mathrm{kg}$ & 2 & 3.5 & $=$ & 6.0 & 0.39 \\
\hline Columbia River & RM 428 & SCA-3B & October 1, 1999 & Arsenic & $\mathrm{mg} / \mathrm{kg}$ & 2 & 3.6 & $=$ & 6.0 & 0.39 \\
\hline Columbia River & RM 417.5 & GHI-5 & October 1, 1999 & Arsenic & $\mathrm{mg} / \mathrm{kg}$ & 2 & 3.8 & $=$ & 6.0 & 0.39 \\
\hline Columbia River & Pr Rapids Dam & B0W3D8 & August 3, 1999 & Arsenic & $\mathrm{mg} / \mathrm{kg}$ & 1 & 4.1 & $=$ & 6.0 & 0.39 \\
\hline Columbia River & RM 428 & SCA-3C & October 1, 1999 & Arsenic & $\mathrm{mg} / \mathrm{kg}$ & 2 & 4.3 & $=$ & 6.0 & 0.39 \\
\hline Columbia River & RM 354.4 & PEW-1sed & July 16,1992 & Arsenic & $\mathrm{mg} / \mathrm{kg}$ & 2 & 4.6 & $=$ & 6.0 & 0.39 \\
\hline Columbia River & Pr Rapids Dam & 4444204 & June 1,2004 & Arsenic & $\mathrm{mg} / \mathrm{kg}$ & 1 & 4.8 & $=$ & 6.0 & 0.39 \\
\hline Columbia River & Pr Rapids Dam & 4444203 & June 1, 2004 & Arsenic & $\mathrm{mg} / \mathrm{kg}$ & 1 & 4.9 & $=$ & 6.0 & 0.39 \\
\hline Columbia River & Wanapum Dam & 4444210 & June 1,2004 & Arsenic & $\mathrm{mg} / \mathrm{kg}$ & 1 & 5.1 & $=$ & 6.0 & 0.39 \\
\hline Columbia River & Vernita Area & B07NF8 & November 20, 1992 & Arsenic & $\mathrm{mg} / \mathrm{kg}$ & 1 & 5.8 & $\mathrm{JN}+$ & 6.0 & 0.39 \\
\hline Lower Crab Creek & RM 5 & 4444202 & June 1, 2004 & Arsenic & $\mathrm{mg} / \mathrm{kg}$ & 1 & 5.9 & $=$ & 6.0 & 0.39 \\
\hline Columbia River & Vernita BRG & B12RY7 & August 28, 2001 & Arsenic & $\mathrm{mg} / \mathrm{kg}$ dry-wt & 1 & 6.5 & $=$ & 6.0 & 0.39 \\
\hline Columbia River & RM 428 & SCA-3A & October 1, 1999 & Arsenic & $\mathrm{mg} / \mathrm{kg}$ & 2 & 6.5 & $=$ & 6.0 & 0.39 \\
\hline Columbia River & Pr Rapids Dam & BOW3F0 & August 3, 1999 & Arsenic & $\mathrm{mg} / \mathrm{kg}$ & 1 & 6.7 & $=$ & 6.0 & 0.39 \\
\hline Columbia River & Pr Rapids Dam & BOYWC2 & August 7,2000 & Arsenic & $\mathrm{mg} / \mathrm{kg}$ & 1 & 7.3 & $=$ & 6.0 & 0.39 \\
\hline Columbia River & Pr Rapids Dam & 4444205 & June 1, 2004 & Arsenic & $\mathrm{mg} / \mathrm{kg}$ & 1 & 7.6 & $=$ & 6.0 & 0.39 \\
\hline Columbia River & Pr Rapids Dam & 4444206 & June 1,2004 & Arsenic & $\mathrm{mg} / \mathrm{kg}$ & 1 & 7.6 & $=$ & 6.0 & 0.39 \\
\hline Columbia River & Pr Rapids Dam & BOYWC4 & August 7,2000 & Arsenic & $\mathrm{mg} / \mathrm{kg}$ & 1 & 8.2 & $=$ & 6.0 & 0.39 \\
\hline Columbia River & Pr Rapids Dam & B12CL9 & July 19, 2001 & Arsenic & $\mathrm{mg} / \mathrm{kg}$ dry-wt & 1 & 8.2 & $=$ & 6.0 & 0.39 \\
\hline Columbia River & Pr Rapids Dam & B0W3D6 & August 3, 1999 & Arsenic & $\mathrm{mg} / \mathrm{kg}$ & 1 & 8.6 & $=$ & 6.0 & 0.39 \\
\hline Columbia River & RM 398.5 & GOO-3C & October 1, 1999 & Arsenic & $\mathrm{mg} / \mathrm{kg}$ & 2 & 9.0 & $=$ & 6.0 & 0.39 \\
\hline Columbia River & Pr Rapids Dam & B19PK9 & July 12, 2004 & Arsenic & $\mathrm{mg} / \mathrm{kg}$ & 1 & 9.1 & $x$ & 6.0 & 0.39 \\
\hline Columbia River & Pr Rapids Dam & B1B7W4 & September 20, 2004 & Arsenic & $\mathrm{mg} / \mathrm{kg}$ & 1 & 9.1 & $\mathrm{x}$ & 6.0 & 0.39 \\
\hline Columbia River & Pr Rapids Dam & B17702 & July 14,2003 & Arsenic & $\mathrm{mg} / \mathrm{kg}$ & 1 & 9.2 & $=$ & 6.0 & 0.39 \\
\hline Columbia River & RM 417.5 & GHI-3 & October 1, 1999 & Arsenic & $\mathrm{mg} / \mathrm{kg}$ & 2 & 9.3 & $=$ & 6.0 & 0.39 \\
\hline Columbia River & Pr Rapids Dam & BOW3N5 & August 3, 1999 & Arsenic & $\mathrm{mg} / \mathrm{kg}$ & 2 & 9.4 & $=$ & 6.0 & 0.39 \\
\hline Columbia River & Vernita Area & B07NF5 & November 20, 1992 & Arsenic & $\mathrm{mg} / \mathrm{kg}$ & 1 & 9.4 & $\mathrm{JN}$ & 6.0 & 0.39 \\
\hline Columbia River & RM 428 & SCA- 5 & October 1, 1999 & Arsenic & $\mathrm{mg} / \mathrm{kg}$ & 1 & 9.4 & $=$ & 6.0 & 0.39 \\
\hline Columbia River & Pr Rapids Dam & B12CM1 & July 19, 2001 & Arsenic & $\mathrm{mg} / \mathrm{kg}$ dry-wt & 1 & 9.6 & $=$ & 6.0 & 0.39 \\
\hline Columbia River & Pr Rapids Dam & B17704 & July 14,2003 & Arsenic & $\mathrm{mg} / \mathrm{kg}$ & 1 & 9.7 & $=$ & 6.0 & 0.39 \\
\hline Columbia River & Pr Rapids Dam & BOW3F2 & August 3, 1999 & Arsenic & $\mathrm{mg} / \mathrm{kg}$ & 1 & 9.9 & $=$ & 6.0 & 0.39 \\
\hline Columbia River & Pr Rapids Dam & BOW3N3 & August 3, 1999 & Arsenic & $\mathrm{mg} / \mathrm{kg}$ & 1 & 10 & $=$ & 6.0 & 0.39 \\
\hline Columbia River & RM 417.5 & GHI-4 & October 1, 1999 & Arsenic & $\mathrm{mg} / \mathrm{kg}$ & 2 & 10 & $=$ & 6.0 & 0.39 \\
\hline Columbia River & RM 428 & SCA-4 & October 1,1999 & Arsenic & $\mathrm{mg} / \mathrm{kg}$ & 2 & 10 & $=$ & 6.0 & 0.39 \\
\hline
\end{tabular}




\section{Table A-8}

Summary of Surface Sediment Results From Segment 1 Exceeding Select Ecological or Human Health Benchmark Values

\begin{tabular}{|c|c|c|c|c|c|c|c|c|c|c|}
\hline Sample Area & Sample Site & Sample ID & Date Collected & Constituent Name & Units & $\begin{array}{c}\text { QA } \\
\text { Category }\end{array}$ & $\begin{array}{l}\text { Analytical } \\
\text { Value }\end{array}$ & Qualifier & $\begin{array}{c}\text { Ecological } \\
\text { Benchmark } \\
\text { Value }\end{array}$ & $\begin{array}{c}\text { Human Health } \\
\text { Benchmark } \\
\text { Value }\end{array}$ \\
\hline Columbia River & RM 398.5 & GOO-1 & October 1, 1999 & Arsenic & $\mathrm{mg} / \mathrm{kg}$ & 2 & 11 & $=$ & 6.0 & 0.39 \\
\hline Columbia River & RM 398.5 & GOO-3B & October 1, 1999 & Arsenic & $\mathrm{mg} / \mathrm{kg}$ & 2 & 11 & $=$ & 6.0 & 0.39 \\
\hline Columbia River & RM 398.5 & GOO-4 & October 1, 1999 & Arsenic & $\mathrm{mg} / \mathrm{kg}$ & 2 & 11 & $=$ & 6.0 & 0.39 \\
\hline Columbia River & Pr Rapids Dam & B19PL1 & July 12, 2004 & Arsenic & $\mathrm{mg} / \mathrm{kg}$ & 1 & 11 & $x$ & 6.0 & 0.39 \\
\hline Columbia River & RM 417.5 & GHI-2 & October 1, 1999 & Arsenic & $\mathrm{mg} / \mathrm{kg}$ & 2 & 12 & $=$ & 6.0 & 0.39 \\
\hline Columbia River & RM 398.5 & GOO-2 & October 1, 1999 & Arsenic & $\mathrm{mg} / \mathrm{kg}$ & 2 & 12 & $=$ & 6.0 & 0.39 \\
\hline Columbia River & RM 398.5 & GOO-3A & October 1, 1999 & Arsenic & $\mathrm{mg} / \mathrm{kg}$ & 2 & 12 & $=$ & 6.0 & 0.39 \\
\hline Columbia River & Vernita Area & B07NF8 & November 20,1992 & Cadmium & $\mathrm{mg} / \mathrm{kg}$ & 1 & 0.62 & B & 0.60 & 37 \\
\hline Columbia River & Wanapum Dam & 4444210 & June 1, 2004 & Cadmium & $\mathrm{mg} / \mathrm{kg}$ & 1 & 0.64 & $=$ & 0.60 & 37 \\
\hline Columbia River & Vernita Area & B07NF7 & November 20, 1992 & Cadmium & $\mathrm{mg} / \mathrm{kg}$ & 1 & 0.65 & B & 0.60 & 37 \\
\hline Columbia River & Pr Rapids Dam & BOCSH1 & September 22, 1994 & Cadmium & $\mathrm{mg} / \mathrm{kg}$ & 1 & 0.80 & L & 0.60 & 37 \\
\hline Columbia River & Wanapum Dam & 4444208 & June 1, 2004 & Cadmium & $\mathrm{mg} / \mathrm{kg}$ & 1 & 1.2 & $=$ & 0.60 & 37 \\
\hline Columbia River & Pr Rapids Dam & 4444203 & June 1, 2004 & Cadmium & $\mathrm{mg} / \mathrm{kg}$ & 1 & 1.6 & $=$ & 0.60 & 37 \\
\hline Columbia River & RM 440 & SS-3-3 & October 1, 1999 & Cadmium & $\mathrm{mg} / \mathrm{kg}$ & 2 & 2.1 & $=$ & 0.60 & 37 \\
\hline Columbia River & Pr Rapids Dam & B0W3D8 & August 3, 1999 & Cadmium & $\mathrm{mg} / \mathrm{kg}$ & 1 & 2.1 & $=$ & 0.60 & 37 \\
\hline Columbia River & RM 440 & SS-4-3 & October 1, 1999 & Cadmium & $\mathrm{mg} / \mathrm{kg}$ & 2 & 2.3 & $=$ & 0.60 & 37 \\
\hline Columbia River & Pr Rapids Dam & BOCSHO & September 22, 1994 & Cadmium & $\mathrm{mg} / \mathrm{kg}$ & 1 & 2.4 & $=$ & 0.60 & 37 \\
\hline Columbia River & RM 440 & SS-1-3 & October 1, 1999 & Cadmium & $\mathrm{mg} / \mathrm{kg}$ & 2 & 2.4 & $=$ & 0.60 & 37 \\
\hline Columbia River & RM 440 & SS-2-3 & October 1, 1999 & Cadmium & $\mathrm{mg} / \mathrm{kg}$ & 2 & 2.4 & $=$ & 0.60 & 37 \\
\hline Columbia River & Pr Rapids Dam & 4444205 & June 1, 2004 & Cadmium & $\mathrm{mg} / \mathrm{kg}$ & 1 & 2.9 & $=$ & 0.60 & 37 \\
\hline Columbia River & Pr Rapids Dam & B0CSG9 & September 22, 1994 & Cadmium & $\mathrm{mg} / \mathrm{kg}$ & 1 & 3.3 & $=$ & 0.60 & 37 \\
\hline Columbia River & Pr Rapids Dam & BOCSH2 & September 22, 1994 & Cadmium & $\mathrm{mg} / \mathrm{kg}$ & 1 & 3.4 & $=$ & 0.60 & 37 \\
\hline Columbia River & RM 417.5 & GHI-1 & October 1, 1999 & Cadmium & $\mathrm{mg} / \mathrm{kg}$ & 2 & 3.5 & $=$ & 0.60 & 37 \\
\hline Columbia River & Pr Rapids Dam & BOW3F0 & August 3, 1999 & Cadmium & $\mathrm{mg} / \mathrm{kg}$ & 1 & 4.0 & $=$ & 0.60 & 37 \\
\hline Columbia River & RM 440 & SS-4-1 & October 1, 1999 & Cadmium & $\mathrm{mg} / \mathrm{kg}$ & 2 & 4.2 & $=$ & 0.60 & 37 \\
\hline Columbia River & Pr Rapids Dam & 4444206 & June 1, 2004 & Cadmium & $\mathrm{mg} / \mathrm{kg}$ & 1 & 4.2 & $=$ & 0.60 & 37 \\
\hline Columbia River & Pr Rapids Dam & BOYWC2 & August 7, 2000 & Cadmium & $\mathrm{mg} / \mathrm{kg}$ & 1 & 4.3 & $=$ & 0.60 & 37 \\
\hline Columbia River & RM 440 & SS-1-1 & October 1, 1999 & Cadmium & $\mathrm{mg} / \mathrm{kg}$ & 2 & 4.6 & $=$ & 0.60 & 37 \\
\hline Columbia River & Pr Rapids Dam & B12CL9 & $7 / 19 / 2001$ & Cadmium & mg/kg dry-wt & 1 & 5.0 & $=$ & 0.60 & 37 \\
\hline Columbia River & RM 405.5 & HAN-5 & October 1, 1999 & Cadmium & $\mathrm{mg} / \mathrm{kg}$ & 2 & 5.1 & $=$ & 0.60 & 37 \\
\hline Columbia River & RM 440 & SS-2-2 & October 1, 1999 & Cadmium & $\mathrm{mg} / \mathrm{kg}$ & 2 & 5.1 & $=$ & 0.60 & 37 \\
\hline Columbia River & Pr Rapids Dam & B1B7W4 & September 20, 2004 & Cadmium & $\mathrm{mg} / \mathrm{kg}$ & 1 & 5.4 & $x$ & 0.60 & 37 \\
\hline Columbia River & RM 440 & SS-2-1 & October 1, 1999 & Cadmium & $\mathrm{mg} / \mathrm{kg}$ & 2 & 5.6 & $=$ & 0.60 & 37 \\
\hline Columbia River & RM 440 & SS-4-2 & October 1, 1999 & Cadmium & $\mathrm{mg} / \mathrm{kg}$ & 2 & 5.6 & $=$ & 0.60 & 37 \\
\hline Columbia River & RM 428 & SCA-3B & October 1, 1999 & Cadmium & $\mathrm{mg} / \mathrm{kg}$ & 2 & 5.7 & $=$ & 0.60 & 37 \\
\hline Columbia River & RM 440 & SS-1-2 & October 1, 1999 & Cadmium & $\mathrm{mg} / \mathrm{kg}$ & 2 & 5.7 & $=$ & 0.60 & 37 \\
\hline Columbia River & RM 440 & SS-3-2 & October 1, 1999 & Cadmium & $\mathrm{mg} / \mathrm{kg}$ & 2 & 5.7 & $=$ & 0.60 & 37 \\
\hline Columbia River & RM 440 & SS-3-1 & October 1, 1999 & Cadmium & $\mathrm{mg} / \mathrm{kg}$ & 2 & 5.9 & $=$ & 0.60 & 37 \\
\hline Columbia River & Pr Rapids Dam & B17702 & July 14, 2003 & Cadmium & $\mathrm{mg} / \mathrm{kg}$ & 1 & 6.0 & $=$ & 0.60 & 37 \\
\hline Columbia River & RM 417.5 & GHI-5 & October 1, 1999 & Cadmium & $\mathrm{mg} / \mathrm{kg}$ & 2 & 6.0 & $=$ & 0.60 & 37 \\
\hline Columbia River & RM 428 & SCA-3C & October 1, 1999 & Cadmium & $\mathrm{mg} / \mathrm{kg}$ & 2 & 6.1 & $=$ & 0.60 & 37 \\
\hline
\end{tabular}




\section{Table A-8}

Summary of Surface Sediment Results From Segment 1 Exceeding Select Ecological or Human Health Benchmark Values

\begin{tabular}{|c|c|c|c|c|c|c|c|c|c|c|}
\hline Sample Area & Sample Site & Sample ID & Date Collected & Constituent Name & Units & $\begin{array}{c}\text { QA } \\
\text { Category }\end{array}$ & $\begin{array}{c}\text { Analytical } \\
\text { Value }\end{array}$ & Qualifier & $\begin{array}{c}\text { Ecological } \\
\text { Benchmark } \\
\text { Value }\end{array}$ & $\begin{array}{c}\text { Human Health } \\
\text { Benchmark } \\
\text { Value }\end{array}$ \\
\hline Columbia River & RM 428 & SCA-3A & October 1, 1999 & Cadmium & $\mathrm{mg} / \mathrm{kg}$ & 2 & 6.5 & $=$ & 0.60 & 37 \\
\hline Columbia River & Pr Rapids Dam & BOW3F2 & August 3, 1999 & Cadmium & $\mathrm{mg} / \mathrm{kg}$ & 1 & 6.7 & $=$ & 0.60 & 37 \\
\hline Columbia River & Pr Rapids Dam & B0W3D6 & August 3, 1999 & Cadmium & $\mathrm{mg} / \mathrm{kg}$ & 1 & 6.9 & $=$ & 0.60 & 37 \\
\hline Columbia River & Pr Rapids Dam & B19PK9 & July 12,2004 & Cadmium & $\mathrm{mg} / \mathrm{kg}$ & 1 & 7.3 & $\mathrm{x}$ & 0.60 & 37 \\
\hline Columbia River & Pr Rapids Dam & BOW3N3 & August 3, 1999 & Cadmium & $\mathrm{mg} / \mathrm{kg}$ & 1 & 7.5 & $=$ & 0.60 & 37 \\
\hline Columbia River & Pr Rapids Dam & B19PL1 & July 12, 2004 & Cadmium & $\mathrm{mg} / \mathrm{kg}$ & 1 & 7.8 & $x$ & 0.60 & 37 \\
\hline Columbia River & Pr Rapids Dam & B12CM1 & July 19, 2001 & Cadmium & $\mathrm{mg} / \mathrm{kg}$ dry-wt & 1 & 8.0 & $=$ & 0.60 & 37 \\
\hline Columbia River & Pr Rapids Dam & BOYWC4 & August 7, 2000 & Cadmium & $\mathrm{mg} / \mathrm{kg}$ & 1 & 8.3 & $=$ & 0.60 & 37 \\
\hline Columbia River & Pr Rapids Dam & BOW3N5 & August 3, 1999 & Cadmium & $\mathrm{mg} / \mathrm{kg}$ & 1 & 8.7 & $=$ & 0.60 & 37 \\
\hline Columbia River & Pr Rapids Dam & B17704 & July 14, 2003 & Cadmium & $\mathrm{mg} / \mathrm{kg}$ & 1 & 8.9 & $=$ & 0.60 & 37 \\
\hline Columbia River & RM 398.5 & GOO-3C & October 1, 1999 & Cadmium & $\mathrm{mg} / \mathrm{kg}$ & 2 & 9.7 & $=$ & 0.60 & 37 \\
\hline Columbia River & RM 417.5 & GHI-2 & October 1, 1999 & Cadmium & $\mathrm{mg} / \mathrm{kg}$ & 2 & 12 & $=$ & 0.60 & 37 \\
\hline Columbia River & RM 398.5 & GOO-2 & October 1, 1999 & Cadmium & $\mathrm{mg} / \mathrm{kg}$ & 2 & 12 & $=$ & 0.60 & 37 \\
\hline Columbia River & RM 398.5 & GOO-1 & October 1, 1999 & Cadmium & $\mathrm{mg} / \mathrm{kg}$ & 2 & 13 & $=$ & 0.60 & 37 \\
\hline Columbia River & RM 398.5 & GOO-4 & October 1, 1999 & Cadmium & $\mathrm{mg} / \mathrm{kg}$ & 2 & 13 & $=$ & 0.60 & 37 \\
\hline Columbia River & RM 428 & SCA-4 & October 1, 1999 & Cadmium & $\mathrm{mg} / \mathrm{kg}$ & 2 & 13 & $=$ & 0.60 & 37 \\
\hline Columbia River & RM 417.5 & GHI-4 & October 1, 1999 & Cadmium & $\mathrm{mg} / \mathrm{kg}$ & 2 & 14 & $=$ & 0.60 & 37 \\
\hline Columbia River & RM 398.5 & GOO-3A & October 1, 1999 & Cadmium & $\mathrm{mg} / \mathrm{kg}$ & 2 & 14 & $=$ & 0.60 & 37 \\
\hline Columbia River & RM 398.5 & GOO-3B & October 1, 1999 & Cadmium & $\mathrm{mg} / \mathrm{kg}$ & 2 & 15 & $=$ & 0.60 & 37 \\
\hline Columbia River & RM 428 & SCA-5 & October 1, 1999 & Cadmium & $\mathrm{mg} / \mathrm{kg}$ & 2 & 15 & $=$ & 0.60 & 37 \\
\hline Columbia River & RM 417.5 & GHI-3 & October 1, 1999 & Cadmium & $\mathrm{mg} / \mathrm{kg}$ & 2 & 16 & $=$ & 0.60 & 37 \\
\hline Columbia River & RM 405.5 & $\mathrm{HAN}-5$ & October 1,1999 & Chromium & $\mathrm{mg} / \mathrm{kg}$ & 2 & 32 & $=$ & 37 & 30 \\
\hline Columbia River & RM 440 & CRE-3 & October 1, 1999 & Chromium & $\mathrm{mg} / \mathrm{kg}$ & 2 & 33 & $=$ & 37 & 30 \\
\hline Columbia River & RM 417.5 & GHI-5 & October 1, 1999 & Chromium & $\mathrm{mg} / \mathrm{kg}$ & 2 & 33 & $=$ & 37 & 30 \\
\hline Columbia River & RM 440 & SS-1-3 & October 1, 1999 & Chromium & $\mathrm{mg} / \mathrm{kg}$ & 2 & 34 & $=$ & 37 & 30 \\
\hline Columbia River & RM 440 & SS-4-3 & October 1, 1999 & Chromium & $\mathrm{mg} / \mathrm{kg}$ & 2 & 34 & $=$ & 37 & 30 \\
\hline Columbia River & RM 440 & SS-3-3 & October 1, 1999 & Chromium & $\mathrm{mg} / \mathrm{kg}$ & 2 & 40 & $=$ & 37 & 30 \\
\hline Columbia River & Pr Rapids Dam & B0W3D8 & August 3, 1999 & Chromium & $\mathrm{mg} / \mathrm{kg}$ & 1 & 42 & $=$ & 37 & 30 \\
\hline Columbia River & RM 354.4 & PEW-1sed & July 16, 1992 & Chromium & $\mathrm{mg} / \mathrm{kg}$ & 2 & 46 & $=$ & 37 & 30 \\
\hline Columbia River & Pr Rapids Dam & B0W3D6 & August 3, 1999 & Chromium & $\mathrm{mg} / \mathrm{kg}$ & 1 & 52 & $=$ & 37 & 30 \\
\hline Columbia River & RM 398.5 & GOO-2 & October 1, 1999 & Chromium & $\mathrm{mg} / \mathrm{kg}$ & 2 & 53 & $=$ & 37 & 30 \\
\hline Columbia River & RM 398.5 & GOO-3B & October 1, 1999 & Chromium & $\mathrm{mg} / \mathrm{kg}$ & 2 & 55 & $=$ & 37 & 30 \\
\hline Columbia River & RM 398.5 & GOO-4 & October 1, 1999 & Chromium & $\mathrm{mg} / \mathrm{kg}$ & 2 & 56 & $=$ & 37 & 30 \\
\hline Columbia River & RM 398.5 & GOO-1 & October 1, 1999 & Chromium & $\mathrm{mg} / \mathrm{kg}$ & 2 & 57 & $=$ & 37 & 30 \\
\hline Columbia River & RM 398.5 & GOO-3A & October 1, 1999 & Chromium & $\mathrm{mg} / \mathrm{kg}$ & 2 & 57 & $=$ & 37 & 30 \\
\hline Columbia River & RM 417.5 & GHI-2 & October 1, 1999 & Chromium & $\mathrm{mg} / \mathrm{kg}$ & 2 & 58 & $=$ & 37 & 30 \\
\hline Columbia River & RM 440 & SS-2-2 & October 1, 1999 & Chromium & $\mathrm{mg} / \mathrm{kg}$ & 2 & 60 & $=$ & 37 & 30 \\
\hline Columbia River & RM 398.5 & GOO-3C & October 1, 1999 & Chromium & $\mathrm{mg} / \mathrm{kg}$ & 2 & 61 & $=$ & 37 & 30 \\
\hline Columbia River & RM 440 & SS-1-2 & October 1, 1999 & Chromium & $\mathrm{mg} / \mathrm{kg}$ & 2 & 63 & $=$ & 37 & 30 \\
\hline Columbia River & RM 440 & SS-2-1 & October 1, 1999 & Chromium & $\mathrm{mg} / \mathrm{kg}$ & 2 & 66 & $=$ & 37 & 30 \\
\hline Columbia River & RM 440 & SS-4-2 & October 1, 1999 & Chromium & $\mathrm{mg} / \mathrm{kg}$ & 2 & 66 & $=$ & 37 & 30 \\
\hline Columbia River & RM 440 & SS-3-2 & October 1, 1999 & Chromium & $\mathrm{mg} / \mathrm{kg}$ & 2 & 67 & $=$ & 37 & 30 \\
\hline
\end{tabular}




\section{Table A-8}

Summary of Surface Sediment Results From Segment 1 Exceeding Select Ecological or Human Health Benchmark Values

\begin{tabular}{|c|c|c|c|c|c|c|c|c|c|c|}
\hline Sample Area & Sample Site & Sample ID & Date Collected & Constituent Name & Units & $\begin{array}{c}\text { QA } \\
\text { Category }\end{array}$ & $\begin{array}{c}\text { Analytical } \\
\text { Value }\end{array}$ & Qualifier & $\begin{array}{c}\text { Ecological } \\
\text { Benchmark } \\
\text { Value }\end{array}$ & $\begin{array}{c}\text { Human Health } \\
\text { Benchmark } \\
\text { Value }\end{array}$ \\
\hline Columbia River & Vernita BRG & B12RY7 & August 28, 2001 & Chromium & $\mathrm{mg} / \mathrm{kg}$ dry-wt & 1 & 66 & $=$ & 37 & 30 \\
\hline Columbia River & RM 440 & SS-1-1 & October 1, 1999 & Chromium & $\mathrm{mg} / \mathrm{kg}$ & 2 & 70 & $=$ & 37 & 30 \\
\hline Columbia River & RM 440 & SS-3-1 & October 1, 1999 & Chromium & $\mathrm{mg} / \mathrm{kg}$ & 2 & 71 & $=$ & 37 & 30 \\
\hline Columbia River & RM 440 & SS-4-1 & October 1, 1999 & Chromium & $\mathrm{mg} / \mathrm{kg}$ & 2 & 71 & $=$ & 37 & 30 \\
\hline Columbia River & RM 428 & SCA-5 & October 1, 1999 & Chromium & $\mathrm{mg} / \mathrm{kg}$ & 2 & 72 & $=$ & 37 & 30 \\
\hline Columbia River & Pr Rapids Dam & B0W3F2 & August 3, 1999 & Chromium & $\mathrm{mg} / \mathrm{kg}$ & 1 & 72 & $=$ & 37 & 30 \\
\hline Columbia River & RM 417.5 & GHI-3 & October 1, 1999 & Chromium & $\mathrm{mg} / \mathrm{kg}$ & 2 & 74 & $=$ & 37 & 30 \\
\hline Columbia River & RM 417.5 & GHI-4 & October 1, 1999 & Chromium & $\mathrm{mg} / \mathrm{kg}$ & 2 & 75 & $=$ & 37 & 30 \\
\hline Columbia River & RM 428 & SCA-4 & October 1, 1999 & Chromium & $\mathrm{mg} / \mathrm{kg}$ & 2 & 77 & $=$ & 37 & 30 \\
\hline Columbia River & Pr Rapids Dam & BOYWC4 & August 7, 2000 & Chromium & $\mathrm{mg} / \mathrm{kg}$ & 1 & 79 & $=$ & 37 & 30 \\
\hline Columbia River & Pr Rapids Dam & B0W3F0 & August 3, 1999 & Chromium & $\mathrm{mg} / \mathrm{kg}$ & 1 & 80 & $=$ & 37 & 30 \\
\hline Columbia River & Pr Rapids Dam & B0W3N5 & August 3, 1999 & Chromium & $\mathrm{mg} / \mathrm{kg}$ & 1 & 80 & $=$ & 37 & 30 \\
\hline Columbia River & Pr Rapids Dam & B12CL9 & July 19, 2001 & Chromium & $\mathrm{mg} / \mathrm{kg}$ dry-wt & 1 & 80 & $=$ & 37 & 30 \\
\hline Columbia River & Pr Rapids Dam & B19PK9 & July 12,2004 & Chromium & $\mathrm{mg} / \mathrm{kg}$ & 1 & 83 & $c X$ & 37 & 30 \\
\hline Columbia River & Pr Rapids Dam & BOW3N3 & August 3, 1999 & Chromium & $\mathrm{mg} / \mathrm{kg}$ & 1 & 84 & $=$ & 37 & 30 \\
\hline Columbia River & Pr Rapids Dam & BOYWC2 & August 7, 2000 & Chromium & $\mathrm{mg} / \mathrm{kg}$ & 1 & 84 & $=$ & 37 & 30 \\
\hline Columbia River & Pr Rapids Dam & B17704 & July 14,2003 & Chromium & $\mathrm{mg} / \mathrm{kg}$ & 1 & 87 & C & 37 & 30 \\
\hline Columbia River & RM 428 & SCA-3B & October 1, 1999 & Chromium & $\mathrm{mg} / \mathrm{kg}$ & 2 & 89 & $=$ & 37 & 30 \\
\hline Columbia River & RM 428 & SCA-3C & October 1, 1999 & Chromium & $\mathrm{mg} / \mathrm{kg}$ & 2 & 89 & $=$ & 37 & 30 \\
\hline Columbia River & Pr Rapids Dam & B1B7W4 & September 20, 2004 & Chromium & $\mathrm{mg} / \mathrm{kg}$ & 1 & 89 & $x$ & 37 & 30 \\
\hline Columbia River & Pr Rapids Dam & B19PL1 & July 12,2004 & Chromium & $\mathrm{mg} / \mathrm{kg}$ & 1 & 90 & $C X$ & 37 & 30 \\
\hline Columbia River & RM 428 & SCA-3A & October 1, 1999 & Chromium & $\mathrm{mg} / \mathrm{kg}$ & 2 & 92 & $=$ & 37 & 30 \\
\hline Columbia River & Pr Rapids Dam & B17702 & July 14,2003 & Chromium & $\mathrm{mg} / \mathrm{kg}$ & 1 & 92 & C & 37 & 30 \\
\hline Columbia River & Pr Rapids Dam & B12CM1 & July 19, 2001 & Chromium & $\mathrm{mg} / \mathrm{kg}$ dry-wt & 1 & 93 & $=$ & 37 & 30 \\
\hline Columbia River & Pr Rapids Dam & $\mathrm{B} 0 \mathrm{~W} 3 \mathrm{~F} 2$ & August 3,1999 & Copper & $\mathrm{mg} / \mathrm{kg}$ & 1 & 34 & $=$ & 32 & 2,960 \\
\hline Columbia River & Pr Rapids Dam & 4444206 & June 1, 2004 & Copper & $\mathrm{mg} / \mathrm{kg}$ & 1 & 37 & $=$ & 32 & 2,960 \\
\hline Columbia River & Pr Rapids Dam & B12CL9 & July 19, 2001 & Copper & $\mathrm{mg} / \mathrm{kg}$ dry-wt & 1 & 39 & $=$ & 32 & 2,960 \\
\hline Columbia River & RM 428 & SCA-3A & October 1, 1999 & Copper & $\mathrm{mg} / \mathrm{kg}$ & 2 & 39 & $=$ & 32 & 2,960 \\
\hline Columbia River & RM 398.5 & GOO-2 & October 1, 1999 & Copper & $\mathrm{mg} / \mathrm{kg}$ & 2 & 40 & $=$ & 32 & 2,960 \\
\hline Columbia River & Pr Rapids Dam & B19PK9 & July 12, 2004 & Copper & $\mathrm{mg} / \mathrm{kg}$ & 1 & 44 & $x$ & 32 & 2,960 \\
\hline Columbia River & Pr Rapids Dam & BOW3N3 & August 3, 1999 & Copper & $\mathrm{mg} / \mathrm{kg}$ & 1 & 46 & $=$ & 32 & 2,960 \\
\hline Columbia River & Pr Rapids Dam & B17702 & July 14, 2003 & Copper & $\mathrm{mg} / \mathrm{kg}$ & 1 & 46 & $=$ & 32 & 2,960 \\
\hline Columbia River & RM 398.5 & GOO-3B & October 1, 1999 & Copper & $\mathrm{mg} / \mathrm{kg}$ & 2 & 48 & $=$ & 32 & 2,960 \\
\hline Columbia River & Pr Rapids Dam & BOW3N5 & August 3, 1999 & Copper & $\mathrm{mg} / \mathrm{kg}$ & 1 & 49 & $=$ & 32 & 2,960 \\
\hline Columbia River & Pr Rapids Dam & B17704 & July 14, 2003 & Copper & $\mathrm{mg} / \mathrm{kg}$ & 1 & 50 & $=$ & 32 & 2,960 \\
\hline Columbia River & RM 398.5 & GOO-4 & October 1, 1999 & Copper & $\mathrm{mg} / \mathrm{kg}$ & 2 & 51 & $=$ & 32 & 2,960 \\
\hline Columbia River & Pr Rapids Dam & B1B7W4 & September 20, 2004 & Copper & $\mathrm{mg} / \mathrm{kg}$ & 1 & 52 & $x$ & 32 & 2,960 \\
\hline Columbia River & RM 417.5 & GHI-2 & October 1, 1999 & Copper & $\mathrm{mg} / \mathrm{kg}$ & 2 & 52 & $=$ & 32 & 2,960 \\
\hline Columbia River & RM 398.5 & GOO-3A & October 1, 1999 & Copper & $\mathrm{mg} / \mathrm{kg}$ & 2 & 53 & $=$ & 32 & 2,960 \\
\hline Columbia River & RM 398.5 & GOO-3C & October 1, 1999 & Copper & $\mathrm{mg} / \mathrm{kg}$ & 2 & 53 & $=$ & 32 & 2,960 \\
\hline Columbia River & Pr Rapids Dam & BOYWC2 & August 7, 2000 & Copper & $\mathrm{mg} / \mathrm{kg}$ & 1 & 55 & $=$ & 32 & 2,960 \\
\hline Columbia River & RM 398.5 & GOO-1 & October 1, 1999 & Copper & $\mathrm{mg} / \mathrm{kg}$ & 2 & 56 & $=$ & 32 & 2,960 \\
\hline
\end{tabular}




\section{Table A-8}

Summary of Surface Sediment Results From Segment 1 Exceeding Select Ecological or Human Health Benchmark Values

\begin{tabular}{|c|c|c|c|c|c|c|c|c|c|c|}
\hline Sample Area & Sample Site & Sample ID & Date Collected & Constituent Name & Units & $\begin{array}{c}\text { QA } \\
\text { Category }\end{array}$ & $\begin{array}{l}\text { Analytical } \\
\text { Value }\end{array}$ & Qualifier & $\begin{array}{c}\text { Ecological } \\
\text { Benchmark } \\
\text { Value }\end{array}$ & $\begin{array}{c}\text { Human Health } \\
\text { Benchmark } \\
\text { Value }\end{array}$ \\
\hline Columbia River & Pr Rapids Dam & B19PL1 & July 12, 2004 & Copper & $\mathrm{mg} / \mathrm{kg}$ & 1 & 58 & $\bar{x}$ & 32 & 2,960 \\
\hline Columbia River & Pr Rapids Dam & B12CM1 & July 19, 2001 & Copper & mg/kg dry-wt & 1 & 59 & $=$ & 32 & 2,960 \\
\hline Columbia River & Pr Rapids Dam & BOYWC4 & August 7, 2000 & Copper & $\mathrm{mg} / \mathrm{kg}$ & 1 & 60 & $=$ & 32 & 2,960 \\
\hline Columbia River & RM 428 & SCA-4 & October 1, 1999 & Copper & $\mathrm{mg} / \mathrm{kg}$ & 2 & 62 & $=$ & 32 & 2,960 \\
\hline Columbia River & RM 417.5 & GHI-4 & October 1, 1999 & Copper & $\mathrm{mg} / \mathrm{kg}$ & 2 & 63 & $=$ & 32 & 2,960 \\
\hline Columbia River & RM 417.5 & GHI-3 & October 1, 1999 & Copper & $\mathrm{mg} / \mathrm{kg}$ & 2 & 70 & $=$ & 32 & 2,960 \\
\hline Columbia River & RM 428 & SCA- 5 & October 1, 1999 & Copper & $\mathrm{mg} / \mathrm{kg}$ & 2 & 70 & $=$ & 32 & 2,960 \\
\hline Wenatchee River & Wenatchee & $45 \mathrm{~A} 070-7$ & September 1,1984 & Dichlorodiphenyldichloroethylene & $\mathrm{mg} / \mathrm{kg}$ dry & & 0.007 & $=$ & 0.0015 & 1.7 \\
\hline Columbia River & RM 354.4 & PEW-1sed & July 16,1992 & Iron & $\mathrm{mg} / \mathrm{kg}$ & 2 & 40,000 & $=$ & 20,000 & 23,463 \\
\hline Columbia River & $\mathrm{RM} 440$ & CRE-3 & October 1, 1999 & Lead & $\mathrm{mg} / \mathrm{kg}$ & 2 & 37 & $=$ & 35 & 250 \\
\hline Columbia River & RM 440 & SS-4-1 & October 1, 1999 & Lead & $\mathrm{mg} / \mathrm{kg}$ & 2 & 40 & $=$ & 35 & 250 \\
\hline Columbia River & Pr Rapids Dam & BOYWC2 & August 7, 2000 & Lead & $\mathrm{mg} / \mathrm{kg}$ & 1 & 42 & $=$ & 35 & 250 \\
\hline Columbia River & Pr Rapids Dam & B1B7W4 & September 20, 2004 & Lead & $\mathrm{mg} / \mathrm{kg}$ & 1 & 44 & $\mathrm{x}$ & 35 & 250 \\
\hline Columbia River & RM 417.5 & GHI-5 & October 1, 1999 & Lead & $\mathrm{mg} / \mathrm{kg}$ & 2 & 44 & $=$ & 35 & 250 \\
\hline Columbia River & RM 405.5 & HAN-5 & October 1, 1999 & Lead & $\mathrm{mg} / \mathrm{kg}$ & 2 & 44 & $=$ & 35 & 250 \\
\hline Columbia River & RM 440 & SS-1-1 & October 1, 1999 & Lead & $\mathrm{mg} / \mathrm{kg}$ & 2 & 44 & $=$ & 35 & 250 \\
\hline Columbia River & RM 440 & SS-2-2 & October 1, 1999 & Lead & $\mathrm{mg} / \mathrm{kg}$ & 2 & 44 & $=$ & 35 & 250 \\
\hline Columbia River & Pr Rapids Dam & B19PL1 & July 12, 2004 & Lead & $\mathrm{mg} / \mathrm{kg}$ & 1 & 44 & $x$ & 35 & 250 \\
\hline Columbia River & RM 440 & SS-1-2 & October 1, 1999 & Lead & $\mathrm{mg} / \mathrm{kg}$ & 2 & 45 & $=$ & 35 & 250 \\
\hline Columbia River & Pr Rapids Dam & BOYWC4 & August 7, 2000 & Lead & $\mathrm{mg} / \mathrm{kg}$ & 1 & 46 & $=$ & 35 & 250 \\
\hline Columbia River & Pr Rapids Dam & B0W3N5 & August 3, 1999 & Lead & $\mathrm{mg} / \mathrm{kg}$ & 1 & 48 & $=$ & 35 & 250 \\
\hline Columbia River & Pr Rapids Dam & B19PK9 & July 12, 2004 & Lead & $\mathrm{mg} / \mathrm{kg}$ & 1 & 49 & $x$ & 35 & 250 \\
\hline Columbia River & RM 440 & SS-2-1 & October 1, 1999 & Lead & $\mathrm{mg} / \mathrm{kg}$ & 2 & 49 & $=$ & 35 & 250 \\
\hline Columbia River & RM 440 & SS-3-1 & October 1, 1999 & Lead & $\mathrm{mg} / \mathrm{kg}$ & 2 & 49 & $=$ & 35 & 250 \\
\hline Columbia River & RM 440 & SS-3-2 & October 1, 1999 & Lead & $\mathrm{mg} / \mathrm{kg}$ & 2 & 49 & $=$ & 35 & 250 \\
\hline Columbia River & RM 440 & SS-4-2 & October 1, 1999 & Lead & $\mathrm{mg} / \mathrm{kg}$ & 2 & 49 & $=$ & 35 & 250 \\
\hline Columbia River & Pr Rapids Dam & B17704 & July 14, 2003 & Lead & $\mathrm{mg} / \mathrm{kg}$ & 1 & 50 & $=$ & 35 & 250 \\
\hline Columbia River & Pr Rapids Dam & BOW3N3 & August 3, 1999 & Lead & $\mathrm{mg} / \mathrm{kg}$ & 1 & 53 & $=$ & 35 & 250 \\
\hline Columbia River & Pr Rapids Dam & B17702 & July 14, 2003 & Lead & $\mathrm{mg} / \mathrm{kg}$ & 1 & 54 & $=$ & 35 & 250 \\
\hline Columbia River & Pr Rapids Dam & B12CM1 & July 19, 2001 & Lead & $\mathrm{mg} / \mathrm{kg}$ dry-wt & 1 & 56 & $=$ & 35 & 250 \\
\hline Columbia River & Pr Rapids Dam & B12CL9 & July 19, 2001 & Lead & $\mathrm{mg} / \mathrm{kg}$ dry-wt & 1 & 56 & $=$ & 35 & 250 \\
\hline Columbia River & RM 428 & SCA-3B & October 1, 1999 & Lead & $\mathrm{mg} / \mathrm{kg}$ & 2 & 57 & $=$ & 35 & 250 \\
\hline Columbia River & Vernita Area & B07NF5 & November 20, 1992 & Lead & $\mathrm{mg} / \mathrm{kg}$ & 1 & 58 & JS & 35 & 250 \\
\hline Columbia River & Pr Rapids Dam & BOW3F0 & August 3, 1999 & Lead & $\mathrm{mg} / \mathrm{kg}$ & 1 & 59 & $=$ & 35 & 250 \\
\hline Columbia River & RM 428 & SCA-3C & October 1, 1999 & Lead & $\mathrm{mg} / \mathrm{kg}$ & 2 & 60 & $=$ & 35 & 250 \\
\hline Columbia River & Pr Rapids Dam & B0W3F2 & August 3, 1999 & Lead & $\mathrm{mg} / \mathrm{kg}$ & 1 & 63 & $=$ & 35 & 250 \\
\hline Columbia River & RM 428 & SCA-3A & October 1, 1999 & Lead & $\mathrm{mg} / \mathrm{kg}$ & 2 & 73 & $=$ & 35 & 250 \\
\hline Columbia River & RM 398.5 & GOO-3C & October 1, 1999 & Lead & $\mathrm{mg} / \mathrm{kg}$ & 2 & 89 & $=$ & 35 & 250 \\
\hline Columbia River & RM 398.5 & GOO-4 & October 1, 1999 & Lead & $\mathrm{mg} / \mathrm{kg}$ & 2 & 89 & $=$ & 35 & 250 \\
\hline Columbia River & RM 398.5 & GOO-1 & October 1, 1999 & Lead & $\mathrm{mg} / \mathrm{kg}$ & 2 & 91 & $=$ & 35 & 250 \\
\hline Columbia River & RM 428 & SCA-4 & October 1, 1999 & Lead & $\mathrm{mg} / \mathrm{kg}$ & 2 & 97 & $=$ & 35 & 250 \\
\hline Columbia River & RM 417.5 & GHI-4 & October 1, 1999 & Lead & $\mathrm{mg} / \mathrm{kg}$ & 2 & 110 & $=$ & 35 & 250 \\
\hline
\end{tabular}




\section{Table A-8}

Summary of Surface Sediment Results From Segment 1 Exceeding Select Ecological or Human Health Benchmark Values

\begin{tabular}{|c|c|c|c|c|c|c|c|c|c|c|}
\hline Sample Area & Sample Site & Sample ID & Date Collected & Constituent Name & Units & $\begin{array}{c}\text { QA } \\
\text { Category }\end{array}$ & $\begin{array}{c}\text { Analytical } \\
\text { Value }\end{array}$ & Qualifier & $\begin{array}{c}\text { Ecological } \\
\text { Benchmark } \\
\text { Value }\end{array}$ & $\begin{array}{l}\text { Human Health } \\
\text { Benchmark } \\
\text { Value }\end{array}$ \\
\hline Columbia River & RM 398.5 & GOO-3A & October 1, 1999 & Lead & $\mathrm{mg} / \mathrm{kg}$ & 2 & 110 & $=$ & 35 & 250 \\
\hline Columbia River & RM 428 & SCA-5 & October 1, 1999 & Lead & $\mathrm{mg} / \mathrm{kg}$ & 2 & 110 & $=$ & 35 & 250 \\
\hline Columbia River & RM 417.5 & GHI-2 & October 1, 1999 & Lead & $\mathrm{mg} / \mathrm{kg}$ & 2 & 120 & $=$ & 35 & 250 \\
\hline Columbia River & RM 417.5 & GHI-3 & October 1, 1999 & Lead & $\mathrm{mg} / \mathrm{kg}$ & 2 & 120 & $=$ & 35 & 250 \\
\hline Columbia River & RM 398.5 & GOO-3B & October 1, 1999 & Lead & $\mathrm{mg} / \mathrm{kg}$ & 2 & 120 & $=$ & 35 & 250 \\
\hline Columbia River & RM 398.5 & GOO-2 & October 1, 1999 & Lead & $\mathrm{mg} / \mathrm{kg}$ & 2 & 140 & $=$ & 35 & 250 \\
\hline Columbia River & RM 354.4 & PEW-1sed & July 16,1992 & Manganese & $\mathrm{mg} / \mathrm{kg}$ & 2 & 660 & $=$ & 460 & 1,762 \\
\hline Columbia River & Pr Rapids Dam & $\mathrm{B} 0 \mathrm{~W} 3 \mathrm{~F} 2$ & August 3,1999 & Mercury & $\mathrm{mg} / \mathrm{kg}$ & 1 & 0.19 & $=$ & 0.18 & 23 \\
\hline Columbia River & Pr Rapids Dam & B17702 & July 14,2003 & Mercury & $\mathrm{mg} / \mathrm{kg}$ & 1 & 0.19 & $=$ & 0.18 & 23 \\
\hline Columbia River & Pr Rapids Dam & BOYWC4 & August 7, 2000 & Mercury & $\mathrm{mg} / \mathrm{kg}$ & 1 & 0.19 & $=$ & 0.18 & 23 \\
\hline Columbia River & Pr Rapids Dam & B19PK9 & July 12, 2004 & Mercury & $\mathrm{mg} / \mathrm{kg}$ & 1 & 0.20 & $c x$ & 0.18 & 23 \\
\hline Columbia River & RM 398.5 & GOO-2 & October 1, 1999 & Mercury & $\mathrm{mg} / \mathrm{kg}$ & 2 & 0.20 & $=$ & 0.18 & 23 \\
\hline Columbia River & Pr Rapids Dam & BOW3N5 & August 3, 1999 & Mercury & $\mathrm{mg} / \mathrm{kg}$ & 1 & 0.21 & $=$ & 0.18 & 23 \\
\hline Columbia River & RM 398.5 & GOO-3A & October 1, 1999 & Mercury & $\mathrm{mg} / \mathrm{kg}$ & 2 & 0.23 & $=$ & 0.18 & 23 \\
\hline Columbia River & Pr Rapids Dam & BOW3N3 & August 3, 1999 & Mercury & $\mathrm{mg} / \mathrm{kg}$ & 1 & 0.24 & $=$ & 0.18 & 23 \\
\hline Columbia River & RM 398.5 & GOO-3B & October 1, 1999 & Mercury & $\mathrm{mg} / \mathrm{kg}$ & 2 & 0.25 & $=$ & 0.18 & 23 \\
\hline Columbia River & RM 417.5 & $\mathrm{GHI}-2$ & October 1, 1999 & Mercury & $\mathrm{mg} / \mathrm{kg}$ & 2 & 0.26 & $=$ & 0.18 & 23 \\
\hline Columbia River & RM 428 & SCA-5 & October 1, 1999 & Mercury & $\mathrm{mg} / \mathrm{kg}$ & 2 & 0.30 & $=$ & 0.18 & 23 \\
\hline Columbia River & RM 417.5 & GHI-4 & October 1, 1999 & Mercury & $\mathrm{mg} / \mathrm{kg}$ & 2 & 0.31 & $=$ & 0.18 & 23 \\
\hline Columbia River & Pr Rapids Dam & B0W3D8 & August 3,1999 & Nickel & $\mathrm{mg} / \mathrm{kg}$ & 1 & 18 & $=$ & 18 & 1,564 \\
\hline Columbia River & Vernita BRG & B12RY7 & August 28, 2001 & Nickel & $\mathrm{mg} / \mathrm{kg}$ dry-wt & 1 & 22 & $=$ & 18 & 1,564 \\
\hline Columbia River & Pr Rapids Dam & B0W3D6 & August 3, 1999 & Nickel & $\mathrm{mg} / \mathrm{kg}$ & 1 & 25 & $=$ & 18 & 1,564 \\
\hline Columbia River & Pr Rapids Dam & 4444206 & June 1, 2004 & Nickel & $\mathrm{mg} / \mathrm{kg}$ & 1 & 27 & $=$ & 18 & 1,564 \\
\hline Columbia River & Pr Rapids Dam & B0W3F0 & August 3, 1999 & Nickel & $\mathrm{mg} / \mathrm{kg}$ & 1 & 35 & $=$ & 18 & 1,564 \\
\hline Columbia River & Pr Rapids Dam & B0W3F2 & August 3, 1999 & Nickel & $\mathrm{mg} / \mathrm{kg}$ & 1 & 35 & $=$ & 18 & 1,564 \\
\hline Columbia River & Pr Rapids Dam & B12CL9 & July 19, 2001 & Nickel & $\mathrm{mg} / \mathrm{kg}$ dry-wt & 1 & 38 & $=$ & 18 & 1,564 \\
\hline Columbia River & Pr Rapids Dam & B19PK9 & July 12,2004 & Nickel & $\mathrm{mg} / \mathrm{kg}$ & 1 & 41 & $x$ & 18 & 1,564 \\
\hline Columbia River & Pr Rapids Dam & B17704 & July 14,2003 & Nickel & $\mathrm{mg} / \mathrm{kg}$ & 1 & 42 & $=$ & 18 & 1,564 \\
\hline Columbia River & Pr Rapids Dam & B0W3N5 & August 3, 1999 & Nickel & $\mathrm{mg} / \mathrm{kg}$ & 1 & 42 & $=$ & 18 & 1,564 \\
\hline Columbia River & Pr Rapids Dam & BOYWC4 & August 7, 2000 & Nickel & $\mathrm{mg} / \mathrm{kg}$ & 1 & 44 & $=$ & 18 & 1,564 \\
\hline Columbia River & Pr Rapids Dam & BOW3N3 & August 3, 1999 & Nickel & $\mathrm{mg} / \mathrm{kg}$ & 1 & 46 & $=$ & 18 & 1,564 \\
\hline Columbia River & Pr Rapids Dam & B12CM1 & July 19, 2001 & Nickel & $\mathrm{mg} / \mathrm{kg}$ dry-wt & 1 & 46 & $=$ & 18 & 1,564 \\
\hline Columbia River & Pr Rapids Dam & B17702 & July 14,2003 & Nickel & $\mathrm{mg} / \mathrm{kg}$ & 1 & 47 & $=$ & 18 & 1,564 \\
\hline Columbia River & Pr Rapids Dam & B19PL1 & July 12,2004 & Nickel & $\mathrm{mg} / \mathrm{kg}$ & 1 & 47 & $\mathrm{x}$ & 18 & 1,564 \\
\hline Columbia River & Pr Rapids Dam & B1B7W4 & September 20, 2004 & Nickel & $\mathrm{mg} / \mathrm{kg}$ & 1 & 49 & $\mathrm{x}$ & 18 & 1,564 \\
\hline Columbia River & Pr Rapids Dam & BOYWC2 & August 7,2000 & Nickel & $\mathrm{mg} / \mathrm{kg}$ & 1 & 53 & $=$ & 18 & 1,564 \\
\hline Columbia River & RM 354.4 & PEW-1sed & July 16,1992 & Thorium & $\mathrm{mg} / \mathrm{kg}$ & 2 & 9.4 & $=$ & 1,300 & 1.3 \\
\hline Columbia River & Vernita Bridge & 27652 & August 28,2001 & Thorium-232 & $\mathrm{pCi} / \mathrm{g}$ dry-wt & 1 & 1.5 & $=$ & 1,300 & 1.3 \\
\hline Columbia River & Vernita Area & B07NF6 & November 20, 1992 & Thorium-232 & $\mathrm{pCi} / \mathrm{g}$ & 1 & 2.5 & $=$ & 1,300 & 1.3 \\
\hline 12989 & Columbia River & Pr Rapids & October 1,1992 & Uranium-234 & pCi/g dry & 1.61 & $=$ & --- & 0,001 & \\
\hline 12992 & Columbia River & Pr Rapids & October 1, 1992 & Uranium-234 & $\mathrm{pCi} / \mathrm{g}$ dry & 1.22 & $=$ & -- & 0,001 & \\
\hline 15827 & Columbia River & Pr Rapids & September 22, 1994 & Uranium-234 & $\mathrm{pCi} / \mathrm{g}$ dry & 1.14 & $=$ & -- & 0,001 & \\
\hline
\end{tabular}




\section{Table A-8}

Summary of Surface Sediment Results From Segment 1 Exceeding Select Ecological or Human Health Benchmark Values

\begin{tabular}{|c|c|c|c|c|c|c|c|c|c|c|}
\hline Sample Area & Sample Site & Sample ID & Date Collected & Constituent Name & Units & $\begin{array}{c}\text { QA } \\
\text { Category }\end{array}$ & $\begin{array}{c}\text { Analytical } \\
\text { Value }\end{array}$ & Qualifier & $\begin{array}{c}\text { Ecological } \\
\text { Benchmark } \\
\text { Value }\end{array}$ & $\begin{array}{c}\text { Human Health } \\
\text { Benchmark } \\
\text { Value }\end{array}$ \\
\hline 18296 & Columbia River & Pr Rapids & August 15, 1996 & Uranium-234 & pCi/g dry & 1.29 & $=$ & -- & 0,001 & \\
\hline 23522 & Columbia River & Pr Rapids & August 3, 1999 & Uranium-234 & $\mathrm{pCi} / \mathrm{g}$ dry & 1.21 & $=$ & -- & 0,001 & \\
\hline 26251 & Columbia River & Pr Rapids & July 19, 2001 & Uranium-234 & $\mathrm{pCi} / \mathrm{g}$ dry & 1.3 & $=$ & -- & 0,001 & \\
\hline 30872 & Columbia River & Pr Rapids & July 14,2003 & Uranium-234 & pCi/g dry & 1.32 & $=$ & -- & 0,001 & \\
\hline Columbia River & Pr Rapids Dam & 26251 & July 19,2001 & Uranium-238 & pCi/g dry-wt & 1 & 1.2 & $=$ & 2,490 & 1.1 \\
\hline Columbia River & Pr Rapids Dam & 30872 & July 14,2003 & Uranium-238 & $\mathrm{pCi} / \mathrm{g}$ dry-wt & 1 & 1.3 & $=$ & 2,490 & 1.1 \\
\hline Columbia River & Pr Rapids Dam & 18296 & August 15, 1996 & Uranium-238 & pCi/g dry-wt & 1 & 1.3 & $=$ & 2,490 & 1.1 \\
\hline Columbia River & Pr Rapids Dam & 12989 & October 1, 1992 & Uranium-238 & pCi/g dry-wt & 1 & 1.3 & $=$ & 2,490 & 1.1 \\
\hline Columbia River & Pr Rapids Dam & BOBRT8 & April 14, 1994 & Uranium-238 & $\mathrm{pCi} / \mathrm{g}$ & 1 & 1.4 & $=$ & 2,490 & 1.1 \\
\hline Columbia River & $\mathrm{RM} 354.4$ & $\mathrm{PEW}-1$ sed & July 16,1992 & Vanadium & $\mathrm{mg} / \mathrm{kg}$ & 2 & 130 & $=$ & -- & 78 \\
\hline Columbia River & Pr Rapids Dam & $\mathrm{BOCSH} 1$ & September 22, 1994 & Zinc- & $\mathrm{mg} / \mathrm{kg}$ & 1 & 130 & $=$ & 120 & 23,463 \\
\hline Columbia River & Vernita BRG & B12RY7 & August 28, 2001 & Zinc & $\mathrm{mg} / \mathrm{kg}$ dry-wt & 1 & 148 & $=$ & 120 & 23,463 \\
\hline Columbia River & Wanapum Dam & 4444208 & June 1, 2004 & Zinc & $\mathrm{mg} / \mathrm{kg}$ & 1 & 149 & $=$ & 120 & 23,463 \\
\hline Columbia River & Vernita Area & B07NF7 & November 20, 1992 & Zinc & $\mathrm{mg} / \mathrm{kg}$ & 1 & 151 & JE & 120 & 23,463 \\
\hline Columbia River & Pr Rapids Dam & 4444203 & June 1,2004 & Zinc & $\mathrm{mg} / \mathrm{kg}$ & 1 & 178 & $=$ & 120 & 23,463 \\
\hline Columbia River & RM 440 & SS-4-1 & October 1, 1999 & Zinc & $\mathrm{mg} / \mathrm{kg}$ & 2 & 180 & $=$ & 120 & 23,463 \\
\hline Columbia River & Pr Rapids Dam & BOCSHO & September 22, 1994 & Zinc & $\mathrm{mg} / \mathrm{kg}$ & 1 & 190 & $=$ & 120 & 23,463 \\
\hline Columbia River & RM 440 & CRE-3 & October 1, 1999 & Zinc & $\mathrm{mg} / \mathrm{kg}$ & 2 & 200 & $=$ & 120 & 23,463 \\
\hline Columbia River & Vernita Area & B07NF8 & November 20, 1992 & Zinc & $\mathrm{mg} / \mathrm{kg}$ & 1 & 205 & $\mathrm{JE}$ & 120 & 23,463 \\
\hline Columbia River & RM 440 & SS-1-1 & October 1, 1999 & Zinc & $\mathrm{mg} / \mathrm{kg}$ & 2 & 220 & $=$ & 120 & 23,463 \\
\hline Columbia River & Vernita Area & B07NF5 & November 20, 1992 & Zinc & $\mathrm{mg} / \mathrm{kg}$ & 1 & 226 & $\mathrm{JE}$ & 120 & 23,463 \\
\hline Columbia River & RM 440 & SS-2-2 & October 1, 1999 & Zinc & $\mathrm{mg} / \mathrm{kg}$ & 2 & 230 & $=$ & 120 & 23,463 \\
\hline Columbia River & RM 417.5 & GHI-5 & October 1, 1999 & Zinc & $\mathrm{mg} / \mathrm{kg}$ & 2 & 240 & $=$ & 120 & 23,463 \\
\hline Columbia River & Pr Rapids Dam & B0W3D8 & August 3, 1999 & Zinc & $\mathrm{mg} / \mathrm{kg}$ & 1 & 242 & $=$ & 120 & 23,463 \\
\hline Columbia River & Pr Rapids Dam & B0CSG9 & September 22, 1994 & Zinc & $\mathrm{mg} / \mathrm{kg}$ & 1 & 250 & $=$ & 120 & 23,463 \\
\hline Columbia River & RM 440 & SS-2-1 & October 1, 1999 & Zinc & $\mathrm{mg} / \mathrm{kg}$ & 2 & 250 & $=$ & 120 & 23,463 \\
\hline Columbia River & RM 440 & SS-3-1 & October 1, 1999 & Zinc & $\mathrm{mg} / \mathrm{kg}$ & 2 & 250 & $=$ & 120 & 23,463 \\
\hline Columbia River & RM 440 & SS-1-2 & October 1, 1999 & Zinc & $\mathrm{mg} / \mathrm{kg}$ & 2 & 260 & $=$ & 120 & 23,463 \\
\hline Columbia River & RM 440 & SS-4-2 & October 1, 1999 & Zinc & $\mathrm{mg} / \mathrm{kg}$ & 2 & 260 & $=$ & 120 & 23,463 \\
\hline Columbia River & RM 405.5 & HAN-5 & October 1, 1999 & Zinc & $\mathrm{mg} / \mathrm{kg}$ & 2 & 290 & $=$ & 120 & 23,463 \\
\hline Columbia River & RM 440 & SS-3-2 & October 1, 1999 & Zinc & $\mathrm{mg} / \mathrm{kg}$ & 2 & 290 & $=$ & 120 & 23,463 \\
\hline Columbia River & Pr Rapids Dam & BOCSH2 & September 22, 1994 & Zinc & $\mathrm{mg} / \mathrm{kg}$ & 1 & 300 & $=$ & 120 & 23,463 \\
\hline Columbia River & Pr Rapids Dam & 4444205 & June 1, 2004 & Zinc & $\mathrm{mg} / \mathrm{kg}$ & 1 & 346 & $=$ & 120 & 23,463 \\
\hline Columbia River & RM 428 & SCA-3B & October 1, 1999 & Zinc & $\mathrm{mg} / \mathrm{kg}$ & 2 & 350 & $=$ & 120 & 23,463 \\
\hline Columbia River & RM 428 & SCA-3C & October 1, 1999 & Zinc & $\mathrm{mg} / \mathrm{kg}$ & 2 & 350 & $=$ & 120 & 23,463 \\
\hline Columbia River & RM 428 & SCA-3A & October 1, 1999 & Zinc & $\mathrm{mg} / \mathrm{kg}$ & 2 & 380 & $=$ & 120 & 23,463 \\
\hline Columbia River & Pr Rapids Dam & 4444206 & June 1,2004 & Zinc & $\mathrm{mg} / \mathrm{kg}$ & 1 & 428 & $=$ & 120 & 23,463 \\
\hline Columbia River & Pr Rapids Dam & B0W3D6 & August 3, 1999 & Zinc & $\mathrm{mg} / \mathrm{kg}$ & 1 & 439 & $=$ & 120 & 23,463 \\
\hline Columbia River & Pr Rapids Dam & BOYWC2 & August 7,2000 & Zinc & $\mathrm{mg} / \mathrm{kg}$ & 1 & 443 & $=$ & 120 & 23,463 \\
\hline Columbia River & Pr Rapids Dam & B1B7W4 & September 20, 2004 & Zinc & $\mathrm{mg} / \mathrm{kg}$ & 1 & 487 & $x$ & 120 & 23,463 \\
\hline Columbia River & Pr Rapids Dam & B17702 & July 14,2003 & Zinc & $\mathrm{mg} / \mathrm{kg}$ & 1 & 495 & $=$ & 120 & 23,463 \\
\hline Columbia River & Pr Rapids Dam & BOW3F0 & August 3, 1999 & Zinc & $\mathrm{mg} / \mathrm{kg}$ & 1 & 503 & $=$ & 120 & 23,463 \\
\hline
\end{tabular}




\section{Table A-8}

Summary of Surface Sediment Results From Segment 1 Exceeding Select Ecological or Human Health Benchmark Values

\begin{tabular}{|c|c|c|c|c|c|c|c|c|c|c|}
\hline Sample Area & Sample Site & Sample ID & Date Collected & Constituent Name & Units & $\begin{array}{c}\text { QA } \\
\text { Category }\end{array}$ & $\begin{array}{c}\text { Analytical } \\
\text { Value }\end{array}$ & Qualifier & $\begin{array}{c}\text { Ecological } \\
\text { Benchmark } \\
\text { Value }\end{array}$ & $\begin{array}{l}\text { Human Health } \\
\text { Benchmark } \\
\text { Value }\end{array}$ \\
\hline Columbia River & Pr Rapids Dam & B12CL9 & July 19, 2001 & Zinc & $\mathrm{mg} / \mathrm{kg}$ dry-wt & 1 & 510 & $\mathrm{~J}$ & 120 & 23,463 \\
\hline Columbia River & Pr Rapids Dam & BOW3N3 & August 3, 1999 & Zinc & $\mathrm{mg} / \mathrm{kg}$ & 1 & 556 & $=$ & 120 & 23,463 \\
\hline Columbia River & RM 398.5 & GOO-3C & October 1, 1999 & Zinc & $\mathrm{mg} / \mathrm{kg}$ & 2 & 560 & $=$ & 120 & 23,463 \\
\hline Columbia River & Pr Rapids Dam & B0W3F2 & August 3, 1999 & Zinc & $\mathrm{mg} / \mathrm{kg}$ & 1 & 578 & $=$ & 120 & 23,463 \\
\hline Columbia River & Pr Rapids Dam & B19PL1 & July 12, 2004 & Zinc & $\mathrm{mg} / \mathrm{kg}$ & 1 & 582 & $x$ & 120 & 23,463 \\
\hline Columbia River & Pr Rapids Dam & B19PK9 & July 12, 2004 & Zinc & $\mathrm{mg} / \mathrm{kg}$ & 1 & 588 & $\mathrm{x}$ & 120 & 23,463 \\
\hline Columbia River & Pr Rapids Dam & B17704 & July 14, 2003 & Zinc & $\mathrm{mg} / \mathrm{kg}$ & 1 & 591 & $=$ & 120 & 23,463 \\
\hline Columbia River & RM 398.5 & GOO-4 & October 1, 1999 & Zinc & $\mathrm{mg} / \mathrm{kg}$ & 2 & 600 & $=$ & 120 & 23,463 \\
\hline Columbia River & RM 428 & SCA-4 & October 1, 1999 & Zinc & $\mathrm{mg} / \mathrm{kg}$ & 2 & 610 & $=$ & 120 & 23,463 \\
\hline Columbia River & Pr Rapids Dam & B12CM1 & July 19, 2001 & Zinc & $\mathrm{mg} / \mathrm{kg}$ dry-wt & 1 & 616 & $\mathrm{~J}$ & 120 & 23,463 \\
\hline Columbia River & Pr Rapids Dam & B0W3N5 & August 3, 1999 & Zinc & $\mathrm{mg} / \mathrm{kg}$ & 1 & 622 & $=$ & 120 & 23,463 \\
\hline Columbia River & RM 417.5 & GHI-4 & October 1, 1999 & Zinc & $\mathrm{mg} / \mathrm{kg}$ & 2 & 670 & $=$ & 120 & 23,463 \\
\hline Columbia River & RM 398.5 & GOO-1 & October 1, 1999 & Zinc & $\mathrm{mg} / \mathrm{kg}$ & 2 & 680 & $=$ & 120 & 23,463 \\
\hline Columbia River & RM 428 & SCA-5 & October 1, 1999 & Zinc & $\mathrm{mg} / \mathrm{kg}$ & 2 & 700 & $=$ & 120 & 23,463 \\
\hline Columbia River & Pr Rapids Dam & B0YWC4 & August 7, 2000 & Zinc & $\mathrm{mg} / \mathrm{kg}$ & 1 & 703 & $=$ & 120 & 23,463 \\
\hline Columbia River & RM 417.5 & GHI-2 & October 1, 1999 & Zinc & $\mathrm{mg} / \mathrm{kg}$ & 2 & 710 & $=$ & 120 & 23,463 \\
\hline Columbia River & RM 417.5 & GHI-3 & October 1, 1999 & Zinc & $\mathrm{mg} / \mathrm{kg}$ & 2 & 720 & $=$ & 120 & 23,463 \\
\hline Columbia River & RM 398.5 & GOO-2 & October 1, 1999 & Zinc & $\mathrm{mg} / \mathrm{kg}$ & 2 & 800 & $=$ & 120 & 23,463 \\
\hline Columbia River & RM 398.5 & GOO-3A & October 1, 1999 & Zinc & $\mathrm{mg} / \mathrm{kg}$ & 2 & 800 & $=$ & 120 & 23,463 \\
\hline Columbia River & RM 398.5 & GOO-3B & October 1, 1999 & Zinc & $\mathrm{mg} / \mathrm{kg}$ & 2 & 850 & $=$ & 120 & 23,463 \\
\hline
\end{tabular}


Table A-9

Summary Statistics for All Categories of Surface Water Samples Collected from Segment 1 of the Columbia River

\begin{tabular}{|c|c|c|c|c|c|c|c|c|c|c|}
\hline $\begin{array}{c}\text { Constituent } \\
\text { Class }\end{array}$ & Constituent Name & Units & $\begin{array}{l}\text { Number } \\
\text { Analyzed }\end{array}$ & $\begin{array}{l}\text { Number } \\
\text { Detected }\end{array}$ & $\begin{array}{c}\text { Frequency of } \\
\text { Detection }\end{array}$ & $\begin{array}{l}\text { Minimum } \\
\text { Nondetect }\end{array}$ & $\begin{array}{l}\text { Maximum } \\
\text { Nondetect }\end{array}$ & $\begin{array}{l}\text { Minimum } \\
\text { Detect }\end{array}$ & $\begin{array}{l}\text { Maximum } \\
\text { Detect }\end{array}$ & $\begin{array}{c}\text { Average } \\
\text { Concentration }\end{array}$ \\
\hline$\overline{C O N V}$ & Alkalinity & $\mathrm{mg} / \mathrm{L}$ & 135 & 135 & $100 \%$ & $\overline{--}$ & $\overline{--}$ & 41 & 77 & 54 \\
\hline CONV & Ammonia & $\mathrm{mg} / \mathrm{L}$ & 76 & 10 & $13 \%$ & 0.010 & 0.040 & 0.010 & 0.034 & 0.0074 \\
\hline CONV & Chloride & $\mathrm{mg} / \mathrm{L}$ & 83 & 83 & $100 \%$ & -- & -- & 0.72 & 3.3 & 1.0 \\
\hline CONV & Cyanide & $\mathrm{mg} / \mathrm{L}$ & 8 & 0 & $0 \%$ & 0.0016 & 0.0016 & -- & -- & 8.00E-04 \\
\hline CONV & Fluoride & $\mathrm{mg} / \mathrm{L}$ & 83 & 78 & $94 \%$ & 0.0060 & 0.040 & 0.020 & 0.20 & 0.093 \\
\hline CONV & Nitrogen in ammonia & $\mathrm{mg} / \mathrm{L}$ & 2 & 2 & $100 \%$ & -- & -- & 0.080 & 0.11 & 0.095 \\
\hline CONV & Nitrogen, Nitrate (as N) & $\mathrm{mg} / \mathrm{L}$ & 94 & 94 & $100 \%$ & -- & -- & 0.050 & 0.22 & 0.11 \\
\hline CONV & Nitrogen, Nitrate-Nitrite & $\mathrm{mg} / \mathrm{L}$ & 224 & 157 & $70 \%$ & 0.010 & 0.10 & 0.037 & 0.30 & 0.10 \\
\hline CONV & Nitrogen, Nitrite & $\mathrm{mg} / \mathrm{L}$ & 83 & 0 & $0 \%$ & 0.0020 & 0.011 & -- & -- & 0.0034 \\
\hline CONV & Orthophosphate & $\mathrm{mg} / \mathrm{L}$ & 76 & 29 & $38 \%$ & 0.0030 & 0.020 & 0.0031 & 0.013 & 0.0034 \\
\hline CONV & $\mathrm{pH}$ & pH Units & 65 & 65 & $100 \%$ & -- & -- & 6.9 & 8.5 & 8.1 \\
\hline CONV & Specific Conductance & umhos/cm & 67 & 67 & $100 \%$ & -- & -- & 107 & 205 & 134 \\
\hline CONV & Sulfate & $\mathrm{mg} / \mathrm{L}$ & 83 & 83 & $100 \%$ & -- & -- & 6.8 & 25 & 9.1 \\
\hline CONV & Temperature & Deg C & 4 & 4 & $100 \%$ & -- & -- & 7.2 & 7.3 & 7.3 \\
\hline CONV & Total Persulfate Nitrogen & $\mathrm{mg} / \mathrm{L}$ & 75 & 75 & $100 \%$ & -- & -- & 0.083 & 0.38 & 0.17 \\
\hline CONV & Total suspended solids & $\mathrm{mg} / \mathrm{L}$ & 75 & 68 & $91 \%$ & 1.0 & 1.0 & 1.0 & 11 & 2.2 \\
\hline METAL & Aluminum & $\mathrm{mg} / \mathrm{L}$ & 11 & 11 & $100 \%$ & -- & -- & 4.65E-04 & 0.033 & 0.010 \\
\hline METAL & Antimony & $\mathrm{mg} / \mathrm{L}$ & 160 & 159 & $99 \%$ & 0.020 & 0.020 & 1.24E-04 & 2.73E-04 & 2.55E-04 \\
\hline METAL & Arsenic & $\mathrm{mg} / \mathrm{L}$ & 173 & 173 & $100 \%$ & -- & -- & $2.60 \mathrm{E}-04$ & 0.0017 & 5.97E-04 \\
\hline METAL & Barium & $\mathrm{mg} / \mathrm{L}$ & 5 & 5 & $100 \%$ & -- & -- & 0.028 & 0.029 & 0.029 \\
\hline METAL & Beryllium & $\mathrm{mg} / \mathrm{L}$ & 141 & 13 & $9 \%$ & 8.00E-06 & 0.0050 & 1.05E-05 & $7.20 \mathrm{E}-05$ & 3.27E-05 \\
\hline METAL & Cadmium & $\mathrm{mg} / \mathrm{L}$ & 159 & 131 & $82 \%$ & 1.00E-05 & 4.00E-05 & 8.82E-06 & 7.84E-05 & 2.14E-05 \\
\hline METAL & Calcium & $\mathrm{mg} / \mathrm{L}$ & 2 & 2 & $100 \%$ & -- & -- & 21 & 29 & 25 \\
\hline METAL & Chromium & $\mathrm{mg} / \mathrm{L}$ & 162 & 115 & $71 \%$ & 1.65E-05 & 0.10 & 1.94E-05 & 0.0022 & $4.85 \mathrm{E}-04$ \\
\hline METAL & Cobalt & $\mathrm{mg} / \mathrm{L}$ & 1 & 0 & $0 \%$ & 0.0030 & 0.0030 & -- & -- & 0.0015 \\
\hline METAL & Copper & $\mathrm{mg} / \mathrm{L}$ & 162 & 161 & $99 \%$ & 10 & 10 & 3.38E-04 & 0.0042 & 0.032 \\
\hline METAL & lodine & $\mathrm{mg} / \mathrm{L}$ & 19 & 19 & $100 \%$ & -- & -- & 4.31E-04 & 9.62E-04 & $6.15 E-04$ \\
\hline METAL & Iron & $\mathrm{mg} / \mathrm{L}$ & 2 & 1 & $50 \%$ & 0.010 & 0.010 & 0.015 & 0.015 & 0.010 \\
\hline METAL & Lead & $\mathrm{mg} / \mathrm{L}$ & 161 & 153 & $95 \%$ & 1.10E-05 & 1.10E-05 & $3.47 \mathrm{E}-06$ & 3.42E-04 & 6.02E-05 \\
\hline METAL & Magnesium & $\mathrm{mg} / \mathrm{L}$ & 2 & 2 & $100 \%$ & -- & -- & 5.2 & 5.2 & 5.2 \\
\hline METAL & Manganese & $\mathrm{mg} / \mathrm{L}$ & 12 & 11 & $92 \%$ & 0.0030 & 0.0030 & $8.52 \mathrm{E}-04$ & 0.0051 & 0.0020 \\
\hline METAL & Mercury & $\mathrm{mg} / \mathrm{L}$ & 70 & 67 & $96 \%$ & 2.00E-06 & 2.00E-06 & 2.89E-07 & 3.32E-06 & $6.74 \mathrm{E}-07$ \\
\hline METAL & Nickel & $\mathrm{mg} / \mathrm{L}$ & 161 & 161 & $100 \%$ & -- & -- & 3.92E-05 & 0.0020 & 4.93E-04 \\
\hline METAL & Phosphorus & $\mathrm{mg} / \mathrm{L}$ & 213 & 132 & $62 \%$ & 0.0040 & 0.010 & 0.0025 & 0.030 & 0.012 \\
\hline METAL & Potassium & $\mathrm{mg} / \mathrm{L}$ & 2 & 2 & $100 \%$ & -- & -- & 0.90 & 1.3 & 1.1 \\
\hline METAL & Selenium & $\mathrm{mg} / \mathrm{L}$ & 160 & 68 & $43 \%$ & 8.00E-05 & 5.50E-04 & 7.52E-05 & 5.65E-04 & 1.93E-04 \\
\hline METAL & Silica & $\mathrm{mg} / \mathrm{L}$ & 1 & 1 & $100 \%$ & -- & -- & 9.9 & 9.9 & 9.9 \\
\hline METAL & Silver & $\mathrm{mg} / \mathrm{L}$ & 162 & 84 & $52 \%$ & 1.20E-06 & $5.00 \mathrm{E}-05$ & 8.33E-07 & 1.73E-05 & $5.79 \mathrm{E}-06$ \\
\hline METAL & Sodium & $\mathrm{mg} / \mathrm{L}$ & 2 & 2 & $100 \%$ & -- & -- & 2.5 & 4.4 & 3.5 \\
\hline METAL & Thallium & $\mathrm{mg} / \mathrm{L}$ & 152 & 139 & $91 \%$ & 3.00E-06 & $5.00 \mathrm{E}-04$ & 8.55E-06 & 3.90E-05 & 2.27E-05 \\
\hline METAL & Thorium & $\mathrm{mg} / \mathrm{L}$ & 6 & 1 & $17 \%$ & $4.23 \mathrm{E}-05$ & 4.23E-05 & 7.30E-05 & 7.30E-05 & 2.98E-05 \\
\hline METAL & Uranium & $\mathrm{mg} / \mathrm{L}$ & 6 & 6 & $100 \%$ & -- & -- & $4.75 \mathrm{E}-04$ & $6.01 \mathrm{E}-04$ & 5.42E-04 \\
\hline
\end{tabular}


Table A-9

Summary Statistics for All Categories of Surface Water Samples Collected from Segment 1 of the Columbia River

\begin{tabular}{|c|c|c|c|c|c|c|c|c|c|c|}
\hline $\begin{array}{l}\text { Constituent } \\
\text { Class }\end{array}$ & Constituent Name & Units & $\begin{array}{l}\text { Number } \\
\text { Analyzed }\end{array}$ & $\begin{array}{l}\text { Number } \\
\text { Detected }\end{array}$ & $\begin{array}{l}\text { Frequency of } \\
\text { Detection }\end{array}$ & $\begin{array}{l}\text { Minimum } \\
\text { Nondetect }\end{array}$ & $\begin{array}{l}\text { Maximum } \\
\text { Nondetect }\end{array}$ & $\begin{array}{l}\text { Minimum } \\
\text { Detect }\end{array}$ & $\begin{array}{l}\text { Maximum } \\
\text { Detect }\end{array}$ & $\begin{array}{c}\text { Average } \\
\text { Concentration }\end{array}$ \\
\hline$\overline{\text { METAL }}$ & Vanadium & $\mathrm{mg} / \mathrm{L}$ & 1 & $\overline{0}$ & $0 \%$ & 0.0030 & 0.0030 & $\overline{--}$ & -- & 0.0015 \\
\hline METAL & Zinc & $\mathrm{mg} / \mathrm{L}$ & 161 & 161 & $100 \%$ & -- & -- & $5.36 \mathrm{E}-04$ & 0.0051 & 0.0019 \\
\hline PEST & Alachlor & $\mathrm{mg} / \mathrm{L}$ & 1 & 0 & $0 \%$ & 7.30E-05 & 7.30E-05 & -- & -- & 3.65E-05 \\
\hline PEST & Ametryn & $\mathrm{mg} / \mathrm{L}$ & 1 & 0 & $0 \%$ & $2.00 \mathrm{E}-05$ & 2.00E-05 & -- & -- & $1.00 \mathrm{E}-05$ \\
\hline PEST & Atraton & $\mathrm{mg} / \mathrm{L}$ & 1 & 0 & $0 \%$ & $3.00 \mathrm{E}-05$ & $3.00 \mathrm{E}-05$ & -- & -- & 1.50E-05 \\
\hline PEST & Atrazine & $\mathrm{mg} / \mathrm{L}$ & 1 & 0 & $0 \%$ & 2.00E-05 & 2.00E-05 & -- & -- & 1.00E-05 \\
\hline PEST & Benefin (Benfluralin) & $\mathrm{mg} / \mathrm{L}$ & 1 & 0 & $0 \%$ & $3.00 \mathrm{E}-05$ & $3.00 \mathrm{E}-05$ & -- & -- & 1.50E-05 \\
\hline PEST & Bromacil (ACN) & $\mathrm{mg} / \mathrm{L}$ & 1 & 0 & $0 \%$ & 8.10E-05 & 8.10E-05 & -- & -- & 4.05E-05 \\
\hline PEST & Butachlor & $\mathrm{mg} / \mathrm{L}$ & 1 & 0 & $0 \%$ & $1.20 \mathrm{E}-04$ & $1.20 \mathrm{E}-04$ & -- & -- & $6.00 \mathrm{E}-05$ \\
\hline PEST & Butylate & $\mathrm{mg} / \mathrm{L}$ & 1 & 0 & $0 \%$ & 4.10E-05 & 4.10E-05 & -- & -- & 2.05E-05 \\
\hline PEST & Carboxin & $\mathrm{mg} / \mathrm{L}$ & 1 & 0 & $0 \%$ & $1.20 \mathrm{E}-04$ & $1.20 \mathrm{E}-04$ & -- & -- & $6.00 \mathrm{E}-05$ \\
\hline PEST & Chlorothalonil & $\mathrm{mg} / \mathrm{L}$ & 1 & 0 & $0 \%$ & $4.90 \mathrm{E}-05$ & $4.90 \mathrm{E}-05$ & -- & -- & 2.45E-05 \\
\hline PEST & Chlorpropham & $\mathrm{mg} / \mathrm{L}$ & 1 & 0 & $0 \%$ & $8.10 \mathrm{E}-05$ & 8.10E-05 & -- & -- & 4.05E-05 \\
\hline PEST & Cyanazine & $\mathrm{mg} / \mathrm{L}$ & 1 & 0 & $0 \%$ & $3.00 \mathrm{E}-05$ & $3.00 \mathrm{E}-05$ & -- & -- & $1.50 \mathrm{E}-05$ \\
\hline PEST & Cycloate & $\mathrm{mg} / \mathrm{L}$ & 1 & 0 & $0 \%$ & 4.10E-05 & 4.10E-05 & -- & -- & 2.05E-05 \\
\hline PEST & Diallate & $\mathrm{mg} / \mathrm{L}$ & 1 & 0 & $0 \%$ & $1.40 \mathrm{E}-04$ & 1.40E-04 & -- & -- & 7.00E-05 \\
\hline PEST & Dichlobenil & $\mathrm{mg} / \mathrm{L}$ & 1 & 0 & $0 \%$ & 4.10E-05 & 4.10E-05 & -- & -- & 2.05E-05 \\
\hline PEST & Diphenamid & $\mathrm{mg} / \mathrm{L}$ & 1 & 0 & $0 \%$ & $6.10 \mathrm{E}-05$ & $6.10 \mathrm{E}-05$ & -- & -- & 3.05E-05 \\
\hline PEST & Diuron & $\mathrm{mg} / \mathrm{L}$ & 1 & 0 & $0 \%$ & 1.20E-04 & 1.20E-04 & -- & -- & 6.00E-05 \\
\hline PEST & Eptam & $\mathrm{mg} / \mathrm{L}$ & 1 & 0 & $0 \%$ & 4.10E-05 & 4.10E-05 & -- & -- & $2.05 E-05$ \\
\hline PEST & Ethalfluralin (Sonalan) & $\mathrm{mg} / \mathrm{L}$ & 1 & 0 & $0 \%$ & 3.00E-05 & 3.00E-05 & -- & -- & $1.50 \mathrm{E}-05$ \\
\hline PEST & Fenarimol & $\mathrm{mg} / \mathrm{L}$ & 1 & 0 & $0 \%$ & $6.10 \mathrm{E}-05$ & $6.10 \mathrm{E}-05$ & -- & -- & 3.05E-05 \\
\hline PEST & Fluridone & $\mathrm{mg} / \mathrm{L}$ & 1 & 0 & $0 \%$ & $1.20 \mathrm{E}-04$ & 1.20E-04 & -- & -- & $6.00 \mathrm{E}-05$ \\
\hline PEST & Hexazinone & $\mathrm{mg} / \mathrm{L}$ & 1 & 0 & $0 \%$ & 3.00E-05 & 3.00E-05 & -- & -- & $1.50 \mathrm{E}-05$ \\
\hline PEST & Metalaxyl & $\mathrm{mg} / \mathrm{L}$ & 1 & 0 & $0 \%$ & $1.20 \mathrm{E}-04$ & $1.20 \mathrm{E}-04$ & -- & -- & $6.00 \mathrm{E}-05$ \\
\hline PEST & Metolachlor & $\mathrm{mg} / \mathrm{L}$ & 1 & 0 & $0 \%$ & 8.10E-05 & 8.10E-05 & -- & -- & 4.05E-05 \\
\hline PEST & Metribuzin & $\mathrm{mg} / \mathrm{L}$ & 1 & 0 & $0 \%$ & $2.00 \mathrm{E}-05$ & $2.00 \mathrm{E}-05$ & -- & -- & $1.00 \mathrm{E}-05$ \\
\hline PEST & MGK264 & $\mathrm{mg} / \mathrm{L}$ & 1 & 0 & $0 \%$ & $1.60 \mathrm{E}-04$ & 1.60E-04 & -- & -- & 8.00E-05 \\
\hline PEST & Molinate & $\mathrm{mg} / \mathrm{L}$ & 1 & 0 & $0 \%$ & 4.10E-05 & 4.10E-05 & -- & -- & $2.05 E-05$ \\
\hline PEST & Napropamide & $\mathrm{mg} / \mathrm{L}$ & 1 & 0 & $0 \%$ & $6.10 \mathrm{E}-05$ & $6.10 \mathrm{E}-05$ & -- & -- & 3.05E-05 \\
\hline PEST & Norflurazon & $\mathrm{mg} / \mathrm{L}$ & 1 & 0 & $0 \%$ & 4.10E-05 & 4.10E-05 & -- & -- & 2.05E-05 \\
\hline PEST & Oxyfluorfen & $\mathrm{mg} / \mathrm{L}$ & 1 & 0 & $0 \%$ & 8.10E-05 & 8.10E-05 & -- & -- & 4.05E-05 \\
\hline PEST & Pebulate & $\mathrm{mg} / \mathrm{L}$ & 1 & 0 & $0 \%$ & 4.10E-05 & 4.10E-05 & -- & -- & 2.05E-05 \\
\hline PEST & Pendimethalin & $\mathrm{mg} / \mathrm{L}$ & 1 & 0 & $0 \%$ & 3.00E-05 & 3.00E-05 & -- & -- & $1.50 \mathrm{E}-05$ \\
\hline PEST & Pramitol (Prometon) & $\mathrm{mg} / \mathrm{L}$ & 1 & 0 & $0 \%$ & $2.00 \mathrm{E}-05$ & 2.00E-05 & -- & -- & $1.00 \mathrm{E}-05$ \\
\hline PEST & Profluralin & $\mathrm{mg} / \mathrm{L}$ & 1 & 0 & $0 \%$ & 4.90E-05 & $4.90 \mathrm{E}-05$ & -- & -- & 2.45E-05 \\
\hline PEST & Prometryn & $\mathrm{mg} / \mathrm{L}$ & 1 & 0 & $0 \%$ & $2.00 \mathrm{E}-05$ & $2.00 \mathrm{E}-05$ & -- & -- & 1.00E-05 \\
\hline PEST & Pronamide & $\mathrm{mg} / \mathrm{L}$ & 1 & 0 & $0 \%$ & 8.10E-05 & 8.10E-05 & -- & -- & 4.05E-05 \\
\hline PEST & Propachlor (Ramrod) & $\mathrm{mg} / \mathrm{L}$ & 1 & 0 & $0 \%$ & 4.90E-05 & 4.90E-05 & -- & -- & 2.45E-05 \\
\hline PEST & Propazine & $\mathrm{mg} / \mathrm{L}$ & 1 & 0 & $0 \%$ & $2.00 \mathrm{E}-05$ & 2.00E-05 & -- & -- & $1.00 \mathrm{E}-05$ \\
\hline PEST & Simazine & $\mathrm{mg} / \mathrm{L}$ & 1 & 0 & $0 \%$ & 2.00E-05 & 2.00E-05 & -- & -- & 1.00E-05 \\
\hline PEST & Tebuthiuron & $\mathrm{mg} / \mathrm{L}$ & 1 & 0 & $0 \%$ & $3.00 \mathrm{E}-05$ & 3.00E-05 & -- & -- & 1.50E-05 \\
\hline
\end{tabular}


Table A-9

Summary Statistics for All Categories of Surface Water Samples Collected from Segment 1 of the Columbia River

\begin{tabular}{|c|c|c|c|c|c|c|c|c|c|c|}
\hline $\begin{array}{c}\text { Constituent } \\
\text { Class }\end{array}$ & Constituent Name & Units & $\begin{array}{l}\text { Number } \\
\text { Analyzed }\end{array}$ & $\begin{array}{l}\text { Number } \\
\text { Detected }\end{array}$ & $\begin{array}{c}\text { Frequency of } \\
\text { Detection }\end{array}$ & $\begin{array}{l}\text { Minimum } \\
\text { Nondetect }\end{array}$ & $\begin{array}{l}\text { Maximum } \\
\text { Nondetect }\end{array}$ & $\begin{array}{l}\text { Minimum } \\
\text { Detect }\end{array}$ & $\begin{array}{l}\text { Maximum } \\
\text { Detect }\end{array}$ & $\begin{array}{c}\text { Average } \\
\text { Concentration }\end{array}$ \\
\hline PEST & Terbacil & $\overline{\mathrm{mg} / \mathrm{L}}$ & 1 & 0 & $0 \%$ & $6.10 \mathrm{E}-05$ & $6.10 \mathrm{E}-05$ & $\overline{--}$ & $\overline{--}$ & 3.05E-05 \\
\hline PEST & Terbutryn & $\mathrm{mg} / \mathrm{L}$ & 1 & 0 & $0 \%$ & $2.00 \mathrm{E}-05$ & $2.00 \mathrm{E}-05$ & -- & -- & $1.00 \mathrm{E}-05$ \\
\hline PEST & Triadimefon & $\mathrm{mg} / \mathrm{L}$ & 1 & 0 & $0 \%$ & 5.30E-05 & $5.30 \mathrm{E}-05$ & -- & -- & $2.65 \mathrm{E}-05$ \\
\hline PEST & Triallate & $\mathrm{mg} / \mathrm{L}$ & 1 & 0 & $0 \%$ & $6.10 \mathrm{E}-05$ & $6.10 \mathrm{E}-05$ & -- & -- & 3.05E-05 \\
\hline PEST & Trifluralin (Treflan) & $\mathrm{mg} / \mathrm{L}$ & 1 & 0 & $0 \%$ & 3.00E-05 & 3.00E-05 & -- & -- & $1.50 E-05$ \\
\hline PEST & Vernolate & $\mathrm{mg} / \mathrm{L}$ & 1 & 0 & $0 \%$ & 4.10E-05 & 4.10E-05 & -- & -- & 2.05E-05 \\
\hline RAD & Antimony-125 & $\mathrm{pCi} / \mathrm{L}$ & 63 & 0 & $0 \%$ & $9.78 \mathrm{E}-06$ & 2.0 & -- & -- & 0.032 \\
\hline RAD & Beryllium-7 & $\mathrm{pCi} / \mathrm{L}$ & 134 & 42 & $31 \%$ & $8.15 E-05$ & 12 & 0.013 & 0.096 & 0.13 \\
\hline RAD & Carbon-14 & $\mathrm{pCi} / \mathrm{L}$ & 6 & 1 & $17 \%$ & 1.5 & 8.0 & 8.8 & 8.8 & 3.0 \\
\hline RAD & Cesium-134 & $\mathrm{pCi} / \mathrm{L}$ & 75 & 0 & $0 \%$ & 3.30E-05 & 1.8 & -- & -- & 0.028 \\
\hline RAD & Cesium-137 & $\mathrm{pCi} / \mathrm{L}$ & 115 & 4 & $3 \%$ & 2.26E-05 & 2.7 & 8.97E-04 & 1.0 & 0.048 \\
\hline RAD & Cobalt- 60 & $\mathrm{pCi} / \mathrm{L}$ & 110 & 2 & $2 \%$ & $9.77 \mathrm{E}-06$ & 1.3 & 0.40 & 1.0 & 0.030 \\
\hline RAD & Europium-152 & $\mathrm{pCi} / \mathrm{L}$ & 18 & 0 & $0 \%$ & 2.83E-05 & 0.0027 & -- & -- & 4.09E-04 \\
\hline RAD & Europium-154 & $\mathrm{pCi} / \mathrm{L}$ & 68 & 0 & $0 \%$ & 1.47E-04 & 0.0091 & -- & -- & 0.0012 \\
\hline RAD & Europium-155 & $\mathrm{pCi} / \mathrm{L}$ & 87 & 0 & $0 \%$ & 4.33E-05 & 3.5 & -- & -- & 0.033 \\
\hline RAD & Gross alpha & $\mathrm{pCi} / \mathrm{L}$ & 80 & 11 & $14 \%$ & 0.025 & 1.2 & 0.48 & 5.6 & 0.46 \\
\hline RAD & Gross beta & $\mathrm{pCi} / \mathrm{L}$ & 69 & 11 & $16 \%$ & 0.0033 & 3.6 & 1.2 & 8.4 & 0.91 \\
\hline RAD & lodine-129 & $\mathrm{pCi} / \mathrm{L}$ & 23 & 23 & $100 \%$ & -- & -- & $6.00 \mathrm{E}-07$ & $2.12 \mathrm{E}-05$ & 7.00E-06 \\
\hline RAD & Lead-212 & $\mathrm{pCi} / \mathrm{L}$ & 1 & 1 & $100 \%$ & -- & -- & 0.011 & 0.011 & 0.011 \\
\hline RAD & Plutonium-238 & $\mathrm{pCi} / \mathrm{L}$ & 24 & 3 & $13 \%$ & 4.63E-07 & $9.05 E-05$ & 9.36E-06 & $1.54 \mathrm{E}-04$ & 1.49E-05 \\
\hline RAD & Plutonium-239/240 & $\mathrm{pCi} / \mathrm{L}$ & 39 & 19 & $49 \%$ & 1.66E-06 & $5.48 \mathrm{E}-05$ & $1.31 \mathrm{E}-05$ & $1.79 \mathrm{E}-04$ & $2.25 \mathrm{E}-05$ \\
\hline RAD & Potassium-40 & $\mathrm{pCi} / \mathrm{L}$ & 137 & 122 & $89 \%$ & 0.0023 & 29 & 0.030 & 60 & 2.5 \\
\hline RAD & Ruthenium-106 & $\mathrm{pCi} / \mathrm{L}$ & 63 & 0 & $0 \%$ & 5.58E-05 & 11 & -- & -- & 0.17 \\
\hline RAD & Strontium-90 & $\mathrm{pCi} / \mathrm{L}$ & 174 & 157 & $90 \%$ & 0.025 & 0.70 & 0.042 & 2.0 & 0.085 \\
\hline RAD & Technetium-99 & $\mathrm{pCi} / \mathrm{L}$ & 41 & 0 & $0 \%$ & 0.0015 & 0.80 & - & - & 0.11 \\
\hline RAD & Thorium-228 & $\mathrm{pCi} / \mathrm{L}$ & 1 & 1 & $100 \%$ & -- & -- & 0.023 & 0.023 & 0.023 \\
\hline RAD & Thorium-230 & $\mathrm{pCi} / \mathrm{L}$ & 2 & 0 & $0 \%$ & 0.0059 & 0.012 & -- & -- & 0.0044 \\
\hline RAD & Thorium-232 & $\mathrm{pCi} / \mathrm{L}$ & 2 & 0 & $0 \%$ & 0.0039 & 0.010 & -- & -- & 0.0036 \\
\hline RAD & Tritium & $\mathrm{pCi} / \mathrm{L}$ & 173 & 169 & $98 \%$ & 1.0 & 34 & 14 & 130 & 35 \\
\hline RAD & Uranium & $\mathrm{pCi} / \mathrm{L}$ & 12 & 11 & $92 \%$ & 0.040 & 0.040 & 0.37 & 0.67 & 0.43 \\
\hline RAD & Uranium-234 & $\mathrm{pCi} / \mathrm{L}$ & 185 & 184 & $99 \%$ & 0.040 & 0.040 & 0.11 & 0.42 & 0.23 \\
\hline RAD & Uranium-235 & $\mathrm{pCi} / \mathrm{L}$ & 154 & 28 & $18 \%$ & $9.14 \mathrm{E}-05$ & 0.020 & 0.0049 & 0.030 & 0.0048 \\
\hline RAD & Uranium-236 & $\mathrm{pCi} / \mathrm{L}$ & 1 & 1 & $100 \%$ & -- & -- & 0.0025 & 0.0025 & 0.0025 \\
\hline RAD & Uranium-238 & $\mathrm{pCi} / \mathrm{L}$ & 185 & 184 & $99 \%$ & 0.010 & 0.010 & 0.065 & 0.38 & 0.18 \\
\hline SVOC & 1,4-Dichlorobenzene & $\mathrm{mg} / \mathrm{L}$ & 26 & 3 & $12 \%$ & $1.10 \mathrm{E}-04$ & $2.50 \mathrm{E}-04$ & $1.20 \mathrm{E}-04$ & 4.40E-04 & $1.11 \mathrm{E}-04$ \\
\hline VOC & 1,1,1-Trichloroethane & $\mathrm{mg} / \mathrm{L}$ & 26 & 0 & $0 \%$ & $7.00 \mathrm{E}-05$ & $3.10 \mathrm{E}-04$ & -- & -- & 1.13E-04 \\
\hline VOC & 1,1,2-Trichloroethane & $\mathrm{mg} / \mathrm{L}$ & 26 & 0 & $0 \%$ & 5.00E-05 & $3.10 \mathrm{E}-04$ & -- & -- & $1.20 \mathrm{E}-04$ \\
\hline VOC & 1,1-Dichloroethane & $\mathrm{mg} / \mathrm{L}$ & 26 & 0 & $0 \%$ & $1.20 \mathrm{E}-04$ & $2.50 \mathrm{E}-04$ & -- & -- & $1.02 \mathrm{E}-04$ \\
\hline VOC & 1,1-Dichloroethene & $\mathrm{mg} / \mathrm{L}$ & 6 & 0 & $0 \%$ & 2.30E-04 & 2.30E-04 & -- & -- & $1.15 \mathrm{E}-04$ \\
\hline VOC & 1,2-Dichloroethane & $\mathrm{mg} / \mathrm{L}$ & 26 & 0 & $0 \%$ & 8.00E-05 & $2.70 \mathrm{E}-04$ & -- & -- & $1.00 \mathrm{E}-04$ \\
\hline VOC & 2-Butanone & $\mathrm{mg} / \mathrm{L}$ & 26 & 0 & $0 \%$ & $1.00 \mathrm{E}-04$ & 7.00E-04 & -- & -- & $2.13 \mathrm{E}-04$ \\
\hline VOC & 4-Methyl-2-Pentanone & $\mathrm{mg} / \mathrm{L}$ & 26 & 0 & $0 \%$ & 1.90E-04 & 0.0013 & -- & -- & $3.22 \mathrm{E}-04$ \\
\hline
\end{tabular}


Table A-9

Summary Statistics for All Categories of Surface Water Samples Collected from Segment 1 of the Columbia River

\begin{tabular}{|c|c|c|c|c|c|c|c|c|c|c|}
\hline $\begin{array}{c}\text { Constituent } \\
\text { Class }\end{array}$ & Constituent Name & Units & $\begin{array}{l}\text { Number } \\
\text { Analyzed }\end{array}$ & $\begin{array}{l}\text { Number } \\
\text { Detected }\end{array}$ & $\begin{array}{l}\text { Frequency of } \\
\text { Detection }\end{array}$ & $\begin{array}{l}\text { Minimum } \\
\text { Nondetect }\end{array}$ & $\begin{array}{l}\text { Maximum } \\
\text { Nondetect }\end{array}$ & $\begin{array}{c}\text { Minimum } \\
\text { Detect }\end{array}$ & $\begin{array}{l}\text { Maximum } \\
\text { Detect }\end{array}$ & $\begin{array}{c}\text { Average } \\
\text { Concentration }\end{array}$ \\
\hline$\overline{\mathrm{VOC}}$ & Acetone & $\mathrm{mg} / \mathrm{L}$ & 26 & 11 & $42 \%$ & $3.00 \mathrm{E}-04$ & 0.0040 & $6.20 \mathrm{E}-04$ & 0.0038 & 0.0014 \\
\hline VOC & Benzene & $\mathrm{mg} / \mathrm{L}$ & 26 & 0 & $0 \%$ & $7.00 \mathrm{E}-05$ & 2.30E-04 & -- & -- & $9.35 \mathrm{E}-05$ \\
\hline VOC & Carbon disulfide & $\mathrm{mg} / \mathrm{L}$ & 26 & 2 & $8 \%$ & $6.00 \mathrm{E}-05$ & 4.30E-04 & 3.30E-04 & 6.10E-04 & 1.64E-04 \\
\hline VOC & Carbon tetrachloride & $\mathrm{mg} / \mathrm{L}$ & 26 & 0 & $0 \%$ & 1.00E-04 & 3.30E-04 & -- & -- & 1.18E-04 \\
\hline VOC & Chlorobenzene & $\mathrm{mg} / \mathrm{L}$ & 2 & 0 & $0 \%$ & $2.80 \mathrm{E}-04$ & 2.80E-04 & -- & -- & 1.40E-04 \\
\hline VOC & Chloroform & $\mathrm{mg} / \mathrm{L}$ & 26 & 0 & $0 \%$ & $7.00 \mathrm{E}-05$ & 2.30E-04 & -- & -- & 8.96E-05 \\
\hline VOC & cis-1,2-dichloroethene & $\mathrm{mg} / \mathrm{L}$ & 26 & 0 & $0 \%$ & $6.00 \mathrm{E}-05$ & $2.40 \mathrm{E}-04$ & -- & -- & 8.62E-05 \\
\hline VOC & Ethylbenzene & $\mathrm{mg} / \mathrm{L}$ & 8 & 0 & $0 \%$ & $1.40 \mathrm{E}-04$ & $2.40 \mathrm{E}-04$ & -- & -- & $9.50 \mathrm{E}-05$ \\
\hline VOC & Ethylcyanide & $\mathrm{mg} / \mathrm{L}$ & 26 & 0 & $0 \%$ & 0.0013 & 0.0026 & -- & -- & 9.85E-04 \\
\hline VOC & Methylene chloride & $\mathrm{mg} / \mathrm{L}$ & 26 & 6 & $23 \%$ & 1.70E-04 & 3.70E-04 & 3.60E-04 & 5.80E-04 & 2.02E-04 \\
\hline VOC & n-Butanol & $\mathrm{mg} / \mathrm{L}$ & 22 & 0 & $0 \%$ & 0.0046 & 0.0066 & -- & -- & 0.0026 \\
\hline VOC & Tetrachloroethene & $\mathrm{mg} / \mathrm{L}$ & 26 & 0 & $0 \%$ & $8.00 \mathrm{E}-05$ & 5.70E-04 & -- & -- & $1.76 \mathrm{E}-04$ \\
\hline VOC & Tetrahydrofuran & $\mathrm{mg} / \mathrm{L}$ & 26 & 0 & $0 \%$ & 0.0015 & 0.0023 & -- & -- & $9.35 \mathrm{E}-04$ \\
\hline VOC & Toluene & $\mathrm{mg} / \mathrm{L}$ & 26 & 8 & $31 \%$ & $1.20 \mathrm{E}-04$ & 3.30E-04 & $2.50 \mathrm{E}-04$ & 5.50E-04 & 2.03E-04 \\
\hline VOC & trans-1,2-Dichloroethene & $\mathrm{mg} / \mathrm{L}$ & 26 & 0 & $0 \%$ & $9.00 \mathrm{E}-05$ & $2.60 \mathrm{E}-04$ & -- & -- & $1.04 \mathrm{E}-04$ \\
\hline VOC & Trichloroethene & $\mathrm{mg} / \mathrm{L}$ & 26 & 0 & $0 \%$ & $9.00 \mathrm{E}-05$ & $2.90 \mathrm{E}-04$ & -- & -- & $9.96 \mathrm{E}-05$ \\
\hline VOC & Vinyl chloride & $\mathrm{mg} / \mathrm{L}$ & 26 & 0 & $0 \%$ & 8.00E-05 & $3.20 \mathrm{E}-04$ & -- & -- & 1.13E-04 \\
\hline VOC & Xylenes (total) & $\mathrm{mg} / \mathrm{L}$ & 26 & 3 & $12 \%$ & $2.80 \mathrm{E}-04$ & $6.60 \mathrm{E}-04$ & $3.20 \mathrm{E}-04$ & $5.20 \mathrm{E}-04$ & 2.93E-04 \\
\hline
\end{tabular}

CONV - Conventional parameter

PEST - Pesticide

RAD - Radionuclide

SVOC - Semivolatile Organic Compound

VOC - Volatile Organic Compound 
Table A-10

Summary Statistics for Category 1 Surface Water Samples Collected from the Methow River (Segment 1)

\begin{tabular}{|c|c|c|c|c|c|c|c|c|c|c|}
\hline $\begin{array}{l}\text { Constituent } \\
\text { Class }\end{array}$ & Constituent Name & Units & $\begin{array}{l}\text { Number } \\
\text { Analyzed }\end{array}$ & $\begin{array}{l}\text { Number } \\
\text { Detected }\end{array}$ & $\begin{array}{l}\text { Frequency of } \\
\text { Detection }\end{array}$ & $\begin{array}{l}\text { Minimum } \\
\text { Nondetect }\end{array}$ & $\begin{array}{l}\text { Maximum } \\
\text { Nondetect }\end{array}$ & $\begin{array}{c}\text { Minimum } \\
\text { Detect }\end{array}$ & $\begin{array}{l}\text { Maximum } \\
\text { Detect }\end{array}$ & $\begin{array}{c}\text { Average } \\
\text { Concentration }\end{array}$ \\
\hline$\overline{\text { CONV }}$ & Ammonia & $\overline{\mathrm{mg} / \mathrm{L}}$ & 1 & 0 & $0 \%$ & 0.040 & 0.040 & -- & -- & 0.020 \\
\hline CONV & Chloride & $\mathrm{mg} / \mathrm{L}$ & 1 & 1 & $100 \%$ & -- & -- & 3.3 & 3.3 & 3.3 \\
\hline CONV & Fluoride & $\mathrm{mg} / \mathrm{L}$ & 1 & 1 & $100 \%$ & -- & -- & 0.20 & 0.20 & 0.20 \\
\hline CONV & Nitrogen in ammonia & $\mathrm{mg} / \mathrm{L}$ & 2 & 2 & $100 \%$ & -- & -- & 0.080 & 0.11 & 0.095 \\
\hline CONV & Nitrogen, Nitrate-Nitrite & $\mathrm{mg} / \mathrm{L}$ & 1 & 1 & $100 \%$ & -- & -- & 0.22 & 0.22 & 0.22 \\
\hline CONV & Nitrogen, Nitrite & $\mathrm{mg} / \mathrm{L}$ & 1 & 0 & $0 \%$ & 0.0060 & 0.0060 & -- & - & 0.0030 \\
\hline CONV & Orthophosphate & $\mathrm{mg} / \mathrm{L}$ & 1 & 0 & $0 \%$ & 0.020 & 0.020 & -- & -- & 0.010 \\
\hline CONV & $\mathrm{pH}$ & $\mathrm{pH}$ Units & 1 & 1 & $100 \%$ & -- & -- & 8.1 & 8.1 & 8.1 \\
\hline CONV & Specific Conductance & umhos/cm & 1 & 1 & $100 \%$ & -- & -- & 205 & 205 & 205 \\
\hline CONV & Sulfate & $\mathrm{mg} / \mathrm{L}$ & 1 & 1 & $100 \%$ & -- & -- & 9.7 & 9.7 & 9.7 \\
\hline METAL & Calcium & $\mathrm{mg} / \mathrm{L}$ & 1 & 1 & $100 \%$ & -- & -- & 29 & 29 & 29 \\
\hline METAL & Iron & $\mathrm{mg} / \mathrm{L}$ & 1 & 0 & $0 \%$ & 0.010 & 0.010 & - & - & 0.0050 \\
\hline METAL & Magnesium & $\mathrm{mg} / \mathrm{L}$ & 1 & 1 & $100 \%$ & -- & -- & 5.2 & 5.2 & 5.2 \\
\hline METAL & Manganese & $\mathrm{mg} / \mathrm{L}$ & 1 & 0 & $0 \%$ & 0.0030 & 0.0030 & -- & -- & 0.0015 \\
\hline METAL & Phosphorus & $\mathrm{mg} / \mathrm{L}$ & 2 & 0 & $0 \%$ & 0.0040 & 0.0060 & -- & -- & 0.0025 \\
\hline METAL & Potassium & $\mathrm{mg} / \mathrm{L}$ & 1 & 1 & $100 \%$ & -- & -- & 0.90 & 0.90 & 0.90 \\
\hline METAL & Silica & $\mathrm{mg} / \mathrm{L}$ & 1 & 1 & $100 \%$ & -- & -- & 9.9 & 9.9 & 9.9 \\
\hline METAL & Sodium & $\mathrm{mg} / \mathrm{L}$ & 1 & 1 & $100 \%$ & -- & -- & 4.4 & 4.4 & 4.4 \\
\hline
\end{tabular}

CONV - Conventional parameter 
Table A-11

Comparison of All Categories of Surface Water Concentrations Collected from Segment 1 of the Columbia River to Ecological and Human Health Benchmark Values

\begin{tabular}{|c|c|c|c|c|c|c|c|c|c|c|c|c|c|c|c|c|}
\hline $\begin{array}{l}\text { Constituent } \\
\text { Class }\end{array}$ & Constituent Name & Units & $\begin{array}{l}\text { Number } \\
\text { Analyzed }\end{array}$ & $\begin{array}{l}\text { Number } \\
\text { Detected }\end{array}$ & $\begin{array}{l}\text { Frequency } \\
\text { of Detection }\end{array}$ & $\underset{\text { Detect }}{\text { Minimum }}$ & $\begin{array}{c}\text { Maximum } \\
\text { Detect }\end{array}$ & $\begin{array}{c}\text { Average } \\
\text { Concentration }\end{array}$ & $\begin{array}{l}\text { Benchmark } \\
\text { Value }\end{array}$ & $\begin{array}{l}\text { Is Max > Eco } \\
\text { Benchmark? }\end{array}$ & $\begin{array}{c}\text { Factor of } \\
\text { Exceedance }\end{array}$ & $\begin{array}{c}\text { Number of } \\
\text { Exceedances }\end{array}$ & $\begin{array}{l}\text { Human Health } \\
\text { Benchmark Value }\end{array}$ & $\begin{array}{l}\text { Is Max }>\mathrm{HH} \\
\text { Benchmark? }\end{array}$ & $\begin{array}{c}\text { Factor of } \\
\text { Exceedance }\end{array}$ & $\begin{array}{c}\text { Number of } \\
\text { Exceedances }\end{array}$ \\
\hline CONV & Alkalinity & $\mathrm{mg} / \mathrm{L}$ & 135 & 135 & $100 \%$ & 41 & 77 & 54 & -- & -- & -- & -- & -- & -- & $\overline{--}$ & -- \\
\hline CONV & Ammonia & $\mathrm{mg} / \mathrm{L}$ & 76 & 10 & $13 \%$ & 0.010 & 0.034 & 0.0074 & -- & -- & -- & -- & -- & -- & -- & -- \\
\hline CONV & Chloride & $\mathrm{mg} / \mathrm{L}$ & 83 & 83 & $100 \%$ & 0.72 & 3.3 & 1.0 & 230 & No & 0.014 & 0 & -- & -- & -- & -- \\
\hline CONV & Fluoride & $\mathrm{mg} / \mathrm{L}$ & 83 & 78 & $94 \%$ & 0.020 & 0.20 & 0.093 & -- & -- & - & -- & -- & -- & -- & -- \\
\hline CONV & Nitrogen in ammonia & $\mathrm{mg} / \mathrm{L}$ & 2 & 2 & $100 \%$ & 0.080 & 0.11 & 0.095 & -- & -- & -- & -- & -- & -- & -- & -- \\
\hline CONV & Nitrogen, Nitrate (as N) & $\mathrm{mg} / \mathrm{L}$ & 94 & 94 & $100 \%$ & 0.050 & 0.22 & 0.11 & -- & -- & $\ldots$ & -- & -- & -- & -- & $\ldots$ \\
\hline CONV & Nitrogen, Nitrate-Nitrite & $\mathrm{mg} / \mathrm{L}$ & 224 & 157 & $70 \%$ & 0.037 & 0.30 & 0.10 & -- & -- & -- & -- & -- & -- & $\ldots$ & -- \\
\hline CONV & Orthophosphate & $\mathrm{mg} / \mathrm{L}$ & 76 & 29 & $38 \%$ & 0.0031 & 0.013 & 0.0034 & -- & -- & -- & -- & -- & -- & -- & -- \\
\hline CONV & $\mathrm{pH}$ & pH Units & 65 & 65 & $100 \%$ & 6.9 & 8.5 & 8.1 & -- & -- & -- & -- & -- & -- & -- & -- \\
\hline CONV & Specific Conductance & umhos $/ \mathrm{cm}$ & 67 & 67 & $100 \%$ & 107 & 205 & 134 & -- & -- & -- & -- & -- & -- & -- & -- \\
\hline CONV & Sulfate & $\mathrm{mg} / \mathrm{L}$ & 83 & 83 & $100 \%$ & 6.8 & 25 & 9.1 & -- & -- & -- & -- & -- & -- & -- & -- \\
\hline CONV & Temperature & Deg C & 4 & 4 & $100 \%$ & 7.2 & 7.3 & 7.3 & -- & -- & -- & -- & -- & -- & -- & -- \\
\hline CONV & Total Persulfate Nitrogen & $\mathrm{mg} / \mathrm{L}$ & 75 & 75 & $100 \%$ & 0.083 & 0.38 & 0.17 & -- & -- & -- & -- & -- & -- & -- & -- \\
\hline $\begin{array}{l}\text { CONV } \\
\text { METAL }\end{array}$ & Total suspended solids & $\mathrm{mg} / \mathrm{L}$ & 75 & 68 & $91 \%$ & 1.0 & 11 & 2.2 & -- & -- & -- & -- & -- & -- & -- & -- \\
\hline & Aluminum & $\mathrm{mg} / \mathrm{L}$ & 11 & 11 & $100 \%$ & $4.65 \mathrm{E}-04$ & 0.033 & 0.010 & 0.087 & No & 0.38 & 0 & -- & -- & -- & -- \\
\hline $\begin{array}{l}\text { METAL } \\
\text { MEALL }\end{array}$ & Antimony & $\mathrm{mg} / \mathrm{L}$ & 160 & 159 & $99 \%$ & $1.24 \mathrm{E}-04$ & $2.73 E-04$ & $2.55 \mathrm{E}-04$ & 1.6 & No & $1.71 \mathrm{E}-04$ & 0 & 0.0056 & No & 0.049 & 0 \\
\hline $\begin{array}{l}\text { MEEAL } \\
\text { METAL }\end{array}$ & $\begin{array}{l}\text { Arsenic } \\
\text { Barium }\end{array}$ & $\mathrm{mg} / \mathrm{L}$ & 173 & 173 & $100 \%$ & 2.60E-04 & 0.0017 & 5.97E-04 & 0.15 & No & 0.012 & 0 & 1.80E-05 & Yes & 96 & 173 \\
\hline METAL & Beryllium & $\mathrm{mg} / \mathrm{L}$ & $\begin{array}{c}5 \\
141\end{array}$ & $\begin{array}{c}5 \\
13\end{array}$ & $\begin{array}{l}100 \% \\
9 \%\end{array}$ & $\begin{array}{c}0.028 \\
1.05 E-05\end{array}$ & $\begin{array}{l}0.029 \\
7.02-05\end{array}$ & $\begin{array}{l}0.029 \\
3275.05\end{array}$ & 0.0040 & Yes & 7.4 & 5 & 1.0 & No & 0.029 & \\
\hline METAL & Cadmium & $\mathrm{mg} / \mathrm{L}$ & 159 & 131 & $82 \%$ & $8.82 \mathrm{E}-06$ & $\begin{array}{l}1.20 \mathrm{E}-05 \\
7.84 \mathrm{E}-05\end{array}$ & $\begin{array}{l}3.27 \mathrm{E}-05 \\
2.14 \mathrm{E}-05\end{array}$ & $\begin{array}{l}0.0053 \\
2.50 E-04\end{array}$ & $\begin{array}{l}\text { No } \\
\text { No }\end{array}$ & 0.014 & 0 & -- & $\ddot{-}$ & $-\overline{-}$ & -- \\
\hline METAL & Calcium & $\mathrm{mg} / \mathrm{L}$ & 2 & 2 & $100 \%$ & 21 & 29 & 25 & 116 & No & 0.25 & 0 & -- & $\ldots$ & $\ldots$ & -- \\
\hline METAL & Chromium & $\mathrm{mg} / \mathrm{L}$ & 162 & 115 & $71 \%$ & $1.94 \mathrm{E}-05$ & 0.0022 & $4.85 \mathrm{E}-04$ & 0.010 & No & 0.22 & 0 & -- & -- & -- & -- \\
\hline METAL & Copper & $\mathrm{mg} / \mathrm{L}$ & 162 & 161 & $99 \%$ & $3.38 \mathrm{E}-04$ & 0.0042 & 0.032 & 0.0060 & No & 0.71 & 0 & -- & -- & -- & -- \\
\hline METAL & Iodine & $\mathrm{mg} / \mathrm{L}$ & 19 & 19 & $100 \%$ & $4.31 \mathrm{E}-04$ & $9.62 \mathrm{E}-04$ & $6.15 \mathrm{E}-04$ & 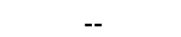 & 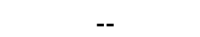 & 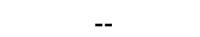 & 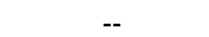 & 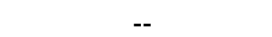 & .- & 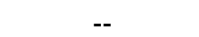 & -- \\
\hline METAL & Iron & $\mathrm{mg} / \mathrm{L}$ & 2 & 1 & $50 \%$ & 0.015 & 0.015 & 0.010 & 1.0 & No & 0.015 & 0 & 0.30 & No & 0.050 & \\
\hline METAL & Lead & $\mathrm{mg} / \mathrm{L}$ & 161 & 153 & $95 \%$ & $3.47 \mathrm{E}-06$ & 3.42E-04 & $6.02 \mathrm{E}-05$ & $8.67 \mathrm{E}-04$ & No & 0.39 & 0 & -- &.- & -- & -- \\
\hline METAL & Magnesium & $\mathrm{mg} / \mathrm{L}$ & 2 & 2 & $100 \%$ & 5.2 & 5.2 & 5.2 & 82 & No & 0.064 & 0 & -- & -- & -- & -- \\
\hline METAL & Manganese & $\mathrm{mg} / \mathrm{L}$ & 12 & 11 & $92 \%$ & $8.52 \mathrm{E}-04$ & 0.0051 & 0.0020 & 0.12 & No & 0.042 & 0 & 0.050 & No & 0.10 & \\
\hline METAL & Mercury & $\mathrm{mg} / \mathrm{L}$ & 70 & 67 & $96 \%$ & 2.89E-07 & $3.32 \mathrm{E}-06$ & $6.74 \mathrm{E}-07$ & $1.20 \mathrm{E}-05$ & No & 0.28 & 0 & -- & -- & -- & -- \\
\hline METAL & Nickel & $\mathrm{mg} / \mathrm{L}$ & 161 & 161 & $100 \%$ & $3.92 \mathrm{E}-05$ & 0.0020 & 4.93E-04 & 0.028 & No & 0.074 & 0 & 0.61 & No & 0.0033 & \\
\hline METAL & Phosphorus & $\mathrm{mg} / \mathrm{L}$ & 213 & 132 & $62 \%$ & 0.0025 & 0.030 & 0.012 & -- & -- & -- & -- & -- & -- & -- & -- \\
\hline METAL & Potassium & $\mathrm{mg} / \mathrm{L}$ & 2 & 2 & $100 \%$ & 0.90 & 1.3 & 1.1 & 53 & No & 0.025 & 0 & -- & -- & -- & -- \\
\hline METAL & Selenium & $\mathrm{mg} / \mathrm{L}$ & 160 & 68 & $43 \%$ & $7.52 \mathrm{E}-05$ & $5.65 \mathrm{E}-04$ & 1.93E-04 & 0.0050 & No & 0.11 & 0 & 0.17 & No & 0.0033 & \\
\hline METAL & Silica & $\mathrm{mg} / \mathrm{L}$ & 1 & 1 & $100 \%$ & 9.9 & 9.9 & 9.9 & -- & -- & -- & -- & -- & -- & -- & -- \\
\hline METAL & Silver & $\mathrm{mg} / \mathrm{L}$ & 162 & 84 & $52 \%$ & $8.33 \mathrm{E}-07$ & $1.73 E-05$ & 5.79E-06 & $1.20 \mathrm{E}-04$ & No & 0.14 & 0 & -- & -- & -- & -- \\
\hline METAL & Sodium & $\mathrm{mg} / \mathrm{L}$ & 2 & 2 & $100 \%$ & 2.5 & 4.4 & 3.5 & 680 & No & 0.0065 & 0 & -- & -- & -- & -- \\
\hline METAL & Thallium & $\mathrm{mg} / \mathrm{L}$ & 152 & 139 & $91 \%$ & $8.55 \mathrm{E}-06$ & $3.90 \mathrm{E}-05$ & 2.27E-05 & 0.040 & No & $9.74 \mathrm{E}-04$ & 0 & 2.40E-04 & No & 0.16 & \\
\hline METAL & Thorium & $\mathrm{mg} / \mathrm{L}$ & 6 & 1 & $17 \%$ & $7.30 \mathrm{E}-05$ & $7.30 \mathrm{E}-05$ & $2.98 \mathrm{E}-05$ & & -- & & - & & 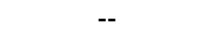 & & -- \\
\hline METAL & Uranium & $\mathrm{mg} / \mathrm{L}$ & 6 & 6 & $100 \%$ & 4.75E-04 & $6.01 E-04$ & 5.42E-04 & 0.0026 & No & 0.23 & 0 & 0.03 & No & 0.020 & -- \\
\hline METAL & Zinc & $\mathrm{mg} / \mathrm{L}$ & 161 & 161 & $100 \%$ & $5.36 \mathrm{E}-04$ & 0.0051 & 0.0019 & 0.055 & No & 0.092 & 0 & 7.4 & No & 6.84E-04 & \\
\hline RAD & Beryllium-7 & $\mathrm{pCi} / \mathrm{L}$ & 134 & 42 & $31 \%$ & 0.013 & 0.096 & 0.13 & -- & -- & -- & -- & -- & -- & -- & -- \\
\hline $\begin{array}{l}\text { RAD } \\
\text { RAD }\end{array}$ & Carbon-14 & pCi/L & 6 & 1 & $17 \%$ & $\begin{array}{c}8.8 \\
0.8\end{array}$ & 8.8 & 3.0 & -- & -- & -- & -- & 2,000 & No & 0.0044 & \\
\hline $\begin{array}{l}\text { RAD } \\
\text { RAD }\end{array}$ & $\begin{array}{l}\text { Cesium-137 } \\
\text { Cobalt-60 }\end{array}$ & $\mathrm{pCi} / \mathrm{L}$ & 115 & 4 & $3 \%$ & 8.97E-04 & $\begin{array}{l}1.0 \\
10\end{array}$ & 0.048 & 43 & No & 0.023 & 0 & 200 & No & 0.0050 & \\
\hline $\begin{array}{l}\text { RAD } \\
\text { RAD }\end{array}$ & $\begin{array}{l}\text { Cobalt-60 } \\
\text { Gross aluha }\end{array}$ & $\mathrm{pCi/L}$ & $\begin{array}{l}110 \\
80\end{array}$ & $\stackrel{2}{11}$ & $\begin{array}{l}2 \% \\
14 \%\end{array}$ & $\begin{array}{l}0.40 \\
0.48\end{array}$ & $\begin{array}{l}1.0 \\
56\end{array}$ & $\begin{array}{l}0.030 \\
0.46\end{array}$ & 3,760 & No & $2.66 \mathrm{E}-04$ & 0 & 100 & No & 0.010 & \\
\hline RAD & $\begin{array}{l}\text { Gross aipna } \\
\text { Gross beta }\end{array}$ & $\begin{array}{l}\mathrm{p} P \mathrm{pl} / \mathrm{L} \\
\mathrm{pCi} / \mathrm{s}\end{array}$ & $\begin{array}{l}80 \\
69\end{array}$ & 11 & $16 \%$ & $\begin{array}{l}0.48 \\
1.2\end{array}$ & $\begin{array}{l}5.6 \\
8.4\end{array}$ & $\begin{array}{l}0.46 \\
0.91\end{array}$ & -- & $\overline{--}$ & -- & -- & -- & -- & -- & -- \\
\hline RAD & Iodine-129 & pCill & 23 & 23 & $100 \%$ & $6.00 \mathrm{E}-07$ & $2.12 \mathrm{E}-05$ & 7.00E-06 & .- & -- & -- & -- & -.- & -- & -- & .. \\
\hline RAD & Lead-212 & $\mathrm{pCi} / \mathrm{L}$ & 1 & 1 & $100 \%$ & 0.011 & 0.011 & 0.011 & -- & -- & -- & -- & -- & -- & -- & .. \\
\hline RAD & Plutonium-238 & $\mathrm{pCi} / \mathrm{L}$ & 24 & 3 & $13 \%$ & $9.36 \mathrm{E}-06$ & $1.54 \mathrm{E}-04$ & 1.49E-05 & -- & - & - & -- & 15 & No & $1.03 E-05$ & 0 \\
\hline RAD & Plutonium-239/240 & $\mathrm{pCi} / \mathrm{L}$ & 39 & 19 & $49 \%$ & $1.31 \mathrm{E}-05$ & $1.79 \mathrm{E}-04$ & $2.25 \mathrm{E}-05$ & -- & -- & - & -- & 15 & No & $1.19 \mathrm{E}-05$ & 0 \\
\hline RAD & $\begin{array}{l}\text { Potassium- } 40 \\
\text { Po }\end{array}$ & $\mathrm{pCi} / \mathrm{L}$ & 137 & 122 & $89 \%$ & 0.030 & 60 & 2.5 & -- & -- & -- & - & IJ & No & I.IVL-OU & - \\
\hline RAD & Strontium-90 & $\mathrm{pCi} / \mathrm{L}$ & 174 & 157 & $90 \%$ & 0.042 & 2.0 & 0.085 & 278 & No & 0.0072 & 0 & 8.0 & No & 0.25 & 0 \\
\hline RAD & Thorium-228 & $\mathrm{pCi} / \mathrm{L}$ & 1 & 1 & $100 \%$ & 0.023 & 0.023 & 0.023 & -- & -- & -- & - & -- & - & -- & - \\
\hline RAD & Tritium & $\mathrm{pCi} / \mathrm{L}$ & 173 & 169 & $98 \%$ & 14 & 130 & 35 & $2.65 \mathrm{E}+08$ & No & $4.91 \mathrm{E}-07$ & 0 & 20,000 & No & 0.0065 & 0 \\
\hline RAD & Uranium & $\mathrm{pCi} / \mathrm{L}$ & 12 & 11 & $92 \%$ & 0.37 & 0.67 & 0.43 & 223 & No & 0.0030 & 0 & -- & -- & -- & -- \\
\hline RAD & Uranium-234 & $\mathrm{pCi} / \mathrm{L}$ & 185 & 184 & $99 \%$ & 0.11 & 0.42 & 0.23 & 202 & No & 0.0021 & 0 & -- & -- & -- & -- \\
\hline
\end{tabular}


Table A-11

Comparison of All Categories of Surface Water Concentrations Collected from Segment 1 of the Columbia River to Ecological and Human Health Benchmark Values

\begin{tabular}{|c|c|c|c|c|c|c|c|c|c|c|c|c|c|c|c|c|}
\hline $\begin{array}{l}\text { Constituent } \\
\text { Class }\end{array}$ & Constituent Name & Units & $\begin{array}{l}\text { Number } \\
\text { Analyzed }\end{array}$ & $\begin{array}{l}\text { Number } \\
\text { Detected }\end{array}$ & $\begin{array}{c}\text { Frequency } \\
\text { of Detection }\end{array}$ & $\begin{array}{c}\text { Minimum } \\
\text { Detect }\end{array}$ & $\begin{array}{c}\text { Maximum } \\
\text { Detect }\end{array}$ & $\begin{array}{c}\text { Average } \\
\text { Concentration }\end{array}$ & $\begin{array}{c}\text { Ecological } \\
\text { Benchmark } \\
\text { Value }\end{array}$ & $\begin{array}{l}\text { Is Max > Eco } \\
\text { Benchmark? }\end{array}$ & $\begin{array}{c}\text { Factor of } \\
\text { Exceedance }\end{array}$ & $\begin{array}{c}\text { Number of } \\
\text { Exceedances }\end{array}$ & $\begin{array}{c}\text { Human Health } \\
\text { Benchmark Value }\end{array}$ & $\begin{array}{l}\text { Is Max }>\mathrm{HH} \\
\text { Benchmark? }\end{array}$ & $\begin{array}{c}\text { Factor of } \\
\text { Exceedance }\end{array}$ & $\begin{array}{c}\text { Number of } \\
\text { Exceedances }\end{array}$ \\
\hline RAD & Uranium-235 & $\mathrm{pCi/L}$ & 154 & 28 & $18 \%$ & 0.0049 & 0.030 & 0.0048 & 217 & No & $1.38 \mathrm{E}-04$ & 0 & $-\overline{-}$ & -- & -- & -- \\
\hline RAD & Uranium-236 & $\mathrm{pC} / \mathrm{L}$ & 1 & 1 & $100 \%$ & 0.0025 & 0.0025 & 0.0025 & -- & -- & -- & -- & -- & -- & -- & -- \\
\hline RAD & Uranium-238 & $\mathrm{pC} / \mathrm{L}$ & 185 & 184 & $99 \%$ & 0.065 & 0.38 & 0.18 & 223 & No & 0.0017 & 0 & 30 & No & 0.013 & 0 \\
\hline SVOC & 1,4-Dichlorobenzene & $\mathrm{mg} / \mathrm{L}$ & 26 & 3 & $12 \%$ & $1.20 \mathrm{E}-04$ & $4.40 \mathrm{E}-04$ & $1.11 \mathrm{E}-04$ & 0.015 & No & 0.029 & 0 & 0.063 & No & 0.0070 & 0 \\
\hline VOC & Acetone & $\mathrm{mg} / \mathrm{L}$ & 26 & 11 & $42 \%$ & $6.20 \mathrm{E}-04$ & 0.0038 & 0.0014 & -- & -- & -- & -- & -- & -- & -- & -- \\
\hline VOC & Carbon disulfide & $\mathrm{mg} / \mathrm{L}$ & 26 & 2 & $8 \%$ & 3.30E-04 & $6.10 \mathrm{E}-04$ & 1.64E-04 & -- & -- & -- & -- & -- & -- & -- & -- \\
\hline VOC & Methylene chloride & $\mathrm{mg} / \mathrm{L}$ & 26 & 6 & $23 \%$ & 3.60E-04 & $5.80 \mathrm{E}-04$ & 2.02E-04 & -- & -- & -- & - & -- & -- & -- & -- \\
\hline $\begin{array}{l}\text { VOC } \\
\text { vOc }\end{array}$ & $\begin{array}{l}\text { Toluene } \\
\text { Xylenes (total) }\end{array}$ & $\mathrm{mg} / \mathrm{L}$ & 26 & $\begin{array}{l}8 \\
3\end{array}$ & $\begin{array}{l}31 \% \\
12 \%\end{array}$ & $\begin{array}{l}2.50 \mathrm{E}-04 \\
320 \mathrm{~F}-44\end{array}$ & $\begin{array}{l}5.50 \mathrm{E}-04 \\
520 \mathrm{E}-04\end{array}$ & $\begin{array}{l}2.03 E-04 \\
2.93 \mathrm{~F}-04\end{array}$ & -- & -- & -- & -- & -- & -- & -- & -- \\
\hline
\end{tabular}

CONV - Conventional parameter

SVOC - Semivolatile Organic Compound

VOC - Volatile Organic Compound 
Table A-12

Summary of Surface Water Results From Segment 1 Exceeding Select Ecological or Human Health Benchmark Values

\begin{tabular}{|c|c|c|c|c|c|c|c|c|c|c|}
\hline Sample Area & Sample Site & Sample ID & Date Collected & Constituent Name & Units & $\begin{array}{c}\text { QA } \\
\text { Category }\end{array}$ & $\begin{array}{l}\text { Analytical } \\
\text { Value }\end{array}$ & Qualifier & $\begin{array}{c}\text { Ecological } \\
\text { Benchmark } \\
\text { Value }\end{array}$ & $\begin{array}{l}\text { Human Health } \\
\text { Benchmark Value }\end{array}$ \\
\hline Columbia River & NORTHPORT WA & 2018080 & December 3, 2001 & Arsenic & $\mathrm{mg} / \mathrm{L}$ & $\overline{1}$ & $2.60 \mathrm{E}-04$ & $\overline{=}$ & $\overline{--}$ & 1.80E-05 \\
\hline Columbia River & NORTHPORT WA & 1408080 & September 10, 2001 & Arsenic & $\mathrm{mg} / \mathrm{L}$ & 1 & $3.20 \mathrm{E}-04$ & $=$ & -- & 1.80E-05 \\
\hline Columbia River & NORTHPORT WA & 1368080 & August 6, 2001 & Arsenic & $\mathrm{mg} / \mathrm{L}$ & 1 & $3.50 \mathrm{E}-04$ & $=$ & -- & 1.80E-05 \\
\hline Columbia River & Vernita3 HRM0.3 & B183P7 & December 9, 2003 & Arsenic & $\mathrm{mg} / \mathrm{L}$ & 1 & $3.62 \mathrm{E}-04$ & $=$ & -- & 1.80E-05 \\
\hline Columbia River & NORTHPORT WA & 2268155 & June 3, 2002 & Arsenic & $\mathrm{mg} / \mathrm{L}$ & 1 & $3.80 \mathrm{E}-04$ & $=$ & -- & $1.80 \mathrm{E}-05$ \\
\hline Columbia River & NORTHPORT WA & 2068080 & January 14, 2002 & Arsenic & $\mathrm{mg} / \mathrm{L}$ & 1 & $3.90 \mathrm{E}-04$ & $=$ & -- & $1.80 \mathrm{E}-05$ \\
\hline Columbia River & NORTHPORT WA & 1498100 & November 5, 2001 & Arsenic & $\mathrm{mg} / \mathrm{L}$ & 1 & 4.00E-04 & $=$ & -- & 1.80E-05 \\
\hline Columbia River & NORTHPORT WA & 2158155 & March 11, 2002 & Arsenic & $\mathrm{mg} / \mathrm{L}$ & 1 & $4.20 \mathrm{E}-04$ & $=$ & -- & 1.80E-05 \\
\hline Columbia River & Vernita2 HRM0.3 & B0XRM9 & March 27, 2000 & Arsenic & $\mathrm{mg} / \mathrm{L}$ & 1 & 4.27E-04 & $=$ & -- & $1.80 \mathrm{E}-05$ \\
\hline Columbia River & NORTHPORT WA & 1286057 & July 9, 2001 & Arsenic & $\mathrm{mg} / \mathrm{L}$ & 1 & 4.40E-04 & $=$ & -- & 1.80E-05 \\
\hline Columbia River & NORTHPORT WA & 2228080 & May 12, 2002 & Arsenic & $\mathrm{mg} / \mathrm{L}$ & 1 & 4.40E-04 & $=$ & -- & 1.80E-05 \\
\hline Columbia River & Vernita1 HRM0.3 & B0XRM8 & March 27, 2000 & Arsenic & $\mathrm{mg} / \mathrm{L}$ & 1 & 4.44E-04 & $=$ & -- & $1.80 \mathrm{E}-05$ \\
\hline Columbia River & Vernita3 HRM0.3 & B10669 & September 14, 2000 & Arsenic & $\mathrm{mg} / \mathrm{L}$ & 1 & 4.48E-04 & $=$ & -- & 1.80E-05 \\
\hline Columbia River & Vernita1 HRM0.3 & B10671 & September 14, 2000 & Arsenic & $\mathrm{mg} / \mathrm{L}$ & 1 & 4.53E-04 & $=$ & -- & 1.80E-05 \\
\hline Columbia River & Vernita4 HRM0.3 & B0X3Y5 & December 7, 1999 & Arsenic & $\mathrm{mg} / \mathrm{L}$ & 1 & 4.57E-04 & $=$ & -- & $1.80 \mathrm{E}-05$ \\
\hline Columbia River & Vernita3 HRM0.3 & BOXRNO & March 27, 2000 & Arsenic & $\mathrm{mg} / \mathrm{L}$ & 1 & 4.64E-04 & $=$ & -- & 1.80E-05 \\
\hline Columbia River & Vernita3 HRM0.3 & B0X3W2 & December 7, 1999 & Arsenic & $\mathrm{mg} / \mathrm{L}$ & 1 & 4.66E-04 & $=$ & -- & 1.80E-05 \\
\hline Columbia River & Vernita1 HRM0.3 & В0X3Y2 & December 7, 1999 & Arsenic & $\mathrm{mg} / \mathrm{L}$ & 1 & $4.66 \mathrm{E}-04$ & $=$ & -- & 1.80E-05 \\
\hline Columbia River & Vernita1 HRM0.3 & B158P2 & September 5, 2002 & Arsenic & $\mathrm{mg} / \mathrm{L}$ & 1 & 4.77E-04 & $=$ & -- & $1.80 \mathrm{E}-05$ \\
\hline Columbia River & Vernita4 HRM0.3 & B158P5 & September 5, 2002 & Arsenic & $\mathrm{mg} / \mathrm{L}$ & 1 & 4.81E-04 & $=$ & -- & 1.80E-05 \\
\hline Columbia River & Vernita4 HRM0.3 & B183P8 & December 9, 2003 & Arsenic & $\mathrm{mg} / \mathrm{L}$ & 1 & 4.82E-04 & $=$ & -- & 1.80E-05 \\
\hline Columbia River & Vernita4 HRM0.3 & B0XRN1 & March 27, 2000 & Arsenic & $\mathrm{mg} / \mathrm{L}$ & 1 & 4.84E-04 & $=$ & -- & 1.80E-05 \\
\hline Columbia River & Vernita3 HRM0.3 & B158P4 & September 5, 2002 & Arsenic & $\mathrm{mg} / \mathrm{L}$ & 1 & 4.87E-04 & $=$ & -- & $1.80 \mathrm{E}-05$ \\
\hline Columbia River & Vernita1 HRM0.3 & B16340 & December 9, 2002 & Arsenic & $\mathrm{mg} / \mathrm{L}$ & 1 & 4.96E-04 & $=$ & -- & 1.80E-05 \\
\hline Columbia River & Vernita4 HRM0.3 & B10691 & September 14, 2000 & Arsenic & $\mathrm{mg} / \mathrm{L}$ & 1 & $4.98 \mathrm{E}-04$ & $=$ & -- & 1.80E-05 \\
\hline Columbia River & Vernita2 HRM0.3 & B183P6 & December 9, 2003 & Arsenic & $\mathrm{mg} / \mathrm{L}$ & 1 & $5.01 \mathrm{E}-04$ & $=$ & -- & $1.80 \mathrm{E}-05$ \\
\hline Columbia River & Vernita2 HRM0.3 & B0X3W1 & December 7, 1999 & Arsenic & $\mathrm{mg} / \mathrm{L}$ & 1 & $5.02 \mathrm{E}-04$ & $=$ & -- & 1.80E-05 \\
\hline Columbia River & Vernita3 HRM0.3 & B11137 & December 4, 2000 & Arsenic & $\mathrm{mg} / \mathrm{L}$ & 1 & $5.05 \mathrm{E}-04$ & $=$ & -- & 1.80E-05 \\
\hline Columbia River & Vernita2 HRM0.3 & В0X3Y3 & December 7, 1999 & Arsenic & $\mathrm{mg} / \mathrm{L}$ & 1 & $5.05 \mathrm{E}-04$ & $=$ & -- & 1.80E-05 \\
\hline Columbia River & Vernita4 HRM0.3 & B0X3W3 & December 7, 1999 & Arsenic & $\mathrm{mg} / \mathrm{L}$ & 1 & $5.06 \mathrm{E}-04$ & $=$ & -- & 1.80E-05 \\
\hline Columbia River & Vernita1 HRM0.3 & $\mathrm{B} 11 \mathrm{H} 49$ & February 27, 2001 & Arsenic & $\mathrm{mg} / \mathrm{L}$ & 1 & 5.10E-04 & $=$ & -- & $1.80 \mathrm{E}-05$ \\
\hline Columbia River & Vernita4 HRM0.3 & B16355 & December 9, 2002 & Arsenic & $\mathrm{mg} / \mathrm{L}$ & 1 & 5.11E-04 & $=$ & -- & $1.80 \mathrm{E}-05$ \\
\hline Columbia River & Vernita2 HRM0.3 & B158P3 & September 5, 2002 & Arsenic & $\mathrm{mg} / \mathrm{L}$ & 1 & $5.12 \mathrm{E}-04$ & $=$ & -- & 1.80E-05 \\
\hline Columbia River & Vernita3 HRM0.3 & В183T9 & December 9, 2003 & Arsenic & $\mathrm{mg} / \mathrm{L}$ & 1 & $5.12 \mathrm{E}-04$ & C & -- & 1.80E-05 \\
\hline Columbia River & Vernita3 HRM0.3 & B16354 & December 9, 2002 & Arsenic & $\mathrm{mg} / \mathrm{L}$ & 1 & 5.17E-04 & $=$ & -- & 1.80E-05 \\
\hline Columbia River & Vernita2 HRM0.3 & B10668 & September 14, 2000 & Arsenic & $\mathrm{mg} / \mathrm{L}$ & 1 & 5.19E-04 & $=$ & -- & $1.80 \mathrm{E}-05$ \\
\hline Columbia River & Vernita3 HRM0.3 & B13LH5 & December 3, 2001 & Arsenic & $\mathrm{mg} / \mathrm{L}$ & 1 & 5.19E-04 & $=$ & -- & 1.80E-05 \\
\hline Columbia River & NORTHPORT WA & 2178180 & April 8, 2002 & Arsenic & $\mathrm{mg} / \mathrm{L}$ & 1 & $5.20 \mathrm{E}-04$ & $=$ & -- & 1.80E-05 \\
\hline Columbia River & Vernita3 HRM0.3 & B0X3Y4 & December 7, 1999 & Arsenic & $\mathrm{mg} / \mathrm{L}$ & 1 & $5.23 \mathrm{E}-04$ & $=$ & -- & 1.80E-05 \\
\hline Columbia River & Vernita2 HRM0.3 & B16331 & December 9, 2002 & Arsenic & $\mathrm{mg} / \mathrm{L}$ & 1 & $5.24 \mathrm{E}-04$ & $=$ & -- & 1.80E-05 \\
\hline Columbia River & Vernita2 HRM0.3 & B16353 & December 9, 2002 & Arsenic & $\mathrm{mg} / \mathrm{L}$ & 1 & $5.24 \mathrm{E}-04$ & $=$ & -- & 1.80E-05 \\
\hline Columbia River & Vernita1 HRM0.3 & B11123 & December 4, 2000 & Arsenic & $\mathrm{mg} / \mathrm{L}$ & 1 & $5.25 \mathrm{E}-04$ & $=$ & -- & $1.80 \mathrm{E}-05$ \\
\hline
\end{tabular}


Table A-12

Summary of Surface Water Results From Segment 1 Exceeding Select Ecological or Human Health Benchmark Values

\begin{tabular}{|c|c|c|c|c|c|c|c|c|c|c|}
\hline Sample Area & Sample Site & Sample ID & Date Collected & Constituent Name & Units & $\begin{array}{c}\text { QA } \\
\text { Category }\end{array}$ & $\begin{array}{l}\text { Analytical } \\
\text { Value }\end{array}$ & Qualifier & $\begin{array}{c}\text { Ecological } \\
\text { Benchmark } \\
\text { Value }\end{array}$ & $\begin{array}{c}\text { Human Health } \\
\text { Benchmark Value }\end{array}$ \\
\hline Columbia River & Vernita4 HRM0.3 & B11138 & December 4, 2000 & Arsenic & $\mathrm{mg} / \mathrm{L}$ & 1 & $5.25 \mathrm{E}-04$ & $=$ & -- & 1.80E-05 \\
\hline Columbia River & Vernita BRG & B14K64 & May 3, 2002 & Arsenic & $\mathrm{mg} / \mathrm{L}$ & 2 & $5.28 \mathrm{E}-04$ & $=$ & -- & $1.80 \mathrm{E}-05$ \\
\hline Columbia River & Vernita2 HRM0.3 & B11114 & December 4, 2000 & Arsenic & $\mathrm{mg} / \mathrm{L}$ & 1 & $5.28 \mathrm{E}-04$ & $=$ & -- & 1.80E-05 \\
\hline Columbia River & Vernita1 HRM0.3 & B10688 & September 14, 2000 & Arsenic & $\mathrm{mg} / \mathrm{L}$ & 1 & $5.29 \mathrm{E}-04$ & $=$ & -- & 1.80E-05 \\
\hline Columbia River & NORTHPORT WA & 1468155 & October 15, 2001 & Arsenic & $\mathrm{mg} / \mathrm{L}$ & 1 & $5.30 \mathrm{E}-04$ & $=$ & -- & 1.80E-05 \\
\hline Columbia River & Vernita2 HRM0.3 & B11H50 & February 27, 2001 & Arsenic & $\mathrm{mg} / \mathrm{L}$ & 1 & $5.32 \mathrm{E}-04$ & $=$ & -- & $1.80 \mathrm{E}-05$ \\
\hline Columbia River & Vernita3 HRM0.3 & B17CM5 & September 4, 2003 & Arsenic & $\mathrm{mg} / \mathrm{L}$ & 1 & 5.32E-04 & $=$ & -- & 1.80E-05 \\
\hline Columbia River & Vernita4 HRM0.3 & B11H52 & February 27, 2001 & Arsenic & $\mathrm{mg} / \mathrm{L}$ & 1 & $5.36 \mathrm{E}-04$ & $=$ & -- & 1.80E-05 \\
\hline Columbia River & Vernita1 HRM0.3 & $\mathrm{B} 158 \mathrm{H} 2$ & September 5, 2002 & Arsenic & $\mathrm{mg} / \mathrm{L}$ & 1 & 5.38E-04 & $=$ & -- & 1.80E-05 \\
\hline Columbia River & Vernita4 HRM0.3 & B17CM6 & September 4, 2003 & Arsenic & $\mathrm{mg} / \mathrm{L}$ & 1 & $5.43 \mathrm{E}-04$ & $=$ & -- & $1.80 \mathrm{E}-05$ \\
\hline Columbia River & Vernita2 HRM0.3 & B11136 & December 4, 2000 & Arsenic & $\mathrm{mg} / \mathrm{L}$ & 1 & $5.45 \mathrm{E}-04$ & $=$ & -- & 1.80E-05 \\
\hline Columbia River & Vernita1 HRM0.3 & B11135 & December 4, 2000 & Arsenic & $\mathrm{mg} / \mathrm{L}$ & 1 & 5.46E-04 & $=$ & -- & $1.80 \mathrm{E}-05$ \\
\hline Columbia River & Vernita2 HRM0.3 & B10689 & September 14, 2000 & Arsenic & $\mathrm{mg} / \mathrm{L}$ & 1 & $5.46 \mathrm{E}-04$ & $=$ & -- & $1.80 \mathrm{E}-05$ \\
\hline Columbia River & Vernita2 HRM0.3 & В183Т8 & December 9, 2003 & Arsenic & $\mathrm{mg} / \mathrm{L}$ & 1 & $5.47 \mathrm{E}-04$ & C & -- & $1.80 \mathrm{E}-05$ \\
\hline Columbia River & Vernita1 HRM0.3 & $\mathrm{B} 0 \times 3 \times 0$ & December 7, 1999 & Arsenic & $\mathrm{mg} / \mathrm{L}$ & 1 & $5.47 \mathrm{E}-04$ & $=$ & -- & 1.80E-05 \\
\hline Columbia River & Vernita2 HRM0.3 & Bow970 & September 10, 1999 & Arsenic & $\mathrm{mg} / \mathrm{L}$ & 1 & 5.47E-04 & $=$ & -- & $1.80 \mathrm{E}-05$ \\
\hline Columbia River & Vernita BRG & B14K65 & May 3, 2002 & Arsenic & $\mathrm{mg} / \mathrm{L}$ & 2 & $5.48 \mathrm{E}-04$ & $=$ & -- & $1.80 \mathrm{E}-05$ \\
\hline Columbia River & Vernita3 HRM0.3 & B11115 & December 4, 2000 & Arsenic & $\mathrm{mg} / \mathrm{L}$ & 1 & $5.48 \mathrm{E}-04$ & $=$ & -- & 1.80E-05 \\
\hline Columbia River & Vernita4 HRM0.3 & В11H30 & February 27, 2001 & Arsenic & $\mathrm{mg} / \mathrm{L}$ & 1 & 5.49E-04 & $=$ & -- & $1.80 \mathrm{E}-05$ \\
\hline Columbia River & Vernita3 HRM0.3 & B0XRJ1 & March 27, 2000 & Arsenic & $\mathrm{mg} / \mathrm{L}$ & 1 & $5.50 \mathrm{E}-04$ & $=$ & -- & 1.80E-05 \\
\hline Columbia River & Vernita1 HRM0.3 & B0V228 & June 11, 1999 & Arsenic & $\mathrm{mg} / \mathrm{L}$ & 1 & $5.51 \mathrm{E}-04$ & $=$ & -- & 1.80E-05 \\
\hline Columbia River & Vernita1 HRM0.3 & B16L95 & March 24, 2003 & Arsenic & $\mathrm{mg} / \mathrm{L}$ & 1 & 5.51E-04 & C & -- & 1.80E-05 \\
\hline Columbia River & Vernita1 HRM0.3 & B0XRJ9 & March 27, 2000 & Arsenic & $\mathrm{mg} / \mathrm{L}$ & 1 & 5.52E-04 & $=$ & -- & $1.80 \mathrm{E}-05$ \\
\hline Columbia River & Vernita4 HRM0.3 & B11116 & December 4, 2000 & Arsenic & $\mathrm{mg} / \mathrm{L}$ & 1 & 5.52E-04 & $=$ & -- & 1.80E-05 \\
\hline Columbia River & Vernita2 HRM0.3 & BOXRJO & March 27, 2000 & Arsenic & $\mathrm{mg} / \mathrm{L}$ & 1 & 5.53E-04 & $=$ & -- & 1.80E-05 \\
\hline Columbia River & Vernita3 HRM0.3 & B13LD3 & December 3, 2001 & Arsenic & $\mathrm{mg} / \mathrm{L}$ & 1 & 5.57E-04 & $=$ & -- & 1.80E-05 \\
\hline Columbia River & Vernita3 HRM0.3 & B16332 & December 9, 2002 & Arsenic & $\mathrm{mg} / \mathrm{L}$ & 1 & $5.60 \mathrm{E}-04$ & $=$ & -- & 1.80E-05 \\
\hline Columbia River & Vernita4 HRM0.3 & B0XRJ2 & March 27, 2000 & Arsenic & $\mathrm{mg} / \mathrm{L}$ & 1 & 5.61E-04 & $=$ & -- & 1.80E-05 \\
\hline Columbia River & Vernita1 HRM0.3 & B17CD6 & September 4, 2003 & Arsenic & $\mathrm{mg} / \mathrm{L}$ & 1 & $5.64 \mathrm{E}-04$ & $=$ & -- & 1.80E-05 \\
\hline Columbia River & Vernita3 HRM0.3 & B11H51 & February 27, 2001 & Arsenic & $\mathrm{mg} / \mathrm{L}$ & 1 & 5.64E-04 & $=$ & -- & $1.80 \mathrm{E}-05$ \\
\hline Columbia River & Vernita3 HRM0.3 & $\mathrm{B} 11 \mathrm{H} 29$ & February 27, 2001 & Arsenic & $\mathrm{mg} / \mathrm{L}$ & 1 & 5.69E-04 & $=$ & -- & $1.80 \mathrm{E}-05$ \\
\hline Columbia River & EB NR Wenatchee & 01258001 & June 20,2001 & Arsenic & $\mathrm{mg} / \mathrm{L}$ & 1 & $5.70 \mathrm{E}-04$ & $=$ & -- & $1.80 \mathrm{E}-05$ \\
\hline Columbia River & Vernita1 HRM0.3 & B12521 & June 14, 2001 & Arsenic & $\mathrm{mg} / \mathrm{L}$ & 1 & $5.70 \mathrm{E}-04$ & $=$ & -- & 1.80E-05 \\
\hline Columbia River & Vernita2 HRM0.3 & $\mathrm{B} 17 \mathrm{CC} 7$ & September 4, 2003 & Arsenic & $\mathrm{mg} / \mathrm{L}$ & 1 & $5.71 \mathrm{E}-04$ & $=$ & -- & $1.80 \mathrm{E}-05$ \\
\hline Columbia River & Vernita1 HRM0.3 & B17CM3 & September 4, 2003 & Arsenic & $\mathrm{mg} / \mathrm{L}$ & 1 & $5.72 \mathrm{E}-04$ & $=$ & -- & 1.80E-05 \\
\hline Columbia River & Vernita2 HRM0.3 & B17CM4 & September 4, 2003 & Arsenic & $\mathrm{mg} / \mathrm{L}$ & 1 & $5.72 \mathrm{E}-04$ & $=$ & -- & $1.80 \mathrm{E}-05$ \\
\hline Columbia River & Vernita1 HRM0.3 & В0YCJ3 & June 19, 2000 & Arsenic & $\mathrm{mg} / \mathrm{L}$ & 1 & 5.73E-04 & $=$ & -- & 1.80E-05 \\
\hline Columbia River & Vernita4 HRM0.3 & B12524 & June 14, 2001 & Arsenic & $\mathrm{mg} / \mathrm{L}$ & 1 & 5.73E-04 & $=$ & -- & 1.80E-05 \\
\hline Columbia River & Vernita1 HRM0.3 & B16352 & December 9, 2002 & Arsenic & $\mathrm{mg} / \mathrm{L}$ & 1 & $5.74 \mathrm{E}-04$ & $=$ & -- & $1.80 \mathrm{E}-05$ \\
\hline Columbia River & Vernita2 HRM0.3 & B11H28 & February 27, 2001 & Arsenic & $\mathrm{mg} / \mathrm{L}$ & 1 & $5.74 \mathrm{E}-04$ & $=$ & -- & 1.80E-05 \\
\hline Columbia River & Vernita3 HRM0.3 & B10690 & September 14, 2000 & Arsenic & $\mathrm{mg} / \mathrm{L}$ & 1 & $5.74 \mathrm{E}-04$ & $=$ & -- & 1.80E-05 \\
\hline Columbia River & Vernita1 HRM0.3 & B183R5 & December 9, 2003 & Arsenic & $\mathrm{mg} / \mathrm{L}$ & 1 & $5.76 \mathrm{E}-04$ & $=$ & -- & 1.80E-05 \\
\hline
\end{tabular}


Table A-12

Summary of Surface Water Results From Segment 1 Exceeding Select Ecological or Human Health Benchmark Values

\begin{tabular}{|c|c|c|c|c|c|c|c|c|c|c|}
\hline Sample Area & Sample Site & Sample ID & Date Collected & Constituent Name & Units & $\begin{array}{c}\text { QA } \\
\text { Category }\end{array}$ & $\begin{array}{l}\text { Analytical } \\
\text { Value }\end{array}$ & Qualifier & $\begin{array}{c}\text { Ecological } \\
\text { Benchmark } \\
\text { Value }\end{array}$ & $\begin{array}{c}\text { Human Health } \\
\text { Benchmark Value }\end{array}$ \\
\hline Columbia River & Vernita3 HRM0.3 & B158F4 & September 5, 2002 & Arsenic & $\mathrm{mg} / \mathrm{L}$ & 1 & 5.77E-04 & $=$ & -- & 1.80E-05 \\
\hline Columbia River & Vernita2 HRM0.3 & B13LD2 & December 3, 2001 & Arsenic & $\mathrm{mg} / \mathrm{L}$ & 1 & 5.79E-04 & $=$ & -- & 1.80E-05 \\
\hline Columbia River & Vernita2 HRM0.3 & B13LH4 & December 3, 2001 & Arsenic & $\mathrm{mg} / \mathrm{L}$ & 1 & 5.83E-04 & $=$ & -- & 1.80E-05 \\
\hline Columbia River & Vernita2 HRM0.3 & B0YCJ4 & June 19, 2000 & Arsenic & $\mathrm{mg} / \mathrm{L}$ & 1 & $5.84 \mathrm{E}-04$ & $=$ & -- & 1.80E-05 \\
\hline Columbia River & Vernita2 HRM0.3 & B16L86 & March 24, 2003 & Arsenic & $\mathrm{mg} / \mathrm{L}$ & 1 & 5.84E-04 & C & -- & 1.80E-05 \\
\hline Columbia River & Vernita4 HRM0.3 & B10670 & September 14, 2000 & Arsenic & $\mathrm{mg} / \mathrm{L}$ & 1 & 5.85E-04 & $=$ & -- & $1.80 \mathrm{E}-05$ \\
\hline Columbia River & Vernita4 HRM0.3 & B17CC9 & September 4, 2003 & Arsenic & $\mathrm{mg} / \mathrm{L}$ & 1 & 5.87E-04 & $=$ & -- & 1.80E-05 \\
\hline Columbia River & Vernita2 HRM0.3 & B16LB8 & March 24, 2003 & Arsenic & $\mathrm{mg} / \mathrm{L}$ & 1 & 5.89E-04 & C & -- & 1.80E-05 \\
\hline Columbia River & Wenatchee @ CHN & 01258000 & June 20, 2001 & Arsenic & $\mathrm{mg} / \mathrm{L}$ & 1 & $5.90 \mathrm{E}-04$ & $=$ & -- & 1.80E-05 \\
\hline Columbia River & Vernita3 HRM0.3 & B17CC8 & September 4, 2003 & Arsenic & $\mathrm{mg} / \mathrm{L}$ & 1 & 5.92E-04 & $=$ & -- & $1.80 \mathrm{E}-05$ \\
\hline Columbia River & Vernita2 HRM0.3 & B171Y2 & June 9, 2003 & Arsenic & $\mathrm{mg} / \mathrm{L}$ & 1 & $5.98 \mathrm{E}-04$ & C & -- & 1.80E-05 \\
\hline Columbia River & Vernita3 HRM0.3 & B16L87 & March 24, 2003 & Arsenic & $\mathrm{mg} / \mathrm{L}$ & 1 & $5.98 \mathrm{E}-04$ & C & -- & $1.80 \mathrm{E}-05$ \\
\hline Columbia River & Vernita1 HRM0.3 & B13LF1 & December 3, 2001 & Arsenic & $\mathrm{mg} / \mathrm{L}$ & 1 & 5.99E-04 & $=$ & -- & $1.80 \mathrm{E}-05$ \\
\hline Columbia River & Vernita4 HRM0.3 & B16L88 & March 24, 2003 & Arsenic & $\mathrm{mg} / \mathrm{L}$ & 1 & $6.00 \mathrm{E}-04$ & C & -- & $1.80 \mathrm{E}-05$ \\
\hline Columbia River & Vernita2 HRM0.3 & B158F3 & September 5, 2002 & Arsenic & $\mathrm{mg} / \mathrm{L}$ & 1 & 6.02E-04 & $=$ & -- & 1.80E-05 \\
\hline Columbia River & Vernita3 HRM0.3 & B0YCJ5 & June 19, 2000 & Arsenic & $\mathrm{mg} / \mathrm{L}$ & 1 & $6.02 \mathrm{E}-04$ & $=$ & -- & $1.80 \mathrm{E}-05$ \\
\hline Columbia River & Vernita1 HRM0.3 & B11H37 & February 27, 2001 & Arsenic & $\mathrm{mg} / \mathrm{L}$ & 1 & 6.04E-04 & $=$ & -- & $1.80 \mathrm{E}-05$ \\
\hline Columbia River & Vernita4 HRM0.3 & B16333 & December 9, 2002 & Arsenic & $\mathrm{mg} / \mathrm{L}$ & 1 & 6.04E-04 & $=$ & -- & 1.80E-05 \\
\hline Columbia River & Vernita3 HRM0.3 & B16LB9 & March 24, 2003 & Arsenic & $\mathrm{mg} / \mathrm{L}$ & 1 & $6.06 \mathrm{E}-04$ & C & -- & $1.80 \mathrm{E}-05$ \\
\hline Columbia River & Vernita BRG & B14L10 & May 17, 2002 & Arsenic & $\mathrm{mg} / \mathrm{L}$ & 2 & 6.07E-04 & $=$ & -- & 1.80E-05 \\
\hline Columbia River & Vernita1 HRM0.3 & B16LB7 & March 24, 2003 & Arsenic & $\mathrm{mg} / \mathrm{L}$ & 1 & $6.08 \mathrm{E}-04$ & C & -- & 1.80E-05 \\
\hline Columbia River & Vernita3 HRM0.3 & B1B749 & September 13, 2004 & Arsenic & $\mathrm{mg} / \mathrm{L}$ & 1 & 6.09E-04 & $x$ & -- & 1.80E-05 \\
\hline Columbia River & Vernita BRG & B14L11 & May 17, 2002 & Arsenic & $\mathrm{mg} / \mathrm{L}$ & 2 & $6.10 \mathrm{E}-04$ & $=$ & -- & $1.80 \mathrm{E}-05$ \\
\hline Columbia River & Vernita4 HRM0.3 & B16LC0 & March 24, 2003 & Arsenic & $\mathrm{mg} / \mathrm{L}$ & 1 & $6.10 \mathrm{E}-04$ & C & -- & 1.80E-05 \\
\hline Columbia River & Vernita4 HRM0.3 & B13LH6 & December 3, 2001 & Arsenic & $\mathrm{mg} / \mathrm{L}$ & 1 & 6.11E-04 & $=$ & -- & 1.80E-05 \\
\hline Columbia River & Vernita4 HRM0.3 & B158F5 & September 5, 2002 & Arsenic & $\mathrm{mg} / \mathrm{L}$ & 1 & $6.12 \mathrm{E}-04$ & $=$ & -- & 1.80E-05 \\
\hline Columbia River & Vernita1 HRM0.3 & B1B747 & September 13, 2004 & Arsenic & $\mathrm{mg} / \mathrm{L}$ & 1 & $6.14 \mathrm{E}-04$ & $\mathrm{x}$ & -- & 1.80E-05 \\
\hline Columbia River & Vernita1 HRM0.3 & B13LH3 & December 3, 2001 & Arsenic & $\mathrm{mg} / \mathrm{L}$ & 1 & $6.18 \mathrm{E}-04$ & $=$ & -- & 1.80E-05 \\
\hline Columbia River & Vernita2 HRM0.3 & B1B748 & September 13, 2004 & Arsenic & $\mathrm{mg} / \mathrm{L}$ & 1 & 6.19E-04 & $x$ & -- & 1.80E-05 \\
\hline Columbia River & Vernita3 HRM0.3 & Bow971 & September 10, 1999 & Arsenic & $\mathrm{mg} / \mathrm{L}$ & 1 & $6.22 \mathrm{E}-04$ & $=$ & -- & 1.80E-05 \\
\hline Columbia River & Vernita BRG & B14PX2 & June 10, 2002 & Arsenic & $\mathrm{mg} / \mathrm{L}$ & 2 & $6.23 \mathrm{E}-04$ & $=$ & -- & $1.80 \mathrm{E}-05$ \\
\hline Columbia River & Vernita2 HRM0.3 & BOV202 & June 11,1999 & Arsenic & $\mathrm{mg} / \mathrm{L}$ & 1 & $6.28 \mathrm{E}-04$ & $=$ & -- & $1.80 \mathrm{E}-05$ \\
\hline Columbia River & Vernita1 HRM0.3 & Bow969 & September 10, 1999 & Arsenic & $\mathrm{mg} / \mathrm{L}$ & 1 & $6.28 \mathrm{E}-04$ & $=$ & -- & 1.80E-05 \\
\hline Columbia River & Vernita4 HRM0.3 & B13LD4 & December 3, 2001 & Arsenic & $\mathrm{mg} / \mathrm{L}$ & 1 & $6.30 \mathrm{E}-04$ & $=$ & -- & $1.80 \mathrm{E}-05$ \\
\hline Columbia River & Vernita BRG & B14PX1 & June 10, 2002 & Arsenic & $\mathrm{mg} / \mathrm{L}$ & 2 & 6.32E-04 & $=$ & -- & 1.80E-05 \\
\hline Columbia River & Vernita2 HRM0.3 & B0YCC6 & June 19, 2000 & Arsenic & $\mathrm{mg} / \mathrm{L}$ & 1 & $6.32 \mathrm{E}-04$ & $=$ & -- & 1.80E-05 \\
\hline Columbia River & Vernita4 HRM0.3 & B0YCJ6 & June 19, 2000 & Arsenic & $\mathrm{mg} / \mathrm{L}$ & 1 & 6.32E-04 & $=$ & -- & 1.80E-05 \\
\hline Columbia River & Vernita2 HRM0.3 & B12522 & June 14, 2001 & Arsenic & $\mathrm{mg} / \mathrm{L}$ & 1 & 6.34E-04 & $=$ & -- & 1.80E-05 \\
\hline Columbia River & Vernita1 HRM0.3 & B0YCD5 & June 19,2000 & Arsenic & $\mathrm{mg} / \mathrm{L}$ & 1 & $6.36 \mathrm{E}-04$ & $=$ & -- & $1.80 \mathrm{E}-05$ \\
\hline Columbia River & Vernita2 HRM0.3 & B124Y0 & June 14, 2001 & Arsenic & $\mathrm{mg} / \mathrm{L}$ & 1 & 6.36E-04 & $=$ & -- & 1.80E-05 \\
\hline Columbia River & Vernita1 HRM0.3 & B14RW4 & June 10, 2002 & Arsenic & $\mathrm{mg} / \mathrm{L}$ & 1 & 6.37E-04 & $x$ & -- & 1.80E-05 \\
\hline Columbia River & Vernita3 HRM0.3 & B0V204 & June 11, 1999 & Arsenic & $\mathrm{mg} / \mathrm{L}$ & 1 & 6.44E-04 & $=$ & -- & 1.80E-05 \\
\hline
\end{tabular}


Table A-12

Summary of Surface Water Results From Segment 1 Exceeding Select Ecological or Human Health Benchmark Values

\begin{tabular}{|c|c|c|c|c|c|c|c|c|c|c|}
\hline Sample Area & Sample Site & Sample ID & Date Collected & Constituent Name & Units & $\begin{array}{c}\text { QA } \\
\text { Category }\end{array}$ & $\begin{array}{l}\text { Analytical } \\
\text { Value }\end{array}$ & Qualifier & $\begin{array}{c}\text { Ecological } \\
\text { Benchmark } \\
\text { Value }\end{array}$ & $\begin{array}{c}\text { Human Health } \\
\text { Benchmark Value }\end{array}$ \\
\hline Columbia River & Vernita1 HRM0.3 & B171Y1 & June 9, 2003 & Arsenic & $\mathrm{mg} / \mathrm{L}$ & 1 & $6.44 \mathrm{E}-04$ & $\mathrm{C}$ & -- & 1.80E-05 \\
\hline Columbia River & Vernita3 HRM0.3 & B12TH8 & September 7, 2001 & Arsenic & $\mathrm{mg} / \mathrm{L}$ & 1 & $6.44 \mathrm{E}-04$ & $=$ & -- & 1.80E-05 \\
\hline Columbia River & Vernita4 HRM0.3 & B171Y4 & June 9, 2003 & Arsenic & $\mathrm{mg} / \mathrm{L}$ & 1 & $6.46 \mathrm{E}-04$ & C & -- & 1.80E-05 \\
\hline Columbia River & Vernita4 HRM0.3 & Bow972 & September 10, 1999 & Arsenic & $\mathrm{mg} / \mathrm{L}$ & 1 & $6.48 \mathrm{E}-04$ & $=$ & -- & 1.80E-05 \\
\hline Columbia River & Vernita BRG2 & B12RP4 & August 27, 2001 & Arsenic & $\mathrm{mg} / \mathrm{L}$ & 1 & $6.50 \mathrm{E}-04$ & $=$ & -- & 1.80E-05 \\
\hline Columbia River & Vernita4 HRM0.3 & B0YCC8 & June 19, 2000 & Arsenic & $\mathrm{mg} / \mathrm{L}$ & 1 & $6.52 \mathrm{E}-04$ & $=$ & -- & $1.80 \mathrm{E}-05$ \\
\hline Columbia River & Vernita4 HRM0.3 & B1B750 & September 13, 2004 & Arsenic & $\mathrm{mg} / \mathrm{L}$ & 1 & $6.53 \mathrm{E}-04$ & $\mathrm{x}$ & -- & 1.80E-05 \\
\hline Columbia River & Vernita1 HRM0.3 & В183Т7 & December 9, 2003 & Arsenic & $\mathrm{mg} / \mathrm{L}$ & 1 & $6.54 \mathrm{E}-04$ & C & -- & 1.80E-05 \\
\hline Columbia River & Vernita4 HRM0.3 & B124Y2 & June 14, 2001 & Arsenic & $\mathrm{mg} / \mathrm{L}$ & 1 & 6.56E-04 & $=$ & -- & 1.80E-05 \\
\hline Columbia River & Vernita3 HRM0.3 & B12523 & June 14, 2001 & Arsenic & $\mathrm{mg} / \mathrm{L}$ & 1 & $6.60 \mathrm{E}-04$ & $=$ & -- & $1.80 \mathrm{E}-05$ \\
\hline Columbia River & Vernita3 HRM0.3 & BOYCC7 & June 19, 2000 & Arsenic & $\mathrm{mg} / \mathrm{L}$ & 1 & $6.61 \mathrm{E}-04$ & $=$ & -- & 1.80E-05 \\
\hline Columbia River & Vernita2 HRM0.3 & B1B6Y0 & September 13, 2004 & Arsenic & $\mathrm{mg} / \mathrm{L}$ & 1 & $6.62 \mathrm{E}-04$ & $\mathrm{x}$ & -- & 1.80E-05 \\
\hline Columbia River & Vernita3 HRM0.3 & B171Y3 & June 9, 2003 & Arsenic & $\mathrm{mg} / \mathrm{L}$ & 1 & $6.64 \mathrm{E}-04$ & C & -- & $1.80 \mathrm{E}-05$ \\
\hline Columbia River & Vernita1 HRM0.3 & B1B6Y9 & September 13, 2004 & Arsenic & $\mathrm{mg} / \mathrm{L}$ & 1 & $6.65 \mathrm{E}-04$ & $\mathrm{x}$ & -- & $1.80 \mathrm{E}-05$ \\
\hline Columbia River & Vernita BRG1 & B12RP0 & August 27, 2001 & Arsenic & $\mathrm{mg} / \mathrm{L}$ & 1 & $6.68 \mathrm{E}-04$ & $=$ & -- & 1.80E-05 \\
\hline Columbia River & Vernita3 HRM0.3 & B124Y1 & June 14, 2001 & Arsenic & $\mathrm{mg} / \mathrm{L}$ & 1 & $6.70 \mathrm{E}-04$ & $=$ & -- & $1.80 \mathrm{E}-05$ \\
\hline Columbia River & Vernita4 HRM0.3 & B183V0 & December 9, 2003 & Arsenic & $\mathrm{mg} / \mathrm{L}$ & 1 & $6.71 \mathrm{E}-04$ & C & -- & $1.80 \mathrm{E}-05$ \\
\hline Columbia River & Vernita4 HRM0.3 & B0V206 & June 11, 1999 & Arsenic & $\mathrm{mg} / \mathrm{L}$ & 1 & $6.79 \mathrm{E}-04$ & $=$ & -- & $1.80 \mathrm{E}-05$ \\
\hline Columbia River & Vernita1 HRM0.3 & B124Y9 & June 14, 2001 & Arsenic & $\mathrm{mg} / \mathrm{L}$ & 1 & 6.79E-04 & $=$ & -- & 1.80E-05 \\
\hline Columbia River & Vernita3 HRM0.3 & B1B6Y1 & September 13, 2004 & Arsenic & $\mathrm{mg} / \mathrm{L}$ & 1 & $6.94 \mathrm{E}-04$ & $\mathrm{x}$ & -- & 1.80E-05 \\
\hline Columbia River & Vernita2 HRM0.3 & B14RW5 & June 10, 2002 & Arsenic & $\mathrm{mg} / \mathrm{L}$ & 1 & $6.95 \mathrm{E}-04$ & $x$ & -- & 1.80E-05 \\
\hline Columbia River & Vernita4 HRM0.3 & B1B6Y2 & September 13, 2004 & Arsenic & $\mathrm{mg} / \mathrm{L}$ & 1 & 7.05E-04 & $x$ & -- & 1.80E-05 \\
\hline Columbia River & Vernita2 HRM0.3 & B12TH7 & September 7, 2001 & Arsenic & $\mathrm{mg} / \mathrm{L}$ & 1 & 7.07E-04 & $=$ & -- & $1.80 \mathrm{E}-05$ \\
\hline Columbia River & Vernita1 HRM0.3 & B12TH6 & September 7, 2001 & Arsenic & $\mathrm{mg} / \mathrm{L}$ & 1 & 7.11E-04 & $=$ & -- & $1.80 \mathrm{E}-05$ \\
\hline Columbia River & Vernita BRG2 & B12RP3 & August 27, 2001 & Arsenic & $\mathrm{mg} / \mathrm{L}$ & 1 & 7.12E-04 & $=$ & -- & 1.80E-05 \\
\hline Columbia River & Vernita3 HRM0.3 & B14RW6 & June 10, 2002 & Arsenic & $\mathrm{mg} / \mathrm{L}$ & 1 & 7.15E-04 & $\mathrm{x}$ & -- & 1.80E-05 \\
\hline Columbia River & Vernita1 HRM0.3 & B12T84 & September 7, 2001 & Arsenic & $\mathrm{mg} / \mathrm{L}$ & 1 & 7.23E-04 & $=$ & -- & 1.80E-05 \\
\hline Columbia River & Vernita3 HRM0.3 & B12T76 & September 7, 2001 & Arsenic & $\mathrm{mg} / \mathrm{L}$ & 1 & $7.24 \mathrm{E}-04$ & $=$ & -- & 1.80E-05 \\
\hline Columbia River & Vernita2 HRM0.3 & B12Т75 & September 7, 2001 & Arsenic & $\mathrm{mg} / \mathrm{L}$ & 1 & $7.28 \mathrm{E}-04$ & $=$ & -- & 1.80E-05 \\
\hline Columbia River & Vernita4 HRM0.3 & B14RT6 & June 10, 2002 & Arsenic & $\mathrm{mg} / \mathrm{L}$ & 1 & 7.30E-04 & $x$ & -- & $1.80 \mathrm{E}-05$ \\
\hline Columbia River & Vernita2 HRM0.3 & B0V203 & June 11, 1999 & Arsenic & $\mathrm{mg} / \mathrm{L}$ & 1 & 7.32E-04 & $=$ & -- & $1.80 \mathrm{E}-05$ \\
\hline Columbia River & Vernita4 HRM0.3 & B12Т77 & September 7, 2001 & Arsenic & $\mathrm{mg} / \mathrm{L}$ & 1 & 7.38E-04 & $=$ & -- & 1.80E-05 \\
\hline Columbia River & Vernita4 HRM0.3 & B0V207 & June 11, 1999 & Arsenic & $\mathrm{mg} / \mathrm{L}$ & 1 & $7.38 \mathrm{E}-04$ & $=$ & -- & 1.80E-05 \\
\hline Columbia River & Vernita1 HRM0.3 & B0V229 & June 11, 1999 & Arsenic & $\mathrm{mg} / \mathrm{L}$ & 1 & $7.42 \mathrm{E}-04$ & $=$ & -- & $1.80 \mathrm{E}-05$ \\
\hline Columbia River & Vernita4 HRM0.3 & В12TH9 & September 7, 2001 & Arsenic & $\mathrm{mg} / \mathrm{L}$ & 1 & 7.52E-04 & $=$ & -- & 1.80E-05 \\
\hline Columbia River & Vernita2 HRM0.3 & B171V0 & June 9, 2003 & Arsenic & $\mathrm{mg} / \mathrm{L}$ & 1 & 7.55E-04 & C & -- & 1.80E-05 \\
\hline Columbia River & Vernita BRG1 & B12RN9 & August 27, 2001 & Arsenic & $\mathrm{mg} / \mathrm{L}$ & 1 & 7.58E-04 & $=$ & -- & 1.80E-05 \\
\hline Columbia River & Vernita1 HRM0.3 & B14RV3 & June 10, 2002 & Arsenic & $\mathrm{mg} / \mathrm{L}$ & 1 & $7.68 \mathrm{E}-04$ & $x$ & -- & 1.80E-05 \\
\hline Columbia River & Vernita1 HRM0.3 & B171V9 & June 9, 2003 & Arsenic & $\mathrm{mg} / \mathrm{L}$ & 1 & 7.69E-04 & C & -- & 1.80E-05 \\
\hline Columbia River & Vernita4 HRM0.3 & B14RW7 & June 10, 2002 & Arsenic & $\mathrm{mg} / \mathrm{L}$ & 1 & 7.76E-04 & $x$ & -- & 1.80E-05 \\
\hline Columbia River & Vernita3 HRM0.3 & B0V205 & June 11, 1999 & Arsenic & $\mathrm{mg} / \mathrm{L}$ & 1 & 8.19E-04 & $=$ & -- & 1.80E-05 \\
\hline Columbia River & Vernita3 HRM0.3 & B171V1 & June 9, 2003 & Arsenic & $\mathrm{mg} / \mathrm{L}$ & 1 & $8.20 \mathrm{E}-04$ & C & -- & 1.80E-05 \\
\hline
\end{tabular}


Table A-12

Summary of Surface Water Results From Segment 1 Exceeding Select Ecological or Human Health Benchmark Values

\begin{tabular}{|c|c|c|c|c|c|c|c|c|c|c|}
\hline Sample Area & Sample Site & Sample ID & Date Collected & Constituent Name & Units & $\begin{array}{c}\text { QA } \\
\text { Category }\end{array}$ & $\begin{array}{l}\text { Analytical } \\
\text { Value }\end{array}$ & Qualifier & $\begin{array}{c}\text { Ecological } \\
\text { Benchmark } \\
\text { Value }\end{array}$ & $\begin{array}{c}\text { Human Health } \\
\text { Benchmark Value }\end{array}$ \\
\hline Columbia River & Vernita2 HRM0.3 & B14RT4 & June 10,2002 & Arsenic & $\mathrm{mg} / \mathrm{L}$ & 1 & $8.22 \mathrm{E}-04$ & $\bar{x}$ & $\overline{--}$ & $1.80 \mathrm{E}-05$ \\
\hline Columbia River & NORTHPORT WA & 2118105 & February 11, 2002 & Arsenic & $\mathrm{mg} / \mathrm{L}$ & 1 & $8.60 \mathrm{E}-04$ & $=$ & -- & $1.80 \mathrm{E}-05$ \\
\hline Columbia River & Vernita4 HRM0.3 & B171V2 & June 9, 2003 & Arsenic & $\mathrm{mg} / \mathrm{L}$ & 1 & 8.66E-04 & C & -- & 1.80E-05 \\
\hline Columbia River & Vernita BRG1 & B13J18 & November 14, 2001 & Arsenic & $\mathrm{mg} / \mathrm{L}$ & 1 & 0.0011 & $=$ & -- & $1.80 \mathrm{E}-05$ \\
\hline Columbia River & Vernita BRG1 & B13J19 & November 14, 2001 & Arsenic & $\mathrm{mg} / \mathrm{L}$ & 1 & 0.0017 & $=$ & -- & 1.80E-05 \\
\hline Columbia River & Vernita BRG1 & B12RP0 & August 27, 2001 & Barium & $\mathrm{mg} / \mathrm{L}$ & 1 & 0.028 & $=$ & 0.0040 & -- \\
\hline Columbia River & Vernita BRG2 & B12RP4 & August 27, 2001 & Barium & $\mathrm{mg} / \mathrm{L}$ & 1 & 0.028 & $=$ & 0.0040 & -- \\
\hline Columbia River & Vernita BRG1 & B12RN9 & August 27, 2001 & Barium & $\mathrm{mg} / \mathrm{L}$ & 1 & 0.028 & $=$ & 0.0040 & -- \\
\hline Columbia River & Wenatchee @ CHN & 01108030 & March 6, 2001 & Barium & $\mathrm{mg} / \mathrm{L}$ & 1 & 0.029 & $=$ & 0.0040 & -- \\
\hline Columbia River & Vernita BRG2 & B12RP3 & August 27, 2001 & Barium & $\mathrm{mg} / \mathrm{L}$ & 1 & 0.029 & $=$ & 0.0040 & -- \\
\hline
\end{tabular}




\section{APPENDIX B \\ SEGMENT 2 \\ SUMMARY STATISTICS, BENCHMARK COMPARISON, AND INDIVIDUAL SAMPLE EXCEEDENCES}


WCH-91

Rev. 0

Columbia River Component Data Evaluation Summary Report

July 2006

B-ii 


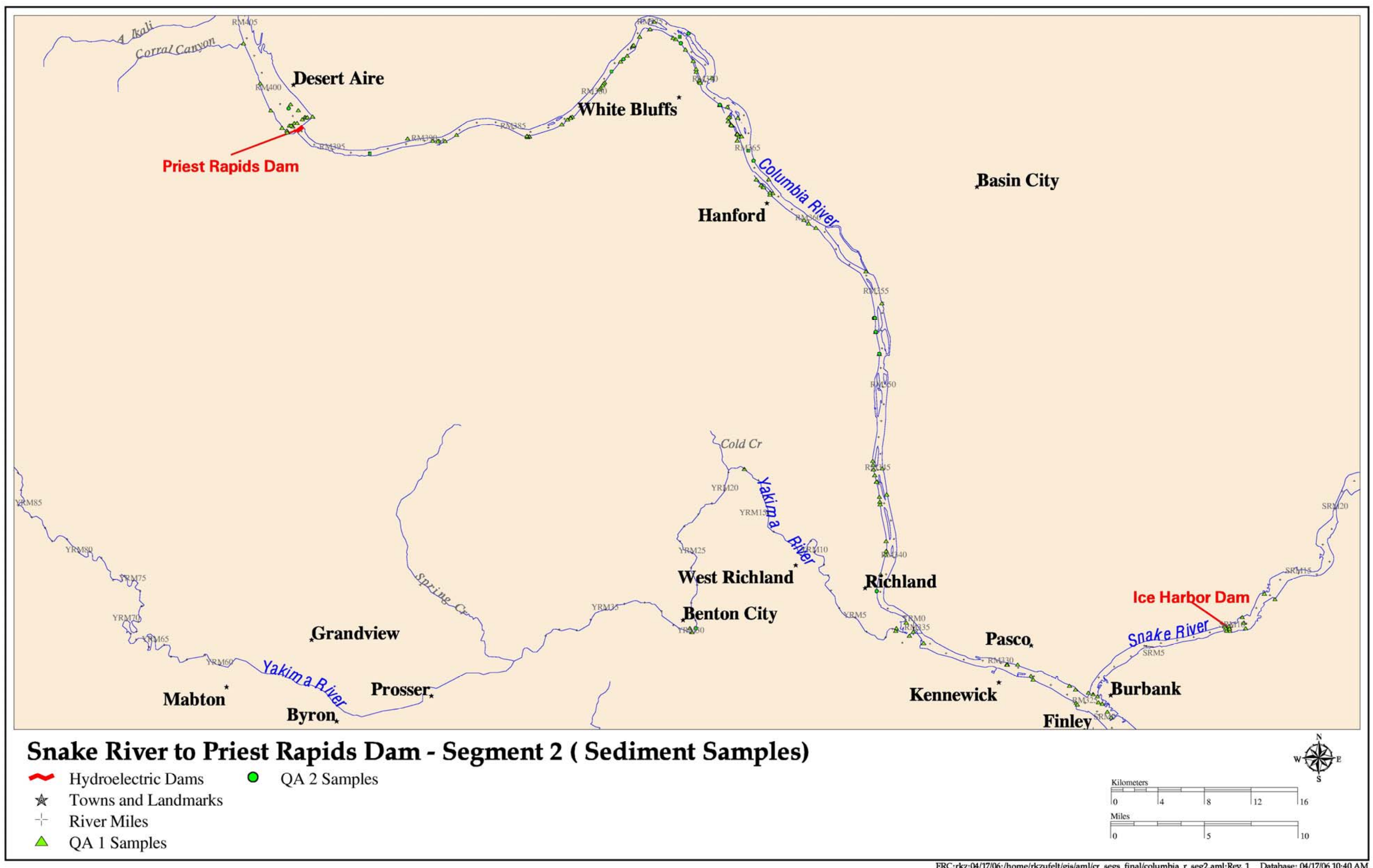




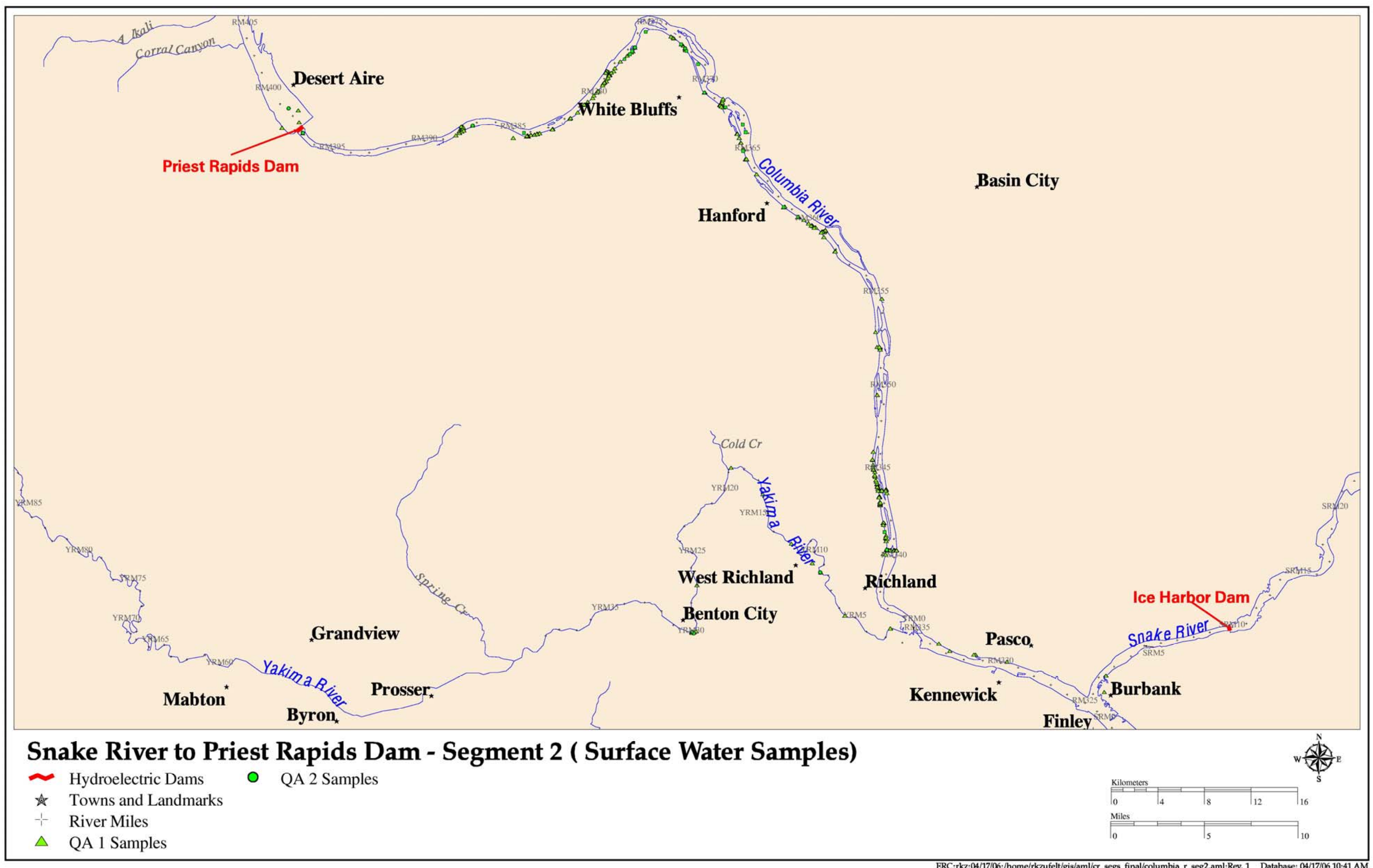




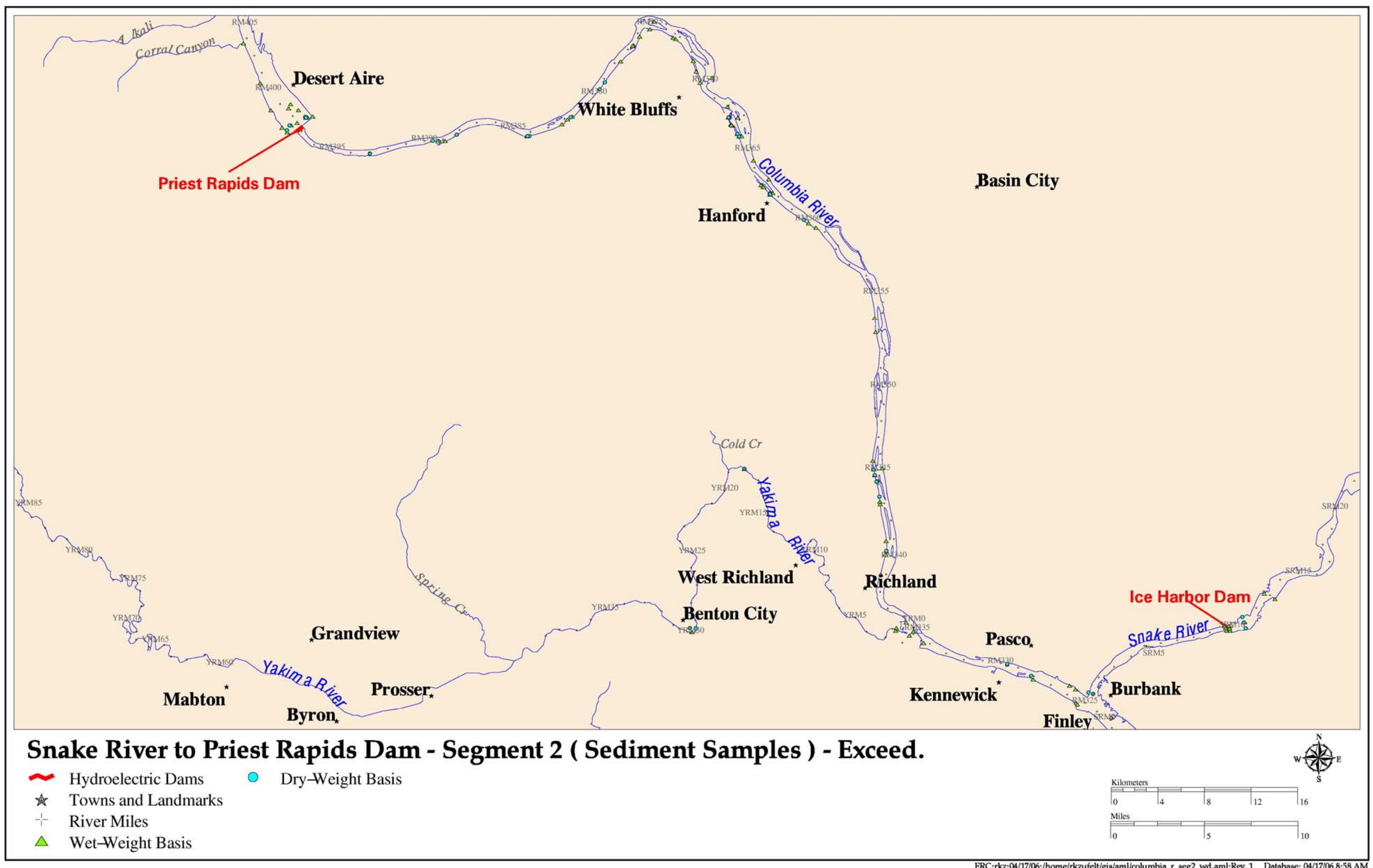




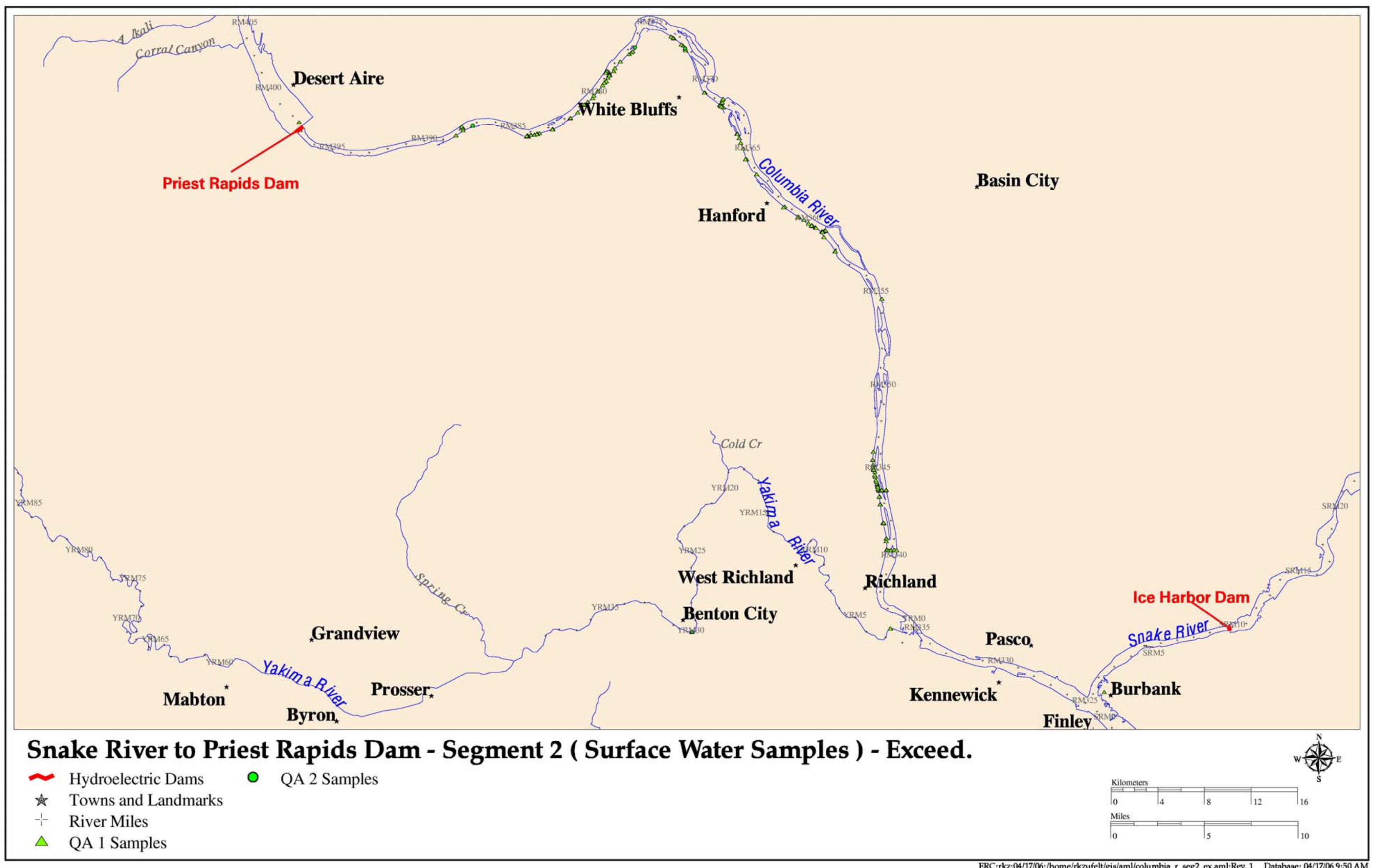


Table B-1

\section{Summary Table of Segment 2 Sediment Samples By Area, Site, Date, and Category Columbia River Component - Existing Sample Data Evaluation and Scoping Report}

\begin{tabular}{|c|c|c|c|c|}
\hline Sample ID & Sample Area & Sample Site & Sample Date & Category \\
\hline 10942 & Columbia River & CGS STA 33 & 10-Apr-91 & 1 \\
\hline 10943 & Columbia River & CGS STA 34 & 10-Apr-91 & 1 \\
\hline 12337 & Columbia River & CGS STA 33 & 09-Apr-92 & 1 \\
\hline 12338 & Columbia River & CGS STA 34 & 09-Apr-92 & 1 \\
\hline 12645 & Columbia River & 100N SHORE HGP & 30-Jun-92 & 1 \\
\hline 12646 & Columbia River & 100N-51 SHORE & 30-Jun-92 & 1 \\
\hline 12913 & Columbia River & 300 FF-5 & 14-Sep-92 & 1 \\
\hline 12914 & Columbia River & 300 FF-5 & 14-Sep-92 & 1 \\
\hline 13163 & Columbia River & F Slough & 12-Nov-92 & 1 \\
\hline 13164 & Columbia River & F Slough & 12-Nov-92 & 1 \\
\hline 13165 & Columbia River & H Slough & 13-Nov-92 & 1 \\
\hline 13166 & Columbia River & F Slough & 16-Nov-92 & 1 \\
\hline 13167 & Columbia River & Spit Tip & 16-Nov-92 & 1 \\
\hline 13168 & Columbia River & Hanford Slough & 17-Nov-92 & 1 \\
\hline 13169 & Columbia River & Horn Area & 17-Nov-92 & 1 \\
\hline 13170 & Columbia River & D Island & 19-Nov-92 & 1 \\
\hline 13171 & Columbia River & D Island & 19-Nov-92 & 1 \\
\hline 13172 & Columbia River & 100-B/C INTAKE & 20-Nov-92 & 1 \\
\hline 13747 & Columbia River & Shot Rock & 02-Apr-93 & 1 \\
\hline 13748 & Columbia River & Shot Rock & 02-Apr-93 & 1 \\
\hline 13749 & Columbia River & Shot Rock & 02-Apr-93 & 1 \\
\hline 13768 & Columbia River & CGS STA 33 & 15-Apr-93 & 1 \\
\hline 13769 & Columbia River & CGS STA 34 & 15-Apr-93 & 1 \\
\hline 15114 & Columbia River & CGS STA 33 & 07-Apr-94 & 1 \\
\hline 15115 & Columbia River & CGS STA 34 & 07-Apr-94 & 1 \\
\hline 15846 & Columbia River & 100-F Area & 26-Sep-94 & 1 \\
\hline 16496 & Columbia River & CGS STA 33 & 06-Apr-95 & 1 \\
\hline 16497 & Columbia River & CGS STA 34 & 06-Apr-95 & 1 \\
\hline 17208 & Columbia River & 100-B Area & 28-Aug-95 & 1 \\
\hline 17209 & Columbia River & 100-K Area & 05-Sep-95 & 1 \\
\hline 17210 & Columbia River & HFD SPR 28-2 & 05-Sep-95 & 1 \\
\hline 17211 & Columbia River & 300 SPR 42-2 & 05-Sep-95 & 1 \\
\hline 17212 & Columbia River & 100-F Area & 12-Sep-95 & 1 \\
\hline 17312 & Columbia River & D Island & 18-Sep-95 & 1 \\
\hline 18445 & Columbia River & CGS STA 33 & 09-Oct-96 & 1 \\
\hline 18446 & Columbia River & CGS STA 34 & 09-Oct-96 & 1 \\
\hline 18470 & Columbia River & F Slough & 20-Aug-96 & 1 \\
\hline 18471 & Columbia River & Wht Bluffs Slg & 20-Aug-96 & 1 \\
\hline 18472 & Columbia River & Richland, WA & 20-Aug-96 & 1 \\
\hline 18473 & Columbia River & Hanford Slough & 20-Aug-96 & 1 \\
\hline 20043 & Columbia River & CGS STA 33 & 29-Oct-97 & 1 \\
\hline 20044 & Columbia River & CGS STA 34 & 29-Oct-97 & 1 \\
\hline 20496 & Columbia River & 100-K Area & 03-Nov-97 & 1 \\
\hline 20757 & Columbia River & $100 F 1 / 10$ & 17-Aug-98 & 1 \\
\hline 21207 & Columbia River & CGS STA 33 & 08-Apr-98 & 1 \\
\hline 21208 & Columbia River & CGS STA 34 & 08-Apr-98 & 1 \\
\hline
\end{tabular}


Table B-1

Summary Table of Segment 2 Sediment Samples By Area, Site, Date, and Category Columbia River Component - Existing Sample Data Evaluation and Scoping Report

\begin{tabular}{|c|c|c|c|c|}
\hline Sample ID & Sample Area & Sample Site & Sample Date & Category \\
\hline 23445 & Columbia River & CGS STA 34 & 19-Oct-99 & 1 \\
\hline 23523 & Columbia River & F Slough & 09-Aug-99 & 1 \\
\hline 23525 & Columbia River & 100F SPR 207-1 & 01-Nov-99 & 1 \\
\hline 23737 & Columbia River & CGS STA 33 & 19-Oct-99 & 1 \\
\hline 24625 & Columbia River & CGS STA 33 & 22-Mar-00 & 1 \\
\hline 24626 & Columbia River & CGS STA 34 & 22-Mar-00 & 1 \\
\hline 24750 & Columbia River & F Slough & 15-Aug-00 & 1 \\
\hline 24751 & Columbia River & HFD SPR 28-2 & 27-Sep-00 & 1 \\
\hline 24752 & Columbia River & 300 SPR 42-2 & 27-Sep-00 & 1 \\
\hline 24753 & Columbia River & 100F SPR 207-1 & 07-Nov-00 & 1 \\
\hline 25010 & Columbia River & 100N SPRINGS & 01-May-00 & 1 \\
\hline 26112 & Columbia River & CGS STA 33 & 21-Mar-01 & 1 \\
\hline 26113 & Columbia River & CGS STA 34 & 21-Mar-01 & 1 \\
\hline 26252 & Columbia River & F Slough & 19-Jul-01 & 1 \\
\hline 26255 & Columbia River & 100F SPR 207-1 & 22-Oct-01 & 1 \\
\hline 26256 & Columbia River & Richland, WA & 23-Jul-01 & 1 \\
\hline 26953 & Columbia River & 100B SPR 38-3 & 22-Oct-01 & 1 \\
\hline 27573 & Columbia River & F Slough & 16-Jul-01 & 1 \\
\hline 27574 & Columbia River & F Slough & 16-Jul-01 & 1 \\
\hline 27651 & Columbia River & 300 SPR 42-2 & 27-Aug-01 & 1 \\
\hline 27687 & Columbia River & 300 SPR 42-2 & 27-Aug-01 & 1 \\
\hline 27701 & Columbia River & 300 SPR 11 & 27-Aug-01 & 1 \\
\hline 27711 & Columbia River & 300 SPR 14 & 27-Aug-01 & 1 \\
\hline 28074 & Columbia River & CGS STA 33 & 29-Mar-02 & 1 \\
\hline 28075 & Columbia River & CGS STA 34 & 29-Mar-02 & 1 \\
\hline 29028 & Columbia River & HFD SPR 28-2 & 07-Oct-02 & 1 \\
\hline 29047 & Columbia River & F Slough & 23-Jul-02 & 1 \\
\hline 29417 & Columbia River & 100N SPRINGS & 24-Jun-02 & 1 \\
\hline 29551 & Columbia River & 100K SPR 63-2 & 16-Sep-02 & 1 \\
\hline 29695 & Columbia River & 100F SPR 207-1 & 29-Oct-02 & 1 \\
\hline 30584 & Columbia River & CGS STA 33 & 13-Mar-03 & 1 \\
\hline 30585 & Columbia River & CGS STA 34 & 13-Mar-03 & 1 \\
\hline 30874 & Columbia River & F Slough & 14-Jul-03 & 1 \\
\hline 30926 & Columbia River & 100K SPR 63-1 & 20-Oct-03 & 1 \\
\hline 30930 & Columbia River & 100F SPR 207-1 & 03-Nov-03 & 1 \\
\hline 30932 & Columbia River & 300 SPR $42-2$ & 13-Oct-03 & 1 \\
\hline 30934 & Columbia River & 100B SPR 37-1 & 20-Oct-03 & 1 \\
\hline 30939 & Columbia River & 300 SPR 42-2 & 13-Oct-03 & 1 \\
\hline 32830 & Columbia River & CGS STA 33 & 25-Mar-04 & 1 \\
\hline 32831 & Columbia River & CGS STA 34 & 25-Mar-04 & 1 \\
\hline 33073 & Columbia River & 300 SPRING 6 & 08-Dec-03 & 1 \\
\hline 33224 & Columbia River & 300 SPR 42-2 & 25-Oct-04 & 1 \\
\hline 34493 & Columbia River & CGS STA 33 & 10-Mar-05 & 1 \\
\hline 34494 & Columbia River & CGS STA 34 & 10-Mar-05 & 1 \\
\hline 4434235 & Columbia River & 300 Area & 01-Jun-04 & 1 \\
\hline 4434236 & Columbia River & RM 343.5 & 01-Jun-04 & 1 \\
\hline
\end{tabular}


Table B-1

Summary Table of Segment 2 Sediment Samples By Area, Site, Date, and Category Columbia River Component - Existing Sample Data Evaluation and Scoping Report

\begin{tabular}{|c|c|c|c|c|}
\hline Sample ID & Sample Area & Sample Site & Sample Date & Category \\
\hline 4434237 & Columbia River & RM 345 & 01-Jun-04 & 1 \\
\hline 4434238 & Columbia River & RM 356.5 & 01-Jun-04 & 1 \\
\hline 4434239 & Columbia River & RM 362 & 01-Jun-04 & 1 \\
\hline 4444201 & Columbia River & RM 354.4 & 01-Jun-04 & 1 \\
\hline 4434245 & Columbia River & RM 366 & 01-Jun-04 & 1 \\
\hline 4434246 & Columbia River & RM 370 & 01-Jun-04 & 1 \\
\hline 4465 & Columbia River & CGS STA 33 & 16-Apr-85 & 2 \\
\hline 4466 & Columbia River & CGS STA 34 & 16-Apr-85 & 2 \\
\hline 4976 & Columbia River & CGS STA 33 & 16-Apr-86 & 2 \\
\hline 4977 & Columbia River & CGS STA 34 & 16-Apr-86 & 2 \\
\hline 6135 & Columbia River & CGS STA 33 & 09-Apr-87 & 2 \\
\hline 6136 & Columbia River & CGS STA 34 & 09-Apr-87 & 2 \\
\hline 6431 & Columbia River & 100-H Area & 25-Jun-87 & 2 \\
\hline 6432 & Columbia River & 100-D Area & 25-Jun-87 & 2 \\
\hline 6433 & Columbia River & 100-D Area & 25-Jun-87 & 2 \\
\hline 6434 & Columbia River & Richland WA & 25-Jun-87 & 2 \\
\hline 6435 & Columbia River & Locke Island & 25-Jun-87 & 2 \\
\hline 7073 & Columbia River & CGS STA 33 & 21-Apr-88 & 2 \\
\hline 7074 & Columbia River & CGS STA 34 & 21-Apr-88 & 2 \\
\hline 72970 & Columbia River & CGS STA 33 & 09-Apr-92 & 1 \\
\hline 72971 & Columbia River & CGS STA 34 & 09-Apr-92 & 1 \\
\hline 7383 & Columbia River & 100-F Area & 06-Sep-88 & 2 \\
\hline $84-33-1$ & Columbia River & CGS STA 33 & $26-O c t-84$ & 1 \\
\hline $84-34-1$ & Columbia River & CGS STA 34 & $26-O c t-84$ & 1 \\
\hline $85-33-1$ & Columbia River & CGS STA 33 & 16-Apr-85 & 1 \\
\hline $85-33-2$ & Columbia River & CGS STA 33 & 10-Oct-85 & 1 \\
\hline $85-34-1$ & Columbia River & CGS STA 34 & 16-Apr-85 & 1 \\
\hline $85-34-2$ & Columbia River & CGS STA 34 & 10-Oct-85 & 1 \\
\hline $86-33-1$ & Columbia River & CGS STA 33 & 16-Apr-86 & 1 \\
\hline $86-33-2$ & Columbia River & CGS STA 33 & 25-Jun-86 & 1 \\
\hline $86-33-3$ & Columbia River & CGS STA 33 & 25-Jun-86 & 1 \\
\hline $86-33-4$ & Columbia River & CGS STA 33 & 21-Oct-86 & 1 \\
\hline $86-34-1$ & Columbia River & CGS STA 34 & 16-Apr-86 & 1 \\
\hline $86-34-2$ & Columbia River & CGS STA 34 & 25-Jun-86 & 1 \\
\hline $86-34-3$ & Columbia River & CGS STA 34 & 25-Jun-86 & 1 \\
\hline $86-34-4$ & Columbia River & CGS STA 34 & 21-Jan-86 & 1 \\
\hline $87-33-1$ & Columbia River & CGS STA 33 & 09-Apr-87 & 1 \\
\hline $87-33-2$ & Columbia River & CGS STA 33 & 14-Oct-87 & 1 \\
\hline $87-34-1$ & Columbia River & CGS STA 34 & 09-Apr-87 & 1 \\
\hline $87-34-2$ & Columbia River & CGS STA 34 & 14-Oct-87 & 1 \\
\hline $88-33-1$ & Columbia River & CGS STA 33 & 21-Apr-88 & 1 \\
\hline $88-33-2$ & Columbia River & CGS STA 33 & $26-$ Oct-88 & 1 \\
\hline $88-34-1$ & Columbia River & CGS STA 34 & 21-Apr-88 & 1 \\
\hline $88-34-2$ & Columbia River & CGS STA 34 & 26-Oct-88 & 1 \\
\hline 89-33-1 & Columbia River & CGS STA 33 & 11-Apr-89 & 1 \\
\hline 89-33-2 & Columbia River & CGS STA 33 & 19-Oct-89 & 1 \\
\hline
\end{tabular}


Table B-1

Summary Table of Segment 2 Sediment Samples By Area, Site, Date, and Category Columbia River Component - Existing Sample Data Evaluation and Scoping Report

\begin{tabular}{|c|c|c|c|c|}
\hline Sample ID & Sample Area & Sample Site & Sample Date & Category \\
\hline $89-34-1$ & Columbia River & CGS STA 34 & 11-Apr-89 & 1 \\
\hline $89-34-2$ & Columbia River & CGS STA 34 & 19-Oct-89 & 1 \\
\hline $90-33-1$ & Columbia River & CGS STA 33 & 19-Apr-90 & 1 \\
\hline $90-33-2$ & Columbia River & CGS STA 33 & $16-$ Oct-90 & 1 \\
\hline $90-34-1$ & Columbia River & CGS STA 34 & 19-Apr-90 & 1 \\
\hline $90-34-2$ & Columbia River & CGS STA 34 & $16-$ Oct-90 & 1 \\
\hline $91-33-1$ & Columbia River & CGS STA 33 & 10-Apr-91 & 1 \\
\hline $91-33-2$ & Columbia River & CGS STA 33 & 03-Oct-91 & 1 \\
\hline $91-34-1$ & Columbia River & CGS STA 34 & 10-Apr-91 & 1 \\
\hline $91-34-2$ & Columbia River & CGS STA 34 & 03-Oct-91 & 1 \\
\hline $93-33-1$ & Columbia River & CGS STA 33 & 15-Apr-93 & 1 \\
\hline $93-33-2$ & Columbia River & CGS STA 33 & 06-Oct-93 & 1 \\
\hline $93-34-1$ & Columbia River & CGS STA 34 & 15-Apr-93 & 1 \\
\hline $93-34-2$ & Columbia River & CGS STA 34 & 06-Oct-93 & 1 \\
\hline 93607 & Columbia River & CGS STA 33 & 20-Oct-92 & 1 \\
\hline 93608 & Columbia River & CGS STA 34 & 20-Oct-92 & 1 \\
\hline $94-33-1$ & Columbia River & CGS STA 33 & 07-Apr-94 & 1 \\
\hline $94-33-2$ & Columbia River & CGS STA 34 & 05-Oct-94 & 1 \\
\hline $94-34-1$ & Columbia River & CGS STA 34 & 07-Apr-94 & 1 \\
\hline $94-34-2$ & Columbia River & CGS STA 33 & 05-Oct-94 & 1 \\
\hline $95-33-1$ & Columbia River & CGS STA 33 & 06-Apr-95 & 1 \\
\hline $95-33-2$ & Columbia River & CGS STA 34 & 25-Oct-95 & 1 \\
\hline $95-34-1$ & Columbia River & CGS STA 34 & 06-Apr-95 & 1 \\
\hline $95-34-2$ & Columbia River & CGS STA 34 & 25-Oct-95 & 1 \\
\hline $96-33-1$ & Columbia River & CGS STA 33 & 09-Oct-96 & 1 \\
\hline $96-34-1$ & Columbia River & CGS STA 34 & 09-Oct-96 & 1 \\
\hline $97-33-1$ & Columbia River & CGS STA 33 & 29-Oct-97 & 1 \\
\hline $97-34-1$ & Columbia River & CGS STA 34 & 29-Oct-97 & 1 \\
\hline 9731 & Columbia River & CGS STA 33 & 19-Apr-90 & 1 \\
\hline 9732 & Columbia River & CGS STA 34 & 19-Apr-90 & 1 \\
\hline $98-33-1$ & Columbia River & CGS STA 33 & 08-Apr-98 & 1 \\
\hline $98-33-2$ & Columbia River & CGS STA 33 & 21-Oct-98 & 1 \\
\hline $98-34-1$ & Columbia River & CGS STA 34 & 08-Apr-98 & 1 \\
\hline $98-34-2$ & Columbia River & CGS STA 34 & 21-Oct-98 & 1 \\
\hline $99-33-1$ & Columbia River & CGS STA 33 & 19-Oct-99 & 1 \\
\hline $99-34-1$ & Columbia River & CGS STA 34 & 19-Oct-99 & 1 \\
\hline B07NB6 & Columbia River & F Slough & 12-Nov-92 & 1 \\
\hline B07NB7 & Columbia River & F Slough & 12-Nov-92 & 1 \\
\hline B07NB8 & Columbia River & F Slough & 12-Nov-92 & 1 \\
\hline B07NB9 & Columbia River & F Slough & 12-Nov-92 & 1 \\
\hline B07NC2 & Columbia River & 100-H Area & 13-Nov-92 & 1 \\
\hline B07NC3 & Columbia River & 100-H Area & 13-Nov-92 & 1 \\
\hline B07NC4 & Columbia River & F Slough & 16-Nov-92 & 1 \\
\hline B07NC5 & Columbia River & F Slough & 16-Nov-92 & 1 \\
\hline B07NC6 & Columbia River & Hanford Tnsite & 16-Nov-92 & 1 \\
\hline B07NC7 & Columbia River & Hanford Tnsite & 16-Nov-92 & 1 \\
\hline
\end{tabular}


Table B-1

Summary Table of Segment 2 Sediment Samples By Area, Site, Date, and Category Columbia River Component - Existing Sample Data Evaluation and Scoping Report

\begin{tabular}{|c|c|c|c|c|}
\hline Sample ID & Sample Area & Sample Site & Sample Date & Category \\
\hline B07NC9 & Columbia River & Horn Area & 17-Nov-92 & 1 \\
\hline B07ND0 & Columbia River & Horn Area & 17-Nov-92 & 1 \\
\hline B07ND1 & Columbia River & 100-B/C Area & 20-Nov-92 & 1 \\
\hline B07ND2 & Columbia River & Hanford Tnsite & 17-Nov-92 & 1 \\
\hline B07ND3 & Columbia River & Hanford Tnsite & 17-Nov-92 & 1 \\
\hline B07ND4 & Columbia River & D Island & 19-Nov-92 & 1 \\
\hline B07ND5 & Columbia River & D Island & 19-Nov-92 & 1 \\
\hline B07ND6 & Columbia River & D Island & 19-Nov-92 & 1 \\
\hline B07ND7 & Columbia River & D Island & 19-Nov-92 & 1 \\
\hline B07ND8 & Columbia River & D Island & 19-Nov-92 & 1 \\
\hline B07ND9 & Columbia River & 100-B/C Area & 20-Nov-92 & 1 \\
\hline B07NF0 & Columbia River & Horn Area & 19-Nov-92 & 1 \\
\hline B07NF1 & Columbia River & 100-D Area & 19-Nov-92 & 1 \\
\hline B07NF2 & Columbia River & 100-D Area & 19-Nov-92 & 1 \\
\hline B07NF9 & Columbia River & 100-K Area & 21-Nov-92 & 1 \\
\hline B07NG0 & Columbia River & 100-K Area & 21-Nov-92 & 1 \\
\hline B07NG1 & Columbia River & 100-K Area & 21-Nov-92 & 1 \\
\hline B07NG2 & Columbia River & 100-K Area & 21-Nov-92 & 1 \\
\hline B07NG3 & Columbia River & 100-H Area & 22-Nov-92 & 1 \\
\hline B07NG5 & Columbia River & 100-H Area & 22-Nov-92 & 1 \\
\hline B07NG6 & Columbia River & 100-H Area & 22-Nov-92 & 1 \\
\hline B07NG7 & Columbia River & 100-H Area & 22-Nov-92 & 1 \\
\hline B07NG9 & Columbia River & F Slough & 23-Nov-92 & 1 \\
\hline B07NHO & Columbia River & F Slough & 23-Nov-92 & 1 \\
\hline B07NH1 & Columbia River & Horn Area & 23-Nov-92 & 1 \\
\hline $\mathrm{B} 07 \mathrm{NH} 2$ & Columbia River & Horn Area & 23-Nov-92 & 1 \\
\hline B07NH3 & Columbia River & Hanford Tnsite & 23-Nov-92 & 1 \\
\hline B07NH4 & Columbia River & Hanford Tnsite & 23-Nov-92 & 1 \\
\hline B07NH5 & Columbia River & D Island & 24-Nov-92 & 1 \\
\hline B07NH6 & Columbia River & D Island & 24-Nov-92 & 1 \\
\hline B07NH7 & Columbia River & D Island & 24-Nov-92 & 1 \\
\hline BOBRV4 & Columbia River & Wht Bluffs Slg & 15-Apr-94 & 1 \\
\hline BOBRV6 & Columbia River & F Slough & 15-Apr-94 & 1 \\
\hline BOBRV8 & Columbia River & Hanford Slough & 15-Apr-94 & 1 \\
\hline BOCSH3 & Columbia River & Wht Bluffs Slg & 24-Sep-94 & 1 \\
\hline B0CSH5 & Columbia River & Hanford Slough & 25-Sep-94 & 1 \\
\hline B0CSH6 & Columbia River & Richland, WA & 25-Sep-94 & 1 \\
\hline BOCSH8 & Columbia River & HFD SPR 28-2 & 02-Sep-94 & 1 \\
\hline B0W3F3 & Columbia River & Wht Bluffs Slg & 09-Aug-99 & 1 \\
\hline B0W3F4 & Columbia River & Wht Bluffs Slg & 09-Aug-99 & 1 \\
\hline B0W3F5 & Columbia River & 100 F SLOUGH & 09-Aug-99 & 1 \\
\hline B0W3F6 & Columbia River & 100 F SLOUGH & 09-Aug-99 & 1 \\
\hline B0W3F7 & Columbia River & Hanford Slough & 09-Aug-99 & 1 \\
\hline B0W3F8 & Columbia River & Hanford Slough & 09-Aug-99 & 1 \\
\hline B0W3H7 & Columbia River & Hanford Slough & 09-Aug-99 & 1 \\
\hline В0W3H8 & Columbia River & RICHLAND-RIVER & 03-Sep-99 & 1 \\
\hline
\end{tabular}


Table B-1

Summary Table of Segment 2 Sediment Samples By Area, Site, Date, and Category Columbia River Component - Existing Sample Data Evaluation and Scoping Report

\begin{tabular}{|c|c|c|c|c|}
\hline Sample ID & Sample Area & Sample Site & Sample Date & Category \\
\hline B0W3L9 & Columbia River & Wht Bluffs Slg & 09-Aug-99 & 1 \\
\hline BOW3M0 & Columbia River & 100 F SLOUGH & 09-Aug-99 & 1 \\
\hline B0W3V1 & Columbia River & 100 F SLOUGH & 09-Aug-99 & 1 \\
\hline B0W3V2 & Columbia River & Hanford Slough & 09-Aug-99 & 1 \\
\hline Bow3W9 & Columbia River & Wht Bluffs Slg & 09-Aug-99 & 1 \\
\hline BOWDL4 & Columbia River & 300 SPR 42-2 & 01-Nov-99 & 1 \\
\hline B0WDL8 & Columbia River & 300 SPR 42-2 & 01-Nov-99 & 1 \\
\hline BOWDP4 & Columbia River & 100-B SPR 38-3 & 25-Oct-99 & 1 \\
\hline B0WDP5 & Columbia River & 100-B SPR 38-3 & 25-Oct-99 & 1 \\
\hline BOWDP8 & Columbia River & HFD SPR UR 28-2 & 01-Nov-99 & 1 \\
\hline BOWDP9 & Columbia River & HFD SPR UR 28-2 & 01-Nov-99 & 1 \\
\hline BOWDRO & Columbia River & HFD SPR DR 28-2 & 01-Nov-99 & 1 \\
\hline BOWDR1 & Columbia River & HFD SPR DR 28-2 & 01-Nov-99 & 1 \\
\hline BOWDR2 & Columbia River & 300 SPR DR 42-2 & 01-Nov-99 & 1 \\
\hline BOWDR3 & Columbia River & 300 SPR DR $42-2$ & 01-Nov-99 & 1 \\
\hline BOWDR8 & Columbia River & 100-F SPR 207-1 & 01-Nov-99 & 1 \\
\hline BOWDT0 & Columbia River & 100-F SPR 207-1 & 01-Nov-99 & 1 \\
\hline BOWFMO & Columbia River & Wht Bluffs Slg & 09-Aug-99 & 1 \\
\hline BOWFM1 & Columbia River & Wht Bluffs Slg & 09-Aug-99 & 1 \\
\hline BOWFM2 & Columbia River & Wht Bluffs Slg & 09-Aug-99 & 1 \\
\hline BOWFM3 & Columbia River & Wht Bluffs Slg & 09-Aug-99 & 1 \\
\hline BOWFM5 & Columbia River & 100 F SLOUGH & 09-Aug-99 & 1 \\
\hline BoWFM6 & Columbia River & 100 F SLOUGH & 09-Aug-99 & 1 \\
\hline BOWFM7 & Columbia River & 100 F SLOUGH & 09-Aug-99 & 1 \\
\hline BOWFM8 & Columbia River & 100 F SLOUGH & 09-Aug-99 & 1 \\
\hline BOWFNO & Columbia River & Hanford Slough & 09-Aug-99 & 1 \\
\hline BOWFN1 & Columbia River & Hanford Slough & 09-Aug-99 & 1 \\
\hline BOWFN2 & Columbia River & Hanford Slough & 09-Aug-99 & 1 \\
\hline BOWFN3 & Columbia River & Hanford Slough & 09-Aug-99 & 1 \\
\hline BOWFN5 & Columbia River & RICHLAND-RIVER & 03-Sep-99 & 1 \\
\hline BOWFN6 & Columbia River & RICHLAND-RIVER & 03-Sep-99 & 1 \\
\hline BOWFN7 & Columbia River & RICHLAND-RIVER & 03-Sep-99 & 1 \\
\hline BOWFN8 & Columbia River & RICHLAND-RIVER & 03-Sep-99 & 1 \\
\hline B0YW84 & Columbia River & RICHLAND-RIVER & 08-Aug-00 & 1 \\
\hline B0YW85 & Columbia River & RICHLAND-RIVER & 08-Aug-00 & 1 \\
\hline BoYW87 & Columbia river & RICHLAND-RIVER & 08-Aug-00 & 1 \\
\hline B0YY64 & Columbia River & Wht Bluffs Slg & 15-Aug-00 & 1 \\
\hline B0YY65 & Columbia River & Wht Bluffs Slg & 15-Aug-00 & 1 \\
\hline B0YY66 & Columbia River & 100 F SLOUGH & 15-Aug-00 & 1 \\
\hline B0YY67 & Columbia River & 100 F SLOUGH & 15-Aug-00 & 1 \\
\hline B0YY68 & Columbia River & Hanford Slough & 15-Aug-00 & 1 \\
\hline B0YY69 & Columbia River & Hanford Slough & 15-Aug-00 & 1 \\
\hline BOYY70 & Columbia river & Hanford Slough & 15-Aug-00 & 1 \\
\hline B0YY71 & Columbia river & Wht Bluffs Slg & 15-Aug-00 & 1 \\
\hline B0YY72 & Columbia river & 100 F SLOUGH & 15-Aug-00 & 1 \\
\hline B10167 & Columbia river & Hanford Slough & 15-Aug-00 & 1 \\
\hline
\end{tabular}


Table B-1

Summary Table of Segment 2 Sediment Samples By Area, Site, Date, and Category Columbia River Component - Existing Sample Data Evaluation and Scoping Report

\begin{tabular}{|c|c|c|c|c|}
\hline Sample ID & Sample Area & Sample Site & Sample Date & Category \\
\hline B10168 & Columbia river & Wht Bluffs Slg & 15-Aug-00 & 1 \\
\hline B10169 & Columbia river & 100 F SLOUGH & 15-Aug-00 & 1 \\
\hline B10905 & Columbia River & 300 SPR 42-2 & 02-Nov-00 & 1 \\
\hline B10908 & Columbia river & 300 SPR 42-2 & 27-Sep-00 & 1 \\
\hline B10915 & Columbia river & 100-B SPR 38-3 & 25-Oct-00 & 1 \\
\hline B10916 & Columbia River & 100-B SPR 38-3 & 20-Nov-00 & 1 \\
\hline B10917 & Columbia river & HFD SPR UR 28-2 & 27-Sep-00 & 1 \\
\hline B10918 & Columbia River & HFD SPR UR 28-2 & 02-Nov-00 & 1 \\
\hline B10919 & Columbia river & HFD SPR DR 28-2 & 27-Sep-00 & 1 \\
\hline B10921 & Columbia River & HFD SPR DR 28-2 & 02-Nov-00 & 1 \\
\hline B10922 & Columbia river & 300 SPR DR 42-2 & 27-Sep-00 & 1 \\
\hline B10923 & Columbia River & 300 SPR DR 42-2 & 02-Nov-00 & 1 \\
\hline B10929 & Columbia river & 100-F SPR 207-1 & 07-Nov-00 & 1 \\
\hline B10931 & Columbia River & 100-F SPR 207-1 & 20-Nov-00 & 1 \\
\hline B12CJ7 & Columbia River & 100 F SLOUGH & 19-Jul-01 & 1 \\
\hline B12CJ9 & Columbia River & Hanford Slough & 19-Jul-01 & 1 \\
\hline B12CK1 & Columbia River & RICHLAND-RIVER & 23-Jul-01 & 1 \\
\hline B12CK8 & Columbia River & Hanford Slough & 19-Jul-01 & 1 \\
\hline B12CK9 & Columbia River & RICHLAND-RIVER & 23-Jul-01 & 1 \\
\hline B12CL5 & Columbia River & Wht Bluffs Slg & 19-Jul-01 & 1 \\
\hline B12CL6 & Columbia River & 100 F SLOUGH & 19-Jul-01 & 1 \\
\hline В12CT0 & Columbia River & 100 F SLOUGH & 19-Jul-01 & 1 \\
\hline B12CT1 & Columbia River & Hanford Slough & 19-Jul-01 & 1 \\
\hline B12CV3 & Columbia River & RICHLAND-RIVER & 23-Jul-01 & 1 \\
\hline B12CV4 & Columbia River & Wht Bluffs Slg & 19-Jul-01 & 1 \\
\hline B12RL7 & Columbia River & 300 SPR 42-2 & 27-Aug-01 & 1 \\
\hline B12RL9 & Columbia River & 300 SPR 42-2 & 27-Aug-01 & 1 \\
\hline B12RM5 & Columbia River & 300 SPR DR 42-2 & 27-Aug-01 & 1 \\
\hline B12RY8 & Columbia River & 300 SPR 11 & 27-Aug-01 & 1 \\
\hline B12RY9 & Columbia River & 300 SPR 11 & 27-Aug-01 & 1 \\
\hline В12Т01 & Columbia River & 300 SPR 14 & 27-Aug-01 & 1 \\
\hline В12Т04 & Columbia River & 300 SPR 42-2 & 27-Aug-01 & 1 \\
\hline В12Т05 & Columbia River & 300 SPR DR 42-2 & 27-Aug-01 & 1 \\
\hline В12Т06 & Columbia River & 300 SPR 11 & 27-Aug-01 & 1 \\
\hline В12Т07 & Columbia River & 300 SPR 14 & 27-Aug-01 & 1 \\
\hline В12Т08 & Columbia River & 300 SPR 14 & 27-Aug-01 & 1 \\
\hline B12X20 & Columbia River & 100-B SPR 38-3 & 22-Oct-01 & 1 \\
\hline B12X22 & Columbia River & 100-B SPR 38-3 & 22-Oct-01 & 1 \\
\hline B12X35 & Columbia River & 100-F SPR 207-1 & 22-Oct-01 & 1 \\
\hline B12X37 & Columbia River & 100-F SPR 207-1 & 22-Oct-01 & 1 \\
\hline B14WF4 & Columbia River & 100 F SLOUGH & 23-Jul-02 & 1 \\
\hline B14WF5 & Columbia River & Hanford Slough & 23-Jul-02 & 1 \\
\hline $\mathrm{B} 14 \mathrm{WHO}$ & Columbia River & RICHLAND-RIVER & 19-Jul-02 & 1 \\
\hline B14WH1 & Columbia River & Wht Bluffs Slg & 23-Jul-02 & 1 \\
\hline B14YL6 & Columbia River & Wht Bluffs Slg & 23-Jul-02 & 1 \\
\hline B14YL7 & Columbia River & Wht Bluffs Slg & 23-Jul-02 & 1 \\
\hline
\end{tabular}


Table B-1

Summary Table of Segment 2 Sediment Samples By Area, Site, Date, and Category Columbia River Component - Existing Sample Data Evaluation and Scoping Report

\begin{tabular}{|c|c|c|c|c|}
\hline Sample ID & Sample Area & Sample Site & Sample Date & Category \\
\hline B14YL8 & Columbia River & 100 F SLOUGH & 23-Jul-02 & 1 \\
\hline B14YL9 & Columbia River & 100 F SLOUGH & 23-Jul-02 & 1 \\
\hline B14YM0 & Columbia River & Hanford Slough & 23-Jul-02 & 1 \\
\hline B14YM1 & Columbia River & Hanford Slough & 23-Jul-02 & 1 \\
\hline B14YM2 & Columbia River & RICHLAND-RIVER & 19-Jul-02 & 1 \\
\hline B14YM3 & Columbia River & RICHLAND-RIVER & 19-Jul-02 & 1 \\
\hline B14YM4 & Columbia River & Hanford Slough & 23-Jul-02 & 1 \\
\hline B14YM5 & Columbia River & RICHLAND-RIVER & 19-Jul-02 & 1 \\
\hline B14YM6 & Columbia River & Wht Bluffs Slg & 23-Jul-02 & 1 \\
\hline B14YM7 & Columbia River & 100 F SLOUGH & 23-Jul-02 & 1 \\
\hline B15C03 & Columbia River & 300 SPR 42-2 & 07-Oct-02 & 1 \\
\hline B15C07 & Columbia River & 300 SPR 42-2 & 07-Oct-02 & 1 \\
\hline B15C40 & Columbia River & 100-B SPR 37-1 & 16-Sep-02 & 1 \\
\hline B15C41 & Columbia River & 100-B SPR 37-1 & 16-Sep-02 & 1 \\
\hline B15C44 & Columbia River & HFD SPR DR 28-2 & 07-Oct-02 & 1 \\
\hline B15C46 & Columbia River & HFD SPR DR 28-2 & 07-Oct-02 & 1 \\
\hline B15C47 & Columbia River & 300 SPR DR 42-2 & 07-Oct-02 & 1 \\
\hline B15C48 & Columbia River & 300 SPR DR 42-2 & 07-Oct-02 & 1 \\
\hline B15C49 & Columbia River & 100-K SPR 63-2 & 16-Sep-02 & 1 \\
\hline B15C51 & Columbia River & 100-K SPR 63-2 & 16-Sep-02 & 1 \\
\hline B15C52 & Columbia River & 100-F SPR 207-1 & 29-Oct-02 & 1 \\
\hline B15C54 & Columbia River & 100-F SPR 207-1 & 29-Oct-02 & 1 \\
\hline B16WW4 & Columbia River & Ringold WW & 14-May-03 & 1 \\
\hline B16WW5 & Columbia River & Ringold WW & 14-May-03 & 1 \\
\hline B16WW6 & Columbia River & ESQUATZEL CANAL & 14-May-03 & 1 \\
\hline B16WW7 & Columbia River & ESQUATZEL CANAL & 14-May-03 & 1 \\
\hline B16WX6 & Columbia River & Ringold WW & 14-May-03 & 1 \\
\hline B16WX7 & Columbia River & Ringold WW & 14-May-03 & 1 \\
\hline B16WX8 & Columbia River & ESQUATZEL CANAL & 14-May-03 & 1 \\
\hline B16WX9 & Columbia River & ESQUATZEL CANAL & 14-May-03 & 1 \\
\hline B170V0 & Columbia River & ESQUATZEL CANAL & 14-May-03 & 1 \\
\hline B170V1 & Columbia River & ESQUATZEL CANAL & 14-May-03 & 1 \\
\hline B170V4 & Columbia River & Ringold WW & 14-May-03 & 1 \\
\hline B170V5 & Columbia River & Ringold WW & 14-May-03 & 1 \\
\hline B176T7 & Columbia River & 100 F SLOUGH & 14-Jul-03 & 1 \\
\hline В176T8 & Columbia River & RICHLAND-RIVER & $16-J u l-03$ & 1 \\
\hline B176T9 & Columbia River & Wht Bluffs Slg & 14-Jul-03 & 1 \\
\hline B176V0 & Columbia River & Hanford Slough & 14-Jul-03 & 1 \\
\hline B176X8 & Columbia River & Wht Bluffs Slg & 14-Jul-03 & 1 \\
\hline B176X9 & Columbia River & Wht Bluffs Slg & 14-Jul-03 & 1 \\
\hline B176Y0 & Columbia River & 100 F SLOUGH & 14-Jul-03 & 1 \\
\hline B176Y1 & Columbia River & 100 F SLOUGH & 14-Jul-03 & 1 \\
\hline B176Y2 & Columbia River & Hanford Slough & 14-Jul-03 & 1 \\
\hline B176Y3 & Columbia River & Hanford Slough & 14-Jul-03 & 1 \\
\hline B176Y4 & Columbia River & RICHLAND-RIVER & 16-Jul-03 & 1 \\
\hline B176Y5 & Columbia River & RICHLAND-RIVER & 16-Jul-03 & 1 \\
\hline
\end{tabular}


Table B-1

Summary Table of Segment 2 Sediment Samples By Area, Site, Date, and Category Columbia River Component - Existing Sample Data Evaluation and Scoping Report

\begin{tabular}{|c|c|c|c|c|}
\hline Sample ID & Sample Area & Sample Site & Sample Date & Category \\
\hline B176Y6 & Columbia River & Hanford Slough & 14-Jul-03 & 1 \\
\hline B176Y7 & Columbia River & RICHLAND-RIVER & 16-Jul-03 & 1 \\
\hline B176Y8 & Columbia River & Wht Bluffs Slg & 14-Jul-03 & 1 \\
\hline B176Y9 & Columbia River & 100 F SLOUGH & 14-Jul-03 & 1 \\
\hline B17J18 & Columbia River & 300 SPR 42-2 & 13-Oct-03 & 1 \\
\hline B17J20 & Columbia River & 300 SPR 42-2 & 13-Oct-03 & 1 \\
\hline B17J51 & Columbia River & 100-B SPR 37-1 & 20-Oct-03 & 1 \\
\hline B17J53 & Columbia River & 100-B SPR 37-1 & 20-Oct-03 & 1 \\
\hline B17J58 & Columbia River & HFD SPR DR 28-2 & 03-Nov-03 & 1 \\
\hline B17J59 & Columbia River & 300 SPR DR 42-2 & 13-Oct-03 & 1 \\
\hline B17J61 & Columbia River & 300 SPR DR 42-2 & 13-Oct-03 & 1 \\
\hline B17J62 & Columbia River & 100-K SPR 63-1 & 20-Oct-03 & 1 \\
\hline B17J64 & Columbia River & 100-K SPR 63-1 & 20-Oct-03 & 1 \\
\hline B17J65 & Columbia River & 100-F SPR 207-1 & 03-Nov-03 & 1 \\
\hline B17J67 & Columbia River & 100-F SPR 207-1 & 03-Nov-03 & 1 \\
\hline B17J99 & Columbia River & 100-H SPR 145-1 & 27-Oct-03 & 1 \\
\hline B17JB1 & Columbia River & 100-H SPR 145-1 & 27-Oct-03 & 1 \\
\hline B17XN6 & Columbia River & 100 B/C AREA UR & 17-Nov-03 & 1 \\
\hline B17XN8 & Columbia River & 100-B SPR 37-1 & 13-Nov-03 & 1 \\
\hline B17XN9 & Columbia River & 100-B SPR 38-3 & 13-Nov-03 & 1 \\
\hline В199H6 & Columbia River & North 300 Area & 02-Aug-04 & 1 \\
\hline B199J0 & Columbia River & Hanford Tnsite & 07-Jun-04 & 1 \\
\hline B199J7 & Columbia River & Ringold & 03-Jun-04 & 1 \\
\hline B19PJ5 & Columbia River & Wht Bluffs Slg & 12-Jul-04 & 1 \\
\hline B19PJ6 & Columbia River & Wht Bluffs Slg & 12-Jul-04 & 1 \\
\hline B19PJ7 & Columbia River & 100 F SLOUGH & 12-Jul-04 & 1 \\
\hline B19PJ8 & Columbia River & 100 F SLOUGH & 12-Jul-04 & 1 \\
\hline B19PJ9 & Columbia River & Hanford Slough & 12-Jul-04 & 1 \\
\hline B19PK0 & Columbia River & Hanford Slough & 12-Jul-04 & 1 \\
\hline B19PK1 & Columbia River & RICHLAND-RIVER & 13-Jul-04 & 1 \\
\hline B19PK2 & Columbia River & RICHLAND-RIVER & 13-Jul-04 & 1 \\
\hline B19PK3 & Columbia River & Hanford Slough & 12-Jul-04 & 1 \\
\hline B19PK4 & Columbia River & RICHLAND-RIVER & 13-Jul-04 & 1 \\
\hline B19PK5 & Columbia River & Wht Bluffs Slg & 12-Jul-04 & 1 \\
\hline B19PK6 & Columbia River & 100 F SLOUGH & 12-Jul-04 & 1 \\
\hline B19TF9 & Columbia River & Wht Bluffs Slg & 12-Jul-04 & 1 \\
\hline В19ТН0 & Columbia River & Hanford Slough & 12-Jul-04 & 1 \\
\hline В19TH1 & Columbia River & RICHLAND-RIVER & 13-Jul-04 & 1 \\
\hline B19TH2 & Columbia River & 100 F SLOUGH & 12-Jul-04 & 1 \\
\hline B1BFM1 & Columbia River & 300 SPR 42-2 & 25-Oct-04 & 1 \\
\hline B1BFN9 & Columbia River & 300 SPR 42-2 & 25-Oct-04 & 1 \\
\hline B1BFP6 & Columbia River & 100-B SPR 37-1 & 11-Oct-04 & 1 \\
\hline B1BFP7 & Columbia River & 100-B SPR 37-1 & 11-Oct-04 & 1 \\
\hline B1BFP8 & Columbia River & HFD SPR UR 28-2 & 25-Oct-04 & 1 \\
\hline B1BFP9 & Columbia River & HFD SPR UR 28-2 & 25-Oct-04 & 1 \\
\hline B1BFR2 & Columbia River & HFD SPR DR 28-2 & 25-Oct-04 & 1 \\
\hline
\end{tabular}


Table B-1

\section{Summary Table of Segment 2 Sediment Samples By Area, Site, Date, and Category Columbia River Component - Existing Sample Data Evaluation and Scoping Report}

\begin{tabular}{|c|c|c|c|c|}
\hline Sample ID & Sample Area & Sample Site & Sample Date & Category \\
\hline B1BFR3 & Columbia River & 300 SPR DR 42-2 & 25-Oct-04 & 1 \\
\hline B1BFR5 & Columbia River & 300 SPR DR 42-2 & 25-Oct-04 & 1 \\
\hline B1BFR9 & Columbia River & 100-F SPR 207-1 & 26-Oct-04 & 1 \\
\hline B1BFT0 & Columbia River & 100-F SPR 207-1 & 26-Oct-04 & 1 \\
\hline B1BH02 & Columbia River & 100-H SPR 145-1 & 26-Oct-04 & 1 \\
\hline В1BH03 & Columbia River & 100-H SPR 145-1 & 26-Oct-04 & 1 \\
\hline B1BH12 & Columbia River & 300 SPR 41-9 & 25-Oct-04 & 1 \\
\hline B1BH14 & Columbia River & RICHLAND SPR & 25-Oct-04 & 1 \\
\hline CGS-89-8081 & Columbia River & CGS STA 33 & 11-Apr-89 & 1 \\
\hline CGS-89-8082 & Columbia River & CGS STA 34 & 11-Apr-89 & 1 \\
\hline CGS-89-8407 & Columbia River & CGS STA 34 & 07-Jun-89 & 1 \\
\hline HR-location 1 & Columbia River & RM 393 & 01-Aug-86 & 3 \\
\hline HR-location 2 & Columbia River & RM 378.5 & 01-Aug-86 & 3 \\
\hline HR-location 3 & Columbia River & RM 373 & 01-Aug-86 & 3 \\
\hline HR-location 4 & Columbia River & RM 365 & 01-Aug-86 & 3 \\
\hline HR004W & Columbia River & 100-B/C Area & 07-Oct-03 & 1 \\
\hline HR006B/C & Columbia River & 100-B/C Area & 07-Oct-03 & 1 \\
\hline HR016N & Columbia River & 100-N Area & 09-Oct-03 & 1 \\
\hline HR019N & Columbia River & 100-N Area & 09-Oct-03 & 1 \\
\hline HR020N & Columbia River & 100-N Area & 09-Oct-03 & 1 \\
\hline HR023D & Columbia River & 100-D Area & 08-Oct-03 & 1 \\
\hline $\mathrm{HR} 027 \mathrm{H}$ & Columbia River & H Reactor Area & 09-Oct-03 & 1 \\
\hline HR032F & Columbia River & F Reactor Area & 09-Oct-03 & 1 \\
\hline HR037-300 & Columbia River & 300 Upstream & 04-Aug-04 & 1 \\
\hline HR046-300 & Columbia River & 300 Area & 04-Aug-04 & 1 \\
\hline HR048-300 & Columbia River & 300 Area & 04-Aug-04 & 1 \\
\hline HR063S & Columbia River & 300 Outfall & 04-Aug-04 & 1 \\
\hline L15057-1 & Columbia River & CGS STA 33 & 21-Mar-01 & 1 \\
\hline L15057-2 & Columbia River & CGS STA 34 & 21-Mar-01 & 1 \\
\hline L16927-1 & Columbia River & CGS STA 33 & 31-Oct-01 & 1 \\
\hline L16927-2 & Columbia River & CGS STA 34 & 31-Oct-01 & 1 \\
\hline L17850-1 & Columbia River & CGS STA 33 & 20-Mar-02 & 1 \\
\hline L17850-2 & Columbia River & CGS STA 34 & 20-Mar-02 & 1 \\
\hline L19569-2 & Columbia River & CGS STA 33 & 29-Oct-02 & 1 \\
\hline L19569-3 & Columbia River & CGS STA 34 & 29-Oct-02 & 1 \\
\hline L20678-1 & Columbia River & CGS STA 33 & 13-Mar-03 & 1 \\
\hline L20678-2 & Columbia River & CGS STA 34 & 13-Mar-03 & 1 \\
\hline L22408-1 & Columbia River & CGS STA 33 & 30-Oct-03 & 1 \\
\hline L22408-2 & Columbia River & CGS STA 34 & 30-Oct-03 & 1 \\
\hline L23195-1 & Columbia River & CGS STA 33 & 25-Mar-04 & 1 \\
\hline L23195-2 & Columbia River & CGS STA 34 & 25-Mar-04 & 1 \\
\hline L23195-3 & Columbia River & CGS STA 33 & 25-Mar-04 & 1 \\
\hline L24794-1 & Columbia River & CGS STA 33 & 07-Dec-04 & 1 \\
\hline L24794-2 & Columbia River & CGS STA 34 & 07-Dec-04 & 1 \\
\hline SD_78-33-1 & Columbia River & CGS STA 33 & 17-May-78 & 2 \\
\hline SD_78-33-2 & Columbia River & CGS STA 33 & 21-Dec-78 & 2 \\
\hline
\end{tabular}


Table B-1

Summary Table of Segment 2 Sediment Samples By Area, Site, Date, and Category Columbia River Component - Existing Sample Data Evaluation and Scoping Report

\begin{tabular}{llccc} 
Sample ID & Sample Area & Sample Site & Sample Date & Category \\
\hline SD_78-34-1 & Columbia River & CGS STA 34 & $17-$ May-78 & 2 \\
SD_78-34-2 & Columbia River & CGS STA 34 & 27-Nov-78 & 2 \\
SD_79-33-1 & Columbia River & CGS STA 33 & $10-J u l-79$ & 2 \\
SD_79-33-2 & Columbia River & CGS STA 33 & $19-$ Nov-79 & 2 \\
SD_79-34-1 & Columbia River & CGS STA 34 & $10-J u l-79$ & 2 \\
SD_79-34-2 & Columbia River & CGS STA 34 & 19-Nov-79 & 2 \\
SD_80-33-1 & Columbia River & CGS STA 33 & 08-May-80 & 2 \\
SD_80-33-2 & Columbia River & CGS STA 33 & 18-Nov-80 & 2 \\
SD_80-34-1 & Columbia River & CGS STA 34 & 18-Nov-80 & 2 \\
SD_81-33-1 & Columbia River & CGS STA 33 & 08-May-81 & 2 \\
SD_81-33-2 & Columbia River & CGS STA 33 & 19-Nov-81 & 2 \\
SD_81-34-1 & Columbia River & CGS STA 34 & 08-May-81 & 2 \\
SD_81-34-2 & Columbia River & CGS STA 34 & 19-Nov-81 & 2 \\
SD_82-33-1 & Columbia River & CGS STA 33 & 05-Nov-82 & 2 \\
SD_82-34-1 & Columbia River & CGS STA 34 & 05-Nov-82 & 2 \\
SD_83-33-1 & Columbia River & CGS STA 33 & 03-May-83 & 2 \\
SD_83-33-2 & Columbia River & CGS STA 33 & 01-Nov-83 & 2 \\
SD_83-34-1 & Columbia River & CGS STA 34 & 03-May-83 & 2 \\
SD_83-34-2 & Columbia River & CGS STA 34 & 01-Nov-83 & 2 \\
\hline
\end{tabular}


Table B-2

Summary Table of Segment 2 Surface Water Samples By Area, Site, Date, and Category Columbia River Component - Existing Sample Data Evaluation and Scoping Report

\begin{tabular}{|c|c|c|c|c|}
\hline Sample Area & Sample Site & Sample ID & Sample Date & Category \\
\hline Columbia River & 100 B/C AREA DR & B180F3 & 37942 & 1 \\
\hline Columbia River & $100 \mathrm{~B} / \mathrm{C}$ AREA DR & B18148 & 37942 & 1 \\
\hline Columbia River & $100 \mathrm{~B} / \mathrm{C}$ AREA DR & B18147 & 37942 & 1 \\
\hline Columbia River & 100 B/C AREA DR & B18146 & 37942 & 1 \\
\hline Columbia River & 100 B/C AREA DR & B18145 & 37942 & 1 \\
\hline Columbia River & $100 \mathrm{~B} / \mathrm{C}$ AREA DR & J00X57 & 37858 & 1 \\
\hline Columbia River & $100 \mathrm{~B} / \mathrm{C}$ AREA DR & B180F4 & 37942 & 1 \\
\hline Columbia River & $100 \mathrm{~B} / \mathrm{C}$ AREA DR & B180F2 & 37942 & 1 \\
\hline Columbia River & $100 \mathrm{~B} / \mathrm{C}$ AREA DR & B180F1 & 37942 & 1 \\
\hline Columbia River & $100 \mathrm{~B} / \mathrm{C}$ AREA DR & J01450 & 37942 & 1 \\
\hline Columbia River & 100 B/C AREA DR & J014J4 & 37942 & 1 \\
\hline Columbia River & $100 \mathrm{~B} / \mathrm{C}$ AREA DR & J014J5 & 37942 & 1 \\
\hline Columbia River & $100 \mathrm{~B} / \mathrm{C}$ AREA DR & $\mathrm{J} 014 \mathrm{~J} 6$ & 37942 & 1 \\
\hline Columbia River & 100 B/C AREA DR & J00X50 & 37858 & 1 \\
\hline Columbia River & 100 B/C AREA UR & J013J1 & 37938 & 1 \\
\hline Columbia River & 100 B/C AREA UR & B18154 & 37938 & 1 \\
\hline Columbia River & 100 B/C AREA UR & J013J3 & 37938 & 1 \\
\hline Columbia River & 100 B/C AREA UR & J00X44 & 37858 & 1 \\
\hline Columbia River & $100 \mathrm{~B} / \mathrm{C}$ AREA UR & J013J0 & 37938 & 1 \\
\hline Columbia River & 100 B/C AREA UR & J013J2 & 37938 & 1 \\
\hline Columbia River & 100 B/C AREA UR & B18155 & 37938 & 1 \\
\hline Columbia River & $100 \mathrm{~B} / \mathrm{C}$ AREA UR & J00X51 & 37858 & 1 \\
\hline Columbia River & 100 B/C AREA UR & B180C8 & 37938 & 1 \\
\hline Columbia River & 100 B/C AREA UR & B18152 & 37938 & 1 \\
\hline Columbia River & $100 \mathrm{~B} / \mathrm{C}$ AREA UR & B180C6 & 37938 & 1 \\
\hline Columbia River & $100 \mathrm{~B} / \mathrm{C}$ AREA UR & B18153 & 37938 & 1 \\
\hline Columbia River & $100 \mathrm{~B} / \mathrm{C}$ AREA UR & B180C7 & 37938 & 1 \\
\hline Columbia River & 100 B/C AREA UR & B180C9 & 37938 & 1 \\
\hline Columbia River & 100 B/C OUTFLL1 & $\mathrm{J} 00 \times 47$ & 37858 & 1 \\
\hline Columbia River & 100 B/C OUTFLL1 & B180D8 & 37942 & 1 \\
\hline Columbia River & 100 B/C OUTFLL1 & B18149 & 37942 & 1 \\
\hline Columbia River & 100 B/C OUTFLL1 & J013X6 & 37942 & 1 \\
\hline Columbia River & 100 B/C OUTFLL1 & J00X54 & 37858 & 1 \\
\hline Columbia River & 100 B/C OUTFLL2 & J013X9 & 37942 & 1 \\
\hline Columbia River & 100 B/C OUTFLL2 & B180J0 & 37942 & 1 \\
\hline Columbia River & 100 B/C OUTFLL2 & B180D9 & 37942 & 1 \\
\hline Columbia River & 100 B/C OUTFLL2 & B18134 & 37942 & 1 \\
\hline Columbia River & 100 B/C OUTFLL2 & J013Y0 & 37942 & 1 \\
\hline Columbia River & 100 B/C OUTFLL2 & B18150 & 37942 & 1 \\
\hline Columbia River & 100 B/C OUTFLL2 & $\mathrm{J} 00 \times 48$ & 37858 & 1 \\
\hline Columbia River & 100 B/C OUTFLL2 & $300 \times 55$ & 37858 & 1 \\
\hline Columbia River & 100 B/C OUTFLL3 & J00X49 & 37858 & 1 \\
\hline Columbia River & 100 B/C OUTFLL3 & B18151 & 37942 & 1 \\
\hline Columbia River & 100 B/C OUTFLL3 & J013Y1 & 37942 & 1 \\
\hline Columbia River & 100 B/C OUTFLL3 & J00X56 & 37858 & 1 \\
\hline Columbia River & 100 B/C OUTFLL3 & B180F0 & 37942 & 1 \\
\hline
\end{tabular}


Table B-2

\section{Summary Table of Segment 2 Surface Water Samples By Area, Site, Date, and Category Columbia River Component - Existing Sample Data Evaluation and Scoping Report}

\begin{tabular}{|c|c|c|c|c|}
\hline Sample Area & Sample Site & Sample ID & Sample Date & Category \\
\hline Columbia River & 100 F-1 HRM19.0 & BOWBN1 & 36424 & 1 \\
\hline Columbia River & 100 F-1 HRM19.0 & B158J1 & 37508 & 1 \\
\hline Columbia River & 100 F-1 HRM19.0 & B12T70 & 37144 & 1 \\
\hline Columbia River & 100 F-1 HRM19.0 & B109C0 & 36787 & 1 \\
\hline Columbia River & 100 F-1 HRM19.0 & B106J0 & 36787 & 1 \\
\hline Columbia River & 100 F-1 HRM19.0 & B158V0 & 37508 & 1 \\
\hline Columbia River & 100 F-1 HRM19.0 & B17CR1 & 37872 & 1 \\
\hline Columbia River & 100 F-1 HRM19.0 & B17HF1 & 37872 & 1 \\
\hline Columbia River & 100 F-1 HRM19.0 & B0WB95 & 36424 & 1 \\
\hline Columbia River & 100 F-1 HRM19.0 & B10755 & 36787 & 1 \\
\hline Columbia River & 100 F-1 HRM19.0 & B12VM7 & 37144 & 1 \\
\hline Columbia River & 100 F-1 HRM19.0 & B0WB02 & 36424 & 1 \\
\hline Columbia River & 100 F-1 HRM19.0 & B17CF8 & 37872 & 1 \\
\hline Columbia River & 100 F-1 HRM19.0 & B12TL4 & 37144 & 1 \\
\hline Columbia River & 100 F-1 HRM19.0 & B106W1 & 36787 & 1 \\
\hline Columbia River & 100 F-1 HRM19.0 & B17CC2 & 37872 & 1 \\
\hline Columbia River & 100 F-1 HRM19.0 & B158D8 & 37508 & 1 \\
\hline Columbia River & 100 F-1 HRM19.0 & B15995 & 37508 & 1 \\
\hline Columbia River & 100 F-1 HRM19.0 & B106T9 & 36787 & 1 \\
\hline Columbia River & 100 F-1 HRM19.0 & B1B708 & 38244 & 1 \\
\hline Columbia River & 100 F-1 HRM19.0 & B1B6X5 & 38244 & 1 \\
\hline Columbia River & 100 F-1 HRM19.0 & B1B775 & 38244 & 1 \\
\hline Columbia River & 100 F-1 HRM19.0 & B1B464 & 38244 & 1 \\
\hline Columbia River & 100 F-10HRM19.0 & B159B0 & 37508 & 1 \\
\hline Columbia River & 100 F-10HRM19.0 & B158K3 & 37508 & 1 \\
\hline Columbia River & 100 F-10HRM19.0 & B106H9 & 36787 & 1 \\
\hline Columbia River & 100 F-10HRM19.0 & B17CR6 & 37872 & 1 \\
\hline Columbia River & 100 F-10HRM19.0 & B106W7 & 36787 & 1 \\
\hline Columbia River & 100 F-10HRM19.0 & B10760 & 36787 & 1 \\
\hline Columbia River & 100 F-10HRM19.0 & B12TL9 & 37144 & 1 \\
\hline Columbia River & 100 F-10HRM19.0 & B17C96 & 37872 & 1 \\
\hline Columbia River & 100 F-10HRM19.0 & B0WB12 & 36424 & 1 \\
\hline Columbia River & 100 F-10HRM19.0 & В12Т54 & 37144 & 1 \\
\hline Columbia River & 100 F-10HRM19.0 & B12VN2 & 37144 & 1 \\
\hline Columbia River & 100 F-10HRM19.0 & $\mathrm{B} 17 \mathrm{CH} 4$ & 37872 & 1 \\
\hline Columbia River & 100 F-10HRM19.0 & B158C2 & 37508 & 1 \\
\hline Columbia River & 100 F-10HRM19.0 & В12TB0 & 37144 & 1 \\
\hline Columbia River & 100 F-10HRM19.0 & B106R9 & 36787 & 1 \\
\hline Columbia River & 100 F-10HRM19.0 & B158V5 & 37508 & 1 \\
\hline Columbia River & 100 F-10HRM19.0 & B17HF0 & 37872 & 1 \\
\hline Columbia River & 100 F-10HRM19.0 & BOWBN2 & 36424 & 1 \\
\hline Columbia River & 100 F-10HRM19.0 & BOWBB4 & 36424 & 1 \\
\hline Columbia River & 100 F-10HRM19.0 & B1B780 & 38244 & 1 \\
\hline Columbia River & 100 F-10HRM19.0 & B1B6V9 & 38244 & 1 \\
\hline Columbia River & 100 F-10HRM19.0 & B1B469 & 38244 & 1 \\
\hline Columbia River & 100 F-10HRM19.0 & B1B714 & 38244 & 1 \\
\hline
\end{tabular}


Table B-2

\section{Summary Table of Segment 2 Surface Water Samples By Area, Site, Date, and Category Columbia River Component - Existing Sample Data Evaluation and Scoping Report}

\begin{tabular}{|c|c|c|c|c|}
\hline Sample Area & Sample Site & Sample ID & Sample Date & Category \\
\hline Columbia River & 100 F-2 HRM19.0 & B17HF2 & 37872 & 1 \\
\hline Columbia River & 100 F-2 HRM19.0 & B106W4 & 36787 & 1 \\
\hline Columbia River & 100 F-2 HRM19.0 & B158D9 & 37508 & 1 \\
\hline Columbia River & 100 F-2 HRM19.0 & BoWB96 & 36424 & 1 \\
\hline Columbia River & 100 F-2 HRM19.0 & B15996 & 37508 & 1 \\
\hline Columbia River & 100 F-2 HRM19.0 & $\mathrm{B} 17 \mathrm{CH} 1$ & 37872 & 1 \\
\hline Columbia River & 100 F-2 HRM19.0 & B158V1 & 37508 & 1 \\
\hline Columbia River & 100 F-2 HRM19.0 & В12Т97 & 37144 & 1 \\
\hline Columbia River & 100 F-2 HRM19.0 & B12TL5 & 37144 & 1 \\
\hline Columbia River & 100 F-2 HRM19.0 & B0WB06 & 36424 & 1 \\
\hline Columbia River & 100 F-2 HRM19.0 & B12T71 & 37144 & 1 \\
\hline Columbia River & 100 F-2 HRM19.0 & B158J7 & 37508 & 1 \\
\hline Columbia River & 100 F-2 HRM19.0 & BOWBN3 & 36424 & 1 \\
\hline Columbia River & 100 F-2 HRM19.0 & B12VM8 & 37144 & 1 \\
\hline Columbia River & 100 F-2 HRM19.0 & B106V0 & 36787 & 1 \\
\hline Columbia River & 100 F-2 HRM19.0 & B17CR2 & 37872 & 1 \\
\hline Columbia River & 100 F-2 HRM19.0 & B10756 & 36787 & 1 \\
\hline Columbia River & 100 F-2 HRM19.0 & B1B6X6 & 38244 & 1 \\
\hline Columbia River & 100 F-2 HRM19.0 & B1B465 & 38244 & 1 \\
\hline Columbia River & 100 F-2 HRM19.0 & B1B711 & 38244 & 1 \\
\hline Columbia River & 100 F-2 HRM19.0 & B1B776 & 38244 & 1 \\
\hline Columbia River & 100 F-3 HRM19.0 & B12VM9 & 37144 & 1 \\
\hline Columbia River & 100 F-3 HRM19.0 & B17HF3 & 37872 & 1 \\
\hline Columbia River & 100 F-3 HRM19.0 & В12T95 & 37144 & 1 \\
\hline Columbia River & 100 F-3 HRM19.0 & B15997 & 37508 & 1 \\
\hline Columbia River & 100 F-3 HRM19.0 & B106W2 & 36787 & 1 \\
\hline Columbia River & 100 F-3 HRM19.0 & B17CR3 & 37872 & 1 \\
\hline Columbia River & 100 F-3 HRM19.0 & B158F0 & 37508 & 1 \\
\hline Columbia River & 100 F-3 HRM19.0 & B10757 & 36787 & 1 \\
\hline Columbia River & 100 F-3 HRM19.0 & B0WB03 & 36424 & 1 \\
\hline Columbia River & 100 F-3 HRM19.0 & BOWBN4 & 36424 & 1 \\
\hline Columbia River & 100 F-3 HRM19.0 & B106V1 & 36787 & 1 \\
\hline Columbia River & 100 F-3 HRM19.0 & B17CF9 & 37872 & 1 \\
\hline Columbia River & 100 F-3 HRM19.0 & B12TL6 & 37144 & 1 \\
\hline Columbia River & 100 F-3 HRM19.0 & В12Т72 & 37144 & 1 \\
\hline Columbia River & 100 F-3 HRM19.0 & B158J3 & 37508 & 1 \\
\hline Columbia River & 100 F-3 HRM19.0 & B17CC4 & 37872 & 1 \\
\hline Columbia River & 100 F-3 HRM19.0 & B0WB97 & 36424 & 1 \\
\hline Columbia River & 100 F-3 HRM19.0 & B106J2 & 36787 & 1 \\
\hline Columbia River & 100 F-3 HRM19.0 & B158V2 & 37508 & 1 \\
\hline Columbia River & 100 F-3 HRM19.0 & B1B709 & 38244 & 1 \\
\hline Columbia River & 100 F-3 HRM19.0 & B1B466 & 38244 & 1 \\
\hline Columbia River & 100 F-3 HRM19.0 & B1B6X7 & 38244 & 1 \\
\hline Columbia River & 100 F-3 HRM19.0 & B1B777 & 38244 & 1 \\
\hline Columbia River & 100 F-4 HRM19.0 & B0WB07 & 36424 & 1 \\
\hline Columbia River & 100 F-4 HRM19.0 & BOWBN5 & 36424 & 1 \\
\hline
\end{tabular}


Table B-2

\section{Summary Table of Segment 2 Surface Water Samples By Area, Site, Date, and Category Columbia River Component - Existing Sample Data Evaluation and Scoping Report}

\begin{tabular}{|c|c|c|c|c|}
\hline Sample Area & Sample Site & Sample ID & Sample Date & Category \\
\hline Columbia River & 100 F-5 HRM19.0 & B12VN0 & 37144 & 1 \\
\hline Columbia River & 100 F-5 HRM19.0 & B10758 & 36787 & 1 \\
\hline Columbia River & 100 F-5 HRM19.0 & B12TL7 & 37144 & 1 \\
\hline Columbia River & 100 F-5 HRM19.0 & B158F1 & 37508 & 1 \\
\hline Columbia River & 100 F-5 HRM19.0 & B0WB99 & 36424 & 1 \\
\hline Columbia River & 100 F-5 HRM19.0 & B15998 & 37508 & 1 \\
\hline Columbia River & 100 F-5 HRM19.0 & B158V3 & 37508 & 1 \\
\hline Columbia River & 100 F-5 HRM19.0 & B17CR4 & 37872 & 1 \\
\hline Columbia River & 100 F-5 HRM19.0 & B0WB08 & 36424 & 1 \\
\hline Columbia River & 100 F-5 HRM19.0 & B158J9 & 37508 & 1 \\
\hline Columbia River & 100 F-5 HRM19.0 & B106W5 & 36787 & 1 \\
\hline Columbia River & 100 F-5 HRM19.0 & $\mathrm{B} 17 \mathrm{CH} 2$ & 37872 & 1 \\
\hline Columbia River & 100 F-5 HRM19.0 & В12Т98 & 37144 & 1 \\
\hline Columbia River & 100 F-5 HRM19.0 & B12Т73 & 37144 & 1 \\
\hline Columbia River & 100 F-5 HRM19.0 & BOWBN6 & 36424 & 1 \\
\hline Columbia River & 100 F-5 HRM19.0 & B17HF4 & 37872 & 1 \\
\hline Columbia River & 100 F-5 HRM19.0 & B106V2 & 36787 & 1 \\
\hline Columbia River & 100 F-5 HRM19.0 & B106J3 & 36787 & 1 \\
\hline Columbia River & 100 F-5 HRM19.0 & B1B467 & 38244 & 1 \\
\hline Columbia River & 100 F-5 HRM19.0 & B1B6X8 & 38244 & 1 \\
\hline Columbia River & 100 F-5 HRM19.0 & B1B712 & 38244 & 1 \\
\hline Columbia River & 100 F-5 HRM19.0 & B1B778 & 38244 & 1 \\
\hline Columbia River & 100 F-6 HRM19.0 & B0WB09 & 36424 & 1 \\
\hline Columbia River & 100 F-6 HRM19.0 & BOWBN7 & 36424 & 1 \\
\hline Columbia River & 100 F-7 HRM19.0 & B158F2 & 37508 & 1 \\
\hline Columbia River & 100 F-7 HRM19.0 & B17CR5 & 37872 & 1 \\
\hline Columbia River & 100 F-7 HRM19.0 & B12VN1 & 37144 & 1 \\
\hline Columbia River & 100 F-7 HRM19.0 & B12Т74 & 37144 & 1 \\
\hline Columbia River & 100 F-7 HRM19.0 & В12Т99 & 37144 & 1 \\
\hline Columbia River & 100 F-7 HRM19.0 & BoWBB1 & 36424 & 1 \\
\hline Columbia River & 100 F-7 HRM19.0 & BOWBN8 & 36424 & 1 \\
\hline Columbia River & 100 F-7 HRM19.0 & B15999 & 37508 & 1 \\
\hline Columbia River & 100 F-7 HRM19.0 & B12TL8 & 37144 & 1 \\
\hline Columbia River & 100 F-7 HRM19.0 & B106V3 & 36787 & 1 \\
\hline Columbia River & 100 F-7 HRM19.0 & B17HF5 & 37872 & 1 \\
\hline Columbia River & 100 F-7 HRM19.0 & B106J4 & 36787 & 1 \\
\hline Columbia River & 100 F-7 HRM19.0 & B0WB10 & 36424 & 1 \\
\hline Columbia River & 100 F-7 HRM19.0 & B158K1 & 37508 & 1 \\
\hline Columbia River & 100 F-7 HRM19.0 & B158V4 & 37508 & 1 \\
\hline Columbia River & 100 F-7 HRM19.0 & $\mathrm{B} 17 \mathrm{CH} 3$ & 37872 & 1 \\
\hline Columbia River & 100 F-7 HRM19.0 & B10759 & 36787 & 1 \\
\hline Columbia River & 100 F-7 HRM19.0 & B106W6 & 36787 & 1 \\
\hline Columbia River & 100 F-7 HRM19.0 & B1B468 & 38244 & 1 \\
\hline Columbia River & 100 F-7 HRM19.0 & B1B713 & 38244 & 1 \\
\hline Columbia River & 100 F-7 HRM19.0 & B1B779 & 38244 & 1 \\
\hline Columbia River & 100 F-8 HRM19.0 & BOWBN9 & 36424 & 1 \\
\hline
\end{tabular}


Table B-2

\section{Summary Table of Segment 2 Surface Water Samples By Area, Site, Date, and Category Columbia River Component - Existing Sample Data Evaluation and Scoping Report}

\begin{tabular}{|c|c|c|c|c|}
\hline Sample Area & Sample Site & Sample ID & Sample Date & Category \\
\hline Columbia River & 100 F-8 HRM19.0 & B0WB11 & 36424 & 1 \\
\hline Columbia River & 100 F-9 HRM19.0 & B0WB13 & 36424 & 1 \\
\hline Columbia River & 100 F-9 HRM19.0 & BOWBPO & 36424 & 1 \\
\hline Columbia River & 100 FSHR HRM18 & B12VN3 & 37144 & 1 \\
\hline Columbia River & 100 FSHR HRM18 & B159B1 & 37508 & 1 \\
\hline Columbia River & 100 FSHR HRM18 & 29024 & 37508 & 1 \\
\hline Columbia River & 100 FSHR HRM18 & B12TT5 & 37144 & 1 \\
\hline Columbia River & 100 FSHR HRM18 & B106J5 & 36787 & 1 \\
\hline Columbia River & 100 FSHR HRM18 & В12ТT3 & 37144 & 1 \\
\hline Columbia River & 100 FSHR HRM18 & B109C1 & 36787 & 1 \\
\hline Columbia River & 100 FSHR HRM18 & B15909 & 37508 & 1 \\
\hline Columbia River & 100 FSHR HRM18 & B12TT4 & 37144 & 1 \\
\hline Columbia River & 100 FSHR HRM18 & B10790 & 36787 & 1 \\
\hline Columbia River & 100 FSHR HRM18 & B17HF6 & 37872 & 1 \\
\hline Columbia River & 100 FSHR HRM18 & B10789 & 36787 & 1 \\
\hline Columbia River & 100 FSHR HRM18 & B10788 & 36787 & 1 \\
\hline Columbia River & 100 FSHR HRM18 & B17CY6 & 37872 & 1 \\
\hline Columbia River & 100 FSHR HRM18 & 33281 & 38244 & 1 \\
\hline Columbia River & 100 FSHR HRM18 & B17CY5 & 37872 & 1 \\
\hline Columbia River & 100 FSHR HRM18 & B15906 & 37508 & 1 \\
\hline Columbia River & 100 FSHR HRM18 & B15908 & 37508 & 1 \\
\hline Columbia River & 100 FSHR HRM18 & B17CY4 & 37872 & 1 \\
\hline Columbia River & 100 FSHR HRM18 & B1B6X9 & 38244 & 1 \\
\hline Columbia River & 100 FSHR HRM18 & B1B7D5 & 38244 & 1 \\
\hline Columbia River & 100 FSHR HRM18 & B1B470 & 38244 & 1 \\
\hline Columbia River & 100 FSHR HRM18 & B1B7D8 & 38244 & 1 \\
\hline Columbia River & 100 FSHR HRM18 & B1B7D7 & 38244 & 1 \\
\hline Columbia River & 100 FSHR HRM22 & B17CY7 & 37872 & 1 \\
\hline Columbia River & 100 FSHR HRM22 & B17CY8 & 37872 & 1 \\
\hline Columbia River & 100 FSHR HRM22 & B17CY9 & 37872 & 1 \\
\hline Columbia River & 100 FSHR HRM22 & B106J6 & 36787 & 1 \\
\hline Columbia River & 100 FSHR HRM22 & B10791 & 36787 & 1 \\
\hline Columbia River & 100 FSHR HRM22 & B10793 & 36787 & 1 \\
\hline Columbia River & 100 FSHR HRM22 & 33228 & 38244 & 1 \\
\hline Columbia River & 100 FSHR HRM22 & B10792 & 36787 & 1 \\
\hline Columbia River & 100 FSHR HRM22 & B17HF7 & 37872 & 1 \\
\hline Columbia River & 100 FSHR HRM22 & 29025 & 37508 & 1 \\
\hline Columbia River & 100 FSHR HRM22 & B1B7D9 & 38244 & 1 \\
\hline Columbia River & 100 FSHR HRM22 & B1B7F2 & 38244 & 1 \\
\hline Columbia River & 100 FSHR HRM22 & B1B7F1 & 38244 & 1 \\
\hline Columbia River & 100 FSHR HRM22 & B1B471 & 38244 & 1 \\
\hline Columbia River & 100 FSHR HRM23 & B12VN5 & 37144 & 1 \\
\hline Columbia River & 100 FSHR HRM23 & B106J7 & 36787 & 1 \\
\hline Columbia River & 100 FSHR HRM23 & B159B3 & 37508 & 1 \\
\hline Columbia River & 100 FSHR HRM23 & B15914 & 37508 & 1 \\
\hline Columbia River & 100 FSHR HRM23 & 29026 & 37508 & 1 \\
\hline
\end{tabular}


Table B-2

\section{Summary Table of Segment 2 Surface Water Samples By Area, Site, Date, and Category Columbia River Component - Existing Sample Data Evaluation and Scoping Report}

\begin{tabular}{|c|c|c|c|c|}
\hline Sample Area & Sample Site & Sample ID & Sample Date & Category \\
\hline Columbia River & 100 FSHR HRM23 & 33196 & 38244 & 1 \\
\hline Columbia River & 100 FSHR HRM23 & B10794 & 36787 & 1 \\
\hline Columbia River & 100 FSHR HRM23 & B10795 & 36787 & 1 \\
\hline Columbia River & 100 FSHR HRM23 & B15916 & 37508 & 1 \\
\hline Columbia River & 100 FSHR HRM23 & B12TV1 & 37144 & 1 \\
\hline Columbia River & 100 FSHR HRM23 & B12TV0 & 37144 & 1 \\
\hline Columbia River & 100 FSHR HRM23 & В12ТT9 & 37144 & 1 \\
\hline Columbia River & 100 FSHR HRM23 & B15917 & 37508 & 1 \\
\hline Columbia River & 100 FSHR HRM23 & B17D00 & 37872 & 1 \\
\hline Columbia River & 100 FSHR HRM23 & B17D01 & 37872 & 1 \\
\hline Columbia River & 100 FSHR HRM23 & B17D02 & 37872 & 1 \\
\hline Columbia River & 100 FSHR HRM23 & B17HF8 & 37872 & 1 \\
\hline Columbia River & 100 FSHR HRM23 & B10796 & 36787 & 1 \\
\hline Columbia River & 100 FSHR HRM23 & B1B7F3 & 38244 & 1 \\
\hline Columbia River & 100 FSHR HRM23 & B1B7F5 & 38244 & 1 \\
\hline Columbia River & 100 FSHR HRM23 & B1B7F6 & 38244 & 1 \\
\hline Columbia River & 100 FSHR HRM23 & B1B472 & 38244 & 1 \\
\hline Columbia River & 100 FSHR HRM24 & B15912 & 37508 & 1 \\
\hline Columbia River & 100 FSHR HRM24 & B159B2 & 37508 & 1 \\
\hline Columbia River & 100 FSHR HRM24 & B15910 & 37508 & 1 \\
\hline Columbia River & 100 FSHR HRM24 & В12Т94 & 37144 & 1 \\
\hline Columbia River & 100 FSHR HRM24 & B15913 & 37508 & 1 \\
\hline Columbia River & 100 N-1 HRM 9.5 & B159B4 & 37504 & 1 \\
\hline Columbia River & 100 N-1 HRM 9.5 & Bow936 & 36413 & 1 \\
\hline Columbia River & 100 N-1 HRM 9.5 & В12Т85 & 37141 & 1 \\
\hline Columbia River & 100 N-1 HRM 9.5 & B158H8 & 37504 & 1 \\
\hline Columbia River & 100 N-1 HRM 9.5 & B158P6 & 37504 & 1 \\
\hline Columbia River & 100 N-1 HRM 9.5 & В12T64 & 37141 & 1 \\
\hline Columbia River & 100 N-1 HRM 9.5 & B0WBF8 & 36413 & 1 \\
\hline Columbia River & 100 N-1 HRM 9.5 & Bow930 & 36413 & 1 \\
\hline Columbia River & 100 N-1 HRM 9.5 & B10692 & 36783 & 1 \\
\hline Columbia River & 100 N-1 HRM 9.5 & B106J9 & 36783 & 1 \\
\hline Columbia River & 100 N-1 HRM 9.5 & B17HF9 & 37868 & 1 \\
\hline Columbia River & 100 N-1 HRM 9.5 & B12VN6 & 37141 & 1 \\
\hline Columbia River & 100 N-1 HRM 9.5 & B17CB6 & 37868 & 1 \\
\hline Columbia River & 100 N-1 HRM 9.5 & B12TJ0 & 37141 & 1 \\
\hline Columbia River & 100 N-1 HRM 9.5 & B158H3 & 37504 & 1 \\
\hline Columbia River & 100 N-1 HRM 9.5 & B10662 & 36783 & 1 \\
\hline Columbia River & 100 N-1 HRM 9.5 & B158D2 & 37504 & 1 \\
\hline Columbia River & 100 N-1 HRM 9.5 & В12Т91 & 37141 & 1 \\
\hline Columbia River & 100 N-1 HRM 9.5 & B17CM7 & 37868 & 1 \\
\hline Columbia River & 100 N-1 HRM 9.5 & B17CD7 & 37868 & 1 \\
\hline Columbia River & 100 N-1 HRM 9.5 & B109B4 & 36783 & 1 \\
\hline Columbia River & 100 N-1 HRM 9.5 & B10672 & 36783 & 1 \\
\hline Columbia River & 100 N-1 HRM 9.5 & B17CF2 & 37868 & 1 \\
\hline Columbia River & 100 N-1 HRM 9.5 & B10677 & 36783 & 1 \\
\hline
\end{tabular}


Table B-2

\section{Summary Table of Segment 2 Surface Water Samples By Area, Site, Date, and Category Columbia River Component - Existing Sample Data Evaluation and Scoping Report}

\begin{tabular}{|c|c|c|c|c|}
\hline Sample Area & Sample Site & Sample ID & Sample Date & Category \\
\hline Columbia River & 100 N-1 HRM 9.5 & Bow973 & 36413 & 1 \\
\hline Columbia River & 100 N-1 HRM 9.5 & B1B6W9 & 38243 & 1 \\
\hline Columbia River & 100 N-1 HRM 9.5 & B1B473 & 38243 & 1 \\
\hline Columbia River & 100 N-1 HRM 9.5 & B1B751 & 38243 & 1 \\
\hline Columbia River & 100 N-1 HRM 9.5 & B1B700 & 38243 & 1 \\
\hline Columbia River & 100 N-1 HRM 9.5 & B1B705 & 38243 & 1 \\
\hline Columbia River & $100 \mathrm{~N}-10 \mathrm{HRM} 9.5$ & B159B9 & 37504 & 1 \\
\hline Columbia River & $100 \mathrm{~N}-10 \mathrm{HRM} 9.5$ & B10667 & 36783 & 1 \\
\hline Columbia River & $100 \mathrm{~N}-10 \mathrm{HRM} 9.5$ & В17HHO & 37868 & 1 \\
\hline Columbia River & 100 N-10HRM 9.5 & BOWBH3 & 36413 & 1 \\
\hline Columbia River & $100 \mathrm{~N}-10 \mathrm{HRM} 9.5$ & B158D7 & 37504 & 1 \\
\hline Columbia River & $100 \mathrm{~N}-10 \mathrm{HRM} 9.5$ & B10683 & 36783 & 1 \\
\hline Columbia River & $100 \mathrm{~N}-10 \mathrm{HRM} 9.5$ & B158R1 & 37504 & 1 \\
\hline Columbia River & $100 \mathrm{~N}-10 \mathrm{HRM} 9.5$ & B10697 & 36783 & 1 \\
\hline Columbia River & 100 N-10HRM 9.5 & Bow978 & 36413 & 1 \\
\hline Columbia River & 100 N-10HRM 9.5 & В12Т69 & 37141 & 1 \\
\hline Columbia River & $100 \mathrm{~N}-10 \mathrm{HRM} 9.5$ & B12VP1 & 37141 & 1 \\
\hline Columbia River & $100 \mathrm{~N}-10 \mathrm{HRM} 9.5$ & B12TD4 & 37141 & 1 \\
\hline Columbia River & $100 \mathrm{~N}-10 \mathrm{HRM} 9.5$ & $\mathrm{~B} 17 \mathrm{CC} 1$ & 37868 & 1 \\
\hline Columbia River & 100 N-10HRM 9.5 & B106J8 & 36783 & 1 \\
\hline Columbia River & $100 \mathrm{~N}-10 \mathrm{HRM} 9.5$ & B17CN2 & 37868 & 1 \\
\hline Columbia River & $100 \mathrm{~N}-10 \mathrm{HRM} 9.5$ & B17CK8 & 37868 & 1 \\
\hline Columbia River & 100 N-10HRM 9.5 & Bow942 & 36413 & 1 \\
\hline Columbia River & $100 \mathrm{~N}-10 \mathrm{HRM} 9.5$ & B158M7 & 37504 & 1 \\
\hline Columbia River & $100 \mathrm{~N}-10 \mathrm{HRM} 9.5$ & B12TJ5 & 37141 & 1 \\
\hline Columbia River & 100 N-10HRM 9.5 & B1B732 & 38243 & 1 \\
\hline Columbia River & $100 \mathrm{~N}-10 \mathrm{HRM} 9.5$ & B1B6X4 & 38243 & 1 \\
\hline Columbia River & $100 \mathrm{~N}-10 \mathrm{HRM} 9.5$ & B1B756 & 38243 & 1 \\
\hline Columbia River & $100 \mathrm{~N}-10 \mathrm{HRM} 9.5$ & B1B478 & 38243 & 1 \\
\hline Columbia River & 100 N-2 HRM 9.5 & B12TJ1 & 37141 & 1 \\
\hline Columbia River & 100 N-2 HRM 9.5 & Bow974 & 36413 & 1 \\
\hline Columbia River & 100 N-2 HRM 9.5 & B109B5 & 36783 & 1 \\
\hline Columbia River & 100 N-2 HRM 9.5 & B17CM8 & 37868 & 1 \\
\hline Columbia River & 100 N-2 HRM 9.5 & B10693 & 36783 & 1 \\
\hline Columbia River & 100 N-2 HRM 9.5 & B17CF4 & 37868 & 1 \\
\hline Columbia River & 100 N-2 HRM 9.5 & B10663 & 36783 & 1 \\
\hline Columbia River & 100 N-2 HRM 9.5 & B12VN7 & 37141 & 1 \\
\hline Columbia River & 100 N-2 HRM 9.5 & В12T92 & 37141 & 1 \\
\hline Columbia River & 100 N-2 HRM 9.5 & BOWBF9 & 36413 & 1 \\
\hline Columbia River & 100 N-2 HRM 9.5 & B158H9 & 37504 & 1 \\
\hline Columbia River & 100 N-2 HRM 9.5 & B159B5 & 37504 & 1 \\
\hline Columbia River & 100 N-2 HRM 9.5 & B158P7 & 37504 & 1 \\
\hline Columbia River & 100 N-2 HRM 9.5 & $\mathrm{~B} 17 \mathrm{HH} 1$ & 37868 & 1 \\
\hline Columbia River & 100 N-2 HRM 9.5 & Bow938 & 36413 & 1 \\
\hline Columbia River & 100 N-2 HRM 9.5 & B106K0 & 36783 & 1 \\
\hline Columbia River & 100 N-2 HRM 9.5 & В12Т65 & 37141 & 1 \\
\hline
\end{tabular}


Table B-2

\section{Summary Table of Segment 2 Surface Water Samples By Area, Site, Date, and Category Columbia River Component - Existing Sample Data Evaluation and Scoping Report}

\begin{tabular}{|c|c|c|c|c|}
\hline Sample Area & Sample Site & Sample ID & Sample Date & Category \\
\hline Columbia River & 100 N-2 HRM 9.5 & B158D3 & 37504 & 1 \\
\hline Columbia River & $100 \mathrm{~N}-2$ HRM 9.5 & В17CB7 & 37868 & 1 \\
\hline Columbia River & $100 \mathrm{~N}-2$ HRM 9.5 & B10679 & 36783 & 1 \\
\hline Columbia River & 100 N-2 HRM 9.5 & B1B474 & 38243 & 1 \\
\hline Columbia River & 100 N-2 HRM 9.5 & B1B706 & 38243 & 1 \\
\hline Columbia River & 100 N-2 HRM 9.5 & B1B752 & 38243 & 1 \\
\hline Columbia River & 100 N-2 HRM 9.5 & B1B6X0 & 38243 & 1 \\
\hline Columbia River & 100 N-3 HRM 9.5 & B158D4 & 37504 & 1 \\
\hline Columbia River & 100 N-3 HRM 9.5 & B10694 & 36783 & 1 \\
\hline Columbia River & 100 N-3 HRM 9.5 & B158P8 & 37504 & 1 \\
\hline Columbia River & 100 N-3 HRM 9.5 & B10684 & 36783 & 1 \\
\hline Columbia River & 100 N-3 HRM 9.5 & B10664 & 36783 & 1 \\
\hline Columbia River & 100 N-3 HRM 9.5 & B158M8 & 37504 & 1 \\
\hline Columbia River & 100 N-3 HRM 9.5 & B106K1 & 36783 & 1 \\
\hline Columbia River & 100 N-3 HRM 9.5 & B17CM9 & 37868 & 1 \\
\hline Columbia River & 100 N-3 HRM 9.5 & Bow975 & 36413 & 1 \\
\hline Columbia River & 100 N-3 HRM 9.5 & B17CL0 & 37868 & 1 \\
\hline Columbia River & 100 N-3 HRM 9.5 & Bow943 & 36413 & 1 \\
\hline Columbia River & 100 N-3 HRM 9.5 & $\mathrm{~B} 17 \mathrm{HH} 2$ & 37868 & 1 \\
\hline Columbia River & 100 N-3 HRM 9.5 & $\mathrm{~B} 12 \mathrm{TJ} 2$ & 37141 & 1 \\
\hline Columbia River & 100 N-3 HRM 9.5 & B12VN8 & 37141 & 1 \\
\hline Columbia River & 100 N-3 HRM 9.5 & BOWBHO & 36413 & 1 \\
\hline Columbia River & 100 N-3 HRM 9.5 & B12TD5 & 37141 & 1 \\
\hline Columbia River & 100 N-3 HRM 9.5 & B12Т66 & 37141 & 1 \\
\hline Columbia River & 100 N-3 HRM 9.5 & В17CB8 & 37868 & 1 \\
\hline Columbia River & 100 N-3 HRM 9.5 & B1B733 & 38243 & 1 \\
\hline Columbia River & 100 N-3 HRM 9.5 & B1B475 & 38243 & 1 \\
\hline Columbia River & 100 N-3 HRM 9.5 & B1B753 & 38243 & 1 \\
\hline Columbia River & 100 N-3 HRM 9.5 & B1B6X1 & 38243 & 1 \\
\hline Columbia River & 100 N-5 HRM 9.5 & B10665 & 36783 & 1 \\
\hline Columbia River & 100 N-5 HRM 9.5 & B106K2 & 36783 & 1 \\
\hline Columbia River & 100 N-5 HRM 9.5 & B158P9 & 37504 & 1 \\
\hline Columbia River & 100 N-5 HRM 9.5 & В12ТJ3 & 37141 & 1 \\
\hline Columbia River & 100 N-5 HRM 9.5 & B10695 & 36783 & 1 \\
\hline Columbia River & 100 N-5 HRM 9.5 & В12Т67 & 37141 & 1 \\
\hline Columbia River & 100 N-5 HRM 9.5 & B10681 & 36783 & 1 \\
\hline Columbia River & 100 N-5 HRM 9.5 & B12VN9 & 37141 & 1 \\
\hline Columbia River & 100 N-5 HRM 9.5 & Bow976 & 36413 & 1 \\
\hline Columbia River & 100 N-5 HRM 9.5 & B17CNO & 37868 & 1 \\
\hline Columbia River & 100 N-5 HRM 9.5 & B159B7 & 37504 & 1 \\
\hline Columbia River & 100 N-5 HRM 9.5 & B158D5 & 37504 & 1 \\
\hline Columbia River & 100 N-5 HRM 9.5 & B158J0 & 37504 & 1 \\
\hline Columbia River & 100 N-5 HRM 9.5 & В17HH3 & 37868 & 1 \\
\hline Columbia River & 100 N-5 HRM 9.5 & В12Т93 & 37141 & 1 \\
\hline Columbia River & 100 N-5 HRM 9.5 & В17Св9 & 37868 & 1 \\
\hline Columbia River & 100 N-5 HRM 9.5 & BOWBH1 & 36413 & 1 \\
\hline
\end{tabular}


Table B-2

\section{Summary Table of Segment 2 Surface Water Samples By Area, Site, Date, and Category Columbia River Component - Existing Sample Data Evaluation and Scoping Report}

\begin{tabular}{|c|c|c|c|c|}
\hline Sample Area & Sample Site & Sample ID & Sample Date & Category \\
\hline Columbia River & 100 N-5 HRM 9.5 & B17CF6 & 37868 & 1 \\
\hline Columbia River & 100 N-5 HRM 9.5 & Bow940 & 36413 & 1 \\
\hline Columbia River & 100 N-5 HRM 9.5 & B1B754 & 38243 & 1 \\
\hline Columbia River & 100 N-5 HRM 9.5 & B1B6X2 & 38243 & 1 \\
\hline Columbia River & 100 N-5 HRM 9.5 & B1B476 & 38243 & 1 \\
\hline Columbia River & 100 N-5 HRM 9.5 & B1B707 & 38243 & 1 \\
\hline Columbia River & 100 N-7 HRM 9.5 & $\mathrm{~B} 17 \mathrm{CN} 1$ & 37868 & 1 \\
\hline Columbia River & 100 N-7 HRM 9.5 & B10696 & 36783 & 1 \\
\hline Columbia River & 100 N-7 HRM 9.5 & B106K3 & 36783 & 1 \\
\hline Columbia River & 100 N-7 HRM 9.5 & B12VP0 & 37141 & 1 \\
\hline Columbia River & 100 N-7 HRM 9.5 & Bow977 & 36413 & 1 \\
\hline Columbia River & 100 N-7 HRM 9.5 & B12TJ4 & 37141 & 1 \\
\hline Columbia River & 100 N-7 HRM 9.5 & B158R0 & 37504 & 1 \\
\hline Columbia River & 100 N-7 HRM 9.5 & В12Т68 & 37141 & 1 \\
\hline Columbia River & 100 N-7 HRM 9.5 & B158D6 & 37504 & 1 \\
\hline Columbia River & 100 N-7 HRM 9.5 & B159B8 & 37504 & 1 \\
\hline Columbia River & 100 N-7 HRM 9.5 & B158M9 & 37504 & 1 \\
\hline Columbia River & 100 N-7 HRM 9.5 & B10686 & 36783 & 1 \\
\hline Columbia River & 100 N-7 HRM 9.5 & B12TD6 & 37141 & 1 \\
\hline Columbia River & 100 N-7 HRM 9.5 & B17CC0 & 37868 & 1 \\
\hline Columbia River & 100 N-7 HRM 9.5 & B17CL2 & 37868 & 1 \\
\hline Columbia River & 100 N-7 HRM 9.5 & Bow945 & 36413 & 1 \\
\hline Columbia River & 100 N-7 HRM 9.5 & B10666 & 36783 & 1 \\
\hline Columbia River & 100 N-7 HRM 9.5 & BOWBH2 & 36413 & 1 \\
\hline Columbia River & 100 N-7 HRM 9.5 & $\mathrm{~B} 17 \mathrm{HH} 4$ & 37868 & 1 \\
\hline Columbia River & 100 N-7 HRM 9.5 & B1B477 & 38243 & 1 \\
\hline Columbia River & 100 N-7 HRM 9.5 & B1B6X3 & 38243 & 1 \\
\hline Columbia River & 100 N-7 HRM 9.5 & B1B734 & 38243 & 1 \\
\hline Columbia River & 100 N-7 HRM 9.5 & B1B755 & 38243 & 1 \\
\hline Columbia River & 100 NSHR HRM8.4 & B10699 & 36783 & 1 \\
\hline Columbia River & 100 NSHR HRM8.4 & B106B0 & 36783 & 1 \\
\hline Columbia River & 100 NSHR HRM8.4 & В17СТ9 & 37868 & 1 \\
\hline Columbia River & 100 NSHR HRM8.4 & B106K4 & 36783 & 1 \\
\hline Columbia River & 100 NSHR HRM8.4 & B10698 & 36783 & 1 \\
\hline Columbia River & 100 NSHR HRM8.4 & B17CV1 & 37868 & 1 \\
\hline Columbia River & 100 NSHR HRM8.4 & B17CV2 & 37868 & 1 \\
\hline Columbia River & 100 NSHR HRM8.4 & BOWBH4 & 36413 & 1 \\
\hline Columbia River & 100 NSHR HRM8.4 & 33229 & 38243 & 1 \\
\hline Columbia River & 100 NSHR HRM8.4 & B159C0 & 37504 & 1 \\
\hline Columbia River & 100 NSHR HRM8.4 & B17HH5 & 37868 & 1 \\
\hline Columbia River & 100 NSHR HRM8.4 & Bow957 & 36413 & 1 \\
\hline Columbia River & 100 NSHR HRM8.4 & Bow965 & 36413 & 1 \\
\hline Columbia River & 100 NSHR HRM8.4 & B12TN4 & 37141 & 1 \\
\hline Columbia River & 100 NSHR HRM8.4 & B12TN3 & 37141 & 1 \\
\hline Columbia River & 100 NSHR HRM8.4 & B12VP2 & 37141 & 1 \\
\hline Columbia River & 100 NSHR HRM8.4 & 31857 & 37868 & 1 \\
\hline
\end{tabular}


Table B-2

\section{Summary Table of Segment 2 Surface Water Samples By Area, Site, Date, and Category Columbia River Component - Existing Sample Data Evaluation and Scoping Report}

\begin{tabular}{|c|c|c|c|c|}
\hline Sample Area & Sample Site & Sample ID & Sample Date & Category \\
\hline Columbia River & 100 NSHR HRM8.4 & B109B7 & 36783 & 1 \\
\hline Columbia River & 100 NSHR HRM8.4 & B12TN2 & 37141 & 1 \\
\hline Columbia River & 100 NSHR HRM8.4 & B158X0 & 37504 & 1 \\
\hline Columbia River & 100 NSHR HRM8.4 & B158W8 & 37504 & 1 \\
\hline Columbia River & 100 NSHR HRM8.4 & B158W9 & 37504 & 1 \\
\hline Columbia River & 100 NSHR HRM8.4 & B1B796 & 38243 & 1 \\
\hline Columbia River & 100 NSHR HRM8.4 & B1B479 & 38243 & 1 \\
\hline Columbia River & 100 NSHR HRM8.4 & B1B793 & 38243 & 1 \\
\hline Columbia River & 100 NSHR HRM8.4 & B1B795 & 38243 & 1 \\
\hline Columbia River & 100 NSHR HRM8.9 & B106B1 & 36783 & 1 \\
\hline Columbia River & 100 NSHR HRM8.9 & B12VP3 & 37141 & 1 \\
\hline Columbia River & 100 NSHR HRM8.9 & B12TN7 & 37141 & 1 \\
\hline Columbia River & 100 NSHR HRM8.9 & B159C1 & 37504 & 1 \\
\hline Columbia River & 100 NSHR HRM8.9 & Bow958 & 36413 & 1 \\
\hline Columbia River & 100 NSHR HRM8.9 & 31858 & 37868 & 1 \\
\hline Columbia River & 100 NSHR HRM8.9 & Bow966 & 36413 & 1 \\
\hline Columbia River & 100 NSHR HRM8.9 & 33230 & 38243 & 1 \\
\hline Columbia River & 100 NSHR HRM8.9 & B12TN6 & 37141 & 1 \\
\hline Columbia River & 100 NSHR HRM8.9 & B158X3 & 37504 & 1 \\
\hline Columbia River & 100 NSHR HRM8.9 & B106B2 & 36783 & 1 \\
\hline Columbia River & 100 NSHR HRM8.9 & B106K5 & 36783 & 1 \\
\hline Columbia River & 100 NSHR HRM8.9 & B158X1 & 37504 & 1 \\
\hline Columbia River & 100 NSHR HRM8.9 & B158X2 & 37504 & 1 \\
\hline Columbia River & 100 NSHR HRM8.9 & B106B3 & 36783 & 1 \\
\hline Columbia River & 100 NSHR HRM8.9 & B12TN5 & 37141 & 1 \\
\hline Columbia River & 100 NSHR HRM8.9 & B109B6 & 36783 & 1 \\
\hline Columbia River & 100 NSHR HRM8.9 & B0WBH5 & 36413 & 1 \\
\hline Columbia River & 100 NSHR HRM8.9 & B17CV3 & 37868 & 1 \\
\hline Columbia River & 100 NSHR HRM8.9 & B17CV5 & 37868 & 1 \\
\hline Columbia River & 100 NSHR HRM8.9 & В17HH6 & 37868 & 1 \\
\hline Columbia River & 100 NSHR HRM8.9 & B17CV6 & 37868 & 1 \\
\hline Columbia River & 100 NSHR HRM8.9 & B1B480 & 38243 & 1 \\
\hline Columbia River & 100 NSHR HRM8.9 & B1B797 & 38243 & 1 \\
\hline Columbia River & 100 NSHR HRM8.9 & B1B799 & 38243 & 1 \\
\hline Columbia River & 100 NSHR HRM8.9 & B1B7B0 & 38243 & 1 \\
\hline Columbia River & 100 NSHR HRM9.2 & B12TN9 & 37141 & 1 \\
\hline Columbia River & 100 NSHR HRM9.2 & B12TN8 & 37141 & 1 \\
\hline Columbia River & 100 NSHR HRM9.2 & B12TP0 & 37141 & 1 \\
\hline Columbia River & 100 NSHR HRM9.2 & B158X6 & 37504 & 1 \\
\hline Columbia River & 100 NSHR HRM9.2 & B158X5 & 37504 & 1 \\
\hline Columbia River & 100 NSHR HRM9.2 & B158X4 & 37504 & 1 \\
\hline Columbia River & 100 NSHR HRM9.2 & B17CV7 & 37868 & 1 \\
\hline Columbia River & 100 NSHR HRM9.2 & B109B8 & 36783 & 1 \\
\hline Columbia River & 100 NSHR HRM9.2 & B17CW0 & 37868 & 1 \\
\hline Columbia River & 100 NSHR HRM9.2 & Bow967 & 36413 & 1 \\
\hline Columbia River & 100 NSHR HRM9.2 & B12VP4 & 37141 & 1 \\
\hline
\end{tabular}


Table B-2

\section{Summary Table of Segment 2 Surface Water Samples By Area, Site, Date, and Category Columbia River Component - Existing Sample Data Evaluation and Scoping Report}

\begin{tabular}{|c|c|c|c|c|}
\hline Sample Area & Sample Site & Sample ID & Sample Date & Category \\
\hline Columbia River & 100 NSHR HRM9.2 & B0W959 & 36413 & 1 \\
\hline Columbia River & 100 NSHR HRM9.2 & B17CV9 & 37868 & 1 \\
\hline Columbia River & 100 NSHR HRM9.2 & B106B4 & 36783 & 1 \\
\hline Columbia River & 100 NSHR HRM9.2 & BOWBH6 & 36413 & 1 \\
\hline Columbia River & 100 NSHR HRM9.2 & В17H 7 & 37868 & 1 \\
\hline Columbia River & 100 NSHR HRM9.2 & B159C2 & 37504 & 1 \\
\hline Columbia River & 100 NSHR HRM9.2 & B106B5 & 36783 & 1 \\
\hline Columbia River & 100 NSHR HRM9.2 & B106K6 & 36783 & 1 \\
\hline Columbia River & 100 NSHR HRM9.2 & 31859 & 37868 & 1 \\
\hline Columbia River & 100 NSHR HRM9.2 & B106B6 & 36783 & 1 \\
\hline Columbia River & 100 NSHR HRM9.2 & B1B481 & 38243 & 1 \\
\hline Columbia River & 100 NSHR HRM9.2 & B1B7B1 & 38243 & 1 \\
\hline Columbia River & 100 NSHR HRM9.2 & B1B7B4 & 38243 & 1 \\
\hline Columbia River & 100 NSHR HRM9.2 & B1B7B3 & 38243 & 1 \\
\hline Columbia River & 100 NSHR HRM9.8 & 31860 & 37868 & 1 \\
\hline Columbia River & 100 NSHR HRM9.8 & B17CW3 & 37868 & 1 \\
\hline Columbia River & 100 NSHR HRM9.8 & B12VP5 & 37141 & 1 \\
\hline Columbia River & 100 NSHR HRM9.8 & B158X9 & 37504 & 1 \\
\hline Columbia River & 100 NSHR HRM9.8 & B12TP2 & 37141 & 1 \\
\hline Columbia River & 100 NSHR HRM9.8 & B106K7 & 36783 & 1 \\
\hline Columbia River & 100 NSHR HRM9.8 & Bow968 & 36413 & 1 \\
\hline Columbia River & 100 NSHR HRM9.8 & B109B9 & 36783 & 1 \\
\hline Columbia River & 100 NSHR HRM9.8 & B17CW4 & 37868 & 1 \\
\hline Columbia River & 100 NSHR HRM9.8 & 33232 & 38243 & 1 \\
\hline Columbia River & 100 NSHR HRM9.8 & $\mathrm{B} 158 \times 7$ & 37504 & 1 \\
\hline Columbia River & 100 NSHR HRM9.8 & B12TP1 & 37141 & 1 \\
\hline Columbia River & 100 NSHR HRM9.8 & B17CW1 & 37868 & 1 \\
\hline Columbia River & 100 NSHR HRM9.8 & B158X8 & 37504 & 1 \\
\hline Columbia River & 100 NSHR HRM9.8 & B106B9 & 36783 & 1 \\
\hline Columbia River & 100 NSHR HRM9.8 & BOWBH7 & 36413 & 1 \\
\hline Columbia River & 100 NSHR HRM9.8 & Bow960 & 36413 & 1 \\
\hline Columbia River & 100 NSHR HRM9.8 & $\mathrm{B} 17 \mathrm{HH} 8$ & 37868 & 1 \\
\hline Columbia River & 100 NSHR HRM9.8 & B106B7 & 36783 & 1 \\
\hline Columbia River & 100 NSHR HRM9.8 & B159C3 & 37504 & 1 \\
\hline Columbia River & 100 NSHR HRM9.8 & B106B8 & 36783 & 1 \\
\hline Columbia River & 100 NSHR HRM9.8 & B12TP3 & 37141 & 1 \\
\hline Columbia River & 100 NSHR HRM9.8 & B1B7B5 & 38243 & 1 \\
\hline Columbia River & 100 NSHR HRM9.8 & B1B482 & 38243 & 1 \\
\hline Columbia River & 100 NSHR HRM9.8 & B1B7B8 & 38243 & 1 \\
\hline Columbia River & 100 NSHR HRM9.8 & B1B7B7 & 38243 & 1 \\
\hline Columbia River & 100-B SPR 37-1 & B15C62 & 37515 & 1 \\
\hline Columbia River & 100-B SPR 37-1 & B13608 & 37186 & 1 \\
\hline Columbia River & 100-B SPR 37-1 & B15MM0 & 37550 & 1 \\
\hline Columbia River & 100-B SPR 37-1 & B15C71 & 37515 & 1 \\
\hline Columbia River & 100-B SPR 37-1 & B15ML9 & 37550 & 1 \\
\hline Columbia River & 100-B SPR 37-1 & B13607 & 37186 & 1 \\
\hline
\end{tabular}


Table B-2

\section{Summary Table of Segment 2 Surface Water Samples By Area, Site, Date, and Category Columbia River Component - Existing Sample Data Evaluation and Scoping Report}

\begin{tabular}{|c|c|c|c|c|}
\hline Sample Area & Sample Site & Sample ID & Sample Date & Category \\
\hline Columbia River & 100-B SPR 37-1 & B13606 & 37186 & 1 \\
\hline Columbia River & 100-B SPR 37-1 & B15MM1 & 37550 & 1 \\
\hline Columbia River & 100-B SPR 37-1 & В180H6 & 37938 & 1 \\
\hline Columbia River & 100-B SPR 37-1 & B1BFT1 & 38271 & 1 \\
\hline Columbia River & 100-B SPR 37-1 & J013J4 & 37938 & 1 \\
\hline Columbia River & 100-B SPR 37-1 & B1BFT7 & 38271 & 1 \\
\hline Columbia River & 100-B SPR 37-1 & B1BFV3 & 38271 & 1 \\
\hline Columbia River & 100-B SPR 37-1 & B18136 & 37938 & 1 \\
\hline Columbia River & 100-B SPR 38-3 & B13609 & 37186 & 1 \\
\hline Columbia River & 100-B SPR 38-3 & B13610 & 37186 & 1 \\
\hline Columbia River & 100-B SPR 38-3 & B13611 & 37186 & 1 \\
\hline Columbia River & 100-B SPR 38-3 & B15MM3 & 37550 & 1 \\
\hline Columbia River & 100-B SPR 38-3 & B0WP24 & 36458 & 1 \\
\hline Columbia River & 100-B SPR 38-3 & B10937 & 36816 & 1 \\
\hline Columbia River & 100-B SPR 38-3 & B15MM2 & 37550 & 1 \\
\hline Columbia River & 100-B SPR 38-3 & B10944 & 36816 & 1 \\
\hline Columbia River & 100-B SPR 38-3 & B15MM4 & 37550 & 1 \\
\hline Columbia River & 100-B SPR 38-3 & B0WP15 & 36458 & 1 \\
\hline Columbia River & 100-B SPR 38-3 & B10FB0 & 36824 & 1 \\
\hline Columbia River & 100-B SPR 38-3 & B10FB1 & 36824 & 1 \\
\hline Columbia River & 100-B SPR 38-3 & B10FB2 & 36824 & 1 \\
\hline Columbia River & 100-B SPR 38-3 & B18137 & 37938 & 1 \\
\hline Columbia River & 100-B SPR 38-3 & J013K3 & 37938 & 1 \\
\hline Columbia River & 100-B SPR 38-3 & J013K9 & 37938 & 1 \\
\hline Columbia River & 100-B SPR 38-3 & B180F9 & 37938 & 1 \\
\hline Columbia River & 100-B SPR 38-3 & $\mathrm{B} 180 \mathrm{H} 7$ & 37938 & 1 \\
\hline Columbia River & 100-B SPR 38-3 & J013P4 & 37938 & 1 \\
\hline Columbia River & 100-B SPR 38-3 & J013K6 & 37938 & 1 \\
\hline Columbia River & 100-B SPR 38-3 & J013P5 & 37938 & 1 \\
\hline Columbia River & 100-B SPR 38-3 & J00X52 & 37858 & 1 \\
\hline Columbia River & 100-B SPR 38-3 & B18158 & 37938 & 1 \\
\hline Columbia River & 100-B SPR 38-3 & B180D3 & 37938 & 1 \\
\hline Columbia River & 100-B SPR 38-3 & J013P3 & 37938 & 1 \\
\hline Columbia River & 100-B SPR 38-3 & B18160 & 37938 & 1 \\
\hline Columbia River & 100-B SPR 38-3 & B18156 & 37938 & 1 \\
\hline Columbia River & 100-B SPR 38-3 & B18157 & 37938 & 1 \\
\hline Columbia River & 100-B SPR 38-3 & B18166 & 37938 & 1 \\
\hline Columbia River & 100-B SPR 38-3 & J013K8 & 37938 & 1 \\
\hline Columbia River & 100-B SPR 38-3 & J013K7 & 37938 & 1 \\
\hline Columbia River & 100-B SPR 38-3 & $\mathrm{J} 00 \times 45$ & 37858 & 1 \\
\hline Columbia River & 100-B SPR 38-3 & B180D0 & 37938 & 1 \\
\hline Columbia River & 100-B SPR 38-3 & J013P6 & 37938 & 1 \\
\hline Columbia River & 100-B SPR 38-3 & J013K4 & 37938 & 1 \\
\hline Columbia River & 100-B SPR 38-3 & B18159 & 37938 & 1 \\
\hline Columbia River & 100-B SPR 38-3 & B180D2 & 37938 & 1 \\
\hline Columbia River & 100-B SPR 38-3 & B180D1 & 37938 & 1 \\
\hline
\end{tabular}


Table B-2

\section{Summary Table of Segment 2 Surface Water Samples By Area, Site, Date, and Category Columbia River Component - Existing Sample Data Evaluation and Scoping Report}

\begin{tabular}{|c|c|c|c|c|}
\hline Sample Area & Sample Site & Sample ID & Sample Date & Category \\
\hline Columbia River & 100-B SPR 38-3 & B180J4 & 37938 & 1 \\
\hline Columbia River & 100-B SPR 39-2 & B0WP27 & 36458 & 1 \\
\hline Columbia River & 100-B SPR 39-2 & B0WP29 & 36458 & 1 \\
\hline Columbia River & 100-B SPR 39-2 & B180D5 & 37938 & 1 \\
\hline Columbia River & 100-B SPR 39-2 & B180D4 & 37938 & 1 \\
\hline Columbia River & 100-B SPR 39-2 & B1BFW0 & 38271 & 1 \\
\hline Columbia River & 100-B SPR 39-2 & В180H8 & 37942 & 1 \\
\hline Columbia River & 100-B SPR 39-2 & J00X53 & 37858 & 1 \\
\hline Columbia River & 100-B SPR 39-2 & B180D7 & 37938 & 1 \\
\hline Columbia River & 100-B SPR 39-2 & J013W4 & 37942 & 1 \\
\hline Columbia River & 100-B SPR 39-2 & B18162 & 37938 & 1 \\
\hline Columbia River & 100-B SPR 39-2 & J00X46 & 37858 & 1 \\
\hline Columbia River & 100-B SPR 39-2 & B18164 & 37938 & 1 \\
\hline Columbia River & 100-B SPR 39-2 & B18165 & 37938 & 1 \\
\hline Columbia River & 100-B SPR 39-2 & J013V7 & 37938 & 1 \\
\hline Columbia River & 100-B SPR 39-2 & J013W1 & 37938 & 1 \\
\hline Columbia River & 100-B SPR 39-2 & B18163 & 37938 & 1 \\
\hline Columbia River & 100-B SPR 39-2 & J013W2 & 37938 & 1 \\
\hline Columbia River & 100-B SPR 39-2 & B1BFT2 & 38271 & 1 \\
\hline Columbia River & 100-B SPR 39-2 & B180D6 & 37938 & 1 \\
\hline Columbia River & 100-B SPR 39-2 & B18138 & 37942 & 1 \\
\hline Columbia River & 100-B SPR 39-2 & B1BFV7 & 38271 & 1 \\
\hline Columbia River & 100-B SPR 39-2 & J013W0 & 37938 & 1 \\
\hline Columbia River & 100-D SPR 102-1 & B10JP7 & 36816 & 1 \\
\hline Columbia River & 100-D SPR 102-1 & B10962 & 36816 & 1 \\
\hline Columbia River & 100-D SPR 102-1 & B15HV8 & 37550 & 1 \\
\hline Columbia River & 100-D SPR 102-1 & B15HV9 & 37550 & 1 \\
\hline Columbia River & 100-D SPR 102-1 & B15HW0 & 37550 & 1 \\
\hline Columbia River & 100-D SPR 102-1 & B15C89 & 37550 & 1 \\
\hline Columbia River & 100-D SPR 102-1 & B10963 & 36816 & 1 \\
\hline Columbia River & 100-D SPR 102-1 & B0WP42 & 36460 & 1 \\
\hline Columbia River & 100-D SPR 102-1 & B15C88 & 37550 & 1 \\
\hline Columbia River & 100-D SPR 102-1 & B0WP43 & 36460 & 1 \\
\hline Columbia River & 100-D SPR 102-1 & B10JR8 & 36816 & 1 \\
\hline Columbia River & 100-D SPR 102-1 & B1BFV0 & 38286 & 1 \\
\hline Columbia River & 100-D SPR 102-1 & B17JP1 & 37921 & 1 \\
\hline Columbia River & 100-D SPR 102-1 & B1BJ55 & 38286 & 1 \\
\hline Columbia River & 100-D SPR 102-1 & B17JP2 & 37921 & 1 \\
\hline Columbia River & 100-D SPR 102-1 & B17JP3 & 37921 & 1 \\
\hline Columbia River & 100-D SPR 102-1 & B17JP4 & 37921 & 1 \\
\hline Columbia River & 100-D SPR 102-1 & B17JP0 & 37921 & 1 \\
\hline Columbia River & 100-D SPR 102-1 & B17JN9 & 37921 & 1 \\
\hline Columbia River & 100-D SPR 102-1 & B17JN8 & 37921 & 1 \\
\hline Columbia River & 100-D SPR 102-1 & B1BFX3 & 38286 & 1 \\
\hline Columbia River & 100-D SPR 102-1 & B1BFX2 & 38286 & 1 \\
\hline Columbia River & 100-D SPR 110-1 & B15HW2 & 37550 & 1 \\
\hline
\end{tabular}


Table B-2

\section{Summary Table of Segment 2 Surface Water Samples By Area, Site, Date, and Category Columbia River Component - Existing Sample Data Evaluation and Scoping Report}

\begin{tabular}{|c|c|c|c|c|}
\hline Sample Area & Sample Site & Sample ID & Sample Date & Category \\
\hline Columbia River & 100-D SPR 110-1 & B10JT0 & 36816 & 1 \\
\hline Columbia River & 100-D SPR 110-1 & B10JP9 & 36816 & 1 \\
\hline Columbia River & 100-D SPR 110-1 & B10960 & 36816 & 1 \\
\hline Columbia River & 100-D SPR 110-1 & B10961 & 36816 & 1 \\
\hline Columbia River & 100-D SPR 110-1 & B0WP40 & 36460 & 1 \\
\hline Columbia River & 100-D SPR 110-1 & B15HW5 & 37550 & 1 \\
\hline Columbia River & 100-D SPR 110-1 & B15HW1 & 37550 & 1 \\
\hline Columbia River & 100-D SPR 110-1 & B10JR9 & 36816 & 1 \\
\hline Columbia River & 100-D SPR 110-1 & B15HW3 & 37550 & 1 \\
\hline Columbia River & 100-D SPR 110-1 & B15HW4 & 37550 & 1 \\
\hline Columbia River & 100-D SPR 110-1 & B15C87 & 37550 & 1 \\
\hline Columbia River & 100-D SPR 110-1 & B0WP41 & 36460 & 1 \\
\hline Columbia River & 100-D SPR 110-1 & B10JP8 & 36816 & 1 \\
\hline Columbia River & 100-D SPR 110-1 & B15C86 & 37550 & 1 \\
\hline Columbia River & 100-D SPR 110-1 & B1BJ56 & 38286 & 1 \\
\hline Columbia River & 100-D SPR 110-1 & B17JN7 & 37921 & 1 \\
\hline Columbia River & 100-D SPR 110-1 & B1BFX1 & 38286 & 1 \\
\hline Columbia River & 100-D SPR 110-1 & B1BFT9 & 38286 & 1 \\
\hline Columbia River & 100-D SPR 110-1 & B1BFX0 & 38286 & 1 \\
\hline Columbia River & 100-D SPR 98-1 & B15HX1 & 37550 & 1 \\
\hline Columbia River & 100-D SPR 98-1 & B135X2 & 37189 & 1 \\
\hline Columbia River & 100-D SPR 98-1 & B135X3 & 37189 & 1 \\
\hline Columbia River & 100-D SPR 98-1 & $\mathrm{B} 15 \mathrm{H} \times 0$ & 37550 & 1 \\
\hline Columbia River & 100-D SPR 98-1 & B15HW9 & 37550 & 1 \\
\hline Columbia River & 100-F SPR 207-1 & B15C92 & 37558 & 1 \\
\hline Columbia River & 100-F SPR 207-1 & B13619 & 37186 & 1 \\
\hline Columbia River & 100-F SPR 207-1 & B13620 & 37186 & 1 \\
\hline Columbia River & 100-F SPR 207-1 & B15C93 & 37558 & 1 \\
\hline Columbia River & 100-F SPR 207-1 & B10967 & 36837 & 1 \\
\hline Columbia River & 100-F SPR 207-1 & B10966 & 36837 & 1 \\
\hline Columbia River & 100-F SPR 207-1 & B13618 & 37186 & 1 \\
\hline Columbia River & 100-F SPR 207-1 & B15MN1 & 37556 & 1 \\
\hline Columbia River & 100-F SPR 207-1 & B10FC4 & 36837 & 1 \\
\hline Columbia River & 100-F SPR 207-1 & B15MN2 & 37556 & 1 \\
\hline Columbia River & 100-F SPR 207-1 & B15MN3 & 37558 & 1 \\
\hline Columbia River & 100-F SPR 207-1 & B10FC2 & 36837 & 1 \\
\hline Columbia River & 100-F SPR 207-1 & B10FC3 & 36837 & 1 \\
\hline Columbia River & 100-F SPR 207-1 & B0WP48 & 36465 & 1 \\
\hline Columbia River & 100-F SPR 207-1 & B0WP49 & 36465 & 1 \\
\hline Columbia River & 100-F SPR 207-1 & B1BJ59 & 38286 & 1 \\
\hline Columbia River & 100-F SPR 207-1 & B1BFV2 & 38286 & 1 \\
\hline Columbia River & 100-F SPR 207-1 & B1BFX7 & 38286 & 1 \\
\hline Columbia River & 100-F SPR 207-1 & B1BFX6 & 38286 & 1 \\
\hline Columbia River & 100-F SPR 211-1 & B13622 & 37186 & 1 \\
\hline Columbia River & 100-F SPR 211-1 & B13621 & 37186 & 1 \\
\hline Columbia River & 100-F SPR 211-1 & B13623 & 37186 & 1 \\
\hline
\end{tabular}


Table B-2

\section{Summary Table of Segment 2 Surface Water Samples By Area, Site, Date, and Category Columbia River Component - Existing Sample Data Evaluation and Scoping Report}

\begin{tabular}{|c|c|c|c|c|}
\hline Sample Area & Sample Site & Sample ID & Sample Date & Category \\
\hline Columbia River & 100-F SPRING & B17LB4 & 37945 & 1 \\
\hline Columbia River & 100-F SPRING & B17LB2 & 37945 & 1 \\
\hline Columbia River & 100-F SPRING & B17LB1 & 37945 & 1 \\
\hline Columbia River & 100-H SPR 144-1 & B15HX3 & 37556 & 1 \\
\hline Columbia River & 100-H SPR 144-1 & $\mathrm{B} 15 \mathrm{H} \times 4$ & 37556 & 1 \\
\hline Columbia River & 100-H SPR 144-1 & $\mathrm{B} 15 \mathrm{H} \times 2$ & 37556 & 1 \\
\hline Columbia River & 100-H SPR 145-1 & $\mathrm{B} 15 \mathrm{H} \times 8$ & 37556 & 1 \\
\hline Columbia River & 100-H SPR 145-1 & B135X8 & 37196 & 1 \\
\hline Columbia River & 100-H SPR 145-1 & B0WP47 & 36460 & 1 \\
\hline Columbia River & 100-H SPR 145-1 & B135X9 & 37196 & 1 \\
\hline Columbia River & 100-H SPR 145-1 & B10964 & 36831 & 1 \\
\hline Columbia River & 100-H SPR 145-1 & BoWP46 & 36460 & 1 \\
\hline Columbia River & 100-H SPR 145-1 & $\mathrm{B} 15 \mathrm{H} \times 5$ & 37556 & 1 \\
\hline Columbia River & 100-H SPR 145-1 & B10965 & 36831 & 1 \\
\hline Columbia River & 100-H SPR 145-1 & $\mathrm{B} 15 \mathrm{H} \times 7$ & 37556 & 1 \\
\hline Columbia River & 100-H SPR 145-1 & B15HX9 & 37556 & 1 \\
\hline Columbia River & 100-H SPR 145-1 & B10JT4 & 36831 & 1 \\
\hline Columbia River & 100-H SPR 145-1 & B10JR3 & 36831 & 1 \\
\hline Columbia River & 100-H SPR 145-1 & B15HYO & 37556 & 1 \\
\hline Columbia River & 100-H SPR 145-1 & B15HX6 & 37556 & 1 \\
\hline Columbia River & 100-H SPR 145-1 & B1BJ57 & 38286 & 1 \\
\hline Columbia River & 100-H SPR 145-1 & B1BFX5 & 38286 & 1 \\
\hline Columbia River & 100-H SPR 145-1 & B1BFX4 & 38286 & 1 \\
\hline Columbia River & 100-H SPR 145-1 & B1BFV1 & 38286 & 1 \\
\hline Columbia River & 100-H SPR 150-1 & В15HY3 & 37556 & 1 \\
\hline Columbia River & 100-H SPR 150-1 & B15HY2 & 37556 & 1 \\
\hline Columbia River & 100-H SPR 150-1 & B15HY1 & 37556 & 1 \\
\hline Columbia River & 100-H SPR 150-1 & B17VK7 & 37945 & 1 \\
\hline Columbia River & 100-H SPR 150-1 & B17VK6 & 37945 & 1 \\
\hline Columbia River & 100-H SPR 150-1 & B17VK5 & 37945 & 1 \\
\hline Columbia River & 100-H SPR 152-2 & B15CB5 & 37556 & 1 \\
\hline Columbia River & 100-H SPR 152-2 & B15CB4 & 37556 & 1 \\
\hline Columbia River & 100-H SPR 152-2 & B135Y4 & 37196 & 1 \\
\hline Columbia River & 100-H SPR 152-2 & B15HY4 & 37556 & 1 \\
\hline Columbia River & 100-H SPR 152-2 & B15HY5 & 37556 & 1 \\
\hline Columbia River & 100-H SPR 152-2 & B135Y5 & 37196 & 1 \\
\hline Columbia River & 100-H SPR 152-2 & B15HY6 & 37556 & 1 \\
\hline Columbia River & 100-H SPR 153-1 & B15HY8 & 37556 & 1 \\
\hline Columbia River & 100-H SPR 153-1 & B135Y6 & 37196 & 1 \\
\hline Columbia River & 100-H SPR 153-1 & B15HY7 & 37556 & 1 \\
\hline Columbia River & 100-H SPR 153-1 & B15HY9 & 37556 & 1 \\
\hline Columbia River & 100-H SPR 153-1 & B135Y7 & 37196 & 1 \\
\hline Columbia River & 100-H SPR 153-1 & B10979 & 36831 & 1 \\
\hline Columbia River & 100-H SPR 153-1 & B0WP44 & 36460 & 1 \\
\hline Columbia River & 100-H SPR 153-1 & B0WP45 & 36460 & 1 \\
\hline Columbia River & 100-H SPR 153-1 & B10980 & 36831 & 1 \\
\hline
\end{tabular}


Table B-2

\section{Summary Table of Segment 2 Surface Water Samples By Area, Site, Date, and Category Columbia River Component - Existing Sample Data Evaluation and Scoping Report}

\begin{tabular}{|c|c|c|c|c|}
\hline Sample Area & Sample Site & Sample ID & Sample Date & Category \\
\hline Columbia River & 100-H SPR 153-1 & B1BH00 & 38286 & 1 \\
\hline Columbia River & 100-H SPR 153-1 & B1BH01 & 38286 & 1 \\
\hline Columbia River & 100-H SPR 153-1 & B1BJ58 & 38286 & 1 \\
\hline Columbia River & 100-H SPR 153-1 & B1BFY7 & 38286 & 1 \\
\hline Columbia River & 100-K SPR 63-1 & B0WP32 & 36458 & 1 \\
\hline Columbia River & 100-K SPR 63-1 & B0WP34 & 36458 & 1 \\
\hline Columbia River & 100-K SPR 63-1 & B10954 & 36816 & 1 \\
\hline Columbia River & 100-K SPR 63-1 & B10953 & 36816 & 1 \\
\hline Columbia River & 100-K SPR 63-1 & B17JR2 & 37914 & 1 \\
\hline Columbia River & 100-K SPR 63-1 & B17JR1 & 37914 & 1 \\
\hline Columbia River & 100-K SPR 63-2 & B15C80 & 37515 & 1 \\
\hline Columbia River & 100-K SPR 63-2 & B15C79 & 37515 & 1 \\
\hline Columbia River & 100-K SPR 68-1 & B17LF3 & 37935 & 1 \\
\hline Columbia River & 100-K SPR 68-1 & B17LC4 & 37935 & 1 \\
\hline Columbia River & 100-K SPR 68-1 & B17LB5 & 37935 & 1 \\
\hline Columbia River & 100-K SPR 77-1 & B10955 & 36824 & 1 \\
\hline Columbia River & 100-K SPR 77-1 & B10956 & 36824 & 1 \\
\hline Columbia River & 100-K SPR 77-1 & B15LM4 & 37550 & 1 \\
\hline Columbia River & 100-K SPR 77-1 & B15LM5 & 37550 & 1 \\
\hline Columbia River & 100-K SPR 77-1 & B15LM6 & 37550 & 1 \\
\hline Columbia River & 100-K SPR 77-1 & B13600 & 37189 & 1 \\
\hline Columbia River & 100-K SPR 77-1 & B15C81 & 37550 & 1 \\
\hline Columbia River & 100-K SPR 77-1 & B15C82 & 37550 & 1 \\
\hline Columbia River & 100-K SPR 77-1 & B13601 & 37189 & 1 \\
\hline Columbia River & 100-K SPR 77-1 & B17JR0 & 37935 & 1 \\
\hline Columbia River & 100-K SPR 77-1 & B17JP9 & 37935 & 1 \\
\hline Columbia River & 100-K SPR 77-1 & B1BFW6 & 38271 & 1 \\
\hline Columbia River & 100-K SPR 77-1 & B1BFW5 & 38271 & 1 \\
\hline Columbia River & 100-K SPR 77-1 & B1BFT5 & 38271 & 1 \\
\hline Columbia River & 100-K SPR 77-1 & B17JP8 & 37935 & 1 \\
\hline Columbia River & 100-K SPR 82-2 & B15LM7 & 37550 & 1 \\
\hline Columbia River & 100-K SPR 82-2 & B15LM8 & 37550 & 1 \\
\hline Columbia River & 100-K SPR 82-2 & B15LM9 & 37550 & 1 \\
\hline Columbia River & 100-K SPR 82-2 & B13604 & 37189 & 1 \\
\hline Columbia River & 100-K SPR 82-2 & B13605 & 37189 & 1 \\
\hline Columbia River & 100-N SPR 8-13 & B15C02 & 37515 & 1 \\
\hline Columbia River & 100-N SPR 8-13 & B10904 & 36816 & 1 \\
\hline Columbia River & 100-N SPR 8-13 & B0WP37 & 36458 & 1 \\
\hline Columbia River & 100-N SPR 8-13 & B15C83 & 37515 & 1 \\
\hline Columbia River & 100-N SPR 8-13 & B10957 & 36816 & 1 \\
\hline Columbia River & 100-N SPR 8-13 & BOWNX6 & 36458 & 1 \\
\hline Columbia River & 100-N SPR 8-13 & B1BFP1 & 38271 & 1 \\
\hline Columbia River & 100-N SPR 8-13 & B18XL7 & 38075 & 1 \\
\hline Columbia River & 100-N SPR 8-13 & B1BFM0 & 38271 & 1 \\
\hline Columbia River & 100-N SPR 8-13 & B18XM0 & 38075 & 1 \\
\hline Columbia River & 100-N SPR 8-13 & B18XL5 & 38075 & 1 \\
\hline
\end{tabular}


Table B-2

\section{Summary Table of Segment 2 Surface Water Samples By Area, Site, Date, and Category Columbia River Component - Existing Sample Data Evaluation and Scoping Report}

\begin{tabular}{|c|c|c|c|c|}
\hline Sample Area & Sample Site & Sample ID & Sample Date & Category \\
\hline Columbia River & 100-N SPR 8-13 & B18XL6 & 38075 & 1 \\
\hline Columbia River & 100-N SPR 8-13 & B1BFW7 & 38271 & 1 \\
\hline Columbia River & 100-N SPR 8-13 & B18XL9 & 38075 & 1 \\
\hline Columbia River & 100-N SPR 8-13 & B18XL8 & 38075 & 1 \\
\hline Columbia River & 100B Area & B0YP01 & 36732 & 1 \\
\hline Columbia River & 100B Area & B0Y265 & 36648 & 1 \\
\hline Columbia River & 100B Area & B11RY4 & 36998 & 1 \\
\hline Columbia River & 100B Area & B0V8F9 & 36269 & 1 \\
\hline Columbia River & 100B Area & B0W108 & 36368 & 1 \\
\hline Columbia River & 100B Area & B11CX6 & 36937 & 1 \\
\hline Columbia River & 100B Area & B134L1 & 37172 & 1 \\
\hline Columbia River & 100B Area & B129N8 & 37081 & 1 \\
\hline Columbia River & 100B Area & BOWPV6 & 36466 & 1 \\
\hline Columbia River & 100B Area & B0XKV5 & 36578 & 1 \\
\hline Columbia River & 100B Area & B10KN4 & 36817 & 1 \\
\hline Columbia River & 100B SPR 37-1 & B15CC3 & 37515 & 1 \\
\hline Columbia River & 100B SPR 37-1 & B15C55 & 37515 & 1 \\
\hline Columbia River & 100B SPR 37-1 & 26215 & 37015 & 1 \\
\hline Columbia River & 100B SPR 37-1 & B11W40 & 37015 & 1 \\
\hline Columbia River & 100B SPR 37-1 & 30858 & 37914 & 1 \\
\hline Columbia River & 100B SPR 37-1 & B17J76 & 37914 & 1 \\
\hline Columbia River & 100B SPR 37-1 & B17J81 & 37914 & 1 \\
\hline Columbia River & 100B SPR 37-1 & B17J68 & 37914 & 1 \\
\hline Columbia River & 100B SPR 37-1 & B17JM8 & 37914 & 1 \\
\hline Columbia River & 100B SPR 38-3 & B12X38 & 37186 & 1 \\
\hline Columbia River & 100B SPR 38-3 & B10932 & 36824 & 1 \\
\hline Columbia River & 100B SPR 38-3 & B0WP11 & 36458 & 1 \\
\hline Columbia River & 100B SPR 38-3 & B135P3 & 37186 & 1 \\
\hline Columbia River & 100B SPR 38-3 & BOWMR9 & 36458 & 1 \\
\hline Columbia River & 100B SPR 38-3 & B10JP0 & 36824 & 1 \\
\hline Columbia River & 100B SPR 39-2 & BoWP12 & 36458 & 1 \\
\hline Columbia River & 100B SPR 39-2 & 30935 & 37914 & 1 \\
\hline Columbia River & 100B SPR 39-2 & BOWMT0 & 36458 & 1 \\
\hline Columbia River & 100B SPR 39-2 & B17J83 & 37914 & 1 \\
\hline Columbia River & 100B SPR 39-2 & B17J70 & 37914 & 1 \\
\hline Columbia River & 100B SPR 39-2 & B17JM9 & 37914 & 1 \\
\hline Columbia River & 100B SPR 39-2 & B17J82 & 37914 & 1 \\
\hline Columbia River & 100BC Area & BoVW80 & 36333 & 1 \\
\hline Columbia River & 100BC Area & BOVWRO & 36339 & 1 \\
\hline Columbia River & 100D Area & B0XKV6 & 36578 & 1 \\
\hline Columbia River & 100D Area & B0Y266 & 36648 & 1 \\
\hline Columbia River & 100D Area & BoW109 & 36368 & 1 \\
\hline Columbia River & 100D Area & BoVW79 & 36332 & 1 \\
\hline Columbia River & 100D Area & BOWPV7 & 36466 & 1 \\
\hline Columbia River & 100D Area & B0YP02 & 36732 & 1 \\
\hline Columbia River & 100D Area & B134L2 & 37172 & 1 \\
\hline
\end{tabular}


Table B-2

\section{Summary Table of Segment 2 Surface Water Samples By Area, Site, Date, and Category Columbia River Component - Existing Sample Data Evaluation and Scoping Report}

\begin{tabular}{|c|c|c|c|c|}
\hline Sample Area & Sample Site & Sample ID & Sample Date & Category \\
\hline Columbia River & 100D Area & B0VWP8 & 36339 & 1 \\
\hline Columbia River & 100D Area & B11RY5 & 36998 & 1 \\
\hline Columbia River & 100D Area & BOV8HO & 36269 & 1 \\
\hline Columbia River & 100D Area & B10KN5 & 36817 & 1 \\
\hline Columbia River & 100D Area & B129N9 & 37081 & 1 \\
\hline Columbia River & 100D Area & $\mathrm{B} 11 \mathrm{C} \times 7$ & 36937 & 1 \\
\hline Columbia River & 100D SPR 102-1 & B0WP18 & 36460 & 1 \\
\hline Columbia River & 100D SPR 102-1 & BOWMT6 & 36460 & 1 \\
\hline Columbia River & 100D SPR 102-1 & B10940 & 36816 & 1 \\
\hline Columbia River & 100D SPR 102-1 & B17J93 & 37921 & 1 \\
\hline Columbia River & 100D SPR 102-1 & B17J94 & 37921 & 1 \\
\hline Columbia River & 100D SPR 102-1 & B17J80 & 37921 & 1 \\
\hline Columbia River & 100D SPR 102-1 & B17JN4 & 37921 & 1 \\
\hline Columbia River & 100D SPR 110-1 & BOWMT5 & 36460 & 1 \\
\hline Columbia River & 100D SPR 110-1 & B10939 & 36816 & 1 \\
\hline Columbia River & 100D SPR 110-1 & B15C65 & 37550 & 1 \\
\hline Columbia River & 100D SPR 110-1 & B11W48 & 37015 & 1 \\
\hline Columbia River & 100D SPR 110-1 & B0WP17 & 36460 & 1 \\
\hline Columbia River & 100D SPR 110-1 & B17J92 & 37921 & 1 \\
\hline Columbia River & 100D SPR 110-1 & B17JN5 & 37921 & 1 \\
\hline Columbia River & 100D SPR 110-1 & B17J91 & 37921 & 1 \\
\hline Columbia River & 100D SPR 110-1 & B17J79 & 37921 & 1 \\
\hline Columbia River & $100 \mathrm{~F} 1 / 10$ & 29018 & 37508 & 1 \\
\hline Columbia River & $100 \mathrm{~F} 10 / 10$ & 29023 & 37508 & 1 \\
\hline Columbia River & $100 \mathrm{~F} 2 / 10$ & 29019 & 37508 & 1 \\
\hline Columbia River & $100 F 3 / 10$ & 29020 & 37508 & 1 \\
\hline Columbia River & $100 F 5 / 10$ & 29021 & 37508 & 1 \\
\hline Columbia River & $100 \mathrm{~F} 7 / 10$ & 29022 & 37508 & 1 \\
\hline Columbia River & 100F Area & B0VWP2 & 36367 & 1 \\
\hline Columbia River & 100F Area & BoVW76 & 36332 & 1 \\
\hline Columbia River & 100F SPR 207-1 & 24572 & 36837 & 1 \\
\hline Columbia River & 100F SPR 207-1 & B11W52 & 37011 & 1 \\
\hline Columbia River & 100F SPR 207-1 & B0WP22 & 36465 & 1 \\
\hline Columbia River & 100F SPR 207-1 & 29692 & 37558 & 1 \\
\hline Columbia River & 100F SPR 207-1 & 23441 & 36465 & 1 \\
\hline Columbia River & 100F SPR 207-1 & B15C69 & 37558 & 1 \\
\hline Columbia River & 100F SPR 207-1 & B12X50 & 37186 & 1 \\
\hline Columbia River & 100F SPR 207-1 & BOWMT9 & 36465 & 1 \\
\hline Columbia River & 100F SPR 207-1 & B10942 & 36837 & 1 \\
\hline Columbia River & 100F SPR 207-1 & 33078 & 37928 & 1 \\
\hline Columbia River & 100F SPR 207-1 & B17RR2 & 37928 & 1 \\
\hline Columbia River & 100F SPR 207-1 & B17RK7 & 37928 & 1 \\
\hline Columbia River & 100F SPR 207-1 & B17RJ6 & 37928 & 1 \\
\hline Columbia River & 100F SPR 207-1 & B17RK8 & 37928 & 1 \\
\hline Columbia River & $100 \mathrm{H}$ Area & B0VWP6 & 36367 & 1 \\
\hline Columbia River & $100 \mathrm{H}$ Area & BoVW78 & 36332 & 1 \\
\hline
\end{tabular}


Table B-2

\section{Summary Table of Segment 2 Surface Water Samples By Area, Site, Date, and Category Columbia River Component - Existing Sample Data Evaluation and Scoping Report}

\begin{tabular}{|c|c|c|c|c|}
\hline Sample Area & Sample Site & Sample ID & Sample Date & Category \\
\hline Columbia River & 100H SPR 145-1 & B10941 & 36831 & 1 \\
\hline Columbia River & $100 \mathrm{H}$ SPR $145-1$ & B11W50 & 37011 & 1 \\
\hline Columbia River & $100 \mathrm{H}$ SPR $145-1$ & BOWMT8 & 36460 & 1 \\
\hline Columbia River & $100 \mathrm{H}$ SPR $145-1$ & 26216 & 37011 & 1 \\
\hline Columbia River & $100 \mathrm{H}$ SPR $145-1$ & 29696 & 37556 & 1 \\
\hline Columbia River & 100H SPR 145-1 & B0WP21 & 36460 & 1 \\
\hline Columbia River & $100 \mathrm{H}$ SPR $145-1$ & $\mathrm{~B} 12 \times 48$ & 37196 & 1 \\
\hline Columbia River & $100 \mathrm{H}$ SPR $145-1$ & 28287 & 37196 & 1 \\
\hline Columbia River & $100 \mathrm{H}$ SPR $145-1$ & 30931 & 37921 & 1 \\
\hline Columbia River & $100 \mathrm{H}$ SPR $145-1$ & B17RK5 & 37904 & 1 \\
\hline Columbia River & $100 \mathrm{H}$ SPR $145-1$ & B17RK6 & 37921 & 1 \\
\hline Columbia River & $100 \mathrm{H}$ SPR $145-1$ & B17RJ4 & 37921 & 1 \\
\hline Columbia River & 100H SPR 145-1 & B17RR3 & 37921 & 1 \\
\hline Columbia River & 100H SPR 150-1 & B10JP6 & 36831 & 1 \\
\hline Columbia River & $100 \mathrm{H}$ SPR $152-2$ & В15CB3 & 37556 & 1 \\
\hline Columbia River & $100 \mathrm{H}$ SPR $152-2$ & B12X85 & 37196 & 1 \\
\hline Columbia River & 100H SPR 153-1 & B10977 & 36831 & 1 \\
\hline Columbia River & 100H SPR 153-1 & 24571 & 36837 & 1 \\
\hline Columbia River & $100 H$ SPR 153-1 & B11W86 & 37011 & 1 \\
\hline Columbia River & $100 \mathrm{H}$ SPR 153-1 & B0WP19 & 36460 & 1 \\
\hline Columbia River & $100 \mathrm{H}$ SPR $153-1$ & 23440 & 36460 & 1 \\
\hline Columbia River & 100H SPR 153-1 & BOWMT7 & 36460 & 1 \\
\hline Columbia River & $100 H$ SPR 153-1 & B17RL8 & 37921 & 1 \\
\hline Columbia River & 100H SPR 153-1 & B17RR4 & 37921 & 1 \\
\hline Columbia River & 100H SPR 153-1 & B17RM0 & 37921 & 1 \\
\hline Columbia River & $100 \mathrm{H}$ SPR 153-1 & B17RL9 & 37921 & 1 \\
\hline Columbia River & 100K Area & B16RN5 & 37775 & 1 \\
\hline Columbia River & 100K Area & BoW112 & 36368 & 1 \\
\hline Columbia River & 100K Area & B0XKW7 & 36578 & 1 \\
\hline Columbia River & 100K Area & B0WPW7 & 36466 & 1 \\
\hline Columbia River & 100K Area & B10KN8 & 36817 & 1 \\
\hline Columbia River & 100K Area & B0VW82 & 36333 & 1 \\
\hline Columbia River & 100K Area & B176L4 & 37866 & 1 \\
\hline Columbia River & 100K Area & B129P9 & 37081 & 1 \\
\hline Columbia River & 100K Area & BOVWR4 & 36339 & 1 \\
\hline Columbia River & 100K Area & B14CV2 & 37355 & 1 \\
\hline Columbia River & 100K Area & B14YJ2 & 37445 & 1 \\
\hline Columbia River & 100K Area & B0TLJ2 & 36187 & 1 \\
\hline Columbia River & 100K Area & B14266 & 37301 & 1 \\
\hline Columbia River & 100K Area & B134L4 & 37172 & 1 \\
\hline Columbia River & 100K Area & B11T05 & 36998 & 1 \\
\hline Columbia River & 100K Area & B15LF6 & 37602 & 1 \\
\hline Columbia River & 100K Area & B0V8J0 & 36269 & 1 \\
\hline Columbia River & 100K Area & B0YP05 & 36732 & 1 \\
\hline Columbia River & 100K Area & B11CY8 & 36937 & 1 \\
\hline Columbia River & 100K Area & B15LH6 & 37901 & 1 \\
\hline
\end{tabular}


Table B-2

\section{Summary Table of Segment 2 Surface Water Samples By Area, Site, Date, and Category Columbia River Component - Existing Sample Data Evaluation and Scoping Report}

\begin{tabular}{|c|c|c|c|c|}
\hline Sample Area & Sample Site & Sample ID & Sample Date & Category \\
\hline Columbia River & 100K Area & B15LH3 & 37670 & 1 \\
\hline Columbia River & 100K Area & B15LH5 & 37810 & 1 \\
\hline Columbia River & 100K Area & B15LH2 & 37538 & 1 \\
\hline Columbia River & 100K Area & B169B4 & 37683 & 1 \\
\hline Columbia River & 100K Area & B15LH4 & 37718 & 1 \\
\hline Columbia River & 100K Area & BOY269 & 36648 & 1 \\
\hline Columbia River & 100K Area & B15LH1 & 37901 & 1 \\
\hline Columbia River & 100K Area & B17R72 & 37956 & 1 \\
\hline Columbia River & 100K SPR 63-1 & B12X41 & 37189 & 1 \\
\hline Columbia River & 100K SPR 63-1 & B10934 & 36816 & 1 \\
\hline Columbia River & 100K SPR 63-1 & BoWMT1 & 36458 & 1 \\
\hline Columbia River & 100K SPR 63-1 & BoWP13 & 36458 & 1 \\
\hline Columbia River & 100K SPR 63-1 & B10JP2 & 36816 & 1 \\
\hline Columbia River & 100K SPR 63-1 & B135P5 & 37189 & 1 \\
\hline Columbia River & 100K SPR 63-2 & B15C58 & 37515 & 1 \\
\hline Columbia River & 100K SPR 63-2 & 30936 & 37914 & 1 \\
\hline Columbia River & 100K SPR 63-2 & 29030 & 37515 & 1 \\
\hline Columbia River & 100K SPR 63-2 & B15CD0 & 37515 & 1 \\
\hline Columbia River & 100K SPR 63-2 & B17J72 & 37914 & 1 \\
\hline Columbia River & 100K SPR 63-2 & B17J84 & 37914 & 1 \\
\hline Columbia River & 100K SPR 63-2 & B17J85 & 37914 & 1 \\
\hline Columbia River & 100K SPR 63-2 & B17JNO & 37914 & 1 \\
\hline Columbia River & 100K SPR 77-1 & B10JP3 & 36824 & 1 \\
\hline Columbia River & 100K SPR 77-1 & B15C60 & 37550 & 1 \\
\hline Columbia River & 100K SPR 77-1 & B135P6 & 37189 & 1 \\
\hline Columbia River & 100K SPR 77-1 & B15CD1 & 37550 & 1 \\
\hline Columbia River & 100K SPR 77-1 & B10936 & 36824 & 1 \\
\hline Columbia River & 100K SPR 77-1 & B12X42 & 37189 & 1 \\
\hline Columbia River & 100K SPR 77-1 & B17JN1 & 37935 & 1 \\
\hline Columbia River & 100K SPR 77-1 & B17J87 & 37935 & 1 \\
\hline Columbia River & 100K SPR 77-1 & B17J86 & 37935 & 1 \\
\hline Columbia River & 100K SPR 77-1 & B17J74 & 37935 & 1 \\
\hline Columbia River & 100K SPR 77-2 & 30937 & 37935 & 1 \\
\hline Columbia River & 100K SPR 77-2 & 33222 & 38271 & 1 \\
\hline Columbia River & 100K SPR 77-2 & B11W43 & 37015 & 1 \\
\hline Columbia River & 100K SPRINGS & 24569 & 36816 & 1 \\
\hline Columbia River & $100 \mathrm{~N}$ Area & B14YJ8 & 37445 & 1 \\
\hline Columbia River & $100 \mathrm{~N}$ Area & B14271 & 37301 & 1 \\
\hline Columbia River & 100 N Area & B14CV5 & 37354 & 1 \\
\hline Columbia River & $100 \mathrm{~N}$ Area & B15LF2 & 37670 & 1 \\
\hline Columbia River & $100 \mathrm{~N}$ Area & B0VW81 & 36333 & 1 \\
\hline Columbia River & $100 \mathrm{~N}$ Area & B15LF0 & 37901 & 1 \\
\hline Columbia River & $100 \mathrm{~N}$ Area & B15LF4 & 37810 & 1 \\
\hline Columbia River & $100 \mathrm{~N}$ Area & B16999 & 37683 & 1 \\
\hline Columbia River & $100 \mathrm{~N}$ Area & B16RN0 & 37775 & 1 \\
\hline Columbia River & $100 \mathrm{~N}$ Area & BOVWR2 & 36339 & 1 \\
\hline
\end{tabular}


Table B-2

\section{Summary Table of Segment 2 Surface Water Samples By Area, Site, Date, and Category Columbia River Component - Existing Sample Data Evaluation and Scoping Report}

\begin{tabular}{|c|c|c|c|c|}
\hline Sample Area & Sample Site & Sample ID & Sample Date & Category \\
\hline Columbia River & $100 \mathrm{~N}$ Area & B15LF5 & 37901 & 1 \\
\hline Columbia River & 100 N Area & B15LD5 & 37602 & 1 \\
\hline Columbia River & $100 \mathrm{~N}$ Area & B15LF1 & 37538 & 1 \\
\hline Columbia River & 100 N Area & B15LF3 & 37718 & 1 \\
\hline Columbia River & $100 \mathrm{~N}$ Area & B176K9 & 37866 & 1 \\
\hline Columbia River & 100 N Area & B1BC58 & 38255 & 1 \\
\hline Columbia River & $100 N$ Area & В19H75 & 38147 & 1 \\
\hline Columbia River & $100 \mathrm{~N}$ Area & B19113 & 38086 & 1 \\
\hline Columbia River & $100 \mathrm{~N}$ Area & B19114 & 38086 & 1 \\
\hline Columbia River & 100 N Area & B1BC59 & 38255 & 1 \\
\hline Columbia River & $100 \mathrm{~N}$ Area & B1BC57 & 38255 & 1 \\
\hline Columbia River & 100 N Area & $\mathrm{B} 19 \mathrm{H} 72$ & 38147 & 1 \\
\hline Columbia River & $100 N$ Area & B19H73 & 38147 & 1 \\
\hline Columbia River & $100 \mathrm{~N}$ Area & $\mathrm{B} 19 \mathrm{H} 74$ & 38147 & 1 \\
\hline Columbia River & $100 \mathrm{~N}$ Area & B17R67 & 37956 & 1 \\
\hline Columbia River & 100 N Area & B1BC56 & 38255 & 1 \\
\hline Columbia River & 100N SPR 1/10 & 31856 & 37868 & 1 \\
\hline Columbia River & 100N SPR 1/10 & 24558 & 36783 & 1 \\
\hline Columbia River & 100N SPR 1/10 & 23428 & 36413 & 1 \\
\hline Columbia River & 100N SPR 10/10 & 30909 & 37868 & 1 \\
\hline Columbia River & 100N SPR 2/10 & 23429 & 36413 & 1 \\
\hline Columbia River & 100N SPR 2/10 & 24559 & 36783 & 1 \\
\hline Columbia River & 100N SPR 2/10 & 30847 & 37868 & 1 \\
\hline Columbia River & 100N SPR 3/10 & 24560 & 36783 & 1 \\
\hline Columbia River & 100N SPR 3/10 & 23430 & 36413 & 1 \\
\hline Columbia River & 100N SPR 3/10 & 30848 & 37868 & 1 \\
\hline Columbia River & 100N SPR 5/10 & 23431 & 36413 & 1 \\
\hline Columbia River & 100N SPR 5/10 & 24562 & 36783 & 1 \\
\hline Columbia River & 100N SPR 5/10 & 30849 & 37868 & 1 \\
\hline Columbia River & 100N SPR 7/10 & 23427 & 36413 & 1 \\
\hline Columbia River & 100N SPR 7/10 & 30850 & 37868 & 1 \\
\hline Columbia River & 100N SPR 7/10 & 24561 & 36783 & 1 \\
\hline Columbia River & 100N SPR 8-13 & BOWNX9 & 36458 & 1 \\
\hline Columbia River & 100N SPR 8-13 & B11W35 & 37015 & 1 \\
\hline Columbia River & 100N SPR 8-13 & BOWMT3 & 36458 & 1 \\
\hline Columbia River & 100N SPR 8-13 & $\mathrm{B} 135 \mathrm{P} 7$ & 37189 & 1 \\
\hline Columbia River & 100N SPR 8-13 & 24570 & 36816 & 1 \\
\hline Columbia River & 100N SPR 8-13 & 33223 & 38271 & 1 \\
\hline Columbia River & 100N SPR 8-13 & 23438 & 36458 & 1 \\
\hline Columbia River & 100N SPR 8-13 & 30938 & 37919 & 1 \\
\hline Columbia River & 100N SPR 8-13 & B12WY1 & 37189 & 1 \\
\hline Columbia River & 100N SPR 8-13 & B15CD3 & 37515 & 1 \\
\hline Columbia River & 100N SPR 8-13 & B10900 & 36816 & 1 \\
\hline Columbia River & 100N SPR 8-13 & 26214 & 37015 & 1 \\
\hline Columbia River & 100N SPR 8-13 & B10910 & 36816 & 1 \\
\hline Columbia River & 100N SPR 8-13 & B15C08 & 37515 & 1 \\
\hline
\end{tabular}


Table B-2

\section{Summary Table of Segment 2 Surface Water Samples By Area, Site, Date, and Category Columbia River Component - Existing Sample Data Evaluation and Scoping Report}

\begin{tabular}{|c|c|c|c|c|}
\hline Sample Area & Sample Site & Sample ID & Sample Date & Category \\
\hline Columbia River & $100 N$ SPR 8-13 & B17J17 & 37914 & 1 \\
\hline Columbia River & 100N SPR 8-13 & B17JN3 & 37914 & 1 \\
\hline Columbia River & 100N SPR 8-13 & B17J88 & 37914 & 1 \\
\hline Columbia River & 100N SPR 8-13 & B17J22 & 37914 & 1 \\
\hline Columbia River & 100N SPR STA 3 & 23128 & 36334 & 1 \\
\hline Columbia River & 100N SPR STA 3 & 23736 & 36458 & 1 \\
\hline Columbia River & 100N SPR STA 3 & 23123 & 36181 & 1 \\
\hline Columbia River & 100N SPR STA 3 & 23124 & 36213 & 1 \\
\hline Columbia River & 100N SPR STA 3 & 23125 & 36244 & 1 \\
\hline Columbia River & 100N SPR STA 3 & 23126 & 36276 & 1 \\
\hline Columbia River & 100N SPR STA 3 & 23127 & 36304 & 1 \\
\hline Columbia River & 100N SPR STA 3 & 23129 & 36363 & 1 \\
\hline Columbia River & 300 SPR 42-2 & B0WP55 & 36465 & 1 \\
\hline Columbia River & 300 SPR 42-2 & BOWNX5 & 36465 & 1 \\
\hline Columbia River & 300 A1 HRM 43.1 & B17CN9 & 37873 & 1 \\
\hline Columbia River & 300 A1 HRM 43.1 & B158R8 & 37509 & 1 \\
\hline Columbia River & 300 A1 HRM 43.1 & BOWBP1 & 36419 & 1 \\
\hline Columbia River & 300 A1 HRM 43.1 & B10743 & 36788 & 1 \\
\hline Columbia River & 300 A1 HRM 43.1 & B12TB6 & 37147 & 1 \\
\hline Columbia River & 300 A1 HRM 43.1 & B12TK2 & 37147 & 1 \\
\hline Columbia River & 300 A1 HRM 43.1 & В12Т61 & 37147 & 1 \\
\hline Columbia River & 300 A1 HRM 43.1 & $\mathrm{~B} 17 \mathrm{CJ} 0$ & 37873 & 1 \\
\hline Columbia River & 300 A1 HRM 43.1 & B158L5 & 37509 & 1 \\
\hline Columbia River & 300 A1 HRM 43.1 & B109C7 & 36788 & 1 \\
\hline Columbia River & 300 A1 HRM 43.1 & B106K9 & 36788 & 1 \\
\hline Columbia River & 300 A1 HRM 43.1 & В17HH9 & 37873 & 1 \\
\hline Columbia River & 300 A1 HRM 43.1 & B158C9 & 37509 & 1 \\
\hline Columbia River & 300 A1 HRM 43.1 & B106X3 & 36788 & 1 \\
\hline Columbia River & 300 A1 HRM 43.1 & B106T6 & 36788 & 1 \\
\hline Columbia River & 300 A1 HRM 43.1 & B12VP6 & 37147 & 1 \\
\hline Columbia River & 300 A1 HRM 43.1 & B0WB83 & 36419 & 1 \\
\hline Columbia River & 300 A1 HRM 43.1 & B159C4 & 37509 & 1 \\
\hline Columbia River & 300 A1 HRM 43.1 & B0WB23 & 36419 & 1 \\
\hline Columbia River & 300 A1 HRM 43.1 & B1B483 & 38245 & 1 \\
\hline Columbia River & 300 A1 HRM 43.1 & B1B763 & 38245 & 1 \\
\hline Columbia River & 300 A1 HRM 43.1 & B1B720 & 38245 & 1 \\
\hline Columbia River & 300 A1 HRM 43.1 & B1B767 & 38245 & 1 \\
\hline Columbia River & 300 A1 HRM 43.1 & B1B6W6 & 38245 & 1 \\
\hline Columbia River & 300 A10 HRM43.1 & B17CP4 & 37873 & 1 \\
\hline Columbia River & 300 A10 HRM43.1 & B158M0 & 37509 & 1 \\
\hline Columbia River & 300 A10 HRM43.1 & В12Т53 & 37147 & 1 \\
\hline Columbia River & 300 A10 HRM43.1 & $\mathrm{B} 17 \mathrm{HJO}$ & 37873 & 1 \\
\hline Columbia River & 300 A10 HRM43.1 & B158C1 & 37509 & 1 \\
\hline Columbia River & 300 A10 HRM43.1 & B158T3 & 37509 & 1 \\
\hline Columbia River & 300 A10 HRM43.1 & B17CK0 & 37873 & 1 \\
\hline Columbia River & 300 A10 HRM43.1 & B0WBP2 & 36419 & 1 \\
\hline
\end{tabular}


Table B-2

\section{Summary Table of Segment 2 Surface Water Samples By Area, Site, Date, and Category Columbia River Component - Existing Sample Data Evaluation and Scoping Report}

\begin{tabular}{|c|c|c|c|c|}
\hline Sample Area & Sample Site & Sample ID & Sample Date & Category \\
\hline Columbia River & 300 A10 HRM43.1 & B159C9 & 37509 & 1 \\
\hline Columbia River & 300 A10 HRM43.1 & В12ТC6 & 37147 & 1 \\
\hline Columbia River & 300 A10 HRM43.1 & B106Y3 & 36788 & 1 \\
\hline Columbia River & 300 A10 HRM43.1 & B106R8 & 36788 & 1 \\
\hline Columbia River & 300 A10 HRM43.1 & B12TK7 & 37147 & 1 \\
\hline Columbia River & 300 A10 HRM43.1 & B0WB88 & 36419 & 1 \\
\hline Columbia River & 300 A10 HRM43.1 & B12VR1 & 37147 & 1 \\
\hline Columbia River & 300 A10 HRM43.1 & B10748 & 36788 & 1 \\
\hline Columbia River & 300 A10 HRM43.1 & B0WB28 & 36419 & 1 \\
\hline Columbia River & 300 A10 HRM43.1 & B106K8 & 36788 & 1 \\
\hline Columbia River & 300 A10 HRM43.1 & B1B6V8 & 38245 & 1 \\
\hline Columbia River & 300 A10 HRM43.1 & B1B725 & 38245 & 1 \\
\hline Columbia River & 300 A10 HRM43.1 & B1B488 & 38245 & 1 \\
\hline Columbia River & 300 A10 HRM43.1 & B1B768 & 38245 & 1 \\
\hline Columbia River & 300 A2 HRM 43.1 & B159C5 & 37509 & 1 \\
\hline Columbia River & 300 A2 HRM 43.1 & B106L0 & 36788 & 1 \\
\hline Columbia River & 300 A2 HRM 43.1 & B106T7 & 36788 & 1 \\
\hline Columbia River & 300 A2 HRM 43.1 & B158L6 & 37509 & 1 \\
\hline Columbia River & 300 A2 HRM 43.1 & B12VP7 & 37147 & 1 \\
\hline Columbia River & 300 A2 HRM 43.1 & $\mathrm{~B} 17 \mathrm{HJ} 1$ & 37873 & 1 \\
\hline Columbia River & 300 A2 HRM 43.1 & B109C8 & 36788 & 1 \\
\hline Columbia River & 300 A2 HRM 43.1 & B158D0 & 37509 & 1 \\
\hline Columbia River & 300 A2 HRM 43.1 & B158R9 & 37509 & 1 \\
\hline Columbia River & 300 A2 HRM 43.1 & B0WB84 & 36419 & 1 \\
\hline Columbia River & 300 A2 HRM 43.1 & В17CP0 & 37873 & 1 \\
\hline Columbia River & 300 A2 HRM 43.1 & B12T62 & 37147 & 1 \\
\hline Columbia River & 300 A2 HRM 43.1 & B12TK3 & 37147 & 1 \\
\hline Columbia River & 300 A2 HRM 43.1 & BOWBP3 & 36419 & 1 \\
\hline Columbia River & 300 A2 HRM 43.1 & $\mathrm{~B} 17 \mathrm{CJ} 2$ & 37873 & 1 \\
\hline Columbia River & 300 A2 HRM 43.1 & B10744 & 36788 & 1 \\
\hline Columbia River & 300 A2 HRM 43.1 & B0WB24 & 36419 & 1 \\
\hline Columbia River & 300 A2 HRM 43.1 & B12TB8 & 37147 & 1 \\
\hline Columbia River & 300 A2 HRM 43.1 & B106X5 & 36788 & 1 \\
\hline Columbia River & 300 A2 HRM 43.1 & B1B6W7 & 38245 & 1 \\
\hline Columbia River & 300 A2 HRM 43.1 & B1B721 & 38245 & 1 \\
\hline Columbia River & 300 A2 HRM 43.1 & B1B484 & 38245 & 1 \\
\hline Columbia River & 300 A2 HRM 43.1 & B1B764 & 38245 & 1 \\
\hline Columbia River & 300 A3 HRM 43.1 & B12VP8 & 37147 & 1 \\
\hline Columbia River & 300 A3 HRM 43.1 & B12TK4 & 37147 & 1 \\
\hline Columbia River & 300 A3 HRM 43.1 & B12ТC0 & 37147 & 1 \\
\hline Columbia River & 300 A3 HRM 43.1 & B106X7 & 36788 & 1 \\
\hline Columbia River & 300 A3 HRM 43.1 & $\mathrm{~B} 17 \mathrm{HJ} 2$ & 37873 & 1 \\
\hline Columbia River & 300 A3 HRM 43.1 & B17CP1 & 37873 & 1 \\
\hline Columbia River & 300 A3 HRM 43.1 & $\mathrm{~B} 17 \mathrm{CJ} 4$ & 37873 & 1 \\
\hline Columbia River & 300 A3 HRM 43.1 & BoWB25 & 36419 & 1 \\
\hline Columbia River & 300 A3 HRM 43.1 & B0WB85 & 36419 & 1 \\
\hline
\end{tabular}


Table B-2

\section{Summary Table of Segment 2 Surface Water Samples By Area, Site, Date, and Category Columbia River Component - Existing Sample Data Evaluation and Scoping Report}

\begin{tabular}{|c|c|c|c|c|}
\hline Sample Area & Sample Site & Sample ID & Sample Date & Category \\
\hline Columbia River & 300 A3 HRM 43.1 & BOWBP4 & 36419 & 1 \\
\hline Columbia River & 300 A3 HRM 43.1 & B106L1 & 36788 & 1 \\
\hline Columbia River & 300 A3 HRM 43.1 & B106T8 & 36788 & 1 \\
\hline Columbia River & 300 A3 HRM 43.1 & B159C6 & 37509 & 1 \\
\hline Columbia River & 300 A3 HRM 43.1 & В12Т63 & 37147 & 1 \\
\hline Columbia River & 300 A3 HRM 43.1 & B10745 & 36788 & 1 \\
\hline Columbia River & 300 A3 HRM 43.1 & В158T0 & 37509 & 1 \\
\hline Columbia River & 300 A3 HRM 43.1 & B158L7 & 37509 & 1 \\
\hline Columbia River & 300 A3 HRM 43.1 & B158D1 & 37509 & 1 \\
\hline Columbia River & 300 A3 HRM 43.1 & B1B765 & 38245 & 1 \\
\hline Columbia River & 300 A3 HRM 43.1 & B1B6W8 & 38245 & 1 \\
\hline Columbia River & 300 A3 HRM 43.1 & B1B722 & 38245 & 1 \\
\hline Columbia River & 300 A3 HRM 43.1 & B1B485 & 38245 & 1 \\
\hline Columbia River & 300 A5 HRM 43.1 & B12TK5 & 37147 & 1 \\
\hline Columbia River & 300 A5 HRM 43.1 & B106X9 & 36788 & 1 \\
\hline Columbia River & 300 A5 HRM 43.1 & B158B9 & 37509 & 1 \\
\hline Columbia River & 300 A5 HRM 43.1 & B159C7 & 37509 & 1 \\
\hline Columbia River & 300 A5 HRM 43.1 & B106L2 & 36788 & 1 \\
\hline Columbia River & 300 A5 HRM 43.1 & B106R6 & 36788 & 1 \\
\hline Columbia River & 300 A5 HRM 43.1 & B0WB86 & 36419 & 1 \\
\hline Columbia River & 300 A5 HRM 43.1 & B12TC2 & 37147 & 1 \\
\hline Columbia River & 300 A5 HRM 43.1 & B0WB26 & 36419 & 1 \\
\hline Columbia River & 300 A5 HRM 43.1 & B158L8 & 37509 & 1 \\
\hline Columbia River & 300 A5 HRM 43.1 & BowC84 & 36419 & 1 \\
\hline Columbia River & 300 A5 HRM 43.1 & В12Т51 & 37147 & 1 \\
\hline Columbia River & 300 A5 HRM 43.1 & B17CP2 & 37873 & 1 \\
\hline Columbia River & 300 A5 HRM 43.1 & B12VP9 & 37147 & 1 \\
\hline Columbia River & 300 A5 HRM 43.1 & $\mathrm{~B} 17 \mathrm{HJ} 3$ & 37873 & 1 \\
\hline Columbia River & 300 A5 HRM 43.1 & B10746 & 36788 & 1 \\
\hline Columbia River & 300 A5 HRM 43.1 & B158T1 & 37509 & 1 \\
\hline Columbia River & 300 A5 HRM 43.1 & B17CJ6 & 37873 & 1 \\
\hline Columbia River & 300 A5 HRM 43.1 & B1B486 & 38245 & 1 \\
\hline Columbia River & 300 A5 HRM 43.1 & B1B723 & 38245 & 1 \\
\hline Columbia River & 300 A5 HRM 43.1 & B1B6V6 & 38245 & 1 \\
\hline Columbia River & 300 A5 HRM 43.1 & B1B766 & 38245 & 1 \\
\hline Columbia River & 300 A7 HRM 43.1 & B159C8 & 37509 & 1 \\
\hline Columbia River & 300 A7 HRM 43.1 & В12ТC4 & 37147 & 1 \\
\hline Columbia River & 300 A7 HRM 43.1 & B106Y1 & 36788 & 1 \\
\hline Columbia River & 300 A7 HRM 43.1 & B106R7 & 36788 & 1 \\
\hline Columbia River & 300 A7 HRM 43.1 & B106L3 & 36788 & 1 \\
\hline Columbia River & 300 A7 HRM 43.1 & B10747 & 36788 & 1 \\
\hline Columbia River & 300 A7 HRM 43.1 & B0WB87 & 36419 & 1 \\
\hline Columbia River & 300 A7 HRM 43.1 & B0WB27 & 36419 & 1 \\
\hline Columbia River & 300 A7 HRM 43.1 & B17CJ8 & 37873 & 1 \\
\hline Columbia River & 300 A7 HRM 43.1 & В17CP3 & 37873 & 1 \\
\hline Columbia River & 300 A7 HRM 43.1 & В12Т52 & 37147 & 1 \\
\hline
\end{tabular}


Table B-2

\section{Summary Table of Segment 2 Surface Water Samples By Area, Site, Date, and Category Columbia River Component - Existing Sample Data Evaluation and Scoping Report}

\begin{tabular}{|c|c|c|c|c|}
\hline Sample Area & Sample Site & Sample ID & Sample Date & Category \\
\hline Columbia River & 300 A7 HRM 43.1 & $\mathrm{~B} 17 \mathrm{HJ} 4$ & 37873 & 1 \\
\hline Columbia River & 300 A7 HRM 43.1 & B12VR0 & 37147 & 1 \\
\hline Columbia River & 300 A7 HRM 43.1 & B0WC85 & 36419 & 1 \\
\hline Columbia River & 300 A7 HRM 43.1 & B12TK6 & 37147 & 1 \\
\hline Columbia River & 300 A7 HRM 43.1 & B158L9 & 37509 & 1 \\
\hline Columbia River & 300 A7 HRM 43.1 & B158C0 & 37509 & 1 \\
\hline Columbia River & 300 A7 HRM 43.1 & B158T2 & 37509 & 1 \\
\hline Columbia River & 300 A7 HRM 43.1 & B1B724 & 38245 & 1 \\
\hline Columbia River & 300 A7 HRM 43.1 & B1B487 & 38245 & 1 \\
\hline Columbia River & 300 A7 HRM 43.1 & B1B6V7 & 38245 & 1 \\
\hline Columbia River & 300 AREA & B0WKP1 & 36433 & 1 \\
\hline Columbia River & 300 AREA & BovWV9 & 36342 & 1 \\
\hline Columbia River & 300 AREA 1/10 & 24563 & 36788 & 1 \\
\hline Columbia River & 300 AREA 1/10 & 30899 & 37873 & 1 \\
\hline Columbia River & 300 AREA 1/10 & 26208 & 37147 & 1 \\
\hline Columbia River & 300 AREA 10/10 & 30851 & 37873 & 1 \\
\hline Columbia River & 300 AREA 10/10 & 26992 & 37147 & 1 \\
\hline Columbia River & 300 AREA 2/10 & 24564 & 36788 & 1 \\
\hline Columbia River & 300 AREA 2/10 & 30900 & 37873 & 1 \\
\hline Columbia River & 300 AREA 2/10 & 26209 & 37147 & 1 \\
\hline Columbia River & 300 AREA 3/10 & 30901 & 37873 & 1 \\
\hline Columbia River & 300 AREA 3/10 & 24565 & 36788 & 1 \\
\hline Columbia River & 300 AREA 3/10 & 26210 & 37147 & 1 \\
\hline Columbia River & 300 AREA 5/10 & 30907 & 37873 & 1 \\
\hline Columbia River & 300 AREA 5/10 & 26212 & 37147 & 1 \\
\hline Columbia River & 300 AREA 5/10 & 24567 & 36788 & 1 \\
\hline Columbia River & 300 AREA 7/10 & 30908 & 37873 & 1 \\
\hline Columbia River & 300 AREA 7/10 & 24566 & 36788 & 1 \\
\hline Columbia River & 300 AREA 7/10 & 26991 & 37147 & 1 \\
\hline Columbia River & 300 AREA OUT13 & B1B7H8 & 38245 & 1 \\
\hline Columbia River & 300 AREA OUT13 & $\mathrm{B} 1 \mathrm{~B} 7 \mathrm{H} 7$ & 38245 & 1 \\
\hline Columbia River & 300 AREA OUT13 & B1BW54 & 38340 & 1 \\
\hline Columbia River & 300 AREA OUT13 & $\mathrm{B} 1 \mathrm{~B} \times 60$ & 38340 & 1 \\
\hline Columbia River & 300 AREA OUT13 & B1B493 & 38245 & 1 \\
\hline Columbia River & 300 AREA OUT13 & B1B7H9 & 38245 & 1 \\
\hline Columbia River & 300 AREA OUT13 & B19JC5 & 38162 & 1 \\
\hline Columbia River & 300 AREA OUT13 & В19HР8 & 38162 & 1 \\
\hline Columbia River & 300 AREA OUT13 & B19JC4 & 38162 & 1 \\
\hline Columbia River & 300 AREA OUT13 & B19JC6 & 38162 & 1 \\
\hline Columbia River & 300 SHR HRM41.5 & B1B7B9 & 38245 & 1 \\
\hline Columbia River & 300 SHR HRM41.5 & B1B7C1 & 38245 & 1 \\
\hline Columbia River & 300 SHR HRM41.5 & B1B7C2 & 38245 & 1 \\
\hline Columbia River & 300 SHR HRM41.5 & B1B489 & 38245 & 1 \\
\hline Columbia River & 300 SHR HRM42.1 & B1B7C5 & 38245 & 1 \\
\hline Columbia River & 300 SHR HRM42.1 & B1B7C6 & 38245 & 1 \\
\hline Columbia River & 300 SHR HRM42.1 & B1B490 & 38245 & 1 \\
\hline
\end{tabular}


Table B-2

\section{Summary Table of Segment 2 Surface Water Samples By Area, Site, Date, and Category Columbia River Component - Existing Sample Data Evaluation and Scoping Report}

\begin{tabular}{|c|c|c|c|c|}
\hline Sample Area & Sample Site & Sample ID & Sample Date & Category \\
\hline Columbia River & 300 SHR HRM42.1 & B1B7C3 & 38245 & 1 \\
\hline Columbia River & 300 SHR HRM42.4 & B1B7H6 & 38245 & 1 \\
\hline Columbia River & 300 SHR HRM42.4 & B1B7H5 & 38245 & 1 \\
\hline Columbia River & 300 SHR HRM42.4 & B1B7H3 & 38245 & 1 \\
\hline Columbia River & 300 SHR HRM42.4 & B1B491 & 38245 & 1 \\
\hline Columbia River & 300 SHR HRM42.9 & B1B492 & 38245 & 1 \\
\hline Columbia River & 300 SHR HRM42.9 & B1B7C9 & 38245 & 1 \\
\hline Columbia River & 300 SHR HRM42.9 & B1B7D0 & 38245 & 1 \\
\hline Columbia River & 300 SHR HRM42.9 & B1B7C7 & 38245 & 1 \\
\hline Columbia River & 300 SPR 11 -1 & B12RP6 & 37130 & 1 \\
\hline Columbia River & 300 SPR $11-1$ & B12RW5 & 37130 & 1 \\
\hline Columbia River & 300 SPR $11-1$ & B12RP5 & 37130 & 1 \\
\hline Columbia River & 300 SPR 11-1 & 27693 & 37130 & 1 \\
\hline Columbia River & 300 SPR 11-2 & 27694 & 37130 & 1 \\
\hline Columbia River & 300 SPR 11-2 & B12RW7 & 37130 & 1 \\
\hline Columbia River & 300 SPR 11-3 & 27695 & 37130 & 1 \\
\hline Columbia River & 300 SPR $11-3$ & B12RW9 & 37130 & 1 \\
\hline Columbia River & 300 SPR 11-4 & В12Т21 & 37130 & 1 \\
\hline Columbia River & 300 SPR 11-4 & 27696 & 37130 & 1 \\
\hline Columbia River & 300 SPR 11-DR-1 & 27697 & 37130 & 1 \\
\hline Columbia River & 300 SPR 11-DR-2 & 27698 & 37130 & 1 \\
\hline Columbia River & 300 SPR 11-DR-3 & 27699 & 37130 & 1 \\
\hline Columbia River & 300 SPR 11-DR-4 & 27700 & 37130 & 1 \\
\hline Columbia River & 300 SPR 14 & 33072 & 37963 & 1 \\
\hline Columbia River & 300 SPR 14-1 & 27707 & 37130 & 1 \\
\hline Columbia River & 300 SPR 14-1 & $\mathrm{B} 12 \mathrm{R} \times 9$ & 37130 & 1 \\
\hline Columbia River & 300 SPR 14-2 & B12RY1 & 37130 & 1 \\
\hline Columbia River & 300 SPR 14-2 & 27708 & 37130 & 1 \\
\hline Columbia River & 300 SPR $14-3$ & 27709 & 37130 & 1 \\
\hline Columbia River & 300 SPR 14-3 & B12RY3 & 37130 & 1 \\
\hline Columbia River & 300 SPR 14-4 & 27710 & 37130 & 1 \\
\hline Columbia River & 300 SPR 14-4 & B12Т25 & 37130 & 1 \\
\hline Columbia River & 300 SPR $41-9$ & B1BH15 & 38285 & 1 \\
\hline Columbia River & 300 SPR $42-2$ & B12RM1 & 37130 & 1 \\
\hline Columbia River & 300 SPR 42-2 & 30857 & 37907 & 1 \\
\hline Columbia River & 300 SPR 42-2 & B12RM8 & 37130 & 1 \\
\hline Columbia River & 300 SPR 42-2 & B12RN5 & 37130 & 1 \\
\hline Columbia River & 300 SPR 42-2 & BOWNY3 & 36465 & 1 \\
\hline Columbia River & 300 SPR 42-2 & B10907 & 36796 & 1 \\
\hline Columbia River & 300 SPR $42-2$ & B10903 & 36796 & 1 \\
\hline Columbia River & 300 SPR 42-2 & B108Y4 & 36796 & 1 \\
\hline Columbia River & 300 SPR 42-2 & B10973 & 36796 & 1 \\
\hline Columbia River & 300 SPR $42-2$ & 27650 & 37130 & 1 \\
\hline Columbia River & 300 SPR 42-2 & BowMV3 & 36465 & 1 \\
\hline Columbia River & 300 SPR $42-2$ & B12RL6 & 37130 & 1 \\
\hline Columbia River & 300 SPR 42-2 & B12RL8 & 37130 & 1 \\
\hline
\end{tabular}


Table B-2

\section{Summary Table of Segment 2 Surface Water Samples By Area, Site, Date, and Category Columbia River Component - Existing Sample Data Evaluation and Scoping Report}

\begin{tabular}{|c|c|c|c|c|}
\hline Sample Area & Sample Site & Sample ID & Sample Date & Category \\
\hline Columbia River & 300 SPR $42-2$ & B10913 & 36796 & 1 \\
\hline Columbia River & 300 SPR 42-2 & BOWNX8 & 36465 & 1 \\
\hline Columbia River & 300 SPR 42-2 & B11W34 & 37021 & 1 \\
\hline Columbia River & 300 SPR $42-2$ & B11W38 & 37021 & 1 \\
\hline Columbia River & 300 SPR $42-2$ & B12RL2 & 37130 & 1 \\
\hline Columbia River & 300 SPR $42-2$ & 33144 & 38285 & 1 \\
\hline Columbia River & 300 SPR $42-2$ & 26956 & 37021 & 1 \\
\hline Columbia River & 300 SPR 42-2 & B15C01 & 37536 & 1 \\
\hline Columbia River & 300 SPR 42-2 & B15C06 & 37536 & 1 \\
\hline Columbia River & 300 SPR $42-2$ & B15C11 & 37536 & 1 \\
\hline Columbia River & 300 SPR $42-2$ & 24574 & 36796 & 1 \\
\hline Columbia River & 300 SPR $42-2$ & B15CD5 & 37536 & 1 \\
\hline Columbia River & 300 SPR 42-2 & B15C99 & 37536 & 1 \\
\hline Columbia River & 300 SPR $42-2$ & B12XK1 & 37152 & 1 \\
\hline Columbia River & 300 SPR 42-2 & B1BJ63 & 38285 & 1 \\
\hline Columbia River & 300 SPR $42-2$ & B1BFY3 & 38285 & 1 \\
\hline Columbia River & 300 SPR $42-2$ & B1BFP4 & 38285 & 1 \\
\hline Columbia River & 300 SPR $42-2$ & B1BFN1 & 38285 & 1 \\
\hline Columbia River & 300 SPR 42-2 & B1BFL9 & 38285 & 1 \\
\hline Columbia River & 300 SPR $42-2$ & B17RH8 & 37907 & 1 \\
\hline Columbia River & 300 SPR $42-2$ & B17RJ0 & 37907 & 1 \\
\hline Columbia River & 300 SPR $42-2$ & B17RJ2 & 37907 & 1 \\
\hline Columbia River & 300 SPR $42-2$ & B17RL4 & 37907 & 1 \\
\hline Columbia River & 300 SPR 42-2 & B17RR6 & 37907 & 1 \\
\hline Columbia River & 300 SPR 6 & 33071 & 37963 & 1 \\
\hline Columbia River & 300 SPR 7-1 & B12RR5 & 37130 & 1 \\
\hline Columbia River & 300 SPR 7-1 & В12Т09 & 37130 & 1 \\
\hline Columbia River & 300 SPR 7-1 & B12Т10 & 37130 & 1 \\
\hline Columbia River & 300 SPR 7-1 & 27662 & 37130 & 1 \\
\hline Columbia River & 300 SPR 7-2 & 27663 & 37130 & 1 \\
\hline Columbia River & 300 SPR 7-2 & B12RR7 & 37130 & 1 \\
\hline Columbia River & 300 SPR 7-3 & 27664 & 37130 & 1 \\
\hline Columbia River & 300 SPR 7-3 & B12RR9 & 37130 & 1 \\
\hline Columbia River & 300 SPR 7-4 & В12Т13 & 37130 & 1 \\
\hline Columbia River & 300 SPR 7-4 & 27665 & 37130 & 1 \\
\hline Columbia River & 300 SPR 7-DR-1 & 27666 & 37130 & 1 \\
\hline Columbia River & 300 SPR 7-DR-2 & 27667 & 37130 & 1 \\
\hline Columbia River & 300 SPR 7-DR-3 & 27668 & 37130 & 1 \\
\hline Columbia River & 300 SPR 7-DR-4 & 27669 & 37130 & 1 \\
\hline Columbia River & 300 SPR 7-SPR 9 & 27670 & 37130 & 1 \\
\hline Columbia River & 300 SPR 7/9 & $\mathrm{B} 12 \mathrm{RT7}$ & 37130 & 1 \\
\hline Columbia River & 300 SPR 9-1 & 27672 & 37130 & 1 \\
\hline Columbia River & 300 SPR 9-1 & B12RP1 & 37130 & 1 \\
\hline Columbia River & 300 SPR 9-1 & B12RP2 & 37130 & 1 \\
\hline Columbia River & 300 SPR 9-1 & B12RT9 & 37130 & 1 \\
\hline Columbia River & 300 SPR 9-2 & B12RV1 & 37130 & 1 \\
\hline
\end{tabular}


Table B-2

\section{Summary Table of Segment 2 Surface Water Samples By Area, Site, Date, and Category Columbia River Component - Existing Sample Data Evaluation and Scoping Report}

\begin{tabular}{|c|c|c|c|c|}
\hline Sample Area & Sample Site & Sample ID & Sample Date & Category \\
\hline Columbia River & 300 SPR 9-2 & 27673 & 37130 & 1 \\
\hline Columbia River & 300 SPR 9-3 & B12RV3 & 37130 & 1 \\
\hline Columbia River & 300 SPR 9-3 & 27674 & 37130 & 1 \\
\hline Columbia River & 300 SPR 9-4 & B12Т17 & 37130 & 1 \\
\hline Columbia River & 300 SPR 9-4 & 27675 & 37130 & 1 \\
\hline Columbia River & 300 SPR 9-DR-1 & 27676 & 37130 & 1 \\
\hline Columbia River & 300 SPR 9-DR-2 & 27677 & 37130 & 1 \\
\hline Columbia River & 300 SPR 9-DR-3 & 27678 & 37130 & 1 \\
\hline Columbia River & 300 SPR 9-DR-4 & 27679 & 37130 & 1 \\
\hline Columbia River & 300 SPR 9-SPR11 & 27680 & 37130 & 1 \\
\hline Columbia River & 300 SPR 9/11 & B12RW1 & 37130 & 1 \\
\hline Columbia River & 300 SPR DR 11-1 & $\mathrm{B} 12 \mathrm{R} \times 1$ & 37130 & 1 \\
\hline Columbia River & 300 SPR DR 11-2 & $\mathrm{B} 12 \mathrm{R} \times 3$ & 37130 & 1 \\
\hline Columbia River & 300 SPR DR 11-3 & B12RX5 & 37130 & 1 \\
\hline Columbia River & 300 SPR DR 11-4 & B12Т23 & 37130 & 1 \\
\hline Columbia River & 300 SPR DR 42-2 & B108Y5 & 36796 & 1 \\
\hline Columbia River & 300 SPR DR 42-2 & B10949 & 36796 & 1 \\
\hline Columbia River & 300 SPR DR 42-2 & B10975 & 36796 & 1 \\
\hline Columbia River & 300 SPR DR 42-2 & B10976 & 36796 & 1 \\
\hline Columbia River & 300 SPR DR 42-2 & 33225 & 38285 & 1 \\
\hline Columbia River & 300 SPR DR 42-2 & B15C75 & 37616 & 1 \\
\hline Columbia River & 300 SPR DR 42-2 & B10974 & 36796 & 1 \\
\hline Columbia River & 300 SPR DR 42-2 & B0WP28 & 36465 & 1 \\
\hline Columbia River & 300 SPR DR 42-2 & B12RN6 & 37130 & 1 \\
\hline Columbia River & 300 SPR DR 42-2 & B0WP57 & 36465 & 1 \\
\hline Columbia River & 300 SPR DR 42-2 & BOWMV4 & 36465 & 1 \\
\hline Columbia River & 300 SPR DR 42-2 & B12RN1 & 37130 & 1 \\
\hline Columbia River & 300 SPR DR 42-2 & B12RN0 & 37130 & 1 \\
\hline Columbia River & 300 SPR DR 42-2 & B12RM9 & 37130 & 1 \\
\hline Columbia River & 300 SPR DR 42-2 & B12RM6 & 37130 & 1 \\
\hline Columbia River & 300 SPR DR 42-2 & B0WP33 & 36465 & 1 \\
\hline Columbia River & 300 SPR DR 42-2 & B0WP56 & 36465 & 1 \\
\hline Columbia River & 300 SPR DR 42-2 & B12RL1 & 37130 & 1 \\
\hline Columbia River & 300 SPR DR 42-2 & 26957 & 37014 & 1 \\
\hline Columbia River & 300 SPR DR 42-2 & B11W57 & 37014 & 1 \\
\hline Columbia River & 300 SPR DR 42-2 & B15CB2 & 37616 & 1 \\
\hline Columbia River & 300 SPR DR 42-2 & B11W85 & 37014 & 1 \\
\hline Columbia River & 300 SPR DR 42-2 & B12XK2 & 37151 & 1 \\
\hline Columbia River & 300 SPR DR 42-2 & 27671 & 37130 & 1 \\
\hline Columbia River & 300 SPR DR 42-2 & 30940 & 37907 & 1 \\
\hline Columbia River & 300 SPR DR 42-2 & B17RR5 & 37907 & 1 \\
\hline Columbia River & 300 SPR DR 42-2 & B1BJ64 & 38285 & 1 \\
\hline Columbia River & 300 SPR DR 42-2 & B1BFY6 & 38285 & 1 \\
\hline Columbia River & 300 SPR DR 42-2 & B1BFY5 & 38285 & 1 \\
\hline Columbia River & 300 SPR DR 42-2 & B1BFY4 & 38285 & 1 \\
\hline Columbia River & 300 SPR DR 42-2 & B1BFV8 & 38285 & 1 \\
\hline
\end{tabular}


Table B-2

\section{Summary Table of Segment 2 Surface Water Samples By Area, Site, Date, and Category Columbia River Component - Existing Sample Data Evaluation and Scoping Report}

\begin{tabular}{|c|c|c|c|c|}
\hline Sample Area & Sample Site & Sample ID & Sample Date & Category \\
\hline Columbia River & 300 SPR DR 42-2 & B17RK1 & 37907 & 1 \\
\hline Columbia River & 300 SPR DR 42-2 & B17RL5 & 37907 & 1 \\
\hline Columbia River & 300 SPR DR 42-2 & B17RL6 & 37907 & 1 \\
\hline Columbia River & 300 SPR DR 42-2 & B17RL7 & 37907 & 1 \\
\hline Columbia River & 300 SPR DR 7-1 & B12RT1 & 37130 & 1 \\
\hline Columbia River & 300 SPR DR 7-2 & B12RT3 & 37130 & 1 \\
\hline Columbia River & 300 SPR DR 7-3 & B12RT5 & 37130 & 1 \\
\hline Columbia River & 300 SPR DR 7-4 & B12T15 & 37130 & 1 \\
\hline Columbia River & 300 SPR DR 9-1 & B12RV5 & 37130 & 1 \\
\hline Columbia River & 300 SPR DR 9-2 & B12RV7 & 37130 & 1 \\
\hline Columbia River & 300 SPR DR 9-3 & B12RV9 & 37130 & 1 \\
\hline Columbia River & 300 SPR DR 9-4 & В12Т19 & 37130 & 1 \\
\hline Columbia River & 300 SR HRM 41.5 & B0WB61 & 36419 & 1 \\
\hline Columbia River & 300 SR HRM 41.5 & 27407 & 37147 & 1 \\
\hline Columbia River & 300 SR HRM 41.5 & BowC86 & 36419 & 1 \\
\hline Columbia River & 300 SR HRM 41.5 & B158Y1 & 37509 & 1 \\
\hline Columbia River & 300 SR HRM 41.5 & B10775 & 36788 & 1 \\
\hline Columbia River & 300 SR HRM 41.5 & B10774 & 36788 & 1 \\
\hline Columbia River & 300 SR HRM 41.5 & B159D0 & 37509 & 1 \\
\hline Columbia River & 300 SR HRM 41.5 & B10773 & 36788 & 1 \\
\hline Columbia River & 300 SR HRM 41.5 & 30910 & 37873 & 1 \\
\hline Columbia River & 300 SR HRM 41.5 & B106L4 & 36788 & 1 \\
\hline Columbia River & 300 SR HRM 41.5 & B158Y2 & 37509 & 1 \\
\hline Columbia River & 300 SR HRM 41.5 & B0WB53 & 36419 & 1 \\
\hline Columbia River & 300 SR HRM 41.5 & $\mathrm{~B} 17 \mathrm{HJ} 5$ & 37873 & 1 \\
\hline Columbia River & 300 SR HRM 41.5 & B17CW5 & 37873 & 1 \\
\hline Columbia River & 300 SR HRM 41.5 & B17CW8 & 37873 & 1 \\
\hline Columbia River & 300 SR HRM 41.5 & B158Y0 & 37509 & 1 \\
\hline Columbia River & 300 SR HRM 41.5 & B12TP7 & 37147 & 1 \\
\hline Columbia River & 300 SR HRM 41.5 & B12TP6 & 37147 & 1 \\
\hline Columbia River & 300 SR HRM 41.5 & B12TP4 & 37147 & 1 \\
\hline Columbia River & 300 SR HRM 41.5 & B109C9 & 36788 & 1 \\
\hline Columbia River & 300 SR HRM 41.5 & 33197 & 38245 & 1 \\
\hline Columbia River & 300 SR HRM 41.5 & B12VR2 & 37147 & 1 \\
\hline Columbia River & 300 SR HRM 42.1 & B12TP8 & 37147 & 1 \\
\hline Columbia River & 300 SR HRM 42.1 & B0WC87 & 36419 & 1 \\
\hline Columbia River & 300 SR HRM 42.1 & 27408 & 37147 & 1 \\
\hline Columbia River & 300 SR HRM 42.1 & B12TR0 & 37147 & 1 \\
\hline Columbia River & 300 SR HRM 42.1 & B10778 & 36788 & 1 \\
\hline Columbia River & 300 SR HRM 42.1 & B10776 & 36788 & 1 \\
\hline Columbia River & 300 SR HRM 42.1 & B0WB62 & 36419 & 1 \\
\hline Columbia River & 300 SR HRM 42.1 & B109D0 & 36788 & 1 \\
\hline Columbia River & 300 SR HRM 42.1 & B12VR3 & 37147 & 1 \\
\hline Columbia River & 300 SR HRM 42.1 & B10777 & 36788 & 1 \\
\hline Columbia River & 300 SR HRM 42.1 & B12TR1 & 37147 & 1 \\
\hline Columbia River & 300 SR HRM 42.1 & 33198 & 38245 & 1 \\
\hline
\end{tabular}


Table B-2

\section{Summary Table of Segment 2 Surface Water Samples By Area, Site, Date, and Category Columbia River Component - Existing Sample Data Evaluation and Scoping Report}

\begin{tabular}{|c|c|c|c|c|}
\hline Sample Area & Sample Site & Sample ID & Sample Date & Category \\
\hline Columbia River & 300 SR HRM 42.1 & B158Y3 & 37509 & 1 \\
\hline Columbia River & 300 SR HRM 42.1 & 30911 & 37873 & 1 \\
\hline Columbia River & 300 SR HRM 42.1 & B0WB54 & 36419 & 1 \\
\hline Columbia River & 300 SR HRM 42.1 & B158Y5 & 37509 & 1 \\
\hline Columbia River & 300 SR HRM 42.1 & B158Y4 & 37509 & 1 \\
\hline Columbia River & 300 SR HRM 42.1 & B106L5 & 36788 & 1 \\
\hline Columbia River & 300 SR HRM 42.1 & B159D1 & 37509 & 1 \\
\hline Columbia River & 300 SR HRM 42.1 & B17CW9 & 37873 & 1 \\
\hline Columbia River & 300 SR HRM 42.1 & $\mathrm{~B} 17 \mathrm{C} \times 2$ & 37873 & 1 \\
\hline Columbia River & 300 SR HRM 42.1 & $\mathrm{~B} 17 \mathrm{HJ} 6$ & 37873 & 1 \\
\hline Columbia River & 300 SR HRM 42.4 & 33199 & 38245 & 1 \\
\hline Columbia River & 300 SR HRM 42.4 & 30912 & 37873 & 1 \\
\hline Columbia River & 300 SR HRM 42.4 & 27409 & 37147 & 1 \\
\hline Columbia River & 300 SR HRM 42.5 & $\mathrm{~B} 17 \mathrm{C} \times 3$ & 37873 & 1 \\
\hline Columbia River & 300 SR HRM 42.5 & $\mathrm{~B} 17 \mathrm{C} \times 6$ & 37873 & 1 \\
\hline Columbia River & 300 SR HRM 42.5 & BowC88 & 36419 & 1 \\
\hline Columbia River & 300 SR HRM 42.5 & B159D2 & 37509 & 1 \\
\hline Columbia River & 300 SR HRM 42.5 & B12TR4 & 37147 & 1 \\
\hline Columbia River & 300 SR HRM 42.5 & B10781 & 36788 & 1 \\
\hline Columbia River & 300 SR HRM 42.5 & B106L6 & 36788 & 1 \\
\hline Columbia River & 300 SR HRM 42.5 & B109D1 & 36788 & 1 \\
\hline Columbia River & 300 SR HRM 42.5 & B0WB63 & 36419 & 1 \\
\hline Columbia River & 300 SR HRM 42.5 & B12VR4 & 37147 & 1 \\
\hline Columbia River & 300 SR HRM 42.5 & B0WB55 & 36419 & 1 \\
\hline Columbia River & 300 SR HRM 42.5 & B10780 & 36788 & 1 \\
\hline Columbia River & 300 SR HRM 42.5 & B10779 & 36788 & 1 \\
\hline Columbia River & 300 SR HRM 42.5 & B158Y6 & 37509 & 1 \\
\hline Columbia River & 300 SR HRM 42.5 & B158Y7 & 37509 & 1 \\
\hline Columbia River & 300 SR HRM 42.5 & B158Y8 & 37509 & 1 \\
\hline Columbia River & 300 SR HRM 42.5 & B12TR2 & 37147 & 1 \\
\hline Columbia River & 300 SR HRM 42.5 & B12TR5 & 37147 & 1 \\
\hline Columbia River & 300 SR HRM 42.5 & $\mathrm{~B} 17 \mathrm{HJ} 7$ & 37873 & 1 \\
\hline Columbia River & 300 SR HRM 42.9 & B17CY0 & 37873 & 1 \\
\hline Columbia River & 300 SR HRM 42.9 & B106L7 & 36788 & 1 \\
\hline Columbia River & 300 SR HRM 42.9 & $\mathrm{~B} 17 \mathrm{C} \times 7$ & 37873 & 1 \\
\hline Columbia River & 300 SR HRM 42.9 & B12TR6 & 37147 & 1 \\
\hline Columbia River & 300 SR HRM 42.9 & B12TR8 & 37147 & 1 \\
\hline Columbia River & 300 SR HRM 42.9 & B12TR9 & 37147 & 1 \\
\hline Columbia River & 300 SR HRM 42.9 & 33200 & 38245 & 1 \\
\hline Columbia River & 300 SR HRM 42.9 & B109D2 & 36788 & 1 \\
\hline Columbia River & 300 SR HRM 42.9 & B10784 & 36788 & 1 \\
\hline Columbia River & 300 SR HRM 42.9 & B0WB56 & 36419 & 1 \\
\hline Columbia River & 300 SR HRM 42.9 & B15900 & 37509 & 1 \\
\hline Columbia River & 300 SR HRM 42.9 & 30913 & 37873 & 1 \\
\hline Columbia River & 300 SR HRM 42.9 & B0WB64 & 36419 & 1 \\
\hline Columbia River & 300 SR HRM 42.9 & B10783 & 36788 & 1 \\
\hline
\end{tabular}


Table B-2

\section{Summary Table of Segment 2 Surface Water Samples By Area, Site, Date, and Category Columbia River Component - Existing Sample Data Evaluation and Scoping Report}

\begin{tabular}{|c|c|c|c|c|}
\hline Sample Area & Sample Site & Sample ID & Sample Date & Category \\
\hline Columbia River & 300 SR HRM 42.9 & B0WC89 & 36419 & 1 \\
\hline Columbia River & 300 SR HRM 42.9 & B10782 & 36788 & 1 \\
\hline Columbia River & 300 SR HRM 42.9 & B159D3 & 37509 & 1 \\
\hline Columbia River & 300 SR HRM 42.9 & 27410 & 37147 & 1 \\
\hline Columbia River & 300 SR HRM 42.9 & B158Y9 & 37509 & 1 \\
\hline Columbia River & 300 SR HRM 42.9 & B15901 & 37509 & 1 \\
\hline Columbia River & 300 SR HRM 42.9 & B12VR5 & 37147 & 1 \\
\hline Columbia River & 300 SR HRM 42.9 & $\mathrm{~B} 17 \mathrm{HJ} 8$ & 37873 & 1 \\
\hline Columbia River & At Road 56 & 0000099 & 36804 & 1 \\
\hline Columbia River & At Road 56 & 0000098 & 36633 & 1 \\
\hline Columbia River & At Road 56 & 0000105 & 37900 & 1 \\
\hline Columbia River & At Road 56 & 0000104 & 37720 & 1 \\
\hline Columbia River & At Road 56 & 0000103 & 37531 & 1 \\
\hline Columbia River & At Road 56 & 0000102 & 37369 & 1 \\
\hline Columbia River & At Road 56 & 0000100 & 37018 & 1 \\
\hline Columbia River & At Road 56 & 0000101 & 37165 & 1 \\
\hline Columbia River & BENTON PMPHSE & 23694 & 36433 & 1 \\
\hline Columbia River & BENTON PMPHSE & BoWD65 & 36433 & 1 \\
\hline Columbia River & Butterfield WTP & 23693 & 36426 & 1 \\
\hline Columbia River & CGS (WNP-2)S26I & 27565 & 36845 & 1 \\
\hline Columbia River & CGS (WNP-2)S26I & 24060 & 36865 & 1 \\
\hline Columbia River & CGS (WNP-2)S26I & 27399 & 37210 & 1 \\
\hline Columbia River & CGS (WNP-2)S26I & 27398 & 37118 & 1 \\
\hline Columbia River & CGS (WNP-2)S26I & 27397 & 37026 & 1 \\
\hline Columbia River & CGS (WNP-2)S26I & 27396 & 36937 & 1 \\
\hline Columbia River & CGS (WNP-2)S26I & 24036 & 36739 & 1 \\
\hline Columbia River & CGS (WNP-2)S26I & 24048 & 36802 & 1 \\
\hline Columbia River & CGS (WNP-2)S26I & 23994 & 36529 & 1 \\
\hline Columbia River & CGS (WNP-2)S26I & 24054 & 36831 & 1 \\
\hline Columbia River & CGS (WNP-2)S26I & 24042 & 36775 & 1 \\
\hline Columbia River & CGS (WNP-2)S26I & 24030 & 36712 & 1 \\
\hline Columbia River & CGS (WNP-2)S26I & 24024 & 36684 & 1 \\
\hline Columbia River & CGS (WNP-2)S26I & 24018 & 36648 & 1 \\
\hline Columbia River & CGS (WNP-2)S26I & 24012 & 36621 & 1 \\
\hline Columbia River & CGS (WNP-2)S26I & 24000 & 36557 & 1 \\
\hline Columbia River & CGS (WNP-2)S26I & 25916 & 37229 & 1 \\
\hline Columbia River & CGS (WNP-2)S26I & 25910 & 37201 & 1 \\
\hline Columbia River & CGS (WNP-2)S26I & 25904 & 37166 & 1 \\
\hline Columbia River & CGS (WNP-2)S26I & 25898 & 37139 & 1 \\
\hline Columbia River & CGS (WNP-2)S26I & 25892 & 37104 & 1 \\
\hline Columbia River & CGS (WNP-2)S26I & 25880 & 37047 & 1 \\
\hline Columbia River & CGS (WNP-2)S26I & 24006 & 36586 & 1 \\
\hline Columbia River & CGS (WNP-2)S26I & 32600 & 38140 & 1 \\
\hline Columbia River & CGS (WNP-2)S26I & 22914 & 36348 & 1 \\
\hline Columbia River & CGS (WNP-2)S26I & 32572 & 37992 & 1 \\
\hline Columbia River & CGS (WNP-2)S26I & 30667 & 37940 & 1 \\
\hline
\end{tabular}


Table B-2

\section{Summary Table of Segment 2 Surface Water Samples By Area, Site, Date, and Category Columbia River Component - Existing Sample Data Evaluation and Scoping Report}

\begin{tabular}{|c|c|c|c|c|}
\hline Sample Area & Sample Site & Sample ID & Sample Date & Category \\
\hline Columbia River & CGS (WNP-2)S26I & 30666 & 37848 & 1 \\
\hline Columbia River & CGS (WNP-2)S26I & 30665 & 37756 & 1 \\
\hline Columbia River & CGS (WNP-2)S26I & 25519 & 36894 & 1 \\
\hline Columbia River & CGS (WNP-2)S26I & 22206 & 36165 & 1 \\
\hline Columbia River & CGS (WNP-2)S26I & 22894 & 36194 & 1 \\
\hline Columbia River & CGS (WNP-2)S26I & 30338 & 37656 & 1 \\
\hline Columbia River & CGS (WNP-2)S26I & 22902 & 36256 & 1 \\
\hline Columbia River & CGS (WNP-2)S26I & 30340 & 37684 & 1 \\
\hline Columbia River & CGS (WNP-2)S26I & 24824 & 36387 & 1 \\
\hline Columbia River & CGS (WNP-2)S26I & 24827 & 36479 & 1 \\
\hline Columbia River & CGS (WNP-2)S26I & 32907 & 38032 & 1 \\
\hline Columbia River & CGS (WNP-2)S26I & 32636 & 38322 & 1 \\
\hline Columbia River & CGS (WNP-2)S26I & 32630 & 38293 & 1 \\
\hline Columbia River & CGS (WNP-2)S26I & 32908 & 38122 & 1 \\
\hline Columbia River & CGS (WNP-2)S26I & 25492 & 36753 & 1 \\
\hline Columbia River & CGS (WNP-2)S26I & 22906 & 36284 & 1 \\
\hline Columbia River & CGS (WNP-2)S26I & 22910 & 36313 & 1 \\
\hline Columbia River & CGS (WNP-2)S26I & 22898 & 36221 & 1 \\
\hline Columbia River & CGS (WNP-2)S26I & 30384 & 37929 & 1 \\
\hline Columbia River & CGS (WNP-2)S26I & 32909 & 38214 & 1 \\
\hline Columbia River & CGS (WNP-2)S26I & 32910 & 38306 & 1 \\
\hline Columbia River & CGS (WNP-2)S26I & 32594 & 38107 & 1 \\
\hline Columbia River & CGS (WNP-2)S26I & 24820 & 36295 & 1 \\
\hline Columbia River & CGS (WNP-2)S26I & 24691 & 36560 & 1 \\
\hline Columbia River & CGS (WNP-2)S26I & 32588 & 38083 & 1 \\
\hline Columbia River & CGS (WNP-2)S26I & 34236 & 38356 & 1 \\
\hline Columbia River & CGS (WNP-2)S26I & 34248 & 38384 & 1 \\
\hline Columbia River & CGS (WNP-2)S26I & 30326 & 37623 & 1 \\
\hline Columbia River & CGS (WNP-2)S26I & 32586 & 38048 & 1 \\
\hline Columbia River & CGS (WNP-2)S26I & 25488 & 36661 & 1 \\
\hline Columbia River & CGS (WNP-2)S26I & 30378 & 37895 & 1 \\
\hline Columbia River & CGS (WNP-2)S26I & 30372 & 37867 & 1 \\
\hline Columbia River & CGS (WNP-2)S26I & 30366 & 37838 & 1 \\
\hline Columbia River & CGS (WNP-2)S26I & 30360 & 37802 & 1 \\
\hline Columbia River & CGS (WNP-2)S26I & 25027 & 36571 & 1 \\
\hline Columbia River & CGS (WNP-2)S26I & 32584 & 38020 & 1 \\
\hline Columbia River & CGS (WNP-2)S26I & 30354 & 37775 & 1 \\
\hline Columbia River & CGS (WNP-2)S26I & 30348 & 37746 & 1 \\
\hline Columbia River & CGS (WNP-2)S26I & 30342 & 37712 & 1 \\
\hline Columbia River & CGS (WNP-2)S26I & 30390 & 37957 & 1 \\
\hline Columbia River & CGS (WNP-2)S26I & 27850 & 37439 & 1 \\
\hline Columbia River & CGS (WNP-2)S26I & 30664 & 37667 & 1 \\
\hline Columbia River & CGS (WNP-2)S26I & 28159 & 37302 & 1 \\
\hline Columbia River & CGS (WNP-2)S26I & 22918 & 36375 & 1 \\
\hline Columbia River & CGS (WNP-2)S26I & 25886 & 37075 & 1 \\
\hline Columbia River & CGS (WNP-2)S26I & 27880 & 37593 & 1 \\
\hline
\end{tabular}


Table B-2

\section{Summary Table of Segment 2 Surface Water Samples By Area, Site, Date, and Category Columbia River Component - Existing Sample Data Evaluation and Scoping Report}

\begin{tabular}{|c|c|c|c|c|}
\hline Sample Area & Sample Site & Sample ID & Sample Date & Category \\
\hline Columbia River & CGS (WNP-2)S26I & 27874 & 37565 & 1 \\
\hline Columbia River & CGS (WNP-2)S26I & 27868 & 37530 & 1 \\
\hline Columbia River & CGS (WNP-2)S26I & 22934 & 36495 & 1 \\
\hline Columbia River & CGS (WNP-2)S26I & 27856 & 37474 & 1 \\
\hline Columbia River & CGS (WNP-2)S26I & 25874 & 37012 & 1 \\
\hline Columbia River & CGS (WNP-2)S26I & 27844 & 37411 & 1 \\
\hline Columbia River & CGS (WNP-2)S26I & 27838 & 37383 & 1 \\
\hline Columbia River & CGS (WNP-2)S26I & 27832 & 37348 & 1 \\
\hline Columbia River & CGS (WNP-2)S26I & 27830 & 37320 & 1 \\
\hline Columbia River & CGS (WNP-2)S26I & 27828 & 37292 & 1 \\
\hline Columbia River & CGS (WNP-2)S26I & 27816 & 37258 & 1 \\
\hline Columbia River & CGS (WNP-2)S26I & 23335 & 36206 & 1 \\
\hline Columbia River & CGS (WNP-2)S26I & 27862 & 37503 & 1 \\
\hline Columbia River & CGS (WNP-2)S26I & 32612 & 38202 & 1 \\
\hline Columbia River & CGS (WNP-2)S26I & 32624 & 38265 & 1 \\
\hline Columbia River & CGS (WNP-2)S26I & 32618 & 38231 & 1 \\
\hline Columbia River & CGS (WNP-2)S26I & 25856 & 36928 & 1 \\
\hline Columbia River & CGS (WNP-2)S26I & 25862 & 36956 & 1 \\
\hline Columbia River & CGS (WNP-2)S26I & 25868 & 36984 & 1 \\
\hline Columbia River & CGS (WNP-2)S26I & 28162 & 37575 & 1 \\
\hline Columbia River & CGS (WNP-2)S26I & 28161 & 37483 & 1 \\
\hline Columbia River & CGS (WNP-2)S26I & 28160 & 37391 & 1 \\
\hline Columbia River & CGS (WNP-2)S26I & 22922 & 36404 & 1 \\
\hline Columbia River & CGS (WNP-2)S26I & 22930 & 36466 & 1 \\
\hline Columbia River & CGS (WNP-2)S26I & 22926 & 36438 & 1 \\
\hline Columbia River & CGS (WNP-2)S26I & 32606 & 38175 & 1 \\
\hline Columbia River & CGS (WNP-2)S26I & L24019-4 & 38175 & 1 \\
\hline Columbia River & CGS (WNP-2)S26I & L22909-4 & 37992 & 1 \\
\hline Columbia River & CGS (WNP-2)S26I & L19364-1 & 37439 & 1 \\
\hline Columbia River & CGS (WNP-2)S26I & L22549-4 & 37929 & 1 \\
\hline Columbia River & CGS (WNP-2)S26I & L22394-4 & 37895 & 1 \\
\hline Columbia River & CGS (WNP-2)S26I & L22152-1 & 37802 & 1 \\
\hline Columbia River & CGS (WNP-2)S26I & L22128-4 & 37867 & 1 \\
\hline Columbia River & CGS (WNP-2)S26I & L24404-4 & 38231 & 1 \\
\hline Columbia River & CGS (WNP-2)S26I & L19598-4 & 37530 & 1 \\
\hline Columbia River & CGS (WNP-2)S26I & L24216-4 & 38202 & 1 \\
\hline Columbia River & CGS (WNP-2)S26I & L23259-4 & 38048 & 1 \\
\hline Columbia River & CGS (WNP-2)S26I & L23933-1 & 38083 & 1 \\
\hline Columbia River & CGS (WNP-2)S26I & L21908-4 & 37838 & 1 \\
\hline Columbia River & CGS (WNP-2)S26I & L21725-4 & 37802 & 1 \\
\hline Columbia River & CGS (WNP-2)S26I & L23867-4 & 38140 & 1 \\
\hline Columbia River & CGS (WNP-2)S26I & L22128-11 & 37867 & 1 \\
\hline Columbia River & CGS (WNP-2)S26I & L20567-4 & 37656 & 1 \\
\hline Columbia River & CGS (WNP-2)S26I & L24571-4 & 38265 & 1 \\
\hline Columbia River & CGS (WNP-2)S26I & L24718-4 & 38293 & 1 \\
\hline Columbia River & CGS (WNP-2)S26I & L24912-4 & 38322 & 1 \\
\hline
\end{tabular}


Table B-2

\section{Summary Table of Segment 2 Surface Water Samples By Area, Site, Date, and Category Columbia River Component - Existing Sample Data Evaluation and Scoping Report}

\begin{tabular}{|c|c|c|c|c|}
\hline Sample Area & Sample Site & Sample ID & Sample Date & Category \\
\hline Columbia River & CGS (WNP-2)S26I & L21455-1 & 37712 & 1 \\
\hline Columbia River & CGS (WNP-2)S26I & L23447-4 & 38083 & 1 \\
\hline Columbia River & CGS (WNP-2)S26I & L23447-11 & 38083 & 1 \\
\hline Columbia River & CGS (WNP-2)S26I & L23066-4 & 38020 & 1 \\
\hline Columbia River & CGS (WNP-2)S26I & L20742-4 & 37684 & 1 \\
\hline Columbia River & CGS (WNP-2)S26I & L24466-1 & 38175 & 1 \\
\hline Columbia River & CGS (WNP-2)S26I & L20357-4 & 37623 & 1 \\
\hline Columbia River & CGS (WNP-2)S26I & L23644-4 & 38107 & 1 \\
\hline Columbia River & CGS (WNP-2)S26I & L20975-4 & 37712 & 1 \\
\hline Columbia River & CGS (WNP-2)S26I & L20817-1 & 37623 & 1 \\
\hline Columbia River & CGS (WNP-2)S26I & L21183-4 & 37747 & 1 \\
\hline Columbia River & CGS (WNP-2)S26I & L21399-4 & 37775 & 1 \\
\hline Columbia River & CGS (WNP-2)S26I & L20975-11 & 37712 & 1 \\
\hline Columbia River & CGS (WNP-2)S26I & L23346-1 & 37992 & 1 \\
\hline Columbia River & CGS (WNP-2)S26I & L17150-4 & 37201 & 1 \\
\hline Columbia River & CGS (WNP-2)S26I & L18452-1 & 37383 & 1 \\
\hline Columbia River & CGS (WNP-2)S26I & L13375-4 & 36775 & 1 \\
\hline Columbia River & CGS (WNP-2)S26I & L12689-4 & 36712 & 1 \\
\hline Columbia River & CGS (WNP-2)S26I & L12542-1 & 36621 & 1 \\
\hline Columbia River & CGS (WNP-2)S26I & L12370-4 & 36684 & 1 \\
\hline Columbia River & CGS (WNP-2)S26I & L13876-1 & 36831 & 1 \\
\hline Columbia River & CGS (WNP-2)S26I & L16959-4 & 37166 & 1 \\
\hline Columbia River & CGS (WNP-2)S26I & L14841-4 & 36928 & 1 \\
\hline Columbia River & CGS (WNP-2)S26I & L17585-4 & 37258 & 1 \\
\hline Columbia River & CGS (WNP-2)S26I & L17761-5 & 37292 & 1 \\
\hline Columbia River & CGS (WNP-2)S26I & L17906-4 & 37320 & 1 \\
\hline Columbia River & CGS (WNP-2)S26I & L18011-1 & 37258 & 1 \\
\hline Columbia River & CGS (WNP-2)S26I & L12074-4 & 36648 & 1 \\
\hline Columbia River & CGS (WNP-2)S26I & L18161-4 & 37348 & 1 \\
\hline Columbia River & CGS (WNP-2)S26I & L16959-1 & 37166 & 1 \\
\hline Columbia River & CGS (WNP-2)S26I & L15619-4 & 37012 & 1 \\
\hline Columbia River & CGS (WNP-2)S26I & L16658-4 & 37139 & 1 \\
\hline Columbia River & CGS (WNP-2)S26I & L19879-4 & 37565 & 1 \\
\hline Columbia River & CGS (WNP-2)S26I & L16688-1 & 37075 & 1 \\
\hline Columbia River & CGS (WNP-2)S26I & L16425-4 & 37104 & 1 \\
\hline Columbia River & CGS (WNP-2)S26I & L16110-4 & 37075 & 1 \\
\hline Columbia River & CGS (WNP-2)S26I & L13520-4 & 36802 & 1 \\
\hline Columbia River & CGS (WNP-2)S26I & L15843-4 & 37047 & 1 \\
\hline Columbia River & CGS (WNP-2)S26I & L14628-4 & 36894 & 1 \\
\hline Columbia River & CGS (WNP-2)S26I & L15575-1 & 36894 & 1 \\
\hline Columbia River & CGS (WNP-2)S26I & L15329-4 & 36984 & 1 \\
\hline Columbia River & CGS (WNP-2)S26I & L15070-4 & 36956 & 1 \\
\hline Columbia River & CGS (WNP-2)S26I & L11471-4 & 36621 & 1 \\
\hline Columbia River & CGS (WNP-2)S26I & L11430-1 & 36529 & 1 \\
\hline Columbia River & CGS (WNP-2)S26I & L11058-4 & 36586 & 1 \\
\hline Columbia River & CGS (WNP-2)S26I & L15893-1 & 36984 & 1 \\
\hline
\end{tabular}


Table B-2

\section{Summary Table of Segment 2 Surface Water Samples By Area, Site, Date, and Category Columbia River Component - Existing Sample Data Evaluation and Scoping Report}

\begin{tabular}{|c|c|c|c|c|}
\hline Sample Area & Sample Site & Sample ID & Sample Date & Category \\
\hline Columbia River & CGS (WNP-2)S26I & L19103-4 & 37474 & 1 \\
\hline Columbia River & CGS (WNP-2)S26I & L10458-4 & 36557 & 1 \\
\hline Columbia River & CGS (WNP-2)S26I & L10018-4 & 36529 & 1 \\
\hline Columbia River & CGS (WNP-2)S26I & L18679-4 & 37411 & 1 \\
\hline Columbia River & CGS (WNP-2)S26I & L18860-1 & 37348 & 1 \\
\hline Columbia River & CGS (WNP-2)S26I & L18886-4 & 37439 & 1 \\
\hline Columbia River & CGS (WNP-2)S26I & L19282-4 & 37502 & 1 \\
\hline Columbia River & CGS (WNP-2)S26I & L24982-1 & 38265 & 1 \\
\hline Columbia River & CGS (WNP-2)S29W & 28154 & 37302 & 1 \\
\hline Columbia River & CGS (WNP-2)S29W & 30644 & 37756 & 1 \\
\hline Columbia River & CGS (WNP-2)S29W & 30658 & 37848 & 1 \\
\hline Columbia River & CGS (WNP-2)S29W & 27567 & 36845 & 1 \\
\hline Columbia River & CGS (WNP-2)S29W & 23337 & 36206 & 1 \\
\hline Columbia River & CGS (WNP-2)S29W & 24822 & 36295 & 1 \\
\hline Columbia River & CGS (WNP-2)S29W & 30659 & 37667 & 1 \\
\hline Columbia River & CGS (WNP-2)S29W & 30660 & 37940 & 1 \\
\hline Columbia River & CGS (WNP-2)S29W & 24828 & 36479 & 1 \\
\hline Columbia River & CGS (WNP-2)S29W & 28153 & 37483 & 1 \\
\hline Columbia River & CGS (WNP-2)S29W & 27391 & 36937 & 1 \\
\hline Columbia River & CGS (WNP-2)S29W & 27039 & 37118 & 1 \\
\hline Columbia River & CGS (WNP-2)S29W & 26186 & 37026 & 1 \\
\hline Columbia River & CGS (WNP-2)S29W & 25491 & 36661 & 1 \\
\hline Columbia River & CGS (WNP-2)S29W & 25494 & 36753 & 1 \\
\hline Columbia River & CGS (WNP-2)S29W & 24825 & 36387 & 1 \\
\hline Columbia River & CGS (WNP-2)S29W & 28139 & 37391 & 1 \\
\hline Columbia River & CGS (WNP-2)S29W & 25030 & 36571 & 1 \\
\hline Columbia River & CGS (WNP-2)S29W & 27392 & 37210 & 1 \\
\hline Columbia River & CGS (WNP-2)S29W & 28155 & 37575 & 1 \\
\hline Columbia River & ESQUATZEL CANAL & B170R3 & 37755 & 1 \\
\hline Columbia River & ESQUATZEL CANAL & B170R2 & 37755 & 1 \\
\hline Columbia River & ESQUATZEL CANAL & B16WV1 & 37755 & 1 \\
\hline Columbia River & ESQUATZEL CANAL & B16WV0 & 37755 & 1 \\
\hline Columbia River & ESQUATZEL CANAL & B16WT9 & 37755 & 1 \\
\hline Columbia River & ESQUATZEL CANAL & B16WT8 & 37755 & 1 \\
\hline Columbia River & ESQUATZEL CANAL & B16WT7 & 37755 & 1 \\
\hline Columbia River & ESQUATZEL CANAL & B16WT6 & 37755 & 1 \\
\hline Columbia River & ESQUATZEL CANAL & B16WN2 & 37755 & 1 \\
\hline Columbia River & ESQUATZEL CANAL & B16WN1 & 37755 & 1 \\
\hline Columbia River & ESQUATZEL CANAL & B16WN0 & 37755 & 1 \\
\hline Columbia River & ESQUATZEL CANAL & B170R4 & 37755 & 1 \\
\hline Columbia River & ESQUATZEL CWR68 & 0000031 & 37531 & 1 \\
\hline Columbia River & ESQUATZEL CWR68 & 0000026 & 36633 & 1 \\
\hline Columbia River & ESQUATZEL CWR68 & 0000032 & 37720 & 1 \\
\hline Columbia River & ESQUATZEL CWR68 & 0000033 & 37900 & 1 \\
\hline Columbia River & ESQUATZEL CWR68 & 0000027 & 36804 & 1 \\
\hline Columbia River & ESQUATZEL CWR68 & 0000030 & 37369 & 1 \\
\hline
\end{tabular}


Table B-2

\section{Summary Table of Segment 2 Surface Water Samples By Area, Site, Date, and Category Columbia River Component - Existing Sample Data Evaluation and Scoping Report}

\begin{tabular}{|c|c|c|c|c|}
\hline Sample Area & Sample Site & Sample ID & Sample Date & Category \\
\hline Columbia River & ESQUATZEL CWR68 & 0000029 & 37165 & 1 \\
\hline Columbia River & ESQUATZEL CWR68 & 0000028 & 37018 & 1 \\
\hline Columbia River & HFD SPR 28-2 & 29079 & 37536 & 1 \\
\hline Columbia River & HFD SPR 28-2 & B15CD8 & 37536 & 1 \\
\hline Columbia River & HFD SPR 28-2 & 23439 & 36465 & 1 \\
\hline Columbia River & HFD SPR 28-2 & 26955 & 37015 & 1 \\
\hline Columbia River & HFD SPR 28-2 & B15C05 & 37536 & 1 \\
\hline Columbia River & HFD SPR 28-2 & B15C00 & 37536 & 1 \\
\hline Columbia River & HFD SPR 28-2 & B0WP50 & 36465 & 1 \\
\hline Columbia River & HFD SPR 28-2 & BOWNY1 & 36465 & 1 \\
\hline Columbia River & HFD SPR 28-2 & BOWMVO & 36465 & 1 \\
\hline Columbia River & HFD SPR 28-2 & BOWNX4 & 36465 & 1 \\
\hline Columbia River & HFD SPR 28-2 & B10912 & 36796 & 1 \\
\hline Columbia River & HFD SPR 28-2 & B15C94 & 37536 & 1 \\
\hline Columbia River & HFD SPR 28-2 & B10968 & 36796 & 1 \\
\hline Columbia River & HFD SPR 28-2 & B108Y6 & 36796 & 1 \\
\hline Columbia River & HFD SPR 28-2 & B10902 & 36796 & 1 \\
\hline Columbia River & HFD SPR 28-2 & B10906 & 36796 & 1 \\
\hline Columbia River & HFD SPR 28-2 & BOWNX7 & 36465 & 1 \\
\hline Columbia River & HFD SPR 28-2 & B11W36 & 37011 & 1 \\
\hline Columbia River & HFD SPR 28-2 & B15C09 & 37536 & 1 \\
\hline Columbia River & HFD SPR 28-2 & 24573 & 36796 & 1 \\
\hline Columbia River & HFD SPR 28-2 & B11W77 & 37011 & 1 \\
\hline Columbia River & HFD SPR 28-2 & B11W30 & 37011 & 1 \\
\hline Columbia River & HFD SPR 28-2 & B11W33 & 37011 & 1 \\
\hline Columbia River & HFD SPR 28-2 & B1BJ61 & 38285 & 1 \\
\hline Columbia River & HFD SPR 28-2 & B1BFX8 & 38285 & 1 \\
\hline Columbia River & HFD SPR 28-2 & B1BFP3 & 38285 & 1 \\
\hline Columbia River & HFD SPR 28-2 & B1BFN0 & 38285 & 1 \\
\hline Columbia River & HFD SPR 28-2 & B1BFL8 & 38285 & 1 \\
\hline Columbia River & HFD SPR DR 28-2 & B10972 & 36796 & 1 \\
\hline Columbia River & HFD SPR DR 28-2 & B11W81 & 37011 & 1 \\
\hline Columbia River & HFD SPR DR 28-2 & B11W80 & 37011 & 1 \\
\hline Columbia River & HFD SPR DR 28-2 & B10946 & 36796 & 1 \\
\hline Columbia River & HFD SPR DR 28-2 & 30898 & 37928 & 1 \\
\hline Columbia River & HFD SPR DR 28-2 & 33185 & 38285 & 1 \\
\hline Columbia River & HFD SPR DR 28-2 & B11W61 & 37011 & 1 \\
\hline Columbia River & HFD SPR DR 28-2 & B10952 & 36796 & 1 \\
\hline Columbia River & HFD SPR DR 28-2 & BoWMV2 & 36465 & 1 \\
\hline Columbia River & HFD SPR DR 28-2 & B11W55 & 37011 & 1 \\
\hline Columbia River & HFD SPR DR 28-2 & B10971 & 36796 & 1 \\
\hline Columbia River & HFD SPR DR 28-2 & B0WP31 & 36465 & 1 \\
\hline Columbia River & HFD SPR DR 28-2 & B108Y7 & 36796 & 1 \\
\hline Columbia River & HFD SPR DR 28-2 & B0WP53 & 36465 & 1 \\
\hline Columbia River & HFD SPR DR 28-2 & B0WP54 & 36465 & 1 \\
\hline Columbia River & HFD SPR DR 28-2 & B15C73 & 37536 & 1 \\
\hline
\end{tabular}


Table B-2

\section{Summary Table of Segment 2 Surface Water Samples By Area, Site, Date, and Category Columbia River Component - Existing Sample Data Evaluation and Scoping Report}

\begin{tabular}{|c|c|c|c|c|}
\hline Sample Area & Sample Site & Sample ID & Sample Date & Category \\
\hline Columbia River & HFD SPR DR 28-2 & B15C78 & 37536 & 1 \\
\hline Columbia River & HFD SPR DR $28-2$ & B15C97 & 37536 & 1 \\
\hline Columbia River & HFD SPR DR 28-2 & B15C98 & 37536 & 1 \\
\hline Columbia River & HFD SPR DR 28-2 & B15CD6 & 37536 & 1 \\
\hline Columbia River & HFD SPR DR 28-2 & B0WP26 & 36465 & 1 \\
\hline Columbia River & HFD SPR DR 28-2 & B1BFY1 & 38285 & 1 \\
\hline Columbia River & HFD SPR DR 28-2 & B17RL2 & 37928 & 1 \\
\hline Columbia River & HFD SPR DR $28-2$ & B1BFW2 & 38285 & 1 \\
\hline Columbia River & HFD SPR DR 28-2 & B17RK4 & 37928 & 1 \\
\hline Columbia River & HFD SPR DR 28-2 & B17RL3 & 37928 & 1 \\
\hline Columbia River & HFD SPR DR 28-2 & B1BFV5 & 38285 & 1 \\
\hline Columbia River & HFD SPR DR 28-2 & B1BJ62 & 38285 & 1 \\
\hline Columbia River & HFD SPR DR 28-2 & B17RR7 & 37928 & 1 \\
\hline Columbia River & HFD SPR DR 28-2 & B17RJ9 & 37928 & 1 \\
\hline Columbia River & HFD SPR DR 28-2 & B1BFY2 & 38285 & 1 \\
\hline Columbia River & HFD SPR UR 28-2 & B10970 & 36796 & 1 \\
\hline Columbia River & HFD SPR UR 28-2 & B10969 & 36796 & 1 \\
\hline Columbia River & HFD SPR UR 28-2 & B10951 & 36796 & 1 \\
\hline Columbia River & HFD SPR UR 28-2 & B10945 & 36796 & 1 \\
\hline Columbia River & HFD SPR UR 28-2 & BoWMV1 & 36465 & 1 \\
\hline Columbia River & HFD SPR UR 28-2 & B0WP25 & 36465 & 1 \\
\hline Columbia River & HFD SPR UR 28-2 & B0WP30 & 36465 & 1 \\
\hline Columbia River & HFD SPR UR 28-2 & B0WP51 & 36465 & 1 \\
\hline Columbia River & HFD SPR UR 28-2 & B0WP52 & 36465 & 1 \\
\hline Columbia River & HFD SPR UR 28-2 & B108Y8 & 36796 & 1 \\
\hline Columbia River & HFD SPR UR 28-2 & B1BJ60 & 38285 & 1 \\
\hline Columbia River & HFD SPR UR 28-2 & B1BFX9 & 38285 & 1 \\
\hline Columbia River & HFD SPR UR 28-2 & B17RL0 & 37928 & 1 \\
\hline Columbia River & HFD SPR UR 28-2 & B17RR8 & 37928 & 1 \\
\hline Columbia River & HFD SPR UR 28-2 & B17RL1 & 37928 & 1 \\
\hline Columbia River & HFD SPR UR 28-2 & B1BFY0 & 38285 & 1 \\
\hline Columbia River & HFD SPR UR 28-2 & B1BFW1 & 38285 & 1 \\
\hline Columbia River & HFD SPR UR 28-2 & B17RJ8 & 37928 & 1 \\
\hline Columbia River & HFD SPR UR 28-2 & B1BFV4 & 38285 & 1 \\
\hline Columbia River & HFD SPR UR 28-2 & B17RK3 & 37928 & 1 \\
\hline Columbia River & HFD TS HRM 26 & B159F0 & 37508 & 1 \\
\hline Columbia River & HFD TS HRM 26 & B12TF7 & 37144 & 1 \\
\hline Columbia River & HFD TS HRM 26 & B10731 & 36787 & 1 \\
\hline Columbia River & HFD TS HRM 26 & B17CM0 & 37872 & 1 \\
\hline Columbia River & HFD TS HRM 26 & B12THO & 37144 & 1 \\
\hline Columbia River & HFD TS HRM 26 & B0WB73 & 36424 & 1 \\
\hline Columbia River & HFD TS HRM 26 & B158N0 & 37508 & 1 \\
\hline Columbia River & HFD TS HRM 26 & B17CL7 & 37872 & 1 \\
\hline Columbia River & HFD TS HRM 26 & B106M4 & 36787 & 1 \\
\hline Columbia River & HFD TS HRM 26 & 29092 & 37508 & 1 \\
\hline Columbia River & HFD TS HRM 26 & B0WB65 & 36424 & 1 \\
\hline
\end{tabular}


Table B-2

\section{Summary Table of Segment 2 Surface Water Samples By Area, Site, Date, and Category Columbia River Component - Existing Sample Data Evaluation and Scoping Report}

\begin{tabular}{|c|c|c|c|c|}
\hline Sample Area & Sample Site & Sample ID & Sample Date & Category \\
\hline Columbia River & HFD TS HRM 26 & B17CL4 & 37872 & 1 \\
\hline Columbia River & HFD TS HRM 26 & B158N9 & 37508 & 1 \\
\hline Columbia River & HFD TS HRM 26 & B12VT2 & 37144 & 1 \\
\hline Columbia River & HFD TS HRM 26 & B10734 & 36787 & 1 \\
\hline Columbia River & HFD TS HRM 26 & B158N6 & 37508 & 1 \\
\hline Columbia River & HFD TS HRM 26 & BowC96 & 36424 & 1 \\
\hline Columbia River & HFD TS HRM 26 & В12TH3 & 37144 & 1 \\
\hline Columbia River & HFD TS HRM 26 & B10728 & 36787 & 1 \\
\hline Columbia River & HFD TS HRM 26 & B1B735 & 38244 & 1 \\
\hline Columbia River & HFD TS HRM 26 & B1B744 & 38244 & 1 \\
\hline Columbia River & HFD TS HRM 26 & B17HK5 & 37872 & 1 \\
\hline Columbia River & HFD TS HRM 26 & B1B495 & 38244 & 1 \\
\hline Columbia River & HFD TS HRM 26 & B1B741 & 38244 & 1 \\
\hline Columbia River & HFD TS HRM 27 & B106M5 & 36787 & 1 \\
\hline Columbia River & HFD TS HRM 27 & B17CL8 & 37872 & 1 \\
\hline Columbia River & HFD TS HRM 27 & B10735 & 36787 & 1 \\
\hline Columbia River & HFD TS HRM 27 & B0WB66 & 36424 & 1 \\
\hline Columbia River & HFD TS HRM 27 & B12TF8 & 37144 & 1 \\
\hline Columbia River & HFD TS HRM 27 & B158P0 & 37508 & 1 \\
\hline Columbia River & HFD TS HRM 27 & B12VT3 & 37144 & 1 \\
\hline Columbia River & HFD TS HRM 27 & B159F1 & 37508 & 1 \\
\hline Columbia River & HFD TS HRM 27 & B12TH1 & 37144 & 1 \\
\hline Columbia River & HFD TS HRM 27 & 29093 & 37508 & 1 \\
\hline Columbia River & HFD TS HRM 27 & B158N2 & 37508 & 1 \\
\hline Columbia River & HFD TS HRM 27 & В12TH4 & 37144 & 1 \\
\hline Columbia River & HFD TS HRM 27 & B17CL5 & 37872 & 1 \\
\hline Columbia River & HFD TS HRM 27 & B158N7 & 37508 & 1 \\
\hline Columbia River & HFD TS HRM 27 & 33277 & 38244 & 1 \\
\hline Columbia River & HFD TS HRM 27 & B109C4 & 36787 & 1 \\
\hline Columbia River & HFD TS HRM 27 & BowC97 & 36424 & 1 \\
\hline Columbia River & HFD TS HRM 27 & B10732 & 36787 & 1 \\
\hline Columbia River & HFD TS HRM 27 & B10729 & 36787 & 1 \\
\hline Columbia River & HFD TS HRM 27 & B0WB74 & 36424 & 1 \\
\hline Columbia River & HFD TS HRM 27 & B17CM1 & 37872 & 1 \\
\hline Columbia River & HFD TS HRM 27 & B1B742 & 38244 & 1 \\
\hline Columbia River & HFD TS HRM 27 & B1B496 & 38244 & 1 \\
\hline Columbia River & HFD TS HRM 27 & B1B745 & 38244 & 1 \\
\hline Columbia River & HFD TS HRM 27 & B1B737 & 38244 & 1 \\
\hline Columbia River & HFD TS HRM 27 & B17HK6 & 37872 & 1 \\
\hline Columbia River & HFD TS HRM 28 & B15904 & 37508 & 1 \\
\hline Columbia River & HFD TS HRM 28 & B159F2 & 37508 & 1 \\
\hline Columbia River & HFD TS HRM 28 & B17CY3 & 37872 & 1 \\
\hline Columbia River & HFD TS HRM 28 & 29094 & 37508 & 1 \\
\hline Columbia River & HFD TS HRM 28 & B10785 & 36787 & 1 \\
\hline Columbia River & HFD TS HRM 28 & В12ТT0 & 37144 & 1 \\
\hline Columbia River & HFD TS HRM 28 & B17CY1 & 37872 & 1 \\
\hline
\end{tabular}


Table B-2

\section{Summary Table of Segment 2 Surface Water Samples By Area, Site, Date, and Category Columbia River Component - Existing Sample Data Evaluation and Scoping Report}

\begin{tabular}{|c|c|c|c|c|}
\hline Sample Area & Sample Site & Sample ID & Sample Date & Category \\
\hline Columbia River & HFD TS HRM 28 & B12TT2 & 37144 & 1 \\
\hline Columbia River & HFD TS HRM 28 & B109C5 & 36787 & 1 \\
\hline Columbia River & HFD TS HRM 28 & B15902 & 37508 & 1 \\
\hline Columbia River & HFD TS HRM 28 & B17CY2 & 37872 & 1 \\
\hline Columbia River & HFD TS HRM 28 & B10786 & 36787 & 1 \\
\hline Columbia River & HFD TS HRM 28 & B12VT4 & 37144 & 1 \\
\hline Columbia River & HFD TS HRM 28 & B10787 & 36787 & 1 \\
\hline Columbia River & HFD TS HRM 28 & B106M6 & 36787 & 1 \\
\hline Columbia River & HFD TS HRM 28 & B15905 & 37508 & 1 \\
\hline Columbia River & HFD TS HRM 28 & 33278 & 38244 & 1 \\
\hline Columbia River & HFD TS HRM 28 & B12TT1 & 37144 & 1 \\
\hline Columbia River & HFD TS HRM 28 & B1B7D4 & 38244 & 1 \\
\hline Columbia River & HFD TS HRM 28 & B17HK7 & 37872 & 1 \\
\hline Columbia River & HFD TS HRM 28 & B1B497 & 38244 & 1 \\
\hline Columbia River & HFD TS HRM 28 & B1B7D1 & 38244 & 1 \\
\hline Columbia River & HFD TS HRM 28 & B1B7D3 & 38244 & 1 \\
\hline Columbia River & HFD TS HRM 29 & B0WB75 & 36424 & 1 \\
\hline Columbia River & HFD TS HRM 29 & BowC98 & 36424 & 1 \\
\hline Columbia River & HFD TS HRM 29 & B0WB67 & 36424 & 1 \\
\hline Columbia River & HFD TS HRM 30 & B158P1 & 37508 & 1 \\
\hline Columbia River & HFD TS HRM 30 & BowC99 & 36424 & 1 \\
\hline Columbia River & HFD TS HRM 30 & B158N4 & 37508 & 1 \\
\hline Columbia River & HFD TS HRM 30 & B10736 & 36787 & 1 \\
\hline Columbia River & HFD TS HRM 30 & B0WB76 & 36424 & 1 \\
\hline Columbia River & HFD TS HRM 30 & B158N8 & 37508 & 1 \\
\hline Columbia River & HFD TS HRM 30 & B12TH5 & 37144 & 1 \\
\hline Columbia River & HFD TS HRM 30 & В12TH2 & 37144 & 1 \\
\hline Columbia River & HFD TS HRM 30 & B10733 & 36787 & 1 \\
\hline Columbia River & HFD TS HRM 30 & B12VT5 & 37144 & 1 \\
\hline Columbia River & HFD TS HRM 30 & B109C6 & 36787 & 1 \\
\hline Columbia River & HFD TS HRM 30 & B10730 & 36787 & 1 \\
\hline Columbia River & HFD TS HRM 30 & 29095 & 37508 & 1 \\
\hline Columbia River & HFD TS HRM 30 & B17CL6 & 37872 & 1 \\
\hline Columbia River & HFD TS HRM 30 & B159F3 & 37508 & 1 \\
\hline Columbia River & HFD TS HRM 30 & B106M7 & 36787 & 1 \\
\hline Columbia River & HFD TS HRM 30 & B17CL9 & 37872 & 1 \\
\hline Columbia River & HFD TS HRM 30 & B0WB68 & 36424 & 1 \\
\hline Columbia River & HFD TS HRM 30 & B17CM2 & 37872 & 1 \\
\hline Columbia River & HFD TS HRM 30 & B12TF9 & 37144 & 1 \\
\hline Columbia River & HFD TS HRM 30 & B1B743 & 38244 & 1 \\
\hline Columbia River & HFD TS HRM 30 & B1B498 & 38244 & 1 \\
\hline Columbia River & HFD TS HRM 30 & B17HK8 & 37872 & 1 \\
\hline Columbia River & HFD TS HRM 30 & B1B746 & 38244 & 1 \\
\hline Columbia River & HFD TS HRM 30 & B1B739 & 38244 & 1 \\
\hline Columbia River & HFD TS1 HRM28.7 & B0WB90 & 36424 & 1 \\
\hline Columbia River & HFD TS1 HRM28.7 & B17CH5 & 37872 & 1 \\
\hline
\end{tabular}


Table B-2

\section{Summary Table of Segment 2 Surface Water Samples By Area, Site, Date, and Category Columbia River Component - Existing Sample Data Evaluation and Scoping Report}

\begin{tabular}{|c|c|c|c|c|}
\hline Sample Area & Sample Site & Sample ID & Sample Date & Category \\
\hline Columbia River & HFD TS1 HRM28.7 & B12VR6 & 37144 & 1 \\
\hline Columbia River & HFD TS1 HRM28.7 & B12TK8 & 37144 & 1 \\
\hline Columbia River & HFD TS1 HRM28.7 & B0WB16 & 36424 & 1 \\
\hline Columbia River & HFD TS1 HRM28.7 & B109C2 & 36787 & 1 \\
\hline Columbia River & HFD TS1 HRM28.7 & B158K5 & 37508 & 1 \\
\hline Columbia River & HFD TS1 HRM28.7 & B106W8 & 36787 & 1 \\
\hline Columbia River & HFD TS1 HRM28.7 & B159D4 & 37508 & 1 \\
\hline Columbia River & HFD TS1 HRM28.7 & B158C3 & 37508 & 1 \\
\hline Columbia River & HFD TS1 HRM28.7 & B158T4 & 37508 & 1 \\
\hline Columbia River & HFD TS1 HRM28.7 & B106T0 & 36787 & 1 \\
\hline Columbia River & HFD TS1 HRM28.7 & В12Т55 & 37144 & 1 \\
\hline Columbia River & HFD TS1 HRM28.7 & B17CP5 & 37872 & 1 \\
\hline Columbia River & HFD TS1 HRM28.7 & B12TB1 & 37144 & 1 \\
\hline Columbia River & HFD TS1 HRM28.7 & BowC90 & 36424 & 1 \\
\hline Columbia River & HFD TS1 HRM28.7 & B10749 & 36787 & 1 \\
\hline Columbia River & HFD TS1 HRM28.7 & B17C97 & 37872 & 1 \\
\hline Columbia River & HFD TS1 HRM28.7 & B106L9 & 36787 & 1 \\
\hline Columbia River & HFD TS1 HRM28.7 & B1B494 & 38244 & 1 \\
\hline Columbia River & HFD TS1 HRM28.7 & B1B769 & 38244 & 1 \\
\hline Columbia River & HFD TS1 HRM28.7 & B1B715 & 38244 & 1 \\
\hline Columbia River & HFD TS1 HRM28.7 & B1B6W0 & 38244 & 1 \\
\hline Columbia River & HFD TS1 HRM28.7 & B17HJ9 & 37872 & 1 \\
\hline Columbia River & HFD TS10HRM28.7 & $\mathrm{B} 17 \mathrm{CH} 8$ & 37872 & 1 \\
\hline Columbia River & HFD TS10HRM28.7 & B17CR0 & 37872 & 1 \\
\hline Columbia River & HFD TS10HRM28.7 & B10754 & 36787 & 1 \\
\hline Columbia River & HFD TS10HRM28.7 & BowC91 & 36424 & 1 \\
\hline Columbia River & HFD TS10HRM28.7 & B159D9 & 37508 & 1 \\
\hline Columbia River & HFD TS10HRM28.7 & B12VT1 & 37144 & 1 \\
\hline Columbia River & HFD TS10HRM28.7 & B12TB4 & 37144 & 1 \\
\hline Columbia River & HFD TS10HRM28.7 & B158L1 & 37508 & 1 \\
\hline Columbia River & HFD TS10HRM28.7 & B12T60 & 37144 & 1 \\
\hline Columbia River & HFD TS10HRM28.7 & B106L8 & 36787 & 1 \\
\hline Columbia River & HFD TS10HRM28.7 & B0WB20 & 36424 & 1 \\
\hline Columbia River & HFD TS10HRM28.7 & B0WB94 & 36424 & 1 \\
\hline Columbia River & HFD TS10HRM28.7 & B106X1 & 36787 & 1 \\
\hline Columbia River & HFD TS10HRM28.7 & B12TL3 & 37144 & 1 \\
\hline Columbia River & HFD TS10HRM28.7 & B17CB2 & 37872 & 1 \\
\hline Columbia River & HFD TS10HRM28.7 & B158C8 & 37508 & 1 \\
\hline Columbia River & HFD TS10HRM28.7 & B158T9 & 37508 & 1 \\
\hline Columbia River & HFD TS10HRM28.7 & B106T5 & 36787 & 1 \\
\hline Columbia River & HFD TS10HRM28.7 & B1B718 & 38244 & 1 \\
\hline Columbia River & HFD TS10HRM28.7 & B17HK4 & 37872 & 1 \\
\hline Columbia River & HFD TS10HRM28.7 & B1B6W5 & 38244 & 1 \\
\hline Columbia River & HFD TS10HRM28.7 & B1B774 & 38244 & 1 \\
\hline Columbia River & HFD TS10HRM28.7 & B1B9N1 & 38244 & 1 \\
\hline Columbia River & HFD TS2 HRM28.7 & B106W9 & 36787 & 1 \\
\hline
\end{tabular}


Table B-2

\section{Summary Table of Segment 2 Surface Water Samples By Area, Site, Date, and Category Columbia River Component - Existing Sample Data Evaluation and Scoping Report}

\begin{tabular}{|c|c|c|c|c|}
\hline Sample Area & Sample Site & Sample ID & Sample Date & Category \\
\hline Columbia River & HFD TS2 HRM28.7 & B158T5 & 37508 & 1 \\
\hline Columbia River & HFD TS2 HRM28.7 & B158K7 & 37508 & 1 \\
\hline Columbia River & HFD TS2 HRM28.7 & B12Т56 & 37144 & 1 \\
\hline Columbia River & HFD TS2 HRM28.7 & B109C3 & 36787 & 1 \\
\hline Columbia River & HFD TS2 HRM28.7 & BowC92 & 36424 & 1 \\
\hline Columbia River & HFD TS2 HRM28.7 & B17CP6 & 37872 & 1 \\
\hline Columbia River & HFD TS2 HRM28.7 & B12VR7 & 37144 & 1 \\
\hline Columbia River & HFD TS2 HRM28.7 & B106M0 & 36787 & 1 \\
\hline Columbia River & HFD TS2 HRM28.7 & B0WB14 & 36424 & 1 \\
\hline Columbia River & HFD TS2 HRM28.7 & B12TB2 & 37144 & 1 \\
\hline Columbia River & HFD TS2 HRM28.7 & $\mathrm{B} 17 \mathrm{CH} 6$ & 37872 & 1 \\
\hline Columbia River & HFD TS2 HRM28.7 & B17C98 & 37872 & 1 \\
\hline Columbia River & HFD TS2 HRM28.7 & B0WB89 & 36424 & 1 \\
\hline Columbia River & HFD TS2 HRM28.7 & B12TK9 & 37144 & 1 \\
\hline Columbia River & HFD TS2 HRM28.7 & B159D5 & 37508 & 1 \\
\hline Columbia River & HFD TS2 HRM28.7 & B158C4 & 37508 & 1 \\
\hline Columbia River & HFD TS2 HRM28.7 & B1B6W1 & 38244 & 1 \\
\hline Columbia River & HFD TS2 HRM28.7 & B1B9M7 & 38244 & 1 \\
\hline Columbia River & HFD TS2 HRM28.7 & B1B770 & 38244 & 1 \\
\hline Columbia River & HFD TS2 HRM28.7 & B17HK0 & 37872 & 1 \\
\hline Columbia River & HFD TS2 HRM28.7 & B1B716 & 38244 & 1 \\
\hline Columbia River & HFD TS3 HRM28.7 & B12TB5 & 37144 & 1 \\
\hline Columbia River & HFD TS3 HRM28.7 & BoWB91 & 36424 & 1 \\
\hline Columbia River & HFD TS3 HRM28.7 & B10751 & 36787 & 1 \\
\hline Columbia River & HFD TS3 HRM28.7 & B158T6 & 37508 & 1 \\
\hline Columbia River & HFD TS3 HRM28.7 & BoWB21 & 36424 & 1 \\
\hline Columbia River & HFD TS3 HRM28.7 & BowC93 & 36424 & 1 \\
\hline Columbia River & HFD TS3 HRM28.7 & B17C99 & 37872 & 1 \\
\hline Columbia River & HFD TS3 HRM28.7 & В12Т57 & 37144 & 1 \\
\hline Columbia River & HFD TS3 HRM28.7 & B106T2 & 36787 & 1 \\
\hline Columbia River & HFD TS3 HRM28.7 & В17CH9 & 37872 & 1 \\
\hline Columbia River & HFD TS3 HRM28.7 & B159D6 & 37508 & 1 \\
\hline Columbia River & HFD TS3 HRM28.7 & B158L3 & 37508 & 1 \\
\hline Columbia River & HFD TS3 HRM28.7 & B17CP7 & 37872 & 1 \\
\hline Columbia River & HFD TS3 HRM28.7 & B158C5 & 37508 & 1 \\
\hline Columbia River & HFD TS3 HRM28.7 & B12TL0 & 37144 & 1 \\
\hline Columbia River & HFD TS3 HRM28.7 & B106X2 & 36787 & 1 \\
\hline Columbia River & HFD TS3 HRM28.7 & B12VR8 & 37144 & 1 \\
\hline Columbia River & HFD TS3 HRM28.7 & B1B771 & 38244 & 1 \\
\hline Columbia River & HFD TS3 HRM28.7 & B17HK1 & 37872 & 1 \\
\hline Columbia River & HFD TS3 HRM28.7 & B1B6W2 & 38244 & 1 \\
\hline Columbia River & HFD TS3 HRM28.7 & B1B719 & 38244 & 1 \\
\hline Columbia River & HFD TS3 HRM28.7 & В1В9M8 & 38244 & 1 \\
\hline Columbia River & HFD TS5 HRM28.7 & B106W3 & 36787 & 1 \\
\hline Columbia River & HFD TS5 HRM28.7 & B159D7 & 37508 & 1 \\
\hline Columbia River & HFD TS5 HRM28.7 & B17CB0 & 37872 & 1 \\
\hline
\end{tabular}


Table B-2

\section{Summary Table of Segment 2 Surface Water Samples By Area, Site, Date, and Category Columbia River Component - Existing Sample Data Evaluation and Scoping Report}

\begin{tabular}{|c|c|c|c|c|}
\hline Sample Area & Sample Site & Sample ID & Sample Date & Category \\
\hline Columbia River & HFD TS5 HRM28.7 & B158T7 & 37508 & 1 \\
\hline Columbia River & HFD TS5 HRM28.7 & B10752 & 36787 & 1 \\
\hline Columbia River & HFD TS5 HRM28.7 & B12TL1 & 37144 & 1 \\
\hline Columbia River & HFD TS5 HRM28.7 & B106T3 & 36787 & 1 \\
\hline Columbia River & HFD TS5 HRM28.7 & B158J5 & 37508 & 1 \\
\hline Columbia River & HFD TS5 HRM28.7 & В12Т96 & 37144 & 1 \\
\hline Columbia River & HFD TS5 HRM28.7 & B158C6 & 37508 & 1 \\
\hline Columbia River & HFD TS5 HRM28.7 & $\mathrm{B} 17 \mathrm{CHO}$ & 37872 & 1 \\
\hline Columbia River & HFD TS5 HRM28.7 & B17CP8 & 37872 & 1 \\
\hline Columbia River & HFD TS5 HRM28.7 & B0WB04 & 36424 & 1 \\
\hline Columbia River & HFD TS5 HRM28.7 & B12VR9 & 37144 & 1 \\
\hline Columbia River & HFD TS5 HRM28.7 & B106M2 & 36787 & 1 \\
\hline Columbia River & HFD TS5 HRM28.7 & BoWB92 & 36424 & 1 \\
\hline Columbia River & HFD TS5 HRM28.7 & В12Т58 & 37144 & 1 \\
\hline Columbia River & HFD TS5 HRM28.7 & BowC94 & 36424 & 1 \\
\hline Columbia River & HFD TS5 HRM28.7 & B1B6W3 & 38244 & 1 \\
\hline Columbia River & HFD TS5 HRM28.7 & B1B710 & 38244 & 1 \\
\hline Columbia River & HFD TS5 HRM28.7 & B1B772 & 38244 & 1 \\
\hline Columbia River & HFD TS5 HRM28.7 & B17HK2 & 37872 & 1 \\
\hline Columbia River & HFD TS5 HRM28.7 & В1В9M9 & 38244 & 1 \\
\hline Columbia River & HFD TS7 HRM28.7 & B106T4 & 36787 & 1 \\
\hline Columbia River & HFD TS7 HRM28.7 & B12Т59 & 37144 & 1 \\
\hline Columbia River & HFD TS7 HRM28.7 & $\mathrm{B} 17 \mathrm{CH} 7$ & 37872 & 1 \\
\hline Columbia River & HFD TS7 HRM28.7 & Bowc95 & 36424 & 1 \\
\hline Columbia River & HFD TS7 HRM28.7 & B10753 & 36787 & 1 \\
\hline Columbia River & HFD TS7 HRM28.7 & B12VT0 & 37144 & 1 \\
\hline Columbia River & HFD TS7 HRM28.7 & B159D8 & 37508 & 1 \\
\hline Columbia River & HFD TS7 HRM28.7 & B106M3 & 36787 & 1 \\
\hline Columbia River & HFD TS7 HRM28.7 & B17CB1 & 37872 & 1 \\
\hline Columbia River & HFD TS7 HRM28.7 & В12TB3 & 37144 & 1 \\
\hline Columbia River & HFD TS7 HRM28.7 & B158K9 & 37508 & 1 \\
\hline Columbia River & HFD TS7 HRM28.7 & B17CP9 & 37872 & 1 \\
\hline Columbia River & HFD TS7 HRM28.7 & B158C7 & 37508 & 1 \\
\hline Columbia River & HFD TS7 HRM28.7 & B106X0 & 36787 & 1 \\
\hline Columbia River & HFD TS7 HRM28.7 & B0WB93 & 36424 & 1 \\
\hline Columbia River & HFD TS7 HRM28.7 & B12TL2 & 37144 & 1 \\
\hline Columbia River & HFD TS7 HRM28.7 & В158T8 & 37508 & 1 \\
\hline Columbia River & HFD TS7 HRM28.7 & B0WB18 & 36424 & 1 \\
\hline Columbia River & HFD TS7 HRM28.7 & B1B773 & 38244 & 1 \\
\hline Columbia River & HFD TS7 HRM28.7 & B1B717 & 38244 & 1 \\
\hline Columbia River & HFD TS7 HRM28.7 & B17HK3 & 37872 & 1 \\
\hline Columbia River & HFD TS7 HRM28.7 & B1B6W4 & 38244 & 1 \\
\hline Columbia River & HFD TS7 HRM28.7 & B1B9N0 & 38244 & 1 \\
\hline Columbia River & HIP DEEP CRK & 23832 & 36510 & 1 \\
\hline Columbia River & Powerline & 24690 & 36560 & 1 \\
\hline Columbia River & REACTOR D AREA & HR024D & 37902 & 1 \\
\hline
\end{tabular}


Table B-2

Summary Table of Segment 2 Surface Water Samples By Area, Site, Date, and Category Columbia River Component - Existing Sample Data Evaluation and Scoping Report

\begin{tabular}{|c|c|c|c|c|}
\hline Sample Area & Sample Site & Sample ID & Sample Date & Category \\
\hline Columbia River & REACTOR H AREA & $\mathrm{HR} 028 \mathrm{H}$ & 37903 & 1 \\
\hline Columbia River & REACTOR K AREA & HR021K & 37902 & 1 \\
\hline Columbia River & REACTOR N AREA & HR015N & 37903 & 1 \\
\hline Columbia River & RICHLAND SPR & B1BH17 & 38285 & 1 \\
\hline Columbia River & Richland WNTK & HR047R & 38203 & 1 \\
\hline Columbia River & Richland WTP & 28062 & 37474 & 1 \\
\hline Columbia River & Richland WTP & 28061 & 37503 & 1 \\
\hline Columbia River & Richland WTP & 28054 & 37320 & 1 \\
\hline Columbia River & Richland WTP & 27818 & 37258 & 1 \\
\hline Columbia River & Richland WTP & 28053 & 37292 & 1 \\
\hline Columbia River & Richland WTP & 24609 & 36831 & 1 \\
\hline Columbia River & Richland WTP & 30573 & 37775 & 1 \\
\hline Columbia River & Richland WTP & 30571 & 37867 & 1 \\
\hline Columbia River & Richland WTP & 22923 & 36404 & 1 \\
\hline Columbia River & Richland WTP & 24607 & 36712 & 1 \\
\hline Columbia River & Richland WTP & 30572 & 37838 & 1 \\
\hline Columbia River & Richland WTP & 22919 & 36375 & 1 \\
\hline Columbia River & Richland WTP & 24608 & 36802 & 1 \\
\hline Columbia River & Richland WTP & 24612 & 36775 & 1 \\
\hline Columbia River & Richland WTP & 28063 & 37411 & 1 \\
\hline Columbia River & Richland WTP & 22935 & 36495 & 1 \\
\hline Columbia River & Richland WTP & 24605 & 36586 & 1 \\
\hline Columbia River & Richland WTP & 24592 & 36557 & 1 \\
\hline Columbia River & Richland WTP & 22931 & 36466 & 1 \\
\hline Columbia River & Richland WTP & 24610 & 36865 & 1 \\
\hline Columbia River & Richland WTP & 28055 & 37593 & 1 \\
\hline Columbia River & Richland WTP & 24606 & 36621 & 1 \\
\hline Columbia River & Richland WTP & 28060 & 37383 & 1 \\
\hline Columbia River & Richland WTP & 30570 & 37746 & 1 \\
\hline Columbia River & Richland WTP & 22927 & 36438 & 1 \\
\hline Columbia River & Richland WTP & 28059 & 37348 & 1 \\
\hline Columbia River & Richland WTP & 28058 & 37530 & 1 \\
\hline Columbia River & Richland WTP & 28057 & 37439 & 1 \\
\hline Columbia River & Richland WTP & 24611 & 36648 & 1 \\
\hline Columbia River & Richland WTP & 28056 & 37565 & 1 \\
\hline Columbia River & Richland WTP & 26101 & 37047 & 1 \\
\hline Columbia River & Richland WTP & 30566 & 37929 & 1 \\
\hline Columbia River & Richland WTP & 22895 & 36194 & 1 \\
\hline Columbia River & Richland WTP & 32902 & 38032 & 1 \\
\hline Columbia River & Richland WTP & 32903 & 38306 & 1 \\
\hline Columbia River & Richland WTP & 26093 & 37229 & 1 \\
\hline Columbia River & Richland WTP & 26095 & 37075 & 1 \\
\hline Columbia River & Richland WTP & 22208 & 36165 & 1 \\
\hline Columbia River & Richland WTP & 25521 & 36894 & 1 \\
\hline Columbia River & Richland WTP & 32811 & 38322 & 1 \\
\hline Columbia River & Richland WTP & 26096 & 37166 & 1 \\
\hline
\end{tabular}


Table B-2

Summary Table of Segment 2 Surface Water Samples By Area, Site, Date, and Category Columbia River Component - Existing Sample Data Evaluation and Scoping Report

\begin{tabular}{|c|c|c|c|c|}
\hline Sample Area & Sample Site & Sample ID & Sample Date & Category \\
\hline Columbia River & Richland WTP & 24613 & 36739 & 1 \\
\hline Columbia River & Richland WTP & 24614 & 36684 & 1 \\
\hline Columbia River & Richland WTP & 34238 & 38356 & 1 \\
\hline Columbia River & Richland WTP & 32901 & 38214 & 1 \\
\hline Columbia River & Richland WTP & 30567 & 37802 & 1 \\
\hline Columbia River & Richland WTP & 26094 & 37201 & 1 \\
\hline Columbia River & Richland WTP & 26097 & 36984 & 1 \\
\hline Columbia River & Richland WTP & 26098 & 37012 & 1 \\
\hline Columbia River & Richland WTP & 26099 & 37139 & 1 \\
\hline Columbia River & Richland WTP & 26100 & 37104 & 1 \\
\hline Columbia River & Richland WTP & 30565 & 37957 & 1 \\
\hline Columbia River & Richland WTP & 30564 & 37684 & 1 \\
\hline Columbia River & Richland WTP & 24101 & 36529 & 1 \\
\hline Columbia River & Richland WTP & 30563 & 37656 & 1 \\
\hline Columbia River & Richland WTP & 32574 & 37992 & 1 \\
\hline Columbia River & Richland WTP & 32810 & 38048 & 1 \\
\hline Columbia River & Richland WTP & 32809 & 38020 & 1 \\
\hline Columbia River & Richland WTP & 30328 & 37623 & 1 \\
\hline Columbia River & Richland WTP & 34473 & 38384 & 1 \\
\hline Columbia River & Richland WTP & 26092 & 36956 & 1 \\
\hline Columbia River & Richland WTP & 30569 & 37712 & 1 \\
\hline Columbia River & Richland WTP & 22907 & 36284 & 1 \\
\hline Columbia River & Richland WTP & 32815 & 38083 & 1 \\
\hline Columbia River & Richland WTP & 22915 & 36348 & 1 \\
\hline Columbia River & Richland WTP & 30568 & 37895 & 1 \\
\hline Columbia River & Richland WTP & 22903 & 36256 & 1 \\
\hline Columbia River & Richland WTP & 32812 & 38293 & 1 \\
\hline Columbia River & Richland WTP & 32816 & 38107 & 1 \\
\hline Columbia River & Richland WTP & 32814 & 38265 & 1 \\
\hline Columbia River & Richland WTP & 32813 & 38175 & 1 \\
\hline Columbia River & Richland WTP & 32817 & 38231 & 1 \\
\hline Columbia River & Richland WTP & 32818 & 38202 & 1 \\
\hline Columbia River & Richland WTP & 32819 & 38140 & 1 \\
\hline Columbia River & Richland WTP & 22911 & 36313 & 1 \\
\hline Columbia River & Richland WTP & 32887 & 38122 & 1 \\
\hline Columbia River & Richland WTP & 22899 & 36221 & 1 \\
\hline Columbia River & RICHLANDHRM44.4 & B121V4 & 37028 & 1 \\
\hline Columbia River & RICHPS HRM 43.5 & B0X409 & 36500 & 1 \\
\hline Columbia River & RICHPS HRM 43.5 & B11148 & 36865 & 1 \\
\hline Columbia River & RICHPS HRM 43.5 & B106N4 & 36788 & 1 \\
\hline Columbia River & RICHPS HRM 43.5 & BOYCLO & 36697 & 1 \\
\hline Columbia River & RICHPS HRM 43.5 & BOYCL4 & 36697 & 1 \\
\hline Columbia River & RICHPS HRM 43.5 & B12TN1 & 37147 & 1 \\
\hline Columbia River & RICHPS HRM 43.5 & B0X413 & 36500 & 1 \\
\hline Columbia River & RICHPS HRM 43.5 & B16KD2 & 37705 & 1 \\
\hline Columbia River & RICHPS HRM 43.5 & B12TM7 & 37147 & 1 \\
\hline
\end{tabular}


Table B-2

\section{Summary Table of Segment 2 Surface Water Samples By Area, Site, Date, and Category} Columbia River Component - Existing Sample Data Evaluation and Scoping Report

\begin{tabular}{|c|c|c|c|c|}
\hline Sample Area & Sample Site & Sample ID & Sample Date & Category \\
\hline Columbia River & RICHPS HRM 43.5 & B16373 & 37600 & 1 \\
\hline Columbia River & RICHPS HRM 43.5 & B16365 & 37600 & 1 \\
\hline Columbia River & RICHPS HRM 43.5 & В12TM3 & 37147 & 1 \\
\hline Columbia River & RICHPS HRM 43.5 & B16369 & 37600 & 1 \\
\hline Columbia River & RICHPS HRM 43.5 & B164K6 & 37600 & 1 \\
\hline Columbia River & RICHPS HRM 43.5 & B0XRP9 & 36613 & 1 \\
\hline Columbia River & RICHPS HRM 43.5 & B14T06 & 37418 & 1 \\
\hline Columbia River & RICHPS HRM 43.5 & 33140 & 38245 & 1 \\
\hline Columbia River & RICHPS HRM 43.5 & B13LK0 & 37229 & 1 \\
\hline Columbia River & RICHPS HRM 43.5 & B0XRP1 & 36613 & 1 \\
\hline Columbia River & RICHPS HRM 43.5 & B12VL9 & 37147 & 1 \\
\hline Columbia River & RICHPS HRM 43.5 & B0YC96 & 36697 & 1 \\
\hline Columbia River & RICHPS HRM 43.5 & B11237 & 36865 & 1 \\
\hline Columbia River & RICHPS HRM 43.5 & B0X454 & 36500 & 1 \\
\hline Columbia River & RICHPS HRM 43.5 & B14RY1 & 37418 & 1 \\
\hline Columbia River & RICHPS HRM 43.5 & B11156 & 36865 & 1 \\
\hline Columbia River & RICHPS HRM 43.5 & B0XRW2 & 36613 & 1 \\
\hline Columbia River & RICHPS HRM 43.5 & B11152 & 36865 & 1 \\
\hline Columbia River & RICHPS HRM 43.5 & B147W6 & 37341 & 1 \\
\hline Columbia River & RICHPS HRM 43.5 & BOYCK6 & 36697 & 1 \\
\hline Columbia River & RICHPS HRM 43.5 & B13LJ6 & 37229 & 1 \\
\hline Columbia River & RICHPS HRM 43.5 & B11H66 & 36948 & 1 \\
\hline Columbia River & RICHPS HRM 43.5 & B13LV0 & 37229 & 1 \\
\hline Columbia River & RICHPS HRM 43.5 & B109D5 & 36788 & 1 \\
\hline Columbia River & RICHPS HRM 43.5 & B124X1 & 37054 & 1 \\
\hline Columbia River & RICHPS HRM 43.5 & В17СТ0 & 37873 & 1 \\
\hline Columbia River & RICHPS HRM 43.5 & B0WBC6 & 36419 & 1 \\
\hline Columbia River & RICHPS HRM 43.5 & B11H62 & 36948 & 1 \\
\hline Columbia River & RICHPS HRM 43.5 & B14RX7 & 37418 & 1 \\
\hline Columbia River & RICHPS HRM 43.5 & B17CT4 & 37873 & 1 \\
\hline Columbia River & RICHPS HRM 43.5 & B0WBB8 & 36419 & 1 \\
\hline Columbia River & RICHPS HRM 43.5 & B11H70 & 36948 & 1 \\
\hline Columbia River & RICHPS HRM 43.5 & B11J36 & 36948 & 1 \\
\hline Columbia River & RICHPS HRM 43.5 & B158W3 & 37509 & 1 \\
\hline Columbia River & RICHPS HRM 43.5 & B0XRP5 & 36613 & 1 \\
\hline Columbia River & RICHPS HRM 43.5 & B16LD0 & 37705 & 1 \\
\hline Columbia River & RICHPS HRM 43.5 & B16LD4 & 37705 & 1 \\
\hline Columbia River & RICHPS HRM 43.5 & B16LD8 & 37705 & 1 \\
\hline Columbia River & RICHPS HRM 43.5 & B158W7 & 37509 & 1 \\
\hline Columbia River & RICHPS HRM 43.5 & B12534 & 37054 & 1 \\
\hline Columbia River & RICHPS HRM 43.5 & B10764 & 36788 & 1 \\
\hline Columbia River & RICHPS HRM 43.5 & B12538 & 37054 & 1 \\
\hline Columbia River & RICHPS HRM 43.5 & B12542 & 37054 & 1 \\
\hline Columbia River & RICHPS HRM 43.5 & B10768 & 36788 & 1 \\
\hline Columbia River & RICHPS HRM 43.5 & B10772 & 36788 & 1 \\
\hline Columbia River & RICHPS HRM 43.5 & B158V9 & 37509 & 1 \\
\hline
\end{tabular}


Table B-2

\section{Summary Table of Segment 2 Surface Water Samples By Area, Site, Date, and Category} Columbia River Component - Existing Sample Data Evaluation and Scoping Report

\begin{tabular}{|c|c|c|c|c|}
\hline Sample Area & Sample Site & Sample ID & Sample Date & Category \\
\hline Columbia River & RICHPS HRM 43.5 & B17212 & 37782 & 1 \\
\hline Columbia River & RICHPS HRM 43.5 & B146X9 & 37341 & 1 \\
\hline Columbia River & RICHPS HRM 43.5 & B0X405 & 36500 & 1 \\
\hline Columbia River & RICHPS HRM 43.5 & B17282 & 37782 & 1 \\
\hline Columbia River & RICHPS HRM 43.5 & B14RY5 & 37418 & 1 \\
\hline Columbia River & RICHPS HRM 43.5 & B13LK4 & 37229 & 1 \\
\hline Columbia River & RICHPS HRM 43.5 & B17208 & 37782 & 1 \\
\hline Columbia River & RICHPS HRM 43.5 & 27403 & 37147 & 1 \\
\hline Columbia River & RICHPS HRM 43.5 & B17204 & 37782 & 1 \\
\hline Columbia River & RICHPS HRM 43.5 & B15987 & 37509 & 1 \\
\hline Columbia River & RICHPS HRM 43.5 & B0WCB3 & 36419 & 1 \\
\hline Columbia River & RICHPS HRM 43.5 & B1BW51 & 38340 & 1 \\
\hline Columbia River & RICHPS HRM 43.5 & B183W8 & 37963 & 1 \\
\hline Columbia River & RICHPS HRM 43.5 & В19HР6 & 38162 & 1 \\
\hline Columbia River & RICHPS HRM 43.5 & B1BX56 & 38340 & 1 \\
\hline Columbia River & RICHPS HRM 43.5 & B18V25 & 38076 & 1 \\
\hline Columbia River & RICHPS HRM 43.5 & B1B784 & 38245 & 1 \\
\hline Columbia River & RICHPS HRM 43.5 & B17HL5 & 37873 & 1 \\
\hline Columbia River & RICHPS HRM 43.5 & B184R5 & 37963 & 1 \\
\hline Columbia River & RICHPS HRM 43.5 & B19JB7 & 38162 & 1 \\
\hline Columbia River & RICHPS HRM 43.5 & B183W0 & 37963 & 1 \\
\hline Columbia River & RICHPS HRM 43.5 & B1B788 & 38245 & 1 \\
\hline Columbia River & RICHPS HRM 43.5 & B18TJ8 & 38076 & 1 \\
\hline Columbia River & RICHPS HRM 43.5 & B183W4 & 37963 & 1 \\
\hline Columbia River & RICHPS HRM 43.5 & B1B456 & 38245 & 1 \\
\hline Columbia River & RICHPS HRM 43.5 & B1B792 & 38245 & 1 \\
\hline Columbia River & RICHPS HRM 43.9 & B0YCK5 & 36697 & 1 \\
\hline Columbia River & RICHPS HRM 43.9 & B12TN0 & 37147 & 1 \\
\hline Columbia River & RICHPS HRM 43.9 & B0YC97 & 36697 & 1 \\
\hline Columbia River & RICHPS HRM 43.9 & B147W5 & 37341 & 1 \\
\hline Columbia River & RICHPS HRM 43.9 & B16LD7 & 37705 & 1 \\
\hline Columbia River & RICHPS HRM 43.9 & B0X408 & 36500 & 1 \\
\hline Columbia River & RICHPS HRM 43.9 & BOYCK9 & 36697 & 1 \\
\hline Columbia River & RICHPS HRM 43.9 & B16372 & 37600 & 1 \\
\hline Columbia River & RICHPS HRM 43.9 & B12TM6 & 37147 & 1 \\
\hline Columbia River & RICHPS HRM 43.9 & B16LD3 & 37705 & 1 \\
\hline Columbia River & RICHPS HRM 43.9 & B0XRP8 & 36613 & 1 \\
\hline Columbia River & RICHPS HRM 43.9 & BOYCL3 & 36697 & 1 \\
\hline Columbia River & RICHPS HRM 43.9 & B13LK3 & 37229 & 1 \\
\hline Columbia River & RICHPS HRM 43.9 & BOWCB2 & 36419 & 1 \\
\hline Columbia River & RICHPS HRM 43.9 & B164K7 & 37600 & 1 \\
\hline Columbia River & RICHPS HRM 43.9 & B12TM2 & 37147 & 1 \\
\hline Columbia River & RICHPS HRM 43.9 & B0WBB7 & 36419 & 1 \\
\hline Columbia River & RICHPS HRM 43.9 & B13LJ9 & 37229 & 1 \\
\hline Columbia River & RICHPS HRM 43.9 & B17203 & 37782 & 1 \\
\hline Columbia River & RICHPS HRM 43.9 & B146Y0 & 37341 & 1 \\
\hline
\end{tabular}


Table B-2

\section{Summary Table of Segment 2 Surface Water Samples By Area, Site, Date, and Category Columbia River Component - Existing Sample Data Evaluation and Scoping Report}

\begin{tabular}{|c|c|c|c|c|}
\hline Sample Area & Sample Site & Sample ID & Sample Date & Category \\
\hline Columbia River & RICHPS HRM 43.9 & B13LJ5 & 37229 & 1 \\
\hline Columbia River & RICHPS HRM 43.9 & B17207 & 37782 & 1 \\
\hline Columbia River & RICHPS HRM 43.9 & B13LV1 & 37229 & 1 \\
\hline Columbia River & RICHPS HRM 43.9 & B16KD3 & 37705 & 1 \\
\hline Columbia River & RICHPS HRM 43.9 & B17211 & 37782 & 1 \\
\hline Columbia River & RICHPS HRM 43.9 & B12VM0 & 37147 & 1 \\
\hline Columbia River & RICHPS HRM 43.9 & B0X453 & 36500 & 1 \\
\hline Columbia River & RICHPS HRM 43.9 & B0XRW3 & 36613 & 1 \\
\hline Columbia River & RICHPS HRM 43.9 & B16LC9 & 37705 & 1 \\
\hline Columbia River & RICHPS HRM 43.9 & BOXRPO & 36613 & 1 \\
\hline Columbia River & RICHPS HRM 43.9 & B0WBC5 & 36419 & 1 \\
\hline Columbia River & RICHPS HRM 43.9 & B0X404 & 36500 & 1 \\
\hline Columbia River & RICHPS HRM 43.9 & B0XRP4 & 36613 & 1 \\
\hline Columbia River & RICHPS HRM 43.9 & B17283 & 37782 & 1 \\
\hline Columbia River & RICHPS HRM 43.9 & 33139 & 38245 & 1 \\
\hline Columbia River & RICHPS HRM 43.9 & B0X412 & 36500 & 1 \\
\hline Columbia River & RICHPS HRM 43.9 & B16368 & 37600 & 1 \\
\hline Columbia River & RICHPS HRM 43.9 & B10767 & 36788 & 1 \\
\hline Columbia River & RICHPS HRM 43.9 & B12541 & 37054 & 1 \\
\hline Columbia River & RICHPS HRM 43.9 & B11H65 & 36948 & 1 \\
\hline Columbia River & RICHPS HRM 43.9 & B12533 & 37054 & 1 \\
\hline Columbia River & RICHPS HRM 43.9 & 27404 & 37147 & 1 \\
\hline Columbia River & RICHPS HRM 43.9 & B158W6 & 37509 & 1 \\
\hline Columbia River & RICHPS HRM 43.9 & B124X2 & 37054 & 1 \\
\hline Columbia River & RICHPS HRM 43.9 & B11H69 & 36948 & 1 \\
\hline Columbia River & RICHPS HRM 43.9 & B11238 & 36865 & 1 \\
\hline Columbia River & RICHPS HRM 43.9 & B11155 & 36865 & 1 \\
\hline Columbia River & RICHPS HRM 43.9 & B11H61 & 36948 & 1 \\
\hline Columbia River & RICHPS HRM 43.9 & B158V8 & 37509 & 1 \\
\hline Columbia River & RICHPS HRM 43.9 & B14RY4 & 37418 & 1 \\
\hline Columbia River & RICHPS HRM 43.9 & B158W2 & 37509 & 1 \\
\hline Columbia River & RICHPS HRM 43.9 & В14T07 & 37418 & 1 \\
\hline Columbia River & RICHPS HRM 43.9 & B106N5 & 36788 & 1 \\
\hline Columbia River & RICHPS HRM 43.9 & B10771 & 36788 & 1 \\
\hline Columbia River & RICHPS HRM 43.9 & B16364 & 37600 & 1 \\
\hline Columbia River & RICHPS HRM 43.9 & B15988 & 37509 & 1 \\
\hline Columbia River & RICHPS HRM 43.9 & $\mathrm{~B} 14 \mathrm{R} \times 6$ & 37418 & 1 \\
\hline Columbia River & RICHPS HRM 43.9 & B11147 & 36865 & 1 \\
\hline Columbia River & RICHPS HRM 43.9 & B14RY0 & 37418 & 1 \\
\hline Columbia River & RICHPS HRM 43.9 & B11J37 & 36948 & 1 \\
\hline Columbia River & RICHPS HRM 43.9 & B10763 & 36788 & 1 \\
\hline Columbia River & RICHPS HRM 43.9 & B12537 & 37054 & 1 \\
\hline Columbia River & RICHPS HRM 43.9 & B11151 & 36865 & 1 \\
\hline Columbia River & RICHPS HRM 43.9 & B1B787 & 38245 & 1 \\
\hline Columbia River & RICHPS HRM 43.9 & B18V26 & 38076 & 1 \\
\hline Columbia River & RICHPS HRM 43.9 & B18TJ7 & 38076 & 1 \\
\hline
\end{tabular}


Table B-2

\section{Summary Table of Segment 2 Surface Water Samples By Area, Site, Date, and Category} Columbia River Component - Existing Sample Data Evaluation and Scoping Report

\begin{tabular}{|c|c|c|c|c|}
\hline Sample Area & Sample Site & Sample ID & Sample Date & Category \\
\hline Columbia River & RICHPS HRM 43.9 & B19JB6 & 38162 & 1 \\
\hline Columbia River & RICHPS HRM 43.9 & B1BW50 & 38340 & 1 \\
\hline Columbia River & RICHPS HRM 43.9 & B184R6 & 37963 & 1 \\
\hline Columbia River & RICHPS HRM 43.9 & B1B783 & 38245 & 1 \\
\hline Columbia River & RICHPS HRM 43.9 & B1BX57 & 38340 & 1 \\
\hline Columbia River & RICHPS HRM 43.9 & B1B791 & 38245 & 1 \\
\hline Columbia River & RICHPS HRM 43.9 & B183V9 & 37963 & 1 \\
\hline Columbia River & RICHPS HRM 43.9 & B1B457 & 38245 & 1 \\
\hline Columbia River & RICHPS HRM 43.9 & B183W3 & 37963 & 1 \\
\hline Columbia River & RICHPS HRM 43.9 & B19HP5 & 38162 & 1 \\
\hline Columbia River & RICHPS HRM 43.9 & B183W7 & 37963 & 1 \\
\hline Columbia River & RICHPS HRM 45.0 & B13LJ4 & 37229 & 1 \\
\hline Columbia River & RICHPS HRM 45.0 & B16LD6 & 37705 & 1 \\
\hline Columbia River & RICHPS HRM 45.0 & В12TM9 & 37147 & 1 \\
\hline Columbia River & RICHPS HRM 45.0 & B10770 & 36788 & 1 \\
\hline Columbia River & RICHPS HRM 45.0 & BOWBC4 & 36419 & 1 \\
\hline Columbia River & RICHPS HRM 45.0 & B158V7 & 37509 & 1 \\
\hline Columbia River & RICHPS HRM 45.0 & B0YC98 & 36697 & 1 \\
\hline Columbia River & RICHPS HRM 45.0 & B12TM5 & 37147 & 1 \\
\hline Columbia River & RICHPS HRM 45.0 & B11150 & 36865 & 1 \\
\hline Columbia River & RICHPS HRM 45.0 & B17202 & 37782 & 1 \\
\hline Columbia River & RICHPS HRM 45.0 & B16LD2 & 37705 & 1 \\
\hline Columbia River & RICHPS HRM 45.0 & B147W4 & 37341 & 1 \\
\hline Columbia River & RICHPS HRM 45.0 & B0X452 & 36500 & 1 \\
\hline Columbia River & RICHPS HRM 45.0 & 33138 & 38245 & 1 \\
\hline Columbia River & RICHPS HRM 45.0 & B11154 & 36865 & 1 \\
\hline Columbia River & RICHPS HRM 45.0 & B0XRP7 & 36613 & 1 \\
\hline Columbia River & RICHPS HRM 45.0 & B10766 & 36788 & 1 \\
\hline Columbia River & RICHPS HRM 45.0 & B14RX9 & 37418 & 1 \\
\hline Columbia River & RICHPS HRM 45.0 & B13LK2 & 37229 & 1 \\
\hline Columbia River & RICHPS HRM 45.0 & B12TM1 & 37147 & 1 \\
\hline Columbia River & RICHPS HRM 45.0 & BoWBB6 & 36419 & 1 \\
\hline Columbia River & RICHPS HRM 45.0 & B0XRP3 & 36613 & 1 \\
\hline Columbia River & RICHPS HRM 45.0 & B109D6 & 36788 & 1 \\
\hline Columbia River & RICHPS HRM 45.0 & B16367 & 37600 & 1 \\
\hline Columbia River & RICHPS HRM 45.0 & B124X3 & 37054 & 1 \\
\hline Columbia River & RICHPS HRM 45.0 & B11146 & 36865 & 1 \\
\hline Columbia River & RICHPS HRM 45.0 & BOXRN9 & 36613 & 1 \\
\hline Columbia River & RICHPS HRM 45.0 & B13LJ8 & 37229 & 1 \\
\hline Columbia River & RICHPS HRM 45.0 & B0XRW4 & 36613 & 1 \\
\hline Columbia River & RICHPS HRM 45.0 & B16KD4 & 37705 & 1 \\
\hline Columbia River & RICHPS HRM 45.0 & B14T08 & 37418 & 1 \\
\hline Columbia River & RICHPS HRM 45.0 & B11239 & 36865 & 1 \\
\hline Columbia River & RICHPS HRM 45.0 & B10762 & 36788 & 1 \\
\hline Columbia River & RICHPS HRM 45.0 & B14RY3 & 37418 & 1 \\
\hline Columbia River & RICHPS HRM 45.0 & B0YCK8 & 36697 & 1 \\
\hline
\end{tabular}


Table B-2

Summary Table of Segment 2 Surface Water Samples By Area, Site, Date, and Category Columbia River Component - Existing Sample Data Evaluation and Scoping Report

\begin{tabular}{|c|c|c|c|c|}
\hline Sample Area & Sample Site & Sample ID & Sample Date & Category \\
\hline Columbia River & RICHPS HRM 45.0 & B106N6 & 36788 & 1 \\
\hline Columbia River & RICHPS HRM 45.0 & B158W5 & 37509 & 1 \\
\hline Columbia River & RICHPS HRM 45.0 & B17210 & 37782 & 1 \\
\hline Columbia River & RICHPS HRM 45.0 & 27405 & 37147 & 1 \\
\hline Columbia River & RICHPS HRM 45.0 & B12532 & 37054 & 1 \\
\hline Columbia River & RICHPS HRM 45.0 & B11H64 & 36948 & 1 \\
\hline Columbia River & RICHPS HRM 45.0 & B15989 & 37509 & 1 \\
\hline Columbia River & RICHPS HRM 45.0 & В17CT2 & 37873 & 1 \\
\hline Columbia River & RICHPS HRM 45.0 & B12540 & 37054 & 1 \\
\hline Columbia River & RICHPS HRM 45.0 & BOYCL2 & 36697 & 1 \\
\hline Columbia River & RICHPS HRM 45.0 & B14RX5 & 37418 & 1 \\
\hline Columbia River & RICHPS HRM 45.0 & B164K8 & 37600 & 1 \\
\hline Columbia River & RICHPS HRM 45.0 & B11J38 & 36948 & 1 \\
\hline Columbia River & RICHPS HRM 45.0 & B12536 & 37054 & 1 \\
\hline Columbia River & RICHPS HRM 45.0 & B146Y1 & 37341 & 1 \\
\hline Columbia River & RICHPS HRM 45.0 & B17284 & 37782 & 1 \\
\hline Columbia River & RICHPS HRM 45.0 & B16363 & 37600 & 1 \\
\hline Columbia River & RICHPS HRM 45.0 & B16371 & 37600 & 1 \\
\hline Columbia River & RICHPS HRM 45.0 & B11H68 & 36948 & 1 \\
\hline Columbia River & RICHPS HRM 45.0 & B158W1 & 37509 & 1 \\
\hline Columbia River & RICHPS HRM 45.0 & B0WCB1 & 36419 & 1 \\
\hline Columbia River & RICHPS HRM 45.0 & B12VM1 & 37147 & 1 \\
\hline Columbia River & RICHPS HRM 45.0 & B0X403 & 36500 & 1 \\
\hline Columbia River & RICHPS HRM 45.0 & B17206 & 37782 & 1 \\
\hline Columbia River & RICHPS HRM 45.0 & B16LC8 & 37705 & 1 \\
\hline Columbia River & RICHPS HRM 45.0 & B0X407 & 36500 & 1 \\
\hline Columbia River & RICHPS HRM 45.0 & B0X411 & 36500 & 1 \\
\hline Columbia River & RICHPS HRM 45.0 & B17CR8 & 37873 & 1 \\
\hline Columbia River & RICHPS HRM 45.0 & B11H60 & 36948 & 1 \\
\hline Columbia River & RICHPS HRM 45.0 & B13LV2 & 37229 & 1 \\
\hline Columbia River & RICHPS HRM 45.0 & BOYCK4 & 36697 & 1 \\
\hline Columbia River & RICHPS HRM 45.0 & B18V27 & 38076 & 1 \\
\hline Columbia River & RICHPS HRM 45.0 & B1B458 & 38245 & 1 \\
\hline Columbia River & RICHPS HRM 45.0 & B183V8 & 37963 & 1 \\
\hline Columbia River & RICHPS HRM 45.0 & B19HP7 & 38162 & 1 \\
\hline Columbia River & RICHPS HRM 45.0 & B18TJ6 & 38076 & 1 \\
\hline Columbia River & RICHPS HRM 45.0 & B17HL7 & 37873 & 1 \\
\hline Columbia River & RICHPS HRM 45.0 & B1B790 & 38245 & 1 \\
\hline Columbia River & RICHPS HRM 45.0 & B1B782 & 38245 & 1 \\
\hline Columbia River & RICHPS HRM 45.0 & B1B786 & 38245 & 1 \\
\hline Columbia River & RICHPS HRM 45.0 & B1BW49 & 38340 & 1 \\
\hline Columbia River & RICHPS HRM 45.0 & B184R7 & 37963 & 1 \\
\hline Columbia River & RICHPS HRM 45.0 & B19JB5 & 38162 & 1 \\
\hline Columbia River & RICHPS HRM 45.0 & B1BX58 & 38340 & 1 \\
\hline Columbia River & RICHPS HRM 45.0 & B183W2 & 37963 & 1 \\
\hline Columbia River & RICHPS HRM 45.0 & B183W6 & 37963 & 1 \\
\hline
\end{tabular}


Table B-2

\section{Summary Table of Segment 2 Surface Water Samples By Area, Site, Date, and Category} Columbia River Component - Existing Sample Data Evaluation and Scoping Report

\begin{tabular}{|c|c|c|c|c|}
\hline Sample Area & Sample Site & Sample ID & Sample Date & Category \\
\hline Columbia River & RICHPS HRM 45.8 & B0X451 & 36500 & 1 \\
\hline Columbia River & RICHPS HRM 45.8 & В12TM8 & 37147 & 1 \\
\hline Columbia River & RICHPS HRM 45.8 & B14RX4 & 37418 & 1 \\
\hline Columbia River & RICHPS HRM 45.8 & B16LD1 & 37705 & 1 \\
\hline Columbia River & RICHPS HRM 45.8 & B17205 & 37782 & 1 \\
\hline Columbia River & RICHPS HRM 45.8 & B12TM4 & 37147 & 1 \\
\hline Columbia River & RICHPS HRM 45.8 & B11145 & 36865 & 1 \\
\hline Columbia River & RICHPS HRM 45.8 & B17285 & 37782 & 1 \\
\hline Columbia River & RICHPS HRM 45.8 & B146Y2 & 37341 & 1 \\
\hline Columbia River & RICHPS HRM 45.8 & B106N7 & 36788 & 1 \\
\hline Columbia River & RICHPS HRM 45.8 & B109D7 & 36788 & 1 \\
\hline Columbia River & RICHPS HRM 45.8 & B0X402 & 36500 & 1 \\
\hline Columbia River & RICHPS HRM 45.8 & B16LC7 & 37705 & 1 \\
\hline Columbia River & RICHPS HRM 45.8 & B17209 & 37782 & 1 \\
\hline Columbia River & RICHPS HRM 45.8 & B16KD5 & 37705 & 1 \\
\hline Columbia River & RICHPS HRM 45.8 & B0X410 & 36500 & 1 \\
\hline Columbia River & RICHPS HRM 45.8 & B13LK1 & 37229 & 1 \\
\hline Columbia River & RICHPS HRM 45.8 & B17201 & 37782 & 1 \\
\hline Columbia River & RICHPS HRM 45.8 & B15990 & 37509 & 1 \\
\hline Columbia River & RICHPS HRM 45.8 & B13LV3 & 37229 & 1 \\
\hline Columbia River & RICHPS HRM 45.8 & 27406 & 37147 & 1 \\
\hline Columbia River & RICHPS HRM 45.8 & B14RX8 & 37418 & 1 \\
\hline Columbia River & RICHPS HRM 45.8 & B16LD5 & 37705 & 1 \\
\hline Columbia River & RICHPS HRM 45.8 & B12TM0 & 37147 & 1 \\
\hline Columbia River & RICHPS HRM 45.8 & B0X406 & 36500 & 1 \\
\hline Columbia River & RICHPS HRM 45.8 & B11H67 & 36948 & 1 \\
\hline Columbia River & RICHPS HRM 45.8 & B0WBC3 & 36419 & 1 \\
\hline Columbia River & RICHPS HRM 45.8 & B12VM2 & 37147 & 1 \\
\hline Columbia River & RICHPS HRM 45.8 & B0YC99 & 36697 & 1 \\
\hline Columbia River & RICHPS HRM 45.8 & B11H63 & 36948 & 1 \\
\hline Columbia River & RICHPS HRM 45.8 & B11J39 & 36948 & 1 \\
\hline Columbia River & RICHPS HRM 45.8 & B0XRP6 & 36613 & 1 \\
\hline Columbia River & RICHPS HRM 45.8 & B16362 & 37600 & 1 \\
\hline Columbia River & RICHPS HRM 45.8 & B11149 & 36865 & 1 \\
\hline Columbia River & RICHPS HRM 45.8 & B164K9 & 37600 & 1 \\
\hline Columbia River & RICHPS HRM 45.8 & B10769 & 36788 & 1 \\
\hline Columbia River & RICHPS HRM 45.8 & B12539 & 37054 & 1 \\
\hline Columbia River & RICHPS HRM 45.8 & 33137 & 38245 & 1 \\
\hline Columbia River & RICHPS HRM 45.8 & B17CR7 & 37873 & 1 \\
\hline Columbia River & RICHPS HRM 45.8 & B0YCK3 & 36697 & 1 \\
\hline Columbia River & RICHPS HRM 45.8 & B12535 & 37054 & 1 \\
\hline Columbia River & RICHPS HRM 45.8 & B12531 & 37054 & 1 \\
\hline Columbia River & RICHPS HRM 45.8 & В17CT1 & 37873 & 1 \\
\hline Columbia River & RICHPS HRM 45.8 & BOYCK7 & 36697 & 1 \\
\hline Columbia River & RICHPS HRM 45.8 & B158W4 & 37509 & 1 \\
\hline Columbia River & RICHPS HRM 45.8 & BOWCBO & 36419 & 1 \\
\hline
\end{tabular}


Table B-2

\section{Summary Table of Segment 2 Surface Water Samples By Area, Site, Date, and Category} Columbia River Component - Existing Sample Data Evaluation and Scoping Report

\begin{tabular}{|c|c|c|c|c|}
\hline Sample Area & Sample Site & Sample ID & Sample Date & Category \\
\hline Columbia River & RICHPS HRM 45.8 & BOYCL1 & 36697 & 1 \\
\hline Columbia River & RICHPS HRM 45.8 & B11H59 & 36948 & 1 \\
\hline Columbia River & RICHPS HRM 45.8 & B14RY2 & 37418 & 1 \\
\hline Columbia River & RICHPS HRM 45.8 & B11153 & 36865 & 1 \\
\hline Columbia River & RICHPS HRM 45.8 & B10761 & 36788 & 1 \\
\hline Columbia River & RICHPS HRM 45.8 & BOXRN8 & 36613 & 1 \\
\hline Columbia River & RICHPS HRM 45.8 & BOXRP2 & 36613 & 1 \\
\hline Columbia River & RICHPS HRM 45.8 & B124X4 & 37054 & 1 \\
\hline Columbia River & RICHPS HRM 45.8 & B10765 & 36788 & 1 \\
\hline Columbia River & RICHPS HRM 45.8 & B0WBB5 & 36419 & 1 \\
\hline Columbia River & RICHPS HRM 45.8 & B158V6 & 37509 & 1 \\
\hline Columbia River & RICHPS HRM 45.8 & B147W3 & 37341 & 1 \\
\hline Columbia River & RICHPS HRM 45.8 & В14T09 & 37418 & 1 \\
\hline Columbia River & RICHPS HRM 45.8 & B158W0 & 37509 & 1 \\
\hline Columbia River & RICHPS HRM 45.8 & B0XRW5 & 36613 & 1 \\
\hline Columbia River & RICHPS HRM 45.8 & B13LJ3 & 37229 & 1 \\
\hline Columbia River & RICHPS HRM 45.8 & B16370 & 37600 & 1 \\
\hline Columbia River & RICHPS HRM 45.8 & B13LJ7 & 37229 & 1 \\
\hline Columbia River & RICHPS HRM 45.8 & B16366 & 37600 & 1 \\
\hline Columbia River & RICHPS HRM 45.8 & B11240 & 36865 & 1 \\
\hline Columbia River & RICHPS HRM 45.8 & B1B789 & 38245 & 1 \\
\hline Columbia River & RICHPS HRM 45.8 & B1B781 & 38245 & 1 \\
\hline Columbia River & RICHPS HRM 45.8 & B1B785 & 38245 & 1 \\
\hline Columbia River & RICHPS HRM 45.8 & B1BW48 & 38340 & 1 \\
\hline Columbia River & RICHPS HRM 45.8 & B18TJ5 & 38076 & 1 \\
\hline Columbia River & RICHPS HRM 45.8 & B183W5 & 37963 & 1 \\
\hline Columbia River & RICHPS HRM 45.8 & B18V28 & 38076 & 1 \\
\hline Columbia River & RICHPS HRM 45.8 & B1BX59 & 38340 & 1 \\
\hline Columbia River & RICHPS HRM 45.8 & B184R8 & 37963 & 1 \\
\hline Columbia River & RICHPS HRM 45.8 & B183W1 & 37963 & 1 \\
\hline Columbia River & RICHPS HRM 45.8 & В19HР3 & 38162 & 1 \\
\hline Columbia River & RICHPS HRM 45.8 & B183V7 & 37963 & 1 \\
\hline Columbia River & RICHPS HRM 45.8 & B1B459 & 38245 & 1 \\
\hline Columbia River & RICHPS HRM 45.8 & B17HL8 & 37873 & 1 \\
\hline Columbia River & RICHPS HRM 45.8 & B19JB4 & 38162 & 1 \\
\hline Columbia River & RICHPS HRM 46.4 & B17575 & 37894 & 1 \\
\hline Columbia River & RICHPS HRM 46.4 & B13VL2 & 37322 & 1 \\
\hline Columbia River & RICHPS HRM 46.4 & B175F9 & 37841 & 1 \\
\hline Columbia River & RICHPS HRM 46.4 & $\mathrm{~B} 172 \mathrm{~F} 2$ & 37790 & 1 \\
\hline Columbia River & RICHPS HRM 46.4 & $\mathrm{~B} 172 \mathrm{~K} 1$ & 37797 & 1 \\
\hline Columbia River & RICHPS HRM 46.4 & B17215 & 37784 & 1 \\
\hline Columbia River & RICHPS HRM 46.4 & B14X12 & 37503 & 1 \\
\hline Columbia River & RICHPS HRM 46.4 & B13VL1 & 37293 & 1 \\
\hline Columbia River & RICHPS HRM 46.4 & B14X13 & 37531 & 1 \\
\hline Columbia River & RICHPS HRM 46.4 & B153V4 & 37503 & 1 \\
\hline Columbia River & RICHPS HRM 46.4 & B11WT4 & 37041 & 1 \\
\hline
\end{tabular}


Table B-2

\section{Summary Table of Segment 2 Surface Water Samples By Area, Site, Date, and Category Columbia River Component - Existing Sample Data Evaluation and Scoping Report}

\begin{tabular}{|c|c|c|c|c|}
\hline Sample Area & Sample Site & Sample ID & Sample Date & Category \\
\hline Columbia River & RICHPS HRM 46.4 & B14X11 & 37475 & 1 \\
\hline Columbia River & RICHPS HRM 46.4 & B13V88 & 37349 & 1 \\
\hline Columbia River & RICHPS HRM 46.4 & B12TJ6 & 37147 & 1 \\
\hline Columbia River & RICHPS HRM 46.4 & B175J6 & 37894 & 1 \\
\hline Columbia River & RICHPS HRM 46.4 & B10BT4 & 36895 & 1 \\
\hline Columbia River & RICHPS HRM 46.4 & B10C19 & 36832 & 1 \\
\hline Columbia River & RICHPS HRM 46.4 & B10C37 & 36888 & 1 \\
\hline Columbia River & RICHPS HRM 46.4 & В10C38 & 36832 & 1 \\
\hline Columbia River & RICHPS HRM 46.4 & В10С39 & 36859 & 1 \\
\hline Columbia River & RICHPS HRM 46.4 & $\mathrm{~B} 171 \mathrm{~K} 7$ & 37777 & 1 \\
\hline Columbia River & RICHPS HRM 46.4 & $\mathrm{~B} 171 \mathrm{~F} 6$ & 37812 & 1 \\
\hline Columbia River & RICHPS HRM 46.4 & B170P8 & 37754 & 1 \\
\hline Columbia River & RICHPS HRM 46.4 & B170X8 & 37762 & 1 \\
\hline Columbia River & RICHPS HRM 46.4 & B124M3 & 37070 & 1 \\
\hline Columbia River & RICHPS HRM 46.4 & B13VJ0 & 37293 & 1 \\
\hline Columbia River & RICHPS HRM 46.4 & B10C40 & 36888 & 1 \\
\hline Columbia River & RICHPS HRM 46.4 & B10C43 & 36888 & 1 \\
\hline Columbia River & RICHPS HRM 46.4 & B13VL0 & 37349 & 1 \\
\hline Columbia River & RICHPS HRM 46.4 & B124Y3 & 37054 & 1 \\
\hline Columbia River & RICHPS HRM 46.4 & B10C44 & 36832 & 1 \\
\hline Columbia River & RICHPS HRM 46.4 & B12525 & 37054 & 1 \\
\hline Columbia River & RICHPS HRM 46.4 & B10C45 & 36859 & 1 \\
\hline Columbia River & RICHPS HRM 46.4 & B10C46 & 36888 & 1 \\
\hline Columbia River & RICHPS HRM 46.4 & B17141 & 37769 & 1 \\
\hline Columbia River & RICHPS HRM 46.4 & B114W1 & 36929 & 1 \\
\hline Columbia River & RICHPS HRM 46.4 & В0THK7 & 36257 & 1 \\
\hline Columbia River & RICHPS HRM 46.4 & B11LW9 & 37070 & 1 \\
\hline Columbia River & RICHPS HRM 46.4 & B11H31 & 36948 & 1 \\
\hline Columbia River & RICHPS HRM 46.4 & B11C10 & 36951 & 1 \\
\hline Columbia River & RICHPS HRM 46.4 & B13LH7 & 37229 & 1 \\
\hline Columbia River & RICHPS HRM 46.4 & $\mathrm{~B} 114 \mathrm{X0}$ & 36985 & 1 \\
\hline Columbia River & RICHPS HRM 46.4 & B114W9 & 36957 & 1 \\
\hline Columbia River & RICHPS HRM 46.4 & B114W8 & 36929 & 1 \\
\hline Columbia River & RICHPS HRM 46.4 & B114W7 & 36985 & 1 \\
\hline Columbia River & RICHPS HRM 46.4 & B11LW7 & 37013 & 1 \\
\hline Columbia River & RICHPS HRM 46.4 & B114W2 & 36957 & 1 \\
\hline Columbia River & RICHPS HRM 46.4 & B11LW6 & 37070 & 1 \\
\hline Columbia River & RICHPS HRM 46.4 & B114W0 & 36985 & 1 \\
\hline Columbia River & RICHPS HRM 46.4 & B114T0 & 36922 & 1 \\
\hline Columbia River & RICHPS HRM 46.4 & B114H9 & 36985 & 1 \\
\hline Columbia River & RICHPS HRM 46.4 & В0ТН77 & 36257 & 1 \\
\hline Columbia River & RICHPS HRM 46.4 & В0ТНHЗ & 36194 & 1 \\
\hline Columbia River & RICHPS HRM 46.4 & B0THK1 & 36257 & 1 \\
\hline Columbia River & RICHPS HRM 46.4 & В0THK2 & 36187 & 1 \\
\hline Columbia River & RICHPS HRM 46.4 & В0ТНКЗ & 36215 & 1 \\
\hline Columbia River & RICHPS HRM 46.4 & B0VCY5 & 36313 & 1 \\
\hline
\end{tabular}


Table B-2

\section{Summary Table of Segment 2 Surface Water Samples By Area, Site, Date, and Category Columbia River Component - Existing Sample Data Evaluation and Scoping Report}

\begin{tabular}{|c|c|c|c|c|}
\hline Sample Area & Sample Site & Sample ID & Sample Date & Category \\
\hline Columbia River & RICHPS HRM 46.4 & B114W3 & 36985 & 1 \\
\hline Columbia River & RICHPS HRM 46.4 & B13124 & 37252 & 1 \\
\hline Columbia River & RICHPS HRM 46.4 & B11LW0 & 37070 & 1 \\
\hline Columbia River & RICHPS HRM 46.4 & B11LT2 & 37013 & 1 \\
\hline Columbia River & RICHPS HRM 46.4 & B11LJ7 & 37070 & 1 \\
\hline Columbia River & RICHPS HRM 46.4 & B11J46 & 36985 & 1 \\
\hline Columbia River & RICHPS HRM 46.4 & B11LW2 & 37041 & 1 \\
\hline Columbia River & RICHPS HRM 46.4 & B17DK3 & 37894 & 1 \\
\hline Columbia River & RICHPS HRM 46.4 & B130P4 & 37259 & 1 \\
\hline Columbia River & RICHPS HRM 46.4 & B13103 & 37196 & 1 \\
\hline Columbia River & RICHPS HRM 46.4 & B13121 & 37252 & 1 \\
\hline Columbia River & RICHPS HRM 46.4 & B11LW8 & 37041 & 1 \\
\hline Columbia River & RICHPS HRM 46.4 & B13123 & 37222 & 1 \\
\hline Columbia River & RICHPS HRM 46.4 & ВОТНК8 & 36187 & 1 \\
\hline Columbia River & RICHPS HRM 46.4 & B13127 & 37252 & 1 \\
\hline Columbia River & RICHPS HRM 46.4 & B13128 & 37196 & 1 \\
\hline Columbia River & RICHPS HRM 46.4 & B13129 & 37222 & 1 \\
\hline Columbia River & RICHPS HRM 46.4 & B13130 & 37252 & 1 \\
\hline Columbia River & RICHPS HRM 46.4 & B13CD2 & 37222 & 1 \\
\hline Columbia River & RICHPS HRM 46.4 & B13LB3 & 37259 & 1 \\
\hline Columbia River & RICHPS HRM 46.4 & B13LD5 & 37229 & 1 \\
\hline Columbia River & RICHPS HRM 46.4 & B11H53 & 36948 & 1 \\
\hline Columbia River & RICHPS HRM 46.4 & B11LW3 & 37070 & 1 \\
\hline Columbia River & RICHPS HRM 46.4 & B13122 & 37196 & 1 \\
\hline Columbia River & RICHPS HRM 46.4 & Bow905 & 36439 & 1 \\
\hline Columbia River & RICHPS HRM 46.4 & B0THK4 & 36257 & 1 \\
\hline Columbia River & RICHPS HRM 46.4 & B0VV14 & 36369 & 1 \\
\hline Columbia River & RICHPS HRM 46.4 & B0VV15 & 36411 & 1 \\
\hline Columbia River & RICHPS HRM 46.4 & B0VV16 & 36439 & 1 \\
\hline Columbia River & RICHPS HRM 46.4 & B10MR1 & 36859 & 1 \\
\hline Columbia River & RICHPS HRM 46.4 & B14WL4 & 37531 & 1 \\
\hline Columbia River & RICHPS HRM 46.4 & B14WX2 & 37475 & 1 \\
\hline Columbia River & RICHPS HRM 46.4 & B14X03 & 37531 & 1 \\
\hline Columbia River & RICHPS HRM 46.4 & B14X04 & 37475 & 1 \\
\hline Columbia River & RICHPS HRM 46.4 & B0VV10 & 36439 & 1 \\
\hline Columbia River & RICHPS HRM 46.4 & B0W395 & 36404 & 1 \\
\hline Columbia River & RICHPS HRM 46.4 & B0VV09 & 36411 & 1 \\
\hline Columbia River & RICHPS HRM 46.4 & B14X06 & 37531 & 1 \\
\hline Columbia River & RICHPS HRM 46.4 & B14X10 & 37531 & 1 \\
\hline Columbia River & RICHPS HRM 46.4 & B179R7 & 37867 & 1 \\
\hline Columbia River & RICHPS HRM 46.4 & B175K5 & 37894 & 1 \\
\hline Columbia River & RICHPS HRM 46.4 & B175K4 & 37867 & 1 \\
\hline Columbia River & RICHPS HRM 46.4 & B175K3 & 37841 & 1 \\
\hline Columbia River & RICHPS HRM 46.4 & B175K2 & 37894 & 1 \\
\hline Columbia River & RICHPS HRM 46.4 & B175J9 & 37894 & 1 \\
\hline Columbia River & RICHPS HRM 46.4 & B175J8 & 37867 & 1 \\
\hline
\end{tabular}


Table B-2

\section{Summary Table of Segment 2 Surface Water Samples By Area, Site, Date, and Category Columbia River Component - Existing Sample Data Evaluation and Scoping Report}

\begin{tabular}{|c|c|c|c|c|}
\hline Sample Area & Sample Site & Sample ID & Sample Date & Category \\
\hline Columbia River & RICHPS HRM 46.4 & B14X05 & 37503 & 1 \\
\hline Columbia River & RICHPS HRM 46.4 & B0V554 & 36285 & 1 \\
\hline Columbia River & RICHPS HRM 46.4 & ВОТНК9 & 36215 & 1 \\
\hline Columbia River & RICHPS HRM 46.4 & BOTHLO & 36257 & 1 \\
\hline Columbia River & RICHPS HRM 46.4 & В0TM59 & 36222 & 1 \\
\hline Columbia River & RICHPS HRM 46.4 & B0TW39 & 36257 & 1 \\
\hline Columbia River & RICHPS HRM 46.4 & B0V4V1 & 36341 & 1 \\
\hline Columbia River & RICHPS HRM 46.4 & B0V530 & 36285 & 1 \\
\hline Columbia River & RICHPS HRM 46.4 & B0V547 & 36341 & 1 \\
\hline Columbia River & RICHPS HRM 46.4 & B0V548 & 36285 & 1 \\
\hline Columbia River & RICHPS HRM 46.4 & B0V549 & 36313 & 1 \\
\hline Columbia River & RICHPS HRM 46.4 & B0VV13 & 36439 & 1 \\
\hline Columbia River & RICHPS HRM 46.4 & B0V553 & 36341 & 1 \\
\hline Columbia River & RICHPS HRM 46.4 & B175J7 & 37841 & 1 \\
\hline Columbia River & RICHPS HRM 46.4 & B0V555 & 36313 & 1 \\
\hline Columbia River & RICHPS HRM 46.4 & B0V556 & 36341 & 1 \\
\hline Columbia River & RICHPS HRM 46.4 & B11100 & 36895 & 1 \\
\hline Columbia River & RICHPS HRM 46.4 & B13VL3 & 37349 & 1 \\
\hline Columbia River & RICHPS HRM 46.4 & BOVL48 & 36341 & 1 \\
\hline Columbia River & RICHPS HRM 46.4 & BOVTN3 & 36439 & 1 \\
\hline Columbia River & RICHPS HRM 46.4 & BOVTX9 & 36369 & 1 \\
\hline Columbia River & RICHPS HRM 46.4 & B0VV07 & 36439 & 1 \\
\hline Columbia River & RICHPS HRM 46.4 & B0VV08 & 36369 & 1 \\
\hline Columbia River & RICHPS HRM 46.4 & B0V550 & 36341 & 1 \\
\hline Columbia River & RICHPS HRM 46.4 & B11LW1 & 37013 & 1 \\
\hline Columbia River & RICHPS HRM 46.4 & B0XP21 & 36621 & 1 \\
\hline Columbia River & RICHPS HRM 46.4 & B14B67 & 37434 & 1 \\
\hline Columbia River & RICHPS HRM 46.4 & B14B64 & 37434 & 1 \\
\hline Columbia River & RICHPS HRM 46.4 & B14B63 & 37405 & 1 \\
\hline Columbia River & RICHPS HRM 46.4 & B14B62 & 37377 & 1 \\
\hline Columbia River & RICHPS HRM 46.4 & B15K08 & 37629 & 1 \\
\hline Columbia River & RICHPS HRM 46.4 & B15К79 & 37559 & 1 \\
\hline Columbia River & RICHPS HRM 46.4 & B0XVR8 & 36706 & 1 \\
\hline Columbia River & RICHPS HRM 46.4 & B15K97 & 37559 & 1 \\
\hline Columbia River & RICHPS HRM 46.4 & B0X7N3 & 36551 & 1 \\
\hline Columbia River & RICHPS HRM 46.4 & B15K98 & 37586 & 1 \\
\hline Columbia River & RICHPS HRM 46.4 & B15K99 & 37629 & 1 \\
\hline Columbia River & RICHPS HRM 46.4 & B15KB2 & 37629 & 1 \\
\hline Columbia River & RICHPS HRM 46.4 & B15KB3 & 37559 & 1 \\
\hline Columbia River & RICHPS HRM 46.4 & B15KB4 & 37586 & 1 \\
\hline Columbia River & RICHPS HRM 46.4 & BOXVF7 & 36706 & 1 \\
\hline Columbia River & RICHPS HRM 46.4 & B12872 & 37166 & 1 \\
\hline Columbia River & RICHPS HRM 46.4 & B15K96 & 37629 & 1 \\
\hline Columbia River & RICHPS HRM 46.4 & В12Т33 & 37166 & 1 \\
\hline Columbia River & RICHPS HRM 46.4 & B15954 & 37531 & 1 \\
\hline Columbia River & RICHPS HRM 46.4 & B12JT2 & 37139 & 1 \\
\hline
\end{tabular}


Table B-2

\section{Summary Table of Segment 2 Surface Water Samples By Area, Site, Date, and Category} Columbia River Component - Existing Sample Data Evaluation and Scoping Report

\begin{tabular}{|c|c|c|c|c|}
\hline Sample Area & Sample Site & Sample ID & Sample Date & Category \\
\hline Columbia River & RICHPS HRM 46.4 & BOWPT0 & 36495 & 1 \\
\hline Columbia River & RICHPS HRM 46.4 & В12Т78 & 37147 & 1 \\
\hline Columbia River & RICHPS HRM 46.4 & B14RM5 & 37434 & 1 \\
\hline Columbia River & RICHPS HRM 46.4 & B14JF8 & 37405 & 1 \\
\hline Columbia River & RICHPS HRM 46.4 & B14B70 & 37434 & 1 \\
\hline Columbia River & RICHPS HRM 46.4 & B0XH28 & 36593 & 1 \\
\hline Columbia River & RICHPS HRM 46.4 & B14B68 & 37377 & 1 \\
\hline Columbia River & RICHPS HRM 46.4 & B0X7N5 & 36621 & 1 \\
\hline Columbia River & RICHPS HRM 46.4 & B0X474 & 36523 & 1 \\
\hline Columbia River & RICHPS HRM 46.4 & B0X790 & 36621 & 1 \\
\hline Columbia River & RICHPS HRM 46.4 & B0X7K4 & 36558 & 1 \\
\hline Columbia River & RICHPS HRM 46.4 & B0X7M5 & 36621 & 1 \\
\hline Columbia River & RICHPS HRM 46.4 & B0X7M6 & 36551 & 1 \\
\hline Columbia River & RICHPS HRM 46.4 & B0X7M7 & 36593 & 1 \\
\hline Columbia River & RICHPS HRM 46.4 & B0X7M8 & 36621 & 1 \\
\hline Columbia River & RICHPS HRM 46.4 & BOXVR9 & 36649 & 1 \\
\hline Columbia River & RICHPS HRM 46.4 & B14B69 & 37405 & 1 \\
\hline Columbia River & RICHPS HRM 46.4 & B16886 & 37657 & 1 \\
\hline Columbia River & RICHPS HRM 46.4 & BOYKY2 & 36804 & 1 \\
\hline Columbia River & RICHPS HRM 46.4 & BOYKY3 & 36748 & 1 \\
\hline Columbia River & RICHPS HRM 46.4 & BOYKY4 & 36776 & 1 \\
\hline Columbia River & RICHPS HRM 46.4 & B0YKY5 & 36804 & 1 \\
\hline Columbia River & RICHPS HRM 46.4 & BOYKY9 & 36804 & 1 \\
\hline Columbia River & RICHPS HRM 46.4 & BOYL00 & 36748 & 1 \\
\hline Columbia River & RICHPS HRM 46.4 & B0YL01 & 36776 & 1 \\
\hline Columbia River & RICHPS HRM 46.4 & B0XVP1 & 36649 & 1 \\
\hline Columbia River & RICHPS HRM 46.4 & B16887 & 37685 & 1 \\
\hline Columbia River & RICHPS HRM 46.4 & B16888 & 37713 & 1 \\
\hline Columbia River & RICHPS HRM 46.4 & B16885 & 37713 & 1 \\
\hline Columbia River & RICHPS HRM 46.4 & B0YW70 & 36776 & 1 \\
\hline Columbia River & RICHPS HRM 46.4 & B103X3 & 36804 & 1 \\
\hline Columbia River & RICHPS HRM 46.4 & B16868 & 37657 & 1 \\
\hline Columbia River & RICHPS HRM 46.4 & B167Y1 & 37720 & 1 \\
\hline Columbia River & RICHPS HRM 46.4 & B14B43 & 37377 & 1 \\
\hline Columbia River & RICHPS HRM 46.4 & B14B61 & 37434 & 1 \\
\hline Columbia River & RICHPS HRM 46.4 & BOYL02 & 36804 & 1 \\
\hline Columbia River & RICHPS HRM 46.4 & B15VN8 & 37586 & 1 \\
\hline Columbia River & RICHPS HRM 46.4 & BOXVTO & 36677 & 1 \\
\hline Columbia River & RICHPS HRM 46.4 & B0XVT1 & 36706 & 1 \\
\hline Columbia River & RICHPS HRM 46.4 & BOXVT4 & 36706 & 1 \\
\hline Columbia River & RICHPS HRM 46.4 & B0XVT5 & 36649 & 1 \\
\hline Columbia River & RICHPS HRM 46.4 & B0XVT6 & 36677 & 1 \\
\hline Columbia River & RICHPS HRM 46.4 & B0XVT7 & 36706 & 1 \\
\hline Columbia River & RICHPS HRM 46.4 & B0Y237 & 36677 & 1 \\
\hline Columbia River & RICHPS HRM 46.4 & B0YKW1 & 36748 & 1 \\
\hline Columbia River & RICHPS HRM 46.4 & B0YB76 & 36706 & 1 \\
\hline
\end{tabular}


Table B-2

\section{Summary Table of Segment 2 Surface Water Samples By Area, Site, Date, and Category Columbia River Component - Existing Sample Data Evaluation and Scoping Report}

\begin{tabular}{|c|c|c|c|c|}
\hline Sample Area & Sample Site & Sample ID & Sample Date & Category \\
\hline Columbia River & RICHPS HRM 46.4 & BOYKL4 & 36804 & 1 \\
\hline Columbia River & RICHPS HRM 46.4 & B161R7 & 37629 & 1 \\
\hline Columbia River & RICHPS HRM 46.4 & B149W0 & 37439 & 1 \\
\hline Columbia River & RICHPS HRM 46.4 & B16JM4 & 37713 & 1 \\
\hline Columbia River & RICHPS HRM 46.4 & B16BY7 & 37685 & 1 \\
\hline Columbia River & RICHPS HRM 46.4 & B16894 & 37713 & 1 \\
\hline Columbia River & RICHPS HRM 46.4 & B16893 & 37685 & 1 \\
\hline Columbia River & RICHPS HRM 46.4 & B16892 & 37657 & 1 \\
\hline Columbia River & RICHPS HRM 46.4 & B0X7N2 & 36621 & 1 \\
\hline Columbia River & RICHPS HRM 46.4 & B15KB5 & 37629 & 1 \\
\hline Columbia River & RICHPS HRM 46.4 & B16PL8 & 37769 & 1 \\
\hline Columbia River & RICHPS HRM 46.4 & B140R2 & 37322 & 1 \\
\hline Columbia River & RICHPS HRM 46.4 & BoWJ22 & 36523 & 1 \\
\hline Columbia River & RICHPS HRM 46.4 & B12870 & 37112 & 1 \\
\hline Columbia River & RICHPS HRM 46.4 & BoWJ91 & 36467 & 1 \\
\hline Columbia River & RICHPS HRM 46.4 & B0WJB8 & 36523 & 1 \\
\hline Columbia River & RICHPS HRM 46.4 & B0WJB9 & 36467 & 1 \\
\hline Columbia River & RICHPS HRM 46.4 & BOWJCO & 36495 & 1 \\
\hline Columbia River & RICHPS HRM 46.4 & BoWJC1 & 36523 & 1 \\
\hline Columbia River & RICHPS HRM 46.4 & BoWJC4 & 36523 & 1 \\
\hline Columbia River & RICHPS HRM 46.4 & B16PJ9 & 37741 & 1 \\
\hline Columbia River & RICHPS HRM 46.4 & В16РМ3 & 37741 & 1 \\
\hline Columbia River & RICHPS HRM 46.4 & B16PL6 & 37812 & 1 \\
\hline Columbia River & RICHPS HRM 46.4 & B13VM0 & 37349 & 1 \\
\hline Columbia River & RICHPS HRM 46.4 & B12869 & 37166 & 1 \\
\hline Columbia River & RICHPS HRM 46.4 & B16P95 & 37812 & 1 \\
\hline Columbia River & RICHPS HRM 46.4 & B0WJC5 & 36467 & 1 \\
\hline Columbia River & RICHPS HRM 46.4 & B0WJC6 & 36495 & 1 \\
\hline Columbia River & RICHPS HRM 46.4 & BoWJC7 & 36523 & 1 \\
\hline Columbia River & RICHPS HRM 46.4 & B14413 & 37349 & 1 \\
\hline Columbia River & RICHPS HRM 46.4 & B16WL4 & 37748 & 1 \\
\hline Columbia River & RICHPS HRM 46.4 & B16WL2 & 37741 & 1 \\
\hline Columbia River & RICHPS HRM 46.4 & B16WF4 & 37769 & 1 \\
\hline Columbia River & RICHPS HRM 46.4 & B16PM5 & 37812 & 1 \\
\hline Columbia River & RICHPS HRM 46.4 & B16PM4 & 37769 & 1 \\
\hline Columbia River & RICHPS HRM 46.4 & B0X7N4 & 36593 & 1 \\
\hline Columbia River & RICHPS HRM 46.4 & B16PL9 & 37812 & 1 \\
\hline Columbia River & RICHPS HRM 46.4 & B16PM2 & 37812 & 1 \\
\hline Columbia River & RICHPS HRM 46.4 & B16PL7 & 37741 & 1 \\
\hline Columbia River & RICHPS HRM 46.4 & B12863 & 37112 & 1 \\
\hline Columbia River & RICHPS HRM 46.4 & B12864 & 37139 & 1 \\
\hline Columbia River & RICHPS HRM 46.4 & B12862 & 37166 & 1 \\
\hline Columbia River & RICHPS HRM 46.4 & B127W4 & 37166 & 1 \\
\hline Columbia River & RICHPS HRM 46.4 & B12865 & 37166 & 1 \\
\hline Columbia River & RICHPS HRM 46.4 & B12871 & 37139 & 1 \\
\hline Columbia River & RICHPS HRM 46.4 & B13VL7 & 37349 & 1 \\
\hline
\end{tabular}


Table B-2

\section{Summary Table of Segment 2 Surface Water Samples By Area, Site, Date, and Category Columbia River Component - Existing Sample Data Evaluation and Scoping Report}

\begin{tabular}{|c|c|c|c|c|}
\hline Sample Area & Sample Site & Sample ID & Sample Date & Category \\
\hline Columbia River & RICHPS HRM 46.4 & B13VL9 & 37322 & 1 \\
\hline Columbia River & RICHPS HRM 46.4 & B12841 & 37112 & 1 \\
\hline Columbia River & RICHPS HRM 46.4 & B13VL8 & 37293 & 1 \\
\hline Columbia River & RICHPS HRM 46.4 & B19B18 & 38176 & 1 \\
\hline Columbia River & RICHPS HRM 46.4 & B19229 & 38146 & 1 \\
\hline Columbia River & RICHPS HRM 46.4 & B18VX3 & 38176 & 1 \\
\hline Columbia River & RICHPS HRM 46.4 & B18VX2 & 38146 & 1 \\
\hline Columbia River & RICHPS HRM 46.4 & B1BCW2 & 38357 & 1 \\
\hline Columbia River & RICHPS HRM 46.4 & B1BD37 & 38288 & 1 \\
\hline Columbia River & RICHPS HRM 46.4 & B18VX1 & 38105 & 1 \\
\hline Columbia River & RICHPS HRM 46.4 & B1BD61 & 38357 & 1 \\
\hline Columbia River & RICHPS HRM 46.4 & B18VX0 & 38176 & 1 \\
\hline Columbia River & RICHPS HRM 46.4 & B1BD62 & 38288 & 1 \\
\hline Columbia River & RICHPS HRM 46.4 & B18VK0 & 38176 & 1 \\
\hline Columbia River & RICHPS HRM 46.4 & B17NV7 & 37994 & 1 \\
\hline Columbia River & RICHPS HRM 46.4 & B1B610 & 38261 & 1 \\
\hline Columbia River & RICHPS HRM 46.4 & B19XY5 & 38230 & 1 \\
\hline Columbia River & RICHPS HRM 46.4 & B19M56 & 38261 & 1 \\
\hline Columbia River & RICHPS HRM 46.4 & B19M55 & 38230 & 1 \\
\hline Columbia River & RICHPS HRM 46.4 & B19M54 & 38203 & 1 \\
\hline Columbia River & RICHPS HRM 46.4 & B19M53 & 38261 & 1 \\
\hline Columbia River & RICHPS HRM 46.4 & B1BD63 & 38314 & 1 \\
\hline Columbia River & RICHPS HRM 46.4 & B19LV6 & 38266 & 1 \\
\hline Columbia River & RICHPS HRM 46.4 & B1BD64 & 38357 & 1 \\
\hline Columbia River & RICHPS HRM 46.4 & B17P33 & 37923 & 1 \\
\hline Columbia River & RICHPS HRM 46.4 & B17P52 & 37923 & 1 \\
\hline Columbia River & RICHPS HRM 46.4 & B17P53 & 37950 & 1 \\
\hline Columbia River & RICHPS HRM 46.4 & B17P57 & 37994 & 1 \\
\hline Columbia River & RICHPS HRM 46.4 & B17P58 & 37923 & 1 \\
\hline Columbia River & RICHPS HRM 46.4 & B17P59 & 37950 & 1 \\
\hline Columbia River & RICHPS HRM 46.4 & B17P60 & 37994 & 1 \\
\hline Columbia River & RICHPS HRM 46.4 & В19М30 & 38203 & 1 \\
\hline Columbia River & RICHPS HRM 46.4 & B18756 & 38021 & 1 \\
\hline Columbia River & RICHPS HRM 46.4 & B1BMR7 & 38322 & 1 \\
\hline Columbia River & RICHPS HRM 46.4 & B1BW19 & 38357 & 1 \\
\hline Columbia River & RICHPS HRM 46.4 & B183J3 & 37994 & 1 \\
\hline Columbia River & RICHPS HRM 46.4 & B18VT6 & 38113 & 1 \\
\hline Columbia River & RICHPS HRM 46.4 & B186X7 & 38078 & 1 \\
\hline Columbia River & RICHPS HRM 46.4 & B18779 & 38078 & 1 \\
\hline Columbia River & RICHPS HRM 46.4 & B18780 & 38021 & 1 \\
\hline Columbia River & RICHPS HRM 46.4 & B18781 & 38048 & 1 \\
\hline Columbia River & RICHPS HRM 46.4 & B18782 & 38078 & 1 \\
\hline Columbia River & RICHPS HRM 46.4 & B17VC0 & 37950 & 1 \\
\hline Columbia River & RICHPS HRM 46.4 & B18K80 & 38078 & 1 \\
\hline Columbia River & RICHPS HRM 46.4 & B18D89 & 38048 & 1 \\
\hline Columbia River & RICHPS1 HRM46.4 & Bov246 & 36325 & 1 \\
\hline
\end{tabular}


Table B-2

\section{Summary Table of Segment 2 Surface Water Samples By Area, Site, Date, and Category Columbia River Component - Existing Sample Data Evaluation and Scoping Report}

\begin{tabular}{|c|c|c|c|c|}
\hline Sample Area & Sample Site & Sample ID & Sample Date & Category \\
\hline Columbia River & RICHPS1 HRM46.4 & B16334 & 37600 & 1 \\
\hline Columbia River & RICHPS1 HRM46.4 & B11124 & 36865 & 1 \\
\hline Columbia River & RICHPS1 HRM46.4 & B124W5 & 37054 & 1 \\
\hline Columbia River & RICHPS1 HRM46.4 & B16KC7 & 37705 & 1 \\
\hline Columbia River & RICHPS1 HRM46.4 & B11129 & 36865 & 1 \\
\hline Columbia River & RICHPS1 HRM46.4 & B0XRJ3 & 36613 & 1 \\
\hline Columbia River & RICHPS1 HRM46.4 & ВОҮСВО & 36697 & 1 \\
\hline Columbia River & RICHPS1 HRM46.4 & B11139 & 36865 & 1 \\
\hline Columbia River & RICHPS1 HRM46.4 & B0WB77 & 36419 & 1 \\
\hline Columbia River & RICHPS1 HRM46.4 & B0YCC9 & 36697 & 1 \\
\hline Columbia River & RICHPS1 HRM46.4 & B106V4 & 36788 & 1 \\
\hline Columbia River & RICHPS1 HRM46.4 & B158M1 & 37509 & 1 \\
\hline Columbia River & RICHPS1 HRM46.4 & B109D3 & 36788 & 1 \\
\hline Columbia River & RICHPS1 HRM46.4 & B11117 & 36865 & 1 \\
\hline Columbia River & RICHPS1 HRM46.4 & B147T2 & 37341 & 1 \\
\hline Columbia River & RICHPS1 HRM46.4 & B164K0 & 37600 & 1 \\
\hline Columbia River & RICHPS1 HRM46.4 & B0WB29 & 36419 & 1 \\
\hline Columbia River & RICHPS1 HRM46.4 & B171Y5 & 37782 & 1 \\
\hline Columbia River & RICHPS1 HRM46.4 & B0V208 & 36325 & 1 \\
\hline Columbia River & RICHPS1 HRM46.4 & BOV209 & 36325 & 1 \\
\hline Columbia River & RICHPS1 HRM46.4 & B0XRV7 & 36613 & 1 \\
\hline Columbia River & RICHPS1 HRM46.4 & B158R2 & 37509 & 1 \\
\hline Columbia River & RICHPS1 HRM46.4 & B11231 & 36865 & 1 \\
\hline Columbia River & RICHPS1 HRM46.4 & B12TC8 & 37147 & 1 \\
\hline Columbia River & RICHPS1 HRM46.4 & B106M9 & 36788 & 1 \\
\hline Columbia River & RICHPS1 HRM46.4 & B147T7 & 37341 & 1 \\
\hline Columbia River & RICHPS1 HRM46.4 & B146X4 & 37341 & 1 \\
\hline Columbia River & RICHPS1 HRM46.4 & B171W5 & 37782 & 1 \\
\hline Columbia River & RICHPS1 HRM46.4 & B0XRK6 & 36613 & 1 \\
\hline Columbia River & RICHPS1 HRM46.4 & BOXRKO & 36613 & 1 \\
\hline Columbia River & RICHPS1 HRM46.4 & B14RW8 & 37418 & 1 \\
\hline Columbia River & RICHPS1 HRM46.4 & B17CN3 & 37873 & 1 \\
\hline Columbia River & RICHPS1 HRM46.4 & B0V230 & 36325 & 1 \\
\hline Columbia River & RICHPS1 HRM46.4 & B0V235 & 36325 & 1 \\
\hline Columbia River & RICHPS1 HRM46.4 & B15981 & 37509 & 1 \\
\hline Columbia River & RICHPS1 HRM46.4 & B171V3 & 37782 & 1 \\
\hline Columbia River & RICHPS1 HRM46.4 & B12505 & 37054 & 1 \\
\hline Columbia River & RICHPS1 HRM46.4 & B0X3X6 & 36500 & 1 \\
\hline Columbia River & RICHPS1 HRM46.4 & B17CD0 & 37873 & 1 \\
\hline Columbia River & RICHPS1 HRM46.4 & B13LF2 & 37229 & 1 \\
\hline Columbia River & RICHPS1 HRM46.4 & B16L96 & 37705 & 1 \\
\hline Columbia River & RICHPS1 HRM46.4 & B13LT5 & 37229 & 1 \\
\hline Columbia River & RICHPS1 HRM46.4 & B17277 & 37782 & 1 \\
\hline Columbia River & RICHPS1 HRM46.4 & B16346 & 37600 & 1 \\
\hline Columbia River & RICHPS1 HRM46.4 & B14T00 & 37418 & 1 \\
\hline Columbia River & RICHPS1 HRM46.4 & B13LF7 & 37229 & 1 \\
\hline
\end{tabular}


Table B-2

\section{Summary Table of Segment 2 Surface Water Samples By Area, Site, Date, and Category Columbia River Component - Existing Sample Data Evaluation and Scoping Report}

\begin{tabular}{|c|c|c|c|c|}
\hline Sample Area & Sample Site & Sample ID & Sample Date & Category \\
\hline Columbia River & RICHPS1 HRM46.4 & B16341 & 37600 & 1 \\
\hline Columbia River & RICHPS1 HRM46.4 & B0X3Y6 & 36500 & 1 \\
\hline Columbia River & RICHPS1 HRM46.4 & BOYCJ7 & 36697 & 1 \\
\hline Columbia River & RICHPS1 HRM46.4 & $\mathrm{B} 0 \times 3 \times 1$ & 36500 & 1 \\
\hline Columbia River & RICHPS1 HRM46.4 & B16L89 & 37705 & 1 \\
\hline Columbia River & RICHPS1 HRM46.4 & B121R8 & 37028 & 1 \\
\hline Columbia River & RICHPS1 HRM46.4 & BOWCB4 & 36419 & 1 \\
\hline Columbia River & RICHPS1 HRM46.4 & B10737 & 36788 & 1 \\
\hline Columbia River & RICHPS1 HRM46.4 & B0X3W4 & 36500 & 1 \\
\hline Columbia River & RICHPS1 HRM46.4 & B11H38 & 36948 & 1 \\
\hline Columbia River & RICHPS1 HRM46.4 & BOXRN2 & 36613 & 1 \\
\hline Columbia River & RICHPS1 HRM46.4 & B11J30 & 36948 & 1 \\
\hline Columbia River & RICHPS1 HRM46.4 & B16LB1 & 37705 & 1 \\
\hline Columbia River & RICHPS1 HRM46.4 & $\mathrm{B} 11 \mathrm{H} 43$ & 36948 & 1 \\
\hline Columbia River & RICHPS1 HRM46.4 & B0X445 & 36500 & 1 \\
\hline Columbia River & RICHPS1 HRM46.4 & BOYCF1 & 36697 & 1 \\
\hline Columbia River & RICHPS1 HRM46.4 & B158F6 & 37509 & 1 \\
\hline Columbia River & RICHPS1 HRM46.4 & B14RT7 & 37418 & 1 \\
\hline Columbia River & RICHPS1 HRM46.4 & B14RV8 & 37418 & 1 \\
\hline Columbia River & RICHPS1 HRM46.4 & B16LC1 & 37705 & 1 \\
\hline Columbia River & RICHPS1 HRM46.4 & B12VL3 & 37147 & 1 \\
\hline Columbia River & RICHPS1 HRM46.4 & B106Y4 & 36788 & 1 \\
\hline Columbia River & RICHPS1 HRM46.4 & B16356 & 37600 & 1 \\
\hline Columbia River & RICHPS1 HRM46.4 & B17CK2 & 37873 & 1 \\
\hline Columbia River & RICHPS1 HRM46.4 & B1B6Y3 & 38245 & 1 \\
\hline Columbia River & RICHPS1 HRM46.4 & B183R6 & 37963 & 1 \\
\hline Columbia River & RICHPS1 HRM46.4 & B183Т1 & 37963 & 1 \\
\hline Columbia River & RICHPS1 HRM46.4 & B1BX50 & 38340 & 1 \\
\hline Columbia River & RICHPS1 HRM46.4 & B1BW42 & 38340 & 1 \\
\hline Columbia River & RICHPS1 HRM46.4 & B1B757 & 38245 & 1 \\
\hline Columbia River & RICHPS1 HRM46.4 & B183P9 & 37963 & 1 \\
\hline Columbia River & RICHPS1 HRM46.4 & B1B726 & 38245 & 1 \\
\hline Columbia River & RICHPS1 HRM46.4 & B184P9 & 37963 & 1 \\
\hline Columbia River & RICHPS1 HRM46.4 & В18TH9 & 38076 & 1 \\
\hline Columbia River & RICHPS1 HRM46.4 & B18V19 & 38076 & 1 \\
\hline Columbia River & RICHPS1 HRM46.4 & B183V1 & 37963 & 1 \\
\hline Columbia River & RICHPS1 HRM46.4 & B18TF5 & 38076 & 1 \\
\hline Columbia River & RICHPS1 HRM46.4 & B1BW37 & 38340 & 1 \\
\hline Columbia River & RICHPS1 HRM46.4 & B19J70 & 38162 & 1 \\
\hline Columbia River & RICHPS1 HRM46.4 & B1B450 & 38245 & 1 \\
\hline Columbia River & RICHPS1 HRM46.4 & B17HK9 & 37873 & 1 \\
\hline Columbia River & RICHPS1 HRM46.4 & B19HN8 & 38162 & 1 \\
\hline Columbia River & RICHPS10HRM46.4 & B124Y8 & 37054 & 1 \\
\hline Columbia River & RICHPS10HRM46.4 & B147V2 & 37341 & 1 \\
\hline Columbia River & RICHPS10HRM46.4 & BOYCK2 & 36697 & 1 \\
\hline Columbia River & RICHPS10HRM46.4 & B0XRV6 & 36613 & 1 \\
\hline
\end{tabular}


Table B-2

\section{Summary Table of Segment 2 Surface Water Samples By Area, Site, Date, and Category Columbia River Component - Existing Sample Data Evaluation and Scoping Report}

\begin{tabular}{|c|c|c|c|c|}
\hline Sample Area & Sample Site & Sample ID & Sample Date & Category \\
\hline Columbia River & RICHPS10HRM46.4 & B158M6 & 37509 & 1 \\
\hline Columbia River & RICHPS10HRM46.4 & B11236 & 36865 & 1 \\
\hline Columbia River & RICHPS10HRM46.4 & B16351 & 37600 & 1 \\
\hline Columbia River & RICHPS10HRM46.4 & B0WCB5 & 36419 & 1 \\
\hline Columbia River & RICHPS10HRM46.4 & B12530 & 37054 & 1 \\
\hline Columbia River & RICHPS10HRM46.4 & B11J35 & 36948 & 1 \\
\hline Columbia River & RICHPS10HRM46.4 & В11H36 & 36948 & 1 \\
\hline Columbia River & RICHPS10HRM46.4 & B16KC6 & 37705 & 1 \\
\hline Columbia River & RICHPS10HRM46.4 & B0YCD4 & 36697 & 1 \\
\hline Columbia River & RICHPS10HRM46.4 & B14T05 & 37418 & 1 \\
\hline Columbia River & RICHPS10HRM46.4 & B0YCB5 & 36697 & 1 \\
\hline Columbia River & RICHPS10HRM46.4 & B13LH2 & 37229 & 1 \\
\hline Columbia River & RICHPS10HRM46.4 & B12VL8 & 37149 & 1 \\
\hline Columbia River & RICHPS10HRM46.4 & B164K5 & 37600 & 1 \\
\hline Columbia River & RICHPS10HRM46.4 & B17CN8 & 37873 & 1 \\
\hline Columbia River & RICHPS10HRM46.4 & B171V8 & 37782 & 1 \\
\hline Columbia River & RICHPS10HRM46.4 & B13LF0 & 37229 & 1 \\
\hline Columbia River & RICHPS10HRM46.4 & B16361 & 37600 & 1 \\
\hline Columbia River & RICHPS10HRM46.4 & BOYCF6 & 36697 & 1 \\
\hline Columbia River & RICHPS10HRM46.4 & B13LJ2 & 37229 & 1 \\
\hline Columbia River & RICHPS10HRM46.4 & B16339 & 37600 & 1 \\
\hline Columbia River & RICHPS10HRM46.4 & B11H58 & 36948 & 1 \\
\hline Columbia River & RICHPS10HRM46.4 & B158R7 & 37509 & 1 \\
\hline Columbia River & RICHPS10HRM46.4 & B11H48 & 36948 & 1 \\
\hline Columbia River & RICHPS10HRM46.4 & B12TK1 & 37147 & 1 \\
\hline Columbia River & RICHPS10HRM46.4 & B17276 & 37782 & 1 \\
\hline Columbia River & RICHPS10HRM46.4 & BoV244 & 36325 & 1 \\
\hline Columbia River & RICHPS10HRM46.4 & B11122 & 36865 & 1 \\
\hline Columbia River & RICHPS10HRM46.4 & B17CK7 & 37873 & 1 \\
\hline Columbia River & RICHPS10HRM46.4 & B13LT4 & 37229 & 1 \\
\hline Columbia River & RICHPS10HRM46.4 & B0V255 & 36325 & 1 \\
\hline Columbia River & RICHPS10HRM46.4 & B106Y9 & 36788 & 1 \\
\hline Columbia River & RICHPS10HRM46.4 & B12510 & 37054 & 1 \\
\hline Columbia River & RICHPS10HRM46.4 & B14RV2 & 37418 & 1 \\
\hline Columbia River & RICHPS10HRM46.4 & B12TD3 & 37147 & 1 \\
\hline Columbia River & RICHPS10HRM46.4 & B106M8 & 36788 & 1 \\
\hline Columbia River & RICHPS10HRM46.4 & B124X0 & 37054 & 1 \\
\hline Columbia River & RICHPS10HRM46.4 & B16LB6 & 37705 & 1 \\
\hline Columbia River & RICHPS10HRM46.4 & B0X450 & 36500 & 1 \\
\hline Columbia River & RICHPS10HRM46.4 & B15986 & 37509 & 1 \\
\hline Columbia River & RICHPS10HRM46.4 & B146X3 & 37341 & 1 \\
\hline Columbia River & RICHPS10HRM46.4 & B16L94 & 37705 & 1 \\
\hline Columbia River & RICHPS10HRM46.4 & B106V9 & 36788 & 1 \\
\hline Columbia River & RICHPS10HRM46.4 & В12Т83 & 37147 & 1 \\
\hline Columbia River & RICHPS10HRM46.4 & B0X3W9 & 36500 & 1 \\
\hline Columbia River & RICHPS10HRM46.4 & B0X401 & 36500 & 1 \\
\hline
\end{tabular}


Table B-2

\section{Summary Table of Segment 2 Surface Water Samples By Area, Site, Date, and Category Columbia River Component - Existing Sample Data Evaluation and Scoping Report}

\begin{tabular}{|c|c|c|c|c|}
\hline Sample Area & Sample Site & Sample ID & Sample Date & Category \\
\hline Columbia River & RICHPS10HRM46.4 & B14RX3 & 37418 & 1 \\
\hline Columbia River & RICHPS10HRM46.4 & B0X3Y1 & 36500 & 1 \\
\hline Columbia River & RICHPS10HRM46.4 & B17CD5 & 37873 & 1 \\
\hline Columbia River & RICHPS10HRM46.4 & $\mathrm{B} 158 \mathrm{H} 1$ & 37509 & 1 \\
\hline Columbia River & RICHPS10HRM46.4 & B0XRN7 & 36613 & 1 \\
\hline Columbia River & RICHPS10HRM46.4 & B0WB82 & 36419 & 1 \\
\hline Columbia River & RICHPS10HRM46.4 & B0XRJ8 & 36613 & 1 \\
\hline Columbia River & RICHPS10HRM46.4 & B17200 & 37782 & 1 \\
\hline Columbia River & RICHPS10HRM46.4 & B11144 & 36865 & 1 \\
\hline Columbia River & RICHPS10HRM46.4 & B10742 & 36788 & 1 \\
\hline Columbia River & RICHPS10HRM46.4 & B0V227 & 36325 & 1 \\
\hline Columbia River & RICHPS10HRM46.4 & B0V226 & 36325 & 1 \\
\hline Columbia River & RICHPS10HRM46.4 & B0WB34 & 36419 & 1 \\
\hline Columbia River & RICHPS10HRM46.4 & B0XRL1 & 36613 & 1 \\
\hline Columbia River & RICHPS10HRM46.4 & $\mathrm{B} 171 \mathrm{X0}$ & 37782 & 1 \\
\hline Columbia River & RICHPS10HRM46.4 & B14RW3 & 37418 & 1 \\
\hline Columbia River & RICHPS10HRM46.4 & B11134 & 36865 & 1 \\
\hline Columbia River & RICHPS10HRM46.4 & B16LC6 & 37705 & 1 \\
\hline Columbia River & RICHPS10HRM46.4 & B1BW47 & 38340 & 1 \\
\hline Columbia River & RICHPS10HRM46.4 & B1B455 & 38245 & 1 \\
\hline Columbia River & RICHPS10HRM46.4 & B18V24 & 38076 & 1 \\
\hline Columbia River & RICHPS10HRM46.4 & B18TJ4 & 38076 & 1 \\
\hline Columbia River & RICHPS10HRM46.4 & B1B731 & 38245 & 1 \\
\hline Columbia River & RICHPS10HRM46.4 & B1B762 & 38245 & 1 \\
\hline Columbia River & RICHPS10HRM46.4 & B183R4 & 37963 & 1 \\
\hline Columbia River & RICHPS10HRM46.4 & В183Т6 & 37963 & 1 \\
\hline Columbia River & RICHPS10HRM46.4 & B184R4 & 37963 & 1 \\
\hline Columbia River & RICHPS10HRM46.4 & B1BX55 & 38340 & 1 \\
\hline Columbia River & RICHPS10HRM46.4 & B183V6 & 37963 & 1 \\
\hline Columbia River & RICHPS10HRM46.4 & B19J75 & 38162 & 1 \\
\hline Columbia River & RICHPS10HRM46.4 & B19HP4 & 38162 & 1 \\
\hline Columbia River & RICHPS10HRM46.4 & B17HL4 & 37873 & 1 \\
\hline Columbia River & RICHPS10HRM46.4 & B1B6Y8 & 38245 & 1 \\
\hline Columbia River & RICHPS2 HRM46.4 & B16L90 & 37705 & 1 \\
\hline Columbia River & RICHPS2 HRM46.4 & B15982 & 37509 & 1 \\
\hline Columbia River & RICHPS2 HRM46.4 & B16347 & 37600 & 1 \\
\hline Columbia River & RICHPS2 HRM46.4 & B0X3W5 & 36500 & 1 \\
\hline Columbia River & RICHPS2 HRM46.4 & B14RW9 & 37418 & 1 \\
\hline Columbia River & RICHPS2 HRM46.4 & B164K1 & 37600 & 1 \\
\hline Columbia River & RICHPS2 HRM46.4 & BOYCJ8 & 36697 & 1 \\
\hline Columbia River & RICHPS2 HRM46.4 & B0WB30 & 36419 & 1 \\
\hline Columbia River & RICHPS2 HRM46.4 & B0X3X7 & 36500 & 1 \\
\hline Columbia River & RICHPS2 HRM46.4 & B17CD1 & 37873 & 1 \\
\hline Columbia River & RICHPS2 HRM46.4 & В12ТC9 & 37147 & 1 \\
\hline Columbia River & RICHPS2 HRM46.4 & B16LB2 & 37705 & 1 \\
\hline Columbia River & RICHPS2 HRM46.4 & B17278 & 37782 & 1 \\
\hline
\end{tabular}


Table B-2

\section{Summary Table of Segment 2 Surface Water Samples By Area, Site, Date, and Category Columbia River Component - Existing Sample Data Evaluation and Scoping Report}

\begin{tabular}{|c|c|c|c|c|}
\hline Sample Area & Sample Site & Sample ID & Sample Date & Category \\
\hline Columbia River & RICHPS2 HRM46.4 & B16LC2 & 37705 & 1 \\
\hline Columbia River & RICHPS2 HRM46.4 & $\mathrm{B} 158 \mathrm{~F} 7$ & 37509 & 1 \\
\hline Columbia River & RICHPS2 HRM46.4 & B12526 & 37054 & 1 \\
\hline Columbia River & RICHPS2 HRM46.4 & В14T01 & 37418 & 1 \\
\hline Columbia River & RICHPS2 HRM46.4 & B11J31 & 36948 & 1 \\
\hline Columbia River & RICHPS2 HRM46.4 & B14RT8 & 37418 & 1 \\
\hline Columbia River & RICHPS2 HRM46.4 & B11232 & 36865 & 1 \\
\hline Columbia River & RICHPS2 HRM46.4 & BowCB6 & 36419 & 1 \\
\hline Columbia River & RICHPS2 HRM46.4 & B121R9 & 37028 & 1 \\
\hline Columbia River & RICHPS2 HRM46.4 & B106Y5 & 36788 & 1 \\
\hline Columbia River & RICHPS2 HRM46.4 & B16357 & 37600 & 1 \\
\hline Columbia River & RICHPS2 HRM46.4 & B146X5 & 37341 & 1 \\
\hline Columbia River & RICHPS2 HRM46.4 & B10738 & 36788 & 1 \\
\hline Columbia River & RICHPS2 HRM46.4 & B124W6 & 37054 & 1 \\
\hline Columbia River & RICHPS2 HRM46.4 & B14RV9 & 37418 & 1 \\
\hline Columbia River & RICHPS2 HRM46.4 & B12506 & 37054 & 1 \\
\hline Columbia River & RICHPS2 HRM46.4 & B12VL4 & 37147 & 1 \\
\hline Columbia River & RICHPS2 HRM46.4 & B11130 & 36865 & 1 \\
\hline Columbia River & RICHPS2 HRM46.4 & B16KC8 & 37705 & 1 \\
\hline Columbia River & RICHPS2 HRM46.4 & B0XRJ4 & 36613 & 1 \\
\hline Columbia River & RICHPS2 HRM46.4 & B13LH8 & 37229 & 1 \\
\hline Columbia River & RICHPS2 HRM46.4 & B12TJ7 & 37147 & 1 \\
\hline Columbia River & RICHPS2 HRM46.4 & BoV236 & 36325 & 1 \\
\hline Columbia River & RICHPS2 HRM46.4 & B171V4 & 37782 & 1 \\
\hline Columbia River & RICHPS2 HRM46.4 & B11118 & 36865 & 1 \\
\hline Columbia River & RICHPS2 HRM46.4 & B11140 & 36865 & 1 \\
\hline Columbia River & RICHPS2 HRM46.4 & B0YCB1 & 36697 & 1 \\
\hline Columbia River & RICHPS2 HRM46.4 & B0XRK7 & 36613 & 1 \\
\hline Columbia River & RICHPS2 HRM46.4 & B171Y6 & 37782 & 1 \\
\hline Columbia River & RICHPS2 HRM46.4 & B158R3 & 37509 & 1 \\
\hline Columbia River & RICHPS2 HRM46.4 & B147T8 & 37341 & 1 \\
\hline Columbia River & RICHPS2 HRM46.4 & Bov211 & 36325 & 1 \\
\hline Columbia River & RICHPS2 HRM46.4 & BOXRV8 & 36613 & 1 \\
\hline Columbia River & RICHPS2 HRM46.4 & BoV210 & 36325 & 1 \\
\hline Columbia River & RICHPS2 HRM46.4 & B124Y4 & 37054 & 1 \\
\hline Columbia River & RICHPS2 HRM46.4 & B17CN4 & 37873 & 1 \\
\hline Columbia River & RICHPS2 HRM46.4 & B11H44 & 36948 & 1 \\
\hline Columbia River & RICHPS2 HRM46.4 & B11H54 & 36948 & 1 \\
\hline Columbia River & RICHPS2 HRM46.4 & B13LD6 & 37229 & 1 \\
\hline Columbia River & RICHPS2 HRM46.4 & B0X3Y7 & 36500 & 1 \\
\hline Columbia River & RICHPS2 HRM46.4 & B13LT6 & 37229 & 1 \\
\hline Columbia River & RICHPS2 HRM46.4 & BOYCF2 & 36697 & 1 \\
\hline Columbia River & RICHPS2 HRM46.4 & В17CK3 & 37873 & 1 \\
\hline Columbia River & RICHPS2 HRM46.4 & BOXRN3 & 36613 & 1 \\
\hline Columbia River & RICHPS2 HRM46.4 & B106V5 & 36788 & 1 \\
\hline Columbia River & RICHPS2 HRM46.4 & B0V247 & 36325 & 1 \\
\hline
\end{tabular}


Table B-2

\section{Summary Table of Segment 2 Surface Water Samples By Area, Site, Date, and Category Columbia River Component - Existing Sample Data Evaluation and Scoping Report}

\begin{tabular}{|c|c|c|c|c|}
\hline Sample Area & Sample Site & Sample ID & Sample Date & Category \\
\hline Columbia River & RICHPS2 HRM46.4 & B0X446 & 36500 & 1 \\
\hline Columbia River & RICHPS2 HRM46.4 & В12Т79 & 37147 & 1 \\
\hline Columbia River & RICHPS2 HRM46.4 & B109D4 & 36788 & 1 \\
\hline Columbia River & RICHPS2 HRM46.4 & B11H32 & 36948 & 1 \\
\hline Columbia River & RICHPS2 HRM46.4 & BOYCDO & 36697 & 1 \\
\hline Columbia River & RICHPS2 HRM46.4 & B158M2 & 37509 & 1 \\
\hline Columbia River & RICHPS2 HRM46.4 & B0WB78 & 36419 & 1 \\
\hline Columbia River & RICHPS2 HRM46.4 & B13LF8 & 37229 & 1 \\
\hline Columbia River & RICHPS2 HRM46.4 & B106N0 & 36788 & 1 \\
\hline Columbia River & RICHPS2 HRM46.4 & B16335 & 37600 & 1 \\
\hline Columbia River & RICHPS2 HRM46.4 & B171W6 & 37782 & 1 \\
\hline Columbia River & RICHPS2 HRM46.4 & B1BW43 & 38340 & 1 \\
\hline Columbia River & RICHPS2 HRM46.4 & B17HLO & 37873 & 1 \\
\hline Columbia River & RICHPS2 HRM46.4 & B1BX51 & 38340 & 1 \\
\hline Columbia River & RICHPS2 HRM46.4 & B184R0 & 37963 & 1 \\
\hline Columbia River & RICHPS2 HRM46.4 & B183V2 & 37963 & 1 \\
\hline Columbia River & RICHPS2 HRM46.4 & B18V20 & 38076 & 1 \\
\hline Columbia River & RICHPS2 HRM46.4 & B1B758 & 38245 & 1 \\
\hline Columbia River & RICHPS2 HRM46.4 & B1B451 & 38245 & 1 \\
\hline Columbia River & RICHPS2 HRM46.4 & B1B727 & 38245 & 1 \\
\hline Columbia River & RICHPS2 HRM46.4 & B183R0 & 37963 & 1 \\
\hline Columbia River & RICHPS2 HRM46.4 & B18TJ0 & 38076 & 1 \\
\hline Columbia River & RICHPS2 HRM46.4 & В183Т2 & 37963 & 1 \\
\hline Columbia River & RICHPS2 HRM46.4 & B1B6Y4 & 38245 & 1 \\
\hline Columbia River & RICHPS2 HRM46.4 & B19HN9 & 38162 & 1 \\
\hline Columbia River & RICHPS2 HRM46.4 & B19J71 & 38162 & 1 \\
\hline Columbia River & RICHPS3 HRM46.4 & B11119 & 36865 & 1 \\
\hline Columbia River & RICHPS3 HRM46.4 & B0WB79 & 36419 & 1 \\
\hline Columbia River & RICHPS3 HRM46.4 & B13LF9 & 37229 & 1 \\
\hline Columbia River & RICHPS3 HRM46.4 & BOXRV9 & 36613 & 1 \\
\hline Columbia River & RICHPS3 HRM46.4 & B13LH9 & 37229 & 1 \\
\hline Columbia River & RICHPS3 HRM46.4 & BOXRN4 & 36613 & 1 \\
\hline Columbia River & RICHPS3 HRM46.4 & B13LT7 & 37229 & 1 \\
\hline Columbia River & RICHPS3 HRM46.4 & B0XRK8 & 36613 & 1 \\
\hline Columbia River & RICHPS3 HRM46.4 & B146X6 & 37341 & 1 \\
\hline Columbia River & RICHPS3 HRM46.4 & B0XRJ5 & 36613 & 1 \\
\hline Columbia River & RICHPS3 HRM46.4 & B13LD7 & 37229 & 1 \\
\hline Columbia River & RICHPS3 HRM46.4 & B0X447 & 36500 & 1 \\
\hline Columbia River & RICHPS3 HRM46.4 & B0YCD1 & 36697 & 1 \\
\hline Columbia River & RICHPS3 HRM46.4 & B14RT9 & 37418 & 1 \\
\hline Columbia River & RICHPS3 HRM46.4 & B124Y5 & 37054 & 1 \\
\hline Columbia River & RICHPS3 HRM46.4 & B14RW0 & 37418 & 1 \\
\hline Columbia River & RICHPS3 HRM46.4 & B0X3X8 & 36500 & 1 \\
\hline Columbia River & RICHPS3 HRM46.4 & $\mathrm{B} 14 \mathrm{R} \times 0$ & 37418 & 1 \\
\hline Columbia River & RICHPS3 HRM46.4 & B0X3W6 & 36500 & 1 \\
\hline Columbia River & RICHPS3 HRM46.4 & В14Т02 & 37418 & 1 \\
\hline
\end{tabular}


Table B-2

\section{Summary Table of Segment 2 Surface Water Samples By Area, Site, Date, and Category Columbia River Component - Existing Sample Data Evaluation and Scoping Report}

\begin{tabular}{|c|c|c|c|c|}
\hline Sample Area & Sample Site & Sample ID & Sample Date & Category \\
\hline Columbia River & RICHPS3 HRM46.4 & BOWCB7 & 36419 & 1 \\
\hline Columbia River & RICHPS3 HRM46.4 & B158F8 & 37509 & 1 \\
\hline Columbia River & RICHPS3 HRM46.4 & B147T9 & 37341 & 1 \\
\hline Columbia River & RICHPS3 HRM46.4 & B106Y6 & 36788 & 1 \\
\hline Columbia River & RICHPS3 HRM46.4 & B11131 & 36865 & 1 \\
\hline Columbia River & RICHPS3 HRM46.4 & B11141 & 36865 & 1 \\
\hline Columbia River & RICHPS3 HRM46.4 & B11233 & 36865 & 1 \\
\hline Columbia River & RICHPS3 HRM46.4 & B11H33 & 36948 & 1 \\
\hline Columbia River & RICHPS3 HRM46.4 & B11H45 & 36948 & 1 \\
\hline Columbia River & RICHPS3 HRM46.4 & B11H55 & 36948 & 1 \\
\hline Columbia River & RICHPS3 HRM46.4 & B11J32 & 36948 & 1 \\
\hline Columbia River & RICHPS3 HRM46.4 & B121T0 & 37028 & 1 \\
\hline Columbia River & RICHPS3 HRM46.4 & B124W7 & 37054 & 1 \\
\hline Columbia River & RICHPS3 HRM46.4 & B0YCB2 & 36697 & 1 \\
\hline Columbia River & RICHPS3 HRM46.4 & B12507 & 37054 & 1 \\
\hline Columbia River & RICHPS3 HRM46.4 & В0X3Y8 & 36500 & 1 \\
\hline Columbia River & RICHPS3 HRM46.4 & B12527 & 37054 & 1 \\
\hline Columbia River & RICHPS3 HRM46.4 & B106V6 & 36788 & 1 \\
\hline Columbia River & RICHPS3 HRM46.4 & В12Т80 & 37147 & 1 \\
\hline Columbia River & RICHPS3 HRM46.4 & B106N1 & 36788 & 1 \\
\hline Columbia River & RICHPS3 HRM46.4 & B12TD0 & 37147 & 1 \\
\hline Columbia River & RICHPS3 HRM46.4 & BOYCJ9 & 36697 & 1 \\
\hline Columbia River & RICHPS3 HRM46.4 & В12ТJ8 & 37147 & 1 \\
\hline Columbia River & RICHPS3 HRM46.4 & BOYCF3 & 36697 & 1 \\
\hline Columbia River & RICHPS3 HRM46.4 & B12VL5 & 37147 & 1 \\
\hline Columbia River & RICHPS3 HRM46.4 & B10739 & 36788 & 1 \\
\hline Columbia River & RICHPS3 HRM46.4 & B16KC9 & 37705 & 1 \\
\hline Columbia River & RICHPS3 HRM46.4 & B0V237 & 36325 & 1 \\
\hline Columbia River & RICHPS3 HRM46.4 & B171W7 & 37782 & 1 \\
\hline Columbia River & RICHPS3 HRM46.4 & B17279 & 37782 & 1 \\
\hline Columbia River & RICHPS3 HRM46.4 & B158M3 & 37509 & 1 \\
\hline Columbia River & RICHPS3 HRM46.4 & B17CD2 & 37873 & 1 \\
\hline Columbia River & RICHPS3 HRM46.4 & B16LC3 & 37705 & 1 \\
\hline Columbia River & RICHPS3 HRM46.4 & B16LB3 & 37705 & 1 \\
\hline Columbia River & RICHPS3 HRM46.4 & B0V248 & 36325 & 1 \\
\hline Columbia River & RICHPS3 HRM46.4 & B16L91 & 37705 & 1 \\
\hline Columbia River & RICHPS3 HRM46.4 & B171Y7 & 37782 & 1 \\
\hline Columbia River & RICHPS3 HRM46.4 & B17CK4 & 37873 & 1 \\
\hline Columbia River & RICHPS3 HRM46.4 & B171V5 & 37782 & 1 \\
\hline Columbia River & RICHPS3 HRM46.4 & B164K2 & 37600 & 1 \\
\hline Columbia River & RICHPS3 HRM46.4 & B17CN5 & 37873 & 1 \\
\hline Columbia River & RICHPS3 HRM46.4 & B0V213 & 36325 & 1 \\
\hline Columbia River & RICHPS3 HRM46.4 & BoV212 & 36325 & 1 \\
\hline Columbia River & RICHPS3 HRM46.4 & B16358 & 37600 & 1 \\
\hline Columbia River & RICHPS3 HRM46.4 & B16348 & 37600 & 1 \\
\hline Columbia River & RICHPS3 HRM46.4 & B0WB31 & 36419 & 1 \\
\hline
\end{tabular}


Table B-2

\section{Summary Table of Segment 2 Surface Water Samples By Area, Site, Date, and Category Columbia River Component - Existing Sample Data Evaluation and Scoping Report}

\begin{tabular}{|c|c|c|c|c|}
\hline Sample Area & Sample Site & Sample ID & Sample Date & Category \\
\hline Columbia River & RICHPS3 HRM46.4 & B15983 & 37509 & 1 \\
\hline Columbia River & RICHPS3 HRM46.4 & B158R4 & 37509 & 1 \\
\hline Columbia River & RICHPS3 HRM46.4 & B16336 & 37600 & 1 \\
\hline Columbia River & RICHPS3 HRM46.4 & B1B6Y5 & 38245 & 1 \\
\hline Columbia River & RICHPS3 HRM46.4 & B17HL1 & 37873 & 1 \\
\hline Columbia River & RICHPS3 HRM46.4 & В183Т3 & 37963 & 1 \\
\hline Columbia River & RICHPS3 HRM46.4 & B184R1 & 37963 & 1 \\
\hline Columbia River & RICHPS3 HRM46.4 & B1B728 & 38245 & 1 \\
\hline Columbia River & RICHPS3 HRM46.4 & B18TJ1 & 38076 & 1 \\
\hline Columbia River & RICHPS3 HRM46.4 & B18V21 & 38076 & 1 \\
\hline Columbia River & RICHPS3 HRM46.4 & B183V3 & 37963 & 1 \\
\hline Columbia River & RICHPS3 HRM46.4 & B1BX52 & 38340 & 1 \\
\hline Columbia River & RICHPS3 HRM46.4 & В19HР0 & 38162 & 1 \\
\hline Columbia River & RICHPS3 HRM46.4 & B19J72 & 38162 & 1 \\
\hline Columbia River & RICHPS3 HRM46.4 & B1BW44 & 38340 & 1 \\
\hline Columbia River & RICHPS3 HRM46.4 & B183R1 & 37963 & 1 \\
\hline Columbia River & RICHPS3 HRM46.4 & B1B452 & 38245 & 1 \\
\hline Columbia River & RICHPS3 HRM46.4 & B1B759 & 38245 & 1 \\
\hline Columbia River & RICHPS4 HRM46.4 & BoV249 & 36325 & 1 \\
\hline Columbia River & RICHPS4 HRM46.4 & B0V214 & 36325 & 1 \\
\hline Columbia River & RICHPS4 HRM46.4 & B0V238 & 36325 & 1 \\
\hline Columbia River & RICHPS4 HRM46.4 & B0V215 & 36325 & 1 \\
\hline Columbia River & RICHPS5 HRM46.4 & B124W8 & 37054 & 1 \\
\hline Columbia River & RICHPS5 HRM46.4 & B171Y8 & 37782 & 1 \\
\hline Columbia River & RICHPS5 HRM46.4 & B11120 & 36865 & 1 \\
\hline Columbia River & RICHPS5 HRM46.4 & B12508 & 37054 & 1 \\
\hline Columbia River & RICHPS5 HRM46.4 & B17280 & 37782 & 1 \\
\hline Columbia River & RICHPS5 HRM46.4 & B11132 & 36865 & 1 \\
\hline Columbia River & RICHPS5 HRM46.4 & B12528 & 37054 & 1 \\
\hline Columbia River & RICHPS5 HRM46.4 & B17CN6 & 37873 & 1 \\
\hline Columbia River & RICHPS5 HRM46.4 & B11234 & 36865 & 1 \\
\hline Columbia River & RICHPS5 HRM46.4 & B11J33 & 36948 & 1 \\
\hline Columbia River & RICHPS5 HRM46.4 & B11142 & 36865 & 1 \\
\hline Columbia River & RICHPS5 HRM46.4 & B17CD3 & 37873 & 1 \\
\hline Columbia River & RICHPS5 HRM46.4 & B121T1 & 37028 & 1 \\
\hline Columbia River & RICHPS5 HRM46.4 & B11H34 & 36948 & 1 \\
\hline Columbia River & RICHPS5 HRM46.4 & B17CK5 & 37873 & 1 \\
\hline Columbia River & RICHPS5 HRM46.4 & B11H56 & 36948 & 1 \\
\hline Columbia River & RICHPS5 HRM46.4 & B11H46 & 36948 & 1 \\
\hline Columbia River & RICHPS5 HRM46.4 & B124Y6 & 37054 & 1 \\
\hline Columbia River & RICHPS5 HRM46.4 & B13LT8 & 37229 & 1 \\
\hline Columbia River & RICHPS5 HRM46.4 & B158F9 & 37509 & 1 \\
\hline Columbia River & RICHPS5 HRM46.4 & B158M4 & 37509 & 1 \\
\hline Columbia River & RICHPS5 HRM46.4 & В14Т03 & 37418 & 1 \\
\hline Columbia River & RICHPS5 HRM46.4 & B158R5 & 37509 & 1 \\
\hline Columbia River & RICHPS5 HRM46.4 & B14RX1 & 37418 & 1 \\
\hline
\end{tabular}


Table B-2

\section{Summary Table of Segment 2 Surface Water Samples By Area, Site, Date, and Category Columbia River Component - Existing Sample Data Evaluation and Scoping Report}

\begin{tabular}{|c|c|c|c|c|}
\hline Sample Area & Sample Site & Sample ID & Sample Date & Category \\
\hline Columbia River & RICHPS5 HRM46.4 & B15984 & 37509 & 1 \\
\hline Columbia River & RICHPS5 HRM46.4 & B14RW1 & 37418 & 1 \\
\hline Columbia River & RICHPS5 HRM46.4 & B16337 & 37600 & 1 \\
\hline Columbia River & RICHPS5 HRM46.4 & B16349 & 37600 & 1 \\
\hline Columbia River & RICHPS5 HRM46.4 & B147V0 & 37341 & 1 \\
\hline Columbia River & RICHPS5 HRM46.4 & B16359 & 37600 & 1 \\
\hline Columbia River & RICHPS5 HRM46.4 & B12TJ9 & 37147 & 1 \\
\hline Columbia River & RICHPS5 HRM46.4 & B164K3 & 37600 & 1 \\
\hline Columbia River & RICHPS5 HRM46.4 & B171W8 & 37782 & 1 \\
\hline Columbia River & RICHPS5 HRM46.4 & B16KD0 & 37705 & 1 \\
\hline Columbia River & RICHPS5 HRM46.4 & B13LJ0 & 37229 & 1 \\
\hline Columbia River & RICHPS5 HRM46.4 & B13LH0 & 37229 & 1 \\
\hline Columbia River & RICHPS5 HRM46.4 & B16L92 & 37705 & 1 \\
\hline Columbia River & RICHPS5 HRM46.4 & B13LD8 & 37229 & 1 \\
\hline Columbia River & RICHPS5 HRM46.4 & B16LB4 & 37705 & 1 \\
\hline Columbia River & RICHPS5 HRM46.4 & B12VL6 & 37147 & 1 \\
\hline Columbia River & RICHPS5 HRM46.4 & B16LC4 & 37705 & 1 \\
\hline Columbia River & RICHPS5 HRM46.4 & B14RV0 & 37418 & 1 \\
\hline Columbia River & RICHPS5 HRM46.4 & B171V6 & 37782 & 1 \\
\hline Columbia River & RICHPS5 HRM46.4 & B12TD1 & 37147 & 1 \\
\hline Columbia River & RICHPS5 HRM46.4 & В12Т81 & 37147 & 1 \\
\hline Columbia River & RICHPS5 HRM46.4 & B146X7 & 37341 & 1 \\
\hline Columbia River & RICHPS5 HRM46.4 & BOYCF4 & 36697 & 1 \\
\hline Columbia River & RICHPS5 HRM46.4 & B0X3W7 & 36500 & 1 \\
\hline Columbia River & RICHPS5 HRM46.4 & B106Y7 & 36788 & 1 \\
\hline Columbia River & RICHPS5 HRM46.4 & B0XRN5 & 36613 & 1 \\
\hline Columbia River & RICHPS5 HRM46.4 & B0WCB8 & 36419 & 1 \\
\hline Columbia River & RICHPS5 HRM46.4 & B0WB32 & 36419 & 1 \\
\hline Columbia River & RICHPS5 HRM46.4 & B106V7 & 36788 & 1 \\
\hline Columbia River & RICHPS5 HRM46.4 & B0X3X9 & 36500 & 1 \\
\hline Columbia River & RICHPS5 HRM46.4 & B106N2 & 36788 & 1 \\
\hline Columbia River & RICHPS5 HRM46.4 & В0X3Y9 & 36500 & 1 \\
\hline Columbia River & RICHPS5 HRM46.4 & BOYCKO & 36697 & 1 \\
\hline Columbia River & RICHPS5 HRM46.4 & B10740 & 36788 & 1 \\
\hline Columbia River & RICHPS5 HRM46.4 & B0V250 & 36325 & 1 \\
\hline Columbia River & RICHPS5 HRM46.4 & B0WB80 & 36419 & 1 \\
\hline Columbia River & RICHPS5 HRM46.4 & B0X448 & 36500 & 1 \\
\hline Columbia River & RICHPS5 HRM46.4 & B0YCD2 & 36697 & 1 \\
\hline Columbia River & RICHPS5 HRM46.4 & B0V239 & 36325 & 1 \\
\hline Columbia River & RICHPS5 HRM46.4 & BoV216 & 36325 & 1 \\
\hline Columbia River & RICHPS5 HRM46.4 & B0XRJ6 & 36613 & 1 \\
\hline Columbia River & RICHPS5 HRM46.4 & BOXRK9 & 36613 & 1 \\
\hline Columbia River & RICHPS5 HRM46.4 & B0YCB3 & 36697 & 1 \\
\hline Columbia River & RICHPS5 HRM46.4 & BoV217 & 36325 & 1 \\
\hline Columbia River & RICHPS5 HRM46.4 & BOXRWO & 36613 & 1 \\
\hline Columbia River & RICHPS5 HRM46.4 & B19J73 & 38162 & 1 \\
\hline
\end{tabular}


Table B-2

\section{Summary Table of Segment 2 Surface Water Samples By Area, Site, Date, and Category Columbia River Component - Existing Sample Data Evaluation and Scoping Report}

\begin{tabular}{|c|c|c|c|c|}
\hline Sample Area & Sample Site & Sample ID & Sample Date & Category \\
\hline Columbia River & RICHPS5 HRM46.4 & B17HL2 & 37873 & 1 \\
\hline Columbia River & RICHPS5 HRM46.4 & B1B6Y6 & 38245 & 1 \\
\hline Columbia River & RICHPS5 HRM46.4 & B18V22 & 38076 & 1 \\
\hline Columbia River & RICHPS5 HRM46.4 & B1B453 & 38245 & 1 \\
\hline Columbia River & RICHPS5 HRM46.4 & B19HP1 & 38162 & 1 \\
\hline Columbia River & RICHPS5 HRM46.4 & В183Т4 & 37963 & 1 \\
\hline Columbia River & RICHPS5 HRM46.4 & B18TJ2 & 38076 & 1 \\
\hline Columbia River & RICHPS5 HRM46.4 & B184R2 & 37963 & 1 \\
\hline Columbia River & RICHPS5 HRM46.4 & B1B760 & 38245 & 1 \\
\hline Columbia River & RICHPS5 HRM46.4 & B1B729 & 38245 & 1 \\
\hline Columbia River & RICHPS5 HRM46.4 & B183V4 & 37963 & 1 \\
\hline Columbia River & RICHPS5 HRM46.4 & B1BW45 & 38340 & 1 \\
\hline Columbia River & RICHPS5 HRM46.4 & B1BX53 & 38340 & 1 \\
\hline Columbia River & RICHPS5 HRM46.4 & B183R2 & 37963 & 1 \\
\hline Columbia River & RICHPS6 HRM46.4 & B0V251 & 36325 & 1 \\
\hline Columbia River & RICHPS6 HRM46.4 & Bov240 & 36325 & 1 \\
\hline Columbia River & RICHPS6 HRM46.4 & B0V218 & 36325 & 1 \\
\hline Columbia River & RICHPS6 HRM46.4 & Bov219 & 36325 & 1 \\
\hline Columbia River & RICHPS7 HRM46.4 & B14RV1 & 37418 & 1 \\
\hline Columbia River & RICHPS7 HRM46.4 & BOXRLO & 36613 & 1 \\
\hline Columbia River & RICHPS7 HRM46.4 & B0X3W8 & 36500 & 1 \\
\hline Columbia River & RICHPS7 HRM46.4 & B14RW2 & 37418 & 1 \\
\hline Columbia River & RICHPS7 HRM46.4 & B147V1 & 37341 & 1 \\
\hline Columbia River & RICHPS7 HRM46.4 & B13LT9 & 37229 & 1 \\
\hline Columbia River & RICHPS7 HRM46.4 & BOXRJ7 & 36613 & 1 \\
\hline Columbia River & RICHPS7 HRM46.4 & B0X400 & 36500 & 1 \\
\hline Columbia River & RICHPS7 HRM46.4 & B146X8 & 37341 & 1 \\
\hline Columbia River & RICHPS7 HRM46.4 & B0X449 & 36500 & 1 \\
\hline Columbia River & RICHPS7 HRM46.4 & B0X3Y0 & 36500 & 1 \\
\hline Columbia River & RICHPS7 HRM46.4 & B16KD1 & 37705 & 1 \\
\hline Columbia River & RICHPS7 HRM46.4 & B17CN7 & 37873 & 1 \\
\hline Columbia River & RICHPS7 HRM46.4 & B17CK 6 & 37873 & 1 \\
\hline Columbia River & RICHPS7 HRM46.4 & B17CD4 & 37873 & 1 \\
\hline Columbia River & RICHPS7 HRM46.4 & B0V220 & 36325 & 1 \\
\hline Columbia River & RICHPS7 HRM46.4 & B17281 & 37782 & 1 \\
\hline Columbia River & RICHPS7 HRM46.4 & B0V221 & 36325 & 1 \\
\hline Columbia River & RICHPS7 HRM46.4 & B171Y9 & 37782 & 1 \\
\hline Columbia River & RICHPS7 HRM46.4 & B171W9 & 37782 & 1 \\
\hline Columbia River & RICHPS7 HRM46.4 & B171V7 & 37782 & 1 \\
\hline Columbia River & RICHPS7 HRM46.4 & B16LC5 & 37705 & 1 \\
\hline Columbia River & RICHPS7 HRM46.4 & BoV241 & 36325 & 1 \\
\hline Columbia River & RICHPS7 HRM46.4 & $\mathrm{B} 158 \mathrm{HO}$ & 37509 & 1 \\
\hline Columbia River & RICHPS7 HRM46.4 & B16L93 & 37705 & 1 \\
\hline Columbia River & RICHPS7 HRM46.4 & $\mathrm{B} 14 \mathrm{R} \times 2$ & 37418 & 1 \\
\hline Columbia River & RICHPS7 HRM46.4 & B164K4 & 37600 & 1 \\
\hline Columbia River & RICHPS7 HRM46.4 & B16360 & 37600 & 1 \\
\hline
\end{tabular}


Table B-2

\section{Summary Table of Segment 2 Surface Water Samples By Area, Site, Date, and Category Columbia River Component - Existing Sample Data Evaluation and Scoping Report}

\begin{tabular}{|c|c|c|c|c|}
\hline Sample Area & Sample Site & Sample ID & Sample Date & Category \\
\hline Columbia River & RICHPS7 HRM46.4 & B16350 & 37600 & 1 \\
\hline Columbia River & RICHPS7 HRM46.4 & B16338 & 37600 & 1 \\
\hline Columbia River & RICHPS7 HRM46.4 & B0V252 & 36325 & 1 \\
\hline Columbia River & RICHPS7 HRM46.4 & B15985 & 37509 & 1 \\
\hline Columbia River & RICHPS7 HRM46.4 & B158R6 & 37509 & 1 \\
\hline Columbia River & RICHPS7 HRM46.4 & B158M5 & 37509 & 1 \\
\hline Columbia River & RICHPS7 HRM46.4 & B0WB33 & 36419 & 1 \\
\hline Columbia River & RICHPS7 HRM46.4 & B0WB81 & 36419 & 1 \\
\hline Columbia River & RICHPS7 HRM46.4 & В14T04 & 37418 & 1 \\
\hline Columbia River & RICHPS7 HRM46.4 & B124Y7 & 37054 & 1 \\
\hline Columbia River & RICHPS7 HRM46.4 & B16LB5 & 37705 & 1 \\
\hline Columbia River & RICHPS7 HRM46.4 & B11133 & 36865 & 1 \\
\hline Columbia River & RICHPS7 HRM46.4 & B124W9 & 37054 & 1 \\
\hline Columbia River & RICHPS7 HRM46.4 & B10741 & 36788 & 1 \\
\hline Columbia River & RICHPS7 HRM46.4 & B106Y8 & 36788 & 1 \\
\hline Columbia River & RICHPS7 HRM46.4 & B12509 & 37054 & 1 \\
\hline Columbia River & RICHPS7 HRM46.4 & B106V8 & 36788 & 1 \\
\hline Columbia River & RICHPS7 HRM46.4 & B12529 & 37054 & 1 \\
\hline Columbia River & RICHPS7 HRM46.4 & B106N3 & 36788 & 1 \\
\hline Columbia River & RICHPS7 HRM46.4 & В12Т82 & 37147 & 1 \\
\hline Columbia River & RICHPS7 HRM46.4 & B0YCK1 & 36697 & 1 \\
\hline Columbia River & RICHPS7 HRM46.4 & B12TD2 & 37147 & 1 \\
\hline Columbia River & RICHPS7 HRM46.4 & B11143 & 36865 & 1 \\
\hline Columbia River & RICHPS7 HRM46.4 & B11J34 & 36948 & 1 \\
\hline Columbia River & RICHPS7 HRM46.4 & $\mathrm{B} 11 \mathrm{H} 57$ & 36948 & 1 \\
\hline Columbia River & RICHPS7 HRM46.4 & B13LJ1 & 37229 & 1 \\
\hline Columbia River & RICHPS7 HRM46.4 & B0YCD3 & 36697 & 1 \\
\hline Columbia River & RICHPS7 HRM46.4 & B0XRN6 & 36613 & 1 \\
\hline Columbia River & RICHPS7 HRM46.4 & B11H35 & 36948 & 1 \\
\hline Columbia River & RICHPS7 HRM46.4 & B13LH1 & 37229 & 1 \\
\hline Columbia River & RICHPS7 HRM46.4 & B0XRW1 & 36613 & 1 \\
\hline Columbia River & RICHPS7 HRM46.4 & B13LD9 & 37229 & 1 \\
\hline Columbia River & RICHPS7 HRM46.4 & B11235 & 36865 & 1 \\
\hline Columbia River & RICHPS7 HRM46.4 & B12VL7 & 37147 & 1 \\
\hline Columbia River & RICHPS7 HRM46.4 & BOWCB9 & 36419 & 1 \\
\hline Columbia River & RICHPS7 HRM46.4 & B12TKO & 37147 & 1 \\
\hline Columbia River & RICHPS7 HRM46.4 & B0YCF5 & 36697 & 1 \\
\hline Columbia River & RICHPS7 HRM46.4 & $\mathrm{B} 11 \mathrm{H} 47$ & 36948 & 1 \\
\hline Columbia River & RICHPS7 HRM46.4 & B11121 & 36865 & 1 \\
\hline Columbia River & RICHPS7 HRM46.4 & B0YCB4 & 36697 & 1 \\
\hline Columbia River & RICHPS7 HRM46.4 & B1B454 & 38245 & 1 \\
\hline Columbia River & RICHPS7 HRM46.4 & B1B730 & 38245 & 1 \\
\hline Columbia River & RICHPS7 HRM46.4 & B19J74 & 38162 & 1 \\
\hline Columbia River & RICHPS7 HRM46.4 & B1BW46 & 38340 & 1 \\
\hline Columbia River & RICHPS7 HRM46.4 & B183R3 & 37963 & 1 \\
\hline Columbia River & RICHPS7 HRM46.4 & B184R3 & 37963 & 1 \\
\hline
\end{tabular}


Table B-2

\section{Summary Table of Segment 2 Surface Water Samples By Area, Site, Date, and Category Columbia River Component - Existing Sample Data Evaluation and Scoping Report}

\begin{tabular}{|c|c|c|c|c|}
\hline Sample Area & Sample Site & Sample ID & Sample Date & Category \\
\hline Columbia River & RICHPS7 HRM46.4 & B183T5 & 37963 & 1 \\
\hline Columbia River & RICHPS7 HRM46.4 & B18TJ3 & 38076 & 1 \\
\hline Columbia River & RICHPS7 HRM46.4 & B1BX54 & 38340 & 1 \\
\hline Columbia River & RICHPS7 HRM46.4 & B183V5 & 37963 & 1 \\
\hline Columbia River & RICHPS7 HRM46.4 & B17HL3 & 37873 & 1 \\
\hline Columbia River & RICHPS7 HRM46.4 & B1B761 & 38245 & 1 \\
\hline Columbia River & RICHPS7 HRM46.4 & B1B6Y7 & 38245 & 1 \\
\hline Columbia River & RICHPS7 HRM46.4 & B18V23 & 38076 & 1 \\
\hline Columbia River & RICHPS7 HRM46.4 & В19HР2 & 38162 & 1 \\
\hline Columbia River & RICHPS8 HRM46.4 & BoV223 & 36325 & 1 \\
\hline Columbia River & RICHPS8 HRM46.4 & BoV242 & 36325 & 1 \\
\hline Columbia River & RICHPS8 HRM46.4 & B0V222 & 36325 & 1 \\
\hline Columbia River & RICHPS8 HRM46.4 & Bov253 & 36325 & 1 \\
\hline Columbia River & RICHPS9 HRM46.4 & B0V243 & 36325 & 1 \\
\hline Columbia River & RICHPS9 HRM46.4 & B0V225 & 36325 & 1 \\
\hline Columbia River & RICHPS9 HRM46.4 & BoV254 & 36325 & 1 \\
\hline Columbia River & RICHPS9 HRM46.4 & B0V224 & 36325 & 1 \\
\hline Columbia River & Ringold WW & B170R8 & 37755 & 1 \\
\hline Columbia River & Ringold WW & В170т0 & 37755 & 1 \\
\hline Columbia River & Ringold WW & B170R9 & 37755 & 1 \\
\hline Columbia River & Ringold WW & B16WM7 & 37755 & 1 \\
\hline Columbia River & Ringold WW & B16WM8 & 37755 & 1 \\
\hline Columbia River & Ringold WW & B16WM9 & 37755 & 1 \\
\hline Columbia River & Ringold WW & B16WT0 & 37755 & 1 \\
\hline Columbia River & Ringold WW & B16WT1 & 37755 & 1 \\
\hline Columbia River & Ringold WW & B16WT2 & 37755 & 1 \\
\hline Columbia River & Ringold WW & B16WT3 & 37755 & 1 \\
\hline Columbia River & Ringold WW & B16WT4 & 37755 & 1 \\
\hline Columbia River & Ringold WW & B16WT5 & 37755 & 1 \\
\hline Columbia River & RIVERVIEW & 29056 & 37399 & 1 \\
\hline Columbia River & RIVERVIEW & 33166 & 38114 & 1 \\
\hline Columbia River & RIVERVIEW & 30879 & 37750 & 1 \\
\hline Columbia River & RL PUMPHSE & 23426 & 36467 & 1 \\
\hline Columbia River & RL PUMPHSE & 23424 & 36285 & 1 \\
\hline Columbia River & RL PUMPHSE 1/10 & 26203 & 37147 & 1 \\
\hline Columbia River & RL PUMPHSE 2/10 & 26204 & 37147 & 1 \\
\hline Columbia River & RL PUMPHSE 3/10 & 26205 & 37147 & 1 \\
\hline Columbia River & RL PUMPHSE 5/10 & 26206 & 37147 & 1 \\
\hline Columbia River & RL PUMPHSE 7/10 & 26207 & 37147 & 1 \\
\hline Columbia River & RL PUMPHSE10/10 & 26990 & 37147 & 1 \\
\hline Columbia River & SB-037-1 & B10F97 & 36824 & 1 \\
\hline Columbia River & SB-037-1 & B10F99 & 36824 & 1 \\
\hline Columbia River & SB-037-1 & B10F98 & 36824 & 1 \\
\hline Columbia River & SD-110-2 & B10JT1 & 36816 & 1 \\
\hline Columbia River & SD-110-2 & B10JR0 & 36816 & 1 \\
\hline Columbia River & SD-98-1 & B10JV0 & 36811 & 1 \\
\hline
\end{tabular}


Table B-2

\section{Summary Table of Segment 2 Surface Water Samples By Area, Site, Date, and Category Columbia River Component - Existing Sample Data Evaluation and Scoping Report}

\begin{tabular}{|c|c|c|c|c|}
\hline Sample Area & Sample Site & Sample ID & Sample Date & Category \\
\hline Columbia River & SD-98-1 & B10JT9 & 36811 & 1 \\
\hline Columbia River & SD-98-1 & B10JR1 & 36811 & 1 \\
\hline Columbia River & SD-98-1 & B10JT2 & 36811 & 1 \\
\hline Columbia River & SF-190-4 & B10FC1 & 36837 & 1 \\
\hline Columbia River & SF-190-4 & B10FB9 & 36837 & 1 \\
\hline Columbia River & SF-190-4 & B10FC0 & 36837 & 1 \\
\hline Columbia River & SF-211-1 & B10FC5 & 36837 & 1 \\
\hline Columbia River & SF-211-1 & B10FC7 & 36837 & 1 \\
\hline Columbia River & SF-211-1 & B10FC6 & 36837 & 1 \\
\hline Columbia River & SH-144-1 & В10JT3 & 36812 & 1 \\
\hline Columbia River & SH-144-1 & B10JR2 & 36812 & 1 \\
\hline Columbia River & SH-150-1 & B10JT6 & 36831 & 1 \\
\hline Columbia River & SH-150-1 & B10JR5 & 36831 & 1 \\
\hline Columbia River & SH-153-1 & B10JR7 & 36831 & 1 \\
\hline Columbia River & SH-153-1 & B10JT8 & 36831 & 1 \\
\hline Columbia River & TOWNSITE 1/10 & 23432 & 36424 & 1 \\
\hline Columbia River & TOWNSITE 1/10 & 29097 & 37508 & 1 \\
\hline Columbia River & TOWNSITE 10/10 & 29091 & 37508 & 1 \\
\hline Columbia River & TOWNSITE 2/10 & 23433 & 36424 & 1 \\
\hline Columbia River & TOWNSITE 2/10 & 29082 & 37508 & 1 \\
\hline Columbia River & TOWNSITE 3/10 & 29083 & 37508 & 1 \\
\hline Columbia River & TOWNSITE 3/10 & 23434 & 36424 & 1 \\
\hline Columbia River & TOWNSITE 5/10 & 29084 & 37508 & 1 \\
\hline Columbia River & TOWNSITE 5/10 & 23436 & 36424 & 1 \\
\hline Columbia River & TOWNSITE 7/10 & 23435 & 36424 & 1 \\
\hline Columbia River & TOWNSITE 7/10 & 29090 & 37508 & 1 \\
\hline Columbia River & Wooded Island & 24689 & 36560 & 1 \\
\hline Columbia River & WSU-Richland & B0VWW8 & 36342 & 1 \\
\hline Columbia River & WSU-Richland & BovwWo & 36342 & 1 \\
\hline Columbia River & 100D Area & B14L06 & 37393 & 2 \\
\hline Columbia River & 100D Area & B14PW8 & 37417 & 2 \\
\hline Columbia River & 100D Area & B14PW7 & 37417 & 2 \\
\hline Columbia River & 100D Area & B14L07 & 37393 & 2 \\
\hline Columbia River & 100D Area & B14K63 & 37379 & 2 \\
\hline Columbia River & 100D Area & B14K61 & 37379 & 2 \\
\hline Columbia River & 100D Area & $\mathrm{B} 14 \mathrm{~K} 60$ & 37379 & 2 \\
\hline Columbia River & 100D Area & B14K62 & 37379 & 2 \\
\hline Columbia River & $100 \mathrm{H}$ Area & B14PX0 & 37417 & 2 \\
\hline Columbia River & $100 \mathrm{H}$ Area & B14L08 & 37393 & 2 \\
\hline Columbia River & $100 \mathrm{H}$ Area & B14L09 & 37393 & 2 \\
\hline Columbia River & $100 \mathrm{H}$ Area & B14PW9 & 37417 & 2 \\
\hline Columbia River & Richland WNTK & 11200288 & 38001 & 2 \\
\hline Columbia River & Richland WNTK & 12500287 & 38001 & 2 \\
\hline Columbia River & HRM 10.5 & $290615 a$ & 37043 & 3 \\
\hline Columbia River & HRM 10.9 & $271813 a$ & 37043 & 3 \\
\hline Columbia River & HRM 11.1 & $182414 a$ & 37043 & 3 \\
\hline
\end{tabular}


Table B-2

Summary Table of Segment 2 Surface Water Samples By Area, Site, Date, and Category Columbia River Component - Existing Sample Data Evaluation and Scoping Report

\begin{tabular}{lcccc}
\hline Sample Area & Sample Site & Sample ID & Sample Date & Category \\
\hline Columbia River & HRM 11.1 & $181215 \mathrm{a}$ & 37043 & 3 \\
Columbia River & HRM 12.5 & $172915 \mathrm{a}$ & 37043 & 3 \\
Columbia River & HRM 15 & $190616 \mathrm{~b}$ & 37043 & 3 \\
Columbia River & HRM 15 & $190616 \mathrm{a}$ & 37043 & 3 \\
Columbia River & HRM 15.4 & $172916 \mathrm{~b}$ & 37043 & 3 \\
Columbia River & HRM 16.3 & $170214 \mathrm{a}$ & 37043 & 3 \\
Columbia River & HRM 18 & $182310 \mathrm{~b}$ & 37043 & 3 \\
Columbia River & HRM 19.3 & $190607 \mathrm{a}$ & 37043 & 3 \\
Columbia River & HRM 20.6 & $182311 \mathrm{a}$ & 37043 & 3 \\
Columbia River & HRM 20.6 & $1906 \mathrm{x} \mathrm{a}$ & 37043 & 3 \\
Columbia River & HRM 21 & $172114 \mathrm{a}$ & 37043 & 3 \\
Columbia River & HRM 22.5 & $2 \mathrm{y} 0915 \mathrm{a}$ & 37043 & 3 \\
Columbia River & HRM 22.5 & $190613 \mathrm{a}$ & 37043 & 3 \\
Columbia River & HRM 22.5 & $2 \mathrm{y} 0915 \mathrm{~b}$ & 37043 & 3 \\
Columbia River & HRM 26 & $182312 \mathrm{a}$ & 37043 & 3 \\
Columbia River & HRM 27 & $190516 \mathrm{a}$ & 37043 & 3 \\
Columbia River & HRM 3.6 & $290711 \mathrm{a}$ & 37043 & 3 \\
Columbia River & HRM 45.6 & $181316 \mathrm{a}$ & 37043 & 3 \\
\hline
\end{tabular}


Table B-3

Summary Statistics for Sediment Collected from Segment 2 of the Columbia River Including All Categories of Data (units reported "as received") Columbia River Component - Existing Sample Data Evaluation and Scoping Report

\begin{tabular}{|c|c|c|c|c|c|c|c|c|c|c|}
\hline $\begin{array}{l}\text { Constituent } \\
\text { Class }\end{array}$ & Constituent Name & Units & $\begin{array}{c}\text { Number } \\
\text { Analyzed }\end{array}$ & $\begin{array}{l}\text { Number } \\
\text { Detected }\end{array}$ & $\begin{array}{l}\text { Frequency of } \\
\text { Detection }\end{array}$ & $\begin{array}{l}\text { MInImum } \\
\text { Nondetect }\end{array}$ & $\begin{array}{l}\text { Maxımum } \\
\text { Nondetect }\end{array}$ & Minimum Detect & $\begin{array}{l}\text { Maximum } \\
\text { Detect }\end{array}$ & $\begin{array}{c}\text { Average } \\
\text { Concentration }\end{array}$ \\
\hline$\overline{\text { CONV }}$ & Ash Content & $\%$ & 2 & 2 & $100 \%$ & $\overline{--}$ & $\overline{--}$ & 91 & 93 & 92 \\
\hline CONV & Nitrogen in ammonia & $\mathrm{mg} / \mathrm{kg}$ & 1 & 1 & $100 \%$ & -- & -- & 15 & 15 & 15 \\
\hline CONV & Percent Solids & $\%$ & 35 & 35 & $100 \%$ & -- & -- & 2.0 & 81 & 62 \\
\hline CONV & Phosphorous in phosphate & $\mathrm{mg} / \mathrm{kg}$ & 1 & 1 & $100 \%$ & -- & -- & 0.060 & 0.060 & 0.060 \\
\hline CONV & Sulfide & $\mathrm{mg} / \mathrm{kg}$ & 1 & 1 & $100 \%$ & -- & -- & 2.4 & 2.4 & 2.4 \\
\hline CONV & Total Organic Carbon & $\%$ & 9 & 9 & $100 \%$ & -- & -- & 0.15 & 1.9 & 0.87 \\
\hline CONV & Total Organic Carbon & $\%$ dry-wt & 4 & 4 & $100 \%$ & .- & -- & 3.1 & 3.4 & 3.2 \\
\hline CONV & Total Organic Carbon & $\mathrm{mg} / \mathrm{kg}$ & 26 & 26 & $100 \%$ & -- & -- & 175 & 16,500 & 4,850 \\
\hline METAL & Aluminum & $\mathrm{mg} / \mathrm{kg}$ & 45 & 45 & $100 \%$ & -- & -- & 2,460 & 11,100 & 6,349 \\
\hline METAL & Antimony & $\mathrm{mg} / \mathrm{kg}$ & 99 & 61 & $62 \%$ & 3.3 & 18 & 0.019 & 6.8 & 1.4 \\
\hline METAL & Arsenic & $\mathrm{mg} / \mathrm{kg}$ & 108 & 104 & $96 \%$ & 4.5 & 4.5 & 0.0082 & 20 & 4.6 \\
\hline METAL & Barium & $\mathrm{mg} / \mathrm{kg}$ & 48 & 47 & $98 \%$ & 49 & 49 & 35 & 869 & 104 \\
\hline METAL & Beryllium & $\mathrm{mg} / \mathrm{kg}$ & 110 & 85 & $77 \%$ & 0.0050 & 0.25 & 0.032 & 2.4 & 0.74 \\
\hline METAL & Cadmium & $\mathrm{mg} / \mathrm{kg}$ & 112 & 73 & $65 \%$ & 0.20 & 3.4 & $3.80 \mathrm{E}-04$ & 5.6 & 0.72 \\
\hline METAL & Calcium & $\mathrm{mg} / \mathrm{kg}$ & 45 & 45 & $100 \%$ & -- & -- & 1,760 & 9,120 & 3,720 \\
\hline METAL & Chromium & $\mathrm{mg} / \mathrm{kg}$ & 120 & 119 & $99 \%$ & 0.10 & 0.10 & 0.056 & 112 & 35 \\
\hline METAL & Cobalt & $\mathrm{mg} / \mathrm{kg}$ & 45 & 44 & $98 \%$ & 6.4 & 6.4 & 2.8 & 10 & 6.5 \\
\hline METAL & Copper & $\mathrm{mg} / \mathrm{kg}$ & 119 & 119 & $100 \%$ & -- & -- & 0.18 & 70 & 19 \\
\hline METAL & Iron & $\mathrm{mg} / \mathrm{kg}$ & 45 & 45 & $100 \%$ & -- & .- & 6.480 & 31,100 & 14,519 \\
\hline METAL & Lead & $\mathrm{mg} / \mathrm{kg}$ & 108 & 108 & $100 \%$ & -- & -- & 0.10 & 76 & 24 \\
\hline METAL & Magnesium & $\mathrm{mg} / \mathrm{kg}$ & 45 & 45 & $100 \%$ & -- & -- & 1,480 & 5,520 & 3,777 \\
\hline METAL & Manganese & $\mathrm{mg} / \mathrm{kg}$ & 48 & 48 & $100 \%$ & -- & .- & 94 & 603 & 215 \\
\hline METAL & Mercury & $\mathrm{mg} / \mathrm{kg}$ & 108 & 67 & $62 \%$ & 0.0015 & 0.080 & $1.60 \mathrm{E}-05$ & 0.68 & 0.034 \\
\hline METAL & Molybdenum & $\mathrm{mg} / \mathrm{kg}$ & 8 & 0 & $0 \%$ & 0.59 & 0.60 & -- & -- & 0.30 \\
\hline METAL & Nickel & $\mathrm{mg} / \mathrm{kg}$ & 112 & 112 & $100 \%$ & -- & -- & 0.64 & 30 & 14 \\
\hline METAL & Potassium & $\mathrm{mg} / \mathrm{kg}$ & 45 & 45 & $100 \%$ & -- & -- & 424 & 1,650 & 845 \\
\hline METAL & Selenium & $\mathrm{mg} / \mathrm{kg}$ & 107 & 29 & $27 \%$ & 0.0027 & 5.0 & 0.0084 & 1.6 & 0.53 \\
\hline METAL & Silver & $\mathrm{mg} / \mathrm{kg}$ & 99 & 58 & $59 \%$ & 0.0012 & 3.0 & 0.0020 & 5.3 & 0.39 \\
\hline METAL & Sodium & $\mathrm{mg} / \mathrm{kg}$ & 45 & 45 & $100 \%$ & -- & -- & 97 & 364 & 207 \\
\hline METAL & Thallium & $\mathrm{mg} / \mathrm{kg}$ & 99 & 63 & $64 \%$ & 0.26 & 1.1 & 0.027 & 2.4 & 0.48 \\
\hline METAL & Thorium & $\mathrm{mg} / \mathrm{Kg}$ & 3 & 3 & $100 \%$ & -- & -- & 7.0 & 7.9 & 7.4 \\
\hline METAL & Uranium & $\mathrm{mg} / \mathrm{Kg}$ & 7 & 7 & $100 \%$ & -- & -- & 1.1 & 2.6 & 1.6 \\
\hline METAL & Vanadium & $\mathrm{mg} / \mathrm{kg}$ & 45 & 45 & $100 \%$ & -- & -- & 6.5 & 86 & 31 \\
\hline METAL & Zinc & $\mathrm{mg} / \mathrm{kg}$ & 112 & 112 & $100 \%$ & -- & -- & 0.18 & 477 & 179 \\
\hline RAD D & Americium-241, Decayed & $\mathrm{pCi} / \mathrm{g}$ & 2 & 2 & $100 \%$ & -- & -- & 0.0030 & 0.24 & 0.12 \\
\hline RAD D & Antimony-125, Decayed & $\mathrm{pCi} / \mathrm{g}$ & 1 & 1 & $100 \%$ & -- & -- & 0.0018 & 0.0018 & 0.0018 \\
\hline RAD_D & Beryllium-7, Decayed & $\mathrm{pCi} / \mathrm{g}$ & 1 & 1 & $100 \%$ & -- & -- & 0.30 & 0.30 & 0.30 \\
\hline RAD D & Cesium-134, Decayed & $\mathrm{pCi} / \mathrm{g}$ & 4 & 4 & $100 \%$ & -- & -- & $2.86 \mathrm{E}-04$ & 0.0011 & $6.44 \mathrm{E}-04$ \\
\hline RAD D & Cesium-137, Decayed & $\mathrm{pCi} / \mathrm{g}$ & 122 & 122 & $100 \%$ & .- & -- & 0.023 & 1.5 & 0.28 \\
\hline RAD_D & Cobalt-57, Decayed & $\mathrm{pCi} / \mathrm{g}$ & 1 & 1 & $100 \%$ & -- & -- & 3.07E-09 & 3.07E-09 & 3.07E-09 \\
\hline RAD D & Cobalt-60, Decayed & $\mathrm{pCi} / \mathrm{g}$ & 40 & 40 & $100 \%$ & -- & -- & 0.0033 & 2.4 & 0.091 \\
\hline RAD D & Europium-152, Decayed & $\mathrm{pCi} / \mathrm{g}$ & 37 & 37 & $100 \%$ & -- & -- & 0.024 & 0.76 & 0.24 \\
\hline RAD_D & Europium-154, Decayed & $\mathrm{pCi} / \mathrm{g}$ & 6 & 6 & $100 \%$ & -- & -- & 0.015 & 0.075 & 0.047 \\
\hline RAD D & Europium-155, Decayed & $\mathrm{pCi} / \mathrm{g}$ & 3 & 3 & $100 \%$ & -- & -- & 0.0071 & 0.034 & 0.024 \\
\hline RAD D & Manganese-54, Decayed & $\mathrm{pCi} / \mathrm{g}$ & 2 & 2 & $100 \%$ & -- & -- & $1.15 \mathrm{E}-08$ & $1.51 \mathrm{E}-06$ & $7.59 \mathrm{E}-07$ \\
\hline
\end{tabular}


Table B-3

Summary Statistics for Sediment Collected from Segment 2 of the Columbia River Including All Categories of Data (units reported "as received") Columbia River Component - Existing Sample Data Evaluation and Scoping Report

\begin{tabular}{|c|c|c|c|c|c|c|c|c|c|c|}
\hline $\begin{array}{c}\text { Constituent } \\
\text { Class }\end{array}$ & Constituent Name & Units & $\begin{array}{l}\text { Number } \\
\text { Analyzed }\end{array}$ & $\begin{array}{l}\text { Number } \\
\text { Detected }\end{array}$ & $\begin{array}{c}\text { Frequency of } \\
\text { Detection }\end{array}$ & $\begin{array}{l}\text { Minimum } \\
\text { Nondetect }\end{array}$ & $\begin{array}{l}\text { Maximum } \\
\text { Nondetect }\end{array}$ & Minimum Detect & $\begin{array}{l}\text { Maximum } \\
\text { Detect }\end{array}$ & $\begin{array}{c}\text { Average } \\
\text { Concentration }\end{array}$ \\
\hline$\overline{\text { RAD_D }}$ & Neptunium-237, Decayed & $\mathrm{pCi} / \mathrm{g}$ & 2 & 2 & $100 \%$ & $\overline{--}$ & $\overline{--}$ & 0.48 & 0.61 & 0.54 \\
\hline RAD_D & Plutonium-238, Decayed & $\mathrm{pCi} / \mathrm{g}$ & 15 & 15 & $100 \%$ & -- & -- & $1.26 \mathrm{E}-04$ & 0.13 & 0.016 \\
\hline RAD_D & Plutonium-239/240, Decayed & $\mathrm{pCi} / \mathrm{g}$ & 34 & 34 & $100 \%$ & -- & -- & 3.98E-04 & 0.071 & 0.0064 \\
\hline RAD_D & Potassium-40, Decayed & $\mathrm{pCi} / \mathrm{g}$ & 132 & 132 & $100 \%$ & -- & -- & 8.4 & 21 & 16 \\
\hline RAD_D & Radium-226, Decayed & $\mathrm{pCi} / \mathrm{g}$ & 37 & 37 & $100 \%$ & -- & -- & 0.42 & 1.7 & 0.77 \\
\hline RAD_D & Radium-228, Decayed & $\mathrm{pCi} / \mathrm{g}$ & 1 & 1 & $100 \%$ & -- & -- & 0.12 & 0.12 & 0.12 \\
\hline RAD_D & Strontium-89, Decayed & $\mathrm{pCi} / \mathrm{g}$ & 3 & 3 & $100 \%$ & -- & -- & 3.43E-42 & $4.80 \mathrm{E}-42$ & $3.89 \mathrm{E}-42$ \\
\hline RAD_D & Strontium-90, Decayed & $\mathrm{pCi} / \mathrm{g}$ & 18 & 18 & $100 \%$ & -- & -- & 0.0020 & 1.0 & 0.090 \\
\hline RAD_D & Thorium-228, Decayed & $\mathrm{pCi} / \mathrm{g}$ & 41 & 41 & $100 \%$ & -- & -- & 0.0041 & 2.1 & 0.13 \\
\hline RAD_D & Thorium-230, Decayed & $\mathrm{pCi} / \mathrm{g}$ & 4 & 4 & $100 \%$ & -- & -- & 0.16 & 5.6 & 2.1 \\
\hline RAD_D & Thorium-232, Decayed & $\mathrm{pCi} / \mathrm{g}$ & 40 & 40 & $100 \%$ & -- & -- & 0.24 & 3.2 & 1.1 \\
\hline RAD_D & Thorium-234, Decayed & $\mathrm{pCi} / \mathrm{g}$ & 2 & 2 & $100 \%$ & -- & -- & $3.70 \mathrm{E}-60$ & $4.37 \mathrm{E}-60$ & 4.03E-60 \\
\hline RAD_D & Tritium, Decayed & $\mathrm{pCi} / \mathrm{g}$ & 1 & 1 & $100 \%$ & -- & -- & 0.038 & 0.038 & 0.038 \\
\hline RAD_D & Uranium-233/234, Decayed & $\mathrm{pCi} / \mathrm{g}$ & 36 & 36 & $100 \%$ & -- & -- & 0.29 & 2.6 & 0.82 \\
\hline RAD_D & Uranium-234, Decayed & $\mathrm{pCi} / \mathrm{g}$ & 90 & 90 & $100 \%$ & -- & -- & 0.071 & 11 & 0.82 \\
\hline RAD_D & Uranium-235, Decayed & $\mathrm{pCi} / \mathrm{g}$ & 79 & 79 & $100 \%$ & -- & -- & 0.0029 & 0.38 & 0.039 \\
\hline RAD_D & Uranium-236, Decayed & $\mathrm{pCi} / \mathrm{g}$ & 4 & 4 & $100 \%$ & -- & -- & $3.11 \mathrm{E}-05$ & 0.039 & 0.026 \\
\hline RAD_D & Uranium-238, Decayed & $\mathrm{pCi} / \mathrm{g}$ & 129 & 129 & $100 \%$ & -- & -- & 0.051 & 10.0 & 0.76 \\
\hline RAD_D & Zirconium/Niobium-95, Decayed & $\mathrm{pCi} / \mathrm{g}$ & 2 & 2 & $100 \%$ & -- & -- & $2.60 \mathrm{E}-36$ & $3.08 \mathrm{E}-36$ & $2.84 \mathrm{E}-36$ \\
\hline
\end{tabular}

CONV - Conventional parameter

RAD D - Decayed radionuclide 
Table B-4

Summary Statistics for Sediment Collected from Segment 2 of the Columbia River Including All Categories of Data (units reported "dry-weight") Columbia River Component - Existing Sample Data Evaluation and Scoping Report

\begin{tabular}{|c|c|c|c|c|c|c|c|c|c|c|}
\hline $\begin{array}{l}\text { Constituent } \\
\text { Class }\end{array}$ & Constituent Name & Units & $\begin{array}{l}\text { Number } \\
\text { Analyzed }\end{array}$ & $\begin{array}{l}\text { Number } \\
\text { Detected }\end{array}$ & $\begin{array}{c}\text { Frequency of } \\
\text { Detection }\end{array}$ & $\begin{array}{l}\text { Minimum } \\
\text { Nondetect }\end{array}$ & $\begin{array}{l}\text { Maximum } \\
\text { Nondetect }\end{array}$ & $\begin{array}{l}\text { Minimum } \\
\text { Detect }\end{array}$ & $\begin{array}{l}\text { Maximum } \\
\text { Detect }\end{array}$ & $\begin{array}{c}\text { Average } \\
\text { Concentration }\end{array}$ \\
\hline$\overline{\text { CONV }}$ & Total Organic Carbon & $\%$ dry-wt & 4 & 4 & $100 \%$ & -- & -- & 3.1 & 3.4 & 3.2 \\
\hline METAL & Aluminum & $\mathrm{mg} / \mathrm{kg}$ dry-wt & 4 & 4 & $100 \%$ & -- & -- & 0.40 & 1.2 & 0.65 \\
\hline METAL & Antimony & $\mathrm{mg} / \mathrm{kg}$ dry-wt & 9 & 9 & $100 \%$ & -- & -- & 0.39 & 0.81 & 0.57 \\
\hline METAL & Arsenic & $\mathrm{mg} / \mathrm{kg}$ dry-wt & 14 & 14 & $100 \%$ & -- & -- & 0.0020 & 7.8 & 3.5 \\
\hline METAL & Barium & $\mathrm{mg} / \mathrm{kg}$ dry-wt & 4 & 4 & $100 \%$ & -- & -- & 0.40 & 1.0 & 0.60 \\
\hline METAL & Beryllium & $\mathrm{mg} / \mathrm{kg}$ dry-wt & 12 & 12 & $100 \%$ & -- & -- & 0.10 & 1.6 & 1.1 \\
\hline METAL & Cadmium & $\mathrm{mg} / \mathrm{kg}$ dry-wt & 18 & 18 & $100 \%$ & -- & -- & 0.20 & 1.6 & 0.61 \\
\hline METAL & Chromium & $\mathrm{mg} / \mathrm{kg}$ dry-wt & 17 & 13 & $76 \%$ & 0.10 & 0.10 & 20 & 88 & 44 \\
\hline METAL & Copper & $\mathrm{mg} / \mathrm{kg}$ dry-wt & 17 & 17 & $100 \%$ & -- & -- & 1.2 & 26 & 14 \\
\hline METAL & Iron & $\mathrm{mg} / \mathrm{kg}$ dry-wt & 4 & 4 & $100 \%$ & -- & -- & 2.2 & 7.6 & 5.8 \\
\hline METAL & Lead & $\mathrm{mg} / \mathrm{kg}$ dry-wt & 18 & 18 & $100 \%$ & -- & -- & 1.4 & 57 & 19 \\
\hline METAL & Manganese & $\mathrm{mg} / \mathrm{kg}$ dry-wt & 4 & 4 & $100 \%$ & -- & -- & 4.0 & 20 & 12 \\
\hline METAL & Mercury & $\mathrm{mg} / \mathrm{kg}$ dry-wt & 16 & 8 & $50 \%$ & 8.96E-04 & 0.060 & 0.0018 & 0.033 & 0.0094 \\
\hline METAL & Nickel & $\mathrm{mg} / \mathrm{kg}$ dry-wt & 9 & 9 & $100 \%$ & -- & -- & 12 & 21 & 18 \\
\hline METAL & Selenium & $\mathrm{mg} / \mathrm{kg}$ dry-wt & 13 & 7 & $54 \%$ & 0.35 & 0.35 & 0.0010 & 1.5 & 0.27 \\
\hline METAL & Silver & $\mathrm{mg} / \mathrm{kg}$ dry-wt & 9 & 8 & $89 \%$ & 0.033 & 0.033 & 0.048 & 0.33 & 0.21 \\
\hline METAL & Thallium & $\mathrm{mg} / \mathrm{kg}$ dry-wt & 9 & 9 & $100 \%$ & -- & -- & 0.42 & 0.77 & 0.58 \\
\hline METAL & Zinc & $\mathrm{mg} / \mathrm{kg}$ dry-wt & 13 & 13 & $100 \%$ & -- & -- & 4.0 & 366 & 132 \\
\hline RAD_D & Americium-241 & $\mathrm{pCi} / \mathrm{g}$ dry-wt & 4 & 4 & $100 \%$ & -- & -- & 0.0020 & 0.0049 & 0.0032 \\
\hline RAD_D & Barium-140 & $\mathrm{pCi} / \mathrm{g}$ dry-wt & 1 & 1 & $100 \%$ & -- & -- & 7.09E-116 & 7.09E-116 & 7.09E-116 \\
\hline RAD_D & Beryllium-7 & pCi/g dry-wt & 11 & 11 & $100 \%$ & -- & -- & $6.83 E-30$ & 0.0031 & 2.84E-04 \\
\hline RAD_D & Bismuth-214 & $\mathrm{pCi} / \mathrm{g}$ dry-wt & 4 & 4 & $100 \%$ & -- & -- & 3.55E-05 & 8.93E-05 & $5.79 \mathrm{E}-05$ \\
\hline RAD_D & Cerium-141 & $\mathrm{pCi} / \mathrm{g}$ dry-wt & 2 & 2 & $100 \%$ & -- & -- & $1.23 \mathrm{E}-46$ & 1.46E-46 & 1.35E-46 \\
\hline RAD_D & Cerium-144 & $\mathrm{pCi} / \mathrm{g}$ dry-wt & 1 & 1 & $100 \%$ & -- & -- & 1.68E-06 & $1.68 \mathrm{E}-06$ & 1.68E-06 \\
\hline RAD_D & Cesium-134 & $\mathrm{pCi} / \mathrm{g}$ dry-wt & 12 & 12 & $100 \%$ & -- & -- & $2.68 \mathrm{E}-05$ & 0.0015 & $3.20 \mathrm{E}-04$ \\
\hline RAD_D & Cesium-137 & pCi/g dry-wt & 129 & 129 & $100 \%$ & -- & -- & 0.021 & 1.2 & 0.15 \\
\hline RAD_D & Cobalt-57 & $\mathrm{pCi} / \mathrm{g}$ dry-wt & 4 & 4 & $100 \%$ & -- & -- & $4.88 \mathrm{E}-12$ & $3.58 \mathrm{E}-04$ & 8.96E-05 \\
\hline RAD_D & Cobalt-58 & $\mathrm{pCi} / \mathrm{g}$ dry-wt & 1 & 1 & $100 \%$ & -- & -- & $2.21 \mathrm{E}-23$ & $2.21 \mathrm{E}-23$ & $2.21 \mathrm{E}-23$ \\
\hline RAD_D & Cobalt-60 & pCi/g dry-wt & 60 & 60 & $100 \%$ & -- & -- & 0.0014 & 0.020 & 0.0059 \\
\hline RAD_D & Europium-152 & $\mathrm{pCi} / \mathrm{g}$ dry-wt & 26 & 26 & $100 \%$ & -- & -- & 0.012 & 0.11 & 0.051 \\
\hline RAD_D & Europium-154 & $\mathrm{pCi} / \mathrm{g}$ dry-wt & 6 & 6 & $100 \%$ & -- & -- & 0.015 & 0.090 & 0.045 \\
\hline RAD_D & Europium-155 & pCi/g dry-wt & 1 & 1 & $100 \%$ & -- & -- & 0.065 & 0.065 & 0.065 \\
\hline RAD_D & lodine-131 & $\mathrm{pCi} / \mathrm{g}$ dry-wt & 2 & 2 & $100 \%$ & -- & -- & 1.07E-180 & 7.44E-180 & $4.25 \mathrm{E}-180$ \\
\hline RAD_D & Iron-59 & $\mathrm{pCi} / \mathrm{g}$ dry-wt & 2 & 2 & $100 \%$ & -- & -- & 2.23E-35 & $4.70 \mathrm{E}-35$ & 3.47E-35 \\
\hline RAD_D & Lead-214 & $\mathrm{pCi} / \mathrm{g}$ dry-wt & 6 & 6 & $100 \%$ & -- & -- & $5.09 \mathrm{E}-04$ & $9.87 \mathrm{E}-04$ & $7.28 \mathrm{E}-04$ \\
\hline RAD_D & Manganese-54 & $\mathrm{pCi} / \mathrm{g}$ dry-wt & 6 & 6 & $100 \%$ & -- & -- & 1.81E-09 & 4.75E-07 & 1.19E-07 \\
\hline RAD_D & Niobium-95 & $\mathrm{pCi} / \mathrm{g}$ dry-wt & 4 & 4 & $100 \%$ & -- & -- & $1.40 \mathrm{E}-43$ & $7.12 \mathrm{E}-43$ & 4.63E-43 \\
\hline RAD_D & Plutonium-238 & $\mathrm{pCi} / \mathrm{g}$ dry-wt & 9 & 9 & $100 \%$ & -- & -- & $9.10 \mathrm{E}-04$ & 0.050 & 0.0084 \\
\hline RAD_D & Plutonium-239/240 & $\mathrm{pCi} / \mathrm{g}$ dry-wt & 26 & 26 & $100 \%$ & -- & -- & 0.0016 & 0.036 & 0.0082 \\
\hline RAD_D & Potassium-40 & pCi/g dry-wt & 170 & 170 & $100 \%$ & -- & -- & 1.46E-07 & 24 & 16 \\
\hline RAD_D & Radium-226 & $\mathrm{pCi} / \mathrm{g}$ dry-wt & 81 & 81 & $100 \%$ & -- & -- & 0.42 & 3.3 & 1.2 \\
\hline RAD_D & Ruthenium-103 & pCi/g dry-wt & 2 & 2 & $100 \%$ & -- & -- & $9.47 \mathrm{E}-40$ & 5.41E-39 & 3.18E-39 \\
\hline RAD_D & Ruthenium-106 & $\mathrm{pCi} / \mathrm{g}$ dry-wt & 3 & 3 & $100 \%$ & -- & -- & $1.28 \mathrm{E}-07$ & $7.88 \mathrm{E}-06$ & 2.71E-06 \\
\hline RAD_D & Strontium-89 & $\mathrm{pCi} / \mathrm{g}$ dry-wt & 4 & 4 & $100 \%$ & -- & -- & 3.05E-45 & 4.77E-42 & $2.07 \mathrm{E}-42$ \\
\hline RAD_D & Strontium-90 & pCi/g dry-wt & 43 & 43 & $100 \%$ & -- & -- & 9.38E-04 & 0.16 & 0.019 \\
\hline RAD_D & Technetium-99 & $\mathrm{pCi} / \mathrm{g}$ dry-wt & 2 & 2 & $100 \%$ & -- & -- & 0.30 & 0.38 & 0.34 \\
\hline
\end{tabular}


Table B-4

Summary Statistics for Sediment Collected from Segment 2 of the Columbia River Including All Categories of Data (units reported "dry-weight")

Columbia River Component - Existing Sample Data Evaluation and Scoping Report

\begin{tabular}{|c|c|c|c|c|c|c|c|c|c|c|}
\hline $\begin{array}{l}\text { Constituent } \\
\text { Class }\end{array}$ & Constituent Name & Units & $\begin{array}{l}\text { Number } \\
\text { Analyzed }\end{array}$ & $\begin{array}{l}\text { Number } \\
\text { Detected }\end{array}$ & $\begin{array}{l}\text { Frequency of } \\
\text { Detection }\end{array}$ & $\begin{array}{l}\text { Minimum } \\
\text { Nondetect }\end{array}$ & $\begin{array}{l}\text { Maximum } \\
\text { Nondetect }\end{array}$ & $\begin{array}{c}\text { Minimum } \\
\text { Detect }\end{array}$ & $\begin{array}{l}\text { Maximum } \\
\text { Detect }\end{array}$ & $\begin{array}{c}\text { Average } \\
\text { Concentration }\end{array}$ \\
\hline$\overline{\text { RAD_D }}$ & Thallium-208 & pCi/g dry-wt & 6 & 6 & $100 \%$ & $\overline{--}$ & $\overline{--}$ & $8.18 \mathrm{E}-27$ & $5.01 \mathrm{E}-26$ & $2.26 \mathrm{E}-26$ \\
\hline RAD_D & Thorium-228 & $\mathrm{pCi} / \mathrm{g}$ dry-wt & 67 & 67 & $100 \%$ & -- & -- & 5.75E-05 & 4.3 & 0.30 \\
\hline RAD_D & Thorium-230 & $\mathrm{pCi} / \mathrm{g}$ dry-wt & 8 & 8 & $100 \%$ & -- & -- & 0.50 & 1.2 & 0.69 \\
\hline RAD_D & Thorium-232 & $\mathrm{pCi} / \mathrm{g}$ dry-wt & 8 & 8 & $100 \%$ & -- & -- & 0.60 & 1.5 & 0.98 \\
\hline RAD D $D$ & Tritium & $\mathrm{pCi} / \mathrm{g}$ dry-wt & 1 & 1 & $100 \%$ & -- & -- & 0.0098 & 0.0098 & 0.0098 \\
\hline RAD_D & Uranium-234 & $\mathrm{pCi} / \mathrm{g}$ dry-wt & 55 & 55 & $100 \%$ & -- & -- & 0.38 & 4.5 & 1.1 \\
\hline RAD_D & Uranium-235 & $\mathrm{pCi} / \mathrm{g}$ dry-wt & 31 & 31 & $100 \%$ & -- & -- & 0.017 & 0.64 & 0.076 \\
\hline RAD D & Uranium-236 & $\mathrm{pCi} / \mathrm{g}$ dry-wt & 2 & 2 & $100 \%$ & -- & -- & 0.040 & 0.050 & 0.045 \\
\hline RAD_D & Uranium-238 & pCi/g dry-wt & 57 & 57 & $100 \%$ & -- & -- & 0.41 & 4.3 & 1.1 \\
\hline RAD D & Zinc-65 & $\mathrm{pCi} / \mathrm{g}$ dry-wt & 7 & 7 & $100 \%$ & -- & -- & $2.57 \mathrm{E}-13$ & $6.86 \mathrm{E}-08$ & $2.25 \mathrm{E}-08$ \\
\hline RAD_D & Zirconium-95 & $\mathrm{pCi} / \mathrm{g}$ dry-wt & 5 & 5 & $100 \%$ & -- & -- & $1.84 \mathrm{E}-36$ & $8.64 \mathrm{E}-24$ & $3.16 E-24$ \\
\hline
\end{tabular}

CONV - Conventional parameter

RAD D - Decayed radionuclide 
Table B-5

of of Segment 2 Detected Sediment Concentrations to Ecological and Human Health Benchmark Values (units reported "as received")

\begin{tabular}{|c|c|c|c|c|c|c|c|c|c|c|c|c|c|c|c|c|c|c|}
\hline $\begin{array}{c}\text { Constitue } \\
\text { Class } \\
\end{array}$ & Constituent Name & Units & $\begin{array}{l}\text { Number } \\
\text { Analyzed }\end{array}$ & $\begin{array}{l}\begin{array}{l}\text { Number } \\
\text { Detected }\end{array} \\
\end{array}$ & $\begin{array}{c}\text { Frequency o } \\
\text { Detection }\end{array}$ & $\begin{array}{c}\begin{array}{c}\text { Tinimum } \\
\text { Detect }\end{array} \\
\end{array}$ & $\begin{array}{c}\text { Maximum } \\
\text { Detect }\end{array}$ & $\begin{array}{c}\text { Average } \\
\text { Concentration }\end{array}$ & $\begin{array}{c}\text { Backgrounc } \\
\text { Value }\end{array}$ & $\begin{array}{c}\text { Is Max> } \\
\text { Background? }\end{array}$ & $\begin{array}{c}\text { Ecological } \\
\text { Benchmark Value } \\
\end{array}$ & $\begin{array}{l}\text { Ts Max > Eco } \\
\text { Benchmark? }\end{array}$ & $\begin{array}{l}\text { Factor of } \\
\text { Exceedance }\end{array}$ & $\begin{array}{c}\text { Number of } \\
\text { Exceedances } \\
\end{array}$ & $\begin{array}{c}\text { Human Health } \\
\text { Benchmark Value }\end{array}$ & $\begin{array}{l}\text { Is Max > PH } \\
\text { Benchmark? }\end{array}$ & $\begin{array}{c}\text { Factor of } \\
\text { Exceedance }\end{array}$ & $\begin{array}{c}\text { Number of } \\
\text { Exceedances }\end{array}$ \\
\hline $\begin{array}{l}\text { CONV } \\
\text { CONV }\end{array}$ & $\begin{array}{l}\text { Ash Content } \\
\text { Nitrogen in ammonia }\end{array}$ & $\begin{array}{c}\% \\
\text { molka }\end{array}$ & 2.0 & 2.0 & $100 \%$ & 91 & 93 & 92 & $\ldots$ & $\ldots$ &.. & $\ldots$ & $\ldots$ & $\ldots$ &.. & $\ldots$ & $-\bar{x}$ lo & - \\
\hline $\begin{array}{l}\text { CONV } \\
\text { CONV }\end{array}$ & $\begin{array}{l}\text { Nitrogenen in ammonia } \\
\text { Percent Solids }\end{array}$ & $\underset{0 \%}{\mathrm{~m} / \mathrm{kg}}$ & $\begin{array}{l}1.0 \\
35\end{array}$ & $\begin{array}{l}1.0 \\
35\end{array}$ & $\begin{array}{l}100 \% \\
100 \%\end{array}$ & $\begin{array}{l}15 \\
2.0\end{array}$ & $\begin{array}{l}15 \\
81\end{array}$ & $\begin{array}{l}15 \\
62\end{array}$ & -- & $\ddot{-}$ & -- & $-\overline{-}$ & -- & $-\overline{-}$ & $\because$ & $\because-$ & -- & -- \\
\hline CONV & Phosphorous in phosphate & mglkg & 1.0 & 1.0 & $100 \%$ & 0.060 & 0.060 & 0.060 & -- & -- & -- & -- & -- & -- & -- & -- & -- & -- \\
\hline $\begin{array}{l}\text { CONV } \\
\text { CONy }\end{array}$ & $\begin{array}{l}\text { Sulfide } \\
\text { Total Organic Carbon }\end{array}$ & $\mathrm{mglkg}$ & 1.0 & 1.0 & $\begin{array}{l}100 \% \\
100 \%\end{array}$ & 2.4 & 2.4 & $\begin{array}{l}2.4 \\
0.87\end{array}$ & -- & -- & -- & -- & - & -- & -- & -- & -- & -- \\
\hline CONV & $\begin{array}{l}\text { Total OOganic Carbon } \\
\text { Total Organic Carbon }\end{array}$ & $\begin{array}{c}\% \\
\mathrm{mg} / \mathrm{kg}\end{array}$ & $\begin{array}{l}9.0 \\
4.0\end{array}$ & $\begin{array}{l}9.0 \\
4.0\end{array}$ & $\begin{array}{l}100 \% \\
100 \%\end{array}$ & $\begin{array}{l}0.15 \\
3.1\end{array}$ & $\begin{array}{l}1.9 \\
3.4\end{array}$ & 0.87 & -- & -- & -- & $-\overline{-}$ & -- & $\overline{-}$ & -- & - & - & -- \\
\hline METAL & Acid Volatile Sulfate & umole/g & 26 & 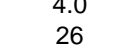 & $\begin{array}{l}100 \% \\
100 \%\end{array}$ & $\begin{array}{l}3.1 \\
175\end{array}$ & $\begin{array}{l}3.4 \\
16,500\end{array}$ & $\begin{array}{r}3.2 \\
4,850\end{array}$ & -- & -- & -- & -- & -. & -- & -- & -- & -. & -- \\
\hline METAL & Aluminum & $\mathrm{mg} / \mathrm{kg}$ & 45 & 45 & $100 \%$ & 2,460 & 11,100 & 6,349 & 11,800 & No & -- & .- & -- & -- & 76,142 & No & 0.15 & 0 \\
\hline METAL & Antimony & $\mathrm{mg} / \mathrm{kg}$ & 99 & 61 & $62 \%$ & 0.019 & 6.8 & 1.4 & 5.0 & Yes & 2.0 & Yes & 3.4 & 3 & 31 & No & 0.22 & 0 \\
\hline METAL & Arsenic & $\mathrm{mg} / \mathrm{kg}$ & 108 & 104 & $96 \%$ & 0.0082 & 20 & 4.6 & 6.5 & Yes & 6.0 & Yes & 3.3 & 25 & 0.39 & Yes & 51 & 96 \\
\hline $\begin{array}{l}\text { METAL } \\
\text { METAA }\end{array}$ & Barium & $\mathrm{mg} / \mathrm{kg}$ & 48 & 47 & $98 \%$ & 35 & 869 & 104 & 132 & $\begin{array}{l}\text { Yes } \\
\text { Yes }\end{array}$ & $\cdots$ & $-\cdots$ & - & $-\cdots$ & 5,375 & No & 0.16 & 0 \\
\hline $\begin{array}{l}\text { METAL } \\
\text { METAL }\end{array}$ & $\begin{array}{l}\text { Beryllium } \\
\text { Cadmium }\end{array}$ & $\begin{array}{l}\mathrm{mg} / \mathrm{kg} \\
\mathrm{m} / \mathrm{kga}\end{array}$ & $\begin{array}{l}110 \\
112\end{array}$ & $\begin{array}{l}85 \\
73 \\
73\end{array}$ & $\begin{array}{l}77 \% \\
65 \% \% \\
65 \%\end{array}$ & $\begin{array}{c}0.032 \\
3.80 \mathrm{E}-04\end{array}$ & $\begin{array}{l}2.4 \\
5.6\end{array}$ & $\begin{array}{l}0.74 \\
0.72\end{array}$ & $\begin{array}{l}1.5 \\
0.81\end{array}$ & $\begin{array}{l}\text { Yes } \\
\text { Yes }\end{array}$ & $\ddot{0}$ & yes & $\ddot{93}$ & $\ddot{4}$ & $\begin{array}{l}154 \\
37\end{array}$ & No & 0.015 & 0 \\
\hline $\begin{array}{l}\text { MEIAL } \\
\text { METAL }\end{array}$ & $\begin{array}{l}\text { Cadmium } \\
\text { Calcium }\end{array}$ & $\begin{array}{l}\mathrm{m} / \mathrm{g} / \mathrm{g} \\
\mathrm{mg} / \mathrm{kg}\end{array}$ & $\begin{array}{l}112 \\
45\end{array}$ & $\begin{array}{l}73 \\
45\end{array}$ & $\begin{array}{l}\begin{array}{l}65 \% \\
100 \%\end{array} \\
\text { S }\end{array}$ & $\begin{array}{c}3.80 E-04 \\
1,760\end{array}$ & $\begin{array}{l}5,6 \\
9,120\end{array}$ & $\begin{array}{l}0.72 \\
3,720\end{array}$ & $\begin{array}{c}0.81 \\
17,200\end{array}$ & $\begin{array}{l}\text { Yes } \\
\text { No }\end{array}$ & 0.60 & Yes & $\begin{array}{l}9.3 \\
--3\end{array}$ & 42 & 37 & No & 0.15 & 0 \\
\hline & Chromium & $\mathrm{mg} / \mathrm{kg}$ & 120 & 119 & $99 \%$ & 0 & 112 & 35 & 19 & & 37 & Yes & 3.0 & 48 & 30 & Yes & 3.7 & 50 \\
\hline METAL & Cobalt & $\mathrm{mg} / \mathrm{kg}$ & 45 & 44 & $98 \%$ & 2.8 & 10 & 6.5 & 16 & No & -- &.- & & & 903 & No & 0.011 & 0 \\
\hline METAL & Copper & $\mathrm{mg} / \mathrm{kg}$ & 119 & 119 & $100 \%$ & 0.18 & 70 & 19 & 22 & Yes & 32 & Yes & 2.2 & 10 & 2,960 & No & 0.024 & 0 \\
\hline METAL & Iron & mglkg & 45 & 45 & $100 \%$ & 6,480 & 31,100 & 14,519 & 32,600 & No & 20,000 & Yes & 1.6 & 2 & 23,463 & Yes & 1.3 & 1 \\
\hline $\begin{array}{l}\text { METAL } \\
\text { METAL }\end{array}$ & $\begin{array}{l}\text { Lead } \\
\text { Magnesium }\end{array}$ & $\begin{array}{l}\mathrm{mg} / \mathrm{kg} \\
\mathrm{m} / \mathrm{kg}\end{array}$ & $\begin{array}{l}108 \\
45\end{array}$ & 108 & $100 \%$ & 0.10 & $\begin{array}{c}76 \\
5520\end{array}$ & $\begin{array}{l}24 \\
2777\end{array}$ & 10 & Yes & 35 & Yes & 2.2 & 24 & 250 & No & 0.30 & 0 \\
\hline $\begin{array}{l}\text { METAL } \\
\text { METAL }\end{array}$ & $\begin{array}{l}\text { Magnesium } \\
\text { Manganese }\end{array}$ & $\begin{array}{l}\mathrm{mg} / \mathrm{kg} \\
\mathrm{m} / \mathrm{kg} g\end{array}$ & $\begin{array}{l}45 \\
48\end{array}$ & $\begin{array}{l}45 \\
48\end{array}$ & $\begin{array}{l}100 \% \\
100 \%\end{array}$ & $\begin{array}{l}1,480 \\
94\end{array}$ & $\begin{array}{l}5,520 \\
603\end{array}$ & $\begin{array}{l}3,777 \\
215\end{array}$ & $\begin{array}{c}7,060 \\
512\end{array}$ & $\begin{array}{l}\text { No } \\
\text { Yes }\end{array}$ & 460 & Yes & 1.3 & 3 & & No & 0.34 & $\ddot{0}$ \\
\hline METAL & $\begin{array}{l}\text { Manganese } \\
\text { Mercury }\end{array}$ & $\begin{array}{l}\text { migkg } \\
\text { mglkg }\end{array}$ & $\begin{array}{l}40 \\
108\end{array}$ & $\begin{array}{l}48 \\
67\end{array}$ & $\begin{array}{l}100 \% \\
62 \%\end{array}$ & $1.60 \mathrm{E}-05$ & 0.68 & 0.034 & $\begin{array}{l}0.33 \\
0.33\end{array}$ & Yes & 0 & $\begin{array}{l}\text { res } \\
\text { Yes }\end{array}$ & $\begin{array}{l}1.5 \\
3.8\end{array}$ & 1 & $\begin{array}{l}1,62 \\
23\end{array}$ & No & 0.029 & 0 \\
\hline METAL & Nickel & $\mathrm{mg} / \mathrm{kg}$ & 112 & 112 & $100 \%$ & 0.64 & 30 & 14 & 19 & Yes & 18 & Yes & 1.7 & 33 & 1,564 & No & 0.019 & 0 \\
\hline METAL & Potassium & mglkg & 45 & 45 & $100 \%$ & 424 & 1,650 & 845 & 2,150 & No & -- & & 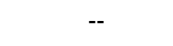 & 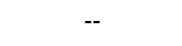 & & & & \\
\hline METAL & Selenium & mglkg & 107 & 29 & $27 \%$ & 0.0084 & 1.6 & 0.53 & 0.78 & Yes & -- & -- & -- & -- & 391 & No & 0.0042 & 0 \\
\hline METAL & Silver & mglkg & 99 & 58 & $59 \%$ & 0.0020 & 5.3 & 0.39 & 0.73 & Yes & 4.5 & Yes & 1.2 & 1 & 391 & No & 0.014 & 0 \\
\hline $\begin{array}{l}\text { METAL } \\
\text { METAL }\end{array}$ & $\begin{array}{l}\text { Sodium } \\
\text { Thallium }\end{array}$ & $\begin{array}{l}\mathrm{mg} / \mathrm{kg} \\
\mathrm{m} / \mathrm{kg} \mathrm{g}\end{array}$ & $\begin{array}{l}45 \\
99\end{array}$ & $\begin{array}{l}45 \\
63\end{array}$ & $\begin{array}{l}100 \% \\
64 \%\end{array}$ & 97 & $\begin{array}{l}364 \\
24\end{array}$ & $\begin{array}{l}207 \\
048\end{array}$ & 690 & No & $\because$ & $=$ & $\because$ & 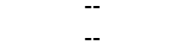 & $\ddot{5}=$ & $\ddot{n}$ & (ت) & $\overline{0}$ \\
\hline $\begin{array}{l}\text { METAL } \\
\text { METAL }\end{array}$ & $\begin{array}{l}\text { Thalum } \\
\text { Thorium }\end{array}$ & $\begin{array}{l}\text { migrg } \\
\text { mglkg }\end{array}$ & 3.0 & $\begin{array}{l}03 \\
3.0\end{array}$ & $\begin{array}{l}\begin{array}{l}10 \% \\
100 \%\end{array} \\
\text { S }\end{array}$ & 7.0 & $\begin{array}{l}2.4 \\
7.9\end{array}$ & $\begin{array}{l}0.48 \\
7.4\end{array}$ & $\overline{1.3}$ & Yes & 1,300 & No & 0.0061 & $\overline{0}$ & $\begin{array}{l}5.2 \\
1.3\end{array}$ & $\begin{array}{l}\text { No } \\
\text { Yes }\end{array}$ & $\begin{array}{l}0.47 \\
6.1\end{array}$ & $\begin{array}{l}0 \\
3\end{array}$ \\
\hline $\begin{array}{l}\text { METAL } \\
\text { METAL }\end{array}$ & $\begin{array}{l}\text { Uranium } \\
\text { Unium }\end{array}$ & $\begin{array}{l}m \text { migrg } \\
\text { mglkg }\end{array}$ & $\begin{array}{l}3.0 \\
7.0\end{array}$ & $\begin{array}{l}3.0 \\
7.0\end{array}$ & $100 \%$ & 1.1 & $\begin{array}{l}2.9 \\
2.6\end{array}$ & $\begin{array}{l}. .4 \\
1.6\end{array}$ & 3.2 & $\begin{array}{l}\text { res } \\
\text { No }\end{array}$ & $\begin{array}{l}1,500 \\
2,490\end{array}$ & No & 0.0010 & 0 & 16 & No & 0.16 & 0 \\
\hline METAL & Vanadium & $\mathrm{mg} / \mathrm{kg}$ & 45 & 45 & $100 \%$ & 6.5 & 86 & 31 & 85 & Yes & $\ldots$ & No & 0.0010 & 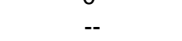 & 78 & Yes & 1.1 & 1 \\
\hline METAL & Zinc & $\mathrm{mg} / \mathrm{kg}$ & 112 & 112 & $100 \%$ & 0.18 & 477 & 179 & 68 & Yes & 120 & Yes & 4.0 & 74 & 23,463 & No & 0.020 & 0 \\
\hline RAD_D & Americium-241 & pcilg & 2.0 & 2.0 & $100 \%$ & 0.0030 & 0.24 & 0.12 & - & -- & 5,150 & No & $4.60 \mathrm{E}-05$ & 0 & 31 & No & 0.0076 & 0 \\
\hline $\begin{array}{l}\text { RAD_D } \\
\text { RAD D }\end{array}$ & $\begin{array}{l}\text { Antimony-125 } \\
\text { Beryllium-7 }\end{array}$ & $\begin{array}{l}\text { pCigg } \\
\text { piciga }\end{array}$ & $\begin{array}{l}1.0 \\
1.0\end{array}$ & $\begin{array}{l}1.0 \\
1.0\end{array}$ & $\begin{array}{l}100 \% \\
100 \%\end{array}$ & $\begin{array}{l}0.0018 \\
0.30\end{array}$ & $\begin{array}{l}0.0018 \\
0.30\end{array}$ & 0.0018 & -- & -- & - & - & - & - & -- & - & -- & -- \\
\hline $\begin{array}{l}\text { RADD } \\
\text { RADD D }\end{array}$ & $\begin{array}{l}\text { Desium-134 } \\
\text { Cesium-r }\end{array}$ & $\begin{array}{l}\text { pCig/ } \\
\text { pCigg }\end{array}$ & $\begin{array}{l}1.0 \\
4.0\end{array}$ & $\begin{array}{l}1.0 \\
4.0\end{array}$ & $100 \%$ & $\begin{array}{c}0.30 \\
2.86 \mathrm{E}-04\end{array}$ & $\begin{array}{l}0.30 \\
0.0011\end{array}$ & $\begin{array}{l}0.30 \\
6.44 E-04\end{array}$ & -- & -- & $\because$ & -- & $\therefore$ & $\therefore$ & $\approx$ & $\therefore$ & $\ldots$ & -- \\
\hline RADDD & Cesium-137 & pcilg & 122 & 122 & $100 \%$ & 0.023 & 1.5 & 0.28 & 1.1 & Yes & & No & $4.73 \mathrm{E}-04$ & 0 & 6.2 & No & 0.24 & 0 \\
\hline RAD_D & Coball-57 & pcilg & 1.0 & 1.0 & $100 \%$ & 3.07E-09 & $3.07 \mathrm{E}-09$ & $3.07 \mathrm{E}-09$ & 1.1 & 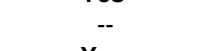 & 然 & & -- & & & & & \\
\hline RAD_D & Cobalt-60 & pcilg & 40 & 40 & $100 \%$ & 0.0033 & 2.4 & 0.091 & 0.0084 & Yes & 1,460 & No & 0.0017 & 0 & 1.4 & Yes & 1.7 & 1 \\
\hline RAD_D & Europium-152 & pCilg & 37 & 37 & $100 \%$ & 0.024 & 0.76 & 0.24 & -- & -- & -- & - & $\cdots$ & -- & 3.3 & No & 0.23 & 0 \\
\hline $\begin{array}{l}\text { RAD_D } \\
\text { RAD }\end{array}$ & $\begin{array}{l}\text { Europium-154 } \\
\text { Euronium-155 }\end{array}$ & $\begin{array}{l}\text { pCigg } \\
\text { ncigag }\end{array}$ & 6.0 & $\begin{array}{l}6.0 \\
30\end{array}$ & & $\begin{array}{l}0.015 \\
0.0071\end{array}$ & $\begin{array}{l}0.075 \\
0.34\end{array}$ & 0.024 & 0.033 & Yes & $\begin{array}{r}2,570 \\
21600\end{array}$ & No & $2.93 E-05$ & 0 & 3.0 & No & 0.025 & 0 \\
\hline $\begin{array}{l}\text { RAD_D } \\
\text { RADD }\end{array}$ & $\begin{array}{l}\text { Europium-155 } \\
\text { Manganese-54 }\end{array}$ & $\begin{array}{c}\text { pCigg } \\
\text { pCigg }\end{array}$ & $\begin{array}{l}3.0 \\
2.0\end{array}$ & $\begin{array}{l}3.0 \\
2.0\end{array}$ & $\begin{array}{l}100 \% \\
100 \%\end{array}$ & $\begin{array}{c}0.0071 \\
1.15 \mathrm{E}-08\end{array}$ & $\begin{array}{c}0.034 \\
1.51 \mathrm{E}-06\end{array}$ & $\begin{array}{l}0.024 \\
7.59 E-07\end{array}$ & $\begin{array}{l}0.054 \\
--.\end{array}$ & № & 31,600 & No & 1.09E-06 & 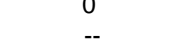 & 125 & № & $2.75 E-04$ & 0 \\
\hline $\begin{array}{l}\text { RAD_D } \\
\text { RAD D }\end{array}$ & $\begin{array}{l}\text { Manganese- } 54 \\
\text { Neptunium-237 }\end{array}$ & $\begin{array}{l}\mathrm{pCClg} \\
\mathrm{pCilg}\end{array}$ & $\begin{array}{l}2.0 \\
2.0\end{array}$ & $\begin{array}{l}2.0 \\
2.0\end{array}$ & $\begin{array}{l}100 \% \\
100 \%\end{array}$ & $\begin{array}{l}1.15 E-08 \\
0.48\end{array}$ & $\begin{array}{l}1.515-06 \\
0.61\end{array}$ & $\begin{array}{l}7.59-567 \\
0.54\end{array}$ & $\therefore$ & $\ldots$ & $\therefore$ & $\therefore$ & $\therefore$ & $\therefore$ & $\therefore$ & $\therefore$ & $\ldots$ & -- \\
\hline RAD_D & Plutonium-238 & $\mathrm{pcilg}$ & 15 & 15 & $100 \%$ & $1.26 \mathrm{E}-04$ & 0.13 & 0.016 & 0.0038 & Yes & -- & -- & -- & -- & 37 & No & 0.0035 & 0 \\
\hline RAD_D & Plutonium-239/240 & $\mathrm{pcilg}$ & 34 & 34 & $100 \%$ & $3.98 \mathrm{E}-04$ & 0.071 & 0.0064 & 0.025 & Yes & 5,860 & No & $1.21 \mathrm{E}-05$ & 0 & 34 & No & 0.0021 & 0 \\
\hline RAD_D & Potassium-40 & pCilg & 132 & 132 & $100 \%$ & 8.4 & 21 & 16 & 17 & Yes & $\therefore$ & 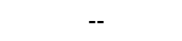 & 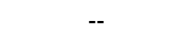 & 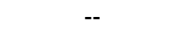 & 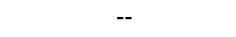 & -- & - & -- \\
\hline RAD_D & Radium-226 & pcilg & 37 & 37 & . & 0.42 & .1. & 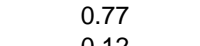 & 0.82 & Yes & 101 & No & 0.017 & 2 & -- & - & - & -- \\
\hline $\begin{array}{l}\text { RAD_D } \\
\text { RADD }\end{array}$ & $\begin{array}{l}\text { Radium-228 } \\
\text { Strontium-89 }\end{array}$ & $\begin{array}{l}\text { pCigg } \\
\text { pCiga }\end{array}$ & $\begin{array}{l}1.0 \\
3.0\end{array}$ & $\begin{array}{l}1.0 \\
3.0\end{array}$ & $\begin{array}{l}100 \% \\
100 \%\end{array}$ & $\begin{array}{c}0.12 \\
3.43 E-42\end{array}$ & $\begin{array}{c}0.12 \\
4.80 E-42\end{array}$ & $\begin{array}{c}0.12 \\
3.89 E-42\end{array}$ & - & 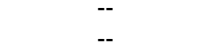 & 88 & No & 0.0014 & 0 & - & - & - & - \\
\hline $\begin{array}{l}\text { RADD } \\
\text { RAD_D }\end{array}$ & $\begin{array}{l}\text { Surtumium-99 } \\
\text { Strontium-90 }\end{array}$ & $\begin{array}{l}\text { pCCig } \\
\text { pCigg }\end{array}$ & $\begin{array}{l}3.0 \\
18\end{array}$ & 18 & $100 \%$ & $\begin{array}{l}3.43 E-42 \\
0.0020\end{array}$ & $\begin{array}{l}4.80-4 L \\
1.0\end{array}$ & $\begin{array}{l}3.89-4 \mathrm{c} \\
0.090\end{array}$ & 0.18 & Yes & 582 & No & 0.0018 & 0 & 4.5 & No & 0.23 & 0 \\
\hline & Thorium-228 & $\mathrm{pC}$ & 41 & 41 & 100 & & 2.1 & 0.13 & -- & -- & - & - & -- & - & -- & -- & -- & \\
\hline RAD_D & Thorium-230 & $\mathrm{pCi}$ & 4.0 & 4.0 & $100 \%$ & 0.16 & 5.6 & 2.1 & $\because$ & $\cdots$ & -- & $\because$ & -- & - & $\because$ & -- & -- & -- \\
\hline RAD_D & Thorium-232 & pCilg & 40 & 40 & $100 \%$ & 0.24 & 3.2 & 1.1 & 1.3 & Yes & 1,300 & No & 0.0025 & 0 & 1.3 & Yes & 2.5 & 9 \\
\hline $\begin{array}{l}\text { RAD_D } \\
\text { RADDD }\end{array}$ & $\begin{array}{l}\text { Thorium-234 } \\
\text { Tritium }\end{array}$ & $\begin{array}{c}\mathrm{pCi} \\
\mathrm{nCi}\end{array}$ & $\begin{array}{l}2.0 \\
1.0\end{array}$ & $\begin{array}{l}2.0 \\
1.0\end{array}$ & $\begin{array}{l}100 \% \\
100 \%\end{array}$ & $\begin{array}{l}3.70 E-60 \\
0.038\end{array}$ & $\begin{array}{r}4.37 E-60 \\
0.038\end{array}$ & $\begin{array}{l}4.03 E-60 \\
0.038\end{array}$ & -- & & 374000 & No & $\ddot{-}$ & & & No & & 0 \\
\hline $\begin{array}{l}\text { RAD_D } \\
\text { RAD_D }\end{array}$ & $\begin{array}{l}\text { Tutium } \\
\text { Uranium-233/234 }\end{array}$ & $\begin{array}{l}\mathrm{pC} \\
\mathrm{pC}\end{array}$ & $\begin{array}{l}1.0 \\
36\end{array}$ & $\begin{array}{l}1.0 \\
36\end{array}$ & $\begin{array}{l}100 \% \\
100 \%\end{array}$ & $\begin{array}{l}0.038 \\
0.29\end{array}$ & $\begin{array}{l}0.038 \\
2.6\end{array}$ & $\begin{array}{l}0.038 \\
0.82\end{array}$ & -- & -- & 374,000 & № & $\begin{array}{l}1.03 E-07 \\
-\end{array}$ & o & 510 & № & $\begin{array}{l}7.54 \mathrm{E}-05 \\
-\end{array}$ & .. \\
\hline RAD_D & Uranium-234 & pcilg & 90 & 90 & $100 \%$ & 0.071 & 11 & 0.82 & 1.1 & Yes & 5,270 & No & 0.0021 & 0 & 1.1 & Yes & 10 & 16 \\
\hline $\begin{array}{l}\text { RAD_D } \\
\text { RAD }\end{array}$ & Uranium-235 & pcilg & 79 & 79 & $100 \%$ & 0.0029 & 0.38 & 0.039 & 0.11 & Yes & 3,730 & No & $1.02 E-04$ & 0 & 0.84 & No & 0.45 & 0 \\
\hline $\begin{array}{l}\text { RAD_D } \\
\text { RAD D }\end{array}$ & $\begin{array}{l}\text { Uranium-236 } \\
\text { Uranium-238 }\end{array}$ & $\begin{array}{l}\text { pCCig } \\
\text { ocilg }\end{array}$ & $\begin{array}{l}4.0 \\
129\end{array}$ & $\begin{array}{l}4.0 \\
129\end{array}$ & $\begin{array}{l}100 \% \\
100 \%\end{array}$ & 3.11E-05 & 0.039 & 0.026 & 32 & Yes & & & & 0 & 11 & & ne & $\ddot{2}$ \\
\hline $\begin{array}{ll}\text { RAD } \\
\text { RADD }\end{array}$ & Zirconium/Niobium-95 & $\begin{array}{l}\mathrm{pll} / \mathrm{g} \\
\mathrm{pCig}\end{array}$ & 2.0 & 2.0 & $\begin{array}{l}100 \% \\
100 \%\end{array}$ & $\begin{array}{c}0.051 \\
2.60 \mathrm{E}-36\end{array}$ & $3.08 \mathrm{E}-36$ & $\begin{array}{l}0.76 \\
2.84 E-36\end{array}$ & 3.2 & res & 2,490 & № & 0.0040 & $\begin{array}{l}0 \\
--\end{array}$ & 1.1 & Yes & 9.1 & 22 \\
\hline
\end{tabular}

CONV - Conventional parameter
RAD_D - Decayed radionuclide 
Table B-6
Comparison of Segment 2 Detected Sediment Concentrations to Ecological and Human Health Benchmark Values (units reported "dry weight") Columbia River Component - Existing Sample Data Evaluation and Scoping Report

\begin{tabular}{|c|c|c|c|c|c|c|c|c|c|c|c|c|c|c|c|c|c|c|}
\hline $\begin{array}{c}\text { Constituent } \\
\text { Class }\end{array}$ & Constituent Name & Units & $\begin{array}{c}\text { Number } \\
\text { Analyzed }\end{array}$ & $\begin{array}{l}\text { Number } \\
\text { Detected }\end{array}$ & $\begin{array}{c}\text { Frequency of } \\
\text { Detection }\end{array}$ & $\begin{array}{c}\text { Minimum } \\
\text { Detect }\end{array}$ & $\begin{array}{c}\text { Maximum } \\
\text { Detect }\end{array}$ & $\begin{array}{c}\text { Average } \\
\text { Concentration }\end{array}$ & $\begin{array}{c}\text { Background } \\
\text { value }\end{array}$ & $\begin{array}{c}\text { Is Max > } \\
\text { Background? }\end{array}$ & $\begin{array}{c}\text { Ecological } \\
\text { Benchmark Value }\end{array}$ & $\begin{array}{l}\text { Is Max > Eco } \\
\text { Benchmark? }\end{array}$ & $\begin{array}{c}\text { Factor of } \\
\text { Exceedance }\end{array}$ & $\begin{array}{c}\text { Number of } \\
\text { Samples that } \\
\text { Exceeded }\end{array}$ & $\begin{array}{c}\text { Human Health } \\
\text { Benchmark } \\
\text { Value } \\
\end{array}$ & $\begin{array}{l}\text { Is Max > HH } \\
\text { Benchmark? }\end{array}$ & $\begin{array}{c}\begin{array}{c}\text { Factor of } \\
\text { Exceedance }\end{array} \\
\text {. }\end{array}$ & $\begin{array}{c}\text { Number of } \\
\text { Samples that } \\
\text { Exceeded }\end{array}$ \\
\hline $\begin{array}{l}\text { CONV } \\
\text { METAI }\end{array}$ & $\begin{array}{l}\text { Total Organic Carbon } \\
\end{array}$ & \% dry-wt & $\begin{array}{l}4 \\
4\end{array}$ & $\begin{array}{l}4 \\
4\end{array}$ & $100 \%$ & 3.12 & 3.4 & $\begin{array}{ll}3.2 \\
0.25\end{array}$ & 11.800 & No & - & - & -- & -- & 76142 & $N$ & $158 E-05$ & 0 \\
\hline $\begin{array}{l}\text { METAL } \\
\text { MEIAL }\end{array}$ & $\begin{array}{l}\text { Auminum } \\
\text { Antimony }\end{array}$ & mglkg dry-wt & $\begin{array}{l}4 \\
9\end{array}$ & $\begin{array}{l}4 \\
9\end{array}$ & $100 \%$ & $\begin{array}{l}0.40 \\
0.39\end{array}$ & $\begin{array}{l}1.2 \\
0.81\end{array}$ & 0.57 & $\begin{array}{c}11,800 \\
5.0\end{array}$ & No & 2.0 & No & 0.40 & 0 & $\begin{array}{l}0,142 \\
31\end{array}$ & No & $\begin{array}{l}1.5 \mathrm{sE}-\mathrm{-ub} \\
0.026\end{array}$ & 0 \\
\hline METAL & Arsenic & $\mathrm{mg} / \mathrm{kg} \mathrm{dr}-\mathrm{wt}$ & 14 & 14 & $100 \%$ & 0.0020 & 7.8 & 3.5 & 6.5 & Yes & 6.0 & Yes & 1.3 & 3 & 0.39 & Yes & 20 & 9 \\
\hline METAL & Barium & mglkg dry-wt & 4 & 4 & $100 \%$ & 0.40 & 1.0 & 0.60 & 132 & No & -- & -- & -- & -- & 5,375 & No & $1.86 \mathrm{E}-04$ & 0 \\
\hline $\begin{array}{l}\text { METAL } \\
\text { METAL }\end{array}$ & $\begin{array}{l}\text { Beryllium } \\
\text { Cadmium }\end{array}$ & $\begin{array}{l}m g / k g ~ d r y-w t \\
m \text { malk drowt }\end{array}$ & ${ }_{12}^{12}$ & 12 & $100 \%$ & 0.10 & 1.6 & 1.1 & 1.5 & Yes & $-\ddot{0}$ & -- & $\ddot{27}$ & $\overline{7}$ & ${ }_{254}^{154}$ & No & 0.010 & 0 \\
\hline & $\begin{array}{l}\text { Cadmium } \\
\text { Chromium }\end{array}$ & $\begin{array}{l}\mathrm{m} / \mathrm{g} / \mathrm{kg} \mathrm{dr}-\mathrm{wt} \\
\mathrm{m} / \mathrm{kg} \mathrm{dr}-\mathrm{wt}\end{array}$ & $\begin{array}{l}18 \\
17\end{array}$ & 18 & $\begin{array}{l}100 \% \\
76 \%\end{array}$ & $\begin{array}{l}0.20 \\
20\end{array}$ & $\begin{array}{l}1.6 \\
88\end{array}$ & $\begin{array}{l}0.61 \\
44\end{array}$ & $\begin{array}{l}0.81 \\
19\end{array}$ & $\begin{array}{l}\text { Yes } \\
\text { Yes }\end{array}$ & $\begin{array}{l}0.60 \\
37\end{array}$ & $\begin{array}{l}\text { Yes } \\
\text { Yyes }\end{array}$ & 2.7 & 7 & 30 & $\begin{array}{l}\text { No } \\
\text { Yes }\end{array}$ & 0.043 & $\begin{array}{c}0 \\
12\end{array}$ \\
\hline $\begin{array}{l}\text { MEIAL } \\
\text { METAL }\end{array}$ & $\begin{array}{l}\text { Cromomium } \\
\text { Copper }\end{array}$ & $\begin{array}{l}\mathrm{m} / \mathrm{g} g \mathrm{~g} \text { dry-wt } \\
\mathrm{m} / \mathrm{kg} \mathrm{dry}-\mathrm{wt}\end{array}$ & $\begin{array}{l}17 \\
17\end{array}$ & 17 & $\begin{array}{l}16 \% \\
100 \%\end{array}$ & $\begin{array}{l}20 \\
1.2\end{array}$ & $\begin{array}{l}00 \\
26\end{array}$ & $\begin{array}{l}44 \\
14\end{array}$ & $\begin{array}{l}19 \\
22\end{array}$ & $\begin{array}{l}\text { Yes } \\
\text { Yes }\end{array}$ & 32 & $\begin{array}{l}\text { Yes } \\
\text { No }\end{array}$ & $\begin{array}{l}2.4 \\
0.83\end{array}$ & $\begin{array}{l}9 \\
0\end{array}$ & $\begin{array}{l}30 \\
2.960\end{array}$ & No & $\begin{array}{c}2.9 \\
0.0088\end{array}$ & ${ }_{0}^{12}$ \\
\hline METAL & Iron & mglkg dry-wt & 4 & 4 & $100 \%$ & 2.2 & 7.6 & 5.8 & 32,600 & No & 20,000 & No & $\begin{array}{ll}3.80 \mathrm{E}-04 \\
04\end{array}$ & 0 & $\begin{array}{l}2,000 \\
23,463\end{array}$ & No & $\begin{array}{l}3.240-04 \\
3.240\end{array}$ & 0 \\
\hline METAL & Lead & mglkg dry-wt & 18 & 18 & $100 \%$ & 1.4 & 57 & 19 & 10 & Yes & 35 & Yes & 1.7 & 1 & 250 & No & 0.23 & 0 \\
\hline METAL & Manganese & mglkg dry-wt & 4 & 4 & $100 \%$ & 4.0 & 20 & 12 & 512 & No & 460 & No & 0.043 & 0 & 1,762 & No & 0.011 & 0 \\
\hline $\begin{array}{l}\text { METAL } \\
\text { METMT }\end{array}$ & Mercury & mglkg dry-wt & 16 & 8 & $50 \%$ & 0.0018 & 0.033 & 0.0094 & 0.33 & No & 0.18 & No & 0.18 & 0 & 23 & No & 0.0014 & 0 \\
\hline $\begin{array}{l}\text { METAL } \\
\text { METAL }\end{array}$ & $\begin{array}{l}\text { Nickel } \\
\text { Selenium }\end{array}$ & $\begin{array}{l}\mathrm{mg} / \mathrm{kg} \mathrm{dry}-\mathrm{wt} \\
\mathrm{m} / \mathrm{kg} \mathrm{d} \text { dr-wt }\end{array}$ & $\begin{array}{c}9 \\
13\end{array}$ & $\begin{array}{l}9 \\
7\end{array}$ & $\begin{array}{l}100 \% \\
55 \% \%\end{array}$ & 12 & ${ }_{15}^{21}$ & $\begin{array}{c}18 \\
0.27\end{array}$ & $\begin{array}{l}19 \\
078\end{array}$ & $\begin{array}{l}\text { Yes } \\
\text { Yes }\end{array}$ & 18 & Yes & 1.2 & 5 & $\begin{array}{l}1,564 \\
391\end{array}$ & No & 0.014 & 0 \\
\hline $\begin{array}{l}\text { METAL } \\
\text { METAL }\end{array}$ & $\begin{array}{l}\text { Selenium } \\
\text { Silver }\end{array}$ & $\begin{array}{l}\mathrm{m} / \mathrm{g} / \mathrm{k} \mathrm{dry}-\mathrm{wt} \\
\mathrm{m} / \mathrm{kg} \mathrm{dr}-\mathrm{wt}\end{array}$ & $\begin{array}{c}13 \\
9\end{array}$ & 8 & $\begin{array}{l}59 \% \\
89 \%\end{array}$ & $\begin{array}{l}0.0010 \\
0.048\end{array}$ & $\begin{array}{l}1.5 \\
0.33\end{array}$ & $\begin{array}{l}0.27 \\
0.21\end{array}$ & $\begin{array}{l}0.78 \\
0.73\end{array}$ & $\begin{array}{l}\text { Yes } \\
\text { No }\end{array}$ & 4.5 & No & 0.072 & $\overline{0}$ & $\begin{array}{l}391 \\
391\end{array}$ & $\begin{array}{l}\text { No } \\
\text { No }\end{array}$ & $\begin{array}{l}0.0039 \\
8.315-04\end{array}$ & $\begin{array}{l}0 \\
0\end{array}$ \\
\hline $\begin{array}{l}\text { METAL } \\
\text { METAL }\end{array}$ & $\begin{array}{l}\text { Slvel } \\
\text { Thallium }\end{array}$ & 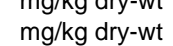 & 9 & 9 & $\begin{array}{l}5 \% \% \\
100 \%\end{array}$ & 0.42 & 0.77 & 0.58 & 0.13 & No & $\begin{array}{l}4.5 \\
-.5\end{array}$ & No & 0.0 .2 & .. & $\begin{array}{l}5.12 \\
5.2\end{array}$ & No & ( & $\begin{array}{lll}0 \\
0\end{array}$ \\
\hline METAL & Zinc & mglkg dry-wt & 13 & 13 & $100 \%$ & 4.0 & 366 & 132 & 68 & Yes & 120 & Yes & 3.1 & 8 & 23,463 & No & 0.016 & 0 \\
\hline RAD_D & Americium-241 & pciig dry-wt & 4 & 4 & $100 \%$ & 0.0020 & 0.0049 & 0.0032 & -- & -- & 5,150 & No & $9.52 \mathrm{E}-07$ & 0 & 31 & No & $1.58 \mathrm{E}-04$ & 0 \\
\hline RAD_D & Barium-140 & pcilg dry-wt & 1 & 1 & $100 \%$ & 7.09E-116 & 7.09E-116 & 7.09E-116 & -- & -- & -- & -- & -- & -- & -- & -- & -- & -- \\
\hline $\begin{array}{l}\text { RAD_D } \\
\text { RAD }\end{array}$ & $\begin{array}{l}\text { Beryllium-7 } \\
\text { Bismuth-214 }\end{array}$ & pcilg dry-wt & 11 & 11 & $100 \%$ & $\begin{array}{l}6.83 E-30 \\
355 E-05\end{array}$ & $\begin{array}{c}0.0031 \\
8.03 E .05\end{array}$ & $\begin{array}{l}2.84 \mathrm{E}-04 \\
570 \mathrm{E}\end{array}$ & -- & -- & -- & -- & -- & & -- & -- & -- & -- \\
\hline $\begin{array}{l}\text { RAD_D } \\
\text { RAD_D }\end{array}$ & $\begin{array}{l}\text { Bismuth-214 } \\
\text { Cerium-141 }\end{array}$ & $\begin{array}{l}\text { pCig/ d dry-wt } \\
\text { pCilg dry-wt }\end{array}$ & $\begin{array}{l}4 \\
2\end{array}$ & $\begin{array}{l}4 \\
2\end{array}$ & $\begin{array}{l}100 \% \\
100 \%\end{array}$ & $\begin{array}{l}3.55 \mathrm{E}-05 \\
1.23 \mathrm{E}-46\end{array}$ & $\begin{array}{l}8.93 E-05 \\
1.46 E-46\end{array}$ & $\begin{array}{l}5.79 E-05 \\
1.35-46\end{array}$ & -- & $\overline{--}$ & - & -- & -- & -- & -- & -- & -- & -- \\
\hline RAD_D & Cerium-144 & pcilg dry-wt & 1 & 1 & $\begin{array}{l}100 \% \\
\end{array}$ & $1.68 \mathrm{E}-06$ & $1.68 \mathrm{E}-06$ & 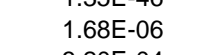 & - & - & - & - & - & - & - & - & - & - \\
\hline RAD_D & Cesium-134 & pcilg dry-wt & 12 & 12 & $100 \%$ & $2.68 \mathrm{E}-05$ & 0.0015 & $3.20 \mathrm{E}-04$ & -- & -- & -- & -- & -- & -- & -- & -- & - & -- \\
\hline RAD-D & $\begin{array}{l}\text { Cesium-137 } \\
\text { Cholt-5 }\end{array}$ & pcilg dry-wt & 129 & 129 & & $\begin{array}{c}0.021 \\
488 \mathrm{~F}-12\end{array}$ & $\begin{array}{c}1.2 \\
358 \mathrm{~F}-04\end{array}$ & $\begin{array}{l}0.15 \\
806-05\end{array}$ & 1.1 & Yes & 3,120 & No & $3.82 \mathrm{E}-04$ & 0 & 6.2 & No & 0.19 & 0 \\
\hline RAD_D & $\begin{array}{l}\text { Cobalt-57 } \\
\text { Cobhlt-58 }\end{array}$ & pcilg dry-wt & 4 & 4 & $100 \%$ & 4.88E-12 & $\begin{array}{l}3.58 \mathrm{E}-04 \\
2.025\end{array}$ & $\begin{array}{l}8.96 E-05 \\
.21-22\end{array}$ & -- & -- & $-\overline{-}$ ( & -- & $\ddot{-}$ & - & -- & -- & -- & -- \\
\hline $\begin{array}{l}\text { RAD D } \\
\text { RAD D }\end{array}$ & $\begin{array}{l}\text { Cobalth-58 } \\
\text { Cobalt-60 }\end{array}$ & $\begin{array}{l}\text { pCig/g dry-wt } \\
\text { pCirg dry-wt }\end{array}$ & $\begin{array}{l}1 \\
60\end{array}$ & $\begin{array}{l}1 \\
60\end{array}$ & $\begin{array}{l}100 \% \\
100 \%\end{array}$ & $\begin{array}{l}2.21 E-23 \\
0.0014\end{array}$ & $\begin{array}{c}2.21 E-23 \\
0.020\end{array}$ & $\begin{array}{c}2.21 E-23 \\
0.0059\end{array}$ & 0.0084 & Yes & $\begin{array}{c}-- \\
1,460\end{array}$ & No & $1.37 E-05$ & $\overline{0}$ & 1.4 & No & 0.014 & $\overline{0}$ \\
\hline RAD_D & Europium-152 & pCilg dry-wt & 26 & 26 & $100 \%$ & 0.012 & 0.11 & 0.051 & 0.0084 & -- & & No.- & & .. & $\begin{array}{l}1.4 \\
3.3 \\
\end{array}$ & No & $\begin{array}{l}0.014 \\
0.034\end{array}$ & 0 \\
\hline RAD_D & Europium-154 & pciig dry-wt & 6 & 6 & $100 \%$ & 0.015 & 0.090 & 0.045 & 0.033 & Yes & 2,570 & No & 3.49E-05 & 0 & 3.0 & No & 0.030 & 0 \\
\hline RAD_D & Europium-155 & pcilg dry-wt & 1 & 1 & $100 \%$ & 0.065 & 0.065 & 0.065 & 0.054 & Yes & 31,600 & No & 2.05E-06 & 0 & 125 & No & 5.17E-04 & 0 \\
\hline RAD & Iodine-131 & pCilg dry-wt & 2 & 2 & $100 \%$ & 1.07E-180 & 7.44E-180 & 4.25E-180 & & $\cdots$ & & & & & - & & & \\
\hline $\begin{array}{l}\text { RAD_D } \\
\text { RAD D }\end{array}$ & $\begin{array}{l}\text { Iron-59 } \\
\text { Lead-214 }\end{array}$ & pcilg dry-wt & $\begin{array}{l}2 \\
6\end{array}$ & $\begin{array}{l}2 \\
6\end{array}$ & $\begin{array}{l}100 \% \\
100 \%\end{array}$ & $\begin{array}{l}2.23 E-35 \\
5\end{array}$ & $\begin{array}{l}4.70 E-35 \\
0.97 E-04\end{array}$ & $\begin{array}{l}3.47 E-35 \\
778 E-04\end{array}$ & -- & -- & - & -- & - & -- & -- & -- & -- & -- \\
\hline $\begin{array}{l}\text { RAD_D } \\
\text { RAD_D }\end{array}$ & Manganese-54 & $\begin{array}{l}\text { pCig/g dry-wt } \\
\text { pCirg dry-wt }\end{array}$ & $\begin{array}{l}6 \\
6\end{array}$ & 6 & $\begin{array}{l}100 \% \\
100 \%\end{array}$ & $\begin{array}{l}5.09 E-04 \\
181 E-09\end{array}$ & $\begin{array}{l}9.87 \mathrm{E}-04 \\
4.75 \mathrm{E}-07\end{array}$ & $\begin{array}{l}7.28 E-04 \\
1.19-0.07\end{array}$ & -- & $\overline{--}$ & - & -- & - & -- & -- & -- & -- & - \\
\hline RADDD & Niobium-95 & pcilg dry-wt & 4 & 4 & $100 \%$ & $1.40 \mathrm{E}-43$ & 7.12E-43 & $\begin{array}{l}\text { I.19E- } \\
4.63 E-43\end{array}$ & -- & -- & -- & $\overline{--}$ & -- & - & -- & -- & $\begin{array}{l}-- \\
-\end{array}$ & -- \\
\hline RAD_D & Plutonium-238 & pCiig dry-wt & 9 & 9 & $100 \%$ & $9.10 \mathrm{E}-04$ & 0.050 & 0.0084 & 0.0038 & Yes & - & -- & -. & .. & 37 & No & 0.0013 & 0 \\
\hline RAD_D & Plutonium-239/240 & pcilg dry-wt & 26 & 170 & & 0 & 0.036 & 0.0082 & 0.025 & Yes & 5,860 & No & $6.14 E-06$ & 0 & 34 & No & 0.0011 & 0 \\
\hline $\begin{array}{l}\text { RAD_D } \\
\text { RAD D }\end{array}$ & $\begin{array}{l}\text { Potassiumm-40 } \\
\text { Radium-226 }\end{array}$ & $\begin{array}{l}\text { pCig/ dry-wt } \\
\text { pCilg dry-wt }\end{array}$ & $\begin{array}{c}170 \\
81\end{array}$ & $\begin{array}{l}170 \\
81\end{array}$ & $\begin{array}{l}100 \% \\
100 \%\end{array}$ & $\begin{array}{l}1.46 \mathrm{E}-07 \\
0.42\end{array}$ & $\begin{array}{l}24 \\
3.3\end{array}$ & $\begin{array}{l}16 \\
1.2\end{array}$ & $\begin{array}{c}17 \\
0.82\end{array}$ & $\begin{array}{l}\text { Yes } \\
\text { Yes }\end{array}$ & 101 & 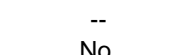 & & & -- & & -- & - \\
\hline $\begin{array}{l}\text { RAD } \\
\text { RADD }\end{array}$ & $\begin{array}{l}\text { Radium-226 } \\
\text { Ruthenium-103 }\end{array}$ & $\begin{array}{l}\text { pCCIg dary-wt } \\
\text { pCigg dry-wt }\end{array}$ & $\begin{array}{c}81 \\
2\end{array}$ & $\begin{array}{l}81 \\
2\end{array}$ & $\begin{array}{l}100 \% \\
100 \%\end{array}$ & $\begin{array}{l}0.42 \\
9.47 E-40\end{array}$ & $5.41 E-39$ & $3.18 E-39$ & 0.82 & Yes & 101 & No & 0.033 & 0 & -- & -- & -- & $-\overline{-}$ y \\
\hline RADDD & Ruthenium-106 & pcilg dry-wt & 3 & 3 & $100 \%$ & $1.28 \mathrm{E}-07$ & $7.88 \mathrm{E}-06$ & $2.71 \mathrm{E}-06$ &.- &.- &.- & 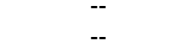 & $\because$ & -- & -- & -- & -- & -- \\
\hline RAD_D & Strontium-89 & pCiig dry-wt & 4 & 4 & $100 \%$ & $3.05 E-45$ & $4.77 E-42$ & $2.07 E-42$ & - & -- & -- & -- & -- & -- & -- & -- & -. & -- \\
\hline RAD_D & Strontiun & pCilg dr & 43 & 43 & $100 \%$ & $9.38 \mathrm{E}-04$ & 0.16 & 0.019 & 0.18 & No & 582 & No & $2.77 E-04$ & 0 & 4.5 & No & 0.036 & 0 \\
\hline $\begin{array}{l}\text { RAD_D } \\
\text { RAD D }\end{array}$ & $\begin{array}{l}\text { Technetium-99 } \\
\text { Thallium-208 }\end{array}$ & $\begin{array}{l}\text { pcig dry-wt } \\
\text { piclad dry-wt }\end{array}$ & $\begin{array}{l}2 \\
6\end{array}$ & $\begin{array}{l}2 \\
6\end{array}$ & $\begin{array}{l}10 \\
10\end{array}$ & $\begin{array}{r}0 \\
8.18\end{array}$ & $\begin{array}{c}0.38 \\
5.01 E-26\end{array}$ & 0 & $-\overline{-}$ & -- & 42,200 & No & $\begin{array}{l}9.00 \mathrm{E}-06 \\
--\end{array}$ & 0 & 15 & No & 0.025 & 0 \\
\hline $\begin{array}{l}\text { RAD } \\
\text { RAD }\end{array}$ & $\begin{array}{l}\text { Thallium-208 } \\
\text { Thorium-228 }\end{array}$ & $\begin{array}{l}\text { pCig/g dry-wt } \\
\text { pCirg dry-wt }\end{array}$ & $\begin{array}{c}6 \\
67\end{array}$ & $\begin{array}{c}6 \\
67\end{array}$ & & $\begin{array}{l}8.18 \mathrm{E}-27 \\
5.75 \mathrm{E}-05\end{array}$ & $\begin{array}{l}5.01 E-26 \\
4.3\end{array}$ & $\begin{array}{l}2.26 E-26 \\
0.30\end{array}$ & $\overline{-}$ & $\overline{-}$ & - & $\overline{-}$ & $\overline{-}$ & - & $\overline{-}$ & $\overline{-}$ & $\overline{-}$ & $\overline{-}$ \\
\hline RAD_D & Thorium-230 & pcilg dry-wt & 8 & 8 & $100 \%$ & 0.50 & 1.2 & 0.69 & -- & .. & -. & -. & -. & .. & - & -- & -- & -- \\
\hline RAD_D & Thorium-232 & pCilg d & 8 & 8 & 10000 & 0.60 & 1.5 & 0.98 & 1.3 & Yes & 1,300 & No & 0.0012 & 0 & 1.3 & Yes & 1.2 & 1 \\
\hline $\begin{array}{l}\text { RAD_D } \\
\text { RAD }\end{array}$ & $\begin{array}{l}\text { Tritium } \\
\text { Ulyanium-234 }\end{array}$ & $\begin{array}{l}\text { pCilg dry-wt } \\
\text { niclodrowt }\end{array}$ & $\stackrel{1}{55}$ & $\frac{1}{55}$ & $\begin{array}{l}100 \% \\
100 \%\end{array}$ & 0.0098 & 0.0098 & 0.0098 & & $y_{0}$ & 374,000 & No & $2.63 \mathrm{E}-08$ & 0 & 510 & No & $1.93 \mathrm{E}-05$ & 0 \\
\hline $\begin{array}{l}\text { RAD D } \\
\text { RAD D }\end{array}$ & $\begin{array}{l}\text { Uranumum-234 } \\
\text { Uranium-235 }\end{array}$ & $\begin{array}{l}\text { pCCIg d dy-wt } \\
\text { pCilg dry-wt }\end{array}$ & $\begin{array}{l}55 \\
31\end{array}$ & $\begin{array}{l}55 \\
31\end{array}$ & $\begin{array}{l}100 \% \\
100 \%\end{array}$ & $\begin{array}{l}0.38 \\
0.017\end{array}$ & $\begin{array}{l}4.5 \\
0.64\end{array}$ & $\begin{array}{l}1.1 \\
0.076\end{array}$ & $\begin{array}{l}1.1 \\
0.11\end{array}$ & $\begin{array}{l}\text { Yes } \\
\text { Yes }\end{array}$ & $\begin{array}{l}5,270 \\
3,730\end{array}$ & No & $8.50 E-04$ & 0 & 1.1 & Yes & 4.1 & $\begin{array}{c}12 \\
0\end{array}$ \\
\hline RADD & Uranium-236 & 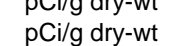 & $\begin{array}{l}31 \\
2\end{array}$ & $\begin{array}{l}11 \\
2\end{array}$ & $100 \%$ & 0.040 & $\begin{array}{l}0.04 \\
0.050\end{array}$ & 0.045 & ... & res & $\begin{array}{l}3,730 \\
-\end{array}$ & № & $1.72 E-04$ & 0 & 0.84 & No & 0.76 & 0 \\
\hline RAD_D & Uranium-238 & pcilg dry-wt & 57 & 57 & $100 \%$ & 0.41 & 4.3 & 1.1 & 3.2 & Yes & 2,490 & No & 0.0017 & 0 & 1.1 & Yes & 3.9 & 13 \\
\hline $\begin{array}{l}\text { RAD-D } \\
\text { RADD }\end{array}$ & $\begin{array}{l}\text { Zinc-65 } \\
\text { Zirconium-95 }\end{array}$ & $\begin{array}{l}\text { pcilg dry-wt } \\
\text { picig div-wt }\end{array}$ & $\begin{array}{l}7 \\
5\end{array}$ & $\begin{array}{l}7 \\
5\end{array}$ & $\begin{array}{l}100 \% \\
100 \%\end{array}$ & $\begin{array}{l}2.57 \mathrm{E}-13 \\
1.84 \mathrm{E}-36\end{array}$ & $\begin{array}{l}6.86 \mathrm{E}-08 \\
8.64 \mathrm{E}-24\end{array}$ & $\begin{array}{l}2.25 E-08 \\
3.16-24\end{array}$ & $\because$ & $\because-$ & $\because$ & -- & $\because$ & -- & -- & -- & $\therefore$ & -- \\
\hline
\end{tabular}

CONV - Conventional parameter
RAD_D - Decayed radionuclide 
Table B-7

Summary of Surface Sediment Results From Segment 2 Exceeding Select Ecological or Human Health Benchmark Values

Columbia River Component - Existing Sample Data Evaluation and Scoping Report

\begin{tabular}{|c|c|c|c|c|c|c|c|c|c|c|}
\hline Sample Area & Sample Site & SampleID & Date Collected & Constituent Name & Units & $\begin{array}{c}\text { QA } \\
\text { Category }\end{array}$ & $\begin{array}{c}\text { Analytical } \\
\text { Value }\end{array}$ & Qualifier & $\begin{array}{c}\text { Ecological } \\
\text { Benchmark } \\
\text { Value }\end{array}$ & $\begin{array}{c}\text { Human Health } \\
\text { Benchmark } \\
\text { Value }\end{array}$ \\
\hline Columbia River & Hanford Tnsite & B07NC6 & November 16, 1992 & Antimony & $\mathrm{mg} / \mathrm{kg}$ & 1 & 3.7 & BJN & 2.0 & 31 \\
\hline Columbia River & Horn Area & B07ND0 & November 17, 1992 & Antimony & $\mathrm{mg} / \mathrm{kg}$ & 1 & 6.8 & BJN & 2.0 & 31 \\
\hline Columbia River & Horn Area & $\mathrm{B} 07 \mathrm{NH} 2$ & November 23, 1992 & Antimony & $\mathrm{mg} / \mathrm{kg}$ & 1 & 4.7 & $\mathrm{JN}$ & 2.0 & 31 \\
\hline Columbia River & 300 Area & 4434235 & June 1,2004 & Arsenic & $\mathrm{mg} / \mathrm{kg}$ & 1 & 5.5 & $=$ & 6.0 & 0.39 \\
\hline Columbia River & RM 370 & 4434246 & June 1, 2004 & Arsenic & $\mathrm{mg} / \mathrm{kg}$ & 1 & 4.6 & $=$ & 6.0 & 0.39 \\
\hline Columbia River & RM 362 & 4434239 & June 1, 2004 & Arsenic & $\mathrm{mg} / \mathrm{kg}$ & 1 & 6.8 & $=$ & 6.0 & 0.39 \\
\hline Columbia River & RM 366 & 4434245 & June 1, 2004 & Arsenic & $\mathrm{mg} / \mathrm{kg}$ & 1 & 20 & $=$ & 6.0 & 0.39 \\
\hline Columbia River & F Slough & B07NB6 & November 12, 1992 & Arsenic & $\mathrm{mg} / \mathrm{kg}$ & 1 & 7.4 & $=$ & 6.0 & 0.39 \\
\hline Columbia River & F Slough & B07NB7 & November 12, 1992 & Arsenic & $\mathrm{mg} / \mathrm{kg}$ & 1 & 6.0 & $=$ & 6.0 & 0.39 \\
\hline Columbia River & F Slough & B07NB8 & November 12, 1992 & Arsenic & $\mathrm{mg} / \mathrm{kg}$ & 1 & 5.4 & $=$ & 6.0 & 0.39 \\
\hline Columbia River & F Slough & B07NB9 & November 12, 1992 & Arsenic & $\mathrm{mg} / \mathrm{kg}$ & 1 & 6.2 & $=$ & 6.0 & 0.39 \\
\hline Columbia River & 100-H Area & B07NC2 & November 13, 1992 & Arsenic & $\mathrm{mg} / \mathrm{kg}$ & 1 & 2.4 & B & 6.0 & 0.39 \\
\hline Columbia River & 100-H Area & B07NC3 & November 13, 1992 & Arsenic & $\mathrm{mg} / \mathrm{kg}$ & 1 & 4.5 & $=$ & 6.0 & 0.39 \\
\hline Columbia River & F Slough & B07NC4 & November 16, 1992 & Arsenic & $\mathrm{mg} / \mathrm{kg}$ & 1 & 5.7 & $=$ & 6.0 & 0.39 \\
\hline Columbia River & F Slough & B07NC5 & November 16, 1992 & Arsenic & $\mathrm{mg} / \mathrm{kg}$ & 1 & 3.0 & $=$ & 6.0 & 0.39 \\
\hline Columbia River & Hanford Tnsite & B07NC6 & November 16, 1992 & Arsenic & $\mathrm{mg} / \mathrm{kg}$ & 1 & 2.5 & $=$ & 6.0 & 0.39 \\
\hline Columbia River & Hanford Tnsite & B07NC7 & November 16, 1992 & Arsenic & $\mathrm{mg} / \mathrm{kg}$ & 1 & 5.6 & $=$ & 6.0 & 0.39 \\
\hline Columbia River & Horn Area & B07NC9 & November 17, 1992 & Arsenic & $\mathrm{mg} / \mathrm{kg}$ & 1 & 6.3 & $=$ & 6.0 & 0.39 \\
\hline Columbia River & Horn Area & B07ND0 & November 17, 1992 & Arsenic & $\mathrm{mg} / \mathrm{kg}$ & 1 & 2.3 & B & 6.0 & 0.39 \\
\hline Columbia River & 100-B/C Area & B07ND1 & November 20, 1992 & Arsenic & $\mathrm{mg} / \mathrm{kg}$ & 1 & 1.9 & $\mathrm{JN}+$ & 6.0 & 0.39 \\
\hline Columbia River & Hanford Tnsite & B07ND2 & November 17, 1992 & Arsenic & $\mathrm{mg} / \mathrm{kg}$ & 1 & 3.4 & $=$ & 6.0 & 0.39 \\
\hline Columbia River & Hanford Tnsite & B07ND3 & November 17, 1992 & Arsenic & $\mathrm{mg} / \mathrm{kg}$ & 1 & 3.2 & $=$ & 6.0 & 0.39 \\
\hline Columbia River & D Island & B07ND4 & November 19, 1992 & Arsenic & $\mathrm{mg} / \mathrm{kg}$ & 1 & 2.9 & B & 6.0 & 0.39 \\
\hline Columbia River & D Island & B07ND5 & November 19, 1992 & Arsenic & $\mathrm{mg} / \mathrm{kg}$ & 1 & 3.5 & $=$ & 6.0 & 0.39 \\
\hline Columbia River & D Island & B07ND6 & November 19, 1992 & Arsenic & $\mathrm{mg} / \mathrm{kg}$ & 1 & 2.7 & $=$ & 6.0 & 0.39 \\
\hline Columbia River & D Island & B07ND7 & November 19, 1992 & Arsenic & $\mathrm{mg} / \mathrm{kg}$ & 1 & 3.1 & $=$ & 6.0 & 0.39 \\
\hline Columbia River & D Island & B07ND8 & November 19, 1992 & Arsenic & $\mathrm{mg} / \mathrm{kg}$ & 1 & 2.2 & $=$ & 6.0 & 0.39 \\
\hline Columbia River & 100-B/C Area & B07ND9 & November 20, 1992 & Arsenic & $\mathrm{mg} / \mathrm{kg}$ & 1 & 1.6 & BJN & 6.0 & 0.39 \\
\hline Columbia River & Horn Area & B07NF0 & November 19, 1992 & Arsenic & $\mathrm{mg} / \mathrm{kg}$ & 1 & 1.9 & B & 6.0 & 0.39 \\
\hline Columbia River & 100-D Area & B07NF1 & November 19, 1992 & Arsenic & $\mathrm{mg} / \mathrm{kg}$ & 1 & 2.0 & B & 6.0 & 0.39 \\
\hline Columbia River & 100-D Area & B07NF2 & November 19, 1992 & Arsenic & $\mathrm{mg} / \mathrm{kg}$ & 1 & 4.4 & $=$ & 6.0 & 0.39 \\
\hline Columbia River & 100-K Area & B07NF9 & November 21, 1992 & Arsenic & $\mathrm{mg} / \mathrm{kg}$ & 1 & 6.2 & $=$ & 6.0 & 0.39 \\
\hline Columbia River & 100-K Area & B07NG0 & November 21, 1992 & Arsenic & $\mathrm{mg} / \mathrm{kg}$ & 1 & 5.1 & $\mathrm{~s}$ & 6.0 & 0.39 \\
\hline Columbia River & 100-K Area & B07NG1 & November 21, 1992 & Arsenic & $\mathrm{mg} / \mathrm{kg}$ & 1 & 6.0 & $=$ & 6.0 & 0.39 \\
\hline Columbia River & 100-K Area & B07NG2 & November 21, 1992 & Arsenic & $\mathrm{mg} / \mathrm{kg}$ & 1 & 2.9 & $=$ & 6.0 & 0.39 \\
\hline Columbia River & 100-H Area & B07NG3 & November 22, 1992 & Arsenic & $\mathrm{mg} / \mathrm{kg}$ & 1 & 4.5 & S & 6.0 & 0.39 \\
\hline Columbia River & 100-H Area & B07NG5 & November 22, 1992 & Arsenic & $\mathrm{mg} / \mathrm{kg}$ & 1 & 4.8 & $\mathrm{~s}$ & 6.0 & 0.39 \\
\hline Columbia River & 100-H Area & B07NG6 & November 22, 1992 & Arsenic & $\mathrm{mg} / \mathrm{kg}$ & 1 & 3.9 & $\mathrm{~s}$ & 6.0 & 0.39 \\
\hline Columbia River & 100-H Area & B07NG7 & November 22, 1992 & Arsenic & $\mathrm{mg} / \mathrm{kg}$ & 1 & 4.3 & JNS & 6.0 & 0.39 \\
\hline Columbia River & F Slough & B07NG9 & November 23, 1992 & Arsenic & $\mathrm{mg} / \mathrm{kg}$ & 1 & 4.9 & $\mathrm{~S}$ & 6.0 & 0.39 \\
\hline Columbia River & F Slough & B07NHO & November 23, 1992 & Arsenic & $\mathrm{mg} / \mathrm{kg}$ & 1 & 4.9 & JS & 6.0 & 0.39 \\
\hline Columbia River & Horn Area & B07NH1 & November 23, 1992 & Arsenic & $\mathrm{mg} / \mathrm{kg}$ & 1 & 6.1 & $=$ & 6.0 & 0.39 \\
\hline
\end{tabular}


Table B-7

Summary of Surface Sediment Results From Segment 2 Exceeding Select Ecological or Human Health Benchmark Values

Columbia River Component - Existing Sample Data Evaluation and Scoping Report

\begin{tabular}{|c|c|c|c|c|c|c|c|c|c|c|}
\hline Sample Area & Sample Site & SampleID & Date Collected & Constituent Name & Units & $\begin{array}{c}\text { QA } \\
\text { Category }\end{array}$ & $\begin{array}{c}\text { Analytical } \\
\text { Value }\end{array}$ & Qualifier & $\begin{array}{c}\text { Ecological } \\
\text { Benchmark } \\
\text { Value }\end{array}$ & $\begin{array}{c}\text { Human Health } \\
\text { Benchmark } \\
\text { Value }\end{array}$ \\
\hline Columbia River & Horn Area & B07NH2 & November 23, 1992 & Arsenic & $\mathrm{mg} / \mathrm{kg}$ & 1 & 6.0 & $\mathrm{~s}$ & 6.0 & 0.39 \\
\hline Columbia River & Hanford Tnsite & B07NH3 & November 23, 1992 & Arsenic & $\mathrm{mg} / \mathrm{kg}$ & 1 & 4.5 & $\mathrm{~s}$ & 6.0 & 0.39 \\
\hline Columbia River & Hanford Tnsite & B07NH4 & November 23, 1992 & Arsenic & $\mathrm{mg} / \mathrm{kg}$ & 1 & 3.5 & $=$ & 6.0 & 0.39 \\
\hline Columbia River & D Island & B07NH5 & November 24, 1992 & Arsenic & $\mathrm{mg} / \mathrm{kg}$ & 1 & 3.0 & $=$ & 6.0 & 0.39 \\
\hline Columbia River & D Island & B07NH6 & November 24, 1992 & Arsenic & $\mathrm{mg} / \mathrm{kg}$ & 1 & 2.8 & $=$ & 6.0 & 0.39 \\
\hline Columbia River & D Island & B07NH7 & November 24, 1992 & Arsenic & $\mathrm{mg} / \mathrm{kg}$ & 1 & 2.2 & BJN & 6.0 & 0.39 \\
\hline Columbia River & Wht Bluffs Slg & B0W3F4 & August 9, 1999 & Arsenic & $\mathrm{mg} / \mathrm{Kg}$ & 1 & 7.1 & $=$ & 6.0 & 0.39 \\
\hline Columbia River & 100 F SLOUGH & B0W3F6 & August 9, 1999 & Arsenic & $\mathrm{mg} / \mathrm{Kg}$ & 1 & 6.6 & $=$ & 6.0 & 0.39 \\
\hline Columbia River & Hanford Slough & B0W3F8 & August 9, 1999 & Arsenic & $\mathrm{mg} / \mathrm{Kg}$ & 1 & 5.8 & $=$ & 6.0 & 0.39 \\
\hline Columbia River & 300 SPR 42-2 & BOWDL4 & November 1, 1999 & Arsenic & $\mathrm{mg} / \mathrm{Kg}$ & 1 & 5.1 & $=$ & 6.0 & 0.39 \\
\hline Columbia River & 100-B SPR 38-3 & B0WDP5 & October 25, 1999 & Arsenic & $\mathrm{mg} / \mathrm{Kg}$ & 1 & 3.4 & $=$ & 6.0 & 0.39 \\
\hline Columbia River & HFD SPR UR 28-2 & BOWDP9 & November 1, 1999 & Arsenic & $\mathrm{mg} / \mathrm{Kg}$ & 1 & 6.6 & $=$ & 6.0 & 0.39 \\
\hline Columbia River & HFD SPR DR 28-2 & BOWDR1 & November 1, 1999 & Arsenic & $\mathrm{mg} / \mathrm{Kg}$ & 1 & 9.6 & $=$ & 6.0 & 0.39 \\
\hline Columbia River & 300 SPR DR 42-2 & BOWDR3 & November 1, 1999 & Arsenic & $\mathrm{mg} / \mathrm{Kg}$ & 1 & 6.0 & $=$ & 6.0 & 0.39 \\
\hline Columbia River & 100-F SPR 207-1 & BOWDTO & November 1, 1999 & Arsenic & $\mathrm{mg} / \mathrm{Kg}$ & 1 & 5.8 & $=$ & 6.0 & 0.39 \\
\hline Columbia River & RICHLAND-RIVER & B0YW85 & August 8, 2000 & Arsenic & $\mathrm{mg} / \mathrm{Kg}$ & 1 & 5.1 & $=$ & 6.0 & 0.39 \\
\hline Columbia River & Wht Bluffs Slg & B0YY65 & August 15, 2000 & Arsenic & $\mathrm{mg} / \mathrm{Kg}$ & 1 & 7.0 & $=$ & 6.0 & 0.39 \\
\hline Columbia River & 100 F SLOUGH & B0YY67 & August 15, 2000 & Arsenic & $\mathrm{mg} / \mathrm{Kg}$ & 1 & 3.3 & $=$ & 6.0 & 0.39 \\
\hline Columbia River & Hanford Slough & B0YY69 & August 15, 2000 & Arsenic & $\mathrm{mg} / \mathrm{Kg}$ & 1 & 4.0 & $=$ & 6.0 & 0.39 \\
\hline Columbia River & 300 SPR 42-2 & B10905 & November 2, 2000 & Arsenic & $\mathrm{mg} / \mathrm{Kg}$ & 1 & 5.9 & $=$ & 6.0 & 0.39 \\
\hline Columbia River & 100-B SPR 38-3 & B10916 & November 20, 2000 & Arsenic & $\mathrm{mg} / \mathrm{Kg}$ & 1 & 2.4 & $=$ & 6.0 & 0.39 \\
\hline Columbia River & HFD SPR UR 28-2 & B10918 & November 2, 2000 & Arsenic & $\mathrm{mg} / \mathrm{Kg}$ & 1 & 5.0 & $=$ & 6.0 & 0.39 \\
\hline Columbia River & HFD SPR DR 28-2 & B10921 & November 2, 2000 & Arsenic & $\mathrm{mg} / \mathrm{Kg}$ & 1 & 7.7 & $=$ & 6.0 & 0.39 \\
\hline Columbia River & 300 SPR DR 42-2 & B10923 & November 2, 2000 & Arsenic & $\mathrm{mg} / \mathrm{Kg}$ & 1 & 5.4 & $=$ & 6.0 & 0.39 \\
\hline Columbia River & 100-F SPR 207-1 & B10931 & November 20, 2000 & Arsenic & $\mathrm{mg} / \mathrm{Kg}$ & 1 & 5.8 & $=$ & 6.0 & 0.39 \\
\hline Columbia River & 100 F SLOUGH & $\mathrm{B} 12 \mathrm{CJ} 7$ & July 19, 2001 & Arsenic & $\mathrm{mg} / \mathrm{kg}$ dry-wt & 1 & 4.3 & $=$ & 6.0 & 0.39 \\
\hline Columbia River & Hanford Slough & B12CJ9 & July 19, 2001 & Arsenic & $\mathrm{mg} / \mathrm{kg}$ dry-wt & 1 & 3.5 & $=$ & 6.0 & 0.39 \\
\hline Columbia River & RICHLAND-RIVER & B12CK1 & July 23, 2001 & Arsenic & $\mathrm{mg} / \mathrm{kg}$ dry-wt & 1 & 7.4 & $=$ & 6.0 & 0.39 \\
\hline Columbia River & 300 SPR 42-2 & B12RL7 & August 27, 2001 & Arsenic & $\mathrm{mg} / \mathrm{kg}$ dry-wt & 1 & 6.6 & $=$ & 6.0 & 0.39 \\
\hline Columbia River & 300 SPR DR 42-2 & B12RM5 & August 27, 2001 & Arsenic & $\mathrm{mg} / \mathrm{kg}$ dry-wt & 1 & 7.8 & $=$ & 6.0 & 0.39 \\
\hline Columbia River & 300 SPR 11 & B12RY8 & August 27, 2001 & Arsenic & $\mathrm{mg} / \mathrm{kg}$ dry-wt & 1 & 3.4 & $=$ & 6.0 & 0.39 \\
\hline Columbia River & 300 SPR 14 & В12Т08 & August 27, 2001 & Arsenic & $\mathrm{mg} / \mathrm{kg}$ dry-wt & 1 & 5.9 & $=$ & 6.0 & 0.39 \\
\hline Columbia River & 100-B SPR 38-3 & B12X22 & October 22, 2001 & Arsenic & $\mathrm{mg} / \mathrm{kg}$ dry-wt & 1 & 2.3 & $=$ & 6.0 & 0.39 \\
\hline Columbia River & 100-F SPR 207-1 & B12X37 & October 22, 2001 & Arsenic & $\mathrm{mg} / \mathrm{kg}$ dry-wt & 1 & 3.9 & $=$ & 6.0 & 0.39 \\
\hline Columbia River & Hanford Slough & B14YM1 & July 23, 2002 & Arsenic & $\mathrm{mg} / \mathrm{kg}$ & 1 & 0.44 & $=$ & 6.0 & 0.39 \\
\hline Columbia River & RICHLAND-RIVER & B14YM3 & July 19, 2002 & Arsenic & $\mathrm{mg} / \mathrm{kg}$ & 1 & 0.45 & $=$ & 6.0 & 0.39 \\
\hline Columbia River & HFD SPR DR 28-2 & B15C46 & October 7, 2002 & Arsenic & $\mathrm{mg} / \mathrm{kg}$ & 1 & 0.65 & $=$ & 6.0 & 0.39 \\
\hline Columbia River & Ringold WW & B16WX6 & May 14, 2003 & Arsenic & $\mathrm{mg} / \mathrm{Kg}$ & 1 & 3.3 & $=$ & 6.0 & 0.39 \\
\hline Columbia River & Ringold WW & B16WX7 & May 14,2003 & Arsenic & $\mathrm{mg} / \mathrm{Kg}$ & 1 & 3.7 & $=$ & 6.0 & 0.39 \\
\hline Columbia River & ESQUATZEL CANAL & B16WX8 & May 14, 2003 & Arsenic & $\mathrm{mg} / \mathrm{Kg}$ & 1 & 2.7 & $=$ & 6.0 & 0.39 \\
\hline Columbia River & ESQUATZEL CANAL & B16WX9 & May 14, 2003 & Arsenic & $\mathrm{mg} / \mathrm{Kg}$ & 1 & 2.7 & $=$ & 6.0 & 0.39 \\
\hline Columbia River & Wht Bluffs Slg & B176X9 & July 14,2003 & Arsenic & $\mathrm{mg} / \mathrm{Kg}$ & 1 & 8.3 & $=$ & 6.0 & 0.39 \\
\hline
\end{tabular}


Table B-7

Summary of Surface Sediment Results From Segment 2 Exceeding Select Ecological or Human Health Benchmark Values Columbia River Component - Existing Sample Data Evaluation and Scoping Report

\begin{tabular}{|c|c|c|c|c|c|c|c|c|c|c|}
\hline Sample Area & Sample Site & SampleID & Date Collected & Constituent Name & Units & $\begin{array}{c}\text { QA } \\
\text { Category }\end{array}$ & $\begin{array}{c}\text { Analytical } \\
\text { Value }\end{array}$ & Qualifier & $\begin{array}{c}\text { Ecological } \\
\text { Benchmark } \\
\text { Value }\end{array}$ & $\begin{array}{c}\text { Human Health } \\
\text { Benchmark } \\
\text { Value }\end{array}$ \\
\hline Columbia River & 100 F SLOUGH & B176Y1 & July 14,2003 & Arsenic & $\mathrm{mg} / \mathrm{Kg}$ & 1 & 4.4 & $=$ & 6.0 & 0.39 \\
\hline Columbia River & Hanford Slough & B176Y3 & July 14, 2003 & Arsenic & $\mathrm{mg} / \mathrm{Kg}$ & 1 & 8.7 & $=$ & 6.0 & 0.39 \\
\hline Columbia River & RICHLAND-RIVER & B176Y5 & July 16, 2003 & Arsenic & $\mathrm{mg} / \mathrm{Kg}$ & 1 & 8.6 & $=$ & 6.0 & 0.39 \\
\hline Columbia River & 300 SPR 42-2 & B17J18 & October 13, 2003 & Arsenic & $\mathrm{mg} / \mathrm{Kg}$ & 1 & 6.2 & $=$ & 6.0 & 0.39 \\
\hline Columbia River & 100-B SPR 37-1 & B17J53 & October 20, 2003 & Arsenic & $\mathrm{mg} / \mathrm{Kg}$ & 1 & 4.8 & C & 6.0 & 0.39 \\
\hline Columbia River & HFD SPR DR 28-2 & B17J58 & November 3, 2003 & Arsenic & $\mathrm{mg} / \mathrm{Kg}$ & 1 & 9.2 & C & 6.0 & 0.39 \\
\hline Columbia River & 300 SPR DR 42-2 & B17J61 & October 13, 2003 & Arsenic & $\mathrm{mg} / \mathrm{Kg}$ & 1 & 5.9 & $=$ & 6.0 & 0.39 \\
\hline Columbia River & 100-K SPR 63-1 & B17J64 & October 20, 2003 & Arsenic & $\mathrm{mg} / \mathrm{Kg}$ & 1 & 5.0 & C & 6.0 & 0.39 \\
\hline Columbia River & 100-F SPR 207-1 & B17J67 & November 3, 2003 & Arsenic & $\mathrm{mg} / \mathrm{Kg}$ & 1 & 7.8 & C & 6.0 & 0.39 \\
\hline Columbia River & 100-H SPR 145-1 & B17JB1 & October 27, 2003 & Arsenic & $\mathrm{mg} / \mathrm{Kg}$ & 1 & 9.6 & C & 6.0 & 0.39 \\
\hline Columbia River & 100 B/C AREA UR & B17XN6 & November 17, 2003 & Arsenic & $\mathrm{mg} / \mathrm{Kg}$ & 1 & 2.7 & C & 6.0 & 0.39 \\
\hline Columbia River & 100-B SPR 37-1 & B17XN8 & November 13, 2003 & Arsenic & $\mathrm{mg} / \mathrm{Kg}$ & 1 & 3.2 & C & 6.0 & 0.39 \\
\hline Columbia River & 100-B SPR 38-3 & B17XN9 & November 13, 2003 & Arsenic & $\mathrm{mg} / \mathrm{Kg}$ & 1 & 6.9 & C & 6.0 & 0.39 \\
\hline Columbia River & Wht Bluffs Slg & B19PJ6 & July 12, 2004 & Arsenic & $\mathrm{mg} / \mathrm{Kg}$ & 1 & 8.1 & $\mathrm{x}$ & 6.0 & 0.39 \\
\hline Columbia River & 100 F SLOUGH & B19PJ8 & July 12,2004 & Arsenic & $\mathrm{mg} / \mathrm{Kg}$ & 1 & 4.8 & $\mathrm{x}$ & 6.0 & 0.39 \\
\hline Columbia River & Hanford Slough & B19PK0 & July 12, 2004 & Arsenic & $\mathrm{mg} / \mathrm{Kg}$ & 1 & 4.5 & $x$ & 6.0 & 0.39 \\
\hline Columbia River & RICHLAND-RIVER & B19PK2 & July 13,2004 & Arsenic & $\mathrm{mg} / \mathrm{Kg}$ & 1 & 6.7 & $\mathrm{x}$ & 6.0 & 0.39 \\
\hline Columbia River & 300 SPR 42-2 & B1BFM1 & October 25, 2004 & Arsenic & $\mathrm{mg} / \mathrm{Kg}$ & 1 & 4.9 & $\mathrm{x}$ & 6.0 & 0.39 \\
\hline Columbia River & 100-B SPR 37-1 & B1BFP7 & October 11, 2004 & Arsenic & $\mathrm{mg} / \mathrm{Kg}$ & 1 & 3.0 & $\mathrm{x}$ & 6.0 & 0.39 \\
\hline Columbia River & HFD SPR UR 28-2 & B1BFP9 & October 25, 2004 & Arsenic & $\mathrm{mg} / \mathrm{Kg}$ & 1 & 11 & $\mathrm{x}$ & 6.0 & 0.39 \\
\hline Columbia River & HFD SPR DR 28-2 & B1BFR2 & October 25, 2004 & Arsenic & $\mathrm{mg} / \mathrm{Kg}$ & 1 & 7.3 & $\mathrm{x}$ & 6.0 & 0.39 \\
\hline Columbia River & 300 SPR DR 42-2 & B1BFR5 & October 25, 2004 & Arsenic & $\mathrm{mg} / \mathrm{Kg}$ & 1 & 4.9 & $\mathrm{x}$ & 6.0 & 0.39 \\
\hline Columbia River & 100-F SPR 207-1 & B1BFT0 & October 26, 2004 & Arsenic & $\mathrm{mg} / \mathrm{Kg}$ & 1 & 5.2 & $x$ & 6.0 & 0.39 \\
\hline Columbia River & 100-H SPR 145-1 & $\mathrm{B} 1 \mathrm{BH} 03$ & October 26, 2004 & Arsenic & $\mathrm{mg} / \mathrm{Kg}$ & 1 & 7.4 & $x$ & 6.0 & 0.39 \\
\hline Columbia River & 300 Area & 4434235 & June 1,2004 & Cadmium & $\mathrm{mg} / \mathrm{kg}$ & 1 & 2.5 & $=$ & 0.60 & 37 \\
\hline Columbia River & RM 345 & 4434237 & June 1, 2004 & Cadmium & mg/kg & 1 & 0.98 & $=$ & 0.60 & 37 \\
\hline Columbia River & RM 362 & 4434239 & June 1, 2004 & Cadmium & $\mathrm{mg} / \mathrm{kg}$ & 1 & 3.8 & $=$ & 0.60 & 37 \\
\hline Columbia River & RM 366 & 4434245 & June 1, 2004 & Cadmium & $\mathrm{mg} / \mathrm{kg}$ & 1 & 1.2 & $=$ & 0.60 & 37 \\
\hline Columbia River & 100-K Area & B07NF9 & November 21, 1992 & Cadmium & $\mathrm{mg} / \mathrm{kg}$ & 1 & 5.6 & $=$ & 0.60 & 37 \\
\hline Columbia River & 100-H Area & B07NG3 & November 22, 1992 & Cadmium & $\mathrm{mg} / \mathrm{kg}$ & 1 & 2.0 & $=$ & 0.60 & 37 \\
\hline Columbia River & 100-H Area & B07NG6 & November 22, 1992 & Cadmium & $\mathrm{mg} / \mathrm{kg}$ & 1 & 1.7 & $=$ & 0.60 & 37 \\
\hline Columbia River & Horn Area & B07NH1 & November 23, 1992 & Cadmium & $\mathrm{mg} / \mathrm{kg}$ & 1 & 1.6 & $=$ & 0.60 & 37 \\
\hline Columbia River & Wht Bluffs Slg & BOCSH3 & September 24, 1994 & Cadmium & $\mathrm{mg} / \mathrm{kg}$ & 1 & 1.2 & $=$ & 0.60 & 37 \\
\hline Columbia River & Richland, WA & BOCSH6 & September 25, 1994 & Cadmium & $\mathrm{mg} / \mathrm{kg}$ & 1 & 1.2 & $=$ & 0.60 & 37 \\
\hline Columbia River & HFD SPR 28-2 & BOCSH8 & September 2, 1994 & Cadmium & $\mathrm{mg} / \mathrm{kg}$ & 1 & 0.80 & L & 0.60 & 37 \\
\hline Columbia River & Wht Bluffs Slg & B0W3F4 & August 9, 1999 & Cadmium & $\mathrm{mg} / \mathrm{Kg}$ & 1 & 1.3 & $=$ & 0.60 & 37 \\
\hline Columbia River & 100 F SLOUGH & B0W3F6 & August 9, 1999 & Cadmium & $\mathrm{mg} / \mathrm{Kg}$ & 1 & 1.5 & $=$ & 0.60 & 37 \\
\hline Columbia River & Hanford Slough & B0W3F8 & August 9, 1999 & Cadmium & $\mathrm{mg} / \mathrm{Kg}$ & 1 & 1.1 & $=$ & 0.60 & 37 \\
\hline Columbia River & 100-B SPR 38-3 & B0WDP5 & October 25, 1999 & Cadmium & $\mathrm{mg} / \mathrm{Kg}$ & 1 & 0.92 & $=$ & 0.60 & 37 \\
\hline Columbia River & HFD SPR UR 28-2 & BOWDP9 & November 1, 1999 & Cadmium & $\mathrm{mg} / \mathrm{Kg}$ & 1 & 0.95 & $=$ & 0.60 & 37 \\
\hline Columbia River & HFD SPR DR 28-2 & BOWDR1 & November 1, 1999 & Cadmium & $\mathrm{mg} / \mathrm{Kg}$ & 1 & 0.83 & $=$ & 0.60 & 37 \\
\hline Columbia River & 300 SPR DR 42-2 & BOWDR3 & November 1, 1999 & Cadmium & $\mathrm{mg} / \mathrm{Kg}$ & 1 & 0.77 & $=$ & 0.60 & 37 \\
\hline
\end{tabular}


Table B-7

Summary of Surface Sediment Results From Segment 2 Exceeding Select Ecological or Human Health Benchmark Values Columbia River Component - Existing Sample Data Evaluation and Scoping Report

\begin{tabular}{|c|c|c|c|c|c|c|c|c|c|c|}
\hline Sample Area & Sample Site & SampleID & Date Collected & Constituent Name & Units & $\begin{array}{c}\text { QA } \\
\text { Category }\end{array}$ & $\begin{array}{c}\text { Analytical } \\
\text { Value }\end{array}$ & Qualifier & $\begin{array}{c}\text { Ecological } \\
\text { Benchmark } \\
\text { Value }\end{array}$ & $\begin{array}{l}\text { Human Health } \\
\text { Benchmark } \\
\text { Value }\end{array}$ \\
\hline Columbia River & 100-F SPR 207-1 & BOWDT0 & November 1, 1999 & Cadmium & $\mathrm{mg} / \mathrm{Kg}$ & $\overline{1}$ & $\overline{1.1}$ & $=$ & 0.60 & 37 \\
\hline Columbia River & RICHLAND-RIVER & B0YW85 & August 8, 2000 & Cadmium & $\mathrm{mg} / \mathrm{Kg}$ & 1 & 1.0 & $=$ & 0.60 & 37 \\
\hline Columbia River & Wht Bluffs Slg & B0YY65 & August 15, 2000 & Cadmium & $\mathrm{mg} / \mathrm{Kg}$ & 1 & 1.8 & $=$ & 0.60 & 37 \\
\hline Columbia River & 100-B SPR 38-3 & B10916 & November 20,2000 & Cadmium & $\mathrm{mg} / \mathrm{Kg}$ & 1 & 0.64 & $=$ & 0.60 & 37 \\
\hline Columbia River & HFD SPR UR 28-2 & B10918 & November 2, 2000 & Cadmium & $\mathrm{mg} / \mathrm{Kg}$ & 1 & 0.95 & $=$ & 0.60 & 37 \\
\hline Columbia River & 300 SPR DR 42-2 & B10923 & November 2, 2000 & Cadmium & $\mathrm{mg} / \mathrm{Kg}$ & 1 & 0.65 & $=$ & 0.60 & 37 \\
\hline Columbia River & 100-F SPR 207-1 & B10931 & November 20, 2000 & Cadmium & $\mathrm{mg} / \mathrm{Kg}$ & 1 & 0.75 & $=$ & 0.60 & 37 \\
\hline Columbia River & 100 F SLOUGH & $\mathrm{B} 12 \mathrm{CJ} 7$ & July 19, 2001 & Cadmium & $\mathrm{mg} / \mathrm{kg}$ dry-wt & 1 & 0.61 & $=$ & 0.60 & 37 \\
\hline Columbia River & RICHLAND-RIVER & B12CK1 & July 23, 2001 & Cadmium & $\mathrm{mg} / \mathrm{kg}$ dry-wt & 1 & 1.5 & $=$ & 0.60 & 37 \\
\hline Columbia River & 300 SPR DR 42-2 & B12RM5 & August 27, 2001 & Cadmium & $\mathrm{mg} / \mathrm{kg}$ dry-wt & 1 & 0.87 & $=$ & 0.60 & 37 \\
\hline Columbia River & 100-B SPR 38-3 & B12X22 & October 22, 2001 & Cadmium & $\mathrm{mg} / \mathrm{kg}$ dry-wt & 1 & 0.77 & $=$ & 0.60 & 37 \\
\hline Columbia River & 100-F SPR 207-1 & B12X37 & October 22, 2001 & Cadmium & $\mathrm{mg} / \mathrm{kg}$ dry-wt & 1 & 0.87 & $=$ & 0.60 & 37 \\
\hline Columbia River & Wht Bluffs Slg & B176X9 & July 14,2003 & Cadmium & $\mathrm{mg} / \mathrm{Kg}$ & 1 & 2.6 & $=$ & 0.60 & 37 \\
\hline Columbia River & 100 F SLOUGH & B176Y1 & July 14,2003 & Cadmium & $\mathrm{mg} / \mathrm{Kg}$ & 1 & 0.67 & $=$ & 0.60 & 37 \\
\hline Columbia River & Hanford Slough & B176Y3 & July 14,2003 & Cadmium & $\mathrm{mg} / \mathrm{Kg}$ & 1 & 3.5 & $=$ & 0.60 & 37 \\
\hline Columbia River & RICHLAND-RIVER & B176Y5 & July 16, 2003 & Cadmium & $\mathrm{mg} / \mathrm{Kg}$ & 1 & 1.00 & $=$ & 0.60 & 37 \\
\hline Columbia River & 100-B SPR 37-1 & B17J53 & October 20, 2003 & Cadmium & $\mathrm{mg} / \mathrm{Kg}$ & 1 & 2.5 & $=$ & 0.60 & 37 \\
\hline Columbia River & HFD SPR DR 28-2 & B17J58 & November 3, 2003 & Cadmium & $\mathrm{mg} / \mathrm{Kg}$ & 1 & 1.1 & $=$ & 0.60 & 37 \\
\hline Columbia River & 100-K SPR 63-1 & B17J64 & October 20, 2003 & Cadmium & $\mathrm{mg} / \mathrm{Kg}$ & 1 & 0.72 & $=$ & 0.60 & 37 \\
\hline Columbia River & $100-F$ SPR 207-1 & B17J67 & November 3, 2003 & Cadmium & $\mathrm{mg} / \mathrm{Kg}$ & 1 & 1.5 & $=$ & 0.60 & 37 \\
\hline Columbia River & 100-H SPR 145-1 & B17JB1 & October 27, 2003 & Cadmium & $\mathrm{mg} / \mathrm{Kg}$ & 1 & 1.1 & $=$ & 0.60 & 37 \\
\hline Columbia River & 100-B SPR 37-1 & B17XN8 & November 13, 2003 & Cadmium & $\mathrm{mg} / \mathrm{Kg}$ & 1 & 0.74 & $=$ & 0.60 & 37 \\
\hline Columbia River & 100-B SPR 38-3 & B17XN9 & November 13, 2003 & Cadmium & $\mathrm{mg} / \mathrm{Kg}$ & 1 & 0.64 & $=$ & 0.60 & 37 \\
\hline Columbia River & Wht Bluffs Slg & B19PJ6 & July 12,2004 & Cadmium & $\mathrm{mg} / \mathrm{Kg}$ & 1 & 1.9 & $x$ & 0.60 & 37 \\
\hline Columbia River & 100 F SLOUGH & B19PJ8 & July 12, 2004 & Cadmium & $\mathrm{mg} / \mathrm{Kg}$ & 1 & 0.70 & $x$ & 0.60 & 37 \\
\hline Columbia River & RICHLAND-RIVER & B19PK2 & July 13,2004 & Cadmium & $\mathrm{mg} / \mathrm{Kg}$ & 1 & 0.83 & $\mathrm{x}$ & 0.60 & 37 \\
\hline Columbia River & HFD SPR UR 28-2 & B1BFP9 & October 25, 2004 & Cadmium & $\mathrm{mg} / \mathrm{Kg}$ & 1 & 1.3 & $\mathrm{x}$ & 0.60 & 37 \\
\hline Columbia River & HFD SPR DR 28-2 & B1BFR2 & October 25, 2004 & Cadmium & $\mathrm{mg} / \mathrm{Kg}$ & 1 & 0.70 & $\mathrm{x}$ & 0.60 & 37 \\
\hline Columbia River & 100-H SPR 145-1 & $\mathrm{B} 1 \mathrm{BH} 03$ & October 26, 2004 & Cadmium & $\mathrm{mg} / \mathrm{Kg}$ & 1 & 0.68 & $\mathrm{x}$ & 0.60 & 37 \\
\hline Columbia River & RM 328 & SRP 3,4 & August 20, 1991 & Cadmium & $\mathrm{mg} / \mathrm{kg}$ dry-wt & 1 & 1.6 & $=$ & 0.60 & 37 \\
\hline Columbia River & CGS STA 33 & 6135 & April 9,1987 & Cobalt-60 & $\mathrm{pCi} / \mathrm{g}$ & 2 & 2.4 & $=$ & 1,460 & 1.4 \\
\hline Columbia River & F Slough & B07NB9 & November 12, 1992 & Chromium & $\mathrm{mg} / \mathrm{kg}$ & 1 & 46 & $=$ & 37 & 30 \\
\hline Columbia River & 100-B/C Area & B07ND1 & November 20, 1992 & Chromium & $\mathrm{mg} / \mathrm{kg}$ & 1 & 48 & $=$ & 37 & 30 \\
\hline Columbia River & 100-B/C Area & B07ND9 & November 20, 1992 & Chromium & $\mathrm{mg} / \mathrm{kg}$ & 1 & 51 & $=$ & 37 & 30 \\
\hline Columbia River & 100-D Area & B07NF1 & November 19, 1992 & Chromium & $\mathrm{mg} / \mathrm{kg}$ & 1 & 47 & $=$ & 37 & 30 \\
\hline Columbia River & 100-K Area & B07NF9 & November 21, 1992 & Chromium & $\mathrm{mg} / \mathrm{kg}$ & 1 & 64 & $=$ & 37 & 30 \\
\hline Columbia River & Horn Area & $\mathrm{B} 07 \mathrm{NH} 2$ & November 23, 1992 & Chromium & $\mathrm{mg} / \mathrm{kg}$ & 1 & 32 & $=$ & 37 & 30 \\
\hline Columbia River & Wht Bluffs Slg & B0W3F4 & August 9, 1999 & Chromium & $\mathrm{mg} / \mathrm{Kg}$ & 1 & 52 & $=$ & 37 & 30 \\
\hline Columbia River & $100 \mathrm{~F}$ SLOUGH & Bow3F6 & August 9, 1999 & Chromium & $\mathrm{mg} / \mathrm{Kg}$ & 1 & 75 & $=$ & 37 & 30 \\
\hline Columbia River & Hanford Slough & B0W3F8 & August 9, 1999 & Chromium & $\mathrm{mg} / \mathrm{Kg}$ & 1 & 59 & $=$ & 37 & 30 \\
\hline Columbia River & 300 SPR 42-2 & BOWDL4 & November 1, 1999 & Chromium & $\mathrm{mg} / \mathrm{Kg}$ & 1 & 47 & $=$ & 37 & 30 \\
\hline Columbia River & 100-B SPR 38-3 & BOWDP5 & October 25, 1999 & Chromium & $\mathrm{mg} / \mathrm{Kg}$ & 1 & 62 & $=$ & 37 & 30 \\
\hline
\end{tabular}


Table B-7

Summary of Surface Sediment Results From Segment 2 Exceeding Select Ecological or Human Health Benchmark Values

Columbia River Component - Existing Sample Data Evaluation and Scoping Report

\begin{tabular}{|c|c|c|c|c|c|c|c|c|c|c|}
\hline Sample Area & Sample Site & SampleID & Date Collected & Constituent Name & Units & $\begin{array}{c}\text { QA } \\
\text { Category }\end{array}$ & $\begin{array}{c}\text { Analytical } \\
\text { Value }\end{array}$ & Qualifier & $\begin{array}{c}\text { Ecological } \\
\text { Benchmark } \\
\text { Value }\end{array}$ & $\begin{array}{l}\text { Human Health } \\
\text { Benchmark } \\
\text { Value }\end{array}$ \\
\hline Columbia River & HFD SPR UR 28-2 & B0WDP9 & November 1, 1999 & Chromium & $\mathrm{mg} / \mathrm{Kg}$ & $\overline{1}$ & 61 & $=$ & 37 & 30 \\
\hline Columbia River & HFD SPR DR 28-2 & BOWDR1 & November 1, 1999 & Chromium & $\mathrm{mg} / \mathrm{Kg}$ & 1 & 84 & $=$ & 37 & 30 \\
\hline Columbia River & 300 SPR DR 42-2 & BOWDR3 & November 1, 1999 & Chromium & $\mathrm{mg} / \mathrm{Kg}$ & 1 & 44 & $=$ & 37 & 30 \\
\hline Columbia River & 100-F SPR 207-1 & BOWDTO & November 1, 1999 & Chromium & $\mathrm{mg} / \mathrm{Kg}$ & 1 & 54 & $=$ & 37 & 30 \\
\hline Columbia River & RICHLAND-RIVER & B0YW85 & August 8, 2000 & Chromium & $\mathrm{mg} / \mathrm{Kg}$ & 1 & 49 & $=$ & 37 & 30 \\
\hline Columbia River & Wht Bluffs Slg & B0YY65 & August 15, 2000 & Chromium & $\mathrm{mg} / \mathrm{Kg}$ & 1 & 59 & $=$ & 37 & 30 \\
\hline Columbia River & Hanford Slough & B0YY69 & August 15,2000 & Chromium & $\mathrm{mg} / \mathrm{Kg}$ & 1 & 60 & $=$ & 37 & 30 \\
\hline Columbia River & 300 SPR 42-2 & B10905 & November 2, 2000 & Chromium & $\mathrm{mg} / \mathrm{Kg}$ & 1 & 54 & $=$ & 37 & 30 \\
\hline Columbia River & 100-B SPR 38-3 & B10916 & November 20, 2000 & Chromium & $\mathrm{mg} / \mathrm{Kg}$ & 1 & 83 & $=$ & 37 & 30 \\
\hline Columbia River & HFD SPR UR 28-2 & B10918 & November 2, 2000 & Chromium & $\mathrm{mg} / \mathrm{Kg}$ & 1 & 76 & $=$ & 37 & 30 \\
\hline Columbia River & HFD SPR DR 28-2 & B10921 & November 2, 2000 & Chromium & $\mathrm{mg} / \mathrm{Kg}$ & 1 & 77 & $=$ & 37 & 30 \\
\hline Columbia River & 300 SPR DR $42-2$ & B10923 & November 2, 2000 & Chromium & $\mathrm{mg} / \mathrm{Kg}$ & 1 & 59 & $=$ & 37 & 30 \\
\hline Columbia River & 100-F SPR 207-1 & B10931 & November 20, 2000 & Chromium & $\mathrm{mg} / \mathrm{Kg}$ & 1 & 72 & $=$ & 37 & 30 \\
\hline Columbia River & 100 F SLOUGH & $\mathrm{B} 12 \mathrm{CJ} 7$ & July 19, 2001 & Chromium & $\mathrm{mg} / \mathrm{kg}$ dry-wt & 1 & 76 & $=$ & 37 & 30 \\
\hline Columbia River & Hanford Slough & B12CJ9 & July 19,2001 & Chromium & $\mathrm{mg} / \mathrm{kg}$ dry-wt & 1 & 65 & $=$ & 37 & 30 \\
\hline Columbia River & RICHLAND-RIVER & B12CK1 & July 23, 2001 & Chromium & $\mathrm{mg} / \mathrm{kg}$ dry-wt & 1 & 53 & $=$ & 37 & 30 \\
\hline Columbia River & 300 SPR 42-2 & B12RL7 & August 27, 2001 & Chromium & $\mathrm{mg} / \mathrm{kg}$ dry-wt & 1 & 78 & $=$ & 37 & 30 \\
\hline Columbia River & 300 SPR DR 42-2 & B12RM5 & August 27, 2001 & Chromium & $\mathrm{mg} / \mathrm{kg}$ dry-wt & 1 & 55 & $=$ & 37 & 30 \\
\hline Columbia River & 300 SPR 11 & B12RY8 & August 27, 2001 & Chromium & $\mathrm{mg} / \mathrm{kg}$ dry-wt & 1 & 34 & $=$ & 37 & 30 \\
\hline Columbia River & 300 SPR 14 & В12T08 & August 27,2001 & Chromium & $\mathrm{mg} / \mathrm{kg}$ dry-wt & 1 & 43 & $=$ & 37 & 30 \\
\hline Columbia River & 100-B SPR 38-3 & B12X22 & October 22, 2001 & Chromium & $\mathrm{mg} / \mathrm{kg}$ dry-wt & 1 & 86 & $=$ & 37 & 30 \\
\hline Columbia River & 100-F SPR 207-1 & B12X37 & October 22, 2001 & Chromium & $\mathrm{mg} / \mathrm{kg}$ dry-wt & 1 & 88 & $=$ & 37 & 30 \\
\hline Columbia River & Wht Bluffs Slg & B176X9 & July 14,2003 & Chromium & $\mathrm{mg} / \mathrm{Kg}$ & 1 & 79 & C & 37 & 30 \\
\hline Columbia River & 100 F SLOUGH & B176Y1 & July 14,2003 & Chromium & $\mathrm{mg} / \mathrm{Kg}$ & 1 & 55 & C & 37 & 30 \\
\hline Columbia River & Hanford Slough & B176Y3 & July 14,2003 & Chromium & $\mathrm{mg} / \mathrm{Kg}$ & 1 & 83 & C & 37 & 30 \\
\hline Columbia River & RICHLAND-RIVER & B176Y5 & July 16,2003 & Chromium & $\mathrm{mg} / \mathrm{Kg}$ & 1 & 65 & C & 37 & 30 \\
\hline Columbia River & 300 SPR 42-2 & B17J18 & October 13, 2003 & Chromium & $\mathrm{mg} / \mathrm{Kg}$ & 1 & 46 & $=$ & 37 & 30 \\
\hline Columbia River & 100-B SPR 37-1 & B17J53 & October 20, 2003 & Chromium & $\mathrm{mg} / \mathrm{Kg}$ & 1 & 112 & C & 37 & 30 \\
\hline Columbia River & HFD SPR DR 28-2 & B17J58 & November 3, 2003 & Chromium & $\mathrm{mg} / \mathrm{Kg}$ & 1 & 81 & C & 37 & 30 \\
\hline Columbia River & 300 SPR DR 42-2 & B17J61 & October 13, 2003 & Chromium & $\mathrm{mg} / \mathrm{Kg}$ & 1 & 51 & $=$ & 37 & 30 \\
\hline Columbia River & 100-K SPR 63-1 & B17J64 & October 20,2003 & Chromium & $\mathrm{mg} / \mathrm{Kg}$ & 1 & 110 & C & 37 & 30 \\
\hline Columbia River & 100-F SPR 207-1 & B17J67 & November 3, 2003 & Chromium & $\mathrm{mg} / \mathrm{Kg}$ & 1 & 74 & C & 37 & 30 \\
\hline Columbia River & 100-H SPR 145-1 & B17JB1 & October 27, 2003 & Chromium & $\mathrm{mg} / \mathrm{Kg}$ & 1 & 53 & C & 37 & 30 \\
\hline Columbia River & 100 B/C AREA UR & B17XN6 & November 17, 2003 & Chromium & $\mathrm{mg} / \mathrm{Kg}$ & 1 & 103 & C & 37 & 30 \\
\hline Columbia River & 100-B SPR 37-1 & B17XN8 & November 13, 2003 & Chromium & $\mathrm{mg} / \mathrm{Kg}$ & 1 & 96 & C & 37 & 30 \\
\hline Columbia River & 100-B SPR 38-3 & B17XN9 & November 13, 2003 & Chromium & $\mathrm{mg} / \mathrm{Kg}$ & 1 & 68 & $\mathrm{C}$ & 37 & 30 \\
\hline Columbia River & Wht Bluffs Slg & B19PJ6 & July 12,2004 & Chromium & $\mathrm{mg} / \mathrm{Kg}$ & 1 & 63 & $c x$ & 37 & 30 \\
\hline Columbia River & 100 F SLOUGH & B19PJ8 & July 12,2004 & Chromium & $\mathrm{mg} / \mathrm{Kg}$ & 1 & 41 & $c x$ & 37 & 30 \\
\hline Columbia River & Hanford Slough & B19PK0 & July 12, 2004 & Chromium & $\mathrm{mg} / \mathrm{Kg}$ & 1 & 59 & $c x$ & 37 & 30 \\
\hline Columbia River & RICHLAND-RIVER & B19PK2 & July 13, 2004 & Chromium & $\mathrm{mg} / \mathrm{Kg}$ & 1 & 78 & $\mathrm{Cx}$ & 37 & 30 \\
\hline Columbia River & 300 SPR 42-2 & B1BFM1 & October 25, 2004 & Chromium & $\mathrm{mg} / \mathrm{Kg}$ & 1 & 50 & $\mathrm{x}$ & 37 & 30 \\
\hline Columbia River & 100-B SPR 37-1 & B1BFP7 & October 11,2004 & Chromium & $\mathrm{mg} / \mathrm{Kg}$ & 1 & 82 & $x$ & 37 & 30 \\
\hline
\end{tabular}


Table B-7

Summary of Surface Sediment Results From Segment 2 Exceeding Select Ecological or Human Health Benchmark Values

Columbia River Component - Existing Sample Data Evaluation and Scoping Report

\begin{tabular}{|c|c|c|c|c|c|c|c|c|c|c|}
\hline Sample Area & Sample Site & SampleID & Date Collected & Constituent Name & Units & $\begin{array}{c}\text { QA } \\
\text { Category }\end{array}$ & $\begin{array}{c}\text { Analytical } \\
\text { Value }\end{array}$ & Qualifier & $\begin{array}{c}\text { Ecological } \\
\text { Benchmark } \\
\text { Value }\end{array}$ & $\begin{array}{l}\text { Human Health } \\
\text { Benchmark } \\
\text { Value }\end{array}$ \\
\hline Columbia River & HFD SPR UR 28-2 & B1BFP9 & October 25,2004 & Chromium & $\mathrm{mg} / \mathrm{Kg}$ & 1 & 45 & $\bar{x}$ & 37 & 30 \\
\hline Columbia River & HFD SPR DR 28-2 & B1BFR2 & October 25, 2004 & Chromium & $\mathrm{mg} / \mathrm{Kg}$ & 1 & 81 & $\mathrm{x}$ & 37 & 30 \\
\hline Columbia River & 300 SPR DR 42-2 & B1BFR5 & October 25, 2004 & Chromium & $\mathrm{mg} / \mathrm{Kg}$ & 1 & 56 & $\mathrm{x}$ & 37 & 30 \\
\hline Columbia River & 100-F SPR 207-1 & B1BFT0 & October 26, 2004 & Chromium & $\mathrm{mg} / \mathrm{Kg}$ & 1 & 62 & $\mathrm{x}$ & 37 & 30 \\
\hline Columbia River & 100-H SPR 145-1 & B1BH03 & October 26, 2004 & Chromium & $\mathrm{mg} / \mathrm{Kg}$ & 1 & 63 & $\mathrm{x}$ & 37 & 30 \\
\hline Columbia River & 100-B/C Area & HR004W & October 7, 2003 & Chromium & $\mathrm{mg} / \mathrm{kg}$ dry-wt & 1 & 86 & $=$ & 37 & 30 \\
\hline Columbia River & 100-B/C Area & $\mathrm{HR} 006 \mathrm{~B} / \mathrm{C}$ & October 7, 2003 & Chromium & $\mathrm{mg} / \mathrm{kg}$ dry-wt & 1 & 36 & $=$ & 37 & 30 \\
\hline Columbia River & 100-N Area & HR016N & October 9, 2003 & Chromium & $\mathrm{mg} / \mathrm{kg}$ dry-wt & 1 & 34 & $=$ & 37 & 30 \\
\hline Columbia River & 300 Area & HR048-300 & August 4, 2004 & Chromium & $\mathrm{mg} / \mathrm{kg}$ & 1 & 36 & $=$ & 37 & 30 \\
\hline Columbia River & F Slough & B07NB6 & November 12,1992 & Copper & $\mathrm{mg} / \mathrm{kg}$ & 1 & 63 & $=$ & 32 & 2,960 \\
\hline Columbia River & F Slough & B07NB7 & November 12, 1992 & Copper & $\mathrm{mg} / \mathrm{kg}$ & 1 & 51 & $=$ & 32 & 2,960 \\
\hline Columbia River & F Slough & B07NB8 & November 12, 1992 & Copper & $\mathrm{mg} / \mathrm{kg}$ & 1 & 37 & $=$ & 32 & 2,960 \\
\hline Columbia River & F Slough & B07NB9 & November 12, 1992 & Copper & $\mathrm{mg} / \mathrm{kg}$ & 1 & 42 & $=$ & 32 & 2,960 \\
\hline Columbia River & 100-H Area & B07NC2 & November 13, 1992 & Copper & $\mathrm{mg} / \mathrm{kg}$ & 1 & 33 & $=$ & 32 & 2,960 \\
\hline Columbia River & F Slough & B07NC4 & November 16, 1992 & Copper & $\mathrm{mg} / \mathrm{kg}$ & 1 & 70 & JE & 32 & 2,960 \\
\hline Columbia River & D Island & B07ND5 & November 19, 1992 & Copper & $\mathrm{mg} / \mathrm{kg}$ & 1 & 34 & $=$ & 32 & 2,960 \\
\hline Columbia River & 100-K Area & B07NF9 & November 21, 1992 & Copper & $\mathrm{mg} / \mathrm{kg}$ & 1 & 43 & $=$ & 32 & 2,960 \\
\hline Columbia River & Wht Bluffs Slg & B176X9 & July 14,2003 & Copper & $\mathrm{mg} / \mathrm{Kg}$ & 1 & 35 & $=$ & 32 & 2,960 \\
\hline Columbia River & Wht Bluffs Slg & B19PJ6 & July 12,2004 & Copper & $\mathrm{mg} / \mathrm{Kg}$ & 1 & 33 & $x$ & 32 & 2,960 \\
\hline Columbia River & FSlough & B07NC5 & November 16, 1992 & Iron & $\mathrm{mg} / \mathrm{kg}$ & 1 & 22,000 & $\star-$ & 20,000 & 23,463 \\
\hline Columbia River & Horn Area & $\mathrm{B} 07 \mathrm{NH} 2$ & November 23, 1992 & Iron & $\mathrm{mg} / \mathrm{kg}$ & 1 & 31,100 & $=$ & 20,000 & 23,463 \\
\hline Columbia River & $\mathrm{RM} 366$ & 4434245 & June 1,2004 & Lead & $\mathrm{mg} / \mathrm{kg}$ & 1 & 76 & $=$ & 35 & 250 \\
\hline Columbia River & F Slough & B07NB6 & November 12, 1992 & Lead & $\mathrm{mg} / \mathrm{kg}$ & 1 & 56 & $=$ & 35 & 250 \\
\hline Columbia River & F Slough & B07NB7 & November 12, 1992 & Lead & $\mathrm{mg} / \mathrm{kg}$ & 1 & 50 & $=$ & 35 & 250 \\
\hline Columbia River & F Slough & B07NB9 & November 12, 1992 & Lead & $\mathrm{mg} / \mathrm{kg}$ & 1 & 44 & $=$ & 35 & 250 \\
\hline Columbia River & 100-H Area & B07NC2 & November 13, 1992 & Lead & $\mathrm{mg} / \mathrm{kg}$ & 1 & 36 & $=$ & 35 & 250 \\
\hline Columbia River & F Slough & B07NC4 & November 16, 1992 & Lead & $\mathrm{mg} / \mathrm{kg}$ & 1 & 50 & $\mathrm{JN}$ & 35 & 250 \\
\hline Columbia River & Hanford Tnsite & B07NC7 & November 16, 1992 & Lead & $\mathrm{mg} / \mathrm{kg}$ & 1 & 39 & JNS & 35 & 250 \\
\hline Columbia River & Hanford Tnsite & B07ND2 & November 17, 1992 & Lead & $\mathrm{mg} / \mathrm{kg}$ & 1 & 43 & $\mathrm{JN}$ & 35 & 250 \\
\hline Columbia River & 100-K Area & B07NF9 & November 21, 1992 & Lead & $\mathrm{mg} / \mathrm{kg}$ & 1 & 59 & $=$ & 35 & 250 \\
\hline Columbia River & 100-K Area & B07NG0 & November 21, 1992 & Lead & $\mathrm{mg} / \mathrm{kg}$ & 1 & 40 & $=$ & 35 & 250 \\
\hline Columbia River & F Slough & B07NG9 & November 23, 1992 & Lead & $\mathrm{mg} / \mathrm{kg}$ & 1 & 50 & $\mathrm{~s}$ & 35 & 250 \\
\hline Columbia River & Horn Area & $\mathrm{B} 07 \mathrm{NH} 1$ & November 23, 1992 & Lead & $\mathrm{mg} / \mathrm{kg}$ & 1 & 60 & $=$ & 35 & 250 \\
\hline Columbia River & Wht Bluffs Slg & B0W3F4 & August 9, 1999 & Lead & $\mathrm{mg} / \mathrm{Kg}$ & 1 & 39 & $=$ & 35 & 250 \\
\hline Columbia River & HFD SPR UR 28-2 & BOWDP9 & November 1, 1999 & Lead & $\mathrm{mg} / \mathrm{Kg}$ & 1 & 39 & $=$ & 35 & 250 \\
\hline Columbia River & RICHLAND-RIVER & B0YW85 & August 8, 2000 & Lead & $\mathrm{mg} / \mathrm{Kg}$ & 1 & 42 & $=$ & 35 & 250 \\
\hline Columbia River & Wht Bluffs Slg & B0YY65 & August 15, 2000 & Lead & $\mathrm{mg} / \mathrm{Kg}$ & 1 & 43 & $=$ & 35 & 250 \\
\hline Columbia River & RICHLAND-RIVER & B12CK1 & July 23,2001 & Lead & $\mathrm{mg} / \mathrm{kg}$ dry-wt & 1 & 57 & $=$ & 35 & 250 \\
\hline Columbia River & Wht Bluffs Slg & B176X9 & July 14,2003 & Lead & $\mathrm{mg} / \mathrm{Kg}$ & 1 & 42 & $=$ & 35 & 250 \\
\hline Columbia River & Hanford Slough & B176Y3 & July 14,2003 & Lead & $\mathrm{mg} / \mathrm{Kg}$ & 1 & 36 & $=$ & 35 & 250 \\
\hline Columbia River & RICHLAND-RIVER & B176Y5 & July 16, 2003 & Lead & $\mathrm{mg} / \mathrm{Kg}$ & 1 & 58 & $=$ & 35 & 250 \\
\hline Columbia River & 100-H SPR 145-1 & B17JB1 & October 27, 2003 & Lead & $\mathrm{mg} / \mathrm{Kg}$ & 1 & 62 & $=$ & 35 & 250 \\
\hline
\end{tabular}


Table B-7

Summary of Surface Sediment Results From Segment 2 Exceeding Select Ecological or Human Health Benchmark Values

Columbia River Component - Existing Sample Data Evaluation and Scoping Report

\begin{tabular}{|c|c|c|c|c|c|c|c|c|c|c|}
\hline Sample Area & Sample Site & SampleID & Date Collected & Constituent Name & Units & $\begin{array}{c}\text { QA } \\
\text { Category }\end{array}$ & $\begin{array}{c}\text { Analytical } \\
\text { Value }\end{array}$ & Qualifier & $\begin{array}{c}\text { Ecological } \\
\text { Benchmark } \\
\text { Value }\end{array}$ & $\begin{array}{c}\text { Human Health } \\
\text { Benchmark } \\
\text { Value }\end{array}$ \\
\hline Columbia River & Wht Bluffs Slg & B19PJ6 & July 12,2004 & Lead & $\mathrm{mg} / \mathrm{Kg}$ & 1 & 58 & $\mathrm{X}$ & 35 & 250 \\
\hline Columbia River & RICHLAND-RIVER & B19PK2 & July 13, 2004 & Lead & $\mathrm{mg} / \mathrm{Kg}$ & 1 & 38 & $\mathrm{x}$ & 35 & 250 \\
\hline Columbia River & HFD SPR UR 28-2 & B1BFP9 & October 25, 2004 & Lead & $\mathrm{mg} / \mathrm{Kg}$ & 1 & 50 & $x$ & 35 & 250 \\
\hline Columbia River & 100-H SPR 145-1 & B1BH03 & October 26, 2004 & Lead & $\mathrm{mg} / \mathrm{Kg}$ & 1 & 48 & $x$ & 35 & 250 \\
\hline Columbia River & $100 \mathrm{~B} / \mathrm{C}$ AREA UR & B17XN6 & November 17,2003 & Manganese & $\mathrm{mg} / \mathrm{Kg}$ & 1 & 511 & $\mathrm{C}$ & 460 & 1,762 \\
\hline Columbia River & 100-B SPR 37-1 & B17XN8 & November 13, 2003 & Manganese & $\mathrm{mg} / \mathrm{Kg}$ & 1 & 603 & C & 460 & 1,762 \\
\hline Columbia River & 100-B SPR 38-3 & B17XN9 & November 13, 2003 & Manganese & $\mathrm{mg} / \mathrm{Kg}$ & 1 & 561 & C & 460 & 1,762 \\
\hline Columbia River & Hanford Tnsite & B07NC7 & November 16,1992 & Mercury & $\mathrm{mg} / \mathrm{kg}$ & 1 & 0.68 & $=$ & 0.18 & 23 \\
\hline Columbia River & $\mathrm{RM} 362$ & 4434239 & June 1,2004 & Nickel & $\mathrm{mg} / \mathrm{kg}$ & 1 & 18 & $=$ & 18 & 1,564 \\
\hline Columbia River & 100-K Area & B07NF9 & November 21, 1992 & Nickel & $\mathrm{mg} / \mathrm{kg}$ & 1 & 19 & $=$ & 18 & 1,564 \\
\hline Columbia River & Horn Area & $\mathrm{B} 07 \mathrm{NH} 2$ & November 23, 1992 & Nickel & $\mathrm{mg} / \mathrm{kg}$ & 1 & 22 & $=$ & 18 & 1,564 \\
\hline Columbia River & 100 F SLOUGH & B0W3F6 & August 9, 1999 & Nickel & $\mathrm{mg} / \mathrm{Kg}$ & 1 & 23 & $=$ & 18 & 1,564 \\
\hline Columbia River & Hanford Slough & B0W3F8 & August 9, 1999 & Nickel & $\mathrm{mg} / \mathrm{Kg}$ & 1 & 23 & $=$ & 18 & 1,564 \\
\hline Columbia River & HFD SPR DR 28-2 & BOWDR1 & November 1, 1999 & Nickel & $\mathrm{mg} / \mathrm{Kg}$ & 1 & 20 & $=$ & 18 & 1,564 \\
\hline Columbia River & 100-F SPR 207-1 & BOWDTO & November 1, 1999 & Nickel & $\mathrm{mg} / \mathrm{Kg}$ & 1 & 19 & $=$ & 18 & 1,564 \\
\hline Columbia River & Wht Bluffs Slg & B0YY65 & August 15, 2000 & Nickel & $\mathrm{mg} / \mathrm{Kg}$ & 1 & 24 & $=$ & 18 & 1,564 \\
\hline Columbia River & Hanford Slough & B0YY69 & August 15, 2000 & Nickel & $\mathrm{mg} / \mathrm{Kg}$ & 1 & 24 & $=$ & 18 & 1,564 \\
\hline Columbia River & 300 SPR 42-2 & B10905 & November 2, 2000 & Nickel & $\mathrm{mg} / \mathrm{Kg}$ & 1 & 21 & $=$ & 18 & 1,564 \\
\hline Columbia River & HFD SPR UR 28-2 & B10918 & November 2, 2000 & Nickel & $\mathrm{mg} / \mathrm{Kg}$ & 1 & 20 & $=$ & 18 & 1,564 \\
\hline Columbia River & HFD SPR DR 28-2 & B10921 & November 2, 2000 & Nickel & $\mathrm{mg} / \mathrm{Kg}$ & 1 & 22 & $=$ & 18 & 1,564 \\
\hline Columbia River & 300 SPR DR 42-2 & B10923 & November 2, 2000 & Nickel & $\mathrm{mg} / \mathrm{Kg}$ & 1 & 20 & $=$ & 18 & 1,564 \\
\hline Columbia River & 100-F SPR 207-1 & B10931 & November 20, 2000 & Nickel & $\mathrm{mg} / \mathrm{Kg}$ & 1 & 26 & $=$ & 18 & 1,564 \\
\hline Columbia River & Hanford Slough & B12CJ9 & July 19, 2001 & Nickel & $\mathrm{mg} / \mathrm{kg}$ dry-wt & 1 & 21 & $=$ & 18 & 1,564 \\
\hline Columbia River & RICHLAND-RIVER & B12CK1 & July 23, 2001 & Nickel & mg/kg dry-wt & 1 & 18 & $=$ & 18 & 1,564 \\
\hline Columbia River & 300 SPR 42-2 & B12RL7 & August 27, 2001 & Nickel & $\mathrm{mg} / \mathrm{kg}$ dry-wt & 1 & 20 & $=$ & 18 & 1,564 \\
\hline Columbia River & 300 SPR DR 42-2 & B12RM5 & August 27, 2001 & Nickel & $\mathrm{mg} / \mathrm{kg}$ dry-wt & 1 & 18 & $=$ & 18 & 1,564 \\
\hline Columbia River & 100-F SPR 207-1 & B12X37 & October 22, 2001 & Nickel & $\mathrm{mg} / \mathrm{kg}$ dry-wt & 1 & 20 & $=$ & 18 & 1,564 \\
\hline Columbia River & Wht Bluffs Slg & B176X9 & July 14, 2003 & Nickel & $\mathrm{mg} / \mathrm{Kg}$ & 1 & 24 & $=$ & 18 & 1,564 \\
\hline Columbia River & Hanford Slough & B176Y3 & July 14, 2003 & Nickel & $\mathrm{mg} / \mathrm{Kg}$ & 1 & 30 & $=$ & 18 & 1,564 \\
\hline Columbia River & RICHLAND-RIVER & B176Y5 & July 16,2003 & Nickel & $\mathrm{mg} / \mathrm{Kg}$ & 1 & 19 & $=$ & 18 & 1,564 \\
\hline Columbia River & 300 SPR 42-2 & B17J18 & October 13, 2003 & Nickel & $\mathrm{mg} / \mathrm{Kg}$ & 1 & 19 & $=$ & 18 & 1,564 \\
\hline Columbia River & HFD SPR DR 28-2 & B17J58 & November 3, 2003 & Nickel & $\mathrm{mg} / \mathrm{Kg}$ & 1 & 26 & $=$ & 18 & 1,564 \\
\hline Columbia River & 100-K SPR 63-1 & B17J64 & October 20, 2003 & Nickel & $\mathrm{mg} / \mathrm{Kg}$ & 1 & 19 & $=$ & 18 & 1,564 \\
\hline Columbia River & 100-F SPR 207-1 & B17J67 & November 3, 2003 & Nickel & $\mathrm{mg} / \mathrm{Kg}$ & 1 & 26 & $=$ & 18 & 1,564 \\
\hline Columbia River & 100-H SPR 145-1 & B17JB1 & October 27, 2003 & Nickel & $\mathrm{mg} / \mathrm{Kg}$ & 1 & 20 & $=$ & 18 & 1,564 \\
\hline Columbia River & 100 B/C AREA UR & B17XN6 & November 17, 2003 & Nickel & $\mathrm{mg} / \mathrm{Kg}$ & 1 & 19 & $=$ & 18 & 1,564 \\
\hline Columbia River & 100-B SPR 38-3 & B17XN9 & November 13, 2003 & Nickel & $\mathrm{mg} / \mathrm{Kg}$ & 1 & 21 & $=$ & 18 & 1,564 \\
\hline Columbia River & Wht Bluffs Slg & B19PJ6 & July 12, 2004 & Nickel & $\mathrm{mg} / \mathrm{Kg}$ & 1 & 22 & $\mathrm{x}$ & 18 & 1,564 \\
\hline Columbia River & Hanford Slough & B19PK0 & July 12,2004 & Nickel & $\mathrm{mg} / \mathrm{Kg}$ & 1 & 22 & $\mathrm{x}$ & 18 & 1,564 \\
\hline Columbia River & RICHLAND-RIVER & B19PK2 & July 13, 2004 & Nickel & $\mathrm{mg} / \mathrm{Kg}$ & 1 & 18 & $\mathrm{x}$ & 18 & 1,564 \\
\hline Columbia River & 100-B SPR 37-1 & B1BFP7 & October 11, 2004 & Nickel & $\mathrm{mg} / \mathrm{Kg}$ & 1 & 20 & $\mathrm{x}$ & 18 & 1,564 \\
\hline Columbia River & HFD SPR UR 28-2 & B1BFP9 & October 25, 2004 & Nickel & $\mathrm{mg} / \mathrm{Kg}$ & 1 & 21 & $\mathrm{x}$ & 18 & 1,564 \\
\hline
\end{tabular}


Table B-7

Summary of Surface Sediment Results From Segment 2 Exceeding Select Ecological or Human Health Benchmark Values Columbia River Component - Existing Sample Data Evaluation and Scoping Report

\begin{tabular}{|c|c|c|c|c|c|c|c|c|c|c|}
\hline Sample Area & Sample Site & SamplelD & Date Collected & Constituent Name & Units & $\begin{array}{c}\text { QA } \\
\text { Category }\end{array}$ & $\begin{array}{c}\text { Analytical } \\
\text { Value }\end{array}$ & Qualifier & $\begin{array}{c}\text { Ecological } \\
\text { Benchmark } \\
\text { Value }\end{array}$ & $\begin{array}{l}\text { Human Health } \\
\text { Benchmark } \\
\text { Value }\end{array}$ \\
\hline Columbia River & HFD SPR DR 28-2 & B1BFR2 & October 25, 2004 & Nickel & $\mathrm{mg} / \mathrm{Kg}$ & 1 & 24 & $\mathrm{X}$ & 18 & 1,564 \\
\hline Columbia River & 100-F SPR 207-1 & B1BFT0 & October 26, 2004 & Nickel & $\mathrm{mg} / \mathrm{Kg}$ & 1 & 21 & $\mathrm{x}$ & 18 & 1,564 \\
\hline Columbia River & 100-H SPR 145-1 & B1BH03 & October 26, 2004 & Nickel & $\mathrm{mg} / \mathrm{Kg}$ & 1 & 19 & $\mathrm{x}$ & 18 & 1,564 \\
\hline Columbia River & 300 Outfall & HR063S & August 4, 2004 & Nickel & $\mathrm{mg} / \mathrm{kg}$ & 1 & 19 & $=$ & 18 & 1,564 \\
\hline Columbia River & D Island & B07NH7 & November 24, 1992 & Silver & $\mathrm{mg} / \mathrm{kg}$ & 1 & 5.3 & $\mathrm{JN}^{*}$ & 4.5 & 391 \\
\hline Columbia River & $100 \mathrm{~B} / \mathrm{C}$ AREA UR & B17XN6 & November 17,2003 & Thorium & $\mathrm{mg} / \mathrm{Kg}$ & 1 & 7.0 & $\mathrm{C}$ & 1,300 & 1.3 \\
\hline Columbia River & 100-B SPR 37-1 & B17XN8 & November 13, 2003 & Thorium & $\mathrm{mg} / \mathrm{Kg}$ & 1 & 7.9 & C & 1,300 & 1.3 \\
\hline Columbia River & 100-B SPR 38-3 & B17XN9 & November 13, 2003 & Thorium & $\mathrm{mg} / \mathrm{Kg}$ & 1 & 7.4 & C & 1,300 & 1.3 \\
\hline Columbia River & 300 SPR $42-2$ & 27651 & August 27,2001 & Thorium-232 & pCi/g dry-wt & 1 & 1.5 & $=$ & 1,300 & 1.3 \\
\hline Columbia River & F Slough & B07NB7 & November 12, 1992 & Thorium-232 & $\mathrm{pCi} / \mathrm{g}$ & 1 & 1.8 & $=$ & 1,300 & 1.3 \\
\hline Columbia River & F Slough & B07NB9 & November 12, 1992 & Thorium-232 & $\mathrm{pCi} / \mathrm{g}$ & 1 & 1.9 & $=$ & 1,300 & 1.3 \\
\hline Columbia River & 100-H Area & B07NC2 & November 13, 1992 & Thorium-232 & $\mathrm{pCi} / \mathrm{g}$ & 1 & 1.8 & $=$ & 1,300 & 1.3 \\
\hline Columbia River & F Slough & B07NC4 & November 16, 1992 & Thorium-232 & $\mathrm{pCi} / \mathrm{g}$ & 1 & 3.2 & $=$ & 1,300 & 1.3 \\
\hline Columbia River & F Slough & B07NC5 & November 16, 1992 & Thorium-232 & $\mathrm{pCi} / \mathrm{g}$ & 1 & 1.5 & $=$ & 1,300 & 1.3 \\
\hline Columbia River & Horn Area & B07NC9 & November 17, 1992 & Thorium-232 & $\mathrm{pCi} / \mathrm{g}$ & 1 & 1.6 & $=$ & 1,300 & 1.3 \\
\hline Columbia River & Hanford Tnsite & B07ND3 & November 17, 1992 & Thorium-232 & $\mathrm{pCi} / \mathrm{g}$ & 1 & 2.1 & $=$ & 1,300 & 1.3 \\
\hline Columbia River & 100-K Area & B07NG2 & November 21, 1992 & Thorium-232 & $\mathrm{pCi} / \mathrm{g}$ & 1 & 1.5 & $=$ & 1,300 & 1.3 \\
\hline Columbia River & Horn Area & B07NH2 & November 23, 1992 & Thorium-232 & $\mathrm{pCi} / \mathrm{g}$ & 1 & 2.3 & $=$ & 1,300 & 1.3 \\
\hline Columbia River & $300 \mathrm{FF}-5$ & 12913 & September 14,1992 & Uranium-234 & $\mathrm{pCi} / \mathrm{g}$ dry-wt & 1 & 1.3 & $=$ & 5,270 & 1.1 \\
\hline Columbia River & 300 FF-5 & 12914 & September 14, 1992 & Uranium-234 & $\mathrm{pCi} / \mathrm{g}$ dry-wt & 1 & 3.5 & $=$ & 5,270 & 1.1 \\
\hline Columbia River & F Slough & 13166 & November 16, 1992 & Uranium-234 & $\mathrm{pCi} / \mathrm{g}$ dry-wt & 1 & 1.5 & $=$ & 5,270 & 1.1 \\
\hline Columbia River & 100-K Area & 17209 & September 5, 1995 & Uranium-234 & $\mathrm{pCi} / \mathrm{g}$ dry-wt & 1 & 2.0 & $=$ & 5,270 & 1.1 \\
\hline Columbia River & 300 SPR 42-2 & 17211 & September 5, 1995 & Uranium-234 & $\mathrm{pCi} / \mathrm{g}$ dry-wt & 1 & 4.5 & $=$ & 5,270 & 1.1 \\
\hline Columbia River & HFD SPR 28-2 & 24751 & September 27, 2000 & Uranium-234 & $\mathrm{pCi} / \mathrm{g}$ dry-wt & 1 & 1.2 & $=$ & 5,270 & 1.1 \\
\hline Columbia River & 300 SPR 42-2 & 24752 & September 27, 2000 & Uranium-234 & $\mathrm{pCi} / \mathrm{g}$ dry-wt & 1 & 2.3 & $=$ & 5,270 & 1.1 \\
\hline Columbia River & 300 SPR 42-2 & 27651 & August 27, 2001 & Uranium-234 & $\mathrm{pCi} / \mathrm{g}$ dry-wt & 1 & 2.7 & $=$ & 5,270 & 1.1 \\
\hline Columbia River & 100F SPR 207-1 & 29695 & October 29, 2002 & Uranium-234 & $\mathrm{pCi} / \mathrm{g}$ dry-wt & 1 & 1.3 & $=$ & 5,270 & 1.1 \\
\hline Columbia River & 300 SPR 42-2 & 30932 & October 13, 2003 & Uranium-234 & $\mathrm{pCi} / \mathrm{g}$ dry-wt & 1 & 2.4 & $=$ & 5,270 & 1.1 \\
\hline Columbia River & 300 SPR 42-2 & 30939 & October 13, 2003 & Uranium-234 & $\mathrm{pCi} / \mathrm{g}$ dry-wt & 1 & 2.2 & $=$ & 5,270 & 1.1 \\
\hline Columbia River & 300 SPR 42-2 & 33224 & October 25, 2004 & Uranium-234 & $\mathrm{pCi} / \mathrm{g}$ dry-wt & 1 & 3.6 & $=$ & 5,270 & 1.1 \\
\hline Columbia River & 300 SPR 42-2 & BOWDL8 & November 1, 1999 & Uranium-234 & $\mathrm{pCi} / \mathrm{g}$ & 1 & 2.6 & $=$ & 5,270 & 1.1 \\
\hline Columbia River & 300 SPR DR 42-2 & BOWDR2 & November 1, 1999 & Uranium-234 & $\mathrm{pCi} / \mathrm{g}$ & 1 & 3.9 & $=$ & 5,270 & 1.1 \\
\hline Columbia river & 300 SPR 42-2 & B10908 & September 27, 2000 & Uranium-234 & $\mathrm{pCi} / \mathrm{g}$ & 1 & 2.0 & $=$ & 5,270 & 1.1 \\
\hline Columbia river & 300 SPR DR 42-2 & B10922 & September 27, 2000 & Uranium-234 & $\mathrm{pCi} / \mathrm{g}$ & 1 & 3.0 & $=$ & 5,270 & 1.1 \\
\hline Columbia River & 300 SPR 42-2 & B12RL9 & August 27, 2001 & Uranium-234 & $\mathrm{pCi} / \mathrm{g}$ & 1 & 2.7 & $=$ & 5,270 & 1.1 \\
\hline Columbia River & 300 SPR 11 & B12RY9 & August 27, 2001 & Uranium-234 & $\mathrm{pCi} / \mathrm{g}$ & 1 & 1.9 & $=$ & 5,270 & 1.1 \\
\hline Columbia River & 300 SPR DR 42-2 & В12T05 & August 27, 2001 & Uranium-234 & $\mathrm{pCi} / \mathrm{g}$ & 1 & 1.5 & $=$ & 5,270 & 1.1 \\
\hline Columbia River & 300 SPR 11 & В12Т06 & August 27, 2001 & Uranium-234 & $\mathrm{pCi} / \mathrm{g}$ & 1 & 1.5 & $=$ & 5,270 & 1.1 \\
\hline Columbia River & Wht Bluffs Slg & B14YM6 & July 23,2002 & Uranium-234 & $\mathrm{pCi} / \mathrm{g}$ & 1 & 1.6 & $=$ & 5,270 & 1.1 \\
\hline Columbia River & 300 SPR DR 42-2 & B15C47 & October 7, 2002 & Uranium-234 & $\mathrm{pCi} / \mathrm{g}$ & 1 & 11 & $=$ & 5,270 & 1.1 \\
\hline Columbia River & 300 SPR 42-2 & B17J20 & October 13, 2003 & Uranium-234 & $\mathrm{pCi} / \mathrm{g}$ & 1 & 1.4 & $=$ & 5,270 & 1.1 \\
\hline Columbia River & 300 SPR DR 42-2 & B17J59 & October 13, 2003 & Uranium-234 & $\mathrm{pCi} / \mathrm{g}$ & 1 & 1.5 & $=$ & 5,270 & 1.1 \\
\hline
\end{tabular}


Table B-7

Summary of Surface Sediment Results From Segment 2 Exceeding Select Ecological or Human Health Benchmark Values Columbia River Component - Existing Sample Data Evaluation and Scoping Report

\begin{tabular}{|c|c|c|c|c|c|c|c|c|c|c|}
\hline Sample Area & Sample Site & SampleID & Date Collected & Constituent Name & Units & $\begin{array}{c}\text { QA } \\
\text { Category }\end{array}$ & $\begin{array}{c}\text { Analytical } \\
\text { Value }\end{array}$ & Qualifier & $\begin{array}{c}\text { Ecological } \\
\text { Benchmark } \\
\text { Value }\end{array}$ & $\begin{array}{l}\text { Human Health } \\
\text { Benchmark } \\
\text { Value }\end{array}$ \\
\hline Columbia River & 300 SPR 42-2 & B1BFN9 & October 25, 2004 & Uranium-234 & $\mathrm{pCi} / \mathrm{g}$ & 1 & 2.4 & $=$ & 5,270 & 1.1 \\
\hline Columbia River & 300 SPR DR 42-2 & B1BFR3 & October 25, 2004 & Uranium-234 & $\mathrm{pCi} / \mathrm{g}$ & 1 & 4.1 & $=$ & 5,270 & 1.1 \\
\hline Columbia River & 300 Upstream & HR037-300 & August 4, 2004 & Uranium-234 & $\mathrm{pCi} / \mathrm{g}$ & 1 & 2.0 & $=$ & 5,270 & 1.1 \\
\hline Columbia River & 300 Area & HR046-300 & August 4, 2004 & Uranium-234 & $\mathrm{pCi} / \mathrm{g}$ & 1 & 3.0 & $=$ & 5,270 & 1.1 \\
\hline Columbia River & $100 \mathrm{~N}$ SHORE HGP & 12645 & June 30,1992 & Uranium-238 & $\mathrm{pCi} / \mathrm{g}$ dry-wt & 1 & 1.6 & $=$ & 2,490 & 1.1 \\
\hline Columbia River & 300 FF-5 & 12913 & September 14, 1992 & Uranium-238 & $\mathrm{pCi} / \mathrm{g}$ dry-wt & 1 & 1.2 & $=$ & 2,490 & 1.1 \\
\hline Columbia River & 300 FF-5 & 12914 & September 14, 1992 & Uranium-238 & $\mathrm{pCi} / \mathrm{g}$ dry-wt & 1 & 3.1 & $=$ & 2,490 & 1.1 \\
\hline Columbia River & F Slough & 13166 & November 16, 1992 & Uranium-238 & $\mathrm{pCi} / \mathrm{g}$ dry-wt & 1 & 1.4 & $=$ & 2,490 & 1.1 \\
\hline Columbia River & 100-K Area & 17209 & September 5, 1995 & Uranium-238 & $\mathrm{pCi} / \mathrm{g}$ dry-wt & 1 & 2.0 & $=$ & 2,490 & 1.1 \\
\hline Columbia River & 300 SPR $42-2$ & 17211 & September 5, 1995 & Uranium-238 & $\mathrm{pCi} / \mathrm{g}$ dry-wt & 1 & 4.3 & $=$ & 2,490 & 1.1 \\
\hline Columbia River & HFD SPR 28-2 & 24751 & September 27, 2000 & Uranium-238 & $\mathrm{pCi} / \mathrm{g}$ dry-wt & 1 & 1.2 & $=$ & 2,490 & 1.1 \\
\hline Columbia River & 300 SPR 42-2 & 24752 & September 27, 2000 & Uranium-238 & $\mathrm{pCi} / \mathrm{g}$ dry-wt & 1 & 2.2 & $=$ & 2,490 & 1.1 \\
\hline Columbia River & 300 SPR 42-2 & 27651 & August 27, 2001 & Uranium-238 & $\mathrm{pCi} / \mathrm{g}$ dry-wt & 1 & 2.5 & $=$ & 2,490 & 1.1 \\
\hline Columbia River & 100F SPR 207-1 & 29695 & October 29, 2002 & Uranium-238 & $\mathrm{pCi} / \mathrm{g}$ dry-wt & 1 & 1.2 & $=$ & 2,490 & 1.1 \\
\hline Columbia River & 300 SPR 42-2 & 30932 & October 13, 2003 & Uranium-238 & $\mathrm{pCi} / \mathrm{g}$ dry-wt & 1 & 2.3 & $=$ & 2,490 & 1.1 \\
\hline Columbia River & 300 SPR 42-2 & 30939 & October 13, 2003 & Uranium-238 & $\mathrm{pCi} / \mathrm{g}$ dry-wt & 1 & 2.1 & $=$ & 2,490 & 1.1 \\
\hline Columbia River & 300 SPR 42-2 & 33224 & October 25, 2004 & Uranium-238 & $\mathrm{pCi} / \mathrm{g}$ dry-wt & 1 & 3.3 & $=$ & 2,490 & 1.1 \\
\hline Columbia River & F Slough & B07NB9 & November 12, 1992 & Uranium-238 & $\mathrm{pCi} / \mathrm{g}$ & 1 & 1.3 & $=$ & 2,490 & 1.1 \\
\hline Columbia River & 100-H Area & B07NC2 & November 13, 1992 & Uranium-238 & $\mathrm{pCi} / \mathrm{g}$ & 1 & 1.2 & $=$ & 2,490 & 1.1 \\
\hline Columbia River & 100-H Area & B07NC3 & November 13, 1992 & Uranium-238 & $\mathrm{pCi} / \mathrm{g}$ & 1 & 1.2 & $=$ & 2,490 & 1.1 \\
\hline Columbia River & F Slough & B07NC4 & November 16, 1992 & Uranium-238 & $\mathrm{pCi} / \mathrm{g}$ & 1 & 2.0 & $=$ & 2,490 & 1.1 \\
\hline Columbia River & Horn Area & B07NH2 & November 23, 1992 & Uranium-238 & $\mathrm{pCi} / \mathrm{g}$ & 1 & 2.0 & $=$ & 2,490 & 1.1 \\
\hline Columbia River & F Slough & BOBRV6 & April 15, 1994 & Uranium-238 & $\mathrm{pCi} / \mathrm{g}$ & 1 & 1.4 & $=$ & 2,490 & 1.1 \\
\hline Columbia River & 300 SPR 42-2 & BOWDL8 & November 1, 1999 & Uranium-238 & $\mathrm{pCi} / \mathrm{g}$ & 1 & 2.2 & $=$ & 2,490 & 1.1 \\
\hline Columbia River & 300 SPR DR 42-2 & BOWDR2 & November 1, 1999 & Uranium-238 & $\mathrm{pCi} / \mathrm{g}$ & 1 & 3.7 & $=$ & 2,490 & 1.1 \\
\hline Columbia river & 300 SPR $42-2$ & B10908 & September 27, 2000 & Uranium-238 & $\mathrm{pCi} / \mathrm{g}$ & 1 & 1.9 & $=$ & 2,490 & 1.1 \\
\hline Columbia river & 300 SPR DR $42-2$ & B10922 & September 27, 2000 & Uranium-238 & $\mathrm{pCi} / \mathrm{g}$ & 1 & 2.6 & $=$ & 2,490 & 1.1 \\
\hline Columbia River & 300 SPR 42-2 & B12RL9 & August 27, 2001 & Uranium-238 & $\mathrm{pCi} / \mathrm{g}$ & 1 & 2.5 & $=$ & 2,490 & 1.1 \\
\hline Columbia River & 300 SPR 11 & B12RY9 & August 27, 2001 & Uranium-238 & $\mathrm{pCi} / \mathrm{g}$ & 1 & 1.8 & $=$ & 2,490 & 1.1 \\
\hline Columbia River & 300 SPR DR 42-2 & B12T05 & August 27, 2001 & Uranium-238 & $\mathrm{pCi} / \mathrm{g}$ & 1 & 1.3 & $=$ & 2,490 & 1.1 \\
\hline Columbia River & 300 SPR 11 & B12T06 & August 27, 2001 & Uranium-238 & $\mathrm{pCi} / \mathrm{g}$ & 1 & 1.3 & $=$ & 2,490 & 1.1 \\
\hline Columbia River & Wht Bluffs Slg & B14YM6 & July 23, 2002 & Uranium-238 & $\mathrm{pCi} / \mathrm{g}$ & 1 & 1.3 & $=$ & 2,490 & 1.1 \\
\hline Columbia River & 300 SPR DR 42-2 & B15C47 & October 7, 2002 & Uranium-238 & $\mathrm{pCi} / \mathrm{g}$ & 1 & 10.0 & $=$ & 2,490 & 1.1 \\
\hline Columbia River & 300 SPR $42-2$ & B17J20 & October 13, 2003 & Uranium-238 & $\mathrm{pCi} / \mathrm{g}$ & 1 & 1.3 & $=$ & 2,490 & 1.1 \\
\hline Columbia River & 300 SPR DR $42-2$ & B17J59 & October 13, 2003 & Uranium-238 & $\mathrm{pCi} / \mathrm{g}$ & 1 & 1.5 & $=$ & 2,490 & 1.1 \\
\hline Columbia River & 300 SPR 42-2 & B1BFN9 & October 25, 2004 & Uranium-238 & $\mathrm{pCi} / \mathrm{g}$ & 1 & 2.4 & $=$ & 2,490 & 1.1 \\
\hline Columbia River & 300 SPR DR 42-2 & B1BFR3 & October 25, 2004 & Uranium-238 & $\mathrm{pCi} / \mathrm{g}$ & 1 & 3.8 & $=$ & 2,490 & 1.1 \\
\hline Columbia River & 300 Upstream & HR037-300 & August 4, 2004 & Uranium-238 & $\mathrm{pCi} / \mathrm{g}$ & 1 & 1.4 & $=$ & 2,490 & 1.1 \\
\hline Columbia River & 300 Area & HR046-300 & August 4, 2004 & Uranium-238 & $\mathrm{pCi} / \mathrm{g}$ & 1 & 3.7 & $=$ & 2,490 & 1.1 \\
\hline Columbia River & Horn Area & B07NH2 & November 23,1992 & Vanadium & $\mathrm{mg} / \mathrm{kg}$ & 1 & 86 & $=$ & - & 78 \\
\hline Columbia River & 300 Area & 4434235 & June 1,2004 & Zinc- & $\mathrm{mg} / \mathrm{kg}$ & 1 & 305 & $=$ & 120 & 23,463 \\
\hline Columbia River & RM 362 & 4434239 & June 1, 2004 & Zinc & $\mathrm{mg} / \mathrm{kg}$ & 1 & 444 & $=$ & 120 & 23,463 \\
\hline
\end{tabular}


Table B-7

Summary of Surface Sediment Results From Segment 2 Exceeding Select Ecological or Human Health Benchmark Values Columbia River Component - Existing Sample Data Evaluation and Scoping Report

\begin{tabular}{|c|c|c|c|c|c|c|c|c|c|c|}
\hline Sample Area & Sample Site & SampleID & Date Collected & Constituent Name & Units & $\begin{array}{c}\text { QA } \\
\text { Category }\end{array}$ & $\begin{array}{c}\text { Analytical } \\
\text { Value }\end{array}$ & Qualifier & $\begin{array}{c}\text { Ecological } \\
\text { Benchmark } \\
\text { Value }\end{array}$ & $\begin{array}{l}\text { Human Health } \\
\text { Benchmark } \\
\text { Value }\end{array}$ \\
\hline Columbia River & RM 366 & 4434245 & June 1,2004 & $\overline{Z i n c}$ & $\mathrm{mg} / \mathrm{kg}$ & $\overline{1}$ & 402 & $=$ & 120 & 23,463 \\
\hline Columbia River & F Slough & B07NB6 & November 12, 1992 & Zinc & $\mathrm{mg} / \mathrm{kg}$ & 1 & 335 & $=$ & 120 & 23,463 \\
\hline Columbia River & F Slough & B07NB7 & November 12, 1992 & Zinc & $\mathrm{mg} / \mathrm{kg}$ & 1 & 309 & $=$ & 120 & 23,463 \\
\hline Columbia River & F Slough & B07NB8 & November 12,1992 & Zinc & $\mathrm{mg} / \mathrm{kg}$ & 1 & 246 & $=$ & 120 & 23,463 \\
\hline Columbia River & F Slough & B07NB9 & November 12, 1992 & Zinc & $\mathrm{mg} / \mathrm{kg}$ & 1 & 195 & $=$ & 120 & 23,463 \\
\hline Columbia River & 100-H Area & B07NC2 & November 13, 1992 & Zinc & $\mathrm{mg} / \mathrm{kg}$ & 1 & 168 & $=$ & 120 & 23,463 \\
\hline Columbia River & 100-H Area & B07NC3 & November 13, 1992 & Zinc & $\mathrm{mg} / \mathrm{kg}$ & 1 & 141 & $=$ & 120 & 23,463 \\
\hline Columbia River & F Slough & B07NC4 & November 16, 1992 & Zinc & $\mathrm{mg} / \mathrm{kg}$ & 1 & 315 & $=$ & 120 & 23,463 \\
\hline Columbia River & Hanford Tnsite & B07NC6 & November 16, 1992 & Zinc & $\mathrm{mg} / \mathrm{kg}$ & 1 & 123 & $=$ & 120 & 23,463 \\
\hline Columbia River & Hanford Tnsite & B07NC7 & November 16, 1992 & Zinc & $\mathrm{mg} / \mathrm{kg}$ & 1 & 219 & $=$ & 120 & 23,463 \\
\hline Columbia River & Horn Area & B07NC9 & November 17, 1992 & Zinc & $\mathrm{mg} / \mathrm{kg}$ & 1 & 279 & $=$ & 120 & 23,463 \\
\hline Columbia River & Horn Area & B07ND0 & November 17, 1992 & Zinc & $\mathrm{mg} / \mathrm{kg}$ & 1 & 221 & $=$ & 120 & 23,463 \\
\hline Columbia River & Hanford Tnsite & B07ND2 & November 17, 1992 & Zinc & $\mathrm{mg} / \mathrm{kg}$ & 1 & 293 & $=$ & 120 & 23,463 \\
\hline Columbia River & Hanford Tnsite & B07ND3 & November 17, 1992 & Zinc & $\mathrm{mg} / \mathrm{kg}$ & 1 & 155 & $=$ & 120 & 23,463 \\
\hline Columbia River & D Island & B07ND4 & November 19, 1992 & Zinc & $\mathrm{mg} / \mathrm{kg}$ & 1 & 192 & $=$ & 120 & 23,463 \\
\hline Columbia River & D Island & B07ND5 & November 19, 1992 & Zinc & $\mathrm{mg} / \mathrm{kg}$ & 1 & 216 & $=$ & 120 & 23,463 \\
\hline Columbia River & D Island & B07ND6 & November 19, 1992 & Zinc & $\mathrm{mg} / \mathrm{kg}$ & 1 & 144 & $=$ & 120 & 23,463 \\
\hline Columbia River & D Island & B07ND7 & November 19, 1992 & Zinc & $\mathrm{mg} / \mathrm{kg}$ & 1 & 203 & $=$ & 120 & 23,463 \\
\hline Columbia River & Horn Area & B07NF0 & November 19, 1992 & Zinc & $\mathrm{mg} / \mathrm{kg}$ & 1 & 377 & $=$ & 120 & 23,463 \\
\hline Columbia River & 100-D Area & B07NF1 & November 19,1992 & Zinc & $\mathrm{mg} / \mathrm{kg}$ & 1 & 221 & $=$ & 120 & 23,463 \\
\hline Columbia River & 100-D Area & B07NF2 & November 19, 1992 & Zinc & $\mathrm{mg} / \mathrm{kg}$ & 1 & 257 & $=$ & 120 & 23,463 \\
\hline Columbia River & 100-K Area & B07NF9 & November 21, 1992 & Zinc & $\mathrm{mg} / \mathrm{kg}$ & 1 & 454 & $=$ & 120 & 23,463 \\
\hline Columbia River & 100-K Area & B07NG0 & November 21, 1992 & Zinc & $\mathrm{mg} / \mathrm{kg}$ & 1 & 378 & $=$ & 120 & 23,463 \\
\hline Columbia River & 100-K Area & B07NG1 & November 21, 1992 & Zinc & $\mathrm{mg} / \mathrm{kg}$ & 1 & 209 & $=$ & 120 & 23,463 \\
\hline Columbia River & 100-H Area & B07NG3 & November 22, 1992 & Zinc & $\mathrm{mg} / \mathrm{kg}$ & 1 & 397 & $=$ & 120 & 23,463 \\
\hline Columbia River & 100-H Area & B07NG5 & November 22, 1992 & Zinc & $\mathrm{mg} / \mathrm{kg}$ & 1 & 253 & $=$ & 120 & 23,463 \\
\hline Columbia River & 100-H Area & B07NG6 & November 22, 1992 & Zinc & $\mathrm{mg} / \mathrm{kg}$ & 1 & 369 & $=$ & 120 & 23,463 \\
\hline Columbia River & 100-H Area & B07NG7 & November 22, 1992 & Zinc & $\mathrm{mg} / \mathrm{kg}$ & 1 & 230 & $=$ & 120 & 23,463 \\
\hline Columbia River & F Slough & B07NG9 & November 23, 1992 & Zinc & $\mathrm{mg} / \mathrm{kg}$ & 1 & 296 & $=$ & 120 & 23,463 \\
\hline Columbia River & Horn Area & B07NH1 & November 23, 1992 & Zinc & $\mathrm{mg} / \mathrm{kg}$ & 1 & 294 & $=$ & 120 & 23,463 \\
\hline Columbia River & D Island & B07NH5 & November 24, 1992 & Zinc & $\mathrm{mg} / \mathrm{kg}$ & 1 & 185 & $=$ & 120 & 23,463 \\
\hline Columbia River & D Island & B07NH6 & November 24, 1992 & Zinc & $\mathrm{mg} / \mathrm{kg}$ & 1 & 209 & $=$ & 120 & 23,463 \\
\hline Columbia River & D Island & B07NH7 & November 24, 1992 & Zinc & $\mathrm{mg} / \mathrm{kg}$ & 1 & 167 & $=$ & 120 & 23,463 \\
\hline Columbia River & Wht Bluffs Slg & BOCSH3 & September 24, 1994 & Zinc & $\mathrm{mg} / \mathrm{kg}$ & 1 & 200 & $=$ & 120 & 23,463 \\
\hline Columbia River & Richland, WA & BOCSH6 & September 25, 1994 & Zinc & $\mathrm{mg} / \mathrm{kg}$ & 1 & 190 & $=$ & 120 & 23,463 \\
\hline Columbia River & HFD SPR 28-2 & BOCSH8 & September 2, 1994 & Zinc & $\mathrm{mg} / \mathrm{kg}$ & 1 & 140 & $=$ & 120 & 23,463 \\
\hline Columbia River & Wht Bluffs Slg & B0W3F4 & August 9, 1999 & Zinc & $\mathrm{mg} / \mathrm{Kg}$ & 1 & 334 & $=$ & 120 & 23,463 \\
\hline Columbia River & $100 \mathrm{~F}$ SLOUGH & Bow3F6 & August 9, 1999 & Zinc & $\mathrm{mg} / \mathrm{Kg}$ & 1 & 275 & $=$ & 120 & 23,463 \\
\hline Columbia River & Hanford Slough & B0W3F8 & August 9, 1999 & Zinc & $\mathrm{mg} / \mathrm{Kg}$ & 1 & 187 & $=$ & 120 & 23,463 \\
\hline Columbia River & 100-B SPR 38-3 & B0WDP5 & October 25, 1999 & Zinc & $\mathrm{mg} / \mathrm{Kg}$ & 1 & 149 & $=$ & 120 & 23,463 \\
\hline Columbia River & HFD SPR UR 28-2 & BOWDP9 & November 1, 1999 & Zinc & $\mathrm{mg} / \mathrm{Kg}$ & 1 & 164 & $=$ & 120 & 23,463 \\
\hline Columbia River & HFD SPR DR 28-2 & BOWDR1 & November 1, 1999 & Zinc & $\mathrm{mg} / \mathrm{Kg}$ & 1 & 154 & $=$ & 120 & 23,463 \\
\hline
\end{tabular}


Table B-7

Summary of Surface Sediment Results From Segment 2 Exceeding Select Ecological or Human Health Benchmark Values Columbia River Component - Existing Sample Data Evaluation and Scoping Report

\begin{tabular}{|c|c|c|c|c|c|c|c|c|c|c|}
\hline Sample Area & Sample Site & SamplelD & Date Collected & Constituent Name & Units & $\begin{array}{c}\text { QA } \\
\text { Category }\end{array}$ & $\begin{array}{c}\text { Analytical } \\
\text { Value }\end{array}$ & Qualifier & $\begin{array}{c}\text { Ecological } \\
\text { Benchmark } \\
\text { Value }\end{array}$ & $\begin{array}{l}\text { Human Health } \\
\text { Benchmark } \\
\text { Value }\end{array}$ \\
\hline Columbia River & 300 SPR DR 42-2 & BOWDR3 & November 1, 1999 & Zinc & $\mathrm{mg} / \mathrm{Kg}$ & 1 & 146 & $=$ & 120 & 23,463 \\
\hline Columbia River & 100-F SPR 207-1 & BOWDTO & November 1, 1999 & Zinc & $\mathrm{mg} / \mathrm{Kg}$ & 1 & 205 & $=$ & 120 & 23,463 \\
\hline Columbia River & RICHLAND-RIVER & B0YW85 & August 8, 2000 & Zinc & $\mathrm{mg} / \mathrm{Kg}$ & 1 & 292 & $=$ & 120 & 23,463 \\
\hline Columbia River & Wht Bluffs Slg & B0YY65 & August 15, 2000 & Zinc & $\mathrm{mg} / \mathrm{Kg}$ & 1 & 360 & $=$ & 120 & 23,463 \\
\hline Columbia River & 100 F SLOUGH & B0YY67 & August 15, 2000 & Zinc & $\mathrm{mg} / \mathrm{Kg}$ & 1 & 219 & $=$ & 120 & 23,463 \\
\hline Columbia River & 100-B SPR 38-3 & B10916 & November 20, 2000 & Zinc & $\mathrm{mg} / \mathrm{Kg}$ & 1 & 137 & $=$ & 120 & 23,463 \\
\hline Columbia River & HFD SPR UR 28-2 & B10918 & November 2, 2000 & Zinc & $\mathrm{mg} / \mathrm{Kg}$ & 1 & 200 & $=$ & 120 & 23,463 \\
\hline Columbia River & HFD SPR DR 28-2 & B10921 & November 2, 2000 & Zinc & $\mathrm{mg} / \mathrm{Kg}$ & 1 & 149 & $=$ & 120 & 23,463 \\
\hline Columbia River & 300 SPR DR 42-2 & B10923 & November 2, 2000 & Zinc & $\mathrm{mg} / \mathrm{Kg}$ & 1 & 161 & $=$ & 120 & 23,463 \\
\hline Columbia River & 100-F SPR 207-1 & B10931 & November 20, 2000 & Zinc & $\mathrm{mg} / \mathrm{Kg}$ & 1 & 155 & $=$ & 120 & 23,463 \\
\hline Columbia River & 100 F SLOUGH & $\mathrm{B} 12 \mathrm{CJ} 7$ & July 19, 2001 & Zinc & $\mathrm{mg} / \mathrm{kg}$ dry-wt & 1 & 212 & $=$ & 120 & 23,463 \\
\hline Columbia River & RICHLAND-RIVER & B12CK1 & July 23, 2001 & Zinc & $\mathrm{mg} / \mathrm{kg}$ dry-wt & 1 & 366 & $=$ & 120 & 23,463 \\
\hline Columbia River & 300 SPR 42-2 & B12RL7 & August 27, 2001 & Zinc & $\mathrm{mg} / \mathrm{kg}$ dry-wt & 1 & 144 & $=$ & 120 & 23,463 \\
\hline Columbia River & 300 SPR DR 42-2 & B12RM5 & August 27, 2001 & Zinc & $\mathrm{mg} / \mathrm{kg}$ dry-wt & 1 & 215 & $=$ & 120 & 23,463 \\
\hline Columbia River & 300 SPR 11 & B12RY8 & August 27, 2001 & Zinc & $\mathrm{mg} / \mathrm{kg}$ dry-wt & 1 & 128 & $=$ & 120 & 23,463 \\
\hline Columbia River & 300 SPR 14 & В12T08 & August 27, 2001 & Zinc & $\mathrm{mg} / \mathrm{kg}$ dry-wt & 1 & 154 & $=$ & 120 & 23,463 \\
\hline Columbia River & 100-B SPR 38-3 & B12X22 & October 22, 2001 & Zinc & $\mathrm{mg} / \mathrm{kg}$ dry-wt & 1 & 141 & $=$ & 120 & 23,463 \\
\hline Columbia River & 100-F SPR 207-1 & B12X37 & October 22, 2001 & Zinc & $\mathrm{mg} / \mathrm{kg}$ dry-wt & 1 & 199 & $=$ & 120 & 23,463 \\
\hline Columbia River & Wht Bluffs Slg & B176X9 & July 14,2003 & Zinc & $\mathrm{mg} / \mathrm{Kg}$ & 1 & 394 & $=$ & 120 & 23,463 \\
\hline Columbia River & 100 F SLOUGH & B176Y1 & July 14,2003 & Zinc & $\mathrm{mg} / \mathrm{Kg}$ & 1 & 222 & $=$ & 120 & 23,463 \\
\hline Columbia River & Hanford Slough & B176Y3 & July 14, 2003 & Zinc & $\mathrm{mg} / \mathrm{Kg}$ & 1 & 477 & $=$ & 120 & 23,463 \\
\hline Columbia River & RICHLAND-RIVER & B176Y5 & July 16, 2003 & Zinc & $\mathrm{mg} / \mathrm{Kg}$ & 1 & 332 & $=$ & 120 & 23,463 \\
\hline Columbia River & 100-B SPR 37-1 & B17J53 & October 20, 2003 & Zinc & $\mathrm{mg} / \mathrm{Kg}$ & 1 & 175 & $=$ & 120 & 23,463 \\
\hline Columbia River & HFD SPR DR 28-2 & B17J58 & November 3, 2003 & Zinc & $\mathrm{mg} / \mathrm{Kg}$ & 1 & 188 & $=$ & 120 & 23,463 \\
\hline Columbia River & 300 SPR DR 42-2 & B17J61 & October 13, 2003 & Zinc & $\mathrm{mg} / \mathrm{Kg}$ & 1 & 128 & $=$ & 120 & 23,463 \\
\hline Columbia River & 100-K SPR 63-1 & B17J64 & October 20, 2003 & Zinc & $\mathrm{mg} / \mathrm{Kg}$ & 1 & 196 & $=$ & 120 & 23,463 \\
\hline Columbia River & 100-F SPR 207-1 & B17J67 & November 3, 2003 & Zinc & $\mathrm{mg} / \mathrm{Kg}$ & 1 & 256 & $=$ & 120 & 23,463 \\
\hline Columbia River & 100-H SPR 145-1 & B17JB1 & October 27, 2003 & Zinc & $\mathrm{mg} / \mathrm{Kg}$ & 1 & 306 & $=$ & 120 & 23,463 \\
\hline Columbia River & 100-B SPR 37-1 & B17XN8 & November 13, 2003 & Zinc & $\mathrm{mg} / \mathrm{Kg}$ & 1 & 151 & $=$ & 120 & 23,463 \\
\hline Columbia River & 100-B SPR 38-3 & B17XN9 & November 13, 2003 & Zinc & $\mathrm{mg} / \mathrm{Kg}$ & 1 & 184 & $=$ & 120 & 23,463 \\
\hline Columbia River & Wht Bluffs Slg & B19PJ6 & July 12,2004 & Zinc & $\mathrm{mg} / \mathrm{Kg}$ & 1 & 380 & $\mathrm{x}$ & 120 & 23,463 \\
\hline Columbia River & 100 F SLOUGH & B19PJ8 & July 12, 2004 & Zinc & $\mathrm{mg} / \mathrm{Kg}$ & 1 & 216 & $\mathrm{x}$ & 120 & 23,463 \\
\hline Columbia River & RICHLAND-RIVER & B19PK2 & July 13,2004 & Zinc & $\mathrm{mg} / \mathrm{Kg}$ & 1 & 242 & $\mathrm{x}$ & 120 & 23,463 \\
\hline Columbia River & HFD SPR UR 28-2 & B1BFP9 & October 25, 2004 & Zinc & $\mathrm{mg} / \mathrm{Kg}$ & 1 & 255 & $\mathrm{x}$ & 120 & 23,463 \\
\hline Columbia River & HFD SPR DR 28-2 & B1BFR2 & October 25, 2004 & Zinc & $\mathrm{mg} / \mathrm{Kg}$ & 1 & 162 & $\mathrm{x}$ & 120 & 23,463 \\
\hline Columbia River & 300 SPR DR 42-2 & B1BFR5 & October 25, 2004 & Zinc & $\mathrm{mg} / \mathrm{Kg}$ & 1 & 144 & $\mathrm{x}$ & 120 & 23,463 \\
\hline Columbia River & 100-F SPR 207-1 & B1BFT0 & October 26, 2004 & Zinc & $\mathrm{mg} / \mathrm{Kg}$ & 1 & 148 & $\mathrm{x}$ & 120 & 23,463 \\
\hline Columbia River & 100-H SPR 145-1 & B1BH03 & October 26, 2004 & Zinc & $\mathrm{mg} / \mathrm{Kg}$ & 1 & 268 & $\mathrm{x}$ & 120 & 23,463 \\
\hline
\end{tabular}




\section{Table B-8}

Summary Statistics for All Categories of Surface Water Samples Collected from Segment 2 of the Columbia River

Columbia River Component - Existing Sample Data Evaluation and Scoping Report

\begin{tabular}{|c|c|c|c|c|c|c|c|c|c|c|}
\hline $\begin{array}{l}\text { Constituent } \\
\text { Class }\end{array}$ & Constituent Name & Units & $\begin{array}{l}\text { Number } \\
\text { Analyzed }\end{array}$ & $\begin{array}{l}\text { Number } \\
\text { Detected }\end{array}$ & $\begin{array}{c}\text { Frequency of } \\
\text { Detection }\end{array}$ & $\begin{array}{l}\text { Minimum } \\
\text { Nondetect }\end{array}$ & $\begin{array}{l}\text { Maximum } \\
\text { Nondetect }\end{array}$ & $\begin{array}{l}\text { Minimum } \\
\text { Detect }\end{array}$ & $\begin{array}{l}\text { Maximum } \\
\text { Detect }\end{array}$ & $\begin{array}{c}\text { Average } \\
\text { Concentration }\end{array}$ \\
\hline$\overline{\text { CONV }}$ & Alkalinity & $\overline{\mathrm{mg} / \mathrm{L}}$ & $\overline{3}$ & 3 & $100 \%$ & $\overline{--}$ & $\overline{--}$ & 56 & 60 & 58 \\
\hline CONV & Ammonia & $\mathrm{mg} / \mathrm{L}$ & 1 & 0 & $0 \%$ & 1.0 & 1.0 & -- & -- & 0.50 \\
\hline CONV & Bicarbonate & $\mathrm{mg} / \mathrm{L}$ & 1 & 1 & $100 \%$ & -- & -- & 57 & 57 & 57 \\
\hline CONV & Bromide & $\mathrm{mg} / \mathrm{L}$ & 8 & 0 & $0 \%$ & 0.25 & 0.25 & -- & -- & 0.13 \\
\hline CONV & Carbonate & $\mathrm{mg} / \mathrm{L}$ & 2 & 2 & $100 \%$ & -- & -- & 39 & 45 & 42 \\
\hline CONV & Chloride & $\mathrm{mg} / \mathrm{L}$ & 515 & 513 & $100 \%$ & 0.035 & 20 & 0.65 & 48 & 2.4 \\
\hline CONV & Cyanide & $\mathrm{mg} / \mathrm{L}$ & 21 & 0 & $0 \%$ & 0.0016 & 0.050 & -- & -- & 0.0020 \\
\hline CONV & Dissolved oxygen & $\mathrm{mg} / \mathrm{L}$ & 5 & 5 & $100 \%$ & -- & -- & 9.3 & 9.8 & 9.7 \\
\hline CONV & Fluoride & $\mathrm{mg} / \mathrm{L}$ & 515 & 488 & $95 \%$ & 0.010 & 0.50 & 0.013 & 2.4 & 0.12 \\
\hline CONV & Hardness & $\mathrm{mg} / \mathrm{L}$ & 1 & 1 & $100 \%$ & -- & -- & 69 & 69 & 69 \\
\hline CONV & Nitrate & $\mathrm{mg} / \mathrm{L}$ & 27 & 26 & $96 \%$ & 0.50 & 0.50 & 0.34 & 7.5 & 2.5 \\
\hline CONV & Nitrite & $\mathrm{mg} / \mathrm{L}$ & 8 & 0 & $0 \%$ & 0.10 & 0.25 & -- & -- & 0.12 \\
\hline CONV & Nitrogen, Nitrate (as N) & $\mathrm{mg} / \mathrm{L}$ & 604 & 603 & $100 \%$ & 0.011 & 0.011 & 0.028 & 295 & 1.6 \\
\hline CONV & Nitrogen, Nitrate-Nitrite & $\mathrm{mg} / \mathrm{L}$ & 91 & 91 & $100 \%$ & -- & -- & 0.067 & 22,000 & 432 \\
\hline CONV & Nitrogen, Nitrite & $\mathrm{mg} / \mathrm{L}$ & 503 & 7 & $1 \%$ & 0.0020 & 0.15 & 0.0081 & 0.023 & 0.0038 \\
\hline CONV & $\begin{array}{l}\text { Orthophosphate } \\
\text { Oxidation Reduction }\end{array}$ & $\mathrm{mg} / \mathrm{L}$ & 1 & 0 & $0 \%$ & 0.10 & 0.10 & -- & -- & 0.050 \\
\hline CONV & Potential & $\mathrm{mV}$ & 13 & 13 & $100 \%$ & -- & -- & 132 & 343 & 231 \\
\hline CONV & $\mathrm{pH}$ & pH Units & 31 & 31 & $100 \%$ & -- & -- & 6.7 & 8.0 & 7.6 \\
\hline CONV & Phosphate & $\mathrm{mg} / \mathrm{L}$ & 9 & 1 & $11 \%$ & 0.25 & 0.25 & 0.010 & 0.010 & 0.11 \\
\hline CONV & Specific Conductance & umhos/cm & 32 & 32 & $100 \%$ & -- & -- & 120 & 426 & 209 \\
\hline CONV & Sulfate & $\mathrm{mg} / \mathrm{L}$ & 515 & 514 & $100 \%$ & 0.11 & 0.11 & 1.1 & 290 & 14 \\
\hline CONV & Temperature & Deg C & 21 & 21 & $100 \%$ & -- & -- & 7.1 & 18 & 13 \\
\hline CONV & Total dissolved solids & $\mathrm{mg} / \mathrm{L}$ & 1 & 0 & $0 \%$ & 150 & 150 & -- & - & 75 \\
\hline CONV & Total suspended solids & $\mathrm{mg} / \mathrm{L}$ & 6 & 6 & $100 \%$ & -- & -- & 7.0 & 14 & 10 \\
\hline METAL & Aluminum & $\mathrm{mg} / \mathrm{L}$ & 109 & 57 & $52 \%$ & 0.012 & 0.096 & 3.39E-04 & 21 & 0.39 \\
\hline METAL & Antimony & $\mathrm{mg} / \mathrm{L}$ & 1058 & 978 & $92 \%$ & 0.0017 & 0.039 & $9.10 \mathrm{E}-05$ & 0.0043 & 0.0010 \\
\hline METAL & Arsenic & $\mathrm{mg} / \mathrm{L}$ & 980 & 979 & $100 \%$ & 0.0020 & 0.0020 & 6.00E-06 & 0.0085 & 8.95E-04 \\
\hline METAL & Barium & $\mathrm{mg} / \mathrm{L}$ & 122 & 121 & $99 \%$ & 0.10 & 0.10 & 0.017 & 0.29 & 0.040 \\
\hline METAL & Beryllium & $\mathrm{mg} / \mathrm{L}$ & 975 & 76 & $8 \%$ & 8.00E-06 & 0.0050 & $9.95 \mathrm{E}-07$ & 0.0018 & 4.55E-05 \\
\hline METAL & Cadmium & $\mathrm{mg} / \mathrm{L}$ & 1057 & 795 & $75 \%$ & 4.44E-06 & 0.0043 & 5.58E-06 & 0.0035 & $1.21 \mathrm{E}-04$ \\
\hline METAL & Calcium & $\mathrm{mg} / \mathrm{L}$ & 84 & 84 & $100 \%$ & -- & -- & 17 & 65 & 31 \\
\hline METAL & Chromium & $\mathrm{mg} / \mathrm{L}$ & 1065 & 843 & $79 \%$ & 1.65E-05 & 0.10 & 2.09E-05 & 0.22 & 0.0053 \\
\hline METAL & Cobalt & $\mathrm{mg} / \mathrm{L}$ & 81 & 3 & $4 \%$ & 8.00E-04 & 0.0050 & 0.0017 & 0.0096 & 0.0020 \\
\hline METAL & Copper & $\mathrm{mg} / \mathrm{L}$ & 1060 & 1001 & $94 \%$ & 8.60E-04 & 10 & $2.02 \mathrm{E}-04$ & 0.037 & 0.0058 \\
\hline METAL & lodine & $\mathrm{mg} / \mathrm{L}$ & 37 & 37 & $100 \%$ & -- & - & $4.28 \mathrm{E}-04$ & 51 & 1.4 \\
\hline METAL & Iron & $\mathrm{mg} / \mathrm{L}$ & 82 & 45 & $55 \%$ & 0.016 & 0.10 & 0.016 & 20 & 0.54 \\
\hline METAL & Lead & $\mathrm{mg} / \mathrm{L}$ & 980 & 900 & $92 \%$ & 4.00E-06 & 0.0020 & $1.11 \mathrm{E}-06$ & 0.066 & $3.14 \mathrm{E}-04$ \\
\hline METAL & Magnesium & $\mathrm{mg} / \mathrm{L}$ & 84 & 84 & $100 \%$ & -- & -- & 4.0 & 18 & 7.0 \\
\hline METAL & Manganese & $\mathrm{mg} / \mathrm{L}$ & 134 & 103 & $77 \%$ & 3.00E-04 & 0.010 & 3.70E-05 & 0.94 & 0.017 \\
\hline METAL & Mercury & $\mathrm{mg} / \mathrm{L}$ & 398 & 397 & $100 \%$ & 5.00E-04 & 5.00E-04 & $2.25 \mathrm{E}-07$ & 4.07E-05 & $1.77 \mathrm{E}-06$ \\
\hline METAL & Molybdenum & $\mathrm{mg} / \mathrm{L}$ & 1 & 1 & $100 \%$ & -- & -- & $6.14 \mathrm{E}-04$ & $6.14 \mathrm{E}-04$ & $6.14 \mathrm{E}-04$ \\
\hline
\end{tabular}




\section{Table B-8}

Summary Statistics for All Categories of Surface Water Samples Collected from Segment 2 of the Columbia River

Columbia River Component - Existing Sample Data Evaluation and Scoping Report

\begin{tabular}{|c|c|c|c|c|c|c|c|c|c|c|}
\hline $\begin{array}{c}\text { Constituent } \\
\text { Class }\end{array}$ & Constituent Name & Units & $\begin{array}{c}\text { Number } \\
\text { Analyzed }\end{array}$ & $\begin{array}{l}\text { Number } \\
\text { Detected }\end{array}$ & $\begin{array}{l}\text { Frequency of } \\
\text { Detection }\end{array}$ & $\begin{array}{l}\text { Minimum } \\
\text { Nondetect }\end{array}$ & $\begin{array}{c}\text { Maximum } \\
\text { Nondetect }\end{array}$ & $\begin{array}{l}\text { Minimum } \\
\text { Detect }\end{array}$ & $\begin{array}{l}\text { Maximum } \\
\text { Detect }\end{array}$ & $\begin{array}{c}\text { Average } \\
\text { Concentration }\end{array}$ \\
\hline METAL & Nickel & $\mathrm{mg} / \mathrm{L}$ & 1061 & 988 & $93 \%$ & 2.83E-05 & 0.040 & $4.10 \mathrm{E}-05$ & 0.032 & 0.0012 \\
\hline METAL & Potassium & $\mathrm{mg} / \mathrm{L}$ & 67 & 33 & $49 \%$ & 1.3 & 3.0 & 0.52 & 12 & 1.9 \\
\hline METAL & Selenium & $\mathrm{mg} / \mathrm{L}$ & 982 & 457 & $47 \%$ & $6.42 \mathrm{E}-05$ & 0.0050 & 6.73E-05 & 0.0039 & $3.78 \mathrm{E}-04$ \\
\hline METAL & Silica & $\mathrm{mg} / \mathrm{L}$ & 1 & 1 & $100 \%$ & -- & -- & 2.0 & 2.0 & 2.0 \\
\hline METAL & Silicon & $\mathrm{mg} / \mathrm{L}$ & 2 & 2 & $100 \%$ & -- & -- & 0.095 & 0.21 & 0.15 \\
\hline METAL & Silver & $\mathrm{mg} / \mathrm{L}$ & 1060 & 355 & $33 \%$ & $1.20 E-06$ & 0.010 & $9.48 \mathrm{E}-07$ & 0.0030 & 1.34E-04 \\
\hline METAL & Sodium & $\mathrm{mg} / \mathrm{L}$ & 84 & 83 & $99 \%$ & 5.0 & 5.0 & 2.0 & 19 & 6.2 \\
\hline METAL & Strontium & $\mathrm{mg} / \mathrm{L}$ & 81 & 81 & $100 \%$ & -- & -- & 0.081 & 0.38 & 0.16 \\
\hline METAL & Thallium & $\mathrm{mg} / \mathrm{L}$ & 899 & 802 & $89 \%$ & $3.00 E-06$ & 0.0020 & 5.18E-06 & 4.07E-04 & $2.21 \mathrm{E}-05$ \\
\hline METAL & Thorium & $\mathrm{mg} / \mathrm{L}$ & 42 & 2 & $5 \%$ & $3.10 \mathrm{E}-06$ & $1.11 \mathrm{E}-04$ & $5.65 \mathrm{E}-06$ & 1.19E-05 & $4.08 \mathrm{E}-05$ \\
\hline METAL & Uranium & $\mathrm{mg} / \mathrm{L}$ & 54 & 54 & $100 \%$ & -- & -- & $1.21 \mathrm{E}-04$ & 0.0087 & 0.0021 \\
\hline METAL & Vanadium & $\mathrm{mg} / \mathrm{L}$ & 81 & 38 & $47 \%$ & $9.00 \mathrm{E}-04$ & 0.0097 & $6.50 \mathrm{E}-04$ & 0.049 & 0.0066 \\
\hline METAL & Zinc & $\mathrm{mg} / \mathrm{L}$ & 1061 & 1038 & $98 \%$ & 1.40E-04 & 0.020 & 3.51E-04 & 0.42 & 0.0045 \\
\hline RAD & Actinium-228 & $\mathrm{pCi} / \mathrm{L}$ & 27 & 0 & $0 \%$ & 5.0 & 26 & -- & -- & 6.2 \\
\hline RAD & Americium-241 & $\mathrm{pCi} / \mathrm{L}$ & 3 & 0 & $0 \%$ & 0.0018 & 0.0052 & -- & -- & 0.0018 \\
\hline RAD & Antimony-124 & $\mathrm{pCi} / \mathrm{L}$ & 1 & 0 & $0 \%$ & 6.1 & 6.1 & -- & -- & 3.0 \\
\hline RAD & Antimony-125 & $\mathrm{pCi} / \mathrm{L}$ & 138 & 0 & $0 \%$ & 4.97E-06 & 15 & -- & -- & 0.60 \\
\hline RAD & Barium-133 & $\mathrm{pCi} / \mathrm{L}$ & 1 & 0 & $0 \%$ & 7.0 & 7.0 & -- & -- & 3.5 \\
\hline RAD & Barium-140 & $\mathrm{pCi} / \mathrm{L}$ & 83 & 2 & $2 \%$ & 0.10 & 506 & 4.0 & 4.5 & 10 \\
\hline RAD & Beryllium-7 & $\mathrm{pCi} / \mathrm{L}$ & 263 & 67 & $25 \%$ & $6.40 \mathrm{E}-07$ & 93 & 0.016 & 41 & 5.1 \\
\hline RAD & Carbon-14 & $\mathrm{pCi} / \mathrm{L}$ & 10 & 4 & $40 \%$ & 1.2 & 7.3 & 9.2 & 608 & 74 \\
\hline RAD & Cerium-139 & $\mathrm{pCi} / \mathrm{L}$ & 1 & 0 & $0 \%$ & 4.6 & 4.6 & -- & -- & 2.3 \\
\hline RAD & Cerium-141 & $\mathrm{pCi} / \mathrm{L}$ & 50 & 0 & $0 \%$ & 2.5 & 25 & -- & -- & 3.1 \\
\hline RAD & Cerium-144 & $\mathrm{pCi} / \mathrm{L}$ & 50 & 0 & $0 \%$ & 8.9 & 39 & -- & -- & 10 \\
\hline RAD & Cesium-134 & $\mathrm{pCi} / \mathrm{L}$ & 218 & 1 & $0 \%$ & $2.46 \mathrm{E}-07$ & 6.6 & 1.0 & 1.0 & 0.55 \\
\hline RAD & Cesium-136 & $\mathrm{pCi} / \mathrm{L}$ & 1 & 0 & $0 \%$ & 8.4 & 8.4 & -- & -- & 4.2 \\
\hline RAD & Cesium-137 & $\mathrm{pCi} / \mathrm{L}$ & 352 & 16 & $5 \%$ & 2.08E-05 & 17 & 0.0020 & 8.5 & 0.49 \\
\hline RAD & Chromium-51 & $\mathrm{pCi} / \mathrm{L}$ & 1 & 0 & $0 \%$ & 52 & 52 & -- & -- & 26 \\
\hline RAD & Cobalt-57 & $\mathrm{pCi} / \mathrm{L}$ & 38 & 0 & $0 \%$ & 1.2 & 4.5 & -- & -- & 1.2 \\
\hline RAD & Cobalt-58 & $\mathrm{pCi} / \mathrm{L}$ & 80 & 0 & $0 \%$ & 0.020 & 9.5 & -- & -- & 1.1 \\
\hline RAD & Cobalt-60 & $\mathrm{pCi} / \mathrm{L}$ & 344 & 2 & $1 \%$ & 1.47E-05 & 22 & 1.0 & 1.2 & 0.50 \\
\hline RAD & Europium-152 & $\mathrm{pCi} / \mathrm{L}$ & 98 & 0 & $0 \%$ & 1.53E-04 & 39 & - & - & 2.8 \\
\hline RAD & Europium-154 & $\mathrm{pCi} / \mathrm{L}$ & 156 & 0 & $0 \%$ & 2.63E-05 & 49 & -- & -- & 0.88 \\
\hline RAD & Europium-155 & $\mathrm{pCi} / \mathrm{L}$ & 152 & 0 & $0 \%$ & 1.46E-04 & 41 & -- & -- & 0.61 \\
\hline RAD & Gross alpha & $\mathrm{pCi} / \mathrm{L}$ & 375 & 150 & $40 \%$ & 0.0065 & 4.0 & 0.20 & 228 & 6.5 \\
\hline RAD & Gross beta & $\mathrm{pCi} / \mathrm{L}$ & 487 & 269 & $55 \%$ & 0.028 & 4.0 & 0.40 & 84 & 5.5 \\
\hline RAD & lodine-129 & $\mathrm{pCi} / \mathrm{L}$ & 53 & 53 & $100 \%$ & -- & -- & $2.55 \mathrm{E}-05$ & 0.41 & 0.054 \\
\hline RAD & Iodine-131 & $\mathrm{pCi} / \mathrm{L}$ & 84 & 3 & $4 \%$ & 0.019 & 1,100 & 1.0 & 1.2 & 10 \\
\hline RAD & Iron-59 & $\mathrm{pCi} / \mathrm{L}$ & 98 & 8 & $8 \%$ & 0.10 & 52 & 1.8 & 3.0 & 2.7 \\
\hline RAD & Lanthanum-140 & $\mathrm{pCi} / \mathrm{L}$ & 51 & 0 & $0 \%$ & 2.0 & 172 & -- & -- & 5.4 \\
\hline RAD & Manganese-54 & $\mathrm{pCi} / \mathrm{L}$ & 82 & 0 & $0 \%$ & 0.010 & 6.1 & -- & -- & 1.1 \\
\hline RAD & Molybdenum-99 & $\mathrm{pCi} / \mathrm{L}$ & 1 & 0 & $0 \%$ & 300 & 300 & -- & -- & 150 \\
\hline
\end{tabular}




\section{Table B-8}

Summary Statistics for All Categories of Surface Water Samples Collected from Segment 2 of the Columbia River

Columbia River Component - Existing Sample Data Evaluation and Scoping Report

\begin{tabular}{|c|c|c|c|c|c|c|c|c|c|c|}
\hline $\begin{array}{c}\text { Constituent } \\
\text { Class }\end{array}$ & Constituent Name & Units & $\begin{array}{c}\text { Number } \\
\text { Analyzed }\end{array}$ & $\begin{array}{l}\text { Number } \\
\text { Detected }\end{array}$ & $\begin{array}{c}\text { Frequency of } \\
\text { Detection }\end{array}$ & $\begin{array}{c}\text { Minimum } \\
\text { Nondetect }\end{array}$ & $\begin{array}{c}\text { Maximum } \\
\text { Nondetect }\end{array}$ & $\begin{array}{l}\text { Minimum } \\
\text { Detect }\end{array}$ & $\begin{array}{l}\text { Maximum } \\
\text { Detect }\end{array}$ & $\begin{array}{c}\text { Average } \\
\text { Concentration } \\
\end{array}$ \\
\hline RAD & Nickel-63 & $\mathrm{pCi} / \mathrm{L}$ & 2 & 0 & $0 \%$ & 2.0 & 3.4 & -- & -- & 1.4 \\
\hline RAD & Niobium-94 & $\mathrm{pCi} / \mathrm{L}$ & 1 & 0 & $0 \%$ & 5.9 & 5.9 & -- & -- & 3.0 \\
\hline RAD & Niobium-95 & $\mathrm{pCi} / \mathrm{L}$ & 50 & 0 & $0 \%$ & 1.3 & 10 & -- & -- & 1.7 \\
\hline RAD & Plutonium-238 & $\mathrm{pCi} / \mathrm{L}$ & 29 & 6 & $21 \%$ & 7.06E-07 & $9.26 \mathrm{E}-04$ & 6.35E-06 & 0.0052 & $3.96 \mathrm{E}-04$ \\
\hline RAD & Plutonium-239/240 & $\mathrm{pCi} / \mathrm{L}$ & 41 & 15 & $37 \%$ & $1.04 \mathrm{E}-07$ & 0.0025 & 6.89E-06 & 1.63E-04 & 7.45E-05 \\
\hline RAD & Potassium-40 & $\mathrm{pCi} / \mathrm{L}$ & 308 & 184 & $60 \%$ & 2.72E-04 & 220 & 0.027 & 126 & 15 \\
\hline RAD & Radium-226 & $\mathrm{pCi} / \mathrm{L}$ & 63 & 10 & $16 \%$ & 9.9 & 192 & 0.022 & 0.058 & 30 \\
\hline RAD & Radium-228 & $\mathrm{pCi} / \mathrm{L}$ & 12 & 4 & $33 \%$ & 0.0059 & 78 & 0.36 & 1.0 & 5.8 \\
\hline RAD & Ruthenium-103 & $\mathrm{pCi} / \mathrm{L}$ & 50 & 0 & $0 \%$ & 1.5 & 14 & -- & -- & 1.9 \\
\hline RAD & Ruthenium-106 & $\mathrm{pCi} / \mathrm{L}$ & 191 & 0 & $0 \%$ & 1.36E-04 & 54 & -- & -- & 5.1 \\
\hline RAD & Selenium-75 & $\mathrm{pCi} / \mathrm{L}$ & 1 & 0 & $0 \%$ & 6.5 & 6.5 & -- & -- & 3.2 \\
\hline RAD & Silver-110 metastable & $\mathrm{pCi} / \mathrm{L}$ & 1 & 0 & $0 \%$ & 5.7 & 5.7 & -- & -- & 2.9 \\
\hline RAD & Sodium-24 & $\mathrm{pCi} / \mathrm{L}$ & 1 & 0 & $0 \%$ & 26,600 & 26,600 & -- & -- & 13,300 \\
\hline RAD & Strontium-85 & $\mathrm{pCi} / \mathrm{L}$ & 1 & 0 & $0 \%$ & 7.8 & 7.8 & -- & -- & 3.9 \\
\hline RAD & Strontium-90 & $\mathrm{pCi} / \mathrm{L}$ & 784 & 636 & $81 \%$ & 2.67E-05 & 1.3 & 0.022 & 109 & 0.44 \\
\hline RAD & Technetium-99 & $\mathrm{pCi} / \mathrm{L}$ & 144 & 64 & $44 \%$ & 0.0041 & 0.70 & 0.27 & 144 & 12 \\
\hline RAD & Tellurium-129M & $\mathrm{pCi} / \mathrm{L}$ & 1 & 0 & $0 \%$ & 68 & 68 & -- & -- & 34 \\
\hline RAD & Thorium-228 & $\mathrm{pCi} / \mathrm{L}$ & 60 & 2 & $3 \%$ & 0.0018 & 500 & 0.080 & 0.084 & 18 \\
\hline RAD & Thorium-230 & $\mathrm{pCi} / \mathrm{L}$ & 6 & 3 & $50 \%$ & 0.0030 & 0.0097 & 0.054 & 2.6 & 0.46 \\
\hline RAD & Thorium-232 & $\mathrm{pCi} / \mathrm{L}$ & 9 & 2 & $22 \%$ & 3.78E-04 & 78 & 0.060 & 0.087 & 8.8 \\
\hline RAD & Tin-113 & $\mathrm{pCi} / \mathrm{L}$ & 1 & 0 & $0 \%$ & 7.1 & 7.1 & -- & -- & 3.5 \\
\hline RAD & Tritium & $\mathrm{pCi} / \mathrm{L}$ & 1136 & 970 & $85 \%$ & 0.060 & 284 & 17 & 112,000 & 1,939 \\
\hline RAD & Uranium & $\mathrm{pCi} / \mathrm{L}$ & 118 & 118 & $100 \%$ & -- & -- & 0.14 & 100 & 5.5 \\
\hline RAD & Uranium-234 & $\mathrm{pCi} / \mathrm{L}$ & 778 & 776 & $100 \%$ & 0.010 & 0.12 & 0.078 & 111 & 1.8 \\
\hline RAD & Uranium-235 & $\mathrm{pCi} / \mathrm{L}$ & 715 & 200 & $28 \%$ & 2.59E-04 & 56 & 0.0065 & 2.9 & 0.48 \\
\hline RAD & Uranium-236 & $\mathrm{pCi} / \mathrm{L}$ & 8 & 8 & $100 \%$ & -- & -- & 0.29 & 1.0 & 0.68 \\
\hline RAD & Uranium-238 & $\mathrm{pCi} / \mathrm{L}$ & 782 & 776 & $99 \%$ & 0.010 & 1,900 & 0.070 & 99 & 3.9 \\
\hline RAD & Yttrium & $\mathrm{pCi} / \mathrm{L}$ & 1 & 0 & $0 \%$ & 6.7 & 6.7 & -- & -- & 3.4 \\
\hline RAD & Zinc-65 & $\mathrm{pCi} / \mathrm{L}$ & 66 & 0 & $0 \%$ & 0.20 & 15 & -- & -- & 2.7 \\
\hline RAD & Zirconium-95 & $\mathrm{pCi} / \mathrm{L}$ & 84 & 5 & $6 \%$ & 0.040 & 16 & 0.90 & 1.5 & 1.9 \\
\hline SVOC & 1,4-Dichlorobenzene & $\mathrm{mg} / \mathrm{L}$ & 120 & 3 & $3 \%$ & 1.10E-04 & $2.50 \mathrm{E}-04$ & $1.90 \mathrm{E}-04$ & $2.50 \mathrm{E}-04$ & 8.16E-05 \\
\hline VOC & 1,1,1-Trichloroethane & $\mathrm{mg} / \mathrm{L}$ & 119 & 0 & $0 \%$ & 7.00E-05 & 3.10E-04 & -- & -- & 1.05E-04 \\
\hline VOC & 1,1,2-Trichloroethane & $\mathrm{mg} / \mathrm{L}$ & 120 & 11 & $9 \%$ & 5.00E-05 & 3.10E-04 & 1.30E-04 & 8.80E-04 & 1.43E-04 \\
\hline VOC & 1,1-Dichloroethane & $\mathrm{mg} / \mathrm{L}$ & 120 & 0 & $0 \%$ & $1.20 \mathrm{E}-04$ & 2.50E-04 & -- & -- & 9.97E-05 \\
\hline VOC & 1,1-Dichloroethene & $\mathrm{mg} / \mathrm{L}$ & 15 & 0 & $0 \%$ & 2.30E-04 & 2.30E-04 & -- & -- & 1.15E-04 \\
\hline VOC & 1,2-Dichloroethane & $\mathrm{mg} / \mathrm{L}$ & 120 & 13 & $11 \%$ & 8.00E-05 & 2.70E-04 & 1.30E-04 & 0.0011 & $1.55 E-04$ \\
\hline VOC & 2-Butanone & $\mathrm{mg} / \mathrm{L}$ & 120 & 0 & $0 \%$ & 1.00E-04 & 8.90E-04 & -- & -- & 2.07E-04 \\
\hline VOC & 4-Methyl-2-Pentanone & $\mathrm{mg} / \mathrm{L}$ & 120 & 0 & $0 \%$ & $1.50 \mathrm{E}-04$ & 0.0013 & -- & -- & 2.87E-04 \\
\hline VOC & Acetone & $\mathrm{mg} / \mathrm{L}$ & 120 & 46 & $38 \%$ & 2.10E-04 & 0.0040 & 6.00E-04 & 0.0062 & 0.0011 \\
\hline VOC & Benzene & $\mathrm{mg} / \mathrm{L}$ & 120 & 1 & $1 \%$ & 7.00E-05 & 2.30E-04 & $1.40 \mathrm{E}-04$ & $1.40 \mathrm{E}-04$ & 7.90E-05 \\
\hline VOC & Carbon disulfide & $\mathrm{mg} / \mathrm{L}$ & 120 & 4 & $3 \%$ & 6.00E-05 & 4.30E-04 & 4.60E-04 & 0.0024 & $1.83 E-04$ \\
\hline VOC & Carbon tetrachloride & $\mathrm{mg} / \mathrm{L}$ & 119 & 1 & $1 \%$ & 1.00E-04 & 3.30E-04 & 0.0041 & 0.0041 & 1.39E-04 \\
\hline
\end{tabular}




\section{Table B-8}

Summary Statistics for All Categories of Surface Water Samples Collected from Segment 2 of the Columbia River

Columbia River Component - Existing Sample Data Evaluation and Scoping Report

\begin{tabular}{|c|c|c|c|c|c|c|c|c|c|c|}
\hline $\begin{array}{l}\text { Constituent } \\
\text { Class }\end{array}$ & Constituent Name & Units & $\begin{array}{l}\text { Number } \\
\text { Analyzed }\end{array}$ & $\begin{array}{l}\text { Number } \\
\text { Detected }\end{array}$ & $\begin{array}{c}\text { Frequency of } \\
\text { Detection }\end{array}$ & $\begin{array}{l}\text { Minimum } \\
\text { Nondetect }\end{array}$ & $\begin{array}{l}\text { Maximum } \\
\text { Nondetect }\end{array}$ & $\begin{array}{l}\text { Minimum } \\
\text { Detect }\end{array}$ & $\begin{array}{l}\text { Maximum } \\
\text { Detect }\end{array}$ & $\begin{array}{c}\text { Average } \\
\text { Concentration }\end{array}$ \\
\hline$\overline{\mathrm{VOC}}$ & Chlorobenzene & $\mathrm{mg} / \mathrm{L}$ & 6 & 0 & $0 \%$ & $2.80 E-04$ & $2.80 \mathrm{E}-04$ & -- & -- & $1.40 \mathrm{E}-04$ \\
\hline VOC & Chloroform & $\mathrm{mg} / \mathrm{L}$ & 120 & 19 & $16 \%$ & 7.00E-05 & 2.30E-04 & 1.90E-04 & 0.0055 & 3.89E-04 \\
\hline VOC & cis-1,2-dichloroethene & $\mathrm{mg} / \mathrm{L}$ & 117 & 4 & $3 \%$ & 6.00E-05 & 2.40E-04 & 3.00E-04 & 4.00E-04 & 8.12E-05 \\
\hline VOC & Ethylbenzene & $\mathrm{mg} / \mathrm{L}$ & 33 & 0 & $0 \%$ & $1.40 \mathrm{E}-04$ & 2.40E-04 & -- & -- & 9.42E-05 \\
\hline VOC & Ethylcyanide & $\mathrm{mg} / \mathrm{L}$ & 120 & 0 & $0 \%$ & $6.80 \mathrm{E}-04$ & 0.0026 & -- & -- & 8.93E-04 \\
\hline VOC & Methylene chloride & $\mathrm{mg} / \mathrm{L}$ & 120 & 13 & $11 \%$ & $1.70 \mathrm{E}-04$ & 4.10E-04 & $2.60 \mathrm{E}-04$ & 0.0010 & 1.87E-04 \\
\hline VOC & n-Butanol & $\mathrm{mg} / \mathrm{L}$ & 108 & 0 & $0 \%$ & 0.0038 & 0.0070 & -- & -- & 0.0025 \\
\hline VOC & Tetrachloroethene & $\mathrm{mg} / \mathrm{L}$ & 120 & 1 & $1 \%$ & 8.00E-05 & $5.70 \mathrm{E}-04$ & 7.00E-04 & 7.00E-04 & 1.60E-04 \\
\hline VOC & Tetrahydrofuran & $\mathrm{mg} / \mathrm{L}$ & 120 & 0 & $0 \%$ & 0.0012 & 0.0023 & -- & -- & 8.95E-04 \\
\hline VOC & Toluene & $\mathrm{mg} / \mathrm{L}$ & 120 & 41 & $34 \%$ & 7.00E-05 & $3.30 \mathrm{E}-04$ & $1.30 \mathrm{E}-04$ & $9.80 \mathrm{E}-04$ & $2.25 \mathrm{E}-04$ \\
\hline VOC & trans-1,2-Dichloroethene & $\mathrm{mg} / \mathrm{L}$ & 120 & 0 & $0 \%$ & $9.00 \mathrm{E}-05$ & $2.60 \mathrm{E}-04$ & -- & -- & $9.83 E-05$ \\
\hline VOC & Trichloroethene & $\mathrm{mg} / \mathrm{L}$ & 120 & 15 & $13 \%$ & $9.00 \mathrm{E}-05$ & 2.90E-04 & 3.10E-04 & 0.0033 & 2.78E-04 \\
\hline VOC & Vinyl chloride & $\mathrm{mg} / \mathrm{L}$ & 120 & 0 & $0 \%$ & 8.00E-05 & $3.20 \mathrm{E}-04$ & -- & -- & 1.13E-04 \\
\hline VOC & Xylenes (total) & $\mathrm{mg} / \mathrm{L}$ & 120 & 15 & $13 \%$ & 2.80E-04 & 7.90E-04 & $3.40 \mathrm{E}-04$ & 0.0012 & $3.14 \mathrm{E}-04$ \\
\hline
\end{tabular}

CONV - Conventional parameter

RAD - Radionuclide

SVOC - Semivolatile Organic Compound

VOC - Volatile Organic Compound 
Comparison of All Categories of Surface Water Concentrations from Segment 2 of the Columbia River to Ecological and Human Health Benchmark Values Columbia River Component - Existing Sample Data Evaluation and Scoping Report

\begin{tabular}{|c|c|c|c|c|c|c|c|c|c|c|c|c|c|c|c|c|}
\hline $\begin{array}{c}\text { Constituent } \\
\text { Class }\end{array}$ & Constituent Name & Units & $\begin{array}{l}\text { Number } \\
\text { Analyzed }\end{array}$ & $\begin{array}{l}\text { Number } \\
\text { Detected }\end{array}$ & $\begin{array}{c}\text { Frequency } \\
\text { of Detection }\end{array}$ & $\underset{\text { Detect }}{\text { Minimum }}$ & $\underset{\text { Detect }}{\text { Maximum }}$ & $\begin{array}{c}\text { Average } \\
\text { Concentration }\end{array}$ & $\begin{array}{l}\text { Benchmark } \\
\text { Value }\end{array}$ & $\begin{array}{l}\text { Is Max > Eco } \\
\text { Benchmark? }\end{array}$ & $\begin{array}{c}\text { Factor of } \\
\text { Exceedance }\end{array}$ & $\begin{array}{c}\text { Number of } \\
\text { Exceedances }\end{array}$ & $\begin{array}{c}\text { Human Health } \\
\text { Benchmark Value }\end{array}$ & $\begin{array}{l}\text { Is Max }>\mathrm{HH} \\
\text { Benchmark? }\end{array}$ & $\begin{array}{c}\text { Factor of } \\
\text { Exceedance }\end{array}$ & $\begin{array}{c}\text { Number of } \\
\text { Exceedances }\end{array}$ \\
\hline$\overline{\text { CONV }}$ & Alkalinity & $\mathrm{mg} / \mathrm{L}$ & 3 & 3 & $100 \%$ & 56 & 60 & 58 & -- & -- & -- & -- & -- & -- & -- & -- \\
\hline CONV & Bicarbonate & $\mathrm{mg} / \mathrm{L}$ & 1 & 1 & $100 \%$ & 57 & 57 & 57 & -- & -- & -- & -- & -- & -- & -- & -- \\
\hline CONV & Carbonate & $\mathrm{mg} / \mathrm{L}$ & 2 & 2 & $100 \%$ & 39 & 45 & 42 & -- &.- &.- & -- & -- & -- &.- &.- \\
\hline CONV & Chloride & $\mathrm{mg} / \mathrm{L}$ & 515 & 513 & $100 \%$ & 0.65 & 48 & 2.4 & 230 & No & 0.21 & 0 & -- & -- & -.- & -.- \\
\hline CONV & Dissolved oxygen & $\mathrm{mg} / \mathrm{L}$ & 5 & 5 & $100 \%$ & 9.3 & 9.8 & 9.7 & -- & -- & -- & -. & -. & -- & -. & -. \\
\hline CONV & Fluoride & $\mathrm{mq} / \mathrm{L}$ & 515 & 488 & $95 \%$ & 0.013 & 2.4 & 0.12 & .- & .- & .- & .- & .- & .- & .. & .- \\
\hline CONV & Hardness & $\mathrm{mq} / \mathrm{L}$ & 1 & 1 & $100 \%$ & 69 & 69 & 69 & -- & -. & -- & .- & .. & .- & .- & -. \\
\hline CONV & Nitrate & $\mathrm{mg} / \mathrm{L}$ & 27 & 26 & $96 \%$ & 0.34 & 7.5 & 2.5 & -- & -- & -- & -- & 10 & No & 0.75 & 0 \\
\hline CONV & Nitrogen, Nitrate (as N) & $\mathrm{mg} / \mathrm{L}$ & 604 & 603 & $100 \%$ & 0.028 & 295 & 1.6 & $\ldots$ & $\ldots$ & $\ldots$ & $\ldots$ & $\ldots$ & $\ldots$ & . & $\ldots$ \\
\hline CONV & Nitrogen, Nitrate-Nitriti & $\mathrm{mg} / \mathrm{L}$ & 91 & 91 & $100 \%$ & 0.067 & 22,000 & 432 & .- & -. & -- & -- & .- & .- & .. & .- \\
\hline CONV & $\begin{array}{l}\text { Nitrogen, Nitrite } \\
\text { Opidation Reduction }\end{array}$ & $\mathrm{mg} / \mathrm{L}$ & 503 & 7 & $1 \%$ & 0.0081 & 0.023 & 0.0038 & - & - & -- & -- & -- & -- & -- & -- \\
\hline CONV & Potential & $\mathrm{mV}$ & 13 & 13 & $100 \%$ & 132 & 343 & 231 & -- & -- & -- & -- & -- & -- & -- & -- \\
\hline CONV & $\mathrm{pH}$ & pH Units & 31 & 31 & $100 \%$ & 6.7 & 8.0 & 7.6 & -- & -- & -- & -- & -- & -- & -- &.- \\
\hline CONV & Phosphate & $\mathrm{mg} / \mathrm{L}$ & 9 & 1 & $11 \%$ & 0.010 & 0.010 & 0.11 & -- & -- & -- & -- & -- & -- & -- & -- \\
\hline CONV & Specific Conductance & umhos $/ \mathrm{cm}$ & 32 & 32 & $100 \%$ & 120 & 426 & 209 & -- & -- & -- & -- & -- & -- & -- & -- \\
\hline CONV & Sulfate & $\mathrm{mg} / \mathrm{L}$ & 515 & 514 & $100 \%$ & 1.1 & 290 & 14 & -- & -- & -- & -- & -- & -- & -- & -- \\
\hline CONV & Temperature & Deg C & 21 & 21 & $100 \%$ & 7.1 & 18 & 13 & -- & -- & -- & -- & -- & -- & -- & -- \\
\hline CONV & Total suspended solids & $\mathrm{mg} / \mathrm{L}$ & 6 & 6 & $100 \%$ & 7.0 & 14 & 10 & -- & -- & -- & -- & -- & -- & -- & -- \\
\hline METAL & Aluminum & $\mathrm{mg} / \mathrm{L}$ & 109 & 57 & $52 \%$ & $3.39 \mathrm{E}-04$ & 21 & 0.39 & 0.087 & Yes & 243 & 18 & -- & -. & $\ldots$ & $\ldots$ \\
\hline METAL & Antimony & $\mathrm{mg} / \mathrm{L}$ & 1058 & 978 & $92 \%$ & $9.10 \mathrm{E}-05$ & 0.0043 & 0.0010 & 1.6 & No & 0.0027 & 0 & 0.0056 & No & 0.77 & 0 \\
\hline METAL & Arsenic & $\mathrm{mg} / \mathrm{L}$ & 980 & 979 & $100 \%$ & $6.00 \mathrm{E}-06$ & 0.0085 & $8.95 E-04$ & 0.15 & No & 0.057 & 0 & 1.80E-05 & Yes & 473 & 978 \\
\hline METAL & Barium & $\mathrm{mg} / \mathrm{L}$ & 122 & 121 & $99 \%$ & 0.017 & 0.29 & 0.040 & 0.0040 & Yes & 73 & 121 & 1.0 & No & 0.29 & 0 \\
\hline METAL & Beryllium & $\mathrm{mg} / \mathrm{L}$ & 975 & 76 & $8 \%$ & $9.95 \mathrm{E}-07$ & 0.0018 & 4.55E-05 & 0.0053 & No & 0.34 & $\begin{array}{lll}0 & \\
0\end{array}$ & $-\ldots$ & $\ldots$ &.- & $\ldots$ \\
\hline METAL & Cadmium & $\mathrm{mg} / \mathrm{L}$ & 1057 & 795 & & $5.58 \mathrm{E}-06$ & 0.0035 & $1.21 \mathrm{E}-04$ & $2.50 \mathrm{E}-04$ & Yes & 14 & 15 & .- & .- & .- & .- \\
\hline METAL & Calcium & $\mathrm{mg} / \mathrm{L}$ & & 84 & $100 \%$ & 17 & 65 & 31 & 116 & No & 0.56 & 0 & -. & .- & $\ldots$ & $\ldots$ \\
\hline METAL & Chromium & $\mathrm{mg} / \mathrm{L}$ & 1065 & 843 & $79 \%$ & $2.09 \mathrm{E}-05$ & 0.22 & 0.0053 & 0.010 & Yes & 22 & 118 & -- & .- & $\ldots$ & $\ldots$ \\
\hline METAL & Cobalt & $\mathrm{mg} / \mathrm{L}$ & 81 & 3 & $4 \%$ & 0.0017 & 0.0096 & 0.0020 & 0.023 & No & 0.42 & 0 & $\ldots$ & $\ldots$ & $\ldots$ & -- \\
\hline $\begin{array}{l}\text { METAL } \\
\text { MET }\end{array}$ & Copper & $\mathrm{mg} / \mathrm{L}$ & 1060 & 1001 & $94 \%$ & $2.02 \mathrm{E}-04$ & 0.037 & 0.0058 & 0.0060 & Yes & 6.3 & 17 & $\ldots$ & $\ldots$ & $\ldots$ & -. \\
\hline $\begin{array}{l}\text { METAL } \\
\text { MEA }\end{array}$ & lodine & $\mathrm{mg} / \mathrm{L}$ & 37 & 37 & $100 \%$ & $4.28 \mathrm{E}-04$ & 51 & 1.4 & .. & -- &.- & $\ldots$ & .- & .- & $\ldots$ & $\ldots$ \\
\hline $\begin{array}{l}\text { METAL } \\
\text { MET }\end{array}$ & Iron & $\mathrm{mg} / \mathrm{L}$ & 82 & 45 & $55 \%$ & 0.016 & 20 & $\begin{array}{l}1.4 \\
0.54\end{array}$ & 1.0 & Yes & 20 & 5 & 0.30 & Yes & 68 & 10 \\
\hline METAL & Lead & $\mathrm{mg} / \mathrm{L}$ & 980 & 900 & $92 \%$ & $1.11 \mathrm{E}-06$ & 0.066 & $3.14 \mathrm{E}-04$ & 8.67E-04 & Yes & 76 & 34 & -- & -- & -- & $\ldots$ \\
\hline METAL & Magnesium & $\mathrm{mg} / \mathrm{L}$ & 84 & 84 & $100 \%$ & 4.0 & 18 & 7.0 & 82 & No & 0.22 & 0 &.- & -- & -- & -- \\
\hline METAL & Manganese & $\mathrm{mg} / \mathrm{L}$ & 134 & 103 & $77 \%$ & 3.70E-05 & 0.94 & 0.017 & 0.12 & Yes & 7.8 & 4 & 0.050 & Yes & 19 & 7 \\
\hline METAL & Mercury & $\mathrm{mg} / \mathrm{L}$ & 398 & 397 & $100 \%$ & 2.25E-07 & 4.07E-05 & $1.77 \mathrm{E}-06$ & 1.20E-05 & Yes & 3.4 & 5 & -- & -. & -- & -- \\
\hline METAL & Molybdenum & $\mathrm{mg} / \mathrm{L}$ & 1 & 1 & $100 \%$ & $6.14 \mathrm{E}-04$ & $6.14 \mathrm{E}-04$ & $6.14 \mathrm{E}-04$ & 0.37 & No & 0.0017 & 0 & -- & -- & -. & -- \\
\hline METAL & Nickel & $\mathrm{mg} / \mathrm{L}$ & 1061 & 988 & $93 \%$ & 4.10E-05 & 0.032 & 0.0012 & 0.028 & Yes & 1.2 & 2 & 0.61 & No & 0.053 & 0 \\
\hline METAL & Potassium & $\mathrm{mg} / \mathrm{L}$ & 67 & 33 & $49 \%$ & 0.52 & 12 & 1.9 & 53 & No & 0.22 & 0 & -- & $\ldots$ & -- & -- \\
\hline METAL & Selenium & $\mathrm{mg} / \mathrm{L}$ & 982 & 457 & $47 \%$ & $6.73 \mathrm{E}-05$ & 0.0039 & $3.78 \mathrm{E}-04$ & 0.0050 & No & 0.79 & 0 & 0.17 & No & 0.023 & 0 \\
\hline METAL & Silica & $\mathrm{mg} / \mathrm{L}$ & 1 & 1 & $100 \%$ & 2.0 & 2.0 & 2.0 & -. & -- & -.- & -- & -- & -. & $\ldots$ & $\ldots$ \\
\hline METAL & Silicon & $\mathrm{mg} / \mathrm{L}$ & 2 & 2 & $100 \%$ & 0.095 & 0.21 & 0.15 & -. & -. & -- & -- & -- & -- & -- & -. \\
\hline METAL & Silver & $\mathrm{mg} / \mathrm{L}$ & 1060 & 355 & $33 \%$ & $9.48 \mathrm{E}-07$ & 0.0030 & 1.34E-04 & $1.20 \mathrm{E}-04$ & Yes & 25 & 7 & -.- & -- & -.- & -. \\
\hline METAL & Sodium & $\mathrm{mg} / \mathrm{L}$ & 84 & 83 & $99 \%$ & 2.0 & 19 & 6.2 & 680 & No & 0.028 & 0 & -. & -- & -. & -. \\
\hline METAL & Strontium & $\mathrm{mg} / \mathrm{L}$ & 81 & 81 & $100 \%$ & 0.081 & 0.38 & 0.16 & 1.5 & No & 0.25 & 0 & $\ldots$ & $\ldots$ & $\ldots$ & -- \\
\hline METAL & Thallium & $\mathrm{mg} / \mathrm{L}$ & 899 & 802 & $89 \%$ & $5.18 \mathrm{E}-06$ & 4.07E-04 & 2.21E-05 & 0.040 & No & 0.010 & 0 & $2.40 \mathrm{E}-04$ & Yes & 1.7 & 2 \\
\hline METAL & Thorium & $\mathrm{mg} / \mathrm{L}$ & 42 & 2 & $5 \%$ & $5.65 \mathrm{E}-06$ & 1.19E-05 & 4.08E-05 & .. & -- & -. & -. & -. & -. & .. & $\ldots$ \\
\hline METAL & Uranium & $\mathrm{mg} / \mathrm{L}$ & 54 & 54 & $100 \%$ & $1.21 \mathrm{E}-04$ & 0.0087 & 0.0021 & 0.0026 & Yes & 33 & 12 & 0.030 & No & 0.29 & 0 \\
\hline $\begin{array}{l}\text { METAL } \\
\text { MET }\end{array}$ & Vanadium & $\mathrm{mg} / \mathrm{L}$ & 81 & 38 & $47 \%$ & $6.50 \mathrm{E}-04$ & 0.049 & 0.0066 & 0.020 & Yes & 2.4 & 4 &.- & .- &.-- & -- \\
\hline METAL & Zinc & $\mathrm{mg} / \mathrm{L}$ & 1061 & 1038 & $98 \%$ & $3.51 \mathrm{E}-04$ & 0.42 & 0.0045 & 0.055 & Yes & $\begin{array}{l}2.4 \\
7.5\end{array}$ & $\begin{array}{c}4 \\
12\end{array}$ & 7.4 & No & 0.056 & 0 \\
\hline RAD & Barium-140 & $\mathrm{pCi} / \mathrm{L}$ & 83 & 2 & $2 \%$ & 4.0 & 4.5 & 10 & -- & -- & $\ldots$ & $\ldots$ & -- & $\ldots$ & .- & -. \\
\hline RAD & Beryllium-7 & $\mathrm{pCi} / \mathrm{L}$ & 263 & 67 & $25 \%$ & 0.016 & 41 & 5.1 & -- & -- & -- & -- & -- & $\ldots$ &.- & -. \\
\hline RAD & Carbon-14 & $\mathrm{pCi} / \mathrm{L}$ & 10 & 4 & $40 \%$ & 9.2 & 608 & 74 & -- & -- & -- & -- & 2,000 & No & 0.30 & 0 \\
\hline RAD & Cesium-134 & $\mathrm{pCi} / \mathrm{L}$ & 218 & 1 & $0 \%$ & 1.0 & 1.0 & 0.55 & -- & -- & -- & -- &.- & 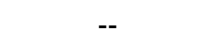 & -- & -- \\
\hline RAD & Cesium-137 & $\mathrm{pCi} / \mathrm{L}$ & 352 & 16 & $5 \%$ & 0.0020 & 8.5 & 0.49 & 43 & No & 0.20 & 0 & 200 & No & 0.043 & 0 \\
\hline RAD & Cobalt-60 & $\mathrm{pCi} / \mathrm{L}$ & 344 & 2 & $1 \%$ & 1.0 & 1.2 & 0.50 & 3,760 & No & $3.19 \mathrm{E}-04$ & 0 & 100 & No & 0.012 & 0 \\
\hline RAD & Gross alpha & $\mathrm{pCi} / \mathrm{L}$ & 375 & 150 & $40 \%$ & 0.20 & 228 & 6.5 & -- & -- & -- & -- & -- & -- & -- & -- \\
\hline
\end{tabular}


Comparison of All Categories of Surface Water Concentrations from Segment 2 of the Columbia River to Ecological and Human Health Benchmark Values Columbia River Component - Existing Sample Data Evaluation and Scoping Report

\begin{tabular}{|c|c|c|c|c|c|c|c|c|c|c|c|c|c|c|c|c|}
\hline $\begin{array}{l}\text { Constituent } \\
\text { Class }\end{array}$ & Constituent Name & Units & $\begin{array}{l}\text { Number } \\
\text { Analyzed }\end{array}$ & $\begin{array}{l}\text { Number } \\
\text { Detected }\end{array}$ & $\begin{array}{l}\text { Frequency } \\
\text { of Detection }\end{array}$ & $\underset{\text { Detect }}{\text { Minimum }}$ & $\begin{array}{c}\text { Maximum } \\
\text { Detect }\end{array}$ & $\begin{array}{c}\text { Average } \\
\text { Concentration }\end{array}$ & $\begin{array}{l}\text { Ecological } \\
\text { Benchmark } \\
\text { Value }\end{array}$ & $\begin{array}{l}\text { Is Max > Eco } \\
\text { Benchmark? }\end{array}$ & $\begin{array}{c}\text { Factor of } \\
\text { Exceedance }\end{array}$ & $\begin{array}{c}\text { Number of } \\
\text { Exceedances }\end{array}$ & $\begin{array}{l}\text { Human Health } \\
\text { Benchmark Value }\end{array}$ & $\begin{array}{l}\text { Is Max }>\mathrm{HH} \\
\text { Benchmark? }\end{array}$ & $\begin{array}{c}\text { Factor of } \\
\text { Exceedance }\end{array}$ & $\begin{array}{c}\text { Number of } \\
\text { Exceedances }\end{array}$ \\
\hline$\overline{\mathrm{RAD}}$ & Gross beta & $\overline{\mathrm{pC} i / \mathrm{L}}$ & 487 & 269 & $55 \%$ & 0.40 & 84 & 5.5 & -- & -- & -- & -- & -- & -- & -- & -- \\
\hline RAD & Iodine-129 & $\mathrm{pCi} / \mathrm{L}$ & 53 & 53 & $100 \%$ & $2.55 \mathrm{E}-05$ & 0.41 & 0.054 & -- & -- & -- & -- & -- & -- & -- & -- \\
\hline RAD & Iodine-131 & $\mathrm{pCi} / \mathrm{L}$ & 84 & 3 & $4 \%$ & 1.0 & 1.2 & 10 & -- & -- & -- & -- & -- & -- & -- & -- \\
\hline RAD & Iron-59 & $\mathrm{pCi} / \mathrm{L}$ & 98 & 8 & $8 \%$ & 1.8 & 3.0 & 2.7 & -- & -- & -- & -- & -- & -- & -- & -- \\
\hline RAD & Plutonium-238 & $\mathrm{pCi} / \mathrm{L}$ & 29 & 6 & $21 \%$ & $6.35 \mathrm{E}-06$ & 0.0052 & $3.96 \mathrm{E}-04$ & -- & -- & -- & -- & 15 & No & $3.47 \mathrm{E}-04$ & 0 \\
\hline RAD & Plutonium-239/240 & $\mathrm{pCi} / \mathrm{L}$ & 41 & 15 & $37 \%$ & $6.89 \mathrm{E}-06$ & 1.63E-04 & 7.45E-05 & -- & -- & -- & -- & 15 & No & $1.09 \mathrm{E}-05$ & 0 \\
\hline RAD & Potassium-40 & $\mathrm{pCi} / \mathrm{L}$ & 308 & 184 & $60 \%$ & 0.027 & 126 & 15 & -- & -- & -- & -- & -- & -- & -- & -- \\
\hline RAD & Radium-226 & $\mathrm{pC} / \mathrm{L}$ & 63 & 10 & $16 \%$ & 0.022 & 0.058 & 30 & 4.1 & No & 0.014 & 0 & -- & -- & -- & -- \\
\hline RAD & Radium-228 & $\mathrm{pCi} / \mathrm{L}$ & 12 & 4 & $33 \%$ & 0.36 & 1.0 & 5.8 & 3.4 & No & 0.31 & 0 & -- & -- & -- & -- \\
\hline RAD & Strontium-90 & $\mathrm{pCi} / \mathrm{L}$ & 784 & 636 & $81 \%$ & 0.022 & 109 & 0.44 & 278 & No & 0.39 & 0 & 8.0 & Yes & 14 & 7 \\
\hline RAD & Technetium-99 & $\mathrm{pCi} / \mathrm{L}$ & 144 & 64 & $44 \%$ & 0.27 & 144 & 12 & 667,000 & No & $2.16 \mathrm{E}-04$ & 0 & 900 & No & 0.16 & 0 \\
\hline RAD & Thorium-228 & $\mathrm{pC} / \mathrm{L}$ & 60 & 2 & $3 \%$ & 0.080 & 0.084 & 18 & -. & -- & -- & -- & -. & -- & -. & -- \\
\hline RAD & Thorium-230 & $\mathrm{pCi} / \mathrm{L}$ & 6 & 3 & $50 \%$ & 0.054 & 2.6 & 0.46 & -- & -- & -- & -- & -- & -- & -.- & -- \\
\hline RAD & Thorium-232 & $\mathrm{pCi} / \mathrm{L}$ & 9 & 2 & $22 \%$ & 0.060 & 0.087 & 8.8 & -- & -- & -- & -- & -- & -- & -. & -. \\
\hline RAD & Tritium & $\mathrm{pCi} / \mathrm{L}$ & 1136 & 970 & $85 \%$ & 17 & 112,000 & 1,939 & $2.65 E+08$ & No & $4.23 \mathrm{E}-04$ & 0 & 20,000 & Yes & 5.6 & 19 \\
\hline RAD & Uranium & $\mathrm{pC} / \mathrm{L}$ & 118 & 118 & $100 \%$ & 0.14 & 100 & 5.5 & 223 & No & 0.45 & 0 & $\ldots$ &.- & $\ldots$ & $\ldots$ \\
\hline RAD & Uranium-234 & $\mathrm{pCi} / \mathrm{L}$ & 778 & 776 & $100 \%$ & 0.078 & 111 & 1.8 & 202 & No & 0.55 & 0 & $\ldots$ & $\ldots$ & $\ldots$ & $\ldots$ \\
\hline RAD & Uranium-235 & $\mathrm{pCi} / \mathrm{L}$ & 715 & 200 & $28 \%$ & 0.0065 & 2.9 & 0.48 & 217 & No & 0.014 & 0 & -- & $\ldots$ & $\ldots$ & $\ldots$ \\
\hline RAD & Uranium-236 & $\mathrm{pCi} / \mathrm{L}$ & 8 & 8 & $100 \%$ & 0.29 & 1.0 & 0.68 & -- & -- & -. & -- & $\ldots$ & $\ldots$ & $\ldots$ & $\ldots$ \\
\hline RAD & Uranium-238 & $\mathrm{pC} / / \mathrm{L}_{-}$ & 782 & 776 & $99 \%$ & 0.070 & 99 & 39 & 223 & No & 0.45 & 0 & $\ldots$ & $\ldots$ & $\ldots$ & .- \\
\hline RAD & Zirconium-95 & $\mathrm{pCi} / \mathrm{L}$ & 84 & 5 & $6 \%$ & 0.90 & 1.5 & 1.9 & 200 & No & 0.40 & -. & $\ldots$ & $\ldots$ & $\ldots$ & -. \\
\hline SVOC & 1,4-Dichlorobenzene & $\mathrm{mg} / \mathrm{L}$ & $\begin{array}{l}04 \\
120\end{array}$ & 3 & $3 \%$ & $1.90 \mathrm{E}-04$ & $2.50 \mathrm{E}-04$ & 8.16E-05 & 0.015 & No & 0.017 & 0 & 0.063 & No & 0.0040 & 0 \\
\hline VOC & 1,1,2-Trichloroethane & $\mathrm{mg} / \mathrm{L}$ & 120 & 11 & $9 \%$ & $1.30 \mathrm{E}-04$ & $8.80 \mathrm{E}-04$ & 1.43E-04 & -- & -- & -- & -- & -- & -- & -- & -- \\
\hline VOC & 1,2-Dichloroethane & $\mathrm{mg} / \mathrm{L}$ & 120 & 13 & $11 \%$ & 1.30E-04 & 0.0011 & $1.55 \mathrm{E}-04$ & -- & -- & -- & -- & -- & -- & -- & -- \\
\hline VOC & Acetone & $\mathrm{mg} / \mathrm{L}$ & 120 & 46 & $38 \%$ & $6.00 \mathrm{E}-04$ & 0.0062 & 0.0011 & -- & -- & -- & -- & -- & -- & -- & -- \\
\hline VOC & Benzene & $\mathrm{mg} / \mathrm{L}$ & 120 & 1 & $1 \%$ & 1.40E-04 & 1.40E-04 & $7.90 \mathrm{E}-05$ & -- & -- & -- & -- & -- & -- & -- & -- \\
\hline VOC & Carbon disulfide & $\mathrm{mg} / \mathrm{L}$ & 120 & 4 & $3 \%$ & $4.60 \mathrm{E}-04$ & 0.0024 & 1.83E-04 & -- & -- & -- & -- & -- & -- & -- & -- \\
\hline VOC & Carbon tetrachloride & $\mathrm{mg} / \mathrm{L}$ & 119 & 1 & $1 \%$ & 0.0041 & 0.0041 & $1.39 \mathrm{E}-04$ & -- & -- & -- & -- & -- & -- & -- & -- \\
\hline VOC & Chloroform & $\mathrm{mg} / \mathrm{L}$ & 120 & 19 & $16 \%$ & $1.90 \mathrm{E}-04$ & 0.0055 & 3.89E-04 & -- & -- & -- & -- & -- & -- & -- & -- \\
\hline VOC & cis-1,2-dichloroethene & $\mathrm{mg} / \mathrm{L}$ & 117 & 4 & $3 \%$ & $3.00 \mathrm{E}-04$ & $4.00 \mathrm{E}-04$ & $8.12 \mathrm{E}-05$ & -- & -- & -- & -. & -. & -- & -. & -. \\
\hline Voc & Methylene chloride & $\mathrm{mg} / \mathrm{L}$ & 120 & 13 & $11 \%$ & $2.60 \mathrm{E}-04$ & 0.0010 & $1.87 E-04$ & -- & -- & -- & $\ldots$ & $\ldots$ & $\ldots$ & $\ldots$ & .- \\
\hline VOC & Tetrachloroethene & $\mathrm{mg} / \mathrm{L}$ & 120 & 1 & $1 \%$ & $7.00 \mathrm{E}-04$ & $7.00 \mathrm{E}-04$ & 1.60E-04 & -- & -- & -- & -- & -. & -- & -. & -. \\
\hline Voc & Toluene & $\mathrm{mg} / \mathrm{L}$ & 120 & 41 & $34 \%$ & $1.30 \mathrm{E}-04$ & $9.80 \mathrm{E}-04$ & $2.25 \mathrm{E}-04$ & $\ldots$ & $\ldots$ & $\ldots$ & $\ldots$ & $\ldots$ & $\ldots$ & $\ldots$ & $\ldots$ \\
\hline VOC & Trichloroethene & $\mathrm{mg} / \mathrm{L}$ & 120 & 15 & $13 \%$ & $3.10 \mathrm{E}-04$ & 0.0033 & $2.78 \mathrm{E}-04$ & -- & -- & -- & -- & -- & -- & -- & -- \\
\hline Voc & Xylenes (total) & $\mathrm{mg} / \mathrm{L}$ & 120 & 15 & $13 \%$ & 3.40E-04 & 0.0012 & 3.14E-04 & -- & -- & -- & -- & -- & -- & -- & -- \\
\hline
\end{tabular}

CONV - Conventional parameter

RAD - Radionuclide

SVOC - Semivolatile Organic Compound 


\section{Table B-10}

Summary of Surface Water Results From Segment 2 Exceeding Select Ecological or Human Health Benchmark Values

Columbia River Component - Existing Sample Data Evaluation and Scoping Report

\begin{tabular}{|c|c|c|c|c|c|c|c|c|c|c|}
\hline Sample Area & Sample Site & Sample ID & Date Collected & Constituent Name & Units & QA Category & $\begin{array}{l}\text { Analytical } \\
\text { Value }\end{array}$ & Qualifier & $\begin{array}{c}\text { Ecological } \\
\text { Benchmark } \\
\text { Value }\end{array}$ & $\begin{array}{l}\text { Human Health } \\
\text { Benchmark Value }\end{array}$ \\
\hline Columbia River & 300 SPR $42-2$ & August 27, 2001 & B12RL6 & Aluminum & $\mathrm{mg} / \mathrm{L}$ & 1 & 0.088 & $=$ & 0.087 & -- \\
\hline Columbia River & 300 SPR 11 -1 & August 27, 2001 & B12RP5 & Aluminum & $\mathrm{mg} / \mathrm{L}$ & 1 & 0.12 & $=$ & 0.087 & -- \\
\hline Columbia River & SD-110-2 & October 17, 2000 & B10JR0 & Aluminum & $\mathrm{mg} / \mathrm{L}$ & 1 & 0.13 & B & 0.087 & -- \\
\hline Columbia River & 100-B SPR 38-3 & October 22, 2001 & B13609 & Aluminum & $\mathrm{mg} / \mathrm{L}$ & 1 & 0.14 & B & 0.087 & -- \\
\hline Columbia River & 100-D SPR 102-1 & October 17, 2000 & B10JP7 & Aluminum & $\mathrm{mg} / \mathrm{L}$ & 1 & 0.15 & B & 0.087 & -- \\
\hline Columbia River & SD-110-2 & October 17, 2000 & B10JT1 & Aluminum & $\mathrm{mg} / \mathrm{L}$ & 1 & 0.18 & B & 0.087 & -- \\
\hline Columbia River & SD-98-1 & October 12, 2000 & B10JR1 & Aluminum & $\mathrm{mg} / \mathrm{L}$ & 1 & 0.27 & $=$ & 0.087 & -- \\
\hline Columbia River & 100-H SPR 150-1 & October 27, 2002 & B15HY1 & Aluminum & $\mathrm{mg} / \mathrm{L}$ & 1 & 0.28 & $=$ & 0.087 & -- \\
\hline Columbia River & 100-H SPR 152-2 & October 27, 2002 & B15HY4 & Aluminum & $\mathrm{mg} / \mathrm{L}$ & 1 & 0.28 & $=$ & 0.087 & -- \\
\hline Columbia River & 100-K SPR 82-2 & October 25, 2001 & B13605 & Aluminum & $\mathrm{mg} / \mathrm{L}$ & 1 & 0.31 & $=$ & 0.087 & -- \\
\hline Columbia River & 100-D SPR 102-1 & October 27, 2003 & B17JP2 & Aluminum & $\mathrm{mg} / \mathrm{L}$ & 1 & 0.32 & $=$ & 0.087 & -- \\
\hline Columbia River & 100-K SPR 77-1 & October 21, 2002 & B15LM4 & Aluminum & $\mathrm{mg} / \mathrm{L}$ & 1 & 0.34 & $=$ & 0.087 & -- \\
\hline Columbia River & 100-D SPR 102-1 & October 21, 2002 & B15HV8 & Aluminum & $\mathrm{mg} / \mathrm{L}$ & 1 & 0.59 & $=$ & 0.087 & -- \\
\hline Columbia River & 100-H SPR 150-1 & November 20, 2003 & B17VK5 & Aluminum & $\mathrm{mg} / \mathrm{L}$ & 1 & 2.1 & $=$ & 0.087 & -- \\
\hline Columbia River & SD-98-1 & October 12, 2000 & B10JT9 & Aluminum & $\mathrm{mg} / \mathrm{L}$ & 1 & 2.3 & $=$ & 0.087 & -- \\
\hline Columbia River & 100-D SPR 98-1 & October 25, 2001 & B135X3 & Aluminum & $\mathrm{mg} / \mathrm{L}$ & 1 & 3.5 & $=$ & 0.087 & -- \\
\hline Columbia River & 100-D SPR 110-1 & October 21, 2002 & B15HW4 & Aluminum & $\mathrm{mg} / \mathrm{L}$ & 1 & 7.9 & $=$ & 0.087 & -- \\
\hline Columbia River & 100-D SPR 110-1 & October 21, 2002 & B15HW1 & Aluminum & $\mathrm{mg} / \mathrm{L}$ & 1 & 21 & $=$ & 0.087 & -- \\
\hline Columbia River & RICHPS1 HRM46.4 & June 14,1999 & B0V209 & Arsenic & $\mathrm{mg} / \mathrm{L}$ & 1 & $2.72 \mathrm{E}-04$ & $=$ & 0.15 & 1.80E-05 \\
\hline Columbia River & 100-K SPR 77-1 & October 25, 2000 & B10955 & Arsenic & $\mathrm{mg} / \mathrm{L}$ & 1 & 3.07E-04 & $=$ & 0.15 & $1.80 \mathrm{E}-05$ \\
\hline Columbia River & 100-K SPR 77-1 & October 25, 2000 & B10956 & Arsenic & $\mathrm{mg} / \mathrm{L}$ & 1 & $3.15 E-04$ & $=$ & 0.15 & $1.80 \mathrm{E}-05$ \\
\hline Columbia River & 100 B/C OUTFLL2 & November 17, 2003 & B180J0 & Arsenic & $\mathrm{mg} / \mathrm{L}$ & 1 & 3.19E-04 & $=$ & 0.15 & $1.80 \mathrm{E}-05$ \\
\hline Columbia River & RICHPS1 HRM46.4 & December 8, 2003 & B183P9 & Arsenic & $\mathrm{mg} / \mathrm{L}$ & 1 & $3.23 E-04$ & $=$ & 0.15 & 1.80E-05 \\
\hline Columbia River & 100K SPR 77-1 & November 10, 2003 & B17J87 & Arsenic & $\mathrm{mg} / \mathrm{L}$ & 1 & 3.48E-04 & $=$ & 0.15 & 1.80E-05 \\
\hline Columbia River & RICHPS3 HRM46.4 & December 8, 2003 & B183V3 & Arsenic & $\mathrm{mg} / \mathrm{L}$ & 1 & 3.67E-04 & C & 0.15 & $1.80 \mathrm{E}-05$ \\
\hline Columbia River & RICHPS HRM 45.0 & December 8, 2003 & B183W2 & Arsenic & $\mathrm{mg} / \mathrm{L}$ & 1 & $3.72 \mathrm{E}-04$ & C & 0.15 & $1.80 \mathrm{E}-05$ \\
\hline Columbia River & RICHPS2 HRM46.4 & December 8, 2003 & B183V2 & Arsenic & $\mathrm{mg} / \mathrm{L}$ & 1 & $3.95 \mathrm{E}-04$ & C & 0.15 & $1.80 \mathrm{E}-05$ \\
\hline Columbia River & 100K SPR 77-1 & November 10, 2003 & B17J86 & Arsenic & $\mathrm{mg} / \mathrm{L}$ & 1 & 3.97E-04 & $=$ & 0.15 & 1.80E-05 \\
\hline Columbia River & RICHPS7 HRM46.4 & December 10, 2002 & B16360 & Arsenic & $\mathrm{mg} / \mathrm{L}$ & 1 & 4.05E-04 & $=$ & 0.15 & $1.80 \mathrm{E}-05$ \\
\hline Columbia River & 100-K SPR 77-1 & October 11, 2004 & B1BFW6 & Arsenic & $\mathrm{mg} / \mathrm{L}$ & 1 & 4.06E-04 & $x$ & 0.15 & $1.80 \mathrm{E}-05$ \\
\hline Columbia River & 100 B/C AREA UR & November 13, 2003 & B180C6 & Arsenic & $\mathrm{mg} / \mathrm{L}$ & 1 & $4.23 \mathrm{E}-04$ & $=$ & 0.15 & $1.80 \mathrm{E}-05$ \\
\hline Columbia River & 100 NSHR HRM9.8 & September 14, 2000 & B106B9 & Arsenic & $\mathrm{mg} / \mathrm{L}$ & 1 & 4.43E-04 & $=$ & 0.15 & $1.80 \mathrm{E}-05$ \\
\hline Columbia River & RICHPS5 HRM46.4 & March 28, 2000 & B0XRN5 & Arsenic & $\mathrm{mg} / \mathrm{L}$ & 1 & 4.47E-04 & $=$ & 0.15 & 1.80E-05 \\
\hline Columbia River & RICHPS HRM 43.9 & March 28, 2000 & B0XRP4 & Arsenic & $\mathrm{mg} / \mathrm{L}$ & 1 & 4.50E-04 & $=$ & 0.15 & $1.80 \mathrm{E}-05$ \\
\hline Columbia River & 100 F-2 HRM19.0 & September 18, 2000 & B10756 & Arsenic & $\mathrm{mg} / \mathrm{L}$ & 1 & $4.52 \mathrm{E}-04$ & $=$ & 0.15 & $1.80 \mathrm{E}-05$ \\
\hline Columbia River & RICHPS7 HRM46.4 & March 28, 2000 & BOXRN6 & Arsenic & $\mathrm{mg} / \mathrm{L}$ & 1 & 4.52E-04 & $=$ & 0.15 & $1.80 \mathrm{E}-05$ \\
\hline Columbia River & RICHPS HRM 45.8 & March 28, 2000 & B0XRP2 & Arsenic & $\mathrm{mg} / \mathrm{L}$ & 1 & 4.56E-04 & $=$ & 0.15 & $1.80 \mathrm{E}-05$ \\
\hline Columbia River & 100 NSHR HRM9.8 & September 14, 2000 & B106B8 & Arsenic & $\mathrm{mg} / \mathrm{L}$ & 1 & $4.58 \mathrm{E}-04$ & $=$ & 0.15 & $1.80 \mathrm{E}-05$ \\
\hline Columbia River & RICHPS HRM 45.0 & March 28, 2000 & B0XRP3 & Arsenic & $\mathrm{mg} / \mathrm{L}$ & 1 & $4.58 \mathrm{E}-04$ & $=$ & 0.15 & $1.80 \mathrm{E}-05$ \\
\hline Columbia River & 300 A2 HRM 43.1 & September 10, 2002 & B158R9 & Arsenic & $\mathrm{mg} / \mathrm{L}$ & 1 & 4.61E-04 & $=$ & 0.15 & $1.80 \mathrm{E}-05$ \\
\hline Columbia River & HFD TS10HRM28.7 & September 18, 2000 & B10754 & Arsenic & $\mathrm{mg} / \mathrm{L}$ & 1 & 4.61E-04 & $=$ & 0.15 & 1.80E-05 \\
\hline Columbia River & RICHPS7 HRM46.4 & September 19, 2000 & B10741 & Arsenic & $\mathrm{mg} / \mathrm{L}$ & 1 & 4.64E-04 & $=$ & 0.15 & 1.80E-05 \\
\hline Columbia River & RICHPS HRM 45.0 & December 6, 1999 & B0X407 & Arsenic & $\mathrm{mg} / \mathrm{L}$ & 1 & 4.66E-04 & $=$ & 0.15 & $1.80 \mathrm{E}-05$ \\
\hline Columbia River & 100 B/C AREA UR & November 13, 2003 & B180C8 & Arsenic & $\mathrm{mg} / \mathrm{L}$ & 1 & 4.69E-04 & $=$ & 0.15 & $1.80 \mathrm{E}-05$ \\
\hline Columbia River & RICHPS2 HRM46.4 & March 28, 2000 & BOXRN3 & Arsenic & $\mathrm{mg} / \mathrm{L}$ & 1 & 4.69E-04 & $=$ & 0.15 & $1.80 \mathrm{E}-05$ \\
\hline
\end{tabular}




\section{Table B-10}

Summary of Surface Water Results From Segment 2 Exceeding Select Ecological or Human Health Benchmark Values

Columbia River Component - Existing Sample Data Evaluation and Scoping Report

\begin{tabular}{|c|c|c|c|c|c|c|c|c|c|c|}
\hline Sample Area & Sample Site & Sample ID & Date Collected & Constituent Name & Units & QA Category & $\begin{array}{l}\text { Analytical } \\
\text { Value }\end{array}$ & Qualifier & $\begin{array}{l}\text { Ecological } \\
\text { Benchmark } \\
\text { Value }\end{array}$ & $\begin{array}{c}\text { Human Health } \\
\text { Benchmark Value }\end{array}$ \\
\hline Columbia River & RICHPS HRM 43.9 & December 6, 1999 & B0X408 & Arsenic & $\mathrm{mg} / \mathrm{L}$ & 1 & 4.73E-04 & $=$ & 0.15 & 1.80E-05 \\
\hline Columbia River & RICHPS5 HRM46.4 & December 6, 1999 & В0Х3Ү9 & Arsenic & $\mathrm{mg} / \mathrm{L}$ & 1 & 4.73E-04 & $=$ & 0.15 & 1.80E-05 \\
\hline Columbia River & 100 N-2 HRM 9.5 & September 14, 2000 & B10693 & Arsenic & $\mathrm{mg} / \mathrm{L}$ & 1 & 4.74E-04 & $=$ & 0.15 & 1.80E-05 \\
\hline Columbia River & RICHPS HRM 43.5 & December 8, 2003 & B183W8 & Arsenic & $\mathrm{mg} / \mathrm{L}$ & 1 & 4.74E-04 & $=$ & 0.15 & 1.80E-05 \\
\hline Columbia River & RICHPS3 HRM46.4 & March 28, 2000 & B0XRN4 & Arsenic & $\mathrm{mg} / \mathrm{L}$ & 1 & 4.74E-04 & $=$ & 0.15 & 1.80E-05 \\
\hline Columbia River & RICHPS3 HRM46.4 & September 19, 2000 & B106V6 & Arsenic & $\mathrm{mg} / \mathrm{L}$ & 1 & $4.76 \mathrm{E}-04$ & $=$ & 0.15 & 1.80E-05 \\
\hline Columbia River & RICHPS5 HRM46.4 & September 19, 2000 & B106V7 & Arsenic & $\mathrm{mg} / \mathrm{L}$ & 1 & 4.76E-04 & $=$ & 0.15 & $1.80 \mathrm{E}-05$ \\
\hline Columbia River & RICHPS2 HRM46.4 & December 10, 2002 & B16357 & Arsenic & $\mathrm{mg} / \mathrm{L}$ & 1 & $4.80 \mathrm{E}-04$ & $=$ & 0.15 & 1.80E-05 \\
\hline Columbia River & RICHPS HRM 45.0 & September 19, 2000 & B10766 & Arsenic & $\mathrm{mg} / \mathrm{L}$ & 1 & 4.83E-04 & $=$ & 0.15 & 1.80E-05 \\
\hline Columbia River & RICHPS2 HRM46.4 & December 8, 2003 & B183R0 & Arsenic & $\mathrm{mg} / \mathrm{L}$ & 1 & 4.85E-04 & $=$ & 0.15 & 1.80E-05 \\
\hline Columbia River & RICHPS HRM 43.5 & December 6, 1999 & B0X413 & Arsenic & $\mathrm{mg} / \mathrm{L}$ & 1 & 4.85E-04 & $=$ & 0.15 & $1.80 \mathrm{E}-05$ \\
\hline Columbia River & 100 B/C AREA UR & November 13, 2003 & B180C7 & Arsenic & $\mathrm{mg} / \mathrm{L}$ & 1 & 4.86E-04 & $=$ & 0.15 & 1.80E-05 \\
\hline Columbia River & 100 NSHR HRM8.9 & September 14, 2000 & B106B3 & Arsenic & $\mathrm{mg} / \mathrm{L}$ & 1 & 4.86E-04 & $=$ & 0.15 & 1.80E-05 \\
\hline Columbia River & RICHPS5 HRM46.4 & September 19, 2000 & B10740 & Arsenic & $\mathrm{mg} / \mathrm{L}$ & 1 & 4.89E-04 & $=$ & 0.15 & 1.80E-05 \\
\hline Columbia River & RICHPS HRM 43.5 & March 28, 2000 & B0XRP5 & Arsenic & $\mathrm{mg} / \mathrm{L}$ & 1 & 4.90E-04 & $=$ & 0.15 & 1.80E-05 \\
\hline Columbia River & RICHPS1 HRM46.4 & March 28, 2000 & BOXRN2 & Arsenic & $\mathrm{mg} / \mathrm{L}$ & 1 & 4.90E-04 & $=$ & 0.15 & $1.80 \mathrm{E}-05$ \\
\hline Columbia River & 300 A3 HRM 43.1 & September 19, 2000 & B10745 & Arsenic & $\mathrm{mg} / \mathrm{L}$ & 1 & 4.91E-04 & $=$ & 0.15 & 1.80E-05 \\
\hline Columbia River & RICHPS2 HRM46.4 & September 19, 2000 & B10738 & Arsenic & $\mathrm{mg} / \mathrm{L}$ & 1 & 4.92E-04 & $=$ & 0.15 & 1.80E-05 \\
\hline Columbia River & 300 A3 HRM 43.1 & September 19, 2000 & B106T8 & Arsenic & $\mathrm{mg} / \mathrm{L}$ & 1 & 4.93E-04 & $=$ & 0.15 & 1.80E-05 \\
\hline Columbia River & RICHPS3 HRM46.4 & September 19, 2000 & B10739 & Arsenic & $\mathrm{mg} / \mathrm{L}$ & 1 & 4.93E-04 & $=$ & 0.15 & $1.80 \mathrm{E}-05$ \\
\hline Columbia River & RICHPS7 HRM46.4 & December 6, 1999 & B0X400 & Arsenic & $\mathrm{mg} / \mathrm{L}$ & 1 & 4.93E-04 & $=$ & 0.15 & 1.80E-05 \\
\hline Columbia River & RICHPS7 HRM46.4 & February 26, 2001 & B11H57 & Arsenic & $\mathrm{mg} / \mathrm{L}$ & 1 & 4.93E-04 & $=$ & 0.15 & 1.80E-05 \\
\hline Columbia River & HFD TS7 HRM28.7 & September 18, 2000 & B10753 & Arsenic & $\mathrm{mg} / \mathrm{L}$ & 1 & 4.94E-04 & $=$ & 0.15 & 1.80E-05 \\
\hline Columbia River & RICHPS7 HRM46.4 & December 5, 2000 & B11143 & Arsenic & $\mathrm{mg} / \mathrm{L}$ & 1 & 4.94E-04 & $=$ & 0.15 & 1.80E-05 \\
\hline Columbia River & 100 NSHR HRM9.2 & September 14, 2000 & B106B5 & Arsenic & $\mathrm{mg} / \mathrm{L}$ & 1 & 4.95E-04 & $=$ & 0.15 & $1.80 \mathrm{E}-05$ \\
\hline Columbia River & 100 F-2 HRM19.0 & September 8, 2003 & B17CR2 & Arsenic & $\mathrm{mg} / \mathrm{L}$ & 1 & 4.96E-04 & $=$ & 0.15 & 1.80E-05 \\
\hline Columbia River & 100 F-7 HRM19.0 & September 9, 2002 & B158V4 & Arsenic & $\mathrm{mg} / \mathrm{L}$ & 1 & 4.96E-04 & $=$ & 0.15 & 1.80E-05 \\
\hline Columbia River & 100 B/C AREA DR & November 17, 2003 & B180F4 & Arsenic & $\mathrm{mg} / \mathrm{L}$ & 1 & 4.97E-04 & $=$ & 0.15 & 1.80E-05 \\
\hline Columbia River & 100 F-2 HRM19.0 & September 9, 2002 & B158V1 & Arsenic & $\mathrm{mg} / \mathrm{L}$ & 1 & 4.97E-04 & $=$ & 0.15 & $1.80 \mathrm{E}-05$ \\
\hline Columbia River & 100 NSHR HRM9.2 & September 14, 2000 & B106B6 & Arsenic & $\mathrm{mg} / \mathrm{L}$ & 1 & 4.97E-04 & $=$ & 0.15 & 1.80E-05 \\
\hline Columbia River & 100 N-5 HRM 9.5 & September 4, 2003 & B17CNO & Arsenic & $\mathrm{mg} / \mathrm{L}$ & 1 & 4.98E-04 & $=$ & 0.15 & 1.80E-05 \\
\hline Columbia River & RICHPS2 HRM46.4 & December 6, 1999 & B0X3Y7 & Arsenic & $\mathrm{mg} / \mathrm{L}$ & 1 & 4.99E-04 & $=$ & 0.15 & 1.80E-05 \\
\hline Columbia River & 300 A2 HRM 43.1 & September 19, 2000 & B10744 & Arsenic & $\mathrm{mg} / \mathrm{L}$ & 1 & 4.99E-04 & $=$ & 0.15 & 1.80E-05 \\
\hline Columbia River & RICHPS3 HRM46.4 & December 5, 2000 & B11141 & Arsenic & $\mathrm{mg} / \mathrm{L}$ & 1 & 4.99E-04 & $=$ & 0.15 & $1.80 \mathrm{E}-05$ \\
\hline Columbia River & 100 F-5 HRM19.0 & September 9, 2002 & B158V3 & Arsenic & $\mathrm{mg} / \mathrm{L}$ & 1 & 5.00E-04 & $=$ & 0.15 & 1.80E-05 \\
\hline Columbia River & RICHPS HRM 43.9 & September 19, 2000 & B10767 & Arsenic & $\mathrm{mg} / \mathrm{L}$ & 1 & 5.00E-04 & $=$ & 0.15 & 1.80E-05 \\
\hline Columbia River & RICHPS HRM 45.0 & September 19, 2000 & B10770 & Arsenic & $\mathrm{mg} / \mathrm{L}$ & 1 & $5.00 \mathrm{E}-04$ & $=$ & 0.15 & 1.80E-05 \\
\hline Columbia River & 300 SR HRM 41.5 & September 10, 2002 & B158Y2 & Arsenic & $\mathrm{mg} / \mathrm{L}$ & 1 & 5.01E-04 & $=$ & 0.15 & $1.80 \mathrm{E}-05$ \\
\hline Columbia River & 300 SR HRM 42.9 & September 19, 2000 & B10784 & Arsenic & $\mathrm{mg} / \mathrm{L}$ & 1 & 5.01E-04 & $=$ & 0.15 & 1.80E-05 \\
\hline Columbia River & RICHPS1 HRM46.4 & December 6, 1999 & B0X3Y6 & Arsenic & $\mathrm{mg} / \mathrm{L}$ & 1 & 5.01E-04 & $=$ & 0.15 & 1.80E-05 \\
\hline Columbia River & 100 F-2 HRM19.0 & September 18, 2000 & B106V0 & Arsenic & $\mathrm{mg} / \mathrm{L}$ & 1 & 5.02E-04 & $=$ & 0.15 & 1.80E-05 \\
\hline Columbia River & RICHPS HRM 45.0 & December 5, 2000 & B11150 & Arsenic & $\mathrm{mg} / \mathrm{L}$ & 1 & 5.02E-04 & $=$ & 0.15 & 1.80E-05 \\
\hline Columbia River & 100 NSHR HRM8.4 & September 14, 2000 & B106B0 & Arsenic & $\mathrm{mg} / \mathrm{L}$ & 1 & 5.03E-04 & $=$ & 0.15 & 1.80E-05 \\
\hline Columbia River & 100 N-3 HRM 9.5 & September 14, 2000 & B10694 & Arsenic & $\mathrm{mg} / \mathrm{L}$ & 1 & 5.04E-04 & $=$ & 0.15 & 1.80E-05 \\
\hline Columbia River & RICHPS3 HRM46.4 & December 5, 2000 & B11119 & Arsenic & $\mathrm{mg} / \mathrm{L}$ & 1 & $5.04 \mathrm{E}-04$ & $=$ & 0.15 & $1.80 \mathrm{E}-05$ \\
\hline
\end{tabular}

107 of 137 


\section{Table B-10}

Summary of Surface Water Results From Segment 2 Exceeding Select Ecological or Human Health Benchmark Values

Columbia River Component - Existing Sample Data Evaluation and Scoping Report

\begin{tabular}{|c|c|c|c|c|c|c|c|c|c|c|}
\hline Sample Area & Sample Site & Sample ID & Date Collected & Constituent Name & Units & QA Category & $\begin{array}{l}\text { Analytical } \\
\text { Value }\end{array}$ & Qualifier & $\begin{array}{l}\text { Ecological } \\
\text { Benchmark } \\
\text { Value }\end{array}$ & $\begin{array}{c}\text { Human Health } \\
\text { Benchmark Value }\end{array}$ \\
\hline Columbia River & HFD TS HRM 26 & September 8, 2003 & B17CM0 & Arsenic & $\mathrm{mg} / \mathrm{L}$ & 1 & $5.05 \mathrm{E}-04$ & $=$ & 0.15 & 1.80E-05 \\
\hline Columbia River & HFD TS5 HRM28.7 & September 18, 2000 & B10752 & Arsenic & $\mathrm{mg} / \mathrm{L}$ & 1 & 5.05E-04 & $=$ & 0.15 & 1.80E-05 \\
\hline Columbia River & 100 N-3 HRM 9.5 & September 14, 2000 & B10664 & Arsenic & $\mathrm{mg} / \mathrm{L}$ & 1 & 5.06E-04 & $=$ & 0.15 & 1.80E-05 \\
\hline Columbia River & RICHPS HRM 45.8 & December 5, 2000 & B11149 & Arsenic & $\mathrm{mg} / \mathrm{L}$ & 1 & 5.07E-04 & $=$ & 0.15 & 1.80E-05 \\
\hline Columbia River & 100 NSHR HRM8.4 & September 14, 2000 & B10699 & Arsenic & $\mathrm{mg} / \mathrm{L}$ & 1 & $5.08 \mathrm{E}-04$ & $=$ & 0.15 & 1.80E-05 \\
\hline Columbia River & $100 \mathrm{~N}$ Area & April 9, 2004 & B19114 & Arsenic & $\mathrm{mg} / \mathrm{L}$ & 1 & $5.08 \mathrm{E}-04$ & $x$ & 0.15 & 1.80E-05 \\
\hline Columbia River & RICHPS HRM 43.5 & February 26, 2001 & B11H66 & Arsenic & $\mathrm{mg} / \mathrm{L}$ & 1 & 5.08E-04 & $=$ & 0.15 & $1.80 \mathrm{E}-05$ \\
\hline Columbia River & 100 FSHR HRM18 & September 18, 2000 & B10790 & Arsenic & $\mathrm{mg} / \mathrm{L}$ & 1 & 5.09E-04 & $=$ & 0.15 & 1.80E-05 \\
\hline Columbia River & 100 N-10HRM 9.5 & September 14, 2000 & B10667 & Arsenic & $\mathrm{mg} / \mathrm{L}$ & 1 & 5.09E-04 & $=$ & 0.15 & 1.80E-05 \\
\hline Columbia River & RICHPS HRM 45.8 & September 19, 2000 & B10765 & Arsenic & $\mathrm{mg} / \mathrm{L}$ & 1 & $5.10 \mathrm{E}-04$ & $=$ & 0.15 & 1.80E-05 \\
\hline Columbia River & RICHPS3 HRM46.4 & December 6, 1999 & B0X3W6 & Arsenic & $\mathrm{mg} / \mathrm{L}$ & 1 & 5.10E-04 & $=$ & 0.15 & $1.80 \mathrm{E}-05$ \\
\hline Columbia River & 100 FSHR HRM18 & September 9, 2002 & B15909 & Arsenic & $\mathrm{mg} / \mathrm{L}$ & 1 & 5.11E-04 & $=$ & 0.15 & 1.80E-05 \\
\hline Columbia River & 100 N-2 HRM 9.5 & September 14, 2000 & B10663 & Arsenic & $\mathrm{mg} / \mathrm{L}$ & 1 & 5.11E-04 & $=$ & 0.15 & 1.80E-05 \\
\hline Columbia River & HFD TS HRM 27 & September 8, 2003 & B17CM1 & Arsenic & $\mathrm{mg} / \mathrm{L}$ & 1 & $5.14 \mathrm{E}-04$ & $=$ & 0.15 & 1.80E-05 \\
\hline Columbia River & RICHPS HRM 43.5 & December 5, 2000 & B11152 & Arsenic & $\mathrm{mg} / \mathrm{L}$ & 1 & $5.14 \mathrm{E}-04$ & $=$ & 0.15 & 1.80E-05 \\
\hline Columbia River & RICHPS7 HRM46.4 & September 10, 2002 & B158R6 & Arsenic & $\mathrm{mg} / \mathrm{L}$ & 1 & $5.14 \mathrm{E}-04$ & $=$ & 0.15 & $1.80 \mathrm{E}-05$ \\
\hline Columbia River & 100 B/C AREA DR & November 17, 2003 & B180F1 & Arsenic & $\mathrm{mg} / \mathrm{L}$ & 1 & 5.15E-04 & $=$ & 0.15 & 1.80E-05 \\
\hline Columbia River & 100 B/C AREA UR & November 13, 2003 & B180C9 & Arsenic & $\mathrm{mg} / \mathrm{L}$ & 1 & 5.15E-04 & $=$ & 0.15 & 1.80E-05 \\
\hline Columbia River & 100 B/C OUTFLL1 & November 17, 2003 & B180D8 & Arsenic & $\mathrm{mg} / \mathrm{L}$ & 1 & 5.15E-04 & $=$ & 0.15 & 1.80E-05 \\
\hline Columbia River & 100 B/C OUTFLL2 & November 17, 2003 & B180D9 & Arsenic & $\mathrm{mg} / \mathrm{L}$ & 1 & 5.15E-04 & $=$ & 0.15 & $1.80 \mathrm{E}-05$ \\
\hline Columbia River & 100-B SPR 38-3 & November 13, 2003 & B180D3 & Arsenic & $\mathrm{mg} / \mathrm{L}$ & 1 & 5.15E-04 & $=$ & 0.15 & 1.80E-05 \\
\hline Columbia River & RICHPS HRM 43.9 & September 10, 2002 & B158W2 & Arsenic & $\mathrm{mg} / \mathrm{L}$ & 1 & 5.15E-04 & $=$ & 0.15 & 1.80E-05 \\
\hline Columbia River & 300 A1 HRM 43.1 & September 10, 2002 & B158R8 & Arsenic & $\mathrm{mg} / \mathrm{L}$ & 1 & $5.16 \mathrm{E}-04$ & $=$ & 0.15 & 1.80E-05 \\
\hline Columbia River & RICHPS2 HRM46.4 & December 5, 2000 & B11140 & Arsenic & $\mathrm{mg} / \mathrm{L}$ & 1 & 5.16E-04 & $=$ & 0.15 & 1.80E-05 \\
\hline Columbia River & 100D Area & May 3, 2002 & B14K61 & Arsenic & $\mathrm{mg} / \mathrm{L}$ & 2 & 5.17E-04 & $=$ & 0.15 & 1.80E-05 \\
\hline Columbia River & HFD TS5 HRM28.7 & September 18, 2000 & B106T3 & Arsenic & $\mathrm{mg} / \mathrm{L}$ & 1 & 5.17E-04 & $=$ & 0.15 & 1.80E-05 \\
\hline Columbia River & RICHPS HRM 43.5 & December 10, 2002 & B16373 & Arsenic & $\mathrm{mg} / \mathrm{L}$ & 1 & 5.17E-04 & $=$ & 0.15 & 1.80E-05 \\
\hline Columbia River & RICHPS HRM 45.0 & December 5, 2000 & B11154 & Arsenic & $\mathrm{mg} / \mathrm{L}$ & 1 & 5.17E-04 & $=$ & 0.15 & 1.80E-05 \\
\hline Columbia River & RICHPS2 HRM46.4 & September 19, 2000 & B106V5 & Arsenic & $\mathrm{mg} / \mathrm{L}$ & 1 & 5.18E-04 & $=$ & 0.15 & $1.80 \mathrm{E}-05$ \\
\hline Columbia River & 100 B/C AREA DR & November 17, 2003 & B180F2 & Arsenic & $\mathrm{mg} / \mathrm{L}$ & 1 & 5.19E-04 & $=$ & 0.15 & 1.80E-05 \\
\hline Columbia River & RICHPS HRM 45.0 & September 10, 2002 & B158W1 & Arsenic & $\mathrm{mg} / \mathrm{L}$ & 1 & 5.19E-04 & $=$ & 0.15 & 1.80E-05 \\
\hline Columbia River & RICHPS5 HRM46.4 & December 5, 2000 & B11142 & Arsenic & $\mathrm{mg} / \mathrm{L}$ & 1 & 5.19E-04 & $=$ & 0.15 & 1.80E-05 \\
\hline Columbia River & 100 B/C AREA DR & November 17, 2003 & B180F3 & Arsenic & $\mathrm{mg} / \mathrm{L}$ & 1 & $5.20 \mathrm{E}-04$ & $=$ & 0.15 & 1.80E-05 \\
\hline Columbia River & 100 N-7 HRM 9.5 & September 14, 2000 & B10696 & Arsenic & $\mathrm{mg} / \mathrm{L}$ & 1 & $5.20 \mathrm{E}-04$ & $=$ & 0.15 & 1.80E-05 \\
\hline Columbia River & 100-B SPR 38-3 & November 13, 2003 & B180J4 & Arsenic & $\mathrm{mg} / \mathrm{L}$ & 1 & 5.20E-04 & $=$ & 0.15 & 1.80E-05 \\
\hline Columbia River & 300 A3 HRM 43.1 & September 10, 2002 & В158T0 & Arsenic & $\mathrm{mg} / \mathrm{L}$ & 1 & 5.20E-04 & $=$ & 0.15 & 1.80E-05 \\
\hline Columbia River & RICHPS HRM 43.9 & December 5, 2000 & B11151 & Arsenic & $\mathrm{mg} / \mathrm{L}$ & 1 & $5.20 \mathrm{E}-04$ & $=$ & 0.15 & 1.80E-05 \\
\hline Columbia River & RICHPS2 HRM46.4 & February 26, 2001 & B11H54 & Arsenic & $\mathrm{mg} / \mathrm{L}$ & 1 & $5.20 \mathrm{E}-04$ & $=$ & 0.15 & $1.80 \mathrm{E}-05$ \\
\hline Columbia River & 100-B SPR 39-2 & November 13, 2003 & B180D5 & Arsenic & $\mathrm{mg} / \mathrm{L}$ & 1 & 5.21E-04 & $=$ & 0.15 & 1.80E-05 \\
\hline Columbia River & RICHPS HRM 43.5 & December 10, 2002 & B16369 & Arsenic & $\mathrm{mg} / \mathrm{L}$ & 1 & 5.21E-04 & $=$ & 0.15 & 1.80E-05 \\
\hline Columbia River & RICHPS7 HRM46.4 & December 5, 2000 & B11121 & Arsenic & $\mathrm{mg} / \mathrm{L}$ & 1 & $5.21 \mathrm{E}-04$ & $=$ & 0.15 & 1.80E-05 \\
\hline Columbia River & 100-B SPR 39-2 & November 13, 2003 & B180D7 & Arsenic & $\mathrm{mg} / \mathrm{L}$ & 1 & $5.22 \mathrm{E}-04$ & $=$ & 0.15 & 1.80E-05 \\
\hline Columbia River & HFD TS7 HRM28.7 & September 8, 2003 & В17CP9 & Arsenic & $\mathrm{mg} / \mathrm{L}$ & 1 & $5.22 \mathrm{E}-04$ & $=$ & 0.15 & 1.80E-05 \\
\hline Columbia River & RICHPS HRM 45.8 & December 10, 2002 & B16366 & Arsenic & $\mathrm{mg} / \mathrm{L}$ & 1 & 5.22E-04 & $=$ & 0.15 & 1.80E-05 \\
\hline Columbia River & 100 FSHR HRM23 & September 9, 2002 & B15917 & Arsenic & $\mathrm{mg} / \mathrm{L}$ & 1 & $5.24 \mathrm{E}-04$ & $=$ & 0.15 & $1.80 \mathrm{E}-05$ \\
\hline
\end{tabular}

108 of 137 


\section{Table B-10}

Summary of Surface Water Results From Segment 2 Exceeding Select Ecological or Human Health Benchmark Values

Columbia River Component - Existing Sample Data Evaluation and Scoping Report

\begin{tabular}{|c|c|c|c|c|c|c|c|c|c|c|}
\hline Sample Area & Sample Site & Sample ID & Date Collected & Constituent Name & Units & QA Category & $\begin{array}{l}\text { Analytical } \\
\text { Value }\end{array}$ & Qualifier & $\begin{array}{l}\text { Ecological } \\
\text { Benchmark } \\
\text { Value }\end{array}$ & $\begin{array}{c}\text { Human Health } \\
\text { Benchmark Value }\end{array}$ \\
\hline Columbia River & RICHPS HRM 43.5 & September 19, 2000 & B10772 & Arsenic & $\mathrm{mg} / \mathrm{L}$ & 1 & $5.24 \mathrm{E}-04$ & $=$ & 0.15 & 1.80E-05 \\
\hline Columbia River & RICHPS HRM 43.5 & December 5, 2000 & B11156 & Arsenic & $\mathrm{mg} / \mathrm{L}$ & 1 & $5.24 \mathrm{E}-04$ & $=$ & 0.15 & 1.80E-05 \\
\hline Columbia River & RICHPS HRM 43.9 & June 12, 2001 & B12537 & Arsenic & $\mathrm{mg} / \mathrm{L}$ & 1 & $5.24 \mathrm{E}-04$ & $=$ & 0.15 & 1.80E-05 \\
\hline Columbia River & 100 F-1 HRM19.0 & September 8, 2003 & B17CR1 & Arsenic & $\mathrm{mg} / \mathrm{L}$ & 1 & 5.25E-04 & $=$ & 0.15 & 1.80E-05 \\
\hline Columbia River & 100-B SPR 39-2 & November 13, 2003 & B180D6 & Arsenic & $\mathrm{mg} / \mathrm{L}$ & 1 & $5.26 \mathrm{E}-04$ & $=$ & 0.15 & 1.80E-05 \\
\hline Columbia River & HFD TS HRM 26 & September 18, 2000 & B10734 & Arsenic & $\mathrm{mg} / \mathrm{L}$ & 1 & $5.26 \mathrm{E}-04$ & $=$ & 0.15 & 1.80E-05 \\
\hline Columbia River & HFD TS3 HRM28.7 & September 18, 2000 & B10751 & Arsenic & $\mathrm{mg} / \mathrm{L}$ & 1 & $5.26 \mathrm{E}-04$ & $=$ & 0.15 & $1.80 \mathrm{E}-05$ \\
\hline Columbia River & RICHPS2 HRM46.4 & September 10, 2002 & B158R3 & Arsenic & $\mathrm{mg} / \mathrm{L}$ & 1 & $5.27 \mathrm{E}-04$ & $=$ & 0.15 & 1.80E-05 \\
\hline Columbia River & 100 N-10HRM 9.5 & September 14, 2000 & B10697 & Arsenic & $\mathrm{mg} / \mathrm{L}$ & 1 & $5.28 \mathrm{E}-04$ & $=$ & 0.15 & 1.80E-05 \\
\hline Columbia River & 100 N-2 HRM 9.5 & September 4, 2003 & B17CB7 & Arsenic & $\mathrm{mg} / \mathrm{L}$ & 1 & $5.28 \mathrm{E}-04$ & $=$ & 0.15 & 1.80E-05 \\
\hline Columbia River & RICHPS HRM 45.8 & December 5, 2000 & B11153 & Arsenic & $\mathrm{mg} / \mathrm{L}$ & 1 & $5.28 \mathrm{E}-04$ & $=$ & 0.15 & $1.80 \mathrm{E}-05$ \\
\hline Columbia River & 100 F-7 HRM19.0 & September 18, 2000 & B10759 & Arsenic & $\mathrm{mg} / \mathrm{L}$ & 1 & $5.29 \mathrm{E}-04$ & $=$ & 0.15 & 1.80E-05 \\
\hline Columbia River & RICHPS HRM 43.9 & February 26, 2001 & B11H65 & Arsenic & $\mathrm{mg} / \mathrm{L}$ & 1 & $5.29 E-04$ & $=$ & 0.15 & 1.80E-05 \\
\hline Columbia River & 100 F-3 HRM19.0 & September 9, 2002 & B158F0 & Arsenic & $\mathrm{mg} / \mathrm{L}$ & 1 & 5.30E-04 & $=$ & 0.15 & 1.80E-05 \\
\hline Columbia River & RICHPS HRM 43.5 & September 10, 2002 & B158W3 & Arsenic & $\mathrm{mg} / \mathrm{L}$ & 1 & $5.30 \mathrm{E}-04$ & $=$ & 0.15 & 1.80E-05 \\
\hline Columbia River & RICHPS1 HRM46.4 & December 5, 2000 & B11139 & Arsenic & $\mathrm{mg} / \mathrm{L}$ & 1 & 5.30E-04 & $=$ & 0.15 & $1.80 \mathrm{E}-05$ \\
\hline Columbia River & RICHPS HRM 43.9 & December 5, 2000 & B11155 & Arsenic & $\mathrm{mg} / \mathrm{L}$ & 1 & 5.31E-04 & $=$ & 0.15 & 1.80E-05 \\
\hline Columbia River & RICHPS5 HRM46.4 & December 10, 2002 & B16359 & Arsenic & $\mathrm{mg} / \mathrm{L}$ & 1 & 5.31E-04 & $=$ & 0.15 & 1.80E-05 \\
\hline Columbia River & 100 F-10HRM19.0 & September 18, 2000 & B106R9 & Arsenic & $\mathrm{mg} / \mathrm{L}$ & 1 & $5.32 \mathrm{E}-04$ & $=$ & 0.15 & 1.80E-05 \\
\hline Columbia River & 100 F-2 HRM19.0 & September 9, 2002 & B158D9 & Arsenic & $\mathrm{mg} / \mathrm{L}$ & 1 & 5.32E-04 & $=$ & 0.15 & $1.80 \mathrm{E}-05$ \\
\hline Columbia River & HFD TS HRM 26 & September 18, 2000 & B10731 & Arsenic & $\mathrm{mg} / \mathrm{L}$ & 1 & 5.32E-04 & $=$ & 0.15 & 1.80E-05 \\
\hline Columbia River & $100 \mathrm{~N}$ Area & June 9, 2004 & B19H73 & Arsenic & $\mathrm{mg} / \mathrm{L}$ & 1 & 5.33E-04 & $x$ & 0.15 & 1.80E-05 \\
\hline Columbia River & RICHPS HRM 43.9 & December 10, 2002 & B16368 & Arsenic & $\mathrm{mg} / \mathrm{L}$ & 1 & 5.33E-04 & $=$ & 0.15 & 1.80E-05 \\
\hline Columbia River & RICHPS3 HRM46.4 & February 26, 2001 & B11H55 & Arsenic & $\mathrm{mg} / \mathrm{L}$ & 1 & 5.33E-04 & $=$ & 0.15 & 1.80E-05 \\
\hline Columbia River & 100 N-5 HRM 9.5 & September 14, 2000 & B10665 & Arsenic & $\mathrm{mg} / \mathrm{L}$ & 1 & 5.34E-04 & $=$ & 0.15 & $1.80 \mathrm{E}-05$ \\
\hline Columbia River & RICHPS2 HRM46.4 & December 5, 2000 & B11118 & Arsenic & $\mathrm{mg} / \mathrm{L}$ & 1 & 5.34E-04 & $=$ & 0.15 & 1.80E-05 \\
\hline Columbia River & HFD TS HRM 27 & September 21, 1999 & B0WB74 & Arsenic & $\mathrm{mg} / \mathrm{L}$ & 1 & 5.35E-04 & $=$ & 0.15 & 1.80E-05 \\
\hline Columbia River & 100 F-1 HRM19.0 & September 9, 2002 & B158V0 & Arsenic & $\mathrm{mg} / \mathrm{L}$ & 1 & 5.35E-04 & $=$ & 0.15 & 1.80E-05 \\
\hline Columbia River & 300 SR HRM 41.5 & September 19, 2000 & B10775 & Arsenic & $\mathrm{mg} / \mathrm{L}$ & 1 & 5.35E-04 & $=$ & 0.15 & $1.80 \mathrm{E}-05$ \\
\hline Columbia River & 100 N-7 HRM 9.5 & September 4, 2003 & B17CN1 & Arsenic & $\mathrm{mg} / \mathrm{L}$ & 1 & 5.36E-04 & $=$ & 0.15 & 1.80E-05 \\
\hline Columbia River & 100-H SPR 153-1 & October 26, 2004 & B1BH01 & Arsenic & $\mathrm{mg} / \mathrm{L}$ & 1 & 5.36E-04 & $\mathrm{x}$ & 0.15 & 1.80E-05 \\
\hline Columbia River & 300 A1 HRM 43.1 & September 19, 2000 & B10743 & Arsenic & $\mathrm{mg} / \mathrm{L}$ & 1 & 5.36E-04 & $=$ & 0.15 & 1.80E-05 \\
\hline Columbia River & 300 SR HRM 42.5 & September 10, 2002 & B158Y8 & Arsenic & $\mathrm{mg} / \mathrm{L}$ & 1 & 5.36E-04 & $=$ & 0.15 & 1.80E-05 \\
\hline Columbia River & 300 A2 HRM 43.1 & September 16, 1999 & B0WB84 & Arsenic & $\mathrm{mg} / \mathrm{L}$ & 1 & 5.37E-04 & $=$ & 0.15 & $1.80 \mathrm{E}-05$ \\
\hline Columbia River & 100 N-1 HRM 9.5 & September 14, 2000 & B10692 & Arsenic & $\mathrm{mg} / \mathrm{L}$ & 1 & 5.37E-04 & $=$ & 0.15 & 1.80E-05 \\
\hline Columbia River & 100-B SPR 38-3 & November 13, 2003 & B180D2 & Arsenic & $\mathrm{mg} / \mathrm{L}$ & 1 & 5.37E-04 & $=$ & 0.15 & 1.80E-05 \\
\hline Columbia River & 100D Area & May 3, 2002 & B14K63 & Arsenic & $\mathrm{mg} / \mathrm{L}$ & 2 & 5.37E-04 & $=$ & 0.15 & 1.80E-05 \\
\hline Columbia River & RICHPS HRM 43.9 & September 19, 2000 & B10771 & Arsenic & $\mathrm{mg} / \mathrm{L}$ & 1 & 5.37E-04 & $=$ & 0.15 & $1.80 \mathrm{E}-05$ \\
\hline Columbia River & 100 N-5 HRM 9.5 & September 4, 2003 & В17СВ9 & Arsenic & $\mathrm{mg} / \mathrm{L}$ & 1 & 5.38E-04 & $=$ & 0.15 & 1.80E-05 \\
\hline Columbia River & 100-D SPR 110-1 & October 26, 2004 & B1BFX0 & Arsenic & $\mathrm{mg} / \mathrm{L}$ & 1 & 5.38E-04 & $\mathrm{x}$ & 0.15 & 1.80E-05 \\
\hline Columbia River & $100 \mathrm{H}$ Area & May 17, 2002 & B14L08 & Arsenic & $\mathrm{mg} / \mathrm{L}$ & 2 & 5.38E-04 & $=$ & 0.15 & 1.80E-05 \\
\hline Columbia River & 300 A5 HRM 43.1 & September 19, 2000 & B106R6 & Arsenic & $\mathrm{mg} / \mathrm{L}$ & 1 & 5.38E-04 & $=$ & 0.15 & 1.80E-05 \\
\hline Columbia River & 300 SR HRM 42.9 & September 10, 2002 & B15901 & Arsenic & $\mathrm{mg} / \mathrm{L}$ & 1 & 5.38E-04 & $=$ & 0.15 & 1.80E-05 \\
\hline Columbia River & HFD TS2 HRM28.7 & September 8, 2003 & B17C98 & Arsenic & $\mathrm{mg} / \mathrm{L}$ & 1 & 5.38E-04 & $=$ & 0.15 & 1.80E-05 \\
\hline Columbia River & RICHPS5 HRM46.4 & December 5, 2000 & B11120 & Arsenic & $\mathrm{mg} / \mathrm{L}$ & 1 & $5.38 \mathrm{E}-04$ & $=$ & 0.15 & $1.80 \mathrm{E}-05$ \\
\hline
\end{tabular}

109 of 137 


\section{Table B-10}

Summary of Surface Water Results From Segment 2 Exceeding Select Ecological or Human Health Benchmark Values

Columbia River Component - Existing Sample Data Evaluation and Scoping Report

\begin{tabular}{|c|c|c|c|c|c|c|c|c|c|c|}
\hline Sample Area & Sample Site & Sample ID & Date Collected & Constituent Name & Units & QA Category & $\begin{array}{l}\text { Analytical } \\
\text { Value }\end{array}$ & Qualifier & $\begin{array}{l}\text { Ecological } \\
\text { Benchmark } \\
\text { Value }\end{array}$ & $\begin{array}{c}\text { Human Health } \\
\text { Benchmark Value }\end{array}$ \\
\hline Columbia River & RICHPS7 HRM46.4 & September 9, 2003 & B17CN7 & Arsenic & $\mathrm{mg} / \mathrm{L}$ & 1 & $5.38 \mathrm{E}-04$ & $=$ & 0.15 & 1.80E-05 \\
\hline Columbia River & 100 FSHR HRM24 & September 9, 2002 & B15913 & Arsenic & $\mathrm{mg} / \mathrm{L}$ & 1 & $5.39 \mathrm{E}-04$ & $=$ & 0.15 & 1.80E-05 \\
\hline Columbia River & RICHPS7 HRM46.4 & December 8, 2003 & B183V5 & Arsenic & $\mathrm{mg} / \mathrm{L}$ & 1 & 5.39E-04 & C & 0.15 & 1.80E-05 \\
\hline Columbia River & 100 F-3 HRM19.0 & September 18, 2000 & B10757 & Arsenic & $\mathrm{mg} / \mathrm{L}$ & 1 & $5.40 \mathrm{E}-04$ & $=$ & 0.15 & 1.80E-05 \\
\hline Columbia River & RICHPS HRM 45.0 & February 26, 2001 & B11H64 & Arsenic & $\mathrm{mg} / \mathrm{L}$ & 1 & $5.40 \mathrm{E}-04$ & $=$ & 0.15 & 1.80E-05 \\
\hline Columbia River & RICHPS3 HRM46.4 & September 10, 2002 & B158R4 & Arsenic & $\mathrm{mg} / \mathrm{L}$ & 1 & $5.40 \mathrm{E}-04$ & $=$ & 0.15 & 1.80E-05 \\
\hline Columbia River & RICHPS3 HRM46.4 & December 10, 2002 & B16358 & Arsenic & $\mathrm{mg} / \mathrm{L}$ & 1 & $5.40 \mathrm{E}-04$ & $=$ & 0.15 & $1.80 \mathrm{E}-05$ \\
\hline Columbia River & RICHPS7 HRM46.4 & September 19, 2000 & B106V8 & Arsenic & $\mathrm{mg} / \mathrm{L}$ & 1 & $5.40 \mathrm{E}-04$ & $=$ & 0.15 & 1.80E-05 \\
\hline Columbia River & RICHPS1 HRM46.4 & December 6, 1999 & B0X3W4 & Arsenic & $\mathrm{mg} / \mathrm{L}$ & 1 & $5.40 \mathrm{E}-04$ & $=$ & 0.15 & 1.80E-05 \\
\hline Columbia River & RICHPS HRM 45.0 & December 10, 2002 & B16367 & Arsenic & $\mathrm{mg} / \mathrm{L}$ & 1 & $5.41 \mathrm{E}-04$ & $=$ & 0.15 & 1.80E-05 \\
\hline Columbia River & RICHPS1 HRM46.4 & December 8, 2003 & B183V1 & Arsenic & $\mathrm{mg} / \mathrm{L}$ & 1 & 5.41E-04 & C & 0.15 & $1.80 \mathrm{E}-05$ \\
\hline Columbia River & 100 F-1 HRM19.0 & September 8, 2003 & B17CC2 & Arsenic & $\mathrm{mg} / \mathrm{L}$ & 1 & $5.42 \mathrm{E}-04$ & $=$ & 0.15 & 1.80E-05 \\
\hline Columbia River & 100 F-10HRM19.0 & September 8, 2003 & B17CR6 & Arsenic & $\mathrm{mg} / \mathrm{L}$ & 1 & $5.42 \mathrm{E}-04$ & $=$ & 0.15 & 1.80E-05 \\
\hline Columbia River & 100 F-5 HRM19.0 & September 18, 2000 & B10758 & Arsenic & $\mathrm{mg} / \mathrm{L}$ & 1 & $5.42 \mathrm{E}-04$ & $=$ & 0.15 & 1.80E-05 \\
\hline Columbia River & 100D Area & May 3, 2002 & B14K60 & Arsenic & $\mathrm{mg} / \mathrm{L}$ & 2 & $5.42 \mathrm{E}-04$ & $=$ & 0.15 & 1.80E-05 \\
\hline Columbia River & HFD TS3 HRM28.7 & September 18, 2000 & B106T2 & Arsenic & $\mathrm{mg} / \mathrm{L}$ & 1 & $5.42 \mathrm{E}-04$ & $=$ & 0.15 & $1.80 \mathrm{E}-05$ \\
\hline Columbia River & 100 B/C OUTFLL3 & November 17, 2003 & B180F0 & Arsenic & $\mathrm{mg} / \mathrm{L}$ & 1 & 5.43E-04 & $=$ & 0.15 & 1.80E-05 \\
\hline Columbia River & 100-D SPR 110-1 & October 26, 2004 & B1BFX1 & Arsenic & $\mathrm{mg} / \mathrm{L}$ & 1 & 5.43E-04 & $\mathrm{x}$ & 0.15 & 1.80E-05 \\
\hline Columbia River & 300 SR HRM 42.5 & September 19, 2000 & B10781 & Arsenic & $\mathrm{mg} / \mathrm{L}$ & 1 & 5.43E-04 & $=$ & 0.15 & 1.80E-05 \\
\hline Columbia River & 100 F-7 HRM19.0 & September 21, 1999 & B0WBB1 & Arsenic & $\mathrm{mg} / \mathrm{L}$ & 1 & 5.43E-04 & $=$ & 0.15 & $1.80 \mathrm{E}-05$ \\
\hline Columbia River & 100 N-7 HRM 9.5 & September 14, 2000 & B10666 & Arsenic & $\mathrm{mg} / \mathrm{L}$ & 1 & 5.44E-04 & $=$ & 0.15 & 1.80E-05 \\
\hline Columbia River & 100 NSHR HRM8.9 & September 14, 2000 & B106B2 & Arsenic & $\mathrm{mg} / \mathrm{L}$ & 1 & $5.44 \mathrm{E}-04$ & $=$ & 0.15 & 1.80E-05 \\
\hline Columbia River & 300 A3 HRM 43.1 & September 16, 1999 & B0WB85 & Arsenic & $\mathrm{mg} / \mathrm{L}$ & 1 & $5.45 \mathrm{E}-04$ & $=$ & 0.15 & 1.80E-05 \\
\hline Columbia River & 100 FSHR HRM18 & September 9, 2002 & B15908 & Arsenic & $\mathrm{mg} / \mathrm{L}$ & 1 & $5.45 \mathrm{E}-04$ & $=$ & 0.15 & 1.80E-05 \\
\hline Columbia River & 100-K SPR 77-1 & October 21, 2002 & B15C82 & Arsenic & $\mathrm{mg} / \mathrm{L}$ & 1 & $5.45 \mathrm{E}-04$ & $=$ & 0.15 & 1.80E-05 \\
\hline Columbia River & RICHPS5 HRM46.4 & September 10, 2002 & B158R5 & Arsenic & $\mathrm{mg} / \mathrm{L}$ & 1 & $5.45 \mathrm{E}-04$ & $=$ & 0.15 & 1.80E-05 \\
\hline Columbia River & 100 F-10HRM19.0 & September 21, 1999 & B0WBB4 & Arsenic & $\mathrm{mg} / \mathrm{L}$ & 1 & $5.46 \mathrm{E}-04$ & $=$ & 0.15 & 1.80E-05 \\
\hline Columbia River & 100 NSHR HRM9.2 & September 4, 2003 & B17CW0 & Arsenic & $\mathrm{mg} / \mathrm{L}$ & 1 & 5.46E-04 & $=$ & 0.15 & 1.80E-05 \\
\hline Columbia River & 300 A5 HRM 43.1 & September 10, 2002 & B158T1 & Arsenic & $\mathrm{mg} / \mathrm{L}$ & 1 & 5.46E-04 & $=$ & 0.15 & $1.80 \mathrm{E}-05$ \\
\hline Columbia River & RICHPS7 HRM46.4 & December 6, 1999 & B0X3W8 & Arsenic & $\mathrm{mg} / \mathrm{L}$ & 1 & 5.46E-04 & $=$ & 0.15 & 1.80E-05 \\
\hline Columbia River & 100-B SPR 38-3 & November 13, 2003 & B180D1 & Arsenic & $\mathrm{mg} / \mathrm{L}$ & 1 & 5.47E-04 & $=$ & 0.15 & 1.80E-05 \\
\hline Columbia River & RICHPS1 HRM46.4 & December 5, 2000 & B11117 & Arsenic & $\mathrm{mg} / \mathrm{L}$ & 1 & 5.47E-04 & $=$ & 0.15 & 1.80E-05 \\
\hline Columbia River & RICHPS3 HRM46.4 & September 9, 2003 & B17CN5 & Arsenic & $\mathrm{mg} / \mathrm{L}$ & 1 & 5.47E-04 & $=$ & 0.15 & 1.80E-05 \\
\hline Columbia River & HFD TS1 HRM28.7 & September 21, 1999 & B0WB90 & Arsenic & $\mathrm{mg} / \mathrm{L}$ & 1 & $5.48 \mathrm{E}-04$ & $=$ & 0.15 & $1.80 \mathrm{E}-05$ \\
\hline Columbia River & 100D Area & May 17, 2002 & B14L07 & Arsenic & $\mathrm{mg} / \mathrm{L}$ & 2 & 5.48E-04 & $=$ & 0.15 & 1.80E-05 \\
\hline Columbia River & RICHPS7 HRM46.4 & December 4, 2001 & B13LJ1 & Arsenic & $\mathrm{mg} / \mathrm{L}$ & 1 & 5.48E-04 & $=$ & 0.15 & 1.80E-05 \\
\hline Columbia River & 100 F-3 HRM19.0 & September 18, 2000 & B106V1 & Arsenic & $\mathrm{mg} / \mathrm{L}$ & 1 & $5.49 \mathrm{E}-04$ & $=$ & 0.15 & 1.80E-05 \\
\hline Columbia River & 300 SR HRM 41.5 & September 19, 2000 & B10774 & Arsenic & $\mathrm{mg} / \mathrm{L}$ & 1 & 5.49E-04 & $=$ & 0.15 & $1.80 \mathrm{E}-05$ \\
\hline Columbia River & RICHPS3 HRM46.4 & June 12, 2001 & B12527 & Arsenic & $\mathrm{mg} / \mathrm{L}$ & 1 & 5.49E-04 & $=$ & 0.15 & 1.80E-05 \\
\hline Columbia River & 100 FSHR HRM23 & September 8, 2003 & B17D02 & Arsenic & $\mathrm{mg} / \mathrm{L}$ & 1 & 5.50E-04 & $=$ & 0.15 & 1.80E-05 \\
\hline Columbia River & 300 A2 HRM 43.1 & September 9, 2003 & В17CР0 & Arsenic & $\mathrm{mg} / \mathrm{L}$ & 1 & 5.50E-04 & $=$ & 0.15 & 1.80E-05 \\
\hline Columbia River & RICHPS2 HRM46.4 & February 26, 2001 & $\mathrm{~B} 11 \mathrm{H} 32$ & Arsenic & $\mathrm{mg} / \mathrm{L}$ & 1 & $5.50 \mathrm{E}-04$ & $=$ & 0.15 & 1.80E-05 \\
\hline Columbia River & 100 N-2 HRM 9.5 & September 10, 1999 & BoW974 & Arsenic & $\mathrm{mg} / \mathrm{L}$ & 1 & 5.51E-04 & $=$ & 0.15 & 1.80E-05 \\
\hline Columbia River & RICHPS5 HRM46.4 & February 26, 2001 & B11H56 & Arsenic & $\mathrm{mg} / \mathrm{L}$ & 1 & 5.51E-04 & $=$ & 0.15 & 1.80E-05 \\
\hline Columbia River & 100 F-3 HRM19.0 & September 9, 2002 & B158V2 & Arsenic & $\mathrm{mg} / \mathrm{L}$ & 1 & $5.52 \mathrm{E}-04$ & $=$ & 0.15 & $1.80 \mathrm{E}-05$ \\
\hline
\end{tabular}

110 of 137 


\section{Table B-10}

Summary of Surface Water Results From Segment 2 Exceeding Select Ecological or Human Health Benchmark Values

Columbia River Component - Existing Sample Data Evaluation and Scoping Report

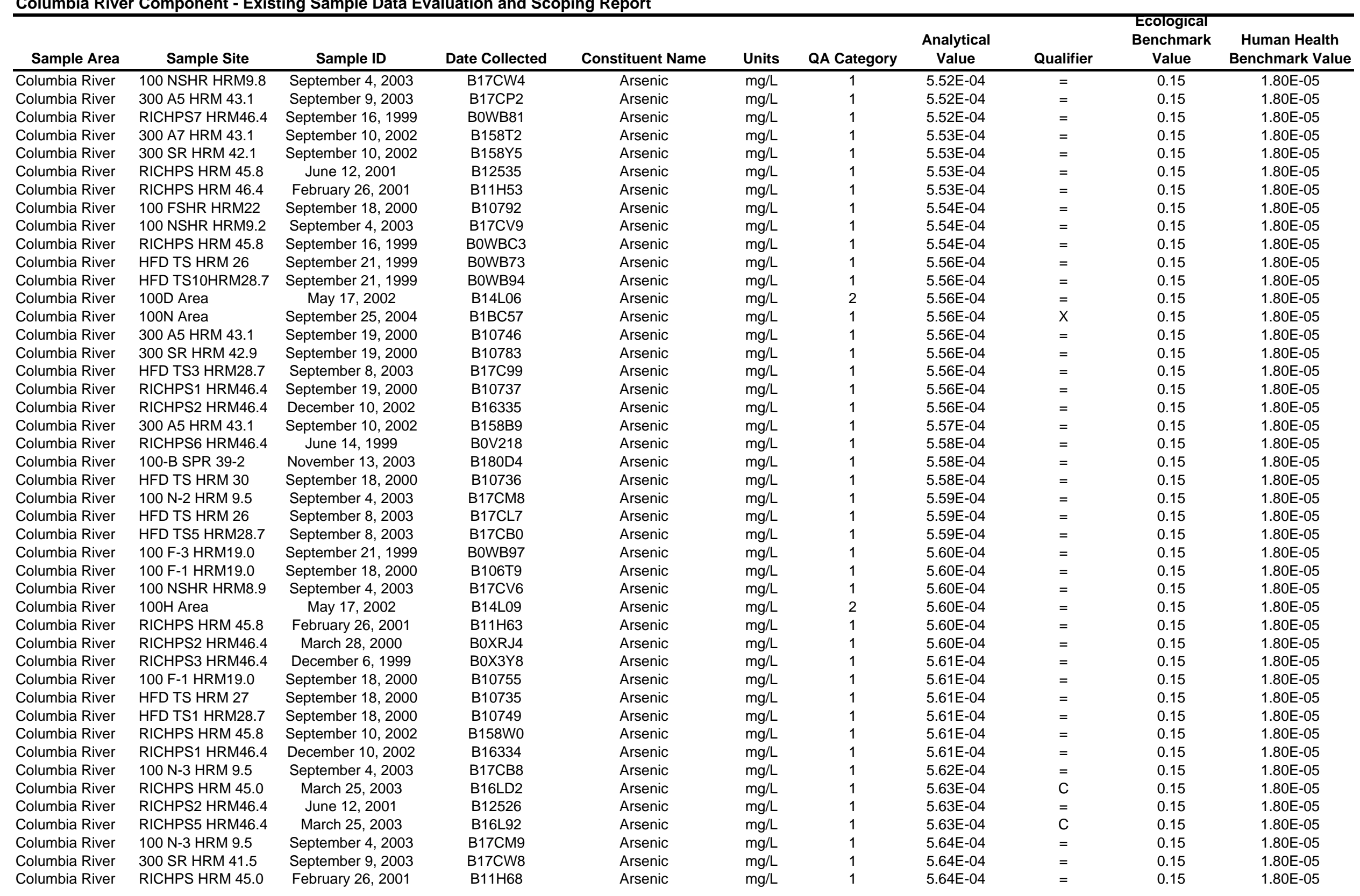




\section{Table B-10}

Summary of Surface Water Results From Segment 2 Exceeding Select Ecological or Human Health Benchmark Values

Columbia River Component - Existing Sample Data Evaluation and Scoping Report

\begin{tabular}{|c|c|c|c|c|c|c|c|c|c|c|}
\hline Sample Area & Sample Site & Sample ID & Date Collected & Constituent Name & Units & QA Category & $\begin{array}{l}\text { Analytical } \\
\text { Value }\end{array}$ & Qualifier & $\begin{array}{l}\text { Ecological } \\
\text { Benchmark } \\
\text { Value }\end{array}$ & $\begin{array}{c}\text { Human Health } \\
\text { Benchmark Value }\end{array}$ \\
\hline Columbia River & 100 FSHR HRM18 & September 8, 2003 & B17CY6 & Arsenic & $\mathrm{mg} / \mathrm{L}$ & 1 & $5.65 \mathrm{E}-04$ & $=$ & 0.15 & 1.80E-05 \\
\hline Columbia River & RICHPS HRM 43.5 & September 19, 2000 & B10768 & Arsenic & $\mathrm{mg} / \mathrm{L}$ & 1 & $5.65 \mathrm{E}-04$ & $=$ & 0.15 & 1.80E-05 \\
\hline Columbia River & RICHPS HRM 43.9 & December 6, 1999 & B0X412 & Arsenic & $\mathrm{mg} / \mathrm{L}$ & 1 & 5.65E-04 & $=$ & 0.15 & 1.80E-05 \\
\hline Columbia River & RICHPS5 HRM46.4 & December 6, 1999 & B0X3W7 & Arsenic & $\mathrm{mg} / \mathrm{L}$ & 1 & 5.66E-04 & $=$ & 0.15 & 1.80E-05 \\
\hline Columbia River & 100 NSHR HRM9.8 & September 4, 2003 & B17CW3 & Arsenic & $\mathrm{mg} / \mathrm{L}$ & 1 & $5.66 \mathrm{E}-04$ & $=$ & 0.15 & 1.80E-05 \\
\hline Columbia River & 100 F-7 HRM19.0 & September 8, 2003 & B17CR5 & Arsenic & $\mathrm{mg} / \mathrm{L}$ & 1 & 5.67E-04 & $=$ & 0.15 & 1.80E-05 \\
\hline Columbia River & 100 FSHR HRM24 & September 9, 2002 & B15912 & Arsenic & $\mathrm{mg} / \mathrm{L}$ & 1 & 5.67E-04 & $=$ & 0.15 & $1.80 \mathrm{E}-05$ \\
\hline Columbia River & 100 F-10HRM19.0 & September 9, 2002 & B158C2 & Arsenic & $\mathrm{mg} / \mathrm{L}$ & 1 & $5.68 \mathrm{E}-04$ & $=$ & 0.15 & 1.80E-05 \\
\hline Columbia River & 100-H SPR 153-1 & November 1, 2000 & B10979 & Arsenic & $\mathrm{mg} / \mathrm{L}$ & 1 & $5.68 \mathrm{E}-04$ & $=$ & 0.15 & 1.80E-05 \\
\hline Columbia River & 100 N-7 HRM 9.5 & September 10, 1999 & BoW977 & Arsenic & $\mathrm{mg} / \mathrm{L}$ & 1 & 5.68E-04 & $=$ & 0.15 & $1.80 \mathrm{E}-05$ \\
\hline Columbia River & 100 NSHR HRM8.4 & September 4, 2003 & B17CV2 & Arsenic & $\mathrm{mg} / \mathrm{L}$ & 1 & 5.69E-04 & $=$ & 0.15 & 1.80E-05 \\
\hline Columbia River & HFD TS5 HRM28.7 & September 8, 2003 & В17CР8 & Arsenic & $\mathrm{mg} / \mathrm{L}$ & 1 & 5.69E-04 & $=$ & 0.15 & 1.80E-05 \\
\hline Columbia River & RICHPS5 HRM46.4 & February 26, 2001 & B11H34 & Arsenic & $\mathrm{mg} / \mathrm{L}$ & 1 & 5.69E-04 & $=$ & 0.15 & 1.80E-05 \\
\hline Columbia River & 100D Area & May 3, 2002 & B14K62 & Arsenic & $\mathrm{mg} / \mathrm{L}$ & 2 & $5.70 \mathrm{E}-04$ & $=$ & 0.15 & 1.80E-05 \\
\hline Columbia River & 300 A3 HRM 43.1 & September 9, 2003 & B17CP1 & Arsenic & $\mathrm{mg} / \mathrm{L}$ & 1 & 5.70E-04 & $=$ & 0.15 & 1.80E-05 \\
\hline Columbia River & 300 SR HRM 41.5 & September 10, 2002 & B158Y1 & Arsenic & $\mathrm{mg} / \mathrm{L}$ & 1 & 5.70E-04 & $=$ & 0.15 & 1.80E-05 \\
\hline Columbia River & RICHPS1 HRM46.4 & December 10, 2002 & B16356 & Arsenic & $\mathrm{mg} / \mathrm{L}$ & 1 & 5.70E-04 & $=$ & 0.15 & $1.80 \mathrm{E}-05$ \\
\hline Columbia River & RICHPS7 HRM46.4 & September 9, 2003 & B17CD4 & Arsenic & $\mathrm{mg} / \mathrm{L}$ & 1 & 5.70E-04 & $=$ & 0.15 & 1.80E-05 \\
\hline Columbia River & 100 F-7 HRM19.0 & September 9, 2002 & B158F2 & Arsenic & $\mathrm{mg} / \mathrm{L}$ & 1 & 5.71E-04 & $=$ & 0.15 & 1.80E-05 \\
\hline Columbia River & 100 FSHR HRM23 & September 18, 2000 & B10796 & Arsenic & $\mathrm{mg} / \mathrm{L}$ & 1 & 5.71E-04 & $=$ & 0.15 & 1.80E-05 \\
\hline Columbia River & 100 N-10HRM 9.5 & September 4, 2003 & B17CN2 & Arsenic & $\mathrm{mg} / \mathrm{L}$ & 1 & 5.71E-04 & $=$ & 0.15 & 1.80E-05 \\
\hline Columbia River & HFD TS1 HRM28.7 & September 18, 2000 & B106T0 & Arsenic & $\mathrm{mg} / \mathrm{L}$ & 1 & 5.71E-04 & $=$ & 0.15 & 1.80E-05 \\
\hline Columbia River & RICHPS3 HRM46.4 & February 26, 2001 & B11H33 & Arsenic & $\mathrm{mg} / \mathrm{L}$ & 1 & 5.71E-04 & $=$ & 0.15 & 1.80E-05 \\
\hline Columbia River & RICHPS HRM 45.8 & December 6, 1999 & B0X410 & Arsenic & $\mathrm{mg} / \mathrm{L}$ & 1 & 5.71E-04 & $=$ & 0.15 & 1.80E-05 \\
\hline Columbia River & 100 F-3 HRM19.0 & September 8, 2003 & B17CC4 & Arsenic & $\mathrm{mg} / \mathrm{L}$ & 1 & $5.72 \mathrm{E}-04$ & $=$ & 0.15 & 1.80E-05 \\
\hline Columbia River & RICHPS HRM 43.5 & December 4, 2001 & B13LK0 & Arsenic & $\mathrm{mg} / \mathrm{L}$ & 1 & $5.72 \mathrm{E}-04$ & $=$ & 0.15 & $1.80 \mathrm{E}-05$ \\
\hline Columbia River & RICHPS HRM 45.0 & December 8, 2003 & B183W6 & Arsenic & $\mathrm{mg} / \mathrm{L}$ & 1 & $5.72 \mathrm{E}-04$ & $=$ & 0.15 & 1.80E-05 \\
\hline Columbia River & 100 FSHR HRM22 & September 8, 2003 & B17CY8 & Arsenic & $\mathrm{mg} / \mathrm{L}$ & 1 & 5.73E-04 & $=$ & 0.15 & 1.80E-05 \\
\hline Columbia River & 100 NSHR HRM9.2 & September 10, 1999 & Bow967 & Arsenic & $\mathrm{mg} / \mathrm{L}$ & 1 & 5.73E-04 & $=$ & 0.15 & 1.80E-05 \\
\hline Columbia River & 100-B SPR 38-3 & November 13, 2003 & B180D0 & Arsenic & $\mathrm{mg} / \mathrm{L}$ & 1 & 5.73E-04 & $=$ & 0.15 & 1.80E-05 \\
\hline Columbia River & 300 SR HRM 42.5 & September 19, 2000 & B10780 & Arsenic & $\mathrm{mg} / \mathrm{L}$ & 1 & 5.73E-04 & $=$ & 0.15 & 1.80E-05 \\
\hline Columbia River & 300 SR HRM 42.5 & September 10, 2002 & B158Y7 & Arsenic & $\mathrm{mg} / \mathrm{L}$ & 1 & 5.73E-04 & $=$ & 0.15 & 1.80E-05 \\
\hline Columbia River & HFD TS5 HRM28.7 & September 21, 1999 & B0WB92 & Arsenic & $\mathrm{mg} / \mathrm{L}$ & 1 & 5.73E-04 & $=$ & 0.15 & 1.80E-05 \\
\hline Columbia River & 100 N-7 HRM 9.5 & September 4, 2003 & B17CC0 & Arsenic & $\mathrm{mg} / \mathrm{L}$ & 1 & $5.74 \mathrm{E}-04$ & $=$ & 0.15 & 1.80E-05 \\
\hline Columbia River & 100 NSHR HRM8.9 & September 5, 2002 & B158X2 & Arsenic & $\mathrm{mg} / \mathrm{L}$ & 1 & $5.74 \mathrm{E}-04$ & $=$ & 0.15 & $1.80 \mathrm{E}-05$ \\
\hline Columbia River & RICHPS7 HRM46.4 & September 10, 2002 & B158H0 & Arsenic & $\mathrm{mg} / \mathrm{L}$ & 1 & $5.74 \mathrm{E}-04$ & $=$ & 0.15 & 1.80E-05 \\
\hline Columbia River & RICHPS HRM 45.0 & December 6, 1999 & B0X411 & Arsenic & $\mathrm{mg} / \mathrm{L}$ & 1 & $5.75 E-04$ & $=$ & 0.15 & 1.80E-05 \\
\hline Columbia River & 100 NSHR HRM8.4 & September 4, 2003 & B17CV1 & Arsenic & $\mathrm{mg} / \mathrm{L}$ & 1 & 5.75E-04 & $=$ & 0.15 & 1.80E-05 \\
\hline Columbia River & 100-B SPR 38-3 & November 13, 2003 & B180F9 & Arsenic & $\mathrm{mg} / \mathrm{L}$ & 1 & $5.75 \mathrm{E}-04$ & $=$ & 0.15 & 1.80E-05 \\
\hline
\end{tabular}




\section{Table B-10}

Summary of Surface Water Results From Segment 2 Exceeding Select Ecological or Human Health Benchmark Values

Columbia River Component - Existing Sample Data Evaluation and Scoping Report

\begin{tabular}{|c|c|c|c|c|c|c|c|c|c|c|}
\hline Sample Area & Sample Site & Sample ID & Date Collected & Constituent Name & Units & QA Category & $\begin{array}{l}\text { Analytical } \\
\text { Value }\end{array}$ & Qualifier & $\begin{array}{l}\text { Ecological } \\
\text { Benchmark } \\
\text { Value }\end{array}$ & $\begin{array}{c}\text { Human Health } \\
\text { Benchmark Value }\end{array}$ \\
\hline Columbia River & RICHPS HRM 43.9 & December 4, 2001 & B13LK3 & Arsenic & $\mathrm{mg} / \mathrm{L}$ & 1 & $5.75 \mathrm{E}-04$ & $=$ & 0.15 & 1.80E-05 \\
\hline Columbia River & RICHPS3 HRM46.4 & December 10, 2002 & B16336 & Arsenic & $\mathrm{mg} / \mathrm{L}$ & 1 & $5.75 \mathrm{E}-04$ & $=$ & 0.15 & 1.80E-05 \\
\hline Columbia River & RICHPS5 HRM46.4 & December 10, 2002 & B16337 & Arsenic & $\mathrm{mg} / \mathrm{L}$ & 1 & $5.75 E-04$ & $=$ & 0.15 & 1.80E-05 \\
\hline Columbia River & 100-K SPR 77-1 & October 11, 2004 & B1BFW5 & Arsenic & $\mathrm{mg} / \mathrm{L}$ & 1 & 5.76E-04 & $x$ & 0.15 & 1.80E-05 \\
\hline Columbia River & 100 N-1 HRM 9.5 & September 4, 2003 & B17CM7 & Arsenic & $\mathrm{mg} / \mathrm{L}$ & 1 & 5.77E-04 & $=$ & 0.15 & 1.80E-05 \\
\hline Columbia River & 100 N-5 HRM 9.5 & September 14, 2000 & B10695 & Arsenic & $\mathrm{mg} / \mathrm{L}$ & 1 & 5.77E-04 & $=$ & 0.15 & 1.80E-05 \\
\hline Columbia River & HFD TS7 HRM28.7 & September 18, 2000 & B106T4 & Arsenic & $\mathrm{mg} / \mathrm{L}$ & 1 & 5.77E-04 & $=$ & 0.15 & $1.80 \mathrm{E}-05$ \\
\hline Columbia River & RICHPS HRM 45.8 & September 19, 2000 & B10769 & Arsenic & $\mathrm{mg} / \mathrm{L}$ & 1 & 5.77E-04 & $=$ & 0.15 & 1.80E-05 \\
\hline Columbia River & RICHPS HRM 45.0 & June 12, 2001 & B12536 & Arsenic & $\mathrm{mg} / \mathrm{L}$ & 1 & $5.78 \mathrm{E}-04$ & $=$ & 0.15 & 1.80E-05 \\
\hline Columbia River & RICHPS5 HRM46.4 & March 28, 2000 & B0XRJ6 & Arsenic & $\mathrm{mg} / \mathrm{L}$ & 1 & $5.79 \mathrm{E}-04$ & $=$ & 0.15 & 1.80E-05 \\
\hline Columbia River & RICHPS7 HRM46.4 & February 26, 2001 & B11H35 & Arsenic & $\mathrm{mg} / \mathrm{L}$ & 1 & 5.79E-04 & $=$ & 0.15 & $1.80 \mathrm{E}-05$ \\
\hline Columbia River & 100 NSHR HRM9.8 & September 10, 1999 & BoW968 & Arsenic & $\mathrm{mg} / \mathrm{L}$ & 1 & $5.80 \mathrm{E}-04$ & $=$ & 0.15 & 1.80E-05 \\
\hline Columbia River & 100 F-3 HRM19.0 & September 8, 2003 & B17CR3 & Arsenic & $\mathrm{mg} / \mathrm{L}$ & 1 & $5.80 \mathrm{E}-04$ & $=$ & 0.15 & 1.80E-05 \\
\hline Columbia River & 100 N-1 HRM 9.5 & September 14, 2000 & B10662 & Arsenic & $\mathrm{mg} / \mathrm{L}$ & 1 & $5.80 \mathrm{E}-04$ & $=$ & 0.15 & 1.80E-05 \\
\hline Columbia River & RICHPS HRM 43.5 & March 25, 2003 & B16LD8 & Arsenic & $\mathrm{mg} / \mathrm{L}$ & 1 & $5.80 \mathrm{E}-04$ & C & 0.15 & 1.80E-05 \\
\hline Columbia River & RICHPS2 HRM46.4 & December 4, 2001 & B13LH8 & Arsenic & $\mathrm{mg} / \mathrm{L}$ & 1 & $5.80 \mathrm{E}-04$ & $=$ & 0.15 & $1.80 \mathrm{E}-05$ \\
\hline Columbia River & RICHPS HRM 45.8 & December 6, 1999 & B0X406 & Arsenic & $\mathrm{mg} / \mathrm{L}$ & 1 & $5.80 \mathrm{E}-04$ & $=$ & 0.15 & 1.80E-05 \\
\hline Columbia River & 100-H SPR 153-1 & November 1, 2000 & B10980 & Arsenic & $\mathrm{mg} / \mathrm{L}$ & 1 & 5.81E-04 & $=$ & 0.15 & 1.80E-05 \\
\hline Columbia River & 300 A2 HRM 43.1 & September 19, 2000 & B106T7 & Arsenic & $\mathrm{mg} / \mathrm{L}$ & 1 & 5.81E-04 & $=$ & 0.15 & 1.80E-05 \\
\hline Columbia River & RICHPS HRM 43.5 & December 6, 1999 & B0X409 & Arsenic & $\mathrm{mg} / \mathrm{L}$ & 1 & 5.82E-04 & $=$ & 0.15 & $1.80 \mathrm{E}-05$ \\
\hline Columbia River & RICHPS HRM 43.9 & September 16, 1999 & B0WBC5 & Arsenic & $\mathrm{mg} / \mathrm{L}$ & 1 & 5.82E-04 & $=$ & 0.15 & 1.80E-05 \\
\hline Columbia River & 100 N-10HRM 9.5 & September 4, 2003 & B17CC1 & Arsenic & $\mathrm{mg} / \mathrm{L}$ & 1 & 5.82E-04 & $=$ & 0.15 & 1.80E-05 \\
\hline Columbia River & 300 A7 HRM 43.1 & September 19, 2000 & B106R7 & Arsenic & $\mathrm{mg} / \mathrm{L}$ & 1 & $5.82 \mathrm{E}-04$ & $=$ & 0.15 & 1.80E-05 \\
\hline Columbia River & RICHPS3 HRM46.4 & March 28, 2000 & B0XRJ5 & Arsenic & $\mathrm{mg} / \mathrm{L}$ & 1 & 5.82E-04 & $=$ & 0.15 & 1.80E-05 \\
\hline Columbia River & 100 N-5 HRM 9.5 & September 10, 1999 & BoW976 & Arsenic & $\mathrm{mg} / \mathrm{L}$ & 1 & $5.82 \mathrm{E}-04$ & $=$ & 0.15 & $1.80 \mathrm{E}-05$ \\
\hline Columbia River & 100 F-1 HRM19.0 & September 21, 1999 & B0WB95 & Arsenic & $\mathrm{mg} / \mathrm{L}$ & 1 & 5.83E-04 & $=$ & 0.15 & 1.80E-05 \\
\hline Columbia River & 100 F-10HRM19.0 & September 18, 2000 & B10760 & Arsenic & $\mathrm{mg} / \mathrm{L}$ & 1 & 5.83E-04 & $=$ & 0.15 & 1.80E-05 \\
\hline Columbia River & 100 FSHR HRM22 & September 18, 2000 & B10793 & Arsenic & $\mathrm{mg} / \mathrm{L}$ & 1 & 5.83E-04 & $=$ & 0.15 & 1.80E-05 \\
\hline Columbia River & 100 NSHR HRM8.9 & September 4, 2003 & B17CV5 & Arsenic & $\mathrm{mg} / \mathrm{L}$ & 1 & 5.83E-04 & $=$ & 0.15 & $1.80 \mathrm{E}-05$ \\
\hline Columbia River & RICHPS HRM 43.5 & March 25, 2003 & B16LD4 & Arsenic & $\mathrm{mg} / \mathrm{L}$ & 1 & 5.83E-04 & C & 0.15 & 1.80E-05 \\
\hline Columbia River & RICHPS HRM 45.0 & December 4, 2001 & B13LK2 & Arsenic & $\mathrm{mg} / \mathrm{L}$ & 1 & 5.83E-04 & $=$ & 0.15 & 1.80E-05 \\
\hline Columbia River & RICHPS1 HRM46.4 & March 28, 2000 & B0XRJ3 & Arsenic & $\mathrm{mg} / \mathrm{L}$ & 1 & 5.83E-04 & $=$ & 0.15 & 1.80E-05 \\
\hline Columbia River & RICHPS2 HRM46.4 & March 25, 2003 & B16L90 & Arsenic & $\mathrm{mg} / \mathrm{L}$ & 1 & 5.83E-04 & C & 0.15 & 1.80E-05 \\
\hline Columbia River & RICHPS3 HRM46.4 & March 25, 2003 & B16LC3 & Arsenic & $\mathrm{mg} / \mathrm{L}$ & 1 & 5.83E-04 & C & 0.15 & $1.80 \mathrm{E}-05$ \\
\hline Columbia River & 100 F-5 HRM19.0 & September 18, 2000 & B106V2 & Arsenic & $\mathrm{mg} / \mathrm{L}$ & 1 & 5.84E-04 & $=$ & 0.15 & 1.80E-05 \\
\hline Columbia River & 100 FSHR HRM18 & September 18, 2000 & B10789 & Arsenic & $\mathrm{mg} / \mathrm{L}$ & 1 & 5.84E-04 & $=$ & 0.15 & 1.80E-05 \\
\hline Columbia River & 300 A3 HRM 43.1 & September 10, 2002 & B158D1 & Arsenic & $\mathrm{mg} / \mathrm{L}$ & 1 & 5.84E-04 & $=$ & 0.15 & 1.80E-05 \\
\hline Columbia River & HFD TS10HRM28.7 & September 18, 2000 & B106T5 & Arsenic & $\mathrm{mg} / \mathrm{L}$ & 1 & 5.84E-04 & $=$ & 0.15 & $1.80 \mathrm{E}-05$ \\
\hline Columbia River & RICHPS HRM 45.0 & September 10, 2002 & B158W5 & Arsenic & $\mathrm{mg} / \mathrm{L}$ & 1 & 5.84E-04 & $=$ & 0.15 & 1.80E-05 \\
\hline Columbia River & RICHPS HRM 46.4 & June 12, 2001 & B12525 & Arsenic & $\mathrm{mg} / \mathrm{L}$ & 1 & 5.84E-04 & $=$ & 0.15 & 1.80E-05 \\
\hline Columbia River & RICHPS5 HRM46.4 & December 8, 2003 & B183V4 & Arsenic & $\mathrm{mg} / \mathrm{L}$ & 1 & 5.84E-04 & C & 0.15 & 1.80E-05 \\
\hline Columbia River & RICHPS3 HRM46.4 & September 16, 1999 & B0WB79 & Arsenic & $\mathrm{mg} / \mathrm{L}$ & 1 & 5.84E-04 & $=$ & 0.15 & 1.80E-05 \\
\hline Columbia River & 300 SR HRM 42.1 & September 16, 1999 & B0WB62 & Arsenic & $\mathrm{mg} / \mathrm{L}$ & 1 & 5.85E-04 & $=$ & 0.15 & 1.80E-05 \\
\hline Columbia River & HFD TS HRM 27 & September 18, 2000 & B10732 & Arsenic & $\mathrm{mg} / \mathrm{L}$ & 1 & 5.85E-04 & $=$ & 0.15 & 1.80E-05 \\
\hline Columbia River & HFD TS3 HRM28.7 & September 8, 2003 & B17CP7 & Arsenic & $\mathrm{mg} / \mathrm{L}$ & 1 & $5.85 \mathrm{E}-04$ & $=$ & 0.15 & $1.80 \mathrm{E}-05$ \\
\hline
\end{tabular}




\section{Table B-10}

Summary of Surface Water Results From Segment 2 Exceeding Select Ecological or Human Health Benchmark Values

Columbia River Component - Existing Sample Data Evaluation and Scoping Report

\begin{tabular}{|c|c|c|c|c|c|c|c|c|c|c|}
\hline Sample Area & Sample Site & Sample ID & Date Collected & Constituent Name & Units & QA Category & $\begin{array}{l}\text { Analytical } \\
\text { Value }\end{array}$ & Qualifier & $\begin{array}{l}\text { Ecological } \\
\text { Benchmark } \\
\text { Value }\end{array}$ & $\begin{array}{c}\text { Human Health } \\
\text { Benchmark Value }\end{array}$ \\
\hline Columbia River & RICHPS HRM 43.9 & March 25, 2003 & B16LD3 & Arsenic & $\mathrm{mg} / \mathrm{L}$ & 1 & $5.85 \mathrm{E}-04$ & C & 0.15 & 1.80E-05 \\
\hline Columbia River & 100 F-2 HRM19.0 & September 21, 1999 & B0WB96 & Arsenic & $\mathrm{mg} / \mathrm{L}$ & 1 & $5.85 \mathrm{E}-04$ & $=$ & 0.15 & 1.80E-05 \\
\hline Columbia River & RICHPS2 HRM46.4 & December 6, 1999 & B0X3W5 & Arsenic & $\mathrm{mg} / \mathrm{L}$ & 1 & 5.85E-04 & $=$ & 0.15 & 1.80E-05 \\
\hline Columbia River & 100 F-7 HRM19.0 & September 18, 2000 & B106V3 & Arsenic & $\mathrm{mg} / \mathrm{L}$ & 1 & 5.86E-04 & $=$ & 0.15 & 1.80E-05 \\
\hline Columbia River & 100 FSHR HRM23 & September 9, 2002 & B15916 & Arsenic & $\mathrm{mg} / \mathrm{L}$ & 1 & $5.86 \mathrm{E}-04$ & $=$ & 0.15 & 1.80E-05 \\
\hline Columbia River & 100 FSHR HRM23 & September 8, 2003 & B17D01 & Arsenic & $\mathrm{mg} / \mathrm{L}$ & 1 & 5.86E-04 & $=$ & 0.15 & 1.80E-05 \\
\hline Columbia River & 100D Area & June 10, 2002 & B14PW7 & Arsenic & $\mathrm{mg} / \mathrm{L}$ & 2 & 5.86E-04 & $=$ & 0.15 & $1.80 \mathrm{E}-05$ \\
\hline Columbia River & RICHPS7 HRM46.4 & December 10, 2002 & B16338 & Arsenic & $\mathrm{mg} / \mathrm{L}$ & 1 & 5.86E-04 & $=$ & 0.15 & 1.80E-05 \\
\hline Columbia River & 100 FSHR HRM23 & September 18, 2000 & B10795 & Arsenic & $\mathrm{mg} / \mathrm{L}$ & 1 & 5.87E-04 & $=$ & 0.15 & 1.80E-05 \\
\hline Columbia River & RICHPS HRM 45.0 & March 28, 2000 & B0XRP7 & Arsenic & $\mathrm{mg} / \mathrm{L}$ & 1 & $5.87 \mathrm{E}-04$ & $=$ & 0.15 & 1.80E-05 \\
\hline Columbia River & RICHPS HRM 45.0 & March 25, 2003 & B16LD6 & Arsenic & $\mathrm{mg} / \mathrm{L}$ & 1 & 5.87E-04 & C & 0.15 & $1.80 \mathrm{E}-05$ \\
\hline Columbia River & RICHPS7 HRM46.4 & March 25, 2003 & B16LC5 & Arsenic & $\mathrm{mg} / \mathrm{L}$ & 1 & 5.87E-04 & C & 0.15 & 1.80E-05 \\
\hline Columbia River & RICHPS HRM 45.0 & September 16, 1999 & BOWBC4 & Arsenic & $\mathrm{mg} / \mathrm{L}$ & 1 & 5.88E-04 & $=$ & 0.15 & 1.80E-05 \\
\hline Columbia River & RICHPS1 HRM46.4 & September 10, 2002 & B158R2 & Arsenic & $\mathrm{mg} / \mathrm{L}$ & 1 & 5.88E-04 & $=$ & 0.15 & 1.80E-05 \\
\hline Columbia River & 100 F-5 HRM19.0 & September 21, 1999 & B0WB99 & Arsenic & $\mathrm{mg} / \mathrm{L}$ & 1 & $5.88 \mathrm{E}-04$ & $=$ & 0.15 & 1.80E-05 \\
\hline Columbia River & 100 FSHR HRM22 & September 8, 2003 & B17CY9 & Arsenic & $\mathrm{mg} / \mathrm{L}$ & 1 & 5.89E-04 & $=$ & 0.15 & $1.80 \mathrm{E}-05$ \\
\hline Columbia River & HFD TS7 HRM28.7 & September 9, 2002 & В158T8 & Arsenic & $\mathrm{mg} / \mathrm{L}$ & 1 & 5.89E-04 & $=$ & 0.15 & 1.80E-05 \\
\hline Columbia River & RICHPS5 HRM46.4 & September 9, 2003 & B17CN6 & Arsenic & $\mathrm{mg} / \mathrm{L}$ & 1 & 5.89E-04 & $=$ & 0.15 & 1.80E-05 \\
\hline Columbia River & RICHPS HRM 45.8 & June 10, 2003 & B17205 & Arsenic & $\mathrm{mg} / \mathrm{L}$ & 1 & $5.90 \mathrm{E}-04$ & C & 0.15 & 1.80E-05 \\
\hline Columbia River & RICHPS5 HRM46.4 & September 9, 2003 & B17CD3 & Arsenic & $\mathrm{mg} / \mathrm{L}$ & 1 & $5.90 \mathrm{E}-04$ & $=$ & 0.15 & $1.80 \mathrm{E}-05$ \\
\hline Columbia River & RICHPS7 HRM46.4 & March 28, 2000 & B0XRJ7 & Arsenic & $\mathrm{mg} / \mathrm{L}$ & 1 & 5.91E-04 & $=$ & 0.15 & 1.80E-05 \\
\hline Columbia River & 300 A1 HRM 43.1 & September 10, 2002 & B158C9 & Arsenic & $\mathrm{mg} / \mathrm{L}$ & 1 & 5.92E-04 & $=$ & 0.15 & 1.80E-05 \\
\hline Columbia River & RICHPS1 HRM46.4 & March 25, 2003 & B16LC1 & Arsenic & $\mathrm{mg} / \mathrm{L}$ & 1 & 5.92E-04 & C & 0.15 & 1.80E-05 \\
\hline Columbia River & RICHPS5 HRM46.4 & September 16, 1999 & B0WB80 & Arsenic & $\mathrm{mg} / \mathrm{L}$ & 1 & 5.92E-04 & $=$ & 0.15 & 1.80E-05 \\
\hline Columbia River & RICHPS5 HRM46.4 & March 25, 2003 & B16LC4 & Arsenic & $\mathrm{mg} / \mathrm{L}$ & 1 & 5.93E-04 & C & 0.15 & $1.80 \mathrm{E}-05$ \\
\hline Columbia River & 100H SPR 153-1 & October 27, 2003 & B17RL9 & Arsenic & $\mathrm{mg} / \mathrm{L}$ & 1 & 5.95E-04 & $=$ & 0.15 & 1.80E-05 \\
\hline Columbia River & HFD TS7 HRM28.7 & September 21, 1999 & B0WB93 & Arsenic & $\mathrm{mg} / \mathrm{L}$ & 1 & 5.95E-04 & $=$ & 0.15 & 1.80E-05 \\
\hline Columbia River & RICHPS HRM 43.5 & March 28, 2000 & B0XRP9 & Arsenic & $\mathrm{mg} / \mathrm{L}$ & 1 & 5.96E-04 & $=$ & 0.15 & 1.80E-05 \\
\hline Columbia River & RICHPS HRM 43.9 & December 8, 2003 & B183W7 & Arsenic & $\mathrm{mg} / \mathrm{L}$ & 1 & 5.96E-04 & $=$ & 0.15 & $1.80 \mathrm{E}-05$ \\
\hline Columbia River & RICHPS2 HRM46.4 & June 10, 2003 & B171Y6 & Arsenic & $\mathrm{mg} / \mathrm{L}$ & 1 & 5.96E-04 & C & 0.15 & 1.80E-05 \\
\hline Columbia River & RICHPS3 HRM46.4 & September 10, 2002 & B158F8 & Arsenic & $\mathrm{mg} / \mathrm{L}$ & 1 & 5.96E-04 & $=$ & 0.15 & 1.80E-05 \\
\hline Columbia River & RICHPS5 HRM46.4 & December 4, 2001 & B13LJ0 & Arsenic & $\mathrm{mg} / \mathrm{L}$ & 1 & 5.96E-04 & $=$ & 0.15 & 1.80E-05 \\
\hline Columbia River & RICHPS HRM 45.8 & March 25, 2003 & B16LD1 & Arsenic & $\mathrm{mg} / \mathrm{L}$ & 1 & 5.97E-04 & C & 0.15 & 1.80E-05 \\
\hline Columbia River & RICHPS2 HRM46.4 & September 10, 2002 & B158F7 & Arsenic & $\mathrm{mg} / \mathrm{L}$ & 1 & 5.97E-04 & $=$ & 0.15 & $1.80 \mathrm{E}-05$ \\
\hline Columbia River & RICHPS5 HRM46.4 & June 20,2000 & BOYCKO & Arsenic & $\mathrm{mg} / \mathrm{L}$ & 1 & 5.97E-04 & $=$ & 0.15 & 1.80E-05 \\
\hline Columbia River & 100 NSHR HRM8.4 & September 10, 1999 & B0W965 & Arsenic & $\mathrm{mg} / \mathrm{L}$ & 1 & 5.98E-04 & $=$ & 0.15 & 1.80E-05 \\
\hline Columbia River & RICHPS HRM 43.5 & September 16, 1999 & B0WBC6 & Arsenic & $\mathrm{mg} / \mathrm{L}$ & 1 & 5.98E-04 & $=$ & 0.15 & 1.80E-05 \\
\hline Columbia River & 100 F-10HRM19.0 & September 9, 2002 & B158V5 & Arsenic & $\mathrm{mg} / \mathrm{L}$ & 1 & 5.98E-04 & $=$ & 0.15 & $1.80 \mathrm{E}-05$ \\
\hline Columbia River & 100-H SPR 153-1 & October 26, 2004 & B1BH00 & Arsenic & $\mathrm{mg} / \mathrm{L}$ & 1 & 5.98E-04 & $\mathrm{x}$ & 0.15 & 1.80E-05 \\
\hline Columbia River & 100D SPR 110-1 & October 27, 2003 & B17J92 & Arsenic & $\mathrm{mg} / \mathrm{L}$ & 1 & 5.98E-04 & $=$ & 0.15 & 1.80E-05 \\
\hline Columbia River & HFD TS10HRM28.7 & September 8, 2003 & B17CR0 & Arsenic & $\mathrm{mg} / \mathrm{L}$ & 1 & 5.98E-04 & $=$ & 0.15 & 1.80E-05 \\
\hline Columbia River & HFD TS7 HRM28.7 & September 8, 2003 & B17CB1 & Arsenic & $\mathrm{mg} / \mathrm{L}$ & 1 & 5.98E-04 & $=$ & 0.15 & 1.80E-05 \\
\hline Columbia River & RICHPS HRM 45.8 & September 10, 2002 & B158W4 & Arsenic & $\mathrm{mg} / \mathrm{L}$ & 1 & 5.98E-04 & $=$ & 0.15 & 1.80E-05 \\
\hline Columbia River & 100-B SPR 39-2 & October 11, 2004 & B1BFW0 & Arsenic & $\mathrm{mg} / \mathrm{L}$ & 1 & 5.99E-04 & $x$ & 0.15 & 1.80E-05 \\
\hline Columbia River & RICHPS HRM 43.9 & March 28, 2000 & B0XRP8 & Arsenic & $\mathrm{mg} / \mathrm{L}$ & 1 & 5.99E-04 & $=$ & 0.15 & $1.80 \mathrm{E}-05$ \\
\hline
\end{tabular}




\section{Table B-10}

Summary of Surface Water Results From Segment 2 Exceeding Select Ecological or Human Health Benchmark Values

Columbia River Component - Existing Sample Data Evaluation and Scoping Report

\begin{tabular}{|c|c|c|c|c|c|c|c|c|c|c|}
\hline Sample Area & Sample Site & Sample ID & Date Collected & Constituent Name & Units & QA Category & $\begin{array}{l}\text { Analytical } \\
\text { Value }\end{array}$ & Qualifier & $\begin{array}{l}\text { Ecological } \\
\text { Benchmark } \\
\text { Value }\end{array}$ & $\begin{array}{c}\text { Human Health } \\
\text { Benchmark Value }\end{array}$ \\
\hline Columbia River & RICHPS HRM 43.9 & February 26, 2001 & B11H69 & Arsenic & $\mathrm{mg} / \mathrm{L}$ & 1 & 5.99E-04 & $=$ & 0.15 & 1.80E-05 \\
\hline Columbia River & RICHPS HRM 43.9 & September 10, 2002 & B158W6 & Arsenic & $\mathrm{mg} / \mathrm{L}$ & 1 & 5.99E-04 & $=$ & 0.15 & 1.80E-05 \\
\hline Columbia River & RICHPS HRM 43.9 & March 25, 2003 & B16LD7 & Arsenic & $\mathrm{mg} / \mathrm{L}$ & 1 & 5.99E-04 & C & 0.15 & 1.80E-05 \\
\hline Columbia River & RICHPS HRM 45.8 & December 8, 2003 & B183W5 & Arsenic & $\mathrm{mg} / \mathrm{L}$ & 1 & 5.99E-04 & $=$ & 0.15 & 1.80E-05 \\
\hline Columbia River & RICHPS HRM 46.4 & February 26, 2001 & B11H31 & Arsenic & $\mathrm{mg} / \mathrm{L}$ & 1 & 5.99E-04 & $=$ & 0.15 & 1.80E-05 \\
\hline Columbia River & RICHPS2 HRM46.4 & June 20, 2000 & B0YCJ8 & Arsenic & $\mathrm{mg} / \mathrm{L}$ & 1 & 5.99E-04 & $=$ & 0.15 & 1.80E-05 \\
\hline Columbia River & RICHPS3 HRM46.4 & December 4, 2001 & B13LD7 & Arsenic & $\mathrm{mg} / \mathrm{L}$ & 1 & 5.99E-04 & $=$ & 0.15 & $1.80 \mathrm{E}-05$ \\
\hline Columbia River & RICHPS3 HRM46.4 & March 25, 2003 & B16L91 & Arsenic & $\mathrm{mg} / \mathrm{L}$ & 1 & 5.99E-04 & C & 0.15 & 1.80E-05 \\
\hline Columbia River & RICHPS1 HRM46.4 & September 16, 1999 & B0WB77 & Arsenic & $\mathrm{mg} / \mathrm{L}$ & 1 & $6.00 \mathrm{E}-04$ & $=$ & 0.15 & 1.80E-05 \\
\hline Columbia River & 100 F-5 HRM19.0 & September 14, 2004 & B1B778 & Arsenic & $\mathrm{mg} / \mathrm{L}$ & 1 & $6.00 \mathrm{E}-04$ & $x$ & 0.15 & 1.80E-05 \\
\hline Columbia River & 300 A1 HRM 43.1 & September 16, 1999 & B0WB83 & Arsenic & $\mathrm{mg} / \mathrm{L}$ & 1 & 6.01E-04 & $=$ & 0.15 & $1.80 \mathrm{E}-05$ \\
\hline Columbia River & $100 \mathrm{H}$ Area & June 10, 2002 & B14PX0 & Arsenic & $\mathrm{mg} / \mathrm{L}$ & 2 & 6.01E-04 & $=$ & 0.15 & 1.80E-05 \\
\hline Columbia River & 300 SR HRM 42.1 & September 10, 2002 & B158Y4 & Arsenic & $\mathrm{mg} / \mathrm{L}$ & 1 & 6.01E-04 & $=$ & 0.15 & 1.80E-05 \\
\hline Columbia River & RICHPS7 HRM46.4 & June 20,2000 & B0YCK1 & Arsenic & $\mathrm{mg} / \mathrm{L}$ & 1 & $6.01 \mathrm{E}-04$ & $=$ & 0.15 & 1.80E-05 \\
\hline Columbia River & HFD TS2 HRM28.7 & September 21, 1999 & B0WB89 & Arsenic & $\mathrm{mg} / \mathrm{L}$ & 1 & 6.02E-04 & $=$ & 0.15 & 1.80E-05 \\
\hline Columbia River & RICHPS HRM 43.5 & June 12, 2001 & B12538 & Arsenic & $\mathrm{mg} / \mathrm{L}$ & 1 & 6.02E-04 & $=$ & 0.15 & $1.80 \mathrm{E}-05$ \\
\hline Columbia River & RICHPS HRM 45.0 & June 20, 2000 & B0YCK8 & Arsenic & $\mathrm{mg} / \mathrm{L}$ & 1 & 6.02E-04 & $=$ & 0.15 & 1.80E-05 \\
\hline Columbia River & RICHPS HRM 45.0 & December 10, 2002 & B16371 & Arsenic & $\mathrm{mg} / \mathrm{L}$ & 1 & 6.02E-04 & $=$ & 0.15 & 1.80E-05 \\
\hline Columbia River & RICHPS HRM 45.8 & December 10, 2002 & B16370 & Arsenic & $\mathrm{mg} / \mathrm{L}$ & 1 & 6.02E-04 & $=$ & 0.15 & 1.80E-05 \\
\hline Columbia River & RICHPS2 HRM46.4 & March 25, 2003 & B16LC2 & Arsenic & $\mathrm{mg} / \mathrm{L}$ & 1 & 6.02E-04 & C & 0.15 & $1.80 \mathrm{E}-05$ \\
\hline Columbia River & RICHPS4 HRM46.4 & June 14, 1999 & B0V214 & Arsenic & $\mathrm{mg} / \mathrm{L}$ & 1 & 6.03E-04 & $=$ & 0.15 & 1.80E-05 \\
\hline Columbia River & RICHPS5 HRM46.4 & June 14, 1999 & B0V216 & Arsenic & $\mathrm{mg} / \mathrm{L}$ & 1 & 6.04E-04 & $=$ & 0.15 & 1.80E-05 \\
\hline Columbia River & 300 A7 HRM 43.1 & September 10, 2002 & B158C0 & Arsenic & $\mathrm{mg} / \mathrm{L}$ & 1 & $6.04 \mathrm{E}-04$ & $=$ & 0.15 & 1.80E-05 \\
\hline Columbia River & RICHPS HRM 45.8 & March 28, 2000 & B0XRP6 & Arsenic & $\mathrm{mg} / \mathrm{L}$ & 1 & 6.04E-04 & $=$ & 0.15 & 1.80E-05 \\
\hline Columbia River & 100 F-1 HRM19.0 & September 9, 2002 & B158D8 & Arsenic & $\mathrm{mg} / \mathrm{L}$ & 1 & 6.05E-04 & $=$ & 0.15 & $1.80 \mathrm{E}-05$ \\
\hline Columbia River & HFD TS HRM 26 & September 14, 2004 & B1B744 & Arsenic & $\mathrm{mg} / \mathrm{L}$ & 1 & 6.05E-04 & $\mathrm{X}$ & 0.15 & 1.80E-05 \\
\hline Columbia River & RICHPS7 HRM46.4 & March 25, 2003 & B16L93 & Arsenic & $\mathrm{mg} / \mathrm{L}$ & 1 & $6.05 E-04$ & C & 0.15 & 1.80E-05 \\
\hline Columbia River & RICHPS2 HRM46.4 & September 16, 1999 & B0WB78 & Arsenic & $\mathrm{mg} / \mathrm{L}$ & 1 & 6.06E-04 & $=$ & 0.15 & 1.80E-05 \\
\hline Columbia River & 100 F-5 HRM19.0 & September 9, 2002 & B158F1 & Arsenic & $\mathrm{mg} / \mathrm{L}$ & 1 & 6.06E-04 & $=$ & 0.15 & $1.80 \mathrm{E}-05$ \\
\hline Columbia River & 100 N-10HRM 9.5 & September 5, 2002 & B158R1 & Arsenic & $\mathrm{mg} / \mathrm{L}$ & 1 & 6.06E-04 & $=$ & 0.15 & 1.80E-05 \\
\hline Columbia River & HFD TS3 HRM28.7 & September 14, 2004 & B1B771 & Arsenic & $\mathrm{mg} / \mathrm{L}$ & 1 & 6.07E-04 & $\mathrm{x}$ & 0.15 & 1.80E-05 \\
\hline Columbia River & RICHPS HRM 43.9 & June 20, 2000 & BOYCK9 & Arsenic & $\mathrm{mg} / \mathrm{L}$ & 1 & 6.07E-04 & $=$ & 0.15 & 1.80E-05 \\
\hline Columbia River & 300 A5 HRM 43.1 & September 16, 1999 & B0WB86 & Arsenic & $\mathrm{mg} / \mathrm{L}$ & 1 & $6.08 \mathrm{E}-04$ & $=$ & 0.15 & 1.80E-05 \\
\hline Columbia River & 100 N-7 HRM 9.5 & September 5, 2002 & B158R0 & Arsenic & $\mathrm{mg} / \mathrm{L}$ & 1 & 6.08E-04 & $=$ & 0.15 & $1.80 \mathrm{E}-05$ \\
\hline Columbia River & 100 N-2 HRM 9.5 & September 5, 2002 & B158P7 & Arsenic & $\mathrm{mg} / \mathrm{L}$ & 1 & 6.09E-04 & $=$ & 0.15 & 1.80E-05 \\
\hline Columbia River & HFD TS HRM 30 & September 18, 2000 & B10733 & Arsenic & $\mathrm{mg} / \mathrm{L}$ & 1 & 6.09E-04 & $=$ & 0.15 & 1.80E-05 \\
\hline Columbia River & HFD TS2 HRM28.7 & September 8, 2003 & B17CP6 & Arsenic & $\mathrm{mg} / \mathrm{L}$ & 1 & 6.09E-04 & $=$ & 0.15 & 1.80E-05 \\
\hline Columbia River & RICHPS5 HRM46.4 & June 12, 2001 & B12528 & Arsenic & $\mathrm{mg} / \mathrm{L}$ & 1 & 6.09E-04 & $=$ & 0.15 & $1.80 \mathrm{E}-05$ \\
\hline Columbia River & HFD TS2 HRM28.7 & September 9, 2002 & B158C4 & Arsenic & $\mathrm{mg} / \mathrm{L}$ & 1 & 6.10E-04 & $=$ & 0.15 & 1.80E-05 \\
\hline Columbia River & HFD TS2 HRM28.7 & September 14, 2004 & B1B770 & Arsenic & $\mathrm{mg} / \mathrm{L}$ & 1 & 6.11E-04 & $\mathrm{x}$ & 0.15 & 1.80E-05 \\
\hline Columbia River & HFD TS3 HRM28.7 & September 9, 2002 & В158T6 & Arsenic & $\mathrm{mg} / \mathrm{L}$ & 1 & $6.11 \mathrm{E}-04$ & $=$ & 0.15 & 1.80E-05 \\
\hline Columbia River & 100 N-5 HRM 9.5 & September 5, 2002 & B158P9 & Arsenic & $\mathrm{mg} / \mathrm{L}$ & 1 & $6.12 \mathrm{E}-04$ & $=$ & 0.15 & 1.80E-05 \\
\hline Columbia River & RICHPS5 HRM46.4 & December 8, 2003 & B183R2 & Arsenic & $\mathrm{mg} / \mathrm{L}$ & 1 & $6.12 \mathrm{E}-04$ & $=$ & 0.15 & 1.80E-05 \\
\hline Columbia River & RICHPS3 HRM46.4 & June 10, 2003 & B171Y7 & Arsenic & $\mathrm{mg} / \mathrm{L}$ & 1 & 6.13E-04 & C & 0.15 & 1.80E-05 \\
\hline Columbia River & HFD TS HRM 30 & September 21, 1999 & B0WB76 & Arsenic & $\mathrm{mg} / \mathrm{L}$ & 1 & 6.14E-04 & $=$ & 0.15 & $1.80 \mathrm{E}-05$ \\
\hline
\end{tabular}




\section{Table B-10}

Summary of Surface Water Results From Segment 2 Exceeding Select Ecological or Human Health Benchmark Values

Columbia River Component - Existing Sample Data Evaluation and Scoping Report

\begin{tabular}{|c|c|c|c|c|c|c|c|c|c|c|}
\hline Sample Area & Sample Site & Sample ID & Date Collected & Constituent Name & Units & QA Category & $\begin{array}{l}\text { Analytical } \\
\text { Value }\end{array}$ & Qualifier & $\begin{array}{l}\text { Ecological } \\
\text { Benchmark } \\
\text { Value }\end{array}$ & $\begin{array}{c}\text { Human Health } \\
\text { Benchmark Value }\end{array}$ \\
\hline Columbia River & RICHPS HRM 45.8 & March 25, 2003 & B16LD5 & Arsenic & $\mathrm{mg} / \mathrm{L}$ & 1 & $6.14 \mathrm{E}-04$ & C & 0.15 & 1.80E-05 \\
\hline Columbia River & $100 \mathrm{H}$ Area & June 10, 2002 & B14PW9 & Arsenic & $\mathrm{mg} / \mathrm{L}$ & 2 & $6.15 \mathrm{E}-04$ & $=$ & 0.15 & 1.80E-05 \\
\hline Columbia River & RICHPS1 HRM46.4 & March 25, 2003 & B16L89 & Arsenic & $\mathrm{mg} / \mathrm{L}$ & 1 & 6.15E-04 & C & 0.15 & 1.80E-05 \\
\hline Columbia River & RICHPS7 HRM46.4 & June 11, 2002 & B14RX2 & Arsenic & $\mathrm{mg} / \mathrm{L}$ & 1 & 6.15E-04 & $x$ & 0.15 & 1.80E-05 \\
\hline Columbia River & 100 NSHR HRM8.4 & September 5, 2002 & B158X0 & Arsenic & $\mathrm{mg} / \mathrm{L}$ & 1 & $6.16 \mathrm{E}-04$ & $=$ & 0.15 & 1.80E-05 \\
\hline Columbia River & RICHPS HRM 45.8 & December 4, 2001 & B13LJ7 & Arsenic & $\mathrm{mg} / \mathrm{L}$ & 1 & $6.16 \mathrm{E}-04$ & $=$ & 0.15 & 1.80E-05 \\
\hline Columbia River & RICHPS HRM 43.9 & December 4, 2001 & B13LJ9 & Arsenic & $\mathrm{mg} / \mathrm{L}$ & 1 & 6.17E-04 & $=$ & 0.15 & $1.80 \mathrm{E}-05$ \\
\hline Columbia River & RICHPS HRM 45.8 & December 8, 2003 & B183W1 & Arsenic & $\mathrm{mg} / \mathrm{L}$ & 1 & 6.17E-04 & C & 0.15 & 1.80E-05 \\
\hline Columbia River & 100 NSHR HRM8.9 & September 10, 1999 & Bow966 & Arsenic & $\mathrm{mg} / \mathrm{L}$ & 1 & $6.18 \mathrm{E}-04$ & $=$ & 0.15 & 1.80E-05 \\
\hline Columbia River & 300 SR HRM 42.9 & September 9, 2003 & B17CY0 & Arsenic & $\mathrm{mg} / \mathrm{L}$ & 1 & $6.19 \mathrm{E}-04$ & $=$ & 0.15 & 1.80E-05 \\
\hline Columbia River & RICHPS2 HRM46.4 & September 15, 2004 & B1B758 & Arsenic & $\mathrm{mg} / \mathrm{L}$ & 1 & 6.19E-04 & $\mathrm{X}$ & 0.15 & $1.80 \mathrm{E}-05$ \\
\hline Columbia River & RICHPS3 HRM46.4 & June 20, 2000 & B0YCJ9 & Arsenic & $\mathrm{mg} / \mathrm{L}$ & 1 & 6.19E-04 & $=$ & 0.15 & 1.80E-05 \\
\hline Columbia River & HFD TS HRM 27 & September 14, 2004 & B1B745 & Arsenic & $\mathrm{mg} / \mathrm{L}$ & 1 & $6.20 \mathrm{E}-04$ & $x$ & 0.15 & 1.80E-05 \\
\hline Columbia River & RICHPS1 HRM46.4 & June 20,2000 & B0YCJ7 & Arsenic & $\mathrm{mg} / \mathrm{L}$ & 1 & $6.20 \mathrm{E}-04$ & $=$ & 0.15 & 1.80E-05 \\
\hline Columbia River & RICHPS10HRM46.4 & December 8, 2003 & B183V6 & Arsenic & $\mathrm{mg} / \mathrm{L}$ & 1 & $6.20 \mathrm{E}-04$ & C & 0.15 & 1.80E-05 \\
\hline Columbia River & RICHPS3 HRM46.4 & December 4, 2001 & B13LH9 & Arsenic & $\mathrm{mg} / \mathrm{L}$ & 1 & $6.20 \mathrm{E}-04$ & $=$ & 0.15 & $1.80 \mathrm{E}-05$ \\
\hline Columbia River & HFD TS7 HRM28.7 & September 14, 2004 & B1B773 & Arsenic & $\mathrm{mg} / \mathrm{L}$ & 1 & $6.21 \mathrm{E}-04$ & $x$ & 0.15 & 1.80E-05 \\
\hline Columbia River & RICHPS HRM 43.5 & June 20, 2000 & BOYCLO & Arsenic & $\mathrm{mg} / \mathrm{L}$ & 1 & 6.21E-04 & $=$ & 0.15 & 1.80E-05 \\
\hline Columbia River & RICHPS HRM 45.8 & June 20,2000 & BOYCK7 & Arsenic & $\mathrm{mg} / \mathrm{L}$ & 1 & $6.21 \mathrm{E}-04$ & $=$ & 0.15 & 1.80E-05 \\
\hline Columbia River & 100D SPR 110-1 & October 27, 2003 & B17J91 & Arsenic & $\mathrm{mg} / \mathrm{L}$ & 1 & $6.22 \mathrm{E}-04$ & $=$ & 0.15 & $1.80 \mathrm{E}-05$ \\
\hline Columbia River & 100 N-1 HRM 9.5 & September 5, 2002 & B158P6 & Arsenic & $\mathrm{mg} / \mathrm{L}$ & 1 & 6.23E-04 & $=$ & 0.15 & 1.80E-05 \\
\hline Columbia River & RICHPS HRM 43.5 & June 10, 2003 & B17208 & Arsenic & $\mathrm{mg} / \mathrm{L}$ & 1 & $6.23 \mathrm{E}-04$ & C & 0.15 & 1.80E-05 \\
\hline Columbia River & 100 N-2 HRM 9.5 & September 13, 2004 & B1B752 & Arsenic & $\mathrm{mg} / \mathrm{L}$ & 1 & $6.24 \mathrm{E}-04$ & $x$ & 0.15 & 1.80E-05 \\
\hline Columbia River & HFD TS HRM 27 & September 14, 2004 & B1B742 & Arsenic & $\mathrm{mg} / \mathrm{L}$ & 1 & $6.24 \mathrm{E}-04$ & $x$ & 0.15 & 1.80E-05 \\
\hline Columbia River & HFD TS3 HRM28.7 & September 14, 2004 & B1B6W2 & Arsenic & $\mathrm{mg} / \mathrm{L}$ & 1 & $6.24 \mathrm{E}-04$ & $x$ & 0.15 & 1.80E-05 \\
\hline Columbia River & RICHPS2 HRM46.4 & September 9, 2003 & B17CD1 & Arsenic & $\mathrm{mg} / \mathrm{L}$ & 1 & $6.24 \mathrm{E}-04$ & $=$ & 0.15 & 1.80E-05 \\
\hline Columbia River & 100 N-3 HRM 9.5 & September 10, 1999 & Bow975 & Arsenic & $\mathrm{mg} / \mathrm{L}$ & 1 & $6.24 \mathrm{E}-04$ & $=$ & 0.15 & 1.80E-05 \\
\hline Columbia River & 100 F-5 HRM19.0 & September 8, 2003 & B17CR4 & Arsenic & $\mathrm{mg} / \mathrm{L}$ & 1 & $6.25 \mathrm{E}-04$ & $=$ & 0.15 & 1.80E-05 \\
\hline Columbia River & 100 FSHR HRM18 & September 8, 2003 & B17CY5 & Arsenic & $\mathrm{mg} / \mathrm{L}$ & 1 & $6.25 \mathrm{E}-04$ & $=$ & 0.15 & $1.80 \mathrm{E}-05$ \\
\hline Columbia River & 100 N-3 HRM 9.5 & September 5, 2002 & B158D4 & Arsenic & $\mathrm{mg} / \mathrm{L}$ & 1 & $6.25 \mathrm{E}-04$ & $=$ & 0.15 & 1.80E-05 \\
\hline Columbia River & HFD TS HRM 30 & September 8, 2003 & B17CM2 & Arsenic & $\mathrm{mg} / \mathrm{L}$ & 1 & $6.25 \mathrm{E}-04$ & $=$ & 0.15 & 1.80E-05 \\
\hline Columbia River & HFD TS10HRM28.7 & September 8, 2003 & B17CB2 & Arsenic & $\mathrm{mg} / \mathrm{L}$ & 1 & $6.25 \mathrm{E}-04$ & $=$ & 0.15 & 1.80E-05 \\
\hline Columbia River & RICHPS2 HRM46.4 & December 4, 2001 & B13LD6 & Arsenic & $\mathrm{mg} / \mathrm{L}$ & 1 & $6.25 \mathrm{E}-04$ & $=$ & 0.15 & 1.80E-05 \\
\hline Columbia River & 100 NSHR HRM9.2 & September 5, 2002 & B158X6 & Arsenic & $\mathrm{mg} / \mathrm{L}$ & 1 & $6.26 \mathrm{E}-04$ & $=$ & 0.15 & 1.80E-05 \\
\hline Columbia River & RICHPS HRM 43.9 & June 11, 2002 & B14RY0 & Arsenic & $\mathrm{mg} / \mathrm{L}$ & 1 & 6.26E-04 & $x$ & 0.15 & 1.80E-05 \\
\hline Columbia River & 100 NSHR HRM9.8 & September 5, 2002 & B158X8 & Arsenic & $\mathrm{mg} / \mathrm{L}$ & 1 & 6.27E-04 & $=$ & 0.15 & 1.80E-05 \\
\hline Columbia River & HFD TS HRM 30 & September 14, 2004 & B1B746 & Arsenic & $\mathrm{mg} / \mathrm{L}$ & 1 & 6.27E-04 & $\mathrm{x}$ & 0.15 & 1.80E-05 \\
\hline Columbia River & RICHPS HRM 45.0 & December 4, 2001 & B13LJ8 & Arsenic & $\mathrm{mg} / \mathrm{L}$ & 1 & $6.27 \mathrm{E}-04$ & $=$ & 0.15 & $1.80 \mathrm{E}-05$ \\
\hline Columbia River & HFD TS HRM 29 & September 21, 1999 & B0WB75 & Arsenic & $\mathrm{mg} / \mathrm{L}$ & 1 & 6.27E-04 & $=$ & 0.15 & 1.80E-05 \\
\hline Columbia River & 100 N-7 HRM 9.5 & September 5, 2002 & B158D6 & Arsenic & $\mathrm{mg} / \mathrm{L}$ & 1 & $6.28 \mathrm{E}-04$ & $=$ & 0.15 & 1.80E-05 \\
\hline Columbia River & RICHPS HRM 43.9 & December 10, 2002 & B16372 & Arsenic & $\mathrm{mg} / \mathrm{L}$ & 1 & $6.28 \mathrm{E}-04$ & $=$ & 0.15 & 1.80E-05 \\
\hline Columbia River & 100 F-10HRM19.0 & September 8, 2003 & B17C96 & Arsenic & $\mathrm{mg} / \mathrm{L}$ & 1 & $6.29 \mathrm{E}-04$ & $=$ & 0.15 & 1.80E-05 \\
\hline Columbia River & 100 NSHR HRM8.9 & September 13, 2004 & B1B7B0 & Arsenic & $\mathrm{mg} / \mathrm{L}$ & 1 & $6.29 \mathrm{E}-04$ & $x$ & 0.15 & 1.80E-05 \\
\hline Columbia River & 100-B SPR 39-2 & October 11, 2004 & B1BFV7 & Arsenic & $\mathrm{mg} / \mathrm{L}$ & 1 & $6.29 \mathrm{E}-04$ & $x$ & 0.15 & 1.80E-05 \\
\hline Columbia River & RICHPS7 HRM46.4 & December 4, 2001 & B13LD9 & Arsenic & $\mathrm{mg} / \mathrm{L}$ & 1 & $6.29 \mathrm{E}-04$ & $=$ & 0.15 & $1.80 \mathrm{E}-05$ \\
\hline
\end{tabular}




\section{Table B-10}

Summary of Surface Water Results From Segment 2 Exceeding Select Ecological or Human Health Benchmark Values

Columbia River Component - Existing Sample Data Evaluation and Scoping Report

\begin{tabular}{|c|c|c|c|c|c|c|c|c|c|c|}
\hline Sample Area & Sample Site & Sample ID & Date Collected & Constituent Name & Units & QA Category & $\begin{array}{l}\text { Analytical } \\
\text { Value }\end{array}$ & Qualifier & $\begin{array}{l}\text { Ecological } \\
\text { Benchmark } \\
\text { Value }\end{array}$ & $\begin{array}{c}\text { Human Health } \\
\text { Benchmark Value }\end{array}$ \\
\hline Columbia River & 100 FSHR HRM23 & September 14, 2004 & B1B7F6 & Arsenic & $\mathrm{mg} / \mathrm{L}$ & 1 & $6.30 \mathrm{E}-04$ & $\bar{x}$ & 0.15 & 1.80E-05 \\
\hline Columbia River & 100 N-1 HRM 9.5 & September 4, 2003 & B17CB6 & Arsenic & $\mathrm{mg} / \mathrm{L}$ & 1 & $6.30 \mathrm{E}-04$ & $=$ & 0.15 & 1.80E-05 \\
\hline Columbia River & HFD TS HRM 27 & September 8, 2003 & B17CL8 & Arsenic & $\mathrm{mg} / \mathrm{L}$ & 1 & $6.30 \mathrm{E}-04$ & $=$ & 0.15 & 1.80E-05 \\
\hline Columbia River & HFD TS3 HRM28.7 & September 9, 2002 & B158C5 & Arsenic & $\mathrm{mg} / \mathrm{L}$ & 1 & 6.30E-04 & $=$ & 0.15 & 1.80E-05 \\
\hline Columbia River & 100 F-2 HRM19.0 & September 14, 2004 & B1B776 & Arsenic & $\mathrm{mg} / \mathrm{L}$ & 1 & $6.31 \mathrm{E}-04$ & $x$ & 0.15 & 1.80E-05 \\
\hline Columbia River & 100 N-3 HRM 9.5 & September 5, 2002 & B158P8 & Arsenic & $\mathrm{mg} / \mathrm{L}$ & 1 & $6.31 \mathrm{E}-04$ & $=$ & 0.15 & 1.80E-05 \\
\hline Columbia River & 100 NSHR HRM9.2 & September 5, 2002 & B158X5 & Arsenic & $\mathrm{mg} / \mathrm{L}$ & 1 & 6.31E-04 & $=$ & 0.15 & $1.80 \mathrm{E}-05$ \\
\hline Columbia River & 100D Area & June 10, 2002 & B14PW8 & Arsenic & $\mathrm{mg} / \mathrm{L}$ & 2 & 6.32E-04 & $=$ & 0.15 & 1.80E-05 \\
\hline Columbia River & RICHPS3 HRM46.4 & September 9, 2003 & B17CD2 & Arsenic & $\mathrm{mg} / \mathrm{L}$ & 1 & $6.32 \mathrm{E}-04$ & $=$ & 0.15 & 1.80E-05 \\
\hline Columbia River & RICHPS7 HRM46.4 & June 12, 2001 & B12529 & Arsenic & $\mathrm{mg} / \mathrm{L}$ & 1 & $6.32 \mathrm{E}-04$ & $=$ & 0.15 & 1.80E-05 \\
\hline Columbia River & 100-H SPR 153-1 & October 27, 1999 & B0WP45 & Arsenic & $\mathrm{mg} / \mathrm{L}$ & 1 & 6.32E-04 & $=$ & 0.15 & $1.80 \mathrm{E}-05$ \\
\hline Columbia River & HFD TS HRM 26 & September 14, 2004 & B1B741 & Arsenic & $\mathrm{mg} / \mathrm{L}$ & 1 & 6.33E-04 & $\mathrm{X}$ & 0.15 & 1.80E-05 \\
\hline Columbia River & HFD TS5 HRM28.7 & September 14, 2004 & B1B6W3 & Arsenic & $\mathrm{mg} / \mathrm{L}$ & 1 & 6.33E-04 & $x$ & 0.15 & 1.80E-05 \\
\hline Columbia River & 100 N-5 HRM 9.5 & September 7, 2001 & В12ТJ3 & Arsenic & $\mathrm{mg} / \mathrm{L}$ & 1 & $6.34 \mathrm{E}-04$ & $=$ & 0.15 & 1.80E-05 \\
\hline Columbia River & HFD TS3 HRM28.7 & September 21, 1999 & B0WB91 & Arsenic & $\mathrm{mg} / \mathrm{L}$ & 1 & $6.35 \mathrm{E}-04$ & $=$ & 0.15 & 1.80E-05 \\
\hline Columbia River & 300 SR HRM 41.5 & September 16, 1999 & B0WB61 & Arsenic & $\mathrm{mg} / \mathrm{L}$ & 1 & 6.35E-04 & $=$ & 0.15 & $1.80 \mathrm{E}-05$ \\
\hline Columbia River & 100 FSHR HRM18 & September 14, 2004 & B1B7D7 & Arsenic & $\mathrm{mg} / \mathrm{L}$ & 1 & $6.35 E-04$ & $x$ & 0.15 & 1.80E-05 \\
\hline Columbia River & 100 NSHR HRM8.9 & September 5, 2002 & B158X3 & Arsenic & $\mathrm{mg} / \mathrm{L}$ & 1 & 6.35E-04 & $=$ & 0.15 & 1.80E-05 \\
\hline Columbia River & HFD TS10HRM28.7 & September 14, 2004 & B1B774 & Arsenic & $\mathrm{mg} / \mathrm{L}$ & 1 & $6.35 \mathrm{E}-04$ & $\mathrm{x}$ & 0.15 & 1.80E-05 \\
\hline Columbia River & RICHPS HRM 43.5 & December 4, 2001 & B13LK4 & Arsenic & $\mathrm{mg} / \mathrm{L}$ & 1 & 6.35E-04 & $=$ & 0.15 & $1.80 \mathrm{E}-05$ \\
\hline Columbia River & RICHPS5 HRM46.4 & September 15, 2004 & B1B760 & Arsenic & $\mathrm{mg} / \mathrm{L}$ & 1 & 6.35E-04 & $\mathrm{X}$ & 0.15 & 1.80E-05 \\
\hline Columbia River & 100 N-2 HRM 9.5 & September 5, 2002 & B158D3 & Arsenic & $\mathrm{mg} / \mathrm{L}$ & 1 & 6.36E-04 & $=$ & 0.15 & 1.80E-05 \\
\hline Columbia River & 100 N-5 HRM 9.5 & September 5, 2002 & B158D5 & Arsenic & $\mathrm{mg} / \mathrm{L}$ & 1 & $6.36 \mathrm{E}-04$ & $=$ & 0.15 & 1.80E-05 \\
\hline Columbia River & 100 NSHR HRM9.8 & September 5, 2002 & B158X9 & Arsenic & $\mathrm{mg} / \mathrm{L}$ & 1 & 6.36E-04 & $=$ & 0.15 & 1.80E-05 \\
\hline Columbia River & HFD TS5 HRM28.7 & September 14, 2004 & B1B772 & Arsenic & $\mathrm{mg} / \mathrm{L}$ & 1 & $6.36 \mathrm{E}-04$ & $x$ & 0.15 & 1.80E-05 \\
\hline Columbia River & RICHPS HRM 43.9 & June 10, 2003 & B17207 & Arsenic & $\mathrm{mg} / \mathrm{L}$ & 1 & 6.36E-04 & C & 0.15 & 1.80E-05 \\
\hline Columbia River & RICHPS HRM 45.8 & June 12, 2001 & B12539 & Arsenic & $\mathrm{mg} / \mathrm{L}$ & 1 & 6.36E-04 & $=$ & 0.15 & 1.80E-05 \\
\hline Columbia River & 100 NSHR HRM8.4 & September 13, 2004 & B1B796 & Arsenic & $\mathrm{mg} / \mathrm{L}$ & 1 & 6.37E-04 & $\mathrm{x}$ & 0.15 & 1.80E-05 \\
\hline Columbia River & HFD TS HRM 30 & September 8, 2003 & B17CL9 & Arsenic & $\mathrm{mg} / \mathrm{L}$ & 1 & 6.37E-04 & $=$ & 0.15 & $1.80 \mathrm{E}-05$ \\
\hline Columbia River & 100 NSHR HRM9.2 & September 13, 2004 & B1B7B4 & Arsenic & $\mathrm{mg} / \mathrm{L}$ & 1 & 6.38E-04 & $\mathrm{X}$ & 0.15 & 1.80E-05 \\
\hline Columbia River & RICHPS5 HRM46.4 & December 4, 2001 & B13LD8 & Arsenic & $\mathrm{mg} / \mathrm{L}$ & 1 & 6.38E-04 & $=$ & 0.15 & 1.80E-05 \\
\hline Columbia River & 100 N-3 HRM 9.5 & September 13, 2004 & B1B753 & Arsenic & $\mathrm{mg} / \mathrm{L}$ & 1 & 6.39E-04 & $x$ & 0.15 & 1.80E-05 \\
\hline Columbia River & 100 N-7 HRM 9.5 & September 13, 2004 & B1B755 & Arsenic & $\mathrm{mg} / \mathrm{L}$ & 1 & 6.39E-04 & $x$ & 0.15 & 1.80E-05 \\
\hline Columbia River & HFD TS HRM 30 & September 14, 2004 & B1B743 & Arsenic & $\mathrm{mg} / \mathrm{L}$ & 1 & 6.39E-04 & $\mathrm{X}$ & 0.15 & $1.80 \mathrm{E}-05$ \\
\hline Columbia River & 100-B SPR 39-2 & November 17, 2003 & B180H8 & Arsenic & $\mathrm{mg} / \mathrm{L}$ & 1 & $6.40 \mathrm{E}-04$ & $=$ & 0.15 & 1.80E-05 \\
\hline Columbia River & RICHPS5 HRM46.4 & June 20,2000 & B0YCD2 & Arsenic & $\mathrm{mg} / \mathrm{L}$ & 1 & 6.40E-04 & $=$ & 0.15 & 1.80E-05 \\
\hline Columbia River & 300 A7 HRM 43.1 & September 16, 1999 & B0WB87 & Arsenic & $\mathrm{mg} / \mathrm{L}$ & 1 & $6.40 \mathrm{E}-04$ & $=$ & 0.15 & 1.80E-05 \\
\hline Columbia River & 100 NSHR HRM8.9 & September 13, 2004 & B1B799 & Arsenic & $\mathrm{mg} / \mathrm{L}$ & 1 & $6.41 \mathrm{E}-04$ & $\mathrm{X}$ & 0.15 & $1.80 \mathrm{E}-05$ \\
\hline Columbia River & HFD TS2 HRM28.7 & September 14, 2004 & B1B6W1 & Arsenic & $\mathrm{mg} / \mathrm{L}$ & 1 & 6.41E-04 & $\mathrm{x}$ & 0.15 & 1.80E-05 \\
\hline Columbia River & RICHPS HRM 43.9 & September 13, 2001 & B12TN0 & Arsenic & $\mathrm{mg} / \mathrm{L}$ & 1 & 6.41E-04 & $=$ & 0.15 & 1.80E-05 \\
\hline Columbia River & 100 N-10HRM 9.5 & September 5, 2002 & B158D7 & Arsenic & $\mathrm{mg} / \mathrm{L}$ & 1 & $6.42 \mathrm{E}-04$ & $=$ & 0.15 & 1.80E-05 \\
\hline Columbia River & HFD TS10HRM28.7 & September 9, 2002 & B158C8 & Arsenic & $\mathrm{mg} / \mathrm{L}$ & 1 & $6.42 \mathrm{E}-04$ & $=$ & 0.15 & 1.80E-05 \\
\hline Columbia River & HFD TS7 HRM28.7 & September 9, 2002 & B158C7 & Arsenic & $\mathrm{mg} / \mathrm{L}$ & 1 & $6.42 \mathrm{E}-04$ & $=$ & 0.15 & 1.80E-05 \\
\hline Columbia River & RICHPS3 HRM46.4 & June 20, 2000 & B0YCD1 & Arsenic & $\mathrm{mg} / \mathrm{L}$ & 1 & 6.43E-04 & $=$ & 0.15 & 1.80E-05 \\
\hline Columbia River & RICHPS3 HRM46.4 & September 15, 2004 & B1B759 & Arsenic & $\mathrm{mg} / \mathrm{L}$ & 1 & 6.43E-04 & $\mathrm{X}$ & 0.15 & $1.80 \mathrm{E}-05$ \\
\hline
\end{tabular}

117 of 137 


\section{Table B-10}

Summary of Surface Water Results From Segment 2 Exceeding Select Ecological or Human Health Benchmark Values

Columbia River Component - Existing Sample Data Evaluation and Scoping Report

\begin{tabular}{|c|c|c|c|c|c|c|c|c|c|c|}
\hline Sample Area & Sample Site & Sample ID & Date Collected & Constituent Name & Units & QA Category & $\begin{array}{l}\text { Analytical } \\
\text { Value }\end{array}$ & Qualifier & $\begin{array}{l}\text { Ecological } \\
\text { Benchmark } \\
\text { Value }\end{array}$ & $\begin{array}{c}\text { Human Health } \\
\text { Benchmark Value }\end{array}$ \\
\hline Columbia River & HFD TS HRM 27 & September 10, 2001 & B12TH1 & Arsenic & $\mathrm{mg} / \mathrm{L}$ & 1 & $6.44 \mathrm{E}-04$ & $=$ & 0.15 & 1.80E-05 \\
\hline Columbia River & 100 NSHR HRM8.4 & September 5, 2002 & B158W9 & Arsenic & $\mathrm{mg} / \mathrm{L}$ & 1 & $6.45 \mathrm{E}-04$ & $=$ & 0.15 & 1.80E-05 \\
\hline Columbia River & RICHPS3 HRM46.4 & December 8, 2003 & B183R1 & Arsenic & $\mathrm{mg} / \mathrm{L}$ & 1 & $6.45 \mathrm{E}-04$ & $=$ & 0.15 & 1.80E-05 \\
\hline Columbia River & HFD TS1 HRM28.7 & September 8, 2003 & B17CP5 & Arsenic & $\mathrm{mg} / \mathrm{L}$ & 1 & 6.46E-04 & $=$ & 0.15 & 1.80E-05 \\
\hline Columbia River & HFD TS HRM 28 & September 14, 2004 & B1B7D3 & Arsenic & $\mathrm{mg} / \mathrm{L}$ & 1 & $6.47 \mathrm{E}-04$ & $x$ & 0.15 & 1.80E-05 \\
\hline Columbia River & 100 F-1 HRM19.0 & September 14, 2004 & B1B775 & Arsenic & $\mathrm{mg} / \mathrm{L}$ & 1 & $6.48 \mathrm{E}-04$ & $x$ & 0.15 & 1.80E-05 \\
\hline Columbia River & HFD TS1 HRM28.7 & September 14, 2004 & B1B6W0 & Arsenic & $\mathrm{mg} / \mathrm{L}$ & 1 & 6.49E-04 & $\mathrm{X}$ & 0.15 & $1.80 \mathrm{E}-05$ \\
\hline Columbia River & HFD TS1 HRM28.7 & September 14, 2004 & B1B769 & Arsenic & $\mathrm{mg} / \mathrm{L}$ & 1 & 6.49E-04 & $\mathrm{X}$ & 0.15 & 1.80E-05 \\
\hline Columbia River & RICHPS HRM 43.5 & September 9, 2003 & B17CT4 & Arsenic & $\mathrm{mg} / \mathrm{L}$ & 1 & $6.49 \mathrm{E}-04$ & $=$ & 0.15 & 1.80E-05 \\
\hline Columbia River & RICHPS2 HRM46.4 & September 9, 2003 & B17CN4 & Arsenic & $\mathrm{mg} / \mathrm{L}$ & 1 & $6.49 \mathrm{E}-04$ & $=$ & 0.15 & 1.80E-05 \\
\hline Columbia River & RICHPS7 HRM46.4 & June 10, 2003 & B171Y9 & Arsenic & $\mathrm{mg} / \mathrm{L}$ & 1 & 6.49E-04 & C & 0.15 & $1.80 \mathrm{E}-05$ \\
\hline Columbia River & 100 F-3 HRM19.0 & September 14, 2004 & B1B777 & Arsenic & $\mathrm{mg} / \mathrm{L}$ & 1 & 6.50E-04 & $\mathrm{X}$ & 0.15 & 1.80E-05 \\
\hline Columbia River & RICHPS HRM 43.5 & June 12, 2001 & B12542 & Arsenic & $\mathrm{mg} / \mathrm{L}$ & 1 & $6.50 \mathrm{E}-04$ & $=$ & 0.15 & 1.80E-05 \\
\hline Columbia River & 100 N-5 HRM 9.5 & September 13, 2004 & B1B754 & Arsenic & $\mathrm{mg} / \mathrm{L}$ & 1 & 6.51E-04 & $\mathrm{X}$ & 0.15 & 1.80E-05 \\
\hline Columbia River & HFD TS7 HRM28.7 & September 14, 2004 & B1B6W4 & Arsenic & $\mathrm{mg} / \mathrm{L}$ & 1 & 6.51E-04 & $x$ & 0.15 & 1.80E-05 \\
\hline Columbia River & RICHPS HRM 43.5 & June 20, 2000 & BOYCL4 & Arsenic & $\mathrm{mg} / \mathrm{L}$ & 1 & 6.52E-04 & $=$ & 0.15 & $1.80 \mathrm{E}-05$ \\
\hline Columbia River & RICHPS5 HRM46.4 & June 10, 2003 & B171Y8 & Arsenic & $\mathrm{mg} / \mathrm{L}$ & 1 & $6.52 \mathrm{E}-04$ & C & 0.15 & 1.80E-05 \\
\hline Columbia River & 100 N-1 HRM 9.5 & September 13, 2004 & B1B751 & Arsenic & $\mathrm{mg} / \mathrm{L}$ & 1 & 6.53E-04 & $\mathrm{x}$ & 0.15 & 1.80E-05 \\
\hline Columbia River & 300 A2 HRM 43.1 & September 13, 2001 & B12TK3 & Arsenic & $\mathrm{mg} / \mathrm{L}$ & 1 & 6.53E-04 & $=$ & 0.15 & 1.80E-05 \\
\hline Columbia River & HFD TS HRM 26 & September 9, 2002 & B158N9 & Arsenic & $\mathrm{mg} / \mathrm{L}$ & 1 & 6.53E-04 & $=$ & 0.15 & $1.80 \mathrm{E}-05$ \\
\hline Columbia River & RICHPS5 HRM46.4 & June 11, 2002 & B14RX1 & Arsenic & $\mathrm{mg} / \mathrm{L}$ & 1 & 6.54E-04 & $\mathrm{X}$ & 0.15 & 1.80E-05 \\
\hline Columbia River & RICHPS HRM 45.0 & September 13, 2001 & В12TM9 & Arsenic & $\mathrm{mg} / \mathrm{L}$ & 1 & $6.55 E-04$ & $=$ & 0.15 & 1.80E-05 \\
\hline Columbia River & 100-D SPR 110-1 & October 27, 1999 & B0WP41 & Arsenic & $\mathrm{mg} / \mathrm{L}$ & 1 & $6.55 \mathrm{E}-04$ & $=$ & 0.15 & 1.80E-05 \\
\hline Columbia River & RICHPS HRM 45.8 & June 20, 2000 & B0YCL1 & Arsenic & $\mathrm{mg} / \mathrm{L}$ & 1 & $6.56 \mathrm{E}-04$ & $=$ & 0.15 & 1.80E-05 \\
\hline Columbia River & RICHPS2 HRM46.4 & June 20, 2000 & BOYCDO & Arsenic & $\mathrm{mg} / \mathrm{L}$ & 1 & $6.56 \mathrm{E}-04$ & $=$ & 0.15 & 1.80E-05 \\
\hline Columbia River & 100 FSHR HRM23 & September 14, 2004 & B1B7F5 & Arsenic & $\mathrm{mg} / \mathrm{L}$ & 1 & 6.57E-04 & $\mathrm{X}$ & 0.15 & 1.80E-05 \\
\hline Columbia River & 300 A7 HRM 43.1 & September 9, 2003 & В17CP3 & Arsenic & $\mathrm{mg} / \mathrm{L}$ & 1 & $6.57 \mathrm{E}-04$ & $=$ & 0.15 & 1.80E-05 \\
\hline Columbia River & RICHPS HRM 43.9 & June 20, 2000 & BOYCL3 & Arsenic & $\mathrm{mg} / \mathrm{L}$ & 1 & 6.57E-04 & $=$ & 0.15 & 1.80E-05 \\
\hline Columbia River & RICHPS HRM 45.0 & June 20, 2000 & B0YCL2 & Arsenic & $\mathrm{mg} / \mathrm{L}$ & 1 & 6.57E-04 & $=$ & 0.15 & $1.80 \mathrm{E}-05$ \\
\hline Columbia River & RICHPS HRM 45.8 & June 11, 2002 & B14RX8 & Arsenic & $\mathrm{mg} / \mathrm{L}$ & 1 & 6.57E-04 & $\mathrm{X}$ & 0.15 & 1.80E-05 \\
\hline Columbia River & HFD TS2 HRM28.7 & September 9, 2002 & B158T5 & Arsenic & $\mathrm{mg} / \mathrm{L}$ & 1 & 6.58E-04 & $=$ & 0.15 & 1.80E-05 \\
\hline Columbia River & HFD TS5 HRM28.7 & September 9, 2002 & B158C6 & Arsenic & $\mathrm{mg} / \mathrm{L}$ & 1 & $6.58 \mathrm{E}-04$ & $=$ & 0.15 & 1.80E-05 \\
\hline Columbia River & RICHPS8 HRM46.4 & June 14, 1999 & B0V222 & Arsenic & $\mathrm{mg} / \mathrm{L}$ & 1 & $6.59 \mathrm{E}-04$ & $=$ & 0.15 & 1.80E-05 \\
\hline Columbia River & HFD TS HRM 26 & September 10, 2001 & B12TH0 & Arsenic & $\mathrm{mg} / \mathrm{L}$ & 1 & $6.60 \mathrm{E}-04$ & $=$ & 0.15 & $1.80 \mathrm{E}-05$ \\
\hline Columbia River & 100 F-7 HRM19.0 & September 10, 2001 & В12Т74 & Arsenic & $\mathrm{mg} / \mathrm{L}$ & 1 & 6.61E-04 & $=$ & 0.15 & 1.80E-05 \\
\hline Columbia River & 100 N-7 HRM 9.5 & September 7, 2001 & B12TJ4 & Arsenic & $\mathrm{mg} / \mathrm{L}$ & 1 & 6.61E-04 & $=$ & 0.15 & 1.80E-05 \\
\hline Columbia River & HFD TS HRM 27 & September 9, 2002 & B158P0 & Arsenic & $\mathrm{mg} / \mathrm{L}$ & 1 & 6.61E-04 & $=$ & 0.15 & 1.80E-05 \\
\hline Columbia River & RICHPS7 HRM46.4 & December 8, 2003 & B183R3 & Arsenic & $\mathrm{mg} / \mathrm{L}$ & 1 & 6.61E-04 & $=$ & 0.15 & $1.80 \mathrm{E}-05$ \\
\hline Columbia River & 100 N-1 HRM 9.5 & September 10, 1999 & BoW973 & Arsenic & $\mathrm{mg} / \mathrm{L}$ & 1 & 6.62E-04 & $=$ & 0.15 & 1.80E-05 \\
\hline Columbia River & 100 N-7 HRM 9.5 & September 13, 2004 & B1B6X3 & Arsenic & $\mathrm{mg} / \mathrm{L}$ & 1 & 6.62E-04 & $\mathrm{x}$ & 0.15 & 1.80E-05 \\
\hline Columbia River & 300 A1 HRM 43.1 & September 13, 2001 & В12Т61 & Arsenic & $\mathrm{mg} / \mathrm{L}$ & 1 & $6.62 \mathrm{E}-04$ & $=$ & 0.15 & 1.80E-05 \\
\hline Columbia River & HFD TS10HRM28.7 & September 10, 2001 & B12Т60 & Arsenic & $\mathrm{mg} / \mathrm{L}$ & 1 & $6.62 \mathrm{E}-04$ & $=$ & 0.15 & 1.80E-05 \\
\hline Columbia River & HFD TS10HRM28.7 & September 9, 2002 & B158T9 & Arsenic & $\mathrm{mg} / \mathrm{L}$ & 1 & $6.62 \mathrm{E}-04$ & $=$ & 0.15 & 1.80E-05 \\
\hline Columbia River & RICHPS HRM 45.0 & June 10, 2003 & B17206 & Arsenic & $\mathrm{mg} / \mathrm{L}$ & 1 & 6.62E-04 & C & 0.15 & 1.80E-05 \\
\hline Columbia River & 100 F-7 HRM19.0 & September 14, 2004 & B1B779 & Arsenic & $\mathrm{mg} / \mathrm{L}$ & 1 & 6.63E-04 & $\mathrm{X}$ & 0.15 & $1.80 \mathrm{E}-05$ \\
\hline
\end{tabular}

118 of 137 


\section{Table B-10}

Summary of Surface Water Results From Segment 2 Exceeding Select Ecological or Human Health Benchmark Values

Columbia River Component - Existing Sample Data Evaluation and Scoping Report

\begin{tabular}{|c|c|c|c|c|c|c|c|c|c|c|}
\hline Sample Area & Sample Site & Sample ID & Date Collected & Constituent Name & Units & QA Category & $\begin{array}{l}\text { Analytical } \\
\text { Value }\end{array}$ & Qualifier & $\begin{array}{l}\text { Ecological } \\
\text { Benchmark } \\
\text { Value }\end{array}$ & $\begin{array}{c}\text { Human Health } \\
\text { Benchmark Value }\end{array}$ \\
\hline Columbia River & RICHPS7 HRM46.4 & September 15, 2004 & B1B6Y7 & Arsenic & $\mathrm{mg} / \mathrm{L}$ & 1 & $6.63 \mathrm{E}-04$ & $\bar{x}$ & 0.15 & 1.80E-05 \\
\hline Columbia River & 100 FSHR HRM18 & September 14, 2004 & B1B7D8 & Arsenic & $\mathrm{mg} / \mathrm{L}$ & 1 & $6.64 \mathrm{E}-04$ & $\mathrm{x}$ & 0.15 & 1.80E-05 \\
\hline Columbia River & 100 NSHR HRM9.8 & September 13, 2004 & B1B7B8 & Arsenic & $\mathrm{mg} / \mathrm{L}$ & 1 & $6.64 \mathrm{E}-04$ & $x$ & 0.15 & 1.80E-05 \\
\hline Columbia River & 300 A3 HRM 43.1 & September 13, 2001 & В12Т63 & Arsenic & $\mathrm{mg} / \mathrm{L}$ & 1 & 6.64E-04 & $=$ & 0.15 & 1.80E-05 \\
\hline Columbia River & 100 N-2 HRM 9.5 & September 13, 2004 & B1B6X0 & Arsenic & $\mathrm{mg} / \mathrm{L}$ & 1 & $6.65 \mathrm{E}-04$ & $x$ & 0.15 & 1.80E-05 \\
\hline Columbia River & HFD TS HRM 27 & September 9, 2002 & B158N7 & Arsenic & $\mathrm{mg} / \mathrm{L}$ & 1 & $6.66 \mathrm{E}-04$ & $=$ & 0.15 & 1.80E-05 \\
\hline Columbia River & RICHPS1 HRM46.4 & June 10, 2003 & B171Y5 & Arsenic & $\mathrm{mg} / \mathrm{L}$ & 1 & 6.66E-04 & C & 0.15 & $1.80 \mathrm{E}-05$ \\
\hline Columbia River & 100 F-5 HRM19.0 & September 14, 2004 & B1B6X8 & Arsenic & $\mathrm{mg} / \mathrm{L}$ & 1 & 6.67E-04 & $\mathrm{X}$ & 0.15 & 1.80E-05 \\
\hline Columbia River & 100 N-1 HRM 9.5 & September 5, 2002 & B158D2 & Arsenic & $\mathrm{mg} / \mathrm{L}$ & 1 & $6.68 \mathrm{E}-04$ & $=$ & 0.15 & 1.80E-05 \\
\hline Columbia River & 100-D SPR 110-1 & October 27, 1999 & BOWP40 & Arsenic & $\mathrm{mg} / \mathrm{L}$ & 1 & $6.68 \mathrm{E}-04$ & $=$ & 0.15 & 1.80E-05 \\
\hline Columbia River & RICHPS10HRM46.4 & September 19, 2000 & B10742 & Arsenic & $\mathrm{mg} / \mathrm{L}$ & 1 & 6.68E-04 & $=$ & 0.15 & $1.80 \mathrm{E}-05$ \\
\hline Columbia River & 100 N-10HRM 9.5 & September 10, 1999 & BoW978 & Arsenic & $\mathrm{mg} / \mathrm{L}$ & 1 & 6.68E-04 & $=$ & 0.15 & 1.80E-05 \\
\hline Columbia River & HFD TS HRM 26 & September 9, 2002 & B158N6 & Arsenic & $\mathrm{mg} / \mathrm{L}$ & 1 & 6.69E-04 & $=$ & 0.15 & 1.80E-05 \\
\hline Columbia River & HFD TS HRM 27 & September 10, 2001 & B12TH4 & Arsenic & $\mathrm{mg} / \mathrm{L}$ & 1 & 6.69E-04 & $=$ & 0.15 & 1.80E-05 \\
\hline Columbia River & RICHPS1 HRM46.4 & June 20, 2000 & BOYCC9 & Arsenic & $\mathrm{mg} / \mathrm{L}$ & 1 & $6.70 \mathrm{E}-04$ & $=$ & 0.15 & 1.80E-05 \\
\hline Columbia River & RICHPS1 HRM46.4 & September 19, 2000 & B106V4 & Arsenic & $\mathrm{mg} / \mathrm{L}$ & 1 & 6.70E-04 & $=$ & 0.15 & $1.80 \mathrm{E}-05$ \\
\hline Columbia River & RICHPS10HRM46.4 & December 6, 1999 & B0X401 & Arsenic & $\mathrm{mg} / \mathrm{L}$ & 1 & $6.70 \mathrm{E}-04$ & $=$ & 0.15 & 1.80E-05 \\
\hline Columbia River & RICHPS1 HRM46.4 & June 14, 1999 & B0V208 & Arsenic & $\mathrm{mg} / \mathrm{L}$ & 1 & 6.72E-04 & $=$ & 0.15 & 1.80E-05 \\
\hline Columbia River & RICHPS2 HRM46.4 & September 15, 2004 & B1B6Y4 & Arsenic & $\mathrm{mg} / \mathrm{L}$ & 1 & $6.72 \mathrm{E}-04$ & $x$ & 0.15 & 1.80E-05 \\
\hline Columbia River & 300 SR HRM 42.9 & September 16, 1999 & B0WB64 & Arsenic & $\mathrm{mg} / \mathrm{L}$ & 1 & 6.73E-04 & $=$ & 0.15 & $1.80 \mathrm{E}-05$ \\
\hline Columbia River & RICHPS HRM 45.0 & September 9, 2003 & B17CT2 & Arsenic & $\mathrm{mg} / \mathrm{L}$ & 1 & 6.73E-04 & $=$ & 0.15 & 1.80E-05 \\
\hline Columbia River & 100 NSHR HRM9.2 & September 13, 2004 & B1B7B3 & Arsenic & $\mathrm{mg} / \mathrm{L}$ & 1 & $6.74 \mathrm{E}-04$ & $x$ & 0.15 & 1.80E-05 \\
\hline Columbia River & 300 A2 HRM 43.1 & September 13, 2001 & В12Т62 & Arsenic & $\mathrm{mg} / \mathrm{L}$ & 1 & $6.74 \mathrm{E}-04$ & $=$ & 0.15 & 1.80E-05 \\
\hline Columbia River & 300 SR HRM 42.9 & September 13, 2001 & B12TR9 & Arsenic & $\mathrm{mg} / \mathrm{L}$ & 1 & $6.74 \mathrm{E}-04$ & $=$ & 0.15 & 1.80E-05 \\
\hline Columbia River & 300 SR HRM 42.9 & September 10, 2002 & B15900 & Arsenic & $\mathrm{mg} / \mathrm{L}$ & 1 & 6.74E-04 & $=$ & 0.15 & $1.80 \mathrm{E}-05$ \\
\hline Columbia River & HFD TS7 HRM28.7 & September 10, 2001 & B12TL2 & Arsenic & $\mathrm{mg} / \mathrm{L}$ & 1 & 6.74E-04 & $=$ & 0.15 & 1.80E-05 \\
\hline Columbia River & RICHPS HRM 43.9 & September 13, 2001 & B12TM6 & Arsenic & $\mathrm{mg} / \mathrm{L}$ & 1 & $6.74 \mathrm{E}-04$ & $=$ & 0.15 & 1.80E-05 \\
\hline Columbia River & RICHPS2 HRM46.4 & June 12, 2001 & B124Y4 & Arsenic & $\mathrm{mg} / \mathrm{L}$ & 1 & $6.74 \mathrm{E}-04$ & $=$ & 0.15 & 1.80E-05 \\
\hline Columbia River & 300 A3 HRM 43.1 & September 15, 2004 & B1B765 & Arsenic & $\mathrm{mg} / \mathrm{L}$ & 1 & 6.75E-04 & $\mathrm{x}$ & 0.15 & $1.80 \mathrm{E}-05$ \\
\hline Columbia River & 100 N-1 HRM 9.5 & September 13, 2004 & B1B6W9 & Arsenic & $\mathrm{mg} / \mathrm{L}$ & 1 & 6.76E-04 & $\mathrm{X}$ & 0.15 & 1.80E-05 \\
\hline Columbia River & 100 N-3 HRM 9.5 & September 13, 2004 & B1B6X1 & Arsenic & $\mathrm{mg} / \mathrm{L}$ & 1 & 6.76E-04 & $\mathrm{x}$ & 0.15 & 1.80E-05 \\
\hline Columbia River & RICHPS3 HRM46.4 & September 15, 2004 & B1B6Y5 & Arsenic & $\mathrm{mg} / \mathrm{L}$ & 1 & $6.76 \mathrm{E}-04$ & $x$ & 0.15 & 1.80E-05 \\
\hline Columbia River & RICHPS7 HRM46.4 & June 20, 2000 & B0YCD3 & Arsenic & $\mathrm{mg} / \mathrm{L}$ & 1 & $6.76 \mathrm{E}-04$ & $=$ & 0.15 & 1.80E-05 \\
\hline Columbia River & HFD TS10HRM28.7 & September 14, 2004 & B1B6W5 & Arsenic & $\mathrm{mg} / \mathrm{L}$ & 1 & 6.77E-04 & $\mathrm{X}$ & 0.15 & $1.80 \mathrm{E}-05$ \\
\hline Columbia River & RICHPS1 HRM46.4 & September 10, 2002 & B158F6 & Arsenic & $\mathrm{mg} / \mathrm{L}$ & 1 & 6.77E-04 & $=$ & 0.15 & 1.80E-05 \\
\hline Columbia River & RICHPS1 HRM46.4 & September 9, 2003 & B17CN3 & Arsenic & $\mathrm{mg} / \mathrm{L}$ & 1 & 6.78E-04 & $=$ & 0.15 & 1.80E-05 \\
\hline Columbia River & RICHPS3 HRM46.4 & June 14, 1999 & B0V212 & Arsenic & $\mathrm{mg} / \mathrm{L}$ & 1 & 6.79E-04 & $=$ & 0.15 & 1.80E-05 \\
\hline Columbia River & RICHPS HRM 43.9 & June 12, 2001 & B12541 & Arsenic & $\mathrm{mg} / \mathrm{L}$ & 1 & 6.79E-04 & $=$ & 0.15 & $1.80 \mathrm{E}-05$ \\
\hline Columbia River & RICHPS5 HRM46.4 & June 12, 2001 & B124Y6 & Arsenic & $\mathrm{mg} / \mathrm{L}$ & 1 & 6.79E-04 & $=$ & 0.15 & 1.80E-05 \\
\hline Columbia River & RICHPS7 HRM46.4 & September 15, 2004 & B1B761 & Arsenic & $\mathrm{mg} / \mathrm{L}$ & 1 & 6.79E-04 & $\mathrm{x}$ & 0.15 & 1.80E-05 \\
\hline Columbia River & 300 A5 HRM 43.1 & September 13, 2001 & B12T51 & Arsenic & $\mathrm{mg} / \mathrm{L}$ & 1 & $6.80 \mathrm{E}-04$ & $=$ & 0.15 & 1.80E-05 \\
\hline Columbia River & RICHPS10HRM46.4 & February 26, 2001 & B11H58 & Arsenic & $\mathrm{mg} / \mathrm{L}$ & 1 & $6.80 \mathrm{E}-04$ & $=$ & 0.15 & 1.80E-05 \\
\hline Columbia River & RICHPS3 HRM46.4 & June 11, 2002 & B14RX0 & Arsenic & $\mathrm{mg} / \mathrm{L}$ & 1 & $6.80 \mathrm{E}-04$ & $x$ & 0.15 & 1.80E-05 \\
\hline Columbia River & RICHPS7 HRM46.4 & September 13, 2001 & B12TK0 & Arsenic & $\mathrm{mg} / \mathrm{L}$ & 1 & 6.80E-04 & $=$ & 0.15 & 1.80E-05 \\
\hline Columbia River & 300 SR HRM 42.5 & September 13, 2001 & B12TR4 & Arsenic & $\mathrm{mg} / \mathrm{L}$ & 1 & 6.81E-04 & $=$ & 0.15 & $1.80 \mathrm{E}-05$ \\
\hline
\end{tabular}

119 of 137 


\section{Table B-10}

Summary of Surface Water Results From Segment 2 Exceeding Select Ecological or Human Health Benchmark Values

Columbia River Component - Existing Sample Data Evaluation and Scoping Report

\begin{tabular}{|c|c|c|c|c|c|c|c|c|c|c|}
\hline Sample Area & Sample Site & Sample ID & Date Collected & Constituent Name & Units & QA Category & $\begin{array}{l}\text { Analytical } \\
\text { Value }\end{array}$ & Qualifier & $\begin{array}{l}\text { Ecological } \\
\text { Benchmark } \\
\text { Value }\end{array}$ & $\begin{array}{c}\text { Human Health } \\
\text { Benchmark Value }\end{array}$ \\
\hline Columbia River & RICHPS HRM 43.5 & September 13, 2001 & B12TN1 & Arsenic & $\mathrm{mg} / \mathrm{L}$ & 1 & $6.81 \mathrm{E}-04$ & $=$ & 0.15 & 1.80E-05 \\
\hline Columbia River & RICHPS5 HRM46.4 & September 13, 2001 & B12TJ9 & Arsenic & $\mathrm{mg} / \mathrm{L}$ & 1 & 6.81E-04 & $=$ & 0.15 & 1.80E-05 \\
\hline Columbia River & 100 F-1 HRM19.0 & September 10, 2001 & B12TL4 & Arsenic & $\mathrm{mg} / \mathrm{L}$ & 1 & $6.82 \mathrm{E}-04$ & $=$ & 0.15 & 1.80E-05 \\
\hline Columbia River & RICHPS HRM 45.8 & December 4, 2001 & B13LK1 & Arsenic & $\mathrm{mg} / \mathrm{L}$ & 1 & 6.82E-04 & $=$ & 0.15 & 1.80E-05 \\
\hline Columbia River & RICHPS5 HRM46.4 & September 15, 2004 & B1B6Y6 & Arsenic & $\mathrm{mg} / \mathrm{L}$ & 1 & $6.82 \mathrm{E}-04$ & $x$ & 0.15 & 1.80E-05 \\
\hline Columbia River & RICHPS3 HRM46.4 & September 13, 2001 & B12TJ8 & Arsenic & $\mathrm{mg} / \mathrm{L}$ & 1 & $6.83 E-04$ & $=$ & 0.15 & 1.80E-05 \\
\hline Columbia River & RICHPS10HRM46.4 & June 14, 1999 & B0V226 & Arsenic & $\mathrm{mg} / \mathrm{L}$ & 1 & 6.83E-04 & $=$ & 0.15 & $1.80 \mathrm{E}-05$ \\
\hline Columbia River & 100 N-10HRM 9.5 & September 13, 2004 & B1B756 & Arsenic & $\mathrm{mg} / \mathrm{L}$ & 1 & 6.84E-04 & $\mathrm{X}$ & 0.15 & 1.80E-05 \\
\hline Columbia River & 100 F-2 HRM19.0 & September 10, 2001 & В12Т71 & Arsenic & $\mathrm{mg} / \mathrm{L}$ & 1 & $6.85 \mathrm{E}-04$ & $=$ & 0.15 & 1.80E-05 \\
\hline Columbia River & RICHPS HRM 43.5 & June 11, 2002 & B14RY1 & Arsenic & $\mathrm{mg} / \mathrm{L}$ & 1 & $6.85 \mathrm{E}-04$ & $x$ & 0.15 & 1.80E-05 \\
\hline Columbia River & RICHPS2 HRM46.4 & June 14, 1999 & B0V210 & Arsenic & $\mathrm{mg} / \mathrm{L}$ & 1 & $6.85 \mathrm{E}-04$ & $=$ & 0.15 & $1.80 \mathrm{E}-05$ \\
\hline Columbia River & 300 SR HRM 41.5 & September 13, 2001 & B12TP6 & Arsenic & $\mathrm{mg} / \mathrm{L}$ & 1 & 6.87E-04 & $=$ & 0.15 & 1.80E-05 \\
\hline Columbia River & 300 A5 HRM 43.1 & September 15, 2004 & B1B6V6 & Arsenic & $\mathrm{mg} / \mathrm{L}$ & 1 & $6.88 \mathrm{E}-04$ & $x$ & 0.15 & 1.80E-05 \\
\hline Columbia River & RICHPS10HRM46.4 & December 5, 2000 & B11144 & Arsenic & $\mathrm{mg} / \mathrm{L}$ & 1 & $6.88 \mathrm{E}-04$ & $=$ & 0.15 & 1.80E-05 \\
\hline Columbia River & 100 F-2 HRM19.0 & September 14, 2004 & B1B6X6 & Arsenic & $\mathrm{mg} / \mathrm{L}$ & 1 & $6.90 \mathrm{E}-04$ & $x$ & 0.15 & 1.80E-05 \\
\hline Columbia River & RICHPS HRM 43.5 & September 13, 2001 & B12TM7 & Arsenic & $\mathrm{mg} / \mathrm{L}$ & 1 & 6.91E-04 & $=$ & 0.15 & $1.80 \mathrm{E}-05$ \\
\hline Columbia River & RICHPS2 HRM46.4 & June 11, 2002 & B14RW9 & Arsenic & $\mathrm{mg} / \mathrm{L}$ & 1 & 6.91E-04 & $\mathrm{X}$ & 0.15 & 1.80E-05 \\
\hline Columbia River & 100 F-1 HRM19.0 & September 14, 2004 & B1B6X5 & Arsenic & $\mathrm{mg} / \mathrm{L}$ & 1 & 6.92E-04 & $\mathrm{x}$ & 0.15 & 1.80E-05 \\
\hline Columbia River & 100 NSHR HRM9.8 & September 13, 2004 & B1B7B7 & Arsenic & $\mathrm{mg} / \mathrm{L}$ & 1 & 6.92E-04 & $x$ & 0.15 & 1.80E-05 \\
\hline Columbia River & 100-D SPR 102-1 & October 26, 2004 & B1BFX3 & Arsenic & $\mathrm{mg} / \mathrm{L}$ & 1 & 6.93E-04 & $\mathrm{X}$ & 0.15 & $1.80 \mathrm{E}-05$ \\
\hline Columbia River & 300 A5 HRM 43.1 & September 15, 2004 & B1B766 & Arsenic & $\mathrm{mg} / \mathrm{L}$ & 1 & 6.93E-04 & $\mathrm{X}$ & 0.15 & 1.80E-05 \\
\hline Columbia River & HFD TS HRM 30 & September 10, 2001 & B12TH2 & Arsenic & $\mathrm{mg} / \mathrm{L}$ & 1 & 6.93E-04 & $=$ & 0.15 & 1.80E-05 \\
\hline Columbia River & HFD TS2 HRM28.7 & September 10, 2001 & B12TK9 & Arsenic & $\mathrm{mg} / \mathrm{L}$ & 1 & 6.93E-04 & $=$ & 0.15 & 1.80E-05 \\
\hline Columbia River & RICHPS2 HRM46.4 & June 14, 1999 & B0V211 & Arsenic & $\mathrm{mg} / \mathrm{L}$ & 1 & 6.93E-04 & $=$ & 0.15 & 1.80E-05 \\
\hline Columbia River & 100 NSHR HRM8.4 & September 13, 2004 & B1B795 & Arsenic & $\mathrm{mg} / \mathrm{L}$ & 1 & 6.94E-04 & $\mathrm{X}$ & 0.15 & $1.80 \mathrm{E}-05$ \\
\hline Columbia River & 300 A5 HRM 43.1 & September 13, 2001 & B12TK5 & Arsenic & $\mathrm{mg} / \mathrm{L}$ & 1 & 6.94E-04 & $=$ & 0.15 & 1.80E-05 \\
\hline Columbia River & HFD TS HRM 28 & September 14, 2004 & B1B7D4 & Arsenic & $\mathrm{mg} / \mathrm{L}$ & 1 & 6.94E-04 & $x$ & 0.15 & 1.80E-05 \\
\hline Columbia River & RICHPS HRM 45.0 & September 13, 2001 & B12TM5 & Arsenic & $\mathrm{mg} / \mathrm{L}$ & 1 & 6.94E-04 & $=$ & 0.15 & 1.80E-05 \\
\hline Columbia River & RICHPS10HRM46.4 & December 10, 2002 & B16361 & Arsenic & $\mathrm{mg} / \mathrm{L}$ & 1 & 6.94E-04 & $=$ & 0.15 & $1.80 \mathrm{E}-05$ \\
\hline Columbia River & 100 FSHR HRM18 & September 14, 2004 & B1B6X9 & Arsenic & $\mathrm{mg} / \mathrm{L}$ & 1 & 6.95E-04 & $\mathrm{X}$ & 0.15 & 1.80E-05 \\
\hline Columbia River & RICHPS HRM 45.8 & September 13, 2001 & B12TM8 & Arsenic & $\mathrm{mg} / \mathrm{L}$ & 1 & 6.96E-04 & $=$ & 0.15 & 1.80E-05 \\
\hline Columbia River & RICHPS HRM 46.4 & June 12, 2001 & B124Y3 & Arsenic & $\mathrm{mg} / \mathrm{L}$ & 1 & 6.96E-04 & $=$ & 0.15 & 1.80E-05 \\
\hline Columbia River & HFD TS HRM 26 & September 10, 2001 & В12ТH3 & Arsenic & $\mathrm{mg} / \mathrm{L}$ & 1 & 6.97E-04 & $=$ & 0.15 & 1.80E-05 \\
\hline Columbia River & RICHPS10HRM46.4 & December 6, 1999 & B0X3W9 & Arsenic & $\mathrm{mg} / \mathrm{L}$ & 1 & 6.97E-04 & $=$ & 0.15 & $1.80 \mathrm{E}-05$ \\
\hline Columbia River & RICHPS10HRM46.4 & June 10, 2003 & B17200 & Arsenic & $\mathrm{mg} / \mathrm{L}$ & 1 & 6.97E-04 & C & 0.15 & 1.80E-05 \\
\hline Columbia River & 100 FSHR HRM22 & September 14, 2004 & B1B7F2 & Arsenic & $\mathrm{mg} / \mathrm{L}$ & 1 & 6.98E-04 & $\mathrm{X}$ & 0.15 & 1.80E-05 \\
\hline Columbia River & 100H SPR 153-1 & October 27, 2003 & B17RM0 & Arsenic & $\mathrm{mg} / \mathrm{L}$ & 1 & $6.98 \mathrm{E}-04$ & $=$ & 0.15 & 1.80E-05 \\
\hline Columbia River & RICHPS1 HRM46.4 & June 11, 2002 & B14RW8 & Arsenic & $\mathrm{mg} / \mathrm{L}$ & 1 & 7.00E-04 & $\mathrm{x}$ & 0.15 & $1.80 \mathrm{E}-05$ \\
\hline Columbia River & HFD TS5 HRM28.7 & September 10, 2001 & B12TL1 & Arsenic & $\mathrm{mg} / \mathrm{L}$ & 1 & 7.01E-04 & $=$ & 0.15 & 1.80E-05 \\
\hline Columbia River & RICHPS HRM 45.0 & June 12, 2001 & B12540 & Arsenic & $\mathrm{mg} / \mathrm{L}$ & 1 & 7.01E-04 & $=$ & 0.15 & 1.80E-05 \\
\hline Columbia River & 100 N-3 HRM 9.5 & September 7, 2001 & B12TJ2 & Arsenic & $\mathrm{mg} / \mathrm{L}$ & 1 & 7.04E-04 & $=$ & 0.15 & 1.80E-05 \\
\hline Columbia River & 300 A1 HRM 43.1 & September 9, 2003 & B17CN9 & Arsenic & $\mathrm{mg} / \mathrm{L}$ & 1 & 7.04E-04 & $=$ & 0.15 & 1.80E-05 \\
\hline Columbia River & 300 A3 HRM 43.1 & September 15, 2004 & B1B6W8 & Arsenic & $\mathrm{mg} / \mathrm{L}$ & 1 & 7.04E-04 & $x$ & 0.15 & 1.80E-05 \\
\hline Columbia River & RICHPS HRM 45.0 & June 11, 2002 & B14RX9 & Arsenic & $\mathrm{mg} / \mathrm{L}$ & 1 & 7.04E-04 & $x$ & 0.15 & 1.80E-05 \\
\hline Columbia River & 300 SR HRM 41.5 & September 13, 2001 & B12TP7 & Arsenic & $\mathrm{mg} / \mathrm{L}$ & 1 & 7.05E-04 & $=$ & 0.15 & $1.80 \mathrm{E}-05$ \\
\hline
\end{tabular}

120 of 137 


\section{Table B-10}

Summary of Surface Water Results From Segment 2 Exceeding Select Ecological or Human Health Benchmark Values

Columbia River Component - Existing Sample Data Evaluation and Scoping Report

\begin{tabular}{|c|c|c|c|c|c|c|c|c|c|c|}
\hline Sample Area & Sample Site & Sample ID & Date Collected & Constituent Name & Units & QA Category & $\begin{array}{l}\text { Analytical } \\
\text { Value }\end{array}$ & Qualifier & $\begin{array}{l}\text { Ecological } \\
\text { Benchmark } \\
\text { Value }\end{array}$ & $\begin{array}{l}\text { Human Health } \\
\text { Benchmark Value }\end{array}$ \\
\hline Columbia River & 100 N-5 HRM 9.5 & September 13, 2004 & B1B6X2 & Arsenic & $\mathrm{mg} / \mathrm{L}$ & 1 & 7.06E-04 & $\mathrm{X}$ & 0.15 & 1.80E-05 \\
\hline Columbia River & RICHPS10HRM46.4 & December 10, 2002 & B16339 & Arsenic & $\mathrm{mg} / \mathrm{L}$ & 1 & 7.06E-04 & $=$ & 0.15 & 1.80E-05 \\
\hline Columbia River & RICHPS3 HRM46.4 & September 13, 2001 & В12Т80 & Arsenic & $\mathrm{mg} / \mathrm{L}$ & 1 & 7.06E-04 & $=$ & 0.15 & 1.80E-05 \\
\hline Columbia River & 300 A1 HRM 43.1 & September 13, 2001 & B12TK2 & Arsenic & $\mathrm{mg} / \mathrm{L}$ & 1 & 7.07E-04 & $=$ & 0.15 & 1.80E-05 \\
\hline Columbia River & 300 A2 HRM 43.1 & September 15, 2004 & B1B6W7 & Arsenic & $\mathrm{mg} / \mathrm{L}$ & 1 & 7.07E-04 & $x$ & 0.15 & $1.80 \mathrm{E}-05$ \\
\hline Columbia River & 300 SR HRM 42.5 & September 13, 2001 & B12TR5 & Arsenic & $\mathrm{mg} / \mathrm{L}$ & 1 & 7.07E-04 & $=$ & 0.15 & 1.80E-05 \\
\hline Columbia River & RICHPS HRM 45.0 & September 15, 2004 & B1B786 & Arsenic & $\mathrm{mg} / \mathrm{L}$ & 1 & 7.07E-04 & $\mathrm{X}$ & 0.15 & $1.80 \mathrm{E}-05$ \\
\hline Columbia River & 100 F-3 HRM19.0 & September 14, 2004 & B1B6X7 & Arsenic & $\mathrm{mg} / \mathrm{L}$ & 1 & 7.09E-04 & $x$ & 0.15 & 1.80E-05 \\
\hline Columbia River & $100 \mathrm{~N}-10 \mathrm{HRM} 9.5$ & September 7, 2001 & B12TJ5 & Arsenic & $\mathrm{mg} / \mathrm{L}$ & 1 & 7.09E-04 & $=$ & 0.15 & 1.80E-05 \\
\hline Columbia River & 100 NSHR HRM9.8 & September 7, 2001 & B12TP3 & Arsenic & $\mathrm{mg} / \mathrm{L}$ & 1 & 7.09E-04 & $=$ & 0.15 & 1.80E-05 \\
\hline Columbia River & 300 A2 HRM 43.1 & September 15, 2004 & B1B764 & Arsenic & $\mathrm{mg} / \mathrm{L}$ & 1 & 7.09E-04 & $x$ & 0.15 & 1.80E-05 \\
\hline Columbia River & 100-D SPR 110-1 & October 17, 2000 & B10961 & Arsenic & $\mathrm{mg} / \mathrm{L}$ & 1 & 7.10E-04 & $=$ & 0.15 & 1.80E-05 \\
\hline Columbia River & 300 A3 HRM 43.1 & September 13, 2001 & B12TK4 & Arsenic & $\mathrm{mg} / \mathrm{L}$ & 1 & 7.11E-04 & $=$ & 0.15 & 1.80E-05 \\
\hline Columbia River & 100-D SPR 102-1 & October 26, 2004 & B1BFX2 & Arsenic & $\mathrm{mg} / \mathrm{L}$ & 1 & $7.12 \mathrm{E}-04$ & $x$ & 0.15 & $1.80 \mathrm{E}-05$ \\
\hline Columbia River & 300 A7 HRM 43.1 & September 13, 2001 & B12TK6 & Arsenic & $\mathrm{mg} / \mathrm{L}$ & 1 & 7.12E-04 & $=$ & 0.15 & 1.80E-05 \\
\hline Columbia River & 300 SR HRM 42.9 & September 13, 2001 & B12TR8 & Arsenic & $\mathrm{mg} / \mathrm{L}$ & 1 & 7.12E-04 & $=$ & 0.15 & $1.80 \mathrm{E}-05$ \\
\hline Columbia River & 100 N-1 HRM 9.5 & September 7, 2001 & B12TJ0 & Arsenic & $\mathrm{mg} / \mathrm{L}$ & 1 & 7.13E-04 & $=$ & 0.15 & 1.80E-05 \\
\hline Columbia River & 100B SPR 39-2 & October 20, 2003 & B17J83 & Arsenic & $\mathrm{mg} / \mathrm{L}$ & 1 & 7.13E-04 & $=$ & 0.15 & 1.80E-05 \\
\hline Columbia River & 300 A7 HRM 43.1 & September 13, 2001 & В12T52 & Arsenic & $\mathrm{mg} / \mathrm{L}$ & 1 & 7.13E-04 & $=$ & 0.15 & 1.80E-05 \\
\hline Columbia River & HFD TS3 HRM28.7 & September 10, 2001 & B12TL0 & Arsenic & $\mathrm{mg} / \mathrm{L}$ & 1 & 7.13E-04 & $=$ & 0.15 & 1.80E-05 \\
\hline Columbia River & 300 A1 HRM 43.1 & September 15, 2004 & B1B6W6 & Arsenic & $\mathrm{mg} / \mathrm{L}$ & 1 & 7.14E-04 & $\mathrm{X}$ & 0.15 & 1.80E-05 \\
\hline Columbia River & RICHPS HRM 43.9 & September 15, 2004 & B1B787 & Arsenic & $\mathrm{mg} / \mathrm{L}$ & 1 & 7.15E-04 & $x$ & 0.15 & 1.80E-05 \\
\hline Columbia River & RICHPS1 HRM46.4 & September 9, 2003 & B17CD0 & Arsenic & $\mathrm{mg} / \mathrm{L}$ & 1 & 7.15E-04 & $=$ & 0.15 & 1.80E-05 \\
\hline Columbia River & HFD TS10HRM28.7 & September 10, 2001 & B12TL3 & Arsenic & $\mathrm{mg} / \mathrm{L}$ & 1 & 7.16E-04 & $=$ & 0.15 & 1.80E-05 \\
\hline Columbia River & 100B SPR 39-2 & October 20, 2003 & B17J82 & Arsenic & $\mathrm{mg} / \mathrm{L}$ & 1 & 7.17E-04 & $=$ & 0.15 & 1.80E-05 \\
\hline Columbia River & 100 FSHR HRM22 & September 14, 2004 & B1B7F1 & Arsenic & $\mathrm{mg} / \mathrm{L}$ & 1 & 7.18E-04 & $x$ & 0.15 & 1.80E-05 \\
\hline Columbia River & RICHPS7 HRM46.4 & September 13, 2001 & В12Т82 & Arsenic & $\mathrm{mg} / \mathrm{L}$ & 1 & 7.18E-04 & $=$ & 0.15 & 1.80E-05 \\
\hline Columbia River & RICHPS7 HRM46.4 & June 14, 1999 & B0V220 & Arsenic & $\mathrm{mg} / \mathrm{L}$ & 1 & 7.19E-04 & $=$ & 0.15 & 1.80E-05 \\
\hline Columbia River & HFD TS5 HRM28.7 & September 10, 2001 & В12Т58 & Arsenic & $\mathrm{mg} / \mathrm{L}$ & 1 & 7.19E-04 & $=$ & 0.15 & 1.80E-05 \\
\hline Columbia River & 100 F-5 HRM19.0 & September 10, 2001 & B12TL7 & Arsenic & $\mathrm{mg} / \mathrm{L}$ & 1 & $7.20 \mathrm{E}-04$ & $=$ & 0.15 & 1.80E-05 \\
\hline Columbia River & RICHPS HRM 43.9 & September 15, 2004 & B1B791 & Arsenic & $\mathrm{mg} / \mathrm{L}$ & 1 & $7.20 \mathrm{E}-04$ & $x$ & 0.15 & $1.80 \mathrm{E}-05$ \\
\hline Columbia River & RICHPS10HRM46.4 & September 10, 2002 & B158R7 & Arsenic & $\mathrm{mg} / \mathrm{L}$ & 1 & $7.20 \mathrm{E}-04$ & $=$ & 0.15 & 1.80E-05 \\
\hline Columbia River & 100 NSHR HRM8.4 & September 7, 2001 & B12TN4 & Arsenic & $\mathrm{mg} / \mathrm{L}$ & 1 & 7.22E-04 & $=$ & 0.15 & 1.80E-05 \\
\hline Columbia River & RICHPS HRM 45.0 & September 15, 2004 & B1B790 & Arsenic & $\mathrm{mg} / \mathrm{L}$ & 1 & $7.22 \mathrm{E}-04$ & $\mathrm{x}$ & 0.15 & $1.80 \mathrm{E}-05$ \\
\hline Columbia River & RICHPS1 HRM46.4 & September 15, 2004 & B1B757 & Arsenic & $\mathrm{mg} / \mathrm{L}$ & 1 & 7.23E-04 & $\mathrm{x}$ & 0.15 & 1.80E-05 \\
\hline Columbia River & RICHPS9 HRM46.4 & June 14, 1999 & B0V224 & Arsenic & $\mathrm{mg} / \mathrm{L}$ & 1 & $7.25 \mathrm{E}-04$ & $=$ & 0.15 & 1.80E-05 \\
\hline Columbia River & HFD TS HRM 30 & September 10, 2001 & B12TH5 & Arsenic & $\mathrm{mg} / \mathrm{L}$ & 1 & 7.26E-04 & $=$ & 0.15 & 1.80E-05 \\
\hline Columbia River & 100 FSHR HRM18 & September 10, 2001 & B12TT5 & Arsenic & $\mathrm{mg} / \mathrm{L}$ & 1 & 7.27E-04 & $=$ & 0.15 & $1.80 \mathrm{E}-05$ \\
\hline Columbia River & 300 A10 HRM43.1 & September 13, 2001 & В12Т53 & Arsenic & $\mathrm{mg} / \mathrm{L}$ & 1 & 7.27E-04 & $=$ & 0.15 & 1.80E-05 \\
\hline Columbia River & 100 F-7 HRM19.0 & September 10, 2001 & B12TL8 & Arsenic & $\mathrm{mg} / \mathrm{L}$ & 1 & $7.28 \mathrm{E}-04$ & $=$ & 0.15 & $1.80 \mathrm{E}-05$ \\
\hline Columbia River & 100 N-2 HRM 9.5 & September 7, 2001 & B12TJ1 & Arsenic & $\mathrm{mg} / \mathrm{L}$ & 1 & $7.28 \mathrm{E}-04$ & $=$ & 0.15 & 1.80E-05 \\
\hline Columbia River & 300 SHR HRM42.9 & September 15, 2004 & B1B7D0 & Arsenic & $\mathrm{mg} / \mathrm{L}$ & 1 & $7.28 \mathrm{E}-04$ & $x$ & 0.15 & 1.80E-05 \\
\hline Columbia River & HFD TS HRM 28 & September 18, 2000 & B10786 & Arsenic & $\mathrm{mg} / \mathrm{L}$ & 1 & $7.28 \mathrm{E}-04$ & $=$ & 0.15 & 1.80E-05 \\
\hline Columbia River & RICHPS10HRM46.4 & February 26, 2001 & B11H36 & Arsenic & $\mathrm{mg} / \mathrm{L}$ & 1 & $7.28 \mathrm{E}-04$ & $=$ & 0.15 & 1.80E-05 \\
\hline Columbia River & RICHPS10HRM46.4 & June 20,2000 & B0YCK2 & Arsenic & $\mathrm{mg} / \mathrm{L}$ & 1 & 7.29E-04 & $=$ & 0.15 & 1.80E-05 \\
\hline
\end{tabular}




\section{Table B-10}

Summary of Surface Water Results From Segment 2 Exceeding Select Ecological or Human Health Benchmark Values

Columbia River Component - Existing Sample Data Evaluation and Scoping Report

\begin{tabular}{|c|c|c|c|c|c|c|c|c|c|c|}
\hline Sample Area & Sample Site & Sample ID & Date Collected & Constituent Name & Units & QA Category & $\begin{array}{l}\text { Analytical } \\
\text { Value }\end{array}$ & Qualifier & $\begin{array}{l}\text { Ecological } \\
\text { Benchmark } \\
\text { Value }\end{array}$ & $\begin{array}{c}\text { Human Health } \\
\text { Benchmark Value }\end{array}$ \\
\hline Columbia River & RICHPS10HRM46.4 & December 5, 2000 & B11122 & Arsenic & $\mathrm{mg} / \mathrm{L}$ & 1 & 7.30E-04 & $=$ & 0.15 & 1.80E-05 \\
\hline Columbia River & RICHPS HRM 43.5 & September 15, 2004 & B1B788 & Arsenic & $\mathrm{mg} / \mathrm{L}$ & 1 & 7.31E-04 & $\mathrm{x}$ & 0.15 & 1.80E-05 \\
\hline Columbia River & 300 SHR HRM41.5 & September 15, 2004 & B1B7C1 & Arsenic & $\mathrm{mg} / \mathrm{L}$ & 1 & 7.32E-04 & $\mathrm{X}$ & 0.15 & 1.80E-05 \\
\hline Columbia River & 300 SPR $11-1$ & August 27, 2001 & B12RP6 & Arsenic & $\mathrm{mg} / \mathrm{L}$ & 1 & 7.32E-04 & $=$ & 0.15 & 1.80E-05 \\
\hline Columbia River & RICHPS HRM 45.8 & September 9, 2003 & В17CT1 & Arsenic & $\mathrm{mg} / \mathrm{L}$ & 1 & 7.32E-04 & $=$ & 0.15 & 1.80E-05 \\
\hline Columbia River & 100 F-2 HRM19.0 & September 10, 2001 & B12TL5 & Arsenic & $\mathrm{mg} / \mathrm{L}$ & 1 & 7.33E-04 & $=$ & 0.15 & 1.80E-05 \\
\hline Columbia River & 300 SR HRM 42.5 & September 9, 2003 & B17CX6 & Arsenic & $\mathrm{mg} / \mathrm{L}$ & 1 & 7.34E-04 & $=$ & 0.15 & $1.80 \mathrm{E}-05$ \\
\hline Columbia River & 100 N-10HRM 9.5 & September 13, 2004 & B1B6X4 & Arsenic & $\mathrm{mg} / \mathrm{L}$ & 1 & 7.35E-04 & $\mathrm{X}$ & 0.15 & 1.80E-05 \\
\hline Columbia River & RICHPS3 HRM46.4 & June 12, 2001 & B124Y5 & Arsenic & $\mathrm{mg} / \mathrm{L}$ & 1 & 7.35E-04 & $=$ & 0.15 & 1.80E-05 \\
\hline Columbia River & HFD TS3 HRM28.7 & September 10, 2001 & B12T57 & Arsenic & $\mathrm{mg} / \mathrm{L}$ & 1 & 7.36E-04 & $=$ & 0.15 & 1.80E-05 \\
\hline Columbia River & 100-D SPR 110-1 & October 17, 2000 & B10960 & Arsenic & $\mathrm{mg} / \mathrm{L}$ & 1 & 7.37E-04 & $=$ & 0.15 & $1.80 \mathrm{E}-05$ \\
\hline Columbia River & 300 SHR HRM41.5 & September 15, 2004 & B1B7C2 & Arsenic & $\mathrm{mg} / \mathrm{L}$ & 1 & 7.37E-04 & $\mathrm{X}$ & 0.15 & 1.80E-05 \\
\hline Columbia River & RICHPS HRM 43.9 & December 8, 2003 & B183W3 & Arsenic & $\mathrm{mg} / \mathrm{L}$ & 1 & 7.37E-04 & C & 0.15 & 1.80E-05 \\
\hline Columbia River & RICHPS5 HRM46.4 & September 10, 2002 & B158F9 & Arsenic & $\mathrm{mg} / \mathrm{L}$ & 1 & 7.37E-04 & $=$ & 0.15 & 1.80E-05 \\
\hline Columbia River & 100 F-3 HRM19.0 & September 10, 2001 & B12TL6 & Arsenic & $\mathrm{mg} / \mathrm{L}$ & 1 & 7.39E-04 & $=$ & 0.15 & 1.80E-05 \\
\hline Columbia River & 100 NSHR HRM8.9 & September 7, 2001 & B12TN7 & Arsenic & $\mathrm{mg} / \mathrm{L}$ & 1 & 7.39E-04 & $=$ & 0.15 & $1.80 \mathrm{E}-05$ \\
\hline Columbia River & HFD TS HRM 28 & September 18, 2000 & B10787 & Arsenic & $\mathrm{mg} / \mathrm{L}$ & 1 & 7.39E-04 & $=$ & 0.15 & 1.80E-05 \\
\hline Columbia River & RICHPS7 HRM46.4 & June 12, 2001 & B124Y7 & Arsenic & $\mathrm{mg} / \mathrm{L}$ & 1 & 7.39E-04 & $=$ & 0.15 & 1.80E-05 \\
\hline Columbia River & 100 FSHR HRM18 & September 10, 2001 & B12TT4 & Arsenic & $\mathrm{mg} / \mathrm{L}$ & 1 & 7.42E-04 & $=$ & 0.15 & 1.80E-05 \\
\hline Columbia River & RICHPS5 HRM46.4 & September 13, 2001 & В12T81 & Arsenic & $\mathrm{mg} / \mathrm{L}$ & 1 & 7.42E-04 & $=$ & 0.15 & $1.80 \mathrm{E}-05$ \\
\hline Columbia River & 100 NSHR HRM9.2 & September 7, 2001 & B12TP0 & Arsenic & $\mathrm{mg} / \mathrm{L}$ & 1 & 7.43E-04 & $=$ & 0.15 & 1.80E-05 \\
\hline Columbia River & 300 A10 HRM43.1 & September 19, 2000 & B10748 & Arsenic & $\mathrm{mg} / \mathrm{L}$ & 1 & 7.44E-04 & $=$ & 0.15 & 1.80E-05 \\
\hline Columbia River & 300 A1 HRM 43.1 & September 15, 2004 & B1B763 & Arsenic & $\mathrm{mg} / \mathrm{L}$ & 1 & $7.45 \mathrm{E}-04$ & $x$ & 0.15 & 1.80E-05 \\
\hline Columbia River & RICHPS8 HRM46.4 & June 14, 1999 & B0V223 & Arsenic & $\mathrm{mg} / \mathrm{L}$ & 1 & 7.45E-04 & $=$ & 0.15 & 1.80E-05 \\
\hline Columbia River & HFD TS HRM 30 & September 9, 2002 & B158P1 & Arsenic & $\mathrm{mg} / \mathrm{L}$ & 1 & 7.46E-04 & $=$ & 0.15 & $1.80 \mathrm{E}-05$ \\
\hline Columbia River & 300 A10 HRM43.1 & September 10, 2002 & В158T3 & Arsenic & $\mathrm{mg} / \mathrm{L}$ & 1 & 7.47E-04 & $=$ & 0.15 & 1.80E-05 \\
\hline Columbia River & 100 N-7 HRM 9.5 & September 7, 2001 & В12Т68 & Arsenic & $\mathrm{mg} / \mathrm{L}$ & 1 & 7.48E-04 & $=$ & 0.15 & 1.80E-05 \\
\hline Columbia River & 300 A7 HRM 43.1 & September 19, 2000 & B10747 & Arsenic & $\mathrm{mg} / \mathrm{L}$ & 1 & 7.49E-04 & $=$ & 0.15 & 1.80E-05 \\
\hline Columbia River & 100 FSHR HRM23 & September 10, 2001 & B12TV0 & Arsenic & $\mathrm{mg} / \mathrm{L}$ & 1 & 7.50E-04 & $=$ & 0.15 & $1.80 \mathrm{E}-05$ \\
\hline Columbia River & RICHPS3 HRM46.4 & June 14, 1999 & B0V213 & Arsenic & $\mathrm{mg} / \mathrm{L}$ & 1 & 7.51E-04 & $=$ & 0.15 & 1.80E-05 \\
\hline Columbia River & HFD TS7 HRM28.7 & September 10, 2001 & В12Т59 & Arsenic & $\mathrm{mg} / \mathrm{L}$ & 1 & 7.52E-04 & $=$ & 0.15 & 1.80E-05 \\
\hline Columbia River & RICHPS10HRM46.4 & June 11, 2002 & B14RX3 & Arsenic & $\mathrm{mg} / \mathrm{L}$ & 1 & $7.52 \mathrm{E}-04$ & $x$ & 0.15 & 1.80E-05 \\
\hline Columbia River & RICHPS2 HRM46.4 & September 13, 2001 & В12Т79 & Arsenic & $\mathrm{mg} / \mathrm{L}$ & 1 & 7.52E-04 & $=$ & 0.15 & 1.80E-05 \\
\hline Columbia River & 100 F-1 HRM19.0 & September 10, 2001 & В12T70 & Arsenic & $\mathrm{mg} / \mathrm{L}$ & 1 & 7.56E-04 & $=$ & 0.15 & $1.80 \mathrm{E}-05$ \\
\hline Columbia River & 100 FSHR HRM23 & September 10, 2001 & B12TV1 & Arsenic & $\mathrm{mg} / \mathrm{L}$ & 1 & 7.56E-04 & $=$ & 0.15 & 1.80E-05 \\
\hline Columbia River & 100 NSHR HRM9.2 & September 7, 2001 & B12TN9 & Arsenic & $\mathrm{mg} / \mathrm{L}$ & 1 & 7.56E-04 & $=$ & 0.15 & 1.80E-05 \\
\hline Columbia River & HFD TS1 HRM28.7 & September 10, 2001 & B12TK8 & Arsenic & $\mathrm{mg} / \mathrm{L}$ & 1 & 7.56E-04 & $=$ & 0.15 & 1.80E-05 \\
\hline Columbia River & RICHPS4 HRM46.4 & June 14, 1999 & B0V215 & Arsenic & $\mathrm{mg} / \mathrm{L}$ & 1 & 7.60E-04 & $=$ & 0.15 & $1.80 \mathrm{E}-05$ \\
\hline Columbia River & RICHPS HRM 43.5 & June 11, 2002 & B14RY5 & Arsenic & $\mathrm{mg} / \mathrm{L}$ & 1 & 7.63E-04 & $\mathrm{x}$ & 0.15 & 1.80E-05 \\
\hline Columbia River & 300 SR HRM 42.1 & September 19, 2000 & B10778 & Arsenic & $\mathrm{mg} / \mathrm{L}$ & 1 & 7.66E-04 & $=$ & 0.15 & 1.80E-05 \\
\hline Columbia River & RICHPS7 HRM46.4 & June 14, 1999 & BoV221 & Arsenic & $\mathrm{mg} / \mathrm{L}$ & 1 & 7.66E-04 & $=$ & 0.15 & 1.80E-05 \\
\hline Columbia River & 100 F-10HRM19.0 & September 10, 2001 & B12TL9 & Arsenic & $\mathrm{mg} / \mathrm{L}$ & 1 & 7.67E-04 & $=$ & 0.15 & 1.80E-05 \\
\hline Columbia River & RICHPS1 HRM46.4 & June 11, 2002 & B14RT7 & Arsenic & $\mathrm{mg} / \mathrm{L}$ & 1 & 7.67E-04 & $x$ & 0.15 & 1.80E-05 \\
\hline Columbia River & RICHPS9 HRM46.4 & June 14, 1999 & B0V225 & Arsenic & $\mathrm{mg} / \mathrm{L}$ & 1 & 7.68E-04 & $=$ & 0.15 & 1.80E-05 \\
\hline Columbia River & 100 F-3 HRM19.0 & September 10, 2001 & В12Т72 & Arsenic & $\mathrm{mg} / \mathrm{L}$ & 1 & 7.68E-04 & $=$ & 0.15 & $1.80 \mathrm{E}-05$ \\
\hline
\end{tabular}




\section{Table B-10}

Summary of Surface Water Results From Segment 2 Exceeding Select Ecological or Human Health Benchmark Values

Columbia River Component - Existing Sample Data Evaluation and Scoping Report

\begin{tabular}{|c|c|c|c|c|c|c|c|c|c|c|}
\hline Sample Area & Sample Site & Sample ID & Date Collected & Constituent Name & Units & QA Category & $\begin{array}{l}\text { Analytical } \\
\text { Value }\end{array}$ & Qualifier & $\begin{array}{l}\text { Ecological } \\
\text { Benchmark } \\
\text { Value }\end{array}$ & $\begin{array}{c}\text { Human Health } \\
\text { Benchmark Value }\end{array}$ \\
\hline Columbia River & 100 F-5 HRM19.0 & September 10, 2001 & B12T73 & Arsenic & $\mathrm{mg} / \mathrm{L}$ & 1 & 7.68E-04 & $=$ & 0.15 & 1.80E-05 \\
\hline Columbia River & HFD TS1 HRM28.7 & September 9, 2002 & B158T4 & Arsenic & $\mathrm{mg} / \mathrm{L}$ & 1 & 7.71E-04 & $=$ & 0.15 & 1.80E-05 \\
\hline Columbia River & RICHPS1 HRM46.4 & September 15, 2004 & B1B6Y3 & Arsenic & $\mathrm{mg} / \mathrm{L}$ & 1 & 7.71E-04 & $x$ & 0.15 & 1.80E-05 \\
\hline Columbia River & RICHPS HRM 45.8 & September 13, 2001 & B12TM4 & Arsenic & $\mathrm{mg} / \mathrm{L}$ & 1 & 7.72E-04 & $=$ & 0.15 & 1.80E-05 \\
\hline Columbia River & RICHPS10HRM46.4 & June 20, 2000 & B0YCD4 & Arsenic & $\mathrm{mg} / \mathrm{L}$ & 1 & $7.72 \mathrm{E}-04$ & $=$ & 0.15 & 1.80E-05 \\
\hline Columbia River & RICHPS HRM 46.4 & December 4, 2001 & B13LH7 & Arsenic & $\mathrm{mg} / \mathrm{L}$ & 1 & $7.75 \mathrm{E}-04$ & $=$ & 0.15 & 1.80E-05 \\
\hline Columbia River & 300 A1 HRM 43.1 & September 15, 2004 & B1B767 & Arsenic & $\mathrm{mg} / \mathrm{L}$ & 1 & 7.76E-04 & $\mathrm{X}$ & 0.15 & $1.80 \mathrm{E}-05$ \\
\hline Columbia River & 300 A7 HRM 43.1 & September 15, 2004 & B1B6V7 & Arsenic & $\mathrm{mg} / \mathrm{L}$ & 1 & 7.76E-04 & $\mathrm{X}$ & 0.15 & 1.80E-05 \\
\hline Columbia River & HFD TS2 HRM28.7 & September 10, 2001 & В12Т56 & Arsenic & $\mathrm{mg} / \mathrm{L}$ & 1 & 7.79E-04 & $=$ & 0.15 & 1.80E-05 \\
\hline Columbia River & RICHPS7 HRM46.4 & June 11, 2002 & B14RV1 & Arsenic & $\mathrm{mg} / \mathrm{L}$ & 1 & 7.79E-04 & $x$ & 0.15 & 1.80E-05 \\
\hline Columbia River & RICHPS10HRM46.4 & June 12, 2001 & B12530 & Arsenic & $\mathrm{mg} / \mathrm{L}$ & 1 & 7.82E-04 & $=$ & 0.15 & $1.80 \mathrm{E}-05$ \\
\hline Columbia River & 100-D SPR 102-1 & October 21, 2002 & B15C89 & Arsenic & $\mathrm{mg} / \mathrm{L}$ & 1 & 7.84E-04 & $=$ & 0.15 & 1.80E-05 \\
\hline Columbia River & HFD TS HRM 30 & September 9, 2002 & B158N8 & Arsenic & $\mathrm{mg} / \mathrm{L}$ & 1 & 7.85E-04 & $=$ & 0.15 & 1.80E-05 \\
\hline Columbia River & 300 A10 HRM43.1 & September 13, 2001 & B12TK7 & Arsenic & $\mathrm{mg} / \mathrm{L}$ & 1 & 7.88E-04 & $=$ & 0.15 & 1.80E-05 \\
\hline Columbia River & RICHPS HRM 45.8 & June 11, 2002 & B14RY2 & Arsenic & $\mathrm{mg} / \mathrm{L}$ & 1 & 7.88E-04 & $x$ & 0.15 & 1.80E-05 \\
\hline Columbia River & 300 SPR 11 -1 & August 27, 2001 & B12RP5 & Arsenic & $\mathrm{mg} / \mathrm{L}$ & 1 & 7.93E-04 & $=$ & 0.15 & $1.80 \mathrm{E}-05$ \\
\hline Columbia River & 300 A10 HRM43.1 & September 19, 2000 & B106R8 & Arsenic & $\mathrm{mg} / \mathrm{L}$ & 1 & 7.94E-04 & $=$ & 0.15 & 1.80E-05 \\
\hline Columbia River & RICHPS HRM 45.8 & September 15, 2004 & B1B785 & Arsenic & $\mathrm{mg} / \mathrm{L}$ & 1 & 7.96E-04 & $\mathrm{x}$ & 0.15 & 1.80E-05 \\
\hline Columbia River & RICHPS3 HRM46.4 & June 11, 2002 & B14RT9 & Arsenic & $\mathrm{mg} / \mathrm{L}$ & 1 & 7.96E-04 & $x$ & 0.15 & 1.80E-05 \\
\hline Columbia River & HFD TS1 HRM28.7 & September 8, 2003 & B17C97 & Arsenic & $\mathrm{mg} / \mathrm{L}$ & 1 & 7.97E-04 & $=$ & 0.15 & $1.80 \mathrm{E}-05$ \\
\hline Columbia River & RICHPS5 HRM46.4 & June 10, 2003 & B171V6 & Arsenic & $\mathrm{mg} / \mathrm{L}$ & 1 & 7.97E-04 & C & 0.15 & 1.80E-05 \\
\hline Columbia River & 300 SR HRM 42.1 & September 19, 2000 & B10777 & Arsenic & $\mathrm{mg} / \mathrm{L}$ & 1 & 7.98E-04 & $=$ & 0.15 & 1.80E-05 \\
\hline Columbia River & RICHPS5 HRM46.4 & June 14, 1999 & BoV217 & Arsenic & $\mathrm{mg} / \mathrm{L}$ & 1 & 7.98E-04 & $=$ & 0.15 & 1.80E-05 \\
\hline Columbia River & 100 N-2 HRM 9.5 & September 7, 2001 & B12T65 & Arsenic & $\mathrm{mg} / \mathrm{L}$ & 1 & 8.03E-04 & $=$ & 0.15 & 1.80E-05 \\
\hline Columbia River & HFD TS1 HRM28.7 & September 9, 2002 & B158C3 & Arsenic & $\mathrm{mg} / \mathrm{L}$ & 1 & 8.03E-04 & $=$ & 0.15 & $1.80 \mathrm{E}-05$ \\
\hline Columbia River & 300 A10 HRM43.1 & September 16, 1999 & B0WB88 & Arsenic & $\mathrm{mg} / \mathrm{L}$ & 1 & 8.03E-04 & $=$ & 0.15 & 1.80E-05 \\
\hline Columbia River & 300 SHR HRM42.4 & September 15, 2004 & B1B7H6 & Arsenic & $\mathrm{mg} / \mathrm{L}$ & 1 & 8.04E-04 & $x$ & 0.15 & 1.80E-05 \\
\hline Columbia River & RICHPS10HRM46.4 & September 10, 2002 & B158H1 & Arsenic & $\mathrm{mg} / \mathrm{L}$ & 1 & 8.05E-04 & $=$ & 0.15 & 1.80E-05 \\
\hline Columbia River & RICHPS2 HRM46.4 & June 11, 2002 & B14RT8 & Arsenic & $\mathrm{mg} / \mathrm{L}$ & 1 & 8.06E-04 & $\mathrm{x}$ & 0.15 & $1.80 \mathrm{E}-05$ \\
\hline Columbia River & 100 F-10HRM19.0 & September 14, 2004 & B1B6V9 & Arsenic & $\mathrm{mg} / \mathrm{L}$ & 1 & 8.08E-04 & $\mathrm{X}$ & 0.15 & 1.80E-05 \\
\hline Columbia River & 100 F-10HRM19.0 & September 14, 2004 & B1B780 & Arsenic & $\mathrm{mg} / \mathrm{L}$ & 1 & 8.08E-04 & $\mathrm{x}$ & 0.15 & 1.80E-05 \\
\hline Columbia River & RICHPS HRM 46.4 & September 13, 2001 & B12TJ6 & Arsenic & $\mathrm{mg} / \mathrm{L}$ & 1 & 8.08E-04 & $=$ & 0.15 & 1.80E-05 \\
\hline Columbia River & RICHPS HRM 45.0 & June 10, 2003 & B17210 & Arsenic & $\mathrm{mg} / \mathrm{L}$ & 1 & 8.12E-04 & C & 0.15 & 1.80E-05 \\
\hline Columbia River & RICHPS10HRM46.4 & March 28, 2000 & BOXRN7 & Arsenic & $\mathrm{mg} / \mathrm{L}$ & 1 & 8.14E-04 & $=$ & 0.15 & $1.80 \mathrm{E}-05$ \\
\hline Columbia River & RICHPS5 HRM46.4 & June 11, 2002 & B14RV0 & Arsenic & $\mathrm{mg} / \mathrm{L}$ & 1 & 8.17E-04 & $x$ & 0.15 & 1.80E-05 \\
\hline Columbia River & 100 N-5 HRM 9.5 & September 7, 2001 & В12T67 & Arsenic & $\mathrm{mg} / \mathrm{L}$ & 1 & 8.20E-04 & $=$ & 0.15 & 1.80E-05 \\
\hline Columbia River & RICHPS10HRM46.4 & September 19, 2000 & B106V9 & Arsenic & $\mathrm{mg} / \mathrm{L}$ & 1 & $8.21 E-04$ & $=$ & 0.15 & 1.80E-05 \\
\hline Columbia River & HFD TS1 HRM28.7 & September 10, 2001 & В12T55 & Arsenic & $\mathrm{mg} / \mathrm{L}$ & 1 & 8.22E-04 & $=$ & 0.15 & $1.80 \mathrm{E}-05$ \\
\hline Columbia River & 300 SHR HRM42.1 & September 15, 2004 & B1B7C6 & Arsenic & $\mathrm{mg} / \mathrm{L}$ & 1 & 8.23E-04 & $\mathrm{x}$ & 0.15 & 1.80E-05 \\
\hline Columbia River & RICHPS HRM 45.8 & September 15, 2004 & B1B789 & Arsenic & $\mathrm{mg} / \mathrm{L}$ & 1 & 8.23E-04 & $\mathrm{x}$ & 0.15 & 1.80E-05 \\
\hline Columbia River & 100-D SPR 102-1 & October 27, 1999 & B0WP42 & Arsenic & $\mathrm{mg} / \mathrm{L}$ & 1 & 8.24E-04 & $=$ & 0.15 & 1.80E-05 \\
\hline Columbia River & 100 NSHR HRM8.9 & September 7, 2001 & B12TN6 & Arsenic & $\mathrm{mg} / \mathrm{L}$ & 1 & $8.25 E-04$ & $=$ & 0.15 & 1.80E-05 \\
\hline Columbia River & RICHPS HRM 43.5 & December 8, 2003 & B183W4 & Arsenic & $\mathrm{mg} / \mathrm{L}$ & 1 & 8.30E-04 & C & 0.15 & 1.80E-05 \\
\hline Columbia River & RICHPS2 HRM46.4 & September 13, 2001 & B12TJ7 & Arsenic & $\mathrm{mg} / \mathrm{L}$ & 1 & 8.34E-04 & $=$ & 0.15 & 1.80E-05 \\
\hline Columbia River & RICHPS7 HRM46.4 & June 10, 2003 & B171V7 & Arsenic & $\mathrm{mg} / \mathrm{L}$ & 1 & 8.35E-04 & C & 0.15 & $1.80 \mathrm{E}-05$ \\
\hline
\end{tabular}




\section{Table B-10}

Summary of Surface Water Results From Segment 2 Exceeding Select Ecological or Human Health Benchmark Values

Columbia River Component - Existing Sample Data Evaluation and Scoping Report

\begin{tabular}{|c|c|c|c|c|c|c|c|c|c|c|}
\hline Sample Area & Sample Site & Sample ID & Date Collected & Constituent Name & Units & QA Category & $\begin{array}{l}\text { Analytical } \\
\text { Value }\end{array}$ & Qualifier & $\begin{array}{l}\text { Ecological } \\
\text { Benchmark } \\
\text { Value }\end{array}$ & $\begin{array}{c}\text { Human Health } \\
\text { Benchmark Value }\end{array}$ \\
\hline Columbia River & 100 N-3 HRM 9.5 & September 7, 2001 & B12T66 & Arsenic & $\mathrm{mg} / \mathrm{L}$ & 1 & 8.36E-04 & $=$ & 0.15 & 1.80E-05 \\
\hline Columbia River & 100 F-10HRM19.0 & September 10, 2001 & B12T54 & Arsenic & $\mathrm{mg} / \mathrm{L}$ & 1 & 8.39E-04 & $=$ & 0.15 & 1.80E-05 \\
\hline Columbia River & RICHPS HRM 45.0 & June 11, 2002 & B14RY3 & Arsenic & $\mathrm{mg} / \mathrm{L}$ & 1 & $8.40 E-04$ & $x$ & 0.15 & 1.80E-05 \\
\hline Columbia River & RICHPS10HRM46.4 & December 4, 2001 & B13LF0 & Arsenic & $\mathrm{mg} / \mathrm{L}$ & 1 & 8.41E-04 & $=$ & 0.15 & 1.80E-05 \\
\hline Columbia River & 100-D SPR 110-1 & October 21, 2002 & B15C87 & Arsenic & $\mathrm{mg} / \mathrm{L}$ & 1 & $8.42 \mathrm{E}-04$ & $=$ & 0.15 & 1.80E-05 \\
\hline Columbia River & RICHPS HRM 43.5 & June 10, 2003 & B17212 & Arsenic & $\mathrm{mg} / \mathrm{L}$ & 1 & $8.42 \mathrm{E}-04$ & C & 0.15 & 1.80E-05 \\
\hline Columbia River & RICHPS HRM 43.5 & September 15, 2004 & B1B792 & Arsenic & $\mathrm{mg} / \mathrm{L}$ & 1 & 8.42E-04 & $\mathrm{X}$ & 0.15 & $1.80 \mathrm{E}-05$ \\
\hline Columbia River & 300 A10 HRM43.1 & September 10, 2002 & B158C1 & Arsenic & $\mathrm{mg} / \mathrm{L}$ & 1 & 8.43E-04 & $=$ & 0.15 & 1.80E-05 \\
\hline Columbia River & 300 SHR HRM42.4 & September 15, 2004 & B1B7H5 & Arsenic & $\mathrm{mg} / \mathrm{L}$ & 1 & 8.43E-04 & $x$ & 0.15 & 1.80E-05 \\
\hline Columbia River & RICHPS HRM 45.8 & June 10, 2003 & B17209 & Arsenic & $\mathrm{mg} / \mathrm{L}$ & 1 & $8.45 \mathrm{E}-04$ & C & 0.15 & 1.80E-05 \\
\hline Columbia River & RICHPS HRM 43.9 & June 10, 2003 & B17211 & Arsenic & $\mathrm{mg} / \mathrm{L}$ & 1 & 8.46E-04 & C & 0.15 & $1.80 \mathrm{E}-05$ \\
\hline Columbia River & RICHPS1 HRM46.4 & June 10, 2003 & B171V3 & Arsenic & $\mathrm{mg} / \mathrm{L}$ & 1 & 8.47E-04 & C & 0.15 & 1.80E-05 \\
\hline Columbia River & 300 SR HRM 42.1 & September 9, 2003 & $\mathrm{~B} 17 \mathrm{C} \times 2$ & Arsenic & $\mathrm{mg} / \mathrm{L}$ & 1 & $8.52 E-04$ & $=$ & 0.15 & 1.80E-05 \\
\hline Columbia River & 100 NSHR HRM9.8 & September 7, 2001 & B12TP2 & Arsenic & $\mathrm{mg} / \mathrm{L}$ & 1 & 8.60E-04 & $=$ & 0.15 & 1.80E-05 \\
\hline Columbia River & RICHPS3 HRM46.4 & June 10, 2003 & B171V5 & Arsenic & $\mathrm{mg} / \mathrm{L}$ & 1 & 8.63E-04 & C & 0.15 & 1.80E-05 \\
\hline Columbia River & RICHPS2 HRM46.4 & June 10, 2003 & B171V4 & Arsenic & $\mathrm{mg} / \mathrm{L}$ & 1 & 8.70E-04 & C & 0.15 & $1.80 \mathrm{E}-05$ \\
\hline Columbia River & 100 NSHR HRM8.4 & September 7, 2001 & B12TN3 & Arsenic & $\mathrm{mg} / \mathrm{L}$ & 1 & 8.73E-04 & $=$ & 0.15 & 1.80E-05 \\
\hline Columbia River & RICHPS HRM 43.9 & June 11, 2002 & B14RY4 & Arsenic & $\mathrm{mg} / \mathrm{L}$ & 1 & 8.73E-04 & $\mathrm{x}$ & 0.15 & 1.80E-05 \\
\hline Columbia River & 100 N-1 HRM 9.5 & September 7, 2001 & B12T64 & Arsenic & $\mathrm{mg} / \mathrm{L}$ & 1 & 8.74E-04 & $=$ & 0.15 & 1.80E-05 \\
\hline Columbia River & RICHPS10HRM46.4 & September 16, 1999 & B0WB82 & Arsenic & $\mathrm{mg} / \mathrm{L}$ & 1 & 8.78E-04 & $=$ & 0.15 & $1.80 \mathrm{E}-05$ \\
\hline Columbia River & 300 SR HRM 42.1 & September 13, 2001 & B12TR1 & Arsenic & $\mathrm{mg} / \mathrm{L}$ & 1 & 8.78E-04 & $=$ & 0.15 & 1.80E-05 \\
\hline Columbia River & RICHPS10HRM46.4 & December 4, 2001 & B13LJ2 & Arsenic & $\mathrm{mg} / \mathrm{L}$ & 1 & 8.81E-04 & $=$ & 0.15 & 1.80E-05 \\
\hline Columbia River & 100D SPR 102-1 & October 27, 2003 & B17J94 & Arsenic & $\mathrm{mg} / \mathrm{L}$ & 1 & 8.92E-04 & $=$ & 0.15 & 1.80E-05 \\
\hline Columbia River & 100-D SPR 102-1 & October 17, 2000 & B10963 & Arsenic & $\mathrm{mg} / \mathrm{L}$ & 1 & 8.94E-04 & $=$ & 0.15 & 1.80E-05 \\
\hline Columbia River & RICHPS10HRM46.4 & December 8, 2003 & B183R4 & Arsenic & $\mathrm{mg} / \mathrm{L}$ & 1 & 8.94E-04 & $=$ & 0.15 & $1.80 \mathrm{E}-05$ \\
\hline Columbia River & HFD TS HRM 28 & September 10, 2001 & B12TT1 & Arsenic & $\mathrm{mg} / \mathrm{L}$ & 1 & 9.01E-04 & $=$ & 0.15 & 1.80E-05 \\
\hline Columbia River & 300 SR HRM 42.5 & September 16, 1999 & B0WB63 & Arsenic & $\mathrm{mg} / \mathrm{L}$ & 1 & $9.02 \mathrm{E}-04$ & $=$ & 0.15 & 1.80E-05 \\
\hline Columbia River & 300 SHR HRM42.1 & September 15, 2004 & B1B7C5 & Arsenic & $\mathrm{mg} / \mathrm{L}$ & 1 & 9.06E-04 & $x$ & 0.15 & 1.80E-05 \\
\hline Columbia River & 100-B SPR 39-2 & October 25, 1999 & B0WP27 & Arsenic & $\mathrm{mg} / \mathrm{L}$ & 1 & 9.07E-04 & $=$ & 0.15 & $1.80 \mathrm{E}-05$ \\
\hline Columbia River & HFD TS HRM 28 & September 10, 2001 & B12TT2 & Arsenic & $\mathrm{mg} / \mathrm{L}$ & 1 & 9.07E-04 & $=$ & 0.15 & 1.80E-05 \\
\hline Columbia River & RICHPS10HRM46.4 & June 10, 2003 & B171V8 & Arsenic & $\mathrm{mg} / \mathrm{L}$ & 1 & 9.10E-04 & C & 0.15 & 1.80E-05 \\
\hline Columbia River & 100 N-10HRM 9.5 & September 7, 2001 & В12Т69 & Arsenic & $\mathrm{mg} / \mathrm{L}$ & 1 & $9.15 E-04$ & $=$ & 0.15 & 1.80E-05 \\
\hline Columbia River & 300 SR HRM 42.1 & September 13, 2001 & B12TR0 & Arsenic & $\mathrm{mg} / \mathrm{L}$ & 1 & $9.26 \mathrm{E}-04$ & $=$ & 0.15 & 1.80E-05 \\
\hline Columbia River & 100-B SPR 39-2 & October 25, 1999 & B0WP29 & Arsenic & $\mathrm{mg} / \mathrm{L}$ & 1 & $9.28 \mathrm{E}-04$ & $=$ & 0.15 & $1.80 \mathrm{E}-05$ \\
\hline Columbia River & RICHPS10HRM46.4 & June 12, 2001 & B124Y8 & Arsenic & $\mathrm{mg} / \mathrm{L}$ & 1 & $9.28 \mathrm{E}-04$ & $=$ & 0.15 & 1.80E-05 \\
\hline Columbia River & 300 SPR 42-2 & October 7, 2002 & B15C01 & Arsenic & $\mathrm{mg} / \mathrm{L}$ & 1 & 9.34E-04 & C & 0.15 & 1.80E-05 \\
\hline Columbia River & 300 A10 HRM43.1 & September 9, 2003 & B17CP4 & Arsenic & $\mathrm{mg} / \mathrm{L}$ & 1 & 9.36E-04 & $=$ & 0.15 & 1.80E-05 \\
\hline Columbia River & 100-D SPR 102-1 & October 27, 1999 & B0WP43 & Arsenic & $\mathrm{mg} / \mathrm{L}$ & 1 & $9.38 \mathrm{E}-04$ & $=$ & 0.15 & $1.80 \mathrm{E}-05$ \\
\hline Columbia River & 100-B SPR 37-1 & September 16, 2002 & B15C62 & Arsenic & $\mathrm{mg} / \mathrm{L}$ & 1 & 9.47E-04 & $=$ & 0.15 & 1.80E-05 \\
\hline Columbia River & RICHPS10HRM46.4 & June 14, 1999 & B0V227 & Arsenic & $\mathrm{mg} / \mathrm{L}$ & 1 & $9.48 \mathrm{E}-04$ & $=$ & 0.15 & 1.80E-05 \\
\hline Columbia River & 300 SPR DR 42-2 & September 27, 2000 & B10975 & Arsenic & $\mathrm{mg} / \mathrm{L}$ & 1 & $9.52 \mathrm{E}-04$ & $=$ & 0.15 & 1.80E-05 \\
\hline Columbia River & 100-B SPR 37-1 & September 16, 2002 & B15C71 & Arsenic & $\mathrm{mg} / \mathrm{L}$ & 1 & 9.53E-04 & $=$ & 0.15 & 1.80E-05 \\
\hline Columbia River & RICHPS10HRM46.4 & June 11, 2002 & B14RV2 & Arsenic & $\mathrm{mg} / \mathrm{L}$ & 1 & $9.61 \mathrm{E}-04$ & $\mathrm{X}$ & 0.15 & 1.80E-05 \\
\hline Columbia River & RICHPS10HRM46.4 & September 9, 2003 & B17CN8 & Arsenic & $\mathrm{mg} / \mathrm{L}$ & 1 & $9.62 \mathrm{E}-04$ & $=$ & 0.15 & 1.80E-05 \\
\hline Columbia River & RICHPS10HRM46.4 & March 28, 2000 & B0XRJ8 & Arsenic & $\mathrm{mg} / \mathrm{L}$ & 1 & $9.75 \mathrm{E}-04$ & $=$ & 0.15 & $1.80 \mathrm{E}-05$ \\
\hline
\end{tabular}




\section{Table B-10}

Summary of Surface Water Results From Segment 2 Exceeding Select Ecological or Human Health Benchmark Values

Columbia River Component - Existing Sample Data Evaluation and Scoping Report

\begin{tabular}{|c|c|c|c|c|c|c|c|c|c|c|}
\hline Sample Area & Sample Site & Sample ID & Date Collected & Constituent Name & Units & QA Category & $\begin{array}{l}\text { Analytical } \\
\text { Value }\end{array}$ & Qualifier & $\begin{array}{l}\text { Ecological } \\
\text { Benchmark } \\
\text { Value }\end{array}$ & $\begin{array}{c}\text { Human Health } \\
\text { Benchmark Value }\end{array}$ \\
\hline Columbia River & 300 SPR DR 42-2 & September 27, 2000 & B10974 & Arsenic & $\mathrm{mg} / \mathrm{L}$ & 1 & $9.87 \mathrm{E}-04$ & $=$ & 0.15 & 1.80E-05 \\
\hline Columbia River & HFD SPR UR 28-2 & October 25, 2004 & B1BFY0 & Arsenic & $\mathrm{mg} / \mathrm{L}$ & 1 & 9.87E-04 & $x$ & 0.15 & 1.80E-05 \\
\hline Columbia River & 100B SPR 37-1 & October 20, 2003 & B17J76 & Arsenic & $\mathrm{mg} / \mathrm{L}$ & 1 & 9.94E-04 & $=$ & 0.15 & 1.80E-05 \\
\hline Columbia River & 300 SPR DR 42-2 & November 1, 1999 & B0WP57 & Arsenic & $\mathrm{mg} / \mathrm{L}$ & 1 & 0.0010 & $=$ & 0.15 & 1.80E-05 \\
\hline Columbia River & 100D SPR 102-1 & October 27, 2003 & B17J93 & Arsenic & $\mathrm{mg} / \mathrm{L}$ & 1 & 0.0010 & $=$ & 0.15 & 1.80E-05 \\
\hline Columbia River & RICHPS10HRM46.4 & September 13, 2001 & В12Т83 & Arsenic & $\mathrm{mg} / \mathrm{L}$ & 1 & 0.0010 & $=$ & 0.15 & 1.80E-05 \\
\hline Columbia River & 300 SHR HRM42.9 & September 15, 2004 & B1B7C9 & Arsenic & $\mathrm{mg} / \mathrm{L}$ & 1 & 0.0010 & $\mathrm{X}$ & 0.15 & $1.80 \mathrm{E}-05$ \\
\hline Columbia River & RICHPS10HRM46.4 & March 25, 2003 & B16L94 & Arsenic & $\mathrm{mg} / \mathrm{L}$ & 1 & 0.0010 & C & 0.15 & 1.80E-05 \\
\hline Columbia River & 100-B SPR 37-1 & November 13, 2003 & В180H6 & Arsenic & $\mathrm{mg} / \mathrm{L}$ & 1 & 0.0011 & $=$ & 0.15 & 1.80E-05 \\
\hline Columbia River & 100B SPR 37-1 & October 20, 2003 & B17J81 & Arsenic & $\mathrm{mg} / \mathrm{L}$ & 1 & 0.0011 & $=$ & 0.15 & 1.80E-05 \\
\hline Columbia River & 100-H SPR 153-1 & October 27, 1999 & B0WP44 & Arsenic & $\mathrm{mg} / \mathrm{L}$ & 1 & 0.0011 & $=$ & 0.15 & $1.80 \mathrm{E}-05$ \\
\hline Columbia River & RICHPS10HRM46.4 & March 25, 2003 & B16LC6 & Arsenic & $\mathrm{mg} / \mathrm{L}$ & 1 & 0.0011 & C & 0.15 & 1.80E-05 \\
\hline Columbia River & RICHPS10HRM46.4 & September 13, 2001 & B12TK1 & Arsenic & $\mathrm{mg} / \mathrm{L}$ & 1 & 0.0011 & $=$ & 0.15 & 1.80E-05 \\
\hline Columbia River & RICHPS10HRM46.4 & September 9, 2003 & B17CD5 & Arsenic & $\mathrm{mg} / \mathrm{L}$ & 1 & 0.0011 & $=$ & 0.15 & 1.80E-05 \\
\hline Columbia River & 300 SPR 9-1 & August 27, 2001 & B12RP2 & Arsenic & $\mathrm{mg} / \mathrm{L}$ & 1 & 0.0011 & $=$ & 0.15 & 1.80E-05 \\
\hline Columbia River & 300 SPR $42-2$ & October 7, 2002 & B15C99 & Arsenic & $\mathrm{mg} / \mathrm{L}$ & 1 & 0.0011 & $=$ & 0.15 & $1.80 \mathrm{E}-05$ \\
\hline Columbia River & 100-B SPR 38-3 & October 25, 1999 & B0WP15 & Arsenic & $\mathrm{mg} / \mathrm{L}$ & 1 & 0.0011 & $=$ & 0.15 & 1.80E-05 \\
\hline Columbia River & 300 SPR DR 42-2 & August 27, 2001 & B12RNO & Arsenic & $\mathrm{mg} / \mathrm{L}$ & 1 & 0.0011 & $=$ & 0.15 & 1.80E-05 \\
\hline Columbia River & RICHPS10HRM46.4 & September 15, 2004 & B1B762 & Arsenic & $\mathrm{mg} / \mathrm{L}$ & 1 & 0.0011 & $x$ & 0.15 & 1.80E-05 \\
\hline Columbia River & RICHPS10HRM46.4 & September 15, 2004 & B1B6Y8 & Arsenic & $\mathrm{mg} / \mathrm{L}$ & 1 & 0.0012 & $\mathrm{X}$ & 0.15 & $1.80 \mathrm{E}-05$ \\
\hline Columbia River & 100-D SPR 102-1 & October 17, 2000 & B10962 & Arsenic & $\mathrm{mg} / \mathrm{L}$ & 1 & 0.0012 & $=$ & 0.15 & 1.80E-05 \\
\hline Columbia River & 300 SPR 42-2 & August 27, 2001 & B12RM8 & Arsenic & $\mathrm{mg} / \mathrm{L}$ & 1 & 0.0012 & $=$ & 0.15 & 1.80E-05 \\
\hline Columbia River & 300 SPR $42-2$ & October 25, 2004 & B1BFY3 & Arsenic & $\mathrm{mg} / \mathrm{L}$ & 1 & 0.0012 & $x$ & 0.15 & 1.80E-05 \\
\hline Columbia River & 300 SPR 9-1 & August 27, 2001 & B12RP1 & Arsenic & $\mathrm{mg} / \mathrm{L}$ & 1 & 0.0012 & $=$ & 0.15 & 1.80E-05 \\
\hline Columbia River & 100-B SPR 38-3 & October 25, 1999 & B0WP24 & Arsenic & $\mathrm{mg} / \mathrm{L}$ & 1 & 0.0012 & $=$ & 0.15 & 1.80E-05 \\
\hline Columbia River & 300 SPR DR 42-2 & August 27, 2001 & B12RM9 & Arsenic & $\mathrm{mg} / \mathrm{L}$ & 1 & 0.0012 & $=$ & 0.15 & 1.80E-05 \\
\hline Columbia River & 300 SPR DR 42-2 & October 25, 2004 & B1BFY4 & Arsenic & $\mathrm{mg} / \mathrm{L}$ & 1 & 0.0012 & $x$ & 0.15 & 1.80E-05 \\
\hline Columbia River & 300 SPR DR 42-2 & October 25, 2004 & B1BFY5 & Arsenic & $\mathrm{mg} / \mathrm{L}$ & 1 & 0.0012 & $x$ & 0.15 & 1.80E-05 \\
\hline Columbia River & 100-B SPR 37-1 & October 11, 2004 & B1BFV3 & Arsenic & $\mathrm{mg} / \mathrm{L}$ & 1 & 0.0012 & $\mathrm{x}$ & 0.15 & $1.80 \mathrm{E}-05$ \\
\hline Columbia River & 300 A10 HRM43.1 & September 15, 2004 & B1B768 & Arsenic & $\mathrm{mg} / \mathrm{L}$ & 1 & 0.0013 & $\mathrm{X}$ & 0.15 & 1.80E-05 \\
\hline Columbia River & 100-B SPR 37-1 & October 11, 2004 & B1BFT7 & Arsenic & $\mathrm{mg} / \mathrm{L}$ & 1 & 0.0013 & $\mathrm{x}$ & 0.15 & 1.80E-05 \\
\hline Columbia River & 300 SPR 42-2 & November 1, 1999 & B0WP55 & Arsenic & $\mathrm{mg} / \mathrm{L}$ & 1 & 0.0013 & $=$ & 0.15 & 1.80E-05 \\
\hline Columbia River & 100-H SPR 152-2 & October 27, 2002 & B15CB4 & Arsenic & $\mathrm{mg} / \mathrm{L}$ & 1 & 0.0013 & $=$ & 0.15 & 1.80E-05 \\
\hline Columbia River & 300 A10 HRM43.1 & September 15, 2004 & B1B6V8 & Arsenic & $\mathrm{mg} / \mathrm{L}$ & 1 & 0.0013 & $\mathrm{X}$ & 0.15 & $1.80 \mathrm{E}-05$ \\
\hline Columbia River & 300 SPR $42-2$ & August 27, 2001 & B12RL6 & Arsenic & $\mathrm{mg} / \mathrm{L}$ & 1 & 0.0013 & $=$ & 0.15 & 1.80E-05 \\
\hline Columbia River & 300 SPR DR 42-2 & October 13, 2003 & B17RL6 & Arsenic & $\mathrm{mg} / \mathrm{L}$ & 1 & 0.0013 & $=$ & 0.15 & 1.80E-05 \\
\hline Columbia River & 100-K SPR 63-1 & October 17, 2000 & B10954 & Arsenic & $\mathrm{mg} / \mathrm{L}$ & 1 & 0.0013 & $=$ & 0.15 & 1.80E-05 \\
\hline Columbia River & 100-K SPR 77-1 & October 21, 2002 & B15C81 & Arsenic & $\mathrm{mg} / \mathrm{L}$ & 1 & 0.0013 & $=$ & 0.15 & $1.80 \mathrm{E}-05$ \\
\hline Columbia River & 100-F SPR 207-1 & October 26, 2004 & B1BFX7 & Arsenic & $\mathrm{mg} / \mathrm{L}$ & 1 & 0.0014 & $\mathrm{x}$ & 0.15 & 1.80E-05 \\
\hline Columbia River & 100-K SPR 63-1 & October 17, 2000 & B10953 & Arsenic & $\mathrm{mg} / \mathrm{L}$ & 1 & 0.0014 & $=$ & 0.15 & 1.80E-05 \\
\hline Columbia River & HFD TS HRM 28 & September 8, 2003 & B17CY3 & Arsenic & $\mathrm{mg} / \mathrm{L}$ & 1 & 0.0014 & $=$ & 0.15 & 1.80E-05 \\
\hline Columbia River & 100K SPR 63-2 & October 20, 2003 & B17J84 & Arsenic & $\mathrm{mg} / \mathrm{L}$ & 1 & 0.0014 & $=$ & 0.15 & 1.80E-05 \\
\hline Columbia River & 300 SPR DR 42-2 & November 1, 1999 & B0WP56 & Arsenic & $\mathrm{mg} / \mathrm{L}$ & 1 & 0.0014 & $=$ & 0.15 & 1.80E-05 \\
\hline Columbia River & 100-D SPR 102-1 & October 21, 2002 & B15C88 & Arsenic & $\mathrm{mg} / \mathrm{L}$ & 1 & 0.0014 & $=$ & 0.15 & 1.80E-05 \\
\hline Columbia River & 300 SPR DR 42-2 & October 13, 2003 & B17RL5 & Arsenic & $\mathrm{mg} / \mathrm{L}$ & 1 & 0.0014 & $=$ & 0.15 & $1.80 \mathrm{E}-05$ \\
\hline
\end{tabular}




\section{Table B-10}

Summary of Surface Water Results From Segment 2 Exceeding Select Ecological or Human Health Benchmark Values

Columbia River Component - Existing Sample Data Evaluation and Scoping Report

\begin{tabular}{|c|c|c|c|c|c|c|c|c|c|c|}
\hline Sample Area & Sample Site & Sample ID & Date Collected & Constituent Name & Units & QA Category & $\begin{array}{l}\text { Analytical } \\
\text { Value }\end{array}$ & Qualifier & $\begin{array}{l}\text { Ecological } \\
\text { Benchmark } \\
\text { Value }\end{array}$ & $\begin{array}{l}\text { Human Health } \\
\text { Benchmark Value }\end{array}$ \\
\hline Columbia River & HFD TS HRM 28 & September 8, 2003 & B17CY2 & Arsenic & $\mathrm{mg} / \mathrm{L}$ & 1 & 0.0014 & $=$ & 0.15 & 1.80E-05 \\
\hline Columbia River & 100-H SPR 152-2 & October 27, 2002 & B15CB5 & Arsenic & $\mathrm{mg} / \mathrm{L}$ & 1 & 0.0015 & $=$ & 0.15 & 1.80E-05 \\
\hline Columbia River & 100K SPR 63-2 & October 20, 2003 & B17J85 & Arsenic & $\mathrm{mg} / \mathrm{L}$ & 1 & 0.0015 & $=$ & 0.15 & 1.80E-05 \\
\hline Columbia River & RICHPS HRM 46.4 & December 4, 2001 & B13LD5 & Arsenic & $\mathrm{mg} / \mathrm{L}$ & 1 & 0.0015 & $=$ & 0.15 & 1.80E-05 \\
\hline Columbia River & 100F SPR 207-1 & November 3, 2003 & B17RK8 & Arsenic & $\mathrm{mg} / \mathrm{L}$ & 1 & 0.0015 & $=$ & 0.15 & $1.80 \mathrm{E}-05$ \\
\hline Columbia River & HFD TS HRM 28 & September 9, 2002 & B15904 & Arsenic & $\mathrm{mg} / \mathrm{L}$ & 1 & 0.0015 & $=$ & 0.15 & 1.80E-05 \\
\hline Columbia River & 100-K SPR 63-1 & October 25, 1999 & B0WP32 & Arsenic & $\mathrm{mg} / \mathrm{L}$ & 1 & 0.0015 & $=$ & 0.15 & $1.80 \mathrm{E}-05$ \\
\hline Columbia River & 100-F SPR 207-1 & November 7, 2000 & B10967 & Arsenic & $\mathrm{mg} / \mathrm{L}$ & 1 & 0.0015 & $=$ & 0.15 & 1.80E-05 \\
\hline Columbia River & 100-K SPR 63-1 & October 25, 1999 & B0WP34 & Arsenic & $\mathrm{mg} / \mathrm{L}$ & 1 & 0.0016 & $=$ & 0.15 & $1.80 \mathrm{E}-05$ \\
\hline Columbia River & 100-B SPR 38-3 & November 13, 2003 & $\mathrm{~B} 180 \mathrm{H} 7$ & Arsenic & $\mathrm{mg} / \mathrm{L}$ & 1 & 0.0016 & $=$ & 0.15 & 1.80E-05 \\
\hline Columbia River & 300 SPR $42-2$ & September 27, 2000 & B10973 & Arsenic & $\mathrm{mg} / \mathrm{L}$ & 1 & 0.0016 & $=$ & 0.15 & 1.80E-05 \\
\hline Columbia River & 300 SPR $42-2$ & October 13, 2003 & B17RL4 & Arsenic & $\mathrm{mg} / \mathrm{L}$ & 1 & 0.0016 & $=$ & 0.15 & 1.80E-05 \\
\hline Columbia River & 100-B SPR 38-3 & October 17, 2000 & B10944 & Arsenic & $\mathrm{mg} / \mathrm{L}$ & 1 & 0.0016 & $=$ & 0.15 & 1.80E-05 \\
\hline Columbia River & HFD TS HRM 28 & September 9, 2002 & B15905 & Arsenic & $\mathrm{mg} / \mathrm{L}$ & 1 & 0.0016 & $=$ & 0.15 & $1.80 \mathrm{E}-05$ \\
\hline Columbia River & 300 SPR $42-2$ & October 25, 2004 & B1BFL9 & Arsenic & $\mathrm{mg} / \mathrm{L}$ & 1 & 0.0017 & $x$ & 0.15 & 1.80E-05 \\
\hline Columbia River & 300 SPR 7-1 & August 27, 2001 & В12T10 & Arsenic & $\mathrm{mg} / \mathrm{L}$ & 1 & 0.0017 & $=$ & 0.15 & $1.80 \mathrm{E}-05$ \\
\hline Columbia River & 300 SPR 7-1 & August 27, 2001 & В12Т09 & Arsenic & $\mathrm{mg} / \mathrm{L}$ & 1 & 0.0018 & $=$ & 0.15 & 1.80E-05 \\
\hline Columbia River & 100-B SPR 38-3 & October 17, 2000 & B10937 & Arsenic & $\mathrm{mg} / \mathrm{L}$ & 1 & 0.0018 & $=$ & 0.15 & $1.80 \mathrm{E}-05$ \\
\hline Columbia River & 100-H SPR 145-1 & November 1, 2000 & B10965 & Arsenic & $\mathrm{mg} / \mathrm{L}$ & 1 & 0.0018 & $=$ & 0.15 & 1.80E-05 \\
\hline Columbia River & 300 SPR 42-2 & October 13, 2003 & B17RH8 & Arsenic & $\mathrm{mg} / \mathrm{L}$ & 1 & 0.0019 & $=$ & 0.15 & 1.80E-05 \\
\hline Columbia River & 100-H SPR 145-1 & October 27, 1999 & B0WP47 & Arsenic & $\mathrm{mg} / \mathrm{L}$ & 1 & 0.0020 & $=$ & 0.15 & 1.80E-05 \\
\hline Columbia River & 100-H SPR 145-1 & October 27, 1999 & B0WP46 & Arsenic & $\mathrm{mg} / \mathrm{L}$ & 1 & 0.0021 & $=$ & 0.15 & 1.80E-05 \\
\hline Columbia River & 100-H SPR 145-1 & October 26, 2004 & B1BFX4 & Arsenic & $\mathrm{mg} / \mathrm{L}$ & 1 & 0.0021 & $x$ & 0.15 & 1.80E-05 \\
\hline Columbia River & 100-K SPR 63-2 & September 16, 2002 & B15C80 & Arsenic & $\mathrm{mg} / \mathrm{L}$ & 1 & 0.0021 & $=$ & 0.15 & 1.80E-05 \\
\hline Columbia River & 100-N SPR 8-13 & October 25, 1999 & BoWNX6 & Arsenic & $\mathrm{mg} / \mathrm{L}$ & 1 & 0.0022 & $=$ & 0.15 & 1.80E-05 \\
\hline Columbia River & 100-H SPR 145-1 & October 26, 2004 & B1BFX5 & Arsenic & $\mathrm{mg} / \mathrm{L}$ & 1 & 0.0022 & $x$ & 0.15 & 1.80E-05 \\
\hline Columbia River & 100-K SPR 63-2 & September 16, 2002 & B15C79 & Arsenic & $\mathrm{mg} / \mathrm{L}$ & 1 & 0.0022 & $=$ & 0.15 & 1.80E-05 \\
\hline Columbia River & 100-N SPR 8-13 & October 11, 2004 & B1BFW7 & Arsenic & $\mathrm{mg} / \mathrm{L}$ & 1 & 0.0022 & $x$ & 0.15 & 1.80E-05 \\
\hline Columbia River & 100-N SPR 8-13 & October 25, 1999 & B0WP37 & Arsenic & $\mathrm{mg} / \mathrm{L}$ & 1 & 0.0022 & $=$ & 0.15 & 1.80E-05 \\
\hline Columbia River & 100-F SPR 207-1 & October 29, 2002 & В15C93 & Arsenic & $\mathrm{mg} / \mathrm{L}$ & 1 & 0.0023 & $=$ & 0.15 & 1.80E-05 \\
\hline Columbia River & 100-D SPR 110-1 & October 21, 2002 & B15C86 & Arsenic & $\mathrm{mg} / \mathrm{L}$ & 1 & 0.0023 & $=$ & 0.15 & $1.80 \mathrm{E}-05$ \\
\hline Columbia River & 100N SPR 8-13 & October 20, 2003 & B17J88 & Arsenic & $\mathrm{mg} / \mathrm{L}$ & 1 & 0.0023 & $=$ & 0.15 & 1.80E-05 \\
\hline Columbia River & 100-F SPR 207-1 & November 7, 2000 & B10966 & Arsenic & $\mathrm{mg} / \mathrm{L}$ & 1 & 0.0024 & $=$ & 0.15 & 1.80E-05 \\
\hline Columbia River & 100-N SPR 8-13 & October 11, 2004 & B1BFM0 & Arsenic & $\mathrm{mg} / \mathrm{L}$ & 1 & 0.0024 & $\mathrm{x}$ & 0.15 & $1.80 \mathrm{E}-05$ \\
\hline Columbia River & 100N SPR 8-13 & October 20, 2003 & B17J17 & Arsenic & $\mathrm{mg} / \mathrm{L}$ & 1 & 0.0024 & $=$ & 0.15 & $1.80 \mathrm{E}-05$ \\
\hline Columbia River & 100-F SPR 207-1 & November 1, 1999 & BOWP49 & Arsenic & $\mathrm{mg} / \mathrm{L}$ & 1 & 0.0024 & $=$ & 0.15 & 1.80E-05 \\
\hline Columbia River & HFD SPR UR 28-2 & November 3, 2003 & B17RL1 & Arsenic & $\mathrm{mg} / \mathrm{L}$ & 1 & 0.0025 & $=$ & 0.15 & 1.80E-05 \\
\hline Columbia River & 100-H SPR 145-1 & November 1, 2000 & B10964 & Arsenic & $\mathrm{mg} / \mathrm{L}$ & 1 & 0.0025 & $=$ & 0.15 & $1.80 \mathrm{E}-05$ \\
\hline Columbia River & HFD SPR UR 28-2 & November 3, 2003 & B17RL0 & Arsenic & $\mathrm{mg} / \mathrm{L}$ & 1 & 0.0026 & $=$ & 0.15 & 1.80E-05 \\
\hline Columbia River & 100-N SPR 8-13 & September 16, 2002 & B15C83 & Arsenic & $\mathrm{mg} / \mathrm{L}$ & 1 & 0.0026 & $=$ & 0.15 & $1.80 \mathrm{E}-05$ \\
\hline Columbia River & HFD SPR UR 28-2 & September 27, 2000 & B10969 & Arsenic & $\mathrm{mg} / \mathrm{L}$ & 1 & 0.0026 & $=$ & 0.15 & $1.80 \mathrm{E}-05$ \\
\hline Columbia River & HFD SPR UR 28-2 & September 27, 2000 & B10970 & Arsenic & $\mathrm{mg} / \mathrm{L}$ & 1 & 0.0026 & $=$ & 0.15 & 1.80E-05 \\
\hline Columbia River & 100-N SPR 8-13 & March 29, 2004 & B18XM0 & Arsenic & $\mathrm{mg} / \mathrm{L}$ & 1 & 0.0027 & $x$ & 0.15 & 1.80E-05 \\
\hline Columbia River & 100-N SPR 8-13 & March 29, 2004 & B18XL7 & Arsenic & $\mathrm{mg} / \mathrm{L}$ & 1 & 0.0027 & $\mathrm{x}$ & 0.15 & 1.80E-05 \\
\hline Columbia River & 300 AREA OUT13 & September 15, 2004 & B1B7H8 & Arsenic & $\mathrm{mg} / \mathrm{L}$ & 1 & 0.0027 & $x$ & 0.15 & 1.80E-05 \\
\hline
\end{tabular}




\section{Table B-10}

Summary of Surface Water Results From Segment 2 Exceeding Select Ecological or Human Health Benchmark Values

Columbia River Component - Existing Sample Data Evaluation and Scoping Report

\begin{tabular}{|c|c|c|c|c|c|c|c|c|c|c|}
\hline Sample Area & Sample Site & Sample ID & Date Collected & Constituent Name & Units & QA Category & $\begin{array}{c}\text { Analytical } \\
\text { Value }\end{array}$ & Qualifier & $\begin{array}{l}\text { Ecological } \\
\text { Benchmark } \\
\text { Value }\end{array}$ & $\begin{array}{c}\text { Human Health } \\
\text { Benchmark Value }\end{array}$ \\
\hline Columbia River & 300 AREA OUT13 & September 15, 2004 & B1B7H9 & Arsenic & $\mathrm{mg} / \mathrm{L}$ & 1 & 0.0028 & $\bar{x}$ & 0.15 & 1.80E-05 \\
\hline Columbia River & 100-F SPR 207-1 & November 1, 1999 & B0WP48 & Arsenic & $\mathrm{mg} / \mathrm{L}$ & 1 & 0.0028 & $=$ & 0.15 & 1.80E-05 \\
\hline Columbia River & HFD SPR UR 28-2 & October 25, 2004 & B1BFX9 & Arsenic & $\mathrm{mg} / \mathrm{L}$ & 1 & 0.0028 & $x$ & 0.15 & 1.80E-05 \\
\hline Columbia River & 100H SPR 145-1 & October 27, 2003 & B17RK6 & Arsenic & $\mathrm{mg} / \mathrm{L}$ & 1 & 0.0029 & $=$ & 0.15 & 1.80E-05 \\
\hline Columbia River & 100-F SPR 207-1 & October 29, 2002 & В15C92 & Arsenic & $\mathrm{mg} / \mathrm{L}$ & 1 & 0.0029 & $=$ & 0.15 & 1.80E-05 \\
\hline Columbia River & 100-N SPR 8-13 & October 17, 2000 & B10904 & Arsenic & $\mathrm{mg} / \mathrm{L}$ & 1 & 0.0029 & $=$ & 0.15 & 1.80E-05 \\
\hline Columbia River & 100-N SPR 8-13 & October 17, 2000 & B10957 & Arsenic & $\mathrm{mg} / \mathrm{L}$ & 1 & 0.0029 & $=$ & 0.15 & $1.80 \mathrm{E}-05$ \\
\hline Columbia River & 300 AREA OUT13 & June 24, 2004 & B19JC6 & Arsenic & $\mathrm{mg} / \mathrm{L}$ & 1 & 0.0030 & $\mathrm{X}$ & 0.15 & 1.80E-05 \\
\hline Columbia River & HFD SPR 28-2 & October 7, 2002 & В15C94 & Arsenic & $\mathrm{mg} / \mathrm{L}$ & 1 & 0.0030 & $=$ & 0.15 & 1.80E-05 \\
\hline Columbia River & 100F SPR 207-1 & November 3, 2003 & B17RK7 & Arsenic & $\mathrm{mg} / \mathrm{L}$ & 1 & 0.0031 & $=$ & 0.15 & 1.80E-05 \\
\hline Columbia River & HFD SPR 28-2 & October 25, 2004 & B1BFX8 & Arsenic & $\mathrm{mg} / \mathrm{L}$ & 1 & 0.0031 & $\mathrm{X}$ & 0.15 & $1.80 \mathrm{E}-05$ \\
\hline Columbia River & 300 AREA OUT13 & June 24, 2004 & B19JC5 & Arsenic & $\mathrm{mg} / \mathrm{L}$ & 1 & 0.0031 & $\mathrm{X}$ & 0.15 & 1.80E-05 \\
\hline Columbia River & HFD SPR DR 28-2 & October 7, 2002 & В15C98 & Arsenic & $\mathrm{mg} / \mathrm{L}$ & 1 & 0.0032 & $=$ & 0.15 & 1.80E-05 \\
\hline Columbia River & HFD SPR 28-2 & October 7, 2002 & В15C00 & Arsenic & $\mathrm{mg} / \mathrm{L}$ & 1 & 0.0032 & C & 0.15 & 1.80E-05 \\
\hline Columbia River & HFD SPR DR 28-2 & October 7, 2002 & B15C97 & Arsenic & $\mathrm{mg} / \mathrm{L}$ & 1 & 0.0032 & C & 0.15 & 1.80E-05 \\
\hline Columbia River & HFD SPR 28-2 & September 27, 2000 & B10902 & Arsenic & $\mathrm{mg} / \mathrm{L}$ & 1 & 0.0034 & $=$ & 0.15 & $1.80 \mathrm{E}-05$ \\
\hline Columbia River & 100-N SPR 8-13 & September 16, 2002 & B15C02 & Arsenic & $\mathrm{mg} / \mathrm{L}$ & 1 & 0.0034 & $=$ & 0.15 & 1.80E-05 \\
\hline Columbia River & HFD SPR 28-2 & September 27, 2000 & B10968 & Arsenic & $\mathrm{mg} / \mathrm{L}$ & 1 & 0.0034 & $=$ & 0.15 & 1.80E-05 \\
\hline Columbia River & HFD SPR DR 28-2 & October 25, 2004 & B1BFY2 & Arsenic & $\mathrm{mg} / \mathrm{L}$ & 1 & 0.0035 & $x$ & 0.15 & 1.80E-05 \\
\hline Columbia River & HFD SPR DR 28-2 & September 27, 2000 & B10972 & Arsenic & $\mathrm{mg} / \mathrm{L}$ & 1 & 0.0035 & $=$ & 0.15 & $1.80 \mathrm{E}-05$ \\
\hline Columbia River & HFD SPR 28-2 & October 25, 2004 & B1BFL8 & Arsenic & $\mathrm{mg} / \mathrm{L}$ & 1 & 0.0036 & $\mathrm{X}$ & 0.15 & 1.80E-05 \\
\hline Columbia River & HFD SPR DR 28-2 & September 27, 2000 & B10971 & Arsenic & $\mathrm{mg} / \mathrm{L}$ & 1 & 0.0036 & $=$ & 0.15 & 1.80E-05 \\
\hline Columbia River & HFD SPR DR 28-2 & October 25, 2004 & B1BFY1 & Arsenic & $\mathrm{mg} / \mathrm{L}$ & 1 & 0.0037 & $x$ & 0.15 & 1.80E-05 \\
\hline Columbia River & HFD SPR DR 28-2 & November 3, 2003 & B17RL3 & Arsenic & $\mathrm{mg} / \mathrm{L}$ & 1 & 0.0037 & $=$ & 0.15 & 1.80E-05 \\
\hline Columbia River & HFD SPR DR 28-2 & April 30, 2001 & B11W81 & Arsenic & $\mathrm{mg} / \mathrm{L}$ & 1 & 0.0039 & $=$ & 0.15 & $1.80 \mathrm{E}-05$ \\
\hline Columbia River & HFD SPR UR 28-2 & November 1, 1999 & B0WP52 & Arsenic & $\mathrm{mg} / \mathrm{L}$ & 1 & 0.0039 & $=$ & 0.15 & 1.80E-05 \\
\hline Columbia River & HFD SPR DR 28-2 & November 3, 2003 & B17RL2 & Arsenic & $\mathrm{mg} / \mathrm{L}$ & 1 & 0.0039 & $=$ & 0.15 & 1.80E-05 \\
\hline Columbia River & HFD SPR 28-2 & April 30, 2001 & B11W77 & Arsenic & $\mathrm{mg} / \mathrm{L}$ & 1 & 0.0040 & $=$ & 0.15 & 1.80E-05 \\
\hline Columbia River & HFD SPR UR 28-2 & November 1, 1999 & B0WP51 & Arsenic & $\mathrm{mg} / \mathrm{L}$ & 1 & 0.0040 & $=$ & 0.15 & $1.80 \mathrm{E}-05$ \\
\hline Columbia River & 300 SPR 42-2 & September 27, 2000 & B10903 & Arsenic & $\mathrm{mg} / \mathrm{L}$ & 1 & 0.0040 & $=$ & 0.15 & 1.80E-05 \\
\hline Columbia River & HFD SPR DR 28-2 & November 1, 1999 & B0WP54 & Arsenic & $\mathrm{mg} / \mathrm{L}$ & 1 & 0.0043 & $=$ & 0.15 & 1.80E-05 \\
\hline Columbia River & ESQUATZEL CANAL & May 14, 2003 & B16WT7 & Arsenic & $\mathrm{mg} / \mathrm{L}$ & 1 & 0.0044 & $x$ & 0.15 & 1.80E-05 \\
\hline Columbia River & ESQUATZEL CANAL & May 14, 2003 & B16WT9 & Arsenic & $\mathrm{mg} / \mathrm{L}$ & 1 & 0.0044 & $x$ & 0.15 & 1.80E-05 \\
\hline Columbia River & ESQUATZEL CANAL & May 14, 2003 & B16WV0 & Arsenic & $\mathrm{mg} / \mathrm{L}$ & 1 & 0.0044 & $\mathrm{X}$ & 0.15 & $1.80 \mathrm{E}-05$ \\
\hline Columbia River & ESQUATZEL CANAL & May 14, 2003 & B16WV1 & Arsenic & $\mathrm{mg} / \mathrm{L}$ & 1 & 0.0045 & $\mathrm{x}$ & 0.15 & 1.80E-05 \\
\hline Columbia River & ESQUATZEL CANAL & May 14, 2003 & B16WT6 & Arsenic & $\mathrm{mg} / \mathrm{L}$ & 1 & 0.0045 & $\mathrm{X}$ & 0.15 & 1.80E-05 \\
\hline Columbia River & HFD SPR DR 28-2 & November 1, 1999 & B0WP53 & Arsenic & $\mathrm{mg} / \mathrm{L}$ & 1 & 0.0046 & $=$ & 0.15 & 1.80E-05 \\
\hline Columbia River & ESQUATZEL CANAL & May 14, 2003 & B16WT8 & Arsenic & $\mathrm{mg} / \mathrm{L}$ & 1 & 0.0047 & $\mathrm{X}$ & 0.15 & $1.80 \mathrm{E}-05$ \\
\hline Columbia River & Ringold WW & May 14, 2003 & B16WT0 & Arsenic & $\mathrm{mg} / \mathrm{L}$ & 1 & 0.0047 & $\mathrm{x}$ & 0.15 & 1.80E-05 \\
\hline Columbia River & Ringold WW & May 14, 2003 & B16WT5 & Arsenic & $\mathrm{mg} / \mathrm{L}$ & 1 & 0.0047 & $\mathrm{x}$ & 0.15 & 1.80E-05 \\
\hline Columbia River & 100-F SPR 207-1 & October 26, 2004 & B1BFX6 & Arsenic & $\mathrm{mg} / \mathrm{L}$ & 1 & 0.0047 & $x$ & 0.15 & 1.80E-05 \\
\hline Columbia River & HFD SPR 28-2 & November 1, 1999 & B0WP50 & Arsenic & $\mathrm{mg} / \mathrm{L}$ & 1 & 0.0048 & $=$ & 0.15 & 1.80E-05 \\
\hline Columbia River & HFD SPR 28-2 & April 30, 2001 & B11W30 & Arsenic & $\mathrm{mg} / \mathrm{L}$ & 1 & 0.0048 & $=$ & 0.15 & 1.80E-05 \\
\hline Columbia River & Ringold WW & May 14, 2003 & B16WT4 & Arsenic & $\mathrm{mg} / \mathrm{L}$ & 1 & 0.0048 & $x$ & 0.15 & 1.80E-05 \\
\hline Columbia River & Ringold WW & May 14, 2003 & B16WT3 & Arsenic & $\mathrm{mg} / \mathrm{L}$ & 1 & 0.0048 & $\mathrm{x}$ & 0.15 & $1.80 \mathrm{E}-05$ \\
\hline
\end{tabular}

127 of 137 


\section{Table B-10}

Summary of Surface Water Results From Segment 2 Exceeding Select Ecological or Human Health Benchmark Values

Columbia River Component - Existing Sample Data Evaluation and Scoping Report

\begin{tabular}{|c|c|c|c|c|c|c|c|c|c|c|}
\hline Sample Area & Sample Site & Sample ID & Date Collected & Constituent Name & Units & QA Category & $\begin{array}{c}\text { Analytical } \\
\text { Value }\end{array}$ & Qualifier & $\begin{array}{l}\text { Ecological } \\
\text { Benchmark } \\
\text { Value }\end{array}$ & $\begin{array}{c}\text { Human Health } \\
\text { Benchmark Value }\end{array}$ \\
\hline Columbia River & HFD SPR DR 28-2 & April 30, 2001 & B11W80 & Arsenic & $\mathrm{mg} / \mathrm{L}$ & 1 & 0.0049 & $=$ & 0.15 & $1.80 \mathrm{E}-05$ \\
\hline Columbia River & Ringold WW & May 14, 2003 & B16WT2 & Arsenic & $\mathrm{mg} / \mathrm{L}$ & 1 & 0.0049 & $\mathrm{x}$ & 0.15 & $1.80 \mathrm{E}-05$ \\
\hline Columbia River & HFD SPR 28-2 & November 1, 1999 & BOWNX4 & Arsenic & $\mathrm{mg} / \mathrm{L}$ & 1 & 0.0049 & $=$ & 0.15 & 1.80E-05 \\
\hline Columbia River & Ringold WW & May 14, 2003 & B16WT1 & Arsenic & mg/L & 1 & 0.0050 & $\mathrm{x}$ & 0.15 & 1.80E-05 \\
\hline Columbia River & 300 SPR $42-2$ & November 1, 1999 & BoWNX5 & Arsenic & $\mathrm{mg} / \mathrm{L}$ & 1 & 0.0082 & $=$ & 0.15 & $1.80 \mathrm{E}-05$ \\
\hline Columbia River & $100 \mathrm{H}$ SPR $145-1$ & October 10, 2003 & B17RK5 & Arsenic & $\mathrm{mg} / \mathrm{L}$ & 1 & 0.0085 & $=$ & 0.15 & $1.80 \mathrm{E}-05$ \\
\hline Columbia River & 100 B/C AREA UR & November 13, 2003 & B180C6 & Barium & $\mathrm{mg} / \mathrm{L}$ & 1 & 0.017 & C & 0.0040 & 1.0 \\
\hline Columbia River & 100-K SPR 77-1 & November 10, 2003 & B17JP9 & Barium & mg/L & 1 & 0.019 & B & 0.0040 & 1.0 \\
\hline Columbia River & 100-K SPR 77-1 & November 10, 2003 & B17JP8 & Barium & $\mathrm{mg} / \mathrm{L}$ & 1 & 0.020 & B & 0.0040 & 1.0 \\
\hline Columbia River & 100-H SPR 145-1 & November 1, 2000 & B10JT4 & Barium & mg/L & 1 & 0.021 & B & 0.0040 & 1.0 \\
\hline Columbia River & 100 B/C AREA UR & November 13, 2003 & B180C7 & Barium & mg/L & 1 & 0.021 & C & 0.0040 & 1.0 \\
\hline Columbia River & 100-H SPR 145-1 & November 1, 2000 & B10JR3 & Barium & $\mathrm{mg} / \mathrm{L}$ & 1 & 0.021 & B & 0.0040 & 1.0 \\
\hline Columbia River & 100 B/C AREA UR & November 13, 2003 & B180C8 & Barium & $\mathrm{mg} / \mathrm{L}$ & 1 & 0.021 & C & 0.0040 & 1.0 \\
\hline Columbia River & 100-H SPR 153-1 & October 27, 2002 & B15HY8 & Barium & $\mathrm{mg} / \mathrm{L}$ & 1 & 0.021 & B & 0.0040 & 1.0 \\
\hline Columbia River & 100 B/C OUTFLL2 & November 17, 2003 & B180J0 & Barium & $\mathrm{mg} / \mathrm{L}$ & 1 & 0.021 & C & 0.0040 & 1.0 \\
\hline Columbia River & 100-H SPR 153-1 & October 27, 2002 & B15HY7 & Barium & mg/L & 1 & 0.022 & B & 0.0040 & 1.0 \\
\hline Columbia River & 100-H SPR 144-1 & October 27, 2002 & B15HX3 & Barium & mg/L & 1 & 0.023 & B & 0.0040 & 1.0 \\
\hline Columbia River & 100-B SPR 39-2 & November 13, 2003 & B180D7 & Barium & mg/L & 1 & 0.023 & C & 0.0040 & 1.0 \\
\hline Columbia River & 100-B SPR 38-3 & November 13, 2003 & B180D3 & Barium & $\mathrm{mg} / \mathrm{L}$ & 1 & 0.023 & C & 0.0040 & 1.0 \\
\hline Columbia River & 100-B SPR 39-2 & November 13, 2003 & B180D6 & Barium & $\mathrm{mg} / \mathrm{L}$ & 1 & 0.023 & C & 0.0040 & 1.0 \\
\hline Columbia River & 100 B/C OUTFLL1 & November 17, 2003 & B180D8 & Barium & $\mathrm{mg} / \mathrm{L}$ & 1 & 0.023 & C & 0.0040 & 1.0 \\
\hline Columbia River & 100-B SPR 39-2 & November 13, 2003 & B180D5 & Barium & $\mathrm{mg} / \mathrm{L}$ & 1 & 0.023 & C & 0.0040 & 1.0 \\
\hline Columbia River & 100-H SPR 152-2 & November 1, 2001 & B135Y4 & Barium & $\mathrm{mg} / \mathrm{L}$ & 1 & 0.023 & $=$ & 0.0040 & 1.0 \\
\hline Columbia River & 100 B/C AREA UR & November 13, 2003 & B180C9 & Barium & mg/L & 1 & 0.024 & C & 0.0040 & 1.0 \\
\hline Columbia River & 100 B/C AREA DR & November 17, 2003 & B180F3 & Barium & $\mathrm{mg} / \mathrm{L}$ & 1 & 0.024 & C & 0.0040 & 1.0 \\
\hline Columbia River & $100 \mathrm{~B} / \mathrm{C}$ AREA DR & November 17, 2003 & B180F1 & Barium & mg/L & 1 & 0.024 & C & 0.0040 & 1.0 \\
\hline Columbia River & $100 \mathrm{~B} / \mathrm{C}$ AREA DR & November 17, 2003 & B180F2 & Barium & mg/L & 1 & 0.024 & C & 0.0040 & 1.0 \\
\hline Columbia River & 100 B/C AREA DR & November 17, 2003 & B180F4 & Barium & $\mathrm{mg} / \mathrm{L}$ & 1 & 0.024 & C & 0.0040 & 1.0 \\
\hline Columbia River & 100 B/C OUTFLL2 & November 17, 2003 & B180D9 & Barium & $\mathrm{mg} / \mathrm{L}$ & 1 & 0.024 & C & 0.0040 & 1.0 \\
\hline Columbia River & 100-B SPR 39-2 & November 13, 2003 & B180D4 & Barium & $\mathrm{mg} / \mathrm{L}$ & 1 & 0.024 & C & 0.0040 & 1.0 \\
\hline Columbia River & 100-H SPR 144-1 & October 27, 2002 & $\mathrm{~B} 15 \mathrm{HX} 2$ & Barium & $\mathrm{mg} / \mathrm{L}$ & 1 & 0.024 & B & 0.0040 & 1.0 \\
\hline Columbia River & 100 B/C OUTFLL3 & November 17, 2003 & B180F0 & Barium & mg/L & 1 & 0.024 & C & 0.0040 & 1.0 \\
\hline Columbia River & 100-B SPR 38-3 & November 13, 2003 & B180F9 & Barium & mg/L & 1 & 0.024 & C & 0.0040 & 1.0 \\
\hline Columbia River & 100-B SPR 38-3 & November 13, 2003 & B180D2 & Barium & $\mathrm{mg} / \mathrm{L}$ & 1 & 0.024 & C & 0.0040 & 1.0 \\
\hline Columbia River & 100-B SPR 38-3 & November 13, 2003 & B180D1 & Barium & mg/L & 1 & 0.024 & C & 0.0040 & 1.0 \\
\hline Columbia River & $100 \mathrm{~N}$ Area & June 9, 2004 & $\mathrm{~B} 19 \mathrm{H} 73$ & Barium & $\mathrm{mg} / \mathrm{L}$ & 1 & 0.025 & $x$ & 0.0040 & 1.0 \\
\hline Columbia River & 100-B SPR 38-3 & November 13, 2003 & B180D0 & Barium & $\mathrm{mg} / \mathrm{L}$ & 1 & 0.025 & C & 0.0040 & 1.0 \\
\hline Columbia River & 100-H SPR 153-1 & November 1, 2001 & B135Y6 & Barium & $\mathrm{mg} / \mathrm{L}$ & 1 & 0.025 & $=$ & 0.0040 & 1.0 \\
\hline Columbia River & 100-H SPR 145-1 & October 27, 2002 & B15HX6 & Barium & $\mathrm{mg} / \mathrm{L}$ & 1 & 0.026 & B & 0.0040 & 1.0 \\
\hline
\end{tabular}




\section{Table B-10}

Summary of Surface Water Results From Segment 2 Exceeding Select Ecological or Human Health Benchmark Values

Columbia River Component - Existing Sample Data Evaluation and Scoping Report

\begin{tabular}{|c|c|c|c|c|c|c|c|c|c|c|}
\hline Sample Area & Sample Site & Sample ID & Date Collected & Constituent Name & Units & QA Category & $\begin{array}{c}\text { Analytical } \\
\text { Value }\end{array}$ & Qualifier & $\begin{array}{l}\text { Ecological } \\
\text { Benchmark } \\
\text { Value }\end{array}$ & $\begin{array}{c}\text { Human Health } \\
\text { Benchmark Value }\end{array}$ \\
\hline Columbia River & 100-H SPR 145-1 & October 27, 2002 & B15HX9 & Barium & $\mathrm{mg} / \mathrm{L}$ & 1 & 0.026 & $\mathrm{~B}$ & 0.0040 & 1.0 \\
\hline Columbia River & 100-K SPR 77-1 & October 25, 2001 & B13601 & Barium & $\mathrm{mg} / \mathrm{L}$ & 1 & 0.026 & $=$ & 0.0040 & 1.0 \\
\hline Columbia River & 100-H SPR 152-2 & October 27, 2002 & B15HY5 & Barium & $\mathrm{mg} / \mathrm{L}$ & 1 & 0.026 & B & 0.0040 & 1.0 \\
\hline Columbia River & 100-K SPR 77-1 & October 21, 2002 & B15LM5 & Barium & mg/L & 1 & 0.026 & B & 0.0040 & 1.0 \\
\hline Columbia River & 100 N Area & April 9, 2004 & B19114 & Barium & $\mathrm{mg} / \mathrm{L}$ & 1 & 0.026 & $x$ & 0.0040 & 1.0 \\
\hline Columbia River & 100-D SPR 110-1 & October 27, 2003 & B17JN7 & Barium & $\mathrm{mg} / \mathrm{L}$ & 1 & 0.027 & $=$ & 0.0040 & 1.0 \\
\hline Columbia River & 100-H SPR 145-1 & October 27, 2002 & B15HX8 & Barium & mg/L & 1 & 0.027 & B & 0.0040 & 1.0 \\
\hline Columbia River & 100-H SPR 150-1 & November 20, 2003 & B17VK6 & Barium & mg/L & 1 & 0.028 & B & 0.0040 & 1.0 \\
\hline Columbia River & 100-K SPR 77-1 & October 25, 2001 & B13600 & Barium & $\mathrm{mg} / \mathrm{L}$ & 1 & 0.028 & $=$ & 0.0040 & 1.0 \\
\hline Columbia River & $100 \mathrm{~N}$ Area & September 25, 2004 & B1BC57 & Barium & mg/L & 1 & 0.028 & $\mathrm{x}$ & 0.0040 & 1.0 \\
\hline Columbia River & 100-H SPR 150-1 & October 27, 2002 & B15HY2 & Barium & mg/L & 1 & 0.028 & B & 0.0040 & 1.0 \\
\hline Columbia River & 100-D SPR 102-1 & October 27, 2003 & B17JN9 & Barium & $\mathrm{mg} / \mathrm{L}$ & 1 & 0.028 & B & 0.0040 & 1.0 \\
\hline Columbia River & 100-H SPR 152-2 & October 27, 2002 & B15HY4 & Barium & $\mathrm{mg} / \mathrm{L}$ & 1 & 0.028 & B & 0.0040 & 1.0 \\
\hline Columbia River & 100-D SPR 102-1 & October 27, 2003 & B17JP0 & Barium & $\mathrm{mg} / \mathrm{L}$ & 1 & 0.029 & B & 0.0040 & 1.0 \\
\hline Columbia River & 100-D SPR 98-1 & October 21, 2002 & B15HX0 & Barium & $\mathrm{mg} / \mathrm{L}$ & 1 & 0.029 & B & 0.0040 & 1.0 \\
\hline Columbia River & 100-D SPR 102-1 & October 27, 2003 & B17JN8 & Barium & mg/L & 1 & 0.030 & $=$ & 0.0040 & 1.0 \\
\hline Columbia River & 100-H SPR 145-1 & November 1, 2001 & B135X9 & Barium & mg/L & 1 & 0.030 & $=$ & 0.0040 & 1.0 \\
\hline Columbia River & 300 SPR 11 -1 & August 27, 2001 & B12RP6 & Barium & mg/L & 1 & 0.031 & $=$ & 0.0040 & 1.0 \\
\hline Columbia River & SD-98-1 & October 12, 2000 & B10JV0 & Barium & $\mathrm{mg} / \mathrm{L}$ & 1 & 0.031 & B & 0.0040 & 1.0 \\
\hline Columbia River & SD-98-1 & October 12, 2000 & B10JT2 & Barium & $\mathrm{mg} / \mathrm{L}$ & 1 & 0.032 & B & 0.0040 & 1.0 \\
\hline Columbia River & 100-D SPR 98-1 & October 21, 2002 & B15HW9 & Barium & $\mathrm{mg} / \mathrm{L}$ & 1 & 0.032 & B & 0.0040 & 1.0 \\
\hline Columbia River & 100-D SPR 98-1 & October 25, 2001 & $\mathrm{~B} 135 \mathrm{X} 2$ & Barium & $\mathrm{mg} / \mathrm{L}$ & 1 & 0.032 & $=$ & 0.0040 & 1.0 \\
\hline Columbia River & 100-H SPR 150-1 & October 27, 2002 & B15HY1 & Barium & $\mathrm{mg} / \mathrm{L}$ & 1 & 0.032 & B & 0.0040 & 1.0 \\
\hline Columbia River & 100-K SPR 63-1 & October 20, 2003 & B17JR1 & Barium & mg/L & 1 & 0.032 & B & 0.0040 & 1.0 \\
\hline Columbia River & 300 SPR $11-1$ & August 27, 2001 & B12RP5 & Barium & $\mathrm{mg} / \mathrm{L}$ & 1 & 0.033 & $=$ & 0.0040 & 1.0 \\
\hline Columbia River & SH-144-1 & October 13, 2000 & B10JR2 & Barium & mg/L & 1 & 0.033 & B & 0.0040 & 1.0 \\
\hline Columbia River & SH-144-1 & October 13, 2000 & B10JT3 & Barium & mg/L & 1 & 0.033 & B & 0.0040 & 1.0 \\
\hline Columbia River & SH-150-1 & November 1, 2000 & B10JT6 & Barium & $\mathrm{mg} / \mathrm{L}$ & 1 & 0.034 & B & 0.0040 & 1.0 \\
\hline Columbia River & SH-150-1 & November 1, 2000 & B10JR5 & Barium & $\mathrm{mg} / \mathrm{L}$ & 1 & 0.034 & B & 0.0040 & 1.0 \\
\hline Columbia River & SD-110-2 & October 17, 2000 & B10JR0 & Barium & $\mathrm{mg} / \mathrm{L}$ & 1 & 0.034 & B & 0.0040 & 1.0 \\
\hline Columbia River & 100-K SPR 68-1 & November 10, 2003 & B17LC4 & Barium & $\mathrm{mg} / \mathrm{L}$ & 1 & 0.035 & B & 0.0040 & 1.0 \\
\hline Columbia River & 100-K SPR 77-1 & October 21, 2002 & B15LM4 & Barium & mg/L & 1 & 0.036 & B & 0.0040 & 1.0 \\
\hline Columbia River & SD-110-2 & October 17, 2000 & B10JT1 & Barium & mg/L & 1 & 0.036 & B & 0.0040 & 1.0 \\
\hline Columbia River & 100-D SPR 102-1 & October 21, 2002 & B15HV9 & Barium & mg/L & 1 & 0.036 & B & 0.0040 & 1.0 \\
\hline Columbia River & SD-98-1 & October 12, 2000 & B10JR1 & Barium & mg/L & 1 & 0.036 & B & 0.0040 & 1.0 \\
\hline Columbia River & 100-D SPR 110-1 & October 17, 2000 & B10JT0 & Barium & $\mathrm{mg} / \mathrm{L}$ & 1 & 0.040 & B & 0.0040 & 1.0 \\
\hline Columbia River & 100-D SPR 110-1 & October 17, 2000 & B10JP8 & Barium & $\mathrm{mg} / \mathrm{L}$ & 1 & 0.041 & B & 0.0040 & 1.0 \\
\hline Columbia River & 100-K SPR 82-2 & October 21, 2002 & B15LM7 & Barium & $\mathrm{mg} / \mathrm{L}$ & 1 & 0.044 & B & 0.0040 & 1.0 \\
\hline Columbia River & 100-N SPR 8-13 & March 29, 2004 & B18XL7 & Barium & $\mathrm{mg} / \mathrm{L}$ & 1 & 0.044 & $\mathrm{x}$ & 0.0040 & 1.0 \\
\hline
\end{tabular}




\section{Table B-10}

Summary of Surface Water Results From Segment 2 Exceeding Select Ecological or Human Health Benchmark Values

Columbia River Component - Existing Sample Data Evaluation and Scoping Report

\begin{tabular}{|c|c|c|c|c|c|c|c|c|c|c|}
\hline Sample Area & Sample Site & Sample ID & Date Collected & Constituent Name & Units & QA Category & $\begin{array}{c}\text { Analytical } \\
\text { Value }\end{array}$ & Qualifier & $\begin{array}{l}\text { Ecological } \\
\text { Benchmark } \\
\text { Value }\end{array}$ & $\begin{array}{c}\text { Human Health } \\
\text { Benchmark Value }\end{array}$ \\
\hline Columbia River & 100-B SPR 38-3 & November 13, 2003 & $\mathrm{~B} 180 \mathrm{H} 7$ & Barium & $\mathrm{mg} / \mathrm{L}$ & 1 & 0.044 & $\mathrm{C}$ & 0.0040 & 1.0 \\
\hline Columbia River & 100-K SPR 82-2 & October 21, 2002 & B15LM8 & Barium & $\mathrm{mg} / \mathrm{L}$ & 1 & 0.044 & B & 0.0040 & 1.0 \\
\hline Columbia River & 100-K SPR 82-2 & October 25, 2001 & B13604 & Barium & $\mathrm{mg} / \mathrm{L}$ & 1 & 0.045 & $=$ & 0.0040 & 1.0 \\
\hline Columbia River & 100-N SPR 8-13 & March 29, 2004 & B18XM0 & Barium & mg/L & 1 & 0.045 & $\mathrm{x}$ & 0.0040 & 1.0 \\
\hline Columbia River & 100-D SPR 102-1 & October 21, 2002 & B15HV8 & Barium & $\mathrm{mg} / \mathrm{L}$ & 1 & 0.046 & B & 0.0040 & 1.0 \\
\hline Columbia River & 100-B SPR 37-1 & November 13, 2003 & $\mathrm{~B} 180 \mathrm{H} 6$ & Barium & $\mathrm{mg} / \mathrm{L}$ & 1 & 0.047 & C & 0.0040 & 1.0 \\
\hline Columbia River & 100-K SPR 82-2 & October 25, 2001 & B13605 & Barium & mg/L & 1 & 0.048 & $=$ & 0.0040 & 1.0 \\
\hline Columbia River & 100-F SPRING & November 20, 2003 & B17LB2 & Barium & mg/L & 1 & 0.049 & B & 0.0040 & 1.0 \\
\hline Columbia River & 100-D SPR 102-1 & October 27, 2003 & B17JP3 & Barium & $\mathrm{mg} / \mathrm{L}$ & 1 & 0.050 & B & 0.0040 & 1.0 \\
\hline Columbia River & 300 SPR 7-1 & August 27, 2001 & B12T10 & Barium & $\mathrm{mg} / \mathrm{L}$ & 1 & 0.050 & $=$ & 0.0040 & 1.0 \\
\hline Columbia River & 300 SPR 7-1 & August 27, 2001 & B12T09 & Barium & mg/L & 1 & 0.051 & $=$ & 0.0040 & 1.0 \\
\hline Columbia River & SF-211-1 & November 7, 2000 & B10FC6 & Barium & $\mathrm{mg} / \mathrm{L}$ & 1 & 0.052 & B & 0.0040 & 1.0 \\
\hline Columbia River & 100-D SPR 102-1 & October 17, 2000 & B10JR8 & Barium & $\mathrm{mg} / \mathrm{L}$ & 1 & 0.052 & B & 0.0040 & 1.0 \\
\hline Columbia River & 100-F SPR 211-1 & October 22, 2001 & B13621 & Barium & $\mathrm{mg} / \mathrm{L}$ & 1 & 0.052 & B & 0.0040 & 1.0 \\
\hline Columbia River & 100-H SPR 150-1 & November 20, 2003 & B17VK5 & Barium & $\mathrm{mg} / \mathrm{L}$ & 1 & 0.053 & B & 0.0040 & 1.0 \\
\hline Columbia River & 100-D SPR 102-1 & October 17, 2000 & B10JP7 & Barium & mg/L & 1 & 0.054 & B & 0.0040 & 1.0 \\
\hline Columbia River & 100-B SPR 37-1 & October 21, 2002 & B15MM0 & Barium & mg/L & 1 & 0.058 & B & 0.0040 & 1.0 \\
\hline Columbia River & 300 SPR 9-1 & August 27, 2001 & B12RP1 & Barium & mg/L & 1 & 0.059 & $=$ & 0.0040 & 1.0 \\
\hline Columbia River & 300 SPR 9-1 & August 27, 2001 & B12RP2 & Barium & $\mathrm{mg} / \mathrm{L}$ & 1 & 0.059 & $=$ & 0.0040 & 1.0 \\
\hline Columbia River & SB-037-1 & October 25, 2000 & B10F98 & Barium & $\mathrm{mg} / \mathrm{L}$ & 1 & 0.062 & B & 0.0040 & 1.0 \\
\hline Columbia River & 100-D SPR 98-1 & October 25, 2001 & B135X3 & Barium & $\mathrm{mg} / \mathrm{L}$ & 1 & 0.066 & $=$ & 0.0040 & 1.0 \\
\hline Columbia River & SD-98-1 & October 12, 2000 & B10JT9 & Barium & $\mathrm{mg} / \mathrm{L}$ & 1 & 0.067 & B & 0.0040 & 1.0 \\
\hline Columbia River & 100-B SPR 38-3 & October 25, 2000 & B10FB1 & Barium & $\mathrm{mg} / \mathrm{L}$ & 1 & 0.070 & B & 0.0040 & 1.0 \\
\hline Columbia River & 100-B SPR 38-3 & October 21, 2002 & B15MM3 & Barium & mg/L & 1 & 0.072 & B & 0.0040 & 1.0 \\
\hline Columbia River & 300 SPR 42-2 & August 27, 2001 & B12RM8 & Barium & $\mathrm{mg} / \mathrm{L}$ & 1 & 0.072 & $=$ & 0.0040 & 1.0 \\
\hline Columbia River & 100-B SPR 37-1 & October 22, 2001 & B13606 & Barium & mg/L & 1 & 0.074 & B & 0.0040 & 1.0 \\
\hline Columbia River & 100-D SPR 110-1 & October 21, 2002 & B15HW2 & Barium & mg/L & 1 & 0.075 & B & 0.0040 & 1.0 \\
\hline Columbia River & 300 SPR DR 42-2 & August 27, 2001 & B12RN0 & Barium & $\mathrm{mg} / \mathrm{L}$ & 1 & 0.078 & $=$ & 0.0040 & 1.0 \\
\hline Columbia River & 100-D SPR 110-1 & October 21, 2002 & B15HW5 & Barium & $\mathrm{mg} / \mathrm{L}$ & 1 & 0.079 & $=$ & 0.0040 & 1.0 \\
\hline Columbia River & 300 SPR $42-2$ & August 27, 2001 & B12RL6 & Barium & $\mathrm{mg} / \mathrm{L}$ & 1 & 0.079 & $=$ & 0.0040 & 1.0 \\
\hline Columbia River & 300 SPR DR 42-2 & August 27, 2001 & B12RM9 & Barium & $\mathrm{mg} / \mathrm{L}$ & 1 & 0.081 & $=$ & 0.0040 & 1.0 \\
\hline Columbia River & 100-B SPR 38-3 & October 22, 2001 & B13609 & Barium & mg/L & 1 & 0.094 & B & 0.0040 & 1.0 \\
\hline Columbia River & 100-D SPR 110-1 & October 21, 2002 & B15HW4 & Barium & mg/L & 1 & 0.15 & $=$ & 0.0040 & 1.0 \\
\hline Columbia River & 100-D SPR 110-1 & October 21, 2002 & B15HW1 & Barium & $\mathrm{mg} / \mathrm{L}$ & 1 & 0.29 & $=$ & 0.0040 & 1.0 \\
\hline Columbia River & 100-N SPR 8-13 & September 16, 2002 & B15C02 & Cadmium & mg/L & 1 & 2.64E-04 & $=$ & $2.50 \mathrm{E}-04$ & -- \\
\hline Columbia River & 100-D SPR 98-1 & October 25, 2001 & B135X3 & Cadmium & $\mathrm{mg} / \mathrm{L}$ & 1 & $4.20 \mathrm{E}-04$ & $=$ & $2.50 \mathrm{E}-04$ & -- \\
\hline Columbia River & 100-D SPR 102-1 & October 21, 2002 & B15C88 & Cadmium & $\mathrm{mg} / \mathrm{L}$ & 1 & $4.52 \mathrm{E}-04$ & $=$ & $2.50 \mathrm{E}-04$ & -- \\
\hline Columbia River & 100-F SPR 207-1 & October 26, 2004 & B1BFX6 & Cadmium & $\mathrm{mg} / \mathrm{L}$ & 1 & 5.59E-04 & $x$ & $2.50 \mathrm{E}-04$ & -- \\
\hline Columbia River & 100-D SPR 110-1 & October 21, 2002 & B15C86 & Cadmium & $\mathrm{mg} / \mathrm{L}$ & 1 & 5.69E-04 & $=$ & $2.50 \mathrm{E}-04$ & -- \\
\hline
\end{tabular}




\section{Table B-10}

Summary of Surface Water Results From Segment 2 Exceeding Select Ecological or Human Health Benchmark Values

Columbia River Component - Existing Sample Data Evaluation and Scoping Report

\begin{tabular}{|c|c|c|c|c|c|c|c|c|c|c|}
\hline Sample Area & Sample Site & Sample ID & Date Collected & Constituent Name & Units & QA Category & $\begin{array}{l}\text { Analytical } \\
\text { Value }\end{array}$ & Qualifier & $\begin{array}{c}\text { Ecological } \\
\text { Benchmark } \\
\text { Value }\end{array}$ & $\begin{array}{l}\text { Human Health } \\
\text { Benchmark Value }\end{array}$ \\
\hline Columbia River & 300 SPR $42-2$ & September 27, 2000 & B10903 & Cadmium & $\mathrm{mg} / \mathrm{L}$ & 1 & $5.71 \mathrm{E}-04$ & $=$ & $2.50 \mathrm{E}-04$ & $\overline{--}$ \\
\hline Columbia River & HFD SPR UR 28-2 & October 25, 2004 & B1BFX9 & Cadmium & $\mathrm{mg} / \mathrm{L}$ & 1 & 6.31E-04 & $\mathrm{x}$ & $2.50 \mathrm{E}-04$ & -- \\
\hline Columbia River & 100-D SPR 110-1 & October 21, 2002 & B15HW4 & Cadmium & $\mathrm{mg} / \mathrm{L}$ & 1 & $6.80 \mathrm{E}-04$ & $=$ & $2.50 \mathrm{E}-04$ & -- \\
\hline Columbia River & $100 \mathrm{H}$ SPR $145-1$ & October 10, 2003 & B17RK5 & Cadmium & $\mathrm{mg} / \mathrm{L}$ & 1 & $6.89 \mathrm{E}-04$ & $=$ & $2.50 \mathrm{E}-04$ & -- \\
\hline Columbia River & 300 SPR 42-2 & November 1, 1999 & BOWNX5 & Cadmium & $\mathrm{mg} / \mathrm{L}$ & 1 & 0.0016 & $=$ & $2.50 \mathrm{E}-04$ & -- \\
\hline Columbia River & 100-H SPR 144-1 & October 27, 2002 & $\mathrm{~B} 15 \mathrm{HX} 2$ & Cadmium & $\mathrm{mg} / \mathrm{L}$ & 1 & 0.0033 & B & $2.50 \mathrm{E}-04$ & -- \\
\hline Columbia River & 100-H SPR 145-1 & October 27, 2002 & B15HX5 & Cadmium & $\mathrm{mg} / \mathrm{L}$ & 1 & 0.0035 & B & $2.50 \mathrm{E}-04$ & -- \\
\hline Columbia River & 100H SPR 153-1 & October 27, 2003 & B17RL9 & Chromium & $\mathrm{mg} / \mathrm{L}$ & 1 & 0.010 & $=$ & 0.010 & -- \\
\hline Columbia River & 100-D SPR 110-1 & October 26, 2004 & B1BFX1 & Chromium & $\mathrm{mg} / \mathrm{L}$ & 1 & 0.011 & $x$ & 0.010 & -- \\
\hline Columbia River & 100-N SPR 8-13 & September 16, 2002 & B15C02 & Chromium & $\mathrm{mg} / \mathrm{L}$ & 1 & 0.011 & $=$ & 0.010 & -- \\
\hline Columbia River & 100F SPR 207-1 & November 3, 2003 & B17RK7 & Chromium & $\mathrm{mg} / \mathrm{L}$ & 1 & 0.011 & C & 0.010 & -- \\
\hline Columbia River & 100-D SPR 110-1 & October 26, 2004 & B1BFX0 & Chromium & $\mathrm{mg} / \mathrm{L}$ & 1 & 0.011 & $x$ & 0.010 & -- \\
\hline Columbia River & 100-H SPR 152-2 & November 1, 2001 & B135Y5 & Chromium & $\mathrm{mg} / \mathrm{L}$ & 1 & 0.011 & $=$ & 0.010 & -- \\
\hline Columbia River & 100-N SPR 8-13 & October 11, 2004 & B1BFW7 & Chromium & $\mathrm{mg} / \mathrm{L}$ & 1 & 0.011 & $x$ & 0.010 & -- \\
\hline Columbia River & 100D SPR 110-1 & October 27, 2003 & B17J91 & Chromium & $\mathrm{mg} / \mathrm{L}$ & 1 & 0.011 & $=$ & 0.010 & -- \\
\hline Columbia River & 100-N SPR 8-13 & October 11, 2004 & B1BFM0 & Chromium & $\mathrm{mg} / \mathrm{L}$ & 1 & 0.011 & $x$ & 0.010 & -- \\
\hline Columbia River & 100-H SPR 152-2 & October 27, 2002 & B15HY4 & Chromium & $\mathrm{mg} / \mathrm{L}$ & 1 & 0.011 & $=$ & 0.010 & -- \\
\hline Columbia River & 100-D SPR 102-1 & October 27, 2003 & B17JN8 & Chromium & $\mathrm{mg} / \mathrm{L}$ & 1 & 0.011 & $=$ & 0.010 & -- \\
\hline Columbia River & 100-N SPR 8-13 & October 17, 2000 & B10904 & Chromium & $\mathrm{mg} / \mathrm{L}$ & 1 & 0.011 & $=$ & 0.010 & -- \\
\hline Columbia River & 100D SPR $110-1$ & October 27, 2003 & B17J92 & Chromium & $\mathrm{mg} / \mathrm{L}$ & 1 & 0.012 & $=$ & 0.010 & -- \\
\hline Columbia River & SF-211-1 & November 7, 2000 & B10FC6 & Chromium & $\mathrm{mg} / \mathrm{L}$ & 1 & 0.012 & $=$ & 0.010 & -- \\
\hline Columbia River & 100-B SPR 39-2 & November 17, 2003 & $\mathrm{~B} 180 \mathrm{H} 8$ & Chromium & $\mathrm{mg} / \mathrm{L}$ & 1 & 0.012 & $\mathrm{C}$ & 0.010 & -- \\
\hline Columbia River & SB-037-1 & October 25, 2000 & B10F98 & Chromium & $\mathrm{mg} / \mathrm{L}$ & 1 & 0.013 & $=$ & 0.010 & -- \\
\hline Columbia River & 100-H SPR 145-1 & November 1, 2000 & B10965 & Chromium & $\mathrm{mg} / \mathrm{L}$ & 1 & 0.013 & $=$ & 0.010 & -- \\
\hline Columbia River & 100-B SPR 38-3 & October 25, 2000 & B10FB1 & Chromium & $\mathrm{mg} / \mathrm{L}$ & 1 & 0.013 & $=$ & 0.010 & -- \\
\hline Columbia River & 100-H SPR 145-1 & November 1, 2000 & B10JT4 & Chromium & $\mathrm{mg} / \mathrm{L}$ & 1 & 0.013 & $=$ & 0.010 & -- \\
\hline Columbia River & 100-D SPR 102-1 & October 27, 2003 & B17JP0 & Chromium & $\mathrm{mg} / \mathrm{L}$ & 1 & 0.013 & $=$ & 0.010 & -- \\
\hline Columbia River & 100-D SPR 102-1 & October 27, 2003 & B17JN9 & Chromium & $\mathrm{mg} / \mathrm{L}$ & $100 \%$ & 0.014 & $=$ & 0.010 & -- \\
\hline Columbia River & 100-H SPR 145-1 & October 27, 2002 & $\mathrm{~B} 15 \mathrm{H} \times 9$ & Chromium & $\mathrm{mg} / \mathrm{L}$ & 1 & 0.014 & $=$ & 0.010 & -- \\
\hline Columbia River & 100-H SPR 145-1 & November 1, 2000 & B10JR3 & Chromium & $\mathrm{mg} / \mathrm{L}$ & 1 & 0.014 & $=$ & 0.010 & -- \\
\hline Columbia River & 100-N SPR 8-13 & March 29, 2004 & B18XL9 & Chromium & $\mathrm{mg} / \mathrm{L}$ & 1 & 0.014 & $x$ & 0.010 & -- \\
\hline Columbia River & 100-F SPR 207-1 & November 7, 2000 & B10FC3 & Chromium & $\mathrm{mg} / \mathrm{L}$ & 1 & 0.014 & $=$ & 0.010 & -- \\
\hline Columbia River & 100-F SPR 207-1 & November 7, 2000 & B10967 & Chromium & $\mathrm{mg} / \mathrm{L}$ & 1 & 0.014 & $=$ & 0.010 & -- \\
\hline Columbia River & 100-N SPR 8-13 & March 29, 2004 & B18XL7 & Chromium & $\mathrm{mg} / \mathrm{L}$ & 1 & 0.014 & $x$ & 0.010 & -- \\
\hline Columbia River & 100-N SPR 8-13 & March 29, 2004 & B18XM0 & Chromium & $\mathrm{mg} / \mathrm{L}$ & 1 & 0.014 & $x$ & 0.010 & -- \\
\hline Columbia River & 100-H SPR 145-1 & October 27, 2002 & $\mathrm{~B} 15 \mathrm{H} \times 8$ & Chromium & $\mathrm{mg} / \mathrm{L}$ & 1 & 0.015 & $=$ & 0.010 & -- \\
\hline Columbia River & 100-F SPR 211-1 & October 22, 2001 & B13621 & Chromium & $\mathrm{mg} / \mathrm{L}$ & 1 & 0.015 & $=$ & 0.010 & -- \\
\hline Columbia River & 100-B SPR 38-3 & October 25, 1999 & B0WP15 & Chromium & $\mathrm{mg} / \mathrm{L}$ & 1 & 0.016 & $=$ & 0.010 & -- \\
\hline Columbia River & 100-H SPR 145-1 & October 27, 2002 & B15HX5 & Chromium & $\mathrm{mg} / \mathrm{L}$ & 1 & 0.016 & $=$ & 0.010 & -- \\
\hline
\end{tabular}




\section{Table B-10}

Summary of Surface Water Results From Segment 2 Exceeding Select Ecological or Human Health Benchmark Values

Columbia River Component - Existing Sample Data Evaluation and Scoping Report

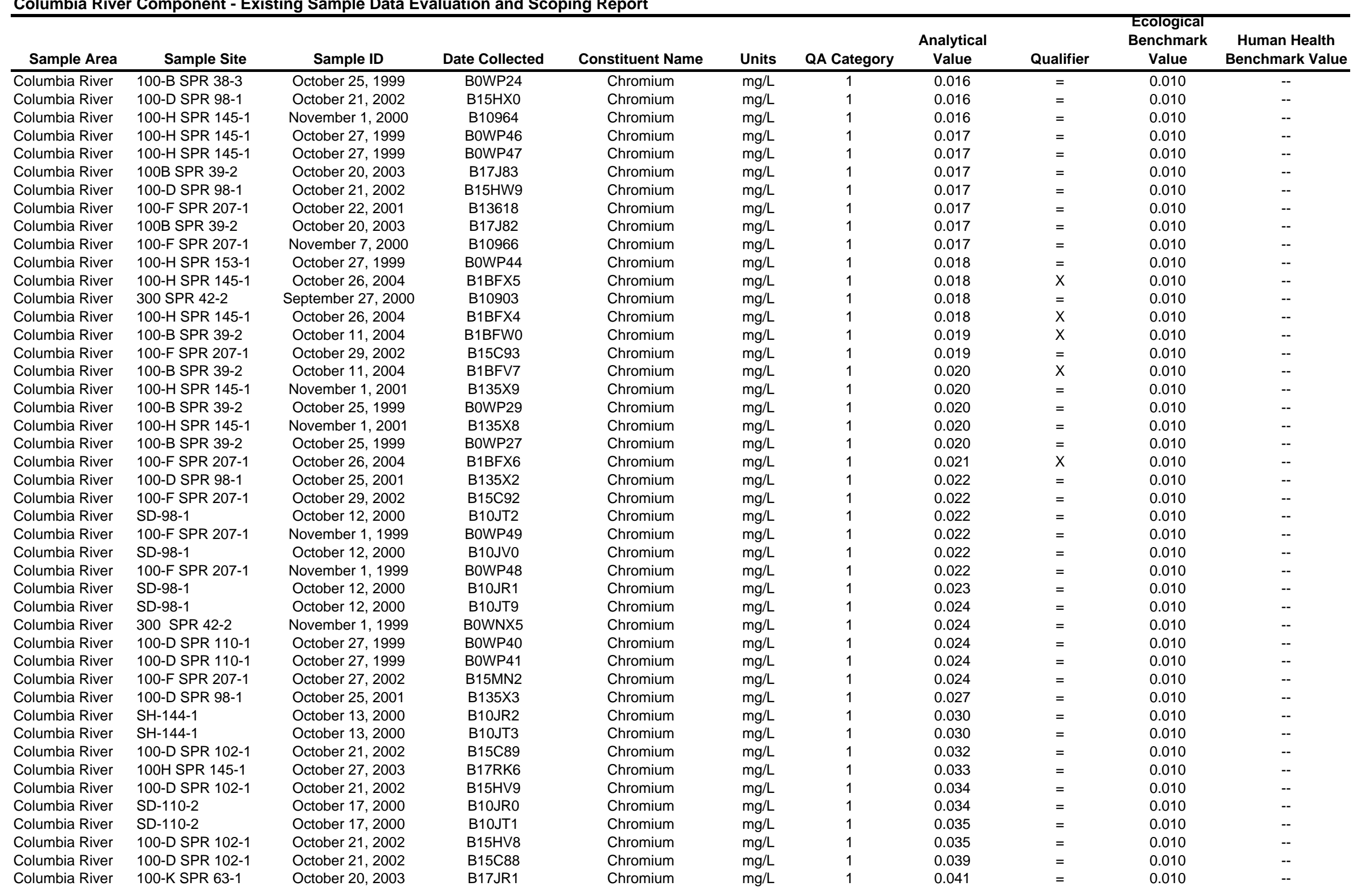




\section{Table B-10}

Summary of Surface Water Results From Segment 2 Exceeding Select Ecological or Human Health Benchmark Values

Columbia River Component - Existing Sample Data Evaluation and Scoping Report

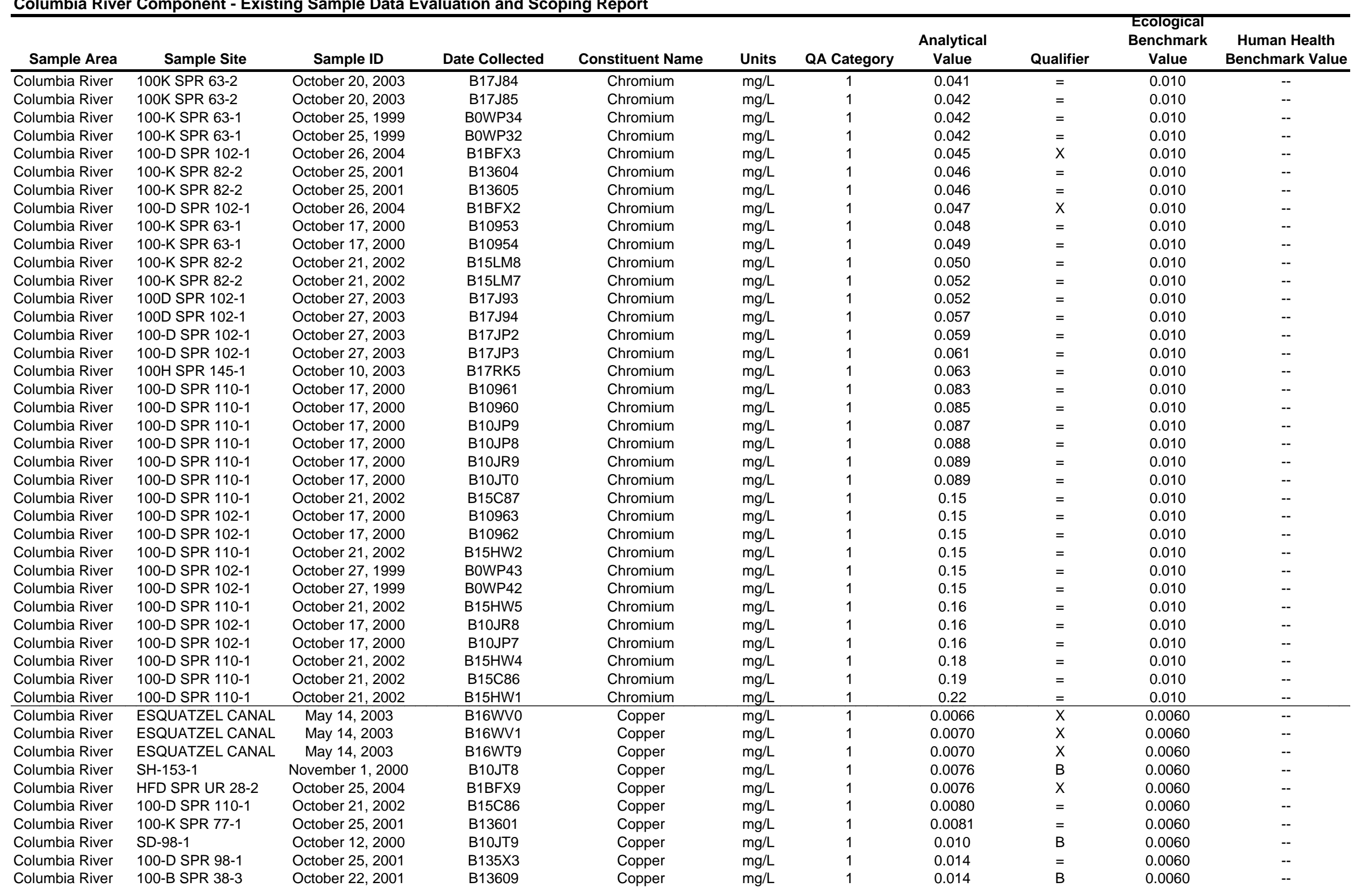




\section{Table B-10}

Summary of Surface Water Results From Segment 2 Exceeding Select Ecological or Human Health Benchmark Values

Columbia River Component - Existing Sample Data Evaluation and Scoping Report

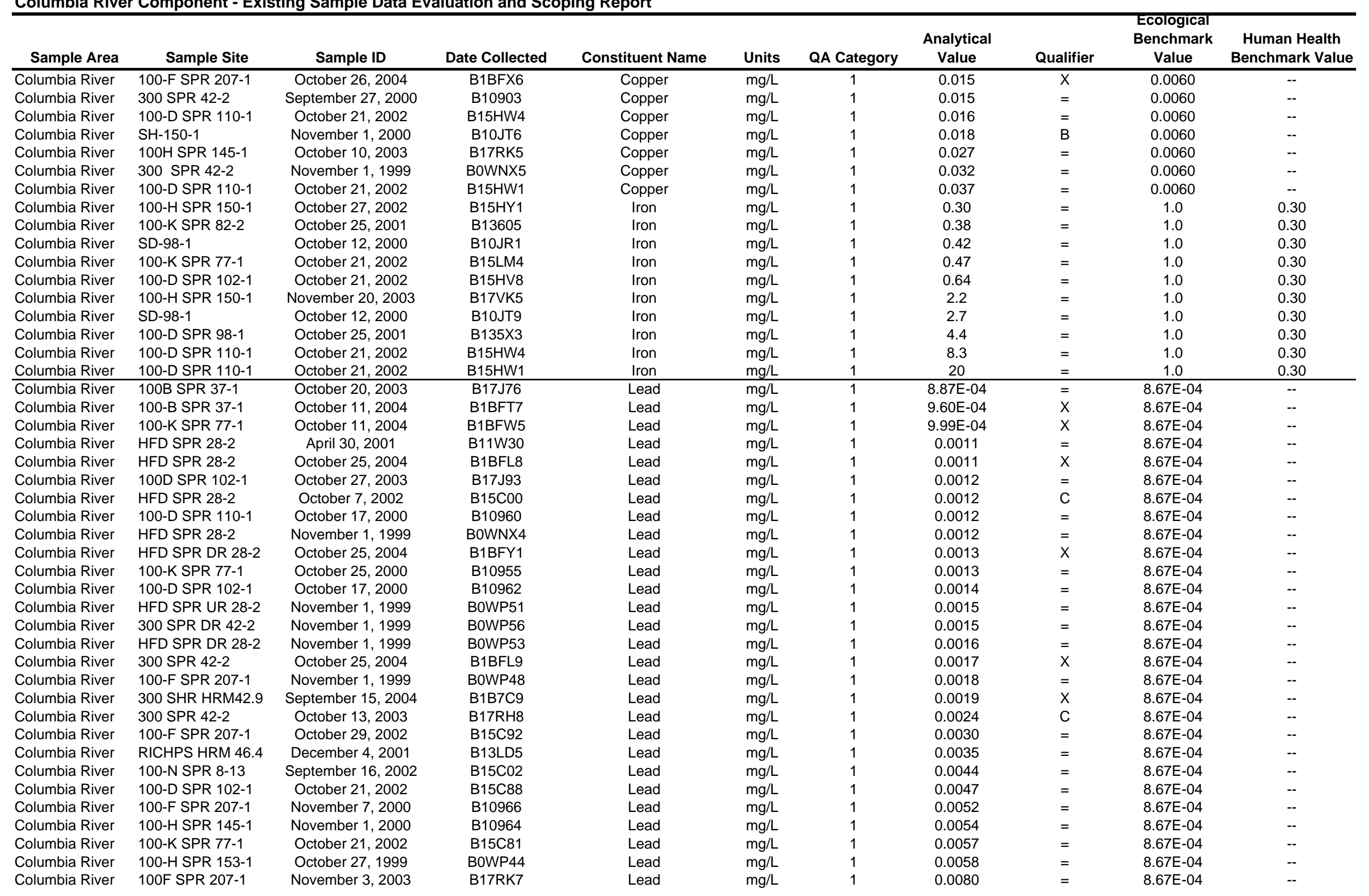




\section{Table B-10}

Summary of Surface Water Results From Segment 2 Exceeding Select Ecological or Human Health Benchmark Values Columbia River Component - Existing Sample Data Evaluation and Scoping Report

\begin{tabular}{|c|c|c|c|c|c|c|c|c|c|c|}
\hline Sample Area & Sample Site & Sample ID & Date Collected & Constituent Name & Units & QA Category & $\begin{array}{c}\text { Analytical } \\
\text { Value }\end{array}$ & Qualifier & $\begin{array}{c}\text { Ecological } \\
\text { Benchmark } \\
\text { Value } \\
\end{array}$ & $\begin{array}{c}\text { Human Health } \\
\text { Benchmark Value } \\
\end{array}$ \\
\hline Columbia River & 100-D SPR 110-1 & October 21, 2002 & B15C86 & Lead & $\mathrm{mg} / \mathrm{L}$ & 1 & 0.0080 & $=$ & $8.67 \mathrm{E}-04$ & $\overline{--}$ \\
\hline Columbia River & 100-F SPR 207-1 & October 26, 2004 & B1BFX6 & Lead & $\mathrm{mg} / \mathrm{L}$ & 1 & 0.015 & $x$ & 8.67E-04 & -- \\
\hline Columbia River & 300 SPR 42-2 & September 27, 2000 & B10903 & Lead & $\mathrm{mg} / \mathrm{L}$ & 1 & 0.016 & $=$ & 8.67E-04 & -- \\
\hline Columbia River & HFD SPR UR 28-2 & October 25, 2004 & B1BFX9 & Lead & $\mathrm{mg} / \mathrm{L}$ & 1 & 0.019 & $x$ & 8.67E-04 & -- \\
\hline Columbia River & 300 SPR 42-2 & November 1, 1999 & BOWNX5 & Lead & $\mathrm{mg} / \mathrm{L}$ & 1 & 0.037 & $=$ & 8.67E-04 & -- \\
\hline Columbia River & $100 \mathrm{H}$ SPR $145-1$ & October 10, 2003 & B17RK5 & Lead & $\mathrm{mg} / \mathrm{L}$ & 1 & 0.066 & $=$ & 8.67E-04 & -- \\
\hline Columbia River & 100-D SPR 102-1 & October 21, 2002 & B15HV8 & Manganese & $\mathrm{mg} / \mathrm{L}$ & 1 & 0.056 & $=$ & 0.12 & 0.050 \\
\hline Columbia River & 100-K SPR 77-1 & October 21, 2002 & B15LM4 & Manganese & $\mathrm{mg} / \mathrm{L}$ & 1 & 0.066 & $=$ & 0.12 & 0.050 \\
\hline Columbia River & 100-H SPR 150-1 & November 20, 2003 & B17VK5 & Manganese & $\mathrm{mg} / \mathrm{L}$ & 1 & 0.075 & $=$ & 0.12 & 0.050 \\
\hline Columbia River & SD-98-1 & October 12, 2000 & B10JT9 & Manganese & $\mathrm{mg} / \mathrm{L}$ & 1 & 0.18 & $=$ & 0.12 & 0.050 \\
\hline Columbia River & 100-D SPR 98-1 & October 25, 2001 & $\mathrm{~B} 135 \times 3$ & Manganese & $\mathrm{mg} / \mathrm{L}$ & 1 & 0.26 & $=$ & 0.12 & 0.050 \\
\hline Columbia River & 100-D SPR 110-1 & October 21, 2002 & B15HW4 & Manganese & $\mathrm{mg} / \mathrm{L}$ & 1 & 0.34 & $=$ & 0.12 & 0.050 \\
\hline Columbia River & 100-D SPR 110-1 & October 21, 2002 & $\mathrm{~B} 15 \mathrm{HW} 1$ & Manganese & $\mathrm{mg} / \mathrm{L}$ & 1 & 0.94 & $=$ & 0.12 & 0.050 \\
\hline Columbia River & 100-K SPR 77-1 & October 21, 2002 & B15C81 & Mercury & $\mathrm{mg} / \mathrm{L}$ & 1 & $1.38 \mathrm{E}-05$ & $=$ & $1.20 \mathrm{E}-05$ & -- \\
\hline Columbia River & 100-D SPR 110-1 & October 21, 2002 & B15C86 & Mercury & $\mathrm{mg} / \mathrm{L}$ & 1 & 2.04E-05 & $=$ & 1.20E-05 & -- \\
\hline Columbia River & 100-D SPR 102-1 & October 21, 2002 & B15C88 & Mercury & $\mathrm{mg} / \mathrm{L}$ & 1 & 2.05E-05 & $=$ & $1.20 \mathrm{E}-05$ & -- \\
\hline Columbia River & 300 SPR 42-2 & November 1, 1999 & BOWNX5 & Mercury & $\mathrm{mg} / \mathrm{L}$ & 1 & $3.46 \mathrm{E}-05$ & $=$ & 1.20E-05 & -- \\
\hline Columbia River & $100 \mathrm{H}$ SPR $145-1$ & October 10, 2003 & B17RK5 & Mercury & $\mathrm{mg} / \mathrm{L}$ & 1 & 4.07E-05 & $x$ & 1.20E-05 & -- \\
\hline Columbia River & 100-D SPR 110-1 & October 21, 2002 & B15HW1 & Nickel & $\mathrm{mg} / \mathrm{L}$ & 1 & 0.029 & B & 0.028 & 610 \\
\hline Columbia River & 300 SPR 42-2 & November 1, 1999 & BoWNX5 & Nickel & $\mathrm{mg} / \mathrm{L}$ & 1 & 0.032 & $=$ & 0.028 & 610 \\
\hline Columbia River & 300 SPR 42-2 & November 1, 1999 & B0WNX5 & Silver & $\mathrm{mg} / \mathrm{L}$ & 1 & $1.45 \mathrm{E}-04$ & $=$ & $1.20 \mathrm{E}-04$ & -- \\
\hline Columbia River & 300 AREA OUT13 & September 15, 2004 & B1B7H8 & Silver & $\mathrm{mg} / \mathrm{L}$ & 1 & $1.62 \mathrm{E}-04$ & $x$ & 1.20E-04 & -- \\
\hline Columbia River & RICHPS5 HRM46.4 & December 6, 1999 & B0X3W7 & Silver & $\mathrm{mg} / \mathrm{L}$ & 1 & $2.51 \mathrm{E}-04$ & $=$ & $1.20 \mathrm{E}-04$ & -- \\
\hline Columbia River & 100-K SPR 77-1 & October 25, 2001 & B13601 & Silver & $\mathrm{mg} / \mathrm{L}$ & 1 & $6.50 \mathrm{E}-04$ & $=$ & $1.20 \mathrm{E}-04$ & -- \\
\hline Columbia River & 100-D SPR 98-1 & October 25, 2001 & $\mathrm{~B} 135 \mathrm{X} 2$ & Silver & $\mathrm{mg} / \mathrm{L}$ & 1 & 7.50E-04 & $=$ & $1.20 \mathrm{E}-04$ & -- \\
\hline Columbia River & 100-H SPR 144-1 & October 27, 2002 & B15HX3 & Silver & $\mathrm{mg} / \mathrm{L}$ & 1 & 0.0018 & B & $1.20 \mathrm{E}-04$ & -- \\
\hline Columbia River & 100-H SPR 145-1 & October 27, 2002 & $\mathrm{~B} 15 \mathrm{H} \times 8$ & Silver & $\mathrm{mg} / \mathrm{L}$ & 1 & 0.0030 & $\mathrm{~B}$ & 1.20E-04 & -- \\
\hline Columbia River & 100H SPR 153-1 & November 7, 2000 & 24571 & Strontium-90 & $\mathrm{pCi} / \mathrm{L}$ & 1 & 8.0 & $=$ & 278 & 8.0 \\
\hline Columbia River & $100 \mathrm{H}$ SPR $153-1$ & October 27, 1999 & 23440 & Strontium-90 & $\mathrm{pCi} / \mathrm{L}$ & 1 & 11 & $=$ & 278 & 8.0 \\
\hline Columbia River & 100H SPR 153-1 & October 27, 2003 & B17RL8 & Strontium-90 & $\mathrm{pCi} / \mathrm{L}$ & 1 & 14 & $=$ & 278 & 8.0 \\
\hline Columbia River & 100H SPR 153-1 & October 27, 1999 & B0WP19 & Strontium-90 & $\mathrm{pCi} / \mathrm{L}$ & 1 & 14 & $=$ & 278 & 8.0 \\
\hline Columbia River & 100H SPR 153-1 & April 30, 2001 & B11W86 & Strontium-90 & $\mathrm{pCi} / \mathrm{L}$ & 1 & 14 & $=$ & 278 & 8.0 \\
\hline Columbia River & 100N SPR STA 3 & October 25, 1999 & 23736 & Strontium-90 & $\mathrm{pCi} / \mathrm{L}$ & 1 & 33 & $=$ & 278 & 8.0 \\
\hline Columbia River & $100 \mathrm{~N}$ Area & June 28, 1999 & BOVWR2 & Strontium-90 & $\mathrm{pCi} / \mathrm{L}$ & 1 & 109 & $=$ & 278 & 8.0 \\
\hline Columbia River & 300 SPR 42-2 & September 27, 2000 & B10903 & Thallium & $\mathrm{mg} / \mathrm{L}$ & 1 & $2.59 \mathrm{E}-04$ & $=$ & 0.040 & $2.40 \mathrm{E}-04$ \\
\hline Columbia River & 300 SPR 42-2 & November 1, 1999 & BoWNX5 & Thallium & $\mathrm{mg} / \mathrm{L}$ & 1 & 4.07E-04 & $=$ & 0.040 & 2.40E-04 \\
\hline Columbia River & 100BC Area & June 22, 1999 & B0VW80 & Tritium & $\mathrm{pCi} / \mathrm{L}$ & 1 & 20,200 & $=$ & $2.65 \mathrm{E}+08$ & 20000 \\
\hline Columbia River & HFD TS HRM 28 & September 9, 2002 & 29094 & Tritium & $\mathrm{pCi} / \mathrm{L}$ & 1 & 21,000 & $=$ & $2.65 E+08$ & 20000 \\
\hline Columbia River & HFD SPR DR 28-2 & October 25, 2004 & B1BFV5 & Tritium & $\mathrm{pCi} / \mathrm{L}$ & 1 & 45,800 & $=$ & $2.65 \mathrm{E}+08$ & 20000 \\
\hline Columbia River & HFD SPR DR 28-2 & October 25, 2004 & 33185 & Tritium & $\mathrm{pCi} / \mathrm{L}$ & 1 & 50,600 & $=$ & $2.65 \mathrm{E}+08$ & 20000 \\
\hline Columbia River & HFD SPR DR 28-2 & October 7, 2002 & B15C73 & Tritium & $\mathrm{pCi} / \mathrm{L}$ & 1 & 53,900 & $=$ & $2.65 \mathrm{E}+08$ & 20000 \\
\hline Columbia River & HFD SPR UR 28-2 & September 27, 2000 & B10945 & Tritium & $\mathrm{pCi} / \mathrm{L}$ & 1 & 53,900 & $=$ & $2.65 \mathrm{E}+08$ & 20000 \\
\hline Columbia River & HFD SPR 28-2 & October 7, 2002 & B15C09 & Tritium & $\mathrm{pCi} / \mathrm{L}$ & 1 & 58,400 & $=$ & $2.65 \mathrm{E}+08$ & 20000 \\
\hline Columbia River & HFD SPR $28-2$ & September 27, 2000 & B10912 & Tritium & $\mathrm{pCi} / \mathrm{L}$ & 1 & 61,100 & $=$ & $2.65 E+08$ & 20000 \\
\hline Columbia River & HFD SPR $28-2$ & November 1, 1999 & 23439 & Tritium & $\mathrm{pCi} / \mathrm{L}$ & 1 & 63,600 & $=$ & $2.65 \mathrm{E}+08$ & 20000 \\
\hline
\end{tabular}




\section{Table B-10}

Summary of Surface Water Results From Segment 2 Exceeding Select Ecological or Human Health Benchmark Values

Columbia River Component - Existing Sample Data Evaluation and Scoping Report

\begin{tabular}{|c|c|c|c|c|c|c|c|c|c|c|}
\hline Sample Area & Sample Site & Sample ID & Date Collected & Constituent Name & Units & QA Category & $\begin{array}{c}\text { Analytical } \\
\text { Value }\end{array}$ & Qualifier & $\begin{array}{l}\text { Ecological } \\
\text { Benchmark } \\
\text { Value }\end{array}$ & $\begin{array}{c}\text { Human Health } \\
\text { Benchmark Value }\end{array}$ \\
\hline Columbia River & HFD SPR 28-2 & October 25, 2004 & B1BFP3 & Tritium & $\mathrm{pCi} / \mathrm{L}$ & 1 & 66,700 & $=$ & $2.65 \mathrm{E}+08$ & 20000 \\
\hline Columbia River & HFD SPR 28-2 & September 27, 2000 & 24573 & Tritium & $\mathrm{pCi} / \mathrm{L}$ & 1 & 70,700 & $=$ & $2.65 \mathrm{E}+08$ & 20000 \\
\hline Columbia River & HFD SPR 28-2 & October 7, 2002 & 29079 & Tritium & $\mathrm{pCi} / \mathrm{L}$ & 1 & 71,600 & $=$ & $2.65 \mathrm{E}+08$ & 20000 \\
\hline Columbia River & HFD SPR UR 28-2 & November 1, 1999 & BOWP25 & Tritium & $\mathrm{pCi} / \mathrm{L}$ & 1 & 75,000 & $=$ & $2.65 E+08$ & 20000 \\
\hline Columbia River & HFD SPR DR 28-2 & September 27, 2000 & B10946 & Tritium & $\mathrm{pCi} / \mathrm{L}$ & 1 & 79,100 & $=$ & $2.65 \mathrm{E}+08$ & 20000 \\
\hline Columbia River & HFD SPR DR 28-2 & November 1, 1999 & B0WP26 & Tritium & $\mathrm{pCi} / \mathrm{L}$ & 1 & 86,100 & $=$ & $2.65 \mathrm{E}+08$ & 20000 \\
\hline Columbia River & HFD SPR 28-2 & April 30, 2001 & B11W36 & Tritium & $\mathrm{pCi} / \mathrm{L}$ & 1 & 102,000 & $=$ & $2.65 \mathrm{E}+08$ & 20000 \\
\hline Columbia River & HFD SPR DR 28-2 & April 30, 2001 & B11W55 & Tritium & $\mathrm{pCi} / \mathrm{L}$ & 1 & 107,000 & $=$ & $2.65 E+08$ & 20000 \\
\hline Columbia River & HFD SPR 28-2 & May 4, 2001 & 26955 & Tritium & $\mathrm{pCi} / \mathrm{L}$ & 1 & 112,000 & $=$ & $2.65 \mathrm{E}+08$ & 20000 \\
\hline Columbia River & ESQUATZEL CANAL & May 14,2003 & B16WT8 & Uranium & $\mathrm{mg} / \mathrm{L}$ & 1 & 0.0058 & $\mathrm{x}$ & 0.0026 & -- \\
\hline Columbia River & ESQUATZEL CANAL & May 14,2003 & B16WT6 & Uranium & mg/L & 1 & 0.0058 & $\mathrm{x}$ & 0.0026 & -- \\
\hline Columbia River & ESQUATZEL CANAL & May 14, 2003 & B16WV0 & Uranium & $\mathrm{mg} / \mathrm{L}$ & 1 & 0.0058 & $x$ & 0.0026 & -- \\
\hline Columbia River & ESQUATZEL CANAL & May 14,2003 & B16WT9 & Uranium & mg/L & 1 & 0.0059 & $x$ & 0.0026 & -- \\
\hline Columbia River & ESQUATZEL CANAL & May 14, 2003 & B16WT7 & Uranium & $\mathrm{mg} / \mathrm{L}$ & 1 & 0.0059 & $x$ & 0.0026 & -- \\
\hline Columbia River & ESQUATZEL CANAL & May 14,2003 & B16WV1 & Uranium & mg/L & 1 & 0.0061 & $\mathrm{x}$ & 0.0026 & -- \\
\hline Columbia River & Ringold WW & May 14,2003 & B16WT0 & Uranium & mg/L & 1 & 0.0083 & $x$ & 0.0026 & -- \\
\hline Columbia River & Ringold WW & May 14,2003 & B16WT4 & Uranium & mg/L & 1 & 0.0086 & $\mathrm{x}$ & 0.0026 & -- \\
\hline Columbia River & Ringold WW & May 14,2003 & B16WT1 & Uranium & mg/L & 1 & 0.0086 & $\mathrm{x}$ & 0.0026 & -- \\
\hline Columbia River & Ringold WW & May 14, 2003 & B16WT3 & Uranium & $\mathrm{mg} / \mathrm{L}$ & 1 & 0.0087 & $x$ & 0.0026 & -- \\
\hline Columbia River & 300 SPR 9-1 & August 27, 2001 & B12RT9 & Uranium-234 & $\mathrm{pCi} / \mathrm{L}$ & 1 & 31 & $=$ & 202 & 30.0 \\
\hline Columbia River & 300 SPR DR 42-2 & September 17, 2001 & B12XK2 & Uranium-234 & $\mathrm{pCi} / \mathrm{L}$ & 1 & 36 & $=$ & 202 & 30.0 \\
\hline Columbia River & 300 SPR DR 42-2 & May 3, 2001 & B11W57 & Uranium-234 & $\mathrm{pCi} / \mathrm{L}$ & 1 & 38 & $=$ & 202 & 30.0 \\
\hline Columbia River & 300 SPR DR 42-2 & August 27, 2001 & B12RN6 & Uranium-234 & $\mathrm{pCi} / \mathrm{L}$ & 1 & 39 & $=$ & 202 & 30.0 \\
\hline Columbia River & 300 SPR DR 42-2 & May 3, 2001 & 26957 & Uranium-234 & $\mathrm{pCi} / \mathrm{L}$ & 1 & 41 & $=$ & 202 & 30.0 \\
\hline Columbia River & 300 SPR 42-2 & October 13, 2003 & 30857 & Uranium-234 & $\mathrm{pCi} / \mathrm{L}$ & 1 & 41 & $=$ & 202 & 30.0 \\
\hline Columbia River & 300 SPR 42-2 & November 1, 1999 & BOWNY3 & Uranium-234 & pCi/L & 1 & 42 & $=$ & 202 & 30.0 \\
\hline Columbia River & 300 SPR DR 42-2 & August 27, 2001 & 27671 & Uranium-234 & $\mathrm{pCi} / \mathrm{L}$ & 1 & 44 & $=$ & 202 & 30.0 \\
\hline Columbia River & 300 SPR DR 42-2 & October 13, 2003 & 30940 & Uranium-234 & $\mathrm{pCi} / \mathrm{L}$ & 1 & 51 & $=$ & 202 & 30.0 \\
\hline Columbia River & 300 SPR DR 42-2 & December 26, 2002 & B15C75 & Uranium-234 & $\mathrm{pCi} / \mathrm{L}$ & 1 & 51 & $=$ & 202 & 30.0 \\
\hline Columbia River & 300 SPR DR 42-2 & August 27, 2001 & B12RM6 & Uranium-234 & $\mathrm{pCi} / \mathrm{L}$ & 1 & 53 & $=$ & 202 & 30.0 \\
\hline Columbia River & 300 SPR DR 42-2 & October 13, 2003 & B17RK1 & Uranium-234 & $\mathrm{pCi} / \mathrm{L}$ & 1 & 57 & $=$ & 202 & 30.0 \\
\hline Columbia River & 300 SPR DR 42-2 & September 27, 2000 & B10949 & Uranium-234 & $\mathrm{pCi} / \mathrm{L}$ & 1 & 69 & $=$ & 202 & 30.0 \\
\hline Columbia River & 300 SPR 42-2 & October 13, 2003 & B17RJ2 & Uranium-234 & $\mathrm{pCi} / \mathrm{L}$ & 1 & 70 & $=$ & 202 & 30.0 \\
\hline Columbia River & 300 SPR DR 42-2 & November 1, 1999 & BOWP28 & Uranium-234 & $\mathrm{pCi} / \mathrm{L}$ & 1 & 111 & $=$ & 202 & 30 \\
\hline Columbia River & 300 SPR DR 42-2 & September 17, 2001 & B12XK2 & Uranium-238 & $\mathrm{pCi} / \mathrm{L}$ & 1 & 34 & $=$ & 223 & 30.0 \\
\hline Columbia River & 300 SPR DR 42-2 & May 3, 2001 & 26957 & Uranium-238 & $\mathrm{pCi} / \mathrm{L}$ & 1 & 37 & $=$ & 223 & 30.0 \\
\hline Columbia River & 300 SPR $42-2$ & November 1, 1999 & BOWNY3 & Uranium-238 & $\mathrm{pCi} / \mathrm{L}$ & 1 & 38 & $=$ & 223 & 30.0 \\
\hline Columbia River & 300 SPR 42-2 & October 13, 2003 & 30857 & Uranium-238 & $\mathrm{pCi} / \mathrm{L}$ & 1 & 40 & $=$ & 223 & 30.0 \\
\hline Columbia River & 300 SPR DR 42-2 & August 27, 2001 & 27671 & Uranium-238 & $\mathrm{pCi} / \mathrm{L}$ & 1 & 44 & $=$ & 223 & 30.0 \\
\hline
\end{tabular}




\section{Table B-10}

Summary of Surface Water Results From Segment 2 Exceeding Select Ecological or Human Health Benchmark Values

Columbia River Component - Existing Sample Data Evaluation and Scoping Report

\begin{tabular}{|c|c|c|c|c|c|c|c|c|c|c|}
\hline Sample Area & Sample Site & Sample ID & Date Collected & Constituent Name & Units & QA Category & $\begin{array}{l}\text { Analytical } \\
\text { Value }\end{array}$ & Qualifier & $\begin{array}{l}\text { Ecological } \\
\text { Benchmark } \\
\text { Value }\end{array}$ & $\begin{array}{l}\text { Human Health } \\
\text { Benchmark Value }\end{array}$ \\
\hline Columbia River & 300 SPR 42-2 & September 27, 2000 & 24574 & Uranium & $\overline{\mathrm{pCi} / \mathrm{L}}$ & 1 & 44 & $=$ & 223 & 30.0 \\
\hline Columbia River & 300 SPR DR 42-2 & October 13, 2003 & 30940 & Uranium-238 & $\mathrm{pCi} / \mathrm{L}$ & 1 & 46 & $=$ & 223 & 30.0 \\
\hline Columbia River & 300 SPR DR $42-2$ & August 27, 2001 & B12RM6 & Uranium-238 & $\mathrm{pCi} / \mathrm{L}$ & 1 & 48 & $=$ & 223 & 30.0 \\
\hline Columbia River & 300 SPR $42-2$ & October 25, 2004 & 33144 & Uranium & $\mathrm{pCi} / \mathrm{L}$ & 1 & 48 & $=$ & 223 & 30.0 \\
\hline Columbia River & 300 SPR DR 42-2 & October 13, 2003 & B17RK1 & Uranium-238 & $\mathrm{pCi} / \mathrm{L}$ & 1 & 52 & $=$ & 223 & 30.0 \\
\hline Columbia River & 300 SPR $42-2$ & May 10,2001 & 26956 & Uranium & $\mathrm{pCi} / \mathrm{L}$ & 1 & 54 & $=$ & 223 & 30.0 \\
\hline Columbia River & 300 SPR DR 42-2 & September 27, 2000 & B10949 & Uranium-238 & $\mathrm{pCi} / \mathrm{L}$ & 1 & 61 & $=$ & 223 & 30.0 \\
\hline Columbia River & 300 SPR $42-2$ & October 13, 2003 & B17RJ2 & Uranium-238 & $\mathrm{pCi} / \mathrm{L}$ & 1 & 66 & $=$ & 223 & 30.0 \\
\hline Columbia River & 300 SPR DR 42-2 & May 3, 2001 & 26957 & Uranium & $\mathrm{pCi} / \mathrm{L}$ & 1 & 82 & $=$ & 223 & 30.0 \\
\hline Columbia River & 300 SPR 42-2 & October 13, 2003 & 30857 & Uranium & $\mathrm{pCi} / \mathrm{L}$ & 1 & 84 & $=$ & 223 & 30.0 \\
\hline Columbia River & 300 SPR DR 42-2 & August 27, 2001 & 27671 & Uranium & $\mathrm{pCi} / \mathrm{L}$ & 1 & 91 & $=$ & 223 & 30.0 \\
\hline Columbia River & 300 SPR DR 42-2 & November 1, 1999 & B0WP28 & Uranium-238 & $\mathrm{pCi} / \mathrm{L}$ & 1 & 99 & $=$ & 223 & 30.0 \\
\hline Columbia River & 300 SPR DR 42-2 & October 13, 2003 & 30940 & Uranium & $\mathrm{pCi} / \mathrm{L}$ & 1 & 100 & $=$ & 223 & 30.0 \\
\hline Columbia River & 100-D SPR 98-1 & October 25,2001 & B135X3 & Vanadium & $\mathrm{mg} / \mathrm{L}$ & 1 & 0.020 & $=$ & 0.020 & -- \\
\hline Columbia River & 100-D SPR 98-1 & October 21, 2002 & B15HW9 & Vanadium & $\mathrm{mg} / \mathrm{L}$ & 1 & 0.021 & $=$ & 0.020 & -- \\
\hline Columbia River & 100-F SPR 207-1 & October 27, 2002 & B15MN2 & Vanadium & $\mathrm{mg} / \mathrm{L}$ & 1 & 0.047 & $=$ & 0.020 & -- \\
\hline Columbia River & 100-D SPR 110-1 & October 21, 2002 & B15HW1 & Vanadium & $\mathrm{mg} / \mathrm{L}$ & 1 & 0.049 & $=$ & 0.020 & -- \\
\hline Columbia River & 300 A10 HRM43.1 & September 13, 2001 & B12Т53 & Zinc & $\mathrm{mg} / \mathrm{L}$ & 1 & 0.057 & $=$ & 0.055 & 7.4 \\
\hline Columbia River & 100-H SPR 150-1 & November 20, 2003 & B17VK5 & Zinc & $\mathrm{mg} / \mathrm{L}$ & 1 & 0.058 & $=$ & 0.055 & 7.4 \\
\hline Columbia River & 100-D SPR 110-1 & October 21, 2002 & B15C86 & Zinc & $\mathrm{mg} / \mathrm{L}$ & 1 & 0.077 & C & 0.055 & 7.4 \\
\hline Columbia River & 100-D SPR 98-1 & October 25, 2001 & B135X3 & Zinc & $\mathrm{mg} / \mathrm{L}$ & 1 & 0.080 & $=$ & 0.055 & 7.4 \\
\hline Columbia River & 100-F SPR 207-1 & October 26, 2004 & B1BFX6 & Zinc & $\mathrm{mg} / \mathrm{L}$ & 1 & 0.11 & $x$ & 0.055 & 7.4 \\
\hline Columbia River & 300 SPR $42-2$ & September 27, 2000 & B10903 & Zinc & $\mathrm{mg} / \mathrm{L}$ & 1 & 0.11 & $=$ & 0.055 & 7.4 \\
\hline Columbia River & HFD SPR UR 28-2 & October 25, 2004 & B1BFX9 & Zinc & $\mathrm{mg} / \mathrm{L}$ & 1 & 0.11 & $\mathrm{x}$ & 0.055 & 7.4 \\
\hline Columbia River & 100-D SPR $110-1$ & October 21, 2002 & B15HW4 & Zinc & $\mathrm{mg} / \mathrm{L}$ & 1 & 0.13 & $=$ & 0.055 & 7.4 \\
\hline Columbia River & 300 SPR 42-2 & November 1, 1999 & BOWNX5 & Zinc & $\mathrm{mg} / \mathrm{L}$ & 1 & 0.23 & $=$ & 0.055 & 7.4 \\
\hline Columbia River & $100 \mathrm{H}$ SPR $145-1$ & October 10, 2003 & B17RK5 & Zinc & $\mathrm{mg} / \mathrm{L}$ & 1 & 0.28 & $=$ & 0.055 & 7.4 \\
\hline Columbia River & 100-D SPR 110-1 & October 21, 2002 & B15HW1 & Zinc & $\mathrm{mg} / \mathrm{L}$ & 1 & 0.42 & $=$ & 0.055 & 7.4 \\
\hline
\end{tabular}




\section{APPENDIX C SEGMENT 3 \\ SUMMARY STATISTICS, BENCHMARK COMPARISON, AND INDIVIDUAL SAMPLE EXCEEDENCES}


WCH-91

Rev. 0

Columbia River Component Data Evaluation Summary Report

July 2006

C-ii 


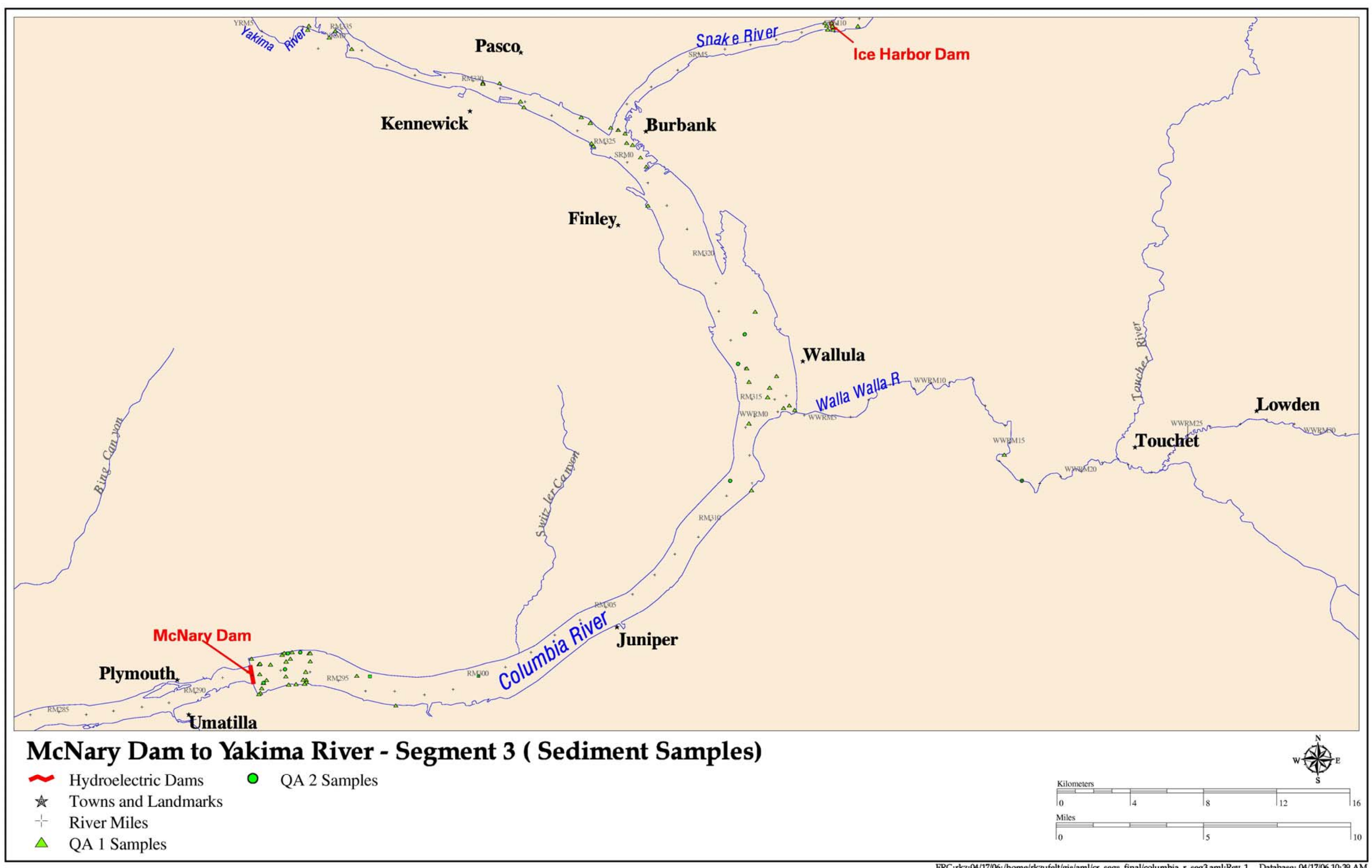




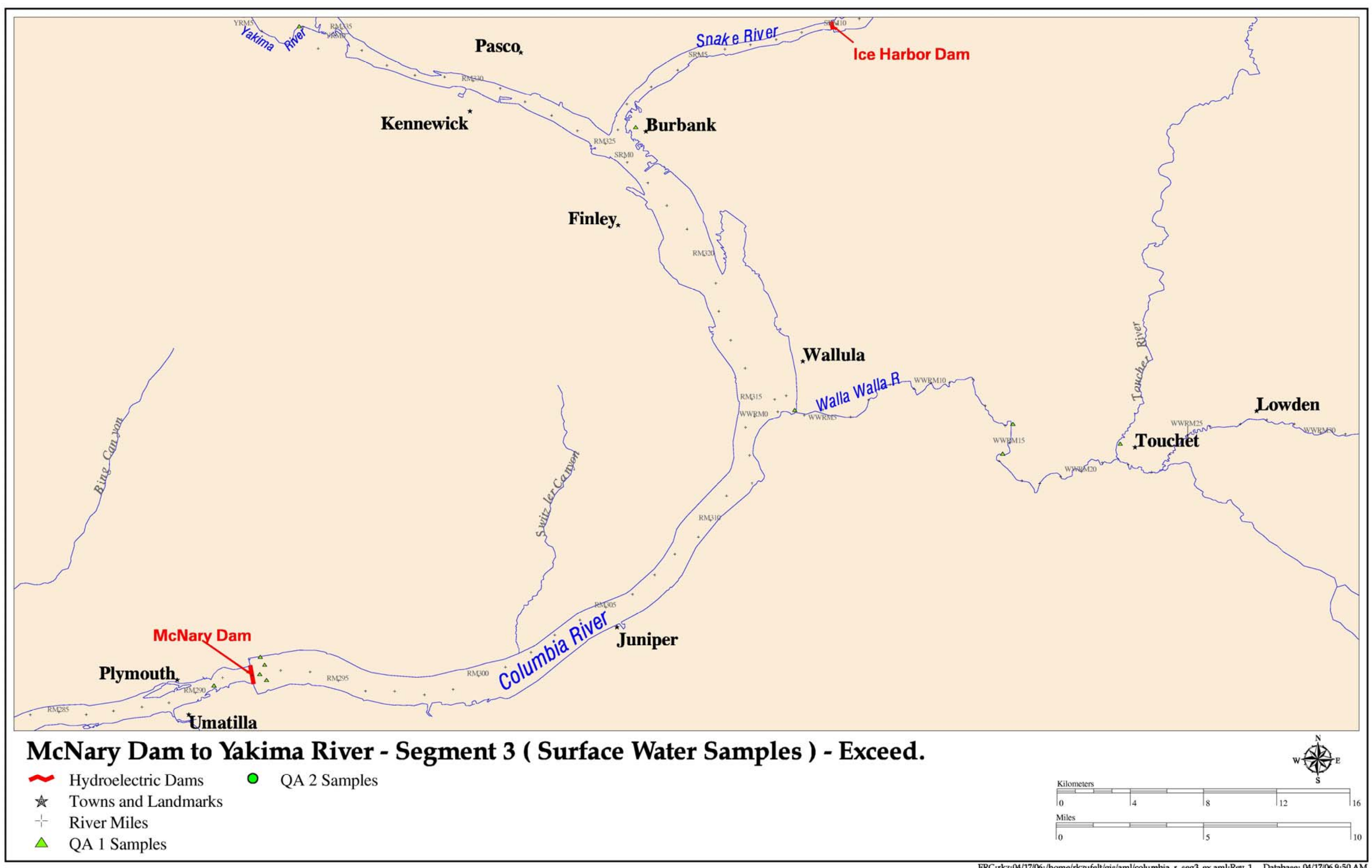




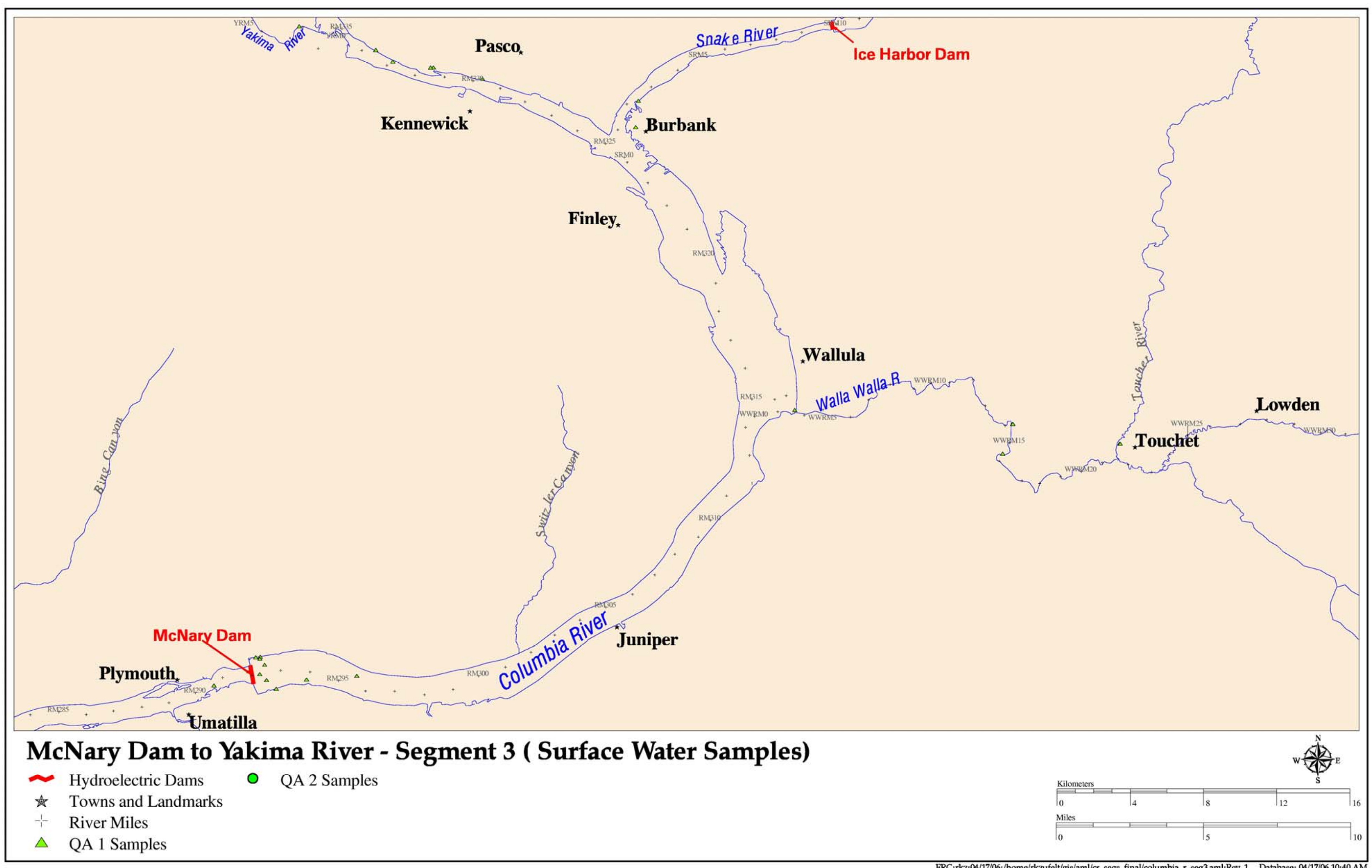




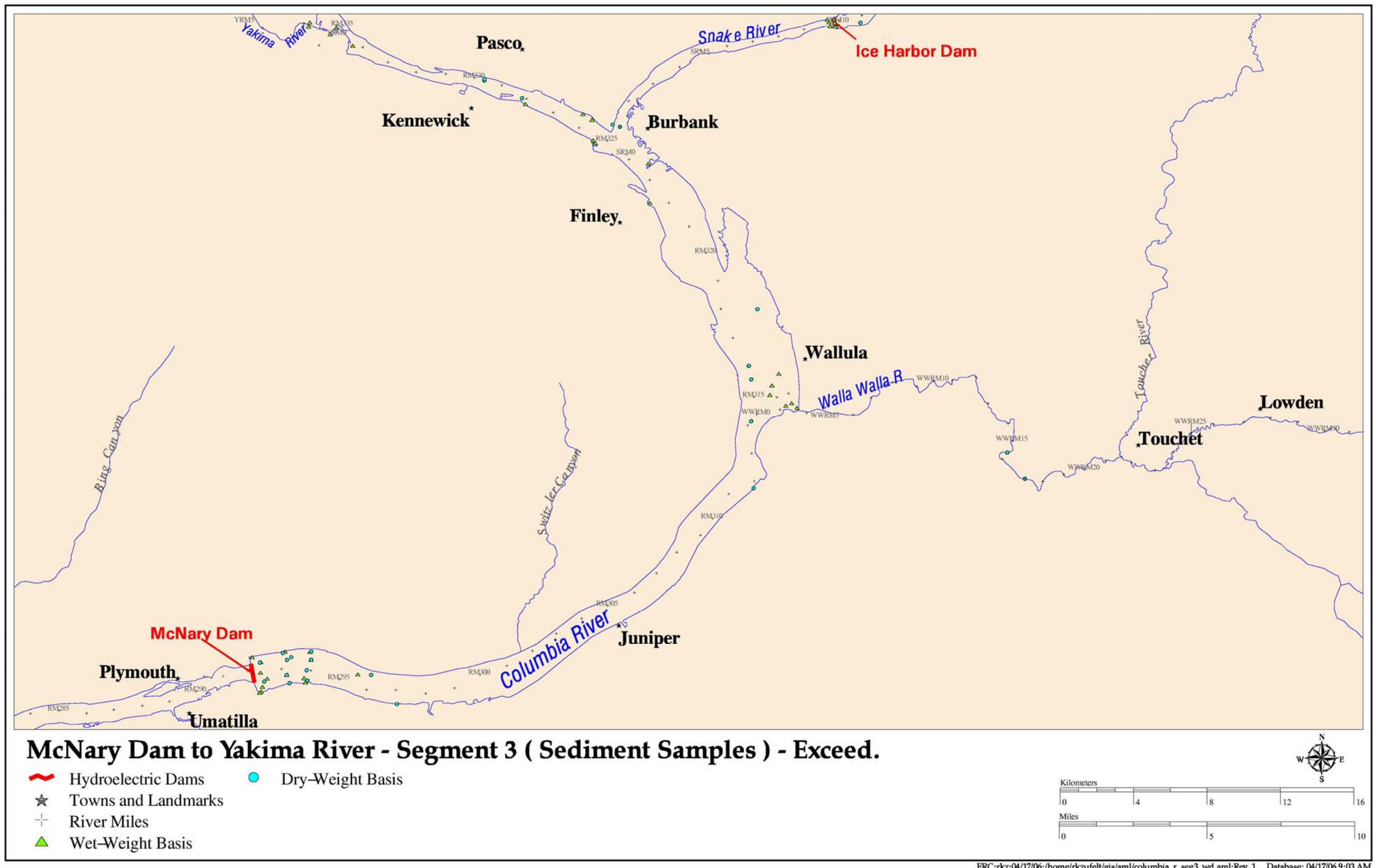


Table C-1

Summary Table of Segment 3 Sediment Samples By Area, Site, Date, and Category

\begin{tabular}{|c|c|c|c|c|}
\hline Sample ID & Sample Area & Sample Site & Sample Date & Category \\
\hline $05-8000$ & Columbia River & Pt of Pasco & 30-Sep-86 & 1 \\
\hline 05-8001 & Columbia River & Pt of Pasco & 30-Sep-86 & 1 \\
\hline $05-8002$ & Columbia River & Pt of Pasco & 30-Sep-86 & 1 \\
\hline $05-8003$ & Columbia River & Pt of Pasco & 30-Sep-86 & 1 \\
\hline 12985 & Columbia River & McNary Dam & 29-Sep-92 & 1 \\
\hline 12986 & Columbia River & McNary Dam & 29-Sep-92 & 1 \\
\hline 12987 & Columbia River & McNary Dam & 29-Sep-92 & 1 \\
\hline 12988 & Columbia River & McNary Dam & 29-Sep-92 & 1 \\
\hline 15828 & Columbia River & McNary Dam & 23-Sep-94 & 1 \\
\hline 15829 & Columbia River & McNary Dam & 23-Sep-94 & 1 \\
\hline 15830 & Columbia River & McNary Dam & 23-Sep-94 & 1 \\
\hline 15831 & Columbia River & McNary Dam & 23-Sep-94 & 1 \\
\hline 168272 & Columbia River & RM 318 & 14-Apr-92 & 1 \\
\hline 168273 & Columbia River & RM 316 & 14-Apr-92 & 1 \\
\hline 168274 & Columbia River & RM 311 & 13-Apr-92 & 1 \\
\hline 168275 & Columbia River & RM 298 & 13-Apr-92 & 1 \\
\hline 16952 & Columbia River & McNary Dam & 28-Jul-95 & 1 \\
\hline 16953 & Columbia River & McNary Dam & 28-Jul-95 & 1 \\
\hline 16954 & Columbia River & McNary Dam & 28-Jul-95 & 1 \\
\hline 16955 & Columbia River & McNary Dam & 28-Jul-95 & 1 \\
\hline 18466 & Columbia River & McNary Dam & 16-Aug-96 & 1 \\
\hline 18467 & Columbia River & McNary Dam & 16-Aug-96 & 1 \\
\hline 18468 & Columbia River & McNary Dam & 16-Aug-96 & 1 \\
\hline 18469 & Columbia River & McNary Dam & 16-Aug-96 & 1 \\
\hline 194274 & Columbia River & Wallula Mill & 10-Sep-91 & 2 \\
\hline 194275 & Columbia River & Wallula Mill & 10-Sep-91 & 2 \\
\hline 194276 & Columbia River & Wallula Mill & 10-Sep-91 & 2 \\
\hline 194277 & Columbia River & Wallula Mill & 10-Sep-91 & 2 \\
\hline 195563 & Columbia River & Wallula Mill & 01-Sep-91 & 2 \\
\hline 195564 & Columbia River & Wallula Mill & 01-Sep-91 & 2 \\
\hline 19679 & Columbia River & McNary Dam & 15-Aug-97 & 1 \\
\hline 19680 & Columbia River & McNary Dam & 15-Aug-97 & 1 \\
\hline 19681 & Columbia River & McNary Dam & 15-Aug-97 & 1 \\
\hline 19682 & Columbia River & McNary Dam & 15-Aug-97 & 1 \\
\hline 20749 & Columbia River & McNary Dam & 11-Aug-98 & 1 \\
\hline 20750 & Columbia River & McNary Dam & 11-Aug-98 & 1 \\
\hline 20751 & Columbia River & McNary Dam & 11-Aug-98 & 1 \\
\hline 20752 & Columbia River & McNary Dam & 11-Aug-98 & 1 \\
\hline 23515 & Columbia River & McNary Dam & 02-Aug-99 & 1 \\
\hline 23516 & Columbia River & McNary Dam & 02-Aug-99 & 1 \\
\hline 23517 & Columbia River & McNary Dam & 02-Aug-99 & 1 \\
\hline 23518 & Columbia River & McNary Dam & 02-Aug-99 & 1 \\
\hline 24743 & Columbia River & McNary Dam & 08-Aug-00 & 1 \\
\hline 24746 & Columbia River & McNary Dam & 08-Aug-00 & 1 \\
\hline 26245 & Columbia River & McNary Dam & 20-Jul-01 & 1 \\
\hline 26248 & Columbia River & McNary Dam & 23-Jul-01 & 1 \\
\hline 29043 & Columbia River & McNary Dam & 19-Jul-02 & 1 \\
\hline
\end{tabular}


Table C-1

Summary Table of Segment 3 Sediment Samples By Area, Site, Date, and Category

\begin{tabular}{|c|c|c|c|c|}
\hline Sample ID & Sample Area & Sample Site & Sample Date & Categ \\
\hline 29044 & Columbia River & McNary Dam & 19-Jul-02 & \\
\hline 30870 & Columbia River & McNary Dam & 16-Jul-03 & \\
\hline 30871 & Columbia River & McNary Dam & 16-Jul-03 & \\
\hline 31809 & Columbia River & McNary Dam & 08-Aug-03 & \\
\hline 31810 & Columbia River & McNary Dam & 08-Aug-03 & \\
\hline 31811 & Columbia River & McNary Dam & 08-Aug-03 & \\
\hline 31812 & Columbia River & McNary Dam & 08-Aug-03 & \\
\hline 31813 & Columbia River & McNary Dam & 08-Aug-03 & \\
\hline 31814 & Columbia River & McNary Dam & 08-Aug-03 & \\
\hline 33603 & Columbia River & McNary Dam & 21-Sep-04 & \\
\hline 33604 & Columbia River & McNary Dam & 21-Sep-04 & \\
\hline 33605 & Columbia River & McNary Dam & 21-Sep-04 & \\
\hline 3504038 & Columbia River & McNary Dam & 01-Jun-04 & \\
\hline $40-8324$ & Columbia River & Pt of Pasco & 30-Sep-86 & \\
\hline $40-8325$ & Columbia River & Pt of Pasco & 30-Sep-86 & \\
\hline $40-8326$ & Columbia River & Pt of Pasco & 30-Sep-86 & \\
\hline $40-8327$ & Columbia River & Pt of Pasco & 30-Sep-86 & \\
\hline 4414200 & Columbia River & McNary Dam & 01-Jun-04 & 1 \\
\hline 4414202 & Columbia River & McNary Dam & 01-Jun-04 & \\
\hline 4414203 & Columbia River & McNary Dam & 01-Jun-04 & \\
\hline 4414210 & Columbia River & RM 316 & 01-Jun-04 & \\
\hline 4414211 & Columbia River & RM 316 & 01-Jun-04 & \\
\hline 4414212 & Columbia River & RM 316 & 01-Jun-04 & \\
\hline 4414213 & Columbia River & Lake Wallula & 01-Jun-04 & 1 \\
\hline 4424215 & Columbia River & Lake Wallula & 01-Jun-04 & \\
\hline 4424216 & Columbia River & Lake Wallula & 01-Jun-04 & \\
\hline 4424220 & Columbia River & Lake Wallula & 01-Jun-04 & \\
\hline 4424224 & Columbia River & Lake Wallula & 01-Jun-04 & \\
\hline 4424225 & Columbia River & Lake Wallula & 01-Jun-04 & \\
\hline 4424226 & Columbia River & Lake Wallula & 01-Jun-04 & 1 \\
\hline 4424227 & Columbia River & Lake Wallula & 01-Jun-04 & \\
\hline 4434234 & Columbia River & RM 339.5 & 01-Jun-04 & \\
\hline 59550 & Columbia River & RM 312 & 23-Oct-91 & \\
\hline 59551 & Columbia River & Wallula Mill & 23-Oct-91 & \\
\hline 59552 & Columbia River & Wallula Mill & 23-Oct-91 & 2 \\
\hline 6428 & Columbia River & McNary Dam & 24-Jun-87 & 2 \\
\hline 6429 & Columbia River & McNary Dam & 24-Jun-87 & \\
\hline 6430 & Columbia River & McNary Dam & 24-Jun-87 & \\
\hline 7401 & Columbia River & McNary Dam & 07-Sep-88 & \\
\hline 8094 & Columbia River & Pt of Pasco & 10-Sep-85 & \\
\hline 8095 & Columbia River & Pt of Pasco & 10-Sep-85 & \\
\hline 8096 & Columbia River & Pt of Pasco & 10-Sep-85 & 2 \\
\hline B0BRS3 & Columbia River & McNary Dam & 11-Apr-94 & \\
\hline B0BRS5 & Columbia River & McNary Dam & 11-Apr-94 & \\
\hline BOBRS7 & Columbia River & McNary Dam & 11-Apr-94 & \\
\hline B0BRS9 & Columbia River & McNary Dam & 11-Apr-94 & \\
\hline B0CSG5 & Columbia River & McNary Dam 1 & 23-Sep-94 & \\
\hline
\end{tabular}


Table C-1

Summary Table of Segment 3 Sediment Samples By Area, Site, Date, and Category

\begin{tabular}{|c|c|c|c|c|}
\hline Sample ID & Sample Area & Sample Site & Sample Date & Category \\
\hline B0CSG6 & Columbia River & McNary Dam 2 & 23-Sep-94 & 1 \\
\hline B0CSG7 & Columbia River & McNary Dam & 23-Sep-94 & 1 \\
\hline B0CSG8 & Columbia River & McNary Dam & 23-Sep-94 & 1 \\
\hline B0W3D3 & Columbia River & McNary Dam & 02-Aug-99 & 1 \\
\hline B0W3D4 & Columbia River & McNary Dam & 02-Aug-99 & 1 \\
\hline BOW3H1 & Columbia River & McNary Dam & 02-Aug-99 & 1 \\
\hline BOW3H2 & Columbia River & McNary Dam & 02-Aug-99 & 1 \\
\hline BOW3H3 & Columbia River & McNary Dam & 02-Aug-99 & 1 \\
\hline BOW3H4 & Columbia River & McNary Dam & 02-Aug-99 & 1 \\
\hline B0W3H5 & Columbia River & McNary Dam & 02-Aug-99 & 1 \\
\hline B0W3H6 & Columbia River & McNary Dam & 02-Aug-99 & 1 \\
\hline BOW3K3 & Columbia River & McNary Dam & 02-Aug-99 & 1 \\
\hline B0W3K5 & Columbia River & McNary Dam & 02-Aug-99 & 1 \\
\hline B0W3K7 & Columbia River & McNary Dam & 02-Aug-99 & 1 \\
\hline B0W3K9 & Columbia River & McNary Dam & 02-Aug-99 & 1 \\
\hline BOW3N8 & Columbia River & McNary Dam & 02-Aug-99 & 1 \\
\hline BOW3N9 & Columbia River & McNary Dam & 02-Aug-99 & 1 \\
\hline BOW3P0 & Columbia River & McNary Dam & 02-Aug-99 & 1 \\
\hline B0W3P1 & Columbia River & McNary Dam & 02-Aug-99 & 1 \\
\hline B0W3P2 & Columbia River & McNary Dam & 02-Aug-99 & 1 \\
\hline B0W3P3 & Columbia River & McNary Dam & 02-Aug-99 & 1 \\
\hline B0W3V6 & Columbia River & McNary Dam & 02-Aug-99 & 1 \\
\hline B0W3V7 & Columbia River & McNary Dam & 02-Aug-99 & 1 \\
\hline B0W3V8 & Columbia River & McNary Dam & 02-Aug-99 & 1 \\
\hline Bow3V9 & Columbia River & McNary Dam & 02-Aug-99 & 1 \\
\hline B0W3W0 & Columbia River & McNary Dam & 02-Aug-99 & 1 \\
\hline B0W3W1 & Columbia River & McNary Dam & 02-Aug-99 & 1 \\
\hline B0WFL6 & Columbia River & MC NARY DAM & 02-Aug-99 & 1 \\
\hline BOWFL7 & Columbia River & MC NARY DAM & 02-Aug-99 & 1 \\
\hline BOWFL8 & Columbia River & MC NARY DAM & 02-Aug-99 & 1 \\
\hline BOYWC9 & Columbia River & McNary Dam & 08-Aug-00 & 1 \\
\hline BOYWDO & Columbia River & McNary Dam & 08-Aug-00 & 1 \\
\hline B0YWD1 & Columbia River & McNary Dam & 08-Aug-00 & 1 \\
\hline B0YWD2 & Columbia River & McNary Dam & 08-Aug-00 & 1 \\
\hline B0YWD3 & Columbia river & McNary Dam & 08-Aug-00 & 1 \\
\hline B0YWD5 & Columbia river & McNary Dam & 08-Aug-00 & 1 \\
\hline B0YXP4 & Columbia river & McNary Dam & 08-Aug-00 & 1 \\
\hline B0YXP5 & Columbia river & McNary Dam & 08-Aug-00 & 1 \\
\hline $\mathrm{B} 12 \mathrm{CJ} 3$ & Columbia River & McNary Dam & 20-Jul-01 & 1 \\
\hline B12CK3 & Columbia River & McNary Dam & 20-Jul-01 & 1 \\
\hline B12CK5 & Columbia River & McNary Dam & 20-Jul-01 & 1 \\
\hline $\mathrm{B} 12 \mathrm{CK} 7$ & Columbia River & McNary Dam & 20-Jul-01 & 1 \\
\hline B12CL1 & Columbia River & McNary Dam & 20-Jul-01 & 1 \\
\hline B12CL2 & Columbia River & McNary Dam & 20-Jul-01 & 1 \\
\hline B12CL3 & Columbia River & McNary Dam & 20-Jul-01 & 1 \\
\hline B12CL4 & Columbia River & McNary Dam & 20-Jul-01 & 1 \\
\hline B12CM7 & Columbia River & McNary Dam & 23-Jul-01 & 1 \\
\hline
\end{tabular}


Table C-1

Summary Table of Segment 3 Sediment Samples By Area, Site, Date, and Category

\begin{tabular}{|c|c|c|c|c|}
\hline Sample ID & Sample Area & Sample Site & Sample Date & Cates \\
\hline B12CM9 & Columbia River & McNary Dam & 23-Jul-01 & \\
\hline B12CNO & Columbia River & McNary Dam & 23-Jul-01 & \\
\hline $\mathrm{B} 12 \mathrm{CN} 2$ & Columbia River & McNary Dam & 23-Jul-01 & \\
\hline В12CT5 & Columbia River & McNary Dam & 20-Jul-01 & \\
\hline В12CT6 & Columbia River & McNary Dam & 20-Jul-01 & \\
\hline В12CT7 & Columbia River & McNary Dam & 20-Jul-01 & \\
\hline В12CT8 & Columbia River & McNary Dam & 23-Jul-01 & \\
\hline В12СТ9 & Columbia River & McNary Dam & 23-Jul-01 & \\
\hline B12CV0 & Columbia River & McNary Dam & 23-Jul-01 & \\
\hline B14WF6 & Columbia River & McNary Dam & 19-Jul-02 & \\
\hline B14WF7 & Columbia River & McNary Dam & 19-Jul-02 & \\
\hline B14YN7 & Columbia River & McNary Dam & 19-Jul-02 & \\
\hline B14YN8 & Columbia River & McNary Dam & 19-Jul-02 & 1 \\
\hline B14YN9 & Columbia River & McNary Dam & 19-Jul-02 & \\
\hline B14YP0 & Columbia River & McNary Dam & 19-Jul-02 & \\
\hline B14YP1 & Columbia River & McNary Dam & 19-Jul-02 & \\
\hline B14YP3 & Columbia River & McNary Dam & 19-Jul-02 & \\
\hline B176V3 & Columbia River & McNary Dam & 16-Jul-03 & \\
\hline B176V4 & Columbia River & McNary Dam & 16-Jul-03 & \\
\hline B17709 & Columbia River & McNary Dam & 16-Jul-03 & \\
\hline B17710 & Columbia River & McNary Dam & 16-Jul-03 & \\
\hline B17711 & Columbia River & McNary Dam & 16-Jul-03 & \\
\hline B17712 & Columbia River & McNary Dam & 16-Jul-03 & \\
\hline B17713 & Columbia River & McNary Dam & 16-Jul-03 & 1 \\
\hline B17715 & Columbia River & McNary Dam & 16-Jul-03 & \\
\hline B17B57 & Columbia River & McNary Dam & 08-Aug-03 & \\
\hline B17B60 & Columbia River & McNary Dam & 08-Aug-03 & \\
\hline B17B65 & Columbia River & McNary Dam & 08-Aug-03 & \\
\hline B17B67 & Columbia River & McNary Dam & 08-Aug-03 & 1 \\
\hline B17BD7 & Columbia River & McNary Dam & 08-Aug-03 & 1 \\
\hline B17BD8 & Columbia River & McNary Dam & 08-Aug-03 & \\
\hline B17BD9 & Columbia River & McNary Dam & 08-Aug-03 & \\
\hline B17BF1 & Columbia River & McNary Dam & 08-Aug-03 & \\
\hline B17BH7 & Columbia River & McNary Dam & 08-Aug-03 & \\
\hline B17BJ1 & Columbia River & McNary Dam & 08-Aug-03 & \\
\hline B17BJ2 & Columbia River & McNary Dam & 08-Aug-03 & 1 \\
\hline B17BJ3 & Columbia River & McNary Dam & 08-Aug-03 & \\
\hline B17BJ4 & Columbia River & McNary Dam & 08-Aug-03 & \\
\hline B17BP1 & Columbia River & McNary Dam & 08-Aug-03 & \\
\hline B17BP2 & Columbia River & McNary Dam & 08-Aug-03 & \\
\hline B17BP3 & Columbia River & McNary Dam & 08-Aug-03 & \\
\hline B17BP4 & Columbia River & McNary Dam & 08-Aug-03 & \\
\hline B17BP5 & Columbia River & McNary Dam & 08-Aug-03 & \\
\hline B17BP6 & Columbia River & McNary Dam & 08-Aug-03 & \\
\hline B199D2 & Columbia River & McNary Dam & 11-Jun-04 & \\
\hline B19PL6 & Columbia River & McNary Dam & 13-Jul-04 & \\
\hline B19PL7 & Columbia River & McNary Dam & 13-Jul-04 & \\
\hline
\end{tabular}


Table C-1

Summary Table of Segment 3 Sediment Samples By Area, Site, Date, and Category

\begin{tabular}{|c|c|c|c|c|}
\hline Sample ID & Sample Area & Sample Site & Sample Date & Categ \\
\hline B19PL8 & Columbia River & McNary Dam & 13-Jul-04 & 1 \\
\hline B19PL9 & Columbia River & McNary Dam & 13-Jul-04 & 1 \\
\hline B19PM0 & Columbia River & McNary Dam & 13-Jul-04 & \\
\hline B19PM2 & Columbia River & McNary Dam & 13-Jul-04 & \\
\hline B19TF5 & Columbia River & McNary Dam & 13-Jul-04 & \\
\hline B19TF6 & Columbia River & McNary Dam & 13-Jul-04 & \\
\hline B1B7N0 & Columbia River & McNary Dam & 21-Sep-04 & \\
\hline B1B7N1 & Columbia River & McNary Dam & 21-Sep-04 & \\
\hline B1B7N2 & Columbia River & McNary Dam & 21-Sep-04 & \\
\hline B1B7N3 & Columbia River & McNary Dam & 21-Sep-04 & \\
\hline B1B7N7 & Columbia River & McNary Dam & 21-Sep-04 & \\
\hline B1B7N8 & Columbia River & McNary Dam & 21-Sep-04 & \\
\hline B1B7N9 & Columbia River & McNary Dam & 21-Sep-04 & \\
\hline B1B7P0 & Columbia River & McNary Dam & 21-Sep-04 & 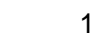 \\
\hline CRUSD128 & Columbia River & RM 274-286 & 01-Nov-91 & \\
\hline MD-04/12/83 & Columbia River & RM 300 & 12-Apr-83 & \\
\hline MD-05/19/77 & Columbia River & RM 300 & 19-May-77 & \\
\hline MD-07/10/78 & Columbia River & RM 300 & 10-Jul-78 & \\
\hline MD-08/29/79 & Columbia River & RM 300 & 29-Aug-79 & 3 \\
\hline MD-09/03/80 & Columbia River & RM 300 & 03-Sep-80 & 3 \\
\hline MD-09/12/83 & Columbia River & RM 300 & 12-Sep-83 & \\
\hline MD-09/20/82 & Columbia River & RM 300 & 20-Sep-82 & \\
\hline MD-09/28/81 & Columbia River & RM 300 & 28-Sep-81 & \\
\hline MD-1/28/1993 & Columbia River & RM 300 & 28-Jan-93 & \\
\hline MD-10/22/1992 & Columbia River & RM 300 & 22-Oct-92 & 3 \\
\hline MD-11/20/1986 & Columbia River & RM 300 & 20-Nov-86 & . \\
\hline MD-11/9/1984 & Columbia River & RM 300 & 09-Nov-84 & \\
\hline MD-12/14/76 & Columbia River & RM 300 & 14-Dec-76 & \\
\hline MD-2/25/1992 & Columbia River & RM 300 & 25-Feb-92 & 3 \\
\hline MD-2/6/1991 & Columbia River & RM 300 & 06-Feb-91 & 3 \\
\hline MD-3/18/1987 & Columbia River & RM 300 & 18-Mar-87 & 3 \\
\hline MD-3/28/1990 & Columbia River & RM 300 & 28-Mar-90 & 3 \\
\hline MD-4/24/1985 & Columbia River & RM 300 & 24-Apr-85 & 3 \\
\hline MD-4/7/1988 & Columbia River & RM 300 & 07-Apr-88 & \\
\hline MD-5/26/1993 & Columbia River & RM 300 & 26-May-93 & \\
\hline MD-5/3/1984 & Columbia River & RM 300 & 03-May-84 & \\
\hline MD-7/21/1989 & Columbia River & RM 300 & 21-Jul-89 & 3 \\
\hline MD-7/29/1985 & Columbia River & RM 300 & 29-Jul-85 & \\
\hline MD-7/3/1991 & Columbia River & RM 300 & 03-Jul-91 & \\
\hline MD-8/27/1986 & Columbia River & RM 300 & 27-Aug-86 & \\
\hline MD-8/5/1987 & Columbia River & RM 300 & 05-Aug-87 & \\
\hline MD-9/5/1990 & Columbia River & RM 300 & 05-Sep-90 & \\
\hline MD-9/8/1988 & Columbia River & RM 300 & 08-Sep-88 & \\
\hline MD-mid river-1 & Columbia River & McNary Dam & 01-Oct-86 & \\
\hline MD-mid river-2 & Columbia River & McNary Dam & 01-Oct-86 & \\
\hline MD-north shore & Columbia River & McNary Dam & 01-Oct-86 & \\
\hline MD-south shore & Columbia River & McNary Dam & 01-Oct-86 & \\
\hline
\end{tabular}


Table C-1

Summary Table of Segment 3 Sediment Samples By Area, Site, Date, and Category

\begin{tabular}{|c|c|c|c|c|}
\hline Sample ID & Sample Area & Sample Site & Sample Date & Categ \\
\hline SRP 1,2 & Columbia River & RM 314-315 & 19-Aug-91 & 1 \\
\hline SRP 3,4 & Columbia River & RM 328 & 20-Aug-91 & \\
\hline SRP 50 & Columbia River & RM 315-316 & 19-Aug-91 & \\
\hline SRP 51 & Columbia River & RM 315-316 & 19-Aug-91 & \\
\hline $\mathrm{B} 12 \mathrm{CH} 3$ & Snake Harbor & ICE HARBOR DAM & 23-Jul-01 & \\
\hline B12CJ0 & Snake Harbor & ICE HARBOR DAM & 23-Jul-01 & \\
\hline $\mathrm{B} 12 \mathrm{CJ} 1$ & Snake Harbor & ICE HARBOR DAM & 23-Jul-01 & \\
\hline В12CT2 & Snake Harbor & ICE HARBOR DAM & 23-Jul-01 & \\
\hline В12CT3 & Snake Harbor & ICE HARBOR DAM & 23-Jul-01 & \\
\hline B12CT4 & Snake Harbor & ICE HARBOR DAM & 23-Jul-01 & \\
\hline 3504002 & Snake River & RM 10 & 01-Jun-04 & \\
\hline 3504003 & Snake River & RM 10 & 01-Jun-04 & \\
\hline 3504026 & Snake River & RM 10 & 01-Jun-04 & \\
\hline 3504027 & Snake River & RM 10 & 01-Jun-04 & \\
\hline 4414205 & Snake River & RM 10 & 01-Jun-04 & \\
\hline 4414206 & Snake River & RM 10 & 01-Jun-04 & \\
\hline 4414207 & Snake River & RM 10 & 01-Jun-04 & \\
\hline 4424217 & Snake River & RM 1 & 01-Jun-04 & \\
\hline 4424218 & Snake River & RM 1 & 01-Jun-04 & \\
\hline 94378145 & Snake River & Lake Sacajawea & 14-Sep-94 & \\
\hline B0W3C4 & Snake River & ICE HARBOR DAM & 16-Aug-99 & \\
\hline B0W3C5 & Snake River & ICE HARBOR DAM & 16-Aug-99 & \\
\hline B0W3C6 & Snake River & ICE HARBOR DAM & 16-Aug-99 & \\
\hline B0W3C7 & Snake River & ICE HARBOR DAM & 16-Aug-99 & \\
\hline B0W3C8 & Snake River & ICE HARBOR DAM & 16-Aug-99 & \\
\hline B0W3C9 & Snake River & ICE HARBOR DAM & 16-Aug-99 & \\
\hline B0W3D0 & Snake River & ICE HARBOR DAM & 16-Aug-99 & \\
\hline B0W3D1 & Snake River & ICE HARBOR DAM & 16-Aug-99 & \\
\hline B0W3D2 & Snake River & ICE HARBOR DAM & 16-Aug-99 & \\
\hline B0W3V3 & Snake River & ICE HARBOR DAM & 16-Aug-99 & \\
\hline B0W3V4 & Snake River & ICE HARBOR DAM & 16-Aug-99 & \\
\hline B0W3V5 & Snake River & ICE HARBOR DAM & 16-Aug-99 & \\
\hline B0WFK5 & Snake River & ICE HARBOR DAM & 16-Aug-99 & \\
\hline BOWFK6 & Snake River & ICE HARBOR DAM & 16-Aug-99 & \\
\hline BOWFK7 & Snake River & ICE HARBOR DAM & 16-Aug-99 & \\
\hline BOWFK8 & Snake River & ICE HARBOR DAM & 16-Aug-99 & \\
\hline $\mathrm{B} 12 \mathrm{CH} 5$ & Snake River & ICE HARBOR DAM & 23-Jul-01 & \\
\hline $\mathrm{B} 12 \mathrm{CH} 7$ & Snake River & ICE HARBOR DAM & 23-Jul-01 & \\
\hline B12CH9 & Snake River & ICE HARBOR DAM & 23-Jul-01 & \\
\hline B1B7T7 & Snake River & ICE HARBOR DAM & 20-Sep-04 & \\
\hline B1B7T8 & Snake River & ICE HARBOR DAM & 20-Sep-04 & \\
\hline B1B7T9 & Snake River & ICE HARBOR DAM & 20-Sep-04 & \\
\hline B1B7W6 & Snake River & ICE HARBOR DAM & 20-Sep-04 & \\
\hline B1B7W7 & Snake River & ICE HARBOR DAM & 20-Sep-04 & \\
\hline B1B7W8 & Snake River & ICE HARBOR DAM & 20-Sep-04 & \\
\hline B1B7W9 & Snake River & ICE HARBOR DAM & 20-Sep-04 & \\
\hline SRP 7A & Snake River & RM 2 & 19-Aug-91 & \\
\hline
\end{tabular}


Table C-1

Summary Table of Segment 3 Sediment Samples By Area, Site, Date, and Category

\begin{tabular}{|c|c|c|c|c|}
\hline Sample ID & Sample Area & Sample Site & Sample Date & Category \\
\hline 2398242-SD & Walla Walla River & Walla Walla, WA & 27-Sep-02 & 1 \\
\hline 2398243 & Walla Walla River & Walla Walla, WA & 27-Sep-02 & 1 \\
\hline 2398244 & Walla Walla River & Walla Walla, WA & 27-Sep-02 & 1 \\
\hline $32 \mathrm{~A} 070-4$ & Walla Walla River & Blw Warm Sprgs & 12-Sep-84 & 2 \\
\hline 4414209 & Walla Walla River & RM 1 & 01-Jun-04 & 1 \\
\hline 92388089 & Walla Walla River & Near mouth & 16-Sep-92 & 1 \\
\hline B16WW0 & Walla Walla River & Walla Walla Riv & 15-May-03 & 1 \\
\hline B16WW1 & Walla Walla River & Walla Walla Riv & 15-May-03 & 1 \\
\hline B16WX2 & Walla Walla River & Walla Walla Riv & 15-May-03 & 1 \\
\hline B16WX3 & Walla Walla River & Walla Walla Riv & 15-May-03 & 1 \\
\hline B170V8 & Walla Walla River & Walla Walla Riv & 15-May-03 & 1 \\
\hline B170V9 & Walla Walla River & Walla Walla Riv & 15-May-03 & 1 \\
\hline Walla 003 & Walla Walla River & Cummins Rd Bdg & 18-Jun-96 & 1 \\
\hline $37 \mathrm{~A} 070-7$ & Yakima River & Below Kiona & 12-Sep-84 & 2 \\
\hline 4434230 & Yakima River & RM 1 & 01-Jun-04 & 1 \\
\hline 4434231 & Yakima River & RM 1 & 01-Jun-04 & 1 \\
\hline 4434233 & Yakima River & RM 1 & 01-Jun-04 & 1 \\
\hline 4434242 & Yakima River & RM 2 & 01-Jun-04 & 1 \\
\hline 4434243 & Yakima River & RM 2 & 01-Jun-04 & 1 \\
\hline 4434244 & Yakima River & RM 1 & 01-Jun-04 & 1 \\
\hline 8140 & Yakima River & RM 18 & 24-Sep-85 & 1 \\
\hline 92388096 & Yakima River & Horn Rapids Dam & 16-Sep-92 & 1 \\
\hline 92388097 & Yakima River & Horn Rapids Dam & 16-Sep-92 & 1 \\
\hline B16WV8 & Yakima River & YAKIMA RIVER & 14-May-03 & 1 \\
\hline B16WV9 & Yakima River & YAKIMA RIVER & 14-May-03 & 1 \\
\hline B16WX0 & Yakima River & YAKIMA RIVER & 14-May-03 & 1 \\
\hline B16WX1 & Yakima River & YAKIMA RIVER & 14-May-03 & 1 \\
\hline B170W0 & Yakima River & YAKIMA RIVER & 14-May-03 & 1 \\
\hline B170W1 & Yakima River & YAKIMA RIVER & 14-May-03 & 1 \\
\hline Bed sediment \#1 & Yakima River & Kiona WA & 31-Aug-88 & 1 \\
\hline Bed sediment \#2 & Yakima River & Kiona WA & 31-Aug-88 & 1 \\
\hline Bed sediment \#3 & Yakima River & Kiona WA & 10-Nov-90 & 1 \\
\hline Yakima sed & Yakima River & Kiona WA & 25-Aug-87 & 1 \\
\hline
\end{tabular}


Table C-2

Rev. 0

Summary Table of Segment 3 Surface Water Samples By Category, Area, Site, Date, and Category

\begin{tabular}{|c|c|c|c|c|}
\hline Sample Area & Sample Site & Sample ID & Sample Date & Category \\
\hline Columbia River & MCNARY DAM & B16WN7 & May 15, 2003 & 1 \\
\hline Columbia River & MCNARY DAM & B16WL5 & May 15,2003 & 1 \\
\hline Columbia River & MCNARY DAM & B16WP0 & May 15, 2003 & 1 \\
\hline Columbia River & MCNARY DAM & B16WL6 & May 15, 2003 & 1 \\
\hline Columbia River & MCNARY DAM & B16WL7 & May 15, 2003 & 1 \\
\hline Columbia River & MCNARY DAM & B170P9 & May 15, 2003 & 1 \\
\hline Columbia River & MCNARY DAM & B170R1 & May 15, 2003 & 1 \\
\hline Columbia River & MCNARY DAM & B16WN6 & May 15, 2003 & 1 \\
\hline Columbia River & MCNARY DAM & B16WN8 & May 15, 2003 & 1 \\
\hline Columbia River & MCNARY DAM & B16WN9 & May 15, 2003 & 1 \\
\hline Columbia River & MCNARY DAM & B16WP1 & May 15, 2003 & 1 \\
\hline Columbia River & McNary Dam & McNary-WA Shore & August 8, 2003 & 1 \\
\hline Columbia River & McNary Dam & B17BH8 & August 8, 2003 & 1 \\
\hline Columbia River & McNary Dam & В17BH9 & August 8, 2003 & 1 \\
\hline Columbia River & MCNARY DAM & B17BJ0 & August 8, 2003 & 1 \\
\hline Columbia River & McNary Dam & McNary OR Shore & August 8, 2003 & 1 \\
\hline Columbia River & McNary Dam & McNary-2/3 OR Shore & August 8, 2003 & 1 \\
\hline Columbia River & McNary MID-RVR & B170R0 & May 15, 2003 & 1 \\
\hline Snake River & HOOD PK-FRKN CS & B16WR8 & May 15, 2003 & 1 \\
\hline Snake River & HOOD PK-FRKN CS & B16WR5 & May 15, 2003 & 1 \\
\hline Snake River & HOOD PK-FRKN CS & B170T1 & May 15, 2003 & 1 \\
\hline Snake River & HOOD PK-FRKN CS & B16WM6 & May 15, 2003 & 1 \\
\hline Snake River & HOOD PK-MID-RVR & B16WM5 & May 15, 2003 & 1 \\
\hline Snake River & HOOD PK-MID-RVR & B16WR4 & May 15, 2003 & 1 \\
\hline Snake River & HOOD PK-MID-RVR & B16WR9 & May 15, 2003 & 1 \\
\hline Snake River & HOOD PK-MID-RVR & В170Т2 & May 15, 2003 & 1 \\
\hline Snake River & HOOD PK-WALLA S & B16WR6 & May 15, 2003 & 1 \\
\hline Snake River & HOOD PK-WALLA S & B16WM4 & May 15, 2003 & 1 \\
\hline Snake River & HOOD PK-WALLA S & В170T3 & May 15, 2003 & 1 \\
\hline Snake River & HOOD PK-WALLA S & B16WR7 & May 15, 2003 & 1 \\
\hline Snake River & Hood Prk BTLNCH & 0000202 & April 9, 2003 & 1 \\
\hline Snake River & Hood Prk BTLNCH & 0000203 & October 6, 2003 & 1 \\
\hline Snake River & Hood Prk BTLNCH & 0000201 & October 2, 2002 & 1 \\
\hline Snake River & Hood Prk BTLNCH & 0000200 & April 23, 2002 & 1 \\
\hline Snake River & Hood Prk BTLNCH & 0000199 & October 1, 2001 & 1 \\
\hline Snake River & Hood Prk BTLNCH & 0000198 & May 7, 2001 & 1 \\
\hline Snake River & Hood Prk BTLNCH & 0000197 & October 5, 2000 & 1 \\
\hline Snake River & Hood Prk BTLNCH & 0000196 & April 17, 2000 & 1 \\
\hline Touchet River & HWY12 Bridge & 3228272 & May 30, 2002 & 1 \\
\hline Touchet River & HWY12 Bridge & 2368894 & September 3, 2002 & 1 \\
\hline Touchet River & HWY12 Bridge & 3078993 & February 11, 2003 & 1 \\
\hline Touchet River & HWY12 Bridge & 3248272 & June 9, 2003 & 1 \\
\hline Touchet River & HWY12 Bridge & 3058983 & January 30, 2003 & 1 \\
\hline Touchet River & HWY12 Bridge & 2518973 & December 16, 2002 & 1 \\
\hline Touchet River & HWY12 Bridge & 2498956 & December 2, 2002 & 1 \\
\hline Touchet River & HWY12 Bridge & 2478956 & November 19, 2002 & 1 \\
\hline Touchet River & HWY12 Bridge & 2388911 & September 16, 2002 & 1 \\
\hline Touchet River & HWY12 Bridge & 2378911 & September 16, 2002 & 1 \\
\hline Touchet River & HWY12 Bridge & 2248088 & June 12, 2002 & 1 \\
\hline Touchet River & HWY12 Bridge & 3138162 & March 24, 2003 & 1 \\
\hline Touchet River & HWY12 Bridge & 2348863 & August 21, 2002 & 1 \\
\hline Touchet River & HWY12 Bridge & 3108993 & March 6, 2003 & 1 \\
\hline Touchet River & HWY12 Bridge & 3098995 & February 24, 2003 & 1 \\
\hline Touchet River & HWY12 Bridge & 3208163 & May 14, 2003 & 1 \\
\hline Touchet River & HWY12 Bridge & 2228163 & May 30, 2002 & 1 \\
\hline Walla Walla River & RM15.6 CMNS BRG & 3058984 & January 30, 2003 & 1 \\
\hline Walla Walla River & RM15.6 CMNS BRG & 2248090 & June 12, 2002 & 1 \\
\hline
\end{tabular}


Summary Table of Segment 3 Surface Water Samples By Category, Area, Site, Date, and Category

\begin{tabular}{|c|c|c|c|c|}
\hline Sample Area & Sample Site & Sample ID & Sample Date & Category \\
\hline Walla Walla River & RM15.6 CMNS BRG & 2228167 & May 30, 2002 & 1 \\
\hline Walla Walla River & RM15.6 CMNS BRG & 2228168 & May 30, 2002 & 1 \\
\hline Walla Walla River & WALLA 1 & 2398242 & September 27, 2002 & 1 \\
\hline Walla Walla River & WALLA 2 & 2398245 & September 27, 2002 & 1 \\
\hline Walla Walla River & WALLA 2 & 2398243-SW & September 27, 2002 & 1 \\
\hline Walla Walla River & Walla Walla Riv & B170T5 & May 15, 2003 & 1 \\
\hline Walla Walla River & Walla Walla Riv & В170T6 & May 15, 2003 & 1 \\
\hline Walla Walla River & Walla Walla Riv & B16WR1 & May 15, 2003 & 1 \\
\hline Walla Walla River & Walla Walla Riv & B16WP8 & May 15, 2003 & 1 \\
\hline Walla Walla River & Walla Walla Riv & B16WM3 & May 15, 2003 & 1 \\
\hline Walla Walla River & Walla Walla Riv & B16WM2 & May 15, 2003 & 1 \\
\hline Walla Walla River & Walla Walla Riv & B16WM1 & May 15, 2003 & 1 \\
\hline Walla Walla River & Walla Walla Riv & B16WP9 & May 15, 2003 & 1 \\
\hline Walla Walla River & Walla Walla Riv & B16WR0 & May 15, 2003 & 1 \\
\hline Walla Walla River & Walla Walla Riv & B16WR2 & May 15, 2003 & 1 \\
\hline Walla Walla River & Walla Walla Riv & B170T4 & May 15, 2003 & 1 \\
\hline Walla Walla River & Walla Walla Riv & B16WR3 & May 15, 2003 & 1 \\
\hline Walla Walla River & WWR (32WAL14.3) & 3208164 & May 14, 2003 & 1 \\
\hline Walla Walla River & WWR (32WAL14.3) & 2348864 & August 21, 2002 & 1 \\
\hline Walla Walla River & WWR (32WAL14.3) & 3108994 & March 6, 2003 & 1 \\
\hline Walla Walla River & WWR (32WAL14.3) & 2368895 & September 3, 2002 & 1 \\
\hline Walla Walla River & WWR (32WAL14.3) & 3098996 & February 24, 2003 & 1 \\
\hline Walla Walla River & WWR (32WAL14.3) & 2248089 & June 11, 2002 & 1 \\
\hline Walla Walla River & WWR (32WAL14.3) & 2208114 & May 14, 2002 & 1 \\
\hline Walla Walla River & WWR (32WAL14.3) & 3138163 & March 24, 2003 & 1 \\
\hline Walla Walla River & WWR (32WAL14.3) & 2388912 & September 16, 2002 & 1 \\
\hline Walla Walla River & WWR (32WAL14.3) & 3078994 & February 10, 2003 & 1 \\
\hline Walla Walla River & WWR (32WAL14.3) & 3248273 & June 9, 2003 & 1 \\
\hline Walla Walla River & WWR (32WAL14.3) & 3228273 & May 30, 2003 & 1 \\
\hline Walla Walla River & WWR (32WAL14.3) & 2478957 & November 19, 2002 & 1 \\
\hline Walla Walla River & WWR (32WAL14.3) & 2518974 & December 16, 2002 & 1 \\
\hline Walla Walla River & WWR (32WAL14.3) & 2498957 & December 3, 2002 & 1 \\
\hline Walla Walla River & WWR (32WAL14.3) & 2228166 & May 30, 2002 & 1 \\
\hline Yakima River & BENTON CITY BRG & B16KJ0 & March 7, 2003 & 1 \\
\hline Yakima River & KIONA WA & 1296007 & July 9, 2001 & 1 \\
\hline Yakima River & KIONA WA & 1308113 & July 25, 2001 & 1 \\
\hline Yakima River & KIONA WA & 308194 & July 25, 2000 & 1 \\
\hline Yakima River & KIONA WA & 2058088 & January 29, 2002 & 1 \\
\hline Yakima River & KIONA WA & 1378090-F & September 12, 2001 & 1 \\
\hline Yakima River & KIONA WA & 1378090 & September 12, 2001 & 1 \\
\hline Yakima River & KIONA WA & 1378089-F & September 12, 2001 & 1 \\
\hline Yakima River & KIONA WA & 1378089 & September 12, 2001 & 1 \\
\hline Yakima River & KIONA WA & 1368084 & August 14, 2001 & 1 \\
\hline Yakima River & KIONA WA & 1468091 & November 14, 2001 & 1 \\
\hline Yakima River & KIONA WA & 1468091-F & November 14, 2001 & 1 \\
\hline Yakima River & KIONA WA & 1468165 & October 10, 2001 & 1 \\
\hline Yakima River & KIONA WA & 1498111 & November 13, 2001 & 1 \\
\hline Yakima River & KIONA WA & 308197 & July 25,2000 & 1 \\
\hline Yakima River & KIONA WA & $2208164-F$ & May 16, 2002 & 1 \\
\hline Yakima River & KIONA WA & 2018090 & December 5, 2001 & 1 \\
\hline Yakima River & KIONA WA & 2138003 & March 25, 2002 & 1 \\
\hline Yakima River & KIONA WA & 2228090 & May 10, 2002 & 1 \\
\hline Yakima River & KIONA WA & 2208164 & May 16, 2002 & 1 \\
\hline Yakima River & KIONA WA & 1408090 & September 4, 2001 & 1 \\
\hline Yakima River & KIONA WA & 2178190 & April 3, 2002 & 1 \\
\hline Yakima River & KIONA WA & 1308113-F & July 25, 2001 & 1 \\
\hline Yakima River & KIONA WA & 2138003-F & March 25, 2002 & 1 \\
\hline
\end{tabular}




\begin{tabular}{|c|c|c|c|c|}
\hline Sample Area & Sample Site & Sample ID & Sample Date & Category \\
\hline Yakima River & KIONA WA & 2268165 & June 12, 2002 & 1 \\
\hline Yakima River & KIONA WA & 2118115 & February 6, 2002 & 1 \\
\hline Yakima River & KIONA WA & 2068090 & January 9, 2002 & 1 \\
\hline Yakima River & KIONA WA & 2058088-F & January 29, 2002 & 1 \\
\hline Yakima River & KIONA WA & 99398291 & September 28, 1999 & 1 \\
\hline Yakima River & KIONA WA & 2158165 & March 6, 2002 & 1 \\
\hline Yakima River & Richland WTP-W & 99398311 & September 29, 1999 & 1 \\
\hline Yakima River & Richland WTP-W & 99398306 & September 29, 1999 & 1 \\
\hline Yakima River & Richland WTP-W & 99398307 & September 29, 1999 & 1 \\
\hline Yakima River & RM12.8, TWIN BR & 308206 & July 26, 2000 & 1 \\
\hline Yakima River & RM12.8, TWIN BR & 99398304 & September 29, 1999 & 1 \\
\hline Yakima River & RM16.2, WANA DM & 308205 & July 26,2000 & 1 \\
\hline Yakima River & RM16.2, WANA DM & 99398310 & September 29, 1999 & 1 \\
\hline Yakima River & RM16.2, WANA DM & 99398303 & September 29, 1999 & 1 \\
\hline Yakima River & RM27.0 KIONA & 308195 & July 25,2000 & 1 \\
\hline Yakima River & RM27.0 KIONA & 99398292 & September 28, 1999 & 1 \\
\hline Yakima River & RM27.0 KIONA & 99398295 & September 28, 1999 & 1 \\
\hline Yakima River & RM5.5 I182 & 308208 & July 26,2000 & 1 \\
\hline Yakima River & RM5.5 I182 & 99398309 & September 29, 1999 & 1 \\
\hline Yakima River & RM5.5 I182 & 308209 & July 26,2000 & 1 \\
\hline Yakima River & VAN GIESEN & 99398308 & September 29, 1999 & 1 \\
\hline Yakima River & VAN GIESEN & 99398294 & September 28, 1999 & 1 \\
\hline Yakima River & VAN GIESEN & 308199 & July 26,2000 & 1 \\
\hline Yakima River & VAN GIESEN & 308207 & July 26,2000 & 1 \\
\hline Yakima River & WANAWISH DAM & 308196 & July 25,2000 & 1 \\
\hline Yakima River & WANAWISH DAM & 99398293 & September 28, 1999 & 1 \\
\hline Yakima River & WANAWISH DAM & 99398298 & September 29, 1999 & 1 \\
\hline Yakima River & WANAWISH DAM & 308198 & July 26, 2000 & 1 \\
\hline Yakima River & WANAWISH DAM & 308204 & July 26,2000 & 1 \\
\hline Yakima River & YAK-6 & 95338432 & August 15, 1995 & 1 \\
\hline Yakima River & YAK-6 & 95178425 & April 26, 1995 & 1 \\
\hline Yakima River & YAK-6 & 95398438 & September 27, 1995 & 1 \\
\hline Yakima River & YAK-6 & 95158431 & April 11, 1995 & 1 \\
\hline Yakima River & YAK-6 & 95128429 & March 21, 1995 & 1 \\
\hline Yakima River & YAK-6 & 95378424 & September 13, 1995 & 1 \\
\hline Yakima River & YAK-6 & 95238422 & June 6, 1995 & 1 \\
\hline Yakima River & YAK-6 & 95358432 & August 29, 1995 & 1 \\
\hline Yakima River & YAK-6 & 95258430 & June 20, 1995 & 1 \\
\hline Yakima River & YAK-6 & 95278424 & July 6, 1995 & 1 \\
\hline Yakima River & YAK-6 & 95318430 & August 2, 1995 & 1 \\
\hline Yakima River & YAK-7 & 95398439 & September 27, 1995 & 1 \\
\hline Yakima River & YAK-7 & 95378425 & September 13, 1995 & 1 \\
\hline Yakima River & YAK-7 & 95358433 & August 29, 1995 & 1 \\
\hline Yakima River & YAK-7 & 95318431 & August 2, 1995 & 1 \\
\hline Yakima River & YAK-7 & 95128419 & March 21, 1995 & 1 \\
\hline Yakima River & YAK-7 & 95158432 & April 11, 1995 & 1 \\
\hline Yakima River & YAK-7 & 95338433 & August 15, 1995 & 1 \\
\hline Yakima River & YAK-7 & 95128430 & March 21, 1995 & 1 \\
\hline Yakima River & YAK-7 & 95278425 & July 6, 1995 & 1 \\
\hline Yakima River & YAK-7 & 95178426 & April 26, 1995 & 1 \\
\hline Yakima River & YAK-7 & 95238423 & June 6, 1995 & 1 \\
\hline Yakima River & YAK-7 & 95258431 & June 20, 1995 & 1 \\
\hline Yakima River & Yakima Rvr & B16WL9 & May 14, 2003 & 1 \\
\hline Yakima River & Yakima Rvr & B16WP5 & May 14, 2003 & 1 \\
\hline Yakima River & Yakima Rvr & B16WP4 & May 14, 2003 & 1 \\
\hline Yakima River & Yakima Rvr & B16WP3 & May 14, 2003 & 1 \\
\hline Yakima River & Yakima Rvr & B16WP2 & May 14, 2003 & 1 \\
\hline
\end{tabular}




\begin{tabular}{|c|c|c|c|c|}
\hline Sample Area & Sample Site & Sample ID & Sample Date & Category \\
\hline Yakima River & Yakima Rvr & B170T7 & May 14, 2003 & 1 \\
\hline Yakima River & Yakima Rvr & В170т9 & May 14, 2003 & 1 \\
\hline Yakima River & Yakima Rvr & B16WM0 & May 14, 2003 & 1 \\
\hline Yakima River & Yakima Rvr & B16WL8 & May 14, 2003 & 1 \\
\hline Yakima River & Yakima Rvr & B16WP7 & May 14, 2003 & 1 \\
\hline Yakima River & Yakima Rvr & В170т8 & May 14, 2003 & 1 \\
\hline Yakima River & Yakima Rvr & B16WP6 & May 14, 2003 & 1 \\
\hline Yakima River & KIONA BRG & 10000007 & May 19, 2004 & 2 \\
\hline Yakima River & KIONA BRG & 10000008 & June 2, 2004 & 2 \\
\hline Yakima River & KIONA BRG & 10000018 & October 20, 2004 & 2 \\
\hline Yakima River & KIONA BRG & 40000011 & August 26, 2003 & 2 \\
\hline Yakima River & KIONA BRG & 10000016 & September 22, 2004 & 2 \\
\hline Yakima River & KIONA BRG & 10000004 & April 7, 2004 & 2 \\
\hline Yakima River & KIONA BRG & 10000006 & May 5, 2004 & 2 \\
\hline Yakima River & KIONA BRG & 40000001 & May 20, 2003 & 2 \\
\hline Yakima River & KIONA BRG & 40000002 & June 3, 2003 & 2 \\
\hline Yakima River & KIONA BRG & 40000004 & July 1, 2003 & 2 \\
\hline Yakima River & KIONA BRG & 10000002 & March 18, 2004 & 2 \\
\hline Yakima River & KIONA BRG & 40000009 & August 12, 2003 & 2 \\
\hline Yakima River & KIONA BRG & 10000003 & March 24, 2004 & 2 \\
\hline Yakima River & KIONA BRG & 40000012 & September 23, 2003 & 2 \\
\hline Yakima River & KIONA BRG & 40000014 & October 1, 2003 & 2 \\
\hline Yakima River & KIONA BRG & 40000016 & October 21, 2003 & 2 \\
\hline Yakima River & KIONA BRG & 40000018 & November 12, 2003 & 2 \\
\hline Yakima River & KIONA BRG & 40000019 & November 24, 2003 & 2 \\
\hline Yakima River & KIONA BRG & 40000020 & December 9, 2003 & 2 \\
\hline Yakima River & KIONA BRG & 40000007 & July 29, 2003 & 2 \\
\hline Yakima River & KIONA BRG & 10000009 & June 15, 2004 & 2 \\
\hline Yakima River & KIONA BRG & 10000011 & July 14, 2004 & 2 \\
\hline Yakima River & KIONA BRG & 10000001 & February 25, 2004 & 2 \\
\hline Yakima River & KIONA BRG & 10000005 & April 21, 2004 & 2 \\
\hline Yakima River & KIONA WA & 132911 & January 14, 2004 & 2 \\
\hline Yakima River & KIONA WA & $134232-F$ & March 8, 2005 & 2 \\
\hline Yakima River & KIONA WA & 132112 & April 9, 2003 & 2 \\
\hline Yakima River & KIONA WA & 134330 & April 5, 2005 & 2 \\
\hline Yakima River & KIONA WA & $134330-F$ & April 5, 2005 & 2 \\
\hline Yakima River & KIONA WA & 133091 & March 10, 2004 & 2 \\
\hline Yakima River & KIONA WA & $133000-\mathrm{F}$ & February 11, 2004 & 2 \\
\hline Yakima River & KIONA WA & 133000 & February 11, 2004 & 2 \\
\hline Yakima River & KIONA WA & $132642-F$ & October 8, 2003 & 2 \\
\hline Yakima River & KIONA WA & 132819-F & December 9, 2003 & 2 \\
\hline Yakima River & KIONA WA & 132819 & December 9, 2003 & 2 \\
\hline Yakima River & KIONA WA & 134232 & March 8, 2005 & 2 \\
\hline Yakima River & KIONA WA & $134132-\mathrm{F}$ & February 8, 2005 & 2 \\
\hline Yakima River & KIONA WA & 133239-F & April 14, 2004 & 2 \\
\hline Yakima River & KIONA WA & $132729-F$ & November 5, 2003 & 2 \\
\hline Yakima River & KIONA WA & 132729 & November 5, 2003 & 2 \\
\hline Yakima River & KIONA WA & 133091-F & March 10, 2004 & 2 \\
\hline Yakima River & KIONA WA & 133239 & April 14, 2004 & 2 \\
\hline Yakima River & KIONA WA & 132911-F & January 14, 2004 & 2 \\
\hline Yakima River & KIONA WA & 130678 & December 5, 2001 & 2 \\
\hline Yakima River & KIONA WA & $131198-F$ & June 12, 2002 & 2 \\
\hline Yakima River & KIONA WA & 131198 & June 12, 2002 & 2 \\
\hline Yakima River & KIONA WA & 131038-F & May 8, 2002 & 2 \\
\hline Yakima River & KIONA WA & 131038 & May 8, 2002 & 2 \\
\hline Yakima River & KIONA WA & 130969-F & April 3, 2002 & 2 \\
\hline Yakima River & KIONA WA & 130969 & April 3, 2002 & 2 \\
\hline
\end{tabular}




\begin{tabular}{|c|c|c|c|c|}
\hline Sample Area & Sample Site & Sample ID & Sample Date & Category \\
\hline Yakima River & KIONA WA & 130879-F & March 6, 2002 & 2 \\
\hline Yakima River & KIONA WA & 130879 & March 6, 2002 & 2 \\
\hline Yakima River & KIONA WA & 130768-F & February 6, 2002 & 2 \\
\hline Yakima River & KIONA WA & 130768 & February 6, 2002 & 2 \\
\hline Yakima River & KIONA WA & 130698-F & January 9, 2002 & 2 \\
\hline Yakima River & KIONA WA & 132171 & May 7, 2003 & 2 \\
\hline Yakima River & KIONA WA & 130678-F & December 5, 2001 & 2 \\
\hline Yakima River & KIONA WA & 131382 & August 7, 2002 & 2 \\
\hline Yakima River & KIONA WA & 130657-F & November 14, 2001 & 2 \\
\hline Yakima River & KIONA WA & 130657 & November 14, 2001 & 2 \\
\hline Yakima River & KIONA WA & $130520-\mathrm{F}$ & October 10, 2001 & 2 \\
\hline Yakima River & KIONA WA & 130520 & October 10, 2001 & 2 \\
\hline Yakima River & KIONA WA & 1286007 & July 10, 2001 & 2 \\
\hline Yakima River & KIONA WA & 1236007 & June 5, 2001 & 2 \\
\hline Yakima River & KIONA WA & 1196007 & May 8, 2001 & 2 \\
\hline Yakima River & KIONA WA & 1146007 & April 3, 2001 & 2 \\
\hline Yakima River & KIONA WA & 1106007 & March 6, 2001 & 2 \\
\hline Yakima River & KIONA WA & 1066007 & February 6, 2001 & 2 \\
\hline Yakima River & KIONA WA & 106014 & March 8, 2000 & 2 \\
\hline Yakima River & KIONA WA & 1036007 & January 16, 2001 & 2 \\
\hline Yakima River & KIONA WA & 130698 & January 9, 2002 & 2 \\
\hline Yakima River & KIONA WA & 131880 & February 5, 2003 & 2 \\
\hline Yakima River & KIONA WA & 132529-F & September 10, 2003 & 2 \\
\hline Yakima River & KIONA WA & 132529 & September 10, 2003 & 2 \\
\hline Yakima River & KIONA WA & $132440-\mathrm{F}$ & August 6, 2003 & 2 \\
\hline Yakima River & KIONA WA & 132440 & August 6, 2003 & 2 \\
\hline Yakima River & KIONA WA & $132373-F$ & July 9, 2003 & 2 \\
\hline Yakima River & KIONA WA & 132373 & July 9, 2003 & 2 \\
\hline Yakima River & KIONA WA & 132261-F & June 4, 2003 & 2 \\
\hline Yakima River & KIONA WA & 132261 & June 4, 2003 & 2 \\
\hline Yakima River & KIONA WA & $132171-F$ & May 7, 2003 & 2 \\
\hline Yakima River & KIONA WA & 134463 & May 3, 2005 & 2 \\
\hline Yakima River & KIONA WA & $132112-F$ & April 9, 2003 & 2 \\
\hline Yakima River & KIONA WA & $131947-F$ & March 5, 2003 & 2 \\
\hline Yakima River & KIONA WA & 131219 & July 10, 2002 & 2 \\
\hline Yakima River & KIONA WA & $131880-F$ & February 5, 2003 & 2 \\
\hline Yakima River & KIONA WA & $131219-F$ & July 10, 2002 & 2 \\
\hline Yakima River & KIONA WA & 131789-F & January 8, 2003 & 2 \\
\hline Yakima River & KIONA WA & 131789 & January 8, 2003 & 2 \\
\hline Yakima River & KIONA WA & $131676-F$ & December 4, 2002 & 2 \\
\hline Yakima River & KIONA WA & 131676 & December 4, 2002 & 2 \\
\hline Yakima River & KIONA WA & 131587-F & November 6, 2002 & 2 \\
\hline Yakima River & KIONA WA & 131587 & November 6, 2002 & 2 \\
\hline Yakima River & KIONA WA & $131494-\mathrm{F}$ & October 9, 2002 & 2 \\
\hline Yakima River & KIONA WA & 131494 & October 9, 2002 & 2 \\
\hline Yakima River & KIONA WA & $131402-F$ & September 11, 2002 & 2 \\
\hline Yakima River & KIONA WA & 131402 & September 11, 2002 & 2 \\
\hline Yakima River & KIONA WA & $131382-F$ & August 7, 2002 & 2 \\
\hline Yakima River & KIONA WA & 132642 & October 8, 2003 & 2 \\
\hline Yakima River & KIONA WA & 131947 & March 5, 2003 & 2 \\
\hline Yakima River & KIONA WA & 133484-F & July 14, 2004 & 2 \\
\hline Yakima River & KIONA WA & 86499 & February 6, 2001 & 2 \\
\hline Yakima River & KIONA WA & 86593 & March 6, 2001 & 2 \\
\hline Yakima River & KIONA WA & 86687 & April 3, 2001 & 2 \\
\hline Yakima River & KIONA WA & $133304-F$ & May 5, 2004 & 2 \\
\hline Yakima River & KIONA WA & 99366015 & September 8, 1999 & 2 \\
\hline Yakima River & KIONA WA & 133304 & May 5, 2004 & 2 \\
\hline
\end{tabular}




\begin{tabular}{|c|c|c|c|c|}
\hline Sample Area & Sample Site & Sample ID & Sample Date & Category \\
\hline Yakima River & KIONA WA & 134463-F & May 3, 2005 & 2 \\
\hline Yakima River & KIONA WA & 85846 & November 7, 2000 & 2 \\
\hline Yakima River & KIONA WA & 133572 & August 4, 2004 & 2 \\
\hline Yakima River & KIONA WA & $133572-\mathrm{F}$ & August 4, 2004 & 2 \\
\hline Yakima River & KIONA WA & 1336007 & August 14, 2001 & 2 \\
\hline Yakima River & KIONA WA & 133664 & September 15, 2004 & 2 \\
\hline Yakima River & KIONA WA & 86778 & May 8, 2001 & 2 \\
\hline Yakima River & KIONA WA & 133664-F & September 15, 2004 & 2 \\
\hline Yakima River & KIONA WA & 133374-F & June 9, 2004 & 2 \\
\hline Yakima River & KIONA WA & 41393 & August 16, 2000 & 2 \\
\hline Yakima River & KIONA WA & 496007 & December 5, 2000 & 2 \\
\hline Yakima River & KIONA WA & 456007 & November 7, 2000 & 2 \\
\hline Yakima River & KIONA WA & 42116 & July 12,2000 & 2 \\
\hline Yakima River & KIONA WA & 42097 & June 7,2000 & 2 \\
\hline Yakima River & KIONA WA & 42078 & May 3, 2000 & 2 \\
\hline Yakima River & KIONA WA & 42041 & April 5, 2000 & 2 \\
\hline Yakima River & KIONA WA & 86429 & January 16, 2001 & 2 \\
\hline Yakima River & KIONA WA & 41429 & September 6, 2000 & 2 \\
\hline Yakima River & KIONA WA & 86340 & December 5, 2000 & 2 \\
\hline Yakima River & KIONA WA & 40813 & July 7, 1999 & 2 \\
\hline Yakima River & KIONA WA & 406007 & October 3, 2000 & 2 \\
\hline Yakima River & KIONA WA & 366014 & September 6, 2000 & 2 \\
\hline Yakima River & KIONA WA & 336014 & August 16, 2000 & 2 \\
\hline Yakima River & KIONA WA & 66014 & February 9, 2000 & 2 \\
\hline Yakima River & KIONA WA & 133484 & July 14, 2004 & 2 \\
\hline Yakima River & KIONA WA & 41554 & October 3, 2000 & 2 \\
\hline Yakima River & KIONA WA & 16014 & January 5, 2000 & 2 \\
\hline Yakima River & KIONA WA & 99156015 & April 14, 1999 & 2 \\
\hline Yakima River & KIONA WA & 99406014 & October 6, 1999 & 2 \\
\hline Yakima River & KIONA WA & 99316015 & August 4, 1999 & 2 \\
\hline Yakima River & KIONA WA & 99446014 & November 3, 1999 & 2 \\
\hline Yakima River & KIONA WA & 186014 & May 3, 2000 & 2 \\
\hline Yakima River & KIONA WA & 99496014 & December 8, 1999 & 2 \\
\hline Yakima River & KIONA WA & 99246015 & June 16, 1999 & 2 \\
\hline Yakima River & KIONA WA & 99026015 & January 13, 1999 & 2 \\
\hline Yakima River & KIONA WA & 133723 & October 5, 2004 & 2 \\
\hline Yakima River & KIONA WA & 134543 & June 7, 2005 & 2 \\
\hline Yakima River & KIONA WA & 133374 & June 9, 2004 & 2 \\
\hline Yakima River & KIONA WA & $134543-F$ & June 7, 2005 & 2 \\
\hline Yakima River & KIONA WA & 1366007 & September 4, 2001 & 2 \\
\hline Yakima River & KIONA WA & 146014 & April 5, 2000 & 2 \\
\hline Yakima River & KIONA WA & 99276015 & July 7, 1999 & 2 \\
\hline Yakima River & KIONA WA & 86894 & June 5, 2001 & 2 \\
\hline Yakima River & KIONA WA & 133723-F & October 5, 2004 & 2 \\
\hline Yakima River & KIONA WA & 133829 & November 2, 2004 & 2 \\
\hline Yakima River & KIONA WA & $133829-F$ & November 2, 2004 & 2 \\
\hline Yakima River & KIONA WA & 133928 & December 7, 2004 & 2 \\
\hline Yakima River & KIONA WA & 133928-F & December 7, 2004 & 2 \\
\hline Yakima River & KIONA WA & 134051 & January 4, 2005 & 2 \\
\hline Yakima River & KIONA WA & 99116015 & March 17, 1999 & 2 \\
\hline Yakima River & KIONA WA & 134132 & February 8, 2005 & 2 \\
\hline Yakima River & KIONA WA & 99056015 & February 3, 1999 & 2 \\
\hline Yakima River & KIONA WA & 286014 & July 12,2000 & 2 \\
\hline Yakima River & KIONA WA & 86993 & July 10, 2001 & 2 \\
\hline Yakima River & KIONA WA & 87080 & August 14, 2001 & 2 \\
\hline Yakima River & KIONA WA & 87125 & September 4, 2001 & 2 \\
\hline Yakima River & KIONA WA & 236014 & June 7, 2000 & 2 \\
\hline
\end{tabular}


Table C-2

Rev. 0

Summary Table of Segment 3 Surface Water Samples By Category, Area, Site, Date, and Category

\begin{tabular}{|c|c|c|c|c|}
\hline Sample Area & Sample Site & Sample ID & Sample Date & Category \\
\hline Yakima River & KIONA WA & 99196015 & May 12, 1999 & 2 \\
\hline Yakima River & KIONA WA & 134051-F & January 4, 2005 & 2 \\
\hline Yakima River & Terrace HGHTSBR & 50000001 & February 25, 2004 & 2 \\
\hline Yakima River & Terrace HGHTSBR & 50000002 & March 18, 2004 & 2 \\
\hline Yakima River & Terrace HGHTSBR & 50000003 & March 24, 2004 & 2 \\
\hline Yakima River & Terrace HGHTSBR & 50000004 & April 8, 2004 & 2 \\
\hline Yakima River & Terrace HGHTSBR & 50000006 & May 6, 2004 & 2 \\
\hline Yakima River & Terrace HGHTSBR & 50000015 & September 7, 2004 & 2 \\
\hline Yakima River & Terrace HGHTSBR & 50000005 & April 22, 2004 & 2 \\
\hline Yakima River & Terrace HGHTSBR & 50000007 & July 29, 2003 & 2 \\
\hline Yakima River & Terrace HGHTSBR & 50000008 & June 3, 2004 & 2 \\
\hline Yakima River & Terrace HGHTSBR & 50000009 & June 14, 2004 & 2 \\
\hline Yakima River & Terrace HGHTSBR & 50000010 & June 29, 2004 & 2 \\
\hline Yakima River & Terrace HGHTSBR & 50000011 & July 13, 2004 & 2 \\
\hline Yakima River & Terrace HGHTSBR & 50000012 & July 29, 2004 & 2 \\
\hline Yakima River & Terrace HGHTSBR & 50000014 & August 26, 2004 & 2 \\
\hline Yakima River & Terrace HGHTSBR & 50000016 & September 22, 2004 & 2 \\
\hline Yakima River & Terrace HGHTSBR & 50000017 & October 6, 2004 & 2 \\
\hline Yakima River & Terrace HGHTSBR & 50000018 & October 19, 2004 & 2 \\
\hline Yakima River & Terrace HGHTSBR & 50000019 & November 2, 2004 & 2 \\
\hline Yakima River & Terrace HGHTSBR & 50000020 & December 9, 2003 & 2 \\
\hline Yakima River & Terrace HGHTSBR & 50000013 & August 11, 2004 & 2 \\
\hline Yakima River & VAN GIESEN & 300000014 & August 26, 2003 & 2 \\
\hline Yakima River & VAN GIESEN & 300000017 & October 7, 2003 & 2 \\
\hline Yakima River & VAN GIESEN & 300000008 & July 8, 2003 & 2 \\
\hline Yakima River & VAN GIESEN & 300000009 & July 22, 2003 & 2 \\
\hline Yakima River & VAN GIESEN & 300000010 & July 29, 2003 & 2 \\
\hline Yakima River & VAN GIESEN & 300000011 & August 5, 2003 & 2 \\
\hline Yakima River & VAN GIESEN & 300000013 & August 19, 2003 & 2 \\
\hline Yakima River & VAN GIESEN & 300000012 & August 12, 2003 & 2 \\
\hline Yakima River & VAN GIESEN & 300000016 & September 30, 2003 & 2 \\
\hline Yakima River & VAN GIESEN & 300000004 & June 3, 2003 & 2 \\
\hline Yakima River & VAN GIESEN & 300000018 & October 14, 2003 & 2 \\
\hline Yakima River & VAN GIESEN & 300000019 & October 21, 2003 & 2 \\
\hline Yakima River & VAN GIESEN & 300000020 & October 28, 2003 & 2 \\
\hline Yakima River & VAN GIESEN & 300000021 & November 12, 2003 & 2 \\
\hline Yakima River & VAN GIESEN & 300000022 & November 24, 2003 & 2 \\
\hline Yakima River & VAN GIESEN & 300000023 & December 9, 2003 & 2 \\
\hline Yakima River & VAN GIESEN & 300000015 & September 23, 2003 & 2 \\
\hline Yakima River & VAN GIESEN & 20000017 & October 6, 2004 & 2 \\
\hline Yakima River & VAN GIESEN & 20000009 & June 15, 2004 & 2 \\
\hline Yakima River & VAN GIESEN & 20000010 & June 30, 2004 & 2 \\
\hline Yakima River & VAN GIESEN & 20000011 & July 14, 2004 & 2 \\
\hline Yakima River & VAN GIESEN & 20000012 & July 29, 2004 & 2 \\
\hline Yakima River & VAN GIESEN & 20000013 & August 11, 2004 & 2 \\
\hline Yakima River & VAN GIESEN & 20000014 & August 25, 2004 & 2 \\
\hline Yakima River & VAN GIESEN & 300000007 & July 1,2003 & 2 \\
\hline Yakima River & VAN GIESEN & 20000016 & September 22, 2004 & 2 \\
\hline Yakima River & VAN GIESEN & 300000006 & June 24, 2003 & 2 \\
\hline Yakima River & VAN GIESEN & 20000018 & October 20, 2004 & 2 \\
\hline Yakima River & VAN GIESEN & 20000019 & November 3, 2004 & 2 \\
\hline Yakima River & VAN GIESEN & 300000001 & April 29, 2003 & 2 \\
\hline Yakima River & VAN GIESEN & 300000002 & May 20, 2003 & 2 \\
\hline Yakima River & VAN GIESEN & 300000003 & May 27, 2003 & 2 \\
\hline Yakima River & VAN GIESEN & 20000001 & February 25, 2004 & 2 \\
\hline Yakima River & VAN GIESEN & 20000015 & September 7, 2004 & 2 \\
\hline
\end{tabular}


Table C-3

Summary Statistics for Sediment Collected from Segment 3 of the Columbia River Including All Categories of Data (units reported "as received")

Constituent

\begin{tabular}{|c|c|c|c|c|c|c|c|c|c|c|}
\hline $\begin{array}{l}\text { Constituent } \\
\text { Class }\end{array}$ & Constituent Name & Units & $\begin{array}{c}\text { Number } \\
\text { Analyzed }\end{array}$ & $\begin{array}{l}\text { Number } \\
\text { Detected }\end{array}$ & $\begin{array}{l}\text { Frequency of } \\
\text { Detection }\end{array}$ & $\begin{array}{l}\text { Minimum } \\
\text { Nondetect }\end{array}$ & $\begin{array}{l}\text { Maximum } \\
\text { Nondetect }\end{array}$ & Minimum Detect & $\begin{array}{l}\text { Maximum } \\
\text { Detect }\end{array}$ & $\begin{array}{c}\text { Average } \\
\text { Concentration }\end{array}$ \\
\hline$\overline{\text { CONV }}$ & Nitrogen in ammonia & $\mathrm{mg} / \mathrm{kg}$ & 4 & 4 & $100 \%$ & -- & --- & 6.0 & 35 & 17 \\
\hline CONV & Phosphorous in phosphate & $\mathrm{mg} / \mathrm{kg}$ & 4 & 4 & $100 \%$ & -- & -- & 0.040 & 0.23 & 0.11 \\
\hline CONV & Sulfide & $\mathrm{mg} / \mathrm{kg}$ & 4 & 2 & $50 \%$ & 1.2 & 1.2 & 1.9 & 3.6 & 1.7 \\
\hline CONV & Total Organic Carbon & $\%$ & 41 & 41 & $100 \%$ & & & 0.11 & 2.1 & 0.93 \\
\hline CONV & Total Organic Carbon & $\mathrm{mg} / \mathrm{kg}$ & 42 & 42 & $100 \%$ & -- & -- & 1.0 & 28,692 & 9,089 \\
\hline METAL & Acid Volatile Sulfate & umole/g & 18 & 15 & $83 \%$ & 0.069 & 0.12 & 0.081 & 4.9 & 1.1 \\
\hline METAL & Aluminum & $\mathrm{mg} / \mathrm{kg}$ & 5 & 5 & $100 \%$ & -- & -- & 5,900 & 68,000 & 20,380 \\
\hline METAL & Antimony & $\mathrm{mg} / \mathrm{kg}$ & 25 & 25 & $100 \%$ & -- & -- & 0.035 & 1.3 & 0.69 \\
\hline METAL & Arsenic & $\mathrm{mg} / \mathrm{kg}$ & 55 & 45 & $82 \%$ & 4.5 & 4.5 & 0.37 & 10 & 5.8 \\
\hline METAL & Barium & $\mathrm{mg} / \mathrm{kg}$ & 5 & 5 & $100 \%$ & -- & -- & 47 & 530 & 154 \\
\hline METAL & Beryllium & $\mathrm{mg} / \mathrm{kg}$ & 29 & 29 & $100 \%$ & -- & -- & 0.085 & 2.1 & 1.4 \\
\hline METAL & Bismuth & $\mathrm{mg} / \mathrm{kg}$ & 1 & 0 & $0 \%$ & 10 & 10 & -- & -- & 5.0 \\
\hline METAL & Boron & $\mathrm{mg} / \mathrm{kg}$ & 1 & 1 & $100 \%$ & -- & -- & 2.3 & 2.3 & 2.3 \\
\hline METAL & Cadmium & $\mathrm{mg} / \mathrm{kg}$ & 58 & 43 & $74 \%$ & 0.090 & 2.0 & 0.067 & 6.0 & 1.1 \\
\hline METAL & Calcium & $\mathrm{mg} / \mathrm{kg}$ & 5 & 5 & $100 \%$ & -- & -- & 2,800 & 26,000 & 8,000 \\
\hline METAL & Cerium & $\mathrm{mg} / \mathrm{kg}$ & 1 & 1 & $100 \%$ & -- & -- & 53 & 53 & 53 \\
\hline METAL & Chromium & $\mathrm{mg} / \mathrm{kg}$ & 59 & 59 & $100 \%$ & -- & -- & 2.6 & 74 & 30 \\
\hline METAL & Cobalt & $\mathrm{mg} / \mathrm{kg}$ & 5 & 5 & $100 \%$ & -- & -- & 5.2 & 20 & 9.0 \\
\hline METAL & Copper & $\mathrm{mg} / \mathrm{kg}$ & 59 & 59 & $100 \%$ & -- & -- & 1.5 & 37 & 20 \\
\hline METAL & Europium & $\mathrm{mg} / \mathrm{kg}$ & 1 & 0 & $0 \%$ & 2.0 & 2.0 & -- & -- & 1.0 \\
\hline METAL & Gallium & $\mathrm{mg} / \mathrm{kg}$ & 1 & 1 & $100 \%$ & -- & -- & 19 & 19 & 19 \\
\hline METAL & Gold & $\mathrm{mg} / \mathrm{kg}$ & 1 & 0 & $0 \%$ & 8.0 & 8.0 & -- & -- & 4.0 \\
\hline METAL & Iron & $\mathrm{mg} / \mathrm{kg}$ & 5 & 5 & $100 \%$ & -- & -- & 12,000 & 51,000 & 23,000 \\
\hline METAL & Lanthanum & $\mathrm{mg} / \mathrm{kg}$ & 1 & 1 & $100 \%$ & -- & -- & 28 & 28 & 28 \\
\hline METAL & Lead & $\mathrm{mg} / \mathrm{kg}$ & 55 & 54 & $98 \%$ & 2.5 & 2.5 & 1.1 & 32 & 14 \\
\hline METAL & Lithium & $\mathrm{mg} / \mathrm{kg}$ & 1 & 1 & $100 \%$ & -- & -- & 26 & 26 & 26 \\
\hline METAL & Magnesium & $\mathrm{mg} / \mathrm{kg}$ & 5 & 5 & $100 \%$ & -- & -- & 2,700 & 14,000 & 5,400 \\
\hline METAL & Manganese & $\mathrm{mg} / \mathrm{kg}$ & 5 & 5 & $100 \%$ & -- & -- & 250 & 1,500 & 532 \\
\hline METAL & Mercury & $\mathrm{mg} / \mathrm{kg}$ & 54 & 36 & $67 \%$ & 0.040 & 0.16 & 0.0032 & 0.17 & 0.061 \\
\hline METAL & Molybdenum & $\mathrm{mg} / \mathrm{kg}$ & 31 & 0 & $0 \%$ & 0.59 & 2.0 & -- & -- & 0.32 \\
\hline METAL & Neodymium & $\mathrm{mg} / \mathrm{kg}$ & 1 & 1 & $100 \%$ & -- & -- & 30 & 30 & 30 \\
\hline METAL & Nickel & $\mathrm{mg} / \mathrm{kg}$ & 59 & 59 & $100 \%$ & -- & -- & 1.3 & 32 & 19 \\
\hline METAL & Niobium & $\mathrm{mg} / \mathrm{kg}$ & 1 & 0 & $0 \%$ & 4.0 & 4.0 & -- & -- & 2.0 \\
\hline METAL & Phosphorus & $\mathrm{mg} / \mathrm{kg}$ & 1 & 1 & $100 \%$ & -- & -- & 1,000 & 1,000 & 1,000 \\
\hline METAL & Potassium & $\mathrm{mg} / \mathrm{kg}$ & 4 & 4 & $100 \%$ & -- & -- & 700 & 1,400 & 1,175 \\
\hline METAL & Scandium & $\mathrm{mg} / \mathrm{kg}$ & 1 & 1 & $100 \%$ & -- & -- & 19 & 19 & 19 \\
\hline METAL & Selenium & $\mathrm{mg} / \mathrm{kg}$ & 54 & 8 & $15 \%$ & 0.40 & 5.0 & 0.015 & 1.8 & 1.6 \\
\hline METAL & Silver & $\mathrm{mg} / \mathrm{kg}$ & 25 & 24 & $96 \%$ & 2.0 & 2.0 & 0.0039 & 0.56 & 0.28 \\
\hline METAL & Sodium & $\mathrm{mg} / \mathrm{kg}$ & 5 & 5 & $100 \%$ & -- & -- & 280 & 16,000 & 3,478 \\
\hline METAL & Strontium & $\mathrm{mg} / \mathrm{kg}$ & 1 & 1 & $100 \%$ & -- & -- & 260 & 260 & 260 \\
\hline METAL & Thallium & $\mathrm{mg} / \mathrm{kg}$ & 24 & 24 & $100 \%$ & -- & -- & 0.035 & 1.1 & 0.57 \\
\hline METAL & Thorium & $\mathrm{mg} / \mathrm{kg}$ & 1 & 1 & $100 \%$ & -- & -- & 8.0 & 8.0 & 8.0 \\
\hline METAL & Tin & $\mathrm{mg} / \mathrm{kg}$ & 1 & 0 & $0 \%$ & 10 & 10 & -- & -- & 5.0 \\
\hline METAL & Titanium & $\mathrm{mg} / \mathrm{kg}$ & 1 & 1 & $100 \%$ & -- & -- & 6,200 & 6,200 & 6,200 \\
\hline METAL & Uranium & $\mathrm{mg} / \mathrm{kg}$ & 5 & 5 & $100 \%$ & -- & -- & 1.1 & 1.5 & 1.3 \\
\hline METAL & Vanadium & $\mathrm{mg} / \mathrm{kg}$ & 5 & 5 & $100 \%$ & -- & -- & 26 & 140 & 61 \\
\hline METAL & Ytterbium & $\mathrm{mg} / \mathrm{kg}$ & 1 & 1 & $100 \%$ & -- & -- & 3.0 & 3.0 & 3.0 \\
\hline
\end{tabular}


Table C-3

Summary Statistics for Sediment Collected from Segment 3 of the Columbia River Including All Categories of Data (units reported "as received")

\begin{tabular}{|c|c|c|c|c|c|c|c|c|c|c|}
\hline $\begin{array}{c}\text { Constituent } \\
\text { Class }\end{array}$ & Constituent Name & Units & $\begin{array}{c}\text { Number } \\
\text { Analyzed }\end{array}$ & $\begin{array}{l}\text { Number } \\
\text { Detected }\end{array}$ & $\begin{array}{l}\text { Frequency of } \\
\text { Detection }\end{array}$ & $\begin{array}{l}\text { Minimum } \\
\text { Nondetect }\end{array}$ & $\begin{array}{l}\text { Maximum } \\
\text { Nondetect }\end{array}$ & Minimum Detect & $\begin{array}{l}\text { Maximum } \\
\text { Detect }\end{array}$ & $\begin{array}{c}\text { Average } \\
\text { Concentration }\end{array}$ \\
\hline$\overline{\mathrm{METAL}}$ & Yttrium & $\mathrm{mg} / \mathrm{kg}$ & 1 & 1 & $100 \%$ & -- & -- & 23 & 23 & 23 \\
\hline METAL & Zinc & $\mathrm{mg} / \mathrm{kg}$ & 59 & 59 & $100 \%$ & -- & -- & 11 & 558 & 142 \\
\hline PCB & Aroclor-1221 & $\mathrm{mg} / \mathrm{kg}$ & 3 & 0 & $0 \%$ & 0.31 & 0.33 & -- & -- & 0.16 \\
\hline PCB & Aroclor-1232 & $\mathrm{mg} / \mathrm{kg}$ & 3 & 0 & $0 \%$ & 0.12 & 0.13 & -- & -- & 0.062 \\
\hline $\mathrm{PCB}$ & Aroclor-1242/1016 & $\mathrm{mg} / \mathrm{kg}$ & 3 & 0 & $0 \%$ & 0.060 & 0.070 & -- & -- & 0.032 \\
\hline PCB & Aroclor-1248 & $\mathrm{mg} / \mathrm{kg}$ & 3 & 0 & $0 \%$ & 0.12 & 0.13 & -- & -- & 0.063 \\
\hline PCB & Aroclor-1254 & $\mathrm{mg} / \mathrm{kg}$ & 8 & 0 & $0 \%$ & 1.20E-04 & 0.13 & -- & -- & 0.048 \\
\hline PCB & Aroclor-1260 & $\mathrm{mg} / \mathrm{kg}$ & 6 & 0 & $0 \%$ & 0.060 & 0.13 & -- & -- & 0.048 \\
\hline PEST & $\begin{array}{l}2,4^{\prime}-\mathrm{DDD} \\
\text {. }\end{array}$ & $\mathrm{mg} / \mathrm{kg}$ & 3 & 0 & $0 \%$ & 0.036 & 0.040 & -- & -- & 0.019 \\
\hline PEST & 2,4'-DDE & $\mathrm{mg} / \mathrm{kg}$ & 3 & 0 & $0 \%$ & 0.036 & 0.040 & -- & -- & 0.019 \\
\hline PEST & 2,4'-DDT & $\mathrm{mg} / \mathrm{kg}$ & 3 & 0 & $0 \%$ & 0.036 & 0.040 & -- & -- & 0.019 \\
\hline PEST & Alachlor & $\mathrm{mg} / \mathrm{kg}$ & 3 & 0 & $0 \%$ & 0.14 & 0.16 & -- & -- & 0.075 \\
\hline PEST & Aldrin & $\mathrm{mg} / \mathrm{kg}$ & 8 & 0 & $0 \%$ & 0.0010 & 0.040 & -- & -- & 0.0082 \\
\hline PEST & Alpha-BHC & $\mathrm{mg} / \mathrm{kg}$ & 6 & 0 & $0 \%$ & 0.0050 & 0.040 & -- & -- & 0.011 \\
\hline PEST & alpha-Chlordane & $\mathrm{mg} / \mathrm{kg}$ & 3 & 0 & $0 \%$ & 0.036 & 0.040 & -- & -- & 0.019 \\
\hline PEST & alpha-Chlordene & $\mathrm{mg} / \mathrm{kg}$ & 3 & 0 & $0 \%$ & 0.036 & 0.040 & -- & -- & 0.019 \\
\hline PEST & Ametryn & $\mathrm{mg} / \mathrm{kg}$ & 3 & 0 & $0 \%$ & 0.060 & 0.066 & -- & -- & 0.032 \\
\hline PEST & Atraton & $\mathrm{mg} / \mathrm{kg}$ & 3 & 0 & $0 \%$ & 0.18 & 0.20 & -- & -- & 0.095 \\
\hline PEST & Atrazine & $\mathrm{mg} / \mathrm{kg}$ & 3 & 0 & $0 \%$ & 0.060 & 0.066 & -- & -- & 0.032 \\
\hline PEST & Azinphos Ethyl & $\mathrm{mg} / \mathrm{kg}$ & 3 & 0 & $0 \%$ & 0.096 & 0.11 & -- & -- & 0.051 \\
\hline PEST & Azinphos Methyl & $\mathrm{mg} / \mathrm{kg}$ & 3 & 0 & $0 \%$ & 0.11 & 0.12 & -- & -- & 0.057 \\
\hline PEST & Benefin (Benfluralin) & $\mathrm{mg} / \mathrm{kg}$ & 3 & 0 & $0 \%$ & 0.090 & 0.099 & -- & -- & 0.048 \\
\hline PEST & Butachlor & $\mathrm{mg} / \mathrm{kg}$ & 3 & 0 & $0 \%$ & 0.21 & 0.23 & -- & -- & 0.11 \\
\hline PEST & Butylate & $\mathrm{mg} / \mathrm{kg}$ & 3 & 0 & $0 \%$ & 0.090 & 0.099 & -- & -- & 0.048 \\
\hline PEST & Carbethoxy malathion & $\mathrm{mg} / \mathrm{kg}$ & 3 & 0 & $0 \%$ & 0.048 & 0.053 & -- & -- & 0.025 \\
\hline PEST & Carboxin & $\mathrm{mg} / \mathrm{kg}$ & 3 & 0 & $0 \%$ & 0.66 & 0.73 & -- & -- & 0.35 \\
\hline PEST & Chlordane & $\mathrm{mg} / \mathrm{kg}$ & 3 & 0 & $0 \%$ & 0.060 & 0.070 & -- & -- & 0.032 \\
\hline PEST & Chlorothalonil & $\mathrm{mg} / \mathrm{kg}$ & 3 & 0 & $0 \%$ & 0.14 & 0.16 & -- & -- & 0.075 \\
\hline PEST & Chlorpropham & $\mathrm{mg} / \mathrm{kg}$ & 3 & 0 & $0 \%$ & 0.30 & 0.33 & -- & -- & 0.16 \\
\hline PEST & Chlorpyrifos & $\mathrm{mg} / \mathrm{kg}$ & 3 & 0 & $0 \%$ & 0.042 & 0.046 & -- & -- & 0.022 \\
\hline PEST & Cyanazine & $\mathrm{mg} / \mathrm{kg}$ & 3 & 0 & $0 \%$ & 0.091 & 0.099 & -- & -- & 0.048 \\
\hline PEST & Cycloate & $\mathrm{mg} / \mathrm{kg}$ & 3 & 0 & $0 \%$ & 0.090 & 0.099 & -- & -- & 0.048 \\
\hline PEST & DDMU & $\mathrm{mg} / \mathrm{kg}$ & 3 & 0 & $0 \%$ & 0.036 & 0.040 & -- & -- & 0.019 \\
\hline PEST & Delta-BHC & $\mathrm{mg} / \mathrm{kg}$ & 6 & 0 & $0 \%$ & 0.0050 & 0.040 & -- & -- & 0.011 \\
\hline PEST & Diazinon & $\mathrm{mg} / \mathrm{kg}$ & 3 & 0 & $0 \%$ & 0.048 & 0.053 & -- & -- & 0.025 \\
\hline PEST & Dichlobenil & $\mathrm{mg} / \mathrm{kg}$ & 3 & 0 & $0 \%$ & 0.072 & 0.079 & -- & -- & 0.038 \\
\hline PEST & Dichlorodiphenyldichloroethane & $\mathrm{mg} / \mathrm{kg}$ & 6 & 2 & $33 \%$ & 0.0050 & 0.038 & 0.0030 & 0.0030 & 0.0054 \\
\hline PEST & Dichlorodiphenyldichloroethylene & $\mathrm{mg} / \mathrm{kg}$ & 8 & 3 & $38 \%$ & $1.00 \mathrm{E}-05$ & 0.0050 & 0.0080 & 0.016 & 0.0078 \\
\hline PEST & Dichlorodiphenyltrichloroethane & $\mathrm{mg} / \mathrm{kg}$ & 7 & 0 & $0 \%$ & 0.0050 & 0.040 & -- & -- & 0.0096 \\
\hline PEST & Dicofol & $\mathrm{mg} / \mathrm{kg}$ & 3 & 0 & $0 \%$ & 0.14 & 0.16 & -- & -- & 0.075 \\
\hline PEST & Dieldrin & $\mathrm{mg} / \mathrm{kg}$ & 8 & 0 & $0 \%$ & 0.0010 & 0.040 & -- & -- & 0.0082 \\
\hline PEST & Dimethoate & $\mathrm{mg} / \mathrm{kg}$ & 3 & 0 & $0 \%$ & 0.048 & 0.053 & -- & -- & 0.025 \\
\hline PEST & Diphenamid & $\mathrm{mg} / \mathrm{kg}$ & 3 & 0 & $0 \%$ & 0.18 & 0.20 & -- & -- & 0.095 \\
\hline PEST & Disulfoton & $\mathrm{mg} / \mathrm{kg}$ & 3 & 0 & $0 \%$ & 0.036 & 0.040 & -- & -- & 0.019 \\
\hline
\end{tabular}


Table C-3

Summary Statistics for Sediment Collected from Segment 3 of the Columbia River Including All Categories of Data (units reported "as received")

\begin{tabular}{|c|c|c|c|c|c|c|c|c|c|c|}
\hline $\begin{array}{l}\text { Constituent } \\
\text { Class }\end{array}$ & Constituent Name & Units & $\begin{array}{c}\text { Number } \\
\text { Analyzed }\end{array}$ & $\begin{array}{l}\text { Number } \\
\text { Detected }\end{array}$ & $\begin{array}{l}\text { Frequency of } \\
\text { Detection }\end{array}$ & $\begin{array}{l}\text { Minimum } \\
\text { Nondetect }\end{array}$ & $\begin{array}{l}\text { Maximum } \\
\text { Nondetect }\end{array}$ & Minimum Detect & $\begin{array}{l}\text { Maximum } \\
\text { Detect }\end{array}$ & $\begin{array}{c}\text { Average } \\
\text { Concentration }\end{array}$ \\
\hline$\overline{\mathrm{PEST}}$ & Diuron & $\mathrm{mg} / \mathrm{kg}$ & 3 & $\overline{0}$ & $0 \%$ & 0.48 & 0.53 & -- & $\overline{--}$ & 0.25 \\
\hline PEST & Endosulfan I & $\mathrm{mg} / \mathrm{kg}$ & 6 & 0 & $0 \%$ & 0.0050 & 0.040 & -- & -- & 0.011 \\
\hline PEST & Endosulfan II & $\mathrm{mg} / \mathrm{kg}$ & 6 & 0 & $0 \%$ & 0.0050 & 0.040 & -- & -- & 0.011 \\
\hline PEST & Endosulfan sulfate & $\mathrm{mg} / \mathrm{kg}$ & 6 & 0 & $0 \%$ & 0.0050 & 0.040 & -- & -- & 0.011 \\
\hline PEST & Endrin aldehyde & $\mathrm{mg} / \mathrm{kg}$ & 3 & 0 & $0 \%$ & 0.036 & 0.040 & -- & -- & 0.019 \\
\hline PEST & Endrin ketone & $\mathrm{mg} / \mathrm{kg}$ & 3 & 0 & $0 \%$ & 0.018 & 0.020 & -- & -- & 0.0095 \\
\hline PEST & Eptam & $\mathrm{mg} / \mathrm{kg}$ & 3 & 0 & $0 \%$ & 0.090 & 0.099 & -- & -- & 0.048 \\
\hline PEST & Ethalfluralin (Sonalan) & $\mathrm{mg} / \mathrm{kg}$ & 3 & 0 & $0 \%$ & 0.090 & 0.099 & -- & -- & 0.048 \\
\hline PEST & Ethyl methylene phosphorodithioate & $\mathrm{mg} / \mathrm{kg}$ & 3 & 0 & $0 \%$ & 0.042 & 0.046 & -- & -- & 0.022 \\
\hline PEST & Fenarimol & $\mathrm{mg} / \mathrm{kg}$ & 3 & 0 & $0 \%$ & 0.18 & 0.20 & -- & -- & 0.095 \\
\hline PEST & Fensulfothion & $\mathrm{mg} / \mathrm{kg}$ & 3 & 0 & $0 \%$ & 0.060 & 0.066 & -- & -- & 0.032 \\
\hline PEST & Fluridone & $\mathrm{mg} / \mathrm{kg}$ & 3 & 0 & $0 \%$ & 0.48 & 0.53 & -- & -- & 0.25 \\
\hline PEST & Fonofos & $\mathrm{mg} / \mathrm{kg}$ & 3 & 0 & $0 \%$ & 0.036 & 0.040 & -- & -- & 0.019 \\
\hline PEST & Gamma-BHC (Lindane) & $\mathrm{mg} / \mathrm{kg}$ & 6 & 1 & $17 \%$ & 0.0050 & 0.040 & 0.0080 & 0.0080 & 0.0089 \\
\hline PEST & gamma-Chlordene & $\mathrm{mg} / \mathrm{kg}$ & 3 & 0 & $0 \%$ & 0.036 & 0.040 & -- & -- & 0.019 \\
\hline PEST & Heptachlor & $\mathrm{mg} / \mathrm{kg}$ & 6 & 0 & $0 \%$ & 0.0050 & 0.040 & -- & -- & 0.011 \\
\hline PEST & Heptachlor epoxide & $\mathrm{mg} / \mathrm{kg}$ & 6 & 0 & $0 \%$ & 0.0050 & 0.040 & -- & -- & 0.011 \\
\hline PEST & Hexazinone & $\mathrm{mg} / \mathrm{kg}$ & 3 & 0 & $0 \%$ & 0.090 & 0.099 & -- & -- & 0.048 \\
\hline PEST & Methoxychlor & $\mathrm{mg} / \mathrm{kg}$ & 5 & 0 & $0 \%$ & 0.0010 & 0.040 & -- & -- & 0.012 \\
\hline PEST & Methyl Chlorpyrifos & $\mathrm{mg} / \mathrm{kg}$ & 3 & 0 & $0 \%$ & 0.042 & 0.046 & -- & -- & 0.022 \\
\hline PEST & Methyl parathion & $\mathrm{mg} / \mathrm{kg}$ & 3 & 0 & $0 \%$ & 0.042 & 0.046 & -- & -- & 0.022 \\
\hline PEST & Metolachlor & $\mathrm{mg} / \mathrm{kg}$ & 3 & 0 & $0 \%$ & 0.18 & 0.20 & -- & -- & 0.095 \\
\hline PEST & Mirex & $\mathrm{mg} / \mathrm{kg}$ & 3 & 0 & $0 \%$ & 0.036 & 0.040 & -- & -- & 0.019 \\
\hline PEST & Molinate & $\mathrm{mg} / \mathrm{kg}$ & 3 & 0 & $0 \%$ & 0.16 & 0.17 & -- & -- & 0.083 \\
\hline PEST & Napropamide & $\mathrm{mg} / \mathrm{kg}$ & 3 & 0 & $0 \%$ & 0.18 & 0.20 & -- & -- & 0.095 \\
\hline PEST & Norflurazon & $\mathrm{mg} / \mathrm{kg}$ & 3 & 0 & $0 \%$ & 0.090 & 0.099 & -- & -- & 0.048 \\
\hline PEST & Oxychlordane & $\mathrm{mg} / \mathrm{kg}$ & 3 & 0 & $0 \%$ & 0.036 & 0.040 & -- & -- & 0.019 \\
\hline PEST & Oxyfluorfen & $\mathrm{mg} / \mathrm{kg}$ & 3 & 0 & $0 \%$ & 0.16 & 0.17 & -- & -- & 0.083 \\
\hline PEST & Parathion & $\mathrm{mg} / \mathrm{kg}$ & 3 & 0 & $0 \%$ & 0.048 & 0.053 & -- & -- & 0.025 \\
\hline PEST & Pebulate & $\mathrm{mg} / \mathrm{kg}$ & 3 & 0 & $0 \%$ & 0.14 & 0.16 & -- & -- & 0.075 \\
\hline PEST & Pendimethalin & $\mathrm{mg} / \mathrm{kg}$ & 3 & 0 & $0 \%$ & 0.090 & 0.099 & -- & -- & 0.048 \\
\hline PEST & Pentachloroanisole & $\mathrm{mg} / \mathrm{kg}$ & 3 & 0 & $0 \%$ & 0.036 & 0.040 & -- & -- & 0.019 \\
\hline PEST & Phorate & $\mathrm{mg} / \mathrm{kg}$ & 3 & 0 & $0 \%$ & 0.042 & 0.046 & -- & -- & 0.022 \\
\hline PEST & Pramitol (Prometon) & $\mathrm{mg} / \mathrm{kg}$ & 3 & 0 & $0 \%$ & 0.060 & 0.096 & -- & -- & 0.037 \\
\hline PEST & Prometryn & $\mathrm{mg} / \mathrm{kg}$ & 3 & 0 & $0 \%$ & 0.060 & 0.066 & -- & -- & 0.032 \\
\hline PEST & Pronamide & $\mathrm{mg} / \mathrm{kg}$ & 3 & 0 & $0 \%$ & 0.18 & 0.20 & -- & -- & 0.095 \\
\hline PEST & Propachlor (Ramrod) & $\mathrm{mg} / \mathrm{kg}$ & 3 & 0 & $0 \%$ & 0.12 & 0.13 & -- & -- & 0.063 \\
\hline PEST & Propazine & $\mathrm{mg} / \mathrm{kg}$ & 3 & 0 & $0 \%$ & 0.060 & 0.096 & -- & -- & 0.037 \\
\hline PEST & Simazine & $\mathrm{mg} / \mathrm{kg}$ & 3 & 0 & $0 \%$ & 0.060 & 0.066 & -- & -- & 0.032 \\
\hline PEST & Terbacil & $\mathrm{mg} / \mathrm{kg}$ & 3 & 0 & $0 \%$ & 0.30 & 0.33 & -- & -- & 0.16 \\
\hline PEST & Terbutryn & $\mathrm{mg} / \mathrm{kg}$ & 3 & 0 & $0 \%$ & 0.060 & 0.066 & -- & -- & 0.032 \\
\hline PEST & Toxaphene & $\mathrm{mg} / \mathrm{kg}$ & 6 & 0 & $0 \%$ & 0.060 & 0.79 & -- & -- & 0.21 \\
\hline PEST & trans-Chlordane & $\mathrm{mg} / \mathrm{kg}$ & 3 & 0 & $0 \%$ & 0.036 & 0.040 & -- & -- & 0.019 \\
\hline PEST & trans-Nonachlor & $\mathrm{mg} / \mathrm{kg}$ & 3 & 0 & $0 \%$ & 0.036 & 0.040 & -- & -- & 0.019 \\
\hline
\end{tabular}


Table C-3

Summary Statistics for Sediment Collected from Segment 3 of the Columbia River Including All Categories of Data (units reported "as received")

Constituent

Class
Clituenta

PEST

PEST

Triadimefo
Triallate

Constituent Name

Triallate

PEST Trifluralin (Treflan)

PEST Vernolate

RAD_D Cerium-141

RAD_D Cesium-134

RAD_D Cesium-137

RAD_D Cobalt-60

RAD_D Europium-152

RAD D Europium-155

RAD D Plutonium-238

RAD D Plutonium-239/240

RAD

RAD D

RAD D

RAD

RAD D

RAD

RAD D

RAD D

RAD D

SVOC

Plutonium-239/240

Radium-226

Ruthenium-106

Strontium-90

Thorium-232

Uranium-234

Uranium-235

Uranium-238

Zirconium/Niobium-95

1,2,4-Trichlorobenzene

1,2-Dichlorobenzene

1,2-Dichlorobenzene

1,4-Dichlorobenzene

2,4,5-Trichloropheno

2,4,6-Trichloropheno

2,4-Dimethylphenol

2,4-Dinitrophenol

2,4-Dinitrotoluene

2-Chloronaphthalene

2-Chlorophenol

2-Methylnaphthalene

2-Nitroaniline

3,3'-Dichlorobenzidine

3-Nitroaniline

4,6-Dinitro-2-methylpheno

4-Bromophenylphenyl ethe

4-Chloroaniline

4-Chlorophenylphenyl ether
4-Methylphenol (cresol, p-)
Units Analyzed Detected

Minimum

Maximum

Nondetect Minimum Detect

Maximum

Detect

$\begin{array}{cccccc}0 \% & 0.16 & 0.17 & -- & -- & \text { Concentration }\end{array}$

0.083

mg

$0 \%$

$\begin{array}{ll}0.16 & 0.17 \\ 0.090 & 0.099\end{array}$

$\mathrm{mg} / \mathrm{kg}$

$\mathrm{pCi} / \mathrm{g}$

$\mathrm{pCi} / \mathrm{g}$

$\mathrm{pCi} / \mathrm{g}$

$\mathrm{pCi} / \mathrm{g}$

$\mathrm{pCi} / \mathrm{g}$

$\mathrm{pCi} / \mathrm{g}$

$\mathrm{pCi} / \mathrm{g}$
$\mathrm{pCi} / \mathrm{g}$

$\mathrm{pCi} / \mathrm{g}$
$\mathrm{pCi} / \mathrm{g}$

$\mathrm{pCi} / \mathrm{g}$

$\mathrm{pCi} / \mathrm{g}$

pCi/g

$\mathrm{pCi} / \mathrm{g}$

$\mathrm{pCi} / \mathrm{g}$

$\mathrm{pCi} / \mathrm{g}$

$\mathrm{mg} / \mathrm{kg}$

$\mathrm{mg} / \mathrm{kg}$

$\mathrm{mg} / \mathrm{kg}$

$\mathrm{mg} / \mathrm{kg}$

$\mathrm{mg} / \mathrm{kg}$

$\mathrm{mg} / \mathrm{kg}$

$\mathrm{mg} / \mathrm{kg}$

$\mathrm{mg} / \mathrm{kg}$

$\mathrm{mg} / \mathrm{kg}$

$\mathrm{mg} / \mathrm{kg}$

$\mathrm{mg} / \mathrm{kg}$

$\mathrm{mg} / \mathrm{kg}$

$\mathrm{mg} / \mathrm{kg}$

$\mathrm{mg} / \mathrm{kg}$

$\mathrm{mg} / \mathrm{kg}$

$\mathrm{mg} / \mathrm{kg}$

$\mathrm{mg} / \mathrm{kg}$

$\mathrm{mg} / \mathrm{kg}$

$\mathrm{mg} / \mathrm{kg}$

$\mathrm{mg} / \mathrm{kg}$

$\mathrm{mg} / \mathrm{kg}$

$0 \%$

$\begin{array}{ll}0.1690 & 0.17 \\ 0.099 & 0.099\end{array}$

0.090

$100 \%$

$100 \%$

$100 \%$

$100 \%$

$100 \%$

$100 \%$

$100 \%$

$100 \%$

$100 \%$

$100 \%$

$100 \%$

$100 \%$

$100 \%$

$100 \%$

$100 \%$

$100 \%$
$100 \%$

$0 \%$

$0 \%$

$0 \%$

$0 \%$

$0 \%$

$0 \%$

$0 \%$

$0 \%$

$0 \%$

$0 \%$

$0 \%$

$0 \%$

$0 \%$

$0 \%$

$0 \%$

$0 \%$

$0 \%$
$0 \%$
$0 \%$
$0 \%$

$0 \%$

0.099
0.099

--
--
--
$1.36 \mathrm{E}-13$
$2.18 \mathrm{E}-96$
$08 \mathrm{E}-04$

$\begin{array}{ll}-- & 0.083 \\ -- & 0.048\end{array}$

0.048
0.048

$\begin{array}{cc}-- & 0.048 \\ 1.36 \mathrm{E}-13 & 1.36 \mathrm{E}-13 \\ 1.80 \mathrm{E}-62 & 6.01 \mathrm{E}-63\end{array}$

$\begin{array}{ccc}1.08 \mathrm{E}-04 & 0.054 & 0.040 \\ 0.027 & 0.0 & 0.28\end{array}$

$\begin{array}{lll}0.027 & 1.0 & 0.28 \\ 0.0024 & 0.081 & 0.023 \\ 0.035 & 0.49 & 0.18\end{array}$

$\begin{array}{lll}0.035 & 0.49 & 0.18 \\ 0.063 & 0.063 & 0.063\end{array}$

$\begin{array}{lll}0.063 & 0.063 & 0.024\end{array}$

$\begin{array}{lll}1.53 \mathrm{E}-04 & 0.0018 & 4.79 \mathrm{E}-04\end{array}$

$\begin{array}{lll}1.53 E-04 & 0.0018 & 4.79 E-04 \\ 7.66 \mathrm{E}-04 & 0.032 & 0.0087\end{array}$

$\begin{array}{ccc}6.7 & 22 & 14 \\ 0.40 & 0.95 & 0.55\end{array}$

$\begin{array}{ccc}0.40 & 0.95 & 0.55 \\ 9.51 \mathrm{E}-05 & 9.51 \mathrm{E}-05 & 9.51 \mathrm{E}-05\end{array}$

$\begin{array}{lll}0.51 \mathrm{E}-05 & 9.51 \mathrm{E}-05 & 9.51 \mathrm{E}-05 \\ 0.0073 & 0.039 & 0.022\end{array}$

$\begin{array}{lll}0.40 & 0.80 & 0.022 \\ 0.094 & 1.9 & 0.56\end{array}$

$\begin{array}{ccc}0.404 & 1.9 & 0.73 \\ 0.094 & 0.12 & 0.028 \\ 0.0092 & 1.6 & 0.65\end{array}$

$\begin{array}{lll}0.0092 & 0.12 & 0.73 \\ 0.21 & 1.6 & 0.65\end{array}$

$\begin{array}{lll}0.21 & 1.6 & 0.65 \\ 3.51 \mathrm{E}-36 & 3.51 \mathrm{E}-36 & 3.51 \mathrm{E}-36\end{array}$

$\begin{array}{lllll}0.010 & 0.010 & - & - & 0.0050 \\ 0.010 & 0.010 & - & - & 0.0050 \\ 0.010 & 0.010 & - & - & 0.0050\end{array}$

$\begin{array}{lllll}0.010 & 0.010 & - & - & 0.0050 \\ 0.010 & 0.010 & - & - & 0.0050 \\ 0.010 & 0.010 & -- & - & 0.0050\end{array}$

$\begin{array}{ll}0.010 & 0.010 \\ 0.010 & 0.010\end{array}$

$\begin{array}{ll}0.010 & 0.010 \\ 0.010 & 0.010\end{array}$

$\begin{array}{ll}0.010 & 0.010 \\ 0.010 & 0.010\end{array}$

$\begin{array}{ll}0.010 & 0.010 \\ 0.010 & 0.010\end{array}$

$\begin{array}{ll}0.010 & 0.010 \\ 0.025 & 0.025\end{array}$

$\begin{array}{ll}0.010 & 0.010 \\ 0.010 & 0.010\end{array}$

$\begin{array}{ll}0.010 & 0.010 \\ 0.010 & 0.010 \\ 0.010 & 0.010\end{array}$

$\begin{array}{ll}0.010 & 0.010 \\ 0.0032 & 0.043\end{array}$

$\begin{array}{ll}0.010 & 0.043 \\ 0.010 & 0.010\end{array}$

$\begin{array}{ll}0.010 & 0.010 \\ 0.020 & 0.020 \\ 0.010 & 0.010\end{array}$

$\begin{array}{ll}0.010 & 0.010 \\ 0.010 & 0.010 \\ 0.025 & 0.025\end{array}$

$\begin{array}{ll}0.010 & 0.025 \\ 0.025 & 0.010 \\ 0.010 & 0.020\end{array}$

$\begin{array}{ll}0.020 & 0.020 \\ 0 & 0.010\end{array}$

$\begin{array}{ll}0.010 & 0.010 \\ & 0.010\end{array}$

0.0050
0.0050

0.0050

0.0050

0.0050

0.0050

0.0050

0.013

0.0050

0.0050

0.0050

0.0050

0.0050

0.0050

0.0050

0.013

0.0050

0.010

0.0050 
Table C-3

Summary Statistics for Sediment Collected from Segment 3 of the Columbia River Including All Categories of Data (units reported "as received")

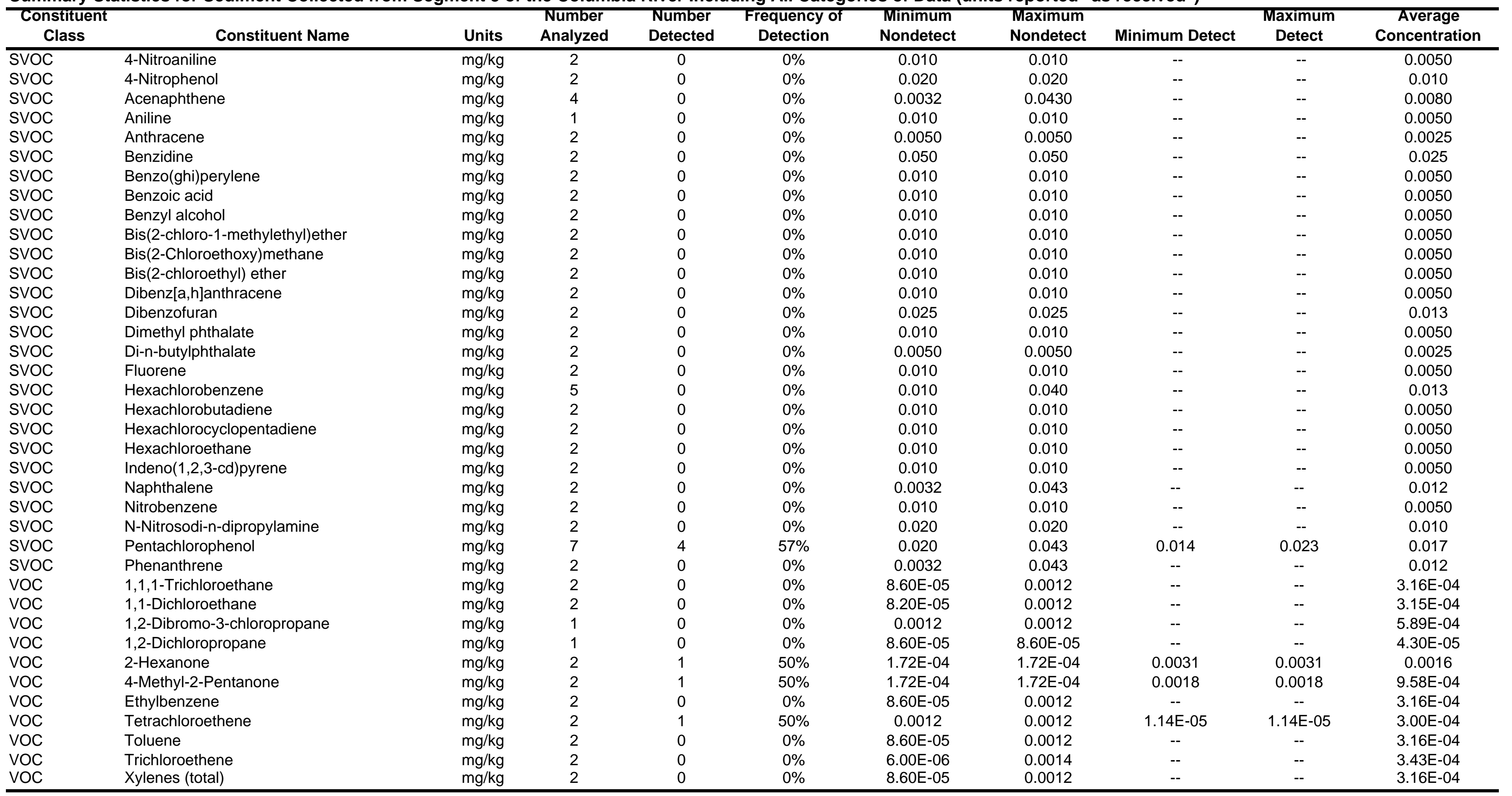

CONV - Conventional parameter

PEST - Pesticide

PCB - Polychlorinated biphenyl

SVOC - Semivolatile Organic Compound

VOC - Volatile Organic Compound 
Table C-4

Summary Statistics for Sediment Collected from Segment 3 of the Columbia River Including All Categories of Data (units reported "dry-weight")

Number Arequency of Minimum waximum "weight")

\begin{tabular}{|c|c|c|c|c|c|c|c|c|c|c|}
\hline $\begin{array}{l}\text { Constituent } \\
\text { Class }\end{array}$ & Constituent Name & Units & $\begin{array}{c}\text { Number } \\
\text { Analyzed }\end{array}$ & $\begin{array}{l}\text { Number } \\
\text { Detected }\end{array}$ & $\begin{array}{l}\text { Frequency of } \\
\text { Detection }\end{array}$ & $\begin{array}{l}\text { Minimum } \\
\text { Nondetect }\end{array}$ & $\begin{array}{l}\text { Maximum } \\
\text { Nondetect }\end{array}$ & $\begin{array}{l}\text { Minimum } \\
\text { Detect }\end{array}$ & $\begin{array}{l}\text { Maximum } \\
\text { Detect }\end{array}$ & $\begin{array}{c}\text { Average } \\
\text { Concentration }\end{array}$ \\
\hline$\overline{\text { CONV }}$ & Total Organic Carbon & $\mathrm{mg} / \mathrm{kg}$ dry-wt & 2 & $\overline{2}$ & $100 \%$ & & & 0.0092 & 1.0 & 0.50 \\
\hline CONV & Total solids & $\mathrm{mg} / \mathrm{kg}$ dry-wt & 1 & 1 & $100 \%$ & & & 50 & 50 & 50 \\
\hline METAL & Aluminum & $\mathrm{mg} / \mathrm{kg}$ dry-wt & 4 & 4 & $100 \%$ & & & 2,900 & 17,000 & 10,000 \\
\hline METAL & Antimony & $\mathrm{mg} / \mathrm{kg}$ dry-wt & 17 & 13 & $76 \%$ & 3.0 & 3.0 & 0.52 & 5.1 & 1.6 \\
\hline METAL & Arsenic & $\mathrm{mg} / \mathrm{kg}$ dry-wt & 23 & 23 & $100 \%$ & & & 1.7 & 12 & 5.5 \\
\hline METAL & Barium & $\mathrm{mg} / \mathrm{kg}$ dry-wt & 4 & 4 & $100 \%$ & & & 53 & 150 & 101 \\
\hline METAL & Beryllium & $\mathrm{mg} / \mathrm{kg}$ dry-wt & 17 & 17 & $100 \%$ & & & 0.17 & 1.8 & 1.2 \\
\hline METAL & Cadmium & $\mathrm{mg} / \mathrm{kg}$ dry-wt & 23 & 21 & $91 \%$ & 0.20 & 0.20 & 0.19 & 4.3 & 1.2 \\
\hline METAL & Calcium & $\mathrm{mg} / \mathrm{kg}$ dry-wt & 4 & 4 & $100 \%$ & & & 3,500 & 5,800 & 4,500 \\
\hline METAL & Chromium & $\mathrm{mg} / \mathrm{kg}$ dry-wt & 23 & 21 & $91 \%$ & 0.10 & 0.10 & 3.7 & 73 & 33 \\
\hline METAL & Cobalt & $\mathrm{mg} / \mathrm{kg}$ dry-wt & 4 & 4 & $100 \%$ & & & 11 & 17 & 13 \\
\hline METAL & Copper & $\mathrm{mg} / \mathrm{kg}$ dry-wt & 23 & 23 & $100 \%$ & & & 7.1 & 39 & 23 \\
\hline METAL & Iron & $\mathrm{mg} / \mathrm{kg}$ dry-wt & 4 & 4 & $100 \%$ & & & 24,000 & 34,000 & 27,250 \\
\hline METAL & Lead & $\mathrm{mg} / \mathrm{kg}$ dry-wt & 23 & 22 & $96 \%$ & 0.10 & 0.10 & 2.1 & 58 & 17 \\
\hline METAL & Magnesium & $\mathrm{mg} / \mathrm{kg}$ dry-wt & 4 & 4 & $100 \%$ & & & 3,200 & 6,700 & 4,825 \\
\hline METAL & Manganese & $\mathrm{mg} / \mathrm{kg}$ dry-wt & 4 & 4 & $100 \%$ & & & 280 & 640 & 490 \\
\hline METAL & Mercury & $\mathrm{mg} / \mathrm{kg}$ dry-wt & 26 & 20 & $77 \%$ & 0.0050 & 0.060 & 0.013 & 0.31 & 0.069 \\
\hline METAL & Nickel & $\mathrm{mg} / \mathrm{kg}$ dry-wt & 17 & 17 & $100 \%$ & & & 6.1 & 32 & 20 \\
\hline METAL & Potassium & $\mathrm{mg} / \mathrm{kg}$ dry-wt & 4 & 4 & $100 \%$ & & & 490 & 2,300 & 1,288 \\
\hline METAL & Selenium & $\mathrm{mg} / \mathrm{kg}$ dry-wt & 17 & 12 & $71 \%$ & 0.20 & 0.35 & 0.32 & 1.3 & 0.59 \\
\hline METAL & Silver & $\mathrm{mg} / \mathrm{kg}$ dry-wt & 17 & 14 & $82 \%$ & 0.30 & 0.30 & 0.29 & 2.5 & 0.65 \\
\hline METAL & Sodium & $\mathrm{mg} / \mathrm{kg}$ dry-wt & 4 & 4 & $100 \%$ & & & 130 & 280 & 193 \\
\hline METAL & Thallium & $\mathrm{mg} / \mathrm{kg}$ dry-wt & 17 & 14 & $82 \%$ & 0.25 & 0.25 & 0.27 & 2.5 & 0.96 \\
\hline METAL & Vanadium & $\mathrm{mg} / \mathrm{kg}$ dry-wt & 4 & 4 & $100 \%$ & & & 50 & 77 & 66 \\
\hline METAL & Zinc & $\mathrm{mg} / \mathrm{kg}$ dry-wt & 23 & 22 & $96 \%$ & 60 & 60 & 45 & 375 & 160 \\
\hline PCB & Aroclor-1016 & $\mathrm{mg} / \mathrm{kg}$ dry-wt & 4 & 0 & $0 \%$ & 0.090 & 0.20 & -- & -- & 0.061 \\
\hline PCB & Aroclor-1221 & $\mathrm{mg} / \mathrm{kg}$ dry-wt & 6 & 0 & $0 \%$ & 0.090 & 0.33 & -- & -- & 0.095 \\
\hline PCB & Aroclor-1232 & $\mathrm{mg} / \mathrm{kg}$ dry-wt & 6 & 0 & $0 \%$ & 0.090 & 0.20 & -- & -- & 0.063 \\
\hline PCB & Aroclor-1242 & $\mathrm{mg} / \mathrm{kg}$ dry-wt & 5 & 0 & $0 \%$ & 0.040 & 0.20 & -- & -- & 0.053 \\
\hline PCB & Aroclor-1242/1016 & $\mathrm{mg} / \mathrm{kg}$ dry-wt & 8 & 0 & $0 \%$ & 0.010 & 0.070 & -- & -- & 0.014 \\
\hline PCB & Aroclor-1248 & $\mathrm{mg} / \mathrm{kg}$ dry-wt & 10 & 0 & $0 \%$ & 0.010 & 0.20 & -- & -- & 0.029 \\
\hline PCB & Aroclor-1254 & $\mathrm{mg} / \mathrm{kg}$ dry-wt & 17 & 3 & $18 \%$ & 0.010 & 0.20 & 0.010 & 0.013 & 0.025 \\
\hline PCB & Aroclor-1260 & $\mathrm{mg} / \mathrm{kg}$ dry-wt & 15 & 3 & $20 \%$ & 0.0015 & 0.20 & 0.0056 & 0.011 & 0.025 \\
\hline PCB & Total polychlorinated biphenyl & $\mathrm{mg} / \mathrm{kg}$ dry-wt & 3 & 0 & $0 \%$ & $1.00 \mathrm{E}-04$ & 0.050 & -- & -- & 0.0085 \\
\hline PEST & 2,4'-DDD & $\mathrm{mg} / \mathrm{kg}$ dry-wt & 1 & 0 & $0 \%$ & 0.010 & 0.010 & -- & -- & 0.0050 \\
\hline PEST & 2,4'-DDE & $\mathrm{mg} / \mathrm{kg}$ dry-wt & 1 & 0 & $0 \%$ & 0.010 & 0.010 & -- & -- & 0.0050 \\
\hline PEST & 2,4'-DDT & $\mathrm{mg} / \mathrm{kg}$ dry-wt & 2 & 1 & $50 \%$ & 0.010 & 0.010 & $7.10 \mathrm{E}-04$ & $7.10 \mathrm{E}-04$ & 0.0029 \\
\hline PEST & Alachlor & $\mathrm{mg} / \mathrm{kg}$ dry-wt & 1 & 0 & $0 \%$ & 0.024 & 0.024 & -- & -- & 0.012 \\
\hline PEST & Aldrin & $\mathrm{mg} / \mathrm{kg}$ dry-wt & 23 & 0 & $0 \%$ & $1.00 \mathrm{E}-04$ & 0.020 & -- & -- & 0.0019 \\
\hline PEST & Alpha-BHC & $\mathrm{mg} / \mathrm{kg}$ dry-wt & 14 & 0 & $0 \%$ & 0.0010 & 0.020 & -- & -- & 0.0028 \\
\hline PEST & alpha-Chlordane & $\mathrm{mg} / \mathrm{kg}$ dry-wt & 5 & 0 & $0 \%$ & 0.0010 & 0.010 & -- & -- & 0.0011 \\
\hline PEST & alpha-Chlordene & $\mathrm{mg} / \mathrm{kg}$ dry-wt & 1 & 0 & $0 \%$ & 0.010 & 0.010 & -- & -- & 0.0050 \\
\hline PEST & Azinphos Ethyl & $\mathrm{mg} / \mathrm{kg}$ dry-wt & 1 & 0 & $0 \%$ & 0.016 & 0.016 & -- & -- & 0.0080 \\
\hline PEST & Azinphos Methyl & $\mathrm{mg} / \mathrm{kg}$ dry-wt & 1 & 0 & $0 \%$ & 0.018 & 0.018 & -- & -- & 0.0090 \\
\hline PEST & Benefin (Benfluralin) & $\mathrm{mg} / \mathrm{kg}$ dry-wt & 1 & 0 & $0 \%$ & 0.015 & 0.015 & -- & -- & 0.0075 \\
\hline PEST & beta-1,2,3,4,5,6-Hexachlorocyclohexane & $\mathrm{mg} / \mathrm{kg}$ dry-wt & 12 & 0 & $0 \%$ & 0.0020 & 0.020 & -- & -- & 0.0031 \\
\hline PEST & Bolstar & $\mathrm{mg} / \mathrm{kg}$ dry-wt & 1 & 0 & $0 \%$ & 0.0070 & 0.0070 & -- & -- & 0.0035 \\
\hline
\end{tabular}


Table C-4

Summary Statistics for Sediment Collected from Segment 3 of the Columbia River Including All Categories of Data (units reported "dry-weight")

\begin{tabular}{|c|c|c|c|c|c|c|c|c|c|c|}
\hline $\begin{array}{l}\text { Constituent } \\
\text { Class }\end{array}$ & Constituent Name & Units & $\begin{array}{l}\text { Number } \\
\text { Analyzed }\end{array}$ & $\begin{array}{l}\text { Number } \\
\text { Detected }\end{array}$ & $\begin{array}{l}\text { Frequency of } \\
\text { Detection }\end{array}$ & $\begin{array}{l}\text { Minimum } \\
\text { Nondetect }\end{array}$ & $\begin{array}{l}\text { Maximum } \\
\text { Nondetect }\end{array}$ & $\begin{array}{c}\text { Minimum } \\
\text { Detect }\end{array}$ & $\begin{array}{l}\text { Maximum } \\
\text { Detect }\end{array}$ & $\begin{array}{c}\text { Average } \\
\text { Concentration }\end{array}$ \\
\hline$\overline{\text { PEST }}$ & Bromacil (ACN) & $\mathrm{mg} / \mathrm{kg}$ dry-wt & 1 & $\overline{0}$ & $0 \%$ & 0.060 & 0.060 & -- & $\overline{--}$ & 0.030 \\
\hline PEST & Butachlor & $\mathrm{mg} / \mathrm{kg}$ dry-wt & 1 & 0 & $0 \%$ & 0.035 & 0.035 & -- & -- & 0.018 \\
\hline PEST & Butylate & $\mathrm{mg} / \mathrm{kg}$ dry-wt & 1 & 0 & $0 \%$ & 0.015 & 0.015 & -- & -- & 0.0075 \\
\hline PEST & Captan & $\mathrm{mg} / \mathrm{kg}$ dry-wt & 1 & 0 & $0 \%$ & 0.030 & 0.030 & -- & -- & 0.015 \\
\hline PEST & Carbethoxy malathion & $\mathrm{mg} / \mathrm{kg}$ dry-wt & 1 & 0 & $0 \%$ & 0.0080 & 0.0080 & -- & -- & 0.0040 \\
\hline PEST & Carbophenothion & $\mathrm{mg} / \mathrm{kg}$ dry-wt & 1 & 0 & $0 \%$ & 0.010 & 0.010 & -- & -- & 0.0050 \\
\hline PEST & Carboxin & $\mathrm{mg} / \mathrm{kg}$ dry-wt & 1 & 0 & $0 \%$ & 0.11 & 0.11 & -- & -- & 0.055 \\
\hline PEST & Chlordane & $\mathrm{mg} / \mathrm{kg}$ dry-wt & 12 & 1 & $8 \%$ & 0.0020 & 0.20 & 0.0030 & 0.0030 & 0.027 \\
\hline PEST & Chlorothalonil & $\mathrm{mg} / \mathrm{kg}$ dry-wt & 1 & 0 & $0 \%$ & 0.024 & 0.024 & -- & -- & 0.012 \\
\hline PEST & Chlorpropham & $\mathrm{mg} / \mathrm{kg}$ dry-wt & 1 & 0 & $0 \%$ & 0.050 & 0.050 & -- & -- & 0.025 \\
\hline PEST & Chlorpyrifos & $\mathrm{mg} / \mathrm{kg}$ dry-wt & 1 & 0 & $0 \%$ & 0.0070 & 0.0070 & -- & -- & 0.0035 \\
\hline PEST & cis-Nonachlor & $\mathrm{mg} / \mathrm{kg}$ dry-wt & 1 & 0 & $0 \%$ & 0.010 & 0.010 & -- & -- & 0.0050 \\
\hline PEST & Coumaphos & $\mathrm{mg} / \mathrm{kg}$ dry-wt & 1 & 0 & $0 \%$ & 0.012 & 0.012 & -- & -- & 0.0060 \\
\hline PEST & Cyanazine & $\mathrm{mg} / \mathrm{kg}$ dry-wt & 1 & 0 & $0 \%$ & 0.015 & 0.015 & -- & -- & 0.0075 \\
\hline PEST & Cycloate & $\mathrm{mg} / \mathrm{kg}$ dry-wt & 1 & 0 & $0 \%$ & 0.015 & 0.015 & -- & -- & 0.0075 \\
\hline PEST & DDMU & $\mathrm{mg} / \mathrm{kg}$ dry-wt & 1 & 0 & $0 \%$ & 0.010 & 0.010 & -- & -- & 0.0050 \\
\hline PEST & Delta-BHC & $\mathrm{mg} / \mathrm{kg}$ dry-wt & 12 & 0 & $0 \%$ & 0.0020 & 0.020 & -- & -- & 0.0031 \\
\hline PEST & Demeton-O & $\mathrm{mg} / \mathrm{kg}$ dry-wt & 1 & 0 & $0 \%$ & 0.0070 & 0.0070 & -- & -- & 0.0035 \\
\hline PEST & Demeton-S & $\mathrm{mg} / \mathrm{kg}$ dry-wt & 1 & 0 & $0 \%$ & 0.0070 & 0.0070 & -- & -- & 0.0035 \\
\hline PEST & Diallate & $\mathrm{mg} / \mathrm{kg}$ dry-wt & 1 & 0 & $0 \%$ & 0.038 & 0.038 & -- & -- & 0.019 \\
\hline PEST & Diazinon & $\mathrm{mg} / \mathrm{kg}$ dry-wt & 1 & 0 & $0 \%$ & 0.0080 & 0.0080 & -- & -- & 0.0040 \\
\hline PEST & Dichlobenil & $\mathrm{mg} / \mathrm{kg}$ dry-wt & 1 & 0 & $0 \%$ & 0.012 & 0.012 & -- & -- & 0.0060 \\
\hline PEST & Dichlorodiphenyldichloroethane & $\mathrm{mg} / \mathrm{kg}$ dry-wt & 26 & 15 & $58 \%$ & 0.0010 & 0.020 & 4.00E-04 & 0.023 & 0.0046 \\
\hline PEST & Dichlorodiphenyldichloroethylene & $\mathrm{mg} / \mathrm{kg}$ dry-wt & 28 & 15 & $54 \%$ & 0.0010 & 0.020 & 7.00E-04 & 0.051 & 0.0058 \\
\hline PEST & Dichlorodiphenyltrichloroethane & $\mathrm{mg} / \mathrm{kg}$ dry-wt & 26 & 6 & $23 \%$ & 0.0010 & 0.020 & $3.00 \mathrm{E}-04$ & 0.035 & 0.0037 \\
\hline PEST & Dichlorvos & $\mathrm{mg} / \mathrm{kg}$ dry-wt & 1 & 0 & $0 \%$ & 0.0080 & 0.0080 & - - & -- & 0.0040 \\
\hline PEST & Dicofol & $\mathrm{mg} / \mathrm{kg}$ dry-wt & 1 & 0 & $0 \%$ & 0.040 & 0.040 & -- & -- & 0.020 \\
\hline PEST & Dieldrin & $\mathrm{mg} / \mathrm{kg}$ dry-wt & 23 & 3 & $13 \%$ & 0.0010 & 0.020 & $2.00 \mathrm{E}-04$ & 0.0050 & 0.0022 \\
\hline PEST & Dimethoate & $\mathrm{mg} / \mathrm{kg}$ dry-wt & 1 & 0 & $0 \%$ & 0.0080 & 0.0080 & -- & -- & 0.0040 \\
\hline PEST & Dioxathion & $\mathrm{mg} / \mathrm{kg}$ dry-wt & 1 & 0 & $0 \%$ & 0.017 & 0.017 & -- & -- & 0.0085 \\
\hline PEST & Diphenamid & $\mathrm{mg} / \mathrm{kg}$ dry-wt & 1 & 0 & $0 \%$ & 0.030 & 0.030 & -- & -- & 0.015 \\
\hline PEST & Disulfoton & $\mathrm{mg} / \mathrm{kg}$ dry-wt & 1 & 0 & $0 \%$ & 0.0060 & 0.0060 & -- & -- & 0.0030 \\
\hline PEST & Diuron & $\mathrm{mg} / \mathrm{kg}$ dry-wt & 1 & 0 & $0 \%$ & 0.060 & 0.060 & -- & -- & 0.030 \\
\hline PEST & Endosulfan I & $\mathrm{mg} / \mathrm{kg}$ dry-wt & 14 & 0 & $0 \%$ & $1.00 \mathrm{E}-04$ & 0.020 & -- & -- & 0.0027 \\
\hline PEST & Endosulfan II & $\mathrm{mg} / \mathrm{kg}$ dry-wt & 12 & 0 & $0 \%$ & 0.0020 & 0.020 & -- & -- & 0.0031 \\
\hline PEST & Endosulfan sulfate & $\mathrm{mg} / \mathrm{kg}$ dry-wt & 12 & 0 & $0 \%$ & 0.0020 & 0.020 & -- & -- & 0.0031 \\
\hline PEST & Endrin & $\mathrm{mg} / \mathrm{kg}$ dry-wt & 14 & 0 & $0 \%$ & $1.00 \mathrm{E}-04$ & 0.020 & -- & -- & 0.0027 \\
\hline PEST & Endrin aldehyde & $\mathrm{mg} / \mathrm{kg}$ dry-wt & 7 & 0 & $0 \%$ & 0.0050 & 0.020 & -- & -- & 0.0046 \\
\hline PEST & Endrin ketone & $\mathrm{mg} / \mathrm{kg}$ dry-wt & 15 & 0 & $0 \%$ & 0.0020 & 0.020 & -- & -- & 0.0030 \\
\hline PEST & Eptam & $\mathrm{mg} / \mathrm{kg}$ dry-wt & 1 & 0 & $0 \%$ & 0.015 & 0.015 & -- & -- & 0.0075 \\
\hline PEST & Ethalfluralin (Sonalan) & $\mathrm{mg} / \mathrm{kg}$ dry-wt & 1 & 0 & $0 \%$ & 0.015 & 0.015 & -- & -- & 0.0075 \\
\hline PEST & Ethyl methylene phosphorodithioate & $\mathrm{mg} / \mathrm{kg}$ dry-wt & 1 & 0 & $0 \%$ & 0.0070 & 0.0070 & -- & -- & 0.0035 \\
\hline PEST & Ethyl O-(p-nitrophenyl) & $\mathrm{mg} / \mathrm{kg}$ dry-wt & 1 & 0 & $0 \%$ & 0.010 & 0.010 & -- & -- & 0.0050 \\
\hline PEST & Fenarimol & $\mathrm{mg} / \mathrm{kg}$ dry-wt & 1 & 0 & $0 \%$ & 0.030 & 0.030 & -- & -- & 0.015 \\
\hline PEST & Fensulfothion & $\mathrm{mg} / \mathrm{kg}$ dry-wt & 1 & 0 & $0 \%$ & 0.010 & 0.010 & -- & -- & 0.0050 \\
\hline PEST & Fenthion & $\mathrm{mg} / \mathrm{kg}$ dry-wt & 1 & 0 & $0 \%$ & 0.0070 & 0.0070 & -- & -- & 0.0035 \\
\hline PEST & Fluridone & $\mathrm{mg} / \mathrm{kg}$ dry-wt & 1 & 0 & $0 \%$ & 0.080 & 0.080 & -- & -- & 0.040 \\
\hline
\end{tabular}


Table C-4

Summary Statistics for Sediment Collected from Segment 3 of the Columbia River Including All Categories of Data (units reported "dry-weight")

\begin{tabular}{|c|c|c|c|c|c|c|c|c|c|c|}
\hline $\begin{array}{l}\text { Constituent } \\
\text { Class }\end{array}$ & Constituent Name & Units & $\begin{array}{c}\text { Number } \\
\text { Analyzed }\end{array}$ & $\begin{array}{l}\text { Number } \\
\text { Detected }\end{array}$ & $\begin{array}{l}\text { Frequency of } \\
\text { Detection }\end{array}$ & $\begin{array}{l}\text { Minimum } \\
\text { Nondetect }\end{array}$ & $\begin{array}{l}\text { Maximum } \\
\text { Nondetect }\end{array}$ & $\begin{array}{c}\text { Minimum } \\
\text { Detect }\end{array}$ & $\begin{array}{l}\text { Maximum } \\
\text { Detect }\end{array}$ & $\begin{array}{c}\text { Average } \\
\text { Concentration }\end{array}$ \\
\hline$\overline{\text { PEST }}$ & Fonofos & $\mathrm{mg} / \mathrm{kg}$ dry-wt & 1 & 0 & $0 \%$ & 0.0060 & 0.0060 & - & - & 0.0030 \\
\hline PEST & Gamma-BHC (Lindane) & $\mathrm{mg} / \mathrm{kg}$ dry-wt & 22 & 1 & $5 \%$ & $1.00 \mathrm{E}-04$ & 0.020 & 8.00E-04 & 8.00E-04 & 0.0019 \\
\hline PEST & gamma-Chlordene & $\mathrm{mg} / \mathrm{kg}$ dry-wt & 1 & 0 & $0 \%$ & 0.010 & 0.010 & -- & -- & 0.0050 \\
\hline PEST & Heptachlor & $\mathrm{mg} / \mathrm{kg}$ dry-wt & 22 & 0 & $0 \%$ & $1.00 \mathrm{E}-04$ & 0.020 & -- & -- & 0.0020 \\
\hline PEST & Heptachlor epoxide & $\mathrm{mg} / \mathrm{kg}$ dry-wt & 22 & 0 & $0 \%$ & $1.00 \mathrm{E}-04$ & 0.020 & -- & -- & 0.0021 \\
\hline PEST & Hexazinone & $\mathrm{mg} / \mathrm{kg}$ dry-wt & 1 & 0 & $0 \%$ & 0.015 & 0.015 & -- & -- & 0.0075 \\
\hline PEST & Metalaxyl & $\mathrm{mg} / \mathrm{kg}$ dry-wt & 1 & 0 & $0 \%$ & 0.068 & 0.068 & -- & -- & 0.034 \\
\hline PEST & Methoxychlor & $\mathrm{mg} / \mathrm{kg}$ dry-wt & 17 & 0 & $0 \%$ & $1.00 \mathrm{E}-04$ & 0.021 & -- & -- & 0.0049 \\
\hline PEST & Methyl Chlorpyrifos & $\mathrm{mg} / \mathrm{kg}$ dry-wt & 1 & 0 & $0 \%$ & 0.0070 & 0.0070 & -- & -- & 0.0035 \\
\hline PEST & Methyl parathion & $\mathrm{mg} / \mathrm{kg}$ dry-wt & 1 & 0 & $0 \%$ & 0.0070 & 0.0070 & -- & -- & 0.0035 \\
\hline PEST & Metribuzin & $\mathrm{mg} / \mathrm{kg}$ dry-wt & 1 & 0 & $0 \%$ & 0.010 & 0.010 & -- & -- & 0.0050 \\
\hline PEST & Mevinphos & $\mathrm{mg} / \mathrm{kg}$ dry-wt & 1 & 0 & $0 \%$ & 0.010 & 0.010 & -- & -- & 0.0050 \\
\hline PEST & Mirex & $\mathrm{mg} / \mathrm{kg}$ dry-wt & 11 & 0 & $0 \%$ & $1.00 \mathrm{E}-04$ & 0.010 & -- & -- & 0.0012 \\
\hline PEST & Molinate & $\mathrm{mg} / \mathrm{kg}$ dry-wt & 1 & 0 & $0 \%$ & 0.026 & 0.026 & -- & -- & 0.013 \\
\hline PEST & Napropamide & $\mathrm{mg} / \mathrm{kg}$ dry-wt & 1 & 0 & $0 \%$ & 0.030 & 0.030 & -- & -- & 0.015 \\
\hline PEST & Norflurazon & $\mathrm{mg} / \mathrm{kg}$ dry-wt & 1 & 0 & $0 \%$ & 0.015 & 0.015 & -- & -- & 0.0075 \\
\hline PEST & Oxychlordane & $\mathrm{mg} / \mathrm{kg}$ dry-wt & 1 & 0 & $0 \%$ & 0.010 & 0.010 & -- & -- & 0.0050 \\
\hline PEST & Oxyfluorfen & $\mathrm{mg} / \mathrm{kg}$ dry-wt & 1 & 0 & $0 \%$ & 0.026 & 0.026 & -- & -- & 0.013 \\
\hline PEST & Parathion & $\mathrm{mg} / \mathrm{kg}$ dry-wt & 1 & 0 & $0 \%$ & 0.0080 & 0.0080 & -- & -- & 0.0040 \\
\hline PEST & Pebulate & $\mathrm{mg} / \mathrm{kg}$ dry-wt & 1 & 0 & $0 \%$ & 0.024 & 0.024 & -- & -- & 0.012 \\
\hline PEST & Pendimethalin & $\mathrm{mg} / \mathrm{kg}$ dry-wt & 1 & 0 & $0 \%$ & 0.015 & 0.015 & -- & -- & 0.0075 \\
\hline PEST & Pentachloroanisole & $\mathrm{mg} / \mathrm{kg}$ dry-wt & 1 & 0 & $0 \%$ & 0.010 & 0.010 & -- & -- & 0.0050 \\
\hline PEST & Perthane & $\mathrm{mg} / \mathrm{kg}$ dry-wt & 2 & 0 & $0 \%$ & 0.0010 & 0.0010 & -- & -- & $5.00 \mathrm{E}-04$ \\
\hline PEST & Phorate & $\mathrm{mg} / \mathrm{kg}$ dry-wt & 1 & 0 & $0 \%$ & 0.0070 & 0.0070 & -- & -- & 0.0035 \\
\hline PEST & Phosmet & $\mathrm{mg} / \mathrm{kg}$ dry-wt & 1 & 0 & $0 \%$ & 0.011 & 0.011 & -- & -- & 0.0055 \\
\hline PEST & Profluralin & $\mathrm{mg} / \mathrm{kg}$ dry-wt & 1 & 0 & $0 \%$ & 0.024 & 0.024 & -- & -- & 0.012 \\
\hline PEST & Pronamide & $\mathrm{mg} / \mathrm{kg}$ dry-wt & 1 & 0 & $0 \%$ & 0.030 & 0.030 & -- & -- & 0.015 \\
\hline PEST & Propachlor (Ramrod) & $\mathrm{mg} / \mathrm{kg}$ dry-wt & 1 & 0 & $0 \%$ & 0.020 & 0.020 & -- & -- & 0.010 \\
\hline PEST & Ronnel & $\mathrm{mg} / \mathrm{kg}$ dry-wt & 1 & 0 & $0 \%$ & 0.0070 & 0.0070 & -- & -- & 0.0035 \\
\hline PEST & Terbacil & $\mathrm{mg} / \mathrm{kg}$ dry-wt & 1 & 0 & $0 \%$ & 0.050 & 0.050 & -- & -- & 0.025 \\
\hline PEST & Tetraethyl dithiopyrophosphate & $\mathrm{mg} / \mathrm{kg}$ dry-wt & 1 & 0 & $0 \%$ & 0.0060 & 0.0060 & -- & -- & 0.0030 \\
\hline PEST & Toxaphene & $\mathrm{mg} / \mathrm{kg}$ dry-wt & 14 & 0 & $0 \%$ & 0.0010 & 0.60 & -- & -- & 0.068 \\
\hline PEST & trans-Chlordane & $\mathrm{mg} / \mathrm{kg}$ dry-wt & 1 & 0 & $0 \%$ & 0.0060 & 0.0060 & -- & -- & 0.0030 \\
\hline PEST & trans-Nonachlor & $\mathrm{mg} / \mathrm{kg}$ dry-wt & 9 & 4 & $44 \%$ & 0.010 & 0.010 & 0.0020 & 0.0030 & 0.0019 \\
\hline PEST & Triadimefon & $\mathrm{mg} / \mathrm{kg}$ dry-wt & 1 & 0 & $0 \%$ & 0.026 & 0.026 & -- & -- & 0.013 \\
\hline PEST & Triallate & $\mathrm{mg} / \mathrm{kg}$ dry-wt & 1 & 0 & $0 \%$ & 0.026 & 0.026 & -- & -- & 0.013 \\
\hline PEST & Tributylphosphorotrithioate & $\mathrm{mg} / \mathrm{kg}$ dry-wt & 1 & 0 & $0 \%$ & 0.014 & 0.014 & -- & -- & 0.0070 \\
\hline PEST & Trifluralin (Treflan) & $\mathrm{mg} / \mathrm{kg}$ dry-wt & 1 & 0 & $0 \%$ & 0.015 & 0.015 & -- & -- & 0.0075 \\
\hline PEST & Vernolate & $\mathrm{mg} / \mathrm{kg}$ dry-wt & 1 & 0 & $0 \%$ & 0.015 & 0.015 & -- & -- & 0.0075 \\
\hline RAD_D & Beryllium-7 & pCi/g dry-wt & 5 & 5 & $100 \%$ & & & $1.45 \mathrm{E}-19$ & $2.26 \mathrm{E}-05$ & $4.52 \mathrm{E}-06$ \\
\hline RAD_D & Cesium-134 & pCi/g dry-wt & 3 & 3 & $100 \%$ & & & $2.11 \mathrm{E}-04$ & 3.54E-04 & 2.72E-04 \\
\hline RAD_D & Cesium-137 & pCi/g dry-wt & 20 & 20 & $100 \%$ & & & 0.040 & 0.81 & 0.33 \\
\hline RAD_D & Cobalt-57 & $\mathrm{pCi} / \mathrm{g}$ dry-wt & 2 & 2 & $100 \%$ & & & 4.42E-09 & 0.0097 & 0.0049 \\
\hline RAD_D & Cobalt- 60 & pCi/g dry-wt & 20 & 20 & $100 \%$ & & & 0.0030 & 0.047 & 0.019 \\
\hline RAD_D & Europium-152 & pCi/g dry-wt & 15 & 15 & $100 \%$ & & & 0.027 & 0.97 & 0.16 \\
\hline RAD_D & Europium-154 & $\mathrm{pCi} / \mathrm{g}$ dry-wt & 4 & 4 & $100 \%$ & & & 0.036 & 0.088 & 0.053 \\
\hline RAD_D & Plutonium-238 & $\mathrm{pCi} / \mathrm{g}$ dry-wt & 8 & 8 & $100 \%$ & & & 0.0020 & 0.012 & 0.0060 \\
\hline
\end{tabular}


Table C-4

Summary Statistics for Sediment Collected from Segment 3 of the Columbia River Including All Categories of Data (units reported "dry-weight")

\begin{tabular}{|c|c|c|c|c|c|c|c|c|c|c|}
\hline $\begin{array}{l}\text { Constituent } \\
\text { Class }\end{array}$ & Constituent Name & Units & $\begin{array}{c}\text { Number } \\
\text { Analyzed }\end{array}$ & $\begin{array}{l}\text { Number } \\
\text { Detected }\end{array}$ & $\begin{array}{l}\text { Frequency of } \\
\text { Detection }\end{array}$ & $\begin{array}{l}\text { Minimum } \\
\text { Nondetect }\end{array}$ & $\begin{array}{l}\text { Maximum } \\
\text { Nondetect }\end{array}$ & $\begin{array}{l}\text { Minimum } \\
\text { Detect }\end{array}$ & $\begin{array}{l}\text { Maximum } \\
\text { Detect }\end{array}$ & $\begin{array}{c}\text { Average } \\
\text { Concentration }\end{array}$ \\
\hline$\overline{\text { RAD_D }}$ & Plutonium-239/240 & pCi/g dry-wt & 46 & $\overline{46}$ & $100 \%$ & & & 0.0020 & 0.024 & 0.0092 \\
\hline RAD_D & Potassium-40 & $\mathrm{pCi} / \mathrm{g}$ dry-wt & 48 & 48 & $100 \%$ & & & 13 & 19 & 15 \\
\hline RAD_D & Radium-226 & $\mathrm{pCi} / \mathrm{g}$ dry-wt & 1 & 1 & $100 \%$ & & & 0.99 & 0.99 & 0.99 \\
\hline RAD_D & Strontium-90 & $\mathrm{pCi} / \mathrm{g}$ dry-wt & 45 & 45 & $100 \%$ & & & 0.0019 & 0.037 & 0.014 \\
\hline RAD_D & Thorium-230 & $\mathrm{pCi} / \mathrm{g}$ dry-wt & 4 & 4 & $100 \%$ & & & 0.63 & 0.88 & 0.77 \\
\hline RAD_D & Thorium-232 & $\mathrm{pCi} / \mathrm{g}$ dry-wt & 4 & 4 & $100 \%$ & & & 0.70 & 0.91 & 0.82 \\
\hline RAD_D & Uranium-234 & $\mathrm{pCi} / \mathrm{g}$ dry-wt & 45 & 45 & $100 \%$ & & & 0.39 & 1.8 & 1.2 \\
\hline RAD_D & Uranium-235 & $\mathrm{pCi} / \mathrm{g}$ dry-wt & 24 & 24 & $100 \%$ & & & 0.020 & 0.070 & 0.041 \\
\hline RAD_D & Uranium-238 & $\mathrm{pCi} / \mathrm{g}$ dry-wt & 45 & 45 & $100 \%$ & & & 0.35 & 1.6 & 1.1 \\
\hline SVOC & 1,2,4-Trichlorobenzene & $\mathrm{mg} / \mathrm{kg}$ dry-wt & 3 & 0 & $0 \%$ & 0.20 & 0.20 & -- & -- & 0.10 \\
\hline SVOC & 1,2-Dichlorobenzene & $\mathrm{mg} / \mathrm{kg}$ dry-wt & 3 & 0 & $0 \%$ & 0.20 & 0.20 & -- & -- & 0.10 \\
\hline sVOC & 1,3-Dichlorobenzene & $\mathrm{mg} / \mathrm{kg}$ dry-wt & 3 & 0 & $0 \%$ & 0.20 & 0.20 & -- & -- & 0.10 \\
\hline sVoc & 1,4-Dichlorobenzene & $\mathrm{mg} / \mathrm{kg}$ dry-wt & 3 & 0 & $0 \%$ & 0.20 & 0.20 & -- & -- & 0.10 \\
\hline sVOc & 1-methylnaphthalene & $\mathrm{mg} / \mathrm{kg}$ dry-wt & 8 & 0 & $0 \%$ & 0.0050 & 0.026 & -- & -- & 0.0067 \\
\hline sVoc & 1-Methylphenanthrene & $\mathrm{mg} / \mathrm{kg}$ dry-wt & 8 & 0 & $0 \%$ & 0.0030 & 0.0070 & -- & -- & 0.0024 \\
\hline sVOC & $2,3,3^{\prime}, 4,4^{\prime}, 5^{\prime}$-Hexachlorobiphenyl & $\mathrm{mg} / \mathrm{kg}$ dry-wt & 2 & 0 & $0 \%$ & 0.0050 & 0.0050 & -- & -- & 0.0025 \\
\hline sVoc & 2,3,4-Trichlorophenol & $\mathrm{mg} / \mathrm{kg}$ dry-wt & 4 & 0 & $0 \%$ & 0.13 & 0.21 & -- & -- & 0.078 \\
\hline SVOC & 2,3,6-Trichlorophenol & $\mathrm{mg} / \mathrm{kg}$ dry-wt & 4 & 0 & $0 \%$ & 0.13 & 0.21 & -- & -- & 0.078 \\
\hline sVOC & 2,4,5-Trichlorophenol & $\mathrm{mg} / \mathrm{kg}$ dry-wt & 5 & 0 & $0 \%$ & 0.041 & 0.21 & -- & -- & 0.066 \\
\hline sVOC & 2,4,6-Trichlorophenol & $\mathrm{mg} / \mathrm{kg}$ dry-wt & 8 & 0 & $0 \%$ & 0.042 & 0.60 & -- & -- & 0.15 \\
\hline sVoc & 2,4-Dichlorophenol & $\mathrm{mg} / \mathrm{kg}$ dry-wt & 7 & 0 & $0 \%$ & 0.13 & 0.21 & -- & -- & 0.087 \\
\hline sVOC & 2,4-Dimethylphenol & $\mathrm{mg} / \mathrm{kg}$ dry-wt & 7 & 0 & $0 \%$ & 0.13 & 0.21 & -- & -- & 0.087 \\
\hline sVOC & 2,4-Dinitrophenol & $\mathrm{mg} / \mathrm{kg}$ dry-wt & 7 & 1 & $14 \%$ & 0.60 & 1.20 & 0.093 & 0.093 & 0.38 \\
\hline sVOC & 2,4-Dinitrotoluene & $\mathrm{mg} / \mathrm{kg}$ dry-wt & 3 & 0 & $0 \%$ & 0.20 & 0.20 & -- & -- & 0.10 \\
\hline sVOC & 2,6-Dimethylnaphthalene & $\mathrm{mg} / \mathrm{kg}$ dry-wt & 8 & 0 & $0 \%$ & 0.0050 & 0.013 & -- & -- & 0.0034 \\
\hline sVOC & 2,6-Dinitrotoluene & $\mathrm{mg} / \mathrm{kg}$ dry-wt & 3 & 0 & $0 \%$ & 0.20 & 0.20 & -- & -- & 0.10 \\
\hline SVOC & 2-Chloronaphthalene & $\mathrm{mg} / \mathrm{kg}$ dry-wt & 3 & 0 & $0 \%$ & 0.20 & 0.20 & -- & -- & 0.10 \\
\hline sVOC & 2-Chlorophenol & $\mathrm{mg} / \mathrm{kg}$ dry-wt & 7 & 0 & $0 \%$ & 0.13 & 0.21 & -- & -- & 0.087 \\
\hline sVOC & 2-Methylnaphthalene & $\mathrm{mg} / \mathrm{kg}$ dry-wt & 16 & 2 & $13 \%$ & 0.0040 & 12 & 0.010 & 0.011 & 0.42 \\
\hline SVOC & 2-Methylphenol (cresol, o-) & $\mathrm{mg} / \mathrm{kg}$ dry-wt & 6 & 1 & $17 \%$ & 0.010 & 0.21 & 0.81 & 0.81 & 0.19 \\
\hline sVOC & 2-Nitrophenol & $\mathrm{mg} / \mathrm{kg}$ dry-wt & 7 & 0 & $0 \%$ & 0.13 & 0.21 & -- & -- & 0.087 \\
\hline sVOC & 3,4,5-trichlorocatechol & $\mathrm{mg} / \mathrm{kg}$ dry-wt & 4 & 0 & $0 \%$ & 0.13 & 0.21 & -- & -- & 0.078 \\
\hline sVOC & 3,4,5-trichloroguaiacol & $\mathrm{mg} / \mathrm{kg}$ dry-wt & 4 & 0 & $0 \%$ & 0.13 & 0.21 & -- & -- & 0.078 \\
\hline SVOC & 4,5,6-Trichloroguaiacol & $\mathrm{mg} / \mathrm{kg}$ dry-wt & 4 & 0 & $0 \%$ & 0.13 & 0.21 & -- & -- & 0.078 \\
\hline SVOC & 4,5-Dichlorocatechol & $\mathrm{mg} / \mathrm{kg}$ dry-wt & 4 & 0 & $0 \%$ & 0.13 & 0.21 & -- & -- & 0.078 \\
\hline sVOC & 4,5-Dichloroguaiacol & $\mathrm{mg} / \mathrm{kg}$ dry-wt & 4 & 0 & $0 \%$ & 0.13 & 0.21 & -- & -- & 0.078 \\
\hline sVoc & 4,6-Dinitro-2-methylphenol & $\mathrm{mg} / \mathrm{kg}$ dry-wt & 3 & 0 & $0 \%$ & 0.60 & 0.60 & -- & -- & 0.30 \\
\hline svoc & 4-Allylguaiacol & $\mathrm{mg} / \mathrm{kg}$ dry-wt & 4 & 0 & $0 \%$ & 0.13 & 0.21 & -- & -- & 0.078 \\
\hline sVoc & 4-Bromophenylphenyl ether & $\mathrm{mg} / \mathrm{kg}$ dry-wt & 3 & 0 & $0 \%$ & 0.20 & 0.20 & -- & -- & 0.10 \\
\hline sVOC & 4-Chloro-3-methylphenol & $\mathrm{mg} / \mathrm{kg}$ dry-wt & 7 & 0 & $0 \%$ & 0.13 & 0.60 & -- & -- & 0.17 \\
\hline sVOC & 4-Chlorocatechol & $\mathrm{mg} / \mathrm{kg}$ dry-wt & 4 & 0 & $0 \%$ & 0.13 & 0.21 & -- & -- & 0.078 \\
\hline sVOC & 4-Chloroguaiacol & $\mathrm{mg} / \mathrm{kg}$ dry-wt & 4 & 0 & $0 \%$ & 0.13 & 0.21 & -- & -- & 0.078 \\
\hline sVoc & 4-Chlorophenylphenyl ether & $\mathrm{mg} / \mathrm{kg}$ dry-wt & 3 & 0 & $0 \%$ & 0.20 & 0.20 & -- & -- & 0.10 \\
\hline sVOC & 4-Methylphenol (cresol, p-) & $\mathrm{mg} / \mathrm{kg}$ dry-wt & 4 & 0 & $0 \%$ & 0.13 & 0.21 & -- & -- & 0.078 \\
\hline sVoc & 4-Nitrophenol & $\mathrm{mg} / \mathrm{kg}$ dry-wt & 7 & 0 & $0 \%$ & 0.13 & 0.60 & -- & -- & 0.17 \\
\hline SVOC & 4-Propenylguaiacol & $\mathrm{mg} / \mathrm{kg}$ dry-wt & 4 & 0 & $0 \%$ & 0.13 & 0.21 & -- & -- & 0.078 \\
\hline SVOC & 5,6-Dichlorovanillin & $\mathrm{mg} / \mathrm{kg}$ dry-wt & 4 & 0 & $0 \%$ & 0.13 & 0.21 & -- & -- & 0.078 \\
\hline
\end{tabular}


Table C-4

Summary Statistics for Sediment Collected from Segment 3 of the Columbia River Including All Categories of Data (units reported "dry-weight")

\begin{tabular}{|c|c|c|c|c|c|c|c|c|c|c|}
\hline $\begin{array}{c}\text { Constituent } \\
\text { Class }\end{array}$ & Constituent Name & Units & $\begin{array}{l}\text { Number } \\
\text { Analyzed }\end{array}$ & $\begin{array}{l}\text { Number } \\
\text { Detected }\end{array}$ & $\begin{array}{l}\text { Frequency of } \\
\text { Detection }\end{array}$ & $\begin{array}{l}\text { Minimum } \\
\text { Nondetect }\end{array}$ & $\begin{array}{l}\text { Maximum } \\
\text { Nondetect }\end{array}$ & $\begin{array}{l}\text { Minimum } \\
\text { Detect }\end{array}$ & $\begin{array}{l}\text { Maximum } \\
\text { Detect }\end{array}$ & $\begin{array}{c}\text { Average } \\
\text { Concentration }\end{array}$ \\
\hline$\overline{\text { SVOC }}$ & 6-chlorovanillin & $\mathrm{mg} / \mathrm{kg}$ dry-wt & 4 & 0 & $0 \%$ & 0.13 & 0.21 & -- & -- & 0.078 \\
\hline SVOC & Acenaphthene & $\mathrm{mg} / \mathrm{kg}$ dry-wt & 17 & 1 & $6 \%$ & 0.0040 & 12 & 0.021 & 0.021 & 0.42 \\
\hline SVOC & Acenaphthylene & $\mathrm{mg} / \mathrm{kg}$ dry-wt & 10 & 0 & $0 \%$ & 0.010 & 12 & -- & -- & 0.63 \\
\hline SVOC & Alpha-terpineol & $\mathrm{mg} / \mathrm{kg}$ dry-wt & 4 & 0 & $0 \%$ & 0.13 & 0.21 & -- & -- & 0.078 \\
\hline sVOC & Anthracene & $\mathrm{mg} / \mathrm{kg}$ dry-wt & 20 & 3 & $15 \%$ & 0.0030 & 12 & 0.0060 & 0.0880 & 0.34 \\
\hline SVOC & Benzo(a)anthracene & $\mathrm{mg} / \mathrm{kg}$ dry-wt & 22 & 2 & $9 \%$ & 0.0020 & 12 & 0.013 & 0.12 & 0.32 \\
\hline SVOC & Benzo(a)pyrene & $\mathrm{mg} / \mathrm{kg}$ dry-wt & 22 & 3 & $14 \%$ & 0.0020 & 1.1 & 0.013 & 0.21 & 0.078 \\
\hline SVOC & Benzo(b)fluoranthene & $\mathrm{mg} / \mathrm{kg}$ dry-wt & 11 & 4 & $36 \%$ & 0.012 & 12 & 0.018 & 0.22 & 0.66 \\
\hline sVOC & Benzo(e)pyrene & $\mathrm{mg} / \mathrm{kg}$ dry-wt & 8 & 0 & $0 \%$ & 0.0040 & 0.0150 & -- & -- & 0.0042 \\
\hline SVOC & Benzo(ghi)perylene & $\mathrm{mg} / \mathrm{kg}$ dry-wt & 8 & 2 & $25 \%$ & 0.012 & 12 & 0.014 & 0.016 & 0.83 \\
\hline SVOC & Benzo(k)fluoranthene & $\mathrm{mg} / \mathrm{kg}$ dry-wt & 10 & 1 & $10 \%$ & 0.012 & 12 & 0.023 & 0.023 & 0.67 \\
\hline SVOC & Benzoic acid & $\mathrm{mg} / \mathrm{kg}$ dry-wt & 4 & 2 & $50 \%$ & 1.1 & 1.2 & 0.20 & 1.1 & 0.61 \\
\hline sVOC & Biphenyl & $\mathrm{mg} / \mathrm{kg}$ dry-wt & 8 & 0 & $0 \%$ & 0.0050 & 0.0070 & -- & -- & 0.0030 \\
\hline SVOC & Bis(2-chloro-1-methylethyl)ether & $\mathrm{mg} / \mathrm{kg}$ dry-wt & 3 & 0 & $0 \%$ & 0.20 & 0.20 & -- & -- & 0.10 \\
\hline SVOC & Bis(2-Chloroethoxy)methane & $\mathrm{mg} / \mathrm{kg}$ dry-wt & 3 & 0 & $0 \%$ & 0.20 & 0.20 & -- & -- & 0.10 \\
\hline SVOC & Bis(2-chloroethyl) ether & $\mathrm{mg} / \mathrm{kg}$ dry-wt & 3 & 0 & $0 \%$ & 0.20 & 0.20 & -- & -- & 0.10 \\
\hline sVOC & Bis(2-ethylhexyl) phthalate & $\mathrm{mg} / \mathrm{kg}$ dry-wt & 9 & 5 & $56 \%$ & 0.20 & 0.23 & 0.053 & 0.78 & 0.27 \\
\hline SVOC & Butylbenzylphthalate & $\mathrm{mg} / \mathrm{kg}$ dry-wt & 5 & 0 & $0 \%$ & 0.0050 & 0.20 & -- & -- & 0.061 \\
\hline sVOC & Chrysene & $\mathrm{mg} / \mathrm{kg}$ dry-wt & 22 & 3 & $14 \%$ & 0.0030 & 12 & 0.013 & 0.130 & 0.32 \\
\hline SVOC & Dibenz[a,h]anthracene & $\mathrm{mg} / \mathrm{kg}$ dry-wt & 16 & 4 & $25 \%$ & 0.0040 & 12 & 0.0080 & 0.018 & 0.42 \\
\hline sVOC & Dibenzofuran & $\mathrm{mg} / \mathrm{kg}$ dry-wt & 5 & 1 & $20 \%$ & 0.012 & 12 & 0.015 & 0.015 & 1.2 \\
\hline SVOC & Diethylphthalate & $\mathrm{mg} / \mathrm{kg}$ dry-wt & 5 & 1 & $20 \%$ & 0.010 & 0.20 & 0.010 & 0.010 & 0.063 \\
\hline SVOC & Dimethyl phthalate & $\mathrm{mg} / \mathrm{kg}$ dry-wt & 3 & 0 & $0 \%$ & 0.20 & 0.20 & -- & -- & 0.10 \\
\hline SVOC & Di-n-butylphthalate & $\mathrm{mg} / \mathrm{kg}$ dry-wt & 3 & 0 & $0 \%$ & 0.20 & 0.20 & -- & -- & 0.10 \\
\hline sVOC & Di-n-octylphthalate & $\mathrm{mg} / \mathrm{kg}$ dry-wt & 5 & 0 & $0 \%$ & 0.0050 & 0.40 & -- & -- & 0.12 \\
\hline SVOC & Fluoranthene & $\mathrm{mg} / \mathrm{kg}$ dry-wt & 22 & 9 & $41 \%$ & 0.0040 & 12 & 0.0040 & 0.200 & 0.31 \\
\hline SVOC & Fluorene & $\mathrm{mg} / \mathrm{kg}$ dry-wt & 16 & 3 & $19 \%$ & 0.0030 & 12 & 0.0070 & 0.022 & 0.40 \\
\hline sVOC & Guaiacol & $\mathrm{mg} / \mathrm{kg}$ dry-wt & 4 & 0 & $0 \%$ & 0.13 & 0.21 & -- & -- & 0.078 \\
\hline sVOC & Hexachlorobenzene & $\mathrm{mg} / \mathrm{kg}$ dry-wt & 13 & 5 & $38 \%$ & 0.001 & 0.20 & 0.0010 & 0.0030 & 0.024 \\
\hline SVOC & Hexachlorobutadiene & $\mathrm{mg} / \mathrm{kg}$ dry-wt & 3 & 0 & $0 \%$ & 0.20 & 0.20 & -- & -- & 0.10 \\
\hline sVOC & Hexachlorocyclopentadiene & $\mathrm{mg} / \mathrm{kg}$ dry-wt & 3 & 0 & $0 \%$ & 0.20 & 0.20 & -- & -- & 0.10 \\
\hline \multirow[t]{2}{*}{ SVOC } & Hexachloroethane & $\mathrm{mg} / \mathrm{kg}$ dry-wt & 3 & 0 & $0 \%$ & 0.20 & 0.20 & -- & -- & 0.10 \\
\hline & Hexadecanoic acid (9Cl) & $\mathrm{mg} / \mathrm{kg}$ dry-wt & 1 & 1 & $100 \%$ & -- & -- & 130 & 130 & 130.00 \\
\hline sVOC & Indeno(1,2,3-cd)pyrene & $\mathrm{mg} / \mathrm{kg}$ dry-wt & 8 & 1 & $13 \%$ & 0.012 & 12 & 0.016 & 0.016 & 0.83 \\
\hline SVOC & Isophorone & $\mathrm{mg} / \mathrm{kg}$ dry-wt & 9 & 5 & $56 \%$ & 0.010 & 0.20 & 0.015 & 0.091 & 0.054 \\
\hline sVOC & Naphthalene & $\mathrm{mg} / \mathrm{kg}$ dry-wt & 19 & 3 & $16 \%$ & 0.006 & 12 & 0.0020 & 0.015 & 0.36 \\
\hline sVOC & Nitrobenzene & $\mathrm{mg} / \mathrm{kg}$ dry-wt & 3 & 0 & $0 \%$ & 0.20 & 0.20 & -- & -- & 0.10 \\
\hline SVOC & N-Nitrosodimethylamine & $\mathrm{mg} / \mathrm{kg}$ dry-wt & 3 & 0 & $0 \%$ & 0.20 & 0.20 & -- & -- & 0.10 \\
\hline SVOC & N-Nitrosodi-n-dipropylamine & $\mathrm{mg} / \mathrm{kg}$ dry-wt & 3 & 0 & $0 \%$ & 0.20 & 0.20 & -- & -- & 0.10 \\
\hline SVOC & N-Nitrosodiphenylamine & $\mathrm{mg} / \mathrm{kg}$ dry-wt & 3 & 0 & $0 \%$ & 0.20 & 0.20 & -- & -- & 0.10 \\
\hline sVOC & Pentachlorophenol & $\mathrm{mg} / \mathrm{kg}$ dry-wt & 13 & 3 & $23 \%$ & 0.13 & 1.40 & 0.013 & 1.200 & 0.33 \\
\hline SVOC & Perylene & $\mathrm{mg} / \mathrm{kg}$ dry-wt & 8 & 3 & $38 \%$ & 0.0040 & 0.0170 & 0.0070 & 0.011 & 0.0068 \\
\hline SVOC & Phenanthrene & $\mathrm{mg} / \mathrm{kg}$ dry-wt & 19 & 4 & $21 \%$ & 0.0030 & 12 & 0.0040 & 0.057 & 0.37 \\
\hline SVOC & Phenol & $\mathrm{mg} / \mathrm{kg}$ dry-wt & 9 & 3 & $33 \%$ & 0.010 & 0.28 & 0.035 & 0.10 & 0.082 \\
\hline sVOC & Pyrene & $\mathrm{mg} / \mathrm{kg}$ dry-wt & 22 & 7 & $32 \%$ & 0.0030 & 12 & 0.0030 & 0.220 & 0.32 \\
\hline SVOC & Tetrachloro-1-1'-biphenyl & $\mathrm{mg} / \mathrm{kg}$ dry-wt & 2 & 0 & $0 \%$ & 0.0050 & 0.0050 & -- & -- & 0.0025 \\
\hline SVOC & Tetrachlorocatechol & $\mathrm{mg} / \mathrm{kg}$ dry-wt & 4 & 0 & $0 \%$ & 0.13 & 0.21 & -- & -- & 0.078 \\
\hline
\end{tabular}


Table C-4

Summary Statistics for Sediment Collected from Segment 3 of the Columbia River Including All Categories of Data (units reported "dry-weight")

Number Frequency of Minimum

Constituent

Constituent Name

Units

Number

Number

Detection

inimum Maximum

Detect

Maximum

Average

SVOC Tetrachloroguaiaco

$\mathrm{mg} / \mathrm{kg}$ dry-w

$4 \quad 0$

$0 \%$

0.13

0.21

$\mathrm{mg} / \mathrm{kg}$ dry-wt

\begin{tabular}{ll}
$0 \%$ & 0.13 \\
$0 \%$ & 0.13 \\
\hline
\end{tabular}

0.21

--

$-$

Trichlorotrimethoxybenzen

CNV - Conventional parameter

PEST - Pesticide

PCB - Polychlorinated biphenyl 
Table C-5

Summary Statistics for Sediment Samples Collected From the Yakima River Tributary of Segment 3 of the Columbia River (units reported "as received")

\begin{tabular}{|c|c|c|c|c|c|c|c|c|c|c|}
\hline $\begin{array}{l}\text { Constituent } \\
\text { Class }\end{array}$ & Constituent Name & Units & $\begin{array}{l}\text { Number } \\
\text { Analyzed }\end{array}$ & $\begin{array}{l}\text { Number } \\
\text { Detected }\end{array}$ & $\begin{array}{l}\text { Frequency of } \\
\text { Detection }\end{array}$ & $\begin{array}{l}\text { Minimum } \\
\text { Nondetect }\end{array}$ & $\begin{array}{l}\text { Maximum } \\
\text { Nondetect }\end{array}$ & $\begin{array}{l}\text { Minimum } \\
\text { Detect }\end{array}$ & $\begin{array}{l}\text { Maximum } \\
\text { Detect }\end{array}$ & $\begin{array}{c}\text { Average } \\
\text { Concentration }\end{array}$ \\
\hline CONV & Percent Solids & $\%$ & 6 & 6 & $100 \%$ & $\overline{--}$ & $\overline{--}$ & 57 & 71 & 65 \\
\hline CONV & Total Organic Carbon & $\%$ & 7 & 7 & $100 \%$ & -- & -- & 0.62 & 2.0 & 0.97 \\
\hline CONV & Total Organic Carbon & $\mathrm{mg} / \mathrm{Kg}$ & 3 & 3 & $100 \%$ & -- & -- & 1.0 & 7,070 & 4,047 \\
\hline CONV & Total solids & $\mathrm{mg} / \mathrm{Kg}$ & 1 & 1 & $100 \%$ & -- & -- & 52 & 52 & 52 \\
\hline METAL & Aluminum & $\mathrm{mg} / \mathrm{Kg}$ & 1 & 1 & $100 \%$ & -- & -- & 68,000 & 68,000 & 68,000 \\
\hline METAL & Antimony & $\mathrm{mg} / \mathrm{Kg}$ & 3 & 3 & $100 \%$ & -- & -- & 0.34 & 0.60 & 0.43 \\
\hline METAL & Arsenic & $\mathrm{mg} / \mathrm{kg}$ & 9 & 4 & $44 \%$ & 4.5 & 4.5 & 2.4 & 6.7 & 3.1 \\
\hline METAL & Barium & $\mathrm{mg} / \mathrm{kg}$ & 1 & 1 & $100 \%$ & -- & -- & 530 & 530 & 530 \\
\hline METAL & Beryllium & $\mathrm{mg} / \mathrm{Kg}$ & 3 & 3 & $100 \%$ & -- & -- & 0.78 & 2.0 & 1.2 \\
\hline METAL & Bismuth & $\mathrm{mg} / \mathrm{kg}$ & 1 & 0 & $0 \%$ & 10 & 10 & -- & -- & 5.0 \\
\hline METAL & Boron & $\mathrm{mg} / \mathrm{kg}$ & 1 & 1 & $100 \%$ & -- & -- & 2.3 & 2.3 & 2.3 \\
\hline METAL & Cadmium & $\mathrm{mg} / \mathrm{kg}$ & 9 & 4 & $44 \%$ & 0.50 & 2.0 & 0.093 & 6.0 & 0.98 \\
\hline METAL & Calcium & $\mathrm{mg} / \mathrm{kg}$ & 1 & 1 & $100 \%$ & -- & -- & 26,000 & 26,000 & 26,000 \\
\hline METAL & Cerium & $\mathrm{mg} / \mathrm{kg}$ & 1 & 1 & $100 \%$ & -- & -- & 53 & 53 & 53 \\
\hline METAL & Chromium & $\mathrm{mg} / \mathrm{kg}$ & 9 & 9 & $100 \%$ & -- & -- & 15 & 61 & 27 \\
\hline METAL & Cobalt & $\mathrm{mg} / \mathrm{kg}$ & 1 & 1 & $100 \%$ & -- & -- & 20 & 20 & 20 \\
\hline METAL & Copper & $\mathrm{mg} / \mathrm{kg}$ & 9 & 9 & $100 \%$ & -- & -- & 12 & 29 & 17 \\
\hline METAL & Europium & $\mathrm{mg} / \mathrm{kg}$ & 1 & 0 & $0 \%$ & 2.0 & 2.0 & -- & -- & 1.0 \\
\hline METAL & Gallium & $\mathrm{mg} / \mathrm{kg}$ & 1 & 1 & $100 \%$ & -- & -- & 19 & 19 & 19 \\
\hline METAL & Gold & $\mathrm{mg} / \mathrm{kg}$ & 1 & 0 & $0 \%$ & 8.0 & 8.0 & -- & -- & 4.0 \\
\hline METAL & Iron & $\mathrm{mg} / \mathrm{kg}$ & 1 & 1 & $100 \%$ & -- & -- & 51,000 & 51,000 & 51,000 \\
\hline METAL & Lanthanum & $\mathrm{mg} / \mathrm{kg}$ & 1 & 1 & $100 \%$ & -- & -- & 28 & 28 & 28 \\
\hline METAL & Lead & $\mathrm{mg} / \mathrm{kg}$ & 9 & 9 & $100 \%$ & -- & -- & 4.9 & 27 & 10 \\
\hline METAL & Lithium & $\mathrm{mg} / \mathrm{kg}$ & 1 & 1 & $100 \%$ & -- & -- & 26 & 26 & 26 \\
\hline METAL & Magnesium & $\mathrm{mg} / \mathrm{Kg}$ & 1 & 1 & $100 \%$ & -- & -- & 14,000 & 14,000 & 14,000 \\
\hline METAL & Manganese & $\mathrm{mg} / \mathrm{kg}$ & 1 & 1 & $100 \%$ & -- & -- & 1,500 & 1,500 & 1,500 \\
\hline METAL & Mercury & $\mathrm{mg} / \mathrm{kg}$ & 9 & 5 & $56 \%$ & 0.040 & 0.040 & 0.025 & 0.17 & 0.049 \\
\hline METAL & Molybdenum & $\mathrm{mg} / \mathrm{kg}$ & 7 & 0 & $0 \%$ & 0.60 & 2.0 & -- & -- & 0.40 \\
\hline METAL & Neodymium & $\mathrm{mg} / \mathrm{kg}$ & 1 & 1 & $100 \%$ & -- & -- & 30 & 30 & 30 \\
\hline METAL & Nickel & $\mathrm{mg} / \mathrm{kg}$ & 9 & 9 & $100 \%$ & -- & -- & 20 & 30 & 24 \\
\hline METAL & Niobium & $\mathrm{mg} / \mathrm{kg}$ & 1 & 0 & $0 \%$ & 4.0 & 4.0 & -- & -- & 2.0 \\
\hline METAL & Phosphorus & $\mathrm{mg} / \mathrm{Kg}$ & 1 & 1 & $100 \%$ & -- & -- & 1,000 & 1,000 & 1,000 \\
\hline METAL & Scandium & $\mathrm{mg} / \mathrm{kg}$ & 1 & 1 & $100 \%$ & -- & -- & 19 & 19 & 19 \\
\hline METAL & Selenium & $\mathrm{mg} / \mathrm{kg}$ & 8 & 0 & $0 \%$ & 0.46 & 5.0 & -- & -- & 1.9 \\
\hline METAL & Silver & $\mathrm{mg} / \mathrm{kg}$ & 3 & 2 & $67 \%$ & 2.0 & 2.0 & 0.31 & 0.35 & 0.55 \\
\hline METAL & Sodium & $\mathrm{mg} / \mathrm{Kg}$ & 1 & 1 & $100 \%$ & -- & -- & 16,000 & 16,000 & 16,000 \\
\hline METAL & Strontium & $\mathrm{mg} / \mathrm{kg}$ & 1 & 1 & $100 \%$ & -- & -- & 260 & 260 & 260 \\
\hline METAL & Thallium & $\mathrm{mg} / \mathrm{Kg}$ & 2 & 2 & $100 \%$ & -- & -- & 0.21 & 0.22 & 0.21 \\
\hline METAL & Thorium & $\mathrm{mg} / \mathrm{kg}$ & 1 & 1 & $100 \%$ & -- & -- & 8.0 & 8.0 & 8.0 \\
\hline METAL & Tin & $\mathrm{mg} / \mathrm{kg}$ & 1 & 0 & $0 \%$ & 10 & 10 & -- & -- & 5.0 \\
\hline METAL & Titanium & $\mathrm{mg} / \mathrm{Kg}$ & 1 & 1 & $100 \%$ & -- & -- & 6,200 & 6,200 & 6,200 \\
\hline METAL & Uranium & $\mathrm{mg} / \mathrm{Kg}$ & 3 & 3 & $100 \%$ & -- & -- & 1.1 & 1.3 & 1.2 \\
\hline METAL & Vanadium & $\mathrm{mg} / \mathrm{kg}$ & 1 & 1 & $100 \%$ & -- & -- & 140 & 140 & 140 \\
\hline METAL & Ytterbium & $\mathrm{mg} / \mathrm{kg}$ & 1 & 1 & $100 \%$ & -- & -- & 3.0 & 3.0 & 3.0 \\
\hline METAL & Yttrium & $\mathrm{mg} / \mathrm{kg}$ & 1 & 1 & $100 \%$ & -- & -- & 23 & 23 & 23 \\
\hline METAL & Zinc & $\mathrm{mg} / \mathrm{kg}$ & 9 & 9 & $100 \%$ & -- & -- & 53 & 558 & 127 \\
\hline РCB & Aroclor-1242 & $\mathrm{mg} / \mathrm{kg}$ & 2 & 0 & $0 \%$ & 0.12 & 0.13 & -- & -- & 0.063 \\
\hline
\end{tabular}


Table C-5

Summary Statistics for Sediment Samples Collected From the Yakima River Tributary of Segment 3 of the Columbia River (units reported "as received")

\begin{tabular}{|c|c|c|c|c|c|c|c|c|c|c|}
\hline $\begin{array}{c}\begin{array}{c}\text { Constituent } \\
\text { Class }\end{array} \\
\end{array}$ & Constituent Name & Units & $\begin{array}{l}\text { Number } \\
\text { Analyzed }\end{array}$ & $\begin{array}{l}\text { Number } \\
\text { Detected }\end{array}$ & $\begin{array}{c}\text { Frequency of } \\
\text { Detection }\end{array}$ & $\begin{array}{l}\text { Minimum } \\
\text { Nondetect }\end{array}$ & $\begin{array}{l}\text { Maximum } \\
\text { Nondetect }\end{array}$ & $\begin{array}{c}\text { Minimum } \\
\text { Detect }\end{array}$ & $\begin{array}{c}\text { Maximum } \\
\text { Detect }\end{array}$ & $\begin{array}{c}\text { Average } \\
\text { Concentration }\end{array}$ \\
\hline$\overline{\mathrm{PCB}}$ & Aroclor-1248 & $\mathrm{mg} / \mathrm{Kg}$ & 2 & 0 & $0 \%$ & 0.12 & 0.13 & $\overline{--}$ & $\overline{--}$ & 0.063 \\
\hline PCB & Aroclor-1254 & $\mathrm{mg} / \mathrm{Kg}$ & 2 & 0 & $0 \%$ & 0.12 & 0.13 & -- & -- & 0.063 \\
\hline PCB & Aroclor-1260 & $\mathrm{mg} / \mathrm{Kg}$ & 2 & 0 & $0 \%$ & 0.12 & 0.13 & -- & -- & 0.063 \\
\hline PEST & 2,4'-DDD & $\mathrm{mg} / \mathrm{Kg}$ & 2 & 0 & $0 \%$ & 0.036 & 0.040 & -- & -- & 0.019 \\
\hline PEST & 2,4'-DDE & $\mathrm{mg} / \mathrm{Kg}$ & 2 & 0 & $0 \%$ & 0.036 & 0.040 & -- & -- & 0.019 \\
\hline PEST & 2,4'-DDT & $\mathrm{mg} / \mathrm{Kg}$ & 2 & 0 & $0 \%$ & 0.036 & 0.040 & -- & -- & 0.019 \\
\hline PEST & Alachlor & $\mathrm{mg} / \mathrm{Kg}$ & 2 & 0 & $0 \%$ & 0.14 & 0.16 & -- & -- & 0.075 \\
\hline PEST & Aldrin & $\mathrm{mg} / \mathrm{Kg}$ & 3 & 0 & $0 \%$ & 0.0010 & 0.040 & -- & -- & 0.013 \\
\hline PEST & Alpha-BHC & $\mathrm{mg} / \mathrm{Kg}$ & 2 & 0 & $0 \%$ & 0.036 & 0.040 & -- & -- & 0.019 \\
\hline PEST & alpha-Chlordane & $\mathrm{mg} / \mathrm{Kg}$ & 2 & 0 & $0 \%$ & 0.036 & 0.040 & -- & -- & 0.019 \\
\hline PEST & alpha-Chlordene & $\mathrm{mg} / \mathrm{Kg}$ & 2 & 0 & $0 \%$ & 0.036 & 0.040 & -- & -- & 0.019 \\
\hline PEST & Ametryn & $\mathrm{mg} / \mathrm{Kg}$ & 2 & 0 & $0 \%$ & 0.060 & 0.066 & -- & -- & 0.032 \\
\hline PEST & Atraton & $\mathrm{mg} / \mathrm{Kg}$ & 2 & 0 & $0 \%$ & 0.18 & 0.20 & -- & -- & 0.095 \\
\hline PEST & Atrazine & $\mathrm{mg} / \mathrm{Kg}$ & 2 & 0 & $0 \%$ & 0.060 & 0.066 & -- & -- & 0.032 \\
\hline PEST & Azinphos Ethyl & $\mathrm{mg} / \mathrm{Kg}$ & 2 & 0 & $0 \%$ & 0.096 & 0.11 & -- & -- & 0.052 \\
\hline PEST & Azinphos Methyl & $\mathrm{mg} / \mathrm{Kg}$ & 2 & 0 & $0 \%$ & 0.11 & 0.12 & -- & -- & 0.058 \\
\hline PEST & Benefin (Benfluralin) & $\mathrm{mg} / \mathrm{Kg}$ & 2 & 0 & $0 \%$ & 0.090 & 0.099 & -- & -- & 0.047 \\
\hline PEST & beta-1,2,3,4,5,6-Hexachlorocyclohexane & $\mathrm{mg} / \mathrm{Kg}$ & 2 & 0 & $0 \%$ & 0.036 & 0.040 & -- & -- & 0.019 \\
\hline PEST & Bromacil (ACN) & $\mathrm{mg} / \mathrm{Kg}$ & 2 & 0 & $0 \%$ & 0.36 & 0.40 & -- & -- & 0.19 \\
\hline PEST & Butachlor & $\mathrm{mg} / \mathrm{Kg}$ & 2 & 0 & $0 \%$ & 0.21 & 0.23 & -- & -- & 0.11 \\
\hline PEST & Butylate & $\mathrm{mg} / \mathrm{Kg}$ & 2 & 0 & $0 \%$ & 0.090 & 0.099 & -- & -- & 0.047 \\
\hline PEST & Carbethoxy malathion & $\mathrm{mg} / \mathrm{Kg}$ & 2 & 0 & $0 \%$ & 0.048 & 0.053 & -- & -- & 0.025 \\
\hline PEST & Carboxin & $\mathrm{mg} / \mathrm{Kg}$ & 2 & 0 & $0 \%$ & 0.66 & 0.73 & -- & -- & 0.35 \\
\hline PEST & Chlorothalonil & $\mathrm{mg} / \mathrm{Kg}$ & 2 & 0 & $0 \%$ & 0.14 & 0.16 & -- & -- & 0.075 \\
\hline PEST & Chlorpropham & $\mathrm{mg} / \mathrm{Kg}$ & 2 & 0 & $0 \%$ & 0.30 & 0.33 & -- & -- & 0.16 \\
\hline PEST & Chlorpyrifos & $\mathrm{mg} / \mathrm{Kg}$ & 2 & 0 & $0 \%$ & 0.042 & 0.046 & -- & -- & 0.022 \\
\hline PEST & Cyanazine & $\mathrm{mg} / \mathrm{Kg}$ & 2 & 0 & $0 \%$ & 0.091 & 0.099 & -- & -- & 0.048 \\
\hline PEST & Cycloate & $\mathrm{mg} / \mathrm{Kg}$ & 2 & 0 & $0 \%$ & 0.090 & 0.099 & -- & -- & 0.047 \\
\hline PEST & DDMU & $\mathrm{mg} / \mathrm{Kg}$ & 2 & 0 & $0 \%$ & 0.036 & 0.040 & -- & -- & 0.019 \\
\hline PEST & Delta-BHC & $\mathrm{mg} / \mathrm{Kg}$ & 2 & 0 & $0 \%$ & 0.036 & 0.040 & -- & -- & 0.019 \\
\hline PEST & Diazinon & $\mathrm{mg} / \mathrm{Kg}$ & 2 & 0 & $0 \%$ & 0.048 & 0.053 & -- & -- & 0.025 \\
\hline PEST & Dichlobenil & $\mathrm{mg} / \mathrm{Kg}$ & 2 & 0 & $0 \%$ & 0.072 & 0.079 & -- & -- & 0.038 \\
\hline PEST & Dichlorodiphenyldichloroethane & $\mathrm{mg} / \mathrm{Kg}$ & 2 & 2 & $100 \%$ & -- & -- & 0.0030 & 0.0030 & 0.0030 \\
\hline PEST & Dichlorodiphenyldichloroethylene & $\mathrm{mg} / \mathrm{Kg}$ & 2 & 2 & $100 \%$ & -- & -- & 0.015 & 0.016 & 0.016 \\
\hline PEST & Dichlorodiphenyltrichloroethane & $\mathrm{mg} / \mathrm{Kg}$ & 2 & 0 & $0 \%$ & 0.036 & 0.040 & -- & -- & 0.019 \\
\hline PEST & Dicofol & $\mathrm{mg} / \mathrm{Kg}$ & 2 & 0 & $0 \%$ & 0.14 & 0.16 & -- & -- & 0.075 \\
\hline PEST & Dieldrin & $\mathrm{mg} / \mathrm{Kg}$ & 3 & 0 & $0 \%$ & 0.0010 & 0.040 & -- & -- & 0.013 \\
\hline PEST & Dimethoate & $\mathrm{mg} / \mathrm{Kg}$ & 2 & 0 & $0 \%$ & 0.048 & 0.053 & -- & -- & 0.025 \\
\hline PEST & Diphenamid & $\mathrm{mg} / \mathrm{Kg}$ & 2 & 0 & $0 \%$ & 0.18 & 0.20 & -- & -- & 0.095 \\
\hline PEST & Disulfoton & $\mathrm{mg} / \mathrm{Kg}$ & 2 & 0 & $0 \%$ & 0.036 & 0.040 & -- & -- & 0.019 \\
\hline PEST & Diuron & $\mathrm{mg} / \mathrm{Kg}$ & 2 & 0 & $0 \%$ & 0.48 & 0.53 & -- & -- & 0.25 \\
\hline PEST & Endosulfan I & $\mathrm{mg} / \mathrm{Kg}$ & 2 & 0 & $0 \%$ & 0.036 & 0.040 & -- & -- & 0.019 \\
\hline PEST & Endosulfan II & $\mathrm{mg} / \mathrm{Kg}$ & 2 & 0 & $0 \%$ & 0.036 & 0.040 & -- & -- & 0.019 \\
\hline PEST & Endosulfan sulfate & $\mathrm{mg} / \mathrm{Kg}$ & 2 & 0 & $0 \%$ & 0.036 & 0.040 & -- & -- & 0.019 \\
\hline PEST & Endrin & $\mathrm{mg} / \mathrm{Kg}$ & 2 & 0 & $0 \%$ & 0.036 & 0.040 & -- & -- & 0.019 \\
\hline PEST & Endrin aldehyde & $\mathrm{mg} / \mathrm{Kg}$ & 2 & 0 & $0 \%$ & 0.036 & 0.040 & -- & -- & 0.019 \\
\hline PEST & Endrin ketone & $\mathrm{mg} / \mathrm{Kg}$ & 2 & 0 & $0 \%$ & 0.018 & 0.020 & -- & -- & 0.0095 \\
\hline
\end{tabular}


Table C-5

Summary Statistics for Sediment Samples Collected From the Yakima River Tributary of Segment 3 of the Columbia River (units reported "as received")

\begin{tabular}{|c|c|c|c|c|c|c|c|c|c|c|}
\hline $\begin{array}{c}\begin{array}{c}\text { Constituent } \\
\text { Class }\end{array} \\
\end{array}$ & Constituent Name & Units & $\begin{array}{l}\text { Number } \\
\text { Analyzed }\end{array}$ & $\begin{array}{l}\text { Number } \\
\text { Detected }\end{array}$ & $\begin{array}{c}\text { Frequency of } \\
\text { Detection }\end{array}$ & $\begin{array}{l}\text { Minimum } \\
\text { Nondetect }\end{array}$ & $\begin{array}{l}\text { Maximum } \\
\text { Nondetect }\end{array}$ & $\begin{array}{c}\text { Minimum } \\
\text { Detect }\end{array}$ & $\begin{array}{c}\text { Maximum } \\
\text { Detect }\end{array}$ & $\begin{array}{c}\text { Average } \\
\text { Concentration }\end{array}$ \\
\hline$\overline{\text { PEST }}$ & Eptam & $\mathrm{mg} / \mathrm{Kg}$ & 2 & 0 & $0 \%$ & 0.090 & 0.099 & $\overline{--}$ & $\overline{--}$ & 0.047 \\
\hline PEST & Ethalfluralin (Sonalan) & $\mathrm{mg} / \mathrm{Kg}$ & 2 & 0 & $0 \%$ & 0.090 & 0.099 & -- & -- & 0.047 \\
\hline PEST & Ethyl methylene phosphorodithioate & $\mathrm{mg} / \mathrm{Kg}$ & 2 & 0 & $0 \%$ & 0.042 & 0.046 & -- & -- & 0.022 \\
\hline PEST & Fenarimol & $\mathrm{mg} / \mathrm{Kg}$ & 2 & 0 & $0 \%$ & 0.18 & 0.20 & -- & -- & 0.095 \\
\hline PEST & Fensulfothion & $\mathrm{mg} / \mathrm{Kg}$ & 2 & 0 & $0 \%$ & 0.060 & 0.066 & -- & -- & 0.032 \\
\hline PEST & Fluridone & $\mathrm{mg} / \mathrm{Kg}$ & 2 & 0 & $0 \%$ & 0.48 & 0.53 & -- & -- & 0.25 \\
\hline PEST & Fonofos & $\mathrm{mg} / \mathrm{Kg}$ & 2 & 0 & $0 \%$ & 0.036 & 0.040 & -- & -- & 0.019 \\
\hline PEST & Gamma-BHC (Lindane) & $\mathrm{mg} / \mathrm{Kg}$ & 2 & 0 & $0 \%$ & 0.036 & 0.040 & -- & -- & 0.019 \\
\hline PEST & gamma-Chlordene & $\mathrm{mg} / \mathrm{Kg}$ & 2 & 0 & $0 \%$ & 0.036 & 0.040 & -- & -- & 0.019 \\
\hline PEST & Heptachlor & $\mathrm{mg} / \mathrm{Kg}$ & 2 & 0 & $0 \%$ & 0.036 & 0.040 & -- & -- & 0.019 \\
\hline PEST & Heptachlor epoxide & $\mathrm{mg} / \mathrm{Kg}$ & 2 & 0 & $0 \%$ & 0.036 & 0.040 & -- & -- & 0.019 \\
\hline PEST & Hexazinone & $\mathrm{mg} / \mathrm{Kg}$ & 2 & 0 & $0 \%$ & 0.090 & 0.099 & -- & -- & 0.047 \\
\hline PEST & Methoxychlor & $\mathrm{mg} / \mathrm{Kg}$ & 3 & 0 & $0 \%$ & 0.0010 & 0.040 & -- & -- & 0.013 \\
\hline PEST & Methyl Chlorpyrifos & $\mathrm{mg} / \mathrm{Kg}$ & 2 & 0 & $0 \%$ & 0.042 & 0.046 & -- & -- & 0.022 \\
\hline PEST & Methyl parathion & $\mathrm{mg} / \mathrm{Kg}$ & 2 & 0 & $0 \%$ & 0.042 & 0.046 & -- & -- & 0.022 \\
\hline PEST & Metolachlor & $\mathrm{mg} / \mathrm{Kg}$ & 2 & 0 & $0 \%$ & 0.18 & 0.20 & -- & -- & 0.095 \\
\hline PEST & Metribuzin & $\mathrm{mg} / \mathrm{Kg}$ & 2 & 0 & $0 \%$ & 0.060 & 0.066 & -- & -- & 0.032 \\
\hline PEST & MGK264 & $\mathrm{mg} / \mathrm{Kg}$ & 2 & 0 & $0 \%$ & 0.42 & 0.46 & -- & -- & 0.22 \\
\hline PEST & Mirex & $\mathrm{mg} / \mathrm{Kg}$ & 2 & 0 & $0 \%$ & 0.036 & 0.040 & -- & -- & 0.019 \\
\hline PEST & Molinate & $\mathrm{mg} / \mathrm{Kg}$ & 2 & 0 & $0 \%$ & 0.16 & 0.17 & -- & -- & 0.083 \\
\hline PEST & Napropamide & $\mathrm{mg} / \mathrm{Kg}$ & 2 & 0 & $0 \%$ & 0.18 & 0.20 & -- & -- & 0.095 \\
\hline PEST & Norflurazon & $\mathrm{mg} / \mathrm{Kg}$ & 2 & 0 & $0 \%$ & 0.090 & 0.099 & -- & -- & 0.047 \\
\hline PEST & Oxychlordane & $\mathrm{mg} / \mathrm{Kg}$ & 2 & 0 & $0 \%$ & 0.036 & 0.040 & -- & -- & 0.019 \\
\hline PEST & Oxyfluorfen & $\mathrm{mg} / \mathrm{Kg}$ & 2 & 0 & $0 \%$ & 0.16 & 0.17 & -- & -- & 0.083 \\
\hline PEST & Parathion & $\mathrm{mg} / \mathrm{Kg}$ & 2 & 0 & $0 \%$ & 0.048 & 0.053 & -- & -- & 0.025 \\
\hline PEST & Pebulate & $\mathrm{mg} / \mathrm{Kg}$ & 2 & 0 & $0 \%$ & 0.14 & 0.16 & -- & -- & 0.075 \\
\hline PEST & Pendimethalin & $\mathrm{mg} / \mathrm{Kg}$ & 2 & 0 & $0 \%$ & 0.090 & 0.099 & -- & -- & 0.047 \\
\hline PEST & Pentachloroanisole & $\mathrm{mg} / \mathrm{Kg}$ & 2 & 0 & $0 \%$ & 0.036 & 0.040 & -- & -- & 0.019 \\
\hline PEST & Phorate & $\mathrm{mg} / \mathrm{Kg}$ & 2 & 0 & $0 \%$ & 0.042 & 0.046 & -- & -- & 0.022 \\
\hline PEST & Pramitol (Prometon) & $\mathrm{mg} / \mathrm{Kg}$ & 2 & 0 & $0 \%$ & 0.060 & 0.066 & -- & -- & 0.032 \\
\hline PEST & Prometryn & $\mathrm{mg} / \mathrm{Kg}$ & 2 & 0 & $0 \%$ & 0.060 & 0.066 & -- & -- & 0.032 \\
\hline PEST & Pronamide & $\mathrm{mg} / \mathrm{Kg}$ & 2 & 0 & $0 \%$ & 0.18 & 0.20 & -- & -- & 0.095 \\
\hline PEST & Propachlor (Ramrod) & $\mathrm{mg} / \mathrm{Kg}$ & 2 & 0 & $0 \%$ & 0.12 & 0.13 & -- & -- & 0.063 \\
\hline PEST & Propazine & $\mathrm{mg} / \mathrm{Kg}$ & 2 & 0 & $0 \%$ & 0.060 & 0.066 & -- & -- & 0.032 \\
\hline PEST & Simazine & $\mathrm{mg} / \mathrm{Kg}$ & 2 & 0 & $0 \%$ & 0.060 & 0.066 & -- & -- & 0.032 \\
\hline PEST & Terbacil & $\mathrm{mg} / \mathrm{Kg}$ & 2 & 0 & $0 \%$ & 0.30 & 0.33 & -- & -- & 0.16 \\
\hline PEST & Terbutryn & $\mathrm{mg} / \mathrm{Kg}$ & 2 & 0 & $0 \%$ & 0.060 & 0.066 & -- & -- & 0.032 \\
\hline PEST & Toxaphene & $\mathrm{mg} / \mathrm{Kg}$ & 2 & 0 & $0 \%$ & 0.72 & 0.79 & -- & -- & 0.38 \\
\hline PEST & trans-Chlordane & $\mathrm{mg} / \mathrm{Kg}$ & 2 & 0 & $0 \%$ & 0.036 & 0.040 & -- & -- & 0.019 \\
\hline PEST & trans-Nonachlor & $\mathrm{mg} / \mathrm{Kg}$ & 2 & 0 & $0 \%$ & 0.036 & 0.040 & -- & -- & 0.019 \\
\hline PEST & Triadimefon & $\mathrm{mg} / \mathrm{Kg}$ & 2 & 0 & $0 \%$ & 0.16 & 0.17 & -- & -- & 0.083 \\
\hline PEST & Triallate & $\mathrm{mg} / \mathrm{Kg}$ & 2 & 0 & $0 \%$ & 0.16 & 0.17 & -- & -- & 0.083 \\
\hline PEST & Trifluralin (Treflan) & $\mathrm{mg} / \mathrm{Kg}$ & 2 & 0 & $0 \%$ & 0.090 & 0.099 & -- & -- & 0.047 \\
\hline PEST & Vernolate & $\mathrm{mg} / \mathrm{Kg}$ & 2 & 0 & $0 \%$ & 0.090 & 0.099 & -- & -- & 0.047 \\
\hline SVOC & 1,2,4-Trichlorobenzene & $\mathrm{mg} / \mathrm{Kg}$ & 1 & 0 & $0 \%$ & 0.010 & 0.010 & -- & -- & 0.0050 \\
\hline SVOC & 1,2-Dichlorobenzene & $\mathrm{mg} / \mathrm{Kg}$ & 1 & 0 & $0 \%$ & 0.010 & 0.010 & -- & -- & 0.0050 \\
\hline SVOC & 1,2-Diphenylhydrazine & $\mathrm{mg} / \mathrm{Kg}$ & 1 & 0 & $0 \%$ & 0.010 & 0.010 & -- & -- & 0.0050 \\
\hline
\end{tabular}


Table C-5

Summary Statistics for Sediment Samples Collected From the Yakima River Tributary of Segment 3 of the Columbia River (units reported "as received")

\begin{tabular}{|c|c|c|c|c|c|c|c|c|c|c|}
\hline $\begin{array}{c}\text { Constituent } \\
\text { Class }\end{array}$ & Constituent Name & Units & $\begin{array}{c}\text { Number } \\
\text { Analyzed }\end{array}$ & $\begin{array}{l}\text { Number } \\
\text { Detected }\end{array}$ & $\begin{array}{l}\text { Frequency of } \\
\text { Detection }\end{array}$ & $\begin{array}{l}\text { Minimum } \\
\text { Nondetect }\end{array}$ & $\begin{array}{l}\text { Maximum } \\
\text { Nondetect }\end{array}$ & $\begin{array}{l}\text { Minimum } \\
\text { Detect }\end{array}$ & $\begin{array}{l}\text { Maximum } \\
\text { Detect }\end{array}$ & $\begin{array}{c}\text { Average } \\
\text { Concentration }\end{array}$ \\
\hline$\overline{\text { SVOC }}$ & 1,3-Dichlorobenzene & $\mathrm{mg} / \mathrm{Kg}$ & 1 & $\overline{0}$ & $0 \%$ & 0.010 & 0.010 & $\overline{--}$ & $\overline{--}$ & 0.0050 \\
\hline sVOC & 1,4-Dichlorobenzene & $\mathrm{mg} / \mathrm{Kg}$ & 1 & 0 & $0 \%$ & 0.010 & 0.010 & -- & -- & 0.0050 \\
\hline SVOC & 2,4,5-Trichlorophenol & $\mathrm{mg} / \mathrm{Kg}$ & 1 & 0 & $0 \%$ & 0.010 & 0.010 & -- & -- & 0.0050 \\
\hline SVOC & 2,4,6-Trichlorophenol & $\mathrm{mg} / \mathrm{Kg}$ & 1 & 0 & $0 \%$ & 0.010 & 0.010 & -- & -- & 0.0050 \\
\hline SVOC & 2,4-Dichlorophenol & $\mathrm{mg} / \mathrm{Kg}$ & 1 & 0 & $0 \%$ & 0.010 & 0.010 & -- & -- & 0.0050 \\
\hline SVOC & 2,4-Dimethylphenol & $\mathrm{mg} / \mathrm{Kg}$ & 1 & 0 & $0 \%$ & 0.010 & 0.010 & -- & -- & 0.0050 \\
\hline SVOC & 2,4-Dinitrophenol & $\mathrm{mg} / \mathrm{Kg}$ & 1 & 0 & $0 \%$ & 0.025 & 0.025 & -- & -- & 0.013 \\
\hline SVOC & 2,4-Dinitrotoluene & $\mathrm{mg} / \mathrm{Kg}$ & 1 & 0 & $0 \%$ & 0.010 & 0.010 & -- & -- & 0.0050 \\
\hline SVOC & 2,6-Dinitrotoluene & $\mathrm{mg} / \mathrm{Kg}$ & 1 & 0 & $0 \%$ & 0.010 & 0.010 & -- & -- & 0.0050 \\
\hline sVOC & 2-Chloronaphthalene & $\mathrm{mg} / \mathrm{Kg}$ & 1 & 0 & $0 \%$ & 0.010 & 0.010 & -- & -- & 0.0050 \\
\hline sVOC & 2-Chlorophenol & $\mathrm{mg} / \mathrm{Kg}$ & 1 & 0 & $0 \%$ & 0.010 & 0.010 & -- & -- & 0.0050 \\
\hline SVOC & 2-Nitroaniline & $\mathrm{mg} / \mathrm{Kg}$ & 1 & 0 & $0 \%$ & 0.010 & 0.010 & -- & -- & 0.0050 \\
\hline sVOC & 2-Nitrophenol & $\mathrm{mg} / \mathrm{Kg}$ & 1 & 0 & $0 \%$ & 0.010 & 0.010 & -- & -- & 0.0050 \\
\hline sVOC & 3,3'-Dichlorobenzidine & $\mathrm{mg} / \mathrm{Kg}$ & 1 & 0 & $0 \%$ & 0.020 & 0.020 & -- & -- & 0.010 \\
\hline sVOC & 3-Nitroaniline & $\mathrm{mg} / \mathrm{Kg}$ & 1 & 0 & $0 \%$ & 0.010 & 0.010 & -- & -- & 0.0050 \\
\hline SVOC & 4,6-Dinitro-2-methylphenol & $\mathrm{mg} / \mathrm{Kg}$ & 1 & 0 & $0 \%$ & 0.025 & 0.025 & -- & -- & 0.013 \\
\hline sVOC & 4-Bromophenylphenyl ether & $\mathrm{mg} / \mathrm{Kg}$ & 1 & 0 & $0 \%$ & 0.010 & 0.010 & -- & -- & 0.0050 \\
\hline sVOC & 4-Chloroaniline & $\mathrm{mg} / \mathrm{Kg}$ & 1 & 0 & $0 \%$ & 0.020 & 0.020 & -- & -- & 0.010 \\
\hline SVOC & 4-Chlorophenylphenyl ether & $\mathrm{mg} / \mathrm{Kg}$ & 1 & 0 & $0 \%$ & 0.010 & 0.010 & -- & -- & 0.0050 \\
\hline SVOC & 4-Methylphenol (cresol, p-) & $\mathrm{mg} / \mathrm{Kg}$ & 1 & 0 & $0 \%$ & 0.010 & 0.010 & -- & -- & 0.0050 \\
\hline sVOC & 4-Nitroaniline & $\mathrm{mg} / \mathrm{Kg}$ & 1 & 0 & $0 \%$ & 0.010 & 0.010 & -- & -- & 0.0050 \\
\hline sVOC & 4-Nitrophenol & $\mathrm{mg} / \mathrm{Kg}$ & 1 & 0 & $0 \%$ & 0.020 & 0.020 & -- & -- & 0.010 \\
\hline sVOC & Acenaphthene & $\mathrm{mg} / \mathrm{Kg}$ & 1 & 0 & $0 \%$ & 0.0050 & 0.0050 & -- & -- & 0.0025 \\
\hline SVOC & Anthracene & $\mathrm{mg} / \mathrm{Kg}$ & 1 & 0 & $0 \%$ & 0.0050 & 0.0050 & -- & -- & 0.0025 \\
\hline sVOC & Benzidine & $\mathrm{mg} / \mathrm{Kg}$ & 1 & 0 & $0 \%$ & 0.050 & 0.050 & -- & -- & 0.025 \\
\hline sVOC & Benzo(ghi)perylene & $\mathrm{mg} / \mathrm{Kg}$ & 1 & 0 & $0 \%$ & 0.010 & 0.010 & -- & -- & 0.0050 \\
\hline sVOC & Benzoic acid & $\mathrm{mg} / \mathrm{Kg}$ & 1 & 0 & $0 \%$ & 0.010 & 0.010 & -- & -- & 0.0050 \\
\hline SVOC & Benzyl alcohol & $\mathrm{mg} / \mathrm{Kg}$ & 1 & 0 & $0 \%$ & 0.010 & 0.010 & -- & -- & 0.0050 \\
\hline svoc & Bis(2-chloro-1-methylethyl)ether & $\mathrm{mg} / \mathrm{Kg}$ & 1 & 0 & $0 \%$ & 0.010 & 0.010 & -- & -- & 0.0050 \\
\hline sVOC & Bis(2-Chloroethoxy)methane & $\mathrm{mg} / \mathrm{Kg}$ & 1 & 0 & $0 \%$ & 0.010 & 0.010 & -- & -- & 0.0050 \\
\hline sVOC & Bis(2-chloroethyl) ether & $\mathrm{mg} / \mathrm{Kg}$ & 1 & 0 & $0 \%$ & 0.010 & 0.010 & -- & -- & 0.0050 \\
\hline SVOC & Dibenz[a,h]anthracene & $\mathrm{mg} / \mathrm{Kg}$ & 1 & 0 & $0 \%$ & 0.010 & 0.010 & -- & -- & 0.0050 \\
\hline sVoc & Dibenzofuran & $\mathrm{mg} / \mathrm{Kg}$ & 1 & 0 & $0 \%$ & 0.025 & 0.025 & -- & -- & 0.013 \\
\hline sVOC & Dimethyl phthalate & $\mathrm{mg} / \mathrm{Kg}$ & 1 & 0 & $0 \%$ & 0.010 & 0.010 & -- & -- & 0.0050 \\
\hline sVOC & Di-n-butylphthalate & $\mathrm{mg} / \mathrm{Kg}$ & 1 & 0 & $0 \%$ & 0.0050 & 0.0050 & -- & -- & 0.0025 \\
\hline SVOC & Fluorene & $\mathrm{mg} / \mathrm{Kg}$ & 1 & 0 & $0 \%$ & 0.010 & 0.010 & -- & -- & 0.0050 \\
\hline sVOC & Hexachlorobenzene & $\mathrm{mg} / \mathrm{Kg}$ & 3 & 0 & $0 \%$ & 0.010 & 0.040 & -- & -- & 0.014 \\
\hline sVOC & Hexachlorobutadiene & $\mathrm{mg} / \mathrm{Kg}$ & 1 & 0 & $0 \%$ & 0.010 & 0.010 & -- & -- & 0.0050 \\
\hline sVOC & Hexachlorocyclopentadiene & $\mathrm{mg} / \mathrm{Kg}$ & 1 & 0 & $0 \%$ & 0.010 & 0.010 & -- & -- & 0.0050 \\
\hline SVOC & Hexachloroethane & $\mathrm{mg} / \mathrm{Kg}$ & 1 & 0 & $0 \%$ & 0.010 & 0.010 & -- & -- & 0.0050 \\
\hline sVOC & Indeno(1,2,3-cd)pyrene & $\mathrm{mg} / \mathrm{Kg}$ & 1 & 0 & $0 \%$ & 0.010 & 0.010 & -- & -- & 0.0050 \\
\hline sVOC & Nitrobenzene & $\mathrm{mg} / \mathrm{Kg}$ & 1 & 0 & $0 \%$ & 0.010 & 0.010 & -- & -- & 0.0050 \\
\hline sVOC & N-Nitrosodi-n-dipropylamine & $\mathrm{mg} / \mathrm{Kg}$ & 1 & 0 & $0 \%$ & 0.020 & 0.020 & -- & -- & 0.010 \\
\hline SVOC & Pentachlorophenol & $\mathrm{mg} / \mathrm{Kg}$ & 3 & 2 & $67 \%$ & 0.020 & 0.020 & 0.016 & 0.023 & 0.016 \\
\hline RAD & Antimony-125 & $\mathrm{pCi} / \mathrm{g}$ & 1 & 0 & $0 \%$ & 0.013 & 0.013 & -- & -- & 0.0067 \\
\hline RAD & Beryllium-7 & $\mathrm{pCi} / \mathrm{g}$ & 2 & 0 & $0 \%$ & 0.016 & 0.23 & -- & -- & 0.063 \\
\hline RAD & Cesium-134 & $\mathrm{pCi} / \mathrm{g}$ & 2 & 0 & $0 \%$ & 0.037 & 0.046 & -- & -- & 0.021 \\
\hline
\end{tabular}


Table C-5

Summary Statistics for Sediment Samples Collected From the Yakima River Tributary of Segment 3 of the Columbia River (units reported "as received")

\begin{tabular}{|c|c|c|c|c|c|c|c|c|c|c|}
\hline $\begin{array}{l}\text { Constituent } \\
\text { Class }\end{array}$ & Constituent Name & Units & $\begin{array}{c}\text { Number } \\
\text { Analyzed }\end{array}$ & $\begin{array}{l}\text { Number } \\
\text { Detected }\end{array}$ & $\begin{array}{l}\text { Frequency of } \\
\text { Detection }\end{array}$ & $\begin{array}{l}\text { Minimum } \\
\text { Nondetect }\end{array}$ & $\begin{array}{l}\text { Maximum } \\
\text { Nondetect }\end{array}$ & $\begin{array}{c}\text { Minimum } \\
\text { Detect }\end{array}$ & $\begin{array}{c}\text { Maximum } \\
\text { Detect }\end{array}$ & $\begin{array}{c}\text { Average } \\
\text { Concentration }\end{array}$ \\
\hline$\overline{A D}$ & Cesium-137 & $\mathrm{pCi} / \mathrm{g}$ & 2 & 0 & $0 \%$ & 0.0099 & 0.031 & -- & -- & 0.010 \\
\hline RAD & Cobalt-60 & $\mathrm{pCi} / \mathrm{g}$ & 2 & 0 & $0 \%$ & 0.0017 & 0.011 & -- & -- & 0.0031 \\
\hline RAD & Europium-152 & $\mathrm{pCi} / \mathrm{g}$ & 1 & 0 & $0 \%$ & 0.020 & 0.020 & -- & -- & 0.010 \\
\hline AD & Europium-154 & $\mathrm{pCi} / \mathrm{g}$ & 1 & 0 & $0 \%$ & 0.0071 & 0.0071 & -- & -- & 0.0036 \\
\hline RAD & Europium-155 & $\mathrm{pCi} / \mathrm{g}$ & 2 & 0 & $0 \%$ & 0.017 & 0.044 & -- & -- & 0.015 \\
\hline RAD & Potassium-40 & $\mathrm{pCi} / \mathrm{g}$ & 2 & 2 & $100 \%$ & -- & -- & 11 & 12 & 11 \\
\hline RAD & Technetium-99 & $\mathrm{pCi} / \mathrm{g}$ & 2 & 0 & $0 \%$ & 0.18 & 0.26 & -- & -- & 0.11 \\
\hline RAD_D & Potassium-40, Decayed & $\mathrm{pCi} / \mathrm{g}$ & 2 & 2 & $100 \%$ & -- & -- & 11 & 11 & 11 \\
\hline
\end{tabular}

CONV - Conventional parameter

PEST - Pesticide

PCB - Polychlorinated bipheny

SVOC - Semivolatile Organic Compound 
Table C-6

Summary Statistics for Sediment Samples Collected From the Yakima River Tributary of Segment 3 of the Columbia River (units reported "dry-weight")

\begin{tabular}{|c|c|c|c|c|c|c|c|c|c|c|}
\hline $\begin{array}{l}\text { Constituent } \\
\text { Class }\end{array}$ & Constituent Name & Units & $\begin{array}{l}\text { Number } \\
\text { Analyzed }\end{array}$ & $\begin{array}{l}\text { Number } \\
\text { Detected }\end{array}$ & $\begin{array}{l}\text { Frequency of } \\
\text { Detection }\end{array}$ & $\begin{array}{l}\text { Minimum } \\
\text { Nondetect }\end{array}$ & $\begin{array}{l}\text { Maximum } \\
\text { Nondetect }\end{array}$ & $\begin{array}{l}\text { Minimum } \\
\text { Detect }\end{array}$ & $\begin{array}{l}\text { Maximum } \\
\text { Detect }\end{array}$ & $\begin{array}{c}\text { Average } \\
\text { Concentration }\end{array}$ \\
\hline CONV & Total Organic Carbon & $\mathrm{mg} / \mathrm{kg}$ dry-wt & 1 & 1 & $100 \%$ & -- & -- & 1.0 & 1.0 & 1.0 \\
\hline CONV & Total solids & $\mathrm{mg} / \mathrm{kg}$ dry-wt & 1 & 1 & $100 \%$ & -- & -- & 50 & 50 & 50 \\
\hline METAL & Arsenic & $\mathrm{mg} / \mathrm{kg}$ dry-wt & 1 & 1 & $100 \%$ & -- & -- & 2.8 & 2.8 & 2.8 \\
\hline METAL & Cadmium & $\mathrm{mg} / \mathrm{kg}$ dry-wt & 1 & 1 & $100 \%$ & -- & -- & 1.4 & 1.4 & 1.4 \\
\hline METAL & Chromium & $\mathrm{mg} / \mathrm{kg}$ dry-wt & 1 & 0 & $0 \%$ & 0.10 & 0.10 & -- & -- & 0.050 \\
\hline METAL & Copper & $\mathrm{mg} / \mathrm{kg}$ dry-wt & 1 & 1 & $100 \%$ & -- & -- & 36 & 36 & 36 \\
\hline METAL & Lead & $\mathrm{mg} / \mathrm{kg}$ dry-wt & 1 & 1 & $100 \%$ & -- & -- & 4.2 & 4.2 & 4.2 \\
\hline METAL & Mercury & $\mathrm{mg} / \mathrm{kg}$ dry-wt & 2 & 2 & $100 \%$ & -- & -- & 0.022 & 0.038 & 0.030 \\
\hline METAL & Zinc & $\mathrm{mg} / \mathrm{kg}$ dry-wt & 1 & 1 & $100 \%$ & -- & -- & 83 & 83 & 83 \\
\hline PCB & Aroclor-1260 & $\mathrm{mg} / \mathrm{kg}$ dry-wt & 2 & 0 & $0 \%$ & 0.0015 & 0.010 & -- & -- & 0.0029 \\
\hline PCB & Total polychlorinated biphenyl & $\mathrm{mg} / \mathrm{kg}$ dry-wt & 2 & 0 & $0 \%$ & $1.00 \mathrm{E}-04$ & 0.0010 & -- & -- & $2.75 \mathrm{E}-04$ \\
\hline PEST & 2,4'-DDT & $\mathrm{mg} / \mathrm{kg}$ dry-wt & 1 & 1 & $100 \%$ & -- & -- & $7.10 \mathrm{E}-04$ & 7.10E-04 & 7.10E-04 \\
\hline PEST & Aldrin & $\mathrm{mg} / \mathrm{kg}$ dry-wt & 3 & 0 & $0 \%$ & $1.00 \mathrm{E}-04$ & $1.00 \mathrm{E}-04$ & -- & -- & $5.00 \mathrm{E}-05$ \\
\hline PEST & Alpha-BHC & $\mathrm{mg} / \mathrm{kg}$ dry-wt & 1 & 0 & $0 \%$ & 0.0010 & 0.0010 & -- & -- & $5.00 \mathrm{E}-04$ \\
\hline PEST & Dichlorodiphenyldichloroethane & $\mathrm{mg} / \mathrm{kg}$ dry-wt & 4 & 4 & $100 \%$ & -- & -- & 0.0010 & 0.023 & 0.0077 \\
\hline PEST & Dichlorodiphenyldichloroethylene & $\mathrm{mg} / \mathrm{kg}$ dry-wt & 4 & 4 & $100 \%$ & -- & -- & 0.0027 & 0.051 & 0.022 \\
\hline PEST & Dichlorodiphenyltrichloroethane & $\mathrm{mg} / \mathrm{kg}$ dry-wt & 4 & 4 & $100 \%$ & -- & -- & 3.00E-04 & 0.035 & 0.012 \\
\hline PEST & Dieldrin & $\mathrm{mg} / \mathrm{kg}$ dry-wt & 3 & 3 & $100 \%$ & -- & -- & 2.00E-04 & 0.0050 & 0.0023 \\
\hline PEST & Endosulfan I & $\mathrm{mg} / \mathrm{kg}$ dry-wt & 2 & 0 & $0 \%$ & $1.00 \mathrm{E}-04$ & $1.00 \mathrm{E}-04$ & -- & -- & $5.00 \mathrm{E}-05$ \\
\hline PEST & Endrin & $\mathrm{mg} / \mathrm{kg}$ dry-wt & 2 & 0 & $0 \%$ & $1.00 \mathrm{E}-04$ & $1.00 \mathrm{E}-04$ & -- & -- & $5.00 \mathrm{E}-05$ \\
\hline PEST & Gamma-BHC (Lindane) & $\mathrm{mg} / \mathrm{kg}$ dry-wt & 1 & 0 & $0 \%$ & $1.00 \mathrm{E}-04$ & $1.00 \mathrm{E}-04$ & -- & -- & $5.00 \mathrm{E}-05$ \\
\hline PEST & Heptachlor & $\mathrm{mg} / \mathrm{kg}$ dry-wt & 2 & 0 & $0 \%$ & $1.00 \mathrm{E}-04$ & $1.00 \mathrm{E}-04$ & -- & -- & $5.00 \mathrm{E}-05$ \\
\hline PEST & Heptachlor epoxide & $\mathrm{mg} / \mathrm{kg}$ dry-wt & 2 & 0 & $0 \%$ & $1.00 \mathrm{E}-04$ & $1.00 \mathrm{E}-04$ & -- & -- & $5.00 \mathrm{E}-05$ \\
\hline PEST & Methoxychlor & $\mathrm{mg} / \mathrm{kg}$ dry-wt & 2 & 0 & $0 \%$ & $1.00 \mathrm{E}-04$ & $1.00 \mathrm{E}-04$ & -- & -- & $5.00 \mathrm{E}-05$ \\
\hline PEST & Mirex & $\mathrm{mg} / \mathrm{kg}$ dry-wt & 2 & 0 & $0 \%$ & $1.00 \mathrm{E}-04$ & $1.00 \mathrm{E}-04$ & -- & -- & $5.00 \mathrm{E}-05$ \\
\hline PEST & Perthane & $\mathrm{mg} / \mathrm{kg}$ dry-wt & 2 & 0 & $0 \%$ & 0.0010 & 0.0010 & -- & -- & $5.00 \mathrm{E}-04$ \\
\hline PEST & Toxaphene & $\mathrm{mg} / \mathrm{kg}$ dry-wt & 2 & 0 & $0 \%$ & 0.0010 & 0.010 & -- & -- & 0.0028 \\
\hline sVOC & 1,2,4-Trichlorobenzene & $\mathrm{mg} / \mathrm{kg}$ dry-wt & 3 & 0 & $0 \%$ & 0.20 & 0.20 & -- & -- & 0.10 \\
\hline SVOC & 1,2-Dichlorobenzene & $\mathrm{mg} / \mathrm{kg}$ dry-wt & 3 & 0 & $0 \%$ & 0.20 & 0.20 & -- & -- & 0.10 \\
\hline SVOC & 1,3-Dichlorobenzene & $\mathrm{mg} / \mathrm{kg}$ dry-wt & 3 & 0 & $0 \%$ & 0.20 & 0.20 & -- & -- & 0.10 \\
\hline sVOC & 1,4-Dichlorobenzene & $\mathrm{mg} / \mathrm{kg}$ dry-wt & 3 & 0 & $0 \%$ & 0.20 & 0.20 & -- & -- & 0.10 \\
\hline SVOC & 2,4,6-Trichlorophenol & $\mathrm{mg} / \mathrm{kg}$ dry-wt & 3 & 0 & $0 \%$ & 0.60 & 0.60 & -- & -- & 0.30 \\
\hline sVOC & 2,4-Dichlorophenol & $\mathrm{mg} / \mathrm{kg}$ dry-wt & 3 & 0 & $0 \%$ & 0.20 & 0.20 & -- & -- & 0.10 \\
\hline SVOC & 2,4-Dimethylphenol & $\mathrm{mg} / \mathrm{kg}$ dry-wt & 3 & 0 & $0 \%$ & 0.20 & 0.20 & -- & -- & 0.10 \\
\hline SVOC & 2,4-Dinitrophenol & $\mathrm{mg} / \mathrm{kg}$ dry-wt & 3 & 0 & $0 \%$ & 0.60 & 0.60 & -- & -- & 0.30 \\
\hline SVOC & 2,4-Dinitrotoluene & $\mathrm{mg} / \mathrm{kg}$ dry-wt & 3 & 0 & $0 \%$ & 0.20 & 0.20 & -- & -- & 0.10 \\
\hline SVOC & 2,6-Dinitrotoluene & $\mathrm{mg} / \mathrm{kg}$ dry-wt & 3 & 0 & $0 \%$ & 0.20 & 0.20 & -- & -- & 0.10 \\
\hline SVOC & 2-Chloronaphthalene & $\mathrm{mg} / \mathrm{kg}$ dry-wt & 3 & 0 & $0 \%$ & 0.20 & 0.20 & -- & -- & 0.10 \\
\hline SVOC & 2-Chlorophenol & $\mathrm{mg} / \mathrm{kg}$ dry-wt & 3 & 0 & $0 \%$ & 0.20 & 0.20 & -- & -- & 0.10 \\
\hline SVOC & 2-Methylnaphthalene & $\mathrm{mg} / \mathrm{kg}$ dry-wt & 1 & 1 & $100 \%$ & -- & -- & 0.010 & 0.010 & 0.010 \\
\hline SVOC & 2-Methylphenol (cresol, o-) & $\mathrm{mg} / \mathrm{kg}$ dry-wt & 1 & 1 & $100 \%$ & -- & -- & 0.81 & 0.81 & 0.81 \\
\hline SVOC & 2-Nitrophenol & $\mathrm{mg} / \mathrm{kg}$ dry-wt & 3 & 0 & $0 \%$ & 0.20 & 0.20 & -- & -- & 0.10 \\
\hline SVOC & 4,6-Dinitro-2-methylphenol & $\mathrm{mg} / \mathrm{kg}$ dry-wt & 3 & 0 & $0 \%$ & 0.60 & 0.60 & -- & -- & 0.30 \\
\hline sVOC & 4-Bromophenylphenyl ether & $\mathrm{mg} / \mathrm{kg}$ dry-wt & 3 & 0 & $0 \%$ & 0.20 & 0.20 & -- & -- & 0.10 \\
\hline sVOC & 4-Chloro-3-methylphenol & $\mathrm{mg} / \mathrm{kg}$ dry-wt & 3 & 0 & $0 \%$ & 0.60 & 0.60 & -- & -- & 0.30 \\
\hline SVOC & 4-Chlorophenylphenyl ether & $\mathrm{mg} / \mathrm{kg}$ dry-wt & 3 & 0 & $0 \%$ & 0.20 & 0.20 & -- & -- & 0.10 \\
\hline
\end{tabular}


Table C-6

Summary Statistics for Sediment Samples Collected From the Yakima River Tributary of Segment 3 of the Columbia River (units reported "dry-weight")

\begin{tabular}{|c|c|c|c|c|c|c|c|c|c|c|}
\hline $\begin{array}{l}\text { Constituent } \\
\text { Class }\end{array}$ & Constituent Name & Units & $\begin{array}{c}\text { Number } \\
\text { Analyzed }\end{array}$ & $\begin{array}{l}\text { Number } \\
\text { Detected }\end{array}$ & $\begin{array}{l}\text { Frequency of } \\
\text { Detection }\end{array}$ & $\begin{array}{c}\text { Minimum } \\
\text { Nondetect }\end{array}$ & $\begin{array}{l}\text { Maximum } \\
\text { Nondetect }\end{array}$ & $\begin{array}{c}\text { Minimum } \\
\text { Detect }\end{array}$ & $\begin{array}{c}\text { Maximum } \\
\text { Detect }\end{array}$ & $\begin{array}{c}\text { Average } \\
\text { Concentration }\end{array}$ \\
\hline$\overline{\text { SVOC }}$ & 4-Nitrophenol & $\mathrm{mg} / \mathrm{kg}$ dry-wt & 3 & $\overline{0}$ & $0 \%$ & 0.60 & 0.60 & $\overline{--}$ & $\overline{--}$ & 0.30 \\
\hline SVOC & Acenaphthene & $\mathrm{mg} / \mathrm{kg}$ dry-wt & 3 & 0 & $0 \%$ & 0.20 & 0.20 & -- & -- & 0.10 \\
\hline sVOC & Acenaphthylene & $\mathrm{mg} / \mathrm{kg}$ dry-wt & 4 & 0 & $0 \%$ & 0.010 & 0.20 & -- & -- & 0.076 \\
\hline sVOC & Anthracene & $\mathrm{mg} / \mathrm{kg}$ dry-wt & 3 & 0 & $0 \%$ & 0.20 & 0.20 & -- & -- & 0.10 \\
\hline sVOC & Benzo(a)anthracene & $\mathrm{mg} / \mathrm{kg}$ dry-wt & 4 & 0 & $0 \%$ & 0.0050 & 0.40 & -- & -- & 0.15 \\
\hline SVOC & Benzo(a)pyrene & $\mathrm{mg} / \mathrm{kg}$ dry-wt & 4 & 0 & $0 \%$ & 0.010 & 0.40 & -- & -- & 0.15 \\
\hline sVOC & Benzo(b)fluoranthene & $\mathrm{mg} / \mathrm{kg}$ dry-wt & 3 & 0 & $0 \%$ & 0.40 & 0.40 & -- & -- & 0.20 \\
\hline SVOC & Benzo(ghi)perylene & $\mathrm{mg} / \mathrm{kg}$ dry-wt & 3 & 0 & $0 \%$ & 0.40 & 0.40 & -- & -- & 0.20 \\
\hline SVOC & Benzo(k)fluoranthene & $\mathrm{mg} / \mathrm{kg}$ dry-wt & 4 & 0 & $0 \%$ & 0.050 & 0.40 & -- & -- & 0.16 \\
\hline SVOC & Bis(2-chloro-1-methylethyl)ether & $\mathrm{mg} / \mathrm{kg}$ dry-wt & 3 & 0 & $0 \%$ & 0.20 & 0.20 & -- & -- & 0.10 \\
\hline SVOC & Bis(2-Chloroethoxy)methane & $\mathrm{mg} / \mathrm{kg}$ dry-wt & 3 & 0 & $0 \%$ & 0.20 & 0.20 & -- & -- & 0.10 \\
\hline SVOC & Bis(2-chloroethyl) ether & $\mathrm{mg} / \mathrm{kg}$ dry-wt & 3 & 0 & $0 \%$ & 0.20 & 0.20 & -- & -- & 0.10 \\
\hline SVOC & Bis(2-ethylhexyl) phthalate & $\mathrm{mg} / \mathrm{kg}$ dry-wt & 4 & 2 & $50 \%$ & 0.20 & 0.20 & 0.053 & 0.61 & 0.22 \\
\hline SVOC & Butylbenzylphthalate & $\mathrm{mg} / \mathrm{kg}$ dry-wt & 4 & 0 & $0 \%$ & 0.0050 & 0.20 & -- & -- & 0.076 \\
\hline SVOC & Chrysene & $\mathrm{mg} / \mathrm{kg}$ dry-wt & 4 & 1 & $25 \%$ & 0.40 & 0.40 & 0.013 & 0.013 & 0.15 \\
\hline SVOC & Dibenz[a,h]anthracene & $\mathrm{mg} / \mathrm{kg}$ dry-wt & 3 & 0 & $0 \%$ & 0.40 & 0.40 & -- & -- & 0.20 \\
\hline SVOC & Diethylphthalate & $\mathrm{mg} / \mathrm{kg}$ dry-wt & 4 & 1 & $25 \%$ & 0.20 & 0.20 & 0.010 & 0.010 & 0.078 \\
\hline SVOC & Dimethyl phthalate & $\mathrm{mg} / \mathrm{kg}$ dry-wt & 3 & 0 & $0 \%$ & 0.20 & 0.20 & -- & -- & 0.10 \\
\hline SVOC & Di-n-butylphthalate & $\mathrm{mg} / \mathrm{kg}$ dry-wt & 3 & 0 & $0 \%$ & 0.20 & 0.20 & -- & -- & 0.10 \\
\hline SVOC & Di-n-octylphthalate & $\mathrm{mg} / \mathrm{kg}$ dry-wt & 4 & 0 & $0 \%$ & 0.0050 & 0.40 & -- & -- & 0.15 \\
\hline SVOC & Fluoranthene & $\mathrm{mg} / \mathrm{kg}$ dry-wt & 4 & 2 & $50 \%$ & 0.20 & 0.20 & 0.0040 & 0.015 & 0.055 \\
\hline SVOC & Fluorene & $\mathrm{mg} / \mathrm{kg}$ dry-wt & 3 & 0 & $0 \%$ & 0.20 & 0.20 & -- & -- & 0.10 \\
\hline SVOC & Hexachlorobenzene & $\mathrm{mg} / \mathrm{kg}$ dry-wt & 3 & 0 & $0 \%$ & 0.20 & 0.20 & -- & -- & 0.10 \\
\hline SVOC & Hexachlorobutadiene & $\mathrm{mg} / \mathrm{kg}$ dry-wt & 3 & 0 & $0 \%$ & 0.20 & 0.20 & -- & -- & 0.10 \\
\hline SVOC & Hexachlorocyclopentadiene & $\mathrm{mg} / \mathrm{kg}$ dry-wt & 3 & 0 & $0 \%$ & 0.20 & 0.20 & -- & -- & 0.10 \\
\hline SVOC & Hexachloroethane & $\mathrm{mg} / \mathrm{kg}$ dry-wt & 3 & 0 & $0 \%$ & 0.20 & 0.20 & -- & -- & 0.10 \\
\hline SVOC & Indeno(1,2,3-cd)pyrene & $\mathrm{mg} / \mathrm{kg}$ dry-wt & 3 & 0 & $0 \%$ & 0.40 & 0.40 & -- & -- & 0.20 \\
\hline SVOC & Isophorone & $\mathrm{mg} / \mathrm{kg}$ dry-wt & 4 & 1 & $25 \%$ & 0.010 & 0.20 & 0.015 & 0.015 & 0.055 \\
\hline SVOC & Naphthalene & $\mathrm{mg} / \mathrm{kg}$ dry-wt & 4 & 3 & $75 \%$ & 0.20 & 0.20 & 0.0020 & 0.015 & 0.031 \\
\hline SVOC & Nitrobenzene & $\mathrm{mg} / \mathrm{kg}$ dry-wt & 3 & 0 & $0 \%$ & 0.20 & 0.20 & -- & -- & 0.10 \\
\hline SVOC & N-Nitrosodimethylamine & $\mathrm{mg} / \mathrm{kg}$ dry-wt & 3 & 0 & $0 \%$ & 0.20 & 0.20 & -- & -- & 0.10 \\
\hline SVOC & N-Nitrosodi-n-dipropylamine & $\mathrm{mg} / \mathrm{kg}$ dry-wt & 3 & 0 & $0 \%$ & 0.20 & 0.20 & -- & -- & 0.10 \\
\hline sVOC & N-Nitrosodiphenylamine & $\mathrm{mg} / \mathrm{kg}$ dry-wt & 3 & 0 & $0 \%$ & 0.20 & 0.20 & -- & -- & 0.10 \\
\hline SVOC & Pentachlorophenol & $\mathrm{mg} / \mathrm{kg}$ dry-wt & 3 & 0 & $0 \%$ & 0.60 & 0.60 & -- & -- & 0.30 \\
\hline SVOC & Phenanthrene & $\mathrm{mg} / \mathrm{kg}$ dry-wt & 4 & 2 & $50 \%$ & 0.20 & 0.20 & 0.0040 & 0.025 & 0.057 \\
\hline sVOC & Phenol & $\mathrm{mg} / \mathrm{kg}$ dry-wt & 4 & 3 & $75 \%$ & 0.20 & 0.20 & 0.035 & 0.10 & 0.075 \\
\hline SVOC & Pyrene & $\mathrm{mg} / \mathrm{kg}$ dry-wt & 4 & 2 & $50 \%$ & 0.20 & 0.20 & 0.0030 & 0.016 & 0.055 \\
\hline
\end{tabular}

CONV - Conventional parameter

PEST - Pesticide

PCB - Polychlorinated biphenyl

SVOC - Semivolatile Organic Compound 
Table C-7

Summary Statistics for Sediment Samples Collected From the Walla Walla River Tributary of Segment 3 of the Columbia River (units reported "as received")

\begin{tabular}{|c|c|c|c|c|c|c|c|c|c|c|}
\hline $\begin{array}{l}\text { Constituent } \\
\text { Class }\end{array}$ & Constituent Name & Units & $\begin{array}{l}\text { Number } \\
\text { Analyzed }\end{array}$ & $\begin{array}{l}\text { Number } \\
\text { Detected }\end{array}$ & $\begin{array}{l}\text { Frequency of } \\
\text { Detection }\end{array}$ & $\begin{array}{l}\text { Minimum } \\
\text { Nondetect }\end{array}$ & $\begin{array}{l}\text { Maximum } \\
\text { Nondetect }\end{array}$ & $\begin{array}{c}\text { Minimum } \\
\text { Detect }\end{array}$ & $\begin{array}{c}\text { Maximum } \\
\text { Detect }\end{array}$ & $\begin{array}{c}\text { Average } \\
\text { Concentration }\end{array}$ \\
\hline$\overline{\text { CONV }}$ & Percent Solids & $\%$ & 1 & 1 & $100 \%$ & $\overline{--}$ & $\overline{--}$ & 68 & 68 & 68 \\
\hline CONV & Total Organic Carbon & $\%$ & 4 & 4 & $100 \%$ & -- & -- & 0.80 & 1.1 & 0.92 \\
\hline CONV & Total Organic Carbon & $\mathrm{mg} / \mathrm{Kg}$ & 3 & 3 & $100 \%$ & -- & -- & 2.0 & 7,880 & 3,297 \\
\hline CONV & Total solids & $\mathrm{mg} / \mathrm{Kg}$ & 1 & 1 & $100 \%$ & -- & -- & 46 & 46 & 46 \\
\hline METAL & Antimony & $\mathrm{mg} / \mathrm{Kg}$ & 2 & 2 & $100 \%$ & -- & -- & 0.31 & 0.38 & 0.35 \\
\hline METAL & Arsenic & $\mathrm{mg} / \mathrm{kg}$ & 3 & 3 & $100 \%$ & -- & -- & 2.4 & 4.6 & 3.3 \\
\hline METAL & Beryllium & $\mathrm{mg} / \mathrm{Kg}$ & 2 & 2 & $100 \%$ & -- & -- & 1.1 & 1.1 & 1.1 \\
\hline METAL & Cadmium & $\mathrm{mg} / \mathrm{kg}$ & 3 & 2 & $67 \%$ & 0.50 & 0.50 & 0.11 & 0.12 & 0.16 \\
\hline METAL & Chromium & $\mathrm{mg} / \mathrm{kg}$ & 3 & 3 & $100 \%$ & -- & -- & 11 & 30 & 23 \\
\hline METAL & Copper & $\mathrm{mg} / \mathrm{kg}$ & 3 & 3 & $100 \%$ & -- & -- & 11 & 15 & 13 \\
\hline METAL & Lead & $\mathrm{mg} / \mathrm{kg}$ & 3 & 3 & $100 \%$ & -- & -- & 5.8 & 9.0 & 7.7 \\
\hline METAL & Mercury & $\mathrm{mg} / \mathrm{kg}$ & 3 & 2 & $67 \%$ & 0.040 & 0.040 & 0.0078 & 0.0099 & 0.013 \\
\hline METAL & Molybdenum & $\mathrm{mg} / \mathrm{kg}$ & 1 & 0 & $0 \%$ & 0.60 & 0.60 & -- & -- & 0.30 \\
\hline METAL & Nickel & $\mathrm{mg} / \mathrm{kg}$ & 3 & 3 & $100 \%$ & -- & -- & 8.9 & 12 & 11 \\
\hline METAL & Selenium & $\mathrm{mg} / \mathrm{kg}$ & 3 & 0 & $0 \%$ & 0.46 & 5.0 & -- & -- & 0.99 \\
\hline METAL & Silver & $\mathrm{mg} / \mathrm{Kg}$ & 2 & 2 & $100 \%$ & -- & -- & 0.41 & 0.46 & 0.44 \\
\hline METAL & Thallium & $\mathrm{mg} / \mathrm{Kg}$ & 2 & 2 & $100 \%$ & -- & -- & 0.26 & 0.27 & 0.27 \\
\hline METAL & Uranium & $\mathrm{mg} / \mathrm{Kg}$ & 2 & 2 & $100 \%$ & -- & -- & 1.5 & 1.5 & 1.5 \\
\hline METAL & Zinc & $\mathrm{mg} / \mathrm{kg}$ & 3 & 3 & $100 \%$ & -- & -- & 46 & 66 & 59 \\
\hline PCB & Aroclor-1242 & $\mathrm{mg} / \mathrm{Kg}$ & 1 & 0 & $0 \%$ & 0.13 & 0.13 & -- & -- & 0.065 \\
\hline РСB & Aroclor-1248 & $\mathrm{mg} / \mathrm{Kg}$ & 1 & 0 & $0 \%$ & 0.13 & 0.13 & -- & -- & 0.065 \\
\hline РCB & Aroclor-1254 & $\mathrm{mg} / \mathrm{Kg}$ & 1 & 0 & $0 \%$ & 0.13 & 0.13 & -- & -- & 0.065 \\
\hline PCB & Aroclor-1260 & $\mathrm{mg} / \mathrm{Kg}$ & 1 & 0 & $0 \%$ & 0.13 & 0.13 & -- & -- & 0.065 \\
\hline PEST & 2,4'-DDD & $\mathrm{mg} / \mathrm{Kg}$ & 1 & 0 & $0 \%$ & 0.038 & 0.038 & -- & -- & 0.019 \\
\hline PEST & 2,4'-DDE & $\mathrm{mg} / \mathrm{Kg}$ & 1 & 0 & $0 \%$ & 0.038 & 0.038 & -- & -- & 0.019 \\
\hline PEST & 2,4'-DDT & $\mathrm{mg} / \mathrm{Kg}$ & 1 & 0 & $0 \%$ & 0.038 & 0.038 & -- & -- & 0.019 \\
\hline PEST & Alachlor & $\mathrm{mg} / \mathrm{Kg}$ & 1 & 0 & $0 \%$ & 0.15 & 0.15 & -- & -- & 0.075 \\
\hline PEST & Aldrin & $\mathrm{mg} / \mathrm{Kg}$ & 2 & 0 & $0 \%$ & 0.0010 & 0.038 & -- & -- & 0.0098 \\
\hline PEST & Alpha-BHC & $\mathrm{mg} / \mathrm{Kg}$ & 1 & 0 & $0 \%$ & 0.038 & 0.038 & -- & -- & 0.019 \\
\hline PEST & alpha-Chlordane & $\mathrm{mg} / \mathrm{Kg}$ & 1 & 0 & $0 \%$ & 0.038 & 0.038 & -- & -- & 0.019 \\
\hline PEST & alpha-Chlordene & $\mathrm{mg} / \mathrm{Kg}$ & 1 & 0 & $0 \%$ & 0.038 & 0.038 & -- & -- & 0.019 \\
\hline PEST & Ametryn & $\mathrm{mg} / \mathrm{Kg}$ & 1 & 0 & $0 \%$ & 0.064 & 0.064 & -- & -- & 0.032 \\
\hline PEST & Atraton & $\mathrm{mg} / \mathrm{Kg}$ & 1 & 0 & $0 \%$ & 0.19 & 0.19 & -- & -- & 0.095 \\
\hline PEST & Atrazine & $\mathrm{mg} / \mathrm{Kg}$ & 1 & 0 & $0 \%$ & 0.064 & 0.064 & -- & -- & 0.032 \\
\hline PEST & Azinphos Ethyl & $\mathrm{mg} / \mathrm{Kg}$ & 1 & 0 & $0 \%$ & 0.10 & 0.10 & -- & -- & 0.050 \\
\hline PEST & Azinphos Methyl & $\mathrm{mg} / \mathrm{Kg}$ & 1 & 0 & $0 \%$ & 0.11 & 0.11 & -- & -- & 0.055 \\
\hline PEST & Benefin (Benfluralin) & $\mathrm{mg} / \mathrm{Kg}$ & 1 & 0 & $0 \%$ & 0.096 & 0.096 & -- & -- & 0.048 \\
\hline PEST & beta-1,2,3,4,5,6-Hexachlorocyclohexane & $\mathrm{mg} / \mathrm{Kg}$ & 1 & 0 & $0 \%$ & 0.038 & 0.038 & -- & -- & 0.019 \\
\hline PEST & Bromacil (ACN) & $\mathrm{mg} / \mathrm{Kg}$ & 1 & 0 & $0 \%$ & 0.38 & 0.38 & -- & -- & 0.19 \\
\hline PEST & Butachlor & $\mathrm{mg} / \mathrm{Kg}$ & 1 & 0 & $0 \%$ & 0.22 & 0.22 & -- & -- & 0.11 \\
\hline PEST & Butylate & $\mathrm{mg} / \mathrm{Kg}$ & 1 & 0 & $0 \%$ & 0.096 & 0.096 & -- & -- & 0.048 \\
\hline PEST & Carbethoxy malathion & $\mathrm{mg} / \mathrm{Kg}$ & 1 & 0 & $0 \%$ & 0.051 & 0.051 & -- & -- & 0.026 \\
\hline PEST & Carboxin & $\mathrm{mg} / \mathrm{Kg}$ & 1 & 0 & $0 \%$ & 0.70 & 0.70 & -- & -- & 0.35 \\
\hline PEST & Chlorothalonil & $\mathrm{mg} / \mathrm{Kg}$ & 1 & 0 & $0 \%$ & 0.15 & 0.15 & -- & -- & 0.075 \\
\hline PEST & Chlorpropham & $\mathrm{mg} / \mathrm{Kg}$ & 1 & 0 & $0 \%$ & 0.32 & 0.32 & -- & -- & 0.16 \\
\hline PEST & Chlorpyrifos & $\mathrm{mg} / \mathrm{Kg}$ & 1 & 0 & $0 \%$ & 0.045 & 0.045 & -- & -- & 0.023 \\
\hline PEST & Cyanazine & $\mathrm{mg} / \mathrm{Kg}$ & 1 & 0 & $0 \%$ & 0.096 & 0.096 & -- & -- & 0.048 \\
\hline
\end{tabular}


Table C-7

Summary Statistics for Sediment Samples Collected From the Walla Walla River Tributary of Segment 3 of the Columbia River (units reported "as received")

\begin{tabular}{|c|c|c|c|c|c|c|c|c|c|c|}
\hline $\begin{array}{l}\text { Constituent } \\
\text { Class }\end{array}$ & Constituent Name & Units & $\begin{array}{l}\text { Number } \\
\text { Analyzed }\end{array}$ & $\begin{array}{l}\text { Number } \\
\text { Detected }\end{array}$ & $\begin{array}{l}\text { Frequency of } \\
\text { Detection }\end{array}$ & $\begin{array}{l}\text { Minimum } \\
\text { Nondetect }\end{array}$ & $\begin{array}{l}\text { Maximum } \\
\text { Nondetect }\end{array}$ & $\begin{array}{c}\text { Minimum } \\
\text { Detect }\end{array}$ & $\begin{array}{c}\text { Maximum } \\
\text { Detect }\end{array}$ & $\begin{array}{c}\text { Average } \\
\text { Concentration }\end{array}$ \\
\hline$\overline{\mathrm{PEST}}$ & Cycloate & $\mathrm{mg} / \mathrm{Kg}$ & 1 & 0 & $0 \%$ & 0.096 & 0.096 & $\overline{--}$ & $\overline{--}$ & 0.048 \\
\hline PEST & DDMU & $\mathrm{mg} / \mathrm{Kg}$ & 1 & 0 & $0 \%$ & 0.038 & 0.038 & -- & -- & 0.019 \\
\hline PEST & Delta-BHC & $\mathrm{mg} / \mathrm{Kg}$ & 1 & 0 & $0 \%$ & 0.038 & 0.038 & -- & -- & 0.019 \\
\hline PEST & Diazinon & $\mathrm{mg} / \mathrm{Kg}$ & 1 & 0 & $0 \%$ & 0.051 & 0.051 & -- & -- & 0.026 \\
\hline PEST & Dichlobenil & $\mathrm{mg} / \mathrm{Kg}$ & 1 & 0 & $0 \%$ & 0.076 & 0.076 & -- & -- & 0.038 \\
\hline PEST & Dichlorodiphenyldichloroethane & $\mathrm{mg} / \mathrm{Kg}$ & 1 & 0 & $0 \%$ & 0.038 & 0.038 & -- & -- & 0.019 \\
\hline PEST & Dichlorodiphenyldichloroethylene & $\mathrm{mg} / \mathrm{Kg}$ & 1 & 1 & $100 \%$ & -- & -- & 0.0080 & 0.0080 & 0.0080 \\
\hline PEST & Dichlorodiphenyltrichloroethane & $\mathrm{mg} / \mathrm{Kg}$ & 1 & 0 & $0 \%$ & 0.038 & 0.038 & -- & -- & 0.019 \\
\hline PEST & Dicofol & $\mathrm{mg} / \mathrm{Kg}$ & 1 & 0 & $0 \%$ & 0.15 & 0.15 & -- & -- & 0.075 \\
\hline PEST & Dieldrin & $\mathrm{mg} / \mathrm{Kg}$ & 2 & 0 & $0 \%$ & 0.0010 & 0.038 & -- & -- & 0.0098 \\
\hline PEST & Dimethoate & $\mathrm{mg} / \mathrm{Kg}$ & 1 & 0 & $0 \%$ & 0.051 & 0.051 & -- & -- & 0.026 \\
\hline PEST & Diphenamid & $\mathrm{mg} / \mathrm{Kg}$ & 1 & 0 & $0 \%$ & 0.19 & 0.19 & -- & -- & 0.095 \\
\hline PEST & Disulfoton & $\mathrm{mg} / \mathrm{Kg}$ & 1 & 0 & $0 \%$ & 0.038 & 0.038 & -- & -- & 0.019 \\
\hline PEST & Diuron & $\mathrm{mg} / \mathrm{Kg}$ & 1 & 0 & $0 \%$ & 0.51 & 0.51 & -- & -- & 0.26 \\
\hline PEST & Endosulfan I & $\mathrm{mg} / \mathrm{Kg}$ & 1 & 0 & $0 \%$ & 0.038 & 0.038 & -- & -- & 0.019 \\
\hline PEST & Endosulfan II & $\mathrm{mg} / \mathrm{Kg}$ & 1 & 0 & $0 \%$ & 0.038 & 0.038 & -- & -- & 0.019 \\
\hline PEST & Endosulfan sulfate & $\mathrm{mg} / \mathrm{Kg}$ & 1 & 0 & $0 \%$ & 0.038 & 0.038 & -- & -- & 0.019 \\
\hline PEST & Endrin & $\mathrm{mg} / \mathrm{Kg}$ & 1 & 0 & $0 \%$ & 0.036 & 0.036 & -- & -- & 0.018 \\
\hline PEST & Endrin aldehyde & $\mathrm{mg} / \mathrm{Kg}$ & 1 & 0 & $0 \%$ & 0.038 & 0.038 & -- & -- & 0.019 \\
\hline PEST & Endrin ketone & $\mathrm{mg} / \mathrm{Kg}$ & 1 & 0 & $0 \%$ & 0.019 & 0.019 & -- & -- & 0.0095 \\
\hline PEST & Eptam & $\mathrm{mg} / \mathrm{Kg}$ & 1 & 0 & $0 \%$ & 0.096 & 0.096 & -- & -- & 0.048 \\
\hline PEST & Ethalfluralin (Sonalan) & $\mathrm{mg} / \mathrm{Kg}$ & 1 & 0 & $0 \%$ & 0.096 & 0.096 & -- & -- & 0.048 \\
\hline PEST & Ethyl methylene phosphorodithioate & $\mathrm{mg} / \mathrm{Kg}$ & 1 & 0 & $0 \%$ & 0.045 & 0.045 & -- & -- & 0.023 \\
\hline PEST & Fenarimol & $\mathrm{mg} / \mathrm{Kg}$ & 1 & 0 & $0 \%$ & 0.19 & 0.19 & -- & -- & 0.095 \\
\hline PEST & Fensulfothion & $\mathrm{mg} / \mathrm{Kg}$ & 1 & 0 & $0 \%$ & 0.064 & 0.064 & -- & -- & 0.032 \\
\hline PEST & Fluridone & $\mathrm{mg} / \mathrm{Kg}$ & 1 & 0 & $0 \%$ & 0.51 & 0.51 & -- & -- & 0.26 \\
\hline PEST & Fonofos & $\mathrm{mg} / \mathrm{Kg}$ & 1 & 0 & $0 \%$ & 0.038 & 0.038 & -- & -- & 0.019 \\
\hline PEST & Gamma-BHC (Lindane) & $\mathrm{mg} / \mathrm{Kg}$ & 1 & 1 & $100 \%$ & -- & -- & 0.0080 & 0.0080 & 0.0080 \\
\hline PEST & gamma-Chlordene & $\mathrm{mg} / \mathrm{Kg}$ & 1 & 0 & $0 \%$ & 0.038 & 0.038 & -- & -- & 0.019 \\
\hline PEST & Heptachlor & $\mathrm{mg} / \mathrm{Kg}$ & 1 & 0 & $0 \%$ & 0.038 & 0.038 & -- & -- & 0.019 \\
\hline PEST & Heptachlor epoxide & $\mathrm{mg} / \mathrm{Kg}$ & 1 & 0 & $0 \%$ & 0.038 & 0.038 & -- & -- & 0.019 \\
\hline PEST & Hexazinone & $\mathrm{mg} / \mathrm{Kg}$ & 1 & 0 & $0 \%$ & 0.096 & 0.096 & -- & -- & 0.048 \\
\hline PEST & Methoxychlor & $\mathrm{mg} / \mathrm{Kg}$ & 2 & 0 & $0 \%$ & 0.0010 & 0.038 & -- & -- & 0.0098 \\
\hline PEST & Methyl Chlorpyrifos & $\mathrm{mg} / \mathrm{Kg}$ & 1 & 0 & $0 \%$ & 0.045 & 0.045 & -- & -- & 0.023 \\
\hline PEST & Methyl parathion & $\mathrm{mg} / \mathrm{Kg}$ & 1 & 0 & $0 \%$ & 0.045 & 0.045 & -- & -- & 0.023 \\
\hline PEST & Metolachlor & $\mathrm{mg} / \mathrm{Kg}$ & 1 & 0 & $0 \%$ & 0.19 & 0.19 & -- & -- & 0.095 \\
\hline PEST & Metribuzin & $\mathrm{mg} / \mathrm{Kg}$ & 1 & 0 & $0 \%$ & 0.064 & 0.064 & -- & -- & 0.032 \\
\hline PEST & MGK264 & $\mathrm{mg} / \mathrm{Kg}$ & 1 & 0 & $0 \%$ & 0.45 & 0.45 & -- & -- & 0.23 \\
\hline PEST & Mirex & $\mathrm{mg} / \mathrm{Kg}$ & 1 & 0 & $0 \%$ & 0.038 & 0.038 & -- & -- & 0.019 \\
\hline PEST & Molinate & $\mathrm{mg} / \mathrm{Kg}$ & 1 & 0 & $0 \%$ & 0.17 & 0.17 & -- & -- & 0.085 \\
\hline PEST & Napropamide & $\mathrm{mg} / \mathrm{Kg}$ & 1 & 0 & $0 \%$ & 0.19 & 0.19 & -- & -- & 0.095 \\
\hline PEST & Norflurazon & $\mathrm{mg} / \mathrm{Kg}$ & 1 & 0 & $0 \%$ & 0.096 & 0.096 & -- & -- & 0.048 \\
\hline PEST & Oxychlordane & $\mathrm{mg} / \mathrm{Kg}$ & 1 & 0 & $0 \%$ & 0.038 & 0.038 & -- & -- & 0.019 \\
\hline PEST & Oxyfluorfen & $\mathrm{mg} / \mathrm{Kg}$ & 1 & 0 & $0 \%$ & 0.17 & 0.17 & -- & -- & 0.085 \\
\hline PEST & Parathion & $\mathrm{mg} / \mathrm{Kg}$ & 1 & 0 & $0 \%$ & 0.051 & 0.051 & -- & -- & 0.026 \\
\hline PEST & Pebulate & $\mathrm{mg} / \mathrm{Kg}$ & 1 & 0 & $0 \%$ & 0.15 & 0.15 & -- & -- & 0.075 \\
\hline PEST & Pendimethalin & $\mathrm{mg} / \mathrm{Kg}$ & 1 & 0 & $0 \%$ & 0.096 & 0.096 & -- & -- & 0.048 \\
\hline
\end{tabular}


Table C-7

Summary Statistics for Sediment Samples Collected From the Walla Walla River Tributary of Segment 3 of the Columbia River (units reported "as received")

\begin{tabular}{|c|c|c|c|c|c|c|c|c|c|c|}
\hline $\begin{array}{l}\text { Constituent } \\
\text { Class }\end{array}$ & Constituent Name & Units & $\begin{array}{l}\text { Number } \\
\text { Analyzed }\end{array}$ & $\begin{array}{l}\text { Number } \\
\text { Detected }\end{array}$ & $\begin{array}{l}\text { Frequency of } \\
\text { Detection }\end{array}$ & $\begin{array}{l}\text { Minimum } \\
\text { Nondetect }\end{array}$ & $\begin{array}{l}\text { Maximum } \\
\text { Nondetect }\end{array}$ & $\begin{array}{c}\text { Minimum } \\
\text { Detect }\end{array}$ & $\begin{array}{c}\text { Maximum } \\
\text { Detect }\end{array}$ & $\begin{array}{c}\text { Average } \\
\text { Concentration }\end{array}$ \\
\hline$\overline{\mathrm{PEST}}$ & Pentachloroanisole & $\mathrm{mg} / \mathrm{Kg}$ & 1 & 0 & $0 \%$ & 0.038 & 0.038 & $\overline{--}$ & $\overline{--}$ & 0.019 \\
\hline PEST & Phorate & $\mathrm{mg} / \mathrm{Kg}$ & 1 & 0 & $0 \%$ & 0.045 & 0.045 & -- & -- & 0.023 \\
\hline PEST & Pramitol (Prometon) & $\mathrm{mg} / \mathrm{Kg}$ & 1 & 0 & $0 \%$ & 0.096 & 0.096 & -- & -- & 0.048 \\
\hline PEST & Prometryn & $\mathrm{mg} / \mathrm{Kg}$ & 1 & 0 & $0 \%$ & 0.064 & 0.064 & -- & -- & 0.032 \\
\hline PEST & Pronamide & $\mathrm{mg} / \mathrm{Kg}$ & 1 & 0 & $0 \%$ & 0.19 & 0.19 & -- & -- & 0.095 \\
\hline PEST & Propachlor (Ramrod) & $\mathrm{mg} / \mathrm{Kg}$ & 1 & 0 & $0 \%$ & 0.13 & 0.13 & -- & -- & 0.065 \\
\hline PEST & Propazine & $\mathrm{mg} / \mathrm{Kg}$ & 1 & 0 & $0 \%$ & 0.096 & 0.096 & -- & -- & 0.048 \\
\hline PEST & Simazine & $\mathrm{mg} / \mathrm{Kg}$ & 1 & 0 & $0 \%$ & 0.064 & 0.064 & -- & -- & 0.032 \\
\hline PEST & Terbacil & $\mathrm{mg} / \mathrm{Kg}$ & 1 & 0 & $0 \%$ & 0.32 & 0.32 & -- & -- & 0.16 \\
\hline PEST & Terbutryn & $\mathrm{mg} / \mathrm{Kg}$ & 1 & 0 & $0 \%$ & 0.064 & 0.064 & -- & -- & 0.032 \\
\hline PEST & Toxaphene & $\mathrm{mg} / \mathrm{Kg}$ & 1 & 0 & $0 \%$ & 0.76 & 0.76 & -- & -- & 0.38 \\
\hline PEST & trans-Chlordane & $\mathrm{mg} / \mathrm{Kg}$ & 1 & 0 & $0 \%$ & 0.038 & 0.038 & -- & -- & 0.019 \\
\hline PEST & trans-Nonachlor & $\mathrm{mg} / \mathrm{Kg}$ & 1 & 0 & $0 \%$ & 0.038 & 0.038 & -- & -- & 0.019 \\
\hline PEST & Triadimefon & $\mathrm{mg} / \mathrm{Kg}$ & 1 & 0 & $0 \%$ & 0.17 & 0.17 & -- & -- & 0.085 \\
\hline PEST & Triallate & $\mathrm{mg} / \mathrm{Kg}$ & 1 & 0 & $0 \%$ & 0.17 & 0.17 & -- & -- & 0.085 \\
\hline PEST & Trifluralin (Treflan) & $\mathrm{mg} / \mathrm{Kg}$ & 1 & 0 & $0 \%$ & 0.096 & 0.096 & -- & -- & 0.048 \\
\hline PEST & Vernolate & $\mathrm{mg} / \mathrm{Kg}$ & 1 & 0 & $0 \%$ & 0.096 & 0.096 & -- & -- & 0.048 \\
\hline SVOC & 1,2,4-Trichlorobenzene & $\mathrm{mg} / \mathrm{Kg}$ & 1 & 0 & $0 \%$ & 0.010 & 0.010 & -- & -- & 0.0050 \\
\hline sVoc & 1,2-Dichlorobenzene & $\mathrm{mg} / \mathrm{Kg}$ & 1 & 0 & $0 \%$ & 0.010 & 0.010 & -- & -- & 0.0050 \\
\hline SVOC & 1,2-Diphenylhydrazine & $\mathrm{mg} / \mathrm{Kg}$ & 1 & 0 & $0 \%$ & 0.010 & 0.010 & -- & -- & 0.0050 \\
\hline sVOC & 1,3-Dichlorobenzene & $\mathrm{mg} / \mathrm{Kg}$ & 1 & 0 & $0 \%$ & 0.010 & 0.010 & -- & -- & 0.0050 \\
\hline sVoc & 1,4-Dichlorobenzene & $\mathrm{mg} / \mathrm{Kg}$ & 1 & 0 & $0 \%$ & 0.010 & 0.010 & -- & -- & 0.0050 \\
\hline sVOC & 2,4,5-Trichlorophenol & $\mathrm{mg} / \mathrm{Kg}$ & 1 & 0 & $0 \%$ & 0.010 & 0.010 & -- & -- & 0.0050 \\
\hline SVOC & 2,4,6-Trichlorophenol & $\mathrm{mg} / \mathrm{Kg}$ & 1 & 0 & $0 \%$ & 0.010 & 0.010 & -- & -- & 0.0050 \\
\hline sVOC & 2,4-Dichlorophenol & $\mathrm{mg} / \mathrm{Kg}$ & 1 & 0 & $0 \%$ & 0.010 & 0.010 & -- & -- & 0.0050 \\
\hline SVOC & 2,4-Dimethylphenol & $\mathrm{mg} / \mathrm{Kg}$ & 1 & 0 & $0 \%$ & 0.010 & 0.010 & -- & -- & 0.0050 \\
\hline sVoc & 2,4-Dinitrophenol & $\mathrm{mg} / \mathrm{Kg}$ & 1 & 0 & $0 \%$ & 0.025 & 0.025 & -- & -- & 0.013 \\
\hline SVOC & 2,4-Dinitrotoluene & $\mathrm{mg} / \mathrm{Kg}$ & 1 & 0 & $0 \%$ & 0.010 & 0.010 & -- & -- & 0.0050 \\
\hline sVOC & 2,6-Dinitrotoluene & $\mathrm{mg} / \mathrm{Kg}$ & 1 & 0 & $0 \%$ & 0.010 & 0.010 & -- & -- & 0.0050 \\
\hline sVoc & 2-Chloronaphthalene & $\mathrm{mg} / \mathrm{Kg}$ & 1 & 0 & $0 \%$ & 0.010 & 0.010 & -- & -- & 0.0050 \\
\hline sVoc & 2-Chlorophenol & $\mathrm{mg} / \mathrm{Kg}$ & 1 & 0 & $0 \%$ & 0.010 & 0.010 & -- & -- & 0.0050 \\
\hline SVOC & 2-Nitroaniline & $\mathrm{mg} / \mathrm{Kg}$ & 1 & 0 & $0 \%$ & 0.010 & 0.010 & -- & -- & 0.0050 \\
\hline sVOC & 2-Nitrophenol & $\mathrm{mg} / \mathrm{Kg}$ & 1 & 0 & $0 \%$ & 0.010 & 0.010 & -- & -- & 0.0050 \\
\hline SVOC & 3,3'-Dichlorobenzidine & $\mathrm{mg} / \mathrm{Kg}$ & 1 & 0 & $0 \%$ & 0.020 & 0.020 & -- & -- & 0.010 \\
\hline sVoc & 3-Nitroaniline & $\mathrm{mg} / \mathrm{Kg}$ & 1 & 0 & $0 \%$ & 0.010 & 0.010 & -- & -- & 0.0050 \\
\hline SVOC & 4,6-Dinitro-2-methylphenol & $\mathrm{mg} / \mathrm{Kg}$ & 1 & 0 & $0 \%$ & 0.025 & 0.025 & -- & -- & 0.013 \\
\hline sVOC & 4-Bromophenylphenyl ether & $\mathrm{mg} / \mathrm{Kg}$ & 1 & 0 & $0 \%$ & 0.010 & 0.010 & -- & -- & 0.0050 \\
\hline sVoc & 4-Chloroaniline & $\mathrm{mg} / \mathrm{Kg}$ & 1 & 0 & $0 \%$ & 0.020 & 0.020 & -- & -- & 0.010 \\
\hline sVOC & 4-Chlorophenylphenyl ether & $\mathrm{mg} / \mathrm{Kg}$ & 1 & 0 & $0 \%$ & 0.010 & 0.010 & -- & -- & 0.0050 \\
\hline sVOC & 4-Methylphenol (cresol, p-) & $\mathrm{mg} / \mathrm{Kg}$ & 1 & 0 & $0 \%$ & 0.010 & 0.010 & -- & -- & 0.0050 \\
\hline sVOC & 4-Nitroaniline & $\mathrm{mg} / \mathrm{Kg}$ & 1 & 0 & $0 \%$ & 0.010 & 0.010 & -- & -- & 0.0050 \\
\hline SVOC & 4-Nitrophenol & $\mathrm{mg} / \mathrm{Kg}$ & 1 & 0 & $0 \%$ & 0.020 & 0.020 & -- & -- & 0.010 \\
\hline svoc & Acenaphthene & $\mathrm{mg} / \mathrm{Kg}$ & 1 & 0 & $0 \%$ & 0.0050 & 0.0050 & -- & -- & 0.0025 \\
\hline sVoc & Aniline & $\mathrm{mg} / \mathrm{Kg}$ & 1 & 0 & $0 \%$ & 0.010 & 0.010 & -- & -- & 0.0050 \\
\hline sVOC & Anthracene & $\mathrm{mg} / \mathrm{Kg}$ & 1 & 0 & $0 \%$ & 0.0050 & 0.0050 & -- & -- & 0.0025 \\
\hline sVOC & Benzidine & $\mathrm{mg} / \mathrm{Kg}$ & 1 & 0 & $0 \%$ & 0.050 & 0.050 & -- & -- & 0.025 \\
\hline sVOC & Benzo(ghi)perylene & $\mathrm{mg} / \mathrm{Kg}$ & 1 & 0 & $0 \%$ & 0.010 & 0.010 & -- & -- & 0.0050 \\
\hline
\end{tabular}


Table C-7

Summary Statistics for Sediment Samples Collected From the Walla Walla River Tributary of Segment 3 of the Columbia River (units reported "as received")

\begin{tabular}{|c|c|c|c|c|c|c|c|c|c|c|}
\hline $\begin{array}{l}\text { Constituent } \\
\text { Class }\end{array}$ & Constituent Name & Units & $\begin{array}{c}\text { Number } \\
\text { Analyzed }\end{array}$ & $\begin{array}{l}\text { Number } \\
\text { Detected }\end{array}$ & $\begin{array}{l}\text { Frequency of } \\
\text { Detection }\end{array}$ & $\begin{array}{c}\text { Minimum } \\
\text { Nondetect }\end{array}$ & $\begin{array}{c}\text { Maximum } \\
\text { Nondetect }\end{array}$ & $\begin{array}{c}\text { Minimum } \\
\text { Detect }\end{array}$ & $\begin{array}{c}\text { Maximum } \\
\text { Detect }\end{array}$ & $\begin{array}{c}\text { Average } \\
\text { Concentration }\end{array}$ \\
\hline$\overline{\text { SVOC }}$ & Benzoic acid & $\mathrm{mg} / \mathrm{Kg}$ & 1 & $\overline{0}$ & $0 \%$ & 0.010 & 0.010 & $\overline{--}$ & -- & 0.0050 \\
\hline sVoc & Benzyl alcohol & $\mathrm{mg} / \mathrm{Kg}$ & 1 & 0 & $0 \%$ & 0.010 & 0.010 & -- & -- & 0.0050 \\
\hline sVOC & Bis(2-chloro-1-methylethyl)ether & $\mathrm{mg} / \mathrm{Kg}$ & 1 & 0 & $0 \%$ & 0.010 & 0.010 & -- & -- & 0.0050 \\
\hline SVOC & Bis(2-Chloroethoxy)methane & $\mathrm{mg} / \mathrm{Kg}$ & 1 & 0 & $0 \%$ & 0.010 & 0.010 & -- & -- & 0.0050 \\
\hline SVOC & Bis(2-chloroethyl) ether & $\mathrm{mg} / \mathrm{Kg}$ & 1 & 0 & $0 \%$ & 0.010 & 0.010 & -- & -- & 0.0050 \\
\hline sVOc & Dibenz[a,h]anthracene & $\mathrm{mg} / \mathrm{Kg}$ & 1 & 0 & $0 \%$ & 0.010 & 0.010 & -- & -- & 0.0050 \\
\hline sVOC & Dibenzofuran & $\mathrm{mg} / \mathrm{Kg}$ & 1 & 0 & $0 \%$ & 0.025 & 0.025 & -- & -- & 0.013 \\
\hline SVOC & Dimethyl phthalate & $\mathrm{mg} / \mathrm{Kg}$ & 1 & 0 & $0 \%$ & 0.010 & 0.010 & -- & -- & 0.0050 \\
\hline sVOC & Di-n-butylphthalate & $\mathrm{mg} / \mathrm{Kg}$ & 1 & 0 & $0 \%$ & 0.0050 & 0.0050 & -- & -- & 0.0025 \\
\hline sVoc & Fluorene & $\mathrm{mg} / \mathrm{Kg}$ & 1 & 0 & $0 \%$ & 0.010 & 0.010 & -- & -- & 0.0050 \\
\hline sVOC & Hexachlorobenzene & $\mathrm{mg} / \mathrm{Kg}$ & 2 & 0 & $0 \%$ & 0.010 & 0.038 & -- & -- & 0.012 \\
\hline sVoc & Hexachlorobutadiene & $\mathrm{mg} / \mathrm{Kg}$ & 1 & 0 & $0 \%$ & 0.010 & 0.010 & -- & -- & 0.0050 \\
\hline sVOC & Hexachlorocyclopentadiene & $\mathrm{mg} / \mathrm{Kg}$ & 1 & 0 & $0 \%$ & 0.010 & 0.010 & -- & -- & 0.0050 \\
\hline sVoc & Hexachloroethane & $\mathrm{mg} / \mathrm{Kg}$ & 1 & 0 & $0 \%$ & 0.010 & 0.010 & -- & -- & 0.0050 \\
\hline sVOC & Indeno(1,2,3-cd)pyrene & $\mathrm{mg} / \mathrm{Kg}$ & 1 & 0 & $0 \%$ & 0.010 & 0.010 & -- & -- & 0.0050 \\
\hline SVOC & Nitrobenzene & $\mathrm{mg} / \mathrm{Kg}$ & 1 & 0 & $0 \%$ & 0.010 & 0.010 & -- & -- & 0.0050 \\
\hline sVOC & N-Nitrosodi-n-dipropylamine & $\mathrm{mg} / \mathrm{Kg}$ & 1 & 0 & $0 \%$ & 0.020 & 0.020 & -- & -- & 0.010 \\
\hline sVOC & Pentachlorophenol & $\mathrm{mg} / \mathrm{Kg}$ & 2 & 1 & $50 \%$ & 0.020 & 0.020 & 0.014 & 0.014 & 0.012 \\
\hline RAD_D & Cesium-137 & $\mathrm{pCi} / \mathrm{g}$ & 2 & 2 & $100 \%$ & -- & -- & 0.031 & 0.050 & 0.041 \\
\hline RAD D & Potassium-40 & $\mathrm{pCi} / \mathrm{g}$ & 2 & 2 & $100 \%$ & -- & -- & 14 & 14 & 14 \\
\hline
\end{tabular}

CONV - Conventional parameter

PEST - Pesticide

PCB - Polychlorinated bipheny

RAD_D - Dect

SVOC - Semivolatile Organic Compound 
Table C-8

Summary Statistics for Sediment Samples Collected From the Walla Walla River Tributary of Segment 3 of the Columbia River (units reported "dry-weight")

\begin{tabular}{|c|c|c|c|c|c|c|c|c|c|c|}
\hline $\begin{array}{l}\text { Constituent } \\
\text { Class }\end{array}$ & Constituent Name & Units & $\begin{array}{l}\text { Number } \\
\text { Analyzed }\end{array}$ & $\begin{array}{l}\text { Number } \\
\text { Detected }\end{array}$ & $\begin{array}{l}\text { Frequency of } \\
\text { Detection }\end{array}$ & $\begin{array}{l}\text { Minimum } \\
\text { Nondetect }\end{array}$ & $\begin{array}{l}\text { Maximum } \\
\text { Nondetect }\end{array}$ & $\begin{array}{l}\text { Minimum } \\
\text { Detect }\end{array}$ & $\begin{array}{l}\text { Maximum } \\
\text { Detect }\end{array}$ & $\begin{array}{c}\text { Average } \\
\text { Concentration }\end{array}$ \\
\hline$\overline{\mathrm{CONV}}$ & Total Organic Carbon & $\mathrm{mg} / \mathrm{kg}$ dry-wt & 1 & $\overline{1}$ & $100 \%$ & -- & -- & 0.0092 & 0.0092 & 0.0092 \\
\hline METAL & Arsenic & $\mathrm{mg} / \mathrm{kg}$ dry-wt & 1 & 1 & $100 \%$ & -- & -- & 1.7 & 1.7 & 1.7 \\
\hline METAL & Cadmium & $\mathrm{mg} / \mathrm{kg}$ dry-wt & 1 & 1 & $100 \%$ & -- & -- & 0.70 & 0.70 & 0.70 \\
\hline METAL & Chromium & $\mathrm{mg} / \mathrm{kg}$ dry-wt & 1 & 0 & $0 \%$ & 0.10 & 0.10 & -- & -- & 0.050 \\
\hline METAL & Copper & $\mathrm{mg} / \mathrm{kg}$ dry-wt & 1 & 1 & $100 \%$ & -- & -- & 12 & 12 & 12 \\
\hline METAL & Lead & $\mathrm{mg} / \mathrm{kg}$ dry-wt & 1 & 0 & $0 \%$ & 0.10 & 0.10 & -- & -- & 0.050 \\
\hline METAL & Mercury & $\mathrm{mg} / \mathrm{kg}$ dry-wt & 4 & 3 & $75 \%$ & 0.0070 & 0.0070 & 0.013 & 0.014 & 0.011 \\
\hline METAL & Zinc & $\mathrm{mg} / \mathrm{kg}$ dry-wt & 1 & 0 & $0 \%$ & 60 & 60 & -- & -- & 30 \\
\hline PCB & Aroclor-1260 & $\mathrm{mg} / \mathrm{kg}$ dry-wt & 1 & 0 & $0 \%$ & 0.010 & 0.010 & -- & -- & 0.0050 \\
\hline PEST & Alpha-BHC & $\mathrm{mg} / \mathrm{kg}$ dry-wt & 1 & 0 & $0 \%$ & 0.0010 & 0.0010 & -- & -- & $5.00 \mathrm{E}-04$ \\
\hline PEST & Chlordane & $\mathrm{mg} / \mathrm{kg}$ dry-wt & 1 & 1 & $100 \%$ & -- & -- & 0.0030 & 0.0030 & 0.0030 \\
\hline PEST & Dichlorodiphenyldichloroethane & $\mathrm{mg} / \mathrm{kg}$ dry-wt & 2 & 1 & $50 \%$ & 0.0010 & 0.0010 & 0.0018 & 0.0018 & 0.0012 \\
\hline PEST & Dichlorodiphenyldichloroethylene & $\mathrm{mg} / \mathrm{kg}$ dry-wt & 2 & 1 & $50 \%$ & 0.0010 & 0.0010 & 0.0076 & 0.0076 & 0.0041 \\
\hline PEST & Dichlorodiphenyltrichloroethane & $\mathrm{mg} / \mathrm{kg}$ dry-wt & 2 & 1 & $50 \%$ & 0.0010 & 0.0010 & 0.0012 & 0.0012 & $8.50 \mathrm{E}-04$ \\
\hline PEST & Gamma-BHC (Lindane) & $\mathrm{mg} / \mathrm{kg}$ dry-wt & 1 & 1 & $100 \%$ & -- & -- & 8.00E-04 & $8.00 \mathrm{E}-04$ & $8.00 \mathrm{E}-04$ \\
\hline SVOC & 2-MethyInaphthalene & $\mathrm{mg} / \mathrm{kg}$ dry-wt & 1 & 0 & $0 \%$ & 0.010 & 0.010 & -- & -- & 0.0050 \\
\hline sVOC & 2-Methylphenol (cresol, o-) & $\mathrm{mg} / \mathrm{kg}$ dry-wt & 1 & 0 & $0 \%$ & 0.010 & 0.010 & -- & -- & 0.0050 \\
\hline SVOC & Acenaphthylene & $\mathrm{mg} / \mathrm{kg}$ dry-wt & 1 & 0 & $0 \%$ & 0.010 & 0.010 & -- & -- & 0.0050 \\
\hline SVOC & Benzo(a)anthracene & $\mathrm{mg} / \mathrm{kg}$ dry-wt & 1 & 0 & $0 \%$ & 0.0050 & 0.0050 & -- & -- & 0.0025 \\
\hline sVoc & Benzo(a)pyrene & $\mathrm{mg} / \mathrm{kg}$ dry-wt & 1 & 0 & $0 \%$ & 0.010 & 0.010 & -- & -- & 0.0050 \\
\hline SVOC & Benzo(k)fluoranthene & $\mathrm{mg} / \mathrm{kg}$ dry-wt & 1 & 0 & $0 \%$ & 0.050 & 0.050 & -- & -- & 0.025 \\
\hline SVOC & Bis(2-ethylhexyl) phthalate & $\mathrm{mg} / \mathrm{kg}$ dry-wt & 1 & 1 & $100 \%$ & -- & -- & 0.058 & 0.058 & 0.058 \\
\hline SVOC & Butylbenzylphthalate & $\mathrm{mg} / \mathrm{kg}$ dry-wt & 1 & 0 & $0 \%$ & 0.0050 & 0.0050 & -- & -- & 0.0025 \\
\hline sVoc & Chrysene & $\mathrm{mg} / \mathrm{kg}$ dry-wt & 1 & 0 & $0 \%$ & 0.0050 & 0.0050 & -- & -- & 0.0025 \\
\hline sVOC & Diethylphthalate & $\mathrm{mg} / \mathrm{kg}$ dry-wt & 1 & 0 & $0 \%$ & 0.010 & 0.010 & -- & -- & 0.0050 \\
\hline SVOC & Di-n-octylphthalate & $\mathrm{mg} / \mathrm{kg}$ dry-wt & 1 & 0 & $0 \%$ & 0.0050 & 0.0050 & -- & -- & 0.0025 \\
\hline SVOC & Fluoranthene & $\mathrm{mg} / \mathrm{kg}$ dry-wt & 1 & 0 & $0 \%$ & 0.0050 & 0.0050 & -- & -- & 0.0025 \\
\hline SVOC & Hexachlorobenzene & $\mathrm{mg} / \mathrm{kg}$ dry-wt & 1 & 1 & $100 \%$ & -- & -- & 0.0020 & 0.0020 & 0.0020 \\
\hline sVOC & Isophorone & $\mathrm{mg} / \mathrm{kg}$ dry-wt & 1 & 0 & $0 \%$ & 0.010 & 0.010 & -- & -- & 0.0050 \\
\hline SVOC & Naphthalene & $\mathrm{mg} / \mathrm{kg}$ dry-wt & 1 & 0 & $0 \%$ & 0.010 & 0.010 & -- & -- & 0.0050 \\
\hline sVoc & Phenanthrene & $\mathrm{mg} / \mathrm{kg}$ dry-wt & 1 & 0 & $0 \%$ & 0.0050 & 0.0050 & -- & -- & 0.0025 \\
\hline sVOC & Phenol & $\mathrm{mg} / \mathrm{kg}$ dry-wt & 1 & 0 & $0 \%$ & 0.010 & 0.010 & -- & -- & 0.0050 \\
\hline SVOC & Pyrene & $\mathrm{mg} / \mathrm{kg}$ dry-wt & 1 & 0 & $0 \%$ & 0.0050 & 0.0050 & -- & -- & 0.0025 \\
\hline
\end{tabular}

CONV - Conventional parameter

PEST - Pesticide

PCB - Polychlorinated biphenyl

SVOC - Semivolatile Organic Compound 
Comparison of Segment 3 Detected Sediment Concentrations to Ecological and Human Health Benchmark Values (units reported "as received")

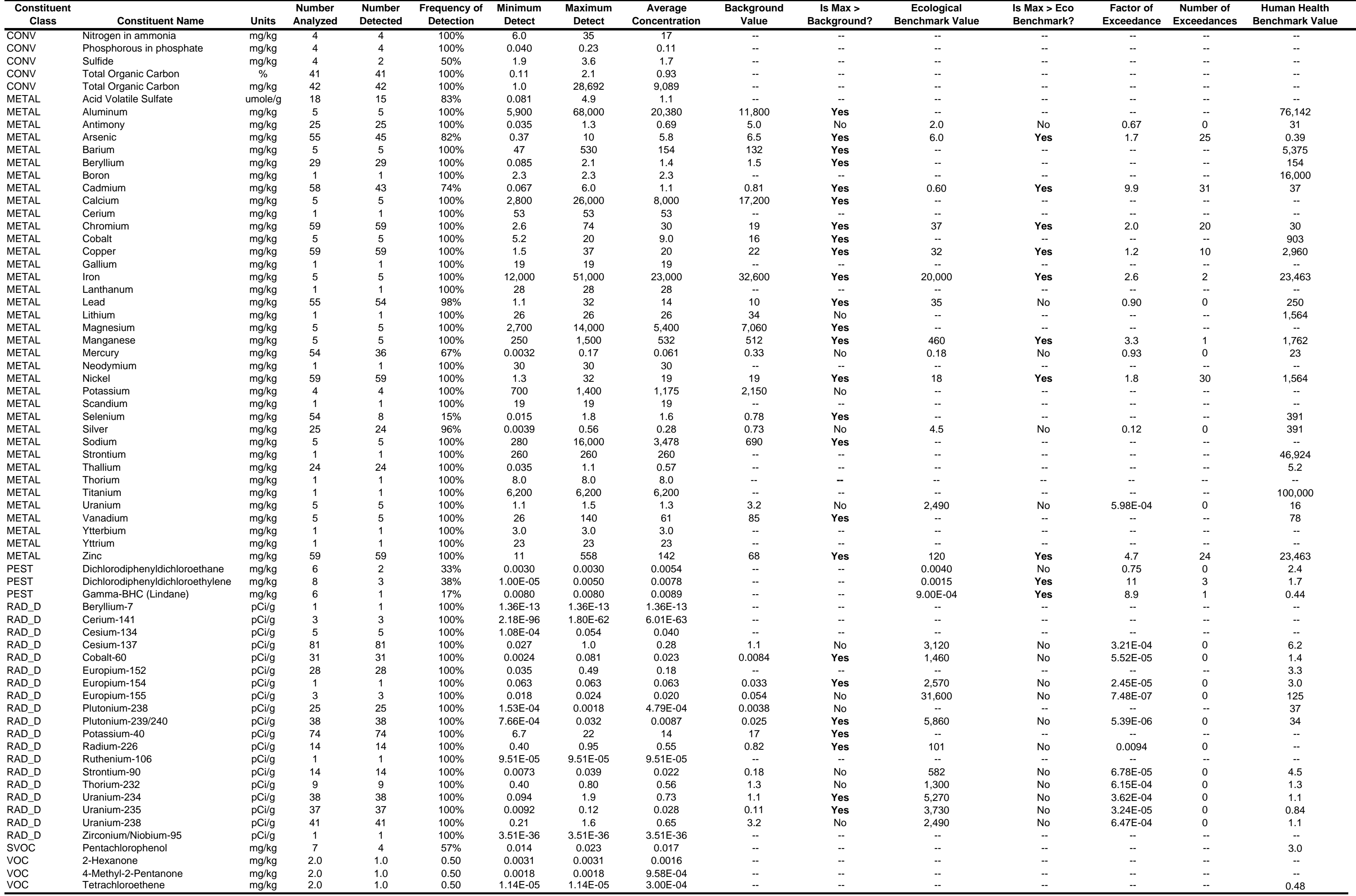

\begin{tabular}{lcc}
$\begin{array}{l}\text { Is Max }>\mathrm{HH} \\
\text { Benchmark? }\end{array}$ & $\begin{array}{c}\text { Factor of } \\
\text { Exceedance }\end{array}$ & $\begin{array}{c}\text { Number of } \\
\text { Exceedances }\end{array}$ \\
\hline
\end{tabular}

CONV - Conventional parameter
PEST - Pesticide 
Comparison of Segment 3 Detected Sediment Concentrations to Ecological and Human Health Benchmark Values (units reported "as received")

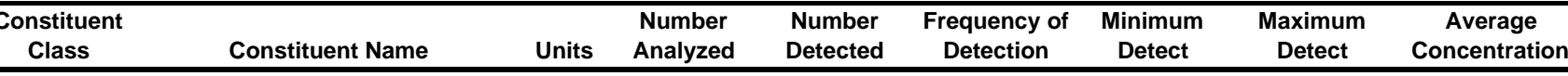

Background
Value

\begin{tabular}{cc} 
Is Max> \\
Background? & $\begin{array}{c}\text { Ecological } \\
\text { Benchmark Value }\end{array}$ \\
\hline
\end{tabular}

Is Max > Eco
Benchmark?

$\begin{array}{ccc}\text { Factor of } & \text { Number of } & \text { Human Health } \\ \text { Exceedance } & \text { Is Max }>\mathrm{HH}\end{array}$

\begin{tabular}{cc} 
Factor of & Number of \\
Exceedance & Exceedances \\
\hline
\end{tabular}

SVoC - Semivolatile Organic Compound

- Volatile Organic Compound 
Table C-10

Comparison of Segment 3 Detected Sediment Concentrations to Ecological and Human Health Benchmark Values (units reported "dry weight")

\begin{tabular}{|c|c|c|c|c|c|c|c|c|c|c|c|c|c|c|c|c|c|c|}
\hline $\begin{array}{l}\text { Constituent } \\
\text { Class }\end{array}$ & Constituent Name & Units & $\begin{array}{l}\text { Number } \\
\text { Analyzed }\end{array}$ & $\begin{array}{l}\text { Number } \\
\text { Detected }\end{array}$ & $\begin{array}{l}\text { Frequency of } \\
\text { Detection }\end{array}$ & $\underset{\substack{\text { Minimum } \\
\text { Detect }}}{ }$ & $\begin{array}{c}\text { Maximum } \\
\text { Detect }\end{array}$ & $\begin{array}{c}\text { Average } \\
\text { Concentration }\end{array}$ & $\begin{array}{c}\text { Background } \\
\text { Value }\end{array}$ & $\begin{array}{c}\text { Is Max > } \\
\text { Background? }\end{array}$ & $\begin{array}{c}\text { Ecological } \\
\text { Benchmark Value }\end{array}$ & $\begin{array}{l}\text { Is Max > Eco } \\
\text { Benchmark? }\end{array}$ & $\begin{array}{c}\text { Factor of } \\
\text { Exceedance }\end{array}$ & $\begin{array}{c}\text { Number of } \\
\text { Exceedances }\end{array}$ & $\begin{array}{c}\text { Benchmark } \\
\text { Value }\end{array}$ & $\begin{array}{l}\text { Is Max }>\mathrm{HH} \\
\text { Benchmark? }\end{array}$ & $\begin{array}{c}\text { Factor of } \\
\text { Exceedance }\end{array}$ & $\begin{array}{c}\text { Number of } \\
\text { Exceedances }\end{array}$ \\
\hline CONV & $\begin{array}{l}\text { Total Organic Carbon } \\
\text { Total }\end{array}$ & $\mathrm{mg} / \mathrm{kg}$ dry-wt & 2 & 2 & $100 \%$ & $\frac{0.0092}{502}$ & $\begin{array}{l}1.0 \\
50\end{array}$ & 0.50 & - & - & - & & - & 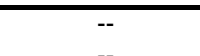 & - & - & 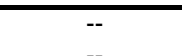 & \\
\hline $\begin{array}{l}\text { CONV } \\
\text { LTEN }\end{array}$ & Total solids & & 1 & 1 & $100 \%$ & & & 50 & & - & - & - & - & -- & -- & - & $\therefore$ & -- \\
\hline METAL & Aluminum & $\mathrm{mg} / \mathrm{kg}$ dry-wt & 4 & 4 & $100 \%$ & 2,900 & 17,000 & 10,000 & 11,800 & Yes & - & a & -- & - & 76,142 & No & 0.22 & 0 \\
\hline $\begin{array}{l}\text { METAL } \\
\text { METAL }\end{array}$ & $\begin{array}{l}\text { Antimony } \\
\text { Arsenic }\end{array}$ & $\mathrm{mg} / \mathrm{kg} \mathrm{d} d \mathrm{dr}-\mathrm{wt}$ & 17 & 13 13 & $6 \%$ & 0.52 & 5.1 & 1.6 & 5.0 & $\begin{array}{l}\text { Yes } \\
\text { yes }\end{array}$ & 2.0 & Yes & 2.6 & 4 & 31 & No & 0.16 & 0 \\
\hline $\begin{array}{l}\text { METAL } \\
\text { METL }\end{array}$ & $\begin{array}{l}\text { Assenic } \\
\text { Barium }\end{array}$ & $\begin{array}{l}\mathrm{mg} / \mathrm{kg} \text { ary } \\
\mathrm{mg} / \mathrm{kg} \text { dry-wt }\end{array}$ & 4 & 4 & $\begin{array}{l}100 \% \\
100 \%\end{array}$ & $\begin{array}{l}1.1 \\
53\end{array}$ & $\begin{array}{l}12 \\
150\end{array}$ & $\begin{array}{l}5.5 \\
101\end{array}$ & $\begin{array}{l}0.5 \\
132\end{array}$ & $\begin{array}{l}\text { Yes } \\
\text { Yes }\end{array}$ & 6.0 & Yes & 2.0 & $\begin{array}{l}9 \\
-\end{array}$ & 5.375 & $\begin{array}{l}\text { Yes } \\
\text { No }\end{array}$ & 31 & 24 \\
\hline METAL & $\begin{array}{l}\text { Beryllium } \\
\text { Berlon }\end{array}$ & 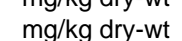 & 17 & 17 & $100 \%$ & 0.17 & 18 & 12 & $\begin{array}{l}1.5 \\
1.5\end{array}$ & Yes & $\ldots$ & $\ldots$ & $\ldots$ & $\ldots$ & 154 & No & 0.012 & 0 \\
\hline METAL & $\begin{array}{l}\text { Cadmium } \\
\text { Cadme }\end{array}$ & $\mathrm{mg} / \mathrm{kg}$ dry-wt & 23 & 21 & $91 \%$ & 0.19 & 4.3 & 1.2 & 0.81 & Yes & 0.60 & Yes & 7.2 & 17 & 37 & No & 0.12 & 0 \\
\hline METAL & Calcium & $\mathrm{mg} / \mathrm{kg}$ dry-wt & 4 & 4 & $100 \%$ & 3,500 & 5,800 & 4,500 & 17,200 & No & & . & & & $\ldots$ & $\ldots$ & 0.12 & $\sigma$ \\
\hline METAL & Chromium & $\mathrm{mg} / \mathrm{kg}$ dry-wt & 23 & 21 & $91 \%$ & 3.7 & 73 & 33 & 19 & Yes & 37 & Yes & 2.0 & 9 & 30 & Yes & 2.4 & 9 \\
\hline METAL & Cobalt & $\mathrm{mg} / \mathrm{kg}$ dry-wt & 4 & 4 & $100 \%$ & 11 & 17 & 13 & 16 & Yes & -- & -- & -- & -- & 903 & No & 0.019 & 0 \\
\hline METAL & Copper & mg/kg dry-wt & 23 & 23 & $100 \%$ & 7.1 & 39 & 23 & 22 & Yes & 32 & Yes & 1.2 & 7 & 2,960 & No & 0.013 & 0 \\
\hline METAL & Iron & $\mathrm{mg} / \mathrm{kg}$ dry-wt & 4 & 4 & $100 \%$ & 24,000 & 34,000 & 27,250 & 32,600 & Yes & 20,000 & Yes & 1.7 & 4 & 23,463 & Yes & 1.4 & 4 \\
\hline $\begin{array}{l}\text { METAL } \\
\text { METAL }\end{array}$ & $\begin{array}{l}\text { Lead } \\
\text { Magnesium }\end{array}$ & $\mathrm{mg} / \mathrm{kg}$ dry-wt & 23 & 22 & $\begin{array}{l}96 \% \\
100 \%\end{array}$ & 2,11 & 58 & $\begin{array}{c}17 \\
4825\end{array}$ & 10 & Yes & 35 & Yes & 1.7 & 4 & 250 & No & 0.23 & 0 \\
\hline $\begin{array}{l}\text { MEIAL } \\
\text { METAL }\end{array}$ & $\begin{array}{l}\text { Magnesulum } \\
\text { Manoanese }\end{array}$ & $\begin{array}{l}\mathrm{mg} / \mathrm{kg} \text { dry-wt } \\
\mathrm{mg} / \mathrm{kg} \text { dry-wt }\end{array}$ & $\begin{array}{l}4 \\
4\end{array}$ & 4 & $\begin{array}{l}100 \% \\
100 \%\end{array}$ & $\begin{array}{l}3,200 \\
3,280\end{array}$ & 6, & $\begin{array}{l}4,825 \\
4,00\end{array}$ & (1, & No & & & & & & & & \\
\hline METL & $\begin{array}{l}\text { Nanganese } \\
\text { Mercury }\end{array}$ & 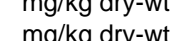 & 26 & 20 & 770 & 280 & 列 & 490 & 032 & res & ${ }^{460}$ & Yes & 1.4 & 2 & 1,762 & No & 0.36 & 0 \\
\hline METAL & Nickel & $\mathrm{mg} / \mathrm{kg}$ dry-wt & 17 & 17 & $100 \%$ & 6.1 & 32 & 20 & 19 & Yos & 0.18 & Yes & 1.1 & 3 & 23 & No & 0.013 & 0 \\
\hline METAL & Potassium & $\mathrm{mg} / \mathrm{kg}$ dry-wt & 4 & 4 & $100 \%$ & 490 & 2,300 & 1,288 & 2,150 & $\begin{array}{l}\text { res } \\
\text { Yes }\end{array}$ & 18 & res & 1.8 & 12 & 1,564 & No & 0.020 & 0 \\
\hline METAL & Selenium & $\mathrm{mg} / \mathrm{kg}$ dry-wt & 17 & 12 & $71 \%$ & 0.32 & 1.3 & 0.59 & 然.78 & Yes & -- & $\ldots$ & $\ldots$ & $\ldots$ & 391 & No & 0.0033 & 0 \\
\hline METAL & & $\mathrm{mg} / \mathrm{kg}$ dry-wt & & & $82 \%$ & 0.29 & 2.5 & 0.65 & 0.73 & Yes & 4.5 & No & 0.56 & 0 & 391 & No & 0.0064 & 0 \\
\hline METAL & Sodium & $\mathrm{mg} / \mathrm{kg}$ dry-wt & 4 & 4 & $100 \%$ & 130 & 280 & 193 & 690 & No & $\ldots$ & No & 0.00 & & & & & \\
\hline METAL & Thallium & $\mathrm{mg} / \mathrm{kg}$ dry-wt & 17 & 14 & $82 \%$ & 0.27 & 2.5 & 0.96 & & -.- & -- &.- &.- &.- & 5.2 & No & 0.48 & 0 \\
\hline METAL & Vanadium & $\mathrm{mg} / \mathrm{kg}$ dry-wt & 4 & 4 & $100 \%$ & 50 & 77 & 66 & 85 & No & -- & -- & - & $\ldots$ & 78 & No & 0.98 & 0 \\
\hline METAL & Zinc & $\mathrm{mg} / \mathrm{kg}$ dry-wt & 23 & 22 & $96 \%$ & 45 & 375 & 160 & 68 & Yes & 120 & Yes & 3.1 & 12 & 23,463 & No & 0.016 & 0 \\
\hline $\begin{array}{l}P C B \\
P P C B\end{array}-100$ & Aroclor-1254 & $\mathrm{mg} / \mathrm{kg}$ dry-wt & 6 & 3 & $50 \%$ & $\begin{array}{l}0.010 \\
0005\end{array}$ & 0.013 & 0.012 & - & - & 0.0070 & Yes & 1.9 & 3 & 0.22 & No & 0.059 & 0 \\
\hline $\begin{array}{l}\text { PCB } \\
\text { PEST }\end{array}$ & $\begin{array}{l}\text { Aroclor-1260 } \\
24^{\prime} \text {-DDT }\end{array}$ & $\begin{array}{l}\mathrm{mg} / \mathrm{kg} \text { dry-wt } \\
\mathrm{ma} / \mathrm{wg} \text { dry-wt }\end{array}$ & $\begin{array}{c}15 \\
2\end{array}$ & 3 & $\begin{array}{l}20 \% \\
50 \%\end{array}$ & $\begin{array}{c}0.0056 \\
710 \mathrm{E}-04\end{array}$ & $\begin{array}{l}0.011 \\
710 E-04\end{array}$ & & $-\overline{-}$ & -- & 0.021 & No & 0.52 & 0 & 0.22 & No & 0.050 & 0 \\
\hline $\begin{array}{l}\text { PEST } \\
\text { PES }\end{array}$ & $\begin{array}{l}\text { 2,4-DDT } \\
\text { Chlordane }\end{array}$ & $\begin{array}{l}\mathrm{mg} / \mathrm{kg} \text { drry-wt } \\
\mathrm{mg} / \mathrm{kg} \text { dry-wt }\end{array}$ & $\begin{array}{c}2 \\
12\end{array}$ & $\begin{array}{l}1 \\
1\end{array}$ & $\begin{array}{l}50 \% \\
8 \%\end{array}$ & $\begin{array}{c}7.10 E-04 \\
0.0030\end{array}$ & $\begin{array}{l}7.10 E-04 \\
0.0030\end{array}$ & $\begin{array}{l}0.0029 \\
0.027\end{array}$ & -- & -- & 0.0032 & No & $0 .-93$ & $\ddot{0}$ & $1 . !$ & No & $4.13 \mathrm{E}-04$ & 0 \\
\hline PEST & Dichlorodiphenyldichloroethane & $\mathrm{mg} / \mathrm{kg}$ dry-wt & 26 & 15 & $58 \%$ & 4.00E-04 & 0.023 & 0.0046 & -- & -- & 0.0040 & Yes & 5.8 & 6 & 2.4 & No & 0.0094 & 0 \\
\hline PEST & Dichlorodiphenyldichloroethylene & $\mathrm{mg} / \mathrm{kg}$ dry-wt & 28 & 15 & $54 \%$ & 7.00E-04 & 0.051 & 0.0058 & -- & -- & 0.0015 & Yes & 34 & 12 & 1.7 & No & 0.030 & 0 \\
\hline PEST & Dichlorodiphenyltrichloroethane & $\mathrm{mg} / \mathrm{kg}$ dry-wt & 26 & 6 & $23 \%$ & 3.00E-04 & 0.035 & 0.0037 &.- & $\ldots$ & 0.0042 & Yes & 8.4 & 2 & 1.7 & No & 0.020 & 0 \\
\hline PEST & Dieldrin & $\mathrm{mg} / \mathrm{kg}$ dry-wt & 23 & 3 & $13 \%$ & 2.00E-04 & 0.0050 & 0.0022 & -- & -- & 0.0019 & Yes & 2.6 & 1 & 0.030 & No & 0.16 & 0 \\
\hline PEST & Gamma-BHC (Lindane) & $\mathrm{mg} / \mathrm{kg}$ dry-wt & 22 & 1 & $5 \%$ & $8.00 E-04$ & $8.00 \mathrm{E}-04$ & 0.0019 & -- & -- & $9.00 E-04$ & No & 0.89 & 0 & 0.44 & No & 0.0018 & 0 \\
\hline $\begin{array}{l}\text { PEST } \\
\text { RAD }\end{array}$ & $\begin{array}{l}\text { trans-Nonachlor } \\
\text { Bentllium-. }\end{array}$ & $\mathrm{mg} / \mathrm{kg}$ dry-wt & 9 & 4 & $44 \%$ & $\begin{array}{l}0.0020 \\
145-19\end{array}$ & $\begin{array}{c}0.0030 \\
226 \mathrm{~F}-05\end{array}$ & $\begin{array}{c}0.0019 \\
4.52 \mathrm{E}-06\end{array}$ & -- & -- & - & -- & - & -- & -- & - & - & - \\
\hline $\begin{array}{l}\text { RADD } \\
\text { RAD D }\end{array}$ & $\begin{array}{l}\text { Berlylumm-7 } \\
\text { Cesium-134 }\end{array}$ & $\begin{array}{l}\text { pClig dry-wt } \\
\text { pCi/g dry-wt }\end{array}$ & $\begin{array}{l}5 \\
3\end{array}$ & $\begin{array}{l}5 \\
3\end{array}$ & $\begin{array}{l}100 \% \\
100 \%\end{array}$ & $\begin{array}{l}1.45 \mathrm{E}-19 \\
2.11 \mathrm{E}-04\end{array}$ & $\begin{array}{l}2.26 \mathrm{E}-05 \\
3.54 \mathrm{E}-04\end{array}$ & $\begin{array}{l}4.52 \mathrm{E}-06 \\
2.72 \mathrm{E}-04\end{array}$ & -- & -- & -- & -- & -- & -- & -- & -- & - & -- \\
\hline RAD_D & Cesium-137 & pCi/g dry-wt & 20 & 20 & $100 \%$ & 0.040 & $\begin{array}{l}0.04 L-04 \\
0.81\end{array}$ & 0.33 & 1.1 & No & 3,120 & No & $2.60 \mathrm{E}-04$ & 0 & 6.2 & No & 0.13 & 0 \\
\hline RAD_D & Cobalt-57 & pCi/g dry-wt & 2 & 2 & $100 \%$ & 4.42E-09 & 0.0097 & 0.0049 & -- & -- & $x_{1}$ & -- & & 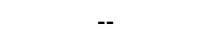 & 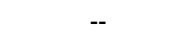 & $\cdots$ & .1. & 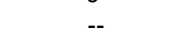 \\
\hline RAD_D & Cobalt-60 & pCilg dry-wt & 20 & 20 & $100 \%$ & 0.0030 & 0.047 & 0.019 & 0.0084 & Yes & 1,460 & No & $3.22 \mathrm{E}-05$ & 0 & 1.4 & No & 0.034 & 0 \\
\hline RAD_D & Europium-152 & pCilg dry-wt & 15 & 15 & $100 \%$ & 0.027 & 0.97 & 0.16 & & & & & & & 3.3 & No & 0.30 & 0 \\
\hline RAD_D & Europium-154 & pCi/g dry-wt & 4 & 4 & $100 \%$ & 0.036 & 0.088 & 0.053 & 0.033 & Yes & 2,570 & No & $3.41 \mathrm{E}-05$ & 0 & 3.0 & No & 0.029 & 0 \\
\hline RAD_D & Plutonium-238 & pcilg dry-wt & 8 & 8 & $100 \%$ & 0.0020 & 0.012 & 0.0060 & 0.0038 & Yes & 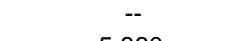 & $\because$ & Mer & 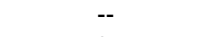 & 37 & No & 3.13E-04 & 0 \\
\hline RAD_D & Plutonium-239/240 & pCi/g dry-wt & 46 & 46 & $100 \%$ & 0.0020 & 0.024 & 0.0092 & 0.025 & No & 5,860 & No & 4.10E-06 & 0 & 34 & No & $7.08 E-04$ & 0 \\
\hline $\begin{array}{l}\text { RAD_D } \\
\text { RAD }\end{array}$ & $\begin{array}{l}\text { Potassium-40 } \\
\text { Padium-26 }\end{array}$ & pCi/g dry-wt & 48 & 48 & $100 \%$ & 13 & 19 & $\begin{array}{r}15 \\
099\end{array}$ & 17 & $\begin{array}{l}\text { Yes } \\
\text { Yes }\end{array}$ & - & -- & م0098 & 0 & 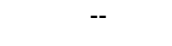 & 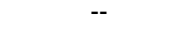 & -- & 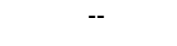 \\
\hline $\begin{array}{l}\text { RADD D } \\
\text { RAD D }\end{array}$ & $\begin{array}{l}\text { Radium-226 } \\
\text { Strontium-90 }\end{array}$ & $\begin{array}{l}\text { pCl/g dry-wt } \\
\text { pCi/q dry-wt }\end{array}$ & $\begin{array}{l}1 \\
45\end{array}$ & $\begin{array}{l}1 \\
45\end{array}$ & $\begin{array}{l}100 \% \\
100 \%\end{array}$ & $\begin{array}{l}0.99 \\
0.0019\end{array}$ & $\begin{array}{l}0.99 \\
0.037\end{array}$ & $\begin{array}{l}0.99 \\
0.014\end{array}-1$ & $\begin{array}{l}0.82 \\
0.18\end{array}$ & $\begin{array}{l}\text { Yes } \\
\text { No }\end{array}$ & ${ }_{602}^{101}$ & No & 0.0908 & 0 & $\ddot{-5}$ & $\ddot{0}$ & -- & - \\
\hline $\begin{array}{l}\text { RADD } \\
\text { RAD D }\end{array}$ & $\begin{array}{l}\text { Strontium-90 } \\
\text { Thorium-230 }\end{array}$ & $\begin{array}{l}\text { pCl/g ary-wt } \\
\text { pCi/g dry-wt }\end{array}$ & $\begin{array}{c}45 \\
4\end{array}$ & ${ }_{4}^{45}$ & $\begin{array}{l}1000 \% \\
1000\end{array}$ & 0.63 & $\begin{array}{ll}.0 .083 \\
0.88\end{array}$ & 0.074 & 0.18 & No & 582 & No & $0.40 E-05$ & 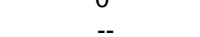 & 4.5 & No. & 0.0083 & 0 \\
\hline RAD_D & Thorium-232 & pCilg dry-wt & 4 & 4 & $100 \%$ & 0.70 & 0.91 & 0.82 & 1.3 & No & 1.300 & No & $7.00 E-04$ & 0 & 1.3 & No & 070 & $\overline{0}$ \\
\hline RAD_D & Uranium-234 & pCilg dry-wt & 45 & 45 & $100 \%$ & 0.39 & 1.8 & 1.2 & 1.1 & Yes & 5,270 & No & $3.43 E-04$ & 0 & 1.1 & Yes & 0 & 30 \\
\hline $\mathrm{RAD}_{-}^{-} \mathrm{D}$ & Uranium-235 & pCilg dry-wt & 24 & 24 & $100 \%$ & 0.020 & 0.070 & 0.041 & 0.11 & No & 3,730 & No & $1.88 \mathrm{E}-05$ & 0 & 0.84 & No & 0.083 & 然 \\
\hline RAD $D$ & Uranium-238 & pCi/g dry-wt & 45 & 45 & $100 \%$ & & 1. & 1.1 & 3.2 & No & 2,490 & No & $6.43 E-04$ & 0 & 1.1 & Yes & 0.000 & 19 \\
\hline svoc & 2,4-Dinitrophenol & $\mathrm{mg} / \mathrm{kg}$ dry-wt & 4 & 1 & $25 \%$ & 0.093 & 0.093 & 0.44 & (1) & $\cdots$ & & & & & 122 & No & $7.61 \mathrm{E}-04$ & $0_{0}$ \\
\hline svoc & 2-Methyinaphthalene & $\mathrm{mg} / \mathrm{kg}$ dry-wt & 16 & 2 & $13 \%$ & 0.010 & 0.011 & 0.42 &.- & -- & 0.020 & No & 0.50 & 0 & - & 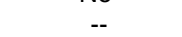 & 年 & \\
\hline svoc & 2-Methylphenol (cresol, o-) & $\mathrm{mg} / \mathrm{kg}$ dry-wt & 6 & 1 & $17 \%$ & 0.81 & 0.81 & 0.19 & -- & -- & & & & & 3,055 & No & $2.65 \mathrm{E}-04$ & 0 \\
\hline SVOC & Acenaphthene & $\mathrm{mg} / \mathrm{kg}$ dry-wt & 6 & 1 & 1 & 0.021 & 0.021 & 0.12 & -- & -- & 0.0067 & Yes & 3.1 & 1 & 3,682 & No & $5.70 \mathrm{E}-06$ & 0 \\
\hline SVOC & Anthracene & $\mathrm{mg} / \mathrm{kg}$ dry-wt & 20 & 3 & $15 \%$ & 0.0060 & 0.0880 & 0.34 & -- & -- & 0.057 & $\begin{array}{l}\text { Yes } \\
\text { Yes }\end{array}$ & 1.50 & 1 & 21,896 & No & 4.20E-06 & 0 \\
\hline $\begin{array}{l}\text { SVOC } \\
\text { sYoc }\end{array}$ & $\begin{array}{l}\text { Benzo(a)anthracene } \\
\text { Benzo(a)pyrene }\end{array}$ & $\begin{array}{l}\mathrm{mg} / \mathrm{kg} \text { dry-wt } \\
\mathrm{ma} / \mathrm{kg} \text { dry-wt }\end{array}$ & 9 & 2 & $\begin{array}{l}22 \% \\
33 \%\end{array}$ & $\begin{array}{l}0.013 \\
0.013\end{array}$ & 0.12 & $\begin{array}{l}0.049 \\
0.060\end{array}$ & -- & -- & $\begin{array}{l}0.032 \\
0.032\end{array}$ & $\begin{array}{l}\text { Yes } \\
\text { Yes }\end{array}$ & 3.8 & $\frac{1}{2}$ & $\begin{array}{l}0.62 \\
0.062\end{array}$ & $\begin{array}{l}\text { No } \\
\text { Yes }\end{array}$ & 0.19 & 0 \\
\hline $\begin{array}{l}\text { SVOC } \\
\text { sVOC }\end{array}$ & $\begin{array}{l}\text { Benzo(a)pyrene } \\
\text { Benzoo(b+kfflluoranthene }\end{array}$ & $\begin{array}{l}\mathrm{mg} / \mathrm{kg} \text { drry-wt } \\
\mathrm{mg} / \mathrm{kg} \text { dry-wt }\end{array}$ & $\begin{array}{l}9 \\
4\end{array}$ & $\begin{array}{l}3 \\
4 \\
3\end{array}$ & $\begin{array}{l}33 \% \\
100 \%\end{array}$ & $\begin{array}{l}0.013 \\
0.018\end{array}$ & 0.21 & 0.060 & -- & 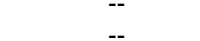 & 0.032 & Yes & 6.6 & 2 & $\begin{array}{c}0.062 \\
-.\end{array}$ & Yes & 3.4 & 2 \\
\hline $\begin{array}{l}\text { svoc } \\
\text { soc }\end{array}$ & $\begin{array}{l}\text { Benzoo(b+r)fifuorantinene } \\
\text { Benzo(ghi)perylene }\end{array}$ & $\begin{array}{l}\mathrm{mg} / \mathrm{kg} \text { dry-wt } \\
\mathrm{mg} / \mathrm{kg} \text { dry-wt }\end{array}$ & $\begin{array}{l}4 \\
8\end{array}$ & $\begin{array}{l}4 \\
2\end{array}$ & $\begin{array}{l}100 \% \\
25 \%\end{array}$ & $\begin{array}{l}.0 .018 \\
0.014\end{array}$ & $\begin{array}{l}0.22 \\
0.016\end{array}$ & $\begin{array}{l}.115 \\
0.83\end{array}$ & -- & -- & 0.17 & No & 0.082 & 0 & 2.400 & No & $5.83 E-06$ & 0 \\
\hline svoc & $\begin{array}{l}\text { Benzo(k)ffluoranthene } \\
\text { Benthe }\end{array}$ & $\mathrm{mg} / \mathrm{kg} \mathrm{drr}-\mathrm{wt}$ & 1 & 1 & $100 \%$ & 0.023 & 0.023 & 0.023 & $\ldots$ & $\ldots$ & 0.027 & No & 0.85 & 0 & 6.2 & No & 0.037 & 0 \\
\hline $\mathrm{OC}$ & Benzoic acid & $\mathrm{mg} / \mathrm{kg}$ dry-wt & 4 & 2 & $50 \%$ & 0.20 & 1.1 & 0.61 & -- & -- & & & & & 100,000 & No & $1.10 \mathrm{E}-05$ & 0 \\
\hline & Bis(2-ethylhexyl) phtha & & 9 & 5 & & 0.053 & 0.78 & 0.27 & $\ldots$ & & 0.64 & Yes & 1.20 & & & & & \\
\hline
\end{tabular}


Table C-10

Comparison of Segment 3 Detected Sediment Concentrations to Ecological and Human Health Benchmark Values (units reported "dry weight")

\begin{tabular}{|c|c|c|c|c|c|c|c|c|c|c|c|c|c|c|c|c|c|c|}
\hline $\begin{array}{l}\text { Constituent } \\
\text { Class }\end{array}$ & Constituent Name & Units & $\begin{array}{c}\text { Number } \\
\text { Analyzed }\end{array}$ & $\begin{array}{l}\text { Number } \\
\text { Detected }\end{array}$ & $\begin{array}{c}\text { Frequency of } \\
\text { Detection }\end{array}$ & $\begin{array}{c}\text { Minimum } \\
\text { Detect }\end{array}$ & $\begin{array}{c}\text { Maximum } \\
\text { Detect }\end{array}$ & $\begin{array}{c}\text { Average } \\
\text { Concentration }\end{array}$ & $\begin{array}{l}\text { Background } \\
\text { Value }\end{array}$ & $\begin{array}{c}\text { Is Max > } \\
\text { Background? }\end{array}$ & $\begin{array}{c}\text { Ecological } \\
\text { Benchmark Value }\end{array}$ & $\begin{array}{l}\text { Is Max > Eco } \\
\text { Benchmark? }\end{array}$ & $\begin{array}{c}\text { Factor of } \\
\text { Exceedance }\end{array}$ & $\begin{array}{c}\text { Number of } \\
\text { Exceedances }\end{array}$ & $\begin{array}{c}\text { Human Health } \\
\text { Benchmark } \\
\text { Value }\end{array}$ & $\begin{array}{l}\text { Is Max }>\mathrm{HH} \\
\text { Benchmark? }\end{array}$ & $\begin{array}{c}\text { Factor of } \\
\text { Exceedance }\end{array}$ & $\begin{array}{c}\text { Number of } \\
\text { Exceedances }\end{array}$ \\
\hline$\overline{\text { svoc }}$ & Chrysene & $\mathrm{mg} / \mathrm{kg}$ dry-wt & 22 & 3 & $14 \%$ & 0.013 & 0.130 & 0.32 & & & 0.057 & Yes & 2.30 & 1 & 62 & No & $2.10 \mathrm{E}-03$ & 0 \\
\hline svoc & Dibenz[a,h]anthracene & $\mathrm{mg} / \mathrm{kg}$ dry-wt & 16 & 4 & $25 \%$ & 0.0080 & 0.018 & 0.42 & -- & -- & 0.033 & No & 0.55 & 0 & 0.062 & No & 0.29 & 0 \\
\hline sVoc & Dibenzofuran & $\mathrm{mg} / \mathrm{kg}$ dry-wt & 1 & 1 & $100 \%$ & 0.015 & 0.015 & 0.015 & -- & -- & 5.1 & No & 0.0029 & 0 & 145 & No & $1.03 E-04$ & 0 \\
\hline sVoc & Diethylphthalate & $\mathrm{mg} / \mathrm{kg}$ dry-wt & 5 & 1 & $20 \%$ & 0.010 & 0.010 & 0.063 & -- & -- & & & & - & 48,882 & No & $2.05 E-07$ & 0 \\
\hline SVOC & Fluoranthene & $\mathrm{mg} / \mathrm{kg}$ dry-wt & 22 & 9 & $41 \%$ & 0.0040 & 0.200 & 0.31 & -- & -- & 0.11 & Yes & 1.80 & 3 & 2,294 & No & $8.72 \mathrm{E}-05$ & 0 \\
\hline sooc & $\begin{array}{l}\text { Fluorene } \\
\text { Hexachlorobenzene }\end{array}$ & g/kg dry-wt & 16 & 3 & $19 \%$ & 0.0070 & 0.022 & 0.40 & - & - & 0.077 & No & 0.29 & 0 & 2,747 & No & $8.01 \mathrm{E}-06$ & 0 \\
\hline $\begin{array}{l}\text { SVOC } \\
\text { sVOC }\end{array}$ & $\begin{array}{l}\text { Hexachlorobenzene } \\
\text { Hexadecanoic acid (9Cl) }\end{array}$ & $\mathrm{mg} / \mathrm{kg}$ dry-wt & 13 & 5 & $\begin{array}{l}38 \% \\
100 \%\end{array}$ & 0.0010 & 0.0030 & 0.024 & $\therefore$ & $\because$ & 0.10 & No & 0.030 & 0 & 0.30 & No & 0.0099 & 0 \\
\hline svoc & $\begin{array}{l}\text { Hexadecanolic accid (ICl) } \\
\text { Indeno(1.2,-cd) pyren }\end{array}$ & $\begin{array}{l}\mathrm{mg} / \mathrm{kg} \text { dry-wt } \\
\mathrm{mg} / \mathrm{kg} \text { dry-wt }\end{array}$ & 1 & 1 & $\begin{array}{l}100 \% \\
100 \%\end{array}$ & $\begin{array}{l}130 \\
0.016\end{array}$ & $\begin{array}{l}10016 \\
0.016\end{array}$ & $\begin{array}{l}10016 \\
0.016\end{array}$ & $\ldots$ & .. & 0.017 & No & 0.94 & 0 & 062 & $\ddot{2}$ & 0006 & $\overline{0}$ \\
\hline sVoc & Isophorone & $\mathrm{mg} / \mathrm{kg}$ dry-wt & 5 & 1 & $20 \%$ & 0.015 & 0.015 & 0.045 & -- & -- & & & & & $\begin{array}{l}0.02 \\
512\end{array}$ & $\begin{array}{l}\text { Noo } \\
\text { No }\end{array}$ & $\begin{array}{l}0.026 \\
2.93-05\end{array}$ & 0 \\
\hline svoc & Naphthalene & $\mathrm{mg} / \mathrm{kg}$ dry-wt & 13 & 3 & $23 \%$ & 0.0020 & 0.015 & 0.48 & -- & -- & 0.18 & No & 0.085 & 0 & 56 & No & $\begin{array}{l}2.93 E-05 \\
2.68 E-04\end{array}$ & 0 \\
\hline sVoc & Pentachlorophenol & $\mathrm{mg} / \mathrm{kg}$ dry-wt & 13 & 3 & $23 \%$ & 0.013 & 1.200 & 0. & -- & -- & -- & -- & -- & -- & 3.0 & No & 0.0044 & 0 \\
\hline SVOC & Perylene & $\mathrm{mg} / \mathrm{kg}$ dry-wt & 8 & 3 & $38 \%$ & & 0.011 & 0.0068 & -- & -- & - & $\cdots$ & - & - & -- & -- & -- & -- \\
\hline $\begin{array}{l}\text { SVOC } \\
\text { SVOC }\end{array}$ & $\begin{array}{l}\text { Phenanthrene } \\
\text { Phenol }\end{array}$ & $\mathrm{mg} / \mathrm{kg}$ dry-wt & 19 & 4 & $21 \%$ & 0.0040 & 0.057 & 0.37 & -- & -- & 0.042 & Yes & 1.40 & 1 & 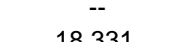 & $\ddot{n}$ & $\approx$ & $\overline{0}$ \\
\hline $\begin{array}{l}\text { SVOC } \\
\text { SVOC }\end{array}$ & $\begin{array}{l}\text { Phenol } \\
\text { Pyrene }\end{array}$ & $\begin{array}{l}\mathrm{mg} / \mathrm{kg} \text { dry-wt } \\
\mathrm{mg} / \mathrm{kg} \text { dry-wt }\end{array}$ & $\begin{array}{c}9 \\
22\end{array}$ & $\begin{array}{l}3 \\
7\end{array}$ & $\begin{array}{l}33 \% \% \\
32 \%\end{array}$ & $\begin{array}{l}0.035 \\
0.0030\end{array}$ & $\begin{array}{l}0.10 \\
0.220\end{array}$ & $\begin{array}{l}0.082 \\
0.32\end{array}$ & -- & -- & $0--$ & yes & 420 & $\overline{3}$ & $\begin{array}{l}18,331 \\
2316\end{array}$ & No & $\begin{array}{l}5.46 E-06 \\
950-05\end{array}$ & $\begin{array}{l}0 \\
0\end{array}$ \\
\hline
\end{tabular}

ConV - Conventional parameter

PCB - Polychlorinated biphenyl

RAD_D - Decayed radionuclide
SvoC - Semivolatili Organic Compound 


\begin{tabular}{|c|c|c|c|c|c|c|c|c|c|c|}
\hline Sample Area & Sample Site & SampleID & Date Collected & Constituent Name & Units & QA Category & $\begin{array}{l}\text { Analytical } \\
\text { Value }\end{array}$ & Qualifier & $\begin{array}{l}\text { Benchmark } \\
\text { Value }\end{array}$ & $\begin{array}{c}\text { Benchma } \\
\text { Value }\end{array}$ \\
\hline Columbia River & RM 328 & SRP 3,4 & August 20, 1991 & Acenaphthene & $\mathrm{mg} / \mathrm{kg}$ dry-wt & 1 & 0.021 & $=$ & 0.0067 & $\overline{3,681}$ \\
\hline Columbia River & PtofPasco & $05-8002$ & September 30,1986 & Anthracene & $\mathrm{mg} / \mathrm{kg}$ dry-wt & 1 & 0.088 & - & 0.057 & 21,896 \\
\hline Columbia River & McNary Dam & B17B57 & August 8,2003 & Antimony & $\mathrm{mg} / \mathrm{kg}$ dry-wt & 1 & 2.4 & $=$ & 2.0 & 31 \\
\hline Columbia River & McNary Dam & B17B60 & August 8, 2003 & Antimony & $\mathrm{mg} / \mathrm{kg}$ dry-wt & 1 & 5.1 & $=$ & 2.0 & 31 \\
\hline Columbia River & McNary Dam & B17B65 & August 8,2003 & Antimony & $\mathrm{mg} / \mathrm{kg}$ dry-wt & 1 & 3.7 & $=$ & 2.0 & 31 \\
\hline Columbia River & McNary Dam & B17B67 & August 8,2003 & Antimony & $\mathrm{mg} / \mathrm{kg}$ dry-wt & 1 & 3.4 & $=$ & 2.0 & 31 \\
\hline Columbia River & Pt of Pasco & $05-8000$ & September 30,1986 & Aroclor-1254 & $\mathrm{mg} / \mathrm{kg}$ dry-wt & 1 & 0.010 & $\mathrm{j}$ & 0.0070 & 0.22 \\
\hline Columbia River & Pt of Pasco & 05-8001 & September 30, 1986 & Aroclor-1254 & $\mathrm{mg} / \mathrm{kg}$ dry-wt & 1 & 0.010 & $\mathrm{~J}$ & 0.0070 & 0.22 \\
\hline Columbia River & $\mathrm{Pt}$ of Pasco & 05-8002 & September 30,1986 & Aroclor-1254 & $\mathrm{mg} / \mathrm{kg}$ dry-wt & 1 & 0.013 & $=$ & 0.0070 & 0.22 \\
\hline Columbia River & RM 318 & 168272 & April 14,1992 & Arsenic & $\mathrm{mg} / \mathrm{kg}$ dry-wt & 1 & 3.3 & $=$ & 6.0 & 0.39 \\
\hline Columbia River & RM 316 & 168273 & April 14, 1992 & Arsenic & $\mathrm{mg} / \mathrm{kg}$ dry-wt & 1 & 2.7 & $=$ & 6.0 & 0.39 \\
\hline Columbia River & RM 298 & 168275 & April 13, 1992 & Arsenic & $\mathrm{mg} / \mathrm{kg}$ dry-wt & 1 & 4.9 & $=$ & 6.0 & 0.39 \\
\hline Walla Walla River & Blw Warm Sprgs & 32A070-4 & September 12, 1984 & Arsenic & $\mathrm{mg} / \mathrm{kg}$ dry-wt & 2 & 1.7 & $=$ & 6.0 & 0.39 \\
\hline Snake River & RM 10 & 3504002 & June 1,2004 & Arsenic & $\mathrm{mg} / \mathrm{kg}$ & 1 & 8.9 & $=$ & 6.0 & 0.39 \\
\hline Snake River & RM 10 & 3504026 & June 1,2004 & Arsenic & $\mathrm{mg} / \mathrm{kg}$ & 1 & 9.2 & $=$ & 6.0 & 0.39 \\
\hline Yakima River & Below Kiona & 37A070-7 & September 12, 1984 & Arsenic & $\mathrm{mg} / \mathrm{kg}$ dry-wt & 2 & 2.8 & $=$ & 6.0 & 0.39 \\
\hline Columbia River & McNary Dam & 4414200 & June 1,2004 & Arsenic & $\mathrm{mg} / \mathrm{kg}$ & 1 & 5.5 & $=$ & 6.0 & 0.39 \\
\hline Columbia River & McNary Dam & 4414202 & June 1, 2004 & Arsenic & $\mathrm{mg} / \mathrm{kg}$ & 1 & 7.1 & $=$ & 6.0 & 0.39 \\
\hline Columbia River & McNary Dam & 4414203 & June 1,2004 & Arsenic & $\mathrm{mg} / \mathrm{kg}$ & 1 & 9.6 & $=$ & 6.0 & 0.39 \\
\hline Snake River & RM 10 & 4414205 & June 1, 2004 & Arsenic & $\mathrm{mg} / \mathrm{kg}$ & 1 & 5.7 & $=$ & 6.0 & 0.39 \\
\hline Snake River & RM 10 & 4414206 & June 1,2004 & Arsenic & $\mathrm{mg} / \mathrm{kg}$ & 1 & 8.0 & $=$ & 6.0 & 0.39 \\
\hline Snake River & RM 10 & 4414207 & June 1,2004 & Arsenic & $\mathrm{mg} / \mathrm{kg}$ & 1 & 8.9 & $=$ & 6.0 & 0.39 \\
\hline Walla Walla River & RM 1 & 4414209 & June 1,2004 & Arsenic & $\mathrm{mg} / \mathrm{kg}$ & 1 & 4.6 & $=$ & 6.0 & 0.39 \\
\hline Columbia River & RM 316 & 4414212 & June 1,2004 & Arsenic & $\mathrm{mg} / \mathrm{kg}$ & 1 & 5.7 & $=$ & 6.0 & 0.39 \\
\hline Columbia River & Lake Wallula & 4414213 & June 1,2004 & Arsenic & $\mathrm{mg} / \mathrm{kg}$ & 1 & 7.2 & $=$ & 6.0 & 0.39 \\
\hline Columbia River & Lake Wallula & 4424215 & June 1, 2004 & Arsenic & $\mathrm{mg} / \mathrm{kg}$ & 1 & 7.4 & $=$ & 6.0 & 0.39 \\
\hline Columbia River & Lake Wallula & 4424220 & June 1,2004 & Arsenic & $\mathrm{mg} / \mathrm{kg}$ & 1 & 4.8 & $=$ & 6.0 & 0.39 \\
\hline Columbia River & Lake Wallula & 4424224 & June 1,2004 & Arsenic & $\mathrm{mg} / \mathrm{kg}$ & 1 & 4.5 & $=$ & 6.0 & 0.39 \\
\hline Columbia River & Lake Wallula & 4424225 & June 1,2004 & Arsenic & $\mathrm{mg} / \mathrm{kg}$ & 1 & 5.8 & $=$ & 6.0 & 0.39 \\
\hline Columbia River & Lake Wallula & 4424226 & June 1, 2004 & Arsenic & $\mathrm{mg} / \mathrm{kg}$ & 1 & 5.1 & $=$ & 6.0 & 0.39 \\
\hline Yakima River & RM 1 & 4434230 & June 1,2004 & Arsenic & $\mathrm{mg} / \mathrm{kg}$ & 1 & 6.7 & $=$ & 6.0 & 0.39 \\
\hline Columbia River & RM 339.5 & 4434234 & June 1, 2004 & Arsenic & $\mathrm{mg} / \mathrm{kg}$ & 1 & 5.3 & $=$ & 6.0 & 0.39 \\
\hline Snake River & ICE HARBOR DAM & Bow3C6 & August 16, 1999 & Arsenic & $\mathrm{mg} / \mathrm{Kg}$ & 1 & 6.3 & $=$ & 6.0 & 0.39 \\
\hline Snake River & ICE HARBOR DAM & B0W3C8 & August 16, 1999 & Arsenic & $\mathrm{mg} / \mathrm{Kg}$ & 1 & 8.6 & $=$ & 6.0 & 0.39 \\
\hline Snake River & ICE HARBOR DAM & BOW3D0 & August 16, 1999 & Arsenic & $\mathrm{mg} / \mathrm{Kg}$ & 1 & 8.2 & $=$ & 6.0 & 0.39 \\
\hline Columbia River & McNary Dam & BOW3D4 & August 2, 1999 & Arsenic & $\mathrm{mg} / \mathrm{kg}$ & 1 & 9.1 & $=$ & 6.0 & 0.39 \\
\hline Columbia River & McNary Dam & BOW3H2 & August 2, 1999 & Arsenic & $\mathrm{mg} / \mathrm{Kg}$ & 1 & 8.9 & $=$ & 6.0 & 0.39 \\
\hline Columbia River & McNary Dam & BOW3H4 & August 2, 1999 & Arsenic & $\mathrm{mg} / \mathrm{Kg}$ & 1 & 6.0 & $=$ & 6.0 & 0.39 \\
\hline Columbia River & McNary Dam & BOW3H6 & August 2, 1999 & Arsenic & $\mathrm{mg} / \mathrm{Kg}$ & 1 & 8.1 & $=$ & 6.0 & 0.39 \\
\hline Columbia River & McNary Dam & BOW3N9 & August 2, 1999 & Arsenic & $\mathrm{mg} / \mathrm{kg}$ & 1 & 9.6 & $=$ & 6.0 & 0.39 \\
\hline Columbia River & McNary Dam & BOW3P1 & August 2, 1999 & Arsenic & $\mathrm{mg} / \mathrm{Kg}$ & 1 & 7.1 & $=$ & 6.0 & 0.39 \\
\hline Columbia River & McNary Dam & BOYWDO & August 8,2000 & Arsenic & $\mathrm{mg} / \mathrm{Kg}$ & 1 & 7.6 & $=$ & 6.0 & 0.39 \\
\hline Columbia River & McNary Dam & BOYWD2 & August 8,2000 & Arsenic & $\mathrm{mg} / \mathrm{Kg}$ & 1 & 6.4 & $=$ & 6.0 & 0.39 \\
\hline Snake River & ICE HARBOR DAM & $\mathrm{B} 12 \mathrm{CH} 5$ & July 23, 2001 & Arsenic & $\mathrm{mg} / \mathrm{kg}$ dry-wt & 1 & 7.4 & $=$ & 6.0 & 0.39 \\
\hline Snake River & ICE HARBOR DAM & $\mathrm{B} 12 \mathrm{CH} 7$ & July 23,2001 & Arsenic & $\mathrm{mg} / \mathrm{kg}$ dry-wt & 1 & 8.2 & $=$ & 6.0 & 0.39 \\
\hline
\end{tabular}




\begin{tabular}{|c|c|c|c|c|c|c|c|c|c|c|}
\hline Sample Area & Sample Site & SamplelD & Date Collected & Constituent Name & Units & QA Category & $\begin{array}{c}\text { Analytical } \\
\text { Value }\end{array}$ & Qualifier & $\begin{array}{c}\text { Ecological } \\
\text { Benchmark } \\
\text { Value }\end{array}$ & $\begin{array}{c}\text { Health } \\
\text { Benchmark } \\
\text { Value }\end{array}$ \\
\hline Snake River & ICE HARBOR DAM & $\mathrm{B} 12 \mathrm{CH} 9$ & July 23,2001 & Arsenic & $\mathrm{mg} / \mathrm{kg}$ dry-wt & 1 & 8.3 & $=$ & 6.0 & 0.39 \\
\hline Columbia River & McNary Dam & $\mathrm{B} 12 \mathrm{CJ3}$ & July 20, 2001 & Arsenic & $\mathrm{mg} / \mathrm{kg}$ dry-wt & 1 & 9.2 & $=$ & 6.0 & 0.39 \\
\hline Columbia River & McNary Dam & B12CK3 & July 20, 2001 & Arsenic & $\mathrm{mg} / \mathrm{kg}$ dry-wt & 1 & 6.6 & $=$ & 6.0 & 0.39 \\
\hline Columbia River & McNary Dam & B12CK7 & July 20, 2001 & Arsenic & $\mathrm{mg} / \mathrm{kg}$ dry-wt & 1 & 12 & $=$ & 6.0 & 0.39 \\
\hline Columbia River & McNary Dam & B12CM7 & July 23,2001 & Arsenic & $\mathrm{mg} / \mathrm{kg}$ dry-wt & 1 & 10 & $=$ & 6.0 & 0.39 \\
\hline Columbia River & McNary Dam & B12CM9 & July 23, 2001 & Arsenic & $\mathrm{mg} / \mathrm{kg}$ dry-wt & 1 & 8.3 & $=$ & 6.0 & 0.39 \\
\hline Columbia River & McNary Dam & B14YN8 & July 19, 2002 & Arsenic & $\mathrm{mg} / \mathrm{kg}$ & 1 & 0.40 & $=$ & 6.0 & 0.39 \\
\hline Yakima River & YAKIMA RIVER & B16WX0 & May 14,2003 & Arsenic & $\mathrm{mg} / \mathrm{Kg}$ & 1 & 2.4 & $=$ & 6.0 & 0.39 \\
\hline Yakima River & YAKIMA RIVER & B16WX1 & May 14, 2003 & Arsenic & $\mathrm{mg} / \mathrm{Kg}$ & 1 & 2.5 & $=$ & 6.0 & 0.39 \\
\hline Walla Walla River & Walla Walla Riv & B16WX2 & May 15, 2003 & Arsenic & $\mathrm{mg} / \mathrm{Kg}$ & 1 & 2.4 & $=$ & 6.0 & 0.39 \\
\hline Walla Walla River & Walla Walla Riv & B16WX3 & May 15, 2003 & Arsenic & $\mathrm{mg} / \mathrm{Kg}$ & 1 & 2.8 & $=$ & 6.0 & 0.39 \\
\hline Columbia River & McNary Dam & B17710 & July 16, 2003 & Arsenic & $\mathrm{mg} / \mathrm{Kg}$ & 1 & 9.9 & $=$ & 6.0 & 0.39 \\
\hline Columbia River & McNary Dam & B17712 & July 16, 2003 & Arsenic & $\mathrm{mg} / \mathrm{Kg}$ & 1 & 8.2 & $=$ & 6.0 & 0.39 \\
\hline Columbia River & McNary Dam & B17B57 & August 8, 2003 & Arsenic & $\mathrm{mg} / \mathrm{kg}$ dry-wt & 1 & 2.1 & $=$ & 6.0 & 0.39 \\
\hline Columbia River & McNary Dam & B17B60 & August 8, 2003 & Arsenic & $\mathrm{mg} / \mathrm{kg}$ dry-wt & 1 & 7.6 & $=$ & 6.0 & 0.39 \\
\hline Columbia River & McNary Dam & B17B65 & August 8,2003 & Arsenic & $\mathrm{mg} / \mathrm{kg}$ dry-wt & 1 & 5.8 & $=$ & 6.0 & 0.39 \\
\hline Columbia River & McNary Dam & B17B67 & August 8,2003 & Arsenic & $\mathrm{mg} / \mathrm{kg}$ dry-wt & 1 & 4.3 & $=$ & 6.0 & 0.39 \\
\hline Columbia River & McNary Dam & B17BJ3 & August 8, 2003 & Arsenic & $\mathrm{mg} / \mathrm{Kg}$ & 1 & 7.6 & $=$ & 6.0 & 0.39 \\
\hline Columbia River & McNary Dam & B19PL7 & July 13, 2004 & Arsenic & $\mathrm{mg} / \mathrm{Kg}$ & 1 & 10 & $x$ & 6.0 & 0.39 \\
\hline Columbia River & McNary Dam & B19PL9 & July 13, 2004 & Arsenic & $\mathrm{mg} / \mathrm{Kg}$ & 1 & 9.3 & $x$ & 6.0 & 0.39 \\
\hline Columbia River & McNary Dam & B1B7N9 & September 21, 2004 & Arsenic & $\mathrm{mg} / \mathrm{Kg}$ & 1 & 9.5 & $x$ & 6.0 & 0.39 \\
\hline Columbia River & RM 328 & SRP 3,4 & August 20,1991 & Arsenic & $\mathrm{mg} / \mathrm{kg}$ dry-wt & 1 & 4.0 & $=$ & 6.0 & 0.39 \\
\hline Columbia River & RM 315-316 & SRP 50 & August 19, 1991 & Arsenic & $\mathrm{mg} / \mathrm{kg}$ dry-wt & 1 & 3.6 & $=$ & 6.0 & 0.39 \\
\hline Columbia River & RM 315-316 & SRP 51 & August 19, 1991 & Arsenic & $\mathrm{mg} / \mathrm{kg}$ dry-wt & 1 & 2.8 & $=$ & 6.0 & 0.39 \\
\hline Snake River & RM 2 & SRP 7A & August 19, 1991 & Arsenic & $\mathrm{mg} / \mathrm{kg}$ dry-wt & 1 & 5.7 & $=$ & 6.0 & 0.39 \\
\hline Yakima River & Kiona WA & Yakima sed & August 25,1987 & Arsenic & $\mathrm{mg} / \mathrm{kg}$ & 1 & 5.4 & $=$ & 6.0 & 0.39 \\
\hline Columbia River & Pt of Pasco & $05-8002$ & September 30,1986 & Benzo(a)anthracene & $\mathrm{mg} / \mathrm{kg}$ dry-wt & 1 & 0.12 & $\mathrm{~J}$ & 0.032 & 0.062 \\
\hline Columbia River & $\mathrm{Pt}$ of Pasco & $05-8000$ & September 30,1986 & Benzo(a)pyrene & $\mathrm{mg} / \mathrm{kg}$ dry-wt & 1 & 0.21 & $=$ & 0.032 & 0.062 \\
\hline Columbia River & Pt of Pasco & 05-8002 & September 30, 1986 & Benzo(a)pyrene & $\mathrm{mg} / \mathrm{kg}$ dry-wt & 1 & 0.11 & $\mathrm{~J}$ & 0.032 & 0.062 \\
\hline Columbia River & Pt of Pasco & $05-8002$ & September 30,1986 & Bis(2-ethylhexyl) phthalate & $\mathrm{mg} / \mathrm{kg}$ dry-wt & 1 & 0.78 & B & 0.64 & 35 \\
\hline Columbia River & RM 318 & 168272 & April 14,1992 & Cadmium & $\mathrm{mg} / \mathrm{kg}$ dry-wt & 1 & $0.79^{-}$ & $\mathrm{P}$ & $0.60^{\circ}$ & 37 \\
\hline Columbia River & RM 316 & 168273 & April 14, 1992 & Cadmium & $\mathrm{mg} / \mathrm{kg}$ dry-wt & 1 & 0.87 & $\mathrm{P}$ & 0.60 & 37 \\
\hline Columbia River & RM 298 & 168275 & April 13, 1992 & Cadmium & $\mathrm{mg} / \mathrm{kg}$ dry-wt & 1 & 3.2 & $=$ & 0.60 & 37 \\
\hline Walla Walla River & Blw Warm Sprgs & $32 \mathrm{~A} 070-4$ & September 12,1984 & Cadmium & $\mathrm{mg} / \mathrm{kg}$ dry-wt & 2 & 0.70 & $=$ & 0.60 & 37 \\
\hline Columbia River & McNary Dam & 3504038 & June 1, 2004 & Cadmium & $\mathrm{mg} / \mathrm{kg}$ & 1 & 0.68 & $=$ & 0.60 & 37 \\
\hline Yakima River & Below Kiona & $37 A 070-7$ & September 12, 1984 & Cadmium & $\mathrm{mg} / \mathrm{kg}$ dry-wt & 2 & 1.4 & $=$ & 0.60 & 37 \\
\hline Columbia River & McNary Dam & 4414200 & June 1, 2004 & Cadmium & $\mathrm{mg} / \mathrm{kg}$ & 1 & 0.62 & $=$ & 0.60 & 37 \\
\hline Columbia River & McNary Dam & 4414202 & June 1,2004 & Cadmium & $\mathrm{mg} / \mathrm{kg}$ & 1 & 1.4 & $=$ & 0.60 & 37 \\
\hline Columbia River & McNary Dam & 4414203 & June 1, 2004 & Cadmium & $\mathrm{mg} / \mathrm{kg}$ & 1 & 2.1 & $=$ & 0.60 & 37 \\
\hline Snake River & RM 10 & 4414206 & June 1, 2004 & Cadmium & $\mathrm{mg} / \mathrm{kg}$ & 1 & 0.62 & $=$ & 0.60 & 37 \\
\hline Columbia River & Lake Wallula & 4414213 & June 1, 2004 & Cadmium & $\mathrm{mg} / \mathrm{kg}$ & 1 & 2.0 & $=$ & 0.60 & 37 \\
\hline Columbia River & Lake Wallula & 4424220 & June 1,2004 & Cadmium & $\mathrm{mg} / \mathrm{kg}$ & 1 & 0.64 & $=$ & 0.60 & 37 \\
\hline Columbia River & Lake Wallula & 4424224 & June 1, 2004 & Cadmium & $\mathrm{mg} / \mathrm{kg}$ & 1 & 1.3 & $=$ & 0.60 & 37 \\
\hline Columbia River & Lake Wallula & 4424225 & June 1, 2004 & Cadmium & $\mathrm{mg} / \mathrm{kg}$ & 1 & 1.4 & $=$ & 0.60 & 37 \\
\hline Columbia River & Lake Wallula & 4424226 & June 1, 2004 & Cadmium & $\mathrm{mg} / \mathrm{kg}$ & 1 & 1.2 & $=$ & 0.60 & 37 \\
\hline
\end{tabular}




\begin{tabular}{|c|c|c|c|c|c|c|c|c|c|c|}
\hline Sample Area & Sample Site & SampleID & Date Collected & Constituent Name & Units & QA Category & $\begin{array}{c}\text { Analytical } \\
\text { Value }\end{array}$ & Qualifier & $\begin{array}{l}\text { Benchmark } \\
\text { Value }\end{array}$ & $\begin{array}{l}\text { Benchmark } \\
\text { Value }\end{array}$ \\
\hline Columbia River & Lake Wallula & 4424227 & June 1,2004 & Cadmium & $\mathrm{mg} / \mathrm{kg}$ & 1 & 2.1 & $=$ & 0.60 & 37 \\
\hline Yakima River & RM 1 & 4434230 & June 1, 2004 & Cadmium & $\mathrm{mg} / \mathrm{kg}$ & 1 & 6.0 & $=$ & 0.60 & 37 \\
\hline Yakima River & RM 1 & 4434233 & June 1, 2004 & Cadmium & $\mathrm{mg} / \mathrm{kg}$ & 1 & 0.63 & $=$ & 0.60 & 37 \\
\hline Columbia River & RM 339.5 & 4434234 & June 1, 2004 & Cadmium & $\mathrm{mg} / \mathrm{kg}$ & 1 & 1.4 & $=$ & 0.60 & 37 \\
\hline Columbia River & McNary Dam 1 & B0CSG5 & September 23, 1994 & Cadmium & $\mathrm{mg} / \mathrm{kg}$ & 1 & 0.66 & $\mathrm{~L}$ & 0.60 & 37 \\
\hline Columbia River & McNary Dam 2 & B0CSG6 & September 23, 1994 & Cadmium & $\mathrm{mg} / \mathrm{kg}$ & 1 & 1.4 & $=$ & 0.60 & 37 \\
\hline Columbia River & McNary Dam & BOCSG7 & September 23, 1994 & Cadmium & $\mathrm{mg} / \mathrm{kg}$ & 1 & 1.6 & $=$ & 0.60 & 37 \\
\hline Columbia River & McNary Dam & BOW3D4 & August 2, 1999 & Cadmium & $\mathrm{mg} / \mathrm{kg}$ & 1 & 3.3 & $=$ & 0.60 & 37 \\
\hline Columbia River & McNary Dam & BOW3H2 & August 2, 1999 & Cadmium & $\mathrm{mg} / \mathrm{Kg}$ & 1 & 1.4 & $=$ & 0.60 & 37 \\
\hline Columbia River & McNary Dam & BOW3H4 & August 2, 1999 & Cadmium & $\mathrm{mg} / \mathrm{Kg}$ & 1 & 1.2 & $=$ & 0.60 & 37 \\
\hline Columbia River & McNary Dam & BOW3H6 & August 2, 1999 & Cadmium & $\mathrm{mg} / \mathrm{Kg}$ & 1 & 1.6 & $=$ & 0.60 & 37 \\
\hline Columbia River & McNary Dam & B0W3P1 & August 2, 1999 & Cadmium & $\mathrm{mg} / \mathrm{kg}$ & 1 & 2.2 & $=$ & 0.60 & 37 \\
\hline Columbia River & McNary Dam & BOYWDO & August 8,2000 & Cadmium & $\mathrm{mg} / \mathrm{kg}$ & 1 & 1.5 & $=$ & 0.60 & 37 \\
\hline Columbia River & McNary Dam & BOYWD2 & August 8,2000 & Cadmium & $\mathrm{mg} / \mathrm{Kg}$ & 1 & 5.4 & $=$ & 0.60 & 37 \\
\hline Snake River & ICE HARBOR DAM & $\mathrm{B} 12 \mathrm{CH} 7$ & July 23, 2001 & Cadmium & $\mathrm{mg} / \mathrm{kg}$ dry-wt & 1 & 1.9 & $=$ & 0.60 & 37 \\
\hline Columbia River & McNary Dam & B12CJ3 & July 20, 2001 & Cadmium & $\mathrm{mg} / \mathrm{kg}$ dry-wt & 1 & 1.6 & $=$ & 0.60 & 37 \\
\hline Columbia River & McNary Dam & B12CK3 & July 20,2001 & Cadmium & $\mathrm{mg} / \mathrm{kg}$ dry-wt & 1 & 0.94 & $=$ & 0.60 & 37 \\
\hline Columbia River & McNary Dam & B12CK5 & July 20, 2001 & Cadmium & $\mathrm{mg} / \mathrm{kg}$ dry-wt & 1 & 0.82 & $=$ & 0.60 & 37 \\
\hline Columbia River & McNary Dam & B12CK7 & July 20, 2001 & Cadmium & $\mathrm{mg} / \mathrm{kg}$ dry-wt & 1 & 2.2 & $=$ & 0.60 & 37 \\
\hline Columbia River & McNary Dam & B12CM7 & July 23, 2001 & Cadmium & $\mathrm{mg} / \mathrm{kg}$ dry-wt & 1 & 4.3 & $=$ & 0.60 & 37 \\
\hline Columbia River & McNary Dam & B12CM9 & July 23,2001 & Cadmium & $\mathrm{mg} / \mathrm{kg}$ dry-wt & 1 & 2.5 & $=$ & 0.60 & 37 \\
\hline Columbia River & McNary Dam & B17710 & July 16,2003 & Cadmium & $\mathrm{mg} / \mathrm{Kg}$ & 1 & 1.6 & $=$ & 0.60 & 37 \\
\hline Columbia River & McNary Dam & B17712 & July 16, 2003 & Cadmium & $\mathrm{mg} / \mathrm{kg}$ & 1 & 1.6 & $=$ & 0.60 & 37 \\
\hline Columbia River & McNary Dam & B17B60 & August 8,2003 & Cadmium & $\mathrm{mg} / \mathrm{kg}$ dry-wt & 1 & 1.9 & $=$ & 0.60 & 37 \\
\hline Columbia River & McNary Dam & B17B65 & August 8, 2003 & Cadmium & $\mathrm{mg} / \mathrm{kg}$ dry-wt & 1 & 1.4 & $=$ & 0.60 & 37 \\
\hline Columbia River & McNary Dam & B17B67 & August 8,2003 & Cadmium & $\mathrm{mg} / \mathrm{kg}$ dry-wt & 1 & 1.0 & $=$ & 0.60 & 37 \\
\hline Columbia River & McNary Dam & B17BJ3 & August 8, 2003 & Cadmium & $\mathrm{mg} / \mathrm{Kg}$ & 1 & 1.5 & $=$ & 0.60 & 37 \\
\hline Columbia River & McNary Dam & B19PL7 & July 13, 2004 & Cadmium & $\mathrm{mg} / \mathrm{kg}$ & 1 & 1.7 & $x$ & 0.60 & 37 \\
\hline Columbia River & McNary Dam & B19PL9 & July 13,2004 & Cadmium & $\mathrm{mg} / \mathrm{Kg}$ & 1 & 2.2 & $x$ & 0.60 & 37 \\
\hline Columbia River & McNary Dam & B1B7N9 & September 21, 2004 & Cadmium & $\mathrm{mg} / \mathrm{kg}$ & 1 & 2.9 & $x$ & 0.60 & 37 \\
\hline Columbia River & RM 328 & SRP 3,4 & August 20, 1991 & Cadmium & $\mathrm{mg} / \mathrm{kg}$ dry-wt & 1 & 1.6 & $=$ & 0.60 & 37 \\
\hline Snake River & ICE HARBOR DAM & Bow $3 \mathrm{C} 6$ & August 16,1999 & Chromium & $\mathrm{mg} / \mathrm{kg}$ & $\overline{1}$ & 47 & $=$ & 37 & 30 \\
\hline Snake River & ICE HARBOR DAM & BOW3C8 & August 16, 1999 & Chromium & $\mathrm{mg} / \mathrm{Kg}$ & 1 & 52 & $=$ & 37 & 30 \\
\hline Snake River & ICE HARBOR DAM & BOW3D0 & August 16, 1999 & Chromium & $\mathrm{mg} / \mathrm{kg}$ & 1 & 51 & $=$ & 37 & 30 \\
\hline Columbia River & McNary Dam & BOW3D4 & August 2, 1999 & Chromium & $\mathrm{mg} / \mathrm{Kg}$ & 1 & 63 & $=$ & 37 & 30 \\
\hline Columbia River & McNary Dam & BOW3H2 & August 2, 1999 & Chromium & $\mathrm{mg} / \mathrm{kg}$ & 1 & 59 & $=$ & 37 & 30 \\
\hline Columbia River & McNary Dam & BOW3H4 & August 2, 1999 & Chromium & $\mathrm{mg} / \mathrm{Kg}$ & 1 & 55 & $=$ & 37 & 30 \\
\hline Columbia River & McNary Dam & BOW3H6 & August 2, 1999 & Chromium & $\mathrm{mg} / \mathrm{kg}$ & 1 & 61 & $=$ & 37 & 30 \\
\hline Columbia River & McNary Dam & BOW3N9 & August 2, 1999 & Chromium & $\mathrm{mg} / \mathrm{Kg}$ & 1 & 61 & $=$ & 37 & 30 \\
\hline Columbia River & McNary Dam & BOW3P1 & August 2, 1999 & Chromium & $\mathrm{mg} / \mathrm{kg}$ & 1 & 55 & $=$ & 37 & 30 \\
\hline Columbia River & McNary Dam & BOYWDO & August 8,2000 & Chromium & $\mathrm{mg} / \mathrm{Kg}$ & 1 & 56 & $=$ & 37 & 30 \\
\hline Columbia River & McNary Dam & BOYWD2 & August 8,2000 & Chromium & $\mathrm{mg} / \mathrm{kg}$ & 1 & 57 & $=$ & 37 & 30 \\
\hline Snake River & ICE HARBOR DAM & $\mathrm{B} 12 \mathrm{CH} 5$ & July 23,2001 & Chromium & $\mathrm{mg} / \mathrm{kg}$ dry-wt & 1 & 56 & $=$ & 37 & 30 \\
\hline Snake River & ICE HARBOR DAM & $\mathrm{B} 12 \mathrm{CH} 7$ & July 23,2001 & Chromium & $\mathrm{mg} / \mathrm{kg}$ dry-wt & 1 & 71 & $=$ & 37 & 30 \\
\hline Snake River & ICE HARBOR DAM & $\mathrm{B} 12 \mathrm{CH} 9$ & July 23,2001 & Chromium & $\mathrm{mg} / \mathrm{kg}$ dry-wt & 1 & 59 & $=$ & 37 & 30 \\
\hline Columbia River & McNary Dam & B12CJ3 & July 20, 2001 & Chromium & $\mathrm{mg} / \mathrm{kg}$ dry-wt & 1 & 71 & $=$ & 37 & 30 \\
\hline
\end{tabular}




\begin{tabular}{|c|c|c|c|c|c|c|c|c|c|c|}
\hline Sample Area & Sample Site & SamplelD & Date Collected & Constituent Name & Units & QA Category & $\begin{array}{l}\text { Analytical } \\
\text { Value }\end{array}$ & Qualifier & $\begin{array}{c}\text { Ecological } \\
\text { Benchmark } \\
\text { Value }\end{array}$ & $\begin{array}{c}\text { Human } \\
\text { Health } \\
\text { Benchmark } \\
\text { Value }\end{array}$ \\
\hline Columbia River & McNary Dam & B12CK3 & July 20, 2001 & Chromium & $\mathrm{mg} / \mathrm{kg}$ dry-wt & 1 & 51 & $=$ & 37 & 30 \\
\hline Columbia River & McNary Dam & B12CK5 & July 20,2001 & Chromium & $\mathrm{mg} / \mathrm{kg}$ dry-wt & 1 & 60 & $=$ & 37 & 30 \\
\hline Columbia River & McNary Dam & $\mathrm{B} 12 \mathrm{CM} 7$ & July 23, 2001 & Chromium & $\mathrm{mg} / \mathrm{kg}$ dry-wt & 1 & 70 & $=$ & 37 & 30 \\
\hline Columbia River & McNary Dam & B12CM9 & July 23,2001 & Chromium & $\mathrm{mg} / \mathrm{kg}$ dry-wt & 1 & 70 & $=$ & 37 & 30 \\
\hline Yakima River & YAKIMA RIVER & B16WX0 & May 14,2003 & Chromium & $\mathrm{mg} / \mathrm{Kg}$ & 1 & 40 & $=$ & 37 & 30 \\
\hline Yakima River & YAKIMA RIVER & B16WX1 & May 14, 2003 & Chromium & $\mathrm{mg} / \mathrm{kg}$ & 1 & 35 & $=$ & 37 & 30 \\
\hline Columbia River & McNary Dam & B17710 & July 16, 2003 & Chromium & $\mathrm{mg} / \mathrm{Kg}$ & 1 & 71 & c & 37 & 30 \\
\hline Columbia River & McNary Dam & B17712 & July 16, 2003 & Chromium & $\mathrm{mg} / \mathrm{Kg}$ & 1 & 74 & c & 37 & 30 \\
\hline Columbia River & McNary Dam & B19PL7 & July 13, 2004 & Chromium & $\mathrm{mg} / \mathrm{kg}$ & 1 & 64 & $c x$ & 37 & 30 \\
\hline Columbia River & McNary Dam & B19PL9 & July 13,2004 & Chromium & $\mathrm{mg} / \mathrm{Kg}$ & 1 & 68 & $\mathrm{CX}$ & 37 & 30 \\
\hline Columbia River & McNary Dam & B1B7N9 & September 21, 2004 & Chromium & $\mathrm{mg} / \mathrm{kg}$ & 1 & 62 & $x$ & 37 & 30 \\
\hline Snake River & ICE HARBOR DAM & B1B7W8 & September 20, 2004 & Chromium & $\mathrm{mg} / \mathrm{Kg}$ & 1 & 51 & $x$ & 37 & 30 \\
\hline Yakima River & Kiona WA & Yakima sed & August 25, 1987 & Chromium & $\mathrm{mg} / \mathrm{kg}$ & 1 & 61 & $=$ & 37 & 30 \\
\hline Columbia River & $\mathrm{Pt}$ of Pasco & $05-8002$ & September 30,1986 & Chrysene & $\mathrm{mg} / \mathrm{kg}$ dry-wt & 1 & 0.13 & j & 0.057 & 62 \\
\hline Yakima River & Below Kiona & $37 \mathrm{~A} 070-7$ & September 12,1984 & Copper & $\mathrm{mg} / \mathrm{kg}$ dry-wt & 2 & 36 & $=$ & 32 & 2,960 \\
\hline Columbia River & McNary Dam & BOW3D4 & August 2, 1999 & Copper & $\mathrm{mg} / \mathrm{Kg}$ & 1 & 34 & $=$ & 32 & 2,960 \\
\hline Columbia River & McNary Dam & BOW3H2 & August 2, 1999 & Copper & $\mathrm{mg} / \mathrm{Kg}$ & 1 & 33 & $=$ & 32 & 2,960 \\
\hline Columbia River & McNary Dam & BOW3N9 & August 2, 1999 & Copper & $\mathrm{mg} / \mathrm{Kg}$ & 1 & 33 & $=$ & 32 & 2,960 \\
\hline Columbia River & McNary Dam & BOYWDO & August 8,2000 & Copper & $\mathrm{mg} / \mathrm{kg}$ & 1 & 37 & $=$ & 32 & 2,960 \\
\hline Snake River & ICE HARBOR DAM & $\mathrm{B} 12 \mathrm{CH} 9$ & July 23,2001 & Copper & $\mathrm{mg} / \mathrm{kg}$ dry-wt & 1 & 33 & $=$ & 32 & 2,960 \\
\hline Columbia River & McNary Dam & $\mathrm{B} 12 \mathrm{CJ} 3$ & July 20,2001 & Copper & $\mathrm{mg} / \mathrm{kg}$ dry-wt & 1 & 36 & $=$ & 32 & 2,960 \\
\hline Columbia River & McNary Dam & B12CK7 & July 20,2001 & Copper & $\mathrm{mg} / \mathrm{kg}$ dry-wt & 1 & 33 & $=$ & 32 & 2,960 \\
\hline Columbia River & McNary Dam & $\mathrm{B} 12 \mathrm{CM} 7$ & July 23, 2001 & Copper & $\mathrm{mg} / \mathrm{kg}$ dry-wt & 1 & 39 & $=$ & 32 & 2,960 \\
\hline Columbia River & McNary Dam & B12CM9 & July 23,2001 & Copper & $\mathrm{mg} / \mathrm{kg}$ dry-wt & 1 & 37 & $=$ & 32 & 2,960 \\
\hline Columbia River & McNary Dam & B17710 & July 16, 2003 & Copper & $\mathrm{mg} / \mathrm{Kg}$ & 1 & 37 & $=$ & 32 & 2,960 \\
\hline Columbia River & McNary Dam & B19PL7 & July 13,2004 & Copper & $\mathrm{mg} / \mathrm{Kg}$ & 1 & 36 & $x$ & 32 & 2,960 \\
\hline Columbia River & McNary Dam & B19PL9 & July 13,2004 & Copper & $\mathrm{mg} / \mathrm{Kg}$ & 1 & 34 & $\mathrm{x}$ & 32 & 2,960 \\
\hline Columbia River & McNary Dam & B1B7N9 & September 21, 2004 & Copper & $\mathrm{mg} / \mathrm{Kg}$ & 1 & 37 & $x$ & 32 & 2,960 \\
\hline Snake River & ICE HARBOR DAM & B1B7W8 & September 20, 2004 & Copper & $\mathrm{mg} / \mathrm{kg}$ & 1 & 34 & $\mathrm{x}$ & 32 & 2,960 \\
\hline Yakima River & Below Kiona & $37 \mathrm{~A} 070-7$ & September 12,1984 & Dichlorodiphenyldichloroethane & $\mathrm{mg} / \mathrm{kg}$ dry-wt & 2 & $0.023^{-}$ & $=$ & 0.0040 & 2.4 \\
\hline Yakima River & RM 18 & 8140 & September 24, 1985 & Dichlorodiphenyldichloroethane & $\mathrm{mg} / \mathrm{kg}$ dry-wt & 1 & 0.0053 & $=$ & 0.0040 & 2.4 \\
\hline Columbia River & RM 274-286 & CRUSD128 & November 1, 1991 & Dichlorodiphenyldichloroethane & $\mathrm{mg} / \mathrm{kg}$ dry-wt & 1 & 0.020 & $\mathrm{~J}$ & 0.0040 & 2.4 \\
\hline Columbia River & McNary Dam & MD-mid river-1 & October 1, 1986 & Dichlorodiphenyldichloroethane & $\mathrm{mg} / \mathrm{kg}$ dry-wt & 3 & 0.010 & $=$ & 0.0040 & 2.4 \\
\hline Columbia River & McNary Dam & MD-mid river-2 & October 1, 1986 & Dichlorodiphenyldichloroethane & $\mathrm{mg} / \mathrm{kg}$ dry-wt & 3 & 0.0090 & $=$ & 0.0040 & 2.4 \\
\hline Columbia River & McNary Dam & MD-north shore & October 1, 1986 & Dichlorodiphenyldichloroethane & $\mathrm{mg} / \mathrm{kg}$ dry-wt & 3 & 0.0090 & D & 0.0040 & 2.4 \\
\hline Yákima River & Below Kiona & $3 \bar{A} \overline{0} \overline{0} 0-\overline{7}$ & September 12,1984 & Dichlorodiphenyldichloroethylene & $\mathrm{mg} / \mathrm{kg}$ dry-wt & 2 & $0.051^{-}$ & $=$ & 0.0015 & 1.7 \\
\hline Yakima River & RM 18 & 8140 & September 24, 1985 & Dichlorodiphenyldichloroethylene & $\mathrm{mg} / \mathrm{kg}$ dry-wt & 1 & 0.030 & $=$ & 0.0015 & 1.7 \\
\hline Walla Walla River & Near mouth & 92388089 & September 16, 1992 & Dichlorodiphenyldichloroethylene & $\mathrm{mg} / \mathrm{Kg}$ & 1 & 0.0080 & $\mathrm{~J}$ & 0.0015 & 1.7 \\
\hline Yakima River & Horn Rapids Dam & 92388096 & September 16, 1992 & Dichlorodiphenyldichloroethylene & $\mathrm{mg} / \mathrm{Kg}$ & 1 & 0.015 & $\mathrm{~J}$ & 0.0015 & 1.7 \\
\hline Yakima River & Horn Rapids Dam & 92388097 & September 16, 1992 & Dichlorodiphenyldichloroethylene & $\mathrm{mg} / \mathrm{Kg}$ & 1 & 0.016 & $\mathrm{~J}$ & 0.0015 & 1.7 \\
\hline Snake River & Lake Sacajawea & 94378145 & September 14, 1994 & Dichlorodiphenyldichloroethylene & $\mathrm{mg} / \mathrm{kg}$ dry-wt & 1 & 0.0020 & $\mathrm{~J}$ & 0.0015 & 1.7 \\
\hline Yakima River & Kiona WA & Bed sediment \#1 & August 31, 1988 & Dichlorodiphenyldichloroethylene & $\mathrm{mg} / \mathrm{kg}$ dry-wt & 1 & 0.0030 & $=$ & 0.0015 & 1.7 \\
\hline Yakima River & Kiona WA & Bed sediment \#2 & August 31, 1988 & Dichlorodiphenyldichloroethylene & $\mathrm{mg} / \mathrm{kg}$ dry-wt & 1 & 0.0027 & $=$ & 0.0015 & 1.7 \\
\hline
\end{tabular}




\begin{tabular}{|c|c|c|c|c|c|c|c|c|c|c|}
\hline Sample Area & Sample Site & SampleID & Date Collected & Constituent Name & Units & QA Category & $\begin{array}{c}\text { Analytical } \\
\text { Value }\end{array}$ & Qualifier & $\begin{array}{c}\text { Ecological } \\
\text { Benchmark } \\
\text { Value }\end{array}$ & $\begin{array}{c}\text { Human } \\
\text { Health } \\
\text { Benchmark } \\
\text { Value }\end{array}$ \\
\hline Columbia River & McNary Dam & MD-mid river-2 & October 1, 1986 & Dichlorodiphenyldichloroethylene & $\mathrm{mg} / \mathrm{kg}$ dry-wt & $\overline{3}$ & 0.0050 & $=$ & 0.0015 & 1.7 \\
\hline Columbia River & McNary Dam & MD-north shore & October 1, 1986 & Dichlorodiphenyldichloroethylene & $\mathrm{mg} / \mathrm{kg}$ dry-wt & 3 & 0.0060 & D & 0.0015 & 1.7 \\
\hline Columbia River & RM 314-315 & SRP 1,2 & August 19, 1991 & Dichlorodiphenyldichloroethylene & $\mathrm{mg} / \mathrm{kg}$ dry-wt & 1 & 0.0024 & $=$ & 0.0015 & 1.7 \\
\hline Columbia River & RM 315-316 & SRP 51 & August 19, 1991 & Dichlorodiphenyldichloroethylene & $\mathrm{mg} / \mathrm{kg}$ dry-wt & 1 & 0.0020 & $=$ & 0.0015 & 1.7 \\
\hline Walla Walla River & Cummins Rd Bdg & Walla 003 & June 18,1996 & Dichlorodiphenyldichloroethylene & $\mathrm{mg} / \mathrm{kg}$ dry-wt & 1 & 0.0076 & $=$ & 0.0015 & 1.7 \\
\hline Yakima River & Below Kiona & $37 \mathrm{~A} 070-7$ & September 12,1984 & Dichlorodiphenyltrichloroethane & $\mathrm{mg} / \mathrm{kg}$ dry-wt & 2 & 0.035 & $=$ & 0.0042 & 1.7 \\
\hline Yakima River & Kiona WA & Bed sediment $\# 2$ & August 31,1988 & Dichlorodiphenyltrichloroethane & $\mathrm{mg} / \mathrm{kg}$ dry-wt & 1 & 0.010 & $=$ & 0.0042 & 1.7 \\
\hline Columbia River & $\mathrm{Pt}$ of Pasco & $05-8000$ & September 30,1986 & Fluoranthene & $\mathrm{mg} / \mathrm{kg}$ dry-wt & $\overline{1}$ & 0.12 & $\mathrm{j}$ & 0.11 & 2,294 \\
\hline Columbia River & Pt of Pasco & 05-8001 & September 30, 1986 & Fluoranthene & $\mathrm{mg} / \mathrm{kg}$ dry-wt & 1 & 0.12 & $\mathrm{~J}$ & 0.11 & 2,294 \\
\hline Columbia River & Pt of Pasco & 05-8002 & September 30, 1986 & Fluoranthene & $\mathrm{mg} / \mathrm{kg}$ dry-wt & 1 & 0.20 & $\mathrm{~J}$ & 0.11 & 2,294 \\
\hline Walla Wailla River & Near mouth & 92388089 & September 16,1992 & Gamma-BHC (Lindane) & $\mathrm{mg} / \mathrm{Kg}$ & 1 & 0.0080 & j & $9.00 \mathrm{E}-04$ & \\
\hline Columbia River & McNary Dam & BOCSG8 & September 23,1994 & $\begin{array}{l}\text { Iron } \\
\text { - }\end{array}$ & $\mathrm{mg} / \mathrm{kg}$ & $\overline{1}$ & 22,000 & $=$ & 20,000 & 23,463 \\
\hline Columbia River & McNary Dam & B17B57 & August 8,2003 & Iron & $\mathrm{mg} / \mathrm{kg}$ dry-wt & 1 & 24,000 & $=$ & 20,000 & 23,463 \\
\hline Columbia River & McNary Dam & B17B60 & August 8, 2003 & Iron & $\mathrm{mg} / \mathrm{kg}$ dry-wt & 1 & 34,000 & $=$ & 20,000 & 23,463 \\
\hline Columbia River & McNary Dam & B17B65 & August 8, 2003 & Iron & $\mathrm{mg} / \mathrm{kg}$ dry-wt & 1 & 27,000 & $=$ & 20,000 & 23,463 \\
\hline Columbia River & McNary Dam & B17B67 & August 8,2003 & Iron & $\mathrm{mg} / \mathrm{kg}$ dry-wt & 1 & 24,000 & $=$ & 20,000 & 23,463 \\
\hline Yakima River & Kiona WA & Yakima sed & August 25,1987 & Iron & $\mathrm{mg} / \mathrm{Kg}$ & 1 & 51,000 & $=$ & 20,000 & 23,463 \\
\hline Columbia River & $\mathrm{Pt}$ of Pasco & $40-8326$ & September 30,1986 & Lead & $\mathrm{mg} / \mathrm{kg}$ dry-wt & $\overline{1}$ & 58 & $=$ & $35^{-}$ & 250 \\
\hline Columbia River & RM 298 & 168275 & April 13, 1992 & Lead & $\mathrm{mg} / \mathrm{kg}$ dry-wt & 1 & 39 & $=$ & 35 & 250 \\
\hline Cölumbia River & McNary Dam & B17B60 & August 8,2003 & Manganese & $\mathrm{mg} / \mathrm{kg}$ dry-wt & 1 & $640^{-}$ & $=$ & 460 & 1,762 \\
\hline Columbia River & McNary Dam & B17B65 & August 8,2003 & Manganese & $\mathrm{mg} / \mathrm{kg}$ dry-wt & 1 & 630 & $=$ & 460 & 1,762 \\
\hline Yakima River & Kiona WA & Yakima sed & August 25, 1987 & Manganese & $\mathrm{mg} / \mathrm{kg}$ & 1 & 1,500 & $=$ & 460 & 1,762 \\
\hline Columbia River & McNary Dam & $\mathrm{B} 12 \mathrm{CM} 7$ & July 23,2001 & Mercury & $\mathrm{mg} / \mathrm{kg}$ dry-wt & 1 & 0.31 & $=$ & 0.18 & 23 \\
\hline Columbia River & McNary Dam & B17B60 & August 8, 2003 & Mercury & $\mathrm{mg} / \mathrm{kg}$ dry-wt & 1 & 0.25 & $=$ & 0.18 & 23 \\
\hline Columbia River & McNary Dam & B17B65 & August 8,2003 & Mercury & $\mathrm{mg} / \mathrm{kg}$ dry-wt & 1 & 0.19 & $=$ & 0.18 & 23 \\
\hline Columbia River & $\mathrm{RM} 298$ & 168275 & April 13,1992 & Nickel- & $\mathrm{mg} / \mathrm{kg}$ dry-wt & $\overline{1}$ & $21^{-}$ & $=$ & 18 & 1,564 \\
\hline Columbia River & McNary Dam & 4414203 & June 1, 2004 & Nickel & $\mathrm{mg} / \mathrm{kg}$ & 1 & 21 & $=$ & 18 & 1,564 \\
\hline Columbia River & Lake Wallula & 4424225 & June 1, 2004 & Nickel & $\mathrm{mg} / \mathrm{kg}$ & 1 & 22 & $=$ & 18 & 1,564 \\
\hline Columbia River & Lake Wallula & 4424226 & June 1, 2004 & Nickel & $\mathrm{mg} / \mathrm{kg}$ & 1 & 25 & $=$ & 18 & 1,564 \\
\hline Columbia River & Lake Wallula & 4424227 & June 1,2004 & Nickel & $\mathrm{mg} / \mathrm{kg}$ & 1 & 20 & $=$ & 18 & 1,564 \\
\hline Yakima River & RM 1 & 4434230 & June 1, 2004 & Nickel & $\mathrm{mg} / \mathrm{kg}$ & 1 & 22 & $=$ & 18 & 1,564 \\
\hline Yakima River & RM 1 & 4434231 & June 1, 2004 & Nickel & $\mathrm{mg} / \mathrm{kg}$ & 1 & 24 & $=$ & 18 & 1,564 \\
\hline Yakima River & RM 1 & 4434233 & June 1, 2004 & Nickel & $\mathrm{mg} / \mathrm{kg}$ & 1 & 20 & $=$ & 18 & 1,564 \\
\hline Yakima River & RM 2 & 4434242 & June 1,2004 & Nickel & $\mathrm{mg} / \mathrm{kg}$ & 1 & 24 & $=$ & 18 & 1,564 \\
\hline Yakima River & RM 2 & 4434243 & June 1, 2004 & Nickel & $\mathrm{mg} / \mathrm{kg}$ & 1 & 27 & $=$ & 18 & 1,564 \\
\hline Yakima River & RM 1 & 4434244 & June 1, 2004 & Nickel & $\mathrm{mg} / \mathrm{kg}$ & 1 & 27 & $=$ & 18 & 1,564 \\
\hline Snake River & ICE HARBOR DAM & BOW3C8 & August 16, 1999 & Nickel & $\mathrm{mg} / \mathrm{Kg}$ & 1 & 22 & $=$ & 18 & 1,564 \\
\hline Snake River & ICE HARBOR DAM & BOW3D0 & August 16, 1999 & Nickel & $\mathrm{mg} / \mathrm{Kg}$ & 1 & 23 & $=$ & 18 & 1,564 \\
\hline Columbia River & McNary Dam & BOW3D4 & August 2, 1999 & Nickel & $\mathrm{mg} / \mathrm{Kg}$ & 1 & 29 & $=$ & 18 & 1,564 \\
\hline Columbia River & McNary Dam & BOW3H2 & August 2, 1999 & Nickel & $\mathrm{mg} / \mathrm{kg}$ & 1 & 29 & $=$ & 18 & 1,564 \\
\hline Columbia River & McNary Dam & BOW3H4 & August 2, 1999 & Nickel & $\mathrm{mg} / \mathrm{Kg}$ & 1 & 23 & $=$ & 18 & 1,564 \\
\hline Columbia River & McNary Dam & BOW3H6 & August 2, 1999 & Nickel & $\mathrm{mg} / \mathrm{Kg}$ & 1 & 30 & $=$ & 18 & 1,564 \\
\hline Columbia River & McNary Dam & BOW3N9 & August 2, 1999 & Nickel & $\mathrm{mg} / \mathrm{Kg}$ & 1 & 29 & $=$ & 18 & 1,564 \\
\hline
\end{tabular}




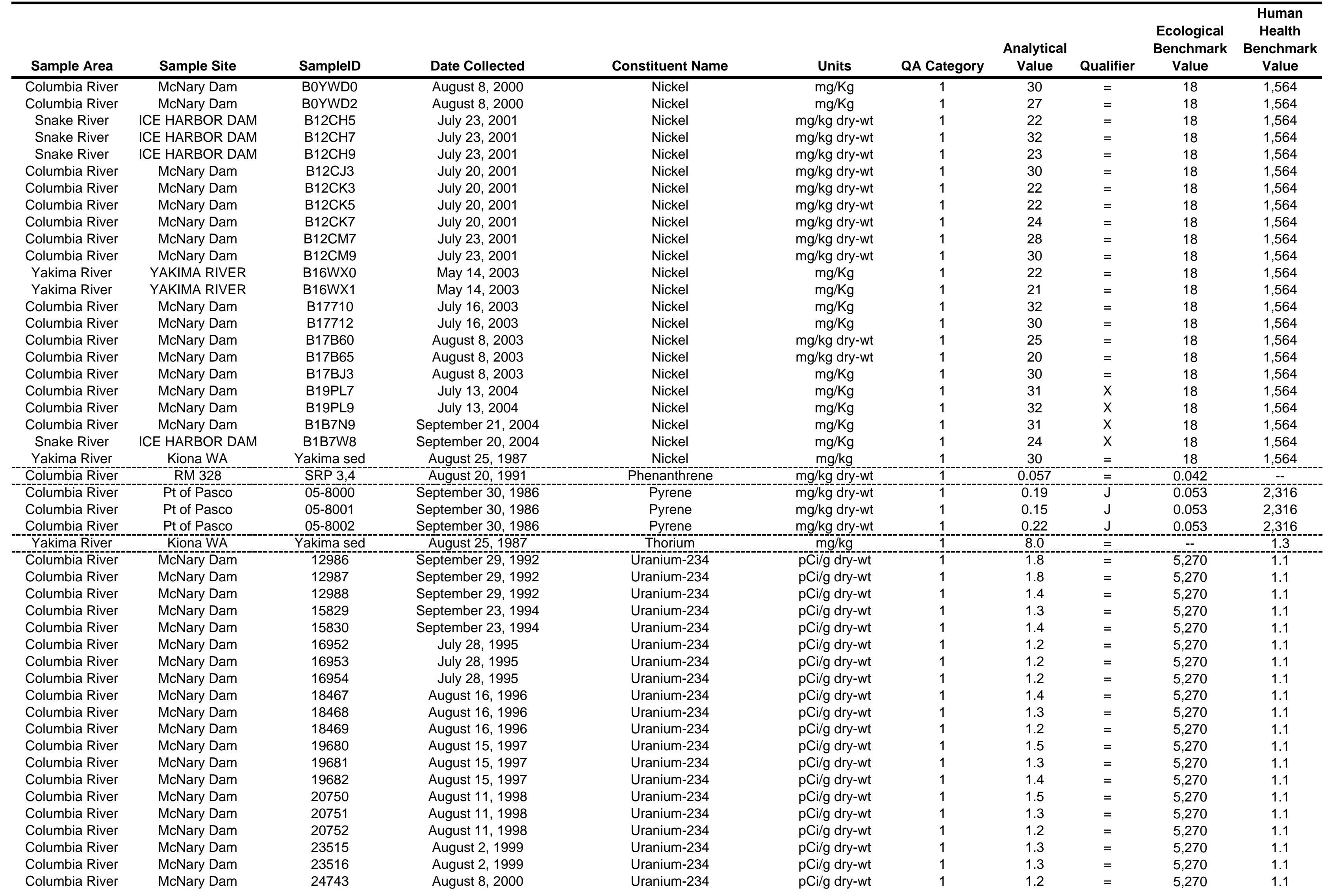




\begin{tabular}{|c|c|c|c|c|c|c|c|c|c|c|}
\hline Sample Area & Sample Site & SampleID & Date Collected & Constituent Name & Units & QA Category & $\begin{array}{c}\text { Analytical } \\
\text { Value }\end{array}$ & Qualifier & $\begin{array}{c}\text { Ecological } \\
\text { Benchmark } \\
\text { Value }\end{array}$ & $\begin{array}{c}\text { Human } \\
\text { Health } \\
\text { Benchmark } \\
\text { Value }\end{array}$ \\
\hline Columbia River & McNary Dam & 24746 & August 8,2000 & Uranium-234 & pCi/g dry-wt & 1 & 1.2 & $=$ & 5,270 & 1.1 \\
\hline Columbia River & McNary Dam & 26248 & July 23,2001 & Uranium-234 & $\mathrm{pCi} / \mathrm{g}$ dry-wt & 1 & 1.2 & $=$ & 5,270 & 1.1 \\
\hline Columbia River & McNary Dam & 29043 & July 19, 2002 & Uranium-234 & $\mathrm{pCi} / \mathrm{g}$ dry-wt & 1 & 1.3 & $=$ & 5,270 & 1.1 \\
\hline Columbia River & McNary Dam & 29044 & July 19, 2002 & Uranium-234 & pCi/g dry-wt & 1 & 1.2 & $=$ & 5,270 & 1.1 \\
\hline Columbia River & McNary Dam & 30870 & July 16, 2003 & Uranium-234 & $\mathrm{pCi} / \mathrm{g}$ dry-wt & 1 & 1.4 & $=$ & 5,270 & 1.1 \\
\hline Columbia River & McNary Dam & 30871 & July 16,2003 & Uranium-234 & $\mathrm{pCi} / \mathrm{g}$ dry-wt & 1 & 1.6 & $=$ & 5,270 & 1.1 \\
\hline Columbia River & McNary Dam & 31810 & August 8, 2003 & Uranium-234 & $\mathrm{pCi} / \mathrm{g}$ dry-wt & 1 & 1.6 & $=$ & 5,270 & 1.1 \\
\hline Columbia River & McNary Dam & 31811 & August 8, 2003 & Uranium-234 & $\mathrm{pCi} / \mathrm{g}$ dry-wt & 1 & 1.5 & $=$ & 5,270 & 1.1 \\
\hline Columbia River & McNary Dam & 33603 & September 21, 2004 & Uranium-234 & $\mathrm{pCi} / \mathrm{g}$ dry-wt & 1 & 1.2 & $=$ & 5,270 & 1.1 \\
\hline Snake River & ICE HARBOR DAM & B1B7T8 & September 20,2004 & Uranium-234 & $\mathrm{pCi} / \mathrm{g}$ & 1 & 1.9 & $=$ & 5,270 & 1.1 \\
\hline Columbia River & McNary Dam & 12986 & September 29,1992 & Uranium-238 & pCi/g dry-wt & 1 & 1.6 & $=$ & 2,490 & 1.1 \\
\hline Columbia River & McNary Dam & 12987 & September 29, 1992 & Uranium-238 & $\mathrm{pCi} / \mathrm{g}$ dry-wt & 1 & 1.6 & $=$ & 2,490 & 1.1 \\
\hline Columbia River & McNary Dam & 12988 & September 29, 1992 & Uranium-238 & $\mathrm{pCi} / \mathrm{g}$ dry-wt & 1 & 1.2 & $=$ & 2,490 & 1.1 \\
\hline Columbia River & McNary Dam & 18467 & August 16, 1996 & Uranium-238 & $\mathrm{pCi} / \mathrm{g}$ dry-wt & 1 & 1.2 & $=$ & 2,490 & 1.1 \\
\hline Columbia River & McNary Dam & 18468 & August 16,1996 & Uranium-238 & $\mathrm{pCi} / \mathrm{g}$ dry-wt & 1 & 1.2 & $=$ & 2,490 & 1.1 \\
\hline Columbia River & McNary Dam & 19680 & August 15, 1997 & Uranium-238 & $\mathrm{pCi} / \mathrm{g}$ dry-wt & 1 & 1.3 & $=$ & 2,490 & 1.1 \\
\hline Columbia River & McNary Dam & 19681 & August 15, 1997 & Uranium-238 & $\mathrm{pCi} / \mathrm{g}$ dry-wt & 1 & 1.2 & $=$ & 2,490 & 1.1 \\
\hline Columbia River & McNary Dam & 19682 & August 15, 1997 & Uranium-238 & $\mathrm{pCi} / \mathrm{g}$ dry-wt & 1 & 1.3 & $=$ & 2,490 & 1.1 \\
\hline Columbia River & McNary Dam & 20751 & August 11,1998 & Uranium-238 & $\mathrm{pCi} / \mathrm{g}$ dry-wt & 1 & 1.2 & $=$ & 2,490 & 1.1 \\
\hline Columbia River & McNary Dam & 23516 & August 2, 1999 & Uranium-238 & $\mathrm{pCi} / \mathrm{g}$ dry-wt & 1 & 1.3 & $=$ & 2,490 & 1.1 \\
\hline Columbia River & McNary Dam & 29044 & July 19, 2002 & Uranium-238 & $\mathrm{pCi} / \mathrm{g}$ dry-wt & 1 & 1.1 & $=$ & 2,490 & 1.1 \\
\hline Columbia River & McNary Dam & 30870 & July 16,2003 & Uranium-238 & $\mathrm{pCi} / \mathrm{g}$ dry-wt & 1 & 1.3 & $=$ & 2,490 & 1.1 \\
\hline Columbia River & McNary Dam & 30871 & July 16,2003 & Uranium-238 & $\mathrm{pCi} / \mathrm{g}$ dry-wt & 1 & 1.2 & $=$ & 2,490 & 1.1 \\
\hline Columbia River & McNary Dam & 31810 & August 8,2003 & Uranium-238 & $\mathrm{pCi} / \mathrm{g}$ dry-wt & 1 & 1.3 & $=$ & 2,490 & 1.1 \\
\hline Columbia River & McNary Dam & 31811 & August 8,2003 & Uranium-238 & $\mathrm{pCi} / \mathrm{g}$ dry-wt & 1 & 1.4 & $=$ & 2,490 & 1.1 \\
\hline Columbia River & McNary Dam & 31812 & August 8,2003 & Uranium-238 & $\mathrm{pCi} / \mathrm{g}$ dry-wt & 1 & 1.4 & $=$ & 2,490 & 1.1 \\
\hline Columbia River & McNary Dam & 33603 & September 21, 2004 & Uranium-238 & $\mathrm{pCi} / \mathrm{g}$ dry-wt & 1 & 1.2 & $=$ & 2,490 & 1.1 \\
\hline Columbia River & McNary Dam & BOBRS5 & April 11, 1994 & Uranium-238 & $\mathrm{pCi} / \mathrm{g}$ & 1 & 1.3 & $=$ & 2,490 & 1.1 \\
\hline Columbia River & McNary Dam & BOBRS7 & April 11, 1994 & Uranium-238 & $\mathrm{pCi} / \mathrm{g}$ & 1 & 1.6 & $=$ & 2,490 & 1.1 \\
\hline Columbia River & McNary Dam & BOBRS9 & April 11, 1994 & Uranium-238 & $\mathrm{pCi} / \mathrm{g}$ & 1 & 1.5 & $=$ & 2,490 & 1.1 \\
\hline Snake River & ICE HARBOR DAM & B1B7T8 & September 20,2004 & Uranium-238 & $\mathrm{pCi} / \mathrm{g}$ & 1 & 1.3 & $=$ & 2,490 & 1.1 \\
\hline Yákima River & Kiona WA & Yakima sed & August 25,1987 & Vanadium & $\mathrm{mg} / \mathrm{kg}$ & 1 & $140^{-}$ & $=$ & --:- & 78 \\
\hline Columbia River & $R M 298$ & 168275 & April 13,1992 & Zinc & $\mathrm{mg} / \mathrm{kg}$ dry-wt & 1 & 284 & $\mathrm{j}$ & 120 & 23,463 \\
\hline Columbia River & McNary Dam & 4414200 & June 1, 2004 & Zinc & $\mathrm{mg} / \mathrm{kg}$ & 1 & 131 & $=$ & 120 & 23,463 \\
\hline Columbia River & McNary Dam & 4414203 & June 1, 2004 & Zinc & $\mathrm{mg} / \mathrm{kg}$ & 1 & 178 & $=$ & 120 & 23,463 \\
\hline Columbia River & Lake Wallula & 4414213 & June 1, 2004 & Zinc & $\mathrm{mg} / \mathrm{kg}$ & 1 & 247 & $=$ & 120 & 23,463 \\
\hline Columbia River & Lake Wallula & 4424224 & June 1, 2004 & Zinc & $\mathrm{mg} / \mathrm{kg}$ & 1 & 124 & $=$ & 120 & 23,463 \\
\hline Columbia River & Lake Wallula & 4424225 & June 1, 2004 & Zinc & $\mathrm{mg} / \mathrm{kg}$ & 1 & 195 & $=$ & 120 & 23,463 \\
\hline Columbia River & Lake Wallula & 4424226 & June 1,2004 & Zinc & $\mathrm{mg} / \mathrm{kg}$ & 1 & 163 & $=$ & 120 & 23,463 \\
\hline Columbia River & Lake Wallula & 4424227 & June 1, 2004 & Zinc & $\mathrm{mg} / \mathrm{kg}$ & 1 & 241 & $=$ & 120 & 23,463 \\
\hline Yakima River & RM 1 & 4434230 & June 1,2004 & Zinc & $\mathrm{mg} / \mathrm{kg}$ & 1 & 558 & $=$ & 120 & 23,463 \\
\hline Columbia River & RM 339.5 & 4434234 & June 1, 2004 & Zinc & $\mathrm{mg} / \mathrm{kg}$ & 1 & 209 & $=$ & 120 & 23,463 \\
\hline Columbia River & McNary Dam & BOCSG7 & September 23, 1994 & Zinc & $\mathrm{mg} / \mathrm{kg}$ & 1 & 150 & $=$ & 120 & 23,463 \\
\hline
\end{tabular}




\begin{tabular}{|c|c|c|c|c|c|c|c|c|c|c|}
\hline Sample Area & Sample Site & SampleID & Date Collected & Constituent Name & Units & QA Category & $\begin{array}{c}\text { Analytical } \\
\text { Value }\end{array}$ & Qualifier & $\begin{array}{c}\text { Ecological } \\
\text { Benchmark } \\
\text { Value }\end{array}$ & $\begin{array}{c}\text { Human } \\
\text { Health } \\
\text { Benchmark } \\
\text { Value }\end{array}$ \\
\hline Columbia River & McNary Dam & BOW3H4 & August 2, 1999 & Zinc & $\mathrm{mg} / \mathrm{Kg}$ & 1 & 208 & $=$ & 120 & 23,463 \\
\hline Columbia River & McNary Dam & BOW3H6 & August 2, 1999 & Zinc & $\mathrm{mg} / \mathrm{Kg}$ & 1 & 241 & $=$ & 120 & 23,463 \\
\hline Columbia River & McNary Dam & B0W3P1 & August 2, 1999 & Zinc & $\mathrm{mg} / \mathrm{Kg}$ & 1 & 282 & $=$ & 120 & 23,463 \\
\hline Columbia River & McNary Dam & BOYWDO & August 8, 2000 & Zinc & $\mathrm{mg} / \mathrm{Kg}$ & 1 & 244 & $=$ & 120 & 23,463 \\
\hline Columbia River & McNary Dam & BOYWD2 & August 8,2000 & Zinc & $\mathrm{mg} / \mathrm{Kg}$ & 1 & 483 & $=$ & 120 & 23,463 \\
\hline Snake River & ICE HARBOR DAM & $\mathrm{B} 12 \mathrm{CH} 5$ & July 23, 2001 & Zinc & $\mathrm{mg} / \mathrm{kg}$ dry-wt & 1 & 136 & $=$ & 120 & 23,463 \\
\hline Snake River & ICE HARBOR DAM & $\mathrm{B} 12 \mathrm{CH} 7$ & July 23, 2001 & Zinc & $\mathrm{mg} / \mathrm{kg}$ dry-wt & 1 & 316 & $=$ & 120 & 23,463 \\
\hline Columbia River & McNary Dam & $\mathrm{B} 12 \mathrm{CJ} 3$ & July 20,2001 & Zinc & $\mathrm{mg} / \mathrm{kg}$ dry-wt & 1 & 280 & $=$ & 120 & 23,463 \\
\hline Columbia River & McNary Dam & B12CK3 & July 20,2001 & Zinc & $\mathrm{mg} / \mathrm{kg}$ dry-wt & 1 & 175 & $=$ & 120 & 23,463 \\
\hline Columbia River & McNary Dam & B12CK5 & July 20, 2001 & Zinc & $\mathrm{mg} / \mathrm{kg}$ dry-wt & 1 & 223 & $=$ & 120 & 23,463 \\
\hline Columbia River & McNary Dam & B12CK7 & July 20, 2001 & Zinc & $\mathrm{mg} / \mathrm{kg}$ dry-wt & 1 & 375 & $=$ & 120 & 23,463 \\
\hline Columbia River & McNary Dam & B12CM7 & July 23,2001 & Zinc & $\mathrm{mg} / \mathrm{kg}$ dry-wt & 1 & 345 & $=$ & 120 & 23,463 \\
\hline Columbia River & McNary Dam & B12CM9 & July 23,2001 & Zinc & $\mathrm{mg} / \mathrm{kg}$ dry-wt & 1 & 322 & $=$ & 120 & 23,463 \\
\hline Columbia River & McNary Dam & B17710 & July 16, 2003 & Zinc & $\mathrm{mg} / \mathrm{Kg}$ & 1 & 247 & $=$ & 120 & 23,463 \\
\hline Columbia River & McNary Dam & B17712 & July 16, 2003 & Zinc & $\mathrm{mg} / \mathrm{Kg}$ & 1 & 275 & $=$ & 120 & 23,463 \\
\hline Columbia River & McNary Dam & B17B60 & August 8,2003 & Zinc & $\mathrm{mg} / \mathrm{kg}$ dry-wt & 1 & 220 & $=$ & 120 & 23,463 \\
\hline Columbia River & McNary Dam & B17B65 & August 8,2003 & Zinc & $\mathrm{mg} / \mathrm{kg}$ dry-wt & 1 & 240 & $=$ & 120 & 23,463 \\
\hline Columbia River & McNary Dam & B17BJ3 & August 8, 2003 & Zinc & $\mathrm{mg} / \mathrm{Kg}$ & 1 & 276 & C & 120 & 23,463 \\
\hline Columbia River & McNary Dam & B19PL7 & July 13, 2004 & Zinc & $\mathrm{mg} / \mathrm{Kg}$ & 1 & 244 & $x$ & 120 & 23,463 \\
\hline
\end{tabular}


Table C-12

Summary Statistics for All Categories of Surface Water Samples Collected from Segment 3 of the Columbia River

\begin{tabular}{|c|c|c|c|c|c|c|c|c|c|c|}
\hline $\begin{array}{c}\text { Constituent } \\
\text { Class }\end{array}$ & Constituent Name & Units & $\begin{array}{l}\text { Number } \\
\text { Analyzed }\end{array}$ & $\begin{array}{l}\text { Number } \\
\text { Detected }\end{array}$ & $\begin{array}{l}\text { Frequency of } \\
\text { Detection }\end{array}$ & $\begin{array}{l}\text { Minimum } \\
\text { Nondetect }\end{array}$ & $\begin{array}{l}\text { Maximum } \\
\text { Nondetect }\end{array}$ & $\begin{array}{l}\text { Minimum } \\
\text { Detect }\end{array}$ & $\begin{array}{l}\text { Maximum } \\
\text { Detect }\end{array}$ & $\begin{array}{c}\text { Average } \\
\text { Concentration }\end{array}$ \\
\hline CONV & Alkalinity & $\mathrm{mg} / \mathrm{L}$ & 23 & 23 & $100 \%$ & $\overline{--}$ & $\overline{--}$ & 99 & 121 & 104 \\
\hline CONV & $\begin{array}{l}\text { Ammonia } \\
\text { Biochemical Oxygen }\end{array}$ & $\mathrm{mg} / \mathrm{L}$ & 103 & 63 & $61 \%$ & 0.010 & 0.010 & 0.010 & 0.090 & 0.020 \\
\hline CONV & Demand & $\mathrm{mg} / \mathrm{L}$ & 9 & 0 & $0 \%$ & 2.0 & 4.0 & -- & -- & 1.6 \\
\hline CONV & Chloride & $\mathrm{mg} / \mathrm{L}$ & 37 & 37 & $100 \%$ & -- & -- & 2.0 & 150 & 13 \\
\hline CONV & Dissolved organic carbon & $\mathrm{mg} / \mathrm{L}$ & 46 & 46 & $100 \%$ & -- & -- & 1.5 & 4.4 & 2.3 \\
\hline CONV & Dissolved oxygen & $\mathrm{mg} / \mathrm{L}$ & 1 & 1 & $100 \%$ & -- & -- & 9.3 & 9.3 & 9.3 \\
\hline CONV & Fluoride & $\mathrm{mg} / \mathrm{L}$ & 12 & 12 & $100 \%$ & -- & -- & 0.11 & 0.21 & 0.16 \\
\hline CONV & Hardness & $\mathrm{mg} / \mathrm{L}$ & 32 & 32 & $100 \%$ & -- & -- & 56 & 118 & 95 \\
\hline CONV & Nitrate & $\mathrm{mg} / \mathrm{L}$ & 48 & 48 & $100 \%$ & -- & -- & 0.20 & 4.6 & 1.9 \\
\hline CONV & Nitrogen, Nitrate (as N) & $\mathrm{mg} / \mathrm{L}$ & 12 & 12 & $100 \%$ & -- & -- & 0.15 & 0.60 & 0.29 \\
\hline CONV & Nitrogen, Nitrate-Nitrite & $\mathrm{mg} / \mathrm{L}$ & 3 & 3 & $100 \%$ & -- & -- & 0.86 & 1.0 & 0.92 \\
\hline CONV & Nitrogen, Nitrite & $\mathrm{mg} / \mathrm{L}$ & 12 & 0 & $0 \%$ & 0.011 & 0.011 & -- & -- & 0.0055 \\
\hline CONV & Orthophosphate & $\mathrm{mg} / \mathrm{L}$ & 98 & 98 & $100 \%$ & -- & -- & 0.032 & 0.14 & 0.080 \\
\hline CONV & $\mathrm{pH}$ & $\mathrm{pH}$ Units & 82 & 82 & $100 \%$ & -- & -- & 7.6 & 9.5 & 8.3 \\
\hline CONV & Phosphorous in phosphate & $\mathrm{mg} / \mathrm{L}$ & 36 & 35 & $97 \%$ & 0.010 & 0.010 & 0.010 & 0.12 & 0.046 \\
\hline CONV & Specific Conductance & umhos/cm & 124 & 124 & $100 \%$ & -- & - & 70 & 1,030 & 219 \\
\hline CONV & Sulfate & $\mathrm{mg} / \mathrm{L}$ & 12 & 12 & $100 \%$ & -- & -- & 4.6 & 14 & 8.8 \\
\hline CONV & $\begin{array}{l}\text { Temperature } \\
\text { Total Non-Volatile }\end{array}$ & Deg C & 19 & 19 & $100 \%$ & -- & -- & 3.8 & 24 & 14 \\
\hline CONV & Suspended Solids & $\mathrm{mg} / \mathrm{L}$ & 16 & 16 & $100 \%$ & -- & -- & 15 & 57 & 29 \\
\hline CONV & Total Organic Carbon & $\%$ & 1 & 1 & $100 \%$ & -- & -- & 0.83 & 0.83 & 0.83 \\
\hline CONV & Total Organic Carbon & $\mathrm{mg} / \mathrm{L}$ & 48 & 48 & $100 \%$ & -- & -- & 1.7 & 5.0 & 2.5 \\
\hline CONV & Total Persulfate Nitrogen & $\mathrm{mg} / \mathrm{L}$ & 103 & 103 & $100 \%$ & -- & -- & 0.43 & 23 & 1.5 \\
\hline CONV & Total suspended solids & $\mathrm{mg} / \mathrm{L}$ & 258 & 258 & $100 \%$ & -- & -- & 1.0 & 405 & 24 \\
\hline CONV & Turbidity & NTU & 163 & 163 & $100 \%$ & -- & -- & 1.2 & 210 & 12 \\
\hline METAL & Antimony & $\mathrm{mg} / \mathrm{L}$ & 24 & 24 & $100 \%$ & -- & -- & 2.89E-05 & 2.13E-04 & $1.02 \mathrm{E}-04$ \\
\hline METAL & Arsenic & $\mathrm{mg} / \mathrm{L}$ & 48 & 48 & $100 \%$ & -- & -- & 5.08E-04 & 0.0028 & 0.0013 \\
\hline METAL & Beryllium & $\mathrm{mg} / \mathrm{L}$ & 24 & 0 & $0 \%$ & $6.60 \mathrm{E}-05$ & $6.60 \mathrm{E}-05$ & -- & -- & 3.30E-05 \\
\hline METAL & Cadmium & $\mathrm{mg} / \mathrm{L}$ & 36 & 3 & $8 \%$ & $2.00 \mathrm{E}-05$ & 1.00E-04 & $2.59 \mathrm{E}-05$ & 3.32E-05 & 1.91E-05 \\
\hline METAL & Chromium & $\mathrm{mg} / \mathrm{L}$ & 39 & 33 & $85 \%$ & 4.70E-05 & $5.00 \mathrm{E}-04$ & 8.82E-05 & 0.0017 & $5.38 \mathrm{E}-04$ \\
\hline METAL & Copper & $\mathrm{mg} / \mathrm{L}$ & 39 & 39 & $100 \%$ & -- & -- & 4.80E-04 & 0.0025 & $9.18 \mathrm{E}-04$ \\
\hline METAL & Lead & $\mathrm{mg} / \mathrm{L}$ & 39 & 28 & $72 \%$ & 1.10E-05 & $1.00 \mathrm{E}-04$ & $1.11 \mathrm{E}-05$ & $6.90 \mathrm{E}-04$ & 8.91E-05 \\
\hline METAL & Mercury & $\mathrm{mg} / \mathrm{L}$ & 37 & 28 & $76 \%$ & 2.00E-06 & 2.00E-06 & 5.83E-07 & $3.20 \mathrm{E}-06$ & 1.35E-06 \\
\hline METAL & Nickel & $\mathrm{mg} / \mathrm{L}$ & 36 & 36 & $100 \%$ & -- & -- & 3.03E-05 & 0.0023 & $6.65 \mathrm{E}-04$ \\
\hline METAL & Phosphorus & $\mathrm{mg} / \mathrm{L}$ & 139 & 139 & $100 \%$ & -- & -- & 0.010 & 3.9 & 0.19 \\
\hline METAL & Selenium & $\mathrm{mg} / \mathrm{L}$ & 24 & 12 & $50 \%$ & 8.00E-05 & 8.00E-05 & 8.01E-05 & 4.66E-04 & 1.37E-04 \\
\hline METAL & Silver & $\mathrm{mg} / \mathrm{L}$ & 43 & 12 & $28 \%$ & $8.50 \mathrm{E}-06$ & 2.00E-05 & $2.00 \mathrm{E}-05$ & 1.00E-04 & 2.07E-05 \\
\hline METAL & Thallium & $\mathrm{mg} / \mathrm{L}$ & 24 & 7 & $29 \%$ & $9.00 \mathrm{E}-06$ & $9.00 \mathrm{E}-06$ & $9.48 \mathrm{E}-06$ & 1.51E-05 & $6.81 \mathrm{E}-06$ \\
\hline METAL & Uranium & $\mathrm{mg} / \mathrm{L}$ & 24 & 24 & $100 \%$ & -- & -- & $3.71 \mathrm{E}-04$ & 0.0016 & $8.48 \mathrm{E}-04$ \\
\hline METAL & Zinc & $\mathrm{mg} / \mathrm{L}$ & 39 & 39 & $100 \%$ & -- & -- & 4.90E-04 & 0.0064 & 0.0024 \\
\hline PCB & Aroclor-1016 & $\mathrm{mg} / \mathrm{L}$ & 2 & 0 & $0 \%$ & $6.30 \mathrm{E}-07$ & $6.60 \mathrm{E}-07$ & -- & -- & $3.23 \mathrm{E}-07$ \\
\hline PCB & Aroclor-1221 & $\mathrm{mg} / \mathrm{L}$ & 2 & 0 & $0 \%$ & $6.30 \mathrm{E}-07$ & $6.60 \mathrm{E}-07$ & -- & -- & 3.23E-07 \\
\hline PCB & Aroclor-1232 & $\mathrm{mg} / \mathrm{L}$ & 2 & 0 & $0 \%$ & $6.30 \mathrm{E}-07$ & $6.60 \mathrm{E}-07$ & -- & -- & $3.23 \mathrm{E}-07$ \\
\hline PCB & Aroclor-1242 & $\mathrm{mg} / \mathrm{L}$ & 2 & 0 & $0 \%$ & $6.30 \mathrm{E}-07$ & $6.60 \mathrm{E}-07$ & -- & -- & $3.23 \mathrm{E}-07$ \\
\hline PCB & Aroclor-1248 & $\mathrm{mg} / \mathrm{L}$ & 2 & 0 & $0 \%$ & $6.30 \mathrm{E}-07$ & $6.60 \mathrm{E}-07$ & -- & -- & 3.23E-07 \\
\hline
\end{tabular}


Table C-12

Summary Statistics for All Categories of Surface Water Samples Collected from Segment 3 of the Columbia River

\begin{tabular}{|c|c|c|c|c|c|c|c|c|c|c|}
\hline $\begin{array}{l}\text { Constituent } \\
\text { Class }\end{array}$ & Constituent Name & Units & $\begin{array}{l}\text { Number } \\
\text { Analyzed }\end{array}$ & $\begin{array}{l}\text { Number } \\
\text { Detected }\end{array}$ & $\begin{array}{l}\text { Frequency of } \\
\text { Detection }\end{array}$ & $\begin{array}{l}\text { Minimum } \\
\text { Nondetect }\end{array}$ & $\begin{array}{l}\text { Maximum } \\
\text { Nondetect }\end{array}$ & $\begin{array}{l}\text { Minimum } \\
\text { Detect }\end{array}$ & $\begin{array}{l}\text { Maximum } \\
\text { Detect }\end{array}$ & $\begin{array}{c}\text { Average } \\
\text { Concentration }\end{array}$ \\
\hline PCB & Aroclor-1254 & $\mathrm{mg} / \mathrm{L}$ & 2 & 0 & $0 \%$ & $6.30 \mathrm{E}-07$ & $6.60 \mathrm{E}-07$ & -- & -- & 3.23E-07 \\
\hline PCB & Aroclor-1260 & $\mathrm{mg} / \mathrm{L}$ & 2 & 0 & $0 \%$ & $6.30 \mathrm{E}-07$ & $6.60 \mathrm{E}-07$ & -- & -- & 3.23E-07 \\
\hline PEST & 2,4'-DDD & $\mathrm{mg} / \mathrm{L}$ & 16 & 0 & $0 \%$ & $6.20 \mathrm{E}-08$ & $6.90 \mathrm{E}-08$ & -- & -- & 3.33E-08 \\
\hline PEST & 2,4'-DDE & $\mathrm{mg} / \mathrm{L}$ & 16 & 0 & $0 \%$ & $6.20 \mathrm{E}-08$ & $6.90 \mathrm{E}-08$ & -- & -- & 3.33E-08 \\
\hline PEST & 2,4'-DDT & $\mathrm{mg} / \mathrm{L}$ & 16 & 0 & $0 \%$ & $6.20 \mathrm{E}-08$ & 1.10E-07 & -- & -- & 3.47E-08 \\
\hline PEST & alpha-Chlordane & $\mathrm{mg} / \mathrm{L}$ & 21 & 0 & $0 \%$ & $6.20 \mathrm{E}-08$ & 6.90E-08 & -- & -- & $3.30 \mathrm{E}-08$ \\
\hline PEST & $\begin{array}{l}\text { cis-Nonachlor } \\
\text { Dichlorodiphenyldichloroeth }\end{array}$ & $\mathrm{mg} / \mathrm{L}$ & 21 & 0 & $0 \%$ & $6.20 \mathrm{E}-08$ & $6.90 \mathrm{E}-08$ & -- & -- & 3.30E-08 \\
\hline PEST & $\begin{array}{l}\text { ane } \\
\text { Dichlorodiphenyldichloroeth }\end{array}$ & $\mathrm{mg} / \mathrm{L}$ & 21 & 4 & $19 \%$ & $6.20 \mathrm{E}-08$ & $9.20 \mathrm{E}-08$ & $6.70 \mathrm{E}-08$ & 1.30E-07 & 4.68E-08 \\
\hline PEST & $\begin{array}{l}\text { ylene } \\
\text { Dichlorodiphenyltrichloroeth }\end{array}$ & $\mathrm{mg} / \mathrm{L}$ & 21 & 18 & $86 \%$ & $6.60 \mathrm{E}-08$ & 6.90E-08 & 7.10E-08 & 1.20E-06 & 2.37E-07 \\
\hline PEST & ane & $\mathrm{mg} / \mathrm{L}$ & 21 & 5 & $24 \%$ & $6.40 \mathrm{E}-08$ & $1.60 \mathrm{E}-07$ & $1.00 \mathrm{E}-07$ & $6.50 \mathrm{E}-07$ & $8.82 \mathrm{E}-08$ \\
\hline PEST & Dieldrin & $\mathrm{mg} / \mathrm{L}$ & 21 & 4 & $19 \%$ & $6.20 \mathrm{E}-08$ & $1.60 \mathrm{E}-07$ & 7.60E-08 & $3.00 \mathrm{E}-07$ & $6.26 \mathrm{E}-08$ \\
\hline PEST & Heptachlor & mg/L & 21 & 0 & $0 \%$ & $6.20 \mathrm{E}-08$ & 6.90E-08 & -- & -- & 3.30E-08 \\
\hline PEST & Heptachlor epoxide & $\mathrm{mg} / \mathrm{L}$ & 21 & 6 & $29 \%$ & $6.20 \mathrm{E}-08$ & 1.10E-07 & 3.30E-08 & $1.20 \mathrm{E}-07$ & 4.00E-08 \\
\hline PEST & Oxychlordane & $\mathrm{mg} / \mathrm{L}$ & 21 & 0 & $0 \%$ & $6.20 \mathrm{E}-08$ & 1.70E-07 & -- & -- & 4.47E-08 \\
\hline PEST & trans-Chlordane & $\mathrm{mg} / \mathrm{L}$ & 21 & 0 & $0 \%$ & $6.20 \mathrm{E}-08$ & 1.70E-07 & -- & -- & 4.47E-08 \\
\hline PEST & trans-Nonachlor & $\mathrm{mg} / \mathrm{L}$ & 21 & 0 & $0 \%$ & $6.20 \mathrm{E}-08$ & 6.90E-08 & -- & -- & $3.30 \mathrm{E}-08$ \\
\hline RAD & Antimony-125 & $\mathrm{pCi} / \mathrm{L}$ & 8 & 1 & $13 \%$ & 0.13 & 5.0 & 0.13 & 0.13 & 1.2 \\
\hline RAD & Beryllium-7 & $\mathrm{pCi} / \mathrm{L}$ & 7 & 0 & $0 \%$ & 0.42 & 23 & -- & -- & 4.5 \\
\hline RAD & Cesium-134 & $\mathrm{pCi} / \mathrm{L}$ & 10 & 1 & $10 \%$ & 0.025 & 2.5 & 0.70 & 0.70 & 0.68 \\
\hline RAD & Cesium-137 & $\mathrm{pCi} / \mathrm{L}$ & 8 & 0 & $0 \%$ & 0.16 & 1.8 & -- & -- & 0.40 \\
\hline RAD & Cobalt- 60 & $\mathrm{pCi} / \mathrm{L}$ & 8 & 1 & $13 \%$ & 0.77 & 4.4 & 4.4 & 4.4 & 1.3 \\
\hline RAD & Europium-152 & $\mathrm{pCi} / \mathrm{L}$ & 8 & 0 & $0 \%$ & 0.079 & 3.1 & -- & -- & 0.60 \\
\hline RAD & Europium-154 & $\mathrm{pCi} / \mathrm{L}$ & 5 & 0 & $0 \%$ & 0.52 & 7.6 & -- & -- & 1.5 \\
\hline RAD & Europium-155 & $\mathrm{pCi} / \mathrm{L}$ & 8 & 0 & $0 \%$ & 1.2 & 5.3 & -- & -- & 1.5 \\
\hline RAD & Plutonium-239/240 & $\mathrm{pCi} / \mathrm{L}$ & 1 & 0 & $0 \%$ & 0.0018 & 0.0018 & -- & -- & 8.85E-04 \\
\hline RAD & Potassium-40 & $\mathrm{pCi} / \mathrm{L}$ & 4 & 0 & $0 \%$ & 6.5 & 23 & -- & -- & 7.3 \\
\hline RAD & Ruthenium-106 & $\mathrm{pCi} / \mathrm{L}$ & 7 & 1 & $14 \%$ & 0.54 & 8.8 & 4.3 & 4.3 & 2.5 \\
\hline RAD & Strontium-90 & $\mathrm{pCi} / \mathrm{L}$ & 18 & 17 & $94 \%$ & 0.046 & 0.046 & 0.055 & 0.15 & 0.089 \\
\hline RAD & Technetium-99 & $\mathrm{pCi} / \mathrm{L}$ & 11 & 0 & $0 \%$ & 0.0039 & 0.44 & -- & -- & 0.13 \\
\hline RAD & Tritium & $\mathrm{pCi} / \mathrm{L}$ & 12 & 12 & $100 \%$ & -- & -- & 13 & 35 & 25 \\
\hline RAD & Uranium-234 & $\mathrm{pCi} / \mathrm{L}$ & 3 & 3 & $100 \%$ & -- & -- & 0.25 & 0.54 & 0.35 \\
\hline RAD & Uranium-235 & $\mathrm{pCi} / \mathrm{L}$ & 3 & 2 & $67 \%$ & 0.0035 & 0.0035 & 0.0035 & 0.019 & 0.0080 \\
\hline RAD & Uranium-238 & $\mathrm{pCi} / \mathrm{L}$ & 3 & 3 & $100 \%$ & -- & -- & 0.19 & 0.41 & 0.26 \\
\hline SVOC & Hexachlorobenzene & $\mathrm{mg} / \mathrm{L}$ & 21 & 3 & $14 \%$ & $6.30 \mathrm{E}-08$ & $1.00 \mathrm{E}-07$ & 1.10E-07 & $1.80 \mathrm{E}-07$ & $5.31 \mathrm{E}-08$ \\
\hline
\end{tabular}

CONV - Conventional parameter

PCB - Polychlorinated Biphenyls

PEST - Pesticide

RAD - Radionuclide

SVOC - Semivolatile Organic Compound 
Table C-13

Summary Statistics for All Categories of Surface Water Samples Collected from the Yakima River (Segment 3)

\begin{tabular}{|c|c|c|c|c|c|c|c|c|c|c|}
\hline $\begin{array}{l}\text { Constituent } \\
\text { Class }\end{array}$ & Constituent Name & Units & $\begin{array}{l}\text { Number } \\
\text { Analyzed }\end{array}$ & $\begin{array}{l}\text { Number } \\
\text { Detected }\end{array}$ & $\begin{array}{l}\text { Frequency of } \\
\text { Detection }\end{array}$ & $\begin{array}{l}\text { Minimum } \\
\text { Nondetect }\end{array}$ & $\begin{array}{l}\text { Maximum } \\
\text { Nondetect }\end{array}$ & $\begin{array}{l}\text { Mínimum } \\
\text { Detect }\end{array}$ & $\begin{array}{l}\text { Maximum } \\
\text { Detect }\end{array}$ & $\begin{array}{c}\text { Average } \\
\text { Concentration }\end{array}$ \\
\hline CONV & Alkalinity & $\mathrm{mg} / \mathrm{L}$ & 22 & 22 & $100 \%$ & $\overline{--}$ & $\overline{--}$ & 99 & 107 & 103 \\
\hline CONV & Ammonia & $\mathrm{mg} / \mathrm{L}$ & 103 & 63 & $61 \%$ & 0.010 & 0.010 & 0.010 & 0.090 & 0.020 \\
\hline CONV & Biochemical Oxygen Deman & $\mathrm{mg} / \mathrm{L}$ & 9 & 0 & $0 \%$ & 2.0 & 4.0 & -- & -- & 1.6 \\
\hline CONV & Chloride & $\mathrm{mg} / \mathrm{L}$ & 28 & 28 & $100 \%$ & -- & -- & 5.5 & 150 & 16 \\
\hline CONV & Dissolved organic carbon & $\mathrm{mg} / \mathrm{L}$ & 14 & 14 & $100 \%$ & -- & -- & 1.5 & 4.4 & 1.9 \\
\hline CONV & Fluoride & $\mathrm{mg} / \mathrm{L}$ & 3 & 3 & $100 \%$ & -- & -- & 0.18 & 0.19 & 0.18 \\
\hline CONV & Hardness & $\mathrm{mg} / \mathrm{L}$ & 31 & 31 & $100 \%$ & -- & -- & 56 & 118 & 95 \\
\hline CONV & Nitrate & $\mathrm{mg} / \mathrm{L}$ & 40 & 40 & $100 \%$ & -- & -- & 0.20 & 4.6 & 2.2 \\
\hline CONV & Nitrogen, Nitrate (as N) & $\mathrm{mg} / \mathrm{L}$ & 3 & 3 & $100 \%$ & -- & -- & 0.60 & 0.60 & 0.60 \\
\hline CONV & Nitrogen, Nitrate-Nitrite & $\mathrm{mg} / \mathrm{L}$ & 3 & 3 & $100 \%$ & -- & -- & 0.86 & 1.0 & 0.92 \\
\hline CONV & Nitrogen, Nitrite & $\mathrm{mg} / \mathrm{L}$ & 3 & 0 & $0 \%$ & 0.011 & 0.011 & -- & -- & 0.0055 \\
\hline CONV & Orthophosphate & $\mathrm{mg} / \mathrm{L}$ & 98 & 98 & $100 \%$ & -- & -- & 0.032 & 0.14 & 0.080 \\
\hline CONV & $\mathrm{pH}$ & pH Units & 80 & 80 & $100 \%$ & -- & -- & 7.6 & 9.5 & 8.3 \\
\hline CONV & Phosphorous in phosphate & $\mathrm{mg} / \mathrm{L}$ & 36 & 35 & $97 \%$ & 0.010 & 0.010 & 0.010 & 0.12 & 0.046 \\
\hline CONV & Specific Conductance & umhos/cm & 86 & 86 & $100 \%$ & -- & -- & 113 & 1,030 & 241 \\
\hline CONV & Sulfate & $\mathrm{mg} / \mathrm{L}$ & 3 & 3 & $100 \%$ & -- & -- & 13 & 14 & 14 \\
\hline CONV & Total Non-Volatile Suspend $\epsilon$ & $\mathrm{mg} / \mathrm{L}$ & 16 & 16 & $100 \%$ & -- & -- & 15 & 57 & 29 \\
\hline CONV & Total Organic Carbon & $\mathrm{mg} / \mathrm{L}$ & 16 & 16 & $100 \%$ & -- & -- & 1.7 & 4.9 & 2.1 \\
\hline CONV & Total Persulfate Nitrogen & $\mathrm{mg} / \mathrm{L}$ & 103 & 103 & $100 \%$ & -- & -- & 0.43 & 23 & 1.5 \\
\hline CONV & Total suspended solids & $\mathrm{mg} / \mathrm{L}$ & 213 & 213 & $100 \%$ & -- & -- & 1.0 & 379 & 20 \\
\hline CONV & Turbidity & NTU & 127 & 127 & $100 \%$ & -- & -- & 1.2 & 170 & 10 \\
\hline METAL & Antimony & $\mathrm{mg} / \mathrm{L}$ & 6 & 6 & $100 \%$ & -- & -- & 5.36E-05 & $7.58 \mathrm{E}-05$ & $6.07 \mathrm{E}-05$ \\
\hline METAL & Arsenic & $\mathrm{mg} / \mathrm{L}$ & 30 & 30 & $100 \%$ & -- & -- & 7.30E-04 & 0.0028 & 0.0015 \\
\hline METAL & Beryllium & $\mathrm{mg} / \mathrm{L}$ & 6 & 0 & $0 \%$ & $6.60 \mathrm{E}-05$ & $6.60 \mathrm{E}-05$ & -- & -- & 3.30E-05 \\
\hline METAL & Cadmium & $\mathrm{mg} / \mathrm{L}$ & 18 & 0 & $0 \%$ & 2.00E-05 & $1.00 \mathrm{E}-04$ & -- & -- & $2.38 \mathrm{E}-05$ \\
\hline METAL & Chromium & $\mathrm{mg} / \mathrm{L}$ & 21 & 16 & $76 \%$ & 5.00E-04 & 5.00E-04 & $2.50 \mathrm{E}-04$ & 0.0017 & 6.52E-04 \\
\hline METAL & Copper & $\mathrm{mg} / \mathrm{L}$ & 21 & 21 & $100 \%$ & -- & -- & $4.80 \mathrm{E}-04$ & 0.0025 & 0.0010 \\
\hline METAL & Lead & $\mathrm{mg} / \mathrm{L}$ & 21 & 13 & $62 \%$ & 2.00E-05 & 1.00E-04 & $1.55 \mathrm{E}-05$ & $6.90 \mathrm{E}-04$ & 8.12E-05 \\
\hline METAL & Mercury & $\mathrm{mg} / \mathrm{L}$ & 19 & 10 & $53 \%$ & $2.00 \mathrm{E}-06$ & 2.00E-06 & 8.08E-07 & $3.20 E-06$ & 1.47E-06 \\
\hline METAL & Nickel & $\mathrm{mg} / \mathrm{L}$ & 18 & 18 & $100 \%$ & -- & -- & $5.20 \mathrm{E}-04$ & 0.0023 & $9.70 \mathrm{E}-04$ \\
\hline METAL & Phosphorus & $\mathrm{mg} / \mathrm{L}$ & 139 & 139 & $100 \%$ & -- & -- & 0.010 & 3.9 & 0.19 \\
\hline METAL & Selenium & $\mathrm{mg} / \mathrm{L}$ & 6 & 3 & $50 \%$ & 8.00E-05 & 8.00E-05 & 2.43E-04 & $2.65 E-04$ & $1.45 E-04$ \\
\hline METAL & Silver & $\mathrm{mg} / \mathrm{L}$ & 25 & 12 & $48 \%$ & 8.50E-06 & 2.00E-05 & $2.00 \mathrm{E}-05$ & $1.00 \mathrm{E}-04$ & $3.26 \mathrm{E}-05$ \\
\hline METAL & Thallium & $\mathrm{mg} / \mathrm{L}$ & 6 & 0 & $0 \%$ & $9.00 \mathrm{E}-06$ & $9.00 \mathrm{E}-06$ & -- & -- & 4.50E-06 \\
\hline METAL & Uranium & $\mathrm{mg} / \mathrm{L}$ & 6 & 6 & $100 \%$ & -- & -- & 0.0015 & 0.0016 & 0.0015 \\
\hline METAL & Zinc & $\mathrm{mg} / \mathrm{L}$ & 21 & 21 & $100 \%$ & -- & -- & 4.90E-04 & 0.0064 & 0.0026 \\
\hline RAD & Antimony-125 & $\mathrm{pCi} / \mathrm{L}$ & 2 & 0 & $0 \%$ & 1.7 & 5.0 & -- & -- & 1.7 \\
\hline RAD & Beryllium-7 & $\mathrm{pCi} / \mathrm{L}$ & 1 & 0 & $0 \%$ & 2.4 & 2.4 & -- & -- & 1.2 \\
\hline RAD & Cesium-134 & $\mathrm{pCi} / \mathrm{L}$ & 2 & 0 & $0 \%$ & 0.025 & 0.98 & -- & -- & 0.25 \\
\hline RAD & Cesium-137 & $\mathrm{pCi} / \mathrm{L}$ & 1 & 0 & $0 \%$ & 0.75 & 0.75 & -- & -- & 0.37 \\
\hline RAD & Cobalt-60 & $\mathrm{pCi} / \mathrm{L}$ & 2 & 0 & $0 \%$ & 0.85 & 0.92 & -- & -- & 0.44 \\
\hline RAD & Europium-152 & $\mathrm{pCi} / \mathrm{L}$ & 3 & 0 & $0 \%$ & 0.079 & 3.1 & -- & -- & 0.84 \\
\hline RAD & Europium-154 & $\mathrm{pCi} / \mathrm{L}$ & 2 & 0 & $0 \%$ & 0.52 & 0.64 & -- & -- & 0.29 \\
\hline RAD & Europium-155 & $\mathrm{pCi} / \mathrm{L}$ & 2 & 0 & $0 \%$ & 2.9 & 3.9 & -- & -- & 1.7 \\
\hline RAD & Potassium-40 & $\mathrm{pCi} / \mathrm{L}$ & 1 & 0 & $0 \%$ & 6.5 & 6.5 & -- & -- & 3.3 \\
\hline RAD & Ruthenium-106 & $\mathrm{pCi} / \mathrm{L}$ & 1 & 0 & $0 \%$ & 4.6 & 4.6 & -- & -- & 2.3 \\
\hline RAD & Strontium-90 & $\mathrm{pCi} / \mathrm{L}$ & 3 & 3 & $100 \%$ & -- & -- & 0.062 & 0.073 & 0.067 \\
\hline
\end{tabular}


Table C-13

Summary Statistics for All Categories of Surface Water Samples Collected from the Yakima River (Segment 3)

\begin{tabular}{|c|c|c|c|c|c|c|c|c|c|c|}
\hline $\begin{array}{c}\text { Constituent } \\
\text { Class }\end{array}$ & Constituent Name & Units & $\begin{array}{l}\text { Number } \\
\text { Analyzed }\end{array}$ & $\begin{array}{l}\text { Number } \\
\text { Detected }\end{array}$ & $\begin{array}{l}\text { Frequency of } \\
\text { Detection }\end{array}$ & $\begin{array}{l}\text { Minimum } \\
\text { Nondetect }\end{array}$ & $\begin{array}{l}\text { Maximum } \\
\text { Nondetect }\end{array}$ & $\begin{array}{l}\text { Minimum } \\
\text { Detect }\end{array}$ & $\begin{array}{l}\text { Maximum } \\
\text { Detect }\end{array}$ & $\begin{array}{c}\text { Average } \\
\text { Concentration }\end{array}$ \\
\hline$\overline{\text { RAD }}$ & Technetium-99 & $\overline{\mathrm{pCi} / \mathrm{L}}$ & 2 & 0 & $0 \%$ & 0.18 & 0.23 & -- & $\overline{--}$ & 0.10 \\
\hline RAD & Tritium & $\mathrm{pCi} / \mathrm{L}$ & 3 & 3 & $100 \%$ & -- & -- & 13 & 14 & 14 \\
\hline RAD & Uranium-234 & $\mathrm{pCi} / \mathrm{L}$ & 1 & 1 & $100 \%$ & -- & -- & 0.54 & 0.54 & 0.54 \\
\hline RAD & Uranium-235 & $\mathrm{pCi} / \mathrm{L}$ & 1 & 1 & $100 \%$ & -- & -- & 0.019 & 0.019 & 0.019 \\
\hline RAD & Uranium-238 & $\mathrm{pCi} / \mathrm{L}$ & 1 & 1 & $100 \%$ & -- & -- & 0.41 & 0.41 & 0.41 \\
\hline
\end{tabular}

CONV - Conventional parameter

RAD - Radionuclide 
Table C-14

Summary Statistics for Category 1 Surface Water Samples Collected from the Snake River (Segment 3 )

\begin{tabular}{|c|c|c|c|c|c|c|c|c|c|c|}
\hline $\begin{array}{l}\text { Constituent } \\
\text { Class }\end{array}$ & Constituent Name & Units & $\begin{array}{l}\text { Number } \\
\text { Analyzed }\end{array}$ & $\begin{array}{l}\text { Number } \\
\text { Detected }\end{array}$ & $\begin{array}{c}\text { Frequency of } \\
\text { Detection }\end{array}$ & $\begin{array}{l}\text { Minimum } \\
\text { Nondetect }\end{array}$ & $\begin{array}{l}\text { Maximum } \\
\text { Nondetect }\end{array}$ & $\begin{array}{l}\text { Minimum } \\
\text { Detect }\end{array}$ & $\begin{array}{l}\text { Maximum } \\
\text { Detect }\end{array}$ & $\begin{array}{c}\text { Average } \\
\text { Concentration }\end{array}$ \\
\hline$\overline{\mathrm{CONV}}$ & Chloride & $\mathrm{mg} / \mathrm{L}$ & 3 & 3 & $100 \%$ & $\overline{--}$ & $\overline{--}$ & 3.3 & 3.4 & 3.3 \\
\hline CONV & Fluoride & $\mathrm{mg} / \mathrm{L}$ & 3 & 3 & $100 \%$ & -- & -- & 0.20 & 0.21 & 0.20 \\
\hline CONV & Nitrate & $\mathrm{mg} / \mathrm{L}$ & 8 & 8 & $100 \%$ & -- & -- & 0.30 & 1.0 & 0.71 \\
\hline CONV & Nitrogen, Nitrate (as N) & mg/L & 3 & 3 & $100 \%$ & -- & -- & 0.17 & 0.18 & 0.18 \\
\hline CONV & Nitrogen, Nitrite & $\mathrm{mg} / \mathrm{L}$ & 3 & 0 & $0 \%$ & 0.011 & 0.011 & -- & -- & 0.0055 \\
\hline CONV & Sulfate & $\mathrm{mg} / \mathrm{L}$ & 3 & 3 & $100 \%$ & -- & -- & 7.9 & 8.0 & 7.9 \\
\hline CONV & Total suspended solids & $\mathrm{mg} / \mathrm{L}$ & 3 & 3 & $100 \%$ & -- & -- & 5.0 & 6.0 & 5.3 \\
\hline METAL & Antimony & mg/L & 6 & 6 & $100 \%$ & -- & -- & 9.11E-05 & 1.68E-04 & $1.28 \mathrm{E}-04$ \\
\hline METAL & Arsenic & $\mathrm{mg} / \mathrm{L}$ & 6 & 6 & $100 \%$ & -- & -- & 0.0011 & 0.0012 & 0.0011 \\
\hline METAL & Beryllium & $\mathrm{mg} / \mathrm{L}$ & 6 & 0 & $0 \%$ & $6.60 \mathrm{E}-05$ & 6.60E-05 & -- & -- & 3.30E-05 \\
\hline METAL & Cadmium & $\mathrm{mg} / \mathrm{L}$ & 6 & 0 & $0 \%$ & $2.30 \mathrm{E}-05$ & 2.30E-05 & -- & -- & 1.15E-05 \\
\hline METAL & Chromium & mg/L & 6 & 6 & $100 \%$ & -- & -- & 1.13E-04 & 5.72E-04 & $4.22 \mathrm{E}-04$ \\
\hline METAL & Copper & mg/L & 6 & 6 & $100 \%$ & -- & -- & 5.01E-04 & 7.84E-04 & 6.36E-04 \\
\hline METAL & Lead & mg/L & 6 & 6 & $100 \%$ & -- & -- & 1.26E-05 & 1.26E-04 & 6.86E-05 \\
\hline METAL & Mercury & $\mathrm{mg} / \mathrm{L}$ & 6 & 6 & $100 \%$ & -- & -- & 1.01E-06 & 1.82E-06 & $1.40 \mathrm{E}-06$ \\
\hline METAL & Nickel & $\mathrm{mg} / \mathrm{L}$ & 6 & 6 & $100 \%$ & -- & -- & 3.03E-05 & 8.01E-04 & 1.87E-04 \\
\hline METAL & Selenium & mg/L & 6 & 2 & $33 \%$ & 8.00E-05 & 8.00E-05 & 1.52E-04 & $2.40 \mathrm{E}-04$ & $9.20 \mathrm{E}-05$ \\
\hline METAL & Silver & mg/L & 6 & 0 & $0 \%$ & 8.50E-06 & 8.50E-06 & -- & -- & 4.25E-06 \\
\hline METAL & Thallium & mg/L & 6 & 0 & $0 \%$ & $9.00 \mathrm{E}-06$ & 9.00E-06 & -- & -- & 4.50E-06 \\
\hline METAL & Uranium & $\mathrm{mg} / \mathrm{L}$ & 6 & 6 & $100 \%$ & -- & -- & 7.09E-04 & 7.72E-04 & 7.39E-04 \\
\hline METAL & Zinc & mg/L & 6 & 6 & $100 \%$ & -- & -- & 6.11E-04 & 0.0015 & 0.0010 \\
\hline RAD & Antimony-125 & $\mathrm{pCi} / \mathrm{L}$ & 1 & 0 & $0 \%$ & 1.4 & 1.4 & -- & -- & 0.70 \\
\hline RAD & Beryllium-7 & $\mathrm{pCi} / \mathrm{L}$ & 2 & 0 & $0 \%$ & 8.5 & 23 & -- & -- & 8.0 \\
\hline RAD & Cesium-134 & $\mathrm{pCi} / \mathrm{L}$ & 1 & 0 & $0 \%$ & 2.5 & 2.5 & -- & -- & 1.3 \\
\hline RAD & Cesium-137 & $\mathrm{pCi} / \mathrm{L}$ & 3 & 0 & $0 \%$ & 0.19 & 1.8 & -- & -- & 0.39 \\
\hline RAD & Cobalt-60 & $\mathrm{pCi} / \mathrm{L}$ & 2 & 0 & $0 \%$ & 0.77 & 0.94 & -- & -- & 0.43 \\
\hline RAD & Europium-152 & $\mathrm{pCi} / \mathrm{L}$ & 1 & 0 & $0 \%$ & 0.28 & 0.28 & -- & -- & 0.14 \\
\hline RAD & Europium-154 & $\mathrm{pCi} / \mathrm{L}$ & 1 & 0 & $0 \%$ & 0.65 & 0.65 & -- & -- & 0.32 \\
\hline RAD & Europium-155 & $\mathrm{pCi} / \mathrm{L}$ & 2 & 0 & $0 \%$ & 2.3 & 4.4 & -- & -- & 1.7 \\
\hline RAD & Potassium-40 & $\mathrm{pCi} / \mathrm{L}$ & 2 & 0 & $0 \%$ & 6.5 & 22 & -- & -- & 7.2 \\
\hline RAD & Ruthenium-106 & $\mathrm{pCi} / \mathrm{L}$ & 1 & 0 & $0 \%$ & 8.8 & 8.8 & -- & -- & 4.4 \\
\hline RAD & Strontium-90 & $\mathrm{pCi} / \mathrm{L}$ & 3 & 3 & $100 \%$ & -- & -- & 0.055 & 0.069 & 0.061 \\
\hline RAD & Technetium-99 & $\mathrm{pCi} / \mathrm{L}$ & 3 & 0 & $0 \%$ & 0.0039 & 0.43 & -- & -- & 0.14 \\
\hline RAD & Tritium & $\mathrm{pCi} / \mathrm{L}$ & 3 & 3 & $100 \%$ & -- & -- & 28 & 29 & 29 \\
\hline
\end{tabular}

CONV - Conventional parameter

RAD - Radionuclide 
Table C-15

Summary Statistics for Category 1 Surface Water Samples Collected from the Walla Walla River (Segment 3)

\begin{tabular}{|c|c|c|c|c|c|c|c|c|c|c|}
\hline $\begin{array}{l}\text { Constituent } \\
\text { Class }\end{array}$ & Constituent Name & Units & $\begin{array}{l}\text { Number } \\
\text { Analyzed }\end{array}$ & $\begin{array}{l}\text { Number } \\
\text { Detected }\end{array}$ & $\begin{array}{l}\text { Frequency of } \\
\text { Detection }\end{array}$ & $\begin{array}{l}\text { Minimum } \\
\text { Nondetect }\end{array}$ & $\begin{array}{l}\text { Maximum } \\
\text { Nondetect }\end{array}$ & $\begin{array}{l}\text { Minimum } \\
\text { Detect }\end{array}$ & $\begin{array}{l}\text { Maximum } \\
\text { Detect }\end{array}$ & $\begin{array}{c}\text { Average } \\
\text { Concentration }\end{array}$ \\
\hline$\overline{\text { CONV }}$ & Alkalinity & $\mathrm{mg} / \mathrm{L}$ & 1 & 1 & $100 \%$ & -- & $\overline{--}$ & 121 & 121 & 121 \\
\hline CONV & Chloride & $\mathrm{mg} / \mathrm{L}$ & 3 & 3 & $100 \%$ & -- & -- & 3.2 & 3.2 & 3.2 \\
\hline CONV & Dissolved organic carbon & $\mathrm{mg} / \mathrm{L}$ & 18 & 18 & $100 \%$ & -- & -- & 1.6 & 3.8 & 2.5 \\
\hline CONV & Dissolved oxygen & $\mathrm{mg} / \mathrm{L}$ & 1 & 1 & $100 \%$ & -- & -- & 9.3 & 9.3 & 9.3 \\
\hline CONV & Fluoride & $\mathrm{mg} / \mathrm{L}$ & 3 & 3 & $100 \%$ & -- & -- & 0.13 & 0.15 & 0.14 \\
\hline CONV & Hardness & $\mathrm{mg} / \mathrm{L}$ & 1 & 1 & $100 \%$ & -- & -- & 109 & 109 & 109 \\
\hline CONV & Nitrogen, Nitrate (as N) & $\mathrm{mg} / \mathrm{L}$ & 3 & 3 & $100 \%$ & -- & -- & 0.23 & 0.23 & 0.23 \\
\hline CONV & Nitrogen, Nitrite & $\mathrm{mg} / \mathrm{L}$ & 3 & 0 & $0 \%$ & 0.011 & 0.011 & -- & -- & 0.0055 \\
\hline CONV & $\mathrm{pH}$ & pH Units & 2 & 2 & $100 \%$ & -- & -- & 8.1 & 8.3 & 8.2 \\
\hline CONV & Specific Conductance & umhos/cm & 22 & 22 & $100 \%$ & -- & -- & 86 & 608 & 209 \\
\hline CONV & Sulfate & $\mathrm{mg} / \mathrm{L}$ & 3 & 3 & $100 \%$ & -- & -- & 4.6 & 4.6 & 4.6 \\
\hline CONV & Temperature & $\operatorname{Deg} C$ & 4 & 4 & $100 \%$ & -- & -- & 7.5 & 21 & 15 \\
\hline CONV & Total Organic Carbon & $\%$ & 1 & 1 & $100 \%$ & -- & -- & 0.83 & 0.83 & 0.83 \\
\hline CONV & Total Organic Carbon & $\mathrm{mg} / \mathrm{L}$ & 18 & 18 & $100 \%$ & -- & -- & 1.9 & 5.0 & 2.8 \\
\hline CONV & Total suspended solids & $\mathrm{mg} / \mathrm{L}$ & 23 & 23 & $100 \%$ & -- & -- & 2.0 & 405 & 55 \\
\hline CONV & Turbidity & NTU & 20 & 20 & $100 \%$ & -- & -- & 2.0 & 210 & 24 \\
\hline METAL & Antimony & $\mathrm{mg} / \mathrm{L}$ & 6 & 6 & $100 \%$ & -- & -- & $2.89 \mathrm{E}-05$ & $4.42 \mathrm{E}-05$ & $3.68 \mathrm{E}-05$ \\
\hline METAL & Arsenic & $\mathrm{mg} / \mathrm{L}$ & 6 & 6 & $100 \%$ & -- & -- & 5.08E-04 & 7.05E-04 & $5.81 \mathrm{E}-04$ \\
\hline METAL & Beryllium & $\mathrm{mg} / \mathrm{L}$ & 6 & 0 & $0 \%$ & $6.60 \mathrm{E}-05$ & $6.60 \mathrm{E}-05$ & -- & -- & 3.30E-05 \\
\hline METAL & Cadmium & $\mathrm{mg} / \mathrm{L}$ & 6 & 0 & $0 \%$ & $2.30 \mathrm{E}-05$ & $2.30 \mathrm{E}-05$ & -- & -- & $1.15 \mathrm{E}-05$ \\
\hline METAL & Chromium & $\mathrm{mg} / \mathrm{L}$ & 6 & 5 & $83 \%$ & 4.70E-05 & 4.70E-05 & 8.82E-05 & 7.50E-04 & $4.14 \mathrm{E}-04$ \\
\hline METAL & Copper & $\mathrm{mg} / \mathrm{L}$ & 6 & 6 & $100 \%$ & -- & -- & 7.02E-04 & 0.0015 & 0.0011 \\
\hline METAL & Lead & $\mathrm{mg} / \mathrm{L}$ & 6 & 3 & $50 \%$ & 1.10E-05 & 1.10E-05 & 2.73E-04 & $3.27 \mathrm{E}-04$ & $1.52 \mathrm{E}-04$ \\
\hline METAL & Mercury & $\mathrm{mg} / \mathrm{L}$ & 6 & 6 & $100 \%$ & -- & -- & 7.59E-07 & 1.52E-06 & 1.17E-06 \\
\hline METAL & Nickel & $\mathrm{mg} / \mathrm{L}$ & 6 & 6 & $100 \%$ & -- & -- & 1.59E-04 & 0.0018 & $5.79 \mathrm{E}-04$ \\
\hline METAL & Selenium & $\mathrm{mg} / \mathrm{L}$ & 6 & 3 & $50 \%$ & 8.00E-05 & 8.00E-05 & 1.32E-04 & 3.97E-04 & $1.48 \mathrm{E}-04$ \\
\hline METAL & Silver & $\mathrm{mg} / \mathrm{L}$ & 6 & 0 & $0 \%$ & 8.50E-06 & $8.50 \mathrm{E}-06$ & -- & -- & $4.25 \mathrm{E}-06$ \\
\hline METAL & Thallium & $\mathrm{mg} / \mathrm{L}$ & 6 & 1 & $17 \%$ & $9.00 \mathrm{E}-06$ & $9.00 \mathrm{E}-06$ & $9.48 \mathrm{E}-06$ & $9.48 \mathrm{E}-06$ & $5.33 \mathrm{E}-06$ \\
\hline METAL & Uranium & $\mathrm{mg} / \mathrm{L}$ & 6 & 6 & $100 \%$ & -- & -- & 3.71E-04 & $4.35 \mathrm{E}-04$ & 4.05E-04 \\
\hline METAL & Zinc & $\mathrm{mg} / \mathrm{L}$ & 6 & 6 & $100 \%$ & -- & -- & 0.0015 & 0.0034 & 0.0024 \\
\hline PCB & Aroclor-1016 & $\mathrm{mg} / \mathrm{L}$ & 1 & 0 & $0 \%$ & $6.30 \mathrm{E}-07$ & $6.30 \mathrm{E}-07$ & -- & -- & $3.15 \mathrm{E}-07$ \\
\hline PCB & Aroclor-1221 & $\mathrm{mg} / \mathrm{L}$ & 1 & 0 & $0 \%$ & 6.30E-07 & 6.30E-07 & -- & -- & $3.15 \mathrm{E}-07$ \\
\hline PCB & Aroclor-1232 & $\mathrm{mg} / \mathrm{L}$ & 1 & 0 & $0 \%$ & 6.30E-07 & 6.30E-07 & -- & -- & 3.15E-07 \\
\hline PCB & Aroclor-1242 & $\mathrm{mg} / \mathrm{L}$ & 1 & 0 & $0 \%$ & $6.30 \mathrm{E}-07$ & 6.30E-07 & -- & -- & $3.15 \mathrm{E}-07$ \\
\hline PCB & Aroclor-1248 & $\mathrm{mg} / \mathrm{L}$ & 1 & 0 & $0 \%$ & 6.30E-07 & 6.30E-07 & -- & -- & $3.15 \mathrm{E}-07$ \\
\hline PCB & Aroclor-1254 & $\mathrm{mg} / \mathrm{L}$ & 1 & 0 & $0 \%$ & $6.30 \mathrm{E}-07$ & $6.30 \mathrm{E}-07$ & -- & -- & $3.15 \mathrm{E}-07$ \\
\hline PCB & Aroclor-1260 & $\mathrm{mg} / \mathrm{L}$ & 1 & 0 & $0 \%$ & 6.30E-07 & 6.30E-07 & -- & -- & $3.15 \mathrm{E}-07$ \\
\hline PEST & 2,4'-DDD & $\mathrm{mg} / \mathrm{L}$ & 8 & 0 & $0 \%$ & $6.50 \mathrm{E}-08$ & $6.90 \mathrm{E}-08$ & -- & -- & 3.34E-08 \\
\hline PEST & 2,4'-DDE & $\mathrm{mg} / \mathrm{L}$ & 8 & 0 & $0 \%$ & $6.50 \mathrm{E}-08$ & $6.90 \mathrm{E}-08$ & -- & -- & $3.34 \mathrm{E}-08$ \\
\hline PEST & 2,4'-DDT & $\mathrm{mg} / \mathrm{L}$ & 8 & 0 & $0 \%$ & $6.50 \mathrm{E}-08$ & $1.10 \mathrm{E}-07$ & -- & -- & 3.61E-08 \\
\hline PEST & alpha-Chlordane & $\mathrm{mg} / \mathrm{L}$ & 11 & 0 & $0 \%$ & $6.30 \mathrm{E}-08$ & $6.90 \mathrm{E}-08$ & -- & -- & $3.29 \mathrm{E}-08$ \\
\hline PEST & cis-Nonachlor & $\mathrm{mg} / \mathrm{L}$ & 11 & 0 & $0 \%$ & $6.30 \mathrm{E}-08$ & $6.90 \mathrm{E}-08$ & -- & -- & $3.29 \mathrm{E}-08$ \\
\hline PEST & Dichlorodiphenyldichloroethane & $\mathrm{mg} / \mathrm{L}$ & 11 & 4 & $36 \%$ & $6.30 \mathrm{E}-08$ & 7.70E-08 & 6.70E-08 & 1.30E-07 & $5.79 \mathrm{E}-08$ \\
\hline PEST & Dichlorodiphenyldichloroethylene & $\mathrm{mg} / \mathrm{L}$ & 11 & 11 & $100 \%$ & -- & -- & 1.50E-07 & 1.20E-06 & 3.39E-07 \\
\hline
\end{tabular}


Table C-15

Summary Statistics for Category 1 Surface Water Samples Collected from the Walla Walla River (Segment 3)

\begin{tabular}{|c|c|c|c|c|c|c|c|c|c|c|}
\hline $\begin{array}{l}\text { Constituent } \\
\text { Class }\end{array}$ & Constituent Name & Units & $\begin{array}{l}\text { Number } \\
\text { Analyzed }\end{array}$ & $\begin{array}{l}\text { Number } \\
\text { Detected }\end{array}$ & $\begin{array}{l}\text { Frequency of } \\
\text { Detection }\end{array}$ & $\begin{array}{l}\text { Minimum } \\
\text { Nondetect }\end{array}$ & $\begin{array}{l}\text { Maximum } \\
\text { Nondetect }\end{array}$ & $\begin{array}{c}\text { Minimum } \\
\text { Detect }\end{array}$ & $\begin{array}{l}\text { Maximum } \\
\text { Detect }\end{array}$ & $\begin{array}{c}\text { Average } \\
\text { Concentration }\end{array}$ \\
\hline PEST & Dichlorodiphenyltrichloroethane & $\mathrm{mg} / \mathrm{L}$ & 11 & 4 & $36 \%$ & $6.50 \mathrm{E}-08$ & $1.60 \mathrm{E}-07$ & $1.00 \mathrm{E}-07$ & $6.50 \mathrm{E}-07$ & $1.27 \mathrm{E}-07$ \\
\hline PEST & Dieldrin & $\mathrm{mg} / \mathrm{L}$ & 11 & 4 & $36 \%$ & $6.30 \mathrm{E}-08$ & $1.60 \mathrm{E}-07$ & $7.60 \mathrm{E}-08$ & $3.00 \mathrm{E}-07$ & $8.22 \mathrm{E}-08$ \\
\hline PEST & Heptachlor & $\mathrm{mg} / \mathrm{L}$ & 11 & 0 & $0 \%$ & $6.30 \mathrm{E}-08$ & $6.90 \mathrm{E}-08$ & -- & -- & $3.29 \mathrm{E}-08$ \\
\hline PEST & Heptachlor epoxide & $\mathrm{mg} / \mathrm{L}$ & 11 & 4 & $36 \%$ & $6.50 \mathrm{E}-08$ & 1.10E-07 & $3.80 \mathrm{E}-08$ & $1.20 \mathrm{E}-07$ & 4.55E-08 \\
\hline PEST & Oxychlordane & $\mathrm{mg} / \mathrm{L}$ & 11 & 0 & $0 \%$ & $6.50 \mathrm{E}-08$ & $1.60 \mathrm{E}-07$ & -- & -- & $4.61 \mathrm{E}-08$ \\
\hline PEST & trans-Chlordane & $\mathrm{mg} / \mathrm{L}$ & 11 & 0 & $0 \%$ & $6.50 \mathrm{E}-08$ & $1.60 \mathrm{E}-07$ & -- & -- & $4.61 \mathrm{E}-08$ \\
\hline PEST & trans-Nonachlor & $\mathrm{mg} / \mathrm{L}$ & 11 & 0 & $0 \%$ & $6.30 \mathrm{E}-08$ & $6.90 \mathrm{E}-08$ & -- & -- & $3.29 \mathrm{E}-08$ \\
\hline RAD & Antimony-125 & $\mathrm{pCi} / \mathrm{L}$ & 2 & 0 & $0 \%$ & 2.8 & 3.2 & -- & -- & 1.5 \\
\hline RAD & Beryllium-7 & $\mathrm{pCi} / \mathrm{L}$ & 2 & 0 & $0 \%$ & 0.42 & 0.70 & -- & -- & 0.28 \\
\hline RAD & Cesium-134 & $\mathrm{pCi} / \mathrm{L}$ & 2 & 0 & $0 \%$ & 0.46 & 1.8 & -- & -- & 0.56 \\
\hline RAD & Cesium-137 & $\mathrm{pCi} / \mathrm{L}$ & 2 & 0 & $0 \%$ & 0.16 & 0.95 & -- & -- & 0.28 \\
\hline RAD & Cobalt- 60 & $\mathrm{pCi} / \mathrm{L}$ & 1 & 0 & $0 \%$ & 2.0 & 2.0 & -- & -- & 1.0 \\
\hline RAD & Europium-152 & $\mathrm{pCi} / \mathrm{L}$ & 1 & 0 & $0 \%$ & 1.1 & 1.1 & -- & -- & 0.57 \\
\hline RAD & Europium-155 & $\mathrm{pCi} / \mathrm{L}$ & 3 & 0 & $0 \%$ & 1.2 & 2.0 & -- & -- & 0.77 \\
\hline RAD & Ruthenium-106 & $\mathrm{pCi} / \mathrm{L}$ & 2 & 0 & $0 \%$ & 0.54 & 6.7 & -- & -- & 1.8 \\
\hline RAD & Strontium-90 & $\mathrm{pCi} / \mathrm{L}$ & 3 & 2 & $67 \%$ & 0.046 & 0.046 & 0.061 & 0.065 & 0.050 \\
\hline RAD & Technetium-99 & $\mathrm{pCi} / \mathrm{L}$ & 3 & 0 & $0 \%$ & 0.18 & 0.44 & -- & -- & 0.14 \\
\hline RAD & Tritium & $\mathrm{pCi} / \mathrm{L}$ & 3 & 3 & $100 \%$ & -- & -- & 19 & 29 & 23 \\
\hline SVOC & Hexachlorobenzene & $\mathrm{mg} / \mathrm{L}$ & 11 & 2 & $18 \%$ & $6.30 \mathrm{E}-08$ & $1.00 \mathrm{E}-07$ & 1.10E-07 & 1.80E-07 & $5.58 \mathrm{E}-08$ \\
\hline
\end{tabular}

CONV - Conventional parameter

PCB- Poylychlorinated biphenyls

PEST - Pesticide

RAD - Radionuclide

SVOC - Semivolatile Organic Compound 
Table C-16

Summary Statistics for Category 1 Surface Water Samples Collected from the Touchet River (Segment 3)

\begin{tabular}{|c|c|c|c|c|c|c|c|c|c|c|}
\hline $\begin{array}{l}\text { Constituent } \\
\text { Class }\end{array}$ & Constituent Name & Units & $\begin{array}{l}\text { Number } \\
\text { Analyzed }\end{array}$ & $\begin{array}{l}\text { Number } \\
\text { Detected }\end{array}$ & $\begin{array}{l}\text { Frequency of } \\
\text { Detection }\end{array}$ & $\begin{array}{l}\text { Minimum } \\
\text { Nondetect }\end{array}$ & $\begin{array}{l}\text { Maximum } \\
\text { Nondetect }\end{array}$ & $\begin{array}{l}\text { Minimum } \\
\text { Detect }\end{array}$ & $\begin{array}{l}\text { Maximum } \\
\text { Detect }\end{array}$ & $\begin{array}{c}\text { Average } \\
\text { Concentration }\end{array}$ \\
\hline CONV & Dissolved organic carbon & $\mathrm{mg} / \mathrm{L}$ & 14 & 14 & $100 \%$ & -- & -- & 1.6 & 3.2 & 2.3 \\
\hline CONV & Specific Conductance & umhos $/ \mathrm{cm}$ & 16 & 16 & $100 \%$ & -- & -- & 70 & 179 & 113 \\
\hline CONV & Temperature & Deg C & 15 & 15 & $100 \%$ & -- & -- & 3.8 & 24 & 14 \\
\hline CONV & Total Organic Carbon & $\mathrm{mg} / \mathrm{L}$ & 14 & 14 & $100 \%$ & -- & -- & 1.8 & 3.7 & 2.6 \\
\hline CONV & Total suspended solids & $\mathrm{mg} / \mathrm{L}$ & 16 & 16 & $100 \%$ & -- & -- & 1.0 & 187 & 40 \\
\hline CONV & Turbidity & NTU & 16 & 16 & $100 \%$ & -- & -- & 1.3 & 72 & 14 \\
\hline PCB & Aroclor-1016 & $\mathrm{mg} / \mathrm{L}$ & 1 & 0 & $0 \%$ & $6.60 \mathrm{E}-07$ & $6.60 \mathrm{E}-07$ & -- & -- & 3.30E-07 \\
\hline PCB & Aroclor-1221 & $\mathrm{mg} / \mathrm{L}$ & 1 & 0 & $0 \%$ & $6.60 \mathrm{E}-07$ & $6.60 \mathrm{E}-07$ & -- & -- & 3.30E-07 \\
\hline PCB & Aroclor-1232 & $\mathrm{mg} / \mathrm{L}$ & 1 & 0 & $0 \%$ & $6.60 \mathrm{E}-07$ & $6.60 \mathrm{E}-07$ & -- & -- & 3.30E-07 \\
\hline PCB & Aroclor-1242 & $\mathrm{mg} / \mathrm{L}$ & 1 & 0 & $0 \%$ & $6.60 \mathrm{E}-07$ & $6.60 \mathrm{E}-07$ & -- & -- & 3.30E-07 \\
\hline PCB & Aroclor-1248 & $\mathrm{mg} / \mathrm{L}$ & 1 & 0 & $0 \%$ & $6.60 \mathrm{E}-07$ & $6.60 \mathrm{E}-07$ & -- & -- & 3.30E-07 \\
\hline PCB & Aroclor-1254 & $\mathrm{mg} / \mathrm{L}$ & 1 & 0 & $0 \%$ & $6.60 \mathrm{E}-07$ & $6.60 \mathrm{E}-07$ & -- & -- & 3.30E-07 \\
\hline PCB & Aroclor-1260 & $\mathrm{mg} / \mathrm{L}$ & 1 & 0 & $0 \%$ & $6.60 \mathrm{E}-07$ & $6.60 \mathrm{E}-07$ & -- & -- & $3.30 \mathrm{E}-07$ \\
\hline PEST & 2,4'-DDD & $\mathrm{mg} / \mathrm{L}$ & 8 & 0 & $0 \%$ & $6.20 \mathrm{E}-08$ & $6.90 \mathrm{E}-08$ & -- & -- & 3.33E-08 \\
\hline PEST & 2,4'-DDE & $\mathrm{mg} / \mathrm{L}$ & 8 & 0 & $0 \%$ & $6.20 \mathrm{E}-08$ & 6.90E-08 & -- & -- & 3.33E-08 \\
\hline PEST & 2,4'-DDT & $\mathrm{mg} / \mathrm{L}$ & 8 & 0 & $0 \%$ & $6.20 \mathrm{E}-08$ & 6.90E-08 & -- & -- & 3.33E-08 \\
\hline PEST & alpha-Chlordane & $\mathrm{mg} / \mathrm{L}$ & 10 & 0 & $0 \%$ & 6.20E-08 & 6.90E-08 & -- & -- & 3.33E-08 \\
\hline PEST & $\begin{array}{l}\text { cis-Nonachlor } \\
\text { Dichlorodiphenyldichloroeth }\end{array}$ & $\mathrm{mg} / \mathrm{L}$ & 10 & 0 & $0 \%$ & $6.20 \mathrm{E}-08$ & $6.90 \mathrm{E}-08$ & -- & -- & 3.33E-08 \\
\hline PEST & $\begin{array}{l}\text { ane } \\
\text { Dichlorodiphenyldichloroeth }\end{array}$ & $\mathrm{mg} / \mathrm{L}$ & 10 & 0 & $0 \%$ & $6.20 \mathrm{E}-08$ & $9.20 \mathrm{E}-08$ & -- & -- & 3.46E-08 \\
\hline PEST & $\begin{array}{l}\text { ylene } \\
\text { Dichlorodiphenyltrichloroeth }\end{array}$ & $\mathrm{mg} / \mathrm{L}$ & 10 & 7 & $70 \%$ & $6.60 \mathrm{E}-08$ & $6.90 \mathrm{E}-08$ & 7.10E-08 & 4.10E-07 & $1.24 \mathrm{E}-07$ \\
\hline PEST & ane & $\mathrm{mg} / \mathrm{L}$ & 10 & 1 & $10 \%$ & $6.40 \mathrm{E}-08$ & 9.30E-08 & $1.20 \mathrm{E}-07$ & $1.20 \mathrm{E}-07$ & 4.60E-08 \\
\hline PEST & Dieldrin & $\mathrm{mg} / \mathrm{L}$ & 10 & 0 & $0 \%$ & $6.20 \mathrm{E}-08$ & 1.60E-07 & -- & -- & 4.10E-08 \\
\hline PEST & Heptachlor & $\mathrm{mg} / \mathrm{L}$ & 10 & 0 & $0 \%$ & $6.20 \mathrm{E}-08$ & $6.90 \mathrm{E}-08$ & -- & -- & 3.33E-08 \\
\hline PEST & Heptachlor epoxide & $\mathrm{mg} / \mathrm{L}$ & 10 & 2 & $20 \%$ & $6.20 \mathrm{E}-08$ & 7.00E-08 & 3.30E-08 & $3.90 \mathrm{E}-08$ & $3.40 \mathrm{E}-08$ \\
\hline PEST & Oxychlordane & $\mathrm{mg} / \mathrm{L}$ & 10 & 0 & $0 \%$ & $6.20 \mathrm{E}-08$ & 1.70E-07 & -- & -- & $4.31 \mathrm{E}-08$ \\
\hline PEST & trans-Chlordane & $\mathrm{mg} / \mathrm{L}$ & 10 & 0 & $0 \%$ & $6.20 \mathrm{E}-08$ & 1.70E-07 & -- & -- & 4.31E-08 \\
\hline PEST & trans-Nonachlor & $\mathrm{mg} / \mathrm{L}$ & 10 & 0 & $0 \%$ & $6.20 \mathrm{E}-08$ & 6.90E-08 & -- & -- & 3.33E-08 \\
\hline SVOC & Hexachlorobenzene & $\mathrm{mg} / \mathrm{L}$ & 10 & 1 & $10 \%$ & $6.40 \mathrm{E}-08$ & 9.70E-08 & $1.60 \mathrm{E}-07$ & 1.60E-07 & $5.02 \mathrm{E}-08$ \\
\hline
\end{tabular}

CONV - Conventional paramete

PCB - Polychlorinated Biphenyls

PEST - Pesticide

SVOC - Semivolatile Organic Compound 
Table C-17

Comparison of All Categories of Surface Water Concentrations Collected from Segment 3 of the Columbia River to Ecological and Human Health Benchmark Values

\begin{tabular}{|c|c|c|c|c|c|c|c|c|c|c|c|c|c|c|c|c|}
\hline $\begin{array}{l}\text { Constituent } \\
\text { Class }\end{array}$ & Constituent Name & Units & $\begin{array}{c}\text { Number } \\
\text { Analyzed }\end{array}$ & $\begin{array}{l}\text { Number } \\
\text { Detected }\end{array}$ & $\begin{array}{c}\text { Frequency } \\
\text { of Detection }\end{array}$ & $\begin{array}{c}\text { Minimum } \\
\text { Detect }\end{array}$ & $\begin{array}{c}\text { Maximum } \\
\text { Detect }\end{array}$ & $\begin{array}{c}\text { Average } \\
\text { Concentration }\end{array}$ & $\begin{array}{c}\text { Benchmark } \\
\text { Value }\end{array}$ & $\begin{array}{l}\text { Is Max > Eco } \\
\text { Benchmark? }\end{array}$ & $\begin{array}{c}\begin{array}{c}\text { Factor of } \\
\text { Exceedance }\end{array} \\
\end{array}$ & $\begin{array}{c}\text { Number of } \\
\text { Exceedances }\end{array}$ & $\begin{array}{c}\text { Human Health } \\
\text { Benchmark Value }\end{array}$ & $\begin{array}{l}\text { Is Max > HH } \\
\text { Benchmark? }\end{array}$ & $\begin{array}{c}\begin{array}{c}\text { Factor of } \\
\text { Exceedance }\end{array} \\
\end{array}$ & $\begin{array}{c}\text { Number of } \\
\text { Exceedances }\end{array}$ \\
\hline CONV & $\begin{array}{l}\text { Alkalinity } \\
\text { Ammonian }\end{array}$ & $\mathrm{mg} / \mathrm{L}$ & 23 & 23 & $100 \%$ & 99 & 121 & 104 & -- & 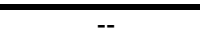 & -- & -- & - & -- & 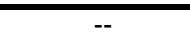 & -- \\
\hline CONV & Ammonia & $\mathrm{mg} / \mathrm{L}$ & 103 & 63 & $61 \%$ & 0.010 & 0.090 & 0.020 & - & -- & -- & -- & -- & -- & - & -- \\
\hline CONV & Chloride & $\mathrm{mg} / \mathrm{L}$ & 37 & 37 & $100 \%$ & 2.0 & 150 & 13 & 230 & No & 0.65 & 0 & -- & -- & -- & -- \\
\hline $\begin{array}{l}\text { CONV } \\
\text { CONV }\end{array}$ & $\begin{array}{l}\text { Dissolved organic carbon } \\
\text { DDisolved oxyean }\end{array}$ & $\mathrm{mg} / \mathrm{L}$ & 46 & 46 & $100 \%$ & 1.5 & $\begin{array}{l}4.4 \\
0.3\end{array}$ & $\begin{array}{l}2.3 \\
0.3\end{array}$ & -- & -- & -- & -- & -- & -- & -- & -- \\
\hline $\begin{array}{l}\text { CONV } \\
\text { CONV }\end{array}$ & $\begin{array}{l}\text { Dissolved oxygen } \\
\text { Fluoride }\end{array}$ & $\begin{array}{l}\mathrm{mg} / \mathrm{L} \\
\mathrm{mg} / \mathrm{L}\end{array}$ & $\begin{array}{c}1 \\
12\end{array}$ & $\begin{array}{c}1 \\
12\end{array}$ & $\begin{array}{l}100 \% \\
100 \%\end{array}$ & $\begin{array}{c}9.3 \\
0.11\end{array}$ & $\begin{array}{l}9.3 \\
0.21\end{array}$ & 9.3 & -- & -- & -- & -- & -- & -- & -- & -- \\
\hline CONV & Hardness & $\mathrm{mg} / \mathrm{L}$ & 32 & 32 & $\begin{array}{l}100 \% \\
100 \%\end{array}$ & $\begin{array}{l}.11 \\
56\end{array}$ & 118 & $\begin{array}{l}.10 \\
95\end{array}$ & -- & $\begin{array}{c}-- \\
-\end{array}$ & $\begin{array}{l}-- \\
-\end{array}$ & $\begin{array}{l}-- \\
-\end{array}$ & -- & -- & -- & -- \\
\hline CONV & Nitrate & $\mathrm{mg} / \mathrm{L}$ & 48 & 48 & $100 \%$ & 0.20 & 4.6 & 1.9 & -- & -- & -- & -- & 10 & No & 0.46 & 0 \\
\hline CONV & Nitrogen, Nitrate (as N) & $\mathrm{mg} / \mathrm{L}$ & 12 & 12 & $100 \%$ & 0.15 & 0.60 & 0.29 & -- & -- & -- & -- & -- & -- & -- & -- \\
\hline CONV & Nitrogen, Nitrate-Nitrite & $\mathrm{mg} / \mathrm{L}$ & 3 & 3 & $100 \%$ & 0.86 & 1.0 & 0.92 & -- & -- & -- & -- & -- & -- & -- & -- \\
\hline CONV & Orthophosphate & $\mathrm{mg} / \mathrm{L}$ & 98 & 98 & $100 \%$ & 0.032 & 0.14 & 0.080 & -- & -- & -- & -- & -- & -- & -- & -- \\
\hline CONV & $\mathrm{pH}$ & $\mathrm{pH}$ Units & 82 & 82 & $100 \%$ & 7.6 & 9.5 & 8.3 & -- & -- & -- & -- & -- & -- & -- & -- \\
\hline CONV & Phosphorous in phosphate & $\mathrm{mg} / \mathrm{L}$ & 36 & 35 & $97 \%$ & 0.010 & 0.12 & 0.046 & -- & -- & -- & - & -- & -- & -- & - \\
\hline CONV & Specific Conductance & umhos $/ \mathrm{cm}$ & 124 & 124 & $100 \%$ & 70 & 1,030 & 219 & -- & -- & -- & -- & $-\cdots$ & -- & - & -- \\
\hline CONV & Sulfate & $\mathrm{mg} / \mathrm{L}$ & 12 & 12 & $100 \%$ & 4.6 & 14 & 8.8 & -- & -- & - & -- & -- & - & -- & -- \\
\hline $\begin{array}{l}\text { CONV } \\
\text { CONV }\end{array}$ & Temperature & Deg C & 19 & 19 & $100 \%$ & 3.8 & 24 & 14 & -- & -- & -- & -- & -- & -- & -- & -- \\
\hline $\begin{array}{l}\text { CONV } \\
\text { CONV }\end{array}$ & $\begin{array}{l}\text { Total Non-Volatile Suspended Solic } \\
\text { Total Organic Carbon }\end{array}$ & $\mathrm{mg} / \mathrm{L}$ & 16 & 16 & $\begin{array}{l}100 \% \\
100 \%\end{array}$ & 15 & 57 & 29 & -- & -- & -- & -- & -- & -- & -- & -- \\
\hline CONV & Total Organic Carbon & $\mathrm{mg} / \mathrm{L}$ & $\begin{array}{l}1 \\
48\end{array}$ & $\begin{array}{l}1 \\
48\end{array}$ & $100 \%$ & $\begin{array}{l}0.83 \\
1.7\end{array}$ & 5.0 & $\begin{array}{l}0.83 \\
2.5\end{array}$ & -- & $\begin{array}{c}-- \\
-\end{array}$ & -- & -- & -- & -- & -- & -- \\
\hline CONV & Total Persulfate Nitrogen & $\mathrm{mg} / \mathrm{L}$ & 103 & 103 & $100 \%$ & 0.43 & 23 & 1.5 & -- & -- & -- & -- & -- & -- & -- & -- \\
\hline CONV & Total suspended solids & $\mathrm{mg} / \mathrm{L}$ & 258 & 258 & $100 \%$ & 1.0 & 405 & 24 & -- & -- & -- & -- & -- & -- & -- & -- \\
\hline CONV & Turbidity & NTU & 163 & 163 & $100 \%$ & 1.2 & 210 & 12 & -- & -- & -- & -- & -- & -- & -- & -- \\
\hline METAL & Antimony & $\mathrm{mg} / \mathrm{L}$ & 24 & 24 & $100 \%$ & $2.89 \mathrm{E}-05$ & $2.13 \mathrm{E}-04$ & $1.02 \mathrm{E}-04$ & 1.6 & No & $1.33 \mathrm{E}-04$ & 0 & 0.0056 & No & 0.038 & 0 \\
\hline METAL & Arsenic & $\mathrm{mg} / \mathrm{L}$ & 48 & 48 & $100 \%$ & $5.08 \mathrm{E}-04$ & 0.0028 & 0.0013 & 0.15 & No & 0.019 & 0 & $1.80 \mathrm{E}-05$ & Yes & 156 & 48 \\
\hline METAL & Cadmium & $\mathrm{mg} / \mathrm{L}$ & 36 & 3 & $8 \%$ & 2.59E-05 & $3.32 \mathrm{E}-05$ & $1.91 \mathrm{E}-05$ & $2.50 \mathrm{E}-04$ & No & 0.13 & 0 & -- & -- & -- & -- \\
\hline METAL & Chromium & $\mathrm{mg} / \mathrm{L}$ & 39 & 33 & $85 \%$ & $8.82 \mathrm{E}-05$ & 0.0017 & $5.38 \mathrm{E}-04$ & 0.010 & No & 0.17 & 0 & -- & -- & -- & -- \\
\hline METAL & Copper & $\mathrm{mg} / \mathrm{L}$ & 39 & 39 & $100 \%$ & $4.80 E-04$ & 0.0025 & $9.18 \mathrm{E}-04$ & 0.0060 & No & 0.42 & 0 & -- & -- & -- & -- \\
\hline $\begin{array}{l}\text { METAL } \\
\text { METAL }\end{array}$ & Lead & $\mathrm{mg} / \mathrm{L}$ & $\begin{array}{l}39 \\
37\end{array}$ & 28 & $72 \%$ & $\begin{array}{l}1.11 \mathrm{E}-05 \\
583 \mathrm{E}-07\end{array}$ & $\begin{array}{l}6.90 \mathrm{E}-04 \\
320 \mathrm{E}-06\end{array}$ & $\begin{array}{l}8.91 \mathrm{E}-05 \\
135 \mathrm{~F}-06\end{array}$ & 8.67E-04 & No & 0.80 & 0 & -- & -- & -- & -- \\
\hline $\begin{array}{l}\text { METAL } \\
\text { METAL }\end{array}$ & $\begin{array}{l}\text { Mercury } \\
\text { Nickel }\end{array}$ & $\begin{array}{l}\mathrm{mg} / \mathrm{L} \\
\mathrm{mg} / \mathrm{L}\end{array}$ & $\begin{array}{l}37 \\
36\end{array}$ & $\begin{array}{l}28 \\
36\end{array}$ & $\begin{array}{l}76 \% \\
100 \%\end{array}$ & $\begin{array}{l}5.83 \mathrm{E}-07 \\
3.03 \mathrm{E}-05\end{array}$ & $\begin{array}{l}3.20 \mathrm{E}-06 \\
0.023\end{array}$ & $\begin{array}{l}1.35 \mathrm{E}-06 \\
6.65 \mathrm{E}-04\end{array}$ & $\begin{array}{l}1.20 \mathrm{E}-05 \\
0.028\end{array}$ & $\begin{array}{l}\text { No } \\
\text { No }\end{array}$ & $\begin{array}{l}0.27 \\
0.085\end{array}$ & $\begin{array}{l}0 \\
0\end{array}$ & 0.61 & No & 0.0038 & $\overline{0}$ \\
\hline METAL & Phosphorus & $\mathrm{mg} / \mathrm{L}$ & 139 & 139 & $100 \%$ & 0.010 & 3.9 & 0.19 & -- & $\cdots$ & & & & 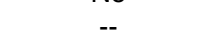 & & -- \\
\hline METAL & Selenium & $\mathrm{mg} / \mathrm{L}$ & 24 & 12 & $50 \%$ & $8.01 \mathrm{E}-05$ & 4.66E-04 & $1.37 \mathrm{E}-04$ & 0.0050 & No & 0.093 & 0 & 0.17 & No & 0.0027 & 0 \\
\hline METAL & Silver & $\mathrm{mg} / \mathrm{L}$ & 43 & 12 & $28 \%$ & $2.00 \mathrm{E}-05$ & $1.00 E-04$ & 2.07E-05 & $1.20 \mathrm{E}-04$ & No & 0.83 & 0 & & & & \\
\hline METAL & Thallium & $\mathrm{mg} / \mathrm{L}$ & 24 & 7 & $29 \%$ & $9.48 \mathrm{E}-06$ & $1.51 \mathrm{E}-05$ & $6.81 \mathrm{E}-06$ & 0.040 & No & $3.78 \mathrm{E}-04$ & 0 & $2.40 \mathrm{E}-04$ & No & 0.063 & 0 \\
\hline METAL & Uranium & $\mathrm{mg} / \mathrm{L}$ & 24 & 24 & $100 \%$ & $3.71 E-04$ & 0.0016 & $8.48 \mathrm{E}-04$ & 0.0026 & No & 0.61 & 0 & 0.030 & No & 0.053 & 0 \\
\hline METAL & Zinc & $\mathrm{mg} / \mathrm{L}$ & 39 & 39 & $100 \%$ & $4.90 \mathrm{E}-04$ & 0.0064 & 0.0024 & 0.055 & No & 0.12 & 0 & 1.4 .0 & No & $8.65 \mathrm{E}-04$ & 0 \\
\hline PEST & Dichlorodiphenyldichloroethane & $\mathrm{mg} / \mathrm{L}$ & 21 & 4 & $19 \%$ & $6.70 \mathrm{E}-08$ & 1.30E-07 & $4.68 \mathrm{E}-08$ & 1.00E-06 & No & 0.13 & 0 & 3.10E-07 & No & 0.42 & 0 \\
\hline $\begin{array}{l}\text { PEST } \\
\text { DEST }-10\end{array}$ & Dichlorodiphenyldichloroethylene & $\mathrm{mg} / \mathrm{L}$ & 21 & 18 & $86 \%$ & $7.10 \mathrm{E}-08$ & $1.20 \mathrm{E}-06$ & 2.37E-07 & 1 & -- & -- & -- & $2.20 \mathrm{E}-07$ & Yes & 5.5 & 8 \\
\hline $\begin{array}{l}\text { PEST } \\
\text { DEST }\end{array}$ & Dichlorodiphenyltrichloroethane & $\mathrm{mg} / \mathrm{L}$ & 21 & 5 & $24 \%$ & $1.00 E-07$ & $6.50 E-07$ & $8.82 \mathrm{E}-08$ & 1.00E-06 & No & 0.65 & 0 & 2.20E-07 & Yes & 3.0 & 1 \\
\hline $\begin{array}{l}\text { PEST } \\
\text { PEST }\end{array}$ & $\begin{array}{l}\text { Dieldrin } \\
\text { Hentanhen enoxide }\end{array}$ & $\mathrm{mg} / \mathrm{L}$ & 21 & 4 & $\begin{array}{l}19 \% \\
29 \%\end{array}$ & $\begin{array}{l}7.60 \mathrm{E}-08 \\
3.30 \mathrm{E}-08\end{array}$ & $\begin{array}{l}3.00 E-07 \\
120 \mathrm{E}-07\end{array}$ & $\begin{array}{l}6.26 \mathrm{E}-08 \\
400 \mathrm{~F}-08\end{array}$ & $1.90 E-06$ & No & 0.16 & 0 & 5.20E-08 & Yes & 5.8 & 4 \\
\hline $\begin{array}{l}\text { PEST } \\
\text { RAD }\end{array}$ & $\begin{array}{l}\text { Heptachlor epoxide } \\
\text { Antimony-125 }\end{array}$ & $\begin{array}{l}\mathrm{mg} / \mathrm{L} \\
\mathrm{pci} / \mathrm{L}\end{array}$ & 21 & 6 & $\begin{array}{l}29 \% \\
13 \%\end{array}$ & $3.30 \mathrm{E}-08$ & $1.20 \mathrm{E}-07$ & $\begin{array}{l}4.00 E-08 \\
12\end{array}$ & $3.80 \mathrm{E}-06$ & No & 0.032 & 0 & $3.90 \mathrm{E}-08$ & Yes & 3.1 & 4 \\
\hline RAD & Cesium-134 & $\mathrm{pCi/L}$ & 10 & 1 & $10 \%$ & 0.70 & 0.70 & $\begin{array}{l}1.2 \\
0.68\end{array}$ & -- & -- & -- & -- & -- & -- & -- & -- \\
\hline RAD & Cobalt-60 & $\mathrm{pCi} / \mathrm{L}$ & 8 & 1 & $13 \%$ & 4.4 & 4.4 & 1.3 & 3,760 & No & 0.0012 & 0 & 100 & No & 0.044 & 0 \\
\hline RAD & Ruthenium-106 & pCi/L & 7 & 1 & $14 \%$ & 4.3 & 4.3 & 2.5 & 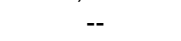 & & & 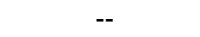 & & & & $\ldots$ \\
\hline RAD & Strontium-90 & $\mathrm{pCi} / \mathrm{L}$ & 18 & 17 & $94 \%$ & 0.055 & 0.15 & 0.089 & 278 & No & $5.47 \mathrm{E}-04$ & 0 & 8.0 & No & 0.019 & 0 \\
\hline RAD & Tritium & $\mathrm{pCi} / \mathrm{L}$ & 12 & 12 & $100 \%$ & 13 & 35 & 25 & $2.65 E+08$ & No & 1.33E-07 & 0 & 20,000 & No & 0.0018 & 0 \\
\hline RAD & Uranium-234 & $\mathrm{pCi} / \mathrm{L}$ & 3 & 3 & $100 \%$ & 0.25 & 0.54 & 0.35 & 202 & No & 0.0027 & 0 & -- & -- & -- & -- \\
\hline RAD & Uranium-235 & $\mathrm{pCi} / \mathrm{L}$ & 3 & 2 & $67 \%$ & 0.0035 & 0.019 & 0.0080 & 217 & No & $8.71 E-05$ & 0 & -- & -- & -- & -- \\
\hline $\begin{array}{l}\text { RAD } \\
\text { SVOC }\end{array}$ & $\begin{array}{l}\text { Uranium-238 } \\
\text { Hexachlorobenzene }\end{array}$ & $\begin{array}{l}\mathrm{pCi} / \mathrm{L} \\
\mathrm{mg} / \mathrm{L}\end{array}$ & $\begin{array}{c}3 \\
21\end{array}$ & $\begin{array}{l}3 \\
3\end{array}$ & $\begin{array}{l}100 \% \\
14 \%\end{array}$ & $\begin{array}{l}0.19 \\
1.10 \mathrm{E}-07\end{array}$ & $\begin{array}{c}0.41 \\
1.80 \mathrm{E}-07\end{array}$ & $\begin{array}{c}0.26 \\
5.31 \mathrm{E}-08\end{array}$ & 223 & No & 0.0018 & 0 & $2.80 \mathrm{E}-07$ & No & $0 .-64$ & $\overline{0}$ \\
\hline
\end{tabular}

Conv - Conventional parameter

PEST - Pesticide

SVOC - Semivolatile Organic Compound 
Table C-18

Summary of Surface Water Results From Segment 3 Exceeding Select Ecological or Human Health Benchmark Values

\begin{tabular}{|c|c|c|c|c|c|c|c|c|c|c|}
\hline Sample Area & Sample Site & Sample ID & Date Collected & Constituent Name & Units & $\begin{array}{c}\text { QA } \\
\text { Category }\end{array}$ & $\begin{array}{l}\text { Analytical } \\
\text { Value }\end{array}$ & Qualifier & $\begin{array}{l}\text { Ecological } \\
\text { Benchmark } \\
\text { Value }\end{array}$ & $\begin{array}{l}\text { Human Health } \\
\text { Benchmark Value }\end{array}$ \\
\hline Walla Walla River & Walla Walla Riv & B16WP9 & May 15,2003 & Arsenic & $\mathrm{mg} / \mathrm{L}$ & 1 & $5.08 \mathrm{E}-04$ & $=$ & 0.15 & $1.80 \mathrm{E}-05$ \\
\hline Walla Walla River & Walla Walla Riv & B16WR0 & May 15, 2003 & Arsenic & $\mathrm{mg} / \mathrm{L}$ & 1 & 5.11E-04 & $=$ & 0.15 & $1.80 \mathrm{E}-05$ \\
\hline Walla Walla River & Walla Walla Riv & B16WR1 & May 15, 2003 & Arsenic & $\mathrm{mg} / \mathrm{L}$ & 1 & $5.67 \mathrm{E}-04$ & $=$ & 0.15 & $1.80 \mathrm{E}-05$ \\
\hline Walla Walla River & Walla Walla Riv & B16WP8 & May 15, 2003 & Arsenic & $\mathrm{mg} / \mathrm{L}$ & 1 & $5.72 \mathrm{E}-04$ & $=$ & 0.15 & $1.80 \mathrm{E}-05$ \\
\hline Walla Walla River & Walla Walla Riv & B16WR2 & May 15, 2003 & Arsenic & $\mathrm{mg} / \mathrm{L}$ & 1 & $6.20 \mathrm{E}-04$ & $=$ & 0.15 & $1.80 \mathrm{E}-05$ \\
\hline Walla Walla River & Walla Walla Riv & B16WR3 & May 15, 2003 & Arsenic & $\mathrm{mg} / \mathrm{L}$ & 1 & 7.05E-04 & $=$ & 0.15 & 1.80E-05 \\
\hline Yakima River & KIONA WA & 133239-F & April 14, 2004 & Arsenic & $\mathrm{mg} / \mathrm{L}$ & 2 & 7.30E-04 & $=$ & 0.15 & $1.80 \mathrm{E}-05$ \\
\hline Columbia River & MCNARY DAM & B16WN7 & May 15, 2003 & Arsenic & $\mathrm{mg} / \mathrm{L}$ & 1 & 8.00E-04 & $=$ & 0.15 & 1.80E-05 \\
\hline Yakima River & KIONA WA & 2158165 & March 6, 2002 & Arsenic & $\mathrm{mg} / \mathrm{L}$ & 1 & $8.20 \mathrm{E}-04$ & $=$ & 0.15 & $1.80 \mathrm{E}-05$ \\
\hline Yakima River & KIONA WA & 2228090 & May 10, 2002 & Arsenic & $\mathrm{mg} / \mathrm{L}$ & 1 & $8.20 \mathrm{E}-04$ & $=$ & 0.15 & 1.80E-05 \\
\hline Columbia River & MCNARY DAM & B16WN6 & May 15, 2003 & Arsenic & $\mathrm{mg} / \mathrm{L}$ & 1 & 8.41E-04 & $=$ & 0.15 & $1.80 \mathrm{E}-05$ \\
\hline Columbia River & MCNARY DAM & B16WP0 & May 15, 2003 & Arsenic & $\mathrm{mg} / \mathrm{L}$ & 1 & 8.43E-04 & $=$ & 0.15 & $1.80 \mathrm{E}-05$ \\
\hline Yakima River & KIONA WA & 2268165 & June 12, 2002 & Arsenic & $\mathrm{mg} / \mathrm{L}$ & 1 & 8.70E-04 & $=$ & 0.15 & $1.80 \mathrm{E}-05$ \\
\hline Columbia River & MCNARY DAM & B16WP1 & May 15, 2003 & Arsenic & $\mathrm{mg} / \mathrm{L}$ & 1 & 8.83E-04 & $=$ & 0.15 & $1.80 \mathrm{E}-05$ \\
\hline Columbia River & MCNARY DAM & B16WN8 & May 15, 2003 & Arsenic & $\mathrm{mg} / \mathrm{L}$ & 1 & 8.88E-04 & $=$ & 0.15 & $1.80 \mathrm{E}-05$ \\
\hline Yakima River & KIONA WA & 2118115 & February 6, 2002 & Arsenic & $\mathrm{mg} / \mathrm{L}$ & 1 & $9.30 \mathrm{E}-04$ & $=$ & 0.15 & $1.80 \mathrm{E}-05$ \\
\hline Yakima River & KIONA WA & 133239 & April 14, 2004 & Arsenic & $\mathrm{mg} / \mathrm{L}$ & 2 & $9.90 \mathrm{E}-04$ & $=$ & 0.15 & $1.80 \mathrm{E}-05$ \\
\hline Columbia River & MCNARY DAM & B16WN9 & May 15, 2003 & Arsenic & $\mathrm{mg} / \mathrm{L}$ & 1 & $9.95 \mathrm{E}-04$ & $=$ & 0.15 & $1.80 \mathrm{E}-05$ \\
\hline Yakima River & KIONA WA & 2178190 & April 3, 2002 & Arsenic & $\mathrm{mg} / \mathrm{L}$ & 1 & 0.0010 & $=$ & 0.15 & $1.80 \mathrm{E}-05$ \\
\hline Snake River & HOOD PK-WALLA S & B16WR6 & May 15, 2003 & Arsenic & $\mathrm{mg} / \mathrm{L}$ & 1 & 0.0011 & $=$ & 0.15 & $1.80 \mathrm{E}-05$ \\
\hline Snake River & HOOD PK-MID-RVR & B16WR4 & May 15, 2003 & Arsenic & $\mathrm{mg} / \mathrm{L}$ & 1 & 0.0011 & $=$ & 0.15 & $1.80 \mathrm{E}-05$ \\
\hline Yakima River & KIONA WA & 132819 & December 9, 2003 & Arsenic & $\mathrm{mg} / \mathrm{L}$ & 2 & 0.0011 & $=$ & 0.15 & $1.80 \mathrm{E}-05$ \\
\hline Yakima River & KIONA WA & 133000 & February 11, 2004 & Arsenic & $\mathrm{mg} / \mathrm{L}$ & 2 & 0.0011 & $=$ & 0.15 & $1.80 \mathrm{E}-05$ \\
\hline Snake River & HOOD PK-FRKN CS & B16WR5 & May 15,2003 & Arsenic & $\mathrm{mg} / \mathrm{L}$ & 1 & 0.0011 & $=$ & 0.15 & $1.80 \mathrm{E}-05$ \\
\hline Snake River & HOOD PK-FRKN CS & B16WR8 & May 15, 2003 & Arsenic & $\mathrm{mg} / \mathrm{L}$ & 1 & 0.0011 & $=$ & 0.15 & $1.80 \mathrm{E}-05$ \\
\hline Snake River & HOOD PK-MID-RVR & B16WR9 & May 15, 2003 & Arsenic & $\mathrm{mg} / \mathrm{L}$ & 1 & 0.0011 & $=$ & 0.15 & $1.80 \mathrm{E}-05$ \\
\hline Yakima River & KIONA WA & 132819-F & December 9, 2003 & Arsenic & $\mathrm{mg} / \mathrm{L}$ & 2 & 0.0012 & $=$ & 0.15 & $1.80 \mathrm{E}-05$ \\
\hline Yakima River & KIONA WA & $133000-F$ & February 11, 2004 & Arsenic & $\mathrm{mg} / \mathrm{L}$ & 2 & 0.0012 & $=$ & 0.15 & $1.80 \mathrm{E}-05$ \\
\hline Snake River & HOOD PK-WALLA S & B16WR7 & May 15,2003 & Arsenic & $\mathrm{mg} / \mathrm{L}$ & 1 & 0.0012 & $=$ & 0.15 & $1.80 \mathrm{E}-05$ \\
\hline Yakima River & KIONA WA & 2018090 & December 5, 2001 & Arsenic & $\mathrm{mg} / \mathrm{L}$ & 1 & 0.0012 & $=$ & 0.15 & $1.80 \mathrm{E}-05$ \\
\hline Yakima River & KIONA WA & 1498111 & November 13, 2001 & Arsenic & $\mathrm{mg} / \mathrm{L}$ & 1 & 0.0013 & $=$ & 0.15 & $1.80 \mathrm{E}-05$ \\
\hline Yakima River & Yakima Rvr & B16WP3 & May 14, 2003 & Arsenic & $\mathrm{mg} / \mathrm{L}$ & 1 & 0.0014 & $=$ & 0.15 & $1.80 \mathrm{E}-05$ \\
\hline Yakima River & KIONA WA & 133374 & June 9,2004 & Arsenic & $\mathrm{mg} / \mathrm{L}$ & 2 & 0.0015 & $=$ & 0.15 & $1.80 \mathrm{E}-05$ \\
\hline Yakima River & Yakima Rvr & B16WP2 & May 14, 2003 & Arsenic & $\mathrm{mg} / \mathrm{L}$ & 1 & 0.0015 & $=$ & 0.15 & $1.80 \mathrm{E}-05$ \\
\hline Yakima River & KIONA WA & 2068090 & January 9, 2002 & Arsenic & $\mathrm{mg} / \mathrm{L}$ & 1 & 0.0015 & $=$ & 0.15 & $1.80 \mathrm{E}-05$ \\
\hline Yakima River & Yakima Rvr & B16WP4 & May 14, 2003 & Arsenic & $\mathrm{mg} / \mathrm{L}$ & 1 & 0.0015 & $=$ & 0.15 & $1.80 \mathrm{E}-05$ \\
\hline Yakima River & Yakima Rvr & B16WP5 & May 14,2003 & Arsenic & $\mathrm{mg} / \mathrm{L}$ & 1 & 0.0015 & $=$ & 0.15 & $1.80 \mathrm{E}-05$ \\
\hline Yakima River & KIONA WA & 133374-F & June 9, 2004 & Arsenic & $\mathrm{mg} / \mathrm{L}$ & 2 & 0.0015 & $=$ & 0.15 & $1.80 \mathrm{E}-05$ \\
\hline Yakima River & Yakima Rvr & B16WP7 & May 14, 2003 & Arsenic & $\mathrm{mg} / \mathrm{L}$ & 1 & 0.0016 & $=$ & 0.15 & $1.80 \mathrm{E}-05$ \\
\hline Yakima River & Yakima Rvr & B16WP6 & May 14, 2003 & Arsenic & $\mathrm{mg} / \mathrm{L}$ & 1 & 0.0017 & $=$ & 0.15 & $1.80 \mathrm{E}-05$ \\
\hline Yakima River & KIONA WA & 1468165 & October 10, 2001 & Arsenic & $\mathrm{mg} / \mathrm{L}$ & 1 & 0.0020 & $=$ & 0.15 & $1.80 \mathrm{E}-05$ \\
\hline Yakima River & KIONA WA & 132642 & October 8, 2003 & Arsenic & $\mathrm{mg} / \mathrm{L}$ & 2 & 0.0020 & $=$ & 0.15 & 1.80E-05 \\
\hline Yakima River & KIONA WA & 1408090 & September 4, 2001 & Arsenic & $\mathrm{mg} / \mathrm{L}$ & 1 & 0.0022 & $=$ & 0.15 & 1.80E-05 \\
\hline Yakima River & KIONA WA & 132642-F & October 8, 2003 & Arsenic & $\mathrm{mg} / \mathrm{L}$ & 2 & 0.0024 & $=$ & 0.15 & $1.80 \mathrm{E}-05$ \\
\hline Yakima River & KIONA WA & 1296007 & July 9, 2001 & Arsenic & $\mathrm{mg} / \mathrm{L}$ & 1 & 0.0024 & $=$ & 0.15 & 1.80E-05 \\
\hline
\end{tabular}


Table C-18

Summary of Surface Water Results From Segment 3 Exceeding Select Ecological or Human Health Benchmark Values

\begin{tabular}{|c|c|c|c|c|c|c|c|c|c|c|}
\hline Sample Area & Sample Site & Sample ID & Date Collected & Constituent Name & Units & $\begin{array}{c}\text { QA } \\
\text { Category }\end{array}$ & $\begin{array}{c}\text { Analytical } \\
\text { Value }\end{array}$ & Qualifier & $\begin{array}{c}\text { Ecological } \\
\text { Benchmark } \\
\text { Value }\end{array}$ & $\begin{array}{c}\text { Human Health } \\
\text { Benchmark Value }\end{array}$ \\
\hline Yakima River & KIONA WA & 133572 & August 4, 2004 & Arsenic & $\mathrm{mg} / \mathrm{L}$ & 2 & 0.0026 & $=$ & 0.15 & $1.80 \mathrm{E}-05$ \\
\hline Yakima River & KIONA WA & 1368084 & August 14, 2001 & Arsenic & $\mathrm{mg} / \mathrm{L}$ & 1 & 0.0027 & $=$ & 0.15 & $1.80 \mathrm{E}-05$ \\
\hline Yakima River & KIONA WA & 133572-F & August 4, 2004 & Arsenic & $\mathrm{mg} / \mathrm{L}$ & 2 & 0.0028 & $=$ & 0.15 & $1.80 \mathrm{E}-05$ \\
\hline Walla Walla River & WWR (32WAL14.3) & 3208164 & May 14,2003 & Dichlorodiphenyldichloroethylene & $\mathrm{mg} / \mathrm{L}$ & 1 & $2.40 \mathrm{E}-07$ & $\mathrm{~J}$ & -- & 2.20E-07 \\
\hline Walla Walla River & RM15.6 CMNS BRG & 2228168 & May 30, 2002 & Dichlorodiphenyldichloroethylene & $\mathrm{mg} / \mathrm{L}$ & 1 & $2.80 \mathrm{E}-07$ & $\mathrm{~J}$ & -- & 2.20E-07 \\
\hline Walla Walla River & RM15.6 CMNS BRG & 2228167 & May 30, 2002 & Dichlorodiphenyldichloroethylene & $\mathrm{mg} / \mathrm{L}$ & 1 & $2.80 \mathrm{E}-07$ & $=$ & -- & 2.20E-07 \\
\hline Walla Walla River & WWR (32WAL14.3) & 3098996 & February 24, 2003 & Dichlorodiphenyldichloroethylene & $\mathrm{mg} / \mathrm{L}$ & 1 & $2.80 \mathrm{E}-07$ & $\mathrm{~J}$ & -- & 2.20E-07 \\
\hline Walla Walla River & WWR (32WAL14.3) & 2368895 & September 3, 2002 & Dichlorodiphenyldichloroethylene & $\mathrm{mg} / \mathrm{L}$ & 1 & $3.10 \mathrm{E}-07$ & $=$ & -- & 2.20E-07 \\
\hline Touchet River & HWY12 Bridge & 3138162 & March 24, 2003 & Dichlorodiphenyldichloroethylene & $\mathrm{mg} / \mathrm{L}$ & 1 & 4.10E-07 & $=$ & -- & 2.20E-07 \\
\hline Walla Walla River & WWR (32WAL14.3) & 3138163 & March 24, 2003 & Dichlorodiphenyldichloroethylene & $\mathrm{mg} / \mathrm{L}$ & 1 & 4.10E-07 & $=$ & -- & 2.20E-07 \\
\hline Walla Walla River & RM15.6 CMNS BRG & 3058984 & January 30,2003 & Dichlorodiphenyldichloroethylene & $\mathrm{mg} / \mathrm{L}$ & 1 & $1.20 \mathrm{E}-06$ & $=$ & -- & 2.20E-07 \\
\hline Walla Walla River & RM15.6 CMNS BRG & 3058984 & January 30,2003 & Dichlorodiphenyltrichloroethane & $\mathrm{mg} / \mathrm{L}$ & 1 & $6.50 \mathrm{E}-07$ & $=$ & $1.00 \mathrm{E}-06$ & $2.20 \mathrm{E}-07$ \\
\hline Walla Walla River & WWR (32WAL14.3) & 3098996 & February 24, 2003 & Dieldrin & $\mathrm{mg} / \mathrm{L}$ & 1 & $7.60 \mathrm{E}-08$ & $\mathrm{~J}$ & $1.90 \mathrm{E}-06$ & $5.20 \mathrm{E}-08$ \\
\hline Walla Walla River & WWR (32WAL14.3) & 2368895 & September 3, 2002 & Dieldrin & $\mathrm{mg} / \mathrm{L}$ & 1 & 8.00E-08 & $=$ & $1.90 \mathrm{E}-06$ & $5.20 \mathrm{E}-08$ \\
\hline Walla Walla River & WWR (32WAL14.3) & 2518974 & December 16, 2002 & Dieldrin & $\mathrm{mg} / \mathrm{L}$ & 1 & 1.10E-07 & $=$ & $1.90 \mathrm{E}-06$ & $5.20 \mathrm{E}-08$ \\
\hline Walla Walla River & RM15.6 CMNS BRG & 3058984 & January 30,2003 & Dieldrin & $\mathrm{mg} / \mathrm{L}$ & 1 & $3.00 \mathrm{E}-07$ & $\mathrm{~J}$ & $1.90 \mathrm{E}-06$ & $5.20 \mathrm{E}-08$ \\
\hline Touchet River & HWY12 Bridge & 2248088 & June 12,2002 & Heptachlor epoxide & $\mathrm{mg} / \mathrm{L}$ & 1 & $3.90 \mathrm{E}-08$ & $\mathrm{~J}$ & $3.80 \mathrm{E}-06$ & $3.90 \mathrm{E}-08$ \\
\hline Walla Walla River & RM15.6 CMNS BRG & 2228168 & May 30, 2002 & Heptachlor epoxide & $\mathrm{mg} / \mathrm{L}$ & 1 & 4.10E-08 & $\mathrm{J}$ & 3.80E-06 & $3.90 \mathrm{E}-08$ \\
\hline Walla Walla River & RM15.6 CMNS BRG & 2248090 & June 12, 2002 & Heptachlor epoxide & $\mathrm{mg} / \mathrm{L}$ & 1 & 4.40E-08 & $\mathrm{J}$ & 3.80E-06 & $3.90 \mathrm{E}-08$ \\
\hline Walla Walla River & RM15.6 CMNS BRG & 3058984 & January 30, 2003 & Heptachlor epoxide & $\mathrm{mg} / \mathrm{L}$ & 1 & $1.20 \mathrm{E}-07$ & $\mathrm{~J}$ & 3.80E-06 & $3.90 \mathrm{E}-08$ \\
\hline
\end{tabular}




\section{APPENDIX D \\ SEGMENT 4 \\ SUMMARY STATISTICS, BENCHMARK COMPARISON, AND INDIVIDUAL SAMPLE EXCEEDENCES}


WCH-91

Rev. 0

Columbia River Component Data Evaluation Summary Report

July 2006 


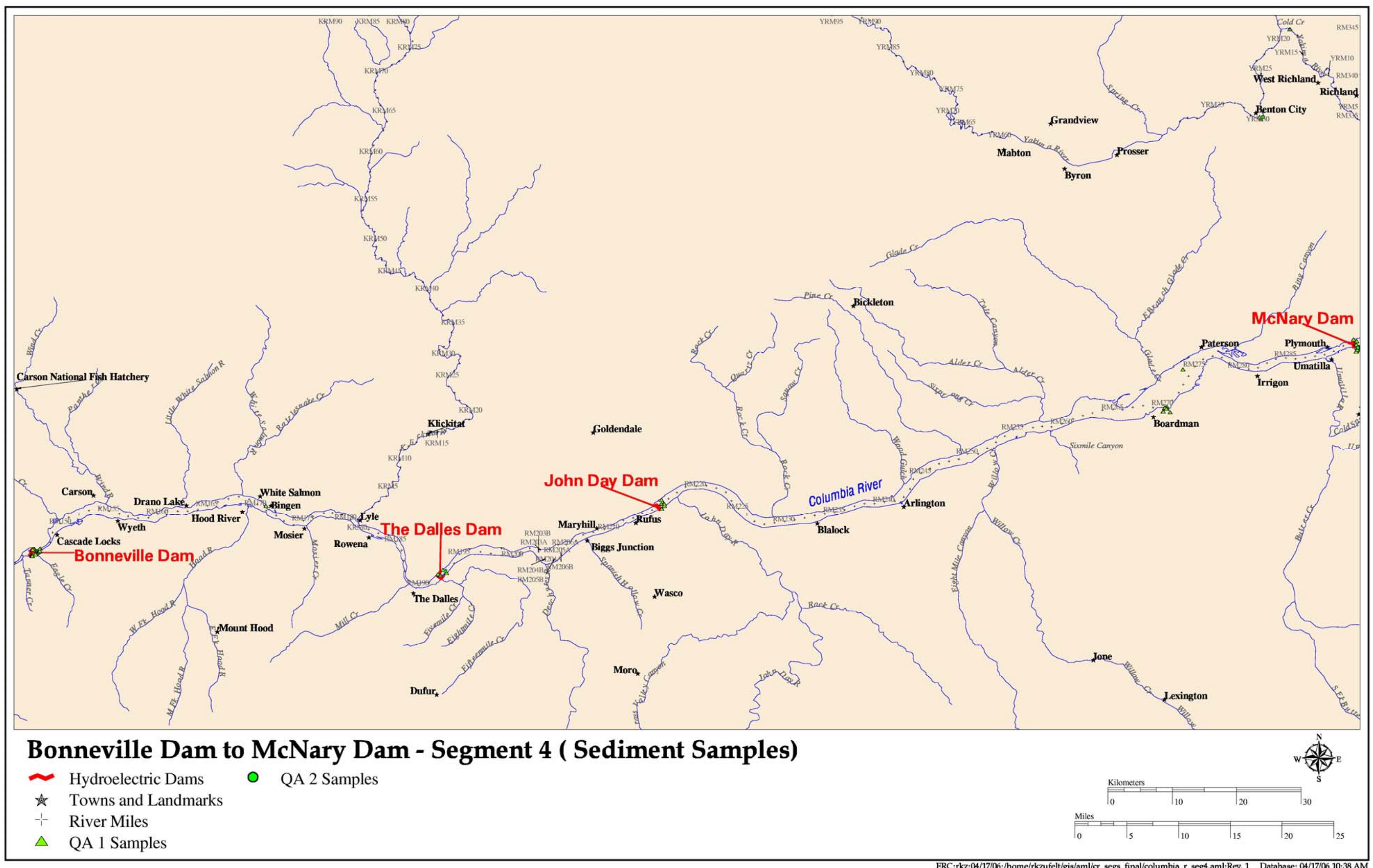




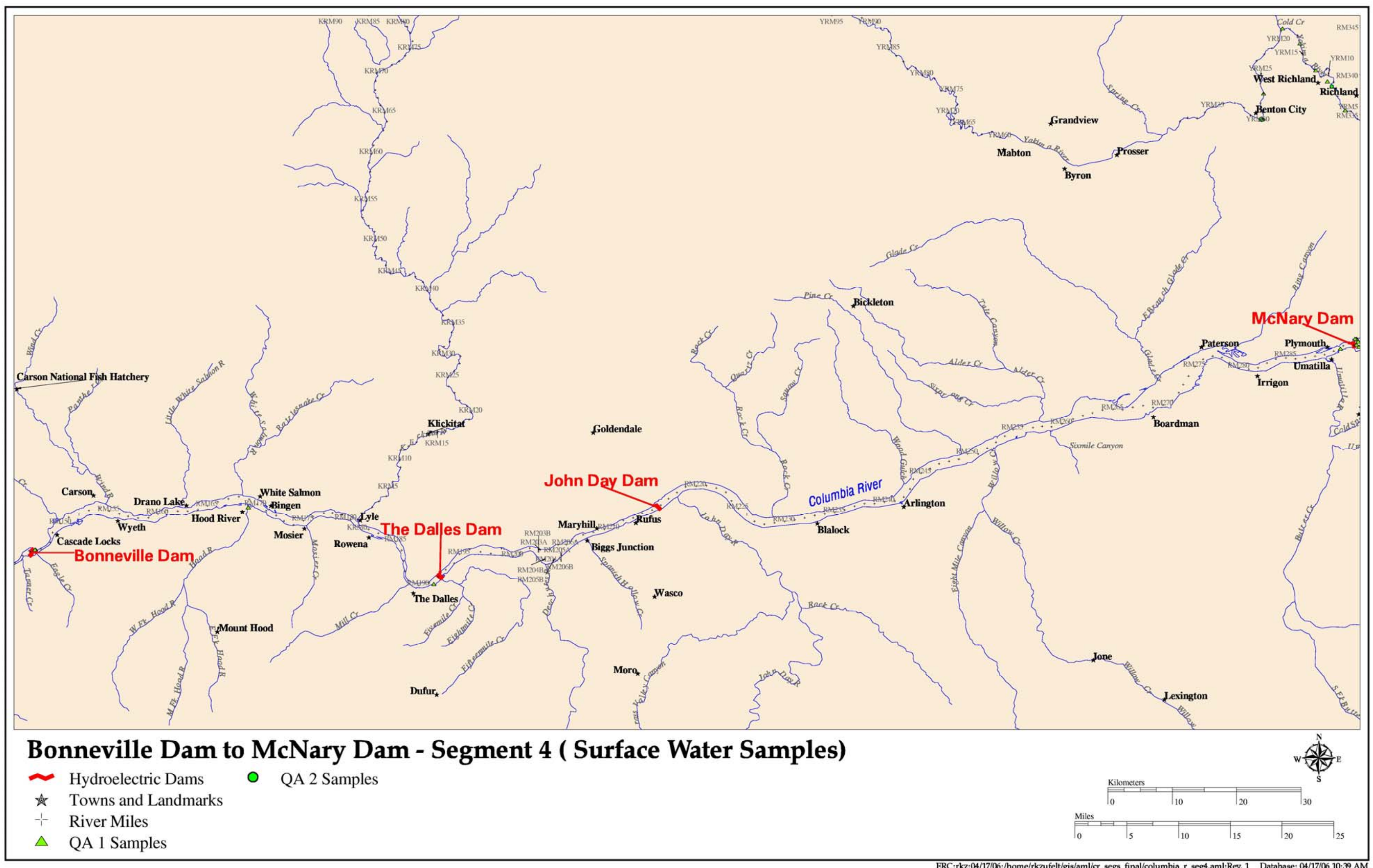




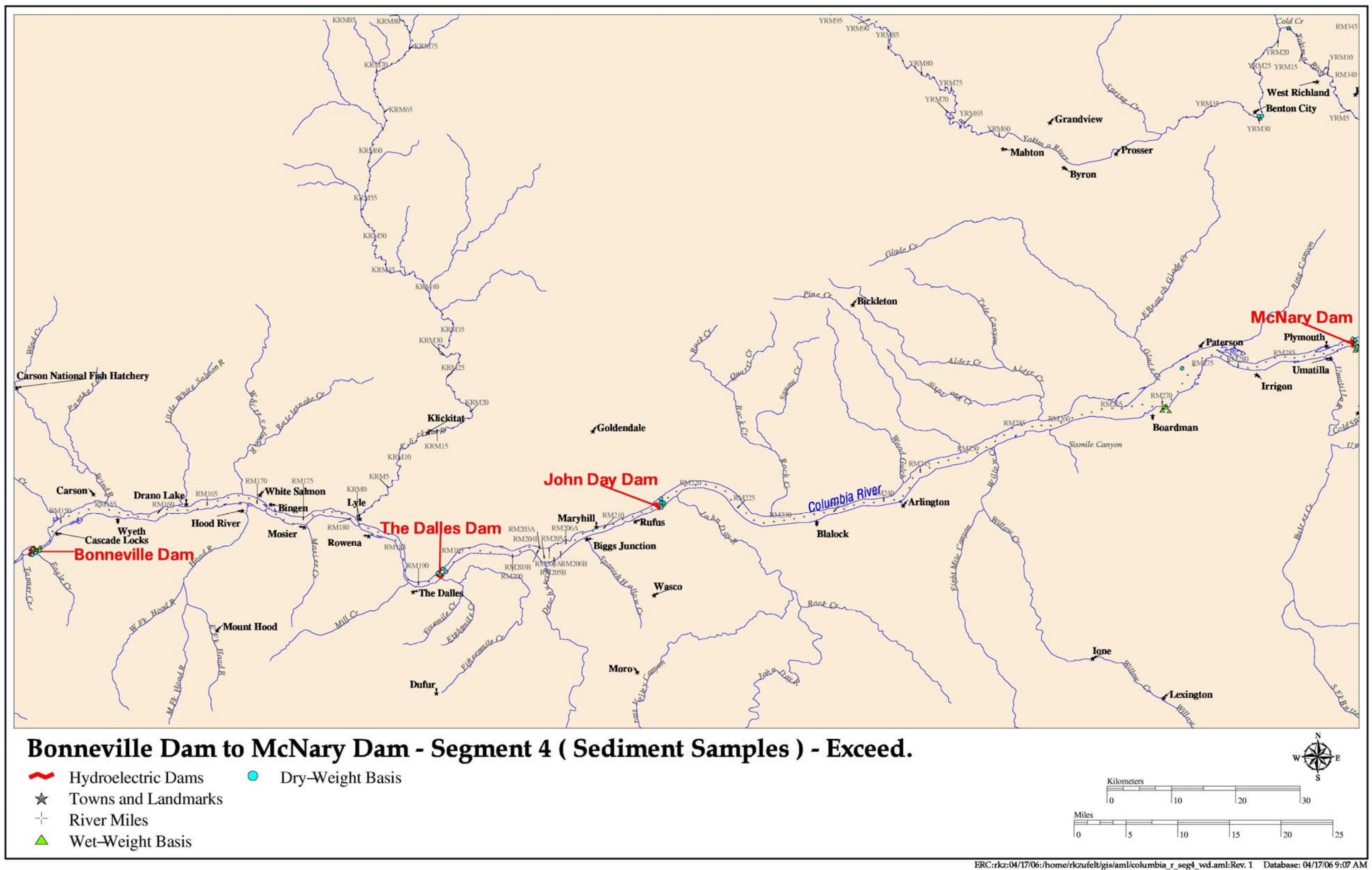




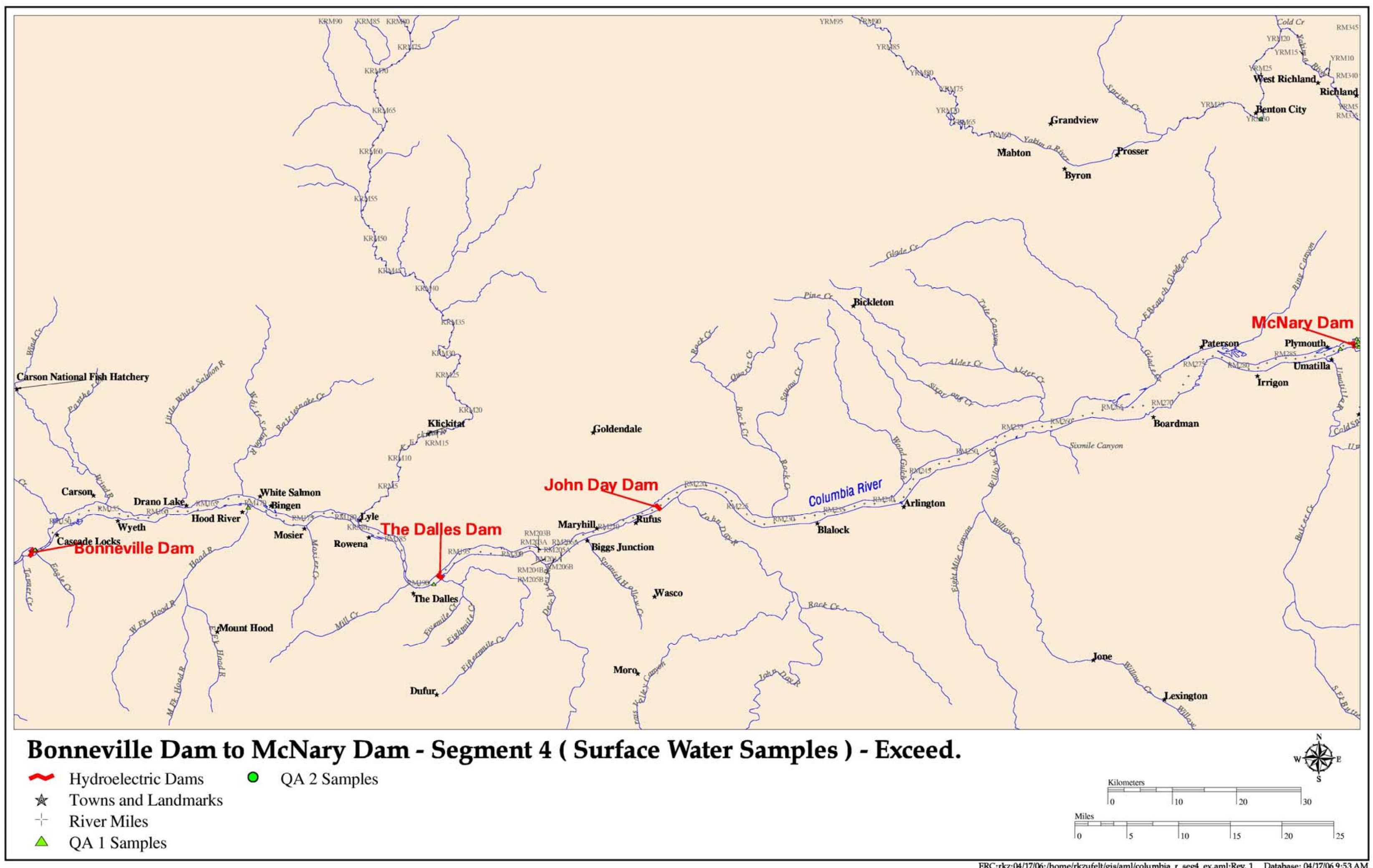


Table D-1

Summary Table of Segment 4 Sediment Samples By Area, Site, Date, and Category

\begin{tabular}{|c|c|c|c|c|}
\hline Sample ID & Sample Area & Sample Site & Sample Date & Category \\
\hline 12225 & Columbia River & Bingen WA & 11-Mar-92 & 1 \\
\hline 31802 & Columbia River & Bonneville Dam & 07-Aug-03 & 1 \\
\hline 31803 & Columbia River & Bonneville Dam & 07-Aug-03 & 1 \\
\hline 31804 & Columbia River & Dalles Dam & 07-Aug-03 & 1 \\
\hline 31805 & Columbia River & Dalles Dam & 07-Aug-03 & 1 \\
\hline 31806 & Columbia River & John Day Dam & 08-Aug-03 & 1 \\
\hline 31807 & Columbia River & John Day Dam & 08-Aug-03 & 1 \\
\hline 31808 & Columbia River & John Day Dam & 08-Aug-03 & 1 \\
\hline 33607 & Columbia River & John Day Dam & 21-Sep-04 & 1 \\
\hline B17B78 & Columbia River & John Day Dam & 08-Aug-03 & 1 \\
\hline B17B81 & Columbia River & John Day Dam & 08-Aug-03 & 1 \\
\hline B17B84 & Columbia River & John Day Dam & 08-Aug-03 & 1 \\
\hline B17B99 & Columbia River & Dalles Dam & 08-Aug-03 & 1 \\
\hline B17BB5 & Columbia River & Bonneville Dam & 07-Aug-03 & 1 \\
\hline B17BF3 & Columbia River & John Day Dam & 08-Aug-03 & 1 \\
\hline B17BF4 & Columbia River & John Day Dam & 08-Aug-03 & 1 \\
\hline B17BF5 & Columbia River & John Day Dam & 08-Aug-03 & 1 \\
\hline B17BF9 & Columbia River & Dalles Dam & 07-Aug-03 & 1 \\
\hline В17BH0 & Columbia River & Dalles Dam & 07-Aug-03 & 1 \\
\hline B17BH2 & Columbia River & Bonneville Dam & 07-Aug-03 & 1 \\
\hline B17BH4 & Columbia River & Bonneville Dam & 07-Aug-03 & 1 \\
\hline B17BJ5 & Columbia River & John Day Dam & 08-Aug-03 & 1 \\
\hline B17BJ6 & Columbia River & John Day Dam & 08-Aug-03 & 1 \\
\hline B17BJ8 & Columbia River & John Day Dam & 08-Aug-03 & 1 \\
\hline B17BJ9 & Columbia River & Dalles Dam & 07-Aug-03 & 1 \\
\hline B17BK1 & Columbia River & Dalles Dam & 07-Aug-03 & 1 \\
\hline B17BK2 & Columbia River & Dalles Dam & 07-Aug-03 & 1 \\
\hline B17BK3 & Columbia River & Bonneville Dam & 07-Aug-03 & 1 \\
\hline B17BK5 & Columbia River & Bonneville Dam & 07-Aug-03 & 1 \\
\hline B17BP8 & Columbia River & John Day Dam & 08-Aug-03 & 1 \\
\hline B17BP9 & Columbia River & John Day Dam & 08-Aug-03 & 1 \\
\hline B17BR0 & Columbia River & John Day Dam & 08-Aug-03 & 1 \\
\hline B17BR3 & Columbia River & Dalles Dam & 07-Aug-03 & 1 \\
\hline B17BR5 & Columbia River & Dalles Dam & 07-Aug-03 & 1 \\
\hline B17BR7 & Columbia River & Bonneville Dam & 07-Aug-03 & 1 \\
\hline B17BR8 & Columbia River & Bonneville Dam & 07-Aug-03 & 1 \\
\hline B1B7N4 & Columbia River & John Day Dam & 21-Sep-04 & 1 \\
\hline B1B7N5 & Columbia River & John Day Dam & 21-Sep-04 & 1 \\
\hline B1B7N6 & Columbia River & John Day Dam & 21-Sep-04 & 1 \\
\hline B1B7P1 & Columbia River & John Day Dam & 21-Sep-04 & 1 \\
\hline B1B7P2 & Columbia River & John Day Dam & 21-Sep-04 & 1 \\
\hline B1B7P3 & Columbia River & John Day Dam & 21-Sep-04 & 1 \\
\hline B1B7P4 & Columbia River & John Day Dam & 21-Sep-04 & 1 \\
\hline B2GC1 & Columbia River & RM 145 & 28-Jul-97 & 1 \\
\hline B2GC2 & Columbia River & RM 145 & 28-Jul-97 & 1 \\
\hline B2GC3 & Columbia River & RM 145 & 28-Jul-97 & 1 \\
\hline BF-BC-03 & Columbia River & Bonneville Dam & 18-Sep-02 & 1 \\
\hline
\end{tabular}


Table D-1

Summary Table of Segment 4 Sediment Samples By Area, Site, Date, and Category

\begin{tabular}{|c|c|c|c|c|}
\hline Sample ID & Sample Area & Sample Site & Sample Date & Category \\
\hline BF-BC-04 & Columbia River & Bonneville Dam & 18-Sep-02 & 1 \\
\hline BF-BC-07 & Columbia River & Bonneville Dam & 18-Sep-02 & 1 \\
\hline BF-BC-14 & Columbia River & Bonneville Dam & 18-Sep-02 & 1 \\
\hline BF-BC-15 & Columbia River & Bonneville Dam & 18-Sep-02 & 1 \\
\hline BF-BC-16 & Columbia River & Bonneville Dam & 18-Sep-02 & 1 \\
\hline BF-BC-17 & Columbia River & Bonneville Dam & 18-Sep-02 & 1 \\
\hline BF-BC-26 & Columbia River & Bonneville Dam & 18-Sep-02 & 1 \\
\hline DD-02/09/65 & Columbia River & RM 192.8 & 09-Feb-65 & 3 \\
\hline DD-02/21/67 & Columbia River & RM 192.8 & 21-Feb-67 & 3 \\
\hline DD-02/24/66 & Columbia River & RM 192.8 & 24-Feb-66 & 3 \\
\hline DD-05/17/67 & Columbia River & RM 192.8 & 17-May-67 & 3 \\
\hline DD-05/31/66 & Columbia River & RM 192.8 & 31-May-66 & 3 \\
\hline DD-07/06/66 & Columbia River & RM 192.8 & 06-Jul-66 & 3 \\
\hline DD-08/31/66 & Columbia River & RM 192.8 & 13-Aug-66 & 3 \\
\hline DD-1/28/1993 & Columbia River & RM 192.8 & 28-Jan-93 & 3 \\
\hline DD-10/13/1977 & Columbia River & RM 192.8 & $13-O c t-77$ & 3 \\
\hline DD-11/13/1991 & Columbia River & RM 192.8 & 13-Nov-91 & 3 \\
\hline DD-11/17/65 & Columbia River & RM 192.8 & 17-Nov-65 & 3 \\
\hline DD-11/19/1975 & Columbia River & RM 192.8 & 19-Nov-75 & 3 \\
\hline DD-11/20/1986 & Columbia River & RM 192.8 & 20-Nov-86 & 3 \\
\hline DD-11/21/1973 & Columbia River & RM 192.8 & 21-Nov-73 & 3 \\
\hline DD-11/28/1969 & Columbia River & RM 192.8 & 28-Nov-69 & 3 \\
\hline DD-11/28/1990 & Columbia River & RM 192.8 & 28-Nov-90 & 3 \\
\hline DD-11/28/67 & Columbia River & RM 192.8 & 28-Nov-67 & 3 \\
\hline DD-11/29/1972 & Columbia River & RM 192.8 & 29-Nov-72 & 3 \\
\hline DD-11/29/66 & Columbia River & RM 192.8 & 29-Nov-66 & 3 \\
\hline DD-11/4/1987 & Columbia River & RM 192.8 & 04-Nov-87 & 3 \\
\hline DD-11/9/1984 & Columbia River & RM 192.8 & 09-Nov-84 & 3 \\
\hline DD-12/10/1981 & Columbia River & RM 192.8 & 10-Dec-81 & 3 \\
\hline DD-12/14/1976 & Columbia River & RM 192.8 & 14-Dec-76 & 3 \\
\hline DD-12/19/1974 & Columbia River & RM 192.8 & 19-Dec-74 & 3 \\
\hline DD-12/19/1989 & Columbia River & RM 192.8 & 19-Dec-89 & 3 \\
\hline DD-12/22/1971 & Columbia River & RM 192.8 & 22-Dec-71 & 3 \\
\hline DD-12/23/1982 & Columbia River & RM 192.8 & 23-Dec-82 & 3 \\
\hline DD-12/30/1970 & Columbia River & RM 192.8 & 30-Dec-70 & 3 \\
\hline DD-12/4/1980 & Columbia River & RM 192.8 & 04-Dec-80 & 3 \\
\hline DD-12/5/1979 & Columbia River & RM 192.8 & 05-Dec-79 & 3 \\
\hline DD-12/6/1988 & Columbia River & RM 192.8 & 06-Dec-88 & 3 \\
\hline DD-2/15/1989 & Columbia River & RM 192.8 & 15-Feb-89 & 3 \\
\hline DD-2/17/1971 & Columbia River & RM 192.8 & 17-Feb-71 & 3 \\
\hline DD-2/20/1969 & Columbia River & RM 192.8 & 20-Feb-69 & 3 \\
\hline DD-2/25/1975 & Columbia River & RM 192.8 & 25-Feb-75 & 3 \\
\hline DD-2/25/1988 & Columbia River & RM 192.8 & 25-Feb-88 & 3 \\
\hline DD-2/25/1992 & Columbia River & RM 192.8 & 25-Feb-92 & 3 \\
\hline DD-2/6/1991 & Columbia River & RM 192.8 & 06-Feb-91 & 3 \\
\hline DD-3/14/1980 & Columbia River & RM 192.8 & 14-Mar-80 & 3 \\
\hline DD-3/17/1987 & Columbia River & RM 192.8 & 17-Mar-87 & 3 \\
\hline
\end{tabular}


Table D-1

Summary Table of Segment 4 Sediment Samples By Area, Site, Date, and Category

\begin{tabular}{|c|c|c|c|c|}
\hline Sample ID & Sample Area & Sample Site & Sample Date & Category \\
\hline DD-3/19/1981 & Columbia River & RM 192.8 & 19-Mar-81 & 3 \\
\hline DD-3/21/1968 & Columbia River & RM 192.8 & 21-Mar-68 & 3 \\
\hline DD-3/22/1974 & Columbia River & RM 192.8 & 22-Mar-74 & 3 \\
\hline DD-3/22/1978 & Columbia River & RM 192.8 & 22-Mar-78 & 3 \\
\hline DD-3/23/1973 & Columbia River & RM 192.8 & 23-Mar-73 & 3 \\
\hline DD-3/25/1982 & Columbia River & RM 192.8 & 25-Mar-82 & 3 \\
\hline DD-3/26/1970 & Columbia River & RM 192.8 & 26-Mar-70 & 3 \\
\hline DD-3/28/1990 & Columbia River & RM 192.8 & 28-Mar-90 & 3 \\
\hline DD-3/3/1972 & Columbia River & RM 192.8 & 03-Mar-72 & 3 \\
\hline DD-3/31/1986 & Columbia River & RM 192.8 & 31-Mar-86 & 3 \\
\hline DD-4/13/1983 & Columbia River & RM 192.8 & 13-Apr-83 & 3 \\
\hline DD-4/24/1985 & Columbia River & RM 192.8 & 24-Apr-85 & 3 \\
\hline DD-5/1/1974 & Columbia River & RM 192.8 & 01-May-74 & 3 \\
\hline DD-5/19/1977 & Columbia River & RM 192.8 & 19-May-77 & 3 \\
\hline DD-5/22/1987 & Columbia River & RM 192.8 & 22-May-87 & 3 \\
\hline DD-5/26/1993 & Columbia River & RM 192.8 & 26-May-93 & 3 \\
\hline DD-5/28/1992 & Columbia River & RM 192.8 & 28-May-92 & 3 \\
\hline DD-5/3/1984 & Columbia River & RM 192.8 & 03-May-84 & 3 \\
\hline DD-5/30/1991 & Columbia River & RM 192.8 & 30-May-91 & 3 \\
\hline DD-6/10/1976 & Columbia River & RM 192.8 & 10-Jun-76 & 3 \\
\hline DD-6/15/1973 & Columbia River & RM 192.8 & 15-Jun-73 & 3 \\
\hline DD-6/19/1979 & Columbia River & RM 192.8 & 19-Jun-79 & 3 \\
\hline DD-6/2/1990 & Columbia River & RM 192.8 & 02-Jun-90 & 3 \\
\hline DD-6/28/1988 & Columbia River & RM 192.8 & 28-Jun-88 & 3 \\
\hline DD-6/30/1971 & Columbia River & RM 192.8 & 30-Jun-71 & 3 \\
\hline DD-7/1/1975 & Columbia River & RM 192.8 & 01-Jul-75 & 3 \\
\hline DD-7/10/1978 & Columbia River & RM 192.8 & 10-Jul-78 & 3 \\
\hline DD-7/13/1970 & Columbia River & RM 192.8 & 13-Jul-70 & 3 \\
\hline DD-7/21/1989 & Columbia River & RM 192.8 & 21-Jul-89 & 3 \\
\hline DD-7/29/1985 & Columbia River & RM 192.8 & 29-Jul-85 & 3 \\
\hline DD-7/8/1980 & Columbia River & RM 192.8 & 08-Jul-80 & 3 \\
\hline DD-8/13/1981 & Columbia River & RM 192.8 & 13-Aug-81 & 3 \\
\hline DD-8/19/1993 & Columbia River & RM 192.8 & 19-Aug-93 & 3 \\
\hline DD-8/23/1974 & Columbia River & RM 192.8 & 23-Aug-74 & 3 \\
\hline DD-8/24/1979 & Columbia River & RM 192.8 & 24-Aug-79 & 3 \\
\hline DD-8/24/1988 & Columbia River & RM 192.8 & 24-Aug-88 & 3 \\
\hline DD-8/28/1986 & Columbia River & RM 192.8 & 28-Aug-86 & 3 \\
\hline DD-8/29/1975 & Columbia River & RM 192.8 & 29-Aug-75 & 3 \\
\hline DD-8/30/1973 & Columbia River & RM 192.8 & 30-Aug-73 & 3 \\
\hline DD-8/30/1978 & Columbia River & RM 192.8 & 30-Aug-78 & 3 \\
\hline DD-8/5/1987 & Columbia River & RM 192.8 & 05-Aug-87 & 3 \\
\hline DD-8/7/1991 & Columbia River & RM 192.8 & 07-Aug-91 & 3 \\
\hline DD-9/12/1983 & Columbia River & RM 192.8 & 12-Sep-83 & 3 \\
\hline DD-9/16/1976 & Columbia River & RM 192.8 & 16-Sep-76 & 3 \\
\hline DD-9/2/1980 & Columbia River & RM 192.8 & 02-Sep-80 & 3 \\
\hline DD-9/20/1982 & Columbia River & RM 192.8 & 20-Sep-82 & 3 \\
\hline DD-9/23/1992 & Columbia River & RM 192.8 & 23-Sep-92 & 3 \\
\hline
\end{tabular}


Table D-1

Summary Table of Segment 4 Sediment Samples By Area, Site, Date, and Category

\begin{tabular}{|c|c|c|c|c|}
\hline Sample ID & Sample Area & Sample Site & Sample Date & Category \\
\hline DD-9/24/1968 & Columbia River & RM 192.8 & 24-Sep-68 & 3 \\
\hline DD-9/26/1969 & Columbia River & RM 192.8 & 26-Sep-69 & 3 \\
\hline DD-9/29/1971 & Columbia River & RM 192.8 & 29-Sep-71 & 3 \\
\hline DD-9/6/1990 & Columbia River & RM 192.8 & 06-Sep-90 & 3 \\
\hline $\mathrm{PH} 2 \# 1$ & Columbia River & RM 145 & 24-Jul-97 & 1 \\
\hline $\mathrm{PH} 2 \# 2$ & Columbia River & RM 145 & 24-Jul-97 & 1 \\
\hline PM-BC-02 & Columbia River & Messner Cove & 17-Mar-99 & 1 \\
\hline PM-BC-03 & Columbia River & Messner Cove & 17-Mar-99 & 1 \\
\hline PM-BC-04 & Columbia River & Messner Cove & 17-Mar-99 & 1 \\
\hline PM-BC-05 & Columbia River & Messner Cove & 17-Mar-99 & 1 \\
\hline PM-BC-06 & Columbia River & Messner Cove & 17-Mar-99 & 1 \\
\hline PM-BC-07 & Columbia River & Messner Cove & 17-Mar-99 & 1 \\
\hline PM-BC-08 & Columbia River & Messner Cove & 17-Mar-99 & 1 \\
\hline PM-BC-09 & Columbia River & Messner Cove & 17-Mar-99 & 1 \\
\hline PM-BC-10 & Columbia River & Messner Cove & 17-Mar-99 & 1 \\
\hline PM-BC-11 & Columbia River & Messner Cove & 17-Mar-99 & 1 \\
\hline PM-GC-01 & Columbia River & Messner Cove & 17-Mar-99 & 1 \\
\hline
\end{tabular}


Table D-2

Summary Table of Segment 4 Surface Water Samples by Area, Site, Date, and Category

\begin{tabular}{|c|c|c|c|c|}
\hline Sample Area & Sample Site & Sample ID & Sample Date & Category \\
\hline Columbia River & Bonneville Dam & 3514105 & December 15, 2003 & CRC1_Seg4 \\
\hline Columbia River & Bonneville Dam & 4154230 & January 12, 2004 & CRC1_Seg4 \\
\hline Columbia River & Bonneville Dam & 3354155 & August 27, 2003 & CRC1_Seg4 \\
\hline Columbia River & Bonneville Dam & 3384080 & September 15, 2003 & CRC1_Seg4 \\
\hline Columbia River & Bonneville Dam & 3454413 & September 24, 2003 & CRC1_Seg4 \\
\hline Columbia River & Bonneville Dam & 4024105 & January 5, 2004 & CRC1_Seg4 \\
\hline Columbia River & Bonneville Dam & 4034250 & January 12, 2004 & CRC1_Seg4 \\
\hline Columbia River & Bonneville Dam & 4314230 & June 22, 2004 & CRC1_Seg4 \\
\hline Columbia River & Bonneville Dam & 4264250 & June 22, 2004 & CRC1_Seg4 \\
\hline Columbia River & Bonneville Dam & 4244130 & June 9, 2004 & CRC1_Seg4 \\
\hline Columbia River & Bonneville Dam & 4224105 & May 24, 2004 & CRC1_Seg4 \\
\hline Columbia River & Bonneville Dam & 3394400 & September 24, 2003 & CRC1_Seg4 \\
\hline Columbia River & DALLES BRDG & 101304 & October 13, 2004 & CRC1_Seg4 \\
\hline Columbia River & DALLES BRDG & 083000 & August 30, 2000 & CRC1_Seg4 \\
\hline Columbia River & DALLES BRDG & 020403 & February 4, 2003 & CRC1_Seg4 \\
\hline Columbia River & DALLES BRDG & 111300 & November 13, 2000 & CRC1_Seg4 \\
\hline Columbia River & DALLES BRDG & 110502 & November 5, 2002 & CRC1_Seg4 \\
\hline Columbia River & DALLES BRDG & 052201 & May 22, 2001 & CRC1_Seg4 \\
\hline Columbia River & DALLES BRDG & 020502 & February 5, 2002 & CRC1_Seg4 \\
\hline Columbia River & DALLES BRDG & 020601 & February 6, 2001 & CRC1_Seg4 \\
\hline Columbia River & DALLES BRDG & 020800 & February 8, 2000 & CRC1_Seg4 \\
\hline Columbia River & DALLES BRDG & 020805 & February 8, 2005 & CRC1_Seg4 \\
\hline Columbia River & DALLES BRDG & 021004 & February 10, 2004 & CRC1_Seg4 \\
\hline Columbia River & DALLES BRDG & 050702 & May 7, 2002 & CRC1_Seg4 \\
\hline Columbia River & DALLES BRDG & 050703 & May 7, 2003 & CRC1_Seg4 \\
\hline Columbia River & DALLES BRDG & 051004 & May 10, 2004 & CRC1_Seg4 \\
\hline Columbia River & DALLES BRDG & 110601 & November 6, 2001 & CRC1_Seg4 \\
\hline Columbia River & DALLES BRDG & 052200 & May 22, 2000 & CRC1_Seg4 \\
\hline Columbia River & DALLES BRDG & 081301 & August 13, 2001 & CRC1_Seg4 \\
\hline Columbia River & DALLES BRDG & 080503 & August 5, 2003 & CRC1_Seg4 \\
\hline Columbia River & DALLES BRDG & 080602 & August 6, 2002 & CRC1_Seg4 \\
\hline Columbia River & DALLES BRDG & 080701 & August 7, 2001 & CRC1_Seg4 \\
\hline Columbia River & DALLES BRDG & 080904 & August 9, 2004 & CRC1_Seg4 \\
\hline Columbia River & DALLES BRDG & 051005 & May 10, 2005 & CRC1_Seg4 \\
\hline Columbia River & FTBRDG DSM I-84 & 279257 & October 2, 2000 & CRC1_Seg4 \\
\hline Columbia River & FTBRDG DSM I-84 & 283919 & December 18, 2000 & CRC1_Seg4 \\
\hline Columbia River & FTBRDG DSM I-84 & 286118 & February 5, 2001 & CRC1_Seg4 \\
\hline Columbia River & FTBRDG DSM I-84 & 249958 & April 26, 1999 & CRC1_Seg4 \\
\hline Columbia River & FTBRDG DSM I-84 & 275833 & August 7, 2000 & CRC1_Seg4 \\
\hline Columbia River & FTBRDG DSM I-84 & 274023 & June 19, 2000 & CRC1_Seg4 \\
\hline Columbia River & FTBRDG DSM I-84 & 270829 & April 24, 2000 & CRC1_Seg4 \\
\hline Columbia River & FTBRDG DSM I-84 & 265415 & February 7, 2000 & CRC1_Seg4 \\
\hline Columbia River & FTBRDG DSM I-84 & 260087 & December 13, 1999 & CRC1_Seg4 \\
\hline Columbia River & FTBRDG DSM I-84 & 257175 & October 18, 1999 & CRC1_Seg4 \\
\hline Columbia River & FTBRDG DSM I-84 & 257169 & October 18, 1999 & CRC1_Seg4 \\
\hline Columbia River & FTBRDG DSM I-84 & 251708 & June 14, 1999 & CRC1_Seg4 \\
\hline Columbia River & FTBRDG DSM I-84 & 303688 & August 13, 2001 & CRC1_Seg4 \\
\hline Columbia River & FTBRDG DSM I-84 & 247183 & February 22, 1999 & CRC1_Seg4 \\
\hline
\end{tabular}


Table D-2

Summary Table of Segment 4 Surface Water Samples by Area, Site, Date, and Category

\begin{tabular}{|c|c|c|c|c|}
\hline Sample Area & Sample Site & Sample ID & Sample Date & Category \\
\hline Columbia River & FTBRDG DSM I-84 & 254819 & August 23, 1999 & CRC1_Seg4 \\
\hline Columbia River & FTBRDG DSM I-84 & 630522 & April 14, 2003 & CRC1_Seg4 \\
\hline Columbia River & FTBRDG DSM I-84 & 706316 & August 9, 2004 & CRC1_Seg4 \\
\hline Columbia River & FTBRDG DSM I-84 & 722716 & February 14, 2005 & CRC1_Seg4 \\
\hline Columbia River & FTBRDG DSM I-84 & 721191 & December 27, 2004 & CRC1_Seg4 \\
\hline Columbia River & FTBRDG DSM I-84 & 296548 & June 4, 2001 & CRC1_Seg4 \\
\hline Columbia River & FTBRDG DSM I-84 & 711932 & October 25, 2004 & CRC1_Seg4 \\
\hline Columbia River & FTBRDG DSM I-84 & 293695 & April 23, 2001 & CRC1_Seg4 \\
\hline Columbia River & FTBRDG DSM I-84 & 611573 & October 28, 2002 & CRC1_Seg4 \\
\hline Columbia River & FTBRDG DSM I-84 & 730580 & March 23, 2005 & CRC1_Seg4 \\
\hline Columbia River & FTBRDG DSM I-84 & 626385 & February 24, 2003 & CRC1_Seg4 \\
\hline Columbia River & FTBRDG DSM I-84 & 737705 & April 25, 2005 & CRC1_Seg4 \\
\hline Columbia River & FTBRDG DSM I-84 & 632874 & June 9, 2003 & CRC1_Seg4 \\
\hline Columbia River & FTBRDG DSM I-84 & 637606 & August 18, 2003 & CRC1_Seg4 \\
\hline Columbia River & FTBRDG DSM I-84 & 640925 & October 13, 2003 & CRC1_Seg4 \\
\hline Columbia River & FTBRDG DSM I-84 & 690799 & December 8, 2003 & CRC1_Seg4 \\
\hline Columbia River & FTBRDG DSM I-84 & 698009 & February 2, 2004 & CRC1_Seg4 \\
\hline Columbia River & FTBRDG DSM I-84 & 701821 & April 19, 2004 & CRC1_Seg4 \\
\hline Columbia River & FTBRDG DSM I-84 & 704280 & June 14, 2004 & CRC1_Seg4 \\
\hline Columbia River & FTBRDG DSM I-84 & 613379 & December 2, 2002 & CRC1_Seg4 \\
\hline Columbia River & FTBRDG DSM I-84 & 605402 & April 1, 2002 & CRC1_Seg4 \\
\hline Columbia River & FTBRDG DSM I-84 & 307595 & October 15, 2001 & CRC1_Seg4 \\
\hline Columbia River & FTBRDG DSM I-84 & 310795 & December 10, 2001 & CRC1_Seg4 \\
\hline Columbia River & FTBRDG DSM I-84 & 603559 & March 4, 2002 & CRC1_Seg4 \\
\hline Columbia River & FTBRDG DSM I-84 & 605342 & April 8, 2002 & CRC1_Seg4 \\
\hline Columbia River & FTBRDG DSM I-84 & 605360 & March 14, 2002 & CRC1_Seg4 \\
\hline Columbia River & FTBRDG DSM I-84 & 605371 & March 22, 2002 & CRC1_Seg4 \\
\hline Columbia River & FTBRDG DSM I-84 & 730520 & March 21, 2005 & CRC1_Seg4 \\
\hline Columbia River & FTBRDG DSM I-84 & 605398 & March 29, 2002 & CRC1_Seg4 \\
\hline Columbia River & FTBRDG DSM I-84 & 605855 & April 19, 2002 & CRC1_Seg4 \\
\hline Columbia River & FTBRDG DSM I-84 & 605926 & April 22, 2002 & CRC1_Seg4 \\
\hline Columbia River & FTBRDG DSM I-84 & 607432 & June 10, 2002 & CRC1_Seg4 \\
\hline Columbia River & FTBRDG DSM I-84 & 608972 & August 5, 2002 & CRC1_Seg4 \\
\hline Columbia River & FTBRDG DSM I-84 & 740442 & June 13, 2005 & CRC1_Seg4 \\
\hline Columbia River & FTBRDG DSM I-84 & 605388 & March 27, 2002 & CRC1_Seg4 \\
\hline Columbia River & RM147 BONN DAM & loc 01 5/04-6/04 & June 1, 2004 & CRC1_Seg4 \\
\hline Columbia River & RM147 BONN DAM & loc 01 12/03-1/04 & January 1, 2004 & CRC1_Seg4 \\
\hline Columbia River & RM147 BONN DAM & loc 01 8/03-9/03 & September 1, 2003 & CRC1_Seg4 \\
\hline Columbia River & Umatilla OR & 1408081 & September 12, 2001 & CRC1_Seg4 \\
\hline Columbia River & Umatilla OR & 1368082 & August 8, 2001 & CRC1_Seg4 \\
\hline Columbia River & Umatilla OR & 1468156 & October 17, 2001 & CRC1_Seg4 \\
\hline Columbia River & Umatilla OR & 1498101 & November 7, 2001 & CRC1_Seg4 \\
\hline Columbia River & Umatilla OR & 2018081 & December 5, 2001 & CRC1_Seg4 \\
\hline Columbia River & Umatilla OR & 2068081 & January 16, 2002 & CRC1_Seg4 \\
\hline Columbia River & Umatilla OR & 2118106 & February 11, 2002 & CRC1_Seg4 \\
\hline Columbia River & Umatilla OR & 2158156 & March 13, 2002 & CRC1_Seg4 \\
\hline Columbia River & Umatilla OR & 2178181 & April 10, 2002 & CRC1_Seg4 \\
\hline Columbia River & Umatilla OR & 2228081 & May 15, 2002 & CRC1_Seg4 \\
\hline
\end{tabular}


Table D-2

Summary Table of Segment 4 Surface Water Samples by Area, Site, Date, and Category

\begin{tabular}{lcccc} 
Sample Area & Sample Site & Sample ID & Sample Date & Category \\
\hline Columbia River & Umatilla OR & 1286070 & July 11, 2001 & CRC1_Seg4 \\
\hline
\end{tabular}


Table D-3

Summary Statistics for Sediment Collected from Segment 4 of the Columbia River Including All Categories of Data (units reported "as received")

\begin{tabular}{|c|c|c|c|c|c|c|c|c|c|c|}
\hline $\begin{array}{l}\text { Constituent } \\
\text { Class }\end{array}$ & Constituent Name & Units & $\begin{array}{l}\text { Number } \\
\text { Analyzed }\end{array}$ & $\begin{array}{l}\text { Number } \\
\text { Detected }\end{array}$ & $\begin{array}{l}\text { Frequency of } \\
\text { Detection }\end{array}$ & $\begin{array}{l}\text { Minimum } \\
\text { Nondetect }\end{array}$ & $\begin{array}{l}\text { Maximum } \\
\text { Nondetect }\end{array}$ & Minimum Detect & $\begin{array}{l}\text { Maximum } \\
\text { Detect }\end{array}$ & $\begin{array}{c}\text { Average } \\
\text { Concentration }\end{array}$ \\
\hline$\overline{\text { CONV }}$ & Percent Solids & $\%$ & 6 & 6 & $100 \%$ & $\overline{--}$ & -- & 37 & 74 & 50 \\
\hline CONV & Total Organic Carbon & $\%$ & 5 & 5 & $100 \%$ & -- & -- & 0.080 & 0.43 & 0.29 \\
\hline CONV & Total Organic Carbon & $\mathrm{mg} / \mathrm{kg}$ & 26 & 26 & $100 \%$ & -- & -- & 480 & 16,200 & 7,184 \\
\hline CONV & Total Volatile Solids & $\%$ & 11 & 11 & $100 \%$ & -- & -- & 1.0 & 4.8 & 2.7 \\
\hline METAL & Acid Volatile Sulfate & $\mathrm{mg} / \mathrm{kg}$ & 5 & 3 & $60 \%$ & 0.30 & 0.30 & 2.0 & 2.3 & 1.3 \\
\hline METAL & Aluminum & $\mathrm{mg} / \mathrm{kg}$ & 4 & 4 & $100 \%$ & -- & -- & 9,500 & 11,900 & 10,668 \\
\hline METAL & Antimony & $\mathrm{mg} / \mathrm{Kg}$ & 19 & 10 & $53 \%$ & 53 & 65 & 0.34 & 65 & 21 \\
\hline METAL & Arsenic & $\mathrm{mg} / \mathrm{Kg}$ & 24 & 13 & $54 \%$ & 18 & 22 & 2.0 & 15 & 8.0 \\
\hline METAL & Barium & $\mathrm{mg} / \mathrm{kg}$ & 4 & 4 & $100 \%$ & -- & -- & 118 & 199 & 156 \\
\hline METAL & Beryllium & $\mathrm{mg} / \mathrm{Kg}$ & 8 & 8 & $100 \%$ & -- & -- & 0.37 & 2.0 & 1.1 \\
\hline METAL & Cadmium & $\mathrm{mg} / \mathrm{Kg}$ & 24 & 18 & $75 \%$ & 0.48 & 1.1 & 0.060 & 2.2 & 0.64 \\
\hline METAL & Calcium & $\mathrm{mg} / \mathrm{kg}$ & 4 & 4 & $100 \%$ & -- & -- & 5,510 & 7,150 & 6,180 \\
\hline METAL & Chromium & $\mathrm{mg} / \mathrm{Kg}$ & 13 & 13 & $100 \%$ & -- & -- & 9.2 & 69 & 29 \\
\hline METAL & Cobalt & $\mathrm{mg} / \mathrm{kg}$ & 4 & 4 & $100 \%$ & -- & -- & 9.9 & 15 & 12 \\
\hline METAL & Copper & $\mathrm{mg} / \mathrm{Kg}$ & 24 & 24 & $100 \%$ & -- & -- & 7.7 & 46 & 19 \\
\hline METAL & Iron & $\mathrm{mg} / \mathrm{kg}$ & 4 & 4 & $100 \%$ & -- & -- & 20,700 & 26,100 & 23,950 \\
\hline METAL & Lead & $\mathrm{mg} / \mathrm{Kg}$ & 27 & 20 & $74 \%$ & 8.7 & 11 & 7.6 & 250 & 20 \\
\hline METAL & Magnesium & $\mathrm{mg} / \mathrm{kg}$ & 4 & 4 & $100 \%$ & -- & -- & 5,100 & 6,980 & 5,803 \\
\hline METAL & Manganese & $\mathrm{mg} / \mathrm{kg}$ & 4 & 4 & $100 \%$ & -- & -- & 399 & 716 & 531 \\
\hline METAL & Mercury & $\mathrm{mg} / \mathrm{Kg}$ & 28 & 13 & $46 \%$ & $1.00 \mathrm{E}-04$ & 0.11 & 1.17E-04 & 0.51 & 0.081 \\
\hline METAL & Nickel & $\mathrm{mg} / \mathrm{Kg}$ & 24 & 23 & $96 \%$ & 3.9 & 3.9 & 4.4 & 33 & 13 \\
\hline METAL & Potassium & $\mathrm{mg} / \mathrm{kg}$ & 4 & 4 & $100 \%$ & -- & -- & 845 & 1,550 & 1,068 \\
\hline METAL & Selenium & $\mathrm{mg} / \mathrm{Kg}$ & 8 & 1 & $13 \%$ & 0.46 & 0.73 & 0.66 & 0.66 & 0.31 \\
\hline METAL & Silver & $\mathrm{mg} / \mathrm{Kg}$ & 24 & 14 & $58 \%$ & 1.7 & 2.1 & 0.060 & 2.2 & 0.59 \\
\hline METAL & Sodium & $\mathrm{mg} / \mathrm{kg}$ & 4 & 4 & $100 \%$ & -- & -- & 263 & 366 & 312 \\
\hline METAL & Thallium & $\mathrm{mg} / \mathrm{Kg}$ & 8 & 8 & $100 \%$ & -- & -- & 0.19 & 0.69 & 0.42 \\
\hline METAL & Tin & $\mathrm{mg} / \mathrm{kg}$ & 4 & 4 & $100 \%$ & -- & -- & 49 & 69 & 57 \\
\hline METAL & Zinc & $\mathrm{mg} / \mathrm{Kg}$ & 24 & 24 & $100 \%$ & -- & -- & 38 & 293 & 112 \\
\hline PCB & Aroclor-1016 & $\mathrm{mg} / \mathrm{kg}$ & 19 & 0 & $0 \%$ & 0.0027 & 0.010 & -- & -- & 0.0032 \\
\hline PCB & Aroclor-1221 & $\mathrm{mg} / \mathrm{kg}$ & 19 & 0 & $0 \%$ & 0.012 & 0.019 & -- & -- & 0.0077 \\
\hline PCB & Aroclor-1232 & $\mathrm{mg} / \mathrm{kg}$ & 19 & 0 & $0 \%$ & 0.0064 & 0.015 & -- & -- & 0.0047 \\
\hline PCB & Aroclor-1242 & $\mathrm{mg} / \mathrm{kg}$ & 19 & 0 & $0 \%$ & 0.0040 & 0.0083 & -- & -- & 0.0027 \\
\hline РСB & Aroclor-1248 & $\mathrm{mg} / \mathrm{kg}$ & 19 & 0 & $0 \%$ & 0.0022 & 0.021 & -- & -- & 0.0042 \\
\hline PCB & Aroclor-1254 & $\mathrm{mg} / \mathrm{kg}$ & 19 & 1 & $5 \%$ & 0.0037 & 0.0064 & 0.019 & 0.019 & 0.0032 \\
\hline РCB & Aroclor-1260 & $\mathrm{mg} / \mathrm{kg}$ & 19 & 0 & $0 \%$ & 0.0032 & 0.0064 & -- & -- & 0.0021 \\
\hline PEST & Aldrin & $\mathrm{mg} / \mathrm{kg}$ & 11 & 0 & $0 \%$ & $8.40 \mathrm{E}-05$ & 1.10E-04 & -- & -- & $4.75 \mathrm{E}-05$ \\
\hline PEST & Alpha-BHC & $\mathrm{mg} / \mathrm{kg}$ & 16 & 0 & $0 \%$ & $8.70 \mathrm{E}-05$ & $2.00 \mathrm{E}-04$ & -- & -- & $6.50 \mathrm{E}-05$ \\
\hline PEST & beta-1,2,3,4,5,6-Hexachlorocyclohexane & $\mathrm{mg} / \mathrm{kg}$ & 11 & 0 & $0 \%$ & $1.60 \mathrm{E}-04$ & $2.20 \mathrm{E}-04$ & -- & -- & $9.14 \mathrm{E}-05$ \\
\hline PEST & Chlordane & $\mathrm{mg} / \mathrm{kg}$ & 11 & 0 & $0 \%$ & 0.0028 & 0.0038 & -- & -- & 0.0016 \\
\hline PEST & Delta-BHC & $\mathrm{mg} / \mathrm{kg}$ & 11 & 0 & $0 \%$ & $8.80 \mathrm{E}-05$ & $1.20 \mathrm{E}-04$ & -- & -- & $5.00 \mathrm{E}-05$ \\
\hline PEST & Dichlorodiphenyldichloroethane & $\mathrm{mg} / \mathrm{kg}$ & 11 & 0 & $0 \%$ & $1.90 \mathrm{E}-04$ & $2.60 \mathrm{E}-04$ & -- & -- & 1.10E-04 \\
\hline PEST & Dichlorodiphenyldichloroethylene & $\mathrm{mg} / \mathrm{kg}$ & 11 & 0 & $0 \%$ & $4.10 \mathrm{E}-04$ & $5.50 \mathrm{E}-04$ & -- & -- & $2.32 \mathrm{E}-04$ \\
\hline PEST & Dichlorodiphenyltrichloroethane & $\mathrm{mg} / \mathrm{kg}$ & 16 & 1 & $6 \%$ & $2.00 \mathrm{E}-04$ & 0.0019 & 0.0020 & 0.0020 & $7.09 \mathrm{E}-04$ \\
\hline PEST & Dieldrin & $\mathrm{mg} / \mathrm{kg}$ & 11 & 0 & $0 \%$ & $8.00 \mathrm{E}-05$ & 1.10E-04 & -- & -- & 4.59E-05 \\
\hline PEST & Endosulfan I & $\mathrm{mg} / \mathrm{kg}$ & 11 & 0 & $0 \%$ & $3.70 \mathrm{E}-04$ & $5.00 \mathrm{E}-04$ & -- & -- & $2.08 \mathrm{E}-04$ \\
\hline PEST & Endosulfan II & $\mathrm{mg} / \mathrm{kg}$ & 11 & 0 & $0 \%$ & $1.90 \mathrm{E}-04$ & $2.50 \mathrm{E}-04$ & -- & -- & 1.07E-04 \\
\hline
\end{tabular}


Table D-3

Summary Statistics for Sediment Collected from Segment 4 of the Columbia River Including All Categories of Data (units reported "as received")

\begin{tabular}{|c|c|c|c|c|c|c|c|c|c|c|}
\hline $\begin{array}{l}\text { Constituent } \\
\text { Class }\end{array}$ & Constituent Name & Units & $\begin{array}{l}\text { Number } \\
\text { Analyzed }\end{array}$ & $\begin{array}{l}\text { Number } \\
\text { Detected }\end{array}$ & $\begin{array}{c}\text { Frequency of } \\
\text { Detection }\end{array}$ & $\begin{array}{l}\text { Minimum } \\
\text { Nondetect }\end{array}$ & $\begin{array}{l}\text { Maximum } \\
\text { Nondetect }\end{array}$ & Minimum Detect & $\begin{array}{l}\text { Maximum } \\
\text { Detect }\end{array}$ & $\begin{array}{c}\text { Average } \\
\text { Concentration }\end{array}$ \\
\hline PEST & Endosulfan sulfate & $\mathrm{mg} / \mathrm{kg}$ & 11 & 0 & $0 \%$ & $2.70 \mathrm{E}-04$ & $3.70 \mathrm{E}-04$ & -- & -- & $1.55 \mathrm{E}-04$ \\
\hline PEST & Endrin & $\mathrm{mg} / \mathrm{kg}$ & 11 & 0 & $0 \%$ & $1.40 \mathrm{E}-04$ & $1.90 \mathrm{E}-04$ & -- & -- & 7.82E-05 \\
\hline PEST & Endrin aldehyde & $\mathrm{mg} / \mathrm{kg}$ & 11 & 0 & $0 \%$ & 0.0010 & 0.0014 & -- & -- & $5.73 \mathrm{E}-04$ \\
\hline PEST & Endrin ketone & $\mathrm{mg} / \mathrm{kg}$ & 11 & 0 & $0 \%$ & 3.60E-04 & 4.90E-04 & -- & -- & 2.05E-04 \\
\hline PEST & Gamma-BHC (Lindane) & $\mathrm{mg} / \mathrm{kg}$ & 16 & 1 & $6 \%$ & $1.90 \mathrm{E}-04$ & $2.60 \mathrm{E}-04$ & $2.00 \mathrm{E}-04$ & $2.00 \mathrm{E}-04$ & 1.13E-04 \\
\hline PEST & Heptachlor & $\mathrm{mg} / \mathrm{kg}$ & 11 & 0 & $0 \%$ & $1.10 \mathrm{E}-04$ & 0.0033 & -- & -- & 2.09E-04 \\
\hline PEST & Heptachlor epoxide & $\mathrm{mg} / \mathrm{kg}$ & 11 & 0 & $0 \%$ & 2.60E-05 & $2.50 \mathrm{E}-04$ & -- & -- & $9.75 \mathrm{E}-05$ \\
\hline PEST & Methoxychlor & $\mathrm{mg} / \mathrm{kg}$ & 11 & 0 & $0 \%$ & $3.90 \mathrm{E}-04$ & 0.0034 & -- & -- & 0.0013 \\
\hline PEST & Toxaphene & $\mathrm{mg} / \mathrm{kg}$ & 11 & 0 & $0 \%$ & 0.049 & 0.067 & -- & -- & 0.028 \\
\hline RAD_D & Cerium-141, Decayed & $\mathrm{pCi} / \mathrm{g}$ & 4 & 4 & $100 \%$ & -- & -- & 3.77E-103 & $1.26 \mathrm{E}-89$ & $3.14 \mathrm{E}-90$ \\
\hline RAD_D & Cesium-137, Decayed & $\mathrm{pCi} / \mathrm{g}$ & 36 & 36 & $100 \%$ & -- & -- & 0.049 & 0.59 & 0.10 \\
\hline RAD_D & Cobalt-60, Decayed & $\mathrm{pCi} / \mathrm{g}$ & 23 & 23 & $100 \%$ & -- & -- & $8.78 \mathrm{E}-04$ & 0.021 & 0.0061 \\
\hline RAD_D & Plutonium-238, Decayed & $\mathrm{pCi} / \mathrm{g}$ & 5 & 5 & $100 \%$ & -- & -- & $1.88 \mathrm{E}-04$ & 0.0011 & 4.94E-04 \\
\hline RAD_D & Plutonium-239/240, Decayed & $\mathrm{pCi} / \mathrm{g}$ & 6 & 6 & $100 \%$ & -- & -- & 0.0045 & 0.018 & 0.0089 \\
\hline RAD_D & Potassium-40, Decayed & $\mathrm{pCi} / \mathrm{g}$ & 95 & 95 & $100 \%$ & -- & -- & 7.2 & 31 & 13 \\
\hline RAD_D & Radium-226, Decayed & $\mathrm{pCi} / \mathrm{g}$ & 25 & 25 & $100 \%$ & -- & -- & 0.50 & 1.9 & 0.81 \\
\hline RAD_D & Ruthenium-106, Decayed & $\mathrm{pCi} / \mathrm{g}$ & 23 & 23 & $100 \%$ & -- & -- & $2.27 \mathrm{E}-13$ & $1.03 \mathrm{E}-08$ & $4.59 \mathrm{E}-10$ \\
\hline RAD_D & Strontium-90, Decayed & $\mathrm{pCi} / \mathrm{g}$ & 1 & 1 & $100 \%$ & -- & -- & 0.028 & 0.028 & 0.028 \\
\hline RAD_D & Thorium-232, Decayed & $\mathrm{pCi} / \mathrm{g}$ & 16 & 16 & $100 \%$ & -- & -- & 0.40 & 1.00 & 0.78 \\
\hline RAD_D & Uranium-234, Decayed & $\mathrm{pCi} / \mathrm{g}$ & 4 & 4 & $100 \%$ & -- & -- & 0.67 & 0.97 & 0.82 \\
\hline RAD_D & Uranium-235, Decayed & $\mathrm{pCi} / \mathrm{g}$ & 4 & 4 & $100 \%$ & -- & -- & 0.010 & 0.034 & 0.022 \\
\hline RAD_D & Uranium-238, Decayed & $\mathrm{pCi} / \mathrm{g}$ & 4 & 4 & $100 \%$ & -- & -- & 0.52 & 0.79 & 0.64 \\
\hline RAD_D & Zinc-65, Decayed & $\mathrm{pCi} / \mathrm{g}$ & 37 & 37 & $100 \%$ & -- & -- & $6.80 \mathrm{E}-19$ & $1.10 \mathrm{E}-12$ & $8.25 \mathrm{E}-14$ \\
\hline RAD_D & Zirconium/Niobium-95, Decayed & $\mathrm{pCi} / \mathrm{g}$ & 34 & 34 & $100 \%$ & -- & -- & 2.77E-126 & $5.25 \mathrm{E}-86$ & $1.54 \mathrm{E}-87$ \\
\hline SVOC & 1,2,4-Trichlorobenzene & $\mathrm{mg} / \mathrm{kg}$ & 11 & 0 & $0 \%$ & 0.0026 & 0.0031 & -- & -- & 0.0014 \\
\hline sVOC & 1,2-Dichlorobenzene & $\mathrm{mg} / \mathrm{kg}$ & 11 & 0 & $0 \%$ & 0.0027 & 0.0032 & -- & -- & 0.0014 \\
\hline SVOC & 1,3-Dichlorobenzene & $\mathrm{mg} / \mathrm{kg}$ & 11 & 0 & $0 \%$ & 0.0041 & 0.0049 & -- & -- & 0.0022 \\
\hline SVOC & 1,4-Dichlorobenzene & $\mathrm{mg} / \mathrm{kg}$ & 11 & 0 & $0 \%$ & 0.0036 & 0.0044 & -- & -- & 0.0020 \\
\hline SVOC & 2,4-Dimethylphenol & $\mathrm{mg} / \mathrm{kg}$ & 16 & 0 & $0 \%$ & 0.0027 & 0.050 & -- & -- & 0.0088 \\
\hline SVOC & 2-MethyInaphthalene & $\mathrm{mg} / \mathrm{kg}$ & 20 & 14 & $70 \%$ & 0.0019 & 0.0030 & 0.0010 & 0.014 & 0.0040 \\
\hline SVOC & 2-Methylphenol (cresol, o-) & $\mathrm{mg} / \mathrm{kg}$ & 16 & 0 & $0 \%$ & 0.0029 & 0.030 & -- & -- & 0.0058 \\
\hline SVOC & 3\&4-Methylphenol (Cresol, $m+p)$ & $\mathrm{mg} / \mathrm{kg}$ & 11 & 2 & $18 \%$ & 0.0016 & 0.0019 & 0.0058 & 0.013 & 0.0024 \\
\hline SVOC & 4-Methylphenol (cresol, p-) & $\mathrm{mg} / \mathrm{kg}$ & 5 & 1 & $20 \%$ & 0.050 & 0.050 & 0.10 & 0.10 & 0.040 \\
\hline SVOC & Acenaphthene & $\mathrm{mg} / \mathrm{kg}$ & 20 & 7 & $35 \%$ & $5.00 \mathrm{E}-04$ & 0.0019 & $9.20 \mathrm{E}-04$ & 0.018 & 0.0019 \\
\hline sVOC & Acenaphthylene & $\mathrm{mg} / \mathrm{kg}$ & 20 & 5 & $25 \%$ & $7.50 \mathrm{E}-04$ & 0.0024 & $2.00 \mathrm{E}-04$ & 0.0060 & 0.0012 \\
\hline SVOC & Anthracene & $\mathrm{mg} / \mathrm{kg}$ & 20 & 5 & $25 \%$ & $6.00 \mathrm{E}-04$ & 0.0022 & $9.00 \mathrm{E}-04$ & 0.013 & 0.0017 \\
\hline SVOC & Benzo(a)anthracene & $\mathrm{mg} / \mathrm{kg}$ & 20 & 6 & $30 \%$ & $7.00 \mathrm{E}-04$ & 0.0014 & 0.0010 & 0.0068 & 0.0014 \\
\hline SVOC & Benzo(a)pyrene & $\mathrm{mg} / \mathrm{kg}$ & 20 & 5 & $25 \%$ & $5.00 \mathrm{E}-04$ & 0.0015 & 0.0020 & 0.0073 & 0.0017 \\
\hline SVOC & Benzo(b)fluoranthene & $\mathrm{mg} / \mathrm{kg}$ & 16 & 5 & $31 \%$ & $8.00 \mathrm{E}-04$ & 0.0021 & 0.0010 & 0.0040 & 0.0016 \\
\hline SVOC & Benzo(b+k)fluoranthene & $\mathrm{mg} / \mathrm{kg}$ & 4 & 3 & $75 \%$ & 0.0015 & 0.0015 & 0.0035 & 0.014 & 0.0069 \\
\hline SVOC & Benzo(ghi)perylene & $\mathrm{mg} / \mathrm{kg}$ & 20 & 6 & $30 \%$ & $7.50 \mathrm{E}-04$ & 0.0015 & $5.00 \mathrm{E}-04$ & 0.0070 & 0.0016 \\
\hline SVOC & Benzo(k)fluoranthene & $\mathrm{mg} / \mathrm{kg}$ & 16 & 3 & $19 \%$ & $6.00 \mathrm{E}-04$ & 0.0019 & 0.0010 & 0.0040 & 0.0011 \\
\hline SVOC & Benzoic acid & $\mathrm{mg} / \mathrm{kg}$ & 15 & 3 & $20 \%$ & 0.0069 & 0.061 & 0.011 & 0.014 & 0.011 \\
\hline SVOC & Benzyl alcohol & $\mathrm{mg} / \mathrm{kg}$ & 11 & 0 & $0 \%$ & 0.0046 & 0.0055 & -- & -- & 0.0025 \\
\hline SVOC & Bis(2-ethylhexyl) phthalate & $\mathrm{mg} / \mathrm{kg}$ & 15 & 15 & $100 \%$ & -- & -- & 0.0087 & 0.029 & 0.021 \\
\hline SVOC & Butylbenzylphthalate & $\mathrm{mg} / \mathrm{kg}$ & 15 & 7 & $47 \%$ & 0.0015 & 0.0098 & 0.0030 & 0.037 & 0.0058 \\
\hline
\end{tabular}


Table D-3

Summary Statistics for Sediment Collected from Segment 4 of the Columbia River Including All Categories of Data (units reported "as received")

\begin{tabular}{|c|c|c|c|c|c|c|c|c|c|c|}
\hline $\begin{array}{l}\text { Constituent } \\
\text { Class }\end{array}$ & Constituent Name & Units & $\begin{array}{l}\text { Number } \\
\text { Analyzed }\end{array}$ & $\begin{array}{l}\text { Number } \\
\text { Detected }\end{array}$ & $\begin{array}{c}\text { Frequency of } \\
\text { Detection }\end{array}$ & $\begin{array}{l}\text { Minimum } \\
\text { Nondetect }\end{array}$ & $\begin{array}{l}\text { Maximum } \\
\text { Nondetect }\end{array}$ & Minimum Detect & $\begin{array}{c}\text { Maximum } \\
\text { Detect }\end{array}$ & $\begin{array}{c}\text { Average } \\
\text { Concentration }\end{array}$ \\
\hline$\overline{\text { SVOC }}$ & Chrysene & $\mathrm{mg} / \mathrm{kg}$ & 20 & 10 & $50 \%$ & $7.50 \mathrm{E}-04$ & 0.0024 & $6.00 \mathrm{E}-04$ & 0.0096 & 0.0026 \\
\hline SVOC & Dibenz[a,h]anthracene & $\mathrm{mg} / \mathrm{kg}$ & 20 & 3 & $15 \%$ & $5.00 \mathrm{E}-04$ & 0.0014 & $6.00 \mathrm{E}-04$ & 0.0030 & 7.36E-04 \\
\hline SVOC & Dibenzofuran & $\mathrm{mg} / \mathrm{kg}$ & 16 & 3 & $19 \%$ & $5.00 \mathrm{E}-04$ & 0.0028 & 0.0010 & 0.0056 & 0.0014 \\
\hline SVOC & Diethylphthalate & $\mathrm{mg} / \mathrm{kg}$ & 11 & 1 & $9 \%$ & 0.0045 & 0.0045 & 0.064 & 0.064 & 0.0079 \\
\hline SVOC & Dimethyl phthalate & $\mathrm{mg} / \mathrm{kg}$ & 11 & 11 & $100 \%$ & -- & -- & 0.0073 & 0.12 & 0.044 \\
\hline SVOC & Di-n-butylphthalate & $\mathrm{mg} / \mathrm{kg}$ & 11 & 10 & $91 \%$ & 0.019 & 0.019 & 0.0094 & 0.065 & 0.018 \\
\hline SVOC & Di-n-octylphthalate & $\mathrm{mg} / \mathrm{kg}$ & 11 & 3 & $27 \%$ & 0.0017 & 0.0017 & 0.0027 & 0.0071 & 0.0020 \\
\hline SVOC & Fluoranthene & $\mathrm{mg} / \mathrm{kg}$ & 20 & 13 & $65 \%$ & 0.0022 & 0.0022 & 0.0010 & 0.022 & 0.0052 \\
\hline SVOC & Fluorene & $\mathrm{mg} / \mathrm{kg}$ & 20 & 6 & $30 \%$ & $5.00 \mathrm{E}-04$ & 0.0023 & $7.00 \mathrm{E}-04$ & 0.010 & 0.0014 \\
\hline SVOC & Hexachlorobenzene & $\mathrm{mg} / \mathrm{kg}$ & 11 & 0 & $0 \%$ & 0.0013 & 0.0016 & -- & -- & 7.27E-04 \\
\hline SVOC & Hexachlorocyclopentadiene & $\mathrm{mg} / \mathrm{kg}$ & 11 & 0 & $0 \%$ & 0.0032 & 0.0038 & -- & -- & 0.0017 \\
\hline SVOC & Hexachloroethane & $\mathrm{mg} / \mathrm{kg}$ & 11 & 0 & $0 \%$ & 0.0025 & 0.0039 & -- & -- & 0.0014 \\
\hline SVOC & Indeno(1,2,3-cd)pyrene & $\mathrm{mg} / \mathrm{kg}$ & 20 & 6 & $30 \%$ & $7.00 \mathrm{E}-04$ & 0.0018 & 0.0010 & 0.0070 & 0.0015 \\
\hline SVOC & Naphthalene & $\mathrm{mg} / \mathrm{kg}$ & 20 & 9 & $45 \%$ & 0.0019 & 0.0030 & 8.00E-04 & 0.0086 & 0.0024 \\
\hline SVOC & N-Nitrosodi-n-dipropylamine & $\mathrm{mg} / \mathrm{kg}$ & 11 & 0 & $0 \%$ & 0.0029 & 0.0034 & -- & -- & 0.0016 \\
\hline SVOC & Pentachlorophenol & $\mathrm{mg} / \mathrm{kg}$ & 16 & 2 & $13 \%$ & 0.0018 & 1.8 & 0.011 & 0.029 & 0.067 \\
\hline SVOC & Phenanthrene & $\mathrm{mg} / \mathrm{kg}$ & 20 & 13 & $65 \%$ & $7.50 \mathrm{E}-04$ & 0.0018 & $9.00 \mathrm{E}-04$ & 0.029 & 0.0045 \\
\hline SVOC & Phenol & $\mathrm{mg} / \mathrm{kg}$ & 20 & 4 & $20 \%$ & 0.0031 & 3.1 & 0.0054 & 0.020 & 0.082 \\
\hline SVOC & Pyrene & $\mathrm{mg} / \mathrm{kg}$ & 20 & 14 & $70 \%$ & 0.0022 & 0.0022 & 0.0010 & 0.027 & 0.0052 \\
\hline
\end{tabular}

CONV - Conventional parameter

PEST - Pesticide

PCB - Polychlorinated bipheny

RAD D - Decayed radionuclide

SVOC - Semivolatile Organic Compound 
Table D-4

Summary Statistics for Sediment Collected from Segment 4 of the Columbia River Including All Categories of Data (units reported "dry-weight")

\begin{tabular}{|c|c|c|c|c|c|c|c|c|c|c|}
\hline $\begin{array}{l}\text { Constituent } \\
\text { Class }\end{array}$ & Constituent Name & Units & $\begin{array}{l}\text { Number } \\
\text { Analyzed }\end{array}$ & $\begin{array}{l}\text { Number } \\
\text { Detected }\end{array}$ & $\begin{array}{l}\text { Frequency of } \\
\text { Detection }\end{array}$ & $\begin{array}{l}\text { Minimum } \\
\text { Nondetect }\end{array}$ & $\begin{array}{l}\text { Maximum } \\
\text { Nondetect }\end{array}$ & $\begin{array}{l}\text { Minimum } \\
\text { Detect }\end{array}$ & $\begin{array}{c}\text { Maximum } \\
\text { Detect }\end{array}$ & $\begin{array}{c}\text { Average } \\
\text { Concentration }\end{array}$ \\
\hline$\overline{M E T A L}$ & Aluminum & $\mathrm{mg} / \mathrm{kg}$ dry-wt & 5 & 5 & $100 \%$ & -- & -- & 5,500 & 22,000 & 15,100 \\
\hline METAL & Antimony & $\mathrm{mg} / \mathrm{kg}$ dry-wt & 5 & 5 & $100 \%$ & -- & -- & 2.6 & 6.1 & 4.6 \\
\hline METAL & Arsenic & $\mathrm{mg} / \mathrm{kg}$ dry-wt & 5 & 5 & $100 \%$ & -- & -- & 1.5 & 9.5 & 6.1 \\
\hline METAL & Barium & $\mathrm{mg} / \mathrm{kg}$ dry-wt & 5 & 5 & $100 \%$ & -- & -- & 72 & 210 & 140 \\
\hline METAL & Beryllium & $\mathrm{mg} / \mathrm{kg}$ dry-wt & 5 & 5 & $100 \%$ & -- & -- & 0.65 & 1.5 & 1.2 \\
\hline METAL & Cadmium & $\mathrm{mg} / \mathrm{kg}$ dry-wt & 5 & 5 & $100 \%$ & -- & -- & 0.65 & 2.5 & 1.9 \\
\hline METAL & Calcium & $\mathrm{mg} / \mathrm{kg}$ dry-wt & 5 & 5 & $100 \%$ & -- & -- & 3,100 & 6,800 & 5,280 \\
\hline METAL & Chromium & $\mathrm{mg} / \mathrm{kg}$ dry-wt & 5 & 5 & $100 \%$ & -- & -- & 9.4 & 30 & 21 \\
\hline METAL & Cobalt & $\mathrm{mg} / \mathrm{kg}$ dry-wt & 5 & 5 & $100 \%$ & -- & -- & 10 & 22 & 18 \\
\hline METAL & Copper & $\mathrm{mg} / \mathrm{kg}$ dry-wt & 5 & 5 & $100 \%$ & -- & -- & 7.5 & 46 & 28 \\
\hline METAL & Iron & $\mathrm{mg} / \mathrm{kg}$ dry-wt & 5 & 5 & $100 \%$ & -- & -- & 22,000 & 44,000 & 36,400 \\
\hline METAL & Lead & $\mathrm{mg} / \mathrm{kg}$ dry-wt & 5 & 5 & $100 \%$ & -- & -- & 4.0 & 27 & 17 \\
\hline METAL & Magnesium & $\mathrm{mg} / \mathrm{kg}$ dry-wt & 5 & 5 & $100 \%$ & -- & -- & 3,100 & 7,500 & 6,140 \\
\hline METAL & Manganese & $\mathrm{mg} / \mathrm{kg}$ dry-wt & 5 & 5 & $100 \%$ & -- & -- & 370 & 840 & 622 \\
\hline METAL & Mercury & $\mathrm{mg} / \mathrm{kg}$ dry-wt & 5 & 5 & $100 \%$ & -- & -- & 0.13 & 0.30 & 0.23 \\
\hline METAL & Nickel & $\mathrm{mg} / \mathrm{kg}$ dry-wt & 5 & 5 & $100 \%$ & -- & -- & 9.7 & 30 & 24 \\
\hline METAL & Potassium & $\mathrm{mg} / \mathrm{kg}$ dry-wt & 5 & 5 & $100 \%$ & -- & -- & 450 & 3,000 & 1,856 \\
\hline METAL & Selenium & $\mathrm{mg} / \mathrm{kg}$ dry-wt & 5 & 5 & $100 \%$ & -- & -- & 0.96 & 1.6 & 1.4 \\
\hline METAL & Silver & $\mathrm{mg} / \mathrm{kg}$ dry-wt & 5 & 5 & $100 \%$ & -- & -- & 1.3 & 3.0 & 2.3 \\
\hline METAL & Sodium & $\mathrm{mg} / \mathrm{kg}$ dry-wt & 5 & 5 & $100 \%$ & -- & -- & 170 & 390 & 314 \\
\hline METAL & Thallium & $\mathrm{mg} / \mathrm{kg}$ dry-wt & 5 & 5 & $100 \%$ & -- & -- & 1.3 & 3.0 & 2.6 \\
\hline METAL & Uranium & $\mathrm{pCi} / \mathrm{g}$ dry-wt & 1 & 1 & $100 \%$ & -- & -- & 2.7 & 2.7 & 2.7 \\
\hline METAL & Vanadium & $\mathrm{mg} / \mathrm{kg}$ dry-wt & 5 & 5 & $100 \%$ & -- & -- & 62 & 120 & 83 \\
\hline METAL & Zinc & $\mathrm{mg} / \mathrm{kg}$ dry-wt & 5 & 5 & $100 \%$ & -- & -- & 64 & 270 & 182 \\
\hline PCB & Aroclor-1016 & $\mathrm{mg} / \mathrm{kg}$ dry-wt & 5 & 0 & $0 \%$ & 0.033 & 0.033 & -- & -- & 0.017 \\
\hline PCB & Aroclor-1221 & $\mathrm{mg} / \mathrm{kg}$ dry-wt & 5 & 0 & $0 \%$ & 0.067 & 0.067 & -- & -- & 0.034 \\
\hline PCB & Aroclor-1232 & $\mathrm{mg} / \mathrm{kg}$ dry-wt & 5 & 0 & $0 \%$ & 0.033 & 0.033 & -- & -- & 0.017 \\
\hline PCB & Aroclor-1242 & $\mathrm{mg} / \mathrm{kg}$ dry-wt & 5 & 0 & $0 \%$ & 0.033 & 0.033 & -- & -- & 0.017 \\
\hline PCB & Aroclor-1248 & $\mathrm{mg} / \mathrm{kg}$ dry-wt & 5 & 0 & $0 \%$ & 0.033 & 0.033 & -- & -- & 0.017 \\
\hline PCB & Aroclor-1254 & $\mathrm{mg} / \mathrm{kg}$ dry-wt & 5 & 0 & $0 \%$ & 0.033 & 0.033 & -- & -- & 0.017 \\
\hline PCB & Aroclor-1260 & $\mathrm{mg} / \mathrm{kg}$ dry-wt & 5 & 0 & $0 \%$ & 0.033 & 0.033 & -- & -- & 0.017 \\
\hline PEST & Aldrin & $\mathrm{mg} / \mathrm{kg}$ dry-wt & 5 & 0 & $0 \%$ & 0.0017 & 0.0017 & -- & -- & 8.50E-04 \\
\hline PEST & Alpha-BHC & $\mathrm{mg} / \mathrm{kg}$ dry-wt & 5 & 0 & $0 \%$ & 0.0017 & 0.0017 & -- & -- & 8.50E-04 \\
\hline PEST & beta-1,2,3,4,5,6-Hexachlorocyclohexane & $\mathrm{mg} / \mathrm{kg}$ dry-wt & 5 & 0 & $0 \%$ & 0.0017 & 0.0017 & -- & -- & $8.50 \mathrm{E}-04$ \\
\hline PEST & Delta-BHC & $\mathrm{mg} / \mathrm{kg}$ dry-wt & 5 & 0 & $0 \%$ & 0.0017 & 0.0017 & -- & -- & $8.50 \mathrm{E}-04$ \\
\hline PEST & Dieldrin & $\mathrm{mg} / \mathrm{kg}$ dry-wt & 5 & 0 & $0 \%$ & 0.0017 & 0.0017 & -- & -- & $8.50 \mathrm{E}-04$ \\
\hline PEST & Endosulfan I & $\mathrm{mg} / \mathrm{kg}$ dry-wt & 5 & 0 & $0 \%$ & 0.0017 & 0.0017 & -- & -- & 8.50E-04 \\
\hline PEST & Endosulfan II & $\mathrm{mg} / \mathrm{kg}$ dry-wt & 5 & 0 & $0 \%$ & 0.0017 & 0.0017 & -- & -- & $8.50 \mathrm{E}-04$ \\
\hline PEST & Endosulfan sulfate & $\mathrm{mg} / \mathrm{kg}$ dry-wt & 5 & 0 & $0 \%$ & 0.0017 & 0.0017 & -- & -- & 8.50E-04 \\
\hline PEST & Endrin & $\mathrm{mg} / \mathrm{kg}$ dry-wt & 5 & 0 & $0 \%$ & 0.0017 & 0.0017 & -- & -- & $8.50 \mathrm{E}-04$ \\
\hline PEST & Endrin aldehyde & $\mathrm{mg} / \mathrm{kg}$ dry-wt & 5 & 0 & $0 \%$ & 0.0017 & 0.0017 & -- & -- & 8.50E-04 \\
\hline PEST & Endrin ketone & $\mathrm{mg} / \mathrm{kg}$ dry-wt & 5 & 0 & $0 \%$ & 0.0017 & 0.0017 & -- & -- & 8.50E-04 \\
\hline PEST & gamma-Chlordane & $\mathrm{mg} / \mathrm{kg}$ dry-wt & 5 & 0 & $0 \%$ & 0.0017 & 0.0017 & -- & -- & $8.50 \mathrm{E}-04$ \\
\hline PEST & Methoxychlor & $\mathrm{mg} / \mathrm{kg}$ dry-wt & 5 & 0 & $0 \%$ & 0.0017 & 0.0017 & -- & -- & 8.50E-04 \\
\hline PEST & Toxaphene & $\mathrm{mg} / \mathrm{kg}$ dry-wt & 5 & 0 & $0 \%$ & 0.083 & 0.083 & -- & -- & 0.042 \\
\hline RAD_D & Beryllium-7 & $\mathrm{pCi} / \mathrm{g}$ dry-wt & 1 & 1 & $100 \%$ & -- & -- & 0.0016 & 0.0016 & 0.0016 \\
\hline
\end{tabular}


Table D-4

Summary Statistics for Sediment Collected from Segment 4 of the Columbia River Including All Categories of Data (units reported "dry-weight")

\begin{tabular}{|c|c|c|c|c|c|c|c|c|c|c|}
\hline $\begin{array}{l}\text { Constituent } \\
\text { Class }\end{array}$ & Constituent Name & Units & $\begin{array}{l}\text { Number } \\
\text { Analyzed }\end{array}$ & $\begin{array}{l}\text { Number } \\
\text { Detected }\end{array}$ & $\begin{array}{l}\text { Frequency of } \\
\text { Detection }\end{array}$ & $\begin{array}{c}\text { Minimum } \\
\text { Nondetect }\end{array}$ & $\begin{array}{l}\text { Maximum } \\
\text { Nondetect }\end{array}$ & $\begin{array}{c}\text { Minimum } \\
\text { Detect }\end{array}$ & $\begin{array}{c}\text { Maximum } \\
\text { Detect }\end{array}$ & $\begin{array}{c}\text { Average } \\
\text { Concentration }\end{array}$ \\
\hline$\overline{R A D \_D}$ & Cesium-134 & pCi/g dry-wt & 1 & 1 & $100 \%$ & -- & $\overline{--}$ & 0.039 & 0.039 & 0.039 \\
\hline RAD_D & Cesium-137 & $\mathrm{pCi} / \mathrm{g}$ dry-wt & 8 & 8 & $100 \%$ & -- & -- & 0.090 & 0.41 & 0.20 \\
\hline RAD_D & Cobalt-60 & $\mathrm{pCi} / \mathrm{g}$ dry-wt & 5 & 5 & $100 \%$ & -- & -- & 0.0077 & 0.038 & 0.016 \\
\hline RAD_D & Europium-152 & $\mathrm{pCi} / \mathrm{g}$ dry-wt & 2 & 2 & $100 \%$ & -- & -- & 0.064 & 0.22 & 0.14 \\
\hline RAD_D & Plutonium-238 & $\mathrm{pCi} / \mathrm{g}$ dry-wt & 5 & 5 & $100 \%$ & -- & -- & $2.66 \mathrm{E}-04$ & 0.020 & 0.0054 \\
\hline RAD_D & Plutonium-239/240 & $\mathrm{pCi} / \mathrm{g}$ dry-wt & 8 & 8 & $100 \%$ & -- & -- & $1.00 \mathrm{E}-03$ & 0.049 & 0.013 \\
\hline RAD_D & Potassium- 40 & $\mathrm{pCi} / \mathrm{g}$ dry-wt & 10 & 10 & $100 \%$ & -- & -- & 8.2 & 16 & 13 \\
\hline RAD_D & Strontium-90 & $\mathrm{pCi} / \mathrm{g}$ dry-wt & 9 & 9 & $100 \%$ & -- & -- & 0.0057 & 0.031 & 0.016 \\
\hline RAD_D & Uranium-234 & $\mathrm{pCi} / \mathrm{g}$ dry-wt & 9 & 9 & $100 \%$ & -- & -- & 0.50 & 1.7 & 1.3 \\
\hline RAD_D & Uranium-235 & $\mathrm{pCi} / \mathrm{g}$ dry-wt & 9 & 9 & $100 \%$ & -- & -- & 0.017 & 0.062 & 0.039 \\
\hline RAD_D & Uranium-236 & $\mathrm{pCi} / \mathrm{g}$ dry-wt & 1 & 1 & $100 \%$ & -- & -- & 1.2 & 1.2 & 1.2 \\
\hline RAD_D & Uranium-238 & $\mathrm{pCi} / \mathrm{g}$ dry-wt & 8 & 8 & $100 \%$ & -- & -- & 0.38 & 1.7 & 1.1 \\
\hline SVOC & 1,2-Dichlorobenzene & $\mathrm{mg} / \mathrm{kg}$ dry-wt & 5 & 0 & $0 \%$ & 0.33 & 0.33 & -- & - & 0.17 \\
\hline sVOC & 1,4-Dichlorobenzene & $\mathrm{mg} / \mathrm{kg}$ dry-wt & 5 & 0 & $0 \%$ & 0.33 & 0.33 & -- & -- & 0.17 \\
\hline sVOC & 2,4-Dichlorophenol & $\mathrm{mg} / \mathrm{kg}$ dry-wt & 5 & 0 & $0 \%$ & 0.33 & 0.33 & -- & -- & 0.17 \\
\hline SVOC & 2,4-Dimethylphenol & $\mathrm{mg} / \mathrm{kg}$ dry-wt & 5 & 0 & $0 \%$ & 0.33 & 0.33 & -- & -- & 0.17 \\
\hline SVOC & 2,4-Dinitrophenol & $\mathrm{mg} / \mathrm{kg}$ dry-wt & 5 & 0 & $0 \%$ & 1.7 & 1.7 & -- & -- & 0.85 \\
\hline SVOC & 2,4-Dinitrotoluene & $\mathrm{mg} / \mathrm{kg}$ dry-wt & 5 & 0 & $0 \%$ & 0.33 & 0.33 & -- & -- & 0.17 \\
\hline SVOC & 2,6-Dinitrotoluene & $\mathrm{mg} / \mathrm{kg}$ dry-wt & 5 & 0 & $0 \%$ & 0.33 & 0.33 & -- & -- & 0.17 \\
\hline sVOC & 2-Chloronaphthalene & $\mathrm{mg} / \mathrm{kg}$ dry-wt & 5 & 0 & $0 \%$ & 0.33 & 0.33 & -- & -- & 0.17 \\
\hline SVOC & 2-Chlorophenol & $\mathrm{mg} / \mathrm{kg}$ dry-wt & 5 & 0 & $0 \%$ & 0.33 & 0.33 & -- & -- & 0.17 \\
\hline SVOC & 2-Methylnaphthalene & $\mathrm{mg} / \mathrm{kg}$ dry-wt & 5 & 0 & $0 \%$ & 0.33 & 0.33 & -- & -- & 0.17 \\
\hline SVOC & 2-Nitroaniline & $\mathrm{mg} / \mathrm{kg}$ dry-wt & 5 & 0 & $0 \%$ & 1.7 & 1.7 & -- & -- & 0.85 \\
\hline SVOC & 2-Nitrophenol & $\mathrm{mg} / \mathrm{kg}$ dry-wt & 5 & 0 & $0 \%$ & 0.33 & 0.33 & -- & -- & 0.17 \\
\hline SVOC & 3-Nitroaniline & $\mathrm{mg} / \mathrm{kg}$ dry-wt & 5 & 0 & $0 \%$ & 1.7 & 1.7 & -- & -- & 0.85 \\
\hline SVOC & 4,6-Dinitro-2-methylphenol & $\mathrm{mg} / \mathrm{kg}$ dry-wt & 5 & 0 & $0 \%$ & 1.7 & 1.7 & -- & -- & 0.85 \\
\hline SVOC & 4-Chloro-3-methylphenol & $\mathrm{mg} / \mathrm{kg}$ dry-wt & 5 & 0 & $0 \%$ & 0.33 & 0.33 & -- & -- & 0.17 \\
\hline sVOC & 4-Chloroaniline & $\mathrm{mg} / \mathrm{kg}$ dry-wt & 5 & 0 & $0 \%$ & 0.33 & 0.33 & -- & -- & 0.17 \\
\hline SVOC & 4-Nitrophenol & $\mathrm{mg} / \mathrm{kg}$ dry-wt & 5 & 0 & $0 \%$ & 1.7 & 1.7 & -- & -- & 0.85 \\
\hline SVOC & Acenaphthene & $\mathrm{mg} / \mathrm{kg}$ dry-wt & 5 & 0 & $0 \%$ & 0.33 & 0.33 & -- & -- & 0.17 \\
\hline SVOC & Aniline & $\mathrm{mg} / \mathrm{kg}$ dry-wt & 5 & 0 & $0 \%$ & 0.83 & 0.83 & -- & -- & 0.42 \\
\hline SVOC & Anthracene & $\mathrm{mg} / \mathrm{kg}$ dry-wt & 5 & 0 & $0 \%$ & 0.33 & 0.33 & -- & -- & 0.17 \\
\hline SVOC & Azobenzene & $\mathrm{mg} / \mathrm{kg}$ dry-wt & 5 & 0 & $0 \%$ & 0.33 & 0.33 & -- & -- & 0.17 \\
\hline SVOC & Benzo(a)pyrene & $\mathrm{mg} / \mathrm{kg}$ dry-wt & 5 & 0 & $0 \%$ & 0.33 & 0.33 & -- & -- & 0.17 \\
\hline SVOC & Benzo(b)fluoranthene & $\mathrm{mg} / \mathrm{kg}$ dry-wt & 5 & 0 & $0 \%$ & 0.33 & 0.33 & -- & -- & 0.17 \\
\hline sVOC & Benzoic acid & $\mathrm{mg} / \mathrm{kg}$ dry-wt & 5 & 0 & $0 \%$ & 1.7 & 1.7 & -- & -- & 0.85 \\
\hline SVOC & Benzyl alcohol & $\mathrm{mg} / \mathrm{kg}$ dry-wt & 5 & 0 & $0 \%$ & 0.33 & 0.33 & -- & -- & 0.17 \\
\hline sVOC & Bis(2-chloro-1-methylethyl)ether & $\mathrm{mg} / \mathrm{kg}$ dry-wt & 5 & 0 & $0 \%$ & 0.33 & 0.33 & -- & -- & 0.17 \\
\hline SVOC & Bis(2-Chloroethoxy)methane & $\mathrm{mg} / \mathrm{kg}$ dry-wt & 5 & 0 & $0 \%$ & 0.33 & 0.33 & -- & -- & 0.17 \\
\hline SVOC & Butylbenzylphthalate & $\mathrm{mg} / \mathrm{kg}$ dry-wt & 5 & 0 & $0 \%$ & 0.33 & 0.33 & -- & -- & 0.17 \\
\hline SVOC & Carbazole & $\mathrm{mg} / \mathrm{kg}$ dry-wt & 5 & 0 & $0 \%$ & 0.33 & 0.33 & -- & -- & 0.17 \\
\hline SVOC & Chrysene & $\mathrm{mg} / \mathrm{kg}$ dry-wt & 5 & 0 & $0 \%$ & 0.33 & 0.33 & -- & -- & 0.17 \\
\hline SVOC & Dibenzofuran & $\mathrm{mg} / \mathrm{kg}$ dry-wt & 5 & 0 & $0 \%$ & 0.33 & 0.33 & -- & -- & 0.17 \\
\hline SVOC & Diethylphthalate & $\mathrm{mg} / \mathrm{kg}$ dry-wt & 5 & 0 & $0 \%$ & 0.33 & 0.33 & -- & -- & 0.17 \\
\hline SVOC & Fluoranthene & $\mathrm{mg} / \mathrm{kg}$ dry-wt & 5 & 0 & $0 \%$ & 0.33 & 0.33 & -- & -- & 0.17 \\
\hline SVOC & Fluorene & $\mathrm{mg} / \mathrm{kg}$ dry-wt & 5 & 0 & $0 \%$ & 0.33 & 0.33 & -- & -- & 0.17 \\
\hline
\end{tabular}


Table D-4

Summary Statistics for Sediment Collected from Segment 4 of the Columbia River Including All Categories of Data (units reported "dry-weight")

\begin{tabular}{|c|c|c|c|c|c|c|c|c|c|c|}
\hline $\begin{array}{l}\text { Constituent } \\
\text { Class }\end{array}$ & Constituent Name & Units & $\begin{array}{l}\text { Number } \\
\text { Analyzed }\end{array}$ & $\begin{array}{l}\text { Number } \\
\text { Detected }\end{array}$ & $\begin{array}{l}\text { Frequency of } \\
\text { Detection }\end{array}$ & $\begin{array}{l}\text { Minimum } \\
\text { Nondetect }\end{array}$ & $\begin{array}{l}\text { Maximum } \\
\text { Nondetect }\end{array}$ & $\begin{array}{l}\text { Minimum } \\
\text { Detect }\end{array}$ & $\begin{array}{c}\text { Maximum } \\
\text { Detect }\end{array}$ & $\begin{array}{c}\text { Average } \\
\text { Concentration }\end{array}$ \\
\hline$\overline{\text { SVOC }}$ & Hexachlorobenzene & $\mathrm{mg} / \mathrm{kg}$ dry-wt & 5 & $\overline{0}$ & $0 \%$ & 0.33 & 0.33 & -- & -- & 0.17 \\
\hline SVOC & Hexachlorobutadiene & $\mathrm{mg} / \mathrm{kg}$ dry-wt & 5 & 0 & $0 \%$ & 0.33 & 0.33 & -- & -- & 0.17 \\
\hline SVOC & Hexachlorocyclopentadiene & $\mathrm{mg} / \mathrm{kg}$ dry-wt & 5 & 0 & $0 \%$ & 0.33 & 0.33 & -- & -- & 0.17 \\
\hline sVOC & Hexachloroethane & $\mathrm{mg} / \mathrm{kg}$ dry-wt & 5 & 0 & $0 \%$ & 0.33 & 0.33 & -- & -- & 0.17 \\
\hline sVOC & Indeno(1,2,3-cd)pyrene & $\mathrm{mg} / \mathrm{kg}$ dry-wt & 5 & 0 & $0 \%$ & 0.33 & 0.33 & -- & -- & 0.17 \\
\hline SVOC & Isophorone & $\mathrm{mg} / \mathrm{kg}$ dry-wt & 5 & 0 & $0 \%$ & 0.33 & 0.33 & -- & -- & 0.17 \\
\hline SVOC & Naphthalene & $\mathrm{mg} / \mathrm{kg}$ dry-wt & 5 & 0 & $0 \%$ & 0.33 & 0.33 & -- & -- & 0.17 \\
\hline sVOC & Nitrobenzene & $\mathrm{mg} / \mathrm{kg}$ dry-wt & 5 & 0 & $0 \%$ & 0.33 & 0.33 & -- & -- & 0.17 \\
\hline sVOC & N-Nitrosodiphenylamine & $\mathrm{mg} / \mathrm{kg}$ dry-wt & 5 & 0 & $0 \%$ & 0.33 & 0.33 & -- & -- & 0.17 \\
\hline SVOC & Pentachlorophenol & $\mathrm{mg} / \mathrm{kg}$ dry-wt & 5 & 0 & $0 \%$ & 1.7 & 1.7 & -- & -- & 0.85 \\
\hline SVOC & Phenanthrene & $\mathrm{mg} / \mathrm{kg}$ dry-wt & 5 & 0 & $0 \%$ & 0.33 & 0.33 & -- & -- & 0.17 \\
\hline sVOC & Phenol & $\mathrm{mg} / \mathrm{kg}$ dry-wt & 5 & 0 & $0 \%$ & 0.33 & 0.33 & -- & -- & 0.17 \\
\hline SVOC & Pyrene & $\mathrm{mg} / \mathrm{kg}$ dry-wt & 5 & 0 & $0 \%$ & 0.33 & 0.33 & -- & -- & 0.17 \\
\hline SVOC & Pyridine & $\mathrm{mg} / \mathrm{kg}$ dry-wt & 5 & 0 & $0 \%$ & 0.33 & 0.33 & -- & -- & 0.2 \\
\hline
\end{tabular}

PEST - Pesticide

PCB - Polychlorinated biphenyl

SVOC 
Table D-5

and Human Health Benchmark Values (units reported "as received)

\begin{tabular}{|c|c|c|c|c|c|c|c|c|c|c|c|c|c|c|c|c|c|c|}
\hline $\begin{array}{l}\text { Constituer } \\
\text { Class } \\
\text { META }\end{array}$ & Constituent Name & Units & $\begin{array}{l}\text { Number } \\
\text { Analyzzed } \\
\end{array}$ & $\begin{array}{l}\text { Number } \\
\text { Detected }\end{array}$ & $\begin{array}{c}\text { Frequency of } \\
\text { Detection }\end{array}$ & 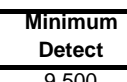 & $\begin{array}{c}\text { Maximum } \\
\text { Detect }\end{array}$ & $\begin{array}{c}\text { Average } \\
\text { Concentration } \\
\end{array}$ & $\begin{array}{c}\begin{array}{c}\text { Background } \\
\text { Value }\end{array} \\
\text { 111000 }\end{array}$ & $\begin{array}{c}\text { Is Max }> \\
\text { Background? } \\
\end{array}$ & $\begin{array}{c}\text { Ecological Benchmark } \\
\text { Value }\end{array}$ & $\begin{array}{l}\begin{array}{l}\text { Is Max }>\text { Eco } \\
\text { Benchmark? }\end{array} \\
\end{array}$ & $\begin{array}{c}\text { Factor of } \\
\text { Exceedance }\end{array}$ & $\begin{array}{l}\text { Number of } \\
\text { Exceedances } \\
\end{array}$ & $\begin{array}{c}\text { Puman Heathh } \\
\text { Benchmark Value } \\
\text { 76112 }\end{array}$ & $\begin{array}{l}\text { Is Max >AH } \\
\text { Benchmark? }\end{array}$ & $\begin{array}{l}\begin{array}{c}\text { Factor of } \\
\text { Exceedance }\end{array} \\
\end{array}$ & $\begin{array}{l}\text { Number of } \\
\text { Exceedances } \\
\end{array}$ \\
\hline METAL & $\begin{array}{l}\text { Aluminum } \\
\text { Antimony }\end{array}$ & 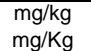 & $\begin{array}{l}4 \\
19\end{array}$ & $\begin{array}{c}4 \\
10\end{array}$ & $\begin{array}{l}100 \% \% \\
53 \%\end{array}$ & $\begin{array}{l}9,500 \\
0.34\end{array}$ & $\begin{array}{l}11,900 \\
65\end{array}$ & $\begin{array}{l}10,668 \\
21\end{array}$ & $\begin{array}{l}11,800 \\
5.0\end{array}$ & $\begin{array}{l}\begin{array}{l}\text { Yes } \\
\text { Yes }\end{array} \\
\text { Yons }\end{array}$ & 2.0 & & & 2 & $\begin{array}{l}76,142 \\
31 \\
31\end{array}$ & $\begin{array}{l}\text { No } \\
\text { Yes }\end{array}$ & 0.16 & 2 \\
\hline METAL & $\begin{array}{l}\text { Alumpenic } \\
\text { Arsen }\end{array}$ & $\begin{array}{l}m g / k g \\
m g / g\end{array}$ & 24 & $\begin{array}{l}10 \\
13\end{array}$ & $54 \%$ & $\begin{array}{l}0.34 \\
2.0\end{array}$ & $\begin{array}{l}65 \\
15 \\
15\end{array}$ & $\begin{array}{l}21 \\
8.0\end{array}$ & $\begin{array}{l}5.0 \\
6.5\end{array}$ & $\begin{array}{l}\text { Yes } \\
\text { Yes }\end{array}$ & $\begin{array}{l}2.0 \\
6.0\end{array}$ & $\begin{array}{l}\text { Yes } \\
\text { Yes }\end{array}$ & $\begin{array}{l}33 \\
2.5 \\
\end{array}$ & ${ }^{2}$ & $\begin{array}{l}31 \\
0.39\end{array}$ & $\begin{array}{l}\text { Yes } \\
\text { Yes }\end{array}$ & $\begin{array}{l}2.1 \\
38\end{array}$ & $\begin{array}{l}2 \\
12\end{array}$ \\
\hline $\begin{array}{l}\text { METAL } \\
\text { METAL }\end{array}$ & $\begin{array}{l}\text { Barium } \\
\text { Bensllium }\end{array}$ & $\begin{array}{l}\mathrm{mg} / \mathrm{kg} \\
\mathrm{mg} / \mathrm{kg}\end{array}$ & $\begin{array}{l}4 \\
8\end{array}$ & $\begin{array}{l}4 \\
8\end{array}$ & $\begin{array}{l}100 \% \\
100 \%\end{array}$ & $\begin{array}{l}118 \\
0,37\end{array}$ & $\begin{array}{l}199 \\
20\end{array}$ & $\begin{array}{l}156 \\
11\end{array}$ & $\begin{array}{l}132 \\
15\end{array}$ & $\begin{array}{l}\text { Yes } \\
\text { Yes }\end{array}$ & - & $\because$ & - & - & 5,375 & No & 0.037 & 0 \\
\hline METAL & $\begin{array}{l}\text { caymum } \\
\text { Cadnium }\end{array}$ & $\begin{array}{l}m g / k g \\
m g / k g\end{array}$ & 24 & 18 & $75 \%$ & 0.060 & $\begin{array}{l}2.0 \\
2.2\end{array}$ & $\begin{array}{l}0.64 \\
0.64\end{array}$ & $\begin{array}{l}0.81 \\
0.81\end{array}$ & Yes & 0.60 & Yes & 3.7 & 8 & & $\begin{array}{l}\text { No } \\
\text { No }\end{array}$ & $\begin{array}{l}0.013 \\
0.059\end{array}$ & $\begin{array}{l}0 \\
0\end{array}$ \\
\hline $\begin{array}{l}\text { METAL } \\
\text { METAL }\end{array}$ & $\begin{array}{l}\text { Caclium } \\
\text { Chromium }\end{array}$ & $\begin{array}{l}\mathrm{mg} / \mathrm{g} g \\
\mathrm{mg} / \mathrm{kg}\end{array}$ & $\begin{array}{c}4 \\
13\end{array}$ & $\begin{array}{c}4 \\
13\end{array}$ & $\begin{array}{l}100 \% \\
100 \%\end{array}$ & $\begin{array}{l}5,510 \\
9.2\end{array}$ & $\begin{array}{l}7,150 \\
69\end{array}$ & $\begin{array}{l}6,180 \\
29\end{array}$ & $\begin{array}{l}17,200 \\
19\end{array}$ & $\begin{array}{l}\text { No } \\
\text { Yes }\end{array}$ & $\ddot{37}$ & Yes & $\ddot{19}$ & $-\overline{3}$ & $\ddot{30}$ & Yes & 23 & \\
\hline $\begin{array}{l}\text { METAL } \\
\text { METAL }\end{array}$ & cobalt & mglkg & 4 & $\begin{array}{c}13 \\
4\end{array}$ & $\begin{array}{l}100 \% \\
\end{array}$ & $\begin{array}{l}9.9 \\
9.9 \\
9.9\end{array}$ & $\begin{array}{l}69 \\
15\end{array}$ & ${ }_{12}^{29}$ & $\begin{array}{l}19 \\
16\end{array}$ & $\begin{array}{l}\text { Yes } \\
\text { No }\end{array}$ & - & Yes & 1.9 & 3 & $\begin{array}{l}30 \\
903\end{array}$ & $\begin{array}{l}\text { Yes } \\
\text { No }\end{array}$ & $\begin{array}{l}2.3 \\
0.016\end{array}$ & $\begin{array}{l}3 \\
0\end{array}$ \\
\hline $\begin{array}{l}\text { MEIAL } \\
\text { METAL }\end{array}$ & $\begin{array}{c}\text { Copper } \\
\text { tron }\end{array}$ & $\mathrm{mg} / \mathrm{kg}$ & 24 & 24 & $100 \%$ & 7.78 & 46 & 19 & 22 & Yes & 32 & Yes & 1.4 & 4 & 2,960 & No & 0.015 & 0 \\
\hline $\begin{array}{l}\text { METAL } \\
\text { METAL }\end{array}$ & $\begin{array}{l}\text { Tron } \\
\text { Lead }\end{array}$ & $\begin{array}{l}\mathrm{mgkg} \\
\mathrm{mg} / \mathrm{kg}\end{array}$ & $\begin{array}{l}4 \\
27\end{array}$ & $\begin{array}{l}4 \\
20\end{array}$ & $\begin{array}{l}100 \% \% \\
74 \%\end{array}$ & $\begin{array}{c}20,100 \\
7.6\end{array}$ & $\begin{array}{c}26,100 \\
250\end{array}$ & $\begin{array}{l}23,250 \\
20\end{array}$ & $\begin{array}{lll}32,600 & \\
10 & \end{array}$ & $\begin{array}{l}\text { No } \\
\text { Yes }\end{array}$ & $\begin{array}{l}2,000 \\
35\end{array}$ & $\begin{array}{l}\text { Yes } \\
\text { Yes }\end{array}$ & $\begin{array}{l}1.3 \\
7.1\end{array}$ & ${ }_{1}^{4}$ & $\begin{array}{l}23,463 \\
250\end{array}$ & $\begin{array}{l}\text { Yes } \\
\text { No }\end{array}$ & $\begin{array}{l}1.1 \\
1.0\end{array}$ & $\begin{array}{l}3 \\
1\end{array}$ \\
\hline $\begin{array}{l}\text { METAL } \\
\text { METAL }\end{array}$ & $\begin{array}{l}\text { Magnesium } \\
\text { Manganese }\end{array}$ & $\begin{array}{l}\mathrm{m} \mathrm{m} / \mathrm{g} / \mathrm{g} \\
\mathrm{mg} / \mathrm{kg}\end{array}$ & $\begin{array}{l}4 \\
4\end{array}$ & $\begin{array}{l}4 \\
4\end{array}$ & $\begin{array}{l}100 \% \% \\
100 \%\end{array}$ & $\begin{array}{l}5,100 \\
399\end{array}$ & $\begin{array}{l}6,980 \\
716\end{array}$ & $\begin{array}{l}5,803 \\
531\end{array}$ & $\begin{array}{l}7,060 \\
512\end{array}$ & $\begin{array}{l}\text { No } \\
\text { Yes }\end{array}$ & 460 & Yes & $\ddot{1.6}$ & 3 & $1 . \overline{7}$ & No & 0.41 & 0 \\
\hline $\begin{array}{l}\text { METAL } \\
\text { METAL }\end{array}$ & Mercury & $\mathrm{mg} / \mathrm{kg}$ & 28 & 13 & $46 \%$ & 1.177E-04 & 0.51 & 0.081 & $\begin{array}{l}0.33 \\
0.33\end{array}$ & Yes & 0.18 & $\begin{array}{l}\text { Yes } \\
\text { Yes }\end{array}$ & $\begin{array}{l}1.0 \\
2.8\end{array}$ & 3 & $\begin{array}{l}1,162 \\
23\end{array}$ & $\begin{array}{l}\text { No } \\
\text { No }\end{array}$ & $\begin{array}{l}0.41 \\
0.022\end{array}$ & $\begin{array}{l}0 \\
0\end{array}$ \\
\hline $\begin{array}{l}\text { MEIAL } \\
\text { METAL }\end{array}$ & $\begin{array}{l}\text { Nickel } \\
\text { Potassium }\end{array}$ & $\begin{array}{l}\mathrm{m} \mathrm{m} / \mathrm{kg} g \\
\mathrm{mg} / \mathrm{kg}\end{array}$ & $\begin{array}{l}24 \\
4\end{array}$ & $\begin{array}{l}23 \\
4\end{array}$ & $\begin{array}{l}96 \% \% \\
100 \%\end{array}$ & $\begin{array}{l}4.4 \\
845\end{array}$ & $\begin{array}{c}33 \\
1.550 \\
\end{array}$ & 13 & 19 & Yes & 18 & Yes & 1.8 & 6 & 1,564 & № & 0.021 & 0 \\
\hline $\begin{array}{l}\text { METAL } \\
\text { META }\end{array}$ & 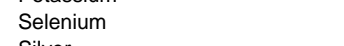 & $\mathrm{mg} / \mathrm{kg}$ & 8 & 1 & $13 \%$ & $\begin{array}{l}0.66 \\
0.66\end{array}$ & $\begin{array}{l}1,656 \\
0.66\end{array}$ & $\begin{array}{l}1,068 \\
0.31\end{array}$ & $\begin{array}{l}2,150 \\
0.78\end{array}$ & $\begin{array}{l}\text { No } \\
\text { No }\end{array}$ & -- & -- & $\because$ & 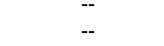 & 391 & No & 0.0017 & $\ddot{0}$ \\
\hline $\begin{array}{l}\text { METAL } \\
\text { METAL }\end{array}$ & $\begin{array}{l}\text { Silver } \\
\text { sodium }\end{array}$ & $\begin{array}{l}\mathrm{mg} / \mathrm{Kg} \\
\mathrm{mg} / \mathrm{kg}\end{array}$ & $\begin{array}{l}24 \\
4\end{array}$ & $\begin{array}{l}14 \\
4\end{array}$ & $\begin{array}{l}58 \% \\
100 \%\end{array}$ & $\begin{array}{l}0.060 \\
203\end{array}$ & $\begin{array}{l}2.2 \\
366 \\
365\end{array}$ & $\begin{array}{l}0.59 \\
312\end{array}$ & $\begin{array}{l}0.73 \\
690\end{array}$ & $\begin{array}{l}\text { Yes } \\
\text { No }\end{array}$ & 4.5 & No & 0.49 & 0 & 391 & № & 0.0056 & 0 \\
\hline $\begin{array}{l}\text { METAL } \\
\text { META }\end{array}$ & Thallium & mg/kg & 8 & 8 & $\begin{array}{l}100 \% \\
100 \%\end{array}$ & 0.19 & 0.69 & 0.42 & - & - & $\therefore$ & $\therefore$ & - & $\cdots$ & 5.2 & No & 0.13 & 0 \\
\hline $\begin{array}{l}\text { MEIAL } \\
\text { METAL }\end{array}$ & Zinc & $\begin{array}{l}\text { Mgkg } \\
\text { mglkg }\end{array}$ & & $\begin{array}{l}4 \\
24\end{array}$ & $\begin{array}{l}100 \% \% \\
100 \%\end{array}$ & 38 & $\begin{array}{l}69 \\
293\end{array}$ & $\begin{array}{l}51 \\
112\end{array}$ & 68 & Yes & $\stackrel{20}{120}$ & Yes & 2.4 & 4 & $\begin{array}{l}4,6,24 \\
23,463 \\
2\end{array}$ & $\begin{array}{l}\text { No } \\
\text { No }\end{array}$ & $\begin{array}{l}0.0015 \\
0.012\end{array}$ & $\begin{array}{l}0 \\
0\end{array}$ \\
\hline $\begin{array}{l}\text { PCB } \\
\text { PEST }\end{array}$ & $\begin{array}{l}\text { Aroclor-1254 } \\
\text { Difhlorofiphenytrichloroethane }\end{array}$ & $\begin{array}{c}\mathrm{mg} / \mathrm{kg} \\
\mathrm{m} / \mathrm{kg}\end{array}$ & 19 & 1 & $\begin{array}{l}5 \% \\
66 \%\end{array}$ & $\begin{array}{l}0.019 \\
0.0002\end{array}$ & $\begin{array}{l}0.019 \\
0.0200\end{array}$ & $\begin{aligned} 0.0032 \\
700504\end{aligned}$ & - & - & $\begin{array}{l}0.0070 \\
0.0042\end{array}$ & Yes & 2.7 & 1 & 0.22 & No & 0.087 & 0 \\
\hline $\begin{array}{l}\text { PEST } \\
\text { RAD D }\end{array}$ & 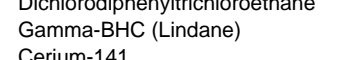 & $\begin{array}{l}\text { ligh/ga } \\
\text { molkg }\end{array}$ & $\begin{array}{l}16 \\
16 \\
4\end{array}$ & 1 & $6 \%$ & $\begin{array}{l}2.00 E-04 \\
3.375-043\end{array}$ & $\begin{array}{l}0.020 \\
2.00-04\end{array}$ & 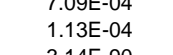 & $\because$ & $\because$ & $\begin{array}{l}9.000 \mathrm{E}-04 \\
9.04\end{array}$ & $\begin{array}{l}\text { No } \\
\text { No }\end{array}$ & $\begin{array}{l}0.088 \\
0.22\end{array}$ & $\begin{array}{l}0 \\
0\end{array}$ & $\begin{array}{l}1.7 \\
0.44\end{array}$ & $\begin{array}{l}\text { No } \\
\text { No }\end{array}$ & $\begin{array}{l}0.0012 \\
4.57 \mathrm{E}-04\end{array}$ & $\begin{array}{l}0 \\
0\end{array}$ \\
\hline $\begin{array}{lll} & -1\end{array}$ & $\begin{array}{l}\text { Cerlum-141 } \\
\text { Cesium-137 }\end{array}$ & $\begin{array}{l}\text { pelig } \\
\text { pCilg }\end{array}$ & $\begin{array}{r}4 \\
36 \\
\end{array}$ & $\begin{array}{r}4 \\
36\end{array}$ & $\begin{array}{l}100 \% \% \\
100 \%\end{array}$ & 0.049 & $\begin{array}{l}1.260-89 \\
0.59\end{array}$ & 0.10 & 1.1 & No & $\widetilde{3,120}$ & No & 1.88E-04 & 0 & 6.2 & No & 0.095 & 0 \\
\hline $\begin{array}{l}\text { RAD D } \\
\text { RAD }\end{array}$ & $\begin{array}{l}\text { Cobalt-60 } \\
\text { Plutonium-238 }\end{array}$ & $\begin{array}{l}\text { pCig } \\
\text { cilig }\end{array}$ & $\begin{array}{l}23 \\
5\end{array}$ & $\begin{array}{l}23 \\
5\end{array}$ & $\begin{array}{l}100 \% \\
100 \%\end{array}$ & $\begin{array}{l}8.78 E-04 \\
188 E-04\end{array}$ & $\begin{array}{l}0.021 \\
0.0011\end{array}$ & $\begin{array}{l}0.0061 \\
4995-04\end{array}$ & $\begin{array}{l}0.0084 \\
0.0038\end{array}$ & $\begin{array}{l}\text { Yes } \\
\text { No }\end{array}$ & 1,460 & No & $1.42 E-05$ & 0 & 1.4 & No & $\begin{array}{c}0.015 \\
2815.05\end{array}$ & 0 \\
\hline RADD & Plutonium-239/240 & pcilg & 6 & 6 & $\begin{array}{l}100 \% \\
100 \%\end{array}$ & $\begin{array}{l}1.00004 \\
0.045\end{array}$ & 0.018 & 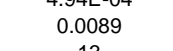 & 0.025 & No & 5,860 & No & $3.07 E-06$ & 0 & 34 & No & $5.31 E-04$ & 0 \\
\hline $\begin{array}{ll}-1 & -1\end{array}$ & Radium-226 & $\begin{array}{l}\text { pelg } \\
\text { pcilgh }\end{array}$ & 25 & 25 & $\begin{array}{l}100 \% \\
100 \%\end{array}$ & $\begin{array}{l}1.52 \\
0.50 \\
0.50\end{array}$ & 1.9 & $\begin{array}{l}1.81 \\
0.81 \\
.0 .12\end{array}$ & $\begin{array}{l}17 \\
0.82\end{array}$ & $\begin{array}{l}\text { res } \\
\text { Yes }\end{array}$ & 101 & No & 0.019 & $\ddot{0}$ & $\because$ & $\ddot{-}$ & $\because$ &.- \\
\hline $\begin{array}{l}\text { RADDD } \\
\text { RADDD }\end{array}$ & $\begin{array}{l}\text { Ruthenium-106 } \\
\text { Strontium-90 }\end{array}$ & $\begin{array}{l}\text { pcilg } \\
\text { pcilg }\end{array}$ & $\begin{array}{c}23 \\
1\end{array}$ & ${ }_{1}^{23}$ & $\begin{array}{l}100 \% \\
100 \%\end{array}$ & $\begin{array}{l}2.276-13 \\
0.028\end{array}$ & $\begin{array}{l}1.03 E-08 \\
0.028\end{array}$ & $\begin{array}{l}4.59 E-10 \\
0.028\end{array}$ & 0.18 & No & $\ldots$ & No & $488 \mathrm{E}-05$ & 0 & 45. & No & 0.0063 & 0 \\
\hline RADDD & Thorium-232 & pcilg & 16 & 16 & $\begin{array}{l}100 \% \% \\
100 \%\end{array}$ & 0.00 & 1.00 & 0.78 & .1.3 & 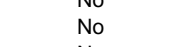 & 1,300 & No & 7.69e-04 -04 & o & $\begin{array}{l}4.5 \\
1.3 \\
-\end{array}$ & 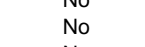 & $\begin{array}{l}0.005 \\
0.77\end{array}$ & 0 \\
\hline $\begin{array}{l}\text { RAD } D \\
\text { RAD }\end{array}$ & $\begin{array}{l}\text { UUarainum-234 } \\
\text { Uraniumm-235 }\end{array}$ & $\begin{array}{l}\text { pcig } \\
\text { pcilg }\end{array}$ & $\begin{array}{l}4 \\
4\end{array}$ & $\begin{array}{l}4 \\
4\end{array}$ & $\begin{array}{l}100 \% \\
100 \%\end{array}$ & $\begin{array}{l}0.67 \\
0.010\end{array}$ & $\begin{array}{l}0.97 \\
0.034\end{array}$ & $\begin{array}{l}0.82 \\
0.022\end{array}$ & 0.11 & $\begin{array}{l}\begin{array}{l}\text { No } \\
\text { No }\end{array} \\
\text { N }\end{array}$ & $\begin{array}{l}5,270 \\
3,730\end{array}$ & $\begin{array}{l}\text { No } \\
\text { No }\end{array}$ & $\begin{array}{l}1.85 E-04 \\
909-06\end{array}$ & $\begin{array}{c}0 \\
0 \\
0\end{array}$ & $\begin{array}{l}1.1 \\
0.84\end{array}$ & $\begin{array}{ll}\text { No } \\
\text { No }\end{array}$ & $\begin{array}{l}0.89 \\
0.040\end{array}$ & $\begin{array}{l}0 \\
0\end{array}$ \\
\hline RADDD & Uranium-238 & $\begin{array}{c}\text { pcilg } \\
\text { ccig }\end{array}$ & ${ }_{4}^{4}$ & ${ }_{4}^{4}$ & $\begin{array}{l}100 \% \\
100 \%\end{array}$ & 0.52 & $\begin{array}{l}0.79 \\
0.79\end{array}$ & $\begin{array}{l}0.022 \\
0.64\end{array}$ & $\begin{array}{l}0.11 \\
3.2\end{array}$ & $\begin{array}{l}\text { No } \\
\text { No }\end{array}$ & $\begin{array}{l}3,430 \\
2,490\end{array}$ & $\begin{array}{l}\text { No } \\
\text { No }\end{array}$ & $\begin{array}{ll}9 & 9.056-06 \\
3.16 E_{-04} & \end{array}$ & 0 & ${ }_{1.1}^{0.04}$ & No & 0.71 & 0 \\
\hline RADD D & zinc-65 & pcilg & 37 & 37 & $100 \%$ & $6.80 E-19$ & $1.10 \mathrm{E}-12$ & $8.25 E-14$ & - & -- & $-\infty$ & $\ldots$ & - & & -- & 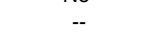 & -- & -- \\
\hline $\begin{array}{l}\text { RADDD } \\
\text { SVOC }\end{array}$ & $\begin{array}{l}\text { lirconouiu//ivobium-95 } \\
\text { 2-Methylnaphthalene }\end{array}$ & $\begin{array}{c}\text { pcilg } \\
\mathrm{mg} / \mathrm{kg}\end{array}$ & $\begin{array}{l}34 \\
20\end{array}$ & $\begin{array}{l}34 \\
14\end{array}$ & $\begin{array}{l}100 \% \\
70 \%\end{array}$ & $\begin{array}{l}2.77-1-1-126 \\
0.0010\end{array}$ & $\begin{array}{l}5.25 E-86 \\
0.014\end{array}$ & $\begin{array}{l}1.544-87 \\
0.0040\end{array}$ & $\because$ & $\because$ & 0.020 & No & 0.69 & ö & $\because$ & $\because$ & $\because$ & - \\
\hline $\begin{array}{l}\text { svoc } \\
\text { svoc }\end{array}$ & 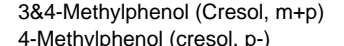 & $\begin{array}{l}\mathrm{mg} / \mathrm{kg} \\
\mathrm{mg} / \mathrm{kg}\end{array}$ & ${ }_{5}^{11}$ & ${ }_{1}^{2}$ & $\begin{array}{l}{ }_{20 \%} \\
20 \%\end{array}$ & $\begin{array}{c}0.0058 \\
0.10\end{array}$ & $\begin{array}{l}0.013 \\
0.10\end{array}$ & 0.040 & $\because$ & $\because$ & $\ldots$ & $\ldots$ & $\ldots$ & $\ddot{-}$ & 306 & No & $3.27=-04$ & 0 \\
\hline svoc & 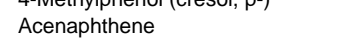 & mglkg & 20 & 7 & $35 \%$ & $\begin{array}{l}0.1100 \\
9.204\end{array}$ & 0.018 & 0.0019 & -. & -. & 0.0067 & Yes & 2.7 & 1 & ${ }_{3,682}$ & No & $\begin{array}{l}3.271--04 \\
4.89 E-06\end{array}$ & $\begin{array}{l}0 \\
0\end{array}$ \\
\hline $\begin{array}{l}\text { svoc } \\
\text { svoc }\end{array}$ & Acenaphthylene & mglkg & 20 & 5 & $25 \%$ & 2.00E- -04 & 0.0060 & 0.0012 & $\therefore$ & -- & 0.0059 & Yes & 1.0 & 1 & 4,800 & No & $1.25 E-06$ & 0 \\
\hline $\begin{array}{l}\text { svoc } \\
\text { svoc }\end{array}$ & $\begin{array}{l}\text { Anthracene } \\
\text { Benzo(a)anthracene }\end{array}$ & $\begin{array}{l}\text { migkg } \\
\text { makga }\end{array}$ & 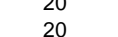 & $\begin{array}{l}5 \\
6\end{array}$ & $\begin{array}{l}25 \% \% \\
{ }_{30}^{20 \%}\end{array}$ & $\begin{array}{l}9.0 .06-04 \\
0.0010\end{array}$ & $\begin{array}{l}0.013 \\
0.0068\end{array}$ & $\begin{array}{l}0.00077 \\
0.0014\end{array}$ & $\because$ & $\because$ & $\begin{array}{l}0.057 \\
0.032\end{array}$ & $\begin{array}{l}\text { No } \\
\text { No }\end{array}$ & $\begin{array}{l}0.23 \\
0.221\end{array}$ & : & $\begin{array}{l}2,1,996 \\
0.62\end{array}$ & $\begin{array}{l}\text { Noo } \\
\text { No }\end{array}$ & $\begin{array}{l}5.944-07 \\
0.01\end{array}$ & 0 \\
\hline $\begin{array}{l}\text { svoc } \\
\text { svoc }\end{array}$ & Benzo(a)pyrene & mglkg & ${ }_{20}$ & 5 & $25 \%$ & 0.0020 & 0.0073 & 0.0017 & - & $\therefore$ & 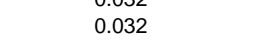 & No & 0.23 & 0 & 0.062 & No & $\begin{array}{ll}0.11 \\
0.12\end{array}$ & o \\
\hline $\begin{array}{l}\text { svoc } \\
\text { svoc }\end{array}$ & 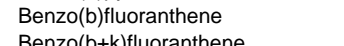 & $\mathrm{mg} / \mathrm{kg}$ & 16 & 5 & $31 \%$ & 0.0010 & 0.0040 & 0.0016 & - & - & 0.24 & No & 0.017 & 0 & 0.62 & No & 0.0064 & 0 \\
\hline $\begin{array}{l}\text { svoc } \\
\text { soma }\end{array}$ & 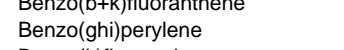 & $\begin{array}{l}\text { mglkg } \\
\text { mglkg }\end{array}$ & $\begin{array}{r}4 \\
20\end{array}$ & 6 & $\begin{array}{l}30 \% \\
30 \%\end{array}$ & $\begin{array}{l}0.0005 .-04 \\
5.005\end{array}$ & $\begin{array}{l}0.014 \\
0.0070\end{array}$ & $\begin{array}{l}0.0016 \\
0.0016\end{array}$ & $\because$ & $\because$ & 0.17 & No & 0.041 & 0 & 2,400 & No & $2.92-.06$ & 0 \\
\hline $\begin{array}{l}\text { oc } \\
\text { oc }\end{array}$ & $\begin{array}{l}\text { Benzo(k)ffluoranthene } \\
\text { Benzocicacid }\end{array}$ & $\begin{array}{l}\mathrm{mg} / \mathrm{kg} \\
\mathrm{mg} / \mathrm{kg}\end{array}$ & $\begin{array}{l}16 \\
15\end{array}$ & $\begin{array}{l}3 \\
3\end{array}$ & $\begin{array}{l}19 \% \\
20 \%\end{array}$ & & $\begin{array}{l}0.0040 \\
0.014\end{array}$ & & $\because$ & - & 0.027 & № & 0.15 & 0 & $\begin{array}{c}6.2 \\
160.000\end{array}$ & No & 1400.07 & 0 \\
\hline svoc & $\begin{array}{l}\text { Benziolcacid } \\
\text { Bis(2-thylhexyl) phthalate }\end{array}$ & $\begin{array}{l}\mathrm{mg/kg} \\
\mathrm{mg} / \mathrm{kg}\end{array}$ & $\begin{array}{l}15 \\
15\end{array}$ & $\begin{array}{l}3 \\
15\end{array}$ & $\begin{array}{l}20 \% \\
100 \%\end{array}$ & $\begin{array}{l}0.0087 \\
0.011\end{array}$ & 0.029 & 0.021 & $\because$ & $\because$ & $0 . \overline{64}$ & No & 0.045 & 0 & $\begin{array}{l}100,000 \\
35\end{array}$ & No & $\begin{array}{l}1.40 E-0 / \\
8.35-04\end{array}$ & $\begin{array}{l}0 \\
0\end{array}$ \\
\hline svoc & Butyl|benzylphthalate & mglkg & 15 & 7 & $47 \%$ & 0.0030 & 0.037 & 0.0058 & $x_{0}+2$ & -- & . & & & & 12,221 & No & 3.03E- -06 & 0 \\
\hline $\begin{array}{l}\text { soc } \\
\text { soce }\end{array}$ & $\begin{array}{l}\text { Chrysene } \\
\text { Dibentantantracene }\end{array}$ & 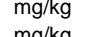 & 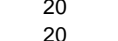 & 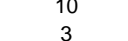 & $\begin{array}{l}50 \% \\
15 \%\end{array}$ & $\begin{array}{l}6.00 E-04 \\
600=04\end{array}-04$ & $\begin{array}{l}0.0096 \\
0.030\end{array}$ & $\begin{array}{c}0.0026 \\
736 E-04\end{array}$ & - & $\because$ & 0.057 & No & 0.17 & $\begin{array}{l}0 \\
0\end{array}$ & 62 & No & 1.55E-04 & 0 \\
\hline svoc & & 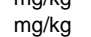 & 16 & ${ }_{3}$ & ${ }_{19 \%}$ & - 0.0010 & - 0.0056 & $\begin{array}{l}0.0014 \\
0.014\end{array}$ & $\therefore$ & $\therefore$ & 5.1 & No & 0.0011 & 0 & (145 & No & $\begin{array}{l}3.866-05 \\
3\end{array}$ & \\
\hline IOC & Diethy|phthalate & mglkg & 11 & 1 & $9 \%$ & 0.064 & 0.064 & 0.0079 & - & - & -- & $\ldots$ & 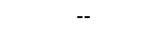 & - & 48,882 & No & $1.31 E-06$ & 0 \\
\hline oc & 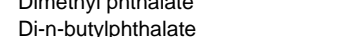 & malkg & $\begin{array}{l}11 \\
11\end{array}$ & $\begin{array}{l}1 \\
10 \\
\end{array}$ & 年10 & - & $0.6 \quad-6$ & 0.6 & $\because$ & $\therefore$ & 0.043 & Yes & $\ddot{15}$ & 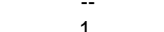 & $\begin{array}{l}8,0,000 \\
6.110\end{array}$ & No & 年.50 1.50 & 0 \\
\hline vVoc & 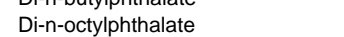 & 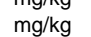 & ${ }_{11}^{1}$ & 3 & ${ }_{27 \%}^{27 \%}$ & 0.0027 & 0.0071 & 0.0020 & $\ldots$ & $\ldots$ & 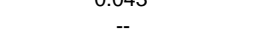 & $\because$ & 热 & $\ldots$ & 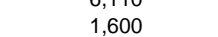 & No & $\begin{array}{l}1 \\
4.446-06 \\
4\end{array}$ & 0 \\
\hline svoc & Fluoranthene & mglkg & 20 & 13 & $65 \%$ & 0.0010 & 0.022 & 0.6 & - & - & 0.11 & No & 0.20 & 0 & 2,294 & No & 9.59E-06 & 0 \\
\hline $\begin{array}{l}\text { svoc } \\
\text { soc }\end{array}$ & 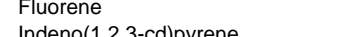 & 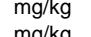 & 年0 & ${ }_{6}^{6}$ & $\begin{array}{l}30 \% \\
300\end{array}$ & $\begin{array}{l}7.006-04 \\
.00010\end{array}$ & $\begin{array}{l}0.010 \\
0.070\end{array}$ & 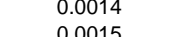 & - & - & $\begin{array}{l}0.077 \\
0.077\end{array}$ & No & $\begin{array}{l}0.13 \\
0.141\end{array}$ & 0 & $\begin{array}{l}2,747 \\
2027\end{array}$ & No & 3.66 3 & 0 \\
\hline oc & $\begin{array}{l}\text { Naphinalene } \\
\text { Noprenter }\end{array}$ & 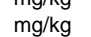 & 20 & $\begin{array}{c}9 \\
9\end{array}$ & $45 \%$ & $\begin{array}{l}8.00 E-04 \\
\text { 8. }\end{array}$ & 0 & 0.0024 & $\therefore$ & $\therefore$ & $\begin{array}{l}0.18 \\
0.18\end{array}$ & No & 0.049 & 0 & 56 & No & 1.54E-04 & 0 \\
\hline svoc & Pentachlorophenol & $\mathrm{mc}$ & 16 & 2 & 13\%\% & 0.011 & 0.0 & (2000 & $\cdots$ & - & & 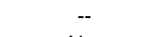 & 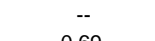 & 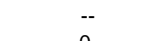 & 3.0 & No & $9.73 \mathrm{E}-03$ & 0 \\
\hline $\begin{array}{l}\text { svec } \\
\text { svoc }\end{array}$ & 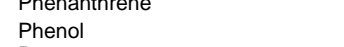 & 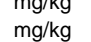 & 20 & $\begin{array}{c}13 \\
4\end{array}$ & $20 \%$ & $\begin{array}{l}9.006-04 \\
0.0054\end{array}$ & $\begin{array}{l}0.029 \\
0.020\end{array}$ & $\begin{array}{l}0.0045 \\
0.082\end{array}$ & $\because$ & - & 0.042 & № & 0.69 & 0 & 18,331 & No & $1.09 E-06$ & 0 \\
\hline & Pyrene & & & & $70 \%$ & 0.0010 & 0.027 & 0.0052 & & & 0.053 & No & 0.51 & 0 & 2,316 & No & $1.177-05$ & 0 \\
\hline
\end{tabular}


Comparison of Segment 4 Detected Sediment Concentrations to Ecological and Human Health Benchmark Values (units reported "dry weight")

\begin{tabular}{|c|c|c|c|c|c|c|c|c|c|c|c|c|c|c|c|c|c|c|}
\hline $\begin{array}{c}\text { Constituent } \\
\text { Class }\end{array}$ & Constituent Name & Units & $\begin{array}{l}\text { Number } \\
\text { Analyzed }\end{array}$ & $\begin{array}{l}\text { Number } \\
\text { Detected }\end{array}$ & $\begin{array}{l}\text { Frequency of } \\
\text { Detection }\end{array}$ & $\begin{array}{c}\text { Minimum } \\
\text { Detect }\end{array}$ & $\begin{array}{c}\text { Maximum } \\
\text { Detect }\end{array}$ & $\begin{array}{c}\text { Average } \\
\text { Concentration }\end{array}$ & $\begin{array}{l}\text { Background } \\
\text { Value }\end{array}$ & $\begin{array}{c}\text { Is Max > } \\
\text { Background? }\end{array}$ & $\begin{array}{c}\text { Ecological } \\
\text { Benchmark Value }\end{array}$ & $\begin{array}{l}\text { Is Max > Eco } \\
\text { Benchmark? }\end{array}$ & $\begin{array}{c}\text { Factor of } \\
\text { Exceedance }\end{array}$ & $\begin{array}{c}\text { Number of } \\
\text { Exceedances }\end{array}$ & $\begin{array}{l}\text { Human Health } \\
\text { Benchmark } \\
\text { Value }\end{array}$ & $\begin{array}{l}\text { Is Max > HH } \\
\text { Benchmark? }\end{array}$ & $\begin{array}{c}\text { Factor of } \\
\text { Exceedance }\end{array}$ & $\begin{array}{l}\text { Number of } \\
\text { Exceedances }\end{array}$ \\
\hline & Aluminum & $\mathrm{mg} / \mathrm{kg}$ dry-wt & 5 & 5 & $100 \%$ & 5,500 & & & 11,800 & Yes & & & & & 76,142 & No & 0.29 & 0 \\
\hline $\begin{array}{l}\text { METAL } \\
\text { METAL }\end{array}$ & $\begin{array}{l}\text { Antimony } \\
\text { Atsenic }\end{array}$ & 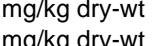 & $\begin{array}{l}5 \\
5\end{array}$ & 5 & $\begin{array}{l}100 \% \\
100 \%\end{array}$ & 2.6 & $\begin{array}{l}6.1 \\
0.5\end{array}$ & $\begin{array}{l}4.6 \\
4.6\end{array}$ & $\begin{array}{l}5.0 \\
6.5\end{array}$ & Yes & 2.0 & $\begin{array}{l}\text { Yes } \\
\text { Yocas }\end{array}$ & 3.1 & 5 & 31 & $\begin{array}{l}\text { No } \\
\text { Noos }\end{array}$ & 0.19 & ${ }_{5}^{0}$ \\
\hline $\begin{array}{l}\text { MEATL } \\
\text { METAL }\end{array}$ & $\begin{array}{l}\text { Assenic } \\
\text { Barium }\end{array}$ & $\begin{array}{l}m g / k g a r y-w t \\
m / k g d r y-w t\end{array}$ & $\begin{array}{l}5 \\
5\end{array}$ & $\begin{array}{l}5 \\
5\end{array}$ & $\begin{array}{l}100 \% \\
100 \%\end{array}$ & $\begin{array}{l}1.5 \\
72\end{array}$ & $\begin{array}{l}9.5 \\
210\end{array}$ & $\begin{array}{l}6.1 \\
140\end{array}$ & $\begin{array}{l}6.5 \\
132\end{array}$ & $\begin{array}{l}\text { Yes } \\
\text { Yes }\end{array}$ & 6.0 & Yes & 1.6 & 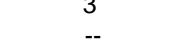 & $\begin{array}{l}0.39 \\
5375\end{array}$ & $\begin{array}{l}\text { Yes } \\
\text { Sol }\end{array}$ & $\begin{array}{l}24 \\
0.039\end{array}$ & $\begin{array}{ll}5 \\
0\end{array}$ \\
\hline METAL & Beryllium & $\begin{array}{l}m / k g \\
m g r y-w t\end{array}$ & 5 & 5 & $100 \%$ & 0.65 & 1.5 & 1.2 & 1.5 & No & .- &.- & .- &.- & 年 & No & 0.0097 & 0 \\
\hline METAL & Cadmium & $\mathrm{mg} / \mathrm{kg}$ dry-wt & 5 & 5 & $100 \%$ & 0.65 & 2.5 & 1.9 & 0.81 & Yes & 0.60 & Yes & 4.2 & 5 & $\begin{array}{l}154 \\
37\end{array}$ & No & 0.068 & 0 \\
\hline METAL & Calcium & $\mathrm{mg} / \mathrm{kg}$ dry-wt & 5 & 5 & $100 \%$ & 3,100 & 6,800 & 5,280 & 17,200 & No & & & & 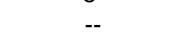 & & & & \\
\hline METAL & Chromium & $\mathrm{mg} / \mathrm{kg}$ dry-wt & 5 & 5 & $100 \%$ & 9.4 & 30 & 21 & 19 & Yes & 37 & No & 0.81 & 0 & 30 & No & 1.00 & 0 \\
\hline $\begin{array}{l}\text { METAL } \\
\text { METII }\end{array}$ & Cobalt & $m g / k g d r y-w t$ & 5 & 5 & $100 \%$ & $\begin{array}{l}10 \\
75\end{array}$ & 22 & $\begin{array}{l}18 \\
28\end{array}$ & 16 & Yes & $\cdots$ & 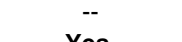 & -- & - & 903 & No & 0.024 & \\
\hline $\begin{array}{l}\text { MEATL } \\
\text { METAL }\end{array}$ & $\begin{array}{l}\text { Copper } \\
\text { Iron }\end{array}$ & $\begin{array}{l}m g / k g d r d y-w t \\
m / k g d r-w t\end{array}$ & $\begin{array}{l}5 \\
5\end{array}$ & $\begin{array}{l}5 \\
5\end{array}$ & $\begin{array}{l}100 \% \\
100 \%\end{array}$ & $\begin{array}{l}7.5 \\
22.000\end{array}$ & $\begin{array}{l}46 \\
44,000\end{array}$ & $\begin{array}{c}28 \\
36,400\end{array}$ & $\begin{array}{l}22 \\
32600\end{array}$ & $\begin{array}{l}\text { Yes } \\
\text { Yes }\end{array}$ & $\begin{array}{l}32 \\
20,000\end{array}$ & $\begin{array}{l}\text { Yys } \\
\text { Yes }\end{array}$ & $\begin{array}{l}1.5 \\
22\end{array}$ & ${ }_{5}^{3}$ & $\begin{array}{l}2,960 \\
23463\end{array}$ & $\begin{array}{l}\text { No } \\
\text { Noc }\end{array}$ & $\begin{array}{l}0.016 \\
0.16\end{array}$ & 0 \\
\hline $\begin{array}{l}\text { METAL } \\
\text { MET }\end{array}$ & Lead & malka growert & 5 & 5 & $\begin{array}{l}100 \% \\
100 \%\end{array}$ & $\begin{array}{ll}22,000 \\
40\end{array}$ & $\begin{array}{l}44,000 \\
27\end{array}$ & $\begin{array}{l}36,400 \\
17\end{array}$ & $\begin{array}{l}32,600 \\
10\end{array}$ & $\begin{array}{l}\text { Yes } \\
\text { Yes }\end{array}$ & 党, & $\begin{array}{l}\text { Nos } \\
\text { No }\end{array}$ & 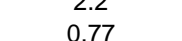 & $\begin{array}{lll}5 & \\
0 & & \end{array}$ & $\begin{array}{l}2,3403 \\
250\end{array}$ & $\begin{array}{l}\text { res } \\
\text { No }\end{array}$ & $\begin{array}{l}0.911 \\
0.11\end{array}$ & $\begin{array}{l}4 \\
0\end{array}$ \\
\hline METAL & Magnesium & $\begin{array}{l}m m g / k g \text { gry-wt } \\
m g / k g d r y-w t\end{array}$ & $\begin{array}{l}5 \\
5\end{array}$ & $\begin{array}{l}5 \\
5\end{array}$ & $\begin{array}{l}100 \% \\
100 \%\end{array}$ & $\begin{array}{l}4.0 \\
3,100\end{array}$ & 7,500 & 6,140 & $\begin{array}{l}10 \\
7,060\end{array}$ & $\begin{array}{l}\text { Yes } \\
\text { Yes }\end{array}$ & & & & & & & & \\
\hline METAL & Manganese & $\mathrm{mg} / \mathrm{kg}$ dry-wt & 5 & 5 & $100 \%$ & 370 & 840 & 622 & 512 & Yes & 460 & Yes & 1.8 & 3 & 1,762 & No & 0.48 & 0 \\
\hline METAL & Mercury & $\mathrm{mg} / \mathrm{kg}$ dry-wt & 5 & 5 & $100 \%$ & 0.13 & 0.30 & 0.23 & 0.33 & No & 0.18 & Yes & 1.7 & 3 & 23 & No & 0.013 & 0 \\
\hline METAL & Nickel & $\mathrm{mg} / \mathrm{kg}$ dry-wt & 5 & 5 & $100 \%$ & 9.7 & 30 & 24 & 19 & Yes & 18 & Yes & 1.7 & 4 & 1,564 & No & 0.019 & 0 \\
\hline $\begin{array}{l}\text { METAL } \\
\text { METAL }\end{array}$ & $\begin{array}{l}\text { Potassium } \\
\text { Selenium }\end{array}$ & $\begin{array}{l}m g / k g d r y-w t \\
m a g k d r-w t\end{array}$ & $\begin{array}{l}5 \\
5\end{array}$ & 5 & $100 \%$ & $\begin{array}{l}450 \\
0.96\end{array}$ & 3,000 & $\begin{array}{c}1,856 \\
14\end{array}$ & 2,150 & Yes & -- & - & -- & - & 201 & N & (20091 & - \\
\hline $\begin{array}{l}\text { METAL } \\
\text { METLL }\end{array}$ & $\begin{array}{l}\text { Silver } \\
\text { Silum }\end{array}$ & $\begin{array}{l}m \mathrm{mgk} / \mathrm{kg} \operatorname{sin-wt} \\
\mathrm{mg} / \mathrm{kg} \text { dry-wt }\end{array}$ & $\begin{array}{l}5 \\
5\end{array}$ & $\begin{array}{l}5 \\
5\end{array}$ & $100 \%$ & $\begin{array}{l}0.96 \\
1.3\end{array}$ & $\begin{array}{l}1.6 \\
3.0\end{array}$ & $\begin{array}{l}2.4 \\
2.3\end{array}$ & $\begin{array}{l}0.78 \\
0.73\end{array}$ & $\begin{array}{l}\text { Yes } \\
\text { Yes }\end{array}$ & 4.5 & No & 0.67 & 0 & $\begin{array}{l}391 \\
391\end{array}$ & $\begin{array}{l}\text { No } \\
\text { No }\end{array}$ & $\begin{array}{l}0.0071 \\
0.0077\end{array}$ & 0 \\
\hline METAL & Sodium & $\mathrm{mg} / \mathrm{kg}$ dry-wt & 5 & 5 & $100 \%$ & 170 & 390 & 314 & 690 & No & -- & -- & -- & -- & - & -- & & 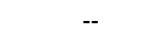 \\
\hline METAL & Thallium & $m g / k g d r y-w t$ & ${ }^{5}$ & ${ }^{5}$ & $100 \%$ & 1.3 & 3.0 & 2.6 & 20 & A & م & $\ddot{-}$ & - & - & 5.2 & No & 0.58 & 0 \\
\hline $\begin{array}{l}\text { MEALA } \\
\text { METAA }\end{array}$ & $\begin{array}{l}\text { Uranulum } \\
\text { Vanndium }\end{array}$ & 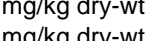 & ${ }_{5}^{1}$ & $\frac{1}{5}$ & $\begin{array}{l}100 \% \\
100 \%\end{array}$ & 20 & $\begin{array}{l}2.7 \\
1.120\end{array}$ & - & (3.2 & $\begin{array}{l}\text { No } \\
\text { Noc }\end{array}$ & 2,490 & No & 0.0011 & 0 & $\begin{array}{l}16 \\
16\end{array}$ & No & 0.17 & 0 \\
\hline METAL & $\begin{array}{l}\text { Vandaumur } \\
\text { Zinc }\end{array}$ & 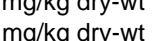 & 5 & 5 & $\begin{array}{l}1000 \% \\
100 \%\end{array}$ & 64 & $\begin{array}{l}120 \\
270\end{array}$ & $\begin{array}{l}183 \\
182\end{array}$ & 要 & $\begin{array}{l}\text { res } \\
\text { Yes }\end{array}$ & 120 & Yes & 2.3 & 3 & 23.463 & $\begin{array}{l}\text { res } \\
\text { No }\end{array}$ & $\begin{array}{l}1.5 \\
0012\end{array}$ & 2 \\
\hline RAD D & $\begin{array}{l}\text { Berllium-7 } \\
\text { Ber }\end{array}$ & pcilg dry-wt & 1 & 1 & $100 \%$ & 0.0016 & 0.0016 & 0.0016 & $\ldots$ & $\ldots$ & $\ldots$ & $\ldots$ & $\cdots$ & - & . & $\ldots$ & 0.012 & 0 \\
\hline RAD_D & Cesium-134 & pCilg dry-wt & 1 & 1 & $100 \%$ & 0.039 & 0.039 & 0.039 & -- & -- & -- & -- & -- & -- & -. & -- & -- & -- \\
\hline & Cesium-137 & pcilg dry-wt & 8 & 8 & $100 \%$ & 0.090 & 0.41 & 0.20 & 1.1 & No & 3,120 & No & 1.32E-04 & 0 & 6.2 & No & 0.066 & 0 \\
\hline $\begin{array}{l}\text { RADDD } \\
\text { RAD D }\end{array}$ & Cobalt-60 & $\begin{array}{l}\text { pCil/g dry-wt } \\
\text { pCila dr-wt }\end{array}$ & $\begin{array}{l}5 \\
2\end{array}$ & $\begin{array}{r}5 \\
2\end{array}$ & $\begin{array}{l}100 \% \\
100 \%\end{array}$ & $\begin{array}{l}0.0077 \\
0.064\end{array}$ & 0.038 & $\begin{array}{l}0.016 \\
0.14\end{array}$ & 0.0084 & Yes & 1,460 & No & 2.58E-05 & 0 & $\begin{array}{l}1.4 \\
.2\end{array}$ & No & 0.027 & $\begin{array}{l}0 \\
0\end{array}$ \\
\hline $\begin{array}{l}\text { RADD } \\
\text { RAD D }\end{array}$ & $\begin{array}{l}\text { Europium-152 } \\
\text { Plutonium-238 }\end{array}$ & $\begin{array}{l}\text { pCCI/I a dry-wt } \\
\text { ocila drowt }\end{array}$ & $\begin{array}{l}2 \\
5\end{array}$ & $\begin{array}{l}2 \\
5\end{array}$ & $\begin{array}{l}100 \% \\
100 \%\end{array}$ & $\begin{array}{c}0.064 \\
2.66 \mathrm{E}-04\end{array}$ & $\begin{array}{l}0.22 \\
0.020\end{array}$ & $\begin{array}{l}0.14 \\
0.054\end{array}$ & 0.0038 & Yes & $\therefore$ & -- &.- & $\ddot{-}$ & $\begin{array}{l}3.3 \\
37 \\
3\end{array}$ & $\begin{array}{l}\text { No } \\
\text { No }\end{array}$ & $\begin{array}{l}0.066 \\
5.26 \mathrm{E}-04\end{array}$ & 0 \\
\hline RAD_D & $\begin{array}{l}\text { Plutonium-239/240 } \\
\text { Panter }\end{array}$ & pCilg dry-wt & 8 & 8 & $100 \%$ & $1.00 \mathrm{E}-03$ & 0.049 & 0.013 & $\begin{array}{l}0.025 \\
0.025\end{array}$ & Yes & 5,860 & No & $8.36 \mathrm{E}-06$ & 0 & 34 & No & $\begin{array}{l}0.0014 \\
0.0014\end{array}$ & 0 \\
\hline RAD_D & Potassium-40 & pCilg dry-wt & 10 & 10 & $100 \%$ & 8.2 & 16 & 13 & 17 & No & & & & & & & & \\
\hline $\mathrm{RAD}_{-}^{-} \mathrm{D}$ & Strontium-90 & pCilg dry-wt & 9 & 9 & $100 \%$ & 0.0057 & 0.031 & 0.016 & 0.18 & No & 582 & No & $5.24 \mathrm{E}-05$ & 0 & 4.5 & No & 0.0068 & 0 \\
\hline RAD_D & Uranium-234 & pCilg dry-wt & 9 & 9 & $100 \%$ & 0.50 & 1.7 & 1.3 & 1.1 & Yes & 5,270 & No & 3.30E-04 & 0 & 1.1 & Yes & 1.6 & 8 \\
\hline $\begin{array}{l}\text { RADD D } \\
\text { RAD D }\end{array}$ & $\begin{array}{l}\text { Uranium-235 } \\
\text { Uranium-236 }\end{array}$ & $\begin{array}{l}\text { pCCi/g dry-wt } \\
\text { pCi/g dry-wt }\end{array}$ & $\begin{array}{l}9 \\
1\end{array}$ & $\begin{array}{l}9 \\
1\end{array}$ & $\begin{array}{l}100 \% \\
100 \%\end{array}$ & 0.017 & $\begin{array}{l}0.062 \\
1.2\end{array}$ & 0.039 & 0.11 & No & $\begin{array}{l}3,730 \\
--\end{array}$ & No & 1.66E-05 & 0 & 0.84 & No & 0.074 & 0 \\
\hline $\begin{array}{l}\text { RAD DD } \\
\text { D. }\end{array}$ & Uranium-238 & 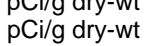 & 8 & 8 & $100 \%$ & 0.38 & $\begin{array}{l}1.2 \\
1.7\end{array}$ & $\begin{array}{l}1.2 \\
1.1\end{array}$ & 3.2 & No & 2,490 & No & $6.95 E-04$ & 0 & 1.1 & Yes & 1.6 & 4 \\
\hline
\end{tabular}

RAD_D - Decayed radionuclide 
Summary of Surface Sediment Results From Segment 4 Exceeding Select Ecological or Human Health Benchmark Values

\begin{tabular}{|c|c|c|c|c|c|c|c|c|c|c|}
\hline Sample Area & Sample Site & SampleID & Date Collected & Constituent Name & Units & QA Category & $\begin{array}{l}\text { Analytical } \\
\text { Value }\end{array}$ & Qualifier & $\begin{array}{c}\text { Ecological } \\
\text { Benchmark } \\
\text { Value }\end{array}$ & $\begin{array}{c}\text { Ruman } \\
\text { Health } \\
\text { Benchmark } \\
\text { Value }\end{array}$ \\
\hline Columbia River & RM 145 & $\mathrm{B} 2 \mathrm{GC} 1$ & July 28,1997 & Acenaphthene & $\mathrm{mg} / \mathrm{kg}$ & 1 & 0.018 & $=$ & 0.0067 & 3,682 \\
\hline Columbia River & RM 145 & $B 2 G C 3$ & July 28,1997 & Acenaphthylene & $\mathrm{mg} / \mathrm{kg}$ & 1 & 0.0060 & $=$ & 0.0059 & 4800 \\
\hline Columbia River & John Day Dam & B17B78 & August 8,2003 & Antimony & $\mathrm{mg} / \mathrm{kg}$ dry-wt & 1 & 5.9 & $=$ & 2.0 & 31 \\
\hline Columbia River & John Day Dam & B17B81 & August 8,2003 & Antimony & $\mathrm{mg} / \mathrm{kg}$ dry-wt & 1 & 6.1 & $=$ & 2.0 & 31 \\
\hline Columbia River & John Day Dam & B17B84 & August 8,2003 & Antimony & $\mathrm{mg} / \mathrm{kg}$ dry-wt & 1 & 5.5 & $=$ & 2.0 & 31 \\
\hline Columbia River & Dalles Dam & B17B99 & August 8,2003 & Antimony & $\mathrm{mg} / \mathrm{kg}$ dry-wt & 1 & 2.6 & $=$ & 2.0 & 31 \\
\hline Columbia River & Bonneville Dam & B17BB5 & August 7, 2003 & Antimony & $\mathrm{mg} / \mathrm{kg}$ dry-wt & 1 & 3.0 & $=$ & 2.0 & 31 \\
\hline Columbia River & Messner Cove & PM-BC-07 & March 17, 1999 & Antimony & $\mathrm{mg} / \mathrm{kg}$ & 1 & 62 & $=$ & 2.0 & 0 \\
\hline Columbia River & Messner Cove & PM-BC-09 & March 17, 1999 & Antimony & $\mathrm{mg} / \mathrm{kg}$ & 1 & 65 & $=$ & 2.0 & 0 \\
\hline Columbia River & Bonnevilie Dam & $B F-B C-07$ & September 18,2002 & Aroclor-1254 & $\mathrm{mg} / \mathrm{kg}$ & 1 & 0.019 & $=$ & 0.0070 & 0.22 \\
\hline Columbia River & John Day Dam & B17B78 & August 8,2003 & Arsenic & $\mathrm{mg} / \mathrm{kg}$ dry-wt & 1 & 9.5 & $=$ & 6.0 & 0.39 \\
\hline Columbia River & John Day Dam & B17B81 & August 8,2003 & Arsenic & $\mathrm{mg} / \mathrm{kg}$ dry-wt & 1 & 8.6 & $=$ & 6.0 & 0.39 \\
\hline Columbia River & John Day Dam & B17B84 & August 8,2003 & Arsenic & $\mathrm{mg} / \mathrm{kg}$ dry-wt & 1 & 7.4 & $=$ & 6.0 & 0.39 \\
\hline Columbia River & Dalles Dam & B17B99 & August 8, 2003 & Arsenic & $\mathrm{mg} / \mathrm{kg}$ dry-wt & 1 & 3.3 & $=$ & 6.0 & 0.39 \\
\hline Columbia River & Bonneville Dam & B17BB5 & August 7, 2003 & Arsenic & $\mathrm{mg} / \mathrm{kg}$ dry-wt & 1 & 1.5 & $=$ & 6.0 & 0.39 \\
\hline Columbia River & Dalles Dam & B17BK1 & August 7,2003 & Arsenic & $\mathrm{mg} / \mathrm{Kg}$ & 1 & 5.6 & $=$ & 6.0 & 0.39 \\
\hline Columbia River & Bonneville Dam & B17BK5 & August 7, 2003 & Arsenic & $\mathrm{mg} / \mathrm{Kg}$ & 1 & 6.0 & $=$ & 6.0 & 0.39 \\
\hline Columbia River & John Day Dam & B1B7P3 & September 21, 2004 & Arsenic & $\mathrm{mg} / \mathrm{Kg}$ & 1 & 10 & $x$ & 6.0 & 0.39 \\
\hline Columbia River & RM 145 & $\mathrm{~B} 2 \mathrm{GC} 1$ & July 28, 1997 & Arsenic & $\mathrm{mg} / \mathrm{kg}$ & 1 & 4.0 & $=$ & 6.0 & 0.39 \\
\hline Columbia River & RM 145 & $\mathrm{~B} 2 \mathrm{GC} 2$ & July 28,1997 & Arsenic & $\mathrm{mg} / \mathrm{kg}$ & 1 & 3.0 & $=$ & 6.0 & 0.39 \\
\hline Columbia River & RM 145 & B2GC3 & July 28,1997 & Arsenic & $\mathrm{mg} / \mathrm{kg}$ & 1 & 5.0 & $=$ & 6.0 & 0.39 \\
\hline Columbia River & Bonneville Dam & BF-BC-03 & September 18, 2002 & Arsenic & $\mathrm{mg} / \mathrm{kg}$ & 1 & 4.6 & $=$ & 6.0 & 0.39 \\
\hline Columbia River & Bonneville Dam & BF-BC-07 & September 18, 2002 & Arsenic & $\mathrm{mg} / \mathrm{kg}$ & 1 & 2.4 & $=$ & 6.0 & 0.39 \\
\hline Columbia River & Bonneville Dam & BF-BC-17 & September 18,2002 & Arsenic & $\mathrm{mg} / \mathrm{kg}$ & 1 & 5.5 & $=$ & 6.0 & 0.39 \\
\hline Columbia River & Bonneville Dam & BF-BC-26 & September 18, 2002 & Arsenic & $\mathrm{mg} / \mathrm{kg}$ & 1 & 5.8 & $=$ & 6.0 & 0.39 \\
\hline Columbia River & RM 145 & $\mathrm{PH} 2 \# 1$ & July 24, 1997 & Arsenic & $\mathrm{mg} / \mathrm{kg}$ & 1 & 2.0 & $=$ & 6.0 & 0.39 \\
\hline Columbia River & RM 145 & $\mathrm{PH} 2 \# 2$ & July 24, 1997 & Arsenic & $\mathrm{mg} / \mathrm{kg}$ & 1 & 15 & $=$ & 6.0 & 0.39 \\
\hline Columbia River & John Day Dam & B17B78 & August 8,2003 & Cadmium & $\mathrm{mg} / \mathrm{kg}$ dry-wt & $\overline{1}$ & 2.5 & $=$ & 0.60 & 37 \\
\hline Columbia River & John Day Dam & B17B81 & August 8,2003 & Cadmium & $\mathrm{mg} / \mathrm{kg}$ dry-wt & 1 & 2.3 & $=$ & 0.60 & 37 \\
\hline Columbia River & John Day Dam & B17B84 & August 8,2003 & Cadmium & $\mathrm{mg} / \mathrm{kg}$ dry-wt & 1 & 2.4 & $=$ & 0.60 & 37 \\
\hline Columbia River & Dalles Dam & B17B99 & August 8,2003 & Cadmium & $\mathrm{mg} / \mathrm{kg}$ dry-wt & 1 & 0.65 & $=$ & 0.60 & 37 \\
\hline Columbia River & Bonneville Dam & B17BB5 & August 7,2003 & Cadmium & $\mathrm{mg} / \mathrm{kg}$ dry-wt & 1 & 1.5 & $=$ & 0.60 & 37 \\
\hline Columbia River & Dalles Dam & B17BK1 & August 7, 2003 & Cadmium & $\mathrm{mg} / \mathrm{kg}$ & 1 & 1.2 & $=$ & 0.60 & 37 \\
\hline Columbia River & Bonneville Dam & B17BK5 & August 7, 2003 & Cadmium & $\mathrm{mg} / \mathrm{Kg}$ & 1 & 0.88 & $=$ & 0.60 & 37 \\
\hline Columbia River & John Day Dam & B1B7P3 & September 21, 2004 & Cadmium & $\mathrm{mg} / \mathrm{Kg}$ & 1 & 2.2 & $x$ & 0.60 & 37 \\
\hline Columbia River & Messner Cove & PM-BC-02 & March 17, 1999 & Cadmium & $\mathrm{mg} / \mathrm{kg}$ & 1 & 0.61 & $=$ & 0.60 & 37 \\
\hline Columbia River & Messner Cove & PM-BC-03 & March 17, 1999 & Cadmium & $\mathrm{mg} / \mathrm{kg}$ & 1 & 0.74 & $=$ & 0.60 & 37 \\
\hline Columbia River & Messner Cove & PM-BC-04 & March 17, 1999 & Cadmium & $\mathrm{mg} / \mathrm{kg}$ & 1 & 0.96 & $=$ & 0.60 & 37 \\
\hline Columbia River & Messner Cove & PM-BC-10 & March 17, 1999 & Cadmium & $\mathrm{mg} / \mathrm{kg}$ & 1 & 1.0 & $=$ & 0.60 & 37 \\
\hline Columbia River & Messner Cove & PM-GC-01 & March 17, 1999 & Cadmium & $\mathrm{mg} / \mathrm{kg}$ & 1 & 0.98 & $=$ & 0.60 & 37 \\
\hline Columbia River & Dalles Dam & 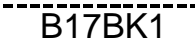 & August 7,2003 & Chromium & $\mathrm{mg} / \mathrm{kg}$ & 1 & 57 & $=$ & 37 & 30 \\
\hline Columbia River & Bonneville Dam & B17BK5 & August 7, 2003 & Chromium & $\mathrm{mg} / \mathrm{Kg}$ & 1 & 57 & $=$ & 37 & 30 \\
\hline Columbia River & John Day Dam & B1B7P3 & September 21, 2004 & Chromium & $\mathrm{mg} / \mathrm{Kg}$ & 1 & 62 & $x$ & 37 & 30 \\
\hline Columbia River & John Day Dam & B17B78 & August 8,2003 & Copper & $\mathrm{mg} / \mathrm{kg}$ dry-wt & 1 & 46 & $=$ & -32 & 2,960 \\
\hline Columbia River & John Day Dam & B17B81 & August 8, 2003 & Copper & $\mathrm{mg} / \mathrm{kg}$ dry-wt & 1 & 35 & $=$ & 32 & 2,960 \\
\hline Columbia River & John Day Dam & B17B84 & August 8, 2003 & Copper & $\mathrm{mg} / \mathrm{kg}$ dry-wt & 1 & 34 & $=$ & 32 & 2,960 \\
\hline Columbia River & Bonneville Dam & B17BK5 & August 7,2003 & Copper & $\mathrm{mg} / \mathrm{Kg}$ & 1 & 32 & $=$ & 32 & 2,960 \\
\hline Columbia River & John Day Dam & B1B7P3 & September 21, 2004 & Copper & $\mathrm{mg} / \mathrm{Kg}$ & 1 & 46 & $\mathrm{x}$ & 32 & 2,960 \\
\hline Columbia River & Bonneville Dam & BF-BC-03 & September 18, 2002 & Copper & $\mathrm{mg} / \mathrm{kg}$ & 1 & 37 & $=$ & 32 & 2,960 \\
\hline Columbia River & Bonneville Dam & BF-BC-26 & September 18, 2002 & Copper & $\mathrm{mg} / \mathrm{kg}$ & 1 & 34 & $=$ & 32 & 2,960 \\
\hline
\end{tabular}


Summary of Surface Sediment Results From Segment 4 Exceeding Select Ecological or Human Health Benchmark Values

\begin{tabular}{|c|c|c|c|c|c|c|c|c|c|c|}
\hline Sample Area & Sample Site & SampleID & Date Collected & Constituent Name & Units & QA Category & $\begin{array}{c}\text { Analytical } \\
\text { Value }\end{array}$ & Qualifier & $\begin{array}{c}\text { Ecological } \\
\text { Benchmark } \\
\text { Value }\end{array}$ & $\begin{array}{c}\text { Human } \\
\text { Health } \\
\text { Benchmark } \\
\text { Value }\end{array}$ \\
\hline Columbia River & Messner Cove & $\overline{\mathrm{PM}-\mathrm{GC}-01}$ & March 17, 1999 & Di-n-butylphthalate & $\mathrm{mg} / \mathrm{kg}$ & 1 & 0.065 & $=$ & 0.043 & 6,110 \\
\hline Columbia River & John Day Dam & B17B78 & August 8,2003 & Iron & $\mathrm{mg} / \mathrm{kg}$ dry-wt & 1 & 44,000 & $=$ & 20,000 & 23,463 \\
\hline Columbia River & John Day Dam & B17B81 & August 8, 2003 & Iron & $\mathrm{mg} / \mathrm{kg}$ dry-wt & 1 & 37,000 & $=$ & 20,000 & 23,463 \\
\hline Columbia River & John Day Dam & B17B84 & August 8, 2003 & Iron & $\mathrm{mg} / \mathrm{kg}$ dry-wt & 1 & 37,000 & $=$ & 20,000 & 23,463 \\
\hline Columbia River & Dalles Dam & B17B99 & August 8,2003 & Iron & $\mathrm{mg} / \mathrm{kg}$ dry-wt & 1 & 22,000 & $=$ & 20,000 & 23,463 \\
\hline Columbia River & Bonneville Dam & B17BB5 & August 7, 2003 & Iron & $\mathrm{mg} / \mathrm{kg}$ dry-wt & 1 & 42,000 & $=$ & 20,000 & 23,463 \\
\hline Columbia River & Bonneville Dam & BF-BC-03 & September 18, 2002 & Iron & $\mathrm{mg} / \mathrm{kg}$ & 1 & 23,800 & $=$ & 20,000 & 23,463 \\
\hline Columbia River & Bonneville Dam & BF-BC-07 & September 18, 2002 & Iron & $\mathrm{mg} / \mathrm{kg}$ & 1 & 20,700 & $=$ & 20,000 & 23,463 \\
\hline Columbia River & Bonneville Dam & BF-BC-17 & September 18,2002 & Iron & $\mathrm{mg} / \mathrm{kg}$ & 1 & 26,100 & $=$ & 20,000 & 23,463 \\
\hline Columbia River & Bonneville Dam & BF-BC-26 & September 18,2002 & Iron & $\mathrm{mg} / \mathrm{kg}$ & 1 & 25,200 & $=$ & 20,000 & 23,463 \\
\hline Columbia River & Mesner Cove & PM-BC-03 & March 17, 1999 & Lead & $\mathrm{mg} / \mathrm{kg}$ & 1 & 0,250 & $=$ & 0,035 & 0,250 \\
\hline Columbia River & John Day Dam & B17B78 & August 8,2003 & Manganese & $\mathrm{mg} / \mathrm{kg}$ dry-wt & 1 & 840 & $=$ & $460^{-}$ & 1,762 \\
\hline Columbia River & John Day Dam & B17B81 & August 8,2003 & Manganese & $\mathrm{mg} / \mathrm{kg}$ dry-wt & 1 & 770 & $=$ & 460 & 1,762 \\
\hline Columbia River & John Day Dam & B17B84 & August 8, 2003 & Manganese & $\mathrm{mg} / \mathrm{kg}$ dry-wt & 1 & 680 & $=$ & 460 & 1,762 \\
\hline Columbia River & Bonneville Dam & BF-BC-03 & September 18, 2002 & Manganese & $\mathrm{mg} / \mathrm{kg}$ & 1 & 510 & $=$ & 460 & 1,762 \\
\hline Columbia River & Bonneville Dam & BF-BC-17 & September 18,2002 & Manganese & $\mathrm{mg} / \mathrm{kg}$ & 1 & 716 & $=$ & 460 & 1,762 \\
\hline Columbia River & Bonneville Dam & BF-BC-26 & September 18,2002 & Manganese & $\mathrm{mg} / \mathrm{kg}$ & 1 & 499 & $=$ & 460 & 1,762 \\
\hline Columbia River & John Day Dam & B17B78 & August 8,2003 & Mercury & $\mathrm{mg} / \mathrm{kg}$ dry-wt & 1 & 0.29 & $=$ & 0.18 & 23 \\
\hline Columbia River & John Day Dam & B17B81 & August 8, 2003 & Mercury & $\mathrm{mg} / \mathrm{kg}$ dry-wt & 1 & 0.30 & $=$ & 0.18 & 23 \\
\hline Columbia River & John Day Dam & B17B84 & August 8,2003 & Mercury & $\mathrm{mg} / \mathrm{kg}$ dry-wt & 1 & 0.28 & $=$ & 0.18 & 23 \\
\hline Columbia River & Bonneville Dam & B17BK5 & August 7, 2003 & Mercury & $\mathrm{mg} / \mathrm{Kg}$ & 1 & 0.51 & c & 0.18 & 23 \\
\hline Columbia River & Bonneville Dam & BF-BC-15 & September 18, 2002 & Mercury & $\mathrm{mg} / \mathrm{kg}$ & 1 & 0.20 & $=$ & 0.18 & 23 \\
\hline Columbia River & Bonneville Dam & BF-BC-17 & September 18,2002 & Mercury & $\mathrm{mg} / \mathrm{kg}$ & 1 & 0.19 & $=$ & 0.18 & 23 \\
\hline Columbia River & John Day Dam & B17B78 & August 8,2003 & Nickel- & $\mathrm{mg} / \mathrm{kg}$ dry-wt & 1 & 29 & $=$ & 18 & 1,564 \\
\hline Columbia River & John Day Dam & B17B81 & August 8,2003 & Nickel & $\mathrm{mg} / \mathrm{kg}$ dry-wt & 1 & 25 & $=$ & 18 & 1,564 \\
\hline Columbia River & John Day Dam & B17B84 & August 8,2003 & Nickel & $\mathrm{mg} / \mathrm{kg}$ dry-wt & 1 & 24 & $=$ & 18 & 1,564 \\
\hline Columbia River & Bonneville Dam & B17BB5 & August 7, 2003 & Nickel & $\mathrm{mg} / \mathrm{kg}$ dry-wt & 1 & 30 & $=$ & 18 & 1,564 \\
\hline Columbia River & Dalles Dam & B17BK1 & August 7, 2003 & Nickel & $\mathrm{mg} / \mathrm{Kg}$ & 1 & 24 & $=$ & 18 & 1,564 \\
\hline Columbia River & Bonneville Dam & B17BK5 & August 7,2003 & Nickel & $\mathrm{mg} / \mathrm{Kg}$ & 1 & 25 & $=$ & 18 & 1,564 \\
\hline Columbia River & John Day Dam & B1B7P3 & September 21, 2004 & Nickel & $\mathrm{mg} / \mathrm{Kg}$ & 1 & 33 & $\mathrm{x}$ & 18 & 1,564 \\
\hline Columbia River & Bonneville Dam & BF-BC-03 & September 18, 2002 & Nickel & $\mathrm{mg} / \mathrm{kg}$ & 1 & 19 & $=$ & 18 & 1,564 \\
\hline Columbia River & Bonneville Dam & BF-BC-17 & September 18,2002 & Nickel & $\mathrm{mg} / \mathrm{kg}$ & 1 & 19 & $=$ & 18 & 1,564 \\
\hline Columbia River & Bonneville Dam & BF-BC-26 & September 18,2002 & Nickel & $\mathrm{mg} / \mathrm{kg}$ & 1 & 19 & $=$ & 18 & 1,564 \\
\hline Columbia River & Bonneville Dam & 31803 & August 7,2003 & Üranium-234 & $\mathrm{pCi} / \mathrm{g}$ dry-wt & 1 & 1.2 & $=$ & 5,270 & 1.1 \\
\hline Columbia River & Dalles Dam & 31804 & August 7, 2003 & Uranium-234 & pCi/g dry-wt & 1 & 1.2 & $=$ & 5,270 & 1.1 \\
\hline Columbia River & Dalles Dam & 31805 & August 7, 2003 & Uranium-234 & $\mathrm{pCi} / \mathrm{g}$ dry-wt & 1 & 1.7 & $=$ & 5,270 & 1.1 \\
\hline Columbia River & John Day Dam & 31806 & August 8,2003 & Uranium-234 & $\mathrm{pCi} / \mathrm{g}$ dry-wt & 1 & 1.2 & $=$ & 5,270 & 1.1 \\
\hline Columbia River & John Day Dam & 31807 & August 8,2003 & Uranium-234 & $\mathrm{pCi} / \mathrm{g}$ dry-wt & 1 & 1.4 & $=$ & 5,270 & 1.1 \\
\hline Columbia River & John Day Dam & 31808 & August 8, 2003 & Uranium-234 & pCi/g dry-wt & 1 & 1.4 & $=$ & 5,270 & 1.1 \\
\hline Columbia River & John Day Dam & 33607 & September 21, 2004 & Uranium-234 & $\mathrm{pCi} / \mathrm{g}$ dry-wt & 1 & 1.4 & $=$ & 5,270 & 1.1 \\
\hline Columbia River & John Day Dam & B17BP9 & August 8,2003 & Uranium-234 & $\mathrm{pCi} / \mathrm{g}$ dry-wt & 1 & 1.4 & $=$ & 5,270 & 1.1 \\
\hline Columbia River & Dalles Dam & 31805 & August 7,2003 & Uranium-238 & $\mathrm{pCi} / \mathrm{g}$ dry-wt & 1 & 1.7 & $=$ & 2,490 & 1.1 \\
\hline Columbia River & John Day Dam & 31807 & August 8, 2003 & Uranium-238 & pCi/g dry-wt & 1 & 1.2 & $=$ & 2,490 & 1.1 \\
\hline Columbia River & John Day Dam & 31808 & August 8,2003 & Uranium-238 & $\mathrm{pCi} / \mathrm{g}$ dry-wt & 1 & 1.2 & $=$ & 2,490 & 1.1 \\
\hline Columbia River & John Day Dam & 33607 & September 21, 2004 & Uranium-238 & $\mathrm{pCi} / \mathrm{g}$ dry-wt & 1 & 1.3 & $=$ & 2,490 & 1.1 \\
\hline Columbia River & John Day Dam & B17B78 & August 8,2003 & Vanadium & $\mathrm{mg} / \mathrm{kg}$ dry-wt & 1 & 87 & $=$ & -- & 78 \\
\hline Columbia River & Bonneville Dam & B17BB5 & August 7,2003 & Vanadium & $\mathrm{mg} / \mathrm{kg}$ dry-wt & 1 & 120 & $=$ & $\ldots$ & 78 \\
\hline Columbia River & John Day Dam & B17B78 & August 8,2003 & Zinc & $\mathrm{mg} / \mathrm{kg}$ dry-wt & 1 & 270 & $=$ & 120 & 23,463 \\
\hline Columbia River & John Day Dam & B17B81 & August 8,2003 & Zinc & $\mathrm{mg} / \mathrm{kg}$ dry-wt & 1 & 250 & $=$ & 120 & 23,463 \\
\hline Columbia River & John Day Dam & B17B84 & August 8,2003 & Zinc & $\mathrm{mg} / \mathrm{kg}$ dry-wt & 1 & 240 & $=$ & 120 & 23,463 \\
\hline
\end{tabular}




\begin{tabular}{|c|c|c|c|c|c|c|c|c|c|c|}
\hline Sample Area & Sample Site & SampleID & Date Collected & Constituent Name & Units & QA Category & $\begin{array}{c}\text { Analytical } \\
\text { Value }\end{array}$ & Qualifier & $\begin{array}{c}\text { Ecological } \\
\text { Benchmark } \\
\text { Value } \\
\end{array}$ & $\begin{array}{c}\text { Human } \\
\text { Health } \\
\text { Benchmark } \\
\text { Value } \\
\end{array}$ \\
\hline Columbia River & Dalles Dam & B17BK1 & August 7,2003 & Zinc & $\mathrm{mg} / \mathrm{Kg}$ & 1 & 179 & $\bar{C}$ & 120 & 23,463 \\
\hline Columbia River & Bonneville Dam & B17BK5 & August 7, 2003 & Zinc & $\mathrm{mg} / \mathrm{Kg}$ & 1 & 175 & C & 120 & 23,463 \\
\hline Columbia River & John Day Dam & B1B7P3 & September 21, 2004 & Zinc & $\mathrm{mg} / \mathrm{Kg}$ & 1 & 293 & $x$ & 120 & 23,463 \\
\hline Columbia River & Bonneville Dam & BF-BC-17 & September 18,2002 & Zinc & $\mathrm{mg} / \mathrm{kg}$ & 1 & 147 & $=$ & 120 & 23,463 \\
\hline
\end{tabular}




\section{Table D-8}

Summary Statistics for All Categories of Surface Water Samples Collected from Segment 4 of the Columbia River

\begin{tabular}{|c|c|c|c|c|c|c|c|c|c|c|}
\hline $\begin{array}{l}\text { Constituent } \\
\text { Class }\end{array}$ & Constituent Name & Units & $\begin{array}{l}\text { Number } \\
\text { Analyzed }\end{array}$ & $\begin{array}{l}\text { Number } \\
\text { Detected }\end{array}$ & $\begin{array}{l}\text { Frequency of } \\
\text { Detection }\end{array}$ & $\begin{array}{l}\text { Minimum } \\
\text { Nondetect }\end{array}$ & $\begin{array}{l}\text { Maximum } \\
\text { Nondetect }\end{array}$ & $\begin{array}{l}\text { Minimum } \\
\text { Detect }\end{array}$ & $\begin{array}{l}\text { Maximum } \\
\text { Detect }\end{array}$ & $\begin{array}{c}\text { Average } \\
\text { Concentration }\end{array}$ \\
\hline$\overline{\text { CONV }}$ & Alkalinity & $\overline{\mathrm{mg} / \mathrm{L}}$ & 22 & 22 & $100 \%$ & $\overline{--}$ & -- & 29 & 109 & 62 \\
\hline CONV & Ammonia & $\mathrm{mg} / \mathrm{L}$ & 40 & 23 & $58 \%$ & 0.020 & 0.020 & 0.020 & 0.050 & 0.020 \\
\hline CONV & Chloride & $\mathrm{mg} / \mathrm{L}$ & 21 & 21 & $100 \%$ & -- & -- & 1.0 & 4.0 & 3.0 \\
\hline CONV & Dissolved organic carbon & $\mathrm{mg} / \mathrm{L}$ & 9 & 9 & $100 \%$ & -- & -- & 1.0 & 2.1 & 1.4 \\
\hline CONV & Fluoride & $\mathrm{mg} / \mathrm{L}$ & 21 & 0 & $0 \%$ & 0.50 & 0.50 & -- & -- & 0.25 \\
\hline CONV & Hardness & $\mathrm{mg} / \mathrm{L}$ & 27 & 27 & $100 \%$ & -- & -- & 17 & 80 & 55 \\
\hline CONV & Nitrate & $\mathrm{mg} / \mathrm{L}$ & 21 & 9 & $43 \%$ & 0.20 & 0.20 & 0.20 & 0.40 & 0.18 \\
\hline CONV & Nitrogen, Kjeldahl total & $\mathrm{mg} / \mathrm{L}$ & 39 & 8 & $21 \%$ & 0.20 & 0.20 & 0.20 & 1.4 & 0.15 \\
\hline CONV & Nitrogen, Nitrate-Nitrite & $\mathrm{mg} / \mathrm{L}$ & 40 & 40 & $100 \%$ & -- & -- & 0.058 & 0.53 & 0.18 \\
\hline CONV & Orthophosphate & $\mathrm{mg} / \mathrm{L}$ & 40 & 40 & $100 \%$ & -- & -- & 0.0060 & 0.061 & 0.019 \\
\hline CONV & $\mathrm{pH}$ & $\mathrm{pH}$ Units & 21 & 21 & $100 \%$ & -- & -- & 7.0 & 8.8 & 8.0 \\
\hline CONV & Phosphorous in phosphate & $\mathrm{mg} / \mathrm{L}$ & 40 & 40 & $100 \%$ & -- & -- & 0.010 & 6.4 & 0.23 \\
\hline CONV & Specific Conductance & umhos/cm & 33 & 33 & $100 \%$ & -- & -- & 44 & 185 & 139 \\
\hline CONV & Sulfate & $\mathrm{mg} / \mathrm{L}$ & 21 & 17 & $81 \%$ & 10 & 20 & 7.0 & 15 & 11 \\
\hline CONV & Total dissolved solids & $\mathrm{mg} / \mathrm{L}$ & 21 & 21 & $100 \%$ & -- & -- & 76 & 118 & 97 \\
\hline CONV & Total Organic Carbon & $\mathrm{mg} / \mathrm{L}$ & 54 & 36 & $67 \%$ & 1.0 & 1.0 & 1.0 & 5.0 & 1.2 \\
\hline CONV & Total solids & $\mathrm{mg} / \mathrm{L}$ & 42 & 42 & $100 \%$ & -- & -- & 40 & 6,300 & 229 \\
\hline CONV & Total suspended solids & $\mathrm{mg} / \mathrm{L}$ & 58 & 57 & $98 \%$ & 1.0 & 1.0 & 1.0 & 5,900 & 118 \\
\hline METAL & Antimony & $\mathrm{mg} / \mathrm{L}$ & 21 & 0 & $0 \%$ & 0.0020 & 0.0030 & -- & -- & 0.0011 \\
\hline METAL & Arsenic & $\mathrm{mg} / \mathrm{L}$ & 32 & 12 & $38 \%$ & 0.0020 & 0.0050 & $4.20 \mathrm{E}-04$ & 0.0021 & 0.0012 \\
\hline METAL & Barium & $\mathrm{mg} / \mathrm{L}$ & 21 & 21 & $100 \%$ & -- & -- & 0.027 & 0.043 & 0.034 \\
\hline METAL & Beryllium & $\mathrm{mg} / \mathrm{L}$ & 21 & 1 & $5 \%$ & 5.00E-05 & 1.00E-04 & $1.00 \mathrm{E}-04$ & 1.00E-04 & 5.12E-05 \\
\hline METAL & Cadmium & $\mathrm{mg} / \mathrm{L}$ & 27 & 1.0 & 0.037 & 1.00E-04 & 0.0050 & 3.00E-04 & 3.00E-04 & 6.26E-04 \\
\hline METAL & Chromium & $\mathrm{mg} / \mathrm{L}$ & 27 & 1 & $4 \%$ & 0.0010 & 0.0020 & 0.0015 & 0.0015 & 6.48E-04 \\
\hline METAL & Copper & $\mathrm{mg} / \mathrm{L}$ & 27 & 0 & $0 \%$ & 0.010 & 0.050 & -- & -- & 0.020 \\
\hline METAL & Iron & $\mathrm{mg} / \mathrm{L}$ & 27 & 27 & $100 \%$ & -- & -- & 0.072 & 0.89 & 0.24 \\
\hline METAL & Lead & $\mathrm{mg} / \mathrm{L}$ & 27 & 1 & $4 \%$ & 0.0020 & 0.010 & 0.0036 & 0.0036 & 0.0020 \\
\hline METAL & Magnesium & $\mathrm{mg} / \mathrm{L}$ & 6 & 6 & $100 \%$ & -- & -- & 1.6 & 2.0 & 1.8 \\
\hline METAL & Manganese & $\mathrm{mg} / \mathrm{L}$ & 21 & 1 & $5 \%$ & 0.020 & 0.020 & 0.022 & 0.022 & 0.011 \\
\hline METAL & Mercury & $\mathrm{mg} / \mathrm{L}$ & 21 & 0 & $0 \%$ & $2.00 \mathrm{E}-04$ & 4.00E-04 & -- & -- & 1.95E-04 \\
\hline METAL & Nickel & $\mathrm{mg} / \mathrm{L}$ & 20 & 2 & $10 \%$ & 0.0020 & 0.0025 & 0.0034 & 0.0039 & 0.0013 \\
\hline METAL & Selenium & $\mathrm{mg} / \mathrm{L}$ & 21 & 0 & $0 \%$ & 0.0020 & 0.0050 & -- & -- & 0.0024 \\
\hline METAL & Silver & $\mathrm{mg} / \mathrm{L}$ & 21 & 0 & $0 \%$ & 5.00E-04 & 0.0050 & -- & -- & 0.0024 \\
\hline METAL & Sodium & $\mathrm{mg} / \mathrm{L}$ & 21 & 21 & $100 \%$ & -- & -- & 3.5 & 11 & 6.7 \\
\hline METAL & Thallium & $\mathrm{mg} / \mathrm{L}$ & 21 & 0 & $0 \%$ & 0.0010 & 0.0020 & -- & -- & $5.24 \mathrm{E}-04$ \\
\hline METAL & $\begin{array}{l}\text { Zinc } \\
\text { Total polychlorinated }\end{array}$ & $\mathrm{mg} / \mathrm{L}$ & 27 & 0 & $0 \%$ & 0.020 & 0.050 & -- & -- & 0.022 \\
\hline PCB & biphenyl & $\mathrm{mg} / \mathrm{L}$ & 3 & 1 & $33 \%$ & 5.90E-07 & $1.70 \mathrm{E}-06$ & $3.70 \mathrm{E}-07$ & $3.70 \mathrm{E}-07$ & 5.05E-07 \\
\hline PEST & Alpha-BHC & $\mathrm{mg} / \mathrm{L}$ & 6 & 0 & $0 \%$ & $4.80 \mathrm{E}-08$ & 8.80E-07 & -- & -- & $2.35 \mathrm{E}-07$ \\
\hline PEST & alpha-Chlordane & $\mathrm{mg} / \mathrm{L}$ & 3 & 2 & $67 \%$ & $2.10 \mathrm{E}-07$ & $2.10 \mathrm{E}-07$ & 3.00E-08 & $1.20 \mathrm{E}-07$ & 8.50E-08 \\
\hline PEST & Atrazine & $\mathrm{mg} / \mathrm{L}$ & 7 & 0 & $0 \%$ & 2.50E-05 & 2.60E-05 & -- & -- & 1.29E-05 \\
\hline PEST & Azinphos Methyl & $\mathrm{mg} / \mathrm{L}$ & 7 & 0 & $0 \%$ & 2.50E-05 & 2.60E-05 & -- & -- & 1.29E-05 \\
\hline PEST & Carbethoxy malathion & $\mathrm{mg} / \mathrm{L}$ & 7 & 0 & $0 \%$ & 2.50E-05 & 2.60E-05 & -- & -- & $1.29 \mathrm{E}-05$ \\
\hline PEST & Chlorpyrifos & $\mathrm{mg} / \mathrm{L}$ & 7 & 0 & $0 \%$ & $2.50 \mathrm{E}-05$ & 2.60E-05 & -- & -- & $1.29 \mathrm{E}-05$ \\
\hline
\end{tabular}




\section{Table D-8}

Summary Statistics for All Categories of Surface Water Samples Collected from Segment 4 of the Columbia River

\begin{tabular}{|c|c|c|c|c|c|c|c|c|c|c|}
\hline $\begin{array}{l}\text { Constituent } \\
\text { Class }\end{array}$ & Constituent Name & Units & $\begin{array}{l}\text { Number } \\
\text { Analyzed }\end{array}$ & $\begin{array}{l}\text { Number } \\
\text { Detected }\end{array}$ & $\begin{array}{l}\text { Frequency of } \\
\text { Detection }\end{array}$ & $\begin{array}{l}\text { Minimum } \\
\text { Nondetect }\end{array}$ & $\begin{array}{l}\text { Maximum } \\
\text { Nondetect }\end{array}$ & $\begin{array}{l}\text { Minimum } \\
\text { Detect }\end{array}$ & $\begin{array}{l}\text { Maximum } \\
\text { Detect }\end{array}$ & $\begin{array}{c}\text { Average } \\
\text { Concentration }\end{array}$ \\
\hline$\overline{\text { PEST }}$ & $\begin{array}{l}\text { Diazinon } \\
\text { Dichlorodiphenyldichloroeth }\end{array}$ & $\overline{\mathrm{mg} / \mathrm{L}}$ & 7 & 0 & $0 \%$ & $2.50 \mathrm{E}-05$ & $2.60 \mathrm{E}-05$ & $\overline{--}$ & $\overline{--}$ & $1.29 \mathrm{E}-05$ \\
\hline PEST & $\begin{array}{l}\text { ane } \\
\text { Dichlorodiphenyldichloroeth }\end{array}$ & $\mathrm{mg} / \mathrm{L}$ & 6 & 6 & $100 \%$ & -- & -- & $3.90 \mathrm{E}-07$ & 2.90E-06 & $1.44 \mathrm{E}-06$ \\
\hline PEST & $\begin{array}{l}\text { ylene } \\
\text { Dichlorodiphenyltrichloroeth }\end{array}$ & $\mathrm{mg} / \mathrm{L}$ & 6 & 6 & $100 \%$ & -- & -- & 2.70E-07 & $1.00 \mathrm{E}-06$ & $6.33 E-07$ \\
\hline PEST & ane & $\mathrm{mg} / \mathrm{L}$ & 6 & 6 & $100 \%$ & -- & -- & 2.40E-08 & $1.20 \mathrm{E}-07$ & 7.30E-08 \\
\hline PEST & Dieldrin & $\mathrm{mg} / \mathrm{L}$ & 6 & 6 & $100 \%$ & -- & -- & 4.60E-08 & $4.90 \mathrm{E}-07$ & $2.24 \mathrm{E}-07$ \\
\hline PEST & Dimethoate & $\mathrm{mg} / \mathrm{L}$ & 7 & 0 & $0 \%$ & 5.00E-05 & $5.20 \mathrm{E}-05$ & -- & -- & 2.54E-05 \\
\hline PEST & Endosulfan I & $\mathrm{mg} / \mathrm{L}$ & 2 & 0 & $0 \%$ & 7.20E-07 & $7.20 \mathrm{E}-07$ & -- & -- & $3.60 \mathrm{E}-07$ \\
\hline PEST & Endrin & $\mathrm{mg} / \mathrm{L}$ & 6 & 0 & $0 \%$ & 9.00E-09 & $2.60 \mathrm{E}-07$ & -- & -- & $6.98 \mathrm{E}-08$ \\
\hline PEST & gamma-Chlordane & $\mathrm{mg} / \mathrm{L}$ & 3 & 2 & $67 \%$ & 2.30E-07 & 2.30E-07 & $3.20 \mathrm{E}-08$ & $9.40 \mathrm{E}-08$ & 8.03E-08 \\
\hline PEST & Heptachlor epoxide & $\mathrm{mg} / \mathrm{L}$ & 6 & 2 & $33 \%$ & $3.20 E-08$ & $2.70 \mathrm{E}-07$ & $6.50 \mathrm{E}-08$ & $6.50 \mathrm{E}-08$ & 7.26E-08 \\
\hline PEST & Methoxychlor & $\mathrm{mg} / \mathrm{L}$ & 6 & 2 & $33 \%$ & 9.00E-09 & 5.30E-07 & 5.40E-08 & $5.40 \mathrm{E}-08$ & $1.08 \mathrm{E}-07$ \\
\hline PEST & Methyl parathion & $\mathrm{mg} / \mathrm{L}$ & 7 & 0 & $0 \%$ & 2.50E-05 & 2.60E-05 & -- & -- & $1.29 \mathrm{E}-05$ \\
\hline PEST & Phosmet & $\mathrm{mg} / \mathrm{L}$ & 7 & 0 & $0 \%$ & 2.50E-05 & 2.60E-05 & -- & -- & 1.29E-05 \\
\hline PEST & Simazine & $\mathrm{mg} / \mathrm{L}$ & 7 & 0 & $0 \%$ & 2.50E-05 & 2.60E-05 & -- & -- & 1.29E-05 \\
\hline RAD & Gross alpha & $\mathrm{pCi} / \mathrm{L}$ & 20 & 3 & $15 \%$ & 0.90 & 3.0 & 1.1 & 2.4 & 0.98 \\
\hline RAD & Gross beta & $\mathrm{pCi} / \mathrm{L}$ & 20 & 4 & $20 \%$ & 0.90 & 5.0 & 1.2 & 6.5 & 2.1 \\
\hline SVOC & Benzo(a)anthracene & $\mathrm{mg} / \mathrm{L}$ & 6 & 4 & $67 \%$ & 3.50E-08 & 3.50E-08 & $1.30 \mathrm{E}-08$ & 3.50E-08 & $2.18 \mathrm{E}-08$ \\
\hline SVOC & Benzo(a)pyrene & $\mathrm{mg} / \mathrm{L}$ & 6 & 4 & $67 \%$ & 2.90E-08 & 2.90E-08 & 4.00E-09 & $1.00 \mathrm{E}-08$ & 9.50E-09 \\
\hline SVOC & Benzo(b)fluoranthene & $\mathrm{mg} / \mathrm{L}$ & 6 & 4 & $67 \%$ & 3.70E-08 & 3.70E-08 & $1.20 \mathrm{E}-08$ & $3.20 \mathrm{E}-08$ & 2.08E-08 \\
\hline SVOC & Benzo(ghi)perylene & $\mathrm{mg} / \mathrm{L}$ & 6 & 4 & $67 \%$ & 5.20E-08 & 5.20E-08 & 8.00E-09 & $1.20 \mathrm{E}-08$ & $1.53 E-08$ \\
\hline SVOC & Benzo(k)fluoranthene & $\mathrm{mg} / \mathrm{L}$ & 6 & 4 & $67 \%$ & 3.10E-08 & 3.10E-08 & $1.40 \mathrm{E}-08$ & 3.20E-08 & 2.05E-08 \\
\hline SVOC & Chrysene & $\mathrm{mg} / \mathrm{L}$ & 6 & 6 & $100 \%$ & -- & -- & $2.30 \mathrm{E}-08$ & 7.80E-08 & 4.80E-08 \\
\hline SVOC & Dibenz[a,h]anthracene & $\mathrm{mg} / \mathrm{L}$ & 3 & 2 & $67 \%$ & 3.80E-08 & 3.80E-08 & 5.00E-09 & 7.00E-09 & 1.03E-08 \\
\hline SVOC & Dibenzo(a,h)pyrene & $\mathrm{mg} / \mathrm{L}$ & 3 & 2 & $67 \%$ & 3.80E-08 & $3.80 \mathrm{E}-08$ & 5.00E-09 & 7.00E-09 & 1.03E-08 \\
\hline SVOC & Hexachlorobenzene & $\mathrm{mg} / \mathrm{L}$ & 6 & 6 & $100 \%$ & -- & -- & 8.90E-08 & $3.50 \mathrm{E}-07$ & 2.23E-07 \\
\hline SVOC & Indeno(1,2,3-cd)pyrene & $\mathrm{mg} / \mathrm{L}$ & 6 & 4 & $67 \%$ & 3.00E-08 & 3.00E-08 & 5.00E-09 & 1.10E-08 & 1.03E-08 \\
\hline
\end{tabular}

CONV - Conventional parameter

PCB - Polychlorinated biphenyls

PEST - Pesticide

RAD - Radionuclide

SVOC - Semivolatile Organic Compound 
Table D-9

Comparison of All Categories of Surface Water Concentrations from Segment 4 of the Columbia River to Ecological and Human Health Benchmark Values

\begin{tabular}{|c|c|c|c|c|c|c|c|c|c|c|c|c|c|c|c|c|}
\hline $\begin{array}{l}\text { Constituent } \\
\text { Class }\end{array}$ & Constituent Name & Units & $\begin{array}{l}\text { Number } \\
\text { Analyzed }\end{array}$ & $\begin{array}{l}\text { Number } \\
\text { Detected }\end{array}$ & $\begin{array}{l}\text { Frequency } \\
\text { of Detection }\end{array}$ & $\begin{array}{c}\text { Minimum } \\
\text { Detect }\end{array}$ & $\begin{array}{c}\text { Maximum } \\
\text { Detect }\end{array}$ & $\begin{array}{c}\text { Average } \\
\text { Concentration }\end{array}$ & $\begin{array}{l}\text { Ecological } \\
\text { Benchmark } \\
\text { Value }\end{array}$ & $\begin{array}{l}\text { Is Max > Eco } \\
\text { Benchmark? }\end{array}$ & $\begin{array}{c}\text { Factor of } \\
\text { Exceedance }\end{array}$ & $\begin{array}{c}\text { Number of } \\
\text { Exceedances }\end{array}$ & $\begin{array}{l}\text { Human Health } \\
\text { Benchmark Value }\end{array}$ & $\begin{array}{l}\text { Is Max }>\mathrm{HH} \\
\text { Benchmark? }\end{array}$ & $\begin{array}{c}\text { Factor of } \\
\text { Exceedance }\end{array}$ & $\begin{array}{c}\text { Number of } \\
\text { Exceedances }\end{array}$ \\
\hline$\overline{\text { CONV }}$ & Alkalinity & $\mathrm{mg} / \mathrm{L}$ & 22 & 22 & $100 \%$ & 29 & 109 & 62 & & & & & & & & \\
\hline CONV & Ammonia & $\mathrm{mg} / \mathrm{L}$ & 40 & 23 & $58 \%$ & 0.020 & 0.050 & 0.020 & -- & -- & -- & -- & -- & -- & -- & -- \\
\hline CONV & Chloride & $\mathrm{mg} / \mathrm{L}$ & 21 & 21 & $100 \%$ & 1.0 & 4.0 & 3.0 & 230 & No & 0.017 & 0 & -- & -- & -- & -- \\
\hline CONV & Dissolved organic carbon & $\mathrm{mg} / \mathrm{L}$ & 9 & 9 & $100 \%$ & 1.0 & 2.1 & 1.4 & -- & -- & -- & -- & -- & -- & -- & -- \\
\hline CONV & $\begin{array}{l}\text { Hardness } \\
\text { Nitrotos }\end{array}$ & $\mathrm{mg} / \mathrm{L}$ & 27 & 27 & $100 \%$ & 17 & 80 & 55 & -- & -- & -- & -- & -- & -- & - & - \\
\hline CONV & Nitrate & $\mathrm{mg} / \mathrm{L}$ & 21 & 9 & $43 \%$ & 0.20 & 0.40 & 0.18 & -- & -- & -- & -- & 10 & No & 0.040 & 0 \\
\hline CONV & Nitrogen, Kjeldahl total & $\mathrm{mg} / \mathrm{L}$ & 39 & 8 & $21 \%$ & 0.20 & 1.4 & 0.15 & -- & -- & -- & -- & -- & -- & -- & -- \\
\hline $\begin{array}{l}\text { CONV } \\
\text { CONV }\end{array}$ & Nitrogen, Nitrate-Nitrite & $\mathrm{mg} / \mathrm{L}$ & 40 & 40 & $100 \%$ & 0.058 & 0.53 & 0.18 & -- & -- & -- & -- & -- & -- & -- & -- \\
\hline & $\begin{array}{l}\text { Orthophosphate } \\
\text { PH }\end{array}$ & $\mathrm{mg} / \mathrm{L}$ & 40 & 40 & $100 \%$ & 0.0060 & $\begin{array}{l}0.061 \\
88\end{array}$ & 0.019 & -- & -- & -- & -- & -- & -- & -- & -- \\
\hline $\begin{array}{l}\text { CONV } \\
\text { CONV }\end{array}$ & $\begin{array}{l}\text { pH } \\
\text { Phosphorous in phosphate }\end{array}$ & $\begin{array}{l}\mathrm{pH} \text { Units } \\
\mathrm{mg} / \mathrm{L}\end{array}$ & ${ }_{40}^{21}$ & ${ }_{40}^{21}$ & $\begin{array}{l}100 \% \\
100 \%\end{array}$ & $\begin{array}{c}7.0 \\
0.010\end{array}$ & $\begin{array}{l}8.8 \\
6.4\end{array}$ & $\begin{array}{c}8.0 \\
0.23\end{array}$ & -- & -- & -- & & -- & -- & -- & - \\
\hline CONV & Specific Conductance & umhos $/ \mathrm{cm}$ & 33 & 33 & $\begin{array}{l}100 \% \\
100 \%\end{array}$ & 44 & 185 & $\begin{array}{l}0.23 \\
139\end{array}$ & -- & -- & -- & -- & -- & -- & -- & -- \\
\hline cONV & Sulfate & $\mathrm{mg} / \mathrm{L}$ & 21 & 17 & $81 \%$ & 7.0 & 15 & 11 & -- & -- & -- & -- & -- & -- & -- & -- \\
\hline CONV & Total dissolved solids & $\mathrm{mg} / \mathrm{L}$ & 21 & 21 & $100 \%$ & 76 & 118 & 97 & -- & -- & -- & -- & -- & -- & -- & -- \\
\hline CONV & Total Organic Carbon & $\mathrm{mg} / \mathrm{L}$ & 54 & 36 & $67 \%$ & 1.0 & 5.0 & 1.2 & -- & -- & -- & -- & -- & -- & -- & -- \\
\hline CONV & Total solids & $\mathrm{mg} / \mathrm{L}$ & 42 & 42 & $100 \%$ & 40 & 6,300 & 229 & -- & -- & -- & -- & -- & -- & -- & -- \\
\hline CONV & Total suspended solids & $\mathrm{mg} / \mathrm{L}$ & 58 & 57 & $98 \%$ & 1.0 & 5,900 & 118 & -- & -- & -- & -- & -- & -- & -- & -- \\
\hline METAL & Arsenic & $\mathrm{mg} / \mathrm{L}$ & 32 & 12 & $38 \%$ & $4.20 \mathrm{E}-04$ & 0.0021 & 0.0012 & 0.15 & No & 0.014 & 0 & $1.80 \mathrm{E}-05$ & Yes & 117 & 12 \\
\hline METAL & Barium & $\mathrm{mg} / \mathrm{L}$ & 21 & 21 & $100 \%$ & 0.027 & 0.043 & 0.034 & 0.0040 & Yes & 11 & 21 & 1.0 & No & 0.043 & 0 \\
\hline $\begin{array}{l}\text { METAL } \\
\text { METAL }\end{array}$ & Beryllium & $\mathrm{mg} / \mathrm{L}$ & 21 & 1 & $5 \%$ & 1.00E-04 & 1.00E-04 & 5.12E-05 & 0.0053 & No & 0.019 & 0 & -- & -- & -- & -- \\
\hline $\begin{array}{l}\text { MEEAL } \\
\text { METAL }\end{array}$ & Cadmium & $\mathrm{mg} / \mathrm{L}$ & 27 & 1 & $4 \%$ & 3.00E-04 & 3.00E-04 & $6.26 \mathrm{E}-04$ & 2.50E-04 & Yes & 1.2 & 1 & -- & -- & -- & -- \\
\hline $\begin{array}{l}\text { METAL } \\
\text { METAL }\end{array}$ & $\begin{array}{l}\text { Crromium } \\
\text { Iron }\end{array}$ & $\begin{array}{l}\mathrm{mgg} / \mathrm{L} \\
\mathrm{mg} / \mathrm{L}\end{array}$ & $\begin{array}{l}27 \\
27\end{array}$ & $\begin{array}{c}1 \\
27\end{array}$ & $\begin{array}{c}4 \% \\
100 \%\end{array}$ & $\begin{array}{l}0.0015 \\
0.072\end{array}$ & $\begin{array}{l}0.0015 \\
0.89\end{array}$ & $\begin{array}{l}6.48 \mathrm{E}-04 \\
0.24\end{array}$ & $\begin{array}{c}0.010 \\
10\end{array}$ & $\begin{array}{l}\text { No } \\
\text { No }\end{array}$ & $\begin{array}{l}0.15 \\
0.89\end{array}$ & $\begin{array}{l}0 \\
0\end{array}$ & 0 & yes & $\ddot{30}$ & $-\overline{6}$ \\
\hline METAL & $\begin{array}{l}\text { Iron } \\
\text { Lead }\end{array}$ & $\begin{array}{l}\mathrm{mgg} / \mathrm{L} \\
\mathrm{m} / \mathrm{L}\end{array}$ & $\begin{array}{l}27 \\
27\end{array}$ & $\begin{array}{l}27 \\
1\end{array}$ & $\begin{array}{c}100 \% \\
4 \%\end{array}$ & $\begin{array}{c}0.072 \\
0.0036\end{array}$ & $\begin{array}{c}0.89 \\
0.0036\end{array}$ & $\begin{array}{c}0.24 \\
0.0020\end{array}$ & $\begin{array}{c}1.0 \\
867 \mathrm{~F}-04\end{array}$ & $\begin{array}{l}\text { No } \\
\text { Yes }\end{array}$ & $\begin{array}{l}0.89 \\
42\end{array}$ & $\begin{array}{l}0 \\
1\end{array}$ & 0.30 & Yes & $\begin{array}{l}3.0 \\
--\end{array}$ & 6 \\
\hline METAL & Magnesium & $\mathrm{mg} / \mathrm{L}$ & $\begin{array}{c}21 \\
6\end{array}$ & $\begin{array}{l}1 \\
6\end{array}$ & $\begin{array}{l}400 \% \\
100 \%\end{array}$ & $\begin{array}{c}0.0036 \\
1.6\end{array}$ & $\begin{array}{c}0.0036 \\
2.0\end{array}$ & $\begin{array}{c}0.020 \\
1.8\end{array}$ & $\begin{array}{c}8.6 / 7-04 \\
82\end{array}$ & $\begin{array}{l}\text { Pes } \\
\text { No }\end{array}$ & $\begin{array}{l}4.2 \\
0.025\end{array}$ & $\begin{array}{l}1 \\
0\end{array}$ & -- & .- & .- & -- \\
\hline METAL & Manganese & $\mathrm{mg} / \mathrm{L}$ & 21 & 1 & $5 \%$ & 0.022 & 0.022 & 0.011 & 0.12 & No & 0.18 & 0 & 0.050 & No & 0.44 & 0 \\
\hline METAL & Nickel & $\mathrm{mg} / \mathrm{L}$ & 20 & 2 & $10 \%$ & 0.0034 & 0.0039 & 0.0013 & 0.028 & No & 0.14 & 0 & 0.61 & No & 0.0064 & 0 \\
\hline METAL & Sodium & $\mathrm{mg} / \mathrm{L}$ & 21 & 21 & $100 \%$ & 3.5 & 11 & 6.7 & 680 & No & 0.016 & 0 & -- & -- & -- & -- \\
\hline PCB & Total polychlorinated biphenyl & $\mathrm{mg} / \mathrm{L}$ & 3 & 1 & $33 \%$ & $3.70 \mathrm{E}-07$ & 3.70E-07 & 5.05E- 07 & $1.40 \mathrm{E}-05$ & No & 0.026 & 0 & $6.40 \mathrm{E}-08$ & Yes & 5.8 & 1 \\
\hline $\begin{array}{l}\text { PEST } \\
\text { DPCT }\end{array}$ & alpha-Chlordane & $\mathrm{mg} / \mathrm{L}$ & 3 & 2 & $67 \%$ & $3.00 \mathrm{E}-08$ & $1.20 \mathrm{E}-07$ & $8.50 \mathrm{E}-08$ & 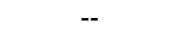 & $\mathrm{c}_{-1}$ & 然 & -- & 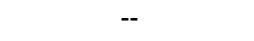 & -- & -- & -- \\
\hline $\begin{array}{l}\text { PEST } \\
\text { PEST }\end{array}$ & 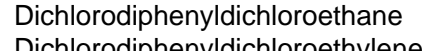 & $\mathrm{mg} / \mathrm{L}$ & 6 & 6 & $100 \%$ & $\begin{array}{l}3.90 \mathrm{E}-07 \\
270 \mathrm{E}-07\end{array}$ & $\begin{array}{l}2.90 E-06 \\
100 E-06\end{array}$ & $\begin{array}{l}1.44 \mathrm{E}-06 \\
633 \mathrm{~F}-07\end{array}$ & 1.00E-06 & Yes & 2.9 & 1 & 3.10E-07 & Yes & 9.4 & 6 \\
\hline $\begin{array}{l}\text { PEST } \\
\text { PEST }\end{array}$ & $\begin{array}{l}\text { Dichlorodiphenyyldichloroethylene } \\
\text { Dichllordinhenytrichlornethana }\end{array}$ & $\mathrm{mg} / \mathrm{L}$ & 6 & 6 & $100 \%$ & $\begin{array}{l}2.70 \mathrm{E}-07 \\
240 \mathrm{E}-08\end{array}$ & $1.00 \mathrm{E}-06$ & $\begin{array}{l}6.33 \mathrm{E}-07 \\
730 \mathrm{~F}-08\end{array}$ & & $\mathrm{No}$ & & $\overline{0}$ & $\begin{array}{l}2.20 \mathrm{E}-07 \\
220 \mathrm{E}-07\end{array}$ & Yes & 4.5 & 6 \\
\hline $\begin{array}{l}\text { PEST } \\
\text { PESI }\end{array}$ & $\begin{array}{l}\text { Dichlorodiliphenyltrichloroethane } \\
\text { Dieldrin }\end{array}$ & $\begin{array}{l}\mathrm{mggh} \\
\mathrm{mg} / \mathrm{L}\end{array}$ & $\begin{array}{l}6 \\
6\end{array}$ & $\begin{array}{l}6 \\
6\end{array}$ & $\begin{array}{l}100 \% \\
100 \%\end{array}$ & $\begin{array}{l}2.40 \mathrm{E}-08 \\
4.60 \mathrm{E}-08\end{array}$ & $\begin{array}{l}1.200-07 \\
4.90 \mathrm{E}-07\end{array}$ & $\begin{array}{l}. .30 \mathrm{E}-08 \\
2.24 \mathrm{E}-07\end{array}$ & $\begin{array}{l}1.00 E-06 \\
1.90 F-06\end{array}$ & No & 0.12 & $\begin{array}{l}0 \\
0\end{array}$ & $\begin{array}{l}2.20 \mathrm{E}-07 \\
520-08\end{array}$ & $\begin{array}{l}\text { No } \\
\text { Yes }\end{array}$ & $\begin{array}{l}0.55 \\
9.4\end{array}$ & 5 \\
\hline PEST & $\begin{array}{l}\text { Delearin } \\
\text { gamma-Chlordane }\end{array}$ & $\mathrm{mg} / \mathrm{L}$ & 3 & 2 & $67 \%$ & $\begin{array}{l}4.60 \mathrm{E}-08 \\
3.20 \mathrm{E}-08\end{array}$ & $9.40 \mathrm{E}-08$ & $\begin{array}{l}2.24-07 \\
8.03 E-08\end{array}$ & $1.90=-00$ & No & 0.20 & -- & 5.20E-08 & res & 9.4 & 5 \\
\hline PEST & Heptachlor epoxide & $\mathrm{mg} / \mathrm{L}$ & 6 & 2 & $33 \%$ & $6.50 \mathrm{E}-08$ & $6.50 \mathrm{E}-08$ & $7.26 \mathrm{E}-08$ & $3.80 \mathrm{E}-06$ & No & 0.017 & 0 & $3.90 \mathrm{E}-08$ & Yes & 1.7 & 2 \\
\hline PEST & Methoxychlor & $\mathrm{mg} / \mathrm{L}$ & 6 & 2 & $33 \%$ & $5.40 \mathrm{E}-08$ & $5.40 \mathrm{E}-08$ & $1.08 \mathrm{E}-07$ & 3.00E-05 & No & 0.0018 & 0 & -- & -- & -- & -- \\
\hline RAD & Gross alpha & $\mathrm{pCi} / \mathrm{L}$ & 20 & 3 & $15 \%$ & 1.1 & 2.4 & 0.98 & -- & -- & -- & -- & -- & -- & -- & -- \\
\hline RAD & Gross beta & $\mathrm{pCi} / \mathrm{L}$ & 20 & 4 & $20 \%$ & 1.2 & 6.5 & 2.1 & -- & -- & -- & -- & -- & -- & -- & -- \\
\hline sVoc & Benzo(a)anthracene & $\mathrm{mg} / \mathrm{L}$ & 6 & 4 & $67 \%$ & $1.30 \mathrm{E}-08$ & $3.50 \mathrm{E}-08$ & $2.18 \mathrm{E}-08$ & $2.70 \mathrm{E}-05$ & No & 0.0013 & 0 & 3.80E-06 & No & 0.0092 & 0 \\
\hline SVOC & Benzo(a)pyrene & $\mathrm{mg} / \mathrm{L}$ & 6 & 4 & $67 \%$ & $4.00 \mathrm{E}-09$ & $1.00 \mathrm{E}-08$ & 9.50E-09 & $1.40 \mathrm{E}-05$ & No & 7.14E-04 & 0 & $3.80 \mathrm{E}-06$ & No & 0.0026 & 0 \\
\hline SVOC & Benzo(b)fluoranthene & $\mathrm{mg} / \mathrm{L}$ & 6 & 4 & $67 \%$ & 1.20E-08 & 3.20E-08 & $2.08 \mathrm{E}-08$ & -- & -- & -- & -- & 3.80E-06 & No & 0.0084 & 0 \\
\hline SVOC & Benzo(ghi)perylene & $\mathrm{mg} / \mathrm{L}$ & 6 & 4 & $67 \%$ & $8.00 \mathrm{E}-09$ & $1.20 \mathrm{E}-08$ & 1.53E-08 & -- & -- & -- & -- & -- & - & م000 & 0 \\
\hline $\begin{array}{l}\text { SVOC } \\
\text { sVOC }\end{array}$ & $\begin{array}{l}\text { Benzo(k)fluoranthene } \\
\text { Chrysene }\end{array}$ & $\mathrm{mg} / \mathrm{L}$ & 6 & 4 & $67 \%$ & $\begin{array}{l}1.40 \mathrm{E}-08 \\
230 \mathrm{E}-08\end{array}$ & $\begin{array}{l}3.20 \mathrm{E}-08 \\
780-08\end{array}$ & $\begin{array}{l}2.05 \mathrm{E}-08 \\
480 \mathrm{~F}-08\end{array}$ & - & - & -- & - & $\begin{array}{l}3.80 \mathrm{E}-06 \\
380 \mathrm{~F}-06\end{array}$ & No & 0.0084 & 0 \\
\hline $\begin{array}{l}\text { SVOC } \\
\text { SVOC }\end{array}$ & $\begin{array}{l}\text { Chrysene } \\
\text { Dibenzla hlanthracene }\end{array}$ & $\mathrm{mg} / \mathrm{L}$ & $\begin{array}{l}6 \\
3\end{array}$ & $\begin{array}{l}6 \\
2\end{array}$ & $\begin{array}{l}100 \% \\
67 \%\end{array}$ & $\begin{array}{l}2.30 \mathrm{E}-08 \\
500-09\end{array}$ & $\begin{array}{l}7.80 \mathrm{E}-08 \\
7700-09\end{array}$ & $\begin{array}{l}4.80 \mathrm{E}-08 \\
1.03 \mathrm{E}-08\end{array}$ & -- & -- & -- & -- & $\begin{array}{l}3.80 \mathrm{E}-06 \\
3.80-06\end{array}$ & $\begin{array}{lll}\text { No } \\
\text { No }\end{array}$ & 0.021 & 0 \\
\hline $\begin{array}{l}\text { SVOC } \\
\text { SVOC }\end{array}$ & $\begin{array}{l}\text { Dibenzl[a,h]anthracene } \\
\text { Dibenzo(a,h)pyrene }\end{array}$ & $\begin{array}{l}\mathrm{mggh} \\
\mathrm{mg} / \mathrm{L}\end{array}$ & $\begin{array}{l}3 \\
3\end{array}$ & $\begin{array}{l}2 \\
2\end{array}$ & $\begin{array}{l}67 \% \\
67 \%\end{array}$ & $\begin{array}{l}5.00 \mathrm{E}-09 \\
5.00 \mathrm{E}-09\end{array}$ & $\begin{array}{l}7.00=-09 \\
7.00 \mathrm{E}-09\end{array}$ & $\begin{array}{l}1.03 E-08 \\
1.03 E-08\end{array}$ & $\because$ & $\therefore$ & -- & -- & $3.80=-06$ & No & 0.0018 & -. \\
\hline sVoc & Hexachlorobenzene & $\mathrm{mg} / \mathrm{L}$ & 6 & 6 & $100 \%$ & $8.90 \mathrm{E}-08$ & $3.50 \mathrm{E}-07$ & 2.23E- 07 & -- & -- & -- & -- & $2.80 \mathrm{E}-07$ & Yes & 1.3 & 2 \\
\hline SVOC & Indeno(1,2,3-cd)pyrene & $\mathrm{mg} / \mathrm{L}$ & 6 & 4 & $67 \%$ & $5.00 \mathrm{E}-09$ & $1.10 \mathrm{E}-08$ & 1.03E-08 & -- & -- & -- & -- & 3.80E-06 & No & 0.0029 & 0 \\
\hline
\end{tabular}

CONV - Conventional parameter

PCB - Polychlorinated Biphenyl

RAD - Radionuclide

SVOC - Semivolatile Organic Compound 


\begin{tabular}{|c|c|c|c|c|c|c|c|c|c|c|}
\hline Sample Area & Sample Site & $\begin{array}{c}\text { Sample } \\
\text { ID }\end{array}$ & Date Collected & Constituent Name & Units & $\begin{array}{c}\text { QA } \\
\text { Category }\end{array}$ & $\begin{array}{l}\text { Analytical } \\
\text { Value }\end{array}$ & Qualifier & $\begin{array}{c}\text { Ecological } \\
\text { Benchmark } \\
\text { Value }\end{array}$ & $\begin{array}{c}\text { Health } \\
\text { Benchmark } \\
\text { Value }\end{array}$ \\
\hline Columbia River & Umatilla OR & 2118106 & February 11,2002 & Arsenic & $\overline{\mathrm{mg} / \mathrm{L}}$ & $\frac{\pi}{1}$ & $4.20 \mathrm{E}-04$ & $=$ & 0.15 & $1.80 \mathrm{E}-05$ \\
\hline Columbia River & Umatilla OR & 2228081 & May 15, 2002 & Arsenic & $\mathrm{mg} / \mathrm{L}$ & 1 & $6.90 \mathrm{E}-04$ & $=$ & 0.15 & $1.80 \mathrm{E}-05$ \\
\hline Columbia River & Umatilla OR & 2158156 & March 13, 2002 & Arsenic & $\mathrm{mg} / \mathrm{L}$ & 1 & 8.80E-04 & $=$ & 0.15 & $1.80 \mathrm{E}-05$ \\
\hline Columbia River & Umatilla OR & 1286070 & July 11,2001 & Arsenic & $\mathrm{mg} / \mathrm{L}$ & 1 & 8.90E-04 & $=$ & 0.15 & $1.80 \mathrm{E}-05$ \\
\hline Columbia River & Umatilla OR & 2018081 & December 5, 2001 & Arsenic & $\mathrm{mg} / \mathrm{L}$ & 1 & $9.90 \mathrm{E}-04$ & $=$ & 0.15 & $1.80 \mathrm{E}-05$ \\
\hline Columbia River & Umatilla OR & 2068081 & January 16, 2002 & Arsenic & $\mathrm{mg} / \mathrm{L}$ & 1 & 0.0010 & $=$ & 0.15 & $1.80 \mathrm{E}-05$ \\
\hline Columbia River & Umatilla OR & 1468156 & October 17, 2001 & Arsenic & $\mathrm{mg} / \mathrm{L}$ & 1 & 0.0011 & $=$ & 0.15 & $1.80 \mathrm{E}-05$ \\
\hline Columbia River & Umatilla OR & 1408081 & September 12, 2001 & Arsenic & $\mathrm{mg} / \mathrm{L}$ & 1 & 0.0012 & $=$ & 0.15 & $1.80 \mathrm{E}-05$ \\
\hline Columbia River & Umatilla OR & 2178181 & April 10, 2002 & Arsenic & $\mathrm{mg} / \mathrm{L}$ & 1 & 0.0014 & $=$ & 0.15 & $1.80 \mathrm{E}-05$ \\
\hline Columbia River & Umatilla OR & 1368082 & August 8,2001 & Arsenic & $\mathrm{mg} / \mathrm{L}$ & 1 & 0.0014 & $=$ & 0.15 & $1.80 \mathrm{E}-05$ \\
\hline Columbia River & Umatilla OR & 1498101 & November 7, 2001 & Arsenic & $\mathrm{mg} / \mathrm{L}$ & 1 & 0.0014 & $=$ & 0.15 & $1.80 \mathrm{E}-05$ \\
\hline Columbia River & DALLES BRDG & 080701 & Auqust 7, 2001 & Arsenic & $\mathrm{mg} / \mathrm{L}$ & 1 & 0.0021 & $=$ & 0.15 & $1.80 \mathrm{E}-05$ \\
\hline Columbia River & DALLES BRDG & 083000 & August 30,2000 & Barium & $\mathrm{mg} / \mathrm{L}$ & 1 & 0.027 & $=$ & 0.0040 & 1.0 \\
\hline Columbia River & DALLES BRDG & 021004 & February 10, 2004 & Barium & $\mathrm{mg} / \mathrm{L}$ & 1 & 0.027 & $=$ & 0.0040 & 1.0 \\
\hline Columbia River & DALLES BRDG & 052200 & May 22, 2000 & Barium & $\mathrm{mg} / \mathrm{L}$ & 1 & 0.028 & $=$ & 0.0040 & 1.0 \\
\hline Columbia River & DALLES BRDG & 020800 & February 8,2000 & Barium & $\mathrm{mg} / \mathrm{L}$ & 1 & 0.029 & $=$ & 0.0040 & 1.0 \\
\hline Columbia River & DALLES BRDG & 051004 & May 10,2004 & Barium & $\mathrm{mg} / \mathrm{L}$ & 1 & 0.029 & $=$ & 0.0040 & 1.0 \\
\hline Columbia River & DALLES BRDG & 080904 & August 9,2004 & Barium & $\mathrm{mg} / \mathrm{L}$ & 1 & 0.030 & $=$ & 0.0040 & 1.0 \\
\hline Columbia River & DALLES BRDG & 111300 & November 13,2000 & Barium & $\mathrm{mg} / \mathrm{L}$ & 1 & 0.030 & $=$ & 0.0040 & 1.0 \\
\hline Columbia River & DALLES BRDG & 110601 & November 6, 2001 & Barium & $\mathrm{mg} / \mathrm{L}$ & 1 & 0.030 & $=$ & 0.0040 & 1.0 \\
\hline Columbia River & DALLES BRDG & 110502 & November 5, 2002 & Barium & $\mathrm{mg} / \mathrm{L}$ & 1 & 0.032 & $=$ & 0.0040 & 1.0 \\
\hline Columbia River & DALLES BRDG & 101304 & October 13, 2004 & Barium & $\mathrm{mg} / \mathrm{L}$ & 1 & 0.032 & $=$ & 0.0040 & 1.0 \\
\hline Columbia River & DALLES BRDG & 050703 & May 7, 2003 & Barium & $\mathrm{mg} / \mathrm{L}$ & 1 & 0.033 & $=$ & 0.0040 & 1.0 \\
\hline Columbia River & DALLES BRDG & 080503 & August 5, 2003 & Barium & $\mathrm{mg} / \mathrm{L}$ & 1 & 0.034 & $=$ & 0.0040 & 1.0 \\
\hline Columbia River & DALLES BRDG & 052201 & May 22, 2001 & Barium & $\mathrm{mg} / \mathrm{L}$ & 1 & 0.034 & $=$ & 0.0040 & 1.0 \\
\hline Columbia River & DALLES BRDG & 080701 & August 7,2001 & Barium & $\mathrm{mg} / \mathrm{L}$ & 1 & 0.035 & $=$ & 0.0040 & 1.0 \\
\hline Columbia River & DALLES BRDG & 020403 & February 4, 2003 & Barium & $\mathrm{mg} / \mathrm{L}$ & 1 & 0.036 & $=$ & 0.0040 & 1.0 \\
\hline Columbia River & DALLES BRDG & 020502 & February 5, 2002 & Barium & $\mathrm{mg} / \mathrm{L}$ & 1 & 0.036 & $=$ & 0.0040 & 1.0 \\
\hline Columbia River & DALLES BRDG & 050702 & May 7,2002 & Barium & $\mathrm{mg} / \mathrm{L}$ & 1 & 0.037 & $=$ & 0.0040 & 1.0 \\
\hline Columbia River & DALLES BRDG & 080602 & August 6, 2002 & Barium & $\mathrm{mg} / \mathrm{L}$ & 1 & 0.038 & $=$ & 0.0040 & 1.0 \\
\hline Columbia River & DALLES BRDG & 020805 & February 8, 2005 & Barium & $\mathrm{mg} / \mathrm{L}$ & 1 & 0.039 & $=$ & 0.0040 & 1.0 \\
\hline Columbia River & DALLES BRDG & 051005 & May 10,2005 & Barium & $\mathrm{mg} / \mathrm{L}$ & 1 & 0.042 & $=$ & 0.0040 & 1.0 \\
\hline Columbia River & DALLES BRDG & 020601 & February 6, 2001 & Barium & $\mathrm{mg} / \mathrm{L}$ & 1 & 0.043 & $=$ & 0.0040 & 1.0 \\
\hline Columbia River & DALLES BRDG & 020805 & February 8,2005 & Cadmium & $\mathrm{mg} / \mathrm{L}$ & $\frac{1}{1}$ & $3.00 \mathrm{E}-04$ & $=$ & $2.50 \mathrm{E}-04$ & $=0$ \\
\hline Columbia River & RM147 BONN DAM & $: 018 / 03-9 /$ & September 1, 2003 & Dichlorodiphenyldichloroethane & $\mathrm{mg} / \mathrm{L}$ & 1 & $3.90 \mathrm{E}-07$ & $=$ & $1.00 \mathrm{E}-06$ & $3.10 \mathrm{E}-07$ \\
\hline Columbia River & Bonneville Dam & 3454413 & September 24,2003 & Dichlorodiphenyldichloroethane & $\mathrm{mg} / \mathrm{L}$ & 1 & 4.90E-07 & $=$ & $1.00 \mathrm{E}-06$ & 3.10E-07 \\
\hline Columbia River & Bonneville Dam & 4314230 & June 22, 2004 & Dichlorodiphenyldichloroethane & $\mathrm{mg} / \mathrm{L}$ & 1 & $9.80 \mathrm{E}-07$ & $=$ & $1.00 \mathrm{E}-06$ & $3.10 \mathrm{E}-07$ \\
\hline Columbia River & RM147 BONN DAM & : $015 / 04-6 /$ & June 1, 2004 & Dichlorodiphenyldichloroethane & $\mathrm{mg} / \mathrm{L}$ & 1 & $9.80 \mathrm{E}-07$ & $=$ & $1.00 \mathrm{E}-06$ & 3.10E-07 \\
\hline Columbia River & Bonneville Dam & 4154230 & January 12, 2004 & Dichlorodiphenyldichloroethane & $\mathrm{mg} / \mathrm{L}$ & 1 & 2.90E-06 & $=$ & $1.00 \mathrm{E}-06$ & 3.10E-07 \\
\hline Columbia River & RM147 BONN DAM & 01 12/03-1 & January 1,2004 & Dichlorodiphenyldichloroethane & $\mathrm{mg} / \mathrm{L}$ & 1 & $2.90 \mathrm{E}-06$ & $=$ & $1.00 \mathrm{E}-06$ & 3.10E-07 \\
\hline Columbia River & RM147 BONN DAM & $: 018 / 03-9 /$ & September 1, 2003 & Dichlorodiphenyldichloroethylene & $\mathrm{mg} / \mathrm{L}$ & 1 & $2.70 \mathrm{E}-07$ & $=$ & -- & $2.20 \mathrm{E}-07$ \\
\hline Columbia River & Bonneville Dam & 3454413 & September 24,2003 & Dichlorodiphenyldichloroethylene & $\mathrm{mg} / \mathrm{L}$ & 1 & 3.30E-07 & $=$ & -- & $2.20 \mathrm{E}-07$ \\
\hline Columbia River & Bonneville Dam & 4314230 & June 22,2004 & Dichlorodiphenyldichloroethylene & $\mathrm{mg} / \mathrm{L}$ & 1 & $6.00 \mathrm{E}-07$ & $=$ & -- & 2.20E-07 \\
\hline Columbia River & RM147 BONN DAM & :01 5/04-6/ & June 1,2004 & Dichlorodiphenyldichloroethylene & $\mathrm{mg} / \mathrm{L}$ & 1 & $6.00 \mathrm{E}-07$ & $=$ & -- & $2.20 \mathrm{E}-07$ \\
\hline Columbia River & Bonneville Dam & 4154230 & January 12, 2004 & Dichlorodiphenyldichloroethylene & $\mathrm{mg} / \mathrm{L}$ & 1 & $1.00 \mathrm{E}-06$ & $=$ & -- & $2.20 \mathrm{E}-07$ \\
\hline Columbia River & RM147 BONN DAM & 01 12/03-1 & January 1, 2004 & Dichlorodiphenyldichloroethylene & $\mathrm{mg} / \mathrm{L}$ & 1 & $1.00 \mathrm{E}-06$ & $=$ & -- & $2.20 \mathrm{E}-07$ \\
\hline Columbia River & Bonneville Dam & 3454413 & September 24,2003 & Dieldrin & $\mathrm{mg} / \mathrm{L}$ & $\frac{1}{1}$ & $5.70 \mathrm{E}-08$ & $=$ & $1.90 \mathrm{E}-06$ & $5.20 \mathrm{E}-08$ \\
\hline Columbia River & Bonneville Dam & 4314230 & June 22, 2004 & Dieldrin & $\mathrm{mg} / \mathrm{L}$ & 1 & $1.30 \mathrm{E}-07$ & $\mathrm{~J}$ & $1.90 \mathrm{E}-06$ & $5.20 \mathrm{E}-08$ \\
\hline Columbia River & RM147 BONN DAM & : $015 / 04-6 /$ & June 1, 2004 & Dieldrin & $\mathrm{mg} / \mathrm{L}$ & 1 & 1.30E-07 & $=$ & 1.90E-06 & $5.20 \mathrm{E}-08$ \\
\hline
\end{tabular}




\begin{tabular}{|c|c|c|c|c|c|c|c|c|c|c|}
\hline Sample Area & Sample Site & $\begin{array}{l}\text { Sample } \\
\text { ID }\end{array}$ & Date Collected & Constituent Name & Units & $\begin{array}{c}\text { QA } \\
\text { Category }\end{array}$ & $\begin{array}{l}\text { Analytical } \\
\text { Value }\end{array}$ & Qualifier & $\begin{array}{c}\text { Ecological } \\
\text { Benchmark } \\
\text { Value }\end{array}$ & $\begin{array}{c}\text { Health } \\
\text { Benchmark } \\
\text { Value }\end{array}$ \\
\hline Columbia River & Bonneville Dam & 4154230 & January 12,2004 & Dieldrin & $\mathrm{mg} / \mathrm{L}$ & $\frac{1}{1}$ & $4.90 \mathrm{E}-07$ & $\mathrm{~J}$ & $1.90 \mathrm{E}-06$ & $5.20 \mathrm{E}-08$ \\
\hline Columbia River & RM147 BONN DAM & 01 12/03-1 & January 1,2004 & Dieldrin & $\mathrm{mg} / \mathrm{L}$ & 1 & 4.90E-07 & $=$ & $1.90 \mathrm{E}-06$ & $5.20 \mathrm{E}-08$ \\
\hline Columbia River & Bonneville Dam & 4314230 & June 22,2004 & Heptachlor epoxide & $\mathrm{mg} / \mathrm{L}$ & 1 & $6.50 \mathrm{E}-08$ & $\mathrm{~J}$ & $3.80 \mathrm{E}-06$ & $3.90 \mathrm{E}-08$ \\
\hline Columbia River & RM147 BONN DAM & : $015 / 04-6 /$ & June 1,2004 & Heptachlor epoxide & $\mathrm{mg} / \mathrm{L}$ & 1 & $6.50 \mathrm{E}-08$ & $=$ & 3.80E-06 & 3.90E-08 \\
\hline Columbia River & Bonneville Dam & 4154230 & January 12,2004 & Hexachlorobenzene & $\mathrm{mg} / \mathrm{L}$ & 1 & $3.50 \mathrm{E}-07$ & $\mathrm{~J}$ & -- & $2.80 \mathrm{E}-07$ \\
\hline Columbia River & RM147 BONN DAM & 01 12/03-1 & January 1, 2004 & Hexachlorobenzene & $\mathrm{mg} / \mathrm{L}$ & 1 & $3.50 \mathrm{E}-07$ & $=$ & -- & $2.80 \mathrm{E}-07$ \\
\hline Columbia River & DALLES BRDG & 052200 & May 22,2000 & Iron & $\mathrm{mg} / \mathrm{L}$ & 1 & 0.33 & $=$ & 1.0 & 0.30 \\
\hline Columbia River & DALLES BRDG & 050703 & May 7,2003 & Iron & $\mathrm{mg} / \mathrm{L}$ & 1 & 0.33 & $=$ & 1.0 & 0.30 \\
\hline Columbia River & DALLES BRDG & 051005 & May 10, 2005 & Iron & $\mathrm{mg} / \mathrm{L}$ & 1 & 0.34 & $=$ & 1.0 & 0.30 \\
\hline Columbia River & DALLES BRDG & 050702 & May 7, 2002 & Iron & $\mathrm{mg} / \mathrm{L}$ & 1 & 0.43 & $=$ & 1.0 & 0.30 \\
\hline Columbia River & FTBRDG DSM I-84 & 605855 & April 19, 2002 & Iron & $\mathrm{mg} / \mathrm{L}$ & 1 & 0.55 & $=$ & 1.0 & 0.30 \\
\hline Columbia River & DALLES BRDG & 080602 & August 6,2002 & Iron & $\mathrm{mg} / \mathrm{L}$ & 1 & 0.89 & $=$ & 1.0 & 0.30 \\
\hline Columbia River & DALLES BRDG & 101304 & October 13,2004 & Lead & $\mathrm{mg} / \mathrm{L}$ & 1 & 0.0036 & $=$ & $8.67 \mathrm{E}-04$ & 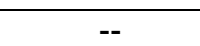 \\
\hline Columbia River & Bonneville Dam & 4314230 & June 22,2004 & Total polychlorinated biphenyl & $\mathrm{mg} / \mathrm{L}$ & 1 & $3.70 \mathrm{E}-07$ & $=$ & $1.40 \mathrm{E}-05$ & $6.40 \mathrm{E}-08$ \\
\hline
\end{tabular}




\section{APPENDIX E SEGMENT 5 \\ SUMMARY STATISTICS, BENCHMARK COMPARISON, AND INDIVIDUAL SAMPLE EXCEEDENCES}


WCH-91

Rev. 0

Columbia River Component Data Evaluation Summary Report

July 2006

E-ii 


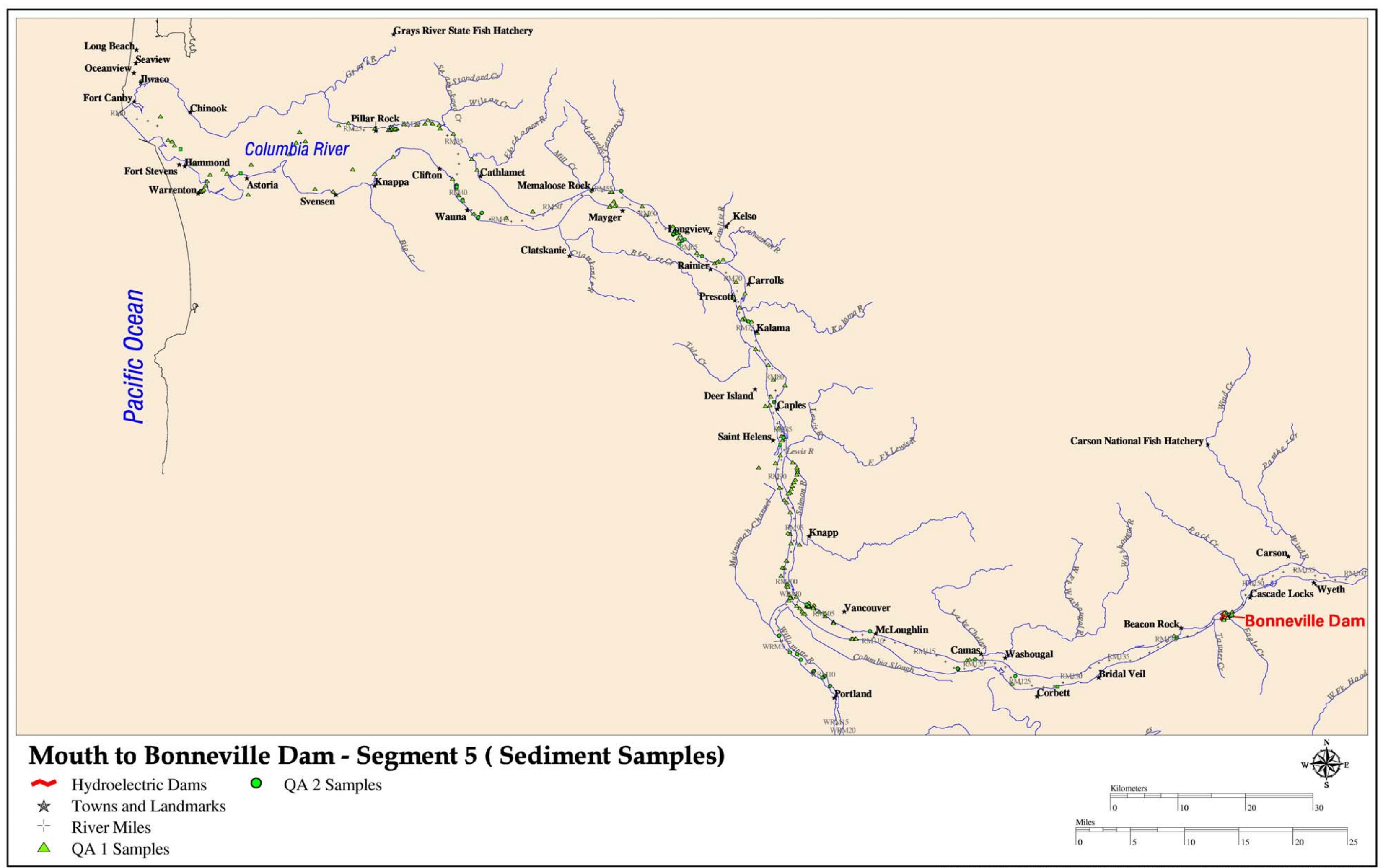




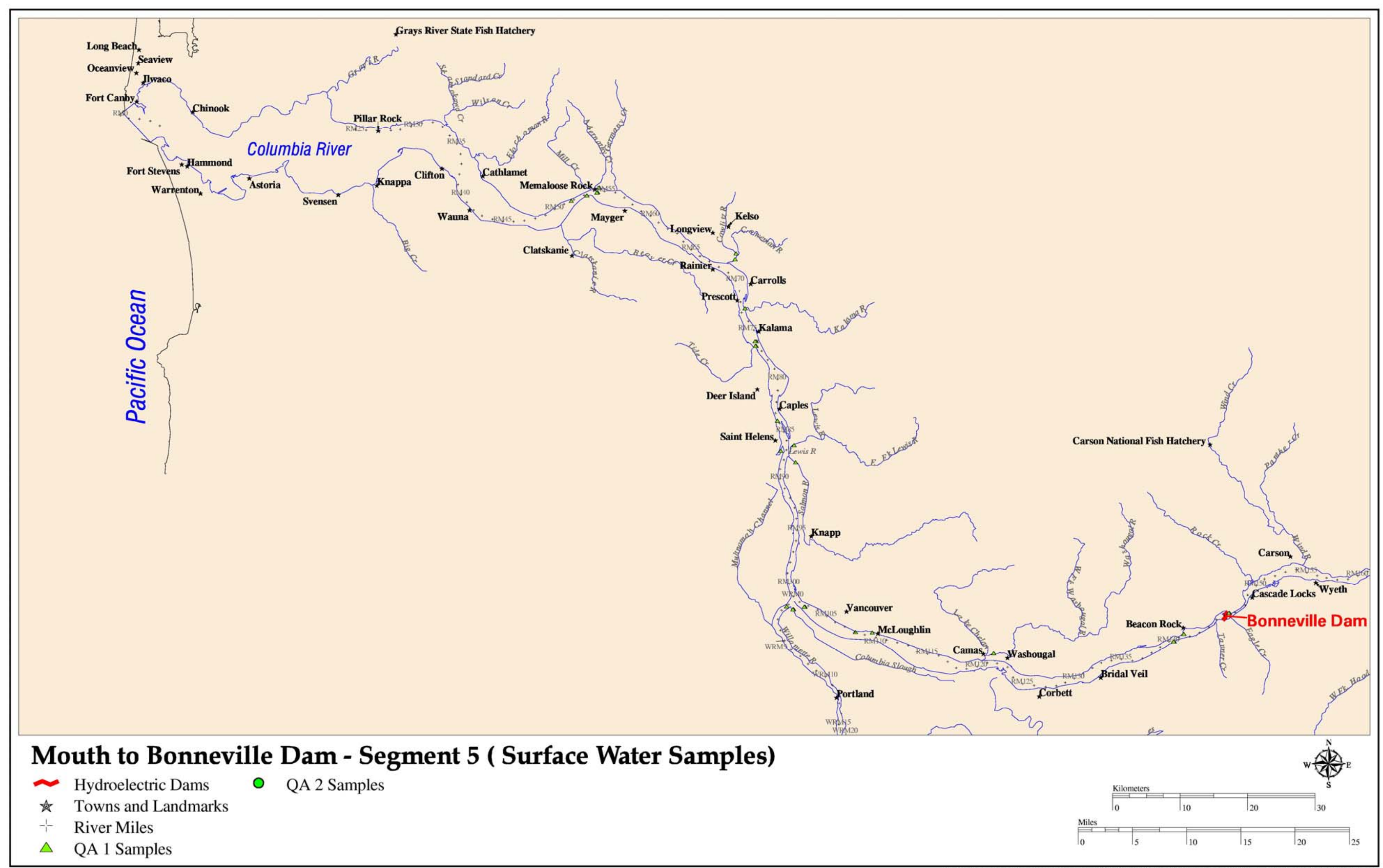




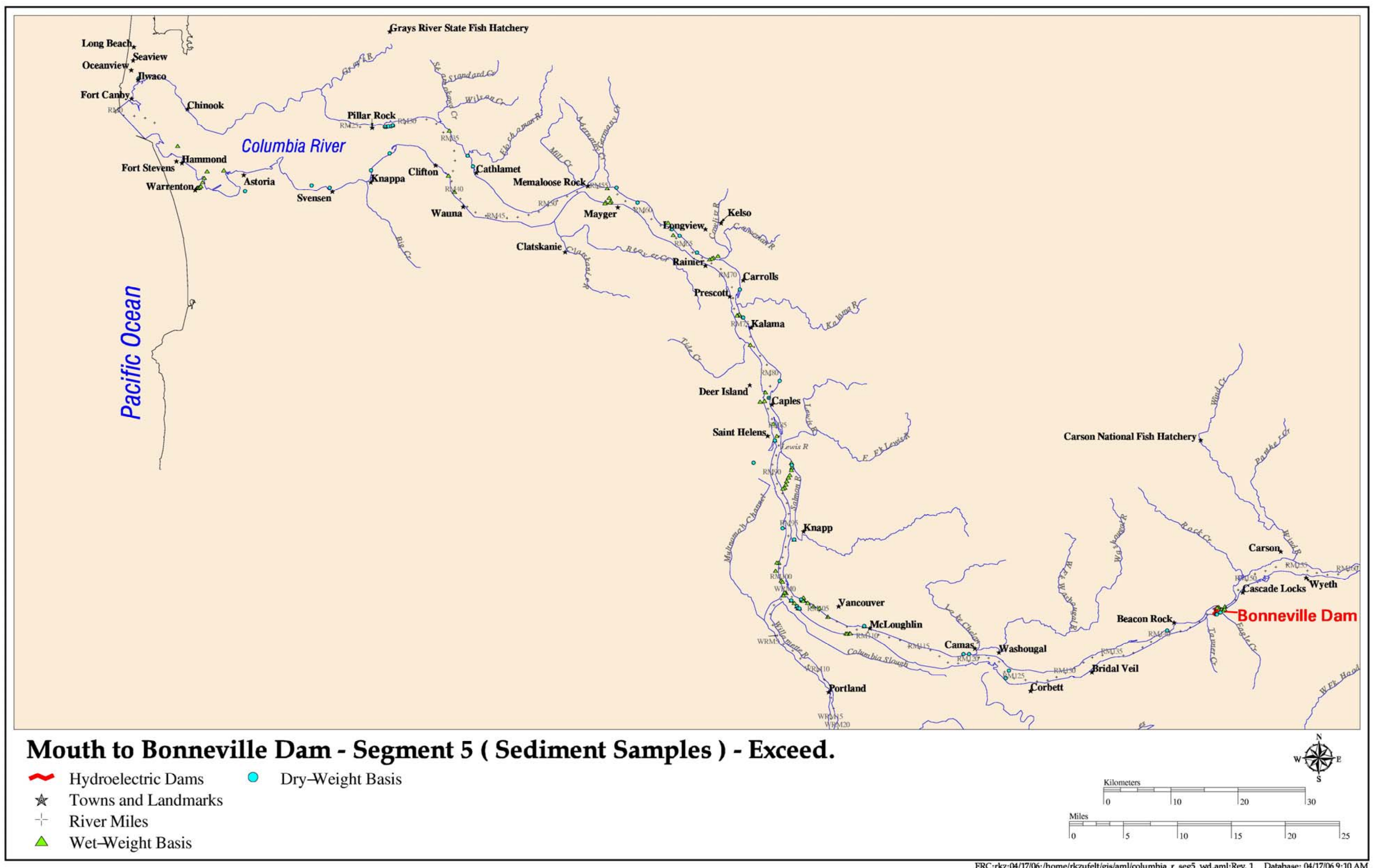




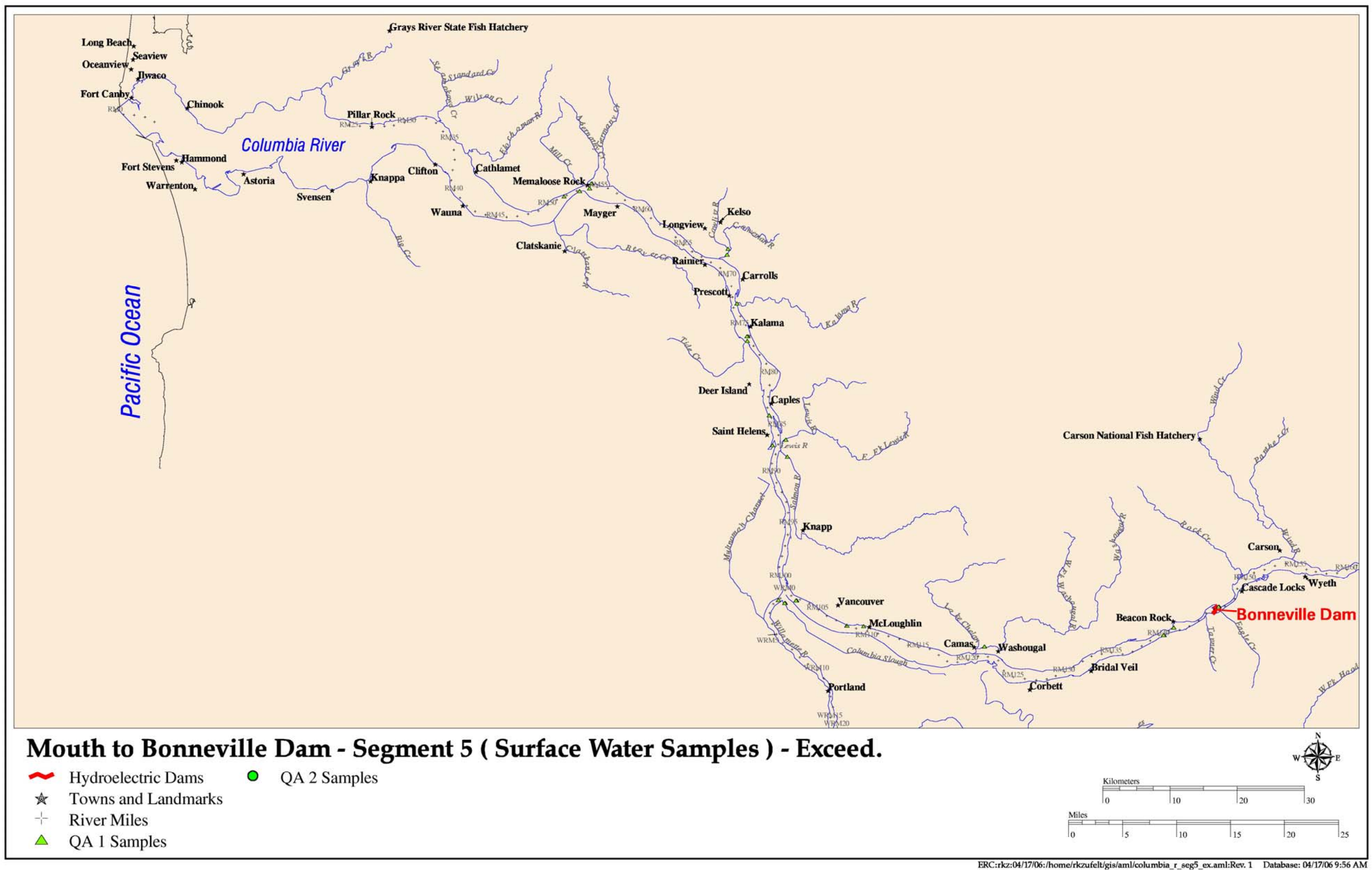


Table E-1

Summary Table of Segment 5 Sediment Samples By Area, Site, Date, and Category

\begin{tabular}{|c|c|c|c|c|}
\hline Sample ID & Sample Area & Sample Site & Sample Date & Category \\
\hline 0205-OS-VC-01 & Columbia River & Oregon Slough & 17-Feb-05 & 1 \\
\hline 0205-OS-VC-02 & Columbia River & Oregon Slough & 17-Feb-05 & 1 \\
\hline 0205-OS-VC-03 & Columbia River & Oregon Slough & 17-Feb-05 & 1 \\
\hline 0205-OS-VC-03A & Columbia River & Oregon Slough & 17-Feb-05 & 1 \\
\hline 0205-OS-VC-04 & Columbia River & Oregon Slough & 17-Feb-05 & 1 \\
\hline 0205-OS-VC-05 & Columbia River & Oregon Slough & 17-Feb-05 & 1 \\
\hline 0205-OS-VC-06 & Columbia River & Oregon Slough & 17-Feb-05 & 1 \\
\hline 0205-OS-VC-06Z & Columbia River & Oregon Slough & 17-Feb-05 & 1 \\
\hline 088020-Diffuser & Columbia River & Longview WA & 23-Feb-90 & 1 \\
\hline 088020-Diffuser-F & Columbia River & Longview WA & 23-Feb-90 & 1 \\
\hline 088021-Dwnstrm & Columbia River & Longview WA & 23-Feb-90 & 1 \\
\hline 088021-Dwnstrm-F & Columbia River & Longview WA & 23-Feb-90 & 1 \\
\hline 088022-Upstrm & Columbia River & Longview WA & 23-Feb-90 & 1 \\
\hline 088022-Upstrm-F & Columbia River & Longview WA & 23-Feb-90 & 1 \\
\hline $1-S$ & Columbia River & RM 14 & 28-Jun-93 & 1 \\
\hline $10-S$ & Columbia River & RM 88 & 28-Jun-93 & 1 \\
\hline $11-S$ & Columbia River & RM 90 & 29-Jun-93 & 1 \\
\hline $12-S$ & Columbia River & RM 95 & 30-Jun-93 & 1 \\
\hline $13-S$ & Columbia River & RM 120 & 01-Jul-93 & 1 \\
\hline $14-S$ & Columbia River & RM 124 & 01-Jul-93 & 1 \\
\hline $15-S$ & Columbia River & RM 141 & 30-Jun-93 & 1 \\
\hline $2-S$ & Columbia River & RM 21 & 27-Jun-93 & 1 \\
\hline $3-S$ & Columbia River & RM 23 & 27-Jun-93 & 1 \\
\hline $4-S$ & Columbia River & RM 26 & 26-Jun-93 & 1 \\
\hline $5-S$ & Columbia River & RM 29 & 26-Jun-93 & 1 \\
\hline $6-S$ & Columbia River & RM 36 & 25-Jun-93 & 1 \\
\hline $7-S$ & Columbia River & RM 59 & 25-Jun-93 & 1 \\
\hline $8-S$ & Columbia River & RM 68 & 24-Jun-93 & 1 \\
\hline $88-398-300$ & Columbia River & Reed Island & 22-Jul-87 & 2 \\
\hline 88-398-301 & Columbia River & Camas Slough & 22-Jul-87 & 2 \\
\hline 88-398-302 & Columbia River & Camas Slough & 22-Jul-87 & 2 \\
\hline 88-398-303 & Columbia River & Vancouver WA & 22-Jul-87 & 2 \\
\hline 88-398-304 & Columbia River & Vancouver WA & 22-Jul-87 & 2 \\
\hline 88-398-305 & Columbia River & Kalama ChemPier & 22-Jul-87 & 2 \\
\hline 88-398-306 & Columbia River & Longview Fibre & 23-Jul-87 & 2 \\
\hline 88-398-307 & Columbia River & Longview WA & 23-Jul-87 & 2 \\
\hline 88-398-308 & Columbia River & Longview WA & 23-Jul-87 & 2 \\
\hline 88-398-309 & Columbia River & Longview WA & 23-Jul-87 & 2 \\
\hline $88-398-310$ & Columbia River & Coal Creek Slg & 23-Jul-87 & 2 \\
\hline 88-398-311 & Columbia River & Ilwaco Boat Bsn & 24-Jul-87 & 2 \\
\hline $9-S$ & Columbia River & RM 81 & 29-Jun-93 & 1 \\
\hline AS-10/20/1988 & Columbia River & RM 13.7 & $20-O c t-88$ & 3 \\
\hline AS-10/7/1992 & Columbia River & RM 13.7 & 07-Oct-92 & 3 \\
\hline AS-11/11/1969 & Columbia River & RM 13.7 & 11-Nov-69 & 3 \\
\hline AS-11/11/1981 & Columbia River & RM 13.7 & 11-Nov-81 & 3 \\
\hline AS-11/14/1972 & Columbia River & RM 13.7 & 14-Nov-72 & 3 \\
\hline AS-11/17/1977 & Columbia River & RM 13.7 & 17-Nov-77 & 3 \\
\hline
\end{tabular}


Table E-1

Summary Table of Segment 5 Sediment Samples By Area, Site, Date, and Category

\begin{tabular}{|c|c|c|c|c|}
\hline Sample ID & Sample Area & Sample Site & Sample Date & Category \\
\hline AS-11/22/1967 & Columbia River & RM 13.7 & 22-Nov-67 & 3 \\
\hline AS-11/25/1965 & Columbia River & RM 13.7 & 25-Nov-65 & 3 \\
\hline AS-11/25/1980 & Columbia River & RM 13.7 & 25-Nov-80 & 3 \\
\hline AS-11/26/1984 & Columbia River & RM 13.7 & 26-Nov-84 & 3 \\
\hline AS-11/29/1966 & Columbia River & RM 13.7 & 29-Nov-66 & 3 \\
\hline AS-11/4/1982 & Columbia River & RM 13.7 & 04-Nov-82 & 3 \\
\hline AS-11/6/1964 & Columbia River & RM 13.7 & $06-N o v-64$ & 3 \\
\hline AS-12/10/1970 & Columbia River & RM 13.7 & 10-Dec-70 & 3 \\
\hline AS-12/10/1990 & Columbia River & RM 13.7 & 10-Dec-90 & 3 \\
\hline AS-12/12/1973 & Columbia River & RM 13.7 & 12-Dec-73 & 3 \\
\hline AS-12/13/1978 & Columbia River & RM 13.7 & 13-Dec-78 & 3 \\
\hline AS-12/14/1989 & Columbia River & RM 13.7 & 14-Dec-89 & 3 \\
\hline AS-12/15/1971 & Columbia River & RM 13.7 & 15-Dec-71 & 3 \\
\hline AS-12/15/1988 & Columbia River & RM 13.7 & 15-Dec-88 & 3 \\
\hline AS-12/16/1991 & Columbia River & RM 13.7 & 16-Dec-91 & 3 \\
\hline AS-12/17/1974 & Columbia River & RM 13.7 & 17-Dec-74 & 3 \\
\hline AS-12/18/1975 & Columbia River & RM 13.7 & 18-Dec-75 & 3 \\
\hline AS-12/18/1986 & Columbia River & RM 13.7 & 18-Dec-86 & 3 \\
\hline AS-12/22/1987 & Columbia River & RM 13.7 & 22-Dec-87 & 3 \\
\hline AS-12/23/1968 & Columbia River & RM 13.7 & 23-Dec-68 & 3 \\
\hline AS-12/23/1985 & Columbia River & RM 13.7 & 23-Dec-85 & 3 \\
\hline AS-12/31/1963 & Columbia River & RM 13.7 & 31-Dec-63 & 3 \\
\hline AS-12/6/1979 & Columbia River & RM 13.7 & 06-Dec-79 & 3 \\
\hline AS-12/7/1976 & Columbia River & RM 13.7 & 07-Dec-76 & 3 \\
\hline AS-12/8/1983 & Columbia River & RM 13.7 & 08-Dec-83 & 3 \\
\hline AS-2/11/1971 & Columbia River & RM 13.7 & 11-Feb-71 & 3 \\
\hline AS-2/12/1965 & Columbia River & RM 13.7 & 12-Feb-65 & 3 \\
\hline AS-2/12/1974 & Columbia River & RM 13.7 & 12-Feb-74 & 3 \\
\hline AS-2/15/1985 & Columbia River & RM 13.7 & 15-Feb-85 & 3 \\
\hline AS-2/17/1972 & Columbia River & RM 13.7 & 17-Feb-72 & 3 \\
\hline AS-2/18/1982 & Columbia River & RM 13.7 & 18-Feb-82 & 3 \\
\hline AS-2/18/1983 & Columbia River & RM 13.7 & 18-Feb-83 & 3 \\
\hline AS-2/19/1981 & Columbia River & RM 13.7 & 19-Feb-81 & 3 \\
\hline AS-2/20/1975 & Columbia River & RM 13.7 & 20-Feb-75 & 3 \\
\hline AS-2/26/1976 & Columbia River & RM 13.7 & 26-Feb-76 & 3 \\
\hline AS-2/27/1980 & Columbia River & RM 13.7 & 27-Feb-80 & 3 \\
\hline AS-2/28/1966 & Columbia River & RM 13.7 & 28-Feb-66 & 3 \\
\hline AS-2/28/1967 & Columbia River & RM 13.7 & 28-Feb-67 & 3 \\
\hline AS-2/3/1984 & Columbia River & RM 13.7 & 03-Feb-84 & 3 \\
\hline AS-2/5/1988 & Columbia River & RM 13.7 & 05-Feb-88 & 3 \\
\hline AS-2/8/1969 & Columbia River & RM 13.7 & 08-Feb-69 & 3 \\
\hline AS-3/10/1989 & Columbia River & RM 13.7 & 10-Mar-89 & 3 \\
\hline AS-3/10/1992 & Columbia River & RM 13.7 & 10-Mar-92 & 3 \\
\hline AS-3/13/1987 & Columbia River & RM 13.7 & 13-Mar-87 & 3 \\
\hline AS-3/20/1964 & Columbia River & RM 13.7 & 20-Mar-64 & 3 \\
\hline AS-3/25/1986 & Columbia River & RM 13.7 & 25-Mar-86 & 3 \\
\hline AS-3/26/1971 & Columbia River & RM 13.7 & 26-Mar-71 & 3 \\
\hline
\end{tabular}


Table E-1

Summary Table of Segment 5 Sediment Samples By Area, Site, Date, and Category

\begin{tabular}{|c|c|c|c|c|}
\hline Sample ID & Sample Area & Sample Site & Sample Date & Category \\
\hline AS-3/26/1973 & Columbia River & RM 13.7 & 26-Mar-73 & 3 \\
\hline AS-3/31/1970 & Columbia River & RM 13.7 & 31-Mar-70 & 3 \\
\hline AS-3/7/1978 & Columbia River & RM 13.7 & 07-Mar-78 & 3 \\
\hline AS-3/8/1979 & Columbia River & RM 13.7 & 08-Mar-79 & 3 \\
\hline AS-3/8/1993 & Columbia River & RM 13.7 & 08-Mar-93 & 3 \\
\hline AS-4/1/1968 & Columbia River & RM 13.7 & 01-Apr-68 & 3 \\
\hline AS-5/10/1984 & Columbia River & RM 13.7 & 10-May-84 & 3 \\
\hline AS-5/11/1966 & Columbia River & RM 13.7 & 11-May-66 & 3 \\
\hline AS-5/12/1988 & Columbia River & RM 13.7 & 12-May-88 & 3 \\
\hline AS-5/16/1983 & Columbia River & RM 13.7 & 16-May-83 & 3 \\
\hline AS-5/17/1969 & Columbia River & RM 13.7 & 17-May-69 & 3 \\
\hline AS-5/19/1987 & Columbia River & RM 13.7 & 19-May-87 & 3 \\
\hline AS-5/20/1980 & Columbia River & RM 13.7 & 20-May-80 & 3 \\
\hline AS-5/23/1972 & Columbia River & RM 13.7 & 23-May-72 & 3 \\
\hline AS-5/24/1967 & Columbia River & RM 13.7 & 24-May-67 & 3 \\
\hline AS-5/25/1989 & Columbia River & RM 13.7 & 25-May-89 & 3 \\
\hline AS-5/26/1977 & Columbia River & RM 13.7 & 26-May-77 & 3 \\
\hline AS-5/26/1982 & Columbia River & RM 13.7 & 26-May-82 & 3 \\
\hline AS-5/27/1963 & Columbia River & RM 13.7 & 27-May-63 & 3 \\
\hline AS-5/28/1981 & Columbia River & RM 13.7 & 28-May-81 & 3 \\
\hline AS-5/7/1985 & Columbia River & RM 13.7 & 07-May-85 & 3 \\
\hline AS-6/1/1978 & Columbia River & RM 13.7 & 01-Jun-78 & 3 \\
\hline AS-6/11/1970 & Columbia River & RM 13.7 & 11-Jun-70 & 3 \\
\hline AS-6/13/1968 & Columbia River & RM 13.7 & 13-Jun-68 & 3 \\
\hline AS-6/17/1966 & Columbia River & RM 13.7 & 17-Jun-66 & 3 \\
\hline AS-6/17/1971 & Columbia River & RM 13.7 & 17-Jun-71 & 3 \\
\hline AS-6/19/1975 & Columbia River & RM 13.7 & 19-Jun-75 & 3 \\
\hline AS-6/25/1965 & Columbia River & RM 13.7 & 25-Jun-65 & 3 \\
\hline AS-6/27/1973 & Columbia River & RM 13.7 & 27-Jun-73 & 3 \\
\hline AS-6/27/1991 & Columbia River & RM 13.7 & 27-Jun-91 & 3 \\
\hline AS-6/28/1990 & Columbia River & RM 13.7 & 28-Jun-90 & 3 \\
\hline AS-6/5/1974 & Columbia River & RM 13.7 & 05-Jun-74 & 3 \\
\hline AS-7/1/1992 & Columbia River & RM 13.7 & 01-Jul-92 & 3 \\
\hline AS-7/15/1980 & Columbia River & RM 13.7 & 15-Jul-80 & 3 \\
\hline AS-7/21/1970 & Columbia River & RM 13.7 & 21-Jul-70 & 3 \\
\hline AS-7/27/1976 & Columbia River & RM 13.7 & 27-Jul-76 & 3 \\
\hline AS-8/10/1982 & Columbia River & RM 13.7 & 10-Aug-82 & 3 \\
\hline AS-8/10/1983 & Columbia River & RM 13.7 & 10-Aug-83 & 3 \\
\hline AS-8/13/1987 & Columbia River & RM 13.7 & 13-Aug-87 & 3 \\
\hline AS-8/19/1981 & Columbia River & RM 13.7 & 19-Aug-81 & 3 \\
\hline AS-8/23/1989 & Columbia River & RM 13.7 & 23-Aug-89 & 3 \\
\hline AS-8/25/1966 & Columbia River & RM 13.7 & 25-Aug-66 & 3 \\
\hline AS-8/25/1975 & Columbia River & RM 13.7 & 25-Aug-75 & 3 \\
\hline AS-8/26/1985 & Columbia River & RM 13.7 & 26-Aug-85 & 3 \\
\hline AS-8/28/1974 & Columbia River & RM 13.7 & 28-Aug-74 & 3 \\
\hline AS-9/13/1978 & Columbia River & RM 13.7 & 13-Sep-78 & 3 \\
\hline AS-9/14/1965 & Columbia River & RM 13.7 & 14-Sep-65 & 3 \\
\hline
\end{tabular}


Table E-1

Summary Table of Segment 5 Sediment Samples By Area, Site, Date, and Category

\begin{tabular}{|c|c|c|c|c|}
\hline Sample ID & Sample Area & Sample Site & Sample Date & Category \\
\hline AS-9/17/1973 & Columbia River & RM 13.7 & 17-Sep-73 & 3 \\
\hline AS-9/18/1979 & Columbia River & RM 13.7 & 18-Sep-79 & 3 \\
\hline AS-9/19/1968 & Columbia River & RM 13.7 & 19-Sep-68 & 3 \\
\hline AS-9/2/1986 & Columbia River & RM 13.7 & 02-Sep-86 & 3 \\
\hline AS-9/25/1969 & Columbia River & RM 13.7 & 25-Sep-69 & 3 \\
\hline AS-9/26/1963 & Columbia River & RM 13.7 & 26-Sep-63 & 3 \\
\hline AS-9/27/1967 & Columbia River & RM 13.7 & 27-Sep-67 & 3 \\
\hline AS-9/8/1971 & Columbia River & RM 13.7 & 08-Sep-71 & 3 \\
\hline AS-9/9/1964 & Columbia River & RM 13.7 & 09-Sep-64 & 3 \\
\hline BACH-BC-01 & Columbia River & Bachelor Slough & 03-Jun-03 & 1 \\
\hline BACH-BC-02 & Columbia River & Bachelor Slough & 03-Jun-03 & 1 \\
\hline BACH-BC-03 & Columbia River & Bachelor Slough & 03-Jun-03 & 1 \\
\hline BACH-BC-04 & Columbia River & Bachelor Slough & 03-Jun-03 & 1 \\
\hline BACH-BC-05 & Columbia River & Bachelor Slough & 03-Jun-03 & 1 \\
\hline BACH-BC-06 & Columbia River & Bachelor Slough & 03-Jun-03 & 1 \\
\hline BACH-BC-07 & Columbia River & Bachelor Slough & 03-Jun-03 & 1 \\
\hline BACH-BC-08 & Columbia River & Bachelor Slough & 03-Jun-03 & 1 \\
\hline BACH-BC-09 & Columbia River & Bachelor Slough & 03-Jun-03 & 1 \\
\hline BACH-GC-10 & Columbia River & Bachelor Slough & 03-Jun-03 & 1 \\
\hline Beav-1 & Columbia River & RM 54 & 08-Sep-97 & 2 \\
\hline CR-BC-1 & Columbia River & RM 6 & 03-Jun-97 & 1 \\
\hline CR-BC-11 & Columbia River & RM 18 & 03-Jun-97 & 1 \\
\hline CR-BC-12 & Columbia River & RM 20 & 03-Jun-97 & 1 \\
\hline CR-BC-13 & Columbia River & RM 20 & 03-Jun-97 & 1 \\
\hline CR-BC-15 & Columbia River & RM 23 & 03-Jun-97 & 1 \\
\hline CR-BC-16 & Columbia River & RM 24 & 03-Jun-97 & 1 \\
\hline CR-BC-17 & Columbia River & RM 27 & 03-Jun-97 & 1 \\
\hline CR-BC-19 & Columbia River & RM 29 & 03-Jun-97 & 1 \\
\hline CR-BC-2 & Columbia River & RM 6 & 03-Jun-97 & 1 \\
\hline CR-BC-20 & Columbia River & RM 32 & 03-Jun-97 & 1 \\
\hline CR-BC-21 & Columbia River & RM 33 & 03-Jun-97 & 1 \\
\hline CR-BC-22 & Columbia River & RM 33 & 03-Jun-97 & 1 \\
\hline CR-BC-24 & Columbia River & RM 39 & 03-Jun-97 & 1 \\
\hline CR-BC-25 & Columbia River & RM 40 & 03-Jun-97 & 1 \\
\hline CR-BC-26 & Columbia River & RM 42 & 03-Jun-97 & 1 \\
\hline CR-BC-29 & Columbia River & RM 46 & 03-Jun-97 & 1 \\
\hline CR-BC-3 & Columbia River & RM 6 & 03-Jun-97 & 1 \\
\hline CR-BC-31 & Columbia River & RM 48 & 03-Jun-97 & 1 \\
\hline CR-BC-34 & Columbia River & RM 56 & 03-Jun-97 & 1 \\
\hline CR-BC-35 & Columbia River & RM 57 & 03-Jun-97 & 1 \\
\hline CR-BC-38 & Columbia River & RM 60 & 03-Jun-97 & 1 \\
\hline CR-BC-4 & Columbia River & RM 9 & 03-Jun-97 & 1 \\
\hline CR-BC-40 & Columbia River & RM 63 & 03-Jun-97 & 1 \\
\hline CR-BC-41 & Columbia River & RM 64 & 03-Jun-97 & 1 \\
\hline CR-BC-44 & Columbia River & RM 66 & 03-Jun-97 & 1 \\
\hline CR-BC-47 & Columbia River & RM 70 & 03-Jun-97 & 1 \\
\hline CR-BC-48 & Columbia River & RM 71 & 03-Jun-97 & 1 \\
\hline
\end{tabular}


Table E-1

Summary Table of Segment 5 Sediment Samples By Area, Site, Date, and Category

\begin{tabular}{|c|c|c|c|c|}
\hline Sample ID & Sample Area & Sample Site & Sample Date & Category \\
\hline CR-BC-49 & Columbia River & RM 73 & 03-Jun-97 & 1 \\
\hline CR-BC-5 & Columbia River & RM 11 & 03-Jun-97 & 1 \\
\hline CR-BC-50 & Columbia River & RM 74 - Kalama & 03-Jun-97 & 1 \\
\hline CR-BC-51 & Columbia River & RM 75 - Kalama & 03-Jun-97 & 1 \\
\hline CR-BC-52 & Columbia River & RM 76 & 03-Jun-97 & 1 \\
\hline CR-BC-53 & Columbia River & RM 79 & 03-Jun-97 & 1 \\
\hline CR-BC-54 & Columbia River & RM 80 & 03-Jun-97 & 1 \\
\hline CR-BC-55 & Columbia River & RM 82 & 03-Jun-97 & 1 \\
\hline CR-BC-56 & Columbia River & RM 83 & 03-Jun-97 & 1 \\
\hline CR-BC-57 & Columbia River & RM 83 & 03-Jun-97 & 1 \\
\hline CR-BC-59 & Columbia River & RM 85 & 03-Jun-97 & 1 \\
\hline CR-BC-60 & Columbia River & RM 85 & 03-Jun-97 & 1 \\
\hline CR-BC-61 & Columbia River & RM 86 & 03-Jun-97 & 1 \\
\hline CR-BC-62 & Columbia River & RM 88 & 03-Jun-97 & 1 \\
\hline CR-BC-63 & Columbia River & RM 89 & 03-Jun-97 & 1 \\
\hline CR-BC-65 & Columbia River & RM 91 & 03-Jun-97 & 1 \\
\hline CR-BC-66 & Columbia River & RM 92 & 03-Jun-97 & 1 \\
\hline CR-BC-67 & Columbia River & RM 93 & 03-Jun-97 & 1 \\
\hline CR-BC-68 & Columbia River & RM 93 & 03-Jun-97 & 1 \\
\hline CR-BC-7 & Columbia River & RM 12 & 03-Jun-97 & 1 \\
\hline CR-BC-70 & Columbia River & RM 96 & 03-Jun-97 & 1 \\
\hline CR-BC-71 & Columbia River & RM 97 & 03-Jun-97 & 1 \\
\hline CR-BC-72 & Columbia River & RM 98 & 03-Jun-97 & 1 \\
\hline CR-BC-73 & Columbia River & RM 99 & 03-Jun-97 & 1 \\
\hline CR-BC-74 & Columbia River & RM 99 & 03-Jun-97 & 1 \\
\hline CR-BC-75 & Columbia River & RM 99 & 03-Jun-97 & 1 \\
\hline CR-BC-76 & Columbia River & RM 100 & 03-Jun-97 & 1 \\
\hline CR-BC-77 & Columbia River & RM 100 & 03-Jun-97 & 1 \\
\hline CR-BC-78 & Columbia River & RM 100 & 03-Jun-97 & 1 \\
\hline CR-BC-79 & Columbia River & RM 102 & 03-Jun-97 & 1 \\
\hline CR-BC-8 & Columbia River & RM 12 & 03-Jun-97 & 1 \\
\hline CR-BC-80 & Columbia River & RM 103 & 03-Jun-97 & 1 \\
\hline CR-BC-81 & Columbia River & RM 103 & 03-Jun-97 & 1 \\
\hline CR-BC-83 & Columbia River & RM 103 & 03-Jun-97 & 1 \\
\hline CR-BC-84 & Columbia River & RM 104 & 03-Jun-97 & 1 \\
\hline CR-BC-85 & Columbia River & RM 104 & 03-Jun-97 & 1 \\
\hline CR-BC-86 & Columbia River & RM 105 & 03-Jun-97 & 1 \\
\hline CR-BC-87 & Columbia River & RM 105 & 03-Jun-97 & 1 \\
\hline CR-BC-88 & Columbia River & RM 106 & 03-Jun-97 & 1 \\
\hline CR-BC-89 & Columbia River & RM 106 & 03-Jun-97 & 1 \\
\hline CR-BC-9 & Columbia River & RM 15 & 03-Jun-97 & 1 \\
\hline CR-GC-15 & Columbia River & CR-GC-15 & 10-May-90 & 2 \\
\hline CR-GC-16 & Columbia River & CR-GC-16 & 10-May-90 & 2 \\
\hline CR-GC-17 & Columbia River & CR-GC-17 & 10-May-90 & 2 \\
\hline CR-GC-18 & Columbia River & CR-GC-18 & 10-May-90 & 2 \\
\hline CR-GC-2 & Columbia River & CR-GC-2 & 09-May-90 & 2 \\
\hline CR-GC-23 & Columbia River & CR-GC-23 & 10-May-90 & 2 \\
\hline
\end{tabular}


Table E-1

Summary Table of Segment 5 Sediment Samples By Area, Site, Date, and Category

\begin{tabular}{|c|c|c|c|c|}
\hline Sample ID & Sample Area & Sample Site & Sample Date & Category \\
\hline CR-GC-24 & Columbia River & CR-GC-24 & 10-May-90 & 2 \\
\hline CR-GC-25 & Columbia River & CR-GC-25 & 10-May-90 & 2 \\
\hline CR-GC-26 & Columbia River & CR-GC-26 & 10-May-90 & 2 \\
\hline CR-GC-4 & Columbia River & CR-GC-4 & 09-May-90 & 2 \\
\hline CR-GC-5 & Columbia River & CR-GC-5 & 09-May-90 & 2 \\
\hline CR-GC-6A & Columbia River & CR-GC-6 & 09-May-90 & 2 \\
\hline CR-GC-6B & Columbia River & CR-GC-6 & 09-May-90 & 2 \\
\hline CR-GC-7 & Columbia River & CR-GC-7 & 09-May-90 & 2 \\
\hline CR-GC6AB & Columbia River & CR-GC-6 & 09-May-90 & 2 \\
\hline CR-S5 .08-1.0m & Columbia River & CR-S5 & 01-Oct-84 & 3 \\
\hline CR-S5 1.8-3.8m & Columbia River & CR-S5 & 01-Oct-84 & 3 \\
\hline CR-VC-10 & Columbia River & CR-VC-10 & 10-May-90 & 2 \\
\hline CR-VC-11 & Columbia River & CR-VC-11 & 10-May-90 & 2 \\
\hline CR-VC-13 & Columbia River & CR-VC-13 & 10-May-90 & 2 \\
\hline CR-VC-14 & Columbia River & CR-VC-14 & 10-May-90 & 2 \\
\hline CR-VC-9 & Columbia River & CR-VC-9 & 10-May-90 & 2 \\
\hline CR-VC12A & Columbia River & CR-VC-12 & 10-May-90 & 2 \\
\hline CR-VC12B & Columbia River & CR-VC-12 & 10-May-90 & 2 \\
\hline CR17/18 & Columbia River & CR17/18 & 10-May-90 & 2 \\
\hline CR23/24 & Columbia River & CR23/24 & 10-May-90 & 2 \\
\hline CR25/26 & Columbia River & CR25/26 & 10-May-90 & 2 \\
\hline CR76-PG-01 & Columbia River & STA CR-BC-76 & 01-Jun-97 & 1 \\
\hline CR76-PG-02 & Columbia River & STA CR-BC-76 & 01-Jun-97 & 1 \\
\hline CR76-PG-03 & Columbia River & STA CR-BC-76 & 01-Jun-97 & 1 \\
\hline CRASD200 & Columbia River & RM 21 & 01-Aug-91 & 1 \\
\hline CRBSD174 & Columbia River & RM 2-6 & 01-Aug-91 & 1 \\
\hline CRCSD151 & Columbia River & RM 111-121 & 01-Aug-91 & 1 \\
\hline CRIM-SG-01 & Columbia River & Crims Island & 15-Jul-03 & 1 \\
\hline CRIM-SG-02 & Columbia River & Crims Island & 15-Jul-03 & 1 \\
\hline CRIM-SG-03 & Columbia River & Crims Island & 15-Jul-03 & 1 \\
\hline CRIM-SG-04 & Columbia River & Crims Island & 15-Jul-03 & 1 \\
\hline CRIM-SG-05 & Columbia River & Crims Island & 15-Jul-03 & 1 \\
\hline CRJSD120 & Columbia River & RM 37-47 & 01-Aug-91 & 1 \\
\hline CRLSD171 & Columbia River & RM 64-72 & 01-Aug-91 & 1 \\
\hline CRM-BC1 & Columbia River & RM 29 & 09-Aug-00 & 1 \\
\hline CRM-BC10 & Columbia River & RM 29 & 09-Aug-00 & 1 \\
\hline CRM-BC11 & Columbia River & RM 29 & 09-Aug-00 & 1 \\
\hline CRM-BC12 & Columbia River & RM 29 & 09-Aug-00 & 1 \\
\hline CRM-BC13 & Columbia River & RM 29 & 09-Aug-00 & 1 \\
\hline CRM-BC14 & Columbia River & RM 29 & 09-Aug-00 & 1 \\
\hline CRM-BC15 & Columbia River & RM 29 & 09-Aug-00 & 1 \\
\hline CRM-BC16 & Columbia River & RM 29 & 09-Aug-00 & 1 \\
\hline CRM-BC17 & Columbia River & RM 31 & 09-Aug-00 & 1 \\
\hline CRM-BC18 & Columbia River & RM 32 & 09-Aug-00 & 1 \\
\hline CRM-BC19 & Columbia River & RM 33 & 09-Aug-00 & 1 \\
\hline CRM-BC2 & Columbia River & RM 29 & 09-Aug-00 & 1 \\
\hline CRM-BC20 & Columbia River & RM 34 & 09-Aug-00 & 1 \\
\hline
\end{tabular}


Table E-1

Summary Table of Segment 5 Sediment Samples By Area, Site, Date, and Category

\begin{tabular}{|c|c|c|c|c|}
\hline Sample ID & Sample Area & Sample Site & Sample Date & Category \\
\hline CRM-BC3 & Columbia River & RM 29 & 09-Aug-00 & 1 \\
\hline CRM-BC4 & Columbia River & RM 29 & 09-Aug-00 & 1 \\
\hline CRM-BC5 & Columbia River & RM 29 & 09-Aug-00 & 1 \\
\hline CRM-BC6 & Columbia River & RM 29 & 09-Aug-00 & 1 \\
\hline CRM-BC7 & Columbia River & RM 29 & 09-Aug-00 & 1 \\
\hline CRM-BC8 & Columbia River & RM 29 & 09-Aug-00 & 1 \\
\hline CRM-BC9 & Columbia River & RM 29 & 09-Aug-00 & 1 \\
\hline CRM-VC-01 & Columbia River & RM 29 & 07-Sep-00 & 1 \\
\hline CRM-VC-02 & Columbia River & RM 29 & 07-Sep-00 & 1 \\
\hline CRM-VC-03 & Columbia River & RM 29 & 07-Sep-00 & 1 \\
\hline CRM-VC-04 & Columbia River & RM 29 & 07-Sep-00 & 1 \\
\hline CRM-VC-05 & Columbia River & RM 29 & 07-Sep-00 & 1 \\
\hline CRMSD214 & Columbia River & RM 21-37 & 01-Aug-91 & 1 \\
\hline CRRSD117 & Columbia River & RM 87-102 & 01-Aug-91 & 1 \\
\hline CRVC12AB & Columbia River & CR-VC-12 & 10-May-90 & 2 \\
\hline KR-BC-01 & Columbia River & RM 73.9-74.8 & 10-Sep-03 & 1 \\
\hline KR-BC-04 & Columbia River & RM 73.9-74.8 & 10-Sep-03 & 1 \\
\hline KR-BC-04A & Columbia River & RM 73.9-74.8 & 10-Sep-03 & 1 \\
\hline OR-VC-01 & Columbia River & Oregon Slough & 19-Jun-01 & 1 \\
\hline OR-VC-02 & Columbia River & Oregon Slough & 19-Jun-01 & 1 \\
\hline OR-VC-03 & Columbia River & Oregon Slough & 19-Jun-01 & 1 \\
\hline OR-VC-04 & Columbia River & Oregon Slough & 19-Jun-01 & 1 \\
\hline RR-1/19/1963 & Columbia River & RM 128.4 & 19-Jan-63 & 3 \\
\hline RR-10/13/1977 & Columbia River & RM 128.4 & 13-Oct-77 & 3 \\
\hline RR-11/13/1991 & Columbia River & RM 128.4 & 13-Nov-91 & 3 \\
\hline RR-11/16/1964 & Columbia River & RM 128.4 & 16-Nov-64 & 3 \\
\hline RR-11/17/1965 & Columbia River & RM 128.4 & 17-Nov-65 & 3 \\
\hline RR-11/20/1986 & Columbia River & RM 128.4 & 20-Nov-86 & 3 \\
\hline RR-11/28/1967 & Columbia River & RM 128.4 & 28-Nov-67 & 3 \\
\hline RR-11/28/1990 & Columbia River & RM 128.4 & 28-Nov-90 & 3 \\
\hline RR-11/29/1966 & Columbia River & RM 128.4 & 29-Nov-66 & 3 \\
\hline RR-11/29/1972 & Columbia River & RM 128.4 & 29-Nov-72 & 3 \\
\hline RR-11/3/1993 & Columbia River & RM 128.4 & 03-Nov-93 & 3 \\
\hline RR-11/4/1987 & Columbia River & RM 128.4 & 04-Nov-87 & 3 \\
\hline RR-11/9/1984 & Columbia River & RM 128.4 & 09-Nov-84 & 3 \\
\hline RR-12/15/1976 & Columbia River & RM 128.4 & 15-Dec-76 & 3 \\
\hline RR-12/19/1989 & Columbia River & RM 128.4 & 19-Dec-89 & 3 \\
\hline RR-12/22/1971 & Columbia River & RM 128.4 & 22-Dec-71 & 3 \\
\hline RR-12/27/1968 & Columbia River & RM 128.4 & 27-Dec-68 & 3 \\
\hline RR-12/27/1985 & Columbia River & RM 128.4 & 27-Dec-85 & 3 \\
\hline RR-12/29/1969 & Columbia River & RM 128.4 & 29-Dec-69 & 3 \\
\hline RR-12/30/1970 & Columbia River & RM 128.4 & 30-Dec-70 & 3 \\
\hline RR-12/31/1963 & Columbia River & RM 128.4 & 31-Dec-63 & 3 \\
\hline RR-12/4/1980 & Columbia River & RM 128.4 & 04-Dec-80 & 3 \\
\hline RR-12/5/1979 & Columbia River & RM 128.4 & 05-Dec-79 & 3 \\
\hline RR-12/6/1988 & Columbia River & RM 128.4 & 06-Dec-88 & 3 \\
\hline RR-2/17/1971 & Columbia River & RM 128.4 & 17-Feb-71 & 3 \\
\hline
\end{tabular}


Table E-1

Summary Table of Segment 5 Sediment Samples By Area, Site, Date, and Category

\begin{tabular}{|c|c|c|c|c|}
\hline Sample ID & Sample Area & Sample Site & Sample Date & Category \\
\hline RR-2/21/1967 & Columbia River & RM 128.4 & 21-Feb-67 & 3 \\
\hline RR-2/24/1966 & Columbia River & RM 128.4 & 24-Feb-66 & 3 \\
\hline RR-2/24/1993 & Columbia River & RM 128.4 & 24-Feb-93 & 3 \\
\hline RR-2/25/1988 & Columbia River & RM 128.4 & 25-Feb-88 & 3 \\
\hline RR-2/25/1992 & Columbia River & RM 128.4 & 25-Feb-92 & 3 \\
\hline RR-2/6/1991 & Columbia River & RM 128.4 & 06-Feb-91 & 3 \\
\hline RR-2/9/1965 & Columbia River & RM 128.4 & 09-Feb-65 & 3 \\
\hline RR-3/14/1980 & Columbia River & RM 128.4 & 14-Mar-80 & 3 \\
\hline RR-3/17/1987 & Columbia River & RM 128.4 & 17-Mar-87 & 3 \\
\hline RR-3/19/1969 & Columbia River & RM 128.4 & 19-Mar-69 & 3 \\
\hline RR-3/19/1970 & Columbia River & RM 128.4 & 19-Mar-70 & 3 \\
\hline RR-3/19/1981 & Columbia River & RM 128.4 & 19-Mar-81 & 3 \\
\hline RR-3/21/1968 & Columbia River & RM 128.4 & 21-Mar-68 & 3 \\
\hline RR-3/22/1978 & Columbia River & RM 128.4 & 22-Mar-78 & 3 \\
\hline RR-3/23/1973 & Columbia River & RM 128.4 & 23-Mar-73 & 3 \\
\hline RR-3/30/1964 & Columbia River & RM 128.4 & 30-Mar-64 & 3 \\
\hline RR-3/30/1990 & Columbia River & RM 128.4 & 30-Mar-90 & 3 \\
\hline RR-3/31/1985 & Columbia River & RM 128.4 & 31-Mar-86 & 3 \\
\hline RR-3/4/1971 & Columbia River & RM 128.4 & 04-Mar-71 & 3 \\
\hline RR-4/24/1985 & Columbia River & RM 128.4 & 24-Apr-85 & 3 \\
\hline RR-5/17/1967 & Columbia River & RM 128.4 & 17-May-67 & 3 \\
\hline RR-5/20/1977 & Columbia River & RM 128.4 & 20-May-77 & 3 \\
\hline RR-5/22/1969 & Columbia River & RM 128.4 & 22-May-69 & 3 \\
\hline RR-5/22/1987 & Columbia River & RM 128.4 & 22-May-87 & 3 \\
\hline RR-5/26/1970 & Columbia River & RM 128.4 & 26-May-70 & 3 \\
\hline RR-5/27/1993 & Columbia River & RM 128.4 & 27-May-93 & 3 \\
\hline RR-5/28/1992 & Columbia River & RM 128.4 & 28-May-92 & 3 \\
\hline RR-5/30/1991 & Columbia River & RM 128.4 & 30-May-91 & 3 \\
\hline RR-5/31/1966 & Columbia River & RM 128.4 & 31-May-66 & 3 \\
\hline RR-6/15/1973 & Columbia River & RM 128.4 & 15-Jun-73 & 3 \\
\hline RR-6/19/1979 & Columbia River & RM 128.4 & 19-Jun-79 & 3 \\
\hline RR-6/2/1990 & Columbia River & RM 128.4 & 02-Jun-90 & 3 \\
\hline RR-6/24/1964 & Columbia River & RM 128.4 & 24-Jun-64 & 3 \\
\hline RR-6/24/1965 & Columbia River & RM 128.4 & 24-Jun-65 & 3 \\
\hline RR-6/27/1968 & Columbia River & RM 128.4 & 27-Jun-68 & 3 \\
\hline RR-6/28/1988 & Columbia River & RM 128.4 & 28-Jun-88 & 3 \\
\hline RR-7/13/1970 & Columbia River & RM 128.4 & 13-Jul-70 & 3 \\
\hline RR-7/16/1971 & Columbia River & RM 128.4 & 16-Jul-71 & 3 \\
\hline RR-7/21/1989 & Columbia River & RM 128.4 & 21-Jul-89 & 3 \\
\hline RR-7/27/1966 & Columbia River & RM 128.4 & 27-Jul-66 & 3 \\
\hline RR-7/29/1985 & Columbia River & RM 128.4 & 29-Jul-85 & 3 \\
\hline RR-7/8/1980 & Columbia River & RM 128.4 & 08-Jul-80 & 3 \\
\hline RR-8/13/1966 & Columbia River & RM 128.4 & 13-Aug-66 & 3 \\
\hline RR-8/13/1981 & Columbia River & RM 128.4 & 13-Aug-81 & 3 \\
\hline RR-8/19/1993 & Columbia River & RM 128.4 & 19-Aug-93 & 3 \\
\hline RR-8/24/1979 & Columbia River & RM 128.4 & 24-Aug-79 & 3 \\
\hline RR-8/24/1988 & Columbia River & RM 128.4 & 24-Aug-88 & 3 \\
\hline
\end{tabular}


Table E-1

Summary Table of Segment 5 Sediment Samples By Area, Site, Date, and Category

\begin{tabular}{|c|c|c|c|c|}
\hline Sample ID & Sample Area & Sample Site & Sample Date & Category \\
\hline RR-8/30/1973 & Columbia River & RM 128.4 & 30-Aug-73 & 3 \\
\hline RR-8/30/1978 & Columbia River & RM 128.4 & 30-Aug-78 & 3 \\
\hline RR-8/5/1987 & Columbia River & RM 128.4 & 05-Aug-87 & 3 \\
\hline RR-8/7/1991 & Columbia River & RM 128.4 & 07-Aug-91 & 3 \\
\hline RR-9/10/1976 & Columbia River & RM 128.4 & 10-Sep-76 & 3 \\
\hline RR-9/12/1983 & Columbia River & RM 128.4 & 12-Sep-83 & 3 \\
\hline RR-9/16/1964 & Columbia River & RM 128.4 & 16-Sep-64 & 3 \\
\hline RR-9/16/1965 & Columbia River & RM 128.4 & 16-Sep-65 & 3 \\
\hline RR-9/20/1982 & Columbia River & RM 128.4 & 20-Sep-82 & 3 \\
\hline RR-9/23/1992 & Columbia River & RM 128.4 & 23-Sep-92 & 3 \\
\hline RR-9/24/1968 & Columbia River & RM 128.4 & 24-Sep-68 & 3 \\
\hline RR-9/26/1967 & Columbia River & RM 128.4 & 26-Sep-67 & 3 \\
\hline RR-9/29/1970 & Columbia River & RM 128.4 & 29-Sep-70 & 3 \\
\hline RR-9/30/1963 & Columbia River & RM 128.4 & 30-Sep-63 & 3 \\
\hline RR-9/30/1971 & Columbia River & RM 128.4 & 30-Sep-71 & 3 \\
\hline RR-9/5/1980 & Columbia River & RM 128.4 & 05-Sep-80 & 3 \\
\hline RR-9/6/1990 & Columbia River & RM 128.4 & 06-Sep-90 & 3 \\
\hline RR-9/9/1969 & Columbia River & RM 128.4 & 09-Sep-69 & 3 \\
\hline SH-VC-4 & Columbia River & RM 86.5 & 04-May-89 & 3 \\
\hline SH-VC-5 & Columbia River & RM 86.5 & 04-May-89 & 3 \\
\hline SH-VC-6 & Columbia River & RM 86.5 & 04-May-89 & 3 \\
\hline V-PG-02 & Columbia River & RM 103 & 20-Jun-01 & 1 \\
\hline V-PG-03 & Columbia River & RM 103 & 20-Jun-01 & 1 \\
\hline V-PG-04 & Columbia River & RM 103 & 20-Jun-01 & 1 \\
\hline V-PG-05 & Columbia River & RM 103 & 20-Jun-01 & 1 \\
\hline V-PG-06 & Columbia River & RM 103 & 20-Jun-01 & 1 \\
\hline V-PG-07 & Columbia River & RM 103 & 20-Jun-01 & 1 \\
\hline V-PG-08 & Columbia River & RM 103 & 20-Jun-01 & 1 \\
\hline V-PG-09 & Columbia River & RM 103 & 20-Jun-01 & 1 \\
\hline V-PG-10 & Columbia River & RM 103 & 20-Jun-01 & 1 \\
\hline V-PG-11 & Columbia River & RM 103 & 20-Jun-01 & 1 \\
\hline V-PG-12 & Columbia River & RM 103 & 20-Jun-01 & 1 \\
\hline V-PG-13 & Columbia River & RM 103 & 20-Jun-01 & 1 \\
\hline V-PG-14 & Columbia River & RM 103 & 20-Jun-01 & 1 \\
\hline V-PG-15 & Columbia River & RM 103 & 20-Jun-01 & 1 \\
\hline V-PG-16 & Columbia River & RM 103 & 20-Jun-01 & 1 \\
\hline V-PG-17 & Columbia River & RM 103 & 20-Jun-01 & 1 \\
\hline V-PG-18 & Columbia River & RM 103 & 20-Jun-01 & 1 \\
\hline V-PG-19 & Columbia River & RM 103 & 20-Jun-01 & 1 \\
\hline V-PG-20 & Columbia River & RM 103 & 20-Jun-01 & 1 \\
\hline V-PG-21 & Columbia River & RM 103 & 20-Jun-01 & 1 \\
\hline V-PG-22 & Columbia River & RM 103 & 20-Jun-01 & 1 \\
\hline V-PG-23 & Columbia River & RM 103 & 20-Jun-01 & 1 \\
\hline V-PG-24 & Columbia River & RM 103 & 20-Jun-01 & 1 \\
\hline V-PG-25 & Columbia River & RM 103 & 20-Jun-01 & 1 \\
\hline V-VC-01A & Columbia River & RM 103 & 20-Jun-01 & 1 \\
\hline V-VC-01B & Columbia River & RM 103 & 20-Jun-01 & 1 \\
\hline
\end{tabular}


Table E-1

Summary Table of Segment 5 Sediment Samples By Area, Site, Date, and Category

\begin{tabular}{|c|c|c|c|c|}
\hline Sample ID & Sample Area & Sample Site & Sample Date & Category \\
\hline Warr-1 & Columbia River & RM 141 & 20-Nov-97 & 2 \\
\hline Will-1 & Columbia River & RM 82 & 10-Sep-97 & 2 \\
\hline OMCR-BC-04 & Cowlitz River & OM Cowlitz Rvr & 10-Sep-03 & 1 \\
\hline OMCR-GC-01 & Cowlitz River & OM Cowlitz Rvr & 10-Sep-03 & 1 \\
\hline OMCR-GC-02 & Cowlitz River & OM Cowlitz Rvr & 10-Sep-03 & 1 \\
\hline OMCR-GC-03 & Cowlitz River & OM Cowlitz Rvr & 10-Sep-03 & 1 \\
\hline SBIO-GC-01 & Skipanon Federal Channel & Warrenton OR & 24-Jun-03 & 1 \\
\hline SBIO-GC-02 & Skipanon Federal Channel & Warrenton OR & 24-Jun-03 & 1 \\
\hline SBIO-GC-03 & Skipanon Federal Channel & Warrenton OR & 24-Jun-03 & 1 \\
\hline SBIO-GC-04 & Skipanon Federal Channel & Warrenton OR & 24-Jun-03 & 1 \\
\hline SKIP-GC-01 & Skipanon Federal channel & Warrenton OR & 12-Sep-01 & 1 \\
\hline SKIP-GC-02 & Skipanon Federal channel & Warrenton OR & 12-Sep-01 & 1 \\
\hline SKIP-GC-03 & Skipanon Federal channel & Warrenton OR & 12-Sep-01 & 1 \\
\hline SKIP-GC-04 & Skipanon Federal channel & Warrenton OR & 12-Sep-01 & 1 \\
\hline SKIP-GC-05 & Skipanon Federal channel & Warrenton OR & 12-Sep-01 & 1 \\
\hline SKIP-GC-06 & Skipanon Federal channel & Warrenton OR & 12-Sep-01 & 1 \\
\hline SKIP-GC-07 & Skipanon Federal channel & Warrenton OR & 12-Sep-01 & 1 \\
\hline WR-BC-1 & Willamette River & RM 0.1 - mouth & 24-Jul-97 & 1 \\
\hline WR-BC-3 & Willamette River & RM 0.4 - mouth & 24-Jul-97 & 1 \\
\hline WR-GC-10 & Willamette River & WR-GC-10 & 03-May-90 & 2 \\
\hline WR-GC-1B & Willamette River & WR-GC-1 & 03-May-90 & 2 \\
\hline WR-GC-1T & Willamette River & WR-GC-1 & 03-May-90 & 2 \\
\hline WR-GC-2 & Willamette River & WR-GC-2 & 03-May-90 & 2 \\
\hline WR-GC-2A & Willamette River & RM 0.1 - mouth & 24-Jul-97 & 2 \\
\hline WR-GC-2B & Willamette River & RM 0.1 - mouth & 24-Jul-97 & 2 \\
\hline WR-GC-3A & Willamette River & WR-GC-3 & 03-May-90 & 2 \\
\hline WR-GC-3B & Willamette River & WR-GC-3 & 03-May-90 & 2 \\
\hline WR-GC-4 & Willamette River & WR-GC-4 & 03-May-90 & 2 \\
\hline WR-GC-5 & Willamette River & WR-GC-5 & 03-May-90 & 2 \\
\hline WR-GC-6 & Willamette River & WR-GC-6 & 03-May-90 & 2 \\
\hline WR-GC-7 & Willamette River & WR-GC-7 & 03-May-90 & 2 \\
\hline WR-GC-8 & Willamette River & WR-GC-8 & 03-May-90 & 2 \\
\hline WR-GC-9 & Willamette River & WR-GC-9 & 03-May-90 & 2 \\
\hline WR-GC3AB & Willamette River & WR-GC-3 & 03-May-90 & 2 \\
\hline
\end{tabular}


Table E-2

Summary Table of Segment 5 Surface Water Samples By Area, Site, Date, and Category

\begin{tabular}{|c|c|c|c|c|}
\hline Sample Area & Sample Site & Sample ID & Sample Date & Category \\
\hline Columbia River & Beacon Rock WA & 1388040 & September 25, 2001 & 1 \\
\hline Columbia River & Beaver Army TML & $14246900-02-01-16$ & February 1, 2002 & 1 \\
\hline Columbia River & Beaver Army TML & $14246900-02-05-16 D$ & February 5, 2002 & 1 \\
\hline Columbia River & Beaver Army TML & 14246900-02-05-16-FD & February 5, 2002 & 1 \\
\hline Columbia River & Beaver Army TML & $14246900-02-05-16-F$ & February 5, 2002 & 1 \\
\hline Columbia River & Beaver Army TML & $14246900-02-05-16$ & February 5, 2002 & 1 \\
\hline Columbia River & Beaver Army TML & 14246900-02-04-09 & February 4, 2002 & 1 \\
\hline Columbia River & Beaver Army TML & 14246900-02-03-12-FD & February 3, 2002 & 1 \\
\hline Columbia River & Beaver Army TML & $14246900-02-03-12-F$ & February 3, 2002 & 1 \\
\hline Columbia River & Beaver Army TML & $14246900-02-03-12$ & February 3, 2002 & 1 \\
\hline Columbia River & Beaver Army TML & 14246900-02-02-14-FD & February 2, 2002 & 1 \\
\hline Columbia River & Beaver Army TML & 14246900-02-02-14-F & February 2, 2002 & 1 \\
\hline Columbia River & Beaver Army TML & $14246900-02-02-14$ & February 2, 2002 & 1 \\
\hline Columbia River & Beaver Army TML & $14246900-99-05-25$ & May 25, 1999 & 1 \\
\hline Columbia River & Beaver Army TML & 14246900-02-01-16-F & February 1, 2002 & 1 \\
\hline Columbia River & Beaver Army TML & 14246900-02-07-16-FD & February 7, 2002 & 1 \\
\hline Columbia River & Beaver Army TML & $14246900-02-01-10$ & February 1, 2002 & 1 \\
\hline Columbia River & Beaver Army TML & 14246900-01-12-18D & January 12, 2001 & 1 \\
\hline Columbia River & Beaver Army TML & 14246900-01-12-18-FD & January 12, 2001 & 1 \\
\hline Columbia River & Beaver Army TML & 14246900-01-12-18-F & January 12, 2001 & 1 \\
\hline Columbia River & Beaver Army TML & $14246900-01-12-18$ & January 12, 2001 & 1 \\
\hline Columbia River & Beaver Army TML & 14246900-01-12-04-FD & January 12, 2001 & 1 \\
\hline Columbia River & Beaver Army TML & 14246900-01-12-04-F & January 12, 2001 & 1 \\
\hline Columbia River & Beaver Army TML & $14246900-01-12-04$ & January 12, 2001 & 1 \\
\hline Columbia River & Beaver Army TML & 14246900-01-11-27-FD & January 11, 2001 & 1 \\
\hline Columbia River & Beaver Army TML & 14246900-01-11-27-F & January 11, 2001 & 1 \\
\hline Columbia River & Beaver Army TML & $14246900-01-11-27$ & January 11, 2001 & 1 \\
\hline Columbia River & Beaver Army TML & 14246900-02-01-16-FD & February 1, 2002 & 1 \\
\hline Columbia River & Beaver Army TML & $14246900-99-02-23$ & February 23, 1999 & 1 \\
\hline Columbia River & Beaver Army TML & 14246900-99-05-11-FD & May 11, 1999 & 1 \\
\hline Columbia River & Beaver Army TML & 14246900-99-05-11-F & May 11, 1999 & 1 \\
\hline Columbia River & Beaver Army TML & $14246900-99-05-11$ & May 11, 1999 & 1 \\
\hline Columbia River & Beaver Army TML & 14246900-99-04-20-FD & April 20, 1999 & 1 \\
\hline Columbia River & Beaver Army TML & 14246900-99-04-20-F & April 20, 1999 & 1 \\
\hline Columbia River & Beaver Army TML & $14246900-99-04-20$ & April 20, 1999 & 1 \\
\hline Columbia River & Beaver Army TML & 14246900-99-03-29D & March 29, 1999 & 1 \\
\hline Columbia River & Beaver Army TML & 14246900-99-03-29-FD & March 29, 1999 & 1 \\
\hline Columbia River & Beaver Army TML & 14246900-99-03-29-F & March 29, 1999 & 1 \\
\hline Columbia River & Beaver Army TML & $14246900-99-03-29$ & March 29, 1999 & 1 \\
\hline Columbia River & Beaver Army TML & 14246900-99-03-08-FD & March 8, 1999 & 1 \\
\hline Columbia River & Beaver Army TML & $14246900-02-07-16$ & February 7, 2002 & 1 \\
\hline Columbia River & Beaver Army TML & 14246900-99-02-23-FD & February 23, 1999 & 1 \\
\hline Columbia River & Beaver Army TML & $14246900-02-07-16-F$ & February 7, 2002 & 1 \\
\hline Columbia River & Beaver Army TML & 14246900-99-01-25-FD & January 25, 1999 & 1 \\
\hline Columbia River & Beaver Army TML & $14246900-99-01-25-\mathrm{F}$ & January 25, 1999 & 1 \\
\hline Columbia River & Beaver Army TML & $14246900-99-01-25$ & January 25, 1999 & 1 \\
\hline Columbia River & Beaver Army TML & 14246900-99-01-05D & January 5, 1999 & 1 \\
\hline Columbia River & Beaver Army TML & 14246900-99-01-05-FD & January 5, 1999 & 1 \\
\hline Columbia River & Beaver Army TML & 14246900-99-01-05-F & January 5, 1999 & 1 \\
\hline Columbia River & Beaver Army TML & 14246900-99-01-05 & January 5, 1999 & 1 \\
\hline
\end{tabular}


Table E-2

Summary Table of Segment 5 Surface Water Samples By Area, Site, Date, and Category

\begin{tabular}{|c|c|c|c|c|}
\hline Sample Area & Sample Site & Sample ID & Sample Date & Category \\
\hline Columbia River & Beaver Army TML & 14246900-99--08-03-F & August 3, 1999 & 1 \\
\hline Columbia River & Beaver Army TML & 14246900-02-09-10-FD & February 9, 2002 & 1 \\
\hline Columbia River & Beaver Army TML & 14246900-02-09-10-F & February 9, 2002 & 1 \\
\hline Columbia River & Beaver Army TML & $14246900-02-09-10$ & February 9, 2002 & 1 \\
\hline Columbia River & Beaver Army TML & 14246900-01-09-24-F & January 9, 2001 & 1 \\
\hline Columbia River & Beaver Army TML & 14246900-99-03-08-F & March 8, 1999 & 1 \\
\hline Columbia River & Beaver Army TML & $14246900-00-05-16-F$ & May 16,2000 & 1 \\
\hline Columbia River & Beaver Army TML & $14246900-00-07-25-\mathrm{F}$ & July 25,2000 & 1 \\
\hline Columbia River & Beaver Army TML & $14246900-00-07-25$ & July 25,2000 & 1 \\
\hline Columbia River & Beaver Army TML & 14246900-00-06-26-FD & June 26, 2000 & 1 \\
\hline Columbia River & Beaver Army TML & $14246900-00-06-26-\mathrm{F}$ & June 26,2000 & 1 \\
\hline Columbia River & Beaver Army TML & $14246900-00-06-26$ & June 26, 2000 & 1 \\
\hline Columbia River & Beaver Army TML & 14246900-00-06-13-FD & June 13, 2000 & 1 \\
\hline Columbia River & Beaver Army TML & $14246900-00-06-13-F$ & June 13, 2000 & 1 \\
\hline Columbia River & Beaver Army TML & $14246900-00-06-13$ & June 13, 2000 & 1 \\
\hline Columbia River & Beaver Army TML & 14246900-00-05-30-FD & May 30, 2000 & 1 \\
\hline Columbia River & Beaver Army TML & 14246900-00-05-30-F & May 30, 2000 & 1 \\
\hline Columbia River & Beaver Army TML & $14246900-00-05-30$ & May 30, 2000 & 1 \\
\hline Columbia River & Beaver Army TML & 14246900-01-09-24D & January 9, 2001 & 1 \\
\hline Columbia River & Beaver Army TML & 14246900-00-05-16-FD & May 16,2000 & 1 \\
\hline Columbia River & Beaver Army TML & $14246900-00-09-12-\mathrm{F}$ & September 12, 2000 & 1 \\
\hline Columbia River & Beaver Army TML & $14246900-00-05-16$ & May 16,2000 & 1 \\
\hline Columbia River & Beaver Army TML & 14246900-00-04-11-FD & April 11, 2000 & 1 \\
\hline Columbia River & Beaver Army TML & 14246900-00-04-11-F & April 11, 2000 & 1 \\
\hline Columbia River & Beaver Army TML & $14246900-00-04-11$ & April 11, 2000 & 1 \\
\hline Columbia River & Beaver Army TML & 14246900-00-02-28-FD & February 28, 2000 & 1 \\
\hline Columbia River & Beaver Army TML & $14246900-00-02-28-\mathrm{F}$ & February 28, 2000 & 1 \\
\hline Columbia River & Beaver Army TML & $14246900-00-02-28$ & February 28, 2000 & 1 \\
\hline Columbia River & Beaver Army TML & 14246900-00-01-18D & January 18, 2000 & 1 \\
\hline Columbia River & Beaver Army TML & 14246900-00-01-18-FD & January 18, 2000 & 1 \\
\hline Columbia River & Beaver Army TML & 14246900-00-01-18-F & January 18, 2000 & 1 \\
\hline Columbia River & Beaver Army TML & $14246900-00-01-18$ & January 18, 2000 & 1 \\
\hline Columbia River & Beaver Army TML & $14246900-00-05-16 \mathrm{D}$ & May 16,2000 & 1 \\
\hline Columbia River & Beaver Army TML & $14246900-01-02-13$ & January 2, 2001 & 1 \\
\hline Columbia River & Beaver Army TML & $14246900-99-03-08$ & March 8, 1999 & 1 \\
\hline Columbia River & Beaver Army TML & $14246900-01-09-24$ & January 9, 2001 & 1 \\
\hline Columbia River & Beaver Army TML & 14246900-01-07-17-F & January 7, 2001 & 1 \\
\hline Columbia River & Beaver Army TML & $14246900-01-07-17$ & January 7, 2001 & 1 \\
\hline Columbia River & Beaver Army TML & 14246900-01-05-15-FD & January 5, 2001 & 1 \\
\hline Columbia River & Beaver Army TML & 14246900-01-05-15-F & January 5, 2001 & 1 \\
\hline Columbia River & Beaver Army TML & $14246900-01-05-15$ & January 5, 2001 & 1 \\
\hline Columbia River & Beaver Army TML & 14246900-01-03-13D & January 3, 2001 & 1 \\
\hline Columbia River & Beaver Army TML & 14246900-01-03-13-FD & January 3, 2001 & 1 \\
\hline Columbia River & Beaver Army TML & 14246900-01-03-13-F & January 3, 2001 & 1 \\
\hline Columbia River & Beaver Army TML & $14246900-01-03-13$ & January 3, 2001 & 1 \\
\hline Columbia River & Beaver Army TML & 14246900-00-07-25-FD & July 25, 2000 & 1 \\
\hline Columbia River & Beaver Army TML & 14246900-01-02-13-F & January 2, 2001 & 1 \\
\hline Columbia River & Beaver Army TML & $14246900-00-09-12$ & September 12, 2000 & 1 \\
\hline Columbia River & Beaver Army TML & 14246900-01-01-16D & January 1, 2001 & 1 \\
\hline Columbia River & Beaver Army TML & 14246900-01-01-16-FD & January 1, 2001 & 1 \\
\hline
\end{tabular}


Table E-2

Summary Table of Segment 5 Surface Water Samples By Area, Site, Date, and Category

\begin{tabular}{|c|c|c|c|c|}
\hline Sample Area & Sample Site & Sample ID & Sample Date & Category \\
\hline Columbia River & Beaver Army TML & 14246900-01-01-16-F & January 1, 2001 & 1 \\
\hline Columbia River & Beaver Army TML & 14246900-01-01-16 & January 1, 2001 & 1 \\
\hline Columbia River & Beaver Army TML & 14246900-00-12-11-FD & December 11, 2000 & 1 \\
\hline Columbia River & Beaver Army TML & 14246900-00-12-11-F & December 11, 2000 & 1 \\
\hline Columbia River & Beaver Army TML & $14246900-00-12-11$ & December 11, 2000 & 1 \\
\hline Columbia River & Beaver Army TML & 14246900-00-11-13-FD & November 13, 2000 & 1 \\
\hline Columbia River & Beaver Army TML & 14246900-00-11-13-F & November 13, 2000 & 1 \\
\hline Columbia River & Beaver Army TML & $14246900-00-11-13$ & November 13, 2000 & 1 \\
\hline Columbia River & Beaver Army TML & 14246900-00-09-12-FD & September 12, 2000 & 1 \\
\hline Columbia River & Beaver Army TML & 14246900-01-09-24-FD & January 9, 2001 & 1 \\
\hline Columbia River & Beaver Army TML & 14246900-01-02-13-FD & January 2, 2001 & 1 \\
\hline Columbia River & Beaver Army TML & 14246900-99-10-19-FD & October 19, 1999 & 1 \\
\hline Columbia River & Beaver Army TML & 14246900-99-05-25-F & May 25, 1999 & 1 \\
\hline Columbia River & Beaver Army TML & 14246900-99-02-23-F & February 23, 1999 & 1 \\
\hline Columbia River & Beaver Army TML & 14246900-99-11-30D & November 30, 1999 & 1 \\
\hline Columbia River & Beaver Army TML & 14246900-99-11-30-FD & November 30, 1999 & 1 \\
\hline Columbia River & Beaver Army TML & $14246900-99-11-30$ & November 30, 1999 & 1 \\
\hline Columbia River & Beaver Army TML & 14246900-99-10-19-F & October 19, 1999 & 1 \\
\hline Columbia River & Beaver Army TML & 14246900-99-10-19 & October 19, 1999 & 1 \\
\hline Columbia River & Beaver Army TML & 14246900-99-09-14-FD & September 14, 1999 & 1 \\
\hline Columbia River & Beaver Army TML & 14246900-99-09-14-F & September 14, 1999 & 1 \\
\hline Columbia River & Beaver Army TML & $14246900-99-09-14$ & September 14, 1999 & 1 \\
\hline Columbia River & Beaver Army TML & 14246900-99-05-25D & May 25, 1999 & 1 \\
\hline Columbia River & Beaver Army TML & 14246900-99-11-30-F & November 30, 1999 & 1 \\
\hline Columbia River & Beaver Army TML & 14246900-99-05-25-FD & May 25, 1999 & 1 \\
\hline Columbia River & Beaver Army TML & 14246900-99-08-03-FD & August 3, 1999 & 1 \\
\hline Columbia River & Beaver Army TML & $14246900-99-06-08$ & June 8, 1999 & 1 \\
\hline Columbia River & Beaver Army TML & 14246900-99-06-08-F & June 8, 1999 & 1 \\
\hline Columbia River & Beaver Army TML & 14246900-99-06-08-FD & June 8, 1999 & 1 \\
\hline Columbia River & Beaver Army TML & 14246900-99-06-22-F & June 22, 1999 & 1 \\
\hline Columbia River & Beaver Army TML & 14246900-99-06-22-FD & June 22, 1999 & 1 \\
\hline Columbia River & Beaver Army TML & $14246900-99-08-03$ & August 3, 1999 & 1 \\
\hline Columbia River & Beaver Army TML & 14246900-99-08-03-F & August 3, 1999 & 1 \\
\hline Columbia River & Beaver Army TML & $14246900-99-06-22$ & June 22, 1999 & 1 \\
\hline Columbia River & Below Longview & 3354167 & August 28, 2003 & 1 \\
\hline Columbia River & Below Longview & 3394412 & September 25, 2003 & 1 \\
\hline Columbia River & Below Longview & 3514117 & December 17, 2003 & 1 \\
\hline Columbia River & Below Longview & 4034262 & January 13, 2004 & 1 \\
\hline Columbia River & Below Longview & 3384092 & September 16, 2003 & 1 \\
\hline Columbia River & Below Longview & 4224117 & May 25, 2004 & 1 \\
\hline Columbia River & Below Longview & 4314243 & June 23, 2004 & 1 \\
\hline Columbia River & Below Longview & 4264262 & June 23, 2004 & 1 \\
\hline Columbia River & Below Longview & 3454426 & September 25, 2003 & 1 \\
\hline Columbia River & Below Longview & 4244142 & June 9, 2004 & 1 \\
\hline Columbia River & Bonneville Dam & 4264251 & June 22, 2004 & 1 \\
\hline Columbia River & Bonneville Dam & 4224106 & May 24, 2004 & 1 \\
\hline Columbia River & Bonneville Dam & 4154232 & January 12, 2004 & 1 \\
\hline Columbia River & Bonneville Dam & 4314231 & June 22, 2004 & 1 \\
\hline Columbia River & Bonneville Dam & 4034251 & January 12, 2004 & 1 \\
\hline Columbia River & Bonneville Dam & 4024106 & January 5, 2004 & 1 \\
\hline
\end{tabular}


Table E-2

Summary Table of Segment 5 Surface Water Samples By Area, Site, Date, and Category

\begin{tabular}{|c|c|c|c|c|}
\hline Sample Area & Sample Site & Sample ID & Sample Date & Category \\
\hline Columbia River & Bonneville Dam & 3514106 & December 15, 2003 & 1 \\
\hline Columbia River & Bonneville Dam & 4244131 & June 9, 2004 & 1 \\
\hline Columbia River & Bonneville Dam & 3454414 & September 24, 2003 & 1 \\
\hline Columbia River & Bonneville Dam & 3384081 & September 15, 2003 & 1 \\
\hline Columbia River & Bonneville Dam & 3354156 & August 27, 2003 & 1 \\
\hline Columbia River & Bonneville Dam & 3394401 & September 24, 2003 & 1 \\
\hline Columbia River & Columbia CTY OR & 1388042 & September 25, 2001 & 1 \\
\hline Columbia River & Columbia Slough & 3454417 & September 25, 2003 & 1 \\
\hline Columbia River & Columbia Slough & 3384084 & September 15, 2003 & 1 \\
\hline Columbia River & Columbia Slough & 4314234 & June 22, 2004 & 1 \\
\hline Columbia River & Columbia Slough & 4264254 & June 22, 2004 & 1 \\
\hline Columbia River & Columbia Slough & 4244134 & June 9, 2004 & 1 \\
\hline Columbia River & Columbia Slough & 4224109 & May 24, 2004 & 1 \\
\hline Columbia River & Columbia Slough & 4034254 & January 12, 2004 & 1 \\
\hline Columbia River & Columbia Slough & 4154236 & January 12, 2004 & 1 \\
\hline Columbia River & Columbia Slough & 3394404 & September 25, 2003 & 1 \\
\hline Columbia River & Columbia Slough & 3354159 & August 27, 2003 & 1 \\
\hline Columbia River & Columbia Slough & 3514109 & December 16, 2003 & 1 \\
\hline Columbia River & County Line PRK & 1388044 & September 25, 2001 & 1 \\
\hline Columbia River & County Line PRK & 1388043 & September 25, 2001 & 1 \\
\hline Columbia River & Kalama WA & 3354164 & August 28, 2003 & 1 \\
\hline Columbia River & Kalama WA & 4264259 & June 23, 2004 & 1 \\
\hline Columbia River & Kalama WA & 4224114 & May 25, 2004 & 1 \\
\hline Columbia River & Kalama WA & 4244139 & June 9, 2004 & 1 \\
\hline Columbia River & Kalama WA & 4314240 & June 23, 2004 & 1 \\
\hline Columbia River & Kalama WA & 4154241 & January 13, 2004 & 1 \\
\hline Columbia River & Kalama WA & 3384089 & September 16, 2003 & 1 \\
\hline Columbia River & Kalama WA & 4034259 & January 13, 2004 & 1 \\
\hline Columbia River & Kalama WA & 4024114 & January 5, 2004 & 1 \\
\hline Columbia River & Kalama WA & 3394409 & September 25, 2003 & 1 \\
\hline Columbia River & Kalama WA & 3514114 & December 17, 2003 & 1 \\
\hline Columbia River & RM102, COL SLGH & loc 05 5/04-6/04 & June 1, 2004 & 1 \\
\hline Columbia River & RM102, COL SLGH & loc 05 8/03-9/03 & September 1, 2003 & 1 \\
\hline Columbia River & RM102, COL SLGH & Ioc 05 12/03-1/04 & January 1, 2004 & 1 \\
\hline Columbia River & RM102, WRVR & loc 06 8/03-9/03 & September 1, 2003 & 1 \\
\hline Columbia River & RM102, WRVR & Ioc 06 12/03-1/04 & January 1, 2004 & 1 \\
\hline Columbia River & RM102, WRVR & loc 06 5/04-6/04 & June 1, 2004 & 1 \\
\hline Columbia River & RM103, WRVR & loc 04 8/03-9/03 & September 1, 2003 & 1 \\
\hline Columbia River & RM103, WRVR & loc 04 5/04-6/04 & June 1, 2004 & 1 \\
\hline Columbia River & RM103, WRVR & Ioc 04 12/03-1/04 & January 1, 2004 & 1 \\
\hline Columbia River & RM121, WASHRVR & Ioc 03 12/03-1/04 & January 1, 2004 & 1 \\
\hline Columbia River & RM121, WASHRVR & loc 03 8/03-9/03 & September 1, 2003 & 1 \\
\hline Columbia River & RM121, WASHRVR & loc 03 5/04-6/04 & June 1, 2004 & 1 \\
\hline Columbia River & RM142 BONN DAM & loc 02 5/04-6/04 & June 1, 2004 & 1 \\
\hline Columbia River & RM142 BONN DAM & Ioc 02 12/03-1/04 & January 1, 2004 & 1 \\
\hline Columbia River & RM142 BONN DAM & loc 02 8/03-9/03 & September 1, 2003 & 1 \\
\hline Columbia River & RM54, LONGVIEW & loc 13 8/03-9/03 & September 1, 2003 & 1 \\
\hline Columbia River & RM54, LONGVIEW & loc 13 5/04-6/04 & June 1, 2004 & 1 \\
\hline Columbia River & RM68, COWLITZ R & Ioc 12 12/03-1/04 & January 1, 2004 & 1 \\
\hline Columbia River & RM68, COWLITZ R & loc 12 5/04-6/04 & June 1, 2004 & 1 \\
\hline
\end{tabular}


Table E-2

Summary Table of Segment 5 Surface Water Samples By Area, Site, Date, and Category

\begin{tabular}{|c|c|c|c|c|}
\hline Sample Area & Sample Site & Sample ID & Sample Date & Category \\
\hline Columbia River & RM68, COWLITZ R & loc 12 8/03-9/03 & September 1, 2003 & 1 \\
\hline Columbia River & RM73, KALAMA R & loc 11 12/3-1/04 & January 1, 2004 & 1 \\
\hline Columbia River & RM73, KALAMA R & loc 11 5/04-6/04 & June 1, 2004 & 1 \\
\hline Columbia River & RM73, KALAMA R & loc 11 8/03-9/03 & September 1, 2003 & 1 \\
\hline Columbia River & RM75, KALAMA R & Ioc 10 5/04-6/04 & June 1, 2004 & 1 \\
\hline Columbia River & RM75, KALAMA R & Ioc 10 12/03-1/04 & January 1, 2004 & 1 \\
\hline Columbia River & RM86, MULT CHNL & loc 09 12/03-1/04 & January 1, 2004 & 1 \\
\hline Columbia River & RM86, MULT CHNL & loc 09 5/04-6/04 & June 1, 2004 & 1 \\
\hline Columbia River & RM86, MULT CHNL & loc 09 8/03-9/03 & September 1, 2003 & 1 \\
\hline Columbia River & RM87, LEWIS R & Ioc 08 12/03-1/04 & January 1, 2004 & 1 \\
\hline Columbia River & RM87, LEWIS R & loc 08 5/04-6/04 & June 1, 2004 & 1 \\
\hline Columbia River & RM87, LEWIS R & loc 08 8/03-9/03 & September 1, 2003 & 1 \\
\hline Columbia River & RM88, LAKE R & Ioc 07 12/03-1/04 & January 1, 2004 & 1 \\
\hline Columbia River & RM88, LAKE R & loc 07 5/04-6/04 & June 1, 2004 & 1 \\
\hline Columbia River & RM88, LAKE R & loc 07 8/03-9/03 & September 1, 2003 & 1 \\
\hline Columbia River & RYAN PT WA & 1388041 & September 25, 2001 & 1 \\
\hline Columbia River & VANCOUV(28A100) & 131985 & March 19, 2003 & 1 \\
\hline Columbia River & VANCOUV(28A100) & $131627-\mathrm{F}$ & November 20, 2002 & 1 \\
\hline Columbia River & VANCOUV(28A100) & 132211 & May 21, 2003 & 1 \\
\hline Columbia River & VANCOUV(28A100) & 132299 & June 18, 2003 & 1 \\
\hline Columbia River & VANCOUV(28A100) & $132084-F$ & April 23, 2003 & 1 \\
\hline Columbia River & VANCOUV(28A100) & 132084 & April 23, 2003 & 1 \\
\hline Columbia River & VANCOUV(28A100) & 131985-F & March 19, 2003 & 1 \\
\hline Columbia River & VANCOUV(28A100) & 132211-F & May 21, 2003 & 1 \\
\hline Columbia River & VANCOUV(28A100) & 131897-F & February 26, 2003 & 1 \\
\hline Columbia River & VANCOUV(28A100) & 131897 & February 26, 2003 & 1 \\
\hline Columbia River & VANCOUV(28A100) & $131807-\mathrm{F}$ & January 29, 2003 & 1 \\
\hline Columbia River & VANCOUV(28A100) & 131807 & January 29, 2003 & 1 \\
\hline Columbia River & VANCOUV(28A100) & $132299-F$ & June 18, 2003 & 1 \\
\hline Columbia River & VANCOUV(28A100) & 131716 & December 11, 2002 & 1 \\
\hline Columbia River & VANCOUV(28A100) & 131627 & November 20, 2002 & 1 \\
\hline Columbia River & VANCOUV(28A100) & $131560-F$ & October 30, 2002 & 1 \\
\hline Columbia River & VANCOUV(28A100) & 131716-F & December 11, 2002 & 1 \\
\hline Columbia River & VANCOUV(28A100) & 131560 & October 30, 2002 & 1 \\
\hline Columbia River & VANCOUV(28A100) & $132390-F$ & July 23, 2003 & 1 \\
\hline Columbia River & VANCOUV(28A100) & 132503 & August 20, 2003 & 1 \\
\hline Columbia River & VANCOUV(28A100) & $132503-F$ & August 20, 2003 & 1 \\
\hline Columbia River & VANCOUV(28A100) & 132569 & September 24, 2003 & 1 \\
\hline Columbia River & VANCOUV(28A100) & 132569-F & September 24, 2003 & 1 \\
\hline Columbia River & VANCOUV(28A100) & 132390 & July 23, 2003 & 1 \\
\hline Columbia River & Willamette RVR & 3394403 & September 25, 2003 & 1 \\
\hline Columbia River & Willamette RVR & 4034253 & January 12, 2004 & 1 \\
\hline Columbia River & Willamette RVR & 3354158 & August 27, 2003 & 1 \\
\hline Columbia River & Willamette RVR & 4154235 & January 12, 2004 & 1 \\
\hline Columbia River & Willamette RVR & 4224108 & May 24, 2004 & 1 \\
\hline Columbia River & Willamette RVR & 3384083 & September 15, 2003 & 1 \\
\hline Columbia River & Willamette RVR & 4314233 & June 22, 2004 & 1 \\
\hline Columbia River & Willamette RVR & 4244133 & June 9, 2004 & 1 \\
\hline Columbia River & Willamette RVR & 3514108 & December 16, 2003 & 1 \\
\hline Columbia River & Willamette RVR & 3454416 & June 25, 2003 & 1 \\
\hline
\end{tabular}


Table E-2

Summary Table of Segment 5 Surface Water Samples By Area, Site, Date, and Category

\begin{tabular}{ccccc}
\hline Sample Area & Sample Site & Sample ID & Sample Date & Category \\
\hline Columbia River & Willamette RVR & 4264253 & June 22, 2004 & 1 \\
Columbia River & Willamette RVR & 4024108 & January 5, 2004 & 1 \\
\hline
\end{tabular}


Table E-3

Summary Statistics for Sediment Collected from Segment $\mathbf{5}$ of the Columbia River Including All Categories of Data (units reported "as received")

\begin{tabular}{|c|c|c|c|c|c|c|c|c|c|c|}
\hline $\begin{array}{l}\text { Constituent } \\
\text { Class }\end{array}$ & Constituent Name & Units & $\begin{array}{c}\text { Number } \\
\text { Analyzed }\end{array}$ & $\begin{array}{l}\text { Number } \\
\text { Detected }\end{array}$ & $\begin{array}{c}\text { Frequency of } \\
\text { Detection }\end{array}$ & $\begin{array}{l}\text { Minimum } \\
\text { Nondetect }\end{array}$ & $\begin{array}{l}\text { Maximum } \\
\text { Nondetect }\end{array}$ & Minimum Detect & $\begin{array}{l}\text { Maximum } \\
\text { Detect }\end{array}$ & $\begin{array}{c}\text { Average } \\
\text { Concentration }\end{array}$ \\
\hline$\overline{\mathrm{CONV}}$ & Cyanide & $\mathrm{mg} / \mathrm{kg}$ & 3 & 0 & $0 \%$ & 0.0071 & 0.0076 & -- & -- & 0.0037 \\
\hline CONV & Nitrogen in ammonia & $\mathrm{mg} / \mathrm{kg}$ & 8 & 8 & $100 \%$ & -- & -- & 55 & 414 & 246 \\
\hline CONV & Percent Solids & $\%$ & 3 & 3 & $100 \%$ & -- & -- & 76 & 78 & 77 \\
\hline CONV & Soluble Fluoride & $\mathrm{mg} / \mathrm{kg}$ & 3 & 3 & $100 \%$ & -- & -- & 1.8 & 8.9 & 4.4 \\
\hline CONV & Total Organic Carbon & $\%$ & 83 & 68 & $82 \%$ & 0.050 & 0.20 & 0.037 & 7.5 & 1.0 \\
\hline CONV & Total Organic Carbon & $\mathrm{mg} / \mathrm{kg}$ & 42 & 42 & $100 \%$ & -- & -- & 370 & 44,300 & 10,216 \\
\hline CONV & Total solids & $\%$ & 15 & 15 & $100 \%$ & -- & -- & 36 & 58 & 46 \\
\hline CONV & Total Volatile Solids & $\%$ & 152 & 152 & $100 \%$ & -- & -- & 0.20 & 11 & 2.0 \\
\hline METAL & Aluminum & $\mathrm{mg} / \mathrm{kg}$ & 3 & 3 & $100 \%$ & -- & -- & 454 & 498 & 483 \\
\hline METAL & Antimony & $\mathrm{mg} / \mathrm{kg}$ & 36 & 25 & $69 \%$ & 0.023 & 2.4 & 0.14 & 3.6 & 0.88 \\
\hline METAL & Arsenic & $\mathrm{mg} / \mathrm{kg}$ & 72 & 72 & $100 \%$ & -- & -- & 0.054 & 11 & 3.3 \\
\hline METAL & Beryllium & $\mathrm{mg} / \mathrm{kg}$ & 3 & 2 & $67 \%$ & 0.043 & 0.043 & 0.054 & 0.077 & 0.051 \\
\hline METAL & Cadmium & $\mathrm{mg} / \mathrm{kg}$ & 71 & 31 & $44 \%$ & 0.018 & 0.80 & 0.038 & 3.3 & 0.52 \\
\hline METAL & Chromium & $\mathrm{mg} / \mathrm{kg}$ & 49 & 49 & $100 \%$ & -- & -- & 0.99 & 35 & 11 \\
\hline METAL & Copper & $\mathrm{mg} / \mathrm{kg}$ & 71 & 71 & $100 \%$ & -- & -- & 1.1 & 76 & 19 \\
\hline METAL & Lead & $\mathrm{mg} / \mathrm{kg}$ & 73 & 73 & $100 \%$ & -- & -- & 0.084 & 140 & 11 \\
\hline METAL & Mercury & $\mathrm{mg} / \mathrm{kg}$ & 72 & 38 & $53 \%$ & $8.40 \mathrm{E}-04$ & 0.50 & 0.0024 & 0.56 & 0.060 \\
\hline METAL & Nickel & $\mathrm{mg} / \mathrm{kg}$ & 71 & 71 & $100 \%$ & -- & -- & 0.57 & 27 & 11 \\
\hline METAL & Selenium & $\mathrm{mg} / \mathrm{kg}$ & 3 & 0 & $0 \%$ & 0.045 & 0.048 & -- & -- & 0.023 \\
\hline METAL & Silver & $\mathrm{mg} / \mathrm{kg}$ & 72 & 28 & $39 \%$ & 0.052 & 0.77 & 0.027 & 2.3 & 0.29 \\
\hline METAL & Thallium & $\mathrm{mg} / \mathrm{kg}$ & 3 & 3 & $100 \%$ & -- & -- & 0.054 & 0.094 & 0.077 \\
\hline METAL & Zinc & $\mathrm{mg} / \mathrm{kg}$ & 72 & 71 & $99 \%$ & 34 & 34 & 2.1 & 200 & 67 \\
\hline PCB & Aroclor-1016 & $\mathrm{mg} / \mathrm{kg}$ & 11 & 0 & $0 \%$ & 0.0017 & 0.0084 & -- & -- & 0.0019 \\
\hline PCB & Aroclor-1221 & $\mathrm{mg} / \mathrm{kg}$ & 11 & 0 & $0 \%$ & 0.0017 & 0.0084 & -- & -- & 0.0019 \\
\hline PCB & Aroclor-1232 & $\mathrm{mg} / \mathrm{kg}$ & 11 & 0 & $0 \%$ & 0.0017 & 0.0084 & -- & -- & 0.0019 \\
\hline PCB & Aroclor-1242 & $\mathrm{mg} / \mathrm{kg}$ & 16 & 1 & $6 \%$ & 0.0017 & 0.010 & 0.019 & 0.019 & 0.0038 \\
\hline PCB & Aroclor-1248 & $\mathrm{mg} / \mathrm{kg}$ & 37 & 6 & $16 \%$ & 0.0017 & 0.0084 & 0.097 & 1.4 & 0.065 \\
\hline PCB & Aroclor-1254 & $\mathrm{mg} / \mathrm{kg}$ & 38 & 1 & $3 \%$ & $9.20 \mathrm{E}-04$ & 0.017 & 0.024 & 0.024 & 0.0048 \\
\hline PCB & Aroclor-1260 & $\mathrm{mg} / \mathrm{kg}$ & 38 & 2 & $5 \%$ & $9.20 \mathrm{E}-04$ & 0.017 & 0.0050 & 0.037 & 0.0052 \\
\hline PEST & Aldrin & $\mathrm{mg} / \mathrm{kg}$ & 34 & 2 & $6 \%$ & 7.54E-05 & 0.0020 & 2.00E-04 & $6.00 \mathrm{E}-04$ & 6.97E-04 \\
\hline PEST & Alpha-BHC & $\mathrm{mg} / \mathrm{kg}$ & 3 & 0 & $0 \%$ & $7.66 \mathrm{E}-04$ & $8.40 \mathrm{E}-04$ & -- & -- & 3.98E-04 \\
\hline PEST & alpha-Chlordane & $\mathrm{mg} / \mathrm{kg}$ & 11 & 0 & $0 \%$ & 7.91E-05 & 0.0084 & -- & -- & 0.0011 \\
\hline PEST & beta-1,2,3,4,5,6-Hexachlorocyclohexane & $\mathrm{mg} / \mathrm{kg}$ & 3 & 0 & $0 \%$ & 7.66E-04 & 8.40E-04 & -- & -- & $3.98 \mathrm{E}-04$ \\
\hline PEST & Bromine & $\mathrm{mg} / \mathrm{kg}$ & 24 & 19 & $79 \%$ & 0.0030 & 0.0030 & 0.0039 & 0.017 & 0.0074 \\
\hline PEST & Chlordane & $\mathrm{mg} / \mathrm{kg}$ & 4 & 0 & $0 \%$ & 0.010 & 0.010 & -- & -- & 0.0050 \\
\hline PEST & Chlorine & $\mathrm{mg} / \mathrm{kg}$ & 24 & 14 & $58 \%$ & 0.030 & 0.030 & 0.031 & 2.4 & 0.34 \\
\hline PEST & Delta-BHC & $\mathrm{mg} / \mathrm{kg}$ & 3 & 0 & $0 \%$ & $7.66 \mathrm{E}-04$ & 8.40E-04 & -- & -- & $3.98 \mathrm{E}-04$ \\
\hline PEST & Dichlorodiphenyldichloroethane & $\mathrm{mg} / \mathrm{kg}$ & 97 & 13 & $13 \%$ & $1.49 \mathrm{E}-04$ & 1.0 & $5.00 \mathrm{E}-04$ & 0.0048 & 0.010 \\
\hline PEST & Dichlorodiphenyldichloroethylene & $\mathrm{mg} / \mathrm{kg}$ & 97 & 20 & $21 \%$ & $1.65 \mathrm{E}-04$ & 1.0 & $4.00 E-04$ & 0.0039 & 0.010 \\
\hline PEST & Dichlorodiphenyltrichloroethane & $\mathrm{mg} / \mathrm{kg}$ & 97 & 3 & $3 \%$ & $1.72 \mathrm{E}-04$ & 1.0 & 3.00E-04 & 0.0030 & 0.010 \\
\hline PEST & Dieldrin & $\mathrm{mg} / \mathrm{kg}$ & 15 & 0 & $0 \%$ & $1.78 \mathrm{E}-04$ & 0.0050 & -- & -- & $5.80 \mathrm{E}-04$ \\
\hline PEST & Endosulfan I & $\mathrm{mg} / \mathrm{kg}$ & 3 & 0 & $0 \%$ & 7.66E-04 & $8.40 \mathrm{E}-04$ & -- & -- & 3.98E-04 \\
\hline PEST & Endosulfan II & $\mathrm{mg} / \mathrm{kg}$ & 3 & 0 & $0 \%$ & 0.0015 & 0.0017 & -- & -- & $8.09 \mathrm{E}-04$ \\
\hline PEST & Endosulfan sulfate & $\mathrm{mg} / \mathrm{kg}$ & 29 & 2 & $7 \%$ & $4.00 \mathrm{E}-04$ & 0.0017 & 0.0023 & 0.0030 & 4.65E-04 \\
\hline PEST & Endrin & $\mathrm{mg} / \mathrm{kg}$ & 3 & 0 & $0 \%$ & 0.0015 & 0.0017 & -- & -- & 8.09E-04 \\
\hline PEST & Endrin ketone & $\mathrm{mg} / \mathrm{kg}$ & 29 & 1 & $3 \%$ & 3.10E-04 & 0.0017 & 0.0047 & 0.0047 & 4.06E-04 \\
\hline PEST & Gamma-BHC (Lindane) & $\mathrm{mg} / \mathrm{kg}$ & 11 & 0 & $0 \%$ & $8.78 \mathrm{E}-05$ & $8.40 \mathrm{E}-04$ & -- & -- & 1.43E-04 \\
\hline PEST & gamma-Chlordane & $\mathrm{mg} / \mathrm{kg}$ & 8 & 0 & $0 \%$ & $8.86 \mathrm{E}-05$ & $1.00 \mathrm{E}-04$ & -- & -- & 4.78E-05 \\
\hline PEST & Heptachlor & $\mathrm{mg} / \mathrm{kg}$ & 16 & 1 & $6 \%$ & 7.32E-05 & 0.0019 & 0.0074 & 0.0074 & $6.99 \mathrm{E}-04$ \\
\hline PEST & Heptachlor epoxide & $\mathrm{mg} / \mathrm{kg}$ & 3 & 0 & $0 \%$ & 7.66E-04 & $8.40 \mathrm{E}-04$ & -- & -- & $3.98 \mathrm{E}-04$ \\
\hline PEST & Methoxychlor & $\mathrm{mg} / \mathrm{kg}$ & 29 & 1 & $3 \%$ & 0.0015 & 0.015 & 0.0055 & 0.0055 & 0.0016 \\
\hline
\end{tabular}


Table E-3

Summary Statistics for Sediment Collected from Segment 5 of the Columbia River Including All Categories of Data (units reported "as received")

\begin{tabular}{|c|c|c|c|c|c|c|c|c|c|c|}
\hline $\begin{array}{l}\text { Constituent } \\
\text { Class }\end{array}$ & Constituent Name & Units & $\begin{array}{c}\text { Number } \\
\text { Analyzed }\end{array}$ & $\begin{array}{l}\text { Number } \\
\text { Detected }\end{array}$ & $\begin{array}{l}\text { Frequency of } \\
\text { Detection }\end{array}$ & $\begin{array}{l}\text { Minimum } \\
\text { Nondetect }\end{array}$ & $\begin{array}{l}\text { Maximum } \\
\text { Nondetect }\end{array}$ & Minimum Detect & $\begin{array}{c}\text { Maximum } \\
\text { Detect }\end{array}$ & $\begin{array}{c}\text { Average } \\
\text { Concentration }\end{array}$ \\
\hline$\overline{\mathrm{PEST}}$ & Toxaphene & $\mathrm{mg} / \mathrm{kg}$ & 3 & $\overline{0}$ & $0 \%$ & 0.015 & 0.017 & -- & -- & 0.0081 \\
\hline PEST & trans-Chlordane & $\mathrm{mg} / \mathrm{kg}$ & 3 & 0 & $0 \%$ & 0.0077 & 0.0084 & -- & -- & 0.0040 \\
\hline RAD_D & Beryllium-7, Decayed & $\mathrm{pCi} / \mathrm{g}$ & 1 & 1 & $100 \%$ & -- & -- & $1.59 \mathrm{E}-28$ & $1.59 \mathrm{E}-28$ & $1.59 \mathrm{E}-28$ \\
\hline RAD_D & Cerium-141, Decayed & $\mathrm{pCi} / \mathrm{g}$ & 9 & 9 & $100 \%$ & -- & -- & $9.05 \mathrm{E}-100$ & 3.77E-89 & $5.59 \mathrm{E}-90$ \\
\hline RAD_D & Cesium-137, Decayed & $\mathrm{pCi} / \mathrm{g}$ & 53 & 53 & $100 \%$ & -- & -- & 0.048 & 0.23 & 0.087 \\
\hline RAD_D & Cobalt-60, Decayed & $\mathrm{pCi} / \mathrm{g}$ & 51 & 51 & $100 \%$ & -- & -- & $8.78 \mathrm{E}-04$ & 0.013 & 0.0056 \\
\hline RAD_D & Potassium-40, Decayed & $\mathrm{pCi} / \mathrm{g}$ & 199 & 199 & $100 \%$ & -- & -- & 2.7 & 30 & 11 \\
\hline RAD_D & Radium-226, Decayed & $\mathrm{pCi} / \mathrm{g}$ & 44 & 44 & $100 \%$ & -- & -- & 0.099 & 1.5 & 0.58 \\
\hline RAD_D & Ruthenium-106, Decayed & $\mathrm{pCi} / \mathrm{g}$ & 70 & 70 & $100 \%$ & -- & -- & $5.77 \mathrm{E}-14$ & $6.58 \mathrm{E}-10$ & $2.01 \mathrm{E}-11$ \\
\hline RAD_D & Thorium-232, Decayed & $\mathrm{pCi} / \mathrm{g}$ & 29 & 29 & $100 \%$ & -- & -- & 0.30 & 1.00 & 0.64 \\
\hline RAD_D & Zinc-65, Decayed & $\mathrm{pCi} / \mathrm{g}$ & 95 & 95 & $100 \%$ & -- & -- & $7.33 \mathrm{E}-20$ & $1.56 \mathrm{E}-12$ & $5.77 \mathrm{E}-14$ \\
\hline RAD_D & Zirconium/Niobium-95, Decayed & $\mathrm{pCi} / \mathrm{g}$ & 67 & 67 & $100 \%$ & -- & -- & 3.95E-132 & 2.43E-76 & $5.44 \mathrm{E}-78$ \\
\hline SVOC & 1,2,4-Trichlorobenzene & $\mathrm{mg} / \mathrm{kg}$ & 11 & 8 & $73 \%$ & 0.0033 & 0.0035 & $4.11 \mathrm{E}-04$ & $7.11 \mathrm{E}-04$ & $8.81 \mathrm{E}-04$ \\
\hline SVOC & 1,2-Dichlorobenzene & $\mathrm{mg} / \mathrm{kg}$ & 11 & 2 & $18 \%$ & $1.76 \mathrm{E}-04$ & 0.0035 & $3.78 \mathrm{E}-04$ & 3.82E-04 & $5.81 \mathrm{E}-04$ \\
\hline SVOC & 1,3-Dichlorobenzene & $\mathrm{mg} / \mathrm{kg}$ & 10 & 0 & $0 \%$ & $3.62 \mathrm{E}-04$ & 0.0035 & -- & -- & $6.44 \mathrm{E}-04$ \\
\hline SVOC & 1,4-Dichlorobenzene & $\mathrm{mg} / \mathrm{kg}$ & 11 & 1 & $9 \%$ & 3.34E-04 & 0.0035 & 4.40E-04 & 4.40E-04 & $6.15 \mathrm{E}-04$ \\
\hline sVOC & 2,4,5-Trichlorophenol & $\mathrm{mg} / \mathrm{kg}$ & 3 & 0 & $0 \%$ & 0.0065 & 0.0070 & -- & -- & 0.0034 \\
\hline SVOC & 2,4,6-Trichlorophenol & $\mathrm{mg} / \mathrm{kg}$ & 3 & 0 & $0 \%$ & 0.0065 & 0.0070 & -- & -- & 0.0034 \\
\hline SVOC & 2,4-Dichlorophenol & $\mathrm{mg} / \mathrm{kg}$ & 3 & 0 & $0 \%$ & 0.0065 & 0.0070 & -- & -- & 0.0034 \\
\hline SVOC & 2,4-Dimethylphenol & $\mathrm{mg} / \mathrm{kg}$ & 11 & 2 & $18 \%$ & $1.65 \mathrm{E}-04$ & 0.0035 & 0.0019 & 0.0020 & $8.61 \mathrm{E}-04$ \\
\hline sVOC & 2,4-Dinitrophenol & $\mathrm{mg} / \mathrm{kg}$ & 3 & 0 & $0 \%$ & 0.033 & 0.035 & -- & -- & 0.017 \\
\hline SVOC & 2,4-Dinitrotoluene & $\mathrm{mg} / \mathrm{kg}$ & 3 & 0 & $0 \%$ & 0.0065 & 0.0070 & -- & -- & 0.0034 \\
\hline SVOC & 2,6-Dinitrotoluene & $\mathrm{mg} / \mathrm{kg}$ & 10 & 7 & $70 \%$ & 0.0065 & 0.0070 & 0.012 & 0.021 & 0.012 \\
\hline sVOC & 2-Chloronaphthalene & $\mathrm{mg} / \mathrm{kg}$ & 3 & 0 & $0 \%$ & 0.0033 & 0.0035 & -- & -- & 0.0017 \\
\hline SVOC & 2-Chlorophenol & $\mathrm{mg} / \mathrm{kg}$ & 3 & 0 & $0 \%$ & 0.0033 & 0.0035 & -- & -- & 0.0017 \\
\hline SVOC & 2-Methylnaphthalene & $\mathrm{mg} / \mathrm{kg}$ & 80 & 33 & $41 \%$ & $2.10 \mathrm{E}-04$ & 0.0071 & $3.60 \mathrm{E}-04$ & 0.41 & 0.0084 \\
\hline SVOC & 2-Methylphenol (cresol, o-) & $\mathrm{mg} / \mathrm{kg}$ & 21 & 4 & $19 \%$ & $3.60 \mathrm{E}-04$ & 0.0099 & 4.59E-04 & 0.015 & 0.0030 \\
\hline SVOC & 2-Nitroaniline & $\mathrm{mg} / \mathrm{kg}$ & 3 & 0 & $0 \%$ & 0.0065 & 0.0070 & -- & -- & 0.0034 \\
\hline sVOC & 2-Nitrophenol & $\mathrm{mg} / \mathrm{kg}$ & 3 & 0 & $0 \%$ & 0.0065 & 0.0070 & -- & -- & 0.0034 \\
\hline SVOC & 3\&4-Methylphenol (Cresol, $m+p)$ & $\mathrm{mg} / \mathrm{kg}$ & 12 & 9 & $75 \%$ & 0.045 & 0.057 & 0.0019 & 0.057 & 0.015 \\
\hline SVOC & 3,3'-Dichlorobenzidine & $\mathrm{mg} / \mathrm{kg}$ & 3 & 0 & $0 \%$ & 0.033 & 0.035 & -- & -- & 0.017 \\
\hline SVOC & 3-Nitroaniline & $\mathrm{mg} / \mathrm{kg}$ & 3 & 0 & $0 \%$ & 0.016 & 0.018 & -- & -- & 0.0083 \\
\hline SVOC & 4,6-Dinitro-2-methylphenol & $\mathrm{mg} / \mathrm{kg}$ & 3 & 0 & $0 \%$ & 0.033 & 0.035 & -- & -- & 0.017 \\
\hline SVOC & 4-Bromophenylphenyl ether & $\mathrm{mg} / \mathrm{kg}$ & 3 & 0 & $0 \%$ & 0.0065 & 0.0070 & -- & -- & 0.0034 \\
\hline SVOC & 4-Chloro-3-methylphenol & $\mathrm{mg} / \mathrm{kg}$ & 3 & 0 & $0 \%$ & 0.0065 & 0.0070 & -- & -- & 0.0034 \\
\hline SVOC & 4-Chloroaniline & $\mathrm{mg} / \mathrm{kg}$ & 3 & 0 & $0 \%$ & 0.0033 & 0.0035 & -- & -- & 0.0017 \\
\hline SVOC & 4-Chlorophenylphenyl ether & $\mathrm{mg} / \mathrm{kg}$ & 3 & 0 & $0 \%$ & 0.0033 & 0.0035 & -- & -- & 0.0017 \\
\hline SVOC & 4-Methylphenol (cresol, p-) & $\mathrm{mg} / \mathrm{kg}$ & 3 & 0 & $0 \%$ & 0.0033 & 0.0035 & -- & -- & 0.0017 \\
\hline SVOC & 4-Nitroaniline & $\mathrm{mg} / \mathrm{kg}$ & 3 & 0 & $0 \%$ & 0.0065 & 0.0070 & -- & -- & 0.0034 \\
\hline SVOC & 4-Nitrophenol & $\mathrm{mg} / \mathrm{kg}$ & 3 & 0 & $0 \%$ & 0.033 & 0.035 & -- & -- & 0.017 \\
\hline sVOC & Acenaphthene & $\mathrm{mg} / \mathrm{kg}$ & 80 & 28 & $35 \%$ & 8.90E-05 & 0.011 & $5.00 E-04$ & 0.11 & 0.0038 \\
\hline SVOC & Acenaphthylene & $\mathrm{mg} / \mathrm{kg}$ & 80 & 30 & $38 \%$ & 1.89E-04 & 0.011 & 2.00E-04 & 0.021 & 0.0021 \\
\hline SVOC & Anthracene & $\mathrm{mg} / \mathrm{kg}$ & 80 & 34 & $43 \%$ & $1.95 \mathrm{E}-04$ & 0.010 & $7.00 \mathrm{E}-04$ & 0.41 & 0.0083 \\
\hline SVOC & Benzo(a)anthracene & $\mathrm{mg} / \mathrm{kg}$ & 76 & 47 & $62 \%$ & $1.36 \mathrm{E}-04$ & 0.0050 & 7.00E-04 & 0.096 & 0.010 \\
\hline SVOC & Benzo(a)pyrene & $\mathrm{mg} / \mathrm{kg}$ & 79 & 52 & $66 \%$ & $6.81 \mathrm{E}-05$ & 0.0070 & $6.00 \mathrm{E}-04$ & 0.10 & 0.013 \\
\hline SVOC & Benzo(b)fluoranthene & $\mathrm{mg} / \mathrm{kg}$ & 29 & 21 & $72 \%$ & $2.46 \mathrm{E}-04$ & 0.0070 & 0.0016 & 0.044 & 0.011 \\
\hline SVOC & Benzo $(b+k)$ fluoranthene & $\mathrm{mg} / \mathrm{kg}$ & 28 & 16 & $57 \%$ & 0.0050 & 0.0050 & $6.00 \mathrm{E}-04$ & 0.12 & 0.013 \\
\hline SVOC & Benzo(ghi)perylene & $\mathrm{mg} / \mathrm{kg}$ & 80 & 52 & $65 \%$ & $1.32 \mathrm{E}-04$ & 0.0070 & 5.00E-04 & 0.077 & 0.0091 \\
\hline SVOC & Benzo(k)fluoranthene & $\mathrm{mg} / \mathrm{kg}$ & 30 & 17 & $57 \%$ & 3.06E-04 & 0.0070 & $5.70 \mathrm{E}-04$ & 0.027 & 0.0048 \\
\hline SVOC & Benzofluoranthene & $\mathrm{mg} / \mathrm{kg}$ & 4 & 3 & $75 \%$ & 0.0056 & 0.0056 & 0.044 & 0.066 & 0.043 \\
\hline SVOC & Benzoic acid & $\mathrm{mg} / \mathrm{kg}$ & 31 & 19 & $61 \%$ & 0.0069 & 0.092 & 0.034 & 0.49 & 0.055 \\
\hline
\end{tabular}


Table E-3

Summary Statistics for Sediment Collected from Segment $\mathbf{5}$ of the Columbia River Including All Categories of Data (units reported "as received")

\begin{tabular}{|c|c|c|c|c|c|c|c|c|c|c|}
\hline $\begin{array}{l}\text { Constituent } \\
\text { Class }\end{array}$ & Constituent Name & Units & $\begin{array}{c}\text { Number } \\
\text { Analyzed }\end{array}$ & $\begin{array}{l}\text { Number } \\
\text { Detected }\end{array}$ & $\begin{array}{l}\text { Frequency of } \\
\text { Detection }\end{array}$ & $\begin{array}{l}\text { Minimum } \\
\text { Nondetect }\end{array}$ & $\begin{array}{l}\text { Maximum } \\
\text { Nondetect }\end{array}$ & Minimum Detect & $\begin{array}{c}\text { Maximum } \\
\text { Detect }\end{array}$ & $\begin{array}{c}\text { Average } \\
\text { Concentration }\end{array}$ \\
\hline$\overline{\text { SVOC }}$ & Benzyl alcohol & $\mathrm{mg} / \mathrm{kg}$ & 16 & 9 & $56 \%$ & 0.0033 & 0.016 & 0.0045 & 0.069 & 0.0099 \\
\hline SVOC & Bis(2-chloro-1-methylethyl)ether & $\mathrm{mg} / \mathrm{kg}$ & 3 & 0 & $0 \%$ & 0.0033 & 0.0035 & -- & -- & 0.0017 \\
\hline SVOC & Bis(2-Chloroethoxy)methane & $\mathrm{mg} / \mathrm{kg}$ & 3 & 0 & $0 \%$ & 0.0033 & 0.0035 & -- & -- & 0.0017 \\
\hline SVOC & Bis(2-chloroethyl) ether & $\mathrm{mg} / \mathrm{kg}$ & 3 & 0 & $0 \%$ & 0.0033 & 0.0035 & -- & -- & 0.0017 \\
\hline sVOC & Bis(2-ethylhexyl) phthalate & $\mathrm{mg} / \mathrm{kg}$ & 45 & 34 & $76 \%$ & 0.0054 & 0.023 & 0.0062 & 0.21 & 0.030 \\
\hline sVOC & Butylbenzylphthalate & $\mathrm{mg} / \mathrm{kg}$ & 27 & 22 & $81 \%$ & 0.0025 & 0.0035 & 0.0032 & 0.033 & 0.0090 \\
\hline SVOC & Chrysene & $\mathrm{mg} / \mathrm{kg}$ & 80 & 54 & $68 \%$ & $1.80 \mathrm{E}-04$ & 0.0050 & $6.00 \mathrm{E}-04$ & 0.13 & 0.016 \\
\hline SVOC & Dibenz[a,h]anthracene & $\mathrm{mg} / \mathrm{kg}$ & 71 & 18 & $25 \%$ & $1.20 \mathrm{E}-04$ & 0.0070 & $9.00 \mathrm{E}-04$ & 0.016 & 0.0022 \\
\hline sVOC & Dibenzofuran & $\mathrm{mg} / \mathrm{kg}$ & 18 & 6 & $33 \%$ & 1.99E-04 & 0.0041 & 0.0010 & 0.0042 & 0.0017 \\
\hline SVOC & Diethylphthalate & $\mathrm{mg} / \mathrm{kg}$ & 25 & 10 & $40 \%$ & 0.0033 & 0.028 & 0.0023 & 0.026 & 0.0062 \\
\hline SVOC & Dimethyl phthalate & $\mathrm{mg} / \mathrm{kg}$ & 11 & 3 & $27 \%$ & 4.44E-04 & 0.0035 & $6.87 \mathrm{E}-04$ & 0.0035 & 0.0010 \\
\hline SVOC & Di-n-butylphthalate & $\mathrm{mg} / \mathrm{kg}$ & 30 & 20 & $67 \%$ & 0.0033 & 0.028 & 0.013 & 0.32 & 0.076 \\
\hline sVOC & Di-n-octylphthalate & $\mathrm{mg} / \mathrm{kg}$ & 21 & 3 & $14 \%$ & 2.93E-04 & 0.0099 & $5.75 \mathrm{E}-04$ & 0.023 & 0.0035 \\
\hline sVOC & Fluoranthene & $\mathrm{mg} / \mathrm{kg}$ & 75 & 55 & $73 \%$ & $6.20 \mathrm{E}-04$ & 0.0050 & 3.21E-04 & 0.087 & 0.015 \\
\hline SVOC & Fluorene & $\mathrm{mg} / \mathrm{kg}$ & 80 & 31 & $39 \%$ & $2.48 \mathrm{E}-04$ & 0.0091 & $6.00 \mathrm{E}-04$ & 0.10 & 0.0049 \\
\hline SVOC & Hexachlorobenzene & $\mathrm{mg} / \mathrm{kg}$ & 3 & 0 & $0 \%$ & 0.0065 & 0.0070 & -- & -- & 0.0034 \\
\hline sVOC & Hexachlorobutadiene & $\mathrm{mg} / \mathrm{kg}$ & 3 & 0 & $0 \%$ & 0.0033 & 0.0035 & -- & -- & 0.0017 \\
\hline SVOC & Hexachlorocyclopentadiene & $\mathrm{mg} / \mathrm{kg}$ & 3 & 0 & $0 \%$ & 0.0065 & 0.0070 & -- & -- & 0.0034 \\
\hline sVOC & Hexachloroethane & $\mathrm{mg} / \mathrm{kg}$ & 11 & 0 & $0 \%$ & 3.76E-04 & 0.0070 & -- & -- & 0.0011 \\
\hline sVOC & Indeno(1,2,3-cd)pyrene & $\mathrm{mg} / \mathrm{kg}$ & 79 & 39 & $49 \%$ & 8.54E-05 & 0.0070 & 7.00E-04 & 0.076 & 0.0069 \\
\hline sVOC & Isophorone & $\mathrm{mg} / \mathrm{kg}$ & 3 & 0 & $0 \%$ & 0.0033 & 0.0035 & -- & -- & 0.0017 \\
\hline SVOC & Naphthalene & $\mathrm{mg} / \mathrm{kg}$ & 80 & 47 & $59 \%$ & $6.20 \mathrm{E}-04$ & 0.0070 & 4.24E-04 & 0.066 & 0.0048 \\
\hline SVOC & Nitrobenzene & $\mathrm{mg} / \mathrm{kg}$ & 3 & 0 & $0 \%$ & 0.0033 & 0.0035 & -- & -- & 0.0017 \\
\hline SVOC & N-Nitrosodi-n-dipropylamine & $\mathrm{mg} / \mathrm{kg}$ & 3 & 0 & $0 \%$ & 0.0033 & 0.0035 & -- & -- & 0.0017 \\
\hline sVOC & N-Nitrosodiphenylamine & $\mathrm{mg} / \mathrm{kg}$ & 11 & 0 & $0 \%$ & $1.38 \mathrm{E}-04$ & 0.0035 & -- & -- & $5.16 \mathrm{E}-04$ \\
\hline SVOC & Pentachlorophenol & $\mathrm{mg} / \mathrm{kg}$ & 21 & 7 & $33 \%$ & 9.59E-04 & 0.035 & 0.0013 & 0.039 & 0.0067 \\
\hline SVOC & Phenanthrene & $\mathrm{mg} / \mathrm{kg}$ & 79 & 52 & $66 \%$ & $2.80 \mathrm{E}-04$ & 0.0092 & $3.81 \mathrm{E}-04$ & 0.33 & 0.017 \\
\hline SVOC & Phenol & $\mathrm{mg} / \mathrm{kg}$ & 21 & 9 & $43 \%$ & 0.0033 & 0.0099 & 0.0033 & 0.013 & 0.0045 \\
\hline sVOC & Pyrene & $\mathrm{mg} / \mathrm{kg}$ & 80 & 52 & $65 \%$ & 4.40E-04 & 0.0050 & $3.62 \mathrm{E}-04$ & 0.17 & 0.019 \\
\hline VOC & 1,1,1-Trichloroethane & $\mathrm{mg} / \mathrm{kg}$ & 3 & 0 & $0 \%$ & $1.53 \mathrm{E}-04$ & $1.56 \mathrm{E}-04$ & -- & -- & $7.71 \mathrm{E}-05$ \\
\hline VOC & 1,1,2,2-Tetrachloroethane & $\mathrm{mg} / \mathrm{kg}$ & 3 & 0 & $0 \%$ & 3.83E-04 & 4.69E-04 & -- & -- & $2.18 \mathrm{E}-04$ \\
\hline VOC & 1,1,2-Trichloroethane & $\mathrm{mg} / \mathrm{kg}$ & 3 & 0 & $0 \%$ & $1.53 \mathrm{E}-04$ & $1.56 \mathrm{E}-04$ & -- & -- & $7.71 \mathrm{E}-05$ \\
\hline VOC & 1,1-Dichloroethane & $\mathrm{mg} / \mathrm{kg}$ & 3 & 0 & $0 \%$ & $1.53 \mathrm{E}-04$ & $1.56 \mathrm{E}-04$ & -- & -- & 7.71E-05 \\
\hline VOC & 1,1-Dichloroethene & $\mathrm{mg} / \mathrm{kg}$ & 3 & 0 & $0 \%$ & 1.53E-04 & 1.56E-04 & -- & -- & $7.71 \mathrm{E}-05$ \\
\hline VOC & 1,2-Dichloroethane & $\mathrm{mg} / \mathrm{kg}$ & 3 & 0 & $0 \%$ & $1.53 \mathrm{E}-04$ & $1.56 \mathrm{E}-04$ & -- & -- & 7.71E-05 \\
\hline VOC & 1,2-Dichloroethene (Total) & $\mathrm{mg} / \mathrm{kg}$ & 3 & 0 & $0 \%$ & $1.53 \mathrm{E}-04$ & $1.56 \mathrm{E}-04$ & -- & -- & $7.71 \mathrm{E}-05$ \\
\hline VOC & 1,2-Dichloropropane & $\mathrm{mg} / \mathrm{kg}$ & 3 & 0 & $0 \%$ & $1.53 \mathrm{E}-04$ & $1.56 \mathrm{E}-04$ & -- & -- & $7.71 \mathrm{E}-05$ \\
\hline VOC & 2-Butanone & $\mathrm{mg} / \mathrm{kg}$ & 3 & 0 & $0 \%$ & 3.83E-04 & 4.69E-04 & -- & -- & $2.18 \mathrm{E}-04$ \\
\hline VOC & 2-Hexanone & $\mathrm{mg} / \mathrm{kg}$ & 3 & 0 & $0 \%$ & 3.83E-04 & 4.69E-04 & -- & -- & $2.18 \mathrm{E}-04$ \\
\hline VOC & 4-Methyl-2-Pentanone & $\mathrm{mg} / \mathrm{kg}$ & 3 & 0 & $0 \%$ & 3.83E-04 & 4.69E-04 & -- & -- & $2.18 \mathrm{E}-04$ \\
\hline VOC & Acetone & $\mathrm{mg} / \mathrm{kg}$ & 3 & 3 & $100 \%$ & -- & -- & 0.0018 & 0.0024 & 0.0021 \\
\hline VOC & Benzene & $\mathrm{mg} / \mathrm{kg}$ & 3 & 0 & $0 \%$ & 1.53E-04 & 1.56E-04 & -- & -- & $7.71 \mathrm{E}-05$ \\
\hline VOC & Bromodichloromethane & $\mathrm{mg} / \mathrm{kg}$ & 3 & 0 & $0 \%$ & $1.53 \mathrm{E}-04$ & $1.56 \mathrm{E}-04$ & -- & -- & $7.71 \mathrm{E}-05$ \\
\hline VOC & Bromoform & $\mathrm{mg} / \mathrm{kg}$ & 3 & 0 & $0 \%$ & $1.53 \mathrm{E}-04$ & 1.56E-04 & -- & -- & $7.71 \mathrm{E}-05$ \\
\hline VOC & Bromomethane & $\mathrm{mg} / \mathrm{kg}$ & 3 & 0 & $0 \%$ & $1.53 \mathrm{E}-04$ & $1.56 \mathrm{E}-04$ & -- & -- & 7.71E-05 \\
\hline VOC & Carbon disulfide & $\mathrm{mg} / \mathrm{kg}$ & 3 & 0 & $0 \%$ & $1.53 \mathrm{E}-04$ & $1.56 \mathrm{E}-04$ & -- & -- & $7.71 \mathrm{E}-05$ \\
\hline VOC & Carbon tetrachloride & $\mathrm{mg} / \mathrm{kg}$ & 3 & 0 & $0 \%$ & $1.53 \mathrm{E}-04$ & $1.56 \mathrm{E}-04$ & -- & -- & 7.71E-05 \\
\hline VOC & Chlorobenzene & $\mathrm{mg} / \mathrm{kg}$ & 3 & 0 & $0 \%$ & 3.83E-04 & $4.69 \mathrm{E}-04$ & -- & -- & $2.18 \mathrm{E}-04$ \\
\hline VOC & Chloroethane & $\mathrm{mg} / \mathrm{kg}$ & 3 & 0 & $0 \%$ & 3.83E-04 & 4.69E-04 & -- & -- & $2.18 \mathrm{E}-04$ \\
\hline VOC & Chloroform & $\mathrm{mg} / \mathrm{kg}$ & 3 & 0 & $0 \%$ & 1.53E-04 & 1.56E-04 & -- & -- & $7.71 \mathrm{E}-05$ \\
\hline VOC & Chloromethane & $\mathrm{mg} / \mathrm{kg}$ & 3 & 0 & $0 \%$ & $1.53 \mathrm{E}-04$ & $1.56 \mathrm{E}-04$ & -- & -- & $7.71 \mathrm{E}-05$ \\
\hline
\end{tabular}


Table E-3

Summary Statistics for Sediment Collected from Segment 5 of the Columbia River Including All Categories of Data (units reported "as received")

\begin{tabular}{|c|c|c|c|c|c|c|c|c|c|c|}
\hline $\begin{array}{c}\text { Constituent } \\
\text { Class }\end{array}$ & Constituent Name & Units & $\begin{array}{c}\text { Number } \\
\text { Analyzed }\end{array}$ & $\begin{array}{l}\text { Number } \\
\text { Detected }\end{array}$ & $\begin{array}{c}\text { Frequency of } \\
\text { Detection }\end{array}$ & $\begin{array}{l}\text { Minimum } \\
\text { Nondetect }\end{array}$ & $\begin{array}{l}\text { Maximum } \\
\text { Nondetect }\end{array}$ & Minimum Detect & $\begin{array}{c}\text { Maximum } \\
\text { Detect }\end{array}$ & $\begin{array}{c}\text { Average } \\
\text { Concentration } \\
\end{array}$ \\
\hline$\overline{\mathrm{VOC}}$ & cis-1,3-Dichloropropene & $\mathrm{mg} / \mathrm{kg}$ & 3 & $\overline{0}$ & $0 \%$ & $3.83 \mathrm{E}-04$ & $4.69 \mathrm{E}-04$ & -- & -- & $2.18 \mathrm{E}-04$ \\
\hline VOC & Dibromochloromethane & $\mathrm{mg} / \mathrm{kg}$ & 3 & 0 & $0 \%$ & $3.83 \mathrm{E}-04$ & $4.69 \mathrm{E}-04$ & -- & -- & $2.18 \mathrm{E}-04$ \\
\hline VOC & Ethylbenzene & $\mathrm{mg} / \mathrm{kg}$ & 3 & 0 & $0 \%$ & $1.53 \mathrm{E}-04$ & $1.56 \mathrm{E}-04$ & -- & -- & $7.71 \mathrm{E}-05$ \\
\hline VOC & Methylene chloride & $\mathrm{mg} / \mathrm{kg}$ & 3 & 3 & $100 \%$ & -- & -- & $9.19 \mathrm{E}-04$ & 0.0011 & $9.76 \mathrm{E}-04$ \\
\hline VOC & Styrene & $\mathrm{mg} / \mathrm{kg}$ & 3 & 0 & $0 \%$ & $1.53 \mathrm{E}-04$ & $1.56 \mathrm{E}-04$ & -- & -- & 7.71E-05 \\
\hline VOC & Tetrachloroethene & $\mathrm{mg} / \mathrm{kg}$ & 3 & 0 & $0 \%$ & $1.53 \mathrm{E}-04$ & $1.56 \mathrm{E}-04$ & -- & -- & $7.71 \mathrm{E}-05$ \\
\hline VOC & Toluene & $\mathrm{mg} / \mathrm{kg}$ & 3 & 0 & $0 \%$ & $1.53 \mathrm{E}-04$ & 1.56E-04 & -- & -- & 7.71E-05 \\
\hline VOC & trans-1,3-Dichloropropene & $\mathrm{mg} / \mathrm{kg}$ & 3 & 0 & $0 \%$ & $3.83 \mathrm{E}-04$ & 4.69E-04 & -- & -- & $2.18 \mathrm{E}-04$ \\
\hline VOC & Trichloroethene & $\mathrm{mg} / \mathrm{kg}$ & 3 & 0 & $0 \%$ & $1.53 \mathrm{E}-04$ & $1.56 \mathrm{E}-04$ & -- & -- & 7.71E-05 \\
\hline VOC & Vinyl acetate & $\mathrm{mg} / \mathrm{kg}$ & 3 & 0 & $0 \%$ & $1.53 \mathrm{E}-04$ & $1.56 \mathrm{E}-04$ & -- & -- & $7.71 \mathrm{E}-05$ \\
\hline VOC & Vinyl chloride & $\mathrm{mg} / \mathrm{kg}$ & 3 & 0 & $0 \%$ & $1.53 \mathrm{E}-04$ & 1.56E-04 & -- & -- & 7.71E-05 \\
\hline VOC & Xylenes (total) & $\mathrm{mg} / \mathrm{kg}$ & 3 & 0 & $0 \%$ & $1.53 \mathrm{E}-04$ & $1.56 \mathrm{E}-04$ & -- & -- & $7.71 \mathrm{E}-05$ \\
\hline
\end{tabular}

CONV - Conventional parameter

PEST - Pesticide

PCB - Polychlorinated bipheny

RAD_D - Decayed radionuclide 
Summary Statistics for Sediment Collected from Segment 5 of the Columbia River Including All Categories of Data (units reported "dry-weight")

\begin{tabular}{|c|c|c|c|c|c|c|c|c|c|c|}
\hline $\begin{array}{l}\text { Constituent } \\
\text { Class }\end{array}$ & Constituent Name & Units & $\begin{array}{l}\text { Number } \\
\text { Analyzed }\end{array}$ & $\begin{array}{l}\text { Number } \\
\text { Detected }\end{array}$ & $\begin{array}{l}\text { Frequency of } \\
\text { Detection }\end{array}$ & $\begin{array}{l}\text { Minimum } \\
\text { Nondetect }\end{array}$ & $\begin{array}{l}\text { Maximum } \\
\text { Nondetect }\end{array}$ & $\begin{array}{c}\text { Minimum } \\
\text { Detect }\end{array}$ & $\begin{array}{c}\text { Maximum } \\
\text { Detect }\end{array}$ & $\begin{array}{c}\text { Average } \\
\text { Concentration }\end{array}$ \\
\hline$\overline{\text { CONV }}$ & Ammonia & $\mathrm{mg} / \mathrm{kg}$ dry-wt & 15 & 15 & $100 \%$ & & & 4.3 & 64 & 28 \\
\hline CONV & Cyanide & $\mathrm{mg} / \mathrm{kg}$ dry-wt & 15 & 1 & $7 \%$ & 0.10 & 0.10 & 0.17 & 0.17 & 0.058 \\
\hline CONV & Nitrogen, Kjeldahl total & $\mathrm{mg} / \mathrm{kg}$ dry-wt & 15 & 15 & $100 \%$ & -- & -- & 650 & 2,000 & 1,367 \\
\hline CONV & Sulfide & $\mathrm{mg} / \mathrm{kg}$ dry-wt & 15 & 15 & $100 \%$ & -- & -- & 1.8 & 134 & 25 \\
\hline METAL & Aluminum & $\mathrm{mg} / \mathrm{kg}$ dry-wt & 27 & 27 & $100 \%$ & -- & -- & 5,500 & 33,300 & 16,304 \\
\hline METAL & Antimony & $\mathrm{mg} / \mathrm{kg}$ dry-wt & 45 & 12 & $27 \%$ & 0.15 & 2.5 & 0.029 & 3.9 & 0.62 \\
\hline METAL & Arsenic & $\mathrm{mg} / \mathrm{kg}$ dry-wt & 48 & 47 & $98 \%$ & 0.67 & 0.67 & 0.80 & 14 & 3.6 \\
\hline METAL & Barium & $\mathrm{mg} / \mathrm{kg}$ dry-wt & 15 & 15 & $100 \%$ & -- & -- & 60 & 186 & 142 \\
\hline METAL & Beryllium & $\mathrm{mg} / \mathrm{kg}$ dry-wt & 27 & 23 & $85 \%$ & 0.10 & 0.10 & 0.10 & 1.2 & 0.57 \\
\hline METAL & Cadmium & $\mathrm{mg} / \mathrm{kg}$ dry-wt & 48 & 37 & $77 \%$ & 0.016 & 0.50 & 0.14 & 1.9 & 0.59 \\
\hline METAL & Chromium & $\mathrm{mg} / \mathrm{kg}$ dry-wt & 38 & 38 & $100 \%$ & -- & -- & 0.17 & 31 & 16 \\
\hline METAL & Copper & $\mathrm{mg} / \mathrm{kg}$ dry-wt & 48 & 47 & $98 \%$ & 0.041 & 0.041 & 6.0 & 75 & 21 \\
\hline METAL & Dibutyltin Dichloride & $\mathrm{mg} / \mathrm{kg}$ dry-wt & 15 & 4 & $27 \%$ & 0.0026 & 0.0026 & 0.0046 & 0.012 & 0.0030 \\
\hline METAL & Iron & $\mathrm{mg} / \mathrm{kg}$ dry-wt & 27 & 27 & $100 \%$ & -- & -- & 12,000 & 39,000 & 19,978 \\
\hline METAL & Lead & $\mathrm{mg} / \mathrm{kg}$ dry-wt & 46 & 34 & $74 \%$ & 0.016 & 18 & 2.5 & 38 & 9.5 \\
\hline METAL & Manganese & $\mathrm{mg} / \mathrm{kg}$ dry-wt & 12 & 12 & $100 \%$ & -- & -- & 110 & 330 & 212 \\
\hline METAL & Mercury & $\mathrm{mg} / \mathrm{kg}$ dry-wt & 48 & 34 & $71 \%$ & 0.0045 & 0.031 & 0.020 & 0.51 & 0.066 \\
\hline METAL & Monobutyltin Trichloride & $\mathrm{mg} / \mathrm{kg}$ dry-wt & 15 & 6 & $40 \%$ & 0.0017 & 0.0034 & 0.0051 & 0.015 & 0.0040 \\
\hline METAL & Nickel & $\mathrm{mg} / \mathrm{kg}$ dry-wt & 48 & 47 & $98 \%$ & 0.026 & 0.026 & 5.0 & 25 & 13 \\
\hline METAL & Selenium & $\mathrm{mg} / \mathrm{kg}$ dry-wt & 27 & 0 & $0 \%$ & 0.50 & 1.6 & -- & -- & 0.45 \\
\hline METAL & Silver & $\mathrm{mg} / \mathrm{kg}$ dry-wt & 48 & 21 & $44 \%$ & 0.012 & 0.60 & 0.030 & 3.1 & 0.20 \\
\hline METAL & Thallium & $\mathrm{mg} / \mathrm{kg}$ dry-wt & 27 & 0 & $0 \%$ & 0.30 & 0.55 & -- & -- & 0.22 \\
\hline METAL & Tin & $\mathrm{mg} / \mathrm{kg}$ dry-wt & 12 & 12 & $100 \%$ & & -- & 6.0 & 21 & 11 \\
\hline METAL & Tributyltin Chloride & $\mathrm{mg} / \mathrm{kg}$ dry-wt & 15 & 11 & $73 \%$ & 0.0032 & 0.0032 & 0.0024 & 0.034 & 0.0071 \\
\hline METAL & Zinc & $\mathrm{mg} / \mathrm{kg}$ dry-wt & 48 & 47 & $98 \%$ & 0.070 & 0.070 & 27 & 155 & 77 \\
\hline PCB & Aroclor-1016 & $\mathrm{mg} / \mathrm{kg}$ dry-wt & 25 & 0 & $0 \%$ & 0.0062 & 0.010 & -- & -- & 0.0045 \\
\hline PCB & Aroclor-1221 & $\mathrm{mg} / \mathrm{kg}$ dry-wt & 25 & 0 & $0 \%$ & 0.0091 & 0.020 & -- & -- & 0.0085 \\
\hline PCB & Aroclor-1232 & $\mathrm{mg} / \mathrm{kg}$ dry-wt & 25 & 0 & $0 \%$ & 0.0060 & 0.010 & -- & -- & 0.0044 \\
\hline PCB & Aroclor-1242 & $\mathrm{mg} / \mathrm{kg}$ dry-wt & 25 & 0 & $0 \%$ & 0.0028 & 0.010 & -- & -- & 0.0037 \\
\hline $\mathrm{PCB}$ & Aroclor-1248 & $\mathrm{mg} / \mathrm{kg}$ dry-wt & 25 & 1 & $4 \%$ & 0.0022 & 0.010 & 0.011 & 0.011 & 0.0037 \\
\hline PCB & Aroclor-1254 & $\mathrm{mg} / \mathrm{kg}$ dry-wt & 25 & 0 & $0 \%$ & 0.0029 & 0.010 & -- & -- & 0.0037 \\
\hline PCB & Aroclor-1260 & $\mathrm{mg} / \mathrm{kg}$ dry-wt & 25 & 0 & $0 \%$ & 0.0030 & 0.010 & -- & -- & 0.0037 \\
\hline PCB & Total polychlorinated biphenyl & $\mathrm{mg} / \mathrm{kg}$ dry-wt & 7 & 0 & $0 \%$ & $5.00 \mathrm{E}-05$ & 50 & -- & -- & 3.6 \\
\hline PEST & 2,4'-DDD & $\mathrm{mg} / \mathrm{kg}$ dry-wt & 17 & 2 & $12 \%$ & $1.60 \mathrm{E}-04$ & $5.00 \mathrm{E}-04$ & 2.00E-04 & 0.0015 & 2.14E-04 \\
\hline PEST & 2,4'-DDE & $\mathrm{mg} / \mathrm{kg}$ dry-wt & 16 & 1 & $6 \%$ & $1.60 \mathrm{E}-04$ & $2.40 \mathrm{E}-04$ & 4.00E-04 & 4.00E-04 & $1.18 \mathrm{E}-04$ \\
\hline PEST & 2,4'-DDT & $\mathrm{mg} / \mathrm{kg}$ dry-wt & 15 & 0 & $0 \%$ & $1.60 \mathrm{E}-04$ & 2.40E-04 & -- & -- & $9.87 \mathrm{E}-05$ \\
\hline PEST & Aldrin & $\mathrm{mg} / \mathrm{kg}$ dry-wt & 25 & 0 & $0 \%$ & 8.40E-05 & $5.00 \mathrm{E}-04$ & -- & -- & $1.69 \mathrm{E}-04$ \\
\hline PEST & Alpha-BHC & $\mathrm{mg} / \mathrm{kg}$ dry-wt & 25 & 0 & $0 \%$ & 8.60E-05 & $5.00 \mathrm{E}-04$ & -- & -- & $1.73 \mathrm{E}-04$ \\
\hline PEST & alpha-Chlordane & $\mathrm{mg} / \mathrm{kg}$ dry-wt & 15 & 0 & $0 \%$ & $5.00 \mathrm{E}-04$ & 5.00E-04 & -- & -- & $2.50 \mathrm{E}-04$ \\
\hline PEST & beta-1,2,3,4,5,6-Hexachlorocyclohexane & $\mathrm{mg} / \mathrm{kg}$ dry-wt & 25 & 0 & $0 \%$ & $1.60 \mathrm{E}-04$ & 5.00E-04 & -- & -- & $1.90 \mathrm{E}-04$ \\
\hline PEST & Chlordane & $\mathrm{mg} / \mathrm{kg}$ dry-wt & 25 & 0 & $0 \%$ & $5.00 \mathrm{E}-04$ & 0.0076 & -- & -- & 0.0011 \\
\hline PEST & Delta-BHC & $\mathrm{mg} / \mathrm{kg}$ dry-wt & 25 & 0 & $0 \%$ & 8.80E-05 & $5.00 \mathrm{E}-04$ & -- & -- & $1.73 \mathrm{E}-04$ \\
\hline PEST & Dichlorodiphenyldichloroethane & $\mathrm{mg} / \mathrm{kg}$ dry-wt & 35 & 11 & $31 \%$ & $1.00 \mathrm{E}-05$ & 10 & $6.00 \mathrm{E}-04$ & 0.0039 & 0.14 \\
\hline PEST & Dichlorodiphenyldichloroethylene & $\mathrm{mg} / \mathrm{kg}$ dry-wt & 33 & 7 & $21 \%$ & $1.00 \mathrm{E}-05$ & 0.010 & $5.00 \mathrm{E}-04$ & 30 & 0.91 \\
\hline PEST & Dichlorodiphenyltrichloroethane & $\mathrm{mg} / \mathrm{kg}$ dry-wt & 33 & 1 & $3 \%$ & $1.00 \mathrm{E}-05$ & 10 & 0.0028 & 0.0028 & 0.15 \\
\hline PEST & Dicofol & $\mathrm{mg} / \mathrm{kg}$ dry-wt & 15 & 0 & $0 \%$ & 0.011 & 0.017 & -- & -- & 0.0069 \\
\hline PEST & Dieldrin & $\mathrm{mg} / \mathrm{kg}$ dry-wt & 26 & 1 & $4 \%$ & 8.00E-05 & 0.0010 & $5.00 \mathrm{E}-04$ & $5.00 \mathrm{E}-04$ & $3.39 \mathrm{E}-04$ \\
\hline PEST & Endosulfan I & $\mathrm{mg} / \mathrm{kg}$ dry-wt & 25 & 0 & $0 \%$ & 1.30E-04 & 5.00E-04 & -- & -- & $2.04 \mathrm{E}-04$ \\
\hline PEST & Endosulfan II & $\mathrm{mg} / \mathrm{kg}$ dry-wt & 25 & 0 & $0 \%$ & $1.90 \mathrm{E}-04$ & 0.0010 & -- & -- & 3.50E-04 \\
\hline PEST & Endosulfan sulfate & $\mathrm{mg} / \mathrm{kg}$ dry-wt & 25 & 0 & $0 \%$ & $2.70 \mathrm{E}-04$ & 0.0010 & -- & -- & $3.59 \mathrm{E}-04$ \\
\hline PEST & Endrin & $\mathrm{mg} / \mathrm{kg}$ dry-wt & 25 & 0 & $0 \%$ & 1.40E-04 & 0.0010 & -- & -- & $3.46 \mathrm{E}-04$ \\
\hline
\end{tabular}


Summary Statistics for Sediment Collected from Segment 5 of the Columbia River Including All Categories of Data (units reported "dry-weight")

\begin{tabular}{|c|c|c|c|c|c|c|c|c|c|c|}
\hline $\begin{array}{l}\text { Constituent } \\
\text { Class }\end{array}$ & Constituent Name & Units & $\begin{array}{l}\text { Number } \\
\text { Analyzed }\end{array}$ & $\begin{array}{l}\text { Number } \\
\text { Detected }\end{array}$ & $\begin{array}{l}\text { Frequency of } \\
\text { Detection }\end{array}$ & $\begin{array}{l}\text { Minimum } \\
\text { Nondetect }\end{array}$ & $\begin{array}{l}\text { Maximum } \\
\text { Nondetect }\end{array}$ & $\begin{array}{c}\text { Minimum } \\
\text { Detect }\end{array}$ & $\begin{array}{l}\text { Maximum } \\
\text { Detect }\end{array}$ & $\begin{array}{c}\text { Average } \\
\text { Concentration }\end{array}$ \\
\hline PEST & Endrin aldehyde & $\mathrm{mg} / \mathrm{kg}$ dry-wt & 25 & $\overline{0}$ & $0 \%$ & $3.30 \mathrm{E}-04$ & 0.0012 & - & $\overline{--}$ & $4.45 \mathrm{E}-04$ \\
\hline PEST & Endrin ketone & $\mathrm{mg} / \mathrm{kg}$ dry-wt & 25 & 0 & $0 \%$ & $2.90 \mathrm{E}-04$ & 0.0010 & -- & -- & $3.71 \mathrm{E}-04$ \\
\hline PEST & Gamma-BHC (Lindane) & $\mathrm{mg} / \mathrm{kg}$ dry-wt & 25 & 0 & $0 \%$ & $1.70 \mathrm{E}-04$ & $5.00 \mathrm{E}-04$ & -- & -- & $1.90 \mathrm{E}-04$ \\
\hline PEST & Heptachlor & $\mathrm{mg} / \mathrm{kg}$ dry-wt & 25 & 0 & $0 \%$ & $1.00 \mathrm{E}-04$ & $5.00 \mathrm{E}-04$ & -- & -- & 1.73E-04 \\
\hline PEST & Heptachlor epoxide & $\mathrm{mg} / \mathrm{kg}$ dry-wt & 25 & 0 & $0 \%$ & $1.60 \mathrm{E}-04$ & $5.00 \mathrm{E}-04$ & -- & -- & $1.88 \mathrm{E}-04$ \\
\hline PEST & Methoxychlor & $\mathrm{mg} / \mathrm{kg}$ dry-wt & 25 & 0 & $0 \%$ & $7.50 \mathrm{E}-04$ & 0.0050 & -- & -- & 0.0019 \\
\hline PEST & Methyl parathion & $\mathrm{mg} / \mathrm{kg}$ dry-wt & 15 & 0 & $0 \%$ & 0.011 & 0.017 & -- & -- & 0.0069 \\
\hline PEST & Toxaphene & $\mathrm{mg} / \mathrm{kg}$ dry-wt & 25 & 0 & $0 \%$ & 0.011 & 0.057 & -- & -- & 0.022 \\
\hline PEST & trans-Chlordane & $\mathrm{mg} / \mathrm{kg}$ dry-wt & 1 & 1 & $100 \%$ & -- & -- & 2.00E-04 & $2.00 \mathrm{E}-04$ & 2.00E-04 \\
\hline PEST & trans-Nonachlor & $\mathrm{mg} / \mathrm{kg}$ dry-wt & 1 & 1 & $100 \%$ & -- & -- & 2.00E-04 & $2.00 \mathrm{E}-04$ & 2.00E-04 \\
\hline RAD_D & Americium-241 & $\mathrm{pCi} / \mathrm{g}$ dry-wt & 1 & 1 & $100 \%$ & -- & -- & 0.068 & 0.068 & 0.068 \\
\hline RAD_D & Cesium-137 & pCi/g dry-wt & 15 & 15 & $100 \%$ & -- & -- & 0.038 & 0.13 & 0.078 \\
\hline RAD_D & Cobalt-60 & $\mathrm{pCi} / \mathrm{g}$ dry-wt & 3 & 3 & $100 \%$ & -- & -- & 0.0025 & 0.0045 & 0.0036 \\
\hline RAD_D & Plutonium-239/240 & pCi/g dry-wt & 7 & 7 & $100 \%$ & -- & -- & 0.0040 & 0.0100 & 0.0069 \\
\hline SVOC & 1,2,4-Trichlorobenzene & $\mathrm{mg} / \mathrm{kg}$ dry-wt & 25 & 0 & $0 \%$ & 0.0014 & 0.020 & -- & -- & 0.0051 \\
\hline SVOC & 1,2-Dichlorobenzene & $\mathrm{mg} / \mathrm{kg}$ dry-wt & 25 & 0 & $0 \%$ & 0.0022 & 0.020 & -- & -- & 0.0053 \\
\hline SVOC & 1,3-Dichlorobenzene & $\mathrm{mg} / \mathrm{kg}$ dry-wt & 25 & 0 & $0 \%$ & 0.0031 & 0.020 & -- & -- & 0.0055 \\
\hline SVOC & 1,4-Dichlorobenzene & $\mathrm{mg} / \mathrm{kg}$ dry-wt & 25 & 0 & $0 \%$ & 0.0025 & 0.040 & -- & -- & 0.010 \\
\hline sVOC & 2,4,5-Trichlorophenol & $\mathrm{mg} / \mathrm{kg}$ dry-wt & 5 & 0 & $0 \%$ & 0.0015 & 0.0016 & -- & -- & $7.80 \mathrm{E}-04$ \\
\hline SVOC & 2,4,6-Trichlorophenol & $\mathrm{mg} / \mathrm{kg}$ dry-wt & 20 & 0 & $0 \%$ & 0.0014 & 0.10 & -- & -- & 0.030 \\
\hline SVOC & 2,4-Dichlorophenol & $\mathrm{mg} / \mathrm{kg}$ dry-wt & 20 & 0 & $0 \%$ & $7.90 \mathrm{E}-04$ & 0.040 & -- & -- & 0.012 \\
\hline SVOC & 2,4-Dimethylphenol & $\mathrm{mg} / \mathrm{kg}$ dry-wt & 25 & 0 & $0 \%$ & 0.0028 & 0.040 & -- & -- & 0.010 \\
\hline sVOC & 2,4-Dinitrophenol & $\mathrm{mg} / \mathrm{kg}$ dry-wt & 20 & 0 & $0 \%$ & 0.0026 & 0.20 & -- & -- & 0.060 \\
\hline SVOC & 2,4-Dinitrotoluene & $\mathrm{mg} / \mathrm{kg}$ dry-wt & 20 & 0 & $0 \%$ & $4.00 \mathrm{E}-05$ & 0.10 & -- & -- & 0.030 \\
\hline sVOC & 2,6-Dinitrotoluene & $\mathrm{mg} / \mathrm{kg}$ dry-wt & 20 & 0 & $0 \%$ & 0.0014 & 0.10 & -- & -- & 0.030 \\
\hline SVOC & 2-Chloronaphthalene & $\mathrm{mg} / \mathrm{kg}$ dry-wt & 20 & 0 & $0 \%$ & $9.00 \mathrm{E}-04$ & 0.020 & -- & -- & 0.0061 \\
\hline sVOC & 2-Chlorophenol & $\mathrm{mg} / \mathrm{kg}$ dry-wt & 20 & 0 & $0 \%$ & 0.0025 & 0.020 & -- & -- & 0.0063 \\
\hline SVOC & 2-Methylnaphthalene & $\mathrm{mg} / \mathrm{kg}$ dry-wt & 37 & 26 & $70 \%$ & 0.0015 & 0.12 & 7.00E-04 & 0.017 & 0.0045 \\
\hline SVOC & 2-Methylphenol (cresol, o-) & $\mathrm{mg} / \mathrm{kg}$ dry-wt & 25 & 0 & $0 \%$ & 0.0016 & 0.020 & -- & -- & 0.0052 \\
\hline SVOC & 2-Nitroaniline & $\mathrm{mg} / \mathrm{kg}$ dry-wt & 20 & 0 & $0 \%$ & 0.0035 & 0.10 & -- & -- & 0.030 \\
\hline sVOC & 2-Nitrophenol & $\mathrm{mg} / \mathrm{kg}$ dry-wt & 20 & 0 & $0 \%$ & 0.0019 & 0.10 & -- & -- & 0.030 \\
\hline SVOC & 2-secButyl-4,6-dinitrophenol (DNBP) & $\mathrm{mg} / \mathrm{kg}$ dry-wt & 5 & 0 & $0 \%$ & 0.0015 & 0.0016 & -- & -- & $7.90 \mathrm{E}-04$ \\
\hline SVOC & 3\&4-Methylphenol (Cresol, m+p) & $\mathrm{mg} / \mathrm{kg}$ dry-wt & 10 & 0 & $0 \%$ & 0.0028 & 0.0043 & -- & -- & 0.0018 \\
\hline SVOC & 3,3'-Dichlorobenzidine & $\mathrm{mg} / \mathrm{kg}$ dry-wt & 20 & 0 & $0 \%$ & 0.0012 & 0.10 & -- & -- & 0.030 \\
\hline SVOC & 3-Nitroaniline & $\mathrm{mg} / \mathrm{kg}$ dry-wt & 20 & 0 & $0 \%$ & 0.0012 & 0.10 & -- & -- & 0.030 \\
\hline SVOC & 4,6-Dinitro-2-methylphenol & $\mathrm{mg} / \mathrm{kg}$ dry-wt & 15 & 0 & $0 \%$ & 0.12 & 0.20 & -- & -- & 0.079 \\
\hline sVOC & 4-Bromophenylphenyl ether & $\mathrm{mg} / \mathrm{kg}$ dry-wt & 20 & 0 & $0 \%$ & 0.0015 & 0.020 & -- & -- & 0.0062 \\
\hline SVOC & 4-Chloro-3-methylphenol & $\mathrm{mg} / \mathrm{kg}$ dry-wt & 15 & 0 & $0 \%$ & 0.025 & 0.040 & -- & -- & 0.016 \\
\hline sVOC & 4-Chloroaniline & $\mathrm{mg} / \mathrm{kg}$ dry-wt & 5 & 0 & $0 \%$ & 0.0017 & 0.0018 & -- & -- & 8.90E-04 \\
\hline SVOC & 4-Chlorophenylphenyl ether & $\mathrm{mg} / \mathrm{kg}$ dry-wt & 20 & 0 & $0 \%$ & 0.0018 & 0.020 & -- & -- & 0.0062 \\
\hline sVOC & 4-Methylphenol (cresol, p-) & $\mathrm{mg} / \mathrm{kg}$ dry-wt & 15 & 1 & $7 \%$ & 0.013 & 0.020 & 0.15 & 0.15 & 0.017 \\
\hline SVOC & 4-Nitroaniline & $\mathrm{mg} / \mathrm{kg}$ dry-wt & 20 & 0 & $0 \%$ & 0.0010 & 0.060 & -- & -- & 0.018 \\
\hline sVOC & 4-Nitrophenol & $\mathrm{mg} / \mathrm{kg}$ dry-wt & 20 & 0 & $0 \%$ & $5.10 \mathrm{E}-05$ & 0.10 & -- & -- & 0.030 \\
\hline SVOC & Acenaphthene & $\mathrm{mg} / \mathrm{kg}$ dry-wt & 40 & 23 & $58 \%$ & $6.70 \mathrm{E}-04$ & 0.84 & 7.20E-04 & 0.094 & 0.023 \\
\hline SVOC & Acenaphthylene & $\mathrm{mg} / \mathrm{kg}$ dry-wt & 38 & 13 & $34 \%$ & $2.60 \mathrm{E}-04$ & 0.84 & 0.0010 & 0.012 & 0.032 \\
\hline SVOC & Anthracene & $\mathrm{mg} / \mathrm{kg}$ dry-wt & 40 & 23 & $58 \%$ & $5.00 \mathrm{E}-04$ & 1.5 & 0.0010 & 0.46 & 0.051 \\
\hline sVOC & Benzo(a)anthracene & $\mathrm{mg} / \mathrm{kg}$ dry-wt & 40 & 27 & $68 \%$ & $6.90 \mathrm{E}-04$ & 0.12 & 0.0050 & 2.2 & 0.076 \\
\hline SVOC & Benzo(a)pyrene & $\mathrm{mg} / \mathrm{kg}$ dry-wt & 40 & 24 & $60 \%$ & $9.00 \mathrm{E}-04$ & 1.5 & 0.0042 & 1.5 & 0.088 \\
\hline SVOC & Benzo(b)fluoranthene & $\mathrm{mg} / \mathrm{kg}$ dry-wt & 25 & 15 & $60 \%$ & $6.20 \mathrm{E}-04$ & $9.30 \mathrm{E}-04$ & 0.0020 & 1.8 & 0.091 \\
\hline SVOC & Benzo(ghi)perylene & $\mathrm{mg} / \mathrm{kg}$ dry-wt & 40 & 18 & $45 \%$ & $3.30 \mathrm{E}-04$ & 1.5 & 0.0060 & 1.1 & 0.065 \\
\hline SVOC & Benzo(k)fluoranthene & $\mathrm{mg} / \mathrm{kg}$ dry-wt & 25 & 16 & $64 \%$ & $7.20 \mathrm{E}-04$ & 0.0011 & 0.0020 & 0.91 & 0.057 \\
\hline
\end{tabular}


Summary Statistics for Sediment Collected from Segment 5 of the Columbia River Including All Categories of Data (units reported "dry-weight")

\begin{tabular}{|c|c|c|c|c|c|c|c|c|c|c|}
\hline $\begin{array}{l}\text { Constituent } \\
\text { Class }\end{array}$ & Constituent Name & Units & $\begin{array}{c}\text { Number } \\
\text { Analyzed }\end{array}$ & $\begin{array}{l}\text { Number } \\
\text { Detected }\end{array}$ & $\begin{array}{l}\text { Frequency of } \\
\text { Detection }\end{array}$ & $\begin{array}{l}\text { Minimum } \\
\text { Nondetect }\end{array}$ & $\begin{array}{l}\text { Maximum } \\
\text { Nondetect }\end{array}$ & $\begin{array}{c}\text { Minimum } \\
\text { Detect }\end{array}$ & $\begin{array}{c}\text { Maximum } \\
\text { Detect }\end{array}$ & $\begin{array}{c}\text { Average } \\
\text { Concentration }\end{array}$ \\
\hline$\overline{\text { SVOC }}$ & Benzoic acid & $\mathrm{mg} / \mathrm{kg}$ dry-wt & 25 & $\overline{0}$ & $0 \%$ & 0.0012 & 0.16 & $\overline{--}$ & -- & 0.016 \\
\hline SVOC & Benzyl alcohol & $\mathrm{mg} / \mathrm{kg}$ dry-wt & 25 & 0 & $0 \%$ & 0.0033 & 0.10 & -- & -- & 0.025 \\
\hline sVoc & Bis(2-chloro-1-methylethyl)ether & $\mathrm{mg} / \mathrm{kg}$ dry-wt & 20 & 0 & $0 \%$ & 0.0030 & 0.020 & -- & -- & 0.0063 \\
\hline SVOC & Bis(2-Chloroethoxy)methane & $\mathrm{mg} / \mathrm{kg}$ dry-wt & 15 & 0 & $0 \%$ & 0.013 & 0.020 & -- & -- & 0.0079 \\
\hline SVOC & Bis(2-chloroethyl) ether & $\mathrm{mg} / \mathrm{kg}$ dry-wt & 20 & 0 & $0 \%$ & 0.0023 & 0.020 & -- & -- & 0.0063 \\
\hline SVOC & Bis(2-ethylhexyl) phthalate & $\mathrm{mg} / \mathrm{kg}$ dry-wt & 38 & 33 & $87 \%$ & 0.011 & 0.025 & 0.012 & 1.9 & 0.098 \\
\hline sVOC & Butylbenzylphthalate & $\mathrm{mg} / \mathrm{kg}$ dry-wt & 37 & 5 & $14 \%$ & 0.0017 & 1.5 & 0.0010 & 0.0020 & 0.056 \\
\hline SVOC & Carbazole & $\mathrm{mg} / \mathrm{kg}$ dry-wt & 15 & 0 & $0 \%$ & 0.013 & 0.020 & -- & -- & 0.0079 \\
\hline sVOC & Chrysene & $\mathrm{mg} / \mathrm{kg}$ dry-wt & 40 & 29 & $73 \%$ & $9.00 \mathrm{E}-04$ & 0.12 & 0.0050 & 4.1 & 0.13 \\
\hline SVOC & Dibenz[a,h]anthracene & $\mathrm{mg} / \mathrm{kg}$ dry-wt & 39 & 14 & $36 \%$ & $5.00 \mathrm{E}-04$ & 1.5 & 0.0015 & 0.40 & 0.059 \\
\hline sVOC & Dibenzofuran & $\mathrm{mg} / \mathrm{kg}$ dry-wt & 37 & 19 & $51 \%$ & 3.90E-04 & 0.84 & 8.10E-04 & 0.028 & 0.021 \\
\hline SVOC & Diethylphthalate & $\mathrm{mg} / \mathrm{kg}$ dry-wt & 36 & 11 & $31 \%$ & 0.0030 & 0.53 & 0.0020 & 0.17 & 0.026 \\
\hline SVOC & Dimethyl phthalate & $\mathrm{mg} / \mathrm{kg}$ dry-wt & 25 & 0 & $0 \%$ & 0.0034 & 0.020 & -- & -- & 0.0056 \\
\hline SVOC & Di-n-butylphthalate & $\mathrm{mg} / \mathrm{kg}$ dry-wt & 37 & 13 & $35 \%$ & 0.013 & 1.5 & 0.0090 & 0.84 & 0.071 \\
\hline sVoc & Di-n-octylphthalate & $\mathrm{mg} / \mathrm{kg}$ dry-wt & 37 & 11 & $30 \%$ & 0.0026 & 0.53 & 0.0040 & 0.072 & 0.018 \\
\hline SVOC & Fluoranthene & $\mathrm{mg} / \mathrm{kg}$ dry-wt & 40 & 29 & $73 \%$ & $6.90 \mathrm{E}-04$ & 1.5 & 5.00E-04 & 2.1 & 0.10 \\
\hline sVOC & Fluorene & $\mathrm{mg} / \mathrm{kg}$ dry-wt & 40 & 27 & $68 \%$ & 7.40E-04 & 0.12 & 7.80E-04 & 0.049 & 0.0081 \\
\hline SVOC & Hexachlorobenzene & $\mathrm{mg} / \mathrm{kg}$ dry-wt & 33 & 0 & $0 \%$ & $6.52 \mathrm{E}-04$ & 0.020 & - & -- & 0.0042 \\
\hline sVoc & Hexachlorobutadiene & $\mathrm{mg} / \mathrm{kg}$ dry-wt & 18 & 0 & $0 \%$ & $3.21 \mathrm{E}-04$ & 0.0035 & -- & -- & 8.74E-04 \\
\hline SVOC & Hexachlorocyclopentadiene & $\mathrm{mg} / \mathrm{kg}$ dry-wt & 20 & 0 & $0 \%$ & 0.0017 & 0.040 & -- & -- & 0.012 \\
\hline SVOC & Hexachloroethane & $\mathrm{mg} / \mathrm{kg}$ dry-wt & 25 & 0 & $0 \%$ & 0.0032 & 0.10 & -- & -- & 0.025 \\
\hline SVOC & Indeno(1,2,3-cd)pyrene & $\mathrm{mg} / \mathrm{kg}$ dry-wt & 40 & 22 & $55 \%$ & 8.10E-04 & 0.84 & 0.0050 & 0.92 & 0.052 \\
\hline sVoc & Isophorone & $\mathrm{mg} / \mathrm{kg}$ dry-wt & 32 & 1 & $3 \%$ & 0.0029 & 1.5 & 0.0040 & 0.0040 & 0.068 \\
\hline SVOC & Naphthalene & $\mathrm{mg} / \mathrm{kg}$ dry-wt & 37 & 19 & $51 \%$ & 0.0021 & 0.84 & 0.0010 & 0.11 & 0.037 \\
\hline SVOC & Nitrobenzene & $\mathrm{mg} / \mathrm{kg}$ dry-wt & 20 & 0 & $0 \%$ & 0.0018 & 0.020 & -- & -- & 0.0062 \\
\hline SVOC & N-Nitrosodi-n-dipropylamine & $\mathrm{mg} / \mathrm{kg}$ dry-wt & 5 & 0 & $0 \%$ & 0.0026 & 0.0028 & -- & -- & 0.0014 \\
\hline sVOC & N-Nitrosodiphenylamine & $\mathrm{mg} / \mathrm{kg}$ dry-wt & 10 & 0 & $0 \%$ & $8.00 \mathrm{E}-04$ & 0.0012 & -- & -- & 4.97E-04 \\
\hline SVOC & Pentachlorophenol & $\mathrm{mg} / \mathrm{kg}$ dry-wt & 25 & 0 & $0 \%$ & 0.0015 & 0.10 & -- & -- & 0.024 \\
\hline sVOC & Phenanthrene & $\mathrm{mg} / \mathrm{kg}$ dry-wt & 40 & 30 & $75 \%$ & 7.10E-04 & 0.0011 & 0.0010 & 0.58 & 0.036 \\
\hline SVOC & Phenol & $\mathrm{mg} / \mathrm{kg}$ dry-wt & 25 & 0 & $0 \%$ & 0.0040 & 0.040 & -- & -- & 0.010 \\
\hline SVOC & Pyrene & $\mathrm{mg} / \mathrm{kg}$ dry-wt & 40 & 29 & $73 \%$ & 6.10E-04 & 0.12 & 0.0050 & 2.5 & 0.11 \\
\hline
\end{tabular}

CONV - Conventional parameter

PEST - Pesticide

PCB - Polychlorinated bipheny

RAD_D - Decayed radionuclide

SVOC - Semivolatile Organic Compoun 
Summary Statistics for Sediment Samples Collected from the Willamette River Tributary to Segment $\mathbf{5}$ of the Columbia River (units reported "as received")

\begin{tabular}{|c|c|c|c|c|c|c|c|c|c|c|}
\hline $\begin{array}{c}\text { Constituent } \\
\text { Class }\end{array}$ & Constituent Name & Units & $\begin{array}{l}\text { Number } \\
\text { Analyzed }\end{array}$ & $\begin{array}{l}\text { Number } \\
\text { Detected }\end{array}$ & $\begin{array}{l}\text { Frequency of } \\
\text { Detection }\end{array}$ & $\begin{array}{l}\text { Minimum } \\
\text { Nondetect }\end{array}$ & $\begin{array}{l}\text { Maximum } \\
\text { Nondetect }\end{array}$ & $\begin{array}{c}\text { Minimum } \\
\text { Detect }\end{array}$ & $\begin{array}{c}\text { Maximum } \\
\text { Detect }\end{array}$ & $\begin{array}{c}\text { Average } \\
\text { Concentration }\end{array}$ \\
\hline$\overline{\text { CONV }}$ & Total Organic Carbon & $\%$ & 13 & 13 & $100 \%$ & $\overline{--}$ & $\overline{--}$ & 0.13 & 4.0 & 1.8 \\
\hline CONV & Total Volatile Solids & $\%$ & 16 & 16 & $100 \%$ & -- & -- & 0.70 & 10 & 5.7 \\
\hline METAL & Arsenic & $\mathrm{mg} / \mathrm{kg}$ & 4 & 4 & $100 \%$ & -- & -- & 1.0 & 2.7 & 1.8 \\
\hline METAL & Cadmium & $\mathrm{mg} / \mathrm{kg}$ & 4 & 4 & $100 \%$ & -- & -- & 0.10 & 0.50 & 0.28 \\
\hline METAL & Chromium & $\mathrm{mg} / \mathrm{kg}$ & 4 & 4 & $100 \%$ & -- & -- & 11 & 18 & 14 \\
\hline METAL & Copper & $\mathrm{mg} / \mathrm{kg}$ & 4 & 4 & $100 \%$ & -- & -- & 8.0 & 16 & 12 \\
\hline METAL & Lead & $\mathrm{mg} / \mathrm{kg}$ & 4 & 4 & $100 \%$ & -- & -- & 3.4 & 11 & 7.3 \\
\hline METAL & Mercury & $\mathrm{mg} / \mathrm{kg}$ & 4 & 4 & $100 \%$ & -- & -- & 0.030 & 0.060 & 0.040 \\
\hline METAL & Nickel & $\mathrm{mg} / \mathrm{kg}$ & 4 & 4 & $100 \%$ & -- & -- & 9.0 & 17 & 12 \\
\hline METAL & Silver & $\mathrm{mg} / \mathrm{kg}$ & 4 & 4 & $100 \%$ & -- & -- & 0.040 & 0.11 & 0.068 \\
\hline METAL & Zinc & $\mathrm{mg} / \mathrm{kg}$ & 4 & 4 & $100 \%$ & -- & -- & 35 & 92 & 67 \\
\hline $\mathrm{PCB}$ & Aroclor-1242 & $\mathrm{mg} / \mathrm{kg}$ & 4 & 0 & $0 \%$ & 0.010 & 0.010 & -- & -- & 0.0050 \\
\hline $\mathrm{PCB}$ & Aroclor-1254 & $\mathrm{mg} / \mathrm{kg}$ & 4 & 0 & $0 \%$ & 0.010 & 0.010 & -- & -- & 0.0050 \\
\hline PCB & Aroclor-1260 & $\mathrm{mg} / \mathrm{kg}$ & 4 & 1 & $25 \%$ & 0.010 & 0.010 & 0.0050 & 0.0050 & 0.0050 \\
\hline PEST & Bromine & $\mathrm{mg} / \mathrm{kg}$ & 10 & 10 & $100 \%$ & -- & -- & 0.0052 & 0.015 & 0.0099 \\
\hline PEST & Chlordane & $\mathrm{mg} / \mathrm{kg}$ & 4 & 0 & $0 \%$ & 0.010 & 0.010 & -- & -- & 0.0050 \\
\hline PEST & Chlorine & $\mathrm{mg} / \mathrm{kg}$ & 10 & 10 & $100 \%$ & -- & -- & 0.12 & 2.4 & 0.77 \\
\hline PEST & Dichlorodiphenyldichloroethane & $\mathrm{mg} / \mathrm{kg}$ & 4 & 2 & $50 \%$ & 0.0020 & 0.0020 & $7.00 \mathrm{E}-04$ & 0.0010 & $9.25 \mathrm{E}-04$ \\
\hline PEST & Dichlorodiphenyldichloroethylene & $\mathrm{mg} / \mathrm{kg}$ & 4 & 2 & $50 \%$ & 0.0020 & 0.0020 & 4.00E-04 & 7.00E-04 & $7.75 \mathrm{E}-04$ \\
\hline PEST & Dichlorodiphenyltrichloroethane & $\mathrm{mg} / \mathrm{kg}$ & 4 & 0 & $0 \%$ & 0.0020 & 0.0020 & -- & -- & 0.0010 \\
\hline PEST & Dieldrin & $\mathrm{mg} / \mathrm{kg}$ & 4 & 0 & $0 \%$ & 0.0020 & 0.0050 & -- & -- & 0.0014 \\
\hline SVOC & 2-Methylnaphthalene & $\mathrm{mg} / \mathrm{kg}$ & 4 & 4 & $100 \%$ & -- & -- & 0.0010 & 0.0040 & 0.0025 \\
\hline sVoc & Acenaphthene & $\mathrm{mg} / \mathrm{kg}$ & 4 & 3 & $75 \%$ & 0.0050 & 0.0050 & $9.00 \mathrm{E}-04$ & 0.0030 & 0.0019 \\
\hline sVOC & Acenaphthylene & $\mathrm{mg} / \mathrm{kg}$ & 4 & 4 & $100 \%$ & -- & -- & $5.00 \mathrm{E}-04$ & 0.0050 & 0.0021 \\
\hline sVoc & Anthracene & $\mathrm{mg} / \mathrm{kg}$ & 4 & 4 & $100 \%$ & -- & -- & 0.0010 & 0.0060 & 0.0023 \\
\hline sVOC & Benzo(a)anthracene & $\mathrm{mg} / \mathrm{kg}$ & 4 & 4 & $100 \%$ & -- & -- & 0.0020 & 0.044 & 0.014 \\
\hline sVoc & Benzo(a)pyrene & $\mathrm{mg} / \mathrm{kg}$ & 4 & 4 & $100 \%$ & -- & -- & 0.0050 & 0.10 & 0.032 \\
\hline sVOC & Benzo $(b+k) f l u o r a n t h e n e$ & $\mathrm{mg} / \mathrm{kg}$ & 4 & 4 & $100 \%$ & -- & -- & 0.0060 & 0.12 & 0.039 \\
\hline SVOC & Benzo(ghi)perylene & $\mathrm{mg} / \mathrm{kg}$ & 4 & 4 & $100 \%$ & -- & -- & 0.0060 & 0.077 & 0.026 \\
\hline sVOC & Chrysene & $\mathrm{mg} / \mathrm{kg}$ & 4 & 4 & $100 \%$ & -- & -- & 0.0020 & 0.052 & 0.016 \\
\hline sVoc & Dibenz[a,h]anthracene & $\mathrm{mg} / \mathrm{kg}$ & 4 & 4 & $100 \%$ & -- & -- & 0.0020 & 0.013 & 0.0058 \\
\hline sVOC & Fluoranthene & $\mathrm{mg} / \mathrm{kg}$ & 4 & 4 & $100 \%$ & -- & -- & 0.0040 & 0.071 & 0.023 \\
\hline sVoc & Fluorene & $\mathrm{mg} / \mathrm{kg}$ & 4 & 4 & $100 \%$ & -- & -- & $7.00 \mathrm{E}-04$ & 0.0030 & 0.0014 \\
\hline sVOC & Indeno(1,2,3-cd)pyrene & $\mathrm{mg} / \mathrm{kg}$ & 4 & 4 & $100 \%$ & -- & -- & 0.0060 & 0.076 & 0.025 \\
\hline sVoc & Naphthalene & $\mathrm{mg} / \mathrm{kg}$ & 4 & 4 & $100 \%$ & -- & -- & 0.0010 & 0.0040 & 0.0020 \\
\hline sVoc & Phenanthrene & $\mathrm{mg} / \mathrm{kg}$ & 4 & 4 & $100 \%$ & -- & -- & 0.0040 & 0.020 & 0.0085 \\
\hline SVOC & Pyrene & $\mathrm{mg} / \mathrm{kg}$ & 4 & 4 & $100 \%$ & -- & -- & 0.0050 & 0.096 & 0.030 \\
\hline
\end{tabular}

CONV - Conventional parameter

PEST - Pesticide

Organic Compoun 
Table E-6

Summary Statistics for Category 1 Sediment Samples Collected from the Kalama River Tributary to Segment 5 of the Columbia River (units reported "as received")

\begin{tabular}{|c|c|c|c|c|c|c|c|c|c|c|}
\hline $\begin{array}{l}\text { Constituent } \\
\text { Class }\end{array}$ & Constituent Name & Units & $\begin{array}{c}\text { Number } \\
\text { Analyzed }\end{array}$ & $\begin{array}{l}\text { Number } \\
\text { Detected }\end{array}$ & $\begin{array}{l}\text { Frequency of } \\
\text { Detection }\end{array}$ & $\begin{array}{l}\text { Minimum } \\
\text { Nondetect }\end{array}$ & $\begin{array}{l}\text { Maximum } \\
\text { Nondetect }\end{array}$ & Minimum Detec & $\mathrm{t}$ Maximum Detect & $\begin{array}{c}\text { Average } \\
\text { Concentration }\end{array}$ \\
\hline $\begin{array}{l}\text { RAD_D } \\
\text { RAD_D }\end{array}$ & $\begin{array}{l}\text { Cesium-134 } \\
\text { lodine-131 }\end{array}$ & $\begin{array}{l}\mathrm{pCi} / \mathrm{g} \\
\mathrm{pCi} / \mathrm{g}\end{array}$ & $\begin{array}{l}2 \\
2\end{array}$ & $\begin{array}{l}2 \\
2\end{array}$ & $\begin{array}{l}100 \% \\
100 \%\end{array}$ & $\begin{array}{l}-- \\
--\end{array}$ & $\begin{array}{l}-- \\
--\end{array}$ & $\begin{array}{c}25 \\
1.31 \mathrm{E}-174\end{array}$ & $\begin{array}{c}25 \\
1.31 \mathrm{E}-174\end{array}$ & $\begin{array}{c}25 \\
1.31 \mathrm{E}-174\end{array}$ \\
\hline
\end{tabular}

RAD_D - Decayed radionuclide 
Table E-7

Summary Statistics for Category 1 Sediment Samples Collected from the Skipanon Federal Channel Tributary to Segment 5 of the Columbia River (units reported "as received")

\begin{tabular}{|c|c|c|c|c|c|c|c|c|c|c|}
\hline $\begin{array}{l}\text { Constituent } \\
\text { Class }\end{array}$ & Constituent Name & Units & $\begin{array}{l}\text { Number } \\
\text { Analyzed }\end{array}$ & $\begin{array}{l}\text { Number } \\
\text { Detected }\end{array}$ & $\begin{array}{l}\text { Frequency of } \\
\text { Detection }\end{array}$ & $\begin{array}{l}\text { Minimum } \\
\text { Nondetect }\end{array}$ & $\begin{array}{l}\text { Maximum } \\
\text { Nondetect }\end{array}$ & Minimum Detect & $\begin{array}{c}\text { Maximum } \\
\text { Detect }\end{array}$ & $\begin{array}{c}\text { Average } \\
\text { Concentration }\end{array}$ \\
\hline$\overline{\mathrm{METAL}}$ & Antimony & $\mathrm{mg} / \mathrm{kg}$ & 7 & 7 & $100 \%$ & -- & $\overline{--}$ & 0.75 & 3.6 & 1.9 \\
\hline METAL & Arsenic & $\mathrm{mg} / \mathrm{kg}$ & 11 & 11 & $100 \%$ & -- & -- & 6.6 & 11 & 9.0 \\
\hline METAL & Cadmium & $\mathrm{mg} / \mathrm{kg}$ & 11 & 8 & $73 \%$ & 0.56 & 0.71 & 0.85 & 3.3 & 1.7 \\
\hline METAL & Chromium & $\mathrm{mg} / \mathrm{kg}$ & 4 & 4 & $100 \%$ & -- & -- & 18 & 32 & 25 \\
\hline METAL & Copper & $\mathrm{mg} / \mathrm{kg}$ & 11 & 11 & $100 \%$ & -- & -- & 32 & 76 & 52 \\
\hline METAL & Lead & $\mathrm{mg} / \mathrm{kg}$ & 11 & 11 & $100 \%$ & -- & -- & 13 & 26 & 21 \\
\hline METAL & Mercury & $\mathrm{mg} / \mathrm{kg}$ & 11 & 11 & $100 \%$ & -- & -- & 0.097 & 0.56 & 0.16 \\
\hline METAL & Nickel & $\mathrm{mg} / \mathrm{kg}$ & 11 & 11 & $100 \%$ & -- & -- & 14 & 25 & 20 \\
\hline METAL & Silver & $\mathrm{mg} / \mathrm{kg}$ & 11 & 7 & $64 \%$ & 0.43 & 0.71 & 0.38 & 1.6 & 0.59 \\
\hline METAL & Zinc & $\mathrm{mg} / \mathrm{kg}$ & 11 & 11 & $100 \%$ & -- & -- & 90 & 200 & 150 \\
\hline PEST & Dichlorodiphenyldichloroethane & $\mathrm{mg} / \mathrm{kg}$ & 11 & 5 & $45 \%$ & $3.40 \mathrm{E}-04$ & 0.0028 & $8.70 \mathrm{E}-04$ & 0.0048 & 0.0018 \\
\hline PEST & Dichlorodiphenyldichloroethylene & $\mathrm{mg} / \mathrm{kg}$ & 11 & 6 & $55 \%$ & $5.30 \mathrm{E}-04$ & 0.0028 & 0.0010 & 0.0039 & 0.0018 \\
\hline PEST & Dichlorodiphenyltrichloroethane & $\mathrm{mg} / \mathrm{kg}$ & 11 & 0 & $0 \%$ & 4.40E-04 & 0.0028 & -- & -- & $6.12 \mathrm{E}-04$ \\
\hline SVOC & 2,6-Dinitrotoluene & $\mathrm{mg} / \mathrm{kg}$ & 7 & 7 & $100 \%$ & -- & -- & 0.012 & 0.021 & 0.016 \\
\hline SVOC & 2-Methylnaphthalene & $\mathrm{mg} / \mathrm{kg}$ & 11 & 0 & $0 \%$ & 3.30E-04 & 0.0071 & -- & -- & 0.0012 \\
\hline SVOC & 3\&4-Methylphenol (Cresol, m+p) & $\mathrm{mg} / \mathrm{kg}$ & 4 & 1 & $25 \%$ & 0.045 & 0.057 & 0.057 & 0.057 & 0.033 \\
\hline SVOC & Acenaphthene & $\mathrm{mg} / \mathrm{kg}$ & 11 & 3 & $27 \%$ & 0.0011 & 0.0022 & 0.0044 & 0.0058 & 0.0020 \\
\hline SVOC & Acenaphthylene & $\mathrm{mg} / \mathrm{kg}$ & 11 & 3 & $27 \%$ & $9.30 \mathrm{E}-04$ & 0.0025 & 0.0034 & 0.0099 & 0.0021 \\
\hline SVOC & Anthracene & $\mathrm{mg} / \mathrm{kg}$ & 11 & 4 & $36 \%$ & $5.40 \mathrm{E}-04$ & $9.00 \mathrm{E}-04$ & 0.0044 & 0.011 & 0.0034 \\
\hline SVOC & Benzo(a)anthracene & $\mathrm{mg} / \mathrm{kg}$ & 11 & 11 & $100 \%$ & -- & -- & 0.0032 & 0.030 & 0.012 \\
\hline SVOC & Benzo(a)pyrene & $\mathrm{mg} / \mathrm{kg}$ & 11 & 10 & $91 \%$ & 0.0029 & 0.0029 & 0.0043 & 0.043 & 0.018 \\
\hline SVOC & Benzo(b)fluoranthene & $\mathrm{mg} / \mathrm{kg}$ & 6 & 6 & $100 \%$ & -- & -- & 0.0048 & 0.020 & 0.012 \\
\hline sVOC & Benzo(ghi)perylene & $\mathrm{mg} / \mathrm{kg}$ & 11 & 8 & $73 \%$ & 4.20E-04 & 0.0022 & 0.0028 & 0.032 & 0.011 \\
\hline SVOC & Benzo(k)fluoranthene & $\mathrm{mg} / \mathrm{kg}$ & 7 & 5 & $71 \%$ & 0.0011 & 0.0012 & 0.0026 & 0.0060 & 0.0031 \\
\hline SVOC & Benzofluoranthene & $\mathrm{mg} / \mathrm{kg}$ & 4 & 3 & $75 \%$ & 0.0056 & 0.0056 & 0.044 & 0.066 & 0.043 \\
\hline SVOC & Benzoic acid & $\mathrm{mg} / \mathrm{kg}$ & 6 & 0 & $0 \%$ & 0.0072 & 0.012 & -- & -- & 0.0047 \\
\hline SVOC & Bis(2-ethylhexyl) phthalate & $\mathrm{mg} / \mathrm{kg}$ & 11 & 11 & $100 \%$ & -- & -- & 0.035 & 0.11 & 0.057 \\
\hline SVOC & Butylbenzylphthalate & $\mathrm{mg} / \mathrm{kg}$ & 7 & 7 & $100 \%$ & -- & -- & 0.0053 & 0.033 & 0.016 \\
\hline SVOC & Chrysene & $\mathrm{mg} / \mathrm{kg}$ & 11 & 11 & $100 \%$ & -- & -- & 0.0058 & 0.038 & 0.020 \\
\hline SVOC & Dibenz[a,h]anthracene & $\mathrm{mg} / \mathrm{kg}$ & 11 & 0 & $0 \%$ & $5.00 \mathrm{E}-04$ & 0.0037 & -- & -- & 7.73E-04 \\
\hline SVOC & Dibenzofuran & $\mathrm{mg} / \mathrm{kg}$ & 7 & 0 & $0 \%$ & 0.0025 & 0.0041 & -- & -- & 0.0016 \\
\hline SVOC & Diethylphthalate & $\mathrm{mg} / \mathrm{kg}$ & 4 & 1 & $25 \%$ & 0.019 & 0.028 & 0.026 & 0.026 & 0.015 \\
\hline SVOC & Di-n-butylphthalate & $\mathrm{mg} / \mathrm{kg}$ & 4 & 0 & $0 \%$ & 0.019 & 0.028 & -- & -- & 0.012 \\
\hline sVOC & Fluoranthene & $\mathrm{mg} / \mathrm{kg}$ & 11 & 11 & $100 \%$ & -- & -- & 0.0070 & 0.068 & 0.028 \\
\hline sVOC & Fluorene & $\mathrm{mg} / \mathrm{kg}$ & 11 & 2 & $18 \%$ & 0.0010 & 0.0028 & 0.0075 & 0.0095 & 0.0022 \\
\hline SVOC & Indeno(1,2,3-cd)pyrene & $\mathrm{mg} / \mathrm{kg}$ & 10 & 4 & $40 \%$ & $5.60 \mathrm{E}-04$ & 0.0029 & 0.0051 & 0.028 & 0.0070 \\
\hline SVOC & Naphthalene & $\mathrm{mg} / \mathrm{kg}$ & 11 & 4 & $36 \%$ & 0.0010 & 0.0017 & 0.0032 & 0.012 & 0.0027 \\
\hline sVOC & Phenanthrene & $\mathrm{mg} / \mathrm{kg}$ & 11 & 11 & $100 \%$ & -- & -- & 0.0035 & 0.032 & 0.012 \\
\hline SVOC & Pyrene & $\mathrm{mg} / \mathrm{kg}$ & 11 & 11 & $100 \%$ & -- & -- & 0.0066 & 0.056 & 0.029 \\
\hline
\end{tabular}

PEST - Pesticide

SVOC - Semivolatile Organic Compound 
Table E-8

Comparison of Segment 5 Detected Sediment Concentrations to Ecological and Human Health Benchmark Values (units reported "as received")

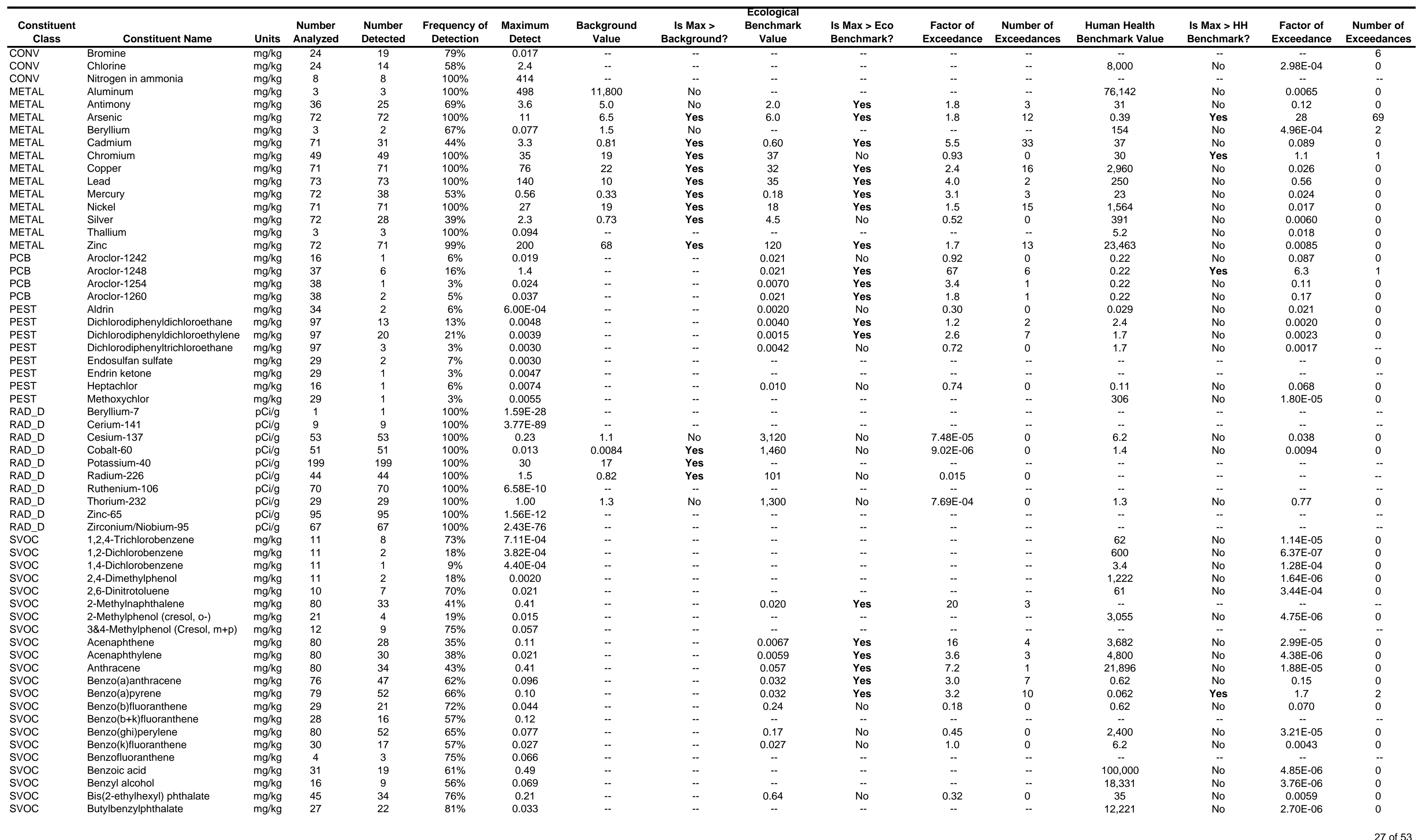


Table E-8

Comparison of Segment 5 Detected Sediment Concentrations to Ecological and Human Health Benchmark Values (units reported "as received")

\begin{tabular}{|c|c|c|c|c|c|c|c|c|c|c|c|c|c|c|c|c|}
\hline $\begin{array}{l}\text { Constituent } \\
\text { Class }\end{array}$ & Constituent Name & Units & $\begin{array}{l}\text { Number } \\
\text { Analyzed }\end{array}$ & $\begin{array}{l}\text { Number } \\
\text { Detected }\end{array}$ & $\begin{array}{l}\text { Frequency of } \\
\text { Detection }\end{array}$ & $\begin{array}{c}\text { Maximum } \\
\text { Detect }\end{array}$ & $\begin{array}{l}\text { Background } \\
\text { Value }\end{array}$ & $\begin{array}{c}\text { Is Max > } \\
\text { Background? }\end{array}$ & $\begin{array}{l}\text { Cecological } \\
\text { Benchmark } \\
\text { Value }\end{array}$ & $\begin{array}{l}\text { Is Max > Eco } \\
\text { Benchmark? }\end{array}$ & $\begin{array}{c}\text { Factor of } \\
\text { Exceedance }\end{array}$ & $\begin{array}{c}\text { Number of } \\
\text { Exceedances }\end{array}$ & $\begin{array}{l}\text { Human Health } \\
\text { Benchmark Value }\end{array}$ & $\begin{array}{l}\text { Is Max > HH } \\
\text { Benchmark? }\end{array}$ & $\begin{array}{c}\text { Factor of } \\
\text { Exceedance }\end{array}$ & $\begin{array}{c}\text { Number of } \\
\text { Exceedances }\end{array}$ \\
\hline SVOC & Chrysene & $\mathrm{mg} / \mathrm{kg}$ & 80 & 54 & $68 \%$ & 0.13 & -- & - & 0.057 & Yes & 2.3 & 4 & 62 & No & 0.0021 & 0 \\
\hline sVoc & Dibenz[a,h]anthracene & $\mathrm{mg} / \mathrm{kg}$ & 71 & 18 & $25 \%$ & 0.016 & -- & -- & 0.033 & No & 0.49 & 0 & 0.062 & No & 0.26 & 0 \\
\hline sVOC & Dibenzofuran & $\mathrm{mg} / \mathrm{kg}$ & 18 & 6 & $33 \%$ & 0.0042 & -- & -- & 5.1 & No & $8.20 \mathrm{E}-04$ & 0 & 145 & No & $2.88 \mathrm{E}-05$ & 0 \\
\hline SVOC & Diethylphthalate & $\mathrm{mg} / \mathrm{kg}$ & 25 & 10 & $40 \%$ & 0.026 & -- & -- & -- & -- & -- & $\ldots$ & 48.882 & No & $5.26 \mathrm{E}-07$ & 0 \\
\hline sVoc & Dimethyl phthalate & $\mathrm{mg} / \mathrm{kg}$ & 11 & 3 & $27 \%$ & 0.0035 & $\ldots$ & -- & $\ldots$ & -- & -- & -- & 80,000 & No & 4.41E-08 & 0 \\
\hline sVoc & Di-n-butyl|phthalate & $\mathrm{mg} / \mathrm{kg}$ & 30 & 20 & $67 \%$ & 0.32 & $\ldots$ & -- & 0.043 & Yes & 7.4 & 10 & 6,110 & No & $5.20 \mathrm{E}-05$ & 0 \\
\hline svoc & Di-n-octylyphthalate & $\mathrm{mg} / \mathrm{kg}$ & 21 & 3 & $14 \%$ & 0.023 & $\ldots$ & -- & -- & -. & -- & -- & 1,600 & No & $1.44 \mathrm{E}-05$ & 0 \\
\hline sVoc & Fluoranthene & $\mathrm{mg} / \mathrm{kg}$ & 75 & 55 & $73 \%$ & 0.087 & -- & -- & 0.11 & No & 0.78 & 0 & 2,294 & No & 3.79E-05 & 0 \\
\hline svoc & Fluorene & $\mathrm{mg} / \mathrm{kg}$ & 80 & 31 & $39 \%$ & 0.10 & -- & -- & 0.077 & Yes & 1.3 & 2 & 2,747 & No & 3.71E-05 & 0 \\
\hline sVoc & Indeno(1,2,3-cd)pyrene & $\mathrm{mg} / \mathrm{kg}$ & 79 & 39 & $49 \%$ & 0.076 & -- & -- & 0.017 & Yes & 4.5 & 8 & 0.62 & No & 0.12 & 0 \\
\hline sVoc & Naphthalene & $\mathrm{mg} / \mathrm{kg}$ & 80 & 47 & $59 \%$ & 0.066 & -- & -- & 0.18 & No & 0.38 & 0 & 56 & No & 0.0012 & 0 \\
\hline sVoc & Pentachlorophenol & $\mathrm{mg} / \mathrm{kg}$ & 21 & 7 & $33 \%$ & 0.039 & -- & -- & & $\ldots$ & & -- & 3.0 & No & 0.013 & 0 \\
\hline sVOC & Phenol & $\mathrm{mg} / \mathrm{kg}$ & 21 & 9 & $43 \%$ & 0.013 & -- & -- & -- & -- & -- &.- & 18,331 & No & $6.98 \mathrm{E}-07$ & 0 \\
\hline sVOC & Pyrene & $\mathrm{mg} / \mathrm{kg}$ & 80 & 52 & $65 \%$ & 0.17 & -- & -- & 0.053 & Yes & 3.1 & 8 & 2,316 & No & 7.12E-05 & 0 \\
\hline VOC & Acetone & $\mathrm{mg} / \mathrm{kg}$ & 3 & 3 & $100 \%$ & 0.0024 & -- & -- & -- & -- & - & -- & 14,000 & No & $1.73 \mathrm{E}-07$ & 0 \\
\hline VOC & Methylene chloride & $\mathrm{mg} / \mathrm{kg}$ & 3 & 3 & $100 \%$ & 0.0011 & -- & -- & -- & -- & -- & -- & 9.1 & No & $1.18 \mathrm{E}-04$ & 0 \\
\hline
\end{tabular}

CoNV - Conventional parameter

PEST - Pesticide

PAB - Polychlorinated biphenyl

RAD_D - Decayed radionuclide 
Table E-9

Comparison of Segment 5 Detected Sediment Concentrations to Ecological and Human Health Benchmark Values (units reported "dry weight")

\begin{tabular}{|c|c|c|c|c|c|c|c|c|c|c|c|c|c|c|c|c|}
\hline $\begin{array}{l}\text { Constituent } \\
\text { Class }\end{array}$ & Constituent Name & Units & $\begin{array}{l}\text { Number } \\
\text { Analyzed }\end{array}$ & $\begin{array}{l}\text { Number } \\
\text { Detected }\end{array}$ & $\begin{array}{l}\text { Frequency of } \\
\text { Detection }\end{array}$ & $\underset{\text { Detect }}{\text { Maximum }}$ & $\begin{array}{l}\text { Background } \\
\text { Value }\end{array}$ & $\begin{array}{c}\text { Is Max > } \\
\text { Background? }\end{array}$ & $\begin{array}{c}\text { Ecological } \\
\text { Benchmark Value }\end{array}$ & $\begin{array}{l}\text { Is Max > Eco } \\
\text { Benchmark? }\end{array}$ & $\begin{array}{c}\text { Factor of } \\
\text { Exceedance }\end{array}$ & $\begin{array}{c}\text { Number of } \\
\text { Exceedances }\end{array}$ & $\begin{array}{l}\text { Human Heatrh } \\
\text { Benchmark } \\
\text { Value }\end{array}$ & $\begin{array}{l}\text { Is Max }>\mathrm{HH} \\
\text { Benchmark? }\end{array}$ & $\begin{array}{c}\text { Factor of } \\
\text { Exceedance }\end{array}$ & $\begin{array}{c}\text { Number of } \\
\text { Exceedances }\end{array}$ \\
\hline CONV & Ammonia & $\mathrm{mg} / \mathrm{kg}$ dry-wt & 15 & 15 & $100 \%$ & 64 & -- & Dache & -- & Dentions: & - Latere & -- & -- & -- & - & - \\
\hline CONV & Cyanide & $\mathrm{mg} / \mathrm{kg}$ dry-wt & 15 & 1 & $7 \%$ & 0.17 & -- & -- & -- & -- & -- & -- & 1,222 & No & $1.41 \mathrm{E}-04$ & 0 \\
\hline CONV & Sulfide & $\mathrm{mg} / \mathrm{kg}$ dry-wt & 15 & 15 & $100 \%$ & 134 & -- & -- & -- & -- & -- & -- & & & & -- \\
\hline METAL & Aluminum & $\mathrm{mg} / \mathrm{kg}$ dry-wt & 27 & 27 & $100 \%$ & 33,300 & 11,800 & Yes & -- & -- & -- & -- & 76,142 & No & 0.44 & 0 \\
\hline METAL & Antimony & $\mathrm{mg} / \mathrm{kg}$ dry-wt & 45 & 12 & $27 \%$ & 3.9 & $\begin{array}{c}1+0 \\
5.0\end{array}$ & No & 2.0 & Yes & 2.0 & 1 & 31 & No & 0.12 & 0 \\
\hline METAL & Arsenic & $\mathrm{mg} / \mathrm{kg}$ dry-wt & 48 & 47 & $98 \%$ & 14 & 6.5 & Yes & 6.0 & Yes & 2.3 & 6 & 0.39 & Yes & 35 & 47 \\
\hline METAL & Barium & $\mathrm{mg} / \mathrm{kg}$ dry-wt & 15 & 15 & $100 \%$ & 186 & 132 & Yes & -- & -- & $\ldots$ & -- & 5,375 & No & 0.035 & 0 \\
\hline METAL & Beryllium & $\mathrm{mg} / \mathrm{kg}$ dry-wt & 27 & 23 & $85 \%$ & 1.2 & 1.5 & No & -- & -- & -- & -- & 154 & No & 0.0078 & 0 \\
\hline METAL & Cadmium & $\mathrm{mg} / \mathrm{kg}$ dry-wt & 48 & 37 & $77 \%$ & 1.9 & 0.81 & Yes & 0.60 & Yes & 3.2 & 16 & 37 & No & 0.051 & 0 \\
\hline METAL & Chromium & $\mathrm{mg} / \mathrm{kg}$ dry-wt & 38 & 38 & $100 \%$ & 31 & 19 & Yes & 37 & No & 0.84 & 0 & 30 & Yes & 1.0 & 1 \\
\hline METAL & Copper & $\mathrm{mg} / \mathrm{kg}$ dry-wt & 48 & 47 & $98 \%$ & 75 & 22 & Yes & 32 & Yes & 2.4 & 6 & 2,960 & No & 0.025 & 0 \\
\hline METAL & Dibutyltin Dichloride & $\mathrm{mg} / \mathrm{kg}$ dry-wt & 15 & 4 & $27 \%$ & 0.012 & & -- & & -- & -- & -- & -- & & -- & -- \\
\hline METAL & Iron & $\mathrm{mg} / \mathrm{kg}$ dry-wt & 27 & 27 & $100 \%$ & 39,000 & 32,600 & Yes & 20,000 & Yes & 2.0 & 12 & 23,463 & Yes & 1.7 & 6 \\
\hline METAL & Lead & $\mathrm{mg} / \mathrm{kg}$ dry-wt & 46 & 34 & $74 \%$ & 38 & 10 & Yes & 35 & Yes & 1.1 & 1 & 250 & No & 0.15 & 0 \\
\hline METAL & Manganese & $\mathrm{mg} / \mathrm{kg}$ dry-wt & 12 & 12 & $100 \%$ & 330 & 512 & No & 460 & No & 0.72 & 0 & 1,762 & No & 0.19 & 0 \\
\hline METAL & Mercury & $\mathrm{mg} / \mathrm{kg}$ dry-wt & 48 & 34 & $71 \%$ & 0.51 & 0.33 & Yes & 0.18 & Yes & 2.9 & 1 & 23 & No & 0.022 & 0 \\
\hline METAL & Monobutyltin Trichloride & $\mathrm{mg} / \mathrm{kg}$ dry-wt & 15 & 6 & $40 \%$ & 0.015 & -- & -- & -- & -- & -- & -- & & -- & & -- \\
\hline METAL & Nickel & $\mathrm{mg} / \mathrm{kg}$ dry-wt & 48 & 47 & $98 \%$ & 25 & 19 & Yes & 18 & Yes & 1.4 & 4 & 1,564 & No & 0.016 & 0 \\
\hline METAL & Silver & $\mathrm{mg} / \mathrm{kg}$ dry-wt & 48 & 21 & $44 \%$ & 3.1 & 0.73 & Yes & 4.5 & No & 0.69 & 0 & 391 & No & 0.0079 & 0 \\
\hline METAL & Tin & $\mathrm{mg} / \mathrm{kg}$ dry-wt & 12 & 12 & $100 \%$ & 21 & -- & -- & -- & -- & -- & -- & 46,924 & No & 4.48E-04 & 0 \\
\hline METAL & Tributyltin Chloride & $\mathrm{mg} / \mathrm{kg}$ dry-wt & 15 & 11 & $73 \%$ & 0.034 & -- & -- & -- & -- & -- & -- & -- & & -- & -- \\
\hline $\begin{array}{l}\text { METAL } \\
\text { PCB }\end{array}$ & Zinc & $\mathrm{mg} / \mathrm{kg}$ dry-wt & 48 & 47 & $98 \%$ & 155 & 68 & Yes & 120 & Yes & 1.3 & 8 & 23,463 & No & 0.0066 & 0 \\
\hline PEST & $\begin{array}{l}\text { Aroclor-1248 } \\
24^{\prime} \text {-DDD }\end{array}$ & $\mathrm{mg} / \mathrm{kg}$ dry-wt & 25 & 1 & $4 \%$ & 0.011 & -- & -- & 0.021 & No & 0.52 & 0 & 0.22 & No & 0.050 & 0 \\
\hline $\begin{array}{l}\text { PEST } \\
\text { PET }\end{array}$ & $\begin{array}{l}2,4-\mathrm{DLD} \\
2,4^{\prime}-\mathrm{DDE}\end{array}$ & $\mathrm{mg} / \mathrm{kg}$ dry-wt & 16 & 1 & $\begin{array}{l}12 \% \\
6 \%\end{array}$ & $\begin{array}{l}0.0015 \\
4.00 \mathrm{E}-04\end{array}$ & -- & -- & -- & -- & -- & -- & $\begin{array}{l}2.4 \\
17\end{array}$ & $\begin{array}{l}\text { No } \\
\text { No }\end{array}$ & $\begin{array}{l}6.16 \mathrm{E}-04 \\
233 \mathrm{~F}-04\end{array}$ & 0 \\
\hline PEST & Dichlorodiphenyldichloroethane & $\mathrm{mg} / \mathrm{kg}$ dry-wt & 35 & 11 & $31 \%$ & 0.0039 & -- & -. & 0.0040 & No & 0.98 & 0 & 2.4 & No & 0.0016 & 0 \\
\hline PEST & Dichlorodiphenyldichloroethylene & $\mathrm{mg} / \mathrm{kg}$ dry-wt & 33 & 7 & $21 \%$ & 0.030 & -- & -. & 0.0015 & Yes & 20 & 2 & 1.7 & No & 0.017 & 0 \\
\hline PEST & Dichlorodiphenyltrichloroethane & $\mathrm{mg} / \mathrm{kg}$ dry-wt & 33 & 1 & $3 \%$ & 0.0028 & -- & -- & 0.0042 & No & 0.67 & 0 & 1.7 & No & 0.0016 & 0 \\
\hline PEST & Dieldrin & $\mathrm{mg} / \mathrm{kg}$ dry-wt & 26 & 1 & $4 \%$ & $5.00 \mathrm{E}-04$ & -- & -- & 0.0019 & No & 0.26 & 0 & 0.030 & No & 0.016 & 0 \\
\hline PEST & trans-Chlordane & $\mathrm{mg} / \mathrm{kg}$ dry-wt & 1 & 1 & $100 \%$ & $2.00 \mathrm{E}-04$ & -- & -- & -. & -- & -- & -- & 1.6 & No & $1.23 \mathrm{E}-04$ & 0 \\
\hline PEST & trans-Nonachlor & $\mathrm{mg} / \mathrm{kg}$ dry-wt & 1 & 1 & $100 \%$ & $2.00 \mathrm{E}-04$ & -- & -- & -- & -- & -- & -- & -- & -- & - & -- \\
\hline RAD_D & Americium-241 & pCi/g dry-wt & 1 & 1 & $100 \%$ & 0.068 & -- & -- & 5,150 & No & $1.31 \mathrm{E}-05$ & 0 & 31 & No & 0.0022 & 0 \\
\hline RAD_D & Cesium-137 & pCi/g dry-wt & 15 & 15 & $100 \%$ & 0.13 & 1.1 & No & 3,120 & No & $4.28 \mathrm{E}-05$ & 0 & 6.2 & No & 0.022 & 0 \\
\hline RAD_D & Cobalt-60 & pCi/g dry-wt & 3 & 3 & $100 \%$ & 0.0045 & 0.0084 & No & 1,460 & No & $3.11 \mathrm{E}-06$ & 0 & 1.4 & No & 0.0032 & 0 \\
\hline RAD_D & Plutonium-239/240 & pCi/g dry-wt & 7 & 7 & $100 \%$ & 0.0100 & 0.025 & No & 5,860 & No & $1.71 \mathrm{E}-06$ & 0 & 34 & No & $2.95 \mathrm{E}-04$ & 0 \\
\hline svoc & 2-Methylnaphthalene & $\mathrm{mg} / \mathrm{kg}$ dry-wt & 37 & 26 & $70 \%$ & 0.017 &.- & $\ldots$ & 0.020 & No & 0.84 & 0 & & & & -- \\
\hline svoc & 4-Methylphenol (cresol, p-) & $\mathrm{mg} / \mathrm{kg}$ dry-wt & 15 & 1 & $7 \%$ & 0.15 & -- & -- & -- & -- & -- & -- & 306 & No & $4.91 \mathrm{E}-04$ & 0 \\
\hline sVoc & Acenaphthene & $\mathrm{mg} / \mathrm{kg}$ dry-wt & 40 & 23 & $58 \%$ & 0.094 & -- & -- & 0.0067 & Yes & 14 & 5 & 3,682 & No & $2.55 \mathrm{E}-05$ & 0 \\
\hline sVoc & Acenaphthylene & $\mathrm{mg} / \mathrm{kg}$ dry-wt & 38 & 13 & $34 \%$ & 0.012 & -- & -- & 0.0059 & Yes & 2.0 & 1 & 4,800 & No & $2.50 \mathrm{E}-06$ & 0 \\
\hline SVOC & Anthracene & $\mathrm{mg} / \mathrm{kg}$ dry-wt & 40 & 23 & $58 \%$ & 0.46 & -- & -- & 0.057 & Yes & 8.1 & 1 & 21,896 & No & $2.10 \mathrm{E}-05$ & 0 \\
\hline sVoc & Benzo(a)anthracene & $\mathrm{mg} / \mathrm{kg}$ dry-wt & 40 & 27 & $68 \%$ & 2.2 & -- & -- & 0.032 & Yes & 69 & 9 & 0.62 & Yes & 3.5 & 1 \\
\hline sVoc & Benzo(a)pyrene & $\mathrm{mg} / \mathrm{kg}$ dry-wt & 40 & 24 & $60 \%$ & 1.5 & -- & -- & 0.032 & Yes & 47 & 7 & 0.062 & Yes & 24 & 1 \\
\hline sVoc & Benzo(b)fluoranthene & $\mathrm{mg} / \mathrm{kg}$ dry-wt & 25 & 15 & $60 \%$ & 1.8 & -- & -- & 0.24 & Yes & 7.5 & 1 & 0.62 & Yes & 2.9 & 1 \\
\hline sVoc & Benzo(ghi)perylene & $\mathrm{mg} / \mathrm{kg}$ dry-wt & 40 & 18 & $45 \%$ & 1.1 & -- & -- & 0.17 & Yes & 6.5 & 1 & 2,400 & No & $4.58 \mathrm{E}-04$ & 0 \\
\hline svoc & Benzo(k)fluoranthene & $\mathrm{mg} / \mathrm{kg}$ dry-wt & 25 & 16 & $64 \%$ & 0.91 & -- & -- & 0.027 & Yes & 34 & 5 & 6.2 & No & 0.15 & 0 \\
\hline sVoc & Bis(2-ethylhexyl) phthalate & $\mathrm{mg} / \mathrm{kg}$ dry-wt & 38 & 33 & $87 \%$ & 1.9 & -- & -- & 0.64 & Yes & 3.0 & 1 & 35 & No & 0.055 & 0 \\
\hline SVOC & Buty|benzylphthalate & $\mathrm{mg} / \mathrm{kg}$ dry-wt & 37 & 5 & $14 \%$ & 0.0020 & -- & -- & & 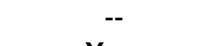 & 70 & -- & 12,221 & No & $1.64 \mathrm{E}-07$ & 0 \\
\hline SVOC & Chrysene & $\mathrm{mg} / \mathrm{kg}$ dry-wt & $\begin{array}{l}40 \\
30\end{array}$ & 29 & $73 \%$ & 4.1 & -- & -- & 0.057 & Yes & 72 & 8 & 62 & No & 0.066 & 0 \\
\hline $\begin{array}{l}\text { SVOC } \\
\text { SVOC }\end{array}$ & $\begin{array}{l}\text { Dibenz[a,h]anthracene } \\
\text { Dibenzofuran }\end{array}$ & $\begin{array}{l}\mathrm{mg} / \mathrm{kg} \text { dry-wt } \\
\mathrm{ma} / \mathrm{kg} \text { dry-wt }\end{array}$ & $\begin{array}{l}39 \\
37\end{array}$ & 14 & $36 \%$ & 0.40 & -- & -- & $\begin{array}{l}0.033 \\
5\end{array}$ & Yes & 12 & 1 & 0.062 & Yes & 6.4 & 1 \\
\hline $\begin{array}{l}\text { SVOC } \\
\text { SVOC }\end{array}$ & $\begin{array}{l}\text { Dibenzofuran } \\
\text { Diethylphthalate }\end{array}$ & $\begin{array}{l}\mathrm{mg} / \mathrm{kg} \text { dry-wt } \\
\mathrm{mg} / \mathrm{kg} \text { dry-wt }\end{array}$ & $\begin{array}{l}37 \\
36\end{array}$ & $\begin{array}{l}19 \\
11\end{array}$ & $\begin{array}{l}51 \% \\
31 \%\end{array}$ & $\begin{array}{l}0.028 \\
0.17\end{array}$ & -- & -- & 5.1 & No & 0.0055 & 0 & $\begin{array}{c}145 \\
4882\end{array}$ & $\begin{array}{l}\text { No } \\
\text { No }\end{array}$ & $\begin{array}{l}1.93 \mathrm{E}-04 \\
3.48 \mathrm{E}-06\end{array}$ & 0 \\
\hline svoc & Di-n-butylphthalate & $\mathrm{mg} / \mathrm{kg}$ dry-wt & 37 & 13 & $35 \%$ & 0.84 & -. & -. & 0.043 & Yes & 20 & 1 & 6,110 & No & $1.37 \mathrm{E}-04$ & 0 \\
\hline sVoc & Di-n-octylphthalate & $\mathrm{mg} / \mathrm{kg}$ dry-wt & 37 & 11 & $30 \%$ & 0.072 & -- & -- & (i) & $\ldots$ & $\ldots$ & $-\ldots$ & 1,600 & No & $4.50 \mathrm{E}-05$ & 0 \\
\hline sVoc & Fluoranthene & $\mathrm{mg} / \mathrm{kg}$ dry-wt & 40 & 29 & $73 \%$ & 2.1 & -. & -- & 0.11 & Yes & 19 & 2 & 2,294 & No & $9.16 \mathrm{E}-04$ & 0 \\
\hline svoc & Fluorene & $\mathrm{mg} / \mathrm{kg}$ dry-wt & 40 & 27 & $68 \%$ & 0.049 & -.- & -- & 0.077 & No & 0.64 & 0 & 2,747 & No & $1.78 \mathrm{E}-05$ & 0 \\
\hline svoc & Indeno(1,2,3-cd)pyrene & $\mathrm{mg} / \mathrm{kg}$ dry-wt & 40 & 22 & $55 \%$ & 0.92 & -- & -- & 0.017 & Yes & 54 & 11 & 0.62 & Yes & 1.5 & 1 \\
\hline svoc & $\begin{array}{l}\text { Isophorone } \\
\text { Pallo }\end{array}$ & $\mathrm{mg} / \mathrm{kg}$ dry-wt & 32 & 1 & $3 \%$ & 0.0040 & -- & -- & & -- &.-- &.- & 512 & No & $7.81 \mathrm{E}-06$ & 0 \\
\hline svoc & Naphthalene & $\mathrm{mg} / \mathrm{kg}$ dry-wt & 37 & 19 & $51 \%$ & 0.11 & -- & - & 0.18 & No & 0.63 & 0 & 56 & No & 0.0020 & 0 \\
\hline SVOC & Phenanthrene & $\mathrm{mg} / \mathrm{kg}$ dry-wt & 40 & 30 & $75 \%$ & 0.58 & -- & -- & 0.042 & Yes & 14 & 6 & -- & -- & -- & -- \\
\hline SVOC & Pyrene & $\mathrm{mg} / \mathrm{kg}$ dry-wt & 40 & 29 & $73 \%$ & 2.5 & -- & -- & 0.053 & Yes & 47 & 20 & 2,316 & No & 0.0011 & 0 \\
\hline
\end{tabular}


Table E-9

Comparison of Segment 5 Detected Sediment Concentrations to Ecological and Human Health Benchmark Values (units reported "dry weight")

\begin{tabular}{|c|c|c|c|c|c|c|c|c|c|c|c|c|c|c|c|c|}
\hline $\begin{array}{l}\text { Constituent } \\
\text { Class }\end{array}$ & Constituent Name & Units & $\begin{array}{l}\text { Number } \\
\text { Analyzed }\end{array}$ & $\begin{array}{l}\text { Number } \\
\text { Detected }\end{array}$ & $\begin{array}{l}\text { Frequency of } \\
\text { Detection }\end{array}$ & $\begin{array}{c}\text { Maximum } \\
\text { Detect }\end{array}$ & $\begin{array}{l}\text { Background } \\
\text { Value }\end{array}$ & $\begin{array}{c}\text { Is Max > } \\
\text { Background? }\end{array}$ & $\begin{array}{c}\text { Ecological } \\
\text { Benchmark Value }\end{array}$ & $\begin{array}{l}\text { Is Max > Eco } \\
\text { Benchmark? }\end{array}$ & $\begin{array}{c}\text { Factor of } \\
\text { Exceedance }\end{array}$ & $\begin{array}{l}\text { Number of } \\
\text { Exceedances }\end{array}$ & $\begin{array}{l}\text { Auman Health } \\
\text { Benchmark } \\
\text { Value }\end{array}$ & $\begin{array}{l}\text { Is Max }>\mathrm{HH} \\
\text { Benchmark? }\end{array}$ & $\begin{array}{c}\text { Factor of } \\
\text { Exceedance }\end{array}$ & $\begin{array}{c}\text { Number of } \\
\text { Exceedances }\end{array}$ \\
\hline
\end{tabular}

CONV - Conventional parameter

PEST - Pesticide
PCB - Polychlorinated biphenyl

PCB - Polychlorinated bipheny

RAD_D - Decayed radionuclide
SVOC - Semivolatile Organic Compound 
Table E-10

Summary of Surface Sediment Results From Segment 5 Exceeding Select Ecological or Human Health Benchmark Values

\begin{tabular}{|c|c|c|c|c|c|c|c|c|c|c|}
\hline Sample Area & Sample Site & SampleID & Date Collected & Constituent Name & Units & QA Category & $\begin{array}{c}\text { Analytical } \\
\text { Value }\end{array}$ & Qualifier & $\begin{array}{c}\text { Eco } \\
\text { Benchmark } \\
\text { Value }\end{array}$ & $\begin{array}{c}\mathrm{HH} \\
\text { Benchmark } \\
\text { Value }\end{array}$ \\
\hline Columbia River & Oregon Slough & 0205-OS-VC-01 & February 17,2005 & 2-Methylnaphthalene & $\mathrm{mg} / \mathrm{kg}$ & 1 & 0.405 & $\bar{E}$ & 0.02 & -- \\
\hline Columbia River & RM 86.5 & SH-VC-4 & May 4, 1989 & 2-Methylnaphthalene & $\mathrm{mg} / \mathrm{kg}$ & 3 & 0.037 & $=$ & 0.02 & -- \\
\hline Columbia River & RM 86.5 & SH-VC-5 & May 4, 1989 & 2-Methylnaphthalene & $\mathrm{mg} / \mathrm{kg}$ & 3 & 0.07 & $=$ & 0.02 & - \\
\hline Columbia River & Oregon Slough & $0205-0 S-v C-01$ & February 17,2005 & Acenaphthene & $\mathrm{mg} / \mathrm{kg}$ & 1 & 0.0257 & $=$ & 0.0067 & 3,682 \\
\hline Columbia River & Oregon Slough & 0205-OS-VC-06Z & February 17, 2005 & Acenaphthene & $\mathrm{mg} / \mathrm{kg}$ & 1 & 0.0109 & $=$ & 0.0067 & 3,682 \\
\hline Columbia River & Vancouver WA & 88-398-304 & July 22, 1987 & Acenaphthene & $\mathrm{mg} / \mathrm{kg}$ dry & 2 & 0.009 & $\mathrm{~J}$ & 0.0067 & 3,682 \\
\hline Columbia River & Longview Fibre & 88-398-306 & July 23, 1987 & Acenaphthene & $\mathrm{mg} / \mathrm{kg}$ dry & 2 & 0.024 & $\mathrm{~J}$ & 0.0067 & 3,682 \\
\hline Columbia River & Longview WA & 88-398-309 & July 23,1987 & Acenaphthene & $\mathrm{mg} / \mathrm{kg}$ dry & 2 & 0.094 & $\mathrm{~J}$ & 0.0067 & 3,682 \\
\hline Columbia River & Ilwaco Boat Bsn & 88-398-311 & July 24, 1987 & Acenaphthene & $\mathrm{mg} / \mathrm{kg}$ dry & 2 & 0.014 & $\mathrm{~J}$ & 0.0067 & 3,682 \\
\hline Columbia River & Crims Island & CRIM-SG-02 & July 15, 2003 & Acenaphthene & $\mathrm{mg} / \mathrm{kg}$ & 1 & 0.11 & $=$ & 0.0067 & 3,682 \\
\hline Columbia River & RM 86.5 & SH-VC-5 & May 4, 1989 & Acenaphthene & $\mathrm{mg} / \mathrm{kg}$ & 3 & 0.019 & $=$ & 0.0067 & 3,682 \\
\hline Columbia River & RM 82 & Will-1 & September 10, 1997 & Acenaphthene & $\mathrm{mg} / \mathrm{kg}$ dry & 2 & 0.014 & $=$ & 0.0067 & 3,682 \\
\hline Columbia River & Ilwaco Boat Bsn & $88-398-311$ & July 24,1987 & Acenaphthylene & $\mathrm{mg} / \mathrm{kg}$ dry & 2 & 0.012 & J & 0.0059 & 4,800 \\
\hline Columbia River & Crims Island & CRIM-SG-03 & July 15, 2003 & Acenaphthylene & $\mathrm{mg} / \mathrm{kg}$ & 1 & 0.00869 & $=$ & 0.0059 & 4,800 \\
\hline Skipanon Federal Channel & Warrenton OR & SBIO-GC-04 & June 24, 2003 & Acenaphthylene & $\mathrm{mg} / \mathrm{kg}$ & 1 & 0.00987 & $=$ & 0.0059 & 4,800 \\
\hline Columbia River & RM 86.5 & SH-VC-5 & May 4, 1989 & Acenaphthylene & $\mathrm{mg} / \mathrm{kg}$ & 3 & 0.021 & $=$ & 0.0059 & 4,800 \\
\hline Columbia River & Longview WA & $88-398-309$ & July 23,1987 & Anthracene & $\mathrm{mg} / \mathrm{kg}$ dry & 2 & 0.46 & j & 0.057 & 21,896 \\
\hline Columbia River & Bachelor Slough & BACH-GC-10 & June 3,2003 & Anthracene & $\mathrm{mg} / \mathrm{kg}$ & 1 & 0.411 & $=$ & 0.057 & 21,896 \\
\hline Columbia River & RM 29 & CRM-BC2 & August 9,2000 & Antimony & $\mathrm{mg} / \mathrm{kg}$ dry & 1 & 3.9 & B & 2 & 0.39 \\
\hline Skipanon Federal channel & Warrenton OR & SKIP-GC-02 & September 12, 2001 & Antimony & $\mathrm{mg} / \mathrm{kg}$ & 1 & 3.6 & $\mathrm{BC}$ & 2 & 0.39 \\
\hline Skipanon Federal channel & Warrenton OR & SKIP-GC-03 & September 12, 2001 & Antimony & $\mathrm{mg} / \mathrm{kg}$ & 1 & 2.8 & BC & 2 & 0.39 \\
\hline Skipanon Federal channel & Warrenton OR & SKIP-GC-04 & September 12, 2001 & Antimony & $\mathrm{mg} / \mathrm{kg}$ & 1 & 2.2 & $\mathrm{BC}$ & 2 & 0.39 \\
\hline Columbia River & RM 103 & V-PG-02 & June 20,2001 & Aroclor- 1248 & $\mathrm{mg} / \mathrm{kg}$ & 1 & 0.22 & $=$ & 0.021 & 0.22 \\
\hline Columbia River & RM 103 & V-PG-03 & June 20, 2001 & Aroclor-1248 & $\mathrm{mg} / \mathrm{kg}$ & 1 & 0.27 & $=$ & 0.021 & 0.22 \\
\hline Columbia River & RM 103 & V-PG-04 & June 20, 2001 & Aroclor-1248 & $\mathrm{mg} / \mathrm{kg}$ & 1 & 0.12 & $=$ & 0.021 & 0.22 \\
\hline Columbia River & RM 103 & V-PG-05 & June 20, 2001 & Aroclor-1248 & $\mathrm{mg} / \mathrm{kg}$ & 1 & 0.26 & $=$ & 0.021 & 0.22 \\
\hline Columbia River & RM 103 & V-PG-06 & June 20, 2001 & Aroclor-1248 & $\mathrm{mg} / \mathrm{kg}$ & 1 & 1.4 & $=$ & 0.021 & 0.22 \\
\hline Columbia River & RM 103 & V-PG-07 & June 20, 2001 & Aroclor-1248 & $\mathrm{mg} / \mathrm{kg}$ & 1 & 0.097 & D & 0.021 & 0.22 \\
\hline Columbia River & RM 100 & CR-BC-76 & June 3,1997 & Aroclor-1254 & $\mathrm{mg} / \mathrm{kg}$ & 1 & 0.024 & $=$ & 0.007 & 0.22 \\
\hline Columbia River & RM 100 & CR-BC-76 & June 3,1997 & Aroclor-1260 & mglkg & 1 & 0.037 & $=$ & 0.021 & 0.22 \\
\hline Columbia River & Oregon Slough & 0205-OS-VC-01 & February 17, 2005 & Arsenic & $\mathrm{mg} / \mathrm{kg}$ dry & 1 & 4.45 & $=$ & 6 & 0.39 \\
\hline Columbia River & Oregon Slough & 0205-OS-VC-03 & February 17, 2005 & Arsenic & $\mathrm{mg} / \mathrm{kg}$ dry & 1 & 2.76 & $=$ & 6 & 0.39 \\
\hline Columbia River & Oregon Slough & 0205-OS-VC-03A & February 17,2005 & Arsenic & $\mathrm{mg} / \mathrm{kg}$ dry & 1 & 3.07 & $=$ & 6 & 0.39 \\
\hline Columbia River & Oregon Slough & 0205-OS-VC-04 & February 17, 2005 & Arsenic & $\mathrm{mg} / \mathrm{kg}$ dry & 1 & 3.14 & $=$ & 6 & 0.39 \\
\hline Columbia River & Oregon Slough & 0205-OS-VC-05 & February 17, 2005 & Arsenic & $\mathrm{mg} / \mathrm{kg}$ dry & 1 & 2.46 & $=$ & 6 & 0.39 \\
\hline Columbia River & Oregon Slough & 0205-OS-VC-06 & February 17, 2005 & Arsenic & $\mathrm{mg} / \mathrm{kg}$ dry & 1 & 2.53 & $=$ & 6 & 0.39 \\
\hline Columbia River & Oregon Slough & 0205-OS-VC-06Z & February 17,2005 & Arsenic & $\mathrm{mg} / \mathrm{kg}$ dry & 1 & 3.85 & $=$ & 6 & 0.39 \\
\hline Columbia River & RM 88 & $10-\mathrm{s}$ & June 28, 1993 & Arsenic & $\mathrm{mg} / \mathrm{kg}$ dry & 1 & 13.6 & $=$ & 6 & 0.39 \\
\hline Columbia River & RM 90 & $11-\mathrm{s}$ & June 29, 1993 & Arsenic & $\mathrm{mg} / \mathrm{kg}$ dry & 1 & 3.6 & $=$ & 6 & 0.39 \\
\hline Columbia River & RM 95 & $12-\mathrm{S}$ & June 30, 1993 & Arsenic & $\mathrm{mg} / \mathrm{kg}$ dry & 1 & 8.6 & $=$ & 6 & 0.39 \\
\hline Columbia River & RM 120 & 13-s & July 1, 1993 & Arsenic & $\mathrm{mg} / \mathrm{kg}$ dry & 1 & 6.2 & $=$ & 6 & 0.39 \\
\hline Columbia River & RM 124 & 14-S & July 1, 1993 & Arsenic & $\mathrm{mg} / \mathrm{kg}$ dry & 1 & 4.4 & E & 6 & 0.39 \\
\hline Columbia River & RM 141 & $15-\mathrm{S}$ & June 30, 1993 & Arsenic & $\mathrm{mg} / \mathrm{kg}$ dry & 1 & 3.7 & $=$ & 6 & 0.39 \\
\hline Columbia River & RM 14 & 1-S & June 28, 1993 & Arsenic & $\mathrm{mg} / \mathrm{kg}$ dry & 1 & 4.6 & $=$ & 6 & 0.39 \\
\hline Columbia River & RM 21 & 2-S & June 27, 1993 & Arsenic & $\mathrm{mg} / \mathrm{kg}$ dry & 1 & 9.7 & $=$ & 6 & 0.39 \\
\hline Columbia River & RM 23 & 3-s & June 27, 1993 & Arsenic & $\mathrm{mg} / \mathrm{kg}$ dry & 1 & 4.7 & $=$ & 6 & 0.39 \\
\hline Columbia River & RM 26 & 4-S & June 26, 1993 & Arsenic & $\mathrm{mg} / \mathrm{kg}$ dry & 1 & 3.9 & $=$ & 6 & 0.39 \\
\hline Columbia River & RM 29 & 5-S & June 26, 1993 & Arsenic & $\mathrm{mg} / \mathrm{kg}$ dry & 1 & 4.3 & $=$ & 6 & 0.39 \\
\hline Columbia River & RM 36 & 6-S & June 25, 1993 & Arsenic & $\mathrm{mg} / \mathrm{kg}$ dry & 1 & 4.5 & $=$ & 6 & 0.39 \\
\hline
\end{tabular}


Table E-10

Summary of Surface Sediment Results From Segment 5 Exceeding Select Ecological or Human Health Benchmark Values

\begin{tabular}{|c|c|c|c|c|c|c|c|c|c|c|}
\hline Sample Area & Sample Site & SampleID & Date Collected & Constituent Name & Units & QA Category & $\begin{array}{c}\text { Analytical } \\
\text { Value }\end{array}$ & Qualifier & $\begin{array}{c}\text { Eco } \\
\text { Benchmark } \\
\text { Value }\end{array}$ & $\begin{array}{c}\text { HH } \\
\text { Benchmark } \\
\text { Value } \\
\end{array}$ \\
\hline Columbia River & RM 59 & $7-\mathrm{S}$ & June 25, 1993 & Arsenic & $\mathrm{mg} / \mathrm{kg}$ dry & 1 & 3.8 & $=$ & 6 & 0.39 \\
\hline Columbia River & Reed Island & $88-398-300$ & July 22, 1987 & Arsenic & $\mathrm{mg} / \mathrm{kg}$ dry & 2 & 2.1 & $=$ & 6 & 0.39 \\
\hline Columbia River & Camas Slough & 88-398-301 & July 22, 1987 & Arsenic & $\mathrm{mg} / \mathrm{kg}$ dry & 2 & 7.2 & $=$ & 6 & 0.39 \\
\hline Columbia River & Camas Slough & 88-398-302 & July 22, 1987 & Arsenic & $\mathrm{mg} / \mathrm{kg}$ dry & 2 & 7.2 & $=$ & 6 & 0.39 \\
\hline Columbia River & Vancouver WA & 88-398-303 & July 22, 1987 & Arsenic & $\mathrm{mg} / \mathrm{kg}$ dry & 2 & 3.8 & $=$ & 6 & 0.39 \\
\hline Columbia River & Vancouver WA & 88-398-304 & July 22, 1987 & Arsenic & $\mathrm{mg} / \mathrm{kg}$ dry & 2 & 3.4 & $=$ & 6 & 0.39 \\
\hline Columbia River & Kalama ChemPier & 88-398-305 & July 22, 1987 & Arsenic & $\mathrm{mg} / \mathrm{kg}$ dry & 2 & 2.4 & $=$ & 6 & 0.39 \\
\hline Columbia River & Longview Fibre & 88-398-306 & July 23, 1987 & Arsenic & $\mathrm{mg} / \mathrm{kg}$ dry & 2 & 0.8 & $=$ & 6 & 0.39 \\
\hline Columbia River & Longview WA & 88-398-307 & July 23, 1987 & Arsenic & $\mathrm{mg} / \mathrm{kg}$ dry & 2 & 1.1 & $=$ & 6 & 0.39 \\
\hline Columbia River & Longview WA & 88-398-308 & July 23, 1987 & Arsenic & $\mathrm{mg} / \mathrm{kg}$ dry & 2 & 1 & $=$ & 6 & 0.39 \\
\hline Columbia River & Longview WA & 88-398-309 & July 23, 1987 & Arsenic & $\mathrm{mg} / \mathrm{kg}$ dry & 2 & 1.1 & $=$ & 6 & 0.39 \\
\hline Columbia River & Coal Creek Slg & $88-398-310$ & July 23, 1987 & Arsenic & $\mathrm{mg} / \mathrm{kg}$ dry & 2 & 1.3 & $=$ & 6 & 0.39 \\
\hline Columbia River & Ilwaco Boat Bsn & 88-398-311 & July 24, 1987 & Arsenic & $\mathrm{mg} / \mathrm{kg}$ dry & 2 & 9.1 & $=$ & 6 & 0.39 \\
\hline Columbia River & RM 68 & 8-S & June 24, 1993 & Arsenic & $\mathrm{mg} / \mathrm{kg}$ dry & 1 & 4.3 & $=$ & 6 & 0.39 \\
\hline Columbia River & RM 81 & 9-S & June 29, 1993 & Arsenic & $\mathrm{mg} / \mathrm{kg}$ dry & 1 & 4.6 & $=$ & 6 & 0.39 \\
\hline Columbia River & Bachelor Slough & BACH-BC-01 & June 3, 2003 & Arsenic & $\mathrm{mg} / \mathrm{kg}$ & 1 & 4.36 & $=$ & 6 & 0.39 \\
\hline Columbia River & Bachelor Slough & BACH-BC-02 & June 3, 2003 & Arsenic & $\mathrm{mg} / \mathrm{kg}$ & 1 & 3.5 & $=$ & 6 & 0.39 \\
\hline Columbia River & Bachelor Slough & BACH-BC-03 & June 3, 2003 & Arsenic & $\mathrm{mg} / \mathrm{kg}$ & 1 & 2.14 & $=$ & 6 & 0.39 \\
\hline Columbia River & Bachelor Slough & BACH-BC-04 & June 3, 2003 & Arsenic & $\mathrm{mg} / \mathrm{kg}$ & 1 & 2.34 & $=$ & 6 & 0.39 \\
\hline Columbia River & Bachelor Slough & BACH-BC-05 & June 3, 2003 & Arsenic & $\mathrm{mg} / \mathrm{kg}$ & 1 & 1.24 & $=$ & 6 & 0.39 \\
\hline Columbia River & Bachelor Slough & BACH-BC-06 & June 3, 2003 & Arsenic & $\mathrm{mg} / \mathrm{kg}$ & 1 & 2.89 & $=$ & 6 & 0.39 \\
\hline Columbia River & Bachelor Slough & BACH-BC-07 & June 3, 2003 & Arsenic & $\mathrm{mg} / \mathrm{kg}$ & 1 & 4.22 & $=$ & 6 & 0.39 \\
\hline Columbia River & Bachelor Slough & BACH-BC-08 & June 3, 2003 & Arsenic & $\mathrm{mg} / \mathrm{kg}$ & 1 & 6.86 & $=$ & 6 & 0.39 \\
\hline Columbia River & Bachelor Slough & BACH-BC-09 & June 3,2003 & Arsenic & $\mathrm{mg} / \mathrm{kg}$ & 1 & 2.97 & $=$ & 6 & 0.39 \\
\hline Columbia River & Bachelor Slough & BACH-GC-10 & June 3, 2003 & Arsenic & $\mathrm{mg} / \mathrm{kg}$ & 1 & 3.22 & $=$ & 6 & 0.39 \\
\hline Columbia River & STA CR-BC-76 & CR76-PG-01 & June 1, 1997 & Arsenic & $\mathrm{mg} / \mathrm{kg}$ & 1 & 1.2 & $=$ & 6 & 0.39 \\
\hline Columbia River & STA CR-BC-76 & CR76-PG-02 & June 1,1997 & Arsenic & $\mathrm{mg} / \mathrm{kg}$ & 1 & 1.2 & $=$ & 6 & 0.39 \\
\hline Columbia River & STA CR-BC-76 & CR76-PG-03 & June 1, 1997 & Arsenic & $\mathrm{mg} / \mathrm{kg}$ & 1 & 1.4 & $=$ & 6 & 0.39 \\
\hline Columbia River & RM 33 & CR-BC-22 & June 3,1997 & Arsenic & $\mathrm{mg} / \mathrm{kg}$ & 1 & 2 & $=$ & 6 & 0.39 \\
\hline Columbia River & RM 39 & CR-BC-24 & June 3, 1997 & Arsenic & $\mathrm{mg} / \mathrm{kg}$ & 1 & 2 & $=$ & 6 & 0.39 \\
\hline Columbia River & RM 40 & CR-BC-25 & June 3,1997 & Arsenic & $\mathrm{mg} / \mathrm{kg}$ & 1 & 1 & $=$ & 6 & 0.39 \\
\hline Columbia River & RM 57 & CR-BC-35 & June 3,1997 & Arsenic & $\mathrm{mg} / \mathrm{kg}$ & 1 & 2 & $=$ & 6 & 0.39 \\
\hline Columbia River & RM 63 & CR-BC-40 & June 3,1997 & Arsenic & $\mathrm{mg} / \mathrm{kg}$ & 1 & 1 & $=$ & 6 & 0.39 \\
\hline Columbia River & RM 64 & CR-BC-41 & June 3, 1997 & Arsenic & $\mathrm{mg} / \mathrm{kg}$ & 1 & 2 & $=$ & 6 & 0.39 \\
\hline Columbia River & RM 11 & CR-BC-5 & June 3,1997 & Arsenic & $\mathrm{mg} / \mathrm{kg}$ & 1 & 3 & $=$ & 6 & 0.39 \\
\hline Columbia River & RM 76 & CR-BC-52 & June 3, 1997 & Arsenic & $\mathrm{mg} / \mathrm{kg}$ & 1 & 2 & $=$ & 6 & 0.39 \\
\hline Columbia River & RM 82 & CR-BC-55 & June 3,1997 & Arsenic & $\mathrm{mg} / \mathrm{kg}$ & 1 & 2 & $=$ & 6 & 0.39 \\
\hline Columbia River & RM 83 & CR-BC-56 & June 3, 1997 & Arsenic & $\mathrm{mg} / \mathrm{kg}$ & 1 & 2 & $=$ & 6 & 0.39 \\
\hline Columbia River & RM 83 & CR-BC-57 & June 3, 1997 & Arsenic & $\mathrm{mg} / \mathrm{kg}$ & 1 & 2 & $=$ & 6 & 0.39 \\
\hline Columbia River & RM 85 & CR-BC-59 & June 3,1997 & Arsenic & $\mathrm{mg} / \mathrm{kg}$ & 1 & 2 & $=$ & 6 & 0.39 \\
\hline Columbia River & RM 86 & CR-BC-61 & June 3, 1997 & Arsenic & $\mathrm{mg} / \mathrm{kg}$ & 1 & 2 & $=$ & 6 & 0.39 \\
\hline Columbia River & RM 12 & CR-BC-7 & June 3, 1997 & Arsenic & $\mathrm{mg} / \mathrm{kg}$ & 1 & 3 & $=$ & 6 & 0.39 \\
\hline Columbia River & RM 99 & CR-BC-73 & June 3, 1997 & Arsenic & $\mathrm{mg} / \mathrm{kg}$ & 1 & 2 & $=$ & 6 & 0.39 \\
\hline Columbia River & RM 99 & CR-BC-74 & June 3,1997 & Arsenic & $\mathrm{mg} / \mathrm{kg}$ & 1 & 2 & $=$ & 6 & 0.39 \\
\hline Columbia River & RM 99 & CR-BC-75 & June 3,1997 & Arsenic & $\mathrm{mg} / \mathrm{kg}$ & 1 & 1 & $=$ & 6 & 0.39 \\
\hline Columbia River & RM 100 & CR-BC-76 & June 3,1997 & Arsenic & $\mathrm{mg} / \mathrm{kg}$ & 1 & 3 & $=$ & 6 & 0.39 \\
\hline Columbia River & RM 103 & CR-BC-80 & June 3,1997 & Arsenic & $\mathrm{mg} / \mathrm{kg}$ & 1 & 2 & $=$ & 6 & 0.39 \\
\hline Columbia River & RM 104 & CR-BC-84 & June 3,1997 & Arsenic & $\mathrm{mg} / \mathrm{kg}$ & 1 & 2 & $=$ & 6 & 0.39 \\
\hline Columbia River & RM 104 & CR-BC-85 & June 3,1997 & Arsenic & $\mathrm{mg} / \mathrm{kg}$ & 1 & 2 & $=$ & 6 & 0.39 \\
\hline
\end{tabular}


Table E-10

Summary of Surface Sediment Results From Segment 5 Exceeding Select Ecological or Human Health Benchmark Values

\begin{tabular}{|c|c|c|c|c|c|c|c|c|c|c|}
\hline Sample Area & Sample Site & SampleID & Date Collected & Constituent Name & Units & QA Category & $\begin{array}{c}\text { Analytical } \\
\text { Value }\end{array}$ & Qualifier & $\begin{array}{c}\text { Eco } \\
\text { Benchmark } \\
\text { Value }\end{array}$ & $\begin{array}{c}\text { HH } \\
\text { Benchmark } \\
\text { Value }\end{array}$ \\
\hline Columbia River & RM 105 & CR-BC-86 & June 3, 1997 & Arsenic & $\mathrm{mg} / \mathrm{kg}$ & 1 & 1 & $=$ & 6 & 0.39 \\
\hline Columbia River & RM 106 & CR-BC-88 & June 3, 1997 & Arsenic & $\mathrm{mg} / \mathrm{kg}$ & 1 & 1 & $=$ & 6 & 0.39 \\
\hline Columbia River & Crims Island & CRIM-SG-01 & July 15, 2003 & Arsenic & $\mathrm{mg} / \mathrm{kg}$ & 1 & 2.28 & $=$ & 6 & 0.39 \\
\hline Columbia River & Crims Island & CRIM-SG-02 & July 15, 2003 & Arsenic & $\mathrm{mg} / \mathrm{kg}$ & 1 & 7.94 & $=$ & 6 & 0.39 \\
\hline Columbia River & Crims Island & CRIM-SG-03 & July 15, 2003 & Arsenic & $\mathrm{mg} / \mathrm{kg}$ & 1 & 9.21 & $=$ & 6 & 0.39 \\
\hline Columbia River & Crims Island & CRIM-SG-04 & July 15, 2003 & Arsenic & $\mathrm{mg} / \mathrm{kg}$ & 1 & 3.02 & $=$ & 6 & 0.39 \\
\hline Columbia River & Crims Island & CRIM-SG-05 & July 15,2003 & Arsenic & $\mathrm{mg} / \mathrm{kg}$ & 1 & 5.81 & $=$ & 6 & 0.39 \\
\hline Columbia River & RM 29 & CRM-BC1 & August 9,2000 & Arsenic & $\mathrm{mg} / \mathrm{kg}$ dry & 1 & 2.1 & $=$ & 6 & 0.39 \\
\hline Columbia River & RM 29 & CRM-BC2 & August 9, 2000 & Arsenic & $\mathrm{mg} / \mathrm{kg}$ dry & 1 & 2 & $=$ & 6 & 0.39 \\
\hline Columbia River & RM 29 & CRM-BC3 & August 9, 2000 & Arsenic & $\mathrm{mg} / \mathrm{kg}$ dry & 1 & 1.8 & $=$ & 6 & 0.39 \\
\hline Columbia River & RM 29 & CRM-BC4 & August 9, 2000 & Arsenic & $\mathrm{mg} / \mathrm{kg}$ dry & 1 & 1.6 & $=$ & 6 & 0.39 \\
\hline Columbia River & RM 29 & CRM-BC5 & August 9, 2000 & Arsenic & $\mathrm{mg} / \mathrm{kg}$ dry & 1 & 1.9 & $=$ & 6 & 0.39 \\
\hline Columbia River & RM 29 & CRM-VC-01 & September 7, 2000 & Arsenic & $\mathrm{mg} / \mathrm{kg}$ dry & 1 & 2.2 & $=$ & 6 & 0.39 \\
\hline Columbia River & RM 29 & CRM-VC-02 & September 7, 2000 & Arsenic & $\mathrm{mg} / \mathrm{kg}$ dry & 1 & 2.1 & $=$ & 6 & 0.39 \\
\hline Columbia River & RM 29 & CRM-VC-03 & September 7, 2000 & Arsenic & $\mathrm{mg} / \mathrm{kg}$ dry & 1 & 2.1 & $=$ & 6 & 0.39 \\
\hline Columbia River & RM 29 & CRM-VC-04 & September 7, 2000 & Arsenic & $\mathrm{mg} / \mathrm{kg}$ dry & 1 & 1.8 & $=$ & 6 & 0.39 \\
\hline Columbia River & RM 29 & CRM-VC-05 & September 7, 2000 & Arsenic & $\mathrm{mg} / \mathrm{kg}$ dry & 1 & 1.9 & $=$ & 6 & 0.39 \\
\hline Columbia River & RM 73.9-74.8 & KR-BC-01 & September 10, 2003 & Arsenic & $\mathrm{mg} / \mathrm{kg}$ & 1 & 2.02 & $=$ & 6 & 0.39 \\
\hline Columbia River & RM 73.9-74.8 & KR-BC-04 & September 10, 2003 & Arsenic & $\mathrm{mg} / \mathrm{kg}$ & 1 & 1.78 & $=$ & 6 & 0.39 \\
\hline Columbia River & RM 73.9-74.8 & KR-BC-04A & September 10, 2003 & Arsenic & $\mathrm{mg} / \mathrm{kg}$ & 1 & 1.82 & $=$ & 6 & 0.39 \\
\hline Cowlitz River & OM Cowlitz Rvr & OMCR-BC-04 & September 10, 2003 & Arsenic & $\mathrm{mg} / \mathrm{kg}$ & 1 & 1.41 & $=$ & 6 & 0.39 \\
\hline Cowlitz River & OM Cowlitz Rvr & OMCR-GC-01 & September 10, 2003 & Arsenic & $\mathrm{mg} / \mathrm{kg}$ & 1 & 2.94 & $=$ & 6 & 0.39 \\
\hline Cowlitz River & OM Cowlitz Rvr & OMCR-GC-02 & September 10, 2003 & Arsenic & $\mathrm{mg} / \mathrm{kg}$ & 1 & 2.28 & $=$ & 6 & 0.39 \\
\hline Cowlitz River & OM Cowlitz Rvr & OMCR-GC-03 & September 10, 2003 & Arsenic & $\mathrm{mg} / \mathrm{kg}$ & 1 & 1.9 & $=$ & 6 & 0.39 \\
\hline Columbia River & Oregon Slough & OR-VC-01 & June 19, 2001 & Arsenic & $\mathrm{mg} / \mathrm{kg}$ & 1 & 0.93 & B & 6 & 0.39 \\
\hline Columbia River & Oregon Slough & OR-VC-02 & June 19, 2001 & Arsenic & $\mathrm{mg} / \mathrm{kg}$ & 1 & 1.6 & $=$ & 6 & 0.39 \\
\hline Columbia River & Oregon Slough & OR-VC-03 & June 19, 2001 & Arsenic & $\mathrm{mg} / \mathrm{kg}$ & 1 & 0.96 & B & 6 & 0.39 \\
\hline Columbia River & Oregon Slough & OR-VC-04 & June 19, 2001 & Arsenic & $\mathrm{mg} / \mathrm{kg}$ & 1 & 1.9 & $=$ & 6 & 0.39 \\
\hline Skipanon Federal Channel & Warrenton OR & SBIO-GC-01 & June 24,2003 & Arsenic & $\mathrm{mg} / \mathrm{kg}$ & 1 & 8.96 & $=$ & 6 & 0.39 \\
\hline Skipanon Federal Channel & Warrenton OR & SBIO-GC-02 & June 24, 2003 & Arsenic & $\mathrm{mg} / \mathrm{kg}$ & 1 & 7.51 & $=$ & 6 & 0.39 \\
\hline Skipanon Federal Channel & Warrenton OR & SBIO-GC-03 & June 24,2003 & Arsenic & $\mathrm{mg} / \mathrm{kg}$ & 1 & 8.95 & $=$ & 6 & 0.39 \\
\hline Skipanon Federal Channel & Warrenton OR & SBIO-GC-04 & June 24, 2003 & Arsenic & $\mathrm{mg} / \mathrm{kg}$ & 1 & 9 & $=$ & 6 & 0.39 \\
\hline Columbia River & RM 86.5 & SH-VC-4 & May 4, 1989 & Arsenic & $\mathrm{mg} / \mathrm{kg}$ dry & 3 & 2.1 & $=$ & 6 & 0.39 \\
\hline Columbia River & RM 86.5 & SH-VC-5 & May 4, 1989 & Arsenic & $\mathrm{mg} / \mathrm{kg}$ dry & 3 & 2.6 & $=$ & 6 & 0.39 \\
\hline Columbia River & RM 86.5 & SH-VC-6 & May 4, 1989 & Arsenic & $\mathrm{mg} / \mathrm{kg}$ dry & 3 & 2.8 & $=$ & 6 & 0.39 \\
\hline Skipanon Federal channel & Warrenton OR & SKIP-GC-01 & September 12, 2001 & Arsenic & $\mathrm{mg} / \mathrm{kg}$ & 1 & 10 & $=$ & 6 & 0.39 \\
\hline Skipanon Federal channel & Warrenton OR & SKIP-GC-02 & September 12, 2001 & Arsenic & $\mathrm{mg} / \mathrm{kg}$ & 1 & 10 & $=$ & 6 & 0.39 \\
\hline Skipanon Federal channel & Warrenton OR & SKIP-GC-03 & September 12, 2001 & Arsenic & $\mathrm{mg} / \mathrm{kg}$ & 1 & 11 & $=$ & 6 & 0.39 \\
\hline Skipanon Federal channel & Warrenton OR & SKIP-GC-04 & September 12, 2001 & Arsenic & $\mathrm{mg} / \mathrm{kg}$ & 1 & 11 & $=$ & 6 & 0.39 \\
\hline Skipanon Federal channel & Warrenton OR & SKIP-GC-05 & September 12, 2001 & Arsenic & $\mathrm{mg} / \mathrm{kg}$ & 1 & 9.6 & $=$ & 6 & 0.39 \\
\hline Skipanon Federal channel & Warrenton OR & SKIP-GC-06 & September 12, 2001 & Arsenic & $\mathrm{mg} / \mathrm{kg}$ & 1 & 6.9 & $=$ & 6 & 0.39 \\
\hline Skipanon Federal channel & Warrenton OR & SKIP-GC-07 & September 12, 2001 & Arsenic & $\mathrm{mg} / \mathrm{kg}$ & 1 & 6.6 & $=$ & 6 & 0.39 \\
\hline Columbia River & RM 103 & $\mathrm{~V}-\mathrm{VC}-01 \mathrm{~A}$ & June 20, 2001 & Arsenic & $\mathrm{mg} / \mathrm{kg}$ & 1 & 0.98 & B & 6 & 0.39 \\
\hline Columbia River & RM 103 & V-VC-01B & June 20, 2001 & Arsenic & $\mathrm{mg} / \mathrm{kg}$ & 1 & 0.86 & B & 6 & 0.39 \\
\hline Willamette River & RM 0.1 - mouth & WR-BC-1 & July 24, 1997 & Arsenic & $\mathrm{mg} / \mathrm{kg}$ & 1 & 1 & $=$ & 6 & 0.39 \\
\hline Willamette River & RM 0.4 - mouth & WR-BC-3 & July 24, 1997 & Arsenic & $\mathrm{mg} / \mathrm{kg}$ & 1 & 2.7 & $=$ & 6 & 0.39 \\
\hline Willamette River & RM 0.1 - mouth & WR-GC-2A & July 24, 1997 & Arsenic & $\mathrm{mg} / \mathrm{kg}$ & 1 & 2.4 & $=$ & 6 & 0.39 \\
\hline Willamette River & RM 0.1 - mouth & WR-GC-2B & July 24, 1997 & Arsenic & $\mathrm{mg} / \mathrm{kg}$ & 1 & 1.1 & $=$ & 6 & 0.39 \\
\hline Columbia River & RM 88 & $10-\mathrm{s}$ & June 28, 1993 & o(a)anthracene & $\mathrm{mg} / \mathrm{kg}$ dry & 1 & 0.04 & $=$ & 0.032 & 0.62 \\
\hline
\end{tabular}


Table E-10

Summary of Surface Sediment Results From Segment 5 Exceeding Select Ecological or Human Health Benchmark Values

\begin{tabular}{|c|c|c|c|c|c|c|c|c|c|c|}
\hline Sample Area & Sample Site & SampleID & Date Collected & Constituent Name & Units & QA Category & $\begin{array}{c}\text { Analytical } \\
\text { Value }\end{array}$ & Qualifier & $\begin{array}{c}\text { Eco } \\
\text { Benchmark } \\
\text { Value }\end{array}$ & $\begin{array}{c}\text { HH } \\
\text { Benchmark } \\
\text { Value }\end{array}$ \\
\hline Columbia River & RM 124 & $14-\mathrm{S}$ & July 1, 1993 & Benzo(a)anthracene & $\mathrm{mg} / \mathrm{kg}$ dry & 1 & 0.037 & $=$ & 0.032 & 0.62 \\
\hline Columbia River & Vancouver WA & 88-398-303 & July 22, 1987 & Benzo(a)anthracene & $\mathrm{mg} / \mathrm{kg}$ dry & 2 & 0.062 & $\mathrm{~J}$ & 0.032 & 0.62 \\
\hline Columbia River & Vancouver WA & 88-398-304 & July 22, 1987 & Benzo(a)anthracene & $\mathrm{mg} / \mathrm{kg}$ dry & 2 & 0.045 & $\mathrm{~J}$ & 0.032 & 0.62 \\
\hline Columbia River & Longview WA & 88-398-307 & July 23,1987 & Benzo(a)anthracene & $\mathrm{mg} / \mathrm{kg}$ dry & 2 & 0.066 & $\mathrm{~J}$ & 0.032 & 0.62 \\
\hline Columbia River & Longview WA & 88-398-309 & July 23, 1987 & Benzo(a)anthracene & $\mathrm{mg} / \mathrm{kg}$ dry & 2 & 2.2 & $=$ & 0.032 & 0.62 \\
\hline Columbia River & Ilwaco Boat Bsn & 88-398-311 & July 24, 1987 & Benzo(a)anthracene & $\mathrm{mg} / \mathrm{kg}$ dry & 2 & 0.18 & $\mathrm{~J}$ & 0.032 & 0.62 \\
\hline Columbia River & RM 81 & 9-S & June 29, 1993 & Benzo(a)anthracene & $\mathrm{mg} / \mathrm{kg}$ dry & 1 & 0.044 & $=$ & 0.032 & 0.62 \\
\hline Columbia River & RM 83 & CR-BC-57 & June 3,1997 & Benzo(a)anthracene & $\mathrm{mg} / \mathrm{kg}$ & 1 & 0.036 & $=$ & 0.032 & 0.62 \\
\hline Columbia River & RM 100 & CR-BC-76 & June 3, 1997 & Benzo(a)anthracene & $\mathrm{mg} / \mathrm{kg}$ & 1 & 0.038 & $=$ & 0.032 & 0.62 \\
\hline Columbia River & Crims Island & CRIM-SG-03 & July 15, 2003 & Benzo(a)anthracene & $\mathrm{mg} / \mathrm{kg}$ & 1 & 0.0493 & $=$ & 0.032 & 0.62 \\
\hline Cowlitz River & OM Cowlitz Rvr & OMCR-GC-01 & September 10, 2003 & Benzo(a)anthracene & $\mathrm{mg} / \mathrm{kg}$ & 1 & 0.0963 & $=$ & 0.032 & 0.62 \\
\hline Columbia River & RM 86.5 & SH-VC-4 & May 4, 1989 & Benzo(a)anthracene & $\mathrm{mg} / \mathrm{kg}$ & 3 & 0.034 & $=$ & 0.032 & 0.62 \\
\hline Columbia River & RM 86.5 & SH-VC-5 & May 4, 1989 & Benzo(a)anthracene & $\mathrm{mg} / \mathrm{kg}$ & 3 & 0.037 & $=$ & 0.032 & 0.62 \\
\hline Columbia River & RM 82 & Will-1 & September 10, 1997 & Benzo(a)anthracene & $\mathrm{mg} / \mathrm{kg}$ dry & 2 & 0.036 & $=$ & 0.032 & 0.62 \\
\hline Willamette River & RM 0.1 - mouth & WR-GC-2A & July 24,1997 & Benzo(a)anthracene & $\mathrm{mg} / \mathrm{kg}$ & 1 & 0.044 & $=$ & 0.032 & 0.62 \\
\hline Columbia River & Oregon Slough & 0205-OS-VC-06Z & February 17,2005 & Benzo(a)pyrene & $\mathrm{mg} / \mathrm{kg}$ & 1 & 0.0368 & $=$ & 0.032 & 0.062 \\
\hline Columbia River & RM 88 & $10-5$ & June 28, 1993 & Benzo(a)pyrene & $\mathrm{mg} / \mathrm{kg}$ dry & 1 & 0.061 & $=$ & 0.032 & 0.062 \\
\hline Columbia River & RM 124 & $14-\mathrm{S}$ & July 1, 1993 & Benzo(a)pyrene & $\mathrm{mg} / \mathrm{kg}$ dry & 1 & 0.035 & $=$ & 0.032 & 0.062 \\
\hline Columbia River & Vancouver WA & 88-398-303 & July 22, 1987 & Benzo(a)pyrene & $\mathrm{mg} / \mathrm{kg}$ dry & 2 & 0.033 & $\mathrm{cJ}$ & 0.032 & 0.062 \\
\hline Columbia River & Vancouver WA & 88-398-304 & July 22, 1987 & Benzo(a)pyrene & $\mathrm{mg} / \mathrm{kg}$ dry & 2 & 0.047 & $\mathrm{CJ}$ & 0.032 & 0.062 \\
\hline Columbia River & Longview WA & 88-398-307 & July 23, 1987 & Benzo(a)pyrene & $\mathrm{mg} / \mathrm{kg}$ dry & 2 & 0.066 & cJ & 0.032 & 0.062 \\
\hline Columbia River & Longview WA & 88-398-309 & July 23, 1987 & Benzo(a)pyrene & $\mathrm{mg} / \mathrm{kg}$ dry & 2 & 1.5 & c & 0.032 & 0.062 \\
\hline Columbia River & RM 83 & CR-BC-57 & June 3,1997 & Benzo(a)pyrene & $\mathrm{mg} / \mathrm{kg}$ & 1 & 0.07 & $=$ & 0.032 & 0.062 \\
\hline Columbia River & RM 100 & CR-BC-76 & June 3, 1997 & Benzo(a)pyrene & $\mathrm{mg} / \mathrm{kg}$ & 1 & 0.037 & $=$ & 0.032 & 0.062 \\
\hline Columbia River & Crims Island & CRIM-SG-02 & July 15, 2003 & Benzo(a)pyrene & $\mathrm{mg} / \mathrm{kg}$ & 1 & 0.0547 & $=$ & 0.032 & 0.062 \\
\hline Columbia River & Crims Island & CRIM-SG-03 & July 15, 2003 & Benzo(a)pyrene & $\mathrm{mg} / \mathrm{kg}$ & 1 & 0.0724 & $=$ & 0.032 & 0.062 \\
\hline Cowlitz River & OM Cowlitz Rvr & OMCR-GC-01 & September 10, 2003 & Benzo(a)pyrene & $\mathrm{mg} / \mathrm{kg}$ & 1 & 0.0423 & $=$ & 0.032 & 0.062 \\
\hline Skipanon Federal Channel & Warrenton OR & SBIO-GC-04 & June 24, 2003 & Benzo(a)pyrene & $\mathrm{mg} / \mathrm{kg}$ & 1 & 0.0434 & $=$ & 0.032 & 0.062 \\
\hline Columbia River & RM 86.5 & SH-VC-4 & May 4, 1989 & Benzo(a)pyrene & $\mathrm{mg} / \mathrm{kg}$ & 3 & 0.036 & $=$ & 0.032 & 0.062 \\
\hline Columbia River & RM 86.5 & SH-VC-5 & May 4, 1989 & Benzo(a)pyrene & $\mathrm{mg} / \mathrm{kg}$ & 3 & 0.05 & $=$ & 0.032 & 0.062 \\
\hline Columbia River & RM 82 & Will-1 & September 10, 1997 & Benzo(a)pyrene & $\mathrm{mg} / \mathrm{kg}$ dry & 2 & 0.063 & $=$ & 0.032 & 0.062 \\
\hline Willamette River & RM 0.1 - mouth & WR-GC-2A & July 24, 1997 & Benzo(a)pyrene & $\mathrm{mg} / \mathrm{kg}$ & 1 & 0.103 & $=$ & 0.032 & 0.062 \\
\hline Columbia River & Longview WA & (88-398-309- & July 23, 1987 & Benzo(b)fluoranthene & $\mathrm{mg} / \mathrm{kg}$ dry & 2 & 1.8 & $c$ & 0.24 & 0.62 \\
\hline Columbia River & Longview WA & $88-398-309$ & July 23,1987 & Benzo(ghi)perylene- & $\mathrm{mg} / \mathrm{kg}$ dry & 2 & 1.1 & c & 0.17 & 2,400 \\
\hline Columbia River & Camas Slough & $88-398-301$ & July 22,1987 & Benzo $(\mathrm{k})$ fluoranthene & $\mathrm{mg} / \mathrm{kg}$ dry & 2 & 0.19 & $\mathrm{CJ}$ & 0.027 & 6.2 \\
\hline Columbia River & Longview WA & 88-398-307 & July 23,1987 & Benzo(k)fluoranthene & $\mathrm{mg} / \mathrm{kg}$ dry & 2 & 0.031 & $\mathrm{cJ}$ & 0.027 & 6.2 \\
\hline Columbia River & Longview WA & 88-398-309 & July 23, 1987 & Benzo(k)fluoranthene & $\mathrm{mg} / \mathrm{kg}$ dry & 2 & 0.91 & c & 0.027 & 6.2 \\
\hline Columbia River & Ilwaco Boat Bsn & 88-398-311 & July 24, 1987 & Benzo(k)fluoranthene & $\mathrm{mg} / \mathrm{kg}$ dry & 2 & 0.12 & $\mathrm{CJ}$ & 0.027 & 6.2 \\
\hline Columbia River & RM 82 & Will-1 & September 10, 1997 & Benzo(k)fluoranthene & $\mathrm{mg} / \mathrm{kg}$ dry & 2 & 0.06 & $=$ & 0.027 & 6.2 \\
\hline Columbia River & Kalama ChemPier & $88-398-305$ & July 22,1987 & is (2-ethylhexyl) phthalate & $\mathrm{mg} / \mathrm{kg}$ dry & 2 & 1.9 & $\mathrm{c}$ & 0.64 & 35 \\
\hline Columbia River & RM 88 & $10-\mathrm{s}$ & June 28,1993 & Cadmium & $\mathrm{mg} / \mathrm{kg}$ dry & 1 & 0.63 & $=$ & 0.6 & 37 \\
\hline Columbia River & RM 90 & $11-\mathrm{s}$ & June 29, 1993 & Cadmium & $\mathrm{mg} / \mathrm{kg}$ dry & 1 & 1.1 & $=$ & 0.6 & 37 \\
\hline Columbia River & RM 95 & $12-\mathrm{s}$ & June 30,1993 & Cadmium & $\mathrm{mg} / \mathrm{kg}$ dry & 1 & 1.9 & $=$ & 0.6 & 37 \\
\hline Columbia River & RM 120 & $13-\mathrm{s}$ & July 1, 1993 & Cadmium & $\mathrm{mg} / \mathrm{kg}$ dry & 1 & 1.3 & $=$ & 0.6 & 37 \\
\hline Columbia River & RM 124 & $14-\mathrm{S}$ & July 1, 1993 & Cadmium & $\mathrm{mg} / \mathrm{kg}$ dry & 1 & 1.9 & $=$ & 0.6 & 37 \\
\hline Columbia River & RM 141 & $15-\mathrm{s}$ & June 30, 1993 & Cadmium & $\mathrm{mg} / \mathrm{kg}$ dry & 1 & 1 & $=$ & 0.6 & 37 \\
\hline Columbia River & RM 14 & $1-\mathrm{s}$ & June 28, 1993 & Cadmium & $\mathrm{mg} / \mathrm{kg}$ dry & 1 & 0.98 & $=$ & 0.6 & 37 \\
\hline Columbia River & RM 21 & 2-s & June 27, 1993 & Cadmium & $\mathrm{mg} / \mathrm{kg}$ dry & 1 & 0.78 & $=$ & 0.6 & 37 \\
\hline Columbia River & RM 23 & 3-s & June 27, 1993 & Cadmium & $\mathrm{mg} / \mathrm{kg}$ dry & 1 & 0.82 & $=$ & 0.6 & 37 \\
\hline
\end{tabular}


Table E-10

Summary of Surface Sediment Results From Segment 5 Exceeding Select Ecological or Human Health Benchmark Values

\begin{tabular}{|c|c|c|c|c|c|c|c|c|c|c|}
\hline Sample Area & Sample Site & SampleID & Date Collected & Constituent Name & Units & QA Category & $\begin{array}{c}\text { Analytical } \\
\text { Value }\end{array}$ & Qualifier & $\begin{array}{c}\text { Eco } \\
\text { Benchmark } \\
\text { Value }\end{array}$ & $\begin{array}{c}\mathrm{HH} \\
\text { Benchmark } \\
\text { Value }\end{array}$ \\
\hline Columbia River & RM 26 & 4-S & June 26,1993 & Cadmium & $\mathrm{mg} / \mathrm{kg}$ dry & 1 & 1 & $=$ & 0.6 & 37 \\
\hline Columbia River & RM 29 & 5-S & June 26, 1993 & Cadmium & $\mathrm{mg} / \mathrm{kg}$ dry & 1 & 1.1 & E & 0.6 & 37 \\
\hline Columbia River & RM 59 & 7-s & June 25, 1993 & Cadmium & $\mathrm{mg} / \mathrm{kg}$ dry & 1 & 0.61 & $=$ & 0.6 & 37 \\
\hline Columbia River & Camas Slough & 88-398-301 & July 22, 1987 & Cadmium & $\mathrm{mg} / \mathrm{kg}$ dry & 2 & 0.9 & $=$ & 0.6 & 37 \\
\hline Columbia River & Camas Slough & 88-398-302 & July 22, 1987 & Cadmium & $\mathrm{mg} / \mathrm{kg}$ dry & 2 & 0.8 & $=$ & 0.6 & 37 \\
\hline Columbia River & RM 68 & 8-S & June 24, 1993 & Cadmium & $\mathrm{mg} / \mathrm{kg}$ dry & 1 & 1.3 & $=$ & 0.6 & 37 \\
\hline Columbia River & RM 81 & 9-S & June 29, 1993 & Cadmium & $\mathrm{mg} / \mathrm{kg}$ dry & 1 & 1.1 & $=$ & 0.6 & 37 \\
\hline Columbia River & Crims Island & CRIM-SG-02 & July 15, 2003 & Cadmium & $\mathrm{mg} / \mathrm{kg}$ & 1 & 0.785 & $\mathrm{~J}$ & 0.6 & 37 \\
\hline Columbia River & Crims Island & CRIM-SG-03 & July 15, 2003 & Cadmium & $\mathrm{mg} / \mathrm{kg}$ & 1 & 1.12 & $=$ & 0.6 & 37 \\
\hline Columbia River & Oregon Slough & OR-VC-04 & June 19, 2001 & Cadmium & $\mathrm{mg} / \mathrm{kg}$ & 1 & 0.66 & B & 0.6 & 37 \\
\hline Skipanon Federal Channel & Warrenton OR & SBIO-GC-04 & June 24, 2003 & Cadmium & $\mathrm{mg} / \mathrm{kg}$ & 1 & 0.852 & $\mathrm{~J}$ & 0.6 & 37 \\
\hline Skipanon Federal channel & Warrenton OR & SKIP-GC-01 & September 12, 2001 & Cadmium & $\mathrm{mg} / \mathrm{kg}$ & 1 & 2.1 & $=$ & 0.6 & 37 \\
\hline Skipanon Federal channel & Warrenton OR & SKIP-GC-02 & September 12, 2001 & Cadmium & $\mathrm{mg} / \mathrm{kg}$ & 1 & 2.4 & $=$ & 0.6 & 37 \\
\hline Skipanon Federal channel & Warrenton OR & SKIP-GC-03 & September 12, 2001 & Cadmium & $\mathrm{mg} / \mathrm{kg}$ & 1 & 2.2 & $=$ & 0.6 & 37 \\
\hline Skipanon Federal channel & Warrenton OR & SKIP-GC-04 & September 12, 2001 & Cadmium & $\mathrm{mg} / \mathrm{kg}$ & 1 & 3.3 & $=$ & 0.6 & 37 \\
\hline Skipanon Federal channel & Warrenton OR & SKIP-GC-05 & September 12, 2001 & Cadmium & $\mathrm{mg} / \mathrm{kg}$ & 1 & 3.2 & $=$ & 0.6 & 37 \\
\hline Skipanon Federal channel & Warrenton OR & SKIP-GC-06 & September 12, 2001 & Cadmium & $\mathrm{mg} / \mathrm{kg}$ & 1 & 2 & $=$ & 0.6 & 37 \\
\hline Skipanon Federal channel & Warrenton OR & SKIP-GC-07 & September 12, 2001 & Cadmium & $\mathrm{mg} / \mathrm{kg}$ & 1 & 1.4 & $=$ & 0.6 & 37 \\
\hline Columbia River- & Crims Island & CRIM-SG-05 & July 15, 2003 & Chromium & $\mathrm{mg} / \mathrm{kg}$ & 1 & 34.5 & $=$ & 37 & 30 \\
\hline Skipanon Federal Channel & Warrenton OR & SBIO-GC-04 & June 24, 2003 & Chromium & $\mathrm{mg} / \mathrm{kg}$ & 1 & 32.3 & $=$ & 37 & 30 \\
\hline Columbia River & RM 36 & $6-\mathrm{S}$ & June 25,1993 & Chromium & $\mathrm{mg} / \mathrm{kg}$ dry & 1 & 31.1 & $=$ & 37 & 30 \\
\hline Columbia River & Camas Slough & - $88-398-302$ & July 22, 1987 & Chrysene & $\mathrm{mg} / \mathrm{kg}$ dry & 2 & 0.058 & $\mathrm{j}$ & 0.057 & 62 \\
\hline Columbia River & Vancouver WA & 88-398-303 & July 22, 1987 & Chrysene & $\mathrm{mg} / \mathrm{kg}$ dry & 2 & 0.065 & $\mathrm{~J}$ & 0.057 & 62 \\
\hline Columbia River & Vancouver WA & 88-398-304 & July 22, 1987 & Chrysene & $\mathrm{mg} / \mathrm{kg}$ dry & 2 & 0.066 & $\mathrm{~J}$ & 0.057 & 62 \\
\hline Columbia River & Longview WA & 88-398-307 & July 23,1987 & Chrysene & $\mathrm{mg} / \mathrm{kg}$ dry & 2 & 0.073 & $\mathrm{j}$ & 0.057 & 62 \\
\hline Columbia River & Longview WA & 88-398-309 & July 23,1987 & Chrysene & $\mathrm{mg} / \mathrm{kg}$ dry & 2 & 4.1 & $=$ & 0.057 & 62 \\
\hline Columbia River & Ilwaco Boat Bsn & 88-398-311 & July 24, 1987 & Chrysene & $\mathrm{mg} / \mathrm{kg}$ dry & 2 & 0.2 & $\mathrm{~J}$ & 0.057 & 62 \\
\hline Columbia River & RM 81 & 9-S & June 29, 1993 & Chrysene & $\mathrm{mg} / \mathrm{kg}$ dry & 1 & 0.1 & $=$ & 0.057 & 62 \\
\hline Columbia River & Crims Island & CRIM-SG-03 & July 15,2003 & Chrysene & $\mathrm{mg} / \mathrm{kg}$ & 1 & 0.0739 & $=$ & 0.057 & 62 \\
\hline Cowlitz River & OM Cowlitz Rvr & OMCR-GC-01 & September 10, 2003 & Chrysene & $\mathrm{mg} / \mathrm{kg}$ & 1 & 0.12 & $=$ & 0.057 & 62 \\
\hline Columbia River & RM 86.5 & SH-VC-4 & May 4, 1989 & Chrysene & $\mathrm{mg} / \mathrm{kg}$ & 3 & 0.125 & $=$ & 0.057 & 62 \\
\hline Columbia River & RM 86.5 & SH-VC-5 & May 4, 1989 & Chrysene & $\mathrm{mg} / \mathrm{kg}$ & 3 & 0.129 & $=$ & 0.057 & 62 \\
\hline Columbia River & RM 82 & Will-1 & September 10, 1997 & Chrysene & $\mathrm{mg} / \mathrm{kg}$ dry & 2 & 0.061 & $=$ & 0.057 & 62 \\
\hline Columbia River & RM 95 & $12-\mathrm{s}$ & June 30,1993 & Copper & $\mathrm{mg} / \mathrm{kg}$ dry & 1 & 32.5 & $=$ & 32 & 2,960 \\
\hline Columbia River & RM 29 & 5-s & June 26, 1993 & Copper & $\mathrm{mg} / \mathrm{kg}$ dry & 1 & 32.2 & $=$ & 32 & 2,960 \\
\hline Columbia River & RM 36 & 6-S & June 25, 1993 & Copper & $\mathrm{mg} / \mathrm{kg}$ dry & 1 & 49.9 & $=$ & 32 & 2,960 \\
\hline Columbia River & Vancouver WA & 88-398-303 & July 22, 1987 & Copper & $\mathrm{mg} / \mathrm{kg}$ dry & 2 & 54 & $=$ & 32 & 2,960 \\
\hline Columbia River & Ilwaco Boat Bsn & 88-398-311 & July 24, 1987 & Copper & $\mathrm{mg} / \mathrm{kg}$ dry & 2 & 75 & $=$ & 32 & 2,960 \\
\hline Columbia River & RM 68 & 8-s & June 24, 1993 & Copper & $\mathrm{mg} / \mathrm{kg}$ dry & 1 & 34 & $=$ & 32 & 2,960 \\
\hline Columbia River & RM 100 & CR-BC-76 & June 3, 1997 & Copper & $\mathrm{mg} / \mathrm{kg}$ & 1 & 33 & $=$ & 32 & 2,960 \\
\hline Columbia River & Crims Island & CRIM-SG-02 & July 15, 2003 & Copper & $\mathrm{mg} / \mathrm{kg}$ & 1 & 39.8 & $=$ & 32 & 2,960 \\
\hline Columbia River & Crims Island & CRIM-SG-05 & July 15, 2003 & Copper & $\mathrm{mg} / \mathrm{kg}$ & 1 & 36.7 & $=$ & 32 & 2,960 \\
\hline Columbia River & CR-S5 & CR-S5 .08-1.0m & October 1, 1984 & Copper & $\mathrm{mg} / \mathrm{kg}$ & 3 & 43 & $=$ & 32 & 2,960 \\
\hline Cowlitz River & OM Cowlitz Rvr & OMCR-GC-01 & September 10, 2003 & Copper & $\mathrm{mg} / \mathrm{kg}$ & 1 & 53.4 & $=$ & 32 & 2,960 \\
\hline Cowlitz River & OM Cowlitz Rvr & OMCR-GC-02 & September 10, 2003 & Copper & $\mathrm{mg} / \mathrm{kg}$ & 1 & 35.1 & $=$ & 32 & 2,960 \\
\hline Skipanon Federal Channel & Warrenton OR & SBIO-GC-01 & June 24,2003 & Copper & $\mathrm{mg} / \mathrm{kg}$ & 1 & 52.3 & $=$ & 32 & 2,960 \\
\hline Skipanon Federal Channel & Warrenton OR & SBIO-GC-02 & June 24, 2003 & Copper & $\mathrm{mg} / \mathrm{kg}$ & 1 & 36.4 & $=$ & 32 & 2,960 \\
\hline Skipanon Federal Channel & Warrenton OR & SBIO-GC-03 & June 24, 2003 & Copper & $\mathrm{mg} / \mathrm{kg}$ & 1 & 56.9 & $=$ & 32 & 2,960 \\
\hline
\end{tabular}


Table E-10

Summary of Surface Sediment Results From Segment 5 Exceeding Select Ecological or Human Health Benchmark Values

\begin{tabular}{|c|c|c|c|c|c|c|c|c|c|c|}
\hline Sample Area & Sample Site & SampleID & Date Collected & Constituent Name & Units & QA Category & $\begin{array}{c}\text { Analytical } \\
\text { Value }\end{array}$ & Qualifier & $\begin{array}{c}\text { Eco } \\
\text { Benchmark } \\
\text { Value }\end{array}$ & $\begin{array}{c}\text { HH } \\
\text { Benchmark } \\
\text { Value }\end{array}$ \\
\hline Skipanon Federal Channel & Warrenton OR & SBIO-GC-04 & June 24,2003 & Copper & $\mathrm{mg} / \mathrm{kg}$ & 1 & 46.2 & $=$ & 32 & 2,960 \\
\hline Skipanon Federal channel & Warrenton OR & SKIP-GC-01 & September 12, 2001 & Copper & $\mathrm{mg} / \mathrm{kg}$ & 1 & 60 & $=$ & 32 & 2,960 \\
\hline Skipanon Federal channel & Warrenton OR & SKIP-GC-02 & September 12, 2001 & Copper & $\mathrm{mg} / \mathrm{kg}$ & 1 & 59 & $=$ & 32 & 2,960 \\
\hline Skipanon Federal channel & Warrenton OR & SKIP-GC-03 & September 12, 2001 & Copper & $\mathrm{mg} / \mathrm{kg}$ & 1 & 76 & $=$ & 32 & 2,960 \\
\hline Skipanon Federal channel & Warrenton OR & SKIP-GC-04 & September 12, 2001 & Copper & $\mathrm{mg} / \mathrm{kg}$ & 1 & 58 & $=$ & 32 & 2,960 \\
\hline Skipanon Federal channel & Warrenton OR & SKIP-GC-05 & September 12, 2001 & Copper & $\mathrm{mg} / \mathrm{kg}$ & 1 & 55 & $=$ & 32 & 2,960 \\
\hline Skipanon Federal channel & Warrenton OR & SKIP-GC-06 & September 12, 2001 & Copper & $\mathrm{mg} / \mathrm{kg}$ & 1 & 41 & $=$ & 32 & 2,960 \\
\hline Columbia River & Longview WA & $88-398-309$ & July 23, 1987 & Dibenz[a,h]anthracene & $\mathrm{mg} / \mathrm{kg}$ dry & 2 & 0.4 & cj & 0.033 & 0.062 \\
\hline Skipanon Federal channel & Warrenton OR- & SKIP-GC-04 & September 12, 2001 & Dichlorodiphenyldichloroethane & $\mathrm{mg} / \mathrm{kg}$ & 1 & 0.0048 & $=$ & 0.004 & 2.4 \\
\hline Skipanon Federal channel & Warrenton OR & SKIP-GC-05 & September 12, 2001 & Dichlorodiphenyldichloroettane & $\mathrm{mg} / \mathrm{kg}$ & 1 & 0.0043 & $=$ & 0.004 & 2.4 \\
\hline Columbia River & Oregon Slough & $0205-$ OS-VC-06 & February 17,2005 & Dichlorodiphenyldichloroethylene & $\mathrm{mg} / \mathrm{kg}$ & 1 & 0.00151 & cJ & 0.0015 & 1.7 \\
\hline Columbia River & RM 100 & CR-BC-76 & June 3,1997 & Dichlorodiphenyldichloroethylene & $\mathrm{mg} / \mathrm{kg}$ & 1 & 0.002 & $=$ & 0.0015 & 1.7 \\
\hline Columbia River & RM 37-47 & CRJSD120 & August 1, 1991 & Dichlorodiphenyldichloroethylene & $\mathrm{mg} / \mathrm{kg}$ dry & 1 & 30 & $\mathrm{~J}$ & 0.0015 & 1.7 \\
\hline Skipanon Federal channel & Warrenton OR & SKIP-GC-01 & September 12, 2001 & Dichlorodiphenyldichloroethylene & $\mathrm{mg} / \mathrm{kg}$ & 1 & 0.0034 & $\mathrm{j}$ & 0.0015 & 1.7 \\
\hline Skipanon Federal channel & Warrenton OR & SKIP-GC-02 & September 12, 2001 & Dichlorodiphenyldichloroethylene & $\mathrm{mg} / \mathrm{kg}$ & 1 & 0.0039 & $\mathrm{~J}$ & 0.0015 & 1.7 \\
\hline Skipanon Federal channel & Warrenton OR & SKIP-GC-04 & September 12, 2001 & Dichlorodiphenyldichloroethylene & $\mathrm{mg} / \mathrm{kg}$ & 1 & 0.0023 & $\mathrm{j}$ & 0.0015 & 1.7 \\
\hline Skipanon Federal channel & Warrenton OR & SKIP-GC-05 & September 12, 2001 & Dichlorodiphenyldichloroethylene & $\mathrm{mg} / \mathrm{kg}$ & 1 & 0.0023 & $\mathrm{j}$ & $\begin{array}{l}0.0015 \\
0.0015\end{array}$ & $\begin{array}{l}1.1 \\
1.7\end{array}$ \\
\hline Skipanon Federal channel & Warrenton OR & SKIP-GC-06 & September 12, 2001 & Dichlorodiphenyldichloroethylene & $\mathrm{mg} / \mathrm{kg}$ & 1 & 0.0019 & $\mathrm{j}$ & 0.0015 & 1.7 \\
\hline Columbia River & RM 82 & Will-1 & September 10, 1997 & Dichlorodiphenyldichloroethylene & $\mathrm{mg} / \mathrm{kg}$ dry & 2 & 0.0018 & $=$ & 0.0015 & 1.7 \\
\hline Columbia River & Oregon Slough & $0205-0 \mathrm{~S}-\mathrm{vC}-01$ & February 17,2005 & Di-n-butylyhthalate & $\mathrm{mg} / \mathrm{kg}$ & 1 & 0.318 & $\mathrm{BE}$ & 0.043 & 6,110 \\
\hline Columbia River & Oregon Slough & 0205-OS-VC-02 & February 17, 2005 & Di-n-butylphthalate & $\mathrm{mg} / \mathrm{kg}$ & 1 & 0.249 & $\mathrm{BE}$ & 0.043 & 6,110 \\
\hline Columbia River & Oregon Slough & 0205-OS-VC-03 & February 17, 2005 & Di-n-butylyhthalate & $\mathrm{mg} / \mathrm{kg}$ & 1 & 0.252 & $\mathrm{BE}$ & 0.043 & 6,110 \\
\hline Columbia River & Oregon Slough & 0205-OS-VC-03A & February 17, 2005 & Di-n-butylyphthalate & $\mathrm{mg} / \mathrm{kg}$ & 1 & 0.217 & $\mathrm{BE}$ & 0.043 & 6,110 \\
\hline Columbia River & Oregon Slough & 0205-OS-VC-04 & February 17, 2005 & Di-n-butylyphthalate & $\mathrm{mg} / \mathrm{kg}$ & 1 & 0.208 & $\mathrm{BE}$ & 0.043 & 6,110 \\
\hline Columbia River & Oregon Slough & 0205-OS-VC-05 & February 17, 2005 & Di-n-butylynthalate & $\mathrm{mg} / \mathrm{kg}$ & 1 & 0.147 & B & 0.043 & 6,110 \\
\hline Columbia River & Oregon Slough & 0205-OS-VC-06 & February 17, 2005 & Di-n-butylyphthalate & $\mathrm{mg} / \mathrm{kg}$ & 1 & 0.215 & $\mathrm{BE}$ & 0.043 & 6,110 \\
\hline Columbia River & Oregon Slough & 0205-OS-VC-06Z & February 17, 2005 & Di-n-butylyphthalate & $\mathrm{mg} / \mathrm{kg}$ & 1 & 0.207 & $\mathrm{BE}$ & 0.043 & 6,110 \\
\hline Columbia River & Camas Slough & 88-398-302 & July 22, 1987 & Di-n-butylphthalate & $\mathrm{mg} / \mathrm{kg}$ dry & 2 & 0.84 & $\mathrm{~J}$ & 0.043 & 6,110 \\
\hline Columbia River & Bachelor Slough & BACH-BC-02 & June 3,2003 & Di-n-butylphthalate & $\mathrm{mg} / \mathrm{kg}$ & 1 & 0.178 & c & 0.043 & 6,110 \\
\hline Columbia River & Crims Island & CRIM-SG-02 & July 15,2003 & Di-n-butylyphthalate & $\mathrm{mg} / \mathrm{kg}$ & 1 & 0.0575 & B & 0.043 & 6,110 \\
\hline Columbia River & Vancouver WA & $88-398-303$ & July 22, 1987 & Fluoranthene- & $\mathrm{mg} / \mathrm{kg}$ dry & 2 & 0.16 & J & 0.11 & 2,294 \\
\hline Columbia River & Longview WA & 88-398-309 & July 23,1987 & Fluoranthene & $\mathrm{mg} / \mathrm{kg}$ dry & 2 & 2.1 & $=$ & 0.11 & $\begin{array}{l}2,294 \\
2,294\end{array}$ \\
\hline Columbia River & RM 86.5 & SH-VC-4 & May 4, 1989 & Fluorene & $\mathrm{mg} / \mathrm{kg}$ & 3 & 0.084 & $=$ & 0.077 & 2,747 \\
\hline Columbia River & RM 86.5 & SH-VC-5 & May 4, 1989 & Fluorene & $\mathrm{mg} / \mathrm{kg}$ & 3 & 0.102 & $=$ & 0.077 & 2,747 \\
\hline Columbia River & Oregon Slough & $0205-O S-V C-06 Z$ & February 17,2005 & Indeno(1,2,3-cd)pyrene & $\mathrm{mg} / \mathrm{kg}$ & 1 & 0.0225 & $=$ & 0.017 & 0.62 \\
\hline Columbia River & RM 88 & $10-\mathrm{S}$ & June 28,1993 & Indeno(1,2,3-cd)pyrene & $\mathrm{mg} / \mathrm{kg}$ dry & 1 & 0.041 & $=$ & 0.017 & 0.62 \\
\hline Columbia River & RM 95 & $12-\mathrm{S}$ & June 30, 1993 & Indeno(1,2,3-cd)pyrene & $\mathrm{mg} / \mathrm{kg}$ dry & 1 & 0.02 & $=$ & 0.017 & 0.62 \\
\hline Columbia River & RM 124 & 14-s & July 1, 1993 & Indeno(1,2,3-cd)pyrene & $\mathrm{mg} / \mathrm{kg}$ dry & 1 & 0.024 & $=$ & 0.017 & 0.62 \\
\hline Columbia River & RM 14 & $1-\mathrm{s}$ & June 28,1993 & Indeno(1,2,3-cd)pyrene & $\mathrm{mg} / \mathrm{kg}$ dry & 1 & 0.033 & $=$ & 0.017 & 0.62 \\
\hline Columbia River & RM 26 & 4-S & June 26, 1993 & Indeno(1,2,3-cd)pyrene & $\mathrm{mg} / \mathrm{kg}$ dry & 1 & 0.022 & $=$ & 0.017 & 0.62 \\
\hline Columbia River & RM 29 & $5-s$ & June 26,1993 & Indeno(1,2,3-cd)pyrene & $\mathrm{mg} / \mathrm{kg}$ dry & 1 & 0.027 & $=$ & 0.017 & 0.62 \\
\hline Columbia River & Vancouver WA & 88-398-304 & July 22, 1987 & Indeno( $(1,2,3$-cd)pyrene & $\mathrm{mg} / \mathrm{kg}$ dry & 2 & 0.033 & $\mathrm{~J}$ & 0.017 & 0.62 \\
\hline Columbia River & Longview WA & 88-398-307 & July 23, 1987 & Indeno(1,2,3-cd)pyrene & $\mathrm{mg} / \mathrm{kg}$ dry & 2 & 0.042 & $\mathrm{~J}$ & 0.017 & 0.62 \\
\hline Columbia River & Longview WA & 88-398-309 & July 23,1987 & Indeno(1,2,3-cd)pyrene & $\mathrm{mg} / \mathrm{kg}$ dry & 2 & 0.92 & $=$ & 0.017 & 0.62 \\
\hline Columbia River & Ilwaco Boat Bsn & 88-398-311 & July 24,1987 & Indeno(1,2,3-cd)pyrene & $\mathrm{mg} / \mathrm{kg}$ dry & 2 & 0.076 & $\mathrm{~J}$ & 0.017 & 0.62 \\
\hline Columbia River & RM 83 & CR-BC-57 & June 3,1997 & Indeno(1,2,3-cd)pyrene & $\mathrm{mg} / \mathrm{kg}$ & 1 & 0.056 & $=$ & 0.017 & 0.62 \\
\hline Columbia River & RM 100 & CR-BC-76 & June 3,1997 & Indeno(1,2,3-cd)pyrene & $\mathrm{mg} / \mathrm{kg}$ & 1 & 0.02 & $=$ & 0.017 & 0.62 \\
\hline Columbia River & Crims Island & CRIM-SG-02 & July 15,2003 & Indeno(1,2,3-cd)pyrene & $\mathrm{mg} / \mathrm{kg}$ & 1 & 0.0217 & $=$ & 0.017 & 0.62 \\
\hline
\end{tabular}


Table E-10

Summary of Surface Sediment Results From Segment 5 Exceeding Select Ecological or Human Health Benchmark Values

\begin{tabular}{|c|c|c|c|c|c|c|c|c|c|c|}
\hline Sample Area & Sample Site & SamplelD & Date Collected & Constituent Name & Units & QA Category & $\begin{array}{c}\text { Analytical } \\
\text { Value }\end{array}$ & Qualifier & $\begin{array}{c}\text { Eco } \\
\text { Benchmark } \\
\text { Value }\end{array}$ & $\begin{array}{c}\text { HH } \\
\text { Benchmark } \\
\text { Value }\end{array}$ \\
\hline Columbia River & Crims Island & CRIM-SG-03 & July 15,2003 & Indeno(1,2,3-cd)pyrene & $\mathrm{mg} / \mathrm{kg}$ & 1 & 0.0475 & $=$ & 0.017 & 0.62 \\
\hline Skipanon Federal Channel & Warrenton OR & SBIO-GC-03 & June 24,2003 & Indeno(1,2,3-cd)pyrene & $\mathrm{mg} / \mathrm{kg}$ & 1 & 0.0177 & $=$ & 0.017 & 0.62 \\
\hline Skipanon Federal Channel & Warrenton OR & SBIO-GC-04 & June 24, 2003 & Indeno(1,2,3-cd)pyrene & $\mathrm{mg} / \mathrm{kg}$ & 1 & 0.0283 & $=$ & 0.017 & 0.62 \\
\hline Columbia River & RM 82 & Will-1 & September 10, 1997 & Indeno(1,2,3-cd)pyrene & $\mathrm{mg} / \mathrm{kg}$ dry & 2 & 0.07 & $=$ & 0.017 & 0.62 \\
\hline Willamette River & RM 0.1 - mouth & WR-GC-2A & July 24,1997 & Indeno(1,2,3-cd)pyrene & $\mathrm{mg} / \mathrm{kg}$ & 1 & 0.076 & $=$ & 0.017 & 0.62 \\
\hline Columbia River & RM 88 & $10-\mathrm{s}$ & June 28,1993 & Iron & $\mathrm{mg} / \mathrm{kg}$ dry & 1 & 27700 & $=$ & 20,000 & 23,463 \\
\hline Columbia River & RM 95 & $12-\mathrm{S}$ & June 30, 1993 & Iron & $\mathrm{mg} / \mathrm{kg}$ dry & 1 & 26900 & $=$ & 20,000 & 23,463 \\
\hline Columbia River & RM 120 & $13-\mathrm{S}$ & July 1, 1993 & Iron & $\mathrm{mg} / \mathrm{kg}$ dry & 1 & 21100 & $=$ & 20,000 & 23,463 \\
\hline Columbia River & RM 14 & 1-S & June 28, 1993 & Iron & $\mathrm{mg} / \mathrm{kg}$ dry & 1 & 21500 & $=$ & 20,000 & 23,463 \\
\hline Columbia River & RM 21 & $2-s$ & June 27,1993 & Iron & $\mathrm{mg} / \mathrm{kg}$ dry & 1 & 21800 & $=$ & 20,000 & 23,463 \\
\hline Columbia River & RM 29 & 5-s & June 26,1993 & Iron & $\mathrm{mg} / \mathrm{kg}$ dry & 1 & 21100 & $=$ & 20,000 & 23,463 \\
\hline Columbia River & RM 36 & $6-5$ & June 25, 1993 & Iron & $\mathrm{mg} / \mathrm{kg}$ dry & 1 & 39000 & $=$ & 20,000 & 23,463 \\
\hline Columbia River & Camas Slough & 88-398-301 & July 22, 1987 & Iron & $\mathrm{mg} / \mathrm{kg}$ dry & 2 & 25000 & $=$ & 20,000 & 23,463 \\
\hline Columbia River & Camas Slough & 88-398-302 & July 22, 1987 & Iron & $\mathrm{mg} / \mathrm{kg}$ dry & 2 & 24000 & $=$ & 20,000 & 23,463 \\
\hline Columbia River & Ilwaco Boat Bsn & 88-398-311 & July 24, 1987 & Iron & $\mathrm{mg} / \mathrm{kg}$ dry & 2 & 28000 & $=$ & 20,000 & 23,463 \\
\hline Columbia River & RM 68 & 8-S & June 24, 1993 & Iron & $\mathrm{mg} / \mathrm{kg}$ dry & 1 & 20600 & $=$ & 20,000 & 23,463 \\
\hline Columbia River & RM 81 & 9-S & June 29,1993 & Iron & $\mathrm{mg} / \mathrm{kg}$ dry & 1 & 21100 & $=$ & 20,000 & 23,463 \\
\hline Columbia River & Camas Slough & $88-398-301$ & July 22, 1987 & Lead & $\mathrm{mg} / \mathrm{kg}$ dry & 2 & 38 & $=$ & 35 & 250 \\
\hline Columbia River & Vancouver WA & 88-398-303 & July 22, 1987 & Lead & $\mathrm{mg} / \mathrm{kg}$ & 2 & 140 & $\mathrm{~J}$ & 35 & 250 \\
\hline Columbia River & Bachelor Slough & BACH-GC-10 & June 3,2003 & Lead & $\mathrm{mg} / \mathrm{kg}$ & 1 & 127 & $=$ & 35 & 250 \\
\hline Columbia River & Oregon Slough & 0205-OS-VC-06 & February 17, 2005 & Mercury & $\mathrm{mg} / \mathrm{kg}$ dry & 1 & 0.513 & $=$ & 0.18 & 23 \\
\hline Columbia River & Crims Island & CRIM-SG-02 & July 15,2003 & Mercury & $\mathrm{mg} / \mathrm{kg}$ & 1 & 0.267 & $=$ & 0.18 & 23 \\
\hline Columbia River & CR-S5 & CR-S5 .08-1.0m & October 1, 1984 & Mercury & $\mathrm{mg} / \mathrm{kg}$ & 3 & 0.21 & $=$ & 0.18 & 23 \\
\hline Skipanon Federal Channel & Warrenton OR & SBIO-GC-04 & June 24,2003 & Mercury & $\mathrm{mg} / \mathrm{kg}$ & 1 & 0.564 & $=$ & 0.18 & 23 \\
\hline Columbia River & RM 88 & $10-\mathrm{s}$ & June 28,1993 & Nickel & $\mathrm{mg} / \mathrm{kg}$ dry & 1 & 18.2 & $=$ & 18 & 1,564 \\
\hline Columbia River & RM 95 & $12-\mathrm{s}$ & June 30,1993 & Nickel & $\mathrm{mg} / \mathrm{kg}$ dry & 1 & 19.4 & $=$ & 18 & 1,564 \\
\hline Columbia River & RM 120 & 13-s & July 1, 1993 & Nickel & $\mathrm{mg} / \mathrm{kg}$ dry & 1 & 18.3 & $=$ & 18 & 1,564 \\
\hline Columbia River & RM 36 & $6-5$ & June 25, 1993 & Nickel & $\mathrm{mg} / \mathrm{kg}$ dry & 1 & 24.8 & $=$ & 18 & 1,564 \\
\hline Columbia River & RM 83 & CR-BC-57 & June 3, 1997 & Nickel & $\mathrm{mg} / \mathrm{kg}$ & 1 & 21 & $=$ & 18 & 1,564 \\
\hline Columbia River & RM 100 & CR-BC-76 & June 3,1997 & Nickel & $\mathrm{mg} / \mathrm{kg}$ & 1 & 22 & $=$ & 18 & 1,564 \\
\hline Columbia River & Crims Island & CRIM-SG-02 & July 15,2003 & Nickel & $\mathrm{mg} / \mathrm{kg}$ & 1 & 22.8 & $=$ & 18 & 1,564 \\
\hline Columbia River & Crims Island & CRIM-SG-03 & July 15,2003 & Nickel & $\mathrm{mg} / \mathrm{kg}$ & 1 & 19.8 & $=$ & 18 & 1,564 \\
\hline Columbia River & Crims Island & CRIM-SG-05 & July 15,2003 & Nickel & $\mathrm{mg} / \mathrm{kg}$ & 1 & 26.7 & $=$ & 18 & 1,564 \\
\hline Columbia River & CR-S5 & CR-S5 .08-1.0m & October 1, 1984 & Nickel & $\mathrm{mg} / \mathrm{kg}$ & 3 & 22 & $=$ & 18 & 1,564 \\
\hline Columbia River & CR-S5 & CR-S5 1.8-3.8m & October 1, 1984 & Nickel & $\mathrm{mg} / \mathrm{kg}$ & 3 & 70 & $=$ & 18 & 1,564 \\
\hline Skipanon Federal Channel & Warrenton OR & SBIO-GC-01 & June 24, 2003 & Nickel & $\mathrm{mg} / \mathrm{kg}$ & 1 & 18.2 & $=$ & 18 & 1,564 \\
\hline Skipanon Federal Channel & Warrenton OR & SBIO-GC-03 & June 24, 2003 & Nickel & $\mathrm{mg} / \mathrm{kg}$ & 1 & 19.9 & $=$ & 18 & 1,564 \\
\hline Skipanon Federal Channel & Warrenton OR & SBIO-GC-04 & June 24,2003 & Nickel & $\mathrm{mg} / \mathrm{kg}$ & 1 & 20.3 & $=$ & 18 & 1,564 \\
\hline Skipanon Federal channel & Warrenton OR & SKIP-GC-01 & September 12, 2001 & Nickel & $\mathrm{mg} / \mathrm{kg}$ & 1 & 20 & $=$ & 18 & 1,564 \\
\hline Skipanon Federal channel & Warrenton OR & SKIP-GC-02 & September 12, 2001 & Nickel & $\mathrm{mg} / \mathrm{kg}$ & 1 & 22 & $=$ & 18 & 1,564 \\
\hline Skipanon Federal channel & Warrenton OR & SKIP-GC-03 & September 12, 2001 & Nickel & $\mathrm{mg} / \mathrm{kg}$ & 1 & 21 & $=$ & 18 & 1,564 \\
\hline Skipanon Federal channel & Warrenton OR & SKIP-GC-04 & September 12, 2001 & Nickel & $\mathrm{mg} / \mathrm{kg}$ & 1 & 21 & $=$ & 18 & 1,564 \\
\hline Skipanon Federal channel & Warrenton OR & SKIP-GC-05 & September 12, 2001 & Nickel & $\mathrm{mg} / \mathrm{kg}$ & 1 & 25 & $=$ & 18 & 1,564 \\
\hline Columbia River & Oregon Slough & 0205-OS-vC-01 & February 17, 2005 & Phenanthrene & $\mathrm{mg} / \mathrm{kg}$ & 1 & 0.23 & $\bar{E}$ & 0.042 & -- \\
\hline Columbia River & Oregon Slough & 0205-OS-VC-06Z & February 17, 2005 & Phenanthrene & $\mathrm{mg} / \mathrm{kg}$ & 1 & 0.0458 & $=$ & 0.042 & -. \\
\hline Columbia River & Camas Slough & $88-398-302$ & July 22, 1987 & Phenanthrene & $\mathrm{mg} / \mathrm{kg}$ dry & 2 & 0.059 & $\mathrm{~J}$ & 0.042 & .- \\
\hline Columbia River & Vancouver WA & 88-398-303 & July 22, 1987 & Phenanthrene & $\mathrm{mg} / \mathrm{kg}$ dry & 2 & 0.065 & $\mathrm{~J}$ & 0.042 & -- \\
\hline Columbia River & Vancouver WA & 88-398-304 & July 22, 1987 & Phenanthrene & $\mathrm{mg} / \mathrm{kg}$ dry & 2 & 0.071 & $\mathrm{~J}$ & 0.042 & -- \\
\hline Columbia River & Longview WA & 88-398-309 & July 23,1987 & Phenanthrene & $\mathrm{mg} / \mathrm{kg}$ dry & 2 & 0.58 & $=$ & 0.042 & -- \\
\hline
\end{tabular}


Table E-10

Summary of Surface Sediment Results From Segment 5 Exceeding Select Ecological or Human Health Benchmark Values

\begin{tabular}{|c|c|c|c|c|c|c|c|c|c|c|}
\hline Sample Area & Sample Site & SampleID & Date Collected & Constituent Name & Units & QA Category & $\begin{array}{c}\text { Analytical } \\
\text { Value }\end{array}$ & Qualifier & $\begin{array}{c}\text { Eco } \\
\text { Benchmark } \\
\text { Value }\end{array}$ & $\begin{array}{c}\text { HH } \\
\text { Benchmark } \\
\text { Value }\end{array}$ \\
\hline Columbia River & Ilwaco Boat Bsn & 88-398-311 & July 24, 1987 & Phenanthrene & $\mathrm{mg} / \mathrm{kg}$ dry & 2 & 0.18 & $\overline{\mathrm{J}}$ & 0.042 & $\overline{--}$ \\
\hline Columbia River & RM 100 & CR-BC-76 & June 3,1997 & Phenanthrene & $\mathrm{mg} / \mathrm{kg}$ & 1 & 0.049 & $=$ & 0.042 & .- \\
\hline Columbia River & RM 86.5 & SH-VC-4 & May 4, 1989 & Phenanthrene & $\mathrm{mg} / \mathrm{kg}$ & 3 & 0.224 & $=$ & 0.042 & .- \\
\hline Columbia River & RM 86.5 & SH-VC-5 & May 4, 1989 & Phenanthrene & $\mathrm{mg} / \mathrm{kg}$ & 3 & 0.332 & $=$ & 0.042 & -- \\
\hline Columbia River & RM 82 & Will-1 & September 10, 1997 & Phenanthrene & $\mathrm{mg} / \mathrm{kg}$ dry & 2 & 0.079 & $=$ & 0.042 & -- \\
\hline Columbia River & Oregon Slough & $0205-\mathrm{OS}-\mathrm{VC}-06 \mathrm{Z}$ & February 17,2005 & Pyrene & $\mathrm{mg} / \mathrm{kg}$ & 1 & 0.0575 & $=$ & 0.053 & 2,316 \\
\hline Columbia River & RM 88 & $10-\mathrm{s}$ & June 28, 1993 & Pyrene & $\mathrm{mg} / \mathrm{kg}$ dry & 1 & 0.093 & $=$ & 0.053 & 2,316 \\
\hline Columbia River & RM 88 & $10-\mathrm{S}$ & June 28, 1993 & Pyrene & $\mathrm{mg} / \mathrm{kg}$ dry & 1 & 0.093 & $=$ & 0.053 & 2,316 \\
\hline Columbia River & Camas Slough & 88-398-302 & July 22, 1987 & Pyrene & $\mathrm{mg} / \mathrm{kg}$ dry & 2 & 0.08 & $\mathrm{~J}$ & 0.053 & 2,316 \\
\hline Columbia River & Camas Slough & 88-398-302 & July 22,1987 & Pyrene & $\mathrm{mg} / \mathrm{kg}$ dry & 2 & 0.08 & $\mathrm{j}$ & 0.053 & 2,316 \\
\hline Columbia River & Vancouver WA & 88-398-303 & July 22, 1987 & Pyrene & $\mathrm{mg} / \mathrm{kg}$ dry & 2 & 0.16 & $\mathrm{~J}$ & 0.053 & 2,316 \\
\hline Columbia River & Vancouver WA & 88-398-303 & July 22, 1987 & Pyrene & $\mathrm{mg} / \mathrm{kg}$ dry & 2 & 0.16 & $\mathrm{~J}$ & 0.053 & 2,316 \\
\hline Columbia River & Vancouver WA & 88-398-304 & July 22, 1987 & Pyrene & $\mathrm{mg} / \mathrm{kg}$ dry & 2 & 0.078 & $\mathrm{j}$ & 0.053 & 2,316 \\
\hline Columbia River & Vancouver WA & 88-398-304 & July 22, 1987 & Pyrene & $\mathrm{mg} / \mathrm{kg}$ dry & 2 & 0.078 & $\mathrm{j}$ & 0.053 & 2,316 \\
\hline Columbia River & Longview WA & 88-398-307 & July 23, 1987 & Pyrene & $\mathrm{mg} / \mathrm{kg}$ dry & 2 & 0.13 & $\mathrm{~J}$ & 0.053 & 2,316 \\
\hline Columbia River & Longview WA & 88-398-307 & July 23, 1987 & Pyrene & $\mathrm{mg} / \mathrm{kg}$ dry & 2 & 0.13 & $\mathrm{~J}$ & 0.053 & 2,316 \\
\hline Columbia River & Longview WA & 88-398-309 & July 23,1987 & Pyrene & $\mathrm{mg} / \mathrm{kg}$ dry & 2 & 2.5 & $=$ & $\begin{array}{l}0.053 \\
0.053\end{array}$ & $\begin{array}{l}2,310 \\
2,316\end{array}$ \\
\hline Columbia River & Longview WA & 88-398-309 & July 23,1987 & Pyrene & $\mathrm{mg} / \mathrm{kg}$ dry & 2 & 2.5 & $=$ & 0.053 & 2,316 \\
\hline Columbia River & Ilwaco Boat Bsn & 88-398-311 & July 24, 1987 & Pyrene & $\mathrm{mg} / \mathrm{kg}$ dry & 2 & 0.59 & $\mathrm{~J}$ & 0.053 & 2,316 \\
\hline Columbia River & Ilwaco Boat Bsn & 88-398-311 & July 24, 1987 & Pyrene & $\mathrm{mg} / \mathrm{kg}$ dry & 2 & 0.59 & $\mathrm{~J}$ & 0.053 & 2,316 \\
\hline Columbia River & RM 68 & 8-S & June 24, 1993 & Pyrene & $\mathrm{mg} / \mathrm{kg}$ dry & 1 & 0.067 & $=$ & 0.053 & 2,316 \\
\hline Columbia River & RM 68 & 8-S & June 24, 1993 & Pyrene & $\mathrm{mg} / \mathrm{kg}$ dry & 1 & 0.067 & $=$ & 0.053 & 2,316 \\
\hline Columbia River & RM 81 & 9-S & June 29, 1993 & Pyrene & $\mathrm{mg} / \mathrm{kg}$ dry & 1 & 0.09 & $=$ & 0.053 & 2,316 \\
\hline Columbia River & RM 81 & 9-S & June 29, 1993 & Pyrene & $\mathrm{mg} / \mathrm{kg}$ dry & 1 & 0.09 & $=$ & 0.053 & 2,316 \\
\hline Columbia River & RM 83 & CR-BC-57 & June 3, 1997 & Pyrene & $\mathrm{mg} / \mathrm{kg}$ & 1 & 0.064 & $=$ & 0.053 & 2,316 \\
\hline Columbia River & RM 100 & CR-BC-76 & June 3,1997 & Pyrene & $\mathrm{mg} / \mathrm{kg}$ & 1 & 0.077 & $=$ & 0.053 & 2,316 \\
\hline Columbia River & Crims Island & CRIM-SG-03 & July 15,2003 & Pyrene & $\mathrm{mg} / \mathrm{kg}$ & 1 & 0.108 & $=$ & 0.053 & 2,316 \\
\hline Skipanon Federal Channel & Warrenton OR & SBIO-GC-01 & June 24, 2003 & Pyrene & $\mathrm{mg} / \mathrm{kg}$ & 1 & 0.0555 & $=$ & 0.053 & 2,316 \\
\hline Columbia River & RM 86.5 & SH-VC-4 & May 4, 1989 & Pyrene & $\mathrm{mg} / \mathrm{kg}$ & 3 & 0.165 & $=$ & 0.053 & 2,316 \\
\hline Columbia River & RM 86.5 & SH-VC-5 & May 4, 1989 & Pyrene & $\mathrm{mg} / \mathrm{kg}$ & 3 & 0.115 & $=$ & 0.053 & 2,316 \\
\hline Columbia River & RM 82 & Will-1 & September 10, 1997 & Pyrene & $\mathrm{mg} / \mathrm{kg}$ dry & 2 & 0.12 & $=$ & 0.053 & 2,316 \\
\hline Columbia River & RM 82 & Will-1 & September 10, 1997 & Pyrene & $\mathrm{mg} / \mathrm{kg}$ dry & 2 & 0.12 & $=$ & 0.053 & 2,316 \\
\hline Willamette River & RM 0.1 - mouth & WR-GC-2A & July 24,1997 & Pyrene & $\mathrm{mg} / \mathrm{kg}$ & 1 & 0.096 & $=$ & 0.053 & 2,316 \\
\hline Columbia River & Oregon Slough & 0205-OS-VC-01 & February 17,2005 & Zinc & $\mathrm{mg} / \mathrm{kg}$ dry & 1 & 121 & $=$ & 120 & 23,463 \\
\hline Columbia River & RM 95 & $12-\mathrm{S}$ & June 30,1993 & Zinc & $\mathrm{mg} / \mathrm{kg}$ dry & 1 & 148 & $=$ & 120 & 23,463 \\
\hline Columbia River & RM 120 & $13-\mathrm{s}$ & July 1, 1993 & Zinc & $\mathrm{mg} / \mathrm{kg}$ dry & 1 & 128 & $=$ & 120 & 23,463 \\
\hline Columbia River & RM 124 & $14-\mathrm{S}$ & July 1,1993 & Zinc & $\mathrm{mg} / \mathrm{kg}$ dry & 1 & 155 & $=$ & 120 & 23,463 \\
\hline Columbia River & Camas Slough & 88-398-301 & July 22, 1987 & Zinc & $\mathrm{mg} / \mathrm{kg}$ dry & 2 & 130 & $=$ & 120 & 23,463 \\
\hline Columbia River & Camas Slough & 88-398-302 & July 22, 1987 & Zinc & $\mathrm{mg} / \mathrm{kg}$ dry & 2 & 130 & $=$ & 120 & 23,463 \\
\hline Columbia River & Ilwaco Boat Bsn & 88-398-311 & July 24,1987 & Zinc & $\mathrm{mg} / \mathrm{kg}$ dry & 2 & 140 & $=$ & 120 & 23,463 \\
\hline Columbia River & RM 68 & $8-\mathrm{s}$ & June 24, 1993 & Zinc & $\mathrm{mg} / \mathrm{kg}$ dry & 1 & 136 & $=$ & 120 & 23,463 \\
\hline Columbia River & Crims Island & CRIM-SG-02 & July 15,2003 & Zinc & $\mathrm{mg} / \mathrm{kg}$ & 1 & 161 & $=$ & 120 & 23,463 \\
\hline Columbia River & Crims Island & CRIM-SG-03 & July 15,2003 & Zinc & $\mathrm{mg} / \mathrm{kg}$ & 1 & 172 & $=$ & 120 & 23,463 \\
\hline Columbia River & CR-S5 & CR-S5 .08-1.0m & October 1, 1984 & Zinc & $\mathrm{mg} / \mathrm{kg}$ & 3 & 170 & $=$ & 120 & 23,463 \\
\hline Columbia River & Oregon Slough & OR-VC-04 & June 19, 2001 & Zinc & $\mathrm{mg} / \mathrm{kg}$ & 1 & 150 & $=$ & 120 & 23,463 \\
\hline Skipanon Federal Channel & Warrenton OR & SBIO-GC-01 & June 24,2003 & Zinc & $\mathrm{mg} / \mathrm{kg}$ & 1 & 129 & $=$ & 120 & 23,463 \\
\hline Skipanon Federal Channel & Warrenton OR & SBIO-GC-03 & June 24, 2003 & Zinc & $\mathrm{mg} / \mathrm{kg}$ & 1 & 149 & $=$ & 120 & 23,463 \\
\hline Skipanon Federal Channel & Warrenton OR & SBIO-GC-04 & June 24,2003 & Zinc & $\mathrm{mg} / \mathrm{kg}$ & 1 & 143 & $=$ & 120 & 23,463 \\
\hline Skipanon Federal channel & Warrenton OR & SKIP-GC-01 & September 12, 2001 & Zinc & $\mathrm{mg} / \mathrm{kg}$ & 1 & 160 & $=$ & 120 & 23,463 \\
\hline
\end{tabular}


Table E-10

Summary of Surface Sediment Results From Segment 5 Exceeding Select Ecological or Human Health Benchmark Values

\begin{tabular}{|c|c|c|c|c|c|c|c|c|c|c|}
\hline Sample Area & Sample Site & SampleID & Date Collected & Constituent Name & Units & QA Category & $\begin{array}{c}\text { Analytical } \\
\text { Value }\end{array}$ & Qualifier & $\begin{array}{c}\text { Eco } \\
\text { Benchmark } \\
\text { Value }\end{array}$ & $\begin{array}{c}\text { HH } \\
\text { Benchmark } \\
\text { Value }\end{array}$ \\
\hline Skipanon Federal channel & Warrenton OR & SKIP-GC-02 & September 12, 2001 & Zinc & $\mathrm{mg} / \mathrm{kg}$ & 1 & 170 & $=$ & 120 & 23,463 \\
\hline Skipanon Federal channel & Warrenton OR & SKIP-GC-03 & September 12, 2001 & Zinc & $\mathrm{mg} / \mathrm{kg}$ & 1 & 180 & $=$ & 120 & 23,463 \\
\hline Skipanon Federal channel & Warrenton OR & SKIP-GC-04 & September 12, 2001 & Zinc & $\mathrm{mg} / \mathrm{kg}$ & 1 & 200 & $=$ & 120 & 23,463 \\
\hline Skipanon Federal channel & Warrenton OR & SKIP-GC-05 & September 12, 2001 & Zinc & $\mathrm{mg} / \mathrm{kg}$ & 1 & 190 & $=$ & 120 & 23,463 \\
\hline Skipanon Federal channel & Warrenton OR & SKIP-GC-06 & September 12, 2001 & Zinc & $\mathrm{mg} / \mathrm{kg}$ & 1 & 130 & $=$ & 120 & 23,463 \\
\hline
\end{tabular}




\section{Table E-11}

Summary Statistics for All Categories of Surface Water Samples Collected from Segment $\mathbf{5}$ of the Columbia River

\begin{tabular}{|c|c|c|c|c|c|c|c|c|c|c|}
\hline $\begin{array}{l}\text { Constituent } \\
\text { Class }\end{array}$ & Constituent Name & Units & $\begin{array}{l}\text { Number } \\
\text { Analyzed }\end{array}$ & $\begin{array}{l}\text { Number } \\
\text { Detected }\end{array}$ & $\begin{array}{c}\text { Frequency of } \\
\text { Detection }\end{array}$ & $\begin{array}{l}\text { Minimum } \\
\text { Nondetect }\end{array}$ & $\begin{array}{l}\text { Maximum } \\
\text { Nondetect }\end{array}$ & $\begin{array}{l}\text { Minimum } \\
\text { Detect }\end{array}$ & $\begin{array}{l}\text { Maximum } \\
\text { Detect }\end{array}$ & $\begin{array}{c}\text { Average } \\
\text { Concentration }\end{array}$ \\
\hline$\overline{\text { CONV }}$ & Alkalinity & $\mathrm{mg} / \mathrm{L}$ & 42 & 42 & $100 \%$ & -- & -- & 22 & 66 & 49 \\
\hline CONV & Ammonia & $\mathrm{mg} / \mathrm{L}$ & 12 & 4 & $33 \%$ & 0.010 & 0.010 & 0.015 & 0.019 & 0.0092 \\
\hline CONV & Chloride & $\mathrm{mg} / \mathrm{L}$ & 50 & 50 & $100 \%$ & -- & -- & 5.6 & 14 & 8.7 \\
\hline CONV & Dissolved organic carbon & $\mathrm{mg} / \mathrm{L}$ & 43 & 43 & $100 \%$ & -- & -- & 1.0 & 3.3 & 1.7 \\
\hline CONV & Dissolved oxygen & $\mathrm{mg} / \mathrm{L}$ & 12 & 12 & $100 \%$ & -- & -- & 8.9 & 12 & 11 \\
\hline CONV & Fluoride & $\mathrm{mg} / \mathrm{L}$ & 50 & 50 & $100 \%$ & -- & -- & 7.8 & 14 & 11 \\
\hline CONV & Hardness & $\mathrm{mg} / \mathrm{L}$ & 6 & 6 & $100 \%$ & -- & -- & 41 & 67 & 59 \\
\hline CONV & Nitrogen in ammonia & $\mathrm{mg} / \mathrm{L}$ & 50 & 38 & $76 \%$ & 0.0020 & 0.015 & 0.0020 & 0.039 & 0.011 \\
\hline CONV & Nitrogen, Kjeldahl total & $\mathrm{mg} / \mathrm{L}$ & 100 & 96 & $96 \%$ & 0.10 & 0.10 & 0.10 & 0.50 & 0.16 \\
\hline CONV & Nitrogen, Nitrate-Nitrite & $\mathrm{mg} / \mathrm{L}$ & 12 & 12 & $100 \%$ & -- & -- & 0.073 & 0.46 & 0.22 \\
\hline CONV & Nitrogen, Nitrite & $\mathrm{mg} / \mathrm{L}$ & 50 & 47 & $94 \%$ & 0.0010 & 0.0010 & 0.0010 & 0.0060 & 0.0031 \\
\hline CONV & Orthophosphate & $\mathrm{mg} / \mathrm{L}$ & 61 & 59 & $97 \%$ & 0.0070 & 0.0070 & 0.0010 & 0.025 & 0.012 \\
\hline CONV & $\mathrm{pH}$ & $\mathrm{pH}$ Units & 53 & 53 & $100 \%$ & -- & -- & 6.6 & 8.5 & 7.8 \\
\hline CONV & Phosphate & $\mathrm{mg} / \mathrm{L}$ & 12 & 12 & $100 \%$ & -- & -- & 0.024 & 0.043 & 0.030 \\
\hline CONV & Specific Conductance & umhos/cm & 100 & 100 & $100 \%$ & -- & -- & 75 & 225 & 132 \\
\hline CONV & Sulfate & $\mathrm{mg} / \mathrm{L}$ & 50 & 50 & $100 \%$ & -- & -- & 0.10 & 0.10 & 0.10 \\
\hline CONV & Total Organic Carbon & $\mathrm{mg} / \mathrm{L}$ & 43 & 43 & $100 \%$ & -- & -- & 1.0 & 3.8 & 1.8 \\
\hline CONV & Total Persulfate Nitrogen & $\mathrm{mg} / \mathrm{L}$ & 12 & 12 & $100 \%$ & -- & -- & 0.16 & 0.51 & 0.30 \\
\hline CONV & Total suspended solids & $\mathrm{mg} / \mathrm{L}$ & 58 & 58 & $100 \%$ & -- & -- & 1.0 & 38 & 9.1 \\
\hline CONV & Turbidity & NTU & 65 & 65 & $100 \%$ & -- & -- & 0.50 & 68 & 10 \\
\hline METAL & Antimony & $\mathrm{mg} / \mathrm{L}$ & 18 & 9 & $50 \%$ & 0.0010 & 0.0010 & 1.00E-04 & 2.00E-04 & 3.06E-04 \\
\hline METAL & Arsenic & $\mathrm{mg} / \mathrm{L}$ & 35 & 27 & $77 \%$ & 0.0010 & 0.0020 & 7.00E-04 & 0.0015 & 9.33E-04 \\
\hline METAL & Barium & $\mathrm{mg} / \mathrm{L}$ & 18 & 18 & $100 \%$ & -- & -- & 0.011 & 0.021 & 0.017 \\
\hline METAL & Beryllium & $\mathrm{mg} / \mathrm{L}$ & 18 & 0 & $0 \%$ & 1.00E-04 & 0.0010 & -- & -- & 2.75E-04 \\
\hline METAL & Boron & $\mathrm{mg} / \mathrm{L}$ & 18 & 16 & $89 \%$ & 0.016 & 0.016 & 0.0081 & 0.020 & 0.012 \\
\hline METAL & Cadmium & $\mathrm{mg} / \mathrm{L}$ & 22 & 1 & $5 \%$ & $2.00 E-05$ & 0.0010 & $1.00 \mathrm{E}-04$ & 1.00E-04 & 2.02E-04 \\
\hline METAL & Calcium & $\mathrm{mg} / \mathrm{L}$ & 50 & 50 & $100 \%$ & -- & -- & 8.3 & 19 & 14 \\
\hline METAL & Chromium & $\mathrm{mg} / \mathrm{L}$ & 12 & 3 & $25 \%$ & $2.50 \mathrm{E}-04$ & $5.00 \mathrm{E}-04$ & 3.60E-04 & 5.50E-04 & 2.61E-04 \\
\hline METAL & Cobalt & $\mathrm{mg} / \mathrm{L}$ & 13 & 4 & $31 \%$ & 0.0010 & 0.0010 & 1.00E-04 & 1.00E-04 & 3.77E-04 \\
\hline METAL & Copper & $\mathrm{mg} / \mathrm{L}$ & 29 & 29 & $100 \%$ & -- & -- & 6.30E-04 & 0.0053 & 0.0013 \\
\hline METAL & Lead & $\mathrm{mg} / \mathrm{L}$ & 12 & 10 & $83 \%$ & 2.00E-05 & 1.00E-04 & 2.80E-05 & 3.80E-04 & 1.31E-04 \\
\hline METAL & Lithium & $\mathrm{mg} / \mathrm{L}$ & 18 & 15 & $83 \%$ & 0.0039 & 0.0039 & 0.0019 & 0.0038 & 0.0027 \\
\hline METAL & Magnesium & $\mathrm{mg} / \mathrm{L}$ & 50 & 50 & $100 \%$ & -- & -- & 2.6 & 5.7 & 4.1 \\
\hline METAL & Mercury & $\mathrm{mg} / \mathrm{L}$ & 6 & 0 & $0 \%$ & 2.00E-06 & 4.00E-06 & -- & -- & 1.33E-06 \\
\hline METAL & Nickel & $\mathrm{mg} / \mathrm{L}$ & 12 & 12 & $100 \%$ & -- & -- & 4.40E-04 & $9.20 \mathrm{E}-04$ & 5.84E-04 \\
\hline METAL & Potassium & $\mathrm{mg} / \mathrm{L}$ & 50 & 50 & $100 \%$ & -- & -- & 2.3 & 7.4 & 3.9 \\
\hline METAL & Selenium & $\mathrm{mg} / \mathrm{L}$ & 18 & 0 & $0 \%$ & 3.00E-04 & 0.0024 & -- & -- & 5.58E-04 \\
\hline METAL & Silica & $\mathrm{mg} / \mathrm{L}$ & 50 & 50 & $100 \%$ & -- & -- & 50 & 112 & 83 \\
\hline METAL & Silver & $\mathrm{mg} / \mathrm{L}$ & 12 & 0 & $0 \%$ & 2.00E-05 & $1.00 \mathrm{E}-04$ & -- & -- & 4.00E-05 \\
\hline METAL & Sodium & $\mathrm{mg} / \mathrm{L}$ & 50 & 50 & $100 \%$ & -- & -- & 3.3 & 9.1 & 5.9 \\
\hline METAL & Uranium & $\mathrm{mg} / \mathrm{L}$ & 18 & 9 & $50 \%$ & 0.0010 & 0.0010 & 4.00E-04 & 8.00E-04 & 5.39E-04 \\
\hline METAL & $\begin{array}{l}\text { Zinc } \\
\text { Total polychlorinated }\end{array}$ & $\mathrm{mg} / \mathrm{L}$ & 29 & 22 & $76 \%$ & 0.0010 & 0.0050 & 0.0010 & 0.0056 & 0.0020 \\
\hline PCB & biphenyl & $\mathrm{mg} / \mathrm{L}$ & 13 & 8 & $62 \%$ & 4.10E-07 & 2.90E-06 & 3.30E-07 & $2.10 \mathrm{E}-05$ & 3.17E-06 \\
\hline PEST & Acetochlor & $\mathrm{mg} / \mathrm{L}$ & 49 & 0 & $0 \%$ & 2.00E-06 & 6.00E-06 & -- & -- & $1.55 \mathrm{E}-06$ \\
\hline
\end{tabular}




\section{Table E-11}

Summary Statistics for All Categories of Surface Water Samples Collected from Segment $\mathbf{5}$ of the Columbia River

\begin{tabular}{|c|c|c|c|c|c|c|c|c|c|c|}
\hline $\begin{array}{l}\text { Constituent } \\
\text { Class }\end{array}$ & Constituent Name & Units & $\begin{array}{l}\text { Number } \\
\text { Analyzed }\end{array}$ & $\begin{array}{l}\text { Number } \\
\text { Detected }\end{array}$ & $\begin{array}{l}\text { Frequency of } \\
\text { Detection }\end{array}$ & $\begin{array}{l}\text { Minimum } \\
\text { Nondetect }\end{array}$ & $\begin{array}{l}\text { Maximum } \\
\text { Nondetect }\end{array}$ & $\begin{array}{l}\text { Minimum } \\
\text { Detect }\end{array}$ & $\begin{array}{l}\text { Maximum } \\
\text { Detect }\end{array}$ & $\begin{array}{c}\text { Average } \\
\text { Concentration }\end{array}$ \\
\hline$\overline{\text { PEST }}$ & Alachlor & $\mathrm{mg} / \mathrm{L}$ & 48 & 31 & $65 \%$ & $2.00 \mathrm{E}-06$ & $1.30 \mathrm{E}-05$ & $1.00 \mathrm{E}-06$ & $1.80 \mathrm{E}-05$ & $5.14 \mathrm{E}-06$ \\
\hline PEST & Alpha-BHC & $\mathrm{mg} / \mathrm{L}$ & 94 & 0 & $0 \%$ & 4.60E-08 & 5.00E-06 & -- & -- & 1.00E-06 \\
\hline PEST & alpha-Chlordane & $\mathrm{mg} / \mathrm{L}$ & 12 & 12 & $100 \%$ & -- & -- & 1.90E-08 & $7.20 \mathrm{E}-07$ & 1.64E-07 \\
\hline PEST & Atrazine & $\mathrm{mg} / \mathrm{L}$ & 43 & 32 & $74 \%$ & 1.00E-06 & 7.00E-06 & 1.00E-06 & $6.50 \mathrm{E}-05$ & 1.17E-05 \\
\hline PEST & Azinphos Methyl & $\mathrm{mg} / \mathrm{L}$ & 49 & 0 & $0 \%$ & $1.00 \mathrm{E}-06$ & $5.00 \mathrm{E}-05$ & -- & -- & $1.08 \mathrm{E}-05$ \\
\hline PEST & Benefin (Benfluralin) & $\mathrm{mg} / \mathrm{L}$ & 49 & 0 & $0 \%$ & 2.00E-06 & $1.00 \mathrm{E}-05$ & -- & -- & 2.63E-06 \\
\hline PEST & Butylate & $\mathrm{mg} / \mathrm{L}$ & 39 & 1 & $3 \%$ & 2.00E-06 & 2.00E-06 & 2.00E-06 & 2.00E-06 & 1.03E-06 \\
\hline PEST & Carbaryl (Sevin) & $\mathrm{mg} / \mathrm{L}$ & 49 & 1 & $2 \%$ & 3.00E-06 & 4.10E-05 & 4.00E-06 & 4.00E-06 & 8.92E-06 \\
\hline PEST & Carbethoxy malathion & $\mathrm{mg} / \mathrm{L}$ & 43 & 0 & $0 \%$ & 5.00E-06 & 2.70E-05 & -- & -- & $6.62 E-06$ \\
\hline PEST & Carbofuran & $\mathrm{mg} / \mathrm{L}$ & 49 & 1 & $2 \%$ & 3.00E-06 & 2.00E-05 & 1.00E-05 & 1.00E-05 & 4.97E-06 \\
\hline PEST & Chlorpyrifos & $\mathrm{mg} / \mathrm{L}$ & 39 & 2 & $5 \%$ & 4.00E-06 & 5.00E-06 & 3.00E-06 & 4.00E-06 & 2.28E-06 \\
\hline PEST & Cyanazine & $\mathrm{mg} / \mathrm{L}$ & 43 & 0 & $0 \%$ & 4.00E-06 & 1.80E-05 & -- & -- & 5.26E-06 \\
\hline PEST & Dachthal (chlorthal) & $\mathrm{mg} / \mathrm{L}$ & 49 & 2 & $4 \%$ & 2.00E-06 & 3.00E-06 & 1.00E-06 & $2.00 \mathrm{E}-06$ & $1.22 \mathrm{E}-06$ \\
\hline PEST & Desethyl atrazine & $\mathrm{mg} / \mathrm{L}$ & 39 & 20 & $51 \%$ & 2.00E-06 & 6.00E-06 & 2.00E-06 & 7.00E-06 & 2.79E-06 \\
\hline PEST & $\begin{array}{l}\text { Diazinon } \\
\text { Dichlorodiphenyldichloroeth }\end{array}$ & $\mathrm{mg} / \mathrm{L}$ & 43 & 0 & $0 \%$ & 2.00E-06 & 5.00E-06 & -- & -- & 1.56E-06 \\
\hline PEST & $\begin{array}{l}\text { ane } \\
\text { Dichlorodiphenyldichloroeth }\end{array}$ & $\mathrm{mg} / \mathrm{L}$ & 45 & 34 & $76 \%$ & $1.20 \mathrm{E}-07$ & 4.40E-07 & 1.90E-07 & 5.60E-06 & 7.48E-07 \\
\hline PEST & $\begin{array}{l}\text { ylene } \\
\text { Dichlorodiphenyltrichloroeth }\end{array}$ & $\mathrm{mg} / \mathrm{L}$ & 93 & 42 & $45 \%$ & 1.40E-07 & $6.00 \mathrm{E}-06$ & 1.50E-08 & $2.10 \mathrm{E}-06$ & 1.46E-06 \\
\hline PEST & ane & $\mathrm{mg} / \mathrm{L}$ & 45 & 39 & $87 \%$ & $3.40 \mathrm{E}-08$ & 3.00E-07 & 1.30E-08 & $1.50 \mathrm{E}-06$ & $1.85 \mathrm{E}-07$ \\
\hline PEST & Dieldrin & $\mathrm{mg} / \mathrm{L}$ & 88 & 39 & $44 \%$ & $1.10 \mathrm{E}-07$ & 5.00E-06 & $1.40 \mathrm{E}-08$ & $5.40 \mathrm{E}-06$ & 8.50E-07 \\
\hline PEST & Disulfoton & $\mathrm{mg} / \mathrm{L}$ & 49 & 0 & $0 \%$ & 1.70E-05 & 2.10E-05 & -- & -- & 9.32E-06 \\
\hline PEST & Endosulfan I & $\mathrm{mg} / \mathrm{L}$ & 15 & 3 & $20 \%$ & $1.80 \mathrm{E}-07$ & $1.30 \mathrm{E}-06$ & 2.70E-07 & 1.90E-06 & 5.63E-07 \\
\hline PEST & Endrin & $\mathrm{mg} / \mathrm{L}$ & 45 & 1 & $2 \%$ & 9.00E-09 & 4.40E-07 & 1.40E-07 & $1.40 \mathrm{E}-07$ & 8.40E-08 \\
\hline PEST & Eptam & $\mathrm{mg} / \mathrm{L}$ & 49 & 12 & $24 \%$ & 2.00E-06 & 5.00E-06 & 1.00E-06 & $9.00 \mathrm{E}-06$ & 1.99E-06 \\
\hline PEST & Ethalfluralin (Sonalan) & $\mathrm{mg} / \mathrm{L}$ & 49 & 0 & $0 \%$ & 4.00E-06 & $9.00 \mathrm{E}-06$ & -- & -- & $3.02 E-06$ \\
\hline PEST & Ethoprop & $\mathrm{mg} / \mathrm{L}$ & 49 & 0 & $0 \%$ & $3.00 \mathrm{E}-06$ & $5.00 \mathrm{E}-06$ & -- & -- & $1.91 \mathrm{E}-06$ \\
\hline PEST & Fonofos & $\mathrm{mg} / \mathrm{L}$ & 49 & 0 & $0 \%$ & 3.00E-06 & 3.00E-06 & -- & -- & 1.50E-06 \\
\hline PEST & Gamma-BHC (Lindane) & $\mathrm{mg} / \mathrm{L}$ & 39 & 0 & $0 \%$ & 4.00E-06 & 4.00E-06 & -- & -- & 2.00E-06 \\
\hline PEST & gamma-Chlordane & $\mathrm{mg} / \mathrm{L}$ & 12 & 9 & $75 \%$ & $1.20 \mathrm{E}-07$ & 3.90E-07 & 2.10E-08 & 8.10E-07 & 1.62E-07 \\
\hline PEST & Heptachlor epoxide & $\mathrm{mg} / \mathrm{L}$ & 45 & 7 & $16 \%$ & 1.10E-08 & 4.80E-07 & 2.50E-08 & $6.70 \mathrm{E}-08$ & $9.40 \mathrm{E}-08$ \\
\hline PEST & Linuron & $\mathrm{mg} / \mathrm{L}$ & 49 & 0 & $0 \%$ & 2.00E-06 & 3.50E-05 & -- & -- & 7.73E-06 \\
\hline PEST & Methoxychlor & $\mathrm{mg} / \mathrm{L}$ & 45 & 9 & $20 \%$ & 9.00E-09 & $9.20 \mathrm{E}-07$ & 2.70E-08 & $3.20 \mathrm{E}-07$ & $1.38 \mathrm{E}-07$ \\
\hline PEST & Methyl parathion & $\mathrm{mg} / \mathrm{L}$ & 49 & 0 & $0 \%$ & 6.00E-06 & 6.00E-06 & -- & -- & 3.00E-06 \\
\hline PEST & Metribuzin & $\mathrm{mg} / \mathrm{L}$ & 49 & 12 & $24 \%$ & 4.00E-06 & $6.00 E-06$ & 3.00E-06 & $2.50 \mathrm{E}-05$ & 4.76E-06 \\
\hline PEST & Molinate & $\mathrm{mg} / \mathrm{L}$ & 49 & 0 & $0 \%$ & 2.00E-06 & 4.00E-06 & -- & -- & $1.59 \mathrm{E}-06$ \\
\hline PEST & Napropamide & $\mathrm{mg} / \mathrm{L}$ & 49 & 2 & $4 \%$ & 3.00E-06 & 7.00E-06 & 3.00E-06 & $6.00 \mathrm{E}-06$ & 2.36E-06 \\
\hline PEST & Parathion & $\mathrm{mg} / \mathrm{L}$ & 43 & 0 & $0 \%$ & 4.00E-06 & $1.00 \mathrm{E}-05$ & -- & -- & 2.77E-06 \\
\hline PEST & Pebulate & $\mathrm{mg} / \mathrm{L}$ & 49 & 0 & $0 \%$ & $2.00 \mathrm{E}-06$ & 4.00E-06 & -- & -- & 1.73E-06 \\
\hline PEST & Pendimethalin & $\mathrm{mg} / \mathrm{L}$ & 45 & 2 & $4 \%$ & 4.00E-06 & $2.20 \mathrm{E}-05$ & 4.00E-06 & 4.00E-06 & 4.36E-06 \\
\hline PEST & Permethrin & $\mathrm{mg} / \mathrm{L}$ & 49 & 0 & $0 \%$ & 5.00E-06 & 6.00E-06 & -- & -- & $2.70 \mathrm{E}-06$ \\
\hline PEST & Phorate & $\mathrm{mg} / \mathrm{L}$ & 49 & 0 & $0 \%$ & 2.00E-06 & 1.10E-05 & -- & -- & 2.84E-06 \\
\hline PEST & Pramitol (Prometon) & $\mathrm{mg} / \mathrm{L}$ & 39 & 2 & $5 \%$ & $1.50 \mathrm{E}-05$ & 1.80E-05 & 3.00E-06 & 8.00E-06 & 8.21E-06 \\
\hline PEST & PRONARIDE & $\mathrm{mg} / \mathrm{L}$ & 49 & 8 & $16 \%$ & 3.00E-06 & 4.00E-06 & 2.00E-06 & 7.00E-06 & 2.27E-06 \\
\hline
\end{tabular}




\section{Table E-11}

Summary Statistics for All Categories of Surface Water Samples Collected from Segment 5 of the Columbia River

\begin{tabular}{|c|c|c|c|c|c|c|c|c|c|c|}
\hline $\begin{array}{l}\text { Constituent } \\
\text { Class }\end{array}$ & Constituent Name & Units & $\begin{array}{l}\text { Number } \\
\text { Analyzed }\end{array}$ & $\begin{array}{l}\text { Number } \\
\text { Detected }\end{array}$ & $\begin{array}{c}\text { Frequency of } \\
\text { Detection }\end{array}$ & $\begin{array}{l}\text { Minimum } \\
\text { Nondetect }\end{array}$ & $\begin{array}{l}\text { Maximum } \\
\text { Nondetect }\end{array}$ & $\begin{array}{c}\text { Minimum } \\
\text { Detect }\end{array}$ & $\begin{array}{l}\text { Maximum } \\
\text { Detect }\end{array}$ & $\begin{array}{c}\text { Average } \\
\text { Concentration }\end{array}$ \\
\hline$\overline{\text { PEST }}$ & Propachlor (Ramrod) & $\mathrm{mg} / \mathrm{L}$ & 39 & 0 & $0 \%$ & $7.00 \mathrm{E}-06$ & $1.00 \mathrm{E}-05$ & -- & -- & $4.12 E-06$ \\
\hline PEST & Propanil & $\mathrm{mg} / \mathrm{L}$ & 49 & 0 & $0 \%$ & 4.00E-06 & 1.10E-05 & -- & -- & 3.43E-06 \\
\hline PEST & Propargite & $\mathrm{mg} / \mathrm{L}$ & 49 & 0 & $0 \%$ & 1.30E-05 & 2.30E-05 & -- & -- & 8.54E-06 \\
\hline PEST & Simazine & $\mathrm{mg} / \mathrm{L}$ & 39 & 18 & $46 \%$ & 5.00E-06 & 1.10E-05 & 3.00E-06 & 1.80E-05 & 5.77E-06 \\
\hline PEST & Tebuthiuron & $\mathrm{mg} / \mathrm{L}$ & 49 & 0 & $0 \%$ & 1.00E-05 & 1.60E-05 & -- & -- & $6.22 \mathrm{E}-06$ \\
\hline PEST & Terbacil & $\mathrm{mg} / \mathrm{L}$ & 49 & 4 & $8 \%$ & 7.00E-06 & 3.40E-05 & 4.00E-06 & $6.00 \mathrm{E}-06$ & $9.17 \mathrm{E}-06$ \\
\hline PEST & Terbufos & $\mathrm{mg} / \mathrm{L}$ & 49 & 0 & $0 \%$ & $1.30 \mathrm{E}-05$ & $1.70 \mathrm{E}-05$ & -- & -- & 7.32E-06 \\
\hline PEST & Thiobencarb & $\mathrm{mg} / \mathrm{L}$ & 49 & 0 & $0 \%$ & 2.00E-06 & 5.00E-06 & -- & -- & 1.61E-06 \\
\hline PEST & Triallate & $\mathrm{mg} / \mathrm{L}$ & 49 & 2 & $4 \%$ & 1.00E-06 & 2.00E-06 & 2.00E-06 & 2.00E-06 & 7.65E-07 \\
\hline PEST & Trifluralin (Treflan) & $\mathrm{mg} / \mathrm{L}$ & 78 & 0 & $0 \%$ & 2.00E-06 & $9.00 \mathrm{E}-06$ & -- & -- & 1.90E-06 \\
\hline SVOC & Benzo(a)anthracene & $\mathrm{mg} / \mathrm{L}$ & 47 & 44 & $94 \%$ & $3.40 \mathrm{E}-08$ & $1.00 \mathrm{E}-07$ & 1.10E-08 & 2.50E-06 & 2.00E-07 \\
\hline SVOC & Benzo(a)pyrene & $\mathrm{mg} / \mathrm{L}$ & 47 & 31 & $66 \%$ & 1.30E-08 & 1.10E-07 & 4.00E-09 & $5.20 \mathrm{E}-07$ & 4.39E-08 \\
\hline SVOC & Benzo(b)fluoranthene & $\mathrm{mg} / \mathrm{L}$ & 47 & 35 & $74 \%$ & 1.60E-08 & $1.20 \mathrm{E}-07$ & 9.00E-09 & $1.20 \mathrm{E}-06$ & $9.76 \mathrm{E}-08$ \\
\hline SVOC & Benzo(ghi)perylene & $\mathrm{mg} / \mathrm{L}$ & 47 & 32 & $68 \%$ & $2.30 \mathrm{E}-08$ & 2.00E-07 & 6.00E-09 & $2.80 \mathrm{E}-07$ & 3.77E-08 \\
\hline SVOC & Benzo(k)fluoranthene & $\mathrm{mg} / \mathrm{L}$ & 47 & 35 & $74 \%$ & $1.40 \mathrm{E}-08$ & 1.10E-07 & 8.00E-09 & 1.10E-06 & $9.40 \mathrm{E}-08$ \\
\hline SVOC & Chrysene & $\mathrm{mg} / \mathrm{L}$ & 47 & 47 & $100 \%$ & -- & -- & 2.50E-08 & $2.50 \mathrm{E}-06$ & 2.39E-07 \\
\hline SVOC & Dibenz[a,h]anthracene & $\mathrm{mg} / \mathrm{L}$ & 34 & 18 & $53 \%$ & 1.70E-08 & $1.60 \mathrm{E}-07$ & 3.00E-09 & 8.30E-08 & 2.06E-08 \\
\hline SVOC & Dibenzo(a,h)pyrene & $\mathrm{mg} / \mathrm{L}$ & 13 & 8 & $62 \%$ & 1.70E-08 & $6.00 \mathrm{E}-08$ & 3.00E-09 & 8.30E-08 & 1.65E-08 \\
\hline SVOC & Hexachlorobenzene & $\mathrm{mg} / \mathrm{L}$ & 45 & 45 & $100 \%$ & -- & -- & 5.70E-08 & 2.30E-06 & 3.33E-07 \\
\hline SVOC & Indeno(1,2,3-cd)pyrene & $\mathrm{mg} / \mathrm{L}$ & 47 & 32 & $68 \%$ & 1.30E-08 & 1.10E-07 & 3.00E-09 & 2.00E-07 & 2.28E-08 \\
\hline
\end{tabular}

CONV - Conventional parameter

PCB - Polychlorinated Biphenyls

PEST - Pesticide

SVOC - Semivolatile Organic Compound 


\begin{tabular}{|c|c|c|c|c|c|c|c|c|c|c|c|c|c|c|c|c|}
\hline $\begin{array}{l}\text { Constituent } \\
\text { Class }\end{array}$ & Constituent Name & Units & $\begin{array}{l}\text { Number } \\
\text { Analyzed }\end{array}$ & $\begin{array}{l}\text { Number } \\
\text { Detected }\end{array}$ & $\begin{array}{c}\text { Frequency } \\
\text { of Detection }\end{array}$ & $\begin{array}{c}\text { Minimum } \\
\text { Detect }\end{array}$ & $\begin{array}{c}\text { Maximum } \\
\text { Detect }\end{array}$ & $\begin{array}{c}\text { Average } \\
\text { Concentration }\end{array}$ & $\begin{array}{l}\text { Ecological } \\
\text { Benchmark } \\
\text { Value }\end{array}$ & $\begin{array}{l}\text { Is Max > Eco } \\
\text { Benchmark? }\end{array}$ & $\begin{array}{c}\text { Factor of } \\
\text { Exceedance }\end{array}$ & $\begin{array}{c}\text { Number of } \\
\text { Exceedances }\end{array}$ & $\begin{array}{l}\text { Human Health } \\
\text { Benchmark Value }\end{array}$ & $\begin{array}{l}\text { Is Max }>\mathrm{HH} \\
\text { Benchmark? }\end{array}$ & $\begin{array}{c}\text { Factor of } \\
\text { Exceedance }\end{array}$ & $\begin{array}{c}\text { Number of } \\
\text { Exceedances }\end{array}$ \\
\hline$\overline{C O N V}$ & Alkalinity & $\mathrm{mg} / \mathrm{L}$ & 42 & 42 & $100 \%$ & 22 & 66 & 49 & -- & -- & -- & -- & -- & $\overline{--}$ & -- & -- \\
\hline CONV & Ammonia & $\mathrm{mg} / \mathrm{L}$ & 12 & 4 & $33 \%$ & 0.015 & 0.019 & 0.0092 & -- & -- & -- & -- & -- & -- & -- & -- \\
\hline CONV & Chloride & $\mathrm{mg} / \mathrm{L}$ & 50 & 50 & $100 \%$ & 5.6 & 14 & 8.7 & 230 & No & 0.061 & 0 & -- & -- & -- & -- \\
\hline CONV & Dissolved organic carbon & $\mathrm{mg} / \mathrm{L}$ & 43 & 43 & $100 \%$ & 1.0 & 3.3 & 1.7 & -. & -- & -. & -- & -- & -- & -- & -- \\
\hline CONV & Dissolved oxygen & $\mathrm{mg} / \mathrm{L}$ & 12 & 12 & $100 \%$ & 8.9 & 12 & 11 & -- & -- & -- & -- & -- & -- & -- & -- \\
\hline CONV & Fluoride & $\mathrm{mg} / \mathrm{L}$ & 50 & 50 & $100 \%$ & 7.8 & 14 & 11 & -- & -- & -- & -- & -- & -- & -- & -- \\
\hline CONV & Hardness & $\mathrm{mg} / \mathrm{L}$ & 6 & 6 & $100 \%$ & 41 & 67 & 59 & -- & -- & -- & -- & -- & -- & -- & -- \\
\hline CONV & Nitrogen in ammonia & $\mathrm{mg} / \mathrm{L}$ & 50 & 38 & $76 \%$ & 0.0020 & 0.039 & 0.011 & -- & -- & -- & -- & -- & -- & -- & -- \\
\hline CONV & Nitrogen, Kjeldahl total & $\mathrm{mg} / \mathrm{L}$ & 100 & 96 & $96 \%$ & 0.10 & 0.50 & 0.16 & -- & -- & -- & -- & -- & -- & -- & .- \\
\hline CONV & Nitrogen, Nitrate-Nitrite & $\mathrm{mg} / \mathrm{L}$ & 12 & 12 & $100 \%$ & 0.073 & 0.46 & 0.22 & -- & -- & -- & -. & -- & -- & -- & .- \\
\hline CONV & Nitrogen, Nitrite & $\mathrm{mg} / \mathrm{L}$ & 50 & 47 & $94 \%$ & 0.0010 & 0.0060 & 0.0031 & -- & -- & -- & -.- & -. & -.- & .- & .. \\
\hline CONV & Orthophosphate & $\mathrm{mg} / \mathrm{L}$ & 61 & 59 & $97 \%$ & 0.0010 & 0.025 & 0.012 & -. & -. & -- & -. & -- & -. & -. & .. \\
\hline CONV & $\mathrm{pH}$ & $\mathrm{pH}$ Units & 53 & 53 & $100 \%$ & 6.6 & 8.5 & 7.8 & .- & .- & -- & .- & .- & .- & .- & -. \\
\hline CONV & Phosphate & $\mathrm{mg} / \mathrm{L}$ & 12 & 12 & $100 \%$ & 0.024 & 0.043 & 0.030 & -. & .- & -. & .- & -. & -. & -. & -. \\
\hline CONV & Specific Conductance & umhos $/ \mathrm{cm}$ & 100 & 100 & $100 \%$ & 75 & 225 & 132 & -. & -- & -- & -- & -- & -. & -. & -- \\
\hline CONV & Sulfate & $\mathrm{mg} / \mathrm{L}$ & 50 & 50 & $100 \%$ & 0.10 & 0.10 & 0.10 & -- & -- & -- & -- & -- & -- & -- & -- \\
\hline CONV & Total Organic Carbon & $\mathrm{mg} / \mathrm{L}$ & 43 & 43 & $100 \%$ & 1.0 & 3.8 & 1.8 & -- & -- & -- & -- & -- & -- & -- & -- \\
\hline CONV & Total Persulfate Nitrogen & $\mathrm{mg} / \mathrm{L}$ & 12 & 12 & $100 \%$ & 0.16 & 0.51 & 0.30 & -- & -- & -- & -- & -- & -- & -- & -- \\
\hline CONV & Total suspended solids & $\mathrm{mg} / \mathrm{L}$ & 58 & 58 & $100 \%$ & 1.0 & 38 & 9.1 & -- & -- & -- & -- & -- & -- & -- & -- \\
\hline CONV & Turbidity & NTU & 65 & 65 & $100 \%$ & 0.50 & 68 & 10 & -- & -- & -- & -- & -- & -- & -- & -- \\
\hline METAL & Antimony & $\mathrm{mg} / \mathrm{L}$ & 18 & 9 & $50 \%$ & 1.00E-04 & $2.00 \mathrm{E}-04$ & $3.06 \mathrm{E}-04$ & 1.6 & No & 1.25E-04 & 0 & 0.0056 & No & 0.036 & 0 \\
\hline METAL & Arsenic & $\mathrm{mg} / \mathrm{L}$ & 35 & 27 & $77 \%$ & 7.00E-04 & 0.0015 & $9.33 \mathrm{E}-04$ & 0.15 & No & 0.010 & 0 & $1.80 \mathrm{E}-05$ & Yes & 83 & 27 \\
\hline METAL & Barium & $\mathrm{mg} / \mathrm{L}$ & 18 & 18 & $100 \%$ & 0.011 & 0.021 & 0.017 & 0.0040 & Yes & 5.2 & 18 & 1.0 & No & 0.021 & 0 \\
\hline METAL & Boron & $\mathrm{mg} / \mathrm{L}$ & 18 & 16 & $89 \%$ & 0.0081 & 0.020 & 0.012 & 0.0016 & Yes & 12 & 16 & -- & -. & -- & -- \\
\hline METAL & Cadmium & $\mathrm{mg} / \mathrm{L}$ & 22 & 1 & $5 \%$ & $1.00 \mathrm{E}-04$ & 1.00E-04 & $2.02 \mathrm{E}-04$ & $2.50 \mathrm{E}-04$ & No & 0.40 & 0 & -- & -- & -- & -- \\
\hline METAL & Calcium & $\mathrm{mg} / \mathrm{L}$ & 50 & 50 & $100 \%$ & 8.3 & 19 & 14 & 116 & No & 0.17 & 0 & -- & -- & -- & -- \\
\hline METAL & Chromium & $\mathrm{mg} / \mathrm{L}$ & 12 & 3 & $25 \%$ & $3.60 \mathrm{E}-04$ & $5.50 \mathrm{E}-04$ & $2.61 \mathrm{E}-04$ & 0.010 & No & 0.055 & 0 & -- & -- & -- & -- \\
\hline METAL & Cobalt & $\mathrm{mg} / \mathrm{L}$ & 13 & 4 & $31 \%$ & 1.00E-04 & 1.00E-04 & 3.77E-04 & 0.023 & No & 0.0043 & 0 & -- & -- & -- & -. \\
\hline METAL & Copper & $\mathrm{mg} / \mathrm{L}$ & 29 & 29 & $100 \%$ & $6.30 \mathrm{E}-04$ & 0.0053 & 0.0013 & 0.0060 & No & 0.89 & 0 & -- & -- & -- & .- \\
\hline METAL & Lead & $\mathrm{mg} / \mathrm{L}$ & 12 & 10 & $83 \%$ & $2.80 \mathrm{E}-05$ & $3.80 \mathrm{E}-04$ & $1.31 \mathrm{E}-04$ & $8.67 E-04$ & No & 0.44 & 0 & -- & -- & -- & .. \\
\hline METAL & Lithium & $\mathrm{mg} / \mathrm{L}$ & 18 & 15 & $83 \%$ & 0.0019 & 0.0038 & 0.0027 & 0.014 & No & 0.27 & 0 & $\ldots$ & $\ldots$ & -- & .. \\
\hline METAL & Magnesium & $\mathrm{mg} / \mathrm{L}$ & 50 & 50 & $100 \%$ & 2.6 & 5.7 & 4.1 & 82 & No & 0.070 & 0 & ..- & -. & -- & .. \\
\hline METAL & Nickel & $\mathrm{mg} / \mathrm{L}$ & 12 & 12 & $100 \%$ & 4. $40 \mathrm{E}-04$ & $9.20 \mathrm{E}-04$ & $5.84 \mathrm{E}-04$ & 0.028 & No & 0.033 & 0 & 0.61 & No & 0.0015 & 0 \\
\hline METAL & Potassium & $\mathrm{mg} / \mathrm{L}$ & 50 & 50 & $100 \%$ & 2.3 & 7.4 & 3.9 & 53 & No & 0.14 & 0 & -- & $\ldots$ & -. & -- \\
\hline METAL & Silica & $\mathrm{mg} / \mathrm{L}$ & 50 & 50 & $100 \%$ & 50 & 112 & 83 & $\ldots$ & $\ldots$ & ... & -. & .. & -. & -- & .- \\
\hline METAL & Sodium & $\mathrm{mg} / \mathrm{L}$ & 50 & 50 & $100 \%$ & 3.3 & 9.1 & 5.9 & 680 & No & 0.013 & 0 & -. & -- & - & -- \\
\hline METAL & Uranium & $\mathrm{mg} / \mathrm{L}$ & 18 & 9 & $50 \%$ & 4.00E-04 & 8.00E-04 & $5.39 \mathrm{E}-04$ & 0.0026 & No & 0.31 & 0 & 0.030 & No & 0.027 & 0 \\
\hline METAL & Zinc & $\mathrm{mg} / \mathrm{L}$ & 29 & 22 & $76 \%$ & 0.0010 & 0.0056 & 0.0020 & 0.055 & No & 0.10 & 0 & 7.4 & No & 7.57E-04 & 0 \\
\hline $\mathrm{PCB}$ & Total polychlorinated biphenyl & $\mathrm{mg} / \mathrm{L}$ & 13 & 8 & $62 \%$ & $3.30 \mathrm{E}-07$ & $2.10 \mathrm{E}-05$ & $3.17 \mathrm{E}-06$ & $1.40 \mathrm{E}-05$ & Yes & 1.5 & 1 & $6.40 \mathrm{E}-08$ & Yes & 328 & 8 \\
\hline PEST & Alachlor & $\mathrm{mg} / \mathrm{L}$ & 48 & 31 & $65 \%$ & 1.00E-06 & $1.80 \mathrm{E}-05$ & $5.14 \mathrm{E}-06$ &.- & -- & -- & -- &.- & -- & -- & -- \\
\hline PEST & alpha-Chlordane & $\mathrm{mg} / \mathrm{L}$ & 12 & 12 & $100 \%$ & $1.90 \mathrm{E}-08$ & $7.20 \mathrm{E}-07$ & $1.64 \mathrm{E}-07$ & -- & -- &.- &.- &.- & -- &.- & -- \\
\hline PEST & Atrazine & $\mathrm{mg} / \mathrm{L}$ & 43 & 32 & $74 \%$ & $1.00 \mathrm{E}-06$ & $6.50 \mathrm{E}-05$ & 1.17E-05 & -- & -- & -- & -- & -- & -- & -- & -- \\
\hline PEST & Butylate & $\mathrm{mg} / \mathrm{L}$ & 39 & 1 & $3 \%$ & $2.00 \mathrm{E}-06$ & 2.00E-06 & $1.03 \mathrm{E}-06$ & -- & -- & -- & -- & -- & -- & -- & -. \\
\hline PEST & Carbaryl (Sevin) & $\mathrm{mg} / \mathrm{L}$ & 49 & 1 & $2 \%$ & 4.00E-06 & 4.00E-06 & 8.92E-06 & -- & -- & -- & -- & -- & -. & -- & .- \\
\hline PEST & Carbofuran & $\mathrm{mg} / \mathrm{L}$ & 49 & 1 & $2 \%$ & $1.00 \mathrm{E}-05$ & $1.00 E-05$ & 4.97E-06 & -- & -- & -- & -- & -- & -- & -. & .- \\
\hline PEST & Chlorpyrifos & $\mathrm{mg} / \mathrm{L}$ & 39 & 2 & $5 \%$ & $3.00 E-06$ & 4.00E-06 & $2.28 \mathrm{E}-06$ & 4.10E-05 & No & 0.098 & 0 & -- & -- & -- & -- \\
\hline PEST & Dachthal (chlorthal) & $\mathrm{mg} / \mathrm{L}$ & 49 & 2 & $4 \%$ & 1.00E-06 & 2.00E-06 & $1.22 \mathrm{E}-06$ & -. & -. & -. & -- & -- & -- & -- & -- \\
\hline PEST & Desethyl atrazine & $\mathrm{mg} / \mathrm{L}$ & 39 & 20 & $51 \%$ & $2.00 \mathrm{E}-06$ & 7.00E-06 & $2.79 \mathrm{E}-06$ & -- & -. & -- & -- & -- & .- & .- & .- \\
\hline PEST & Dichlorodiphenyldichloroethane & $\mathrm{mg} / \mathrm{L}$ & 45 & 34 & $76 \%$ & $1.90 \mathrm{E}-07$ & $5.60 \mathrm{E}-06$ & 7.48E-07 & $1.00 E-06$ & Yes & 5.6 & 4 & $3.10 E-07$ & Yes & 18 & 27 \\
\hline PEST & Dichlorodiphenyldichloroethylent & $\mathrm{mg} / \mathrm{L}$ & 93 & 42 & $45 \%$ & $1.50 \mathrm{E}-08$ & $2.10 \mathrm{E}-06$ & 1.46E-06 & -- & -- & $\ldots$ & -- & $2.20 \mathrm{E}-07$ & Yes & 9.5 & 29 \\
\hline PEST & Dichlorodiphenyltrichloroethane & $\mathrm{mg} / \mathrm{L}$ & 45 & 39 & $87 \%$ & $1.30 \mathrm{E}-08$ & 1.50E-06 & $1.85 \mathrm{E}-07$ & 1.00E-06 & Yes & 1.5 & 3 & 2.20E-07 & Yes & 6.8 & 8 \\
\hline PEST & Dieldrin & $\mathrm{mg} / \mathrm{L}$ & 88 & 39 & $44 \%$ & $1.40 \mathrm{E}-08$ & 5.40E-06 & 8.50E-07 & $1.90 \mathrm{E}-06$ & Yes & 2.8 & 3 & 5.20E-08 & Yes & 104 & 37 \\
\hline PEST & Endosulfan I & $\mathrm{mg} / \mathrm{L}$ & 15 & 3 & $20 \%$ & $2.70 \mathrm{E}-07$ & $1.90 \mathrm{E}-06$ & 5.63E- 07 & 1.- & -. &.- & -- & -2.00 & -. & $\ldots$ & -. \\
\hline PEST & Endrin & $\mathrm{mg} / \mathrm{L}$ & 45 & 1 & $2 \%$ & 1.40E-07 & 1.40E-07 & 8.40E-08 & $2.30 \mathrm{E}-06$ & No & 0.061 & 0 & $5.90 \mathrm{E}-05$ & No & 0.0024 & 0 \\
\hline PEST & Eptam & $\mathrm{mg} / \mathrm{L}$ & 49 & 12 & $24 \%$ & $1.00 \mathrm{E}-06$ & 9.00E-06 & 1.99E-06 & -- & .- & -. & -- & -- & -. & -- & -- \\
\hline PEST & gamma-Chlordane & $\mathrm{mg} / \mathrm{L}$ & 12 & 9 & $75 \%$ & $2.10 \mathrm{E}-08$ & 8.10E-07 & $1.62 \mathrm{E}-07$ & -. & -- & -- & -. & .- & .- & .- & .- \\
\hline PEST & Heptachlor epoxide & $\mathrm{mg} / \mathrm{L}$ & 45 & 7 & $16 \%$ & $2.50 \mathrm{E}-08$ & $6.70 \mathrm{E}-08$ & $9.40 \mathrm{E}-08$ & $3.80 \mathrm{E}-06$ & No & 0.018 & 0 & $3.90 \mathrm{E}-08$ & Yes & 1.7 & 3 \\
\hline PEST & Methoxychlor & $\mathrm{mg} / \mathrm{L}$ & 45 & 9 & $20 \%$ & $2.70 \mathrm{E}-08$ & 3.20E-07 & $1.38 \mathrm{E}-07$ & $3.00 \mathrm{E}-05$ & No & 0.011 & 0 & & -- & -- & -- \\
\hline PEST & & $\mathrm{mg} / \mathrm{L}$ & 49 & 12 & $24 \%$ & $3.00 \mathrm{E}-06$ & 2.50E-05 & 4.76E-06 & & -- & -- & -- & -- & -- & -- & -- \\
\hline
\end{tabular}


Appendix E - Tables

Comparison of All Categories of Surface Water Concentrations Collected from Segment 5 of the Columbia River to Ecological and Human Health Benchmark Values

\begin{tabular}{|c|c|c|c|c|c|c|c|c|c|c|c|c|c|c|c|c|}
\hline $\begin{array}{l}\text { Constituent } \\
\text { Class }\end{array}$ & Constituent Name & Units & $\begin{array}{l}\text { Number } \\
\text { Analyzed }\end{array}$ & $\begin{array}{l}\text { Number } \\
\text { Detected }\end{array}$ & $\begin{array}{l}\text { Frequency } \\
\text { of Detection }\end{array}$ & $\begin{array}{c}\text { Minimum } \\
\text { Detect }\end{array}$ & $\begin{array}{l}\text { Maximum } \\
\text { Detect }\end{array}$ & $\begin{array}{c}\text { Average } \\
\text { Concentration }\end{array}$ & $\begin{array}{l}\text { Ecological } \\
\text { Benchmark } \\
\text { Value }\end{array}$ & $\begin{array}{l}\text { Is Max > Eco } \\
\text { Benchmark? }\end{array}$ & $\begin{array}{c}\text { Factor of } \\
\text { Exceedance }\end{array}$ & $\begin{array}{l}\text { Number of } \\
\text { Exceedances }\end{array}$ & $\begin{array}{l}\text { Human Health } \\
\text { Benchmark Value }\end{array}$ & $\begin{array}{l}\text { Is Max > HH } \\
\text { Benchmark? }\end{array}$ & $\begin{array}{c}\text { Factor of } \\
\text { Exceedance }\end{array}$ & $\begin{array}{c}\text { Number of } \\
\text { Exceedances }\end{array}$ \\
\hline$\overline{\text { PEST }}$ & Napropamide & $\mathrm{mg} / \mathrm{L}$ & 49 & 2 & $4 \%$ & $3.00 \mathrm{E}-06$ & $6.00 \mathrm{E}-06$ & $2.36 \mathrm{E}-06$ & -- & -- & -- & -- & -- & -- & -- & -- \\
\hline PEST & Pendimethalin & $\mathrm{mg} / \mathrm{L}$ & 45 & 2 & $4 \%$ & $4.00 E-06$ & 4.00E-06 & $4.36 \mathrm{E}-06$ & -- & -- & -- & -- & -- & -- & -- & -- \\
\hline PEST & Pramitol (Prometon) & $\mathrm{mg} / \mathrm{L}$ & 39 & 2 & $5 \%$ & $3.00 E-06$ & $8.00 E-06$ & $8.21 \mathrm{E}-06$ & -- & -- & -- & -- & -- & -- & -- & -- \\
\hline PEST & PRONARIDE & $\mathrm{mg} / \mathrm{L}$ & 49 & 8 & $16 \%$ & $2.00 E-06$ & $7.00 E-06$ & $2.27 \mathrm{E}-06$ & -- & -- & -- & -- & -- & -- & -- & -- \\
\hline PEST & Simazine & $\mathrm{mg} / \mathrm{L}$ & 39 & 18 & $46 \%$ & $3.00 E-06$ & $1.80 \mathrm{E}-05$ & $5.77 \mathrm{E}-06$ & -- & -- & -- & -- & -- & -- & -- & -- \\
\hline PEST & Terbacil & $\mathrm{mg} / \mathrm{L}$ & 49 & 4 & $8 \%$ & 4.00E-06 & $6.00 E-06$ & $9.17 \mathrm{E}-06$ & -- & -- & -- & -- & -- & -- & -- & -- \\
\hline PEST & Triallate & $\mathrm{mg} / \mathrm{L}$ & 49 & 2 & $4 \%$ & $2.00 E-06$ & $2.00 E-06$ & $7.65 \mathrm{E}-07$ & -- & -- & -- & -- & -- & -- & -- & -- \\
\hline svoc & Benzo(a)anthracene & $\mathrm{mg} / \mathrm{L}$ & 47 & 44 & $94 \%$ & 1.10E-08 & $2.50 \mathrm{E}-06$ & $2.00 \mathrm{E}-07$ & $2.70 \mathrm{E}-05$ & No & 0.093 & 0 & $3.80 \mathrm{E}-06$ & No & 0.66 & 0 \\
\hline svoc & Benzo(a)pyrene & $\mathrm{mg} / \mathrm{L}$ & 47 & 31 & $66 \%$ & 4.00E-09 & $5.20 \mathrm{E}-07$ & 4.39E-08 & $1.40 \mathrm{E}-05$ & No & 0.037 & 0 & 3.80E-06 & No & 0.14 & 0 \\
\hline SVOC & Benzo(b)fluoranthene & $\mathrm{mg} / \mathrm{L}$ & 47 & 35 & $74 \%$ & $9.00 \mathrm{E}-09$ & 1.20E-06 & $9.76 \mathrm{E}-08$ & -- & -- & -- & -- & 3.80E-06 & No & 0.32 & 0 \\
\hline sVOC & Benzo(ghi)perylene & $\mathrm{mg} / \mathrm{L}$ & 47 & 32 & $68 \%$ & $6.00 \mathrm{E}-09$ & $2.80 \mathrm{E}-07$ & $3.77 \mathrm{E}-08$ & -- & -- & -- & -- & -- & -- & -- & -- \\
\hline sVoc & Benzo(k)fluoranthene & $\mathrm{mg} / \mathrm{L}$ & 47 & 35 & $74 \%$ & 8.00E-09 & 1.10E-06 & $9.40 \mathrm{E}-08$ & -. & -. & -- & -- & $3.80 \mathrm{E}-06$ & No & 0.29 & 0 \\
\hline svoc & Chrysene & $\mathrm{mg} / \mathrm{L}$ & 47 & 47 & $100 \%$ & $2.50 \mathrm{E}-08$ & $2.50 \mathrm{E}-06$ & $2.39 \mathrm{E}-07$ & -- & -- & -- & -- & 3.80E-06 & No & 0.66 & 0 \\
\hline svoc & Dibenz[a,h]anthracene & $\mathrm{mg} / \mathrm{L}$ & 34 & 18 & $53 \%$ & $3.00 E-09$ & $8.30 \mathrm{E}-08$ & $2.06 \mathrm{E}-08$ & -- & -- & -- & -- & $3.80 \mathrm{E}-06$ & No & 0.022 & 0 \\
\hline svoc & Dibenzo(a,h)pyrene & $\mathrm{mg} / \mathrm{L}$ & 13 & 8 & $62 \%$ & 3.00E-09 & $8.30 \mathrm{E}-08$ & $1.65 \mathrm{E}-08$ & -- & -- & -- & -- & & -- & & -- \\
\hline svoc & Hexachlorobenzene & $\mathrm{mg} / \mathrm{L}$ & 45 & 45 & $100 \%$ & $5.70 \mathrm{E}-08$ & $2.30 \mathrm{E}-06$ & 3.33E-07 & -- & -- & -- & -- & 2.80E-07 & Yes & 8. & 14 \\
\hline syoc & Indeno(1, 3-cd) burene & $\mathrm{ma} / \mathrm{L}$ & 47 & 32 & $68 \%$ & $3.00 \mathrm{E}-09$ & $200 E-07$ & $228 \mathrm{E}-08$ & $\ldots$ & $\ldots$ & $\ldots$ & $\ldots$ & $380 \mathrm{E}-06$ & $\mathrm{No}$ & 0.053 & 0 \\
\hline
\end{tabular}

CONV - Conventional parameter

PCB - Polychlorina

SVOC - Semivolatile Organic Compound 
Table E-13

Summary of Surface Water Results From Segment 5 Exceeding Select Ecological or Human Health Benchmark Values

\begin{tabular}{|c|c|c|c|c|c|c|c|c|c|c|}
\hline Sample Area & Sample Site & Sample ID & Date Collected & Constituent Name & Units & $\begin{array}{c}\text { QA } \\
\text { Category }\end{array}$ & $\begin{array}{c}\text { Analytical } \\
\text { Value }\end{array}$ & Qualifier & $\begin{array}{c}\text { Ecological } \\
\text { Benchmark } \\
\text { Value }\end{array}$ & $\begin{array}{c}\text { Human } \\
\text { Health } \\
\text { Benchmark } \\
\text { Value }\end{array}$ \\
\hline Columbia River & Beaver Army TML & 14246900-01-11-27-F & January 11,2001 & Arsenic & $\mathrm{mg} / \mathrm{L}$ & CRC1_Seg5 & $7.00 \mathrm{E}-04$ & $=$ & 0.15 & $1.80 \mathrm{E}-05$ \\
\hline Columbia River & Beaver Army TML & $14246900-02-02-14-F$ & February 2, 2002 & Arsenic & $\mathrm{mg} / \mathrm{L}$ & CRC1_Seg5 & 7.00E-04 & $=$ & 0.15 & $1.80 \mathrm{E}-05$ \\
\hline Columbia River & Beaver Army TML & 14246900-02-05-16-F & February 5, 2002 & Arsenic & $\mathrm{mg} / \mathrm{L}$ & CRC1_Seg5 & 7.00E-04 & $=$ & 0.15 & 1.80E-05 \\
\hline Columbia River & Beaver Army TML & 14246900-02-05-16-FD & February 5, 2002 & Arsenic & $\mathrm{mg} / \mathrm{L}$ & CRC1_Seg5 & 7.00E-04 & $=$ & 0.15 & $1.80 \mathrm{E}-05$ \\
\hline Columbia River & VANCOUV(28A100) & 132084 & April 23, 2003 & Arsenic & $\mathrm{mg} / \mathrm{L}$ & CRC1_Seg5 & 7.40E-04 & $=$ & 0.15 & $1.80 \mathrm{E}-05$ \\
\hline Columbia River & Beaver Army TML & $14246900-01-02-13-F$ & January 2, 2001 & Arsenic & $\mathrm{mg} / \mathrm{L}$ & CRC1_Seg5 & 8.00E-04 & $=$ & 0.15 & 1.80E-05 \\
\hline Columbia River & Beaver Army TML & 14246900-01-05-15-F & January 5, 2001 & Arsenic & $\mathrm{mg} / \mathrm{L}$ & CRC1_Seg5 & 8.00E-04 & $=$ & 0.15 & $1.80 \mathrm{E}-05$ \\
\hline Columbia River & Beaver Army TML & 14246900-02-09-10-F & February 9, 2002 & Arsenic & $\mathrm{mg} / \mathrm{L}$ & CRC1_Seg5 & 8.00E-04 & $=$ & 0.15 & $1.80 \mathrm{E}-05$ \\
\hline Columbia River & VANCOUV(28A100) & 132503 & August 20, 2003 & Arsenic & $\mathrm{mg} / \mathrm{L}$ & CRC1_Seg5 & 8.10E-04 & $=$ & 0.15 & $1.80 \mathrm{E}-05$ \\
\hline Columbia River & VANCOUV(28A100) & 132299 & June 18, 2003 & Arsenic & $\mathrm{mg} / \mathrm{L}$ & CRC1_Seg5 & 8.30E-04 & $=$ & 0.15 & $1.80 \mathrm{E}-05$ \\
\hline Columbia River & VANCOUV(28A100) & 131716 & December 11, 2002 & Arsenic & $\mathrm{mg} / \mathrm{L}$ & CRC1_Seg5 & 8.50E-04 & $=$ & 0.15 & $1.80 \mathrm{E}-05$ \\
\hline Columbia River & VANCOUV(28A100) & 132084-F & April 23, 2003 & Arsenic & $\mathrm{mg} / \mathrm{L}$ & CRC1_Seg5 & 8.90E-04 & $=$ & 0.15 & $1.80 \mathrm{E}-05$ \\
\hline Columbia River & Beaver Army TML & 14246900-01-09-24-FD & January 9, 2001 & Arsenic & $\mathrm{mg} / \mathrm{L}$ & CRC1_Seg5 & $9.00 \mathrm{E}-04$ & $=$ & 0.15 & $1.80 \mathrm{E}-05$ \\
\hline Columbia River & VANCOUV(28A100) & 131716-F & December 11, 2002 & Arsenic & $\mathrm{mg} / \mathrm{L}$ & CRC1_Seg5 & $9.40 \mathrm{E}-04$ & $=$ & 0.15 & $1.80 \mathrm{E}-05$ \\
\hline Columbia River & VANCOUV(28A100) & $132503-F$ & August 20, 2003 & Arsenic & $\mathrm{mg} / \mathrm{L}$ & CRC1_Seg5 & 9.50E-04 & $=$ & 0.15 & $1.80 \mathrm{E}-05$ \\
\hline Columbia River & VANCOUV(28A100) & 132299-F & June 18, 2003 & Arsenic & $\mathrm{mg} / \mathrm{L}$ & CRC1_Seg5 & 9.90E-04 & $=$ & 0.15 & 1.80E-05 \\
\hline Columbia River & Beaver Army TML & $14246900-01-09-24-F$ & January 9, 2001 & Arsenic & $\mathrm{mg} / \mathrm{L}$ & CRC1_Seg5 & 0.0010 & $=$ & 0.15 & 1.80E-05 \\
\hline Columbia River & Beaver Army TML & 14246900-99-06-08-F & June 8, 1999 & Arsenic & $\mathrm{mg} / \mathrm{L}$ & CRC1_Seg5 & 0.0010 & $=$ & 0.15 & $1.80 \mathrm{E}-05$ \\
\hline Columbia River & VANCOUV(28A100) & 131560 & October 30, 2002 & Arsenic & $\mathrm{mg} / \mathrm{L}$ & CRC1_Seg5 & 0.0010 & $=$ & 0.15 & $1.80 \mathrm{E}-05$ \\
\hline Columbia River & VANCOUV(28A100) & 131897 & February 26, 2003 & Arsenic & $\mathrm{mg} / \mathrm{L}$ & CRC1_Seg5 & 0.0011 & $=$ & 0.15 & 1.80E-05 \\
\hline Columbia River & VANCOUV(28A100) & 131560-F & October 30, 2002 & Arsenic & $\mathrm{mg} / \mathrm{L}$ & CRC1_Seg5 & 0.0011 & $=$ & 0.15 & $1.80 \mathrm{E}-05$ \\
\hline Columbia River & Columbia CTY OR & 1388042 & September 25, 2001 & Arsenic & $\mathrm{mg} / \mathrm{L}$ & CRC1_Seg5 & 0.0011 & $=$ & 0.15 & $1.80 \mathrm{E}-05$ \\
\hline Columbia River & VANCOUV(28A100) & 131897-F & February 26, 2003 & Arsenic & $\mathrm{mg} / \mathrm{L}$ & CRC1_Seg5 & 0.0011 & $=$ & 0.15 & $1.80 \mathrm{E}-05$ \\
\hline Columbia River & RYAN PT WA & 1388041 & September 25, 2001 & Arsenic & $\mathrm{mg} / \mathrm{L}$ & CRC1_Seg5 & 0.0012 & $=$ & 0.15 & 1.80E-05 \\
\hline Columbia River & County Line PRK & 1388043 & September 25, 2001 & Arsenic & $\mathrm{mg} / \mathrm{L}$ & CRC1_Seg5 & 0.0012 & $=$ & 0.15 & 1.80E-05 \\
\hline Columbia River & County Line PRK & 1388044 & September 25, 2001 & Arsenic & $\mathrm{mg} / \mathrm{L}$ & CRC1_Seg5 & 0.0012 & $=$ & 0.15 & 1.80E-05 \\
\hline Columbia River & Beacon Rock WA & 1388040 & September 25, 2001 & Arsenic & $\mathrm{mg} / \mathrm{L}$ & CRC1_Seg5 & 0.0015 & $=$ & 0.15 & $1.80 \mathrm{E}-05$ \\
\hline Columbia River & Beaver Army TML & 14246900-99-11-30-FD & November 30, 1999 & Barium & $\mathrm{mg} / \mathrm{L}$ & CRC1_Seg5 & 0.011 & $=$ & 0.0040 & 1.0 \\
\hline Columbia River & Beaver Army TML & 14246900-99-11-30-F & November 30, 1999 & Barium & $\mathrm{mg} / \mathrm{L}$ & CRC1_Seg5 & 0.011 & $=$ & 0.0040 & 1.0 \\
\hline Columbia River & Beaver Army TML & 14246900-01-11-27-F & January 11, 2001 & Barium & $\mathrm{mg} / \mathrm{L}$ & CRC1_Seg5 & 0.012 & $=$ & 0.0040 & 1.0 \\
\hline Columbia River & Beaver Army TML & 14246900-99-06-08-F & June 8, 1999 & Barium & $\mathrm{mg} / \mathrm{L}$ & CRC1_Seg5 & 0.015 & $=$ & 0.0040 & 1.0 \\
\hline Columbia River & Beaver Army TML & 14246900-00-01-18-F & January 18,2000 & Barium & $\mathrm{mg} / \mathrm{L}$ & CRC1_Seg5 & 0.015 & $=$ & 0.0040 & 1.0 \\
\hline Columbia River & Beaver Army TML & $14246900-02-02-14-F$ & February 2, 2002 & Barium & $\mathrm{mg} / \mathrm{L}$ & CRC1_Seg5 & 0.017 & $=$ & 0.0040 & 1.0 \\
\hline Columbia River & Beaver Army TML & 14246900-00-05-16-F & May 16,2000 & Barium & $\mathrm{mg} / \mathrm{L}$ & CRC1_Seg5 & 0.017 & $=$ & 0.0040 & 1.0 \\
\hline Columbia River & Beaver Army TML & 14246900-00-05-16-FD & May 16, 2000 & Barium & $\mathrm{mg} / \mathrm{L}$ & CRC1_Seg5 & 0.017 & $=$ & 0.0040 & 1.0 \\
\hline Columbia River & Beaver Army TML & 14246900-02-05-16-FD & February 5, 2002 & Barium & $\mathrm{mg} / \mathrm{L}$ & CRC1_Seg5 & 0.017 & $=$ & 0.0040 & 1.0 \\
\hline Columbia River & Beaver Army TML & 14246900-01-05-15-F & January 5, 2001 & Barium & $\mathrm{mg} / \mathrm{L}$ & CRC1_Seg5 & 0.018 & $=$ & 0.0040 & 1.0 \\
\hline Columbia River & Beaver Army TML & 14246900-02-05-16-F & February 5, 2002 & Barium & $\mathrm{mg} / \mathrm{L}$ & CRC1_Seg5 & 0.018 & $=$ & 0.0040 & 1.0 \\
\hline Columbia River & Beaver Army TML & 14246900-99-03-08-F & March 8, 1999 & Barium & $\mathrm{mg} / \mathrm{L}$ & CRC1_Seg5 & 0.018 & $=$ & 0.0040 & 1.0 \\
\hline Columbia River & Beaver Army TML & 14246900-99--08-03-F & August 3, 1999 & Barium & $\mathrm{mg} / \mathrm{L}$ & CRC1_Seg5 & 0.018 & $=$ & 0.0040 & 1.0 \\
\hline Columbia River & Beaver Army TML & 14246900-02-09-10-F & February 9, 2002 & Barium & $\mathrm{mg} / \mathrm{L}$ & CRC1_Seg5 & 0.020 & $=$ & 0.0040 & 1.0 \\
\hline Columbia River & Beaver Army TML & 14246900-01-09-24-FD & January 9, 2001 & Barium & $\mathrm{mg} / \mathrm{L}$ & CRC1_Seg5 & 0.020 & $=$ & 0.0040 & 1.0 \\
\hline Columbia River & Beaver Army TML & $14246900-01-09-24-F$ & January 9, 2001 & Barium & $\mathrm{mg} / \mathrm{L}$ & CRC1_Seg5 & 0.020 & $=$ & 0.0040 & 1.0 \\
\hline Columbia River & Beaver Army TML & $14246900-00-09-12-F$ & September 12, 2000 & Barium & $\mathrm{mg} / \mathrm{L}$ & CRC1_Seg5 & 0.021 & $=$ & 0.0040 & 1.0 \\
\hline Columbia River & Beaver Army TML & 14246900-01-02-13-F & January 2, 2001 & Barium & $\mathrm{mg} / \mathrm{L}$ & CRC1_Seg5 & 0.021 & $=$ & 0.0040 & 1.0 \\
\hline Columbia River & Beaver Army TML & 14246900-00-05-16-FD & May 16,2000 & Boron & $\mathrm{mg} / \mathrm{L}$ & CRC1_Seg5 & 0.0081 & E & 0.0016 & -- \\
\hline
\end{tabular}


Table E-13

Summary of Surface Water Results From Segment 5 Exceeding Select Ecological or Human Health Benchmark Values

\begin{tabular}{|c|c|c|c|c|c|c|c|c|c|c|}
\hline Sample Area & Sample Site & Sample ID & Date Collected & Constituent Name & Units & $\begin{array}{c}\text { QA } \\
\text { Category }\end{array}$ & $\begin{array}{c}\text { Analytical } \\
\text { Value }\end{array}$ & Qualifier & $\begin{array}{c}\text { Ecological } \\
\text { Benchmark } \\
\text { Value }\end{array}$ & $\begin{array}{c}\text { Human } \\
\text { Health } \\
\text { Benchmark } \\
\text { Value }\end{array}$ \\
\hline Columbia River & Beaver Army TML & $14246900-99-11-30-\mathrm{F}$ & November 30, 1999 & Boron & $\mathrm{mg} / \mathrm{L}$ & CRC1_Seg5 & 0.0083 & $\mathrm{E}$ & 0.0016 & -- \\
\hline Columbia River & Beaver Army TML & 14246900-02-05-16-FD & February 5, 2002 & Boron & $\mathrm{mg} / \mathrm{L}$ & CRC1_Seg5 & 0.0085 & $=$ & 0.0016 & -- \\
\hline Columbia River & Beaver Army TML & 14246900-99--08-03-F & August 3, 1999 & Boron & $\mathrm{mg} / \mathrm{L}$ & CRC1_Seg5 & 0.0088 & $\mathrm{E}$ & 0.0016 & -- \\
\hline Columbia River & Beaver Army TML & 14246900-02-05-16-F & February 5, 2002 & Boron & $\mathrm{mg} / \mathrm{L}$ & CRC1_Seg5 & 0.0089 & $=$ & 0.0016 & -- \\
\hline Columbia River & Beaver Army TML & 14246900-99-03-08-F & March 8, 1999 & Boron & $\mathrm{mg} / \mathrm{L}$ & CRC1_Seg5 & 0.011 & $\mathrm{E}$ & 0.0016 & -- \\
\hline Columbia River & Beaver Army TML & 14246900-99-11-30-FD & November 30, 1999 & Boron & $\mathrm{mg} / \mathrm{L}$ & CRC1_Seg5 & 0.011 & E & 0.0016 & -- \\
\hline Columbia River & Beaver Army TML & 14246900-01-05-15-F & January 5, 2001 & Boron & $\mathrm{mg} / \mathrm{L}$ & CRC1_Seg5 & 0.011 & $=$ & 0.0016 & -- \\
\hline Columbia River & Beaver Army TML & 14246900-02-09-10-F & February 9, 2002 & Boron & $\mathrm{mg} / \mathrm{L}$ & CRC1_Seg5 & 0.012 & $=$ & 0.0016 & -- \\
\hline Columbia River & Beaver Army TML & 14246900-00-01-18-F & January 18,2000 & Boron & $\mathrm{mg} / \mathrm{L}$ & CRC1_Seg5 & 0.013 & E & 0.0016 & -- \\
\hline Columbia River & Beaver Army TML & 14246900-02-02-14-F & February 2, 2002 & Boron & $\mathrm{mg} / \mathrm{L}$ & CRC1_Seg5 & 0.013 & $=$ & 0.0016 & -- \\
\hline Columbia River & Beaver Army TML & $14246900-00-09-12-F$ & September 12, 2000 & Boron & $\mathrm{mg} / \mathrm{L}$ & CRC1_Seg5 & 0.015 & $\mathrm{E}$ & 0.0016 & -- \\
\hline Columbia River & Beaver Army TML & 14246900-01-11-27-F & January 11, 2001 & Boron & $\mathrm{mg} / \mathrm{L}$ & CRC1_Seg5 & 0.015 & $=$ & 0.0016 & -- \\
\hline Columbia River & Beaver Army TML & 14246900-01-02-13-F & January 2, 2001 & Boron & $\mathrm{mg} / \mathrm{L}$ & CRC1_Seg5 & 0.016 & $=$ & 0.0016 & -- \\
\hline Columbia River & Beaver Army TML & 14246900-01-09-24-FD & January 9, 2001 & Boron & $\mathrm{mg} / \mathrm{L}$ & CRC1_Seg5 & 0.016 & $=$ & 0.0016 & -- \\
\hline Columbia River & Beaver Army TML & 14246900-01-09-24-F & January 9, 2001 & Boron & $\mathrm{mg} / \mathrm{L}$ & CRC1_Seg5 & 0.020 & $=$ & 0.0016 & -- \\
\hline Columbia River & Willamette RVR & 3454416 & June 25,2003 & Dichlorodiphenyldichloroethane & $\mathrm{mg} / \mathrm{L}$ & CRC1_Seg5 & $3.40 \mathrm{E}-07$ & $=$ & $1.00 \mathrm{E}-06$ & $3.10 \mathrm{E}-07$ \\
\hline Columbia River & RM142 BONN DAM & loc 02 8/03-9/03 & September 1, 2003 & Dichlorodiphenyldichloroethane & $\mathrm{mg} / \mathrm{L}$ & CRC1_Seg5 & $3.90 \mathrm{E}-07$ & $=$ & 1.00E-06 & 3.10E-07 \\
\hline Columbia River & RM102, COL SLGH & loc 05 8/03-9/03 & September 1, 2003 & Dichlorodiphenyldichloroethane & $\mathrm{mg} / \mathrm{L}$ & CRC1_Seg5 & 4.60E-07 & $=$ & $1.00 \mathrm{E}-06$ & $3.10 \mathrm{E}-07$ \\
\hline Columbia River & Columbia Slough & 4314234 & June 22, 2004 & Dichlorodiphenyldichloroethane & $\mathrm{mg} / \mathrm{L}$ & CRC1_Seg5 & 4.70E-07 & $\mathrm{J}$ & $1.00 \mathrm{E}-06$ & $3.10 \mathrm{E}-07$ \\
\hline Columbia River & RM102, COL SLGH & loc 05 5/04-6/04 & June 1, 2004 & Dichlorodiphenyldichloroethane & $\mathrm{mg} / \mathrm{L}$ & CRC1_Seg5 & 4.70E-07 & $=$ & $1.00 \mathrm{E}-06$ & 3.10E-07 \\
\hline Columbia River & Bonneville Dam & 3454414 & September 24, 2003 & Dichlorodiphenyldichloroethane & $\mathrm{mg} / \mathrm{L}$ & CRC1_Seg5 & 5.30E-07 & $=$ & $1.00 \mathrm{E}-06$ & 3.10E-07 \\
\hline Columbia River & Columbia Slough & 3454417 & September 25, 2003 & Dichlorodiphenyldichloroethane & $\mathrm{mg} / \mathrm{L}$ & CRC1_Seg5 & $5.80 \mathrm{E}-07$ & $=$ & 1.00E-06 & 3.10E-07 \\
\hline Columbia River & RM86, MULT CHNL & loc 09 5/04-6/04 & June 1, 2004 & Dichlorodiphenyldichloroethane & $\mathrm{mg} / \mathrm{L}$ & CRC1_Seg5 & 5.90E-07 & $=$ & $1.00 \mathrm{E}-06$ & 3.10E-07 \\
\hline Columbia River & Below Longview & 4314243 & June 23, 2004 & Dichlorodiphenyldichloroethane & $\mathrm{mg} / \mathrm{L}$ & CRC1_Seg5 & $6.20 \mathrm{E}-07$ & $\mathrm{JN}$ & 1.00E-06 & 3.10E-07 \\
\hline Columbia River & RM88, LAKE R & loc 07 5/04-6/04 & June 1, 2004 & Dichlorodiphenyldichloroethane & $\mathrm{mg} / \mathrm{L}$ & CRC1_Seg5 & $6.20 \mathrm{E}-07$ & $=$ & $1.00 \mathrm{E}-06$ & 3.10E-07 \\
\hline Columbia River & RM54, LONGVIEW & loc 13 5/04-6/04 & June 1, 2004 & Dichlorodiphenyldichloroethane & $\mathrm{mg} / \mathrm{L}$ & CRC1_Seg5 & $6.20 \mathrm{E}-07$ & $=$ & 1.00E-06 & 3.10E-07 \\
\hline Columbia River & RM88, LAKE R & loc 07 12/03-1/04 & January 1, 2004 & Dichlorodiphenyldichloroethane & $\mathrm{mg} / \mathrm{L}$ & CRC1_Seg5 & 6.60E-07 & $=$ & 1.00E-06 & 3.10E-07 \\
\hline Columbia River & Kalama WA & 4154241 & January 13, 2004 & Dichlorodiphenyldichloroethane & $\mathrm{mg} / \mathrm{L}$ & CRC1_Seg5 & $6.70 \mathrm{E}-07$ & $\mathrm{~J}$ & $1.00 \mathrm{E}-06$ & $3.10 \mathrm{E}-07$ \\
\hline Columbia River & RM75, KALAMA R & loc 10 12/03-1/04 & January 1, 2004 & Dichlorodiphenyldichloroethane & $\mathrm{mg} / \mathrm{L}$ & CRC1_Seg5 & $6.70 \mathrm{E}-07$ & $=$ & $1.00 \mathrm{E}-06$ & 3.10E-07 \\
\hline Columbia River & RM102, WRVR & loc 06 8/03-9/03 & September 1, 2003 & Dichlorodiphenyldichloroethane & $\mathrm{mg} / \mathrm{L}$ & CRC1_Seg5 & $6.90 \mathrm{E}-07$ & $=$ & $1.00 \mathrm{E}-06$ & 3.10E-07 \\
\hline Columbia River & Willamette RVR & 4154235 & January 12, 2004 & Dichlorodiphenyldichloroethane & $\mathrm{mg} / \mathrm{L}$ & CRC1_Seg5 & 7.50E-07 & $\mathrm{J}$ & 1.00E-06 & 3.10E-07 \\
\hline Columbia River & RM103, WRVR & loc 04 12/03-1/04 & January 1, 2004 & Dichlorodiphenyldichloroethane & $\mathrm{mg} / \mathrm{L}$ & CRC1_Seg5 & 7.50E-07 & $=$ & 1.00E-06 & 3.10E-07 \\
\hline Columbia River & RM102, WRVR & loc 06 12/03-1/04 & January 1, 2004 & Dichlorodiphenyldichloroethane & $\mathrm{mg} / \mathrm{L}$ & CRC1_Seg5 & $7.60 \mathrm{E}-07$ & $=$ & 1.00E-06 & 3.10E-07 \\
\hline Columbia River & Bonneville Dam & 4314231 & June 22, 2004 & Dichlorodiphenyldichloroethane & $\mathrm{mg} / \mathrm{L}$ & CRC1_Seg5 & 8.80E-07 & $=$ & $1.00 \mathrm{E}-06$ & 3.10E-07 \\
\hline Columbia River & RM142 BONN DAM & loc 02 5/04-6/04 & June 1, 2004 & Dichlorodiphenyldichloroethane & $\mathrm{mg} / \mathrm{L}$ & CRC1_Seg5 & 8.80E-07 & $=$ & 1.00E-06 & 3.10E-07 \\
\hline Columbia River & Willamette RVR & 4314233 & June 22, 2004 & Dichlorodiphenyldichloroethane & $\mathrm{mg} / \mathrm{L}$ & CRC1_Seg5 & $9.40 \mathrm{E}-07$ & $=$ & 1.00E-06 & 3.10E-07 \\
\hline Columbia River & RM103, WRVR & loc 04 5/04-6/04 & June 1, 2004 & Dichlorodiphenyldichloroethane & $\mathrm{mg} / \mathrm{L}$ & CRC1_Seg5 & $9.40 \mathrm{E}-07$ & $=$ & $1.00 \mathrm{E}-06$ & $3.10 \mathrm{E}-07$ \\
\hline Columbia River & RM86, MULT CHNL & loc 09 8/03-9/03 & September 1, 2003 & Dichlorodiphenyldichloroethane & $\mathrm{mg} / \mathrm{L}$ & CRC1_Seg5 & $9.40 \mathrm{E}-07$ & $=$ & $1.00 \mathrm{E}-06$ & 3.10E-07 \\
\hline Columbia River & Columbia Slough & 4154236 & January 12, 2004 & Dichlorodiphenyldichloroethane & $\mathrm{mg} / \mathrm{L}$ & CRC1_Seg5 & $2.00 \mathrm{E}-06$ & $\mathrm{~J}$ & $1.00 \mathrm{E}-06$ & 3.10E-07 \\
\hline Columbia River & RM102, COL SLGH & loc 05 12/03-1/04 & January 1, 2004 & Dichlorodiphenyldichloroethane & $\mathrm{mg} / \mathrm{L}$ & CRC1_Seg5 & 2.00E-06 & $=$ & 1.00E-06 & 3.10E-07 \\
\hline Columbia River & Bonneville Dam & 4154232 & January 12, 2004 & Dichlorodiphenyldichloroethane & $\mathrm{mg} / \mathrm{L}$ & CRC1_Seg5 & 5.60E-06 & $=$ & 1.00E-06 & 3.10E-07 \\
\hline Columbia River & RM142 BONN DAM & loc 02 12/03-1/04 & January 1, 2004 & Dichlorodiphenyldichloroethane & $\mathrm{mg} / \mathrm{L}$ & CRC1_Seg5 & 5.60E-06 & $=$ & $1.00 \mathrm{E}-06$ & $3.10 \mathrm{E}-07$ \\
\hline Columbia River & RM142 BONN DAM & loc $028 / 03-9 / 03$ & September 1, 2003 & Dichlorodiphenyldichloroethylene & $\mathrm{mg} / \mathrm{L}$ & CRC1_Seg5 & $2.60 \mathrm{E}-07$ & $=$ & -- & $2.20 \mathrm{E}-07$ \\
\hline Columbia River & RM102, COL SLGH & loc 05 8/03-9/03 & September 1, 2003 & Dichlorodiphenyldichloroethylene & $\mathrm{mg} / \mathrm{L}$ & CRC1_Seg5 & 2.70E-07 & $=$ & -- & 2.20E-07 \\
\hline Columbia River & RM88, LAKE R & loc 07 12/03-1/04 & January 1, 2004 & Dichlorodiphenyldichloroethylene & $\mathrm{mg} / \mathrm{L}$ & CRC1_Seg5 & 2.70E-07 & $=$ & -- & $2.20 \mathrm{E}-07$ \\
\hline
\end{tabular}


Table E-13

\begin{tabular}{|c|c|c|c|c|c|c|c|c|c|c|}
\hline Sample Area & Sample Site & Sample ID & Date Collected & Constituent Name & Units & $\begin{array}{c}\text { QA } \\
\text { Category }\end{array}$ & $\begin{array}{l}\text { Analytical } \\
\text { Value }\end{array}$ & Qualifier & $\begin{array}{c}\text { Ecological } \\
\text { Benchmark } \\
\text { Value }\end{array}$ & $\begin{array}{c}\text { Human } \\
\text { Health } \\
\text { Benchmark } \\
\text { Value }\end{array}$ \\
\hline Columbia River & RM68, COWLITZ R & Ioc 12 8/03-9/03 & September 1, 2003 & Dichlorodiphenyldichloroethylene & $\mathrm{mg} / \mathrm{L}$ & CRC1_Seg5 & $2.70 \mathrm{E}-07$ & $\overline{=}$ & $\overline{--}$ & $2.20 \mathrm{E}-07$ \\
\hline Columbia River & Columbia Slough & 4314234 & June 22, 2004 & Dichlorodiphenyldichloroethylene & $\mathrm{mg} / \mathrm{L}$ & CRC1_Seg5 & 3.00E-07 & $\mathrm{J}$ & -- & $2.20 \mathrm{E}-07$ \\
\hline Columbia River & RM102, COL SLGH & Ioc 05 5/04-6/04 & June 1, 2004 & Dichlorodiphenyldichloroethylene & $\mathrm{mg} / \mathrm{L}$ & CRC1_Seg5 & 3.00E-07 & $=$ & -- & $2.20 \mathrm{E}-07$ \\
\hline Columbia River & RM86, MULT CHNL & loc 09 12/03-1/04 & January 1, 2004 & Dichlorodiphenyldichloroethylene & $\mathrm{mg} / \mathrm{L}$ & CRC1_Seg5 & 3.10E-07 & $=$ & -- & $2.20 \mathrm{E}-07$ \\
\hline Columbia River & Willamette RVR & 4154235 & January 12, 2004 & Dichlorodiphenyldichloroethylene & $\mathrm{mg} / \mathrm{L}$ & CRC1_Seg5 & 3.30E-07 & $=$ & -- & $2.20 \mathrm{E}-07$ \\
\hline Columbia River & RM103, WRVR & loc 04 12/03-1/04 & January 1, 2004 & Dichlorodiphenyldichloroethylene & $\mathrm{mg} / \mathrm{L}$ & CRC1_Seg5 & 3.30E-07 & $=$ & -- & $2.20 \mathrm{E}-07$ \\
\hline Columbia River & Columbia Slough & 3454417 & September 25, 2003 & Dichlorodiphenyldichloroethylene & $\mathrm{mg} / \mathrm{L}$ & CRC1_Seg5 & $3.40 \mathrm{E}-07$ & $=$ & -- & $2.20 \mathrm{E}-07$ \\
\hline Columbia River & RM86, MULT CHNL & loc 09 5/04-6/04 & June 1, 2004 & Dichlorodiphenyldichloroethylene & $\mathrm{mg} / \mathrm{L}$ & CRC1_Seg5 & 3.40E-07 & $=$ & -- & $2.20 \mathrm{E}-07$ \\
\hline Columbia River & Bonneville Dam & 3454414 & September 24, 2003 & Dichlorodiphenyldichloroethylene & $\mathrm{mg} / \mathrm{L}$ & CRC1_Seg5 & $3.50 \mathrm{E}-07$ & $=$ & -- & $2.20 \mathrm{E}-07$ \\
\hline Columbia River & RM88, LAKE R & loc 07 5/04-6/04 & June 1, 2004 & Dichlorodiphenyldichloroethylene & $\mathrm{mg} / \mathrm{L}$ & CRC1_Seg5 & $3.50 \mathrm{E}-07$ & $=$ & -- & $2.20 \mathrm{E}-07$ \\
\hline Columbia River & RM86, MULT CHNL & loc 09 8/03-9/03 & September 1, 2003 & Dichlorodiphenyldichloroethylene & $\mathrm{mg} / \mathrm{L}$ & CRC1_Seg5 & 3.60E-07 & $=$ & -- & $2.20 \mathrm{E}-07$ \\
\hline Columbia River & RM73, KALAMA R & loc 11 8/03-9/03 & September 1, 2003 & Dichlorodiphenyldichloroethylene & $\mathrm{mg} / \mathrm{L}$ & CRC1_Seg5 & 3.60E-07 & $=$ & -- & $2.20 \mathrm{E}-07$ \\
\hline Columbia River & Below Longview & 4314243 & June 23, 2004 & Dichlorodiphenyldichloroethylene & $\mathrm{mg} / \mathrm{L}$ & CRC1_Seg5 & 3.70E-07 & $=$ & -- & $2.20 \mathrm{E}-07$ \\
\hline Columbia River & RM54, LONGVIEW & loc 13 5/04-6/04 & June 1, 2004 & Dichlorodiphenyldichloroethylene & $\mathrm{mg} / \mathrm{L}$ & CRC1_Seg5 & 3.70E-07 & $=$ & -- & $2.20 \mathrm{E}-07$ \\
\hline Columbia River & RM102, WRVR & loc 06 8/03-9/03 & September 1, 2003 & Dichlorodiphenyldichloroethylene & $\mathrm{mg} / \mathrm{L}$ & CRC1_Seg5 & 3.90E-07 & $=$ & -- & $2.20 \mathrm{E}-07$ \\
\hline Columbia River & Kalama WA & 4154241 & January 13, 2004 & Dichlorodiphenyldichloroethylene & $\mathrm{mg} / \mathrm{L}$ & CRC1_Seg5 & $5.20 \mathrm{E}-07$ & $=$ & -- & $2.20 \mathrm{E}-07$ \\
\hline Columbia River & RM75, KALAMA R & loc 10 12/03-1/04 & January 1, 2004 & Dichlorodiphenyldichloroethylene & $\mathrm{mg} / \mathrm{L}$ & CRC1_Seg5 & $5.20 \mathrm{E}-07$ & $=$ & -- & $2.20 \mathrm{E}-07$ \\
\hline Columbia River & Bonneville Dam & 4314231 & June 22, 2004 & Dichlorodiphenyldichloroethylene & $\mathrm{mg} / \mathrm{L}$ & CRC1_Seg5 & $6.00 \mathrm{E}-07$ & $=$ & -- & $2.20 \mathrm{E}-07$ \\
\hline Columbia River & RM142 BONN DAM & loc $025 / 04-6 / 04$ & June 1, 2004 & Dichlorodiphenyldichloroethylene & $\mathrm{mg} / \mathrm{L}$ & CRC1_Seg5 & $6.00 \mathrm{E}-07$ & $=$ & -- & $2.20 \mathrm{E}-07$ \\
\hline Columbia River & Willamette RVR & 4314233 & June 22, 2004 & Dichlorodiphenyldichloroethylene & $\mathrm{mg} / \mathrm{L}$ & CRC1_Seg5 & $6.40 \mathrm{E}-07$ & $=$ & -- & $2.20 \mathrm{E}-07$ \\
\hline Columbia River & RM103, WRVR & loc 04 5/04-6/04 & June 1, 2004 & Dichlorodiphenyldichloroethylene & $\mathrm{mg} / \mathrm{L}$ & CRC1_Seg5 & $6.40 \mathrm{E}-07$ & $=$ & -- & $2.20 \mathrm{E}-07$ \\
\hline Columbia River & RM102, WRVR & $\operatorname{loc} 0612 / 03-1 / 04$ & January 1, 2004 & Dichlorodiphenyldichloroethylene & $\mathrm{mg} / \mathrm{L}$ & CRC1_Seg5 & $1.40 \mathrm{E}-06$ & $=$ & -- & $2.20 \mathrm{E}-07$ \\
\hline Columbia River & Columbia Slough & 4154236 & January 12, 2004 & Dichlorodiphenyldichloroethylene & $\mathrm{mg} / \mathrm{L}$ & CRC1_Seg5 & 1.60E-06 & $=$ & -- & $2.20 \mathrm{E}-07$ \\
\hline Columbia River & RM102, COL SLGH & loc 05 12/03-1/04 & January 1, 2004 & Dichlorodiphenyldichloroethylene & $\mathrm{mg} / \mathrm{L}$ & CRC1_Seg5 & 1.60E-06 & $=$ & -- & $2.20 \mathrm{E}-07$ \\
\hline Columbia River & Bonneville Dam & 4154232 & January 12, 2004 & Dichlorodiphenyldichloroethylene & $\mathrm{mg} / \mathrm{L}$ & CRC1_Seg5 & $2.10 \mathrm{E}-06$ & $=$ & -- & $2.20 \mathrm{E}-07$ \\
\hline Columbia River & RM142 BONN DAM & $\operatorname{loc} 02$ 12/03-1/04 & January 1,2004 & Dichlorodiphenyldichloroethylene & $\mathrm{mg} / \mathrm{L}$ & CRC1_Seg5 & $2.10 \mathrm{E}-06$ & $=$ & -- & $2.20 \mathrm{E}-07$ \\
\hline Columbia River & Bonneville Dam & 4154232 & January 12, 2004 & Dichlorodiphenyltrichloroethane & $\mathrm{mg} / \mathrm{L}$ & CRC1_Seg5 & $2.70 \mathrm{E}-07$ & $\mathrm{~J}$ & 1.00E-06 & $2.20 \mathrm{E}-07$ \\
\hline Columbia River & RM142 BONN DAM & loc 02 12/03-1/04 & January 1, 2004 & Dichlorodiphenyltrichloroethane & $\mathrm{mg} / \mathrm{L}$ & CRC1_Seg5 & 2.70E-07 & $=$ & $1.00 \mathrm{E}-06$ & $2.20 \mathrm{E}-07$ \\
\hline Columbia River & RM86, MULT CHNL & loc 09 12/03-1/04 & January 1, 2004 & Dichlorodiphenyltrichloroethane & $\mathrm{mg} / \mathrm{L}$ & CRC1_Seg5 & $2.70 \mathrm{E}-07$ & $=$ & $1.00 \mathrm{E}-06$ & $2.20 \mathrm{E}-07$ \\
\hline Columbia River & Kalama WA & 4154241 & January 13, 2004 & Dichlorodiphenyltrichloroethane & $\mathrm{mg} / \mathrm{L}$ & CRC1_Seg5 & $3.20 \mathrm{E}-07$ & $=$ & $1.00 \mathrm{E}-06$ & $2.20 \mathrm{E}-07$ \\
\hline Columbia River & RM75, KALAMA R & Ioc 10 12/03-1/04 & January 1, 2004 & Dichlorodiphenyltrichloroethane & $\mathrm{mg} / \mathrm{L}$ & CRC1_Seg5 & $3.20 \mathrm{E}-07$ & $=$ & 1.00E-06 & $2.20 \mathrm{E}-07$ \\
\hline Columbia River & Columbia Slough & 4154236 & January 12, 2004 & Dichlorodiphenyltrichloroethane & $\mathrm{mg} / \mathrm{L}$ & CRC1_Seg5 & 1.50E-06 & $\mathrm{J}$ & $1.00 \mathrm{E}-06$ & $2.20 \mathrm{E}-07$ \\
\hline Columbia River & RM102, COL SLGH & loc 05 12/03-1/04 & January 1, 2004 & Dichlorodiphenyltrichloroethane & $\mathrm{mg} / \mathrm{L}$ & CRC1_Seg5 & 1.50E-06 & $=$ & $1.00 \mathrm{E}-06$ & $2.20 \mathrm{E}-07$ \\
\hline Columbia River & RM102, WRVR & loc 06 12/03-1/04 & January 1, 2004 & Dichlorodiphenyltrichloroethane & $\mathrm{mg} / \mathrm{L}$ & CRC1_Seg5 & $1.50 \mathrm{E}-06$ & $=$ & 1.00E-06 & $2.20 \mathrm{E}-07$ \\
\hline Columbia River & Below Longview & 3454426 & September 25, 2003 & Dieldrin & $\mathrm{mg} / \mathrm{L}$ & CRC1_Seg5 & $5.20 \mathrm{E}-08$ & $=$ & $1.90 \mathrm{E}-06$ & $5.20 \mathrm{E}-08$ \\
\hline Columbia River & RM88, LAKE R & loc 07 8/03-9/03 & September 1, 2003 & Dieldrin & $\mathrm{mg} / \mathrm{L}$ & CRC1_Seg5 & 5.60E-08 & $=$ & $1.90 \mathrm{E}-06$ & $5.20 \mathrm{E}-08$ \\
\hline Columbia River & RM103, WRVR & loc 04 8/03-9/03 & September 1, 2003 & Dieldrin & $\mathrm{mg} / \mathrm{L}$ & CRC1_Seg5 & 5.70E-08 & $=$ & $1.90 \mathrm{E}-06$ & $5.20 \mathrm{E}-08$ \\
\hline Columbia River & RM142 BONN DAM & loc 02 8/03-9/03 & September 1, 2003 & Dieldrin & $\mathrm{mg} / \mathrm{L}$ & CRC1_Seg5 & $6.40 \mathrm{E}-08$ & $=$ & 1.90E-06 & $5.20 \mathrm{E}-08$ \\
\hline Columbia River & RM102, WRVR & loc 06 5/04-6/04 & June 1, 2004 & Dieldrin & $\mathrm{mg} / \mathrm{L}$ & CRC1_Seg5 & 6.70E-08 & $=$ & $1.90 \mathrm{E}-06$ & $5.20 \mathrm{E}-08$ \\
\hline Columbia River & Willamette RVR & 3454416 & June 25, 2003 & Dieldrin & $\mathrm{mg} / \mathrm{L}$ & CRC1_Seg5 & 7.00E-08 & $=$ & 1.90E-06 & 5.20E-08 \\
\hline Columbia River & Bonneville Dam & 3454414 & September 24, 2003 & Dieldrin & $\mathrm{mg} / \mathrm{L}$ & CRC1_Seg5 & 8.60E-08 & $=$ & $1.90 \mathrm{E}-06$ & $5.20 \mathrm{E}-08$ \\
\hline Columbia River & Willamette RVR & 4154235 & January 12, 2004 & Dieldrin & $\mathrm{mg} / \mathrm{L}$ & CRC1_Seg5 & 8.60E-08 & $\mathrm{J}$ & $1.90 \mathrm{E}-06$ & $5.20 \mathrm{E}-08$ \\
\hline Columbia River & RM103, WRVR & loc 04 12/03-1/04 & January 1, 2004 & Dieldrin & $\mathrm{mg} / \mathrm{L}$ & CRC1_Seg5 & 8.60E-08 & $=$ & $1.90 \mathrm{E}-06$ & $5.20 \mathrm{E}-08$ \\
\hline Columbia River & Bonneville Dam & 4314231 & June 22, 2004 & Dieldrin & $\mathrm{mg} / \mathrm{L}$ & CRC1_Seg5 & $1.00 \mathrm{E}-07$ & $\mathrm{~J}$ & $1.90 \mathrm{E}-06$ & $5.20 \mathrm{E}-08$ \\
\hline Columbia River & RM142 BONN DAM & loc 02 5/04-6/04 & June 1, 2004 & Dieldrin & $\mathrm{mg} / \mathrm{L}$ & CRC1_Seg5 & $1.00 \mathrm{E}-07$ & $=$ & 1.90E-06 & 5.20E-08 \\
\hline
\end{tabular}


Table E-13

Summary of Surface Water Results From Segment 5 Exceeding Select Ecological or Human Health Benchmark Values

\begin{tabular}{|c|c|c|c|c|c|c|c|c|c|c|}
\hline Sample Area & Sample Site & Sample ID & Date Collected & Constituent Name & Units & $\begin{array}{c}\text { QA } \\
\text { Category }\end{array}$ & $\begin{array}{c}\text { Analytical } \\
\text { Value }\end{array}$ & Qualifier & $\begin{array}{c}\text { Ecological } \\
\text { Benchmark } \\
\text { Value }\end{array}$ & $\begin{array}{c}\text { Human } \\
\text { Health } \\
\text { Benchmark } \\
\text { Value }\end{array}$ \\
\hline Columbia River & RM87, LEWIS R & loc 08 5/04-6/04 & June 1, 2004 & Dieldrin & $\mathrm{mg} / \mathrm{L}$ & CRC1_Seg5 & $1.10 \mathrm{E}-07$ & $=$ & $1.90 \mathrm{E}-06$ & $5.20 \mathrm{E}-08$ \\
\hline Columbia River & Columbia Slough & 4314234 & June 22, 2004 & Dieldrin & $\mathrm{mg} / \mathrm{L}$ & CRC1_Seg5 & $1.20 \mathrm{E}-07$ & $\mathrm{~J}$ & 1.90E-06 & $5.20 \mathrm{E}-08$ \\
\hline Columbia River & RM102, COL SLGH & loc 05 5/04-6/04 & June 1, 2004 & Dieldrin & $\mathrm{mg} / \mathrm{L}$ & CRC1_Seg5 & $1.20 \mathrm{E}-07$ & $=$ & 1.90E-06 & 5.20E-08 \\
\hline Columbia River & Willamette RVR & 4314233 & June 22, 2004 & Dieldrin & $\mathrm{mg} / \mathrm{L}$ & CRC1_Seg5 & $1.30 \mathrm{E}-07$ & $\mathrm{~J}$ & 1.90E-06 & $5.20 E-08$ \\
\hline Columbia River & Below Longview & 4314243 & June 23, 2004 & Dieldrin & $\mathrm{mg} / \mathrm{L}$ & CRC1_Seg5 & $1.30 \mathrm{E}-07$ & $\mathrm{~J}$ & $1.90 \mathrm{E}-06$ & $5.20 \mathrm{E}-08$ \\
\hline Columbia River & RM103, WRVR & loc 04 5/04-6/04 & June 1, 2004 & Dieldrin & $\mathrm{mg} / \mathrm{L}$ & CRC1_Seg5 & $1.30 \mathrm{E}-07$ & $=$ & $1.90 \mathrm{E}-06$ & 5.20E-08 \\
\hline Columbia River & RM73, KALAMA R & loc 11 12/3-1/04 & January 1, 2004 & Dieldrin & $\mathrm{mg} / \mathrm{L}$ & CRC1_Seg5 & $1.30 \mathrm{E}-07$ & $=$ & 1.90E-06 & 5.20E-08 \\
\hline Columbia River & RM54, LONGVIEW & loc 13 5/04-6/04 & June 1, 2004 & Dieldrin & $\mathrm{mg} / \mathrm{L}$ & CRC1_Seg5 & $1.30 \mathrm{E}-07$ & $=$ & 1.90E-06 & 5.20E-08 \\
\hline Columbia River & RM102, COL SLGH & loc 05 8/03-9/03 & September 1, 2003 & Dieldrin & $\mathrm{mg} / \mathrm{L}$ & CRC1_Seg5 & $1.40 \mathrm{E}-07$ & $=$ & $1.90 \mathrm{E}-06$ & 5.20E-08 \\
\hline Columbia River & RM102, WRVR & loc 06 8/03-9/03 & September 1, 2003 & Dieldrin & $\mathrm{mg} / \mathrm{L}$ & CRC1_Seg5 & $1.50 \mathrm{E}-07$ & $=$ & 1.90E-06 & 5.20E-08 \\
\hline Columbia River & RM87, LEWIS R & Ioc 08 12/03-1/04 & January 1, 2004 & Dieldrin & $\mathrm{mg} / \mathrm{L}$ & CRC1_Seg5 & 1.70E-07 & $=$ & 1.90E-06 & 5.20E-08 \\
\hline Columbia River & Columbia Slough & 3454417 & September 25, 2003 & Dieldrin & $\mathrm{mg} / \mathrm{L}$ & CRC1_Seg5 & $1.80 \mathrm{E}-07$ & $=$ & 1.90E-06 & $5.20 \mathrm{E}-08$ \\
\hline Columbia River & RM121, WASHRVR & loc 03 5/04-6/04 & June 1, 2004 & Dieldrin & $\mathrm{mg} / \mathrm{L}$ & CRC1_Seg5 & $1.80 \mathrm{E}-07$ & $=$ & 1.90E-06 & 5.20E-08 \\
\hline Columbia River & RM88, LAKE R & loc 07 5/04-6/04 & June 1, 2004 & Dieldrin & $\mathrm{mg} / \mathrm{L}$ & CRC1_Seg5 & $2.20 \mathrm{E}-07$ & $=$ & $1.90 \mathrm{E}-06$ & $5.20 \mathrm{E}-08$ \\
\hline Columbia River & RM86, MULT CHNL & loc 09 8/03-9/03 & September 1, 2003 & Dieldrin & $\mathrm{mg} / \mathrm{L}$ & CRC1_Seg5 & $2.40 \mathrm{E}-07$ & $=$ & 1.90E-06 & $5.20 \mathrm{E}-08$ \\
\hline Columbia River & RM121, WASHRVR & Ioc 03 12/03-1/04 & January 1, 2004 & Dieldrin & $\mathrm{mg} / \mathrm{L}$ & CRC1_Seg5 & $2.50 \mathrm{E}-07$ & $=$ & $1.90 \mathrm{E}-06$ & 5.20E-08 \\
\hline Columbia River & RM86, MULT CHNL & loc 09 5/04-6/04 & June 1, 2004 & Dieldrin & $\mathrm{mg} / \mathrm{L}$ & CRC1_Seg5 & 3.60E-07 & $=$ & 1.90E-06 & 5.20E-08 \\
\hline Columbia River & RM88, LAKE R & loc 07 12/03-1/04 & January 1, 2004 & Dieldrin & $\mathrm{mg} / \mathrm{L}$ & CRC1_Seg5 & 4.50E-07 & $=$ & $1.90 \mathrm{E}-06$ & $5.20 \mathrm{E}-08$ \\
\hline Columbia River & Kalama WA & 4154241 & January 13, 2004 & Dieldrin & $\mathrm{mg} / \mathrm{L}$ & CRC1_Seg5 & 5.00E-07 & $=$ & 1.90E-06 & 5.20E-08 \\
\hline Columbia River & RM75, KALAMA R & Ioc 10 12/03-1/04 & January 1, 2004 & Dieldrin & $\mathrm{mg} / \mathrm{L}$ & CRC1_Seg5 & 5.00E-07 & $=$ & $1.90 \mathrm{E}-06$ & 5.20E-08 \\
\hline Columbia River & Bonneville Dam & 4154232 & January 12, 2004 & Dieldrin & $\mathrm{mg} / \mathrm{L}$ & CRC1_Seg5 & $5.70 \mathrm{E}-07$ & $\mathrm{~J}$ & 1.90E-06 & $5.20 \mathrm{E}-08$ \\
\hline Columbia River & RM142 BONN DAM & Ioc 02 12/03-1/04 & January 1, 2004 & Dieldrin & $\mathrm{mg} / \mathrm{L}$ & CRC1_Seg5 & $5.70 \mathrm{E}-07$ & $=$ & 1.90E-06 & 5.20E-08 \\
\hline Columbia River & RM86, MULT CHNL & loc 09 12/03-1/04 & January 1, 2004 & Dieldrin & $\mathrm{mg} / \mathrm{L}$ & CRC1_Seg5 & $7.20 \mathrm{E}-07$ & $=$ & $1.90 \mathrm{E}-06$ & $5.20 \mathrm{E}-08$ \\
\hline Columbia River & RM102, WRVR & loc 06 12/03-1/04 & January 1, 2004 & Dieldrin & $\mathrm{mg} / \mathrm{L}$ & CRC1_Seg5 & $2.50 \mathrm{E}-06$ & $=$ & $1.90 \mathrm{E}-06$ & $5.20 \mathrm{E}-08$ \\
\hline Columbia River & Columbia Slough & 4154236 & January 12, 2004 & Dieldrin & $\mathrm{mg} / \mathrm{L}$ & CRC1_Seg5 & 5.40E-06 & $=$ & $1.90 \mathrm{E}-06$ & 5.20E-08 \\
\hline Columbia River & RM102, COL SLGH & Ioc 05 12/03-1/04 & January 1, 2004 & Dieldrin & $\mathrm{mg} / \mathrm{L}$ & CRC1_Seg5 & 5.40E-06 & $=$ & 1.90E-06 & 5.20E-08 \\
\hline Columbia River & Willamette RVR & 4314233 & June 22, 2004 & Heptachlor epoxide & $\mathrm{mg} / \mathrm{L}$ & CRC1_Seg5 & $4.50 \mathrm{E}-08$ & $\mathrm{~J}$ & 3.80E-06 & $3.90 \mathrm{E}-08$ \\
\hline Columbia River & RM103, WRVR & loc 04 5/04-6/04 & June 1, 2004 & Heptachlor epoxide & $\mathrm{mg} / \mathrm{L}$ & CRC1_Seg5 & 4.50E-08 & $=$ & 3.80E-06 & 3.90E-08 \\
\hline Columbia River & RM88, LAKE R & loc 07 5/04-6/04 & June 1, 2004 & Heptachlor epoxide & $\mathrm{mg} / \mathrm{L}$ & CRC1_Seg5 & $6.70 \mathrm{E}-08$ & $=$ & 3.80E-06 & $3.90 \mathrm{E}-08$ \\
\hline Columbia River & RM68, COWLITZ R & Ioc $128 / 03-9 / 03$ & September 1, 2003 & Hexachlorobenzene & $\mathrm{mg} / \mathrm{L}$ & CRC1_Seg5 & $3.40 \mathrm{E}-07$ & $=$ & -- & 2.80E-07 \\
\hline Columbia River & RM73, KALAMA R & loc 11 12/3-1/04 & January 1, 2004 & Hexachlorobenzene & $\mathrm{mg} / \mathrm{L}$ & CRC1_Seg5 & $3.60 \mathrm{E}-07$ & $=$ & -- & 2.80E-07 \\
\hline Columbia River & RM121, WASHRVR & loc 03 12/03-1/04 & January 1, 2004 & Hexachlorobenzene & $\mathrm{mg} / \mathrm{L}$ & CRC1_Seg5 & $3.70 \mathrm{E}-07$ & $=$ & -- & $2.80 \mathrm{E}-07$ \\
\hline Columbia River & RM68, COWLITZ R & loc 12 12/03-1/04 & January 1, 2004 & Hexachlorobenzene & $\mathrm{mg} / \mathrm{L}$ & CRC1_Seg5 & 4.00E-07 & $=$ & -- & $2.80 \mathrm{E}-07$ \\
\hline Columbia River & RM86, MULT CHNL & loc 09 5/04-6/04 & June 1, 2004 & Hexachlorobenzene & $\mathrm{mg} / \mathrm{L}$ & CRC1_Seg5 & 4.10E-07 & $=$ & -- & 2.80E-07 \\
\hline Columbia River & RM86, MULT CHNL & Ioc 09 12/03-1/04 & January 1, 2004 & Hexachlorobenzene & $\mathrm{mg} / \mathrm{L}$ & CRC1_Seg5 & 4.80E-07 & $=$ & -- & 2.80E-07 \\
\hline Columbia River & Kalama WA & 4154241 & January 13, 2004 & Hexachlorobenzene & $\mathrm{mg} / \mathrm{L}$ & CRC1_Seg5 & $5.90 \mathrm{E}-07$ & $\mathrm{~J}$ & -- & $2.80 \mathrm{E}-07$ \\
\hline Columbia River & RM75, KALAMA R & Ioc 10 12/03-1/04 & January 1, 2004 & Hexachlorobenzene & $\mathrm{mg} / \mathrm{L}$ & CRC1_Seg5 & $5.90 \mathrm{E}-07$ & $=$ & -- & 2.80E-07 \\
\hline Columbia River & RM73, KALAMA R & loc 11 8/03-9/03 & September 1, 2003 & Hexachlorobenzene & $\mathrm{mg} / \mathrm{L}$ & CRC1_Seg5 & $6.90 \mathrm{E}-07$ & $=$ & -- & $2.80 \mathrm{E}-07$ \\
\hline Columbia River & Bonneville Dam & 4154232 & January 12, 2004 & Hexachlorobenzene & $\mathrm{mg} / \mathrm{L}$ & CRC1_Seg5 & 8.00E-07 & $\mathrm{J}$ & -- & 2.80E-07 \\
\hline Columbia River & RM142 BONN DAM & Ioc 02 12/03-1/04 & January 1, 2004 & Hexachlorobenzene & $\mathrm{mg} / \mathrm{L}$ & CRC1_Seg5 & 8.00E-07 & $=$ & -- & 2.80E-07 \\
\hline Columbia River & Columbia Slough & 4154236 & January 12, 2004 & Hexachlorobenzene & $\mathrm{mg} / \mathrm{L}$ & CRC1_Seg5 & 1.10E-06 & $\mathrm{J}$ & -- & $2.80 \mathrm{E}-07$ \\
\hline Columbia River & RM102, COL SLGH & loc 05 12/03-1/04 & January 1, 2004 & Hexachlorobenzene & $\mathrm{mg} / \mathrm{L}$ & CRC1_Seg5 & 1.10E-06 & $=$ & -- & $2.80 \mathrm{E}-07$ \\
\hline Columbia River & RM102, WRVR & loc 06 12/03-1/04 & January 1, 2004 & Hexachlorobenzene & $\mathrm{mg} / \mathrm{L}$ & CRC1_Seg5 & $2.30 \mathrm{E}-06$ & $=$ & -- & 2.80E-07 \\
\hline Columbia River & Bonneville Dam & 4314231 & June 22,2004 & al polychlorinated biphe & $\mathrm{mg} / \mathrm{L}$ & CRC1_Seg5 & 3.30E-07 & $=$ & $1.40 \mathrm{E}-05$ & $6.40 \mathrm{E}-08$ \\
\hline Columbia River & Willamette RVR & 4314233 & June 22, 2004 & Total polychlorinated biphenyl & $\mathrm{mg} / \mathrm{L}$ & CRC1_Seg5 & 3.60E-07 & $=$ & 1.40E-05 & $6.40 \mathrm{E}-08$ \\
\hline
\end{tabular}


Table E-13

Summary of Surface Water Results From Segment 5 Exceeding Select Ecological or Human Health Benchmark Values

\begin{tabular}{|c|c|c|c|c|c|c|c|c|c|c|}
\hline Sample Area & Sample Site & Sample ID & Date Collected & Constituent Name & Units & $\begin{array}{c}\text { QA } \\
\text { Category }\end{array}$ & $\begin{array}{c}\text { Analytical } \\
\text { Value }\end{array}$ & Qualifier & $\begin{array}{c}\text { Ecological } \\
\text { Benchmark } \\
\text { Value }\end{array}$ & $\begin{array}{c}\text { Human } \\
\text { Health } \\
\text { Benchmark } \\
\text { Value }\end{array}$ \\
\hline Columbia River & Below Longview & 3454426 & September 25, 2003 & Total polychlorinated biphenyl & $\mathrm{mg} / \mathrm{L}$ & CRC1_Seg5 & $3.90 \mathrm{E}-07$ & $=$ & $1.40 \mathrm{E}-05$ & $6.40 \mathrm{E}-08$ \\
\hline Columbia River & Kalama WA & 4314240 & June 23, 2004 & Total polychlorinated biphenyl & $\mathrm{mg} / \mathrm{L}$ & CRC1_Seg5 & 5.90E-07 & $=$ & 1.40E-05 & $6.40 \mathrm{E}-08$ \\
\hline Columbia River & Below Longview & 4314243 & June 23, 2004 & Total polychlorinated biphenyl & $\mathrm{mg} / \mathrm{L}$ & CRC1_Seg5 & $6.00 \mathrm{E}-07$ & $=$ & 1.40E-05 & $6.40 \mathrm{E}-08$ \\
\hline Columbia River & Columbia Slough & 3454417 & September 25, 2003 & Total polychlorinated biphenyl & $\mathrm{mg} / \mathrm{L}$ & CRC1_Seg5 & $2.20 \mathrm{E}-06$ & $\mathrm{JN}$ & $1.40 \mathrm{E}-05$ & $6.40 \mathrm{E}-08$ \\
\hline Columbia River & Columbia Slough & 4314234 & June 22, 2004 & Total polychlorinated biphenyl & $\mathrm{mg} / \mathrm{L}$ & CRC1_Seg5 & 1.30E-05 & $=$ & $1.40 \mathrm{E}-05$ & $6.40 \mathrm{E}-08$ \\
\hline Columbia River & Columbia Slough & 4154236 & January 12, 2004 & Total polychlorinated biphenyl & $\mathrm{mg} / \mathrm{L}$ & CRC1_Seg5 & 2.10E-05 & $\mathrm{JN}$ & 1.40E-05 & $6.40 \mathrm{E}-08$ \\
\hline
\end{tabular}




\section{APPENDIX F}

\section{BIOTA SUMMARY STATISTIC TABLES}


WCH-91

Rev. 0

Columbia River Component Data Evaluation Summary Report

July 2006

F-ii 
Table F-1

Summary Statistics for Asiatic Clam Shells Collected from Segment 1 of the Columbia River

\begin{tabular}{|c|c|c|c|c|c|c|c|c|c|c|}
\hline $\begin{array}{l}\text { Constituent } \\
\text { Class }\end{array}$ & Constituent Name & Units & $\begin{array}{l}\text { Number } \\
\text { Analyzed }\end{array}$ & $\begin{array}{l}\text { Number } \\
\text { Detected }\end{array}$ & $\begin{array}{c}\text { Frequency of } \\
\text { Detection }\end{array}$ & $\begin{array}{c}\text { Minimum } \\
\text { Nondetect }\end{array}$ & $\begin{array}{l}\text { Maximum } \\
\text { Nondetect }\end{array}$ & $\begin{array}{c}\text { Minimum } \\
\text { Detect }\end{array}$ & $\begin{array}{l}\text { Maximum } \\
\text { Detect }\end{array}$ & $\begin{array}{c}\text { Average } \\
\text { Concentration }\end{array}$ \\
\hline RAD & Antimony-125 & $\mathrm{pCi} / \mathrm{g}$ & 1 & 0 & $0 \%$ & 0.026 & 0.026 & -- & -- & 0.013 \\
\hline RAD & Beryllium-7 & $\mathrm{pCi} / \mathrm{g}$ & 2 & 0 & $0 \%$ & 3.6 & 22 & -- & -- & 6.5 \\
\hline RAD & Cesium-134 & $\mathrm{pCi} / \mathrm{g}$ & 5 & 0 & $0 \%$ & 0.0035 & 0.047 & -- & -- & 0.0093 \\
\hline RAD & Cesium-137 & $\mathrm{pCi} / \mathrm{g}$ & 2 & 0 & $0 \%$ & 8.84E-04 & 0.0056 & -- & -- & 0.0016 \\
\hline RAD & Cobalt-60 & $\mathrm{pCi} / \mathrm{g}$ & 4 & 0 & $0 \%$ & 0.0037 & 0.029 & -- & -- & 0.0070 \\
\hline RAD & Europium-152 & $\mathrm{pCi} / \mathrm{g}$ & 2 & 0 & $0 \%$ & 0.0094 & 0.018 & -- & -- & 0.0068 \\
\hline RAD & Europium-154 & $\mathrm{pCi} / \mathrm{g}$ & 3 & 0 & $0 \%$ & 0.0027 & 0.042 & -- & -- & 0.011 \\
\hline RAD & Europium-155 & $\mathrm{pCi} / \mathrm{g}$ & 3 & 0 & $0 \%$ & 0.033 & 0.073 & -- & -- & 0.024 \\
\hline RAD & Potassium-40 & $\mathrm{pCi} / \mathrm{g}$ & 1 & 1 & $100 \%$ & -- & -- & 0.60 & 0.60 & 0.60 \\
\hline RAD & Ruthenium-106 & $\mathrm{pCi} / \mathrm{g}$ & 1 & 0 & $0 \%$ & 0.11 & 0.11 & -- & -- & 0.054 \\
\hline RAD & Strontium-90 & $\mathrm{pCi} / \mathrm{g}$ & 9 & 9 & $100 \%$ & -- & -- & 0.19 & 0.37 & 0.26 \\
\hline RAD & Technetium-99 & $\mathrm{pCi} / \mathrm{g}$ & 4 & 1 & $25 \%$ & 0.14 & 0.41 & 0.64 & 0.64 & 0.25 \\
\hline
\end{tabular}

RAD - Radionuclide 
Table F-2

Summary Statistics for Asiatic Clam Soft Tissue Collected from Segment 1 of the Columbia River

\begin{tabular}{|c|c|c|c|c|c|c|c|c|c|c|}
\hline $\begin{array}{l}\text { Constituent } \\
\text { Class }\end{array}$ & Constituent Name & Units & $\begin{array}{l}\text { Number } \\
\text { Analyzed }\end{array}$ & $\begin{array}{l}\text { Number } \\
\text { Detected }\end{array}$ & $\begin{array}{c}\text { Frequency of } \\
\text { Detection }\end{array}$ & $\begin{array}{l}\text { Minimum } \\
\text { Nondetect }\end{array}$ & $\begin{array}{l}\text { Maximum } \\
\text { Nondetect }\end{array}$ & $\begin{array}{l}\text { Minimum } \\
\text { Detect }\end{array}$ & $\begin{array}{l}\text { Maximum } \\
\text { Detect }\end{array}$ & $\begin{array}{c}\text { Average } \\
\text { Concentration }\end{array}$ \\
\hline$\overline{\text { METAL }}$ & Aluminum & $\mathrm{mg} / \mathrm{kg}$ & 14 & 14 & $100 \%$ & -- & -- & 239 & 861 & 456 \\
\hline METAL & Antimony & $\mathrm{mg} / \mathrm{kg}$ & 14 & 11 & $79 \%$ & 0.010 & 0.019 & 0.011 & 0.049 & 0.018 \\
\hline METAL & Arsenic & $\mathrm{mg} / \mathrm{kg}$ & 14 & 14 & $100 \%$ & -- & -- & 4.9 & 9.0 & 7.0 \\
\hline METAL & Barium & $\mathrm{mg} / \mathrm{kg}$ & 9 & 9 & $100 \%$ & -- & -- & 8.2 & 25 & 12 \\
\hline METAL & Beryllium & $\mathrm{mg} / \mathrm{kg}$ & 14 & 5 & $36 \%$ & 0.0040 & 0.020 & 0.060 & 0.12 & 0.036 \\
\hline METAL & Cadmium & $\mathrm{mg} / \mathrm{kg}$ & 14 & 14 & $100 \%$ & -- & -- & 1.5 & 5.0 & 2.2 \\
\hline METAL & Chromium & $\mathrm{mg} / \mathrm{kg}$ & 14 & 14 & $100 \%$ & -- & -- & 1.2 & 5.8 & 2.7 \\
\hline METAL & Copper & $\mathrm{mg} / \mathrm{kg}$ & 14 & 14 & $100 \%$ & -- & -- & 25 & 63 & 38 \\
\hline METAL & Lead & $\mathrm{mg} / \mathrm{kg}$ & 14 & 14 & $100 \%$ & -- & -- & 0.44 & 2.0 & 0.89 \\
\hline METAL & Manganese & $\mathrm{mg} / \mathrm{kg}$ & 14 & 14 & $100 \%$ & -- & -- & 18 & 62 & 33 \\
\hline METAL & Mercury & $\mathrm{mg} / \mathrm{kg}$ & 14 & 12 & $86 \%$ & 0.050 & 0.050 & 0.031 & 0.085 & 0.057 \\
\hline METAL & Nickel & $\mathrm{mg} / \mathrm{kg}$ & 14 & 14 & $100 \%$ & -- & -- & 0.63 & 2.2 & 1.1 \\
\hline METAL & Selenium & $\mathrm{mg} / \mathrm{kg}$ & 14 & 14 & $100 \%$ & -- & -- & 2.0 & 3.2 & 2.4 \\
\hline METAL & Silver & $\mathrm{mg} / \mathrm{kg}$ & 14 & 14 & $100 \%$ & -- & -- & 0.040 & 0.086 & 0.055 \\
\hline METAL & Thallium & $\mathrm{mg} / \mathrm{kg}$ & 14 & 14 & $100 \%$ & -- & -- & 0.049 & 0.19 & 0.099 \\
\hline METAL & Thorium & $\mathrm{mg} / \mathrm{kg}$ & 14 & 14 & $100 \%$ & -- & -- & 0.023 & 0.30 & 0.081 \\
\hline METAL & Uranium & $\mathrm{mg} / \mathrm{kg}$ & 26 & 26 & $100 \%$ & -- & -- & 0.11 & 0.24 & 0.17 \\
\hline METAL & Zinc & $\mathrm{mg} / \mathrm{kg}$ & 14 & 14 & $100 \%$ & -- & -- & 90 & 182 & 117 \\
\hline
\end{tabular}


Table F-3

Summary Statistics for Clam Shells Collected from Segment 1 of the Columbia River

\begin{tabular}{|c|c|c|c|c|c|c|c|c|c|c|}
\hline $\begin{array}{c}\text { Constituent } \\
\text { Class }\end{array}$ & Constituent Name & Units & $\begin{array}{l}\text { Number } \\
\text { Analyzed }\end{array}$ & $\begin{array}{l}\text { Number } \\
\text { Detected }\end{array}$ & $\begin{array}{c}\text { Frequency of } \\
\text { Detection }\end{array}$ & $\begin{array}{l}\text { Minimum } \\
\text { Nondetect }\end{array}$ & $\begin{array}{l}\text { Maximum } \\
\text { Nondetect }\end{array}$ & $\begin{array}{c}\text { Minimum } \\
\text { Detect }\end{array}$ & $\begin{array}{c}\text { Maximum } \\
\text { Detect }\end{array}$ & $\begin{array}{c}\text { Average } \\
\text { Concentration }\end{array}$ \\
\hline$\overline{\text { METAL }}$ & Beryllium & $\mathrm{mg} / \mathrm{kg}$ & 2 & 0 & $0 \%$ & 0.20 & 0.20 & $\overline{--}$ & $\overline{--}$ & 0.10 \\
\hline METAL & Chromium & $\mathrm{mg} / \mathrm{kg}$ & 2 & 1 & $50 \%$ & 0.50 & 0.50 & 0.54 & 0.54 & 0.40 \\
\hline METAL & Copper & $\mathrm{mg} / \mathrm{kg}$ & 2 & 2 & $100 \%$ & -- & -- & 3.1 & 4.0 & 3.6 \\
\hline METAL & Nickel & $\mathrm{mg} / \mathrm{kg}$ & 2 & 0 & $0 \%$ & 2.0 & 2.0 & -- & -- & 1.0 \\
\hline RAD & Cesium-137 & $\mathrm{pCi} / \mathrm{g}$ & 1 & 1 & $100 \%$ & -- & -- & 0.011 & 0.011 & 0.011 \\
\hline RAD & Cobalt- 60 & $\mathrm{pCi} / \mathrm{g}$ & 1 & 0 & $0 \%$ & 0.0030 & 0.0030 & -- & -- & 0.0015 \\
\hline RAD & Plutonium-238 & $\mathrm{pCi} / \mathrm{g}$ & 1 & 0 & $0 \%$ & 0.085 & 0.085 & -- & -- & 0.043 \\
\hline RAD & Plutonium-239/240 & $\mathrm{pCi} / \mathrm{g}$ & 1 & 0 & $0 \%$ & 0.090 & 0.090 & -- & -- & 0.045 \\
\hline RAD & Potassium-40 & $\mathrm{pCi} / \mathrm{g}$ & 1 & 1 & $100 \%$ & -- & -- & 0.69 & 0.69 & 0.69 \\
\hline RAD & Strontium-90 & $\mathrm{pCi} / \mathrm{g}$ & 2 & 2 & $100 \%$ & -- & -- & 0.20 & 0.40 & 0.30 \\
\hline RAD & Uranium-234 & $\mathrm{pCi} / \mathrm{g}$ & 1 & 1 & $100 \%$ & -- & -- & 0.28 & 0.28 & 0.28 \\
\hline RAD & Uranium-235 & $\mathrm{pCi} / \mathrm{g}$ & 1 & 1 & $100 \%$ & -- & -- & 0.024 & 0.024 & 0.024 \\
\hline RAD & Uranium-238 & $\mathrm{pCi} / \mathrm{g}$ & 1 & 1 & $100 \%$ & -- & -- & 0.14 & 0.14 & 0.14 \\
\hline
\end{tabular}

RAD - Radionuclide 
Table F-4

Summary Statistics for Bass Fillet with Skin Off Collected from Segment 1 of the Columbia River

\begin{tabular}{|c|c|c|c|c|c|c|c|c|c|c|}
\hline $\begin{array}{c}\text { Constituent } \\
\text { Class }\end{array}$ & Constituent Name & Units & $\begin{array}{l}\text { Number } \\
\text { Analyzed }\end{array}$ & $\begin{array}{l}\text { Number } \\
\text { Detected }\end{array}$ & $\begin{array}{c}\text { Frequency of } \\
\text { Detection }\end{array}$ & $\begin{array}{l}\text { Minimum } \\
\text { Nondetect }\end{array}$ & $\begin{array}{l}\text { Maximum } \\
\text { Nondetect }\end{array}$ & $\begin{array}{l}\text { Minimum } \\
\text { Detect }\end{array}$ & $\begin{array}{c}\text { Maximum } \\
\text { Detect }\end{array}$ & $\begin{array}{c}\text { Average } \\
\text { Concentration }\end{array}$ \\
\hline$\overline{\mathrm{RAD}}$ & Antimony-125 & $\mathrm{pCi} / \mathrm{g}$ & 3 & 0 & $0 \%$ & 0.016 & 0.039 & $\overline{--}$ & -- & 0.014 \\
\hline RAD & Beryllium-7 & $\mathrm{pCi} / \mathrm{g}$ & 2 & 0 & $0 \%$ & 0.053 & 0.12 & -- & -- & 0.043 \\
\hline RAD & Cesium-134 & $\mathrm{pCi} / \mathrm{g}$ & 2 & 0 & $0 \%$ & 0.017 & 0.019 & -- & -- & 0.0088 \\
\hline RAD & Cesium-137 & $\mathrm{pCi} / \mathrm{g}$ & 5 & 0 & $0 \%$ & 8.80E-04 & 0.015 & -- & -- & 0.0040 \\
\hline RAD & Cobalt- 60 & $\mathrm{pCi} / \mathrm{g}$ & 4 & 0 & $0 \%$ & 0.0039 & 0.025 & -- & -- & 0.0055 \\
\hline RAD & Europium-154 & $\mathrm{pCi} / \mathrm{g}$ & 2 & 0 & $0 \%$ & 0.011 & 0.031 & -- & -- & 0.011 \\
\hline RAD & Europium-155 & $\mathrm{pCi} / \mathrm{g}$ & 4 & 0 & $0 \%$ & 0.0035 & 0.044 & -- & -- & 0.0088 \\
\hline RAD & Potassium-40 & $\mathrm{pCi} / \mathrm{g}$ & 5 & 5 & $100 \%$ & -- & -- & 4.1 & 4.7 & 4.4 \\
\hline RAD & Ruthenium-106 & $\mathrm{pCi} / \mathrm{g}$ & 1 & 0 & $0 \%$ & $9.55 E-04$ & $9.55 E-04$ & -- & -- & 4.78E-04 \\
\hline RAD & Uranium-234 & $\mathrm{pCi} / \mathrm{g}$ & 1 & 0 & $0 \%$ & 4.71E-04 & 4.71E-04 & -- & -- & $2.36 \mathrm{E}-04$ \\
\hline RAD & Uranium-235 & $\mathrm{pCi} / \mathrm{g}$ & 1 & 0 & $0 \%$ & 7.97E-04 & 7.97E-04 & -- & -- & 3.99E-04 \\
\hline
\end{tabular}

RAD - Radionuclide 
Table F-5

Summary Statistics for Bass Liver Collected from Segment 1 of the Columbia River

\begin{tabular}{|c|c|c|c|c|c|c|c|c|c|c|}
\hline $\begin{array}{l}\text { Constituent } \\
\text { Class }\end{array}$ & Constituent Name & Units & $\begin{array}{l}\text { Number } \\
\text { Analyzed }\end{array}$ & $\begin{array}{l}\text { Number } \\
\text { Detected }\end{array}$ & $\begin{array}{c}\text { Frequency of } \\
\text { Detection }\end{array}$ & $\begin{array}{l}\text { Minimum } \\
\text { Nondetect }\end{array}$ & $\begin{array}{l}\text { Maximum } \\
\text { Nondetect }\end{array}$ & $\begin{array}{l}\text { Minimum } \\
\text { Detect }\end{array}$ & $\begin{array}{l}\text { Maximum } \\
\text { Detect }\end{array}$ & $\begin{array}{c}\text { Average } \\
\text { Concentration }\end{array}$ \\
\hline CONV & Percent Solids & $\%$ & 2 & 2 & $100 \%$ & $\overline{--}$ & $\overline{--}$ & 28 & 28 & 28 \\
\hline METAL & Aluminum & mg/kg & 2 & 2 & $100 \%$ & -- & -- & 0.25 & 0.32 & 0.28 \\
\hline METAL & Antimony & $\mathrm{mg} / \mathrm{kg}$ & 2 & 0 & $0 \%$ & 0.0018 & 0.0018 & -- & -- & 8.85E-04 \\
\hline METAL & Arsenic & $\mathrm{mg} / \mathrm{kg}$ & 2 & 2 & $100 \%$ & -- & -- & 0.017 & 0.022 & 0.020 \\
\hline METAL & Beryllium & $\mathrm{mg} / \mathrm{kg}$ & 2 & 0 & $0 \%$ & 0.0021 & 0.0021 & -- & -- & 0.0010 \\
\hline METAL & Cadmium & $\mathrm{mg} / \mathrm{kg}$ & 2 & 2 & $100 \%$ & -- & -- & 0.0064 & 0.015 & 0.011 \\
\hline METAL & Chromium & $\mathrm{mg} / \mathrm{kg}$ & 2 & 2 & $100 \%$ & -- & -- & 0.061 & 0.061 & 0.061 \\
\hline METAL & Copper & $\mathrm{mg} / \mathrm{kg}$ & 2 & 2 & $100 \%$ & -- & -- & 0.17 & 0.19 & 0.18 \\
\hline METAL & Lead & $\mathrm{mg} / \mathrm{kg}$ & 2 & 1 & $50 \%$ & 9.95E-04 & 9.95E-04 & 0.0012 & 0.0012 & 8.41E-04 \\
\hline METAL & Manganese & $\mathrm{mg} / \mathrm{kg}$ & 2 & 2 & $100 \%$ & -- & -- & 0.057 & 0.065 & 0.061 \\
\hline METAL & Nickel & $\mathrm{mg} / \mathrm{kg}$ & 2 & 1 & $50 \%$ & 6.94E-04 & 6.94E-04 & 8.79E-04 & 8.79E-04 & $6.13 E-04$ \\
\hline METAL & Selenium & $\mathrm{mg} / \mathrm{kg}$ & 2 & 2 & $100 \%$ & -- & -- & 0.11 & 0.13 & 0.12 \\
\hline METAL & Silver & $\mathrm{mg} / \mathrm{kg}$ & 2 & 0 & $0 \%$ & 0.0010 & 0.0010 & -- & -- & 5.06E-04 \\
\hline METAL & Thallium & $\mathrm{mg} / \mathrm{kg}$ & 2 & 2 & $100 \%$ & -- & -- & 0.0024 & 0.0039 & 0.0031 \\
\hline METAL & Thorium & $\mathrm{mg} / \mathrm{kg}$ & 2 & 0 & $0 \%$ & 5.83E-04 & 5.97E-04 & -- & -- & 2.95E-04 \\
\hline METAL & Uranium & $\mathrm{mg} / \mathrm{kg}$ & 2 & 0 & $0 \%$ & 0.0015 & 0.0015 & -- & -- & 7.45E-04 \\
\hline METAL & Zinc & $\mathrm{mg} / \mathrm{kg}$ & 2 & 2 & $100 \%$ & -- & -- & 1.6 & 1.8 & 1.7 \\
\hline
\end{tabular}


Table F-6

Summary Statistics for Whole Body Bass Collected from Segment 1 of the Columbia River

\begin{tabular}{|c|c|c|c|c|c|c|c|c|c|c|}
\hline $\begin{array}{l}\text { Constituent } \\
\text { Class }\end{array}$ & Constituent Name & Units & $\begin{array}{l}\text { Number } \\
\text { Analyzed }\end{array}$ & $\begin{array}{l}\text { Number } \\
\text { Detected }\end{array}$ & $\begin{array}{l}\text { Frequency of } \\
\text { Detection }\end{array}$ & $\begin{array}{l}\text { Minimum } \\
\text { Nondetect }\end{array}$ & $\begin{array}{l}\text { Maximum } \\
\text { Nondetect }\end{array}$ & $\begin{array}{c}\text { Minimum } \\
\text { Detect }\end{array}$ & $\begin{array}{l}\text { Maximum } \\
\text { Detect }\end{array}$ & $\begin{array}{c}\text { Average } \\
\text { Concentration }\end{array}$ \\
\hline$\overline{\mathrm{RAD}}$ & Strontium-90 & $\mathrm{pCi} / \mathrm{g}$ & 5 & 2 & $40 \%$ & 0.015 & 0.043 & 0.018 & 0.018 & 0.016 \\
\hline RAD & Uranium-234 & $\mathrm{pCi} / \mathrm{g}$ & 2 & 1 & $50 \%$ & 0.0013 & 0.0013 & 0.0023 & 0.0023 & 0.0015 \\
\hline RAD & Uranium-235 & $\mathrm{pCi} / \mathrm{g}$ & 1 & 0 & $0 \%$ & 2.68E-05 & 2.68E-05 & -- & -- & 1.34E-05 \\
\hline RAD & Uranium-238 & $\mathrm{pCi} / \mathrm{g}$ & 2 & 0 & $0 \%$ & 8.14E-04 & 0.0011 & -- & -- & 4.89E-04 \\
\hline
\end{tabular}

RAD - Radionuclide 


\section{Table F-7}

Summary Statistics for Smallmouth Bass Fillet Skin On Collected from Segment 1 of the Columbia River

\begin{tabular}{|c|c|c|c|c|c|c|c|c|c|c|}
\hline $\begin{array}{l}\text { Constituent } \\
\text { Class }\end{array}$ & Constituent Name & Units & $\begin{array}{l}\text { Number } \\
\text { Analyzed }\end{array}$ & $\begin{array}{l}\text { Number } \\
\text { Detected }\end{array}$ & $\begin{array}{l}\text { Frequency of } \\
\text { Detection }\end{array}$ & $\begin{array}{l}\text { Minimum } \\
\text { Nondetect }\end{array}$ & $\begin{array}{c}\text { Maximum } \\
\text { Nondetect }\end{array}$ & $\begin{array}{c}\text { Minimum } \\
\text { Detect }\end{array}$ & $\begin{array}{l}\text { Maximum } \\
\text { Detect }\end{array}$ & $\begin{array}{c}\text { Average } \\
\text { Concentration }\end{array}$ \\
\hline$\overline{\text { CONV }}$ & Percent Lipids & $\%$ & 3 & 3 & $100 \%$ & -- & -- & 0.70 & 1.4 & 1.1 \\
\hline PCB & Aroclor-1016 & $\mathrm{mg} / \mathrm{Kg}$ & 3 & 0 & $0 \%$ & 0.0027 & 0.0028 & -- & -- & 0.0014 \\
\hline PCB & Aroclor-1221 & $\mathrm{mg} / \mathrm{Kg}$ & 3 & 0 & $0 \%$ & 0.0027 & 0.0028 & -- & -- & 0.0014 \\
\hline PCB & Aroclor-1232 & $\mathrm{mg} / \mathrm{Kg}$ & 3 & 0 & $0 \%$ & 0.0027 & 0.0028 & -- & -- & 0.0014 \\
\hline PCB & Aroclor-1242 & $\mathrm{mg} / \mathrm{Kg}$ & 3 & 0 & $0 \%$ & 0.0027 & 0.0028 & -- & -- & 0.0014 \\
\hline PCB & Aroclor-1248 & $\mathrm{mg} / \mathrm{Kg}$ & 3 & 1 & $33 \%$ & 0.0027 & 0.0028 & 0.0029 & 0.0029 & 0.0019 \\
\hline PCB & Aroclor-1254 & $\mathrm{mg} / \mathrm{Kg}$ & 3 & 2 & $67 \%$ & 0.0028 & 0.0028 & 0.0022 & 0.0095 & 0.0044 \\
\hline PCB & Aroclor-1260 & $\mathrm{mg} / \mathrm{Kg}$ & 3 & 1 & $33 \%$ & 0.0027 & 0.0028 & 0.0019 & 0.0019 & 0.0016 \\
\hline PCB & Aroclor-1262 & $\mathrm{mg} / \mathrm{Kg}$ & 3 & 0 & $0 \%$ & 0.0027 & 0.0028 & -- & -- & 0.0014 \\
\hline PCB & Aroclor-1268 & $\mathrm{mg} / \mathrm{Kg}$ & 3 & 0 & $0 \%$ & 0.0027 & 0.0028 & -- & -- & 0.0014 \\
\hline PEST & 2,4'-DDD & $\mathrm{mg} / \mathrm{Kg}$ & 3 & 3 & $100 \%$ & -- & -- & $6.80 \mathrm{E}-04$ & 0.0022 & 0.0015 \\
\hline PEST & 2,4'-DDE & $\mathrm{mg} / \mathrm{Kg}$ & 3 & 0 & $0 \%$ & $5.40 \mathrm{E}-04$ & 0.0011 & -- & -- & $3.78 \mathrm{E}-04$ \\
\hline PEST & 2,4'-DDT & $\mathrm{mg} / \mathrm{Kg}$ & 3 & 0 & $0 \%$ & $5.20 \mathrm{E}-04$ & 5.40E-04 & -- & -- & 2.67E-04 \\
\hline PEST & Dichlorodiphenyldichloroethane & $\mathrm{mg} / \mathrm{Kg}$ & 3 & 3 & $100 \%$ & -- & -- & 0.0034 & 0.014 & 0.0095 \\
\hline PEST & Dichlorodiphenyldichloroethylene & $\mathrm{mg} / \mathrm{Kg}$ & 3 & 3 & $100 \%$ & -- & -- & 0.059 & 0.15 & 0.099 \\
\hline PEST & Dichlorodiphenyltrichloroethane & $\mathrm{mg} / \mathrm{Kg}$ & 3 & 3 & $100 \%$ & -- & -- & $8.30 \mathrm{E}-04$ & 0.0030 & 0.0018 \\
\hline
\end{tabular}

PEST - Pesticide

PCB - Polychlorinated biphenyl

SVOC - Semivolatile Organic Compound 
Table F-8

Summary Statistics for Whole Body Smallmouth Bass Collected from Segment 1 of the Columbia River

\begin{tabular}{|c|c|c|c|c|c|c|c|c|c|c|}
\hline $\begin{array}{l}\text { Constituent } \\
\text { Class }\end{array}$ & Constituent Name & Units & $\begin{array}{l}\text { Number } \\
\text { Analyzed }\end{array}$ & $\begin{array}{l}\text { Number } \\
\text { Detected }\end{array}$ & $\begin{array}{l}\text { Frequency of } \\
\text { Detection }\end{array}$ & $\begin{array}{l}\text { Minimum } \\
\text { Nondetect }\end{array}$ & $\begin{array}{l}\text { Maximum } \\
\text { Nondetect }\end{array}$ & $\begin{array}{l}\text { Minimum } \\
\text { Detect }\end{array}$ & $\begin{array}{l}\text { Maximum } \\
\text { Detect }\end{array}$ & $\begin{array}{c}\text { Average } \\
\text { Concentration }\end{array}$ \\
\hline$\overline{\text { CONV }}$ & Percent Lipids & $\%$ & 1 & 1 & $100 \%$ & $\overline{--}$ & $\overline{--}$ & 8.0 & 8.0 & 8.0 \\
\hline CONV & Percent moisture (wet sample) & $\mathrm{mg} / \mathrm{Kg}$ & 1 & 1 & $100 \%$ & -- & -- & 67 & 67 & 67 \\
\hline DIOXI & TCDD-eq & $\mathrm{mg} / \mathrm{Kg}$ & 1 & 1 & $100 \%$ & -- & -- & 6.0 & 6.0 & 6.0 \\
\hline METAL & Aluminum & $\mathrm{mg} / \mathrm{Kg}$ & 1 & 1 & $100 \%$ & -- & -- & 10 & 10 & 10 \\
\hline METAL & Arsenic & $\mathrm{mg} / \mathrm{Kg}$ & 1 & 0 & $0 \%$ & 0.30 & 0.30 & -- & -- & 0.15 \\
\hline METAL & Barium & $\mathrm{mg} / \mathrm{Kg}$ & 1 & 1 & $100 \%$ & -- & -- & 0.52 & 0.52 & 0.52 \\
\hline METAL & Beryllium & $\mathrm{mg} / \mathrm{Kg}$ & 1 & 0 & $0 \%$ & 0.059 & 0.059 & -- & -- & 0.030 \\
\hline METAL & Boron & $\mathrm{mg} / \mathrm{Kg}$ & 1 & 0 & $0 \%$ & 1.2 & 1.2 & -- & -- & 0.59 \\
\hline METAL & Cadmium & $\mathrm{mg} / \mathrm{Kg}$ & 1 & 0 & $0 \%$ & 0.059 & 0.059 & -- & -- & 0.030 \\
\hline METAL & Chromium & $\mathrm{mg} / \mathrm{Kg}$ & 1 & 1 & $100 \%$ & -- & -- & 0.50 & 0.50 & 0.50 \\
\hline METAL & Copper & $\mathrm{mg} / \mathrm{Kg}$ & 1 & 1 & $100 \%$ & -- & -- & 0.92 & 0.92 & 0.92 \\
\hline METAL & Iron & $\mathrm{mg} / \mathrm{Kg}$ & 1 & 1 & $100 \%$ & -- & -- & 16 & 16 & 16 \\
\hline METAL & Lead & $\mathrm{mg} / \mathrm{Kg}$ & 1 & 0 & $0 \%$ & 0.12 & 0.12 & -- & -- & 0.059 \\
\hline METAL & Magnesium & $\mathrm{mg} / \mathrm{Kg}$ & 1 & 1 & $100 \%$ & -- & -- & 343 & 343 & 343 \\
\hline METAL & Manganese & $\mathrm{mg} / \mathrm{Kg}$ & 1 & 1 & $100 \%$ & -- & -- & 0.54 & 0.54 & 0.54 \\
\hline METAL & Mercury & $\mathrm{mg} / \mathrm{Kg}$ & 1 & 1 & $100 \%$ & -- & -- & 0.15 & 0.15 & 0.15 \\
\hline METAL & Molybdenum & $\mathrm{mg} / \mathrm{Kg}$ & 1 & 0 & $0 \%$ & 0.30 & 0.30 & -- & -- & 0.15 \\
\hline METAL & Nickel & $\mathrm{mg} / \mathrm{Kg}$ & 1 & 0 & $0 \%$ & 0.30 & 0.30 & -- & -- & 0.15 \\
\hline METAL & Selenium & $\mathrm{mg} / \mathrm{Kg}$ & 1 & 1 & $100 \%$ & -- & -- & 0.70 & 0.70 & 0.70 \\
\hline METAL & Strontium & $\mathrm{mg} / \mathrm{Kg}$ & 1 & 1 & $100 \%$ & -- & -- & 9.5 & 9.5 & 9.5 \\
\hline METAL & Vanadium & $\mathrm{mg} / \mathrm{Kg}$ & 1 & 0 & $0 \%$ & 0.30 & 0.30 & -- & -- & 0.15 \\
\hline METAL & Zinc & $\mathrm{mg} / \mathrm{Kg}$ & 1 & 1 & $100 \%$ & -- & -- & 14 & 14 & 14 \\
\hline PCB & Total polychlorinated biphenyl & $\mathrm{mg} / \mathrm{Kg}$ & 1 & 1 & $100 \%$ & -- & -- & 0.30 & 0.30 & 0.30 \\
\hline PEST & 2,4'-DDD & $\mathrm{mg} / \mathrm{Kg}$ & 1 & 0 & $0 \%$ & 0.010 & 0.010 & -- & -- & 0.0050 \\
\hline PEST & 2,4'-DDE & $\mathrm{mg} / \mathrm{Kg}$ & 1 & 0 & $0 \%$ & 0.010 & 0.010 & -- & -- & 0.0050 \\
\hline PEST & 2,4'-DDT & $\mathrm{mg} / \mathrm{Kg}$ & 1 & 0 & $0 \%$ & 0.010 & 0.010 & -- & -- & 0.0050 \\
\hline PEST & Alpha-BHC & $\mathrm{mg} / \mathrm{Kg}$ & 1 & 0 & $0 \%$ & 0.010 & 0.010 & -- & -- & 0.0050 \\
\hline PEST & alpha-Chlordane & $\mathrm{mg} / \mathrm{Kg}$ & 1 & 0 & $0 \%$ & 0.010 & 0.010 & -- & -- & 0.0050 \\
\hline PEST & Beta-BHC & $\mathrm{mg} / \mathrm{Kg}$ & 1 & 0 & $0 \%$ & 0.010 & 0.010 & -- & -- & 0.0050 \\
\hline PEST & cis-Nonachlor & $\mathrm{mg} / \mathrm{Kg}$ & 1 & 0 & $0 \%$ & 0.010 & 0.010 & -- & -- & 0.0050 \\
\hline PEST & Delta-BHC & $\mathrm{mg} / \mathrm{Kg}$ & 1 & 0 & $0 \%$ & 0.010 & 0.010 & -- & -- & 0.0050 \\
\hline PEST & Dichlorodiphenyldichloroethane & $\mathrm{mg} / \mathrm{Kg}$ & 1 & 1 & $100 \%$ & -- & -- & 0.13 & 0.13 & 0.13 \\
\hline PEST & Dichlorodiphenyldichloroethylene & $\mathrm{mg} / \mathrm{Kg}$ & 1 & 1 & $100 \%$ & -- & -- & 0.51 & 0.51 & 0.51 \\
\hline PEST & Dichlorodiphenyltrichloroethane & $\mathrm{mg} / \mathrm{Kg}$ & 1 & 1 & $100 \%$ & -- & -- & 0.017 & 0.017 & 0.017 \\
\hline PEST & Dieldrin & $\mathrm{mg} / \mathrm{Kg}$ & 1 & 0 & $0 \%$ & 0.010 & 0.010 & -- & -- & 0.0050 \\
\hline PEST & Endrin & $\mathrm{mg} / \mathrm{Kg}$ & 1 & 0 & $0 \%$ & 0.010 & 0.010 & -- & -- & 0.0050 \\
\hline PEST & Gamma-BHC (Lindane) & $\mathrm{mg} / \mathrm{Kg}$ & 1 & 0 & $0 \%$ & 0.010 & 0.010 & -- & -- & 0.0050 \\
\hline PEST & gamma-Chlordane & $\mathrm{mg} / \mathrm{Kg}$ & 1 & 0 & $0 \%$ & 0.010 & 0.010 & -- & -- & 0.0050 \\
\hline PEST & Heptachlor epoxide & $\mathrm{mg} / \mathrm{Kg}$ & 1 & 0 & $0 \%$ & 0.010 & 0.010 & -- & -- & 0.0050 \\
\hline PEST & Mirex & $\mathrm{mg} / \mathrm{Kg}$ & 1 & 0 & $0 \%$ & 0.010 & 0.010 & -- & -- & 0.0050 \\
\hline PEST & Oxychlordane & $\mathrm{mg} / \mathrm{Kg}$ & 1 & 0 & $0 \%$ & 0.010 & 0.010 & -- & -- & 0.0050 \\
\hline PEST & Toxaphene & $\mathrm{mg} / \mathrm{Kg}$ & 1 & 0 & $0 \%$ & 0.030 & 0.030 & -- & -- & 0.015 \\
\hline PEST & trans-Nonachlor & $\mathrm{mg} / \mathrm{Kg}$ & 1 & 0 & $0 \%$ & 0.010 & 0.010 & -- & -- & 0.0050 \\
\hline SVOC & Hexachlorobenzene & $\mathrm{mg} / \mathrm{Kg}$ & 1 & 0 & $0 \%$ & 0.010 & 0.010 & -- & -- & 0.0050 \\
\hline
\end{tabular}

PEST - Pesticide

PCB - Polychlorinated biphenyl

SVOC - Semivolatile Organic Compound 
Table F-9

Summary Statistics for Carp Fillet Collected from Segment 1 of the Columbia River

\begin{tabular}{|c|c|c|c|c|c|c|c|c|c|c|}
\hline $\begin{array}{c}\text { Constituent } \\
\text { Class }\end{array}$ & Constituent Name & Units & $\begin{array}{l}\text { Number } \\
\text { Analyzed }\end{array}$ & $\begin{array}{l}\text { Number } \\
\text { Detected }\end{array}$ & $\begin{array}{c}\text { Frequency of } \\
\text { Detection }\end{array}$ & $\begin{array}{l}\text { Minimum } \\
\text { Nondetect }\end{array}$ & $\begin{array}{l}\text { Maximum } \\
\text { Nondetect }\end{array}$ & $\begin{array}{l}\text { Minimum } \\
\text { Detect }\end{array}$ & $\begin{array}{c}\text { Maximum } \\
\text { Detect }\end{array}$ & $\begin{array}{c}\text { Average } \\
\text { Concentration }\end{array}$ \\
\hline$\overline{\mathrm{RAD}}$ & Antimony-125 & $\mathrm{pCi} / \mathrm{g}$ & 2 & $\overline{0}$ & $0 \%$ & 0.0041 & 0.060 & -- & $\overline{--}$ & 0.016 \\
\hline RAD & Beryllium-7 & $\mathrm{pCi} / \mathrm{g}$ & 7 & 0 & $0 \%$ & 0.0039 & 0.12 & -- & -- & 0.026 \\
\hline RAD & Cesium-134 & $\mathrm{pCi} / \mathrm{g}$ & 7 & 0 & $0 \%$ & 1.81E-04 & 0.015 & -- & -- & 0.0032 \\
\hline RAD & Cesium-137 & $\mathrm{pCi} / \mathrm{g}$ & 8 & 0 & $0 \%$ & 0.0041 & 0.033 & -- & -- & 0.0067 \\
\hline RAD & Cobalt-60 & $\mathrm{pCi} / \mathrm{g}$ & 5 & 0 & $0 \%$ & 0.0014 & 0.018 & -- & -- & 0.0035 \\
\hline RAD & Europium-154 & $\mathrm{pCi} / \mathrm{g}$ & 7 & 0 & $0 \%$ & 0.0065 & 0.066 & -- & -- & 0.015 \\
\hline RAD & Europium-155 & $\mathrm{pCi} / \mathrm{g}$ & 5 & 1 & $20 \%$ & 0.0041 & 0.018 & 0.014 & 0.014 & 0.0069 \\
\hline RAD & Potassium-40 & $\mathrm{pCi} / \mathrm{g}$ & 13 & 13 & $100 \%$ & -- & -- & 2.0 & 4.2 & 2.9 \\
\hline RAD & Ruthenium-106 & $\mathrm{pCi} / \mathrm{g}$ & 5 & 0 & $0 \%$ & 0.013 & 0.10 & -- & -- & 0.025 \\
\hline RAD & Uranium-234 & $\mathrm{pCi} / \mathrm{g}$ & 4 & 0 & $0 \%$ & 4.11E-04 & 0.0042 & -- & -- & 0.0013 \\
\hline RAD & Uranium-235 & $\mathrm{pCi} / \mathrm{g}$ & 4 & 0 & $0 \%$ & 2.23E-05 & 7.26E-04 & -- & -- & $1.03 E-04$ \\
\hline RAD & Uranium-238 & $\mathrm{pCi} / \mathrm{g}$ & 1 & 0 & $0 \%$ & 4.53E-04 & 4.53E-04 & -- & -- & 2.27E-04 \\
\hline
\end{tabular}

RAD - Radionuclide 
Table F-10

Summary Statistics for Carp Liver Collected from Segment 1 of the Columbia River

\begin{tabular}{|c|c|c|c|c|c|c|c|c|c|c|}
\hline $\begin{array}{l}\text { Constituent } \\
\text { Class }\end{array}$ & Constituent Name & Units & $\begin{array}{l}\text { Number } \\
\text { Analyzed }\end{array}$ & $\begin{array}{l}\text { Number } \\
\text { Detected }\end{array}$ & $\begin{array}{c}\text { Frequency of } \\
\text { Detection }\end{array}$ & $\begin{array}{l}\text { Minimum } \\
\text { Nondetect }\end{array}$ & $\begin{array}{l}\text { Maximum } \\
\text { Nondetect }\end{array}$ & $\begin{array}{l}\text { Minimum } \\
\text { Detect }\end{array}$ & $\begin{array}{l}\text { Maximum } \\
\text { Detect }\end{array}$ & $\begin{array}{c}\text { Average } \\
\text { Concentration }\end{array}$ \\
\hline$\overline{\text { CONV }}$ & Percent Solids & $\%$ & 5 & 5 & $100 \%$ & $\overline{--}$ & -- & 20 & 35 & 28 \\
\hline METAL & Aluminum & mg/kg & 5 & 5 & $100 \%$ & -- & -- & 0.12 & 0.53 & 0.29 \\
\hline METAL & Antimony & $\mathrm{mg} / \mathrm{kg}$ & 5 & 1 & $20 \%$ & 0.0016 & 0.0022 & 0.0013 & 0.0013 & 0.0010 \\
\hline METAL & Arsenic & $\mathrm{mg} / \mathrm{kg}$ & 5 & 5 & $100 \%$ & -- & -- & 0.0086 & 0.029 & 0.019 \\
\hline METAL & Beryllium & $\mathrm{mg} / \mathrm{kg}$ & 5 & 0 & $0 \%$ & 0.0015 & 0.0026 & -- & -- & 0.0010 \\
\hline METAL & Cadmium & $\mathrm{mg} / \mathrm{kg}$ & 5 & 5 & $100 \%$ & -- & -- & 0.071 & 2.3 & 0.58 \\
\hline METAL & Chromium & $\mathrm{mg} / \mathrm{kg}$ & 5 & 5 & $100 \%$ & -- & -- & 0.028 & 0.046 & 0.036 \\
\hline METAL & Copper & $\mathrm{mg} / \mathrm{kg}$ & 5 & 5 & $100 \%$ & -- & -- & 1.6 & 8.9 & 3.6 \\
\hline METAL & Lead & $\mathrm{mg} / \mathrm{kg}$ & 5 & 5 & $100 \%$ & -- & -- & 0.0024 & 0.0084 & 0.0053 \\
\hline METAL & Manganese & $\mathrm{mg} / \mathrm{kg}$ & 5 & 5 & $100 \%$ & -- & -- & 0.099 & 0.30 & 0.16 \\
\hline METAL & Nickel & $\mathrm{mg} / \mathrm{kg}$ & 5 & 5 & $100 \%$ & -- & -- & 5.85E-04 & 0.0037 & 0.0023 \\
\hline METAL & Selenium & $\mathrm{mg} / \mathrm{kg}$ & 5 & 5 & $100 \%$ & -- & -- & 0.094 & 0.16 & 0.13 \\
\hline METAL & Silver & $\mathrm{mg} / \mathrm{kg}$ & 5 & 5 & $100 \%$ & -- & -- & 0.012 & 0.066 & 0.026 \\
\hline METAL & Thallium & $\mathrm{mg} / \mathrm{kg}$ & 5 & 2 & $40 \%$ & 0.0014 & 0.0017 & 0.0018 & 0.0020 & 0.0012 \\
\hline METAL & Thorium & $\mathrm{mg} / \mathrm{kg}$ & 5 & 1 & $20 \%$ & 5.44E-04 & 7.31E-04 & 0.0019 & 0.0019 & 6.38E-04 \\
\hline METAL & Uranium & $\mathrm{mg} / \mathrm{kg}$ & 5 & 3 & $60 \%$ & 0.0015 & 0.0017 & 0.0017 & 0.0019 & 0.0014 \\
\hline METAL & Zinc & $\mathrm{mg} / \mathrm{kg}$ & 5 & 5 & $100 \%$ & -- & -- & 12 & 28 & 20 \\
\hline
\end{tabular}


Table F-11

Summary Statistics for Whole Body Carp Collected from Segment 1 of the Columbia River

\begin{tabular}{|c|c|c|c|c|c|c|c|c|c|c|}
\hline $\begin{array}{l}\text { Constituent } \\
\text { Class }\end{array}$ & Constituent Name & Units & $\begin{array}{l}\text { Number } \\
\text { Analyzed }\end{array}$ & $\begin{array}{l}\text { Number } \\
\text { Detected }\end{array}$ & $\begin{array}{l}\text { Frequency of } \\
\text { Detection }\end{array}$ & $\begin{array}{l}\text { Minimum } \\
\text { Nondetect }\end{array}$ & $\begin{array}{l}\text { Maximum } \\
\text { Nondetect }\end{array}$ & $\begin{array}{c}\text { Minimum } \\
\text { Detect }\end{array}$ & $\begin{array}{l}\text { Maximum } \\
\text { Detect }\end{array}$ & $\begin{array}{c}\text { Average } \\
\text { Concentration }\end{array}$ \\
\hline$\overline{\text { CONV }}$ & $\begin{array}{l}\text { Percent Lipids } \\
\text { Percent moisture }\end{array}$ & $\%$ & 2 & 2 & $100 \%$ & -- & -- & 5.2 & 6.5 & 5.8 \\
\hline CONV & (wet sample) & $\mathrm{mg} / \mathrm{Kg}$ & 2 & 2 & $100 \%$ & -- & -- & 71 & 72 & 72 \\
\hline DIOXI & TCDD-eq & $\mathrm{mg} / \mathrm{Kg}$ & 2 & 2 & $100 \%$ & -- & -- & 6.0 & 8.0 & 7.0 \\
\hline METAL & Aluminum & $\mathrm{mg} / \mathrm{Kg}$ & 2 & 2 & $100 \%$ & -- & -- & 21 & 43 & 32 \\
\hline METAL & Arsenic & $\mathrm{mg} / \mathrm{Kg}$ & 2 & 0 & $0 \%$ & 0.27 & 0.28 & -- & -- & 0.14 \\
\hline METAL & Barium & $\mathrm{mg} / \mathrm{Kg}$ & 2 & 2 & $100 \%$ & -- & -- & 1.6 & 2.6 & 2.1 \\
\hline METAL & Beryllium & $\mathrm{mg} / \mathrm{Kg}$ & 2 & 0 & $0 \%$ & 0.055 & 0.057 & -- & -- & 0.028 \\
\hline METAL & Boron & $\mathrm{mg} / \mathrm{Kg}$ & 2 & 0 & $0 \%$ & 1.1 & 1.1 & -- & -- & 0.56 \\
\hline METAL & Cadmium & $\mathrm{mg} / \mathrm{Kg}$ & 2 & 2 & $100 \%$ & -- & -- & 0.33 & 0.51 & 0.42 \\
\hline METAL & Chromium & $\mathrm{mg} / \mathrm{Kg}$ & 2 & 2 & $100 \%$ & -- & -- & 1.5 & 2.9 & 2.2 \\
\hline METAL & Copper & $\mathrm{mg} / \mathrm{Kg}$ & 2 & 2 & $100 \%$ & -- & -- & 1.5 & 3.3 & 2.4 \\
\hline METAL & Iron & $\mathrm{mg} / \mathrm{Kg}$ & 2 & 2 & $100 \%$ & -- & -- & 50 & 88 & 69 \\
\hline METAL & Lead & $\mathrm{mg} / \mathrm{Kg}$ & 2 & 2 & $100 \%$ & -- & -- & 0.22 & 0.34 & 0.28 \\
\hline METAL & Magnesium & $\mathrm{mg} / \mathrm{Kg}$ & 2 & 2 & $100 \%$ & -- & -- & 296 & 379 & 338 \\
\hline METAL & Manganese & $\mathrm{mg} / \mathrm{Kg}$ & 2 & 2 & $100 \%$ & -- & -- & 3.8 & 4.5 & 4.2 \\
\hline METAL & Mercury & $\mathrm{mg} / \mathrm{Kg}$ & 2 & 2 & $100 \%$ & -- & -- & 0.081 & 0.10 & 0.092 \\
\hline METAL & Molybdenum & $\mathrm{mg} / \mathrm{Kg}$ & 2 & 0 & $0 \%$ & 0.27 & 0.28 & -- & -- & 0.14 \\
\hline METAL & Nickel & $\mathrm{mg} / \mathrm{Kg}$ & 2 & 1 & $50 \%$ & 0.27 & 0.27 & 0.58 & 0.58 & 0.36 \\
\hline METAL & Selenium & $\mathrm{mg} / \mathrm{Kg}$ & 2 & 2 & $100 \%$ & -- & -- & 0.42 & 0.59 & 0.50 \\
\hline METAL & Strontium & $\mathrm{mg} / \mathrm{Kg}$ & 2 & 2 & $100 \%$ & -- & -- & 14 & 18 & 16 \\
\hline METAL & Vanadium & $\mathrm{mg} / \mathrm{Kg}$ & 2 & 0 & $0 \%$ & 0.27 & 0.28 & -- & -- & 0.14 \\
\hline METAL & Zinc & $\mathrm{mg} / \mathrm{Kg}$ & 2 & 2 & $100 \%$ & -- & -- & 71 & 76 & 73 \\
\hline PCB & $\begin{array}{l}\text { Total polychlorinated } \\
\text { biphenyl }\end{array}$ & $\mathrm{mg} / \mathrm{Kg}$ & 2 & 2 & $100 \%$ & -- & -- & 0.20 & 0.45 & 0.33 \\
\hline PEST & $2,4^{\prime}-\mathrm{DDD}$ & $\mathrm{mg} / \mathrm{Kg}$ & 2 & 0 & $0 \%$ & 0.010 & 0.010 & -- & -- & 0.0050 \\
\hline PEST & 2,4'-DDE & $\mathrm{mg} / \mathrm{Kg}$ & 2 & 0 & $0 \%$ & 0.010 & 0.010 & -- & -- & 0.0050 \\
\hline PEST & 2,4'-DDT & $\mathrm{mg} / \mathrm{Kg}$ & 2 & 1 & $50 \%$ & 0.010 & 0.010 & 0.043 & 0.043 & 0.024 \\
\hline PEST & Alpha-BHC & $\mathrm{mg} / \mathrm{Kg}$ & 2 & 0 & $0 \%$ & 0.010 & 0.010 & -- & -- & 0.0050 \\
\hline PEST & alpha-Chlordane & $\mathrm{mg} / \mathrm{Kg}$ & 2 & 0 & $0 \%$ & 0.010 & 0.010 & -- & -- & 0.0050 \\
\hline PEST & Beta-BHC & $\mathrm{mg} / \mathrm{Kg}$ & 2 & 0 & $0 \%$ & 0.010 & 0.010 & -- & -- & 0.0050 \\
\hline PEST & cis-Nonachlor & $\mathrm{mg} / \mathrm{Kg}$ & 2 & 0 & $0 \%$ & 0.010 & 0.010 & -- & -- & 0.0050 \\
\hline PEST & $\begin{array}{l}\text { Delta-BHC } \\
\text { Dichlorodiphenyldichl }\end{array}$ & $\mathrm{mg} / \mathrm{Kg}$ & 2 & 0 & $0 \%$ & 0.010 & 0.010 & -- & -- & 0.0050 \\
\hline PEST & $\begin{array}{l}\text { oroethane } \\
\text { Dichlorodiphenyldichl }\end{array}$ & $\mathrm{mg} / \mathrm{Kg}$ & 2 & 2 & $100 \%$ & -- & -- & 0.094 & 0.20 & 0.15 \\
\hline PEST & $\begin{array}{l}\text { oroethylene } \\
\text { Dichlorodiphenyltrichl }\end{array}$ & $\mathrm{mg} / \mathrm{Kg}$ & 2 & 2 & $100 \%$ & -- & -- & 0.56 & 1.1 & 0.83 \\
\hline PEST & oroethane & $\mathrm{mg} / \mathrm{Kg}$ & 2 & 0 & $0 \%$ & 0.010 & 0.010 & -- & -- & 0.0050 \\
\hline PEST & Dieldrin & $\mathrm{mg} / \mathrm{Kg}$ & 2 & 1 & $50 \%$ & 0.010 & 0.010 & 0.012 & 0.012 & 0.0085 \\
\hline PEST & Endrin & $\mathrm{mg} / \mathrm{Kg}$ & 2 & 0 & $0 \%$ & 0.010 & 0.010 & -- & -- & 0.0050 \\
\hline
\end{tabular}


Table F-11

Summary Statistics for Whole Body Carp Collected from Segment 1 of the Columbia River

\begin{tabular}{|c|c|c|c|c|c|c|c|c|c|c|}
\hline $\begin{array}{l}\text { Constituent } \\
\text { Class }\end{array}$ & Constituent Name & Units & $\begin{array}{l}\text { Number } \\
\text { Analyzed }\end{array}$ & $\begin{array}{l}\text { Number } \\
\text { Detected }\end{array}$ & $\begin{array}{c}\text { Frequency of } \\
\text { Detection }\end{array}$ & $\begin{array}{l}\text { Minimum } \\
\text { Nondetect }\end{array}$ & $\begin{array}{l}\text { Maximum } \\
\text { Nondetect }\end{array}$ & $\begin{array}{l}\text { Minimum } \\
\text { Detect }\end{array}$ & $\begin{array}{l}\text { Maximum } \\
\text { Detect }\end{array}$ & $\begin{array}{c}\text { Average } \\
\text { Concentration }\end{array}$ \\
\hline PEST & $\begin{array}{l}\text { Gamma-BHC } \\
\text { (Lindane) }\end{array}$ & $\mathrm{mg} / \mathrm{Kg}$ & 2 & 0 & $0 \%$ & 0.010 & 0.010 & -- & -- & 0.0050 \\
\hline PEST & gamma-Chlordane & $\mathrm{mg} / \mathrm{Kg}$ & 2 & 0 & $0 \%$ & 0.010 & 0.010 & -- & -- & 0.0050 \\
\hline PEST & Heptachlor epoxide & $\mathrm{mg} / \mathrm{Kg}$ & 2 & 0 & $0 \%$ & 0.010 & 0.010 & -- & -- & 0.0050 \\
\hline PEST & Mirex & $\mathrm{mg} / \mathrm{Kg}$ & 2 & 0 & $0 \%$ & 0.010 & 0.010 & -- & -- & 0.0050 \\
\hline PEST & Oxychlordane & $\mathrm{mg} / \mathrm{Kg}$ & 2 & 0 & $0 \%$ & 0.010 & 0.010 & -- & -- & 0.0050 \\
\hline PEST & Toxaphene & $\mathrm{mg} / \mathrm{Kg}$ & 2 & 0 & $0 \%$ & 0.030 & 0.030 & -- & -- & 0.015 \\
\hline PEST & trans-Nonachlor & $\mathrm{mg} / \mathrm{Kg}$ & 2 & 0 & $0 \%$ & 0.010 & 0.010 & -- & -- & 0.0050 \\
\hline RAD & Strontium-90 & $\mathrm{pCi} / \mathrm{g}$ & 12 & 11 & $92 \%$ & 0.052 & 0.052 & 0.031 & 0.10 & 0.063 \\
\hline RAD & Uranium-234 & $\mathrm{pCi} / \mathrm{g}$ & 2 & 2 & $100 \%$ & -- & -- & 0.0084 & 0.027 & 0.017 \\
\hline RAD & Uranium-235 & $\mathrm{pCi} / \mathrm{g}$ & 2 & 0 & $0 \%$ & 4.14E-04 & 0.0012 & -- & -- & 3.99E-04 \\
\hline RAD & Uranium-238 & $\mathrm{pCi} / \mathrm{g}$ & 2 & 2 & $100 \%$ & -- & -- & 0.0058 & 0.023 & 0.014 \\
\hline SVOC & Hexachlorobenzene & $\mathrm{mg} / \mathrm{Kg}$ & 2 & 0 & $0 \%$ & 0.010 & 0.010 & -- & -- & 0.0050 \\
\hline
\end{tabular}

PEST - Pesticide

PCB - Polychlorinated biphenyl

RAD - Radionuclide

SVOC - Semivolatile Organic Compound 
Table F-12

Summary Statistics for Sucker Fillet Collected from Segment 1 of the Columbia River

\begin{tabular}{|c|c|c|c|c|c|c|c|c|c|c|}
\hline $\begin{array}{l}\text { Constituent } \\
\text { Class }\end{array}$ & Constituent Name & Units & $\begin{array}{l}\text { Number } \\
\text { Analyzed }\end{array}$ & $\begin{array}{l}\text { Number } \\
\text { Detected }\end{array}$ & $\begin{array}{l}\text { Frequency of } \\
\text { Detection }\end{array}$ & $\begin{array}{l}\text { Minimum } \\
\text { Nondetect }\end{array}$ & $\begin{array}{c}\text { Maximum } \\
\text { Nondetect }\end{array}$ & $\begin{array}{c}\text { Minimum } \\
\text { Detect }\end{array}$ & $\begin{array}{l}\text { Maximum } \\
\text { Detect }\end{array}$ & $\begin{array}{c}\text { Average } \\
\text { Concentration }\end{array}$ \\
\hline$\overline{\mathrm{RAD}}$ & Antimony-125 & $\mathrm{pCi} / \mathrm{g}$ & 2 & 0 & $0 \%$ & 0.0050 & 0.0077 & $\overline{--}$ & $\overline{--}$ & 0.0032 \\
\hline RAD & Beryllium-7 & $\mathrm{pCi} / \mathrm{g}$ & 4 & 0 & $0 \%$ & 0.022 & 0.075 & -- & -- & 0.029 \\
\hline RAD & Cesium-134 & $\mathrm{pCi} / \mathrm{g}$ & 1 & 0 & $0 \%$ & 0.0013 & 0.0013 & -- & -- & $6.70 \mathrm{E}-04$ \\
\hline RAD & Cesium-137 & $\mathrm{pCi} / \mathrm{g}$ & 4 & 0 & $0 \%$ & 0.011 & 0.020 & -- & -- & 0.0077 \\
\hline RAD & Cobalt-60 & $\mathrm{pCi} / \mathrm{g}$ & 4 & 0 & $0 \%$ & 0.0022 & 0.016 & -- & -- & 0.0039 \\
\hline RAD & Europium-154 & $\mathrm{pCi} / \mathrm{g}$ & 3 & 0 & $0 \%$ & 0.0084 & 0.047 & -- & -- & 0.013 \\
\hline RAD & Europium-155 & $\mathrm{pCi} / \mathrm{g}$ & 6 & 0 & $0 \%$ & 0.020 & 0.064 & -- & -- & 0.021 \\
\hline RAD & Potassium-40 & $\mathrm{pCi} / \mathrm{g}$ & 7 & 7 & $100 \%$ & -- & -- & 2.9 & 4.1 & 3.5 \\
\hline RAD & Ruthenium-106 & $\mathrm{pCi} / \mathrm{g}$ & 4 & 0 & $0 \%$ & 0.021 & 0.36 & -- & -- & 0.065 \\
\hline
\end{tabular}

RAD - Radionuclide 
Table F-13

Summary Statistics for Whole Body Sucker Collected from Segment 1 of the Columbia River

\begin{tabular}{|c|c|c|c|c|c|c|c|c|c|c|}
\hline $\begin{array}{c}\text { Constituent } \\
\text { Class }\end{array}$ & Constituent Name & Units & $\begin{array}{c}\text { Number } \\
\text { Analyzed }\end{array}$ & $\begin{array}{l}\text { Number } \\
\text { Detected }\end{array}$ & $\begin{array}{c}\text { Frequency of } \\
\text { Detection }\end{array}$ & $\begin{array}{l}\text { Minimum } \\
\text { Nondetect }\end{array}$ & $\begin{array}{l}\text { Maximum } \\
\text { Nondetect }\end{array}$ & $\begin{array}{c}\text { Minimum } \\
\text { Detect }\end{array}$ & $\begin{array}{c}\text { Maximum } \\
\text { Detect }\end{array}$ & $\begin{array}{c}\text { Average } \\
\text { Concentration }\end{array}$ \\
\hline$\overline{\text { METAL }}$ & Aluminum & $\mathrm{mg} / \mathrm{kg}$ & 5 & 5 & $100 \%$ & -- & $\overline{--}$ & 15 & 529 & 348 \\
\hline METAL & Antimony & $\mathrm{mg} / \mathrm{kg}$ & 5 & 5 & $100 \%$ & -- & -- & 0.011 & 0.024 & 0.019 \\
\hline METAL & Arsenic & $\mathrm{mg} / \mathrm{kg}$ & 5 & 5 & $100 \%$ & -- & -- & 0.14 & 0.54 & 0.34 \\
\hline METAL & Barium & $\mathrm{mg} / \mathrm{kg}$ & 5 & 5 & $100 \%$ & -- & -- & 7.2 & 17 & 13 \\
\hline METAL & Beryllium & $\mathrm{mg} / \mathrm{kg}$ & 5 & 0 & $0 \%$ & 0.020 & 0.020 & -- & -- & 0.010 \\
\hline METAL & Cadmium & $\mathrm{mg} / \mathrm{kg}$ & 5 & 5 & $100 \%$ & -- & -- & 0.078 & 0.18 & 0.14 \\
\hline METAL & Chromium & $\mathrm{mg} / \mathrm{kg}$ & 5 & 5 & $100 \%$ & -- & -- & 1.1 & 2.1 & 1.6 \\
\hline METAL & Copper & $\mathrm{mg} / \mathrm{kg}$ & 5 & 5 & $100 \%$ & -- & -- & 2.2 & 3.2 & 2.7 \\
\hline METAL & Lead & $\mathrm{mg} / \mathrm{kg}$ & 5 & 5 & $100 \%$ & -- & -- & 0.070 & 0.94 & 0.59 \\
\hline METAL & Manganese & $\mathrm{mg} / \mathrm{kg}$ & 5 & 5 & $100 \%$ & -- & -- & 24 & 58 & 42 \\
\hline METAL & Mercury & $\mathrm{mg} / \mathrm{kg}$ & 5 & 5 & $100 \%$ & -- & -- & 0.040 & 0.089 & 0.064 \\
\hline METAL & Nickel & $\mathrm{mg} / \mathrm{kg}$ & 5 & 5 & $100 \%$ & -- & -- & 1.3 & 2.2 & 1.7 \\
\hline METAL & Selenium & $\mathrm{mg} / \mathrm{kg}$ & 5 & 5 & $100 \%$ & -- & -- & 1.2 & 1.8 & 1.5 \\
\hline METAL & Silver & $\mathrm{mg} / \mathrm{kg}$ & 5 & 3 & $60 \%$ & 0.010 & 0.010 & 0.012 & 0.013 & 0.0094 \\
\hline METAL & Thallium & $\mathrm{mg} / \mathrm{kg}$ & 5 & 5 & $100 \%$ & -- & -- & 0.056 & 0.076 & 0.066 \\
\hline METAL & Thorium & $\mathrm{mg} / \mathrm{kg}$ & 5 & 4 & $80 \%$ & 0.020 & 0.020 & 0.023 & 0.11 & 0.067 \\
\hline METAL & Uranium & $\mathrm{mg} / \mathrm{kg}$ & 5 & 5 & $100 \%$ & -- & -- & 0.015 & 0.065 & 0.045 \\
\hline METAL & Zinc & $\mathrm{mg} / \mathrm{kg}$ & 5 & 5 & $100 \%$ & -- & -- & 88 & 109 & 100 \\
\hline RAD & Strontium-90 & $\mathrm{pCi} / \mathrm{g}$ & 8 & 4 & $50 \%$ & 0.011 & 0.026 & 0.031 & 0.079 & 0.028 \\
\hline
\end{tabular}

RAD - Radionuclide 
Table F-14

Summary Statistics for Crayfish Hepatopancreas Collected from Segment 1 of the Columbia River

\begin{tabular}{|c|c|c|c|c|c|c|c|c|c|c|}
\hline $\begin{array}{l}\text { Constituent } \\
\text { Class }\end{array}$ & Constituent Name & Units & $\begin{array}{l}\text { Number } \\
\text { Analyzed }\end{array}$ & $\begin{array}{l}\text { Number } \\
\text { Detected }\end{array}$ & $\begin{array}{l}\text { Frequency of } \\
\text { Detection }\end{array}$ & $\begin{array}{l}\text { Minimum } \\
\text { Nondetect }\end{array}$ & $\begin{array}{l}\text { Maximum } \\
\text { Nondetect }\end{array}$ & $\begin{array}{c}\text { Minimum } \\
\text { Detect }\end{array}$ & $\begin{array}{l}\text { Maximum } \\
\text { Detect }\end{array}$ & $\begin{array}{c}\text { Average } \\
\text { Concentration }\end{array}$ \\
\hline$\overline{M E T A L}$ & Aluminum & $\mathrm{mg} / \mathrm{kg}$ & 15 & 13 & $87 \%$ & 5.0 & 5.0 & 2.8 & 36 & 13 \\
\hline METAL & Antimony & $\mathrm{mg} / \mathrm{kg}$ & 15 & 15 & $100 \%$ & -- & -- & 0.032 & 0.78 & 0.17 \\
\hline METAL & Arsenic & $\mathrm{mg} / \mathrm{kg}$ & 15 & 14 & $93 \%$ & 0.10 & 0.10 & 1.1 & 6.9 & 3.9 \\
\hline METAL & Barium & $\mathrm{mg} / \mathrm{kg}$ & 15 & 15 & $100 \%$ & -- & -- & 1.7 & 17 & 9.0 \\
\hline METAL & Beryllium & $\mathrm{mg} / \mathrm{kg}$ & 15 & 0 & $0 \%$ & 0.019 & 0.020 & -- & -- & 0.0096 \\
\hline METAL & Cadmium & $\mathrm{mg} / \mathrm{kg}$ & 15 & 15 & $100 \%$ & -- & -- & 2.2 & 14 & 5.3 \\
\hline METAL & Chromium & $\mathrm{mg} / \mathrm{kg}$ & 15 & 15 & $100 \%$ & -- & -- & 0.25 & 0.76 & 0.47 \\
\hline METAL & Copper & $\mathrm{mg} / \mathrm{kg}$ & 15 & 15 & $100 \%$ & -- & -- & 193 & 2,480 & 972 \\
\hline METAL & Lead & $\mathrm{mg} / \mathrm{kg}$ & 15 & 15 & $100 \%$ & -- & -- & 0.074 & 0.59 & 0.19 \\
\hline METAL & Manganese & $\mathrm{mg} / \mathrm{kg}$ & 15 & 15 & $100 \%$ & -- & -- & 28 & 414 & 130 \\
\hline METAL & Mercury & $\mathrm{mg} / \mathrm{kg}$ & 15 & 13 & $87 \%$ & 0.050 & 0.050 & 0.046 & 0.34 & 0.090 \\
\hline METAL & Nickel & $\mathrm{mg} / \mathrm{kg}$ & 15 & 15 & $100 \%$ & -- & -- & 0.99 & 3.4 & 1.9 \\
\hline METAL & Selenium & $\mathrm{mg} / \mathrm{kg}$ & 15 & 15 & $100 \%$ & -- & -- & 1.4 & 9.6 & 4.6 \\
\hline METAL & Silver & $\mathrm{mg} / \mathrm{kg}$ & 15 & 15 & $100 \%$ & -- & -- & 1.5 & 8.6 & 4.9 \\
\hline METAL & Thallium & $\mathrm{mg} / \mathrm{kg}$ & 15 & 15 & $100 \%$ & -- & -- & 0.076 & 0.36 & 0.20 \\
\hline METAL & Thorium & $\mathrm{mg} / \mathrm{kg}$ & 15 & 2 & $13 \%$ & 0.020 & 0.021 & 0.049 & 0.58 & 0.051 \\
\hline METAL & Uranium & $\mathrm{mg} / \mathrm{kg}$ & 15 & 15 & $100 \%$ & -- & -- & 0.12 & 1.0 & 0.37 \\
\hline METAL & Zinc & $\mathrm{mg} / \mathrm{kg}$ & 15 & 15 & $100 \%$ & -- & -- & 64 & 319 & 179 \\
\hline
\end{tabular}


Table F-15

Summary Statistics for Whole Body Crayfish Collected from Segment 1 of the Columbia River

\begin{tabular}{|c|c|c|c|c|c|c|c|c|c|c|}
\hline $\begin{array}{l}\text { Constituent } \\
\text { Class }\end{array}$ & Constituent Name & Units & $\begin{array}{l}\text { Number } \\
\text { Analyzed }\end{array}$ & $\begin{array}{l}\text { Number } \\
\text { Detected }\end{array}$ & $\begin{array}{c}\text { Frequency of } \\
\text { Detection }\end{array}$ & $\begin{array}{l}\text { Minimum } \\
\text { Nondetect }\end{array}$ & $\begin{array}{l}\text { Maximum } \\
\text { Nondetect }\end{array}$ & $\begin{array}{l}\text { Minimum } \\
\text { Detect }\end{array}$ & $\begin{array}{l}\text { Maximum } \\
\text { Detect }\end{array}$ & $\begin{array}{c}\text { Average } \\
\text { Concentration }\end{array}$ \\
\hline$\overline{\mathrm{METAL}}$ & Aluminum & $\mathrm{mg} / \mathrm{kg}$ & 5 & 5 & $100 \%$ & $\overline{--}$ & -- & 113 & 307 & 218 \\
\hline METAL & Antimony & $\mathrm{mg} / \mathrm{kg}$ & 5 & 5 & $100 \%$ & -- & -- & 0.015 & 0.083 & 0.035 \\
\hline METAL & Arsenic & $\mathrm{mg} / \mathrm{kg}$ & 5 & 3 & $60 \%$ & 0.10 & 0.10 & 0.23 & 0.41 & 0.20 \\
\hline METAL & Barium & $\mathrm{mg} / \mathrm{kg}$ & 5 & 5 & $100 \%$ & -- & -- & 144 & 557 & 299 \\
\hline METAL & Beryllium & $\mathrm{mg} / \mathrm{kg}$ & 5 & 0 & $0 \%$ & 0.020 & 0.020 & -- & -- & 0.010 \\
\hline METAL & Cadmium & $\mathrm{mg} / \mathrm{kg}$ & 5 & 5 & $100 \%$ & -- & -- & 0.12 & 0.34 & 0.21 \\
\hline METAL & Chromium & $\mathrm{mg} / \mathrm{kg}$ & 5 & 5 & $100 \%$ & -- & -- & 0.61 & 1.0 & 0.81 \\
\hline METAL & Copper & $\mathrm{mg} / \mathrm{kg}$ & 5 & 5 & $100 \%$ & -- & -- & 43 & 74 & 65 \\
\hline METAL & Lead & $\mathrm{mg} / \mathrm{kg}$ & 5 & 5 & $100 \%$ & -- & -- & 0.22 & 0.84 & 0.45 \\
\hline METAL & Manganese & $\mathrm{mg} / \mathrm{kg}$ & 5 & 5 & $100 \%$ & -- & -- & 62 & 460 & 166 \\
\hline METAL & Mercury & $\mathrm{mg} / \mathrm{kg}$ & 5 & 1 & $20 \%$ & 0.050 & 0.050 & 0.066 & 0.066 & 0.033 \\
\hline METAL & Nickel & $\mathrm{mg} / \mathrm{kg}$ & 5 & 5 & $100 \%$ & -- & -- & 3.0 & 4.5 & 3.6 \\
\hline METAL & Selenium & $\mathrm{mg} / \mathrm{kg}$ & 5 & 5 & $100 \%$ & -- & -- & 0.41 & 1.3 & 0.91 \\
\hline METAL & Silver & $\mathrm{mg} / \mathrm{kg}$ & 5 & 5 & $100 \%$ & -- & -- & 0.040 & 0.10 & 0.073 \\
\hline METAL & Thallium & $\mathrm{mg} / \mathrm{kg}$ & 5 & 5 & $100 \%$ & -- & -- & 0.057 & 0.12 & 0.083 \\
\hline METAL & Thorium & $\mathrm{mg} / \mathrm{kg}$ & 5 & 4 & $80 \%$ & 0.020 & 0.020 & 0.024 & 0.046 & 0.031 \\
\hline METAL & Uranium & $\mathrm{mg} / \mathrm{kg}$ & 5 & 5 & $100 \%$ & -- & -- & 0.057 & 0.16 & 0.11 \\
\hline METAL & Zinc & $\mathrm{mg} / \mathrm{kg}$ & 5 & 5 & $100 \%$ & -- & -- & 64 & 98 & 81 \\
\hline RAD & Antimony-125 & $\mathrm{pCi} / \mathrm{g}$ & 3 & 0 & $0 \%$ & 0.040 & 0.064 & -- & -- & 0.025 \\
\hline RAD & Beryllium-7 & $\mathrm{pCi} / \mathrm{g}$ & 3 & 0 & $0 \%$ & 0.0080 & 0.23 & -- & -- & 0.054 \\
\hline RAD & Cesium-134 & $\mathrm{pCi} / \mathrm{g}$ & 6 & 0 & $0 \%$ & 0.0031 & 0.13 & -- & -- & 0.017 \\
\hline RAD & Cesium-137 & $\mathrm{pCi} / \mathrm{g}$ & 4 & 0 & $0 \%$ & 0.0035 & 0.082 & -- & -- & 0.017 \\
\hline RAD & Cobalt-60 & $\mathrm{pCi} / \mathrm{g}$ & 4 & 0 & $0 \%$ & 0.031 & 0.086 & -- & -- & 0.029 \\
\hline RAD & Europium-152 & $\mathrm{pCi} / \mathrm{g}$ & 1 & 0 & $0 \%$ & 0.076 & 0.076 & -- & -- & 0.038 \\
\hline RAD & Europium-154 & $\mathrm{pCi} / \mathrm{g}$ & 3 & 0 & $0 \%$ & 0.0078 & 0.12 & -- & -- & 0.039 \\
\hline RAD & Europium-155 & $\mathrm{pCi} / \mathrm{g}$ & 6 & 0 & $0 \%$ & 0.017 & 0.094 & -- & -- & 0.025 \\
\hline RAD & Potassium-40 & $\mathrm{pCi} / \mathrm{g}$ & 5 & 2 & $40 \%$ & 0.48 & 0.87 & 1.4 & 1.6 & 0.81 \\
\hline RAD & Ruthenium-106 & $\mathrm{pCi} / \mathrm{g}$ & 2 & 0 & $0 \%$ & 0.29 & 0.53 & -- & -- & 0.20 \\
\hline RAD & Strontium-90 & $\mathrm{pCi} / \mathrm{g}$ & 6 & 6 & $100 \%$ & -- & -- & 0.092 & 0.14 & 0.11 \\
\hline RAD & Technetium-99 & $\mathrm{pCi} / \mathrm{g}$ & 6 & 0 & $0 \%$ & $2.70 \mathrm{E}-04$ & 0.12 & -- & -- & 0.044 \\
\hline
\end{tabular}

RAD - Radionuclide 
Table F-16

Summary Statistics for Chinook Salmon Fillet Skin On Collected from Segment 1 of the Columbia River

\begin{tabular}{|c|c|c|c|c|c|c|c|c|c|c|}
\hline $\begin{array}{l}\text { Constituent } \\
\text { Class }\end{array}$ & Constituent Name & Units & $\begin{array}{l}\text { Number } \\
\text { Analyzed }\end{array}$ & $\begin{array}{l}\text { Number } \\
\text { Detected }\end{array}$ & $\begin{array}{l}\text { Frequency of } \\
\text { Detection }\end{array}$ & $\begin{array}{l}\text { Minimum } \\
\text { Nondetect }\end{array}$ & $\begin{array}{l}\text { Maximum } \\
\text { Nondetect }\end{array}$ & $\begin{array}{l}\text { Minimum } \\
\text { Detect }\end{array}$ & $\begin{array}{l}\text { Maximum } \\
\text { Detect }\end{array}$ & $\begin{array}{c}\text { Average } \\
\text { Concentration }\end{array}$ \\
\hline$\overline{\mathrm{DIOXI}}$ & $1,2,3,4,6,7,8$-Heptachlorodibenzodioxin & $\mathrm{mg} / \mathrm{kg}$ & 3 & 3 & $100 \%$ & $\overline{--}$ & -- & $3.00 \mathrm{E}-08$ & $7.00 \mathrm{E}-08$ & $4.67 \mathrm{E}-08$ \\
\hline DIOXI & $1,2,3,4,6,7,8$-Heptachlorodibenzofuran & $\mathrm{mg} / \mathrm{kg}$ & 3 & 0 & $0 \%$ & $2.00 \mathrm{E}-08$ & $2.00 \mathrm{E}-08$ & -- & -- & 1.00E-08 \\
\hline DIOXI & 1,2,3,4,7,8,9-Heptachlorodibenzofuran & $\mathrm{mg} / \mathrm{kg}$ & 3 & 0 & $0 \%$ & $2.00 \mathrm{E}-08$ & $2.00 \mathrm{E}-08$ & -- & -- & $1.00 \mathrm{E}-08$ \\
\hline DIOXI & $1,2,3,4,7,8$-Hexachlorodibenzofuran & $\mathrm{mg} / \mathrm{kg}$ & 3 & 0 & $0 \%$ & $2.00 \mathrm{E}-08$ & 4.00E-08 & -- & -- & 1.33E-08 \\
\hline DIOXI & 1,2,3,4,7,8-Hexachlorodibenzo-p-dioxin & $\mathrm{mg} / \mathrm{kg}$ & 3 & 0 & $0 \%$ & $2.00 \mathrm{E}-08$ & $5.00 \mathrm{E}-08$ & -- & -- & 1.67E-08 \\
\hline DIOXI & $1,2,3,6,7,8$-Hexachlorodibenzofuran & $\mathrm{mg} / \mathrm{kg}$ & 3 & 0 & $0 \%$ & $2.00 \mathrm{E}-08$ & 4.00E-08 & -- & -- & 1.33E-08 \\
\hline DIOXI & $1,2,3,6,7,8$-Hexachlorodibenzo-p-dioxin & $\mathrm{mg} / \mathrm{kg}$ & 3 & 0 & $0 \%$ & $2.00 \mathrm{E}-08$ & $5.00 \mathrm{E}-08$ & -- & -- & 1.67E-08 \\
\hline DIOXI & 1,2,3,7,8,9-Hexachlorodibenzofuran & $\mathrm{mg} / \mathrm{kg}$ & 3 & 0 & $0 \%$ & $2.00 \mathrm{E}-08$ & 4.00E-08 & -- & -- & 1.33E-08 \\
\hline DIOXI & 1,2,3,7,8,9-Hexachlorodibenzo-p-dioxin & $\mathrm{mg} / \mathrm{kg}$ & 3 & 0 & $0 \%$ & $2.00 \mathrm{E}-08$ & $5.00 \mathrm{E}-08$ & -- & -- & $1.67 \mathrm{E}-08$ \\
\hline DIOXI & 1,2,3,7,8-Pentachlorodibenzofuran & $\mathrm{mg} / \mathrm{kg}$ & 3 & 1 & $33 \%$ & $1.00 \mathrm{E}-08$ & $2.00 \mathrm{E}-08$ & $8.00 \mathrm{E}-08$ & $8.00 \mathrm{E}-08$ & 3.17E-08 \\
\hline DIOXI & $1,2,3,7,8$-Pentachlorodibenzo-p-dioxin & $\mathrm{mg} / \mathrm{kg}$ & 3 & 0 & $0 \%$ & $2.00 \mathrm{E}-08$ & $5.00 \mathrm{E}-08$ & -- & -- & 1.50E-08 \\
\hline DIOXI & 2,3,4,6,7,8-Hexachlorodibenzofuran & $\mathrm{mg} / \mathrm{kg}$ & 3 & 0 & $0 \%$ & $2.00 \mathrm{E}-08$ & 4.00E-08 & -- & -- & 1.33E-08 \\
\hline DIOXI & 2,3,4,7,8-Pentachlorodibenzofuran & $\mathrm{mg} / \mathrm{kg}$ & 3 & 1 & $33 \%$ & $1.00 \mathrm{E}-08$ & $2.00 \mathrm{E}-08$ & $1.60 \mathrm{E}-07$ & $1.60 \mathrm{E}-07$ & $5.83 E-08$ \\
\hline DIOXI & 2,3,7,8-Tetrachlorodibenzofuran & $\mathrm{mg} / \mathrm{kg}$ & 3 & 3 & $100 \%$ & -- & -- & $8.50 \mathrm{E}-07$ & $1.38 \mathrm{E}-06$ & 1.05E-06 \\
\hline DIOXI & 2,3,7,8-Tetrachlorodibenzo-p-dioxin & $\mathrm{mg} / \mathrm{kg}$ & 3 & 0 & $0 \%$ & $2.00 \mathrm{E}-08$ & 4.00E-08 & -- & -- & 1.33E-08 \\
\hline DIOXI & Octachlorodibenzofuran & $\mathrm{mg} / \mathrm{kg}$ & 3 & 0 & $0 \%$ & $2.00 \mathrm{E}-08$ & $5.00 \mathrm{E}-08$ & -- & -- & $1.67 \mathrm{E}-08$ \\
\hline DIOXI & Octachlorodibenzo-p-dioxin & $\mathrm{mg} / \mathrm{kg}$ & 3 & 1 & $33 \%$ & $1.00 \mathrm{E}-08$ & $3.00 \mathrm{E}-08$ & $1.50 \mathrm{E}-07$ & $1.50 \mathrm{E}-07$ & $5.67 \mathrm{E}-08$ \\
\hline METAL & Aluminum & $\mathrm{mg} / \mathrm{kg}$ & 3 & 1 & $33 \%$ & 1.0 & 1.0 & 1.1 & 1.1 & 0.70 \\
\hline METAL & Antimony & $\mathrm{mg} / \mathrm{kg}$ & 3 & 0 & $0 \%$ & 0.050 & 0.050 & -- & -- & 0.025 \\
\hline METAL & Arsenic & $\mathrm{mg} / \mathrm{kg}$ & 3 & 3 & $100 \%$ & -- & -- & 0.73 & 0.79 & 0.76 \\
\hline METAL & Barium & $\mathrm{mg} / \mathrm{kg}$ & 3 & 0 & $0 \%$ & 0.20 & 0.20 & -- & -- & 0.10 \\
\hline METAL & Beryllium & $\mathrm{mg} / \mathrm{kg}$ & 3 & 0 & $0 \%$ & 0.0040 & 0.0040 & -- & -- & 0.0020 \\
\hline METAL & Cadmium & $\mathrm{mg} / \mathrm{kg}$ & 3 & 0 & $0 \%$ & 0.0040 & 0.0040 & -- & -- & 0.0020 \\
\hline METAL & Chromium & $\mathrm{mg} / \mathrm{kg}$ & 3 & 0 & $0 \%$ & 0.10 & 0.10 & -- & -- & 0.050 \\
\hline METAL & Cobalt & $\mathrm{mg} / \mathrm{kg}$ & 3 & 3 & $100 \%$ & -- & -- & 0.0032 & 0.0035 & 0.0034 \\
\hline METAL & Copper & mg/kg & 3 & 3 & $100 \%$ & -- & -- & 0.53 & 0.71 & 0.61 \\
\hline METAL & Lead & $\mathrm{mg} / \mathrm{kg}$ & 3 & 0 & $0 \%$ & 0.010 & 0.010 & -- & -- & 0.0050 \\
\hline METAL & Manganese & $\mathrm{mg} / \mathrm{kg}$ & 3 & 3 & $100 \%$ & -- & -- & 0.070 & 0.070 & 0.070 \\
\hline METAL & Mercury & $\mathrm{mg} / \mathrm{kg}$ & 3 & 3 & $100 \%$ & -- & -- & 0.060 & 0.14 & 0.11 \\
\hline METAL & Nickel & $\mathrm{mg} / \mathrm{kg}$ & 3 & 1 & $33 \%$ & 0.030 & 0.030 & 0.32 & 0.32 & 0.12 \\
\hline METAL & Selenium & $\mathrm{mg} / \mathrm{kg}$ & 3 & 3 & $100 \%$ & -- & -- & 0.29 & 0.30 & 0.30 \\
\hline METAL & Silver & $\mathrm{mg} / \mathrm{kg}$ & 3 & 0 & $0 \%$ & 0.10 & 0.10 & -- & -- & 0.050 \\
\hline METAL & Thallium & $\mathrm{mg} / \mathrm{kg}$ & 3 & 0 & $0 \%$ & 0.050 & 0.050 & -- & -- & 0.025 \\
\hline METAL & Vanadium & $\mathrm{mg} / \mathrm{kg}$ & 3 & 0 & $0 \%$ & 0.010 & 0.010 & -- & -- & 0.0050 \\
\hline METAL & Zinc & $\mathrm{mg} / \mathrm{kg}$ & 3 & 3 & $100 \%$ & -- & -- & 6.1 & 6.8 & 6.4 \\
\hline PCB & 2,2',3,3',4,4',5-Heptachloro-1,1'-biphenyl & $\mathrm{mg} / \mathrm{kg}$ & 3 & 3 & $100 \%$ & -- & -- & $6.62 \mathrm{E}-05$ & 8.37E-05 & $7.23 \mathrm{E}-05$ \\
\hline РСВ & 2,2',3,4,4',5,5'-Heptachlorobiphenyl & $\mathrm{mg} / \mathrm{kg}$ & 3 & 3 & $100 \%$ & -- & -- & 1.97E-04 & $2.68 \mathrm{E}-04$ & $2.26 \mathrm{E}-04$ \\
\hline PCB & 2,3,3',4,4',5'-Hexachlorobiphenyl & $\mathrm{mg} / \mathrm{kg}$ & 3 & 3 & $100 \%$ & -- & -- & $1.36 \mathrm{E}-05$ & $1.78 \mathrm{E}-05$ & 1.50E-05 \\
\hline РСB & 2,3,3',4,4',5-Hexachlorobiphenyl (BZ 156) & $\mathrm{mg} / \mathrm{kg}$ & 3 & 3 & $100 \%$ & -- & -- & $5.68 \mathrm{E}-05$ & $7.32 \mathrm{E}-05$ & $6.33 \mathrm{E}-05$ \\
\hline PCB & 2,3,3',4,4'-Pentachlorobiphenyl & $\mathrm{mg} / \mathrm{kg}$ & 3 & 3 & $100 \%$ & -- & -- & $4.27 \mathrm{E}-04$ & $6.30 \mathrm{E}-04$ & $5.14 \mathrm{E}-04$ \\
\hline PCB & 2,3',4,4',5,5'-Hexachlorobiphenyl (BZ 167) & $\mathrm{mg} / \mathrm{kg}$ & 3 & 3 & $100 \%$ & -- & -- & $2.86 \mathrm{E}-05$ & $3.57 \mathrm{E}-05$ & 3.12E-05 \\
\hline РСB & 2',3,4,4',5-Pentachloro-1,1'-biphenyl (BZ 123) & $\mathrm{mg} / \mathrm{kg}$ & 3 & 3 & $100 \%$ & -- & -- & 1.13E-05 & $1.52 \mathrm{E}-05$ & 1.39E-05 \\
\hline PCB & 2,3,4,4',5-Pentachlorobiphenyl (BZ 114) & $\mathrm{mg} / \mathrm{kg}$ & 3 & 3 & $100 \%$ & -- & -- & $3.11 \mathrm{E}-05$ & 4.47E-05 & 3.63E-05 \\
\hline PCB & 2,3',4,4',5-Pentachlorobiphenyl (BZ 118) & $\mathrm{mg} / \mathrm{kg}$ & 3 & 3 & $100 \%$ & -- & -- & $8.84 \mathrm{E}-04$ & 0.0013 & 0.0011 \\
\hline РСB & 2,3,4,5,3',4',5'-Heptachlorobiphenyl & $\mathrm{mg} / \mathrm{kg}$ & 3 & 3 & $100 \%$ & -- & -- & $2.32 \mathrm{E}-06$ & $3.05 \mathrm{E}-06$ & 2.70E-06 \\
\hline РСB & 3,3',4,4',5,5'-Hexachlorobiphenyl (BZ 169) & $\mathrm{mg} / \mathrm{kg}$ & 3 & 0 & $0 \%$ & $1.00 \mathrm{E}-06$ & $1.00 \mathrm{E}-06$ & -- & -- & $5.00 \mathrm{E}-07$ \\
\hline PCB & 3,3',4,4',5-Pentachlorobiphenyl (BZ 126) & $\mathrm{mg} / \mathrm{kg}$ & 3 & 2 & $67 \%$ & $5.00 \mathrm{E}-07$ & 5.00E-07 & $3.24 \mathrm{E}-06$ & $3.58 \mathrm{E}-06$ & 2.36E-06 \\
\hline РСB & 3,3',4,4'-Tetrachlorobiphenyl (BZ 77) & $\mathrm{mg} / \mathrm{kg}$ & 3 & 3 & $100 \%$ & -- & -- & $3.55 \mathrm{E}-05$ & $5.74 \mathrm{E}-05$ & 4.42E-05 \\
\hline PCB & Aroclor-1016 & $\mathrm{mg} / \mathrm{kg}$ & 2 & 0 & $0 \%$ & 0.020 & 0.020 & -- & -- & 0.010 \\
\hline PCB & Aroclor-1221 & $\mathrm{mg} / \mathrm{kg}$ & 3 & 0 & $0 \%$ & 0.020 & 0.020 & -- & -- & 0.010 \\
\hline РСB & Aroclor-1232 & $\mathrm{mg} / \mathrm{kg}$ & 3 & 0 & $0 \%$ & 0.020 & 0.020 & -- & -- & 0.010 \\
\hline РСB & Aroclor-1248 & $\mathrm{mg} / \mathrm{kg}$ & 3 & 0 & $0 \%$ & 0.020 & 0.020 & -- & -- & 0.010 \\
\hline
\end{tabular}


Table F-16

Summary Statistics for Chinook Salmon Fillet Skin On Collected from Segment 1 of the Columbia River

\begin{tabular}{|c|c|c|c|c|c|c|c|c|c|c|}
\hline $\begin{array}{l}\text { Constituent } \\
\text { Class }\end{array}$ & Constituent Name & Units & $\begin{array}{l}\text { Number } \\
\text { Analyzed }\end{array}$ & $\begin{array}{l}\text { Number } \\
\text { Detected }\end{array}$ & $\begin{array}{l}\text { Frequency of } \\
\text { Detection }\end{array}$ & $\begin{array}{l}\text { Minimum } \\
\text { Nondetect }\end{array}$ & $\begin{array}{l}\text { Maximum } \\
\text { Nondetect }\end{array}$ & $\begin{array}{l}\text { Minimum } \\
\text { Detect }\end{array}$ & $\begin{array}{l}\text { Maximum } \\
\text { Detect }\end{array}$ & $\begin{array}{c}\text { Average } \\
\text { Concentration }\end{array}$ \\
\hline$\overline{\mathrm{PCB}}$ & Aroclor-1254 & $\mathrm{mg} / \mathrm{kg}$ & 3 & 3 & $100 \%$ & $\overline{--}$ & $\overline{--}$ & 0.024 & 0.035 & 0.029 \\
\hline РСB & Aroclor-1260 & $\mathrm{mg} / \mathrm{kg}$ & 3 & 0 & $0 \%$ & 0.020 & 0.020 & -- & -- & 0.010 \\
\hline PEST & 2,4'-DDD & $\mathrm{mg} / \mathrm{kg}$ & 3 & 0 & $0 \%$ & 0.0020 & 0.0020 & -- & -- & 0.0010 \\
\hline PEST & 2,4'-DDE & $\mathrm{mg} / \mathrm{kg}$ & 3 & 0 & $0 \%$ & 0.0020 & 0.0020 & -- & -- & 0.0010 \\
\hline PEST & 2,4'-DDT & $\mathrm{mg} / \mathrm{kg}$ & 3 & 0 & $0 \%$ & 0.0020 & 0.0020 & -- & -- & 0.0010 \\
\hline PEST & Aldrin & $\mathrm{mg} / \mathrm{kg}$ & 3 & 0 & $0 \%$ & 0.0020 & 0.0020 & -- & -- & 0.0010 \\
\hline PEST & Alpha-BHC & $\mathrm{mg} / \mathrm{kg}$ & 3 & 0 & $0 \%$ & 0.0020 & 0.0020 & -- & -- & 0.0010 \\
\hline PEST & alpha-Chlordane & $\mathrm{mg} / \mathrm{kg}$ & 3 & 0 & $0 \%$ & 0.0020 & 0.0020 & -- & -- & 0.0010 \\
\hline PEST & alpha-Chlordene & $\mathrm{mg} / \mathrm{kg}$ & 3 & 0 & $0 \%$ & 0.0020 & 0.0020 & -- & -- & 0.0010 \\
\hline PEST & Beta-BHC & $\mathrm{mg} / \mathrm{kg}$ & 3 & 0 & $0 \%$ & 0.0020 & 0.0020 & -- & -- & 0.0010 \\
\hline PEST & cis-Nonachlor & $\mathrm{mg} / \mathrm{kg}$ & 3 & 0 & $0 \%$ & 0.0020 & 0.0020 & -- & -- & 0.0010 \\
\hline PEST & DDMU & $\mathrm{mg} / \mathrm{kg}$ & 3 & 0 & $0 \%$ & 0.0020 & 0.0020 & -- & -- & 0.0010 \\
\hline PEST & Delta-BHC & $\mathrm{mg} / \mathrm{kg}$ & 3 & 0 & $0 \%$ & 0.0020 & 0.0020 & -- & -- & 0.0010 \\
\hline PEST & Dichlorodiphenyldichloroethane & $\mathrm{mg} / \mathrm{kg}$ & 3 & 3 & $100 \%$ & -- & -- & 0.0033 & 0.0057 & 0.0042 \\
\hline PEST & Dichlorodiphenyldichloroethylene & $\mathrm{mg} / \mathrm{kg}$ & 3 & 3 & $100 \%$ & -- & -- & 0.0081 & 0.012 & 0.0095 \\
\hline PEST & Dichlorodiphenyltrichloroethane & $\mathrm{mg} / \mathrm{kg}$ & 3 & 2 & $67 \%$ & 0.0020 & 0.0020 & 0.0018 & 0.0042 & 0.0023 \\
\hline PEST & Gamma-BHC (Lindane) & $\mathrm{mg} / \mathrm{kg}$ & 3 & 0 & $0 \%$ & 0.0020 & 0.0020 & -- & -- & 0.0010 \\
\hline PEST & gamma-Chlordane & $\mathrm{mg} / \mathrm{kg}$ & 3 & 0 & $0 \%$ & 0.0020 & 0.0020 & -- & -- & 0.0010 \\
\hline PEST & gamma-Chlordene & $\mathrm{mg} / \mathrm{kg}$ & 3 & 0 & $0 \%$ & 0.0020 & 0.0020 & -- & -- & 0.0010 \\
\hline PEST & Heptachlor & $\mathrm{mg} / \mathrm{kg}$ & 3 & 0 & $0 \%$ & 0.0020 & 0.0020 & -- & -- & 0.0010 \\
\hline PEST & Heptachlor epoxide & $\mathrm{mg} / \mathrm{kg}$ & 3 & 0 & $0 \%$ & 0.0020 & 0.0020 & -- & -- & 0.0010 \\
\hline PEST & Mirex & $\mathrm{mg} / \mathrm{kg}$ & 3 & 0 & $0 \%$ & 0.0020 & 0.0020 & -- & -- & 0.0010 \\
\hline PEST & Oxychlordane & $\mathrm{mg} / \mathrm{kg}$ & 3 & 0 & $0 \%$ & 0.0020 & 0.0020 & -- & -- & 0.0010 \\
\hline PEST & Pentachloroanisole & $\mathrm{mg} / \mathrm{kg}$ & 3 & 0 & $0 \%$ & 0.0020 & 0.0020 & -- & -- & 0.0010 \\
\hline PEST & Toxaphene & $\mathrm{mg} / \mathrm{kg}$ & 3 & 0 & $0 \%$ & 0.059 & 0.060 & -- & -- & 0.030 \\
\hline PEST & trans-Nonachlor & $\mathrm{mg} / \mathrm{kg}$ & 3 & 3 & $100 \%$ & -- & -- & 0.0024 & 0.0041 & 0.0032 \\
\hline SVOC & 1,2,4-Trichlorobenzene & $\mathrm{mg} / \mathrm{kg}$ & 3 & 0 & $0 \%$ & 0.0094 & 0.010 & -- & -- & 0.0048 \\
\hline SVOC & 1,2-Dichlorobenzene & $\mathrm{mg} / \mathrm{kg}$ & 3 & 0 & $0 \%$ & 0.0094 & 0.010 & -- & -- & 0.0048 \\
\hline SVOC & 1,2-Diphenylhydrazine & $\mathrm{mg} / \mathrm{kg}$ & 3 & 0 & $0 \%$ & 0.0094 & 0.010 & -- & -- & 0.0048 \\
\hline SVOC & 1,3-Dichlorobenzene & $\mathrm{mg} / \mathrm{kg}$ & 3 & 0 & $0 \%$ & 0.0094 & 0.010 & -- & -- & 0.0048 \\
\hline SVOC & 1,4-Dichlorobenzene & $\mathrm{mg} / \mathrm{kg}$ & 3 & 0 & $0 \%$ & 0.0094 & 0.010 & -- & -- & 0.0048 \\
\hline SVOC & 1-methylnaphthalene & $\mathrm{mg} / \mathrm{kg}$ & 3 & 0 & $0 \%$ & 0.0094 & 0.010 & -- & -- & 0.0048 \\
\hline SVOC & 2,4-Dinitrotoluene & $\mathrm{mg} / \mathrm{kg}$ & 3 & 0 & $0 \%$ & 0.15 & 0.16 & -- & -- & 0.077 \\
\hline SVOC & 2,6-Dinitrotoluene & $\mathrm{mg} / \mathrm{kg}$ & 3 & 0 & $0 \%$ & 0.038 & 0.040 & -- & -- & 0.019 \\
\hline SVOC & 2-Chloronaphthalene & $\mathrm{mg} / \mathrm{kg}$ & 3 & 0 & $0 \%$ & 0.019 & 0.020 & -- & -- & 0.0097 \\
\hline SVOC & 2-Methylnaphthalene & $\mathrm{mg} / \mathrm{kg}$ & 3 & 0 & $0 \%$ & 0.0094 & 0.010 & -- & -- & 0.0048 \\
\hline SVOC & 4-Bromophenylphenyl ether & $\mathrm{mg} / \mathrm{kg}$ & 3 & 0 & $0 \%$ & 0.0094 & 0.010 & -- & -- & 0.0048 \\
\hline SVOC & 4-Chlorophenylphenyl ether & $\mathrm{mg} / \mathrm{kg}$ & 3 & 0 & $0 \%$ & 0.0094 & 0.010 & -- & -- & 0.0048 \\
\hline SVOC & Acenaphthene & $\mathrm{mg} / \mathrm{kg}$ & 3 & 0 & $0 \%$ & 0.019 & 0.020 & -- & -- & 0.0097 \\
\hline SVOC & Acenaphthylene & $\mathrm{mg} / \mathrm{kg}$ & 3 & 0 & $0 \%$ & 0.0094 & 0.010 & -- & -- & 0.0048 \\
\hline SVOC & Anthracene & $\mathrm{mg} / \mathrm{kg}$ & 3 & 0 & $0 \%$ & 0.0094 & 0.010 & -- & -- & 0.0048 \\
\hline SVOC & Benzo(a)anthracene & $\mathrm{mg} / \mathrm{kg}$ & 3 & 0 & $0 \%$ & 0.0094 & 0.010 & -- & -- & 0.0048 \\
\hline SVOC & Benzo(a)pyrene & $\mathrm{mg} / \mathrm{kg}$ & 3 & 0 & $0 \%$ & 0.0094 & 0.010 & -- & -- & 0.0048 \\
\hline SVOC & Benzo(b)fluoranthene & $\mathrm{mg} / \mathrm{kg}$ & 3 & 0 & $0 \%$ & 0.0094 & 0.010 & -- & -- & 0.0048 \\
\hline SVOC & Benzo(ghi)perylene & $\mathrm{mg} / \mathrm{kg}$ & 3 & 0 & $0 \%$ & 0.019 & 0.020 & -- & -- & 0.0097 \\
\hline SVOC & Benzo(k)fluoranthene & $\mathrm{mg} / \mathrm{kg}$ & 3 & 0 & $0 \%$ & 0.0094 & 0.010 & -- & -- & 0.0048 \\
\hline SVOC & Bis(2-chloro-1-methylethyl)ether & $\mathrm{mg} / \mathrm{kg}$ & 3 & 0 & $0 \%$ & 0.019 & 0.020 & -- & -- & 0.0097 \\
\hline SVOC & Chrysene & $\mathrm{mg} / \mathrm{kg}$ & 3 & 0 & $0 \%$ & 0.0094 & 0.010 & -- & -- & 0.0048 \\
\hline SVOC & Dibenz[a,h]anthracene & $\mathrm{mg} / \mathrm{kg}$ & 3 & 0 & $0 \%$ & 0.019 & 0.020 & -- & -- & 0.0097 \\
\hline SVOC & Dibenzofuran & $\mathrm{mg} / \mathrm{kg}$ & 3 & 0 & $0 \%$ & 0.0094 & 0.010 & -- & -- & 0.0048 \\
\hline SVOC & Fluoranthene & $\mathrm{mg} / \mathrm{kg}$ & 3 & 0 & $0 \%$ & 0.019 & 0.020 & -- & -- & 0.0097 \\
\hline SVOC & Fluorene & $\mathrm{mg} / \mathrm{kg}$ & 3 & 0 & $0 \%$ & 0.0083 & 0.0099 & -- & -- & 0.0046 \\
\hline
\end{tabular}


Table F-16

Summary Statistics for Chinook Salmon Fillet Skin On Collected from Segment 1 of the Columbia River

\begin{tabular}{|c|c|c|c|c|c|c|c|c|c|c|}
\hline $\begin{array}{l}\text { Constituent } \\
\text { Class }\end{array}$ & Constituent Name & Units & $\begin{array}{l}\text { Number } \\
\text { Analyzed }\end{array}$ & $\begin{array}{l}\text { Number } \\
\text { Detected }\end{array}$ & $\begin{array}{l}\text { Frequency of } \\
\text { Detection }\end{array}$ & $\begin{array}{c}\text { Minimum } \\
\text { Nondetect }\end{array}$ & $\begin{array}{l}\text { Maximum } \\
\text { Nondetect }\end{array}$ & $\begin{array}{l}\text { Minimum } \\
\text { Detect }\end{array}$ & $\begin{array}{l}\text { Maximum } \\
\text { Detect }\end{array}$ & $\begin{array}{c}\text { Average } \\
\text { Concentration }\end{array}$ \\
\hline$\overline{S V O C}$ & Hexachlorobenzene & $\mathrm{mg} / \mathrm{kg}$ & 3 & 0 & $0 \%$ & 0.019 & 0.020 & -- & -- & 0.0097 \\
\hline SVOC & Hexachlorobutadiene & $\mathrm{mg} / \mathrm{kg}$ & 3 & 0 & $0 \%$ & 0.0094 & 0.010 & -- & -- & 0.0048 \\
\hline SVOC & Hexachloroethane & $\mathrm{mg} / \mathrm{kg}$ & 3 & 0 & $0 \%$ & 0.0094 & 0.010 & -- & -- & 0.0048 \\
\hline SVOC & Indeno(1,2,3-cd)pyrene & $\mathrm{mg} / \mathrm{kg}$ & 3 & 0 & $0 \%$ & 0.019 & 0.020 & -- & -- & 0.0097 \\
\hline SVOC & Naphthalene & $\mathrm{mg} / \mathrm{kg}$ & 3 & 0 & $0 \%$ & 0.015 & 0.025 & -- & -- & 0.0097 \\
\hline SVOC & Nitrobenzene & $\mathrm{mg} / \mathrm{kg}$ & 3 & 0 & $0 \%$ & 0.0094 & 0.010 & -- & -- & 0.0048 \\
\hline SVOC & Phenanthrene & $\mathrm{mg} / \mathrm{kg}$ & 3 & 0 & $0 \%$ & 0.0094 & 0.010 & -- & -- & 0.0048 \\
\hline SVOC & Pyrene & $\mathrm{mg} / \mathrm{kg}$ & 3 & 0 & $0 \%$ & 0.019 & 0.020 & -- & -- & 0.0097 \\
\hline VOC & Retene & $\mathrm{mg} / \mathrm{kg}$ & 3 & 0 & $0 \%$ & 0.019 & 0.020 & -- & -- & 0.0097 \\
\hline
\end{tabular}

DIOXI - Dioxins and Furans

PEST - Pesticide

PCB - Polychlorinated bipheny

SVOC - Semivolatile Organic Compound

VOC - Volatile Organic Compound 
Table F-17

Summary Statistics for Whole Body Chinook Salmon Collected from Segment 1 of the Columbia River

\begin{tabular}{|c|c|c|c|c|c|c|c|c|c|c|}
\hline $\begin{array}{l}\text { Constituent } \\
\text { Class }\end{array}$ & Constituent Name & Units & $\begin{array}{l}\text { Number } \\
\text { Analyzed }\end{array}$ & $\begin{array}{l}\text { Number } \\
\text { Detected }\end{array}$ & $\begin{array}{c}\text { Frequency of } \\
\text { Detection }\end{array}$ & $\begin{array}{l}\text { Minimum } \\
\text { Nondetect }\end{array}$ & $\begin{array}{l}\text { Maximum } \\
\text { Nondetect }\end{array}$ & $\begin{array}{c}\text { Minimum } \\
\text { Detect }\end{array}$ & $\begin{array}{c}\text { Maximum } \\
\text { Detect }\end{array}$ & $\begin{array}{c}\text { Average } \\
\text { Concentration }\end{array}$ \\
\hline$\overline{D I O X I}$ & $1,2,3,4,6,7,8$-Heptachlorodibenzodioxin & $\mathrm{mg} / \mathrm{kg}$ & 3 & 2 & $67 \%$ & $2.00 \mathrm{E}-08$ & $2.00 \mathrm{E}-08$ & $5.00 \mathrm{E}-08$ & $6.00 \mathrm{E}-08$ & $4.00 \mathrm{E}-08$ \\
\hline DIOXI & $1,2,3,4,6,7,8$-Heptachlorodibenzofuran & $\mathrm{mg} / \mathrm{kg}$ & 3 & 0 & $0 \%$ & $1.00 \mathrm{E}-08$ & $2.00 \mathrm{E}-08$ & -- & -- & 6.67E-09 \\
\hline DIOXI & $1,2,3,4,7,8,9$-Heptachlorodibenzofuran & $\mathrm{mg} / \mathrm{kg}$ & 3 & 0 & $0 \%$ & $1.00 \mathrm{E}-08$ & $2.00 \mathrm{E}-08$ & -- & -- & $6.67 \mathrm{E}-09$ \\
\hline DIOXI & 1,2,3,4,7,8-Hexachlorodibenzofuran & $\mathrm{mg} / \mathrm{kg}$ & 3 & 0 & $0 \%$ & $1.00 \mathrm{E}-08$ & $2.00 \mathrm{E}-08$ & -- & -- & 8.33E-09 \\
\hline DIOXI & $1,2,3,4,7,8$-Hexachlorodibenzo-p-dioxin & $\mathrm{mg} / \mathrm{kg}$ & 3 & 0 & $0 \%$ & $1.00 \mathrm{E}-08$ & $3.00 \mathrm{E}-08$ & -- & -- & $1.00 \mathrm{E}-08$ \\
\hline DIOXI & 1,2,3,6,7,8-Hexachlorodibenzofuran & $\mathrm{mg} / \mathrm{kg}$ & 3 & 0 & $0 \%$ & $1.00 \mathrm{E}-08$ & $2.00 \mathrm{E}-08$ & -- & -- & 8.33E-09 \\
\hline DIOXI & $1,2,3,6,7,8$-Hexachlorodibenzo-p-dioxin & $\mathrm{mg} / \mathrm{kg}$ & 3 & 0 & $0 \%$ & $1.00 \mathrm{E}-08$ & $3.00 \mathrm{E}-08$ & -- & -- & $1.00 \mathrm{E}-08$ \\
\hline DIOXI & 1,2,3,7,8,9-Hexachlorodibenzofuran & $\mathrm{mg} / \mathrm{kg}$ & 3 & 0 & $0 \%$ & $1.00 \mathrm{E}-08$ & $2.00 \mathrm{E}-08$ & -- & -- & 8.33E-09 \\
\hline DIOXI & $1,2,3,7,8,9$-Hexachlorodibenzo-p-dioxin & $\mathrm{mg} / \mathrm{kg}$ & 3 & 0 & $0 \%$ & $1.00 \mathrm{E}-08$ & $3.00 \mathrm{E}-08$ & -- & -- & $1.00 \mathrm{E}-08$ \\
\hline DIOXI & 1,2,3,7,8-Pentachlorodibenzofuran & $\mathrm{mg} / \mathrm{kg}$ & 3 & 0 & $0 \%$ & $2.00 \mathrm{E}-08$ & $3.00 \mathrm{E}-08$ & -- & -- & 1.17E-08 \\
\hline DIOXI & $1,2,3,7,8$-Pentachlorodibenzo-p-dioxin & $\mathrm{mg} / \mathrm{kg}$ & 3 & 0 & $0 \%$ & $2.00 \mathrm{E}-08$ & $3.00 \mathrm{E}-08$ & -- & -- & $1.33 \mathrm{E}-08$ \\
\hline DIOXI & 2,3,4,6,7,8-Hexachlorodibenzofuran & $\mathrm{mg} / \mathrm{kg}$ & 3 & 0 & $0 \%$ & $1.00 \mathrm{E}-08$ & $2.00 \mathrm{E}-08$ & -- & -- & 8.33E-09 \\
\hline DIOXI & 2,3,4,7,8-Pentachlorodibenzofuran & $\mathrm{mg} / \mathrm{kg}$ & 3 & 2 & $67 \%$ & $3.00 \mathrm{E}-08$ & $3.00 \mathrm{E}-08$ & $1.30 \mathrm{E}-07$ & $1.50 \mathrm{E}-07$ & $9.83 \mathrm{E}-08$ \\
\hline DIOXI & 2,3,7,8-Tetrachlorodibenzofuran & $\mathrm{mg} / \mathrm{kg}$ & 3 & 2 & $67 \%$ & $1.00 \mathrm{E}-06$ & $1.00 \mathrm{E}-06$ & 8.40E-07 & $1.05 \mathrm{E}-06$ & 7.97E-07 \\
\hline DIOXI & 2,3,7,8-Tetrachlorodibenzo-p-dioxin & $\mathrm{mg} / \mathrm{kg}$ & 3 & 0 & $0 \%$ & $2.00 \mathrm{E}-08$ & $3.00 \mathrm{E}-08$ & -- & -- & 1.17E-08 \\
\hline DIOXI & Octachlorodibenzofuran & $\mathrm{mg} / \mathrm{kg}$ & 3 & 0 & $0 \%$ & $3.00 \mathrm{E}-08$ & $6.00 \mathrm{E}-08$ & -- & -- & $2.17 \mathrm{E}-08$ \\
\hline DIOXI & Octachlorodibenzo-p-dioxin & $\mathrm{mg} / \mathrm{kg}$ & 3 & 0 & $0 \%$ & $2.00 \mathrm{E}-08$ & $5.00 \mathrm{E}-08$ & -- & -- & $1.67 \mathrm{E}-08$ \\
\hline METAL & Aluminum & $\mathrm{mg} / \mathrm{kg}$ & 3 & 0 & $0 \%$ & 1.0 & 1.0 & -- & -- & 0.50 \\
\hline METAL & Antimony & $\mathrm{mg} / \mathrm{kg}$ & 3 & 0 & $0 \%$ & 0.050 & 0.050 & -- & -- & 0.025 \\
\hline METAL & Arsenic & $\mathrm{mg} / \mathrm{kg}$ & 3 & 3 & $100 \%$ & -- & -- & 0.84 & 0.99 & 0.93 \\
\hline METAL & Barium & $\mathrm{mg} / \mathrm{kg}$ & 3 & 0 & $0 \%$ & 0.20 & 0.20 & -- & -- & 0.10 \\
\hline METAL & Beryllium & $\mathrm{mg} / \mathrm{kg}$ & 3 & 0 & $0 \%$ & 0.0040 & 0.0040 & -- & -- & 0.0020 \\
\hline METAL & Cadmium & $\mathrm{mg} / \mathrm{kg}$ & 3 & 3 & $100 \%$ & -- & -- & 0.0057 & 0.010 & 0.0073 \\
\hline METAL & Chromium & $\mathrm{mg} / \mathrm{kg}$ & 3 & 2 & $67 \%$ & 0.10 & 0.10 & 0.14 & 0.15 & 0.11 \\
\hline METAL & Cobalt & $\mathrm{mg} / \mathrm{kg}$ & 3 & 3 & $100 \%$ & -- & -- & 0.070 & 0.19 & 0.14 \\
\hline METAL & Copper & $\mathrm{mg} / \mathrm{kg}$ & 3 & 3 & $100 \%$ & -- & -- & 1.1 & 14 & 6.1 \\
\hline METAL & Lead & $\mathrm{mg} / \mathrm{kg}$ & 3 & 3 & $100 \%$ & -- & -- & 0.010 & 1.2 & 0.47 \\
\hline METAL & Manganese & $\mathrm{mg} / \mathrm{kg}$ & 3 & 3 & $100 \%$ & -- & -- & 0.24 & 0.29 & 0.27 \\
\hline METAL & Mercury & $\mathrm{mg} / \mathrm{kg}$ & 3 & 3 & $100 \%$ & -- & -- & 0.050 & 0.14 & 0.093 \\
\hline METAL & Nickel & $\mathrm{mg} / \mathrm{kg}$ & 3 & 3 & $100 \%$ & -- & -- & 0.060 & 0.24 & 0.14 \\
\hline METAL & Selenium & $\mathrm{mg} / \mathrm{kg}$ & 3 & 2 & $67 \%$ & 0.50 & 0.50 & 0.39 & 0.53 & 0.39 \\
\hline METAL & Silver & $\mathrm{mg} / \mathrm{kg}$ & 3 & 0 & $0 \%$ & 0.10 & 0.10 & -- & -- & 0.050 \\
\hline METAL & Thallium & $\mathrm{mg} / \mathrm{kg}$ & 3 & 0 & $0 \%$ & 0.050 & 0.050 & -- & -- & 0.025 \\
\hline METAL & Vanadium & $\mathrm{mg} / \mathrm{kg}$ & 3 & 3 & $100 \%$ & -- & -- & 0.010 & 0.020 & 0.017 \\
\hline METAL & Zinc & $\mathrm{mg} / \mathrm{kg}$ & 3 & 3 & $100 \%$ & -- & -- & 24 & 27 & 26 \\
\hline PCB & 2,2',3,3',4,4',5-Heptachloro-1,1'-biphenyl & $\mathrm{mg} / \mathrm{kg}$ & 3 & 3 & $100 \%$ & -- & -- & 7.91E-05 & 1.11E-04 & $9.09 \mathrm{E}-05$ \\
\hline РСB & 2,2',3,4,4',5,5'-Heptachlorobiphenyl & $\mathrm{mg} / \mathrm{kg}$ & 3 & 3 & $100 \%$ & -- & -- & $2.28 \mathrm{E}-04$ & $3.50 \mathrm{E}-04$ & $2.76 \mathrm{E}-04$ \\
\hline PCB & 2,3,3',4,4',5'-Hexachlorobiphenyl & $\mathrm{mg} / \mathrm{kg}$ & 3 & 3 & $100 \%$ & -- & -- & 1.79E-05 & 1.96E-05 & 1.87E-05 \\
\hline РСB & 2,3,3',4,4',5-Hexachlorobiphenyl (BZ 156) & $\mathrm{mg} / \mathrm{kg}$ & 3 & 3 & $100 \%$ & -- & -- & $7.62 \mathrm{E}-05$ & $8.28 \mathrm{E}-05$ & $7.89 \mathrm{E}-05$ \\
\hline PCB & 2,3,3',4,4'-Pentachlorobiphenyl & $\mathrm{mg} / \mathrm{kg}$ & 3 & 3 & $100 \%$ & -- & -- & 4.63E-04 & 5.91E-04 & $5.44 \mathrm{E}-04$ \\
\hline PCB & 2,3',4,4',5,5'-Hexachlorobiphenyl (BZ 167) & $\mathrm{mg} / \mathrm{kg}$ & 3 & 3 & $100 \%$ & -- & -- & 3.12E-05 & $3.57 \mathrm{E}-05$ & 3.34E-05 \\
\hline РCB & 2',3,4,4',5-Pentachloro-1,1'-biphenyl (BZ 123) & $\mathrm{mg} / \mathrm{kg}$ & 3 & 3 & $100 \%$ & -- & -- & $1.34 \mathrm{E}-05$ & $1.40 \mathrm{E}-05$ & $1.37 \mathrm{E}-05$ \\
\hline РCB & 2,3,4,4',5-Pentachlorobiphenyl (BZ 114) & $\mathrm{mg} / \mathrm{kg}$ & 3 & 3 & $100 \%$ & -- & -- & 4.12E-05 & 4.19E-05 & 4.16E-05 \\
\hline PCB & 2,3',4,4',5-Pentachlorobiphenyl (BZ 118) & $\mathrm{mg} / \mathrm{kg}$ & 3 & 3 & $100 \%$ & -- & -- & $9.78 \mathrm{E}-04$ & 0.0012 & 0.0011 \\
\hline РСB & 2,3,4,5,3',4',5'-Heptachlorobiphenyl & $\mathrm{mg} / \mathrm{kg}$ & 3 & 2 & $67 \%$ & $1.00 \mathrm{E}-06$ & $1.00 \mathrm{E}-06$ & 2.92E-06 & 4.33E-06 & $2.58 \mathrm{E}-06$ \\
\hline РСB & 3,3',4,4',5,5'-Hexachlorobiphenyl (BZ 169) & $\mathrm{mg} / \mathrm{kg}$ & 3 & 0 & $0 \%$ & $1.00 \mathrm{E}-06$ & $1.00 \mathrm{E}-06$ & -- & -- & $5.00 \mathrm{E}-07$ \\
\hline PCB & 3,3',4,4',5-Pentachlorobiphenyl (BZ 126) & $\mathrm{mg} / \mathrm{kg}$ & 3 & 3 & $100 \%$ & -- & -- & 5.00E-07 & 4.31E-06 & $2.75 \mathrm{E}-06$ \\
\hline РСB & 3,3',4,4'-Tetrachlorobiphenyl (BZ 77) & $\mathrm{mg} / \mathrm{kg}$ & 3 & 3 & $100 \%$ & -- & -- & 3.07E-05 & $4.88 \mathrm{E}-05$ & 4.19E-05 \\
\hline РCB & Aroclor-1016 & $\mathrm{mg} / \mathrm{kg}$ & 3 & 0 & $0 \%$ & 0.019 & 0.020 & -- & -- & 0.0098 \\
\hline РCB & Aroclor-1221 & $\mathrm{mg} / \mathrm{kg}$ & 3 & 0 & $0 \%$ & 0.019 & 0.020 & -- & -- & 0.0098 \\
\hline РCB & Aroclor-1232 & $\mathrm{mg} / \mathrm{kg}$ & 3 & 0 & $0 \%$ & 0.019 & 0.020 & -- & -- & 0.0098 \\
\hline PCB & Aroclor-1248 & $\mathrm{mg} / \mathrm{kg}$ & 3 & 0 & $0 \%$ & 0.019 & 0.020 & -- & -- & 0.0098 \\
\hline
\end{tabular}


Table F-17

Summary Statistics for Whole Body Chinook Salmon Collected from Segment 1 of the Columbia River

\begin{tabular}{|c|c|c|c|c|c|c|c|c|c|c|}
\hline $\begin{array}{l}\text { Constituent } \\
\text { Class }\end{array}$ & Constituent Name & Units & $\begin{array}{l}\text { Number } \\
\text { Analyzed }\end{array}$ & $\begin{array}{l}\text { Number } \\
\text { Detected }\end{array}$ & $\begin{array}{l}\text { Frequency of } \\
\text { Detection }\end{array}$ & $\begin{array}{l}\text { Minimum } \\
\text { Nondetect }\end{array}$ & $\begin{array}{l}\text { Maximum } \\
\text { Nondetect }\end{array}$ & $\begin{array}{l}\text { Minimum } \\
\text { Detect }\end{array}$ & $\begin{array}{l}\text { Maximum } \\
\text { Detect }\end{array}$ & $\begin{array}{c}\text { Average } \\
\text { Concentration }\end{array}$ \\
\hline$\overline{\mathrm{PCB}}$ & Aroclor-1254 & $\mathrm{mg} / \mathrm{kg}$ & 3 & 3 & $100 \%$ & $\overline{--}$ & $\overline{--}$ & 0.023 & 0.030 & 0.026 \\
\hline РСB & Aroclor-1260 & $\mathrm{mg} / \mathrm{kg}$ & 3 & 0 & $0 \%$ & 0.019 & 0.020 & -- & -- & 0.0098 \\
\hline PEST & 2,4'-DDD & $\mathrm{mg} / \mathrm{kg}$ & 3 & 3 & $100 \%$ & -- & -- & $9.60 \mathrm{E}-04$ & 0.0015 & 0.0012 \\
\hline PEST & 2,4'-DDE & $\mathrm{mg} / \mathrm{kg}$ & 3 & 0 & $0 \%$ & 0.0019 & 0.0020 & -- & -- & $9.83 E-04$ \\
\hline PEST & 2,4'-DDT & $\mathrm{mg} / \mathrm{kg}$ & 3 & 0 & $0 \%$ & 0.0019 & 0.0020 & -- & -- & $9.83 \mathrm{E}-04$ \\
\hline PEST & Aldrin & $\mathrm{mg} / \mathrm{kg}$ & 3 & 0 & $0 \%$ & 0.0019 & 0.0020 & -- & -- & $9.83 \mathrm{E}-04$ \\
\hline PEST & Alpha-BHC & $\mathrm{mg} / \mathrm{kg}$ & 3 & 0 & $0 \%$ & 0.0019 & 0.0020 & -- & -- & $9.83 E-04$ \\
\hline PEST & alpha-Chlordane & $\mathrm{mg} / \mathrm{kg}$ & 3 & 2 & $67 \%$ & 0.0020 & 0.0020 & 0.0014 & 0.0016 & 0.0013 \\
\hline PEST & alpha-Chlordene & $\mathrm{mg} / \mathrm{kg}$ & 3 & 0 & $0 \%$ & 0.0019 & 0.0020 & -- & -- & $9.83 \mathrm{E}-04$ \\
\hline PEST & Beta-BHC & $\mathrm{mg} / \mathrm{kg}$ & 3 & 0 & $0 \%$ & 0.0019 & 0.0020 & -- & -- & $9.83 E-04$ \\
\hline PEST & cis-Nonachlor & $\mathrm{mg} / \mathrm{kg}$ & 3 & 0 & $0 \%$ & 0.0019 & 0.0020 & -- & -- & 9.83E-04 \\
\hline PEST & DDMU & $\mathrm{mg} / \mathrm{kg}$ & 3 & 0 & $0 \%$ & 0.0019 & 0.0020 & -- & -- & $9.83 \mathrm{E}-04$ \\
\hline PEST & Delta-BHC & $\mathrm{mg} / \mathrm{kg}$ & 3 & 0 & $0 \%$ & 0.0019 & 0.0020 & -- & -- & $9.83 \mathrm{E}-04$ \\
\hline PEST & Dichlorodiphenyldichloroethane & $\mathrm{mg} / \mathrm{kg}$ & 3 & 3 & $100 \%$ & -- & -- & 0.0036 & 0.0069 & 0.0055 \\
\hline PEST & Dichlorodiphenyldichloroethylene & $\mathrm{mg} / \mathrm{kg}$ & 3 & 3 & $100 \%$ & -- & -- & 0.0082 & 0.014 & 0.010 \\
\hline PEST & Dichlorodiphenyltrichloroethane & $\mathrm{mg} / \mathrm{kg}$ & 3 & 0 & $0 \%$ & 0.0019 & 0.0020 & -- & -- & $9.83 \mathrm{E}-04$ \\
\hline PEST & Endosulfan sulfate & $\mathrm{mg} / \mathrm{kg}$ & 3 & 0 & $0 \%$ & 0.0019 & 0.0020 & -- & -- & $9.83 E-04$ \\
\hline PEST & Gamma-BHC (Lindane) & $\mathrm{mg} / \mathrm{kg}$ & 3 & 0 & $0 \%$ & 0.0019 & 0.0020 & -- & -- & $9.83 \mathrm{E}-04$ \\
\hline PEST & gamma-Chlordane & $\mathrm{mg} / \mathrm{kg}$ & 3 & 0 & $0 \%$ & 0.0019 & 0.0020 & -- & -- & $9.83 E-04$ \\
\hline PEST & gamma-Chlordene & $\mathrm{mg} / \mathrm{kg}$ & 3 & 0 & $0 \%$ & 0.0019 & 0.0020 & -- & -- & $9.83 E-04$ \\
\hline PEST & Heptachlor & $\mathrm{mg} / \mathrm{kg}$ & 3 & 0 & $0 \%$ & 0.0019 & 0.0020 & -- & -- & $9.83 \mathrm{E}-04$ \\
\hline PEST & Heptachlor epoxide & $\mathrm{mg} / \mathrm{kg}$ & 3 & 0 & $0 \%$ & 0.0019 & 0.0020 & -- & -- & $9.83 \mathrm{E}-04$ \\
\hline PEST & Mirex & $\mathrm{mg} / \mathrm{kg}$ & 3 & 0 & $0 \%$ & 0.0019 & 0.0020 & -- & -- & $9.83 E-04$ \\
\hline PEST & Oxychlordane & $\mathrm{mg} / \mathrm{kg}$ & 3 & 0 & $0 \%$ & 0.0019 & 0.0020 & -- & -- & $9.83 \mathrm{E}-04$ \\
\hline PEST & Pentachloroanisole & $\mathrm{mg} / \mathrm{kg}$ & 3 & 0 & $0 \%$ & 0.0019 & 0.0020 & -- & -- & $9.83 \mathrm{E}-04$ \\
\hline PEST & Toxaphene & $\mathrm{mg} / \mathrm{kg}$ & 3 & 0 & $0 \%$ & 0.058 & 0.060 & -- & -- & 0.030 \\
\hline PEST & trans-Nonachlor & $\mathrm{mg} / \mathrm{kg}$ & 3 & 3 & $100 \%$ & -- & -- & 0.0022 & 0.0046 & 0.0031 \\
\hline SVOC & 1,2,4-Trichlorobenzene & $\mathrm{mg} / \mathrm{kg}$ & 3 & 0 & $0 \%$ & 0.0093 & 0.0098 & -- & -- & 0.0048 \\
\hline SVOC & 1,2-Dichlorobenzene & $\mathrm{mg} / \mathrm{kg}$ & 3 & 0 & $0 \%$ & 0.0093 & 0.0098 & -- & -- & 0.0048 \\
\hline SVOC & 1,2-Diphenylhydrazine & $\mathrm{mg} / \mathrm{kg}$ & 3 & 0 & $0 \%$ & 0.0093 & 0.0098 & -- & -- & 0.0048 \\
\hline SVOC & 1,3-Dichlorobenzene & $\mathrm{mg} / \mathrm{kg}$ & 3 & 0 & $0 \%$ & 0.0093 & 0.0098 & -- & -- & 0.0048 \\
\hline SVOC & 1,4-Dichlorobenzene & $\mathrm{mg} / \mathrm{kg}$ & 3 & 0 & $0 \%$ & 0.0093 & 0.0098 & -- & -- & 0.0048 \\
\hline SVOC & 1-methylnaphthalene & $\mathrm{mg} / \mathrm{kg}$ & 3 & 0 & $0 \%$ & 0.0093 & 0.0098 & -- & -- & 0.0048 \\
\hline SVOC & 2,4-Dinitrotoluene & $\mathrm{mg} / \mathrm{kg}$ & 3 & 0 & $0 \%$ & 0.019 & 0.020 & -- & -- & 0.0097 \\
\hline SVOC & 2,6-Dinitrotoluene & $\mathrm{mg} / \mathrm{kg}$ & 3 & 0 & $0 \%$ & 0.037 & 0.039 & -- & -- & 0.019 \\
\hline SVOC & 2-Chloronaphthalene & $\mathrm{mg} / \mathrm{kg}$ & 3 & 0 & $0 \%$ & 0.019 & 0.020 & -- & -- & 0.0097 \\
\hline SVOC & 2-Methylnaphthalene & $\mathrm{mg} / \mathrm{kg}$ & 3 & 0 & $0 \%$ & 0.0093 & 0.0098 & -- & -- & 0.0048 \\
\hline SVOC & 4-Bromophenylphenyl ether & $\mathrm{mg} / \mathrm{kg}$ & 3 & 0 & $0 \%$ & 0.0093 & 0.0098 & -- & -- & 0.0048 \\
\hline SVOC & 4-Chlorophenylphenyl ether & $\mathrm{mg} / \mathrm{kg}$ & 3 & 0 & $0 \%$ & 0.0093 & 0.0098 & -- & -- & 0.0048 \\
\hline SVOC & Acenaphthene & $\mathrm{mg} / \mathrm{kg}$ & 3 & 0 & $0 \%$ & 0.019 & 0.020 & -- & -- & 0.0097 \\
\hline SVOC & Acenaphthylene & $\mathrm{mg} / \mathrm{kg}$ & 3 & 0 & $0 \%$ & 0.0093 & 0.0098 & -- & -- & 0.0048 \\
\hline SVOC & Anthracene & $\mathrm{mg} / \mathrm{kg}$ & 3 & 0 & $0 \%$ & 0.0093 & 0.0098 & -- & -- & 0.0048 \\
\hline SVOC & Benzo(a)anthracene & $\mathrm{mg} / \mathrm{kg}$ & 3 & 0 & $0 \%$ & 0.0093 & 0.0098 & -- & -- & 0.0048 \\
\hline SVOC & Benzo(a)pyrene & $\mathrm{mg} / \mathrm{kg}$ & 3 & 0 & $0 \%$ & 0.0093 & 0.0098 & -- & -- & 0.0048 \\
\hline SVOC & Benzo(b)fluoranthene & $\mathrm{mg} / \mathrm{kg}$ & 3 & 0 & $0 \%$ & 0.0093 & 0.0098 & -- & -- & 0.0048 \\
\hline SVOC & Benzo(ghi)perylene & $\mathrm{mg} / \mathrm{kg}$ & 3 & 0 & $0 \%$ & 0.019 & 0.020 & -- & -- & 0.0097 \\
\hline SVOC & Benzo(k)fluoranthene & $\mathrm{mg} / \mathrm{kg}$ & 3 & 0 & $0 \%$ & 0.0093 & 0.0098 & -- & -- & 0.0048 \\
\hline SVOC & Bis(2-chloro-1-methylethyl)ether & $\mathrm{mg} / \mathrm{kg}$ & 3 & 0 & $0 \%$ & 0.019 & 0.020 & -- & -- & 0.0097 \\
\hline SVOC & Chrysene & $\mathrm{mg} / \mathrm{kg}$ & 3 & 0 & $0 \%$ & 0.0093 & 0.0098 & -- & -- & 0.0048 \\
\hline SVOC & Dibenz[a,h]anthracene & $\mathrm{mg} / \mathrm{kg}$ & 3 & 0 & $0 \%$ & 0.019 & 0.020 & -- & -- & 0.0097 \\
\hline SVOC & Dibenzofuran & $\mathrm{mg} / \mathrm{kg}$ & 3 & 0 & $0 \%$ & 0.0093 & 0.0098 & -- & -- & 0.0048 \\
\hline SVOC & Fluoranthene & $\mathrm{mg} / \mathrm{kg}$ & 3 & 0 & $0 \%$ & 0.019 & 0.020 & -- & -- & 0.0097 \\
\hline
\end{tabular}


Table F-17

Summary Statistics for Whole Body Chinook Salmon Collected from Segment 1 of the Columbia River

\begin{tabular}{|c|c|c|c|c|c|c|c|c|c|c|}
\hline $\begin{array}{c}\text { Constituent } \\
\text { Class }\end{array}$ & Constituent Name & Units & $\begin{array}{l}\text { Number } \\
\text { Analyzed }\end{array}$ & $\begin{array}{l}\text { Number } \\
\text { Detected }\end{array}$ & $\begin{array}{c}\text { Frequency of } \\
\text { Detection }\end{array}$ & $\begin{array}{c}\text { Minimum } \\
\text { Nondetect }\end{array}$ & $\begin{array}{l}\text { Maximum } \\
\text { Nondetect }\end{array}$ & $\begin{array}{c}\text { Minimum } \\
\text { Detect }\end{array}$ & $\begin{array}{l}\text { Maximum } \\
\text { Detect }\end{array}$ & $\begin{array}{c}\text { Average } \\
\text { Concentration }\end{array}$ \\
\hline SVOC & Fluorene & $\mathrm{mg} / \mathrm{kg}$ & 3 & 0 & $0 \%$ & 0.0048 & 0.0098 & -- & -- & 0.0033 \\
\hline SVOC & Hexachlorobenzene & $\mathrm{mg} / \mathrm{kg}$ & 3 & 0 & $0 \%$ & 0.019 & 0.020 & -- & -- & 0.0097 \\
\hline SVOC & Hexachlorobutadiene & $\mathrm{mg} / \mathrm{kg}$ & 3 & 0 & $0 \%$ & 0.0093 & 0.0098 & -- & -- & 0.0048 \\
\hline SVOC & Hexachloroethane & $\mathrm{mg} / \mathrm{kg}$ & 3 & 0 & $0 \%$ & 0.0093 & 0.0098 & -- & -- & 0.0048 \\
\hline SVOC & Indeno(1,2,3-cd)pyrene & $\mathrm{mg} / \mathrm{kg}$ & 3 & 0 & $0 \%$ & 0.019 & 0.020 & -- & -- & 0.0097 \\
\hline SVOC & Naphthalene & $\mathrm{mg} / \mathrm{kg}$ & 3 & 0 & $0 \%$ & 0.012 & 0.028 & -- & -- & 0.010 \\
\hline SVOC & Nitrobenzene & $\mathrm{mg} / \mathrm{kg}$ & 3 & 0 & $0 \%$ & 0.0093 & 0.0098 & -- & -- & 0.0048 \\
\hline SVOC & Phenanthrene & $\mathrm{mg} / \mathrm{kg}$ & 3 & 0 & $0 \%$ & 0.0093 & 0.0098 & -- & -- & 0.0048 \\
\hline SVOC & Pyrene & $\mathrm{mg} / \mathrm{kg}$ & 3 & 0 & $0 \%$ & 0.019 & 0.020 & -- & -- & 0.0097 \\
\hline VOC & Retene & $\mathrm{mg} / \mathrm{kg}$ & 3 & 0 & $0 \%$ & 0.019 & 0.020 & -- & -- & 0.0097 \\
\hline
\end{tabular}

DIOXI - Dioxins and Furans

PEST - Pesticide

PCB - Polychlorinated biphenyl

SVOC - Semivolatile Organic Compound

VOC - Volatile Organic Compound 
Table F-18

Summary Statistics for Whole Body Juvenile Chinook Salmon Collected from Segment 1 of the Columbia River

\begin{tabular}{|c|c|c|c|c|c|c|c|c|c|c|}
\hline $\begin{array}{l}\text { Constituent } \\
\text { Class }\end{array}$ & Constituent Name & Units & $\begin{array}{l}\text { Number } \\
\text { Analyzed }\end{array}$ & $\begin{array}{l}\text { Number } \\
\text { Detected }\end{array}$ & $\begin{array}{c}\text { Frequency of } \\
\text { Detection }\end{array}$ & $\begin{array}{l}\text { Minimum } \\
\text { Nondetect }\end{array}$ & $\begin{array}{l}\text { Maximum } \\
\text { Nondetect }\end{array}$ & $\begin{array}{c}\text { Minimum } \\
\text { Detect }\end{array}$ & $\begin{array}{l}\text { Maximum } \\
\text { Detect }\end{array}$ & $\begin{array}{c}\text { Average } \\
\text { Concentration }\end{array}$ \\
\hline CONV & Percent Solids & $\%$ & 60 & 60 & $100 \%$ & -- & $\overline{--}$ & 5.1 & 43 & 16 \\
\hline METAL & Aluminum & $\mathrm{mg} / \mathrm{kg}$ & 10 & 10 & $100 \%$ & -- & -- & 0.19 & 1.3 & 0.67 \\
\hline METAL & Antimony & $\mathrm{mg} / \mathrm{kg}$ & 10 & 10 & $100 \%$ & -- & -- & 0.047 & 0.21 & 0.092 \\
\hline METAL & Arsenic & $\mathrm{mg} / \mathrm{kg}$ & 10 & 7 & $70 \%$ & 0.0021 & 0.0039 & 0.0056 & 0.020 & 0.0092 \\
\hline METAL & Beryllium & $\mathrm{mg} / \mathrm{kg}$ & 10 & 1 & $10 \%$ & 3.77E-04 & 0.0013 & 0.0054 & 0.0054 & $9.42 \mathrm{E}-04$ \\
\hline METAL & Cadmium & $\mathrm{mg} / \mathrm{kg}$ & 10 & 10 & $100 \%$ & -- & -- & 0.0011 & 0.0094 & 0.0049 \\
\hline METAL & Chromium & $\mathrm{mg} / \mathrm{kg}$ & 60 & 60 & $100 \%$ & -- & -- & 0.020 & 0.12 & 0.044 \\
\hline METAL & Copper & $\mathrm{mg} / \mathrm{kg}$ & 10 & 10 & $100 \%$ & -- & -- & 0.023 & 0.10 & 0.064 \\
\hline METAL & Lead & $\mathrm{mg} / \mathrm{kg}$ & 10 & 10 & $100 \%$ & -- & -- & 7.70E-04 & 0.0080 & 0.0026 \\
\hline METAL & Manganese & $\mathrm{mg} / \mathrm{kg}$ & 10 & 10 & $100 \%$ & -- & -- & 0.021 & 0.13 & 0.075 \\
\hline METAL & Nickel & $\mathrm{mg} / \mathrm{kg}$ & 10 & 10 & $100 \%$ & -- & -- & 6.84E-04 & 0.016 & 0.0065 \\
\hline METAL & Selenium & $\mathrm{mg} / \mathrm{kg}$ & 10 & 10 & $100 \%$ & -- & -- & 0.018 & 0.068 & 0.047 \\
\hline METAL & Silver & $\mathrm{mg} / \mathrm{kg}$ & 10 & 5 & $50 \%$ & 3.60E-04 & $6.52 \mathrm{E}-04$ & 4.13E-04 & 0.0048 & 8.62E-04 \\
\hline METAL & Thallium & $\mathrm{mg} / \mathrm{kg}$ & 10 & 10 & $100 \%$ & -- & -- & 9.90E-04 & 0.0082 & 0.0029 \\
\hline METAL & Thorium & $\mathrm{mg} / \mathrm{kg}$ & 10 & 8 & $80 \%$ & 3.57E-04 & 3.78E-04 & 2.50E-04 & 0.011 & 0.0018 \\
\hline METAL & Uranium & $\mathrm{mg} / \mathrm{kg}$ & 60 & 1 & $2 \%$ & $2.70 \mathrm{E}-04$ & 0.0019 & 0.0078 & 0.0078 & $4.68 \mathrm{E}-04$ \\
\hline METAL & Zinc & $\mathrm{mg} / \mathrm{kg}$ & 10 & 10 & $100 \%$ & -- & -- & 0.64 & 1.8 & 1.3 \\
\hline
\end{tabular}


Table F-19

Summary Statistics for Mountain Whitefish Fillet Skin On Collected from Segment 1 of the Columbia River

\begin{tabular}{|c|c|c|c|c|c|c|c|c|c|c|}
\hline $\begin{array}{l}\text { Constituent } \\
\text { Class }\end{array}$ & Constituent Name & Units & $\begin{array}{l}\text { Number } \\
\text { Analyzed }\end{array}$ & $\begin{array}{l}\text { Number } \\
\text { Detected }\end{array}$ & $\begin{array}{l}\text { Frequency of } \\
\text { Detection }\end{array}$ & $\begin{array}{l}\text { Minimum } \\
\text { Nondetect }\end{array}$ & $\begin{array}{l}\text { Maximum } \\
\text { Nondetect }\end{array}$ & $\begin{array}{l}\text { Minimum } \\
\text { Detect }\end{array}$ & $\begin{array}{l}\text { Maximum } \\
\text { Detect }\end{array}$ & $\begin{array}{c}\text { Average } \\
\text { Concentration }\end{array}$ \\
\hline CONV & Percent Lipids & $\%$ & 3 & 3 & $100 \%$ & $\overline{--}$ & $\overline{--}$ & 1.6 & 3.1 & 2.5 \\
\hline РCB & Aroclor-1016 & $\mathrm{mg} / \mathrm{Kg}$ & 3 & 0 & $0 \%$ & 0.0026 & 0.0028 & -- & -- & 0.0014 \\
\hline PCB & Aroclor-1221 & $\mathrm{mg} / \mathrm{Kg}$ & 3 & 0 & $0 \%$ & 0.0026 & 0.0028 & -- & -- & 0.0014 \\
\hline PCB & Aroclor-1232 & $\mathrm{mg} / \mathrm{Kg}$ & 3 & 0 & $0 \%$ & 0.0026 & 0.0028 & -- & -- & 0.0014 \\
\hline PCB & Aroclor-1242 & $\mathrm{mg} / \mathrm{Kg}$ & 3 & 0 & $0 \%$ & 0.0026 & 0.0028 & -- & -- & 0.0014 \\
\hline PCB & Aroclor-1248 & $\mathrm{mg} / \mathrm{Kg}$ & 3 & 2 & $67 \%$ & 0.0028 & 0.0028 & 0.0025 & 0.0035 & 0.0025 \\
\hline PCB & Aroclor-1254 & $\mathrm{mg} / \mathrm{Kg}$ & 3 & 3 & $100 \%$ & -- & -- & 0.0029 & 0.0098 & 0.0064 \\
\hline PCB & Aroclor-1260 & $\mathrm{mg} / \mathrm{Kg}$ & 3 & 3 & $100 \%$ & -- & -- & 0.0021 & 0.0062 & 0.0035 \\
\hline PCB & Aroclor-1262 & $\mathrm{mg} / \mathrm{Kg}$ & 3 & 0 & $0 \%$ & 0.0026 & 0.0028 & -- & -- & 0.0014 \\
\hline PCB & Aroclor-1268 & $\mathrm{mg} / \mathrm{Kg}$ & 3 & 0 & $0 \%$ & 0.0026 & 0.0028 & -- & -- & 0.0014 \\
\hline PEST & 2,4'-DDD & $\mathrm{mg} / \mathrm{Kg}$ & 3 & 3 & $100 \%$ & -- & -- & $6.40 \mathrm{E}-04$ & 0.0022 & 0.0014 \\
\hline PEST & 2,4'-DDE & $\mathrm{mg} / \mathrm{Kg}$ & 3 & 0 & $0 \%$ & 5.30E-04 & 0.0012 & -- & -- & 3.82E-04 \\
\hline PEST & 2,4'-DDT & $\mathrm{mg} / \mathrm{Kg}$ & 3 & 3 & $100 \%$ & -- & -- & 7.60E-04 & 8.00E-04 & 7.73E-04 \\
\hline PEST & Dichlorodiphenyldichloroethane & $\mathrm{mg} / \mathrm{Kg}$ & 3 & 3 & $100 \%$ & -- & -- & 0.0049 & 0.016 & 0.012 \\
\hline PEST & Dichlorodiphenyldichloroethylene & $\mathrm{mg} / \mathrm{Kg}$ & 3 & 3 & $100 \%$ & -- & -- & 0.073 & 0.12 & 0.10 \\
\hline PEST & Dichlorodiphenyltrichloroethane & $\mathrm{mg} / \mathrm{Kg}$ & 3 & 3 & $100 \%$ & -- & -- & 0.0028 & 0.0037 & 0.0032 \\
\hline
\end{tabular}

PEST - Pesticide

PCB - Polychlorinated biphenyl 
Table F-20

Summary Statistics for Whitefish Fillet Collected from Segment 1 of the Columbia River

\begin{tabular}{|c|c|c|c|c|c|c|c|c|c|c|}
\hline $\begin{array}{l}\text { Constituent } \\
\text { Class }\end{array}$ & Constituent Name & Units & $\begin{array}{l}\text { Number } \\
\text { Analyzed }\end{array}$ & $\begin{array}{l}\text { Number } \\
\text { Detected }\end{array}$ & $\begin{array}{l}\text { Frequency of } \\
\text { Detection }\end{array}$ & $\begin{array}{l}\text { Minimum } \\
\text { Nondetect }\end{array}$ & $\begin{array}{l}\text { Maximum } \\
\text { Nondetect }\end{array}$ & $\begin{array}{c}\text { Minimum } \\
\text { Detect }\end{array}$ & $\begin{array}{l}\text { Maximum } \\
\text { Detect }\end{array}$ & $\begin{array}{c}\text { Average } \\
\text { Concentration }\end{array}$ \\
\hline$\overline{\mathrm{RAD}}$ & Beryllium-7 & $\mathrm{pCi} / \mathrm{g}$ & 5 & 0 & $0 \%$ & 0.0042 & 0.19 & -- & -- & 0.048 \\
\hline RAD & Cesium-134 & $\mathrm{pCi} / \mathrm{g}$ & 3 & 1 & $33 \%$ & 0.0079 & 0.016 & 0.024 & 0.024 & 0.012 \\
\hline RAD & Cesium-137 & $\mathrm{pCi} / \mathrm{g}$ & 7 & 3 & $43 \%$ & 0.0082 & 0.026 & 0.022 & 0.056 & 0.020 \\
\hline RAD & Cobalt-60 & $\mathrm{pCi} / \mathrm{g}$ & 4 & 0 & $0 \%$ & 3.69E-04 & 0.019 & -- & -- & 0.0042 \\
\hline RAD & Europium-154 & $\mathrm{pCi} / \mathrm{g}$ & 6 & 0 & $0 \%$ & 0.015 & 0.16 & -- & -- & 0.027 \\
\hline RAD & Europium-155 & $\mathrm{pCi} / \mathrm{g}$ & 4 & 0 & $0 \%$ & 0.0027 & 0.019 & -- & -- & 0.0058 \\
\hline RAD & Potassium-40 & $\mathrm{pCi} / \mathrm{g}$ & 9 & 9 & $100 \%$ & -- & -- & 2.1 & 4.1 & 3.1 \\
\hline RAD & Ruthenium-106 & $\mathrm{pCi} / \mathrm{g}$ & 1 & 0 & $0 \%$ & 0.0036 & 0.0036 & -- & -- & 0.0018 \\
\hline RAD & Strontium-90 & $\mathrm{pCi} / \mathrm{g}$ & 3 & 1 & $33 \%$ & 4.64E-04 & 0.0030 & 0.0035 & 0.0035 & 0.0017 \\
\hline
\end{tabular}

RAD - Radionuclide 
Table F-21

Summary Statistics for Whitefish Liver Collected from Segment 1 of the Columbia River

\begin{tabular}{|c|c|c|c|c|c|c|c|c|c|c|}
\hline $\begin{array}{c}\text { Constituent } \\
\text { Class }\end{array}$ & Constituent Name & Units & $\begin{array}{l}\text { Number } \\
\text { Analyzed }\end{array}$ & $\begin{array}{l}\text { Number } \\
\text { Detected }\end{array}$ & $\begin{array}{c}\text { Frequency of } \\
\text { Detection }\end{array}$ & $\begin{array}{l}\text { Minimum } \\
\text { Nondetect }\end{array}$ & $\begin{array}{l}\text { Maximum } \\
\text { Nondetect }\end{array}$ & $\begin{array}{l}\text { Minimum } \\
\text { Detect }\end{array}$ & $\begin{array}{l}\text { Maximum } \\
\text { Detect }\end{array}$ & $\begin{array}{c}\text { Average } \\
\text { Concentration }\end{array}$ \\
\hline METAL & Aluminum & $\mathrm{mg} / \mathrm{kg}$ & 1 & 1 & $100 \%$ & -- & -- & 2.6 & 2.6 & 2.6 \\
\hline METAL & Antimony & $\mathrm{mg} / \mathrm{kg}$ & 1 & 0 & $0 \%$ & 0.019 & 0.019 & -- & -- & 0.0095 \\
\hline METAL & Arsenic & $\mathrm{mg} / \mathrm{kg}$ & 1 & 1 & $100 \%$ & -- & -- & 1.1 & 1.1 & 1.1 \\
\hline METAL & Beryllium & $\mathrm{mg} / \mathrm{kg}$ & 1 & 1 & $100 \%$ & -- & -- & 0.063 & 0.063 & 0.063 \\
\hline METAL & Cadmium & $\mathrm{mg} / \mathrm{kg}$ & 1 & 1 & $100 \%$ & -- & -- & 1.6 & 1.6 & 1.6 \\
\hline METAL & Chromium & $\mathrm{mg} / \mathrm{kg}$ & 1 & 1 & $100 \%$ & -- & -- & 0.061 & 0.061 & 0.061 \\
\hline METAL & Copper & $\mathrm{mg} / \mathrm{kg}$ & 1 & 1 & $100 \%$ & -- & -- & 34 & 34 & 34 \\
\hline METAL & Lead & $\mathrm{mg} / \mathrm{kg}$ & 1 & 0 & $0 \%$ & 0.030 & 0.030 & -- & -- & 0.015 \\
\hline METAL & Manganese & $\mathrm{mg} / \mathrm{kg}$ & 1 & 1 & $100 \%$ & -- & -- & 4.4 & 4.4 & 4.4 \\
\hline METAL & Mercury & $\mathrm{mg} / \mathrm{kg}$ & 1 & 1 & $100 \%$ & -- & -- & 0.49 & 0.49 & 0.49 \\
\hline METAL & Nickel & $\mathrm{mg} / \mathrm{kg}$ & 1 & 1 & $100 \%$ & -- & -- & 0.069 & 0.069 & 0.069 \\
\hline METAL & Selenium & $\mathrm{mg} / \mathrm{kg}$ & 1 & 1 & $100 \%$ & -- & -- & 6.0 & 6.0 & 6.0 \\
\hline METAL & Silver & $\mathrm{mg} / \mathrm{kg}$ & 1 & 1 & $100 \%$ & -- & -- & 0.34 & 0.34 & 0.34 \\
\hline METAL & Thallium & $\mathrm{mg} / \mathrm{kg}$ & 1 & 1 & $100 \%$ & -- & -- & 1.3 & 1.3 & 1.3 \\
\hline METAL & Thorium & $\mathrm{mg} / \mathrm{kg}$ & 1 & 0 & $0 \%$ & 0.021 & 0.021 & -- & -- & 0.011 \\
\hline METAL & Uranium & $\mathrm{mg} / \mathrm{kg}$ & 1 & 0 & $0 \%$ & 0.031 & 0.031 & -- & -- & 0.015 \\
\hline METAL & Zinc & $\mathrm{mg} / \mathrm{kg}$ & 1 & 1 & $100 \%$ & -- & -- & 105 & 105 & 105 \\
\hline
\end{tabular}


Table F-22

Summary Statistics for Wholebody Whitefish Collected from Segment 1 of the Columbia River

\begin{tabular}{|c|c|c|c|c|c|c|c|c|c|c|}
\hline $\begin{array}{l}\text { Constituent } \\
\text { Class }\end{array}$ & Constituent Name & Units & $\begin{array}{l}\text { Number } \\
\text { Analyzed }\end{array}$ & $\begin{array}{l}\text { Number } \\
\text { Detected }\end{array}$ & $\begin{array}{c}\text { Frequency of } \\
\text { Detection }\end{array}$ & $\begin{array}{l}\text { Minimum } \\
\text { Nondetect }\end{array}$ & $\begin{array}{l}\text { Maximum } \\
\text { Nondetect }\end{array}$ & $\begin{array}{c}\text { Minimum } \\
\text { Detect }\end{array}$ & $\begin{array}{l}\text { Maximum } \\
\text { Detect }\end{array}$ & $\begin{array}{c}\text { Average } \\
\text { Concentration }\end{array}$ \\
\hline$\overline{\mathrm{RAD}}$ & Strontium-90 & $\mathrm{pCi} / \mathrm{g}$ & 8 & 7 & $88 \%$ & 0.0073 & 0.0073 & 0.035 & 0.071 & 0.044 \\
\hline RAD & Uranium-234 & $\mathrm{pCi} / \mathrm{g}$ & 7 & 7 & $100 \%$ & -- & -- & 0.0030 & 0.060 & 0.015 \\
\hline RAD & Uranium-235 & $\mathrm{pCi} / \mathrm{g}$ & 4 & 1 & $25 \%$ & $1.75 \mathrm{E}-04$ & 0.0010 & 0.0033 & 0.0033 & 0.0011 \\
\hline RAD & Uranium-238 & $\mathrm{pCi} / \mathrm{g}$ & 7 & 7 & $100 \%$ & -- & -- & 0.0039 & 0.050 & 0.012 \\
\hline
\end{tabular}

RAD - radionuclide 
Table F-23

Summary Statistics for Whole Body Northern Squawfish Collected from Segment 1 of the Columbia River

\begin{tabular}{|c|c|c|c|c|c|c|c|c|c|c|}
\hline $\begin{array}{l}\text { Constituent } \\
\text { Class }\end{array}$ & Constituent Name & Units & $\begin{array}{l}\text { Number } \\
\text { Analyzed }\end{array}$ & $\begin{array}{l}\text { Number } \\
\text { Detected }\end{array}$ & $\begin{array}{c}\text { Frequency of } \\
\text { Detection }\end{array}$ & $\begin{array}{l}\text { Minimum } \\
\text { Nondetect }\end{array}$ & $\begin{array}{l}\text { Maximum } \\
\text { Nondetect }\end{array}$ & $\begin{array}{c}\text { Minimum } \\
\text { Detect }\end{array}$ & $\begin{array}{l}\text { Maximum } \\
\text { Detect }\end{array}$ & $\begin{array}{c}\text { Average } \\
\text { Concentration }\end{array}$ \\
\hline$\overline{\text { CONV }}$ & Percent Lipids & $\%$ & 1 & 1 & $100 \%$ & -- & -- & 4.1 & 4.1 & 4.1 \\
\hline CONV & Percent moisture (wet sample) & $\mathrm{mg} / \mathrm{Kg}$ & 1 & 1 & $100 \%$ & -- & -- & 72 & 72 & 72 \\
\hline DIOXI & TCDD-eq & $\mathrm{mg} / \mathrm{Kg}$ & 1 & 1 & $100 \%$ & -- & -- & 6.0 & 6.0 & 6.0 \\
\hline METAL & Aluminum & $\mathrm{mg} / \mathrm{Kg}$ & 1 & 1 & $100 \%$ & -- & -- & 67 & 67 & 67 \\
\hline METAL & Arsenic & $\mathrm{mg} / \mathrm{Kg}$ & 1 & 0 & $0 \%$ & 0.26 & 0.26 & -- & -- & 0.13 \\
\hline METAL & Barium & $\mathrm{mg} / \mathrm{Kg}$ & 1 & 1 & $100 \%$ & -- & -- & 3.4 & 3.4 & 3.4 \\
\hline METAL & Beryllium & $\mathrm{mg} / \mathrm{Kg}$ & 1 & 0 & $0 \%$ & 0.051 & 0.051 & -- & -- & 0.026 \\
\hline METAL & Boron & $\mathrm{mg} / \mathrm{Kg}$ & 1 & 0 & $0 \%$ & 1.0 & 1.0 & -- & -- & 0.52 \\
\hline METAL & Cadmium & $\mathrm{mg} / \mathrm{Kg}$ & 1 & 1 & $100 \%$ & -- & -- & 0.14 & 0.14 & 0.14 \\
\hline METAL & Chromium & $\mathrm{mg} / \mathrm{Kg}$ & 1 & 1 & $100 \%$ & -- & -- & 3.4 & 3.4 & 3.4 \\
\hline METAL & Copper & $\mathrm{mg} / \mathrm{Kg}$ & 1 & 1 & $100 \%$ & -- & -- & 2.3 & 2.3 & 2.3 \\
\hline METAL & Iron & $\mathrm{mg} / \mathrm{Kg}$ & 1 & 1 & $100 \%$ & -- & -- & 149 & 149 & 149 \\
\hline METAL & Lead & $\mathrm{mg} / \mathrm{Kg}$ & 1 & 0 & $0 \%$ & 0.10 & 0.10 & -- & -- & 0.051 \\
\hline METAL & Magnesium & $\mathrm{mg} / \mathrm{Kg}$ & 1 & 1 & $100 \%$ & -- & -- & 335 & 335 & 335 \\
\hline METAL & Manganese & $\mathrm{mg} / \mathrm{Kg}$ & 1 & 1 & $100 \%$ & -- & -- & 5.8 & 5.8 & 5.8 \\
\hline METAL & Mercury & $\mathrm{mg} / \mathrm{Kg}$ & 1 & 1 & $100 \%$ & -- & -- & 0.30 & 0.30 & 0.30 \\
\hline METAL & Molybdenum & $\mathrm{mg} / \mathrm{Kg}$ & 1 & 0 & $0 \%$ & 0.26 & 0.26 & -- & -- & 0.13 \\
\hline METAL & Nickel & $\mathrm{mg} / \mathrm{Kg}$ & 1 & 1 & $100 \%$ & -- & -- & 0.59 & 0.59 & 0.59 \\
\hline METAL & Selenium & $\mathrm{mg} / \mathrm{Kg}$ & 1 & 1 & $100 \%$ & -- & -- & 0.41 & 0.41 & 0.41 \\
\hline METAL & Strontium & $\mathrm{mg} / \mathrm{Kg}$ & 1 & 1 & $100 \%$ & -- & -- & 15 & 15 & 15 \\
\hline METAL & Vanadium & $\mathrm{mg} / \mathrm{Kg}$ & 1 & 0 & $0 \%$ & 0.26 & 0.26 & -- & -- & 0.13 \\
\hline METAL & Zinc & $\mathrm{mg} / \mathrm{Kg}$ & 1 & 1 & $100 \%$ & -- & -- & 20 & 20 & 20 \\
\hline PCB & Total polychlorinated biphenyl & $\mathrm{mg} / \mathrm{Kg}$ & 1 & 1 & $100 \%$ & -- & -- & 1.3 & 1.3 & 1.3 \\
\hline PEST & 2,4'-DDD & $\mathrm{mg} / \mathrm{Kg}$ & 1 & 0 & $0 \%$ & 0.010 & 0.010 & -- & -- & 0.0050 \\
\hline PEST & 2,4'-DDE & $\mathrm{mg} / \mathrm{Kg}$ & 1 & 0 & $0 \%$ & 0.010 & 0.010 & -- & -- & 0.0050 \\
\hline PEST & 2,4'-DDT & $\mathrm{mg} / \mathrm{Kg}$ & 1 & 0 & $0 \%$ & 0.010 & 0.010 & -- & -- & 0.0050 \\
\hline PEST & Alpha-BHC & $\mathrm{mg} / \mathrm{Kg}$ & 1 & 0 & $0 \%$ & 0.010 & 0.010 & -- & -- & 0.0050 \\
\hline PEST & alpha-Chlordane & $\mathrm{mg} / \mathrm{Kg}$ & 1 & 0 & $0 \%$ & 0.010 & 0.010 & -- & -- & 0.0050 \\
\hline PEST & Beta-BHC & $\mathrm{mg} / \mathrm{Kg}$ & 1 & 0 & $0 \%$ & 0.010 & 0.010 & -- & -- & 0.0050 \\
\hline PEST & cis-Nonachlor & $\mathrm{mg} / \mathrm{Kg}$ & 1 & 0 & $0 \%$ & 0.010 & 0.010 & -- & -- & 0.0050 \\
\hline PEST & Delta-BHC & $\mathrm{mg} / \mathrm{Kg}$ & 1 & 0 & $0 \%$ & 0.010 & 0.010 & -- & -- & 0.0050 \\
\hline PEST & Dichlorodiphenyldichloroethane & $\mathrm{mg} / \mathrm{Kg}$ & 1 & 1 & $100 \%$ & -- & -- & 0.067 & 0.067 & 0.067 \\
\hline PEST & Dichlorodiphenyldichloroethylene & $\mathrm{mg} / \mathrm{Kg}$ & 1 & 1 & $100 \%$ & -- & -- & 0.28 & 0.28 & 0.28 \\
\hline PEST & Dichlorodiphenyltrichloroethane & $\mathrm{mg} / \mathrm{Kg}$ & 1 & 0 & $0 \%$ & 0.010 & 0.010 & -- & -- & 0.0050 \\
\hline PEST & Dieldrin & $\mathrm{mg} / \mathrm{Kg}$ & 1 & 0 & $0 \%$ & 0.010 & 0.010 & -- & -- & 0.0050 \\
\hline PEST & Endrin & $\mathrm{mg} / \mathrm{Kg}$ & 1 & 0 & $0 \%$ & 0.010 & 0.010 & -- & -- & 0.0050 \\
\hline PEST & Gamma-BHC (Lindane) & $\mathrm{mg} / \mathrm{Kg}$ & 1 & 0 & $0 \%$ & 0.010 & 0.010 & -- & -- & 0.0050 \\
\hline PEST & gamma-Chlordane & $\mathrm{mg} / \mathrm{Kg}$ & 1 & 0 & $0 \%$ & 0.010 & 0.010 & -- & -- & 0.0050 \\
\hline PEST & Heptachlor epoxide & $\mathrm{mg} / \mathrm{Kg}$ & 1 & 0 & $0 \%$ & 0.010 & 0.010 & -- & -- & 0.0050 \\
\hline PEST & Mirex & $\mathrm{mg} / \mathrm{Kg}$ & 1 & 0 & $0 \%$ & 0.010 & 0.010 & -- & -- & 0.0050 \\
\hline PEST & Oxychlordane & $\mathrm{mg} / \mathrm{Kg}$ & 1 & 0 & $0 \%$ & 0.010 & 0.010 & -- & -- & 0.0050 \\
\hline PEST & Toxaphene & $\mathrm{mg} / \mathrm{Kg}$ & 1 & 0 & $0 \%$ & 0.030 & 0.030 & -- & -- & 0.015 \\
\hline PEST & trans-Nonachlor & $\mathrm{mg} / \mathrm{Kg}$ & 1 & 0 & $0 \%$ & 0.010 & 0.010 & -- & -- & 0.0050 \\
\hline SVOC & Hexachlorobenzene & $\mathrm{mg} / \mathrm{Kg}$ & 1 & 0 & $0 \%$ & 0.010 & 0.010 & -- & -- & 0.0050 \\
\hline
\end{tabular}

PEST - Pesticide

PCB - Polychlorinated biphenyl

SVOC - Semivolatile Organic Compound 


\section{Table F-24}

Summary Statistics for Sculpin Liver Collected from Segment 1 of the Columbia River

\begin{tabular}{|c|c|c|c|c|c|c|c|c|c|c|}
\hline $\begin{array}{l}\text { Constituent } \\
\text { Class }\end{array}$ & Constituent Name & Units & $\begin{array}{l}\text { Number } \\
\text { Analyzed }\end{array}$ & $\begin{array}{l}\text { Number } \\
\text { Detected }\end{array}$ & $\begin{array}{l}\text { Frequency of } \\
\text { Detection }\end{array}$ & $\begin{array}{l}\text { Minimum } \\
\text { Nondetect }\end{array}$ & $\begin{array}{c}\text { Maximum } \\
\text { Nondetect }\end{array}$ & $\begin{array}{c}\text { Minimum } \\
\text { Detect }\end{array}$ & $\begin{array}{l}\text { Maximum } \\
\text { Detect }\end{array}$ & $\begin{array}{c}\text { Average } \\
\text { Concentration }\end{array}$ \\
\hline$\overline{\text { METAL }}$ & Aluminum & $\mathrm{mg} / \mathrm{kg}$ & 13 & 13 & $100 \%$ & -- & -- & 1.9 & 17 & 6.0 \\
\hline METAL & Antimony & $\mathrm{mg} / \mathrm{kg}$ & 15 & 11 & $73 \%$ & 0.015 & 0.019 & 0.024 & 0.35 & 0.052 \\
\hline METAL & Arsenic & $\mathrm{mg} / \mathrm{kg}$ & 15 & 12 & $80 \%$ & 0.10 & 0.10 & 0.50 & 2.3 & 1.1 \\
\hline METAL & Barium & $\mathrm{mg} / \mathrm{kg}$ & 13 & 13 & $100 \%$ & -- & -- & 0.053 & 0.80 & 0.24 \\
\hline METAL & Beryllium & $\mathrm{mg} / \mathrm{kg}$ & 15 & 0 & $0 \%$ & 0.019 & 0.15 & -- & -- & 0.018 \\
\hline METAL & Cadmium & $\mathrm{mg} / \mathrm{kg}$ & 15 & 15 & $100 \%$ & -- & -- & 1.8 & 15 & 5.8 \\
\hline METAL & Chromium & $\mathrm{mg} / \mathrm{kg}$ & 15 & 15 & $100 \%$ & -- & -- & 0.14 & 7.9 & 1.6 \\
\hline METAL & Copper & $\mathrm{mg} / \mathrm{kg}$ & 15 & 15 & $100 \%$ & -- & -- & 4.9 & 44 & 18 \\
\hline METAL & Lead & $\mathrm{mg} / \mathrm{kg}$ & 15 & 11 & $73 \%$ & 0.034 & 0.034 & 0.046 & 0.23 & 0.090 \\
\hline METAL & Manganese & $\mathrm{mg} / \mathrm{kg}$ & 13 & 13 & $100 \%$ & -- & -- & 0.75 & 6.8 & 2.2 \\
\hline METAL & Mercury & $\mathrm{mg} / \mathrm{kg}$ & 15 & 15 & $100 \%$ & -- & -- & 0.078 & 0.63 & 0.23 \\
\hline METAL & Nickel & $\mathrm{mg} / \mathrm{kg}$ & 15 & 15 & $100 \%$ & -- & -- & 0.068 & 0.32 & 0.15 \\
\hline METAL & Selenium & $\mathrm{mg} / \mathrm{kg}$ & 15 & 15 & $100 \%$ & -- & -- & 2.6 & 15 & 7.1 \\
\hline METAL & Silver & $\mathrm{mg} / \mathrm{kg}$ & 15 & 4 & $27 \%$ & 0.010 & 0.045 & 0.026 & 0.065 & 0.026 \\
\hline METAL & Thallium & $\mathrm{mg} / \mathrm{kg}$ & 15 & 15 & $100 \%$ & -- & -- & 0.18 & 0.39 & 0.27 \\
\hline METAL & Thorium & $\mathrm{mg} / \mathrm{kg}$ & 13 & 3 & $23 \%$ & 0.020 & 0.021 & 0.041 & 0.27 & 0.045 \\
\hline METAL & Uranium & $\mathrm{mg} / \mathrm{kg}$ & 13 & 2 & $15 \%$ & 0.010 & 0.031 & 0.017 & 0.018 & 0.015 \\
\hline METAL & Zinc & $\mathrm{mg} / \mathrm{kg}$ & 15 & 15 & $100 \%$ & -- & -- & 82 & 232 & 157 \\
\hline
\end{tabular}


Table F-25

Summary Statistics for Whole Body Sculpin Collected from Segment 1 of the Columbia River

\begin{tabular}{|c|c|c|c|c|c|c|c|c|c|c|}
\hline $\begin{array}{l}\text { Constituent } \\
\text { Class }\end{array}$ & Constituent Name & Units & $\begin{array}{l}\text { Number } \\
\text { Analyzed }\end{array}$ & $\begin{array}{l}\text { Number } \\
\text { Detected }\end{array}$ & $\begin{array}{l}\text { Frequency of } \\
\text { Detection }\end{array}$ & $\begin{array}{c}\text { Minimum } \\
\text { Nondetect }\end{array}$ & $\begin{array}{c}\text { Maximum } \\
\text { Nondetect }\end{array}$ & $\begin{array}{c}\text { Minimum } \\
\text { Detect }\end{array}$ & $\begin{array}{l}\text { Maximum } \\
\text { Detect }\end{array}$ & $\begin{array}{c}\text { Average } \\
\text { Concentration }\end{array}$ \\
\hline$\overline{\mathrm{RAD}}$ & Antimony-125 & $\mathrm{pCi} / \mathrm{g}$ & 3 & 0 & $0 \%$ & 0.0039 & 0.050 & $\overline{--}$ & -- & 0.011 \\
\hline RAD & Beryllium-7 & $\mathrm{pCi} / \mathrm{g}$ & 5 & 0 & $0 \%$ & 0.024 & 1.2 & -- & -- & 0.19 \\
\hline RAD & Cesium-134 & $\mathrm{pCi} / \mathrm{g}$ & 4 & 0 & $0 \%$ & 0.0088 & 0.029 & -- & -- & 0.0085 \\
\hline RAD & Cesium-137 & $\mathrm{pCi} / \mathrm{g}$ & 4 & 0 & $0 \%$ & 2.42E-05 & 0.0089 & -- & -- & 0.0024 \\
\hline RAD & Cobalt-60 & $\mathrm{pCi} / \mathrm{g}$ & 6 & 0 & $0 \%$ & 0.0021 & 0.068 & -- & -- & 0.013 \\
\hline RAD & Europium-152 & $\mathrm{pCi} / \mathrm{g}$ & 4 & 0 & $0 \%$ & 0.0028 & 0.028 & -- & -- & 0.0061 \\
\hline RAD & Europium-154 & $\mathrm{pCi} / \mathrm{g}$ & 2 & 0 & $0 \%$ & 0.0030 & 0.011 & -- & -- & 0.0035 \\
\hline RAD & Europium-155 & $\mathrm{pCi} / \mathrm{g}$ & 4 & 0 & $0 \%$ & 0.0060 & 0.11 & -- & -- & 0.019 \\
\hline RAD & Potassium-40 & $\mathrm{pCi} / \mathrm{g}$ & 7 & 7 & $100 \%$ & -- & -- & 2.5 & 3.6 & 3.1 \\
\hline RAD & Ruthenium-106 & $\mathrm{pCi} / \mathrm{g}$ & 3 & 0 & $0 \%$ & 0.037 & 0.082 & -- & -- & 0.033 \\
\hline RAD & Strontium-90 & $\mathrm{pCi} / \mathrm{g}$ & 10 & 1 & $10 \%$ & 7.61E-04 & 0.033 & 0.015 & 0.015 & 0.0073 \\
\hline RAD & Technetium-99 & $\mathrm{pCi} / \mathrm{g}$ & 8 & 2 & $25 \%$ & 0.053 & 0.20 & 0.15 & 0.33 & 0.10 \\
\hline
\end{tabular}

RAD - Radionuclide 
Table F-26

Summary Statistics for White Sturgeon Fillet Collected from Segment 1 of the Columbia River

\begin{tabular}{|c|c|c|c|c|c|c|c|c|c|c|}
\hline $\begin{array}{l}\text { Constituent } \\
\text { Class }\end{array}$ & Constituent Name & Units & $\begin{array}{c}\text { Number } \\
\text { Analyzed }\end{array}$ & $\begin{array}{l}\text { Number } \\
\text { Detected }\end{array}$ & $\begin{array}{l}\text { Frequency of } \\
\text { Detection }\end{array}$ & $\begin{array}{l}\text { Minimum } \\
\text { Nondetect }\end{array}$ & $\begin{array}{c}\text { Maximum } \\
\text { Nondetect }\end{array}$ & $\begin{array}{l}\text { Minimum } \\
\text { Detect }\end{array}$ & $\begin{array}{l}\text { Maximum } \\
\text { Detect }\end{array}$ & $\begin{array}{c}\text { Average } \\
\text { Concentration }\end{array}$ \\
\hline RAD & Beryllium-7 & $\mathrm{pCi} / \mathrm{g}$ & 5 & 0 & $0 \%$ & 0.0042 & 0.19 & -- & -- & 0.048 \\
\hline RAD & Cesium-134 & $\mathrm{pCi} / \mathrm{g}$ & 3 & 1 & $33 \%$ & 0.0079 & 0.016 & 0.024 & 0.024 & 0.012 \\
\hline RAD & Cesium-137 & $\mathrm{pCi} / \mathrm{g}$ & 7 & 3 & $43 \%$ & 0.0082 & 0.026 & 0.022 & 0.056 & 0.020 \\
\hline RAD & Cobalt-60 & $\mathrm{pCi} / \mathrm{g}$ & 4 & 0 & $0 \%$ & 3.69E-04 & 0.019 & -- & -- & 0.0042 \\
\hline RAD & Europium-154 & $\mathrm{pCi} / \mathrm{g}$ & 6 & 0 & $0 \%$ & 0.015 & 0.16 & -- & -- & 0.027 \\
\hline RAD & Europium-155 & $\mathrm{pCi} / \mathrm{g}$ & 4 & 0 & $0 \%$ & 0.0027 & 0.019 & -- & -- & 0.0058 \\
\hline RAD & Potassium-40 & $\mathrm{pCi} / \mathrm{g}$ & 9 & 9 & $100 \%$ & -- & -- & 2.1 & 4.1 & 3.1 \\
\hline RAD & Ruthenium-106 & $\mathrm{pCi} / \mathrm{g}$ & 1 & 0 & $0 \%$ & 0.0036 & 0.0036 & -- & -- & 0.0018 \\
\hline RAD & Strontium-90 & $\mathrm{pCi} / \mathrm{g}$ & 3 & 1 & $33 \%$ & $4.64 \mathrm{E}-04$ & 0.0030 & 0.0035 & 0.0035 & 0.0017 \\
\hline
\end{tabular}

RAD- radionuclide 
Table F-27

Summary Statistics for Anadonta Shells Collected from Segment 2 of the Columbia River

\begin{tabular}{|c|c|c|c|c|c|c|c|c|c|c|}
\hline $\begin{array}{c}\text { Constituent } \\
\text { Class }\end{array}$ & $\begin{array}{c}\text { Constituent } \\
\text { Name }\end{array}$ & Units & $\begin{array}{l}\text { Number } \\
\text { Analyzed }\end{array}$ & $\begin{array}{l}\text { Number } \\
\text { Detected }\end{array}$ & $\begin{array}{c}\text { Frequency of } \\
\text { Detection }\end{array}$ & $\begin{array}{l}\text { Minimum } \\
\text { Nondetect }\end{array}$ & $\begin{array}{l}\text { Maximum } \\
\text { Nondetect }\end{array}$ & $\begin{array}{c}\text { Minimum } \\
\text { Detect }\end{array}$ & $\begin{array}{l}\text { Maximum } \\
\text { Detect }\end{array}$ & $\begin{array}{c}\text { Average } \\
\text { Concentration }\end{array}$ \\
\hline$\overline{\mathrm{RAD}}$ & Strontium-90 & $\mathrm{pCi} / \mathrm{g}$ & 1 & 1 & $100 \%$ & $\overline{--}$ & $\overline{--}$ & 0.41 & 0.41 & 0.41 \\
\hline RAD & Technetium-99 & $\mathrm{pCi} / \mathrm{g}$ & 1 & 1 & $100 \%$ & -- & -- & 0.61 & 0.61 & 0.61 \\
\hline
\end{tabular}

RAD - Radionuclide 
Table F-28

Summary Statistics for Anadonta Soft Tissue Collected from Segment 2 of the Columbia River

\begin{tabular}{|c|c|c|c|c|c|c|c|c|c|c|}
\hline $\begin{array}{c}\text { Constituent } \\
\text { Class }\end{array}$ & $\begin{array}{c}\text { Constituent } \\
\text { Name }\end{array}$ & Units & $\begin{array}{l}\text { Number } \\
\text { Analyzed }\end{array}$ & $\begin{array}{l}\text { Number } \\
\text { Detected }\end{array}$ & $\begin{array}{c}\text { Frequency of } \\
\text { Detection }\end{array}$ & $\begin{array}{l}\text { Minimum } \\
\text { Nondetect }\end{array}$ & $\begin{array}{l}\text { Maximum } \\
\text { Nondetect }\end{array}$ & $\begin{array}{l}\text { Minimum } \\
\text { Detect }\end{array}$ & $\begin{array}{c}\text { Maximum } \\
\text { Detect }\end{array}$ & $\begin{array}{c}\text { Average } \\
\text { Concentration }\end{array}$ \\
\hline METAL & Antimony & $\mathrm{mg} / \mathrm{kg}$ & 3 & 3 & $100 \%$ & $\overline{--}$ & $\overline{--}$ & 0.037 & 0.053 & 0.047 \\
\hline METAL & Arsenic & $\mathrm{mg} / \mathrm{kg}$ & 3 & 3 & $100 \%$ & -- & -- & 8.0 & 9.3 & 8.7 \\
\hline METAL & Beryllium & $\mathrm{mg} / \mathrm{kg}$ & 3 & 0 & $0 \%$ & 0.019 & 0.019 & -- & -- & 0.0094 \\
\hline METAL & Cadmium & $\mathrm{mg} / \mathrm{kg}$ & 3 & 3 & $100 \%$ & -- & -- & 8.9 & 10 & 9.5 \\
\hline METAL & Chromium & $\mathrm{mg} / \mathrm{kg}$ & 3 & 3 & $100 \%$ & -- & -- & 8.5 & 22 & 14 \\
\hline METAL & Copper & $\mathrm{mg} / \mathrm{kg}$ & 3 & 3 & $100 \%$ & -- & -- & 7.0 & 9.6 & 8.2 \\
\hline METAL & Lead & $\mathrm{mg} / \mathrm{kg}$ & 3 & 3 & $100 \%$ & -- & -- & 0.95 & 1.2 & 1.1 \\
\hline METAL & Nickel & $\mathrm{mg} / \mathrm{kg}$ & 3 & 3 & $100 \%$ & -- & -- & 2.1 & 3.0 & 2.5 \\
\hline METAL & Selenium & $\mathrm{mg} / \mathrm{kg}$ & 3 & 3 & $100 \%$ & -- & -- & 0.72 & 2.4 & 1.6 \\
\hline METAL & Silver & $\mathrm{mg} / \mathrm{kg}$ & 3 & 3 & $100 \%$ & -- & -- & 0.41 & 0.80 & 0.66 \\
\hline METAL & Thallium & $\mathrm{mg} / \mathrm{kg}$ & 3 & 3 & $100 \%$ & -- & -- & 0.080 & 0.10 & 0.093 \\
\hline METAL & Zinc & $\mathrm{mg} / \mathrm{kg}$ & 3 & 3 & $100 \%$ & -- & -- & 538 & 1,360 & 912 \\
\hline
\end{tabular}


Table F-29

Summary Statistics for Asiatic Clam Shells Collected from Segment 2 of the Columbia River

\begin{tabular}{|c|c|c|c|c|c|c|c|c|c|c|}
\hline $\begin{array}{l}\text { Constituent } \\
\text { Class }\end{array}$ & Constituent Name & Units & $\begin{array}{l}\text { Number } \\
\text { Analyzed }\end{array}$ & $\begin{array}{l}\text { Number } \\
\text { Detected }\end{array}$ & $\begin{array}{c}\text { Frequency of } \\
\text { Detection }\end{array}$ & $\begin{array}{l}\text { Minimum } \\
\text { Nondetect }\end{array}$ & $\begin{array}{l}\text { Maximum } \\
\text { Nondetect }\end{array}$ & $\begin{array}{l}\text { Minimum } \\
\text { Detect }\end{array}$ & $\begin{array}{l}\text { Maximum } \\
\text { Detect }\end{array}$ & $\begin{array}{c}\text { Average } \\
\text { Concentration }\end{array}$ \\
\hline$\overline{\mathrm{RAD}}$ & Strontium-90 & $\mathrm{pCi} / \mathrm{g}$ & 76 & 74 & $97 \%$ & 0.075 & 0.13 & 0.16 & 383 & 6.4 \\
\hline RAD & Technetium-99 & $\mathrm{pCi} / \mathrm{g}$ & 23 & 3 & $13 \%$ & 0.0048 & 0.68 & 0.47 & 3.4 & 0.28 \\
\hline
\end{tabular}

RAD - Radionuclide 
Table F-30

Summary Statistics for Asiatic Clam Soft Tissue Collected from Segment 2 of the Columbia River

\begin{tabular}{|c|c|c|c|c|c|c|c|c|c|c|}
\hline $\begin{array}{c}\text { Constituent } \\
\text { Class }\end{array}$ & Constituent Name & Units & $\begin{array}{l}\text { Number } \\
\text { Analyzed }\end{array}$ & $\begin{array}{l}\text { Number } \\
\text { Detected }\end{array}$ & $\begin{array}{c}\text { Frequency of } \\
\text { Detection }\end{array}$ & $\begin{array}{l}\text { Minimum } \\
\text { Nondetect }\end{array}$ & $\begin{array}{l}\text { Maximum } \\
\text { Nondetect }\end{array}$ & $\begin{array}{l}\text { Minimum } \\
\text { Detect }\end{array}$ & $\begin{array}{c}\text { Maximum } \\
\text { Detect }\end{array}$ & $\begin{array}{c}\text { Average } \\
\text { Concentration }\end{array}$ \\
\hline$\overline{\text { METAL }}$ & Aluminum & $\mathrm{mg} / \mathrm{kg}$ & 92 & 92 & $100 \%$ & $\overline{--}$ & -- & 20 & 3,070 & 385 \\
\hline METAL & Antimony & $\mathrm{mg} / \mathrm{kg}$ & 150 & 103 & $69 \%$ & 0.010 & 0.019 & 0.010 & 0.094 & 0.019 \\
\hline METAL & Arsenic & $\mathrm{mg} / \mathrm{kg}$ & 150 & 150 & $100 \%$ & -- & -- & 3.8 & 9.3 & 6.7 \\
\hline METAL & Barium & $\mathrm{mg} / \mathrm{kg}$ & 68 & 68 & $100 \%$ & -- & -- & 6.0 & 18 & 9.7 \\
\hline METAL & Beryllium & $\mathrm{mg} / \mathrm{kg}$ & 150 & 28 & $19 \%$ & 0.0040 & 0.020 & 0.021 & 0.78 & 0.039 \\
\hline METAL & Cadmium & $\mathrm{mg} / \mathrm{kg}$ & 150 & 150 & $100 \%$ & -- & -- & 0.81 & 4.3 & 2.1 \\
\hline METAL & Chromium & $\mathrm{mg} / \mathrm{kg}$ & 178 & 178 & $100 \%$ & -- & -- & 0.69 & 70 & 8.6 \\
\hline METAL & Copper & $\mathrm{mg} / \mathrm{kg}$ & 150 & 150 & $100 \%$ & -- & -- & 18 & 82 & 40 \\
\hline METAL & Lead & $\mathrm{mg} / \mathrm{kg}$ & 150 & 150 & $100 \%$ & -- & -- & 0.26 & 3.6 & 0.90 \\
\hline METAL & Manganese & $\mathrm{mg} / \mathrm{kg}$ & 92 & 92 & $100 \%$ & -- & -- & 4.8 & 97 & 28 \\
\hline METAL & Mercury & $\mathrm{mg} / \mathrm{kg}$ & 92 & 79 & $86 \%$ & 0.050 & 0.050 & 0.018 & 0.28 & 0.054 \\
\hline METAL & Nickel & $\mathrm{mg} / \mathrm{kg}$ & 150 & 150 & $100 \%$ & -- & -- & 0.20 & 3.7 & 0.97 \\
\hline METAL & Selenium & $\mathrm{mg} / \mathrm{kg}$ & 178 & 178 & $100 \%$ & -- & -- & 1.6 & 5.0 & 2.4 \\
\hline METAL & Silver & $\mathrm{mg} / \mathrm{kg}$ & 150 & 144 & $96 \%$ & 0.045 & 0.045 & 0.034 & 0.17 & 0.061 \\
\hline METAL & Thallium & $\mathrm{mg} / \mathrm{kg}$ & 150 & 150 & $100 \%$ & -- & -- & 0.024 & 0.26 & 0.11 \\
\hline METAL & Thorium & $\mathrm{mg} / \mathrm{kg}$ & 92 & 87 & $95 \%$ & 0.020 & 0.021 & 0.011 & 1.5 & 0.11 \\
\hline METAL & Uranium & $\mathrm{mg} / \mathrm{kg}$ & 132 & 132 & $100 \%$ & -- & -- & 0.071 & 9.7 & 0.84 \\
\hline METAL & Zinc & $\mathrm{mg} / \mathrm{kg}$ & 150 & 150 & $100 \%$ & -- & -- & 74 & 221 & 113 \\
\hline RAD & Beryllium-7 & $\mathrm{pCi} / \mathrm{g}$ & 1 & 0 & $0 \%$ & 0.0078 & 0.0078 & -- & -- & 0.0039 \\
\hline RAD & Cesium-134 & $\mathrm{pCi} / \mathrm{g}$ & 1 & 0 & $0 \%$ & 0.0039 & 0.0039 & -- & -- & 0.0020 \\
\hline RAD & Cesium-137 & $\mathrm{pCi} / \mathrm{g}$ & 1 & 0 & $0 \%$ & 0.0079 & 0.0079 & -- & -- & 0.0039 \\
\hline RAD & Europium-152 & $\mathrm{pCi} / \mathrm{g}$ & 1 & 0 & $0 \%$ & 0.11 & 0.11 & -- & -- & 0.055 \\
\hline RAD & Europium-154 & $\mathrm{pCi} / \mathrm{g}$ & 1 & 0 & $0 \%$ & 0.051 & 0.051 & -- & -- & 0.025 \\
\hline RAD & Potassium-40 & $\mathrm{pCi} / \mathrm{g}$ & 1 & 0 & $0 \%$ & 0.051 & 0.051 & -- & -- & 0.026 \\
\hline RAD & Technetium-99 & $\mathrm{pCi} / \mathrm{g}$ & 1 & 1 & $100 \%$ & -- & -- & 0.16 & 0.16 & 0.16 \\
\hline
\end{tabular}

RAD - Radionuclide 
Table F-31

Summary Statistics for Clam Shells Collected from Segment 2 of the Columbia River

\begin{tabular}{|c|c|c|c|c|c|c|c|c|c|c|}
\hline $\begin{array}{l}\text { Constituent } \\
\text { Class }\end{array}$ & Constituent Name & Units & $\begin{array}{l}\text { Number } \\
\text { Analyzed }\end{array}$ & $\begin{array}{l}\text { Number } \\
\text { Detected }\end{array}$ & $\begin{array}{c}\text { Frequency of } \\
\text { Detection }\end{array}$ & $\begin{array}{l}\text { Minimum } \\
\text { Nondetect }\end{array}$ & $\begin{array}{l}\text { Maximum } \\
\text { Nondetect }\end{array}$ & $\begin{array}{c}\text { Minimum } \\
\text { Detect }\end{array}$ & $\begin{array}{l}\text { Maximum } \\
\text { Detect }\end{array}$ & $\begin{array}{c}\text { Average } \\
\text { Concentration }\end{array}$ \\
\hline METAL & Antimony & $\mathrm{mg} / \mathrm{kg}$ & 2 & 1 & $50 \%$ & 0.015 & 0.015 & 0.019 & 0.019 & 0.013 \\
\hline METAL & Arsenic & $\mathrm{mg} / \mathrm{kg}$ & 2 & 2 & $100 \%$ & -- & -- & 0.11 & 0.51 & 0.31 \\
\hline METAL & Beryllium & $\mathrm{mg} / \mathrm{kg}$ & 3 & 0 & $0 \%$ & 0.0050 & 0.15 & -- & -- & 0.051 \\
\hline METAL & Cadmium & $\mathrm{mg} / \mathrm{kg}$ & 2 & 2 & $100 \%$ & -- & -- & 0.066 & 0.33 & 0.20 \\
\hline METAL & Chromium & $\mathrm{mg} / \mathrm{kg}$ & 3 & 2 & $67 \%$ & 0.10 & 0.10 & 0.25 & 1.9 & 0.74 \\
\hline METAL & Copper & $\mathrm{mg} / \mathrm{kg}$ & 3 & 3 & $100 \%$ & -- & -- & 3.0 & 12 & 8.4 \\
\hline METAL & Lead & $\mathrm{mg} / \mathrm{kg}$ & 2 & 2 & $100 \%$ & -- & -- & 0.21 & 2.7 & 1.4 \\
\hline METAL & Mercury & $\mathrm{mg} / \mathrm{kg}$ & 2 & 2 & $100 \%$ & -- & -- & 0.0014 & 0.0024 & 0.0019 \\
\hline METAL & Nickel & $\mathrm{mg} / \mathrm{kg}$ & 2 & 2 & $100 \%$ & -- & -- & 12 & 12 & 12 \\
\hline METAL & Selenium & $\mathrm{mg} / \mathrm{kg}$ & 2 & 0 & $0 \%$ & 1.0 & 1.0 & -- & -- & 0.50 \\
\hline METAL & Silver & $\mathrm{mg} / \mathrm{kg}$ & 2 & 0 & $0 \%$ & 0.045 & 0.045 & -- & -- & 0.023 \\
\hline METAL & Thallium & $\mathrm{mg} / \mathrm{kg}$ & 2 & 2 & $100 \%$ & -- & -- & 0.048 & 0.12 & 0.086 \\
\hline METAL & Zinc & $\mathrm{mg} / \mathrm{kg}$ & 2 & 2 & $100 \%$ & -- & -- & 2.0 & 27 & 14 \\
\hline RAD & Plutonium-238 & $\mathrm{pCi} / \mathrm{g}$ & 1 & 1 & $100 \%$ & -- & -- & 0.068 & 0.068 & 0.068 \\
\hline RAD & Plutonium-239/240 & $\mathrm{pCi} / \mathrm{g}$ & 1 & 1 & $100 \%$ & -- & -- & 0.012 & 0.012 & 0.012 \\
\hline RAD & Potassium-40 & pCi/g dry & 2 & 2 & $100 \%$ & -- & -- & 0.50 & 0.57 & 0.54 \\
\hline RAD & Strontium-90 & $\mathrm{pCi} / \mathrm{g}$ & 2 & 2 & $100 \%$ & -- & -- & 0.10 & 0.23 & 0.17 \\
\hline RAD & Strontium-90 & $\mathrm{pCi} / \mathrm{g}$ dry & 2 & 1 & $50 \%$ & 0.13 & 0.13 & 1.5 & 1.5 & 0.78 \\
\hline RAD & Uranium-234 & $\mathrm{pCi} / \mathrm{g}$ & 1 & 1 & $100 \%$ & -- & -- & 0.20 & 0.20 & 0.20 \\
\hline RAD & Uranium-238 & $\mathrm{pCi} / \mathrm{g}$ & 1 & 1 & $100 \%$ & -- & -- & 0.090 & 0.090 & 0.090 \\
\hline
\end{tabular}

RAD - Radionuclide 
Table F-32

Summary Statistics for Clam Soft Tissue Collected from Segment 2 of the Columbia River

\begin{tabular}{|c|c|c|c|c|c|c|c|c|c|c|}
\hline $\begin{array}{l}\text { Constituent } \\
\text { Class }\end{array}$ & Constituent Name & Units & $\begin{array}{l}\text { Number } \\
\text { Analyzed }\end{array}$ & $\begin{array}{l}\text { Number } \\
\text { Detected }\end{array}$ & $\begin{array}{l}\text { Frequency of } \\
\text { Detection }\end{array}$ & $\begin{array}{l}\text { Minimum } \\
\text { Nondetect }\end{array}$ & $\begin{array}{l}\text { Maximum } \\
\text { Nondetect }\end{array}$ & $\begin{array}{c}\text { Minimum } \\
\text { Detect }\end{array}$ & $\begin{array}{l}\text { Maximum } \\
\text { Detect }\end{array}$ & $\begin{array}{c}\text { Average } \\
\text { Concentration }\end{array}$ \\
\hline$\overline{M E T A L}$ & Aluminum & $\mathrm{mg} / \mathrm{kg}$ & 1 & 1 & $100 \%$ & $\overline{--}$ & -- & 450 & 450 & 450 \\
\hline METAL & Antimony & $\mathrm{mg} / \mathrm{kg}$ & 3 & 1 & $33 \%$ & 0.015 & 0.015 & 0.020 & 0.020 & 0.012 \\
\hline METAL & Arsenic & $\mathrm{mg} / \mathrm{kg}$ & 3 & 3 & $100 \%$ & -- & -- & 4.3 & 16 & 9.3 \\
\hline METAL & Barium & $\mathrm{mg} / \mathrm{kg}$ & 1 & 1 & $100 \%$ & -- & -- & 15 & 15 & 15 \\
\hline METAL & Beryllium & $\mathrm{mg} / \mathrm{kg}$ & 3 & 1 & $33 \%$ & 0.15 & 0.15 & 0.14 & 0.14 & 0.097 \\
\hline METAL & Cadmium & $\mathrm{mg} / \mathrm{kg}$ & 3 & 3 & $100 \%$ & -- & -- & 2.5 & 5.6 & 3.5 \\
\hline METAL & Chromium & $\mathrm{mg} / \mathrm{kg}$ & 3 & 3 & $100 \%$ & -- & -- & 2.0 & 20 & 8.3 \\
\hline METAL & Copper & $\mathrm{mg} / \mathrm{kg}$ & 3 & 3 & $100 \%$ & -- & -- & 22 & 92 & 50 \\
\hline METAL & Lead & $\mathrm{mg} / \mathrm{kg}$ & 3 & 3 & $100 \%$ & -- & -- & 0.47 & 3.0 & 1.5 \\
\hline METAL & Manganese & $\mathrm{mg} / \mathrm{kg}$ & 1 & 1 & $100 \%$ & -- & -- & 25 & 25 & 25 \\
\hline METAL & Mercury & $\mathrm{mg} / \mathrm{kg}$ & 3 & 3 & $100 \%$ & -- & -- & 0.030 & 0.050 & 0.041 \\
\hline METAL & Nickel & $\mathrm{mg} / \mathrm{kg}$ & 3 & 3 & $100 \%$ & -- & -- & 0.74 & 1.5 & 1.1 \\
\hline METAL & Selenium & $\mathrm{mg} / \mathrm{kg}$ & 3 & 3 & $100 \%$ & -- & -- & 2.4 & 3.9 & 2.9 \\
\hline METAL & Silver & $\mathrm{mg} / \mathrm{kg}$ & 3 & 3 & $100 \%$ & -- & -- & 0.066 & 0.13 & 0.092 \\
\hline METAL & Thallium & $\mathrm{mg} / \mathrm{kg}$ & 3 & 3 & $100 \%$ & -- & -- & 0.16 & 0.36 & 0.25 \\
\hline METAL & Thorium & $\mathrm{mg} / \mathrm{kg}$ & 1 & 1 & $100 \%$ & -- & -- & 0.058 & 0.058 & 0.058 \\
\hline METAL & Uranium & $\mathrm{mg} / \mathrm{kg}$ & 1 & 1 & $100 \%$ & -- & -- & 0.19 & 0.19 & 0.19 \\
\hline METAL & Zinc & $\mathrm{mg} / \mathrm{kg}$ & 3 & 3 & $100 \%$ & -- & -- & 92 & 134 & 111 \\
\hline
\end{tabular}


Table F-33

Summary Statistics for Whole Body Clam Collected from Segment 2 of the Columbia River

\begin{tabular}{|c|c|c|c|c|c|c|c|c|c|c|}
\hline $\begin{array}{c}\text { Constituent } \\
\text { Class }\end{array}$ & Constituent Name & Units & $\begin{array}{l}\text { Number } \\
\text { Analyzed }\end{array}$ & $\begin{array}{l}\text { Number } \\
\text { Detected }\end{array}$ & $\begin{array}{c}\text { Frequency of } \\
\text { Detection }\end{array}$ & $\begin{array}{l}\text { Minimum } \\
\text { Nondetect }\end{array}$ & $\begin{array}{l}\text { Maximum } \\
\text { Nondetect }\end{array}$ & $\begin{array}{c}\text { Minimum } \\
\text { Detect }\end{array}$ & $\begin{array}{c}\text { Maximum } \\
\text { Detect }\end{array}$ & $\begin{array}{c}\text { Average } \\
\text { Concentration }\end{array}$ \\
\hline$\overline{M E T A L}$ & Beryllium & $\mathrm{mg} / \mathrm{kg}$ & 2 & 1 & $50 \%$ & 0.0050 & 0.0050 & 0.017 & 0.017 & 0.0098 \\
\hline METAL & Chromium & $\mathrm{mg} / \mathrm{kg}$ & 2 & 0 & $0 \%$ & 0.10 & 0.10 & -- & -- & 0.050 \\
\hline METAL & Copper & $\mathrm{mg} / \mathrm{kg}$ & 2 & 2 & $100 \%$ & -- & -- & 7.3 & 9.5 & 8.4 \\
\hline RAD & Americium-241 & $\mathrm{pCi} / \mathrm{g}$ & 2 & 1 & $50 \%$ & 0.085 & 0.085 & 0.022 & 0.022 & 0.032 \\
\hline RAD & Plutonium-238 & $\mathrm{pCi} / \mathrm{g}$ & 3 & 3 & $100 \%$ & -- & -- & 0.029 & 0.075 & 0.058 \\
\hline RAD & Plutonium-239/240 & $\mathrm{pCi} / \mathrm{g}$ & 3 & 2 & $67 \%$ & 0.090 & 0.090 & 0.0060 & 0.023 & 0.025 \\
\hline RAD & Strontium-90 & $\mathrm{pCi} / \mathrm{g}$ & 3 & 3 & $100 \%$ & -- & -- & 0.022 & 0.35 & 0.15 \\
\hline RAD & Uranium-234 & $\mathrm{pCi} / \mathrm{g}$ & 2 & 2 & $100 \%$ & -- & -- & 0.19 & 0.37 & 0.28 \\
\hline RAD & Uranium-235 & $\mathrm{pCi} / \mathrm{g}$ & 2 & 2 & $100 \%$ & -- & -- & 0.014 & 0.036 & 0.025 \\
\hline RAD & Uranium-238 & $\mathrm{pCi} / \mathrm{g}$ & 2 & 2 & $100 \%$ & -- & -- & 0.10 & 0.33 & 0.21 \\
\hline
\end{tabular}

RAD - Radionuclide 
Table F-34

Summary Statistics for Bass Fillet with Skin Off Collected from Segment 2 of the Columbia River

\begin{tabular}{|c|c|c|c|c|c|c|c|c|c|c|}
\hline $\begin{array}{l}\text { Constituent } \\
\text { Class }\end{array}$ & Constituent Name & Units & $\begin{array}{l}\text { Number } \\
\text { Analyzed }\end{array}$ & $\begin{array}{l}\text { Number } \\
\text { Detected }\end{array}$ & $\begin{array}{c}\text { Frequency of } \\
\text { Detection }\end{array}$ & $\begin{array}{l}\text { Minimum } \\
\text { Nondetect }\end{array}$ & $\begin{array}{l}\text { Maximum } \\
\text { Nondetect }\end{array}$ & $\begin{array}{l}\text { Minimum } \\
\text { Detect }\end{array}$ & $\begin{array}{l}\text { Maximum } \\
\text { Detect }\end{array}$ & $\begin{array}{c}\text { Average } \\
\text { Concentration }\end{array}$ \\
\hline$\overline{\mathrm{RAD}}$ & Antimony-125 & $\mathrm{pCi} / \mathrm{g}$ & 13 & 0 & $0 \%$ & 0.0013 & 0.033 & $\overline{--}$ & $\overline{--}$ & 0.0036 \\
\hline RAD & Beryllium-7 & $\mathrm{pCi} / \mathrm{g}$ & 18 & 0 & $0 \%$ & 7.12E-04 & 0.15 & -- & -- & 0.033 \\
\hline RAD & Cesium-134 & $\mathrm{pCi} / \mathrm{g}$ & 13 & 0 & $0 \%$ & 4.56E-04 & 0.021 & -- & -- & 0.0028 \\
\hline RAD & Cesium-137 & $\mathrm{pCi} / \mathrm{g}$ & 27 & 3 & $11 \%$ & 0.0022 & 0.032 & 0.016 & 0.021 & 0.0069 \\
\hline RAD & Cobalt-58 & $\mathrm{pCi} / \mathrm{g}$ & 1 & 0 & $0 \%$ & 0.0011 & 0.0011 & -- & -- & 5.65E-04 \\
\hline RAD & Cobalt-60 & $\mathrm{pCi} / \mathrm{g}$ & 19 & 1 & $5 \%$ & 5.34E-04 & 0.052 & 0.018 & 0.018 & 0.0060 \\
\hline RAD & Europium-152 & $\mathrm{pCi} / \mathrm{g}$ & 4 & 0 & $0 \%$ & $4.22 \mathrm{E}-04$ & 0.028 & -- & -- & 0.0047 \\
\hline RAD & Europium-154 & $\mathrm{pCi} / \mathrm{g}$ & 15 & 0 & $0 \%$ & 0.0028 & 0.074 & -- & -- & 0.0092 \\
\hline RAD & Europium-155 & $\mathrm{pCi} / \mathrm{g}$ & 12 & 0 & $0 \%$ & 0.0019 & 0.033 & -- & -- & 0.0066 \\
\hline RAD & Potassium-40 & $\mathrm{pCi} / \mathrm{g}$ & 31 & 31 & $100 \%$ & -- & -- & 1.7 & 3.9 & 3.1 \\
\hline RAD & Ruthenium-106 & $\mathrm{pCi} / \mathrm{g}$ & 10 & 0 & $0 \%$ & 0.0069 & 0.29 & -- & -- & 0.034 \\
\hline RAD & Uranium-234 & $\mathrm{pCi} / \mathrm{g}$ & 2 & 2 & $100 \%$ & -- & -- & 0.0095 & 0.031 & 0.020 \\
\hline RAD & Uranium-235 & $\mathrm{pCi} / \mathrm{g}$ & 1 & 1 & $100 \%$ & -- & -- & 0.032 & 0.032 & 0.032 \\
\hline RAD & Uranium-238 & $\mathrm{pCi} / \mathrm{g}$ & 2 & 1 & $50 \%$ & 0.0023 & 0.0023 & 0.045 & 0.045 & 0.023 \\
\hline
\end{tabular}

RAD - Radionuclide 
Table F-35

Summary Statistics for Bass Kidney Collected from Segment 2 of the Columbia River

\begin{tabular}{|c|c|c|c|c|c|c|c|c|c|c|}
\hline $\begin{array}{l}\text { Constituent } \\
\text { Class }\end{array}$ & Constituent Name & Units & $\begin{array}{l}\text { Number } \\
\text { Analyzed }\end{array}$ & $\begin{array}{l}\text { Number } \\
\text { Detected }\end{array}$ & $\begin{array}{c}\text { Frequency of } \\
\text { Detection }\end{array}$ & $\begin{array}{c}\text { Minimum } \\
\text { Nondetect }\end{array}$ & $\begin{array}{l}\text { Maximum } \\
\text { Nondetect }\end{array}$ & $\begin{array}{c}\text { Minimum } \\
\text { Detect }\end{array}$ & $\begin{array}{l}\text { Maximum } \\
\text { Detect }\end{array}$ & $\begin{array}{c}\text { Average } \\
\text { Concentration }\end{array}$ \\
\hline$\overline{\text { METAL }}$ & Antimony & $\mathrm{mg} / \mathrm{kg}$ & 14 & 7 & $50 \%$ & 0.015 & 0.015 & 0.020 & 0.040 & 0.018 \\
\hline METAL & Arsenic & $\mathrm{mg} / \mathrm{kg}$ & 14 & 14 & $100 \%$ & -- & -- & 0.24 & 0.60 & 0.46 \\
\hline METAL & Beryllium & $\mathrm{mg} / \mathrm{kg}$ & 14 & 0 & $0 \%$ & 0.15 & 0.15 & -- & -- & 0.075 \\
\hline METAL & Cadmium & $\mathrm{mg} / \mathrm{kg}$ & 14 & 14 & $100 \%$ & -- & -- & 0.20 & 2.3 & 0.83 \\
\hline METAL & Chromium & $\mathrm{mg} / \mathrm{kg}$ & 14 & 8 & $57 \%$ & 0.20 & 0.20 & 0.21 & 0.55 & 0.24 \\
\hline METAL & Copper & $\mathrm{mg} / \mathrm{kg}$ & 14 & 14 & $100 \%$ & -- & -- & 2.6 & 8.3 & 5.5 \\
\hline METAL & Lead & $\mathrm{mg} / \mathrm{kg}$ & 14 & 11 & $79 \%$ & 0.036 & 0.036 & 0.044 & 1.4 & 0.19 \\
\hline METAL & Mercury & $\mathrm{mg} / \mathrm{kg}$ & 14 & 14 & $100 \%$ & -- & -- & 0.085 & 0.57 & 0.22 \\
\hline METAL & Nickel & $\mathrm{mg} / \mathrm{kg}$ & 14 & 14 & $100 \%$ & -- & -- & 0.060 & 0.54 & 0.18 \\
\hline METAL & Selenium & $\mathrm{mg} / \mathrm{kg}$ & 14 & 14 & $100 \%$ & -- & -- & 4.0 & 9.0 & 6.1 \\
\hline METAL & Silver & $\mathrm{mg} / \mathrm{kg}$ & 14 & 0 & $0 \%$ & 0.045 & 0.045 & -- & -- & 0.023 \\
\hline METAL & Thallium & $\mathrm{mg} / \mathrm{kg}$ & 14 & 14 & $100 \%$ & -- & -- & 0.10 & 0.26 & 0.18 \\
\hline METAL & Zinc & $\mathrm{mg} / \mathrm{kg}$ & 14 & 14 & $100 \%$ & -- & -- & 47 & 193 & 72 \\
\hline
\end{tabular}


Table F-36

Summary Statistics for Bass Liver Collected from Segment 2 of the Columbia River

\begin{tabular}{|c|c|c|c|c|c|c|c|c|c|c|}
\hline $\begin{array}{c}\text { Constituent } \\
\text { Class }\end{array}$ & Constituent Name & Units & $\begin{array}{l}\text { Number } \\
\text { Analyzed }\end{array}$ & $\begin{array}{l}\text { Number } \\
\text { Detected }\end{array}$ & $\begin{array}{c}\text { Frequency of } \\
\text { Detection }\end{array}$ & $\begin{array}{l}\text { Minimum } \\
\text { Nondetect }\end{array}$ & $\begin{array}{l}\text { Maximum } \\
\text { Nondetect }\end{array}$ & $\begin{array}{l}\text { Minimum } \\
\text { Detect }\end{array}$ & $\begin{array}{c}\text { Maximum } \\
\text { Detect }\end{array}$ & $\begin{array}{c}\text { Average } \\
\text { Concentration }\end{array}$ \\
\hline CONV & Percent Solids & $\%$ & 7 & 7 & $100 \%$ & $\overline{--}$ & $\overline{--}$ & 19 & 32 & 24 \\
\hline METAL & Aluminum & $\mathrm{mg} / \mathrm{kg}$ & 7 & 7 & $100 \%$ & -- & -- & 0.075 & 0.48 & 0.19 \\
\hline METAL & Antimony & $\mathrm{mg} / \mathrm{kg}$ & 22 & 12 & $55 \%$ & 0.0012 & 0.015 & 0.015 & 0.14 & 0.028 \\
\hline METAL & Arsenic & $\mathrm{mg} / \mathrm{kg}$ & 22 & 22 & $100 \%$ & -- & -- & 0.014 & 0.66 & 0.32 \\
\hline METAL & Beryllium & $\mathrm{mg} / \mathrm{kg}$ & 22 & 0 & $0 \%$ & 0.0014 & 0.15 & -- & -- & 0.051 \\
\hline METAL & Cadmium & $\mathrm{mg} / \mathrm{kg}$ & 22 & 22 & $100 \%$ & -- & -- & 0.029 & 11 & 1.8 \\
\hline METAL & Chromium & $\mathrm{mg} / \mathrm{kg}$ & 22 & 13 & $59 \%$ & 0.20 & 0.20 & 0.023 & 0.24 & 0.11 \\
\hline METAL & Copper & $\mathrm{mg} / \mathrm{kg}$ & 22 & 22 & $100 \%$ & -- & -- & 0.050 & 35 & 7.3 \\
\hline METAL & Lead & $\mathrm{mg} / \mathrm{kg}$ & 22 & 3 & $14 \%$ & $6.81 E-04$ & 0.036 & 0.0039 & 2.0 & 0.11 \\
\hline METAL & Manganese & $\mathrm{mg} / \mathrm{kg}$ & 7 & 7 & $100 \%$ & -- & -- & 0.067 & 0.16 & 0.100 \\
\hline METAL & Mercury & $\mathrm{mg} / \mathrm{kg}$ & 15 & 15 & $100 \%$ & -- & -- & 0.14 & 1.1 & 0.37 \\
\hline METAL & Nickel & $\mathrm{mg} / \mathrm{kg}$ & 22 & 13 & $59 \%$ & 4.87E-04 & 0.020 & 9.11E-04 & 0.11 & 0.029 \\
\hline METAL & Selenium & $\mathrm{mg} / \mathrm{kg}$ & 22 & 22 & $100 \%$ & -- & -- & 0.071 & 12 & 5.4 \\
\hline METAL & Silver & $\mathrm{mg} / \mathrm{kg}$ & 22 & 1 & $5 \%$ & 7.01E-04 & 0.045 & 0.11 & 0.11 & 0.019 \\
\hline METAL & Thallium & $\mathrm{mg} / \mathrm{kg}$ & 22 & 22 & $100 \%$ & -- & -- & 0.0029 & 0.55 & 0.22 \\
\hline METAL & Thorium & $\mathrm{mg} / \mathrm{kg}$ & 7 & 0 & $0 \%$ & 4.09E-04 & $6.75 \mathrm{E}-04$ & -- & -- & 2.53E-04 \\
\hline METAL & Uranium & $\mathrm{mg} / \mathrm{kg}$ & 7 & 0 & $0 \%$ & 0.0010 & 0.0017 & -- & -- & 6.39E-04 \\
\hline METAL & Zinc & $\mathrm{mg} / \mathrm{kg}$ & 22 & 22 & $100 \%$ & -- & -- & 1.00 & 103 & 50 \\
\hline
\end{tabular}


Table F-37

Summary Statistics for Whole Body Bass Collected from Segment 2 of the Columbia River

\begin{tabular}{|c|c|c|c|c|c|c|c|c|c|c|}
\hline $\begin{array}{c}\text { Constituent } \\
\text { Class }\end{array}$ & Constituent Name & Units & $\begin{array}{l}\text { Number } \\
\text { Analyzed }\end{array}$ & $\begin{array}{l}\text { Number } \\
\text { Detected }\end{array}$ & $\begin{array}{c}\text { Frequency of } \\
\text { Detection }\end{array}$ & $\begin{array}{l}\text { Minimum } \\
\text { Nondetect }\end{array}$ & $\begin{array}{l}\text { Maximum } \\
\text { Nondetect }\end{array}$ & $\begin{array}{c}\text { Minimum } \\
\text { Detect }\end{array}$ & $\begin{array}{l}\text { Maximum } \\
\text { Detect }\end{array}$ & $\begin{array}{c}\text { Average } \\
\text { Concentration }\end{array}$ \\
\hline RAD & Strontium-90 & $\mathrm{pCi} / \mathrm{g}$ & 22 & 12 & $55 \%$ & 0.0042 & 0.053 & 0.019 & 0.062 & 0.024 \\
\hline RAD & Uranium-234 & $\mathrm{pCi} / \mathrm{g}$ & 5 & 0 & $0 \%$ & $6.50 \mathrm{E}-04$ & 0.0018 & -- & -- & $6.18 \mathrm{E}-04$ \\
\hline RAD & Uranium-235 & $\mathrm{pCi} / \mathrm{g}$ & 2 & 0 & $0 \%$ & 4.66E-04 & 4.92E-04 & -- & -- & $2.40 \mathrm{E}-04$ \\
\hline RAD & Uranium-238 & $\mathrm{pCi} / \mathrm{g}$ & 3 & 0 & $0 \%$ & 9.62E-04 & 0.0012 & -- & -- & $5.24 \mathrm{E}-04$ \\
\hline
\end{tabular}

RAD - Radionuclide 
Table F-38

Summary Statistics for Whole Body Smallmouth Bass Collected from Segment 2 of the Columbia River

\begin{tabular}{|c|c|c|c|c|c|c|c|c|c|c|}
\hline $\begin{array}{l}\text { Constituent } \\
\text { Class }\end{array}$ & Constituent Name & Units & $\begin{array}{l}\text { Number } \\
\text { Analyzed }\end{array}$ & $\begin{array}{l}\text { Number } \\
\text { Detected }\end{array}$ & $\begin{array}{l}\text { Frequency of } \\
\text { Detection }\end{array}$ & $\begin{array}{l}\text { Minimum } \\
\text { Nondetect }\end{array}$ & $\begin{array}{c}\text { Maximum } \\
\text { Nondetect }\end{array}$ & $\begin{array}{c}\text { Minimum } \\
\text { Detect }\end{array}$ & $\begin{array}{l}\text { Maximum } \\
\text { Detect }\end{array}$ & $\begin{array}{c}\text { Average } \\
\text { Concentration }\end{array}$ \\
\hline$\overline{M E T A L}$ & Aluminum & $\mathrm{mg} / \mathrm{kg}$ & 3 & 3 & $100 \%$ & $\overline{--}$ & $\overline{--}$ & 0.99 & 1.5 & 1.3 \\
\hline METAL & Antimony & $\mathrm{mg} / \mathrm{kg}$ & 3 & 0 & $0 \%$ & 0.050 & 0.050 & -- & -- & 0.025 \\
\hline METAL & Arsenic & $\mathrm{mg} / \mathrm{kg}$ & 3 & 3 & $100 \%$ & -- & -- & 0.16 & 0.17 & 0.17 \\
\hline METAL & Barium & $\mathrm{mg} / \mathrm{kg}$ & 3 & 3 & $100 \%$ & -- & -- & 0.54 & 0.96 & 0.73 \\
\hline METAL & Beryllium & $\mathrm{mg} / \mathrm{kg}$ & 3 & 0 & $0 \%$ & 0.0040 & 0.0040 & -- & -- & 0.0020 \\
\hline METAL & Cadmium & $\mathrm{mg} / \mathrm{kg}$ & 3 & 3 & $100 \%$ & -- & -- & 0.0048 & 0.010 & 0.0083 \\
\hline METAL & Chromium & $\mathrm{mg} / \mathrm{kg}$ & 3 & 2 & $67 \%$ & 0.10 & 0.10 & 0.13 & 0.23 & 0.14 \\
\hline METAL & Cobalt & $\mathrm{mg} / \mathrm{kg}$ & 3 & 3 & $100 \%$ & -- & -- & 0.11 & 0.18 & 0.14 \\
\hline METAL & Copper & $\mathrm{mg} / \mathrm{kg}$ & 3 & 3 & $100 \%$ & -- & -- & 0.50 & 0.55 & 0.52 \\
\hline METAL & Lead & $\mathrm{mg} / \mathrm{kg}$ & 3 & 3 & $100 \%$ & -- & -- & 0.010 & 0.13 & 0.063 \\
\hline METAL & Manganese & $\mathrm{mg} / \mathrm{kg}$ & 3 & 3 & $100 \%$ & -- & -- & 2.0 & 2.4 & 2.3 \\
\hline METAL & Mercury & $\mathrm{mg} / \mathrm{kg}$ & 3 & 3 & $100 \%$ & -- & -- & 0.22 & 0.35 & 0.30 \\
\hline METAL & Nickel & $\mathrm{mg} / \mathrm{kg}$ & 3 & 3 & $100 \%$ & -- & -- & 0.050 & 0.090 & 0.070 \\
\hline METAL & Selenium & $\mathrm{mg} / \mathrm{kg}$ & 3 & 3 & $100 \%$ & -- & -- & 0.48 & 0.70 & 0.59 \\
\hline METAL & Silver & $\mathrm{mg} / \mathrm{kg}$ & 3 & 0 & $0 \%$ & 0.10 & 0.10 & -- & -- & 0.050 \\
\hline METAL & Thallium & $\mathrm{mg} / \mathrm{kg}$ & 3 & 0 & $0 \%$ & 0.050 & 0.050 & -- & -- & 0.025 \\
\hline METAL & Vanadium & $\mathrm{mg} / \mathrm{kg}$ & 3 & 3 & $100 \%$ & -- & -- & 0.010 & 0.080 & 0.057 \\
\hline METAL & Zinc & $\mathrm{mg} / \mathrm{kg}$ & 3 & 3 & $100 \%$ & -- & -- & 15 & 18 & 16 \\
\hline PCB & Aroclor-1016 & $\mathrm{mg} / \mathrm{kg}$ & 3 & 0 & $0 \%$ & 0.018 & 0.020 & -- & -- & 0.0097 \\
\hline PCB & Aroclor-1221 & $\mathrm{mg} / \mathrm{kg}$ & 3 & 0 & $0 \%$ & 0.018 & 0.020 & -- & -- & 0.0097 \\
\hline PCB & Aroclor-1232 & $\mathrm{mg} / \mathrm{kg}$ & 3 & 0 & $0 \%$ & 0.018 & 0.020 & -- & -- & 0.0097 \\
\hline PCB & Aroclor-1248 & $\mathrm{mg} / \mathrm{kg}$ & 3 & 0 & $0 \%$ & 0.018 & 0.020 & -- & -- & 0.0097 \\
\hline PCB & Aroclor-1254 & $\mathrm{mg} / \mathrm{kg}$ & 3 & 3 & $100 \%$ & -- & -- & 0.046 & 0.094 & 0.066 \\
\hline PCB & Aroclor-1260 & $\mathrm{mg} / \mathrm{kg}$ & 3 & 3 & $100 \%$ & -- & -- & 0.080 & 0.19 & 0.13 \\
\hline PEST & 2,4'-DDD & $\mathrm{mg} / \mathrm{kg}$ & 3 & 3 & $100 \%$ & -- & -- & 0.018 & 0.027 & 0.021 \\
\hline PEST & 2,4'-DDE & $\mathrm{mg} / \mathrm{kg}$ & 3 & 3 & $100 \%$ & -- & -- & 0.010 & 0.014 & 0.012 \\
\hline PEST & 2,4'-DDT & $\mathrm{mg} / \mathrm{kg}$ & 3 & 3 & $100 \%$ & -- & -- & 0.012 & 0.020 & 0.017 \\
\hline PEST & Aldrin & $\mathrm{mg} / \mathrm{kg}$ & 3 & 0 & $0 \%$ & 0.0018 & 0.0020 & -- & -- & 9.67E-04 \\
\hline PEST & Alpha-BHC & $\mathrm{mg} / \mathrm{kg}$ & 3 & 0 & $0 \%$ & 0.0018 & 0.0020 & -- & -- & $9.67 \mathrm{E}-04$ \\
\hline PEST & alpha-Chlordane & $\mathrm{mg} / \mathrm{kg}$ & 3 & 2 & $67 \%$ & 0.0020 & 0.0020 & 0.0036 & 0.0047 & 0.0031 \\
\hline PEST & alpha-Chlordene & $\mathrm{mg} / \mathrm{kg}$ & 3 & 0 & $0 \%$ & 0.0018 & 0.0020 & -- & -- & 9.67E-04 \\
\hline PEST & Beta-BHC & $\mathrm{mg} / \mathrm{kg}$ & 3 & 0 & $0 \%$ & 0.0018 & 0.0020 & -- & -- & 9.67E-04 \\
\hline PEST & cis-Nonachlor & $\mathrm{mg} / \mathrm{kg}$ & 3 & 1 & $33 \%$ & 0.0018 & 0.0020 & 0.0014 & 0.0014 & 0.0011 \\
\hline PEST & DDMU & $\mathrm{mg} / \mathrm{kg}$ & 3 & 3 & $100 \%$ & -- & -- & 0.033 & 0.055 & 0.041 \\
\hline PEST & Delta-BHC & $\mathrm{mg} / \mathrm{kg}$ & 3 & 0 & $0 \%$ & 0.0018 & 0.0020 & -- & -- & 9.67E-04 \\
\hline PEST & Dichlorodiphenyldichloroethane & $\mathrm{mg} / \mathrm{kg}$ & 3 & 3 & $100 \%$ & -- & -- & 0.040 & 0.063 & 0.054 \\
\hline PEST & Dichlorodiphenyldichloroethylene & $\mathrm{mg} / \mathrm{kg}$ & 3 & 3 & $100 \%$ & -- & -- & 0.97 & 1.7 & 1.3 \\
\hline PEST & Dichlorodiphenyltrichloroethane & $\mathrm{mg} / \mathrm{kg}$ & 3 & 3 & $100 \%$ & -- & -- & 0.044 & 0.080 & 0.062 \\
\hline PEST & Endosulfan sulfate & $\mathrm{mg} / \mathrm{kg}$ & 3 & 3 & $100 \%$ & -- & -- & 0.0024 & 0.0056 & 0.0038 \\
\hline PEST & Gamma-BHC (Lindane) & $\mathrm{mg} / \mathrm{kg}$ & 3 & 0 & $0 \%$ & 0.0018 & 0.0020 & -- & -- & 9.67E-04 \\
\hline PEST & gamma-Chlordane & $\mathrm{mg} / \mathrm{kg}$ & 3 & 0 & $0 \%$ & 0.0018 & 0.0020 & -- & -- & 9.67E-04 \\
\hline PEST & gamma-Chlordene & $\mathrm{mg} / \mathrm{kg}$ & 3 & 0 & $0 \%$ & 0.0018 & 0.0020 & -- & -- & 9.67E-04 \\
\hline PEST & Heptachlor & $\mathrm{mg} / \mathrm{kg}$ & 3 & 0 & $0 \%$ & 0.0018 & 0.0020 & -- & -- & 9.67E-04 \\
\hline PEST & Heptachlor epoxide & $\mathrm{mg} / \mathrm{kg}$ & 3 & 0 & $0 \%$ & 0.0018 & 0.0020 & -- & -- & $9.67 \mathrm{E}-04$ \\
\hline PEST & Mirex & $\mathrm{mg} / \mathrm{kg}$ & 3 & 0 & $0 \%$ & 0.0018 & 0.0020 & -- & -- & 9.67E-04 \\
\hline PEST & Oxychlordane & $\mathrm{mg} / \mathrm{kg}$ & 3 & 3 & $100 \%$ & -- & -- & 0.0014 & 0.0026 & 0.0018 \\
\hline PEST & Pentachloroanisole & $\mathrm{mg} / \mathrm{kg}$ & 3 & 1 & $33 \%$ & 0.0018 & 0.0020 & $9.90 \mathrm{E}-04$ & $9.90 \mathrm{E}-04$ & $9.63 \mathrm{E}-04$ \\
\hline
\end{tabular}




\section{Table F-38}

Summary Statistics for Whole Body Smallmouth Bass Collected from Segment 2 of the Columbia River

\begin{tabular}{|c|c|c|c|c|c|c|c|c|c|c|}
\hline $\begin{array}{l}\text { Constituent } \\
\text { Class }\end{array}$ & Constituent Name & Units & $\begin{array}{l}\text { Number } \\
\text { Analyzed }\end{array}$ & $\begin{array}{l}\text { Number } \\
\text { Detected }\end{array}$ & $\begin{array}{l}\text { Frequency of } \\
\text { Detection }\end{array}$ & $\begin{array}{l}\text { Minimum } \\
\text { Nondetect }\end{array}$ & $\begin{array}{l}\text { Maximum } \\
\text { Nondetect }\end{array}$ & $\begin{array}{l}\text { Minimum } \\
\text { Detect }\end{array}$ & $\begin{array}{l}\text { Maximum } \\
\text { Detect }\end{array}$ & $\begin{array}{c}\text { Average } \\
\text { Concentration }\end{array}$ \\
\hline PEST & Toxaphene & $\mathrm{mg} / \mathrm{kg}$ & 3 & 0 & $0 \%$ & 0.055 & 0.060 & -- & -- & 0.029 \\
\hline PEST & trans-Nonachlor & $\mathrm{mg} / \mathrm{kg}$ & 3 & 3 & $100 \%$ & -- & -- & 0.0071 & 0.014 & 0.010 \\
\hline SVOC & 1,2,4-Trichlorobenzene & $\mathrm{mg} / \mathrm{kg}$ & 3 & 0 & $0 \%$ & 0.0086 & 0.0090 & -- & -- & 0.0044 \\
\hline SVOC & 1,2-Dichlorobenzene & $\mathrm{mg} / \mathrm{kg}$ & 3 & 0 & $0 \%$ & 0.0086 & 0.0090 & -- & -- & 0.0044 \\
\hline SVOC & 1,2-Diphenylhydrazine & $\mathrm{mg} / \mathrm{kg}$ & 3 & 0 & $0 \%$ & 0.0086 & 0.0090 & -- & -- & 0.0044 \\
\hline SVOC & 1,3-Dichlorobenzene & $\mathrm{mg} / \mathrm{kg}$ & 3 & 0 & $0 \%$ & 0.0086 & 0.0090 & -- & -- & 0.0044 \\
\hline SVOC & 1,4-Dichlorobenzene & $\mathrm{mg} / \mathrm{kg}$ & 3 & 0 & $0 \%$ & 0.0086 & 0.0090 & -- & -- & 0.0044 \\
\hline SVOC & 1-methylnaphthalene & $\mathrm{mg} / \mathrm{kg}$ & 3 & 0 & $0 \%$ & 0.0086 & 0.0090 & -- & -- & 0.0044 \\
\hline SVOC & 2,4-Dinitrotoluene & $\mathrm{mg} / \mathrm{kg}$ & 3 & 0 & $0 \%$ & 0.017 & 0.018 & -- & -- & 0.0087 \\
\hline SVOC & 2,6-Dinitrotoluene & $\mathrm{mg} / \mathrm{kg}$ & 3 & 0 & $0 \%$ & 0.034 & 0.036 & -- & -- & 0.018 \\
\hline SVOC & 2-Chloronaphthalene & $\mathrm{mg} / \mathrm{kg}$ & 3 & 0 & $0 \%$ & 0.017 & 0.018 & -- & -- & 0.0087 \\
\hline SVOC & 2-Methylnaphthalene & $\mathrm{mg} / \mathrm{kg}$ & 3 & 0 & $0 \%$ & 0.0086 & 0.0090 & -- & -- & 0.0044 \\
\hline SVOC & 4-Bromophenylphenyl ether & $\mathrm{mg} / \mathrm{kg}$ & 3 & 0 & $0 \%$ & 0.0086 & 0.0090 & -- & -- & 0.0044 \\
\hline SVOC & 4-Chlorophenylphenyl ether & $\mathrm{mg} / \mathrm{kg}$ & 3 & 0 & $0 \%$ & 0.0086 & 0.0090 & -- & -- & 0.0044 \\
\hline SVOC & Acenaphthene & $\mathrm{mg} / \mathrm{kg}$ & 3 & 0 & $0 \%$ & 0.017 & 0.018 & -- & -- & 0.0087 \\
\hline SVOC & Acenaphthylene & $\mathrm{mg} / \mathrm{kg}$ & 3 & 0 & $0 \%$ & 0.0086 & 0.0090 & -- & -- & 0.0044 \\
\hline SVOC & Anthracene & $\mathrm{mg} / \mathrm{kg}$ & 3 & 0 & $0 \%$ & 0.0086 & 0.0090 & -- & -- & 0.0044 \\
\hline SVOC & Benzo(a)anthracene & $\mathrm{mg} / \mathrm{kg}$ & 3 & 0 & $0 \%$ & 0.0086 & 0.0090 & -- & -- & 0.0044 \\
\hline SVOC & Benzo(a)pyrene & $\mathrm{mg} / \mathrm{kg}$ & 3 & 0 & $0 \%$ & 0.0086 & 0.0090 & -- & -- & 0.0044 \\
\hline SVOC & Benzo(b)fluoranthene & $\mathrm{mg} / \mathrm{kg}$ & 3 & 0 & $0 \%$ & 0.0086 & 0.0090 & -- & -- & 0.0044 \\
\hline SVOC & Benzo(ghi)perylene & $\mathrm{mg} / \mathrm{kg}$ & 3 & 0 & $0 \%$ & 0.017 & 0.018 & -- & -- & 0.0087 \\
\hline SVOC & Benzo(k)fluoranthene & $\mathrm{mg} / \mathrm{kg}$ & 3 & 0 & $0 \%$ & 0.0086 & 0.0090 & -- & -- & 0.0044 \\
\hline SVOC & Bis(2-chloro-1-methylethyl)ether & $\mathrm{mg} / \mathrm{kg}$ & 3 & 0 & $0 \%$ & 0.017 & 0.018 & -- & -- & 0.0087 \\
\hline SVOC & Chrysene & $\mathrm{mg} / \mathrm{kg}$ & 3 & 0 & $0 \%$ & 0.0086 & 0.0090 & -- & -- & 0.0044 \\
\hline SVOC & Dibenz[a,h]anthracene & $\mathrm{mg} / \mathrm{kg}$ & 3 & 0 & $0 \%$ & 0.017 & 0.018 & -- & -- & 0.0087 \\
\hline SVOC & Dibenzofuran & $\mathrm{mg} / \mathrm{kg}$ & 3 & 0 & $0 \%$ & 0.0086 & 0.0090 & -- & -- & 0.0044 \\
\hline SVOC & Fluoranthene & $\mathrm{mg} / \mathrm{kg}$ & 3 & 0 & $0 \%$ & 0.017 & 0.018 & -- & -- & 0.0087 \\
\hline SVOC & Fluorene & $\mathrm{mg} / \mathrm{kg}$ & 3 & 0 & $0 \%$ & 0.0046 & 0.0097 & -- & -- & 0.0032 \\
\hline SVOC & Hexachlorobenzene & $\mathrm{mg} / \mathrm{kg}$ & 3 & 0 & $0 \%$ & 0.017 & 0.018 & -- & -- & 0.0087 \\
\hline SVOC & Hexachlorobutadiene & $\mathrm{mg} / \mathrm{kg}$ & 3 & 0 & $0 \%$ & 0.0086 & 0.0090 & -- & -- & 0.0044 \\
\hline SVOC & Hexachloroethane & $\mathrm{mg} / \mathrm{kg}$ & 3 & 0 & $0 \%$ & 0.0086 & 0.0090 & -- & -- & 0.0044 \\
\hline SVOC & Indeno(1,2,3-cd)pyrene & $\mathrm{mg} / \mathrm{kg}$ & 3 & 0 & $0 \%$ & 0.017 & 0.018 & -- & -- & 0.0087 \\
\hline SVOC & Naphthalene & $\mathrm{mg} / \mathrm{kg}$ & 3 & 0 & $0 \%$ & 0.0094 & 0.024 & -- & -- & 0.0084 \\
\hline SVOC & Nitrobenzene & $\mathrm{mg} / \mathrm{kg}$ & 3 & 0 & $0 \%$ & 0.0086 & 0.0090 & -- & -- & 0.0044 \\
\hline SVOC & Phenanthrene & $\mathrm{mg} / \mathrm{kg}$ & 3 & 1 & $33 \%$ & 0.0086 & 0.0086 & 0.016 & 0.016 & 0.0082 \\
\hline SVOC & Pyrene & $\mathrm{mg} / \mathrm{kg}$ & 3 & 0 & $0 \%$ & 0.017 & 0.018 & -- & -- & 0.0087 \\
\hline VOC & Retene & $\mathrm{mg} / \mathrm{kg}$ & 3 & 0 & $0 \%$ & 0.017 & 0.018 & -- & -- & 0.0087 \\
\hline
\end{tabular}

PEST - Pesticide

PCB - Polychlorinated biphenyl

SVOC - Semivolatile Organic Compound

VOC - Volatile Organic Compound 
Table F-39

Summary Statistics for Carp Fillet Collected from Segment 2 of the Columbia River

\begin{tabular}{|c|c|c|c|c|c|c|c|c|c|c|}
\hline $\begin{array}{l}\text { Constituent } \\
\text { Class }\end{array}$ & Constituent Name & Units & $\begin{array}{l}\text { Number } \\
\text { Analyzed }\end{array}$ & $\begin{array}{l}\text { Number } \\
\text { Detected }\end{array}$ & $\begin{array}{l}\text { Frequency of } \\
\text { Detection }\end{array}$ & $\begin{array}{l}\text { Minimum } \\
\text { Nondetect }\end{array}$ & $\begin{array}{l}\text { Maximum } \\
\text { Nondetect }\end{array}$ & $\begin{array}{c}\text { Minimum } \\
\text { Detect }\end{array}$ & $\begin{array}{l}\text { Maximum } \\
\text { Detect }\end{array}$ & $\begin{array}{c}\text { Average } \\
\text { Concentration }\end{array}$ \\
\hline$\overline{\mathrm{RAD}}$ & Actinium-228 & $\mathrm{pCi} / \mathrm{g}$ & 4 & 0 & $0 \%$ & 0.043 & 0.17 & $\overline{--}$ & - & 0.062 \\
\hline RAD & Antimony-125 & $\mathrm{pCi} / \mathrm{g}$ & 22 & 0 & $0 \%$ & 3.00E-04 & 0.091 & -- & -- & 0.0072 \\
\hline RAD & Barium-140 & $\mathrm{pCi} / \mathrm{g}$ & 6 & 0 & $0 \%$ & 0.18 & 0.77 & -- & -- & 0.19 \\
\hline RAD & Beryllium-7 & $\mathrm{pCi} / \mathrm{g}$ & 24 & 0 & $0 \%$ & 0.0052 & 0.43 & -- & -- & 0.052 \\
\hline RAD & Cerium-141 & $\mathrm{pCi} / \mathrm{g}$ & 6 & 0 & $0 \%$ & 0.029 & 0.13 & -- & -- & 0.029 \\
\hline RAD & Cerium-144 & $\mathrm{pCi} / \mathrm{g}$ & 6 & 0 & $0 \%$ & 0.072 & 0.29 & -- & -- & 0.071 \\
\hline RAD & Cesium-134 & $\mathrm{pCi} / \mathrm{g}$ & 27 & 0 & $0 \%$ & $3.25 E-04$ & 0.036 & -- & -- & 0.0040 \\
\hline RAD & Cesium-137 & $\mathrm{pCi} / \mathrm{g}$ & 37 & 0 & $0 \%$ & 2.80E-04 & 0.043 & -- & -- & 0.0048 \\
\hline RAD & Cobalt-57 & $\mathrm{pCi} / \mathrm{g}$ & 6 & 0 & $0 \%$ & 0.0091 & 0.036 & -- & -- & 0.0098 \\
\hline RAD & Cobalt-58 & $\mathrm{pCi} / \mathrm{g}$ & 9 & 0 & $0 \%$ & 5.15E-04 & 0.046 & -- & -- & 0.0090 \\
\hline RAD & Cobalt-60 & $\mathrm{pCi} / \mathrm{g}$ & 29 & 0 & $0 \%$ & 3.58E-04 & 0.042 & -- & -- & 0.0047 \\
\hline RAD & Europium-152 & $\mathrm{pCi} / \mathrm{g}$ & 11 & 0 & $0 \%$ & 0.0039 & 0.10 & -- & -- & 0.018 \\
\hline RAD & Europium-154 & $\mathrm{pCi} / \mathrm{g}$ & 19 & 0 & $0 \%$ & 3.10E-04 & 0.069 & -- & -- & 0.0085 \\
\hline RAD & Europium-155 & $\mathrm{pCi} / \mathrm{g}$ & 24 & 0 & $0 \%$ & 0.0012 & 0.035 & -- & -- & 0.0052 \\
\hline RAD & Iodine-131 & $\mathrm{pCi} / \mathrm{g}$ & 6 & 0 & $0 \%$ & 0.13 & 0.84 & -- & -- & 0.17 \\
\hline RAD & Iron-59 & $\mathrm{pCi} / \mathrm{g}$ & 11 & 0 & $0 \%$ & 0.0039 & 0.14 & -- & -- & 0.023 \\
\hline RAD & Lanthanum-140 & $\mathrm{pCi} / \mathrm{g}$ & 6 & 0 & $0 \%$ & 0.055 & 0.29 & -- & -- & 0.066 \\
\hline RAD & Manganese-54 & $\mathrm{pCi} / \mathrm{g}$ & 13 & 0 & $0 \%$ & 3.72E-04 & 0.034 & -- & -- & 0.0063 \\
\hline RAD & Niobium-95 & $\mathrm{pCi} / \mathrm{g}$ & 6 & 0 & $0 \%$ & 0.015 & 0.058 & -- & -- & 0.016 \\
\hline RAD & Potassium-40 & $\mathrm{pCi} / \mathrm{g}$ & 56 & 55 & $98 \%$ & 1.5 & 1.5 & 0.27 & 4.0 & 2.8 \\
\hline RAD & Radium-226 & $\mathrm{pCi} / \mathrm{g}$ & 7 & 0 & $0 \%$ & 0.024 & 0.97 & -- & -- & 0.22 \\
\hline RAD & Ruthenium-103 & $\mathrm{pCi} / \mathrm{g}$ & 6 & 0 & $0 \%$ & 0.018 & 0.058 & -- & -- & 0.017 \\
\hline RAD & Ruthenium-106 & $\mathrm{pCi} / \mathrm{g}$ & 23 & 0 & $0 \%$ & 0.0020 & 0.32 & -- & -- & 0.034 \\
\hline RAD & Thorium-228 & $\mathrm{pCi} / \mathrm{g}$ & 9 & 0 & $0 \%$ & 0.0014 & 0.82 & -- & -- & 0.12 \\
\hline RAD & Uranium-234 & $\mathrm{pCi} / \mathrm{g}$ & 2 & 1 & $50 \%$ & 0.0035 & 0.0035 & 0.0082 & 0.0082 & 0.0050 \\
\hline RAD & Uranium-235 & $\mathrm{pCi} / \mathrm{g}$ & 11 & 0 & $0 \%$ & 1.23E-05 & 0.28 & -- & -- & 0.030 \\
\hline RAD & Uranium-238 & $\mathrm{pCi} / \mathrm{g}$ & 8 & 2 & $25 \%$ & $3.72 \mathrm{E}-04$ & 0.0042 & 0.014 & 0.017 & 0.0046 \\
\hline RAD & Zinc-65 & $\mathrm{pCi} / \mathrm{g}$ & 9 & 0 & $0 \%$ & $3.20 \mathrm{E}-04$ & 0.10 & -- & -- & 0.021 \\
\hline RAD & Zirconium-95 & $\mathrm{pCi} / \mathrm{g}$ & 6 & 0 & $0 \%$ & 0.026 & 0.085 & -- & -- & 0.025 \\
\hline
\end{tabular}

RAD - Radionuclide 
Table F-40

Summary Statistics for Carp Kidney Collected from Segment 2 of the Columbia River

\begin{tabular}{|c|c|c|c|c|c|c|c|c|c|c|}
\hline $\begin{array}{l}\text { Constituent } \\
\text { Class }\end{array}$ & Constituent Name & Units & $\begin{array}{l}\text { Number } \\
\text { Analyzed }\end{array}$ & $\begin{array}{l}\text { Number } \\
\text { Detected }\end{array}$ & $\begin{array}{c}\text { Frequency of } \\
\text { Detection }\end{array}$ & $\begin{array}{l}\text { Minimum } \\
\text { Nondetect }\end{array}$ & $\begin{array}{l}\text { Maximum } \\
\text { Nondetect }\end{array}$ & $\begin{array}{c}\text { Minimum } \\
\text { Detect }\end{array}$ & $\begin{array}{l}\text { Maximum } \\
\text { Detect }\end{array}$ & $\begin{array}{c}\text { Average } \\
\text { Concentration }\end{array}$ \\
\hline METAL & Antimony & $\mathrm{mg} / \mathrm{kg}$ & 14 & 11 & $79 \%$ & 0.015 & 0.015 & 0.018 & 0.15 & 0.039 \\
\hline METAL & Arsenic & $\mathrm{mg} / \mathrm{kg}$ & 14 & 14 & $100 \%$ & -- & -- & 0.30 & 2.2 & 1.2 \\
\hline METAL & Beryllium & $\mathrm{mg} / \mathrm{kg}$ & 14 & 0 & $0 \%$ & 0.15 & 0.15 & -- & -- & 0.075 \\
\hline METAL & Cadmium & $\mathrm{mg} / \mathrm{kg}$ & 14 & 14 & $100 \%$ & -- & -- & 15 & 322 & 142 \\
\hline METAL & Chromium & $\mathrm{mg} / \mathrm{kg}$ & 14 & 14 & $100 \%$ & -- & -- & 0.21 & 0.50 & 0.34 \\
\hline METAL & Copper & $\mathrm{mg} / \mathrm{kg}$ & 14 & 14 & $100 \%$ & -- & -- & 5.2 & 19 & 11 \\
\hline METAL & Lead & $\mathrm{mg} / \mathrm{kg}$ & 14 & 14 & $100 \%$ & -- & -- & 0.81 & 4.7 & 1.8 \\
\hline METAL & Mercury & $\mathrm{mg} / \mathrm{kg}$ & 14 & 14 & $100 \%$ & -- & -- & 0.20 & 0.87 & 0.50 \\
\hline METAL & Nickel & $\mathrm{mg} / \mathrm{kg}$ & 14 & 14 & $100 \%$ & -- & -- & 0.38 & 1.6 & 1.0 \\
\hline METAL & Selenium & $\mathrm{mg} / \mathrm{kg}$ & 14 & 14 & $100 \%$ & -- & -- & 4.6 & 11 & 7.6 \\
\hline METAL & Silver & $\mathrm{mg} / \mathrm{kg}$ & 14 & 10 & $71 \%$ & 0.045 & 0.045 & 0.045 & 0.18 & 0.080 \\
\hline METAL & Thallium & $\mathrm{mg} / \mathrm{kg}$ & 14 & 14 & $100 \%$ & -- & -- & 0.21 & 0.95 & 0.49 \\
\hline METAL & Zinc & $\mathrm{mg} / \mathrm{kg}$ & 14 & 14 & $100 \%$ & -- & -- & 355 & 1,690 & 949 \\
\hline
\end{tabular}


Table F-41

Summary Statistics for Carp Liver Collected from Segment 2 of the Columbia River

\begin{tabular}{|c|c|c|c|c|c|c|c|c|c|c|}
\hline $\begin{array}{l}\text { Constituent } \\
\text { Class }\end{array}$ & Constituent Name & Units & $\begin{array}{l}\text { Number } \\
\text { Analyzed }\end{array}$ & $\begin{array}{l}\text { Number } \\
\text { Detected }\end{array}$ & $\begin{array}{l}\text { Frequency of } \\
\text { Detection }\end{array}$ & $\begin{array}{l}\text { Minimum } \\
\text { Nondetect }\end{array}$ & $\begin{array}{l}\text { Maximum } \\
\text { Nondetect }\end{array}$ & $\begin{array}{l}\text { Minimum } \\
\text { Detect }\end{array}$ & $\begin{array}{l}\text { Maximum } \\
\text { Detect }\end{array}$ & $\begin{array}{c}\text { Average } \\
\text { Concentration }\end{array}$ \\
\hline CONV & Percent Solids & $\%$ & 10 & 10 & $100 \%$ & $\overline{--}$ & $\overline{--}$ & 21 & 27 & 24 \\
\hline METAL & Aluminum & $\mathrm{mg} / \mathrm{kg}$ & 20 & 19 & $95 \%$ & 1.0 & 1.0 & 0.12 & 36 & 4.5 \\
\hline METAL & Antimony & $\mathrm{mg} / \mathrm{kg}$ & 35 & 26 & $74 \%$ & 0.0013 & 0.010 & 0.0014 & 0.097 & 0.036 \\
\hline METAL & Arsenic & $\mathrm{mg} / \mathrm{kg}$ & 35 & 35 & $100 \%$ & -- & -- & 0.0093 & 2.9 & 0.60 \\
\hline METAL & Barium & $\mathrm{mg} / \mathrm{kg}$ & 10 & 9 & $90 \%$ & 0.10 & 0.10 & 0.10 & 0.82 & 0.29 \\
\hline METAL & Beryllium & $\mathrm{mg} / \mathrm{kg}$ & 35 & 0 & $0 \%$ & 0.0016 & 0.15 & -- & -- & 0.035 \\
\hline METAL & Cadmium & $\mathrm{mg} / \mathrm{kg}$ & 35 & 35 & $100 \%$ & -- & -- & 0.54 & 209 & 37 \\
\hline METAL & Chromium & $\mathrm{mg} / \mathrm{kg}$ & 35 & 31 & $89 \%$ & 0.20 & 0.20 & 0.023 & 12 & 0.73 \\
\hline METAL & Copper & $\mathrm{mg} / \mathrm{kg}$ & 35 & 35 & $100 \%$ & -- & -- & 1.7 & 324 & 85 \\
\hline METAL & Lead & $\mathrm{mg} / \mathrm{kg}$ & 35 & 35 & $100 \%$ & -- & -- & 0.0021 & 1.4 & 0.33 \\
\hline METAL & Manganese & $\mathrm{mg} / \mathrm{kg}$ & 20 & 20 & $100 \%$ & -- & -- & 0.078 & 10 & 3.4 \\
\hline METAL & Mercury & $\mathrm{mg} / \mathrm{kg}$ & 20 & 20 & $100 \%$ & -- & -- & 0.088 & 0.79 & 0.27 \\
\hline METAL & Nickel & $\mathrm{mg} / \mathrm{kg}$ & 35 & 29 & $83 \%$ & 6.60E-04 & 0.050 & 7.04E-04 & 0.61 & 0.092 \\
\hline METAL & Selenium & $\mathrm{mg} / \mathrm{kg}$ & 35 & 35 & $100 \%$ & -- & -- & 0.077 & 7.4 & 3.5 \\
\hline METAL & Silver & $\mathrm{mg} / \mathrm{kg}$ & 35 & 34 & $97 \%$ & 0.010 & 0.010 & 0.0085 & 3.6 & 0.82 \\
\hline METAL & Thallium & $\mathrm{mg} / \mathrm{kg}$ & 35 & 30 & $86 \%$ & 0.0010 & 0.0014 & 0.0013 & 0.24 & 0.081 \\
\hline METAL & Thorium & $\mathrm{mg} / \mathrm{kg}$ & 20 & 3 & $15 \%$ & 4.41E-04 & 0.020 & 8.09E-04 & 0.028 & 0.0061 \\
\hline METAL & Uranium & $\mathrm{mg} / \mathrm{kg}$ & 20 & 18 & $90 \%$ & 0.0014 & 0.0014 & 0.0019 & 0.38 & 0.068 \\
\hline METAL & Zinc & $\mathrm{mg} / \mathrm{kg}$ & 35 & 35 & $100 \%$ & -- & -- & 6.4 & 1,900 & 668 \\
\hline
\end{tabular}


Table F-42

Summary Statistics for Whole Body Carp Collected from Segment 2 of the Columbia River

\begin{tabular}{|c|c|c|c|c|c|c|c|c|c|c|}
\hline $\begin{array}{l}\text { Constituent } \\
\text { Class }\end{array}$ & Constituent Name & Units & $\begin{array}{l}\text { Number } \\
\text { Analyzed }\end{array}$ & $\begin{array}{l}\text { Number } \\
\text { Detected }\end{array}$ & $\begin{array}{l}\text { Frequency of } \\
\text { Detection }\end{array}$ & $\begin{array}{c}\text { Minimum } \\
\text { Nondetect }\end{array}$ & $\begin{array}{l}\text { Maximum } \\
\text { Nondetect }\end{array}$ & $\begin{array}{l}\text { Minimum } \\
\text { Detect }\end{array}$ & $\begin{array}{l}\text { Maximum } \\
\text { Detect }\end{array}$ & $\begin{array}{c}\text { Average } \\
\text { Concentration }\end{array}$ \\
\hline$\overline{\text { CONV }}$ & Percent Lipids & $\%$ & 2 & 2 & $100 \%$ & $\overline{--}$ & $\overline{--}$ & 4.4 & 4.9 & 4.6 \\
\hline CONV & Percent moisture (wet sample) & $\mathrm{mg} / \mathrm{Kg}$ & 2 & 2 & $100 \%$ & -- & -- & 73 & 73 & 73 \\
\hline DIOXI & TCDD-eq & $\mathrm{mg} / \mathrm{Kg}$ & 2 & 2 & $100 \%$ & -- & -- & 3.0 & 6.0 & 4.5 \\
\hline METAL & Aluminum & $\mathrm{mg} / \mathrm{Kg}$ & 2 & 2 & $100 \%$ & -- & -- & 67 & 81 & 74 \\
\hline METAL & Arsenic & $\mathrm{mg} / \mathrm{Kg}$ & 2 & 1 & $50 \%$ & 0.24 & 0.24 & 0.32 & 0.32 & 0.22 \\
\hline METAL & Barium & $\mathrm{mg} / \mathrm{Kg}$ & 2 & 2 & $100 \%$ & -- & -- & 4.3 & 5.3 & 4.8 \\
\hline METAL & Beryllium & $\mathrm{mg} / \mathrm{Kg}$ & 2 & 0 & $0 \%$ & 0.048 & 0.051 & -- & -- & 0.025 \\
\hline METAL & Boron & $\mathrm{mg} / \mathrm{Kg}$ & 2 & 0 & $0 \%$ & 0.97 & 1.0 & -- & -- & 0.50 \\
\hline METAL & Cadmium & $\mathrm{mg} / \mathrm{Kg}$ & 2 & 2 & $100 \%$ & -- & -- & 0.30 & 0.39 & 0.35 \\
\hline METAL & Chromium & $\mathrm{mg} / \mathrm{Kg}$ & 2 & 2 & $100 \%$ & -- & -- & 3.7 & 3.7 & 3.7 \\
\hline METAL & Copper & $\mathrm{mg} / \mathrm{Kg}$ & 2 & 2 & $100 \%$ & -- & -- & 1.5 & 1.6 & 1.5 \\
\hline METAL & Iron & $\mathrm{mg} / \mathrm{Kg}$ & 2 & 2 & $100 \%$ & -- & -- & 119 & 137 & 128 \\
\hline METAL & Lead & $\mathrm{mg} / \mathrm{Kg}$ & 2 & 1 & $50 \%$ & 0.10 & 0.10 & 0.14 & 0.14 & 0.098 \\
\hline METAL & Magnesium & $\mathrm{mg} / \mathrm{Kg}$ & 2 & 2 & $100 \%$ & -- & -- & 339 & 352 & 346 \\
\hline METAL & Manganese & $\mathrm{mg} / \mathrm{Kg}$ & 2 & 2 & $100 \%$ & -- & -- & 4.8 & 4.9 & 4.8 \\
\hline METAL & Mercury & $\mathrm{mg} / \mathrm{Kg}$ & 2 & 1 & $50 \%$ & 0.050 & 0.050 & 0.081 & 0.081 & 0.053 \\
\hline METAL & Molybdenum & $\mathrm{mg} / \mathrm{Kg}$ & 2 & 0 & $0 \%$ & 0.24 & 0.26 & -- & -- & 0.12 \\
\hline METAL & Nickel & $\mathrm{mg} / \mathrm{Kg}$ & 2 & 2 & $100 \%$ & -- & -- & 0.50 & 0.75 & 0.63 \\
\hline METAL & Selenium & $\mathrm{mg} / \mathrm{Kg}$ & 2 & 2 & $100 \%$ & -- & -- & 0.89 & 1.1 & 1.00 \\
\hline METAL & Strontium & $\mathrm{mg} / \mathrm{Kg}$ & 2 & 2 & $100 \%$ & -- & -- & 19 & 21 & 20 \\
\hline METAL & Vanadium & $\mathrm{mg} / \mathrm{Kg}$ & 2 & 2 & $100 \%$ & -- & -- & 0.30 & 0.35 & 0.32 \\
\hline METAL & Zinc & $\mathrm{mg} / \mathrm{Kg}$ & 2 & 2 & $100 \%$ & -- & -- & 72 & 105 & 88 \\
\hline PCB & Total polychlorinated biphenyl & $\mathrm{mg} / \mathrm{Kg}$ & 1 & 0 & $0 \%$ & 0.030 & 0.030 & -- & -- & 0.015 \\
\hline PEST & 2,4'-DDD & $\mathrm{mg} / \mathrm{Kg}$ & 2 & 0 & $0 \%$ & 0.010 & 0.010 & -- & -- & 0.0050 \\
\hline PEST & 2,4'-DDE & $\mathrm{mg} / \mathrm{Kg}$ & 2 & 0 & $0 \%$ & 0.010 & 0.010 & -- & -- & 0.0050 \\
\hline PEST & $2,4^{\prime}-\mathrm{DDT}$ & $\mathrm{mg} / \mathrm{Kg}$ & 2 & 0 & $0 \%$ & 0.010 & 0.010 & -- & -- & 0.0050 \\
\hline PEST & Alpha-BHC & $\mathrm{mg} / \mathrm{Kg}$ & 2 & 0 & $0 \%$ & 0.010 & 0.010 & -- & -- & 0.0050 \\
\hline PEST & alpha-Chlordane & $\mathrm{mg} / \mathrm{Kg}$ & 2 & 0 & $0 \%$ & 0.010 & 0.010 & -- & -- & 0.0050 \\
\hline PEST & Beta-BHC & $\mathrm{mg} / \mathrm{Kg}$ & 2 & 0 & $0 \%$ & 0.010 & 0.010 & -- & -- & 0.0050 \\
\hline PEST & cis-Nonachlor & $\mathrm{mg} / \mathrm{Kg}$ & 2 & 0 & $0 \%$ & 0.010 & 0.010 & -- & -- & 0.0050 \\
\hline PEST & Delta-BHC & $\mathrm{mg} / \mathrm{Kg}$ & 2 & 0 & $0 \%$ & 0.010 & 0.010 & -- & -- & 0.0050 \\
\hline PEST & Dichlorodiphenyldichloroethane & $\mathrm{mg} / \mathrm{Kg}$ & 2 & 2 & $100 \%$ & -- & -- & 0.038 & 0.070 & 0.054 \\
\hline PEST & Dichlorodiphenyldichloroethylene & $\mathrm{mg} / \mathrm{Kg}$ & 2 & 2 & $100 \%$ & -- & -- & 0.18 & 0.35 & 0.27 \\
\hline PEST & Dichlorodiphenyltrichloroethane & $\mathrm{mg} / \mathrm{Kg}$ & 2 & 0 & $0 \%$ & 0.010 & 0.010 & -- & -- & 0.0050 \\
\hline PEST & Dieldrin & $\mathrm{mg} / \mathrm{Kg}$ & 2 & 0 & $0 \%$ & 0.010 & 0.010 & -- & -- & 0.0050 \\
\hline PEST & Endrin & $\mathrm{mg} / \mathrm{Kg}$ & 2 & 0 & $0 \%$ & 0.010 & 0.010 & -- & -- & 0.0050 \\
\hline PEST & Gamma-BHC (Lindane) & $\mathrm{mg} / \mathrm{Kg}$ & 2 & 0 & $0 \%$ & 0.010 & 0.010 & -- & -- & 0.0050 \\
\hline PEST & gamma-Chlordane & $\mathrm{mg} / \mathrm{Kg}$ & 2 & 0 & $0 \%$ & 0.010 & 0.010 & -- & -- & 0.0050 \\
\hline PEST & Heptachlor epoxide & $\mathrm{mg} / \mathrm{Kg}$ & 2 & 0 & $0 \%$ & 0.010 & 0.010 & -- & -- & 0.0050 \\
\hline PEST & Mirex & $\mathrm{mg} / \mathrm{Kg}$ & 2 & 0 & $0 \%$ & 0.010 & 0.010 & -- & -- & 0.0050 \\
\hline PEST & Oxychlordane & $\mathrm{mg} / \mathrm{Kg}$ & 2 & 0 & $0 \%$ & 0.010 & 0.010 & -- & -- & 0.0050 \\
\hline PEST & Toxaphene & $\mathrm{mg} / \mathrm{Kg}$ & 2 & 0 & $0 \%$ & 0.030 & 0.030 & -- & -- & 0.015 \\
\hline PEST & trans-Nonachlor & $\mathrm{mg} / \mathrm{Kg}$ & 2 & 0 & $0 \%$ & 0.010 & 0.010 & -- & -- & 0.0050 \\
\hline RAD & Cesium-137 & $\mathrm{pCi} / \mathrm{g}$ & 1 & 1 & $100 \%$ & -- & -- & 0.0051 & 0.0051 & 0.0051 \\
\hline RAD & Cobalt-60 & $\mathrm{pCi} / \mathrm{g}$ & 1 & 0 & $0 \%$ & 0.0022 & 0.0022 & -- & -- & 0.0011 \\
\hline RAD & Manganese-54 & $\mathrm{pCi} / \mathrm{g}$ & 1 & 0 & $0 \%$ & 2.00E-04 & 2.00E-04 & -- & -- & 1.00E-04 \\
\hline RAD & Potassium-40 & $\mathrm{pCi} / \mathrm{g}$ & 1 & 1 & $100 \%$ & -- & -- & 3.5 & 3.5 & 3.5 \\
\hline
\end{tabular}


Table F-42

Summary Statistics for Whole Body Carp Collected from Segment 2 of the Columbia River

\begin{tabular}{|c|c|c|c|c|c|c|c|c|c|c|}
\hline $\begin{array}{l}\text { Constituent } \\
\text { Class }\end{array}$ & Constituent Name & Units & $\begin{array}{l}\text { Number } \\
\text { Analyzed }\end{array}$ & $\begin{array}{l}\text { Number } \\
\text { Detected }\end{array}$ & $\begin{array}{c}\text { Frequency of } \\
\text { Detection }\end{array}$ & $\begin{array}{c}\text { Minimum } \\
\text { Nondetect }\end{array}$ & $\begin{array}{c}\text { Maximum } \\
\text { Nondetect }\end{array}$ & $\begin{array}{c}\text { Minimum } \\
\text { Detect }\end{array}$ & $\begin{array}{c}\text { Maximum } \\
\text { Detect }\end{array}$ & $\begin{array}{c}\text { Average } \\
\text { Concentration }\end{array}$ \\
\hline RAD & Strontium-90 & $\mathrm{pCi} / \mathrm{g}$ & 38 & 28 & $74 \%$ & 0.0052 & 0.048 & 0.016 & 1.5 & 0.16 \\
\hline RAD & Uranium & $\mathrm{pCi} / \mathrm{g}$ & 2 & 2 & $100 \%$ & -- & -- & 0.064 & 0.077 & 0.071 \\
\hline RAD & Uranium-234 & $\mathrm{pCi} / \mathrm{g}$ & 9 & 5 & $56 \%$ & 8.29E-04 & 0.0030 & 0.0099 & 0.036 & 0.012 \\
\hline RAD & Uranium-235 & $\mathrm{pCi} / \mathrm{g}$ & 6 & 2 & $33 \%$ & $6.29 \mathrm{E}-04$ & 0.0018 & 0.0030 & 0.0050 & 0.0017 \\
\hline RAD & Uranium-238 & $\mathrm{pCi} / \mathrm{g}$ & 9 & 8 & $89 \%$ & 2.33E-04 & 2.33E-04 & 0.0056 & 0.026 & 0.014 \\
\hline SVOC & Hexachlorobenzene & $\mathrm{mg} / \mathrm{Kg}$ & 2 & 0 & $0 \%$ & 0.010 & 0.010 & -- & -- & 0.0050 \\
\hline
\end{tabular}

PEST - Pesticide

PCB - Polychlorinated biphenyl

RAD - Radionuclide

SVOC - Semivolatile Organic Compound 
Table F-43

Summary Statistics for Largescale Sucker Fillet Skin On Collected from Segment 2 of the Columbia River

\begin{tabular}{|c|c|c|c|c|c|c|c|c|c|c|}
\hline $\begin{array}{l}\text { Constituent } \\
\text { Class }\end{array}$ & Constituent Name & Units & $\begin{array}{l}\text { Number } \\
\text { Analyzed }\end{array}$ & $\begin{array}{l}\text { Number } \\
\text { Detected }\end{array}$ & $\begin{array}{c}\text { Frequency of } \\
\text { Detection }\end{array}$ & $\begin{array}{l}\text { Minimum } \\
\text { Nondetect }\end{array}$ & $\begin{array}{l}\text { Maximum } \\
\text { Nondetect }\end{array}$ & $\begin{array}{c}\text { Minimum } \\
\text { Detect }\end{array}$ & $\begin{array}{c}\text { Maximum } \\
\text { Detect }\end{array}$ & $\begin{array}{c}\text { Average } \\
\text { Concentration }\end{array}$ \\
\hline$\overline{D I O X I}$ & $1,2,3,4,6,7,8$-Heptachlorodibenzodioxin & $\mathrm{mg} / \mathrm{kg}$ & 3 & 2 & $67 \%$ & $1.00 \mathrm{E}-08$ & $1.00 \mathrm{E}-08$ & $5.00 \mathrm{E}-08$ & $1.20 \mathrm{E}-07$ & $5.83 \mathrm{E}-08$ \\
\hline DIOXI & 1,2,3,4,6,7,8-Heptachlorodibenzofuran & $\mathrm{mg} / \mathrm{kg}$ & 3 & 1 & $33 \%$ & $1.00 \mathrm{E}-08$ & $2.00 \mathrm{E}-08$ & $6.00 \mathrm{E}-08$ & $6.00 \mathrm{E}-08$ & $2.50 \mathrm{E}-08$ \\
\hline DIOXI & 1,2,3,4,7,8,9-Heptachlorodibenzofuran & $\mathrm{mg} / \mathrm{kg}$ & 3 & 0 & $0 \%$ & $1.00 \mathrm{E}-08$ & $2.00 \mathrm{E}-08$ & -- & -- & 8.33E-09 \\
\hline DIOXI & 1,2,3,4,7,8-Hexachlorodibenzofuran & $\mathrm{mg} / \mathrm{kg}$ & 3 & 1 & $33 \%$ & $1.00 \mathrm{E}-08$ & $1.00 \mathrm{E}-08$ & 3.00E-08 & 3.00E-08 & 1.33E-08 \\
\hline DIOXI & 1,2,3,4,7,8-Hexachlorodibenzo-p-dioxin & $\mathrm{mg} / \mathrm{kg}$ & 3 & 0 & $0 \%$ & $1.00 \mathrm{E}-08$ & $2.00 \mathrm{E}-08$ & -- & -- & 8.33E-09 \\
\hline DIOXI & 1,2,3,6,7,8-Hexachlorodibenzofuran & $\mathrm{mg} / \mathrm{kg}$ & 3 & 0 & $0 \%$ & $1.00 \mathrm{E}-08$ & $1.00 \mathrm{E}-08$ & -- & -- & 5.00E-09 \\
\hline DIOXI & 1,2,3,6,7,8-Hexachlorodibenzo-p-dioxin & $\mathrm{mg} / \mathrm{kg}$ & 3 & 1 & $33 \%$ & $2.00 \mathrm{E}-08$ & $2.00 \mathrm{E}-08$ & 3.00E-08 & 3.00E-08 & 1.67E-08 \\
\hline DIOXI & 1,2,3,7,8,9-Hexachlorodibenzofuran & $\mathrm{mg} / \mathrm{kg}$ & 3 & 1 & $33 \%$ & $1.00 \mathrm{E}-08$ & 1.00E-08 & 2.00E-08 & $2.00 \mathrm{E}-08$ & 1.00E-08 \\
\hline DIOXI & 1,2,3,7,8,9-Hexachlorodibenzo-p-dioxin & $\mathrm{mg} / \mathrm{kg}$ & 3 & 2 & $67 \%$ & $2.00 \mathrm{E}-08$ & $2.00 \mathrm{E}-08$ & $3.00 \mathrm{E}-08$ & $5.00 \mathrm{E}-08$ & $3.00 \mathrm{E}-08$ \\
\hline DIOXI & 1,2,3,7,8-Pentachlorodibenzofuran & $\mathrm{mg} / \mathrm{kg}$ & 3 & 0 & $0 \%$ & $1.00 \mathrm{E}-08$ & $2.00 \mathrm{E}-08$ & -- & -- & 8.33E-09 \\
\hline DIOXI & 1,2,3,7,8-Pentachlorodibenzo-p-dioxin & $\mathrm{mg} / \mathrm{kg}$ & 3 & 2 & $67 \%$ & $1.00 \mathrm{E}-08$ & $1.00 \mathrm{E}-08$ & $2.00 \mathrm{E}-08$ & 4.00E-08 & 2.17E-08 \\
\hline DIOXI & $2,3,4,6,7,8$-Hexachlorodibenzofuran & $\mathrm{mg} / \mathrm{kg}$ & 3 & 0 & $0 \%$ & $1.00 \mathrm{E}-08$ & $1.00 \mathrm{E}-08$ & -- & -- & 5.00E-09 \\
\hline DIOXI & 2,3,4,7,8-Pentachlorodibenzofuran & $\mathrm{mg} / \mathrm{kg}$ & 3 & 2 & $67 \%$ & $2.00 \mathrm{E}-08$ & $2.00 \mathrm{E}-08$ & $5.00 \mathrm{E}-08$ & $5.00 \mathrm{E}-08$ & 3.67E-08 \\
\hline DIOXI & 2,3,7,8-Tetrachlorodibenzofuran & $\mathrm{mg} / \mathrm{kg}$ & 3 & 3 & $100 \%$ & -- & -- & 1.02E-06 & $1.45 \mathrm{E}-06$ & $1.21 \mathrm{E}-06$ \\
\hline DIOXI & 2,3,7,8-Tetrachlorodibenzo-p-dioxin & $\mathrm{mg} / \mathrm{kg}$ & 3 & 1 & $33 \%$ & $1.00 \mathrm{E}-08$ & $2.00 \mathrm{E}-08$ & 4.00E-08 & 4.00E-08 & 1.83E-08 \\
\hline DIOXI & Octachlorodibenzofuran & $\mathrm{mg} / \mathrm{kg}$ & 3 & 2 & $67 \%$ & $2.00 \mathrm{E}-08$ & $2.00 \mathrm{E}-08$ & 8.00E-08 & 1.10E-07 & 6.67E-08 \\
\hline DIOXI & Octachlorodibenzo-p-dioxin & $\mathrm{mg} / \mathrm{kg}$ & 3 & 3 & $100 \%$ & -- & -- & $2.30 \mathrm{E}-07$ & 1.96E-06 & 8.53E-07 \\
\hline METAL & Aluminum & $\mathrm{mg} / \mathrm{kg}$ & 3 & 1 & $33 \%$ & 1.0 & 1.0 & 3.8 & 3.8 & 1.6 \\
\hline METAL & Antimony & $\mathrm{mg} / \mathrm{kg}$ & 3 & 0 & $0 \%$ & 0.050 & 0.050 & -- & -- & 0.025 \\
\hline METAL & Arsenic & $\mathrm{mg} / \mathrm{kg}$ & 3 & 3 & $100 \%$ & -- & -- & 0.050 & 0.080 & 0.060 \\
\hline METAL & Barium & $\mathrm{mg} / \mathrm{kg}$ & 3 & 3 & $100 \%$ & -- & -- & 0.80 & 0.97 & 0.91 \\
\hline METAL & Beryllium & $\mathrm{mg} / \mathrm{kg}$ & 3 & 0 & $0 \%$ & 0.0040 & 0.0040 & -- & -- & 0.0020 \\
\hline METAL & Cadmium & $\mathrm{mg} / \mathrm{kg}$ & 3 & 3 & $100 \%$ & -- & -- & 0.010 & 0.020 & 0.013 \\
\hline METAL & Chromium & $\mathrm{mg} / \mathrm{kg}$ & 3 & 3 & $100 \%$ & -- & -- & 0.36 & 0.38 & 0.37 \\
\hline METAL & Cobalt & $\mathrm{mg} / \mathrm{kg}$ & 3 & 3 & $100 \%$ & -- & -- & 0.020 & 0.24 & 0.15 \\
\hline METAL & Copper & $\mathrm{mg} / \mathrm{kg}$ & 3 & 3 & $100 \%$ & -- & -- & 0.43 & 0.44 & 0.44 \\
\hline METAL & Lead & $\mathrm{mg} / \mathrm{kg}$ & 3 & 2 & $67 \%$ & 0.010 & 0.010 & 0.12 & 0.13 & 0.085 \\
\hline METAL & Manganese & $\mathrm{mg} / \mathrm{kg}$ & 3 & 3 & $100 \%$ & -- & -- & 3.7 & 5.4 & 4.5 \\
\hline METAL & Mercury & $\mathrm{mg} / \mathrm{kg}$ & 3 & 3 & $100 \%$ & -- & -- & 0.15 & 0.22 & 0.18 \\
\hline METAL & Nickel & $\mathrm{mg} / \mathrm{kg}$ & 3 & 2 & $67 \%$ & 0.030 & 0.030 & 0.39 & 0.50 & 0.30 \\
\hline METAL & Selenium & $\mathrm{mg} / \mathrm{kg}$ & 3 & 3 & $100 \%$ & -- & -- & 0.25 & 0.30 & 0.28 \\
\hline METAL & Silver & $\mathrm{mg} / \mathrm{kg}$ & 3 & 0 & $0 \%$ & 0.10 & 0.10 & -- & -- & 0.050 \\
\hline METAL & Thallium & $\mathrm{mg} / \mathrm{kg}$ & 3 & 0 & $0 \%$ & 0.050 & 0.050 & -- & -- & 0.025 \\
\hline METAL & Vanadium & $\mathrm{mg} / \mathrm{kg}$ & 3 & 0 & $0 \%$ & 0.010 & 0.010 & -- & -- & 0.0050 \\
\hline METAL & Zinc & $\mathrm{mg} / \mathrm{kg}$ & 3 & 3 & $100 \%$ & -- & -- & 24 & 26 & 25 \\
\hline PCB & 2,2',3,3',4,4',5-Heptachloro-1,1'-biphenyl & $\mathrm{mg} / \mathrm{kg}$ & 3 & 3 & $100 \%$ & -- & -- & 9.99E-04 & 0.0025 & 0.0018 \\
\hline РСВ & 2,2',3,4,4',5,5'-Heptachlorobiphenyl & $\mathrm{mg} / \mathrm{kg}$ & 3 & 3 & $100 \%$ & -- & -- & 0.0032 & 0.0077 & 0.0055 \\
\hline PCB & 2,3,3',4,4',5'-Hexachlorobiphenyl & $\mathrm{mg} / \mathrm{kg}$ & 3 & 3 & $100 \%$ & -- & -- & 1.37E-04 & 2.72E-04 & $2.09 \mathrm{E}-04$ \\
\hline РСВ & 2,3,3',4,4',5-Hexachlorobiphenyl (BZ 156) & $\mathrm{mg} / \mathrm{kg}$ & 3 & 3 & $100 \%$ & -- & -- & 7.14E-04 & 0.0014 & 0.0011 \\
\hline PCB & 2,3,3',4,4'-Pentachlorobiphenyl & $\mathrm{mg} / \mathrm{kg}$ & 3 & 3 & $100 \%$ & -- & -- & 0.0016 & 0.0030 & 0.0023 \\
\hline РСВ & 2,3',4,4',5,5'-Hexachlorobiphenyl (BZ 167) & $\mathrm{mg} / \mathrm{kg}$ & 3 & 3 & $100 \%$ & -- & -- & 2.84E-04 & $6.01 \mathrm{E}-04$ & 4.54E-04 \\
\hline PCB & 2',3,4,4',5-Pentachloro-1,1'-biphenyl (BZ 123) & $\mathrm{mg} / \mathrm{kg}$ & 3 & 3 & $100 \%$ & -- & -- & $5.86 \mathrm{E}-05$ & 1.31E-04 & $9.52 \mathrm{E}-05$ \\
\hline РCB & 2,3,4,4',5-Pentachlorobiphenyl (BZ 114) & $\mathrm{mg} / \mathrm{kg}$ & 3 & 3 & $100 \%$ & -- & -- & 1.32E-04 & 2.75E-04 & $2.10 \mathrm{E}-04$ \\
\hline PCB & 2,3',4,4',5-Pentachlorobiphenyl (BZ 118) & $\mathrm{mg} / \mathrm{kg}$ & 3 & 3 & $100 \%$ & -- & -- & 0.0034 & 0.0066 & 0.0051 \\
\hline РСВ & 2,3,4,5,3',4',5'-Heptachlorobiphenyl & $\mathrm{mg} / \mathrm{kg}$ & 3 & 3 & $100 \%$ & -- & -- & $6.10 \mathrm{E}-05$ & 1.11E-04 & 8.94E-05 \\
\hline PCB & 3,3',4,4',5,5'-Hexachlorobiphenyl (BZ 169) & $\mathrm{mg} / \mathrm{kg}$ & 3 & 0 & $0 \%$ & $1.26 \mathrm{E}-06$ & 2.43E-06 & -- & -- & 8.57E-07 \\
\hline РСВ & 3,3',4,4',5-Pentachlorobiphenyl (BZ 126) & $\mathrm{mg} / \mathrm{kg}$ & 3 & 3 & $100 \%$ & -- & -- & 6.97E-06 & 1.28E-05 & 9.42E-06 \\
\hline PCB & 3,3',4,4'-Tetrachlorobiphenyl (BZ 77) & $\mathrm{mg} / \mathrm{kg}$ & 3 & 3 & $100 \%$ & -- & -- & 3.17E-05 & 4.15E-05 & 3.51E-05 \\
\hline РСВ & Aroclor-1016 & $\mathrm{mg} / \mathrm{kg}$ & 3 & 0 & $0 \%$ & 0.019 & 0.020 & -- & -- & 0.0097 \\
\hline PCB & Aroclor-1221 & $\mathrm{mg} / \mathrm{kg}$ & 3 & 0 & $0 \%$ & 0.019 & 0.020 & -- & -- & 0.0097 \\
\hline
\end{tabular}


Table F-43

Summary Statistics for Largescale Sucker Fillet Skin On Collected from Segment 2 of the Columbia River

\begin{tabular}{|c|c|c|c|c|c|c|c|c|c|c|}
\hline $\begin{array}{l}\text { Constituent } \\
\text { Class }\end{array}$ & Constituent Name & Units & $\begin{array}{l}\text { Number } \\
\text { Analyzed }\end{array}$ & $\begin{array}{l}\text { Number } \\
\text { Detected }\end{array}$ & $\begin{array}{l}\text { Frequency of } \\
\text { Detection }\end{array}$ & $\begin{array}{l}\text { Minimum } \\
\text { Nondetect }\end{array}$ & $\begin{array}{l}\text { Maximum } \\
\text { Nondetect }\end{array}$ & $\begin{array}{l}\text { Minimum } \\
\text { Detect }\end{array}$ & $\begin{array}{c}\text { Maximum } \\
\text { Detect }\end{array}$ & $\begin{array}{c}\text { Average } \\
\text { Concentration }\end{array}$ \\
\hline$\overline{\mathrm{PCB}}$ & Aroclor-1232 & $\mathrm{mg} / \mathrm{kg}$ & 3 & 0 & $0 \%$ & 0.019 & 0.020 & $\overline{--}$ & -- & 0.0097 \\
\hline РCB & Aroclor-1248 & $\mathrm{mg} / \mathrm{kg}$ & 3 & 0 & $0 \%$ & 0.019 & 0.020 & -- & -- & 0.0097 \\
\hline РСB & Aroclor-1254 & $\mathrm{mg} / \mathrm{kg}$ & 3 & 3 & $100 \%$ & -- & -- & 0.019 & 0.041 & 0.031 \\
\hline РСB & Aroclor-1260 & $\mathrm{mg} / \mathrm{kg}$ & 3 & 3 & $100 \%$ & -- & -- & 0.028 & 0.054 & 0.038 \\
\hline PEST & 2,4'-DDD & $\mathrm{mg} / \mathrm{kg}$ & 3 & 3 & $100 \%$ & -- & -- & 0.0020 & 0.0042 & 0.0030 \\
\hline PEST & 2,4'-DDE & $\mathrm{mg} / \mathrm{kg}$ & 3 & 3 & $100 \%$ & -- & -- & 0.0032 & 0.0050 & 0.0040 \\
\hline PEST & 2,4'-DDT & $\mathrm{mg} / \mathrm{kg}$ & 3 & 0 & $0 \%$ & 0.0019 & 0.0020 & -- & -- & $9.67 \mathrm{E}-04$ \\
\hline PEST & Aldrin & $\mathrm{mg} / \mathrm{kg}$ & 3 & 0 & $0 \%$ & 0.0019 & 0.0020 & -- & -- & $9.67 \mathrm{E}-04$ \\
\hline PEST & Alpha-BHC & $\mathrm{mg} / \mathrm{kg}$ & 3 & 0 & $0 \%$ & 0.0019 & 0.0020 & -- & -- & $9.67 \mathrm{E}-04$ \\
\hline PEST & alpha-Chlordane & $\mathrm{mg} / \mathrm{kg}$ & 3 & 0 & $0 \%$ & 0.0019 & 0.0020 & -- & -- & $9.67 \mathrm{E}-04$ \\
\hline PEST & alpha-Chlordene & $\mathrm{mg} / \mathrm{kg}$ & 3 & 0 & $0 \%$ & 0.0019 & 0.0020 & -- & -- & $9.67 \mathrm{E}-04$ \\
\hline PEST & Beta-BHC & $\mathrm{mg} / \mathrm{kg}$ & 3 & 0 & $0 \%$ & 0.0019 & 0.0020 & -- & -- & $9.67 \mathrm{E}-04$ \\
\hline PEST & cis-Nonachlor & $\mathrm{mg} / \mathrm{kg}$ & 3 & 0 & $0 \%$ & 0.0019 & 0.0020 & -- & -- & $9.67 \mathrm{E}-04$ \\
\hline PEST & DDMU & $\mathrm{mg} / \mathrm{kg}$ & 3 & 3 & $100 \%$ & -- & -- & 0.0055 & 0.0094 & 0.0074 \\
\hline PEST & Delta-BHC & $\mathrm{mg} / \mathrm{kg}$ & 3 & 0 & $0 \%$ & 0.0019 & 0.0020 & -- & -- & $9.67 \mathrm{E}-04$ \\
\hline PEST & Dichlorodiphenyldichloroethane & $\mathrm{mg} / \mathrm{kg}$ & 3 & 2 & $67 \%$ & 0.0020 & 0.0020 & 0.035 & 0.042 & 0.026 \\
\hline PEST & Dichlorodiphenyldichloroethylene & $\mathrm{mg} / \mathrm{kg}$ & 3 & 3 & $100 \%$ & -- & -- & 0.26 & 0.39 & 0.32 \\
\hline PEST & Dichlorodiphenyltrichloroethane & $\mathrm{mg} / \mathrm{kg}$ & 3 & 3 & $100 \%$ & -- & -- & 0.0063 & 0.029 & 0.020 \\
\hline PEST & Gamma-BHC (Lindane) & $\mathrm{mg} / \mathrm{kg}$ & 3 & 0 & $0 \%$ & 0.0019 & 0.0020 & -- & -- & $9.67 \mathrm{E}-04$ \\
\hline PEST & gamma-Chlordane & $\mathrm{mg} / \mathrm{kg}$ & 3 & 0 & $0 \%$ & 0.0019 & 0.0020 & -- & -- & $9.67 \mathrm{E}-04$ \\
\hline PEST & gamma-Chlordene & $\mathrm{mg} / \mathrm{kg}$ & 3 & 0 & $0 \%$ & 0.0019 & 0.0020 & -- & -- & $9.67 \mathrm{E}-04$ \\
\hline PEST & Heptachlor & $\mathrm{mg} / \mathrm{kg}$ & 3 & 0 & $0 \%$ & 0.0019 & 0.0020 & -- & -- & $9.67 \mathrm{E}-04$ \\
\hline PEST & Heptachlor epoxide & $\mathrm{mg} / \mathrm{kg}$ & 1 & 0 & $0 \%$ & 0.0019 & 0.0019 & -- & -- & $9.50 \mathrm{E}-04$ \\
\hline PEST & Mirex & $\mathrm{mg} / \mathrm{kg}$ & 3 & 0 & $0 \%$ & 0.0019 & 0.0020 & -- & -- & $9.67 \mathrm{E}-04$ \\
\hline PEST & Oxychlordane & $\mathrm{mg} / \mathrm{kg}$ & 3 & 0 & $0 \%$ & 0.0019 & 0.0020 & -- & -- & $9.67 \mathrm{E}-04$ \\
\hline PEST & Pentachloroanisole & $\mathrm{mg} / \mathrm{kg}$ & 3 & 0 & $0 \%$ & 0.0019 & 0.0020 & -- & -- & $9.67 \mathrm{E}-04$ \\
\hline PEST & Toxaphene & $\mathrm{mg} / \mathrm{kg}$ & 3 & 0 & $0 \%$ & 0.058 & 0.059 & -- & -- & 0.029 \\
\hline PEST & trans-Nonachlor & $\mathrm{mg} / \mathrm{kg}$ & 3 & 0 & $0 \%$ & 0.0019 & 0.0020 & -- & -- & $9.67 \mathrm{E}-04$ \\
\hline SVOC & 1,2,4-Trichlorobenzene & $\mathrm{mg} / \mathrm{kg}$ & 3 & 0 & $0 \%$ & 0.0048 & 0.0096 & -- & -- & 0.0040 \\
\hline SVOC & 1,2-Dichlorobenzene & $\mathrm{mg} / \mathrm{kg}$ & 3 & 0 & $0 \%$ & 0.0048 & 0.0096 & -- & -- & 0.0040 \\
\hline SVOC & 1,2-Diphenylhydrazine & $\mathrm{mg} / \mathrm{kg}$ & 3 & 0 & $0 \%$ & 0.0048 & 0.0096 & -- & -- & 0.0040 \\
\hline SVOC & 1,3-Dichlorobenzene & $\mathrm{mg} / \mathrm{kg}$ & 3 & 0 & $0 \%$ & 0.0048 & 0.0096 & -- & -- & 0.0040 \\
\hline SVOC & 1,4-Dichlorobenzene & $\mathrm{mg} / \mathrm{kg}$ & 3 & 0 & $0 \%$ & 0.0048 & 0.0096 & -- & -- & 0.0040 \\
\hline SVOC & 1-methylnaphthalene & $\mathrm{mg} / \mathrm{kg}$ & 3 & 0 & $0 \%$ & 0.0048 & 0.0096 & -- & -- & 0.0040 \\
\hline SVOC & 2,4-Dinitrotoluene & $\mathrm{mg} / \mathrm{kg}$ & 3 & 0 & $0 \%$ & 0.0096 & 0.019 & -- & -- & 0.0079 \\
\hline SVOC & 2,6-Dinitrotoluene & $\mathrm{mg} / \mathrm{kg}$ & 3 & 0 & $0 \%$ & 0.019 & 0.038 & -- & -- & 0.016 \\
\hline SVOC & 2-Chloronaphthalene & $\mathrm{mg} / \mathrm{kg}$ & 3 & 0 & $0 \%$ & 0.0096 & 0.019 & -- & -- & 0.0079 \\
\hline SVOC & 2-MethyInaphthalene & $\mathrm{mg} / \mathrm{kg}$ & 3 & 0 & $0 \%$ & 0.0048 & 0.0096 & -- & -- & 0.0040 \\
\hline SVOC & 4-Bromophenylphenyl ether & $\mathrm{mg} / \mathrm{kg}$ & 3 & 0 & $0 \%$ & 0.0048 & 0.0096 & -- & -- & 0.0040 \\
\hline SVOC & 4-Chlorophenylphenyl ether & $\mathrm{mg} / \mathrm{kg}$ & 3 & 0 & $0 \%$ & 0.0048 & 0.0096 & -- & -- & 0.0040 \\
\hline SVOC & Acenaphthene & $\mathrm{mg} / \mathrm{kg}$ & 3 & 0 & $0 \%$ & 0.0096 & 0.019 & -- & -- & 0.0079 \\
\hline SVOC & Acenaphthylene & $\mathrm{mg} / \mathrm{kg}$ & 3 & 0 & $0 \%$ & 0.0048 & 0.0096 & -- & -- & 0.0040 \\
\hline SVOC & Anthracene & $\mathrm{mg} / \mathrm{kg}$ & 3 & 0 & $0 \%$ & 0.0048 & 0.0096 & -- & -- & 0.0040 \\
\hline SVOC & Benzo(a)anthracene & $\mathrm{mg} / \mathrm{kg}$ & 3 & 0 & $0 \%$ & 0.0048 & 0.0096 & -- & -- & 0.0040 \\
\hline SVOC & Benzo(a)pyrene & $\mathrm{mg} / \mathrm{kg}$ & 3 & 0 & $0 \%$ & 0.0048 & 0.0096 & -- & -- & 0.0040 \\
\hline SVOC & Benzo(b)fluoranthene & $\mathrm{mg} / \mathrm{kg}$ & 3 & 0 & $0 \%$ & 0.0048 & 0.0096 & -- & -- & 0.0040 \\
\hline SVOC & Benzo(ghi)perylene & $\mathrm{mg} / \mathrm{kg}$ & 3 & 0 & $0 \%$ & 0.0096 & 0.019 & -- & -- & 0.0079 \\
\hline SVOC & Benzo(k)fluoranthene & $\mathrm{mg} / \mathrm{kg}$ & 3 & 0 & $0 \%$ & 0.0048 & 0.0096 & -- & -- & 0.0040 \\
\hline SVOC & Bis(2-chloro-1-methylethyl)ether & $\mathrm{mg} / \mathrm{kg}$ & 2 & 0 & $0 \%$ & 0.019 & 0.019 & -- & -- & 0.0095 \\
\hline SVOC & Chrysene & $\mathrm{mg} / \mathrm{kg}$ & 3 & 0 & $0 \%$ & 0.0048 & 0.0096 & -- & -- & 0.0040 \\
\hline
\end{tabular}


Table F-43

Summary Statistics for Largescale Sucker Fillet Skin On Collected from Segment 2 of the Columbia River

\begin{tabular}{|c|c|c|c|c|c|c|c|c|c|c|}
\hline $\begin{array}{l}\text { Constituent } \\
\text { Class }\end{array}$ & Constituent Name & Units & $\begin{array}{l}\text { Number } \\
\text { Analyzed }\end{array}$ & $\begin{array}{l}\text { Number } \\
\text { Detected }\end{array}$ & $\begin{array}{l}\text { Frequency of } \\
\text { Detection }\end{array}$ & $\begin{array}{l}\text { Minimum } \\
\text { Nondetect }\end{array}$ & $\begin{array}{c}\text { Maximum } \\
\text { Nondetect }\end{array}$ & $\begin{array}{l}\text { Minimum } \\
\text { Detect }\end{array}$ & $\begin{array}{l}\text { Maximum } \\
\text { Detect }\end{array}$ & $\begin{array}{c}\text { Average } \\
\text { Concentration }\end{array}$ \\
\hline$\overline{\text { SVOC }}$ & Dibenz[a,h]anthracene & $\mathrm{mg} / \mathrm{kg}$ & 3 & 0 & $0 \%$ & 0.0096 & 0.019 & $\overline{--}$ & $\overline{--}$ & 0.0079 \\
\hline SVOC & Dibenzofuran & $\mathrm{mg} / \mathrm{kg}$ & 3 & 0 & $0 \%$ & 0.0048 & 0.0096 & -- & -- & 0.0040 \\
\hline SVOC & Fluoranthene & $\mathrm{mg} / \mathrm{kg}$ & 3 & 0 & $0 \%$ & 0.0096 & 0.019 & -- & -- & 0.0079 \\
\hline SVOC & Fluorene & $\mathrm{mg} / \mathrm{kg}$ & 3 & 0 & $0 \%$ & 0.0048 & 0.0096 & -- & -- & 0.0040 \\
\hline SVOC & Hexachlorobenzene & $\mathrm{mg} / \mathrm{kg}$ & 3 & 0 & $0 \%$ & 0.0096 & 0.019 & -- & -- & 0.0079 \\
\hline SVOC & Hexachlorobutadiene & $\mathrm{mg} / \mathrm{kg}$ & 3 & 0 & $0 \%$ & 0.0048 & 0.0096 & -- & -- & 0.0040 \\
\hline SVOC & Hexachloroethane & $\mathrm{mg} / \mathrm{kg}$ & 3 & 0 & $0 \%$ & 0.0048 & 0.0096 & -- & -- & 0.0040 \\
\hline SVOC & Indeno(1,2,3-cd)pyrene & $\mathrm{mg} / \mathrm{kg}$ & 3 & 0 & $0 \%$ & 0.0096 & 0.019 & -- & -- & 0.0079 \\
\hline SVOC & Naphthalene & $\mathrm{mg} / \mathrm{kg}$ & 3 & 0 & $0 \%$ & 0.018 & 0.027 & -- & -- & 0.011 \\
\hline SVOC & Nitrobenzene & $\mathrm{mg} / \mathrm{kg}$ & 3 & 0 & $0 \%$ & 0.0048 & 0.0096 & -- & -- & 0.0040 \\
\hline SVOC & Phenanthrene & $\mathrm{mg} / \mathrm{kg}$ & 3 & 0 & $0 \%$ & 0.0048 & 0.0096 & -- & -- & 0.0040 \\
\hline SVOC & Pyrene & $\mathrm{mg} / \mathrm{kg}$ & 3 & 0 & $0 \%$ & 0.0096 & 0.019 & -- & -- & 0.0079 \\
\hline VOC & Retene & $\mathrm{mg} / \mathrm{kg}$ & 3 & 0 & $0 \%$ & 0.0096 & 0.019 & -- & -- & 0.0079 \\
\hline
\end{tabular}

DIOXI - Dioxins and Furans

PEST - Pesticide

PCB - Polychlorinated bipheny

SVOC - Semivolatile Organic Compound

VOC - Volatile Organic Compound 
Table F-44

Summary Statistics for Whole Body Largescale Sucker Collected from Segment 2 of the Columbia River

\begin{tabular}{|c|c|c|c|c|c|c|c|c|c|c|}
\hline $\begin{array}{l}\text { Constituent } \\
\text { Class }\end{array}$ & Constituent Name & Units & $\begin{array}{l}\text { Number } \\
\text { Analyzed }\end{array}$ & $\begin{array}{l}\text { Number } \\
\text { Detected }\end{array}$ & $\begin{array}{c}\text { Frequency of } \\
\text { Detection }\end{array}$ & $\begin{array}{l}\text { Minimum } \\
\text { Nondetect }\end{array}$ & $\begin{array}{l}\text { Maximum } \\
\text { Nondetect }\end{array}$ & $\begin{array}{c}\text { Minimum } \\
\text { Detect }\end{array}$ & $\begin{array}{l}\text { Maximum } \\
\text { Detect }\end{array}$ & $\begin{array}{c}\text { Average } \\
\text { Concentration }\end{array}$ \\
\hline$\overline{D I O X I}$ & $1,2,3,4,6,7,8$-Heptachlorodibenzodioxin & $\mathrm{mg} / \mathrm{kg}$ & 3 & 3 & $100 \%$ & -- & -- & $1.10 \mathrm{E}-07$ & $1.30 \mathrm{E}-07$ & $1.20 \mathrm{E}-07$ \\
\hline DIOXI & 1,2,3,4,6,7,8-Heptachlorodibenzofuran & $\mathrm{mg} / \mathrm{kg}$ & 3 & 2 & $67 \%$ & 1.00E-08 & 1.00E-08 & $3.00 \mathrm{E}-08$ & 3.00E-08 & 2.17E-08 \\
\hline DIOXI & 1,2,3,4,7,8,9-Heptachlorodibenzofuran & $\mathrm{mg} / \mathrm{kg}$ & 3 & 0 & $0 \%$ & 1.00E-08 & $2.00 \mathrm{E}-08$ & -- & -- & $6.67 \mathrm{E}-09$ \\
\hline DIOXI & $1,2,3,4,7,8$-Hexachlorodibenzofuran & $\mathrm{mg} / \mathrm{kg}$ & 3 & 1 & $33 \%$ & 1.00E-08 & 2.00E-08 & $2.00 \mathrm{E}-08$ & $2.00 \mathrm{E}-08$ & 1.17E-08 \\
\hline DIOXI & $1,2,3,4,7,8$-Hexachlorodibenzo-p-dioxin & $\mathrm{mg} / \mathrm{kg}$ & 3 & 1 & $33 \%$ & 1.00E-08 & 2.00E-08 & $1.00 \mathrm{E}-08$ & 1.00E-08 & 8.33E-09 \\
\hline DIOXI & 1,2,3,6,7,8-Hexachlorodibenzofuran & $\mathrm{mg} / \mathrm{kg}$ & 3 & 1 & $33 \%$ & 1.00E-08 & 2.00E-08 & $1.00 \mathrm{E}-08$ & 1.00E-08 & 8.33E-09 \\
\hline DIOXI & 1,2,3,6,7,8-Hexachlorodibenzo-p-dioxin & $\mathrm{mg} / \mathrm{kg}$ & 3 & 2 & $67 \%$ & $1.00 \mathrm{E}-08$ & $1.00 \mathrm{E}-08$ & $4.00 \mathrm{E}-08$ & 4.00E-08 & 2.83E-08 \\
\hline DIOXI & 1,2,3,7,8,9-Hexachlorodibenzofuran & $\mathrm{mg} / \mathrm{kg}$ & 3 & 0 & $0 \%$ & $1.00 \mathrm{E}-08$ & $2.00 \mathrm{E}-08$ & -- & -- & $6.67 \mathrm{E}-09$ \\
\hline DIOXI & 1,2,3,7,8,9-Hexachlorodibenzo-p-dioxin & $\mathrm{mg} / \mathrm{kg}$ & 3 & 0 & $0 \%$ & 1.00E-08 & $2.00 \mathrm{E}-08$ & -- & -- & $6.67 \mathrm{E}-09$ \\
\hline DIOXI & 1,2,3,7,8-Pentachlorodibenzofuran & $\mathrm{mg} / \mathrm{kg}$ & 3 & 0 & $0 \%$ & 2.00E-08 & 2.00E-08 & -- & -- & 1.00E-08 \\
\hline DIOXI & $1,2,3,7,8$-Pentachlorodibenzo-p-dioxin & $\mathrm{mg} / \mathrm{kg}$ & 3 & 3 & $100 \%$ & -- & -- & $4.00 \mathrm{E}-08$ & $9.00 \mathrm{E}-08$ & 5.67E-08 \\
\hline DIOXI & 2,3,4,6,7,8-Hexachlorodibenzofuran & $\mathrm{mg} / \mathrm{kg}$ & 3 & 0 & $0 \%$ & 1.00E-08 & 2.00E-08 & -- & -- & 6.67E-09 \\
\hline DIOXI & 2,3,4,7,8-Pentachlorodibenzofuran & $\mathrm{mg} / \mathrm{kg}$ & 3 & 1 & $33 \%$ & 2.00E-08 & 2.00E-08 & $1.20 \mathrm{E}-07$ & $1.20 \mathrm{E}-07$ & 4.67E-08 \\
\hline DIOXI & 2,3,7,8-Tetrachlorodibenzofuran & $\mathrm{mg} / \mathrm{kg}$ & 3 & 3 & $100 \%$ & -- & -- & 1.37E-06 & $2.88 \mathrm{E}-06$ & 2.03E-06 \\
\hline DIOXI & 2,3,7,8-Tetrachlorodibenzo-p-dioxin & $\mathrm{mg} / \mathrm{kg}$ & 3 & 2 & $67 \%$ & 1.00E-08 & 1.00E-08 & $4.00 \mathrm{E}-08$ & 1.00E-07 & 4.83E-08 \\
\hline DIOXI & Octachlorodibenzofuran & $\mathrm{mg} / \mathrm{kg}$ & 3 & 1 & $33 \%$ & $1.00 \mathrm{E}-08$ & $1.00 \mathrm{E}-08$ & 4.00E-08 & $4.00 \mathrm{E}-08$ & 1.67E-08 \\
\hline DIOXI & Octachlorodibenzo-p-dioxin & $\mathrm{mg} / \mathrm{kg}$ & 3 & 3 & $100 \%$ & -- & -- & $4.50 \mathrm{E}-07$ & $6.40 \mathrm{E}-07$ & 5.43E-07 \\
\hline METAL & Aluminum & $\mathrm{mg} / \mathrm{kg}$ & 3 & 3 & $100 \%$ & -- & -- & 85 & 130 & 100 \\
\hline METAL & Antimony & $\mathrm{mg} / \mathrm{kg}$ & 3 & 0 & $0 \%$ & 0.050 & 0.050 & -- & -- & 0.025 \\
\hline METAL & Arsenic & $\mathrm{mg} / \mathrm{kg}$ & 3 & 3 & $100 \%$ & -- & -- & 0.20 & 0.26 & 0.22 \\
\hline METAL & Barium & $\mathrm{mg} / \mathrm{kg}$ & 3 & 3 & $100 \%$ & -- & -- & 4.1 & 4.7 & 4.4 \\
\hline METAL & Beryllium & $\mathrm{mg} / \mathrm{kg}$ & 3 & 2 & $67 \%$ & 0.0040 & 0.0040 & 0.0050 & 0.0054 & 0.0041 \\
\hline METAL & Cadmium & $\mathrm{mg} / \mathrm{kg}$ & 3 & 3 & $100 \%$ & -- & -- & 0.18 & 0.24 & 0.21 \\
\hline METAL & Chromium & $\mathrm{mg} / \mathrm{kg}$ & 3 & 3 & $100 \%$ & -- & -- & 0.50 & 0.52 & 0.51 \\
\hline METAL & Cobalt & $\mathrm{mg} / \mathrm{kg}$ & 3 & 3 & $100 \%$ & -- & -- & 0.17 & 0.21 & 0.19 \\
\hline METAL & Copper & $\mathrm{mg} / \mathrm{kg}$ & 3 & 3 & $100 \%$ & -- & -- & 1.5 & 5.6 & 2.9 \\
\hline METAL & Lead & $\mathrm{mg} / \mathrm{kg}$ & 3 & 3 & $100 \%$ & -- & -- & 0.50 & 1.1 & 0.74 \\
\hline METAL & Manganese & $\mathrm{mg} / \mathrm{kg}$ & 3 & 3 & $100 \%$ & -- & -- & 18 & 21 & 19 \\
\hline METAL & Mercury & $\mathrm{mg} / \mathrm{kg}$ & 3 & 3 & $100 \%$ & -- & -- & 0.080 & 0.14 & 0.11 \\
\hline METAL & Nickel & $\mathrm{mg} / \mathrm{kg}$ & 3 & 3 & $100 \%$ & -- & -- & 3.0 & 11 & 5.8 \\
\hline METAL & Selenium & $\mathrm{mg} / \mathrm{kg}$ & 3 & 3 & $100 \%$ & -- & -- & 0.33 & 0.39 & 0.36 \\
\hline METAL & Silver & $\mathrm{mg} / \mathrm{kg}$ & 3 & 0 & $0 \%$ & 0.10 & 0.10 & -- & -- & 0.050 \\
\hline METAL & Thallium & $\mathrm{mg} / \mathrm{kg}$ & 3 & 0 & $0 \%$ & 0.050 & 0.050 & -- & -- & 0.025 \\
\hline METAL & Vanadium & $\mathrm{mg} / \mathrm{kg}$ & 3 & 3 & $100 \%$ & -- & -- & 0.32 & 0.42 & 0.36 \\
\hline METAL & Zinc & $\mathrm{mg} / \mathrm{kg}$ & 3 & 3 & $100 \%$ & -- & -- & 33 & 38 & 36 \\
\hline PCB & 2,2',3,3',4,4',5-Heptachloro-1,1'-biphenyl & $\mathrm{mg} / \mathrm{kg}$ & 3 & 3 & $100 \%$ & -- & -- & 0.0016 & 0.0052 & 0.0037 \\
\hline РCB & 2,2',3,4,4',5,5'-Heptachlorobiphenyl & $\mathrm{mg} / \mathrm{kg}$ & 3 & 3 & $100 \%$ & -- & -- & 0.0047 & 0.017 & 0.011 \\
\hline РCB & 2,3,3',4,4', 5'-Hexachlorobiphenyl & $\mathrm{mg} / \mathrm{kg}$ & 3 & 3 & $100 \%$ & -- & -- & 2.03E-04 & $5.25 \mathrm{E}-04$ & $3.49 \mathrm{E}-04$ \\
\hline РCB & 2,3,3',4,4',5-Hexachlorobiphenyl (BZ 156) & $\mathrm{mg} / \mathrm{kg}$ & 3 & 3 & $100 \%$ & -- & -- & 0.0010 & 0.0027 & 0.0018 \\
\hline PCB & 2,3,3',4,4'-Pentachlorobiphenyl & $\mathrm{mg} / \mathrm{kg}$ & 3 & 3 & $100 \%$ & -- & -- & 0.0027 & 0.0065 & 0.0042 \\
\hline РCB & 2,3',4,4',5,5'-Hexachlorobiphenyl (BZ 167) & $\mathrm{mg} / \mathrm{kg}$ & 3 & 3 & $100 \%$ & -- & -- & $4.52 E-04$ & 0.0012 & 8.02E-04 \\
\hline PCB & 2',3,4,4',5-Pentachloro-1,1'-biphenyl (BZ 123) & $\mathrm{mg} / \mathrm{kg}$ & 3 & 3 & $100 \%$ & -- & -- & $1.12 \mathrm{E}-04$ & $2.46 \mathrm{E}-04$ & 1.63E-04 \\
\hline PCB & 2,3,4,4',5-Pentachlorobiphenyl (BZ 114) & $\mathrm{mg} / \mathrm{kg}$ & 3 & 3 & $100 \%$ & -- & -- & $2.32 \mathrm{E}-04$ & $5.41 \mathrm{E}-04$ & 3.67E-04 \\
\hline PCB & 2,3',4,4',5-Pentachlorobiphenyl (BZ 118) & $\mathrm{mg} / \mathrm{kg}$ & 3 & 3 & $100 \%$ & -- & -- & 0.0060 & 0.014 & 0.0093 \\
\hline PCB & 2,3,4,5,3',4',5'-Heptachlorobiphenyl & $\mathrm{mg} / \mathrm{kg}$ & 3 & 3 & $100 \%$ & -- & -- & 7.73E-05 & $1.98 \mathrm{E}-04$ & 1.52E-04 \\
\hline PCB & 3,3',4,4',5,5'-Hexachlorobiphenyl (BZ 169) & $\mathrm{mg} / \mathrm{kg}$ & 3 & 0 & $0 \%$ & 1.33E-06 & 2.30E-06 & -- & -- & $8.48 \mathrm{E}-07$ \\
\hline РСВ & 3,3',4,4',5-Pentachlorobiphenyl (BZ 126) & $\mathrm{mg} / \mathrm{kg}$ & 3 & 3 & $100 \%$ & -- & -- & 1.34E-05 & 2.12E-05 & 1.63E-05 \\
\hline PCB & 3,3',4,4'-Tetrachlorobiphenyl (BZ 77) & $\mathrm{mg} / \mathrm{kg}$ & 3 & 3 & $100 \%$ & -- & -- & $5.38 \mathrm{E}-05$ & 1.07E-04 & 7.38E-05 \\
\hline РСВ & Aroclor-1016 & $\mathrm{mg} / \mathrm{kg}$ & 3 & 0 & $0 \%$ & 0.019 & 0.020 & -- & -- & 0.0098 \\
\hline PCB & Aroclor-1221 & $\mathrm{mg} / \mathrm{kg}$ & 3 & 0 & $0 \%$ & 0.019 & 0.020 & -- & -- & 0.0098 \\
\hline
\end{tabular}


Table F-44

Summary Statistics for Whole Body Largescale Sucker Collected from Segment 2 of the Columbia River

\begin{tabular}{|c|c|c|c|c|c|c|c|c|c|c|}
\hline $\begin{array}{l}\text { Constituent } \\
\text { Class }\end{array}$ & Constituent Name & Units & $\begin{array}{l}\text { Number } \\
\text { Analyzed }\end{array}$ & $\begin{array}{l}\text { Number } \\
\text { Detected }\end{array}$ & $\begin{array}{c}\text { Frequency of } \\
\text { Detection }\end{array}$ & $\begin{array}{l}\text { Minimum } \\
\text { Nondetect }\end{array}$ & $\begin{array}{l}\text { Maximum } \\
\text { Nondetect }\end{array}$ & $\begin{array}{l}\text { Minimum } \\
\text { Detect }\end{array}$ & $\begin{array}{l}\text { Maximum } \\
\text { Detect }\end{array}$ & $\begin{array}{c}\text { Average } \\
\text { Concentration }\end{array}$ \\
\hline$\overline{\mathrm{PCB}}$ & Aroclor-1232 & $\mathrm{mg} / \mathrm{kg}$ & 3 & 0 & $0 \%$ & 0.019 & 0.020 & -- & -- & 0.0098 \\
\hline PCB & Aroclor-1248 & $\mathrm{mg} / \mathrm{kg}$ & 3 & 0 & $0 \%$ & 0.019 & 0.020 & -- & -- & 0.0098 \\
\hline РCB & Aroclor-1254 & $\mathrm{mg} / \mathrm{kg}$ & 3 & 3 & $100 \%$ & -- & -- & 0.038 & 0.065 & 0.048 \\
\hline РСВ & Aroclor-1260 & $\mathrm{mg} / \mathrm{kg}$ & 3 & 3 & $100 \%$ & -- & -- & 0.032 & 0.10 & 0.077 \\
\hline PEST & 2,4'-DDD & $\mathrm{mg} / \mathrm{kg}$ & 3 & 3 & $100 \%$ & -- & -- & 0.0036 & 0.015 & 0.0079 \\
\hline PEST & 2,4'-DDE & $\mathrm{mg} / \mathrm{kg}$ & 3 & 3 & $100 \%$ & -- & -- & 0.0045 & 0.012 & 0.0073 \\
\hline PEST & 2,4'-DDT & $\mathrm{mg} / \mathrm{kg}$ & 3 & 0 & $0 \%$ & 0.0019 & 0.0020 & -- & -- & $9.83 \mathrm{E}-04$ \\
\hline PEST & Aldrin & $\mathrm{mg} / \mathrm{kg}$ & 3 & 0 & $0 \%$ & 0.0019 & 0.0020 & -- & -- & $9.83 \mathrm{E}-04$ \\
\hline PEST & Alpha-BHC & $\mathrm{mg} / \mathrm{kg}$ & 3 & 0 & $0 \%$ & 0.0019 & 0.0020 & -- & -- & $9.83 \mathrm{E}-04$ \\
\hline PEST & alpha-Chlordane & $\mathrm{mg} / \mathrm{kg}$ & 3 & 0 & $0 \%$ & 0.0019 & 0.0020 & -- & -- & $9.83 E-04$ \\
\hline PEST & alpha-Chlordene & $\mathrm{mg} / \mathrm{kg}$ & 3 & 0 & $0 \%$ & 0.0019 & 0.0020 & -- & -- & $9.83 \mathrm{E}-04$ \\
\hline PEST & Beta-BHC & $\mathrm{mg} / \mathrm{kg}$ & 3 & 0 & $0 \%$ & 0.0019 & 0.0020 & -- & -- & $9.83 \mathrm{E}-04$ \\
\hline PEST & cis-Nonachlor & $\mathrm{mg} / \mathrm{kg}$ & 3 & 0 & $0 \%$ & 0.0019 & 0.0020 & -- & -- & $9.83 \mathrm{E}-04$ \\
\hline PEST & DDMU & $\mathrm{mg} / \mathrm{kg}$ & 3 & 3 & $100 \%$ & -- & -- & 0.010 & 0.024 & 0.015 \\
\hline PEST & Delta-BHC & $\mathrm{mg} / \mathrm{kg}$ & 3 & 0 & $0 \%$ & 0.0019 & 0.0020 & -- & -- & $9.83 \mathrm{E}-04$ \\
\hline PEST & Dichlorodiphenyldichloroethane & $\mathrm{mg} / \mathrm{kg}$ & 3 & 2 & $67 \%$ & 0.0020 & 0.0020 & 0.038 & 0.13 & 0.056 \\
\hline PEST & Dichlorodiphenyldichloroethylene & $\mathrm{mg} / \mathrm{kg}$ & 3 & 3 & $100 \%$ & -- & -- & 0.36 & 0.83 & 0.54 \\
\hline PEST & Dichlorodiphenyltrichloroethane & $\mathrm{mg} / \mathrm{kg}$ & 3 & 3 & $100 \%$ & -- & -- & 0.014 & 0.052 & 0.029 \\
\hline PEST & Gamma-BHC (Lindane) & $\mathrm{mg} / \mathrm{kg}$ & 3 & 0 & $0 \%$ & 0.0019 & 0.0020 & -- & -- & $9.83 \mathrm{E}-04$ \\
\hline PEST & gamma-Chlordane & $\mathrm{mg} / \mathrm{kg}$ & 3 & 0 & $0 \%$ & 0.0019 & 0.0020 & -- & -- & $9.83 \mathrm{E}-04$ \\
\hline PEST & gamma-Chlordene & $\mathrm{mg} / \mathrm{kg}$ & 3 & 0 & $0 \%$ & 0.0019 & 0.0020 & -- & -- & $9.83 \mathrm{E}-04$ \\
\hline PEST & Heptachlor & $\mathrm{mg} / \mathrm{kg}$ & 3 & 0 & $0 \%$ & 0.0019 & 0.0020 & -- & -- & $9.83 \mathrm{E}-04$ \\
\hline PEST & Mirex & $\mathrm{mg} / \mathrm{kg}$ & 3 & 0 & $0 \%$ & 0.0019 & 0.0020 & -- & -- & $9.83 \mathrm{E}-04$ \\
\hline PEST & Oxychlordane & $\mathrm{mg} / \mathrm{kg}$ & 3 & 0 & $0 \%$ & 0.0019 & 0.0020 & -- & -- & $9.83 \mathrm{E}-04$ \\
\hline PEST & Pentachloroanisole & $\mathrm{mg} / \mathrm{kg}$ & 3 & 0 & $0 \%$ & 0.0019 & 0.0020 & -- & -- & $9.83 \mathrm{E}-04$ \\
\hline PEST & Toxaphene & $\mathrm{mg} / \mathrm{kg}$ & 3 & 0 & $0 \%$ & 0.056 & 0.059 & -- & -- & 0.029 \\
\hline PEST & trans-Nonachlor & $\mathrm{mg} / \mathrm{kg}$ & 3 & 3 & $100 \%$ & -- & -- & 0.0012 & 0.0024 & 0.0016 \\
\hline SVOC & 1,2,4-Trichlorobenzene & $\mathrm{mg} / \mathrm{kg}$ & 3 & 0 & $0 \%$ & 0.0086 & 0.0099 & -- & -- & 0.0047 \\
\hline SVOC & 1,2-Dichlorobenzene & $\mathrm{mg} / \mathrm{kg}$ & 3 & 0 & $0 \%$ & 0.0086 & 0.0099 & -- & -- & 0.0047 \\
\hline SVOC & 1,2-Diphenylhydrazine & $\mathrm{mg} / \mathrm{kg}$ & 3 & 0 & $0 \%$ & 0.0086 & 0.0099 & -- & -- & 0.0047 \\
\hline SVOC & 1,3-Dichlorobenzene & $\mathrm{mg} / \mathrm{kg}$ & 3 & 0 & $0 \%$ & 0.0086 & 0.0099 & -- & -- & 0.0047 \\
\hline SVOC & 1,4-Dichlorobenzene & $\mathrm{mg} / \mathrm{kg}$ & 3 & 0 & $0 \%$ & 0.0086 & 0.0099 & -- & -- & 0.0047 \\
\hline SVOC & 1-methylnaphthalene & $\mathrm{mg} / \mathrm{kg}$ & 3 & 0 & $0 \%$ & 0.0086 & 0.0099 & -- & -- & 0.0047 \\
\hline SVOC & 2,4-Dinitrotoluene & $\mathrm{mg} / \mathrm{kg}$ & 3 & 0 & $0 \%$ & 0.017 & 0.020 & -- & -- & 0.0095 \\
\hline SVOC & 2,6-Dinitrotoluene & $\mathrm{mg} / \mathrm{kg}$ & 3 & 0 & $0 \%$ & 0.034 & 0.040 & -- & -- & 0.019 \\
\hline SVOC & 2-Chloronaphthalene & $\mathrm{mg} / \mathrm{kg}$ & 3 & 0 & $0 \%$ & 0.017 & 0.020 & -- & -- & 0.0095 \\
\hline SVOC & 2-Methylnaphthalene & $\mathrm{mg} / \mathrm{kg}$ & 3 & 0 & $0 \%$ & 0.0086 & 0.0099 & -- & -- & 0.0047 \\
\hline SVOC & 4-Bromophenylphenyl ether & $\mathrm{mg} / \mathrm{kg}$ & 3 & 0 & $0 \%$ & 0.0086 & 0.0099 & -- & -- & 0.0047 \\
\hline SVOC & 4-Chlorophenylphenyl ether & $\mathrm{mg} / \mathrm{kg}$ & 3 & 0 & $0 \%$ & 0.0086 & 0.0099 & -- & -- & 0.0047 \\
\hline SVOC & Acenaphthene & $\mathrm{mg} / \mathrm{kg}$ & 3 & 0 & $0 \%$ & 0.017 & 0.020 & -- & -- & 0.0095 \\
\hline SVOC & Acenaphthylene & $\mathrm{mg} / \mathrm{kg}$ & 3 & 0 & $0 \%$ & 0.0086 & 0.0099 & -- & -- & 0.0047 \\
\hline SVOC & Anthracene & $\mathrm{mg} / \mathrm{kg}$ & 3 & 0 & $0 \%$ & 0.0086 & 0.0099 & -- & -- & 0.0047 \\
\hline SVOC & Benzo(a)anthracene & $\mathrm{mg} / \mathrm{kg}$ & 3 & 0 & $0 \%$ & 0.0086 & 0.0099 & -- & -- & 0.0047 \\
\hline SVOC & Benzo(a)pyrene & $\mathrm{mg} / \mathrm{kg}$ & 3 & 0 & $0 \%$ & 0.0086 & 0.0099 & -- & -- & 0.0047 \\
\hline SVOC & Benzo(b)fluoranthene & $\mathrm{mg} / \mathrm{kg}$ & 3 & 0 & $0 \%$ & 0.0086 & 0.0099 & -- & -- & 0.0047 \\
\hline SVOC & Benzo(ghi)perylene & $\mathrm{mg} / \mathrm{kg}$ & 3 & 0 & $0 \%$ & 0.017 & 0.020 & -- & -- & 0.0095 \\
\hline SVOC & Benzo(k)fluoranthene & $\mathrm{mg} / \mathrm{kg}$ & 3 & 0 & $0 \%$ & 0.0086 & 0.0099 & -- & -- & 0.0047 \\
\hline SVOC & Bis(2-chloro-1-methylethyl)ether & $\mathrm{mg} / \mathrm{kg}$ & 3 & 0 & $0 \%$ & 0.017 & 0.020 & -- & -- & 0.0095 \\
\hline SVOC & Chrysene & $\mathrm{mg} / \mathrm{kg}$ & 3 & 0 & $0 \%$ & 0.0086 & 0.0099 & -- & -- & 0.0047 \\
\hline SVOC & Dibenz[a,h]anthracene & $\mathrm{mg} / \mathrm{kg}$ & 3 & 0 & $0 \%$ & 0.017 & 0.020 & -- & -- & 0.0095 \\
\hline
\end{tabular}


Table F-44

Summary Statistics for Whole Body Largescale Sucker Collected from Segment 2 of the Columbia River

\begin{tabular}{|c|c|c|c|c|c|c|c|c|c|c|}
\hline $\begin{array}{l}\text { Constituent } \\
\text { Class }\end{array}$ & Constituent Name & Units & $\begin{array}{l}\text { Number } \\
\text { Analyzed }\end{array}$ & $\begin{array}{l}\text { Number } \\
\text { Detected }\end{array}$ & $\begin{array}{l}\text { Frequency of } \\
\text { Detection }\end{array}$ & $\begin{array}{l}\text { Minimum } \\
\text { Nondetect }\end{array}$ & $\begin{array}{l}\text { Maximum } \\
\text { Nondetect }\end{array}$ & $\begin{array}{l}\text { Minimum } \\
\text { Detect }\end{array}$ & $\begin{array}{l}\text { Maximum } \\
\text { Detect }\end{array}$ & $\begin{array}{c}\text { Average } \\
\text { Concentration }\end{array}$ \\
\hline SVOC & Dibenzofuran & $\mathrm{mg} / \mathrm{kg}$ & 3 & 0 & $0 \%$ & 0.0086 & 0.0099 & -- & -- & 0.0047 \\
\hline SVOC & Fluoranthene & $\mathrm{mg} / \mathrm{kg}$ & 3 & 0 & $0 \%$ & 0.017 & 0.020 & -- & -- & 0.0095 \\
\hline SVOC & Fluorene & $\mathrm{mg} / \mathrm{kg}$ & 3 & 0 & $0 \%$ & 0.0048 & 0.0048 & -- & -- & 0.0024 \\
\hline SVOC & Hexachlorobenzene & $\mathrm{mg} / \mathrm{kg}$ & 3 & 0 & $0 \%$ & 0.017 & 0.020 & -- & -- & 0.0095 \\
\hline SVOC & Hexachlorobutadiene & $\mathrm{mg} / \mathrm{kg}$ & 3 & 0 & $0 \%$ & 0.0086 & 0.0099 & -- & -- & 0.0047 \\
\hline SVOC & Hexachloroethane & $\mathrm{mg} / \mathrm{kg}$ & 3 & 0 & $0 \%$ & 0.0086 & 0.0099 & -- & -- & 0.0047 \\
\hline SVOC & Indeno(1,2,3-cd)pyrene & $\mathrm{mg} / \mathrm{kg}$ & 3 & 0 & $0 \%$ & 0.017 & 0.020 & -- & -- & 0.0095 \\
\hline SVOC & Naphthalene & $\mathrm{mg} / \mathrm{kg}$ & 3 & 0 & $0 \%$ & 0.019 & 0.021 & -- & -- & 0.010 \\
\hline SVOC & Nitrobenzene & $\mathrm{mg} / \mathrm{kg}$ & 3 & 0 & $0 \%$ & 0.0086 & 0.0099 & -- & -- & 0.0047 \\
\hline SVOC & Phenanthrene & $\mathrm{mg} / \mathrm{kg}$ & 3 & 0 & $0 \%$ & 0.0086 & 0.0099 & -- & -- & 0.0047 \\
\hline SVOC & Pyrene & $\mathrm{mg} / \mathrm{kg}$ & 3 & 0 & $0 \%$ & 0.017 & 0.020 & -- & -- & 0.0095 \\
\hline VOC & Retene & $\mathrm{mg} / \mathrm{kg}$ & 3 & 0 & $0 \%$ & 0.017 & 0.020 & -- & -- & 0.0095 \\
\hline
\end{tabular}

DIOXI - Dioxins and Furans

PEST - Pesticide

PCB - Polychlorinated bipheny

SVOC - Semivolatile Organic Compound

VOC - Volatile Organic Compound 
Table F-45

Summary Statistics for Sculpin Liver Collected from Segment 2 of the Columbia River

\begin{tabular}{|c|c|c|c|c|c|c|c|c|c|c|}
\hline $\begin{array}{l}\text { Constituent } \\
\text { Class }\end{array}$ & Constituent Name & Units & $\begin{array}{c}\text { Number } \\
\text { Analyzed }\end{array}$ & $\begin{array}{l}\text { Number } \\
\text { Detected }\end{array}$ & $\begin{array}{l}\text { Frequency of } \\
\text { Detection }\end{array}$ & $\begin{array}{l}\text { Minimum } \\
\text { Nondetect }\end{array}$ & $\begin{array}{l}\text { Maximum } \\
\text { Nondetect }\end{array}$ & $\begin{array}{c}\text { Minimum } \\
\text { Detect }\end{array}$ & $\begin{array}{c}\text { Maximum } \\
\text { Detect }\end{array}$ & $\begin{array}{c}\text { Average } \\
\text { Concentration }\end{array}$ \\
\hline METAL & Aluminum & $\mathrm{mg} / \mathrm{kg}$ & 30 & 30 & $100 \%$ & $\overline{--}$ & $\overline{--}$ & 1.7 & 32 & 8.3 \\
\hline METAL & Antimony & $\mathrm{mg} / \mathrm{kg}$ & 33 & 27 & $82 \%$ & 0.010 & 0.015 & 0.011 & 0.16 & 0.043 \\
\hline METAL & Arsenic & $\mathrm{mg} / \mathrm{kg}$ & 33 & 25 & $76 \%$ & 0.10 & 0.10 & 0.41 & 2.5 & 0.81 \\
\hline METAL & Barium & $\mathrm{mg} / \mathrm{kg}$ & 33 & 30 & $91 \%$ & 0.10 & 0.10 & 0.076 & 0.63 & 0.30 \\
\hline METAL & Beryllium & $\mathrm{mg} / \mathrm{kg}$ & 33 & 3 & $9 \%$ & 0.020 & 0.15 & 0.022 & 0.30 & 0.026 \\
\hline METAL & Cadmium & $\mathrm{mg} / \mathrm{kg}$ & 36 & 36 & $100 \%$ & -- & -- & 0.72 & 25 & 6.8 \\
\hline METAL & Chromium & $\mathrm{mg} / \mathrm{kg}$ & 36 & 36 & $100 \%$ & -- & -- & 0.21 & 4.4 & 0.74 \\
\hline METAL & Copper & $\mathrm{mg} / \mathrm{kg}$ & 33 & 33 & $100 \%$ & -- & -- & 6.0 & 46 & 21 \\
\hline METAL & Lead & $\mathrm{mg} / \mathrm{kg}$ & 36 & 36 & $100 \%$ & -- & -- & 0.036 & 0.27 & 0.13 \\
\hline METAL & Manganes $€$ & $\mathrm{mg} / \mathrm{kg}$ & 33 & 33 & $100 \%$ & -- & -- & 0.72 & 12 & 3.4 \\
\hline METAL & Mercury & $\mathrm{mg} / \mathrm{kg}$ & 36 & 36 & $100 \%$ & -- & -- & 0.087 & 0.74 & 0.25 \\
\hline METAL & Nickel & $\mathrm{mg} / \mathrm{kg}$ & 36 & 32 & $89 \%$ & 0.045 & 0.050 & 0.074 & 0.39 & 0.18 \\
\hline METAL & Selenium & $\mathrm{mg} / \mathrm{kg}$ & 36 & 36 & $100 \%$ & -- & -- & 3.0 & 11 & 6.5 \\
\hline METAL & Silver & $\mathrm{mg} / \mathrm{kg}$ & 36 & 29 & $81 \%$ & 0.010 & 0.045 & 0.010 & 0.13 & 0.029 \\
\hline METAL & Thallium & $\mathrm{mg} / \mathrm{kg}$ & 33 & 33 & $100 \%$ & -- & -- & 0.062 & 0.36 & 0.15 \\
\hline METAL & Thorium & $\mathrm{mg} / \mathrm{kg}$ & 33 & 2 & $6 \%$ & 0.020 & 0.021 & 0.021 & 0.026 & 0.011 \\
\hline METAL & Uranium & $\mathrm{mg} / \mathrm{kg}$ & 33 & 7 & $21 \%$ & 0.010 & 0.031 & 0.010 & 0.048 & 0.010 \\
\hline METAL & Zinc & $\mathrm{mg} / \mathrm{kg}$ & 33 & 33 & $100 \%$ & -- & -- & 81 & 396 & 160 \\
\hline
\end{tabular}




\section{Table F-46}

Summary Statistics for Whole Body Sculpin Collected from Segment 2 of the Columbia River

\begin{tabular}{|c|c|c|c|c|c|c|c|c|c|c|}
\hline $\begin{array}{l}\text { Constituent } \\
\text { Class }\end{array}$ & Constituent Name & Units & $\begin{array}{l}\text { Number } \\
\text { Analyzed }\end{array}$ & $\begin{array}{l}\text { Number } \\
\text { Detected }\end{array}$ & $\begin{array}{l}\text { Frequency of } \\
\text { Detection }\end{array}$ & $\begin{array}{l}\text { Minimum } \\
\text { Nondetect }\end{array}$ & $\begin{array}{l}\text { Maximum } \\
\text { Nondetect }\end{array}$ & $\begin{array}{l}\text { Minimum } \\
\text { Detect }\end{array}$ & $\begin{array}{l}\text { Maximum } \\
\text { Detect }\end{array}$ & $\begin{array}{c}\text { Average } \\
\text { Concentration }\end{array}$ \\
\hline$\overline{\text { METAL }}$ & Aluminum & $\mathrm{mg} / \mathrm{kg}$ & $\overline{9}$ & 9 & $100 \%$ & -- & -- & 11 & 243 & 103 \\
\hline METAL & Antimony & $\mathrm{mg} / \mathrm{kg}$ & 9 & 2 & $22 \%$ & 0.010 & 0.019 & 0.012 & 0.016 & 0.0075 \\
\hline METAL & Arsenic & $\mathrm{mg} / \mathrm{kg}$ & 9 & 7 & $78 \%$ & 0.097 & 0.10 & 0.11 & 0.68 & 0.29 \\
\hline METAL & Barium & $\mathrm{mg} / \mathrm{kg}$ & 9 & 9 & $100 \%$ & -- & -- & 5.2 & 25 & 12 \\
\hline METAL & Beryllium & $\mathrm{mg} / \mathrm{kg}$ & 9 & 1 & $11 \%$ & 0.020 & 0.020 & 0.053 & 0.053 & 0.015 \\
\hline METAL & Cadmium & $\mathrm{mg} / \mathrm{kg}$ & 9 & 9 & $100 \%$ & -- & -- & 0.028 & 0.78 & 0.35 \\
\hline METAL & Chromium & $\mathrm{mg} / \mathrm{kg}$ & 9 & 9 & $100 \%$ & -- & -- & 0.74 & 1.8 & 1.2 \\
\hline METAL & Copper & $\mathrm{mg} / \mathrm{kg}$ & 9 & 9 & $100 \%$ & -- & -- & 2.2 & 5.1 & 3.0 \\
\hline METAL & Lead & $\mathrm{mg} / \mathrm{kg}$ & 9 & 9 & $100 \%$ & -- & -- & 0.097 & 0.64 & 0.30 \\
\hline METAL & Manganes $€$ & $\mathrm{mg} / \mathrm{kg}$ & 9 & 9 & $100 \%$ & -- & -- & 3.2 & 19 & 9.9 \\
\hline METAL & Mercury & $\mathrm{mg} / \mathrm{kg}$ & 9 & 9 & $100 \%$ & -- & -- & 0.061 & 0.22 & 0.099 \\
\hline METAL & Nickel & $\mathrm{mg} / \mathrm{kg}$ & 9 & 9 & $100 \%$ & -- & -- & 0.76 & 3.1 & 1.5 \\
\hline METAL & Selenium & $\mathrm{mg} / \mathrm{kg}$ & 9 & 9 & $100 \%$ & -- & -- & 1.7 & 2.6 & 2.2 \\
\hline METAL & Silver & $\mathrm{mg} / \mathrm{kg}$ & 9 & 0 & $0 \%$ & 0.010 & 0.045 & -- & -- & 0.0069 \\
\hline METAL & Thallium & $\mathrm{mg} / \mathrm{kg}$ & 9 & 9 & $100 \%$ & -- & -- & 0.049 & 0.21 & 0.12 \\
\hline METAL & Thorium & $\mathrm{mg} / \mathrm{kg}$ & 9 & 2 & $22 \%$ & 0.020 & 0.021 & 0.020 & 0.031 & 0.013 \\
\hline METAL & Uranium & $\mathrm{mg} / \mathrm{kg}$ & 9 & 7 & $78 \%$ & 0.010 & 0.031 & 0.013 & 0.040 & 0.025 \\
\hline METAL & Zinc & $\mathrm{mg} / \mathrm{kg}$ & 9 & 9 & $100 \%$ & -- & -- & 62 & 144 & 104 \\
\hline RAD & Antimony-125 & $\mathrm{pCi} / \mathrm{g}$ & 2 & 0 & $0 \%$ & 0.085 & 0.35 & -- & -- & 0.11 \\
\hline RAD & Beryllium-7 & $\mathrm{pCi} / \mathrm{g}$ & 4 & 0 & $0 \%$ & 0.23 & 1.1 & -- & -- & 0.23 \\
\hline RAD & Cesium-134 & $\mathrm{pCi} / \mathrm{g}$ & 4 & 0 & $0 \%$ & 0.020 & 0.19 & -- & -- & 0.036 \\
\hline RAD & Cesium-137 & $\mathrm{pCi} / \mathrm{g}$ & 1 & 0 & $0 \%$ & 0.013 & 0.013 & -- & -- & 0.0064 \\
\hline RAD & Cobalt-60 & $\mathrm{pCi} / \mathrm{g}$ & 4 & 0 & $0 \%$ & 0.021 & 0.052 & -- & -- & 0.018 \\
\hline RAD & Europium-152 & $\mathrm{pCi} / \mathrm{g}$ & 1 & 0 & $0 \%$ & 0.060 & 0.060 & -- & -- & 0.030 \\
\hline RAD & Europium-154 & $\mathrm{pCi} / \mathrm{g}$ & 2 & 0 & $0 \%$ & 0.12 & 0.31 & -- & -- & 0.11 \\
\hline RAD & Europium-155 & $\mathrm{pCi} / \mathrm{g}$ & 2 & 0 & $0 \%$ & 0.0091 & 0.026 & -- & -- & 0.0088 \\
\hline RAD & Potassium-40 & $\mathrm{pCi} / \mathrm{g}$ & 5 & 3 & $60 \%$ & 0.28 & 2.4 & 2.4 & 3.4 & 1.9 \\
\hline RAD & Ruthenium-106 & $\mathrm{pCi} / \mathrm{g}$ & 3 & 0 & $0 \%$ & 0.13 & 1.4 & -- & -- & 0.30 \\
\hline RAD & Strontium-90 & $\mathrm{pCi} / \mathrm{g}$ & 21 & 3 & $14 \%$ & 0.0052 & 0.079 & 0.048 & 0.75 & 0.055 \\
\hline RAD & Technetium-99 & $\mathrm{pCi} / \mathrm{g}$ & 17 & 5 & $29 \%$ & 0.035 & 0.25 & 0.27 & 0.73 & 0.17 \\
\hline
\end{tabular}

RAD - Radionuclide 
Table F-47

Summary Statistics for Sucker Fillet Collected from Segment 2 of the Columbia River

\begin{tabular}{|c|c|c|c|c|c|c|c|c|c|c|}
\hline $\begin{array}{l}\text { Constituent } \\
\text { Class }\end{array}$ & Constituent Name & Units & $\begin{array}{l}\text { Number } \\
\text { Analyzed }\end{array}$ & $\begin{array}{l}\text { Number } \\
\text { Detected }\end{array}$ & $\begin{array}{l}\text { Frequency of } \\
\text { Detection }\end{array}$ & $\begin{array}{l}\text { Minimum } \\
\text { Nondetect }\end{array}$ & $\begin{array}{l}\text { Maximum } \\
\text { Nondetect }\end{array}$ & $\begin{array}{l}\text { Minimum } \\
\text { Detect }\end{array}$ & $\begin{array}{l}\text { Maximum } \\
\text { Detect }\end{array}$ & $\begin{array}{c}\text { Average } \\
\text { Concentration }\end{array}$ \\
\hline$\overline{\mathrm{RAD}}$ & Actinium-228 & $\mathrm{pCi} / \mathrm{g}$ & 6 & 0 & $0 \%$ & $1.59 \mathrm{E}-04$ & 0.15 & $\overline{--}$ & $\overline{--}$ & 0.026 \\
\hline RAD & Antimony-124 & $\mathrm{pCi} / \mathrm{g}$ & 1 & 0 & $0 \%$ & 0.018 & 0.018 & -- & -- & 0.0089 \\
\hline RAD & Antimony-125 & $\mathrm{pCi} / \mathrm{g}$ & 1 & 0 & $0 \%$ & 0.028 & 0.028 & -- & -- & 0.014 \\
\hline RAD & Barium-133 & $\mathrm{pCi} / \mathrm{g}$ & 1 & 0 & $0 \%$ & 0.012 & 0.012 & -- & -- & 0.0062 \\
\hline RAD & Barium-140 & $\mathrm{pCi} / \mathrm{g}$ & 8 & 0 & $0 \%$ & $6.46 \mathrm{E}-04$ & 0.88 & -- & -- & 0.16 \\
\hline RAD & Beryllium-7 & $\mathrm{pCi} / \mathrm{g}$ & 11 & 0 & $0 \%$ & 4.13E-04 & 0.39 & -- & -- & 0.068 \\
\hline RAD & Cerium-139 & $\mathrm{pCi} / \mathrm{g}$ & 1 & 0 & $0 \%$ & 0.0091 & 0.0091 & -- & -- & 0.0046 \\
\hline RAD & Cerium-141 & $\mathrm{pCi} / \mathrm{g}$ & 8 & 0 & $0 \%$ & $8.12 \mathrm{E}-05$ & 0.093 & -- & -- & 0.019 \\
\hline RAD & Cerium-144 & $\mathrm{pCi} / \mathrm{g}$ & 8 & 0 & $0 \%$ & $2.10 \mathrm{E}-04$ & 0.18 & -- & -- & 0.038 \\
\hline RAD & Cesium-134 & $\mathrm{pCi} / \mathrm{g}$ & 17 & 0 & $0 \%$ & 3.96E-05 & 0.037 & -- & -- & 0.0044 \\
\hline RAD & Cesium-136 & $\mathrm{pCi} / \mathrm{g}$ & 1 & 0 & $0 \%$ & 0.097 & 0.097 & -- & -- & 0.048 \\
\hline RAD & Cesium-137 & $\mathrm{pCi} / \mathrm{g}$ & 26 & 6 & $23 \%$ & 4.04E-05 & 0.035 & 0.0072 & 0.036 & 0.0084 \\
\hline RAD & Chromium-51 & $\mathrm{pCi} / \mathrm{g}$ & 1 & 0 & $0 \%$ & 0.23 & 0.23 & -- & -- & 0.12 \\
\hline RAD & Cobalt-57 & $\mathrm{pCi} / \mathrm{g}$ & 8 & 0 & $0 \%$ & $2.71 \mathrm{E}-05$ & 0.025 & -- & -- & 0.0051 \\
\hline RAD & Cobalt-58 & $\mathrm{pCi} / \mathrm{g}$ & 16 & 0 & $0 \%$ & $4.75 \mathrm{E}-05$ & 0.039 & -- & -- & 0.0046 \\
\hline RAD & Cobalt-60 & $\mathrm{pCi} / \mathrm{g}$ & 18 & 2 & $11 \%$ & $3.87 \mathrm{E}-05$ & 0.028 & 0.028 & 0.059 & 0.0088 \\
\hline RAD & Europium-152 & $\mathrm{pCi} / \mathrm{g}$ & 8 & 0 & $0 \%$ & $1.00 \mathrm{E}-04$ & 0.089 & -- & -- & 0.017 \\
\hline RAD & Europium-154 & $\mathrm{pCi} / \mathrm{g}$ & 3 & 0 & $0 \%$ & 0.0026 & 0.053 & -- & -- & 0.012 \\
\hline RAD & Europium-155 & $\mathrm{pCi} / \mathrm{g}$ & 5 & 0 & $0 \%$ & 0.0022 & 0.038 & -- & -- & 0.0083 \\
\hline RAD & Iodine-131 & $\mathrm{pCi} / \mathrm{g}$ & 8 & 0 & $0 \%$ & $4.60 \mathrm{E}-04$ & 0.72 & -- & -- & 0.15 \\
\hline RAD & Iron-59 & $\mathrm{pCi} / \mathrm{g}$ & 12 & 0 & $0 \%$ & $9.64 \mathrm{E}-05$ & 0.12 & -- & -- & 0.017 \\
\hline RAD & Lanthanum-140 & $\mathrm{pCi} / \mathrm{g}$ & 8 & 0 & $0 \%$ & 1.99E-04 & 0.25 & -- & -- & 0.050 \\
\hline RAD & Manganese-54 & $\mathrm{pCi} / \mathrm{g}$ & 19 & 0 & $0 \%$ & $3.85 \mathrm{E}-05$ & 0.036 & -- & -- & 0.0040 \\
\hline RAD & Niobium-94 & $\mathrm{pCi} / \mathrm{g}$ & 1 & 0 & $0 \%$ & 0.010 & 0.010 & -- & -- & 0.0051 \\
\hline RAD & Niobium-95 & $\mathrm{pCi} / \mathrm{g}$ & 8 & 0 & $0 \%$ & $5.56 \mathrm{E}-05$ & 0.042 & -- & -- & 0.0092 \\
\hline RAD & Potassium-40 & $\mathrm{pCi} / \mathrm{g}$ & 27 & 27 & $100 \%$ & -- & -- & 0.0042 & 4.1 & 3.2 \\
\hline RAD & Radium-226 & $\mathrm{pCi} / \mathrm{g}$ & 10 & 0 & $0 \%$ & $6.42 \mathrm{E}-04$ & 0.65 & -- & -- & 0.12 \\
\hline RAD & Ruthenium-103 & $\mathrm{pCi} / \mathrm{g}$ & 8 & 0 & $0 \%$ & $6.24 \mathrm{E}-05$ & 0.052 & -- & -- & 0.011 \\
\hline RAD & Ruthenium-106 & $\mathrm{pCi} / \mathrm{g}$ & 8 & 0 & $0 \%$ & 3.35E-04 & 0.36 & -- & -- & 0.063 \\
\hline RAD & Selenium-75 & $\mathrm{pCi} / \mathrm{g}$ & 1 & 0 & $0 \%$ & 0.014 & 0.014 & -- & -- & 0.0072 \\
\hline RAD & Silver-110 metastable & $\mathrm{pCi} / \mathrm{g}$ & 1 & 0 & $0 \%$ & 0.011 & 0.011 & -- & -- & 0.0054 \\
\hline RAD & Strontium-85 & $\mathrm{pCi} / \mathrm{g}$ & 1 & 0 & $0 \%$ & 0.024 & 0.024 & -- & -- & 0.012 \\
\hline RAD & Strontium-90 & $\mathrm{pCi} / \mathrm{g}$ & 5 & 0 & $0 \%$ & $5.76 \mathrm{E}-04$ & 0.0020 & -- & -- & $5.72 \mathrm{E}-04$ \\
\hline RAD & Tellurium-129M & $\mathrm{pCi} / \mathrm{g}$ & 1 & 0 & $0 \%$ & 0.25 & 0.25 & -- & -- & 0.13 \\
\hline RAD & Thorium-228 & $\mathrm{pCi} / \mathrm{g}$ & 13 & 0 & $0 \%$ & $7.90 \mathrm{E}-04$ & 0.61 & -- & -- & 0.057 \\
\hline RAD & Thorium-232 & $\mathrm{pCi} / \mathrm{g}$ & 1 & 0 & $0 \%$ & 0.048 & 0.048 & -- & -- & 0.024 \\
\hline RAD & Tin-113 & $\mathrm{pCi} / \mathrm{g}$ & 1 & 0 & $0 \%$ & 0.016 & 0.016 & -- & -- & 0.0078 \\
\hline RAD & Uranium-235 & $\mathrm{pCi} / \mathrm{g}$ & 6 & 0 & $0 \%$ & $2.11 \mathrm{E}-04$ & 0.20 & -- & -- & 0.036 \\
\hline RAD & Uranium-238 & $\mathrm{pCi} / \mathrm{g}$ & 1 & 0 & $0 \%$ & 1.2 & 1.2 & -- & -- & 0.61 \\
\hline RAD & Yttrium & $\mathrm{pCi} / \mathrm{g}$ & 1 & 0 & $0 \%$ & 0.012 & 0.012 & -- & -- & 0.0060 \\
\hline RAD & Zinc-65 & $\mathrm{pCi} / \mathrm{g}$ & 15 & 1 & $7 \%$ & 8.87E-05 & 0.078 & 0.010 & 0.010 & 0.0099 \\
\hline RAD & Zirconium-95 & $\mathrm{pCi} / \mathrm{g}$ & 8 & 0 & $0 \%$ & $1.01 \mathrm{E}-04$ & 0.079 & -- & -- & 0.016 \\
\hline
\end{tabular}

RAD - Radionuclide 
Table F-48

Summary Statistics for Sucker Kidney Collected from Segment 2 of the Columbia River

\begin{tabular}{|c|c|c|c|c|c|c|c|c|c|c|}
\hline $\begin{array}{l}\text { Constituent } \\
\text { Class }\end{array}$ & Constituent Name & Units & $\begin{array}{l}\text { Number } \\
\text { Analyzed }\end{array}$ & $\begin{array}{l}\text { Number } \\
\text { Detected }\end{array}$ & $\begin{array}{c}\text { Frequency of } \\
\text { Detection }\end{array}$ & $\begin{array}{l}\text { Minimum } \\
\text { Nondetect }\end{array}$ & $\begin{array}{l}\text { Maximum } \\
\text { Nondetect }\end{array}$ & $\begin{array}{c}\text { Minimum } \\
\text { Detect }\end{array}$ & $\begin{array}{l}\text { Maximum } \\
\text { Detect }\end{array}$ & $\begin{array}{c}\text { Average } \\
\text { Concentration }\end{array}$ \\
\hline METAL & Antimony & $\mathrm{mg} / \mathrm{kg}$ & 15 & 6 & $40 \%$ & 0.015 & 0.015 & 0.021 & 0.12 & 0.027 \\
\hline METAL & Arsenic & $\mathrm{mg} / \mathrm{kg}$ & 15 & 15 & $100 \%$ & -- & -- & 0.17 & 1.6 & 0.75 \\
\hline METAL & Beryllium & $\mathrm{mg} / \mathrm{kg}$ & 15 & 0 & $0 \%$ & 0.15 & 0.15 & -- & -- & 0.075 \\
\hline METAL & Cadmium & $\mathrm{mg} / \mathrm{kg}$ & 15 & 15 & $100 \%$ & -- & -- & 0.060 & 69 & 28 \\
\hline METAL & Chromium & $\mathrm{mg} / \mathrm{kg}$ & 15 & 14 & $93 \%$ & 0.20 & 0.20 & 0.22 & 1.2 & 0.44 \\
\hline METAL & Copper & $\mathrm{mg} / \mathrm{kg}$ & 15 & 15 & $100 \%$ & -- & -- & 2.5 & 7.8 & 5.2 \\
\hline METAL & Lead & $\mathrm{mg} / \mathrm{kg}$ & 15 & 15 & $100 \%$ & -- & -- & 0.060 & 0.76 & 0.28 \\
\hline METAL & Mercury & $\mathrm{mg} / \mathrm{kg}$ & 15 & 15 & $100 \%$ & -- & -- & 0.035 & 0.76 & 0.23 \\
\hline METAL & Nickel & $\mathrm{mg} / \mathrm{kg}$ & 15 & 15 & $100 \%$ & -- & -- & 0.11 & 7.3 & 2.8 \\
\hline METAL & Selenium & $\mathrm{mg} / \mathrm{kg}$ & 15 & 15 & $100 \%$ & -- & -- & 1.9 & 7.2 & 4.3 \\
\hline METAL & Silver & $\mathrm{mg} / \mathrm{kg}$ & 15 & 1 & $7 \%$ & 0.045 & 0.045 & 0.055 & 0.055 & 0.025 \\
\hline METAL & Thallium & $\mathrm{mg} / \mathrm{kg}$ & 15 & 15 & $100 \%$ & -- & -- & 0.016 & 0.11 & 0.073 \\
\hline METAL & Zinc & $\mathrm{mg} / \mathrm{kg}$ & 15 & 15 & $100 \%$ & -- & -- & 41 & 378 & 136 \\
\hline
\end{tabular}


Table F-49

Summary Statistics for Sucker Liver Collected from Segment 2 of the Columbia River

\begin{tabular}{|c|c|c|c|c|c|c|c|c|c|c|}
\hline $\begin{array}{l}\text { Constituent } \\
\text { Class }\end{array}$ & Constituent Name & Units & $\begin{array}{l}\text { Number } \\
\text { Analyzed }\end{array}$ & $\begin{array}{l}\text { Number } \\
\text { Detected }\end{array}$ & $\begin{array}{c}\text { Frequency of } \\
\text { Detection }\end{array}$ & $\begin{array}{l}\text { Minimum } \\
\text { Nondetect }\end{array}$ & $\begin{array}{l}\text { Maximum } \\
\text { Nondetect }\end{array}$ & $\begin{array}{c}\text { Minimum } \\
\text { Detect }\end{array}$ & $\begin{array}{l}\text { Maximum } \\
\text { Detect }\end{array}$ & $\begin{array}{c}\text { Average } \\
\text { Concentration }\end{array}$ \\
\hline METAL & Antimony & $\mathrm{mg} / \mathrm{kg}$ & 15 & 13 & $87 \%$ & 0.015 & 0.015 & 0.015 & 0.15 & 0.039 \\
\hline METAL & Arsenic & $\mathrm{mg} / \mathrm{kg}$ & 15 & 15 & $100 \%$ & -- & -- & 0.16 & 1.5 & 0.56 \\
\hline METAL & Beryllium & $\mathrm{mg} / \mathrm{kg}$ & 15 & 0 & $0 \%$ & 0.15 & 0.15 & -- & -- & 0.075 \\
\hline METAL & Cadmium & $\mathrm{mg} / \mathrm{kg}$ & 15 & 14 & $93 \%$ & 0.020 & 0.020 & 0.027 & 8.1 & 1.00 \\
\hline METAL & Chromium & $\mathrm{mg} / \mathrm{kg}$ & 15 & 4 & $27 \%$ & 0.20 & 0.20 & 0.23 & 0.31 & 0.14 \\
\hline METAL & Copper & $\mathrm{mg} / \mathrm{kg}$ & 15 & 15 & $100 \%$ & -- & -- & 3.3 & 26 & 8.1 \\
\hline METAL & Lead & $\mathrm{mg} / \mathrm{kg}$ & 15 & 6 & $40 \%$ & 0.036 & 0.036 & 0.038 & 0.41 & 0.063 \\
\hline METAL & Mercury & $\mathrm{mg} / \mathrm{kg}$ & 15 & 15 & $100 \%$ & -- & -- & 0.11 & 1.2 & 0.37 \\
\hline METAL & Nickel & $\mathrm{mg} / \mathrm{kg}$ & 15 & 15 & $100 \%$ & -- & -- & 0.028 & 0.67 & 0.26 \\
\hline METAL & Selenium & $\mathrm{mg} / \mathrm{kg}$ & 15 & 14 & $93 \%$ & 1.0 & 1.0 & 1.0 & 4.4 & 1.6 \\
\hline METAL & Silver & $\mathrm{mg} / \mathrm{kg}$ & 15 & 1 & $7 \%$ & 0.045 & 0.045 & 0.096 & 0.096 & 0.027 \\
\hline METAL & Thallium & $\mathrm{mg} / \mathrm{kg}$ & 15 & 15 & $100 \%$ & -- & -- & 0.040 & 0.13 & 0.086 \\
\hline METAL & Zinc & $\mathrm{mg} / \mathrm{kg}$ & 15 & 15 & $100 \%$ & -- & -- & 17 & 72 & 30 \\
\hline
\end{tabular}


Table F-50

Summary Statistics for Whole Body Sucker Collected from Segment 2 of the Columbia River

\begin{tabular}{|c|c|c|c|c|c|c|c|c|c|c|}
\hline $\begin{array}{c}\text { Constituent } \\
\text { Class }\end{array}$ & Constituent Name & Units & $\begin{array}{c}\text { Number } \\
\text { Analyzed }\end{array}$ & $\begin{array}{l}\text { Number } \\
\text { Detected }\end{array}$ & $\begin{array}{c}\text { Frequency of } \\
\text { Detection }\end{array}$ & $\begin{array}{l}\text { Minimum } \\
\text { Nondetect }\end{array}$ & $\begin{array}{l}\text { Maximum } \\
\text { Nondetect }\end{array}$ & $\begin{array}{l}\text { Minimum } \\
\text { Detect }\end{array}$ & $\begin{array}{l}\text { Maximum } \\
\text { Detect }\end{array}$ & $\begin{array}{c}\text { Average } \\
\text { Concentration }\end{array}$ \\
\hline$\overline{\text { METAL }}$ & Aluminum & $\mathrm{mg} / \mathrm{kg}$ & 5 & 5 & $100 \%$ & -- & $\overline{--}$ & 232 & 655 & 393 \\
\hline METAL & Antimony & $\mathrm{mg} / \mathrm{kg}$ & 5 & 1 & $20 \%$ & 0.010 & 0.019 & 0.022 & 0.022 & 0.0093 \\
\hline METAL & Arsenic & $\mathrm{mg} / \mathrm{kg}$ & 5 & 2 & $40 \%$ & 0.097 & 0.10 & 0.14 & 0.48 & 0.15 \\
\hline METAL & Barium & $\mathrm{mg} / \mathrm{kg}$ & 7 & 7 & $100 \%$ & -- & -- & 1.2 & 22 & 12 \\
\hline METAL & Beryllium & $\mathrm{mg} / \mathrm{kg}$ & 5 & 2 & $40 \%$ & 0.020 & 0.020 & 0.038 & 0.054 & 0.024 \\
\hline METAL & Cadmium & $\mathrm{mg} / \mathrm{kg}$ & 7 & 7 & $100 \%$ & -- & -- & 0.015 & 0.14 & 0.099 \\
\hline METAL & Chromium & $\mathrm{mg} / \mathrm{kg}$ & 7 & 7 & $100 \%$ & -- & -- & 0.14 & 4.3 & 1.7 \\
\hline METAL & Copper & $\mathrm{mg} / \mathrm{kg}$ & 5 & 5 & $100 \%$ & -- & -- & 0.27 & 3.3 & 2.0 \\
\hline METAL & Lead & $\mathrm{mg} / \mathrm{kg}$ & 7 & 7 & $100 \%$ & -- & -- & 0.045 & 1.0 & 0.41 \\
\hline METAL & Manganese & $\mathrm{mg} / \mathrm{kg}$ & 7 & 7 & $100 \%$ & -- & -- & 3.7 & 63 & 35 \\
\hline METAL & Mercury & $\mathrm{mg} / \mathrm{kg}$ & 7 & 4 & $57 \%$ & 0.050 & 0.050 & 0.031 & 0.12 & 0.045 \\
\hline METAL & Nickel & $\mathrm{mg} / \mathrm{kg}$ & 7 & 7 & $100 \%$ & -- & -- & 0.12 & 1.5 & 1.0 \\
\hline METAL & Selenium & $\mathrm{mg} / \mathrm{kg}$ & 7 & 6 & $86 \%$ & 0.20 & 0.20 & 0.35 & 2.5 & 1.4 \\
\hline METAL & Silver & $\mathrm{mg} / \mathrm{kg}$ & 7 & 2 & $29 \%$ & 0.010 & 0.045 & 0.013 & 0.018 & 0.015 \\
\hline METAL & Thallium & $\mathrm{mg} / \mathrm{kg}$ & 5 & 5 & $100 \%$ & -- & -- & 0.0081 & 0.15 & 0.058 \\
\hline METAL & Thorium & $\mathrm{mg} / \mathrm{kg}$ & 7 & 6 & $86 \%$ & 0.020 & 0.020 & 0.022 & 1.8 & 0.32 \\
\hline METAL & Uranium & $\mathrm{mg} / \mathrm{kg}$ & 7 & 5 & $71 \%$ & 0.010 & 0.010 & 0.032 & 0.18 & 0.051 \\
\hline METAL & Zinc & $\mathrm{mg} / \mathrm{kg}$ & 5 & 5 & $100 \%$ & -- & -- & 8.6 & 92 & 53 \\
\hline RAD & Cesium-134 & $\mathrm{pCi} / \mathrm{g}$ & 1 & 0 & $0 \%$ & 0.0028 & 0.0028 & -- & -- & 0.0014 \\
\hline RAD & Cesium-137 & $\mathrm{pCi} / \mathrm{g}$ & 5 & 3 & $60 \%$ & 0.0040 & 0.0060 & 0.0068 & 0.0080 & 0.0056 \\
\hline RAD & Cobalt-58 & $\mathrm{pCi} / \mathrm{g}$ & 2 & 1 & $50 \%$ & 0.0030 & 0.0030 & 0.0070 & 0.0070 & 0.0043 \\
\hline RAD & Cobalt- 60 & $\mathrm{pCi} / \mathrm{g}$ & 4 & 0 & $0 \%$ & $6.00 \mathrm{E}-04$ & 0.0050 & -- & -- & 0.0014 \\
\hline RAD & Iron-59 & $\mathrm{pCi} / \mathrm{g}$ & 3 & 1 & $33 \%$ & 0.0060 & 0.012 & 0.036 & 0.036 & 0.015 \\
\hline RAD & Manganese-54 & $\mathrm{pCi} / \mathrm{g}$ & 3 & 0 & $0 \%$ & $2.00 \mathrm{E}-04$ & 0.0010 & -- & -- & $2.67 \mathrm{E}-04$ \\
\hline RAD & Potassium-40 & $\mathrm{pCi} / \mathrm{g}$ & 5 & 5 & $100 \%$ & -- & -- & 3.0 & 3.6 & 3.3 \\
\hline RAD & Strontium-90 & $\mathrm{pCi} / \mathrm{g}$ & 6 & 6 & $100 \%$ & -- & -- & 0.032 & 0.053 & 0.042 \\
\hline RAD & Uranium-234 & $\mathrm{pCi} / \mathrm{g}$ & 6 & 6 & $100 \%$ & -- & -- & 0.012 & 0.080 & 0.031 \\
\hline RAD & Uranium-235 & $\mathrm{pCi} / \mathrm{g}$ & 5 & 4 & $80 \%$ & $3.91 \mathrm{E}-04$ & $3.91 \mathrm{E}-04$ & 0.0012 & 0.0036 & 0.0018 \\
\hline RAD & Uranium-238 & $\mathrm{pCi} / \mathrm{g}$ & 6 & 6 & $100 \%$ & -- & -- & 0.0085 & 0.056 & 0.023 \\
\hline
\end{tabular}

RAD - Radionuclide 
Table F-51

Summary Statistics for Crayfish Hepatopancreas Collected from Segment 2 of the Columbia River

\begin{tabular}{|c|c|c|c|c|c|c|c|c|c|c|}
\hline $\begin{array}{l}\text { Constituent } \\
\text { Class }\end{array}$ & Constituent Name & Units & $\begin{array}{l}\text { Number } \\
\text { Analyzed }\end{array}$ & $\begin{array}{l}\text { Number } \\
\text { Detected }\end{array}$ & $\begin{array}{l}\text { Frequency of } \\
\text { Detection }\end{array}$ & $\begin{array}{l}\text { Minimum } \\
\text { Nondetect }\end{array}$ & $\begin{array}{l}\text { Maximum } \\
\text { Nondetect }\end{array}$ & $\begin{array}{l}\text { Minimum } \\
\text { Detect }\end{array}$ & $\begin{array}{l}\text { Maximum } \\
\text { Detect }\end{array}$ & $\begin{array}{c}\text { Average } \\
\text { Concentration }\end{array}$ \\
\hline$\overline{\text { METAL }}$ & Aluminum & $\mathrm{mg} / \mathrm{kg}$ & 8 & 6 & $75 \%$ & 5.0 & 5.0 & 6.9 & 26 & 10 \\
\hline METAL & Antimony & $\mathrm{mg} / \mathrm{kg}$ & 8 & 8 & $100 \%$ & -- & -- & 0.041 & 0.39 & 0.20 \\
\hline METAL & Arsenic & $\mathrm{mg} / \mathrm{kg}$ & 8 & 8 & $100 \%$ & -- & -- & 2.5 & 18 & 8.9 \\
\hline METAL & Barium & $\mathrm{mg} / \mathrm{kg}$ & 8 & 8 & $100 \%$ & -- & -- & 2.1 & 11 & 7.1 \\
\hline METAL & Beryllium & $\mathrm{mg} / \mathrm{kg}$ & 8 & 6 & $75 \%$ & 0.020 & 0.020 & 0.12 & 0.35 & 0.15 \\
\hline METAL & Cadmium & $\mathrm{mg} / \mathrm{kg}$ & 8 & 8 & $100 \%$ & -- & -- & 6.3 & 35 & 17 \\
\hline METAL & Chromium & $\mathrm{mg} / \mathrm{kg}$ & 8 & 8 & $100 \%$ & -- & -- & 0.53 & 3.1 & 1.1 \\
\hline METAL & Copper & $\mathrm{mg} / \mathrm{kg}$ & 8 & 8 & $100 \%$ & -- & -- & 514 & 3,750 & 1,550 \\
\hline METAL & Lead & $\mathrm{mg} / \mathrm{kg}$ & 8 & 8 & $100 \%$ & -- & -- & 0.094 & 0.67 & 0.36 \\
\hline METAL & Manganese & $\mathrm{mg} / \mathrm{kg}$ & 8 & 8 & $100 \%$ & -- & -- & 114 & 477 & 304 \\
\hline METAL & Mercury & $\mathrm{mg} / \mathrm{kg}$ & 8 & 8 & $100 \%$ & -- & -- & 0.094 & 0.36 & 0.16 \\
\hline METAL & Nickel & $\mathrm{mg} / \mathrm{kg}$ & 8 & 8 & $100 \%$ & -- & -- & 0.75 & 12 & 6.0 \\
\hline METAL & Selenium & $\mathrm{mg} / \mathrm{kg}$ & 8 & 8 & $100 \%$ & -- & -- & 1.7 & 12 & 6.3 \\
\hline METAL & Silver & $\mathrm{mg} / \mathrm{kg}$ & 8 & 8 & $100 \%$ & -- & -- & 2.7 & 14 & 8.5 \\
\hline METAL & Thallium & $\mathrm{mg} / \mathrm{kg}$ & 8 & 8 & $100 \%$ & -- & -- & 0.10 & 0.69 & 0.35 \\
\hline METAL & Thorium & $\mathrm{mg} / \mathrm{kg}$ & 8 & 3 & $38 \%$ & 0.020 & 0.021 & 0.023 & 0.053 & 0.021 \\
\hline METAL & Uranium & $\mathrm{mg} / \mathrm{kg}$ & 8 & 8 & $100 \%$ & -- & -- & 0.089 & 1.4 & 0.63 \\
\hline METAL & Zinc & $\mathrm{mg} / \mathrm{kg}$ & 8 & 8 & $100 \%$ & -- & -- & 113 & 680 & 339 \\
\hline
\end{tabular}


Table F-52

Summary Statistics for Whole Body Crayfish Collected from Segment 2 of the Columbia River

\begin{tabular}{|c|c|c|c|c|c|c|c|c|c|c|}
\hline $\begin{array}{l}\text { Constituent } \\
\text { Class }\end{array}$ & Constituent Name & Units & $\begin{array}{l}\text { Number } \\
\text { Analyzed }\end{array}$ & $\begin{array}{l}\text { Number } \\
\text { Detected }\end{array}$ & $\begin{array}{c}\text { Frequency of } \\
\text { Detection }\end{array}$ & $\begin{array}{l}\text { Minimum } \\
\text { Nondetect }\end{array}$ & $\begin{array}{l}\text { Maximum } \\
\text { Nondetect }\end{array}$ & $\begin{array}{l}\text { Minimum } \\
\text { Detect }\end{array}$ & $\begin{array}{l}\text { Maximum } \\
\text { Detect }\end{array}$ & $\begin{array}{c}\text { Average } \\
\text { Concentration }\end{array}$ \\
\hline METAL & Aluminum & $\mathrm{mg} / \mathrm{kg}$ & 2 & 2 & $100 \%$ & $\overline{--}$ & -- & 75 & 101 & 88 \\
\hline METAL & Antimony & $\mathrm{mg} / \mathrm{kg}$ & 2 & 2 & $100 \%$ & -- & -- & 0.016 & 0.023 & 0.019 \\
\hline METAL & Arsenic & $\mathrm{mg} / \mathrm{kg}$ & 2 & 0 & $0 \%$ & 0.10 & 0.10 & -- & -- & 0.050 \\
\hline METAL & Barium & $\mathrm{mg} / \mathrm{kg}$ & 2 & 2 & $100 \%$ & -- & -- & 255 & 434 & 345 \\
\hline METAL & Beryllium & $\mathrm{mg} / \mathrm{kg}$ & 2 & 0 & $0 \%$ & 0.020 & 0.020 & -- & -- & 0.010 \\
\hline METAL & Cadmium & $\mathrm{mg} / \mathrm{kg}$ & 2 & 2 & $100 \%$ & -- & -- & 0.12 & 0.23 & 0.18 \\
\hline METAL & Chromium & $\mathrm{mg} / \mathrm{kg}$ & 2 & 2 & $100 \%$ & -- & -- & 0.55 & 0.61 & 0.58 \\
\hline METAL & Copper & $\mathrm{mg} / \mathrm{kg}$ & 2 & 2 & $100 \%$ & -- & -- & 58 & 86 & 72 \\
\hline METAL & Lead & $\mathrm{mg} / \mathrm{kg}$ & 2 & 2 & $100 \%$ & -- & -- & 0.19 & 0.27 & 0.23 \\
\hline METAL & Manganese & $\mathrm{mg} / \mathrm{kg}$ & 2 & 2 & $100 \%$ & -- & -- & 125 & 146 & 136 \\
\hline METAL & Mercury & $\mathrm{mg} / \mathrm{kg}$ & 2 & 0 & $0 \%$ & 0.050 & 0.050 & -- & -- & 0.025 \\
\hline METAL & Nickel & $\mathrm{mg} / \mathrm{kg}$ & 2 & 2 & $100 \%$ & -- & -- & 4.5 & 4.8 & 4.6 \\
\hline METAL & Selenium & $\mathrm{mg} / \mathrm{kg}$ & 2 & 2 & $100 \%$ & -- & -- & 0.49 & 0.81 & 0.65 \\
\hline METAL & Silver & $\mathrm{mg} / \mathrm{kg}$ & 2 & 2 & $100 \%$ & -- & -- & 0.067 & 0.100 & 0.083 \\
\hline METAL & Thallium & $\mathrm{mg} / \mathrm{kg}$ & 2 & 2 & $100 \%$ & -- & -- & 0.075 & 0.080 & 0.077 \\
\hline METAL & Thorium & $\mathrm{mg} / \mathrm{kg}$ & 2 & 1 & $50 \%$ & 0.020 & 0.020 & 0.61 & 0.61 & 0.31 \\
\hline METAL & Uranium & $\mathrm{mg} / \mathrm{kg}$ & 2 & 2 & $100 \%$ & -- & -- & 0.052 & 0.072 & 0.062 \\
\hline METAL & Zinc & $\mathrm{mg} / \mathrm{kg}$ & 2 & 2 & $100 \%$ & -- & -- & 73 & 89 & 81 \\
\hline RAD & Antimony-125 & $\mathrm{pCi} / \mathrm{g}$ & 2 & 0 & $0 \%$ & 0.21 & 0.24 & -- & -- & 0.11 \\
\hline RAD & Beryllium-7 & $\mathrm{pCi} / \mathrm{g}$ & 3 & 0 & $0 \%$ & 0.47 & 1.1 & -- & -- & 0.41 \\
\hline RAD & Cesium-134 & $\mathrm{pCi} / \mathrm{g}$ & 4 & 0 & $0 \%$ & 0.017 & 0.079 & -- & -- & 0.020 \\
\hline RAD & Cesium-137 & $\mathrm{pCi} / \mathrm{g}$ & 4 & 0 & $0 \%$ & 0.0082 & 0.080 & -- & -- & 0.021 \\
\hline RAD & Cobalt-60 & $\mathrm{pCi} / \mathrm{g}$ & 4 & 0 & $0 \%$ & 0.021 & 0.11 & -- & -- & 0.030 \\
\hline RAD & Europium-154 & $\mathrm{pCi} / \mathrm{g}$ & 4 & 0 & $0 \%$ & 0.021 & 0.051 & -- & -- & 0.020 \\
\hline RAD & Europium-155 & $\mathrm{pCi} / \mathrm{g}$ & 2 & 0 & $0 \%$ & 0.070 & 0.086 & -- & -- & 0.039 \\
\hline RAD & Potassium-40 & $\mathrm{pCi} / \mathrm{g}$ & 5 & 2 & $40 \%$ & 0.50 & 3.3 & 2.7 & 7.1 & 2.6 \\
\hline RAD & Ruthenium-106 & $\mathrm{pCi} / \mathrm{g}$ & 3 & 0 & $0 \%$ & 0.051 & 2.0 & -- & -- & 0.44 \\
\hline RAD & Strontium-90 & $\mathrm{pCi} / \mathrm{g}$ & 6 & 6 & $100 \%$ & -- & -- & 0.079 & 0.27 & 0.12 \\
\hline RAD & Technetium-99 & $\mathrm{pCi} / \mathrm{g}$ & 6 & 0 & $0 \%$ & 0.031 & 0.13 & -- & -- & 0.037 \\
\hline
\end{tabular}

RAD - Radionuclide 
Table F-53

Summary Statistics for Whole Body Juvenile Chinook Salmon Collected from Segment 2 of the Columbia River

\begin{tabular}{|c|c|c|c|c|c|c|c|c|c|c|}
\hline $\begin{array}{l}\text { Constituent } \\
\text { Class }\end{array}$ & Constituent Name & Units & $\begin{array}{l}\text { Number } \\
\text { Analyzed }\end{array}$ & $\begin{array}{l}\text { Number } \\
\text { Detected }\end{array}$ & $\begin{array}{c}\text { Frequency of } \\
\text { Detection }\end{array}$ & $\begin{array}{l}\text { Minimum } \\
\text { Nondetect }\end{array}$ & $\begin{array}{l}\text { Maximum } \\
\text { Nondetect }\end{array}$ & $\begin{array}{l}\text { Minimum } \\
\text { Detect }\end{array}$ & $\begin{array}{l}\text { Maximum } \\
\text { Detect }\end{array}$ & $\begin{array}{c}\text { Average } \\
\text { Concentration }\end{array}$ \\
\hline CONV & Percent Lipids & $\%$ & 104 & 104 & $100 \%$ & $\overline{--}$ & $\overline{--}$ & 8.2 & 19 & 15 \\
\hline METAL & Aluminum & $\mathrm{mg} / \mathrm{kg}$ & 20 & 20 & $100 \%$ & -- & -- & 0.28 & 1.1 & 0.58 \\
\hline METAL & Antimony & $\mathrm{mg} / \mathrm{kg}$ & 20 & 20 & $100 \%$ & -- & -- & 0.039 & 3.0 & 0.46 \\
\hline METAL & Arsenic & $\mathrm{mg} / \mathrm{kg}$ & 20 & 12 & $60 \%$ & 0.0019 & 0.0036 & 0.0056 & 0.028 & 0.0070 \\
\hline METAL & Beryllium & $\mathrm{mg} / \mathrm{kg}$ & 20 & 0 & $0 \%$ & $6.44 \mathrm{E}-04$ & 0.0013 & -- & -- & 5.03E-04 \\
\hline METAL & Cadmium & $\mathrm{mg} / \mathrm{kg}$ & 20 & 20 & $100 \%$ & -- & -- & 0.0011 & 0.0067 & 0.0034 \\
\hline METAL & Chromium & $\mathrm{mg} / \mathrm{kg}$ & 104 & 104 & $100 \%$ & -- & -- & 0.014 & 0.064 & 0.043 \\
\hline METAL & Copper & $\mathrm{mg} / \mathrm{kg}$ & 20 & 20 & $100 \%$ & -- & -- & 0.031 & 0.083 & 0.060 \\
\hline METAL & Lead & $\mathrm{mg} / \mathrm{kg}$ & 20 & 20 & $100 \%$ & -- & -- & 0.0012 & 0.0068 & 0.0023 \\
\hline METAL & Manganese & $\mathrm{mg} / \mathrm{kg}$ & 20 & 20 & $100 \%$ & -- & -- & 0.027 & 0.14 & 0.072 \\
\hline METAL & Nickel & $\mathrm{mg} / \mathrm{kg}$ & 20 & 20 & $100 \%$ & -- & -- & 0.0023 & 0.020 & 0.0083 \\
\hline METAL & Selenium & $\mathrm{mg} / \mathrm{kg}$ & 20 & 20 & $100 \%$ & -- & -- & 0.020 & 0.087 & 0.047 \\
\hline METAL & Silver & $\mathrm{mg} / \mathrm{kg}$ & 20 & 7 & $35 \%$ & 4.00E-04 & $6.12 E-04$ & 3.74E-04 & 7.18E-04 & 3.67E-04 \\
\hline METAL & Thallium & $\mathrm{mg} / \mathrm{kg}$ & 20 & 20 & $100 \%$ & -- & -- & 0.0013 & 0.0048 & 0.0029 \\
\hline METAL & Thorium & $\mathrm{mg} / \mathrm{kg}$ & 20 & 8 & $40 \%$ & 2.18E-04 & 3.57E-04 & 2.88E-04 & 7.35E-04 & 2.91E-04 \\
\hline METAL & Uranium & $\mathrm{mg} / \mathrm{kg}$ & 104 & 0 & $0 \%$ & 3.53E-04 & 9.06E-04 & -- & -- & 3.38E-04 \\
\hline METAL & Zinc & $\mathrm{mg} / \mathrm{kg}$ & 20 & 20 & $100 \%$ & -- & -- & 0.55 & 1.8 & 1.3 \\
\hline
\end{tabular}


Table F-54

Summary Statistics for Mountain Whitefish Fillet Skin On Collected from Segment 2 of the Columbia River

\begin{tabular}{|c|c|c|c|c|c|c|c|c|c|c|}
\hline $\begin{array}{l}\text { Constituent } \\
\text { Class }\end{array}$ & Constituent Name & Units & $\begin{array}{l}\text { Number } \\
\text { Analyzed }\end{array}$ & $\begin{array}{l}\text { Number } \\
\text { Detected }\end{array}$ & $\begin{array}{l}\text { Frequency of } \\
\text { Detection }\end{array}$ & $\begin{array}{l}\text { Minimum } \\
\text { Nondetect }\end{array}$ & $\begin{array}{l}\text { Maximum } \\
\text { Nondetect }\end{array}$ & $\begin{array}{c}\text { Minimum } \\
\text { Detect }\end{array}$ & $\begin{array}{l}\text { Maximum } \\
\text { Detect }\end{array}$ & $\begin{array}{c}\text { Average } \\
\text { Concentration }\end{array}$ \\
\hline$\overline{D I O X I}$ & $1,2,3,4,6,7,8$-Heptachlorodibenzodioxin & $\mathrm{mg} / \mathrm{kg}$ & 3 & 2 & $67 \%$ & $1.00 \mathrm{E}-08$ & $1.00 \mathrm{E}-08$ & $1.50 \mathrm{E}-07$ & $1.50 \mathrm{E}-07$ & $1.02 \mathrm{E}-07$ \\
\hline DIOXI & $1,2,3,4,6,7,8$-Heptachlorodibenzofuran & $\mathrm{mg} / \mathrm{kg}$ & 3 & 0 & $0 \%$ & 2.00E-08 & 3.00E-08 & -- & -- & 1.17E-08 \\
\hline DIOXI & 1,2,3,4,7,8,9-Heptachlorodibenzofuran & $\mathrm{mg} / \mathrm{kg}$ & 3 & 0 & $0 \%$ & 2.00E-08 & $3.00 \mathrm{E}-08$ & -- & -- & 1.17E-08 \\
\hline DIOXI & $1,2,3,4,7,8$-Hexachlorodibenzofuran & $\mathrm{mg} / \mathrm{kg}$ & 3 & 3 & $100 \%$ & -- & -- & $3.00 \mathrm{E}-08$ & 4.00E-08 & 3.67E-08 \\
\hline DIOXI & $1,2,3,4,7,8$-Hexachlorodibenzo-p-dioxin & $\mathrm{mg} / \mathrm{kg}$ & 3 & 3 & $100 \%$ & -- & -- & $3.00 \mathrm{E}-08$ & 4.00E-08 & 3.33E-08 \\
\hline DIOXI & 1,2,3,6,7,8-Hexachlorodibenzofuran & $\mathrm{mg} / \mathrm{kg}$ & 3 & 2 & $67 \%$ & $1.00 \mathrm{E}-08$ & $1.00 \mathrm{E}-08$ & $2.00 \mathrm{E}-08$ & $3.00 \mathrm{E}-08$ & 1.83E-08 \\
\hline DIOXI & $1,2,3,6,7,8$-Hexachlorodibenzo-p-dioxin & $\mathrm{mg} / \mathrm{kg}$ & 3 & 3 & $100 \%$ & -- & -- & $1.20 \mathrm{E}-07$ & $1.30 \mathrm{E}-07$ & 1.23E-07 \\
\hline DIOXI & 1,2,3,7,8,9-Hexachlorodibenzofuran & $\mathrm{mg} / \mathrm{kg}$ & 3 & 1 & $33 \%$ & $1.00 \mathrm{E}-08$ & $1.00 \mathrm{E}-08$ & 2.00E-08 & $2.00 \mathrm{E}-08$ & $1.00 \mathrm{E}-08$ \\
\hline DIOXI & $1,2,3,7,8,9-$ Hexachlorodibenzo-p-dioxin & $\mathrm{mg} / \mathrm{kg}$ & 3 & 2 & $67 \%$ & $1.00 \mathrm{E}-08$ & $1.00 \mathrm{E}-08$ & $6.00 \mathrm{E}-08$ & $6.00 \mathrm{E}-08$ & 4.17E-08 \\
\hline DIOXI & 1,2,3,7,8-Pentachlorodibenzofuran & $\mathrm{mg} / \mathrm{kg}$ & 3 & 3 & $100 \%$ & -- & -- & $1.20 \mathrm{E}-07$ & 1.40E-07 & $1.30 \mathrm{E}-07$ \\
\hline DIOXI & 1,2,3,7,8-Pentachlorodibenzo-p-dioxin & $\mathrm{mg} / \mathrm{kg}$ & 3 & 3 & $100 \%$ & -- & -- & $1.70 \mathrm{E}-07$ & $1.80 \mathrm{E}-07$ & $1.73 \mathrm{E}-07$ \\
\hline DIOXI & 2,3,4,6,7,8-Hexachlorodibenzofuran & $\mathrm{mg} / \mathrm{kg}$ & 3 & 0 & $0 \%$ & $1.00 \mathrm{E}-08$ & $1.00 \mathrm{E}-08$ & -- & -- & $5.00 \mathrm{E}-09$ \\
\hline DIOXI & 2,3,4,7,8-Pentachlorodibenzofuran & $\mathrm{mg} / \mathrm{kg}$ & 3 & 3 & $100 \%$ & -- & -- & $2.60 \mathrm{E}-07$ & 3.50E-07 & 2.93E-07 \\
\hline DIOXI & 2,3,7,8-Tetrachlorodibenzofuran & $\mathrm{mg} / \mathrm{kg}$ & 3 & 3 & $100 \%$ & -- & -- & 1.31E-05 & 1.38E-05 & 1.34E-05 \\
\hline DIOXI & 2,3,7,8-Tetrachlorodibenzo-p-dioxin & $\mathrm{mg} / \mathrm{kg}$ & 3 & 3 & $100 \%$ & -- & -- & $2.00 \mathrm{E}-07$ & 2.10E-07 & 2.07E-07 \\
\hline DIOXI & Octachlorodibenzofuran & $\mathrm{mg} / \mathrm{kg}$ & 3 & 2 & $67 \%$ & $1.00 \mathrm{E}-08$ & $1.00 \mathrm{E}-08$ & 4.00E-08 & $5.00 \mathrm{E}-08$ & 3.17E-08 \\
\hline DIOXI & Octachlorodibenzo-p-dioxin & $\mathrm{mg} / \mathrm{kg}$ & 3 & 3 & $100 \%$ & -- & -- & $1.90 \mathrm{E}-07$ & $2.80 \mathrm{E}-07$ & $2.27 \mathrm{E}-07$ \\
\hline METAL & Aluminum & $\mathrm{mg} / \mathrm{kg}$ & 3 & 2 & $67 \%$ & 1.0 & 1.0 & 1.3 & 2.2 & 1.3 \\
\hline METAL & Antimony & $\mathrm{mg} / \mathrm{kg}$ & 3 & 0 & $0 \%$ & 0.050 & 0.050 & -- & -- & 0.025 \\
\hline METAL & Arsenic & $\mathrm{mg} / \mathrm{kg}$ & 3 & 3 & $100 \%$ & -- & -- & 0.12 & 0.14 & 0.13 \\
\hline METAL & Barium & $\mathrm{mg} / \mathrm{kg}$ & 3 & 3 & $100 \%$ & -- & -- & 0.26 & 0.38 & 0.33 \\
\hline METAL & Beryllium & $\mathrm{mg} / \mathrm{kg}$ & 3 & 0 & $0 \%$ & 0.0040 & 0.0040 & -- & -- & 0.0020 \\
\hline METAL & Cadmium & $\mathrm{mg} / \mathrm{kg}$ & 3 & 3 & $100 \%$ & -- & -- & 0.0087 & 0.010 & 0.0094 \\
\hline METAL & Chromium & $\mathrm{mg} / \mathrm{kg}$ & 3 & 3 & $100 \%$ & -- & -- & 0.21 & 0.45 & 0.32 \\
\hline METAL & Cobalt & $\mathrm{mg} / \mathrm{kg}$ & 3 & 3 & $100 \%$ & -- & -- & 0.040 & 0.060 & 0.050 \\
\hline METAL & Copper & $\mathrm{mg} / \mathrm{kg}$ & 3 & 3 & $100 \%$ & -- & -- & 0.50 & 0.56 & 0.52 \\
\hline METAL & Lead & $\mathrm{mg} / \mathrm{kg}$ & 3 & 3 & $100 \%$ & -- & -- & 0.010 & 0.020 & 0.017 \\
\hline METAL & Manganese & $\mathrm{mg} / \mathrm{kg}$ & 3 & 3 & $100 \%$ & -- & -- & 0.68 & 0.74 & 0.71 \\
\hline METAL & Mercury & $\mathrm{mg} / \mathrm{kg}$ & 3 & 0 & $0 \%$ & 0.10 & 0.10 & -- & -- & 0.050 \\
\hline METAL & Nickel & $\mathrm{mg} / \mathrm{kg}$ & 3 & 3 & $100 \%$ & -- & -- & 0.040 & 0.12 & 0.093 \\
\hline METAL & Selenium & $\mathrm{mg} / \mathrm{kg}$ & 3 & 3 & $100 \%$ & -- & -- & 0.44 & 0.50 & 0.47 \\
\hline METAL & Silver & $\mathrm{mg} / \mathrm{kg}$ & 3 & 0 & $0 \%$ & 0.10 & 0.10 & -- & -- & 0.050 \\
\hline METAL & Thallium & $\mathrm{mg} / \mathrm{kg}$ & 3 & 0 & $0 \%$ & 0.050 & 0.050 & -- & -- & 0.025 \\
\hline METAL & Vanadium & $\mathrm{mg} / \mathrm{kg}$ & 3 & 2 & $67 \%$ & 0.010 & 0.010 & 0.010 & 0.020 & 0.012 \\
\hline METAL & Zinc & $\mathrm{mg} / \mathrm{kg}$ & 3 & 3 & $100 \%$ & -- & -- & 13 & 15 & 14 \\
\hline PCB & 2,2',3,3',4,4',5-Heptachloro-1,1'-biphenyl & $\mathrm{mg} / \mathrm{kg}$ & 3 & 3 & $100 \%$ & -- & -- & 0.0043 & 0.0098 & 0.0062 \\
\hline PCB & $2,2^{\prime}, 3,4,4^{\prime}, 5,5^{\prime}$-Heptachlorobiphenyl & $\mathrm{mg} / \mathrm{kg}$ & 3 & 3 & $100 \%$ & -- & -- & 0.011 & 0.020 & 0.014 \\
\hline PCB & $2,3,3^{\prime}, 4,4^{\prime}, 5^{\prime}-$ Hexachlorobiphenyl & $\mathrm{mg} / \mathrm{kg}$ & 3 & 3 & $100 \%$ & -- & -- & $6.45 \mathrm{E}-04$ & 0.0030 & 0.0014 \\
\hline PCB & 2,3,3',4,4',5-Hexachlorobiphenyl (BZ 156) & $\mathrm{mg} / \mathrm{kg}$ & 3 & 3 & $100 \%$ & -- & -- & 0.0035 & 0.015 & 0.0074 \\
\hline PCB & 2,3,3',4,4'-Pentachlorobiphenyl & $\mathrm{mg} / \mathrm{kg}$ & 3 & 3 & $100 \%$ & -- & -- & 0.0085 & 0.045 & 0.021 \\
\hline PCB & 2,3',4,4',5,5'-Hexachlorobiphenyl (BZ 167) & $\mathrm{mg} / \mathrm{kg}$ & 3 & 3 & $100 \%$ & -- & -- & $9.81 \mathrm{E}-04$ & 0.0041 & 0.0020 \\
\hline PCB & 2',3,4,4',5-Pentachloro-1,1'-biphenyl (BZ 123) & $\mathrm{mg} / \mathrm{kg}$ & 3 & 3 & $100 \%$ & -- & -- & 2.89E-04 & 0.0015 & 7.20E-04 \\
\hline PCB & 2,3,4,4',5-Pentachlorobiphenyl (BZ 114) & $\mathrm{mg} / \mathrm{kg}$ & 3 & 3 & $100 \%$ & -- & -- & $7.86 \mathrm{E}-04$ & 0.0031 & 0.0016 \\
\hline PCB & 2,3',4,4',5-Pentachlorobiphenyl (BZ 118) & $\mathrm{mg} / \mathrm{kg}$ & 3 & 3 & $100 \%$ & -- & -- & 0.015 & 0.11 & 0.049 \\
\hline PCB & $2,3,4,5,3^{\prime}, 4^{\prime}, 5^{\prime}$-Heptachlorobiphenyl & $\mathrm{mg} / \mathrm{kg}$ & 3 & 3 & $100 \%$ & -- & -- & 1.57E-04 & 4.05E-04 & $2.40 \mathrm{E}-04$ \\
\hline PCB & 3,3',4,4',5,5'-Hexachlorobiphenyl (BZ 169) & $\mathrm{mg} / \mathrm{kg}$ & 3 & 0 & $0 \%$ & $6.40 \mathrm{E}-06$ & $1.58 \mathrm{E}-05$ & -- & -- & 4.89E-06 \\
\hline PCB & 3,3',4,4',5-Pentachlorobiphenyl (BZ 126) & $\mathrm{mg} / \mathrm{kg}$ & 3 & 3 & $100 \%$ & -- & -- & $3.22 \mathrm{E}-05$ & $9.56 \mathrm{E}-05$ & 5.63E-05 \\
\hline PCB & 3,3',4,4'-Tetrachlorobiphenyl (BZ 77) & $\mathrm{mg} / \mathrm{kg}$ & 3 & 3 & $100 \%$ & -- & -- & 3.19E-04 & 8.75E-04 & $5.16 \mathrm{E}-04$ \\
\hline
\end{tabular}


Table F-54

Summary Statistics for Mountain Whitefish Fillet Skin On Collected from Segment 2 of the Columbia River

\begin{tabular}{|c|c|c|c|c|c|c|c|c|c|c|}
\hline $\begin{array}{l}\text { Constituent } \\
\text { Class }\end{array}$ & Constituent Name & Units & $\begin{array}{l}\text { Number } \\
\text { Analyzed }\end{array}$ & $\begin{array}{l}\text { Number } \\
\text { Detected }\end{array}$ & $\begin{array}{l}\text { Frequency of } \\
\text { Detection }\end{array}$ & $\begin{array}{l}\text { Minimum } \\
\text { Nondetect }\end{array}$ & $\begin{array}{l}\text { Maximum } \\
\text { Nondetect }\end{array}$ & $\begin{array}{c}\text { Minimum } \\
\text { Detect }\end{array}$ & $\begin{array}{l}\text { Maximum } \\
\text { Detect }\end{array}$ & $\begin{array}{c}\text { Average } \\
\text { Concentration }\end{array}$ \\
\hline$\overline{\mathrm{PCB}}$ & Aroclor-1016 & $\mathrm{mg} / \mathrm{kg}$ & 3 & 0 & $0 \%$ & 0.017 & 0.019 & $\overline{--}$ & $\overline{--}$ & 0.0092 \\
\hline PCB & Aroclor-1221 & $\mathrm{mg} / \mathrm{kg}$ & 3 & 0 & $0 \%$ & 0.017 & 0.019 & -- & -- & 0.0092 \\
\hline PCB & Aroclor-1232 & $\mathrm{mg} / \mathrm{kg}$ & 3 & 0 & $0 \%$ & 0.017 & 0.019 & -- & -- & 0.0092 \\
\hline PCB & Aroclor-1248 & $\mathrm{mg} / \mathrm{kg}$ & 3 & 0 & $0 \%$ & 0.017 & 0.019 & -- & -- & 0.0092 \\
\hline PCB & Aroclor-1254 & $\mathrm{mg} / \mathrm{kg}$ & 3 & 3 & $100 \%$ & -- & -- & 0.065 & 0.93 & 0.36 \\
\hline PCB & Aroclor-1260 & $\mathrm{mg} / \mathrm{kg}$ & 3 & 3 & $100 \%$ & -- & -- & 0.086 & 0.17 & 0.12 \\
\hline PEST & 2,4'-DDD & $\mathrm{mg} / \mathrm{kg}$ & 3 & 3 & $100 \%$ & -- & -- & 0.0099 & 0.012 & 0.011 \\
\hline PEST & $2,4^{\prime}-\mathrm{DDE}$ & $\mathrm{mg} / \mathrm{kg}$ & 3 & 3 & $100 \%$ & -- & -- & 0.011 & 0.056 & 0.027 \\
\hline PEST & 2,4'-DDT & $\mathrm{mg} / \mathrm{kg}$ & 3 & 0 & $0 \%$ & 0.0017 & 0.0019 & -- & -- & $9.17 \mathrm{E}-04$ \\
\hline PEST & Aldrin & $\mathrm{mg} / \mathrm{kg}$ & 3 & 1 & $33 \%$ & 0.0017 & 0.0019 & 0.0060 & 0.0060 & 0.0026 \\
\hline PEST & Alpha-BHC & $\mathrm{mg} / \mathrm{kg}$ & 3 & 0 & $0 \%$ & 0.0017 & 0.0019 & -- & -- & $9.17 \mathrm{E}-04$ \\
\hline PEST & alpha-Chlordane & $\mathrm{mg} / \mathrm{kg}$ & 3 & 0 & $0 \%$ & 0.0017 & 0.0019 & -- & -- & $9.17 \mathrm{E}-04$ \\
\hline PEST & alpha-Chlordene & $\mathrm{mg} / \mathrm{kg}$ & 3 & 0 & $0 \%$ & 0.0017 & 0.0019 & -- & -- & $9.17 \mathrm{E}-04$ \\
\hline PEST & Beta-BHC & $\mathrm{mg} / \mathrm{kg}$ & 3 & 0 & $0 \%$ & 0.0017 & 0.0019 & -- & -- & $9.17 \mathrm{E}-04$ \\
\hline PEST & cis-Nonachlor & $\mathrm{mg} / \mathrm{kg}$ & 3 & 3 & $100 \%$ & -- & -- & 0.0063 & 0.017 & 0.010 \\
\hline PEST & DDMU & $\mathrm{mg} / \mathrm{kg}$ & 3 & 3 & $100 \%$ & -- & -- & 0.023 & 0.040 & 0.030 \\
\hline PEST & Delta-BHC & $\mathrm{mg} / \mathrm{kg}$ & 3 & 0 & $0 \%$ & 0.0017 & 0.0019 & -- & -- & 9.17E-04 \\
\hline PEST & Dichlorodiphenyldichloroethane & $\mathrm{mg} / \mathrm{kg}$ & 3 & 3 & $100 \%$ & -- & -- & 0.076 & 0.096 & 0.083 \\
\hline PEST & Dichlorodiphenyldichloroethylene & $\mathrm{mg} / \mathrm{kg}$ & 3 & 3 & $100 \%$ & -- & -- & 0.60 & 0.71 & 0.65 \\
\hline PEST & Dichlorodiphenyltrichloroethane & $\mathrm{mg} / \mathrm{kg}$ & 3 & 3 & $100 \%$ & -- & -- & 0.0040 & 0.013 & 0.0093 \\
\hline PEST & Endosulfan sulfate & $\mathrm{mg} / \mathrm{kg}$ & 3 & 0 & $0 \%$ & 0.0017 & 0.0019 & -- & -- & 9.17E-04 \\
\hline PEST & Gamma-BHC (Lindane) & $\mathrm{mg} / \mathrm{kg}$ & 3 & 0 & $0 \%$ & 0.0017 & 0.0019 & -- & -- & $9.17 \mathrm{E}-04$ \\
\hline PEST & gamma-Chlordane & $\mathrm{mg} / \mathrm{kg}$ & 3 & 0 & $0 \%$ & 0.0017 & 0.0019 & -- & -- & $9.17 \mathrm{E}-04$ \\
\hline PEST & gamma-Chlordene & $\mathrm{mg} / \mathrm{kg}$ & 3 & 0 & $0 \%$ & 0.0017 & 0.0019 & -- & -- & 9.17E-04 \\
\hline PEST & Heptachlor & $\mathrm{mg} / \mathrm{kg}$ & 3 & 0 & $0 \%$ & 0.0017 & 0.0019 & -- & -- & $9.17 \mathrm{E}-04$ \\
\hline PEST & Heptachlor epoxide & $\mathrm{mg} / \mathrm{kg}$ & 3 & 0 & $0 \%$ & 0.0017 & 0.0019 & -- & -- & $9.17 \mathrm{E}-04$ \\
\hline PEST & Mirex & $\mathrm{mg} / \mathrm{kg}$ & 3 & 3 & $100 \%$ & -- & -- & 0.0058 & 0.013 & 0.0088 \\
\hline PEST & Oxychlordane & $\mathrm{mg} / \mathrm{kg}$ & 3 & 0 & $0 \%$ & 0.0017 & 0.0019 & -- & -- & $9.17 \mathrm{E}-04$ \\
\hline PEST & Pentachloroanisole & $\mathrm{mg} / \mathrm{kg}$ & 3 & 0 & $0 \%$ & 0.0017 & 0.0019 & -- & -- & $9.17 \mathrm{E}-04$ \\
\hline PEST & Toxaphene & $\mathrm{mg} / \mathrm{kg}$ & 3 & 0 & $0 \%$ & 0.052 & 0.058 & -- & -- & 0.028 \\
\hline PEST & trans-Nonachlor & $\mathrm{mg} / \mathrm{kg}$ & 3 & 2 & $67 \%$ & 0.0017 & 0.0017 & 0.0017 & 0.0037 & 0.0021 \\
\hline SVOC & 1,2,4-Trichlorobenzene & $\mathrm{mg} / \mathrm{kg}$ & 3 & 0 & $0 \%$ & 0.0092 & 0.0098 & -- & -- & 0.0048 \\
\hline SVOC & 1,2-Dichlorobenzene & $\mathrm{mg} / \mathrm{kg}$ & 3 & 0 & $0 \%$ & 0.0092 & 0.0098 & -- & -- & 0.0048 \\
\hline SVOC & 1,2-Diphenylhydrazine & $\mathrm{mg} / \mathrm{kg}$ & 3 & 0 & $0 \%$ & 0.0092 & 0.0098 & -- & -- & 0.0048 \\
\hline SVOC & 1,3-Dichlorobenzene & $\mathrm{mg} / \mathrm{kg}$ & 3 & 0 & $0 \%$ & 0.0092 & 0.0098 & -- & -- & 0.0048 \\
\hline SVOC & 1,4-Dichlorobenzene & $\mathrm{mg} / \mathrm{kg}$ & 3 & 0 & $0 \%$ & 0.0092 & 0.0098 & -- & -- & 0.0048 \\
\hline SVOC & 1-methylnaphthalene & $\mathrm{mg} / \mathrm{kg}$ & 3 & 0 & $0 \%$ & 0.0092 & 0.0098 & -- & -- & 0.0048 \\
\hline SVOC & 2,4-Dinitrotoluene & $\mathrm{mg} / \mathrm{kg}$ & 3 & 0 & $0 \%$ & 0.018 & 0.020 & -- & -- & 0.0095 \\
\hline SVOC & 2,6-Dinitrotoluene & $\mathrm{mg} / \mathrm{kg}$ & 3 & 0 & $0 \%$ & 0.037 & 0.039 & -- & -- & 0.019 \\
\hline SVOC & 2-Chloronaphthalene & $\mathrm{mg} / \mathrm{kg}$ & 3 & 0 & $0 \%$ & 0.018 & 0.020 & -- & -- & 0.0095 \\
\hline SVOC & 2-Methylnaphthalene & $\mathrm{mg} / \mathrm{kg}$ & 3 & 0 & $0 \%$ & 0.0092 & 0.0098 & -- & -- & 0.0048 \\
\hline SVOC & 4-Bromophenylphenyl ether & $\mathrm{mg} / \mathrm{kg}$ & 3 & 0 & $0 \%$ & 0.0092 & 0.0098 & -- & -- & 0.0048 \\
\hline SVOC & 4-Chlorophenylphenyl ether & $\mathrm{mg} / \mathrm{kg}$ & 3 & 0 & $0 \%$ & 0.0092 & 0.0098 & -- & -- & 0.0048 \\
\hline SVOC & Acenaphthene & $\mathrm{mg} / \mathrm{kg}$ & 3 & 0 & $0 \%$ & 0.018 & 0.020 & -- & -- & 0.0095 \\
\hline SVOC & Acenaphthylene & $\mathrm{mg} / \mathrm{kg}$ & 3 & 0 & $0 \%$ & 0.0092 & 0.0098 & -- & -- & 0.0048 \\
\hline SVOC & Anthracene & $\mathrm{mg} / \mathrm{kg}$ & 3 & 0 & $0 \%$ & 0.0092 & 0.0098 & -- & -- & 0.0048 \\
\hline SVOC & Benzo(a)anthracene & $\mathrm{mg} / \mathrm{kg}$ & 3 & 0 & $0 \%$ & 0.0092 & 0.0098 & -- & -- & 0.0048 \\
\hline SVOC & Benzo(a)pyrene & $\mathrm{mg} / \mathrm{kg}$ & 3 & 0 & $0 \%$ & 0.0092 & 0.0098 & -- & -- & 0.0048 \\
\hline
\end{tabular}


Table F-54

Summary Statistics for Mountain Whitefish Fillet Skin On Collected from Segment 2 of the Columbia River

\begin{tabular}{|c|c|c|c|c|c|c|c|c|c|c|}
\hline $\begin{array}{l}\text { Constituent } \\
\text { Class }\end{array}$ & Constituent Name & Units & $\begin{array}{c}\text { Number } \\
\text { Analyzed }\end{array}$ & $\begin{array}{l}\text { Number } \\
\text { Detected }\end{array}$ & $\begin{array}{l}\text { Frequency of } \\
\text { Detection }\end{array}$ & $\begin{array}{l}\text { Minimum } \\
\text { Nondetect }\end{array}$ & $\begin{array}{c}\text { Maximum } \\
\text { Nondetect }\end{array}$ & $\begin{array}{c}\text { Minimum } \\
\text { Detect }\end{array}$ & $\begin{array}{c}\text { Maximum } \\
\text { Detect }\end{array}$ & $\begin{array}{c}\text { Average } \\
\text { Concentration }\end{array}$ \\
\hline$\overline{\text { SVOC }}$ & Benzo(b)fluoranthene & $\mathrm{mg} / \mathrm{kg}$ & 3 & $\overline{0}$ & $0 \%$ & 0.0092 & 0.0098 & $\overline{--}$ & $\overline{--}$ & 0.0048 \\
\hline SVOC & Benzo(ghi)perylene & $\mathrm{mg} / \mathrm{kg}$ & 3 & 0 & $0 \%$ & 0.018 & 0.020 & -- & -- & 0.0095 \\
\hline SVOC & Benzo(k)fluoranthene & $\mathrm{mg} / \mathrm{kg}$ & 3 & 0 & $0 \%$ & 0.0092 & 0.0098 & -- & _- & 0.0048 \\
\hline SVOC & Bis(2-chloro-1-methylethyl)ether & $\mathrm{mg} / \mathrm{kg}$ & 3 & 0 & $0 \%$ & 0.018 & 0.020 & -- & -- & 0.0095 \\
\hline SVOC & Chrysene & $\mathrm{mg} / \mathrm{kg}$ & 3 & 0 & $0 \%$ & 0.0092 & 0.0098 & -- & -- & 0.0048 \\
\hline SVOC & Dibenz[a,h]anthracene & $\mathrm{mg} / \mathrm{kg}$ & 3 & 0 & $0 \%$ & 0.018 & 0.020 & -- & -- & 0.0095 \\
\hline SVOC & Dibenzofuran & $\mathrm{mg} / \mathrm{kg}$ & 3 & 0 & $0 \%$ & 0.0092 & 0.0098 & -- & -- & 0.0048 \\
\hline SVOC & Fluoranthene & $\mathrm{mg} / \mathrm{kg}$ & 3 & 0 & $0 \%$ & 0.018 & 0.020 & -- & -- & 0.0095 \\
\hline SVOC & Fluorene & $\mathrm{mg} / \mathrm{kg}$ & 3 & 0 & $0 \%$ & 0.0095 & 0.0099 & -- & -- & 0.0049 \\
\hline SVOC & Hexachlorobenzene & $\mathrm{mg} / \mathrm{kg}$ & 3 & 0 & $0 \%$ & 0.018 & 0.020 & -- & -- & 0.0095 \\
\hline SVOC & Hexachlorobutadiene & $\mathrm{mg} / \mathrm{kg}$ & 3 & 0 & $0 \%$ & 0.0092 & 0.0098 & -- & -- & 0.0048 \\
\hline SVOC & Hexachloroethane & $\mathrm{mg} / \mathrm{kg}$ & 3 & 0 & $0 \%$ & 0.0092 & 0.0098 & -- & -- & 0.0048 \\
\hline SVOC & Indeno(1,2,3-cd)pyrene & $\mathrm{mg} / \mathrm{kg}$ & 3 & 0 & $0 \%$ & 0.018 & 0.020 & -- & -- & 0.0095 \\
\hline SVOC & Naphthalene & $\mathrm{mg} / \mathrm{kg}$ & 3 & 0 & $0 \%$ & 0.025 & 0.026 & -- & -- & 0.013 \\
\hline SVOC & Nitrobenzene & $\mathrm{mg} / \mathrm{kg}$ & 3 & 0 & $0 \%$ & 0.0092 & 0.0098 & -- & -- & 0.0048 \\
\hline SVOC & Phenanthrene & $\mathrm{mg} / \mathrm{kg}$ & 3 & 0 & $0 \%$ & 0.0092 & 0.0098 & -- & -- & 0.0048 \\
\hline SVOC & Pyrene & $\mathrm{mg} / \mathrm{kg}$ & 3 & 0 & $0 \%$ & 0.018 & 0.020 & -- & -- & 0.0095 \\
\hline VOC & Retene & $\mathrm{mg} / \mathrm{kg}$ & 3 & 0 & $0 \%$ & 0.018 & 0.020 & -- & -- & 0.0095 \\
\hline
\end{tabular}

DIOXI - Dioxins and Furans

PEST - Pesticide

PCB - Polychlorinated biphenyl

SVOC - Semivolatile Organic Compound

VOC - Volatile Organic Compound 
Table F-55

Summary Statistics for Whole Body Mountain Whitefish Collected from Segment 2 of the Columbia River

\begin{tabular}{|c|c|c|c|c|c|c|c|c|c|c|}
\hline $\begin{array}{l}\text { Constituent } \\
\text { Class }\end{array}$ & Constituent Name & Units & $\begin{array}{l}\text { Number } \\
\text { Analyzed }\end{array}$ & $\begin{array}{l}\text { Number } \\
\text { Detected }\end{array}$ & $\begin{array}{l}\text { Frequency of } \\
\text { Detection }\end{array}$ & $\begin{array}{l}\text { Minimum } \\
\text { Nondetect }\end{array}$ & $\begin{array}{l}\text { Maximum } \\
\text { Nondetect }\end{array}$ & $\begin{array}{c}\text { Minimum } \\
\text { Detect }\end{array}$ & $\begin{array}{l}\text { Maximum } \\
\text { Detect }\end{array}$ & $\begin{array}{c}\text { Average } \\
\text { Concentration }\end{array}$ \\
\hline$\overline{D I O X I}$ & $1,2,3,4,6,7,8$-Heptachlorodibenzodioxin & $\mathrm{mg} / \mathrm{kg}$ & 3 & 3 & $100 \%$ & -- & -- & $1.30 \mathrm{E}-07$ & $3.30 \mathrm{E}-07$ & $2.53 \mathrm{E}-07$ \\
\hline DIOXI & $1,2,3,4,6,7,8$-Heptachlorodibenzofuran & $\mathrm{mg} / \mathrm{kg}$ & 3 & 0 & $0 \%$ & $1.00 \mathrm{E}-08$ & $1.30 \mathrm{E}-07$ & -- & -- & 2.67E-08 \\
\hline DIOXI & 1,2,3,4,7,8,9-Heptachlorodibenzofuran & $\mathrm{mg} / \mathrm{kg}$ & 3 & 1 & $33 \%$ & 2.00E-08 & 1.30E-07 & 4.00E-08 & 4.00E-08 & 3.83E-08 \\
\hline DIOXI & $1,2,3,4,7,8$-Hexachlorodibenzofuran & $\mathrm{mg} / \mathrm{kg}$ & 3 & 2 & $67 \%$ & $2.00 \mathrm{E}-08$ & $2.00 \mathrm{E}-08$ & 3.00E-08 & $6.00 \mathrm{E}-08$ & 3.33E-08 \\
\hline DIOXI & $1,2,3,4,7,8$-Hexachlorodibenzo-p-dioxin & $\mathrm{mg} / \mathrm{kg}$ & 3 & 0 & $0 \%$ & $1.00 \mathrm{E}-08$ & $9.00 \mathrm{E}-08$ & -- & -- & $2.00 \mathrm{E}-08$ \\
\hline DIOXI & 1,2,3,6,7,8-Hexachlorodibenzofuran & $\mathrm{mg} / \mathrm{kg}$ & 3 & 2 & $67 \%$ & $2.00 \mathrm{E}-08$ & $2.00 \mathrm{E}-08$ & 2.00E-08 & 4.00E-08 & 2.33E-08 \\
\hline DIOXI & $1,2,3,6,7,8$-Hexachlorodibenzo-p-dioxin & $\mathrm{mg} / \mathrm{kg}$ & 3 & 1 & $33 \%$ & $1.00 \mathrm{E}-08$ & $5.00 \mathrm{E}-08$ & $1.10 \mathrm{E}-07$ & 1.10E-07 & 4.67E-08 \\
\hline DIOXI & 1,2,3,7,8,9-Hexachlorodibenzofuran & $\mathrm{mg} / \mathrm{kg}$ & 3 & 2 & $67 \%$ & $1.00 \mathrm{E}-08$ & $1.00 \mathrm{E}-08$ & 2.00E-08 & 2.00E-08 & $1.50 \mathrm{E}-08$ \\
\hline DIOXI & $1,2,3,7,8,9-$ Hexachlorodibenzo-p-dioxin & $\mathrm{mg} / \mathrm{kg}$ & 3 & 2 & $67 \%$ & $2.00 \mathrm{E}-08$ & $2.00 \mathrm{E}-08$ & $5.00 \mathrm{E}-08$ & 1.10E-07 & $5.67 \mathrm{E}-08$ \\
\hline DIOXI & 1,2,3,7,8-Pentachlorodibenzofuran & $\mathrm{mg} / \mathrm{kg}$ & 3 & 3 & $100 \%$ & -- & -- & 1.10E-07 & $2.60 \mathrm{E}-07$ & $1.60 \mathrm{E}-07$ \\
\hline DIOXI & 1,2,3,7,8-Pentachlorodibenzo-p-dioxin & $\mathrm{mg} / \mathrm{kg}$ & 3 & 3 & $100 \%$ & -- & -- & $2.00 \mathrm{E}-08$ & $1.90 \mathrm{E}-07$ & $1.27 \mathrm{E}-07$ \\
\hline DIOXI & 2,3,4,6,7,8-Hexachlorodibenzofuran & $\mathrm{mg} / \mathrm{kg}$ & 3 & 2 & $67 \%$ & $7.00 \mathrm{E}-08$ & $7.00 \mathrm{E}-08$ & $3.00 \mathrm{E}-08$ & 4.00E-08 & $3.50 \mathrm{E}-08$ \\
\hline DIOXI & 2,3,4,7,8-Pentachlorodibenzofuran & $\mathrm{mg} / \mathrm{kg}$ & 3 & 2 & $67 \%$ & 3.60E-07 & 3.60E-07 & $2.50 \mathrm{E}-07$ & $2.90 \mathrm{E}-07$ & $2.40 \mathrm{E}-07$ \\
\hline DIOXI & 2,3,7,8-Tetrachlorodibenzofuran & $\mathrm{mg} / \mathrm{kg}$ & 3 & 3 & $100 \%$ & -- & -- & $9.00 \mathrm{E}-06$ & 1.17E-05 & 1.06E-05 \\
\hline DIOXI & 2,3,7,8-Tetrachlorodibenzo-p-dioxin & $\mathrm{mg} / \mathrm{kg}$ & 3 & 2 & $67 \%$ & 2.10E-07 & 2.10E-07 & $1.50 \mathrm{E}-07$ & $1.60 \mathrm{E}-07$ & $1.38 \mathrm{E}-07$ \\
\hline DIOXI & Octachlorodibenzofuran & $\mathrm{mg} / \mathrm{kg}$ & 3 & 3 & $100 \%$ & -- & -- & 4.00E-08 & $2.00 \mathrm{E}-07$ & 1.23E-07 \\
\hline DIOXI & Octachlorodibenzo-p-dioxin & $\mathrm{mg} / \mathrm{kg}$ & 3 & 2 & $67 \%$ & $1.00 \mathrm{E}-08$ & $1.00 \mathrm{E}-08$ & 2.30E-07 & 7.60E-07 & $3.32 \mathrm{E}-07$ \\
\hline METAL & Aluminum & $\mathrm{mg} / \mathrm{kg}$ & 3 & 3 & $100 \%$ & -- & -- & 14 & 17 & 16 \\
\hline METAL & Antimony & $\mathrm{mg} / \mathrm{kg}$ & 3 & 0 & $0 \%$ & 0.050 & 0.050 & -- & -- & 0.025 \\
\hline METAL & Arsenic & $\mathrm{mg} / \mathrm{kg}$ & 3 & 3 & $100 \%$ & -- & -- & 0.13 & 0.15 & 0.14 \\
\hline METAL & Barium & $\mathrm{mg} / \mathrm{kg}$ & 3 & 3 & $100 \%$ & -- & -- & 0.57 & 0.73 & 0.66 \\
\hline METAL & Beryllium & $\mathrm{mg} / \mathrm{kg}$ & 3 & 0 & $0 \%$ & 0.0040 & 0.0040 & -- & -- & 0.0020 \\
\hline METAL & Cadmium & $\mathrm{mg} / \mathrm{kg}$ & 3 & 3 & $100 \%$ & -- & -- & 0.040 & 0.050 & 0.047 \\
\hline METAL & Chromium & $\mathrm{mg} / \mathrm{kg}$ & 3 & 3 & $100 \%$ & -- & -- & 0.13 & 0.18 & 0.15 \\
\hline METAL & Cobalt & $\mathrm{mg} / \mathrm{kg}$ & 3 & 3 & $100 \%$ & -- & -- & 0.090 & 0.12 & 0.10 \\
\hline METAL & Copper & $\mathrm{mg} / \mathrm{kg}$ & 3 & 3 & $100 \%$ & -- & -- & 0.61 & 0.70 & 0.66 \\
\hline METAL & Lead & $\mathrm{mg} / \mathrm{kg}$ & 3 & 3 & $100 \%$ & -- & -- & 0.040 & 0.070 & 0.057 \\
\hline METAL & Manganese & $\mathrm{mg} / \mathrm{kg}$ & 3 & 3 & $100 \%$ & -- & -- & 2.9 & 3.2 & 3.0 \\
\hline METAL & Mercury & $\mathrm{mg} / \mathrm{kg}$ & 3 & 0 & $0 \%$ & 0.10 & 0.10 & -- & -- & 0.050 \\
\hline METAL & Nickel & $\mathrm{mg} / \mathrm{kg}$ & 3 & 3 & $100 \%$ & -- & -- & 0.070 & 0.080 & 0.077 \\
\hline METAL & Selenium & $\mathrm{mg} / \mathrm{kg}$ & 3 & 3 & $100 \%$ & -- & -- & 1.0 & 1.1 & 1.1 \\
\hline METAL & Silver & $\mathrm{mg} / \mathrm{kg}$ & 3 & 0 & $0 \%$ & 0.10 & 0.10 & -- & -- & 0.050 \\
\hline METAL & Thallium & $\mathrm{mg} / \mathrm{kg}$ & 3 & 0 & $0 \%$ & 0.050 & 0.050 & -- & -- & 0.025 \\
\hline METAL & Vanadium & $\mathrm{mg} / \mathrm{kg}$ & 3 & 3 & $100 \%$ & -- & -- & 0.060 & 0.080 & 0.070 \\
\hline METAL & Zinc & $\mathrm{mg} / \mathrm{kg}$ & 3 & 3 & $100 \%$ & -- & -- & 24 & 25 & 25 \\
\hline PCB & 2,2',3,3',4,4',5-Heptachloro-1,1'-biphenyl & $\mathrm{mg} / \mathrm{kg}$ & 3 & 3 & $100 \%$ & -- & -- & 0.0026 & 0.0036 & 0.0032 \\
\hline PCB & $2,2^{\prime}, 3,4,4^{\prime}, 5,5^{\prime}$-Heptachlorobiphenyl & $\mathrm{mg} / \mathrm{kg}$ & 3 & 3 & $100 \%$ & -- & -- & 0.0061 & 0.0097 & 0.0076 \\
\hline PCB & $2,3,3^{\prime}, 4,4^{\prime}, 5^{\prime}-$ Hexachlorobiphenyl & $\mathrm{mg} / \mathrm{kg}$ & 3 & 3 & $100 \%$ & -- & -- & 3.53E-04 & $5.70 \mathrm{E}-04$ & $4.55 E-04$ \\
\hline PCB & 2,3,3',4,4',5-Hexachlorobiphenyl (BZ 156) & $\mathrm{mg} / \mathrm{kg}$ & 3 & 3 & $100 \%$ & -- & -- & 0.0019 & 0.0030 & 0.0024 \\
\hline PCB & 2,3,3',4,4'-Pentachlorobiphenyl & $\mathrm{mg} / \mathrm{kg}$ & 3 & 3 & $100 \%$ & -- & -- & 0.0046 & 0.0073 & 0.0058 \\
\hline PCB & 2,3',4,4',5,5'-Hexachlorobiphenyl (BZ 167) & $\mathrm{mg} / \mathrm{kg}$ & 3 & 3 & $100 \%$ & -- & -- & $5.40 \mathrm{E}-04$ & 7.04E-04 & $6.21 \mathrm{E}-04$ \\
\hline PCB & 2',3,4,4',5-Pentachloro-1,1'-biphenyl (BZ 123) & $\mathrm{mg} / \mathrm{kg}$ & 3 & 3 & $100 \%$ & -- & -- & 1.95E-04 & $2.45 \mathrm{E}-04$ & 2.15E-04 \\
\hline PCB & 2,3,4,4',5-Pentachlorobiphenyl (BZ 114) & $\mathrm{mg} / \mathrm{kg}$ & 3 & 3 & $100 \%$ & -- & -- & 4.45E-04 & 7.06E-04 & $5.81 \mathrm{E}-04$ \\
\hline PCB & 2,3',4,4',5-Pentachlorobiphenyl (BZ 118) & $\mathrm{mg} / \mathrm{kg}$ & 3 & 3 & $100 \%$ & -- & -- & 0.0087 & 0.012 & 0.011 \\
\hline PCB & $2,3,4,5,3^{\prime}, 4^{\prime}, 5^{\prime}$-Heptachlorobiphenyl & $\mathrm{mg} / \mathrm{kg}$ & 3 & 3 & $100 \%$ & -- & -- & $9.33 \mathrm{E}-05$ & $1.31 \mathrm{E}-04$ & 1.12E-04 \\
\hline PCB & 3,3',4,4',5,5'-Hexachlorobiphenyl (BZ 169) & $\mathrm{mg} / \mathrm{kg}$ & 3 & 0 & $0 \%$ & $5.09 \mathrm{E}-06$ & $5.24 \mathrm{E}-06$ & -- & -- & $2.57 \mathrm{E}-06$ \\
\hline PCB & 3,3',4,4',5-Pentachlorobiphenyl (BZ 126) & $\mathrm{mg} / \mathrm{kg}$ & 3 & 3 & $100 \%$ & -- & -- & $1.02 \mathrm{E}-05$ & $1.56 \mathrm{E}-05$ & $1.28 \mathrm{E}-05$ \\
\hline PCB & 3,3',4,4'-Tetrachlorobiphenyl (BZ 77) & $\mathrm{mg} / \mathrm{kg}$ & 3 & 3 & $100 \%$ & -- & -- & $2.78 \mathrm{E}-04$ & 3.07E-04 & 2.92E-04 \\
\hline
\end{tabular}


Table F-55

Summary Statistics for Whole Body Mountain Whitefish Collected from Segment 2 of the Columbia River

\begin{tabular}{|c|c|c|c|c|c|c|c|c|c|c|}
\hline $\begin{array}{l}\text { Constituent } \\
\text { Class }\end{array}$ & Constituent Name & Units & $\begin{array}{c}\text { Number } \\
\text { Analyzed }\end{array}$ & $\begin{array}{l}\text { Number } \\
\text { Detected }\end{array}$ & $\begin{array}{l}\text { Frequency of } \\
\text { Detection }\end{array}$ & $\begin{array}{c}\text { Minimum } \\
\text { Nondetect }\end{array}$ & $\begin{array}{c}\text { Maximum } \\
\text { Nondetect }\end{array}$ & $\begin{array}{c}\text { Minimum } \\
\text { Detect }\end{array}$ & $\begin{array}{l}\text { Maximum } \\
\text { Detect }\end{array}$ & $\begin{array}{c}\text { Average } \\
\text { Concentration }\end{array}$ \\
\hline$\overline{\mathrm{PCB}}$ & Aroclor-1016 & $\mathrm{mg} / \mathrm{kg}$ & 3 & $\overline{0}$ & $0 \%$ & 0.016 & 0.019 & $\overline{--}$ & $\overline{--}$ & 0.0088 \\
\hline PCB & Aroclor-1221 & $\mathrm{mg} / \mathrm{kg}$ & 3 & 0 & $0 \%$ & 0.016 & 0.019 & -- & -- & 0.0088 \\
\hline PCB & Aroclor-1232 & $\mathrm{mg} / \mathrm{kg}$ & 3 & 0 & $0 \%$ & 0.016 & 0.019 & -- & -- & 0.0088 \\
\hline PCB & Aroclor-1248 & $\mathrm{mg} / \mathrm{kg}$ & 3 & 0 & $0 \%$ & 0.016 & 0.019 & -- & -- & 0.0088 \\
\hline PCB & Aroclor-1254 & $\mathrm{mg} / \mathrm{kg}$ & 3 & 3 & $100 \%$ & -- & -- & 0.11 & 0.14 & 0.13 \\
\hline PCB & Aroclor-1260 & $\mathrm{mg} / \mathrm{kg}$ & 3 & 3 & $100 \%$ & -- & -- & 0.055 & 0.086 & 0.071 \\
\hline PEST & 2,4'-DDD & $\mathrm{mg} / \mathrm{kg}$ & 3 & 3 & $100 \%$ & -- & -- & 0.017 & 0.018 & 0.017 \\
\hline PEST & 2,4'-DDE & $\mathrm{mg} / \mathrm{kg}$ & 3 & 3 & $100 \%$ & -- & -- & 0.014 & 0.019 & 0.016 \\
\hline PEST & 2,4'-DDT & $\mathrm{mg} / \mathrm{kg}$ & 3 & 0 & $0 \%$ & 0.0016 & 0.0019 & -- & -- & 8.83E-04 \\
\hline PEST & Aldrin & $\mathrm{mg} / \mathrm{kg}$ & 3 & 3 & $100 \%$ & -- & -- & 0.0016 & 0.0018 & 0.0017 \\
\hline PEST & Alpha-BHC & $\mathrm{mg} / \mathrm{kg}$ & 3 & 3 & $100 \%$ & -- & -- & 0.0013 & 0.0018 & 0.0015 \\
\hline PEST & alpha-Chlordane & $\mathrm{mg} / \mathrm{kg}$ & 3 & 0 & $0 \%$ & 0.0016 & 0.0019 & -- & -- & 8.83E-04 \\
\hline PEST & alpha-Chlordene & $\mathrm{mg} / \mathrm{kg}$ & 3 & 0 & $0 \%$ & 0.0016 & 0.0019 & -- & -- & 8.83E-04 \\
\hline PEST & Beta-BHC & $\mathrm{mg} / \mathrm{kg}$ & 3 & 0 & $0 \%$ & 0.0016 & 0.0019 & -- & -- & 8.83E-04 \\
\hline PEST & cis-Nonachlor & $\mathrm{mg} / \mathrm{kg}$ & 3 & 3 & $100 \%$ & -- & -- & 0.011 & 0.017 & 0.014 \\
\hline PEST & DDMU & $\mathrm{mg} / \mathrm{kg}$ & 3 & 3 & $100 \%$ & -- & -- & 0.025 & 0.031 & 0.029 \\
\hline PEST & Delta-BHC & $\mathrm{mg} / \mathrm{kg}$ & 3 & 0 & $0 \%$ & 0.0016 & 0.0019 & -- & -- & 8.83E-04 \\
\hline PEST & Dichlorodiphenyldichloroethane & $\mathrm{mg} / \mathrm{kg}$ & 3 & 3 & $100 \%$ & -- & -- & 0.093 & 0.11 & 0.099 \\
\hline PEST & Dichlorodiphenyldichloroethylene & $\mathrm{mg} / \mathrm{kg}$ & 3 & 3 & $100 \%$ & -- & -- & 0.52 & 0.74 & 0.64 \\
\hline PEST & Dichlorodiphenyltrichloroethane & $\mathrm{mg} / \mathrm{kg}$ & 3 & 3 & $100 \%$ & -- & -- & 0.0029 & 0.0064 & 0.0042 \\
\hline PEST & Endosulfan sulfate & $\mathrm{mg} / \mathrm{kg}$ & 3 & 0 & $0 \%$ & 0.0016 & 0.0019 & -- & -- & 8.83E-04 \\
\hline PEST & Gamma-BHC (Lindane) & $\mathrm{mg} / \mathrm{kg}$ & 3 & 0 & $0 \%$ & 0.0016 & 0.0019 & -- & -- & 8.83E-04 \\
\hline PEST & gamma-Chlordane & $\mathrm{mg} / \mathrm{kg}$ & 3 & 0 & $0 \%$ & 0.0016 & 0.0019 & -- & -- & 8.83E-04 \\
\hline PEST & gamma-Chlordene & $\mathrm{mg} / \mathrm{kg}$ & 3 & 0 & $0 \%$ & 0.0016 & 0.0019 & -- & -- & 8.83E-04 \\
\hline PEST & Heptachlor & $\mathrm{mg} / \mathrm{kg}$ & 3 & 0 & $0 \%$ & 0.0016 & 0.0019 & -- & -- & 8.83E-04 \\
\hline PEST & Heptachlor epoxide & $\mathrm{mg} / \mathrm{kg}$ & 3 & 0 & $0 \%$ & 0.0016 & 0.0019 & -- & -- & 8.83E-04 \\
\hline PEST & Mirex & $\mathrm{mg} / \mathrm{kg}$ & 3 & 3 & $100 \%$ & -- & -- & 0.0039 & 0.0060 & 0.0051 \\
\hline PEST & Oxychlordane & $\mathrm{mg} / \mathrm{kg}$ & 3 & 0 & $0 \%$ & 0.0016 & 0.0019 & -- & -- & 8.83E-04 \\
\hline PEST & Pentachloroanisole & $\mathrm{mg} / \mathrm{kg}$ & 3 & 1 & $33 \%$ & 0.0016 & 0.0019 & 0.0016 & 0.0016 & 0.0011 \\
\hline PEST & Toxaphene & $\mathrm{mg} / \mathrm{kg}$ & 3 & 0 & $0 \%$ & 0.048 & 0.057 & -- & -- & 0.027 \\
\hline PEST & trans-Nonachlor & $\mathrm{mg} / \mathrm{kg}$ & 3 & 3 & $100 \%$ & -- & -- & 0.0027 & 0.0044 & 0.0037 \\
\hline SVOC & 1,2,4-Trichlorobenzene & $\mathrm{mg} / \mathrm{kg}$ & 3 & 0 & $0 \%$ & 0.0089 & 0.0099 & -- & -- & 0.0047 \\
\hline SVOC & 1,2-Dichlorobenzene & $\mathrm{mg} / \mathrm{kg}$ & 3 & 0 & $0 \%$ & 0.0089 & 0.0099 & -- & -- & 0.0047 \\
\hline SVOC & 1,2-Diphenylhydrazine & $\mathrm{mg} / \mathrm{kg}$ & 3 & 0 & $0 \%$ & 0.0089 & 0.0099 & -- & -- & 0.0047 \\
\hline SVOC & 1,3-Dichlorobenzene & $\mathrm{mg} / \mathrm{kg}$ & 3 & 0 & $0 \%$ & 0.0089 & 0.0099 & -- & -- & 0.0047 \\
\hline SVOC & 1,4-Dichlorobenzene & $\mathrm{mg} / \mathrm{kg}$ & 3 & 0 & $0 \%$ & 0.0089 & 0.0099 & -- & -- & 0.0047 \\
\hline SVOC & 1-methylnaphthalene & $\mathrm{mg} / \mathrm{kg}$ & 3 & 0 & $0 \%$ & 0.0089 & 0.0099 & -- & -- & 0.0047 \\
\hline SVOC & 2,4-Dinitrotoluene & $\mathrm{mg} / \mathrm{kg}$ & 3 & 0 & $0 \%$ & 0.018 & 0.020 & -- & -- & 0.0095 \\
\hline SVOC & 2,6-Dinitrotoluene & $\mathrm{mg} / \mathrm{kg}$ & 3 & 0 & $0 \%$ & 0.036 & 0.040 & -- & -- & 0.019 \\
\hline SVOC & 2-Chloronaphthalene & $\mathrm{mg} / \mathrm{kg}$ & 3 & 0 & $0 \%$ & 0.018 & 0.020 & -- & -- & 0.0095 \\
\hline SVOC & 2-Methylnaphthalene & $\mathrm{mg} / \mathrm{kg}$ & 3 & 0 & $0 \%$ & 0.0089 & 0.0099 & -- & -- & 0.0047 \\
\hline SVOC & 4-Bromophenylphenyl ether & $\mathrm{mg} / \mathrm{kg}$ & 3 & 0 & $0 \%$ & 0.0089 & 0.0099 & -- & -- & 0.0047 \\
\hline SVOC & 4-Chlorophenylphenyl ether & $\mathrm{mg} / \mathrm{kg}$ & 3 & 0 & $0 \%$ & 0.0089 & 0.0099 & -- & -- & 0.0047 \\
\hline SVOC & Acenaphthene & $\mathrm{mg} / \mathrm{kg}$ & 3 & 0 & $0 \%$ & 0.019 & 0.021 & -- & -- & 0.010 \\
\hline SVOC & Acenaphthylene & $\mathrm{mg} / \mathrm{kg}$ & 3 & 0 & $0 \%$ & 0.0089 & 0.0099 & -- & -- & 0.0047 \\
\hline SVOC & Anthracene & $\mathrm{mg} / \mathrm{kg}$ & 3 & 0 & $0 \%$ & 0.0089 & 0.0099 & -- & -- & 0.0047 \\
\hline SVOC & Benzo(a)anthracene & $\mathrm{mg} / \mathrm{kg}$ & 3 & 0 & $0 \%$ & 0.0089 & 0.0099 & -- & -- & 0.0047 \\
\hline SVOC & Benzo(a)pyrene & $\mathrm{mg} / \mathrm{kg}$ & 3 & 0 & $0 \%$ & 0.0089 & 0.0099 & -- & -- & 0.0047 \\
\hline
\end{tabular}


Table F-55

Summary Statistics for Whole Body Mountain Whitefish Collected from Segment 2 of the Columbia River

\begin{tabular}{|c|c|c|c|c|c|c|c|c|c|c|}
\hline $\begin{array}{c}\text { Constituent } \\
\text { Class }\end{array}$ & Constituent Name & Units & $\begin{array}{l}\text { Number } \\
\text { Analyzed }\end{array}$ & $\begin{array}{l}\text { Number } \\
\text { Detected }\end{array}$ & $\begin{array}{l}\text { Frequency of } \\
\text { Detection }\end{array}$ & $\begin{array}{c}\text { Minimum } \\
\text { Nondetect }\end{array}$ & $\begin{array}{c}\text { Maximum } \\
\text { Nondetect }\end{array}$ & $\begin{array}{c}\text { Minimum } \\
\text { Detect }\end{array}$ & $\begin{array}{c}\text { Maximum } \\
\text { Detect }\end{array}$ & $\begin{array}{c}\text { Average } \\
\text { Concentration }\end{array}$ \\
\hline$\overline{\text { SVOC }}$ & Benzo(b)fluoranthene & $\mathrm{mg} / \mathrm{kg}$ & 3 & 0 & $0 \%$ & 0.0089 & 0.0099 & -- & -- & 0.0047 \\
\hline SVOC & Benzo(ghi)perylene & $\mathrm{mg} / \mathrm{kg}$ & 3 & 0 & $0 \%$ & 0.018 & 0.020 & -- & -- & 0.0095 \\
\hline SVOC & Benzo(k)fluoranthene & $\mathrm{mg} / \mathrm{kg}$ & 3 & 0 & $0 \%$ & 0.0089 & 0.0099 & -- & -- & 0.0047 \\
\hline SVOC & Bis(2-chloro-1-methylethyl)ether & $\mathrm{mg} / \mathrm{kg}$ & 3 & 0 & $0 \%$ & 0.018 & 0.020 & -- & -- & 0.0095 \\
\hline SVOC & Chrysene & $\mathrm{mg} / \mathrm{kg}$ & 3 & 0 & $0 \%$ & 0.0089 & 0.0099 & -- & -- & 0.0047 \\
\hline SVOC & Dibenz[a,h]anthracene & $\mathrm{mg} / \mathrm{kg}$ & 3 & 0 & $0 \%$ & 0.018 & 0.020 & -- & -- & 0.0095 \\
\hline SVOC & Dibenzofuran & $\mathrm{mg} / \mathrm{kg}$ & 3 & 0 & $0 \%$ & 0.0089 & 0.0099 & -- & -- & 0.0047 \\
\hline SVOC & Fluoranthene & $\mathrm{mg} / \mathrm{kg}$ & 3 & 0 & $0 \%$ & 0.018 & 0.020 & -- & -- & 0.0095 \\
\hline SVOC & Fluorene & $\mathrm{mg} / \mathrm{kg}$ & 3 & 0 & $0 \%$ & 0.0091 & 0.010 & -- & -- & 0.0048 \\
\hline SVOC & Hexachlorobenzene & $\mathrm{mg} / \mathrm{kg}$ & 3 & 0 & $0 \%$ & 0.018 & 0.020 & -- & -- & 0.0095 \\
\hline SVOC & Hexachlorobutadiene & $\mathrm{mg} / \mathrm{kg}$ & 3 & 0 & $0 \%$ & 0.0089 & 0.0099 & -- & -- & 0.0047 \\
\hline SVOC & Hexachloroethane & $\mathrm{mg} / \mathrm{kg}$ & 3 & 0 & $0 \%$ & 0.0089 & 0.0099 & -- & -- & 0.0047 \\
\hline SVOC & Indeno(1,2,3-cd)pyrene & $\mathrm{mg} / \mathrm{kg}$ & 3 & 0 & $0 \%$ & 0.018 & 0.020 & -- & -- & 0.0095 \\
\hline SVOC & Naphthalene & $\mathrm{mg} / \mathrm{kg}$ & 3 & 0 & $0 \%$ & 0.022 & 0.027 & -- & -- & 0.013 \\
\hline SVOC & Nitrobenzene & $\mathrm{mg} / \mathrm{kg}$ & 3 & 0 & $0 \%$ & 0.0089 & 0.0099 & -- & -- & 0.0047 \\
\hline SVOC & Phenanthrene & $\mathrm{mg} / \mathrm{kg}$ & 3 & 0 & $0 \%$ & 0.0089 & 0.0099 & -- & -- & 0.0047 \\
\hline SVOC & Pyrene & $\mathrm{mg} / \mathrm{kg}$ & 3 & 0 & $0 \%$ & 0.018 & 0.020 & -- & -- & 0.0095 \\
\hline VOC & Retene & $\mathrm{mg} / \mathrm{kg}$ & 3 & 0 & $0 \%$ & 0.018 & 0.020 & -- & -- & 0.0095 \\
\hline
\end{tabular}

DIOXI - Dioxins and Furans

PEST - Pesticide

PCB - Polychlorinated biphenyl

SVOC - Semivolatile Organic Compound

VOC - Volatile Organic Compound 
Table F-56

Summary Statistics for Whitefish Fillet Collected from Segment 2 of the Columbia River

\begin{tabular}{|c|c|c|c|c|c|c|c|c|c|c|}
\hline $\begin{array}{l}\text { Constituent } \\
\text { Class }\end{array}$ & Constituent Name & Units & $\begin{array}{l}\text { Number } \\
\text { Analyzed }\end{array}$ & $\begin{array}{l}\text { Number } \\
\text { Detected }\end{array}$ & $\begin{array}{c}\text { Frequency of } \\
\text { Detection }\end{array}$ & $\begin{array}{l}\text { Minimum } \\
\text { Nondetect }\end{array}$ & $\begin{array}{l}\text { Maximum } \\
\text { Nondetect }\end{array}$ & $\begin{array}{c}\text { Minimum } \\
\text { Detect }\end{array}$ & $\begin{array}{l}\text { Maximum } \\
\text { Detect }\end{array}$ & $\begin{array}{c}\text { Average } \\
\text { Concentration }\end{array}$ \\
\hline RAD & Antimony-125 & $\mathrm{pCi} / \mathrm{g}$ & 11 & 0 & $0 \%$ & 0.0014 & 0.065 & -- & -- & 0.0082 \\
\hline RAD & Beryllium-7 & $\mathrm{pCi} / \mathrm{g}$ & 14 & 0 & $0 \%$ & 0.0063 & 0.31 & -- & -- & 0.053 \\
\hline RAD & Cesium-134 & $\mathrm{pCi} / \mathrm{g}$ & 15 & 0 & $0 \%$ & 8.65E-04 & 0.016 & -- & -- & 0.0034 \\
\hline RAD & Cesium-137 & $\mathrm{pCi} / \mathrm{g}$ & 26 & 1 & $4 \%$ & 6.82E-04 & 0.036 & 0.020 & 0.020 & 0.0081 \\
\hline RAD & Cobalt-60 & $\mathrm{pCi} / \mathrm{g}$ & 24 & 3 & $13 \%$ & 0.0048 & 0.041 & 0.024 & 0.037 & 0.010 \\
\hline RAD & Europium-152 & $\mathrm{pCi} / \mathrm{g}$ & 2 & 0 & $0 \%$ & 3.13E-04 & 0.0046 & -- & -- & 0.0012 \\
\hline RAD & Europium-154 & $\mathrm{pCi} / \mathrm{g}$ & 18 & 1 & $6 \%$ & 0.0056 & 0.088 & 0.066 & 0.066 & 0.017 \\
\hline RAD & Europium-155 & $\mathrm{pCi} / \mathrm{g}$ & 21 & 1 & $5 \%$ & 7.79E-04 & 0.070 & 0.030 & 0.030 & 0.013 \\
\hline RAD & Manganese-54 & $\mathrm{pCi} / \mathrm{g}$ & 1 & 0 & $0 \%$ & 0.0022 & 0.0022 & -- & -- & 0.0011 \\
\hline RAD & Potassium-40 & $\mathrm{pCi} / \mathrm{g}$ & 35 & 34 & $97 \%$ & 2.6 & 2.6 & 2.2 & 5.1 & 3.6 \\
\hline RAD & Ruthenium-106 & $\mathrm{pCi} / \mathrm{g}$ & 10 & 0 & $0 \%$ & 0.0089 & 0.17 & -- & -- & 0.030 \\
\hline RAD & Strontium-90 & $\mathrm{pCi} / \mathrm{g}$ & 9 & 0 & $0 \%$ & 3.97E-04 & 0.0048 & -- & -- & 8.00E-04 \\
\hline RAD & Technetium-99 & $\mathrm{pCi} / \mathrm{g}$ & 1 & 0 & $0 \%$ & 0.047 & 0.047 & -- & -- & 0.024 \\
\hline RAD & Uranium-234 & $\mathrm{pCi} / \mathrm{g}$ & 1 & 0 & $0 \%$ & 0.0012 & 0.0012 & -- & -- & 5.80E-04 \\
\hline RAD & Uranium-235 & $\mathrm{pCi} / \mathrm{g}$ & 1 & 0 & $0 \%$ & 4.58E-04 & 4.58E-04 & -- & -- & $2.29 \mathrm{E}-04$ \\
\hline RAD & Uranium-238 & $\mathrm{pCi} / \mathrm{g}$ & 2 & 0 & $0 \%$ & 4.05E-04 & 5.38E-04 & -- & -- & 2.36E-04 \\
\hline RAD & Zinc-65 & $\mathrm{pCi} / \mathrm{g}$ & 1 & 0 & $0 \%$ & 7.91E-04 & 7.91E-04 & -- & -- & 3.96E-04 \\
\hline RAD & Zirconium/Niobium-95 & $\mathrm{pCi} / \mathrm{g}$ & 1 & 0 & $0 \%$ & 0.0078 & 0.0078 & -- & -- & 0.0039 \\
\hline
\end{tabular}

RAD - Radionuclide 
Table F-57

Summary Statistics for Whitefish Liver Collected from Segment 2 of the Columbia River

\begin{tabular}{|c|c|c|c|c|c|c|c|c|c|c|}
\hline $\begin{array}{l}\text { Constituent } \\
\text { Class }\end{array}$ & Constituent Name & Units & $\begin{array}{l}\text { Number } \\
\text { Analyzed }\end{array}$ & $\begin{array}{l}\text { Number } \\
\text { Detected }\end{array}$ & $\begin{array}{l}\text { Frequency of } \\
\text { Detection }\end{array}$ & $\begin{array}{l}\text { Minimum } \\
\text { Nondetect }\end{array}$ & $\begin{array}{l}\text { Maximum } \\
\text { Nondetect }\end{array}$ & $\begin{array}{c}\text { Minimum } \\
\text { Detect }\end{array}$ & $\begin{array}{l}\text { Maximum } \\
\text { Detect }\end{array}$ & $\begin{array}{c}\text { Average } \\
\text { Concentration }\end{array}$ \\
\hline$\overline{M E T A L}$ & Aluminum & $\mathrm{mg} / \mathrm{kg}$ & 5 & 5 & $100 \%$ & $\overline{--}$ & $\overline{--}$ & 1.3 & 6.6 & 3.6 \\
\hline METAL & Antimony & $\mathrm{mg} / \mathrm{kg}$ & 5 & 3 & $60 \%$ & 0.019 & 0.019 & 0.020 & 0.023 & 0.017 \\
\hline METAL & Arsenic & $\mathrm{mg} / \mathrm{kg}$ & 5 & 5 & $100 \%$ & -- & -- & 0.48 & 0.86 & 0.71 \\
\hline METAL & Beryllium & $\mathrm{mg} / \mathrm{kg}$ & 5 & 4 & $80 \%$ & 0.019 & 0.019 & 0.038 & 0.054 & 0.037 \\
\hline METAL & Cadmium & $\mathrm{mg} / \mathrm{kg}$ & 5 & 5 & $100 \%$ & -- & -- & 1.9 & 5.5 & 3.1 \\
\hline METAL & Chromium & $\mathrm{mg} / \mathrm{kg}$ & 5 & 5 & $100 \%$ & -- & -- & 0.11 & 1.9 & 0.50 \\
\hline METAL & Copper & $\mathrm{mg} / \mathrm{kg}$ & 5 & 5 & $100 \%$ & -- & -- & 11 & 23 & 15 \\
\hline METAL & Lead & $\mathrm{mg} / \mathrm{kg}$ & 5 & 5 & $100 \%$ & -- & -- & 0.058 & 0.15 & 0.092 \\
\hline METAL & Manganese & $\mathrm{mg} / \mathrm{kg}$ & 5 & 5 & $100 \%$ & -- & -- & 4.6 & 12 & 7.5 \\
\hline METAL & Nickel & $\mathrm{mg} / \mathrm{kg}$ & 5 & 5 & $100 \%$ & -- & -- & 0.055 & 0.37 & 0.13 \\
\hline METAL & Selenium & $\mathrm{mg} / \mathrm{kg}$ & 5 & 5 & $100 \%$ & -- & -- & 9.3 & 14 & 11 \\
\hline METAL & Silver & $\mathrm{mg} / \mathrm{kg}$ & 5 & 4 & $80 \%$ & 0.045 & 0.045 & 0.077 & 0.36 & 0.14 \\
\hline METAL & Thallium & $\mathrm{mg} / \mathrm{kg}$ & 5 & 5 & $100 \%$ & -- & -- & 0.86 & 1.7 & 1.2 \\
\hline METAL & Thorium & $\mathrm{mg} / \mathrm{kg}$ & 5 & 0 & $0 \%$ & 0.021 & 0.021 & -- & -- & 0.011 \\
\hline METAL & Uranium & $\mathrm{mg} / \mathrm{kg}$ & 5 & 2 & $40 \%$ & 0.031 & 0.031 & 0.035 & 0.037 & 0.024 \\
\hline METAL & Zinc & $\mathrm{mg} / \mathrm{kg}$ & 5 & 5 & $100 \%$ & -- & -- & 86 & 110 & 95 \\
\hline
\end{tabular}


Table F-58

Summary Statistics for Wholebody Whitefish Collected from Segment 2 of the Columbia River

\begin{tabular}{|c|c|c|c|c|c|c|c|c|c|c|}
\hline $\begin{array}{l}\text { Constituent } \\
\text { Class }\end{array}$ & Constituent Name & Units & $\begin{array}{l}\text { Number } \\
\text { Analyzed }\end{array}$ & $\begin{array}{l}\text { Number } \\
\text { Detected }\end{array}$ & $\begin{array}{c}\text { Frequency of } \\
\text { Detection }\end{array}$ & $\begin{array}{l}\text { Minimum } \\
\text { Nondetect }\end{array}$ & $\begin{array}{l}\text { Maximum } \\
\text { Nondetect }\end{array}$ & $\begin{array}{c}\text { Minimum } \\
\text { Detect }\end{array}$ & $\begin{array}{l}\text { Maximum } \\
\text { Detect }\end{array}$ & $\begin{array}{c}\text { Average } \\
\text { Concentration }\end{array}$ \\
\hline$\overline{\mathrm{RAD}}$ & Strontium-90 & $\mathrm{pCi} / \mathrm{g}$ & 28 & 12 & $43 \%$ & 0.0041 & 0.026 & 0.0061 & 0.46 & 0.028 \\
\hline RAD & Uranium-234 & $\mathrm{pCi} / \mathrm{g}$ & 11 & 9 & $82 \%$ & $1.56 \mathrm{E}-05$ & 0.0015 & 0.0029 & 0.022 & 0.0089 \\
\hline RAD & Uranium-235 & $\mathrm{pCi} / \mathrm{g}$ & 10 & 3 & $30 \%$ & $1.50 \mathrm{E}-04$ & 7.43E-04 & 8.33E-04 & 0.0018 & 4.93E-04 \\
\hline RAD & Uranium-238 & $\mathrm{pCi} / \mathrm{g}$ & 11 & 9 & $82 \%$ & 4.16E-04 & 0.0010 & 0.0014 & 0.017 & 0.0066 \\
\hline
\end{tabular}

RAD - radionuclide 
Table F-59

Summary Statistics for Northern Squawfish Fillet Collected from Segment 2 of the Columbia River

\begin{tabular}{|c|c|c|c|c|c|c|c|c|c|c|}
\hline $\begin{array}{l}\text { Constituent } \\
\text { Class }\end{array}$ & Constituent Name & Units & $\begin{array}{c}\text { Number } \\
\text { Analyzed }\end{array}$ & $\begin{array}{l}\text { Number } \\
\text { Detected }\end{array}$ & $\begin{array}{l}\text { Frequency of } \\
\text { Detection }\end{array}$ & $\begin{array}{l}\text { Minimum } \\
\text { Nondetect }\end{array}$ & $\begin{array}{c}\text { Maximum } \\
\text { Nondetect }\end{array}$ & $\begin{array}{c}\text { Minimum } \\
\text { Detect }\end{array}$ & $\begin{array}{l}\text { Maximum } \\
\text { Detect }\end{array}$ & $\begin{array}{c}\text { Average } \\
\text { Concentration }\end{array}$ \\
\hline RAD & Cesium-137 & $\mathrm{pCi} / \mathrm{g}$ & 1 & 0 & $0 \%$ & 0.011 & 0.011 & -- & -- & 0.0056 \\
\hline RAD & Cobalt-58 & $\mathrm{pCi} / \mathrm{g}$ & 1 & 0 & $0 \%$ & 0.0051 & 0.0051 & -- & -- & 0.0025 \\
\hline RAD & Cobalt-60 & $\mathrm{pCi} / \mathrm{g}$ & 1 & 0 & $0 \%$ & 0.0078 & 0.0078 & -- & -- & 0.0039 \\
\hline RAD & Iron-59 & $\mathrm{pCi} / \mathrm{g}$ & 1 & 0 & $0 \%$ & 0.0097 & 0.0097 & -- & -- & 0.0049 \\
\hline RAD & Manganese-54 & $\mathrm{pCi} / \mathrm{g}$ & 1 & 0 & $0 \%$ & 0.0047 & 0.0047 & -- & -- & 0.0024 \\
\hline RAD & Potassium-40 & $\mathrm{pCi} / \mathrm{g}$ & 2 & 2 & $100 \%$ & -- & -- & 2.4 & 3.8 & 3.1 \\
\hline
\end{tabular}

RAD - Radionuclide 
Table F-60

Summary Statistics for Whole Body Northern Squawfish Collected from Segment 2 of the Columbia River

\begin{tabular}{|c|c|c|c|c|c|c|c|c|c|c|}
\hline $\begin{array}{c}\text { Constituent } \\
\text { Class }\end{array}$ & Constituent Name & Units & $\begin{array}{l}\text { Number } \\
\text { Analyzed }\end{array}$ & $\begin{array}{l}\text { Number } \\
\text { Detected }\end{array}$ & $\begin{array}{c}\text { Frequency of } \\
\text { Detection }\end{array}$ & $\begin{array}{l}\text { Minimum } \\
\text { Nondetect }\end{array}$ & $\begin{array}{l}\text { Maximum } \\
\text { Nondetect }\end{array}$ & $\begin{array}{c}\text { Minimum } \\
\text { Detect }\end{array}$ & $\begin{array}{l}\text { Maximum } \\
\text { Detect }\end{array}$ & $\begin{array}{c}\text { Average } \\
\text { Concentration }\end{array}$ \\
\hline$\overline{R A D}$ & Strontium-90 & $\mathrm{pCi} / \mathrm{g}$ & 1 & 1 & $100 \%$ & -- & $\overline{--}$ & 0.20 & 0.20 & 0.20 \\
\hline
\end{tabular}

RAD - Radionuclide 
Table F-61

Summary Statistics for Trout Fillet Collected from Segment 2 of the Columbia River

\begin{tabular}{|c|c|c|c|c|c|c|c|c|c|c|}
\hline $\begin{array}{l}\text { Constituent } \\
\text { Class }\end{array}$ & Constituent Name & Units & $\begin{array}{l}\text { Number } \\
\text { Analyzed }\end{array}$ & $\begin{array}{l}\text { Number } \\
\text { Detected }\end{array}$ & $\begin{array}{l}\text { Frequency of } \\
\text { Detection }\end{array}$ & $\begin{array}{l}\text { Minimum } \\
\text { Nondetect }\end{array}$ & $\begin{array}{l}\text { Maximum } \\
\text { Nondetect }\end{array}$ & $\begin{array}{c}\text { Minimum } \\
\text { Detect }\end{array}$ & $\begin{array}{l}\text { Maximum } \\
\text { Detect }\end{array}$ & $\begin{array}{c}\text { Average } \\
\text { Concentration }\end{array}$ \\
\hline$\overline{\mathrm{RAD}}$ & Potassium-40 & $\mathrm{pCi} / \mathrm{g}$ & 1 & 1 & $100 \%$ & -- & -- & 3.0 & 3.0 & 3.0 \\
\hline RAD & Strontium-90 & $\mathrm{pCi} / \mathrm{g}$ & 1 & 0 & $0 \%$ & 0.0030 & 0.0030 & -- & -- & 0.0015 \\
\hline
\end{tabular}

RAD - radionuclide 


\section{Table F-62}

Summary Statistics for Steelhead Fillet Collected from Segment 2 of the Columbia River

\begin{tabular}{|c|c|c|c|c|c|c|c|c|c|c|}
\hline $\begin{array}{l}\text { Constituent } \\
\text { Class }\end{array}$ & Constituent Name & Units & $\begin{array}{l}\text { Number } \\
\text { Analyzed }\end{array}$ & $\begin{array}{l}\text { Number } \\
\text { Detected }\end{array}$ & $\begin{array}{c}\text { Frequency of } \\
\text { Detection }\end{array}$ & $\begin{array}{c}\text { Minimum } \\
\text { Nondetect }\end{array}$ & $\begin{array}{c}\text { Maximum } \\
\text { Nondetect }\end{array}$ & $\begin{array}{l}\text { Minimum } \\
\text { Detect }\end{array}$ & $\begin{array}{c}\text { Maximum } \\
\text { Detect }\end{array}$ & $\begin{array}{c}\text { Average } \\
\text { Concentration }\end{array}$ \\
\hline$\overline{\mathrm{RAD}}$ & Actinium-228 & $\mathrm{pCi} / \mathrm{g}$ & 5 & 0 & $0 \%$ & 0.088 & 0.18 & -- & -- & 0.071 \\
\hline RAD & Barium-140 & $\mathrm{pCi} / \mathrm{g}$ & 6 & 0 & $0 \%$ & 0.19 & 0.88 & -- & -- & 0.23 \\
\hline RAD & Beryllium-7 & $\mathrm{pCi} / \mathrm{g}$ & 6 & 0 & $0 \%$ & 0.15 & 0.46 & -- & -- & 0.16 \\
\hline RAD & Cerium-141 & $\mathrm{pCi} / \mathrm{g}$ & 6 & 0 & $0 \%$ & 0.043 & 0.10 & -- & -- & 0.033 \\
\hline RAD & Cerium-144 & $\mathrm{pCi} / \mathrm{g}$ & 6 & 0 & $0 \%$ & 0.075 & 0.22 & -- & -- & 0.085 \\
\hline RAD & Cesium-134 & $\mathrm{pCi} / \mathrm{g}$ & 7 & 0 & $0 \%$ & 0.0063 & 0.044 & -- & -- & 0.013 \\
\hline RAD & Cesium-137 & $\mathrm{pCi} / \mathrm{g}$ & 13 & 0 & $0 \%$ & 0.0024 & 0.045 & -- & -- & 0.0095 \\
\hline RAD & Cobalt-57 & $\mathrm{pCi} / \mathrm{g}$ & 6 & 0 & $0 \%$ & 0.0098 & 0.029 & -- & -- & 0.011 \\
\hline RAD & Cobalt-58 & $\mathrm{pCi} / \mathrm{g}$ & 10 & 0 & $0 \%$ & $6.08 \mathrm{E}-04$ & 0.061 & -- & -- & 0.012 \\
\hline RAD & Cobalt- 60 & $\mathrm{pCi} / \mathrm{g}$ & 9 & 0 & $0 \%$ & 0.0021 & 0.047 & -- & -- & 0.012 \\
\hline RAD & Europium-152 & $\mathrm{pCi} / \mathrm{g}$ & 6 & 0 & $0 \%$ & 0.027 & 0.12 & -- & -- & 0.040 \\
\hline RAD & lodine-131 & $\mathrm{pCi} / \mathrm{g}$ & 6 & 0 & $0 \%$ & 0.096 & 0.94 & -- & -- & 0.18 \\
\hline RAD & Iron-59 & $\mathrm{pCi} / \mathrm{g}$ & 13 & 0 & $0 \%$ & 0.0025 & 0.13 & -- & -- & 0.022 \\
\hline RAD & Lanthanum-140 & $\mathrm{pCi} / \mathrm{g}$ & 6 & 0 & $0 \%$ & 0.057 & 0.38 & -- & -- & 0.077 \\
\hline RAD & Manganese-54 & $\mathrm{pCi} / \mathrm{g}$ & 12 & 0 & $0 \%$ & $7.58 \mathrm{E}-04$ & 0.047 & -- & -- & 0.0089 \\
\hline RAD & Niobium-95 & $\mathrm{pCi} / \mathrm{g}$ & 6 & 0 & $0 \%$ & 0.018 & 0.066 & -- & -- & 0.021 \\
\hline RAD & Potassium-40 & $\mathrm{pCi} / \mathrm{g}$ & 13 & 13 & $100 \%$ & -- & -- & 3.2 & 4.5 & 3.6 \\
\hline RAD & Radium-226 & $\mathrm{pCi} / \mathrm{g}$ & 8 & 0 & $0 \%$ & 0.047 & 0.95 & -- & -- & 0.26 \\
\hline RAD & Ruthenium-103 & $\mathrm{pCi} / \mathrm{g}$ & 6 & 0 & $0 \%$ & 0.021 & 0.077 & -- & -- & 0.023 \\
\hline RAD & Ruthenium-106 & $\mathrm{pCi} / \mathrm{g}$ & 6 & 0 & $0 \%$ & 0.12 & 0.37 & -- & -- & 0.13 \\
\hline RAD & Thorium-228 & $\mathrm{pCi} / \mathrm{g}$ & 10 & 0 & $0 \%$ & 0.0039 & 0.77 & -- & -- & 0.17 \\
\hline RAD & Uranium-235 & $\mathrm{pCi} / \mathrm{g}$ & 5 & 0 & $0 \%$ & 0.17 & 0.22 & -- & -- & 0.097 \\
\hline RAD & Zinc-65 & $\mathrm{pCi} / \mathrm{g}$ & 9 & 0 & $0 \%$ & 0.011 & 0.098 & -- & -- & 0.026 \\
\hline RAD & Zirconium-95 & $\mathrm{pCi} / \mathrm{g}$ & 6 & 0 & $0 \%$ & 0.028 & 0.12 & -- & -- & 0.035 \\
\hline
\end{tabular}

RAD - Radionuclide 
Table F-63

Summary Statistics for White Sturgeon Fillet Collected from Segment 2 of the Columbia River

\begin{tabular}{|c|c|c|c|c|c|c|c|c|c|c|}
\hline $\begin{array}{l}\text { Constituent } \\
\text { Class }\end{array}$ & Constituent Name & Units & $\begin{array}{c}\text { Number } \\
\text { Analyzed }\end{array}$ & $\begin{array}{l}\text { Number } \\
\text { Detected }\end{array}$ & $\begin{array}{l}\text { Frequency of } \\
\text { Detection }\end{array}$ & $\begin{array}{l}\text { Minimum } \\
\text { Nondetect }\end{array}$ & $\begin{array}{c}\text { Maximum } \\
\text { Nondetect }\end{array}$ & $\begin{array}{c}\text { Minimum } \\
\text { Detect }\end{array}$ & $\begin{array}{c}\text { Maximum } \\
\text { Detect }\end{array}$ & $\begin{array}{c}\text { Average } \\
\text { Concentration }\end{array}$ \\
\hline DIOXI & $1,2,3,4,6,7,8$-Heptachlorodibenzodioxin & $\mathrm{mg} / \mathrm{kg}$ & 2 & 2 & $100 \%$ & $\overline{--}$ & $\overline{--}$ & $5.00 \mathrm{E}-08$ & $2.70 \mathrm{E}-07$ & $1.60 \mathrm{E}-07$ \\
\hline DIOXI & 1,2,3,4,6,7,8-Heptachlorodibenzofuran & $\mathrm{mg} / \mathrm{kg}$ & 2 & 0 & $0 \%$ & 8.00E-08 & $1.00 \mathrm{E}-07$ & -- & -- & $4.50 \mathrm{E}-08$ \\
\hline DIOXI & 1,2,3,4,7,8,9-Heptachlorodibenzofuran & $\mathrm{mg} / \mathrm{kg}$ & 2 & 0 & $0 \%$ & 8.00E-08 & $1.00 \mathrm{E}-07$ & -- & -- & 4.50E-08 \\
\hline DIOXI & 1,2,3,4,7,8-Hexachlorodibenzofuran & $\mathrm{mg} / \mathrm{kg}$ & 2 & 1 & $50 \%$ & $3.00 \mathrm{E}-08$ & $3.00 \mathrm{E}-08$ & 4.00E-08 & 4.00E-08 & $2.75 \mathrm{E}-08$ \\
\hline DIOXI & 1,2,3,4,7,8-Hexachlorodibenzo-p-dioxin & $\mathrm{mg} / \mathrm{kg}$ & 2 & 0 & $0 \%$ & $2.00 \mathrm{E}-08$ & 4.00E-08 & -- & -- & $1.50 \mathrm{E}-08$ \\
\hline DIOXI & 1,2,3,6,7,8-Hexachlorodibenzofuran & $\mathrm{mg} / \mathrm{kg}$ & 2 & 1 & $50 \%$ & $3.00 \mathrm{E}-08$ & $3.00 \mathrm{E}-08$ & $5.00 \mathrm{E}-08$ & 5.00E-08 & $3.25 \mathrm{E}-08$ \\
\hline DIOXI & 1,2,3,6,7,8-Hexachlorodibenzo-p-dioxin & $\mathrm{mg} / \mathrm{kg}$ & 2 & 2 & $100 \%$ & -- & -- & $9.00 \mathrm{E}-08$ & 1.40E-07 & 1.15E-07 \\
\hline DIOXI & 1,2,3,7,8,9-Hexachlorodibenzofuran & $\mathrm{mg} / \mathrm{kg}$ & 2 & 0 & $0 \%$ & $3.00 \mathrm{E}-08$ & $3.00 \mathrm{E}-08$ & -- & -- & $1.50 \mathrm{E}-08$ \\
\hline DIOXI & 1,2,3,7,8,9-Hexachlorodibenzo-p-dioxin & $\mathrm{mg} / \mathrm{kg}$ & 2 & 1 & $50 \%$ & $2.00 \mathrm{E}-08$ & $2.00 \mathrm{E}-08$ & $6.00 \mathrm{E}-08$ & $6.00 \mathrm{E}-08$ & $3.50 \mathrm{E}-08$ \\
\hline DIOXI & 1,2,3,7,8-Pentachlorodibenzofuran & $\mathrm{mg} / \mathrm{kg}$ & 2 & 2 & $100 \%$ & -- & -- & $1.50 \mathrm{E}-07$ & 1.60E-07 & $1.55 \mathrm{E}-07$ \\
\hline DIOXI & 1,2,3,7,8-Pentachlorodibenzo-p-dioxin & $\mathrm{mg} / \mathrm{kg}$ & 2 & 2 & $100 \%$ & -- & -- & $5.00 \mathrm{E}-08$ & 5.00E-08 & $5.00 \mathrm{E}-08$ \\
\hline DIOXI & 2,3,4,6,7,8-Hexachlorodibenzofuran & $\mathrm{mg} / \mathrm{kg}$ & 2 & 2 & $100 \%$ & -- & -- & 4.00E-08 & 4.00E-08 & 4.00E-08 \\
\hline DIOXI & 2,3,4,7,8-Pentachlorodibenzofuran & $\mathrm{mg} / \mathrm{kg}$ & 2 & 2 & $100 \%$ & -- & -- & $2.30 \mathrm{E}-07$ & $2.30 \mathrm{E}-07$ & $2.30 \mathrm{E}-07$ \\
\hline DIOXI & 2,3,7,8-Tetrachlorodibenzofuran & $\mathrm{mg} / \mathrm{kg}$ & 2 & 2 & $100 \%$ & -- & -- & 2.11E-05 & 2.35E-05 & 2.23E-05 \\
\hline DIOXI & 2,3,7,8-Tetrachlorodibenzo-p-dioxin & $\mathrm{mg} / \mathrm{kg}$ & 2 & 2 & $100 \%$ & -- & -- & $2.30 \mathrm{E}-07$ & $2.40 \mathrm{E}-07$ & $2.35 \mathrm{E}-07$ \\
\hline DIOXI & Octachlorodibenzofuran & $\mathrm{mg} / \mathrm{kg}$ & 2 & 2 & $100 \%$ & -- & -- & $1.90 \mathrm{E}-07$ & $2.50 \mathrm{E}-07$ & $2.20 \mathrm{E}-07$ \\
\hline DIOXI & Octachlorodibenzo-p-dioxin & $\mathrm{mg} / \mathrm{kg}$ & 2 & 2 & $100 \%$ & -- & -- & $3.00 \mathrm{E}-08$ & 1.15E-06 & $5.90 \mathrm{E}-07$ \\
\hline METAL & Aluminum & $\mathrm{mg} / \mathrm{kg}$ & 2 & 0 & $0 \%$ & 1.0 & 1.0 & -- & -- & 0.50 \\
\hline METAL & Antimony & $\mathrm{mg} / \mathrm{kg}$ & 2 & 0 & $0 \%$ & 0.050 & 0.050 & -- & -- & 0.025 \\
\hline METAL & Arsenic & $\mathrm{mg} / \mathrm{kg}$ & 2 & 2 & $100 \%$ & -- & -- & 0.26 & 0.26 & 0.26 \\
\hline METAL & Barium & $\mathrm{mg} / \mathrm{kg}$ & 2 & 1 & $50 \%$ & 0.20 & 0.20 & 0.50 & 0.50 & 0.30 \\
\hline METAL & Beryllium & $\mathrm{mg} / \mathrm{kg}$ & 2 & 0 & $0 \%$ & 0.0040 & 0.0040 & -- & -- & 0.0020 \\
\hline METAL & Cadmium & $\mathrm{mg} / \mathrm{kg}$ & 2 & 1 & $50 \%$ & 0.0040 & 0.0040 & 0.0049 & 0.0049 & 0.0035 \\
\hline METAL & Chromium & $\mathrm{mg} / \mathrm{kg}$ & 2 & 2 & $100 \%$ & -- & -- & 0.12 & 0.16 & 0.14 \\
\hline METAL & Cobalt & $\mathrm{mg} / \mathrm{kg}$ & 2 & 2 & $100 \%$ & -- & -- & 0.080 & 0.080 & 0.080 \\
\hline METAL & Copper & $\mathrm{mg} / \mathrm{kg}$ & 2 & 2 & $100 \%$ & -- & -- & 0.23 & 0.23 & 0.23 \\
\hline METAL & Lead & $\mathrm{mg} / \mathrm{kg}$ & 2 & 0 & $0 \%$ & 0.010 & 0.010 & -- & -- & 0.0050 \\
\hline METAL & Manganese & $\mathrm{mg} / \mathrm{kg}$ & 2 & 2 & $100 \%$ & -- & -- & 0.14 & 0.14 & 0.14 \\
\hline METAL & Mercury & $\mathrm{mg} / \mathrm{kg}$ & 2 & 2 & $100 \%$ & -- & -- & 0.33 & 0.38 & 0.36 \\
\hline METAL & Nickel & $\mathrm{mg} / \mathrm{kg}$ & 2 & 1 & $50 \%$ & 0.030 & 0.030 & 0.040 & 0.040 & 0.028 \\
\hline METAL & Selenium & $\mathrm{mg} / \mathrm{kg}$ & 2 & 2 & $100 \%$ & -- & -- & 0.61 & 0.65 & 0.63 \\
\hline METAL & Silver & $\mathrm{mg} / \mathrm{kg}$ & 2 & 0 & $0 \%$ & 0.10 & 0.10 & -- & -- & 0.050 \\
\hline METAL & Thallium & $\mathrm{mg} / \mathrm{kg}$ & 2 & 0 & $0 \%$ & 0.050 & 0.050 & -- & -- & 0.025 \\
\hline METAL & Vanadium & $\mathrm{mg} / \mathrm{kg}$ & 2 & 0 & $0 \%$ & 0.010 & 0.010 & -- & -- & 0.0050 \\
\hline METAL & Zinc & $\mathrm{mg} / \mathrm{kg}$ & 2 & 2 & $100 \%$ & -- & -- & 4.4 & 4.5 & 4.5 \\
\hline PCB & 2,2',3,3',4,4',5-Heptachloro-1,1'-biphenyl & $\mathrm{mg} / \mathrm{kg}$ & 2 & 2 & $100 \%$ & -- & -- & 0.0068 & 0.0068 & 0.0068 \\
\hline PCB & 2,2',3,4,4',5,5'-Heptachlorobiphenyl & $\mathrm{mg} / \mathrm{kg}$ & 2 & 2 & $100 \%$ & -- & -- & 0.023 & 0.023 & 0.023 \\
\hline РСB & 2,3,3',4,4',5'-Hexachlorobiphenyl & $\mathrm{mg} / \mathrm{kg}$ & 2 & 2 & $100 \%$ & -- & -- & $7.29 \mathrm{E}-04$ & 7.56E-04 & 7.43E-04 \\
\hline РCB & 2,3,3',4,4',5-Hexachlorobiphenyl (BZ 156) & $\mathrm{mg} / \mathrm{kg}$ & 2 & 2 & $100 \%$ & -- & -- & 0.0035 & 0.0035 & 0.0035 \\
\hline PCB & 2,3,3',4,4'-Pentachlorobiphenyl & $\mathrm{mg} / \mathrm{kg}$ & 2 & 2 & $100 \%$ & -- & -- & 0.0093 & 0.0099 & 0.0096 \\
\hline РСВ & 2,3',4,4',5,5'-Hexachlorobiphenyl (BZ 167) & $\mathrm{mg} / \mathrm{kg}$ & 2 & 2 & $100 \%$ & -- & -- & 1.67E-04 & 1.74E-04 & $1.71 \mathrm{E}-04$ \\
\hline PCB & 2',3,4,4',5-Pentachloro-1,1'-biphenyl (BZ 123) & $\mathrm{mg} / \mathrm{kg}$ & 2 & 2 & $100 \%$ & -- & -- & $1.52 \mathrm{E}-04$ & 1.73E-04 & $1.63 \mathrm{E}-04$ \\
\hline РСВ & 2,3,4,4',5-Pentachlorobiphenyl (BZ 114) & $\mathrm{mg} / \mathrm{kg}$ & 2 & 2 & $100 \%$ & -- & -- & 7.39E-04 & 7.63E-04 & 7.51E-04 \\
\hline РCB & 2,3',4,4',5-Pentachlorobiphenyl (BZ 118) & $\mathrm{mg} / \mathrm{kg}$ & 2 & 2 & $100 \%$ & -- & -- & 0.014 & 0.014 & 0.014 \\
\hline PCB & 2,3,4,5,3',4',5'-Heptachlorobiphenyl & $\mathrm{mg} / \mathrm{kg}$ & 2 & 2 & $100 \%$ & -- & -- & 3.03E-04 & 3.09E-04 & 3.06E-04 \\
\hline РСВ & 3,3',4,4',5,5'-Hexachlorobiphenyl (BZ 169) & $\mathrm{mg} / \mathrm{kg}$ & 2 & 0 & $0 \%$ & $3.78 \mathrm{E}-06$ & 4.24E-06 & -- & -- & 2.01E-06 \\
\hline РCB & 3,3',4,4',5-Pentachlorobiphenyl (BZ 126) & $\mathrm{mg} / \mathrm{kg}$ & 2 & 0 & $0 \%$ & 2.07E-06 & 2.09E-06 & -- & -- & $1.04 \mathrm{E}-06$ \\
\hline PCB & 3,3',4,4'-Tetrachlorobiphenyl (BZ 77) & $\mathrm{mg} / \mathrm{kg}$ & 2 & 2 & $100 \%$ & -- & -- & 5.93E-05 & $6.25 \mathrm{E}-05$ & 6.09E-05 \\
\hline РСВ & Aroclor-1016 & $\mathrm{mg} / \mathrm{kg}$ & 2 & 0 & $0 \%$ & 0.019 & 0.019 & -- & -- & 0.0095 \\
\hline
\end{tabular}


Table F-63

Summary Statistics for White Sturgeon Fillet Collected from Segment 2 of the Columbia River

\begin{tabular}{|c|c|c|c|c|c|c|c|c|c|c|}
\hline $\begin{array}{l}\text { Constituent } \\
\text { Class }\end{array}$ & Constituent Name & Units & $\begin{array}{l}\text { Number } \\
\text { Analyzed }\end{array}$ & $\begin{array}{l}\text { Number } \\
\text { Detected }\end{array}$ & $\begin{array}{c}\text { Frequency of } \\
\text { Detection }\end{array}$ & $\begin{array}{c}\text { Minimum } \\
\text { Nondetect }\end{array}$ & $\begin{array}{l}\text { Maximum } \\
\text { Nondetect }\end{array}$ & $\begin{array}{l}\text { Minimum } \\
\text { Detect }\end{array}$ & $\begin{array}{l}\text { Maximum } \\
\text { Detect }\end{array}$ & $\begin{array}{c}\text { Average } \\
\text { Concentration }\end{array}$ \\
\hline$\overline{\mathrm{PCB}}$ & Aroclor-1221 & $\mathrm{mg} / \mathrm{kg}$ & 2 & 0 & $0 \%$ & 0.019 & 0.019 & $\overline{--}$ & $\overline{--}$ & 0.0095 \\
\hline PCB & Aroclor-1232 & $\mathrm{mg} / \mathrm{kg}$ & 2 & 0 & $0 \%$ & 0.019 & 0.019 & -- & -- & 0.0095 \\
\hline РСВ & Aroclor-1248 & $\mathrm{mg} / \mathrm{kg}$ & 2 & 0 & $0 \%$ & 0.019 & 0.019 & -- & -- & 0.0095 \\
\hline РСВ & Aroclor-1254 & $\mathrm{mg} / \mathrm{kg}$ & 2 & 2 & $100 \%$ & -- & -- & 0.17 & 0.21 & 0.19 \\
\hline РСВ & Aroclor-1260 & $\mathrm{mg} / \mathrm{kg}$ & 2 & 2 & $100 \%$ & -- & -- & 0.19 & 0.21 & 0.20 \\
\hline PEST & 2,4'-DDD & $\mathrm{mg} / \mathrm{kg}$ & 2 & 2 & $100 \%$ & -- & -- & 0.022 & 0.025 & 0.024 \\
\hline PEST & 2,4'-DDE & $\mathrm{mg} / \mathrm{kg}$ & 2 & 2 & $100 \%$ & -- & -- & 0.020 & 0.020 & 0.020 \\
\hline PEST & 2,4'-DDT & $\mathrm{mg} / \mathrm{kg}$ & 2 & 0 & $0 \%$ & 0.0019 & 0.0019 & -- & -- & $9.50 \mathrm{E}-04$ \\
\hline PEST & Aldrin & $\mathrm{mg} / \mathrm{kg}$ & 2 & 2 & $100 \%$ & -- & -- & $9.30 \mathrm{E}-04$ & $9.40 \mathrm{E}-04$ & $9.35 \mathrm{E}-04$ \\
\hline PEST & Alpha-BHC & $\mathrm{mg} / \mathrm{kg}$ & 2 & 0 & $0 \%$ & 0.0019 & 0.0019 & -- & -- & $9.50 \mathrm{E}-04$ \\
\hline PEST & alpha-Chlordane & $\mathrm{mg} / \mathrm{kg}$ & 2 & 2 & $100 \%$ & -- & -- & 0.0028 & 0.0037 & 0.0033 \\
\hline PEST & alpha-Chlordene & $\mathrm{mg} / \mathrm{kg}$ & 2 & 0 & $0 \%$ & 0.0019 & 0.0019 & -- & -- & $9.50 \mathrm{E}-04$ \\
\hline PEST & Beta-BHC & $\mathrm{mg} / \mathrm{kg}$ & 2 & 0 & $0 \%$ & 0.0019 & 0.0019 & -- & -- & $9.50 \mathrm{E}-04$ \\
\hline PEST & cis-Nonachlor & $\mathrm{mg} / \mathrm{kg}$ & 2 & 2 & $100 \%$ & -- & -- & 0.027 & 0.028 & 0.028 \\
\hline PEST & DDMU & $\mathrm{mg} / \mathrm{kg}$ & 2 & 2 & $100 \%$ & -- & -- & 0.045 & 0.046 & 0.046 \\
\hline PEST & Delta-BHC & $\mathrm{mg} / \mathrm{kg}$ & 2 & 0 & $0 \%$ & 0.0019 & 0.0019 & -- & -- & $9.50 \mathrm{E}-04$ \\
\hline PEST & Dichlorodiphenyldichloroethane & $\mathrm{mg} / \mathrm{kg}$ & 2 & 2 & $100 \%$ & -- & -- & 0.33 & 0.36 & 0.35 \\
\hline PEST & Dichlorodiphenyldichloroethylene & $\mathrm{mg} / \mathrm{kg}$ & 2 & 2 & $100 \%$ & -- & -- & 1.3 & 1.4 & 1.4 \\
\hline PEST & Dichlorodiphenyltrichloroethane & $\mathrm{mg} / \mathrm{kg}$ & 2 & 2 & $100 \%$ & -- & -- & 0.014 & 0.019 & 0.017 \\
\hline PEST & Endosulfan sulfate & $\mathrm{mg} / \mathrm{kg}$ & 2 & 0 & $0 \%$ & 0.0019 & 0.0019 & -- & -- & $9.50 \mathrm{E}-04$ \\
\hline PEST & Gamma-BHC (Lindane) & $\mathrm{mg} / \mathrm{kg}$ & 2 & 0 & $0 \%$ & 0.0019 & 0.0019 & -- & -- & $9.50 \mathrm{E}-04$ \\
\hline PEST & gamma-Chlordane & $\mathrm{mg} / \mathrm{kg}$ & 2 & 2 & $100 \%$ & -- & -- & 0.0069 & 0.0071 & 0.0070 \\
\hline PEST & gamma-Chlordene & $\mathrm{mg} / \mathrm{kg}$ & 2 & 0 & $0 \%$ & 0.0019 & 0.0019 & -- & -- & $9.50 \mathrm{E}-04$ \\
\hline PEST & Heptachlor & $\mathrm{mg} / \mathrm{kg}$ & 2 & 0 & $0 \%$ & 0.0019 & 0.0019 & -- & -- & $9.50 \mathrm{E}-04$ \\
\hline PEST & Heptachlor epoxide & $\mathrm{mg} / \mathrm{kg}$ & 2 & 0 & $0 \%$ & 0.0019 & 0.0019 & -- & -- & $9.50 \mathrm{E}-04$ \\
\hline PEST & Mirex & $\mathrm{mg} / \mathrm{kg}$ & 2 & 0 & $0 \%$ & 0.0019 & 0.0019 & -- & -- & $9.50 \mathrm{E}-04$ \\
\hline PEST & Oxychlordane & $\mathrm{mg} / \mathrm{kg}$ & 2 & 2 & $100 \%$ & -- & -- & 0.0023 & 0.0027 & 0.0025 \\
\hline PEST & Pentachloroanisole & $\mathrm{mg} / \mathrm{kg}$ & 2 & 0 & $0 \%$ & 0.0019 & 0.0019 & -- & -- & $9.50 \mathrm{E}-04$ \\
\hline PEST & Toxaphene & $\mathrm{mg} / \mathrm{kg}$ & 2 & 0 & $0 \%$ & 0.056 & 0.056 & -- & -- & 0.028 \\
\hline PEST & trans-Nonachlor & $\mathrm{mg} / \mathrm{kg}$ & 2 & 2 & $100 \%$ & -- & -- & 0.014 & 0.014 & 0.014 \\
\hline RAD & Antimony-125 & $\mathrm{pCi} / \mathrm{g}$ & 11 & 0 & $0 \%$ & 0.0014 & 0.065 & -- & -- & 0.0082 \\
\hline RAD & Beryllium-7 & $\mathrm{pCi} / \mathrm{g}$ & 14 & 0 & $0 \%$ & 0.0063 & 0.31 & -- & -- & 0.053 \\
\hline RAD & Cesium-134 & $\mathrm{pCi} / \mathrm{g}$ & 15 & 0 & $0 \%$ & 8.65E-04 & 0.016 & -- & -- & 0.0034 \\
\hline RAD & Cesium-137 & $\mathrm{pCi} / \mathrm{g}$ & 26 & 1 & $4 \%$ & $6.82 \mathrm{E}-04$ & 0.036 & 0.020 & 0.020 & 0.0081 \\
\hline RAD & Cobalt-60 & $\mathrm{pCi} / \mathrm{g}$ & 24 & 3 & $13 \%$ & 0.0048 & 0.041 & 0.024 & 0.037 & 0.010 \\
\hline RAD & Europium-152 & $\mathrm{pCi} / \mathrm{g}$ & 2 & 0 & $0 \%$ & 3.13E-04 & 0.0046 & -- & -- & 0.0012 \\
\hline RAD & Europium-154 & $\mathrm{pCi} / \mathrm{g}$ & 18 & 1 & $6 \%$ & 0.0056 & 0.088 & 0.066 & 0.066 & 0.017 \\
\hline RAD & Europium-155 & $\mathrm{pCi} / \mathrm{g}$ & 21 & 1 & $5 \%$ & 7.79E-04 & 0.070 & 0.030 & 0.030 & 0.013 \\
\hline RAD & Manganese-54 & $\mathrm{pCi} / \mathrm{g}$ & 1 & 0 & $0 \%$ & 0.0022 & 0.0022 & -- & -- & 0.0011 \\
\hline RAD & Potassium-40 & $\mathrm{pCi} / \mathrm{g}$ & 35 & 34 & $97 \%$ & 2.6 & 2.6 & 2.2 & 5.1 & 3.6 \\
\hline RAD & Ruthenium-106 & $\mathrm{pCi} / \mathrm{g}$ & 10 & 0 & $0 \%$ & 0.0089 & 0.17 & -- & -- & 0.030 \\
\hline RAD & Strontium-90 & $\mathrm{pCi} / \mathrm{g}$ & 9 & 0 & $0 \%$ & $3.97 \mathrm{E}-04$ & 0.0048 & -- & -- & 8.00E-04 \\
\hline RAD & Technetium-99 & $\mathrm{pCi} / \mathrm{g}$ & 1 & 0 & $0 \%$ & 0.047 & 0.047 & -- & -- & 0.024 \\
\hline RAD & Uranium-234 & $\mathrm{pCi} / \mathrm{g}$ & 1 & 0 & $0 \%$ & 0.0012 & 0.0012 & -- & -- & $5.80 \mathrm{E}-04$ \\
\hline RAD & Uranium-235 & $\mathrm{pCi} / \mathrm{g}$ & 1 & 0 & $0 \%$ & $4.58 \mathrm{E}-04$ & $4.58 \mathrm{E}-04$ & -- & -- & $2.29 \mathrm{E}-04$ \\
\hline RAD & Uranium-238 & $\mathrm{pCi} / \mathrm{g}$ & 2 & 0 & $0 \%$ & 4.05E-04 & $5.38 \mathrm{E}-04$ & -- & -- & $2.36 \mathrm{E}-04$ \\
\hline RAD & Zinc-65 & $\mathrm{pCi} / \mathrm{g}$ & 1 & 0 & $0 \%$ & 7.91E-04 & $7.91 \mathrm{E}-04$ & -- & -- & $3.96 \mathrm{E}-04$ \\
\hline RAD & Zirconium/Niobium-95 & $\mathrm{pCi} / \mathrm{g}$ & 1 & 0 & $0 \%$ & 0.0078 & 0.0078 & -- & -- & 0.0039 \\
\hline SVOC & 1,2,4-Trichlorobenzene & $\mathrm{mg} / \mathrm{kg}$ & 2 & 0 & $0 \%$ & 0.0088 & 0.0096 & -- & -- & 0.0046 \\
\hline
\end{tabular}


Table F-63

Summary Statistics for White Sturgeon Fillet Collected from Segment 2 of the Columbia River

\begin{tabular}{|c|c|c|c|c|c|c|c|c|c|c|}
\hline $\begin{array}{l}\text { Constituent } \\
\text { Class }\end{array}$ & Constituent Name & Units & $\begin{array}{c}\text { Number } \\
\text { Analyzed }\end{array}$ & $\begin{array}{l}\text { Number } \\
\text { Detected }\end{array}$ & $\begin{array}{l}\text { Frequency of } \\
\text { Detection }\end{array}$ & $\begin{array}{l}\text { Minimum } \\
\text { Nondetect }\end{array}$ & $\begin{array}{l}\text { Maximum } \\
\text { Nondetect }\end{array}$ & $\begin{array}{l}\text { Minimum } \\
\text { Detect }\end{array}$ & $\begin{array}{c}\text { Maximum } \\
\text { Detect }\end{array}$ & $\begin{array}{c}\text { Average } \\
\text { Concentration }\end{array}$ \\
\hline SVOC & 1,2-Dichlorobenzene & $\mathrm{mg} / \mathrm{kg}$ & 2 & 0 & $0 \%$ & 0.0088 & 0.0096 & $\overline{--}$ & $\overline{--}$ & 0.0046 \\
\hline SVOC & 1,2-Diphenylhydrazine & $\mathrm{mg} / \mathrm{kg}$ & 2 & 0 & $0 \%$ & 0.0088 & 0.0096 & -- & -- & 0.0046 \\
\hline SVOC & 1,3-Dichlorobenzene & $\mathrm{mg} / \mathrm{kg}$ & 2 & 0 & $0 \%$ & 0.0088 & 0.0096 & -- & -- & 0.0046 \\
\hline SVOC & 1,4-Dichlorobenzene & $\mathrm{mg} / \mathrm{kg}$ & 2 & 0 & $0 \%$ & 0.0088 & 0.0096 & -- & -- & 0.0046 \\
\hline SVOC & 1-methylnaphthalene & $\mathrm{mg} / \mathrm{kg}$ & 2 & 0 & $0 \%$ & 0.0088 & 0.0096 & -- & -- & 0.0046 \\
\hline SVOC & 2,4-Dinitrotoluene & $\mathrm{mg} / \mathrm{kg}$ & 2 & 0 & $0 \%$ & 0.018 & 0.019 & -- & -- & 0.0093 \\
\hline SVOC & 2,6-Dinitrotoluene & $\mathrm{mg} / \mathrm{kg}$ & 2 & 0 & $0 \%$ & 0.035 & 0.038 & -- & -- & 0.018 \\
\hline SVOC & 2-Chloronaphthalene & $\mathrm{mg} / \mathrm{kg}$ & 2 & 0 & $0 \%$ & 0.018 & 0.019 & -- & -- & 0.0093 \\
\hline SVOC & 2-Methylnaphthalene & $\mathrm{mg} / \mathrm{kg}$ & 2 & 0 & $0 \%$ & 0.0088 & 0.0096 & -- & -- & 0.0046 \\
\hline SVOC & 4-Bromophenylphenyl ether & $\mathrm{mg} / \mathrm{kg}$ & 2 & 0 & $0 \%$ & 0.0088 & 0.0096 & -- & -- & 0.0046 \\
\hline SVOC & 4-Chlorophenylphenyl ether & $\mathrm{mg} / \mathrm{kg}$ & 2 & 0 & $0 \%$ & 0.0088 & 0.0096 & -- & -- & 0.0046 \\
\hline SVOC & Acenaphthene & $\mathrm{mg} / \mathrm{kg}$ & 2 & 0 & $0 \%$ & 0.018 & 0.019 & -- & -- & 0.0093 \\
\hline SVOC & Acenaphthylene & $\mathrm{mg} / \mathrm{kg}$ & 2 & 0 & $0 \%$ & 0.0088 & 0.0096 & -- & -- & 0.0046 \\
\hline SVOC & Anthracene & $\mathrm{mg} / \mathrm{kg}$ & 2 & 0 & $0 \%$ & 0.0088 & 0.0096 & -- & -- & 0.0046 \\
\hline SVOC & Benzo(a)anthracene & $\mathrm{mg} / \mathrm{kg}$ & 2 & 0 & $0 \%$ & 0.0088 & 0.0096 & -- & -- & 0.0046 \\
\hline SVOC & Benzo(a)pyrene & $\mathrm{mg} / \mathrm{kg}$ & 2 & 0 & $0 \%$ & 0.0088 & 0.0096 & -- & -- & 0.0046 \\
\hline SVOC & Benzo(b)fluoranthene & $\mathrm{mg} / \mathrm{kg}$ & 2 & 0 & $0 \%$ & 0.0088 & 0.0096 & -- & -- & 0.0046 \\
\hline SVOC & Benzo(ghi)perylene & $\mathrm{mg} / \mathrm{kg}$ & 2 & 0 & $0 \%$ & 0.018 & 0.019 & -- & -- & 0.0093 \\
\hline SVOC & Benzo(k)fluoranthene & $\mathrm{mg} / \mathrm{kg}$ & 2 & 0 & $0 \%$ & 0.0088 & 0.0096 & -- & -- & 0.0046 \\
\hline SVOC & Bis(2-chloro-1-methylethyl)ether & $\mathrm{mg} / \mathrm{kg}$ & 2 & 0 & $0 \%$ & 0.018 & 0.019 & -- & -- & 0.0093 \\
\hline SVOC & Chrysene & $\mathrm{mg} / \mathrm{kg}$ & 2 & 0 & $0 \%$ & 0.0088 & 0.0096 & -- & -- & 0.0046 \\
\hline SVOC & Dibenz[a,h]anthracene & $\mathrm{mg} / \mathrm{kg}$ & 2 & 0 & $0 \%$ & 0.018 & 0.019 & -- & -- & 0.0093 \\
\hline SVOC & Dibenzofuran & $\mathrm{mg} / \mathrm{kg}$ & 2 & 0 & $0 \%$ & 0.0088 & 0.0096 & -- & -- & 0.0046 \\
\hline SVOC & Fluoranthene & $\mathrm{mg} / \mathrm{kg}$ & 2 & 0 & $0 \%$ & 0.018 & 0.019 & -- & -- & 0.0093 \\
\hline SVOC & Fluorene & $\mathrm{mg} / \mathrm{kg}$ & 2 & 0 & $0 \%$ & 0.0049 & 0.024 & -- & -- & 0.0072 \\
\hline SVOC & Hexachlorobenzene & $\mathrm{mg} / \mathrm{kg}$ & 2 & 0 & $0 \%$ & 0.018 & 0.019 & -- & -- & 0.0093 \\
\hline SVOC & Hexachlorobutadiene & $\mathrm{mg} / \mathrm{kg}$ & 2 & 0 & $0 \%$ & 0.0088 & 0.0096 & -- & -- & 0.0046 \\
\hline SVOC & Hexachloroethane & $\mathrm{mg} / \mathrm{kg}$ & 2 & 0 & $0 \%$ & 0.0088 & 0.0096 & -- & -- & 0.0046 \\
\hline SVOC & Indeno(1,2,3-cd)pyrene & $\mathrm{mg} / \mathrm{kg}$ & 2 & 0 & $0 \%$ & 0.018 & 0.019 & -- & -- & 0.0093 \\
\hline SVOC & Naphthalene & $\mathrm{mg} / \mathrm{kg}$ & 2 & 0 & $0 \%$ & 0.0088 & 0.0096 & -- & -- & 0.0046 \\
\hline SVOC & Nitrobenzene & $\mathrm{mg} / \mathrm{kg}$ & 2 & 0 & $0 \%$ & 0.0088 & 0.0096 & -- & -- & 0.0046 \\
\hline SVOC & Phenanthrene & $\mathrm{mg} / \mathrm{kg}$ & 2 & 0 & $0 \%$ & 0.0088 & 0.0096 & -- & -- & 0.0046 \\
\hline SVOC & Pyrene & $\mathrm{mg} / \mathrm{kg}$ & 2 & 0 & $0 \%$ & 0.018 & 0.019 & -- & -- & 0.0093 \\
\hline VOC & Retene & $\mathrm{mg} / \mathrm{kg}$ & 2 & 0 & $0 \%$ & 0.018 & 0.019 & -- & -- & 0.0093 \\
\hline
\end{tabular}

DIOXI - Dioxins and Furans

PEST - Pesticide

PCB - Polychlorinated biphenyl

RAD- radionuclide

SVOC - Semivolatile Organic Compound

VOC - Volatile Organic Compound 
Table F-64

Summary Statistics for Whole Body White Sturgeon Collected from Segment 2 of the Columbia River

\begin{tabular}{|c|c|c|c|c|c|c|c|c|c|c|}
\hline $\begin{array}{l}\text { Constituent } \\
\text { Class }\end{array}$ & Constituent Name & Units & $\begin{array}{l}\text { Number } \\
\text { Analyzed }\end{array}$ & $\begin{array}{l}\text { Number } \\
\text { Detected }\end{array}$ & $\begin{array}{c}\text { Frequency of } \\
\text { Detection }\end{array}$ & $\begin{array}{l}\text { Minimum } \\
\text { Nondetect }\end{array}$ & $\begin{array}{l}\text { Maximum } \\
\text { Nondetect }\end{array}$ & $\begin{array}{c}\text { Minimum } \\
\text { Detect }\end{array}$ & $\begin{array}{l}\text { Maximum } \\
\text { Detect }\end{array}$ & $\begin{array}{c}\text { Average } \\
\text { Concentration }\end{array}$ \\
\hline$\overline{D I O X I}$ & $1,2,3,4,6,7,8$-Heptachlorodibenzodioxin & $\mathrm{mg} / \mathrm{kg}$ & 2 & 2 & $100 \%$ & -- & -- & $3.80 \mathrm{E}-07$ & $7.50 \mathrm{E}-07$ & $5.65 \mathrm{E}-07$ \\
\hline DIOXI & 1,2,3,4,6,7,8-Heptachlorodibenzofuran & $\mathrm{mg} / \mathrm{kg}$ & 2 & 1 & $50 \%$ & 1.10E-07 & 1.10E-07 & $2.10 \mathrm{E}-07$ & 2.10E-07 & 1.33E-07 \\
\hline DIOXI & 1,2,3,4,7,8,9-Heptachlorodibenzofuran & $\mathrm{mg} / \mathrm{kg}$ & 2 & 0 & $0 \%$ & 1.10E-07 & 1.70E-07 & -- & -- & $7.00 \mathrm{E}-08$ \\
\hline DIOXI & $1,2,3,4,7,8$-Hexachlorodibenzofuran & $\mathrm{mg} / \mathrm{kg}$ & 2 & 2 & $100 \%$ & -- & -- & $3.00 \mathrm{E}-08$ & $1.20 \mathrm{E}-07$ & $7.50 \mathrm{E}-08$ \\
\hline DIOXI & 1,2,3,4,7,8-Hexachlorodibenzo-p-dioxin & $\mathrm{mg} / \mathrm{kg}$ & 2 & 0 & $0 \%$ & 4.00E-08 & $5.00 \mathrm{E}-08$ & -- & -- & $2.25 \mathrm{E}-08$ \\
\hline DIOXI & $1,2,3,6,7,8$-Hexachlorodibenzofuran & $\mathrm{mg} / \mathrm{kg}$ & 2 & 1 & $50 \%$ & 5.00E-08 & $5.00 \mathrm{E}-08$ & $3.00 \mathrm{E}-08$ & $3.00 \mathrm{E}-08$ & 2.75E-08 \\
\hline DIOXI & 1,2,3,6,7,8-Hexachlorodibenzo-p-dioxin & $\mathrm{mg} / \mathrm{kg}$ & 2 & 2 & $100 \%$ & -- & -- & 7.00E-08 & $3.40 \mathrm{E}-07$ & 2.05E-07 \\
\hline DIOXI & 1,2,3,7,8,9-Hexachlorodibenzofuran & $\mathrm{mg} / \mathrm{kg}$ & 2 & 1 & $50 \%$ & $3.00 \mathrm{E}-08$ & $3.00 \mathrm{E}-08$ & $5.00 \mathrm{E}-08$ & $5.00 \mathrm{E}-08$ & $3.25 \mathrm{E}-08$ \\
\hline DIOXI & 1,2,3,7,8,9-Hexachlorodibenzo-p-dioxin & $\mathrm{mg} / \mathrm{kg}$ & 2 & 1 & $50 \%$ & 4.00E-08 & 4.00E-08 & $5.00 \mathrm{E}-08$ & $5.00 \mathrm{E}-08$ & $3.50 \mathrm{E}-08$ \\
\hline DIOXI & 1,2,3,7,8-Pentachlorodibenzofuran & $\mathrm{mg} / \mathrm{kg}$ & 2 & 2 & $100 \%$ & -- & -- & $1.20 \mathrm{E}-07$ & 4.70E-07 & 2.95E-07 \\
\hline DIOXI & 1,2,3,7,8-Pentachlorodibenzo-p-dioxin & $\mathrm{mg} / \mathrm{kg}$ & 2 & 2 & $100 \%$ & -- & -- & $3.00 \mathrm{E}-08$ & $9.00 \mathrm{E}-08$ & $6.00 \mathrm{E}-08$ \\
\hline DIOXI & 2,3,4,6,7,8-Hexachlorodibenzofuran & $\mathrm{mg} / \mathrm{kg}$ & 2 & 1 & $50 \%$ & 3.00E-08 & $3.00 \mathrm{E}-08$ & $5.00 \mathrm{E}-08$ & $5.00 \mathrm{E}-08$ & 3.25E-08 \\
\hline DIOXI & 2,3,4,7,8-Pentachlorodibenzofuran & $\mathrm{mg} / \mathrm{kg}$ & 2 & 2 & $100 \%$ & -- & -- & $1.30 \mathrm{E}-07$ & $5.30 \mathrm{E}-07$ & 3.30E-07 \\
\hline DIOXI & 2,3,7,8-Tetrachlorodibenzofuran & $\mathrm{mg} / \mathrm{kg}$ & 2 & 2 & $100 \%$ & -- & -- & $9.03 \mathrm{E}-06$ & 4.73E-05 & 2.82E-05 \\
\hline DIOXI & 2,3,7,8-Tetrachlorodibenzo-p-dioxin & $\mathrm{mg} / \mathrm{kg}$ & 2 & 2 & $100 \%$ & -- & -- & $6.00 \mathrm{E}-08$ & 1.25E-06 & $6.55 \mathrm{E}-07$ \\
\hline DIOXI & Octachlorodibenzofuran & $\mathrm{mg} / \mathrm{kg}$ & 2 & 2 & $100 \%$ & -- & -- & 3.30E-07 & $9.50 \mathrm{E}-07$ & $6.40 \mathrm{E}-07$ \\
\hline DIOXI & Octachlorodibenzo-p-dioxin & $\mathrm{mg} / \mathrm{kg}$ & 2 & 2 & $100 \%$ & -- & -- & $1.53 \mathrm{E}-06$ & $3.52 \mathrm{E}-06$ & $2.53 \mathrm{E}-06$ \\
\hline METAL & Aluminum & $\mathrm{mg} / \mathrm{kg}$ & 2 & 1 & $50 \%$ & 1.0 & 1.0 & 1.6 & 1.6 & 1.1 \\
\hline METAL & Antimony & $\mathrm{mg} / \mathrm{kg}$ & 2 & 0 & $0 \%$ & 0.050 & 0.050 & -- & -- & 0.025 \\
\hline METAL & Arsenic & $\mathrm{mg} / \mathrm{kg}$ & 2 & 2 & $100 \%$ & -- & -- & 0.20 & 0.36 & 0.28 \\
\hline METAL & Barium & $\mathrm{mg} / \mathrm{kg}$ & 2 & 2 & $100 \%$ & -- & -- & 0.95 & 2.1 & 1.5 \\
\hline METAL & Beryllium & $\mathrm{mg} / \mathrm{kg}$ & 2 & 0 & $0 \%$ & 0.0040 & 0.0040 & -- & -- & 0.0020 \\
\hline METAL & Cadmium & $\mathrm{mg} / \mathrm{kg}$ & 2 & 2 & $100 \%$ & -- & -- & 0.010 & 0.040 & 0.025 \\
\hline METAL & Chromium & $\mathrm{mg} / \mathrm{kg}$ & 2 & 2 & $100 \%$ & -- & -- & 0.11 & 0.39 & 0.25 \\
\hline METAL & Cobalt & $\mathrm{mg} / \mathrm{kg}$ & 2 & 2 & $100 \%$ & -- & -- & 0.070 & 0.33 & 0.20 \\
\hline METAL & Copper & $\mathrm{mg} / \mathrm{kg}$ & 2 & 2 & $100 \%$ & -- & -- & 0.26 & 1.1 & 0.68 \\
\hline METAL & Lead & $\mathrm{mg} / \mathrm{kg}$ & 2 & 2 & $100 \%$ & -- & -- & 0.030 & 0.030 & 0.030 \\
\hline METAL & Manganese & $\mathrm{mg} / \mathrm{kg}$ & 2 & 2 & $100 \%$ & -- & -- & 0.32 & 0.85 & 0.59 \\
\hline METAL & Mercury & $\mathrm{mg} / \mathrm{kg}$ & 2 & 1 & $50 \%$ & 0.10 & 0.10 & 0.13 & 0.13 & 0.090 \\
\hline METAL & Nickel & $\mathrm{mg} / \mathrm{kg}$ & 2 & 2 & $100 \%$ & -- & -- & 0.060 & 0.17 & 0.12 \\
\hline METAL & Selenium & $\mathrm{mg} / \mathrm{kg}$ & 2 & 1 & $50 \%$ & 0.50 & 0.50 & 1.1 & 1.1 & 0.68 \\
\hline METAL & Silver & $\mathrm{mg} / \mathrm{kg}$ & 2 & 0 & $0 \%$ & 0.10 & 0.10 & -- & -- & 0.050 \\
\hline METAL & Thallium & $\mathrm{mg} / \mathrm{kg}$ & 2 & 0 & $0 \%$ & 0.050 & 0.050 & -- & -- & 0.025 \\
\hline METAL & Vanadium & $\mathrm{mg} / \mathrm{kg}$ & 2 & 1 & $50 \%$ & 0.010 & 0.010 & 0.040 & 0.040 & 0.023 \\
\hline METAL & Zinc & $\mathrm{mg} / \mathrm{kg}$ & 2 & 2 & $100 \%$ & -- & -- & 2.4 & 8.5 & 5.5 \\
\hline PCB & 2,2',3,3',4,4',5-Heptachloro-1,1'-biphenyl & $\mathrm{mg} / \mathrm{kg}$ & 2 & 2 & $100 \%$ & -- & -- & 0.0020 & 0.0024 & 0.0022 \\
\hline PCB & 2,2',3,4,4',5,5'-Heptachlorobiphenyl & $\mathrm{mg} / \mathrm{kg}$ & 2 & 2 & $100 \%$ & -- & -- & 0.0069 & 0.011 & 0.0091 \\
\hline PCB & 2,3,3',4,4',5'-Hexachlorobiphenyl & $\mathrm{mg} / \mathrm{kg}$ & 2 & 2 & $100 \%$ & -- & -- & $2.00 \mathrm{E}-04$ & $2.79 \mathrm{E}-04$ & $2.40 \mathrm{E}-04$ \\
\hline PCB & 2,3,3',4,4',5-Hexachlorobiphenyl (BZ 156) & $\mathrm{mg} / \mathrm{kg}$ & 2 & 2 & $100 \%$ & -- & -- & 0.0011 & 0.0016 & 0.0013 \\
\hline PCB & 2,3,3',4,4'-Pentachlorobiphenyl & $\mathrm{mg} / \mathrm{kg}$ & 2 & 2 & $100 \%$ & -- & -- & 0.0030 & 0.0039 & 0.0034 \\
\hline РCB & 2,3',4,4',5,5'-Hexachlorobiphenyl (BZ 167) & $\mathrm{mg} / \mathrm{kg}$ & 2 & 2 & $100 \%$ & -- & -- & 2.12E-05 & 3.31E-05 & $2.72 \mathrm{E}-05$ \\
\hline PCB & 2',3,4,4',5-Pentachloro-1,1'-biphenyl (BZ 123) & $\mathrm{mg} / \mathrm{kg}$ & 2 & 2 & $100 \%$ & -- & -- & 4.75E-05 & $6.18 \mathrm{E}-05$ & 5.47E-05 \\
\hline РCB & 2,3,4,4',5-Pentachlorobiphenyl (BZ 114) & $\mathrm{mg} / \mathrm{kg}$ & 2 & 2 & $100 \%$ & -- & -- & 2.19E-04 & 3.35E-04 & $2.77 \mathrm{E}-04$ \\
\hline PCB & 2,3',4,4',5-Pentachlorobiphenyl (BZ 118) & $\mathrm{mg} / \mathrm{kg}$ & 2 & 2 & $100 \%$ & -- & -- & 0.0036 & 0.0036 & 0.0036 \\
\hline РСB & 2,3,4,5,3',4',5'-Heptachlorobiphenyl & $\mathrm{mg} / \mathrm{kg}$ & 2 & 2 & $100 \%$ & -- & -- & 8.72E-05 & $1.21 \mathrm{E}-04$ & 1.04E-04 \\
\hline PCB & 3,3',4,4',5,5'-Hexachlorobiphenyl (BZ 169) & $\mathrm{mg} / \mathrm{kg}$ & 2 & 0 & $0 \%$ & 1.05E-06 & 2.12E-06 & -- & -- & 7.93E-07 \\
\hline PCB & 3,3',4,4',5-Pentachlorobiphenyl (BZ 126) & $\mathrm{mg} / \mathrm{kg}$ & 2 & 0 & $0 \%$ & $1.22 \mathrm{E}-06$ & 1.53E-06 & -- & -- & $6.88 \mathrm{E}-07$ \\
\hline PCB & 3,3',4,4'-Tetrachlorobiphenyl (BZ 77) & $\mathrm{mg} / \mathrm{kg}$ & 2 & 2 & $100 \%$ & -- & -- & 1.70E-05 & 2.79E-05 & $2.25 \mathrm{E}-05$ \\
\hline PCB & Aroclor-1016 & $\mathrm{mg} / \mathrm{kg}$ & 2 & 0 & $0 \%$ & 0.018 & 0.019 & -- & -- & 0.0093 \\
\hline PCB & Aroclor-1221 & $\mathrm{mg} / \mathrm{kg}$ & 2 & 0 & $0 \%$ & 0.018 & 0.019 & -- & -- & 0.0093 \\
\hline
\end{tabular}


Table F-64

Summary Statistics for Whole Body White Sturgeon Collected from Segment 2 of the Columbia River

\begin{tabular}{|c|c|c|c|c|c|c|c|c|c|c|}
\hline $\begin{array}{l}\text { Constituent } \\
\text { Class }\end{array}$ & Constituent Name & Units & $\begin{array}{l}\text { Number } \\
\text { Analyzed }\end{array}$ & $\begin{array}{l}\text { Number } \\
\text { Detected }\end{array}$ & $\begin{array}{c}\text { Frequency of } \\
\text { Detection }\end{array}$ & $\begin{array}{l}\text { Minimum } \\
\text { Nondetect }\end{array}$ & $\begin{array}{l}\text { Maximum } \\
\text { Nondetect }\end{array}$ & $\begin{array}{l}\text { Minimum } \\
\text { Detect }\end{array}$ & $\begin{array}{l}\text { Maximum } \\
\text { Detect }\end{array}$ & $\begin{array}{c}\text { Average } \\
\text { Concentration }\end{array}$ \\
\hline$\overline{\mathrm{PCB}}$ & Aroclor-1232 & $\mathrm{mg} / \mathrm{kg}$ & 2 & 0 & $0 \%$ & 0.018 & 0.019 & -- & -- & 0.0093 \\
\hline PCB & Aroclor-1248 & $\mathrm{mg} / \mathrm{kg}$ & 2 & 0 & $0 \%$ & 0.018 & 0.019 & -- & -- & 0.0093 \\
\hline РCB & Aroclor-1254 & $\mathrm{mg} / \mathrm{kg}$ & 2 & 2 & $100 \%$ & -- & -- & 0.083 & 0.090 & 0.087 \\
\hline РСВ & Aroclor-1260 & $\mathrm{mg} / \mathrm{kg}$ & 2 & 2 & $100 \%$ & -- & -- & 0.063 & 0.087 & 0.075 \\
\hline PEST & 2,4'-DDD & $\mathrm{mg} / \mathrm{kg}$ & 2 & 2 & $100 \%$ & -- & -- & 0.018 & 0.022 & 0.020 \\
\hline PEST & 2,4'-DDE & $\mathrm{mg} / \mathrm{kg}$ & 2 & 2 & $100 \%$ & -- & -- & 0.0079 & 0.0083 & 0.0081 \\
\hline PEST & 2,4'-DDT & $\mathrm{mg} / \mathrm{kg}$ & 2 & 0 & $0 \%$ & 0.0018 & 0.0019 & -- & -- & $9.25 \mathrm{E}-04$ \\
\hline PEST & Aldrin & $\mathrm{mg} / \mathrm{kg}$ & 2 & 1 & $50 \%$ & 0.0018 & 0.0018 & 0.0011 & 0.0011 & 0.0010 \\
\hline PEST & Alpha-BHC & $\mathrm{mg} / \mathrm{kg}$ & 2 & 0 & $0 \%$ & 0.0018 & 0.0019 & -- & -- & $9.25 \mathrm{E}-04$ \\
\hline PEST & alpha-Chlordane & $\mathrm{mg} / \mathrm{kg}$ & 2 & 2 & $100 \%$ & -- & -- & 0.0022 & 0.0043 & 0.0033 \\
\hline PEST & alpha-Chlordene & $\mathrm{mg} / \mathrm{kg}$ & 2 & 0 & $0 \%$ & 0.0018 & 0.0019 & -- & -- & $9.25 \mathrm{E}-04$ \\
\hline PEST & Beta-BHC & $\mathrm{mg} / \mathrm{kg}$ & 2 & 0 & $0 \%$ & 0.0018 & 0.0019 & -- & -- & $9.25 \mathrm{E}-04$ \\
\hline PEST & cis-Nonachlor & $\mathrm{mg} / \mathrm{kg}$ & 2 & 2 & $100 \%$ & -- & -- & 0.012 & 0.012 & 0.012 \\
\hline PEST & DDMU & $\mathrm{mg} / \mathrm{kg}$ & 2 & 2 & $100 \%$ & -- & -- & 0.013 & 0.016 & 0.015 \\
\hline PEST & Delta-BHC & $\mathrm{mg} / \mathrm{kg}$ & 2 & 0 & $0 \%$ & 0.0018 & 0.0019 & -- & -- & $9.25 \mathrm{E}-04$ \\
\hline PEST & Dichlorodiphenyldichloroethane & $\mathrm{mg} / \mathrm{kg}$ & 2 & 2 & $100 \%$ & -- & -- & 0.19 & 0.20 & 0.20 \\
\hline PEST & Dichlorodiphenyldichloroethylene & $\mathrm{mg} / \mathrm{kg}$ & 2 & 2 & $100 \%$ & -- & -- & 0.54 & 0.64 & 0.59 \\
\hline PEST & Dichlorodiphenyltrichloroethane & $\mathrm{mg} / \mathrm{kg}$ & 2 & 2 & $100 \%$ & -- & -- & 0.0039 & 0.0070 & 0.0055 \\
\hline PEST & Endosulfan sulfate & $\mathrm{mg} / \mathrm{kg}$ & 2 & 0 & $0 \%$ & 0.0018 & 0.0019 & -- & -- & $9.25 \mathrm{E}-04$ \\
\hline PEST & Gamma-BHC (Lindane) & $\mathrm{mg} / \mathrm{kg}$ & 2 & 0 & $0 \%$ & 0.0018 & 0.0019 & -- & -- & $9.25 \mathrm{E}-04$ \\
\hline PEST & gamma-Chlordane & $\mathrm{mg} / \mathrm{kg}$ & 2 & 2 & $100 \%$ & -- & -- & 0.0044 & 0.0072 & 0.0058 \\
\hline PEST & gamma-Chlordene & $\mathrm{mg} / \mathrm{kg}$ & 2 & 0 & $0 \%$ & 0.0018 & 0.0019 & -- & -- & $9.25 \mathrm{E}-04$ \\
\hline PEST & Heptachlor & $\mathrm{mg} / \mathrm{kg}$ & 2 & 0 & $0 \%$ & 0.0018 & 0.0019 & -- & -- & $9.25 \mathrm{E}-04$ \\
\hline PEST & Heptachlor epoxide & $\mathrm{mg} / \mathrm{kg}$ & 2 & 0 & $0 \%$ & 0.0018 & 0.0019 & -- & -- & $9.25 \mathrm{E}-04$ \\
\hline PEST & Mirex & $\mathrm{mg} / \mathrm{kg}$ & 2 & 0 & $0 \%$ & 0.0018 & 0.0019 & -- & -- & $9.25 \mathrm{E}-04$ \\
\hline PEST & Oxychlordane & $\mathrm{mg} / \mathrm{kg}$ & 2 & 1 & $50 \%$ & 0.0018 & 0.0018 & 0.0016 & 0.0016 & 0.0013 \\
\hline PEST & Pentachloroanisole & $\mathrm{mg} / \mathrm{kg}$ & 2 & 0 & $0 \%$ & 0.0018 & 0.0019 & -- & -- & $9.25 \mathrm{E}-04$ \\
\hline PEST & Toxaphene & $\mathrm{mg} / \mathrm{kg}$ & 2 & 0 & $0 \%$ & 0.055 & 0.056 & -- & -- & 0.028 \\
\hline PEST & trans-Nonachlor & $\mathrm{mg} / \mathrm{kg}$ & 2 & 2 & $100 \%$ & -- & -- & 0.0046 & 0.0093 & 0.0070 \\
\hline SVOC & 1,2,4-Trichlorobenzene & $\mathrm{mg} / \mathrm{kg}$ & 2 & 0 & $0 \%$ & 0.0094 & 0.0097 & -- & -- & 0.0048 \\
\hline SVOC & 1,2-Dichlorobenzene & $\mathrm{mg} / \mathrm{kg}$ & 2 & 0 & $0 \%$ & 0.0094 & 0.0097 & -- & -- & 0.0048 \\
\hline SVOC & 1,2-Diphenylhydrazine & $\mathrm{mg} / \mathrm{kg}$ & 2 & 0 & $0 \%$ & 0.0094 & 0.0097 & -- & -- & 0.0048 \\
\hline SVOC & 1,3-Dichlorobenzene & $\mathrm{mg} / \mathrm{kg}$ & 2 & 0 & $0 \%$ & 0.0094 & 0.0097 & -- & -- & 0.0048 \\
\hline SVOC & 1,4-Dichlorobenzene & $\mathrm{mg} / \mathrm{kg}$ & 2 & 0 & $0 \%$ & 0.0094 & 0.0097 & -- & -- & 0.0048 \\
\hline SVOC & 1-methylnaphthalene & $\mathrm{mg} / \mathrm{kg}$ & 2 & 0 & $0 \%$ & 0.0094 & 0.0097 & -- & -- & 0.0048 \\
\hline SVOC & 2,4-Dinitrotoluene & $\mathrm{mg} / \mathrm{kg}$ & 2 & 0 & $0 \%$ & 0.019 & 0.019 & -- & -- & 0.0095 \\
\hline SVOC & 2,6-Dinitrotoluene & $\mathrm{mg} / \mathrm{kg}$ & 2 & 0 & $0 \%$ & 0.038 & 0.039 & -- & -- & 0.019 \\
\hline SVOC & 2-Chloronaphthalene & $\mathrm{mg} / \mathrm{kg}$ & 2 & 0 & $0 \%$ & 0.019 & 0.019 & -- & -- & 0.0095 \\
\hline SVOC & 2-MethyInaphthalene & $\mathrm{mg} / \mathrm{kg}$ & 2 & 0 & $0 \%$ & 0.0094 & 0.0097 & -- & -- & 0.0048 \\
\hline SVOC & 4-Bromophenylphenyl ether & $\mathrm{mg} / \mathrm{kg}$ & 2 & 0 & $0 \%$ & 0.0094 & 0.0097 & -- & -- & 0.0048 \\
\hline SVOC & 4-Chlorophenylphenyl ether & $\mathrm{mg} / \mathrm{kg}$ & 2 & 0 & $0 \%$ & 0.0094 & 0.0097 & -- & -- & 0.0048 \\
\hline SVOC & Acenaphthene & $\mathrm{mg} / \mathrm{kg}$ & 2 & 0 & $0 \%$ & 0.019 & 0.019 & -- & -- & 0.0095 \\
\hline SVOC & Acenaphthylene & $\mathrm{mg} / \mathrm{kg}$ & 2 & 0 & $0 \%$ & 0.0094 & 0.0097 & -- & -- & 0.0048 \\
\hline SVOC & Anthracene & $\mathrm{mg} / \mathrm{kg}$ & 2 & 0 & $0 \%$ & 0.0094 & 0.0097 & -- & -- & 0.0048 \\
\hline SVOC & Benzo(a)anthracene & $\mathrm{mg} / \mathrm{kg}$ & 2 & 0 & $0 \%$ & 0.0094 & 0.0097 & -- & -- & 0.0048 \\
\hline SVOC & Benzo(a)pyrene & $\mathrm{mg} / \mathrm{kg}$ & 2 & 0 & $0 \%$ & 0.0094 & 0.0097 & -- & -- & 0.0048 \\
\hline SVOC & Benzo(b)fluoranthene & $\mathrm{mg} / \mathrm{kg}$ & 2 & 0 & $0 \%$ & 0.0094 & 0.0097 & -- & -- & 0.0048 \\
\hline SVOC & Benzo(ghi)perylene & $\mathrm{mg} / \mathrm{kg}$ & 2 & 0 & $0 \%$ & 0.019 & 0.019 & -- & -- & 0.0095 \\
\hline SVOC & Benzo(k)fluoranthene & $\mathrm{mg} / \mathrm{kg}$ & 2 & 0 & $0 \%$ & 0.0094 & 0.0097 & -- & -- & 0.0048 \\
\hline SVOC & Bis(2-chloro-1-methylethyl)ether & $\mathrm{mg} / \mathrm{kg}$ & 2 & 0 & $0 \%$ & 0.019 & 0.019 & -- & -- & 0.0095 \\
\hline
\end{tabular}


Table F-64

Summary Statistics for Whole Body White Sturgeon Collected from Segment 2 of the Columbia River

\begin{tabular}{|c|c|c|c|c|c|c|c|c|c|c|}
\hline $\begin{array}{l}\text { Constituent } \\
\text { Class }\end{array}$ & Constituent Name & Units & $\begin{array}{l}\text { Number } \\
\text { Analyzed }\end{array}$ & $\begin{array}{l}\text { Number } \\
\text { Detected }\end{array}$ & $\begin{array}{c}\text { Frequency of } \\
\text { Detection }\end{array}$ & $\begin{array}{l}\text { Minimum } \\
\text { Nondetect }\end{array}$ & $\begin{array}{c}\text { Maximum } \\
\text { Nondetect }\end{array}$ & $\begin{array}{c}\text { Minimum } \\
\text { Detect }\end{array}$ & $\begin{array}{c}\text { Maximum } \\
\text { Detect }\end{array}$ & $\begin{array}{c}\text { Average } \\
\text { Concentration }\end{array}$ \\
\hline SVOC & Chrysene & $\mathrm{mg} / \mathrm{kg}$ & 2 & 0 & $0 \%$ & 0.0094 & 0.0097 & -- & -- & 0.0048 \\
\hline SVOC & Dibenz[a,h]anthracene & $\mathrm{mg} / \mathrm{kg}$ & 2 & 0 & $0 \%$ & 0.019 & 0.019 & -- & -- & 0.0095 \\
\hline SVOC & Dibenzofuran & $\mathrm{mg} / \mathrm{kg}$ & 2 & 0 & $0 \%$ & 0.0094 & 0.0097 & -- & -- & 0.0048 \\
\hline SVOC & Fluoranthene & $\mathrm{mg} / \mathrm{kg}$ & 2 & 0 & $0 \%$ & 0.019 & 0.019 & -- & -- & 0.0095 \\
\hline SVOC & Fluorene & $\mathrm{mg} / \mathrm{kg}$ & 2 & 0 & $0 \%$ & 0.0093 & 0.0093 & -- & -- & 0.0047 \\
\hline SVOC & Hexachlorobenzene & $\mathrm{mg} / \mathrm{kg}$ & 2 & 0 & $0 \%$ & 0.019 & 0.019 & -- & -- & 0.0095 \\
\hline SVOC & Hexachlorobutadiene & $\mathrm{mg} / \mathrm{kg}$ & 2 & 0 & $0 \%$ & 0.0094 & 0.0097 & -- & -- & 0.0048 \\
\hline SVOC & Hexachloroethane & $\mathrm{mg} / \mathrm{kg}$ & 2 & 0 & $0 \%$ & 0.0094 & 0.0097 & -- & -- & 0.0048 \\
\hline SVOC & Indeno(1,2,3-cd)pyrene & $\mathrm{mg} / \mathrm{kg}$ & 2 & 0 & $0 \%$ & 0.019 & 0.019 & -- & -- & 0.0095 \\
\hline SVOC & Naphthalene & $\mathrm{mg} / \mathrm{kg}$ & 2 & 0 & $0 \%$ & 0.0094 & 0.0097 & -- & -- & 0.0048 \\
\hline SVOC & Nitrobenzene & $\mathrm{mg} / \mathrm{kg}$ & 2 & 0 & $0 \%$ & 0.0094 & 0.0097 & -- & -- & 0.0048 \\
\hline SVOC & Phenanthrene & $\mathrm{mg} / \mathrm{kg}$ & 2 & 0 & $0 \%$ & 0.0094 & 0.0097 & -- & -- & 0.0048 \\
\hline SVOC & Pyrene & $\mathrm{mg} / \mathrm{kg}$ & 2 & 0 & $0 \%$ & 0.019 & 0.019 & -- & -- & 0.0095 \\
\hline VOC & Retene & $\mathrm{mg} / \mathrm{kg}$ & 2 & 0 & $0 \%$ & 0.019 & 0.019 & -- & -- & 0.0095 \\
\hline
\end{tabular}

DIOXI - Dioxins and Furans

PEST - Pesticide

PCB - Polychlorinated biphenyl

SVOC - Semivolatile Organic Compound

VOC - Volatile Organic Compound 
Table F-65

Summary Statistics for Bass Fillet with Skin Off Collected from Segment 3 of the Columbia River

\begin{tabular}{|c|c|c|c|c|c|c|c|c|c|c|}
\hline $\begin{array}{l}\text { Constituent } \\
\text { Class }\end{array}$ & Constituent Name & Units & $\begin{array}{l}\text { Number } \\
\text { Analyzed }\end{array}$ & $\begin{array}{l}\text { Number } \\
\text { Detected }\end{array}$ & $\begin{array}{l}\text { Frequency of } \\
\text { Detection }\end{array}$ & $\begin{array}{l}\text { Minimum } \\
\text { Nondetect }\end{array}$ & $\begin{array}{l}\text { Maximum } \\
\text { Nondetect }\end{array}$ & $\begin{array}{c}\text { Minimum } \\
\text { Detect }\end{array}$ & $\begin{array}{l}\text { Maximum } \\
\text { Detect }\end{array}$ & $\begin{array}{c}\text { Average } \\
\text { Concentration }\end{array}$ \\
\hline CONV & Percent Lipids & $\%$ & 11 & 11 & $100 \%$ & $\overline{--}$ & $\overline{--}$ & 0.14 & 1.4 & 0.48 \\
\hline METAL & Mercury & $\mathrm{mg} / \mathrm{Kg}$ & 11 & 11 & $100 \%$ & -- & -- & 0.058 & 0.28 & 0.19 \\
\hline
\end{tabular}


Table F-66

Summary Statistics for Whole Body Largemouth Bass Collected from Segment 3 of the Columbia River

\begin{tabular}{|c|c|c|c|c|c|c|c|c|c|c|}
\hline $\begin{array}{l}\text { Constituent } \\
\text { Class }\end{array}$ & Constituent Name & Units & $\begin{array}{c}\text { Number } \\
\text { Analyzed }\end{array}$ & $\begin{array}{l}\text { Number } \\
\text { Detected }\end{array}$ & $\begin{array}{l}\text { Frequency of } \\
\text { Detection }\end{array}$ & $\begin{array}{c}\text { Minimum } \\
\text { Nondetect }\end{array}$ & $\begin{array}{l}\text { Maximum } \\
\text { Nondetect }\end{array}$ & $\begin{array}{c}\text { Minimum } \\
\text { Detect }\end{array}$ & $\begin{array}{c}\text { Maximum } \\
\text { Detect }\end{array}$ & $\begin{array}{c}\text { Average } \\
\text { Concentration }\end{array}$ \\
\hline$\overline{\text { CONV }}$ & Percent Lipids & $\%$ & 2 & 2 & $100 \%$ & -- & -- & 3.5 & 5.9 & 4.7 \\
\hline CONV & Percent moisture (wet sample) & $\mathrm{mg} / \mathrm{Kg}$ & 2 & 2 & $100 \%$ & -- & -- & 72 & 72 & 72 \\
\hline DIOXI & TCDD-eq & $\mathrm{mg} / \mathrm{Kg}$ & 2 & 2 & $100 \%$ & -- & -- & 5.0 & 6.0 & 5.5 \\
\hline METAL & Aluminum & $\mathrm{mg} / \mathrm{Kg}$ & 2 & 2 & $100 \%$ & -- & -- & 11 & 17 & 14 \\
\hline METAL & Arsenic & $\mathrm{mg} / \mathrm{Kg}$ & 2 & 0 & $0 \%$ & 0.24 & 0.27 & -- & -- & 0.13 \\
\hline METAL & Barium & $\mathrm{mg} / \mathrm{Kg}$ & 2 & 2 & $100 \%$ & -- & -- & 0.34 & 0.53 & 0.44 \\
\hline METAL & Beryllium & $\mathrm{mg} / \mathrm{Kg}$ & 2 & 0 & $0 \%$ & 0.047 & 0.055 & -- & -- & 0.025 \\
\hline METAL & Boron & $\mathrm{mg} / \mathrm{Kg}$ & 2 & 0 & $0 \%$ & 0.94 & 1.1 & -- & -- & 0.51 \\
\hline METAL & Cadmium & $\mathrm{mg} / \mathrm{Kg}$ & 2 & 0 & $0 \%$ & 0.047 & 0.055 & -- & -- & 0.025 \\
\hline METAL & Chromium & $\mathrm{mg} / \mathrm{Kg}$ & 2 & 2 & $100 \%$ & -- & -- & 0.66 & 1.4 & 1.0 \\
\hline METAL & Copper & $\mathrm{mg} / \mathrm{Kg}$ & 2 & 2 & $100 \%$ & -- & -- & 0.47 & 0.61 & 0.54 \\
\hline METAL & Iron & $\mathrm{mg} / \mathrm{Kg}$ & 2 & 2 & $100 \%$ & -- & -- & 15 & 19 & 17 \\
\hline METAL & Lead & $\mathrm{mg} / \mathrm{Kg}$ & 2 & 0 & $0 \%$ & 0.094 & 0.11 & -- & -- & 0.051 \\
\hline METAL & Magnesium & $\mathrm{mg} / \mathrm{Kg}$ & 2 & 2 & $100 \%$ & -- & -- & 299 & 417 & 358 \\
\hline METAL & Manganese & $\mathrm{mg} / \mathrm{Kg}$ & 2 & 2 & $100 \%$ & -- & -- & 1.0 & 1.0 & 1.0 \\
\hline METAL & Mercury & $\mathrm{mg} / \mathrm{Kg}$ & 2 & 2 & $100 \%$ & -- & -- & 0.059 & 0.18 & 0.12 \\
\hline METAL & Molybdenum & $\mathrm{mg} / \mathrm{Kg}$ & 2 & 0 & $0 \%$ & 0.24 & 0.27 & -- & -- & 0.13 \\
\hline METAL & Nickel & $\mathrm{mg} / \mathrm{Kg}$ & 2 & 0 & $0 \%$ & 0.24 & 0.27 & -- & -- & 0.13 \\
\hline METAL & Selenium & $\mathrm{mg} / \mathrm{Kg}$ & 2 & 2 & $100 \%$ & -- & -- & 0.80 & 0.81 & 0.81 \\
\hline METAL & Strontium & $\mathrm{mg} / \mathrm{Kg}$ & 2 & 2 & $100 \%$ & -- & -- & 12 & 24 & 18 \\
\hline METAL & Vanadium & $\mathrm{mg} / \mathrm{Kg}$ & 2 & 0 & $0 \%$ & 0.24 & 0.27 & - & -- & 0.13 \\
\hline METAL & Zinc & $\mathrm{mg} / \mathrm{Kg}$ & 2 & 2 & $100 \%$ & -- & -- & 15 & 19 & 17 \\
\hline PCB & Total polychlorinated biphenyl & $\mathrm{mg} / \mathrm{Kg}$ & 2 & 1 & $50 \%$ & 0.030 & 0.030 & 0.042 & 0.042 & 0.029 \\
\hline PEST & 2,4'-DDD & $\mathrm{mg} / \mathrm{Kg}$ & 2 & 0 & $0 \%$ & 0.010 & 0.010 & -- & -- & 0.0050 \\
\hline PEST & 2,4'-DDE & $\mathrm{mg} / \mathrm{Kg}$ & 2 & 0 & $0 \%$ & 0.010 & 0.010 & -- & -- & 0.0050 \\
\hline PEST & 2,4'-DDT & $\mathrm{mg} / \mathrm{Kg}$ & 2 & 0 & $0 \%$ & 0.010 & 0.010 & -- & -- & 0.0050 \\
\hline PEST & Alpha-BHC & $\mathrm{mg} / \mathrm{Kg}$ & 2 & 0 & $0 \%$ & 0.010 & 0.010 & -- & -- & 0.0050 \\
\hline PEST & alpha-Chlordane & $\mathrm{mg} / \mathrm{Kg}$ & 2 & 0 & $0 \%$ & 0.010 & 0.010 & -- & -- & 0.0050 \\
\hline PEST & Beta-BHC & $\mathrm{mg} / \mathrm{Kg}$ & 2 & 0 & $0 \%$ & 0.010 & 0.010 & -- & -- & 0.0050 \\
\hline PEST & cis-Nonachlor & $\mathrm{mg} / \mathrm{Kg}$ & 2 & 0 & $0 \%$ & 0.010 & 0.010 & -- & -- & 0.0050 \\
\hline PEST & Delta-BHC & $\mathrm{mg} / \mathrm{Kg}$ & 2 & 0 & $0 \%$ & 0.010 & 0.010 & -- & -- & 0.0050 \\
\hline PEST & Dichlorodiphenyldichloroethane & $\mathrm{mg} / \mathrm{Kg}$ & 2 & 2 & $100 \%$ & -- & -- & 0.023 & 0.040 & 0.032 \\
\hline PEST & Dichlorodiphenyldichloroethylene & $\mathrm{mg} / \mathrm{Kg}$ & 2 & 2 & $100 \%$ & -- & -- & 0.17 & 0.25 & 0.21 \\
\hline PEST & Dichlorodiphenyltrichloroethane & $\mathrm{mg} / \mathrm{Kg}$ & 2 & 0 & $0 \%$ & 0.010 & 0.010 & -- & -- & 0.0050 \\
\hline PEST & Dieldrin & $\mathrm{mg} / \mathrm{Kg}$ & 2 & 1 & $50 \%$ & 0.010 & 0.010 & 0.012 & 0.012 & 0.0085 \\
\hline PEST & Endrin & $\mathrm{mg} / \mathrm{Kg}$ & 2 & 0 & $0 \%$ & 0.010 & 0.010 & -- & -- & 0.0050 \\
\hline PEST & Gamma-BHC (Lindane) & $\mathrm{mg} / \mathrm{Kg}$ & 2 & 0 & $0 \%$ & 0.010 & 0.010 & -- & -- & 0.0050 \\
\hline PEST & gamma-Chlordane & $\mathrm{mg} / \mathrm{Kg}$ & 2 & 0 & $0 \%$ & 0.010 & 0.010 & -- & -- & 0.0050 \\
\hline PEST & Heptachlor epoxide & $\mathrm{mg} / \mathrm{kg}$ & 2 & 0 & $0 \%$ & 0.010 & 0.010 & -- & -- & 0.0050 \\
\hline PEST & Mirex & $\mathrm{mg} / \mathrm{Kg}$ & 2 & 0 & $0 \%$ & 0.010 & 0.010 & -- & -- & 0.0050 \\
\hline PEST & Oxychlordane & $\mathrm{mg} / \mathrm{Kg}$ & 2 & 0 & $0 \%$ & 0.010 & 0.010 & -- & -- & 0.0050 \\
\hline PEST & Toxaphene & $\mathrm{mg} / \mathrm{Kg}$ & 2 & 0 & $0 \%$ & 0.030 & 0.030 & -- & -- & 0.015 \\
\hline PEST & trans-Nonachlor & $\mathrm{mg} / \mathrm{Kg}$ & 2 & 0 & $0 \%$ & 0.010 & 0.010 & -- & -- & 0.0050 \\
\hline SVOC & Hexachlorobenzene & $\mathrm{mg} / \mathrm{Kg}$ & 2 & 0 & $0 \%$ & 0.010 & 0.010 & -- & -- & 0.0050 \\
\hline
\end{tabular}

DIOXI - Dioxins and Furans

PEST - Pesticide

PCB - Polychlorinated biphenyl

SVOC - Semivolatile Organic Compound 


\section{Table F-67}

Summary Statistics for Smallmouth Bass Fillet Collected from Segment 3 of the Columbia River

\begin{tabular}{|c|c|c|c|c|c|c|c|c|c|c|}
\hline $\begin{array}{l}\text { Constituent } \\
\text { Class }\end{array}$ & Constituent Name & Units & $\begin{array}{l}\text { Number } \\
\text { Analyzed }\end{array}$ & $\begin{array}{l}\text { Number } \\
\text { Detected }\end{array}$ & $\begin{array}{c}\text { Frequency of } \\
\text { Detection }\end{array}$ & $\begin{array}{c}\text { Minimum } \\
\text { Nondetect }\end{array}$ & $\begin{array}{l}\text { Maximum } \\
\text { Nondetect }\end{array}$ & $\begin{array}{l}\text { Minimum } \\
\text { Detect }\end{array}$ & $\begin{array}{l}\text { Maximum } \\
\text { Detect }\end{array}$ & $\begin{array}{c}\text { Average } \\
\text { Concentration }\end{array}$ \\
\hline$\overline{\text { CONV }}$ & Percent Lipids & $\%$ & 5 & 5 & $100 \%$ & $\overline{--}$ & $\overline{--}$ & 0.78 & 5.3 & 1.8 \\
\hline PCB & Aroclor-1016 & $\mathrm{mg} / \mathrm{Kg}$ & 4 & 0 & $0 \%$ & 0.0046 & 0.0055 & -- & -- & 0.0025 \\
\hline PCB & Aroclor-1221 & $\mathrm{mg} / \mathrm{Kg}$ & 4 & 0 & $0 \%$ & 0.0046 & 0.0055 & -- & -- & 0.0025 \\
\hline PCB & Aroclor-1232 & $\mathrm{mg} / \mathrm{Kg}$ & 4 & 0 & $0 \%$ & 0.0046 & 0.0055 & -- & -- & 0.0025 \\
\hline PCB & Aroclor-1242 & $\mathrm{mg} / \mathrm{Kg}$ & 4 & 0 & $0 \%$ & 0.0046 & 0.0055 & -- & -- & 0.0025 \\
\hline PCB & Aroclor-1248 & $\mathrm{mg} / \mathrm{Kg}$ & 4 & 0 & $0 \%$ & 0.0046 & 0.0055 & -- & -- & 0.0025 \\
\hline PCB & Aroclor-1254 & $\mathrm{mg} / \mathrm{Kg}$ & 5 & 5 & $100 \%$ & -- & -- & 0.0032 & 0.058 & 0.016 \\
\hline PCB & Aroclor-1260 & $\mathrm{mg} / \mathrm{Kg}$ & 5 & 3 & $60 \%$ & 0.0046 & 0.0055 & 0.0026 & 0.031 & 0.0083 \\
\hline PCB & Aroclor-1262 & $\mathrm{mg} / \mathrm{Kg}$ & 4 & 0 & $0 \%$ & 0.0046 & 0.0055 & -- & -- & 0.0025 \\
\hline PCB & Aroclor-1268 & $\mathrm{mg} / \mathrm{Kg}$ & 4 & 0 & $0 \%$ & 0.0046 & 0.0055 & -- & -- & 0.0025 \\
\hline PEST & 2,4'-DDD & $\mathrm{mg} / \mathrm{Kg}$ & 5 & 1 & $20 \%$ & $4.60 \mathrm{E}-04$ & 5.50E-04 & 0.0028 & 0.0028 & 7.62E-04 \\
\hline PEST & 2,4'-DDE & $\mathrm{mg} / \mathrm{Kg}$ & 5 & 2 & $40 \%$ & $4.60 \mathrm{E}-04$ & 5.50E-04 & $5.40 \mathrm{E}-04$ & 0.0035 & $9.59 \mathrm{E}-04$ \\
\hline PEST & 2,4'-DDT & $\mathrm{mg} / \mathrm{Kg}$ & 5 & 1 & $20 \%$ & 4.60E-04 & $5.50 \mathrm{E}-04$ & 0.0017 & 0.0017 & $5.42 \mathrm{E}-04$ \\
\hline PEST & alpha-Chlordane & $\mathrm{mg} / \mathrm{Kg}$ & 5 & 1 & $20 \%$ & $4.60 \mathrm{E}-04$ & 5.50E-04 & 0.0013 & 0.0013 & $4.62 \mathrm{E}-04$ \\
\hline PEST & cis-Nonachlor & $\mathrm{mg} / \mathrm{Kg}$ & 5 & 1 & $20 \%$ & 4.60E-04 & $5.50 \mathrm{E}-04$ & 0.0019 & 0.0019 & $5.82 \mathrm{E}-04$ \\
\hline PEST & Dichlorodiphenyldichloroethane & $\mathrm{mg} / \mathrm{Kg}$ & 5 & 5 & $100 \%$ & -- & -- & 0.0016 & 0.020 & 0.0056 \\
\hline PEST & Dichlorodiphenyldichloroethylene & $\mathrm{mg} / \mathrm{Kg}$ & 5 & 5 & $100 \%$ & -- & -- & 0.025 & 0.29 & 0.079 \\
\hline PEST & Dichlorodiphenyltrichloroethane & $\mathrm{mg} / \mathrm{Kg}$ & 5 & 3 & $60 \%$ & 5.10E-04 & 5.50E-04 & $5.30 \mathrm{E}-04$ & 0.0018 & $6.82 \mathrm{E}-04$ \\
\hline PEST & Dieldrin & $\mathrm{mg} / \mathrm{Kg}$ & 5 & 1 & $20 \%$ & 4.60E-04 & $5.50 \mathrm{E}-04$ & 0.0016 & 0.0016 & $5.22 \mathrm{E}-04$ \\
\hline PEST & Heptachlor & $\mathrm{mg} / \mathrm{Kg}$ & 5 & 0 & $0 \%$ & 4.60E-04 & $5.50 \mathrm{E}-04$ & -- & -- & 2.53E-04 \\
\hline PEST & Heptachlor epoxide & $\mathrm{mg} / \mathrm{Kg}$ & 5 & 1 & $20 \%$ & 4.60E-04 & $5.50 \mathrm{E}-04$ & 0.0016 & 0.0016 & $5.22 \mathrm{E}-04$ \\
\hline PEST & Oxychlordane & $\mathrm{mg} / \mathrm{Kg}$ & 5 & 1 & $20 \%$ & 4.60E-04 & 5.50E-04 & 0.0017 & 0.0017 & $5.42 \mathrm{E}-04$ \\
\hline PEST & Toxaphene & $\mathrm{mg} / \mathrm{Kg}$ & 5 & 5 & $100 \%$ & -- & -- & 0.0077 & 0.050 & 0.018 \\
\hline PEST & trans-Chlordane & $\mathrm{mg} / \mathrm{Kg}$ & 5 & 1 & $20 \%$ & 4.60E-04 & 5.50E-04 & $6.20 \mathrm{E}-04$ & $6.20 \mathrm{E}-04$ & 3.26E-04 \\
\hline PEST & trans-Nonachlor & $\mathrm{mg} / \mathrm{Kg}$ & 5 & 5 & $100 \%$ & -- & -- & $6.40 \mathrm{E}-04$ & 0.0072 & 0.0020 \\
\hline SVOC & Hexachlorobenzene & $\mathrm{mg} / \mathrm{Kg}$ & 5 & 5 & $100 \%$ & -- & -- & $7.00 \mathrm{E}-04$ & 0.0058 & 0.0018 \\
\hline
\end{tabular}

PEST - Pesticide

PCB - Polychlorinated biphenyl

SVOC - Semivolatile Organic Compound 


\section{Table F-68}

Summary Statistics for Smallmouth Bass Fillet Skin On Collected from Segment 3 of the Columbia River

\begin{tabular}{|c|c|c|c|c|c|c|c|c|c|c|}
\hline $\begin{array}{l}\text { Constituent } \\
\text { Class }\end{array}$ & Constituent Name & Units & $\begin{array}{l}\text { Number } \\
\text { Analyzed }\end{array}$ & $\begin{array}{l}\text { Number } \\
\text { Detected }\end{array}$ & $\begin{array}{l}\text { Frequency of } \\
\text { Detection }\end{array}$ & $\begin{array}{l}\text { Minimum } \\
\text { Nondetect }\end{array}$ & $\begin{array}{l}\text { Maximum } \\
\text { Nondetect }\end{array}$ & $\begin{array}{l}\text { Minimum } \\
\text { Detect }\end{array}$ & $\begin{array}{l}\text { Maximum } \\
\text { Detect }\end{array}$ & $\begin{array}{c}\text { Average } \\
\text { Concentration }\end{array}$ \\
\hline$\overline{\text { METAL }}$ & Aluminum & $\mathrm{mg} / \mathrm{kg}$ & 3 & 3 & $100 \%$ & $\overline{--}$ & -- & 5.0 & 8.1 & 6.5 \\
\hline METAL & Antimony & $\mathrm{mg} / \mathrm{kg}$ & 3 & 0 & $0 \%$ & 0.050 & 0.050 & -- & -- & 0.025 \\
\hline METAL & Arsenic & $\mathrm{mg} / \mathrm{kg}$ & 3 & 3 & $100 \%$ & -- & -- & 0.11 & 0.17 & 0.14 \\
\hline METAL & Barium & $\mathrm{mg} / \mathrm{kg}$ & 3 & 3 & $100 \%$ & -- & -- & 0.36 & 0.60 & 0.49 \\
\hline METAL & Beryllium & $\mathrm{mg} / \mathrm{kg}$ & 3 & 0 & $0 \%$ & 0.0040 & 0.0040 & -- & -- & 0.0020 \\
\hline METAL & Cadmium & $\mathrm{mg} / \mathrm{kg}$ & 3 & 0 & $0 \%$ & 0.0040 & 0.0040 & -- & -- & 0.0020 \\
\hline METAL & Chromium & $\mathrm{mg} / \mathrm{kg}$ & 3 & 3 & $100 \%$ & -- & -- & 0.22 & 0.33 & 0.28 \\
\hline METAL & Cobalt & $\mathrm{mg} / \mathrm{kg}$ & 3 & 3 & $100 \%$ & -- & -- & 0.010 & 0.010 & 0.010 \\
\hline METAL & Copper & $\mathrm{mg} / \mathrm{kg}$ & 3 & 3 & $100 \%$ & -- & -- & 0.50 & 0.56 & 0.53 \\
\hline METAL & Lead & $\mathrm{mg} / \mathrm{kg}$ & 3 & 2 & $67 \%$ & 0.010 & 0.010 & 0.020 & 0.050 & 0.025 \\
\hline METAL & Manganese & $\mathrm{mg} / \mathrm{kg}$ & 3 & 3 & $100 \%$ & -- & -- & 0.74 & 1.1 & 0.88 \\
\hline METAL & Mercury & $\mathrm{mg} / \mathrm{kg}$ & 3 & 3 & $100 \%$ & -- & -- & 0.38 & 0.47 & 0.41 \\
\hline METAL & Nickel & $\mathrm{mg} / \mathrm{kg}$ & 3 & 3 & $100 \%$ & -- & -- & 0.12 & 0.34 & 0.20 \\
\hline METAL & Selenium & $\mathrm{mg} / \mathrm{kg}$ & 3 & 3 & $100 \%$ & -- & -- & 0.45 & 0.52 & 0.49 \\
\hline METAL & Silver & $\mathrm{mg} / \mathrm{kg}$ & 3 & 0 & $0 \%$ & 0.10 & 0.10 & -- & -- & 0.050 \\
\hline METAL & Thallium & $\mathrm{mg} / \mathrm{kg}$ & 3 & 0 & $0 \%$ & 0.050 & 0.050 & -- & -- & 0.025 \\
\hline METAL & Vanadium & $\mathrm{mg} / \mathrm{kg}$ & 3 & 1 & $33 \%$ & 0.010 & 0.010 & 0.010 & 0.010 & 0.0067 \\
\hline METAL & Zinc & $\mathrm{mg} / \mathrm{kg}$ & 3 & 3 & $100 \%$ & -- & -- & 9.2 & 10 & 9.7 \\
\hline PCB & Aroclor-1016 & $\mathrm{mg} / \mathrm{kg}$ & 3 & 0 & $0 \%$ & 0.018 & 0.023 & -- & -- & 0.010 \\
\hline PCB & Aroclor-1221 & $\mathrm{mg} / \mathrm{kg}$ & 3 & 0 & $0 \%$ & 0.018 & 0.023 & -- & -- & 0.010 \\
\hline PCB & Aroclor-1232 & $\mathrm{mg} / \mathrm{kg}$ & 3 & 0 & $0 \%$ & 0.018 & 0.023 & -- & -- & 0.010 \\
\hline PCB & Aroclor-1248 & $\mathrm{mg} / \mathrm{kg}$ & 3 & 0 & $0 \%$ & 0.018 & 0.023 & -- & -- & 0.010 \\
\hline PCB & Aroclor-1254 & $\mathrm{mg} / \mathrm{kg}$ & 3 & 3 & $100 \%$ & -- & -- & 0.038 & 0.083 & 0.055 \\
\hline PCB & Aroclor-1260 & $\mathrm{mg} / \mathrm{kg}$ & 3 & 3 & $100 \%$ & -- & -- & 0.068 & 0.22 & 0.12 \\
\hline PEST & 2,4'-DDD & $\mathrm{mg} / \mathrm{kg}$ & 3 & 3 & $100 \%$ & -- & -- & 0.011 & 0.020 & 0.015 \\
\hline PEST & 2,4'-DDE & $\mathrm{mg} / \mathrm{kg}$ & 3 & 3 & $100 \%$ & -- & -- & 0.0052 & 0.0094 & 0.0067 \\
\hline PEST & 2,4'-DDT & $\mathrm{mg} / \mathrm{kg}$ & 3 & 3 & $100 \%$ & -- & -- & 0.0057 & 0.018 & 0.0099 \\
\hline PEST & Aldrin & $\mathrm{mg} / \mathrm{kg}$ & 3 & 0 & $0 \%$ & 0.0018 & 0.0023 & -- & -- & 0.0010 \\
\hline PEST & Alpha-BHC & $\mathrm{mg} / \mathrm{kg}$ & 3 & 0 & $0 \%$ & 0.0018 & 0.0023 & -- & -- & 0.0010 \\
\hline PEST & alpha-Chlordane & $\mathrm{mg} / \mathrm{kg}$ & 3 & 1 & $33 \%$ & 0.0018 & 0.0023 & 0.0041 & 0.0041 & 0.0021 \\
\hline PEST & alpha-Chlordene & $\mathrm{mg} / \mathrm{kg}$ & 3 & 0 & $0 \%$ & 0.0018 & 0.0023 & -- & -- & 0.0010 \\
\hline PEST & Beta-BHC & $\mathrm{mg} / \mathrm{kg}$ & 3 & 0 & $0 \%$ & 0.0018 & 0.0023 & -- & -- & 0.0010 \\
\hline PEST & cis-Nonachlor & $\mathrm{mg} / \mathrm{kg}$ & 3 & 0 & $0 \%$ & 0.0018 & 0.0023 & -- & -- & 0.0010 \\
\hline PEST & DDMU & $\mathrm{mg} / \mathrm{kg}$ & 3 & 3 & $100 \%$ & -- & -- & 0.020 & 0.056 & 0.032 \\
\hline PEST & Delta-BHC & $\mathrm{mg} / \mathrm{kg}$ & 3 & 0 & $0 \%$ & 0.0018 & 0.0023 & -- & -- & 0.0010 \\
\hline PEST & Dichlorodiphenyldichloroethane & $\mathrm{mg} / \mathrm{kg}$ & 3 & 3 & $100 \%$ & -- & -- & 0.030 & 0.085 & 0.051 \\
\hline PEST & Dichlorodiphenyldichloroethylene & $\mathrm{mg} / \mathrm{kg}$ & 3 & 3 & $100 \%$ & -- & -- & 0.48 & 1.2 & 0.77 \\
\hline PEST & Dichlorodiphenyltrichloroethane & $\mathrm{mg} / \mathrm{kg}$ & 3 & 3 & $100 \%$ & -- & -- & 0.023 & 0.048 & 0.034 \\
\hline PEST & Endosulfan sulfate & $\mathrm{mg} / \mathrm{kg}$ & 3 & 3 & $100 \%$ & -- & -- & 0.0015 & 0.0030 & 0.0021 \\
\hline PEST & Gamma-BHC (Lindane) & $\mathrm{mg} / \mathrm{kg}$ & 3 & 0 & $0 \%$ & 0.0018 & 0.0023 & -- & -- & 0.0010 \\
\hline PEST & gamma-Chlordane & $\mathrm{mg} / \mathrm{kg}$ & 3 & 1 & $33 \%$ & 0.0018 & 0.0023 & 0.014 & 0.014 & 0.0054 \\
\hline PEST & gamma-Chlordene & $\mathrm{mg} / \mathrm{kg}$ & 3 & 0 & $0 \%$ & 0.0018 & 0.0023 & -- & -- & 0.0010 \\
\hline PEST & Heptachlor & $\mathrm{mg} / \mathrm{kg}$ & 3 & 0 & $0 \%$ & 0.0018 & 0.0023 & -- & -- & 0.0010 \\
\hline PEST & Heptachlor epoxide & $\mathrm{mg} / \mathrm{kg}$ & 3 & 0 & $0 \%$ & 0.0018 & 0.0023 & -- & -- & 0.0010 \\
\hline PEST & Mirex & $\mathrm{mg} / \mathrm{kg}$ & 3 & 0 & $0 \%$ & 0.0018 & 0.0023 & -- & -- & 0.0010 \\
\hline PEST & Oxychlordane & $\mathrm{mg} / \mathrm{kg}$ & 3 & 1 & $33 \%$ & 0.0018 & 0.0023 & 0.0016 & 0.0016 & 0.0012 \\
\hline
\end{tabular}


Table F-68

Summary Statistics for Smallmouth Bass Fillet Skin On Collected from Segment 3 of the Columbia River

\begin{tabular}{|c|c|c|c|c|c|c|c|c|c|c|}
\hline $\begin{array}{c}\text { Constituent } \\
\text { Class }\end{array}$ & Constituent Name & Units & $\begin{array}{l}\text { Number } \\
\text { Analyzed }\end{array}$ & $\begin{array}{l}\text { Number } \\
\text { Detected }\end{array}$ & $\begin{array}{c}\text { Frequency of } \\
\text { Detection }\end{array}$ & $\begin{array}{c}\text { Minimum } \\
\text { Nondetect }\end{array}$ & $\begin{array}{l}\text { Maximum } \\
\text { Nondetect }\end{array}$ & $\begin{array}{c}\text { Minimum } \\
\text { Detect }\end{array}$ & $\begin{array}{l}\text { Maximum } \\
\text { Detect }\end{array}$ & $\begin{array}{c}\text { Average } \\
\text { Concentration }\end{array}$ \\
\hline PEST & Pentachloroanisole & $\mathrm{mg} / \mathrm{kg}$ & 3 & 1 & $33 \%$ & 0.0018 & 0.0023 & 7.90E-04 & 7.90E-04 & 9.47E-04 \\
\hline PEST & Toxaphene & $\mathrm{mg} / \mathrm{kg}$ & 3 & 0 & $0 \%$ & 0.054 & 0.068 & -- & -- & 0.030 \\
\hline PEST & trans-Nonachlor & $\mathrm{mg} / \mathrm{kg}$ & 3 & 3 & $100 \%$ & -- & -- & 0.0026 & 0.0086 & 0.0047 \\
\hline SVOC & 1,2,4-Trichlorobenzene & $\mathrm{mg} / \mathrm{kg}$ & 3 & 0 & $0 \%$ & 0.0057 & 0.0084 & -- & -- & 0.0038 \\
\hline SVOC & 1,2-Dichlorobenzene & $\mathrm{mg} / \mathrm{kg}$ & 3 & 0 & $0 \%$ & 0.0057 & 0.0084 & -- & -- & 0.0038 \\
\hline SVOC & 1,2-Diphenylhydrazine & $\mathrm{mg} / \mathrm{kg}$ & 3 & 0 & $0 \%$ & 0.0057 & 0.0084 & -- & -- & 0.0038 \\
\hline SVOC & 1,3-Dichlorobenzene & $\mathrm{mg} / \mathrm{kg}$ & 3 & 0 & $0 \%$ & 0.0057 & 0.0084 & -- & -- & 0.0038 \\
\hline SVOC & 1,4-Dichlorobenzene & $\mathrm{mg} / \mathrm{kg}$ & 3 & 0 & $0 \%$ & 0.0057 & 0.0084 & -- & -- & 0.0038 \\
\hline SVOC & 1-methylnaphthalene & $\mathrm{mg} / \mathrm{kg}$ & 3 & 0 & $0 \%$ & 0.0057 & 0.0084 & -- & -- & 0.0038 \\
\hline SVOC & 2,4-Dinitrotoluene & $\mathrm{mg} / \mathrm{kg}$ & 3 & 0 & $0 \%$ & 0.011 & 0.017 & -- & -- & 0.0075 \\
\hline SVOC & 2,6-Dinitrotoluene & $\mathrm{mg} / \mathrm{kg}$ & 3 & 0 & $0 \%$ & 0.023 & 0.034 & -- & -- & 0.015 \\
\hline SVOC & 2-Chloronaphthalene & $\mathrm{mg} / \mathrm{kg}$ & 3 & 0 & $0 \%$ & 0.011 & 0.017 & -- & -- & 0.0075 \\
\hline SVOC & 2-Methylnaphthalene & $\mathrm{mg} / \mathrm{kg}$ & 3 & 0 & $0 \%$ & 0.0057 & 0.0084 & -- & -- & 0.0038 \\
\hline SVOC & 4-Bromophenylphenyl ether & $\mathrm{mg} / \mathrm{kg}$ & 3 & 0 & $0 \%$ & 0.0057 & 0.0084 & -- & -- & 0.0038 \\
\hline SVOC & 4-Chlorophenylphenyl ether & $\mathrm{mg} / \mathrm{kg}$ & 3 & 0 & $0 \%$ & 0.0057 & 0.0084 & -- & -- & 0.0038 \\
\hline SVOC & Acenaphthene & $\mathrm{mg} / \mathrm{kg}$ & 3 & 0 & $0 \%$ & 0.011 & 0.017 & -- & -- & 0.0075 \\
\hline SVOC & Acenaphthylene & $\mathrm{mg} / \mathrm{kg}$ & 3 & 0 & $0 \%$ & 0.0057 & 0.0084 & -- & -- & 0.0038 \\
\hline SVOC & Anthracene & $\mathrm{mg} / \mathrm{kg}$ & 3 & 0 & $0 \%$ & 0.0057 & 0.0084 & -- & -- & 0.0038 \\
\hline SVOC & Benzo(a)anthracene & $\mathrm{mg} / \mathrm{kg}$ & 3 & 0 & $0 \%$ & 0.0057 & 0.0084 & -- & -- & 0.0038 \\
\hline SVOC & Benzo(a)pyrene & $\mathrm{mg} / \mathrm{kg}$ & 3 & 0 & $0 \%$ & 0.0057 & 0.0084 & -- & -- & 0.0038 \\
\hline SVOC & Benzo(b)fluoranthene & $\mathrm{mg} / \mathrm{kg}$ & 3 & 0 & $0 \%$ & 0.0057 & 0.0084 & -- & -- & 0.0038 \\
\hline SVOC & Benzo(ghi)perylene & $\mathrm{mg} / \mathrm{kg}$ & 3 & 0 & $0 \%$ & 0.011 & 0.017 & -- & -- & 0.0075 \\
\hline SVOC & Benzo(k)fluoranthene & $\mathrm{mg} / \mathrm{kg}$ & 3 & 0 & $0 \%$ & 0.0057 & 0.0084 & -- & -- & 0.0038 \\
\hline SVOC & Bis(2-chloro-1-methylethyl)ether & $\mathrm{mg} / \mathrm{kg}$ & 3 & 0 & $0 \%$ & 0.011 & 0.017 & -- & -- & 0.0075 \\
\hline SVOC & Chrysene & $\mathrm{mg} / \mathrm{kg}$ & 3 & 0 & $0 \%$ & 0.0057 & 0.0084 & -- & -- & 0.0038 \\
\hline SVOC & Dibenz[a,h]anthracene & $\mathrm{mg} / \mathrm{kg}$ & 3 & 0 & $0 \%$ & 0.011 & 0.017 & -- & -- & 0.0075 \\
\hline SVOC & Dibenzofuran & $\mathrm{mg} / \mathrm{kg}$ & 3 & 0 & $0 \%$ & 0.0057 & 0.0084 & -- & -- & 0.0038 \\
\hline SVOC & Fluoranthene & $\mathrm{mg} / \mathrm{kg}$ & 3 & 0 & $0 \%$ & 0.011 & 0.017 & -- & -- & 0.0075 \\
\hline SVOC & Fluorene & $\mathrm{mg} / \mathrm{kg}$ & 3 & 0 & $0 \%$ & 0.0092 & 0.0099 & -- & -- & 0.0048 \\
\hline SVOC & Hexachlorobenzene & $\mathrm{mg} / \mathrm{kg}$ & 3 & 0 & $0 \%$ & 0.011 & 0.017 & -- & -- & 0.0075 \\
\hline SVOC & Hexachlorobutadiene & $\mathrm{mg} / \mathrm{kg}$ & 3 & 0 & $0 \%$ & 0.0057 & 0.0084 & -- & -- & 0.0038 \\
\hline SVOC & Hexachloroethane & $\mathrm{mg} / \mathrm{kg}$ & 3 & 0 & $0 \%$ & 0.0057 & 0.0084 & -- & -- & 0.0038 \\
\hline SVOC & Indeno(1,2,3-cd)pyrene & $\mathrm{mg} / \mathrm{kg}$ & 3 & 0 & $0 \%$ & 0.011 & 0.017 & -- & -- & 0.0075 \\
\hline SVOC & Naphthalene & $\mathrm{mg} / \mathrm{kg}$ & 3 & 0 & $0 \%$ & 0.010 & 0.017 & -- & -- & 0.0063 \\
\hline SVOC & Nitrobenzene & $\mathrm{mg} / \mathrm{kg}$ & 3 & 0 & $0 \%$ & 0.0057 & 0.0084 & -- & -- & 0.0038 \\
\hline SVOC & Phenanthrene & $\mathrm{mg} / \mathrm{kg}$ & 3 & 0 & $0 \%$ & 0.0057 & 0.0099 & -- & -- & 0.0040 \\
\hline SVOC & Pyrene & $\mathrm{mg} / \mathrm{kg}$ & 3 & 0 & $0 \%$ & 0.011 & 0.017 & -- & -- & 0.0075 \\
\hline VOC & Retene & $\mathrm{mg} / \mathrm{kg}$ & 3 & 0 & $0 \%$ & 0.011 & 0.017 & -- & -- & 0.0075 \\
\hline
\end{tabular}

PEST - Pesticide

PCB - Polychlorinated biphenyl

SVOC - Semivolatile Organic Compound

VOC - Volatile Organic Compound 


\section{Table F-69}

Summary Statistics for Carp Fillet Collected from Segment 3 of the Columbia River

\begin{tabular}{|c|c|c|c|c|c|c|c|c|c|c|}
\hline $\begin{array}{l}\text { Constituent } \\
\text { Class }\end{array}$ & Constituent Name & Units & $\begin{array}{l}\text { Number } \\
\text { Analyzed }\end{array}$ & $\begin{array}{l}\text { Number } \\
\text { Detected }\end{array}$ & $\begin{array}{l}\text { Frequency of } \\
\text { Detection }\end{array}$ & $\begin{array}{c}\text { Minimum } \\
\text { Nondetect }\end{array}$ & $\begin{array}{l}\text { Maximum } \\
\text { Nondetect }\end{array}$ & $\begin{array}{c}\text { Minimum } \\
\text { Detect }\end{array}$ & $\begin{array}{l}\text { Maximum } \\
\text { Detect }\end{array}$ & $\begin{array}{c}\text { Average } \\
\text { Concentration }\end{array}$ \\
\hline CONV & Percent Lipids & $\%$ & 4 & 4 & $100 \%$ & $\overline{--}$ & $\overline{--}$ & 2.4 & 7.0 & 4.6 \\
\hline PCB & Aroclor-1016 & $\mathrm{mg} / \mathrm{Kg}$ & 4 & 0 & $0 \%$ & 0.0049 & 0.0055 & -- & -- & 0.0026 \\
\hline PCB & Aroclor-1221 & $\mathrm{mg} / \mathrm{Kg}$ & 4 & 0 & $0 \%$ & 0.0049 & 0.0055 & -- & -- & 0.0026 \\
\hline PCB & Aroclor-1232 & $\mathrm{mg} / \mathrm{Kg}$ & 4 & 0 & $0 \%$ & 0.0049 & 0.0055 & -- & -- & 0.0026 \\
\hline PCB & Aroclor-1242 & $\mathrm{mg} / \mathrm{Kg}$ & 4 & 0 & $0 \%$ & 0.0049 & 0.0055 & -- & -- & 0.0026 \\
\hline PCB & Aroclor-1248 & $\mathrm{mg} / \mathrm{Kg}$ & 4 & 0 & $0 \%$ & 0.0049 & 0.0055 & -- & -- & 0.0026 \\
\hline PCB & Aroclor-1254 & $\mathrm{mg} / \mathrm{Kg}$ & 4 & 4 & $100 \%$ & -- & -- & 0.029 & 0.14 & 0.081 \\
\hline PCB & Aroclor-1260 & $\mathrm{mg} / \mathrm{Kg}$ & 4 & 4 & $100 \%$ & -- & -- & 0.039 & 0.27 & 0.14 \\
\hline PCB & Aroclor-1262 & $\mathrm{mg} / \mathrm{Kg}$ & 4 & 0 & $0 \%$ & 0.0049 & 0.0055 & -- & -- & 0.0026 \\
\hline PCB & Aroclor-1268 & $\mathrm{mg} / \mathrm{Kg}$ & 4 & 0 & $0 \%$ & 0.0049 & 0.0055 & -- & -- & 0.0026 \\
\hline PEST & 2,4'-DDD & $\mathrm{mg} / \mathrm{Kg}$ & 4 & 4 & $100 \%$ & -- & -- & 9.40E-04 & 0.0068 & 0.0036 \\
\hline PEST & 2,4'-DDE & $\mathrm{mg} / \mathrm{Kg}$ & 4 & 4 & $100 \%$ & -- & -- & 0.0018 & 0.012 & 0.0065 \\
\hline PEST & 2,4'-DDT & $\mathrm{mg} / \mathrm{Kg}$ & 4 & 4 & $100 \%$ & -- & -- & 0.0011 & 0.0033 & 0.0022 \\
\hline PEST & alpha-Chlordane & $\mathrm{mg} / \mathrm{Kg}$ & 4 & 4 & $100 \%$ & -- & -- & 0.0018 & 0.0048 & 0.0031 \\
\hline PEST & $\begin{array}{l}\text { cis-Nonachlor } \\
\text { Dichlorodiphenyldichl }\end{array}$ & $\mathrm{mg} / \mathrm{Kg}$ & 4 & 4 & $100 \%$ & -- & -- & 0.0018 & 0.0045 & 0.0030 \\
\hline PEST & $\begin{array}{l}\text { oroethane } \\
\text { Dichlorodiphenyldichl }\end{array}$ & $\mathrm{mg} / \mathrm{Kg}$ & 4 & 4 & $100 \%$ & -- & -- & 0.021 & 0.091 & 0.051 \\
\hline PEST & $\begin{array}{l}\text { oroethylene } \\
\text { Dichlorodiphenyltrichl }\end{array}$ & $\mathrm{mg} / \mathrm{Kg}$ & 4 & 4 & $100 \%$ & -- & -- & 0.30 & 0.93 & 0.55 \\
\hline PEST & oroethane & $\mathrm{mg} / \mathrm{Kg}$ & 4 & 4 & $100 \%$ & -- & -- & $7.40 \mathrm{E}-04$ & 0.0014 & $9.88 \mathrm{E}-04$ \\
\hline PEST & Dieldrin & $\mathrm{mg} / \mathrm{Kg}$ & 4 & 4 & $100 \%$ & -- & -- & 0.0011 & 0.0029 & 0.0018 \\
\hline PEST & Heptachlor & $\mathrm{mg} / \mathrm{Kg}$ & 4 & 0 & $0 \%$ & 4.90E-04 & 5.50E-04 & -- & -- & 2.61E-04 \\
\hline PEST & Heptachlor epoxide & $\mathrm{mg} / \mathrm{Kg}$ & 4 & 4 & $100 \%$ & -- & -- & 8.60E-04 & 0.0021 & 0.0013 \\
\hline PEST & Oxychlordane & $\mathrm{mg} / \mathrm{Kg}$ & 4 & 4 & $100 \%$ & -- & -- & 7.00E-04 & 0.0015 & $9.80 \mathrm{E}-04$ \\
\hline PEST & Toxaphene & $\mathrm{mg} / \mathrm{Kg}$ & 4 & 4 & $100 \%$ & -- & -- & 0.046 & 0.065 & 0.055 \\
\hline PEST & trans-Chlordane & $\mathrm{mg} / \mathrm{Kg}$ & 4 & 4 & $100 \%$ & -- & -- & 0.0015 & 0.0039 & 0.0023 \\
\hline PEST & trans-Nonachlor & $\mathrm{mg} / \mathrm{Kg}$ & 4 & 4 & $100 \%$ & -- & -- & 7.30E-04 & 0.016 & 0.0076 \\
\hline SVOC & Hexachlorobenzene & $\mathrm{mg} / \mathrm{Kg}$ & 4 & 4 & $100 \%$ & -- & -- & 0.0053 & 0.0095 & 0.0065 \\
\hline
\end{tabular}

PEST - Pesticide

PCB - Polychlorinated biphenyl

SVOC - Semivolatile Organic Compound 


\section{Table F-70}

Summary Statistics for Channel Catfish Fillet Collected from Segment 3 of the Columbia River

\begin{tabular}{|c|c|c|c|c|c|c|c|c|c|c|}
\hline $\begin{array}{c}\text { Constituent } \\
\text { Class }\end{array}$ & Constituent Name & Units & $\begin{array}{l}\text { Number } \\
\text { Analyzed }\end{array}$ & $\begin{array}{l}\text { Number } \\
\text { Detected }\end{array}$ & $\begin{array}{c}\text { Frequency of } \\
\text { Detection }\end{array}$ & $\begin{array}{l}\text { Minimum } \\
\text { Nondetect }\end{array}$ & $\begin{array}{l}\text { Maximum } \\
\text { Nondetect }\end{array}$ & $\begin{array}{c}\text { Minimum } \\
\text { Detect }\end{array}$ & $\begin{array}{c}\text { Maximum } \\
\text { Detect }\end{array}$ & $\begin{array}{c}\text { Average } \\
\text { Concentration }\end{array}$ \\
\hline$\overline{\text { CONV }}$ & Percent Lipids & $\%$ & 1 & 1 & $100 \%$ & $\overline{--}$ & -- & 4.2 & 4.2 & 4.2 \\
\hline PCB & Aroclor-1016 & $\mathrm{mg} / \mathrm{Kg}$ & 1 & 0 & $0 \%$ & 0.0055 & 0.0055 & -- & -- & 0.0028 \\
\hline PCB & Aroclor-1221 & $\mathrm{mg} / \mathrm{Kg}$ & 1 & 0 & $0 \%$ & 0.0055 & 0.0055 & -- & -- & 0.0028 \\
\hline PCB & Aroclor-1232 & $\mathrm{mg} / \mathrm{Kg}$ & 1 & 0 & $0 \%$ & 0.0055 & 0.0055 & -- & -- & 0.0028 \\
\hline PCB & Aroclor-1242 & $\mathrm{mg} / \mathrm{Kg}$ & 1 & 0 & $0 \%$ & 0.0055 & 0.0055 & -- & -- & 0.0028 \\
\hline PCB & Aroclor-1248 & $\mathrm{mg} / \mathrm{Kg}$ & 1 & 0 & $0 \%$ & 0.0055 & 0.0055 & -- & -- & 0.0028 \\
\hline PCB & Aroclor-1254 & $\mathrm{mg} / \mathrm{Kg}$ & 1 & 1 & $100 \%$ & -- & -- & 0.029 & 0.029 & 0.029 \\
\hline PCB & Aroclor-1260 & $\mathrm{mg} / \mathrm{Kg}$ & 1 & 1 & $100 \%$ & -- & -- & 0.024 & 0.024 & 0.024 \\
\hline PCB & Aroclor-1262 & $\mathrm{mg} / \mathrm{Kg}$ & 1 & 0 & $0 \%$ & 0.0055 & 0.0055 & -- & -- & 0.0028 \\
\hline PCB & Aroclor-1268 & $\mathrm{mg} / \mathrm{Kg}$ & 1 & 0 & $0 \%$ & 0.0055 & 0.0055 & -- & -- & 0.0028 \\
\hline PEST & 2,4'-DDD & $\mathrm{mg} / \mathrm{Kg}$ & 1 & 1 & $100 \%$ & -- & -- & 0.0012 & 0.0012 & 0.0012 \\
\hline PEST & 2,4'-DDE & $\mathrm{mg} / \mathrm{Kg}$ & 1 & 1 & $100 \%$ & -- & -- & 8.20E-04 & 8.20E-04 & 8.20E-04 \\
\hline PEST & 2,4'-DDT & $\mathrm{mg} / \mathrm{Kg}$ & 1 & 0 & $0 \%$ & 5.50E-04 & 5.50E-04 & -- & -- & 2.75E-04 \\
\hline PEST & alpha-Chlordane & $\mathrm{mg} / \mathrm{Kg}$ & 1 & 1 & $100 \%$ & -- & -- & 0.0024 & 0.0024 & 0.0024 \\
\hline PEST & cis-Nonachlor & $\mathrm{mg} / \mathrm{Kg}$ & 1 & 1 & $100 \%$ & -- & -- & 0.0017 & 0.0017 & 0.0017 \\
\hline PEST & Dichlorodiphenyldichloroethane & $\mathrm{mg} / \mathrm{Kg}$ & 1 & 1 & $100 \%$ & -- & -- & 0.023 & 0.023 & 0.023 \\
\hline PEST & Dichlorodiphenyldichloroethylene & $\mathrm{mg} / \mathrm{Kg}$ & 1 & 1 & $100 \%$ & -- & -- & 0.22 & 0.22 & 0.22 \\
\hline PEST & Dichlorodiphenyltrichloroethane & $\mathrm{mg} / \mathrm{Kg}$ & 1 & 1 & $100 \%$ & -- & -- & 0.0083 & 0.0083 & 0.0083 \\
\hline PEST & Dieldrin & $\mathrm{mg} / \mathrm{Kg}$ & 1 & 1 & $100 \%$ & -- & -- & 0.0020 & 0.0020 & 0.0020 \\
\hline PEST & Heptachlor & $\mathrm{mg} / \mathrm{Kg}$ & 1 & 0 & $0 \%$ & 5.50E-04 & 5.50E-04 & -- & -- & $2.75 E-04$ \\
\hline PEST & Heptachlor epoxide & $\mathrm{mg} / \mathrm{Kg}$ & 1 & 1 & $100 \%$ & -- & -- & $6.80 \mathrm{E}-04$ & 6.80E-04 & $6.80 \mathrm{E}-04$ \\
\hline PEST & Oxychlordane & $\mathrm{mg} / \mathrm{Kg}$ & 1 & 1 & $100 \%$ & -- & -- & 0.0011 & 0.0011 & 0.0011 \\
\hline PEST & Toxaphene & $\mathrm{mg} / \mathrm{Kg}$ & 1 & 1 & $100 \%$ & -- & -- & 0.072 & 0.072 & 0.072 \\
\hline PEST & trans-Chlordane & $\mathrm{mg} / \mathrm{Kg}$ & 1 & 1 & $100 \%$ & -- & -- & 0.0019 & 0.0019 & 0.0019 \\
\hline PEST & trans-Nonachlor & $\mathrm{mg} / \mathrm{Kg}$ & 1 & 1 & $100 \%$ & -- & -- & 0.0039 & 0.0039 & 0.0039 \\
\hline SVOC & Hexachlorobenzene & $\mathrm{mg} / \mathrm{Kg}$ & 1 & 1 & $100 \%$ & -- & -- & 0.0025 & 0.0025 & 0.0025 \\
\hline
\end{tabular}

PEST - Pesticide

PCB - Polychlorinated biphenyl

SVOC - Semivolatile Organic Compound 
Table F-71

Summary Statistics for Bridgelip Sucker Fillet Collected from Segment 3 of the Columbia River

\begin{tabular}{|c|c|c|c|c|c|c|c|c|c|c|}
\hline $\begin{array}{l}\text { Constituent } \\
\text { Class }\end{array}$ & Constituent Name & Units & $\begin{array}{l}\text { Number } \\
\text { Analyzed }\end{array}$ & $\begin{array}{l}\text { Number } \\
\text { Detected }\end{array}$ & $\begin{array}{l}\text { Frequency of } \\
\text { Detection }\end{array}$ & $\begin{array}{c}\text { Minimum } \\
\text { Nondetect }\end{array}$ & $\begin{array}{l}\text { Maximum } \\
\text { Nondetect }\end{array}$ & $\underset{\text { Detect }}{\text { Minimum }}$ & $\begin{array}{c}\text { Maximum } \\
\text { Detect }\end{array}$ & $\begin{array}{c}\text { Average } \\
\text { Concentration }\end{array}$ \\
\hline$\overline{\text { CONV }}$ & Percent Lipids & $\%$ & 5 & 5 & $100 \%$ & $\overline{--}$ & $\overline{--}$ & 1.4 & 4.4 & 2.7 \\
\hline PCB & Aroclor-1016 & $\mathrm{mg} / \mathrm{Kg}$ & 5 & 0 & $0 \%$ & 0.0026 & 0.0055 & -- & -- & 0.0021 \\
\hline PCB & Aroclor-1221 & $\mathrm{mg} / \mathrm{Kg}$ & 5 & 0 & $0 \%$ & 0.0026 & 0.0055 & -- & -- & 0.0021 \\
\hline PCB & Aroclor-1232 & $\mathrm{mg} / \mathrm{Kg}$ & 5 & 0 & $0 \%$ & 0.0026 & 0.0055 & -- & -- & 0.0021 \\
\hline PCB & Aroclor-1242 & $\mathrm{mg} / \mathrm{Kg}$ & 5 & 0 & $0 \%$ & 0.0026 & 0.0055 & -- & -- & 0.0021 \\
\hline PCB & Aroclor-1248 & $\mathrm{mg} / \mathrm{Kg}$ & 5 & 0 & $0 \%$ & 0.0026 & 0.0055 & -- & -- & 0.0021 \\
\hline PCB & Aroclor-1254 & $\mathrm{mg} / \mathrm{Kg}$ & 5 & 5 & $100 \%$ & -- & -- & 0.0054 & 0.016 & 0.010 \\
\hline PCB & Aroclor-1260 & $\mathrm{mg} / \mathrm{Kg}$ & 5 & 5 & $100 \%$ & -- & -- & 0.0031 & 0.011 & 0.0066 \\
\hline PCB & Aroclor-1262 & $\mathrm{mg} / \mathrm{Kg}$ & 5 & 0 & $0 \%$ & 0.0040 & 0.0055 & -- & -- & 0.0024 \\
\hline PCB & Aroclor-1268 & $\mathrm{mg} / \mathrm{Kg}$ & 5 & 0 & $0 \%$ & 0.0040 & 0.0055 & -- & -- & 0.0024 \\
\hline PEST & 2,4'-DDD & $\mathrm{mg} / \mathrm{Kg}$ & 5 & 5 & $100 \%$ & -- & -- & $5.80 \mathrm{E}-04$ & $9.40 \mathrm{E}-04$ & $7.22 \mathrm{E}-04$ \\
\hline PEST & 2,4'-DDE & $\mathrm{mg} / \mathrm{Kg}$ & 5 & 3 & $60 \%$ & $4.90 \mathrm{E}-04$ & $5.50 \mathrm{E}-04$ & 5.50E-04 & 0.0014 & $6.18 \mathrm{E}-04$ \\
\hline PEST & 2,4'-DDT & $\mathrm{mg} / \mathrm{Kg}$ & 5 & 1 & $20 \%$ & 4.00E-04 & $5.50 \mathrm{E}-04$ & 8.00E-04 & 8.00E-04 & 3.56E-04 \\
\hline PEST & alpha-Chlordane & $\mathrm{mg} / \mathrm{Kg}$ & 5 & 3 & $60 \%$ & $4.90 \mathrm{E}-04$ & $5.50 \mathrm{E}-04$ & 5.20E-04 & $8.00 \mathrm{E}-04$ & $5.04 \mathrm{E}-04$ \\
\hline PEST & cis-Nonachlor & $\mathrm{mg} / \mathrm{Kg}$ & 5 & 3 & $60 \%$ & 4.90E-04 & $5.50 \mathrm{E}-04$ & 4.60E-04 & $6.90 \mathrm{E}-04$ & $4.54 \mathrm{E}-04$ \\
\hline PEST & Dichlorodiphenyldichloroethane & $\mathrm{mg} / \mathrm{Kg}$ & 5 & 5 & $100 \%$ & -- & -- & 0.0042 & 0.0067 & 0.0055 \\
\hline PEST & Dichlorodiphenyldichloroethylene & $\mathrm{mg} / \mathrm{Kg}$ & 5 & 5 & $100 \%$ & -- & -- & 0.042 & 0.17 & 0.087 \\
\hline PEST & Dichlorodiphenyltrichloroethane & $\mathrm{mg} / \mathrm{Kg}$ & 5 & 5 & $100 \%$ & -- & -- & 0.0015 & 0.0044 & 0.0024 \\
\hline PEST & Dieldrin & $\mathrm{mg} / \mathrm{Kg}$ & 5 & 3 & $60 \%$ & $4.90 \mathrm{E}-04$ & $5.50 \mathrm{E}-04$ & $5.50 \mathrm{E}-04$ & 0.0010 & $5.70 \mathrm{E}-04$ \\
\hline PEST & Heptachlor & $\mathrm{mg} / \mathrm{Kg}$ & 5 & 0 & $0 \%$ & $4.00 \mathrm{E}-04$ & $5.50 \mathrm{E}-04$ & -- & -- & $2.40 \mathrm{E}-04$ \\
\hline PEST & Heptachlor epoxide & $\mathrm{mg} / \mathrm{Kg}$ & 5 & 1 & $20 \%$ & 4.40E-04 & $5.50 \mathrm{E}-04$ & $6.50 \mathrm{E}-04$ & $6.50 \mathrm{E}-04$ & 3.30E-04 \\
\hline PEST & Oxychlordane & $\mathrm{mg} / \mathrm{Kg}$ & 5 & 1 & $20 \%$ & 4.40E-04 & $5.50 \mathrm{E}-04$ & $6.20 \mathrm{E}-04$ & $6.20 \mathrm{E}-04$ & 3.24E-04 \\
\hline PEST & Toxaphene & $\mathrm{mg} / \mathrm{Kg}$ & 5 & 5 & $100 \%$ & -- & -- & 0.013 & 0.027 & 0.021 \\
\hline PEST & trans-Chlordane & $\mathrm{mg} / \mathrm{Kg}$ & 5 & 1 & $20 \%$ & 4.40E-04 & $5.50 \mathrm{E}-04$ & 4.40E-04 & 4.40E-04 & $2.88 \mathrm{E}-04$ \\
\hline PEST & trans-Nonachlor & $\mathrm{mg} / \mathrm{Kg}$ & 5 & 4 & $80 \%$ & $9.80 \mathrm{E}-04$ & $9.80 \mathrm{E}-04$ & 0.0011 & 0.0037 & 0.0019 \\
\hline SVOC & Hexachlorobenzene & $\mathrm{mg} / \mathrm{Kg}$ & 5 & 5 & $100 \%$ & -- & -- & 0.0011 & 0.0034 & 0.0018 \\
\hline
\end{tabular}

PEST - Pesticide

PCB - Polychlorinated biphenyl

SVOC - Semivolatile Organic Compound 


\section{Table F-72}

Summary Statistics for Whole Body Bridgelip Sucker Collected from Segment 3 of the Columbia River

\begin{tabular}{|c|c|c|c|c|c|c|c|c|c|c|}
\hline $\begin{array}{l}\text { Constituent } \\
\text { Class }\end{array}$ & Constituent Name & Units & $\begin{array}{l}\text { Number } \\
\text { Analyzed }\end{array}$ & $\begin{array}{l}\text { Number } \\
\text { Detected }\end{array}$ & $\begin{array}{c}\text { Frequency of } \\
\text { Detection }\end{array}$ & $\begin{array}{c}\text { Minimum } \\
\text { Nondetect }\end{array}$ & $\begin{array}{c}\text { Maximum } \\
\text { Nondetect }\end{array}$ & $\begin{array}{c}\text { Minimum } \\
\text { Detect }\end{array}$ & $\begin{array}{l}\text { Maximum } \\
\text { Detect }\end{array}$ & $\begin{array}{c}\text { Average } \\
\text { Concentration }\end{array}$ \\
\hline$\overline{\mathrm{DIOXI}}$ & 1,2,3,4,6,7,8-Heptachlorodibenzodioxin & $\mathrm{mg} / \mathrm{kg}$ & 3 & $\overline{3}$ & $100 \%$ & -- & -- & $3.60 \mathrm{E}-07$ & $4.20 \mathrm{E}-07$ & $3.97 \mathrm{E}-07$ \\
\hline DIOXI & $1,2,3,4,6,7,8$-Heptachlorodibenzofuran & $\mathrm{mg} / \mathrm{kg}$ & 3 & 2 & $67 \%$ & 1.00E-08 & 1.00E-08 & 1.10E-07 & $1.20 \mathrm{E}-07$ & 7.83E-08 \\
\hline DIOXI & $1,2,3,4,7,8,9$-Heptachlorodibenzofuran & $\mathrm{mg} / \mathrm{kg}$ & 3 & 0 & $0 \%$ & $1.00 \mathrm{E}-08$ & $1.00 \mathrm{E}-08$ & -- & -- & $5.00 \mathrm{E}-09$ \\
\hline DIOXI & $1,2,3,4,7,8$-Hexachlorodibenzofuran & $\mathrm{mg} / \mathrm{kg}$ & 3 & 3 & $100 \%$ & -- & -- & $2.00 \mathrm{E}-08$ & $2.00 \mathrm{E}-08$ & $2.00 \mathrm{E}-08$ \\
\hline DIOXI & 1,2,3,4,7,8-Hexachlorodibenzo-p-dioxin & $\mathrm{mg} / \mathrm{kg}$ & 3 & 3 & $100 \%$ & -- & -- & $1.20 \mathrm{E}-07$ & $1.30 \mathrm{E}-07$ & 1.23E-07 \\
\hline DIOXI & 1,2,3,6,7,8-Hexachlorodibenzofuran & $\mathrm{mg} / \mathrm{kg}$ & 3 & 3 & $100 \%$ & -- & -- & $3.00 \mathrm{E}-08$ & $4.00 \mathrm{E}-08$ & 3.33E-08 \\
\hline DIOXI & $1,2,3,6,7,8$-Hexachlorodibenzo-p-dioxin & $\mathrm{mg} / \mathrm{kg}$ & 3 & 3 & $100 \%$ & -- & -- & $1.40 \mathrm{E}-07$ & $1.70 \mathrm{E}-07$ & $1.50 \mathrm{E}-07$ \\
\hline DIOXI & 1,2,3,7,8,9-Hexachlorodibenzofuran & $\mathrm{mg} / \mathrm{kg}$ & 3 & 3 & $100 \%$ & -- & -- & $5.00 \mathrm{E}-08$ & $6.00 \mathrm{E}-08$ & $5.67 \mathrm{E}-08$ \\
\hline DIOXI & 1,2,3,7,8,9-Hexachlorodibenzo-p-dioxin & $\mathrm{mg} / \mathrm{kg}$ & 3 & 3 & $100 \%$ & -- & -- & $5.00 \mathrm{E}-08$ & $6.00 \mathrm{E}-08$ & 5.33E-08 \\
\hline DIOXI & $1,2,3,7,8$-Pentachlorodibenzofuran & $\mathrm{mg} / \mathrm{kg}$ & 3 & 3 & $100 \%$ & -- & -- & $5.00 \mathrm{E}-08$ & $6.00 \mathrm{E}-08$ & $5.33 \mathrm{E}-08$ \\
\hline DIOXI & $1,2,3,7,8$-Pentachlorodibenzo-p-dioxin & $\mathrm{mg} / \mathrm{kg}$ & 3 & 3 & $100 \%$ & -- & -- & $1.80 \mathrm{E}-07$ & $1.80 \mathrm{E}-07$ & $1.80 \mathrm{E}-07$ \\
\hline DIOXI & $2,3,4,6,7,8$-Hexachlorodibenzofuran & $\mathrm{mg} / \mathrm{kg}$ & 3 & 3 & $100 \%$ & -- & -- & 1.30E-07 & $1.50 \mathrm{E}-07$ & 1.40E-07 \\
\hline DIOXI & $2,3,4,7,8$-Pentachlorodibenzofuran & $\mathrm{mg} / \mathrm{kg}$ & 3 & 3 & $100 \%$ & -- & -- & $1.10 \mathrm{E}-07$ & 1.20E-07 & 1.17E-07 \\
\hline DIOXI & $2,3,7,8$-Tetrachlorodibenzofuran & $\mathrm{mg} / \mathrm{kg}$ & 3 & 3 & $100 \%$ & -- & -- & $7.60 \mathrm{E}-07$ & $1.11 \mathrm{E}-06$ & $9.60 \mathrm{E}-07$ \\
\hline DIOXI & 2,3,7,8-Tetrachlorodibenzo-p-dioxin & $\mathrm{mg} / \mathrm{kg}$ & 3 & 3 & $100 \%$ & -- & -- & $6.00 \mathrm{E}-08$ & $8.00 \mathrm{E}-08$ & $7.00 \mathrm{E}-08$ \\
\hline DIOXI & Octachlorodibenzofuran & $\mathrm{mg} / \mathrm{kg}$ & 3 & 3 & $100 \%$ & -- & -- & $5.00 \mathrm{E}-08$ & $8.00 \mathrm{E}-08$ & $6.67 \mathrm{E}-08$ \\
\hline DIOXI & Octachlorodibenzo-p-dioxin & $\mathrm{mg} / \mathrm{kg}$ & 3 & 3 & $100 \%$ & -- & -- & $7.50 \mathrm{E}-07$ & $9.80 \mathrm{E}-07$ & 8.93E-07 \\
\hline METAL & Aluminum & $\mathrm{mg} / \mathrm{kg}$ & 3 & 3 & $100 \%$ & -- & -- & 22 & 64 & 37 \\
\hline METAL & Antimony & $\mathrm{mg} / \mathrm{kg}$ & 3 & 0 & $0 \%$ & 0.050 & 0.050 & -- & -- & 0.025 \\
\hline METAL & Arsenic & $\mathrm{mg} / \mathrm{kg}$ & 3 & 3 & $100 \%$ & -- & -- & 0.26 & 0.30 & 0.28 \\
\hline METAL & Barium & $\mathrm{mg} / \mathrm{kg}$ & 3 & 3 & $100 \%$ & -- & -- & 1.9 & 2.1 & 2.0 \\
\hline METAL & Beryllium & $\mathrm{mg} / \mathrm{kg}$ & 3 & 2 & $67 \%$ & 0.0040 & 0.0040 & 0.0060 & 0.0080 & 0.0053 \\
\hline METAL & Cadmium & $\mathrm{mg} / \mathrm{kg}$ & 3 & 3 & $100 \%$ & -- & -- & 0.020 & 0.030 & 0.027 \\
\hline METAL & Chromium & $\mathrm{mg} / \mathrm{kg}$ & 3 & 3 & $100 \%$ & -- & -- & 0.12 & 0.23 & 0.18 \\
\hline METAL & Cobalt & $\mathrm{mg} / \mathrm{kg}$ & 3 & 3 & $100 \%$ & -- & -- & 0.080 & 0.11 & 0.090 \\
\hline METAL & Copper & $\mathrm{mg} / \mathrm{kg}$ & 3 & 3 & $100 \%$ & -- & -- & 0.88 & 1.8 & 1.2 \\
\hline METAL & Lead & $\mathrm{mg} / \mathrm{kg}$ & 3 & 2 & $67 \%$ & 0.070 & 0.070 & 0.030 & 0.040 & 0.035 \\
\hline METAL & Manganese & $\mathrm{mg} / \mathrm{kg}$ & 3 & 3 & $100 \%$ & -- & -- & 17 & 20 & 18 \\
\hline METAL & Mercury & $\mathrm{mg} / \mathrm{kg}$ & 3 & 1 & $33 \%$ & 0.040 & 0.040 & 0.050 & 0.050 & 0.030 \\
\hline METAL & Nickel & $\mathrm{mg} / \mathrm{kg}$ & 3 & 3 & $100 \%$ & -- & -- & 0.27 & 0.60 & 0.39 \\
\hline METAL & Selenium & $\mathrm{mg} / \mathrm{kg}$ & 3 & 3 & $100 \%$ & -- & -- & 0.28 & 0.28 & 0.28 \\
\hline METAL & Silver & $\mathrm{mg} / \mathrm{kg}$ & 3 & 0 & $0 \%$ & 0.10 & 0.10 & -- & -- & 0.050 \\
\hline METAL & Thallium & $\mathrm{mg} / \mathrm{kg}$ & 3 & 0 & $0 \%$ & 0.050 & 0.050 & -- & -- & 0.025 \\
\hline METAL & Vanadium & $\mathrm{mg} / \mathrm{kg}$ & 3 & 3 & $100 \%$ & -- & -- & 0.14 & 0.26 & 0.19 \\
\hline METAL & Zinc & $\mathrm{mg} / \mathrm{kg}$ & 3 & 3 & $100 \%$ & -- & -- & 20 & 21 & 20 \\
\hline PCB & 2,2',3,3',4,4',5-Heptachloro-1,1'-biphenyl & $\mathrm{mg} / \mathrm{kg}$ & 3 & 3 & $100 \%$ & -- & -- & $7.42 \mathrm{E}-04$ & 0.0013 & $9.44 \mathrm{E}-04$ \\
\hline РСB & 2,2',3,4,4',5,5'-Heptachlorobiphenyl & $\mathrm{mg} / \mathrm{kg}$ & 3 & 3 & $100 \%$ & -- & -- & 0.0021 & 0.0041 & 0.0028 \\
\hline РCB & 2,3,3',4,4',5'-Hexachlorobiphenyl & $\mathrm{mg} / \mathrm{kg}$ & 3 & 0 & $0 \%$ & 4.42E-06 & $6.78 \mathrm{E}-06$ & -- & -- & $2.78 \mathrm{E}-06$ \\
\hline PCB & $2,3,3^{\prime}, 4,44^{\prime}, 5-$ Hexachlorobiphenyl (BZ 156) & $\mathrm{mg} / \mathrm{kg}$ & 3 & 3 & $100 \%$ & -- & -- & $2.76 \mathrm{E}-04$ & $3.48 \mathrm{E}-04$ & $3.02 \mathrm{E}-04$ \\
\hline PCB & 2,3,3',4,4'-Pentachlorobiphenyl & $\mathrm{mg} / \mathrm{kg}$ & 3 & 3 & $100 \%$ & -- & -- & $5.31 \mathrm{E}-04$ & $5.86 \mathrm{E}-04$ & $5.65 \mathrm{E}-04$ \\
\hline РСB & 2,3',4,4',5,5'-Hexachlorobiphenyl (BZ 167) & $\mathrm{mg} / \mathrm{kg}$ & 3 & 3 & $100 \%$ & -- & -- & $1.29 \mathrm{E}-04$ & $1.88 \mathrm{E}-04$ & 1.50E-04 \\
\hline PCB & 2',3,4,4',5-Pentachloro-1,1'-biphenyl (BZ 123) & $\mathrm{mg} / \mathrm{kg}$ & 3 & 3 & $100 \%$ & -- & -- & $2.59 \mathrm{E}-05$ & $3.87 \mathrm{E}-05$ & 3.04E-05 \\
\hline PCB & 2,3,4,4',5-Pentachlorobiphenyl (BZ 114) & $\mathrm{mg} / \mathrm{kg}$ & 3 & 3 & $100 \%$ & -- & -- & 4.30E-05 & 4.99E-05 & $4.69 \mathrm{E}-05$ \\
\hline РCB & 2,3',4,4',5-Pentachlorobiphenyl (BZ 118) & $\mathrm{mg} / \mathrm{kg}$ & 3 & 3 & $100 \%$ & -- & -- & 0.0010 & 0.0013 & 0.0011 \\
\hline PCB & $2,3,4,5,3^{\prime}, 4^{\prime}, 5^{\prime}$-Heptachlorobiphenyl & $\mathrm{mg} / \mathrm{kg}$ & 3 & 3 & $100 \%$ & .- & -- & $2.92 \mathrm{E}-05$ & 4.45E-05 & $3.46 \mathrm{E}-05$ \\
\hline PCB & $3,3^{\prime}, 4,4^{\prime}, 5,5^{\prime}$-Hexachlorobiphenyl (BZ 169) & $\mathrm{mg} / \mathrm{kg}$ & 3 & 1 & $33 \%$ & 7.30E-07 & 8.20E-07 & 5.81E-06 & $5.81 \mathrm{E}-06$ & $2.20 \mathrm{E}-06$ \\
\hline РСB & 3,3',4,4',5-Pentachlorobiphenyl (BZ 126) & $\mathrm{mg} / \mathrm{kg}$ & 3 & 1 & $33 \%$ & 2.09E-06 & 2.30E-06 & $1.21 \mathrm{E}-05$ & $1.21 \mathrm{E}-05$ & 4.77E-06 \\
\hline PCB & 3,3',4,4'-Tetrachlorobiphenyl (BZ 77) & $\mathrm{mg} / \mathrm{kg}$ & 3 & 3 & $100 \%$ & -- & -- & $3.11 \mathrm{E}-05$ & $3.65 \mathrm{E}-05$ & $3.42 \mathrm{E}-05$ \\
\hline РСB & Aroclor-1016 & $\mathrm{mg} / \mathrm{kg}$ & 3 & 0 & $0 \%$ & 0.021 & 0.023 & -- & -- & 0.011 \\
\hline PCB & Aroclor-1221 & $\mathrm{mg} / \mathrm{kg}$ & 3 & 0 & $0 \%$ & 0.021 & 0.023 & -- & -- & 0.011 \\
\hline PCB & Aroclor-1232 & $\mathrm{mg} / \mathrm{kg}$ & 3 & 0 & $0 \%$ & 0.021 & 0.023 & -- & -- & 0.011 \\
\hline
\end{tabular}




\section{Table F-72}

Summary Statistics for Whole Body Bridgelip Sucker Collected from Segment 3 of the Columbia River

\begin{tabular}{|c|c|c|c|c|c|c|c|c|c|c|}
\hline $\begin{array}{l}\text { Constituent } \\
\text { Class }\end{array}$ & Constituent Name & Units & $\begin{array}{l}\text { Number } \\
\text { Analyzed }\end{array}$ & $\begin{array}{l}\text { Number } \\
\text { Detected }\end{array}$ & $\begin{array}{l}\text { Frequency of } \\
\text { Detection }\end{array}$ & $\begin{array}{c}\text { Minimum } \\
\text { Nondetect }\end{array}$ & $\begin{array}{c}\text { Maximum } \\
\text { Nondetect }\end{array}$ & $\begin{array}{c}\text { Minimum } \\
\text { Detect }\end{array}$ & $\begin{array}{l}\text { Maximum } \\
\text { Detect }\end{array}$ & $\begin{array}{c}\text { Average } \\
\text { Concentration }\end{array}$ \\
\hline$\overline{\mathrm{PCB}}$ & Aroclor-1248 & $\mathrm{mg} / \mathrm{kg}$ & 3 & 0 & $0 \%$ & 0.021 & 0.023 & $\overline{--}$ & $\overline{--}$ & 0.011 \\
\hline PCB & Aroclor-1254 & $\mathrm{mg} / \mathrm{kg}$ & 3 & 3 & $100 \%$ & -- & -- & 0.018 & 0.032 & 0.025 \\
\hline PCB & Aroclor-1260 & $\mathrm{mg} / \mathrm{kg}$ & 3 & 3 & $100 \%$ & -- & -- & 0.027 & 0.049 & 0.034 \\
\hline PEST & 2,4'-DDD & $\mathrm{mg} / \mathrm{kg}$ & 3 & 3 & $100 \%$ & -- & -- & 0.0087 & 0.011 & 0.0099 \\
\hline PEST & 2,4'-DDE & $\mathrm{mg} / \mathrm{kg}$ & 3 & 3 & $100 \%$ & -- & -- & 0.0059 & 0.0079 & 0.0068 \\
\hline PEST & 2,4'-DDT & $\mathrm{mg} / \mathrm{kg}$ & 3 & 0 & $0 \%$ & 0.0028 & 0.0035 & -- & -- & 0.0015 \\
\hline PEST & Aldrin & $\mathrm{mg} / \mathrm{kg}$ & 3 & 0 & $0 \%$ & 0.0028 & 0.0035 & -- & -- & 0.0015 \\
\hline PEST & Alpha-BHC & $\mathrm{mg} / \mathrm{kg}$ & 3 & 0 & $0 \%$ & 0.0028 & 0.0035 & -- & -- & 0.0015 \\
\hline PEST & alpha-Chlordane & $\mathrm{mg} / \mathrm{kg}$ & 3 & 0 & $0 \%$ & 0.0028 & 0.0035 & -- & -- & 0.0015 \\
\hline PEST & alpha-Chlordene & $\mathrm{mg} / \mathrm{kg}$ & 3 & 0 & $0 \%$ & 0.0028 & 0.0035 & -- & -- & 0.0015 \\
\hline PEST & Beta-BHC & $\mathrm{mg} / \mathrm{kg}$ & 3 & 0 & $0 \%$ & 0.0028 & 0.0035 & -- & -- & 0.0015 \\
\hline PEST & cis-Nonachlor & $\mathrm{mg} / \mathrm{kg}$ & 3 & 0 & $0 \%$ & 0.0028 & 0.0035 & -- & -- & 0.0015 \\
\hline PEST & DDMU & $\mathrm{mg} / \mathrm{kg}$ & 3 & 3 & $100 \%$ & -- & -- & 0.010 & 0.021 & 0.016 \\
\hline PEST & Delta-BHC & $\mathrm{mg} / \mathrm{kg}$ & 3 & 0 & $0 \%$ & 0.0028 & 0.0035 & -- & -- & 0.0015 \\
\hline PEST & Dichlorodiphenyldichloroethane & $\mathrm{mg} / \mathrm{kg}$ & 3 & 3 & $100 \%$ & -- & -- & 0.052 & 0.072 & 0.065 \\
\hline PEST & Dichlorodiphenyldichloroethylene & $\mathrm{mg} / \mathrm{kg}$ & 3 & 3 & $100 \%$ & -- & -- & 0.31 & 0.56 & 0.40 \\
\hline PEST & Dichlorodiphenyltrichloroethane & $\mathrm{mg} / \mathrm{kg}$ & 3 & 3 & $100 \%$ & -- & -- & 0.037 & 0.052 & 0.046 \\
\hline PEST & Endosulfan sulfate & $\mathrm{mg} / \mathrm{kg}$ & 3 & 3 & $100 \%$ & -- & -- & 0.0035 & 0.0054 & 0.0046 \\
\hline PEST & Gamma-BHC (Lindane) & $\mathrm{mg} / \mathrm{kg}$ & 3 & 0 & $0 \%$ & 0.0028 & 0.0035 & -- & -- & 0.0015 \\
\hline PEST & gamma-Chlordane & $\mathrm{mg} / \mathrm{kg}$ & 3 & 0 & $0 \%$ & 0.0028 & 0.0035 & -- & -- & 0.0015 \\
\hline PEST & gamma-Chlordene & $\mathrm{mg} / \mathrm{kg}$ & 3 & 0 & $0 \%$ & 0.0028 & 0.0035 & -- & -- & 0.0015 \\
\hline PEST & Heptachlor & $\mathrm{mg} / \mathrm{kg}$ & 3 & 0 & $0 \%$ & 0.0028 & 0.0035 & -- & -- & 0.0015 \\
\hline PEST & Heptachlor epoxide & $\mathrm{mg} / \mathrm{kg}$ & 3 & 0 & $0 \%$ & 0.0028 & 0.0035 & -- & -- & 0.0015 \\
\hline PEST & Mirex & $\mathrm{mg} / \mathrm{kg}$ & 3 & 0 & $0 \%$ & 0.0028 & 0.0035 & -- & -- & 0.0015 \\
\hline PEST & Oxychlordane & $\mathrm{mg} / \mathrm{kg}$ & 3 & 0 & $0 \%$ & 0.0028 & 0.0035 & -- & -- & 0.0015 \\
\hline PEST & Pentachloroanisole & $\mathrm{mg} / \mathrm{kg}$ & 3 & 0 & $0 \%$ & 0.0028 & 0.0035 & -- & -- & 0.0015 \\
\hline PEST & Toxaphene & $\mathrm{mg} / \mathrm{kg}$ & 3 & 0 & $0 \%$ & 0.080 & 0.085 & -. & -- & 0.041 \\
\hline PEST & trans-Nonachlor & $\mathrm{mg} / \mathrm{kg}$ & 3 & 0 & $0 \%$ & 0.0028 & 0.0035 & -- & -- & 0.0015 \\
\hline SVOC & 1,2,4-Trichlorobenzene & $\mathrm{mg} / \mathrm{kg}$ & 3 & 0 & $0 \%$ & 0.0063 & 0.010 & -- & -- & 0.0039 \\
\hline SVOC & 1,2-Dichlorobenzene & $\mathrm{mg} / \mathrm{kg}$ & 3 & 0 & $0 \%$ & 0.0063 & 0.010 & -- & -- & 0.0039 \\
\hline SVOC & 1,2-Diphenylhydrazine & $\mathrm{mg} / \mathrm{kg}$ & 3 & 1 & $33 \%$ & 0.0063 & 0.0072 & 0.014 & 0.014 & 0.0069 \\
\hline SVOC & 1,3-Dichlorobenzene & $\mathrm{mg} / \mathrm{kg}$ & 3 & 0 & $0 \%$ & 0.0063 & 0.010 & -- & -- & 0.0039 \\
\hline sVOC & 1,4-Dichlorobenzene & $\mathrm{mg} / \mathrm{kg}$ & 3 & 0 & $0 \%$ & 0.0063 & 0.010 & -. & -- & 0.0039 \\
\hline SVOC & 1-methylnaphthalene & $\mathrm{mg} / \mathrm{kg}$ & 3 & 1 & $33 \%$ & 0.0063 & 0.010 & 0.0075 & 0.0075 & 0.0052 \\
\hline sVOC & 2,4-Dinitrotoluene & $\mathrm{mg} / \mathrm{kg}$ & 3 & 0 & $0 \%$ & 0.013 & 0.020 & -- & -- & 0.0080 \\
\hline sVOC & 2,6-Dinitrotoluene & $\mathrm{mg} / \mathrm{kg}$ & 3 & 0 & $0 \%$ & 0.025 & 0.040 & -. & -- & 0.016 \\
\hline SVOC & 2-Chloronaphthalene & $\mathrm{mg} / \mathrm{kg}$ & 3 & 0 & $0 \%$ & 0.013 & 0.020 & -- & -- & 0.0080 \\
\hline SVOC & 2-Methylnaphthalene & $\mathrm{mg} / \mathrm{kg}$ & 3 & 3 & $100 \%$ & -- & -- & 0.012 & 0.020 & 0.016 \\
\hline sVoc & 4-Bromophenylphenyl ether & $\mathrm{mg} / \mathrm{kg}$ & 3 & 0 & $0 \%$ & 0.0063 & 0.010 & -- & -- & 0.0039 \\
\hline SVOC & 4-Chlorophenylphenyl ether & $\mathrm{mg} / \mathrm{kg}$ & 3 & 0 & $0 \%$ & 0.0063 & 0.010 & -- & -- & 0.0039 \\
\hline SVOC & Acenaphthene & $\mathrm{mg} / \mathrm{kg}$ & 3 & 0 & $0 \%$ & 0.013 & 0.020 & -- & -- & 0.0080 \\
\hline sVOC & Acenaphthylene & $\mathrm{mg} / \mathrm{kg}$ & 3 & 0 & $0 \%$ & 0.0063 & 0.010 & -. & -- & 0.0039 \\
\hline SVOC & Anthracene & $\mathrm{mg} / \mathrm{kg}$ & 3 & 0 & $0 \%$ & 0.0063 & 0.010 & -- & -- & 0.0039 \\
\hline SVOC & Benzo(a)anthracene & $\mathrm{mg} / \mathrm{kg}$ & 3 & 0 & $0 \%$ & 0.0063 & 0.010 & -- & -- & 0.0039 \\
\hline SVOC & Benzo(a)pyrene & $\mathrm{mg} / \mathrm{kg}$ & 3 & 0 & $0 \%$ & 0.0063 & 0.010 & -- & -- & 0.0039 \\
\hline SVOC & Benzo(b)fluoranthene & $\mathrm{mg} / \mathrm{kg}$ & 3 & 0 & $0 \%$ & 0.0063 & 0.010 & -- & -- & 0.0039 \\
\hline sVOC & Benzo(ghi)perylene & $\mathrm{mg} / \mathrm{kg}$ & 3 & 0 & $0 \%$ & 0.013 & 0.020 & -. & -- & 0.0080 \\
\hline SVOC & Benzo $(k)$ fluoranthene & $\mathrm{mg} / \mathrm{kg}$ & 3 & 0 & $0 \%$ & 0.0063 & 0.010 & -- & -- & 0.0039 \\
\hline SVOC & Bis(2-chloro-1-methylethyl)ether & $\mathrm{mg} / \mathrm{kg}$ & 3 & 0 & $0 \%$ & 0.013 & 0.020 & -- & -- & 0.0080 \\
\hline SVOC & Chrysene & $\mathrm{mg} / \mathrm{kg}$ & 3 & 0 & $0 \%$ & 0.0063 & 0.010 & -- & -- & 0.0039 \\
\hline sVOC & Dibenz[a,h]anthracene & $\mathrm{mg} / \mathrm{kg}$ & 3 & 0 & $0 \%$ & 0.013 & 0.020 & -- & -- & 0.0080 \\
\hline
\end{tabular}


Table F-72

Summary Statistics for Whole Body Bridgelip Sucker Collected from Segment 3 of the Columbia River

\begin{tabular}{|c|c|c|c|c|c|c|c|c|c|c|}
\hline $\begin{array}{l}\text { Constituent } \\
\text { Class }\end{array}$ & Constituent Name & Units & $\begin{array}{c}\text { Number } \\
\text { Analyzed }\end{array}$ & $\begin{array}{l}\text { Number } \\
\text { Detected }\end{array}$ & $\begin{array}{c}\text { Frequency of } \\
\text { Detection }\end{array}$ & $\begin{array}{l}\text { Minimum } \\
\text { Nondetect }\end{array}$ & $\begin{array}{l}\text { Maximum } \\
\text { Nondetect }\end{array}$ & $\begin{array}{c}\text { Minimum } \\
\text { Detect }\end{array}$ & $\begin{array}{l}\text { Maximum } \\
\text { Detect }\end{array}$ & $\begin{array}{c}\text { Average } \\
\text { Concentration }\end{array}$ \\
\hline$\overline{\text { SVOC }}$ & Dibenzofuran & $\mathrm{mg} / \mathrm{kg}$ & 3 & 0 & $0 \%$ & 0.0063 & 0.010 & -- & -- & 0.0039 \\
\hline SVOC & Fluoranthene & $\mathrm{mg} / \mathrm{kg}$ & 3 & 0 & $0 \%$ & 0.013 & 0.020 & -- & -- & 0.0080 \\
\hline SVOC & Fluorene & $\mathrm{mg} / \mathrm{kg}$ & 3 & 0 & $0 \%$ & 0.0046 & 0.024 & -- & -- & 0.0056 \\
\hline SVOC & Hexachlorobenzene & $\mathrm{mg} / \mathrm{kg}$ & 3 & 0 & $0 \%$ & 0.013 & 0.020 & -- & -- & 0.0080 \\
\hline SVOC & Hexachlorobutadiene & $\mathrm{mg} / \mathrm{kg}$ & 3 & 0 & $0 \%$ & 0.0063 & 0.010 & -- & -- & 0.0039 \\
\hline SVOC & Hexachloroethane & $\mathrm{mg} / \mathrm{kg}$ & 3 & 0 & $0 \%$ & 0.0063 & 0.010 & -- & -- & 0.0039 \\
\hline SVOC & Indeno(1,2,3-cd)pyrene & $\mathrm{mg} / \mathrm{kg}$ & 3 & 0 & $0 \%$ & 0.013 & 0.020 & -- & -- & 0.0080 \\
\hline SVOC & Naphthalene & $\mathrm{mg} / \mathrm{kg}$ & 3 & 0 & $0 \%$ & 0.014 & 0.029 & -- & -- & 0.010 \\
\hline SVOC & Nitrobenzene & $\mathrm{mg} / \mathrm{kg}$ & 3 & 0 & $0 \%$ & 0.0063 & 0.010 & -- & -- & 0.0039 \\
\hline SVOC & Phenanthrene & $\mathrm{mg} / \mathrm{kg}$ & 3 & 0 & $0 \%$ & 0.0063 & 0.010 & -- & -- & 0.0039 \\
\hline SVOC & Pyrene & $\mathrm{mg} / \mathrm{kg}$ & 3 & 0 & $0 \%$ & 0.013 & 0.020 & -- & -- & 0.0080 \\
\hline VOC & Retene & $\mathrm{mg} / \mathrm{kg}$ & 3 & 0 & $0 \%$ & 0.013 & 0.020 & -- & -- & 0.0080 \\
\hline
\end{tabular}

DIOXI - Dioxins and Furans

PEST - Pesticide

PCB - Polychlorinated bipheny

SVOC - Semivolatile Organic Compound

VOC - Volatile Organic Compound 


\section{Table F-73}

Summary Statistics for Northern Squawfish Fillet Collected from Segment 3 of the Columbia River

\begin{tabular}{|c|c|c|c|c|c|c|c|c|c|c|}
\hline $\begin{array}{l}\text { Constituent } \\
\text { Class }\end{array}$ & Constituent Name & Units & $\begin{array}{l}\text { Number } \\
\text { Analyzed }\end{array}$ & $\begin{array}{l}\text { Number } \\
\text { Detected }\end{array}$ & $\begin{array}{l}\text { Frequency of } \\
\text { Detection }\end{array}$ & $\begin{array}{l}\text { Minimum } \\
\text { Nondetect }\end{array}$ & $\begin{array}{l}\text { Maximum } \\
\text { Nondetect }\end{array}$ & $\begin{array}{c}\text { Minimum } \\
\text { Detect }\end{array}$ & $\begin{array}{l}\text { Maximum } \\
\text { Detect }\end{array}$ & $\begin{array}{c}\text { Average } \\
\text { Concentration }\end{array}$ \\
\hline$\overline{\text { CONV }}$ & Percent Lipids & $\%$ & 2 & 2 & $100 \%$ & $\overline{--}$ & $\overline{--}$ & 1.0 & 1.3 & 1.2 \\
\hline PCB & Aroclor-1016 & $\mathrm{mg} / \mathrm{Kg}$ & 2 & 0 & $0 \%$ & 0.0054 & 0.0055 & -- & -- & 0.0027 \\
\hline PCB & Aroclor-1221 & $\mathrm{mg} / \mathrm{Kg}$ & 2 & 0 & $0 \%$ & 0.0054 & 0.0055 & -- & -- & 0.0027 \\
\hline PCB & Aroclor-1232 & $\mathrm{mg} / \mathrm{Kg}$ & 2 & 0 & $0 \%$ & 0.0054 & 0.0055 & -- & -- & 0.0027 \\
\hline PCB & Aroclor-1242 & $\mathrm{mg} / \mathrm{Kg}$ & 2 & 0 & $0 \%$ & 0.0054 & 0.0055 & -- & -- & 0.0027 \\
\hline PCB & Aroclor-1248 & $\mathrm{mg} / \mathrm{Kg}$ & 2 & 0 & $0 \%$ & 0.0054 & 0.0055 & -- & -- & 0.0027 \\
\hline PCB & Aroclor-1254 & $\mathrm{mg} / \mathrm{Kg}$ & 2 & 2 & $100 \%$ & -- & -- & 0.0062 & 0.0074 & 0.0068 \\
\hline PCB & Aroclor-1260 & $\mathrm{mg} / \mathrm{Kg}$ & 2 & 2 & $100 \%$ & -- & -- & 0.0065 & 0.044 & 0.025 \\
\hline PCB & Aroclor-1262 & $\mathrm{mg} / \mathrm{Kg}$ & 2 & 0 & $0 \%$ & 0.0054 & 0.0055 & -- & -- & 0.0027 \\
\hline PCB & Aroclor-1268 & $\mathrm{mg} / \mathrm{Kg}$ & 2 & 0 & $0 \%$ & 0.0054 & 0.0055 & -- & -- & 0.0027 \\
\hline PEST & 2,4'-DDD & $\mathrm{mg} / \mathrm{Kg}$ & 2 & 0 & $0 \%$ & $5.40 \mathrm{E}-04$ & $5.50 \mathrm{E}-04$ & -- & -- & 2.73E-04 \\
\hline PEST & 2,4'-DDE & $\mathrm{mg} / \mathrm{Kg}$ & 2 & 0 & $0 \%$ & $5.40 \mathrm{E}-04$ & $5.50 \mathrm{E}-04$ & -- & -- & 2.73E-04 \\
\hline PEST & 2,4'-DDT & $\mathrm{mg} / \mathrm{Kg}$ & 2 & 0 & $0 \%$ & $5.40 \mathrm{E}-04$ & 0.0011 & -- & -- & 4.10E-04 \\
\hline PEST & alpha-Chlordane & $\mathrm{mg} / \mathrm{Kg}$ & 2 & 0 & $0 \%$ & $5.40 \mathrm{E}-04$ & $5.50 \mathrm{E}-04$ & -- & -- & 2.73E-04 \\
\hline PEST & cis-Nonachlor & $\mathrm{mg} / \mathrm{Kg}$ & 2 & 0 & $0 \%$ & $5.40 \mathrm{E}-04$ & $5.50 \mathrm{E}-04$ & -- & -- & 2.73E-04 \\
\hline PEST & Dichlorodiphenyldichloroethane & $\mathrm{mg} / \mathrm{Kg}$ & 2 & 2 & $100 \%$ & -- & -- & 0.0021 & 0.0025 & 0.0023 \\
\hline PEST & Dichlorodiphenyldichloroethylene & $\mathrm{mg} / \mathrm{Kg}$ & 2 & 2 & $100 \%$ & -- & -- & 0.048 & 0.064 & 0.056 \\
\hline PEST & Dichlorodiphenyltrichloroethane & $\mathrm{mg} / \mathrm{Kg}$ & 2 & 0 & $0 \%$ & $5.40 \mathrm{E}-04$ & $5.50 \mathrm{E}-04$ & -- & -- & 2.73E-04 \\
\hline PEST & Dieldrin & $\mathrm{mg} / \mathrm{Kg}$ & 2 & 0 & $0 \%$ & $5.40 \mathrm{E}-04$ & $5.50 \mathrm{E}-04$ & -- & -- & 2.73E-04 \\
\hline PEST & Heptachlor & $\mathrm{mg} / \mathrm{Kg}$ & 2 & 0 & $0 \%$ & $5.40 \mathrm{E}-04$ & $5.50 \mathrm{E}-04$ & -- & -- & 2.73E-04 \\
\hline PEST & Heptachlor epoxide & $\mathrm{mg} / \mathrm{Kg}$ & 2 & 0 & $0 \%$ & $5.40 \mathrm{E}-04$ & $5.50 \mathrm{E}-04$ & -- & -- & 2.73E-04 \\
\hline PEST & Oxychlordane & $\mathrm{mg} / \mathrm{Kg}$ & 2 & 0 & $0 \%$ & $5.40 \mathrm{E}-04$ & $5.50 \mathrm{E}-04$ & -- & -- & 2.73E-04 \\
\hline PEST & Toxaphene & $\mathrm{mg} / \mathrm{Kg}$ & 2 & 2 & $100 \%$ & -- & -- & 0.015 & 0.039 & 0.027 \\
\hline PEST & trans-Chlordane & $\mathrm{mg} / \mathrm{Kg}$ & 2 & 0 & $0 \%$ & $5.40 \mathrm{E}-04$ & $5.50 \mathrm{E}-04$ & -- & -- & 2.73E-04 \\
\hline PEST & trans-Nonachlor & $\mathrm{mg} / \mathrm{Kg}$ & 2 & 2 & $100 \%$ & -- & -- & 0.0014 & 0.0018 & 0.0016 \\
\hline SVOC & Hexachlorobenzene & $\mathrm{mg} / \mathrm{Kg}$ & 2 & 2 & $100 \%$ & -- & -- & 0.0012 & 0.0013 & 0.0013 \\
\hline
\end{tabular}

PEST - Pesticide

PCB - Polychlorinated biphenyl

SVOC - Semivolatile Organic Compound 
Table F-74

Summary Statistics for White Sturgeon Fillet Collected from Segment 3 of the Columbia River

\begin{tabular}{|c|c|c|c|c|c|c|c|c|c|c|}
\hline $\begin{array}{l}\text { Constituent } \\
\text { Class }\end{array}$ & Constituent Name & Units & $\begin{array}{l}\text { Number } \\
\text { Analyzed }\end{array}$ & $\begin{array}{l}\text { Number } \\
\text { Detected }\end{array}$ & $\begin{array}{l}\text { Frequency of } \\
\text { Detection }\end{array}$ & $\begin{array}{l}\text { Minimum } \\
\text { Nondetect }\end{array}$ & $\begin{array}{l}\text { Maximum } \\
\text { Nondetect }\end{array}$ & $\begin{array}{l}\text { Minimum } \\
\text { Detect }\end{array}$ & $\begin{array}{l}\text { Maximum } \\
\text { Detect }\end{array}$ & $\begin{array}{c}\text { Average } \\
\text { Concentration }\end{array}$ \\
\hline$\overline{\mathrm{DIOXI}}$ & $1,2,3,4,6,7,8$-Heptachlorodibenzodioxin & $\mathrm{mg} / \mathrm{kg}$ & 4 & 3 & $75 \%$ & $9.00 \mathrm{E}-08$ & $9.00 \mathrm{E}-08$ & $3.80 \mathrm{E}-07$ & $6.80 \mathrm{E}-07$ & $3.91 \mathrm{E}-07$ \\
\hline DIOXI & 1,2,3,4,6,7,8-Heptachlorodibenzofuran & $\mathrm{mg} / \mathrm{kg}$ & 4 & 3 & $75 \%$ & $1.00 \mathrm{E}-07$ & 1.00E-07 & 1.30E-07 & $2.10 \mathrm{E}-07$ & 1.33E-07 \\
\hline DIOXI & 1,2,3,4,7,8,9-Heptachlorodibenzofuran & $\mathrm{mg} / \mathrm{kg}$ & 4 & 0 & $0 \%$ & 3.00E-08 & 1.00E-07 & -- & -- & 2.75E-08 \\
\hline DIOXI & $1,2,3,4,7,8$-Hexachlorodibenzofuran & $\mathrm{mg} / \mathrm{kg}$ & 4 & 3 & $75 \%$ & 4.00E-08 & 4.00E-08 & 5.00E-08 & 8.00E-08 & $5.00 \mathrm{E}-08$ \\
\hline DIOXI & 1,2,3,4,7,8-Hexachlorodibenzo-p-dioxin & $\mathrm{mg} / \mathrm{kg}$ & 4 & 3 & $75 \%$ & $5.00 \mathrm{E}-08$ & 5.00E-08 & 3.00E-08 & $5.00 \mathrm{E}-08$ & 3.63E-08 \\
\hline DIOXI & $1,2,3,6,7,8$-Hexachlorodibenzofuran & $\mathrm{mg} / \mathrm{kg}$ & 4 & 3 & $75 \%$ & 4.00E-08 & 4.00E-08 & 7.00E-08 & 1.10E-07 & 7.00E-08 \\
\hline DIOXI & 1,2,3,6,7,8-Hexachlorodibenzo-p-dioxin & $\mathrm{mg} / \mathrm{kg}$ & 4 & 3 & $75 \%$ & $5.00 \mathrm{E}-08$ & 5.00E-08 & 1.60E-07 & $2.80 \mathrm{E}-07$ & 1.81E-07 \\
\hline DIOXI & $1,2,3,7,8,9$-Hexachlorodibenzofuran & $\mathrm{mg} / \mathrm{kg}$ & 4 & 0 & $0 \%$ & 2.00E-08 & 4.00E-08 & -- & -- & $1.50 \mathrm{E}-08$ \\
\hline DIOXI & 1,2,3,7,8,9-Hexachlorodibenzo-p-dioxin & $\mathrm{mg} / \mathrm{kg}$ & 4 & 1 & $25 \%$ & $3.00 \mathrm{E}-08$ & $5.00 \mathrm{E}-08$ & $6.00 \mathrm{E}-08$ & $6.00 \mathrm{E}-08$ & $2.88 \mathrm{E}-08$ \\
\hline DIOXI & 1,2,3,7,8-Pentachlorodibenzofuran & $\mathrm{mg} / \mathrm{kg}$ & 4 & 4 & $100 \%$ & -- & -- & $2.80 \mathrm{E}-07$ & 4.60E-07 & 3.60E-07 \\
\hline DIOXI & 1,2,3,7,8-Pentachlorodibenzo-p-dioxin & $\mathrm{mg} / \mathrm{kg}$ & 4 & 2 & $50 \%$ & 1.00E-08 & $2.00 \mathrm{E}-08$ & 5.00E-08 & 7.00E-08 & $3.38 \mathrm{E}-08$ \\
\hline DIOXI & $2,3,4,6,7,8$-Hexachlorodibenzofuran & $\mathrm{mg} / \mathrm{kg}$ & 4 & 3 & $75 \%$ & 3.00E-08 & 3.00E-08 & 3.00E-08 & $5.00 \mathrm{E}-08$ & 3.63E-08 \\
\hline DIOXI & 2,3,4,7,8-Pentachlorodibenzofuran & $\mathrm{mg} / \mathrm{kg}$ & 4 & 4 & $100 \%$ & -- & -- & $2.80 \mathrm{E}-07$ & 4.00E-07 & $3.40 \mathrm{E}-07$ \\
\hline DIOXI & $2,3,7,8$-Tetrachlorodibenzofuran & $\mathrm{mg} / \mathrm{kg}$ & 4 & 4 & $100 \%$ & -- & -- & 1.69E-05 & $5.41 \mathrm{E}-05$ & 3.92E-05 \\
\hline DIOXI & 2,3,7,8-Tetrachlorodibenzo-p-dioxin & $\mathrm{mg} / \mathrm{kg}$ & 4 & 4 & $100 \%$ & -- & -- & 4.40E-07 & 1.39E-06 & $9.78 \mathrm{E}-07$ \\
\hline DIOXI & Octachlorodibenzofuran & $\mathrm{mg} / \mathrm{kg}$ & 4 & 2 & $50 \%$ & $2.00 \mathrm{E}-08$ & 4.00E-08 & 3.20E-07 & 3.60E-07 & 1.78E-07 \\
\hline DIOXI & Octachlorodibenzo-p-dioxin & $\mathrm{mg} / \mathrm{kg}$ & 4 & 4 & $100 \%$ & -- & -- & $7.20 \mathrm{E}-07$ & 1.87E-06 & 1.37E-06 \\
\hline METAL & Aluminum & $\mathrm{mg} / \mathrm{kg}$ & 4 & 1 & $25 \%$ & 1.0 & 1.0 & 2.1 & 2.1 & 0.90 \\
\hline METAL & Antimony & $\mathrm{mg} / \mathrm{kg}$ & 4 & 0 & $0 \%$ & 0.050 & 0.050 & -- & -- & 0.025 \\
\hline METAL & Arsenic & $\mathrm{mg} / \mathrm{kg}$ & 4 & 4 & $100 \%$ & -- & -- & 0.26 & 0.39 & 0.35 \\
\hline METAL & Barium & $\mathrm{mg} / \mathrm{kg}$ & 4 & 2 & $50 \%$ & 0.20 & 0.20 & 0.34 & 0.45 & 0.25 \\
\hline METAL & Beryllium & $\mathrm{mg} / \mathrm{kg}$ & 4 & 1 & $25 \%$ & 0.0040 & 0.0040 & 0.0040 & 0.0040 & 0.0025 \\
\hline METAL & Cadmium & $\mathrm{mg} / \mathrm{kg}$ & 4 & 0 & $0 \%$ & 0.0040 & 0.0040 & -- & -- & 0.0020 \\
\hline METAL & Chromium & $\mathrm{mg} / \mathrm{kg}$ & 4 & 2 & $50 \%$ & 0.10 & 0.10 & 0.15 & 0.15 & 0.10 \\
\hline METAL & Cobalt & $\mathrm{mg} / \mathrm{kg}$ & 4 & 4 & $100 \%$ & -- & -- & 0.0078 & 0.14 & 0.054 \\
\hline METAL & Copper & $\mathrm{mg} / \mathrm{kg}$ & 4 & 4 & $100 \%$ & -- & -- & 0.20 & 0.34 & 0.25 \\
\hline METAL & Lead & $\mathrm{mg} / \mathrm{kg}$ & 4 & 2 & $50 \%$ & 0.010 & 0.010 & 0.010 & 0.010 & 0.0075 \\
\hline METAL & Manganese & $\mathrm{mg} / \mathrm{kg}$ & 4 & 4 & $100 \%$ & -- & -- & 0.19 & 0.39 & 0.28 \\
\hline METAL & Mercury & $\mathrm{mg} / \mathrm{kg}$ & 4 & 4 & $100 \%$ & -- & -- & 0.10 & 0.11 & 0.11 \\
\hline METAL & Nickel & $\mathrm{mg} / \mathrm{kg}$ & 4 & 1 & $25 \%$ & 0.030 & 0.030 & 0.040 & 0.040 & 0.021 \\
\hline METAL & Selenium & $\mathrm{mg} / \mathrm{kg}$ & 4 & 4 & $100 \%$ & -- & -- & 1.3 & 2.7 & 1.7 \\
\hline METAL & Silver & $\mathrm{mg} / \mathrm{kg}$ & 4 & 0 & $0 \%$ & 0.10 & 0.10 & -- & -- & 0.050 \\
\hline METAL & Thallium & $\mathrm{mg} / \mathrm{kg}$ & 4 & 0 & $0 \%$ & 0.050 & 0.050 & -- & -- & 0.025 \\
\hline METAL & Vanadium & $\mathrm{mg} / \mathrm{kg}$ & 4 & 1 & $25 \%$ & 0.010 & 0.020 & 0.010 & 0.010 & 0.0075 \\
\hline METAL & Zinc & $\mathrm{mg} / \mathrm{kg}$ & 4 & 4 & $100 \%$ & -- & -- & 3.1 & 3.6 & 3.3 \\
\hline PCB & 2,2',3,3',4,4',5-Heptachloro-1,1'-biphenyl & $\mathrm{mg} / \mathrm{kg}$ & 4 & 4 & $100 \%$ & -- & -- & 0.0015 & 0.0039 & 0.0031 \\
\hline РCB & $2,2^{\prime}, 3,4,4^{\prime}, 5,5^{\prime}$-Heptachlorobiphenyl & $\mathrm{mg} / \mathrm{kg}$ & 4 & 4 & $100 \%$ & -- & -- & 0.0047 & 0.011 & 0.0087 \\
\hline РCB & 2,3,3',4,4',5'-Hexachlorobiphenyl & $\mathrm{mg} / \mathrm{kg}$ & 4 & 4 & $100 \%$ & -- & -- & 1.35E-04 & $3.26 \mathrm{E}-04$ & 2.36E-04 \\
\hline PCB & $2,3,3^{\prime}, 4,4,5-$ Hexachlorobiphenyl (BZ 156) & $\mathrm{mg} / \mathrm{kg}$ & 4 & 4 & $100 \%$ & -- & -- & 7.32E-04 & 0.0017 & 0.0013 \\
\hline РCB & 2,3,3',4,4'-Pentachlorobiphenyl & $\mathrm{mg} / \mathrm{kg}$ & 4 & 4 & $100 \%$ & -- & -- & 0.0017 & 0.0036 & 0.0028 \\
\hline PCB & $2,3^{\prime}, 4,4^{\prime}, 5,5^{\prime}$-Hexachlorobiphenyl (BZ 167) & $\mathrm{mg} / \mathrm{kg}$ & 4 & 4 & $100 \%$ & -- & -- & 2.43E-05 & $7.85 \mathrm{E}-05$ & 3.93E-05 \\
\hline РCB & 2',3,4,4',5-Pentachloro-1,1'-biphenyl (BZ 123) & $\mathrm{mg} / \mathrm{kg}$ & 4 & 4 & $100 \%$ & -- & -- & 3.04E-05 & 9.39E-05 & 4.94E-05 \\
\hline PCB & 2,3,4,4',5-Pentachlorobiphenyl (BZ 114) & $\mathrm{mg} / \mathrm{kg}$ & 4 & 4 & $100 \%$ & -- & -- & 1.37E-04 & $3.38 \mathrm{E}-04$ & $2.52 \mathrm{E}-04$ \\
\hline PCB & 2,3',4,4',5-Pentachlorobiphenyl (BZ 118) & $\mathrm{mg} / \mathrm{kg}$ & 4 & 4 & $100 \%$ & -- & -- & 0.0020 & 0.0050 & 0.0031 \\
\hline PCB & 2,3,4,5,3',4',5'-Heptachlorobiphenyl & $\mathrm{mg} / \mathrm{kg}$ & 4 & 4 & $100 \%$ & -- & -- & 5.31E-05 & $1.38 \mathrm{E}-04$ & 1.10E-04 \\
\hline РCB & 3,3',4,4',5,5'-Hexachlorobiphenyl (BZ 169) & $\mathrm{mg} / \mathrm{kg}$ & 4 & 0 & $0 \%$ & 1.33E-06 & 2.16E-06 & -- & -- & 7.91E-07 \\
\hline PCB & 3,3',4,4',5-Pentachlorobiphenyl (BZ 126) & $\mathrm{mg} / \mathrm{kg}$ & 4 & 0 & $0 \%$ & 1.27E-06 & $1.72 \mathrm{E}-06$ & -- & -- & $7.55 \mathrm{E}-07$ \\
\hline РCB & 3,3',4,4'-Tetrachlorobiphenyl (BZ 77) & $\mathrm{mg} / \mathrm{kg}$ & 4 & 4 & $100 \%$ & -- & -- & 7.53E-06 & $3.08 \mathrm{E}-05$ & 1.57E-05 \\
\hline PCB & Aroclor-1016 & $\mathrm{mg} / \mathrm{kg}$ & 4 & 0 & $0 \%$ & 0.019 & 0.020 & -- & -- & 0.0098 \\
\hline PCB & Aroclor-1221 & $\mathrm{mg} / \mathrm{kg}$ & 4 & 0 & $0 \%$ & 0.019 & 0.020 & -- & -- & 0.0098 \\
\hline
\end{tabular}


Table F-74

Summary Statistics for White Sturgeon Fillet Collected from Segment 3 of the Columbia River

\begin{tabular}{|c|c|c|c|c|c|c|c|c|c|c|}
\hline $\begin{array}{l}\text { Constituent } \\
\text { Class }\end{array}$ & Constituent Name & Units & $\begin{array}{l}\text { Number } \\
\text { Analyzed }\end{array}$ & $\begin{array}{l}\text { Number } \\
\text { Detected }\end{array}$ & $\begin{array}{c}\text { Frequency of } \\
\text { Detection }\end{array}$ & $\begin{array}{l}\text { Minimum } \\
\text { Nondetect }\end{array}$ & $\begin{array}{l}\text { Maximum } \\
\text { Nondetect }\end{array}$ & $\begin{array}{l}\text { Minimum } \\
\text { Detect }\end{array}$ & $\begin{array}{l}\text { Maximum } \\
\text { Detect }\end{array}$ & $\begin{array}{c}\text { Average } \\
\text { Concentration }\end{array}$ \\
\hline$\overline{\mathrm{PCB}}$ & Aroclor-1232 & $\mathrm{mg} / \mathrm{kg}$ & 4 & 0 & $0 \%$ & 0.019 & 0.020 & -- & -- & 0.0098 \\
\hline PCB & Aroclor-1248 & $\mathrm{mg} / \mathrm{kg}$ & 4 & 0 & $0 \%$ & 0.019 & 0.020 & -- & -- & 0.0098 \\
\hline РCB & Aroclor-1254 & $\mathrm{mg} / \mathrm{kg}$ & 4 & 4 & $100 \%$ & -- & -- & 0.049 & 0.11 & 0.077 \\
\hline РСВ & Aroclor-1260 & $\mathrm{mg} / \mathrm{kg}$ & 4 & 4 & $100 \%$ & -- & -- & 0.048 & 0.10 & 0.086 \\
\hline PEST & 2,4'-DDD & $\mathrm{mg} / \mathrm{kg}$ & 4 & 4 & $100 \%$ & -- & -- & 0.015 & 0.018 & 0.017 \\
\hline PEST & 2,4'-DDE & $\mathrm{mg} / \mathrm{kg}$ & 4 & 3 & $75 \%$ & 0.0019 & 0.0019 & 0.0046 & 0.0080 & 0.0051 \\
\hline PEST & 2,4'-DDT & $\mathrm{mg} / \mathrm{kg}$ & 4 & 0 & $0 \%$ & 0.0019 & 0.0020 & -- & -- & $9.75 \mathrm{E}-04$ \\
\hline PEST & Aldrin & $\mathrm{mg} / \mathrm{kg}$ & 4 & 4 & $100 \%$ & -- & -- & 0.0010 & 0.0013 & 0.0012 \\
\hline PEST & Alpha-BHC & $\mathrm{mg} / \mathrm{kg}$ & 4 & 0 & $0 \%$ & 0.0019 & 0.0020 & -- & -- & $9.75 \mathrm{E}-04$ \\
\hline PEST & alpha-Chlordane & $\mathrm{mg} / \mathrm{kg}$ & 4 & 4 & $100 \%$ & -- & -- & 0.0030 & 0.0088 & 0.0060 \\
\hline PEST & alpha-Chlordene & $\mathrm{mg} / \mathrm{kg}$ & 4 & 0 & $0 \%$ & 0.0019 & 0.0020 & -- & -- & $9.75 \mathrm{E}-04$ \\
\hline PEST & Beta-BHC & $\mathrm{mg} / \mathrm{kg}$ & 4 & 0 & $0 \%$ & 0.0019 & 0.0020 & -- & -- & $9.75 \mathrm{E}-04$ \\
\hline PEST & cis-Nonachlor & $\mathrm{mg} / \mathrm{kg}$ & 4 & 4 & $100 \%$ & -- & -- & 0.0072 & 0.015 & 0.012 \\
\hline PEST & DDMU & $\mathrm{mg} / \mathrm{kg}$ & 4 & 4 & $100 \%$ & -- & -- & 0.0041 & 0.015 & 0.011 \\
\hline PEST & Delta-BHC & $\mathrm{mg} / \mathrm{kg}$ & 4 & 0 & $0 \%$ & 0.0019 & 0.0020 & -- & -- & $9.75 \mathrm{E}-04$ \\
\hline PEST & Dichlorodiphenyldichloroethane & $\mathrm{mg} / \mathrm{kg}$ & 4 & 4 & $100 \%$ & -- & -- & 0.13 & 0.16 & 0.15 \\
\hline PEST & Dichlorodiphenyldichloroethylene & $\mathrm{mg} / \mathrm{kg}$ & 4 & 4 & $100 \%$ & -- & -- & 0.42 & 0.67 & 0.58 \\
\hline PEST & Dichlorodiphenyltrichloroethane & $\mathrm{mg} / \mathrm{kg}$ & 4 & 4 & $100 \%$ & -- & -- & 0.0051 & 0.0099 & 0.0066 \\
\hline PEST & Endosulfan sulfate & $\mathrm{mg} / \mathrm{kg}$ & 4 & 0 & $0 \%$ & 0.0019 & 0.0020 & -- & -- & $9.75 \mathrm{E}-04$ \\
\hline PEST & Gamma-BHC (Lindane) & $\mathrm{mg} / \mathrm{kg}$ & 4 & 0 & $0 \%$ & 0.0019 & 0.0020 & -- & -- & $9.75 \mathrm{E}-04$ \\
\hline PEST & gamma-Chlordane & $\mathrm{mg} / \mathrm{kg}$ & 4 & 4 & $100 \%$ & -- & -- & 0.0059 & 0.0074 & 0.0068 \\
\hline PEST & gamma-Chlordene & $\mathrm{mg} / \mathrm{kg}$ & 4 & 0 & $0 \%$ & 0.0019 & 0.0020 & -- & -- & $9.75 \mathrm{E}-04$ \\
\hline PEST & Heptachlor & $\mathrm{mg} / \mathrm{kg}$ & 4 & 0 & $0 \%$ & 0.0019 & 0.0020 & -- & -- & $9.75 \mathrm{E}-04$ \\
\hline PEST & Heptachlor epoxide & $\mathrm{mg} / \mathrm{kg}$ & 4 & 0 & $0 \%$ & 0.0019 & 0.0020 & -- & -- & $9.75 \mathrm{E}-04$ \\
\hline PEST & Mirex & $\mathrm{mg} / \mathrm{kg}$ & 4 & 0 & $0 \%$ & 0.0019 & 0.0020 & -- & -- & $9.75 \mathrm{E}-04$ \\
\hline PEST & Oxychlordane & $\mathrm{mg} / \mathrm{kg}$ & 4 & 3 & $75 \%$ & 0.0020 & 0.0020 & 7.60E-04 & 0.0018 & 0.0012 \\
\hline PEST & Pentachloroanisole & $\mathrm{mg} / \mathrm{kg}$ & 4 & 0 & $0 \%$ & 0.0019 & 0.0020 & -- & -- & $9.75 \mathrm{E}-04$ \\
\hline PEST & Toxaphene & $\mathrm{mg} / \mathrm{kg}$ & 4 & 0 & $0 \%$ & 0.057 & 0.059 & -- & -- & 0.029 \\
\hline PEST & trans-Nonachlor & $\mathrm{mg} / \mathrm{kg}$ & 4 & 4 & $100 \%$ & -- & -- & 0.0051 & 0.013 & 0.010 \\
\hline SVOC & 1,2,4-Trichlorobenzene & $\mathrm{mg} / \mathrm{kg}$ & 4 & 0 & $0 \%$ & 0.0086 & 0.0098 & -- & -- & 0.0047 \\
\hline SVOC & 1,2-Dichlorobenzene & $\mathrm{mg} / \mathrm{kg}$ & 4 & 0 & $0 \%$ & 0.0086 & 0.0098 & -- & -- & 0.0047 \\
\hline SVOC & 1,2-Diphenylhydrazine & $\mathrm{mg} / \mathrm{kg}$ & 4 & 0 & $0 \%$ & 0.0086 & 0.0098 & -- & -- & 0.0047 \\
\hline SVOC & 1,3-Dichlorobenzene & $\mathrm{mg} / \mathrm{kg}$ & 4 & 0 & $0 \%$ & 0.0086 & 0.0098 & -- & -- & 0.0047 \\
\hline SVOC & 1,4-Dichlorobenzene & $\mathrm{mg} / \mathrm{kg}$ & 4 & 0 & $0 \%$ & 0.0086 & 0.0098 & -- & -- & 0.0047 \\
\hline SVOC & 1-methylnaphthalene & $\mathrm{mg} / \mathrm{kg}$ & 4 & 0 & $0 \%$ & 0.0086 & 0.0098 & -- & -- & 0.0047 \\
\hline SVOC & 2,4-Dinitrotoluene & $\mathrm{mg} / \mathrm{kg}$ & 4 & 0 & $0 \%$ & 0.017 & 0.020 & -- & -- & 0.0094 \\
\hline SVOC & 2,6-Dinitrotoluene & $\mathrm{mg} / \mathrm{kg}$ & 4 & 0 & $0 \%$ & 0.034 & 0.039 & -- & -- & 0.019 \\
\hline SVOC & 2-Chloronaphthalene & $\mathrm{mg} / \mathrm{kg}$ & 4 & 0 & $0 \%$ & 0.017 & 0.020 & -- & -- & 0.0094 \\
\hline SVOC & 2-MethyInaphthalene & $\mathrm{mg} / \mathrm{kg}$ & 4 & 0 & $0 \%$ & 0.0086 & 0.0098 & -- & -- & 0.0047 \\
\hline SVOC & 4-Bromophenylphenyl ether & $\mathrm{mg} / \mathrm{kg}$ & 4 & 0 & $0 \%$ & 0.0086 & 0.0098 & -- & -- & 0.0047 \\
\hline SVOC & 4-Chlorophenylphenyl ether & $\mathrm{mg} / \mathrm{kg}$ & 4 & 0 & $0 \%$ & 0.0086 & 0.0098 & -- & -- & 0.0047 \\
\hline SVOC & Acenaphthene & $\mathrm{mg} / \mathrm{kg}$ & 4 & 0 & $0 \%$ & 0.017 & 0.020 & -- & -- & 0.0094 \\
\hline SVOC & Acenaphthylene & $\mathrm{mg} / \mathrm{kg}$ & 4 & 0 & $0 \%$ & 0.0086 & 0.0098 & -- & -- & 0.0047 \\
\hline SVOC & Anthracene & $\mathrm{mg} / \mathrm{kg}$ & 4 & 0 & $0 \%$ & 0.0086 & 0.0098 & -- & -- & 0.0047 \\
\hline SVOC & Benzo(a)anthracene & $\mathrm{mg} / \mathrm{kg}$ & 4 & 0 & $0 \%$ & 0.0086 & 0.0098 & -- & -- & 0.0047 \\
\hline SVOC & Benzo(a)pyrene & $\mathrm{mg} / \mathrm{kg}$ & 4 & 0 & $0 \%$ & 0.0086 & 0.0098 & -- & -- & 0.0047 \\
\hline SVOC & Benzo(b)fluoranthene & $\mathrm{mg} / \mathrm{kg}$ & 4 & 0 & $0 \%$ & 0.0086 & 0.0098 & -- & -- & 0.0047 \\
\hline SVOC & Benzo(ghi)perylene & $\mathrm{mg} / \mathrm{kg}$ & 4 & 0 & $0 \%$ & 0.017 & 0.020 & -- & -- & 0.0094 \\
\hline SVOC & Benzo(k)fluoranthene & $\mathrm{mg} / \mathrm{kg}$ & 4 & 0 & $0 \%$ & 0.0086 & 0.0098 & -- & -- & 0.0047 \\
\hline SVOC & Bis(2-chloro-1-methylethyl)ether & $\mathrm{mg} / \mathrm{kg}$ & 4 & 0 & $0 \%$ & 0.017 & 0.020 & -- & -- & 0.0094 \\
\hline
\end{tabular}


Table F-74

Summary Statistics for White Sturgeon Fillet Collected from Segment 3 of the Columbia River

\begin{tabular}{|c|c|c|c|c|c|c|c|c|c|c|}
\hline $\begin{array}{c}\text { Constituent } \\
\text { Class }\end{array}$ & Constituent Name & Units & $\begin{array}{c}\text { Number } \\
\text { Analyzed }\end{array}$ & $\begin{array}{l}\text { Number } \\
\text { Detected }\end{array}$ & $\begin{array}{c}\text { Frequency of } \\
\text { Detection }\end{array}$ & $\begin{array}{l}\text { Minimum } \\
\text { Nondetect }\end{array}$ & $\begin{array}{l}\text { Maximum } \\
\text { Nondetect }\end{array}$ & $\begin{array}{l}\text { Minimum } \\
\text { Detect }\end{array}$ & $\begin{array}{l}\text { Maximum } \\
\text { Detect }\end{array}$ & $\begin{array}{c}\text { Average } \\
\text { Concentration }\end{array}$ \\
\hline$\overline{\text { SVOC }}$ & Chrysene & $\mathrm{mg} / \mathrm{kg}$ & 4 & 0 & $0 \%$ & 0.0086 & 0.0098 & $\overline{--}$ & $\overline{--}$ & 0.0047 \\
\hline SVOC & Dibenz $[\mathrm{a}, \mathrm{h}]$ anthracene & $\mathrm{mg} / \mathrm{kg}$ & 4 & 0 & $0 \%$ & 0.017 & 0.020 & -- & -- & 0.0094 \\
\hline SVOC & Dibenzofuran & $\mathrm{mg} / \mathrm{kg}$ & 4 & 0 & $0 \%$ & 0.0086 & 0.0098 & -- & -- & 0.0047 \\
\hline SVOC & Fluoranthene & $\mathrm{mg} / \mathrm{kg}$ & 4 & 0 & $0 \%$ & 0.017 & 0.020 & -- & -- & 0.0094 \\
\hline SVOC & Fluorene & $\mathrm{mg} / \mathrm{kg}$ & 4 & 0 & $0 \%$ & 0.0048 & 0.025 & -- & -- & 0.0059 \\
\hline SVOC & Hexachlorobenzene & $\mathrm{mg} / \mathrm{kg}$ & 4 & 1 & $25 \%$ & 0.019 & 0.020 & 0.023 & 0.023 & 0.013 \\
\hline SVOC & Hexachlorobutadiene & $\mathrm{mg} / \mathrm{kg}$ & 4 & 0 & $0 \%$ & 0.0086 & 0.0098 & -- & -- & 0.0047 \\
\hline SVOC & Hexachloroethane & $\mathrm{mg} / \mathrm{kg}$ & 4 & 0 & $0 \%$ & 0.0086 & 0.0098 & -- & -- & 0.0047 \\
\hline SVOC & Indeno(1,2,3-cd)pyrene & $\mathrm{mg} / \mathrm{kg}$ & 4 & 0 & $0 \%$ & 0.017 & 0.020 & -- & -- & 0.0094 \\
\hline SVOC & Naphthalene & $\mathrm{mg} / \mathrm{kg}$ & 4 & 0 & $0 \%$ & 0.0086 & 0.0098 & -- & -- & 0.0047 \\
\hline SVOC & Nitrobenzene & $\mathrm{mg} / \mathrm{kg}$ & 4 & 0 & $0 \%$ & 0.0086 & 0.0098 & -- & -- & 0.0047 \\
\hline SVOC & Phenanthrene & $\mathrm{mg} / \mathrm{kg}$ & 4 & 0 & $0 \%$ & 0.0086 & 0.0098 & -- & -- & 0.0047 \\
\hline SVOC & Pyrene & $\mathrm{mg} / \mathrm{kg}$ & 4 & 0 & $0 \%$ & 0.017 & 0.020 & -- & -- & 0.0094 \\
\hline VOC & Retene & $\mathrm{mg} / \mathrm{kg}$ & 4 & 0 & $0 \%$ & 0.017 & 0.020 & -- & -- & 0.0094 \\
\hline
\end{tabular}

DIOXI - Dioxins and Furans

PEST - Pesticide

PCB - Polychlorinated biphenyl

SVOC - Semivolatile Organic Compound

VOC - Volatile Organic Compound 
Table F-75

Summary Statistics for Whole Body White Sturgeon Collected from Segment 3 of the Columbia River

\begin{tabular}{|c|c|c|c|c|c|c|c|c|c|c|}
\hline $\begin{array}{l}\text { Constituent } \\
\text { Class }\end{array}$ & Constituent Name & Units & $\begin{array}{l}\text { Number } \\
\text { Analyzed }\end{array}$ & $\begin{array}{l}\text { Number } \\
\text { Detected }\end{array}$ & $\begin{array}{c}\text { Frequency of } \\
\text { Detection }\end{array}$ & $\begin{array}{l}\text { Minimum } \\
\text { Nondetect }\end{array}$ & $\begin{array}{l}\text { Maximum } \\
\text { Nondetect }\end{array}$ & $\begin{array}{l}\text { Minimum } \\
\text { Detect }\end{array}$ & $\begin{array}{l}\text { Maximum } \\
\text { Detect }\end{array}$ & $\begin{array}{c}\text { Average } \\
\text { Concentration }\end{array}$ \\
\hline DIOXI & $1,2,3,4,6,7,8$-Heptachlorodibenzodioxin & $\mathrm{mg} / \mathrm{kg}$ & 3 & 2 & $67 \%$ & $1.00 \mathrm{E}-08$ & $1.00 \mathrm{E}-08$ & $2.60 \mathrm{E}-07$ & $8.40 \mathrm{E}-07$ & $3.68 \mathrm{E}-07$ \\
\hline DIOXI & $1,2,3,4,6,7,8$-Heptachlorodibenzofuran & $\mathrm{mg} / \mathrm{kg}$ & 3 & 2 & $67 \%$ & $2.00 \mathrm{E}-08$ & $2.00 \mathrm{E}-08$ & $9.00 \mathrm{E}-08$ & $2.00 \mathrm{E}-07$ & 1.00E-07 \\
\hline DIOXI & 1,2,3,4,7,8,9-Heptachlorodibenzofuran & $\mathrm{mg} / \mathrm{kg}$ & 3 & 0 & $0 \%$ & 1.00E-08 & 3.00E-08 & -- & -- & 1.00E-08 \\
\hline DIOXI & 1,2,3,4,7,8-Hexachlorodibenzofuran & $\mathrm{mg} / \mathrm{kg}$ & 3 & 2 & $67 \%$ & $1.00 \mathrm{E}-08$ & 1.00E-08 & 4.00E-08 & $1.50 \mathrm{E}-07$ & $6.50 \mathrm{E}-08$ \\
\hline DIOXI & $1,2,3,4,7,8$-Hexachlorodibenzo-p-dioxin & $\mathrm{mg} / \mathrm{kg}$ & 3 & 0 & $0 \%$ & 1.00E-08 & 3.00E-08 & -- & -- & 8.33E-09 \\
\hline DIOXI & $1,2,3,6,7,8$-Hexachlorodibenzofuran & $\mathrm{mg} / \mathrm{kg}$ & 3 & 3 & $100 \%$ & -- & -- & 8.00E-08 & $1.50 \mathrm{E}-07$ & 1.07E-07 \\
\hline DIOXI & $1,2,3,6,7,8$-Hexachlorodibenzo-p-dioxin & $\mathrm{mg} / \mathrm{kg}$ & 3 & 2 & $67 \%$ & 1.00E-08 & 1.00E-08 & 1.50E-07 & 4.30E-07 & 1.95E-07 \\
\hline DIOXI & 1,2,3,7,8,9-Hexachlorodibenzofuran & $\mathrm{mg} / \mathrm{kg}$ & 3 & 0 & $0 \%$ & $1.00 \mathrm{E}-08$ & 2.00E-08 & -- & -- & 6.67E-09 \\
\hline DIOXI & 1,2,3,7,8,9-Hexachlorodibenzo-p-dioxin & $\mathrm{mg} / \mathrm{kg}$ & 3 & 1 & $33 \%$ & 1.00E-08 & 3.00E-08 & 4.00E-08 & 4.00E-08 & 2.00E-08 \\
\hline DIOXI & 1,2,3,7,8-Pentachlorodibenzofuran & $\mathrm{mg} / \mathrm{kg}$ & 3 & 3 & $100 \%$ & -- & -- & 2.70E-07 & $3.80 \mathrm{E}-07$ & 3.33E-07 \\
\hline DIOXI & 1,2,3,7,8-Pentachlorodibenzo-p-dioxin & $\mathrm{mg} / \mathrm{kg}$ & 3 & 0 & $0 \%$ & 1.00E-08 & 1.00E-08 & -- & -- & 5.00E-09 \\
\hline DIOXI & 2,3,4,6,7,8-Hexachlorodibenzofuran & $\mathrm{mg} / \mathrm{kg}$ & 3 & 2 & $67 \%$ & $1.00 \mathrm{E}-08$ & 1.00E-08 & 2.00E-08 & 7.00E-08 & 3.17E-08 \\
\hline DIOXI & 2,3,4,7,8-Pentachlorodibenzofuran & $\mathrm{mg} / \mathrm{kg}$ & 3 & 3 & $100 \%$ & -- & -- & $2.20 \mathrm{E}-07$ & 4.40E-07 & 3.57E-07 \\
\hline DIOXI & $2,3,7,8$-Tetrachlorodibenzofuran & $\mathrm{mg} / \mathrm{kg}$ & 3 & 3 & $100 \%$ & -- & -- & 7.97E-06 & 4.16E-05 & 2.01E-05 \\
\hline DIOXI & 2,3,7,8-Tetrachlorodibenzo-p-dioxin & $\mathrm{mg} / \mathrm{kg}$ & 3 & 3 & $100 \%$ & -- & -- & 2.80E-07 & 1.19E-06 & $7.80 \mathrm{E}-07$ \\
\hline DIOXI & Octachlorodibenzofuran & $\mathrm{mg} / \mathrm{kg}$ & 3 & 2 & $67 \%$ & $2.00 \mathrm{E}-08$ & 2.00E-08 & 1.00E-07 & $2.30 \mathrm{E}-07$ & 1.13E-07 \\
\hline DIOXI & Octachlorodibenzo-p-dioxin & $\mathrm{mg} / \mathrm{kg}$ & 3 & 3 & $100 \%$ & -- & -- & $6.10 \mathrm{E}-07$ & $2.46 \mathrm{E}-06$ & 1.35E-06 \\
\hline METAL & Aluminum & $\mathrm{mg} / \mathrm{kg}$ & 3 & 3 & $100 \%$ & -- & -- & 6.8 & 100 & 56 \\
\hline METAL & Antimony & $\mathrm{mg} / \mathrm{kg}$ & 3 & 0 & $0 \%$ & 0.050 & 0.050 & -- & -- & 0.025 \\
\hline METAL & Arsenic & $\mathrm{mg} / \mathrm{kg}$ & 3 & 3 & $100 \%$ & -- & -- & 0.24 & 0.36 & 0.30 \\
\hline METAL & Barium & $\mathrm{mg} / \mathrm{kg}$ & 3 & 3 & $100 \%$ & -- & -- & 1.6 & 2.8 & 2.2 \\
\hline METAL & Beryllium & $\mathrm{mg} / \mathrm{kg}$ & 3 & 2 & $67 \%$ & 0.0040 & 0.0040 & 0.0057 & 0.0069 & 0.0049 \\
\hline METAL & Cadmium & $\mathrm{mg} / \mathrm{kg}$ & 3 & 3 & $100 \%$ & -- & -- & 0.010 & 0.070 & 0.040 \\
\hline METAL & Chromium & $\mathrm{mg} / \mathrm{kg}$ & 3 & 3 & $100 \%$ & -- & -- & 0.31 & 0.64 & 0.48 \\
\hline METAL & Cobalt & $\mathrm{mg} / \mathrm{kg}$ & 3 & 3 & $100 \%$ & -- & -- & 0.18 & 0.33 & 0.27 \\
\hline METAL & Copper & $\mathrm{mg} / \mathrm{kg}$ & 3 & 3 & $100 \%$ & -- & -- & 0.81 & 1.8 & 1.2 \\
\hline METAL & Lead & $\mathrm{mg} / \mathrm{kg}$ & 3 & 3 & $100 \%$ & -- & -- & 0.020 & 0.21 & 0.12 \\
\hline METAL & Manganese & $\mathrm{mg} / \mathrm{kg}$ & 3 & 3 & $100 \%$ & -- & -- & 1.2 & 5.4 & 3.9 \\
\hline METAL & Mercury & $\mathrm{mg} / \mathrm{kg}$ & 3 & 2 & $67 \%$ & 0.10 & 0.10 & 0.11 & 0.22 & 0.13 \\
\hline METAL & Nickel & $\mathrm{mg} / \mathrm{kg}$ & 3 & 3 & $100 \%$ & -- & -- & 0.21 & 2.0 & 0.86 \\
\hline METAL & Selenium & $\mathrm{mg} / \mathrm{kg}$ & 3 & 3 & $100 \%$ & -- & -- & 0.65 & 0.92 & 0.78 \\
\hline METAL & Silver & $\mathrm{mg} / \mathrm{kg}$ & 3 & 0 & $0 \%$ & 0.10 & 0.10 & -- & -- & 0.050 \\
\hline METAL & Thallium & $\mathrm{mg} / \mathrm{kg}$ & 3 & 0 & $0 \%$ & 0.050 & 0.050 & -- & -- & 0.025 \\
\hline METAL & Vanadium & $\mathrm{mg} / \mathrm{kg}$ & 3 & 3 & $100 \%$ & -- & -- & 0.070 & 0.52 & 0.28 \\
\hline METAL & Zinc & $\mathrm{mg} / \mathrm{kg}$ & 3 & 3 & $100 \%$ & -- & -- & 7.5 & 12 & 9.2 \\
\hline РCB & 2,2',3,3',4,4',5-Heptachloro-1,1'-biphenyl & $\mathrm{mg} / \mathrm{kg}$ & 3 & 3 & $100 \%$ & -- & -- & 0.0016 & 0.0028 & 0.0023 \\
\hline РCB & $2,2^{\prime}, 3,4,4^{\prime}, 5,5^{\prime}$-Heptachlorobiphenyl & $\mathrm{mg} / \mathrm{kg}$ & 3 & 3 & $100 \%$ & -- & -- & 0.0052 & 0.010 & 0.0075 \\
\hline PCB & $2,3,3^{\prime}, 4,4^{\prime}, 5^{\prime}$-Hexachlorobiphenyl & $\mathrm{mg} / \mathrm{kg}$ & 3 & 3 & $100 \%$ & -- & -- & $1.80 \mathrm{E}-04$ & 3.10E-04 & 2.29E-04 \\
\hline PCB & $2,3,3^{\prime}, 4,4^{\prime}, 5$-Hexachlorobiphenyl (BZ 156) & $\mathrm{mg} / \mathrm{kg}$ & 3 & 3 & $100 \%$ & -- & -- & 0.0010 & 0.0018 & 0.0013 \\
\hline PCB & $2,3,3^{\prime}, 4,4^{\prime}$-Pentachlorobiphenyl & $\mathrm{mg} / \mathrm{kg}$ & 3 & 3 & $100 \%$ & -- & -- & 0.0025 & 0.0034 & 0.0028 \\
\hline РCB & 2,3',4,4',5,5'-Hexachlorobiphenyl (BZ 167) & $\mathrm{mg} / \mathrm{kg}$ & 3 & 3 & $100 \%$ & -- & -- & 3.22E-05 & $5.00 \mathrm{E}-05$ & 3.83E-05 \\
\hline РСВ & 2',3,4,4',5-Pentachloro-1,1'-biphenyl (BZ 123) & $\mathrm{mg} / \mathrm{kg}$ & 3 & 3 & $100 \%$ & -- & -- & 4.41E-05 & $6.58 \mathrm{E}-05$ & 5.39E-05 \\
\hline PCB & 2,3,4,4',5-Pentachlorobiphenyl (BZ 114) & $\mathrm{mg} / \mathrm{kg}$ & 3 & 3 & $100 \%$ & -- & -- & $1.65 \mathrm{E}-04$ & $2.55 \mathrm{E}-04$ & 2.15E-04 \\
\hline РСВ & 2,3',4,4',5-Pentachlorobiphenyl (BZ 118) & $\mathrm{mg} / \mathrm{kg}$ & 3 & 3 & $100 \%$ & -- & -- & 0.0027 & 0.0048 & 0.0037 \\
\hline PCB & $2,3,4,5,3^{\prime}, 4^{\prime}, 5^{\prime}$-Heptachlorobiphenyl & $\mathrm{mg} / \mathrm{kg}$ & 3 & 3 & $100 \%$ & -- & -- & $6.56 \mathrm{E}-05$ & $1.12 \mathrm{E}-04$ & 8.84E-05 \\
\hline РСВ & 3,3',4,4',5,5'-Hexachlorobiphenyl (BZ 169) & $\mathrm{mg} / \mathrm{kg}$ & 3 & 0 & $0 \%$ & 1.19E-06 & 1.49E-06 & -- & -- & $6.65 \mathrm{E}-07$ \\
\hline РCB & 3,3',4,4',5-Pentachlorobiphenyl (BZ 126) & $\mathrm{mg} / \mathrm{kg}$ & 3 & 0 & $0 \%$ & $1.52 \mathrm{E}-06$ & $1.78 \mathrm{E}-06$ & -- & -- & $8.28 \mathrm{E}-07$ \\
\hline РСВ & 3,3',4,4'-Tetrachlorobiphenyl (BZ 77) & $\mathrm{mg} / \mathrm{kg}$ & 3 & 3 & $100 \%$ & -- & -- & 1.24E-05 & $1.72 \mathrm{E}-05$ & 1.52E-05 \\
\hline PCB & Aroclor-1016 & $\mathrm{mg} / \mathrm{kg}$ & 3 & 0 & $0 \%$ & 0.019 & 0.019 & -- & -- & 0.0095 \\
\hline РСВ & Aroclor-1221 & $\mathrm{mg} / \mathrm{kg}$ & 3 & 0 & $0 \%$ & 0.019 & 0.019 & -- & -- & 0.0095 \\
\hline
\end{tabular}


Table F-75

Summary Statistics for Whole Body White Sturgeon Collected from Segment 3 of the Columbia River

\begin{tabular}{|c|c|c|c|c|c|c|c|c|c|c|}
\hline $\begin{array}{l}\text { Constituent } \\
\text { Class }\end{array}$ & Constituent Name & Units & $\begin{array}{l}\text { Number } \\
\text { Analyzed }\end{array}$ & $\begin{array}{l}\text { Number } \\
\text { Detected }\end{array}$ & $\begin{array}{l}\text { Frequency of } \\
\text { Detection }\end{array}$ & $\begin{array}{l}\text { Minimum } \\
\text { Nondetect }\end{array}$ & $\begin{array}{l}\text { Maximum } \\
\text { Nondetect }\end{array}$ & $\begin{array}{l}\text { Minimum } \\
\text { Detect }\end{array}$ & $\begin{array}{c}\text { Maximum } \\
\text { Detect }\end{array}$ & $\begin{array}{c}\text { Average } \\
\text { Concentration }\end{array}$ \\
\hline$\overline{\mathrm{PCB}}$ & Aroclor-1232 & $\mathrm{mg} / \mathrm{kg}$ & 3 & 0 & $0 \%$ & 0.019 & 0.019 & $\overline{--}$ & -- & 0.0095 \\
\hline РCB & Aroclor-1248 & $\mathrm{mg} / \mathrm{kg}$ & 3 & 0 & $0 \%$ & 0.019 & 0.019 & -- & -- & 0.0095 \\
\hline РСB & Aroclor-1254 & $\mathrm{mg} / \mathrm{kg}$ & 3 & 3 & $100 \%$ & -- & -- & 0.060 & 0.079 & 0.068 \\
\hline РСB & Aroclor-1260 & $\mathrm{mg} / \mathrm{kg}$ & 3 & 3 & $100 \%$ & -- & -- & 0.041 & 0.078 & 0.059 \\
\hline PEST & 2,4'-DDD & $\mathrm{mg} / \mathrm{kg}$ & 3 & 3 & $100 \%$ & -- & -- & 0.0093 & 0.016 & 0.013 \\
\hline PEST & 2,4'-DDE & $\mathrm{mg} / \mathrm{kg}$ & 3 & 3 & $100 \%$ & -- & -- & 0.0051 & 0.0060 & 0.0055 \\
\hline PEST & 2,4'-DDT & $\mathrm{mg} / \mathrm{kg}$ & 3 & 0 & $0 \%$ & 0.0019 & 0.0019 & -- & -- & $9.50 \mathrm{E}-04$ \\
\hline PEST & Aldrin & $\mathrm{mg} / \mathrm{kg}$ & 3 & 3 & $100 \%$ & -- & -- & $9.70 \mathrm{E}-04$ & 0.0020 & 0.0014 \\
\hline PEST & Alpha-BHC & $\mathrm{mg} / \mathrm{kg}$ & 3 & 0 & $0 \%$ & 0.0019 & 0.0019 & -- & -- & $9.50 \mathrm{E}-04$ \\
\hline PEST & alpha-Chlordane & $\mathrm{mg} / \mathrm{kg}$ & 3 & 3 & $100 \%$ & -- & -- & 0.0023 & 0.0092 & 0.0051 \\
\hline PEST & alpha-Chlordene & $\mathrm{mg} / \mathrm{kg}$ & 3 & 0 & $0 \%$ & 0.0019 & 0.0019 & -- & -- & $9.50 \mathrm{E}-04$ \\
\hline PEST & Beta-BHC & $\mathrm{mg} / \mathrm{kg}$ & 3 & 0 & $0 \%$ & 0.0019 & 0.0019 & -- & -- & $9.50 \mathrm{E}-04$ \\
\hline PEST & cis-Nonachlor & $\mathrm{mg} / \mathrm{kg}$ & 3 & 3 & $100 \%$ & -- & -- & 0.0083 & 0.013 & 0.010 \\
\hline PEST & DDMU & $\mathrm{mg} / \mathrm{kg}$ & 3 & 3 & $100 \%$ & -- & -- & 0.0038 & 0.0095 & 0.0069 \\
\hline PEST & Delta-BHC & $\mathrm{mg} / \mathrm{kg}$ & 3 & 0 & $0 \%$ & 0.0019 & 0.0019 & -- & -- & $9.50 \mathrm{E}-04$ \\
\hline PEST & Dichlorodiphenyldichloroethane & $\mathrm{mg} / \mathrm{kg}$ & 3 & 3 & $100 \%$ & -- & -- & 0.10 & 0.13 & 0.12 \\
\hline PEST & Dichlorodiphenyldichloroethylene & $\mathrm{mg} / \mathrm{kg}$ & 3 & 3 & $100 \%$ & -- & -- & 0.40 & 0.56 & 0.50 \\
\hline PEST & Dichlorodiphenyltrichloroethane & $\mathrm{mg} / \mathrm{kg}$ & 3 & 3 & $100 \%$ & -- & -- & 0.0056 & 0.022 & 0.011 \\
\hline PEST & Endosulfan sulfate & $\mathrm{mg} / \mathrm{kg}$ & 3 & 0 & $0 \%$ & 0.0019 & 0.0019 & -- & -- & $9.50 \mathrm{E}-04$ \\
\hline PEST & Gamma-BHC (Lindane) & $\mathrm{mg} / \mathrm{kg}$ & 3 & 0 & $0 \%$ & 0.0019 & 0.0019 & -- & -- & $9.50 \mathrm{E}-04$ \\
\hline PEST & gamma-Chlordane & $\mathrm{mg} / \mathrm{kg}$ & 3 & 3 & $100 \%$ & -- & -- & 0.0034 & 0.017 & 0.0088 \\
\hline PEST & gamma-Chlordene & $\mathrm{mg} / \mathrm{kg}$ & 3 & 0 & $0 \%$ & 0.0019 & 0.0019 & -- & -- & $9.50 \mathrm{E}-04$ \\
\hline PEST & Heptachlor & $\mathrm{mg} / \mathrm{kg}$ & 3 & 0 & $0 \%$ & 0.0019 & 0.0019 & -- & -- & $9.50 \mathrm{E}-04$ \\
\hline PEST & Heptachlor epoxide & $\mathrm{mg} / \mathrm{kg}$ & 3 & 0 & $0 \%$ & 0.0019 & 0.0019 & -- & -- & $9.50 \mathrm{E}-04$ \\
\hline PEST & Mirex & $\mathrm{mg} / \mathrm{kg}$ & 3 & 0 & $0 \%$ & 0.0019 & 0.0019 & -- & -- & $9.50 \mathrm{E}-04$ \\
\hline PEST & Oxychlordane & $\mathrm{mg} / \mathrm{kg}$ & 3 & 3 & $100 \%$ & -- & -- & $9.50 \mathrm{E}-04$ & 0.0025 & 0.0017 \\
\hline PEST & Pentachloroanisole & $\mathrm{mg} / \mathrm{kg}$ & 3 & 0 & $0 \%$ & 0.0019 & 0.0019 & -- & -- & $9.50 \mathrm{E}-04$ \\
\hline PEST & Toxaphene & $\mathrm{mg} / \mathrm{kg}$ & 3 & 0 & $0 \%$ & 0.057 & 0.058 & -- & -- & 0.029 \\
\hline PEST & trans-Nonachlor & $\mathrm{mg} / \mathrm{kg}$ & 3 & 3 & $100 \%$ & -- & -- & 0.0074 & 0.011 & 0.0086 \\
\hline SVOC & 1,2,4-Trichlorobenzene & $\mathrm{mg} / \mathrm{kg}$ & 3 & 0 & $0 \%$ & 0.0086 & 0.0094 & -- & -- & 0.0045 \\
\hline SVOC & 1,2-Dichlorobenzene & $\mathrm{mg} / \mathrm{kg}$ & 3 & 0 & $0 \%$ & 0.0086 & 0.0094 & -- & -- & 0.0045 \\
\hline SVOC & 1,2-Diphenylhydrazine & $\mathrm{mg} / \mathrm{kg}$ & 3 & 0 & $0 \%$ & 0.0086 & 0.0094 & -- & -- & 0.0045 \\
\hline SVOC & 1,3-Dichlorobenzene & $\mathrm{mg} / \mathrm{kg}$ & 3 & 0 & $0 \%$ & 0.0086 & 0.0094 & -- & -- & 0.0045 \\
\hline SVOC & 1,4-Dichlorobenzene & $\mathrm{mg} / \mathrm{kg}$ & 3 & 0 & $0 \%$ & 0.0086 & 0.0094 & -- & -- & 0.0045 \\
\hline SVOC & 1-methylnaphthalene & $\mathrm{mg} / \mathrm{kg}$ & 3 & 0 & $0 \%$ & 0.0086 & 0.0094 & -- & -- & 0.0045 \\
\hline SVOC & 2,4-Dinitrotoluene & $\mathrm{mg} / \mathrm{kg}$ & 3 & 0 & $0 \%$ & 0.017 & 0.019 & -- & -- & 0.0090 \\
\hline SVOC & 2,6-Dinitrotoluene & $\mathrm{mg} / \mathrm{kg}$ & 3 & 0 & $0 \%$ & 0.034 & 0.038 & -- & -- & 0.018 \\
\hline SVOC & 2-Chloronaphthalene & $\mathrm{mg} / \mathrm{kg}$ & 3 & 0 & $0 \%$ & 0.017 & 0.019 & -- & -- & 0.0090 \\
\hline SVOC & 2-MethyInaphthalene & $\mathrm{mg} / \mathrm{kg}$ & 3 & 0 & $0 \%$ & 0.0086 & 0.0094 & -- & -- & 0.0045 \\
\hline SVOC & 4-Bromophenylphenyl ether & $\mathrm{mg} / \mathrm{kg}$ & 3 & 0 & $0 \%$ & 0.0086 & 0.0094 & -- & -- & 0.0045 \\
\hline SVOC & 4-Chlorophenylphenyl ether & $\mathrm{mg} / \mathrm{kg}$ & 3 & 0 & $0 \%$ & 0.0086 & 0.0094 & -- & -- & 0.0045 \\
\hline SVOC & Acenaphthene & $\mathrm{mg} / \mathrm{kg}$ & 3 & 0 & $0 \%$ & 0.017 & 0.019 & -- & -- & 0.0090 \\
\hline SVOC & Acenaphthylene & $\mathrm{mg} / \mathrm{kg}$ & 3 & 0 & $0 \%$ & 0.0086 & 0.0094 & -- & -- & 0.0045 \\
\hline SVOC & Anthracene & $\mathrm{mg} / \mathrm{kg}$ & 3 & 0 & $0 \%$ & 0.0086 & 0.0094 & -- & -- & 0.0045 \\
\hline SVOC & Benzo(a)anthracene & $\mathrm{mg} / \mathrm{kg}$ & 3 & 0 & $0 \%$ & 0.0086 & 0.0094 & -- & -- & 0.0045 \\
\hline SVOC & Benzo(a)pyrene & $\mathrm{mg} / \mathrm{kg}$ & 3 & 0 & $0 \%$ & 0.0086 & 0.0094 & -- & -- & 0.0045 \\
\hline SVOC & Benzo(b)fluoranthene & $\mathrm{mg} / \mathrm{kg}$ & 3 & 0 & $0 \%$ & 0.0086 & 0.0094 & -- & -- & 0.0045 \\
\hline SVOC & Benzo(ghi)perylene & $\mathrm{mg} / \mathrm{kg}$ & 3 & 0 & $0 \%$ & 0.017 & 0.019 & -- & -- & 0.0090 \\
\hline SVOC & Benzo(k)fluoranthene & $\mathrm{mg} / \mathrm{kg}$ & 3 & 0 & $0 \%$ & 0.0086 & 0.0094 & -- & -- & 0.0045 \\
\hline SVOC & Bis(2-chloro-1-methylethyl)ether & $\mathrm{mg} / \mathrm{kg}$ & 3 & 0 & $0 \%$ & 0.017 & 0.019 & -- & -- & 0.0090 \\
\hline
\end{tabular}


Table F-75

Summary Statistics for Whole Body White Sturgeon Collected from Segment 3 of the Columbia River

\begin{tabular}{|c|c|c|c|c|c|c|c|c|c|c|}
\hline $\begin{array}{l}\text { Constituent } \\
\text { Class }\end{array}$ & Constituent Name & Units & $\begin{array}{l}\text { Number } \\
\text { Analyzed }\end{array}$ & $\begin{array}{l}\text { Number } \\
\text { Detected }\end{array}$ & $\begin{array}{c}\text { Frequency of } \\
\text { Detection }\end{array}$ & $\begin{array}{l}\text { Minimum } \\
\text { Nondetect }\end{array}$ & $\begin{array}{l}\text { Maximum } \\
\text { Nondetect }\end{array}$ & $\begin{array}{l}\text { Minimum } \\
\text { Detect }\end{array}$ & $\begin{array}{l}\text { Maximum } \\
\text { Detect }\end{array}$ & $\begin{array}{c}\text { Average } \\
\text { Concentration }\end{array}$ \\
\hline SVOC & Chrysene & $\mathrm{mg} / \mathrm{kg}$ & 3 & 0 & $0 \%$ & 0.0086 & 0.0094 & $\overline{--}$ & -- & 0.0045 \\
\hline SVOC & Dibenz[a,h]anthracene & $\mathrm{mg} / \mathrm{kg}$ & 3 & 0 & $0 \%$ & 0.017 & 0.019 & -- & -- & 0.0090 \\
\hline SVOC & Dibenzofuran & $\mathrm{mg} / \mathrm{kg}$ & 3 & 0 & $0 \%$ & 0.0086 & 0.0094 & -- & -- & 0.0045 \\
\hline SVOC & Fluoranthene & $\mathrm{mg} / \mathrm{kg}$ & 3 & 0 & $0 \%$ & 0.017 & 0.019 & -- & -- & 0.0090 \\
\hline SVOC & Fluorene & $\mathrm{mg} / \mathrm{kg}$ & 3 & 0 & $0 \%$ & 0.0077 & 0.0082 & -- & -- & 0.0040 \\
\hline SVOC & Hexachlorobenzene & $\mathrm{mg} / \mathrm{kg}$ & 3 & 1 & $33 \%$ & 0.017 & 0.018 & 0.020 & 0.020 & 0.013 \\
\hline SVOC & Hexachlorobutadiene & $\mathrm{mg} / \mathrm{kg}$ & 3 & 0 & $0 \%$ & 0.0086 & 0.0094 & -- & -- & 0.0045 \\
\hline SVOC & Hexachloroethane & $\mathrm{mg} / \mathrm{kg}$ & 3 & 0 & $0 \%$ & 0.0086 & 0.0094 & -- & -- & 0.0045 \\
\hline SVOC & Indeno(1,2,3-cd)pyrene & $\mathrm{mg} / \mathrm{kg}$ & 3 & 0 & $0 \%$ & 0.017 & 0.019 & -- & -- & 0.0090 \\
\hline SVOC & Naphthalene & $\mathrm{mg} / \mathrm{kg}$ & 3 & 0 & $0 \%$ & 0.0086 & 0.0094 & -- & -- & 0.0045 \\
\hline SVOC & Nitrobenzene & $\mathrm{mg} / \mathrm{kg}$ & 3 & 0 & $0 \%$ & 0.0086 & 0.0094 & -- & -- & 0.0045 \\
\hline SVOC & Phenanthrene & $\mathrm{mg} / \mathrm{kg}$ & 3 & 0 & $0 \%$ & 0.0086 & 0.0094 & -- & -- & 0.0045 \\
\hline SVOC & Pyrene & $\mathrm{mg} / \mathrm{kg}$ & 3 & 0 & $0 \%$ & 0.017 & 0.019 & -- & -- & 0.0090 \\
\hline VOC & Retene & $\mathrm{mg} / \mathrm{kg}$ & 3 & 0 & $0 \%$ & 0.017 & 0.019 & -- & -- & 0.0090 \\
\hline
\end{tabular}

DIOXI - Dioxins and Furans

PEST - Pesticide

PCB - Polychlorinated biphenyl

RAD- radionuclide

SVOC - Semivolatile Organic Compound

VOC - Volatile Organic Compound 
Table F-76

Summary Statistics for Whole Body Clam Collected from Segment 4 of the Columbia River

\begin{tabular}{|c|c|c|c|c|c|c|c|c|c|c|}
\hline $\begin{array}{l}\text { Constituent } \\
\text { Class }\end{array}$ & Constituent Name & Units & $\begin{array}{l}\text { Number } \\
\text { Analyzed }\end{array}$ & $\begin{array}{l}\text { Number } \\
\text { Detected }\end{array}$ & $\begin{array}{l}\text { Frequency of } \\
\text { Detection }\end{array}$ & $\begin{array}{l}\text { Minimum } \\
\text { Nondetect }\end{array}$ & $\begin{array}{l}\text { Maximum } \\
\text { Nondetect }\end{array}$ & $\begin{array}{l}\text { Minimum } \\
\text { Detect }\end{array}$ & $\begin{array}{c}\text { Maximum } \\
\text { Detect }\end{array}$ & $\begin{array}{c}\text { Average } \\
\text { Concentration }\end{array}$ \\
\hline RAD & Strontium-90 & $\mathrm{pCi} / \mathrm{g}$ & 1 & 1 & $100 \%$ & -- & -- & 0.38 & 0.38 & 0.38 \\
\hline
\end{tabular}

RAD - Radionuclide 
Table F-77

Summary Statistics for Channel Catfish Fillet Skin On Collected from Segment 4 of the Columbia River

\begin{tabular}{|c|c|c|c|c|c|c|c|c|c|c|}
\hline $\begin{array}{l}\text { Constituent } \\
\text { Class }\end{array}$ & Constituent Name & Units & $\begin{array}{l}\text { Number } \\
\text { Analyzed }\end{array}$ & $\begin{array}{l}\text { Number } \\
\text { Detected }\end{array}$ & $\begin{array}{l}\text { Frequency of } \\
\text { Detection }\end{array}$ & $\begin{array}{l}\text { Minimum } \\
\text { Nondetect }\end{array}$ & $\begin{array}{l}\text { Maximum } \\
\text { Nondetect }\end{array}$ & $\begin{array}{l}\text { Minimum } \\
\text { Detect }\end{array}$ & $\begin{array}{l}\text { Maximum } \\
\text { Detect }\end{array}$ & $\begin{array}{c}\text { Average } \\
\text { Concentration }\end{array}$ \\
\hline$\overline{\mathrm{DIOXI}}$ & $1,2,3,4,6,7,8$-Heptachlorodibenzodioxin & $\mathrm{mg} / \mathrm{kg}$ & 2 & 2 & $100 \%$ & $\overline{--}$ & $\overline{--}$ & $4.70 \mathrm{E}-07$ & $1.24 \mathrm{E}-06$ & $8.55 \mathrm{E}-07$ \\
\hline DIOXI & $1,2,3,4,6,7,8$-Heptachlorodibenzofuran & $\mathrm{mg} / \mathrm{kg}$ & 2 & 0 & $0 \%$ & $2.00 \mathrm{E}-08$ & $5.00 \mathrm{E}-08$ & -- & -- & 1.75E-08 \\
\hline DIOXI & 1,2,3,4,7,8,9-Heptachlorodibenzofuran & $\mathrm{mg} / \mathrm{kg}$ & 2 & 0 & $0 \%$ & $2.00 \mathrm{E}-08$ & $5.00 \mathrm{E}-08$ & -- & -- & $1.75 \mathrm{E}-08$ \\
\hline DIOXI & $1,2,3,4,7,8$-Hexachlorodibenzofuran & $\mathrm{mg} / \mathrm{kg}$ & 2 & 1 & $50 \%$ & $2.00 \mathrm{E}-08$ & $2.00 \mathrm{E}-08$ & $9.00 \mathrm{E}-08$ & $9.00 \mathrm{E}-08$ & $5.00 \mathrm{E}-08$ \\
\hline DIOXI & 1,2,3,4,7,8-Hexachlorodibenzo-p-dioxin & $\mathrm{mg} / \mathrm{kg}$ & 2 & 2 & $100 \%$ & -- & -- & 1.40E-07 & 3.00E-07 & $2.20 \mathrm{E}-07$ \\
\hline DIOXI & $1,2,3,6,7,8$-Hexachlorodibenzofuran & $\mathrm{mg} / \mathrm{kg}$ & 2 & 2 & $100 \%$ & -- & -- & $3.00 \mathrm{E}-08$ & $5.00 \mathrm{E}-08$ & $4.00 \mathrm{E}-08$ \\
\hline DIOXI & $1,2,3,6,7,8$-Hexachlorodibenzo-p-dioxin & $\mathrm{mg} / \mathrm{kg}$ & 2 & 2 & $100 \%$ & -- & -- & $5.90 \mathrm{E}-07$ & 1.10E-06 & 8.45E-07 \\
\hline DIOXI & 1,2,3,7,8,9-Hexachlorodibenzofuran & $\mathrm{mg} / \mathrm{kg}$ & 2 & 0 & $0 \%$ & $2.00 \mathrm{E}-08$ & $2.00 \mathrm{E}-08$ & -- & -- & $1.00 \mathrm{E}-08$ \\
\hline DIOXI & 1,2,3,7,8,9-Hexachlorodibenzo-p-dioxin & $\mathrm{mg} / \mathrm{kg}$ & 2 & 2 & $100 \%$ & -- & -- & $1.40 \mathrm{E}-07$ & 1.70E-07 & $1.55 \mathrm{E}-07$ \\
\hline DIOXI & 1,2,3,7,8-Pentachlorodibenzofuran & $\mathrm{mg} / \mathrm{kg}$ & 2 & 2 & $100 \%$ & -- & -- & $2.10 \mathrm{E}-07$ & 3.10E-07 & $2.60 \mathrm{E}-07$ \\
\hline DIOXI & $1,2,3,7,8$-Pentachlorodibenzo-p-dioxin & $\mathrm{mg} / \mathrm{kg}$ & 2 & 2 & $100 \%$ & -- & -- & 4.90E-07 & $5.70 \mathrm{E}-07$ & $5.30 \mathrm{E}-07$ \\
\hline DIOXI & 2,3,4,6,7,8-Hexachlorodibenzofuran & $\mathrm{mg} / \mathrm{kg}$ & 2 & 2 & $100 \%$ & -- & -- & $5.00 \mathrm{E}-08$ & $5.00 \mathrm{E}-08$ & $5.00 \mathrm{E}-08$ \\
\hline DIOXI & 2,3,4,7,8-Pentachlorodibenzofuran & $\mathrm{mg} / \mathrm{kg}$ & 2 & 2 & $100 \%$ & -- & -- & $5.20 \mathrm{E}-07$ & $6.60 \mathrm{E}-07$ & $5.90 \mathrm{E}-07$ \\
\hline DIOXI & 2,3,7,8-Tetrachlorodibenzofuran & $\mathrm{mg} / \mathrm{kg}$ & 2 & 2 & $100 \%$ & -- & -- & $2.17 \mathrm{E}-06$ & 3.37E-06 & 2.77E-06 \\
\hline DIOXI & 2,3,7,8-Tetrachlorodibenzo-p-dioxin & $\mathrm{mg} / \mathrm{kg}$ & 2 & 2 & $100 \%$ & -- & -- & $1.00 \mathrm{E}-06$ & $1.41 \mathrm{E}-06$ & $1.21 \mathrm{E}-06$ \\
\hline DIOXI & Octachlorodibenzofuran & $\mathrm{mg} / \mathrm{kg}$ & 2 & 2 & $100 \%$ & -- & -- & $3.00 \mathrm{E}-08$ & $9.00 \mathrm{E}-08$ & $6.00 \mathrm{E}-08$ \\
\hline DIOXI & Octachlorodibenzo-p-dioxin & $\mathrm{mg} / \mathrm{kg}$ & 2 & 2 & $100 \%$ & -- & -- & 3.70E-07 & $1.25 \mathrm{E}-06$ & $8.10 \mathrm{E}-07$ \\
\hline METAL & Aluminum & $\mathrm{mg} / \mathrm{kg}$ & 2 & 1 & $50 \%$ & 1.0 & 1.0 & 42 & 42 & 21 \\
\hline METAL & Antimony & $\mathrm{mg} / \mathrm{kg}$ & 2 & 0 & $0 \%$ & 0.050 & 0.050 & -- & -- & 0.025 \\
\hline METAL & Arsenic & $\mathrm{mg} / \mathrm{kg}$ & 2 & 1 & $50 \%$ & 0.050 & 0.050 & 0.33 & 0.33 & 0.18 \\
\hline METAL & Barium & $\mathrm{mg} / \mathrm{kg}$ & 2 & 1 & $50 \%$ & 0.20 & 0.20 & 0.28 & 0.28 & 0.19 \\
\hline METAL & Beryllium & $\mathrm{mg} / \mathrm{kg}$ & 2 & 0 & $0 \%$ & 0.0040 & 0.0040 & -- & -- & 0.0020 \\
\hline METAL & Cadmium & $\mathrm{mg} / \mathrm{kg}$ & 2 & 0 & $0 \%$ & 0.0040 & 0.0040 & -- & -- & 0.0020 \\
\hline METAL & Chromium & $\mathrm{mg} / \mathrm{kg}$ & 2 & 2 & $100 \%$ & -- & -- & 0.11 & 0.13 & 0.12 \\
\hline METAL & Cobalt & $\mathrm{mg} / \mathrm{kg}$ & 2 & 2 & $100 \%$ & -- & -- & 0.070 & 0.090 & 0.080 \\
\hline METAL & Copper & $\mathrm{mg} / \mathrm{kg}$ & 2 & 2 & $100 \%$ & -- & -- & 0.35 & 0.36 & 0.36 \\
\hline METAL & Lead & $\mathrm{mg} / \mathrm{kg}$ & 2 & 1 & $50 \%$ & 0.010 & 0.010 & 0.010 & 0.010 & 0.0075 \\
\hline METAL & Manganese & $\mathrm{mg} / \mathrm{kg}$ & 2 & 2 & $100 \%$ & -- & -- & 0.13 & 0.13 & 0.13 \\
\hline METAL & Mercury & $\mathrm{mg} / \mathrm{kg}$ & 2 & 2 & $100 \%$ & -- & -- & 0.23 & 0.24 & 0.24 \\
\hline METAL & Nickel & $\mathrm{mg} / \mathrm{kg}$ & 2 & 0 & $0 \%$ & 0.030 & 0.030 & -- & -- & 0.015 \\
\hline METAL & Selenium & $\mathrm{mg} / \mathrm{kg}$ & 2 & 0 & $0 \%$ & 0.50 & 0.50 & -- & -- & 0.25 \\
\hline METAL & Silver & $\mathrm{mg} / \mathrm{kg}$ & 2 & 0 & $0 \%$ & 0.10 & 0.10 & -- & -- & 0.050 \\
\hline METAL & Thallium & $\mathrm{mg} / \mathrm{kg}$ & 2 & 0 & $0 \%$ & 0.050 & 0.050 & -- & -- & 0.025 \\
\hline METAL & Vanadium & $\mathrm{mg} / \mathrm{kg}$ & 2 & 0 & $0 \%$ & 0.010 & 0.010 & -- & -- & 0.0050 \\
\hline METAL & Zinc & $\mathrm{mg} / \mathrm{kg}$ & 2 & 2 & $100 \%$ & -- & -- & 7.8 & 9.9 & 8.9 \\
\hline PCB & 2,2',3,3',4,4',5-Heptachloro-1,1'-biphenyl & $\mathrm{mg} / \mathrm{kg}$ & 2 & 2 & $100 \%$ & -- & -- & 0.0017 & 0.0039 & 0.0028 \\
\hline РСВ & 2,2',3,4,4',5,5'-Heptachlorobiphenyl & $\mathrm{mg} / \mathrm{kg}$ & 2 & 2 & $100 \%$ & -- & -- & 0.0043 & 0.010 & 0.0073 \\
\hline PCB & 2,3,3',4,4',5'-Hexachlorobiphenyl & $\mathrm{mg} / \mathrm{kg}$ & 2 & 2 & $100 \%$ & -- & -- & $1.65 \mathrm{E}-04$ & $3.56 \mathrm{E}-04$ & 2.61E-04 \\
\hline РСB & 2,3,3',4,4',5-Hexachlorobiphenyl (BZ 156) & $\mathrm{mg} / \mathrm{kg}$ & 2 & 2 & $100 \%$ & -- & -- & $9.06 \mathrm{E}-04$ & 0.0019 & 0.0014 \\
\hline PCB & 2,3,3',4,4'-Pentachlorobiphenyl & $\mathrm{mg} / \mathrm{kg}$ & 2 & 2 & $100 \%$ & -- & -- & 0.0020 & 0.0039 & 0.0030 \\
\hline PCB & 2,3',4,4',5,5'-Hexachlorobiphenyl (BZ 167) & $\mathrm{mg} / \mathrm{kg}$ & 2 & 2 & $100 \%$ & -- & -- & $3.46 \mathrm{E}-04$ & 7.51E-04 & 5.49E-04 \\
\hline РСB & 2',3,4,4',5-Pentachloro-1,1'-biphenyl (BZ 123) & $\mathrm{mg} / \mathrm{kg}$ & 2 & 2 & $100 \%$ & -- & -- & $9.17 \mathrm{E}-05$ & $1.82 \mathrm{E}-04$ & 1.37E-04 \\
\hline PCB & 2,3,4,4',5-Pentachlorobiphenyl (BZ 114) & $\mathrm{mg} / \mathrm{kg}$ & 2 & 2 & $100 \%$ & -- & -- & $1.94 \mathrm{E}-04$ & 4.16E-04 & 3.05E-04 \\
\hline PCB & 2,3',4,4',5-Pentachlorobiphenyl (BZ 118) & $\mathrm{mg} / \mathrm{kg}$ & 2 & 2 & $100 \%$ & -- & -- & 0.0046 & 0.0091 & 0.0068 \\
\hline РСB & 2,3,4,5,3',4',5'-Heptachlorobiphenyl & $\mathrm{mg} / \mathrm{kg}$ & 2 & 2 & $100 \%$ & -- & -- & $5.83 \mathrm{E}-05$ & 1.34E-04 & $9.62 \mathrm{E}-05$ \\
\hline РСB & 3,3',4,4',5,5'-Hexachlorobiphenyl (BZ 169) & $\mathrm{mg} / \mathrm{kg}$ & 2 & 0 & $0 \%$ & 1.79E-06 & $2.57 \mathrm{E}-06$ & -- & -- & $1.09 \mathrm{E}-06$ \\
\hline PCB & 3,3',4,4',5-Pentachlorobiphenyl (BZ 126) & $\mathrm{mg} / \mathrm{kg}$ & 2 & 1 & $50 \%$ & 1.71E-06 & $1.71 \mathrm{E}-06$ & 1.37E-05 & 1.37E-05 & $7.28 \mathrm{E}-06$ \\
\hline РСB & 3,3',4,4'-Tetrachlorobiphenyl (BZ 77) & $\mathrm{mg} / \mathrm{kg}$ & 2 & 2 & $100 \%$ & -- & -- & $3.58 \mathrm{E}-05$ & 3.67E-05 & 3.63E-05 \\
\hline PCB & Aroclor-1016 & $\mathrm{mg} / \mathrm{kg}$ & 2 & 0 & $0 \%$ & 0.019 & 0.020 & -- & -- & 0.0098 \\
\hline PCB & Aroclor-1221 & $\mathrm{mg} / \mathrm{kg}$ & 2 & 0 & $0 \%$ & 0.019 & 0.020 & -- & -- & 0.0098 \\
\hline РСB & Aroclor-1232 & $\mathrm{mg} / \mathrm{kg}$ & 2 & 0 & $0 \%$ & 0.019 & 0.020 & -- & -- & 0.0098 \\
\hline РСB & Aroclor-1248 & $\mathrm{mg} / \mathrm{kg}$ & 2 & 0 & $0 \%$ & 0.019 & 0.020 & -- & -- & 0.0098 \\
\hline
\end{tabular}


Table F-77

Summary Statistics for Channel Catfish Fillet Skin On Collected from Segment 4 of the Columbia River

\begin{tabular}{|c|c|c|c|c|c|c|c|c|c|c|}
\hline $\begin{array}{l}\text { Constituent } \\
\text { Class }\end{array}$ & Constituent Name & Units & $\begin{array}{l}\text { Number } \\
\text { Analyzed }\end{array}$ & $\begin{array}{l}\text { Number } \\
\text { Detected }\end{array}$ & $\begin{array}{l}\text { Frequency of } \\
\text { Detection }\end{array}$ & $\begin{array}{l}\text { Minimum } \\
\text { Nondetect }\end{array}$ & $\begin{array}{l}\text { Maximum } \\
\text { Nondetect }\end{array}$ & $\begin{array}{l}\text { Minimum } \\
\text { Detect }\end{array}$ & $\begin{array}{l}\text { Maximum } \\
\text { Detect }\end{array}$ & $\begin{array}{c}\text { Average } \\
\text { Concentration }\end{array}$ \\
\hline$\overline{\mathrm{PCB}}$ & Aroclor-1254 & $\mathrm{mg} / \mathrm{kg}$ & 2 & 2 & $100 \%$ & -- & $\overline{--}$ & 0.036 & 0.063 & 0.050 \\
\hline PCB & Aroclor-1260 & $\mathrm{mg} / \mathrm{kg}$ & 2 & 2 & $100 \%$ & -- & -- & 0.037 & 0.088 & 0.063 \\
\hline PEST & 2,4'-DDD & $\mathrm{mg} / \mathrm{kg}$ & 2 & 2 & $100 \%$ & -- & -- & 0.0064 & 0.0094 & 0.0079 \\
\hline PEST & 2,4'-DDE & $\mathrm{mg} / \mathrm{kg}$ & 2 & 1 & $50 \%$ & 0.0020 & 0.0020 & 0.0096 & 0.0096 & 0.0053 \\
\hline PEST & 2,4'-DDT & $\mathrm{mg} / \mathrm{kg}$ & 2 & 0 & $0 \%$ & 0.0019 & 0.0020 & -- & -- & $9.75 \mathrm{E}-04$ \\
\hline PEST & Aldrin & $\mathrm{mg} / \mathrm{kg}$ & 2 & 0 & $0 \%$ & 0.0019 & 0.0020 & -- & -- & $9.75 \mathrm{E}-04$ \\
\hline PEST & Alpha-BHC & $\mathrm{mg} / \mathrm{kg}$ & 2 & 0 & $0 \%$ & 0.0019 & 0.0020 & -- & -- & $9.75 \mathrm{E}-04$ \\
\hline PEST & alpha-Chlordane & $\mathrm{mg} / \mathrm{kg}$ & 2 & 1 & $50 \%$ & 0.0020 & 0.0020 & 0.0032 & 0.0032 & 0.0021 \\
\hline PEST & alpha-Chlordene & $\mathrm{mg} / \mathrm{kg}$ & 2 & 0 & $0 \%$ & 0.0019 & 0.0020 & -- & -- & $9.75 \mathrm{E}-04$ \\
\hline PEST & Beta-BHC & $\mathrm{mg} / \mathrm{kg}$ & 2 & 0 & $0 \%$ & 0.0019 & 0.0020 & -- & -- & $9.75 \mathrm{E}-04$ \\
\hline PEST & cis-Nonachlor & $\mathrm{mg} / \mathrm{kg}$ & 2 & 2 & $100 \%$ & -- & -- & 0.0020 & 0.0052 & 0.0036 \\
\hline PEST & DDMU & $\mathrm{mg} / \mathrm{kg}$ & 2 & 2 & $100 \%$ & -- & -- & 0.0065 & 0.011 & 0.0088 \\
\hline PEST & Delta-BHC & $\mathrm{mg} / \mathrm{kg}$ & 2 & 0 & $0 \%$ & 0.0019 & 0.0020 & -- & -- & $9.75 \mathrm{E}-04$ \\
\hline PEST & Dichlorodiphenyldichloroethane & $\mathrm{mg} / \mathrm{kg}$ & 2 & 2 & $100 \%$ & -- & -- & 0.042 & 0.068 & 0.055 \\
\hline PEST & Dichlorodiphenyldichloroethylene & $\mathrm{mg} / \mathrm{kg}$ & 2 & 2 & $100 \%$ & -- & -- & 0.33 & 0.52 & 0.43 \\
\hline PEST & Dichlorodiphenyltrichloroethane & $\mathrm{mg} / \mathrm{kg}$ & 2 & 2 & $100 \%$ & -- & -- & 0.0019 & 0.0074 & 0.0047 \\
\hline PEST & Endosulfan sulfate & $\mathrm{mg} / \mathrm{kg}$ & 2 & 0 & $0 \%$ & 0.0019 & 0.0020 & -- & -- & $9.75 \mathrm{E}-04$ \\
\hline PEST & Gamma-BHC (Lindane) & $\mathrm{mg} / \mathrm{kg}$ & 2 & 0 & $0 \%$ & 0.0019 & 0.0020 & -- & -- & $9.75 \mathrm{E}-04$ \\
\hline PEST & gamma-Chlordane & $\mathrm{mg} / \mathrm{kg}$ & 2 & 2 & $100 \%$ & -- & -- & 0.0021 & 0.0036 & 0.0029 \\
\hline PEST & gamma-Chlordene & $\mathrm{mg} / \mathrm{kg}$ & 2 & 0 & $0 \%$ & 0.0019 & 0.0020 & -- & -- & $9.75 \mathrm{E}-04$ \\
\hline PEST & Heptachlor & $\mathrm{mg} / \mathrm{kg}$ & 2 & 0 & $0 \%$ & 0.0019 & 0.0020 & -- & -- & $9.75 \mathrm{E}-04$ \\
\hline PEST & Heptachlor epoxide & $\mathrm{mg} / \mathrm{kg}$ & 2 & 0 & $0 \%$ & 0.0019 & 0.0020 & -- & -- & $9.75 \mathrm{E}-04$ \\
\hline PEST & Mirex & $\mathrm{mg} / \mathrm{kg}$ & 2 & 2 & $100 \%$ & -- & -- & 0.0028 & 0.0059 & 0.0044 \\
\hline PEST & Oxychlordane & $\mathrm{mg} / \mathrm{kg}$ & 2 & 2 & $100 \%$ & -- & -- & 0.0015 & 0.0026 & 0.0021 \\
\hline PEST & Pentachloroanisole & $\mathrm{mg} / \mathrm{kg}$ & 2 & 0 & $0 \%$ & 0.0019 & 0.0020 & -- & -- & 9.75E-04 \\
\hline PEST & Toxaphene & $\mathrm{mg} / \mathrm{kg}$ & 2 & 0 & $0 \%$ & 0.058 & 0.059 & -- & -- & 0.029 \\
\hline PEST & trans-Nonachlor & $\mathrm{mg} / \mathrm{kg}$ & 2 & 2 & $100 \%$ & -- & -- & 0.0053 & 0.011 & 0.0082 \\
\hline SVOC & 1,2,4-Trichlorobenzene & $\mathrm{mg} / \mathrm{kg}$ & 2 & 0 & $0 \%$ & 0.0091 & 0.0093 & -- & -- & 0.0046 \\
\hline SVOC & 1,2-Dichlorobenzene & $\mathrm{mg} / \mathrm{kg}$ & 2 & 0 & $0 \%$ & 0.0091 & 0.0093 & -- & -- & 0.0046 \\
\hline SVOC & 1,2-Diphenylhydrazine & $\mathrm{mg} / \mathrm{kg}$ & 2 & 0 & $0 \%$ & 0.0091 & 0.0093 & -- & -- & 0.0046 \\
\hline SVOC & 1,3-Dichlorobenzene & $\mathrm{mg} / \mathrm{kg}$ & 2 & 0 & $0 \%$ & 0.0091 & 0.0093 & -- & -- & 0.0046 \\
\hline SVOC & 1,4-Dichlorobenzene & $\mathrm{mg} / \mathrm{kg}$ & 2 & 0 & $0 \%$ & 0.0091 & 0.0093 & -- & -- & 0.0046 \\
\hline SVOC & 1-methylnaphthalene & $\mathrm{mg} / \mathrm{kg}$ & 2 & 0 & $0 \%$ & 0.0091 & 0.0093 & -- & -- & 0.0046 \\
\hline SVOC & 2,4-Dinitrotoluene & $\mathrm{mg} / \mathrm{kg}$ & 2 & 0 & $0 \%$ & 0.018 & 0.019 & -- & -- & 0.0093 \\
\hline SVOC & 2,6-Dinitrotoluene & $\mathrm{mg} / \mathrm{kg}$ & 2 & 0 & $0 \%$ & 0.036 & 0.037 & -- & -- & 0.018 \\
\hline SVOC & 2-Chloronaphthalene & $\mathrm{mg} / \mathrm{kg}$ & 2 & 0 & $0 \%$ & 0.018 & 0.019 & -- & -- & 0.0093 \\
\hline SVOC & 2-Methylnaphthalene & $\mathrm{mg} / \mathrm{kg}$ & 2 & 0 & $0 \%$ & 0.0091 & 0.0093 & -- & -- & 0.0046 \\
\hline SVOC & 4-Bromophenylphenyl ether & $\mathrm{mg} / \mathrm{kg}$ & 2 & 0 & $0 \%$ & 0.0091 & 0.0093 & -- & -- & 0.0046 \\
\hline SVOC & 4-Chlorophenylphenyl ether & $\mathrm{mg} / \mathrm{kg}$ & 2 & 0 & $0 \%$ & 0.0091 & 0.0093 & -- & -- & 0.0046 \\
\hline SVOC & Acenaphthene & $\mathrm{mg} / \mathrm{kg}$ & 2 & 0 & $0 \%$ & 0.018 & 0.019 & -- & -- & 0.0093 \\
\hline SVOC & Acenaphthylene & $\mathrm{mg} / \mathrm{kg}$ & 2 & 0 & $0 \%$ & 0.0091 & 0.0093 & -- & -- & 0.0046 \\
\hline SVOC & Anthracene & $\mathrm{mg} / \mathrm{kg}$ & 2 & 0 & $0 \%$ & 0.0091 & 0.0093 & -- & -- & 0.0046 \\
\hline SVOC & Benzo(a)anthracene & $\mathrm{mg} / \mathrm{kg}$ & 2 & 0 & $0 \%$ & 0.0091 & 0.0093 & -- & -- & 0.0046 \\
\hline SVOC & Benzo(a)pyrene & $\mathrm{mg} / \mathrm{kg}$ & 2 & 0 & $0 \%$ & 0.0091 & 0.0093 & -- & -- & 0.0046 \\
\hline SVOC & Benzo(b)fluoranthene & $\mathrm{mg} / \mathrm{kg}$ & 2 & 0 & $0 \%$ & 0.0091 & 0.0093 & -- & -- & 0.0046 \\
\hline SVOC & Benzo(ghi)perylene & $\mathrm{mg} / \mathrm{kg}$ & 2 & 0 & $0 \%$ & 0.018 & 0.019 & -- & -- & 0.0093 \\
\hline SVOC & Benzo(k)fluoranthene & $\mathrm{mg} / \mathrm{kg}$ & 2 & 0 & $0 \%$ & 0.0091 & 0.0093 & -- & -- & 0.0046 \\
\hline SVOC & Bis(2-chloro-1-methylethyl)ether & $\mathrm{mg} / \mathrm{kg}$ & 2 & 0 & $0 \%$ & 0.018 & 0.019 & -- & -- & 0.0093 \\
\hline SVOC & Chrysene & $\mathrm{mg} / \mathrm{kg}$ & 2 & 0 & $0 \%$ & 0.0091 & 0.0093 & -- & -- & 0.0046 \\
\hline SVOC & Dibenz[a,h]anthracene & $\mathrm{mg} / \mathrm{kg}$ & 2 & 0 & $0 \%$ & 0.018 & 0.019 & -- & -- & 0.0093 \\
\hline SVOC & Dibenzofuran & $\mathrm{mg} / \mathrm{kg}$ & 2 & 0 & $0 \%$ & 0.0091 & 0.0093 & -- & -- & 0.0046 \\
\hline SVOC & Fluoranthene & $\mathrm{mg} / \mathrm{kg}$ & 2 & 0 & $0 \%$ & 0.018 & 0.019 & -- & -- & 0.0093 \\
\hline
\end{tabular}


Table F-77

Summary Statistics for Channel Catfish Fillet Skin On Collected from Segment 4 of the Columbia River

\begin{tabular}{|c|c|c|c|c|c|c|c|c|c|c|}
\hline $\begin{array}{c}\text { Constituent } \\
\text { Class }\end{array}$ & Constituent Name & Units & $\begin{array}{c}\text { Number } \\
\text { Analyzed }\end{array}$ & $\begin{array}{l}\text { Number } \\
\text { Detected }\end{array}$ & $\begin{array}{c}\text { Frequency of } \\
\text { Detection }\end{array}$ & $\begin{array}{l}\text { Minimum } \\
\text { Nondetect }\end{array}$ & $\begin{array}{l}\text { Maximum } \\
\text { Nondetect }\end{array}$ & $\begin{array}{c}\text { Minimum } \\
\text { Detect }\end{array}$ & $\begin{array}{c}\text { Maximum } \\
\text { Detect }\end{array}$ & $\begin{array}{c}\text { Average } \\
\text { Concentration }\end{array}$ \\
\hline$\overline{\text { SVOC }}$ & Fluorene & $\mathrm{mg} / \mathrm{kg}$ & 2 & 0 & $0 \%$ & 0.024 & 0.024 & -- & -- & 0.012 \\
\hline SVOC & Hexachlorobenzene & $\mathrm{mg} / \mathrm{kg}$ & 2 & 0 & $0 \%$ & 0.018 & 0.019 & -- & -- & 0.0093 \\
\hline SVOC & Hexachlorobutadiene & $\mathrm{mg} / \mathrm{kg}$ & 2 & 0 & $0 \%$ & 0.0091 & 0.0093 & -- & -- & 0.0046 \\
\hline SVOC & Hexachloroethane & $\mathrm{mg} / \mathrm{kg}$ & 2 & 0 & $0 \%$ & 0.0091 & 0.0093 & -- & -- & 0.0046 \\
\hline SVOC & Indeno(1,2,3-cd)pyrene & $\mathrm{mg} / \mathrm{kg}$ & 2 & 0 & $0 \%$ & 0.018 & 0.019 & -- & -- & 0.0093 \\
\hline SVOC & Naphthalene & $\mathrm{mg} / \mathrm{kg}$ & 2 & 0 & $0 \%$ & 0.024 & 0.027 & -- & -- & 0.013 \\
\hline SVOC & Nitrobenzene & $\mathrm{mg} / \mathrm{kg}$ & 2 & 0 & $0 \%$ & 0.0091 & 0.0093 & -- & -- & 0.0046 \\
\hline SVOC & Phenanthrene & $\mathrm{mg} / \mathrm{kg}$ & 2 & 0 & $0 \%$ & 0.0091 & 0.0093 & -- & -- & 0.0046 \\
\hline SVOC & Pyrene & $\mathrm{mg} / \mathrm{kg}$ & 2 & 0 & $0 \%$ & 0.018 & 0.019 & -- & -- & 0.0093 \\
\hline VOC & Retene & $\mathrm{mg} / \mathrm{kg}$ & 2 & 0 & $0 \%$ & 0.018 & 0.019 & -- & -- & 0.0093 \\
\hline
\end{tabular}

DIOXI - Dioxins and Furans

PEST - Pesticide

PCB - Polychlorinated biphenyl

SVOC - Semivolatile Organic Compound

VOC - Volatile Organic Compound 
Table F-78

Summary Statistics for Whole Body Channel Catfish Collected from Segment 4 of the Columbia River

\begin{tabular}{|c|c|c|c|c|c|c|c|c|c|c|}
\hline $\begin{array}{l}\text { Constituent } \\
\text { Class }\end{array}$ & Constituent Name & Units & $\begin{array}{c}\text { Number } \\
\text { Analyzed }\end{array}$ & $\begin{array}{l}\text { Number } \\
\text { Detected }\end{array}$ & $\begin{array}{c}\text { Frequency of } \\
\text { Detection }\end{array}$ & $\begin{array}{l}\text { Minimum } \\
\text { Nondetect }\end{array}$ & $\begin{array}{l}\text { Maximum } \\
\text { Nondetect }\end{array}$ & $\begin{array}{l}\text { Minimum } \\
\text { Detect }\end{array}$ & $\begin{array}{l}\text { Maximum } \\
\text { Detect }\end{array}$ & $\begin{array}{c}\text { Average } \\
\text { Concentration }\end{array}$ \\
\hline$\overline{D I O X I}$ & $1,2,3,4,6,7,8$-Heptachlorodibenzodioxin & $\mathrm{mg} / \mathrm{kg}$ & 3 & 3 & $100 \%$ & -- & $\overline{--}$ & $5.90 \mathrm{E}-07$ & $7.30 \mathrm{E}-07$ & $6.47 \mathrm{E}-07$ \\
\hline DIOXI & 1,2,3,4,6,7,8-Heptachlorodibenzofuran & $\mathrm{mg} / \mathrm{kg}$ & 3 & 0 & $0 \%$ & $1.00 \mathrm{E}-08$ & $3.00 \mathrm{E}-08$ & -- & -- & $1.00 \mathrm{E}-08$ \\
\hline DIOXI & 1,2,3,4,7,8,9-Heptachlorodibenzofuran & $\mathrm{mg} / \mathrm{kg}$ & 3 & 0 & $0 \%$ & 1.00E-08 & 3.00E-08 & -- & -- & $1.00 \mathrm{E}-08$ \\
\hline DIOXI & 1,2,3,4,7,8-Hexachlorodibenzofuran & $\mathrm{mg} / \mathrm{kg}$ & 3 & 3 & $100 \%$ & -- & -- & $3.00 \mathrm{E}-08$ & 8.00E-08 & $5.00 \mathrm{E}-08$ \\
\hline DIOXI & 1,2,3,4,7,8-Hexachlorodibenzo-p-dioxin & $\mathrm{mg} / \mathrm{kg}$ & 3 & 3 & $100 \%$ & -- & -- & $2.00 \mathrm{E}-07$ & $2.20 \mathrm{E}-07$ & 2.07E-07 \\
\hline DIOXI & 1,2,3,6,7,8-Hexachlorodibenzofuran & $\mathrm{mg} / \mathrm{kg}$ & 3 & 1 & $33 \%$ & $1.00 \mathrm{E}-08$ & $2.00 \mathrm{E}-08$ & $6.00 \mathrm{E}-08$ & $6.00 \mathrm{E}-08$ & $2.50 \mathrm{E}-08$ \\
\hline DIOXI & 1,2,3,6,7,8-Hexachlorodibenzo-p-dioxin & $\mathrm{mg} / \mathrm{kg}$ & 3 & 3 & $100 \%$ & -- & -- & $7.00 \mathrm{E}-07$ & 8.50E-07 & 7.67E-07 \\
\hline DIOXI & 1,2,3,7,8,9-Hexachlorodibenzofuran & $\mathrm{mg} / \mathrm{kg}$ & 3 & 0 & $0 \%$ & $1.00 \mathrm{E}-08$ & $2.00 \mathrm{E}-08$ & -- & -- & 8.33E-09 \\
\hline DIOXI & 1,2,3,7,8,9-Hexachlorodibenzo-p-dioxin & $\mathrm{mg} / \mathrm{kg}$ & 3 & 3 & $100 \%$ & -- & -- & $1.20 \mathrm{E}-07$ & $1.40 \mathrm{E}-07$ & $1.30 \mathrm{E}-07$ \\
\hline DIOXI & 1,2,3,7,8-Pentachlorodibenzofuran & $\mathrm{mg} / \mathrm{kg}$ & 3 & 3 & $100 \%$ & -- & -- & 1.40E-07 & $2.90 \mathrm{E}-07$ & 2.03E-07 \\
\hline DIOXI & 1,2,3,7,8-Pentachlorodibenzo-p-dioxin & $\mathrm{mg} / \mathrm{kg}$ & 3 & 3 & $100 \%$ & -- & -- & $3.70 \mathrm{E}-07$ & $5.50 \mathrm{E}-07$ & 4.70E-07 \\
\hline DIOXI & 2,3,4,6,7,8-Hexachlorodibenzofuran & $\mathrm{mg} / \mathrm{kg}$ & 3 & 2 & $67 \%$ & $1.00 \mathrm{E}-08$ & $1.00 \mathrm{E}-08$ & $6.00 \mathrm{E}-08$ & $6.00 \mathrm{E}-08$ & 4.17E-08 \\
\hline DIOXI & 2,3,4,7,8-Pentachlorodibenzofuran & $\mathrm{mg} / \mathrm{kg}$ & 3 & 3 & $100 \%$ & -- & -- & $3.30 \mathrm{E}-07$ & $5.50 \mathrm{E}-07$ & 4.47E-07 \\
\hline DIOXI & 2,3,7,8-Tetrachlorodibenzofuran & $\mathrm{mg} / \mathrm{kg}$ & 3 & 3 & $100 \%$ & -- & -- & 1.79E-06 & $3.06 \mathrm{E}-06$ & 2.46E-06 \\
\hline DIOXI & 2,3,7,8-Tetrachlorodibenzo-p-dioxin & $\mathrm{mg} / \mathrm{kg}$ & 3 & 3 & $100 \%$ & -- & -- & $6.60 \mathrm{E}-07$ & 1.09E-06 & 8.73E-07 \\
\hline DIOXI & Octachlorodibenzofuran & $\mathrm{mg} / \mathrm{kg}$ & 3 & 2 & $67 \%$ & $2.00 \mathrm{E}-08$ & $2.00 \mathrm{E}-08$ & 1.00E-08 & 4.00E-08 & $2.00 \mathrm{E}-08$ \\
\hline DIOXI & Octachlorodibenzo-p-dioxin & $\mathrm{mg} / \mathrm{kg}$ & 3 & 3 & $100 \%$ & -- & -- & $5.70 \mathrm{E}-07$ & $8.60 \mathrm{E}-07$ & $7.40 \mathrm{E}-07$ \\
\hline METAL & Aluminum & $\mathrm{mg} / \mathrm{kg}$ & 3 & 3 & $100 \%$ & -- & -- & 1.7 & 9.0 & 4.3 \\
\hline METAL & Antimony & $\mathrm{mg} / \mathrm{kg}$ & 3 & 0 & $0 \%$ & 0.050 & 0.050 & -- & -- & 0.025 \\
\hline METAL & Arsenic & $\mathrm{mg} / \mathrm{kg}$ & 3 & 3 & $100 \%$ & -- & -- & 0.27 & 0.43 & 0.33 \\
\hline METAL & Barium & $\mathrm{mg} / \mathrm{kg}$ & 3 & 3 & $100 \%$ & -- & -- & 0.49 & 0.58 & 0.55 \\
\hline METAL & Beryllium & $\mathrm{mg} / \mathrm{kg}$ & 3 & 0 & $0 \%$ & 0.0040 & 0.0040 & -- & -- & 0.0020 \\
\hline METAL & Cadmium & $\mathrm{mg} / \mathrm{kg}$ & 3 & 3 & $100 \%$ & -- & -- & 0.010 & 0.010 & 0.010 \\
\hline METAL & Chromium & $\mathrm{mg} / \mathrm{kg}$ & 3 & 3 & $100 \%$ & -- & -- & 0.21 & 0.25 & 0.23 \\
\hline METAL & Cobalt & $\mathrm{mg} / \mathrm{kg}$ & 3 & 3 & $100 \%$ & -- & -- & 0.11 & 0.19 & 0.16 \\
\hline METAL & Copper & $\mathrm{mg} / \mathrm{kg}$ & 3 & 3 & $100 \%$ & -- & -- & 0.41 & 0.58 & 0.50 \\
\hline METAL & Lead & $\mathrm{mg} / \mathrm{kg}$ & 3 & 3 & $100 \%$ & -- & -- & 0.010 & 0.020 & 0.013 \\
\hline METAL & Manganese & $\mathrm{mg} / \mathrm{kg}$ & 3 & 3 & $100 \%$ & -- & -- & 1.2 & 1.8 & 1.6 \\
\hline METAL & Mercury & $\mathrm{mg} / \mathrm{kg}$ & 3 & 3 & $100 \%$ & -- & -- & 0.17 & 0.31 & 0.22 \\
\hline METAL & Nickel & $\mathrm{mg} / \mathrm{kg}$ & 3 & 3 & $100 \%$ & -- & -- & 0.090 & 0.090 & 0.090 \\
\hline METAL & Selenium & $\mathrm{mg} / \mathrm{kg}$ & 3 & 3 & $100 \%$ & -- & -- & 0.51 & 0.62 & 0.58 \\
\hline METAL & Silver & $\mathrm{mg} / \mathrm{kg}$ & 3 & 0 & $0 \%$ & 0.10 & 0.10 & -- & -- & 0.050 \\
\hline METAL & Thallium & $\mathrm{mg} / \mathrm{kg}$ & 3 & 0 & $0 \%$ & 0.050 & 0.050 & -- & -- & 0.025 \\
\hline METAL & Vanadium & $\mathrm{mg} / \mathrm{kg}$ & 3 & 3 & $100 \%$ & -- & -- & 0.040 & 0.090 & 0.067 \\
\hline METAL & Zinc & $\mathrm{mg} / \mathrm{kg}$ & 3 & 3 & $100 \%$ & -- & -- & 17 & 19 & 18 \\
\hline PCB & 2,2',3,3',4,4',5-Heptachloro-1,1'-biphenyl & $\mathrm{mg} / \mathrm{kg}$ & 3 & 3 & $100 \%$ & -- & -- & 9.97E-04 & 0.0030 & 0.0020 \\
\hline РСВ & 2,2',3,4,4',5,5'-Heptachlorobiphenyl & $\mathrm{mg} / \mathrm{kg}$ & 3 & 3 & $100 \%$ & -- & -- & 0.0032 & 0.0092 & 0.0062 \\
\hline PCB & 2,3,3',4,4',5'-Hexachlorobiphenyl & $\mathrm{mg} / \mathrm{kg}$ & 3 & 3 & $100 \%$ & -- & -- & $9.88 \mathrm{E}-05$ & 2.81E-04 & 1.95E-04 \\
\hline РСВ & 2,3,3',4,4',5-Hexachlorobiphenyl (BZ 156) & $\mathrm{mg} / \mathrm{kg}$ & 3 & 3 & $100 \%$ & -- & -- & $5.21 \mathrm{E}-04$ & 0.0015 & 0.0010 \\
\hline PCB & 2,3,3',4,4'-Pentachlorobiphenyl & $\mathrm{mg} / \mathrm{kg}$ & 3 & 3 & $100 \%$ & -- & -- & 0.0013 & 0.0033 & 0.0023 \\
\hline РСВ & 2,3',4,4',5,5'-Hexachlorobiphenyl (BZ 167) & $\mathrm{mg} / \mathrm{kg}$ & 3 & 3 & $100 \%$ & -- & -- & 2.23E-04 & $5.64 \mathrm{E}-04$ & 3.97E-04 \\
\hline PCB & 2',3,4,4',5-Pentachloro-1,1'-biphenyl (BZ 123) & $\mathrm{mg} / \mathrm{kg}$ & 3 & 3 & $100 \%$ & -- & -- & 5.87E-05 & $1.51 \mathrm{E}-04$ & 1.07E-04 \\
\hline РCB & 2,3,4,4',5-Pentachlorobiphenyl (BZ 114) & $\mathrm{mg} / \mathrm{kg}$ & 3 & 3 & $100 \%$ & -- & -- & 1.19E-04 & $3.28 \mathrm{E}-04$ & 2.27E-04 \\
\hline PCB & 2,3',4,4',5-Pentachlorobiphenyl (BZ 118) & $\mathrm{mg} / \mathrm{kg}$ & 3 & 3 & $100 \%$ & -- & -- & 0.0029 & 0.0071 & 0.0049 \\
\hline РСВ & 2,3,4,5,3',4',5'-Heptachlorobiphenyl & $\mathrm{mg} / \mathrm{kg}$ & 3 & 3 & $100 \%$ & -- & -- & 3.30E-05 & 1.01E-04 & 6.71E-05 \\
\hline PCB & 3,3',4,4',5,5'-Hexachlorobiphenyl (BZ 169) & $\mathrm{mg} / \mathrm{kg}$ & 3 & 0 & $0 \%$ & $1.82 \mathrm{E}-06$ & 2.69E-06 & -- & -- & 1.12E-06 \\
\hline РСВ & 3,3',4,4',5-Pentachlorobiphenyl (BZ 126) & $\mathrm{mg} / \mathrm{kg}$ & 3 & 2 & $67 \%$ & 1.74E-06 & 1.74E-06 & $6.80 \mathrm{E}-06$ & 3.01E-05 & $1.26 \mathrm{E}-05$ \\
\hline PCB & 3,3',4,4'-Tetrachlorobiphenyl (BZ 77) & $\mathrm{mg} / \mathrm{kg}$ & 3 & 3 & $100 \%$ & -- & -- & 2.21E-05 & 3.67E-05 & $2.85 \mathrm{E}-05$ \\
\hline РСВ & Aroclor-1016 & $\mathrm{mg} / \mathrm{kg}$ & 3 & 0 & $0 \%$ & 0.016 & 0.020 & -- & -- & 0.0088 \\
\hline PCB & Aroclor-1221 & $\mathrm{mg} / \mathrm{kg}$ & 3 & 0 & $0 \%$ & 0.016 & 0.020 & -- & -- & 0.0088 \\
\hline
\end{tabular}


Table F-78

Summary Statistics for Whole Body Channel Catfish Collected from Segment 4 of the Columbia River

\begin{tabular}{|c|c|c|c|c|c|c|c|c|c|c|}
\hline $\begin{array}{c}\text { Constituent } \\
\text { Class }\end{array}$ & Constituent Name & Units & $\begin{array}{c}\text { Number } \\
\text { Analyzed }\end{array}$ & $\begin{array}{l}\text { Number } \\
\text { Detected }\end{array}$ & $\begin{array}{c}\text { Frequency of } \\
\text { Detection }\end{array}$ & $\begin{array}{l}\text { Minimum } \\
\text { Nondetect }\end{array}$ & $\begin{array}{l}\text { Maximum } \\
\text { Nondetect }\end{array}$ & $\begin{array}{c}\text { Minimum } \\
\text { Detect }\end{array}$ & $\begin{array}{c}\text { Maximum } \\
\text { Detect }\end{array}$ & $\begin{array}{c}\text { Average } \\
\text { Concentration }\end{array}$ \\
\hline PCB & Aroclor-1232 & $\mathrm{mg} / \mathrm{kg}$ & 3 & 0 & $0 \%$ & 0.016 & 0.020 & -- & -- & 0.0088 \\
\hline PCB & Aroclor-1248 & $\mathrm{mg} / \mathrm{kg}$ & 3 & 0 & $0 \%$ & 0.016 & 0.020 & -- & -- & 0.0088 \\
\hline PCB & Aroclor-1254 & $\mathrm{mg} / \mathrm{kg}$ & 3 & 3 & $100 \%$ & -- & -- & 0.025 & 0.041 & 0.033 \\
\hline PCB & Aroclor-1260 & $\mathrm{mg} / \mathrm{kg}$ & 3 & 3 & $100 \%$ & -- & -- & 0.032 & 0.077 & 0.052 \\
\hline PEST & 2,4'-DDD & $\mathrm{mg} / \mathrm{kg}$ & 3 & 3 & $100 \%$ & -- & -- & 0.0052 & 0.0076 & 0.0064 \\
\hline PEST & 2,4'-DDE & $\mathrm{mg} / \mathrm{kg}$ & 3 & 3 & $100 \%$ & -- & -- & 0.0038 & 0.0081 & 0.0061 \\
\hline PEST & 2,4'-DDT & $\mathrm{mg} / \mathrm{kg}$ & 3 & 0 & $0 \%$ & 0.0016 & 0.0020 & -- & -- & 8.83E-04 \\
\hline PEST & Aldrin & $\mathrm{mg} / \mathrm{kg}$ & 3 & 0 & $0 \%$ & 0.0016 & 0.0020 & -- & -- & 8.83E-04 \\
\hline PEST & Alpha-BHC & $\mathrm{mg} / \mathrm{kg}$ & 3 & 0 & $0 \%$ & 0.0016 & 0.0020 & -- & -- & 8.83E-04 \\
\hline PEST & alpha-Chlordane & $\mathrm{mg} / \mathrm{kg}$ & 3 & 1 & $33 \%$ & 0.0016 & 0.0020 & $9.40 \mathrm{E}-04$ & $9.40 \mathrm{E}-04$ & $9.13 \mathrm{E}-04$ \\
\hline PEST & alpha-Chlordene & $\mathrm{mg} / \mathrm{kg}$ & 3 & 0 & $0 \%$ & 0.0016 & 0.0020 & -- & -- & 8.83E-04 \\
\hline PEST & Beta-BHC & $\mathrm{mg} / \mathrm{kg}$ & 3 & 0 & $0 \%$ & 0.0016 & 0.0020 & -- & -- & 8.83E-04 \\
\hline PEST & cis-Nonachlor & $\mathrm{mg} / \mathrm{kg}$ & 3 & 3 & $100 \%$ & -- & -- & 0.0013 & 0.0032 & 0.0025 \\
\hline PEST & DDMU & $\mathrm{mg} / \mathrm{kg}$ & 3 & 3 & $100 \%$ & -- & -- & 0.0053 & 0.010 & 0.0077 \\
\hline PEST & Delta-BHC & $\mathrm{mg} / \mathrm{kg}$ & 3 & 0 & $0 \%$ & 0.0016 & 0.0020 & -- & -- & 8.83E-04 \\
\hline PEST & Dichlorodiphenyldichloroethane & $\mathrm{mg} / \mathrm{kg}$ & 3 & 3 & $100 \%$ & -- & -- & 0.037 & 0.066 & 0.051 \\
\hline PEST & Dichlorodiphenyldichloroethylene & $\mathrm{mg} / \mathrm{kg}$ & 3 & 3 & $100 \%$ & -- & -- & 0.28 & 0.53 & 0.41 \\
\hline PEST & Dichlorodiphenyltrichloroethane & $\mathrm{mg} / \mathrm{kg}$ & 3 & 3 & $100 \%$ & -- & -- & 8.20E-04 & 0.0032 & 0.0019 \\
\hline PEST & Endosulfan sulfate & $\mathrm{mg} / \mathrm{kg}$ & 3 & 0 & $0 \%$ & 0.0016 & 0.0020 & -- & -- & 8.83E-04 \\
\hline PEST & Gamma-BHC (Lindane) & $\mathrm{mg} / \mathrm{kg}$ & 3 & 0 & $0 \%$ & 0.0016 & 0.0020 & -- & -- & 8.83E-04 \\
\hline PEST & gamma-Chlordane & $\mathrm{mg} / \mathrm{kg}$ & 3 & 3 & $100 \%$ & -- & -- & 0.0015 & 0.0029 & 0.0022 \\
\hline PEST & gamma-Chlordene & $\mathrm{mg} / \mathrm{kg}$ & 3 & 0 & $0 \%$ & 0.0016 & 0.0020 & -- & -- & 8.83E-04 \\
\hline PEST & Heptachlor & $\mathrm{mg} / \mathrm{kg}$ & 3 & 0 & $0 \%$ & 0.0016 & 0.0020 & -- & -- & 8.83E-04 \\
\hline PEST & Heptachlor epoxide & $\mathrm{mg} / \mathrm{kg}$ & 3 & 0 & $0 \%$ & 0.0016 & 0.0020 & -- & -- & 8.83E-04 \\
\hline PEST & Mirex & $\mathrm{mg} / \mathrm{kg}$ & 3 & 3 & $100 \%$ & -- & -- & 0.0028 & 0.0076 & 0.0056 \\
\hline PEST & Oxychlordane & $\mathrm{mg} / \mathrm{kg}$ & 3 & 2 & $67 \%$ & 0.0016 & 0.0016 & 0.0021 & 0.0027 & 0.0019 \\
\hline PEST & Pentachloroanisole & $\mathrm{mg} / \mathrm{kg}$ & 3 & 0 & $0 \%$ & 0.0016 & 0.0020 & -- & -- & 8.83E-04 \\
\hline PEST & Toxaphene & $\mathrm{mg} / \mathrm{kg}$ & 3 & 0 & $0 \%$ & 0.049 & 0.060 & -- & -- & 0.027 \\
\hline PEST & trans-Nonachlor & $\mathrm{mg} / \mathrm{kg}$ & 3 & 3 & $100 \%$ & -- & -- & 0.0043 & 0.0087 & 0.0065 \\
\hline SVOC & 1,2,4-Trichlorobenzene & $\mathrm{mg} / \mathrm{kg}$ & 3 & 0 & $0 \%$ & 0.0094 & 0.0098 & -- & -- & 0.0048 \\
\hline SVOC & 1,2-Dichlorobenzene & $\mathrm{mg} / \mathrm{kg}$ & 3 & 0 & $0 \%$ & 0.0094 & 0.0098 & -- & -- & 0.0048 \\
\hline SVOC & 1,2-Diphenylhydrazine & $\mathrm{mg} / \mathrm{kg}$ & 3 & 0 & $0 \%$ & 0.0094 & 0.0098 & -- & -- & 0.0048 \\
\hline SVOC & 1,3-Dichlorobenzene & $\mathrm{mg} / \mathrm{kg}$ & 3 & 0 & $0 \%$ & 0.0094 & 0.0098 & -- & -- & 0.0048 \\
\hline SVOC & 1,4-Dichlorobenzene & $\mathrm{mg} / \mathrm{kg}$ & 3 & 0 & $0 \%$ & 0.0094 & 0.0098 & -- & -- & 0.0048 \\
\hline SVOC & 1-methylnaphthalene & $\mathrm{mg} / \mathrm{kg}$ & 3 & 0 & $0 \%$ & 0.0094 & 0.0098 & -- & -- & 0.0048 \\
\hline SVOC & 2,4-Dinitrotoluene & $\mathrm{mg} / \mathrm{kg}$ & 3 & 0 & $0 \%$ & 0.019 & 0.020 & -- & -- & 0.0097 \\
\hline SVOC & 2,6-Dinitrotoluene & $\mathrm{mg} / \mathrm{kg}$ & 3 & 0 & $0 \%$ & 0.038 & 0.039 & -- & -- & 0.019 \\
\hline SVOC & 2-Chloronaphthalene & $\mathrm{mg} / \mathrm{kg}$ & 3 & 0 & $0 \%$ & 0.019 & 0.020 & -- & -- & 0.0097 \\
\hline SVOC & 2-MethyInaphthalene & $\mathrm{mg} / \mathrm{kg}$ & 3 & 0 & $0 \%$ & 0.0094 & 0.0098 & -- & -- & 0.0048 \\
\hline SVOC & 4-Bromophenylphenyl ether & $\mathrm{mg} / \mathrm{kg}$ & 3 & 0 & $0 \%$ & 0.0094 & 0.0098 & -- & -- & 0.0048 \\
\hline SVOC & 4-Chlorophenylphenyl ether & $\mathrm{mg} / \mathrm{kg}$ & 3 & 0 & $0 \%$ & 0.0094 & 0.0098 & -- & -- & 0.0048 \\
\hline SVOC & Acenaphthene & $\mathrm{mg} / \mathrm{kg}$ & 3 & 0 & $0 \%$ & 0.019 & 0.020 & -- & -- & 0.0097 \\
\hline SVOC & Acenaphthylene & $\mathrm{mg} / \mathrm{kg}$ & 3 & 0 & $0 \%$ & 0.0094 & 0.0098 & -- & -- & 0.0048 \\
\hline SVOC & Anthracene & $\mathrm{mg} / \mathrm{kg}$ & 3 & 0 & $0 \%$ & 0.0094 & 0.0098 & -- & -- & 0.0048 \\
\hline SVOC & Benzo(a)anthracene & $\mathrm{mg} / \mathrm{kg}$ & 3 & 0 & $0 \%$ & 0.0094 & 0.0098 & -- & -- & 0.0048 \\
\hline SVOC & Benzo(a)pyrene & $\mathrm{mg} / \mathrm{kg}$ & 3 & 0 & $0 \%$ & 0.0094 & 0.049 & -- & -- & 0.011 \\
\hline SVOC & Benzo(b)fluoranthene & $\mathrm{mg} / \mathrm{kg}$ & 3 & 0 & $0 \%$ & 0.0094 & 0.049 & -- & -- & 0.011 \\
\hline SVOC & Benzo(ghi)perylene & $\mathrm{mg} / \mathrm{kg}$ & 3 & 1 & $33 \%$ & 0.019 & 0.098 & 0.015 & 0.015 & 0.025 \\
\hline SVOC & Benzo(k)fluoranthene & $\mathrm{mg} / \mathrm{kg}$ & 3 & 0 & $0 \%$ & 0.0094 & 0.049 & -- & -- & 0.011 \\
\hline SVOC & Bis(2-chloro-1-methylethyl)ether & $\mathrm{mg} / \mathrm{kg}$ & 3 & 0 & $0 \%$ & 0.019 & 0.020 & -- & -- & 0.0097 \\
\hline
\end{tabular}


Table F-78

Summary Statistics for Whole Body Channel Catfish Collected from Segment 4 of the Columbia River

\begin{tabular}{|c|c|c|c|c|c|c|c|c|c|c|}
\hline $\begin{array}{l}\text { Constituent } \\
\text { Class }\end{array}$ & Constituent Name & Units & $\begin{array}{l}\text { Number } \\
\text { Analyzed }\end{array}$ & $\begin{array}{l}\text { Number } \\
\text { Detected }\end{array}$ & $\begin{array}{l}\text { Frequency of } \\
\text { Detection }\end{array}$ & $\begin{array}{l}\text { Minimum } \\
\text { Nondetect }\end{array}$ & $\begin{array}{c}\text { Maximum } \\
\text { Nondetect }\end{array}$ & $\begin{array}{c}\text { Minimum } \\
\text { Detect }\end{array}$ & $\begin{array}{l}\text { Maximum } \\
\text { Detect }\end{array}$ & $\begin{array}{c}\text { Average } \\
\text { Concentration }\end{array}$ \\
\hline$\overline{\text { SVOC }}$ & Chrysene & $\mathrm{mg} / \mathrm{kg}$ & 3 & 0 & $0 \%$ & 0.0094 & 0.0098 & $\overline{--}$ & $\overline{--}$ & 0.0048 \\
\hline SVOC & Dibenz[a,h]anthracene & $\mathrm{mg} / \mathrm{kg}$ & 3 & 0 & $0 \%$ & 0.019 & 0.098 & -- & -- & 0.023 \\
\hline SVOC & Dibenzofuran & $\mathrm{mg} / \mathrm{kg}$ & 3 & 0 & $0 \%$ & 0.0094 & 0.0098 & -- & -- & 0.0048 \\
\hline SVOC & Fluoranthene & $\mathrm{mg} / \mathrm{kg}$ & 3 & 0 & $0 \%$ & 0.019 & 0.020 & -- & -- & 0.0097 \\
\hline SVOC & Fluorene & $\mathrm{mg} / \mathrm{kg}$ & 3 & 0 & $0 \%$ & 0.0095 & 0.17 & -- & -- & 0.054 \\
\hline SVOC & Hexachlorobenzene & $\mathrm{mg} / \mathrm{kg}$ & 3 & 0 & $0 \%$ & 0.019 & 0.020 & -- & -- & 0.0097 \\
\hline SVOC & Hexachlorobutadiene & $\mathrm{mg} / \mathrm{kg}$ & 3 & 0 & $0 \%$ & 0.0094 & 0.0098 & -- & -- & 0.0048 \\
\hline SVOC & Hexachloroethane & $\mathrm{mg} / \mathrm{kg}$ & 3 & 0 & $0 \%$ & 0.0094 & 0.0098 & -- & -- & 0.0048 \\
\hline SVOC & Indeno(1,2,3-cd)pyrene & $\mathrm{mg} / \mathrm{kg}$ & 3 & 0 & $0 \%$ & 0.019 & 0.098 & -- & -- & 0.023 \\
\hline SVOC & Naphthalene & $\mathrm{mg} / \mathrm{kg}$ & 3 & 0 & $0 \%$ & 0.020 & 0.024 & -- & -- & 0.011 \\
\hline SVOC & Nitrobenzene & $\mathrm{mg} / \mathrm{kg}$ & 3 & 0 & $0 \%$ & 0.0094 & 0.0098 & -- & -- & 0.0048 \\
\hline SVOC & Phenanthrene & $\mathrm{mg} / \mathrm{kg}$ & 3 & 0 & $0 \%$ & 0.0094 & 0.0098 & -- & -- & 0.0048 \\
\hline SVOC & Pyrene & $\mathrm{mg} / \mathrm{kg}$ & 3 & 0 & $0 \%$ & 0.019 & 0.020 & -- & -- & 0.0097 \\
\hline VOC & Retene & $\mathrm{mg} / \mathrm{kg}$ & 3 & 0 & $0 \%$ & 0.019 & 0.020 & -- & -- & 0.0097 \\
\hline
\end{tabular}

DIOXI - Dioxins and Furans

PEST - Pesticide

PCB - Polychlorinated bipheny

SVOC - Semivolatile Organic Compound

VOC - Volatile Organic Compound 
Table F-79

Summary Statistics for Largescale Sucker Fillet Skin On Collected from Segment 4 of the Columbia River

\begin{tabular}{|c|c|c|c|c|c|c|c|c|c|c|}
\hline $\begin{array}{l}\text { Constituent } \\
\text { Class }\end{array}$ & Constituent Name & Units & $\begin{array}{l}\text { Number } \\
\text { Analyzed }\end{array}$ & $\begin{array}{l}\text { Number } \\
\text { Detected }\end{array}$ & $\begin{array}{c}\text { Frequency of } \\
\text { Detection }\end{array}$ & $\begin{array}{l}\text { Minimum } \\
\text { Nondetect }\end{array}$ & $\begin{array}{l}\text { Maximum } \\
\text { Nondetect }\end{array}$ & $\begin{array}{l}\text { Minimum } \\
\text { Detect }\end{array}$ & $\begin{array}{l}\text { Maximum } \\
\text { Detect }\end{array}$ & $\begin{array}{c}\text { Average } \\
\text { Concentration }\end{array}$ \\
\hline$\overline{D I O X I}$ & 1,2,3,4,6,7,8-Heptachlorodibenzodioxin & $\mathrm{mg} / \mathrm{kg}$ & 7 & 6 & $86 \%$ & $1.10 \mathrm{E}-07$ & $1.10 \mathrm{E}-07$ & $1.10 \mathrm{E}-07$ & $2.00 \mathrm{E}-07$ & $1.32 \mathrm{E}-07$ \\
\hline DIOXI & 1,2,3,4,6,7,8-Heptachlorodibenzofuran & $\mathrm{mg} / \mathrm{kg}$ & 7 & 5 & $71 \%$ & $1.00 \mathrm{E}-08$ & 1.30E-07 & $7.00 \mathrm{E}-08$ & $1.60 \mathrm{E}-07$ & $8.29 \mathrm{E}-08$ \\
\hline DIOXI & 1,2,3,4,7,8,9-Heptachlorodibenzofuran & $\mathrm{mg} / \mathrm{kg}$ & 7 & 2 & $29 \%$ & 1.00E-08 & 1.30E-07 & 4.00E-08 & 1.30E-07 & 4.07E-08 \\
\hline DIOXI & 1,2,3,4,7,8-Hexachlorodibenzofuran & $\mathrm{mg} / \mathrm{kg}$ & 7 & 3 & $43 \%$ & $1.00 \mathrm{E}-08$ & 8.00E-08 & 3.00E-08 & 8.00E-08 & $3.29 \mathrm{E}-08$ \\
\hline DIOXI & 1,2,3,4,7,8-Hexachlorodibenzo-p-dioxin & $\mathrm{mg} / \mathrm{kg}$ & 7 & 5 & $71 \%$ & 8.00E-08 & 1.10E-07 & $9.00 \mathrm{E}-08$ & $1.50 \mathrm{E}-07$ & $9.50 \mathrm{E}-08$ \\
\hline DIOXI & 1,2,3,6,7,8-Hexachlorodibenzofuran & $\mathrm{mg} / \mathrm{kg}$ & 7 & 4 & $57 \%$ & $1.00 \mathrm{E}-08$ & 1.00E-07 & 3.00E-08 & 8.00E-08 & 4.07E-08 \\
\hline DIOXI & 1,2,3,6,7,8-Hexachlorodibenzo-p-dioxin & $\mathrm{mg} / \mathrm{kg}$ & 7 & 3 & $43 \%$ & $1.00 \mathrm{E}-08$ & 1.10E-07 & $6.00 \mathrm{E}-08$ & 1.10E-07 & $5.21 \mathrm{E}-08$ \\
\hline DIOXI & 1,2,3,7,8,9-Hexachlorodibenzofuran & $\mathrm{mg} / \mathrm{kg}$ & 7 & 5 & $71 \%$ & $1.00 \mathrm{E}-08$ & 8.00E-08 & 8.00E-08 & 1.70E-07 & 8.64E-08 \\
\hline DIOXI & 1,2,3,7,8,9-Hexachlorodibenzo-p-dioxin & $\mathrm{mg} / \mathrm{kg}$ & 7 & 3 & $43 \%$ & $1.00 \mathrm{E}-08$ & 1.10E-07 & 5.00E-08 & 8.00E-08 & 4.36E-08 \\
\hline DIOXI & 1,2,3,7,8-Pentachlorodibenzofuran & $\mathrm{mg} / \mathrm{kg}$ & 7 & 4 & $57 \%$ & $1.00 \mathrm{E}-08$ & 4.00E-08 & $3.00 \mathrm{E}-08$ & $6.00 \mathrm{E}-08$ & $3.00 \mathrm{E}-08$ \\
\hline DIOXI & 1,2,3,7,8-Pentachlorodibenzo-p-dioxin & $\mathrm{mg} / \mathrm{kg}$ & 7 & 5 & $71 \%$ & $3.00 \mathrm{E}-08$ & 4.00E-08 & 1.10E-07 & 1.60E-07 & 1.01E-07 \\
\hline DIOXI & 2,3,4,6,7,8-Hexachlorodibenzofuran & $\mathrm{mg} / \mathrm{kg}$ & 7 & 5 & $71 \%$ & $1.00 \mathrm{E}-08$ & 8.00E-08 & 1.40E-07 & $2.10 \mathrm{E}-07$ & $1.22 \mathrm{E}-07$ \\
\hline DIOXI & 2,3,4,7,8-Pentachlorodibenzofuran & $\mathrm{mg} / \mathrm{kg}$ & 7 & 4 & $57 \%$ & $1.00 \mathrm{E}-08$ & 4.00E-08 & $3.00 \mathrm{E}-08$ & 7.00E-08 & $2.86 \mathrm{E}-08$ \\
\hline DIOXI & 2,3,7,8-Tetrachlorodibenzofuran & $\mathrm{mg} / \mathrm{kg}$ & 7 & 7 & $100 \%$ & -- & -- & $1.50 \mathrm{E}-07$ & $5.80 \mathrm{E}-07$ & 2.67E-07 \\
\hline DIOXI & 2,3,7,8-Tetrachlorodibenzo-p-dioxin & $\mathrm{mg} / \mathrm{kg}$ & 7 & 4 & $57 \%$ & $1.00 \mathrm{E}-08$ & $6.00 \mathrm{E}-08$ & 1.00E-08 & 7.00E-08 & 3.71E-08 \\
\hline DIOXI & Octachlorodibenzofuran & $\mathrm{mg} / \mathrm{kg}$ & 7 & 5 & $71 \%$ & 8.00E-08 & $1.20 \mathrm{E}-07$ & $2.00 \mathrm{E}-08$ & $2.20 \mathrm{E}-07$ & 8.00E-08 \\
\hline DIOXI & Octachlorodibenzo-p-dioxin & $\mathrm{mg} / \mathrm{kg}$ & 7 & 5 & $71 \%$ & $1.50 \mathrm{E}-07$ & $1.90 \mathrm{E}-07$ & 1.00E-08 & 3.10E-07 & 1.53E-07 \\
\hline METAL & Aluminum & $\mathrm{mg} / \mathrm{kg}$ & 7 & 5 & $71 \%$ & 5.0 & 5.0 & 1.0 & 4.6 & 2.5 \\
\hline METAL & Antimony & $\mathrm{mg} / \mathrm{kg}$ & 7 & 0 & $0 \%$ & 0.050 & 0.050 & -- & -- & 0.025 \\
\hline METAL & Arsenic & $\mathrm{mg} / \mathrm{kg}$ & 7 & 7 & $100 \%$ & -- & -- & 0.050 & 0.10 & 0.081 \\
\hline METAL & Barium & $\mathrm{mg} / \mathrm{kg}$ & 7 & 7 & $100 \%$ & -- & -- & 0.46 & 1.1 & 0.67 \\
\hline METAL & Beryllium & $\mathrm{mg} / \mathrm{kg}$ & 7 & 2 & $29 \%$ & 0.0040 & 0.0060 & 0.0044 & 0.0045 & 0.0028 \\
\hline METAL & Cadmium & $\mathrm{mg} / \mathrm{kg}$ & 7 & 2 & $29 \%$ & 0.0040 & 0.0040 & 0.0040 & 0.0057 & 0.0028 \\
\hline METAL & Chromium & $\mathrm{mg} / \mathrm{kg}$ & 7 & 1 & $14 \%$ & 0.10 & 0.10 & 0.13 & 0.13 & 0.061 \\
\hline METAL & Cobalt & $\mathrm{mg} / \mathrm{kg}$ & 7 & 7 & $100 \%$ & -- & -- & 0.010 & 0.040 & 0.019 \\
\hline METAL & Copper & $\mathrm{mg} / \mathrm{kg}$ & 7 & 7 & $100 \%$ & -- & -- & 0.48 & 0.81 & 0.59 \\
\hline METAL & Lead & $\mathrm{mg} / \mathrm{kg}$ & 7 & 5 & $71 \%$ & 0.010 & 0.010 & 0.010 & 0.040 & 0.014 \\
\hline METAL & Manganese & $\mathrm{mg} / \mathrm{kg}$ & 7 & 7 & $100 \%$ & -- & -- & 1.5 & 2.5 & 2.0 \\
\hline METAL & Mercury & $\mathrm{mg} / \mathrm{kg}$ & 7 & 7 & $100 \%$ & -- & -- & 0.070 & 0.28 & 0.19 \\
\hline METAL & Nickel & $\mathrm{mg} / \mathrm{kg}$ & 7 & 7 & $100 \%$ & -- & -- & 0.050 & 0.16 & 0.097 \\
\hline METAL & Selenium & $\mathrm{mg} / \mathrm{kg}$ & 7 & 7 & $100 \%$ & -- & -- & 0.20 & 0.31 & 0.25 \\
\hline METAL & Silver & $\mathrm{mg} / \mathrm{kg}$ & 7 & 0 & $0 \%$ & 0.10 & 0.20 & -- & -- & 0.057 \\
\hline METAL & Thallium & $\mathrm{mg} / \mathrm{kg}$ & 7 & 0 & $0 \%$ & 0.050 & 0.050 & -- & -- & 0.025 \\
\hline METAL & Vanadium & $\mathrm{mg} / \mathrm{kg}$ & 7 & 5 & $71 \%$ & 0.020 & 0.020 & 0.010 & 0.020 & 0.011 \\
\hline METAL & Zinc & $\mathrm{mg} / \mathrm{kg}$ & 7 & 7 & $100 \%$ & -- & -- & 16 & 21 & 18 \\
\hline PCB & 2,2',3,3',4,4',5-Heptachloro-1,1'-biphenyl & $\mathrm{mg} / \mathrm{kg}$ & 7 & 7 & $100 \%$ & -- & -- & $1.81 \mathrm{E}-04$ & $6.82 \mathrm{E}-04$ & 3.77E-04 \\
\hline РCB & 2,2',3,4,4',5,5'-Heptachlorobiphenyl & $\mathrm{mg} / \mathrm{kg}$ & 7 & 7 & $100 \%$ & -- & -- & $5.49 \mathrm{E}-04$ & 0.0023 & 0.0012 \\
\hline PCB & 2,3,3',4,4',5'-Hexachlorobiphenyl & $\mathrm{mg} / \mathrm{kg}$ & 7 & 3 & $43 \%$ & 1.14E-06 & 2.07E-06 & 2.34E-05 & 4.31E-05 & 1.37E-05 \\
\hline РCB & 2,3,3',4,4',5-Hexachlorobiphenyl (BZ 156) & $\mathrm{mg} / \mathrm{kg}$ & 7 & 7 & $100 \%$ & -- & -- & 1.18E-04 & $3.40 \mathrm{E}-04$ & $1.89 \mathrm{E}-04$ \\
\hline РCB & $2,3,3^{\prime}, 4,4^{\prime}$-Pentachlorobiphenyl & $\mathrm{mg} / \mathrm{kg}$ & 7 & 7 & $100 \%$ & -- & -- & $2.72 \mathrm{E}-04$ & 6.07E-04 & 4.26E-04 \\
\hline РCB & 2,3',4,4',5,5'-Hexachlorobiphenyl (BZ 167) & $\mathrm{mg} / \mathrm{kg}$ & 7 & 7 & $100 \%$ & -- & -- & 5.54E-05 & 1.71E-04 & 8.98E-05 \\
\hline PCB & 2',3,4,4',5-Pentachloro-1,1'-biphenyl (BZ 123) & $\mathrm{mg} / \mathrm{kg}$ & 7 & 7 & $100 \%$ & -- & -- & 1.10E-05 & $2.58 \mathrm{E}-05$ & 1.75E-05 \\
\hline PCB & 2,3,4,4',5-Pentachlorobiphenyl (BZ 114) & $\mathrm{mg} / \mathrm{kg}$ & 7 & 7 & $100 \%$ & -- & -- & 2.44E-05 & 5.53E-05 & 3.60E-05 \\
\hline РСВ & 2,3',4,4',5-Pentachlorobiphenyl (BZ 118) & $\mathrm{mg} / \mathrm{kg}$ & 7 & 7 & $100 \%$ & -- & -- & 6.33E-04 & 0.0013 & 9.43E-04 \\
\hline PCB & 2,3,4,5,3',4',5'-Heptachlorobiphenyl & $\mathrm{mg} / \mathrm{kg}$ & 7 & 7 & $100 \%$ & -- & -- & 7.53E-06 & $2.89 \mathrm{E}-05$ & $1.58 \mathrm{E}-05$ \\
\hline PCB & 3,3',4,4',5,5'-Hexachlorobiphenyl (BZ 169) & $\mathrm{mg} / \mathrm{kg}$ & 7 & 0 & $0 \%$ & $5.00 \mathrm{E}-07$ & 1.50E-06 & -- & -- & 4.57E-07 \\
\hline РCB & 3,3',4,4',5-Pentachlorobiphenyl (BZ 126) & $\mathrm{mg} / \mathrm{kg}$ & 7 & 3 & $43 \%$ & $1.38 \mathrm{E}-06$ & 2.35E-06 & 2.32E-06 & $3.72 \mathrm{E}-06$ & $1.85 \mathrm{E}-06$ \\
\hline PCB & 3,3',4,4'-Tetrachlorobiphenyl (BZ 77) & $\mathrm{mg} / \mathrm{kg}$ & 7 & 7 & $100 \%$ & -- & -- & 1.21E-05 & 2.36E-05 & 1.73E-05 \\
\hline РСВ & Aroclor-1016 & $\mathrm{mg} / \mathrm{kg}$ & 7 & 0 & $0 \%$ & 0.018 & 0.021 & -- & -- & 0.0096 \\
\hline PCB & Aroclor-1221 & $\mathrm{mg} / \mathrm{kg}$ & 7 & 0 & $0 \%$ & 0.018 & 0.021 & -- & -- & 0.0096 \\
\hline
\end{tabular}


Table F-79

Summary Statistics for Largescale Sucker Fillet Skin On Collected from Segment 4 of the Columbia River

\begin{tabular}{|c|c|c|c|c|c|c|c|c|c|c|}
\hline $\begin{array}{l}\text { Constituent } \\
\text { Class }\end{array}$ & Constituent Name & Units & $\begin{array}{l}\text { Number } \\
\text { Analyzed }\end{array}$ & $\begin{array}{l}\text { Number } \\
\text { Detected }\end{array}$ & $\begin{array}{c}\text { Frequency of } \\
\text { Detection }\end{array}$ & $\begin{array}{l}\text { Minimum } \\
\text { Nondetect }\end{array}$ & $\begin{array}{c}\text { Maximum } \\
\text { Nondetect }\end{array}$ & $\begin{array}{l}\text { Minimum } \\
\text { Detect }\end{array}$ & $\begin{array}{l}\text { Maximum } \\
\text { Detect }\end{array}$ & $\begin{array}{c}\text { Average } \\
\text { Concentration }\end{array}$ \\
\hline$\overline{\mathrm{PCB}}$ & Aroclor-1232 & $\mathrm{mg} / \mathrm{kg}$ & 7 & 0 & $0 \%$ & 0.018 & 0.021 & $\overline{--}$ & -- & 0.0096 \\
\hline PCB & Aroclor-1248 & $\mathrm{mg} / \mathrm{kg}$ & 7 & 0 & $0 \%$ & 0.018 & 0.021 & -- & -- & 0.0096 \\
\hline PCB & Aroclor-1254 & $\mathrm{mg} / \mathrm{kg}$ & 7 & 2 & $29 \%$ & 0.018 & 0.020 & 0.010 & 0.014 & 0.010 \\
\hline PCB & Aroclor-1260 & $\mathrm{mg} / \mathrm{kg}$ & 7 & 3 & $43 \%$ & 0.019 & 0.021 & 0.011 & 0.030 & 0.013 \\
\hline PEST & 2,4'-DDD & $\mathrm{mg} / \mathrm{kg}$ & 7 & 0 & $0 \%$ & 0.0019 & 0.0024 & -- & -- & 0.0011 \\
\hline PEST & 2,4'-DDE & $\mathrm{mg} / \mathrm{kg}$ & 7 & 0 & $0 \%$ & 0.0019 & 0.0024 & -- & -- & 0.0011 \\
\hline PEST & 2,4'-DDT & $\mathrm{mg} / \mathrm{kg}$ & 7 & 0 & $0 \%$ & 0.0019 & 0.0024 & -- & -- & 0.0011 \\
\hline PEST & Aldrin & $\mathrm{mg} / \mathrm{kg}$ & 7 & 0 & $0 \%$ & 0.0019 & 0.0024 & -- & -- & 0.0011 \\
\hline PEST & Alpha-BHC & $\mathrm{mg} / \mathrm{kg}$ & 7 & 0 & $0 \%$ & 0.0019 & 0.0024 & -- & -- & 0.0011 \\
\hline PEST & alpha-Chlordane & $\mathrm{mg} / \mathrm{kg}$ & 7 & 0 & $0 \%$ & 0.0019 & 0.0024 & -- & -- & 0.0011 \\
\hline PEST & alpha-Chlordene & $\mathrm{mg} / \mathrm{kg}$ & 7 & 0 & $0 \%$ & 0.0019 & 0.0024 & -- & -- & 0.0011 \\
\hline PEST & Beta-BHC & $\mathrm{mg} / \mathrm{kg}$ & 7 & 0 & $0 \%$ & 0.0019 & 0.0024 & -- & -- & 0.0011 \\
\hline PEST & cis-Nonachlor & $\mathrm{mg} / \mathrm{kg}$ & 7 & 0 & $0 \%$ & 0.0019 & 0.0024 & -- & -- & 0.0011 \\
\hline PEST & DDMU & $\mathrm{mg} / \mathrm{kg}$ & 7 & 0 & $0 \%$ & 0.0019 & 0.0024 & -- & -- & 0.0011 \\
\hline PEST & Delta-BHC & $\mathrm{mg} / \mathrm{kg}$ & 7 & 0 & $0 \%$ & 0.0019 & 0.0024 & -- & -- & 0.0011 \\
\hline PEST & Dichlorodiphenyldichloroethane & $\mathrm{mg} / \mathrm{kg}$ & 7 & 4 & $57 \%$ & 0.0019 & 0.0021 & 0.0027 & 0.0097 & 0.0038 \\
\hline PEST & Dichlorodiphenyldichloroethylene & $\mathrm{mg} / \mathrm{kg}$ & 7 & 7 & $100 \%$ & -- & -- & 0.014 & 0.10 & 0.044 \\
\hline PEST & Dichlorodiphenyltrichloroethane & $\mathrm{mg} / \mathrm{kg}$ & 7 & 4 & $57 \%$ & 0.0019 & 0.0022 & 0.0024 & 0.0038 & 0.0022 \\
\hline PEST & Endosulfan sulfate & $\mathrm{mg} / \mathrm{kg}$ & 7 & 0 & $0 \%$ & 0.0019 & 0.0024 & -- & -- & 0.0011 \\
\hline PEST & Gamma-BHC (Lindane) & $\mathrm{mg} / \mathrm{kg}$ & 7 & 0 & $0 \%$ & 0.0019 & 0.0024 & -- & -- & 0.0011 \\
\hline PEST & gamma-Chlordane & $\mathrm{mg} / \mathrm{kg}$ & 7 & 0 & $0 \%$ & 0.0019 & 0.0024 & -- & -- & 0.0011 \\
\hline PEST & gamma-Chlordene & $\mathrm{mg} / \mathrm{kg}$ & 7 & 0 & $0 \%$ & 0.0019 & 0.0024 & -- & -- & 0.0011 \\
\hline PEST & Heptachlor & $\mathrm{mg} / \mathrm{kg}$ & 7 & 0 & $0 \%$ & 0.0019 & 0.0024 & -- & -- & 0.0011 \\
\hline PEST & Heptachlor epoxide & $\mathrm{mg} / \mathrm{kg}$ & 7 & 0 & $0 \%$ & 0.0019 & 0.0024 & -- & -- & 0.0011 \\
\hline PEST & Mirex & $\mathrm{mg} / \mathrm{kg}$ & 7 & 0 & $0 \%$ & 0.0019 & 0.0024 & -- & -- & 0.0011 \\
\hline PEST & Oxychlordane & $\mathrm{mg} / \mathrm{kg}$ & 7 & 0 & $0 \%$ & 0.0019 & 0.0024 & -- & -- & 0.0011 \\
\hline PEST & Pentachloroanisole & $\mathrm{mg} / \mathrm{kg}$ & 7 & 0 & $0 \%$ & 0.0019 & 0.0024 & -- & -- & 0.0011 \\
\hline PEST & Toxaphene & $\mathrm{mg} / \mathrm{kg}$ & 7 & 0 & $0 \%$ & 0.054 & 0.068 & -- & -- & 0.030 \\
\hline PEST & trans-Nonachlor & $\mathrm{mg} / \mathrm{kg}$ & 7 & 0 & $0 \%$ & 0.0019 & 0.0024 & -- & -- & 0.0011 \\
\hline SVOC & 1,2,4-Trichlorobenzene & $\mathrm{mg} / \mathrm{kg}$ & 7 & 0 & $0 \%$ & 0.0047 & 0.024 & -- & -- & 0.0063 \\
\hline SVOC & 1,2-Dichlorobenzene & $\mathrm{mg} / \mathrm{kg}$ & 7 & 0 & $0 \%$ & 0.0047 & 0.024 & -- & -- & 0.0063 \\
\hline SVOC & 1,2-Diphenylhydrazine & $\mathrm{mg} / \mathrm{kg}$ & 7 & 0 & $0 \%$ & 0.0047 & 0.024 & -- & -- & 0.0063 \\
\hline SVOC & 1,3-Dichlorobenzene & $\mathrm{mg} / \mathrm{kg}$ & 7 & 0 & $0 \%$ & 0.0047 & 0.024 & -- & -- & 0.0063 \\
\hline SVOC & 1,4-Dichlorobenzene & $\mathrm{mg} / \mathrm{kg}$ & 7 & 0 & $0 \%$ & 0.0047 & 0.024 & -- & -- & 0.0063 \\
\hline SVOC & 1-methylnaphthalene & $\mathrm{mg} / \mathrm{kg}$ & 7 & 0 & $0 \%$ & 0.0047 & 0.024 & -- & -- & 0.0063 \\
\hline SVOC & 2,4-Dinitrotoluene & $\mathrm{mg} / \mathrm{kg}$ & 7 & 0 & $0 \%$ & 0.0094 & 0.047 & -- & -- & 0.013 \\
\hline SVOC & 2,6-Dinitrotoluene & $\mathrm{mg} / \mathrm{kg}$ & 7 & 0 & $0 \%$ & 0.019 & 0.095 & -- & -- & 0.025 \\
\hline SVOC & 2-Chloronaphthalene & $\mathrm{mg} / \mathrm{kg}$ & 7 & 0 & $0 \%$ & 0.0094 & 0.047 & -- & -- & 0.013 \\
\hline SVOC & 2-Methylnaphthalene & $\mathrm{mg} / \mathrm{kg}$ & 7 & 2 & $29 \%$ & 0.0054 & 0.024 & 0.0064 & 0.0075 & 0.0077 \\
\hline SVOC & 4-Bromophenylphenyl ether & $\mathrm{mg} / \mathrm{kg}$ & 7 & 0 & $0 \%$ & 0.0047 & 0.024 & -- & -- & 0.0063 \\
\hline SVOC & 4-Chlorophenylphenyl ether & $\mathrm{mg} / \mathrm{kg}$ & 7 & 0 & $0 \%$ & 0.0047 & 0.024 & -- & -- & 0.0063 \\
\hline SVOC & Acenaphthene & $\mathrm{mg} / \mathrm{kg}$ & 7 & 0 & $0 \%$ & 0.0094 & 0.047 & -- & -- & 0.013 \\
\hline SVOC & Acenaphthylene & $\mathrm{mg} / \mathrm{kg}$ & 7 & 0 & $0 \%$ & 0.0047 & 0.024 & -- & -- & 0.0063 \\
\hline SVOC & Anthracene & $\mathrm{mg} / \mathrm{kg}$ & 7 & 0 & $0 \%$ & 0.0047 & 0.024 & -- & -- & 0.0063 \\
\hline SVOC & Benzo(a)anthracene & $\mathrm{mg} / \mathrm{kg}$ & 7 & 0 & $0 \%$ & 0.0047 & 0.024 & -- & -- & 0.0063 \\
\hline SVOC & Benzo(a)pyrene & $\mathrm{mg} / \mathrm{kg}$ & 7 & 0 & $0 \%$ & 0.0047 & 0.024 & -- & -- & 0.0063 \\
\hline SVOC & Benzo(b)fluoranthene & $\mathrm{mg} / \mathrm{kg}$ & 7 & 0 & $0 \%$ & 0.0047 & 0.024 & -- & -- & 0.0063 \\
\hline SVOC & Benzo(ghi)perylene & $\mathrm{mg} / \mathrm{kg}$ & 7 & 0 & $0 \%$ & 0.0094 & 0.047 & -- & -- & 0.013 \\
\hline SVOC & Benzo(k)fluoranthene & $\mathrm{mg} / \mathrm{kg}$ & 7 & 0 & $0 \%$ & 0.0047 & 0.024 & -- & -- & 0.0063 \\
\hline SVOC & Bis(2-chloro-1-methylethyl)ether & $\mathrm{mg} / \mathrm{kg}$ & 7 & 0 & $0 \%$ & 0.0094 & 0.047 & -- & -- & 0.013 \\
\hline
\end{tabular}


Table F-79

Summary Statistics for Largescale Sucker Fillet Skin On Collected from Segment 4 of the Columbia River

\begin{tabular}{|c|c|c|c|c|c|c|c|c|c|c|}
\hline $\begin{array}{l}\text { Constituent } \\
\text { Class }\end{array}$ & Constituent Name & Units & $\begin{array}{l}\text { Number } \\
\text { Analyzed }\end{array}$ & $\begin{array}{l}\text { Number } \\
\text { Detected }\end{array}$ & $\begin{array}{c}\text { Frequency of } \\
\text { Detection }\end{array}$ & $\begin{array}{l}\text { Minimum } \\
\text { Nondetect }\end{array}$ & $\begin{array}{l}\text { Maximum } \\
\text { Nondetect }\end{array}$ & $\begin{array}{l}\text { Minimum } \\
\text { Detect }\end{array}$ & $\begin{array}{l}\text { Maximum } \\
\text { Detect }\end{array}$ & $\begin{array}{c}\text { Average } \\
\text { Concentration }\end{array}$ \\
\hline$\overline{\text { SVOC }}$ & Chrysene & $\mathrm{mg} / \mathrm{kg}$ & 7 & 0 & $0 \%$ & 0.0047 & 0.024 & $\overline{--}$ & $\overline{--}$ & 0.0063 \\
\hline SVOC & Dibenz[a,h]anthracene & $\mathrm{mg} / \mathrm{kg}$ & 7 & 0 & $0 \%$ & 0.0094 & 0.047 & -- & -- & 0.013 \\
\hline SVOC & Dibenzofuran & $\mathrm{mg} / \mathrm{kg}$ & 7 & 0 & $0 \%$ & 0.0047 & 0.024 & -- & -- & 0.0063 \\
\hline SVOC & Fluoranthene & $\mathrm{mg} / \mathrm{kg}$ & 7 & 0 & $0 \%$ & 0.0094 & 0.047 & -- & -- & 0.013 \\
\hline SVOC & Fluorene & $\mathrm{mg} / \mathrm{kg}$ & 7 & 0 & $0 \%$ & 0.0092 & 0.0099 & -- & -- & 0.0047 \\
\hline SVOC & Hexachlorobenzene & $\mathrm{mg} / \mathrm{kg}$ & 7 & 0 & $0 \%$ & 0.0094 & 0.045 & -- & -- & 0.011 \\
\hline SVOC & Hexachlorobutadiene & $\mathrm{mg} / \mathrm{kg}$ & 7 & 0 & $0 \%$ & 0.0047 & 0.024 & -- & -- & 0.0063 \\
\hline SVOC & Hexachloroethane & $\mathrm{mg} / \mathrm{kg}$ & 7 & 0 & $0 \%$ & 0.0047 & 0.024 & -- & -- & 0.0063 \\
\hline SVOC & Indeno(1,2,3-cd)pyrene & $\mathrm{mg} / \mathrm{kg}$ & 7 & 0 & $0 \%$ & 0.0094 & 0.047 & -- & -- & 0.013 \\
\hline SVOC & Naphthalene & $\mathrm{mg} / \mathrm{kg}$ & 7 & 0 & $0 \%$ & 0.0064 & 0.028 & -- & -- & 0.0082 \\
\hline SVOC & Nitrobenzene & $\mathrm{mg} / \mathrm{kg}$ & 7 & 0 & $0 \%$ & 0.0047 & 0.024 & -- & -- & 0.0063 \\
\hline SVOC & Phenanthrene & $\mathrm{mg} / \mathrm{kg}$ & 7 & 0 & $0 \%$ & 0.0047 & 0.024 & -- & -- & 0.0063 \\
\hline SVOC & Pyrene & $\mathrm{mg} / \mathrm{kg}$ & 7 & 0 & $0 \%$ & 0.0094 & 0.047 & -- & -- & 0.013 \\
\hline VOC & Retene & $\mathrm{mg} / \mathrm{kg}$ & 7 & 0 & $0 \%$ & 0.0094 & 0.047 & -- & -- & 0.013 \\
\hline
\end{tabular}

DIOXI - Dioxins and Furans

PEST - Pesticide

PCB - Polychlorinated biphenyl

SVOC - Semivolatile Organic Compound

VOC - Volatile Organic Compound 
Table F-80

Summary Statistics for Whole Body Largescale Sucker Collected from Segment 4 of the Columbia River

\begin{tabular}{|c|c|c|c|c|c|c|c|c|c|c|}
\hline $\begin{array}{l}\text { Constituent } \\
\text { Class }\end{array}$ & Constituent Name & Units & $\begin{array}{l}\text { Number } \\
\text { Analyzed }\end{array}$ & $\begin{array}{l}\text { Number } \\
\text { Detected }\end{array}$ & $\begin{array}{c}\text { Frequency of } \\
\text { Detection }\end{array}$ & $\begin{array}{l}\text { Minimum } \\
\text { Nondetect }\end{array}$ & $\begin{array}{l}\text { Maximum } \\
\text { Nondetect }\end{array}$ & $\begin{array}{c}\text { Minimum } \\
\text { Detect }\end{array}$ & $\begin{array}{l}\text { Maximum } \\
\text { Detect }\end{array}$ & $\begin{array}{c}\text { Average } \\
\text { Concentration }\end{array}$ \\
\hline$\overline{D I O X I}$ & $1,2,3,4,6,7,8$-Heptachlorodibenzodioxin & $\mathrm{mg} / \mathrm{kg}$ & 11 & 9 & $82 \%$ & $1.00 \mathrm{E}-08$ & $2.00 \mathrm{E}-08$ & $1.50 \mathrm{E}-07$ & $4.60 \mathrm{E}-07$ & $2.20 \mathrm{E}-07$ \\
\hline DIOXI & 1,2,3,4,6,7,8-Heptachlorodibenzofuran & $\mathrm{mg} / \mathrm{kg}$ & 11 & 10 & $91 \%$ & 2.00E-08 & $2.00 \mathrm{E}-08$ & $6.00 \mathrm{E}-08$ & 1.20E-07 & 8.64E-08 \\
\hline DIOXI & 1,2,3,4,7,8,9-Heptachlorodibenzofuran & $\mathrm{mg} / \mathrm{kg}$ & 11 & 1 & $9 \%$ & $1.00 \mathrm{E}-08$ & $4.00 \mathrm{E}-08$ & $3.00 \mathrm{E}-08$ & $3.00 \mathrm{E}-08$ & 1.00E-08 \\
\hline DIOXI & $1,2,3,4,7,8$-Hexachlorodibenzofuran & $\mathrm{mg} / \mathrm{kg}$ & 11 & 7 & $64 \%$ & 1.00E-08 & $2.00 \mathrm{E}-08$ & $2.00 \mathrm{E}-08$ & $3.00 \mathrm{E}-08$ & 1.77E-08 \\
\hline DIOXI & $1,2,3,4,7,8$-Hexachlorodibenzo-p-dioxin & $\mathrm{mg} / \mathrm{kg}$ & 11 & 10 & $91 \%$ & 2.00E-08 & 2.00E-08 & $3.00 \mathrm{E}-08$ & 1.40E-07 & $9.64 \mathrm{E}-08$ \\
\hline DIOXI & 1,2,3,6,7,8-Hexachlorodibenzofuran & $\mathrm{mg} / \mathrm{kg}$ & 11 & 6 & $55 \%$ & 1.00E-08 & 2.00E-08 & $2.00 \mathrm{E}-08$ & 4.00E-08 & 2.05E-08 \\
\hline DIOXI & 1,2,3,6,7,8-Hexachlorodibenzo-p-dioxin & $\mathrm{mg} / \mathrm{kg}$ & 11 & 7 & $64 \%$ & $1.00 \mathrm{E}-08$ & $3.00 \mathrm{E}-08$ & $5.00 \mathrm{E}-08$ & $2.10 \mathrm{E}-07$ & 8.18E-08 \\
\hline DIOXI & 1,2,3,7,8,9-Hexachlorodibenzofuran & $\mathrm{mg} / \mathrm{kg}$ & 11 & 6 & $55 \%$ & 1.00E-08 & 2.00E-08 & $5.00 \mathrm{E}-08$ & 8.00E-08 & 3.59E-08 \\
\hline DIOXI & 1,2,3,7,8,9-Hexachlorodibenzo-p-dioxin & $\mathrm{mg} / \mathrm{kg}$ & 11 & 9 & $82 \%$ & $2.00 \mathrm{E}-08$ & $3.00 \mathrm{E}-08$ & $3.00 \mathrm{E}-08$ & 7.00E-08 & 4.14E-08 \\
\hline DIOXI & 1,2,3,7,8-Pentachlorodibenzofuran & $\mathrm{mg} / \mathrm{kg}$ & 11 & 10 & $91 \%$ & 2.00E-08 & 2.00E-08 & $3.00 \mathrm{E}-08$ & $6.00 \mathrm{E}-08$ & 4.09E-08 \\
\hline DIOXI & 1,2,3,7,8-Pentachlorodibenzo-p-dioxin & $\mathrm{mg} / \mathrm{kg}$ & 11 & 11 & $100 \%$ & -- & -- & $5.00 \mathrm{E}-08$ & $2.30 \mathrm{E}-07$ & 1.43E-07 \\
\hline DIOXI & 2,3,4,6,7,8-Hexachlorodibenzofuran & $\mathrm{mg} / \mathrm{kg}$ & 11 & 10 & $91 \%$ & 2.00E-08 & 2.00E-08 & $1.00 \mathrm{E}-08$ & 1.70E-07 & 1.20E-07 \\
\hline DIOXI & 2,3,4,7,8-Pentachlorodibenzofuran & $\mathrm{mg} / \mathrm{kg}$ & 11 & 11 & $100 \%$ & -- & -- & $3.00 \mathrm{E}-08$ & $1.90 \mathrm{E}-07$ & 8.27E-08 \\
\hline DIOXI & 2,3,7,8-Tetrachlorodibenzofuran & $\mathrm{mg} / \mathrm{kg}$ & 11 & 11 & $100 \%$ & -- & -- & $2.80 \mathrm{E}-07$ & $3.62 \mathrm{E}-06$ & $9.91 \mathrm{E}-07$ \\
\hline DIOXI & 2,3,7,8-Tetrachlorodibenzo-p-dioxin & $\mathrm{mg} / \mathrm{kg}$ & 11 & 7 & $64 \%$ & $1.00 \mathrm{E}-08$ & $2.00 \mathrm{E}-08$ & $5.00 \mathrm{E}-08$ & $2.10 \mathrm{E}-07$ & 7.00E-08 \\
\hline DIOXI & Octachlorodibenzofuran & $\mathrm{mg} / \mathrm{kg}$ & 11 & 7 & $64 \%$ & $1.00 \mathrm{E}-08$ & $4.00 \mathrm{E}-08$ & $6.00 \mathrm{E}-08$ & 1.10E-07 & $5.45 \mathrm{E}-08$ \\
\hline DIOXI & Octachlorodibenzo-p-dioxin & $\mathrm{mg} / \mathrm{kg}$ & 11 & 9 & $82 \%$ & 4.00E-08 & 4.00E-08 & $3.50 \mathrm{E}-07$ & 1.02E-06 & 5.79E-07 \\
\hline METAL & Aluminum & $\mathrm{mg} / \mathrm{kg}$ & 11 & 8 & $73 \%$ & 5.0 & 20 & 42 & 190 & 59 \\
\hline METAL & Antimony & $\mathrm{mg} / \mathrm{kg}$ & 11 & 0 & $0 \%$ & 0.050 & 0.050 & -- & -- & 0.025 \\
\hline METAL & Arsenic & $\mathrm{mg} / \mathrm{kg}$ & 11 & 11 & $100 \%$ & -- & -- & 0.080 & 0.32 & 0.16 \\
\hline METAL & Barium & $\mathrm{mg} / \mathrm{kg}$ & 11 & 11 & $100 \%$ & -- & -- & 0.87 & 3.3 & 1.9 \\
\hline METAL & Beryllium & $\mathrm{mg} / \mathrm{kg}$ & 11 & 3 & $27 \%$ & 0.0040 & 0.010 & 0.0069 & 0.0086 & 0.0045 \\
\hline METAL & Cadmium & $\mathrm{mg} / \mathrm{kg}$ & 11 & 11 & $100 \%$ & -- & -- & 0.010 & 0.10 & 0.028 \\
\hline METAL & Chromium & $\mathrm{mg} / \mathrm{kg}$ & 11 & 11 & $100 \%$ & -- & -- & 0.13 & 0.60 & 0.26 \\
\hline METAL & Cobalt & $\mathrm{mg} / \mathrm{kg}$ & 11 & 11 & $100 \%$ & -- & -- & 0.060 & 0.26 & 0.13 \\
\hline METAL & Copper & $\mathrm{mg} / \mathrm{kg}$ & 11 & 11 & $100 \%$ & -- & -- & 0.79 & 1.4 & 1.1 \\
\hline METAL & Lead & $\mathrm{mg} / \mathrm{kg}$ & 11 & 11 & $100 \%$ & -- & -- & 0.030 & 0.22 & 0.094 \\
\hline METAL & Manganese & $\mathrm{mg} / \mathrm{kg}$ & 11 & 11 & $100 \%$ & -- & -- & 6.4 & 19 & 11 \\
\hline METAL & Mercury & $\mathrm{mg} / \mathrm{kg}$ & 11 & 10 & $91 \%$ & 0.10 & 0.10 & 0.050 & 0.20 & 0.094 \\
\hline METAL & Nickel & $\mathrm{mg} / \mathrm{kg}$ & 11 & 11 & $100 \%$ & -- & -- & 0.050 & 0.51 & 0.34 \\
\hline METAL & Selenium & $\mathrm{mg} / \mathrm{kg}$ & 11 & 9 & $82 \%$ & 0.50 & 0.50 & 0.25 & 0.34 & 0.28 \\
\hline METAL & Silver & $\mathrm{mg} / \mathrm{kg}$ & 11 & 0 & $0 \%$ & 0.10 & 0.10 & -- & -- & 0.050 \\
\hline METAL & Thallium & $\mathrm{mg} / \mathrm{kg}$ & 11 & 0 & $0 \%$ & 0.050 & 0.050 & -- & -- & 0.025 \\
\hline METAL & Vanadium & $\mathrm{mg} / \mathrm{kg}$ & 11 & 11 & $100 \%$ & -- & -- & 0.10 & 0.61 & 0.28 \\
\hline METAL & Zinc & $\mathrm{mg} / \mathrm{kg}$ & 11 & 11 & $100 \%$ & -- & -- & 16 & 27 & 21 \\
\hline PCB & 2,2',3,3',4,4',5-Heptachloro-1,1'-biphenyl & $\mathrm{mg} / \mathrm{kg}$ & 11 & 11 & $100 \%$ & -- & -- & $3.18 \mathrm{E}-04$ & 0.0015 & 7.74E-04 \\
\hline РCB & 2,2',3,4,4',5,5'-Heptachlorobiphenyl & $\mathrm{mg} / \mathrm{kg}$ & 11 & 11 & $100 \%$ & -- & -- & $9.05 \mathrm{E}-04$ & 0.0043 & 0.0023 \\
\hline РCB & 2,3,3',4,4', 5'-Hexachlorobiphenyl & $\mathrm{mg} / \mathrm{kg}$ & 11 & 5 & $45 \%$ & 8.70E-07 & $9.50 \mathrm{E}-06$ & 7.14E-05 & 8.97E-05 & 3.83E-05 \\
\hline РCB & 2,3,3',4,4',5-Hexachlorobiphenyl (BZ 156) & $\mathrm{mg} / \mathrm{kg}$ & 11 & 11 & $100 \%$ & -- & -- & 1.53E-04 & 6.79E-04 & 3.93E-04 \\
\hline PCB & 2,3,3',4,4'-Pentachlorobiphenyl & $\mathrm{mg} / \mathrm{kg}$ & 11 & 11 & $100 \%$ & -- & -- & 3.35E-04 & 0.0013 & 8.06E-04 \\
\hline РCB & 2,3',4,4',5,5'-Hexachlorobiphenyl (BZ 167) & $\mathrm{mg} / \mathrm{kg}$ & 11 & 11 & $100 \%$ & -- & -- & 6.69E-05 & $3.33 \mathrm{E}-04$ & $1.80 \mathrm{E}-04$ \\
\hline PCB & 2',3,4,4',5-Pentachloro-1,1'-biphenyl (BZ 123) & $\mathrm{mg} / \mathrm{kg}$ & 11 & 11 & $100 \%$ & -- & -- & $1.21 \mathrm{E}-05$ & 5.26E-05 & 3.12E-05 \\
\hline PCB & 2,3,4,4',5-Pentachlorobiphenyl (BZ 114) & $\mathrm{mg} / \mathrm{kg}$ & 11 & 11 & $100 \%$ & -- & -- & $2.62 \mathrm{E}-05$ & $9.60 \mathrm{E}-05$ & $6.14 \mathrm{E}-05$ \\
\hline PCB & 2,3',4,4',5-Pentachlorobiphenyl (BZ 118) & $\mathrm{mg} / \mathrm{kg}$ & 11 & 11 & $100 \%$ & -- & -- & $7.12 \mathrm{E}-04$ & 0.0027 & 0.0016 \\
\hline РCB & 2,3,4,5,3',4',5'-Heptachlorobiphenyl & $\mathrm{mg} / \mathrm{kg}$ & 11 & 11 & $100 \%$ & -- & -- & 1.39E-05 & 8.59E-05 & 3.67E-05 \\
\hline PCB & 3,3',4,4',5,5'-Hexachlorobiphenyl (BZ 169) & $\mathrm{mg} / \mathrm{kg}$ & 11 & 0 & $0 \%$ & 8.00E-07 & 3.83E-06 & -- & -- & 8.44E-07 \\
\hline РСВ & 3,3',4,4',5-Pentachlorobiphenyl (BZ 126) & $\mathrm{mg} / \mathrm{kg}$ & 11 & 3 & $27 \%$ & 2.60E-07 & $6.06 \mathrm{E}-06$ & 7.15E-06 & 8.07E-06 & $2.78 \mathrm{E}-06$ \\
\hline PCB & 3,3',4,4'-Tetrachlorobiphenyl (BZ 77) & $\mathrm{mg} / \mathrm{kg}$ & 11 & 11 & $100 \%$ & -- & -- & 2.87E-05 & 5.56E-05 & 3.91E-05 \\
\hline РСВ & Aroclor-1016 & $\mathrm{mg} / \mathrm{kg}$ & 11 & 0 & $0 \%$ & 0.017 & 0.022 & -- & -- & 0.0098 \\
\hline PCB & Aroclor-1221 & $\mathrm{mg} / \mathrm{kg}$ & 11 & 0 & $0 \%$ & 0.017 & 0.022 & -- & -- & 0.0098 \\
\hline
\end{tabular}


Table F-80

Summary Statistics for Whole Body Largescale Sucker Collected from Segment 4 of the Columbia River

\begin{tabular}{|c|c|c|c|c|c|c|c|c|c|c|}
\hline $\begin{array}{l}\text { Constituent } \\
\text { Class }\end{array}$ & Constituent Name & Units & $\begin{array}{l}\text { Number } \\
\text { Analyzed }\end{array}$ & $\begin{array}{l}\text { Number } \\
\text { Detected }\end{array}$ & $\begin{array}{c}\text { Frequency of } \\
\text { Detection }\end{array}$ & $\begin{array}{l}\text { Minimum } \\
\text { Nondetect }\end{array}$ & $\begin{array}{l}\text { Maximum } \\
\text { Nondetect }\end{array}$ & $\begin{array}{l}\text { Minimum } \\
\text { Detect }\end{array}$ & $\begin{array}{l}\text { Maximum } \\
\text { Detect }\end{array}$ & $\begin{array}{c}\text { Average } \\
\text { Concentration }\end{array}$ \\
\hline$\overline{\mathrm{PCB}}$ & Aroclor-1232 & $\mathrm{mg} / \mathrm{kg}$ & 11 & 0 & $0 \%$ & 0.017 & 0.022 & -- & -- & 0.0098 \\
\hline PCB & Aroclor-1248 & $\mathrm{mg} / \mathrm{kg}$ & 11 & 0 & $0 \%$ & 0.017 & 0.022 & -- & -- & 0.0098 \\
\hline РCB & Aroclor-1254 & $\mathrm{mg} / \mathrm{kg}$ & 11 & 7 & $64 \%$ & 0.018 & 0.021 & 0.023 & 0.058 & 0.028 \\
\hline РСВ & Aroclor-1260 & $\mathrm{mg} / \mathrm{kg}$ & 11 & 8 & $73 \%$ & 0.017 & 0.020 & 0.012 & 0.093 & 0.030 \\
\hline PEST & 2,4'-DDD & $\mathrm{mg} / \mathrm{kg}$ & 11 & 5 & $45 \%$ & 0.0017 & 0.0027 & 0.0078 & 0.016 & 0.0050 \\
\hline PEST & 2,4'-DDE & $\mathrm{mg} / \mathrm{kg}$ & 11 & 8 & $73 \%$ & 0.0017 & 0.0020 & 0.0031 & 0.0058 & 0.0037 \\
\hline PEST & 2,4'-DDT & $\mathrm{mg} / \mathrm{kg}$ & 11 & 0 & $0 \%$ & 0.0017 & 0.0027 & -- & -- & 0.0011 \\
\hline PEST & Aldrin & $\mathrm{mg} / \mathrm{kg}$ & 11 & 0 & $0 \%$ & 0.0017 & 0.0027 & -- & -- & 0.0011 \\
\hline PEST & Alpha-BHC & $\mathrm{mg} / \mathrm{kg}$ & 11 & 0 & $0 \%$ & 0.0017 & 0.0027 & -- & -- & 0.0011 \\
\hline PEST & alpha-Chlordane & $\mathrm{mg} / \mathrm{kg}$ & 11 & 2 & $18 \%$ & 0.0017 & 0.0027 & 9.30E-04 & 0.0011 & 0.0011 \\
\hline PEST & alpha-Chlordene & $\mathrm{mg} / \mathrm{kg}$ & 11 & 0 & $0 \%$ & 0.0017 & 0.0027 & -- & -- & 0.0011 \\
\hline PEST & Beta-BHC & $\mathrm{mg} / \mathrm{kg}$ & 11 & 0 & $0 \%$ & 0.0017 & 0.0027 & -- & -- & 0.0011 \\
\hline PEST & cis-Nonachlor & $\mathrm{mg} / \mathrm{kg}$ & 11 & 2 & $18 \%$ & 0.0017 & 0.0027 & 0.0019 & 0.0019 & 0.0013 \\
\hline PEST & DDMU & $\mathrm{mg} / \mathrm{kg}$ & 11 & 5 & $45 \%$ & 0.0017 & 0.0027 & 0.0048 & 0.040 & 0.0091 \\
\hline PEST & Delta-BHC & $\mathrm{mg} / \mathrm{kg}$ & 11 & 0 & $0 \%$ & 0.0017 & 0.0027 & -- & -- & 0.0011 \\
\hline PEST & Dichlorodiphenyldichloroethane & $\mathrm{mg} / \mathrm{kg}$ & 11 & 11 & $100 \%$ & -- & -- & 0.0016 & 0.084 & 0.028 \\
\hline PEST & Dichlorodiphenyldichloroethylene & $\mathrm{mg} / \mathrm{kg}$ & 11 & 11 & $100 \%$ & -- & -- & 0.028 & 0.97 & 0.27 \\
\hline PEST & Dichlorodiphenyltrichloroethane & $\mathrm{mg} / \mathrm{kg}$ & 11 & 10 & $91 \%$ & 0.0020 & 0.0020 & 0.0011 & 0.072 & 0.017 \\
\hline PEST & Endosulfan sulfate & $\mathrm{mg} / \mathrm{kg}$ & 11 & 1 & $9 \%$ & 0.0017 & 0.0027 & 0.0065 & 0.0065 & 0.0016 \\
\hline PEST & Gamma-BHC (Lindane) & $\mathrm{mg} / \mathrm{kg}$ & 11 & 0 & $0 \%$ & 0.0017 & 0.0027 & -- & -- & 0.0011 \\
\hline PEST & gamma-Chlordane & $\mathrm{mg} / \mathrm{kg}$ & 11 & 2 & $18 \%$ & 0.0017 & 0.0027 & 0.0014 & 0.0016 & 0.0012 \\
\hline PEST & gamma-Chlordene & $\mathrm{mg} / \mathrm{kg}$ & 11 & 0 & $0 \%$ & 0.0017 & 0.0027 & -- & -- & 0.0011 \\
\hline PEST & Heptachlor & $\mathrm{mg} / \mathrm{kg}$ & 11 & 0 & $0 \%$ & 0.0017 & 0.0027 & -- & -- & 0.0011 \\
\hline PEST & Heptachlor epoxide & $\mathrm{mg} / \mathrm{kg}$ & 11 & 0 & $0 \%$ & 0.0017 & 0.0027 & -- & -- & 0.0011 \\
\hline PEST & Mirex & $\mathrm{mg} / \mathrm{kg}$ & 11 & 2 & $18 \%$ & 0.0017 & 0.0027 & $9.60 \mathrm{E}-04$ & 0.0012 & 0.0012 \\
\hline PEST & Oxychlordane & $\mathrm{mg} / \mathrm{kg}$ & 11 & 0 & $0 \%$ & 0.0017 & 0.0027 & -- & -- & 0.0011 \\
\hline PEST & Pentachloroanisole & $\mathrm{mg} / \mathrm{kg}$ & 11 & 0 & $0 \%$ & 0.0017 & 0.0027 & -- & -- & 0.0011 \\
\hline PEST & Toxaphene & $\mathrm{mg} / \mathrm{kg}$ & 11 & 0 & $0 \%$ & 0.051 & 0.081 & -- & -- & 0.034 \\
\hline PEST & trans-Nonachlor & $\mathrm{mg} / \mathrm{kg}$ & 11 & 2 & $18 \%$ & 0.0017 & 0.0027 & 0.0027 & 0.0030 & 0.0015 \\
\hline SVOC & 1,2,4-Trichlorobenzene & $\mathrm{mg} / \mathrm{kg}$ & 11 & 0 & $0 \%$ & 0.0047 & 0.010 & -- & -- & 0.0031 \\
\hline SVOC & 1,2-Dichlorobenzene & $\mathrm{mg} / \mathrm{kg}$ & 11 & 0 & $0 \%$ & 0.0047 & 0.010 & -- & -- & 0.0031 \\
\hline SVOC & 1,2-Diphenylhydrazine & $\mathrm{mg} / \mathrm{kg}$ & 11 & 0 & $0 \%$ & 0.0047 & 0.095 & -- & -- & 0.0072 \\
\hline SVOC & 1,3-Dichlorobenzene & $\mathrm{mg} / \mathrm{kg}$ & 11 & 0 & $0 \%$ & 0.0047 & 0.010 & -- & -- & 0.0031 \\
\hline SVOC & 1,4-Dichlorobenzene & $\mathrm{mg} / \mathrm{kg}$ & 11 & 0 & $0 \%$ & 0.0047 & 0.010 & -- & -- & 0.0031 \\
\hline SVOC & 1-methylnaphthalene & $\mathrm{mg} / \mathrm{kg}$ & 11 & 2 & $18 \%$ & 0.0048 & 0.010 & 0.0049 & 0.0085 & 0.0039 \\
\hline SVOC & 2,4-Dinitrotoluene & $\mathrm{mg} / \mathrm{kg}$ & 11 & 0 & $0 \%$ & 0.0093 & 0.020 & -- & -- & 0.0063 \\
\hline SVOC & 2,6-Dinitrotoluene & $\mathrm{mg} / \mathrm{kg}$ & 11 & 0 & $0 \%$ & 0.019 & 0.040 & -- & -- & 0.013 \\
\hline SVOC & 2-Chloronaphthalene & $\mathrm{mg} / \mathrm{kg}$ & 11 & 0 & $0 \%$ & 0.0048 & 0.020 & -- & -- & 0.0056 \\
\hline SVOC & 2-Methylnaphthalene & $\mathrm{mg} / \mathrm{kg}$ & 11 & 7 & $64 \%$ & 0.0048 & 0.012 & 0.0076 & 0.018 & 0.0084 \\
\hline SVOC & 4-Bromophenylphenyl ether & $\mathrm{mg} / \mathrm{kg}$ & 11 & 0 & $0 \%$ & 0.0047 & 0.095 & -- & -- & 0.0072 \\
\hline SVOC & 4-Chlorophenylphenyl ether & $\mathrm{mg} / \mathrm{kg}$ & 11 & 0 & $0 \%$ & 0.0047 & 0.010 & -- & -- & 0.0031 \\
\hline SVOC & Acenaphthene & $\mathrm{mg} / \mathrm{kg}$ & 11 & 1 & $9 \%$ & 0.0093 & 0.020 & 0.037 & 0.037 & 0.0092 \\
\hline SVOC & Acenaphthylene & $\mathrm{mg} / \mathrm{kg}$ & 11 & 2 & $18 \%$ & 0.0047 & 0.010 & 0.0028 & 0.0065 & 0.0036 \\
\hline SVOC & Anthracene & $\mathrm{mg} / \mathrm{kg}$ & 11 & 0 & $0 \%$ & 0.0047 & 0.095 & -- & -- & 0.0072 \\
\hline SVOC & Benzo(a)anthracene & $\mathrm{mg} / \mathrm{kg}$ & 11 & 0 & $0 \%$ & 0.0047 & 0.099 & -- & -- & 0.012 \\
\hline SVOC & Benzo(a)pyrene & $\mathrm{mg} / \mathrm{kg}$ & 11 & 0 & $0 \%$ & 0.0047 & 0.099 & -- & -- & 0.016 \\
\hline SVOC & Benzo(b)fluoranthene & $\mathrm{mg} / \mathrm{kg}$ & 11 & 0 & $0 \%$ & 0.0047 & 0.20 & -- & -- & 0.029 \\
\hline SVOC & Benzo(ghi)perylene & $\mathrm{mg} / \mathrm{kg}$ & 11 & 0 & $0 \%$ & 0.0093 & 0.20 & -- & -- & 0.031 \\
\hline SVOC & Benzo(k)fluoranthene & $\mathrm{mg} / \mathrm{kg}$ & 11 & 0 & $0 \%$ & 0.0047 & 0.20 & -- & -- & 0.029 \\
\hline SVOC & Bis(2-chloro-1-methylethyl)ether & $\mathrm{mg} / \mathrm{kg}$ & 11 & 0 & $0 \%$ & 0.0093 & 0.020 & -- & -- & 0.0063 \\
\hline
\end{tabular}


Table F-80

Summary Statistics for Whole Body Largescale Sucker Collected from Segment 4 of the Columbia River

\begin{tabular}{|c|c|c|c|c|c|c|c|c|c|c|}
\hline $\begin{array}{c}\text { Constituent } \\
\text { Class }\end{array}$ & Constituent Name & Units & $\begin{array}{c}\text { Number } \\
\text { Analyzed }\end{array}$ & $\begin{array}{l}\text { Number } \\
\text { Detected }\end{array}$ & $\begin{array}{c}\text { Frequency of } \\
\text { Detection }\end{array}$ & $\begin{array}{l}\text { Minimum } \\
\text { Nondetect }\end{array}$ & $\begin{array}{l}\text { Maximum } \\
\text { Nondetect }\end{array}$ & $\begin{array}{l}\text { Minimum } \\
\text { Detect }\end{array}$ & $\begin{array}{c}\text { Maximum } \\
\text { Detect }\end{array}$ & $\begin{array}{c}\text { Average } \\
\text { Concentration }\end{array}$ \\
\hline$\overline{\text { SVOC }}$ & Chrysene & $\mathrm{mg} / \mathrm{kg}$ & 11 & 0 & $0 \%$ & 0.0047 & 0.099 & $\overline{--}$ & $\overline{--}$ & 0.012 \\
\hline SVOC & Dibenz[a,h]anthracene & $\mathrm{mg} / \mathrm{kg}$ & 11 & 0 & $0 \%$ & 0.0093 & 0.20 & -- & -- & 0.031 \\
\hline SVOC & Dibenzofuran & $\mathrm{mg} / \mathrm{kg}$ & 11 & 0 & $0 \%$ & 0.0047 & 0.010 & -- & -- & 0.0031 \\
\hline SVOC & Fluoranthene & $\mathrm{mg} / \mathrm{kg}$ & 11 & 0 & $0 \%$ & 0.0093 & 0.19 & -- & -- & 0.014 \\
\hline SVOC & Fluorene & $\mathrm{mg} / \mathrm{kg}$ & 11 & 0 & $0 \%$ & 0.0042 & 0.023 & -- & -- & 0.0056 \\
\hline SVOC & Hexachlorobenzene & $\mathrm{mg} / \mathrm{kg}$ & 11 & 0 & $0 \%$ & 0.0093 & 0.19 & -- & -- & 0.014 \\
\hline SVOC & Hexachlorobutadiene & $\mathrm{mg} / \mathrm{kg}$ & 11 & 0 & $0 \%$ & 0.0047 & 0.010 & -- & -- & 0.0031 \\
\hline SVOC & Hexachloroethane & $\mathrm{mg} / \mathrm{kg}$ & 11 & 0 & $0 \%$ & 0.0047 & 0.13 & -- & -- & 0.0088 \\
\hline SVOC & Indeno(1,2,3-cd)pyrene & $\mathrm{mg} / \mathrm{kg}$ & 11 & 0 & $0 \%$ & 0.0093 & 0.20 & -- & -- & 0.031 \\
\hline SVOC & Naphthalene & $\mathrm{mg} / \mathrm{kg}$ & 11 & 0 & $0 \%$ & 0.0062 & 0.034 & -- & -- & 0.0093 \\
\hline SVOC & Nitrobenzene & $\mathrm{mg} / \mathrm{kg}$ & 11 & 0 & $0 \%$ & 0.0047 & 0.010 & -- & -- & 0.0031 \\
\hline SVOC & Phenanthrene & $\mathrm{mg} / \mathrm{kg}$ & 11 & 1 & $9 \%$ & 0.0048 & 0.095 & 0.0048 & 0.0048 & 0.0077 \\
\hline SVOC & Pyrene & $\mathrm{mg} / \mathrm{kg}$ & 11 & 2 & $18 \%$ & 0.0093 & 0.020 & 0.0055 & 0.0065 & 0.0065 \\
\hline VOC & Retene & $\mathrm{mg} / \mathrm{kg}$ & 11 & 2 & $18 \%$ & 0.0093 & 0.20 & 0.019 & 0.039 & 0.019 \\
\hline
\end{tabular}

DIOXI - Dioxins and Furans

PEST - Pesticide

PCB - Polychlorinated biphenyl

SVOC - Semivolatile Organic Compound

VOC - Volatile Organic Compound 
Table F-81

Summary Statistics for Coho Salmon Eggs and Ovum Collected from Segment 4 of the Columbia River

\begin{tabular}{|c|c|c|c|c|c|c|c|c|c|c|}
\hline $\begin{array}{l}\text { Constituent } \\
\text { Class }\end{array}$ & Constituent Name & Units & $\begin{array}{l}\text { Number } \\
\text { Analyzed }\end{array}$ & $\begin{array}{l}\text { Number } \\
\text { Detected }\end{array}$ & $\begin{array}{l}\text { Frequency of } \\
\text { Detection }\end{array}$ & $\begin{array}{l}\text { Minimum } \\
\text { Nondetect }\end{array}$ & $\begin{array}{l}\text { Maximum } \\
\text { Nondetect }\end{array}$ & $\begin{array}{l}\text { Minimum } \\
\text { Detect }\end{array}$ & $\begin{array}{l}\text { Maximum } \\
\text { Detect }\end{array}$ & $\begin{array}{c}\text { Average } \\
\text { Concentration }\end{array}$ \\
\hline$\overline{\mathrm{DIOXI}}$ & $1,2,3,4,6,7,8$-Heptachlorodibenzodioxin & $\mathrm{mg} / \mathrm{kg}$ & 3 & 3 & $100 \%$ & $\overline{--}$ & -- & $8.00 \mathrm{E}-08$ & $2.10 \mathrm{E}-07$ & $1.43 \mathrm{E}-07$ \\
\hline DIOXI & $1,2,3,4,6,7,8$-Heptachlorodibenzofuran & $\mathrm{mg} / \mathrm{kg}$ & 3 & 1 & $33 \%$ & $1.00 \mathrm{E}-08$ & $1.00 \mathrm{E}-08$ & $1.90 \mathrm{E}-07$ & $1.90 \mathrm{E}-07$ & 6.67E-08 \\
\hline DIOXI & 1,2,3,4,7,8,9-Heptachlorodibenzofuran & $\mathrm{mg} / \mathrm{kg}$ & 3 & 2 & $67 \%$ & $1.00 \mathrm{E}-08$ & $1.00 \mathrm{E}-08$ & $4.00 \mathrm{E}-08$ & $5.00 \mathrm{E}-08$ & 3.17E-08 \\
\hline DIOXI & $1,2,3,4,7,8$-Hexachlorodibenzofuran & $\mathrm{mg} / \mathrm{kg}$ & 3 & 2 & $67 \%$ & $1.00 \mathrm{E}-08$ & $1.00 \mathrm{E}-08$ & $2.00 \mathrm{E}-08$ & $5.00 \mathrm{E}-08$ & $2.50 \mathrm{E}-08$ \\
\hline DIOXI & 1,2,3,4,7,8-Hexachlorodibenzo-p-dioxin & $\mathrm{mg} / \mathrm{kg}$ & 3 & 1 & $33 \%$ & $1.00 \mathrm{E}-08$ & $1.00 \mathrm{E}-08$ & $1.00 \mathrm{E}-08$ & $1.00 \mathrm{E}-08$ & 6.67E-09 \\
\hline DIOXI & $1,2,3,6,7,8$-Hexachlorodibenzofuran & $\mathrm{mg} / \mathrm{kg}$ & 3 & 1 & $33 \%$ & $1.00 \mathrm{E}-08$ & $1.00 \mathrm{E}-08$ & $3.00 \mathrm{E}-08$ & $3.00 \mathrm{E}-08$ & 1.33E-08 \\
\hline DIOXI & $1,2,3,6,7,8$-Hexachlorodibenzo-p-dioxin & $\mathrm{mg} / \mathrm{kg}$ & 3 & 2 & $67 \%$ & $1.00 \mathrm{E}-08$ & $1.00 \mathrm{E}-08$ & $6.00 \mathrm{E}-08$ & $8.00 \mathrm{E}-08$ & 4.83E-08 \\
\hline DIOXI & 1,2,3,7,8,9-Hexachlorodibenzofuran & $\mathrm{mg} / \mathrm{kg}$ & 3 & 2 & $67 \%$ & $1.00 \mathrm{E}-08$ & $1.00 \mathrm{E}-08$ & $3.00 \mathrm{E}-08$ & $3.00 \mathrm{E}-08$ & 2.17E-08 \\
\hline DIOXI & 1,2,3,7,8,9-Hexachlorodibenzo-p-dioxin & $\mathrm{mg} / \mathrm{kg}$ & 3 & 0 & $0 \%$ & $1.00 \mathrm{E}-08$ & $1.00 \mathrm{E}-08$ & -- & -- & $5.00 \mathrm{E}-09$ \\
\hline DIOXI & 1,2,3,7,8-Pentachlorodibenzofuran & $\mathrm{mg} / \mathrm{kg}$ & 3 & 1 & $33 \%$ & $1.00 \mathrm{E}-08$ & $1.00 \mathrm{E}-08$ & $3.00 \mathrm{E}-08$ & $3.00 \mathrm{E}-08$ & 1.33E-08 \\
\hline DIOXI & $1,2,3,7,8$-Pentachlorodibenzo-p-dioxin & $\mathrm{mg} / \mathrm{kg}$ & 3 & 1 & $33 \%$ & $1.00 \mathrm{E}-08$ & $1.00 \mathrm{E}-08$ & $1.00 \mathrm{E}-07$ & $1.00 \mathrm{E}-07$ & 3.67E-08 \\
\hline DIOXI & 2,3,4,6,7,8-Hexachlorodibenzofuran & $\mathrm{mg} / \mathrm{kg}$ & 3 & 1 & $33 \%$ & $1.00 \mathrm{E}-08$ & $1.00 \mathrm{E}-08$ & $4.00 \mathrm{E}-08$ & $4.00 \mathrm{E}-08$ & 1.67E-08 \\
\hline DIOXI & 2,3,4,7,8-Pentachlorodibenzofuran & $\mathrm{mg} / \mathrm{kg}$ & 3 & 2 & $67 \%$ & $1.00 \mathrm{E}-08$ & $1.00 \mathrm{E}-08$ & $1.00 \mathrm{E}-07$ & $1.00 \mathrm{E}-07$ & $6.83 \mathrm{E}-08$ \\
\hline DIOXI & 2,3,7,8-Tetrachlorodibenzofuran & $\mathrm{mg} / \mathrm{kg}$ & 3 & 3 & $100 \%$ & -- & -- & $2.90 \mathrm{E}-07$ & $6.60 \mathrm{E}-07$ & 5.13E-07 \\
\hline DIOXI & 2,3,7,8-Tetrachlorodibenzo-p-dioxin & $\mathrm{mg} / \mathrm{kg}$ & 3 & 2 & $67 \%$ & $1.00 \mathrm{E}-08$ & $1.00 \mathrm{E}-08$ & $5.00 \mathrm{E}-08$ & $5.00 \mathrm{E}-08$ & 3.50E-08 \\
\hline DIOXI & Octachlorodibenzofuran & $\mathrm{mg} / \mathrm{kg}$ & 3 & 2 & $67 \%$ & $1.00 \mathrm{E}-08$ & $1.00 \mathrm{E}-08$ & $1.80 \mathrm{E}-07$ & $2.20 \mathrm{E}-07$ & 1.35E-07 \\
\hline DIOXI & Octachlorodibenzo-p-dioxin & $\mathrm{mg} / \mathrm{kg}$ & 3 & 3 & $100 \%$ & -- & -- & $1.32 \mathrm{E}-06$ & $2.53 \mathrm{E}-06$ & 1.77E-06 \\
\hline METAL & Aluminum & $\mathrm{mg} / \mathrm{kg}$ & 3 & 1 & $33 \%$ & 1.0 & 1.0 & 1.6 & 1.6 & 0.87 \\
\hline METAL & Antimony & $\mathrm{mg} / \mathrm{kg}$ & 3 & 0 & $0 \%$ & 0.050 & 0.050 & -- & -- & 0.025 \\
\hline METAL & Arsenic & $\mathrm{mg} / \mathrm{kg}$ & 3 & 3 & $100 \%$ & -- & -- & 0.31 & 0.36 & 0.33 \\
\hline METAL & Barium & $\mathrm{mg} / \mathrm{kg}$ & 3 & 0 & $0 \%$ & 0.20 & 0.20 & -- & -- & 0.10 \\
\hline METAL & Beryllium & $\mathrm{mg} / \mathrm{kg}$ & 3 & 0 & $0 \%$ & 0.0040 & 0.0040 & -- & -- & 0.0020 \\
\hline METAL & Cadmium & $\mathrm{mg} / \mathrm{kg}$ & 3 & 0 & $0 \%$ & 0.0040 & 0.0040 & -- & -- & 0.0020 \\
\hline METAL & Chromium & $\mathrm{mg} / \mathrm{kg}$ & 3 & 0 & $0 \%$ & 0.10 & 0.10 & -- & -- & 0.050 \\
\hline METAL & Cobalt & $\mathrm{mg} / \mathrm{kg}$ & 3 & 3 & $100 \%$ & -- & -- & 0.010 & 0.010 & 0.010 \\
\hline METAL & Copper & $\mathrm{mg} / \mathrm{kg}$ & 3 & 3 & $100 \%$ & -- & -- & 4.1 & 5.0 & 4.4 \\
\hline METAL & Lead & $\mathrm{mg} / \mathrm{kg}$ & 3 & 0 & $0 \%$ & 0.010 & 0.010 & -- & -- & 0.0050 \\
\hline METAL & Manganese & $\mathrm{mg} / \mathrm{kg}$ & 3 & 3 & $100 \%$ & -- & -- & 0.62 & 0.79 & 0.70 \\
\hline METAL & Mercury & $\mathrm{mg} / \mathrm{kg}$ & 3 & 0 & $0 \%$ & 0.10 & 0.10 & -- & -- & 0.050 \\
\hline METAL & Nickel & $\mathrm{mg} / \mathrm{kg}$ & 3 & 3 & $100 \%$ & -- & -- & 0.030 & 0.14 & 0.080 \\
\hline METAL & Selenium & $\mathrm{mg} / \mathrm{kg}$ & 3 & 3 & $100 \%$ & -- & -- & 1.1 & 1.3 & 1.2 \\
\hline METAL & Silver & $\mathrm{mg} / \mathrm{kg}$ & 3 & 0 & $0 \%$ & 0.10 & 0.10 & -- & -- & 0.050 \\
\hline METAL & Thallium & $\mathrm{mg} / \mathrm{kg}$ & 3 & 0 & $0 \%$ & 0.050 & 0.050 & -- & -- & 0.025 \\
\hline METAL & Vanadium & $\mathrm{mg} / \mathrm{kg}$ & 3 & 3 & $100 \%$ & -- & -- & 0.010 & 0.040 & 0.023 \\
\hline METAL & Zinc & $\mathrm{mg} / \mathrm{kg}$ & 3 & 3 & $100 \%$ & -- & -- & 30 & 34 & 31 \\
\hline PCB & 2,2',3,3',4,4',5-Heptachloro-1,1'-biphenyl & $\mathrm{mg} / \mathrm{kg}$ & 3 & 3 & $100 \%$ & -- & -- & $1.45 \mathrm{E}-04$ & $1.89 \mathrm{E}-04$ & $1.64 \mathrm{E}-04$ \\
\hline РСВ & 2,2',3,4,4',5,5'-Heptachlorobiphenyl & $\mathrm{mg} / \mathrm{kg}$ & 3 & 3 & $100 \%$ & -- & -- & 4.44E-04 & $5.78 \mathrm{E}-04$ & 5.05E-04 \\
\hline PCB & 2,3,3',4,4',5'-Hexachlorobiphenyl & $\mathrm{mg} / \mathrm{kg}$ & 3 & 3 & $100 \%$ & -- & -- & $1.77 \mathrm{E}-05$ & $2.34 \mathrm{E}-05$ & 2.01E-05 \\
\hline РСB & 2,3,3',4,4',5-Hexachlorobiphenyl (BZ 156) & $\mathrm{mg} / \mathrm{kg}$ & 3 & 3 & $100 \%$ & -- & -- & 8.23E-05 & $1.00 \mathrm{E}-04$ & 8.91E-05 \\
\hline PCB & 2,3,3',4,4'-Pentachlorobiphenyl & $\mathrm{mg} / \mathrm{kg}$ & 3 & 3 & $100 \%$ & -- & -- & $2.71 \mathrm{E}-04$ & $3.48 \mathrm{E}-04$ & 3.17E-04 \\
\hline PCB & 2,3',4,4',5,5'-Hexachlorobiphenyl (BZ 167) & $\mathrm{mg} / \mathrm{kg}$ & 3 & 3 & $100 \%$ & -- & -- & 3.53E-05 & 3.83E-05 & 3.67E-05 \\
\hline РСB & 2',3,4,4',5-Pentachloro-1,1'-biphenyl (BZ 123) & $\mathrm{mg} / \mathrm{kg}$ & 3 & 3 & $100 \%$ & -- & -- & $1.12 \mathrm{E}-05$ & $1.31 \mathrm{E}-05$ & $1.24 \mathrm{E}-05$ \\
\hline PCB & 2,3,4,4',5-Pentachlorobiphenyl (BZ 114) & $\mathrm{mg} / \mathrm{kg}$ & 3 & 3 & $100 \%$ & -- & -- & $2.39 \mathrm{E}-05$ & $3.44 \mathrm{E}-05$ & 2.91E-05 \\
\hline PCB & 2,3',4,4',5-Pentachlorobiphenyl (BZ 118) & $\mathrm{mg} / \mathrm{kg}$ & 3 & 3 & $100 \%$ & -- & -- & $6.77 \mathrm{E}-04$ & 7.59E-04 & $7.22 \mathrm{E}-04$ \\
\hline РСB & 2,3,4,5,3',4',5'-Heptachlorobiphenyl & $\mathrm{mg} / \mathrm{kg}$ & 3 & 3 & $100 \%$ & -- & -- & $5.12 \mathrm{E}-06$ & $6.79 \mathrm{E}-06$ & $5.80 \mathrm{E}-06$ \\
\hline РСB & 3,3',4,4',5,5'-Hexachlorobiphenyl (BZ 169) & $\mathrm{mg} / \mathrm{kg}$ & 3 & 0 & $0 \%$ & $1.00 \mathrm{E}-06$ & $1.27 \mathrm{E}-06$ & -- & -- & $5.48 \mathrm{E}-07$ \\
\hline PCB & 3,3',4,4',5-Pentachlorobiphenyl (BZ 126) & $\mathrm{mg} / \mathrm{kg}$ & 3 & 0 & $0 \%$ & 7.70E-07 & $1.08 \mathrm{E}-06$ & -- & -- & 4.77E-07 \\
\hline РСB & 3,3',4,4'-Tetrachlorobiphenyl (BZ 77) & $\mathrm{mg} / \mathrm{kg}$ & 3 & 3 & $100 \%$ & -- & -- & $1.01 \mathrm{E}-05$ & 1.07E-05 & $1.04 \mathrm{E}-05$ \\
\hline PCB & Aroclor-1016 & $\mathrm{mg} / \mathrm{kg}$ & 3 & 0 & $0 \%$ & 0.019 & 0.019 & -- & -- & 0.0095 \\
\hline PCB & Aroclor-1221 & $\mathrm{mg} / \mathrm{kg}$ & 3 & 0 & $0 \%$ & 0.019 & 0.019 & -- & -- & 0.0095 \\
\hline РСB & Aroclor-1232 & $\mathrm{mg} / \mathrm{kg}$ & 3 & 0 & $0 \%$ & 0.019 & 0.019 & -- & -- & 0.0095 \\
\hline РСB & Aroclor-1248 & $\mathrm{mg} / \mathrm{kg}$ & 3 & 0 & $0 \%$ & 0.019 & 0.019 & -- & -- & 0.0095 \\
\hline
\end{tabular}


Table F-81

Summary Statistics for Coho Salmon Eggs and Ovum Collected from Segment 4 of the Columbia River

\begin{tabular}{|c|c|c|c|c|c|c|c|c|c|c|}
\hline $\begin{array}{l}\text { Constituent } \\
\text { Class }\end{array}$ & Constituent Name & Units & $\begin{array}{l}\text { Number } \\
\text { Analyzed }\end{array}$ & $\begin{array}{l}\text { Number } \\
\text { Detected }\end{array}$ & $\begin{array}{l}\text { Frequency of } \\
\text { Detection }\end{array}$ & $\begin{array}{l}\text { Minimum } \\
\text { Nondetect }\end{array}$ & $\begin{array}{l}\text { Maximum } \\
\text { Nondetect }\end{array}$ & $\begin{array}{l}\text { Minimum } \\
\text { Detect }\end{array}$ & $\begin{array}{l}\text { Maximum } \\
\text { Detect }\end{array}$ & $\begin{array}{c}\text { Average } \\
\text { Concentration }\end{array}$ \\
\hline$\overline{\mathrm{PCB}}$ & Aroclor-1254 & $\mathrm{mg} / \mathrm{kg}$ & 3 & 3 & $100 \%$ & $\overline{--}$ & $\overline{--}$ & 0.011 & 0.017 & 0.015 \\
\hline РСB & Aroclor-1260 & $\mathrm{mg} / \mathrm{kg}$ & 3 & 0 & $0 \%$ & 0.019 & 0.019 & -- & -- & 0.0095 \\
\hline PEST & 2,4'-DDD & $\mathrm{mg} / \mathrm{kg}$ & 3 & 0 & $0 \%$ & 0.0019 & 0.0019 & -- & -- & $9.50 \mathrm{E}-04$ \\
\hline PEST & 2,4'-DDE & $\mathrm{mg} / \mathrm{kg}$ & 3 & 0 & $0 \%$ & 0.0019 & 0.0019 & -- & -- & $9.50 \mathrm{E}-04$ \\
\hline PEST & 2,4'-DDT & $\mathrm{mg} / \mathrm{kg}$ & 3 & 0 & $0 \%$ & 0.0019 & 0.0019 & -- & -- & $9.50 \mathrm{E}-04$ \\
\hline PEST & Aldrin & $\mathrm{mg} / \mathrm{kg}$ & 3 & 0 & $0 \%$ & 0.0019 & 0.0019 & -- & -- & $9.50 \mathrm{E}-04$ \\
\hline PEST & Alpha-BHC & $\mathrm{mg} / \mathrm{kg}$ & 3 & 0 & $0 \%$ & 0.0019 & 0.0019 & -- & -- & $9.50 \mathrm{E}-04$ \\
\hline PEST & alpha-Chlordane & $\mathrm{mg} / \mathrm{kg}$ & 3 & 0 & $0 \%$ & 0.0019 & 0.0019 & -- & -- & $9.50 \mathrm{E}-04$ \\
\hline PEST & alpha-Chlordene & $\mathrm{mg} / \mathrm{kg}$ & 3 & 0 & $0 \%$ & 0.0019 & 0.0019 & -- & -- & $9.50 \mathrm{E}-04$ \\
\hline PEST & Beta-BHC & $\mathrm{mg} / \mathrm{kg}$ & 3 & 0 & $0 \%$ & 0.0019 & 0.0019 & -- & -- & $9.50 \mathrm{E}-04$ \\
\hline PEST & cis-Nonachlor & $\mathrm{mg} / \mathrm{kg}$ & 3 & 0 & $0 \%$ & 0.0019 & 0.0019 & -- & -- & $9.50 \mathrm{E}-04$ \\
\hline PEST & DDMU & $\mathrm{mg} / \mathrm{kg}$ & 3 & 0 & $0 \%$ & 0.0019 & 0.0019 & -- & -- & $9.50 \mathrm{E}-04$ \\
\hline PEST & Delta-BHC & $\mathrm{mg} / \mathrm{kg}$ & 3 & 0 & $0 \%$ & 0.0019 & 0.0019 & -- & -- & $9.50 \mathrm{E}-04$ \\
\hline PEST & Dichlorodiphenyldichloroethane & $\mathrm{mg} / \mathrm{kg}$ & 3 & 3 & $100 \%$ & -- & -- & 0.0027 & 0.0029 & 0.0028 \\
\hline PEST & Dichlorodiphenyldichloroethylene & $\mathrm{mg} / \mathrm{kg}$ & 3 & 3 & $100 \%$ & -- & -- & 0.031 & 0.033 & 0.032 \\
\hline PEST & Dichlorodiphenyltrichloroethane & $\mathrm{mg} / \mathrm{kg}$ & 3 & 0 & $0 \%$ & 0.0019 & 0.0019 & -- & -- & $9.50 \mathrm{E}-04$ \\
\hline PEST & Endosulfan sulfate & $\mathrm{mg} / \mathrm{kg}$ & 3 & 0 & $0 \%$ & 0.0019 & 0.0019 & -- & -- & $9.50 \mathrm{E}-04$ \\
\hline PEST & Gamma-BHC (Lindane) & $\mathrm{mg} / \mathrm{kg}$ & 3 & 0 & $0 \%$ & 0.0019 & 0.0019 & -- & -- & $9.50 \mathrm{E}-04$ \\
\hline PEST & gamma-Chlordane & $\mathrm{mg} / \mathrm{kg}$ & 3 & 0 & $0 \%$ & 0.0019 & 0.0019 & -- & -- & $9.50 \mathrm{E}-04$ \\
\hline PEST & gamma-Chlordene & $\mathrm{mg} / \mathrm{kg}$ & 3 & 0 & $0 \%$ & 0.0019 & 0.0019 & -- & -- & $9.50 \mathrm{E}-04$ \\
\hline PEST & Heptachlor & $\mathrm{mg} / \mathrm{kg}$ & 3 & 0 & $0 \%$ & 0.0019 & 0.0019 & -- & -- & $9.50 \mathrm{E}-04$ \\
\hline PEST & Heptachlor epoxide & $\mathrm{mg} / \mathrm{kg}$ & 3 & 0 & $0 \%$ & 0.0019 & 0.0019 & -- & -- & $9.50 \mathrm{E}-04$ \\
\hline PEST & Mirex & $\mathrm{mg} / \mathrm{kg}$ & 3 & 0 & $0 \%$ & 0.0019 & 0.0019 & -- & -- & $9.50 \mathrm{E}-04$ \\
\hline PEST & Oxychlordane & $\mathrm{mg} / \mathrm{kg}$ & 3 & 0 & $0 \%$ & 0.0019 & 0.0019 & -- & -- & $9.50 \mathrm{E}-04$ \\
\hline PEST & Pentachloroanisole & $\mathrm{mg} / \mathrm{kg}$ & 3 & 0 & $0 \%$ & 0.0019 & 0.0019 & -- & -- & $9.50 \mathrm{E}-04$ \\
\hline PEST & Toxaphene & $\mathrm{mg} / \mathrm{kg}$ & 3 & 0 & $0 \%$ & 0.056 & 0.057 & -- & -- & 0.028 \\
\hline PEST & trans-Nonachlor & $\mathrm{mg} / \mathrm{kg}$ & 3 & 0 & $0 \%$ & 0.0019 & 0.0019 & -- & -- & $9.50 \mathrm{E}-04$ \\
\hline SVOC & 1,2,4-Trichlorobenzene & $\mathrm{mg} / \mathrm{kg}$ & 3 & 0 & $0 \%$ & 0.0096 & 0.010 & -- & -- & 0.0049 \\
\hline SVOC & 1,2-Dichlorobenzene & $\mathrm{mg} / \mathrm{kg}$ & 3 & 0 & $0 \%$ & 0.0096 & 0.010 & -- & -- & 0.0049 \\
\hline SVOC & 1,2-Diphenylhydrazine & $\mathrm{mg} / \mathrm{kg}$ & 3 & 0 & $0 \%$ & 0.0096 & 0.010 & -- & -- & 0.0049 \\
\hline SVOC & 1,3-Dichlorobenzene & $\mathrm{mg} / \mathrm{kg}$ & 3 & 0 & $0 \%$ & 0.0096 & 0.010 & -- & -- & 0.0049 \\
\hline SVOC & 1,4-Dichlorobenzene & $\mathrm{mg} / \mathrm{kg}$ & 3 & 0 & $0 \%$ & 0.0096 & 0.010 & -- & -- & 0.0049 \\
\hline SVOC & 1-methylnaphthalene & $\mathrm{mg} / \mathrm{kg}$ & 3 & 0 & $0 \%$ & 0.0096 & 0.010 & -- & -- & 0.0049 \\
\hline SVOC & 2,4-Dinitrotoluene & $\mathrm{mg} / \mathrm{kg}$ & 3 & 0 & $0 \%$ & 0.019 & 0.020 & -- & -- & 0.0097 \\
\hline SVOC & 2,6-Dinitrotoluene & $\mathrm{mg} / \mathrm{kg}$ & 3 & 0 & $0 \%$ & 0.038 & 0.040 & -- & -- & 0.019 \\
\hline SVOC & 2-Chloronaphthalene & $\mathrm{mg} / \mathrm{kg}$ & 3 & 0 & $0 \%$ & 0.019 & 0.020 & -- & -- & 0.0097 \\
\hline SVOC & 2-Methylnaphthalene & $\mathrm{mg} / \mathrm{kg}$ & 3 & 0 & $0 \%$ & 0.0096 & 0.010 & -- & -- & 0.0049 \\
\hline SVOC & 4-Bromophenylphenyl ether & $\mathrm{mg} / \mathrm{kg}$ & 3 & 0 & $0 \%$ & 0.0096 & 0.010 & -- & -- & 0.0049 \\
\hline SVOC & 4-Chlorophenylphenyl ether & $\mathrm{mg} / \mathrm{kg}$ & 3 & 0 & $0 \%$ & 0.0096 & 0.010 & -- & -- & 0.0049 \\
\hline SVOC & Acenaphthene & $\mathrm{mg} / \mathrm{kg}$ & 3 & 0 & $0 \%$ & 0.019 & 0.020 & -- & -- & 0.0097 \\
\hline SVOC & Acenaphthylene & $\mathrm{mg} / \mathrm{kg}$ & 3 & 0 & $0 \%$ & 0.0096 & 0.010 & -- & -- & 0.0049 \\
\hline SVOC & Anthracene & $\mathrm{mg} / \mathrm{kg}$ & 3 & 0 & $0 \%$ & 0.0096 & 0.010 & -- & -- & 0.0049 \\
\hline SVOC & Benzo(a)anthracene & $\mathrm{mg} / \mathrm{kg}$ & 3 & 0 & $0 \%$ & 0.0096 & 0.010 & -- & -- & 0.0049 \\
\hline SVOC & Benzo(a)pyrene & $\mathrm{mg} / \mathrm{kg}$ & 3 & 0 & $0 \%$ & 0.0096 & 0.010 & -- & -- & 0.0049 \\
\hline SVOC & Benzo(b)fluoranthene & $\mathrm{mg} / \mathrm{kg}$ & 3 & 0 & $0 \%$ & 0.0096 & 0.010 & -- & -- & 0.0049 \\
\hline SVOC & Benzo(ghi)perylene & $\mathrm{mg} / \mathrm{kg}$ & 3 & 0 & $0 \%$ & 0.019 & 0.020 & -- & -- & 0.0097 \\
\hline SVOC & Benzo(k)fluoranthene & $\mathrm{mg} / \mathrm{kg}$ & 3 & 0 & $0 \%$ & 0.0096 & 0.010 & -- & -- & 0.0049 \\
\hline SVOC & Bis(2-chloro-1-methylethyl)ether & $\mathrm{mg} / \mathrm{kg}$ & 3 & 0 & $0 \%$ & 0.019 & 0.020 & -- & -- & 0.0097 \\
\hline SVOC & Chrysene & $\mathrm{mg} / \mathrm{kg}$ & 3 & 0 & $0 \%$ & 0.0096 & 0.010 & -- & -- & 0.0049 \\
\hline SVOC & Dibenz[a,h]anthracene & $\mathrm{mg} / \mathrm{kg}$ & 3 & 0 & $0 \%$ & 0.019 & 0.020 & -- & -- & 0.0097 \\
\hline SVOC & Dibenzofuran & $\mathrm{mg} / \mathrm{kg}$ & 3 & 0 & $0 \%$ & 0.0096 & 0.010 & -- & -- & 0.0049 \\
\hline SVOC & Fluoranthene & $\mathrm{mg} / \mathrm{kg}$ & 3 & 0 & $0 \%$ & 0.019 & 0.020 & -- & -- & 0.0097 \\
\hline
\end{tabular}


Table F-81

Summary Statistics for Coho Salmon Eggs and Ovum Collected from Segment 4 of the Columbia River

\begin{tabular}{|c|c|c|c|c|c|c|c|c|c|c|}
\hline $\begin{array}{l}\text { Constituent } \\
\text { Class }\end{array}$ & Constituent Name & Units & $\begin{array}{l}\text { Number } \\
\text { Analyzed }\end{array}$ & $\begin{array}{l}\text { Number } \\
\text { Detected }\end{array}$ & $\begin{array}{l}\text { Frequency of } \\
\text { Detection }\end{array}$ & $\begin{array}{c}\text { Minimum } \\
\text { Nondetect }\end{array}$ & $\begin{array}{l}\text { Maximum } \\
\text { Nondetect }\end{array}$ & $\begin{array}{c}\text { Minimum } \\
\text { Detect }\end{array}$ & $\begin{array}{c}\text { Maximum } \\
\text { Detect }\end{array}$ & $\begin{array}{c}\text { Average } \\
\text { Concentration }\end{array}$ \\
\hline$\overline{\text { SVOC }}$ & Fluorene & $\mathrm{mg} / \mathrm{kg}$ & 3 & 0 & $0 \%$ & 0.0048 & 0.0094 & -- & -- & 0.0039 \\
\hline SVOC & Hexachlorobenzene & $\mathrm{mg} / \mathrm{kg}$ & 3 & 0 & $0 \%$ & 0.019 & 0.020 & -- & -- & 0.0097 \\
\hline SVOC & Hexachlorobutadiene & $\mathrm{mg} / \mathrm{kg}$ & 3 & 0 & $0 \%$ & 0.0096 & 0.010 & -- & -- & 0.0049 \\
\hline SVOC & Hexachloroethane & $\mathrm{mg} / \mathrm{kg}$ & 3 & 0 & $0 \%$ & 0.0096 & 0.010 & -- & -- & 0.0049 \\
\hline SVOC & Indeno(1,2,3-cd)pyrene & $\mathrm{mg} / \mathrm{kg}$ & 3 & 0 & $0 \%$ & 0.019 & 0.020 & -- & -- & 0.0097 \\
\hline SVOC & Naphthalene & $\mathrm{mg} / \mathrm{kg}$ & 3 & 0 & $0 \%$ & 0.021 & 0.024 & -- & -- & 0.011 \\
\hline SVOC & Nitrobenzene & $\mathrm{mg} / \mathrm{kg}$ & 3 & 0 & $0 \%$ & 0.0096 & 0.010 & -- & -- & 0.0049 \\
\hline SVOC & Phenanthrene & $\mathrm{mg} / \mathrm{kg}$ & 3 & 0 & $0 \%$ & 0.0096 & 0.010 & -- & -- & 0.0049 \\
\hline SVOC & Pyrene & $\mathrm{mg} / \mathrm{kg}$ & 3 & 0 & $0 \%$ & 0.019 & 0.020 & -- & -- & 0.0097 \\
\hline VOC & Retene & $\mathrm{mg} / \mathrm{kg}$ & 3 & 0 & $0 \%$ & 0.019 & 0.020 & -- & -- & 0.0097 \\
\hline
\end{tabular}

DIOXI - Dioxins and Furans

PEST - Pesticide

PCB - Polychlorinated biphenyl

SVOC - Semivolatile Organic Compound

VOC - Volatile Organic Compound 
Table F-82

Summary Statistics for Coho Salmon Fillet Skin On Collected from Segment 4 of the Columbia River

\begin{tabular}{|c|c|c|c|c|c|c|c|c|c|c|}
\hline $\begin{array}{c}\text { Constituent } \\
\text { Class }\end{array}$ & Constituent Name & Units & $\begin{array}{l}\text { Number } \\
\text { Analyzed }\end{array}$ & $\begin{array}{l}\text { Number } \\
\text { Detected }\end{array}$ & $\begin{array}{c}\text { Frequency of } \\
\text { Detection }\end{array}$ & $\begin{array}{l}\text { Minimum } \\
\text { Nondetect }\end{array}$ & $\begin{array}{l}\text { Maximum } \\
\text { Nondetect }\end{array}$ & $\begin{array}{c}\text { Minimum } \\
\text { Detect }\end{array}$ & $\begin{array}{c}\text { Maximum } \\
\text { Detect }\end{array}$ & $\begin{array}{c}\text { Average } \\
\text { Concentration }\end{array}$ \\
\hline$\overline{\mathrm{DIOXI}}$ & $1,2,3,4,6,7,8$-Heptachlorodibenzodioxin & $\mathrm{mg} / \mathrm{kg}$ & 3 & 2 & $67 \%$ & $1.00 \mathrm{E}-08$ & $1.00 \mathrm{E}-08$ & $1.00 \mathrm{E}-07$ & $1.80 \mathrm{E}-07$ & $9.50 \mathrm{E}-08$ \\
\hline DIOXI & $1,2,3,4,6,7,8$-Heptachlorodibenzofuran & $\mathrm{mg} / \mathrm{kg}$ & 3 & 2 & $67 \%$ & $1.00 \mathrm{E}-08$ & $1.00 \mathrm{E}-08$ & 3.00E-08 & $5.00 \mathrm{E}-08$ & 2.83E-08 \\
\hline DIOXI & 1,2,3,4,7,8,9-Heptachlorodibenzofuran & $\mathrm{mg} / \mathrm{kg}$ & 3 & 0 & $0 \%$ & $1.00 \mathrm{E}-08$ & 1.00E-08 & -- & -- & $5.00 \mathrm{E}-09$ \\
\hline DIOXI & 1,2,3,4,7,8-Hexachlorodibenzofuran & $\mathrm{mg} / \mathrm{kg}$ & 3 & 0 & $0 \%$ & $1.00 \mathrm{E}-08$ & $1.00 \mathrm{E}-08$ & -- & -- & $5.00 \mathrm{E}-09$ \\
\hline DIOXI & $1,2,3,4,7,8$-Hexachlorodibenzo-p-dioxin & $\mathrm{mg} / \mathrm{kg}$ & 3 & 0 & $0 \%$ & $1.00 \mathrm{E}-08$ & $1.00 \mathrm{E}-08$ & -- & -- & $5.00 \mathrm{E}-09$ \\
\hline DIOXI & 1,2,3,6,7,8-Hexachlorodibenzofuran & $\mathrm{mg} / \mathrm{kg}$ & 3 & 0 & $0 \%$ & $1.00 \mathrm{E}-08$ & $1.00 \mathrm{E}-08$ & -- & -- & $5.00 \mathrm{E}-09$ \\
\hline DIOXI & 1,2,3,6,7,8-Hexachlorodibenzo-p-dioxin & $\mathrm{mg} / \mathrm{kg}$ & 3 & 2 & $67 \%$ & $1.00 \mathrm{E}-08$ & $1.00 \mathrm{E}-08$ & $5.00 \mathrm{E}-08$ & $5.00 \mathrm{E}-08$ & $3.50 \mathrm{E}-08$ \\
\hline DIOXI & 1,2,3,7,8,9-Hexachlorodibenzofuran & $\mathrm{mg} / \mathrm{kg}$ & 3 & 0 & $0 \%$ & $1.00 \mathrm{E}-08$ & $1.00 \mathrm{E}-08$ & -- & -- & $5.00 \mathrm{E}-09$ \\
\hline DIOXI & 1,2,3,7,8,9-Hexachlorodibenzo-p-dioxin & $\mathrm{mg} / \mathrm{kg}$ & 3 & 1 & $33 \%$ & $1.00 \mathrm{E}-08$ & 1.00E-08 & 3.00E-08 & $3.00 \mathrm{E}-08$ & 1.33E-08 \\
\hline DIOXI & 1,2,3,7,8-Pentachlorodibenzofuran & $\mathrm{mg} / \mathrm{kg}$ & 3 & 0 & $0 \%$ & $1.00 \mathrm{E}-08$ & $1.00 \mathrm{E}-08$ & -- & -- & $5.00 \mathrm{E}-09$ \\
\hline DIOXI & 1,2,3,7,8-Pentachlorodibenzo-p-dioxin & $\mathrm{mg} / \mathrm{kg}$ & 3 & 2 & $67 \%$ & $1.00 \mathrm{E}-08$ & $1.00 \mathrm{E}-08$ & $5.00 \mathrm{E}-08$ & $6.00 \mathrm{E}-08$ & $3.83 \mathrm{E}-08$ \\
\hline DIOXI & $2,3,4,6,7,8$-Hexachlorodibenzofuran & $\mathrm{mg} / \mathrm{kg}$ & 3 & 0 & $0 \%$ & $1.00 \mathrm{E}-08$ & $1.00 \mathrm{E}-08$ & -- & -- & $5.00 \mathrm{E}-09$ \\
\hline DIOXI & 2,3,4,7,8-Pentachlorodibenzofuran & $\mathrm{mg} / \mathrm{kg}$ & 3 & 2 & $67 \%$ & $1.00 \mathrm{E}-08$ & $1.00 \mathrm{E}-08$ & 5.00E-08 & $9.00 \mathrm{E}-08$ & 4.83E-08 \\
\hline DIOXI & 2,3,7,8-Tetrachlorodibenzofuran & $\mathrm{mg} / \mathrm{kg}$ & 3 & 3 & $100 \%$ & -- & -- & 3.50E-07 & $5.40 \mathrm{E}-07$ & 4.57E-07 \\
\hline DIOXI & 2,3,7,8-Tetrachlorodibenzo-p-dioxin & $\mathrm{mg} / \mathrm{kg}$ & 3 & 1 & $33 \%$ & $1.00 \mathrm{E}-08$ & 1.00E-08 & 4.00E-08 & 4.00E-08 & $1.67 \mathrm{E}-08$ \\
\hline DIOXI & Octachlorodibenzofuran & $\mathrm{mg} / \mathrm{kg}$ & 3 & 0 & $0 \%$ & $2.00 \mathrm{E}-08$ & 2.00E-08 & -- & -- & $1.00 \mathrm{E}-08$ \\
\hline DIOXI & Octachlorodibenzo-p-dioxin & $\mathrm{mg} / \mathrm{kg}$ & 3 & 3 & $100 \%$ & -- & -- & $9.00 \mathrm{E}-08$ & $8.50 \mathrm{E}-07$ & 3.70E-07 \\
\hline METAL & Aluminum & $\mathrm{mg} / \mathrm{kg}$ & 3 & 0 & $0 \%$ & 1.0 & 1.0 & -- & -- & 0.50 \\
\hline METAL & Antimony & $\mathrm{mg} / \mathrm{kg}$ & 3 & 0 & $0 \%$ & 0.050 & 0.050 & -- & -- & 0.025 \\
\hline METAL & Arsenic & $\mathrm{mg} / \mathrm{kg}$ & 3 & 3 & $100 \%$ & -- & -- & 0.45 & 0.59 & 0.54 \\
\hline METAL & Barium & $\mathrm{mg} / \mathrm{kg}$ & 3 & 1 & $33 \%$ & 0.20 & 0.20 & 0.27 & 0.27 & 0.16 \\
\hline METAL & Beryllium & $\mathrm{mg} / \mathrm{kg}$ & 3 & 0 & $0 \%$ & 0.0040 & 0.0040 & -- & -- & 0.0020 \\
\hline METAL & Cadmium & $\mathrm{mg} / \mathrm{kg}$ & 3 & 0 & $0 \%$ & 0.0040 & 0.0040 & -- & -- & 0.0020 \\
\hline METAL & Chromium & $\mathrm{mg} / \mathrm{kg}$ & 3 & 3 & $100 \%$ & -- & -- & 0.12 & 0.19 & 0.14 \\
\hline METAL & Cobalt & $\mathrm{mg} / \mathrm{kg}$ & 3 & 3 & $100 \%$ & -- & -- & 0.11 & 0.13 & 0.12 \\
\hline METAL & Copper & $\mathrm{mg} / \mathrm{kg}$ & 3 & 3 & $100 \%$ & -- & -- & 0.68 & 3.6 & 1.7 \\
\hline METAL & Lead & $\mathrm{mg} / \mathrm{kg}$ & 3 & 2 & $67 \%$ & 0.010 & 0.010 & 0.010 & 0.22 & 0.078 \\
\hline METAL & Manganese & $\mathrm{mg} / \mathrm{kg}$ & 3 & 3 & $100 \%$ & -- & -- & 0.11 & 0.32 & 0.19 \\
\hline METAL & Mercury & $\mathrm{mg} / \mathrm{kg}$ & 3 & 3 & $100 \%$ & -- & -- & 0.10 & 0.12 & 0.11 \\
\hline METAL & Nickel & $\mathrm{mg} / \mathrm{kg}$ & 3 & 2 & $67 \%$ & 0.030 & 0.030 & 0.040 & 0.10 & 0.052 \\
\hline METAL & Selenium & $\mathrm{mg} / \mathrm{kg}$ & 3 & 3 & $100 \%$ & -- & -- & 0.27 & 0.31 & 0.29 \\
\hline METAL & Silver & $\mathrm{mg} / \mathrm{kg}$ & 3 & 0 & $0 \%$ & 0.10 & 0.10 & -- & -- & 0.050 \\
\hline METAL & Thallium & $\mathrm{mg} / \mathrm{kg}$ & 3 & 0 & $0 \%$ & 0.050 & 0.050 & -- & -- & 0.025 \\
\hline METAL & Vanadium & $\mathrm{mg} / \mathrm{kg}$ & 3 & 1 & $33 \%$ & 0.010 & 0.010 & 0.010 & 0.010 & 0.0067 \\
\hline METAL & Zinc & $\mathrm{mg} / \mathrm{kg}$ & 3 & 3 & $100 \%$ & -- & -- & 6.7 & 7.8 & 7.2 \\
\hline PCB & 2,2',3,3',4,4',5-Heptachloro-1,1'-biphenyl & $\mathrm{mg} / \mathrm{kg}$ & 3 & 3 & $100 \%$ & -- & -- & 1.95E-04 & $2.18 \mathrm{E}-04$ & $2.09 \mathrm{E}-04$ \\
\hline PCB & $2,2^{\prime}, 3,4,4^{\prime}, 5,5^{\prime}-$ Heptachlorobiphenyl & $\mathrm{mg} / \mathrm{kg}$ & 3 & 3 & $100 \%$ & -- & -- & $6.42 \mathrm{E}-04$ & $7.27 \mathrm{E}-04$ & $6.89 \mathrm{E}-04$ \\
\hline PCB & 2,3,3',4,4',5'-Hexachlorobiphenyl & $\mathrm{mg} / \mathrm{kg}$ & 3 & 3 & $100 \%$ & -- & -- & 2.05E-05 & $2.59 \mathrm{E}-05$ & $2.24 \mathrm{E}-05$ \\
\hline PCB & 2,3,3',4,4',5-Hexachlorobiphenyl (BZ 156) & $\mathrm{mg} / \mathrm{kg}$ & 3 & 3 & $100 \%$ & -- & -- & 9.51E-05 & $1.21 \mathrm{E}-04$ & $1.08 \mathrm{E}-04$ \\
\hline PCB & 2,3,3',4,4'-Pentachlorobiphenyl & $\mathrm{mg} / \mathrm{kg}$ & 3 & 3 & $100 \%$ & -- & -- & 2.67E-04 & 3.13E-04 & $2.97 \mathrm{E}-04$ \\
\hline PCB & 2,3',4,4',5,5'-Hexachlorobiphenyl (BZ 167) & $\mathrm{mg} / \mathrm{kg}$ & 3 & 3 & $100 \%$ & -- & -- & 4.48E-05 & $5.07 \mathrm{E}-05$ & $4.68 \mathrm{E}-05$ \\
\hline PCB & 2',3,4,4',5-Pentachloro-1,1'-biphenyl (BZ 123) & $\mathrm{mg} / \mathrm{kg}$ & 3 & 3 & $100 \%$ & -- & -- & $9.42 \mathrm{E}-06$ & 1.50E-05 & $1.18 \mathrm{E}-05$ \\
\hline РСB & 2,3,4,4',5-Pentachlorobiphenyl (BZ 114) & $\mathrm{mg} / \mathrm{kg}$ & 3 & 3 & $100 \%$ & -- & -- & 2.39E-05 & 3.19E-05 & $2.82 \mathrm{E}-05$ \\
\hline PCB & 2,3',4,4',5-Pentachlorobiphenyl (BZ 118) & $\mathrm{mg} / \mathrm{kg}$ & 3 & 3 & $100 \%$ & -- & -- & $6.74 \mathrm{E}-04$ & 7.44E-04 & 7.19E-04 \\
\hline PCB & 2,3,4,5,3',4',5'-Heptachlorobiphenyl & $\mathrm{mg} / \mathrm{kg}$ & 3 & 3 & $100 \%$ & -- & -- & 7.93E-06 & $9.08 \mathrm{E}-06$ & 8.60E-06 \\
\hline РСB & 3,3',4,4',5,5'-Hexachlorobiphenyl (BZ 169) & $\mathrm{mg} / \mathrm{kg}$ & 3 & 0 & $0 \%$ & $1.00 \mathrm{E}-06$ & 1.06E-06 & -- & -- & $5.15 \mathrm{E}-07$ \\
\hline PCB & 3,3',4,4',5-Pentachlorobiphenyl (BZ 126) & $\mathrm{mg} / \mathrm{kg}$ & 3 & 0 & $0 \%$ & $8.50 \mathrm{E}-07$ & $1.99 \mathrm{E}-06$ & -- & -- & 7.10E-07 \\
\hline PCB & 3,3',4,4'-Tetrachlorobiphenyl (BZ 77) & $\mathrm{mg} / \mathrm{kg}$ & 3 & 3 & $100 \%$ & -- & -- & 8.03E-06 & 8.35E-06 & 8.15E-06 \\
\hline РCB & Aroclor-1016 & $\mathrm{mg} / \mathrm{kg}$ & 3 & 0 & $0 \%$ & 0.018 & 0.020 & -- & -- & 0.0097 \\
\hline PCB & Aroclor-1221 & $\mathrm{mg} / \mathrm{kg}$ & 3 & 0 & $0 \%$ & 0.018 & 0.020 & -- & -- & 0.0097 \\
\hline PCB & Aroclor-1232 & $\mathrm{mg} / \mathrm{kg}$ & 3 & 0 & $0 \%$ & 0.018 & 0.020 & -- & -- & 0.0097 \\
\hline PCB & Aroclor-1248 & $\mathrm{mg} / \mathrm{kg}$ & 3 & 0 & $0 \%$ & 0.018 & 0.020 & -- & -- & 0.0097 \\
\hline
\end{tabular}


Table F-82

Summary Statistics for Coho Salmon Fillet Skin On Collected from Segment 4 of the Columbia River

\begin{tabular}{|c|c|c|c|c|c|c|c|c|c|c|}
\hline $\begin{array}{l}\text { Constituent } \\
\text { Class }\end{array}$ & Constituent Name & Units & $\begin{array}{l}\text { Number } \\
\text { Analyzed }\end{array}$ & $\begin{array}{l}\text { Number } \\
\text { Detected }\end{array}$ & $\begin{array}{c}\text { Frequency of } \\
\text { Detection }\end{array}$ & $\begin{array}{l}\text { Minimum } \\
\text { Nondetect }\end{array}$ & $\begin{array}{l}\text { Maximum } \\
\text { Nondetect }\end{array}$ & $\begin{array}{l}\text { Minimum } \\
\text { Detect }\end{array}$ & $\begin{array}{l}\text { Maximum } \\
\text { Detect }\end{array}$ & $\begin{array}{c}\text { Average } \\
\text { Concentration }\end{array}$ \\
\hline$\overline{\mathrm{PCB}}$ & Aroclor-1254 & $\mathrm{mg} / \mathrm{kg}$ & 3 & 3 & $100 \%$ & $\overline{--}$ & $\overline{--}$ & 0.012 & 0.019 & 0.016 \\
\hline PCB & Aroclor-1260 & $\mathrm{mg} / \mathrm{kg}$ & 3 & 0 & $0 \%$ & 0.018 & 0.020 & -- & -- & 0.0097 \\
\hline PEST & 2,4'-DDD & $\mathrm{mg} / \mathrm{kg}$ & 3 & 0 & $0 \%$ & 0.0018 & 0.0020 & -- & -- & $9.67 \mathrm{E}-04$ \\
\hline PEST & 2,4'-DDE & $\mathrm{mg} / \mathrm{kg}$ & 3 & 0 & $0 \%$ & 0.0018 & 0.0020 & -- & -- & $9.67 \mathrm{E}-04$ \\
\hline PEST & 2,4'-DDT & $\mathrm{mg} / \mathrm{kg}$ & 3 & 0 & $0 \%$ & 0.0018 & 0.0020 & -- & -- & $9.67 \mathrm{E}-04$ \\
\hline PEST & Aldrin & $\mathrm{mg} / \mathrm{kg}$ & 3 & 0 & $0 \%$ & 0.0018 & 0.0020 & -- & -- & $9.67 \mathrm{E}-04$ \\
\hline PEST & Alpha-BHC & $\mathrm{mg} / \mathrm{kg}$ & 3 & 0 & $0 \%$ & 0.0018 & 0.0020 & -- & -- & $9.67 \mathrm{E}-04$ \\
\hline PEST & alpha-Chlordane & $\mathrm{mg} / \mathrm{kg}$ & 3 & 0 & $0 \%$ & 0.0018 & 0.0020 & -- & -- & $9.67 \mathrm{E}-04$ \\
\hline PEST & alpha-Chlordene & $\mathrm{mg} / \mathrm{kg}$ & 3 & 0 & $0 \%$ & 0.0018 & 0.0020 & -- & -- & $9.67 \mathrm{E}-04$ \\
\hline PEST & Beta-BHC & $\mathrm{mg} / \mathrm{kg}$ & 3 & 0 & $0 \%$ & 0.0018 & 0.0020 & -- & -- & $9.67 \mathrm{E}-04$ \\
\hline PEST & cis-Nonachlor & $\mathrm{mg} / \mathrm{kg}$ & 3 & 0 & $0 \%$ & 0.0018 & 0.0020 & -- & -- & $9.67 \mathrm{E}-04$ \\
\hline PEST & DDMU & $\mathrm{mg} / \mathrm{kg}$ & 3 & 0 & $0 \%$ & 0.0018 & 0.0020 & -- & -- & $9.67 \mathrm{E}-04$ \\
\hline PEST & Delta-BHC & $\mathrm{mg} / \mathrm{kg}$ & 3 & 0 & $0 \%$ & 0.0018 & 0.0020 & -- & -- & $9.67 \mathrm{E}-04$ \\
\hline PEST & Dichlorodiphenyldichloroethane & $\mathrm{mg} / \mathrm{kg}$ & 3 & 3 & $100 \%$ & -- & -- & 0.0025 & 0.0029 & 0.0027 \\
\hline PEST & Dichlorodiphenyldichloroethylene & $\mathrm{mg} / \mathrm{kg}$ & 3 & 3 & $100 \%$ & -- & -- & 0.029 & 0.035 & 0.033 \\
\hline PEST & Dichlorodiphenyltrichloroethane & $\mathrm{mg} / \mathrm{kg}$ & 3 & 1 & $33 \%$ & 0.0018 & 0.0020 & 0.0040 & 0.0040 & 0.0020 \\
\hline PEST & Endosulfan sulfate & $\mathrm{mg} / \mathrm{kg}$ & 2 & 0 & $0 \%$ & 0.0018 & 0.0020 & -- & -- & $9.50 \mathrm{E}-04$ \\
\hline PEST & Gamma-BHC (Lindane) & $\mathrm{mg} / \mathrm{kg}$ & 3 & 0 & $0 \%$ & 0.0018 & 0.0020 & -- & -- & $9.67 \mathrm{E}-04$ \\
\hline PEST & gamma-Chlordane & $\mathrm{mg} / \mathrm{kg}$ & 3 & 0 & $0 \%$ & 0.0018 & 0.0020 & -- & -- & $9.67 \mathrm{E}-04$ \\
\hline PEST & gamma-Chlordene & $\mathrm{mg} / \mathrm{kg}$ & 3 & 0 & $0 \%$ & 0.0018 & 0.0020 & -- & -- & $9.67 \mathrm{E}-04$ \\
\hline PEST & Heptachlor & $\mathrm{mg} / \mathrm{kg}$ & 3 & 0 & $0 \%$ & 0.0018 & 0.0020 & -- & -- & $9.67 \mathrm{E}-04$ \\
\hline PEST & Heptachlor epoxide & $\mathrm{mg} / \mathrm{kg}$ & 3 & 0 & $0 \%$ & 0.0018 & 0.0020 & -- & -- & $9.67 \mathrm{E}-04$ \\
\hline PEST & Mirex & $\mathrm{mg} / \mathrm{kg}$ & 3 & 0 & $0 \%$ & 0.0018 & 0.0020 & -- & -- & $9.67 \mathrm{E}-04$ \\
\hline PEST & Oxychlordane & $\mathrm{mg} / \mathrm{kg}$ & 3 & 0 & $0 \%$ & 0.0018 & 0.0020 & -- & -- & $9.67 \mathrm{E}-04$ \\
\hline PEST & Pentachloroanisole & $\mathrm{mg} / \mathrm{kg}$ & 3 & 0 & $0 \%$ & 0.0018 & 0.0020 & -- & -- & $9.67 \mathrm{E}-04$ \\
\hline PEST & Toxaphene & $\mathrm{mg} / \mathrm{kg}$ & 3 & 0 & $0 \%$ & 0.055 & 0.060 & -- & -- & 0.029 \\
\hline PEST & trans-Nonachlor & $\mathrm{mg} / \mathrm{kg}$ & 3 & 0 & $0 \%$ & 0.0018 & 0.0020 & -- & -- & $9.67 \mathrm{E}-04$ \\
\hline SVOC & 1,2,4-Trichlorobenzene & $\mathrm{mg} / \mathrm{kg}$ & 3 & 0 & $0 \%$ & 0.0095 & 0.0097 & -- & -- & 0.0048 \\
\hline SVOC & 1,2-Dichlorobenzene & $\mathrm{mg} / \mathrm{kg}$ & 3 & 0 & $0 \%$ & 0.0095 & 0.0097 & -- & -- & 0.0048 \\
\hline SVOC & 1,2-Diphenylhydrazine & $\mathrm{mg} / \mathrm{kg}$ & 3 & 0 & $0 \%$ & 0.0095 & 0.0097 & -- & -- & 0.0048 \\
\hline SVOC & 1,3-Dichlorobenzene & $\mathrm{mg} / \mathrm{kg}$ & 3 & 0 & $0 \%$ & 0.0095 & 0.0097 & -- & -- & 0.0048 \\
\hline SVOC & 1,4-Dichlorobenzene & $\mathrm{mg} / \mathrm{kg}$ & 3 & 0 & $0 \%$ & 0.0095 & 0.0097 & -- & -- & 0.0048 \\
\hline SVOC & 1-methylnaphthalene & $\mathrm{mg} / \mathrm{kg}$ & 3 & 0 & $0 \%$ & 0.0095 & 0.0097 & -- & -- & 0.0048 \\
\hline SVOC & 2,4-Dinitrotoluene & $\mathrm{mg} / \mathrm{kg}$ & 3 & 0 & $0 \%$ & 0.019 & 0.15 & -- & -- & 0.031 \\
\hline SVOC & 2,6-Dinitrotoluene & $\mathrm{mg} / \mathrm{kg}$ & 3 & 0 & $0 \%$ & 0.038 & 0.039 & -- & -- & 0.019 \\
\hline SVOC & 2-Chloronaphthalene & $\mathrm{mg} / \mathrm{kg}$ & 3 & 0 & $0 \%$ & 0.019 & 0.019 & -- & -- & 0.0095 \\
\hline SVOC & 2-MethyInaphthalene & $\mathrm{mg} / \mathrm{kg}$ & 3 & 0 & $0 \%$ & 0.0095 & 0.0097 & -- & -- & 0.0048 \\
\hline SVOC & 4-Bromophenylphenyl ether & $\mathrm{mg} / \mathrm{kg}$ & 3 & 0 & $0 \%$ & 0.0095 & 0.0097 & -- & -- & 0.0048 \\
\hline SVOC & 4-Chlorophenylphenyl ether & $\mathrm{mg} / \mathrm{kg}$ & 3 & 0 & $0 \%$ & 0.0095 & 0.0097 & -- & -- & 0.0048 \\
\hline SVOC & Acenaphthene & $\mathrm{mg} / \mathrm{kg}$ & 3 & 0 & $0 \%$ & 0.019 & 0.019 & -- & -- & 0.0095 \\
\hline SVOC & Acenaphthylene & $\mathrm{mg} / \mathrm{kg}$ & 3 & 0 & $0 \%$ & 0.0095 & 0.0097 & -- & -- & 0.0048 \\
\hline SVOC & Anthracene & $\mathrm{mg} / \mathrm{kg}$ & 3 & 0 & $0 \%$ & 0.0095 & 0.0097 & -- & -- & 0.0048 \\
\hline SVOC & Benzo(a)anthracene & $\mathrm{mg} / \mathrm{kg}$ & 3 & 0 & $0 \%$ & 0.0095 & 0.0097 & -- & -- & 0.0048 \\
\hline SVOC & Benzo(a)pyrene & $\mathrm{mg} / \mathrm{kg}$ & 3 & 0 & $0 \%$ & 0.0095 & 0.0097 & -- & -- & 0.0048 \\
\hline SVOC & Benzo(b)fluoranthene & $\mathrm{mg} / \mathrm{kg}$ & 3 & 0 & $0 \%$ & 0.0095 & 0.0097 & -- & -- & 0.0048 \\
\hline SVOC & Benzo(ghi)perylene & $\mathrm{mg} / \mathrm{kg}$ & 3 & 0 & $0 \%$ & 0.019 & 0.019 & -- & -- & 0.0095 \\
\hline SVOC & Benzo(k)fluoranthene & $\mathrm{mg} / \mathrm{kg}$ & 3 & 0 & $0 \%$ & 0.0095 & 0.0097 & -- & -- & 0.0048 \\
\hline SVOC & Bis(2-chloro-1-methylethyl)ether & $\mathrm{mg} / \mathrm{kg}$ & 3 & 0 & $0 \%$ & 0.019 & 0.019 & -- & -- & 0.0095 \\
\hline SVOC & Chrysene & $\mathrm{mg} / \mathrm{kg}$ & 3 & 0 & $0 \%$ & 0.0095 & 0.0097 & -- & -- & 0.0048 \\
\hline SVOC & Dibenz $[\mathrm{a}, \mathrm{h}]$ anthracene & $\mathrm{mg} / \mathrm{kg}$ & 3 & 0 & $0 \%$ & 0.019 & 0.019 & -- & -- & 0.0095 \\
\hline SVOC & Dibenzofuran & $\mathrm{mg} / \mathrm{kg}$ & 3 & 0 & $0 \%$ & 0.0095 & 0.0097 & -- & -- & 0.0048 \\
\hline SVOC & Fluoranthene & $\mathrm{mg} / \mathrm{kg}$ & 3 & 0 & $0 \%$ & 0.019 & 0.019 & -- & -- & 0.0095 \\
\hline
\end{tabular}


Table F-82

Summary Statistics for Coho Salmon Fillet Skin On Collected from Segment 4 of the Columbia River

\begin{tabular}{|c|c|c|c|c|c|c|c|c|c|c|}
\hline $\begin{array}{c}\text { Constituent } \\
\text { Class }\end{array}$ & Constituent Name & Units & $\begin{array}{l}\text { Number } \\
\text { Analyzed }\end{array}$ & $\begin{array}{l}\text { Number } \\
\text { Detected }\end{array}$ & $\begin{array}{c}\text { Frequency of } \\
\text { Detection }\end{array}$ & $\begin{array}{l}\text { Minimum } \\
\text { Nondetect }\end{array}$ & $\begin{array}{l}\text { Maximum } \\
\text { Nondetect }\end{array}$ & $\begin{array}{c}\text { Minimum } \\
\text { Detect }\end{array}$ & $\begin{array}{l}\text { Maximum } \\
\text { Detect }\end{array}$ & $\begin{array}{c}\text { Average } \\
\text { Concentration }\end{array}$ \\
\hline SVOC & Fluorene & $\mathrm{mg} / \mathrm{kg}$ & 3 & 0 & $0 \%$ & 0.0047 & 0.0086 & -- & -- & 0.0031 \\
\hline SVOC & Hexachlorobenzene & $\mathrm{mg} / \mathrm{kg}$ & 3 & 0 & $0 \%$ & 0.019 & 0.019 & -- & -- & 0.0095 \\
\hline SVOC & Hexachlorobutadiene & $\mathrm{mg} / \mathrm{kg}$ & 3 & 0 & $0 \%$ & 0.0095 & 0.0097 & -- & -- & 0.0048 \\
\hline SVOC & Hexachloroethane & $\mathrm{mg} / \mathrm{kg}$ & 3 & 0 & $0 \%$ & 0.0095 & 0.0097 & -- & -- & 0.0048 \\
\hline SVOC & Indeno(1,2,3-cd)pyrene & $\mathrm{mg} / \mathrm{kg}$ & 3 & 0 & $0 \%$ & 0.019 & 0.019 & -- & -- & 0.0095 \\
\hline SVOC & Naphthalene & $\mathrm{mg} / \mathrm{kg}$ & 3 & 0 & $0 \%$ & 0.017 & 0.026 & -- & -- & 0.011 \\
\hline SVOC & Nitrobenzene & $\mathrm{mg} / \mathrm{kg}$ & 3 & 0 & $0 \%$ & 0.0095 & 0.0097 & -- & -- & 0.0048 \\
\hline SVOC & Phenanthrene & $\mathrm{mg} / \mathrm{kg}$ & 3 & 0 & $0 \%$ & 0.0095 & 0.0097 & -- & -- & 0.0048 \\
\hline SVOC & Pyrene & $\mathrm{mg} / \mathrm{kg}$ & 3 & 0 & $0 \%$ & 0.019 & 0.019 & -- & -- & 0.0095 \\
\hline VOC & Retene & $\mathrm{mg} / \mathrm{kg}$ & 3 & 0 & $0 \%$ & 0.019 & 0.019 & -- & -- & 0.0095 \\
\hline
\end{tabular}

DIOXI - Dioxins and Furans

PEST - Pesticide

PCB - Polychlorinated biphenyl

SVOC - Semivolatile Organic Compound

VOC - Volatile Organic Compound 
Table F-83

Summary Statistics for Whole Body Coho Salmon Collected from Segment 4 of the Columbia River

\begin{tabular}{|c|c|c|c|c|c|c|c|c|c|c|}
\hline $\begin{array}{l}\text { Constituent } \\
\text { Class }\end{array}$ & Constituent Name & Units & $\begin{array}{l}\text { Number } \\
\text { Analyzed }\end{array}$ & $\begin{array}{l}\text { Number } \\
\text { Detected }\end{array}$ & $\begin{array}{c}\text { Frequency of } \\
\text { Detection }\end{array}$ & $\begin{array}{l}\text { Minimum } \\
\text { Nondetect }\end{array}$ & $\begin{array}{l}\text { Maximum } \\
\text { Nondetect }\end{array}$ & $\begin{array}{c}\text { Minimum } \\
\text { Detect }\end{array}$ & $\begin{array}{c}\text { Maximum } \\
\text { Detect }\end{array}$ & $\begin{array}{c}\text { Average } \\
\text { Concentration }\end{array}$ \\
\hline$\overline{D I O X I}$ & $1,2,3,4,6,7,8$-Heptachlorodibenzodioxin & $\mathrm{mg} / \mathrm{kg}$ & 2 & 1 & $50 \%$ & $1.00 \mathrm{E}-08$ & $1.00 \mathrm{E}-08$ & $6.00 \mathrm{E}-08$ & $6.00 \mathrm{E}-08$ & $3.25 \mathrm{E}-08$ \\
\hline DIOXI & $1,2,3,4,6,7,8$-Heptachlorodibenzofuran & $\mathrm{mg} / \mathrm{kg}$ & 2 & 0 & $0 \%$ & $1.00 \mathrm{E}-08$ & $3.00 \mathrm{E}-08$ & -- & -- & $1.00 \mathrm{E}-08$ \\
\hline DIOXI & $1,2,3,4,7,8,9$-Heptachlorodibenzofuran & $\mathrm{mg} / \mathrm{kg}$ & 2 & 0 & $0 \%$ & $1.00 \mathrm{E}-08$ & $3.00 \mathrm{E}-08$ & -- & -- & $1.00 \mathrm{E}-08$ \\
\hline DIOXI & 1,2,3,4,7,8-Hexachlorodibenzofuran & $\mathrm{mg} / \mathrm{kg}$ & 2 & 0 & $0 \%$ & $1.00 \mathrm{E}-08$ & $2.00 \mathrm{E}-08$ & -- & -- & 7.50E-09 \\
\hline DIOXI & $1,2,3,4,7,8$-Hexachlorodibenzo-p-dioxin & $\mathrm{mg} / \mathrm{kg}$ & 2 & 0 & $0 \%$ & $1.00 \mathrm{E}-08$ & 2.00E-08 & -- & -- & $7.50 \mathrm{E}-09$ \\
\hline DIOXI & 1,2,3,6,7,8-Hexachlorodibenzofuran & $\mathrm{mg} / \mathrm{kg}$ & 2 & 0 & $0 \%$ & $1.00 \mathrm{E}-08$ & $2.00 \mathrm{E}-08$ & -- & -- & 7.50E-09 \\
\hline DIOXI & $1,2,3,6,7,8$-Hexachlorodibenzo-p-dioxin & $\mathrm{mg} / \mathrm{kg}$ & 2 & 0 & $0 \%$ & $1.00 \mathrm{E}-08$ & $2.00 \mathrm{E}-08$ & -- & -- & $7.50 \mathrm{E}-09$ \\
\hline DIOXI & 1,2,3,7,8,9-Hexachlorodibenzofuran & $\mathrm{mg} / \mathrm{kg}$ & 2 & 0 & $0 \%$ & $1.00 \mathrm{E}-08$ & $2.00 \mathrm{E}-08$ & -- & -- & 7.50E-09 \\
\hline DIOXI & $1,2,3,7,8,9$-Hexachlorodibenzo-p-dioxin & $\mathrm{mg} / \mathrm{kg}$ & 2 & 1 & $50 \%$ & $2.00 \mathrm{E}-08$ & $2.00 \mathrm{E}-08$ & $2.00 \mathrm{E}-08$ & $2.00 \mathrm{E}-08$ & $1.50 \mathrm{E}-08$ \\
\hline DIOXI & 1,2,3,7,8-Pentachlorodibenzofuran & $\mathrm{mg} / \mathrm{kg}$ & 2 & 0 & $0 \%$ & $2.00 \mathrm{E}-08$ & $3.00 \mathrm{E}-08$ & -- & -- & $1.25 \mathrm{E}-08$ \\
\hline DIOXI & $1,2,3,7,8$-Pentachlorodibenzo-p-dioxin & $\mathrm{mg} / \mathrm{kg}$ & 2 & 0 & $0 \%$ & $1.00 \mathrm{E}-08$ & $6.00 \mathrm{E}-08$ & -- & -- & $1.75 \mathrm{E}-08$ \\
\hline DIOXI & 2,3,4,6,7,8-Hexachlorodibenzofuran & $\mathrm{mg} / \mathrm{kg}$ & 2 & 0 & $0 \%$ & $1.00 \mathrm{E}-08$ & $2.00 \mathrm{E}-08$ & -- & -- & 7.50E-09 \\
\hline DIOXI & 2,3,4,7,8-Pentachlorodibenzofuran & $\mathrm{mg} / \mathrm{kg}$ & 2 & 1 & $50 \%$ & $2.00 \mathrm{E}-08$ & $2.00 \mathrm{E}-08$ & 8.00E-08 & $8.00 \mathrm{E}-08$ & $4.50 \mathrm{E}-08$ \\
\hline DIOXI & 2,3,7,8-Tetrachlorodibenzofuran & $\mathrm{mg} / \mathrm{kg}$ & 2 & 2 & $100 \%$ & -- & -- & 4.80E-07 & 4.90E-07 & 4.85E-07 \\
\hline DIOXI & 2,3,7,8-Tetrachlorodibenzo-p-dioxin & $\mathrm{mg} / \mathrm{kg}$ & 2 & 0 & $0 \%$ & $1.00 \mathrm{E}-08$ & $5.00 \mathrm{E}-08$ & -- & -- & $1.50 \mathrm{E}-08$ \\
\hline DIOXI & Octachlorodibenzofuran & $\mathrm{mg} / \mathrm{kg}$ & 2 & 0 & $0 \%$ & $3.00 \mathrm{E}-08$ & $6.00 \mathrm{E}-08$ & -- & -- & $2.25 \mathrm{E}-08$ \\
\hline DIOXI & Octachlorodibenzo-p-dioxin & $\mathrm{mg} / \mathrm{kg}$ & 2 & 1 & $50 \%$ & $1.00 \mathrm{E}-08$ & $1.00 \mathrm{E}-08$ & 2.50E-07 & $2.50 \mathrm{E}-07$ & $1.28 \mathrm{E}-07$ \\
\hline METAL & Aluminum & $\mathrm{mg} / \mathrm{kg}$ & 2 & 0 & $0 \%$ & 1.0 & 1.0 & -- & -- & 0.50 \\
\hline METAL & Antimony & $\mathrm{mg} / \mathrm{kg}$ & 2 & 0 & $0 \%$ & 0.050 & 0.050 & -- & -- & 0.025 \\
\hline METAL & Arsenic & $\mathrm{mg} / \mathrm{kg}$ & 2 & 2 & $100 \%$ & -- & -- & 0.49 & 0.55 & 0.52 \\
\hline METAL & Barium & $\mathrm{mg} / \mathrm{kg}$ & 2 & 0 & $0 \%$ & 0.20 & 0.20 & -- & -- & 0.10 \\
\hline METAL & Beryllium & $\mathrm{mg} / \mathrm{kg}$ & 2 & 0 & $0 \%$ & 0.0040 & 0.0040 & -- & -- & 0.0020 \\
\hline METAL & Cadmium & $\mathrm{mg} / \mathrm{kg}$ & 2 & 2 & $100 \%$ & -- & -- & 0.010 & 0.020 & 0.015 \\
\hline METAL & Chromium & $\mathrm{mg} / \mathrm{kg}$ & 2 & 2 & $100 \%$ & -- & -- & 0.12 & 0.13 & 0.13 \\
\hline METAL & Cobalt & $\mathrm{mg} / \mathrm{kg}$ & 2 & 2 & $100 \%$ & -- & -- & 0.11 & 0.11 & 0.11 \\
\hline METAL & Copper & $\mathrm{mg} / \mathrm{kg}$ & 2 & 2 & $100 \%$ & -- & -- & 0.72 & 0.96 & 0.84 \\
\hline METAL & Lead & $\mathrm{mg} / \mathrm{kg}$ & 2 & 2 & $100 \%$ & -- & -- & 0.010 & 0.010 & 0.010 \\
\hline METAL & Manganese & $\mathrm{mg} / \mathrm{kg}$ & 2 & 2 & $100 \%$ & -- & -- & 0.39 & 0.56 & 0.48 \\
\hline METAL & Mercury & $\mathrm{mg} / \mathrm{kg}$ & 2 & 2 & $100 \%$ & -- & -- & 0.070 & 0.11 & 0.090 \\
\hline METAL & Nickel & $\mathrm{mg} / \mathrm{kg}$ & 2 & 2 & $100 \%$ & -- & -- & 0.050 & 0.050 & 0.050 \\
\hline METAL & Selenium & $\mathrm{mg} / \mathrm{kg}$ & 2 & 2 & $100 \%$ & -- & -- & 0.33 & 0.34 & 0.34 \\
\hline METAL & Silver & $\mathrm{mg} / \mathrm{kg}$ & 2 & 0 & $0 \%$ & 0.10 & 0.10 & -- & -- & 0.050 \\
\hline METAL & Thallium & $\mathrm{mg} / \mathrm{kg}$ & 2 & 0 & $0 \%$ & 0.050 & 0.050 & -- & -- & 0.025 \\
\hline METAL & Vanadium & $\mathrm{mg} / \mathrm{kg}$ & 2 & 2 & $100 \%$ & -- & -- & 0.030 & 0.030 & 0.030 \\
\hline METAL & Zinc & $\mathrm{mg} / \mathrm{kg}$ & 2 & 2 & $100 \%$ & -- & -- & 31 & 32 & 32 \\
\hline PCB & 2,2',3,3',4,4',5-Heptachloro-1,1'-biphenyl & $\mathrm{mg} / \mathrm{kg}$ & 2 & 2 & $100 \%$ & -- & -- & 1.83E-04 & $1.90 \mathrm{E}-04$ & 1.87E-04 \\
\hline РСB & 2,2',3,4,4',5,5'-Heptachlorobiphenyl & $\mathrm{mg} / \mathrm{kg}$ & 2 & 2 & $100 \%$ & -- & -- & 5.76E-04 & $6.21 \mathrm{E}-04$ & $5.99 \mathrm{E}-04$ \\
\hline PCB & 2,3,3',4,4',5'-Hexachlorobiphenyl & $\mathrm{mg} / \mathrm{kg}$ & 2 & 2 & $100 \%$ & -- & -- & 2.00E-05 & 2.17E-05 & $2.09 \mathrm{E}-05$ \\
\hline РСB & 2,3,3',4,4',5-Hexachlorobiphenyl (BZ 156) & $\mathrm{mg} / \mathrm{kg}$ & 2 & 2 & $100 \%$ & -- & -- & 9.03E-05 & $1.05 \mathrm{E}-04$ & $9.77 \mathrm{E}-05$ \\
\hline PCB & 2,3,3',4,4'-Pentachlorobiphenyl & $\mathrm{mg} / \mathrm{kg}$ & 2 & 2 & $100 \%$ & -- & -- & 2.91E-04 & $3.30 \mathrm{E}-04$ & $3.11 \mathrm{E}-04$ \\
\hline PCB & 2,3',4,4',5,5'-Hexachlorobiphenyl (BZ 167) & $\mathrm{mg} / \mathrm{kg}$ & 2 & 2 & $100 \%$ & -- & -- & 4.16E-05 & 4.82E-05 & 4.49E-05 \\
\hline РCB & 2',3,4,4',5-Pentachloro-1,1'-biphenyl (BZ 123) & $\mathrm{mg} / \mathrm{kg}$ & 2 & 2 & $100 \%$ & -- & -- & $9.64 \mathrm{E}-06$ & $1.12 \mathrm{E}-05$ & $1.04 \mathrm{E}-05$ \\
\hline РCB & 2,3,4,4',5-Pentachlorobiphenyl (BZ 114) & $\mathrm{mg} / \mathrm{kg}$ & 2 & 2 & $100 \%$ & -- & -- & 2.35E-05 & $3.07 \mathrm{E}-05$ & $2.71 \mathrm{E}-05$ \\
\hline PCB & 2,3',4,4',5-Pentachlorobiphenyl (BZ 118) & $\mathrm{mg} / \mathrm{kg}$ & 2 & 2 & $100 \%$ & -- & -- & $6.84 \mathrm{E}-04$ & 7.74E-04 & $7.29 \mathrm{E}-04$ \\
\hline РСB & 2,3,4,5,3',4',5'-Heptachlorobiphenyl & $\mathrm{mg} / \mathrm{kg}$ & 2 & 2 & $100 \%$ & -- & -- & $7.58 \mathrm{E}-06$ & 7.93E-06 & 7.76E-06 \\
\hline PCB & $3,3^{\prime}, 4,4^{\prime}, 5,5^{\prime}-$ Hexachlorobiphenyl (BZ 169) & $\mathrm{mg} / \mathrm{kg}$ & 2 & 0 & $0 \%$ & 1.00E-06 & 1.00E-06 & -- & -- & 5.00E-07 \\
\hline PCB & 3,3',4,4',5-Pentachlorobiphenyl (BZ 126) & $\mathrm{mg} / \mathrm{kg}$ & 2 & 2 & $100 \%$ & -- & -- & 3.11E-06 & 3.30E-06 & $3.21 \mathrm{E}-06$ \\
\hline РСB & 3,3',4,4'-Tetrachlorobiphenyl (BZ 77) & $\mathrm{mg} / \mathrm{kg}$ & 2 & 2 & $100 \%$ & -- & -- & $9.66 \mathrm{E}-06$ & 1.13E-05 & $1.05 \mathrm{E}-05$ \\
\hline PCB & Aroclor-1016 & $\mathrm{mg} / \mathrm{kg}$ & 2 & 0 & $0 \%$ & 0.019 & 0.020 & -- & -- & 0.0098 \\
\hline PCB & Aroclor-1221 & $\mathrm{mg} / \mathrm{kg}$ & 2 & 0 & $0 \%$ & 0.019 & 0.020 & -- & -- & 0.0098 \\
\hline PCB & Aroclor-1232 & $\mathrm{mg} / \mathrm{kg}$ & 2 & 0 & $0 \%$ & 0.019 & 0.020 & -- & -- & 0.0098 \\
\hline PCB & Aroclor-1248 & $\mathrm{mg} / \mathrm{kg}$ & 2 & 0 & $0 \%$ & 0.019 & 0.020 & -- & -- & 0.0098 \\
\hline
\end{tabular}


Table F-83

Summary Statistics for Whole Body Coho Salmon Collected from Segment 4 of the Columbia River

\begin{tabular}{|c|c|c|c|c|c|c|c|c|c|c|}
\hline $\begin{array}{l}\text { Constituent } \\
\text { Class }\end{array}$ & Constituent Name & Units & $\begin{array}{l}\text { Number } \\
\text { Analyzed }\end{array}$ & $\begin{array}{l}\text { Number } \\
\text { Detected }\end{array}$ & $\begin{array}{l}\text { Frequency of } \\
\text { Detection }\end{array}$ & $\begin{array}{l}\text { Minimum } \\
\text { Nondetect }\end{array}$ & $\begin{array}{l}\text { Maximum } \\
\text { Nondetect }\end{array}$ & $\begin{array}{l}\text { Minimum } \\
\text { Detect }\end{array}$ & $\begin{array}{l}\text { Maximum } \\
\text { Detect }\end{array}$ & $\begin{array}{c}\text { Average } \\
\text { Concentration }\end{array}$ \\
\hline$\overline{\mathrm{PCB}}$ & Aroclor-1254 & $\mathrm{mg} / \mathrm{kg}$ & 2 & 2 & $100 \%$ & $\overline{--}$ & $\overline{--}$ & 0.019 & 0.019 & 0.019 \\
\hline РСB & Aroclor-1260 & $\mathrm{mg} / \mathrm{kg}$ & 2 & 0 & $0 \%$ & 0.019 & 0.020 & -- & -- & 0.0098 \\
\hline PEST & 2,4'-DDD & $\mathrm{mg} / \mathrm{kg}$ & 2 & 0 & $0 \%$ & 0.0019 & 0.0020 & -- & -- & $9.75 \mathrm{E}-04$ \\
\hline PEST & 2,4'-DDE & $\mathrm{mg} / \mathrm{kg}$ & 2 & 0 & $0 \%$ & 0.0019 & 0.0020 & -- & -- & $9.75 \mathrm{E}-04$ \\
\hline PEST & 2,4'-DDT & $\mathrm{mg} / \mathrm{kg}$ & 2 & 0 & $0 \%$ & 0.0019 & 0.0020 & -- & -- & $9.75 \mathrm{E}-04$ \\
\hline PEST & Aldrin & $\mathrm{mg} / \mathrm{kg}$ & 2 & 0 & $0 \%$ & 0.0019 & 0.0020 & -- & -- & $9.75 \mathrm{E}-04$ \\
\hline PEST & Alpha-BHC & $\mathrm{mg} / \mathrm{kg}$ & 2 & 0 & $0 \%$ & 0.0019 & 0.0020 & -- & -- & $9.75 \mathrm{E}-04$ \\
\hline PEST & alpha-Chlordane & $\mathrm{mg} / \mathrm{kg}$ & 2 & 0 & $0 \%$ & 0.0019 & 0.0020 & -- & -- & $9.75 \mathrm{E}-04$ \\
\hline PEST & alpha-Chlordene & $\mathrm{mg} / \mathrm{kg}$ & 2 & 0 & $0 \%$ & 0.0019 & 0.0020 & -- & -- & $9.75 \mathrm{E}-04$ \\
\hline PEST & Beta-BHC & $\mathrm{mg} / \mathrm{kg}$ & 2 & 0 & $0 \%$ & 0.0019 & 0.0020 & -- & -- & $9.75 \mathrm{E}-04$ \\
\hline PEST & cis-Nonachlor & $\mathrm{mg} / \mathrm{kg}$ & 2 & 0 & $0 \%$ & 0.0019 & 0.0020 & -- & -- & 9.75E-04 \\
\hline PEST & DDMU & $\mathrm{mg} / \mathrm{kg}$ & 2 & 0 & $0 \%$ & 0.0019 & 0.0020 & -- & -- & $9.75 \mathrm{E}-04$ \\
\hline PEST & Delta-BHC & $\mathrm{mg} / \mathrm{kg}$ & 2 & 0 & $0 \%$ & 0.0019 & 0.0020 & -- & -- & $9.75 \mathrm{E}-04$ \\
\hline PEST & Dichlorodiphenyldichloroethane & $\mathrm{mg} / \mathrm{kg}$ & 2 & 2 & $100 \%$ & -- & -- & 0.0018 & 0.0021 & 0.0020 \\
\hline PEST & Dichlorodiphenyldichloroethylene & $\mathrm{mg} / \mathrm{kg}$ & 2 & 2 & $100 \%$ & -- & -- & 0.031 & 0.037 & 0.034 \\
\hline PEST & Dichlorodiphenyltrichloroethane & $\mathrm{mg} / \mathrm{kg}$ & 2 & 1 & $50 \%$ & 0.0020 & 0.0020 & 0.0040 & 0.0040 & 0.0025 \\
\hline PEST & Gamma-BHC (Lindane) & $\mathrm{mg} / \mathrm{kg}$ & 2 & 0 & $0 \%$ & 0.0019 & 0.0020 & -- & -- & $9.75 \mathrm{E}-04$ \\
\hline PEST & gamma-Chlordane & $\mathrm{mg} / \mathrm{kg}$ & 2 & 0 & $0 \%$ & 0.0019 & 0.0020 & -- & -- & 9.75E-04 \\
\hline PEST & gamma-Chlordene & $\mathrm{mg} / \mathrm{kg}$ & 2 & 0 & $0 \%$ & 0.0019 & 0.0020 & -- & -- & $9.75 \mathrm{E}-04$ \\
\hline PEST & Heptachlor & $\mathrm{mg} / \mathrm{kg}$ & 2 & 0 & $0 \%$ & 0.0019 & 0.0020 & -- & -- & $9.75 \mathrm{E}-04$ \\
\hline PEST & Heptachlor epoxide & $\mathrm{mg} / \mathrm{kg}$ & 2 & 0 & $0 \%$ & 0.0019 & 0.0020 & -- & -- & $9.75 \mathrm{E}-04$ \\
\hline PEST & Mirex & $\mathrm{mg} / \mathrm{kg}$ & 2 & 0 & $0 \%$ & 0.0019 & 0.0020 & -- & -- & $9.75 \mathrm{E}-04$ \\
\hline PEST & Oxychlordane & $\mathrm{mg} / \mathrm{kg}$ & 2 & 0 & $0 \%$ & 0.0019 & 0.0020 & -- & -- & $9.75 \mathrm{E}-04$ \\
\hline PEST & Pentachloroanisole & $\mathrm{mg} / \mathrm{kg}$ & 2 & 0 & $0 \%$ & 0.0019 & 0.0020 & -- & -- & $9.75 \mathrm{E}-04$ \\
\hline PEST & Toxaphene & $\mathrm{mg} / \mathrm{kg}$ & 2 & 0 & $0 \%$ & 0.058 & 0.059 & -- & -- & 0.029 \\
\hline PEST & trans-Nonachlor & $\mathrm{mg} / \mathrm{kg}$ & 2 & 0 & $0 \%$ & 0.0019 & 0.0020 & -- & -- & $9.75 \mathrm{E}-04$ \\
\hline SVOC & 1,2,4-Trichlorobenzene & $\mathrm{mg} / \mathrm{kg}$ & 2 & 0 & $0 \%$ & 0.0092 & 0.0096 & -- & -- & 0.0047 \\
\hline SVOC & 1,2-Dichlorobenzene & $\mathrm{mg} / \mathrm{kg}$ & 2 & 0 & $0 \%$ & 0.0092 & 0.0096 & -- & -- & 0.0047 \\
\hline SVOC & 1,2-Diphenylhydrazine & $\mathrm{mg} / \mathrm{kg}$ & 2 & 0 & $0 \%$ & 0.0092 & 0.0096 & -- & -- & 0.0047 \\
\hline SVOC & 1,3-Dichlorobenzene & $\mathrm{mg} / \mathrm{kg}$ & 2 & 0 & $0 \%$ & 0.0092 & 0.0096 & -- & -- & 0.0047 \\
\hline SVOC & 1,4-Dichlorobenzene & $\mathrm{mg} / \mathrm{kg}$ & 2 & 0 & $0 \%$ & 0.0092 & 0.0096 & -- & -- & 0.0047 \\
\hline SVOC & 1-methylnaphthalene & $\mathrm{mg} / \mathrm{kg}$ & 2 & 0 & $0 \%$ & 0.0092 & 0.0096 & -- & -- & 0.0047 \\
\hline SVOC & 2,4-Dinitrotoluene & $\mathrm{mg} / \mathrm{kg}$ & 2 & 0 & $0 \%$ & 0.15 & 0.15 & -- & -- & 0.075 \\
\hline SVOC & 2,6-Dinitrotoluene & $\mathrm{mg} / \mathrm{kg}$ & 2 & 0 & $0 \%$ & 0.037 & 0.039 & -- & -- & 0.019 \\
\hline SVOC & 2-Chloronaphthalene & $\mathrm{mg} / \mathrm{kg}$ & 2 & 0 & $0 \%$ & 0.019 & 0.019 & -- & -- & 0.0095 \\
\hline SVOC & 2-Methylnaphthalene & $\mathrm{mg} / \mathrm{kg}$ & 2 & 0 & $0 \%$ & 0.0092 & 0.0096 & -- & -- & 0.0047 \\
\hline SVOC & 4-Bromophenylphenyl ether & $\mathrm{mg} / \mathrm{kg}$ & 2 & 0 & $0 \%$ & 0.0092 & 0.0096 & -- & -- & 0.0047 \\
\hline SVOC & 4-Chlorophenylphenyl ether & $\mathrm{mg} / \mathrm{kg}$ & 2 & 0 & $0 \%$ & 0.0092 & 0.0096 & -- & -- & 0.0047 \\
\hline SVOC & Acenaphthene & $\mathrm{mg} / \mathrm{kg}$ & 2 & 0 & $0 \%$ & 0.019 & 0.019 & -- & -- & 0.0095 \\
\hline SVOC & Acenaphthylene & $\mathrm{mg} / \mathrm{kg}$ & 2 & 0 & $0 \%$ & 0.0092 & 0.0096 & -- & -- & 0.0047 \\
\hline SVOC & Anthracene & $\mathrm{mg} / \mathrm{kg}$ & 2 & 0 & $0 \%$ & 0.0092 & 0.0096 & -- & -- & 0.0047 \\
\hline SVOC & Benzo(a)anthracene & $\mathrm{mg} / \mathrm{kg}$ & 2 & 0 & $0 \%$ & 0.0092 & 0.0096 & -- & -- & 0.0047 \\
\hline SVOC & Benzo(a)pyrene & $\mathrm{mg} / \mathrm{kg}$ & 2 & 0 & $0 \%$ & 0.0092 & 0.0096 & -- & -- & 0.0047 \\
\hline SVOC & Benzo(b)fluoranthene & $\mathrm{mg} / \mathrm{kg}$ & 2 & 0 & $0 \%$ & 0.0092 & 0.0096 & -- & -- & 0.0047 \\
\hline SVOC & Benzo(ghi)perylene & $\mathrm{mg} / \mathrm{kg}$ & 2 & 0 & $0 \%$ & 0.019 & 0.019 & -- & -- & 0.0095 \\
\hline SVOC & Benzo(k)fluoranthene & $\mathrm{mg} / \mathrm{kg}$ & 2 & 0 & $0 \%$ & 0.0092 & 0.0096 & -- & -- & 0.0047 \\
\hline SVOC & Bis(2-chloro-1-methylethyl)ether & $\mathrm{mg} / \mathrm{kg}$ & 2 & 0 & $0 \%$ & 0.019 & 0.019 & -- & -- & 0.0095 \\
\hline SVOC & Chrysene & $\mathrm{mg} / \mathrm{kg}$ & 2 & 0 & $0 \%$ & 0.0092 & 0.0096 & -- & -- & 0.0047 \\
\hline SVOC & Dibenz[a,h]anthracene & $\mathrm{mg} / \mathrm{kg}$ & 2 & 0 & $0 \%$ & 0.019 & 0.019 & -- & -- & 0.0095 \\
\hline SVOC & Dibenzofuran & $\mathrm{mg} / \mathrm{kg}$ & 2 & 0 & $0 \%$ & 0.0092 & 0.0096 & -- & -- & 0.0047 \\
\hline SVOC & Fluoranthene & $\mathrm{mg} / \mathrm{kg}$ & 2 & 0 & $0 \%$ & 0.019 & 0.019 & -- & -- & 0.0095 \\
\hline SVOC & Fluorene & $\mathrm{mg} / \mathrm{kg}$ & 2 & 0 & $0 \%$ & 0.0087 & 0.0094 & -- & -- & 0.0045 \\
\hline
\end{tabular}


Table F-83

Summary Statistics for Whole Body Coho Salmon Collected from Segment 4 of the Columbia River

\begin{tabular}{|c|c|c|c|c|c|c|c|c|c|c|}
\hline $\begin{array}{l}\text { Constituent } \\
\text { Class }\end{array}$ & Constituent Name & Units & $\begin{array}{l}\text { Number } \\
\text { Analyzed }\end{array}$ & $\begin{array}{l}\text { Number } \\
\text { Detected }\end{array}$ & $\begin{array}{l}\text { Frequency of } \\
\text { Detection }\end{array}$ & $\begin{array}{c}\text { Minimum } \\
\text { Nondetect }\end{array}$ & $\begin{array}{l}\text { Maximum } \\
\text { Nondetect }\end{array}$ & $\begin{array}{l}\text { Minimum } \\
\text { Detect }\end{array}$ & $\begin{array}{l}\text { Maximum } \\
\text { Detect }\end{array}$ & $\begin{array}{c}\text { Average } \\
\text { Concentration }\end{array}$ \\
\hline$\overline{S V O C}$ & Hexachlorobenzene & $\mathrm{mg} / \mathrm{kg}$ & 2 & 0 & $0 \%$ & 0.019 & 0.019 & -- & -- & 0.0095 \\
\hline SVOC & Hexachlorobutadiene & $\mathrm{mg} / \mathrm{kg}$ & 2 & 0 & $0 \%$ & 0.0092 & 0.0096 & -- & -- & 0.0047 \\
\hline SVOC & Hexachloroethane & $\mathrm{mg} / \mathrm{kg}$ & 2 & 0 & $0 \%$ & 0.0092 & 0.0096 & -- & -- & 0.0047 \\
\hline SVOC & Indeno(1,2,3-cd)pyrene & $\mathrm{mg} / \mathrm{kg}$ & 2 & 0 & $0 \%$ & 0.019 & 0.019 & -- & -- & 0.0095 \\
\hline SVOC & Naphthalene & $\mathrm{mg} / \mathrm{kg}$ & 2 & 0 & $0 \%$ & 0.018 & 0.019 & -- & -- & 0.0093 \\
\hline SVOC & Nitrobenzene & $\mathrm{mg} / \mathrm{kg}$ & 2 & 0 & $0 \%$ & 0.0092 & 0.0096 & -- & -- & 0.0047 \\
\hline SVOC & Phenanthrene & $\mathrm{mg} / \mathrm{kg}$ & 2 & 0 & $0 \%$ & 0.0092 & 0.0096 & -- & -- & 0.0047 \\
\hline SVOC & Pyrene & $\mathrm{mg} / \mathrm{kg}$ & 2 & 0 & $0 \%$ & 0.019 & 0.019 & -- & -- & 0.0095 \\
\hline VOC & Retene & $\mathrm{mg} / \mathrm{kg}$ & 2 & 0 & $0 \%$ & 0.019 & 0.019 & -- & -- & 0.0095 \\
\hline
\end{tabular}

DIOXI - Dioxins and Furans

PEST - Pesticide

PCB - Polychlorinated bipheny

SVOC - Semivolatile Organic Compound

VOC - Volatile Organic Compound 
Table F-84

Summary Statistics for Chinook Salmon Eggs and Ovum Collected from Segment 4 of the Columbia River

\begin{tabular}{|c|c|c|c|c|c|c|c|c|c|c|}
\hline $\begin{array}{l}\text { Constituent } \\
\text { Class }\end{array}$ & Constituent Name & Units & $\begin{array}{l}\text { Number } \\
\text { Analyzed }\end{array}$ & $\begin{array}{l}\text { Number } \\
\text { Detected }\end{array}$ & $\begin{array}{l}\text { Frequency of } \\
\text { Detection }\end{array}$ & $\begin{array}{l}\text { Minimum } \\
\text { Nondetect }\end{array}$ & $\begin{array}{l}\text { Maximum } \\
\text { Nondetect }\end{array}$ & $\begin{array}{l}\text { Minimum } \\
\text { Detect }\end{array}$ & $\begin{array}{l}\text { Maximum } \\
\text { Detect }\end{array}$ & $\begin{array}{c}\text { Average } \\
\text { Concentration }\end{array}$ \\
\hline$\overline{\mathrm{DIOXI}}$ & $1,2,3,4,6,7,8$-Heptachlorodibenzodioxin & $\mathrm{mg} / \mathrm{kg}$ & 4 & 3 & $75 \%$ & $1.30 \mathrm{E}-07$ & $1.30 \mathrm{E}-07$ & $5.00 \mathrm{E}-08$ & $1.40 \mathrm{E}-07$ & $9.63 \mathrm{E}-08$ \\
\hline DIOXI & $1,2,3,4,6,7,8$-Heptachlorodibenzofuran & $\mathrm{mg} / \mathrm{kg}$ & 4 & 1 & $25 \%$ & $2.00 \mathrm{E}-08$ & $3.00 \mathrm{E}-08$ & $6.00 \mathrm{E}-08$ & $6.00 \mathrm{E}-08$ & 2.50E-08 \\
\hline DIOXI & 1,2,3,4,7,8,9-Heptachlorodibenzofuran & $\mathrm{mg} / \mathrm{kg}$ & 4 & 0 & $0 \%$ & $2.00 \mathrm{E}-08$ & $3.00 \mathrm{E}-08$ & -- & -- & 1.25E-08 \\
\hline DIOXI & $1,2,3,4,7,8$-Hexachlorodibenzofuran & $\mathrm{mg} / \mathrm{kg}$ & 4 & 0 & $0 \%$ & $1.00 \mathrm{E}-08$ & 4.00E-08 & -- & -- & $1.25 \mathrm{E}-08$ \\
\hline DIOXI & 1,2,3,4,7,8-Hexachlorodibenzo-p-dioxin & $\mathrm{mg} / \mathrm{kg}$ & 4 & 2 & $50 \%$ & $1.00 \mathrm{E}-08$ & $2.00 \mathrm{E}-08$ & $1.00 \mathrm{E}-07$ & $1.20 \mathrm{E}-07$ & $5.88 \mathrm{E}-08$ \\
\hline DIOXI & $1,2,3,6,7,8$-Hexachlorodibenzofuran & $\mathrm{mg} / \mathrm{kg}$ & 4 & 1 & $25 \%$ & $2.00 \mathrm{E}-08$ & 4.00E-08 & $2.00 \mathrm{E}-08$ & $2.00 \mathrm{E}-08$ & 1.63E-08 \\
\hline DIOXI & $1,2,3,6,7,8$-Hexachlorodibenzo-p-dioxin & $\mathrm{mg} / \mathrm{kg}$ & 4 & 2 & $50 \%$ & $1.00 \mathrm{E}-08$ & $2.00 \mathrm{E}-08$ & $6.00 \mathrm{E}-08$ & $9.00 \mathrm{E}-08$ & 4.13E-08 \\
\hline DIOXI & 1,2,3,7,8,9-Hexachlorodibenzofuran & $\mathrm{mg} / \mathrm{kg}$ & 4 & 0 & $0 \%$ & $1.00 \mathrm{E}-08$ & 4.00E-08 & -- & -- & 1.25E-08 \\
\hline DIOXI & 1,2,3,7,8,9-Hexachlorodibenzo-p-dioxin & $\mathrm{mg} / \mathrm{kg}$ & 4 & 2 & $50 \%$ & $1.00 \mathrm{E}-08$ & $5.00 \mathrm{E}-08$ & $3.00 \mathrm{E}-08$ & $3.00 \mathrm{E}-08$ & $2.25 \mathrm{E}-08$ \\
\hline DIOXI & 1,2,3,7,8-Pentachlorodibenzofuran & $\mathrm{mg} / \mathrm{kg}$ & 4 & 0 & $0 \%$ & $2.00 \mathrm{E}-08$ & $3.00 \mathrm{E}-08$ & -- & -- & $1.38 \mathrm{E}-08$ \\
\hline DIOXI & $1,2,3,7,8$-Pentachlorodibenzo-p-dioxin & $\mathrm{mg} / \mathrm{kg}$ & 4 & 3 & $75 \%$ & $4.00 \mathrm{E}-08$ & $4.00 \mathrm{E}-08$ & $1.40 \mathrm{E}-07$ & $1.50 \mathrm{E}-07$ & 1.15E-07 \\
\hline DIOXI & 2,3,4,6,7,8-Hexachlorodibenzofuran & $\mathrm{mg} / \mathrm{kg}$ & 4 & 3 & $75 \%$ & $2.00 \mathrm{E}-08$ & $2.00 \mathrm{E}-08$ & 1.30E-07 & 1.40E-07 & 1.05E-07 \\
\hline DIOXI & 2,3,4,7,8-Pentachlorodibenzofuran & $\mathrm{mg} / \mathrm{kg}$ & 4 & 2 & $50 \%$ & $3.00 \mathrm{E}-08$ & $3.00 \mathrm{E}-08$ & $6.00 \mathrm{E}-08$ & $9.00 \mathrm{E}-08$ & 4.50E-08 \\
\hline DIOXI & 2,3,7,8-Tetrachlorodibenzofuran & $\mathrm{mg} / \mathrm{kg}$ & 4 & 4 & $100 \%$ & -- & -- & $3.60 \mathrm{E}-07$ & 4.60E-07 & $4.28 \mathrm{E}-07$ \\
\hline DIOXI & 2,3,7,8-Tetrachlorodibenzo-p-dioxin & $\mathrm{mg} / \mathrm{kg}$ & 4 & 1 & $25 \%$ & $1.00 \mathrm{E}-08$ & $4.00 \mathrm{E}-08$ & $3.00 \mathrm{E}-08$ & $3.00 \mathrm{E}-08$ & $1.88 \mathrm{E}-08$ \\
\hline DIOXI & Octachlorodibenzofuran & $\mathrm{mg} / \mathrm{kg}$ & 4 & 0 & $0 \%$ & $3.00 \mathrm{E}-08$ & $6.00 \mathrm{E}-08$ & -- & -- & 2.13E-08 \\
\hline DIOXI & Octachlorodibenzo-p-dioxin & $\mathrm{mg} / \mathrm{kg}$ & 4 & 4 & $100 \%$ & -- & -- & $2.40 \mathrm{E}-07$ & $3.90 \mathrm{E}-07$ & 3.25E-07 \\
\hline METAL & Aluminum & $\mathrm{mg} / \mathrm{kg}$ & 4 & 0 & $0 \%$ & 1.0 & 1.0 & -- & -- & 0.50 \\
\hline METAL & Antimony & $\mathrm{mg} / \mathrm{kg}$ & 4 & 0 & $0 \%$ & 0.050 & 0.050 & -- & -- & 0.025 \\
\hline METAL & Arsenic & $\mathrm{mg} / \mathrm{kg}$ & 4 & 4 & $100 \%$ & -- & -- & 0.24 & 0.53 & 0.45 \\
\hline METAL & Barium & $\mathrm{mg} / \mathrm{kg}$ & 4 & 0 & $0 \%$ & 0.20 & 0.20 & -- & -- & 0.10 \\
\hline METAL & Beryllium & $\mathrm{mg} / \mathrm{kg}$ & 4 & 0 & $0 \%$ & 0.0040 & 0.0040 & -- & -- & 0.0020 \\
\hline METAL & Cadmium & $\mathrm{mg} / \mathrm{kg}$ & 4 & 3 & $75 \%$ & 0.0040 & 0.0040 & 0.020 & 0.030 & 0.021 \\
\hline METAL & Chromium & $\mathrm{mg} / \mathrm{kg}$ & 4 & 2 & $50 \%$ & 0.10 & 0.10 & 0.11 & 0.11 & 0.080 \\
\hline METAL & Cobalt & $\mathrm{mg} / \mathrm{kg}$ & 4 & 4 & $100 \%$ & -- & -- & 0.030 & 0.060 & 0.048 \\
\hline METAL & Copper & $\mathrm{mg} / \mathrm{kg}$ & 4 & 4 & $100 \%$ & -- & -- & 5.8 & 6.6 & 6.3 \\
\hline METAL & Lead & $\mathrm{mg} / \mathrm{kg}$ & 4 & 1 & $25 \%$ & 0.010 & 0.010 & 0.010 & 0.010 & 0.0063 \\
\hline METAL & Manganese & $\mathrm{mg} / \mathrm{kg}$ & 4 & 4 & $100 \%$ & -- & -- & 0.96 & 1.6 & 1.4 \\
\hline METAL & Mercury & $\mathrm{mg} / \mathrm{kg}$ & 4 & 0 & $0 \%$ & 0.050 & 0.090 & -- & -- & 0.036 \\
\hline METAL & Nickel & $\mathrm{mg} / \mathrm{kg}$ & 4 & 4 & $100 \%$ & -- & -- & 0.050 & 0.12 & 0.078 \\
\hline METAL & Selenium & $\mathrm{mg} / \mathrm{kg}$ & 4 & 4 & $100 \%$ & -- & -- & 2.4 & 5.5 & 3.9 \\
\hline METAL & Silver & $\mathrm{mg} / \mathrm{kg}$ & 4 & 0 & $0 \%$ & 0.10 & 0.10 & -- & -- & 0.050 \\
\hline METAL & Thallium & $\mathrm{mg} / \mathrm{kg}$ & 4 & 0 & $0 \%$ & 0.050 & 0.050 & -- & -- & 0.025 \\
\hline METAL & Vanadium & $\mathrm{mg} / \mathrm{kg}$ & 4 & 1 & $25 \%$ & 0.010 & 0.010 & 0.010 & 0.010 & 0.0063 \\
\hline METAL & Zinc & $\mathrm{mg} / \mathrm{kg}$ & 4 & 4 & $100 \%$ & -- & -- & 36 & 40 & 39 \\
\hline PCB & 2,2',3,3',4,4',5-Heptachloro-1,1'-biphenyl & $\mathrm{mg} / \mathrm{kg}$ & 4 & 4 & $100 \%$ & -- & -- & $2.94 \mathrm{E}-05$ & $5.14 \mathrm{E}-05$ & 4.44E-05 \\
\hline РСВ & 2,2',3,4,4',5,5'-Heptachlorobiphenyl & $\mathrm{mg} / \mathrm{kg}$ & 4 & 4 & $100 \%$ & -- & -- & $9.47 \mathrm{E}-05$ & $1.38 \mathrm{E}-04$ & $1.26 \mathrm{E}-04$ \\
\hline PCB & 2,3,3',4,4',5'-Hexachlorobiphenyl & $\mathrm{mg} / \mathrm{kg}$ & 4 & 4 & $100 \%$ & -- & -- & $4.31 \mathrm{E}-06$ & 1.10E-05 & 7.49E-06 \\
\hline РСB & 2,3,3',4,4',5-Hexachlorobiphenyl (BZ 156) & $\mathrm{mg} / \mathrm{kg}$ & 4 & 4 & $100 \%$ & -- & -- & 1.23E-05 & $2.61 \mathrm{E}-05$ & $2.21 \mathrm{E}-05$ \\
\hline PCB & 2,3,3',4,4'-Pentachlorobiphenyl & $\mathrm{mg} / \mathrm{kg}$ & 4 & 4 & $100 \%$ & -- & -- & $1.08 \mathrm{E}-04$ & $1.90 \mathrm{E}-04$ & 1.63E-04 \\
\hline PCB & 2,3',4,4',5,5'-Hexachlorobiphenyl (BZ 167) & $\mathrm{mg} / \mathrm{kg}$ & 4 & 4 & $100 \%$ & -- & -- & 9.93E-06 & $1.78 \mathrm{E}-05$ & 1.53E-05 \\
\hline РСB & 2',3,4,4',5-Pentachloro-1,1'-biphenyl (BZ 123) & $\mathrm{mg} / \mathrm{kg}$ & 4 & 4 & $100 \%$ & -- & -- & $4.85 \mathrm{E}-06$ & $1.06 \mathrm{E}-05$ & 7.23E-06 \\
\hline PCB & 2,3,4,4',5-Pentachlorobiphenyl (BZ 114) & $\mathrm{mg} / \mathrm{kg}$ & 4 & 4 & $100 \%$ & -- & -- & $9.76 \mathrm{E}-06$ & 2.03E-05 & 1.53E-05 \\
\hline РСB & 2,3',4,4',5-Pentachlorobiphenyl (BZ 118) & $\mathrm{mg} / \mathrm{kg}$ & 4 & 4 & $100 \%$ & -- & -- & $2.54 \mathrm{E}-04$ & 4.40E-04 & 3.74E-04 \\
\hline РСB & 2,3,4,5,3',4',5'-Heptachlorobiphenyl & $\mathrm{mg} / \mathrm{kg}$ & 4 & 3 & $75 \%$ & $1.00 \mathrm{E}-06$ & $1.00 \mathrm{E}-06$ & $1.26 \mathrm{E}-06$ & $1.66 \mathrm{E}-06$ & $1.22 \mathrm{E}-06$ \\
\hline РСB & 3,3',4,4',5,5'-Hexachlorobiphenyl (BZ 169) & $\mathrm{mg} / \mathrm{kg}$ & 4 & 0 & $0 \%$ & $1.00 \mathrm{E}-06$ & $1.00 \mathrm{E}-06$ & -- & -- & $5.00 \mathrm{E}-07$ \\
\hline PCB & 3,3',4,4',5-Pentachlorobiphenyl (BZ 126) & $\mathrm{mg} / \mathrm{kg}$ & 4 & 2 & $50 \%$ & $5.00 \mathrm{E}-07$ & $9.00 \mathrm{E}-07$ & $2.22 \mathrm{E}-06$ & $2.28 \mathrm{E}-06$ & 1.30E-06 \\
\hline РСB & 3,3',4,4'-Tetrachlorobiphenyl (BZ 77) & $\mathrm{mg} / \mathrm{kg}$ & 4 & 4 & $100 \%$ & -- & -- & $4.64 \mathrm{E}-06$ & $1.06 \mathrm{E}-05$ & 8.63E-06 \\
\hline PCB & Aroclor-1016 & $\mathrm{mg} / \mathrm{kg}$ & 4 & 0 & $0 \%$ & 0.019 & 0.038 & -- & -- & 0.014 \\
\hline PCB & Aroclor-1221 & $\mathrm{mg} / \mathrm{kg}$ & 4 & 0 & $0 \%$ & 0.019 & 0.038 & -- & -- & 0.014 \\
\hline РСB & Aroclor-1232 & $\mathrm{mg} / \mathrm{kg}$ & 4 & 0 & $0 \%$ & 0.019 & 0.038 & -- & -- & 0.014 \\
\hline РСB & Aroclor-1248 & $\mathrm{mg} / \mathrm{kg}$ & 4 & 0 & $0 \%$ & 0.019 & 0.038 & -- & -- & 0.014 \\
\hline
\end{tabular}


Table F-84

Summary Statistics for Chinook Salmon Eggs and Ovum Collected from Segment 4 of the Columbia River

\begin{tabular}{|c|c|c|c|c|c|c|c|c|c|c|}
\hline $\begin{array}{l}\text { Constituent } \\
\text { Class }\end{array}$ & Constituent Name & Units & $\begin{array}{l}\text { Number } \\
\text { Analyzed }\end{array}$ & $\begin{array}{l}\text { Number } \\
\text { Detected }\end{array}$ & $\begin{array}{l}\text { Frequency of } \\
\text { Detection }\end{array}$ & $\begin{array}{l}\text { Minimum } \\
\text { Nondetect }\end{array}$ & $\begin{array}{l}\text { Maximum } \\
\text { Nondetect }\end{array}$ & $\begin{array}{l}\text { Minimum } \\
\text { Detect }\end{array}$ & $\begin{array}{l}\text { Maximum } \\
\text { Detect }\end{array}$ & $\begin{array}{c}\text { Average } \\
\text { Concentration }\end{array}$ \\
\hline$\overline{\mathrm{PCB}}$ & Aroclor-1254 & $\mathrm{mg} / \mathrm{kg}$ & 4 & 4 & $100 \%$ & $\overline{--}$ & $\overline{--}$ & 0.012 & 0.020 & 0.016 \\
\hline РСB & Aroclor-1260 & $\mathrm{mg} / \mathrm{kg}$ & 4 & 0 & $0 \%$ & 0.019 & 0.038 & -- & -- & 0.014 \\
\hline PEST & 2,4'-DDD & $\mathrm{mg} / \mathrm{kg}$ & 4 & 0 & $0 \%$ & 0.0019 & 0.0038 & -- & -- & 0.0014 \\
\hline PEST & 2,4'-DDE & $\mathrm{mg} / \mathrm{kg}$ & 4 & 0 & $0 \%$ & 0.0019 & 0.0038 & -- & -- & 0.0014 \\
\hline PEST & 2,4'-DDT & $\mathrm{mg} / \mathrm{kg}$ & 4 & 0 & $0 \%$ & 0.0019 & 0.0038 & -- & -- & 0.0014 \\
\hline PEST & Aldrin & $\mathrm{mg} / \mathrm{kg}$ & 4 & 0 & $0 \%$ & 0.0019 & 0.0038 & -- & -- & 0.0014 \\
\hline PEST & Alpha-BHC & $\mathrm{mg} / \mathrm{kg}$ & 4 & 0 & $0 \%$ & 0.0019 & 0.0038 & -- & -- & 0.0014 \\
\hline PEST & alpha-Chlordane & $\mathrm{mg} / \mathrm{kg}$ & 4 & 1 & $25 \%$ & 0.0019 & 0.0038 & 0.0017 & 0.0017 & 0.0016 \\
\hline PEST & alpha-Chlordene & $\mathrm{mg} / \mathrm{kg}$ & 4 & 0 & $0 \%$ & 0.0019 & 0.0038 & -- & -- & 0.0014 \\
\hline PEST & Beta-BHC & $\mathrm{mg} / \mathrm{kg}$ & 4 & 0 & $0 \%$ & 0.0019 & 0.0038 & -- & -- & 0.0014 \\
\hline PEST & cis-Nonachlor & $\mathrm{mg} / \mathrm{kg}$ & 4 & 0 & $0 \%$ & 0.0019 & 0.0038 & -- & -- & 0.0014 \\
\hline PEST & DDMU & $\mathrm{mg} / \mathrm{kg}$ & 4 & 0 & $0 \%$ & 0.0019 & 0.0038 & -- & -- & 0.0014 \\
\hline PEST & Delta-BHC & $\mathrm{mg} / \mathrm{kg}$ & 4 & 0 & $0 \%$ & 0.0019 & 0.0038 & -- & -- & 0.0014 \\
\hline PEST & Dichlorodiphenyldichloroethane & $\mathrm{mg} / \mathrm{kg}$ & 4 & 4 & $100 \%$ & -- & -- & 0.0025 & 0.0037 & 0.0030 \\
\hline PEST & Dichlorodiphenyldichloroethylene & $\mathrm{mg} / \mathrm{kg}$ & 4 & 4 & $100 \%$ & -- & -- & 0.0066 & 0.010 & 0.0090 \\
\hline PEST & Dichlorodiphenyltrichloroethane & $\mathrm{mg} / \mathrm{kg}$ & 4 & 3 & $75 \%$ & 0.0019 & 0.0019 & 0.0040 & 0.0042 & 0.0033 \\
\hline PEST & Endosulfan sulfate & $\mathrm{mg} / \mathrm{kg}$ & 1 & 0 & $0 \%$ & 0.0019 & 0.0019 & -- & -- & $9.50 \mathrm{E}-04$ \\
\hline PEST & Gamma-BHC (Lindane) & $\mathrm{mg} / \mathrm{kg}$ & 4 & 1 & $25 \%$ & 0.0019 & 0.0038 & 0.0019 & 0.0019 & 0.0014 \\
\hline PEST & gamma-Chlordane & $\mathrm{mg} / \mathrm{kg}$ & 4 & 0 & $0 \%$ & 0.0019 & 0.0038 & -- & -- & 0.0014 \\
\hline PEST & gamma-Chlordene & $\mathrm{mg} / \mathrm{kg}$ & 4 & 0 & $0 \%$ & 0.0019 & 0.0038 & -- & -- & 0.0014 \\
\hline PEST & Heptachlor & $\mathrm{mg} / \mathrm{kg}$ & 4 & 0 & $0 \%$ & 0.0019 & 0.0038 & -- & -- & 0.0014 \\
\hline PEST & Heptachlor epoxide & $\mathrm{mg} / \mathrm{kg}$ & 4 & 0 & $0 \%$ & 0.0019 & 0.0038 & -- & -- & 0.0014 \\
\hline PEST & Mirex & $\mathrm{mg} / \mathrm{kg}$ & 4 & 0 & $0 \%$ & 0.0019 & 0.0038 & -- & -- & 0.0014 \\
\hline PEST & Oxychlordane & $\mathrm{mg} / \mathrm{kg}$ & 4 & 0 & $0 \%$ & 0.0019 & 0.0038 & -- & -- & 0.0014 \\
\hline PEST & Pentachloroanisole & $\mathrm{mg} / \mathrm{kg}$ & 4 & 0 & $0 \%$ & 0.0019 & 0.0038 & -- & -- & 0.0014 \\
\hline PEST & Toxaphene & $\mathrm{mg} / \mathrm{kg}$ & 4 & 0 & $0 \%$ & 0.056 & 0.11 & -- & -- & 0.042 \\
\hline PEST & trans-Nonachlor & $\mathrm{mg} / \mathrm{kg}$ & 4 & 2 & $50 \%$ & 0.0038 & 0.0038 & 0.0029 & 0.0032 & 0.0025 \\
\hline SVOC & 1,2,4-Trichlorobenzene & $\mathrm{mg} / \mathrm{kg}$ & 4 & 0 & $0 \%$ & 0.0096 & 0.037 & -- & -- & 0.010 \\
\hline SVOC & 1,2-Dichlorobenzene & $\mathrm{mg} / \mathrm{kg}$ & 4 & 0 & $0 \%$ & 0.0096 & 0.037 & -- & -- & 0.010 \\
\hline SVOC & 1,2-Diphenylhydrazine & $\mathrm{mg} / \mathrm{kg}$ & 4 & 0 & $0 \%$ & 0.0096 & 0.037 & -- & -- & 0.010 \\
\hline SVOC & 1,3-Dichlorobenzene & $\mathrm{mg} / \mathrm{kg}$ & 4 & 0 & $0 \%$ & 0.0096 & 0.037 & -- & -- & 0.010 \\
\hline SVOC & 1,4-Dichlorobenzene & $\mathrm{mg} / \mathrm{kg}$ & 4 & 0 & $0 \%$ & 0.0096 & 0.037 & -- & -- & 0.010 \\
\hline SVOC & 1-methylnaphthalene & $\mathrm{mg} / \mathrm{kg}$ & 4 & 0 & $0 \%$ & 0.0096 & 0.037 & -- & -- & 0.010 \\
\hline SVOC & 2,4-Dinitrotoluene & $\mathrm{mg} / \mathrm{kg}$ & 4 & 0 & $0 \%$ & 0.019 & 0.074 & -- & -- & 0.020 \\
\hline SVOC & 2,6-Dinitrotoluene & $\mathrm{mg} / \mathrm{kg}$ & 4 & 0 & $0 \%$ & 0.038 & 0.15 & -- & -- & 0.041 \\
\hline SVOC & 2-Chloronaphthalene & $\mathrm{mg} / \mathrm{kg}$ & 4 & 0 & $0 \%$ & 0.019 & 0.074 & -- & -- & 0.020 \\
\hline SVOC & 2-Methylnaphthalene & $\mathrm{mg} / \mathrm{kg}$ & 4 & 0 & $0 \%$ & 0.0096 & 0.037 & -- & -- & 0.011 \\
\hline SVOC & 4-Bromophenylphenyl ether & $\mathrm{mg} / \mathrm{kg}$ & 4 & 0 & $0 \%$ & 0.0096 & 0.037 & -- & -- & 0.010 \\
\hline SVOC & 4-Chlorophenylphenyl ether & $\mathrm{mg} / \mathrm{kg}$ & 4 & 0 & $0 \%$ & 0.0096 & 0.037 & -- & -- & 0.010 \\
\hline SVOC & Acenaphthene & $\mathrm{mg} / \mathrm{kg}$ & 4 & 1 & $25 \%$ & 0.019 & 0.074 & 0.026 & 0.026 & 0.024 \\
\hline SVOC & Acenaphthylene & $\mathrm{mg} / \mathrm{kg}$ & 4 & 0 & $0 \%$ & 0.0096 & 0.037 & -- & -- & 0.010 \\
\hline SVOC & Anthracene & $\mathrm{mg} / \mathrm{kg}$ & 4 & 0 & $0 \%$ & 0.0096 & 0.037 & -- & -- & 0.010 \\
\hline SVOC & Benzo(a)anthracene & $\mathrm{mg} / \mathrm{kg}$ & 4 & 0 & $0 \%$ & 0.0096 & 0.037 & -- & -- & 0.010 \\
\hline SVOC & Benzo(a)pyrene & $\mathrm{mg} / \mathrm{kg}$ & 4 & 0 & $0 \%$ & 0.0096 & 0.037 & -- & -- & 0.010 \\
\hline SVOC & Benzo(b)fluoranthene & $\mathrm{mg} / \mathrm{kg}$ & 4 & 0 & $0 \%$ & 0.0096 & 0.037 & -- & -- & 0.010 \\
\hline SVOC & Benzo(ghi)perylene & $\mathrm{mg} / \mathrm{kg}$ & 4 & 0 & $0 \%$ & 0.019 & 0.074 & -- & -- & 0.020 \\
\hline SVOC & Benzo(k)fluoranthene & $\mathrm{mg} / \mathrm{kg}$ & 4 & 0 & $0 \%$ & 0.0096 & 0.037 & -- & -- & 0.010 \\
\hline SVOC & Bis(2-chloro-1-methylethyl)ether & $\mathrm{mg} / \mathrm{kg}$ & 4 & 0 & $0 \%$ & 0.019 & 0.074 & -- & -- & 0.020 \\
\hline SVOC & Chrysene & $\mathrm{mg} / \mathrm{kg}$ & 4 & 0 & $0 \%$ & 0.0096 & 0.037 & -- & -- & 0.010 \\
\hline SVOC & Dibenz[a,h]anthracene & $\mathrm{mg} / \mathrm{kg}$ & 4 & 0 & $0 \%$ & 0.019 & 0.074 & -- & -- & 0.020 \\
\hline SVOC & Dibenzofuran & $\mathrm{mg} / \mathrm{kg}$ & 4 & 0 & $0 \%$ & 0.0096 & 0.037 & -- & -- & 0.010 \\
\hline SVOC & Fluoranthene & $\mathrm{mg} / \mathrm{kg}$ & 4 & 0 & $0 \%$ & 0.019 & 0.074 & -- & -- & 0.020 \\
\hline
\end{tabular}


Table F-84

Summary Statistics for Chinook Salmon Eggs and Ovum Collected from Segment 4 of the Columbia River

\begin{tabular}{|c|c|c|c|c|c|c|c|c|c|c|}
\hline $\begin{array}{l}\text { Constituent } \\
\text { Class }\end{array}$ & Constituent Name & Units & $\begin{array}{l}\text { Number } \\
\text { Analyzed }\end{array}$ & $\begin{array}{l}\text { Number } \\
\text { Detected }\end{array}$ & $\begin{array}{l}\text { Frequency of } \\
\text { Detection }\end{array}$ & $\begin{array}{c}\text { Minimum } \\
\text { Nondetect }\end{array}$ & $\begin{array}{l}\text { Maximum } \\
\text { Nondetect }\end{array}$ & $\begin{array}{c}\text { Minimum } \\
\text { Detect }\end{array}$ & $\begin{array}{c}\text { Maximum } \\
\text { Detect }\end{array}$ & $\begin{array}{c}\text { Average } \\
\text { Concentration }\end{array}$ \\
\hline$\overline{\text { SVOC }}$ & Fluorene & $\mathrm{mg} / \mathrm{kg}$ & 4 & 0 & $0 \%$ & 0.0045 & 0.026 & -- & -- & 0.0056 \\
\hline SVOC & Hexachlorobenzene & $\mathrm{mg} / \mathrm{kg}$ & 4 & 0 & $0 \%$ & 0.019 & 0.074 & -- & -- & 0.020 \\
\hline SVOC & Hexachlorobutadiene & $\mathrm{mg} / \mathrm{kg}$ & 4 & 0 & $0 \%$ & 0.0096 & 0.037 & -- & -- & 0.010 \\
\hline SVOC & Hexachloroethane & $\mathrm{mg} / \mathrm{kg}$ & 4 & 0 & $0 \%$ & 0.0096 & 0.037 & -- & -- & 0.010 \\
\hline SVOC & Indeno(1,2,3-cd)pyrene & $\mathrm{mg} / \mathrm{kg}$ & 4 & 0 & $0 \%$ & 0.019 & 0.074 & -- & -- & 0.020 \\
\hline SVOC & Naphthalene & $\mathrm{mg} / \mathrm{kg}$ & 4 & 0 & $0 \%$ & 0.044 & 0.11 & -- & -- & 0.031 \\
\hline SVOC & Nitrobenzene & $\mathrm{mg} / \mathrm{kg}$ & 4 & 0 & $0 \%$ & 0.0096 & 0.037 & -- & -- & 0.010 \\
\hline SVOC & Phenanthrene & $\mathrm{mg} / \mathrm{kg}$ & 4 & 0 & $0 \%$ & 0.0096 & 0.037 & -- & -- & 0.010 \\
\hline SVOC & Pyrene & $\mathrm{mg} / \mathrm{kg}$ & 4 & 0 & $0 \%$ & 0.019 & 0.074 & -- & -- & 0.020 \\
\hline VOC & Retene & $\mathrm{mg} / \mathrm{kg}$ & 4 & 0 & $0 \%$ & 0.019 & 0.074 & -- & -- & 0.020 \\
\hline
\end{tabular}

DIOXI - Dioxins and Furans

PEST - Pesticide

PCB - Polychlorinated biphenyl

SVOC - Semivolatile Organic Compound

VOC - Volatile Organic Compound 
Table F-85

Summary Statistics for Chinook Salmon Fillet Skin On Collected from Segment 4 of the Columbia River

\begin{tabular}{|c|c|c|c|c|c|c|c|c|c|c|}
\hline $\begin{array}{l}\text { Constituent } \\
\text { Class }\end{array}$ & Constituent Name & Units & $\begin{array}{l}\text { Number } \\
\text { Analyzed }\end{array}$ & $\begin{array}{l}\text { Number } \\
\text { Detected }\end{array}$ & $\begin{array}{l}\text { Frequency of } \\
\text { Detection }\end{array}$ & $\begin{array}{l}\text { Minimum } \\
\text { Nondetect }\end{array}$ & $\begin{array}{l}\text { Maximum } \\
\text { Nondetect }\end{array}$ & $\begin{array}{l}\text { Minimum } \\
\text { Detect }\end{array}$ & $\begin{array}{l}\text { Maximum } \\
\text { Detect }\end{array}$ & $\begin{array}{c}\text { Average } \\
\text { Concentration }\end{array}$ \\
\hline$\overline{\mathrm{DIOXI}}$ & $1,2,3,4,6,7,8$-Heptachlorodibenzodioxin & $\mathrm{mg} / \mathrm{kg}$ & 19 & 12 & $63 \%$ & $1.00 \mathrm{E}-08$ & $6.00 \mathrm{E}-08$ & $3.00 \mathrm{E}-08$ & $5.70 \mathrm{E}-07$ & $9.08 \mathrm{E}-08$ \\
\hline DIOXI & $1,2,3,4,6,7,8$-Heptachlorodibenzofuran & $\mathrm{mg} / \mathrm{kg}$ & 19 & 3 & $16 \%$ & $1.00 \mathrm{E}-08$ & $7.00 \mathrm{E}-08$ & $2.00 \mathrm{E}-08$ & $2.00 \mathrm{E}-07$ & 2.50E-08 \\
\hline DIOXI & 1,2,3,4,7,8,9-Heptachlorodibenzofuran & $\mathrm{mg} / \mathrm{kg}$ & 19 & 2 & $11 \%$ & $1.00 \mathrm{E}-08$ & $7.00 \mathrm{E}-08$ & $2.00 \mathrm{E}-08$ & $2.00 \mathrm{E}-08$ & $1.21 \mathrm{E}-08$ \\
\hline DIOXI & $1,2,3,4,7,8$-Hexachlorodibenzofuran & $\mathrm{mg} / \mathrm{kg}$ & 19 & 0 & $0 \%$ & $1.00 \mathrm{E}-08$ & $6.00 \mathrm{E}-08$ & -- & -- & 9.47E-09 \\
\hline DIOXI & 1,2,3,4,7,8-Hexachlorodibenzo-p-dioxin & $\mathrm{mg} / \mathrm{kg}$ & 19 & 3 & $16 \%$ & $1.00 \mathrm{E}-08$ & $4.00 \mathrm{E}-08$ & $2.00 \mathrm{E}-08$ & 1.30E-07 & 1.74E-08 \\
\hline DIOXI & $1,2,3,6,7,8$-Hexachlorodibenzofuran & $\mathrm{mg} / \mathrm{kg}$ & 19 & 6 & $32 \%$ & $1.00 \mathrm{E}-08$ & $6.00 \mathrm{E}-08$ & $3.00 \mathrm{E}-08$ & $5.00 \mathrm{E}-08$ & 1.92E-08 \\
\hline DIOXI & $1,2,3,6,7,8$-Hexachlorodibenzo-p-dioxin & $\mathrm{mg} / \mathrm{kg}$ & 19 & 9 & $47 \%$ & $1.00 \mathrm{E}-08$ & $2.00 \mathrm{E}-08$ & $2.00 \mathrm{E}-08$ & $1.20 \mathrm{E}-07$ & $3.68 \mathrm{E}-08$ \\
\hline DIOXI & 1,2,3,7,8,9-Hexachlorodibenzofuran & $\mathrm{mg} / \mathrm{kg}$ & 19 & 2 & $11 \%$ & $1.00 \mathrm{E}-08$ & $6.00 \mathrm{E}-08$ & $6.00 \mathrm{E}-08$ & $7.00 \mathrm{E}-08$ & 1.50E-08 \\
\hline DIOXI & 1,2,3,7,8,9-Hexachlorodibenzo-p-dioxin & $\mathrm{mg} / \mathrm{kg}$ & 19 & 6 & $32 \%$ & $1.00 \mathrm{E}-08$ & $4.00 \mathrm{E}-08$ & $2.00 \mathrm{E}-08$ & $6.00 \mathrm{E}-08$ & 1.92E-08 \\
\hline DIOXI & 1,2,3,7,8-Pentachlorodibenzofuran & $\mathrm{mg} / \mathrm{kg}$ & 19 & 6 & $32 \%$ & $1.00 \mathrm{E}-08$ & $4.00 \mathrm{E}-08$ & $8.00 \mathrm{E}-08$ & $1.40 \mathrm{E}-07$ & 4.26E-08 \\
\hline DIOXI & $1,2,3,7,8$-Pentachlorodibenzo-p-dioxin & $\mathrm{mg} / \mathrm{kg}$ & 19 & 5 & $26 \%$ & $1.00 \mathrm{E}-08$ & $5.00 \mathrm{E}-08$ & $6.00 \mathrm{E}-08$ & $1.80 \mathrm{E}-07$ & 3.76E-08 \\
\hline DIOXI & 2,3,4,6,7,8-Hexachlorodibenzofuran & $\mathrm{mg} / \mathrm{kg}$ & 19 & 6 & $32 \%$ & $1.00 \mathrm{E}-08$ & $6.00 \mathrm{E}-08$ & $4.00 \mathrm{E}-08$ & $1.60 \mathrm{E}-07$ & 3.61E-08 \\
\hline DIOXI & 2,3,4,7,8-Pentachlorodibenzofuran & $\mathrm{mg} / \mathrm{kg}$ & 19 & 11 & $58 \%$ & $1.00 \mathrm{E}-08$ & 4.00E-08 & $5.00 \mathrm{E}-08$ & $1.80 \mathrm{E}-07$ & $7.50 \mathrm{E}-08$ \\
\hline DIOXI & 2,3,7,8-Tetrachlorodibenzofuran & $\mathrm{mg} / \mathrm{kg}$ & 19 & 19 & $100 \%$ & -- & -- & $3.00 \mathrm{E}-08$ & $8.20 \mathrm{E}-07$ & $5.98 \mathrm{E}-07$ \\
\hline DIOXI & 2,3,7,8-Tetrachlorodibenzo-p-dioxin & $\mathrm{mg} / \mathrm{kg}$ & 19 & 3 & $16 \%$ & $1.00 \mathrm{E}-08$ & 4.00E-08 & $2.00 \mathrm{E}-08$ & $4.00 \mathrm{E}-08$ & 1.39E-08 \\
\hline DIOXI & Octachlorodibenzofuran & $\mathrm{mg} / \mathrm{kg}$ & 19 & 3 & $16 \%$ & $1.00 \mathrm{E}-08$ & $1.70 \mathrm{E}-07$ & $8.00 \mathrm{E}-08$ & $1.30 \mathrm{E}-07$ & 3.53E-08 \\
\hline DIOXI & Octachlorodibenzo-p-dioxin & $\mathrm{mg} / \mathrm{kg}$ & 19 & 12 & $63 \%$ & $1.00 \mathrm{E}-08$ & $4.00 \mathrm{E}-08$ & $1.10 \mathrm{E}-07$ & $2.68 \mathrm{E}-06$ & 3.20E-07 \\
\hline METAL & Aluminum & $\mathrm{mg} / \mathrm{kg}$ & 19 & 3 & $16 \%$ & 1.0 & 1.0 & 1.2 & 1.9 & 0.65 \\
\hline METAL & Antimony & $\mathrm{mg} / \mathrm{kg}$ & 19 & 0 & $0 \%$ & 0.050 & 0.050 & -- & -- & 0.025 \\
\hline METAL & Arsenic & $\mathrm{mg} / \mathrm{kg}$ & 19 & 19 & $100 \%$ & -- & -- & 0.52 & 1.2 & 0.84 \\
\hline METAL & Barium & $\mathrm{mg} / \mathrm{kg}$ & 19 & 0 & $0 \%$ & 0.20 & 0.20 & -- & -- & 0.10 \\
\hline METAL & Beryllium & $\mathrm{mg} / \mathrm{kg}$ & 19 & 0 & $0 \%$ & 0.0040 & 0.0040 & -- & -- & 0.0020 \\
\hline METAL & Cadmium & $\mathrm{mg} / \mathrm{kg}$ & 19 & 9 & $47 \%$ & 0.0040 & 0.0040 & 0.0078 & 0.010 & 0.0056 \\
\hline METAL & Chromium & $\mathrm{mg} / \mathrm{kg}$ & 19 & 5 & $26 \%$ & 0.10 & 0.10 & 0.14 & 0.49 & 0.11 \\
\hline METAL & Cobalt & $\mathrm{mg} / \mathrm{kg}$ & 19 & 18 & $95 \%$ & 0.0010 & 0.0010 & 0.0029 & 0.18 & 0.033 \\
\hline METAL & Copper & $\mathrm{mg} / \mathrm{kg}$ & 19 & 19 & $100 \%$ & -- & -- & 0.55 & 2.1 & 0.77 \\
\hline METAL & Lead & $\mathrm{mg} / \mathrm{kg}$ & 19 & 5 & $26 \%$ & 0.010 & 0.010 & 0.010 & 0.14 & 0.015 \\
\hline METAL & Manganese & $\mathrm{mg} / \mathrm{kg}$ & 19 & 19 & $100 \%$ & -- & -- & 0.070 & 0.11 & 0.087 \\
\hline METAL & Mercury & $\mathrm{mg} / \mathrm{kg}$ & 19 & 14 & $74 \%$ & 0.050 & 0.11 & 0.050 & 0.51 & 0.11 \\
\hline METAL & Nickel & $\mathrm{mg} / \mathrm{kg}$ & 19 & 11 & $58 \%$ & 0.030 & 0.030 & 0.030 & 0.37 & 0.083 \\
\hline METAL & Selenium & $\mathrm{mg} / \mathrm{kg}$ & 19 & 19 & $100 \%$ & -- & -- & 0.28 & 0.43 & 0.34 \\
\hline METAL & Silver & $\mathrm{mg} / \mathrm{kg}$ & 19 & 0 & $0 \%$ & 0.10 & 0.10 & -- & -- & 0.050 \\
\hline METAL & Thallium & $\mathrm{mg} / \mathrm{kg}$ & 19 & 0 & $0 \%$ & 0.050 & 0.050 & -- & -- & 0.025 \\
\hline METAL & Vanadium & $\mathrm{mg} / \mathrm{kg}$ & 19 & 1 & $5 \%$ & 0.010 & 0.010 & 0.010 & 0.010 & 0.0053 \\
\hline METAL & Zinc & $\mathrm{mg} / \mathrm{kg}$ & 19 & 19 & $100 \%$ & -- & -- & 5.6 & 7.1 & 6.3 \\
\hline PCB & 2,2',3,3',4,4',5-Heptachloro-1,1'-biphenyl & $\mathrm{mg} / \mathrm{kg}$ & 19 & 19 & $100 \%$ & -- & -- & $3.84 \mathrm{E}-05$ & $1.69 \mathrm{E}-04$ & 8.05E-05 \\
\hline РСВ & 2,2',3,4,4',5,5'-Heptachlorobiphenyl & $\mathrm{mg} / \mathrm{kg}$ & 19 & 19 & $100 \%$ & -- & -- & $1.22 \mathrm{E}-04$ & $5.30 \mathrm{E}-04$ & 2.57E-04 \\
\hline PCB & 2,3,3',4,4',5'-Hexachlorobiphenyl & $\mathrm{mg} / \mathrm{kg}$ & 19 & 19 & $100 \%$ & -- & -- & $4.06 \mathrm{E}-06$ & $1.89 \mathrm{E}-05$ & 9.99E-06 \\
\hline РСB & 2,3,3',4,4',5-Hexachlorobiphenyl (BZ 156) & $\mathrm{mg} / \mathrm{kg}$ & 19 & 19 & $100 \%$ & -- & -- & $1.55 \mathrm{E}-05$ & $7.25 \mathrm{E}-05$ & $3.69 \mathrm{E}-05$ \\
\hline PCB & 2,3,3',4,4'-Pentachlorobiphenyl & $\mathrm{mg} / \mathrm{kg}$ & 19 & 19 & $100 \%$ & -- & -- & $8.01 \mathrm{E}-05$ & $2.58 \mathrm{E}-04$ & $1.90 \mathrm{E}-04$ \\
\hline PCB & 2,3',4,4',5,5'-Hexachlorobiphenyl (BZ 167) & $\mathrm{mg} / \mathrm{kg}$ & 19 & 19 & $100 \%$ & -- & -- & $1.01 \mathrm{E}-05$ & $3.41 \mathrm{E}-05$ & $2.29 \mathrm{E}-05$ \\
\hline РСB & 2',3,4,4',5-Pentachloro-1,1'-biphenyl (BZ 123) & $\mathrm{mg} / \mathrm{kg}$ & 19 & 19 & $100 \%$ & -- & -- & 3.33E-06 & $1.42 \mathrm{E}-05$ & 8.46E-06 \\
\hline PCB & 2,3,4,4',5-Pentachlorobiphenyl (BZ 114) & $\mathrm{mg} / \mathrm{kg}$ & 19 & 19 & $100 \%$ & -- & -- & $8.28 \mathrm{E}-06$ & 0.020 & 0.0011 \\
\hline PCB & 2,3',4,4',5-Pentachlorobiphenyl (BZ 118) & $\mathrm{mg} / \mathrm{kg}$ & 19 & 19 & $100 \%$ & -- & -- & 1.96E-04 & 0.042 & 0.0026 \\
\hline РСB & 2,3,4,5,3',4',5'-Heptachlorobiphenyl & $\mathrm{mg} / \mathrm{kg}$ & 19 & 15 & $79 \%$ & $1.00 \mathrm{E}-06$ & $1.72 \mathrm{E}-06$ & $1.55 \mathrm{E}-06$ & $5.66 \mathrm{E}-06$ & 2.66E-06 \\
\hline РСB & 3,3',4,4',5,5'-Hexachlorobiphenyl (BZ 169) & $\mathrm{mg} / \mathrm{kg}$ & 19 & 0 & $0 \%$ & $1.00 \mathrm{E}-06$ & $2.57 \mathrm{E}-06$ & -- & -- & $5.87 \mathrm{E}-07$ \\
\hline РСB & 3,3',4,4',5-Pentachlorobiphenyl (BZ 126) & $\mathrm{mg} / \mathrm{kg}$ & 19 & 11 & $58 \%$ & $5.00 \mathrm{E}-07$ & $2.22 \mathrm{E}-06$ & 8.70E-07 & 3.63E-06 & 1.63E-06 \\
\hline РСB & 3,3',4,4'-Tetrachlorobiphenyl (BZ 77) & $\mathrm{mg} / \mathrm{kg}$ & 19 & 18 & $95 \%$ & $7.60 \mathrm{E}-07$ & $7.60 \mathrm{E}-07$ & 4.07E-06 & $1.31 \mathrm{E}-05$ & 7.46E-06 \\
\hline PCB & Aroclor-1016 & $\mathrm{mg} / \mathrm{kg}$ & 19 & 0 & $0 \%$ & 0.017 & 0.038 & -- & -- & 0.011 \\
\hline PCB & Aroclor-1221 & $\mathrm{mg} / \mathrm{kg}$ & 19 & 0 & $0 \%$ & 0.017 & 0.038 & -- & -- & 0.011 \\
\hline РСB & Aroclor-1232 & $\mathrm{mg} / \mathrm{kg}$ & 19 & 0 & $0 \%$ & 0.017 & 0.038 & -- & -- & 0.011 \\
\hline РСB & Aroclor-1248 & $\mathrm{mg} / \mathrm{kg}$ & 19 & 0 & $0 \%$ & 0.017 & 0.038 & -- & -- & 0.011 \\
\hline
\end{tabular}


Table F-85

Summary Statistics for Chinook Salmon Fillet Skin On Collected from Segment 4 of the Columbia River

\begin{tabular}{|c|c|c|c|c|c|c|c|c|c|c|}
\hline $\begin{array}{l}\text { Constituent } \\
\text { Class }\end{array}$ & Constituent Name & Units & $\begin{array}{l}\text { Number } \\
\text { Analyzed }\end{array}$ & $\begin{array}{l}\text { Number } \\
\text { Detected }\end{array}$ & $\begin{array}{l}\text { Frequency of } \\
\text { Detection }\end{array}$ & $\begin{array}{l}\text { Minimum } \\
\text { Nondetect }\end{array}$ & $\begin{array}{l}\text { Maximum } \\
\text { Nondetect }\end{array}$ & $\begin{array}{l}\text { Minimum } \\
\text { Detect }\end{array}$ & $\begin{array}{l}\text { Maximum } \\
\text { Detect }\end{array}$ & $\begin{array}{c}\text { Average } \\
\text { Concentration }\end{array}$ \\
\hline$\overline{\mathrm{PCB}}$ & Aroclor-1254 & $\mathrm{mg} / \mathrm{kg}$ & 19 & 19 & $100 \%$ & -- & -- & 0.0086 & 0.024 & 0.016 \\
\hline PCB & Aroclor-1260 & $\mathrm{mg} / \mathrm{kg}$ & 19 & 0 & $0 \%$ & 0.017 & 0.038 & -- & -- & 0.011 \\
\hline PEST & 2,4'-DDD & $\mathrm{mg} / \mathrm{kg}$ & 19 & 0 & $0 \%$ & 0.0017 & 0.0038 & -- & -- & 0.0011 \\
\hline PEST & 2,4'-DDE & $\mathrm{mg} / \mathrm{kg}$ & 19 & 0 & $0 \%$ & 0.0017 & 0.0038 & -- & -- & 0.0011 \\
\hline PEST & 2,4'-DDT & $\mathrm{mg} / \mathrm{kg}$ & 19 & 0 & $0 \%$ & 0.0017 & 0.0038 & -- & -- & 0.0011 \\
\hline PEST & Aldrin & $\mathrm{mg} / \mathrm{kg}$ & 19 & 0 & $0 \%$ & 0.0017 & 0.0038 & -- & -- & 0.0011 \\
\hline PEST & Alpha-BHC & $\mathrm{mg} / \mathrm{kg}$ & 19 & 0 & $0 \%$ & 0.0017 & 0.0038 & -- & -- & 0.0011 \\
\hline PEST & alpha-Chlordane & $\mathrm{mg} / \mathrm{kg}$ & 19 & 2 & $11 \%$ & 0.0017 & 0.0038 & 0.0012 & 0.0017 & 0.0012 \\
\hline PEST & alpha-Chlordene & $\mathrm{mg} / \mathrm{kg}$ & 19 & 0 & $0 \%$ & 0.0017 & 0.0038 & -- & -- & 0.0011 \\
\hline PEST & Beta-BHC & $\mathrm{mg} / \mathrm{kg}$ & 19 & 0 & $0 \%$ & 0.0017 & 0.0038 & -- & -- & 0.0011 \\
\hline PEST & cis-Nonachlor & $\mathrm{mg} / \mathrm{kg}$ & 19 & 2 & $11 \%$ & 0.0017 & 0.0038 & 0.0011 & 0.0012 & 0.0011 \\
\hline PEST & DDMU & $\mathrm{mg} / \mathrm{kg}$ & 19 & 3 & $16 \%$ & 0.0017 & 0.0038 & 0.0012 & 0.0024 & 0.0012 \\
\hline PEST & Delta-BHC & $\mathrm{mg} / \mathrm{kg}$ & 19 & 0 & $0 \%$ & 0.0017 & 0.0038 & -- & -- & 0.0011 \\
\hline PEST & Dichlorodiphenyldichloroethane & $\mathrm{mg} / \mathrm{kg}$ & 19 & 18 & $95 \%$ & 0.0020 & 0.0020 & 0.0014 & 0.0033 & 0.0024 \\
\hline PEST & Dichlorodiphenyldichloroethylene & $\mathrm{mg} / \mathrm{kg}$ & 19 & 19 & $100 \%$ & -- & -- & 0.0042 & 0.018 & 0.010 \\
\hline PEST & Dichlorodiphenyltrichloroethane & $\mathrm{mg} / \mathrm{kg}$ & 19 & 12 & $63 \%$ & 0.0019 & 0.0020 & 0.0017 & 0.0058 & 0.0027 \\
\hline PEST & Endosulfan sulfate & $\mathrm{mg} / \mathrm{kg}$ & 7 & 0 & $0 \%$ & 0.0017 & 0.0020 & -- & -- & $9.57 \mathrm{E}-04$ \\
\hline PEST & Gamma-BHC (Lindane) & $\mathrm{mg} / \mathrm{kg}$ & 19 & 0 & $0 \%$ & 0.0017 & 0.0038 & -- & -- & 0.0011 \\
\hline PEST & gamma-Chlordane & $\mathrm{mg} / \mathrm{kg}$ & 19 & 0 & $0 \%$ & 0.0017 & 0.0038 & -- & -- & 0.0011 \\
\hline PEST & gamma-Chlordene & $\mathrm{mg} / \mathrm{kg}$ & 19 & 0 & $0 \%$ & 0.0017 & 0.0038 & -- & -- & 0.0011 \\
\hline PEST & Heptachlor & $\mathrm{mg} / \mathrm{kg}$ & 19 & 0 & $0 \%$ & 0.0017 & 0.0038 & -- & -- & 0.0011 \\
\hline PEST & Heptachlor epoxide & $\mathrm{mg} / \mathrm{kg}$ & 13 & 0 & $0 \%$ & 0.0017 & 0.0038 & -- & -- & 0.0012 \\
\hline PEST & Mirex & $\mathrm{mg} / \mathrm{kg}$ & 19 & 0 & $0 \%$ & 0.0017 & 0.0038 & -- & -- & 0.0011 \\
\hline PEST & Oxychlordane & $\mathrm{mg} / \mathrm{kg}$ & 19 & 0 & $0 \%$ & 0.0017 & 0.0038 & -- & -- & 0.0011 \\
\hline PEST & Pentachloroanisole & $\mathrm{mg} / \mathrm{kg}$ & 19 & 0 & $0 \%$ & 0.0017 & 0.0038 & -- & -- & 0.0011 \\
\hline PEST & Toxaphene & $\mathrm{mg} / \mathrm{kg}$ & 19 & 0 & $0 \%$ & 0.051 & 0.11 & -- & -- & 0.033 \\
\hline PEST & trans-Nonachlor & $\mathrm{mg} / \mathrm{kg}$ & 19 & 15 & $79 \%$ & 0.0019 & 0.0038 & 0.0012 & 0.0044 & 0.0027 \\
\hline SVOC & 1,2,4-Trichlorobenzene & $\mathrm{mg} / \mathrm{kg}$ & 19 & 0 & $0 \%$ & 0.0078 & 0.010 & -- & -- & 0.0046 \\
\hline SVOC & 1,2-Dichlorobenzene & $\mathrm{mg} / \mathrm{kg}$ & 19 & 0 & $0 \%$ & 0.0078 & 0.010 & -- & -- & 0.0046 \\
\hline SVOC & 1,2-Diphenylhydrazine & $\mathrm{mg} / \mathrm{kg}$ & 19 & 0 & $0 \%$ & 0.0078 & 0.010 & -- & -- & 0.0046 \\
\hline SVOC & 1,3-Dichlorobenzene & $\mathrm{mg} / \mathrm{kg}$ & 19 & 0 & $0 \%$ & 0.0078 & 0.010 & -- & -- & 0.0046 \\
\hline SVOC & 1,4-Dichlorobenzene & $\mathrm{mg} / \mathrm{kg}$ & 19 & 0 & $0 \%$ & 0.0078 & 0.010 & -- & -- & 0.0046 \\
\hline SVOC & 1-methylnaphthalene & $\mathrm{mg} / \mathrm{kg}$ & 19 & 0 & $0 \%$ & 0.0078 & 0.010 & -- & -- & 0.0047 \\
\hline SVOC & 2,4-Dinitrotoluene & $\mathrm{mg} / \mathrm{kg}$ & 19 & 0 & $0 \%$ & 0.016 & 0.16 & -- & -- & 0.019 \\
\hline SVOC & 2,6-Dinitrotoluene & $\mathrm{mg} / \mathrm{kg}$ & 19 & 0 & $0 \%$ & 0.031 & 0.040 & -- & -- & 0.019 \\
\hline SVOC & 2-Chloronaphthalene & $\mathrm{mg} / \mathrm{kg}$ & 19 & 0 & $0 \%$ & 0.016 & 0.020 & -- & -- & 0.0093 \\
\hline SVOC & 2-Methylnaphthalene & $\mathrm{mg} / \mathrm{kg}$ & 19 & 1 & $5 \%$ & 0.0078 & 0.021 & 0.011 & 0.011 & 0.0054 \\
\hline SVOC & 4-Bromophenylphenyl ether & $\mathrm{mg} / \mathrm{kg}$ & 19 & 0 & $0 \%$ & 0.0078 & 0.010 & -- & -- & 0.0046 \\
\hline SVOC & 4-Chlorophenylphenyl ether & $\mathrm{mg} / \mathrm{kg}$ & 19 & 0 & $0 \%$ & 0.0078 & 0.010 & -- & -- & 0.0046 \\
\hline SVOC & Acenaphthene & $\mathrm{mg} / \mathrm{kg}$ & 19 & 0 & $0 \%$ & 0.016 & 0.020 & -- & -- & 0.0093 \\
\hline SVOC & Acenaphthylene & $\mathrm{mg} / \mathrm{kg}$ & 19 & 0 & $0 \%$ & 0.0078 & 0.010 & -- & -- & 0.0046 \\
\hline SVOC & Anthracene & $\mathrm{mg} / \mathrm{kg}$ & 19 & 0 & $0 \%$ & 0.0078 & 0.010 & -- & -- & 0.0046 \\
\hline SVOC & Benzo(a)anthracene & $\mathrm{mg} / \mathrm{kg}$ & 19 & 0 & $0 \%$ & 0.0078 & 0.010 & -- & -- & 0.0046 \\
\hline SVOC & Benzo(a)pyrene & $\mathrm{mg} / \mathrm{kg}$ & 19 & 0 & $0 \%$ & 0.0083 & 0.010 & -- & -- & 0.0047 \\
\hline SVOC & Benzo(b)fluoranthene & $\mathrm{mg} / \mathrm{kg}$ & 19 & 0 & $0 \%$ & 0.0083 & 0.010 & -- & -- & 0.0047 \\
\hline SVOC & Benzo(ghi)perylene & $\mathrm{mg} / \mathrm{kg}$ & 19 & 0 & $0 \%$ & 0.017 & 0.020 & -- & -- & 0.0094 \\
\hline SVOC & Benzo(k)fluoranthene & $\mathrm{mg} / \mathrm{kg}$ & 19 & 0 & $0 \%$ & 0.0083 & 0.010 & -- & -- & 0.0047 \\
\hline SVOC & Bis(2-chloro-1-methylethyl)ether & $\mathrm{mg} / \mathrm{kg}$ & 19 & 0 & $0 \%$ & 0.016 & 0.020 & -- & -- & 0.0093 \\
\hline SVOC & Chrysene & $\mathrm{mg} / \mathrm{kg}$ & 19 & 0 & $0 \%$ & 0.0078 & 0.010 & -- & -- & 0.0046 \\
\hline SVOC & Dibenz[a,h]anthracene & $\mathrm{mg} / \mathrm{kg}$ & 19 & 0 & $0 \%$ & 0.017 & 0.020 & -- & -- & 0.0094 \\
\hline SVOC & Dibenzofuran & $\mathrm{mg} / \mathrm{kg}$ & 19 & 0 & $0 \%$ & 0.0078 & 0.010 & -- & -- & 0.0046 \\
\hline SVOC & Fluoranthene & $\mathrm{mg} / \mathrm{kg}$ & 19 & 0 & $0 \%$ & 0.016 & 0.020 & -- & -- & 0.0093 \\
\hline
\end{tabular}


Table F-85

Summary Statistics for Chinook Salmon Fillet Skin On Collected from Segment 4 of the Columbia River

\begin{tabular}{|c|c|c|c|c|c|c|c|c|c|c|}
\hline $\begin{array}{l}\text { Constituent } \\
\text { Class }\end{array}$ & Constituent Name & Units & $\begin{array}{l}\text { Number } \\
\text { Analyzed }\end{array}$ & $\begin{array}{l}\text { Number } \\
\text { Detected }\end{array}$ & $\begin{array}{l}\text { Frequency of } \\
\text { Detection }\end{array}$ & $\begin{array}{c}\text { Minimum } \\
\text { Nondetect }\end{array}$ & $\begin{array}{l}\text { Maximum } \\
\text { Nondetect }\end{array}$ & $\begin{array}{c}\text { Minimum } \\
\text { Detect }\end{array}$ & $\begin{array}{c}\text { Maximum } \\
\text { Detect }\end{array}$ & $\begin{array}{c}\text { Average } \\
\text { Concentration }\end{array}$ \\
\hline$\overline{\text { SVOC }}$ & Fluorene & $\mathrm{mg} / \mathrm{kg}$ & 19 & 0 & $0 \%$ & 0.0046 & 0.037 & -- & -- & 0.0050 \\
\hline SVOC & Hexachlorobenzene & $\mathrm{mg} / \mathrm{kg}$ & 19 & 0 & $0 \%$ & 0.016 & 0.020 & -- & -- & 0.0093 \\
\hline SVOC & Hexachlorobutadiene & $\mathrm{mg} / \mathrm{kg}$ & 19 & 0 & $0 \%$ & 0.0078 & 0.010 & -- & -- & 0.0046 \\
\hline SVOC & Hexachloroethane & $\mathrm{mg} / \mathrm{kg}$ & 19 & 0 & $0 \%$ & 0.0078 & 0.010 & -- & -- & 0.0046 \\
\hline SVOC & Indeno(1,2,3-cd)pyrene & $\mathrm{mg} / \mathrm{kg}$ & 19 & 0 & $0 \%$ & 0.017 & 0.020 & -- & -- & 0.0094 \\
\hline SVOC & Naphthalene & $\mathrm{mg} / \mathrm{kg}$ & 19 & 0 & $0 \%$ & 0.013 & 0.067 & -- & -- & 0.016 \\
\hline SVOC & Nitrobenzene & $\mathrm{mg} / \mathrm{kg}$ & 19 & 0 & $0 \%$ & 0.0078 & 0.010 & -- & -- & 0.0046 \\
\hline SVOC & Phenanthrene & $\mathrm{mg} / \mathrm{kg}$ & 19 & 0 & $0 \%$ & 0.0078 & 0.013 & -- & -- & 0.0048 \\
\hline SVOC & Pyrene & $\mathrm{mg} / \mathrm{kg}$ & 19 & 0 & $0 \%$ & 0.016 & 0.020 & -- & -- & 0.0093 \\
\hline VOC & Retene & $\mathrm{mg} / \mathrm{kg}$ & 19 & 0 & $0 \%$ & 0.016 & 0.020 & -- & -- & 0.0093 \\
\hline
\end{tabular}

DIOXI - Dioxins and Furans

PEST - Pesticide

PCB - Polychlorinated biphenyl

SVOC - Semivolatile Organic Compound

VOC - Volatile Organic Compound 
Table F-86

Summary Statistics for Whole Body Chinook Salmon Collected from Segment 4 of the Columbia River

\begin{tabular}{|c|c|c|c|c|c|c|c|c|c|c|}
\hline $\begin{array}{l}\text { Constituent } \\
\text { Class }\end{array}$ & Constituent Name & Units & $\begin{array}{l}\text { Number } \\
\text { Analyzed }\end{array}$ & $\begin{array}{l}\text { Number } \\
\text { Detected }\end{array}$ & $\begin{array}{c}\text { Frequency of } \\
\text { Detection }\end{array}$ & $\begin{array}{l}\text { Minimum } \\
\text { Nondetect }\end{array}$ & $\begin{array}{l}\text { Maximum } \\
\text { Nondetect }\end{array}$ & $\begin{array}{l}\text { Minimum } \\
\text { Detect }\end{array}$ & $\begin{array}{c}\text { Maximum } \\
\text { Detect }\end{array}$ & $\begin{array}{c}\text { Average } \\
\text { Concentration }\end{array}$ \\
\hline$\overline{\mathrm{DIOXI}}$ & $1,2,3,4,6,7,8$-Heptachlorodibenzodioxin & $\mathrm{mg} / \mathrm{kg}$ & 18 & 9 & $50 \%$ & $1.00 \mathrm{E}-08$ & $8.00 \mathrm{E}-08$ & $8.00 \mathrm{E}-08$ & $2.00 \mathrm{E}-07$ & $7.72 \mathrm{E}-08$ \\
\hline DIOXI & $1,2,3,4,6,7,8$-Heptachlorodibenzofuran & $\mathrm{mg} / \mathrm{kg}$ & 18 & 7 & $39 \%$ & $1.00 \mathrm{E}-08$ & $3.00 \mathrm{E}-08$ & $3.00 \mathrm{E}-08$ & 1.10E-07 & 3.19E-08 \\
\hline DIOXI & 1,2,3,4,7,8,9-Heptachlorodibenzofuran & $\mathrm{mg} / \mathrm{kg}$ & 18 & 0 & $0 \%$ & 1.00E-08 & $6.00 \mathrm{E}-08$ & -- & -- & 1.00E-08 \\
\hline DIOXI & 1,2,3,4,7,8-Hexachlorodibenzofuran & $\mathrm{mg} / \mathrm{kg}$ & 18 & 3 & $17 \%$ & $1.00 \mathrm{E}-08$ & $5.00 \mathrm{E}-08$ & $3.00 \mathrm{E}-08$ & $6.00 \mathrm{E}-08$ & 1.64E-08 \\
\hline DIOXI & $1,2,3,4,7,8$-Hexachlorodibenzo-p-dioxin & $\mathrm{mg} / \mathrm{kg}$ & 18 & 6 & $33 \%$ & $1.00 \mathrm{E}-08$ & 4.00E-08 & $2.00 \mathrm{E}-08$ & 1.40E-07 & $3.11 \mathrm{E}-08$ \\
\hline DIOXI & $1,2,3,6,7,8$-Hexachlorodibenzofuran & $\mathrm{mg} / \mathrm{kg}$ & 18 & 7 & $39 \%$ & $1.00 \mathrm{E}-08$ & $5.00 \mathrm{E}-08$ & $2.00 \mathrm{E}-08$ & $5.00 \mathrm{E}-08$ & $2.11 \mathrm{E}-08$ \\
\hline DIOXI & 1,2,3,6,7,8-Hexachlorodibenzo-p-dioxin & $\mathrm{mg} / \mathrm{kg}$ & 18 & 6 & $33 \%$ & 1.00E-08 & 4.00E-08 & $2.00 \mathrm{E}-08$ & 1.30E-07 & 3.06E-08 \\
\hline DIOXI & 1,2,3,7,8,9-Hexachlorodibenzofuran & $\mathrm{mg} / \mathrm{kg}$ & 18 & 2 & $11 \%$ & $1.00 \mathrm{E}-08$ & $5.00 \mathrm{E}-08$ & $1.00 \mathrm{E}-08$ & $7.00 \mathrm{E}-08$ & $1.42 \mathrm{E}-08$ \\
\hline DIOXI & 1,2,3,7,8,9-Hexachlorodibenzo-p-dioxin & $\mathrm{mg} / \mathrm{kg}$ & 18 & 4 & $22 \%$ & $1.00 \mathrm{E}-08$ & 4.00E-08 & $3.00 \mathrm{E}-08$ & $5.00 \mathrm{E}-08$ & 1.75E-08 \\
\hline DIOXI & 1,2,3,7,8-Pentachlorodibenzofuran & $\mathrm{mg} / \mathrm{kg}$ & 18 & 9 & $50 \%$ & $1.00 \mathrm{E}-08$ & 4.00E-08 & $4.00 \mathrm{E}-08$ & 1.40E-07 & $5.86 \mathrm{E}-08$ \\
\hline DIOXI & $1,2,3,7,8$-Pentachlorodibenzo-p-dioxin & $\mathrm{mg} / \mathrm{kg}$ & 18 & 5 & $28 \%$ & $1.00 \mathrm{E}-08$ & $6.00 \mathrm{E}-08$ & $8.00 \mathrm{E}-08$ & $2.10 \mathrm{E}-07$ & $5.08 \mathrm{E}-08$ \\
\hline DIOXI & $2,3,4,6,7,8$-Hexachlorodibenzofuran & $\mathrm{mg} / \mathrm{kg}$ & 18 & 6 & $33 \%$ & $1.00 \mathrm{E}-08$ & $5.00 \mathrm{E}-08$ & $3.00 \mathrm{E}-08$ & 1.70E-07 & 3.97E-08 \\
\hline DIOXI & 2,3,4,7,8-Pentachlorodibenzofuran & $\mathrm{mg} / \mathrm{kg}$ & 18 & 10 & $56 \%$ & $2.00 \mathrm{E}-08$ & 4.00E-08 & $6.00 \mathrm{E}-08$ & 1.80E-07 & 8.42E-08 \\
\hline DIOXI & 2,3,7,8-Tetrachlorodibenzofuran & $\mathrm{mg} / \mathrm{kg}$ & 18 & 18 & $100 \%$ & -- & -- & 4.30E-07 & 1.35E-06 & $6.94 \mathrm{E}-07$ \\
\hline DIOXI & 2,3,7,8-Tetrachlorodibenzo-p-dioxin & $\mathrm{mg} / \mathrm{kg}$ & 18 & 2 & $11 \%$ & $1.00 \mathrm{E}-08$ & 4.00E-08 & $4.00 \mathrm{E}-08$ & 4.00E-08 & 1.39E-08 \\
\hline DIOXI & Octachlorodibenzofuran & $\mathrm{mg} / \mathrm{kg}$ & 18 & 3 & $17 \%$ & 1.00E-08 & $9.00 \mathrm{E}-08$ & $2.00 \mathrm{E}-08$ & 1.70E-07 & 3.36E-08 \\
\hline DIOXI & Octachlorodibenzo-p-dioxin & $\mathrm{mg} / \mathrm{kg}$ & 18 & 10 & $56 \%$ & $1.00 \mathrm{E}-08$ & $6.00 \mathrm{E}-08$ & $2.10 \mathrm{E}-07$ & $6.90 \mathrm{E}-07$ & 1.98E-07 \\
\hline METAL & Aluminum & $\mathrm{mg} / \mathrm{kg}$ & 18 & 1 & $6 \%$ & 1.0 & 1.0 & 1.4 & 1.4 & 0.55 \\
\hline METAL & Antimony & $\mathrm{mg} / \mathrm{kg}$ & 18 & 0 & $0 \%$ & 0.050 & 0.050 & -- & -- & 0.025 \\
\hline METAL & Arsenic & $\mathrm{mg} / \mathrm{kg}$ & 18 & 18 & $100 \%$ & -- & -- & 0.56 & 1.0 & 0.78 \\
\hline METAL & Barium & $\mathrm{mg} / \mathrm{kg}$ & 18 & 1 & $6 \%$ & 0.20 & 0.20 & 0.29 & 0.29 & 0.11 \\
\hline METAL & Beryllium & $\mathrm{mg} / \mathrm{kg}$ & 18 & 0 & $0 \%$ & 0.0040 & 0.0060 & -- & -- & 0.0021 \\
\hline METAL & Cadmium & $\mathrm{mg} / \mathrm{kg}$ & 18 & 18 & $100 \%$ & -- & -- & 0.0046 & 0.14 & 0.063 \\
\hline METAL & Chromium & $\mathrm{mg} / \mathrm{kg}$ & 18 & 12 & $67 \%$ & 0.10 & 0.10 & 0.11 & 0.19 & 0.12 \\
\hline METAL & Cobalt & $\mathrm{mg} / \mathrm{kg}$ & 18 & 18 & $100 \%$ & -- & -- & 0.040 & 0.21 & 0.12 \\
\hline METAL & Copper & $\mathrm{mg} / \mathrm{kg}$ & 18 & 18 & $100 \%$ & -- & -- & 1.1 & 6.6 & 2.3 \\
\hline METAL & Lead & $\mathrm{mg} / \mathrm{kg}$ & 18 & 18 & $100 \%$ & -- & -- & 0.010 & 0.52 & 0.11 \\
\hline METAL & Manganese & $\mathrm{mg} / \mathrm{kg}$ & 18 & 18 & $100 \%$ & -- & -- & 0.24 & 0.41 & 0.35 \\
\hline METAL & Mercury & $\mathrm{mg} / \mathrm{kg}$ & 18 & 9 & $50 \%$ & 0.050 & 0.11 & 0.060 & 0.19 & 0.067 \\
\hline METAL & Nickel & $\mathrm{mg} / \mathrm{kg}$ & 18 & 15 & $83 \%$ & 0.030 & 0.030 & 0.030 & 0.63 & 0.16 \\
\hline METAL & Selenium & $\mathrm{mg} / \mathrm{kg}$ & 18 & 18 & $100 \%$ & -- & -- & 0.38 & 0.61 & 0.52 \\
\hline METAL & Silver & $\mathrm{mg} / \mathrm{kg}$ & 18 & 0 & $0 \%$ & 0.10 & 0.20 & -- & -- & 0.053 \\
\hline METAL & Thallium & $\mathrm{mg} / \mathrm{kg}$ & 18 & 0 & $0 \%$ & 0.050 & 0.050 & -- & -- & 0.025 \\
\hline METAL & Vanadium & $\mathrm{mg} / \mathrm{kg}$ & 18 & 16 & $89 \%$ & 0.010 & 0.010 & 0.010 & 0.040 & 0.017 \\
\hline METAL & Zinc & $\mathrm{mg} / \mathrm{kg}$ & 18 & 18 & $100 \%$ & -- & -- & 20 & 34 & 27 \\
\hline PCB & 2,2',3,3',4,4',5-Heptachloro-1,1'-biphenyl & $\mathrm{mg} / \mathrm{kg}$ & 18 & 18 & $100 \%$ & -- & -- & 4.37E-05 & $3.54 \mathrm{E}-04$ & $9.58 \mathrm{E}-05$ \\
\hline PCB & 2,2',3,4,4',5,5'-Heptachlorobiphenyl & $\mathrm{mg} / \mathrm{kg}$ & 18 & 18 & $100 \%$ & -- & -- & $1.38 \mathrm{E}-04$ & 0.0012 & $3.08 \mathrm{E}-04$ \\
\hline PCB & 2,3,3',4,4',5'-Hexachlorobiphenyl & $\mathrm{mg} / \mathrm{kg}$ & 18 & 18 & $100 \%$ & -- & -- & $5.33 \mathrm{E}-06$ & 0.042 & 0.0023 \\
\hline PCB & 2,3,3',4,4',5-Hexachlorobiphenyl (BZ 156) & $\mathrm{mg} / \mathrm{kg}$ & 18 & 18 & $100 \%$ & -- & -- & $7.72 \mathrm{E}-06$ & $1.95 \mathrm{E}-04$ & 4.86E-05 \\
\hline РСB & 2,3,3',4,4'-Pentachlorobiphenyl & $\mathrm{mg} / \mathrm{kg}$ & 18 & 18 & $100 \%$ & -- & -- & 1.14E-04 & 0.034 & 0.0021 \\
\hline PCB & 2,3',4,4',5,5'-Hexachlorobiphenyl (BZ 167) & $\mathrm{mg} / \mathrm{kg}$ & 18 & 18 & $100 \%$ & -- & -- & $1.28 \mathrm{E}-05$ & 0.042 & 0.0024 \\
\hline PCB & 2',3,4,4',5-Pentachloro-1,1'-biphenyl (BZ 123) & $\mathrm{mg} / \mathrm{kg}$ & 18 & 18 & $100 \%$ & -- & -- & 4.95E-06 & 0.037 & 0.0021 \\
\hline РСB & 2,3,4,4',5-Pentachlorobiphenyl (BZ 114) & $\mathrm{mg} / \mathrm{kg}$ & 18 & 18 & $100 \%$ & -- & -- & 1.14E-05 & 0.020 & 0.0011 \\
\hline PCB & 2,3',4,4',5-Pentachlorobiphenyl (BZ 118) & $\mathrm{mg} / \mathrm{kg}$ & 18 & 18 & $100 \%$ & -- & -- & $1.00 \mathrm{E}-06$ & 0.0015 & $5.21 \mathrm{E}-04$ \\
\hline PCB & 2,3,4,5,3',4',5'-Heptachlorobiphenyl & $\mathrm{mg} / \mathrm{kg}$ & 18 & 16 & $89 \%$ & $1.00 \mathrm{E}-06$ & $1.77 \mathrm{E}-06$ & $7.60 \mathrm{E}-07$ & 1.42E-05 & 3.33E-06 \\
\hline РСВ & 3,3',4,4',5,5'-Hexachlorobiphenyl (BZ 169) & $\mathrm{mg} / \mathrm{kg}$ & 18 & 0 & $0 \%$ & $1.00 \mathrm{E}-06$ & 0.042 & -- & -- & 0.0012 \\
\hline PCB & 3,3',4,4',5-Pentachlorobiphenyl (BZ 126) & $\mathrm{mg} / \mathrm{kg}$ & 18 & 11 & $61 \%$ & $5.00 \mathrm{E}-07$ & $1.88 \mathrm{E}-06$ & $5.00 \mathrm{E}-07$ & 4.74E-04 & 2.83E-05 \\
\hline PCB & 3,3',4,4'-Tetrachlorobiphenyl (BZ 77) & $\mathrm{mg} / \mathrm{kg}$ & 18 & 18 & $100 \%$ & -- & -- & $5.14 \mathrm{E}-06$ & 0.037 & 0.0035 \\
\hline PCB & Aroclor-1016 & $\mathrm{mg} / \mathrm{kg}$ & 18 & 0 & $0 \%$ & 0.019 & 0.042 & -- & -- & 0.011 \\
\hline PCB & Aroclor-1221 & $\mathrm{mg} / \mathrm{kg}$ & 18 & 0 & $0 \%$ & 2.77E-05 & 0.034 & -- & -- & 0.0094 \\
\hline PCB & Aroclor-1232 & $\mathrm{mg} / \mathrm{kg}$ & 18 & 0 & $0 \%$ & 1.00E-06 & 0.034 & -- & -- & 0.0091 \\
\hline PCB & Aroclor-1248 & $\mathrm{mg} / \mathrm{kg}$ & 18 & 0 & $0 \%$ & $1.78 \mathrm{E}-05$ & 0.034 & -- & -- & 0.0091 \\
\hline
\end{tabular}


Table F-86

Summary Statistics for Whole Body Chinook Salmon Collected from Segment 4 of the Columbia River

\begin{tabular}{|c|c|c|c|c|c|c|c|c|c|c|}
\hline $\begin{array}{l}\text { Constituent } \\
\text { Class }\end{array}$ & Constituent Name & Units & $\begin{array}{l}\text { Number } \\
\text { Analyzed }\end{array}$ & $\begin{array}{l}\text { Number } \\
\text { Detected }\end{array}$ & $\begin{array}{l}\text { Frequency of } \\
\text { Detection }\end{array}$ & $\begin{array}{l}\text { Minimum } \\
\text { Nondetect }\end{array}$ & $\begin{array}{l}\text { Maximum } \\
\text { Nondetect }\end{array}$ & $\begin{array}{l}\text { Minimum } \\
\text { Detect }\end{array}$ & $\begin{array}{l}\text { Maximum } \\
\text { Detect }\end{array}$ & $\begin{array}{c}\text { Average } \\
\text { Concentration }\end{array}$ \\
\hline$\overline{\mathrm{PCB}}$ & Aroclor-1254 & $\mathrm{mg} / \mathrm{kg}$ & 18 & 18 & $100 \%$ & $\overline{--}$ & $\overline{--}$ & $1.23 \mathrm{E}-05$ & 0.047 & 0.015 \\
\hline РСB & Aroclor-1260 & $\mathrm{mg} / \mathrm{kg}$ & 18 & 0 & $0 \%$ & $1.09 \mathrm{E}-05$ & 0.034 & -- & -- & 0.0091 \\
\hline PEST & 2,4'-DDD & $\mathrm{mg} / \mathrm{kg}$ & 18 & 1 & $6 \%$ & 0.0019 & 0.0042 & 0.0012 & 0.0012 & 0.0011 \\
\hline PEST & 2,4'-DDE & $\mathrm{mg} / \mathrm{kg}$ & 18 & 1 & $6 \%$ & 0.0019 & 0.0042 & 0.0014 & 0.0014 & 0.0012 \\
\hline PEST & 2,4'-DDT & $\mathrm{mg} / \mathrm{kg}$ & 18 & 0 & $0 \%$ & 0.0019 & 0.0042 & -- & -- & 0.0011 \\
\hline PEST & Aldrin & $\mathrm{mg} / \mathrm{kg}$ & 18 & 0 & $0 \%$ & 0.0019 & 0.0042 & -- & -- & 0.0011 \\
\hline PEST & Alpha-BHC & $\mathrm{mg} / \mathrm{kg}$ & 18 & 0 & $0 \%$ & 0.0019 & 0.0042 & -- & -- & 0.0011 \\
\hline PEST & alpha-Chlordane & $\mathrm{mg} / \mathrm{kg}$ & 18 & 6 & $33 \%$ & 0.0019 & 0.0042 & 0.0012 & 0.0023 & 0.0013 \\
\hline PEST & alpha-Chlordene & $\mathrm{mg} / \mathrm{kg}$ & 18 & 0 & $0 \%$ & 0.0019 & 0.0042 & -- & -- & 0.0011 \\
\hline PEST & Beta-BHC & $\mathrm{mg} / \mathrm{kg}$ & 18 & 0 & $0 \%$ & 0.0019 & 0.0042 & -- & -- & 0.0011 \\
\hline PEST & cis-Nonachlor & $\mathrm{mg} / \mathrm{kg}$ & 18 & 3 & $17 \%$ & 0.0019 & 0.0042 & 0.0012 & 0.0019 & 0.0012 \\
\hline PEST & DDMU & $\mathrm{mg} / \mathrm{kg}$ & 18 & 4 & $22 \%$ & 0.0019 & 0.0042 & 0.0014 & 0.0028 & 0.0014 \\
\hline PEST & Delta-BHC & $\mathrm{mg} / \mathrm{kg}$ & 18 & 0 & $0 \%$ & 0.0019 & 0.0042 & -- & -- & 0.0011 \\
\hline PEST & Dichlorodiphenyldichloroethane & $\mathrm{mg} / \mathrm{kg}$ & 18 & 16 & $89 \%$ & 0.0020 & 0.0042 & 0.0014 & 0.0041 & 0.0027 \\
\hline PEST & Dichlorodiphenyldichloroethylene & $\mathrm{mg} / \mathrm{kg}$ & 18 & 18 & $100 \%$ & -- & -- & 0.0045 & 0.053 & 0.014 \\
\hline PEST & Dichlorodiphenyltrichloroethane & $\mathrm{mg} / \mathrm{kg}$ & 18 & 13 & $72 \%$ & 0.0019 & 0.0020 & 0.0020 & 0.0070 & 0.0034 \\
\hline PEST & Endosulfan sulfate & $\mathrm{mg} / \mathrm{kg}$ & 4 & 0 & $0 \%$ & 0.0019 & 0.0019 & -- & -- & $9.50 \mathrm{E}-04$ \\
\hline PEST & Gamma-BHC (Lindane) & $\mathrm{mg} / \mathrm{kg}$ & 18 & 0 & $0 \%$ & 0.0019 & 0.0042 & -- & -- & 0.0011 \\
\hline PEST & gamma-Chlordane & $\mathrm{mg} / \mathrm{kg}$ & 18 & 0 & $0 \%$ & 0.0019 & 0.0042 & -- & -- & 0.0011 \\
\hline PEST & gamma-Chlordene & $\mathrm{mg} / \mathrm{kg}$ & 18 & 0 & $0 \%$ & 0.0019 & 0.0042 & -- & -- & 0.0011 \\
\hline PEST & Heptachlor & $\mathrm{mg} / \mathrm{kg}$ & 18 & 0 & $0 \%$ & 0.0019 & 0.0042 & -- & -- & 0.0011 \\
\hline PEST & Heptachlor epoxide & $\mathrm{mg} / \mathrm{kg}$ & 10 & 0 & $0 \%$ & 0.0019 & 0.0042 & -- & -- & 0.0012 \\
\hline PEST & Mirex & $\mathrm{mg} / \mathrm{kg}$ & 18 & 0 & $0 \%$ & 0.0019 & 0.0042 & -- & -- & 0.0011 \\
\hline PEST & Oxychlordane & $\mathrm{mg} / \mathrm{kg}$ & 18 & 0 & $0 \%$ & 0.0019 & 0.0042 & -- & -- & 0.0011 \\
\hline PEST & Pentachloroanisole & $\mathrm{mg} / \mathrm{kg}$ & 18 & 0 & $0 \%$ & 0.0019 & 0.0042 & -- & -- & 0.0011 \\
\hline PEST & Toxaphene & $\mathrm{mg} / \mathrm{kg}$ & 18 & 0 & $0 \%$ & 0.056 & 0.12 & -- & -- & 0.034 \\
\hline PEST & trans-Nonachlor & $\mathrm{mg} / \mathrm{kg}$ & 18 & 14 & $78 \%$ & 0.0020 & 0.0042 & 0.0020 & 0.0065 & 0.0030 \\
\hline SVOC & 1,2,4-Trichlorobenzene & $\mathrm{mg} / \mathrm{kg}$ & 18 & 0 & $0 \%$ & 0.0087 & 0.040 & -- & -- & 0.0055 \\
\hline SVOC & 1,2-Dichlorobenzene & $\mathrm{mg} / \mathrm{kg}$ & 18 & 0 & $0 \%$ & 0.0087 & 0.040 & -- & -- & 0.0055 \\
\hline SVOC & 1,2-Diphenylhydrazine & $\mathrm{mg} / \mathrm{kg}$ & 18 & 0 & $0 \%$ & 0.0087 & 0.040 & -- & -- & 0.0055 \\
\hline SVOC & 1,3-Dichlorobenzene & $\mathrm{mg} / \mathrm{kg}$ & 18 & 0 & $0 \%$ & 0.0087 & 0.040 & -- & -- & 0.0055 \\
\hline SVOC & 1,4-Dichlorobenzene & $\mathrm{mg} / \mathrm{kg}$ & 18 & 0 & $0 \%$ & 0.0087 & 0.040 & -- & -- & 0.0055 \\
\hline SVOC & 1-methylnaphthalene & $\mathrm{mg} / \mathrm{kg}$ & 18 & 0 & $0 \%$ & 0.0087 & 0.040 & -- & -- & 0.0055 \\
\hline SVOC & 2,4-Dinitrotoluene & $\mathrm{mg} / \mathrm{kg}$ & 18 & 0 & $0 \%$ & 0.017 & 0.15 & -- & -- & 0.022 \\
\hline SVOC & 2,6-Dinitrotoluene & $\mathrm{mg} / \mathrm{kg}$ & 18 & 0 & $0 \%$ & 0.035 & 0.16 & -- & -- & 0.022 \\
\hline SVOC & 2-Chloronaphthalene & $\mathrm{mg} / \mathrm{kg}$ & 18 & 0 & $0 \%$ & 0.017 & 0.081 & -- & -- & 0.011 \\
\hline SVOC & 2-Methylnaphthalene & $\mathrm{mg} / \mathrm{kg}$ & 18 & 2 & $11 \%$ & 0.0087 & 0.040 & 0.0096 & 0.010 & 0.0063 \\
\hline SVOC & 4-Bromophenylphenyl ether & $\mathrm{mg} / \mathrm{kg}$ & 18 & 0 & $0 \%$ & 0.0087 & 0.040 & -- & -- & 0.0055 \\
\hline SVOC & 4-Chlorophenylphenyl ether & $\mathrm{mg} / \mathrm{kg}$ & 18 & 0 & $0 \%$ & 0.0087 & 0.040 & -- & -- & 0.0055 \\
\hline SVOC & Acenaphthene & $\mathrm{mg} / \mathrm{kg}$ & 18 & 0 & $0 \%$ & 0.017 & 0.081 & -- & -- & 0.011 \\
\hline SVOC & Acenaphthylene & $\mathrm{mg} / \mathrm{kg}$ & 18 & 0 & $0 \%$ & 0.0087 & 0.040 & -- & -- & 0.0055 \\
\hline SVOC & Anthracene & $\mathrm{mg} / \mathrm{kg}$ & 18 & 0 & $0 \%$ & 0.0087 & 0.040 & -- & -- & 0.0055 \\
\hline SVOC & Benzo(a)anthracene & $\mathrm{mg} / \mathrm{kg}$ & 18 & 0 & $0 \%$ & 0.0087 & 0.040 & -- & -- & 0.0055 \\
\hline SVOC & Benzo(a)pyrene & $\mathrm{mg} / \mathrm{kg}$ & 18 & 0 & $0 \%$ & 0.0087 & 0.040 & -- & -- & 0.0055 \\
\hline SVOC & Benzo(b)fluoranthene & $\mathrm{mg} / \mathrm{kg}$ & 18 & 0 & $0 \%$ & 0.0087 & 0.040 & -- & -- & 0.0055 \\
\hline SVOC & Benzo(ghi)perylene & $\mathrm{mg} / \mathrm{kg}$ & 18 & 0 & $0 \%$ & 0.017 & 0.081 & -- & -- & 0.011 \\
\hline SVOC & Benzo(k)fluoranthene & $\mathrm{mg} / \mathrm{kg}$ & 18 & 0 & $0 \%$ & 0.0087 & 0.040 & -- & -- & 0.0055 \\
\hline SVOC & Bis(2-chloro-1-methylethyl)ether & $\mathrm{mg} / \mathrm{kg}$ & 18 & 0 & $0 \%$ & 0.017 & 0.081 & -- & -- & 0.011 \\
\hline SVOC & Chrysene & $\mathrm{mg} / \mathrm{kg}$ & 18 & 0 & $0 \%$ & 0.0087 & 0.040 & -- & -- & 0.0055 \\
\hline SVOC & Dibenz[a,h]anthracene & $\mathrm{mg} / \mathrm{kg}$ & 18 & 0 & $0 \%$ & 0.017 & 0.081 & -- & -- & 0.011 \\
\hline SVOC & Dibenzofuran & $\mathrm{mg} / \mathrm{kg}$ & 18 & 0 & $0 \%$ & 0.0087 & 0.040 & -- & -- & 0.0055 \\
\hline SVOC & Fluoranthene & $\mathrm{mg} / \mathrm{kg}$ & 18 & 0 & $0 \%$ & 0.017 & 0.081 & -- & -- & 0.011 \\
\hline
\end{tabular}


Table F-86

Summary Statistics for Whole Body Chinook Salmon Collected from Segment 4 of the Columbia River

\begin{tabular}{|c|c|c|c|c|c|c|c|c|c|c|}
\hline $\begin{array}{l}\text { Constituent } \\
\text { Class }\end{array}$ & Constituent Name & Units & $\begin{array}{l}\text { Number } \\
\text { Analyzed }\end{array}$ & $\begin{array}{l}\text { Number } \\
\text { Detected }\end{array}$ & $\begin{array}{l}\text { Frequency of } \\
\text { Detection }\end{array}$ & $\begin{array}{c}\text { Minimum } \\
\text { Nondetect }\end{array}$ & $\begin{array}{l}\text { Maximum } \\
\text { Nondetect }\end{array}$ & $\begin{array}{c}\text { Minimum } \\
\text { Detect }\end{array}$ & $\begin{array}{c}\text { Maximum } \\
\text { Detect }\end{array}$ & $\begin{array}{c}\text { Average } \\
\text { Concentration }\end{array}$ \\
\hline$\overline{\text { SVOC }}$ & Fluorene & $\mathrm{mg} / \mathrm{kg}$ & 18 & 0 & $0 \%$ & 0.0046 & 0.025 & -- & -- & 0.0049 \\
\hline SVOC & Hexachlorobenzene & $\mathrm{mg} / \mathrm{kg}$ & 18 & 0 & $0 \%$ & 0.017 & 0.081 & -- & -- & 0.011 \\
\hline SVOC & Hexachlorobutadiene & $\mathrm{mg} / \mathrm{kg}$ & 18 & 0 & $0 \%$ & 0.0087 & 0.040 & -- & -- & 0.0055 \\
\hline SVOC & Hexachloroethane & $\mathrm{mg} / \mathrm{kg}$ & 18 & 0 & $0 \%$ & 0.0087 & 0.040 & -- & -- & 0.0055 \\
\hline SVOC & Indeno(1,2,3-cd)pyrene & $\mathrm{mg} / \mathrm{kg}$ & 18 & 0 & $0 \%$ & 0.017 & 0.081 & -- & -- & 0.011 \\
\hline SVOC & Naphthalene & $\mathrm{mg} / \mathrm{kg}$ & 18 & 0 & $0 \%$ & 0.015 & 0.17 & -- & -- & 0.021 \\
\hline SVOC & Nitrobenzene & $\mathrm{mg} / \mathrm{kg}$ & 18 & 0 & $0 \%$ & 0.0087 & 0.040 & -- & -- & 0.0056 \\
\hline SVOC & Phenanthrene & $\mathrm{mg} / \mathrm{kg}$ & 18 & 0 & $0 \%$ & 0.0087 & 0.040 & -- & -- & 0.0056 \\
\hline SVOC & Pyrene & $\mathrm{mg} / \mathrm{kg}$ & 18 & 0 & $0 \%$ & 0.017 & 0.081 & -- & -- & 0.011 \\
\hline VOC & Retene & $\mathrm{mg} / \mathrm{kg}$ & 18 & 0 & $0 \%$ & 0.017 & 0.081 & -- & -- & 0.011 \\
\hline
\end{tabular}

DIOXI - Dioxins and Furans

PEST - Pesticide

PCB - Polychlorinated biphenyl

SVOC - Semivolatile Organic Compound

VOC - Volatile Organic Compound 
Table F-87

Summary Statistics for Mountain Whitefish Fillet Skin On Collected from Segment 4 of the Columbia River

\begin{tabular}{|c|c|c|c|c|c|c|c|c|c|c|}
\hline $\begin{array}{c}\text { Constituent } \\
\text { Class }\end{array}$ & Constituent Name & Units & $\begin{array}{l}\text { Number } \\
\text { Analyzed }\end{array}$ & $\begin{array}{l}\text { Number } \\
\text { Detected }\end{array}$ & $\begin{array}{c}\text { Frequency of } \\
\text { Detection }\end{array}$ & $\begin{array}{l}\text { Minimum } \\
\text { Nondetect }\end{array}$ & $\begin{array}{l}\text { Maximum } \\
\text { Nondetect }\end{array}$ & $\begin{array}{c}\text { Minimum } \\
\text { Detect }\end{array}$ & $\begin{array}{l}\text { Maximum } \\
\text { Detect }\end{array}$ & $\begin{array}{c}\text { Average } \\
\text { Concentration }\end{array}$ \\
\hline$\overline{\mathrm{DIOXI}}$ & $1,2,3,4,6,7,8$-Heptachlorodibenzodioxin & $\mathrm{mg} / \mathrm{kg}$ & 7 & 7 & $100 \%$ & -- & -- & $1.90 \mathrm{E}-07$ & $2.90 \mathrm{E}-07$ & $2.49 \mathrm{E}-07$ \\
\hline DIOXI & 1,2,3,4,6,7,8-Heptachlorodibenzofuran & $\mathrm{mg} / \mathrm{kg}$ & 7 & 6 & $86 \%$ & $1.00 \mathrm{E}-08$ & 1.00E-08 & $7.00 \mathrm{E}-08$ & 1.10E-07 & $7.50 \mathrm{E}-08$ \\
\hline DIOXI & 1,2,3,4,7,8,9-Heptachlorodibenzofuran & $\mathrm{mg} / \mathrm{kg}$ & 7 & 4 & $57 \%$ & $1.00 \mathrm{E}-08$ & 1.00E-08 & $2.00 \mathrm{E}-08$ & $6.00 \mathrm{E}-08$ & $2.79 \mathrm{E}-08$ \\
\hline DIOXI & $1,2,3,4,7,8$-Hexachlorodibenzofuran & $\mathrm{mg} / \mathrm{kg}$ & 7 & 5 & $71 \%$ & $1.00 \mathrm{E}-08$ & 1.00E-08 & $2.00 \mathrm{E}-08$ & $5.00 \mathrm{E}-08$ & $2.86 \mathrm{E}-08$ \\
\hline DIOXI & 1,2,3,4,7,8-Hexachlorodibenzo-p-dioxin & $\mathrm{mg} / \mathrm{kg}$ & 7 & 6 & $86 \%$ & 1.00E-08 & 1.00E-08 & 1.00E-07 & 1.40E-07 & 9.93E-08 \\
\hline DIOXI & 1,2,3,6,7,8-Hexachlorodibenzofuran & $\mathrm{mg} / \mathrm{kg}$ & 7 & 7 & $100 \%$ & -- & -- & $3.00 \mathrm{E}-08$ & $6.00 \mathrm{E}-08$ & 4.43E-08 \\
\hline DIOXI & $1,2,3,6,7,8$-Hexachlorodibenzo-p-dioxin & $\mathrm{mg} / \mathrm{kg}$ & 7 & 7 & $100 \%$ & -- & -- & $1.00 \mathrm{E}-07$ & 2.00E-07 & 1.49E-07 \\
\hline DIOXI & 1,2,3,7,8,9-Hexachlorodibenzofuran & $\mathrm{mg} / \mathrm{kg}$ & 7 & 7 & $100 \%$ & -- & -- & $6.00 \mathrm{E}-08$ & $1.60 \mathrm{E}-07$ & $9.43 \mathrm{E}-08$ \\
\hline DIOXI & 1,2,3,7,8,9-Hexachlorodibenzo-p-dioxin & $\mathrm{mg} / \mathrm{kg}$ & 7 & 7 & $100 \%$ & -- & -- & 3.00E-08 & $1.20 \mathrm{E}-07$ & $6.86 \mathrm{E}-08$ \\
\hline DIOXI & 1,2,3,7,8-Pentachlorodibenzofuran & $\mathrm{mg} / \mathrm{kg}$ & 7 & 7 & $100 \%$ & -- & -- & 4.00E-08 & 1.00E-07 & 6.86E-08 \\
\hline DIOXI & 1,2,3,7,8-Pentachlorodibenzo-p-dioxin & $\mathrm{mg} / \mathrm{kg}$ & 7 & 7 & $100 \%$ & -- & -- & 1.50E-07 & 2.10E-07 & 1.71E-07 \\
\hline DIOXI & 2,3,4,6,7,8-Hexachlorodibenzofuran & $\mathrm{mg} / \mathrm{kg}$ & 7 & 7 & $100 \%$ & -- & -- & $1.30 \mathrm{E}-07$ & 1.70E-07 & $1.44 \mathrm{E}-07$ \\
\hline DIOXI & 2,3,4,7,8-Pentachlorodibenzofuran & $\mathrm{mg} / \mathrm{kg}$ & 7 & 6 & $86 \%$ & $1.00 \mathrm{E}-08$ & 1.00E-08 & $4.00 \mathrm{E}-08$ & 1.10E-07 & $6.64 \mathrm{E}-08$ \\
\hline DIOXI & 2,3,7,8-Tetrachlorodibenzofuran & $\mathrm{mg} / \mathrm{kg}$ & 7 & 7 & $100 \%$ & -- & -- & $1.40 \mathrm{E}-07$ & 7.00E-07 & 4.30E-07 \\
\hline DIOXI & 2,3,7,8-Tetrachlorodibenzo-p-dioxin & $\mathrm{mg} / \mathrm{kg}$ & 7 & 6 & $86 \%$ & 1.00E-08 & 1.00E-08 & $5.00 \mathrm{E}-08$ & 8.00E-08 & 5.36E-08 \\
\hline DIOXI & Octachlorodibenzofuran & $\mathrm{mg} / \mathrm{kg}$ & 7 & 6 & $86 \%$ & $1.00 \mathrm{E}-08$ & 1.00E-08 & $2.00 \mathrm{E}-08$ & 1.00E-07 & 4.93E-08 \\
\hline DIOXI & Octachlorodibenzo-p-dioxin & $\mathrm{mg} / \mathrm{kg}$ & 7 & 7 & $100 \%$ & -- & -- & $2.10 \mathrm{E}-07$ & $5.20 \mathrm{E}-07$ & 3.33E-07 \\
\hline METAL & Aluminum & $\mathrm{mg} / \mathrm{kg}$ & 7 & 3 & $43 \%$ & 5.0 & 20 & 1.3 & 3.1 & 4.4 \\
\hline METAL & Antimony & $\mathrm{mg} / \mathrm{kg}$ & 7 & 0 & $0 \%$ & 0.050 & 0.050 & -- & -- & 0.025 \\
\hline METAL & Arsenic & $\mathrm{mg} / \mathrm{kg}$ & 7 & 7 & $100 \%$ & -- & -- & 0.050 & 0.11 & 0.079 \\
\hline METAL & Barium & $\mathrm{mg} / \mathrm{kg}$ & 7 & 5 & $71 \%$ & 0.20 & 0.20 & 0.23 & 0.40 & 0.25 \\
\hline METAL & Beryllium & $\mathrm{mg} / \mathrm{kg}$ & 7 & 0 & $0 \%$ & 0.0040 & 0.0040 & -- & -- & 0.0020 \\
\hline METAL & Cadmium & $\mathrm{mg} / \mathrm{kg}$ & 7 & 0 & $0 \%$ & 0.0040 & 0.0040 & -- & -- & 0.0020 \\
\hline METAL & Chromium & $\mathrm{mg} / \mathrm{kg}$ & 7 & 2 & $29 \%$ & 0.10 & 0.10 & 0.11 & 0.12 & 0.069 \\
\hline METAL & Cobalt & $\mathrm{mg} / \mathrm{kg}$ & 7 & 7 & $100 \%$ & -- & -- & 0.020 & 0.040 & 0.027 \\
\hline METAL & Copper & $\mathrm{mg} / \mathrm{kg}$ & 7 & 7 & $100 \%$ & -- & -- & 0.53 & 0.83 & 0.65 \\
\hline METAL & Lead & $\mathrm{mg} / \mathrm{kg}$ & 7 & 4 & $57 \%$ & 0.010 & 0.010 & 0.010 & 0.020 & 0.0093 \\
\hline METAL & Manganese & $\mathrm{mg} / \mathrm{kg}$ & 7 & 7 & $100 \%$ & -- & -- & 0.54 & 0.90 & 0.70 \\
\hline METAL & Mercury & $\mathrm{mg} / \mathrm{kg}$ & 7 & 7 & $100 \%$ & -- & -- & 0.040 & 0.090 & 0.066 \\
\hline METAL & Nickel & $\mathrm{mg} / \mathrm{kg}$ & 7 & 7 & $100 \%$ & -- & -- & 0.040 & 0.15 & 0.083 \\
\hline METAL & Selenium & $\mathrm{mg} / \mathrm{kg}$ & 7 & 7 & $100 \%$ & -- & -- & 0.30 & 0.55 & 0.42 \\
\hline METAL & Silver & $\mathrm{mg} / \mathrm{kg}$ & 7 & 0 & $0 \%$ & 0.10 & 0.10 & -- & -- & 0.050 \\
\hline METAL & Thallium & $\mathrm{mg} / \mathrm{kg}$ & 7 & 0 & $0 \%$ & 0.050 & 0.050 & -- & -- & 0.025 \\
\hline METAL & Vanadium & $\mathrm{mg} / \mathrm{kg}$ & 7 & 7 & $100 \%$ & -- & -- & 0.020 & 0.050 & 0.031 \\
\hline METAL & Zinc & $\mathrm{mg} / \mathrm{kg}$ & 7 & 7 & $100 \%$ & -- & -- & 15 & 20 & 17 \\
\hline PCB & 2,2',3,3',4,4',5-Heptachloro-1,1'-biphenyl & $\mathrm{mg} / \mathrm{kg}$ & 7 & 7 & $100 \%$ & -- & -- & $2.32 \mathrm{E}-04$ & $6.38 \mathrm{E}-04$ & 3.34E-04 \\
\hline PCB & $2,2^{\prime}, 3,4,4^{\prime}, 5,5^{\prime}-$ Heptachlorobiphenyl & $\mathrm{mg} / \mathrm{kg}$ & 7 & 7 & $100 \%$ & -- & -- & $6.51 \mathrm{E}-04$ & 0.0019 & $9.34 \mathrm{E}-04$ \\
\hline PCB & 2,3,3',4,4',5'-Hexachlorobiphenyl & $\mathrm{mg} / \mathrm{kg}$ & 7 & 5 & $71 \%$ & 7.30E-07 & 1.12E-06 & $5.78 \mathrm{E}-05$ & 7.23E-05 & 4.68E-05 \\
\hline PCB & 2,3,3',4,4',5-Hexachlorobiphenyl (BZ 156) & $\mathrm{mg} / \mathrm{kg}$ & 7 & 7 & $100 \%$ & -- & -- & 5.23E-05 & $3.92 \mathrm{E}-04$ & $2.47 \mathrm{E}-04$ \\
\hline PCB & 2,3,3',4,4'-Pentachlorobiphenyl & $\mathrm{mg} / \mathrm{kg}$ & 7 & 7 & $100 \%$ & -- & -- & $1.48 \mathrm{E}-04$ & 0.0011 & 7.15E-04 \\
\hline PCB & 2,3',4,4',5,5'-Hexachlorobiphenyl (BZ 167) & $\mathrm{mg} / \mathrm{kg}$ & 7 & 7 & $100 \%$ & -- & -- & $2.12 \mathrm{E}-05$ & $1.33 \mathrm{E}-04$ & $9.02 \mathrm{E}-05$ \\
\hline PCB & 2',3,4,4',5-Pentachloro-1,1'-biphenyl (BZ 123) & $\mathrm{mg} / \mathrm{kg}$ & 7 & 7 & $100 \%$ & -- & -- & 7.53E-06 & 2.85E-05 & 2.07E-05 \\
\hline PCB & 2,3,4,4',5-Pentachlorobiphenyl (BZ 114) & $\mathrm{mg} / \mathrm{kg}$ & 7 & 7 & $100 \%$ & -- & -- & 1.30E-05 & $8.45 E-05$ & 5.56E-05 \\
\hline PCB & 2,3',4,4',5-Pentachlorobiphenyl (BZ 118) & $\mathrm{mg} / \mathrm{kg}$ & 7 & 7 & $100 \%$ & -- & -- & 2.63E-04 & 0.0024 & 0.0015 \\
\hline PCB & 2,3,4,5,3',4',5'-Heptachlorobiphenyl & $\mathrm{mg} / \mathrm{kg}$ & 7 & 7 & $100 \%$ & -- & -- & 6.11E-06 & 1.99E-05 & 1.27E-05 \\
\hline PCB & 3,3',4,4',5,5'-Hexachlorobiphenyl (BZ 169) & $\mathrm{mg} / \mathrm{kg}$ & 7 & 1 & $14 \%$ & 5.00E-07 & 3.00E-06 & 4.38E-06 & 4.38E-06 & 1.18E-06 \\
\hline PCB & 3,3',4,4',5-Pentachlorobiphenyl (BZ 126) & $\mathrm{mg} / \mathrm{kg}$ & 7 & 4 & $57 \%$ & $6.10 \mathrm{E}-07$ & $6.90 \mathrm{E}-07$ & 5.10E-06 & 6.49E-06 & $3.60 \mathrm{E}-06$ \\
\hline PCB & 3,3',4,4'-Tetrachlorobiphenyl (BZ 77) & $\mathrm{mg} / \mathrm{kg}$ & 7 & 7 & $100 \%$ & -- & -- & 1.37E-05 & 5.61E-05 & $2.50 \mathrm{E}-05$ \\
\hline
\end{tabular}


Table F-87

Summary Statistics for Mountain Whitefish Fillet Skin On Collected from Segment 4 of the Columbia River

\begin{tabular}{|c|c|c|c|c|c|c|c|c|c|c|}
\hline $\begin{array}{l}\text { Constituent } \\
\text { Class }\end{array}$ & Constituent Name & Units & $\begin{array}{l}\text { Number } \\
\text { Analyzed }\end{array}$ & $\begin{array}{l}\text { Number } \\
\text { Detected }\end{array}$ & $\begin{array}{c}\text { Frequency of } \\
\text { Detection }\end{array}$ & $\begin{array}{l}\text { Minimum } \\
\text { Nondetect }\end{array}$ & $\begin{array}{l}\text { Maximum } \\
\text { Nondetect }\end{array}$ & $\begin{array}{c}\text { Minimum } \\
\text { Detect }\end{array}$ & $\begin{array}{l}\text { Maximum } \\
\text { Detect }\end{array}$ & $\begin{array}{c}\text { Average } \\
\text { Concentration }\end{array}$ \\
\hline PCB & Aroclor-1016 & $\mathrm{mg} / \mathrm{kg}$ & 7 & 0 & $0 \%$ & 0.016 & 0.019 & -- & -- & 0.0088 \\
\hline PCB & Aroclor-1221 & $\mathrm{mg} / \mathrm{kg}$ & 7 & 0 & $0 \%$ & 0.016 & 0.019 & -- & -- & 0.0088 \\
\hline PCB & Aroclor-1232 & $\mathrm{mg} / \mathrm{kg}$ & 7 & 0 & $0 \%$ & 0.016 & 0.019 & -- & -- & 0.0088 \\
\hline PCB & Aroclor-1248 & $\mathrm{mg} / \mathrm{kg}$ & 7 & 0 & $0 \%$ & 0.016 & 0.019 & -- & -- & 0.0088 \\
\hline PCB & Aroclor-1254 & $\mathrm{mg} / \mathrm{kg}$ & 7 & 4 & $57 \%$ & 0.016 & 0.019 & 0.021 & 0.030 & 0.019 \\
\hline PCB & Aroclor-1260 & $\mathrm{mg} / \mathrm{kg}$ & 7 & 2 & $29 \%$ & 0.017 & 0.019 & 0.0085 & 0.022 & 0.011 \\
\hline PEST & 2,4'-DDD & $\mathrm{mg} / \mathrm{kg}$ & 7 & 0 & $0 \%$ & 0.0017 & 0.0024 & -- & -- & $9.93 \mathrm{E}-04$ \\
\hline PEST & 2,4'-DDE & $\mathrm{mg} / \mathrm{kg}$ & 7 & 1 & $14 \%$ & 0.0017 & 0.0024 & 0.0029 & 0.0029 & 0.0013 \\
\hline PEST & 2,4'-DDT & $\mathrm{mg} / \mathrm{kg}$ & 7 & 0 & $0 \%$ & 0.0017 & 0.0024 & -- & -- & 9.93E-04 \\
\hline PEST & Aldrin & $\mathrm{mg} / \mathrm{kg}$ & 7 & 0 & $0 \%$ & 0.0017 & 0.0024 & -- & -- & 9.93E-04 \\
\hline PEST & Alpha-BHC & $\mathrm{mg} / \mathrm{kg}$ & 7 & 0 & $0 \%$ & 0.0017 & 0.0024 & -- & -- & 9.93E-04 \\
\hline PEST & alpha-Chlordane & $\mathrm{mg} / \mathrm{kg}$ & 7 & 0 & $0 \%$ & 0.0017 & 0.0024 & -- & -- & $9.93 \mathrm{E}-04$ \\
\hline PEST & alpha-Chlordene & $\mathrm{mg} / \mathrm{kg}$ & 7 & 0 & $0 \%$ & 0.0017 & 0.0024 & -- & -- & $9.93 E-04$ \\
\hline PEST & Beta-BHC & $\mathrm{mg} / \mathrm{kg}$ & 7 & 0 & $0 \%$ & 0.0017 & 0.0024 & -- & -- & 9.93E-04 \\
\hline PEST & cis-Nonachlor & $\mathrm{mg} / \mathrm{kg}$ & 7 & 0 & $0 \%$ & 0.0017 & 0.0024 & -- & -- & 9.93E-04 \\
\hline PEST & DDMU & $\mathrm{mg} / \mathrm{kg}$ & 7 & 0 & $0 \%$ & 0.0017 & 0.0024 & -- & -- & $9.93 \mathrm{E}-04$ \\
\hline PEST & Delta-BHC & $\mathrm{mg} / \mathrm{kg}$ & 7 & 0 & $0 \%$ & 0.0017 & 0.0024 & -- & -- & $9.93 \mathrm{E}-04$ \\
\hline PEST & Dichlorodiphenyldichloroethane & $\mathrm{mg} / \mathrm{kg}$ & 7 & 0 & $0 \%$ & 0.0017 & 0.0024 & -- & -- & $9.93 \mathrm{E}-04$ \\
\hline PEST & Dichlorodiphenyldichloroethylene & $\mathrm{mg} / \mathrm{kg}$ & 7 & 7 & $100 \%$ & -- & -- & 0.0066 & 0.030 & 0.014 \\
\hline PEST & Dichlorodiphenyltrichloroethane & $\mathrm{mg} / \mathrm{kg}$ & 7 & 3 & $43 \%$ & 0.0017 & 0.0019 & 0.0026 & 0.0028 & 0.0017 \\
\hline PEST & Endosulfan sulfate & $\mathrm{mg} / \mathrm{kg}$ & 7 & 0 & $0 \%$ & 0.0017 & 0.0024 & -- & -- & $9.93 \mathrm{E}-04$ \\
\hline PEST & Gamma-BHC (Lindane) & $\mathrm{mg} / \mathrm{kg}$ & 7 & 0 & $0 \%$ & 0.0017 & 0.0024 & -- & -- & 9.93E-04 \\
\hline PEST & gamma-Chlordane & $\mathrm{mg} / \mathrm{kg}$ & 7 & 0 & $0 \%$ & 0.0017 & 0.0024 & -- & -- & 9.93E-04 \\
\hline PEST & gamma-Chlordene & $\mathrm{mg} / \mathrm{kg}$ & 7 & 0 & $0 \%$ & 0.0017 & 0.0024 & -- & -- & 9.93E-04 \\
\hline PEST & Heptachlor & $\mathrm{mg} / \mathrm{kg}$ & 7 & 0 & $0 \%$ & 0.0017 & 0.0024 & -- & -- & $9.93 \mathrm{E}-04$ \\
\hline PEST & Heptachlor epoxide & $\mathrm{mg} / \mathrm{kg}$ & 7 & 0 & $0 \%$ & 0.0017 & 0.0024 & -- & -- & $9.93 \mathrm{E}-04$ \\
\hline PEST & Mirex & $\mathrm{mg} / \mathrm{kg}$ & 7 & 0 & $0 \%$ & 0.0017 & 0.0024 & -- & -- & 9.93E-04 \\
\hline PEST & Oxychlordane & $\mathrm{mg} / \mathrm{kg}$ & 7 & 0 & $0 \%$ & 0.0017 & 0.0024 & -- & -- & $9.93 \mathrm{E}-04$ \\
\hline PEST & Pentachloroanisole & $\mathrm{mg} / \mathrm{kg}$ & 7 & 0 & $0 \%$ & 0.0017 & 0.0024 & -- & -- & 9.93E-04 \\
\hline PEST & Toxaphene & $\mathrm{mg} / \mathrm{kg}$ & 7 & 0 & $0 \%$ & 0.050 & 0.073 & -- & -- & 0.030 \\
\hline PEST & trans-Nonachlor & $\mathrm{mg} / \mathrm{kg}$ & 7 & 0 & $0 \%$ & 0.0017 & 0.0024 & -- & -- & $9.93 E-04$ \\
\hline SVOC & 1,2,4-Trichlorobenzene & $\mathrm{mg} / \mathrm{kg}$ & 7 & 0 & $0 \%$ & 0.012 & 0.025 & -- & -- & 0.0093 \\
\hline SVOC & 1,2-Dichlorobenzene & $\mathrm{mg} / \mathrm{kg}$ & 7 & 0 & $0 \%$ & 0.012 & 0.025 & -- & -- & 0.0093 \\
\hline SVOC & 1,2-Diphenylhydrazine & $\mathrm{mg} / \mathrm{kg}$ & 7 & 0 & $0 \%$ & 0.012 & 0.025 & -- & -- & 0.0093 \\
\hline SVOC & 1,3-Dichlorobenzene & $\mathrm{mg} / \mathrm{kg}$ & 7 & 0 & $0 \%$ & 0.012 & 0.025 & -- & -- & 0.0093 \\
\hline SVOC & 1,4-Dichlorobenzene & $\mathrm{mg} / \mathrm{kg}$ & 7 & 0 & $0 \%$ & 0.012 & 0.025 & -- & -- & 0.0093 \\
\hline SVOC & 1-methylnaphthalene & $\mathrm{mg} / \mathrm{kg}$ & 7 & 0 & $0 \%$ & 0.012 & 0.025 & -- & -- & 0.0093 \\
\hline SVOC & 2,4-Dinitrotoluene & $\mathrm{mg} / \mathrm{kg}$ & 7 & 0 & $0 \%$ & 0.024 & 0.049 & -- & -- & 0.019 \\
\hline SVOC & 2,6-Dinitrotoluene & $\mathrm{mg} / \mathrm{kg}$ & 7 & 0 & $0 \%$ & 0.047 & 0.098 & -- & -- & 0.037 \\
\hline SVOC & 2-Chloronaphthalene & $\mathrm{mg} / \mathrm{kg}$ & 7 & 0 & $0 \%$ & 0.022 & 0.046 & -- & -- & 0.014 \\
\hline SVOC & 2-Methylnaphthalene & $\mathrm{mg} / \mathrm{kg}$ & 7 & 0 & $0 \%$ & 0.012 & 0.025 & -- & -- & 0.0093 \\
\hline SVOC & 4-Bromophenylphenyl ether & $\mathrm{mg} / \mathrm{kg}$ & 7 & 0 & $0 \%$ & 0.012 & 0.025 & -- & -- & 0.0093 \\
\hline SVOC & 4-Chlorophenylphenyl ether & $\mathrm{mg} / \mathrm{kg}$ & 7 & 0 & $0 \%$ & 0.012 & 0.025 & -- & -- & 0.0093 \\
\hline SVOC & Acenaphthene & $\mathrm{mg} / \mathrm{kg}$ & 7 & 0 & $0 \%$ & 0.024 & 0.049 & -- & -- & 0.019 \\
\hline SVOC & Acenaphthylene & $\mathrm{mg} / \mathrm{kg}$ & 7 & 0 & $0 \%$ & 0.012 & 0.025 & -- & -- & 0.0093 \\
\hline SVOC & Anthracene & $\mathrm{mg} / \mathrm{kg}$ & 7 & 0 & $0 \%$ & 0.012 & 0.025 & -- & -- & 0.0093 \\
\hline SVOC & Benzo(a)anthracene & $\mathrm{mg} / \mathrm{kg}$ & 7 & 0 & $0 \%$ & 0.012 & 0.025 & -- & -- & 0.0093 \\
\hline SVOC & Benzo(a)pyrene & $\mathrm{mg} / \mathrm{kg}$ & 7 & 0 & $0 \%$ & 0.012 & 0.025 & -- & -- & 0.0093 \\
\hline
\end{tabular}


Table F-87

Summary Statistics for Mountain Whitefish Fillet Skin On Collected from Segment 4 of the Columbia River

\begin{tabular}{|c|c|c|c|c|c|c|c|c|c|c|}
\hline $\begin{array}{l}\text { Constituent } \\
\text { Class }\end{array}$ & Constituent Name & Units & $\begin{array}{c}\text { Number } \\
\text { Analyzed }\end{array}$ & $\begin{array}{l}\text { Number } \\
\text { Detected }\end{array}$ & $\begin{array}{l}\text { Frequency of } \\
\text { Detection }\end{array}$ & $\begin{array}{l}\text { Minimum } \\
\text { Nondetect }\end{array}$ & $\begin{array}{c}\text { Maximum } \\
\text { Nondetect }\end{array}$ & $\begin{array}{c}\text { Minimum } \\
\text { Detect }\end{array}$ & $\begin{array}{c}\text { Maximum } \\
\text { Detect }\end{array}$ & $\begin{array}{c}\text { Average } \\
\text { Concentration }\end{array}$ \\
\hline$\overline{\text { SVOC }}$ & Benzo(b)fluoranthene & $\mathrm{mg} / \mathrm{kg}$ & 7 & $\overline{0}$ & $0 \%$ & 0.012 & 0.049 & $\overline{--}$ & $\overline{--}$ & 0.014 \\
\hline SVOC & Benzo(ghi)perylene & $\mathrm{mg} / \mathrm{kg}$ & 7 & 0 & $0 \%$ & 0.024 & 0.049 & -- & -- & 0.019 \\
\hline SVOC & Benzo(k)fluoranthene & $\mathrm{mg} / \mathrm{kg}$ & 7 & 0 & $0 \%$ & 0.012 & 0.049 & -- & -- & 0.014 \\
\hline SVOC & Bis(2-chloro-1-methylethyl)ether & $\mathrm{mg} / \mathrm{kg}$ & 7 & 0 & $0 \%$ & 0.024 & 0.049 & -- & -- & 0.019 \\
\hline SVOC & Chrysene & $\mathrm{mg} / \mathrm{kg}$ & 7 & 0 & $0 \%$ & 0.012 & 0.025 & -- & -- & 0.0093 \\
\hline SVOC & Dibenz[a,h]anthracene & $\mathrm{mg} / \mathrm{kg}$ & 7 & 0 & $0 \%$ & 0.024 & 0.049 & -- & -- & 0.019 \\
\hline SVOC & Dibenzofuran & $\mathrm{mg} / \mathrm{kg}$ & 7 & 0 & $0 \%$ & 0.012 & 0.025 & -- & -- & 0.0093 \\
\hline SVOC & Fluoranthene & $\mathrm{mg} / \mathrm{kg}$ & 7 & 0 & $0 \%$ & 0.024 & 0.049 & -- & -- & 0.019 \\
\hline SVOC & Fluorene & $\mathrm{mg} / \mathrm{kg}$ & 7 & 0 & $0 \%$ & 0.0051 & 0.0099 & -- & -- & 0.0043 \\
\hline SVOC & Hexachlorobenzene & $\mathrm{mg} / \mathrm{kg}$ & 7 & 0 & $0 \%$ & 0.024 & 0.049 & -- & -- & 0.019 \\
\hline SVOC & Hexachlorobutadiene & $\mathrm{mg} / \mathrm{kg}$ & 7 & 0 & $0 \%$ & 0.012 & 0.025 & -- & -- & 0.0093 \\
\hline SVOC & Hexachloroethane & $\mathrm{mg} / \mathrm{kg}$ & 7 & 0 & $0 \%$ & 0.012 & 0.025 & -- & -- & 0.0093 \\
\hline SVOC & Indeno(1,2,3-cd)pyrene & $\mathrm{mg} / \mathrm{kg}$ & 7 & 0 & $0 \%$ & 0.024 & 0.049 & -- & -- & 0.019 \\
\hline SVOC & Naphthalene & $\mathrm{mg} / \mathrm{kg}$ & 7 & 0 & $0 \%$ & 0.013 & 0.025 & -- & -- & 0.0098 \\
\hline SVOC & Nitrobenzene & $\mathrm{mg} / \mathrm{kg}$ & 7 & 0 & $0 \%$ & 0.012 & 0.025 & -- & -- & 0.0093 \\
\hline SVOC & Phenanthrene & $\mathrm{mg} / \mathrm{kg}$ & 7 & 0 & $0 \%$ & 0.012 & 0.025 & -- & -- & 0.0093 \\
\hline SVOC & Pyrene & $\mathrm{mg} / \mathrm{kg}$ & 7 & 0 & $0 \%$ & 0.024 & 0.049 & -- & -- & 0.019 \\
\hline VOC & Retene & $\mathrm{mg} / \mathrm{kg}$ & 7 & 0 & $0 \%$ & 0.024 & 0.049 & -- & -- & 0.019 \\
\hline
\end{tabular}

DIOXI - Dioxins and Furans

PEST - Pesticide

PCB - Polychlorinated biphenyl

SVOC - Semivolatile Organic Compound

VOC - Volatile Organic Compound 
Table F-88

Summary Statistics for Whole Body Mountain Whitefish Collected from Segment 4 of the Columbia River

\begin{tabular}{|c|c|c|c|c|c|c|c|c|c|c|}
\hline $\begin{array}{l}\text { Constituent } \\
\text { Class }\end{array}$ & Constituent Name & Units & $\begin{array}{l}\text { Number } \\
\text { Analyzed }\end{array}$ & $\begin{array}{l}\text { Number } \\
\text { Detected }\end{array}$ & $\begin{array}{c}\text { Frequency of } \\
\text { Detection }\end{array}$ & $\begin{array}{l}\text { Minimum } \\
\text { Nondetect }\end{array}$ & $\begin{array}{l}\text { Maximum } \\
\text { Nondetect }\end{array}$ & $\begin{array}{c}\text { Minimum } \\
\text { Detect }\end{array}$ & $\begin{array}{l}\text { Maximum } \\
\text { Detect }\end{array}$ & $\begin{array}{c}\text { Average } \\
\text { Concentration }\end{array}$ \\
\hline$\overline{D I O X I}$ & $1,2,3,4,6,7,8$-Heptachlorodibenzodioxin & $\mathrm{mg} / \mathrm{kg}$ & 5 & 5 & $100 \%$ & -- & -- & $2.60 \mathrm{E}-07$ & $4.10 \mathrm{E}-07$ & $3.42 \mathrm{E}-07$ \\
\hline DIOXI & 1,2,3,4,6,7,8-Heptachlorodibenzofuran & $\mathrm{mg} / \mathrm{kg}$ & 5 & 4 & $80 \%$ & $6.00 \mathrm{E}-08$ & $6.00 \mathrm{E}-08$ & $9.00 \mathrm{E}-08$ & 1.00E-07 & $8.20 \mathrm{E}-08$ \\
\hline DIOXI & 1,2,3,4,7,8,9-Heptachlorodibenzofuran & $\mathrm{mg} / \mathrm{kg}$ & 5 & 1 & $20 \%$ & $1.00 \mathrm{E}-08$ & 8.00E-08 & $2.00 \mathrm{E}-08$ & 2.00E-08 & $2.00 \mathrm{E}-08$ \\
\hline DIOXI & $1,2,3,4,7,8$-Hexachlorodibenzofuran & $\mathrm{mg} / \mathrm{kg}$ & 5 & 3 & $60 \%$ & $6.00 \mathrm{E}-08$ & $9.00 \mathrm{E}-08$ & $3.00 \mathrm{E}-08$ & 4.00E-08 & $3.50 \mathrm{E}-08$ \\
\hline DIOXI & $1,2,3,4,7,8$-Hexachlorodibenzo-p-dioxin & $\mathrm{mg} / \mathrm{kg}$ & 5 & 5 & $100 \%$ & -- & -- & 1.10E-07 & $1.50 \mathrm{E}-07$ & 1.24E-07 \\
\hline DIOXI & 1,2,3,6,7,8-Hexachlorodibenzofuran & $\mathrm{mg} / \mathrm{kg}$ & 5 & 3 & $60 \%$ & $6.00 \mathrm{E}-08$ & $9.00 \mathrm{E}-08$ & $3.00 \mathrm{E}-08$ & 4.00E-08 & $3.70 \mathrm{E}-08$ \\
\hline DIOXI & $1,2,3,6,7,8$-Hexachlorodibenzo-p-dioxin & $\mathrm{mg} / \mathrm{kg}$ & 5 & 5 & $100 \%$ & -- & -- & $1.40 \mathrm{E}-07$ & $2.70 \mathrm{E}-07$ & 2.06E-07 \\
\hline DIOXI & 1,2,3,7,8,9-Hexachlorodibenzofuran & $\mathrm{mg} / \mathrm{kg}$ & 5 & 4 & $80 \%$ & $1.00 \mathrm{E}-08$ & $1.00 \mathrm{E}-08$ & $6.00 \mathrm{E}-08$ & $1.50 \mathrm{E}-07$ & $7.90 \mathrm{E}-08$ \\
\hline DIOXI & $1,2,3,7,8,9-$ Hexachlorodibenzo-p-dioxin & $\mathrm{mg} / \mathrm{kg}$ & 5 & 4 & $80 \%$ & 7.00E-08 & 7.00E-08 & $5.00 \mathrm{E}-08$ & $9.00 \mathrm{E}-08$ & $6.30 \mathrm{E}-08$ \\
\hline DIOXI & 1,2,3,7,8-Pentachlorodibenzofuran & $\mathrm{mg} / \mathrm{kg}$ & 5 & 4 & $80 \%$ & 4.00E-08 & 4.00E-08 & $6.00 \mathrm{E}-08$ & 8.00E-08 & $5.80 \mathrm{E}-08$ \\
\hline DIOXI & 1,2,3,7,8-Pentachlorodibenzo-p-dioxin & $\mathrm{mg} / \mathrm{kg}$ & 5 & 4 & $80 \%$ & $3.00 \mathrm{E}-08$ & $3.00 \mathrm{E}-08$ & $1.80 \mathrm{E}-07$ & $2.40 \mathrm{E}-07$ & $1.69 \mathrm{E}-07$ \\
\hline DIOXI & 2,3,4,6,7,8-Hexachlorodibenzofuran & $\mathrm{mg} / \mathrm{kg}$ & 5 & 5 & $100 \%$ & -- & -- & $1.30 \mathrm{E}-07$ & $1.70 \mathrm{E}-07$ & $1.48 \mathrm{E}-07$ \\
\hline DIOXI & 2,3,4,7,8-Pentachlorodibenzofuran & $\mathrm{mg} / \mathrm{kg}$ & 5 & 5 & $100 \%$ & -- & -- & $7.00 \mathrm{E}-08$ & $1.00 \mathrm{E}-07$ & 8.60E-08 \\
\hline DIOXI & 2,3,7,8-Tetrachlorodibenzofuran & $\mathrm{mg} / \mathrm{kg}$ & 5 & 5 & $100 \%$ & -- & -- & 2.30E-07 & $1.24 \mathrm{E}-06$ & 7.60E-07 \\
\hline DIOXI & 2,3,7,8-Tetrachlorodibenzo-p-dioxin & $\mathrm{mg} / \mathrm{kg}$ & 5 & 3 & $60 \%$ & $1.00 \mathrm{E}-08$ & $5.00 \mathrm{E}-08$ & 7.00E-08 & $9.00 \mathrm{E}-08$ & $5.40 \mathrm{E}-08$ \\
\hline DIOXI & Octachlorodibenzofuran & $\mathrm{mg} / \mathrm{kg}$ & 5 & 2 & $40 \%$ & $1.00 \mathrm{E}-08$ & $9.00 \mathrm{E}-08$ & $2.00 \mathrm{E}-08$ & 4.00E-08 & $2.90 \mathrm{E}-08$ \\
\hline DIOXI & Octachlorodibenzo-p-dioxin & $\mathrm{mg} / \mathrm{kg}$ & 5 & 5 & $100 \%$ & -- & -- & $2.70 \mathrm{E}-07$ & $5.70 \mathrm{E}-07$ & 4.04E-07 \\
\hline METAL & Aluminum & $\mathrm{mg} / \mathrm{kg}$ & 5 & 4 & $80 \%$ & 20 & 20 & 7.2 & 22 & 12 \\
\hline METAL & Antimony & $\mathrm{mg} / \mathrm{kg}$ & 5 & 0 & $0 \%$ & 0.050 & 0.050 & -- & -- & 0.025 \\
\hline METAL & Arsenic & $\mathrm{mg} / \mathrm{kg}$ & 5 & 5 & $100 \%$ & -- & -- & 0.13 & 0.18 & 0.15 \\
\hline METAL & Barium & $\mathrm{mg} / \mathrm{kg}$ & 5 & 5 & $100 \%$ & -- & -- & 0.50 & 0.93 & 0.71 \\
\hline METAL & Beryllium & $\mathrm{mg} / \mathrm{kg}$ & 5 & 0 & $0 \%$ & 0.0040 & 0.0040 & -- & -- & 0.0020 \\
\hline METAL & Cadmium & $\mathrm{mg} / \mathrm{kg}$ & 5 & 4 & $80 \%$ & 0.0040 & 0.0040 & 0.0042 & 0.010 & 0.0057 \\
\hline METAL & Chromium & $\mathrm{mg} / \mathrm{kg}$ & 5 & 4 & $80 \%$ & 0.10 & 0.10 & 0.10 & 0.11 & 0.096 \\
\hline METAL & Cobalt & $\mathrm{mg} / \mathrm{kg}$ & 5 & 5 & $100 \%$ & -- & -- & 0.050 & 0.10 & 0.076 \\
\hline METAL & Copper & $\mathrm{mg} / \mathrm{kg}$ & 5 & 5 & $100 \%$ & -- & -- & 0.95 & 5.0 & 1.9 \\
\hline METAL & Lead & $\mathrm{mg} / \mathrm{kg}$ & 5 & 5 & $100 \%$ & -- & -- & 0.010 & 0.040 & 0.016 \\
\hline METAL & Manganese & $\mathrm{mg} / \mathrm{kg}$ & 5 & 5 & $100 \%$ & -- & -- & 2.1 & 4.6 & 3.5 \\
\hline METAL & Mercury & $\mathrm{mg} / \mathrm{kg}$ & 5 & 5 & $100 \%$ & -- & -- & 0.040 & 0.11 & 0.060 \\
\hline METAL & Nickel & $\mathrm{mg} / \mathrm{kg}$ & 5 & 5 & $100 \%$ & -- & -- & 0.22 & 1.3 & 0.47 \\
\hline METAL & Selenium & $\mathrm{mg} / \mathrm{kg}$ & 5 & 5 & $100 \%$ & -- & -- & 0.58 & 1.6 & 0.89 \\
\hline METAL & Silver & $\mathrm{mg} / \mathrm{kg}$ & 5 & 0 & $0 \%$ & 0.10 & 0.10 & -- & -- & 0.050 \\
\hline METAL & Thallium & $\mathrm{mg} / \mathrm{kg}$ & 5 & 0 & $0 \%$ & 0.050 & 0.050 & -- & -- & 0.025 \\
\hline METAL & Vanadium & $\mathrm{mg} / \mathrm{kg}$ & 5 & 5 & $100 \%$ & -- & -- & 0.13 & 0.28 & 0.21 \\
\hline METAL & Zinc & $\mathrm{mg} / \mathrm{kg}$ & 5 & 5 & $100 \%$ & -- & -- & 24 & 40 & 31 \\
\hline PCB & 2,2',3,3',4,4',5-Heptachloro-1,1'-biphenyl & $\mathrm{mg} / \mathrm{kg}$ & 5 & 5 & $100 \%$ & -- & -- & $2.74 \mathrm{E}-04$ & $8.20 \mathrm{E}-04$ & 4.76E-04 \\
\hline PCB & $2,2^{\prime}, 3,4,4^{\prime}, 5,5^{\prime}$-Heptachlorobiphenyl & $\mathrm{mg} / \mathrm{kg}$ & 5 & 5 & $100 \%$ & -- & -- & 7.01E-04 & 0.0025 & 0.0013 \\
\hline PCB & $2,3,3^{\prime}, 4,4^{\prime}, 5^{\prime}-$ Hexachlorobiphenyl & $\mathrm{mg} / \mathrm{kg}$ & 5 & 3 & $60 \%$ & $6.80 \mathrm{E}-07$ & 2.19E-06 & 7.38E-05 & 8.49E-05 & 4.68E-05 \\
\hline PCB & 2,3,3',4,4',5-Hexachlorobiphenyl (BZ 156) & $\mathrm{mg} / \mathrm{kg}$ & 5 & 5 & $100 \%$ & -- & -- & $1.31 \mathrm{E}-04$ & 4.67E-04 & 3.30E-04 \\
\hline PCB & 2,3,3',4,4'-Pentachlorobiphenyl & $\mathrm{mg} / \mathrm{kg}$ & 5 & 5 & $100 \%$ & -- & -- & $3.10 \mathrm{E}-04$ & 0.0016 & 0.0011 \\
\hline PCB & 2,3',4,4',5,5'-Hexachlorobiphenyl (BZ 167) & $\mathrm{mg} / \mathrm{kg}$ & 5 & 5 & $100 \%$ & -- & -- & 5.55E-05 & 1.72E-04 & 1.27E-04 \\
\hline PCB & 2',3,4,4',5-Pentachloro-1,1'-biphenyl (BZ 123) & $\mathrm{mg} / \mathrm{kg}$ & 5 & 5 & $100 \%$ & -- & -- & 9.99E-06 & 4.94E-05 & 3.24E-05 \\
\hline PCB & 2,3,4,4',5-Pentachlorobiphenyl (BZ 114) & $\mathrm{mg} / \mathrm{kg}$ & 5 & 5 & $100 \%$ & -- & -- & 2.22E-05 & 1.15E-04 & 8.79E-05 \\
\hline PCB & 2,3',4,4',5-Pentachlorobiphenyl (BZ 118) & $\mathrm{mg} / \mathrm{kg}$ & 5 & 5 & $100 \%$ & -- & -- & $2.74 \mathrm{E}-05$ & 0.0033 & 0.0016 \\
\hline PCB & $2,3,4,5,3^{\prime}, 4^{\prime}, 5^{\prime}$-Heptachlorobiphenyl & $\mathrm{mg} / \mathrm{kg}$ & 5 & 5 & $100 \%$ & -- & -- & $1.36 \mathrm{E}-05$ & $2.74 \mathrm{E}-05$ & 1.83E-05 \\
\hline PCB & 3,3',4,4',5,5'-Hexachlorobiphenyl (BZ 169) & $\mathrm{mg} / \mathrm{kg}$ & 5 & 1 & $20 \%$ & $5.40 \mathrm{E}-07$ & $1.85 \mathrm{E}-06$ & $9.90 \mathrm{E}-07$ & $9.90 \mathrm{E}-07$ & 7.13E-07 \\
\hline PCB & 3,3',4,4',5-Pentachlorobiphenyl (BZ 126) & $\mathrm{mg} / \mathrm{kg}$ & 5 & 3 & $60 \%$ & 8.10E-07 & $1.73 \mathrm{E}-06$ & $7.18 \mathrm{E}-06$ & $9.86 \mathrm{E}-06$ & $5.18 \mathrm{E}-06$ \\
\hline PCB & 3,3',4,4'-Tetrachlorobiphenyl (BZ 77) & $\mathrm{mg} / \mathrm{kg}$ & 5 & 5 & $100 \%$ & -- & -- & 2.17E-05 & 1.22E-04 & 5.15E-05 \\
\hline
\end{tabular}


Table F-88

Summary Statistics for Whole Body Mountain Whitefish Collected from Segment 4 of the Columbia River

\begin{tabular}{|c|c|c|c|c|c|c|c|c|c|c|}
\hline $\begin{array}{c}\text { Constituent } \\
\text { Class }\end{array}$ & Constituent Name & Units & $\begin{array}{l}\text { Number } \\
\text { Analyzed }\end{array}$ & $\begin{array}{l}\text { Number } \\
\text { Detected }\end{array}$ & $\begin{array}{c}\text { Frequency of } \\
\text { Detection }\end{array}$ & $\begin{array}{c}\text { Minimum } \\
\text { Nondetect }\end{array}$ & $\begin{array}{l}\text { Maximum } \\
\text { Nondetect }\end{array}$ & $\begin{array}{c}\text { Minimum } \\
\text { Detect }\end{array}$ & $\begin{array}{l}\text { Maximum } \\
\text { Detect }\end{array}$ & $\begin{array}{c}\text { Average } \\
\text { Concentration }\end{array}$ \\
\hline PCB & Aroclor-1016 & $\mathrm{mg} / \mathrm{kg}$ & 5 & 0 & $0 \%$ & 0.018 & 0.022 & -- & -- & 0.010 \\
\hline PCB & Aroclor-1221 & $\mathrm{mg} / \mathrm{kg}$ & 5 & 0 & $0 \%$ & 0.018 & 0.022 & -- & -- & 0.010 \\
\hline PCB & Aroclor-1232 & $\mathrm{mg} / \mathrm{kg}$ & 5 & 0 & $0 \%$ & 0.018 & 0.022 & -- & -- & 0.010 \\
\hline PCB & Aroclor-1248 & $\mathrm{mg} / \mathrm{kg}$ & 5 & 0 & $0 \%$ & 0.018 & 0.022 & -- & -- & 0.010 \\
\hline PCB & Aroclor-1254 & $\mathrm{mg} / \mathrm{kg}$ & 5 & 3 & $60 \%$ & 0.021 & 0.022 & 0.036 & 0.046 & 0.030 \\
\hline PCB & Aroclor-1260 & $\mathrm{mg} / \mathrm{kg}$ & 5 & 2 & $40 \%$ & 0.018 & 0.020 & 0.026 & 0.030 & 0.017 \\
\hline PEST & 2,4'-DDD & $\mathrm{mg} / \mathrm{kg}$ & 5 & 0 & $0 \%$ & 0.0018 & 0.0028 & -- & -- & 0.0011 \\
\hline PEST & 2,4'-DDE & $\mathrm{mg} / \mathrm{kg}$ & 5 & 1 & $20 \%$ & 0.0018 & 0.0028 & 0.0027 & 0.0027 & 0.0014 \\
\hline PEST & 2,4'-DDT & $\mathrm{mg} / \mathrm{kg}$ & 5 & 0 & $0 \%$ & 0.0018 & 0.0028 & -- & -- & 0.0011 \\
\hline PEST & Aldrin & $\mathrm{mg} / \mathrm{kg}$ & 5 & 0 & $0 \%$ & 0.0018 & 0.0028 & -- & -- & 0.0011 \\
\hline PEST & Alpha-BHC & $\mathrm{mg} / \mathrm{kg}$ & 5 & 0 & $0 \%$ & 0.0018 & 0.0028 & -- & -- & 0.0011 \\
\hline PEST & alpha-Chlordane & $\mathrm{mg} / \mathrm{kg}$ & 5 & 0 & $0 \%$ & 0.0018 & 0.0028 & -- & -- & 0.0011 \\
\hline PEST & alpha-Chlordene & $\mathrm{mg} / \mathrm{kg}$ & 5 & 0 & $0 \%$ & 0.0018 & 0.0028 & -- & -- & 0.0011 \\
\hline PEST & Beta-BHC & $\mathrm{mg} / \mathrm{kg}$ & 5 & 0 & $0 \%$ & 0.0018 & 0.0028 & -- & -- & 0.0011 \\
\hline PEST & cis-Nonachlor & $\mathrm{mg} / \mathrm{kg}$ & 5 & 0 & $0 \%$ & 0.0018 & 0.0028 & -- & -- & 0.0011 \\
\hline PEST & DDMU & $\mathrm{mg} / \mathrm{kg}$ & 5 & 0 & $0 \%$ & 0.0018 & 0.0028 & -- & -- & 0.0011 \\
\hline PEST & Delta-BHC & $\mathrm{mg} / \mathrm{kg}$ & 5 & 0 & $0 \%$ & 0.0018 & 0.0028 & -- & -- & 0.0011 \\
\hline PEST & Dichlorodiphenyldichloroethane & $\mathrm{mg} / \mathrm{kg}$ & 5 & 0 & $0 \%$ & 0.0018 & 0.0028 & -- & -- & 0.0011 \\
\hline PEST & Dichlorodiphenyldichloroethylene & $\mathrm{mg} / \mathrm{kg}$ & 5 & 5 & $100 \%$ & -- & -- & 0.013 & 0.047 & 0.026 \\
\hline PEST & Dichlorodiphenyltrichloroethane & $\mathrm{mg} / \mathrm{kg}$ & 5 & 2 & $40 \%$ & 0.0018 & 0.0020 & 0.0051 & 0.0054 & 0.0027 \\
\hline PEST & Endosulfan sulfate & $\mathrm{mg} / \mathrm{kg}$ & 5 & 0 & $0 \%$ & 0.0018 & 0.0028 & -- & -- & 0.0011 \\
\hline PEST & Gamma-BHC (Lindane) & $\mathrm{mg} / \mathrm{kg}$ & 5 & 0 & $0 \%$ & 0.0018 & 0.0028 & -- & -- & 0.0011 \\
\hline PEST & gamma-Chlordane & $\mathrm{mg} / \mathrm{kg}$ & 5 & 0 & $0 \%$ & 0.0018 & 0.0028 & -- & -- & 0.0011 \\
\hline PEST & gamma-Chlordene & $\mathrm{mg} / \mathrm{kg}$ & 5 & 0 & $0 \%$ & 0.0018 & 0.0028 & -- & -- & 0.0011 \\
\hline PEST & Heptachlor & $\mathrm{mg} / \mathrm{kg}$ & 5 & 0 & $0 \%$ & 0.0018 & 0.0028 & -- & -- & 0.0011 \\
\hline PEST & Heptachlor epoxide & $\mathrm{mg} / \mathrm{kg}$ & 5 & 0 & $0 \%$ & 0.0018 & 0.0028 & -- & -- & 0.0011 \\
\hline PEST & Mirex & $\mathrm{mg} / \mathrm{kg}$ & 5 & 0 & $0 \%$ & 0.0018 & 0.0028 & -- & -- & 0.0011 \\
\hline PEST & Oxychlordane & $\mathrm{mg} / \mathrm{kg}$ & 5 & 0 & $0 \%$ & 0.0018 & 0.0028 & -- & -- & 0.0011 \\
\hline PEST & Pentachloroanisole & $\mathrm{mg} / \mathrm{kg}$ & 5 & 0 & $0 \%$ & 0.0018 & 0.0028 & -- & -- & 0.0011 \\
\hline PEST & Toxaphene & $\mathrm{mg} / \mathrm{kg}$ & 5 & 0 & $0 \%$ & 0.055 & 0.083 & -- & -- & 0.033 \\
\hline PEST & trans-Nonachlor & $\mathrm{mg} / \mathrm{kg}$ & 5 & 0 & $0 \%$ & 0.0018 & 0.0028 & -- & -- & 0.0011 \\
\hline SVOC & 1,2,4-Trichlorobenzene & $\mathrm{mg} / \mathrm{kg}$ & 5 & 0 & $0 \%$ & 0.0042 & 0.0049 & -- & -- & 0.0023 \\
\hline SVOC & 1,2-Dichlorobenzene & $\mathrm{mg} / \mathrm{kg}$ & 5 & 0 & $0 \%$ & 0.0043 & 0.0049 & -- & -- & 0.0023 \\
\hline SVOC & 1,2-Diphenylhydrazine & $\mathrm{mg} / \mathrm{kg}$ & 5 & 0 & $0 \%$ & 0.0042 & 0.0049 & -- & -- & 0.0023 \\
\hline SVOC & 1,3-Dichlorobenzene & $\mathrm{mg} / \mathrm{kg}$ & 5 & 0 & $0 \%$ & 0.0042 & 0.0049 & -- & -- & 0.0023 \\
\hline SVOC & 1,4-Dichlorobenzene & $\mathrm{mg} / \mathrm{kg}$ & 5 & 0 & $0 \%$ & 0.0042 & 0.0049 & -- & -- & 0.0023 \\
\hline SVOC & 1-methylnaphthalene & $\mathrm{mg} / \mathrm{kg}$ & 5 & 0 & $0 \%$ & 0.0042 & 0.0049 & -- & -- & 0.0023 \\
\hline SVOC & 2,4-Dinitrotoluene & $\mathrm{mg} / \mathrm{kg}$ & 5 & 0 & $0 \%$ & 0.0084 & 0.0098 & -- & -- & 0.0046 \\
\hline SVOC & 2,6-Dinitrotoluene & $\mathrm{mg} / \mathrm{kg}$ & 5 & 1 & $20 \%$ & 0.017 & 0.019 & 0.026 & 0.026 & 0.013 \\
\hline SVOC & 2-Chloronaphthalene & $\mathrm{mg} / \mathrm{kg}$ & 5 & 0 & $0 \%$ & 0.0042 & 0.0098 & -- & -- & 0.0033 \\
\hline SVOC & 2-Methylnaphthalene & $\mathrm{mg} / \mathrm{kg}$ & 5 & 3 & $60 \%$ & 0.0047 & 0.0049 & 0.0073 & 0.0089 & 0.0057 \\
\hline SVOC & 4-Bromophenylphenyl ether & $\mathrm{mg} / \mathrm{kg}$ & 5 & 0 & $0 \%$ & 0.0042 & 0.0049 & -- & -- & 0.0023 \\
\hline SVOC & 4-Chlorophenylphenyl ether & $\mathrm{mg} / \mathrm{kg}$ & 5 & 0 & $0 \%$ & 0.0042 & 0.0049 & -- & -- & 0.0023 \\
\hline SVOC & Acenaphthene & $\mathrm{mg} / \mathrm{kg}$ & 5 & 0 & $0 \%$ & 0.0084 & 0.0098 & -- & -- & 0.0046 \\
\hline SVOC & Acenaphthylene & $\mathrm{mg} / \mathrm{kg}$ & 5 & 0 & $0 \%$ & 0.0042 & 0.0049 & -- & -- & 0.0023 \\
\hline SVOC & Anthracene & $\mathrm{mg} / \mathrm{kg}$ & 5 & 0 & $0 \%$ & 0.0042 & 0.0049 & -- & -- & 0.0023 \\
\hline SVOC & Benzo(a)anthracene & $\mathrm{mg} / \mathrm{kg}$ & 5 & 0 & $0 \%$ & 0.0042 & 0.0049 & -- & -- & 0.0023 \\
\hline SVOC & Benzo(a)pyrene & $\mathrm{mg} / \mathrm{kg}$ & 5 & 0 & $0 \%$ & 0.0047 & 0.096 & -- & -- & 0.028 \\
\hline
\end{tabular}


Table F-88

Summary Statistics for Whole Body Mountain Whitefish Collected from Segment 4 of the Columbia River

\begin{tabular}{|c|c|c|c|c|c|c|c|c|c|c|}
\hline $\begin{array}{l}\text { Constituent } \\
\text { Class }\end{array}$ & Constituent Name & Units & $\begin{array}{c}\text { Number } \\
\text { Analyzed }\end{array}$ & $\begin{array}{l}\text { Number } \\
\text { Detected }\end{array}$ & $\begin{array}{l}\text { Frequency of } \\
\text { Detection }\end{array}$ & $\begin{array}{l}\text { Minimum } \\
\text { Nondetect }\end{array}$ & $\begin{array}{c}\text { Maximum } \\
\text { Nondetect }\end{array}$ & $\begin{array}{c}\text { Minimum } \\
\text { Detect }\end{array}$ & $\begin{array}{c}\text { Maximum } \\
\text { Detect }\end{array}$ & $\begin{array}{c}\text { Average } \\
\text { Concentration }\end{array}$ \\
\hline$\overline{\text { SVOC }}$ & Benzo(b)fluoranthene & $\mathrm{mg} / \mathrm{kg}$ & 5 & $\overline{0}$ & $0 \%$ & 0.0047 & 0.19 & $\overline{--}$ & $\overline{--}$ & 0.056 \\
\hline SVOC & Benzo(ghi)perylene & $\mathrm{mg} / \mathrm{kg}$ & 5 & 0 & $0 \%$ & 0.0094 & 0.19 & -- & -- & 0.057 \\
\hline SVOC & Benzo(k)fluoranthene & $\mathrm{mg} / \mathrm{kg}$ & 5 & 0 & $0 \%$ & 0.0047 & 0.19 & -- & _- & 0.056 \\
\hline SVOC & Bis(2-chloro-1-methylethyl)ether & $\mathrm{mg} / \mathrm{kg}$ & 5 & 0 & $0 \%$ & 0.0084 & 0.0098 & -- & -- & 0.0046 \\
\hline SVOC & Chrysene & $\mathrm{mg} / \mathrm{kg}$ & 5 & 0 & $0 \%$ & 0.0042 & 0.0049 & -- & -- & 0.0023 \\
\hline SVOC & Dibenz[a,h]anthracene & $\mathrm{mg} / \mathrm{kg}$ & 5 & 0 & $0 \%$ & 0.0094 & 0.19 & -- & -- & 0.057 \\
\hline SVOC & Dibenzofuran & $\mathrm{mg} / \mathrm{kg}$ & 5 & 0 & $0 \%$ & 0.0042 & 0.0049 & -- & -- & 0.0023 \\
\hline SVOC & Fluoranthene & $\mathrm{mg} / \mathrm{kg}$ & 5 & 0 & $0 \%$ & 0.0084 & 0.0098 & -- & -- & 0.0046 \\
\hline SVOC & Fluorene & $\mathrm{mg} / \mathrm{kg}$ & 5 & 0 & $0 \%$ & 0.0092 & 0.010 & -- & -- & 0.0048 \\
\hline SVOC & Hexachlorobenzene & $\mathrm{mg} / \mathrm{kg}$ & 5 & 0 & $0 \%$ & 0.0084 & 0.0098 & -- & -- & 0.0046 \\
\hline SVOC & Hexachlorobutadiene & $\mathrm{mg} / \mathrm{kg}$ & 5 & 0 & $0 \%$ & 0.0042 & 0.0049 & -- & -- & 0.0023 \\
\hline SVOC & Hexachloroethane & $\mathrm{mg} / \mathrm{kg}$ & 5 & 0 & $0 \%$ & 0.0042 & 0.0049 & -- & -- & 0.0023 \\
\hline SVOC & Indeno(1,2,3-cd)pyrene & $\mathrm{mg} / \mathrm{kg}$ & 5 & 0 & $0 \%$ & 0.0094 & 0.19 & -- & -- & 0.057 \\
\hline SVOC & Naphthalene & $\mathrm{mg} / \mathrm{kg}$ & 5 & 0 & $0 \%$ & 0.010 & 0.030 & -- & -- & 0.011 \\
\hline SVOC & Nitrobenzene & $\mathrm{mg} / \mathrm{kg}$ & 5 & 0 & $0 \%$ & 0.0042 & 0.0049 & -- & -- & 0.0023 \\
\hline SVOC & Phenanthrene & $\mathrm{mg} / \mathrm{kg}$ & 5 & 0 & $0 \%$ & 0.0044 & 0.0059 & -- & -- & 0.0025 \\
\hline SVOC & Pyrene & $\mathrm{mg} / \mathrm{kg}$ & 5 & 0 & $0 \%$ & 0.0084 & 0.0098 & -- & -- & 0.0046 \\
\hline VOC & Retene & $\mathrm{mg} / \mathrm{kg}$ & 5 & 1 & $20 \%$ & 0.0084 & 0.0098 & 0.020 & 0.020 & 0.0077 \\
\hline
\end{tabular}

DIOXI - Dioxins and Furans

PEST - Pesticide

PCB - Polychlorinated biphenyl

SVOC - Semivolatile Organic Compound

VOC - Volatile Organic Compound 
Table F-89

Summary Statistics for Rainbow Trout Fillet Skin On Collected from Segment 4 of the Columbia River

\begin{tabular}{|c|c|c|c|c|c|c|c|c|c|c|}
\hline $\begin{array}{l}\text { Constituent } \\
\text { Class }\end{array}$ & Constituent Name & Units & $\begin{array}{l}\text { Number } \\
\text { Analyzed }\end{array}$ & $\begin{array}{l}\text { Number } \\
\text { Detected }\end{array}$ & $\begin{array}{c}\text { Frequency of } \\
\text { Detection }\end{array}$ & $\begin{array}{l}\text { Minimum } \\
\text { Nondetect }\end{array}$ & $\begin{array}{l}\text { Maximum } \\
\text { Nondetect }\end{array}$ & $\begin{array}{c}\text { Minimum } \\
\text { Detect }\end{array}$ & $\begin{array}{l}\text { Maximum } \\
\text { Detect }\end{array}$ & $\begin{array}{c}\text { Average } \\
\text { Concentration }\end{array}$ \\
\hline$\overline{D I O X I}$ & $1,2,3,4,6,7,8$-Heptachlorodibenzodioxin & $\mathrm{mg} / \mathrm{kg}$ & 4 & 4 & $100 \%$ & -- & -- & $1.90 \mathrm{E}-07$ & $3.00 \mathrm{E}-07$ & $2.40 \mathrm{E}-07$ \\
\hline DIOXI & 1,2,3,4,6,7,8-Heptachlorodibenzofuran & $\mathrm{mg} / \mathrm{kg}$ & 4 & 3 & $75 \%$ & 7.00E-08 & 7.00E-08 & $7.00 \mathrm{E}-08$ & 1.50E-07 & 8.38E-08 \\
\hline DIOXI & 1,2,3,4,7,8,9-Heptachlorodibenzofuran & $\mathrm{mg} / \mathrm{kg}$ & 4 & 0 & $0 \%$ & $1.00 \mathrm{E}-08$ & 7.00E-08 & -- & -- & 1.50E-08 \\
\hline DIOXI & $1,2,3,4,7,8$-Hexachlorodibenzofuran & $\mathrm{mg} / \mathrm{kg}$ & 4 & 1 & $25 \%$ & 1.00E-08 & $5.00 \mathrm{E}-08$ & $2.00 \mathrm{E}-08$ & $2.00 \mathrm{E}-08$ & $1.38 \mathrm{E}-08$ \\
\hline DIOXI & $1,2,3,4,7,8$-Hexachlorodibenzo-p-dioxin & $\mathrm{mg} / \mathrm{kg}$ & 4 & 3 & $75 \%$ & 1.00E-08 & 1.00E-08 & $1.00 \mathrm{E}-07$ & $1.30 \mathrm{E}-07$ & 8.38E-08 \\
\hline DIOXI & 1,2,3,6,7,8-Hexachlorodibenzofuran & $\mathrm{mg} / \mathrm{kg}$ & 4 & 1 & $25 \%$ & 1.00E-08 & $5.00 \mathrm{E}-08$ & $4.00 \mathrm{E}-08$ & 4.00E-08 & $1.88 \mathrm{E}-08$ \\
\hline DIOXI & 1,2,3,6,7,8-Hexachlorodibenzo-p-dioxin & $\mathrm{mg} / \mathrm{kg}$ & 4 & 3 & $75 \%$ & $5.00 \mathrm{E}-08$ & $5.00 \mathrm{E}-08$ & $1.60 \mathrm{E}-07$ & $2.10 \mathrm{E}-07$ & 1.41E-07 \\
\hline DIOXI & 1,2,3,7,8,9-Hexachlorodibenzofuran & $\mathrm{mg} / \mathrm{kg}$ & 4 & 2 & $50 \%$ & $1.00 \mathrm{E}-08$ & $5.00 \mathrm{E}-08$ & $6.00 \mathrm{E}-08$ & $6.00 \mathrm{E}-08$ & $3.75 \mathrm{E}-08$ \\
\hline DIOXI & 1,2,3,7,8,9-Hexachlorodibenzo-p-dioxin & $\mathrm{mg} / \mathrm{kg}$ & 4 & 4 & $100 \%$ & -- & -- & $4.00 \mathrm{E}-08$ & 7.00E-08 & 5.50E-08 \\
\hline DIOXI & 1,2,3,7,8-Pentachlorodibenzofuran & $\mathrm{mg} / \mathrm{kg}$ & 4 & 3 & $75 \%$ & 1.00E-08 & 1.00E-08 & $4.00 \mathrm{E}-08$ & $6.00 \mathrm{E}-08$ & 3.63E-08 \\
\hline DIOXI & 1,2,3,7,8-Pentachlorodibenzo-p-dioxin & $\mathrm{mg} / \mathrm{kg}$ & 4 & 3 & $75 \%$ & $2.00 \mathrm{E}-08$ & $2.00 \mathrm{E}-08$ & $1.60 \mathrm{E}-07$ & $1.90 \mathrm{E}-07$ & 1.30E-07 \\
\hline DIOXI & 2,3,4,6,7,8-Hexachlorodibenzofuran & $\mathrm{mg} / \mathrm{kg}$ & 4 & 4 & $100 \%$ & -- & -- & $1.40 \mathrm{E}-07$ & $1.90 \mathrm{E}-07$ & 1.53E-07 \\
\hline DIOXI & 2,3,4,7,8-Pentachlorodibenzofuran & $\mathrm{mg} / \mathrm{kg}$ & 4 & 3 & $75 \%$ & 1.00E-08 & 1.00E-08 & $6.00 \mathrm{E}-08$ & 7.00E-08 & $4.88 \mathrm{E}-08$ \\
\hline DIOXI & 2,3,7,8-Tetrachlorodibenzofuran & $\mathrm{mg} / \mathrm{kg}$ & 4 & 4 & $100 \%$ & -- & -- & $2.40 \mathrm{E}-07$ & $2.80 \mathrm{E}-07$ & 2.63E-07 \\
\hline DIOXI & 2,3,7,8-Tetrachlorodibenzo-p-dioxin & $\mathrm{mg} / \mathrm{kg}$ & 4 & 1 & $25 \%$ & $1.00 \mathrm{E}-08$ & $6.00 \mathrm{E}-08$ & $6.00 \mathrm{E}-08$ & $6.00 \mathrm{E}-08$ & $2.50 \mathrm{E}-08$ \\
\hline DIOXI & Octachlorodibenzofuran & $\mathrm{mg} / \mathrm{kg}$ & 4 & 3 & $75 \%$ & $5.00 \mathrm{E}-08$ & $5.00 \mathrm{E}-08$ & $3.00 \mathrm{E}-08$ & $6.00 \mathrm{E}-08$ & $3.88 \mathrm{E}-08$ \\
\hline DIOXI & Octachlorodibenzo-p-dioxin & $\mathrm{mg} / \mathrm{kg}$ & 4 & 3 & $75 \%$ & $9.00 \mathrm{E}-08$ & $9.00 \mathrm{E}-08$ & $2.30 \mathrm{E}-07$ & $6.90 \mathrm{E}-07$ & 3.36E-07 \\
\hline METAL & Aluminum & $\mathrm{mg} / \mathrm{kg}$ & 4 & 3 & $75 \%$ & 5.0 & 5.0 & 1.0 & 1.4 & 1.5 \\
\hline METAL & Antimony & $\mathrm{mg} / \mathrm{kg}$ & 4 & 0 & $0 \%$ & 0.050 & 0.050 & -- & -- & 0.025 \\
\hline METAL & Arsenic & $\mathrm{mg} / \mathrm{kg}$ & 4 & 0 & $0 \%$ & 0.050 & 0.050 & -- & -- & 0.025 \\
\hline METAL & Barium & $\mathrm{mg} / \mathrm{kg}$ & 4 & 4 & $100 \%$ & -- & -- & 0.29 & 0.72 & 0.56 \\
\hline METAL & Beryllium & $\mathrm{mg} / \mathrm{kg}$ & 4 & 2 & $50 \%$ & 0.0040 & 0.0060 & 0.0077 & 0.0090 & 0.0054 \\
\hline METAL & Cadmium & $\mathrm{mg} / \mathrm{kg}$ & 4 & 1 & $25 \%$ & 0.0040 & 0.0040 & 0.0050 & 0.0050 & 0.0028 \\
\hline METAL & Chromium & $\mathrm{mg} / \mathrm{kg}$ & 4 & 2 & $50 \%$ & 0.10 & 0.10 & 0.12 & 0.12 & 0.085 \\
\hline METAL & Cobalt & $\mathrm{mg} / \mathrm{kg}$ & 4 & 4 & $100 \%$ & -- & -- & 0.030 & 0.030 & 0.030 \\
\hline METAL & Copper & $\mathrm{mg} / \mathrm{kg}$ & 4 & 4 & $100 \%$ & -- & -- & 0.45 & 0.60 & 0.52 \\
\hline METAL & Lead & $\mathrm{mg} / \mathrm{kg}$ & 4 & 0 & $0 \%$ & 0.010 & 0.010 & -- & -- & 0.0050 \\
\hline METAL & Manganese & $\mathrm{mg} / \mathrm{kg}$ & 4 & 4 & $100 \%$ & -- & -- & 0.37 & 0.44 & 0.41 \\
\hline METAL & Mercury & $\mathrm{mg} / \mathrm{kg}$ & 4 & 4 & $100 \%$ & -- & -- & 0.040 & 0.14 & 0.078 \\
\hline METAL & Nickel & $\mathrm{mg} / \mathrm{kg}$ & 4 & 4 & $100 \%$ & -- & -- & 0.040 & 0.060 & 0.050 \\
\hline METAL & Selenium & $\mathrm{mg} / \mathrm{kg}$ & 4 & 4 & $100 \%$ & -- & -- & 0.18 & 0.24 & 0.21 \\
\hline METAL & Silver & $\mathrm{mg} / \mathrm{kg}$ & 4 & 0 & $0 \%$ & 0.10 & 0.20 & -- & -- & 0.088 \\
\hline METAL & Thallium & $\mathrm{mg} / \mathrm{kg}$ & 4 & 0 & $0 \%$ & 0.050 & 0.050 & -- & -- & 0.025 \\
\hline METAL & Vanadium & $\mathrm{mg} / \mathrm{kg}$ & 4 & 3 & $75 \%$ & 0.020 & 0.020 & 0.020 & 0.020 & 0.018 \\
\hline METAL & Zinc & $\mathrm{mg} / \mathrm{kg}$ & 4 & 4 & $100 \%$ & -- & -- & 13 & 19 & 15 \\
\hline PCB & 2,2',3,3',4,4',5-Heptachloro-1,1'-biphenyl & $\mathrm{mg} / \mathrm{kg}$ & 4 & 4 & $100 \%$ & -- & -- & $1.14 \mathrm{E}-05$ & 2.07E-04 & 1.34E-04 \\
\hline РCB & 2,2',3,4,4',5,5'-Heptachlorobiphenyl & $\mathrm{mg} / \mathrm{kg}$ & 4 & 4 & $100 \%$ & -- & -- & $4.28 \mathrm{E}-04$ & 5.91E-04 & 4.87E-04 \\
\hline PCB & 2,3,3',4,4',5'-Hexachlorobiphenyl & $\mathrm{mg} / \mathrm{kg}$ & 4 & 4 & $100 \%$ & -- & -- & 2.67E-05 & 3.89E-05 & 3.22E-05 \\
\hline РCB & 2,3,3',4,4',5-Hexachlorobiphenyl (BZ 156) & $\mathrm{mg} / \mathrm{kg}$ & 4 & 4 & $100 \%$ & -- & -- & $1.55 \mathrm{E}-04$ & $2.20 \mathrm{E}-04$ & 1.83E-04 \\
\hline PCB & 2,3,3',4,4'-Pentachlorobiphenyl & $\mathrm{mg} / \mathrm{kg}$ & 4 & 4 & $100 \%$ & -- & -- & 4.49E-04 & 0.042 & 0.011 \\
\hline РCB & 2,3',4,4',5,5'-Hexachlorobiphenyl (BZ 167) & $\mathrm{mg} / \mathrm{kg}$ & 4 & 4 & $100 \%$ & -- & -- & $2.22 \mathrm{E}-05$ & 8.88E-05 & 6.17E-05 \\
\hline PCB & 2',3,4,4',5-Pentachloro-1,1'-biphenyl (BZ 123) & $\mathrm{mg} / \mathrm{kg}$ & 4 & 4 & $100 \%$ & -- & -- & 1.63E-05 & 1.98E-05 & 1.79E-05 \\
\hline PCB & 2,3,4,4',5-Pentachlorobiphenyl (BZ 114) & $\mathrm{mg} / \mathrm{kg}$ & 4 & 4 & $100 \%$ & -- & -- & 3.49E-05 & 4.97E-05 & 4.06E-05 \\
\hline PCB & 2,3',4,4',5-Pentachlorobiphenyl (BZ 118) & $\mathrm{mg} / \mathrm{kg}$ & 4 & 4 & $100 \%$ & -- & -- & 0.0010 & 0.0014 & 0.0011 \\
\hline РCB & 2,3,4,5,3',4',5'-Heptachlorobiphenyl & $\mathrm{mg} / \mathrm{kg}$ & 4 & 2 & $50 \%$ & 9.82E-06 & 4.06E-05 & $6.98 \mathrm{E}-06$ & $7.41 \mathrm{E}-06$ & 9.90E-06 \\
\hline PCB & 3,3',4,4',5,5'-Hexachlorobiphenyl (BZ 169) & $\mathrm{mg} / \mathrm{kg}$ & 4 & 0 & $0 \%$ & $6.50 \mathrm{E}-07$ & $5.44 \mathrm{E}-04$ & -- & -- & $6.83 \mathrm{E}-05$ \\
\hline РСВ & 3,3',4,4',5-Pentachlorobiphenyl (BZ 126) & $\mathrm{mg} / \mathrm{kg}$ & 4 & 4 & $100 \%$ & -- & -- & $3.24 \mathrm{E}-06$ & 4.77E-06 & 4.17E-06 \\
\hline PCB & 3,3',4,4'-Tetrachlorobiphenyl (BZ 77) & $\mathrm{mg} / \mathrm{kg}$ & 4 & 4 & $100 \%$ & -- & -- & $1.21 \mathrm{E}-05$ & 1.58E-05 & 1.36E-05 \\
\hline РСВ & Aroclor-1016 & $\mathrm{mg} / \mathrm{kg}$ & 4 & 0 & $0 \%$ & 0.018 & 0.019 & -- & -- & 0.0093 \\
\hline PCB & Aroclor-1221 & $\mathrm{mg} / \mathrm{kg}$ & 4 & 0 & $0 \%$ & 0.018 & 0.019 & -- & -- & 0.0093 \\
\hline
\end{tabular}


Table F-89

Summary Statistics for Rainbow Trout Fillet Skin On Collected from Segment 4 of the Columbia River

\begin{tabular}{|c|c|c|c|c|c|c|c|c|c|c|}
\hline $\begin{array}{l}\text { Constituent } \\
\text { Class }\end{array}$ & Constituent Name & Units & $\begin{array}{l}\text { Number } \\
\text { Analyzed }\end{array}$ & $\begin{array}{l}\text { Number } \\
\text { Detected }\end{array}$ & $\begin{array}{l}\text { Frequency of } \\
\text { Detection }\end{array}$ & $\begin{array}{l}\text { Minimum } \\
\text { Nondetect }\end{array}$ & $\begin{array}{c}\text { Maximum } \\
\text { Nondetect }\end{array}$ & $\begin{array}{l}\text { Minimum } \\
\text { Detect }\end{array}$ & $\begin{array}{c}\text { Maximum } \\
\text { Detect }\end{array}$ & $\begin{array}{c}\text { Average } \\
\text { Concentration }\end{array}$ \\
\hline$\overline{\mathrm{PCB}}$ & Aroclor-1232 & $\mathrm{mg} / \mathrm{kg}$ & 4 & 0 & $0 \%$ & 0.018 & 0.019 & $\overline{--}$ & -- & 0.0093 \\
\hline РCB & Aroclor-1248 & $\mathrm{mg} / \mathrm{kg}$ & 4 & 0 & $0 \%$ & 0.018 & 0.019 & -- & -- & 0.0093 \\
\hline РСB & Aroclor-1254 & $\mathrm{mg} / \mathrm{kg}$ & 4 & 4 & $100 \%$ & -- & -- & 0.0099 & 0.020 & 0.015 \\
\hline РСB & Aroclor-1260 & $\mathrm{mg} / \mathrm{kg}$ & 4 & 0 & $0 \%$ & 0.018 & 0.019 & -- & -- & 0.0093 \\
\hline PEST & 2,4'-DDD & $\mathrm{mg} / \mathrm{kg}$ & 4 & 0 & $0 \%$ & 0.0018 & 0.0019 & -- & -- & $9.25 \mathrm{E}-04$ \\
\hline PEST & 2,4'-DDE & $\mathrm{mg} / \mathrm{kg}$ & 4 & 0 & $0 \%$ & 0.0018 & 0.0019 & -- & -- & $9.25 \mathrm{E}-04$ \\
\hline PEST & 2,4'-DDT & $\mathrm{mg} / \mathrm{kg}$ & 4 & 0 & $0 \%$ & 0.0018 & 0.0019 & -- & -- & $9.25 \mathrm{E}-04$ \\
\hline PEST & Aldrin & $\mathrm{mg} / \mathrm{kg}$ & 4 & 0 & $0 \%$ & 0.0018 & 0.0019 & -- & -- & $9.25 \mathrm{E}-04$ \\
\hline PEST & Alpha-BHC & $\mathrm{mg} / \mathrm{kg}$ & 4 & 0 & $0 \%$ & 0.0018 & 0.0019 & -- & -- & $9.25 \mathrm{E}-04$ \\
\hline PEST & alpha-Chlordane & $\mathrm{mg} / \mathrm{kg}$ & 4 & 0 & $0 \%$ & 0.0018 & 0.0019 & -- & -- & $9.25 \mathrm{E}-04$ \\
\hline PEST & alpha-Chlordene & $\mathrm{mg} / \mathrm{kg}$ & 4 & 0 & $0 \%$ & 0.0018 & 0.0019 & -- & -- & $9.25 \mathrm{E}-04$ \\
\hline PEST & Beta-BHC & $\mathrm{mg} / \mathrm{kg}$ & 4 & 0 & $0 \%$ & 0.0018 & 0.0019 & -- & -- & $9.25 \mathrm{E}-04$ \\
\hline PEST & cis-Nonachlor & $\mathrm{mg} / \mathrm{kg}$ & 4 & 0 & $0 \%$ & 0.0018 & 0.0019 & -- & -- & $9.25 \mathrm{E}-04$ \\
\hline PEST & DDMU & $\mathrm{mg} / \mathrm{kg}$ & 4 & 0 & $0 \%$ & 0.0018 & 0.0019 & -- & -- & $9.25 \mathrm{E}-04$ \\
\hline PEST & Delta-BHC & $\mathrm{mg} / \mathrm{kg}$ & 4 & 0 & $0 \%$ & 0.0018 & 0.0019 & -- & -- & $9.25 \mathrm{E}-04$ \\
\hline PEST & Dichlorodiphenyldichloroethane & $\mathrm{mg} / \mathrm{kg}$ & 4 & 0 & $0 \%$ & 0.0018 & 0.0019 & -- & -- & $9.25 \mathrm{E}-04$ \\
\hline PEST & Dichlorodiphenyldichloroethylene & $\mathrm{mg} / \mathrm{kg}$ & 4 & 4 & $100 \%$ & -- & -- & 0.0041 & 0.0065 & 0.0051 \\
\hline PEST & Dichlorodiphenyltrichloroethane & $\mathrm{mg} / \mathrm{kg}$ & 4 & 3 & $75 \%$ & 0.0019 & 0.0019 & 0.0027 & 0.0037 & 0.0026 \\
\hline PEST & Endosulfan sulfate & $\mathrm{mg} / \mathrm{kg}$ & 1 & 0 & $0 \%$ & 0.0019 & 0.0019 & -- & -- & $9.50 \mathrm{E}-04$ \\
\hline PEST & Gamma-BHC (Lindane) & $\mathrm{mg} / \mathrm{kg}$ & 4 & 0 & $0 \%$ & 0.0018 & 0.0019 & -- & -- & $9.25 \mathrm{E}-04$ \\
\hline PEST & gamma-Chlordane & $\mathrm{mg} / \mathrm{kg}$ & 4 & 0 & $0 \%$ & 0.0018 & 0.0019 & -- & -- & $9.25 \mathrm{E}-04$ \\
\hline PEST & gamma-Chlordene & $\mathrm{mg} / \mathrm{kg}$ & 4 & 0 & $0 \%$ & 0.0018 & 0.0019 & -- & -- & $9.25 \mathrm{E}-04$ \\
\hline PEST & Heptachlor & $\mathrm{mg} / \mathrm{kg}$ & 4 & 0 & $0 \%$ & 0.0018 & 0.0019 & -- & -- & $9.25 \mathrm{E}-04$ \\
\hline PEST & Heptachlor epoxide & $\mathrm{mg} / \mathrm{kg}$ & 4 & 0 & $0 \%$ & 0.0018 & 0.0019 & -- & -- & $9.25 \mathrm{E}-04$ \\
\hline PEST & Mirex & $\mathrm{mg} / \mathrm{kg}$ & 4 & 0 & $0 \%$ & 0.0018 & 0.0019 & -- & -- & $9.25 \mathrm{E}-04$ \\
\hline PEST & Oxychlordane & $\mathrm{mg} / \mathrm{kg}$ & 4 & 0 & $0 \%$ & 0.0018 & 0.0019 & -- & -- & $9.25 \mathrm{E}-04$ \\
\hline PEST & Pentachloroanisole & $\mathrm{mg} / \mathrm{kg}$ & 4 & 0 & $0 \%$ & 0.0018 & 0.0019 & -- & -- & $9.25 \mathrm{E}-04$ \\
\hline PEST & Toxaphene & $\mathrm{mg} / \mathrm{kg}$ & 4 & 0 & $0 \%$ & 0.055 & 0.057 & -- & -- & 0.028 \\
\hline PEST & trans-Nonachlor & $\mathrm{mg} / \mathrm{kg}$ & 4 & 0 & $0 \%$ & 0.0018 & 0.0019 & -- & -- & $9.25 \mathrm{E}-04$ \\
\hline SVOC & 1,2,4-Trichlorobenzene & $\mathrm{mg} / \mathrm{kg}$ & 4 & 0 & $0 \%$ & 0.0048 & 0.0051 & -- & -- & 0.0024 \\
\hline SVOC & 1,2-Dichlorobenzene & $\mathrm{mg} / \mathrm{kg}$ & 4 & 0 & $0 \%$ & 0.0048 & 0.0051 & -- & -- & 0.0024 \\
\hline SVOC & 1,2-Diphenylhydrazine & $\mathrm{mg} / \mathrm{kg}$ & 4 & 0 & $0 \%$ & 0.0048 & 0.0051 & -- & -- & 0.0024 \\
\hline SVOC & 1,3-Dichlorobenzene & $\mathrm{mg} / \mathrm{kg}$ & 4 & 0 & $0 \%$ & 0.0048 & 0.0051 & -- & -- & 0.0024 \\
\hline SVOC & 1,4-Dichlorobenzene & $\mathrm{mg} / \mathrm{kg}$ & 4 & 0 & $0 \%$ & 0.0048 & 0.0051 & -- & -- & 0.0024 \\
\hline SVOC & 1-methylnaphthalene & $\mathrm{mg} / \mathrm{kg}$ & 4 & 0 & $0 \%$ & 0.0048 & 0.0051 & -- & -- & 0.0024 \\
\hline SVOC & 2,4-Dinitrotoluene & $\mathrm{mg} / \mathrm{kg}$ & 4 & 0 & $0 \%$ & 0.0096 & 0.010 & -- & -- & 0.0049 \\
\hline SVOC & 2,6-Dinitrotoluene & $\mathrm{mg} / \mathrm{kg}$ & 4 & 0 & $0 \%$ & 0.019 & 0.020 & -- & -- & 0.0096 \\
\hline SVOC & 2-Chloronaphthalene & $\mathrm{mg} / \mathrm{kg}$ & 4 & 0 & $0 \%$ & 0.0096 & 0.010 & -- & -- & 0.0049 \\
\hline SVOC & 2-MethyInaphthalene & $\mathrm{mg} / \mathrm{kg}$ & 4 & 0 & $0 \%$ & 0.0048 & 0.010 & -- & -- & 0.0031 \\
\hline SVOC & 4-Bromophenylphenyl ether & $\mathrm{mg} / \mathrm{kg}$ & 4 & 0 & $0 \%$ & 0.0048 & 0.0051 & -- & -- & 0.0024 \\
\hline SVOC & 4-Chlorophenylphenyl ether & $\mathrm{mg} / \mathrm{kg}$ & 4 & 0 & $0 \%$ & 0.0048 & 0.0051 & -- & -- & 0.0024 \\
\hline SVOC & Acenaphthene & $\mathrm{mg} / \mathrm{kg}$ & 4 & 0 & $0 \%$ & 0.0096 & 0.010 & -- & -- & 0.0049 \\
\hline SVOC & Acenaphthylene & $\mathrm{mg} / \mathrm{kg}$ & 4 & 0 & $0 \%$ & 0.0048 & 0.0051 & -- & -- & 0.0024 \\
\hline SVOC & Anthracene & $\mathrm{mg} / \mathrm{kg}$ & 4 & 0 & $0 \%$ & 0.0048 & 0.0051 & -- & -- & 0.0024 \\
\hline SVOC & Benzo(a)anthracene & $\mathrm{mg} / \mathrm{kg}$ & 4 & 0 & $0 \%$ & 0.0048 & 0.0051 & -- & -- & 0.0024 \\
\hline SVOC & Benzo(a)pyrene & $\mathrm{mg} / \mathrm{kg}$ & 4 & 0 & $0 \%$ & 0.0048 & 0.0051 & -- & -- & 0.0024 \\
\hline SVOC & Benzo(b)fluoranthene & $\mathrm{mg} / \mathrm{kg}$ & 4 & 0 & $0 \%$ & 0.0048 & 0.0051 & -- & -- & 0.0024 \\
\hline SVOC & Benzo(ghi)perylene & $\mathrm{mg} / \mathrm{kg}$ & 4 & 0 & $0 \%$ & 0.0096 & 0.010 & -- & -- & 0.0049 \\
\hline SVOC & Benzo(k)fluoranthene & $\mathrm{mg} / \mathrm{kg}$ & 4 & 0 & $0 \%$ & 0.0048 & 0.0051 & -- & -- & 0.0024 \\
\hline SVOC & Bis(2-chloro-1-methylethyl)ether & $\mathrm{mg} / \mathrm{kg}$ & 4 & 0 & $0 \%$ & 0.0096 & 0.010 & -- & -- & 0.0049 \\
\hline
\end{tabular}


Table F-89

Summary Statistics for Rainbow Trout Fillet Skin On Collected from Segment 4 of the Columbia River

\begin{tabular}{|c|c|c|c|c|c|c|c|c|c|c|}
\hline $\begin{array}{l}\text { Constituent } \\
\text { Class }\end{array}$ & Constituent Name & Units & $\begin{array}{l}\text { Number } \\
\text { Analyzed }\end{array}$ & $\begin{array}{l}\text { Number } \\
\text { Detected }\end{array}$ & $\begin{array}{c}\text { Frequency of } \\
\text { Detection }\end{array}$ & $\begin{array}{l}\text { Minimum } \\
\text { Nondetect }\end{array}$ & $\begin{array}{l}\text { Maximum } \\
\text { Nondetect }\end{array}$ & $\begin{array}{c}\text { Minimum } \\
\text { Detect }\end{array}$ & $\begin{array}{l}\text { Maximum } \\
\text { Detect }\end{array}$ & $\begin{array}{c}\text { Average } \\
\text { Concentration }\end{array}$ \\
\hline SVOC & Chrysene & $\mathrm{mg} / \mathrm{kg}$ & 4 & 0 & $0 \%$ & 0.0048 & 0.0051 & -- & -- & 0.0024 \\
\hline SVOC & Dibenz[a,h]anthracene & $\mathrm{mg} / \mathrm{kg}$ & 4 & 0 & $0 \%$ & 0.0096 & 0.010 & -- & -- & 0.0049 \\
\hline SVOC & Dibenzofuran & $\mathrm{mg} / \mathrm{kg}$ & 4 & 0 & $0 \%$ & 0.0048 & 0.0051 & -- & -- & 0.0024 \\
\hline SVOC & Fluoranthene & $\mathrm{mg} / \mathrm{kg}$ & 4 & 0 & $0 \%$ & 0.0096 & 0.010 & -- & -- & 0.0049 \\
\hline SVOC & Fluorene & $\mathrm{mg} / \mathrm{kg}$ & 4 & 0 & $0 \%$ & 0.0049 & 0.0099 & -- & -- & 0.0037 \\
\hline SVOC & Hexachlorobenzene & $\mathrm{mg} / \mathrm{kg}$ & 4 & 0 & $0 \%$ & 0.0096 & 0.010 & -- & -- & 0.0049 \\
\hline SVOC & Hexachlorobutadiene & $\mathrm{mg} / \mathrm{kg}$ & 4 & 0 & $0 \%$ & 0.0048 & 0.0051 & -- & -- & 0.0024 \\
\hline SVOC & Hexachloroethane & $\mathrm{mg} / \mathrm{kg}$ & 4 & 0 & $0 \%$ & 0.0048 & 0.0051 & -- & -- & 0.0024 \\
\hline SVOC & Indeno(1,2,3-cd)pyrene & $\mathrm{mg} / \mathrm{kg}$ & 4 & 0 & $0 \%$ & 0.0096 & 0.010 & -- & -- & 0.0049 \\
\hline SVOC & Naphthalene & $\mathrm{mg} / \mathrm{kg}$ & 4 & 0 & $0 \%$ & 0.017 & 0.024 & -- & -- & 0.010 \\
\hline SVOC & Nitrobenzene & $\mathrm{mg} / \mathrm{kg}$ & 4 & 0 & $0 \%$ & 0.0048 & 0.0051 & -- & -- & 0.0024 \\
\hline SVOC & Phenanthrene & $\mathrm{mg} / \mathrm{kg}$ & 4 & 0 & $0 \%$ & 0.0048 & 0.0051 & -- & -- & 0.0024 \\
\hline SVOC & Pyrene & $\mathrm{mg} / \mathrm{kg}$ & 4 & 0 & $0 \%$ & 0.0096 & 0.010 & -- & -- & 0.0049 \\
\hline VOC & Retene & $\mathrm{mg} / \mathrm{kg}$ & 4 & 0 & $0 \%$ & 0.0096 & 0.010 & -- & -- & 0.0049 \\
\hline
\end{tabular}

DIOXI - Dioxins and Furans

PEST - Pesticide

PCB - Polychlorinated biphenyl

SVOC - Semivolatile Organic Compound

VOC - Volatile Organic Compound 
Table F-90

Summary Statistics for Whole Body Rainbow Trout Collected from Segment 4 of the Columbia River

\begin{tabular}{|c|c|c|c|c|c|c|c|c|c|c|}
\hline $\begin{array}{l}\text { Constituent } \\
\text { Class }\end{array}$ & Constituent Name & Units & $\begin{array}{l}\text { Number } \\
\text { Analyzed }\end{array}$ & $\begin{array}{l}\text { Number } \\
\text { Detected }\end{array}$ & $\begin{array}{l}\text { Frequency of } \\
\text { Detection }\end{array}$ & $\begin{array}{l}\text { Minimum } \\
\text { Nondetect }\end{array}$ & $\begin{array}{l}\text { Maximum } \\
\text { Nondetect }\end{array}$ & $\begin{array}{c}\text { Minimum } \\
\text { Detect }\end{array}$ & $\begin{array}{l}\text { Maximum } \\
\text { Detect }\end{array}$ & $\begin{array}{c}\text { Average } \\
\text { Concentration }\end{array}$ \\
\hline$\overline{\mathrm{DIOXI}}$ & $1,2,3,4,6,7,8$-Heptachlorodibenzodioxin & $\mathrm{mg} / \mathrm{kg}$ & 6 & 5 & $83 \%$ & $2.00 \mathrm{E}-08$ & $2.00 \mathrm{E}-08$ & $1.60 \mathrm{E}-07$ & $3.20 \mathrm{E}-07$ & $1.98 \mathrm{E}-07$ \\
\hline DIOXI & 1,2,3,4,6,7,8-Heptachlorodibenzofuran & $\mathrm{mg} / \mathrm{kg}$ & 6 & 3 & $50 \%$ & $2.00 \mathrm{E}-08$ & $9.00 \mathrm{E}-08$ & 7.00E-08 & 8.00E-08 & 4.83E-08 \\
\hline DIOXI & 1,2,3,4,7,8,9-Heptachlorodibenzofuran & $\mathrm{mg} / \mathrm{kg}$ & 6 & 2 & $33 \%$ & 1.00E-08 & $9.00 \mathrm{E}-08$ & $2.00 \mathrm{E}-08$ & $3.00 \mathrm{E}-08$ & $2.08 \mathrm{E}-08$ \\
\hline DIOXI & $1,2,3,4,7,8$-Hexachlorodibenzofuran & $\mathrm{mg} / \mathrm{kg}$ & 6 & 1 & $17 \%$ & $1.00 \mathrm{E}-08$ & 1.20E-07 & $4.00 \mathrm{E}-08$ & 4.00E-08 & 2.17E-08 \\
\hline DIOXI & 1,2,3,4,7,8-Hexachlorodibenzo-p-dioxin & $\mathrm{mg} / \mathrm{kg}$ & 6 & 3 & $50 \%$ & $2.00 \mathrm{E}-08$ & 1.00E-07 & 1.10E-07 & $1.20 \mathrm{E}-07$ & $6.92 \mathrm{E}-08$ \\
\hline DIOXI & $1,2,3,6,7,8$-Hexachlorodibenzofuran & $\mathrm{mg} / \mathrm{kg}$ & 6 & 3 & $50 \%$ & 1.00E-08 & 1.20E-07 & $3.00 \mathrm{E}-08$ & $5.00 \mathrm{E}-08$ & 3.33E-08 \\
\hline DIOXI & $1,2,3,6,7,8$-Hexachlorodibenzo-p-dioxin & $\mathrm{mg} / \mathrm{kg}$ & 6 & 4 & $67 \%$ & 2.00E-08 & 1.00E-07 & $7.00 \mathrm{E}-08$ & $2.60 \mathrm{E}-07$ & 1.10E-07 \\
\hline DIOXI & 1,2,3,7,8,9-Hexachlorodibenzofuran & $\mathrm{mg} / \mathrm{kg}$ & 6 & 3 & $50 \%$ & 1.00E-08 & 1.20E-07 & $6.00 \mathrm{E}-08$ & $9.00 \mathrm{E}-08$ & 5.17E-08 \\
\hline DIOXI & 1,2,3,7,8,9-Hexachlorodibenzo-p-dioxin & $\mathrm{mg} / \mathrm{kg}$ & 6 & 4 & $67 \%$ & $3.00 \mathrm{E}-08$ & $1.00 \mathrm{E}-07$ & $4.00 \mathrm{E}-08$ & 1.00E-07 & $5.42 \mathrm{E}-08$ \\
\hline DIOXI & 1,2,3,7,8-Pentachlorodibenzofuran & $\mathrm{mg} / \mathrm{kg}$ & 6 & 4 & $67 \%$ & 3.00E-08 & $3.00 \mathrm{E}-08$ & 4.00E-08 & $9.00 \mathrm{E}-08$ & $5.00 \mathrm{E}-08$ \\
\hline DIOXI & 1,2,3,7,8-Pentachlorodibenzo-p-dioxin & $\mathrm{mg} / \mathrm{kg}$ & 6 & 3 & $50 \%$ & 4.00E-08 & $5.00 \mathrm{E}-08$ & $1.40 \mathrm{E}-07$ & $2.20 \mathrm{E}-07$ & 1.07E-07 \\
\hline DIOXI & $2,3,4,6,7,8$-Hexachlorodibenzofuran & $\mathrm{mg} / \mathrm{kg}$ & 6 & 3 & $50 \%$ & 1.00E-08 & 1.20E-07 & $1.30 \mathrm{E}-07$ & $1.60 \mathrm{E}-07$ & 8.33E-08 \\
\hline DIOXI & 2,3,4,7,8-Pentachlorodibenzofuran & $\mathrm{mg} / \mathrm{kg}$ & 6 & 3 & $50 \%$ & $3.00 \mathrm{E}-08$ & $5.00 \mathrm{E}-08$ & $4.00 \mathrm{E}-08$ & $1.20 \mathrm{E}-07$ & $5.25 \mathrm{E}-08$ \\
\hline DIOXI & $2,3,7,8$-Tetrachlorodibenzofuran & $\mathrm{mg} / \mathrm{kg}$ & 6 & 6 & $100 \%$ & -- & -- & $4.00 \mathrm{E}-08$ & 4.80E-07 & 2.68E-07 \\
\hline DIOXI & 2,3,7,8-Tetrachlorodibenzo-p-dioxin & $\mathrm{mg} / \mathrm{kg}$ & 6 & 4 & $67 \%$ & $1.00 \mathrm{E}-08$ & $2.00 \mathrm{E}-08$ & $6.00 \mathrm{E}-08$ & 1.00E-07 & $5.25 \mathrm{E}-08$ \\
\hline DIOXI & Octachlorodibenzofuran & $\mathrm{mg} / \mathrm{kg}$ & 6 & 3 & $50 \%$ & $6.00 \mathrm{E}-08$ & 1.00E-07 & $2.00 \mathrm{E}-08$ & 5.00E-08 & 3.50E-08 \\
\hline DIOXI & Octachlorodibenzo-p-dioxin & $\mathrm{mg} / \mathrm{kg}$ & 6 & 6 & $100 \%$ & -- & -- & $2.00 \mathrm{E}-07$ & 2.97E-06 & 8.60E-07 \\
\hline METAL & Aluminum & $\mathrm{mg} / \mathrm{kg}$ & 6 & 4 & $67 \%$ & 5.0 & 20 & 7.4 & 150 & 32 \\
\hline METAL & Antimony & $\mathrm{mg} / \mathrm{kg}$ & 6 & 0 & $0 \%$ & 0.050 & 0.050 & -- & -- & 0.025 \\
\hline METAL & Arsenic & $\mathrm{mg} / \mathrm{kg}$ & 6 & 3 & $50 \%$ & 0.050 & 0.050 & 0.050 & 0.56 & 0.18 \\
\hline METAL & Barium & $\mathrm{mg} / \mathrm{kg}$ & 6 & 6 & $100 \%$ & -- & -- & 0.22 & 4.0 & 1.1 \\
\hline METAL & Beryllium & $\mathrm{mg} / \mathrm{kg}$ & 6 & 1 & $17 \%$ & 0.0040 & 0.0040 & 0.010 & 0.010 & 0.0033 \\
\hline METAL & Cadmium & $\mathrm{mg} / \mathrm{kg}$ & 6 & 4 & $67 \%$ & 0.0040 & 0.0040 & 0.0046 & 0.050 & 0.013 \\
\hline METAL & Chromium & $\mathrm{mg} / \mathrm{kg}$ & 6 & 4 & $67 \%$ & 0.10 & 0.10 & 0.10 & 0.16 & 0.097 \\
\hline METAL & Cobalt & $\mathrm{mg} / \mathrm{kg}$ & 6 & 6 & $100 \%$ & -- & -- & 0.050 & 0.20 & 0.090 \\
\hline METAL & Copper & $\mathrm{mg} / \mathrm{kg}$ & 6 & 6 & $100 \%$ & -- & -- & 1.1 & 5.0 & 2.0 \\
\hline METAL & Lead & $\mathrm{mg} / \mathrm{kg}$ & 6 & 5 & $83 \%$ & 0.010 & 0.010 & 0.010 & 0.080 & 0.021 \\
\hline METAL & Manganese & $\mathrm{mg} / \mathrm{kg}$ & 6 & 6 & $100 \%$ & -- & -- & 1.5 & 9.7 & 3.3 \\
\hline METAL & Mercury & $\mathrm{mg} / \mathrm{kg}$ & 6 & 4 & $67 \%$ & 0.040 & 0.040 & 0.030 & 0.38 & 0.10 \\
\hline METAL & Nickel & $\mathrm{mg} / \mathrm{kg}$ & 6 & 6 & $100 \%$ & -- & -- & 0.060 & 1.1 & 0.28 \\
\hline METAL & Selenium & $\mathrm{mg} / \mathrm{kg}$ & 6 & 6 & $100 \%$ & -- & -- & 0.24 & 0.79 & 0.43 \\
\hline METAL & Silver & $\mathrm{mg} / \mathrm{kg}$ & 6 & 0 & $0 \%$ & 0.10 & 0.10 & -- & -- & 0.050 \\
\hline METAL & Thallium & $\mathrm{mg} / \mathrm{kg}$ & 6 & 0 & $0 \%$ & 0.050 & 0.050 & -- & -- & 0.025 \\
\hline METAL & Vanadium & $\mathrm{mg} / \mathrm{kg}$ & 6 & 6 & $100 \%$ & -- & -- & 0.040 & 0.77 & 0.19 \\
\hline METAL & Zinc & $\mathrm{mg} / \mathrm{kg}$ & 6 & 6 & $100 \%$ & -- & -- & 23 & 39 & 31 \\
\hline PCB & 2,2',3,3',4,4',5-Heptachloro-1,1'-biphenyl & $\mathrm{mg} / \mathrm{kg}$ & 6 & 6 & $100 \%$ & -- & -- & 3.45E-05 & $2.48 \mathrm{E}-04$ & 1.67E-04 \\
\hline PCB & $2,2^{\prime}, 3,4,4^{\prime}, 5,5^{\prime}$-Heptachlorobiphenyl & $\mathrm{mg} / \mathrm{kg}$ & 6 & 6 & $100 \%$ & -- & -- & 1.13E-04 & $6.94 \mathrm{E}-04$ & 4.80E-04 \\
\hline РCB & 2,3,3',4,4',5'-Hexachlorobiphenyl & $\mathrm{mg} / \mathrm{kg}$ & 6 & 5 & $83 \%$ & 5.00E-07 & $5.00 \mathrm{E}-07$ & 4.67E-06 & 4.71E-05 & 2.65E-05 \\
\hline PCB & 2,3,3',4,4',5-Hexachlorobiphenyl (BZ 156) & $\mathrm{mg} / \mathrm{kg}$ & 6 & 6 & $100 \%$ & -- & -- & $1.31 \mathrm{E}-05$ & $2.59 \mathrm{E}-04$ & 1.43E-04 \\
\hline РCB & 2,3,3',4,4'-Pentachlorobiphenyl & $\mathrm{mg} / \mathrm{kg}$ & 6 & 6 & $100 \%$ & -- & -- & 4.19E-05 & 7.89E-04 & 4.40E-04 \\
\hline PCB & 2,3',4,4',5,5'-Hexachlorobiphenyl (BZ 167) & $\mathrm{mg} / \mathrm{kg}$ & 6 & 6 & $100 \%$ & -- & -- & 7.64E-06 & 1.05E-04 & $6.18 \mathrm{E}-05$ \\
\hline PCB & 2',3,4,4',5-Pentachloro-1,1'-biphenyl (BZ 123) & $\mathrm{mg} / \mathrm{kg}$ & 6 & 6 & $100 \%$ & -- & -- & $2.16 \mathrm{E}-06$ & $2.85 \mathrm{E}-05$ & 1.39E-05 \\
\hline PCB & 2,3,4,4',5-Pentachlorobiphenyl (BZ 114) & $\mathrm{mg} / \mathrm{kg}$ & 6 & 6 & $100 \%$ & -- & -- & $3.62 \mathrm{E}-06$ & $6.05 \mathrm{E}-05$ & 3.34E-05 \\
\hline PCB & 2,3',4,4',5-Pentachlorobiphenyl (BZ 118) & $\mathrm{mg} / \mathrm{kg}$ & 6 & 6 & $100 \%$ & -- & -- & 7.76E-05 & 0.0017 & $9.40 \mathrm{E}-04$ \\
\hline PCB & $2,3,4,5,3^{\prime}, 4^{\prime}, 5^{\prime}$-Heptachlorobiphenyl & $\mathrm{mg} / \mathrm{kg}$ & 6 & 5 & $83 \%$ & $5.00 \mathrm{E}-07$ & $5.00 \mathrm{E}-07$ & $2.51 \mathrm{E}-06$ & 1.11E-05 & 7.19E-06 \\
\hline PCB & 3,3',4,4',5,5'-Hexachlorobiphenyl (BZ 169) & $\mathrm{mg} / \mathrm{kg}$ & 6 & 0 & $0 \%$ & $5.00 \mathrm{E}-07$ & 1.03E-06 & -- & -- & 4.45E-07 \\
\hline PCB & 3,3',4,4',5-Pentachlorobiphenyl (BZ 126) & $\mathrm{mg} / \mathrm{kg}$ & 6 & 5 & $83 \%$ & 5.00E-07 & $5.00 \mathrm{E}-07$ & $1.35 \mathrm{E}-06$ & 6.15E-06 & 3.62E-06 \\
\hline PCB & 3,3',4,4'-Tetrachlorobiphenyl (BZ 77) & $\mathrm{mg} / \mathrm{kg}$ & 6 & 6 & $100 \%$ & -- & -- & $2.17 \mathrm{E}-06$ & 2.05E-05 & 1.24E-05 \\
\hline PCB & Aroclor-1016 & $\mathrm{mg} / \mathrm{kg}$ & 6 & 0 & $0 \%$ & 0.018 & 0.020 & -- & -- & 0.0094 \\
\hline PCB & Aroclor-1221 & $\mathrm{mg} / \mathrm{kg}$ & 6 & 0 & $0 \%$ & 0.018 & 0.020 & -- & -- & 0.0094 \\
\hline
\end{tabular}


Table F-90

Summary Statistics for Whole Body Rainbow Trout Collected from Segment 4 of the Columbia River

\begin{tabular}{|c|c|c|c|c|c|c|c|c|c|c|}
\hline $\begin{array}{l}\text { Constituent } \\
\text { Class }\end{array}$ & Constituent Name & Units & $\begin{array}{l}\text { Number } \\
\text { Analyzed }\end{array}$ & $\begin{array}{l}\text { Number } \\
\text { Detected }\end{array}$ & $\begin{array}{c}\text { Frequency of } \\
\text { Detection }\end{array}$ & $\begin{array}{l}\text { Minimum } \\
\text { Nondetect }\end{array}$ & $\begin{array}{l}\text { Maximum } \\
\text { Nondetect }\end{array}$ & $\begin{array}{l}\text { Minimum } \\
\text { Detect }\end{array}$ & $\begin{array}{l}\text { Maximum } \\
\text { Detect }\end{array}$ & $\begin{array}{c}\text { Average } \\
\text { Concentration }\end{array}$ \\
\hline$\overline{\mathrm{PCB}}$ & Aroclor-1232 & $\mathrm{mg} / \mathrm{kg}$ & 6 & 0 & $0 \%$ & 0.018 & 0.020 & $\overline{--}$ & $\overline{--}$ & 0.0094 \\
\hline РСВ & Aroclor-1248 & $\mathrm{mg} / \mathrm{kg}$ & 6 & 0 & $0 \%$ & 0.018 & 0.020 & -- & -- & 0.0094 \\
\hline PCB & Aroclor-1254 & $\mathrm{mg} / \mathrm{kg}$ & 6 & 4 & $67 \%$ & 0.018 & 0.019 & 0.0069 & 0.030 & 0.015 \\
\hline РСВ & Aroclor-1260 & $\mathrm{mg} / \mathrm{kg}$ & 6 & 0 & $0 \%$ & 0.018 & 0.020 & -- & -- & 0.0094 \\
\hline PEST & 2,4'-DDD & $\mathrm{mg} / \mathrm{kg}$ & 6 & 0 & $0 \%$ & 0.0018 & 0.0024 & -- & -- & $9.83 \mathrm{E}-04$ \\
\hline PEST & 2,4'-DDE & $\mathrm{mg} / \mathrm{kg}$ & 6 & 2 & $33 \%$ & 0.0019 & 0.0024 & 0.0016 & 0.0017 & 0.0012 \\
\hline PEST & $2,4^{\prime}-\mathrm{DDT}$ & $\mathrm{mg} / \mathrm{kg}$ & 6 & 0 & $0 \%$ & 0.0018 & 0.0024 & -- & -- & $9.83 \mathrm{E}-04$ \\
\hline PEST & Aldrin & $\mathrm{mg} / \mathrm{kg}$ & 6 & 0 & $0 \%$ & 0.0018 & 0.0020 & -- & -- & $9.42 \mathrm{E}-04$ \\
\hline PEST & Alpha-BHC & $\mathrm{mg} / \mathrm{kg}$ & 6 & 0 & $0 \%$ & 0.0018 & 0.0024 & -- & -- & $9.83 \mathrm{E}-04$ \\
\hline PEST & alpha-Chlordane & $\mathrm{mg} / \mathrm{kg}$ & 6 & 0 & $0 \%$ & 0.0018 & 0.0024 & -- & -- & $9.83 \mathrm{E}-04$ \\
\hline PEST & alpha-Chlordene & $\mathrm{mg} / \mathrm{kg}$ & 6 & 0 & $0 \%$ & 0.0018 & 0.0024 & -- & -- & $9.83 \mathrm{E}-04$ \\
\hline PEST & Beta-BHC & $\mathrm{mg} / \mathrm{kg}$ & 6 & 0 & $0 \%$ & 0.0018 & 0.0024 & -- & -- & $9.83 \mathrm{E}-04$ \\
\hline PEST & cis-Nonachlor & $\mathrm{mg} / \mathrm{kg}$ & 6 & 0 & $0 \%$ & 0.0018 & 0.0024 & -- & -- & $9.83 \mathrm{E}-04$ \\
\hline PEST & DDMU & $\mathrm{mg} / \mathrm{kg}$ & 5 & 0 & $0 \%$ & 0.0018 & 0.0020 & -- & -- & $9.40 \mathrm{E}-04$ \\
\hline PEST & Delta-BHC & $\mathrm{mg} / \mathrm{kg}$ & 6 & 0 & $0 \%$ & 0.0018 & 0.0024 & -- & -- & $9.83 \mathrm{E}-04$ \\
\hline PEST & Dichlorodiphenyldichloroethane & $\mathrm{mg} / \mathrm{kg}$ & 6 & 1 & $17 \%$ & 0.0018 & 0.0024 & 0.0018 & 0.0018 & 0.0011 \\
\hline PEST & Dichlorodiphenyldichloroethylene & $\mathrm{mg} / \mathrm{kg}$ & 6 & 6 & $100 \%$ & -- & -- & 0.0029 & 0.017 & 0.011 \\
\hline PEST & Dichlorodiphenyltrichloroethane & $\mathrm{mg} / \mathrm{kg}$ & 6 & 0 & $0 \%$ & 0.0018 & 0.0024 & -- & -- & $9.83 \mathrm{E}-04$ \\
\hline PEST & Endosulfan sulfate & $\mathrm{mg} / \mathrm{kg}$ & 4 & 0 & $0 \%$ & 0.0018 & 0.0024 & -- & -- & $9.88 \mathrm{E}-04$ \\
\hline PEST & Gamma-BHC (Lindane) & $\mathrm{mg} / \mathrm{kg}$ & 6 & 0 & $0 \%$ & 0.0018 & 0.0024 & -- & -- & $9.83 \mathrm{E}-04$ \\
\hline PEST & gamma-Chlordane & $\mathrm{mg} / \mathrm{kg}$ & 6 & 0 & $0 \%$ & 0.0018 & 0.0024 & -- & -- & 9.83E-04 \\
\hline PEST & gamma-Chlordene & $\mathrm{mg} / \mathrm{kg}$ & 6 & 0 & $0 \%$ & 0.0018 & 0.0024 & -- & -- & $9.83 \mathrm{E}-04$ \\
\hline PEST & Heptachlor & $\mathrm{mg} / \mathrm{kg}$ & 6 & 0 & $0 \%$ & 0.0018 & 0.0020 & -- & -- & $9.42 \mathrm{E}-04$ \\
\hline PEST & Heptachlor epoxide & $\mathrm{mg} / \mathrm{kg}$ & 4 & 0 & $0 \%$ & 0.0018 & 0.0024 & -- & -- & $9.88 \mathrm{E}-04$ \\
\hline PEST & Mirex & $\mathrm{mg} / \mathrm{kg}$ & 6 & 0 & $0 \%$ & 0.0018 & 0.0024 & -- & -- & $9.83 \mathrm{E}-04$ \\
\hline PEST & Oxychlordane & $\mathrm{mg} / \mathrm{kg}$ & 6 & 0 & $0 \%$ & 0.0018 & 0.0024 & -- & -- & 9.83E-04 \\
\hline PEST & Pentachloroanisole & $\mathrm{mg} / \mathrm{kg}$ & 6 & 0 & $0 \%$ & 0.0018 & 0.0024 & -- & -- & $9.83 \mathrm{E}-04$ \\
\hline PEST & Toxaphene & $\mathrm{mg} / \mathrm{kg}$ & 6 & 0 & $0 \%$ & 0.053 & 0.071 & -- & -- & 0.030 \\
\hline PEST & trans-Nonachlor & $\mathrm{mg} / \mathrm{kg}$ & 6 & 0 & $0 \%$ & 0.0018 & 0.0024 & -- & -- & $9.83 \mathrm{E}-04$ \\
\hline SVOC & 1,2,4-Trichlorobenzene & $\mathrm{mg} / \mathrm{kg}$ & 6 & 0 & $0 \%$ & 0.0049 & 0.012 & -- & -- & 0.0037 \\
\hline SVOC & 1,2-Dichlorobenzene & $\mathrm{mg} / \mathrm{kg}$ & 6 & 0 & $0 \%$ & 0.0049 & 0.012 & -- & -- & 0.0037 \\
\hline SVOC & 1,2-Diphenylhydrazine & $\mathrm{mg} / \mathrm{kg}$ & 6 & 0 & $0 \%$ & 0.0049 & 0.012 & -- & -- & 0.0037 \\
\hline SVOC & 1,3-Dichlorobenzene & $\mathrm{mg} / \mathrm{kg}$ & 6 & 0 & $0 \%$ & 0.0049 & 0.012 & -- & -- & 0.0037 \\
\hline SVOC & 1,4-Dichlorobenzene & $\mathrm{mg} / \mathrm{kg}$ & 6 & 0 & $0 \%$ & 0.0049 & 0.012 & -- & -- & 0.0037 \\
\hline SVOC & 1-methylnaphthalene & $\mathrm{mg} / \mathrm{kg}$ & 6 & 0 & $0 \%$ & 0.0049 & 0.012 & -- & -- & 0.0037 \\
\hline SVOC & 2,4-Dinitrotoluene & $\mathrm{mg} / \mathrm{kg}$ & 6 & 0 & $0 \%$ & 0.0098 & 0.023 & -- & -- & 0.0074 \\
\hline SVOC & 2,6-Dinitrotoluene & $\mathrm{mg} / \mathrm{kg}$ & 6 & 0 & $0 \%$ & 0.020 & 0.046 & -- & -- & 0.015 \\
\hline SVOC & 2-Chloronaphthalene & $\mathrm{mg} / \mathrm{kg}$ & 6 & 0 & $0 \%$ & 0.0049 & 0.023 & -- & -- & 0.0070 \\
\hline SVOC & 2-Methylnaphthalene & $\mathrm{mg} / \mathrm{kg}$ & 6 & 1 & $17 \%$ & 0.0073 & 0.012 & 0.0059 & 0.0059 & 0.0048 \\
\hline SVOC & 4-Bromophenylphenyl ether & $\mathrm{mg} / \mathrm{kg}$ & 6 & 0 & $0 \%$ & 0.0049 & 0.012 & -- & -- & 0.0037 \\
\hline SVOC & 4-Chlorophenylphenyl ether & $\mathrm{mg} / \mathrm{kg}$ & 6 & 0 & $0 \%$ & 0.0049 & 0.012 & -- & -- & 0.0037 \\
\hline SVOC & Acenaphthene & $\mathrm{mg} / \mathrm{kg}$ & 6 & 0 & $0 \%$ & 0.0098 & 0.023 & -- & -- & 0.0074 \\
\hline SVOC & Acenaphthylene & $\mathrm{mg} / \mathrm{kg}$ & 6 & 0 & $0 \%$ & 0.0049 & 0.012 & -- & -- & 0.0037 \\
\hline SVOC & Anthracene & $\mathrm{mg} / \mathrm{kg}$ & 6 & 0 & $0 \%$ & 0.0049 & 0.012 & -- & -- & 0.0037 \\
\hline SVOC & Benzo(a)anthracene & $\mathrm{mg} / \mathrm{kg}$ & 6 & 0 & $0 \%$ & 0.0049 & 0.012 & -- & -- & 0.0037 \\
\hline SVOC & Benzo(a)pyrene & $\mathrm{mg} / \mathrm{kg}$ & 6 & 0 & $0 \%$ & 0.0080 & 0.098 & -- & -- & 0.015 \\
\hline SVOC & Benzo(b)fluoranthene & $\mathrm{mg} / \mathrm{kg}$ & 6 & 0 & $0 \%$ & 0.0080 & 0.20 & -- & -- & 0.023 \\
\hline SVOC & Benzo(ghi)perylene & $\mathrm{mg} / \mathrm{kg}$ & 6 & 0 & $0 \%$ & 0.016 & 0.20 & -- & -- & 0.030 \\
\hline SVOC & Benzo(k)fluoranthene & $\mathrm{mg} / \mathrm{kg}$ & 6 & 0 & $0 \%$ & 0.0080 & 0.20 & -- & -- & 0.023 \\
\hline SVOC & Bis(2-chloro-1-methylethyl)ether & $\mathrm{mg} / \mathrm{kg}$ & 6 & 0 & $0 \%$ & 0.0098 & 0.023 & -- & -- & 0.0074 \\
\hline
\end{tabular}


Table F-90

Summary Statistics for Whole Body Rainbow Trout Collected from Segment 4 of the Columbia River

\begin{tabular}{|c|c|c|c|c|c|c|c|c|c|c|}
\hline $\begin{array}{l}\text { Constituent } \\
\text { Class }\end{array}$ & Constituent Name & Units & $\begin{array}{l}\text { Number } \\
\text { Analyzed }\end{array}$ & $\begin{array}{l}\text { Number } \\
\text { Detected }\end{array}$ & $\begin{array}{c}\text { Frequency of } \\
\text { Detection }\end{array}$ & $\begin{array}{l}\text { Minimum } \\
\text { Nondetect }\end{array}$ & $\begin{array}{l}\text { Maximum } \\
\text { Nondetect }\end{array}$ & $\begin{array}{c}\text { Minimum } \\
\text { Detect }\end{array}$ & $\begin{array}{l}\text { Maximum } \\
\text { Detect }\end{array}$ & $\begin{array}{c}\text { Average } \\
\text { Concentration }\end{array}$ \\
\hline SVOC & Chrysene & $\mathrm{mg} / \mathrm{kg}$ & 6 & 0 & $0 \%$ & 0.0049 & 0.012 & -- & -- & 0.0037 \\
\hline SVOC & Dibenz[a,h]anthracene & $\mathrm{mg} / \mathrm{kg}$ & 6 & 0 & $0 \%$ & 0.016 & 0.20 & -- & -- & 0.030 \\
\hline SVOC & Dibenzofuran & $\mathrm{mg} / \mathrm{kg}$ & 6 & 0 & $0 \%$ & 0.0049 & 0.012 & -- & -- & 0.0037 \\
\hline SVOC & Fluoranthene & $\mathrm{mg} / \mathrm{kg}$ & 6 & 0 & $0 \%$ & 0.0098 & 0.023 & -- & -- & 0.0074 \\
\hline SVOC & Fluorene & $\mathrm{mg} / \mathrm{kg}$ & 6 & 0 & $0 \%$ & 0.0050 & 0.048 & -- & -- & 0.0084 \\
\hline SVOC & Hexachlorobenzene & $\mathrm{mg} / \mathrm{kg}$ & 6 & 0 & $0 \%$ & 0.0098 & 0.023 & -- & -- & 0.0074 \\
\hline SVOC & Hexachlorobutadiene & $\mathrm{mg} / \mathrm{kg}$ & 6 & 0 & $0 \%$ & 0.0049 & 0.012 & -- & -- & 0.0037 \\
\hline SVOC & Hexachloroethane & $\mathrm{mg} / \mathrm{kg}$ & 6 & 0 & $0 \%$ & 0.0049 & 0.012 & -- & -- & 0.0037 \\
\hline SVOC & Indeno(1,2,3-cd)pyrene & $\mathrm{mg} / \mathrm{kg}$ & 6 & 0 & $0 \%$ & 0.016 & 0.20 & -- & -- & 0.030 \\
\hline SVOC & Naphthalene & $\mathrm{mg} / \mathrm{kg}$ & 6 & 0 & $0 \%$ & 0.012 & 0.033 & -- & -- & 0.012 \\
\hline SVOC & Nitrobenzene & $\mathrm{mg} / \mathrm{kg}$ & 6 & 0 & $0 \%$ & 0.0049 & 0.012 & -- & -- & 0.0037 \\
\hline SVOC & Phenanthrene & $\mathrm{mg} / \mathrm{kg}$ & 6 & 0 & $0 \%$ & 0.0050 & 0.012 & -- & -- & 0.0038 \\
\hline SVOC & Pyrene & $\mathrm{mg} / \mathrm{kg}$ & 6 & 0 & $0 \%$ & 0.0098 & 0.023 & -- & -- & 0.0074 \\
\hline VOC & Retene & $\mathrm{mg} / \mathrm{kg}$ & 6 & 1 & $17 \%$ & 0.010 & 0.023 & 0.013 & 0.013 & 0.0088 \\
\hline
\end{tabular}

DIOXI - Dioxins and Furans

PEST - Pesticide

PCB - Polychlorinated biphenyl

SVOC - Semivolatile Organic Compound

VOC - Volatile Organic Compound 
Table F-91

Summary Statistics for Steelhead Eggs and Ovum Collected from Segment 4 of the Columbia River

\begin{tabular}{|c|c|c|c|c|c|c|c|c|c|c|}
\hline $\begin{array}{l}\text { Constituent } \\
\text { Class }\end{array}$ & Constituent Name & Units & $\begin{array}{l}\text { Number } \\
\text { Analyzed }\end{array}$ & $\begin{array}{l}\text { Number } \\
\text { Detected }\end{array}$ & $\begin{array}{c}\text { Frequency of } \\
\text { Detection }\end{array}$ & $\begin{array}{l}\text { Minimum } \\
\text { Nondetect }\end{array}$ & $\begin{array}{l}\text { Maximum } \\
\text { Nondetect }\end{array}$ & $\begin{array}{c}\text { Minimum } \\
\text { Detect }\end{array}$ & $\begin{array}{l}\text { Maximum } \\
\text { Detect }\end{array}$ & $\begin{array}{c}\text { Average } \\
\text { Concentration }\end{array}$ \\
\hline DIOXI & 1,2,3,4,6,7,8-Heptachlorodibenzodioxin & $\mathrm{mg} / \mathrm{kg}$ & 1 & 1 & $100 \%$ & $\overline{--}$ & $\overline{--}$ & $7.00 \mathrm{E}-08$ & $7.00 \mathrm{E}-08$ & $7.00 \mathrm{E}-08$ \\
\hline DIOXI & 1,2,3,4,6,7,8-Heptachlorodibenzofuran & $\mathrm{mg} / \mathrm{kg}$ & 1 & 0 & $0 \%$ & $2.00 \mathrm{E}-08$ & $2.00 \mathrm{E}-08$ & -- & -- & $1.00 \mathrm{E}-08$ \\
\hline DIOXI & 1,2,3,4,7,8,9-Heptachlorodibenzofuran & $\mathrm{mg} / \mathrm{kg}$ & 1 & 0 & $0 \%$ & 2.00E-08 & $2.00 \mathrm{E}-08$ & -- & -- & $1.00 \mathrm{E}-08$ \\
\hline DIOXI & $1,2,3,4,7,8$-Hexachlorodibenzofuran & $\mathrm{mg} / \mathrm{kg}$ & 1 & 0 & $0 \%$ & 2.00E-08 & $2.00 \mathrm{E}-08$ & -- & -- & $1.00 \mathrm{E}-08$ \\
\hline DIOXI & $1,2,3,4,7,8$-Hexachlorodibenzo-p-dioxin & $\mathrm{mg} / \mathrm{kg}$ & 1 & 0 & $0 \%$ & $2.00 \mathrm{E}-08$ & $2.00 \mathrm{E}-08$ & -- & -- & $1.00 \mathrm{E}-08$ \\
\hline DIOXI & $1,2,3,6,7,8$-Hexachlorodibenzofuran & $\mathrm{mg} / \mathrm{kg}$ & 1 & 0 & $0 \%$ & $2.00 \mathrm{E}-08$ & $2.00 \mathrm{E}-08$ & -- & -- & $1.00 \mathrm{E}-08$ \\
\hline DIOXI & $1,2,3,6,7,8$-Hexachlorodibenzo-p-dioxin & $\mathrm{mg} / \mathrm{kg}$ & 1 & 0 & $0 \%$ & 2.00E-08 & $2.00 \mathrm{E}-08$ & -- & -- & $1.00 \mathrm{E}-08$ \\
\hline DIOXI & 1,2,3,7,8,9-Hexachlorodibenzofuran & $\mathrm{mg} / \mathrm{kg}$ & 1 & 0 & $0 \%$ & $2.00 \mathrm{E}-08$ & $2.00 \mathrm{E}-08$ & -- & -- & $1.00 \mathrm{E}-08$ \\
\hline DIOXI & 1,2,3,7,8,9-Hexachlorodibenzo-p-dioxin & $\mathrm{mg} / \mathrm{kg}$ & 1 & 0 & $0 \%$ & $2.00 \mathrm{E}-08$ & $2.00 \mathrm{E}-08$ & -- & -- & $1.00 \mathrm{E}-08$ \\
\hline DIOXI & 1,2,3,7,8-Pentachlorodibenzofuran & $\mathrm{mg} / \mathrm{kg}$ & 1 & 0 & $0 \%$ & 2.00E-08 & 2.00E-08 & -- & -- & 1.00E-08 \\
\hline DIOXI & 1,2,3,7,8-Pentachlorodibenzo-p-dioxin & $\mathrm{mg} / \mathrm{kg}$ & 1 & 0 & $0 \%$ & $3.00 \mathrm{E}-08$ & $3.00 \mathrm{E}-08$ & -- & -- & $1.50 \mathrm{E}-08$ \\
\hline DIOXI & $2,3,4,6,7,8$-Hexachlorodibenzofuran & $\mathrm{mg} / \mathrm{kg}$ & 1 & 0 & $0 \%$ & 2.00E-08 & $2.00 \mathrm{E}-08$ & -- & -- & $1.00 \mathrm{E}-08$ \\
\hline DIOXI & $2,3,4,7,8$-Pentachlorodibenzofuran & $\mathrm{mg} / \mathrm{kg}$ & 1 & 0 & $0 \%$ & $2.00 \mathrm{E}-08$ & $2.00 \mathrm{E}-08$ & -- & -- & $1.00 \mathrm{E}-08$ \\
\hline DIOXI & $2,3,7,8$-Tetrachlorodibenzofuran & $\mathrm{mg} / \mathrm{kg}$ & 1 & 1 & $100 \%$ & -- & -- & $2.20 \mathrm{E}-07$ & $2.20 \mathrm{E}-07$ & $2.20 \mathrm{E}-07$ \\
\hline DIOXI & 2,3,7,8-Tetrachlorodibenzo-p-dioxin & $\mathrm{mg} / \mathrm{kg}$ & 1 & 0 & $0 \%$ & $3.00 \mathrm{E}-08$ & $3.00 \mathrm{E}-08$ & -- & -- & $1.50 \mathrm{E}-08$ \\
\hline DIOXI & Octachlorodibenzofuran & $\mathrm{mg} / \mathrm{kg}$ & 1 & 0 & $0 \%$ & $3.00 \mathrm{E}-08$ & $3.00 \mathrm{E}-08$ & -- & -- & $1.50 \mathrm{E}-08$ \\
\hline DIOXI & Octachlorodibenzo-p-dioxin & $\mathrm{mg} / \mathrm{kg}$ & 1 & 1 & $100 \%$ & -- & -- & $3.80 \mathrm{E}-07$ & 3.80E-07 & $3.80 \mathrm{E}-07$ \\
\hline METAL & Aluminum & $\mathrm{mg} / \mathrm{kg}$ & 1 & 1 & $100 \%$ & -- & -- & 4.5 & 4.5 & 4.5 \\
\hline METAL & Antimony & $\mathrm{mg} / \mathrm{kg}$ & 1 & 0 & $0 \%$ & 0.050 & 0.050 & -- & -- & 0.025 \\
\hline METAL & Arsenic & $\mathrm{mg} / \mathrm{kg}$ & 1 & 0 & $0 \%$ & 0.050 & 0.050 & -- & -- & 0.025 \\
\hline METAL & Barium & $\mathrm{mg} / \mathrm{kg}$ & 1 & 0 & $0 \%$ & 0.20 & 0.20 & -- & -- & 0.10 \\
\hline METAL & Beryllium & $\mathrm{mg} / \mathrm{kg}$ & 1 & 0 & $0 \%$ & 0.0040 & 0.0040 & -- & -- & 0.0020 \\
\hline METAL & Cadmium & $\mathrm{mg} / \mathrm{kg}$ & 1 & 1 & $100 \%$ & -- & -- & 0.030 & 0.030 & 0.030 \\
\hline METAL & Chromium & $\mathrm{mg} / \mathrm{kg}$ & 1 & 1 & $100 \%$ & -- & -- & 0.22 & 0.22 & 0.22 \\
\hline METAL & Cobalt & $\mathrm{mg} / \mathrm{kg}$ & 1 & 1 & $100 \%$ & -- & -- & 0.17 & 0.17 & 0.17 \\
\hline METAL & Copper & $\mathrm{mg} / \mathrm{kg}$ & 1 & 1 & $100 \%$ & -- & -- & 18 & 18 & 18 \\
\hline METAL & Lead & $\mathrm{mg} / \mathrm{kg}$ & 1 & 1 & $100 \%$ & -- & -- & 0.040 & 0.040 & 0.040 \\
\hline METAL & Manganese & $\mathrm{mg} / \mathrm{kg}$ & 1 & 1 & $100 \%$ & -- & -- & 2.2 & 2.2 & 2.2 \\
\hline METAL & Mercury & $\mathrm{mg} / \mathrm{kg}$ & 1 & 0 & $0 \%$ & 0.040 & 0.040 & -- & -- & 0.020 \\
\hline METAL & Nickel & $\mathrm{mg} / \mathrm{kg}$ & 1 & 1 & $100 \%$ & -- & -- & 0.51 & 0.51 & 0.51 \\
\hline METAL & Selenium & $\mathrm{mg} / \mathrm{kg}$ & 1 & 1 & $100 \%$ & -- & -- & 4.5 & 4.5 & 4.5 \\
\hline METAL & Silver & $\mathrm{mg} / \mathrm{kg}$ & 1 & 0 & $0 \%$ & 0.10 & 0.10 & -- & -- & 0.050 \\
\hline METAL & Thallium & $\mathrm{mg} / \mathrm{kg}$ & 1 & 0 & $0 \%$ & 0.050 & 0.050 & -- & -- & 0.025 \\
\hline METAL & Vanadium & $\mathrm{mg} / \mathrm{kg}$ & 1 & 1 & $100 \%$ & -- & -- & 0.11 & 0.11 & 0.11 \\
\hline METAL & Zinc & $\mathrm{mg} / \mathrm{kg}$ & 1 & 1 & $100 \%$ & -- & -- & 76 & 76 & 76 \\
\hline PCB & 2,2',3,3',4,4',5-Heptachloro-1,1'-biphenyl & $\mathrm{mg} / \mathrm{kg}$ & 1 & 1 & $100 \%$ & -- & -- & 5.45E-05 & 5.45E-05 & $5.45 \mathrm{E}-05$ \\
\hline PCB & $2,2^{\prime}, 3,4,4^{\prime}, 5,5^{\prime}-$ Heptachlorobiphenyl & $\mathrm{mg} / \mathrm{kg}$ & 1 & 1 & $100 \%$ & -- & -- & $1.60 \mathrm{E}-04$ & $1.60 \mathrm{E}-04$ & $1.60 \mathrm{E}-04$ \\
\hline PCB & 2,3,3',4,4',5'-Hexachlorobiphenyl & $\mathrm{mg} / \mathrm{kg}$ & 1 & 1 & $100 \%$ & -- & -- & 7.66E-06 & 7.66E-06 & 7.66E-06 \\
\hline PCB & 2,3,3',4,4',5-Hexachlorobiphenyl (BZ 156) & $\mathrm{mg} / \mathrm{kg}$ & 1 & 1 & $100 \%$ & -- & -- & 2.80E-05 & 2.80E-05 & 2.80E-05 \\
\hline PCB & 2,3,3',4,4'-Pentachlorobiphenyl & $\mathrm{mg} / \mathrm{kg}$ & 1 & 1 & $100 \%$ & -- & -- & 1.53E-04 & 1.53E-04 & 1.53E-04 \\
\hline PCB & 2,3',4,4',5,5'-Hexachlorobiphenyl (BZ 167) & $\mathrm{mg} / \mathrm{kg}$ & 1 & 1 & $100 \%$ & -- & -- & $1.81 \mathrm{E}-05$ & $1.81 \mathrm{E}-05$ & $1.81 \mathrm{E}-05$ \\
\hline PCB & 2',3,4,4',5-Pentachloro-1,1'-biphenyl (BZ 123) & $\mathrm{mg} / \mathrm{kg}$ & 1 & 1 & $100 \%$ & -- & -- & $5.75 \mathrm{E}-06$ & 5.75E-06 & $5.75 E-06$ \\
\hline PCB & 2,3,4,4',5-Pentachlorobiphenyl (BZ 114) & $\mathrm{mg} / \mathrm{kg}$ & 1 & 1 & $100 \%$ & -- & -- & 1.46E-05 & 1.46E-05 & 1.46E-05 \\
\hline PCB & 2,3',4,4',5-Pentachlorobiphenyl (BZ 118) & $\mathrm{mg} / \mathrm{kg}$ & 1 & 1 & $100 \%$ & -- & -- & $3.51 \mathrm{E}-04$ & $3.51 \mathrm{E}-04$ & $3.51 \mathrm{E}-04$ \\
\hline PCB & 2,3,4,5,3',4',5'-Heptachlorobiphenyl & $\mathrm{mg} / \mathrm{kg}$ & 1 & 0 & $0 \%$ & $1.00 \mathrm{E}-06$ & $1.00 \mathrm{E}-06$ & -- & -- & $5.00 \mathrm{E}-07$ \\
\hline PCB & 3,3',4,4',5,5'-Hexachlorobiphenyl (BZ 169) & $\mathrm{mg} / \mathrm{kg}$ & 1 & 0 & $0 \%$ & 1.00E-06 & 1.00E-06 & -- & -- & 5.00E-07 \\
\hline PCB & 3,3',4,4',5-Pentachlorobiphenyl (BZ 126) & $\mathrm{mg} / \mathrm{kg}$ & 1 & 1 & $100 \%$ & -- & -- & $2.12 \mathrm{E}-06$ & 2.12E-06 & 2.12E-06 \\
\hline PCB & 3,3',4,4'-Tetrachlorobiphenyl (BZ 77) & $\mathrm{mg} / \mathrm{kg}$ & 1 & 1 & $100 \%$ & -- & -- & 7.98E-06 & 7.98E-06 & 7.98E-06 \\
\hline
\end{tabular}


Table F-91

Summary Statistics for Steelhead Eggs and Ovum Collected from Segment 4 of the Columbia River

\begin{tabular}{|c|c|c|c|c|c|c|c|c|c|c|}
\hline $\begin{array}{c}\text { Constituent } \\
\text { Class }\end{array}$ & Constituent Name & Units & $\begin{array}{l}\text { Number } \\
\text { Analyzed }\end{array}$ & $\begin{array}{l}\text { Number } \\
\text { Detected }\end{array}$ & $\begin{array}{c}\text { Frequency of } \\
\text { Detection }\end{array}$ & $\begin{array}{c}\text { Minimum } \\
\text { Nondetect }\end{array}$ & $\begin{array}{l}\text { Maximum } \\
\text { Nondetect }\end{array}$ & $\begin{array}{c}\text { Minimum } \\
\text { Detect }\end{array}$ & $\begin{array}{l}\text { Maximum } \\
\text { Detect }\end{array}$ & $\begin{array}{c}\text { Average } \\
\text { Concentration }\end{array}$ \\
\hline PCB & Aroclor-1016 & $\mathrm{mg} / \mathrm{kg}$ & 1 & 0 & $0 \%$ & 0.020 & 0.020 & -- & -- & 0.010 \\
\hline PCB & Aroclor-1221 & $\mathrm{mg} / \mathrm{kg}$ & 1 & 0 & $0 \%$ & 0.020 & 0.020 & -- & -- & 0.010 \\
\hline PCB & Aroclor-1232 & $\mathrm{mg} / \mathrm{kg}$ & 1 & 0 & $0 \%$ & 0.020 & 0.020 & -- & -- & 0.010 \\
\hline PCB & Aroclor-1248 & $\mathrm{mg} / \mathrm{kg}$ & 1 & 0 & $0 \%$ & 0.020 & 0.020 & -- & -- & 0.010 \\
\hline PCB & Aroclor-1254 & $\mathrm{mg} / \mathrm{kg}$ & 1 & 1 & $100 \%$ & -- & -- & 0.015 & 0.015 & 0.015 \\
\hline PCB & Aroclor-1260 & $\mathrm{mg} / \mathrm{kg}$ & 1 & 0 & $0 \%$ & 0.020 & 0.020 & -- & -- & 0.010 \\
\hline PEST & 2,4'-DDD & $\mathrm{mg} / \mathrm{kg}$ & 1 & 0 & $0 \%$ & 0.0020 & 0.0020 & -- & -- & 0.0010 \\
\hline PEST & 2,4'-DDE & $\mathrm{mg} / \mathrm{kg}$ & 1 & 0 & $0 \%$ & 0.0020 & 0.0020 & -- & -- & 0.0010 \\
\hline PEST & 2,4'-DDT & $\mathrm{mg} / \mathrm{kg}$ & 1 & 0 & $0 \%$ & 0.0020 & 0.0020 & -- & -- & 0.0010 \\
\hline PEST & Aldrin & $\mathrm{mg} / \mathrm{kg}$ & 1 & 0 & $0 \%$ & 0.0020 & 0.0020 & -- & -- & 0.0010 \\
\hline PEST & Alpha-BHC & $\mathrm{mg} / \mathrm{kg}$ & 1 & 0 & $0 \%$ & 0.0020 & 0.0020 & -- & -- & 0.0010 \\
\hline PEST & alpha-Chlordane & $\mathrm{mg} / \mathrm{kg}$ & 1 & 1 & $100 \%$ & -- & -- & 0.0013 & 0.0013 & 0.0013 \\
\hline PEST & alpha-Chlordene & $\mathrm{mg} / \mathrm{kg}$ & 1 & 0 & $0 \%$ & 0.0020 & 0.0020 & -- & -- & 0.0010 \\
\hline PEST & Beta-BHC & $\mathrm{mg} / \mathrm{kg}$ & 1 & 0 & $0 \%$ & 0.0020 & 0.0020 & -- & -- & 0.0010 \\
\hline PEST & cis-Nonachlor & $\mathrm{mg} / \mathrm{kg}$ & 1 & 0 & $0 \%$ & 0.0020 & 0.0020 & -- & -- & 0.0010 \\
\hline PEST & DDMU & $\mathrm{mg} / \mathrm{kg}$ & 1 & 0 & $0 \%$ & 0.0020 & 0.0020 & -- & -- & 0.0010 \\
\hline PEST & Delta-BHC & $\mathrm{mg} / \mathrm{kg}$ & 1 & 0 & $0 \%$ & 0.0020 & 0.0020 & -- & -- & 0.0010 \\
\hline PEST & Dichlorodiphenyldichloroethane & $\mathrm{mg} / \mathrm{kg}$ & 1 & 1 & $100 \%$ & -- & -- & 0.0033 & 0.0033 & 0.0033 \\
\hline PEST & Dichlorodiphenyldichloroethylene & $\mathrm{mg} / \mathrm{kg}$ & 1 & 1 & $100 \%$ & -- & -- & 0.0065 & 0.0065 & 0.0065 \\
\hline PEST & Dichlorodiphenyltrichloroethane & $\mathrm{mg} / \mathrm{kg}$ & 1 & 0 & $0 \%$ & 0.0020 & 0.0020 & -- & -- & 0.0010 \\
\hline PEST & Endosulfan sulfate & $\mathrm{mg} / \mathrm{kg}$ & 1 & 0 & $0 \%$ & 0.0020 & 0.0020 & -- & -- & 0.0010 \\
\hline PEST & Gamma-BHC (Lindane) & $\mathrm{mg} / \mathrm{kg}$ & 1 & 0 & $0 \%$ & 0.0020 & 0.0020 & -- & -- & 0.0010 \\
\hline PEST & gamma-Chlordane & $\mathrm{mg} / \mathrm{kg}$ & 1 & 0 & $0 \%$ & 0.0020 & 0.0020 & -- & -- & 0.0010 \\
\hline PEST & gamma-Chlordene & $\mathrm{mg} / \mathrm{kg}$ & 1 & 0 & $0 \%$ & 0.0020 & 0.0020 & -- & -- & 0.0010 \\
\hline PEST & Heptachlor & $\mathrm{mg} / \mathrm{kg}$ & 1 & 0 & $0 \%$ & 0.0020 & 0.0020 & -- & -- & 0.0010 \\
\hline PEST & Heptachlor epoxide & $\mathrm{mg} / \mathrm{kg}$ & 1 & 0 & $0 \%$ & 0.0020 & 0.0020 & -- & -- & 0.0010 \\
\hline PEST & Mirex & $\mathrm{mg} / \mathrm{kg}$ & 1 & 0 & $0 \%$ & 0.0020 & 0.0020 & -- & -- & 0.0010 \\
\hline PEST & Oxychlordane & $\mathrm{mg} / \mathrm{kg}$ & 1 & 0 & $0 \%$ & 0.0020 & 0.0020 & -- & -- & 0.0010 \\
\hline PEST & Pentachloroanisole & $\mathrm{mg} / \mathrm{kg}$ & 1 & 0 & $0 \%$ & 0.0020 & 0.0020 & -- & -- & 0.0010 \\
\hline PEST & Toxaphene & $\mathrm{mg} / \mathrm{kg}$ & 1 & 0 & $0 \%$ & 0.060 & 0.060 & -- & -- & 0.030 \\
\hline PEST & trans-Nonachlor & $\mathrm{mg} / \mathrm{kg}$ & 1 & 1 & $100 \%$ & -- & -- & 0.0010 & 0.0010 & 0.0010 \\
\hline SVOC & 1,2,4-Trichlorobenzene & $\mathrm{mg} / \mathrm{kg}$ & 1 & 0 & $0 \%$ & 0.0092 & 0.0092 & -- & -- & 0.0046 \\
\hline SVOC & 1,2-Dichlorobenzene & $\mathrm{mg} / \mathrm{kg}$ & 1 & 0 & $0 \%$ & 0.0092 & 0.0092 & -- & -- & 0.0046 \\
\hline SVOC & 1,2-Diphenylhydrazine & $\mathrm{mg} / \mathrm{kg}$ & 1 & 0 & $0 \%$ & 0.0092 & 0.0092 & -- & -- & 0.0046 \\
\hline SVOC & 1,3-Dichlorobenzene & $\mathrm{mg} / \mathrm{kg}$ & 1 & 0 & $0 \%$ & 0.0092 & 0.0092 & -- & -- & 0.0046 \\
\hline SVOC & 1,4-Dichlorobenzene & $\mathrm{mg} / \mathrm{kg}$ & 1 & 0 & $0 \%$ & 0.0092 & 0.0092 & -- & -- & 0.0046 \\
\hline SVOC & 1-methylnaphthalene & $\mathrm{mg} / \mathrm{kg}$ & 1 & 0 & $0 \%$ & 0.0092 & 0.0092 & -- & -- & 0.0046 \\
\hline SVOC & 2,4-Dinitrotoluene & $\mathrm{mg} / \mathrm{kg}$ & 1 & 0 & $0 \%$ & 0.018 & 0.018 & -- & -- & 0.0090 \\
\hline SVOC & 2,6-Dinitrotoluene & $\mathrm{mg} / \mathrm{kg}$ & 1 & 0 & $0 \%$ & 0.037 & 0.037 & -- & -- & 0.019 \\
\hline SVOC & 2-Chloronaphthalene & $\mathrm{mg} / \mathrm{kg}$ & 1 & 0 & $0 \%$ & 0.018 & 0.018 & -- & -- & 0.0090 \\
\hline SVOC & 2-Methylnaphthalene & $\mathrm{mg} / \mathrm{kg}$ & 1 & 0 & $0 \%$ & 0.0092 & 0.0092 & -- & -- & 0.0046 \\
\hline SVOC & 4-Bromophenylphenyl ether & $\mathrm{mg} / \mathrm{kg}$ & 1 & 0 & $0 \%$ & 0.0092 & 0.0092 & -- & -- & 0.0046 \\
\hline SVOC & 4-Chlorophenylphenyl ether & $\mathrm{mg} / \mathrm{kg}$ & 1 & 0 & $0 \%$ & 0.0092 & 0.0092 & -- & -- & 0.0046 \\
\hline SVOC & Acenaphthene & $\mathrm{mg} / \mathrm{kg}$ & 1 & 0 & $0 \%$ & 0.018 & 0.018 & -- & -- & 0.0090 \\
\hline SVOC & Acenaphthylene & $\mathrm{mg} / \mathrm{kg}$ & 1 & 0 & $0 \%$ & 0.0092 & 0.0092 & -- & -- & 0.0046 \\
\hline SVOC & Anthracene & $\mathrm{mg} / \mathrm{kg}$ & 1 & 0 & $0 \%$ & 0.0092 & 0.0092 & -- & -- & 0.0046 \\
\hline SVOC & Benzo(a)anthracene & $\mathrm{mg} / \mathrm{kg}$ & 1 & 0 & $0 \%$ & 0.0092 & 0.0092 & -- & -- & 0.0046 \\
\hline SVOC & Benzo(a)pyrene & $\mathrm{mg} / \mathrm{kg}$ & 1 & 0 & $0 \%$ & 0.0092 & 0.0092 & -- & -- & 0.0046 \\
\hline
\end{tabular}


Table F-91

Summary Statistics for Steelhead Eggs and Ovum Collected from Segment 4 of the Columbia River

\begin{tabular}{|c|c|c|c|c|c|c|c|c|c|c|}
\hline $\begin{array}{c}\text { Constituent } \\
\text { Class }\end{array}$ & Constituent Name & Units & $\begin{array}{l}\text { Number } \\
\text { Analyzed }\end{array}$ & $\begin{array}{l}\text { Number } \\
\text { Detected }\end{array}$ & $\begin{array}{c}\text { Frequency of } \\
\text { Detection }\end{array}$ & $\begin{array}{l}\text { Minimum } \\
\text { Nondetect }\end{array}$ & $\begin{array}{l}\text { Maximum } \\
\text { Nondetect }\end{array}$ & $\begin{array}{c}\text { Minimum } \\
\text { Detect }\end{array}$ & $\begin{array}{l}\text { Maximum } \\
\text { Detect }\end{array}$ & $\begin{array}{c}\text { Average } \\
\text { Concentration }\end{array}$ \\
\hline$\overline{\text { SVOC }}$ & Benzo(b)fluoranthene & $\mathrm{mg} / \mathrm{kg}$ & 1 & 0 & $0 \%$ & 0.0092 & 0.0092 & $\overline{--}$ & $\overline{--}$ & 0.0046 \\
\hline SVOC & Benzo(ghi)perylene & $\mathrm{mg} / \mathrm{kg}$ & 1 & 0 & $0 \%$ & 0.018 & 0.018 & -- & -- & 0.0090 \\
\hline SVOC & Benzo(k)fluoranthene & $\mathrm{mg} / \mathrm{kg}$ & 1 & 0 & $0 \%$ & 0.0092 & 0.0092 & -- & -- & 0.0046 \\
\hline SVOC & Bis(2-chloro-1-methylethyl)ether & $\mathrm{mg} / \mathrm{kg}$ & 1 & 0 & $0 \%$ & 0.018 & 0.018 & -- & -- & 0.0090 \\
\hline SVOC & Chrysene & $\mathrm{mg} / \mathrm{kg}$ & 1 & 0 & $0 \%$ & 0.0092 & 0.0092 & -- & -- & 0.0046 \\
\hline SVOC & Dibenz[a,h]anthracene & $\mathrm{mg} / \mathrm{kg}$ & 1 & 0 & $0 \%$ & 0.018 & 0.018 & -- & -- & 0.0090 \\
\hline SVOC & Dibenzofuran & $\mathrm{mg} / \mathrm{kg}$ & 1 & 0 & $0 \%$ & 0.0092 & 0.0092 & -- & -- & 0.0046 \\
\hline SVOC & Fluoranthene & $\mathrm{mg} / \mathrm{kg}$ & 1 & 0 & $0 \%$ & 0.018 & 0.018 & -- & -- & 0.0090 \\
\hline SVOC & Fluorene & $\mathrm{mg} / \mathrm{kg}$ & 1 & 0 & $0 \%$ & 0.0053 & 0.0053 & -- & -- & 0.0027 \\
\hline SVOC & Hexachlorobenzene & $\mathrm{mg} / \mathrm{kg}$ & 1 & 0 & $0 \%$ & 0.018 & 0.018 & -- & -- & 0.0090 \\
\hline SVOC & Hexachlorobutadiene & $\mathrm{mg} / \mathrm{kg}$ & 1 & 0 & $0 \%$ & 0.0092 & 0.0092 & -- & -- & 0.0046 \\
\hline SVOC & Hexachloroethane & $\mathrm{mg} / \mathrm{kg}$ & 1 & 0 & $0 \%$ & 0.0092 & 0.0092 & -- & -- & 0.0046 \\
\hline SVOC & Indeno(1,2,3-cd)pyrene & $\mathrm{mg} / \mathrm{kg}$ & 1 & 0 & $0 \%$ & 0.018 & 0.018 & -- & -- & 0.0090 \\
\hline SVOC & Naphthalene & $\mathrm{mg} / \mathrm{kg}$ & 1 & 0 & $0 \%$ & 0.022 & 0.022 & -- & -- & 0.011 \\
\hline SVOC & Nitrobenzene & $\mathrm{mg} / \mathrm{kg}$ & 1 & 0 & $0 \%$ & 0.0092 & 0.0092 & -- & -- & 0.0046 \\
\hline SVOC & Phenanthrene & $\mathrm{mg} / \mathrm{kg}$ & 1 & 0 & $0 \%$ & 0.0092 & 0.0092 & -- & -- & 0.0046 \\
\hline SVOC & Pyrene & $\mathrm{mg} / \mathrm{kg}$ & 1 & 0 & $0 \%$ & 0.018 & 0.018 & -- & -- & 0.0090 \\
\hline VOC & Retene & $\mathrm{mg} / \mathrm{kg}$ & 1 & 0 & $0 \%$ & 0.018 & 0.018 & -- & -- & 0.0090 \\
\hline
\end{tabular}

DIOXI - Dioxins and Furans

PEST - Pesticide

PCB - Polychlorinated biphenyl

SVOC - Semivolatile Organic Compound

VOC - Volatile Organic Compound 
Table F-92

Summary Statistics for Steelhead Fillet Skin On Collected from Segment 4 of the Columbia River

\begin{tabular}{|c|c|c|c|c|c|c|c|c|c|c|}
\hline $\begin{array}{l}\text { Constituent } \\
\text { Class }\end{array}$ & Constituent Name & Units & $\begin{array}{l}\text { Number } \\
\text { Analyzed }\end{array}$ & $\begin{array}{l}\text { Number } \\
\text { Detected }\end{array}$ & $\begin{array}{c}\text { Frequency of } \\
\text { Detection }\end{array}$ & $\begin{array}{l}\text { Minimum } \\
\text { Nondetect }\end{array}$ & $\begin{array}{l}\text { Maximum } \\
\text { Nondetect }\end{array}$ & $\begin{array}{l}\text { Minimum } \\
\text { Detect }\end{array}$ & $\begin{array}{l}\text { Maximum } \\
\text { Detect }\end{array}$ & $\begin{array}{c}\text { Average } \\
\text { Concentration }\end{array}$ \\
\hline DIOXI & $1,2,3,4,6,7,8$-Heptachlorodibenzodioxin & $\mathrm{mg} / \mathrm{kg}$ & 12 & 5 & $42 \%$ & $1.00 \mathrm{E}-08$ & $4.00 \mathrm{E}-08$ & $6.00 \mathrm{E}-08$ & $1.40 \mathrm{E}-07$ & $4.54 \mathrm{E}-08$ \\
\hline DIOXI & $1,2,3,4,6,7,8$-Heptachlorodibenzofuran & $\mathrm{mg} / \mathrm{kg}$ & 12 & 2 & $17 \%$ & $1.00 \mathrm{E}-08$ & $4.00 \mathrm{E}-08$ & 4.00E-08 & $5.00 \mathrm{E}-08$ & $1.88 \mathrm{E}-08$ \\
\hline DIOXI & 1,2,3,4,7,8,9-Heptachlorodibenzofuran & $\mathrm{mg} / \mathrm{kg}$ & 12 & 0 & $0 \%$ & $1.00 \mathrm{E}-08$ & $4.00 \mathrm{E}-08$ & -- & -- & $1.38 \mathrm{E}-08$ \\
\hline DIOXI & 1,2,3,4,7,8-Hexachlorodibenzofuran & $\mathrm{mg} / \mathrm{kg}$ & 12 & 3 & $25 \%$ & $1.00 \mathrm{E}-08$ & $5.00 \mathrm{E}-08$ & $3.00 \mathrm{E}-08$ & $3.00 \mathrm{E}-08$ & 1.63E-08 \\
\hline DIOXI & $1,2,3,4,7,8$-Hexachlorodibenzo-p-dioxin & $\mathrm{mg} / \mathrm{kg}$ & 12 & 2 & $17 \%$ & $1.00 \mathrm{E}-08$ & $3.00 \mathrm{E}-08$ & $2.00 \mathrm{E}-08$ & $2.00 \mathrm{E}-08$ & $1.29 \mathrm{E}-08$ \\
\hline DIOXI & $1,2,3,6,7,8$-Hexachlorodibenzofuran & $\mathrm{mg} / \mathrm{kg}$ & 12 & 1 & $8 \%$ & $1.00 \mathrm{E}-08$ & $4.00 \mathrm{E}-08$ & $3.00 \mathrm{E}-08$ & $3.00 \mathrm{E}-08$ & $1.21 \mathrm{E}-08$ \\
\hline DIOXI & $1,2,3,6,7,8$-Hexachlorodibenzo-p-dioxin & $\mathrm{mg} / \mathrm{kg}$ & 12 & 2 & $17 \%$ & 1.00E-08 & $3.00 \mathrm{E}-08$ & 4.00E-08 & $5.00 \mathrm{E}-08$ & $1.71 \mathrm{E}-08$ \\
\hline DIOXI & 1,2,3,7,8,9-Hexachlorodibenzofuran & $\mathrm{mg} / \mathrm{kg}$ & 12 & 1 & $8 \%$ & $1.00 \mathrm{E}-08$ & $5.00 \mathrm{E}-08$ & 1.00E-08 & 1.00E-08 & $1.08 \mathrm{E}-08$ \\
\hline DIOXI & 1,2,3,7,8,9-Hexachlorodibenzo-p-dioxin & $\mathrm{mg} / \mathrm{kg}$ & 12 & 2 & $17 \%$ & $1.00 \mathrm{E}-08$ & $3.00 \mathrm{E}-08$ & 2.00E-08 & 4.00E-08 & 1.42E-08 \\
\hline DIOXI & 1,2,3,7,8-Pentachlorodibenzofuran & $\mathrm{mg} / \mathrm{kg}$ & 12 & 4 & $33 \%$ & $1.00 \mathrm{E}-08$ & $4.00 \mathrm{E}-08$ & $3.00 \mathrm{E}-08$ & $1.20 \mathrm{E}-07$ & $3.46 \mathrm{E}-08$ \\
\hline DIOXI & 1,2,3,7,8-Pentachlorodibenzo-p-dioxin & $\mathrm{mg} / \mathrm{kg}$ & 12 & 8 & $67 \%$ & 1.00E-08 & 4.00E-08 & $5.00 \mathrm{E}-08$ & $1.20 \mathrm{E}-07$ & $6.33 \mathrm{E}-08$ \\
\hline DIOXI & 2,3,4,6,7,8-Hexachlorodibenzofuran & $\mathrm{mg} / \mathrm{kg}$ & 12 & 2 & $17 \%$ & $1.00 \mathrm{E}-08$ & $5.00 \mathrm{E}-08$ & $3.00 \mathrm{E}-08$ & $5.00 \mathrm{E}-08$ & $1.58 \mathrm{E}-08$ \\
\hline DIOXI & 2,3,4,7,8-Pentachlorodibenzofuran & $\mathrm{mg} / \mathrm{kg}$ & 12 & 8 & $67 \%$ & $1.00 \mathrm{E}-08$ & 4.00E-08 & $7.00 \mathrm{E}-08$ & $3.20 \mathrm{E}-07$ & 1.65E-07 \\
\hline DIOXI & $2,3,7,8$-Tetrachlorodibenzofuran & $\mathrm{mg} / \mathrm{kg}$ & 12 & 12 & $100 \%$ & -- & -- & $1.80 \mathrm{E}-07$ & 4.50E-07 & $3.42 \mathrm{E}-07$ \\
\hline DIOXI & 2,3,7,8-Tetrachlorodibenzo-p-dioxin & $\mathrm{mg} / \mathrm{kg}$ & 12 & 1 & $8 \%$ & $1.00 \mathrm{E}-08$ & $5.00 \mathrm{E}-08$ & 4.00E-08 & 4.00E-08 & $1.46 \mathrm{E}-08$ \\
\hline DIOXI & Octachlorodibenzofuran & $\mathrm{mg} / \mathrm{kg}$ & 12 & 1 & $8 \%$ & $3.00 \mathrm{E}-08$ & 8.00E-08 & $6.00 \mathrm{E}-08$ & $6.00 \mathrm{E}-08$ & $2.75 \mathrm{E}-08$ \\
\hline DIOXI & Octachlorodibenzo-p-dioxin & $\mathrm{mg} / \mathrm{kg}$ & 12 & 8 & $67 \%$ & $2.00 \mathrm{E}-08$ & $6.00 \mathrm{E}-08$ & 1.10E-07 & $7.80 \mathrm{E}-07$ & $1.90 \mathrm{E}-07$ \\
\hline METAL & Aluminum & $\mathrm{mg} / \mathrm{kg}$ & 12 & 4 & $33 \%$ & 1.0 & 1.0 & 1.1 & 1.6 & 0.78 \\
\hline METAL & Antimony & $\mathrm{mg} / \mathrm{kg}$ & 12 & 0 & $0 \%$ & 0.050 & 0.050 & -- & -- & 0.025 \\
\hline METAL & Arsenic & $\mathrm{mg} / \mathrm{kg}$ & 12 & 12 & $100 \%$ & -- & -- & 0.47 & 1.5 & 0.69 \\
\hline METAL & Barium & $\mathrm{mg} / \mathrm{kg}$ & 12 & 1 & $8 \%$ & 0.20 & 0.20 & 0.32 & 0.32 & 0.12 \\
\hline METAL & Beryllium & $\mathrm{mg} / \mathrm{kg}$ & 12 & 0 & $0 \%$ & 0.0040 & 0.0040 & -- & -- & 0.0020 \\
\hline METAL & Cadmium & $\mathrm{mg} / \mathrm{kg}$ & 12 & 11 & $92 \%$ & 0.0040 & 0.0040 & 0.0046 & 0.0087 & 0.0057 \\
\hline METAL & Chromium & $\mathrm{mg} / \mathrm{kg}$ & 12 & 4 & $33 \%$ & 0.10 & 0.10 & 0.15 & 0.21 & 0.093 \\
\hline METAL & Cobalt & $\mathrm{mg} / \mathrm{kg}$ & 12 & 12 & $100 \%$ & -- & -- & 0.0059 & 0.19 & 0.066 \\
\hline METAL & Copper & $\mathrm{mg} / \mathrm{kg}$ & 12 & 12 & $100 \%$ & -- & -- & 0.54 & 0.82 & 0.65 \\
\hline METAL & Lead & $\mathrm{mg} / \mathrm{kg}$ & 12 & 3 & $25 \%$ & 0.010 & 0.010 & 0.010 & 0.020 & 0.0071 \\
\hline METAL & Manganese & $\mathrm{mg} / \mathrm{kg}$ & 12 & 12 & $100 \%$ & -- & -- & 0.10 & 0.20 & 0.13 \\
\hline METAL & Mercury & $\mathrm{mg} / \mathrm{kg}$ & 12 & 12 & $100 \%$ & -- & -- & 0.070 & 0.18 & 0.11 \\
\hline METAL & Nickel & $\mathrm{mg} / \mathrm{kg}$ & 12 & 9 & $75 \%$ & 0.030 & 0.030 & 0.030 & 0.070 & 0.037 \\
\hline METAL & Selenium & $\mathrm{mg} / \mathrm{kg}$ & 12 & 11 & $92 \%$ & 0.50 & 0.50 & 0.31 & 0.40 & 0.35 \\
\hline METAL & Silver & $\mathrm{mg} / \mathrm{kg}$ & 12 & 0 & $0 \%$ & 0.10 & 0.10 & -- & -- & 0.050 \\
\hline METAL & Thallium & $\mathrm{mg} / \mathrm{kg}$ & 12 & 0 & $0 \%$ & 0.050 & 0.050 & -- & -- & 0.025 \\
\hline METAL & Vanadium & $\mathrm{mg} / \mathrm{kg}$ & 12 & 6 & $50 \%$ & 0.010 & 0.020 & 0.010 & 0.020 & 0.010 \\
\hline METAL & Zinc & $\mathrm{mg} / \mathrm{kg}$ & 12 & 12 & $100 \%$ & -- & -- & 6.3 & 9.3 & 7.5 \\
\hline PCB & 2,2',3,3',4,4',5-Heptachloro-1,1'-biphenyl & $\mathrm{mg} / \mathrm{kg}$ & 12 & 12 & $100 \%$ & -- & -- & $6.04 \mathrm{E}-05$ & $1.28 \mathrm{E}-04$ & $9.00 \mathrm{E}-05$ \\
\hline PCB & $2,2^{\prime}, 3,4,4^{\prime}, 5,5^{\prime}$-Heptachlorobiphenyl & $\mathrm{mg} / \mathrm{kg}$ & 12 & 12 & $100 \%$ & -- & -- & $1.98 \mathrm{E}-04$ & $3.84 \mathrm{E}-04$ & 2.80E-04 \\
\hline PCB & $2,3,3^{\prime}, 4,4^{\prime}, 5^{\prime}$-Hexachlorobiphenyl & $\mathrm{mg} / \mathrm{kg}$ & 12 & 12 & $100 \%$ & -- & -- & 8.40E-06 & $2.54 \mathrm{E}-05$ & 1.35E-05 \\
\hline PCB & $2,3,3^{\prime}, 4,4^{\prime}, 5$-Hexachlorobiphenyl (BZ 156) & $\mathrm{mg} / \mathrm{kg}$ & 12 & 12 & $100 \%$ & .- & .- & 3.35E-05 & $8.05 \mathrm{E}-05$ & 4.79E-05 \\
\hline PCB & $2,3,3^{\prime}, 4,4^{\prime}$-Pentachlorobiphenyl & $\mathrm{mg} / \mathrm{kg}$ & 12 & 12 & $100 \%$ & -- & -- & $1.52 \mathrm{E}-04$ & $3.02 \mathrm{E}-04$ & 2.10E-04 \\
\hline PCB & $2,3^{\prime}, 4,4^{\prime}, 5,5^{\prime}-$ Hexachlorobiphenyl (BZ 167) & $\mathrm{mg} / \mathrm{kg}$ & 12 & 12 & $100 \%$ & -- & -- & 2.17E-05 & 4.55E-05 & 3.07E-05 \\
\hline PCB & 2',3,4,4',5-Pentachloro-1,1'-biphenyl (BZ 123) & $\mathrm{mg} / \mathrm{kg}$ & 12 & 12 & $100 \%$ & -- & -- & $6.50 \mathrm{E}-06$ & 1.21E-05 & 8.53E-06 \\
\hline PCB & 2,3,4,4',5-Pentachlorobiphenyl (BZ 114) & $\mathrm{mg} / \mathrm{kg}$ & 12 & 12 & $100 \%$ & -- & -- & $1.56 \mathrm{E}-05$ & 3.02E-05 & 2.13E-05 \\
\hline PCB & 2,3',4,4',5-Pentachlorobiphenyl (BZ 118) & $\mathrm{mg} / \mathrm{kg}$ & 12 & 12 & $100 \%$ & -- & -- & 3.67E-04 & $7.24 \mathrm{E}-04$ & $5.11 \mathrm{E}-04$ \\
\hline PCB & $2,3,4,5,3^{\prime}, 4^{\prime}, 5^{\prime}-$ Heptachlorobiphenyl & $\mathrm{mg} / \mathrm{kg}$ & 12 & 12 & $100 \%$ & -- & -- & $2.26 \mathrm{E}-06$ & $6.20 \mathrm{E}-06$ & 3.93E-06 \\
\hline PCB & 3,3',4,4',5,5'-Hexachlorobiphenyl (BZ 169) & $\mathrm{mg} / \mathrm{kg}$ & 12 & 0 & $0 \%$ & $1.00 \mathrm{E}-06$ & $3.94 \mathrm{E}-06$ & -- & -- & $7.50 \mathrm{E}-07$ \\
\hline PCB & 3,3',4,4',5-Pentachlorobiphenyl (BZ 126) & $\mathrm{mg} / \mathrm{kg}$ & 12 & 10 & $83 \%$ & $5.00 \mathrm{E}-07$ & 5.00E-07 & $1.84 \mathrm{E}-06$ & $5.58 \mathrm{E}-06$ & 3.67E-06 \\
\hline PCB & 3,3',4,4'-Tetrachlorobiphenyl (BZ 77) & $\mathrm{mg} / \mathrm{kg}$ & 12 & 12 & $100 \%$ & -- & -- & 8.15E-06 & 1.83E-05 & 1.27E-05 \\
\hline PCB & Aroclor-1016 & $\mathrm{mg} / \mathrm{kg}$ & 12 & 0 & $0 \%$ & 0.016 & 0.020 & -- & -- & 0.0096 \\
\hline PCB & Aroclor-1221 & $\mathrm{mg} / \mathrm{kg}$ & 12 & 0 & $0 \%$ & 0.016 & 0.020 & -- & -- & 0.0096 \\
\hline
\end{tabular}


Table F-92

Summary Statistics for Steelhead Fillet Skin On Collected from Segment 4 of the Columbia River

\begin{tabular}{|c|c|c|c|c|c|c|c|c|c|c|}
\hline $\begin{array}{l}\text { Constituent } \\
\text { Class }\end{array}$ & Constituent Name & Units & $\begin{array}{l}\text { Number } \\
\text { Analyzed }\end{array}$ & $\begin{array}{l}\text { Number } \\
\text { Detected }\end{array}$ & $\begin{array}{c}\text { Frequency of } \\
\text { Detection }\end{array}$ & $\begin{array}{l}\text { Minimum } \\
\text { Nondetect }\end{array}$ & $\begin{array}{l}\text { Maximum } \\
\text { Nondetect }\end{array}$ & $\begin{array}{c}\text { Minimum } \\
\text { Detect }\end{array}$ & $\begin{array}{l}\text { Maximum } \\
\text { Detect }\end{array}$ & $\begin{array}{c}\text { Average } \\
\text { Concentration }\end{array}$ \\
\hline$\overline{\mathrm{PCB}}$ & Aroclor-1232 & $\mathrm{mg} / \mathrm{kg}$ & 12 & 0 & $0 \%$ & 0.016 & 0.020 & -- & -- & 0.0096 \\
\hline РCB & Aroclor-1248 & $\mathrm{mg} / \mathrm{kg}$ & 12 & 0 & $0 \%$ & 0.016 & 0.020 & -- & -- & 0.0096 \\
\hline РCB & Aroclor-1254 & $\mathrm{mg} / \mathrm{kg}$ & 12 & 12 & $100 \%$ & -- & -- & 0.0084 & 0.019 & 0.014 \\
\hline РCB & Aroclor-1260 & $\mathrm{mg} / \mathrm{kg}$ & 12 & 3 & $25 \%$ & 0.019 & 0.020 & 0.0058 & 0.0085 & 0.0091 \\
\hline PEST & 2,4'-DDD & $\mathrm{mg} / \mathrm{kg}$ & 12 & 0 & $0 \%$ & 0.0016 & 0.0020 & -- & -- & $9.63 \mathrm{E}-04$ \\
\hline PEST & 2,4'-DDE & $\mathrm{mg} / \mathrm{kg}$ & 12 & 3 & $25 \%$ & 0.0016 & 0.0020 & $7.80 \mathrm{E}-04$ & 0.0012 & $9.59 \mathrm{E}-04$ \\
\hline PEST & 2,4'-DDT & $\mathrm{mg} / \mathrm{kg}$ & 12 & 0 & $0 \%$ & 0.0016 & 0.0020 & -- & -- & $9.63 \mathrm{E}-04$ \\
\hline PEST & Aldrin & $\mathrm{mg} / \mathrm{kg}$ & 12 & 0 & $0 \%$ & 0.0016 & 0.0020 & -- & -- & $9.63 \mathrm{E}-04$ \\
\hline PEST & Alpha-BHC & $\mathrm{mg} / \mathrm{kg}$ & 12 & 0 & $0 \%$ & 0.0016 & 0.0020 & -- & -- & $9.63 \mathrm{E}-04$ \\
\hline PEST & alpha-Chlordane & $\mathrm{mg} / \mathrm{kg}$ & 12 & 5 & $42 \%$ & 0.0016 & 0.0020 & 0.0012 & 0.0018 & 0.0011 \\
\hline PEST & alpha-Chlordene & $\mathrm{mg} / \mathrm{kg}$ & 12 & 0 & $0 \%$ & 0.0016 & 0.0020 & -- & -- & $9.63 \mathrm{E}-04$ \\
\hline PEST & Beta-BHC & $\mathrm{mg} / \mathrm{kg}$ & 12 & 0 & $0 \%$ & 0.0016 & 0.0020 & -- & -- & 9.63E-04 \\
\hline PEST & cis-Nonachlor & $\mathrm{mg} / \mathrm{kg}$ & 12 & 2 & $17 \%$ & 0.0019 & 0.0020 & 0.0015 & 0.0017 & 0.0011 \\
\hline PEST & DDMU & $\mathrm{mg} / \mathrm{kg}$ & 12 & 2 & $17 \%$ & 0.0019 & 0.0020 & 0.0013 & 0.0019 & 0.0011 \\
\hline PEST & Delta-BHC & $\mathrm{mg} / \mathrm{kg}$ & 12 & 0 & $0 \%$ & 0.0016 & 0.0020 & -- & -- & $9.63 \mathrm{E}-04$ \\
\hline PEST & Dichlorodiphenyldichloroethane & $\mathrm{mg} / \mathrm{kg}$ & 12 & 12 & $100 \%$ & -- & -- & 0.0017 & 0.0090 & 0.0035 \\
\hline PEST & Dichlorodiphenyldichloroethylene & $\mathrm{mg} / \mathrm{kg}$ & 12 & 12 & $100 \%$ & -- & -- & 0.0051 & 0.011 & 0.0071 \\
\hline PEST & Dichlorodiphenyltrichloroethane & $\mathrm{mg} / \mathrm{kg}$ & 12 & 9 & $75 \%$ & 0.0020 & 0.0020 & 0.0012 & 0.0045 & 0.0020 \\
\hline PEST & Endosulfan sulfate & $\mathrm{mg} / \mathrm{kg}$ & 12 & 0 & $0 \%$ & 0.0016 & 0.0020 & -- & -- & $9.63 \mathrm{E}-04$ \\
\hline PEST & Gamma-BHC (Lindane) & $\mathrm{mg} / \mathrm{kg}$ & 12 & 0 & $0 \%$ & 0.0016 & 0.0020 & -- & -- & $9.63 \mathrm{E}-04$ \\
\hline PEST & gamma-Chlordane & $\mathrm{mg} / \mathrm{kg}$ & 12 & 0 & $0 \%$ & 0.0016 & 0.0020 & -- & -- & $9.63 \mathrm{E}-04$ \\
\hline PEST & gamma-Chlordene & $\mathrm{mg} / \mathrm{kg}$ & 12 & 0 & $0 \%$ & 0.0016 & 0.0020 & -- & -- & $9.63 \mathrm{E}-04$ \\
\hline PEST & Heptachlor & $\mathrm{mg} / \mathrm{kg}$ & 12 & 0 & $0 \%$ & 0.0016 & 0.0020 & -- & -- & $9.63 \mathrm{E}-04$ \\
\hline PEST & Heptachlor epoxide & $\mathrm{mg} / \mathrm{kg}$ & 12 & 0 & $0 \%$ & 0.0016 & 0.0020 & -- & -- & $9.63 \mathrm{E}-04$ \\
\hline PEST & Mirex & $\mathrm{mg} / \mathrm{kg}$ & 12 & 0 & $0 \%$ & 0.0016 & 0.0020 & -- & -- & $9.63 \mathrm{E}-04$ \\
\hline PEST & Oxychlordane & $\mathrm{mg} / \mathrm{kg}$ & 12 & 0 & $0 \%$ & 0.0016 & 0.0020 & -- & -- & $9.63 \mathrm{E}-04$ \\
\hline PEST & Pentachloroanisole & $\mathrm{mg} / \mathrm{kg}$ & 12 & 0 & $0 \%$ & 0.0016 & 0.0020 & -- & -- & $9.63 \mathrm{E}-04$ \\
\hline PEST & Toxaphene & $\mathrm{mg} / \mathrm{kg}$ & 12 & 0 & $0 \%$ & 0.048 & 0.060 & -- & -- & 0.029 \\
\hline PEST & trans-Nonachlor & $\mathrm{mg} / \mathrm{kg}$ & 12 & 7 & $58 \%$ & 0.0019 & 0.0020 & 0.0012 & 0.0024 & 0.0013 \\
\hline SVOC & 1,2,4-Trichlorobenzene & $\mathrm{mg} / \mathrm{kg}$ & 12 & 0 & $0 \%$ & 0.0084 & 0.0099 & -- & -- & 0.0048 \\
\hline SVOC & 1,2-Dichlorobenzene & $\mathrm{mg} / \mathrm{kg}$ & 12 & 0 & $0 \%$ & 0.0084 & 0.0099 & -- & -- & 0.0048 \\
\hline SVOC & 1,2-Diphenylhydrazine & $\mathrm{mg} / \mathrm{kg}$ & 11 & 0 & $0 \%$ & 0.0092 & 0.10 & -- & -- & 0.0089 \\
\hline SVOC & 1,3-Dichlorobenzene & $\mathrm{mg} / \mathrm{kg}$ & 12 & 0 & $0 \%$ & 0.0084 & 0.0099 & -- & -- & 0.0048 \\
\hline SVOC & 1,4-Dichlorobenzene & $\mathrm{mg} / \mathrm{kg}$ & 12 & 0 & $0 \%$ & 0.0084 & 0.0099 & -- & -- & 0.0048 \\
\hline SVOC & 1-methylnaphthalene & $\mathrm{mg} / \mathrm{kg}$ & 12 & 0 & $0 \%$ & 0.0084 & 0.0099 & -- & -- & 0.0048 \\
\hline SVOC & 2,4-Dinitrotoluene & $\mathrm{mg} / \mathrm{kg}$ & 10 & 0 & $0 \%$ & 0.018 & 0.020 & -- & -- & 0.0096 \\
\hline SVOC & 2,6-Dinitrotoluene & $\mathrm{mg} / \mathrm{kg}$ & 12 & 0 & $0 \%$ & 0.034 & 0.040 & -- & -- & 0.019 \\
\hline SVOC & 2-Chloronaphthalene & $\mathrm{mg} / \mathrm{kg}$ & 12 & 0 & $0 \%$ & 0.017 & 0.020 & -- & -- & 0.0095 \\
\hline SVOC & 2-Methylnaphthalene & $\mathrm{mg} / \mathrm{kg}$ & 12 & 0 & $0 \%$ & 0.0092 & 0.011 & -- & -- & 0.0049 \\
\hline SVOC & 4-Bromophenylphenyl ether & $\mathrm{mg} / \mathrm{kg}$ & 12 & 0 & $0 \%$ & 0.0084 & 0.0099 & -- & -- & 0.0048 \\
\hline SVOC & 4-Chlorophenylphenyl ether & $\mathrm{mg} / \mathrm{kg}$ & 12 & 0 & $0 \%$ & 0.0084 & 0.0099 & -- & -- & 0.0048 \\
\hline SVOC & Acenaphthene & $\mathrm{mg} / \mathrm{kg}$ & 12 & 0 & $0 \%$ & 0.017 & 0.020 & -- & -- & 0.0095 \\
\hline SVOC & Acenaphthylene & $\mathrm{mg} / \mathrm{kg}$ & 12 & 0 & $0 \%$ & 0.0084 & 0.0099 & -- & -- & 0.0048 \\
\hline SVOC & Anthracene & $\mathrm{mg} / \mathrm{kg}$ & 12 & 0 & $0 \%$ & 0.0084 & 0.0099 & -- & -- & 0.0048 \\
\hline SVOC & Benzo(a)anthracene & $\mathrm{mg} / \mathrm{kg}$ & 12 & 0 & $0 \%$ & 0.0084 & 0.0099 & -- & -- & 0.0048 \\
\hline SVOC & Benzo(a)pyrene & $\mathrm{mg} / \mathrm{kg}$ & 11 & 0 & $0 \%$ & 0.0092 & 0.0099 & -- & -- & 0.0048 \\
\hline SVOC & Benzo(b)fluoranthene & $\mathrm{mg} / \mathrm{kg}$ & 12 & 0 & $0 \%$ & 0.0084 & 0.0099 & -- & -- & 0.0048 \\
\hline SVOC & Benzo(ghi)perylene & $\mathrm{mg} / \mathrm{kg}$ & 12 & 0 & $0 \%$ & 0.017 & 0.020 & -- & -- & 0.0095 \\
\hline SVOC & Benzo(k)fluoranthene & $\mathrm{mg} / \mathrm{kg}$ & 12 & 0 & $0 \%$ & 0.0084 & 0.0099 & -- & -- & 0.0048 \\
\hline SVOC & Bis(2-chloro-1-methylethyl)ether & $\mathrm{mg} / \mathrm{kg}$ & 12 & 0 & $0 \%$ & 0.017 & 0.020 & -- & -- & 0.0095 \\
\hline
\end{tabular}


Table F-92

Summary Statistics for Steelhead Fillet Skin On Collected from Segment 4 of the Columbia River

\begin{tabular}{|c|c|c|c|c|c|c|c|c|c|c|}
\hline $\begin{array}{l}\text { Constituent } \\
\text { Class }\end{array}$ & Constituent Name & Units & $\begin{array}{c}\text { Number } \\
\text { Analyzed }\end{array}$ & $\begin{array}{l}\text { Number } \\
\text { Detected }\end{array}$ & $\begin{array}{l}\text { Frequency of } \\
\text { Detection }\end{array}$ & $\begin{array}{c}\text { Minimum } \\
\text { Nondetect }\end{array}$ & $\begin{array}{c}\text { Maximum } \\
\text { Nondetect }\end{array}$ & $\begin{array}{l}\text { Minimum } \\
\text { Detect }\end{array}$ & $\begin{array}{c}\text { Maximum } \\
\text { Detect }\end{array}$ & $\begin{array}{c}\text { Average } \\
\text { Concentration }\end{array}$ \\
\hline SVOC & Chrysene & $\mathrm{mg} / \mathrm{kg}$ & 12 & 0 & $0 \%$ & 0.0084 & 0.0099 & -- & $\overline{--}$ & 0.0048 \\
\hline SVOC & Dibenz[a,h]anthracene & $\mathrm{mg} / \mathrm{kg}$ & 12 & 0 & $0 \%$ & 0.017 & 0.020 & -- & -- & 0.0095 \\
\hline SVOC & Dibenzofuran & $\mathrm{mg} / \mathrm{kg}$ & 12 & 0 & $0 \%$ & 0.0084 & 0.0099 & -- & -- & 0.0048 \\
\hline SVOC & Fluoranthene & $\mathrm{mg} / \mathrm{kg}$ & 12 & 0 & $0 \%$ & 0.017 & 0.020 & -- & -- & 0.0095 \\
\hline SVOC & Fluorene & $\mathrm{mg} / \mathrm{kg}$ & 12 & 0 & $0 \%$ & 0.0078 & 0.027 & -- & -- & 0.0084 \\
\hline SVOC & Hexachlorobenzene & $\mathrm{mg} / \mathrm{kg}$ & 12 & 0 & $0 \%$ & 0.0092 & 0.020 & -- & -- & 0.0091 \\
\hline SVOC & Hexachlorobutadiene & $\mathrm{mg} / \mathrm{kg}$ & 12 & 0 & $0 \%$ & 0.0084 & 0.0099 & -- & -- & 0.0048 \\
\hline SVOC & Hexachloroethane & $\mathrm{mg} / \mathrm{kg}$ & 12 & 0 & $0 \%$ & 0.0084 & 0.0099 & -- & -- & 0.0048 \\
\hline SVOC & Indeno(1,2,3-cd)pyrene & $\mathrm{mg} / \mathrm{kg}$ & 12 & 0 & $0 \%$ & 0.017 & 0.020 & -- & -- & 0.0095 \\
\hline SVOC & Naphthalene & $\mathrm{mg} / \mathrm{kg}$ & 12 & 0 & $0 \%$ & 0.0095 & 0.030 & -- & -- & 0.0076 \\
\hline SVOC & Nitrobenzene & $\mathrm{mg} / \mathrm{kg}$ & 12 & 0 & $0 \%$ & 0.0084 & 0.0099 & -- & -- & 0.0048 \\
\hline SVOC & Phenanthrene & $\mathrm{mg} / \mathrm{kg}$ & 12 & 0 & $0 \%$ & 0.0084 & 0.0099 & -- & -- & 0.0048 \\
\hline SVOC & Pyrene & $\mathrm{mg} / \mathrm{kg}$ & 12 & 0 & $0 \%$ & 0.017 & 0.020 & -- & -- & 0.0095 \\
\hline VOC & Retene & $\mathrm{mg} / \mathrm{kg}$ & 12 & 0 & $0 \%$ & 0.017 & 0.020 & -- & -- & 0.0095 \\
\hline
\end{tabular}

DIOXI - Dioxins and Furans

PEST - Pesticide

PCB - Polychlorinated biphenyl

SVOC - Semivolatile Organic Compound

VOC - Volatile Organic Compound 
Table F-93

Summary Statistics for Whole Body Steelhead Collected from Segment 4 of the Columbia River

\begin{tabular}{|c|c|c|c|c|c|c|c|c|c|c|}
\hline $\begin{array}{l}\text { Constituent } \\
\text { Class }\end{array}$ & Constituent Name & Units & $\begin{array}{l}\text { Number } \\
\text { Analyzed }\end{array}$ & $\begin{array}{l}\text { Number } \\
\text { Detected }\end{array}$ & $\begin{array}{l}\text { Frequency of } \\
\text { Detection }\end{array}$ & $\begin{array}{l}\text { Minimum } \\
\text { Nondetect }\end{array}$ & $\begin{array}{l}\text { Maximum } \\
\text { Nondetect }\end{array}$ & $\begin{array}{c}\text { Minimum } \\
\text { Detect }\end{array}$ & $\begin{array}{l}\text { Maximum } \\
\text { Detect }\end{array}$ & $\begin{array}{c}\text { Average } \\
\text { Concentration }\end{array}$ \\
\hline$\overline{D I O X I}$ & $1,2,3,4,6,7,8$-Heptachlorodibenzodioxin & $\mathrm{mg} / \mathrm{kg}$ & 12 & 5 & $42 \%$ & $2.00 \mathrm{E}-08$ & $9.00 \mathrm{E}-08$ & $6.00 \mathrm{E}-08$ & $2.00 \mathrm{E}-07$ & $5.88 \mathrm{E}-08$ \\
\hline DIOXI & 1,2,3,4,6,7,8-Heptachlorodibenzofuran & $\mathrm{mg} / \mathrm{kg}$ & 12 & 0 & $0 \%$ & $1.00 \mathrm{E}-08$ & 7.00E-08 & -- & -- & $1.71 \mathrm{E}-08$ \\
\hline DIOXI & $1,2,3,4,7,8,9$-Heptachlorodibenzofuran & $\mathrm{mg} / \mathrm{kg}$ & 12 & 0 & $0 \%$ & $1.00 \mathrm{E}-08$ & 7.00E-08 & -- & -- & $1.71 \mathrm{E}-08$ \\
\hline DIOXI & 1,2,3,4,7,8-Hexachlorodibenzofuran & $\mathrm{mg} / \mathrm{kg}$ & 12 & 2 & $17 \%$ & $1.00 \mathrm{E}-08$ & 4.00E-08 & 4.00E-08 & 4.00E-08 & $1.54 \mathrm{E}-08$ \\
\hline DIOXI & 1,2,3,4,7,8-Hexachlorodibenzo-p-dioxin & $\mathrm{mg} / \mathrm{kg}$ & 12 & 1 & $8 \%$ & $1.00 \mathrm{E}-08$ & 4.00E-08 & 3.00E-08 & 3.00E-08 & 1.13E-08 \\
\hline DIOXI & 1,2,3,6,7,8-Hexachlorodibenzofuran & $\mathrm{mg} / \mathrm{kg}$ & 12 & 3 & $25 \%$ & $1.00 \mathrm{E}-08$ & 4.00E-08 & 4.00E-08 & 7.00E-08 & 2.17E-08 \\
\hline DIOXI & 1,2,3,6,7,8-Hexachlorodibenzo-p-dioxin & $\mathrm{mg} / \mathrm{kg}$ & 12 & 4 & $33 \%$ & 1.00E-08 & 4.00E-08 & 3.00E-08 & 7.00E-08 & $2.58 \mathrm{E}-08$ \\
\hline DIOXI & 1,2,3,7,8,9-Hexachlorodibenzofuran & $\mathrm{mg} / \mathrm{kg}$ & 12 & 0 & $0 \%$ & $1.00 \mathrm{E}-08$ & 4.00E-08 & -- & -- & $1.08 \mathrm{E}-08$ \\
\hline DIOXI & 1,2,3,7,8,9-Hexachlorodibenzo-p-dioxin & $\mathrm{mg} / \mathrm{kg}$ & 12 & 3 & $25 \%$ & $1.00 \mathrm{E}-08$ & 4.00E-08 & 3.00E-08 & 4.00E-08 & 1.71E-08 \\
\hline DIOXI & 1,2,3,7,8-Pentachlorodibenzofuran & $\mathrm{mg} / \mathrm{kg}$ & 12 & 4 & $33 \%$ & 1.00E-08 & 5.00E-08 & $6.00 \mathrm{E}-08$ & 1.90E-07 & 5.04E-08 \\
\hline DIOXI & 1,2,3,7,8-Pentachlorodibenzo-p-dioxin & $\mathrm{mg} / \mathrm{kg}$ & 12 & 3 & $25 \%$ & $1.00 \mathrm{E}-08$ & $5.00 \mathrm{E}-08$ & $9.00 \mathrm{E}-08$ & 1.90E-07 & 4.33E-08 \\
\hline DIOXI & 2,3,4,6,7,8-Hexachlorodibenzofuran & $\mathrm{mg} / \mathrm{kg}$ & 12 & 3 & $25 \%$ & $1.00 \mathrm{E}-08$ & 4.00E-08 & 3.00E-08 & 8.00E-08 & 2.33E-08 \\
\hline DIOXI & 2,3,4,7,8-Pentachlorodibenzofuran & $\mathrm{mg} / \mathrm{kg}$ & 12 & 8 & $67 \%$ & 1.00E-08 & 5.00E-08 & 1.30E-07 & 3.70E-07 & 1.65E-07 \\
\hline DIOXI & 2,3,7,8-Tetrachlorodibenzofuran & $\mathrm{mg} / \mathrm{kg}$ & 12 & 12 & $100 \%$ & -- & -- & $2.50 \mathrm{E}-07$ & $6.00 \mathrm{E}-07$ & 3.86E-07 \\
\hline DIOXI & 2,3,7,8-Tetrachlorodibenzo-p-dioxin & $\mathrm{mg} / \mathrm{kg}$ & 12 & 3 & $25 \%$ & $1.00 \mathrm{E}-08$ & $5.00 \mathrm{E}-08$ & $2.00 \mathrm{E}-08$ & $6.00 \mathrm{E}-08$ & 1.83E-08 \\
\hline DIOXI & Octachlorodibenzofuran & $\mathrm{mg} / \mathrm{kg}$ & 12 & 1 & $8 \%$ & 3.00E-08 & $2.50 \mathrm{E}-07$ & 4.30E-07 & 4.30E-07 & 7.75E-08 \\
\hline DIOXI & Octachlorodibenzo-p-dioxin & $\mathrm{mg} / \mathrm{kg}$ & 12 & 6 & $50 \%$ & 2.00E-08 & 7.00E-08 & 8.00E-08 & 2.56E-06 & 5.93E-07 \\
\hline METAL & Aluminum & $\mathrm{mg} / \mathrm{kg}$ & 12 & 2 & $17 \%$ & 1.0 & 1.0 & 0.67 & 0.77 & 0.54 \\
\hline METAL & Antimony & $\mathrm{mg} / \mathrm{kg}$ & 12 & 0 & $0 \%$ & 0.050 & 0.050 & -- & -- & 0.025 \\
\hline METAL & Arsenic & $\mathrm{mg} / \mathrm{kg}$ & 12 & 12 & $100 \%$ & -- & -- & 0.43 & 1.2 & 0.69 \\
\hline METAL & Barium & $\mathrm{mg} / \mathrm{kg}$ & 12 & 1 & $8 \%$ & 0.20 & 0.20 & 0.22 & 0.22 & 0.11 \\
\hline METAL & Beryllium & $\mathrm{mg} / \mathrm{kg}$ & 12 & 0 & $0 \%$ & 0.0040 & 0.0040 & -- & -- & 0.0020 \\
\hline METAL & Cadmium & $\mathrm{mg} / \mathrm{kg}$ & 12 & 12 & $100 \%$ & -- & -- & 0.020 & 0.070 & 0.048 \\
\hline METAL & Chromium & $\mathrm{mg} / \mathrm{kg}$ & 12 & 8 & $67 \%$ & 0.10 & 0.10 & 0.11 & 0.24 & 0.12 \\
\hline METAL & Cobalt & $\mathrm{mg} / \mathrm{kg}$ & 12 & 12 & $100 \%$ & -- & -- & 0.050 & 0.19 & 0.12 \\
\hline METAL & Copper & $\mathrm{mg} / \mathrm{kg}$ & 12 & 12 & $100 \%$ & -- & -- & 2.0 & 6.8 & 3.5 \\
\hline METAL & Lead & $\mathrm{mg} / \mathrm{kg}$ & 12 & 6 & $50 \%$ & 0.010 & 0.010 & 0.010 & 0.35 & 0.048 \\
\hline METAL & Manganese & $\mathrm{mg} / \mathrm{kg}$ & 12 & 12 & $100 \%$ & -- & -- & 0.24 & 0.64 & 0.41 \\
\hline METAL & Mercury & $\mathrm{mg} / \mathrm{kg}$ & 12 & 10 & $83 \%$ & 0.050 & 0.10 & 0.050 & 0.12 & 0.080 \\
\hline METAL & Nickel & $\mathrm{mg} / \mathrm{kg}$ & 12 & 11 & $92 \%$ & 0.030 & 0.030 & 0.050 & 17 & 1.5 \\
\hline METAL & Selenium & $\mathrm{mg} / \mathrm{kg}$ & 12 & 12 & $100 \%$ & -- & -- & 0.48 & 0.94 & 0.73 \\
\hline METAL & Silver & $\mathrm{mg} / \mathrm{kg}$ & 12 & 0 & $0 \%$ & 0.10 & 0.10 & -- & -- & 0.050 \\
\hline METAL & Thallium & $\mathrm{mg} / \mathrm{kg}$ & 12 & 0 & $0 \%$ & 0.050 & 0.050 & -- & -- & 0.025 \\
\hline METAL & Vanadium & $\mathrm{mg} / \mathrm{kg}$ & 12 & 12 & $100 \%$ & -- & -- & 0.010 & 0.14 & 0.058 \\
\hline METAL & Zinc & $\mathrm{mg} / \mathrm{kg}$ & 12 & 12 & $100 \%$ & -- & -- & 18 & 28 & 22 \\
\hline PCB & 2,2',3,3',4,4',5-Heptachloro-1,1'-biphenyl & $\mathrm{mg} / \mathrm{kg}$ & 12 & 12 & $100 \%$ & -- & -- & 7.61E-05 & 1.42E-04 & 1.07E-04 \\
\hline PCB & 2,2',3,4,4',5,5'-Heptachlorobiphenyl & $\mathrm{mg} / \mathrm{kg}$ & 12 & 12 & $100 \%$ & -- & -- & 2.36E-04 & 4.05E-04 & 3.23E-04 \\
\hline PCB & 2,3,3',4,4',5'-Hexachlorobiphenyl & $\mathrm{mg} / \mathrm{kg}$ & 12 & 12 & $100 \%$ & -- & -- & 1.03E-05 & 2.47E-05 & $1.65 \mathrm{E}-05$ \\
\hline PCB & 2,3,3',4,4',5-Hexachlorobiphenyl (BZ 156) & $\mathrm{mg} / \mathrm{kg}$ & 12 & 12 & $100 \%$ & -- & -- & 3.32E-05 & 8.09E-05 & 5.70E-05 \\
\hline PCB & 2,3,3',4,4'-Pentachlorobiphenyl & $\mathrm{mg} / \mathrm{kg}$ & 12 & 12 & $100 \%$ & -- & -- & 1.67E-04 & 3.30E-04 & $2.52 E-04$ \\
\hline PCB & $\begin{array}{l}\text { 2,3',4,4',5,5'-Hexachlorobiphenyl (BZ 167) } \\
2^{\prime}, 3,4,4^{\prime}, 5 \text {-Pentachloro-1,1'-biphenyl (BZ }\end{array}$ & $\mathrm{mg} / \mathrm{kg}$ & 12 & 12 & $100 \%$ & -- & -- & $2.23 \mathrm{E}-05$ & 4.77E-05 & 3.59E-05 \\
\hline PCB & 123) & $\mathrm{mg} / \mathrm{kg}$ & 12 & 12 & $100 \%$ & -- & -- & 7.07E-06 & 1.61E-05 & 1.14E-05 \\
\hline PCB & 2,3,4,4',5-Pentachlorobiphenyl (BZ 114) & $\mathrm{mg} / \mathrm{kg}$ & 12 & 12 & $100 \%$ & -- & -- & 1.82E-05 & 3.27E-05 & 2.55E-05 \\
\hline PCB & 2,3',4,4',5-Pentachlorobiphenyl (BZ 118) & $\mathrm{mg} / \mathrm{kg}$ & 12 & 12 & $100 \%$ & -- & -- & 4.09E-04 & 7.88E-04 & 6.14E-04 \\
\hline
\end{tabular}


Table F-93

Summary Statistics for Whole Body Steelhead Collected from Segment 4 of the Columbia River

\begin{tabular}{|c|c|c|c|c|c|c|c|c|c|c|}
\hline $\begin{array}{l}\text { Constituent } \\
\text { Class }\end{array}$ & Constituent Name & Units & $\begin{array}{l}\text { Number } \\
\text { Analyzed }\end{array}$ & $\begin{array}{l}\text { Number } \\
\text { Detected }\end{array}$ & $\begin{array}{l}\text { Frequency of } \\
\text { Detection }\end{array}$ & $\begin{array}{l}\text { Minimum } \\
\text { Nondetect }\end{array}$ & $\begin{array}{l}\text { Maximum } \\
\text { Nondetect }\end{array}$ & $\begin{array}{c}\text { Minimum } \\
\text { Detect }\end{array}$ & $\begin{array}{l}\text { Maximum } \\
\text { Detect }\end{array}$ & $\begin{array}{c}\text { Average } \\
\text { Concentration }\end{array}$ \\
\hline$\overline{\mathrm{PCB}}$ & $2,3,4,5,3^{\prime}, 4^{\prime}, 5^{\prime}-$ Heptachlorobiphenyl & $\mathrm{mg} / \mathrm{kg}$ & 12 & 10 & $83 \%$ & $1.00 \mathrm{E}-06$ & $1.00 \mathrm{E}-06$ & $2.47 \mathrm{E}-06$ & $8.15 \mathrm{E}-06$ & $3.73 \mathrm{E}-06$ \\
\hline PCB & 3,3',4,4',5,5'-Hexachlorobiphenyl (BZ 169) & $\mathrm{mg} / \mathrm{kg}$ & 12 & 0 & $0 \%$ & 1.00E-06 & 3.24E-06 & -- & -- & 7.64E-07 \\
\hline PCB & 3,3',4,4',5-Pentachlorobiphenyl (BZ 126) & $\mathrm{mg} / \mathrm{kg}$ & 12 & 9 & $75 \%$ & $6.70 \mathrm{E}-07$ & 9.50E-07 & $2.54 \mathrm{E}-06$ & 9.03E-06 & 4.07E-06 \\
\hline PCB & 3,3',4,4'-Tetrachlorobiphenyl (BZ 77) & $\mathrm{mg} / \mathrm{kg}$ & 12 & 12 & $100 \%$ & -- & -- & $9.55 \mathrm{E}-06$ & 2.22E-05 & $1.50 \mathrm{E}-05$ \\
\hline PCB & Aroclor-1016 & $\mathrm{mg} / \mathrm{kg}$ & 12 & 0 & $0 \%$ & 0.018 & 0.020 & -- & -- & 0.0095 \\
\hline PCB & Aroclor-1221 & $\mathrm{mg} / \mathrm{kg}$ & 12 & 0 & $0 \%$ & 0.018 & 0.020 & -- & -- & 0.0095 \\
\hline PCB & Aroclor-1232 & $\mathrm{mg} / \mathrm{kg}$ & 12 & 0 & $0 \%$ & 0.018 & 0.020 & -- & -- & 0.0095 \\
\hline PCB & Aroclor-1248 & $\mathrm{mg} / \mathrm{kg}$ & 12 & 0 & $0 \%$ & 0.018 & 0.020 & -- & -- & 0.0095 \\
\hline PCB & Aroclor-1254 & $\mathrm{mg} / \mathrm{kg}$ & 12 & 12 & $100 \%$ & -- & -- & 0.0089 & 0.024 & 0.016 \\
\hline PCB & Aroclor-1260 & $\mathrm{mg} / \mathrm{kg}$ & 12 & 3 & $25 \%$ & 0.019 & 0.020 & 0.0064 & 0.0096 & 0.0093 \\
\hline PEST & 2,4'-DDD & $\mathrm{mg} / \mathrm{kg}$ & 12 & 0 & $0 \%$ & 0.0018 & 0.0020 & -- & -- & $9.54 \mathrm{E}-04$ \\
\hline PEST & 2,4'-DDE & $\mathrm{mg} / \mathrm{kg}$ & 12 & 3 & $25 \%$ & 0.0018 & 0.0020 & 0.0012 & 0.0015 & 0.0011 \\
\hline PEST & $2,4^{\prime}-\mathrm{DDT}$ & $\mathrm{mg} / \mathrm{kg}$ & 12 & 0 & $0 \%$ & 0.0018 & 0.0020 & -- & -- & $9.54 \mathrm{E}-04$ \\
\hline PEST & Aldrin & $\mathrm{mg} / \mathrm{kg}$ & 12 & 0 & $0 \%$ & 0.0018 & 0.0020 & -- & -- & $9.54 \mathrm{E}-04$ \\
\hline PEST & Alpha-BHC & $\mathrm{mg} / \mathrm{kg}$ & 12 & 0 & $0 \%$ & 0.0018 & 0.0020 & -- & -- & 9.54E-04 \\
\hline PEST & alpha-Chlordane & $\mathrm{mg} / \mathrm{kg}$ & 12 & 3 & $25 \%$ & 0.0018 & 0.0020 & 0.0016 & 0.0023 & 0.0012 \\
\hline PEST & alpha-Chlordene & $\mathrm{mg} / \mathrm{kg}$ & 12 & 0 & $0 \%$ & 0.0018 & 0.0020 & -- & -- & 9.54E-04 \\
\hline PEST & Beta-BHC & $\mathrm{mg} / \mathrm{kg}$ & 12 & 0 & $0 \%$ & 0.0018 & 0.0020 & -- & -- & $9.54 \mathrm{E}-04$ \\
\hline PEST & cis-Nonachlor & $\mathrm{mg} / \mathrm{kg}$ & 12 & 4 & $33 \%$ & 0.0018 & 0.0020 & 0.0014 & 0.0021 & 0.0012 \\
\hline PEST & DDMU & $\mathrm{mg} / \mathrm{kg}$ & 12 & 4 & $33 \%$ & 0.0018 & 0.0020 & 0.0014 & 0.0018 & 0.0012 \\
\hline PEST & Delta-BHC & $\mathrm{mg} / \mathrm{kg}$ & 12 & 0 & $0 \%$ & 0.0018 & 0.0020 & -- & -- & $9.54 \mathrm{E}-04$ \\
\hline PEST & Dichlorodiphenyldichloroethane & $\mathrm{mg} / \mathrm{kg}$ & 12 & 12 & $100 \%$ & -- & -- & 0.0021 & 0.011 & 0.0045 \\
\hline PEST & Dichlorodiphenyldichloroethylene & $\mathrm{mg} / \mathrm{kg}$ & 12 & 12 & $100 \%$ & -- & -- & 0.0053 & 0.015 & 0.0087 \\
\hline PEST & Dichlorodiphenyltrichloroethane & $\mathrm{mg} / \mathrm{kg}$ & 12 & 8 & $67 \%$ & 0.0019 & 0.0020 & 0.0014 & 0.0060 & 0.0024 \\
\hline PEST & Endosulfan sulfate & $\mathrm{mg} / \mathrm{kg}$ & 9 & 0 & $0 \%$ & 0.0018 & 0.0020 & -- & -- & $9.44 \mathrm{E}-04$ \\
\hline PEST & Gamma-BHC (Lindane) & $\mathrm{mg} / \mathrm{kg}$ & 12 & 0 & $0 \%$ & 0.0018 & 0.0020 & -- & -- & $9.54 \mathrm{E}-04$ \\
\hline PEST & gamma-Chlordane & $\mathrm{mg} / \mathrm{kg}$ & 12 & 0 & $0 \%$ & 0.0018 & 0.0020 & -- & -- & $9.54 \mathrm{E}-04$ \\
\hline PEST & gamma-Chlordene & $\mathrm{mg} / \mathrm{kg}$ & 12 & 0 & $0 \%$ & 0.0018 & 0.0020 & -- & -- & 9.54E-04 \\
\hline PEST & Heptachlor & $\mathrm{mg} / \mathrm{kg}$ & 12 & 0 & $0 \%$ & 0.0018 & 0.0020 & -- & -- & 9.54E-04 \\
\hline PEST & Heptachlor epoxide & $\mathrm{mg} / \mathrm{kg}$ & 9 & 0 & $0 \%$ & 0.0018 & 0.0020 & -- & -- & $9.44 \mathrm{E}-04$ \\
\hline PEST & Mirex & $\mathrm{mg} / \mathrm{kg}$ & 12 & 0 & $0 \%$ & 0.0018 & 0.0020 & -- & -- & $9.54 \mathrm{E}-04$ \\
\hline PEST & Oxychlordane & $\mathrm{mg} / \mathrm{kg}$ & 12 & 0 & $0 \%$ & 0.0018 & 0.0020 & -- & -- & $9.54 \mathrm{E}-04$ \\
\hline PEST & Pentachloroanisole & $\mathrm{mg} / \mathrm{kg}$ & 12 & 2 & $17 \%$ & 0.0018 & 0.0020 & $9.10 \mathrm{E}-04$ & 0.0017 & 0.0010 \\
\hline PEST & Toxaphene & $\mathrm{mg} / \mathrm{kg}$ & 12 & 0 & $0 \%$ & 0.053 & 0.060 & -- & -- & 0.029 \\
\hline PEST & trans-Nonachlor & $\mathrm{mg} / \mathrm{kg}$ & 12 & 9 & $75 \%$ & 0.0018 & 0.0020 & 0.0014 & 0.0028 & 0.0018 \\
\hline SVOC & 1,2,4-Trichlorobenzene & $\mathrm{mg} / \mathrm{kg}$ & 12 & 0 & $0 \%$ & 0.0072 & 0.010 & -- & -- & 0.0047 \\
\hline SVOC & 1,2-Dichlorobenzene & $\mathrm{mg} / \mathrm{kg}$ & 12 & 0 & $0 \%$ & 0.0072 & 0.010 & -- & -- & 0.0047 \\
\hline SVOC & 1,2-Diphenylhydrazine & $\mathrm{mg} / \mathrm{kg}$ & 11 & 0 & $0 \%$ & 0.0072 & 0.010 & -- & -- & 0.0047 \\
\hline SVOC & 1,3-Dichlorobenzene & $\mathrm{mg} / \mathrm{kg}$ & 12 & 0 & $0 \%$ & 0.0072 & 0.010 & -- & -- & 0.0047 \\
\hline SVOC & 1,4-Dichlorobenzene & $\mathrm{mg} / \mathrm{kg}$ & 12 & 0 & $0 \%$ & 0.0072 & 0.010 & -- & -- & 0.0047 \\
\hline SVOC & 1-methylnaphthalene & $\mathrm{mg} / \mathrm{kg}$ & 12 & 0 & $0 \%$ & 0.0072 & 0.010 & -- & -- & 0.0047 \\
\hline SVOC & 2,4-Dinitrotoluene & $\mathrm{mg} / \mathrm{kg}$ & 11 & 0 & $0 \%$ & 0.014 & 0.020 & -- & -- & 0.0094 \\
\hline SVOC & 2,6-Dinitrotoluene & $\mathrm{mg} / \mathrm{kg}$ & 12 & 0 & $0 \%$ & 0.029 & 0.040 & -- & -- & 0.019 \\
\hline SVOC & 2-Chloronaphthalene & $\mathrm{mg} / \mathrm{kg}$ & 12 & 0 & $0 \%$ & 0.014 & 0.020 & -- & -- & 0.0094 \\
\hline SVOC & 2-MethyInaphthalene & $\mathrm{mg} / \mathrm{kg}$ & 12 & 0 & $0 \%$ & 0.0072 & 0.010 & -- & -- & 0.0047 \\
\hline SVOC & 4-Bromophenylphenyl ether & $\mathrm{mg} / \mathrm{kg}$ & 12 & 0 & $0 \%$ & 0.0072 & 0.010 & -- & -- & 0.0047 \\
\hline
\end{tabular}




\section{Table F-93}

Summary Statistics for Whole Body Steelhead Collected from Segment 4 of the Columbia River

\begin{tabular}{|c|c|c|c|c|c|c|c|c|c|c|}
\hline $\begin{array}{l}\text { Constituent } \\
\text { Class }\end{array}$ & Constituent Name & Units & $\begin{array}{l}\text { Number } \\
\text { Analyzed }\end{array}$ & $\begin{array}{l}\text { Number } \\
\text { Detected }\end{array}$ & $\begin{array}{l}\text { Frequency of } \\
\text { Detection }\end{array}$ & $\begin{array}{c}\text { Minimum } \\
\text { Nondetect }\end{array}$ & $\begin{array}{l}\text { Maximum } \\
\text { Nondetect }\end{array}$ & $\begin{array}{l}\text { Minimum } \\
\text { Detect }\end{array}$ & $\begin{array}{l}\text { Maximum } \\
\text { Detect }\end{array}$ & $\begin{array}{c}\text { Average } \\
\text { Concentration }\end{array}$ \\
\hline SVOC & 4-Chlorophenylphenyl ether & $\mathrm{mg} / \mathrm{kg}$ & 12 & 0 & $0 \%$ & 0.0072 & 0.010 & -- & -- & 0.0047 \\
\hline SVOC & Acenaphthene & $\mathrm{mg} / \mathrm{kg}$ & 12 & 0 & $0 \%$ & 0.014 & 0.020 & -- & -- & 0.0094 \\
\hline SVOC & Acenaphthylene & $\mathrm{mg} / \mathrm{kg}$ & 12 & 0 & $0 \%$ & 0.0072 & 0.010 & -- & -- & 0.0047 \\
\hline SVOC & Anthracene & $\mathrm{mg} / \mathrm{kg}$ & 12 & 0 & $0 \%$ & 0.0072 & 0.010 & -- & -- & 0.0047 \\
\hline SVOC & Benzo(a)anthracene & $\mathrm{mg} / \mathrm{kg}$ & 12 & 0 & $0 \%$ & 0.0072 & 0.010 & -- & -- & 0.0047 \\
\hline SVOC & Benzo(a)pyrene & $\mathrm{mg} / \mathrm{kg}$ & 12 & 0 & $0 \%$ & 0.0072 & 0.010 & -- & -- & 0.0047 \\
\hline SVOC & Benzo(b)fluoranthene & $\mathrm{mg} / \mathrm{kg}$ & 12 & 0 & $0 \%$ & 0.0072 & 0.010 & -- & -- & 0.0047 \\
\hline SVOC & Benzo(ghi)perylene & $\mathrm{mg} / \mathrm{kg}$ & 12 & 0 & $0 \%$ & 0.014 & 0.020 & -- & -- & 0.0094 \\
\hline SVOC & Benzo(k)fluoranthene & $\mathrm{mg} / \mathrm{kg}$ & 12 & 0 & $0 \%$ & 0.0072 & 0.010 & -- & -- & 0.0047 \\
\hline SVOC & Bis(2-chloro-1-methylethyl)ether & $\mathrm{mg} / \mathrm{kg}$ & 12 & 0 & $0 \%$ & 0.014 & 0.020 & -- & -- & 0.0094 \\
\hline SVOC & Chrysene & $\mathrm{mg} / \mathrm{kg}$ & 12 & 0 & $0 \%$ & 0.0072 & 0.010 & -- & -- & 0.0047 \\
\hline SVOC & Dibenz[a,h]anthracene & $\mathrm{mg} / \mathrm{kg}$ & 12 & 0 & $0 \%$ & 0.014 & 0.020 & -- & -- & 0.0094 \\
\hline SVOC & Dibenzofuran & $\mathrm{mg} / \mathrm{kg}$ & 12 & 0 & $0 \%$ & 0.0072 & 0.010 & -- & -- & 0.0047 \\
\hline SVOC & Fluoranthene & $\mathrm{mg} / \mathrm{kg}$ & 12 & 0 & $0 \%$ & 0.014 & 0.020 & -- & -- & 0.0094 \\
\hline SVOC & Fluorene & $\mathrm{mg} / \mathrm{kg}$ & 12 & 0 & $0 \%$ & 0.0048 & 0.0095 & -- & -- & 0.0040 \\
\hline SVOC & Hexachlorobenzene & $\mathrm{mg} / \mathrm{kg}$ & 12 & 0 & $0 \%$ & 0.014 & 0.020 & -- & -- & 0.0094 \\
\hline SVOC & Hexachlorobutadiene & $\mathrm{mg} / \mathrm{kg}$ & 12 & 0 & $0 \%$ & 0.0072 & 0.010 & -- & -- & 0.0047 \\
\hline SVOC & Hexachloroethane & $\mathrm{mg} / \mathrm{kg}$ & 12 & 0 & $0 \%$ & 0.0072 & 0.010 & -- & -- & 0.0047 \\
\hline SVOC & Indeno(1,2,3-cd)pyrene & $\mathrm{mg} / \mathrm{kg}$ & 12 & 0 & $0 \%$ & 0.014 & 0.020 & -- & -- & 0.0094 \\
\hline SVOC & Naphthalene & $\mathrm{mg} / \mathrm{kg}$ & 12 & 0 & $0 \%$ & 0.0072 & 0.070 & -- & -- & 0.010 \\
\hline SVOC & Nitrobenzene & $\mathrm{mg} / \mathrm{kg}$ & 12 & 0 & $0 \%$ & 0.0072 & 0.010 & -- & -- & 0.0047 \\
\hline SVOC & Phenanthrene & $\mathrm{mg} / \mathrm{kg}$ & 12 & 0 & $0 \%$ & 0.0072 & 0.010 & -- & -- & 0.0047 \\
\hline SVOC & Pyrene & $\mathrm{mg} / \mathrm{kg}$ & 12 & 0 & $0 \%$ & 0.014 & 0.020 & -- & -- & 0.0094 \\
\hline VOC & Retene & $\mathrm{mg} / \mathrm{kg}$ & 12 & 0 & $0 \%$ & 0.014 & 0.020 & -- & -- & 0.0094 \\
\hline
\end{tabular}

DIOXI - Dioxins and Furans

PEST - Pesticide

PCB - Polychlorinated biphenyl

SVOC - Semivolatile Organic Compound

VOC - Volatile Organic Compound 
Table F-94

Summary Statistics for Walleye Fillet Skin On Collected from Segment 4 of the Columbia River

\begin{tabular}{|c|c|c|c|c|c|c|c|c|c|c|}
\hline $\begin{array}{l}\text { Constituent } \\
\text { Class }\end{array}$ & Constituent Name & Units & $\begin{array}{l}\text { Number } \\
\text { Analyzed }\end{array}$ & $\begin{array}{l}\text { Number } \\
\text { Detected }\end{array}$ & $\begin{array}{c}\text { Frequency of } \\
\text { Detection }\end{array}$ & $\begin{array}{l}\text { Minimum } \\
\text { Nondetect }\end{array}$ & $\begin{array}{l}\text { Maximum } \\
\text { Nondetect }\end{array}$ & $\begin{array}{c}\text { Minimum } \\
\text { Detect }\end{array}$ & $\begin{array}{l}\text { Maximum } \\
\text { Detect }\end{array}$ & $\begin{array}{c}\text { Average } \\
\text { Concentration }\end{array}$ \\
\hline$\overline{D I O X I}$ & $1,2,3,4,6,7,8$-Heptachlorodibenzodioxin & $\mathrm{mg} / \mathrm{kg}$ & 3 & 2 & $67 \%$ & $1.00 \mathrm{E}-08$ & $1.00 \mathrm{E}-08$ & $9.00 \mathrm{E}-08$ & $1.00 \mathrm{E}-07$ & $6.50 \mathrm{E}-08$ \\
\hline DIOXI & 1,2,3,4,6,7,8-Heptachlorodibenzofuran & $\mathrm{mg} / \mathrm{kg}$ & 3 & 2 & $67 \%$ & 1.00E-08 & 1.00E-08 & $5.00 \mathrm{E}-08$ & 7.00E-08 & 4.17E-08 \\
\hline DIOXI & 1,2,3,4,7,8,9-Heptachlorodibenzofuran & $\mathrm{mg} / \mathrm{kg}$ & 3 & 0 & $0 \%$ & 1.00E-08 & $1.00 \mathrm{E}-08$ & -- & -- & $5.00 \mathrm{E}-09$ \\
\hline DIOXI & $1,2,3,4,7,8$-Hexachlorodibenzofuran & $\mathrm{mg} / \mathrm{kg}$ & 3 & 0 & $0 \%$ & 1.00E-08 & 1.00E-08 & -- & -- & $5.00 \mathrm{E}-09$ \\
\hline DIOXI & $1,2,3,4,7,8$-Hexachlorodibenzo-p-dioxin & $\mathrm{mg} / \mathrm{kg}$ & 3 & 3 & $100 \%$ & -- & -- & $8.00 \mathrm{E}-08$ & $9.00 \mathrm{E}-08$ & 8.67E-08 \\
\hline DIOXI & 1,2,3,6,7,8-Hexachlorodibenzofuran & $\mathrm{mg} / \mathrm{kg}$ & 3 & 3 & $100 \%$ & -- & -- & $2.00 \mathrm{E}-08$ & $3.00 \mathrm{E}-08$ & 2.33E-08 \\
\hline DIOXI & 1,2,3,6,7,8-Hexachlorodibenzo-p-dioxin & $\mathrm{mg} / \mathrm{kg}$ & 3 & 3 & $100 \%$ & -- & -- & $4.00 \mathrm{E}-08$ & $5.00 \mathrm{E}-08$ & 4.67E-08 \\
\hline DIOXI & 1,2,3,7,8,9-Hexachlorodibenzofuran & $\mathrm{mg} / \mathrm{kg}$ & 3 & 3 & $100 \%$ & -- & -- & $5.00 \mathrm{E}-08$ & $6.00 \mathrm{E}-08$ & 5.33E-08 \\
\hline DIOXI & 1,2,3,7,8,9-Hexachlorodibenzo-p-dioxin & $\mathrm{mg} / \mathrm{kg}$ & 3 & 2 & $67 \%$ & 1.00E-08 & 1.00E-08 & $2.00 \mathrm{E}-08$ & $2.00 \mathrm{E}-08$ & 1.50E-08 \\
\hline DIOXI & 1,2,3,7,8-Pentachlorodibenzofuran & $\mathrm{mg} / \mathrm{kg}$ & 3 & 3 & $100 \%$ & -- & -- & $3.00 \mathrm{E}-08$ & 4.00E-08 & 3.33E-08 \\
\hline DIOXI & 1,2,3,7,8-Pentachlorodibenzo-p-dioxin & $\mathrm{mg} / \mathrm{kg}$ & 3 & 2 & $67 \%$ & $1.00 \mathrm{E}-08$ & $1.00 \mathrm{E}-08$ & 1.10E-07 & $1.20 \mathrm{E}-07$ & 7.83E-08 \\
\hline DIOXI & 2,3,4,6,7,8-Hexachlorodibenzofuran & $\mathrm{mg} / \mathrm{kg}$ & 3 & 3 & $100 \%$ & -- & -- & $1.20 \mathrm{E}-07$ & $1.30 \mathrm{E}-07$ & 1.23E-07 \\
\hline DIOXI & 2,3,4,7,8-Pentachlorodibenzofuran & $\mathrm{mg} / \mathrm{kg}$ & 3 & 3 & $100 \%$ & -- & -- & $2.00 \mathrm{E}-08$ & 4.00E-08 & $3.00 \mathrm{E}-08$ \\
\hline DIOXI & 2,3,7,8-Tetrachlorodibenzofuran & $\mathrm{mg} / \mathrm{kg}$ & 3 & 3 & $100 \%$ & -- & -- & $6.00 \mathrm{E}-07$ & $7.50 \mathrm{E}-07$ & $6.67 \mathrm{E}-07$ \\
\hline DIOXI & 2,3,7,8-Tetrachlorodibenzo-p-dioxin & $\mathrm{mg} / \mathrm{kg}$ & 3 & 3 & $100 \%$ & -- & -- & $7.00 \mathrm{E}-08$ & 8.00E-08 & 7.67E-08 \\
\hline DIOXI & Octachlorodibenzofuran & $\mathrm{mg} / \mathrm{kg}$ & 3 & 0 & $0 \%$ & $1.00 \mathrm{E}-08$ & $1.00 \mathrm{E}-08$ & -- & -- & 5.00E-09 \\
\hline DIOXI & Octachlorodibenzo-p-dioxin & $\mathrm{mg} / \mathrm{kg}$ & 3 & 2 & $67 \%$ & 1.00E-08 & $1.00 \mathrm{E}-08$ & $1.30 \mathrm{E}-07$ & $1.60 \mathrm{E}-07$ & $9.83 \mathrm{E}-08$ \\
\hline METAL & Aluminum & $\mathrm{mg} / \mathrm{kg}$ & 3 & 3 & $100 \%$ & -- & -- & 2.3 & 2.7 & 2.5 \\
\hline METAL & Antimony & $\mathrm{mg} / \mathrm{kg}$ & 3 & 0 & $0 \%$ & 0.050 & 0.050 & -- & -- & 0.025 \\
\hline METAL & Arsenic & $\mathrm{mg} / \mathrm{kg}$ & 3 & 3 & $100 \%$ & -- & -- & 0.29 & 0.40 & 0.36 \\
\hline METAL & Barium & $\mathrm{mg} / \mathrm{kg}$ & 3 & 3 & $100 \%$ & -- & -- & 0.23 & 0.24 & 0.24 \\
\hline METAL & Beryllium & $\mathrm{mg} / \mathrm{kg}$ & 3 & 0 & $0 \%$ & 0.0040 & 0.0040 & -- & -- & 0.0020 \\
\hline METAL & Cadmium & $\mathrm{mg} / \mathrm{kg}$ & 3 & 0 & $0 \%$ & 0.0040 & 0.0040 & -- & -- & 0.0020 \\
\hline METAL & Chromium & $\mathrm{mg} / \mathrm{kg}$ & 3 & 2 & $67 \%$ & 0.10 & 0.10 & 0.11 & 0.11 & 0.090 \\
\hline METAL & Cobalt & $\mathrm{mg} / \mathrm{kg}$ & 3 & 3 & $100 \%$ & -- & -- & 0.0080 & 0.0090 & 0.0083 \\
\hline METAL & Copper & $\mathrm{mg} / \mathrm{kg}$ & 3 & 3 & $100 \%$ & -- & -- & 0.50 & 0.61 & 0.56 \\
\hline METAL & Lead & $\mathrm{mg} / \mathrm{kg}$ & 3 & 0 & $0 \%$ & 0.010 & 0.010 & -- & -- & 0.0050 \\
\hline METAL & Manganese & $\mathrm{mg} / \mathrm{kg}$ & 3 & 3 & $100 \%$ & -- & -- & 0.35 & 0.38 & 0.36 \\
\hline METAL & Mercury & $\mathrm{mg} / \mathrm{kg}$ & 3 & 3 & $100 \%$ & -- & -- & 0.16 & 0.20 & 0.17 \\
\hline METAL & Nickel & $\mathrm{mg} / \mathrm{kg}$ & 3 & 3 & $100 \%$ & -- & -- & 0.25 & 0.28 & 0.26 \\
\hline METAL & Selenium & $\mathrm{mg} / \mathrm{kg}$ & 3 & 3 & $100 \%$ & -- & -- & 0.38 & 0.40 & 0.39 \\
\hline METAL & Silver & $\mathrm{mg} / \mathrm{kg}$ & 3 & 0 & $0 \%$ & 0.10 & 0.10 & -- & -- & 0.050 \\
\hline METAL & Thallium & $\mathrm{mg} / \mathrm{kg}$ & 3 & 1 & $33 \%$ & 0.050 & 0.050 & 0.060 & 0.060 & 0.037 \\
\hline METAL & Vanadium & $\mathrm{mg} / \mathrm{kg}$ & 3 & 0 & $0 \%$ & 0.010 & 0.010 & -- & -- & 0.0050 \\
\hline METAL & Zinc & $\mathrm{mg} / \mathrm{kg}$ & 3 & 3 & $100 \%$ & -- & -- & 8.5 & 9.0 & 8.7 \\
\hline PCB & 2,2',3,3',4,4',5-Heptachloro-1,1'-biphenyl & $\mathrm{mg} / \mathrm{kg}$ & 3 & 3 & $100 \%$ & -- & -- & 1.57E-04 & 2.87E-04 & $2.20 \mathrm{E}-04$ \\
\hline РCB & 2,2',3,4,4',5,5'-Heptachlorobiphenyl & $\mathrm{mg} / \mathrm{kg}$ & 3 & 3 & $100 \%$ & -- & -- & $4.68 \mathrm{E}-04$ & 0.0010 & 7.26E-04 \\
\hline PCB & 2,3,3',4,4',5'-Hexachlorobiphenyl & $\mathrm{mg} / \mathrm{kg}$ & 3 & 0 & $0 \%$ & 7.30E-07 & $1.41 \mathrm{E}-06$ & -- & -- & 5.70E-07 \\
\hline РCB & 2,3,3',4,4',5-Hexachlorobiphenyl (BZ 156) & $\mathrm{mg} / \mathrm{kg}$ & 3 & 3 & $100 \%$ & -- & -- & $1.08 \mathrm{E}-04$ & 1.49E-04 & $1.22 \mathrm{E}-04$ \\
\hline PCB & 2,3,3',4,4'-Pentachlorobiphenyl & $\mathrm{mg} / \mathrm{kg}$ & 3 & 3 & $100 \%$ & -- & -- & $3.10 \mathrm{E}-04$ & $3.78 \mathrm{E}-04$ & 3.35E-04 \\
\hline РCB & 2,3',4,4',5,5'-Hexachlorobiphenyl (BZ 167) & $\mathrm{mg} / \mathrm{kg}$ & 3 & 3 & $100 \%$ & -- & -- & 4.09E-05 & $4.42 \mathrm{E}-05$ & 4.26E-05 \\
\hline PCB & 2',3,4,4',5-Pentachloro-1,1'-biphenyl (BZ 123) & $\mathrm{mg} / \mathrm{kg}$ & 3 & 3 & $100 \%$ & -- & -- & $1.18 \mathrm{E}-05$ & 1.57E-05 & 1.39E-05 \\
\hline PCB & 2,3,4,4',5-Pentachlorobiphenyl (BZ 114) & $\mathrm{mg} / \mathrm{kg}$ & 3 & 3 & $100 \%$ & -- & -- & $2.49 \mathrm{E}-05$ & 3.05E-05 & $2.68 \mathrm{E}-05$ \\
\hline PCB & 2,3',4,4',5-Pentachlorobiphenyl (BZ 118) & $\mathrm{mg} / \mathrm{kg}$ & 3 & 3 & $100 \%$ & -- & -- & $6.18 \mathrm{E}-04$ & $7.40 \mathrm{E}-04$ & 6.63E-04 \\
\hline РCB & 2,3,4,5,3',4',5'-Heptachlorobiphenyl & $\mathrm{mg} / \mathrm{kg}$ & 3 & 3 & $100 \%$ & -- & -- & $6.36 \mathrm{E}-06$ & $7.41 \mathrm{E}-06$ & $6.83 \mathrm{E}-06$ \\
\hline РCB & 3,3',4,4',5,5'-Hexachlorobiphenyl (BZ 169) & $\mathrm{mg} / \mathrm{kg}$ & 3 & 0 & $0 \%$ & $5.00 \mathrm{E}-07$ & $7.50 \mathrm{E}-07$ & -- & -- & $2.92 \mathrm{E}-07$ \\
\hline РСВ & 3,3',4,4',5-Pentachlorobiphenyl (BZ 126) & $\mathrm{mg} / \mathrm{kg}$ & 3 & 0 & $0 \%$ & $6.70 \mathrm{E}-07$ & 7.00E-07 & -- & -- & 3.45E-07 \\
\hline PCB & 3,3',4,4'-Tetrachlorobiphenyl (BZ 77) & $\mathrm{mg} / \mathrm{kg}$ & 3 & 3 & $100 \%$ & -- & -- & $1.20 \mathrm{E}-05$ & 1.44E-05 & 1.35E-05 \\
\hline РСВ & Aroclor-1016 & $\mathrm{mg} / \mathrm{kg}$ & 3 & 0 & $0 \%$ & 0.0020 & 0.020 & -- & -- & 0.0068 \\
\hline PCB & Aroclor-1221 & $\mathrm{mg} / \mathrm{kg}$ & 3 & 0 & $0 \%$ & 0.0020 & 0.020 & -- & -- & 0.0068 \\
\hline
\end{tabular}


Table F-94

Summary Statistics for Walleye Fillet Skin On Collected from Segment 4 of the Columbia River

\begin{tabular}{|c|c|c|c|c|c|c|c|c|c|c|}
\hline $\begin{array}{l}\text { Constituent } \\
\text { Class }\end{array}$ & Constituent Name & Units & $\begin{array}{c}\text { Number } \\
\text { Analyzed }\end{array}$ & $\begin{array}{l}\text { Number } \\
\text { Detected }\end{array}$ & $\begin{array}{l}\text { Frequency of } \\
\text { Detection }\end{array}$ & $\begin{array}{l}\text { Minimum } \\
\text { Nondetect }\end{array}$ & $\begin{array}{l}\text { Maximum } \\
\text { Nondetect }\end{array}$ & $\begin{array}{c}\text { Minimum } \\
\text { Detect }\end{array}$ & $\begin{array}{c}\text { Maximum } \\
\text { Detect }\end{array}$ & $\begin{array}{c}\text { Average } \\
\text { Concentration }\end{array}$ \\
\hline$\overline{\mathrm{PCB}}$ & Aroclor-1232 & $\mathrm{mg} / \mathrm{kg}$ & 3 & 0 & $0 \%$ & 0.0020 & 0.020 & $\overline{--}$ & $\overline{--}$ & 0.0068 \\
\hline PCB & Aroclor-1248 & $\mathrm{mg} / \mathrm{kg}$ & 3 & 0 & $0 \%$ & 0.0020 & 0.020 & -- & -- & 0.0068 \\
\hline PCB & Aroclor-1254 & $\mathrm{mg} / \mathrm{kg}$ & 3 & 3 & $100 \%$ & -- & -- & 0.012 & 0.014 & 0.013 \\
\hline PCB & Aroclor-1260 & $\mathrm{mg} / \mathrm{kg}$ & 3 & 0 & $0 \%$ & 0.019 & 0.020 & -- & -- & 0.0098 \\
\hline PEST & 2,4'-DDD & $\mathrm{mg} / \mathrm{kg}$ & 3 & 0 & $0 \%$ & 0.0024 & 0.0025 & -- & -- & 0.0012 \\
\hline PEST & 2,4'-DDE & $\mathrm{mg} / \mathrm{kg}$ & 3 & 0 & $0 \%$ & 0.0024 & 0.0028 & -- & -- & 0.0013 \\
\hline PEST & 2,4'-DDT & $\mathrm{mg} / \mathrm{kg}$ & 3 & 0 & $0 \%$ & 0.0024 & 0.0025 & -- & -- & 0.0012 \\
\hline PEST & Aldrin & $\mathrm{mg} / \mathrm{kg}$ & 3 & 0 & $0 \%$ & 0.0019 & 0.0020 & -- & -- & $9.83 \mathrm{E}-04$ \\
\hline PEST & Alpha-BHC & $\mathrm{mg} / \mathrm{kg}$ & 3 & 0 & $0 \%$ & 0.0024 & 0.0025 & -- & -- & 0.0012 \\
\hline PEST & alpha-Chlordane & $\mathrm{mg} / \mathrm{kg}$ & 3 & 0 & $0 \%$ & 0.0024 & 0.0025 & -- & -- & 0.0012 \\
\hline PEST & alpha-Chlordene & $\mathrm{mg} / \mathrm{kg}$ & 3 & 0 & $0 \%$ & 0.0024 & 0.0025 & -- & -- & 0.0012 \\
\hline PEST & Beta-BHC & $\mathrm{mg} / \mathrm{kg}$ & 3 & 0 & $0 \%$ & 0.0024 & 0.0025 & -- & -- & 0.0012 \\
\hline PEST & cis-Nonachlor & $\mathrm{mg} / \mathrm{kg}$ & 3 & 0 & $0 \%$ & 0.0024 & 0.0025 & -- & -- & 0.0012 \\
\hline PEST & Delta-BHC & $\mathrm{mg} / \mathrm{kg}$ & 3 & 0 & $0 \%$ & 0.0024 & 0.0025 & -- & -- & 0.0012 \\
\hline PEST & Dichlorodiphenyldichloroethane & $\mathrm{mg} / \mathrm{kg}$ & 3 & 3 & $100 \%$ & -- & -- & 0.0063 & 0.0071 & 0.0067 \\
\hline PEST & Dichlorodiphenyldichloroethylene & $\mathrm{mg} / \mathrm{kg}$ & 3 & 3 & $100 \%$ & -- & -- & 0.044 & 0.052 & 0.047 \\
\hline PEST & Dichlorodiphenyltrichloroethane & $\mathrm{mg} / \mathrm{kg}$ & 3 & 2 & $67 \%$ & 0.0025 & 0.0025 & 0.0020 & 0.0027 & 0.0020 \\
\hline PEST & Endosulfan sulfate & $\mathrm{mg} / \mathrm{kg}$ & 3 & 0 & $0 \%$ & 0.0024 & 0.0025 & -- & -- & 0.0012 \\
\hline PEST & Gamma-BHC (Lindane) & $\mathrm{mg} / \mathrm{kg}$ & 3 & 0 & $0 \%$ & 0.0024 & 0.0025 & -- & -- & 0.0012 \\
\hline PEST & gamma-Chlordane & $\mathrm{mg} / \mathrm{kg}$ & 3 & 0 & $0 \%$ & 0.0024 & 0.0025 & -- & -- & 0.0012 \\
\hline PEST & gamma-Chlordene & $\mathrm{mg} / \mathrm{kg}$ & 3 & 0 & $0 \%$ & 0.0024 & 0.0025 & -- & -- & 0.0012 \\
\hline PEST & Heptachlor & $\mathrm{mg} / \mathrm{kg}$ & 3 & 0 & $0 \%$ & 0.0019 & 0.0020 & -- & -- & $9.83 \mathrm{E}-04$ \\
\hline PEST & Heptachlor epoxide & $\mathrm{mg} / \mathrm{kg}$ & 3 & 0 & $0 \%$ & 0.0024 & 0.0025 & -- & -- & 0.0012 \\
\hline PEST & Mirex & $\mathrm{mg} / \mathrm{kg}$ & 3 & 0 & $0 \%$ & 0.0024 & 0.0025 & -- & -- & 0.0012 \\
\hline PEST & Oxychlordane & $\mathrm{mg} / \mathrm{kg}$ & 3 & 0 & $0 \%$ & 0.0024 & 0.0025 & -- & -- & 0.0012 \\
\hline PEST & Pentachloroanisole & $\mathrm{mg} / \mathrm{kg}$ & 3 & 0 & $0 \%$ & 0.0024 & 0.0025 & -- & -- & 0.0012 \\
\hline PEST & Toxaphene & $\mathrm{mg} / \mathrm{kg}$ & 3 & 0 & $0 \%$ & 0.073 & 0.074 & -- & -- & 0.037 \\
\hline PEST & trans-Nonachlor & $\mathrm{mg} / \mathrm{kg}$ & 3 & 0 & $0 \%$ & 0.0024 & 0.0025 & -- & -- & 0.0012 \\
\hline SVOC & 1,2,4-Trichlorobenzene & $\mathrm{mg} / \mathrm{kg}$ & 3 & 0 & $0 \%$ & 0.0052 & 0.0096 & -- & -- & 0.0034 \\
\hline SVOC & 1,2-Dichlorobenzene & $\mathrm{mg} / \mathrm{kg}$ & 3 & 0 & $0 \%$ & 0.0052 & 0.0096 & -- & -- & 0.0034 \\
\hline SVOC & 1,2-Diphenylhydrazine & $\mathrm{mg} / \mathrm{kg}$ & 3 & 0 & $0 \%$ & 0.0052 & 0.0096 & -- & -- & 0.0034 \\
\hline SVOC & 1,3-Dichlorobenzene & $\mathrm{mg} / \mathrm{kg}$ & 3 & 0 & $0 \%$ & 0.0052 & 0.0096 & -- & -- & 0.0034 \\
\hline SVOC & 1,4-Dichlorobenzene & $\mathrm{mg} / \mathrm{kg}$ & 3 & 0 & $0 \%$ & 0.0052 & 0.0096 & -- & -- & 0.0034 \\
\hline SVOC & 1-methylnaphthalene & $\mathrm{mg} / \mathrm{kg}$ & 3 & 0 & $0 \%$ & 0.0052 & 0.0096 & -- & -- & 0.0034 \\
\hline SVOC & 2,4-Dinitrotoluene & $\mathrm{mg} / \mathrm{kg}$ & 3 & 0 & $0 \%$ & 0.010 & 0.019 & -- & -- & 0.0067 \\
\hline SVOC & 2,6-Dinitrotoluene & $\mathrm{mg} / \mathrm{kg}$ & 3 & 0 & $0 \%$ & 0.021 & 0.038 & -- & -- & 0.013 \\
\hline SVOC & 2-Chloronaphthalene & $\mathrm{mg} / \mathrm{kg}$ & 3 & 0 & $0 \%$ & 0.010 & 0.019 & -- & -- & 0.0067 \\
\hline SVOC & 2-Methylnaphthalene & $\mathrm{mg} / \mathrm{kg}$ & 3 & 2 & $67 \%$ & 0.0096 & 0.0096 & 0.0060 & 0.0076 & 0.0061 \\
\hline SVOC & 4-Bromophenylphenyl ether & $\mathrm{mg} / \mathrm{kg}$ & 3 & 0 & $0 \%$ & 0.0052 & 0.0096 & -- & -- & 0.0034 \\
\hline SVOC & 4-Chlorophenylphenyl ether & $\mathrm{mg} / \mathrm{kg}$ & 3 & 0 & $0 \%$ & 0.0052 & 0.0096 & -- & -- & 0.0034 \\
\hline SVOC & Acenaphthene & $\mathrm{mg} / \mathrm{kg}$ & 3 & 0 & $0 \%$ & 0.010 & 0.019 & -- & -- & 0.0067 \\
\hline SVOC & Acenaphthylene & $\mathrm{mg} / \mathrm{kg}$ & 3 & 0 & $0 \%$ & 0.0052 & 0.0096 & -- & -- & 0.0034 \\
\hline SVOC & Anthracene & $\mathrm{mg} / \mathrm{kg}$ & 3 & 0 & $0 \%$ & 0.0052 & 0.0096 & -- & -- & 0.0034 \\
\hline SVOC & Benzo(a)anthracene & $\mathrm{mg} / \mathrm{kg}$ & 3 & 0 & $0 \%$ & 0.0052 & 0.0096 & -- & -- & 0.0034 \\
\hline SVOC & Benzo(a)pyrene & $\mathrm{mg} / \mathrm{kg}$ & 3 & 0 & $0 \%$ & 0.0052 & 0.0096 & -- & -- & 0.0034 \\
\hline SVOC & Benzo(b)fluoranthene & $\mathrm{mg} / \mathrm{kg}$ & 3 & 0 & $0 \%$ & 0.0052 & 0.0096 & -- & -- & 0.0034 \\
\hline SVOC & Benzo(ghi)perylene & $\mathrm{mg} / \mathrm{kg}$ & 3 & 0 & $0 \%$ & 0.010 & 0.019 & -- & -- & 0.0067 \\
\hline SVOC & Benzo(k)fluoranthene & $\mathrm{mg} / \mathrm{kg}$ & 3 & 0 & $0 \%$ & 0.0052 & 0.0096 & -- & -- & 0.0034 \\
\hline SVOC & Bis(2-chloro-1-methylethyl)ether & $\mathrm{mg} / \mathrm{kg}$ & 3 & 0 & $0 \%$ & 0.010 & 0.019 & -- & -- & 0.0067 \\
\hline SVOC & Chrysene & $\mathrm{mg} / \mathrm{kg}$ & 3 & 0 & $0 \%$ & 0.0052 & 0.0096 & -- & -- & 0.0034 \\
\hline
\end{tabular}


Table F-94

Summary Statistics for Walleye Fillet Skin On Collected from Segment 4 of the Columbia River

\begin{tabular}{|c|c|c|c|c|c|c|c|c|c|c|}
\hline $\begin{array}{c}\text { Constituent } \\
\text { Class }\end{array}$ & Constituent Name & Units & $\begin{array}{c}\text { Number } \\
\text { Analyzed }\end{array}$ & $\begin{array}{l}\text { Number } \\
\text { Detected }\end{array}$ & $\begin{array}{c}\text { Frequency of } \\
\text { Detection }\end{array}$ & $\begin{array}{l}\text { Minimum } \\
\text { Nondetect }\end{array}$ & $\begin{array}{l}\text { Maximum } \\
\text { Nondetect }\end{array}$ & $\begin{array}{l}\text { Minimum } \\
\text { Detect }\end{array}$ & $\begin{array}{l}\text { Maximum } \\
\text { Detect }\end{array}$ & $\begin{array}{c}\text { Average } \\
\text { Concentration }\end{array}$ \\
\hline$\overline{\text { SVOC }}$ & Dibenz[a,h]anthracene & $\mathrm{mg} / \mathrm{kg}$ & 3 & 0 & $0 \%$ & 0.010 & 0.019 & $\overline{--}$ & $\overline{--}$ & 0.0067 \\
\hline SVOC & Dibenzofuran & $\mathrm{mg} / \mathrm{kg}$ & 3 & 0 & $0 \%$ & 0.0052 & 0.0096 & -- & -- & 0.0034 \\
\hline SVOC & Fluoranthene & $\mathrm{mg} / \mathrm{kg}$ & 3 & 0 & $0 \%$ & 0.010 & 0.019 & -- & -- & 0.0067 \\
\hline SVOC & Fluorene & $\mathrm{mg} / \mathrm{kg}$ & 3 & 0 & $0 \%$ & 0.0063 & 0.010 & -- & -- & 0.0039 \\
\hline SVOC & Hexachlorobenzene & $\mathrm{mg} / \mathrm{kg}$ & 3 & 0 & $0 \%$ & 0.010 & 0.019 & -- & -- & 0.0067 \\
\hline SVOC & Hexachlorobutadiene & $\mathrm{mg} / \mathrm{kg}$ & 3 & 0 & $0 \%$ & 0.0052 & 0.0096 & -- & -- & 0.0034 \\
\hline SVOC & Hexachloroethane & $\mathrm{mg} / \mathrm{kg}$ & 3 & 0 & $0 \%$ & 0.0052 & 0.0096 & -- & -- & 0.0034 \\
\hline SVOC & Indeno(1,2,3-cd)pyrene & $\mathrm{mg} / \mathrm{kg}$ & 3 & 0 & $0 \%$ & 0.010 & 0.019 & -- & -- & 0.0067 \\
\hline SVOC & Naphthalene & $\mathrm{mg} / \mathrm{kg}$ & 3 & 0 & $0 \%$ & 0.0053 & 0.013 & -- & -- & 0.0047 \\
\hline SVOC & Nitrobenzene & $\mathrm{mg} / \mathrm{kg}$ & 3 & 0 & $0 \%$ & 0.0052 & 0.0096 & -- & -- & 0.0034 \\
\hline SVOC & Phenanthrene & $\mathrm{mg} / \mathrm{kg}$ & 3 & 0 & $0 \%$ & 0.0052 & 0.0096 & -- & -- & 0.0034 \\
\hline SVOC & Pyrene & $\mathrm{mg} / \mathrm{kg}$ & 3 & 0 & $0 \%$ & 0.010 & 0.019 & -- & -- & 0.0067 \\
\hline VOC & Retene & $\mathrm{mg} / \mathrm{kg}$ & 3 & 0 & $0 \%$ & 0.010 & 0.019 & -- & -- & 0.0067 \\
\hline
\end{tabular}

DIOXI - Dioxins and Furans

PEST - Pesticide

PCB - Polychlorinated bipheny

SVOC - Semivolatile Organic Compound

VOC - Volatile Organic Compound 
Table F-95

Summary Statistics for Whole Body Walleye Collected from Segment 4 of the Columbia River

\begin{tabular}{|c|c|c|c|c|c|c|c|c|c|c|}
\hline $\begin{array}{l}\text { Constituent } \\
\text { Class }\end{array}$ & Constituent Name & Units & $\begin{array}{l}\text { Number } \\
\text { Analyzed }\end{array}$ & $\begin{array}{l}\text { Number } \\
\text { Detected }\end{array}$ & $\begin{array}{c}\text { Frequency of } \\
\text { Detection }\end{array}$ & $\begin{array}{l}\text { Minimum } \\
\text { Nondetect }\end{array}$ & $\begin{array}{l}\text { Maximum } \\
\text { Nondetect }\end{array}$ & $\begin{array}{c}\text { Minimum } \\
\text { Detect }\end{array}$ & $\begin{array}{l}\text { Maximum } \\
\text { Detect }\end{array}$ & $\begin{array}{c}\text { Average } \\
\text { Concentration }\end{array}$ \\
\hline$\overline{D I O X I}$ & $1,2,3,4,6,7,8$-Heptachlorodibenzodioxin & $\mathrm{mg} / \mathrm{kg}$ & 3 & 3 & $100 \%$ & -- & -- & $1.10 \mathrm{E}-07$ & $2.80 \mathrm{E}-07$ & $1.73 \mathrm{E}-07$ \\
\hline DIOXI & 1,2,3,4,6,7,8-Heptachlorodibenzofuran & $\mathrm{mg} / \mathrm{kg}$ & 3 & 1 & $33 \%$ & 2.00E-08 & 2.00E-08 & 1.10E-07 & 1.10E-07 & 4.33E-08 \\
\hline DIOXI & 1,2,3,4,7,8,9-Heptachlorodibenzofuran & $\mathrm{mg} / \mathrm{kg}$ & 3 & 1 & $33 \%$ & $2.00 \mathrm{E}-08$ & $2.00 \mathrm{E}-08$ & 4.00E-08 & 4.00E-08 & $2.00 \mathrm{E}-08$ \\
\hline DIOXI & $1,2,3,4,7,8$-Hexachlorodibenzofuran & $\mathrm{mg} / \mathrm{kg}$ & 3 & 3 & $100 \%$ & -- & -- & $3.00 \mathrm{E}-08$ & 4.00E-08 & 3.33E-08 \\
\hline DIOXI & $1,2,3,4,7,8$-Hexachlorodibenzo-p-dioxin & $\mathrm{mg} / \mathrm{kg}$ & 3 & 3 & $100 \%$ & -- & -- & $2.00 \mathrm{E}-08$ & $1.30 \mathrm{E}-07$ & $6.33 \mathrm{E}-08$ \\
\hline DIOXI & 1,2,3,6,7,8-Hexachlorodibenzofuran & $\mathrm{mg} / \mathrm{kg}$ & 3 & 2 & $67 \%$ & 1.00E-08 & 1.00E-08 & $3.00 \mathrm{E}-08$ & $5.00 \mathrm{E}-08$ & 2.83E-08 \\
\hline DIOXI & 1,2,3,6,7,8-Hexachlorodibenzo-p-dioxin & $\mathrm{mg} / \mathrm{kg}$ & 3 & 3 & $100 \%$ & -- & -- & $1.20 \mathrm{E}-07$ & $2.00 \mathrm{E}-07$ & 1.63E-07 \\
\hline DIOXI & 1,2,3,7,8,9-Hexachlorodibenzofuran & $\mathrm{mg} / \mathrm{kg}$ & 3 & 1 & $33 \%$ & $1.00 \mathrm{E}-08$ & 1.00E-08 & $1.10 \mathrm{E}-07$ & 1.10E-07 & 4.00E-08 \\
\hline DIOXI & 1,2,3,7,8,9-Hexachlorodibenzo-p-dioxin & $\mathrm{mg} / \mathrm{kg}$ & 3 & 3 & $100 \%$ & -- & -- & $3.00 \mathrm{E}-08$ & 8.00E-08 & $5.67 \mathrm{E}-08$ \\
\hline DIOXI & 1,2,3,7,8-Pentachlorodibenzofuran & $\mathrm{mg} / \mathrm{kg}$ & 3 & 3 & $100 \%$ & -- & -- & $1.20 \mathrm{E}-07$ & $1.50 \mathrm{E}-07$ & 1.37E-07 \\
\hline DIOXI & 1,2,3,7,8-Pentachlorodibenzo-p-dioxin & $\mathrm{mg} / \mathrm{kg}$ & 3 & 3 & $100 \%$ & -- & -- & $1.90 \mathrm{E}-07$ & $2.70 \mathrm{E}-07$ & $2.37 \mathrm{E}-07$ \\
\hline DIOXI & 2,3,4,6,7,8-Hexachlorodibenzofuran & $\mathrm{mg} / \mathrm{kg}$ & 3 & 3 & $100 \%$ & -- & -- & $4.00 \mathrm{E}-08$ & $1.60 \mathrm{E}-07$ & 8.00E-08 \\
\hline DIOXI & 2,3,4,7,8-Pentachlorodibenzofuran & $\mathrm{mg} / \mathrm{kg}$ & 3 & 3 & $100 \%$ & -- & -- & $1.90 \mathrm{E}-07$ & $2.40 \mathrm{E}-07$ & 2.10E-07 \\
\hline DIOXI & 2,3,7,8-Tetrachlorodibenzofuran & $\mathrm{mg} / \mathrm{kg}$ & 3 & 3 & $100 \%$ & -- & -- & $3.82 \mathrm{E}-06$ & 5.53E-06 & 4.55E-06 \\
\hline DIOXI & 2,3,7,8-Tetrachlorodibenzo-p-dioxin & $\mathrm{mg} / \mathrm{kg}$ & 3 & 3 & $100 \%$ & -- & -- & $3.60 \mathrm{E}-07$ & 4.20E-07 & 3.87E-07 \\
\hline DIOXI & Octachlorodibenzofuran & $\mathrm{mg} / \mathrm{kg}$ & 3 & 1 & $33 \%$ & $1.00 \mathrm{E}-08$ & $1.00 \mathrm{E}-08$ & $1.00 \mathrm{E}-07$ & $1.00 \mathrm{E}-07$ & 3.67E-08 \\
\hline DIOXI & Octachlorodibenzo-p-dioxin & $\mathrm{mg} / \mathrm{kg}$ & 3 & 3 & $100 \%$ & -- & -- & $1.50 \mathrm{E}-07$ & $3.20 \mathrm{E}-07$ & 2.10E-07 \\
\hline METAL & Aluminum & $\mathrm{mg} / \mathrm{kg}$ & 3 & 1 & $33 \%$ & 1.0 & 1.0 & 6.2 & 6.2 & 2.4 \\
\hline METAL & Antimony & $\mathrm{mg} / \mathrm{kg}$ & 3 & 0 & $0 \%$ & 0.050 & 0.050 & -- & -- & 0.025 \\
\hline METAL & Arsenic & $\mathrm{mg} / \mathrm{kg}$ & 3 & 3 & $100 \%$ & -- & -- & 0.48 & 0.50 & 0.49 \\
\hline METAL & Barium & $\mathrm{mg} / \mathrm{kg}$ & 3 & 3 & $100 \%$ & -- & -- & 0.51 & 0.85 & 0.67 \\
\hline METAL & Beryllium & $\mathrm{mg} / \mathrm{kg}$ & 3 & 0 & $0 \%$ & 0.0040 & 0.0040 & -- & -- & 0.0020 \\
\hline METAL & Cadmium & $\mathrm{mg} / \mathrm{kg}$ & 3 & 3 & $100 \%$ & -- & -- & 0.0070 & 0.0079 & 0.0074 \\
\hline METAL & Chromium & $\mathrm{mg} / \mathrm{kg}$ & 3 & 3 & $100 \%$ & -- & -- & 0.10 & 0.12 & 0.11 \\
\hline METAL & Cobalt & $\mathrm{mg} / \mathrm{kg}$ & 3 & 3 & $100 \%$ & -- & -- & 0.010 & 0.080 & 0.050 \\
\hline METAL & Copper & $\mathrm{mg} / \mathrm{kg}$ & 3 & 3 & $100 \%$ & -- & -- & 0.73 & 5.7 & 2.5 \\
\hline METAL & Lead & $\mathrm{mg} / \mathrm{kg}$ & 3 & 2 & $67 \%$ & 0.010 & 0.010 & 0.060 & 0.48 & 0.18 \\
\hline METAL & Manganese & $\mathrm{mg} / \mathrm{kg}$ & 3 & 3 & $100 \%$ & -- & -- & 0.89 & 0.97 & 0.94 \\
\hline METAL & Mercury & $\mathrm{mg} / \mathrm{kg}$ & 3 & 3 & $100 \%$ & -- & -- & 0.12 & 0.21 & 0.17 \\
\hline METAL & Nickel & $\mathrm{mg} / \mathrm{kg}$ & 3 & 3 & $100 \%$ & -- & -- & 0.12 & 0.39 & 0.25 \\
\hline METAL & Selenium & $\mathrm{mg} / \mathrm{kg}$ & 3 & 3 & $100 \%$ & -- & -- & 0.41 & 0.54 & 0.47 \\
\hline METAL & Silver & $\mathrm{mg} / \mathrm{kg}$ & 3 & 0 & $0 \%$ & 0.10 & 0.10 & -- & -- & 0.050 \\
\hline METAL & Thallium & $\mathrm{mg} / \mathrm{kg}$ & 3 & 0 & $0 \%$ & 0.050 & 0.050 & -- & -- & 0.025 \\
\hline METAL & Vanadium & $\mathrm{mg} / \mathrm{kg}$ & 3 & 3 & $100 \%$ & -- & -- & 0.010 & 0.020 & 0.013 \\
\hline METAL & Zinc & $\mathrm{mg} / \mathrm{kg}$ & 3 & 3 & $100 \%$ & -- & -- & 13 & 15 & 14 \\
\hline PCB & 2,2',3,3',4,4',5-Heptachloro-1,1'-biphenyl & $\mathrm{mg} / \mathrm{kg}$ & 3 & 3 & $100 \%$ & -- & -- & $9.65 \mathrm{E}-04$ & 0.0028 & 0.0019 \\
\hline РCB & 2,2',3,4,4',5,5'-Heptachlorobiphenyl & $\mathrm{mg} / \mathrm{kg}$ & 3 & 3 & $100 \%$ & -- & -- & 0.0028 & 0.0069 & 0.0049 \\
\hline РCB & 2,3,3',4,4', 5'-Hexachlorobiphenyl & $\mathrm{mg} / \mathrm{kg}$ & 3 & 2 & $67 \%$ & 1.67E-06 & $1.67 \mathrm{E}-06$ & 2.17E-04 & 4.53E-04 & $2.24 \mathrm{E}-04$ \\
\hline РCB & 2,3,3',4,4',5-Hexachlorobiphenyl (BZ 156) & $\mathrm{mg} / \mathrm{kg}$ & 3 & 3 & $100 \%$ & -- & -- & $8.00 \mathrm{E}-04$ & 0.0023 & 0.0014 \\
\hline PCB & 2,3,3',4,4'-Pentachlorobiphenyl & $\mathrm{mg} / \mathrm{kg}$ & 3 & 3 & $100 \%$ & -- & -- & 0.0012 & 0.0053 & 0.0031 \\
\hline РCB & 2,3',4,4',5,5'-Hexachlorobiphenyl (BZ 167) & $\mathrm{mg} / \mathrm{kg}$ & 3 & 3 & $100 \%$ & -- & -- & $3.22 \mathrm{E}-04$ & $6.71 \mathrm{E}-04$ & $4.52 \mathrm{E}-04$ \\
\hline PCB & 2',3,4,4',5-Pentachloro-1,1'-biphenyl (BZ 123) & $\mathrm{mg} / \mathrm{kg}$ & 3 & 3 & $100 \%$ & -- & -- & $5.13 \mathrm{E}-05$ & $2.29 \mathrm{E}-04$ & $1.20 \mathrm{E}-04$ \\
\hline PCB & 2,3,4,4',5-Pentachlorobiphenyl (BZ 114) & $\mathrm{mg} / \mathrm{kg}$ & 3 & 3 & $100 \%$ & -- & -- & $1.26 \mathrm{E}-04$ & 5.06E-04 & $2.88 \mathrm{E}-04$ \\
\hline PCB & 2,3',4,4',5-Pentachlorobiphenyl (BZ 118) & $\mathrm{mg} / \mathrm{kg}$ & 3 & 3 & $100 \%$ & -- & -- & 0.0026 & 0.010 & 0.0060 \\
\hline PCB & 2,3,4,5,3',4',5'-Heptachlorobiphenyl & $\mathrm{mg} / \mathrm{kg}$ & 3 & 3 & $100 \%$ & -- & -- & $5.32 \mathrm{E}-05$ & 9.54E-05 & $6.92 \mathrm{E}-05$ \\
\hline PCB & 3,3',4,4',5,5'-Hexachlorobiphenyl (BZ 169) & $\mathrm{mg} / \mathrm{kg}$ & 3 & 0 & $0 \%$ & 2.35E-06 & 4.91E-06 & -- & -- & 1.63E-06 \\
\hline РСВ & 3,3',4,4',5-Pentachlorobiphenyl (BZ 126) & $\mathrm{mg} / \mathrm{kg}$ & 3 & 2 & $67 \%$ & 1.00E-06 & 1.00E-06 & 8.89E-06 & 1.79E-05 & $9.10 \mathrm{E}-06$ \\
\hline PCB & 3,3',4,4'-Tetrachlorobiphenyl (BZ 77) & $\mathrm{mg} / \mathrm{kg}$ & 3 & 3 & $100 \%$ & -- & -- & 7.43E-05 & $9.10 \mathrm{E}-05$ & 8.19E-05 \\
\hline РСВ & Aroclor-1016 & $\mathrm{mg} / \mathrm{kg}$ & 3 & 0 & $0 \%$ & 0.018 & 0.019 & -- & -- & 0.0093 \\
\hline PCB & Aroclor-1221 & $\mathrm{mg} / \mathrm{kg}$ & 3 & 0 & $0 \%$ & 0.018 & 0.019 & -- & -- & 0.0093 \\
\hline
\end{tabular}


Table F-95

Summary Statistics for Whole Body Walleye Collected from Segment 4 of the Columbia River

\begin{tabular}{|c|c|c|c|c|c|c|c|c|c|c|}
\hline $\begin{array}{l}\text { Constituent } \\
\text { Class }\end{array}$ & Constituent Name & Units & $\begin{array}{l}\text { Number } \\
\text { Analyzed }\end{array}$ & $\begin{array}{l}\text { Number } \\
\text { Detected }\end{array}$ & $\begin{array}{c}\text { Frequency of } \\
\text { Detection }\end{array}$ & $\begin{array}{l}\text { Minimum } \\
\text { Nondetect }\end{array}$ & $\begin{array}{l}\text { Maximum } \\
\text { Nondetect }\end{array}$ & $\begin{array}{l}\text { Minimum } \\
\text { Detect }\end{array}$ & $\begin{array}{l}\text { Maximum } \\
\text { Detect }\end{array}$ & $\begin{array}{c}\text { Average } \\
\text { Concentration }\end{array}$ \\
\hline$\overline{\mathrm{PCB}}$ & Aroclor-1232 & $\mathrm{mg} / \mathrm{kg}$ & 3 & 0 & $0 \%$ & 0.018 & 0.019 & -- & -- & 0.0093 \\
\hline PCB & Aroclor-1248 & $\mathrm{mg} / \mathrm{kg}$ & 3 & 0 & $0 \%$ & 0.018 & 0.019 & -- & -- & 0.0093 \\
\hline PCB & Aroclor-1254 & $\mathrm{mg} / \mathrm{kg}$ & 3 & 3 & $100 \%$ & -- & -- & 0.054 & 0.098 & 0.074 \\
\hline PCB & Aroclor-1260 & $\mathrm{mg} / \mathrm{kg}$ & 3 & 3 & $100 \%$ & -- & -- & 0.047 & 0.061 & 0.052 \\
\hline PEST & 2,4'-DDD & $\mathrm{mg} / \mathrm{kg}$ & 3 & 3 & $100 \%$ & -- & -- & 0.0058 & 0.0073 & 0.0066 \\
\hline PEST & 2,4'-DDE & $\mathrm{mg} / \mathrm{kg}$ & 3 & 3 & $100 \%$ & -- & -- & 0.0068 & 0.012 & 0.0089 \\
\hline PEST & 2,4'-DDT & $\mathrm{mg} / \mathrm{kg}$ & 3 & 0 & $0 \%$ & 0.0018 & 0.0024 & -- & -- & 0.0010 \\
\hline PEST & Aldrin & $\mathrm{mg} / \mathrm{kg}$ & 3 & 0 & $0 \%$ & 0.0018 & 0.0019 & -- & -- & $9.33 \mathrm{E}-04$ \\
\hline PEST & Alpha-BHC & $\mathrm{mg} / \mathrm{kg}$ & 3 & 0 & $0 \%$ & 0.0018 & 0.0024 & -- & -- & 0.0010 \\
\hline PEST & alpha-Chlordane & $\mathrm{mg} / \mathrm{kg}$ & 3 & 3 & $100 \%$ & -- & -- & 0.0035 & 0.0058 & 0.0043 \\
\hline PEST & alpha-Chlordene & $\mathrm{mg} / \mathrm{kg}$ & 3 & 0 & $0 \%$ & 0.0018 & 0.0024 & -- & -- & 0.0010 \\
\hline PEST & Beta-BHC & $\mathrm{mg} / \mathrm{kg}$ & 3 & 0 & $0 \%$ & 0.0018 & 0.0024 & -- & -- & 0.0010 \\
\hline PEST & cis-Nonachlor & $\mathrm{mg} / \mathrm{kg}$ & 3 & 2 & $67 \%$ & 0.0024 & 0.0024 & 0.0035 & 0.0043 & 0.0030 \\
\hline PEST & DDMU & $\mathrm{mg} / \mathrm{kg}$ & 2 & 2 & $100 \%$ & -- & -- & 0.0079 & 0.0083 & 0.0081 \\
\hline PEST & Delta-BHC & $\mathrm{mg} / \mathrm{kg}$ & 3 & 0 & $0 \%$ & 0.0018 & 0.0024 & -- & -- & 0.0010 \\
\hline PEST & Dichlorodiphenyldichloroethane & $\mathrm{mg} / \mathrm{kg}$ & 3 & 3 & $100 \%$ & -- & -- & 0.050 & 0.057 & 0.053 \\
\hline PEST & Dichlorodiphenyldichloroethylene & $\mathrm{mg} / \mathrm{kg}$ & 3 & 3 & $100 \%$ & -- & -- & 0.35 & 0.44 & 0.41 \\
\hline PEST & Dichlorodiphenyltrichloroethane & $\mathrm{mg} / \mathrm{kg}$ & 3 & 3 & $100 \%$ & -- & -- & 0.0066 & 0.012 & 0.0092 \\
\hline PEST & Endosulfan sulfate & $\mathrm{mg} / \mathrm{kg}$ & 3 & 0 & $0 \%$ & 0.0018 & 0.0024 & -- & -- & 0.0010 \\
\hline PEST & Gamma-BHC (Lindane) & $\mathrm{mg} / \mathrm{kg}$ & 3 & 0 & $0 \%$ & 0.0018 & 0.0024 & -- & -- & 0.0010 \\
\hline PEST & gamma-Chlordane & $\mathrm{mg} / \mathrm{kg}$ & 3 & 3 & $100 \%$ & -- & -- & 0.0036 & 0.0043 & 0.0040 \\
\hline PEST & gamma-Chlordene & $\mathrm{mg} / \mathrm{kg}$ & 3 & 0 & $0 \%$ & 0.0018 & 0.0024 & -- & -- & 0.0010 \\
\hline PEST & Heptachlor & $\mathrm{mg} / \mathrm{kg}$ & 3 & 0 & $0 \%$ & 0.0018 & 0.0019 & -- & -- & $9.33 \mathrm{E}-04$ \\
\hline PEST & Heptachlor epoxide & $\mathrm{mg} / \mathrm{kg}$ & 3 & 0 & $0 \%$ & 0.0018 & 0.0024 & -- & -- & 0.0010 \\
\hline PEST & Mirex & $\mathrm{mg} / \mathrm{kg}$ & 3 & 2 & $67 \%$ & 0.0024 & 0.0024 & 0.0030 & 0.0041 & 0.0028 \\
\hline PEST & Oxychlordane & $\mathrm{mg} / \mathrm{kg}$ & 3 & 3 & $100 \%$ & -- & -- & 0.0016 & 0.0024 & 0.0019 \\
\hline PEST & Pentachloroanisole & $\mathrm{mg} / \mathrm{kg}$ & 3 & 1 & $33 \%$ & 0.0018 & 0.0019 & 0.0012 & 0.0012 & 0.0010 \\
\hline PEST & Toxaphene & $\mathrm{mg} / \mathrm{kg}$ & 3 & 0 & $0 \%$ & 0.055 & 0.072 & -- & -- & 0.031 \\
\hline PEST & trans-Nonachlor & $\mathrm{mg} / \mathrm{kg}$ & 3 & 3 & $100 \%$ & -- & -- & 0.0030 & 0.0086 & 0.0066 \\
\hline SVOC & 1,2,4-Trichlorobenzene & $\mathrm{mg} / \mathrm{kg}$ & 3 & 0 & $0 \%$ & 0.0050 & 0.0099 & -- & -- & 0.0041 \\
\hline SVOC & 1,2-Dichlorobenzene & $\mathrm{mg} / \mathrm{kg}$ & 3 & 0 & $0 \%$ & 0.0050 & 0.0099 & -- & -- & 0.0041 \\
\hline SVOC & 1,2-Diphenylhydrazine & $\mathrm{mg} / \mathrm{kg}$ & 3 & 0 & $0 \%$ & 0.0050 & 0.0099 & -- & -- & 0.0041 \\
\hline SVOC & 1,3-Dichlorobenzene & $\mathrm{mg} / \mathrm{kg}$ & 3 & 0 & $0 \%$ & 0.0050 & 0.0099 & -- & -- & 0.0041 \\
\hline SVOC & 1,4-Dichlorobenzene & $\mathrm{mg} / \mathrm{kg}$ & 3 & 0 & $0 \%$ & 0.0050 & 0.0099 & -- & -- & 0.0041 \\
\hline SVOC & 1-methylnaphthalene & $\mathrm{mg} / \mathrm{kg}$ & 3 & 1 & $33 \%$ & 0.0096 & 0.0099 & 0.0077 & 0.0077 & 0.0058 \\
\hline SVOC & 2,4-Dinitrotoluene & $\mathrm{mg} / \mathrm{kg}$ & 3 & 0 & $0 \%$ & 0.010 & 0.020 & -- & -- & 0.0082 \\
\hline SVOC & 2,6-Dinitrotoluene & $\mathrm{mg} / \mathrm{kg}$ & 3 & 0 & $0 \%$ & 0.020 & 0.040 & -- & -- & 0.016 \\
\hline SVOC & 2-Chloronaphthalene & $\mathrm{mg} / \mathrm{kg}$ & 3 & 0 & $0 \%$ & 0.010 & 0.020 & -- & -- & 0.0082 \\
\hline SVOC & 2-Methylnaphthalene & $\mathrm{mg} / \mathrm{kg}$ & 3 & 1 & $33 \%$ & 0.0096 & 0.0099 & 0.016 & 0.016 & 0.0086 \\
\hline SVOC & 4-Bromophenylphenyl ether & $\mathrm{mg} / \mathrm{kg}$ & 3 & 0 & $0 \%$ & 0.0050 & 0.0099 & -- & -- & 0.0041 \\
\hline SVOC & 4-Chlorophenylphenyl ether & $\mathrm{mg} / \mathrm{kg}$ & 3 & 0 & $0 \%$ & 0.0050 & 0.0099 & -- & -- & 0.0041 \\
\hline SVOC & Acenaphthene & $\mathrm{mg} / \mathrm{kg}$ & 3 & 0 & $0 \%$ & 0.010 & 0.020 & -- & -- & 0.0082 \\
\hline SVOC & Acenaphthylene & $\mathrm{mg} / \mathrm{kg}$ & 3 & 0 & $0 \%$ & 0.0050 & 0.0099 & -- & -- & 0.0041 \\
\hline SVOC & Anthracene & $\mathrm{mg} / \mathrm{kg}$ & 3 & 0 & $0 \%$ & 0.0050 & 0.0099 & -- & -- & 0.0041 \\
\hline SVOC & Benzo(a)anthracene & $\mathrm{mg} / \mathrm{kg}$ & 3 & 0 & $0 \%$ & 0.0050 & 0.0099 & -- & -- & 0.0041 \\
\hline SVOC & Benzo(a)pyrene & $\mathrm{mg} / \mathrm{kg}$ & 3 & 0 & $0 \%$ & 0.0050 & 0.0099 & -- & -- & 0.0041 \\
\hline SVOC & Benzo(b)fluoranthene & $\mathrm{mg} / \mathrm{kg}$ & 3 & 0 & $0 \%$ & 0.0050 & 0.0099 & -- & -- & 0.0041 \\
\hline SVOC & Benzo(ghi)perylene & $\mathrm{mg} / \mathrm{kg}$ & 3 & 0 & $0 \%$ & 0.010 & 0.020 & -- & -- & 0.0082 \\
\hline SVOC & Benzo(k)fluoranthene & $\mathrm{mg} / \mathrm{kg}$ & 3 & 0 & $0 \%$ & 0.0050 & 0.0099 & -- & -- & 0.0041 \\
\hline SVOC & Bis(2-chloro-1-methylethyl)ether & $\mathrm{mg} / \mathrm{kg}$ & 3 & 0 & $0 \%$ & 0.010 & 0.020 & -- & -- & 0.0082 \\
\hline
\end{tabular}


Table F-95

Summary Statistics for Whole Body Walleye Collected from Segment 4 of the Columbia River

\begin{tabular}{|c|c|c|c|c|c|c|c|c|c|c|}
\hline $\begin{array}{l}\text { Constituent } \\
\text { Class }\end{array}$ & Constituent Name & Units & $\begin{array}{l}\text { Number } \\
\text { Analyzed }\end{array}$ & $\begin{array}{l}\text { Number } \\
\text { Detected }\end{array}$ & $\begin{array}{c}\text { Frequency of } \\
\text { Detection }\end{array}$ & $\begin{array}{l}\text { Minimum } \\
\text { Nondetect }\end{array}$ & $\begin{array}{l}\text { Maximum } \\
\text { Nondetect }\end{array}$ & $\begin{array}{c}\text { Minimum } \\
\text { Detect }\end{array}$ & $\begin{array}{l}\text { Maximum } \\
\text { Detect }\end{array}$ & $\begin{array}{c}\text { Average } \\
\text { Concentration }\end{array}$ \\
\hline SVOC & Chrysene & $\mathrm{mg} / \mathrm{kg}$ & 3 & 0 & $0 \%$ & 0.0050 & 0.0099 & $\overline{--}$ & $\overline{--}$ & 0.0041 \\
\hline SVOC & Dibenz[a,h]anthracene & $\mathrm{mg} / \mathrm{kg}$ & 3 & 0 & $0 \%$ & 0.010 & 0.020 & -- & -- & 0.0082 \\
\hline SVOC & Dibenzofuran & $\mathrm{mg} / \mathrm{kg}$ & 3 & 0 & $0 \%$ & 0.0050 & 0.0099 & -- & -- & 0.0041 \\
\hline SVOC & Fluoranthene & $\mathrm{mg} / \mathrm{kg}$ & 3 & 0 & $0 \%$ & 0.010 & 0.020 & -- & -- & 0.0082 \\
\hline SVOC & Fluorene & $\mathrm{mg} / \mathrm{kg}$ & 3 & 0 & $0 \%$ & 0.0088 & 0.0099 & -- & -- & 0.0046 \\
\hline SVOC & Hexachlorobenzene & $\mathrm{mg} / \mathrm{kg}$ & 3 & 0 & $0 \%$ & 0.010 & 0.020 & -- & -- & 0.0082 \\
\hline SVOC & Hexachlorobutadiene & $\mathrm{mg} / \mathrm{kg}$ & 3 & 0 & $0 \%$ & 0.0050 & 0.0099 & -- & -- & 0.0041 \\
\hline SVOC & Hexachloroethane & $\mathrm{mg} / \mathrm{kg}$ & 3 & 0 & $0 \%$ & 0.0050 & 0.0099 & -- & -- & 0.0041 \\
\hline SVOC & Indeno(1,2,3-cd)pyrene & $\mathrm{mg} / \mathrm{kg}$ & 3 & 0 & $0 \%$ & 0.010 & 0.020 & -- & -- & 0.0082 \\
\hline SVOC & Naphthalene & $\mathrm{mg} / \mathrm{kg}$ & 3 & 0 & $0 \%$ & 0.016 & 0.028 & -- & -- & 0.011 \\
\hline SVOC & Nitrobenzene & $\mathrm{mg} / \mathrm{kg}$ & 3 & 0 & $0 \%$ & 0.0050 & 0.0099 & -- & -- & 0.0041 \\
\hline SVOC & Phenanthrene & $\mathrm{mg} / \mathrm{kg}$ & 3 & 0 & $0 \%$ & 0.0050 & 0.0099 & -- & -- & 0.0041 \\
\hline SVOC & Pyrene & $\mathrm{mg} / \mathrm{kg}$ & 3 & 0 & $0 \%$ & 0.010 & 0.020 & -- & -- & 0.0082 \\
\hline VOC & Retene & $\mathrm{mg} / \mathrm{kg}$ & 3 & 0 & $0 \%$ & 0.010 & 0.020 & -- & -- & 0.0082 \\
\hline
\end{tabular}

DIOXI - Dioxins and Furans

PEST - Pesticide

PCB - Polychlorinated biphenyl

SVOC - Semivolatile Organic Compound

VOC - Volatile Organic Compound 
Table F-96

Summary Statistics for White Sturgeon Fillet Collected from Segment 4 of the Columbia River

\begin{tabular}{|c|c|c|c|c|c|c|c|c|c|c|}
\hline $\begin{array}{l}\text { Constituent } \\
\text { Class }\end{array}$ & Constituent Name & Units & $\begin{array}{l}\text { Number } \\
\text { Analyzed }\end{array}$ & $\begin{array}{l}\text { Number } \\
\text { Detected }\end{array}$ & $\begin{array}{c}\text { Frequency of } \\
\text { Detection }\end{array}$ & $\begin{array}{l}\text { Minimum } \\
\text { Nondetect }\end{array}$ & $\begin{array}{l}\text { Maximum } \\
\text { Nondetect }\end{array}$ & $\begin{array}{c}\text { Minimum } \\
\text { Detect }\end{array}$ & $\begin{array}{l}\text { Maximum } \\
\text { Detect }\end{array}$ & $\begin{array}{c}\text { Average } \\
\text { Concentration }\end{array}$ \\
\hline$\overline{D I O X I}$ & $1,2,3,4,6,7,8$-Heptachlorodibenzodioxin & $\mathrm{mg} / \mathrm{kg}$ & 10 & 10 & $100 \%$ & -- & -- & $1.70 \mathrm{E}-07$ & $4.50 \mathrm{E}-07$ & $2.87 \mathrm{E}-07$ \\
\hline DIOXI & 1,2,3,4,6,7,8-Heptachlorodibenzofuran & $\mathrm{mg} / \mathrm{kg}$ & 10 & 10 & $100 \%$ & -- & -- & $7.00 \mathrm{E}-08$ & 1.30E-07 & 9.60E-08 \\
\hline DIOXI & 1,2,3,4,7,8,9-Heptachlorodibenzofuran & $\mathrm{mg} / \mathrm{kg}$ & 10 & 4 & $40 \%$ & $1.00 \mathrm{E}-08$ & $6.00 \mathrm{E}-08$ & $2.00 \mathrm{E}-08$ & 7.00E-08 & 2.15E-08 \\
\hline DIOXI & $1,2,3,4,7,8$-Hexachlorodibenzofuran & $\mathrm{mg} / \mathrm{kg}$ & 10 & 9 & $90 \%$ & $5.00 \mathrm{E}-08$ & $5.00 \mathrm{E}-08$ & $2.00 \mathrm{E}-08$ & $6.00 \mathrm{E}-08$ & 4.05E-08 \\
\hline DIOXI & $1,2,3,4,7,8$-Hexachlorodibenzo-p-dioxin & $\mathrm{mg} / \mathrm{kg}$ & 10 & 10 & $100 \%$ & -- & -- & $9.00 \mathrm{E}-08$ & $1.60 \mathrm{E}-07$ & 1.25E-07 \\
\hline DIOXI & 1,2,3,6,7,8-Hexachlorodibenzofuran & $\mathrm{mg} / \mathrm{kg}$ & 10 & 9 & $90 \%$ & $5.00 \mathrm{E}-08$ & $5.00 \mathrm{E}-08$ & $4.00 \mathrm{E}-08$ & $9.00 \mathrm{E}-08$ & $6.25 \mathrm{E}-08$ \\
\hline DIOXI & 1,2,3,6,7,8-Hexachlorodibenzo-p-dioxin & $\mathrm{mg} / \mathrm{kg}$ & 10 & 8 & $80 \%$ & 3.00E-08 & 8.00E-08 & 8.00E-08 & $2.60 \mathrm{E}-07$ & $1.29 \mathrm{E}-07$ \\
\hline DIOXI & 1,2,3,7,8,9-Hexachlorodibenzofuran & $\mathrm{mg} / \mathrm{kg}$ & 10 & 8 & $80 \%$ & 1.00E-08 & $5.00 \mathrm{E}-08$ & $6.00 \mathrm{E}-08$ & $1.30 \mathrm{E}-07$ & 7.70E-08 \\
\hline DIOXI & 1,2,3,7,8,9-Hexachlorodibenzo-p-dioxin & $\mathrm{mg} / \mathrm{kg}$ & 10 & 8 & $80 \%$ & 1.00E-08 & 8.00E-08 & $5.00 \mathrm{E}-08$ & 1.00E-07 & $6.45 \mathrm{E}-08$ \\
\hline DIOXI & 1,2,3,7,8-Pentachlorodibenzofuran & $\mathrm{mg} / \mathrm{kg}$ & 10 & 10 & $100 \%$ & -- & -- & $8.00 \mathrm{E}-08$ & $3.30 \mathrm{E}-07$ & 2.33E-07 \\
\hline DIOXI & 1,2,3,7,8-Pentachlorodibenzo-p-dioxin & $\mathrm{mg} / \mathrm{kg}$ & 10 & 8 & $80 \%$ & $1.00 \mathrm{E}-08$ & 1.00E-08 & $1.20 \mathrm{E}-07$ & $1.80 \mathrm{E}-07$ & 1.23E-07 \\
\hline DIOXI & 2,3,4,6,7,8-Hexachlorodibenzofuran & $\mathrm{mg} / \mathrm{kg}$ & 10 & 10 & $100 \%$ & -- & -- & $1.20 \mathrm{E}-07$ & $1.80 \mathrm{E}-07$ & $1.47 \mathrm{E}-07$ \\
\hline DIOXI & 2,3,4,7,8-Pentachlorodibenzofuran & $\mathrm{mg} / \mathrm{kg}$ & 10 & 10 & $100 \%$ & -- & -- & $6.00 \mathrm{E}-08$ & $2.90 \mathrm{E}-07$ & 1.95E-07 \\
\hline DIOXI & 2,3,7,8-Tetrachlorodibenzofuran & $\mathrm{mg} / \mathrm{kg}$ & 10 & 10 & $100 \%$ & -- & -- & $6.23 \mathrm{E}-06$ & $2.26 \mathrm{E}-05$ & 1.50E-05 \\
\hline DIOXI & 2,3,7,8-Tetrachlorodibenzo-p-dioxin & $\mathrm{mg} / \mathrm{kg}$ & 10 & 10 & $100 \%$ & -- & -- & $1.30 \mathrm{E}-07$ & $5.20 \mathrm{E}-07$ & 3.53E-07 \\
\hline DIOXI & Octachlorodibenzofuran & $\mathrm{mg} / \mathrm{kg}$ & 10 & 7 & $70 \%$ & $1.00 \mathrm{E}-08$ & 4.00E-08 & $4.00 \mathrm{E}-08$ & $8.00 \mathrm{E}-08$ & 4.70E-08 \\
\hline DIOXI & Octachlorodibenzo-p-dioxin & $\mathrm{mg} / \mathrm{kg}$ & 10 & 10 & $100 \%$ & -- & -- & $2.90 \mathrm{E}-07$ & $7.00 \mathrm{E}-07$ & 4.65E-07 \\
\hline METAL & Aluminum & $\mathrm{mg} / \mathrm{kg}$ & 10 & 0 & $0 \%$ & 5.0 & 20 & -- & -- & 6.3 \\
\hline METAL & Antimony & $\mathrm{mg} / \mathrm{kg}$ & 10 & 0 & $0 \%$ & 0.050 & 0.050 & -- & -- & 0.025 \\
\hline METAL & Arsenic & $\mathrm{mg} / \mathrm{kg}$ & 10 & 10 & $100 \%$ & -- & -- & 0.18 & 0.64 & 0.33 \\
\hline METAL & Barium & $\mathrm{mg} / \mathrm{kg}$ & 10 & 5 & $50 \%$ & 0.20 & 0.20 & 0.27 & 0.63 & 0.26 \\
\hline METAL & Beryllium & $\mathrm{mg} / \mathrm{kg}$ & 10 & 0 & $0 \%$ & 0.0040 & 0.0040 & -- & -- & 0.0020 \\
\hline METAL & Cadmium & $\mathrm{mg} / \mathrm{kg}$ & 10 & 0 & $0 \%$ & 0.0040 & 0.0040 & -- & -- & 0.0020 \\
\hline METAL & Chromium & $\mathrm{mg} / \mathrm{kg}$ & 10 & 0 & $0 \%$ & 0.10 & 0.10 & -- & -- & 0.050 \\
\hline METAL & Cobalt & $\mathrm{mg} / \mathrm{kg}$ & 10 & 9 & $90 \%$ & 0.0010 & 0.0010 & 0.0013 & 0.0089 & 0.0049 \\
\hline METAL & Copper & $\mathrm{mg} / \mathrm{kg}$ & 10 & 7 & $70 \%$ & 0.25 & 0.29 & 0.21 & 0.41 & 0.23 \\
\hline METAL & Lead & $\mathrm{mg} / \mathrm{kg}$ & 10 & 4 & $40 \%$ & 0.010 & 0.010 & 0.010 & 0.020 & 0.0080 \\
\hline METAL & Manganese & $\mathrm{mg} / \mathrm{kg}$ & 10 & 10 & $100 \%$ & -- & -- & 0.24 & 0.31 & 0.27 \\
\hline METAL & Mercury & $\mathrm{mg} / \mathrm{kg}$ & 10 & 7 & $70 \%$ & 0.030 & 0.040 & 0.050 & 0.28 & 0.091 \\
\hline METAL & Nickel & $\mathrm{mg} / \mathrm{kg}$ & 10 & 5 & $50 \%$ & 0.030 & 0.030 & 0.030 & 0.060 & 0.033 \\
\hline METAL & Selenium & $\mathrm{mg} / \mathrm{kg}$ & 10 & 10 & $100 \%$ & -- & -- & 0.31 & 1.9 & 0.93 \\
\hline METAL & Silver & $\mathrm{mg} / \mathrm{kg}$ & 10 & 0 & $0 \%$ & 0.10 & 0.10 & -- & -- & 0.050 \\
\hline METAL & Thallium & $\mathrm{mg} / \mathrm{kg}$ & 10 & 0 & $0 \%$ & 0.050 & 0.050 & -- & -- & 0.025 \\
\hline METAL & Vanadium & $\mathrm{mg} / \mathrm{kg}$ & 10 & 4 & $40 \%$ & 0.010 & 0.020 & 0.010 & 0.010 & 0.0095 \\
\hline METAL & Zinc & $\mathrm{mg} / \mathrm{kg}$ & 10 & 10 & $100 \%$ & -- & -- & 2.9 & 4.7 & 3.6 \\
\hline PCB & 2,2',3,3',4,4',5-Heptachloro-1,1'-biphenyl & $\mathrm{mg} / \mathrm{kg}$ & 10 & 10 & $100 \%$ & -- & -- & 3.56E-04 & 0.0014 & 8.83E-04 \\
\hline РCB & 2,2',3,4,4',5,5'-Heptachlorobiphenyl & $\mathrm{mg} / \mathrm{kg}$ & 10 & 10 & $100 \%$ & -- & -- & 0.0012 & 0.0045 & 0.0030 \\
\hline PCB & 2,3,3',4,4',5'-Hexachlorobiphenyl & $\mathrm{mg} / \mathrm{kg}$ & 10 & 10 & $100 \%$ & -- & -- & $2.31 \mathrm{E}-05$ & 1.69E-04 & $9.84 \mathrm{E}-05$ \\
\hline РCB & 2,3,3',4,4',5-Hexachlorobiphenyl (BZ 156) & $\mathrm{mg} / \mathrm{kg}$ & 10 & 10 & $100 \%$ & -- & -- & $1.35 \mathrm{E}-04$ & 8.39E-04 & 4.93E-04 \\
\hline PCB & 2,3,3',4,4'-Pentachlorobiphenyl & $\mathrm{mg} / \mathrm{kg}$ & 10 & 10 & $100 \%$ & -- & -- & $3.50 \mathrm{E}-04$ & 0.0028 & 0.0015 \\
\hline РCB & 2,3',4,4',5,5'-Hexachlorobiphenyl (BZ 167) & $\mathrm{mg} / \mathrm{kg}$ & 10 & 3 & $30 \%$ & $1.24 \mathrm{E}-06$ & 1.10E-05 & $9.44 \mathrm{E}-06$ & 2.15E-05 & $6.85 \mathrm{E}-06$ \\
\hline PCB & 2',3,4,4',5-Pentachloro-1,1'-biphenyl (BZ 123) & $\mathrm{mg} / \mathrm{kg}$ & 10 & 10 & $100 \%$ & -- & -- & $4.44 \mathrm{E}-06$ & 2.87E-05 & $1.75 \mathrm{E}-05$ \\
\hline PCB & 2,3,4,4',5-Pentachlorobiphenyl (BZ 114) & $\mathrm{mg} / \mathrm{kg}$ & 10 & 10 & $100 \%$ & -- & -- & $2.77 \mathrm{E}-05$ & 2.13E-04 & $1.24 \mathrm{E}-04$ \\
\hline PCB & 2,3',4,4',5-Pentachlorobiphenyl (BZ 118) & $\mathrm{mg} / \mathrm{kg}$ & 10 & 10 & $100 \%$ & -- & -- & $2.72 \mathrm{E}-04$ & 0.0027 & 0.0016 \\
\hline РCB & 2,3,4,5,3',4',5'-Heptachlorobiphenyl & $\mathrm{mg} / \mathrm{kg}$ & 10 & 10 & $100 \%$ & -- & -- & 1.47E-05 & 5.27E-05 & 3.49E-05 \\
\hline PCB & 3,3',4,4',5,5'-Hexachlorobiphenyl (BZ 169) & $\mathrm{mg} / \mathrm{kg}$ & 10 & 0 & $0 \%$ & $5.00 \mathrm{E}-07$ & $2.00 \mathrm{E}-06$ & -- & -- & 4.34E-07 \\
\hline РСВ & 3,3',4,4',5-Pentachlorobiphenyl (BZ 126) & $\mathrm{mg} / \mathrm{kg}$ & 10 & 1 & $10 \%$ & 7.40E-07 & 2.85E-06 & $2.57 \mathrm{E}-06$ & 2.57E-06 & 1.07E-06 \\
\hline PCB & 3,3',4,4'-Tetrachlorobiphenyl (BZ 77) & $\mathrm{mg} / \mathrm{kg}$ & 10 & 10 & $100 \%$ & -- & -- & 1.10E-06 & $2.25 \mathrm{E}-05$ & 1.10E-05 \\
\hline РСB & Aroclor-1016 & $\mathrm{mg} / \mathrm{kg}$ & 10 & 0 & $0 \%$ & 0.017 & 0.020 & -- & -- & 0.0093 \\
\hline PCB & Aroclor-1221 & $\mathrm{mg} / \mathrm{kg}$ & 10 & 0 & $0 \%$ & 0.017 & 0.020 & -- & -- & 0.0093 \\
\hline
\end{tabular}


Table F-96

Summary Statistics for White Sturgeon Fillet Collected from Segment 4 of the Columbia River

\begin{tabular}{|c|c|c|c|c|c|c|c|c|c|c|}
\hline $\begin{array}{l}\text { Constituent } \\
\text { Class }\end{array}$ & Constituent Name & Units & $\begin{array}{l}\text { Number } \\
\text { Analyzed }\end{array}$ & $\begin{array}{l}\text { Number } \\
\text { Detected }\end{array}$ & $\begin{array}{c}\text { Frequency of } \\
\text { Detection }\end{array}$ & $\begin{array}{l}\text { Minimum } \\
\text { Nondetect }\end{array}$ & $\begin{array}{l}\text { Maximum } \\
\text { Nondetect }\end{array}$ & $\begin{array}{l}\text { Minimum } \\
\text { Detect }\end{array}$ & $\begin{array}{l}\text { Maximum } \\
\text { Detect }\end{array}$ & $\begin{array}{c}\text { Average } \\
\text { Concentration }\end{array}$ \\
\hline$\overline{\mathrm{PCB}}$ & Aroclor-1232 & $\mathrm{mg} / \mathrm{kg}$ & 10 & 0 & $0 \%$ & 0.017 & 0.020 & -- & -- & 0.0093 \\
\hline PCB & Aroclor-1248 & $\mathrm{mg} / \mathrm{kg}$ & 10 & 0 & $0 \%$ & 0.017 & 0.020 & -- & -- & 0.0093 \\
\hline РCB & Aroclor-1254 & $\mathrm{mg} / \mathrm{kg}$ & 10 & 10 & $100 \%$ & -- & -- & 0.010 & 0.16 & 0.047 \\
\hline РСВ & Aroclor-1260 & $\mathrm{mg} / \mathrm{kg}$ & 10 & 10 & $100 \%$ & -- & -- & 2.32E-04 & 0.046 & 0.032 \\
\hline PEST & 2,4'-DDD & $\mathrm{mg} / \mathrm{kg}$ & 10 & 10 & $100 \%$ & -- & -- & 0.0022 & 0.013 & 0.0067 \\
\hline PEST & 2,4'-DDE & $\mathrm{mg} / \mathrm{kg}$ & 10 & 9 & $90 \%$ & 0.0020 & 0.0020 & 0.0026 & 0.0064 & 0.0041 \\
\hline PEST & 2,4'-DDT & $\mathrm{mg} / \mathrm{kg}$ & 10 & 8 & $80 \%$ & 0.0018 & 0.0020 & 0.0023 & 0.0095 & 0.0034 \\
\hline PEST & Aldrin & $\mathrm{mg} / \mathrm{kg}$ & 10 & 0 & $0 \%$ & 0.0017 & 0.0020 & -- & -- & $9.30 \mathrm{E}-04$ \\
\hline PEST & Alpha-BHC & $\mathrm{mg} / \mathrm{kg}$ & 10 & 0 & $0 \%$ & 0.0017 & 0.0020 & -- & -- & $9.30 \mathrm{E}-04$ \\
\hline PEST & alpha-Chlordane & $\mathrm{mg} / \mathrm{kg}$ & 10 & 4 & $40 \%$ & 0.0017 & 0.0020 & 0.0023 & 0.0080 & 0.0022 \\
\hline PEST & alpha-Chlordene & $\mathrm{mg} / \mathrm{kg}$ & 10 & 0 & $0 \%$ & 0.0017 & 0.0020 & -- & -- & $9.30 \mathrm{E}-04$ \\
\hline PEST & Beta-BHC & $\mathrm{mg} / \mathrm{kg}$ & 10 & 0 & $0 \%$ & 0.0017 & 0.0020 & -- & -- & $9.30 \mathrm{E}-04$ \\
\hline PEST & cis-Nonachlor & $\mathrm{mg} / \mathrm{kg}$ & 10 & 7 & $70 \%$ & 0.0017 & 0.0020 & 0.0039 & 0.0080 & 0.0042 \\
\hline PEST & DDMU & $\mathrm{mg} / \mathrm{kg}$ & 10 & 10 & $100 \%$ & -- & -- & 0.0020 & 0.018 & 0.0066 \\
\hline PEST & Delta-BHC & $\mathrm{mg} / \mathrm{kg}$ & 10 & 0 & $0 \%$ & 0.0017 & 0.0020 & -- & -- & $9.30 \mathrm{E}-04$ \\
\hline PEST & Dichlorodiphenyldichloroethane & $\mathrm{mg} / \mathrm{kg}$ & 10 & 10 & $100 \%$ & -- & -- & 0.016 & 0.074 & 0.045 \\
\hline PEST & Dichlorodiphenyldichloroethylene & $\mathrm{mg} / \mathrm{kg}$ & 10 & 10 & $100 \%$ & -- & -- & 0.10 & 0.59 & 0.36 \\
\hline PEST & Dichlorodiphenyltrichloroethane & $\mathrm{mg} / \mathrm{kg}$ & 10 & 9 & $90 \%$ & 0.0020 & 0.0020 & 0.0014 & 0.0053 & 0.0030 \\
\hline PEST & Endosulfan sulfate & $\mathrm{mg} / \mathrm{kg}$ & 10 & 0 & $0 \%$ & 0.0017 & 0.0020 & -- & -- & $9.30 \mathrm{E}-04$ \\
\hline PEST & Gamma-BHC (Lindane) & $\mathrm{mg} / \mathrm{kg}$ & 10 & 0 & $0 \%$ & 0.0017 & 0.0020 & -- & -- & $9.30 \mathrm{E}-04$ \\
\hline PEST & gamma-Chlordane & $\mathrm{mg} / \mathrm{kg}$ & 10 & 8 & $80 \%$ & 0.0018 & 0.0020 & 0.0022 & 0.0081 & 0.0033 \\
\hline PEST & gamma-Chlordene & $\mathrm{mg} / \mathrm{kg}$ & 10 & 0 & $0 \%$ & 0.0017 & 0.0020 & -- & -- & $9.30 \mathrm{E}-04$ \\
\hline PEST & Heptachlor & $\mathrm{mg} / \mathrm{kg}$ & 10 & 0 & $0 \%$ & 0.0017 & 0.0020 & -- & -- & $9.30 \mathrm{E}-04$ \\
\hline PEST & Heptachlor epoxide & $\mathrm{mg} / \mathrm{kg}$ & 10 & 1 & $10 \%$ & 0.0018 & 0.0020 & 0.0012 & 0.0012 & $9.65 \mathrm{E}-04$ \\
\hline PEST & Mirex & $\mathrm{mg} / \mathrm{kg}$ & 10 & 0 & $0 \%$ & 0.0017 & 0.0020 & -- & -- & $9.30 \mathrm{E}-04$ \\
\hline PEST & Oxychlordane & $\mathrm{mg} / \mathrm{kg}$ & 10 & 0 & $0 \%$ & 0.0018 & 0.0020 & -- & -- & $9.40 \mathrm{E}-04$ \\
\hline PEST & Pentachloroanisole & $\mathrm{mg} / \mathrm{kg}$ & 10 & 0 & $0 \%$ & 0.0017 & 0.0020 & -- & -- & $9.30 \mathrm{E}-04$ \\
\hline PEST & Toxaphene & $\mathrm{mg} / \mathrm{kg}$ & 10 & 0 & $0 \%$ & 0.052 & 0.059 & -- & -- & 0.028 \\
\hline PEST & trans-Nonachlor & $\mathrm{mg} / \mathrm{kg}$ & 10 & 9 & $90 \%$ & 0.0020 & 0.0020 & 0.0035 & 0.0096 & 0.0054 \\
\hline SVOC & 1,2,4-Trichlorobenzene & $\mathrm{mg} / \mathrm{kg}$ & 10 & 0 & $0 \%$ & 0.0046 & 0.025 & -- & -- & 0.0034 \\
\hline SVOC & 1,2-Dichlorobenzene & $\mathrm{mg} / \mathrm{kg}$ & 10 & 0 & $0 \%$ & 0.0046 & 0.025 & -- & -- & 0.0034 \\
\hline SVOC & 1,2-Diphenylhydrazine & $\mathrm{mg} / \mathrm{kg}$ & 10 & 0 & $0 \%$ & 0.0046 & 0.025 & -- & -- & 0.0034 \\
\hline SVOC & 1,3-Dichlorobenzene & $\mathrm{mg} / \mathrm{kg}$ & 10 & 0 & $0 \%$ & 0.0046 & 0.025 & -- & -- & 0.0034 \\
\hline SVOC & 1,4-Dichlorobenzene & $\mathrm{mg} / \mathrm{kg}$ & 10 & 0 & $0 \%$ & 0.0046 & 0.025 & -- & -- & 0.0034 \\
\hline SVOC & 1-methylnaphthalene & $\mathrm{mg} / \mathrm{kg}$ & 10 & 1 & $10 \%$ & 0.0046 & 0.025 & 0.010 & 0.010 & 0.0042 \\
\hline SVOC & 2,4-Dinitrotoluene & $\mathrm{mg} / \mathrm{kg}$ & 10 & 0 & $0 \%$ & 0.0093 & 0.051 & -- & -- & 0.0069 \\
\hline SVOC & 2,6-Dinitrotoluene & $\mathrm{mg} / \mathrm{kg}$ & 10 & 0 & $0 \%$ & 0.019 & 0.10 & -- & -- & 0.014 \\
\hline SVOC & 2-Chloronaphthalene & $\mathrm{mg} / \mathrm{kg}$ & 10 & 0 & $0 \%$ & 0.0093 & 0.025 & -- & -- & 0.0056 \\
\hline SVOC & 2-Methylnaphthalene & $\mathrm{mg} / \mathrm{kg}$ & 10 & 1 & $10 \%$ & 0.0046 & 0.025 & 0.025 & 0.025 & 0.0061 \\
\hline SVOC & 4-Bromophenylphenyl ether & $\mathrm{mg} / \mathrm{kg}$ & 10 & 0 & $0 \%$ & 0.0046 & 0.025 & -- & -- & 0.0034 \\
\hline SVOC & 4-Chlorophenylphenyl ether & $\mathrm{mg} / \mathrm{kg}$ & 10 & 0 & $0 \%$ & 0.0046 & 0.025 & -- & -- & 0.0034 \\
\hline SVOC & Acenaphthene & $\mathrm{mg} / \mathrm{kg}$ & 10 & 0 & $0 \%$ & 0.0093 & 0.051 & -- & -- & 0.0069 \\
\hline SVOC & Acenaphthylene & $\mathrm{mg} / \mathrm{kg}$ & 10 & 0 & $0 \%$ & 0.0046 & 0.025 & -- & -- & 0.0034 \\
\hline SVOC & Anthracene & $\mathrm{mg} / \mathrm{kg}$ & 10 & 0 & $0 \%$ & 0.0046 & 0.025 & -- & -- & 0.0034 \\
\hline SVOC & Benzo(a)anthracene & $\mathrm{mg} / \mathrm{kg}$ & 10 & 0 & $0 \%$ & 0.0046 & 0.025 & -- & -- & 0.0044 \\
\hline SVOC & Benzo(a)pyrene & $\mathrm{mg} / \mathrm{kg}$ & 10 & 0 & $0 \%$ & 0.0047 & 0.025 & -- & -- & 0.0053 \\
\hline SVOC & Benzo(b)fluoranthene & $\mathrm{mg} / \mathrm{kg}$ & 10 & 0 & $0 \%$ & 0.0047 & 0.051 & -- & -- & 0.0066 \\
\hline SVOC & Benzo(ghi)perylene & $\mathrm{mg} / \mathrm{kg}$ & 10 & 0 & $0 \%$ & 0.0094 & 0.051 & -- & -- & 0.011 \\
\hline SVOC & Benzo(k)fluoranthene & $\mathrm{mg} / \mathrm{kg}$ & 10 & 0 & $0 \%$ & 0.0047 & 0.051 & -- & -- & 0.0066 \\
\hline SVOC & Bis(2-chloro-1-methylethyl)ether & $\mathrm{mg} / \mathrm{kg}$ & 10 & 0 & $0 \%$ & 0.0093 & 0.051 & -- & -- & 0.0069 \\
\hline
\end{tabular}


Table F-96

Summary Statistics for White Sturgeon Fillet Collected from Segment 4 of the Columbia River

\begin{tabular}{|c|c|c|c|c|c|c|c|c|c|c|}
\hline $\begin{array}{l}\text { Constituent } \\
\text { Class }\end{array}$ & Constituent Name & Units & $\begin{array}{l}\text { Number } \\
\text { Analyzed }\end{array}$ & $\begin{array}{l}\text { Number } \\
\text { Detected }\end{array}$ & $\begin{array}{c}\text { Frequency of } \\
\text { Detection }\end{array}$ & $\begin{array}{l}\text { Minimum } \\
\text { Nondetect }\end{array}$ & $\begin{array}{l}\text { Maximum } \\
\text { Nondetect }\end{array}$ & $\begin{array}{l}\text { Minimum } \\
\text { Detect }\end{array}$ & $\begin{array}{l}\text { Maximum } \\
\text { Detect }\end{array}$ & $\begin{array}{c}\text { Average } \\
\text { Concentration }\end{array}$ \\
\hline SVOC & Chrysene & $\mathrm{mg} / \mathrm{kg}$ & 10 & 0 & $0 \%$ & 0.0046 & 0.025 & $\overline{--}$ & -- & 0.0044 \\
\hline SVOC & Dibenz[a,h]anthracene & $\mathrm{mg} / \mathrm{kg}$ & 10 & 0 & $0 \%$ & 0.0094 & 0.051 & -- & -- & 0.011 \\
\hline SVOC & Dibenzofuran & $\mathrm{mg} / \mathrm{kg}$ & 10 & 0 & $0 \%$ & 0.0046 & 0.025 & -- & -- & 0.0034 \\
\hline SVOC & Fluoranthene & $\mathrm{mg} / \mathrm{kg}$ & 10 & 0 & $0 \%$ & 0.0093 & 0.051 & -- & -- & 0.0069 \\
\hline SVOC & Fluorene & $\mathrm{mg} / \mathrm{kg}$ & 10 & 0 & $0 \%$ & 0.0074 & 0.0099 & -- & -- & 0.0046 \\
\hline SVOC & Hexachlorobenzene & $\mathrm{mg} / \mathrm{kg}$ & 10 & 1 & $10 \%$ & 0.0093 & 0.051 & 0.0099 & 0.0099 & 0.0074 \\
\hline SVOC & Hexachlorobutadiene & $\mathrm{mg} / \mathrm{kg}$ & 10 & 0 & $0 \%$ & 0.0046 & 0.025 & -- & -- & 0.0034 \\
\hline SVOC & Hexachloroethane & $\mathrm{mg} / \mathrm{kg}$ & 10 & 0 & $0 \%$ & 0.0046 & 0.025 & -- & -- & 0.0034 \\
\hline SVOC & Indeno(1,2,3-cd)pyrene & $\mathrm{mg} / \mathrm{kg}$ & 10 & 0 & $0 \%$ & 0.0094 & 0.051 & -- & -- & 0.011 \\
\hline SVOC & Naphthalene & $\mathrm{mg} / \mathrm{kg}$ & 10 & 0 & $0 \%$ & 0.019 & 0.060 & -- & -- & 0.014 \\
\hline SVOC & Nitrobenzene & $\mathrm{mg} / \mathrm{kg}$ & 10 & 0 & $0 \%$ & 0.0046 & 0.025 & -- & -- & 0.0034 \\
\hline SVOC & Phenanthrene & $\mathrm{mg} / \mathrm{kg}$ & 10 & 0 & $0 \%$ & 0.0046 & 0.025 & -- & -- & 0.0038 \\
\hline SVOC & Pyrene & $\mathrm{mg} / \mathrm{kg}$ & 10 & 0 & $0 \%$ & 0.0093 & 0.051 & -- & -- & 0.0089 \\
\hline VOC & Retene & $\mathrm{mg} / \mathrm{kg}$ & 10 & 0 & $0 \%$ & 0.0093 & 0.051 & -- & -- & 0.0089 \\
\hline
\end{tabular}

DIOXI - Dioxins and Furans

PEST - Pesticide

PCB - Polychlorinated biphenyl

SVOC - Semivolatile Organic Compound

VOC - Volatile Organic Compound 
Table F-97

Summary Statistics for Whole Body White Sturgeon Collected from Segment 4 of the Columbia River

\begin{tabular}{|c|c|c|c|c|c|c|c|c|c|c|}
\hline $\begin{array}{l}\text { Constituent } \\
\text { Class }\end{array}$ & Constituent Name & Units & $\begin{array}{l}\text { Number } \\
\text { Analyzed }\end{array}$ & $\begin{array}{l}\text { Number } \\
\text { Detected }\end{array}$ & $\begin{array}{l}\text { Frequency of } \\
\text { Detection }\end{array}$ & $\begin{array}{l}\text { Minimum } \\
\text { Nondetect }\end{array}$ & $\begin{array}{l}\text { Maximum } \\
\text { Nondetect }\end{array}$ & $\begin{array}{l}\text { Minimum } \\
\text { Detect }\end{array}$ & $\begin{array}{l}\text { Maximum } \\
\text { Detect }\end{array}$ & $\begin{array}{c}\text { Average } \\
\text { Concentration }\end{array}$ \\
\hline$\overline{\mathrm{DIOXI}}$ & $1,2,3,4,6,7,8$-Heptachlorodibenzodioxin & $\mathrm{mg} / \mathrm{kg}$ & 3 & 3 & $100 \%$ & $\overline{--}$ & $\overline{--}$ & $3.40 \mathrm{E}-07$ & $4.90 \mathrm{E}-07$ & $4.27 \mathrm{E}-07$ \\
\hline DIOXI & 1,2,3,4,6,7,8-Heptachlorodibenzofuran & $\mathrm{mg} / \mathrm{kg}$ & 3 & 2 & $67 \%$ & $3.00 \mathrm{E}-08$ & $3.00 \mathrm{E}-08$ & 1.30E-07 & 1.70E-07 & 1.05E-07 \\
\hline DIOXI & 1,2,3,4,7,8,9-Heptachlorodibenzofuran & $\mathrm{mg} / \mathrm{kg}$ & 3 & 1 & $33 \%$ & $3.00 \mathrm{E}-08$ & $3.00 \mathrm{E}-08$ & 5.00E-08 & $5.00 \mathrm{E}-08$ & $2.67 \mathrm{E}-08$ \\
\hline DIOXI & $1,2,3,4,7,8$-Hexachlorodibenzofuran & $\mathrm{mg} / \mathrm{kg}$ & 3 & 3 & $100 \%$ & -- & -- & 4.00E-08 & $6.00 \mathrm{E}-08$ & $5.00 \mathrm{E}-08$ \\
\hline DIOXI & 1,2,3,4,7,8-Hexachlorodibenzo-p-dioxin & $\mathrm{mg} / \mathrm{kg}$ & 3 & 2 & $67 \%$ & 1.00E-08 & 1.00E-08 & 1.20E-07 & 1.30E-07 & 8.50E-08 \\
\hline DIOXI & $1,2,3,6,7,8$-Hexachlorodibenzofuran & $\mathrm{mg} / \mathrm{kg}$ & 3 & 2 & $67 \%$ & 2.00E-08 & 2.00E-08 & 7.00E-08 & $1.00 \mathrm{E}-07$ & $6.00 \mathrm{E}-08$ \\
\hline DIOXI & $1,2,3,6,7,8$-Hexachlorodibenzo-p-dioxin & $\mathrm{mg} / \mathrm{kg}$ & 3 & 2 & $67 \%$ & $2.00 \mathrm{E}-08$ & $2.00 \mathrm{E}-08$ & $2.40 \mathrm{E}-07$ & $2.50 \mathrm{E}-07$ & 1.67E-07 \\
\hline DIOXI & 1,2,3,7,8,9-Hexachlorodibenzofuran & $\mathrm{mg} / \mathrm{kg}$ & 3 & 3 & $100 \%$ & -- & -- & 5.00E-08 & $7.00 \mathrm{E}-08$ & $6.00 \mathrm{E}-08$ \\
\hline DIOXI & 1,2,3,7,8,9-Hexachlorodibenzo-p-dioxin & $\mathrm{mg} / \mathrm{kg}$ & 3 & 2 & $67 \%$ & $2.00 \mathrm{E}-08$ & $2.00 \mathrm{E}-08$ & 5.00E-08 & $7.00 \mathrm{E}-08$ & 4.33E-08 \\
\hline DIOXI & 1,2,3,7,8-Pentachlorodibenzofuran & $\mathrm{mg} / \mathrm{kg}$ & 3 & 3 & $100 \%$ & -- & -- & $2.90 \mathrm{E}-07$ & $3.60 \mathrm{E}-07$ & 3.27E-07 \\
\hline DIOXI & 1,2,3,7,8-Pentachlorodibenzo-p-dioxin & $\mathrm{mg} / \mathrm{kg}$ & 3 & 1 & $33 \%$ & 1.00E-08 & $1.00 \mathrm{E}-08$ & 1.70E-07 & 1.70E-07 & $6.00 \mathrm{E}-08$ \\
\hline DIOXI & $2,3,4,6,7,8$-Hexachlorodibenzofuran & $\mathrm{mg} / \mathrm{kg}$ & 3 & 2 & $67 \%$ & 2.00E-08 & 2.00E-08 & 1.90E-07 & $1.90 \mathrm{E}-07$ & 1.30E-07 \\
\hline DIOXI & 2,3,4,7,8-Pentachlorodibenzofuran & $\mathrm{mg} / \mathrm{kg}$ & 3 & 3 & $100 \%$ & -- & -- & $2.50 \mathrm{E}-07$ & $3.50 \mathrm{E}-07$ & $2.87 \mathrm{E}-07$ \\
\hline DIOXI & $2,3,7,8$-Tetrachlorodibenzofuran & $\mathrm{mg} / \mathrm{kg}$ & 3 & 3 & $100 \%$ & -- & -- & 1.57E-05 & 2.10E-05 & $1.84 \mathrm{E}-05$ \\
\hline DIOXI & 2,3,7,8-Tetrachlorodibenzo-p-dioxin & $\mathrm{mg} / \mathrm{kg}$ & 3 & 3 & $100 \%$ & -- & -- & 4.50E-07 & 8.90E-07 & 6.13E-07 \\
\hline DIOXI & Octachlorodibenzofuran & $\mathrm{mg} / \mathrm{kg}$ & 3 & 1 & $33 \%$ & 1.00E-08 & 2.00E-08 & $1.80 \mathrm{E}-07$ & $1.80 \mathrm{E}-07$ & $6.50 \mathrm{E}-08$ \\
\hline DIOXI & Octachlorodibenzo-p-dioxin & $\mathrm{mg} / \mathrm{kg}$ & 3 & 3 & $100 \%$ & -- & -- & $6.00 \mathrm{E}-07$ & 1.26E-06 & 8.50E-07 \\
\hline METAL & Aluminum & $\mathrm{mg} / \mathrm{kg}$ & 3 & 3 & $100 \%$ & -- & -- & 3.4 & 180 & 69 \\
\hline METAL & Antimony & $\mathrm{mg} / \mathrm{kg}$ & 3 & 0 & $0 \%$ & 0.050 & 0.50 & -- & -- & 0.10 \\
\hline METAL & Arsenic & $\mathrm{mg} / \mathrm{kg}$ & 3 & 2 & $67 \%$ & 0.50 & 0.50 & 0.59 & 0.65 & 0.50 \\
\hline METAL & Barium & $\mathrm{mg} / \mathrm{kg}$ & 3 & 3 & $100 \%$ & -- & -- & 1.1 & 2.7 & 1.9 \\
\hline METAL & Beryllium & $\mathrm{mg} / \mathrm{kg}$ & 3 & 2 & $67 \%$ & 0.060 & 0.060 & 0.0080 & 0.0092 & 0.016 \\
\hline METAL & Cadmium & $\mathrm{mg} / \mathrm{kg}$ & 3 & 3 & $100 \%$ & -- & -- & 0.020 & 0.090 & 0.043 \\
\hline METAL & Chromium & $\mathrm{mg} / \mathrm{kg}$ & 3 & 2 & $67 \%$ & 1.0 & 1.0 & 0.14 & 0.27 & 0.30 \\
\hline METAL & Cobalt & $\mathrm{mg} / \mathrm{kg}$ & 3 & 3 & $100 \%$ & -- & -- & 0.10 & 0.42 & 0.28 \\
\hline METAL & Copper & $\mathrm{mg} / \mathrm{kg}$ & 3 & 3 & $100 \%$ & -- & -- & 0.76 & 1.5 & 1.0 \\
\hline METAL & Lead & $\mathrm{mg} / \mathrm{kg}$ & 3 & 3 & $100 \%$ & -- & -- & 0.10 & 0.33 & 0.18 \\
\hline METAL & Manganese & $\mathrm{mg} / \mathrm{kg}$ & 3 & 3 & $100 \%$ & -- & -- & 1.0 & 6.7 & 3.0 \\
\hline METAL & Mercury & $\mathrm{mg} / \mathrm{kg}$ & 3 & 3 & $100 \%$ & -- & -- & 0.070 & 0.24 & 0.17 \\
\hline METAL & Nickel & $\mathrm{mg} / \mathrm{kg}$ & 3 & 3 & $100 \%$ & -- & -- & 0.050 & 0.33 & 0.15 \\
\hline METAL & Selenium & $\mathrm{mg} / \mathrm{kg}$ & 3 & 2 & $67 \%$ & 1.0 & 1.0 & 0.42 & 0.62 & 0.51 \\
\hline METAL & Silver & $\mathrm{mg} / \mathrm{kg}$ & 3 & 0 & $0 \%$ & 0.20 & 2.0 & -- & -- & 0.40 \\
\hline METAL & Thallium & $\mathrm{mg} / \mathrm{kg}$ & 3 & 0 & $0 \%$ & 0.050 & 0.50 & -- & -- & 0.10 \\
\hline METAL & Vanadium & $\mathrm{mg} / \mathrm{kg}$ & 3 & 3 & $100 \%$ & -- & -- & 0.050 & 0.67 & 0.27 \\
\hline METAL & Zinc & $\mathrm{mg} / \mathrm{kg}$ & 3 & 3 & $100 \%$ & -- & -- & 8.9 & 9.6 & 9.1 \\
\hline PCB & 2,2',3,3',4,4',5-Heptachloro-1,1'-biphenyl & $\mathrm{mg} / \mathrm{kg}$ & 3 & 3 & $100 \%$ & -- & -- & 0.0015 & 0.0044 & 0.0034 \\
\hline PCB & $2,2^{\prime}, 3,4,4^{\prime}, 5,5^{\prime}$-Heptachlorobiphenyl & $\mathrm{mg} / \mathrm{kg}$ & 3 & 3 & $100 \%$ & -- & -- & 0.0048 & 0.016 & 0.012 \\
\hline РCB & 2,3,3',4,4',5'-Hexachlorobiphenyl & $\mathrm{mg} / \mathrm{kg}$ & 3 & 3 & $100 \%$ & -- & -- & 1.45E-04 & 4.00E-04 & 3.12E-04 \\
\hline PCB & 2,3,3',4,4',5-Hexachlorobiphenyl (BZ 156) & $\mathrm{mg} / \mathrm{kg}$ & 3 & 3 & $100 \%$ & -- & -- & 7.73E-04 & 0.0022 & 0.0017 \\
\hline РCB & 2,3,3',4,4'-Pentachlorobiphenyl & $\mathrm{mg} / \mathrm{kg}$ & 3 & 3 & $100 \%$ & -- & -- & 0.0020 & 0.0056 & 0.0044 \\
\hline PCB & 2,3',4,4',5,5'-Hexachlorobiphenyl (BZ 167) & $\mathrm{mg} / \mathrm{kg}$ & 3 & 3 & $100 \%$ & -- & -- & 1.48E-05 & $1.30 \mathrm{E}-04$ & 5.99E-05 \\
\hline PCB & 2',3,4,4',5-Pentachloro-1,1'-biphenyl (BZ 123) & $\mathrm{mg} / \mathrm{kg}$ & 3 & 3 & $100 \%$ & -- & -- & 1.54E-05 & 3.94E-05 & 2.87E-05 \\
\hline PCB & 2,3,4,4',5-Pentachlorobiphenyl (BZ 114) & $\mathrm{mg} / \mathrm{kg}$ & 3 & 3 & $100 \%$ & -- & -- & 1.60E-04 & 4.71E-04 & 3.51E-04 \\
\hline PCB & 2,3',4,4',5-Pentachlorobiphenyl (BZ 118) & $\mathrm{mg} / \mathrm{kg}$ & 3 & 3 & $100 \%$ & -- & -- & 0.0022 & 0.0072 & 0.0052 \\
\hline PCB & 2,3,4,5,3',4',5'-Heptachlorobiphenyl & $\mathrm{mg} / \mathrm{kg}$ & 3 & 3 & $100 \%$ & -- & -- & $6.04 \mathrm{E}-05$ & $1.64 \mathrm{E}-04$ & $1.28 \mathrm{E}-04$ \\
\hline PCB & 3,3',4,4',5,5'-Hexachlorobiphenyl (BZ 169) & $\mathrm{mg} / \mathrm{kg}$ & 3 & 1 & $33 \%$ & 4.01E-06 & 4.70E-06 & 1.67E-06 & 1.67E-06 & 2.01E-06 \\
\hline PCB & 3,3',4,4',5-Pentachlorobiphenyl (BZ 126) & $\mathrm{mg} / \mathrm{kg}$ & 3 & 0 & $0 \%$ & $5.00 \mathrm{E}-07$ & 4.18E-06 & -- & -- & $1.22 \mathrm{E}-06$ \\
\hline PCB & 3,3',4,4'-Tetrachlorobiphenyl (BZ 77) & $\mathrm{mg} / \mathrm{kg}$ & 3 & 3 & $100 \%$ & -- & -- & 8.18E-06 & 1.64E-05 & 1.14E-05 \\
\hline PCB & Aroclor-1016 & $\mathrm{mg} / \mathrm{kg}$ & 3 & 0 & $0 \%$ & 0.019 & 0.020 & -- & -- & 0.0098 \\
\hline PCB & Aroclor-1221 & $\mathrm{mg} / \mathrm{kg}$ & 3 & 0 & $0 \%$ & 0.019 & 0.020 & -- & -- & 0.0098 \\
\hline
\end{tabular}


Table F-97

Summary Statistics for Whole Body White Sturgeon Collected from Segment 4 of the Columbia River

\begin{tabular}{|c|c|c|c|c|c|c|c|c|c|c|}
\hline $\begin{array}{l}\text { Constituent } \\
\text { Class }\end{array}$ & Constituent Name & Units & $\begin{array}{l}\text { Number } \\
\text { Analyzed }\end{array}$ & $\begin{array}{l}\text { Number } \\
\text { Detected }\end{array}$ & $\begin{array}{c}\text { Frequency of } \\
\text { Detection }\end{array}$ & $\begin{array}{l}\text { Minimum } \\
\text { Nondetect }\end{array}$ & $\begin{array}{l}\text { Maximum } \\
\text { Nondetect }\end{array}$ & $\begin{array}{l}\text { Minimum } \\
\text { Detect }\end{array}$ & $\begin{array}{l}\text { Maximum } \\
\text { Detect }\end{array}$ & $\begin{array}{c}\text { Average } \\
\text { Concentration }\end{array}$ \\
\hline$\overline{\mathrm{PCB}}$ & Aroclor-1232 & $\mathrm{mg} / \mathrm{kg}$ & 3 & 0 & $0 \%$ & 0.019 & 0.020 & $\overline{--}$ & $\overline{--}$ & 0.0098 \\
\hline РСВ & Aroclor-1248 & $\mathrm{mg} / \mathrm{kg}$ & 3 & 0 & $0 \%$ & 0.019 & 0.020 & -- & -- & 0.0098 \\
\hline PCB & Aroclor-1254 & $\mathrm{mg} / \mathrm{kg}$ & 3 & 3 & $100 \%$ & -- & -- & 0.038 & 0.12 & 0.082 \\
\hline PCB & Aroclor-1260 & $\mathrm{mg} / \mathrm{kg}$ & 3 & 3 & $100 \%$ & -- & -- & 0.053 & 0.16 & 0.12 \\
\hline PEST & 2,4'-DDD & $\mathrm{mg} / \mathrm{kg}$ & 3 & 3 & $100 \%$ & -- & -- & 0.0049 & 0.0077 & 0.0066 \\
\hline PEST & 2,4'-DDE & $\mathrm{mg} / \mathrm{kg}$ & 3 & 3 & $100 \%$ & -- & -- & 0.0052 & 0.012 & 0.0089 \\
\hline PEST & $2,4^{\prime}-\mathrm{DDT}$ & $\mathrm{mg} / \mathrm{kg}$ & 3 & 2 & $67 \%$ & 0.0020 & 0.0020 & 0.0032 & 0.0063 & 0.0035 \\
\hline PEST & Aldrin & $\mathrm{mg} / \mathrm{kg}$ & 3 & 0 & $0 \%$ & 0.0019 & 0.0020 & -- & -- & $9.83 \mathrm{E}-04$ \\
\hline PEST & Alpha-BHC & $\mathrm{mg} / \mathrm{kg}$ & 3 & 0 & $0 \%$ & 0.0019 & 0.0020 & -- & -- & $9.83 \mathrm{E}-04$ \\
\hline PEST & alpha-Chlordane & $\mathrm{mg} / \mathrm{kg}$ & 3 & 2 & $67 \%$ & 0.0020 & 0.0020 & 0.0035 & 0.0036 & 0.0027 \\
\hline PEST & alpha-Chlordene & $\mathrm{mg} / \mathrm{kg}$ & 3 & 0 & $0 \%$ & 0.0019 & 0.0020 & -- & -- & $9.83 \mathrm{E}-04$ \\
\hline PEST & Beta-BHC & $\mathrm{mg} / \mathrm{kg}$ & 3 & 0 & $0 \%$ & 0.0019 & 0.0020 & -- & -- & $9.83 \mathrm{E}-04$ \\
\hline PEST & cis-Nonachlor & $\mathrm{mg} / \mathrm{kg}$ & 3 & 3 & $100 \%$ & -- & -- & 0.0051 & 0.0081 & 0.0069 \\
\hline PEST & DDMU & $\mathrm{mg} / \mathrm{kg}$ & 3 & 1 & $33 \%$ & 0.0019 & 0.0020 & 0.011 & 0.011 & 0.0043 \\
\hline PEST & Delta-BHC & $\mathrm{mg} / \mathrm{kg}$ & 3 & 0 & $0 \%$ & 0.0019 & 0.0020 & -- & -- & $9.83 \mathrm{E}-04$ \\
\hline PEST & Dichlorodiphenyldichloroethane & $\mathrm{mg} / \mathrm{kg}$ & 3 & 3 & $100 \%$ & -- & -- & 0.078 & 0.14 & 0.10 \\
\hline PEST & Dichlorodiphenyldichloroethylene & $\mathrm{mg} / \mathrm{kg}$ & 3 & 3 & $100 \%$ & -- & -- & 0.40 & 1.1 & 0.75 \\
\hline PEST & Dichlorodiphenyltrichloroethane & $\mathrm{mg} / \mathrm{kg}$ & 3 & 3 & $100 \%$ & -- & -- & 0.016 & 0.038 & 0.025 \\
\hline PEST & Endosulfan sulfate & $\mathrm{mg} / \mathrm{kg}$ & 3 & 0 & $0 \%$ & 0.0019 & 0.0020 & -- & -- & 9.83E-04 \\
\hline PEST & Gamma-BHC (Lindane) & $\mathrm{mg} / \mathrm{kg}$ & 3 & 0 & $0 \%$ & 0.0019 & 0.0020 & -- & -- & $9.83 \mathrm{E}-04$ \\
\hline PEST & gamma-Chlordane & $\mathrm{mg} / \mathrm{kg}$ & 3 & 3 & $100 \%$ & -- & -- & 0.0019 & 0.010 & 0.0057 \\
\hline PEST & gamma-Chlordene & $\mathrm{mg} / \mathrm{kg}$ & 3 & 0 & $0 \%$ & 0.0019 & 0.0020 & -- & -- & 9.83E-04 \\
\hline PEST & Heptachlor & $\mathrm{mg} / \mathrm{kg}$ & 3 & 0 & $0 \%$ & 0.0019 & 0.0020 & -- & -- & $9.83 \mathrm{E}-04$ \\
\hline PEST & Heptachlor epoxide & $\mathrm{mg} / \mathrm{kg}$ & 3 & 0 & $0 \%$ & 0.0019 & 0.0020 & -- & -- & $9.83 \mathrm{E}-04$ \\
\hline PEST & Mirex & $\mathrm{mg} / \mathrm{kg}$ & 3 & 0 & $0 \%$ & 0.0019 & 0.0020 & -- & -- & $9.83 \mathrm{E}-04$ \\
\hline PEST & Oxychlordane & $\mathrm{mg} / \mathrm{kg}$ & 3 & 0 & $0 \%$ & 0.0019 & 0.0020 & -- & -- & 9.83E-04 \\
\hline PEST & Pentachloroanisole & $\mathrm{mg} / \mathrm{kg}$ & 3 & 0 & $0 \%$ & 0.0019 & 0.0020 & -- & -- & $9.83 \mathrm{E}-04$ \\
\hline PEST & Toxaphene & $\mathrm{mg} / \mathrm{kg}$ & 3 & 0 & $0 \%$ & 0.057 & 0.060 & -- & -- & 0.029 \\
\hline PEST & trans-Nonachlor & $\mathrm{mg} / \mathrm{kg}$ & 3 & 1 & $33 \%$ & 0.0019 & 0.0020 & 0.018 & 0.018 & 0.0067 \\
\hline SVOC & 1,2,4-Trichlorobenzene & $\mathrm{mg} / \mathrm{kg}$ & 3 & 0 & $0 \%$ & 0.0046 & 0.046 & -- & -- & 0.013 \\
\hline SVOC & 1,2-Dichlorobenzene & $\mathrm{mg} / \mathrm{kg}$ & 3 & 0 & $0 \%$ & 0.0046 & 0.046 & -- & -- & 0.013 \\
\hline SVOC & 1,2-Diphenylhydrazine & $\mathrm{mg} / \mathrm{kg}$ & 3 & 0 & $0 \%$ & 0.0046 & 0.046 & -- & -- & 0.013 \\
\hline SVOC & 1,3-Dichlorobenzene & $\mathrm{mg} / \mathrm{kg}$ & 3 & 0 & $0 \%$ & 0.0046 & 0.046 & -- & -- & 0.013 \\
\hline SVOC & 1,4-Dichlorobenzene & $\mathrm{mg} / \mathrm{kg}$ & 3 & 0 & $0 \%$ & 0.0046 & 0.046 & -- & -- & 0.013 \\
\hline SVOC & 1-methylnaphthalene & $\mathrm{mg} / \mathrm{kg}$ & 3 & 0 & $0 \%$ & 0.0048 & 0.046 & -- & -- & 0.013 \\
\hline SVOC & 2,4-Dinitrotoluene & $\mathrm{mg} / \mathrm{kg}$ & 3 & 0 & $0 \%$ & 0.0092 & 0.092 & -- & -- & 0.025 \\
\hline SVOC & 2,6-Dinitrotoluene & $\mathrm{mg} / \mathrm{kg}$ & 3 & 0 & $0 \%$ & 0.018 & 0.18 & -- & -- & 0.050 \\
\hline SVOC & 2-Chloronaphthalene & $\mathrm{mg} / \mathrm{kg}$ & 3 & 0 & $0 \%$ & 0.0092 & 0.092 & -- & -- & 0.025 \\
\hline SVOC & 2-Methylnaphthalene & $\mathrm{mg} / \mathrm{kg}$ & 3 & 0 & $0 \%$ & 0.010 & 0.046 & -- & -- & 0.014 \\
\hline SVOC & 4-Bromophenylphenyl ether & $\mathrm{mg} / \mathrm{kg}$ & 3 & 0 & $0 \%$ & 0.0046 & 0.046 & -- & -- & 0.013 \\
\hline SVOC & 4-Chlorophenylphenyl ether & $\mathrm{mg} / \mathrm{kg}$ & 3 & 0 & $0 \%$ & 0.0046 & 0.046 & -- & -- & 0.013 \\
\hline SVOC & Acenaphthene & $\mathrm{mg} / \mathrm{kg}$ & 3 & 0 & $0 \%$ & 0.0092 & 0.092 & -- & -- & 0.025 \\
\hline SVOC & Acenaphthylene & $\mathrm{mg} / \mathrm{kg}$ & 3 & 0 & $0 \%$ & 0.0046 & 0.046 & -- & -- & 0.013 \\
\hline SVOC & Anthracene & $\mathrm{mg} / \mathrm{kg}$ & 3 & 0 & $0 \%$ & 0.0046 & 0.046 & -- & -- & 0.013 \\
\hline SVOC & Benzo(a)anthracene & $\mathrm{mg} / \mathrm{kg}$ & 3 & 0 & $0 \%$ & 0.0046 & 0.046 & -- & -- & 0.013 \\
\hline SVOC & Benzo(a)pyrene & $\mathrm{mg} / \mathrm{kg}$ & 3 & 0 & $0 \%$ & 0.0046 & 0.046 & -- & -- & 0.013 \\
\hline SVOC & Benzo(b)fluoranthene & $\mathrm{mg} / \mathrm{kg}$ & 3 & 0 & $0 \%$ & 0.0046 & 0.046 & -- & -- & 0.013 \\
\hline SVOC & Benzo(ghi)perylene & $\mathrm{mg} / \mathrm{kg}$ & 3 & 0 & $0 \%$ & 0.0092 & 0.092 & -- & -- & 0.025 \\
\hline SVOC & Benzo(k)fluoranthene & $\mathrm{mg} / \mathrm{kg}$ & 3 & 0 & $0 \%$ & 0.0046 & 0.046 & -- & -- & 0.013 \\
\hline SVOC & Bis(2-chloro-1-methylethyl)ether & $\mathrm{mg} / \mathrm{kg}$ & 3 & 0 & $0 \%$ & 0.0092 & 0.092 & -- & -- & 0.025 \\
\hline
\end{tabular}


Table F-97

Summary Statistics for Whole Body White Sturgeon Collected from Segment 4 of the Columbia River

\begin{tabular}{|c|c|c|c|c|c|c|c|c|c|c|}
\hline $\begin{array}{l}\text { Constituent } \\
\text { Class }\end{array}$ & Constituent Name & Units & $\begin{array}{l}\text { Number } \\
\text { Analyzed }\end{array}$ & $\begin{array}{l}\text { Number } \\
\text { Detected }\end{array}$ & $\begin{array}{c}\text { Frequency of } \\
\text { Detection }\end{array}$ & $\begin{array}{l}\text { Minimum } \\
\text { Nondetect }\end{array}$ & $\begin{array}{l}\text { Maximum } \\
\text { Nondetect }\end{array}$ & $\begin{array}{l}\text { Minimum } \\
\text { Detect }\end{array}$ & $\begin{array}{l}\text { Maximum } \\
\text { Detect }\end{array}$ & $\begin{array}{c}\text { Average } \\
\text { Concentration }\end{array}$ \\
\hline$\overline{S V O C}$ & Chrysene & $\mathrm{mg} / \mathrm{kg}$ & 3 & 0 & $0 \%$ & 0.0046 & 0.046 & -- & -- & 0.013 \\
\hline SVOC & Dibenz[a,h]anthracene & $\mathrm{mg} / \mathrm{kg}$ & 3 & 0 & $0 \%$ & 0.0092 & 0.092 & -- & -- & 0.025 \\
\hline SVOC & Dibenzofuran & $\mathrm{mg} / \mathrm{kg}$ & 3 & 0 & $0 \%$ & 0.0046 & 0.046 & -- & -- & 0.013 \\
\hline SVOC & Fluoranthene & $\mathrm{mg} / \mathrm{kg}$ & 3 & 0 & $0 \%$ & 0.0092 & 0.092 & -- & -- & 0.025 \\
\hline SVOC & Fluorene & $\mathrm{mg} / \mathrm{kg}$ & 3 & 0 & $0 \%$ & 0.0049 & 0.0094 & -- & -- & 0.0032 \\
\hline SVOC & Hexachlorobenzene & $\mathrm{mg} / \mathrm{kg}$ & 3 & 0 & $0 \%$ & 0.0092 & 0.092 & -- & -- & 0.025 \\
\hline SVOC & Hexachlorobutadiene & $\mathrm{mg} / \mathrm{kg}$ & 3 & 0 & $0 \%$ & 0.0046 & 0.046 & -- & -- & 0.013 \\
\hline SVOC & Hexachloroethane & $\mathrm{mg} / \mathrm{kg}$ & 3 & 0 & $0 \%$ & 0.0046 & 0.046 & -- & -- & 0.013 \\
\hline SVOC & Indeno(1,2,3-cd)pyrene & $\mathrm{mg} / \mathrm{kg}$ & 3 & 0 & $0 \%$ & 0.0092 & 0.092 & -- & -- & 0.025 \\
\hline SVOC & Naphthalene & $\mathrm{mg} / \mathrm{kg}$ & 3 & 0 & $0 \%$ & 0.025 & 0.046 & -- & -- & 0.016 \\
\hline SVOC & Nitrobenzene & $\mathrm{mg} / \mathrm{kg}$ & 3 & 0 & $0 \%$ & 0.0046 & 0.046 & -- & -- & 0.013 \\
\hline SVOC & Phenanthrene & $\mathrm{mg} / \mathrm{kg}$ & 3 & 0 & $0 \%$ & 0.0046 & 0.046 & -- & -- & 0.013 \\
\hline SVOC & Pyrene & $\mathrm{mg} / \mathrm{kg}$ & 3 & 0 & $0 \%$ & 0.0092 & 0.092 & -- & -- & 0.025 \\
\hline VOC & Retene & $\mathrm{mg} / \mathrm{kg}$ & 3 & 0 & $0 \%$ & 0.0092 & 0.092 & -- & -- & 0.025 \\
\hline
\end{tabular}

DIOXI - Dioxins and Furans

PEST - Pesticide

PCB - Polychlorinated bipheny

RAD- radionuclide

SVOC - Semivolatile Organic Compound

VOC - Volatile Organic Compound 
Table F-98

Summary Statistics for Chinook Salmon Fillet Skin On Collected from Segment 5 of the Columbia River

\begin{tabular}{|c|c|c|c|c|c|c|c|c|c|c|}
\hline $\begin{array}{l}\text { Constituent } \\
\text { Class }\end{array}$ & Constituent Name & Units & $\begin{array}{l}\text { Number } \\
\text { Analyzed }\end{array}$ & $\begin{array}{l}\text { Number } \\
\text { Detected }\end{array}$ & $\begin{array}{c}\text { Frequency of } \\
\text { Detection }\end{array}$ & $\begin{array}{l}\text { Minimum } \\
\text { Nondetect }\end{array}$ & $\begin{array}{l}\text { Maximum } \\
\text { Nondetect }\end{array}$ & $\begin{array}{c}\text { Minimum } \\
\text { Detect }\end{array}$ & $\begin{array}{c}\text { Maximum } \\
\text { Detect }\end{array}$ & $\begin{array}{c}\text { Average } \\
\text { Concentration }\end{array}$ \\
\hline$\overline{D I O X I}$ & $1,2,3,4,6,7,8$-Heptachlorodibenzodioxin & $\mathrm{mg} / \mathrm{kg}$ & 3 & 2 & $67 \%$ & $2.00 \mathrm{E}-08$ & $2.00 \mathrm{E}-08$ & $7.00 \mathrm{E}-08$ & $8.00 \mathrm{E}-08$ & $5.33 \mathrm{E}-08$ \\
\hline DIOXI & $1,2,3,4,6,7,8$-Heptachlorodibenzofuran & $\mathrm{mg} / \mathrm{kg}$ & 3 & 2 & $67 \%$ & $1.00 \mathrm{E}-08$ & 1.00E-08 & 2.00E-08 & $2.00 \mathrm{E}-08$ & $1.50 \mathrm{E}-08$ \\
\hline DIOXI & $1,2,3,4,7,8,9$-Heptachlorodibenzofuran & $\mathrm{mg} / \mathrm{kg}$ & 3 & 0 & $0 \%$ & $1.00 \mathrm{E}-08$ & $2.00 \mathrm{E}-08$ & -- & -- & 8.33E-09 \\
\hline DIOXI & 1,2,3,4,7,8-Hexachlorodibenzofuran & $\mathrm{mg} / \mathrm{kg}$ & 3 & 1 & $33 \%$ & $1.00 \mathrm{E}-08$ & $2.00 \mathrm{E}-08$ & 3.00E-08 & $3.00 \mathrm{E}-08$ & $1.50 \mathrm{E}-08$ \\
\hline DIOXI & $1,2,3,4,7,8$-Hexachlorodibenzo-p-dioxin & $\mathrm{mg} / \mathrm{kg}$ & 3 & 2 & $67 \%$ & $1.00 \mathrm{E}-08$ & $1.00 \mathrm{E}-08$ & $2.00 \mathrm{E}-08$ & $3.00 \mathrm{E}-08$ & 1.83E-08 \\
\hline DIOXI & 1,2,3,6,7,8-Hexachlorodibenzofuran & $\mathrm{mg} / \mathrm{kg}$ & 3 & 3 & $100 \%$ & -- & -- & 3.00E-08 & 4.00E-08 & 3.33E-08 \\
\hline DIOXI & $1,2,3,6,7,8$-Hexachlorodibenzo-p-dioxin & $\mathrm{mg} / \mathrm{kg}$ & 3 & 2 & $67 \%$ & $1.00 \mathrm{E}-08$ & $1.00 \mathrm{E}-08$ & $6.00 \mathrm{E}-08$ & $8.00 \mathrm{E}-08$ & 4.83E-08 \\
\hline DIOXI & 1,2,3,7,8,9-Hexachlorodibenzofuran & $\mathrm{mg} / \mathrm{kg}$ & 3 & 0 & $0 \%$ & $1.00 \mathrm{E}-08$ & $2.00 \mathrm{E}-08$ & -- & -- & 8.33E-09 \\
\hline DIOXI & $1,2,3,7,8,9$-Hexachlorodibenzo-p-dioxin & $\mathrm{mg} / \mathrm{kg}$ & 3 & 2 & $67 \%$ & $1.00 \mathrm{E}-08$ & $1.00 \mathrm{E}-08$ & 3.00E-08 & $4.00 \mathrm{E}-08$ & $2.50 \mathrm{E}-08$ \\
\hline DIOXI & 1,2,3,7,8-Pentachlorodibenzofuran & $\mathrm{mg} / \mathrm{kg}$ & 3 & 3 & $100 \%$ & -- & -- & 1.20E-07 & $1.40 \mathrm{E}-07$ & $1.27 \mathrm{E}-07$ \\
\hline DIOXI & $1,2,3,7,8$-Pentachlorodibenzo-p-dioxin & $\mathrm{mg} / \mathrm{kg}$ & 3 & 2 & $67 \%$ & $1.00 \mathrm{E}-08$ & $1.00 \mathrm{E}-08$ & 7.00E-08 & $1.10 \mathrm{E}-07$ & $6.17 \mathrm{E}-08$ \\
\hline DIOXI & 2,3,4,6,7,8-Hexachlorodibenzofuran & $\mathrm{mg} / \mathrm{kg}$ & 3 & 1 & $33 \%$ & $2.00 \mathrm{E}-08$ & $2.00 \mathrm{E}-08$ & 4.00E-08 & $4.00 \mathrm{E}-08$ & $2.00 \mathrm{E}-08$ \\
\hline DIOXI & 2,3,4,7,8-Pentachlorodibenzofuran & $\mathrm{mg} / \mathrm{kg}$ & 3 & 3 & $100 \%$ & -- & -- & $1.50 \mathrm{E}-07$ & 1.70E-07 & $1.60 \mathrm{E}-07$ \\
\hline DIOXI & 2,3,7,8-Tetrachlorodibenzofuran & $\mathrm{mg} / \mathrm{kg}$ & 3 & 3 & $100 \%$ & -- & -- & 5.30E-07 & $6.10 \mathrm{E}-07$ & $5.80 \mathrm{E}-07$ \\
\hline DIOXI & 2,3,7,8-Tetrachlorodibenzo-p-dioxin & $\mathrm{mg} / \mathrm{kg}$ & 3 & 0 & $0 \%$ & $1.00 \mathrm{E}-08$ & $2.00 \mathrm{E}-08$ & -- & -- & 8.33E-09 \\
\hline DIOXI & Octachlorodibenzofuran & $\mathrm{mg} / \mathrm{kg}$ & 3 & 0 & $0 \%$ & $3.00 \mathrm{E}-08$ & $4.00 \mathrm{E}-08$ & -- & -- & $1.67 \mathrm{E}-08$ \\
\hline DIOXI & Octachlorodibenzo-p-dioxin & $\mathrm{mg} / \mathrm{kg}$ & 3 & 1 & $33 \%$ & $2.00 \mathrm{E}-08$ & $2.00 \mathrm{E}-08$ & 1.00E-07 & $1.00 \mathrm{E}-07$ & $4.00 \mathrm{E}-08$ \\
\hline METAL & Aluminum & $\mathrm{mg} / \mathrm{kg}$ & 3 & 1 & $33 \%$ & 1.0 & 1.0 & 1.3 & 1.3 & 0.77 \\
\hline METAL & Antimony & $\mathrm{mg} / \mathrm{kg}$ & 3 & 0 & $0 \%$ & 0.050 & 0.050 & -- & -- & 0.025 \\
\hline METAL & Arsenic & $\mathrm{mg} / \mathrm{kg}$ & 3 & 3 & $100 \%$ & -- & -- & 0.76 & 1.2 & 0.95 \\
\hline METAL & Barium & $\mathrm{mg} / \mathrm{kg}$ & 3 & 0 & $0 \%$ & 0.20 & 0.20 & -- & -- & 0.10 \\
\hline METAL & Beryllium & $\mathrm{mg} / \mathrm{kg}$ & 3 & 0 & $0 \%$ & 0.0040 & 0.0040 & -- & -- & 0.0020 \\
\hline METAL & Cadmium & $\mathrm{mg} / \mathrm{kg}$ & 3 & 3 & $100 \%$ & -- & -- & 0.010 & 0.010 & 0.010 \\
\hline METAL & Chromium & $\mathrm{mg} / \mathrm{kg}$ & 3 & 2 & $67 \%$ & 0.10 & 0.10 & 0.45 & 0.48 & 0.33 \\
\hline METAL & Cobalt & $\mathrm{mg} / \mathrm{kg}$ & 3 & 3 & $100 \%$ & -- & -- & 0.0033 & 0.0048 & 0.0038 \\
\hline METAL & Copper & $\mathrm{mg} / \mathrm{kg}$ & 3 & 3 & $100 \%$ & -- & -- & 0.64 & 0.67 & 0.65 \\
\hline METAL & Lead & $\mathrm{mg} / \mathrm{kg}$ & 3 & 0 & $0 \%$ & 0.010 & 0.010 & -- & -- & 0.0050 \\
\hline METAL & Manganese & $\mathrm{mg} / \mathrm{kg}$ & 3 & 3 & $100 \%$ & -- & -- & 0.070 & 0.090 & 0.080 \\
\hline METAL & Mercury & $\mathrm{mg} / \mathrm{kg}$ & 3 & 0 & $0 \%$ & 0.090 & 0.12 & -- & -- & 0.055 \\
\hline METAL & Nickel & $\mathrm{mg} / \mathrm{kg}$ & 3 & 0 & $0 \%$ & 0.030 & 0.030 & -- & -- & 0.015 \\
\hline METAL & Selenium & $\mathrm{mg} / \mathrm{kg}$ & 3 & 3 & $100 \%$ & -- & -- & 0.33 & 0.36 & 0.35 \\
\hline METAL & Silver & $\mathrm{mg} / \mathrm{kg}$ & 3 & 0 & $0 \%$ & 0.10 & 0.10 & -- & -- & 0.050 \\
\hline METAL & Thallium & $\mathrm{mg} / \mathrm{kg}$ & 3 & 0 & $0 \%$ & 0.050 & 0.050 & -- & -- & 0.025 \\
\hline METAL & Vanadium & $\mathrm{mg} / \mathrm{kg}$ & 3 & 0 & $0 \%$ & 0.010 & 0.010 & -- & -- & 0.0050 \\
\hline METAL & Zinc & $\mathrm{mg} / \mathrm{kg}$ & 3 & 3 & $100 \%$ & -- & -- & 6.2 & 6.5 & 6.3 \\
\hline PCB & 2,2',3,3',4,4',5-Heptachloro-1,1'-biphenyl & $\mathrm{mg} / \mathrm{kg}$ & 3 & 3 & $100 \%$ & -- & -- & $5.84 \mathrm{E}-05$ & $8.02 \mathrm{E}-05$ & $6.94 \mathrm{E}-05$ \\
\hline РСB & 2,2',3,4,4',5,5'-Heptachlorobiphenyl & $\mathrm{mg} / \mathrm{kg}$ & 3 & 3 & $100 \%$ & -- & -- & 2.10E-04 & $2.71 \mathrm{E}-04$ & 2.43E-04 \\
\hline PCB & 2,3,3',4,4',5'-Hexachlorobiphenyl & $\mathrm{mg} / \mathrm{kg}$ & 3 & 3 & $100 \%$ & -- & -- & 1.05E-05 & 1.52E-05 & $1.28 \mathrm{E}-05$ \\
\hline РСB & 2,3,3',4,4',5-Hexachlorobiphenyl (BZ 156) & $\mathrm{mg} / \mathrm{kg}$ & 3 & 3 & $100 \%$ & -- & -- & 3.22E-05 & 4.65E-05 & $3.91 \mathrm{E}-05$ \\
\hline PCB & 2,3,3',4,4'-Pentachlorobiphenyl & $\mathrm{mg} / \mathrm{kg}$ & 3 & 3 & $100 \%$ & -- & -- & 2.01E-04 & $2.61 \mathrm{E}-04$ & $2.28 \mathrm{E}-04$ \\
\hline PCB & 2,3',4,4',5,5'-Hexachlorobiphenyl (BZ 167) & $\mathrm{mg} / \mathrm{kg}$ & 3 & 3 & $100 \%$ & -- & -- & 2.69E-05 & $2.84 \mathrm{E}-05$ & $2.75 \mathrm{E}-05$ \\
\hline РCB & 2',3,4,4',5-Pentachloro-1,1'-biphenyl (BZ 123) & $\mathrm{mg} / \mathrm{kg}$ & 3 & 3 & $100 \%$ & -- & -- & 1.11E-05 & $1.24 \mathrm{E}-05$ & $1.18 \mathrm{E}-05$ \\
\hline РCB & 2,3,4,4',5-Pentachlorobiphenyl (BZ 114) & $\mathrm{mg} / \mathrm{kg}$ & 3 & 3 & $100 \%$ & -- & -- & 2.04E-05 & $2.56 \mathrm{E}-05$ & 2.23E-05 \\
\hline PCB & 2,3',4,4',5-Pentachlorobiphenyl (BZ 118) & $\mathrm{mg} / \mathrm{kg}$ & 3 & 3 & $100 \%$ & -- & -- & 4.80E-04 & $5.90 \mathrm{E}-04$ & $5.25 \mathrm{E}-04$ \\
\hline РСB & 2,3,4,5,3',4',5'-Heptachlorobiphenyl & $\mathrm{mg} / \mathrm{kg}$ & 3 & 2 & $67 \%$ & $1.00 \mathrm{E}-06$ & $1.00 \mathrm{E}-06$ & $2.45 \mathrm{E}-06$ & $2.70 \mathrm{E}-06$ & $1.88 \mathrm{E}-06$ \\
\hline PCB & $3,3^{\prime}, 4,4^{\prime}, 5,5^{\prime}-$ Hexachlorobiphenyl (BZ 169) & $\mathrm{mg} / \mathrm{kg}$ & 3 & 0 & $0 \%$ & 1.14E-06 & $1.60 \mathrm{E}-06$ & -- & -- & $6.60 \mathrm{E}-07$ \\
\hline PCB & 3,3',4,4',5-Pentachlorobiphenyl (BZ 126) & $\mathrm{mg} / \mathrm{kg}$ & 3 & 3 & $100 \%$ & -- & -- & 8.50E-07 & 3.43E-06 & 2.44E-06 \\
\hline РСB & 3,3',4,4'-Tetrachlorobiphenyl (BZ 77) & $\mathrm{mg} / \mathrm{kg}$ & 3 & 3 & $100 \%$ & -- & -- & $9.87 \mathrm{E}-06$ & $1.32 \mathrm{E}-05$ & $1.11 \mathrm{E}-05$ \\
\hline PCB & Aroclor-1016 & $\mathrm{mg} / \mathrm{kg}$ & 3 & 0 & $0 \%$ & 0.018 & 0.020 & -- & -- & 0.0097 \\
\hline PCB & Aroclor-1221 & $\mathrm{mg} / \mathrm{kg}$ & 3 & 0 & $0 \%$ & 0.018 & 0.020 & -- & -- & 0.0097 \\
\hline PCB & Aroclor-1232 & $\mathrm{mg} / \mathrm{kg}$ & 3 & 0 & $0 \%$ & 0.018 & 0.020 & -- & -- & 0.0097 \\
\hline PCB & Aroclor-1248 & $\mathrm{mg} / \mathrm{kg}$ & 3 & 0 & $0 \%$ & 0.018 & 0.020 & -- & -- & 0.0097 \\
\hline
\end{tabular}


Table F-98

Summary Statistics for Chinook Salmon Fillet Skin On Collected from Segment 5 of the Columbia River

\begin{tabular}{|c|c|c|c|c|c|c|c|c|c|c|}
\hline $\begin{array}{l}\text { Constituent } \\
\text { Class }\end{array}$ & Constituent Name & Units & $\begin{array}{l}\text { Number } \\
\text { Analyzed }\end{array}$ & $\begin{array}{l}\text { Number } \\
\text { Detected }\end{array}$ & $\begin{array}{l}\text { Frequency of } \\
\text { Detection }\end{array}$ & $\begin{array}{l}\text { Minimum } \\
\text { Nondetect }\end{array}$ & $\begin{array}{l}\text { Maximum } \\
\text { Nondetect }\end{array}$ & $\begin{array}{l}\text { Minimum } \\
\text { Detect }\end{array}$ & $\begin{array}{l}\text { Maximum } \\
\text { Detect }\end{array}$ & $\begin{array}{c}\text { Average } \\
\text { Concentration }\end{array}$ \\
\hline$\overline{\mathrm{PCB}}$ & Aroclor-1254 & $\mathrm{mg} / \mathrm{kg}$ & 3 & 3 & $100 \%$ & $\overline{--}$ & $\overline{--}$ & 0.015 & 0.019 & 0.017 \\
\hline РСB & Aroclor-1260 & $\mathrm{mg} / \mathrm{kg}$ & 3 & 0 & $0 \%$ & 0.018 & 0.020 & -- & -- & 0.0097 \\
\hline PEST & 2,4'-DDD & $\mathrm{mg} / \mathrm{kg}$ & 3 & 0 & $0 \%$ & 0.0018 & 0.0020 & -- & -- & $9.67 \mathrm{E}-04$ \\
\hline PEST & 2,4'-DDE & $\mathrm{mg} / \mathrm{kg}$ & 3 & 1 & $33 \%$ & 0.0018 & 0.0020 & 0.0014 & 0.0014 & 0.0011 \\
\hline PEST & 2,4'-DDT & $\mathrm{mg} / \mathrm{kg}$ & 3 & 0 & $0 \%$ & 0.0018 & 0.0020 & -- & -- & $9.67 \mathrm{E}-04$ \\
\hline PEST & Aldrin & $\mathrm{mg} / \mathrm{kg}$ & 3 & 0 & $0 \%$ & 0.0018 & 0.0020 & -- & -- & $9.67 \mathrm{E}-04$ \\
\hline PEST & Alpha-BHC & $\mathrm{mg} / \mathrm{kg}$ & 3 & 0 & $0 \%$ & 0.0018 & 0.0020 & -- & -- & $9.67 \mathrm{E}-04$ \\
\hline PEST & alpha-Chlordane & $\mathrm{mg} / \mathrm{kg}$ & 3 & 0 & $0 \%$ & 0.0018 & 0.0020 & -- & -- & $9.67 \mathrm{E}-04$ \\
\hline PEST & alpha-Chlordene & $\mathrm{mg} / \mathrm{kg}$ & 3 & 0 & $0 \%$ & 0.0018 & 0.0020 & -- & -- & $9.67 \mathrm{E}-04$ \\
\hline PEST & Beta-BHC & $\mathrm{mg} / \mathrm{kg}$ & 3 & 0 & $0 \%$ & 0.0018 & 0.0020 & -- & -- & $9.67 \mathrm{E}-04$ \\
\hline PEST & cis-Nonachlor & $\mathrm{mg} / \mathrm{kg}$ & 3 & 1 & $33 \%$ & 0.0018 & 0.0020 & 0.0013 & 0.0013 & 0.0011 \\
\hline PEST & DDMU & $\mathrm{mg} / \mathrm{kg}$ & 3 & 1 & $33 \%$ & 0.0018 & 0.0020 & 0.0014 & 0.0014 & 0.0011 \\
\hline PEST & Delta-BHC & $\mathrm{mg} / \mathrm{kg}$ & 3 & 0 & $0 \%$ & 0.0018 & 0.0020 & -- & -- & $9.67 \mathrm{E}-04$ \\
\hline PEST & Dichlorodiphenyldichloroethane & $\mathrm{mg} / \mathrm{kg}$ & 3 & 3 & $100 \%$ & -- & -- & 0.0015 & 0.0030 & 0.0022 \\
\hline PEST & Dichlorodiphenyldichloroethylene & $\mathrm{mg} / \mathrm{kg}$ & 3 & 3 & $100 \%$ & -- & -- & 0.0088 & 0.013 & 0.011 \\
\hline PEST & Dichlorodiphenyltrichloroethane & $\mathrm{mg} / \mathrm{kg}$ & 3 & 3 & $100 \%$ & -- & -- & 0.0040 & 0.0053 & 0.0046 \\
\hline PEST & Endosulfan sulfate & $\mathrm{mg} / \mathrm{kg}$ & 1 & 0 & $0 \%$ & 0.0018 & 0.0018 & -- & -- & $9.00 \mathrm{E}-04$ \\
\hline PEST & Gamma-BHC (Lindane) & $\mathrm{mg} / \mathrm{kg}$ & 3 & 0 & $0 \%$ & 0.0018 & 0.0020 & -- & -- & $9.67 \mathrm{E}-04$ \\
\hline PEST & gamma-Chlordane & $\mathrm{mg} / \mathrm{kg}$ & 3 & 0 & $0 \%$ & 0.0018 & 0.0020 & -- & -- & $9.67 \mathrm{E}-04$ \\
\hline PEST & gamma-Chlordene & $\mathrm{mg} / \mathrm{kg}$ & 3 & 0 & $0 \%$ & 0.0018 & 0.0020 & -- & -- & $9.67 \mathrm{E}-04$ \\
\hline PEST & Heptachlor & $\mathrm{mg} / \mathrm{kg}$ & 3 & 0 & $0 \%$ & 0.0018 & 0.0020 & -- & -- & $9.67 \mathrm{E}-04$ \\
\hline PEST & Heptachlor epoxide & $\mathrm{mg} / \mathrm{kg}$ & 1 & 0 & $0 \%$ & 0.0018 & 0.0018 & -- & -- & $9.00 \mathrm{E}-04$ \\
\hline PEST & Mirex & $\mathrm{mg} / \mathrm{kg}$ & 3 & 0 & $0 \%$ & 0.0018 & 0.0020 & -- & -- & $9.67 \mathrm{E}-04$ \\
\hline PEST & Oxychlordane & $\mathrm{mg} / \mathrm{kg}$ & 3 & 0 & $0 \%$ & 0.0018 & 0.0020 & -- & -- & $9.67 \mathrm{E}-04$ \\
\hline PEST & Pentachloroanisole & $\mathrm{mg} / \mathrm{kg}$ & 3 & 0 & $0 \%$ & 0.0018 & 0.0020 & -- & -- & $9.67 \mathrm{E}-04$ \\
\hline PEST & Toxaphene & $\mathrm{mg} / \mathrm{kg}$ & 3 & 0 & $0 \%$ & 0.055 & 0.060 & -- & -- & 0.029 \\
\hline PEST & trans-Nonachlor & $\mathrm{mg} / \mathrm{kg}$ & 3 & 3 & $100 \%$ & -- & -- & 0.0024 & 0.0029 & 0.0027 \\
\hline SVOC & 1,2,4-Trichlorobenzene & $\mathrm{mg} / \mathrm{kg}$ & 3 & 0 & $0 \%$ & 0.0098 & 0.0099 & -- & -- & 0.0049 \\
\hline SVOC & 1,2-Dichlorobenzene & $\mathrm{mg} / \mathrm{kg}$ & 3 & 0 & $0 \%$ & 0.0098 & 0.0099 & -- & -- & 0.0049 \\
\hline SVOC & 1,2-Diphenylhydrazine & $\mathrm{mg} / \mathrm{kg}$ & 3 & 0 & $0 \%$ & 0.0098 & 0.0099 & -- & -- & 0.0049 \\
\hline SVOC & 1,3-Dichlorobenzene & $\mathrm{mg} / \mathrm{kg}$ & 3 & 0 & $0 \%$ & 0.0098 & 0.0099 & -- & -- & 0.0049 \\
\hline SVOC & 1,4-Dichlorobenzene & $\mathrm{mg} / \mathrm{kg}$ & 3 & 0 & $0 \%$ & 0.0098 & 0.0099 & -- & -- & 0.0049 \\
\hline SVOC & 1-methylnaphthalene & $\mathrm{mg} / \mathrm{kg}$ & 3 & 0 & $0 \%$ & 0.0098 & 0.0099 & -- & -- & 0.0049 \\
\hline SVOC & 2,4-Dinitrotoluene & $\mathrm{mg} / \mathrm{kg}$ & 3 & 0 & $0 \%$ & 0.020 & 0.020 & -- & -- & 0.010 \\
\hline SVOC & 2,6-Dinitrotoluene & $\mathrm{mg} / \mathrm{kg}$ & 3 & 0 & $0 \%$ & 0.039 & 0.039 & -- & -- & 0.020 \\
\hline SVOC & 2-Chloronaphthalene & $\mathrm{mg} / \mathrm{kg}$ & 3 & 0 & $0 \%$ & 0.020 & 0.020 & -- & -- & 0.010 \\
\hline SVOC & 2-Methylnaphthalene & $\mathrm{mg} / \mathrm{kg}$ & 3 & 0 & $0 \%$ & 0.0098 & 0.011 & -- & -- & 0.0051 \\
\hline SVOC & 4-Bromophenylphenyl ether & $\mathrm{mg} / \mathrm{kg}$ & 3 & 0 & $0 \%$ & 0.0098 & 0.0099 & -- & -- & 0.0049 \\
\hline SVOC & 4-Chlorophenylphenyl ether & $\mathrm{mg} / \mathrm{kg}$ & 3 & 0 & $0 \%$ & 0.0098 & 0.0099 & -- & -- & 0.0049 \\
\hline SVOC & Acenaphthene & $\mathrm{mg} / \mathrm{kg}$ & 3 & 0 & $0 \%$ & 0.020 & 0.020 & -- & -- & 0.010 \\
\hline SVOC & Acenaphthylene & $\mathrm{mg} / \mathrm{kg}$ & 3 & 0 & $0 \%$ & 0.0098 & 0.0099 & -- & -- & 0.0049 \\
\hline SVOC & Anthracene & $\mathrm{mg} / \mathrm{kg}$ & 3 & 0 & $0 \%$ & 0.0098 & 0.0099 & -- & -- & 0.0049 \\
\hline SVOC & Benzo(a)anthracene & $\mathrm{mg} / \mathrm{kg}$ & 3 & 0 & $0 \%$ & 0.0095 & 0.0099 & -- & -- & 0.0049 \\
\hline SVOC & Benzo(a)pyrene & $\mathrm{mg} / \mathrm{kg}$ & 3 & 0 & $0 \%$ & 0.0095 & 0.0099 & -- & -- & 0.0049 \\
\hline SVOC & Benzo(b)fluoranthene & $\mathrm{mg} / \mathrm{kg}$ & 3 & 0 & $0 \%$ & 0.0095 & 0.0099 & -- & -- & 0.0049 \\
\hline SVOC & Benzo(ghi)perylene & $\mathrm{mg} / \mathrm{kg}$ & 3 & 0 & $0 \%$ & 0.019 & 0.020 & -- & -- & 0.0098 \\
\hline SVOC & Benzo(k)fluoranthene & $\mathrm{mg} / \mathrm{kg}$ & 3 & 0 & $0 \%$ & 0.0095 & 0.0099 & -- & -- & 0.0049 \\
\hline SVOC & Bis(2-chloro-1-methylethyl)ether & $\mathrm{mg} / \mathrm{kg}$ & 3 & 0 & $0 \%$ & 0.020 & 0.020 & -- & -- & 0.010 \\
\hline SVOC & Chrysene & $\mathrm{mg} / \mathrm{kg}$ & 3 & 0 & $0 \%$ & 0.0095 & 0.0099 & -- & -- & 0.0049 \\
\hline SVOC & Dibenz[a,h]anthracene & $\mathrm{mg} / \mathrm{kg}$ & 3 & 0 & $0 \%$ & 0.019 & 0.020 & -- & -- & 0.0098 \\
\hline SVOC & Dibenzofuran & $\mathrm{mg} / \mathrm{kg}$ & 3 & 0 & $0 \%$ & 0.0098 & 0.0099 & -- & -- & 0.0049 \\
\hline SVOC & Fluoranthene & $\mathrm{mg} / \mathrm{kg}$ & 3 & 0 & $0 \%$ & 0.020 & 0.020 & -- & -- & 0.010 \\
\hline
\end{tabular}


Table F-98

Summary Statistics for Chinook Salmon Fillet Skin On Collected from Segment 5 of the Columbia River

\begin{tabular}{|c|c|c|c|c|c|c|c|c|c|c|}
\hline $\begin{array}{l}\text { Constituent } \\
\text { Class }\end{array}$ & Constituent Name & Units & $\begin{array}{l}\text { Number } \\
\text { Analyzed }\end{array}$ & $\begin{array}{l}\text { Number } \\
\text { Detected }\end{array}$ & $\begin{array}{l}\text { Frequency of } \\
\text { Detection }\end{array}$ & $\begin{array}{c}\text { Minimum } \\
\text { Nondetect }\end{array}$ & $\begin{array}{l}\text { Maximum } \\
\text { Nondetect }\end{array}$ & $\begin{array}{c}\text { Minimum } \\
\text { Detect }\end{array}$ & $\begin{array}{c}\text { Maximum } \\
\text { Detect }\end{array}$ & $\begin{array}{c}\text { Average } \\
\text { Concentration }\end{array}$ \\
\hline$\overline{\text { SVOC }}$ & Fluorene & $\mathrm{mg} / \mathrm{kg}$ & 3 & 0 & $0 \%$ & 0.0052 & 0.040 & -- & -- & 0.0091 \\
\hline SVOC & Hexachlorobenzene & $\mathrm{mg} / \mathrm{kg}$ & 3 & 0 & $0 \%$ & 0.020 & 0.020 & -- & -- & 0.010 \\
\hline SVOC & Hexachlorobutadiene & $\mathrm{mg} / \mathrm{kg}$ & 3 & 0 & $0 \%$ & 0.0098 & 0.0099 & -- & -- & 0.0049 \\
\hline SVOC & Hexachloroethane & $\mathrm{mg} / \mathrm{kg}$ & 3 & 0 & $0 \%$ & 0.0098 & 0.0099 & -- & -- & 0.0049 \\
\hline SVOC & Indeno(1,2,3-cd)pyrene & $\mathrm{mg} / \mathrm{kg}$ & 3 & 0 & $0 \%$ & 0.019 & 0.020 & -- & -- & 0.0098 \\
\hline SVOC & Naphthalene & $\mathrm{mg} / \mathrm{kg}$ & 3 & 0 & $0 \%$ & 0.020 & 0.040 & -- & -- & 0.016 \\
\hline SVOC & Nitrobenzene & $\mathrm{mg} / \mathrm{kg}$ & 3 & 0 & $0 \%$ & 0.0098 & 0.0099 & -- & -- & 0.0049 \\
\hline SVOC & Phenanthrene & $\mathrm{mg} / \mathrm{kg}$ & 3 & 0 & $0 \%$ & 0.0098 & 0.0099 & -- & -- & 0.0049 \\
\hline SVOC & Pyrene & $\mathrm{mg} / \mathrm{kg}$ & 3 & 0 & $0 \%$ & 0.019 & 0.020 & -- & -- & 0.0098 \\
\hline VOC & Retene & $\mathrm{mg} / \mathrm{kg}$ & 3 & 0 & $0 \%$ & 0.019 & 0.020 & -- & -- & 0.0098 \\
\hline
\end{tabular}

DIOXI - Dioxins and Furans

PEST - Pesticide

PCB - Polychlorinated bipheny

SVOC - Semivolatile Organic Compound

VOC - Volatile Organic Compound 
Table F-99

Summary Statistics for Whole Body Chinook Salmon Collected from Segment 5 of the Columbia River

\begin{tabular}{|c|c|c|c|c|c|c|c|c|c|c|}
\hline $\begin{array}{c}\text { Constituent } \\
\text { Class }\end{array}$ & Constituent Name & Units & $\begin{array}{l}\text { Number } \\
\text { Analyzed }\end{array}$ & $\begin{array}{l}\text { Number } \\
\text { Detected }\end{array}$ & $\begin{array}{c}\text { Frequency of } \\
\text { Detection }\end{array}$ & $\begin{array}{l}\text { Minimum } \\
\text { Nondetect }\end{array}$ & $\begin{array}{l}\text { Maximum } \\
\text { Nondetect }\end{array}$ & $\begin{array}{c}\text { Minimum } \\
\text { Detect }\end{array}$ & $\begin{array}{c}\text { Maximum } \\
\text { Detect }\end{array}$ & $\begin{array}{c}\text { Average } \\
\text { Concentration }\end{array}$ \\
\hline$\overline{\mathrm{DIOXI}}$ & $1,2,3,4,6,7,8$-Heptachlorodibenzodioxin & $\mathrm{mg} / \mathrm{kg}$ & 3 & 2 & $67 \%$ & $6.00 \mathrm{E}-08$ & $6.00 \mathrm{E}-08$ & $1.00 \mathrm{E}-07$ & $1.50 \mathrm{E}-07$ & $9.33 \mathrm{E}-08$ \\
\hline DIOXI & $1,2,3,4,6,7,8$-Heptachlorodibenzofuran & $\mathrm{mg} / \mathrm{kg}$ & 3 & 1 & $33 \%$ & $3.00 \mathrm{E}-08$ & 4.00E-08 & 4.00E-08 & 4.00E-08 & $2.50 \mathrm{E}-08$ \\
\hline DIOXI & 1,2,3,4,7,8,9-Heptachlorodibenzofuran & $\mathrm{mg} / \mathrm{kg}$ & 3 & 0 & $0 \%$ & $1.00 \mathrm{E}-08$ & 4.00E-08 & -- & -- & 1.33E-08 \\
\hline DIOXI & 1,2,3,4,7,8-Hexachlorodibenzofuran & $\mathrm{mg} / \mathrm{kg}$ & 3 & 1 & $33 \%$ & $5.00 \mathrm{E}-08$ & $6.00 \mathrm{E}-08$ & 3.00E-08 & $3.00 \mathrm{E}-08$ & $2.83 \mathrm{E}-08$ \\
\hline DIOXI & $1,2,3,4,7,8$-Hexachlorodibenzo-p-dioxin & $\mathrm{mg} / \mathrm{kg}$ & 3 & 0 & $0 \%$ & $1.00 \mathrm{E}-08$ & 4.00E-08 & -- & -- & 1.33E-08 \\
\hline DIOXI & 1,2,3,6,7,8-Hexachlorodibenzofuran & $\mathrm{mg} / \mathrm{kg}$ & 3 & 1 & $33 \%$ & $5.00 \mathrm{E}-08$ & $6.00 \mathrm{E}-08$ & 3.00E-08 & $3.00 \mathrm{E}-08$ & 2.83E-08 \\
\hline DIOXI & 1,2,3,6,7,8-Hexachlorodibenzo-p-dioxin & $\mathrm{mg} / \mathrm{kg}$ & 3 & 2 & $67 \%$ & $3.00 \mathrm{E}-08$ & 3.00E-08 & 7.00E-08 & 8.00E-08 & $5.50 \mathrm{E}-08$ \\
\hline DIOXI & 1,2,3,7,8,9-Hexachlorodibenzofuran & $\mathrm{mg} / \mathrm{kg}$ & 3 & 2 & $67 \%$ & $6.00 \mathrm{E}-08$ & $6.00 \mathrm{E}-08$ & 2.00E-08 & $5.00 \mathrm{E}-08$ & 3.33E-08 \\
\hline DIOXI & 1,2,3,7,8,9-Hexachlorodibenzo-p-dioxin & $\mathrm{mg} / \mathrm{kg}$ & 3 & 1 & $33 \%$ & $3.00 \mathrm{E}-08$ & 4.00E-08 & $6.00 \mathrm{E}-08$ & $6.00 \mathrm{E}-08$ & 3.17E-08 \\
\hline DIOXI & 1,2,3,7,8-Pentachlorodibenzofuran & $\mathrm{mg} / \mathrm{kg}$ & 3 & 1 & $33 \%$ & 4.00E-08 & $5.00 \mathrm{E}-08$ & 1.10E-07 & 1.10E-07 & $5.17 \mathrm{E}-08$ \\
\hline DIOXI & 1,2,3,7,8-Pentachlorodibenzo-p-dioxin & $\mathrm{mg} / \mathrm{kg}$ & 3 & 1 & $33 \%$ & $2.00 \mathrm{E}-08$ & $3.00 \mathrm{E}-08$ & 8.00E-08 & $8.00 \mathrm{E}-08$ & $3.50 \mathrm{E}-08$ \\
\hline DIOXI & $2,3,4,6,7,8$-Hexachlorodibenzofuran & $\mathrm{mg} / \mathrm{kg}$ & 3 & 1 & $33 \%$ & $5.00 \mathrm{E}-08$ & $6.00 \mathrm{E}-08$ & 3.00E-08 & $3.00 \mathrm{E}-08$ & 2.83E-08 \\
\hline DIOXI & 2,3,4,7,8-Pentachlorodibenzofuran & $\mathrm{mg} / \mathrm{kg}$ & 3 & 2 & $67 \%$ & $5.00 \mathrm{E}-08$ & $5.00 \mathrm{E}-08$ & 1.40E-07 & 1.50E-07 & $1.05 \mathrm{E}-07$ \\
\hline DIOXI & 2,3,7,8-Tetrachlorodibenzofuran & $\mathrm{mg} / \mathrm{kg}$ & 3 & 3 & $100 \%$ & -- & -- & 5.90E-07 & $6.90 \mathrm{E}-07$ & 6.33E-07 \\
\hline DIOXI & 2,3,7,8-Tetrachlorodibenzo-p-dioxin & $\mathrm{mg} / \mathrm{kg}$ & 3 & 0 & $0 \%$ & $1.00 \mathrm{E}-08$ & $3.00 \mathrm{E}-08$ & -- & -- & 8.33E-09 \\
\hline DIOXI & Octachlorodibenzofuran & $\mathrm{mg} / \mathrm{kg}$ & 3 & 1 & $33 \%$ & $6.00 \mathrm{E}-08$ & 7.00E-08 & 3.00E-08 & $3.00 \mathrm{E}-08$ & $3.17 \mathrm{E}-08$ \\
\hline DIOXI & Octachlorodibenzo-p-dioxin & $\mathrm{mg} / \mathrm{kg}$ & 3 & 3 & $100 \%$ & -- & -- & $2.50 \mathrm{E}-07$ & $3.00 \mathrm{E}-07$ & 2.73E-07 \\
\hline METAL & Aluminum & $\mathrm{mg} / \mathrm{kg}$ & 3 & 2 & $67 \%$ & 1.0 & 1.0 & 1.0 & 1.7 & 1.1 \\
\hline METAL & Antimony & $\mathrm{mg} / \mathrm{kg}$ & 3 & 0 & $0 \%$ & 0.050 & 0.050 & -- & -- & 0.025 \\
\hline METAL & Arsenic & $\mathrm{mg} / \mathrm{kg}$ & 3 & 3 & $100 \%$ & -- & -- & 0.85 & 0.86 & 0.86 \\
\hline METAL & Barium & $\mathrm{mg} / \mathrm{kg}$ & 3 & 0 & $0 \%$ & 0.20 & 0.20 & -- & -- & 0.10 \\
\hline METAL & Beryllium & $\mathrm{mg} / \mathrm{kg}$ & 3 & 0 & $0 \%$ & 0.0040 & 0.0040 & -- & -- & 0.0020 \\
\hline METAL & Cadmium & $\mathrm{mg} / \mathrm{kg}$ & 3 & 3 & $100 \%$ & -- & -- & 0.13 & 0.14 & 0.13 \\
\hline METAL & Chromium & $\mathrm{mg} / \mathrm{kg}$ & 3 & 3 & $100 \%$ & -- & -- & 0.47 & 0.50 & 0.49 \\
\hline METAL & Cobalt & $\mathrm{mg} / \mathrm{kg}$ & 3 & 3 & $100 \%$ & -- & -- & 0.090 & 0.13 & 0.11 \\
\hline METAL & Copper & $\mathrm{mg} / \mathrm{kg}$ & 3 & 3 & $100 \%$ & -- & -- & 1.3 & 1.5 & 1.4 \\
\hline METAL & Lead & $\mathrm{mg} / \mathrm{kg}$ & 3 & 0 & $0 \%$ & 0.010 & 0.010 & -- & -- & 0.0050 \\
\hline METAL & Manganese & $\mathrm{mg} / \mathrm{kg}$ & 3 & 3 & $100 \%$ & -- & -- & 0.32 & 0.38 & 0.35 \\
\hline METAL & Mercury & $\mathrm{mg} / \mathrm{kg}$ & 3 & 0 & $0 \%$ & 0.090 & 0.11 & -- & -- & 0.052 \\
\hline METAL & Nickel & $\mathrm{mg} / \mathrm{kg}$ & 3 & 3 & $100 \%$ & -- & -- & 0.34 & 0.75 & 0.53 \\
\hline METAL & Selenium & $\mathrm{mg} / \mathrm{kg}$ & 3 & 3 & $100 \%$ & -- & -- & 0.51 & 0.64 & 0.57 \\
\hline METAL & Silver & $\mathrm{mg} / \mathrm{kg}$ & 3 & 0 & $0 \%$ & 0.10 & 0.10 & -- & -- & 0.050 \\
\hline METAL & Thallium & $\mathrm{mg} / \mathrm{kg}$ & 3 & 0 & $0 \%$ & 0.050 & 0.050 & -- & -- & 0.025 \\
\hline METAL & Vanadium & $\mathrm{mg} / \mathrm{kg}$ & 3 & 3 & $100 \%$ & -- & -- & 0.010 & 0.020 & 0.013 \\
\hline METAL & Zinc & $\mathrm{mg} / \mathrm{kg}$ & 3 & 3 & $100 \%$ & -- & -- & 20 & 20 & 20 \\
\hline PCB & 2,2',3,3',4,4',5-Heptachloro-1,1'-biphenyl & $\mathrm{mg} / \mathrm{kg}$ & 3 & 3 & $100 \%$ & -- & -- & $6.62 \mathrm{E}-05$ & $7.42 \mathrm{E}-05$ & $6.97 \mathrm{E}-05$ \\
\hline PCB & $2,2^{\prime}, 3,4,4^{\prime}, 5,5^{\prime}-$ Heptachlorobiphenyl & $\mathrm{mg} / \mathrm{kg}$ & 3 & 3 & $100 \%$ & -- & -- & 2.35E-04 & $2.51 \mathrm{E}-04$ & $2.42 \mathrm{E}-04$ \\
\hline PCB & 2,3,3',4,4',5'-Hexachlorobiphenyl & $\mathrm{mg} / \mathrm{kg}$ & 3 & 3 & $100 \%$ & -- & -- & 1.15E-05 & $1.59 \mathrm{E}-05$ & 1.31E-05 \\
\hline PCB & 2,3,3',4,4',5-Hexachlorobiphenyl (BZ 156) & $\mathrm{mg} / \mathrm{kg}$ & 3 & 3 & $100 \%$ & -- & -- & 4.24E-05 & 4.59E-05 & 4.36E-05 \\
\hline PCB & 2,3,3',4,4'-Pentachlorobiphenyl & $\mathrm{mg} / \mathrm{kg}$ & 3 & 3 & $100 \%$ & -- & -- & 2.33E-04 & $2.57 \mathrm{E}-04$ & $2.44 \mathrm{E}-04$ \\
\hline PCB & 2,3',4,4',5,5'-Hexachlorobiphenyl (BZ 167) & $\mathrm{mg} / \mathrm{kg}$ & 3 & 3 & $100 \%$ & -- & -- & 2.75E-05 & $3.09 \mathrm{E}-05$ & 2.87E-05 \\
\hline PCB & 2',3,4,4',5-Pentachloro-1,1'-biphenyl (BZ 123) & $\mathrm{mg} / \mathrm{kg}$ & 3 & 3 & $100 \%$ & -- & -- & 7.82E-06 & 1.27E-05 & $1.07 \mathrm{E}-05$ \\
\hline РСB & 2,3,4,4',5-Pentachlorobiphenyl (BZ 114) & $\mathrm{mg} / \mathrm{kg}$ & 3 & 3 & $100 \%$ & -- & -- & $2.12 \mathrm{E}-05$ & $2.18 \mathrm{E}-05$ & $2.14 \mathrm{E}-05$ \\
\hline PCB & 2,3',4,4',5-Pentachlorobiphenyl (BZ 118) & $\mathrm{mg} / \mathrm{kg}$ & 3 & 3 & $100 \%$ & -- & -- & 5.31E-04 & $5.55 \mathrm{E}-04$ & $5.45 \mathrm{E}-04$ \\
\hline PCB & 2,3,4,5,3',4',5'-Heptachlorobiphenyl & $\mathrm{mg} / \mathrm{kg}$ & 3 & 1 & $33 \%$ & $1.00 \mathrm{E}-06$ & 1.00E-06 & 2.89E-06 & $2.89 \mathrm{E}-06$ & 1.30E-06 \\
\hline РСB & 3,3',4,4',5,5'-Hexachlorobiphenyl (BZ 169) & $\mathrm{mg} / \mathrm{kg}$ & 3 & 0 & $0 \%$ & $1.09 \mathrm{E}-06$ & 1.53E-06 & -- & -- & $6.27 \mathrm{E}-07$ \\
\hline PCB & 3,3',4,4',5-Pentachlorobiphenyl (BZ 126) & $\mathrm{mg} / \mathrm{kg}$ & 3 & 2 & $67 \%$ & $6.40 \mathrm{E}-07$ & $6.40 \mathrm{E}-07$ & 3.10E-06 & $3.42 \mathrm{E}-06$ & $2.28 \mathrm{E}-06$ \\
\hline PCB & 3,3',4,4'-Tetrachlorobiphenyl (BZ 77) & $\mathrm{mg} / \mathrm{kg}$ & 3 & 3 & $100 \%$ & -- & -- & 1.07E-05 & $1.26 \mathrm{E}-05$ & 1.17E-05 \\
\hline РCB & Aroclor-1016 & $\mathrm{mg} / \mathrm{kg}$ & 3 & 0 & $0 \%$ & 0.019 & 0.020 & -- & -- & 0.0097 \\
\hline PCB & Aroclor-1221 & $\mathrm{mg} / \mathrm{kg}$ & 3 & 0 & $0 \%$ & 0.019 & 0.020 & -- & -- & 0.0097 \\
\hline PCB & Aroclor-1232 & $\mathrm{mg} / \mathrm{kg}$ & 3 & 0 & $0 \%$ & 0.019 & 0.020 & -- & -- & 0.0097 \\
\hline PCB & Aroclor-1248 & $\mathrm{mg} / \mathrm{kg}$ & 3 & 0 & $0 \%$ & 0.019 & 0.020 & -- & -- & 0.0097 \\
\hline
\end{tabular}


Table F-99

Summary Statistics for Whole Body Chinook Salmon Collected from Segment 5 of the Columbia River

\begin{tabular}{|c|c|c|c|c|c|c|c|c|c|c|}
\hline $\begin{array}{l}\text { Constituent } \\
\text { Class }\end{array}$ & Constituent Name & Units & $\begin{array}{l}\text { Number } \\
\text { Analyzed }\end{array}$ & $\begin{array}{l}\text { Number } \\
\text { Detected }\end{array}$ & $\begin{array}{l}\text { Frequency of } \\
\text { Detection }\end{array}$ & $\begin{array}{l}\text { Minimum } \\
\text { Nondetect }\end{array}$ & $\begin{array}{l}\text { Maximum } \\
\text { Nondetect }\end{array}$ & $\begin{array}{l}\text { Minimum } \\
\text { Detect }\end{array}$ & $\begin{array}{l}\text { Maximum } \\
\text { Detect }\end{array}$ & $\begin{array}{c}\text { Average } \\
\text { Concentration }\end{array}$ \\
\hline$\overline{\mathrm{PCB}}$ & Aroclor-1254 & $\mathrm{mg} / \mathrm{kg}$ & 3 & 3 & $100 \%$ & $\overline{--}$ & $\overline{--}$ & 0.017 & 0.018 & 0.018 \\
\hline РСB & Aroclor-1260 & $\mathrm{mg} / \mathrm{kg}$ & 3 & 0 & $0 \%$ & 0.019 & 0.020 & -- & -- & 0.0097 \\
\hline PEST & 2,4'-DDD & $\mathrm{mg} / \mathrm{kg}$ & 3 & 0 & $0 \%$ & 0.0019 & 0.0020 & -- & -- & $9.67 \mathrm{E}-04$ \\
\hline PEST & 2,4'-DDE & $\mathrm{mg} / \mathrm{kg}$ & 3 & 0 & $0 \%$ & 0.0019 & 0.0020 & -- & -- & $9.67 \mathrm{E}-04$ \\
\hline PEST & 2,4'-DDT & $\mathrm{mg} / \mathrm{kg}$ & 3 & 0 & $0 \%$ & 0.0019 & 0.0020 & -- & -- & $9.67 \mathrm{E}-04$ \\
\hline PEST & Aldrin & $\mathrm{mg} / \mathrm{kg}$ & 3 & 0 & $0 \%$ & 0.0019 & 0.0020 & -- & -- & $9.67 \mathrm{E}-04$ \\
\hline PEST & Alpha-BHC & $\mathrm{mg} / \mathrm{kg}$ & 3 & 0 & $0 \%$ & 0.0019 & 0.0020 & -- & -- & $9.67 \mathrm{E}-04$ \\
\hline PEST & alpha-Chlordane & $\mathrm{mg} / \mathrm{kg}$ & 3 & 0 & $0 \%$ & 0.0019 & 0.0020 & -- & -- & $9.67 \mathrm{E}-04$ \\
\hline PEST & alpha-Chlordene & $\mathrm{mg} / \mathrm{kg}$ & 3 & 0 & $0 \%$ & 0.0019 & 0.0020 & -- & -- & $9.67 \mathrm{E}-04$ \\
\hline PEST & Beta-BHC & $\mathrm{mg} / \mathrm{kg}$ & 3 & 0 & $0 \%$ & 0.0019 & 0.0020 & -- & -- & $9.67 \mathrm{E}-04$ \\
\hline PEST & cis-Nonachlor & $\mathrm{mg} / \mathrm{kg}$ & 3 & 0 & $0 \%$ & 0.0019 & 0.0020 & -- & -- & 9.67E-04 \\
\hline PEST & DDMU & $\mathrm{mg} / \mathrm{kg}$ & 3 & 0 & $0 \%$ & 0.0019 & 0.0020 & -- & -- & $9.67 \mathrm{E}-04$ \\
\hline PEST & Delta-BHC & $\mathrm{mg} / \mathrm{kg}$ & 3 & 0 & $0 \%$ & 0.0019 & 0.0020 & -- & -- & $9.67 \mathrm{E}-04$ \\
\hline PEST & Dichlorodiphenyldichloroethane & $\mathrm{mg} / \mathrm{kg}$ & 3 & 3 & $100 \%$ & -- & -- & 0.0021 & 0.0034 & 0.0026 \\
\hline PEST & Dichlorodiphenyldichloroethylene & $\mathrm{mg} / \mathrm{kg}$ & 3 & 3 & $100 \%$ & -- & -- & 0.013 & 0.015 & 0.014 \\
\hline PEST & Dichlorodiphenyltrichloroethane & $\mathrm{mg} / \mathrm{kg}$ & 3 & 3 & $100 \%$ & -- & -- & 0.0042 & 0.0067 & 0.0054 \\
\hline PEST & Endosulfan sulfate & $\mathrm{mg} / \mathrm{kg}$ & 3 & 0 & $0 \%$ & 0.0019 & 0.0020 & -- & -- & $9.67 \mathrm{E}-04$ \\
\hline PEST & Gamma-BHC (Lindane) & $\mathrm{mg} / \mathrm{kg}$ & 3 & 0 & $0 \%$ & 0.0019 & 0.0020 & -- & -- & $9.67 \mathrm{E}-04$ \\
\hline PEST & gamma-Chlordane & $\mathrm{mg} / \mathrm{kg}$ & 3 & 0 & $0 \%$ & 0.0019 & 0.0020 & -- & -- & $9.67 \mathrm{E}-04$ \\
\hline PEST & gamma-Chlordene & $\mathrm{mg} / \mathrm{kg}$ & 3 & 0 & $0 \%$ & 0.0019 & 0.0020 & -- & -- & $9.67 \mathrm{E}-04$ \\
\hline PEST & Heptachlor & $\mathrm{mg} / \mathrm{kg}$ & 3 & 0 & $0 \%$ & 0.0019 & 0.0020 & -- & -- & $9.67 \mathrm{E}-04$ \\
\hline PEST & Heptachlor epoxide & $\mathrm{mg} / \mathrm{kg}$ & 3 & 0 & $0 \%$ & 0.0019 & 0.0020 & -- & -- & $9.67 \mathrm{E}-04$ \\
\hline PEST & Mirex & $\mathrm{mg} / \mathrm{kg}$ & 3 & 0 & $0 \%$ & 0.0019 & 0.0020 & -- & -- & $9.67 \mathrm{E}-04$ \\
\hline PEST & Oxychlordane & $\mathrm{mg} / \mathrm{kg}$ & 3 & 0 & $0 \%$ & 0.0019 & 0.0020 & -- & -- & $9.67 \mathrm{E}-04$ \\
\hline PEST & Pentachloroanisole & $\mathrm{mg} / \mathrm{kg}$ & 3 & 0 & $0 \%$ & 0.0019 & 0.0020 & -- & -- & $9.67 \mathrm{E}-04$ \\
\hline PEST & Toxaphene & $\mathrm{mg} / \mathrm{kg}$ & 3 & 0 & $0 \%$ & 0.057 & 0.059 & -- & -- & 0.029 \\
\hline PEST & trans-Nonachlor & $\mathrm{mg} / \mathrm{kg}$ & 3 & 3 & $100 \%$ & -- & -- & 0.0031 & 0.0033 & 0.0032 \\
\hline SVOC & 1,2,4-Trichlorobenzene & $\mathrm{mg} / \mathrm{kg}$ & 3 & 0 & $0 \%$ & 0.0093 & 0.0096 & -- & -- & 0.0047 \\
\hline SVOC & 1,2-Dichlorobenzene & $\mathrm{mg} / \mathrm{kg}$ & 3 & 0 & $0 \%$ & 0.0093 & 0.0096 & -- & -- & 0.0047 \\
\hline SVOC & 1,2-Diphenylhydrazine & $\mathrm{mg} / \mathrm{kg}$ & 3 & 0 & $0 \%$ & 0.0093 & 0.0096 & -- & -- & 0.0047 \\
\hline SVOC & 1,3-Dichlorobenzene & $\mathrm{mg} / \mathrm{kg}$ & 3 & 0 & $0 \%$ & 0.0093 & 0.0096 & -- & -- & 0.0047 \\
\hline SVOC & 1,4-Dichlorobenzene & $\mathrm{mg} / \mathrm{kg}$ & 3 & 0 & $0 \%$ & 0.0093 & 0.0096 & -- & -- & 0.0047 \\
\hline SVOC & 1-methylnaphthalene & $\mathrm{mg} / \mathrm{kg}$ & 3 & 0 & $0 \%$ & 0.0093 & 0.0096 & -- & -- & 0.0047 \\
\hline SVOC & 2,4-Dinitrotoluene & $\mathrm{mg} / \mathrm{kg}$ & 3 & 0 & $0 \%$ & 0.019 & 0.019 & -- & -- & 0.0095 \\
\hline SVOC & 2,6-Dinitrotoluene & $\mathrm{mg} / \mathrm{kg}$ & 3 & 0 & $0 \%$ & 0.037 & 0.038 & -- & -- & 0.019 \\
\hline SVOC & 2-Chloronaphthalene & $\mathrm{mg} / \mathrm{kg}$ & 3 & 0 & $0 \%$ & 0.019 & 0.019 & -- & -- & 0.0095 \\
\hline SVOC & 2-Methylnaphthalene & $\mathrm{mg} / \mathrm{kg}$ & 3 & 0 & $0 \%$ & 0.0093 & 0.0096 & -- & -- & 0.0047 \\
\hline SVOC & 4-Bromophenylphenyl ether & $\mathrm{mg} / \mathrm{kg}$ & 3 & 0 & $0 \%$ & 0.0093 & 0.0096 & -- & -- & 0.0047 \\
\hline SVOC & 4-Chlorophenylphenyl ether & $\mathrm{mg} / \mathrm{kg}$ & 3 & 0 & $0 \%$ & 0.0093 & 0.0096 & -- & -- & 0.0047 \\
\hline SVOC & Acenaphthene & $\mathrm{mg} / \mathrm{kg}$ & 3 & 0 & $0 \%$ & 0.019 & 0.019 & -- & -- & 0.0095 \\
\hline SVOC & Acenaphthylene & $\mathrm{mg} / \mathrm{kg}$ & 3 & 0 & $0 \%$ & 0.0093 & 0.0096 & -- & -- & 0.0047 \\
\hline SVOC & Anthracene & $\mathrm{mg} / \mathrm{kg}$ & 3 & 0 & $0 \%$ & 0.0093 & 0.0096 & -- & -- & 0.0047 \\
\hline SVOC & Benzo(a)anthracene & $\mathrm{mg} / \mathrm{kg}$ & 3 & 0 & $0 \%$ & 0.0096 & 0.0097 & -- & -- & 0.0048 \\
\hline SVOC & Benzo(a)pyrene & $\mathrm{mg} / \mathrm{kg}$ & 3 & 0 & $0 \%$ & 0.0096 & 0.0097 & -- & -- & 0.0048 \\
\hline SVOC & Benzo(b)fluoranthene & $\mathrm{mg} / \mathrm{kg}$ & 3 & 0 & $0 \%$ & 0.0096 & 0.0097 & -- & -- & 0.0048 \\
\hline SVOC & Benzo(ghi)perylene & $\mathrm{mg} / \mathrm{kg}$ & 3 & 0 & $0 \%$ & 0.019 & 0.019 & -- & -- & 0.0095 \\
\hline SVOC & Benzo(k)fluoranthene & $\mathrm{mg} / \mathrm{kg}$ & 3 & 0 & $0 \%$ & 0.0096 & 0.0097 & -- & -- & 0.0048 \\
\hline SVOC & Bis(2-chloro-1-methylethyl)ether & $\mathrm{mg} / \mathrm{kg}$ & 3 & 0 & $0 \%$ & 0.019 & 0.019 & -- & -- & 0.0095 \\
\hline SVOC & Chrysene & $\mathrm{mg} / \mathrm{kg}$ & 3 & 0 & $0 \%$ & 0.0096 & 0.0097 & -- & -- & 0.0048 \\
\hline SVOC & Dibenz[a,h]anthracene & $\mathrm{mg} / \mathrm{kg}$ & 3 & 0 & $0 \%$ & 0.019 & 0.019 & -- & -- & 0.0095 \\
\hline SVOC & Dibenzofuran & $\mathrm{mg} / \mathrm{kg}$ & 3 & 0 & $0 \%$ & 0.0093 & 0.0096 & -- & -- & 0.0047 \\
\hline SVOC & Fluoranthene & $\mathrm{mg} / \mathrm{kg}$ & 3 & 0 & $0 \%$ & 0.019 & 0.019 & -- & -- & 0.0095 \\
\hline
\end{tabular}


Table F-99

Summary Statistics for Whole Body Chinook Salmon Collected from Segment 5 of the Columbia River

\begin{tabular}{|c|c|c|c|c|c|c|c|c|c|c|}
\hline $\begin{array}{c}\text { Constituent } \\
\text { Class }\end{array}$ & Constituent Name & Units & $\begin{array}{l}\text { Number } \\
\text { Analyzed }\end{array}$ & $\begin{array}{l}\text { Number } \\
\text { Detected }\end{array}$ & $\begin{array}{l}\text { Frequency of } \\
\text { Detection }\end{array}$ & $\begin{array}{l}\text { Minimum } \\
\text { Nondetect }\end{array}$ & $\begin{array}{l}\text { Maximum } \\
\text { Nondetect }\end{array}$ & $\underset{\text { Detect }}{\text { Minimum }}$ & $\begin{array}{c}\text { Maximum } \\
\text { Detect }\end{array}$ & $\begin{array}{c}\text { Average } \\
\text { Concentration }\end{array}$ \\
\hline$\overline{\text { SVOC }}$ & Fluorene & $\mathrm{mg} / \mathrm{kg}$ & 3 & 0 & $0 \%$ & 0.0047 & 0.012 & -- & -- & 0.0044 \\
\hline SVOC & Hexachlorobenzene & $\mathrm{mg} / \mathrm{kg}$ & 3 & 0 & $0 \%$ & 0.019 & 0.019 & -- & -- & 0.0095 \\
\hline SVOC & Hexachlorobutadiene & $\mathrm{mg} / \mathrm{kg}$ & 3 & 0 & $0 \%$ & 0.0093 & 0.0096 & -- & -- & 0.0047 \\
\hline SVOC & Hexachloroethane & $\mathrm{mg} / \mathrm{kg}$ & 3 & 0 & $0 \%$ & 0.0093 & 0.0096 & -- & -- & 0.0047 \\
\hline SVOC & Indeno(1,2,3-cd)pyrene & $\mathrm{mg} / \mathrm{kg}$ & 3 & 0 & $0 \%$ & 0.019 & 0.019 & -- & -- & 0.0095 \\
\hline SVOC & Naphthalene & $\mathrm{mg} / \mathrm{kg}$ & 3 & 0 & $0 \%$ & 0.037 & 0.055 & -- & -- & 0.024 \\
\hline SVOC & Nitrobenzene & $\mathrm{mg} / \mathrm{kg}$ & 3 & 0 & $0 \%$ & 0.0093 & 0.0096 & -- & -- & 0.0047 \\
\hline SVOC & Phenanthrene & $\mathrm{mg} / \mathrm{kg}$ & 3 & 0 & $0 \%$ & 0.0093 & 0.0096 & -- & -- & 0.0047 \\
\hline SVOC & Pyrene & $\mathrm{mg} / \mathrm{kg}$ & 3 & 0 & $0 \%$ & 0.019 & 0.019 & -- & -- & 0.0095 \\
\hline VOC & Retene & $\mathrm{mg} / \mathrm{kg}$ & 3 & 0 & $0 \%$ & 0.019 & 0.019 & -- & -- & 0.0095 \\
\hline
\end{tabular}

DIOXI - Dioxins and Furans

PEST - Pesticide

PCB - Polychlorinated biphenyl

SVOC - Semivolatile Organic Compound

VOC - Volatile Organic Compound 
Table F-100

Summary Statistics for Whole Body Eulachon Collected from Segment 5 of the Columbia River

\begin{tabular}{|c|c|c|c|c|c|c|c|c|c|c|}
\hline $\begin{array}{l}\text { Constituent } \\
\text { Class }\end{array}$ & Constituent Name & Units & $\begin{array}{l}\text { Number } \\
\text { Analyzed }\end{array}$ & $\begin{array}{l}\text { Number } \\
\text { Detected }\end{array}$ & $\begin{array}{c}\text { Frequency of } \\
\text { Detection }\end{array}$ & $\begin{array}{l}\text { Minimum } \\
\text { Nondetect }\end{array}$ & $\begin{array}{l}\text { Maximum } \\
\text { Nondetect }\end{array}$ & $\begin{array}{l}\text { Minimum } \\
\text { Detect }\end{array}$ & $\begin{array}{l}\text { Maximum } \\
\text { Detect }\end{array}$ & $\begin{array}{c}\text { Average } \\
\text { Concentration }\end{array}$ \\
\hline$\overline{\mathrm{DIOXI}}$ & $1,2,3,4,6,7,8$-Heptachlorodibenzodioxin & $\mathrm{mg} / \mathrm{kg}$ & 3 & 3 & $100 \%$ & -- & -- & $4.00 \mathrm{E}-07$ & $6.00 \mathrm{E}-07$ & $4.87 \mathrm{E}-07$ \\
\hline DIOXI & $1,2,3,4,6,7,8$-Heptachlorodibenzofuran & $\mathrm{mg} / \mathrm{kg}$ & 3 & 2 & $67 \%$ & $1.00 \mathrm{E}-08$ & $1.00 \mathrm{E}-08$ & $2.40 \mathrm{E}-07$ & $2.60 \mathrm{E}-07$ & $1.68 \mathrm{E}-07$ \\
\hline DIOXI & 1,2,3,4,7,8,9-Heptachlorodibenzofuran & $\mathrm{mg} / \mathrm{kg}$ & 3 & 2 & $67 \%$ & $9.00 \mathrm{E}-08$ & $9.00 \mathrm{E}-08$ & $2.00 \mathrm{E}-08$ & 1.10E-07 & 5.83E-08 \\
\hline DIOXI & 1,2,3,4,7,8-Hexachlorodibenzofuran & $\mathrm{mg} / \mathrm{kg}$ & 3 & 2 & $67 \%$ & $9.00 \mathrm{E}-08$ & $9.00 \mathrm{E}-08$ & $3.00 \mathrm{E}-08$ & $9.00 \mathrm{E}-08$ & $5.50 \mathrm{E}-08$ \\
\hline DIOXI & $1,2,3,4,7,8$-Hexachlorodibenzo-p-dioxin & $\mathrm{mg} / \mathrm{kg}$ & 3 & 1 & $33 \%$ & $1.00 \mathrm{E}-08$ & $1.00 \mathrm{E}-07$ & $1.70 \mathrm{E}-07$ & 1.70E-07 & $7.50 \mathrm{E}-08$ \\
\hline DIOXI & 1,2,3,6,7,8-Hexachlorodibenzofuran & $\mathrm{mg} / \mathrm{kg}$ & 3 & 2 & $67 \%$ & $9.00 \mathrm{E}-08$ & $9.00 \mathrm{E}-08$ & $5.00 \mathrm{E}-08$ & $1.00 \mathrm{E}-07$ & $6.50 \mathrm{E}-08$ \\
\hline DIOXI & 1,2,3,6,7,8-Hexachlorodibenzo-p-dioxin & $\mathrm{mg} / \mathrm{kg}$ & 3 & 2 & $67 \%$ & 1.00E-07 & 1.00E-07 & $2.20 \mathrm{E}-07$ & $2.60 \mathrm{E}-07$ & 1.77E-07 \\
\hline DIOXI & 1,2,3,7,8,9-Hexachlorodibenzofuran & $\mathrm{mg} / \mathrm{kg}$ & 3 & 3 & $100 \%$ & -- & -- & $7.00 \mathrm{E}-08$ & $2.00 \mathrm{E}-07$ & 1.40E-07 \\
\hline DIOXI & 1,2,3,7,8,9-Hexachlorodibenzo-p-dioxin & $\mathrm{mg} / \mathrm{kg}$ & 3 & 2 & $67 \%$ & $1.00 \mathrm{E}-07$ & $1.00 \mathrm{E}-07$ & $1.00 \mathrm{E}-07$ & 1.70E-07 & 1.07E-07 \\
\hline DIOXI & 1,2,3,7,8-Pentachlorodibenzofuran & $\mathrm{mg} / \mathrm{kg}$ & 3 & 3 & $100 \%$ & -- & -- & $8.00 \mathrm{E}-08$ & $1.50 \mathrm{E}-07$ & 1.10E-07 \\
\hline DIOXI & 1,2,3,7,8-Pentachlorodibenzo-p-dioxin & $\mathrm{mg} / \mathrm{kg}$ & 3 & 3 & $100 \%$ & -- & -- & $2.10 \mathrm{E}-07$ & $2.80 \mathrm{E}-07$ & $2.40 \mathrm{E}-07$ \\
\hline DIOXI & 2,3,4,6,7,8-Hexachlorodibenzofuran & $\mathrm{mg} / \mathrm{kg}$ & 3 & 3 & $100 \%$ & -- & -- & $1.50 \mathrm{E}-07$ & $2.10 \mathrm{E}-07$ & $1.87 \mathrm{E}-07$ \\
\hline DIOXI & 2,3,4,7,8-Pentachlorodibenzofuran & $\mathrm{mg} / \mathrm{kg}$ & 3 & 3 & $100 \%$ & -- & -- & $1.00 \mathrm{E}-07$ & $1.50 \mathrm{E}-07$ & 1.30E-07 \\
\hline DIOXI & 2,3,7,8-Tetrachlorodibenzofuran & $\mathrm{mg} / \mathrm{kg}$ & 3 & 3 & $100 \%$ & -- & -- & $5.80 \mathrm{E}-07$ & $7.80 \mathrm{E}-07$ & $6.70 \mathrm{E}-07$ \\
\hline DIOXI & 2,3,7,8-Tetrachlorodibenzo-p-dioxin & $\mathrm{mg} / \mathrm{kg}$ & 3 & 2 & $67 \%$ & $5.00 \mathrm{E}-08$ & $5.00 \mathrm{E}-08$ & $8.00 \mathrm{E}-08$ & $1.00 \mathrm{E}-07$ & $6.83 E-08$ \\
\hline DIOXI & Octachlorodibenzofuran & $\mathrm{mg} / \mathrm{kg}$ & 3 & 3 & $100 \%$ & -- & -- & $1.00 \mathrm{E}-07$ & $2.70 \mathrm{E}-07$ & $2.10 \mathrm{E}-07$ \\
\hline DIOXI & Octachlorodibenzo-p-dioxin & $\mathrm{mg} / \mathrm{kg}$ & 3 & 3 & $100 \%$ & -- & -- & $1.25 \mathrm{E}-06$ & $1.71 \mathrm{E}-06$ & $1.49 \mathrm{E}-06$ \\
\hline METAL & Aluminum & $\mathrm{mg} / \mathrm{kg}$ & 3 & 1 & $33 \%$ & 20 & 20 & 6.5 & 6.5 & 8.8 \\
\hline METAL & Antimony & $\mathrm{mg} / \mathrm{kg}$ & 3 & 0 & $0 \%$ & 0.050 & 0.050 & -- & -- & 0.025 \\
\hline METAL & Arsenic & $\mathrm{mg} / \mathrm{kg}$ & 3 & 3 & $100 \%$ & -- & -- & 0.86 & 0.92 & 0.89 \\
\hline METAL & Barium & $\mathrm{mg} / \mathrm{kg}$ & 3 & 1 & $33 \%$ & 0.20 & 0.20 & 0.35 & 0.35 & 0.18 \\
\hline METAL & Beryllium & $\mathrm{mg} / \mathrm{kg}$ & 3 & 0 & $0 \%$ & 0.0040 & 0.0040 & -- & -- & 0.0020 \\
\hline METAL & Cadmium & $\mathrm{mg} / \mathrm{kg}$ & 3 & 3 & $100 \%$ & -- & -- & 0.0085 & 0.010 & 0.0092 \\
\hline METAL & Chromium & $\mathrm{mg} / \mathrm{kg}$ & 3 & 0 & $0 \%$ & 0.10 & 0.10 & -- & -- & 0.050 \\
\hline METAL & Cobalt & $\mathrm{mg} / \mathrm{kg}$ & 3 & 3 & $100 \%$ & -- & -- & 0.0063 & 0.0079 & 0.0071 \\
\hline METAL & Copper & $\mathrm{mg} / \mathrm{kg}$ & 3 & 3 & $100 \%$ & -- & -- & 0.91 & 0.96 & 0.94 \\
\hline METAL & Lead & $\mathrm{mg} / \mathrm{kg}$ & 3 & 3 & $100 \%$ & -- & -- & 0.37 & 0.68 & 0.49 \\
\hline METAL & Manganese & $\mathrm{mg} / \mathrm{kg}$ & 3 & 3 & $100 \%$ & -- & -- & 0.47 & 0.52 & 0.50 \\
\hline METAL & Mercury & $\mathrm{mg} / \mathrm{kg}$ & 3 & 0 & $0 \%$ & 0.030 & 0.040 & -- & -- & 0.017 \\
\hline METAL & Nickel & $\mathrm{mg} / \mathrm{kg}$ & 3 & 3 & $100 \%$ & -- & -- & 0.030 & 0.060 & 0.047 \\
\hline METAL & Selenium & $\mathrm{mg} / \mathrm{kg}$ & 3 & 3 & $100 \%$ & -- & -- & 0.27 & 0.30 & 0.29 \\
\hline METAL & Silver & $\mathrm{mg} / \mathrm{kg}$ & 3 & 0 & $0 \%$ & 0.10 & 0.10 & -- & -- & 0.050 \\
\hline METAL & Thallium & $\mathrm{mg} / \mathrm{kg}$ & 3 & 0 & $0 \%$ & 0.050 & 0.050 & -- & -- & 0.025 \\
\hline METAL & Vanadium & $\mathrm{mg} / \mathrm{kg}$ & 3 & 2 & $67 \%$ & 0.020 & 0.020 & 0.020 & 0.020 & 0.017 \\
\hline METAL & Zinc & $\mathrm{mg} / \mathrm{kg}$ & 3 & 3 & $100 \%$ & -- & -- & 13 & 14 & 14 \\
\hline PCB & 2,2',3,3',4,4',5-Heptachloro-1,1'-biphenyl & $\mathrm{mg} / \mathrm{kg}$ & 3 & 3 & $100 \%$ & -- & -- & $5.78 \mathrm{E}-05$ & $6.44 \mathrm{E}-05$ & 6.17E-05 \\
\hline PCB & 2,2',3,4,4',5,5'-Heptachlorobiphenyl & $\mathrm{mg} / \mathrm{kg}$ & 3 & 3 & $100 \%$ & -- & -- & $1.69 \mathrm{E}-04$ & 1.83E-04 & $1.78 \mathrm{E}-04$ \\
\hline PCB & 2,3,3',4,4',5'-Hexachlorobiphenyl & $\mathrm{mg} / \mathrm{kg}$ & 3 & 3 & $100 \%$ & -- & -- & $8.12 \mathrm{E}-06$ & $9.47 \mathrm{E}-06$ & 8.74E-06 \\
\hline PCB & 2,3,3',4,4',5-Hexachlorobiphenyl (BZ 156) & $\mathrm{mg} / \mathrm{kg}$ & 3 & 3 & $100 \%$ & -- & -- & $2.78 \mathrm{E}-05$ & 3.04E-05 & 2.89E-05 \\
\hline РСB & 2,3,3',4,4'-Pentachlorobiphenyl & $\mathrm{mg} / \mathrm{kg}$ & 3 & 3 & $100 \%$ & -- & -- & $1.45 \mathrm{E}-04$ & $1.50 \mathrm{E}-04$ & $1.48 \mathrm{E}-04$ \\
\hline PCB & 2,3',4,4',5,5'-Hexachlorobiphenyl (BZ 167) & $\mathrm{mg} / \mathrm{kg}$ & 3 & 3 & $100 \%$ & -- & -- & $1.72 \mathrm{E}-05$ & $1.84 \mathrm{E}-05$ & 1.79E-05 \\
\hline PCB & 2',3,4,4',5-Pentachloro-1,1'-biphenyl (BZ 123) & $\mathrm{mg} / \mathrm{kg}$ & 3 & 3 & $100 \%$ & -- & -- & $1.00 \mathrm{E}-05$ & 1.17E-05 & $1.08 \mathrm{E}-05$ \\
\hline РСB & 2,3,4,4',5-Pentachlorobiphenyl (BZ 114) & $\mathrm{mg} / \mathrm{kg}$ & 3 & 3 & $100 \%$ & -- & -- & $1.04 \mathrm{E}-05$ & 1.13E-05 & 1.09E-05 \\
\hline PCB & 2,3',4,4',5-Pentachlorobiphenyl (BZ 118) & $\mathrm{mg} / \mathrm{kg}$ & 3 & 3 & $100 \%$ & -- & -- & $2.85 \mathrm{E}-04$ & $2.91 \mathrm{E}-04$ & $2.88 \mathrm{E}-04$ \\
\hline PCB & 2,3,4,5,3',4',5'-Heptachlorobiphenyl & $\mathrm{mg} / \mathrm{kg}$ & 3 & 3 & $100 \%$ & -- & -- & 2.33E-06 & $2.46 \mathrm{E}-06$ & 2.37E-06 \\
\hline РСВ & 3,3',4,4',5,5'-Hexachlorobiphenyl (BZ 169) & $\mathrm{mg} / \mathrm{kg}$ & 3 & 0 & $0 \%$ & $5.00 \mathrm{E}-07$ & $5.50 \mathrm{E}-07$ & -- & -- & $2.58 \mathrm{E}-07$ \\
\hline PCB & 3,3',4,4',5-Pentachlorobiphenyl (BZ 126) & $\mathrm{mg} / \mathrm{kg}$ & 3 & 0 & $0 \%$ & $1.68 \mathrm{E}-06$ & $2.14 \mathrm{E}-06$ & -- & -- & $9.52 \mathrm{E}-07$ \\
\hline PCB & 3,3',4,4'-Tetrachlorobiphenyl (BZ 77) & $\mathrm{mg} / \mathrm{kg}$ & 3 & 3 & $100 \%$ & -- & -- & $8.08 \mathrm{E}-06$ & 8.31E-06 & 8.19E-06 \\
\hline РСB & Aroclor-1016 & $\mathrm{mg} / \mathrm{kg}$ & 3 & 0 & $0 \%$ & 0.037 & 0.038 & -- & -- & 0.019 \\
\hline PCB & Aroclor-1221 & $\mathrm{mg} / \mathrm{kg}$ & 3 & 0 & $0 \%$ & 0.037 & 0.038 & -- & -- & 0.019 \\
\hline PCB & Aroclor-1232 & $\mathrm{mg} / \mathrm{kg}$ & 3 & 0 & $0 \%$ & 0.037 & 0.038 & -- & -- & 0.019 \\
\hline PCB & Aroclor-1248 & $\mathrm{mg} / \mathrm{kg}$ & 3 & 0 & $0 \%$ & 0.037 & 0.038 & -- & -- & 0.019 \\
\hline
\end{tabular}


Table F-100

Summary Statistics for Whole Body Eulachon Collected from Segment 5 of the Columbia River

\begin{tabular}{|c|c|c|c|c|c|c|c|c|c|c|}
\hline $\begin{array}{l}\text { Constituent } \\
\text { Class }\end{array}$ & Constituent Name & Units & $\begin{array}{l}\text { Number } \\
\text { Analyzed }\end{array}$ & $\begin{array}{l}\text { Number } \\
\text { Detected }\end{array}$ & $\begin{array}{l}\text { Frequency of } \\
\text { Detection }\end{array}$ & $\begin{array}{l}\text { Minimum } \\
\text { Nondetect }\end{array}$ & $\begin{array}{l}\text { Maximum } \\
\text { Nondetect }\end{array}$ & $\begin{array}{l}\text { Minimum } \\
\text { Detect }\end{array}$ & $\begin{array}{l}\text { Maximum } \\
\text { Detect }\end{array}$ & $\begin{array}{c}\text { Average } \\
\text { Concentration }\end{array}$ \\
\hline$\overline{\mathrm{PCB}}$ & Aroclor-1254 & $\mathrm{mg} / \mathrm{kg}$ & 3 & 0 & $0 \%$ & 0.037 & 0.038 & $\overline{--}$ & $\overline{--}$ & 0.019 \\
\hline РСB & Aroclor-1260 & $\mathrm{mg} / \mathrm{kg}$ & 3 & 0 & $0 \%$ & 0.037 & 0.038 & -- & -- & 0.019 \\
\hline PEST & 2,4'-DDD & $\mathrm{mg} / \mathrm{kg}$ & 3 & 0 & $0 \%$ & 0.0037 & 0.0038 & -- & -- & 0.0019 \\
\hline PEST & 2,4'-DDE & $\mathrm{mg} / \mathrm{kg}$ & 3 & 0 & $0 \%$ & 0.0037 & 0.0038 & -- & -- & 0.0019 \\
\hline PEST & 2,4'-DDT & $\mathrm{mg} / \mathrm{kg}$ & 3 & 0 & $0 \%$ & 0.0037 & 0.0038 & -- & -- & 0.0019 \\
\hline PEST & Aldrin & $\mathrm{mg} / \mathrm{kg}$ & 3 & 0 & $0 \%$ & 0.0037 & 0.0038 & -- & -- & 0.0019 \\
\hline PEST & Alpha-BHC & $\mathrm{mg} / \mathrm{kg}$ & 3 & 0 & $0 \%$ & 0.0037 & 0.0038 & -- & -- & 0.0019 \\
\hline PEST & alpha-Chlordane & $\mathrm{mg} / \mathrm{kg}$ & 3 & 0 & $0 \%$ & 0.0037 & 0.0038 & -- & -- & 0.0019 \\
\hline PEST & alpha-Chlordene & $\mathrm{mg} / \mathrm{kg}$ & 3 & 0 & $0 \%$ & 0.0037 & 0.0038 & -- & -- & 0.0019 \\
\hline PEST & Beta-BHC & $\mathrm{mg} / \mathrm{kg}$ & 3 & 0 & $0 \%$ & 0.0037 & 0.0038 & -- & -- & 0.0019 \\
\hline PEST & cis-Nonachlor & $\mathrm{mg} / \mathrm{kg}$ & 3 & 0 & $0 \%$ & 0.0037 & 0.0038 & -- & -- & 0.0019 \\
\hline PEST & DDMU & $\mathrm{mg} / \mathrm{kg}$ & 3 & 0 & $0 \%$ & 0.0037 & 0.0038 & -- & -- & 0.0019 \\
\hline PEST & Delta-BHC & $\mathrm{mg} / \mathrm{kg}$ & 3 & 0 & $0 \%$ & 0.0037 & 0.0038 & -- & -- & 0.0019 \\
\hline PEST & Dichlorodiphenyldichloroethane & $\mathrm{mg} / \mathrm{kg}$ & 3 & 0 & $0 \%$ & 0.0037 & 0.0038 & -- & -- & 0.0019 \\
\hline PEST & Dichlorodiphenyldichloroethylene & $\mathrm{mg} / \mathrm{kg}$ & 3 & 3 & $100 \%$ & -- & -- & 0.0098 & 0.011 & 0.011 \\
\hline PEST & Dichlorodiphenyltrichloroethane & $\mathrm{mg} / \mathrm{kg}$ & 3 & 0 & $0 \%$ & 0.0037 & 0.0038 & -- & -- & 0.0019 \\
\hline PEST & Endosulfan sulfate & $\mathrm{mg} / \mathrm{kg}$ & 3 & 0 & $0 \%$ & 0.0037 & 0.0038 & -- & -- & 0.0019 \\
\hline PEST & Gamma-BHC (Lindane) & $\mathrm{mg} / \mathrm{kg}$ & 3 & 0 & $0 \%$ & 0.0037 & 0.0038 & -- & -- & 0.0019 \\
\hline PEST & gamma-Chlordane & $\mathrm{mg} / \mathrm{kg}$ & 3 & 0 & $0 \%$ & 0.0037 & 0.0038 & -- & -- & 0.0019 \\
\hline PEST & gamma-Chlordene & $\mathrm{mg} / \mathrm{kg}$ & 3 & 0 & $0 \%$ & 0.0037 & 0.0038 & -- & -- & 0.0019 \\
\hline PEST & Heptachlor & $\mathrm{mg} / \mathrm{kg}$ & 3 & 0 & $0 \%$ & 0.0037 & 0.0038 & -- & -- & 0.0019 \\
\hline PEST & Heptachlor epoxide & $\mathrm{mg} / \mathrm{kg}$ & 3 & 0 & $0 \%$ & 0.0037 & 0.0038 & -- & -- & 0.0019 \\
\hline PEST & Mirex & $\mathrm{mg} / \mathrm{kg}$ & 3 & 0 & $0 \%$ & 0.0037 & 0.0038 & -- & -- & 0.0019 \\
\hline PEST & Oxychlordane & $\mathrm{mg} / \mathrm{kg}$ & 3 & 0 & $0 \%$ & 0.0037 & 0.0038 & -- & -- & 0.0019 \\
\hline PEST & Pentachloroanisole & $\mathrm{mg} / \mathrm{kg}$ & 3 & 0 & $0 \%$ & 0.0037 & 0.0038 & -- & -- & 0.0019 \\
\hline PEST & Toxaphene & $\mathrm{mg} / \mathrm{kg}$ & 3 & 0 & $0 \%$ & 0.11 & 0.11 & -- & -- & 0.055 \\
\hline PEST & trans-Nonachlor & $\mathrm{mg} / \mathrm{kg}$ & 3 & 0 & $0 \%$ & 0.0037 & 0.0038 & -- & -- & 0.0019 \\
\hline SVOC & 1,2,4-Trichlorobenzene & $\mathrm{mg} / \mathrm{kg}$ & 3 & 0 & $0 \%$ & 0.0073 & 0.0083 & -- & -- & 0.0039 \\
\hline SVOC & 1,2-Dichlorobenzene & $\mathrm{mg} / \mathrm{kg}$ & 3 & 0 & $0 \%$ & 0.0073 & 0.0083 & -- & -- & 0.0039 \\
\hline SVOC & 1,2-Diphenylhydrazine & $\mathrm{mg} / \mathrm{kg}$ & 3 & 0 & $0 \%$ & 0.15 & 0.17 & -- & -- & 0.078 \\
\hline SVOC & 1,3-Dichlorobenzene & $\mathrm{mg} / \mathrm{kg}$ & 3 & 0 & $0 \%$ & 0.0073 & 0.0083 & -- & -- & 0.0039 \\
\hline SVOC & 1,4-Dichlorobenzene & $\mathrm{mg} / \mathrm{kg}$ & 3 & 0 & $0 \%$ & 0.0073 & 0.0083 & -- & -- & 0.0039 \\
\hline SVOC & 1-methylnaphthalene & $\mathrm{mg} / \mathrm{kg}$ & 3 & 0 & $0 \%$ & 0.0073 & 0.0083 & -- & -- & 0.0039 \\
\hline SVOC & 2,4-Dinitrotoluene & $\mathrm{mg} / \mathrm{kg}$ & 3 & 0 & $0 \%$ & 0.29 & 0.33 & -- & -- & 0.15 \\
\hline SVOC & 2,6-Dinitrotoluene & $\mathrm{mg} / \mathrm{kg}$ & 3 & 0 & $0 \%$ & 0.59 & 0.66 & -- & -- & 0.31 \\
\hline SVOC & 2-Chloronaphthalene & $\mathrm{mg} / \mathrm{kg}$ & 3 & 0 & $0 \%$ & 0.15 & 0.17 & -- & -- & 0.078 \\
\hline SVOC & 2-Methylnaphthalene & $\mathrm{mg} / \mathrm{kg}$ & 3 & 1 & $33 \%$ & 0.0078 & 0.0086 & 0.011 & 0.011 & 0.0064 \\
\hline SVOC & 4-Bromophenylphenyl ether & $\mathrm{mg} / \mathrm{kg}$ & 3 & 0 & $0 \%$ & 0.15 & 0.17 & -- & -- & 0.078 \\
\hline SVOC & 4-Chlorophenylphenyl ether & $\mathrm{mg} / \mathrm{kg}$ & 3 & 0 & $0 \%$ & 0.15 & 0.17 & -- & -- & 0.078 \\
\hline SVOC & Acenaphthene & $\mathrm{mg} / \mathrm{kg}$ & 3 & 0 & $0 \%$ & 0.29 & 0.33 & -- & -- & 0.15 \\
\hline SVOC & Acenaphthylene & $\mathrm{mg} / \mathrm{kg}$ & 3 & 0 & $0 \%$ & 0.15 & 0.17 & -- & -- & 0.078 \\
\hline SVOC & Anthracene & $\mathrm{mg} / \mathrm{kg}$ & 3 & 0 & $0 \%$ & 0.15 & 0.17 & -- & -- & 0.078 \\
\hline SVOC & Benzo(a)anthracene & $\mathrm{mg} / \mathrm{kg}$ & 3 & 0 & $0 \%$ & 0.15 & 0.17 & -- & -- & 0.078 \\
\hline SVOC & Benzo(a)pyrene & $\mathrm{mg} / \mathrm{kg}$ & 3 & 0 & $0 \%$ & 0.15 & 0.17 & -- & -- & 0.078 \\
\hline SVOC & Benzo(b)fluoranthene & $\mathrm{mg} / \mathrm{kg}$ & 3 & 0 & $0 \%$ & 0.29 & 0.33 & -- & -- & 0.15 \\
\hline SVOC & Benzo(ghi)perylene & $\mathrm{mg} / \mathrm{kg}$ & 3 & 0 & $0 \%$ & 0.29 & 0.33 & -- & -- & 0.15 \\
\hline SVOC & Benzo(k)fluoranthene & $\mathrm{mg} / \mathrm{kg}$ & 3 & 0 & $0 \%$ & 0.29 & 0.33 & -- & -- & 0.15 \\
\hline SVOC & Bis(2-chloro-1-methylethyl)ether & $\mathrm{mg} / \mathrm{kg}$ & 3 & 0 & $0 \%$ & 0.015 & 0.017 & -- & -- & 0.0078 \\
\hline SVOC & Chrysene & $\mathrm{mg} / \mathrm{kg}$ & 3 & 0 & $0 \%$ & 0.15 & 0.17 & -- & -- & 0.078 \\
\hline SVOC & Dibenz[a,h]anthracene & $\mathrm{mg} / \mathrm{kg}$ & 3 & 0 & $0 \%$ & 0.29 & 0.33 & -- & -- & 0.15 \\
\hline SVOC & Dibenzofuran & $\mathrm{mg} / \mathrm{kg}$ & 3 & 0 & $0 \%$ & 0.15 & 0.17 & -- & -- & 0.078 \\
\hline SVOC & Fluoranthene & $\mathrm{mg} / \mathrm{kg}$ & 3 & 0 & $0 \%$ & 0.15 & 0.33 & -- & -- & 0.13 \\
\hline
\end{tabular}


Table F-100

Summary Statistics for Whole Body Eulachon Collected from Segment 5 of the Columbia River

\begin{tabular}{|c|c|c|c|c|c|c|c|c|c|c|}
\hline $\begin{array}{l}\text { Constituent } \\
\text { Class }\end{array}$ & Constituent Name & Units & $\begin{array}{l}\text { Number } \\
\text { Analyzed }\end{array}$ & $\begin{array}{l}\text { Number } \\
\text { Detected }\end{array}$ & $\begin{array}{c}\text { Frequency of } \\
\text { Detection }\end{array}$ & $\begin{array}{l}\text { Minimum } \\
\text { Nondetect }\end{array}$ & $\begin{array}{l}\text { Maximum } \\
\text { Nondetect }\end{array}$ & $\begin{array}{c}\text { Minimum } \\
\text { Detect }\end{array}$ & $\begin{array}{l}\text { Maximum } \\
\text { Detect }\end{array}$ & $\begin{array}{c}\text { Average } \\
\text { Concentration }\end{array}$ \\
\hline$\overline{\text { SVOC }}$ & Fluorene & $\mathrm{mg} / \mathrm{kg}$ & 3 & $\overline{0}$ & $0 \%$ & 0.0093 & 0.025 & -- & -- & 0.0074 \\
\hline SVOC & Hexachlorobenzene & $\mathrm{mg} / \mathrm{kg}$ & 3 & 0 & $0 \%$ & 0.29 & 0.33 & -- & -- & 0.15 \\
\hline SVOC & Hexachlorobutadiene & $\mathrm{mg} / \mathrm{kg}$ & 3 & 0 & $0 \%$ & 0.0073 & 0.0083 & -- & -- & 0.0039 \\
\hline SVOC & Hexachloroethane & $\mathrm{mg} / \mathrm{kg}$ & 3 & 0 & $0 \%$ & 0.0073 & 0.0083 & -- & -- & 0.0039 \\
\hline SVOC & Indeno(1,2,3-cd)pyrene & $\mathrm{mg} / \mathrm{kg}$ & 3 & 0 & $0 \%$ & 0.29 & 0.33 & -- & -- & 0.15 \\
\hline SVOC & Naphthalene & $\mathrm{mg} / \mathrm{kg}$ & 3 & 0 & $0 \%$ & 0.030 & 0.040 & -- & -- & 0.018 \\
\hline SVOC & Nitrobenzene & $\mathrm{mg} / \mathrm{kg}$ & 3 & 0 & $0 \%$ & 0.0075 & 0.017 & -- & -- & 0.0055 \\
\hline SVOC & Phenanthrene & $\mathrm{mg} / \mathrm{kg}$ & 3 & 1 & $33 \%$ & 0.15 & 0.17 & 0.021 & 0.021 & 0.060 \\
\hline SVOC & Pyrene & $\mathrm{mg} / \mathrm{kg}$ & 3 & 0 & $0 \%$ & 0.29 & 0.33 & -- & -- & 0.15 \\
\hline VOC & Retene & $\mathrm{mg} / \mathrm{kg}$ & 3 & 0 & $0 \%$ & 0.29 & 0.33 & -- & -- & 0.15 \\
\hline
\end{tabular}

DIOXI - Dioxins and Furans

PEST - Pesticide

PCB - Polychlorinated biphenyl

SVOC - Semivolatile Organic Compound

VOC - Volatile Organic Compound 
Table F-101

Summary Statistics for Pacific Lamprey Fillet Skin On Collected from Segment 5 of the Columbia River

\begin{tabular}{|c|c|c|c|c|c|c|c|c|c|c|}
\hline $\begin{array}{l}\text { Constituent } \\
\text { Class }\end{array}$ & Constituent Name & Units & $\begin{array}{l}\text { Number } \\
\text { Analyzed }\end{array}$ & $\begin{array}{l}\text { Number } \\
\text { Detected }\end{array}$ & $\begin{array}{c}\text { Frequency of } \\
\text { Detection }\end{array}$ & $\begin{array}{l}\text { Minimum } \\
\text { Nondetect }\end{array}$ & $\begin{array}{l}\text { Maximum } \\
\text { Nondetect }\end{array}$ & $\begin{array}{c}\text { Minimum } \\
\text { Detect }\end{array}$ & $\begin{array}{c}\text { Maximum } \\
\text { Detect }\end{array}$ & $\begin{array}{c}\text { Average } \\
\text { Concentration }\end{array}$ \\
\hline$\overline{D I O X I}$ & $1,2,3,4,6,7,8$-Heptachlorodibenzodioxin & $\mathrm{mg} / \mathrm{kg}$ & 3 & 2 & $67 \%$ & $1.00 \mathrm{E}-07$ & $1.00 \mathrm{E}-07$ & $2.50 \mathrm{E}-07$ & $2.80 \mathrm{E}-07$ & 1.93E-07 \\
\hline DIOXI & 1,2,3,4,6,7,8-Heptachlorodibenzofuran & $\mathrm{mg} / \mathrm{kg}$ & 3 & 0 & $0 \%$ & $1.00 \mathrm{E}-08$ & 7.00E-08 & -- & -- & 1.67E-08 \\
\hline DIOXI & 1,2,3,4,7,8,9-Heptachlorodibenzofuran & $\mathrm{mg} / \mathrm{kg}$ & 3 & 0 & $0 \%$ & $1.00 \mathrm{E}-08$ & 7.00E-08 & -- & -- & 1.67E-08 \\
\hline DIOXI & 1,2,3,4,7,8-Hexachlorodibenzofuran & $\mathrm{mg} / \mathrm{kg}$ & 3 & 2 & $67 \%$ & $1.00 \mathrm{E}-08$ & $1.00 \mathrm{E}-08$ & $1.20 \mathrm{E}-07$ & $1.20 \mathrm{E}-07$ & 8.17E-08 \\
\hline DIOXI & 1,2,3,4,7,8-Hexachlorodibenzo-p-dioxin & $\mathrm{mg} / \mathrm{kg}$ & 3 & 2 & $67 \%$ & 4.00E-08 & 4.00E-08 & $1.00 \mathrm{E}-07$ & 1.10E-07 & 7.67E-08 \\
\hline DIOXI & 1,2,3,6,7,8-Hexachlorodibenzofuran & $\mathrm{mg} / \mathrm{kg}$ & 3 & 3 & $100 \%$ & -- & -- & 8.00E-08 & 1.00E-07 & 8.67E-08 \\
\hline DIOXI & 1,2,3,6,7,8-Hexachlorodibenzo-p-dioxin & $\mathrm{mg} / \mathrm{kg}$ & 3 & 3 & $100 \%$ & -- & -- & 1.60E-07 & 2.10E-07 & $1.80 \mathrm{E}-07$ \\
\hline DIOXI & 1,2,3,7,8,9-Hexachlorodibenzofuran & $\mathrm{mg} / \mathrm{kg}$ & 3 & 2 & $67 \%$ & 4.00E-08 & 4.00E-08 & 5.00E-08 & $6.00 \mathrm{E}-08$ & 4.33E-08 \\
\hline DIOXI & 1,2,3,7,8,9-Hexachlorodibenzo-p-dioxin & $\mathrm{mg} / \mathrm{kg}$ & 3 & 2 & $67 \%$ & 4.00E-08 & 4.00E-08 & $5.00 \mathrm{E}-08$ & $7.00 \mathrm{E}-08$ & 4.67E-08 \\
\hline DIOXI & 1,2,3,7,8-Pentachlorodibenzofuran & $\mathrm{mg} / \mathrm{kg}$ & 3 & 1 & $33 \%$ & $2.00 \mathrm{E}-08$ & 4.00E-08 & $3.20 \mathrm{E}-07$ & $3.20 \mathrm{E}-07$ & 1.17E-07 \\
\hline DIOXI & 1,2,3,7,8-Pentachlorodibenzo-p-dioxin & $\mathrm{mg} / \mathrm{kg}$ & 3 & 2 & $67 \%$ & 4.00E-08 & 4.00E-08 & 1.30E-07 & 1.70E-07 & 1.07E-07 \\
\hline DIOXI & $2,3,4,6,7,8$-Hexachlorodibenzofuran & $\mathrm{mg} / \mathrm{kg}$ & 3 & 3 & $100 \%$ & -- & -- & 8.00E-08 & 2.00E-07 & 1.60E-07 \\
\hline DIOXI & 2,3,4,7,8-Pentachlorodibenzofuran & $\mathrm{mg} / \mathrm{kg}$ & 3 & 3 & $100 \%$ & -- & -- & $1.00 \mathrm{E}-07$ & 1.30E-07 & 1.13E-07 \\
\hline DIOXI & 2,3,7,8-Tetrachlorodibenzofuran & $\mathrm{mg} / \mathrm{kg}$ & 3 & 3 & $100 \%$ & -- & -- & 1.17E-06 & $1.71 \mathrm{E}-06$ & 1.41E-06 \\
\hline DIOXI & 2,3,7,8-Tetrachlorodibenzo-p-dioxin & $\mathrm{mg} / \mathrm{kg}$ & 3 & 1 & $33 \%$ & $1.00 \mathrm{E}-08$ & $3.00 \mathrm{E}-08$ & $6.00 \mathrm{E}-08$ & $6.00 \mathrm{E}-08$ & 2.67E-08 \\
\hline DIOXI & Octachlorodibenzofuran & $\mathrm{mg} / \mathrm{kg}$ & 3 & 0 & $0 \%$ & $2.00 \mathrm{E}-08$ & $1.10 \mathrm{E}-07$ & -- & -- & $2.67 \mathrm{E}-08$ \\
\hline DIOXI & Octachlorodibenzo-p-dioxin & $\mathrm{mg} / \mathrm{kg}$ & 3 & 3 & $100 \%$ & -- & -- & $3.00 \mathrm{E}-07$ & $4.30 \mathrm{E}-07$ & 3.70E-07 \\
\hline METAL & Aluminum & $\mathrm{mg} / \mathrm{kg}$ & 3 & 0 & $0 \%$ & 1.0 & 1.0 & -- & -- & 0.50 \\
\hline METAL & Antimony & $\mathrm{mg} / \mathrm{kg}$ & 3 & 0 & $0 \%$ & 0.050 & 0.050 & -- & -- & 0.025 \\
\hline METAL & Arsenic & $\mathrm{mg} / \mathrm{kg}$ & 3 & 3 & $100 \%$ & -- & -- & 0.28 & 0.36 & 0.31 \\
\hline METAL & Barium & $\mathrm{mg} / \mathrm{kg}$ & 3 & 0 & $0 \%$ & 0.20 & 0.20 & -- & -- & 0.10 \\
\hline METAL & Beryllium & $\mathrm{mg} / \mathrm{kg}$ & 3 & 0 & $0 \%$ & 0.0040 & 0.0040 & -- & -- & 0.0020 \\
\hline METAL & Cadmium & $\mathrm{mg} / \mathrm{kg}$ & 3 & 3 & $100 \%$ & -- & -- & 0.010 & 0.030 & 0.020 \\
\hline METAL & Chromium & $\mathrm{mg} / \mathrm{kg}$ & 3 & 1 & $33 \%$ & 0.10 & 0.10 & 0.14 & 0.14 & 0.080 \\
\hline METAL & Cobalt & $\mathrm{mg} / \mathrm{kg}$ & 3 & 3 & $100 \%$ & -- & -- & 0.010 & 0.070 & 0.030 \\
\hline METAL & Copper & $\mathrm{mg} / \mathrm{kg}$ & 3 & 3 & $100 \%$ & -- & -- & 1.1 & 1.4 & 1.2 \\
\hline METAL & Lead & $\mathrm{mg} / \mathrm{kg}$ & 3 & 0 & $0 \%$ & 0.010 & 0.010 & -- & -- & 0.0050 \\
\hline METAL & Manganese & $\mathrm{mg} / \mathrm{kg}$ & 3 & 3 & $100 \%$ & -- & -- & 0.36 & 0.38 & 0.37 \\
\hline METAL & Mercury & $\mathrm{mg} / \mathrm{kg}$ & 3 & 0 & $0 \%$ & 0.10 & 0.11 & -- & -- & 0.052 \\
\hline METAL & Nickel & $\mathrm{mg} / \mathrm{kg}$ & 3 & 0 & $0 \%$ & 0.030 & 0.030 & -- & -- & 0.015 \\
\hline METAL & Selenium & $\mathrm{mg} / \mathrm{kg}$ & 3 & 3 & $100 \%$ & -- & -- & 0.41 & 0.45 & 0.43 \\
\hline METAL & Silver & $\mathrm{mg} / \mathrm{kg}$ & 3 & 0 & $0 \%$ & 0.10 & 0.10 & -- & -- & 0.050 \\
\hline METAL & Thallium & $\mathrm{mg} / \mathrm{kg}$ & 3 & 0 & $0 \%$ & 0.050 & 0.050 & -- & -- & 0.025 \\
\hline METAL & Vanadium & $\mathrm{mg} / \mathrm{kg}$ & 3 & 2 & $67 \%$ & 0.010 & 0.010 & 0.010 & 0.010 & 0.0083 \\
\hline METAL & Zinc & $\mathrm{mg} / \mathrm{kg}$ & 3 & 3 & $100 \%$ & -- & -- & 19 & 21 & 20 \\
\hline PCB & 2,2',3,3',4,4',5-Heptachloro-1,1'-biphenyl & $\mathrm{mg} / \mathrm{kg}$ & 3 & 3 & $100 \%$ & -- & -- & 4.17E-04 & 4.83E-04 & $4.58 \mathrm{E}-04$ \\
\hline РСВ & 2,2',3,4,4',5,5'-Heptachlorobiphenyl & $\mathrm{mg} / \mathrm{kg}$ & 3 & 3 & $100 \%$ & -- & -- & 0.0015 & 0.0017 & 0.0016 \\
\hline PCB & 2,3,3',4,4',5'-Hexachlorobiphenyl & $\mathrm{mg} / \mathrm{kg}$ & 3 & 3 & $100 \%$ & -- & -- & 4.50E-05 & $5.68 \mathrm{E}-05$ & $5.06 \mathrm{E}-05$ \\
\hline РСВ & 2,3,3',4,4',5-Hexachlorobiphenyl (BZ 156) & $\mathrm{mg} / \mathrm{kg}$ & 3 & 3 & $100 \%$ & -- & -- & $2.56 \mathrm{E}-04$ & $2.98 \mathrm{E}-04$ & $2.74 \mathrm{E}-04$ \\
\hline PCB & 2,3,3',4,4'-Pentachlorobiphenyl & $\mathrm{mg} / \mathrm{kg}$ & 3 & 3 & $100 \%$ & -- & -- & 0.0011 & 0.0014 & 0.0012 \\
\hline РСВ & 2,3',4,4',5,5'-Hexachlorobiphenyl (BZ 167) & $\mathrm{mg} / \mathrm{kg}$ & 3 & 3 & $100 \%$ & -- & -- & 1.63E-04 & 1.70E-04 & $1.68 \mathrm{E}-04$ \\
\hline PCB & 2',3,4,4',5-Pentachloro-1,1'-biphenyl (BZ 123) & $\mathrm{mg} / \mathrm{kg}$ & 3 & 3 & $100 \%$ & -- & -- & 4.70E-05 & 7.00E-05 & 5.83E-05 \\
\hline РCB & 2,3,4,4',5-Pentachlorobiphenyl (BZ 114) & $\mathrm{mg} / \mathrm{kg}$ & 3 & 3 & $100 \%$ & -- & -- & 1.30E-04 & 1.45E-04 & $1.37 \mathrm{E}-04$ \\
\hline PCB & 2,3',4,4',5-Pentachlorobiphenyl (BZ 118) & $\mathrm{mg} / \mathrm{kg}$ & 3 & 3 & $100 \%$ & -- & -- & 0.0034 & 0.0038 & 0.0036 \\
\hline РСВ & 2,3,4,5,3',4',5'-Heptachlorobiphenyl & $\mathrm{mg} / \mathrm{kg}$ & 3 & 3 & $100 \%$ & -- & -- & 1.94E-05 & 2.03E-05 & 1.98E-05 \\
\hline PCB & 3,3',4,4',5,5'-Hexachlorobiphenyl (BZ 169) & $\mathrm{mg} / \mathrm{kg}$ & 3 & 0 & $0 \%$ & $1.00 \mathrm{E}-06$ & $1.00 \mathrm{E}-06$ & -- & -- & $5.00 \mathrm{E}-07$ \\
\hline РСВ & 3,3',4,4',5-Pentachlorobiphenyl (BZ 126) & $\mathrm{mg} / \mathrm{kg}$ & 3 & 3 & $100 \%$ & -- & -- & 1.35E-05 & 1.86E-05 & 1.56E-05 \\
\hline PCB & 3,3',4,4'-Tetrachlorobiphenyl (BZ 77) & $\mathrm{mg} / \mathrm{kg}$ & 3 & 3 & $100 \%$ & -- & -- & 2.21E-05 & 2.38E-05 & $2.30 \mathrm{E}-05$ \\
\hline РСВ & Aroclor-1016 & $\mathrm{mg} / \mathrm{kg}$ & 3 & 0 & $0 \%$ & 0.019 & 0.020 & -- & -- & 0.0097 \\
\hline PCB & Aroclor-1221 & $\mathrm{mg} / \mathrm{kg}$ & 3 & 0 & $0 \%$ & 0.019 & 0.020 & -- & -- & 0.0097 \\
\hline
\end{tabular}


Table F-101

Summary Statistics for Pacific Lamprey Fillet Skin On Collected from Segment 5 of the Columbia River

\begin{tabular}{|c|c|c|c|c|c|c|c|c|c|c|}
\hline $\begin{array}{l}\text { Constituent } \\
\text { Class }\end{array}$ & Constituent Name & Units & $\begin{array}{l}\text { Number } \\
\text { Analyzed }\end{array}$ & $\begin{array}{l}\text { Number } \\
\text { Detected }\end{array}$ & $\begin{array}{c}\text { Frequency of } \\
\text { Detection }\end{array}$ & $\begin{array}{l}\text { Minimum } \\
\text { Nondetect }\end{array}$ & $\begin{array}{l}\text { Maximum } \\
\text { Nondetect }\end{array}$ & $\begin{array}{l}\text { Minimum } \\
\text { Detect }\end{array}$ & $\begin{array}{l}\text { Maximum } \\
\text { Detect }\end{array}$ & $\begin{array}{c}\text { Average } \\
\text { Concentration }\end{array}$ \\
\hline$\overline{\mathrm{PCB}}$ & Aroclor-1232 & $\mathrm{mg} / \mathrm{kg}$ & 3 & 0 & $0 \%$ & 0.019 & 0.020 & -- & -- & 0.0097 \\
\hline PCB & Aroclor-1248 & $\mathrm{mg} / \mathrm{kg}$ & 3 & 0 & $0 \%$ & 0.019 & 0.020 & -- & -- & 0.0097 \\
\hline РCB & Aroclor-1254 & $\mathrm{mg} / \mathrm{kg}$ & 3 & 3 & $100 \%$ & -- & -- & 0.080 & 0.10 & 0.087 \\
\hline РСВ & Aroclor-1260 & $\mathrm{mg} / \mathrm{kg}$ & 3 & 0 & $0 \%$ & 0.019 & 0.020 & -- & -- & 0.0097 \\
\hline PEST & 2,4'-DDD & $\mathrm{mg} / \mathrm{kg}$ & 3 & 0 & $0 \%$ & 0.0019 & 0.0020 & -- & -- & $9.67 \mathrm{E}-04$ \\
\hline PEST & 2,4'-DDE & $\mathrm{mg} / \mathrm{kg}$ & 3 & 3 & $100 \%$ & -- & -- & 0.0043 & 0.0048 & 0.0045 \\
\hline PEST & 2,4'-DDT & $\mathrm{mg} / \mathrm{kg}$ & 3 & 0 & $0 \%$ & 0.0019 & 0.0020 & -- & -- & $9.67 \mathrm{E}-04$ \\
\hline PEST & Aldrin & $\mathrm{mg} / \mathrm{kg}$ & 3 & 0 & $0 \%$ & 0.0019 & 0.0020 & -- & -- & $9.67 \mathrm{E}-04$ \\
\hline PEST & Alpha-BHC & $\mathrm{mg} / \mathrm{kg}$ & 3 & 0 & $0 \%$ & 0.0019 & 0.0020 & -- & -- & $9.67 \mathrm{E}-04$ \\
\hline PEST & alpha-Chlordane & $\mathrm{mg} / \mathrm{kg}$ & 3 & 3 & $100 \%$ & -- & -- & 0.0073 & 0.011 & 0.0092 \\
\hline PEST & alpha-Chlordene & $\mathrm{mg} / \mathrm{kg}$ & 3 & 0 & $0 \%$ & 0.0019 & 0.0020 & -- & -- & $9.67 \mathrm{E}-04$ \\
\hline PEST & Beta-BHC & $\mathrm{mg} / \mathrm{kg}$ & 3 & 0 & $0 \%$ & 0.0019 & 0.0020 & -- & -- & $9.67 \mathrm{E}-04$ \\
\hline PEST & cis-Nonachlor & $\mathrm{mg} / \mathrm{kg}$ & 3 & 3 & $100 \%$ & -- & -- & 0.0085 & 0.011 & 0.0097 \\
\hline PEST & DDMU & $\mathrm{mg} / \mathrm{kg}$ & 3 & 3 & $100 \%$ & -- & -- & 0.0036 & 0.0056 & 0.0045 \\
\hline PEST & Delta-BHC & $\mathrm{mg} / \mathrm{kg}$ & 3 & 0 & $0 \%$ & 0.0019 & 0.0020 & -- & -- & $9.67 \mathrm{E}-04$ \\
\hline PEST & Dichlorodiphenyldichloroethane & $\mathrm{mg} / \mathrm{kg}$ & 3 & 3 & $100 \%$ & -- & -- & 0.0048 & 0.0093 & 0.0077 \\
\hline PEST & Dichlorodiphenyldichloroethylene & $\mathrm{mg} / \mathrm{kg}$ & 3 & 3 & $100 \%$ & -- & -- & 0.046 & 0.055 & 0.050 \\
\hline PEST & Dichlorodiphenyltrichloroethane & $\mathrm{mg} / \mathrm{kg}$ & 3 & 3 & $100 \%$ & -- & -- & 0.028 & 0.038 & 0.031 \\
\hline PEST & Gamma-BHC (Lindane) & $\mathrm{mg} / \mathrm{kg}$ & 3 & 0 & $0 \%$ & 0.0019 & 0.0020 & -- & -- & $9.67 \mathrm{E}-04$ \\
\hline PEST & gamma-Chlordane & $\mathrm{mg} / \mathrm{kg}$ & 3 & 2 & $67 \%$ & 0.0020 & 0.0020 & 0.0013 & 0.0025 & 0.0016 \\
\hline PEST & gamma-Chlordene & $\mathrm{mg} / \mathrm{kg}$ & 3 & 0 & $0 \%$ & 0.0019 & 0.0020 & -- & -- & $9.67 \mathrm{E}-04$ \\
\hline PEST & Heptachlor & $\mathrm{mg} / \mathrm{kg}$ & 3 & 0 & $0 \%$ & 0.0019 & 0.0020 & -- & -- & $9.67 \mathrm{E}-04$ \\
\hline PEST & Heptachlor epoxide & $\mathrm{mg} / \mathrm{kg}$ & 3 & 0 & $0 \%$ & 0.0019 & 0.0020 & -- & -- & $9.67 \mathrm{E}-04$ \\
\hline PEST & Mirex & $\mathrm{mg} / \mathrm{kg}$ & 3 & 0 & $0 \%$ & 0.0019 & 0.0020 & -- & -- & $9.67 \mathrm{E}-04$ \\
\hline PEST & Oxychlordane & $\mathrm{mg} / \mathrm{kg}$ & 3 & 0 & $0 \%$ & 0.0019 & 0.0020 & -- & -- & $9.67 \mathrm{E}-04$ \\
\hline PEST & Pentachloroanisole & $\mathrm{mg} / \mathrm{kg}$ & 3 & 3 & $100 \%$ & -- & -- & 0.0015 & 0.0017 & 0.0016 \\
\hline PEST & Toxaphene & $\mathrm{mg} / \mathrm{kg}$ & 3 & 0 & $0 \%$ & 0.057 & 0.060 & -- & -- & 0.029 \\
\hline PEST & trans-Nonachlor & $\mathrm{mg} / \mathrm{kg}$ & 3 & 3 & $100 \%$ & -- & -- & 0.021 & 0.024 & 0.022 \\
\hline SVOC & 1,2,4-Trichlorobenzene & $\mathrm{mg} / \mathrm{kg}$ & 3 & 0 & $0 \%$ & 0.024 & 0.025 & -- & -- & 0.012 \\
\hline SVOC & 1,2-Dichlorobenzene & $\mathrm{mg} / \mathrm{kg}$ & 3 & 0 & $0 \%$ & 0.024 & 0.025 & -- & -- & 0.012 \\
\hline SVOC & 1,2-Diphenylhydrazine & $\mathrm{mg} / \mathrm{kg}$ & 3 & 0 & $0 \%$ & 0.024 & 0.025 & -- & -- & 0.012 \\
\hline SVOC & 1,3-Dichlorobenzene & $\mathrm{mg} / \mathrm{kg}$ & 3 & 0 & $0 \%$ & 0.024 & 0.025 & -- & -- & 0.012 \\
\hline SVOC & 1,4-Dichlorobenzene & $\mathrm{mg} / \mathrm{kg}$ & 3 & 0 & $0 \%$ & 0.024 & 0.025 & -- & -- & 0.012 \\
\hline SVOC & 1-methylnaphthalene & $\mathrm{mg} / \mathrm{kg}$ & 3 & 0 & $0 \%$ & 0.024 & 0.026 & -- & -- & 0.013 \\
\hline SVOC & 2,4-Dinitrotoluene & $\mathrm{mg} / \mathrm{kg}$ & 3 & 0 & $0 \%$ & 0.049 & 0.050 & -- & -- & 0.025 \\
\hline SVOC & 2,6-Dinitrotoluene & $\mathrm{mg} / \mathrm{kg}$ & 3 & 0 & $0 \%$ & 0.097 & 0.10 & -- & -- & 0.049 \\
\hline SVOC & 2-Chloronaphthalene & $\mathrm{mg} / \mathrm{kg}$ & 3 & 0 & $0 \%$ & 0.049 & 0.050 & -- & -- & 0.025 \\
\hline SVOC & 2-Methylnaphthalene & $\mathrm{mg} / \mathrm{kg}$ & 3 & 1 & $33 \%$ & 0.049 & 0.052 & 0.077 & 0.077 & 0.043 \\
\hline SVOC & 4-Bromophenylphenyl ether & $\mathrm{mg} / \mathrm{kg}$ & 3 & 0 & $0 \%$ & 0.024 & 0.025 & -- & -- & 0.012 \\
\hline SVOC & 4-Chlorophenylphenyl ether & $\mathrm{mg} / \mathrm{kg}$ & 3 & 0 & $0 \%$ & 0.024 & 0.025 & -- & -- & 0.012 \\
\hline SVOC & Acenaphthene & $\mathrm{mg} / \mathrm{kg}$ & 3 & 0 & $0 \%$ & 0.049 & 0.050 & -- & -- & 0.025 \\
\hline SVOC & Acenaphthylene & $\mathrm{mg} / \mathrm{kg}$ & 3 & 0 & $0 \%$ & 0.024 & 0.025 & -- & -- & 0.012 \\
\hline SVOC & Anthracene & $\mathrm{mg} / \mathrm{kg}$ & 3 & 0 & $0 \%$ & 0.024 & 0.025 & -- & -- & 0.012 \\
\hline SVOC & Benzo(a)anthracene & $\mathrm{mg} / \mathrm{kg}$ & 3 & 0 & $0 \%$ & 0.024 & 0.025 & -- & -- & 0.012 \\
\hline SVOC & Benzo(a)pyrene & $\mathrm{mg} / \mathrm{kg}$ & 3 & 0 & $0 \%$ & 0.024 & 0.025 & -- & -- & 0.012 \\
\hline SVOC & Benzo(b)fluoranthene & $\mathrm{mg} / \mathrm{kg}$ & 3 & 0 & $0 \%$ & 0.024 & 0.025 & -- & -- & 0.012 \\
\hline SVOC & Benzo(ghi)perylene & $\mathrm{mg} / \mathrm{kg}$ & 3 & 0 & $0 \%$ & 0.049 & 0.050 & -- & -- & 0.025 \\
\hline SVOC & Benzo(k)fluoranthene & $\mathrm{mg} / \mathrm{kg}$ & 3 & 0 & $0 \%$ & 0.024 & 0.025 & -- & -- & 0.012 \\
\hline SVOC & Bis(2-chloro-1-methylethyl)ether & $\mathrm{mg} / \mathrm{kg}$ & 3 & 0 & $0 \%$ & 0.049 & 0.050 & -- & -- & 0.025 \\
\hline SVOC & Chrysene & $\mathrm{mg} / \mathrm{kg}$ & 3 & 0 & $0 \%$ & 0.024 & 0.025 & -- & -- & 0.012 \\
\hline
\end{tabular}


Table F-101

Summary Statistics for Pacific Lamprey Fillet Skin On Collected from Segment 5 of the Columbia River

\begin{tabular}{|c|c|c|c|c|c|c|c|c|c|c|}
\hline $\begin{array}{l}\text { Constituent } \\
\text { Class }\end{array}$ & Constituent Name & Units & $\begin{array}{l}\text { Number } \\
\text { Analyzed }\end{array}$ & $\begin{array}{l}\text { Number } \\
\text { Detected }\end{array}$ & $\begin{array}{c}\text { Frequency of } \\
\text { Detection }\end{array}$ & $\begin{array}{l}\text { Minimum } \\
\text { Nondetect }\end{array}$ & $\begin{array}{l}\text { Maximum } \\
\text { Nondetect }\end{array}$ & $\begin{array}{l}\text { Minimum } \\
\text { Detect }\end{array}$ & $\begin{array}{l}\text { Maximum } \\
\text { Detect }\end{array}$ & $\begin{array}{c}\text { Average } \\
\text { Concentration }\end{array}$ \\
\hline$\overline{\text { SVOC }}$ & Dibenz[a,h]anthracene & $\mathrm{mg} / \mathrm{kg}$ & 3 & 0 & $0 \%$ & 0.049 & 0.050 & $\overline{--}$ & $\overline{--}$ & 0.025 \\
\hline SVOC & Dibenzofuran & $\mathrm{mg} / \mathrm{kg}$ & 3 & 0 & $0 \%$ & 0.024 & 0.025 & -- & -- & 0.012 \\
\hline SVOC & Fluoranthene & $\mathrm{mg} / \mathrm{kg}$ & 3 & 0 & $0 \%$ & 0.049 & 0.050 & -- & -- & 0.025 \\
\hline SVOC & Fluorene & $\mathrm{mg} / \mathrm{kg}$ & 3 & 0 & $0 \%$ & 0.0084 & 0.0097 & -- & -- & 0.0046 \\
\hline SVOC & Hexachlorobenzene & $\mathrm{mg} / \mathrm{kg}$ & 3 & 0 & $0 \%$ & 0.049 & 0.050 & -- & -- & 0.025 \\
\hline SVOC & Hexachlorobutadiene & $\mathrm{mg} / \mathrm{kg}$ & 3 & 0 & $0 \%$ & 0.024 & 0.025 & -- & -- & 0.012 \\
\hline SVOC & Hexachloroethane & $\mathrm{mg} / \mathrm{kg}$ & 3 & 0 & $0 \%$ & 0.024 & 0.025 & -- & -- & 0.012 \\
\hline SVOC & Indeno $(1,2,3-c d)$ pyrene & $\mathrm{mg} / \mathrm{kg}$ & 3 & 0 & $0 \%$ & 0.049 & 0.050 & -- & -- & 0.025 \\
\hline SVOC & Naphthalene & $\mathrm{mg} / \mathrm{kg}$ & 3 & 0 & $0 \%$ & 0.054 & 0.085 & -- & -- & 0.033 \\
\hline SVOC & Nitrobenzene & $\mathrm{mg} / \mathrm{kg}$ & 3 & 0 & $0 \%$ & 0.024 & 0.025 & -- & -- & 0.012 \\
\hline SVOC & Phenanthrene & $\mathrm{mg} / \mathrm{kg}$ & 3 & 0 & $0 \%$ & 0.024 & 0.025 & -- & -- & 0.012 \\
\hline SVOC & Pyrene & $\mathrm{mg} / \mathrm{kg}$ & 3 & 0 & $0 \%$ & 0.049 & 0.050 & -- & -- & 0.025 \\
\hline VOC & Retene & $\mathrm{mg} / \mathrm{kg}$ & 3 & 0 & $0 \%$ & 0.049 & 0.050 & -- & -- & 0.025 \\
\hline
\end{tabular}

DIOXI - Dioxins and Furans

PEST - Pesticide

PCB - Polychlorinated bipheny

SVOC - Semivolatile Organic Compound

VOC - Volatile Organic Compound 
Table F-102

Summary Statistics for Whole Body Pacific Lamprey Collected from Segment 5 of the Columbia River

\begin{tabular}{|c|c|c|c|c|c|c|c|c|c|c|}
\hline $\begin{array}{l}\text { Constituent } \\
\text { Class }\end{array}$ & Constituent Name & Units & $\begin{array}{l}\text { Number } \\
\text { Analyzed }\end{array}$ & $\begin{array}{l}\text { Number } \\
\text { Detected }\end{array}$ & $\begin{array}{c}\text { Frequency of } \\
\text { Detection }\end{array}$ & $\begin{array}{l}\text { Minimum } \\
\text { Nondetect }\end{array}$ & $\begin{array}{l}\text { Maximum } \\
\text { Nondetect }\end{array}$ & $\begin{array}{c}\text { Minimum } \\
\text { Detect }\end{array}$ & $\begin{array}{l}\text { Maximum } \\
\text { Detect }\end{array}$ & $\begin{array}{c}\text { Average } \\
\text { Concentration }\end{array}$ \\
\hline$\overline{D I O X I}$ & $1,2,3,4,6,7,8$-Heptachlorodibenzodioxin & $\mathrm{mg} / \mathrm{kg}$ & 9 & 9 & $100 \%$ & -- & -- & $2.10 \mathrm{E}-07$ & $2.80 \mathrm{E}-07$ & $2.40 \mathrm{E}-07$ \\
\hline DIOXI & 1,2,3,4,6,7,8-Heptachlorodibenzofuran & $\mathrm{mg} / \mathrm{kg}$ & 9 & 5 & $56 \%$ & 1.00E-08 & $6.00 \mathrm{E}-08$ & $7.00 \mathrm{E}-08$ & 1.00E-07 & 5.61E-08 \\
\hline DIOXI & 1,2,3,4,7,8,9-Heptachlorodibenzofuran & $\mathrm{mg} / \mathrm{kg}$ & 9 & 3 & $33 \%$ & $1.00 \mathrm{E}-08$ & $6.00 \mathrm{E}-08$ & 1.00E-08 & $3.00 \mathrm{E}-08$ & 1.56E-08 \\
\hline DIOXI & $1,2,3,4,7,8$-Hexachlorodibenzofuran & $\mathrm{mg} / \mathrm{kg}$ & 9 & 8 & $89 \%$ & 1.00E-08 & 1.00E-08 & $7.00 \mathrm{E}-08$ & $1.90 \mathrm{E}-07$ & 1.09E-07 \\
\hline DIOXI & $1,2,3,4,7,8$-Hexachlorodibenzo-p-dioxin & $\mathrm{mg} / \mathrm{kg}$ & 9 & 4 & $44 \%$ & 1.00E-08 & 3.00E-08 & $4.00 \mathrm{E}-08$ & $1.30 \mathrm{E}-07$ & 4.83E-08 \\
\hline DIOXI & 1,2,3,6,7,8-Hexachlorodibenzofuran & $\mathrm{mg} / \mathrm{kg}$ & 9 & 7 & $78 \%$ & 1.00E-08 & 2.00E-08 & $8.00 \mathrm{E}-08$ & $1.60 \mathrm{E}-07$ & 1.01E-07 \\
\hline DIOXI & 1,2,3,6,7,8-Hexachlorodibenzo-p-dioxin & $\mathrm{mg} / \mathrm{kg}$ & 9 & 7 & $78 \%$ & $2.00 \mathrm{E}-08$ & $3.00 \mathrm{E}-08$ & $1.60 \mathrm{E}-07$ & $2.50 \mathrm{E}-07$ & 1.53E-07 \\
\hline DIOXI & 1,2,3,7,8,9-Hexachlorodibenzofuran & $\mathrm{mg} / \mathrm{kg}$ & 9 & 4 & $44 \%$ & 2.00E-08 & 7.00E-08 & $6.00 \mathrm{E}-08$ & $6.00 \mathrm{E}-08$ & 3.61E-08 \\
\hline DIOXI & 1,2,3,7,8,9-Hexachlorodibenzo-p-dioxin & $\mathrm{mg} / \mathrm{kg}$ & 9 & 5 & $56 \%$ & 1.00E-08 & $3.00 \mathrm{E}-08$ & $3.00 \mathrm{E}-08$ & 7.00E-08 & 3.56E-08 \\
\hline DIOXI & 1,2,3,7,8-Pentachlorodibenzofuran & $\mathrm{mg} / \mathrm{kg}$ & 9 & 9 & $100 \%$ & -- & -- & $1.80 \mathrm{E}-07$ & 4.70E-07 & 3.18E-07 \\
\hline DIOXI & 1,2,3,7,8-Pentachlorodibenzo-p-dioxin & $\mathrm{mg} / \mathrm{kg}$ & 9 & 5 & $56 \%$ & 2.00E-08 & 3.00E-08 & $7.00 \mathrm{E}-08$ & $1.50 \mathrm{E}-07$ & 7.67E-08 \\
\hline DIOXI & 2,3,4,6,7,8-Hexachlorodibenzofuran & $\mathrm{mg} / \mathrm{kg}$ & 9 & 6 & $67 \%$ & 1.00E-08 & 3.00E-08 & $1.00 \mathrm{E}-07$ & $1.90 \mathrm{E}-07$ & 1.03E-07 \\
\hline DIOXI & 2,3,4,7,8-Pentachlorodibenzofuran & $\mathrm{mg} / \mathrm{kg}$ & 9 & 5 & $56 \%$ & 1.00E-08 & 1.40E-07 & 1.10E-07 & $1.60 \mathrm{E}-07$ & 8.56E-08 \\
\hline DIOXI & 2,3,7,8-Tetrachlorodibenzofuran & $\mathrm{mg} / \mathrm{kg}$ & 9 & 9 & $100 \%$ & -- & -- & $1.10 \mathrm{E}-06$ & $3.18 \mathrm{E}-06$ & 2.00E-06 \\
\hline DIOXI & 2,3,7,8-Tetrachlorodibenzo-p-dioxin & $\mathrm{mg} / \mathrm{kg}$ & 9 & 3 & $33 \%$ & $2.00 \mathrm{E}-08$ & 4.00E-08 & $5.00 \mathrm{E}-08$ & 7.00E-08 & $2.89 \mathrm{E}-08$ \\
\hline DIOXI & Octachlorodibenzofuran & $\mathrm{mg} / \mathrm{kg}$ & 9 & 6 & $67 \%$ & $2.00 \mathrm{E}-08$ & $9.00 \mathrm{E}-08$ & $2.00 \mathrm{E}-08$ & $9.00 \mathrm{E}-08$ & 4.61E-08 \\
\hline DIOXI & Octachlorodibenzo-p-dioxin & $\mathrm{mg} / \mathrm{kg}$ & 9 & 8 & $89 \%$ & $2.00 \mathrm{E}-08$ & $2.00 \mathrm{E}-08$ & $3.00 \mathrm{E}-07$ & $9.90 \mathrm{E}-07$ & 4.33E-07 \\
\hline METAL & Aluminum & $\mathrm{mg} / \mathrm{kg}$ & 9 & 4 & $44 \%$ & 1.0 & 1.0 & 1.9 & 2.2 & 1.2 \\
\hline METAL & Antimony & $\mathrm{mg} / \mathrm{kg}$ & 9 & 0 & $0 \%$ & 0.050 & 0.050 & -- & -- & 0.025 \\
\hline METAL & Arsenic & $\mathrm{mg} / \mathrm{kg}$ & 9 & 9 & $100 \%$ & -- & -- & 0.15 & 0.37 & 0.26 \\
\hline METAL & Barium & $\mathrm{mg} / \mathrm{kg}$ & 9 & 0 & $0 \%$ & 0.20 & 0.20 & -- & -- & 0.10 \\
\hline METAL & Beryllium & $\mathrm{mg} / \mathrm{kg}$ & 9 & 0 & $0 \%$ & 0.0040 & 0.0040 & -- & -- & 0.0020 \\
\hline METAL & Cadmium & $\mathrm{mg} / \mathrm{kg}$ & 9 & 9 & $100 \%$ & -- & -- & 0.050 & 0.15 & 0.11 \\
\hline METAL & Chromium & $\mathrm{mg} / \mathrm{kg}$ & 9 & 4 & $44 \%$ & 0.10 & 0.10 & 0.10 & 0.29 & 0.10 \\
\hline METAL & Cobalt & $\mathrm{mg} / \mathrm{kg}$ & 9 & 9 & $100 \%$ & -- & -- & 0.010 & 0.39 & 0.091 \\
\hline METAL & Copper & $\mathrm{mg} / \mathrm{kg}$ & 9 & 9 & $100 \%$ & -- & -- & 3.7 & 5.5 & 4.5 \\
\hline METAL & Lead & $\mathrm{mg} / \mathrm{kg}$ & 9 & 3 & $33 \%$ & 0.010 & 0.010 & 0.010 & 0.060 & 0.014 \\
\hline METAL & Manganese & $\mathrm{mg} / \mathrm{kg}$ & 9 & 9 & $100 \%$ & -- & -- & 0.24 & 0.53 & 0.39 \\
\hline METAL & Mercury & $\mathrm{mg} / \mathrm{kg}$ & 9 & 7 & $78 \%$ & 0.10 & 0.10 & 0.090 & 0.25 & 0.11 \\
\hline METAL & Nickel & $\mathrm{mg} / \mathrm{kg}$ & 9 & 4 & $44 \%$ & 0.030 & 0.030 & 0.030 & 0.56 & 0.11 \\
\hline METAL & Selenium & $\mathrm{mg} / \mathrm{kg}$ & 9 & 9 & $100 \%$ & -- & -- & 0.51 & 0.75 & 0.58 \\
\hline METAL & Silver & $\mathrm{mg} / \mathrm{kg}$ & 9 & 0 & $0 \%$ & 0.10 & 0.10 & -- & -- & 0.050 \\
\hline METAL & Thallium & $\mathrm{mg} / \mathrm{kg}$ & 9 & 0 & $0 \%$ & 0.050 & 0.050 & -- & -- & 0.025 \\
\hline METAL & Vanadium & $\mathrm{mg} / \mathrm{kg}$ & 9 & 9 & $100 \%$ & -- & -- & 0.030 & 0.040 & 0.037 \\
\hline METAL & Zinc & $\mathrm{mg} / \mathrm{kg}$ & 9 & 9 & $100 \%$ & -- & -- & 18 & 27 & 22 \\
\hline PCB & 2,2',3,3',4,4',5-Heptachloro-1,1'-biphenyl & $\mathrm{mg} / \mathrm{kg}$ & 9 & 9 & $100 \%$ & -- & -- & $2.56 \mathrm{E}-04$ & 7.04E-04 & $4.29 \mathrm{E}-04$ \\
\hline РCB & 2,2',3,4,4',5,5'-Heptachlorobiphenyl & $\mathrm{mg} / \mathrm{kg}$ & 9 & 9 & $100 \%$ & -- & -- & $9.04 \mathrm{E}-04$ & 0.0024 & 0.0015 \\
\hline PCB & 2,3,3',4,4',5'-Hexachlorobiphenyl & $\mathrm{mg} / \mathrm{kg}$ & 9 & 6 & $67 \%$ & 1.96E-06 & $3.28 \mathrm{E}-06$ & $3.70 \mathrm{E}-05$ & 9.39E-05 & 4.06E-05 \\
\hline РCB & 2,3,3',4,4',5-Hexachlorobiphenyl (BZ 156) & $\mathrm{mg} / \mathrm{kg}$ & 9 & 9 & $100 \%$ & -- & -- & $2.00 \mathrm{E}-04$ & 4.64E-04 & 3.18E-04 \\
\hline PCB & 2,3,3',4,4'-Pentachlorobiphenyl & $\mathrm{mg} / \mathrm{kg}$ & 9 & 9 & $100 \%$ & -- & -- & 8.06E-04 & 0.0020 & 0.0012 \\
\hline РCB & 2,3',4,4',5,5'-Hexachlorobiphenyl (BZ 167) & $\mathrm{mg} / \mathrm{kg}$ & 9 & 9 & $100 \%$ & -- & -- & $1.25 \mathrm{E}-04$ & $2.76 \mathrm{E}-04$ & $1.92 \mathrm{E}-04$ \\
\hline PCB & 2',3,4,4',5-Pentachloro-1,1'-biphenyl (BZ 123) & $\mathrm{mg} / \mathrm{kg}$ & 9 & 9 & $100 \%$ & -- & -- & $3.27 \mathrm{E}-05$ & 6.83E-05 & 4.83E-05 \\
\hline PCB & 2,3,4,4',5-Pentachlorobiphenyl (BZ 114) & $\mathrm{mg} / \mathrm{kg}$ & 9 & 9 & $100 \%$ & -- & -- & $1.00 \mathrm{E}-04$ & 2.06E-04 & 1.43E-04 \\
\hline PCB & 2,3',4,4',5-Pentachlorobiphenyl (BZ 118) & $\mathrm{mg} / \mathrm{kg}$ & 9 & 9 & $100 \%$ & -- & -- & 0.0024 & 0.0055 & 0.0035 \\
\hline PCB & 2,3,4,5,3',4',5'-Heptachlorobiphenyl & $\mathrm{mg} / \mathrm{kg}$ & 9 & 9 & $100 \%$ & -- & -- & 1.37E-05 & 2.84E-05 & 2.09E-05 \\
\hline PCB & 3,3',4,4',5,5'-Hexachlorobiphenyl (BZ 169) & $\mathrm{mg} / \mathrm{kg}$ & 9 & 0 & $0 \%$ & 1.00E-06 & 9.96E-06 & -- & -- & $1.45 \mathrm{E}-06$ \\
\hline РСВ & 3,3',4,4',5-Pentachlorobiphenyl (BZ 126) & $\mathrm{mg} / \mathrm{kg}$ & 9 & 9 & $100 \%$ & -- & -- & 1.09E-05 & 3.11E-05 & 2.19E-05 \\
\hline PCB & 3,3',4,4'-Tetrachlorobiphenyl (BZ 77) & $\mathrm{mg} / \mathrm{kg}$ & 9 & 9 & $100 \%$ & -- & -- & $1.81 \mathrm{E}-05$ & 3.74E-05 & 2.74E-05 \\
\hline РСВ & Aroclor-1016 & $\mathrm{mg} / \mathrm{kg}$ & 9 & 0 & $0 \%$ & 0.019 & 0.029 & -- & -- & 0.011 \\
\hline PCB & Aroclor-1221 & $\mathrm{mg} / \mathrm{kg}$ & 9 & 0 & $0 \%$ & 0.019 & 0.029 & -- & -- & 0.011 \\
\hline
\end{tabular}


Table F-102

Summary Statistics for Whole Body Pacific Lamprey Collected from Segment 5 of the Columbia River

\begin{tabular}{|c|c|c|c|c|c|c|c|c|c|c|}
\hline $\begin{array}{l}\text { Constituent } \\
\text { Class }\end{array}$ & Constituent Name & Units & $\begin{array}{l}\text { Number } \\
\text { Analyzed }\end{array}$ & $\begin{array}{l}\text { Number } \\
\text { Detected }\end{array}$ & $\begin{array}{c}\text { Frequency of } \\
\text { Detection }\end{array}$ & $\begin{array}{l}\text { Minimum } \\
\text { Nondetect }\end{array}$ & $\begin{array}{l}\text { Maximum } \\
\text { Nondetect }\end{array}$ & $\begin{array}{l}\text { Minimum } \\
\text { Detect }\end{array}$ & $\begin{array}{l}\text { Maximum } \\
\text { Detect }\end{array}$ & $\begin{array}{c}\text { Average } \\
\text { Concentration }\end{array}$ \\
\hline$\overline{\mathrm{PCB}}$ & Aroclor-1232 & $\mathrm{mg} / \mathrm{kg}$ & 9 & 0 & $0 \%$ & 0.019 & 0.029 & $\overline{--}$ & $\overline{--}$ & 0.011 \\
\hline РСВ & Aroclor-1248 & $\mathrm{mg} / \mathrm{kg}$ & 9 & 0 & $0 \%$ & 0.019 & 0.029 & -- & -- & 0.011 \\
\hline PCB & Aroclor-1254 & $\mathrm{mg} / \mathrm{kg}$ & 9 & 9 & $100 \%$ & -- & -- & 0.060 & 0.15 & 0.092 \\
\hline PCB & Aroclor-1260 & $\mathrm{mg} / \mathrm{kg}$ & 9 & 3 & $33 \%$ & 0.019 & 0.020 & 0.013 & 0.016 & 0.011 \\
\hline PEST & 2,4'-DDD & $\mathrm{mg} / \mathrm{kg}$ & 9 & 4 & $44 \%$ & 0.0020 & 0.0036 & 0.0015 & 0.0048 & 0.0024 \\
\hline PEST & 2,4'-DDE & $\mathrm{mg} / \mathrm{kg}$ & 9 & 8 & $89 \%$ & 0.0020 & 0.0020 & 0.0033 & 0.0085 & 0.0046 \\
\hline PEST & $2,4^{\prime}-\mathrm{DDT}$ & $\mathrm{mg} / \mathrm{kg}$ & 9 & 2 & $22 \%$ & 0.0019 & 0.0036 & 0.0022 & 0.0030 & 0.0015 \\
\hline PEST & Aldrin & $\mathrm{mg} / \mathrm{kg}$ & 9 & 0 & $0 \%$ & 0.0019 & 0.0036 & -- & -- & 0.0012 \\
\hline PEST & Alpha-BHC & $\mathrm{mg} / \mathrm{kg}$ & 9 & 0 & $0 \%$ & 0.0019 & 0.0036 & -- & -- & 0.0012 \\
\hline PEST & alpha-Chlordane & $\mathrm{mg} / \mathrm{kg}$ & 9 & 6 & $67 \%$ & 0.0026 & 0.0036 & 0.0036 & 0.018 & 0.0069 \\
\hline PEST & alpha-Chlordene & $\mathrm{mg} / \mathrm{kg}$ & 9 & 0 & $0 \%$ & 0.0019 & 0.0036 & -- & -- & 0.0012 \\
\hline PEST & Beta-BHC & $\mathrm{mg} / \mathrm{kg}$ & 9 & 0 & $0 \%$ & 0.0019 & 0.0036 & -- & -- & 0.0012 \\
\hline PEST & cis-Nonachlor & $\mathrm{mg} / \mathrm{kg}$ & 9 & 6 & $67 \%$ & 0.0026 & 0.0036 & 0.0066 & 0.015 & 0.0075 \\
\hline PEST & DDMU & $\mathrm{mg} / \mathrm{kg}$ & 9 & 6 & $67 \%$ & 0.0026 & 0.0036 & 0.0037 & 0.0069 & 0.0039 \\
\hline PEST & Delta-BHC & $\mathrm{mg} / \mathrm{kg}$ & 9 & 0 & $0 \%$ & 0.0019 & 0.0036 & -- & -- & 0.0012 \\
\hline PEST & Dichlorodiphenyldichloroethane & $\mathrm{mg} / \mathrm{kg}$ & 9 & 9 & $100 \%$ & -- & -- & 0.0032 & 0.025 & 0.012 \\
\hline PEST & Dichlorodiphenyldichloroethylene & $\mathrm{mg} / \mathrm{kg}$ & 9 & 9 & $100 \%$ & -- & -- & 0.035 & 0.077 & 0.053 \\
\hline PEST & Dichlorodiphenyltrichloroethane & $\mathrm{mg} / \mathrm{kg}$ & 9 & 9 & $100 \%$ & -- & -- & 0.0063 & 0.029 & 0.016 \\
\hline PEST & Endosulfan sulfate & $\mathrm{mg} / \mathrm{kg}$ & 3 & 0 & $0 \%$ & 0.0026 & 0.0036 & -- & -- & 0.0016 \\
\hline PEST & Gamma-BHC (Lindane) & $\mathrm{mg} / \mathrm{kg}$ & 9 & 0 & $0 \%$ & 0.0019 & 0.0036 & -- & -- & 0.0012 \\
\hline PEST & gamma-Chlordane & $\mathrm{mg} / \mathrm{kg}$ & 9 & 4 & $44 \%$ & 0.0020 & 0.0036 & 0.0015 & 0.0031 & 0.0019 \\
\hline PEST & gamma-Chlordene & $\mathrm{mg} / \mathrm{kg}$ & 9 & 0 & $0 \%$ & 0.0019 & 0.0036 & -- & -- & 0.0012 \\
\hline PEST & Heptachlor & $\mathrm{mg} / \mathrm{kg}$ & 9 & 0 & $0 \%$ & 0.0019 & 0.0036 & -- & -- & 0.0012 \\
\hline PEST & Heptachlor epoxide & $\mathrm{mg} / \mathrm{kg}$ & 6 & 0 & $0 \%$ & 0.0019 & 0.0036 & -- & -- & 0.0013 \\
\hline PEST & Mirex & $\mathrm{mg} / \mathrm{kg}$ & 9 & 0 & $0 \%$ & 0.0019 & 0.0036 & -- & -- & 0.0012 \\
\hline PEST & Oxychlordane & $\mathrm{mg} / \mathrm{kg}$ & 9 & 3 & $33 \%$ & 0.0019 & 0.0036 & 0.0046 & 0.0057 & 0.0026 \\
\hline PEST & Pentachloroanisole & $\mathrm{mg} / \mathrm{kg}$ & 9 & 6 & $67 \%$ & 0.0026 & 0.0036 & 0.0011 & 0.0016 & 0.0014 \\
\hline PEST & Toxaphene & $\mathrm{mg} / \mathrm{kg}$ & 8 & 0 & $0 \%$ & 0.056 & 0.11 & -- & -- & 0.034 \\
\hline PEST & trans-Nonachlor & $\mathrm{mg} / \mathrm{kg}$ & 9 & 6 & $67 \%$ & 0.0026 & 0.0036 & 0.016 & 0.025 & 0.014 \\
\hline SVOC & 1,2,4-Trichlorobenzene & $\mathrm{mg} / \mathrm{kg}$ & 9 & 0 & $0 \%$ & 0.0050 & 0.025 & -- & -- & 0.0065 \\
\hline SVOC & 1,2-Dichlorobenzene & $\mathrm{mg} / \mathrm{kg}$ & 9 & 0 & $0 \%$ & 0.0050 & 0.025 & -- & -- & 0.0065 \\
\hline SVOC & 1,2-Diphenylhydrazine & $\mathrm{mg} / \mathrm{kg}$ & 9 & 0 & $0 \%$ & 0.0050 & 0.025 & -- & -- & 0.0065 \\
\hline SVOC & 1,3-Dichlorobenzene & $\mathrm{mg} / \mathrm{kg}$ & 9 & 0 & $0 \%$ & 0.0050 & 0.025 & -- & -- & 0.0065 \\
\hline SVOC & 1,4-Dichlorobenzene & $\mathrm{mg} / \mathrm{kg}$ & 9 & 0 & $0 \%$ & 0.0050 & 0.025 & -- & -- & 0.0065 \\
\hline SVOC & 1-methylnaphthalene & $\mathrm{mg} / \mathrm{kg}$ & 9 & 4 & $44 \%$ & 0.0091 & 0.025 & 0.013 & 0.019 & 0.012 \\
\hline SVOC & 2,4-Dinitrotoluene & $\mathrm{mg} / \mathrm{kg}$ & 9 & 0 & $0 \%$ & 0.010 & 0.050 & -- & -- & 0.013 \\
\hline SVOC & 2,6-Dinitrotoluene & $\mathrm{mg} / \mathrm{kg}$ & 9 & 0 & $0 \%$ & 0.020 & 0.10 & -- & -- & 0.026 \\
\hline SVOC & 2-Chloronaphthalene & $\mathrm{mg} / \mathrm{kg}$ & 9 & 0 & $0 \%$ & 0.010 & 0.050 & -- & -- & 0.013 \\
\hline SVOC & 2-Methylnaphthalene & $\mathrm{mg} / \mathrm{kg}$ & 9 & 4 & $44 \%$ & 0.0091 & 0.044 & 0.031 & 0.039 & 0.022 \\
\hline SVOC & 4-Bromophenylphenyl ether & $\mathrm{mg} / \mathrm{kg}$ & 9 & 0 & $0 \%$ & 0.0050 & 0.025 & -- & -- & 0.0065 \\
\hline SVOC & 4-Chlorophenylphenyl ether & $\mathrm{mg} / \mathrm{kg}$ & 9 & 0 & $0 \%$ & 0.0050 & 0.025 & -- & -- & 0.0065 \\
\hline SVOC & Acenaphthene & $\mathrm{mg} / \mathrm{kg}$ & 9 & 0 & $0 \%$ & 0.010 & 0.050 & -- & -- & 0.013 \\
\hline SVOC & Acenaphthylene & $\mathrm{mg} / \mathrm{kg}$ & 9 & 0 & $0 \%$ & 0.0050 & 0.025 & -- & -- & 0.0065 \\
\hline SVOC & Anthracene & $\mathrm{mg} / \mathrm{kg}$ & 9 & 0 & $0 \%$ & 0.0050 & 0.025 & -- & -- & 0.0065 \\
\hline SVOC & Benzo(a)anthracene & $\mathrm{mg} / \mathrm{kg}$ & 9 & 0 & $0 \%$ & 0.0050 & 0.025 & -- & -- & 0.0065 \\
\hline SVOC & Benzo(a)pyrene & $\mathrm{mg} / \mathrm{kg}$ & 9 & 0 & $0 \%$ & 0.0050 & 0.049 & -- & -- & 0.0087 \\
\hline SVOC & Benzo(b)fluoranthene & $\mathrm{mg} / \mathrm{kg}$ & 9 & 0 & $0 \%$ & 0.0050 & 0.049 & -- & -- & 0.0087 \\
\hline SVOC & Benzo(ghi)perylene & $\mathrm{mg} / \mathrm{kg}$ & 9 & 0 & $0 \%$ & 0.010 & 0.098 & -- & -- & 0.017 \\
\hline SVOC & Benzo(k)fluoranthene & $\mathrm{mg} / \mathrm{kg}$ & 9 & 0 & $0 \%$ & 0.0050 & 0.049 & -- & -- & 0.0087 \\
\hline SVOC & Bis(2-chloro-1-methylethyl)ether & $\mathrm{mg} / \mathrm{kg}$ & 9 & 0 & $0 \%$ & 0.010 & 0.050 & -- & -- & 0.013 \\
\hline
\end{tabular}


Table F-102

Summary Statistics for Whole Body Pacific Lamprey Collected from Segment 5 of the Columbia River

\begin{tabular}{|c|c|c|c|c|c|c|c|c|c|c|}
\hline $\begin{array}{l}\text { Constituent } \\
\text { Class }\end{array}$ & Constituent Name & Units & $\begin{array}{l}\text { Number } \\
\text { Analyzed }\end{array}$ & $\begin{array}{l}\text { Number } \\
\text { Detected }\end{array}$ & $\begin{array}{l}\text { Frequency of } \\
\text { Detection }\end{array}$ & $\begin{array}{l}\text { Minimum } \\
\text { Nondetect }\end{array}$ & $\begin{array}{l}\text { Maximum } \\
\text { Nondetect }\end{array}$ & $\begin{array}{c}\text { Minimum } \\
\text { Detect }\end{array}$ & $\begin{array}{l}\text { Maximum } \\
\text { Detect }\end{array}$ & $\begin{array}{c}\text { Average } \\
\text { Concentration }\end{array}$ \\
\hline$\overline{\text { SVOC }}$ & Chrysene & $\mathrm{mg} / \mathrm{kg}$ & 9 & 0 & $0 \%$ & 0.0050 & 0.025 & $\overline{--}$ & $\overline{--}$ & 0.0065 \\
\hline SVOC & Dibenz[a,h]anthracene & $\mathrm{mg} / \mathrm{kg}$ & 9 & 0 & $0 \%$ & 0.010 & 0.098 & -- & -- & 0.017 \\
\hline SVOC & Dibenzofuran & $\mathrm{mg} / \mathrm{kg}$ & 9 & 0 & $0 \%$ & 0.0050 & 0.025 & -- & -- & 0.0065 \\
\hline SVOC & Fluoranthene & $\mathrm{mg} / \mathrm{kg}$ & 9 & 1 & $11 \%$ & 0.010 & 0.050 & 0.017 & 0.017 & 0.014 \\
\hline SVOC & Fluorene & $\mathrm{mg} / \mathrm{kg}$ & 9 & 0 & $0 \%$ & 0.0080 & 0.0099 & -- & -- & 0.0046 \\
\hline SVOC & Hexachlorobenzene & $\mathrm{mg} / \mathrm{kg}$ & 9 & 1 & $11 \%$ & 0.010 & 0.050 & 0.012 & 0.012 & 0.013 \\
\hline SVOC & Hexachlorobutadiene & $\mathrm{mg} / \mathrm{kg}$ & 9 & 0 & $0 \%$ & 0.0050 & 0.025 & -- & -- & 0.0065 \\
\hline SVOC & Hexachloroethane & $\mathrm{mg} / \mathrm{kg}$ & 9 & 0 & $0 \%$ & 0.0050 & 0.025 & -- & -- & 0.0065 \\
\hline SVOC & Indeno(1,2,3-cd)pyrene & $\mathrm{mg} / \mathrm{kg}$ & 9 & 0 & $0 \%$ & 0.010 & 0.098 & -- & -- & 0.017 \\
\hline SVOC & Naphthalene & $\mathrm{mg} / \mathrm{kg}$ & 9 & 0 & $0 \%$ & 0.0091 & 0.059 & -- & -- & 0.016 \\
\hline SVOC & Nitrobenzene & $\mathrm{mg} / \mathrm{kg}$ & 9 & 0 & $0 \%$ & 0.0050 & 0.025 & -- & -- & 0.0065 \\
\hline SVOC & Phenanthrene & $\mathrm{mg} / \mathrm{kg}$ & 9 & 3 & $33 \%$ & 0.0073 & 0.025 & 0.013 & 0.015 & 0.0096 \\
\hline SVOC & Pyrene & $\mathrm{mg} / \mathrm{kg}$ & 9 & 0 & $0 \%$ & 0.010 & 0.050 & -- & -- & 0.013 \\
\hline VOC & Retene & $\mathrm{mg} / \mathrm{kg}$ & 9 & 0 & $0 \%$ & 0.010 & 0.050 & -- & -- & 0.013 \\
\hline
\end{tabular}

DIOXI - Dioxins and Furans

PEST - Pesticide

PCB - Polychlorinated biphenyl

SVOC - Semivolatile Organic Compound

VOC - Volatile Organic Compound 
Table F-103

Summary Statistics for Whole Body Starry Flounder Collected from Segment 5 of the Columbia River

\begin{tabular}{|c|c|c|c|c|c|c|c|c|c|c|}
\hline $\begin{array}{l}\text { Constituent } \\
\text { Class }\end{array}$ & Constituent Name & Units & $\begin{array}{c}\text { Number } \\
\text { Analyzed }\end{array}$ & $\begin{array}{l}\text { Number } \\
\text { Detected }\end{array}$ & $\begin{array}{l}\text { Frequency of } \\
\text { Detection }\end{array}$ & $\begin{array}{l}\text { Minimum } \\
\text { Nondetect }\end{array}$ & $\begin{array}{l}\text { Maximum } \\
\text { Nondetect }\end{array}$ & $\begin{array}{l}\text { Minimum } \\
\text { Detect }\end{array}$ & $\begin{array}{l}\text { Maximum } \\
\text { Detect }\end{array}$ & $\begin{array}{c}\text { Average } \\
\text { Concentration }\end{array}$ \\
\hline$\overline{\text { CONV }}$ & Percent Lipids & $\%$ & 1 & 1 & $100 \%$ & -- & -- & 4.3 & 4.3 & 4.3 \\
\hline PEST & Hexabromodiphenyl ethe & $\mathrm{mg} / \mathrm{Kg}$ & 1 & 1 & $100 \%$ & -- & -- & 8.40E-04 & $8.40 \mathrm{E}-04$ & $8.40 \mathrm{E}-04$ \\
\hline PEST & Pentabromodiphenyl ethe & $\mathrm{mg} / \mathrm{Kg}$ & 1 & 1 & $100 \%$ & -- & -- & 0.0081 & 0.0081 & 0.0081 \\
\hline PEST & Tetrabromodiphenyl ethe & $\mathrm{mg} / \mathrm{Kg}$ & 1 & 1 & $100 \%$ & -- & -- & 0.021 & 0.021 & 0.021 \\
\hline
\end{tabular}

PEST - Pesticide 